
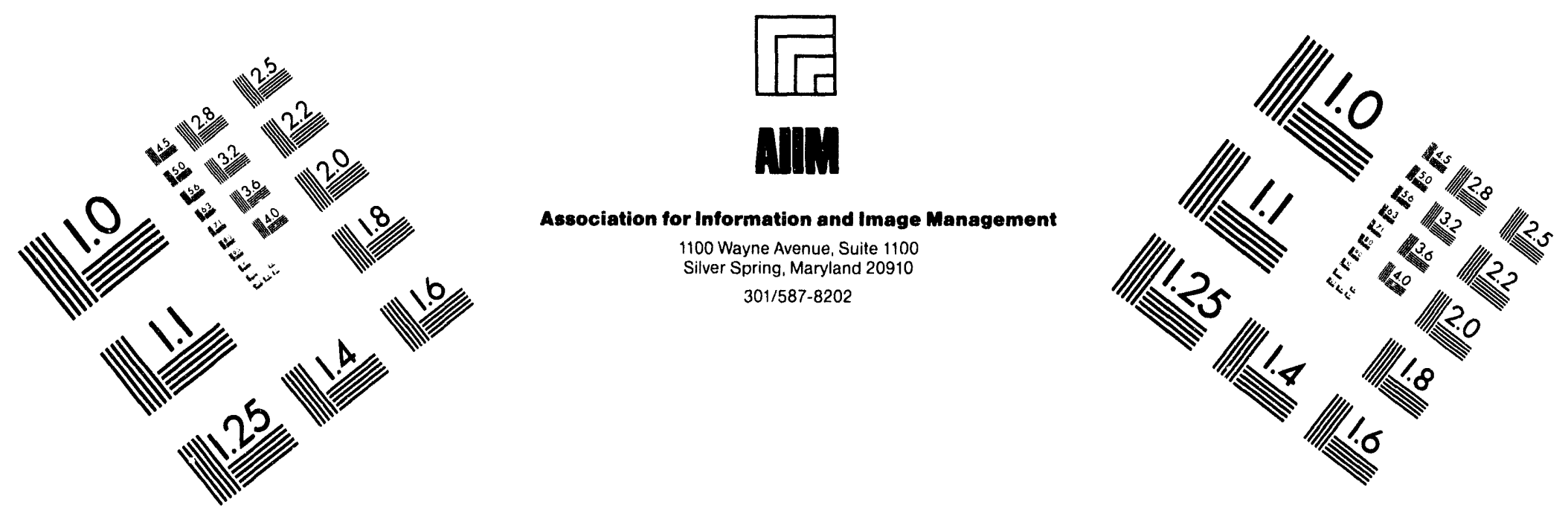

\title{
Centimeter
}

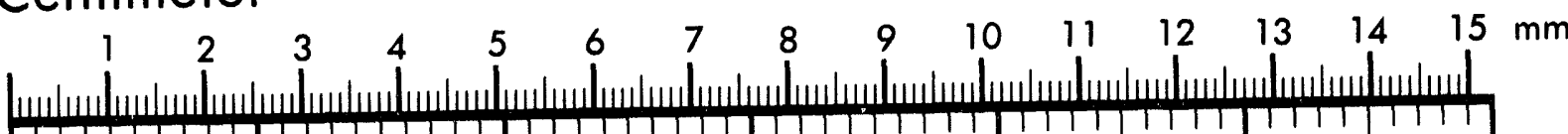

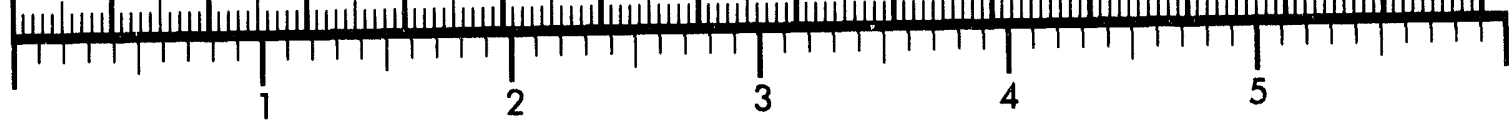
Inches
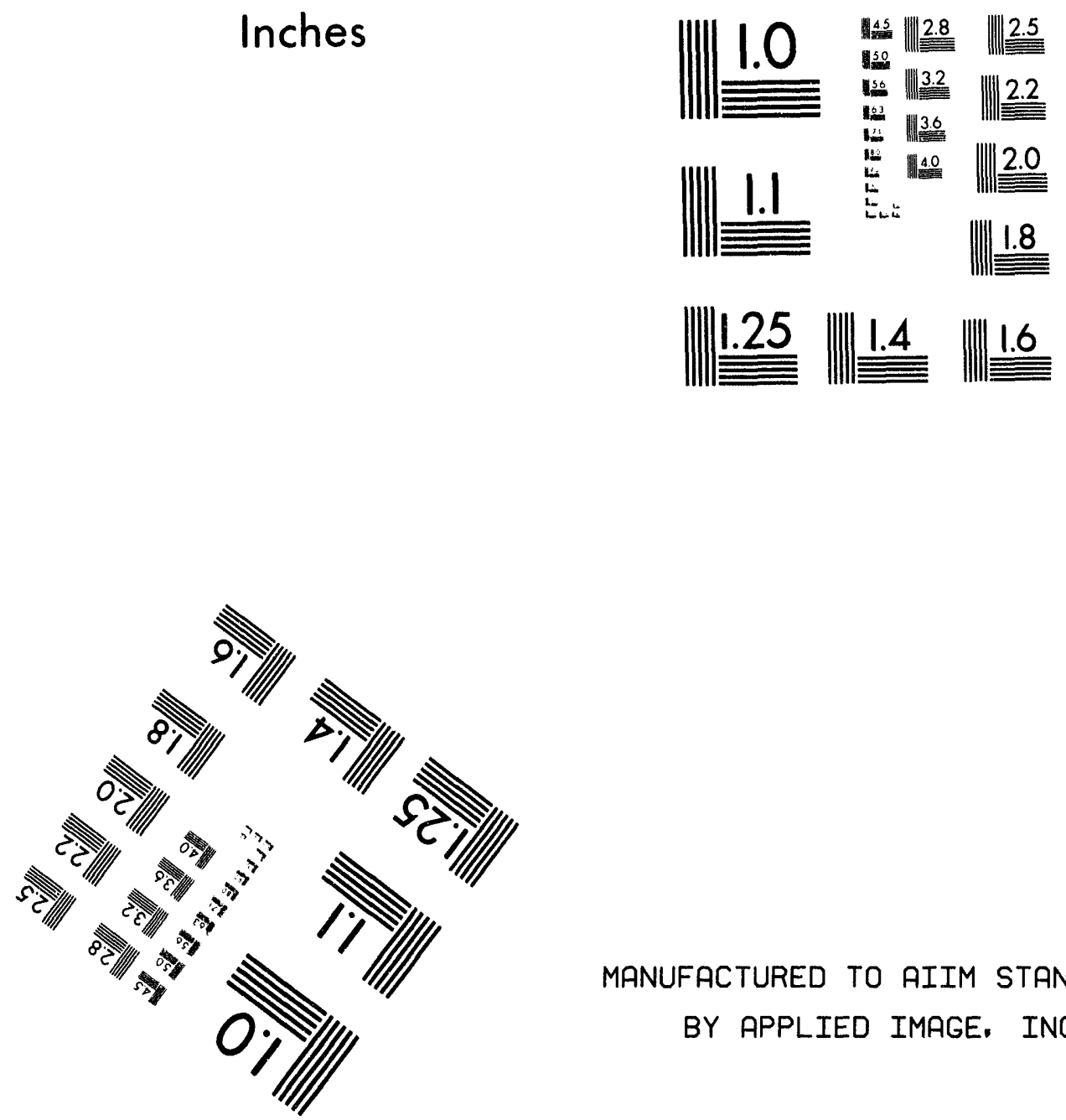

MANUFACTURED TO AIIM STANDARDS BY APPLIED IMAGE. INC.

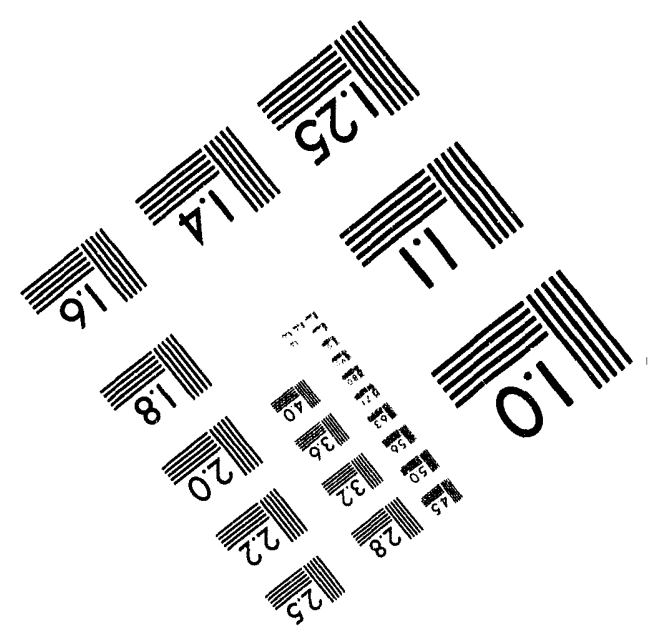



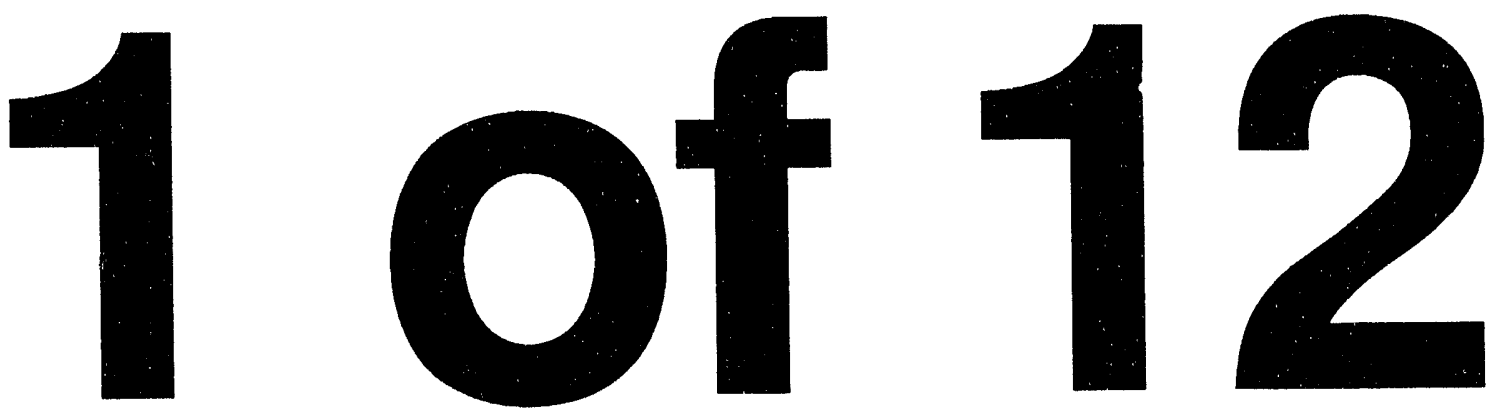


\title{
NRTSC
}

NUCLEAR REACTOR TECHNOLOGY

AND SCIENTIFIC COMPUTATIONS

\author{
WS R C - TR - $92-534$ \\ Task No.: 92-104-1
}

KEYWORDS:

ONSET OF FLOW INSTABILITY

ONSET OF SIGNIFICANT VOID

DEMAND CURVES

LOSS OF COOLANT ACCIDENT

ANNULUS

RETENTION:

PERMANENT

\section{NUCLEATE BOILING PRESSURE DROP IN AN ANNULUS, BOOK 8 (U)}

ISSUED: NOVEMBER, 1992

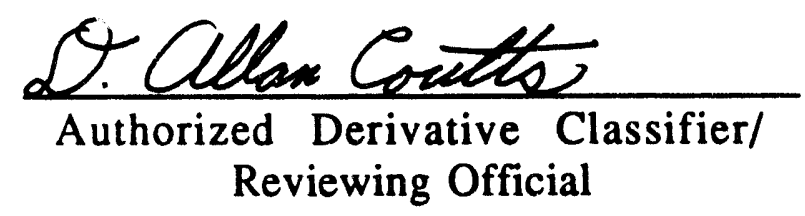

SRL SAVANNAH RIVER TECHNOLOGY CENTER, AIKEN, SC 29808 Westinghouse Savannah River Corporation Prepared for the U.S. Department of Energy under Contract DE-AC09-89SR18035 


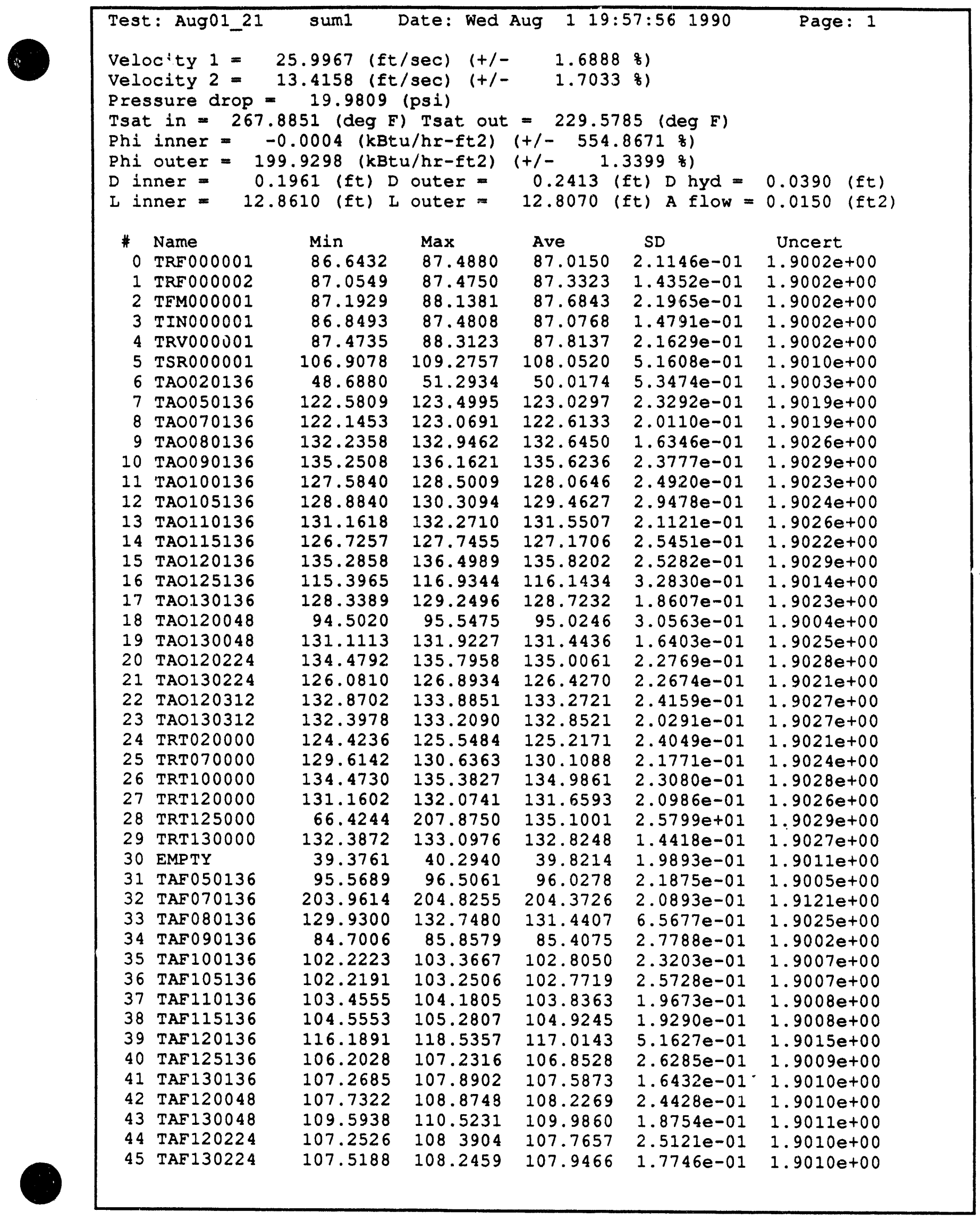




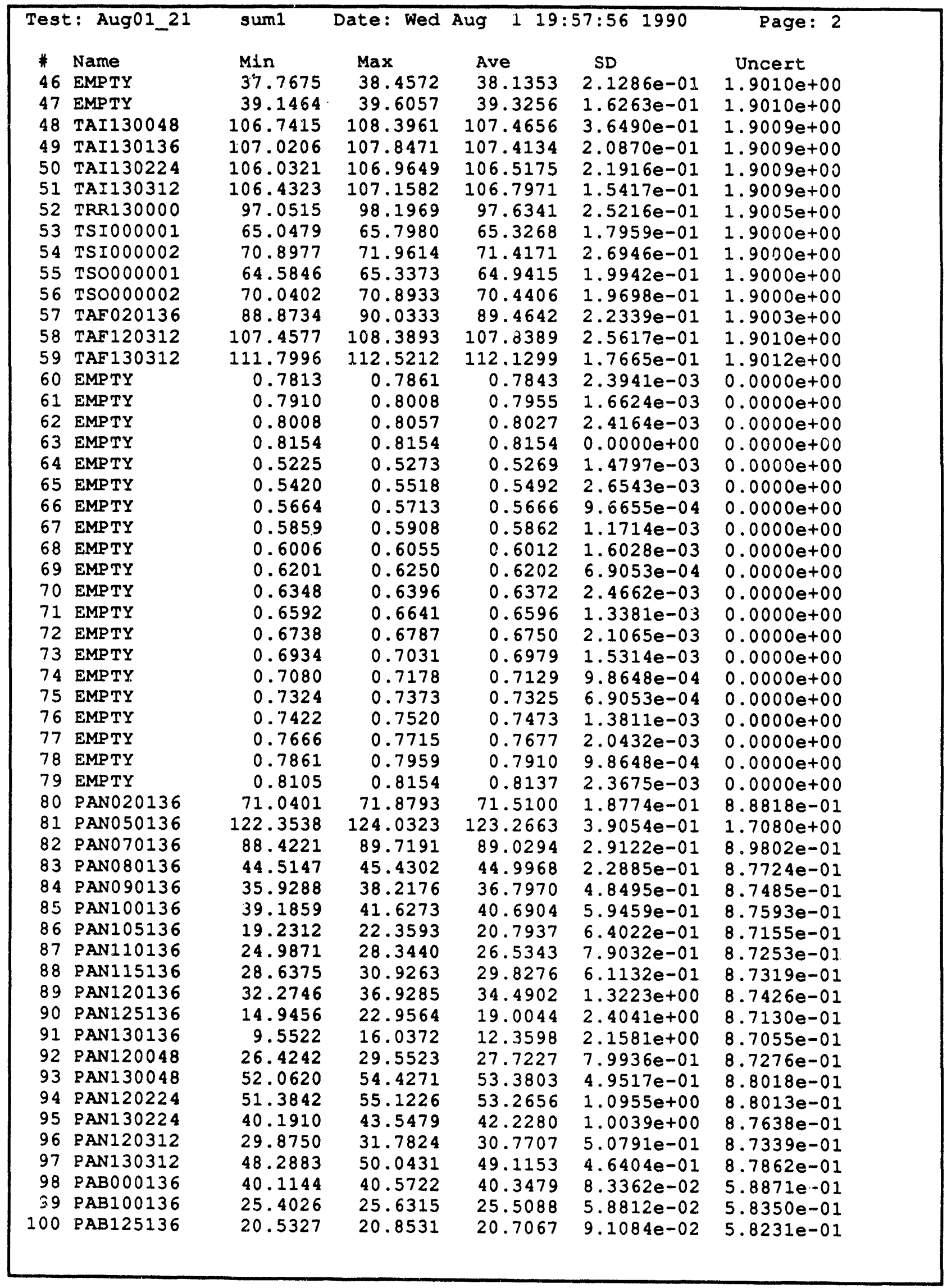




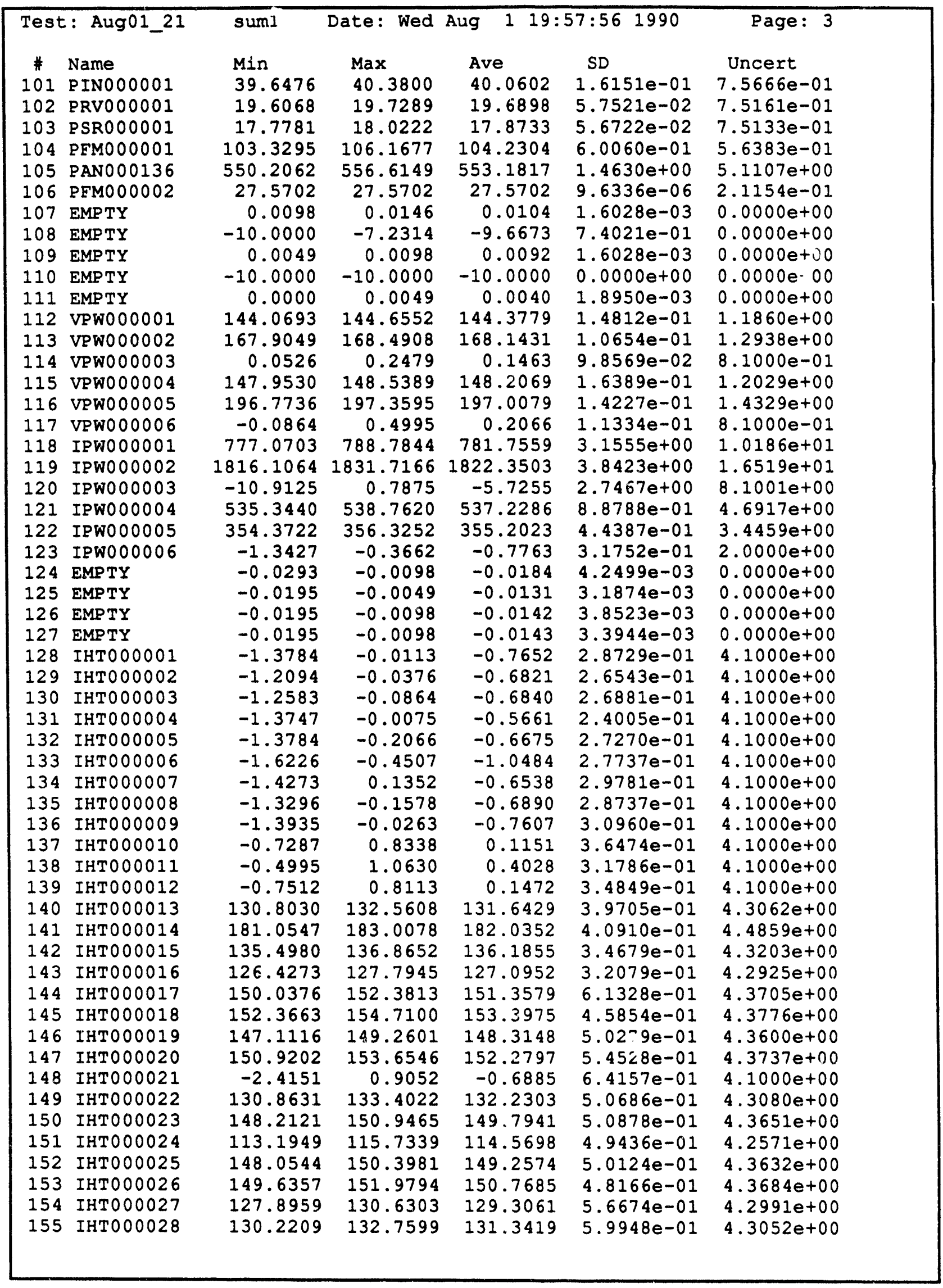



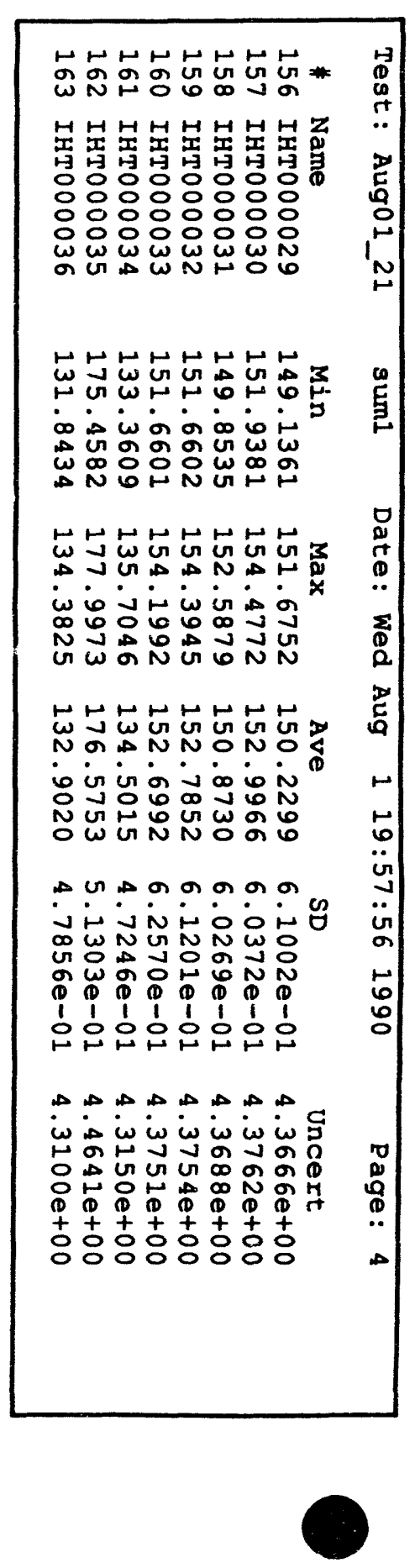


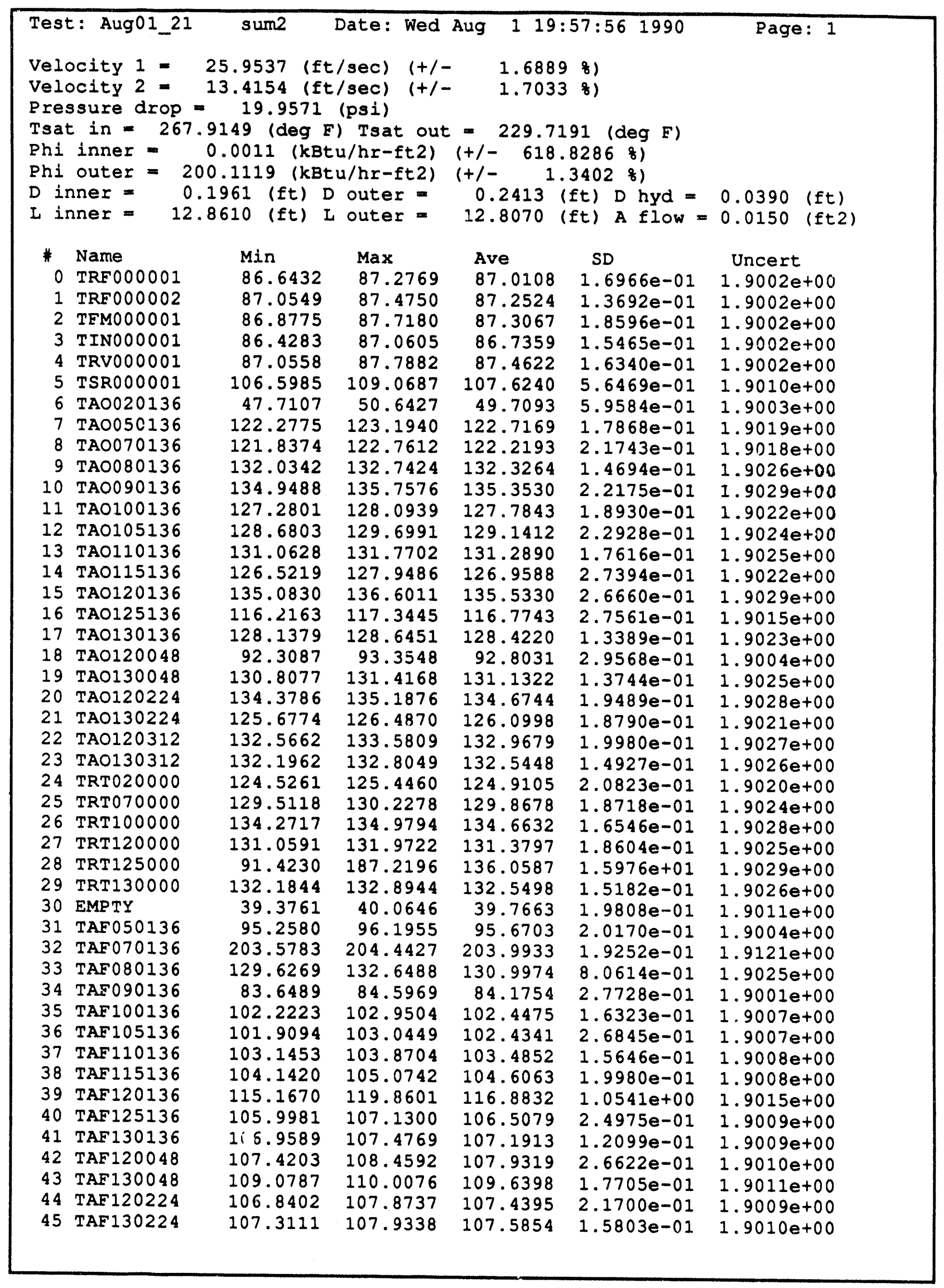




\begin{tabular}{|c|c|c|c|c|c|c|}
\hline \multicolumn{2}{|c|}{ Test: Aug01_21 } & sum2 & Date: Wed & Aug & $: 56 \quad 1990$ & Page: 2 \\
\hline$*$ & Name & Min & $\operatorname{Max}$ & Ave & SD & Uncert \\
\hline 46 & EMPTY & 37.5374 & 38.4572 & 38.0894 & $1.9708 e-01$ & $1.9010 e+00$ \\
\hline 47 & EMPTY & 39.1464 & 39.6057 & 39.3531 & $1.2491 \mathrm{e}-01$ & $1.9010 e+00$ \\
\hline 48 & TAI130048 & 106.5347 & 107.6730 & 107.0149 & $3.1041 e-01$ & $1.9009 e+00$ \\
\hline 49 & TAI130136 & 106.7116 & 107.4351 & 107.1207 & $1.7575 e-01$ & $1.9009 e+00$ \\
\hline 50 & TAI130224 & 105.7206 & 106.9655 & 106.2605 & $3.1973 e-01$ & $1.9009 \mathrm{e}+00$ \\
\hline 51 & TAI130312 & 106.0177 & 106.8473 & 106.4700 & $1.8459 e-01$ & $1.9009 e+00$ \\
\hline 52 & TRR130000 & 96.6354 & 97.6758 & 97.1106 & $3.0229 e-01$ & $1.9005 e+00$ \\
\hline 53 & TSI000001 & 65.0479 & 65.5831 & 65.2755 & $1.3775 e-01$ & $1.9000 e+00$ \\
\hline 54 & TSI000002 & 70.7919 & 71.8539 & 71.3406 & $2.6910 e-01$ & $1.9000 e+00$ \\
\hline 55 & TSO000001 & 64.5852 & 65.3368 & 64.9159 & $1.6792 e-01$ & $1.9000 \mathrm{e}+00$ \\
\hline 56 & TSO000002 & 70.0397 & 70.7853 & 70.4321 & $1.5742 e-01$ & $1.9000 e+00$ \\
\hline 57 & TAF020136 & 88.7676 & 89.5067 & 89.1097 & $2.0183 e-01$ & $1.9002 \mathrm{e}+00$ \\
\hline 58 & TAF120312 & 106.9407 & 108.1827 & 107.5041 & $3.0077 e-01$ & $1.9009 e+00$ \\
\hline 59 & TAF 130312 & 111.2862 & 112.1102 & 111.7309 & $1.7828 e-01$ & $1.9012 \mathrm{e}+00$ \\
\hline 60 & ЕМРTY & 0.7715 & 0.7764 & 0.7759 & $1.4797 e-03$ & $0.0000 e+00$ \\
\hline 61 & EMPTY & 0.7861 & 0.7910 & 0.7863 & $9.6655 e-04$ & $0.0000 e+00$ \\
\hline 62 & EMPTY & 0.7910 & 0.7959 & 0.7957 & $9.6655 e-04$ & $0.0000 e+00$ \\
\hline 63 & EMPTY & 0.8057 & 0.8154 & 0.8104 & $1.3811 e-03$ & $0.0000 e+00$ \\
\hline 64 & EMPTY & 0.5176 & 0.5273 & 0.5221 & $1.6624 \mathrm{e}-03$ & $0.0000 e+00$ \\
\hline 65 & EMPTY & 0.5420 & 0.5469 & 0.5459 & $1.9730 e-03$ & $0.0000 e+00$ \\
\hline 66 & EMPTY & 0.5566 & 0.5664 & 0.5615 & $9.8648 e-04$ & $0.0000 e+00$ \\
\hline 67 & EMPTY & 0.5811 & 0.5859 & 0.5813 & $9.6655 e-04$ & $0.0000 e+00$ \\
\hline 68 & EMPTY & 0.5957 & 0.6006 & 0.5960 & $1.1714 e-03$ & $0.0000 e+00$ \\
\hline 69 & EMPTY & 0.6152 & 0.6201 & 0.6153 & $6.9053 e-04$ & $0.0000 e+00$ \\
\hline 70 & EMPTY & 0.6299 & 0.6348 & 0.6318 & $2.4164 e-03$ & $0.0000 e+00$ \\
\hline 71 & EMPTY & 0.6543 & 0.6592 & 0.6548 & $1.4797 e-03$ & $0.0000 e+00$ \\
\hline 72 & EMPTY & 0.6689 & 0.6738 & 0.6692 & $1.1714 e-03$ & $0.0000 e+00$ \\
\hline 73 & EMPTY & 0.6885 & 0.6934 & 0.6927 & $1.7115 e-03$ & $0.0000 e+00$ \\
\hline 74 & EMPTY & 0.7080 & 0.7080 & 0.7080 & $0.0000 e+00$ & $0.0000 e+00$ \\
\hline 75 & EMPTY & 0.7275 & 0.7324 & 0.7276 & $6.9053 e-04$ & $0.0000 e+00$ \\
\hline 76 & EMPTY & 0.7422 & 0.7471 & 0.7424 & $9.6655 e-04$ & $0.0000 e+00$ \\
\hline 77 & EMPTY & 0.7617 & 0.7666 & 0.7621 & $1.3381 e-03$ & $0.0000 e+00$ \\
\hline 78 & EMPTY & 0.7861 & 0.7861 & 0.7861 & $0.0000 e+00$ & $0.0000 e+00$ \\
\hline 79 & EMPTY & 0.8057 & 0.8105 & 0.8066 & $1.9730 e-03$ & $0.0000 e+00$ \\
\hline 80 & PAN020136 & 70.6586 & 71.9556 & 71.2186 & $3.1687 e-01$ & $8.8803 e-01$ \\
\hline 81 & PAN050136 & 122.5064 & 124.0323 & 123.2510 & $4.0033 e-01$ & $1.7080 e+00$ \\
\hline 82 & PAN070136 & 88.4984 & 89.4139 & 88.9226 & $2.0341 e-01$ & $8.9795 e-01$ \\
\hline 83 & PAN080136 & 44.7436 & 45.5828 & 45.2181 & $1.6333 e-01$ & $8.7731 e-01$ \\
\hline 84 & PAN090136 & 36.2340 & 38.0650 & 36.9008 & $3.6306 e-01$ & $8.7488 e-01$ \\
\hline 85 & PAN100136 & 39.4147 & 41.0932 & 40.1167 & $4.0518 e-01$ & $8.7576 e-01$ \\
\hline 86 & PAN 105136 & 18.6972 & 22.1304 & 20.4016 & $8.0986 e-01$ & $8.7149 e-01$ \\
\hline 87 & PAN110136 & 24.9871 & 28.8781 & 26.9082 & $1.0056 e+00$ & $8.7260 e-01$ \\
\hline 88 & PAN115136 & 29.0189 & 32.1470 & 30.3281 & $9.1324 e-01$ & $8.7330 e-01$ \\
\hline 89 & PAN120136 & 32.4272 & 37.3863 & 34.1713 & $1.2759 \mathrm{e}+00$ & $8.7418 e-01$ \\
\hline 90 & PAN125136 & 13.1908 & 22.2698 & 18.3300 & $2.1306 \mathrm{e}+00$ & $8.7121 e-01$ \\
\hline 91 & PAN130136 & 9.7048 & 16.2661 & 12.5719 & $1.9447 \mathrm{e}+00$ & $8.7057 e-01$ \\
\hline 92 & PAN120048 & 25.8139 & 28.9419 & 27.4557 & $5.9642 e-01$ & $8.7270 e-01$ \\
\hline 93 & PAN130048 & 52.4434 & 54.3508 & 53.4582 & $4.9258 e-01$ & $8.8021 e-01$ \\
\hline 94 & PAN120224 & 51.8420 & 55.8855 & 53.1374 & $1.0536 \mathrm{e}+00$ & $8.8008 e-01$ \\
\hline 95 & PAN130224 & 39.9621 & 43.4716 & 42.2646 & $9.6968 \mathrm{e}-01$ & $8.7639 e-01$ \\
\hline 96 & PAN120312 & 29.5699 & 32.0876 & 30.6395 & $6.7072 e-01$ & $8.7337 e-01$ \\
\hline 97 & PAN130312 & 48.3646 & 50.5771 & 49.4907 & $5.2668 e-01$ & $8.7875 e-01$ \\
\hline 98 & PAB000136 & 40.2060 & 40.5264 & 40.3680 & $9.4861 \mathrm{e}-02$ & $5.8871 e-01$ \\
\hline 99 & PAB100136 & 25.4942 & 25.6773 & 25.5729 & $5.8520 e-02$ & $5.8351 e-01$ \\
\hline 100 & PAB125136 & 20.6243 & 20.8989 & 20.7607 & $9.1314 \mathrm{e}-02$ & $5.8232 e-01$ \\
\hline
\end{tabular}




\begin{tabular}{|c|c|c|c|c|c|c|}
\hline \multicolumn{2}{|c|}{ Test: Aug01_21 } & sum2 & Date: Wed & \multicolumn{2}{|c|}{$119: 57: 56 \quad 1990$} & Page: 3 \\
\hline \# & Name & Min & $\operatorname{Max}$ & Ave & SD & Uncert \\
\hline 101 & PINO00001 & 39.8917 & 40.6241 & 40.1969 & $1.7827 e-01$ & $7.5670 e-01$ \\
\hline 102 & PRV000001 & 19.6068 & 19.7289 & 19.6337 & $5.1081 e-02$ & $7.5160 e-01$ \\
\hline 103 & PSR000001 & 17.9001 & 18.0222 & 17.9319 & $5.4088 e-02$ & $7.5134 e-01$ \\
\hline 104 & PFM000001 & 102.5056 & 104.7944 & 103.8917 & $4.7085 e-01$ & $5.6344 e-01$ \\
\hline $\begin{array}{l}105 \\
106\end{array}$ & $\begin{array}{l}\text { PANO00136 } \\
\text { PFM000002 }\end{array}$ & $\begin{array}{r}549.7485 \\
27.5702\end{array}$ & $\begin{array}{r}555.2416 \\
27.5702\end{array}$ & $\begin{array}{r}552.5225 \\
27.5702\end{array}$ & $\begin{array}{l}1.3125 e+00 \\
9.6336 e-06\end{array}$ & \\
\hline 107 & EMPTY & 0.0098 & 0.0146 & 0.0104 & $1.6028 e-03$ & $\begin{array}{l}2.1154 e-01 \\
0.0000 e+00\end{array}$ \\
\hline 108 & EMPTY & -10.0000 & -7.8076 & -9.7779 & $5.0794 e-01$ & $0.0000 e+00$ \\
\hline 109 & EMPTY & 0.0049 & 0.0146 & 0.0091 & $1.9754 \mathrm{e}-03$ & $0.0000 e+00$ \\
\hline 110 & EMPTY & -10.0000 & -10.0000 & -10.0000 & $0.0000 e+00$ & $0.0000 e+00$ \\
\hline 111 & EMPTY & 0.0000 & 0.0049 & 0.0044 & $1.4797 e-03$ & $0.0000 e+00$ \\
\hline 112 & VPW000001 & 144.0693 & 144.8505 & 144.3740 & $1.9395 e-01$ & $1.1860 \mathrm{e}+00$ \\
\hline 113 & VPW000002 & 167.9049 & 168.2955 & 168.1119 & $1.2731 e-01$ & $1.2936 e+00$ \\
\hline 114 & VPW000003 & -0.1427 & 0.2479 & 0.1307 & $1.0440 e-01$ & $8.1000 e-01$ \\
\hline 115 & VPW000004 & 147.9530 & 148.5389 & 148.1679 & $1.4363 e-01$ & $1.2027 e+00$ \\
\hline 116 & VPW000005 & 196.3830 & 197.3595 & 196.9532 & $1.6667 e-01$ & $1.4327 e+00$ \\
\hline 117 & VPW000006 & -0.0864 & 0.3042 & 0.1753 & $1.0885 e-01$ & $8.1000 e-01$ \\
\hline 118 & IPW000001 & 763.4039 & 771.2133 & 767.6992 & $2.1961 e+00$ & $1.0119 e+01$ \\
\hline 119 & IPW000002 & 1831.7166 & 1853.1805 & 1839.5607 & $5.4266 \mathrm{e}+00$ & $1.6637 e+01$ \\
\hline 120 & IPW000003 & 16.3875 & 20.2875 & 19.0395 & $1.2308 \mathrm{e}+00$ & $8.1014 e+00$ \\
\hline 121 & IPW000004 & 531.9260 & 536.3206 & 533.4200 & $9.6861 e-01$ & $4.6645 e+00$ \\
\hline 122 & IPW000005 & 355.3487 & 357.7899 & 356.8231 & $5.2649 e-01$ & $3.4563 \mathrm{e}+00$ \\
\hline 123 & IPW000006 & -0.3662 & 1.5869 & 0.6104 & $3.8206 e-01$ & $2.0000 e+00$ \\
\hline 124 & EMPTY & -0.0293 & -0.0146 & -0.0196 & $3.6232 e-03$ & $0.0000 e+00$ \\
\hline 125 & EMPTY & -0.0195 & -0.0098 & -0.0133 & $2.7971 e-03$ & $0.0000 e+00$ \\
\hline 126 & EMPTY & -0.0195 & -0.0098 & -0.0154 & $3.1752 e-03$ & $0.0000 e+00$ \\
\hline 127 & EMPTY & -0.0195 & -0.0049 & -0.0137 & $3.2718 e-03$ & $0.0000 e+00$ \\
\hline 128 & IHTO00001 & -1.3784 & -0.2066 & -0.7613 & $2.8553 e-01$ & $4.1000 e+00$ \\
\hline 129 & IHT000002 & -1.2094 & -0.2329 & -0.7251 & $2.5645 e-01$ & $4.1000 e+00$ \\
\hline 130 & IHT000003 & -1.2583 & -0.0864 & -0.7153 & $2.8534 e-01$ & $4.1000 e+00$ \\
\hline 131 & IHTO00004 & -1.1794 & 0.1878 & -0.6247 & $2.8553 e-01$ & $4.1000 e+00$ \\
\hline 132 & IHT000005 & -1.3784 & -0.0113 & -0.7222 & $2.7554 e-01$ & $4.1000 e+00$ \\
\hline 133 & IHT000006 & -1.6226 & -0.4507 & -1.0562 & $2.8523 e-01$ & $4.1000 e+00$ \\
\hline 134 & IHT000007 & -1.2320 & -0.0601 & -0.6460 & $2.9791 e-01$ & $4.1000 e+00$ \\
\hline 135 & IHT000008 & -1.3296 & -0.3531 & -0.6851 & $2.5034 e-01$ & $4.1000 e+00$ \\
\hline 136 & IHT000009 & -1.3935 & -0.2216 & -0.7333 & $2.7307 e-01$ & $4.1000 e+00$ \\
\hline 137 & IHTO00010 & -0.9240 & 0.6385 & -0.0216 & $4.0605 e-01$ & $4.1000 e+00$ \\
\hline 138 & IHT000011 & -0.3042 & 1.0630 & 0.3130 & $3.6881 e-01$ & $4.1000 e+00$ \\
\hline 139 & IHT000012 & -0.7512 & 0.8113 & 0.0496 & $4.0289 e-01$ & $4.1000 \mathrm{e}+00$ \\
\hline 140 & IHT000013 & 130.8030 & 132.3655 & 131.5062 & $4.2864 e-01$ & $4.3057 e+00$ \\
\hline 141 & IHTO00014 & 180.8594 & 182.6172 & 181.8125 & $4.4405 e-01$ & $4.4850 e+00$ \\
\hline 142 & IHT000015 & 135.3027 & 137.0605 & 136.1269 & $4.0103 e-01$ & $4.3201 e+00$ \\
\hline 143 & IHT000016 & 126.4273 & 127.9898 & 127.0601 & $4.1490 e-01$ & $4.2924 e+00$ \\
\hline 144 & IHT000017 & 149.6470 & 152.5766 & 151.3735 & $7.4070 e-01$ & $4.3705 e+00$ \\
\hline 145 & IHTO00018 & 152.5616 & 154.5147 & 153.5069 & $4.9025 e-01$ & $4.3779 e+00$ \\
\hline 146 & IHT000019 & 147.5022 & 149.2601 & 148.2797 & $4.6936 e-01$ & $4.3599 e+00$ \\
\hline 147 & IHTOOOO20 & 151.5061 & 153.4593 & 152.3969 & $4.5191 e-01$ & $4.3741 e+00$ \\
\hline 148 & IHTO00021 & -1.6339 & 0.3193 & -0.6104 & $5.1684 e-01$ & $4.1000 e+00$ \\
\hline 149 & IHT000022 & 131.4491 & 133.2069 & 132.3632 & $4.0710 e-01$ & $4.3084 e+00$ \\
\hline 150 & IHT000023 & 149.1887 & 150.7512 & 149.8956 & $4.3205 e-01$ & $4.3654 e+00$ \\
\hline 151 & IHTO00024 & 113.9761 & 115.3433 & 114.6480 & $4.1037 e-01$ & $4.2573 e+00$ \\
\hline 152 & IHT000025 & 148.4450 & 150.2028 & 149.3746 & $4.7933 e-01$ & $4.3636 e+00$ \\
\hline 153 & IHT000026 & 150.0263 & 151.9794 & 150.9482 & $4.7848 e-01$ & 4. $3690 \mathrm{e}+00$ \\
\hline 154 & IHT000027 & 128.4818 & 130.4350 & 129.5131 & $4.9133 e-01$ & $4.2997 e+00$ \\
\hline 155 & IHTO00028 & 130.0255 & 132.5646 & 131.3068 & $7.6733 e-01$ & $4.3051 e+00$ \\
\hline
\end{tabular}




\begin{tabular}{|c|c|c|c|c|c|c|}
\hline \multicolumn{2}{|c|}{ Test: Aug01_21 } & sum2 & Date: Wed & 119 & $57: 56 \quad 1990$ & Page: 4 \\
\hline $\begin{array}{l}\# \\
156 \\
157 \\
158 \\
159 \\
160 \\
161 \\
162 \\
163\end{array}$ & 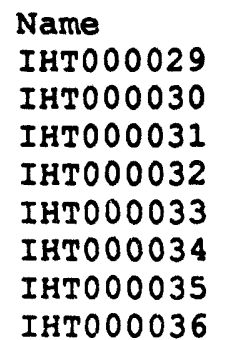 & $\begin{array}{c}\text { M1n } \\
148.7455 \\
151.5475 \\
149.4629 \\
151.4649 \\
151.2695 \\
133.1656 \\
175.2629 \\
131.6481\end{array}$ & $\begin{array}{c}\text { Max } \\
151.4799 \\
154.6725 \\
152.7832 \\
154.9805 \\
154.9805 \\
136.4859 \\
178.1926 \\
134.5778\end{array}$ & $\begin{array}{l}\text { Ave } \\
150.1401 \\
152.9029 \\
150.7949 \\
152.7422 \\
152.6484 \\
134.4351 \\
176.4542 \\
132.8082\end{array}$ & $\begin{array}{l}\text { SD } \\
6.9700 e-01 \\
6.7820 e-01 \\
7.0331 e-01 \\
7.0940 e-01 \\
7.1617 e-01 \\
6.2046 e-01 \\
5.6255 e-01 \\
5.8048 e-01\end{array}$ & $\begin{array}{l}\text { Uncert } \\
4.3663 e+00 \\
4.3758 e+00 \\
4.3685 e+00 \\
4.3753 e+00 \\
4.3749 e+00 \\
4.3148 e+00 \\
4.4636 e+00 \\
4.3097 e+00\end{array}$ \\
\hline
\end{tabular}




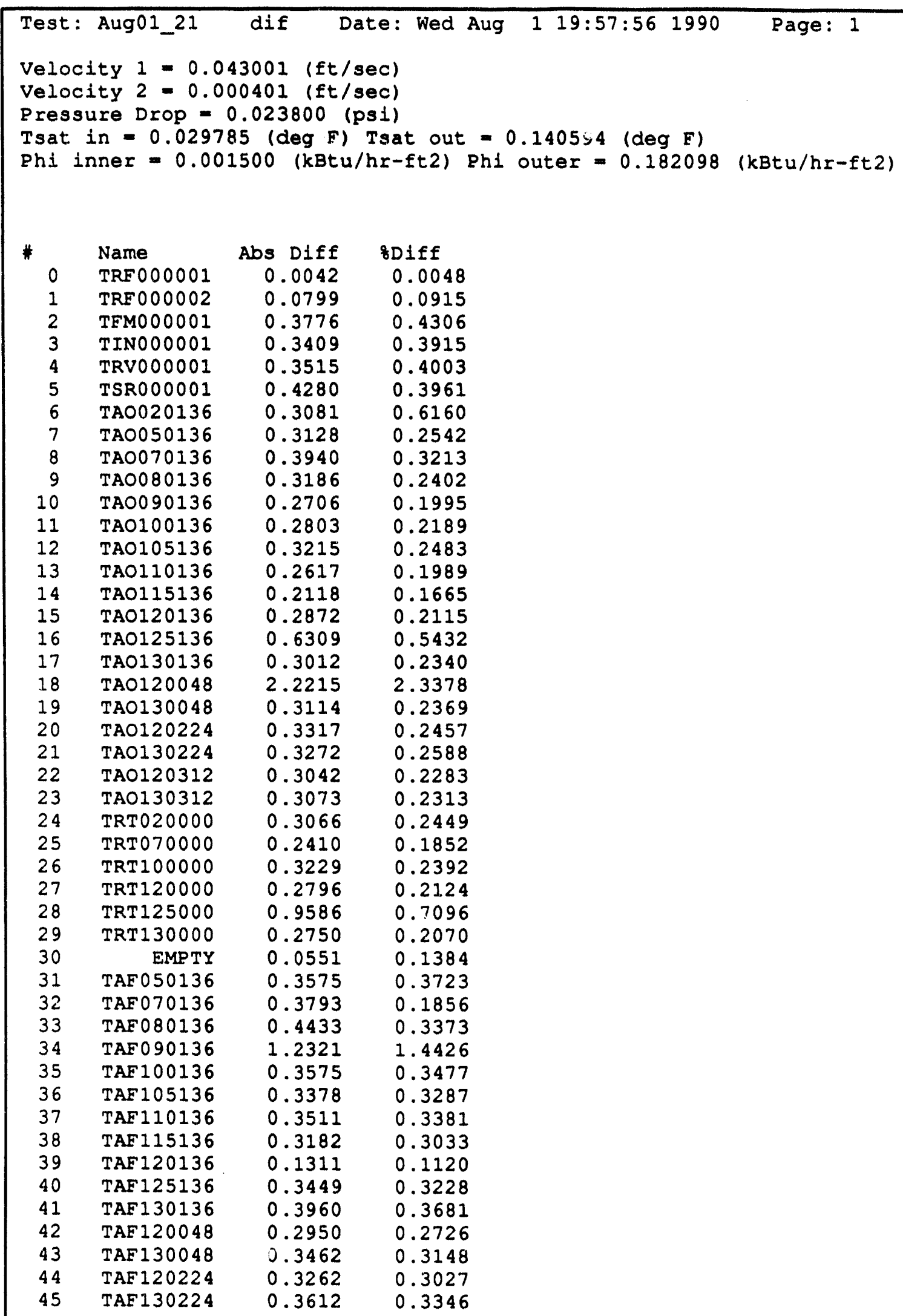




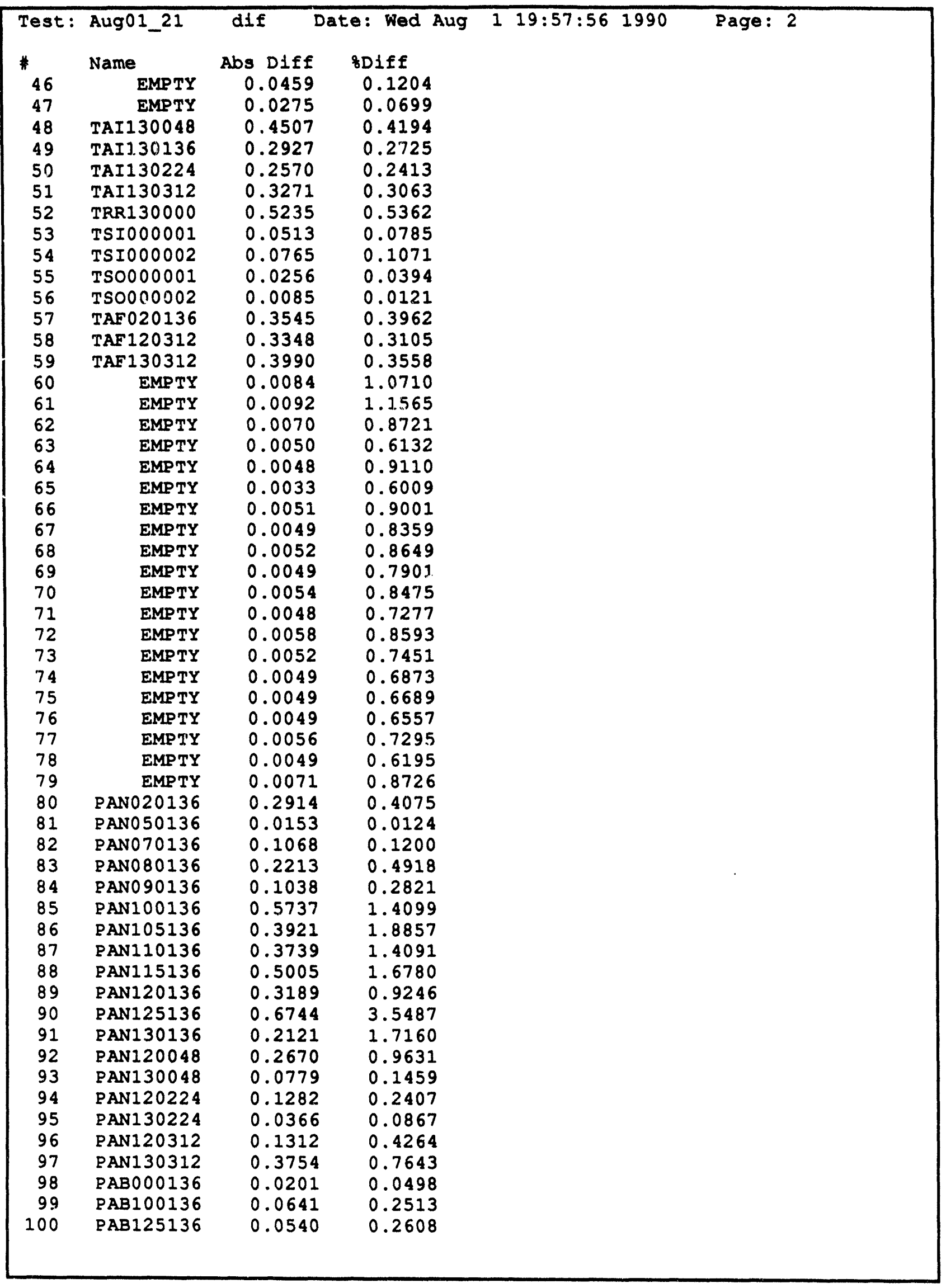





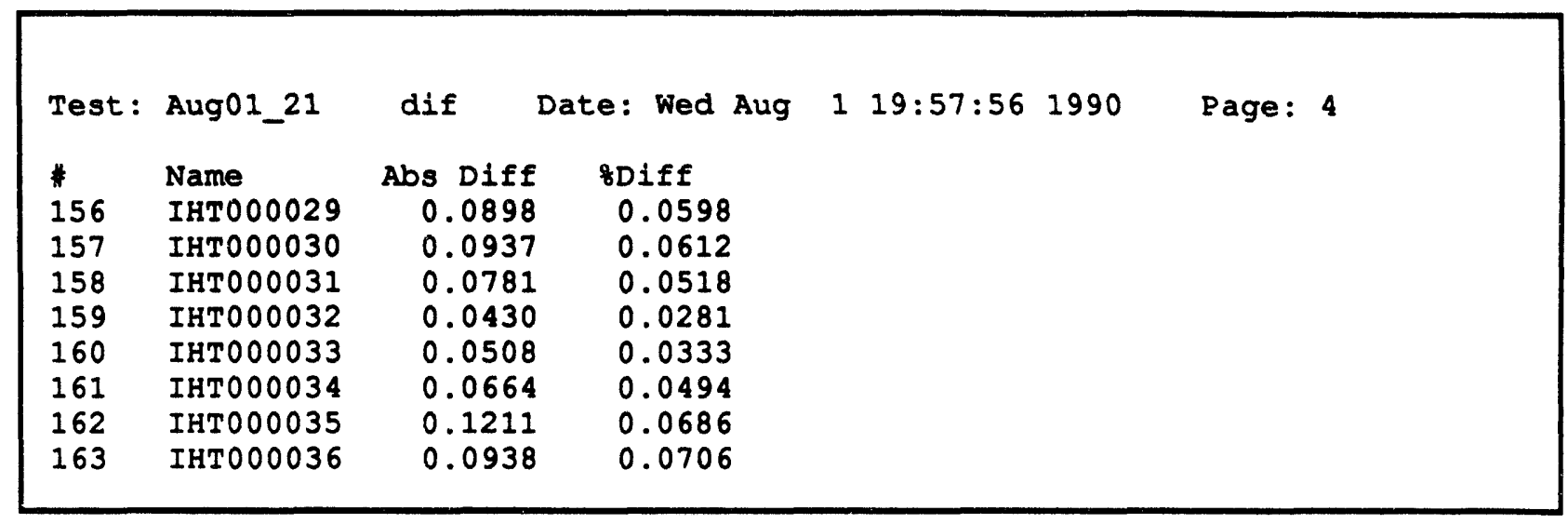




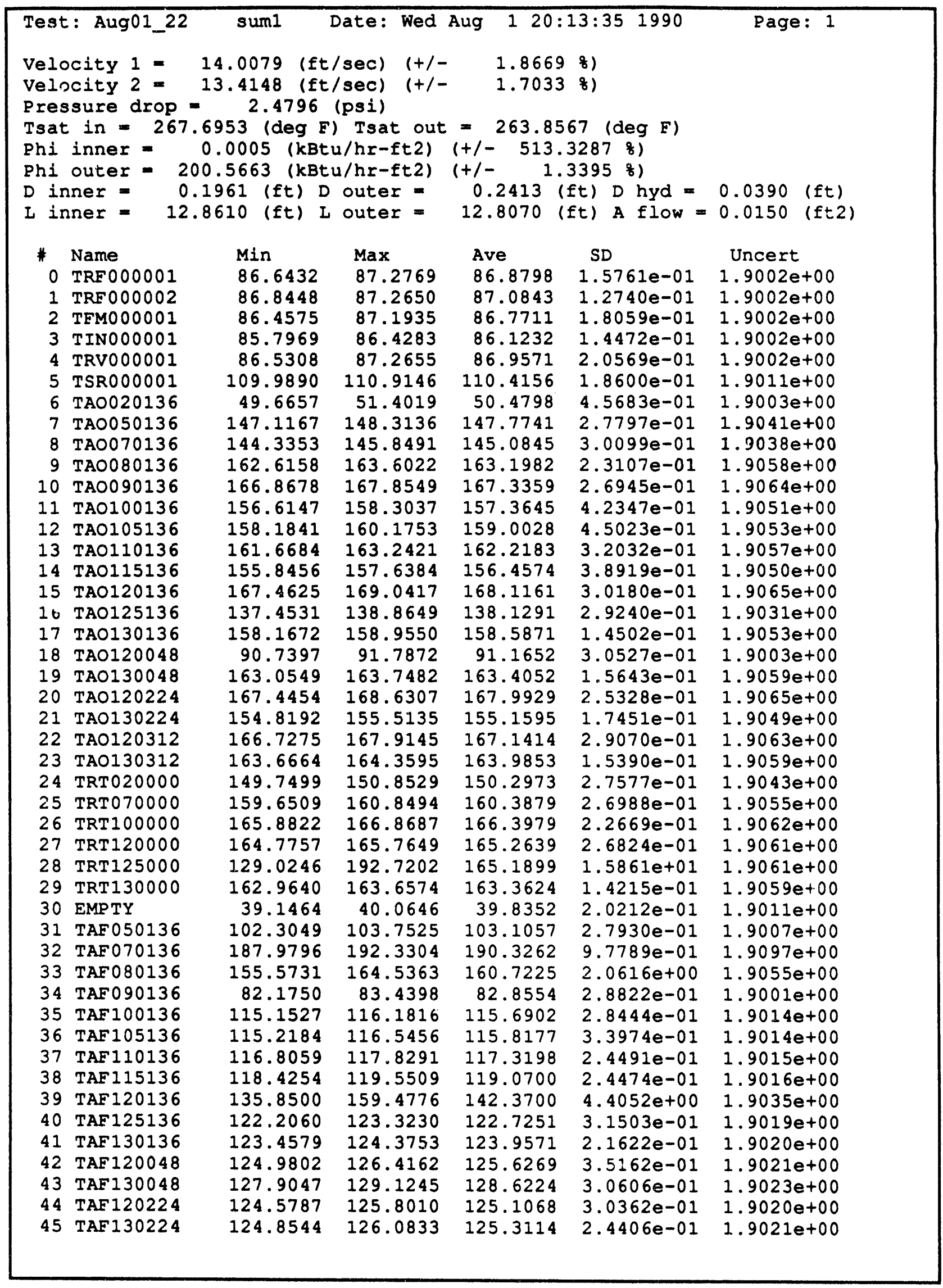




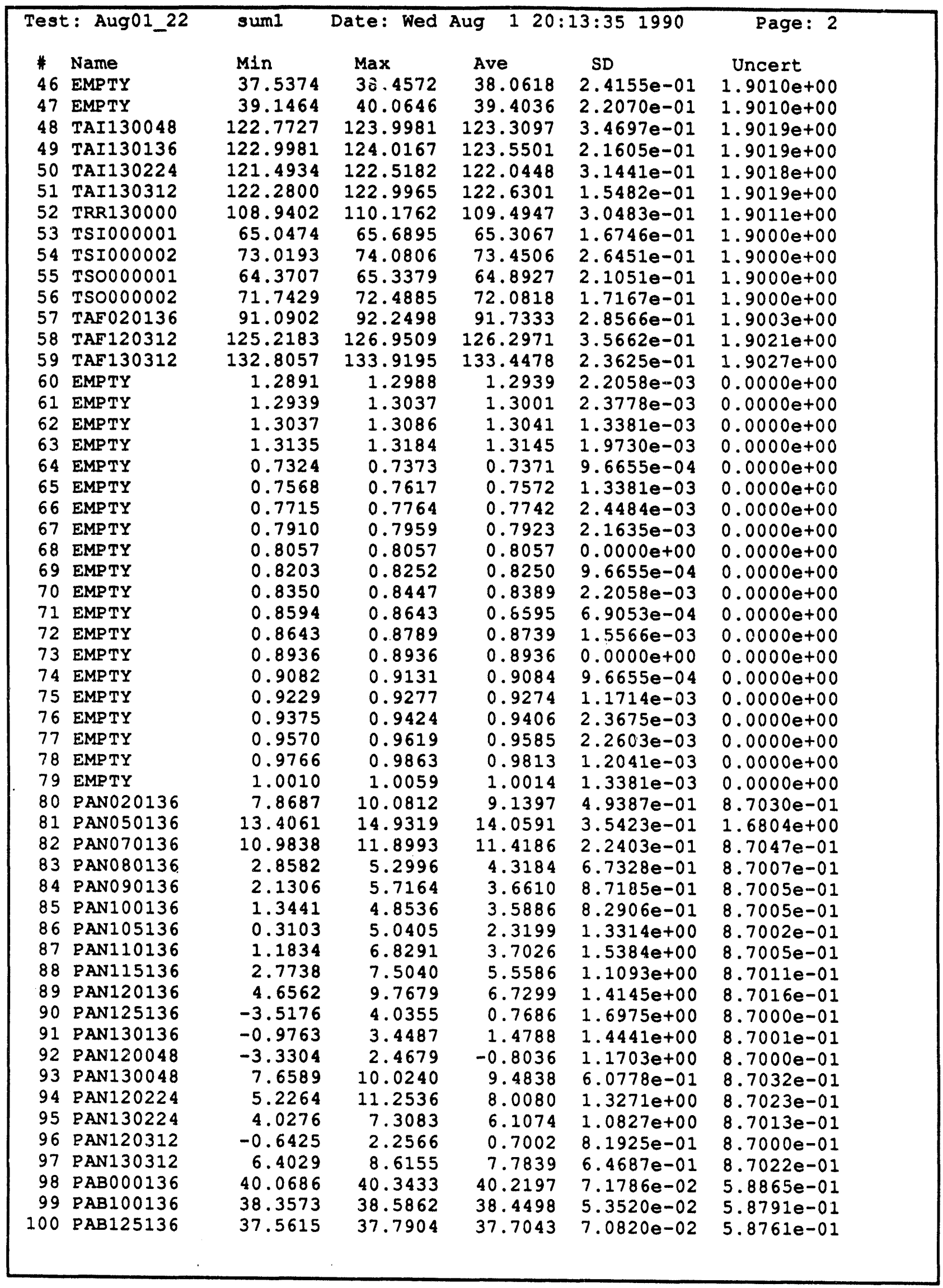




\begin{tabular}{|c|c|c|c|c|c|c|c|}
\hline \multicolumn{2}{|c|}{ Test: Aug01_22 } & sum1 & Date: Wed & Aug 120 & $13: 351990$ & Page: 3 & \\
\hline \# & Name & Min & $\operatorname{Max}$ & Ave & SD & Uncert & \\
\hline 101 & PIN000001 & 39.6476 & 40.2579 & 39.9698 & $1.3454 e-01$ & $7.5663 e-01$ & \\
\hline 102 & PRV000001 & 19.4847 & 19.8509 & 19.6849 & $7.2993 e-02$ & $7.5161 e-01$ & \\
\hline 103 & PSR000001 & 36.8210 & 37.0652 & 36.9260 & $4.9385 e-02$ & $7.5566 e-01$ & \\
\hline 104 & PFM000001 & 29.8127 & 30.4536 & 30.0800 & $1.3822 e-01$ & $5.0562 e-01$ & \\
\hline 105 & PANO00136 & 65.4345 & 71.8432 & 68.6480 & $1.4431 e+00$ & $4.9230 \mathrm{e}+00$ & \\
\hline 106 & PFM000002 & 27.5702 & 27.5702 & 27.5702 & $9.6336 e-06$ & $2.1154 e-01$ & \\
\hline 107 & EMPTY & 0.0049 & 0.0098 & 0.0067 & $2.3941 e-03$ & $0.0000 e+00$ & \\
\hline 108 & EMPTY & -10.0000 & 9.9951 & -8.8132 & $3.1332 e+00$ & $0.0000 e+00$ & \\
\hline 109 & EMPTY & 0.0049 & 0.0098 & 0.0050 & $6.9053 e-04$ & $0.0000 e+00$ & \\
\hline 110 & EMPTY & -10.0000 & -10.0000 & -10.0000 & $0.0000 e+00$ & $0.0000 e+00$ & \\
\hline 111 & EMPTY & 0.0000 & 0.0049 & 0.0002 & $9.6655 e-04$ & $0.0000 e+00$ & \\
\hline 112 & VPW000001 & 144.4599 & 145.0458 & 144.7334 & $1.6741 \mathrm{e}-01$ & $1.1875 e+00$ & \\
\hline 113 & VPW0 00002 & 168.1002 & 168.4908 & 168.3189 & $1.2871 e-01$ & $1.2946 e+00$ & \\
\hline 114 & VPW000003 & 0.0526 & 0.4432 & 0.1581 & $1.0595 e-01$ & $8.1000 e-01$ & \\
\hline 115 & VRW000004 & 148.1483 & 148.7342 & 148.4257 & $1.3720 e-01$ & $1.2038 e+00$ & \\
\hline 116 & VPW0 00005 & 196.9689 & 197.5549 & 197.2774 & $1.4812 e-01$ & $1.4343 e+00$ & \\
\hline 117 & VPW000006 & 0.1089 & 0.3042 & 0.2027 & $9.8569 e-02$ & $8.1000 e-01$ & \\
\hline 118 & IPW0 00001 & 773.1656 & 777.0703 & 775.4693 & $1.4051 \mathrm{e}+00$ & $1.0156 e+01$ & \\
\hline 119 & IPW0 00002 & 1825.8628 & 1831.7166 & 1829.2970 & $1.5550 \mathrm{e}+00$ & $1.6567 \mathrm{e}+01$ & \\
\hline 120 & IPW000003 & 4.6875 & 8.5875 & 6.6375 & $1.4204 \mathrm{e}+00$ & $8.1002 e+00$ & \\
\hline 121 & IPWO 00004 & 537.7854 & 540.2268 & 539.4258 & $5.1975 e-01$ & $4.7074 \mathrm{e}+00$ & \\
\hline 122 & IPW000005 & 356.8134 & 357.7899 & 357.3016 & $3.6907 e-01$ & $3.4594 \mathrm{e}+00$ & \\
\hline 123 & IPW000006 & 0.6104 & 1.5869 & 1.0205 & $3.6057 e-01$ & $2.0000 e+00$ & \\
\hline 124 & EMPTY & -0.0293 & -0.0098 & -0.0177 & $3.8066 e-03$ & $0.0000 e+00$ & \\
\hline 125 & EMPTY & -0.0146 & -0.0049 & -0.0122 & $2.8334 e-03$ & $0.0000 e+00$ & \\
\hline 126 & EMPTY & -0.6244 & -0.0098 & -0.0145 & $3.6858 e-03$ & $0.0000 e+00$ & \\
\hline 127 & EMPTY & -0.0195 & -0.0049 & -0.0139 & $3.3249 e-03$ & $0.0000 \mathrm{e}+00$ & \\
\hline 128 & IHT000001 & -1.3784 & -0.2066 & -0.7613 & $2.3794 e-01$ & $4.1000 e+00$ & \\
\hline 129 & IHTO00002 & -1.4048 & -0.2329 & -0.6860 & $2.3157 e-01$ & $4.1000 e+00$ & \\
\hline 130 & IHT000003 & -1.2583 & -0.0864 & -0.7075 & $2.5170 e-01$ & $4.1000 e+00$ & \\
\hline 131 & IHT000004 & -1.1794 & -0.2028 & -0.6208 & $2.4645 e-01$ & $4.1000 e+00$ & \\
\hline 132 & IHTO00005 & -1.1831 & -0.2066 & -0.6480 & $2.4897 e-01$ & $4.1000 e+00$ & \\
\hline 133 & IHTO00006 & -1.6226 & -0.4507 & -0.9468 & $2.5331 e-01$ & $4.1000 e+00$ & \\
\hline 134 & IHT000007 & -1.2320 & -0.0601 & -0.5991 & $2.5438 e-01$ & $4.1000 e+00$ & \\
\hline 135 & IHTO00008 & -1.3296 & -0.1578 & -0.6070 & $2.4404 e-01$ & $4.1000 e+00$ & \\
\hline 136 & IHTO00009 & -1.3935 & -0.2216 & -0.6747 & $2.4465 e-01$ & $4.1000 e+00$ & \\
\hline 137 & IHTOOOOIO & -0.7287 & 0.8338 & 0.0174 & $4.1515 e-01$ & $4.1000 e+00$ & \\
\hline 138 & IHTO00011 & -0.3042 & 1.2583 & 0.3520 & $4.1151 e-01$ & $4.1000 e+00$ & \\
\hline 139 & IHTO00012 & -0.5559 & 1.0066 & 0.0926 & $4.3304 e-01$ & $4.1000 e+00$ & \\
\hline 140 & IHTO00013 & 130.6077 & 132.3655 & 131.3812 & $4.3755 e-01$ & $4.3054 e+00$ & \\
\hline 141 & IHTO00014 & 180.8594 & 182.4219 & 181.5898 & $4.1910 e-01$ & $4.4841 e+00$ & \\
\hline 142 & IHTO00015 & 135.1074 & 137.2558 & 136.0840 & $4.2131 e-01$ & $4.3199 e+00$ & \\
\hline 143 & IHT000016 & 126.0367 & 127.9898 & 127.0991 & $4.2711 e-01$ & $4.2925 e+00$ & \\
\hline 144 & IHTO00017 & 150.6235 & 153.1626 & 151.5376 & $5.0600 e-01$ & $4.3711 e+00$ & \\
\hline 145 & IHTO00018 & 152.3663 & 154.9053 & 153.3350 & $5.5658 e-01$ & $4.3773 e+00$ & \\
\hline 146 & IHTO00019 & 146.9163 & 149.2601 & 147.9476 & $5.9978 e-01$ & $4.3588 e+00$ & \\
\hline 147 & IHT000020 & 151.3108 & 153.6546 & 152.3187 & $5.3722 e-01$ & $4.3738 e+00$ & \\
\hline 148 & IHT000021 & -1.8292 & 1.1005 & -0.5284 & $5.9792 e-01$ & $4.1000 e+00$ & \\
\hline 149 & IHT000022 & 131.2537 & 133.9881 & 132.4296 & $5.7520 e-01$ & $4.3086 e+00$ & \\
\hline 150 & IHTO00023 & 148.4075 & 151.1418 & 149.7042 & $5.3635 e-01$ & $4.3648 e+00$ & \\
\hline 151 & IHTO00024 & 113.1949 & 115.7339 & 114.4526 & $5.147 \mathrm{se}-01$ & $4.2568 e+00$ & \\
\hline 152 & IHTOO0025 & 147.8591 & 150.3981 & 149.1442 & $5.5114 e-01$ & $4.3628 e+00$ & \\
\hline 153 & IHT000026 & 149.4403 & 152.1747 & 150.5966 & $5.9430 e-01$ & $4.3678 e+00$ & \\
\hline 154 & IHTO00027 & 128.0912 & 130.8256 & 129.3295 & $5.9137 e-01$ & $4.2991 e+00$ & \\
\hline 155 & IHTO00028 & 130.2209 & 132.3693 & 131.3615 & $5.6821 e-01$ & $4.3053 e+00$ & \\
\hline
\end{tabular}




\begin{tabular}{|c|c|c|c|c|c|}
\hline Test: Aug01_22 & suml & Date: Wed & 120 & $13: 351990$ & Page: 4 \\
\hline 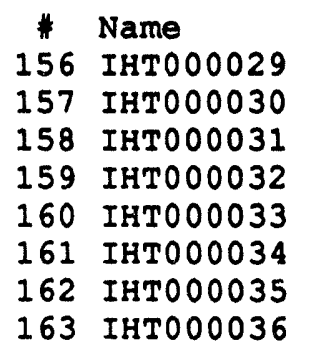 & $\begin{array}{l}\text { Min } \\
148.9408 \\
151.7428 \\
149.6582 \\
151.4649 \\
151.4648 \\
133.5562 \\
175.2629 \\
131.8434\end{array}$ & $\begin{array}{c}\text { Max } \\
150.8939 \\
153.8912 \\
151.8066 \\
154.0039 \\
153.8086 \\
135.3140 \\
177.2160 \\
133.4059\end{array}$ & $\begin{array}{l}\text { Ave } \\
149.9057 \\
152.7818 \\
150.6816 \\
152.6094 \\
152.4805 \\
134.3023 \\
176.2355 \\
132.6286\end{array}$ & $\begin{array}{l}\text { SD } \\
5.6553 e-01 \\
5.8851 e-01 \\
5.3315 e-01 \\
5.6636 e-01 \\
5.5943 e-01 \\
4.4589 e-01 \\
4.9204 e-01 \\
4.2952 e-01\end{array}$ & $\begin{array}{c}\text { Uncert } \\
4.3655 \mathrm{e}+00 \\
4.3754 \mathrm{e}+00 \\
4.3681 \mathrm{e}+00 \\
4.3748 \mathrm{e}+00 \\
4.3744 \mathrm{e}+00 \\
4.3144 \mathrm{e}+00 \\
4.4627 \mathrm{e}+00 \\
4.3092 \mathrm{e}+00\end{array}$ \\
\hline
\end{tabular}




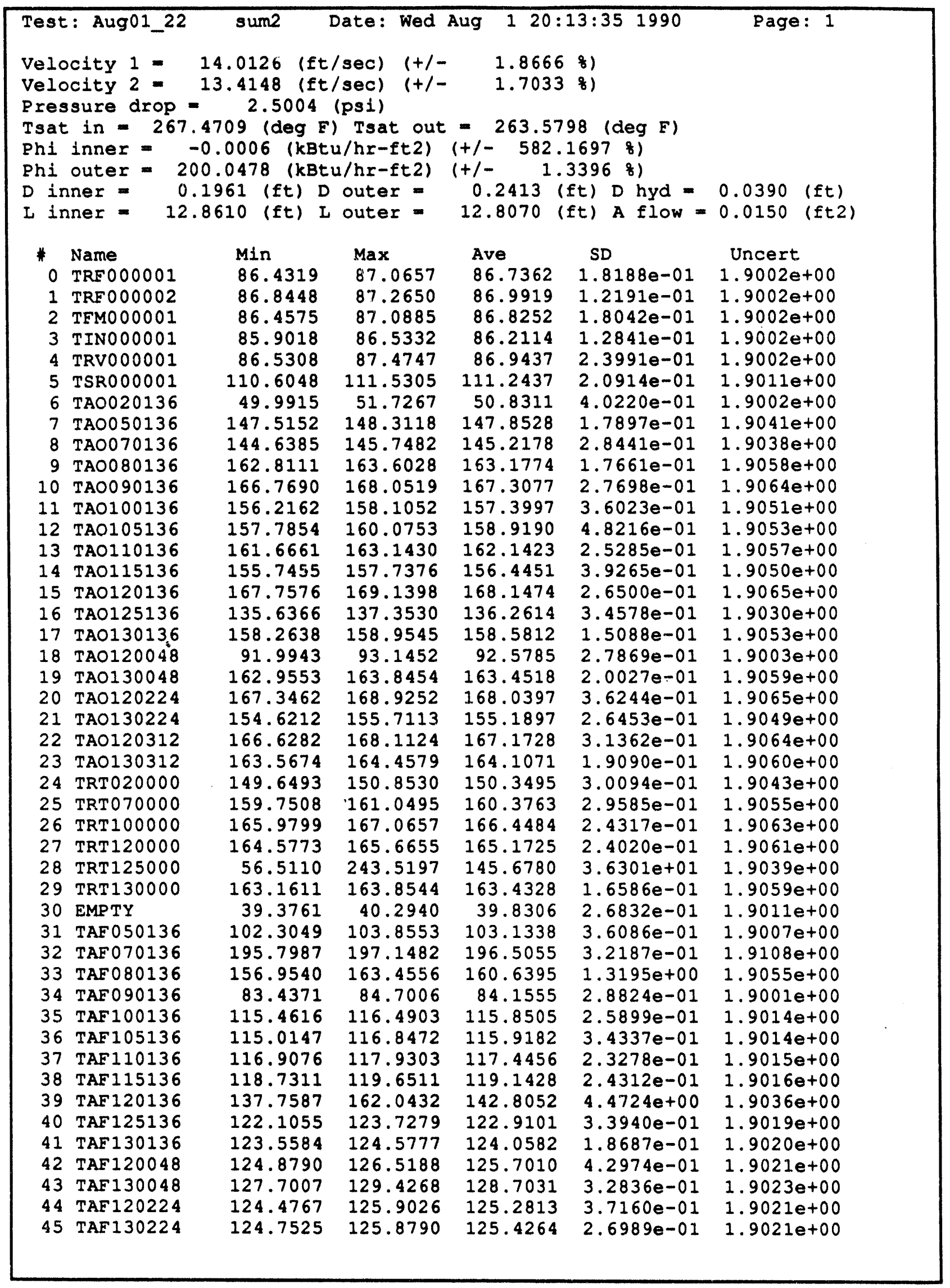




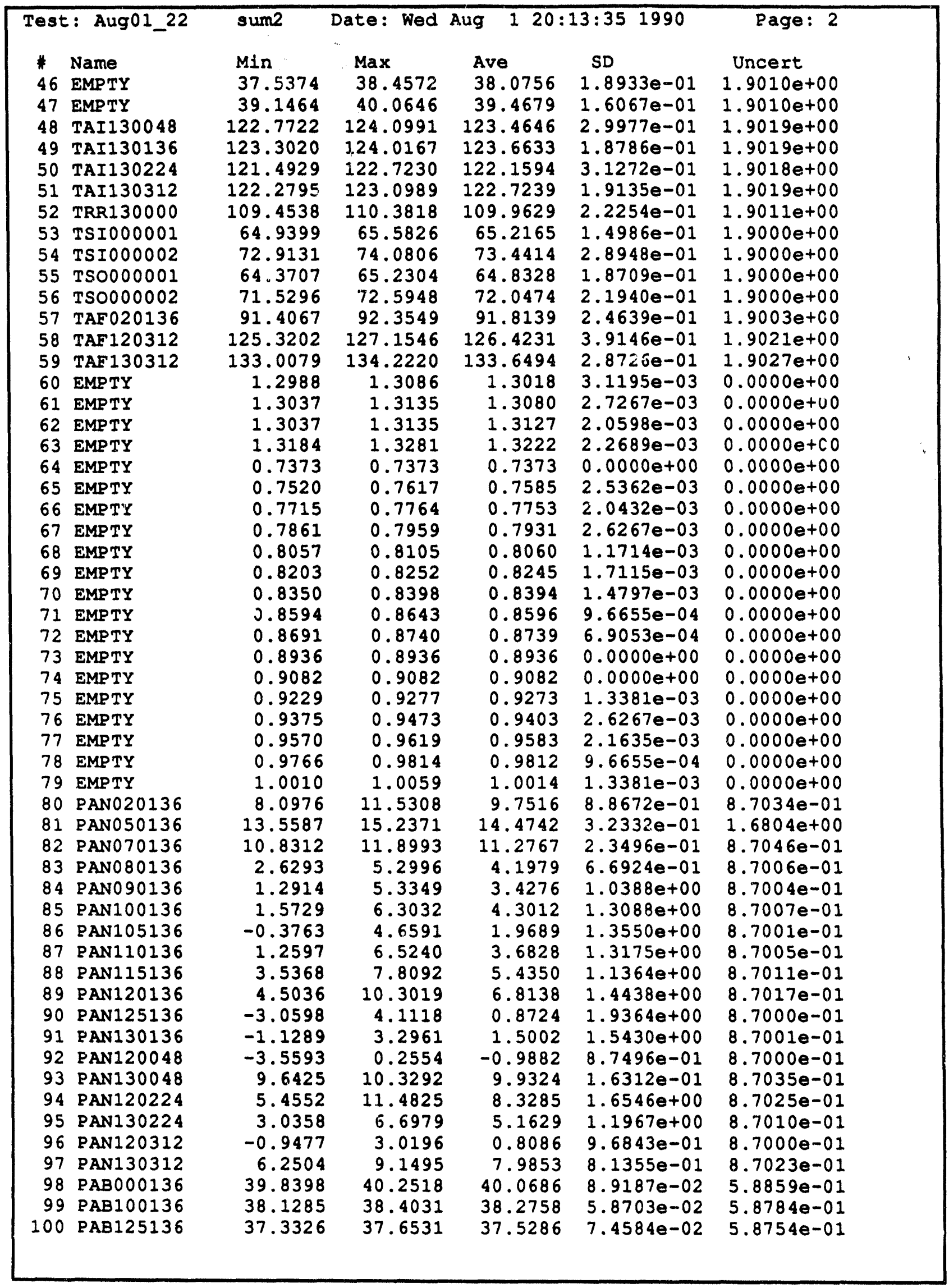




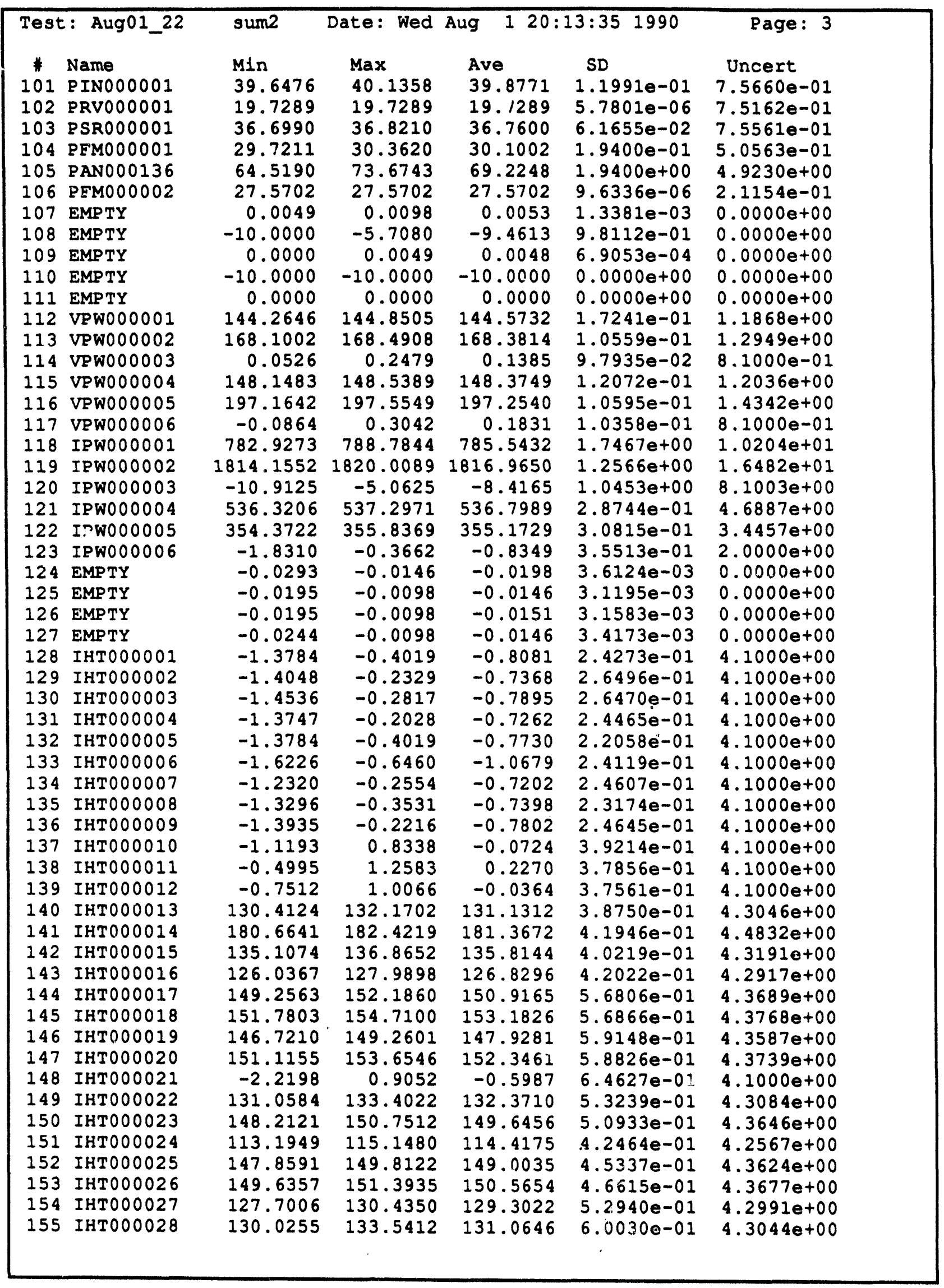




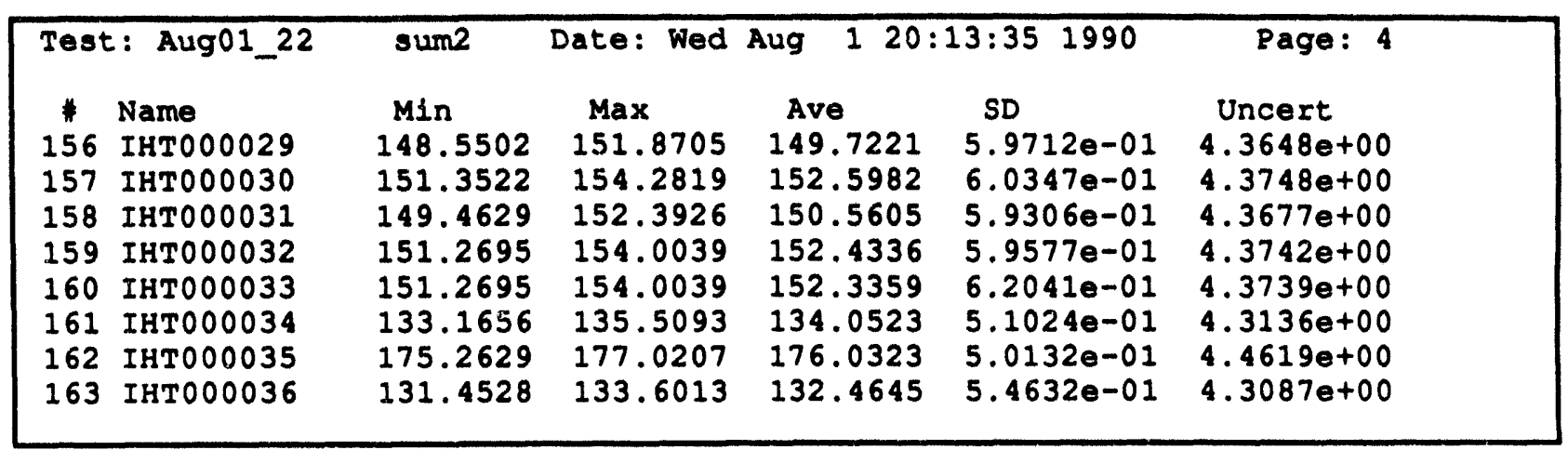




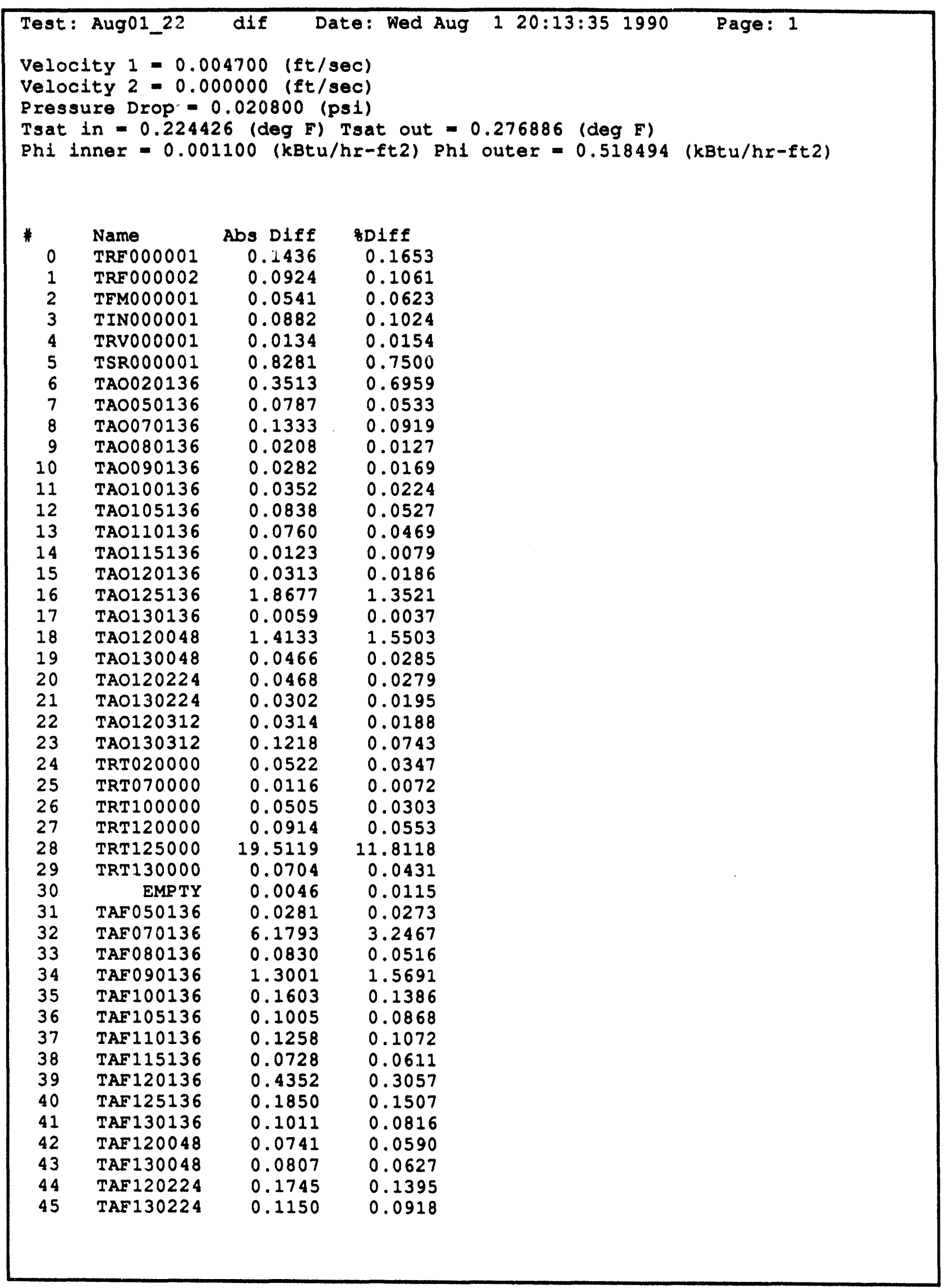




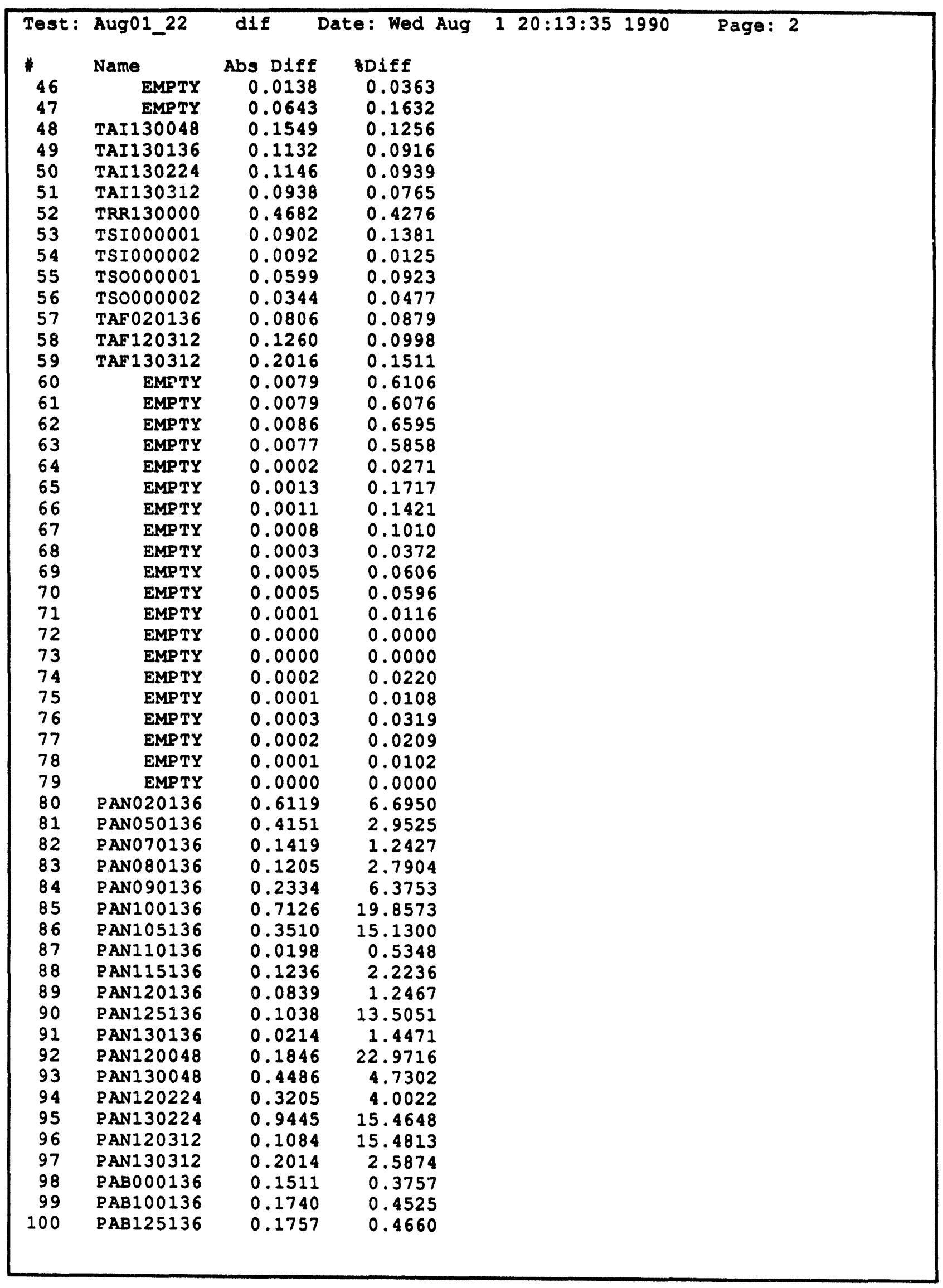




\begin{tabular}{|c|c|c|c|c|c|}
\hline Test: & Aug01_22 & dif & ate: Wed Aug & $120: 13: 351990$ & Page: 3 \\
\hline * & Name & Abs Diff & $8 D i f f$ & & \\
\hline 101 & PINO00001 & 0.0927 & 0.2319 & & \\
\hline 102 & PRV000001 & 0.0440 & 0.2235 & & \\
\hline 103 & PSRO00001 & 0.1660 & 0.4495 & & \\
\hline 104 & PFM000001 & 0.0202 & 0.0672 & & \\
\hline 105 & PAN000136 & 0.5768 & 0.8402 & & \\
\hline 106 & PEMO00002 & 0.0000 & 0.0000 & & \\
\hline 107 & EMPTY & 0.0014 & 20.8955 & & \\
\hline 108 & EMPTY & 0.6481 & 7.3537 & & \\
\hline 109 & EMPTY & 0.0002 & 4.0000 & & \\
\hline 110 & EMPTY & 0.0000 & 0.0000 & & \\
\hline 111 & EMPTY & 0.0002 & 100.0000 & & \\
\hline 112 & VPW000001 & 0.1602 & 0.1107 & & \\
\hline 113 & VPW000002 & 0.0625 & 0.0371 & & \\
\hline 114 & VPW000003 & 0.0196 & 12.3972 & & \\
\hline 115 & VPW000004 & 0.0508 & 0.0342 & & \\
\hline 116 & VPW000005 & 0.0234 & 0.0119 & & \\
\hline 117 & VEW000006 & 0.0196 & 9.6695 & & \\
\hline 118 & IPW000001 & 10.0739 & 1.2991 & & \\
\hline 119 & IPW0 00002 & 12.3320 & 0.6741 & & \\
\hline 120 & IPW000003 & 15.0540 & 226.8022 & & \\
\hline 121 & IPW000004 & 2.6269 & 0.4870 & & \\
\hline 122 & IPWO000005 & 2.1287 & 0.5958 & & \\
\hline 123 & IPW000006 & 1.8554 & 181.8129 & & \\
\hline 124 & ЕMPTY & 0.0021 & 11.8644 & & \\
\hline 125 & EMPTY & 0.0024 & 19.6721 & & \\
\hline 126 & EMPTY & 0.0006 & 4.1379 & & \\
\hline 127 & EMPTY & 0.0007 & 5.0360 & & \\
\hline 128 & IHT000001 & 0.0468 & 6.1474 & & \\
\hline 129 & IHT000002 & 0.0508 & 7.4053 & & \\
\hline 130 & IHT 000003 & 0.0820 & 11.5901 & & \\
\hline 131 & IHT000004 & 0.1054 & 16.9781 & & \\
\hline 132 & IHT000005 & 0.1250 & 19.2901 & & \\
\hline 133 & IHT000006 & 0.1211 & 12.7904 & & \\
\hline 134 & IHT000007 & 0.1211 & 20.2137 & & \\
\hline 135 & IHT000008 & 0.1328 & 21.8781 & & \\
\hline 136 & IHT000009 & 0.1055 & 15.6366 & & \\
\hline 137 & IHT000010 & 0.0898 & 516.0920 & & \\
\hline 138 & IHT000011 & 0.1250 & 35.5114 & & \\
\hline 139 & IHTO00012 & 0.1290 & 139.3089 & & \\
\hline 140 & IHTO00013 & 0.2500 & 0.1903 & & \\
\hline 141 & IHTO00014 & 0.2226 & 0.1226 & & \\
\hline 142 & IHTO00015 & 0.2696 & 0.1981 & & \\
\hline 143 & IHT000016 & 0.2695 & 0.2120 & & \\
\hline 144 & IHTO00017 & 0.6211 & 0.4099 & & \\
\hline 145 & IHT000018 & 0.1524 & 0.0994 & & \\
\hline 146 & IHTO00019 & 0.0195 & 0.0132 & & \\
\hline 147 & IHT000020 & 0.0274 & 0.0180 & & \\
\hline 148 & IHTO00021 & 0.0703 & 13.3043 & & \\
\hline 149 & IHT000022 & 0.0586 & 0.0442 & & \\
\hline 150 & IHT000023 & 0.0586 & 0.0391 & & \\
\hline 151 & IHTO00024 & 0.0351 & 0.0307 & & \\
\hline 152 & IHT000025 & 0.1407 & 0.0943 & & \\
\hline 153 & IHT000026 & 0.0312 & 0.0207 & & \\
\hline 154 & IHT000027 & 0.0273 & 0.0211 & & \\
\hline 155 & IHT000028 & 0.2969 & 0.2260 & & \\
\hline
\end{tabular}




\begin{tabular}{|c|c|c|c|c|c|}
\hline Test: & Aug01_22 & dif & Date: Wed Aug & $120: 13: 351990$ & Page: 4 \\
\hline $\begin{array}{l}* \\
156 \\
157 \\
158 \\
159 \\
160 \\
161 \\
162 \\
163\end{array}$ & 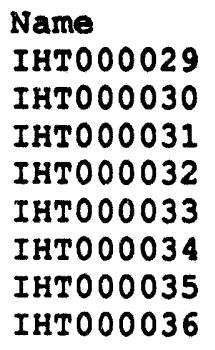 & $\begin{array}{r}\text { Abs Diff } \\
0.1836 \\
0.1836 \\
0.1211 \\
0.1758 \\
0.1446 \\
0.2500 \\
0.2032 \\
0.1641\end{array}$ & $\begin{array}{l}\text { 8Diff } \\
0.1225 \\
0.1202 \\
0.0804 \\
0.1152 \\
0.0948 \\
0.1861 \\
0.1153 \\
0.1237\end{array}$ & & \\
\hline
\end{tabular}




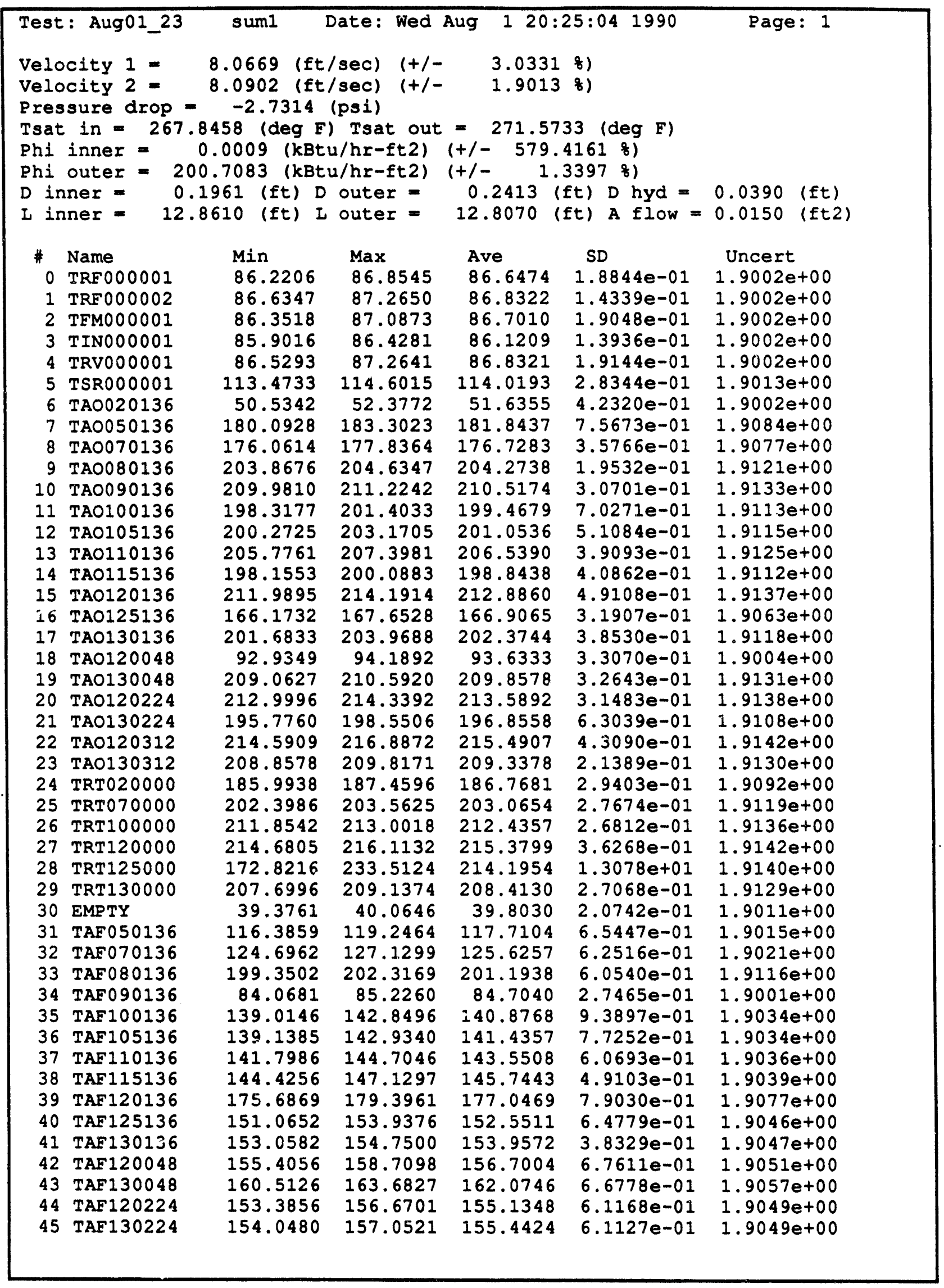




\begin{tabular}{|c|c|c|c|c|c|c|}
\hline & $\therefore$ Aug01_23 & sum1 & Date: Wed & Aug & $5: 041990$ & Page: \\
\hline$\pi$ & Name & Min & $\operatorname{Max}$ & Ave & SD & Uncert \\
\hline 46 & EMPTY & 37.7675 & 38.4572 & 38.0572 & $2.2666 \mathrm{e}-01$ & $1.9010 \mathrm{e}+00$ \\
\hline 47 & EMPTY & 39.3761 & 3352 & 5276 & $1.1925 e-01$ & $1.9011 e+00$ \\
\hline 48 & TAI130048 & 150.3606 & 152.0603 & 151.1045 & $3.7036 e-01$ & $1.9044 \mathrm{e}+00$ \\
\hline 49 & TAI130136 & 150.0952 & 150.8951 & 150.4912 & $1.9676 e-01$ & $1.9044 \mathrm{e}+00$ \\
\hline 50 & TAI130224 & 148.7684 & 149.7723 & 149.3149 & $2.6829 e-01$ & $1.9042 e+00$ \\
\hline 51 & TAI130312 & 148.5057 & 149.6083 & 149.0830 & $2.4188 e-01$ & $1.9042 \mathrm{e}+00$ \\
\hline 52 & TRR130000 & 131.6838 & 132.6964 & 132.2430 & $2.1190 e-01$ & $1.9026 e+00$ \\
\hline 53 & TSI000001 & 64.8323 & 65.7947 & 65.3844 & $1.9005 e-01$ & $1.9000 e+00$ \\
\hline 54 & TSIO0 02 & 77.2515 & 78.2032 & 77.7128 & $2.4991 e-01$ & $1.9000 e+00$ \\
\hline 55 & TSO000001 & 64.3713 & 65.3385 & $6 \div .8396$ & $2.3758 e-01$ & $1.9000 e+00$ \\
\hline 56 & TSO000002 & 991 & 75.2497 & 74.8163 & $2.1122 e-01$ & $1.9000 e+00$ \\
\hline 57 & TAF020136 & 525 & 98.9757 & 97.5534 & $5.0939 e-01$ & $1.9005 e+00$ \\
\hline 58 & TAF 120312 & 156.6525 & 159.5372 & 158.3595 & $6.3395 e-01$ & $1.9053 e+00$ \\
\hline 59 & TAF 130312 & 167.3539 & 170.7039 & 169.0927 & $6.6769 e-01$ & $1.9066 \mathrm{e}+00$ \\
\hline 60 & EMPTY & .1484 & 2.1777 & 2.1630 & $6.6534 e-03$ & $0 e+00$ \\
\hline 61 & EMPTY & 2.1582 & 2.1729 & 2.1611 & $4.0673 e-03$ & $0 e+00$ \\
\hline 62 & EMPTY & 2.1533 & 2.1680 & 2.1595 & $3.5307 e-03$ & $0 e+00$ \\
\hline 63 & EMPTY & 2.1582 & 2.1729 & 2.1646 & $3.4793 e-03$ & $e+00$ \\
\hline 64 & EMPTY & 1.1035 & 1.1084 & 1.1047 & 2.10 & $e+00$ \\
\hline 65 & EMPTY & 1.1182 & 1.1279 & 1.1241 & $2.2689 e-03$ & $0 e+00$ \\
\hline 66 & EMPTY & 1.1328 & 1.1475 & 1.1380 & $2.8608 \epsilon$ & $e+00$ \\
\hline 67 & EMPTY & 1.1523 & 1.1572 & 1.1537 & $2.2146 e-03$ & $0 e+00$ \\
\hline 68 & EMPTY & 1.1621 & 1.1719 & 1.1649 & $2.9742 e-03$ & De+0o \\
\hline 69 & EMPTY & 1.1768 & 1.1865 & 1.1796 & 3.5691 & $\mathrm{De}+00$ \\
\hline 70 & EMPTY & 1.1914 & 1.1963 & 1.1933 & 2.3941 & $0 e+00$ \\
\hline 71 & EMPTY & 1.2109 & 1.2158 & 1.2126 & $2.3365 e-03$ & $0 e+00$ \\
\hline 72 & EMPTY & 1.2207 & 1.2305 & 1.2236 & $2.9594 e-03$ & $10 e+00$ \\
\hline 73 & EMPTY & 1.2354 & 1.2451 & 1.2421 & 2.5894 & $0 e+00$ \\
\hline 74 & EMPTY & 1.2500 & 1.2598 & 1.2544 & $2.8334 e-03$ & $e+00$ \\
\hline 75 & EMPTY & 1.2646 & 1.2744 & 1.2703 & $2.8523 \mathrm{e}$ & $2+00$ \\
\hline 76 & EMPTY & 1.2793 & 1.2842 & 1.2817 & $2.4662 e-03$ & $0 e+00$ \\
\hline 77 & EMPTY & 1.2939 & 1.3037 & 1.2982 & $2.1249 e-03$ & $0.0000 e+00$ \\
\hline 78 & EMPTY & 1.3135 & 1.3232 & 1.3185 & $1.8429 e-03$ & ee+00 \\
\hline 79 & EMPTY & 1.3330 & 1.3428 & 1.3381 & $1.6972 e-03$ & ee+00 \\
\hline 80 & PAN020136 & -13.4173 & -3.4228 & -8.6902 & $2.1082 \mathrm{e}$ & $7 e-01$ \\
\hline 81 & PAN050136 & -24.4357 & -13.7546 & -18.8327 & $3.0082 e$ & $1.6807 \mathrm{e}+00$ \\
\hline 82 & PAN070136 & -11.9044 & -4.1987 & -8.8145 & $2.6785 e+00$ & $28 e-01$ \\
\hline 83 & PAN080136 & -14.0791 & -5.6104 & -12.3136 & $2.7531 \mathrm{e}+00$ & $8.7054 e-01$ \\
\hline 84 & PAN090136 & -13.2045 & 2.8172 & -7.6106 & $3.7728 e+00$ & $1 e-01$ \\
\hline 85 & PAN100136 & -12.0837 & 3.0225 & -6.4288 & $3.7108 \mathrm{e}+00$ & $e-01$ \\
\hline 86 & PAN 105136 & -7.7768 & 8.85 & -3.4082 & $3.5197 \mathrm{e}$ & 8.7 \\
\hline 87 & RAN110136 & -7.4 & 10.1861 & -0.3394 & $4.6110 \mathrm{e}$ & .01 \\
\hline 88 & PAN115136 & -7.6022 & 5.5967 & -2.7880 & $3.0066 \mathrm{e}+00$ & $8.7003 e-01$ \\
\hline 9 & PAN120136 & -6.0250 & 10.5308 & 0.4112 & $4.3693 e+00$ & $=-01$ \\
\hline 90 & PAN125136 & -6.1116 & 8.4606 & -1.9780 & $3.7317 e+00$ & $8.7001 e-01$ \\
\hline 91 & PAN13 & -8.0717 & 7.56 & -1.1305 & 4.0 & 8. \\
\hline 92 & PAN120048 & -12.1042 & 3.3071 & -8.3002 & $4.1329 \mathrm{e}$ & $5 e-01$ \\
\hline 93 & PAN130048 & -11.4909 & 5.9804 & -1.7665 & $3.1347 e+00$ & $8.7001 e-01$ \\
\hline 94 & PAN120224 & -12.5501 & 8.9648 & -4.2692 & $4.4738 e+00$ & $e-01$ \\
\hline 95 & PAN1 30224 & -13.4437 & 4.4091 & -0.6492 & $3.4154 \mathrm{e}+00$ & $0 e-01$ \\
\hline 96 & PAN120312 & -12.4681 & 0.1967 & -8.7236 & $3.9637 e+00$ & $8.7027 e-01$ \\
\hline 97 & PAN130312 & -9.9240 & 2.0542 & -2.4075 & $2.8956 \mathrm{e}+00$ & \\
\hline 98 & PAB000136 & 40.1602 & 40.5722 & 40.3213 & $9.6661 \mathrm{e}-02$ & $5.8869 e-01$ \\
\hline 99 & PAB100136 & 42.1568 & 42.79 & 42.5779 & $1.4593 \mathrm{e}-01$ & 5.8 \\
\hline 100 & PAB125136 & 42.5054 & 43.1462 & 42.9100 & $1.5291 \mathrm{e}-01$ & $5.8984 e-01$ \\
\hline
\end{tabular}




\begin{tabular}{|c|c|c|c|c|c|c|}
\hline \multicolumn{2}{|c|}{ Test: Aug01_23 } & sum1 & \multicolumn{3}{|c|}{ Date: Wed Aug $120: 25: 041990$} & \multirow[t]{2}{*}{ Page: 3} \\
\hline \# & Name & Min & $\operatorname{Max}$ & Ave & SD & \\
\hline 101 & PIN000001 & 39.7696 & 40.3800 & 40.0699 & $1.3997 e-01$ & $7.5666 \mathrm{e}-01$ \\
\hline 102 & PRV000001 & 19.7289 & 19.8509 & 19.7484 & $4.5206 e-02$ & $7.5162 e-01$ \\
\hline 103 & PSR0000001 & 42.5583 & 42.6804 & 42.5852 & $5.1081 e-02$ & $7.5752 \mathrm{e}-01$ \\
\hline 104 & PFM000001 & 9.5795 & 10.0373 & 9.8780 & $9.5772 e-02$ & $5.0061 e-01$ \\
\hline 105 & PANO00136 & -81.5076 & -65.0281 & -75.6208 & $3.2501 e+00$ & $4.9236 e+00$ \\
\hline 106 & PFM000002 & 9.8172 & 10.0185 & 9.9356 & $5.3645 e-02$ & $2.0154 e-01$ \\
\hline 107 & EMPTY & 0.0049 & 0.0049 & 0.0049 & $0.0000 e+00$ & $0.0000 e+00$ \\
\hline 108 & EMPTY & -10.0000 & -1.6553 & -9.1734 & $1.3957 e+00$ & $0.0000 e+00$ \\
\hline 109 & EMPTY & 0.0000 & 0.0049 & 0.0039 & $1.9730 e-03$ & $0.0000 e+00$ \\
\hline 110 & EMPTY & -10.0000 & -10.0000 & -10.0000 & $0.0000 e+00$ & $0.0000 e+00$ \\
\hline 111 & EMPTY & 0.0000 & 0.0000 & 0.0000 & $0.0000 e+00$ & $0.0000 e+00$ \\
\hline 112 & VPW000001 & 144.4599 & 145.0458 & 144.7490 & $1.3805 e-01$ & $1.1876 \mathrm{e}+00$ \\
\hline 113 & VRW000002 & 168.4908 & 168.8815 & 168.6431 & $1.0654 e-01$ & $1.2961 \mathrm{e}+00$ \\
\hline 114 & VPW000003 & 0.0526 & 0.4432 & 0.1424 & $1.0595 e-01$ & $8.1000 e-01$ \\
\hline 115 & VPW000004 & 148.5389 & 148.9295 & 148.7382 & $1.0052 e-01$ & $1.2052 \mathrm{e}+00$ \\
\hline 116 & VPW000005 & 197.3595 & 197.7502 & 197.5704 & $9.5357 e-02$ & $1.4357 e+00$ \\
\hline 117 & VPW000006 & -0.0864 & 0.4995 & 0.1792 & $1.0992 e-01$ & $8.1000 e-01$ \\
\hline 118 & IPW 000001 & 763.4039 & 769.2609 & 766.5275 & $1.3082 \mathrm{e}+00$ & $1.0113 e+01$ \\
\hline 119 & IPW000002 & 1837.5704 & 1843.4242 & 1841.0831 & $1.5769 e+00$ & $1.6648 e+01$ \\
\hline 120 & IPW000003 & 12.4875 & 16.3875 & 14.4765 & $1.2760 \mathrm{e}+00$ & $8.1008 e+00$ \\
\hline 121 & IPW000004 & 534.3674 & 536.3206 & 535.3243 & $3.6858 e-01$ & $4.6781 e+00$ \\
\hline 122 & IPW000005 & 353.8840 & 355.3487 & 354.4796 & $3.3143 e-01$ & $3.4412 e+00$ \\
\hline 123 & IPW000006 & -0.3662 & 1.0987 & 0.2295 & $3.4583 e-01$ & $2.0000 e+00$ \\
\hline 124 & EMPTY & -0.0293 & -0.0146 & -0.0198 & $3.4751 e-03$ & $0.0000 e+00$ \\
\hline 125 & EMPTY & -0.0195 & -0.0098 & -0.0139 & $2.6762 e-03$ & $0.0000 e+00$ \\
\hline 126 & EMPTY & -0.0244 & -0.0098 & -0.0146 & $3.8206 e-03$ & $0.0000 e+00$ \\
\hline 127 & EMPTY & -0.0195 & -0.0098 & -0.0147 & $3.6232 e-03$ & $0.0000 e+00$ \\
\hline 128 & IHT000001 & -1.3784 & -0.2066 & -0.7417 & $2.8675 e-01$ & $4.1000 e+00$ \\
\hline 129 & IHT000002 & -1.2094 & -0.2329 & -0.6430 & $2.6543 e-01$ & $4.1000 e+00$ \\
\hline 130 & IHT000003 & -1.2583 & -0.0864 & -0.6606 & $2.9373 e-01$ & $4.1000 e+00$ \\
\hline 131 & IHTO00004 & -1.1794 & -0.0075 & -0.5934 & $2.7052 e-01$ & $4.1000 e+00$ \\
\hline 132 & IHT000005 & -1.1831 & -0.2066 & -0.6831 & $2.6809 e-01$ & $4.1000 e+00$ \\
\hline 133 & IHT000006 & -1.4273 & -0.2554 & -0.9195 & $2.8179 \mathrm{e}-01$ & $4.1000 e+00$ \\
\hline 134 & IHT000007 & -1.2320 & 0.1352 & -0.5757 & $2.7835 e-01$ & $4.1000 e+00$ \\
\hline 135 & IHT000008 & -1.1343 & 0.0376 & -0.6187 & $2.7554 e-01$ & $4.1000 e+00$ \\
\hline 136 & IHT000009 & -1.1982 & -0.0263 & -0.6552 & $2.9074 e-01$ & $4.1000 \mathrm{e}+00$ \\
\hline 137 & IHTO00010 & -0.9240 & 0.8338 & -0.0412 & $3.8509 e-01$ & $4.1000 e+00$ \\
\hline 138 & IHT000011 & -0.6949 & 1.0630 & 0.2817 & $3.8863 e-01$ & $4.1000 e+00$ \\
\hline 139 & IHT000012 & -0.9465 & 0.8113 & 0.0340 & $3.6908 e-01$ & $4.1000 e+00$ \\
\hline 140 & IHT000013 & 130.0218 & 131.9749 & 131.0140 & $4.0786 e-01$ & $4.3042 e+00$ \\
\hline 141 & IHTO00014 & 180.0781 & 182.2266 & 180.9727 & $4.0643 e-01$ & $4.4816 e+00$ \\
\hline 142 & IHT000015 & 134.9121 & 136.8652 & 135.6816 & $3.9736 \mathrm{e}-01$ & $4.3187 e+00$ \\
\hline 143 & IHT000016 & 125.8414 & 127.7945 & 126.5405 & $3.9674 e-01$ & $4.2908 e+00$ \\
\hline 144 & IHTO00017 & 149.2563 & 151.4048 & 150.2524 & $4.7062 e-01$ & $4.3666 e+00$ \\
\hline 145 & IHT000018 & 151.7803 & 153.7335 & 152.7842 & $4.3227 e-01$ & $4.3754 e+00$ \\
\hline 146 & IHT000019 & 146.5257 & 148.6741 & 147.4437 & $4.6897 e-01$ & $4.3571 e+00$ \\
\hline 147 & IHTO00020 & 151.1155 & 153.2640 & 151.9711 & $4.1739 e-01$ & $4.3726 e+00$ \\
\hline 148 & IHT000021 & -1.8292 & 0.7099 & -0.7667 & $5.0711 e-01$ & $4.1000 \mathrm{e}+00$ \\
\hline 149 & IHT000022 & 131.2537 & 133.2069 & 131.9100 & $4.4601 e-01$ & $4.3070 \mathrm{e}+00$ \\
\hline 150 & IHT000023 & 148.4075 & 150.3606 & 149.3761 & $4.1938 e-01$ & $4.3636 \mathrm{e}+00$ \\
\hline 151 & IHTO00024 & 113.1949 & 114.9527 & 114.0816 & $4.1425 e-01$ & $4.2558 \mathrm{e}+00$ \\
\hline 152 & IHT000025 & 147.8591 & 149.6169 & 148.7379 & $4.1806 e-01$ & $4.3615 e+00$ \\
\hline 153 & IHTO00026 & 149.2450 & 151.3935 & 150.2099 & $4.2206 \mathrm{e}-01$ & $4.3665 e+00$ \\
\hline 154 & IHTO00027 & 127.8959 & 130.0443 & 128.8217 & $4.4784 e-01$ & $4.2976 \mathrm{e}+00$ \\
\hline 155 & IHTO00028 & 129.4396 & 131.9787 & 130.5450 & $4.7615 e-01$ & $4.3028 e+00$ \\
\hline
\end{tabular}




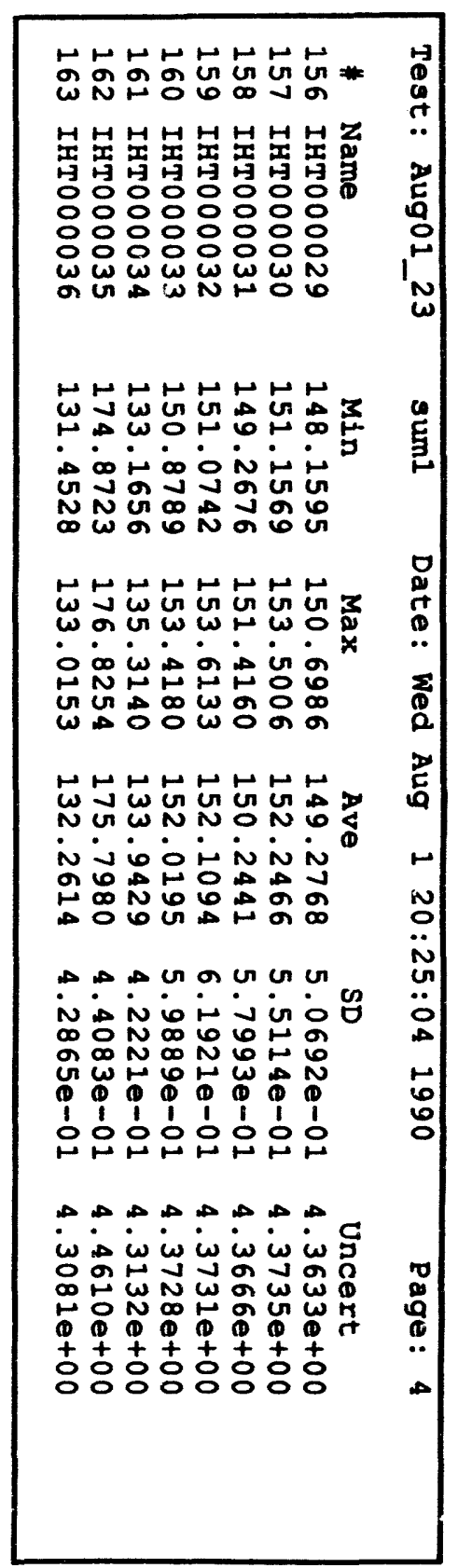




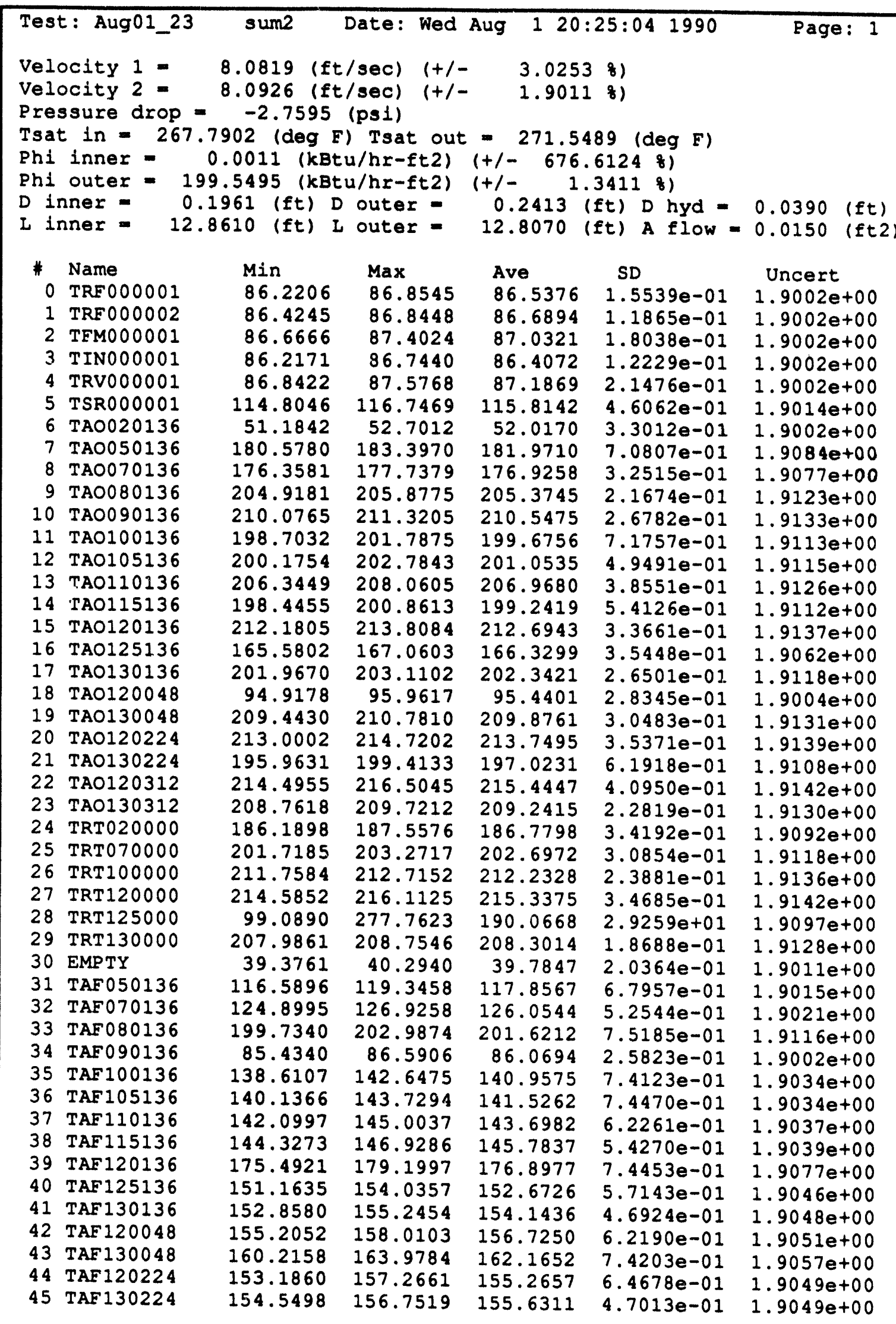




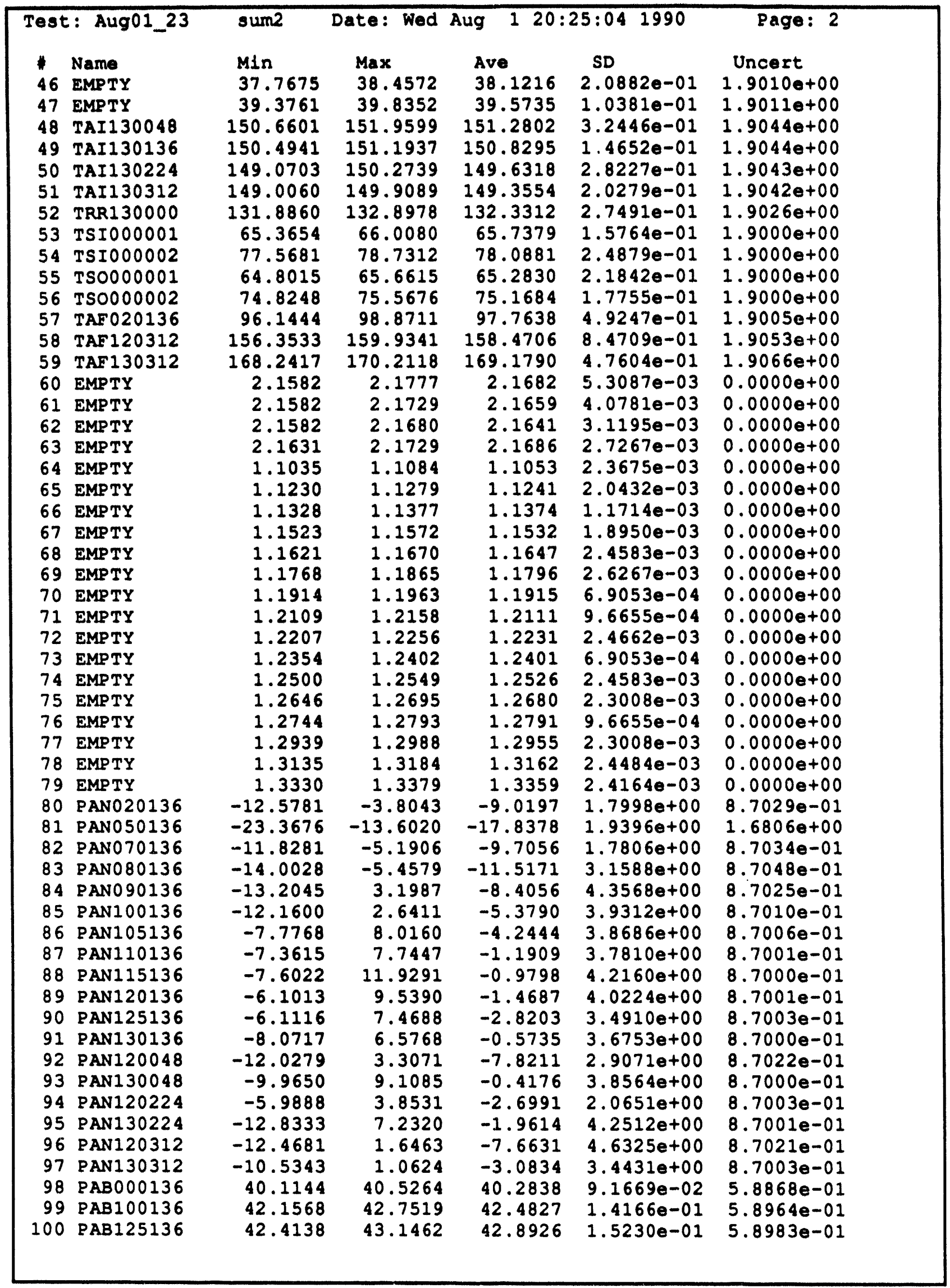




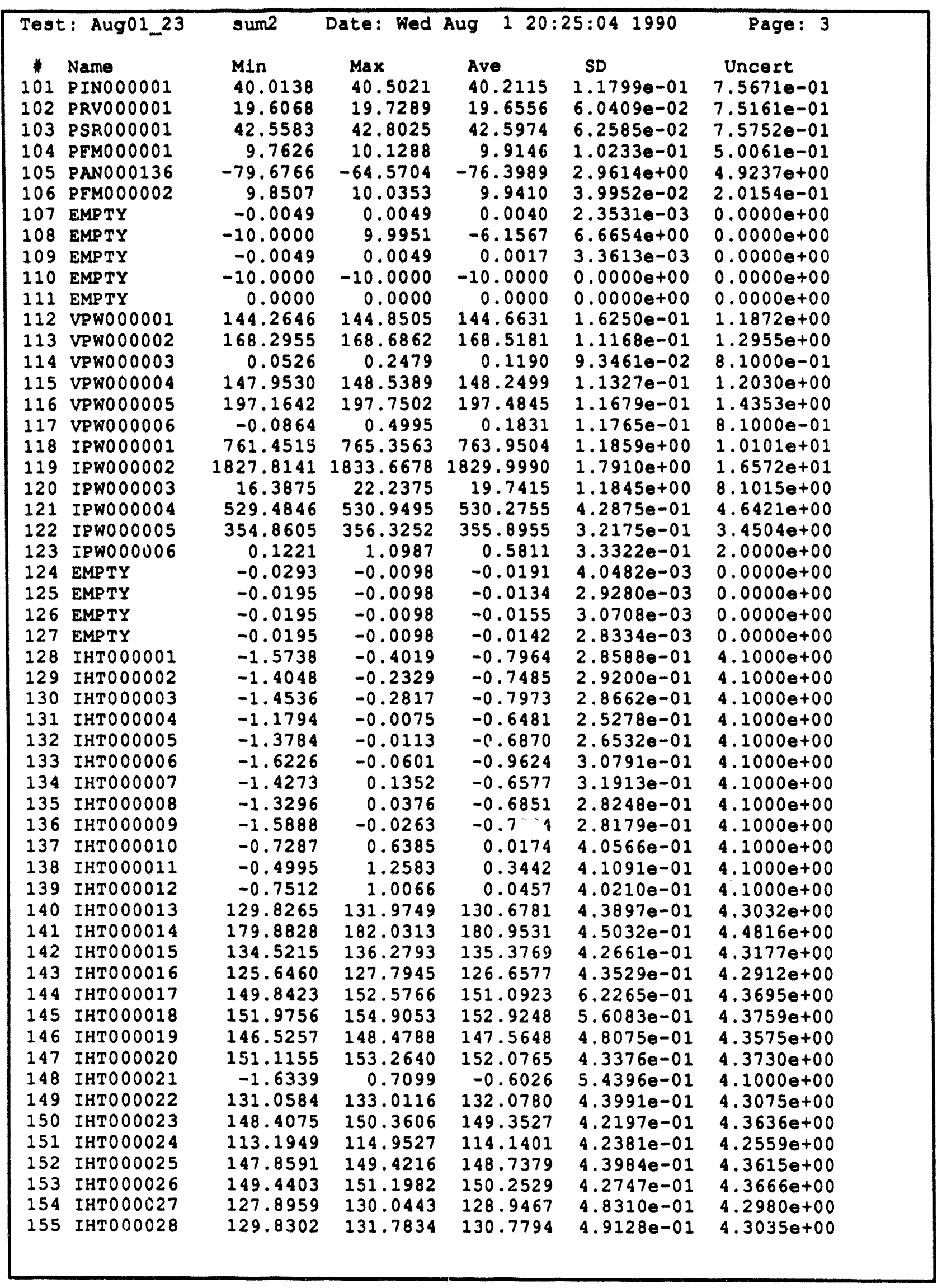




\begin{tabular}{|c|c|c|c|c|c|c|}
\hline \multicolumn{2}{|c|}{ Test: Aug01_23 } & sum2 & Date: Wed & \multicolumn{2}{|c|}{ Aug $120: 25: 041990$} & $\begin{array}{l}\text { Page: } 4 \\
\text { Uncert }\end{array}$ \\
\hline $\begin{array}{l}1 \\
156 \\
157 \\
158 \\
159 \\
160 \\
161 \\
162 \\
163\end{array}$ & 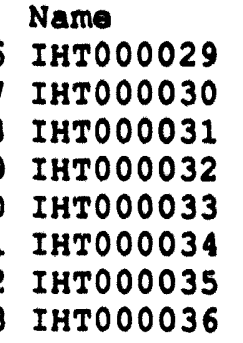 & $\begin{array}{c}\operatorname{Min} \\
148.3549 \\
151.3522 \\
149.2676 \\
151.2695 \\
151.0742 \\
132.7749 \\
174.6770 \\
131.2575\end{array}$ & $\begin{array}{l}\operatorname{Max} \\
150.5033 \\
153.5006 \\
151.4160 \\
153.4180 \\
153.4180 \\
134.3374 \\
176.6301 \\
132.8200\end{array}$ & $\begin{array}{l}\text { Ave } \\
149.3706 \\
152.2935 \\
150.2637 \\
152.1367 \\
152.0586 \\
133.4702 \\
175.6261 \\
131.8161\end{array}$ & $\begin{array}{l}\text { SD } \\
5.0686 e-01 \\
5.3480 e-01 \\
5.0724 e-01 \\
5.3983 e-01 \\
5.6629 e-01 \\
3.7259 e-01 \\
4.4470 e-01 \\
3.7644 e-01\end{array}$ & $\begin{array}{c}\text { Uncert } \\
4.3636 e+00 \\
4.3737 e+00 \\
4.3667 e+00 \\
4.3732 e+00 \\
4.3729 e+00 \\
4.3118 e+00 \\
4.4603 e+00 \\
4.3067 e+00\end{array}$ \\
\hline
\end{tabular}




\begin{tabular}{|c|c|c|c|c|}
\hline \multicolumn{5}{|c|}{$\begin{array}{l}\text { Velocity } 1=0.014999 \text { (ft/sec) } \\
\text { Velocity } 2=0.002399 \text { (ft/sec) } \\
\text { Pressure Drop }=0.028100 \text { (psi) } \\
\text { Tsat in }=0.055603 \text { (deg F) Tsat out }=0.024414 \text { (deg F) } \\
\text { Ph1 inner }=0.000200 \text { (kBtu/hr-ft2) Phi outer }=1.158798 \text { (kBtu/hr-ft2) }\end{array}$} \\
\hline $\begin{array}{r}0 \\
0 \\
1 \\
2 \\
3 \\
4 \\
5 \\
6 \\
7 \\
8 \\
9 \\
10 \\
11 \\
12 \\
13 \\
14 \\
15 \\
16 \\
17 \\
18 \\
19 \\
20 \\
21 \\
22 \\
23 \\
24 \\
25 \\
26 \\
27 \\
28 \\
29 \\
30 \\
31 \\
32 \\
33 \\
34 \\
35 \\
36 \\
37 \\
38 \\
39 \\
40 \\
41 \\
42 \\
43 \\
44 \\
45\end{array}$ & $\begin{array}{l}\text { Name } \\
\text { TRF000001 } \\
\text { TRFO00002 } \\
\text { TFMO00001 } \\
\text { TINO00001 } \\
\text { TRVO00001 } \\
\text { TSRO00001 } \\
\text { TAO020136 } \\
\text { TAO050136 } \\
\text { TAO070136 } \\
\text { TAO080136 } \\
\text { TAO090136 } \\
\text { TAO100136 } \\
\text { TAO105136 } \\
\text { TAO110136 } \\
\text { TAO115136 } \\
\text { TAO120136 } \\
\text { TAO125136 } \\
\text { TAO130136 } \\
\text { TAO120048 } \\
\text { TAO130048 } \\
\text { TAO120224 } \\
\text { TAO130224 } \\
\text { TAO120312 } \\
\text { TAO130312 } \\
\text { TRT020000 } \\
\text { TRT070000 } \\
\text { TRT100000 } \\
\text { TRT120000 } \\
\text { TRT125000 } \\
\text { TRT130000 } \\
\text { EMPTY } \\
\text { TAF050136 } \\
\text { TAF070136 } \\
\text { TAF080136 } \\
\text { TAF090136 } \\
\text { TAF100136 } \\
\text { TAF105136 } \\
\text { TAF110136 } \\
\text { TAF115136 } \\
\text { TAF120136 } \\
\text { TAF125136 } \\
\text { TAF130136 } \\
\text { TAF120048 } \\
\text { TAF130048 } \\
\text { TAF120224 } \\
\text { TAF130224 }\end{array}$ & $\begin{array}{l}\text { AbS Diff } \\
0.1098 \\
0.1428 \\
0.3311 \\
0.2863 \\
0.3548 \\
1.7949 \\
0.3815 \\
0.1273 \\
0.1975 \\
1.1007 \\
0.0301 \\
0.2077 \\
0.0001 \\
0.4290 \\
0.3981 \\
0.1917 \\
0.5766 \\
0.0323 \\
1.8068 \\
0.0183 \\
0.1603 \\
0.1673 \\
0.0460 \\
0.0963 \\
0.0117 \\
0.3682 \\
0.2029 \\
0.0424 \\
24.1286 \\
0.1116 \\
0.0183 \\
0.1463 \\
0.4287 \\
0.4274 \\
1.3654 \\
0.0807 \\
0.0905 \\
0.1474 \\
0.0394 \\
0.1492 \\
0.1215 \\
0.1864 \\
0.0246 \\
0.0906 \\
0.1309 \\
0.1887\end{array}$ & $\begin{array}{l}\text { DDff } \\
0.1267 \\
0.1645 \\
0.3819 \\
0.3324 \\
0.4086 \\
1.5742 \\
0.7388 \\
0.0700 \\
0.1118 \\
0.5388 \\
0.0143 \\
0.1041 \\
0.0001 \\
0.2077 \\
0.2002 \\
0.0900 \\
0.3455 \\
0.0160 \\
1.9297 \\
0.0087 \\
0.0750 \\
0.0850 \\
0.0213 \\
0.0460 \\
0.0063 \\
0.1813 \\
0.0955 \\
0.0197 \\
11.2648 \\
0.0535 \\
0.0460 \\
0.1243 \\
0.3412 \\
0.2124 \\
1.6120 \\
0.0573 \\
0.0640 \\
0.1027 \\
0.0270 \\
0.0843 \\
0.0796 \\
0.1211 \\
0.0157 \\
0.0559 \\
0.0844 \\
0.1214\end{array}$ & \\
\hline
\end{tabular}




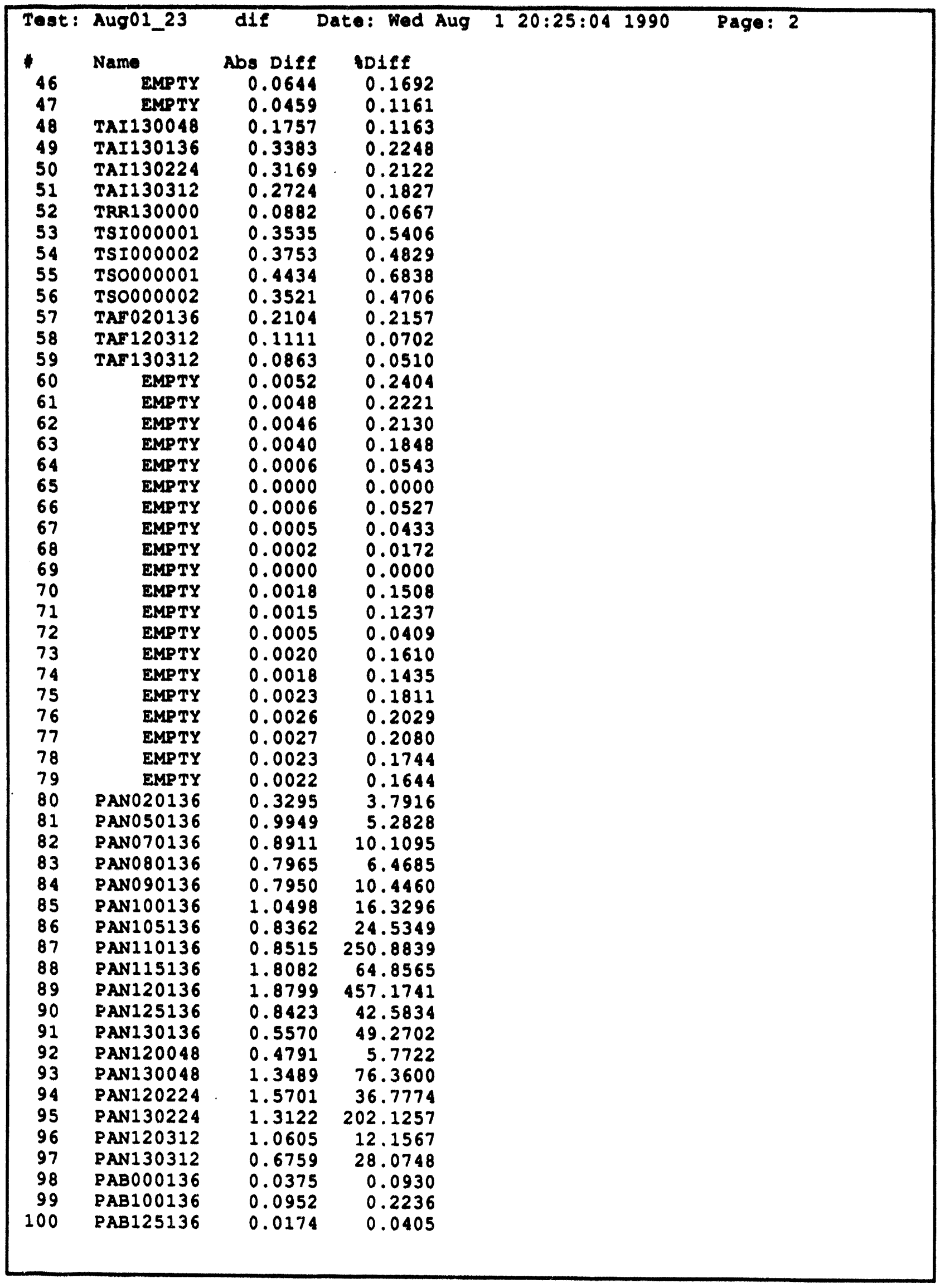




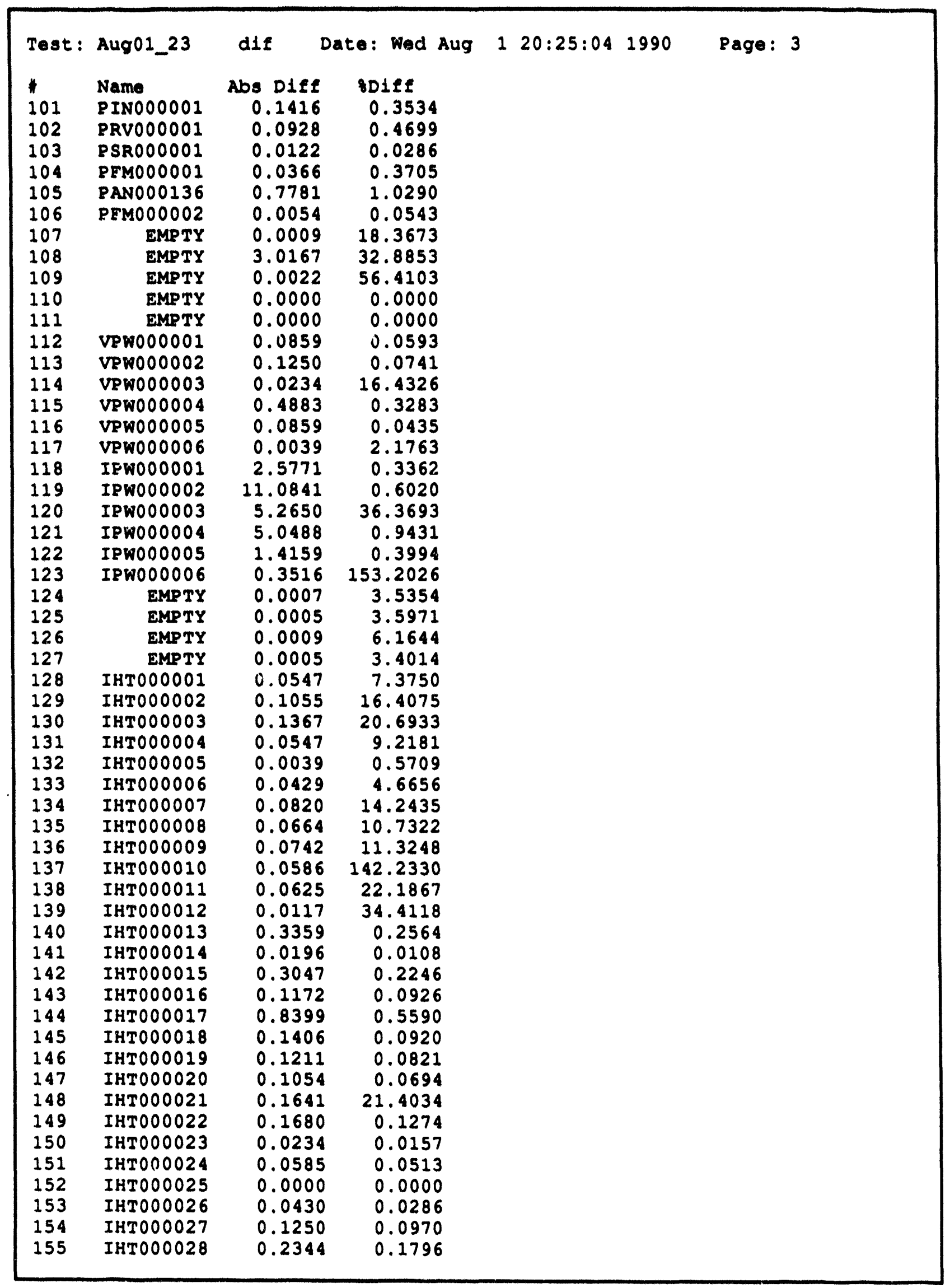




\begin{tabular}{|c|c|c|c|c|c|}
\hline $\begin{array}{l}\text { Test: } \\
\\
156 \\
156 \\
157 \\
158 \\
159 \\
160 \\
161 \\
162 \\
163\end{array}$ & $\begin{array}{l}\text { Aug01_23 } \\
\text { Name } \\
\text { IHTO00029 } \\
\text { IHTOOO030 } \\
\text { IHTOOO031 } \\
\text { IHTOOOO32 } \\
\text { IHTOOO033 } \\
\text { IHTOOOO34 } \\
\text { IHTOOOO35 } \\
\text { IHTOOOO36 }\end{array}$ & $\begin{array}{l}\text { dif } \\
\text { Abs D1ef } \\
0.0938 \\
0.0469 \\
0.0196 \\
0.0273 \\
0.0391 \\
0.4727 \\
0.1719 \\
0.4453\end{array}$ & 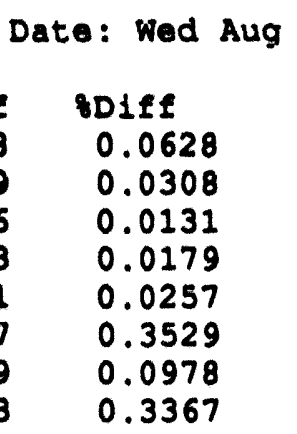 & $120: 25: 041990$ & Page: 4 \\
\hline
\end{tabular}




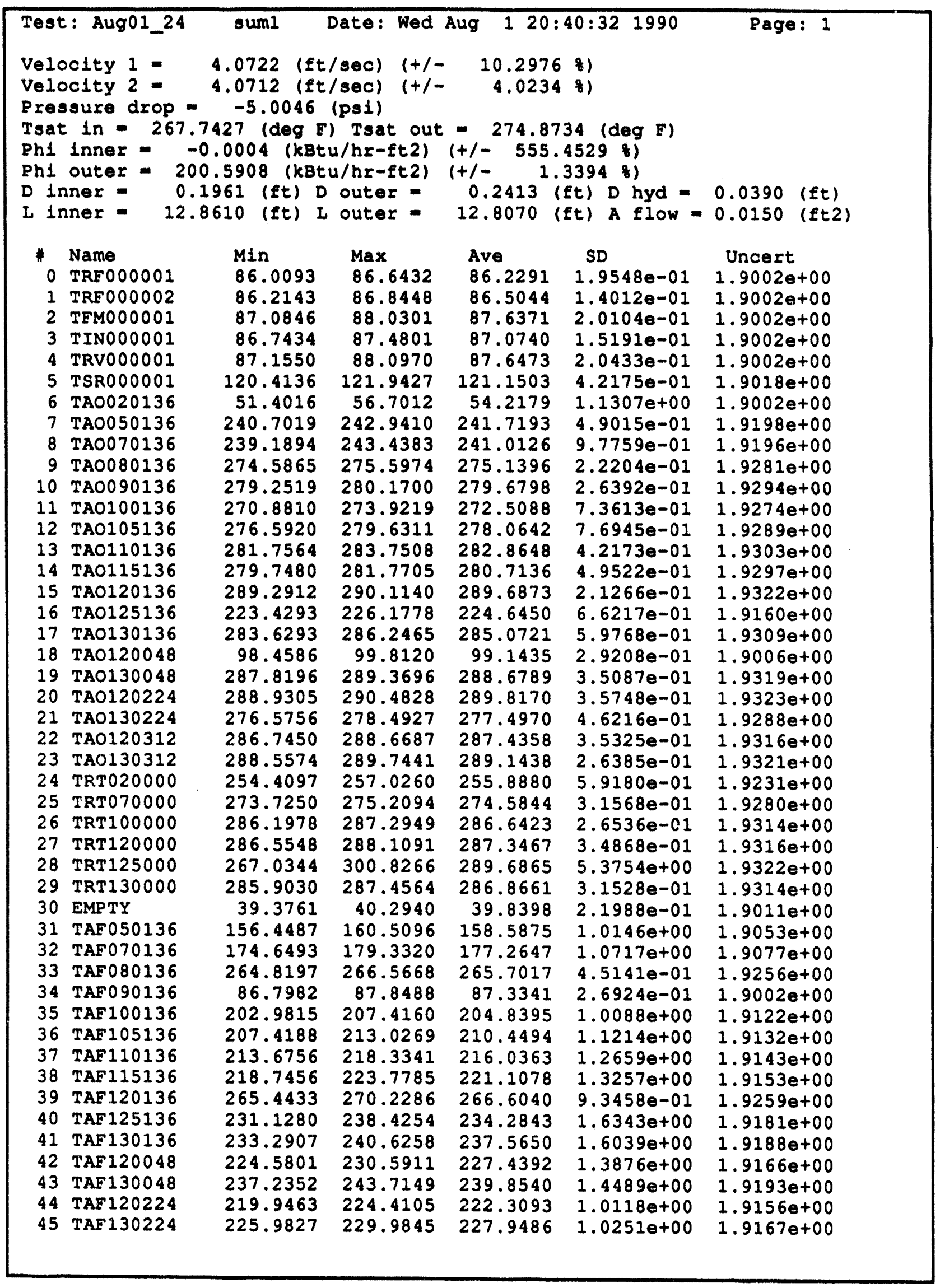




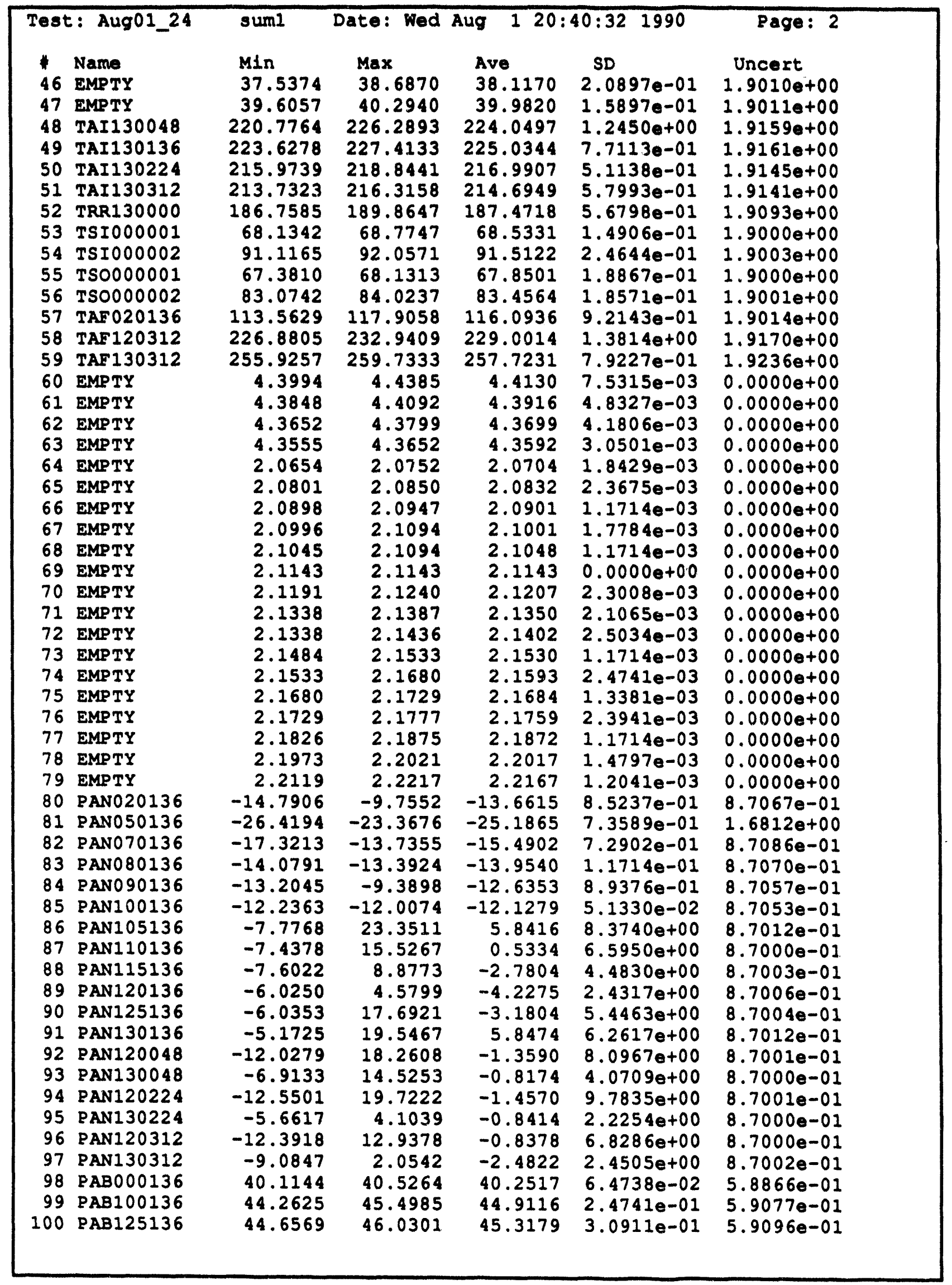




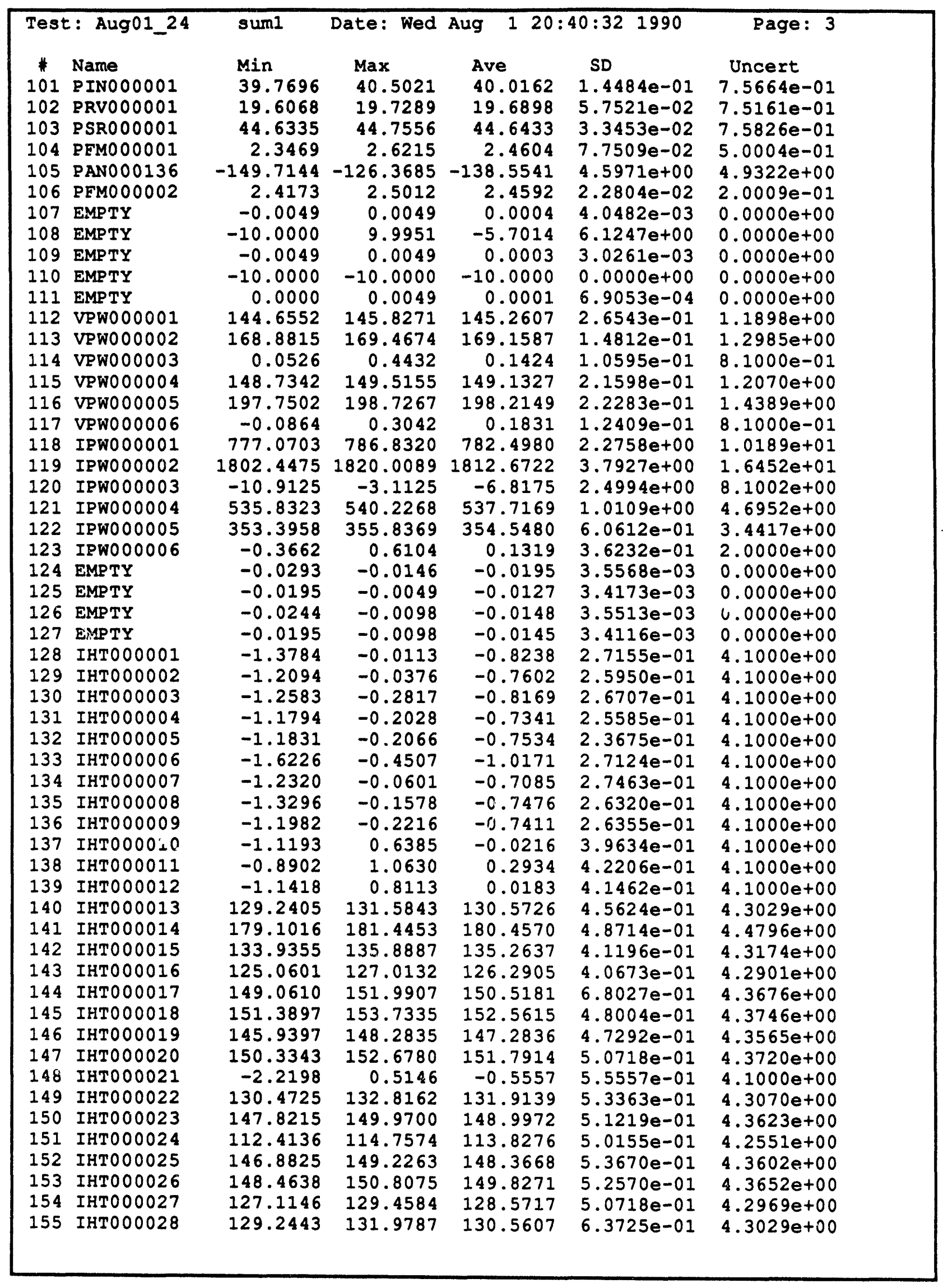




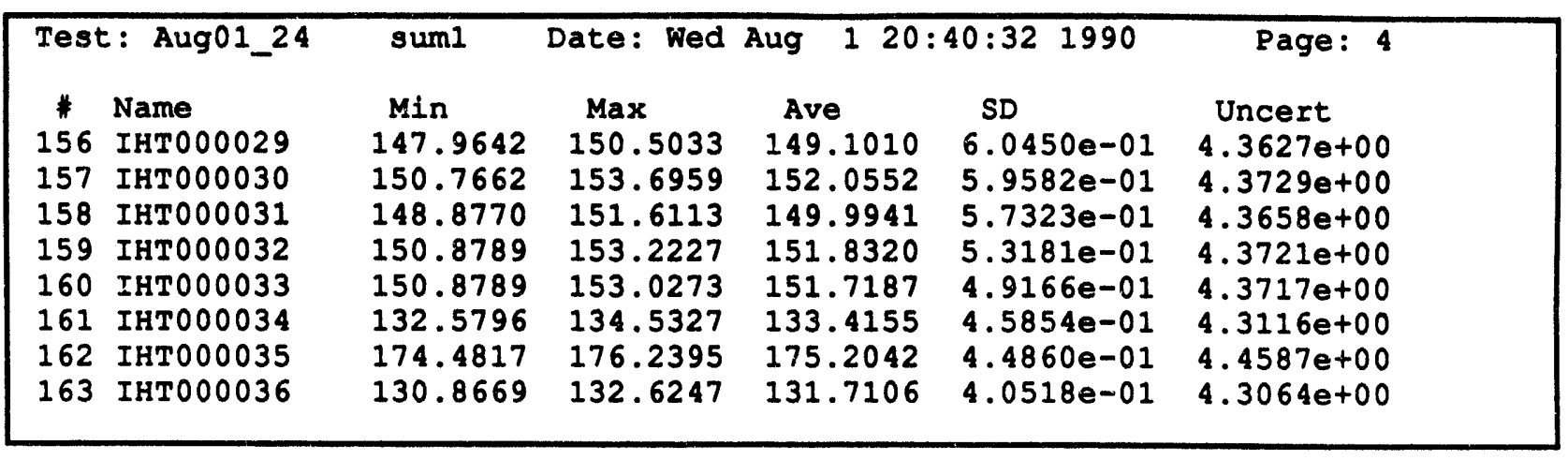




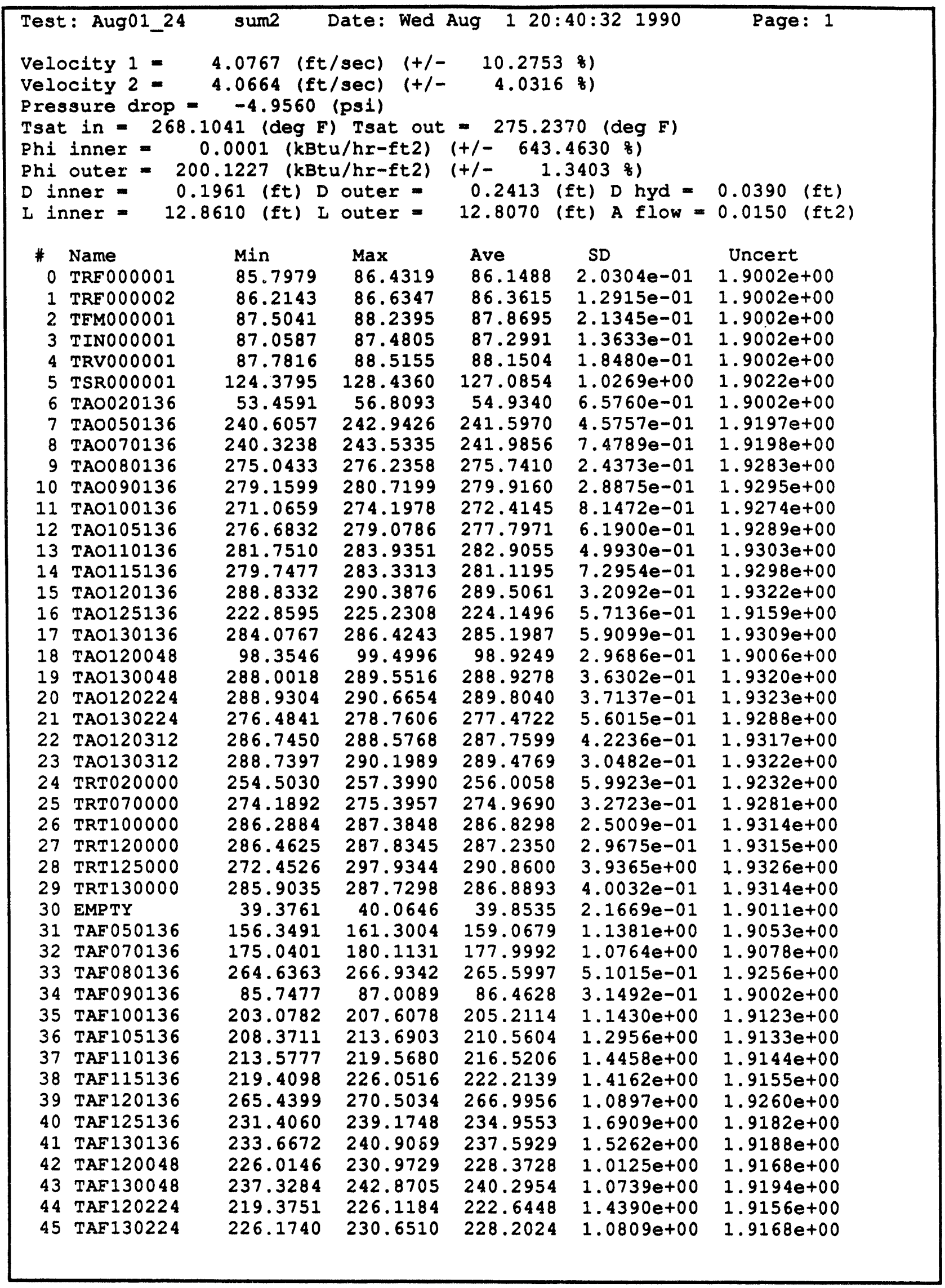




\begin{tabular}{|c|c|c|c|c|c|c|}
\hline \multicolumn{2}{|c|}{ Test: Aug01_24 } & & Date: Wed & \multicolumn{2}{|c|}{ Aug $120: 40: 321990$} & $\begin{array}{l}\text { Page: } 2 \\
\text { Uncert }\end{array}$ \\
\hline 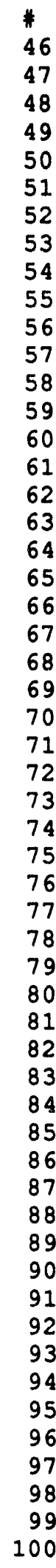 & 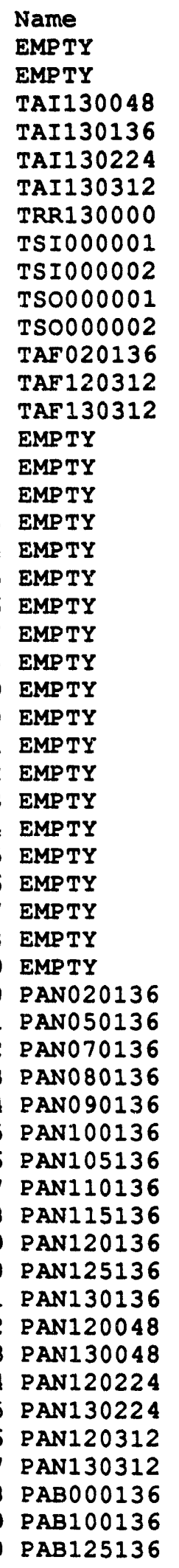 & 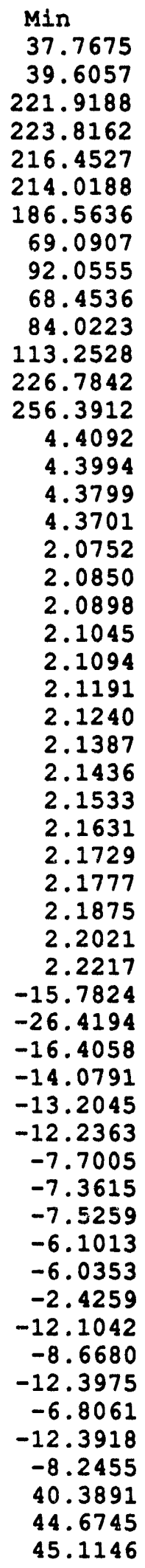 & 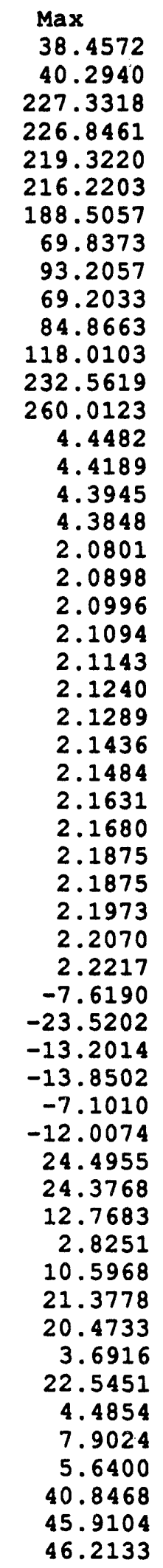 & 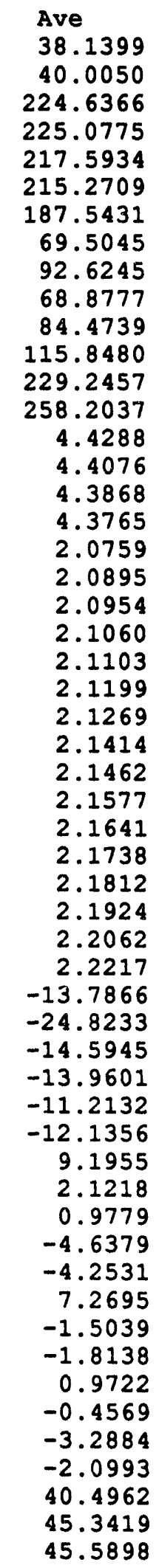 & $\begin{array}{l}\text { SD } \\
2.1230 e-01 \\
1.3760 e-01 \\
1.4047 e+00 \\
6.2313 e-01 \\
5.5559 e-01 \\
4.8402 e-01 \\
4.0500 e-01 \\
1.9217 e-01 \\
2.8660 e-01 \\
2.0293 e-01 \\
1.9412 e-01 \\
1.0599 e+00 \\
1.3508 e+00 \\
8.6153 e-01 \\
9.0675 e-03 \\
5.0724 e-03 \\
4.0781 e-03 \\
3.4527 e-03 \\
1.7115 e-03 \\
1.3381 e-03 \\
1.9754 e-03 \\
2.2603 e-03 \\
1.8950 e-03 \\
1.8082 e-03 \\
2.4344 e-03 \\
2.4484 e-03 \\
2.4583 e-03 \\
1.7784 e-03 \\
1.9730 e-03 \\
2.6100 e-03 \\
2.4662 e-03 \\
1.3951 e-03 \\
1.8082 e-03 \\
0.0000 e+00 \\
1.4661 e+00 \\
6.5700 e-01 \\
7.3725 e-01 \\
6.9207 e-02 \\
1.6700 e+00 \\
4.9803 e-02 \\
6.4780 e+00 \\
7.0059 e+00 \\
4.7215 e+00 \\
2.4263 e+00 \\
3.7100 e+00 \\
5.8203 e+00 \\
8.6838 e+00 \\
3.0562 e+00 \\
9.4620 e+00 \\
2.6437 e+00 \\
6.2000 e+00 \\
2.8495 e+00 \\
9.5041 e-02 \\
2.5731 e-01 \\
2.7897 e-01\end{array}$ & $\begin{array}{l}\text { Uncert } \\
1.9010 e+00 \\
1.9011 e+00 \\
1.9160 e+00 \\
1.9161 e+00 \\
1.9146 e+00 \\
1.9142 e+00 \\
1.9093 e+00 \\
1.9000 e+00 \\
1.9003 e+00 \\
1.9000 e+00 \\
1.9001 e+00 \\
1.9014 e+00 \\
1.9170 e+00 \\
1.9237 e+00 \\
0.0000 e+00 \\
0.0000 e+00 \\
0.0000 e+00 \\
0.0000 e+00 \\
0.0000 e+00 \\
0.0000 e+00 \\
0.0000 e+00 \\
0.0000 e+00 \\
0.0000 e+00 \\
0.0000 e+00 \\
0.0000 e+00 \\
0.0000 e+00 \\
0.0000 e+00 \\
0.0000 e+00 \\
0.0000 e+00 \\
0.0000 e+00 \\
0.0000 e+00 \\
0.0000 e+00 \\
0.0000 e+00 \\
0.0000 e+00 \\
8.7068 e-01 \\
1.6811 e+00 \\
8.7076 e-01 \\
8.7070 e-01 \\
8.7045 e-01 \\
8.7053 e-01 \\
8.7030 e-01 \\
8.7002 e-01 \\
8.7000 e-01 \\
8.7008 e-01 \\
8.7006 e-01 \\
8.7019 e-01 \\
8.7001 e-01 \\
8.7001 e-01 \\
8.7000 e-01 \\
8.7000 e-01 \\
8.7004 e-01 \\
5.8002 e-01 \\
5.91097 e-01 \\
5.901\end{array}$ \\
\hline
\end{tabular}




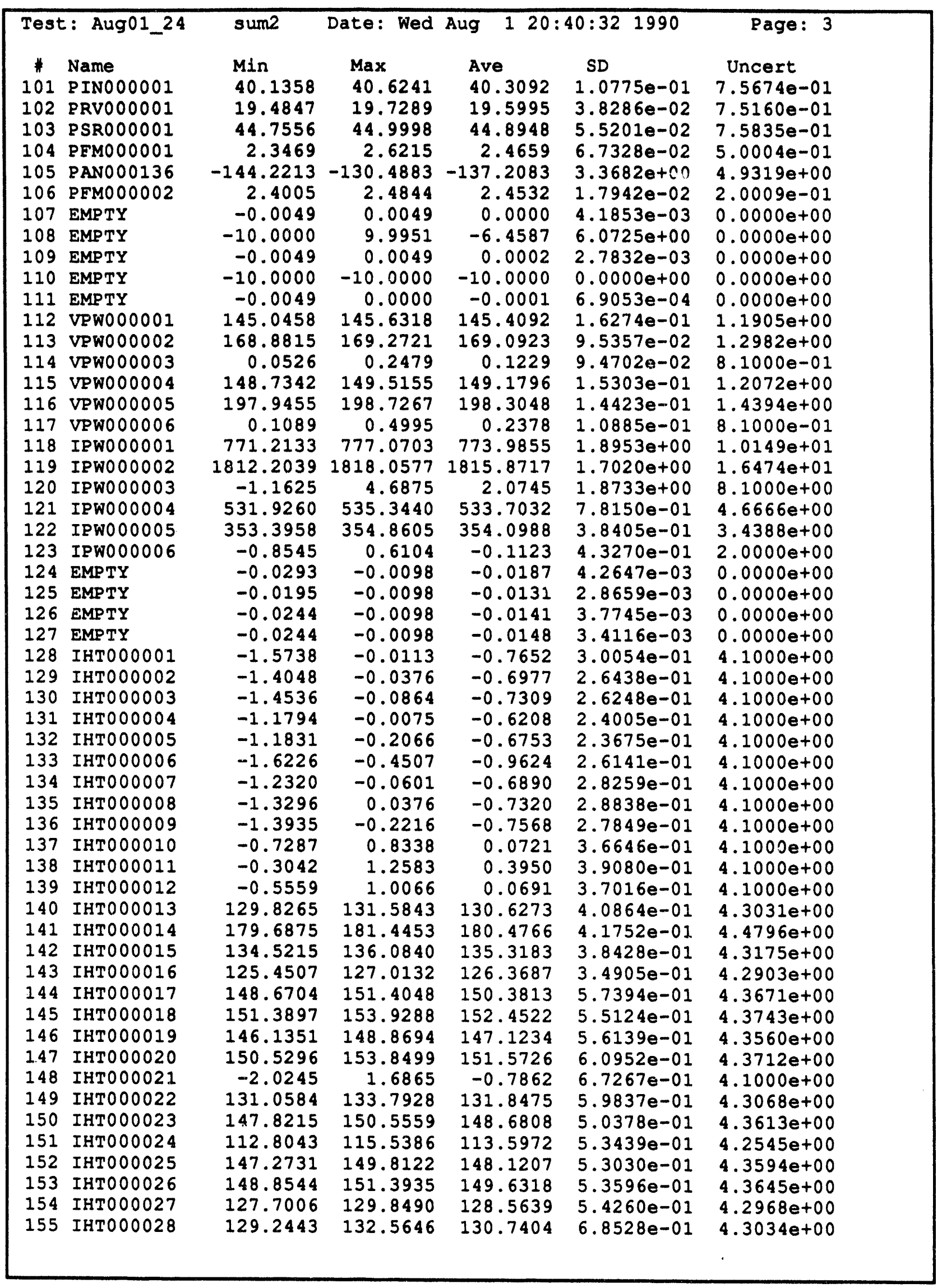




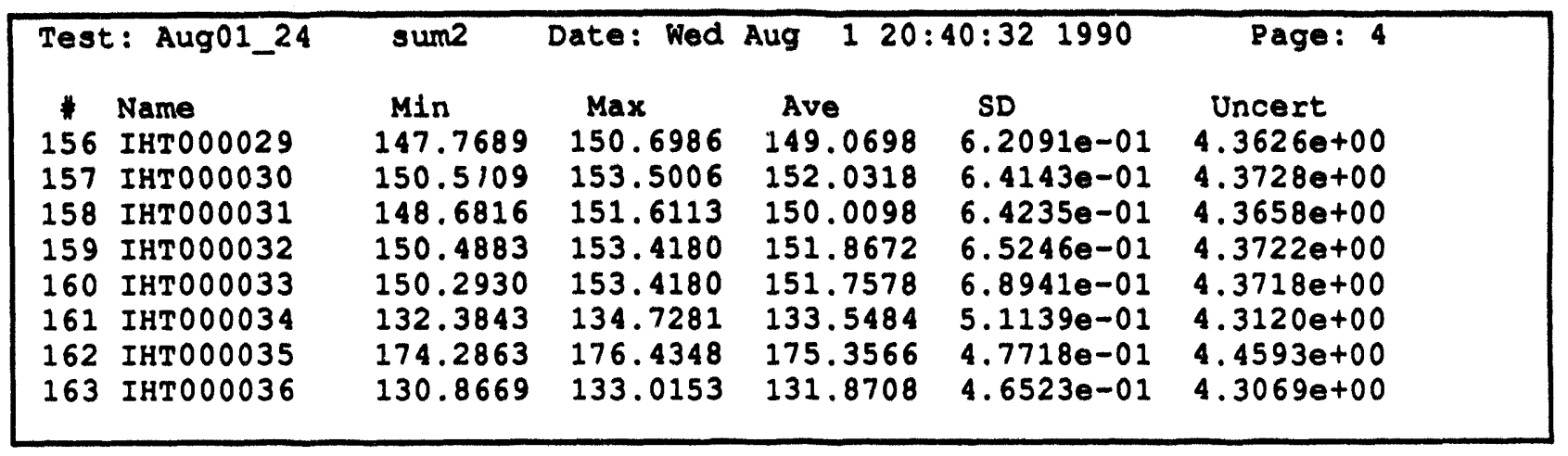




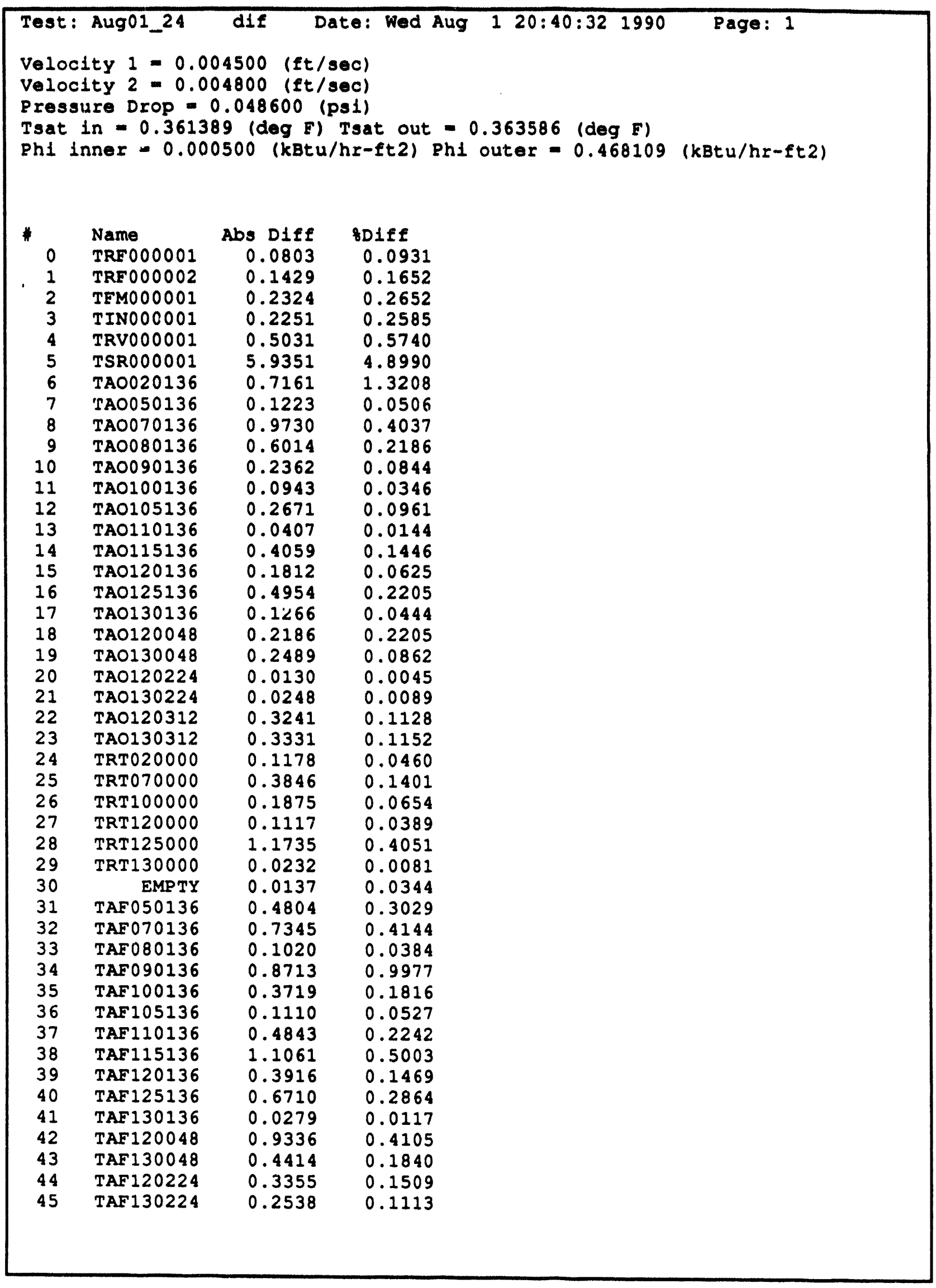




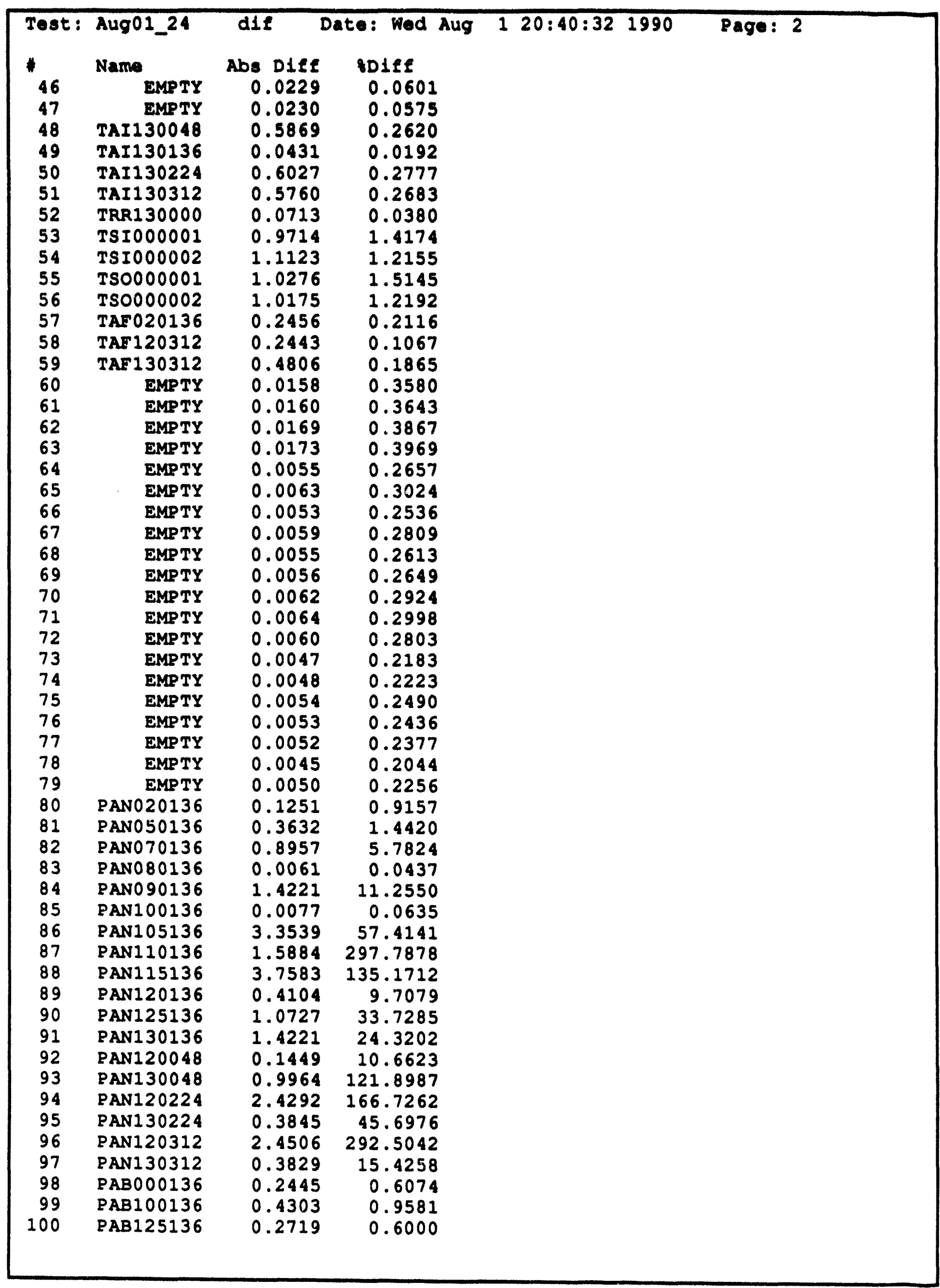




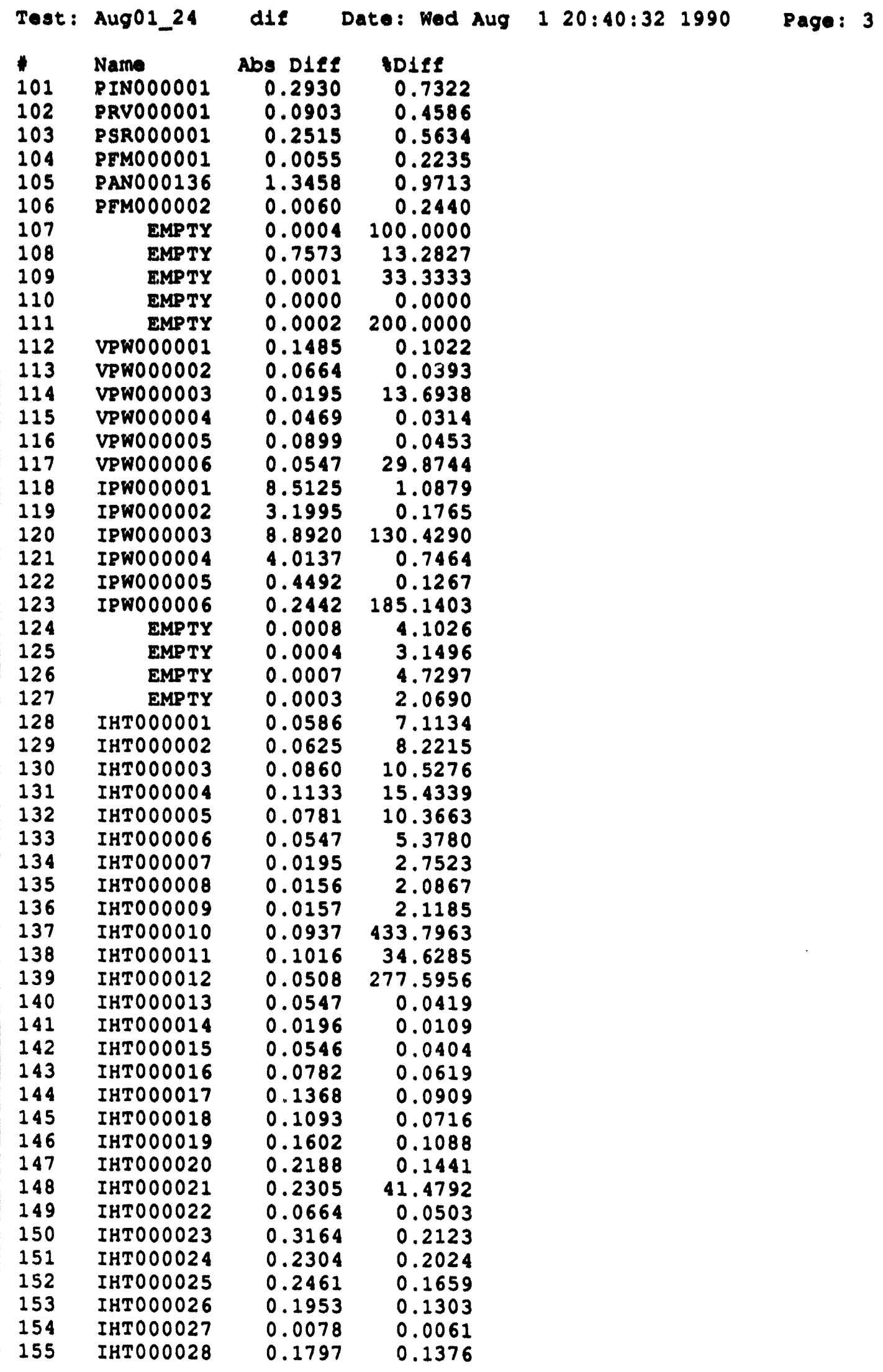




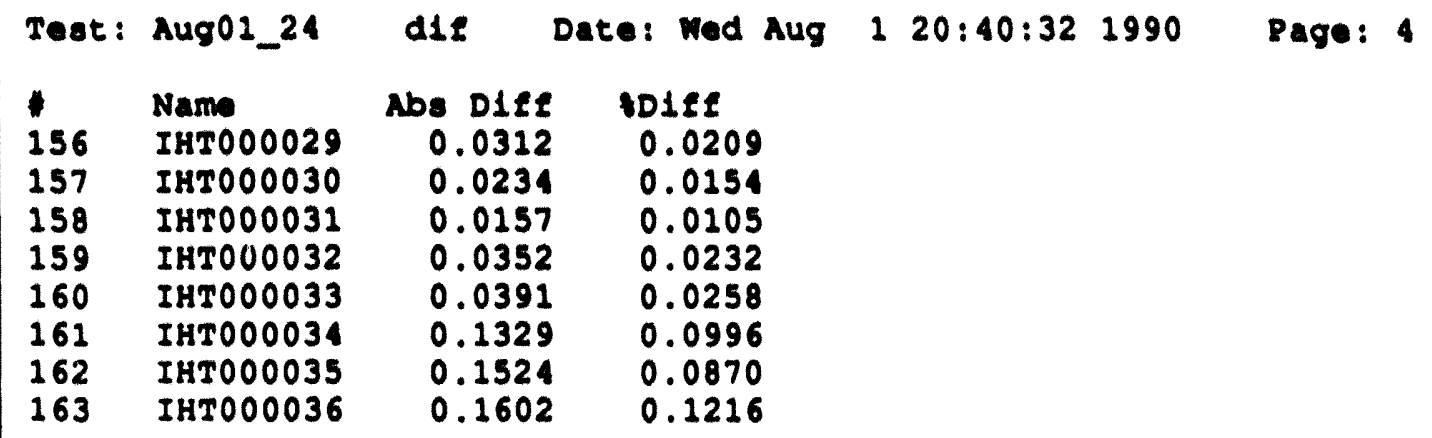




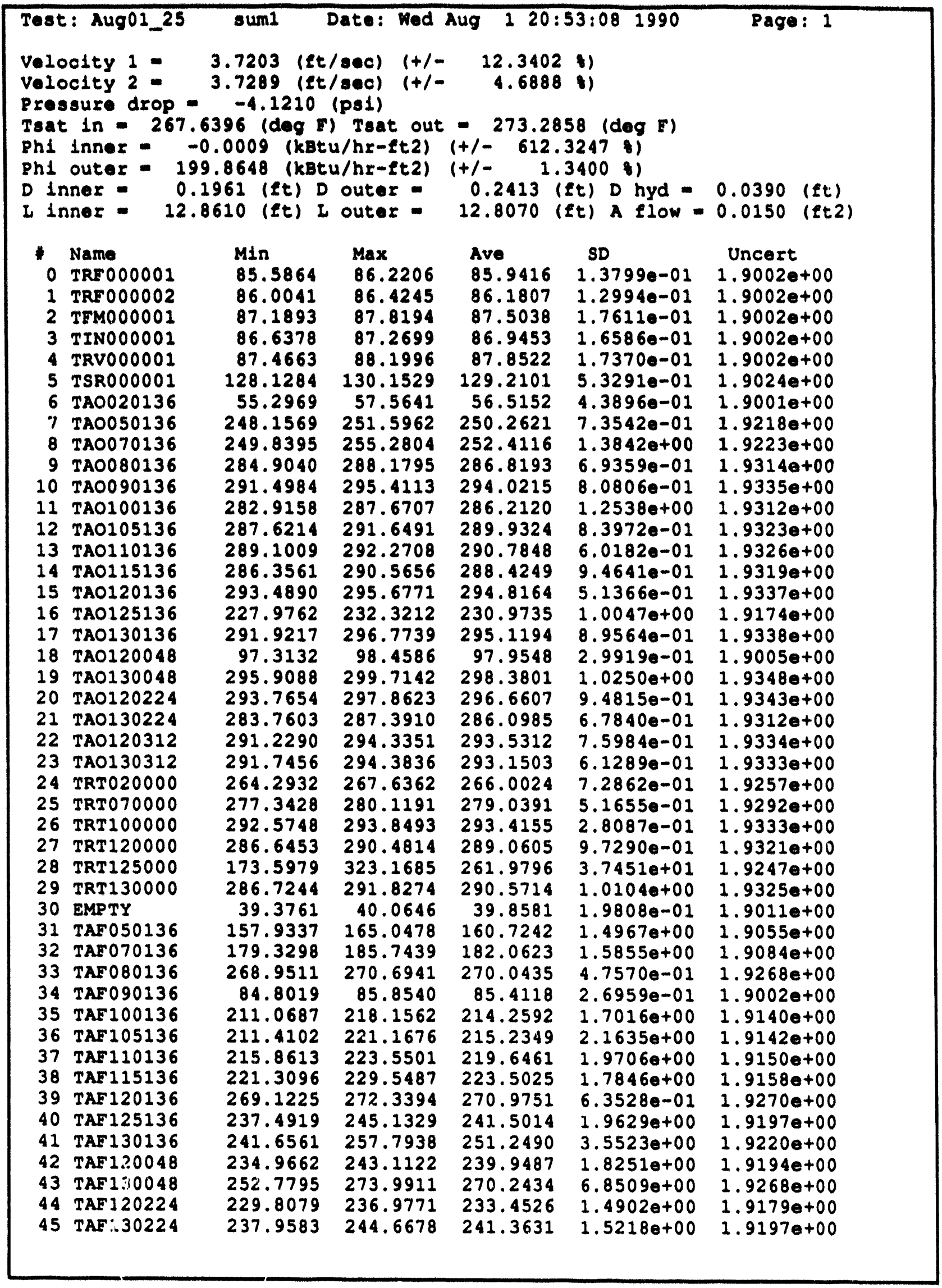




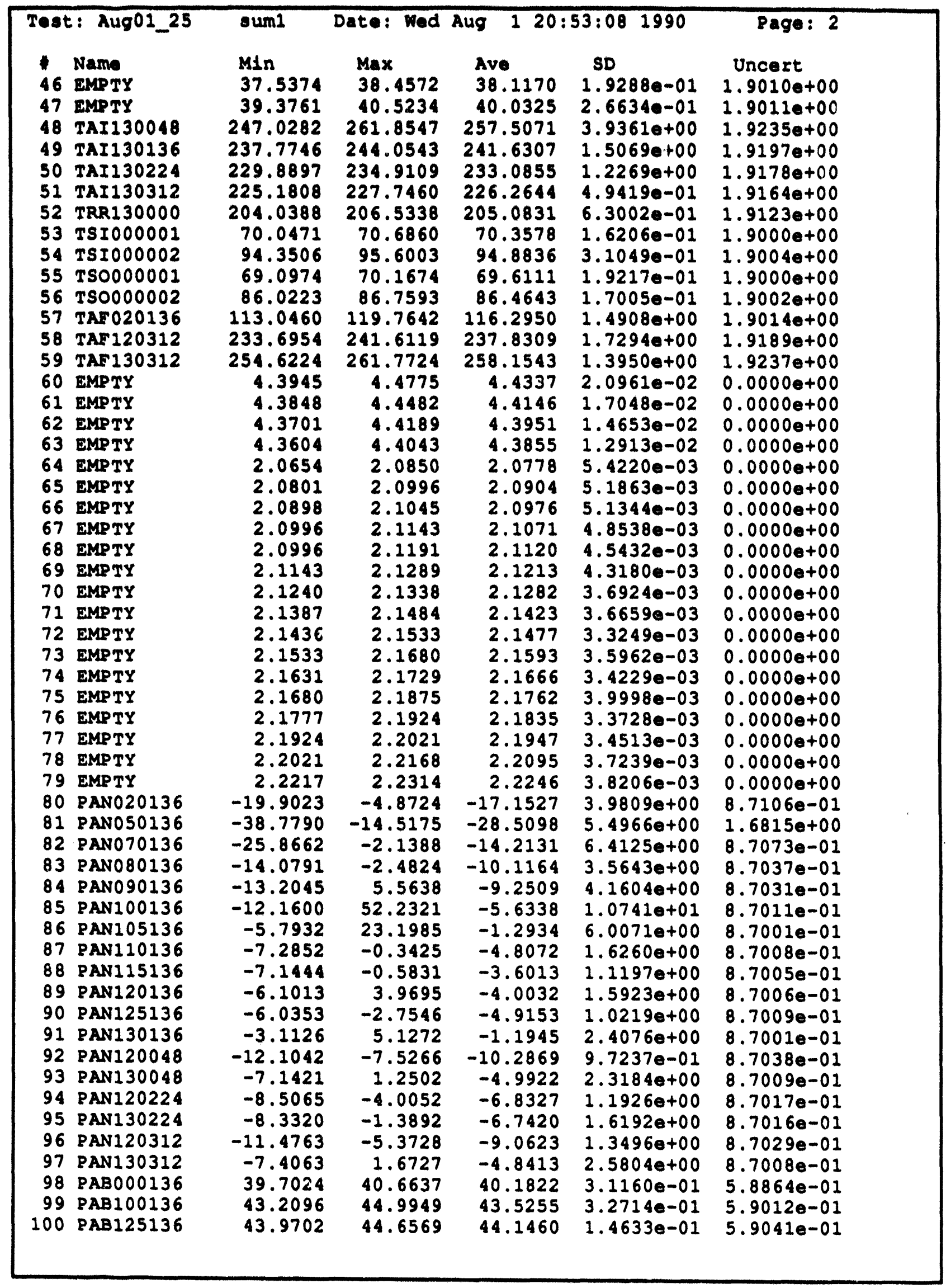




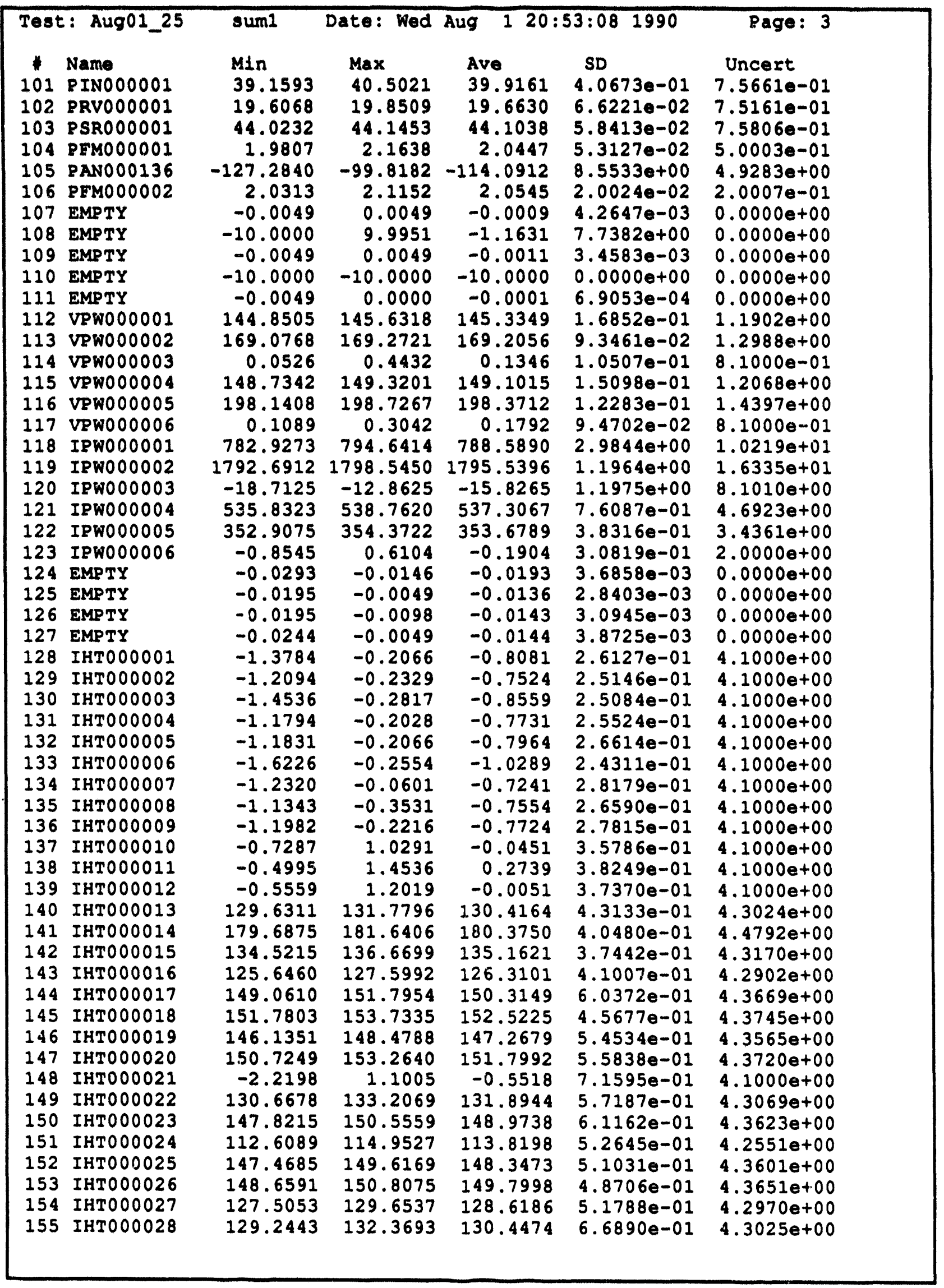




\begin{tabular}{|c|c|c|c|c|c|c|}
\hline $\begin{array}{l}1 \\
156 \\
157 \\
158 \\
159 \\
160 \\
161 \\
162 \\
163\end{array}$ & $\begin{array}{l}\text { Name } \\
\text { IHTO00029 } \\
\text { IHTO00030 } \\
\text { IHTO00031 } \\
\text { IHTO0O032 } \\
\text { IHTO0O033 } \\
\text { IHTO00034 } \\
\text { IHTOOOOO35 } \\
\text { IHTOOO0036 }\end{array}$ & $\begin{array}{c}\operatorname{Min} \\
147.3783 \\
150.3756 \\
148.6816 \\
150.4883 \\
150.2930 \\
132.3843 \\
174.2863 \\
130.4763\end{array}$ & $\begin{array}{l}\operatorname{Max} \\
151.0892 \\
153.8912 \\
151.6113 \\
153.4180 \\
153.4180 \\
134.5327 \\
176.2395 \\
132.6247\end{array}$ & $\begin{array}{l}\text { Ave } \\
148.9331 \\
151.9263 \\
149.9512 \\
151.7969 \\
151.7031 \\
133.3413 \\
175.2863 \\
131.7106\end{array}$ & $\begin{array}{l}\text { SD } \\
6.7654 e-01 \\
6.5722 e-01 \\
6.3165 e-01 \\
6.4868 e-01 \\
6.7114 e-01 \\
5.0878 e-01 \\
4.9227 e-01 \\
4.7750 e-01\end{array}$ & $\begin{array}{c}\text { Uncert } \\
4.3621 e+00 \\
4.3724 \theta+00 \\
4.3656 e+00 \\
4.3720 e+00 \\
4.3717 e+00 \\
4.3114 e+00 \\
4.4590 e+00 \\
4.3064 e+00\end{array}$ \\
\hline
\end{tabular}




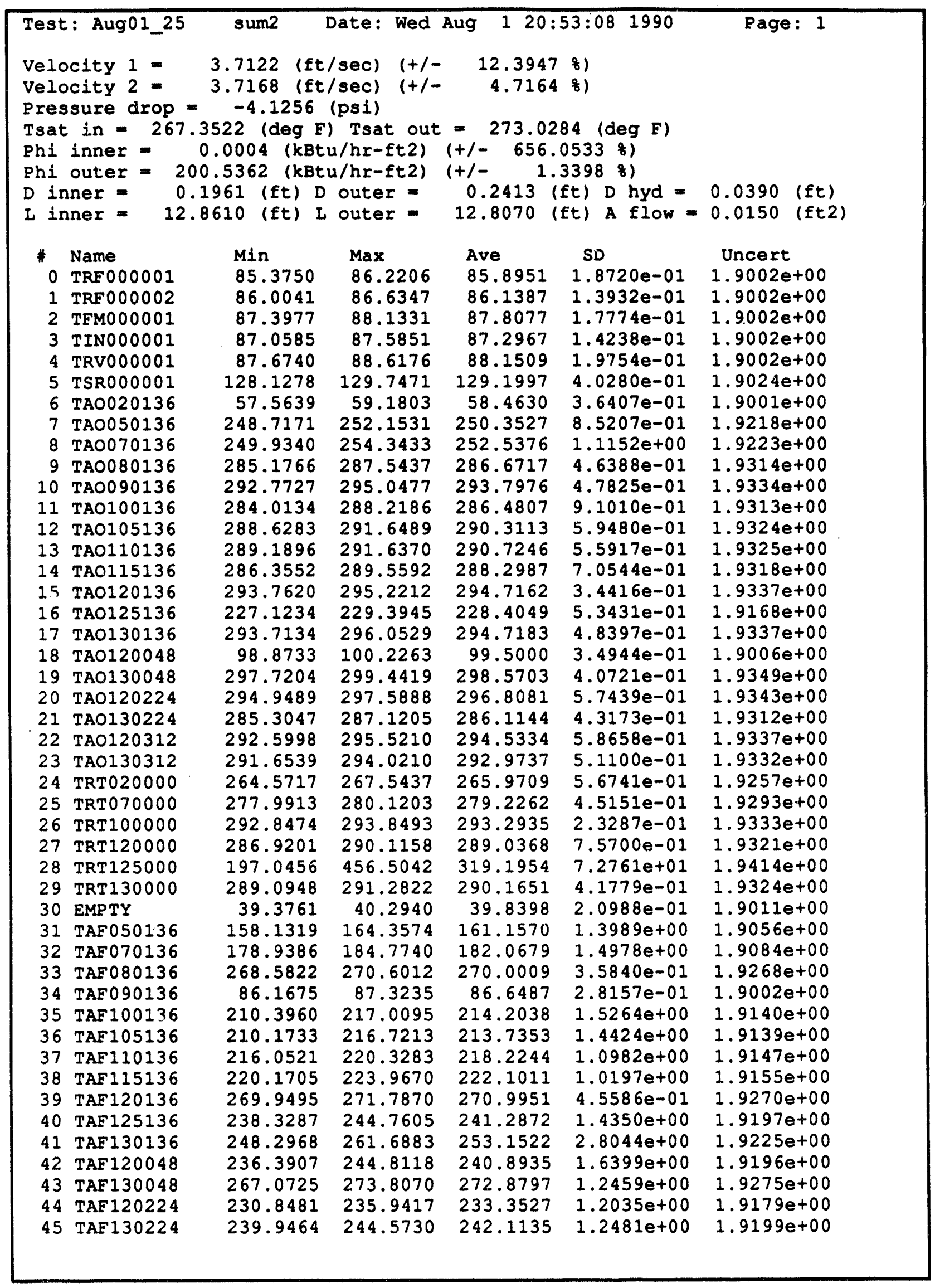




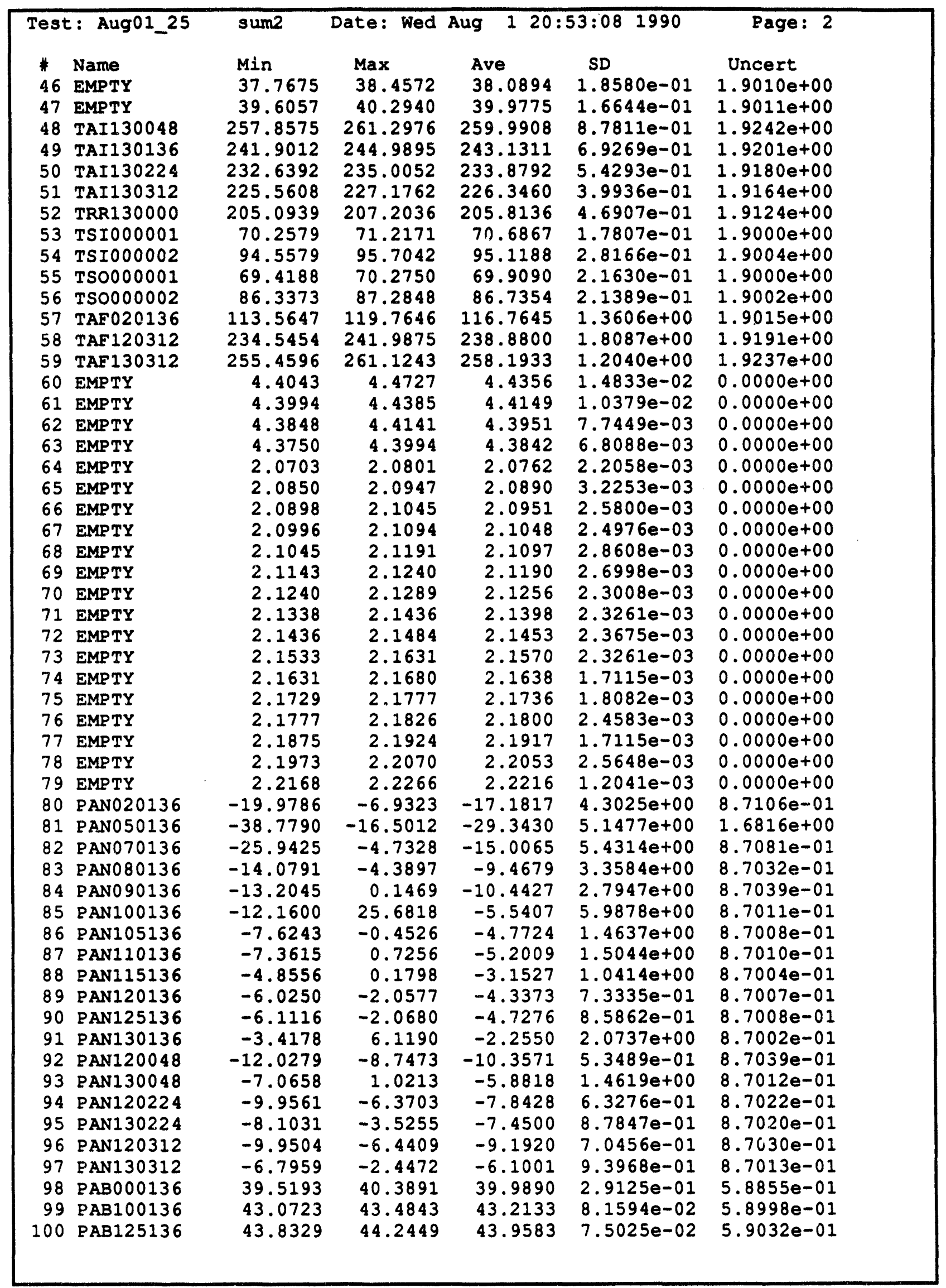




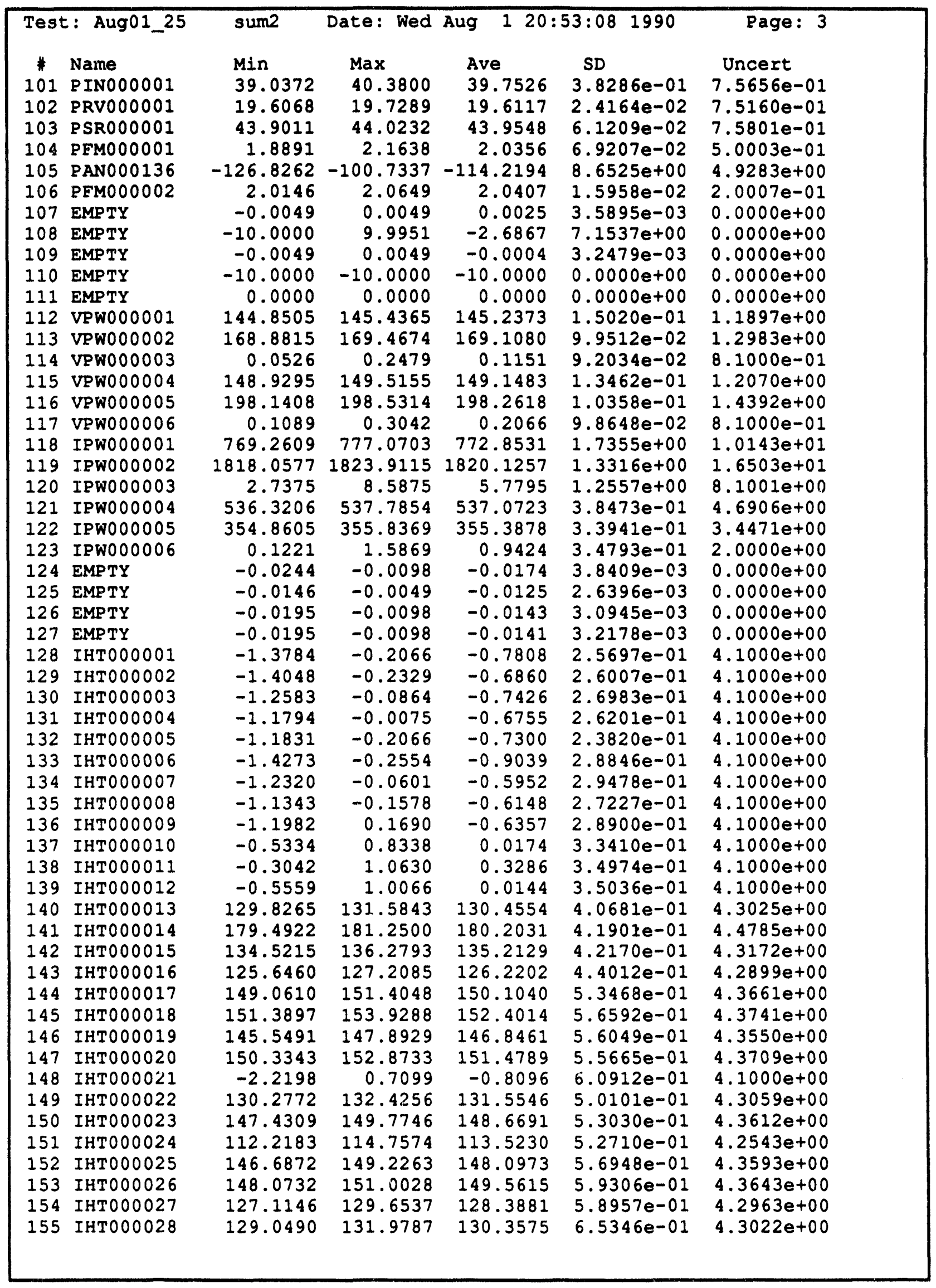




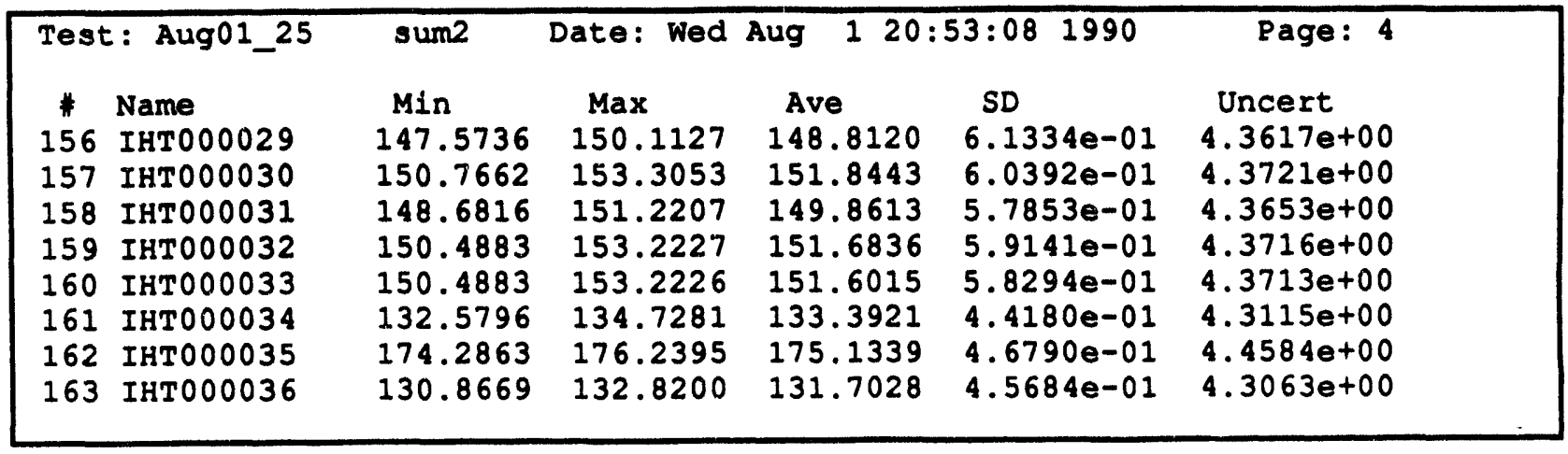




\begin{tabular}{|c|c|c|c|c|}
\hline \multicolumn{5}{|c|}{$\begin{array}{l}\text { Velocity } 1=0.008100 \text { (ft/sec) } \\
\text { Velocity } 2=0.012100 \text { (ft/sec) } \\
\text { Pressure Drop }=0.004600 \text { (psi) } \\
\text { Tsat in }=0.287384 \text { (deg F) Tsat out } \\
\text { Phi inner }=0.001300 \text { (kBtu/hr-ft2) }\end{array}$} \\
\hline $\begin{array}{l}* \\
0 \\
1 \\
2 \\
3 \\
4 \\
5 \\
6 \\
7 \\
8 \\
9 \\
10 \\
11 \\
12 \\
13 \\
14 \\
15 \\
16 \\
17 \\
18 \\
19 \\
20 \\
21 \\
22 \\
23 \\
24 \\
25 \\
26 \\
27 \\
28 \\
29 \\
30 \\
31 \\
32 \\
33 \\
34 \\
35 \\
36 \\
37 \\
38 \\
39 \\
40 \\
41 \\
42 \\
43 \\
44 \\
45\end{array}$ & $\begin{array}{l}\text { Name } \\
\text { TRF000001 } \\
\text { TRF000002 } \\
\text { TFM000001 } \\
\text { TIN000001 } \\
\text { TRV000001 } \\
\text { TSR000001 } \\
\text { TAO020136 } \\
\text { TAO050136 } \\
\text { TAO070136 } \\
\text { TAO080136 } \\
\text { TAO090136 } \\
\text { TAO100136 } \\
\text { TAO105136 } \\
\text { TAO110136 } \\
\text { TAO115136 } \\
\text { TAO120136 } \\
\text { TAO125136 } \\
\text { TAO130136 } \\
\text { TAO120048 } \\
\text { TAO130048 } \\
\text { TAO120224 } \\
\text { TAO130224 } \\
\text { TAO120312 } \\
\text { TAO130312 } \\
\text { TRT020000 } \\
\text { TRT070000 } \\
\text { TRT100000 } \\
\text { TRT120000 } \\
\text { TRT125000 } \\
\text { TRT130000 } \\
\text { EMPTY } \\
\text { TAF050136 } \\
\text { TAF070136 } \\
\text { TAF080136 } \\
\text { TAF090136 } \\
\text { TAF100136 } \\
\text { TAF105136 } \\
\text { TAF110136 } \\
\text { TAF115136 } \\
\text { TAF120136 } \\
\text { TAF125136 } \\
\text { TAF130136 } \\
\text { TAF120048 } \\
\text { TAF130048 } \\
\text { TAF120224 } \\
\text { TAF130224 }\end{array}$ & $\begin{array}{l}\text { Abs Diff } \\
0.0465 \\
0.0420 \\
0.3039 \\
0.3514 \\
0.2987 \\
0.0104 \\
1.9478 \\
0.0906 \\
0.1260 \\
0.1476 \\
0.2239 \\
0.2687 \\
0.3789 \\
0.0602 \\
0.1262 \\
0.1002 \\
2.5686 \\
0.4011 \\
1.5452 \\
0.1902 \\
0.1474 \\
0.0159 \\
1.0022 \\
0.1766 \\
0.0315 \\
0.1871 \\
0.1220 \\
0.0237 \\
57.2158 \\
0.4063 \\
0.0183 \\
0.4328 \\
0.0056 \\
0.0426 \\
1.2369 \\
0.0554 \\
1.4996 \\
1.4217 \\
1.4014 \\
0.0200 \\
0.2142 \\
1.9032 \\
0.9448 \\
2.6363 \\
0.0999 \\
0.7504\end{array}$ & $\begin{array}{l}\text { 8DIff } \\
0.0541 \\
0.0487 \\
0.3473 \\
0.4042 \\
0.3400 \\
0.0080 \\
3.4465 \\
0.0362 \\
0.0499 \\
0.0515 \\
0.0762 \\
0.0939 \\
0.1307 \\
0.0207 \\
0.0438 \\
0.0340 \\
1.1121 \\
0.1359 \\
1.5775 \\
0.0637 \\
0.0497 \\
0.0056 \\
0.3414 \\
0.0602 \\
0.0119 \\
0.0671 \\
0.0416 \\
0.0082 \\
21.8398 \\
0.1398 \\
0.0459 \\
0.2693 \\
0.0031 \\
0.0158 \\
1.4482 \\
0.0259 \\
0.6967 \\
0.6473 \\
0.6270 \\
0.0074 \\
0.0887 \\
0.7575 \\
0.3937 \\
0.9755 \\
0.0428 \\
0.3109\end{array}$ & \\
\hline
\end{tabular}




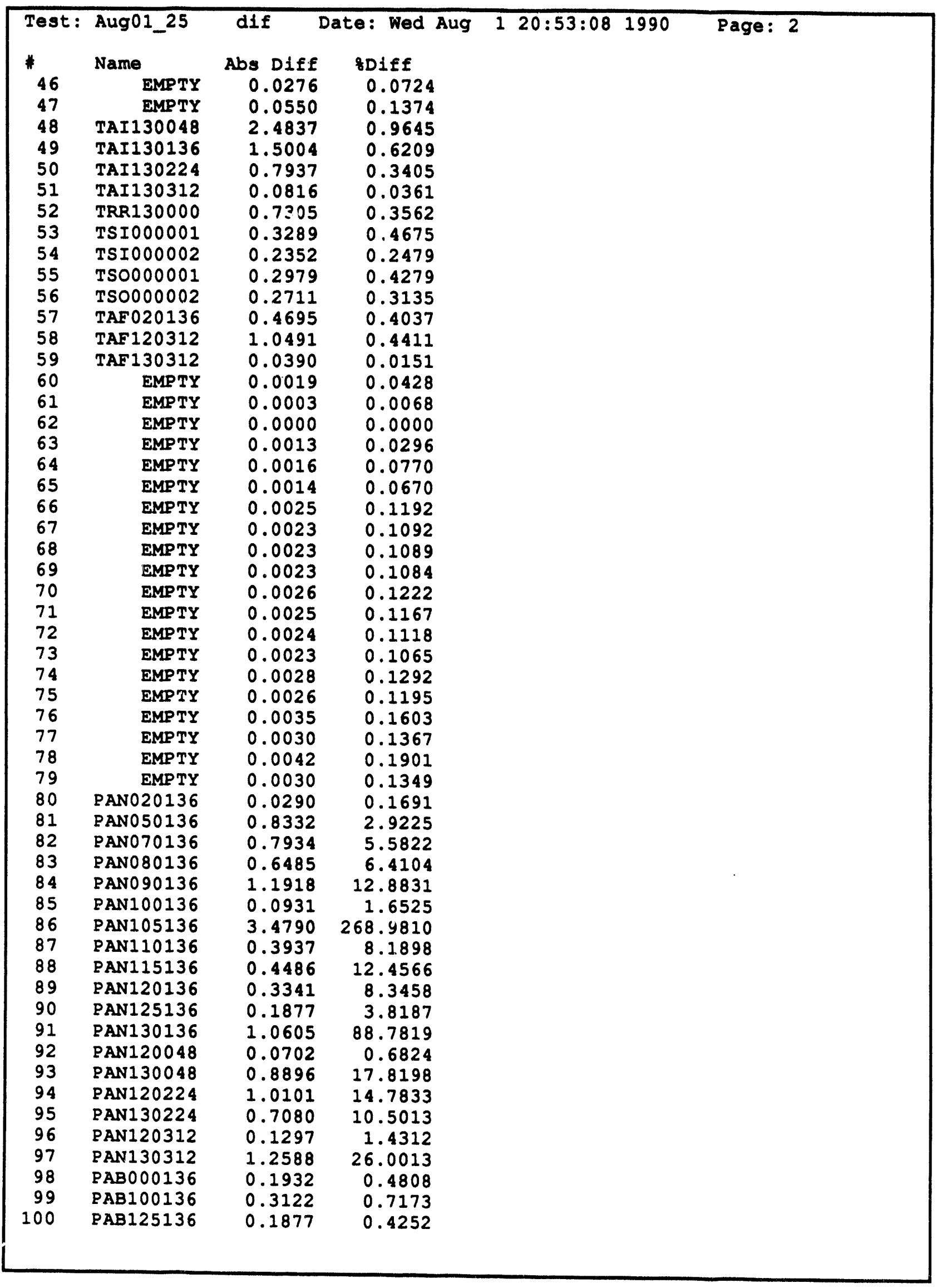




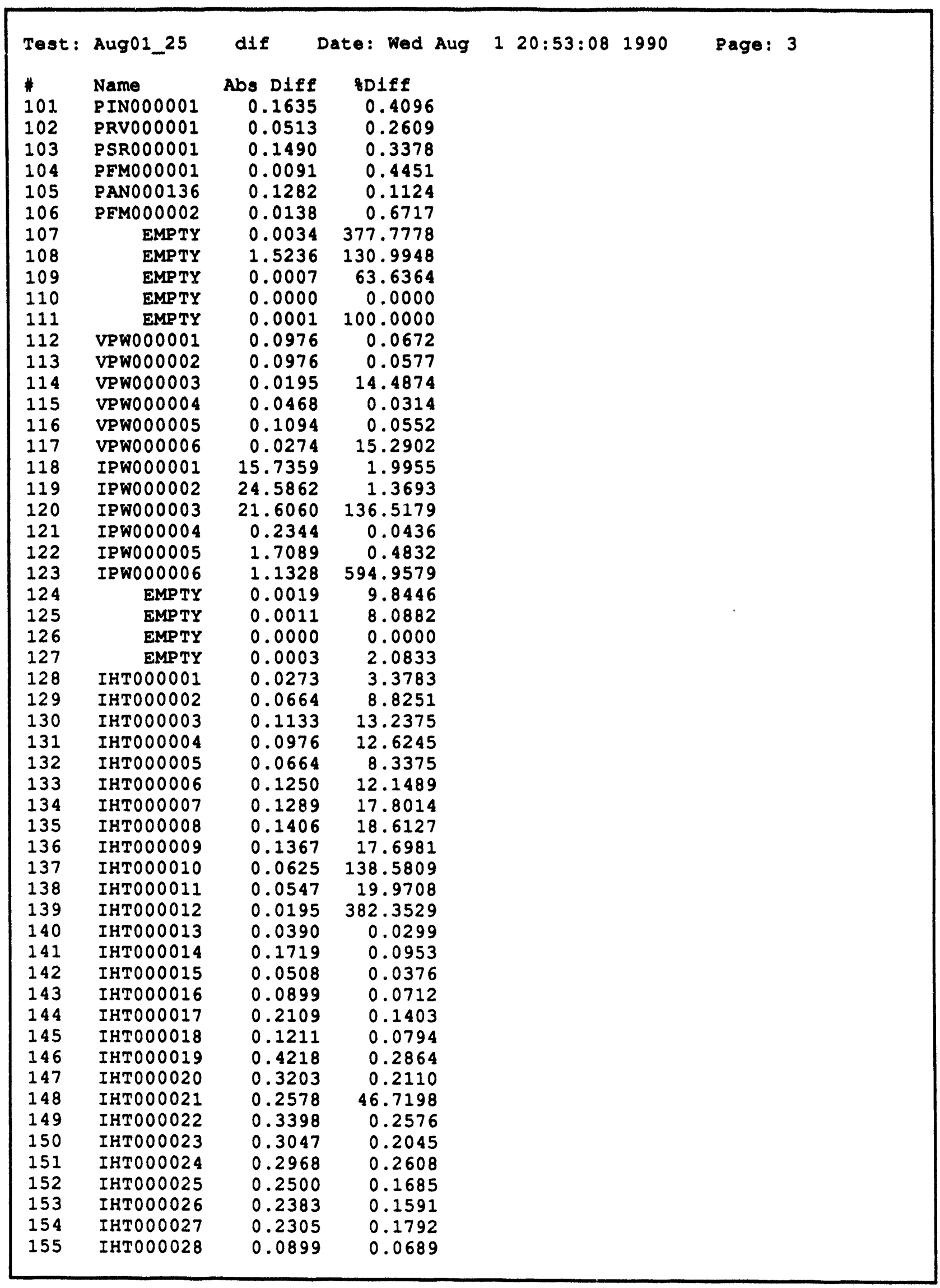




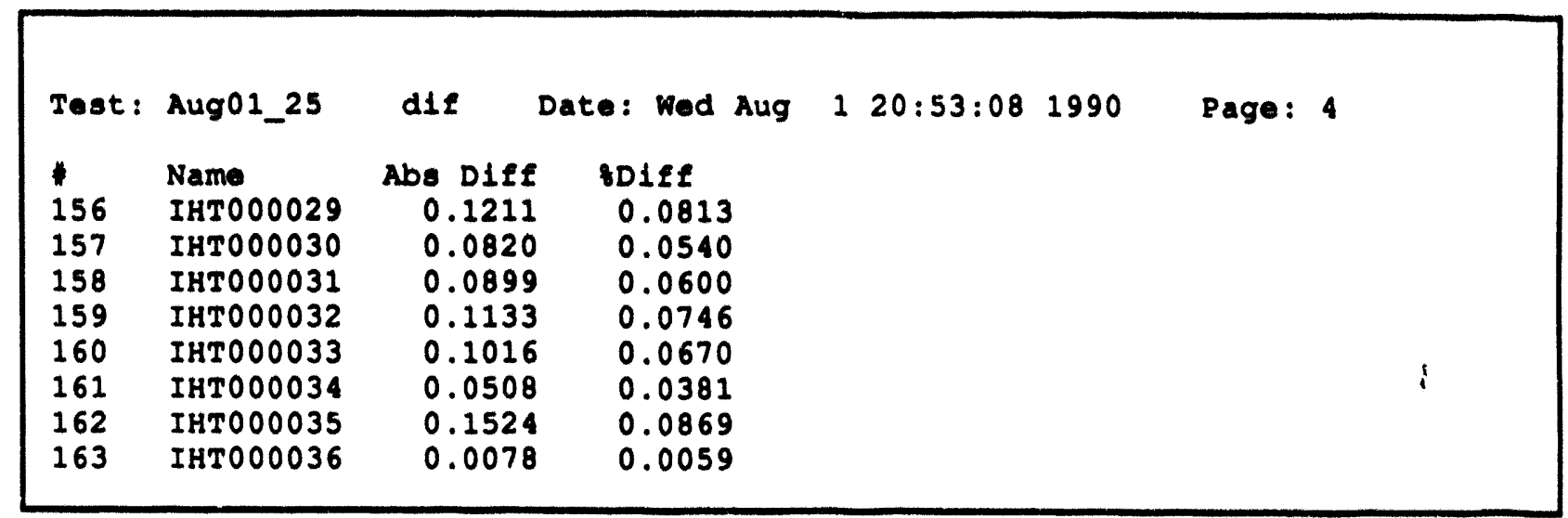




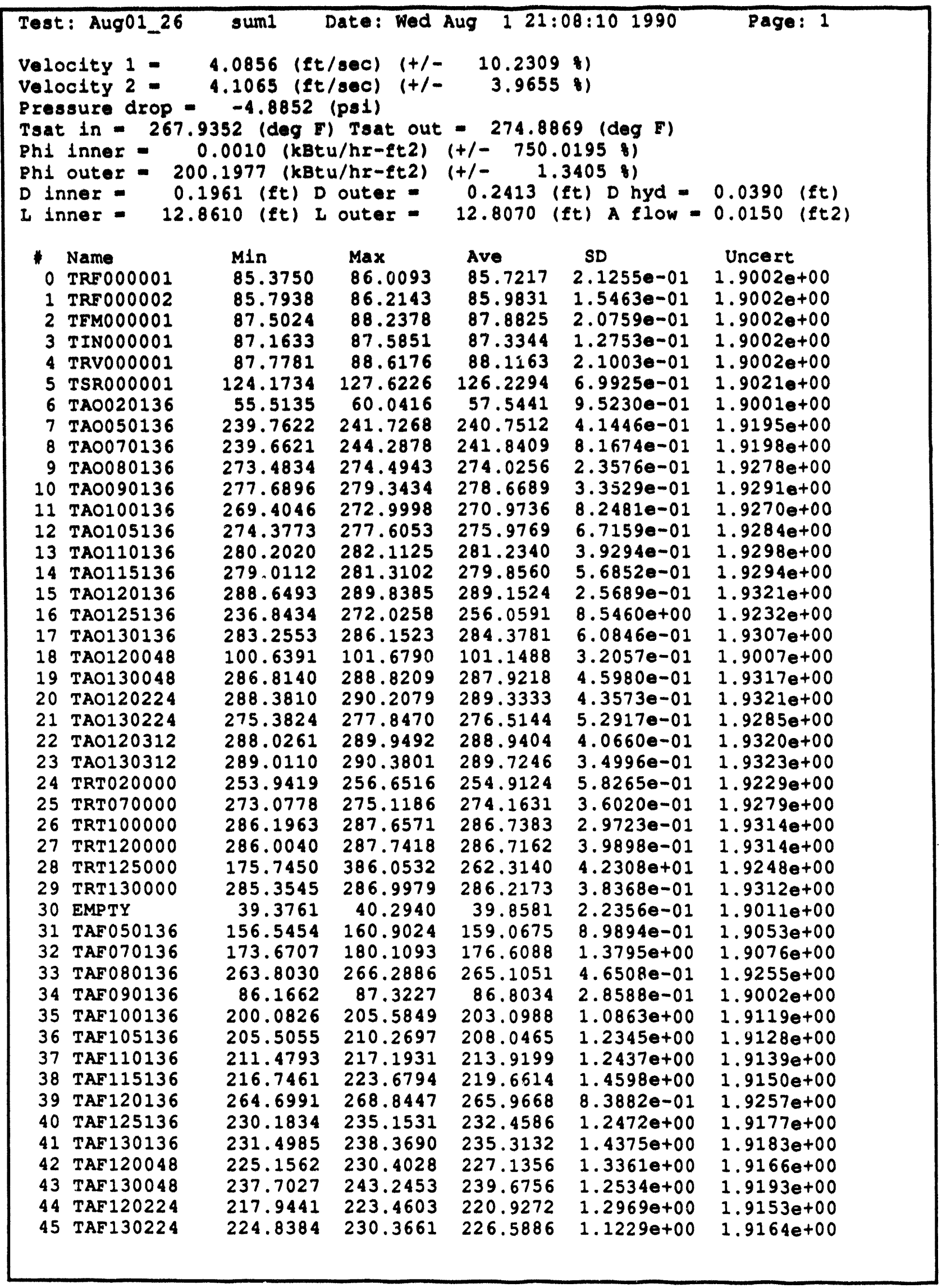




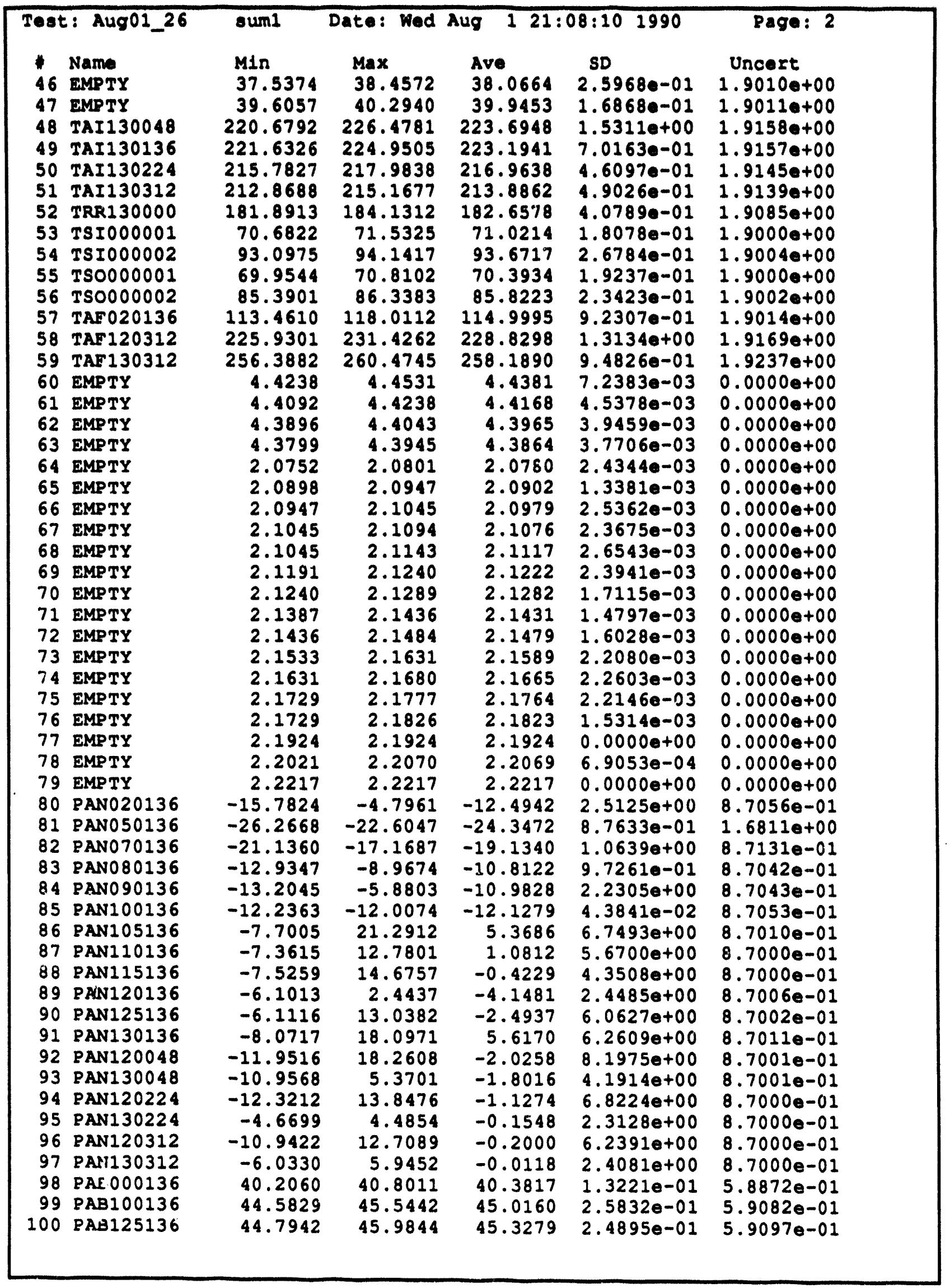




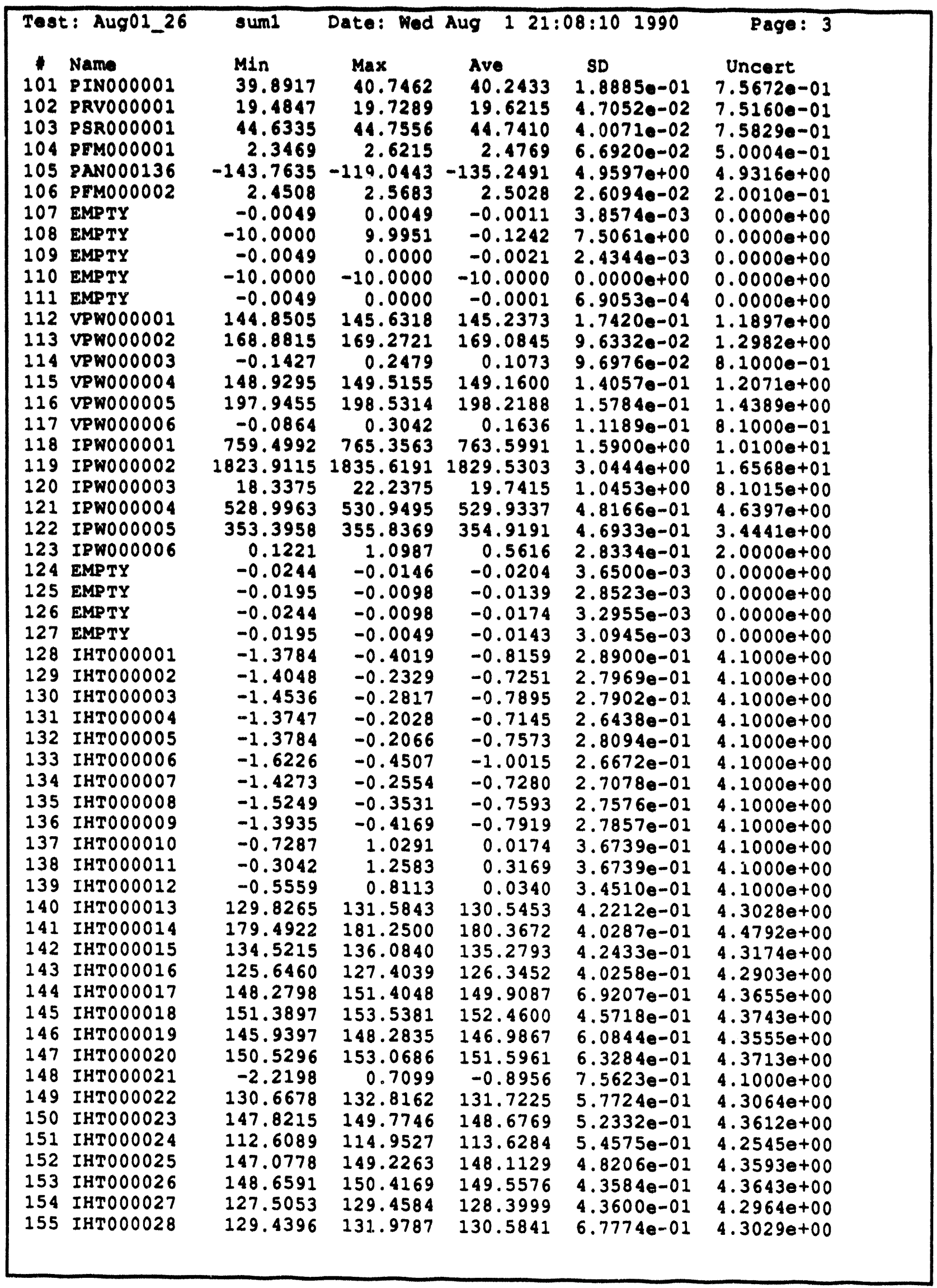




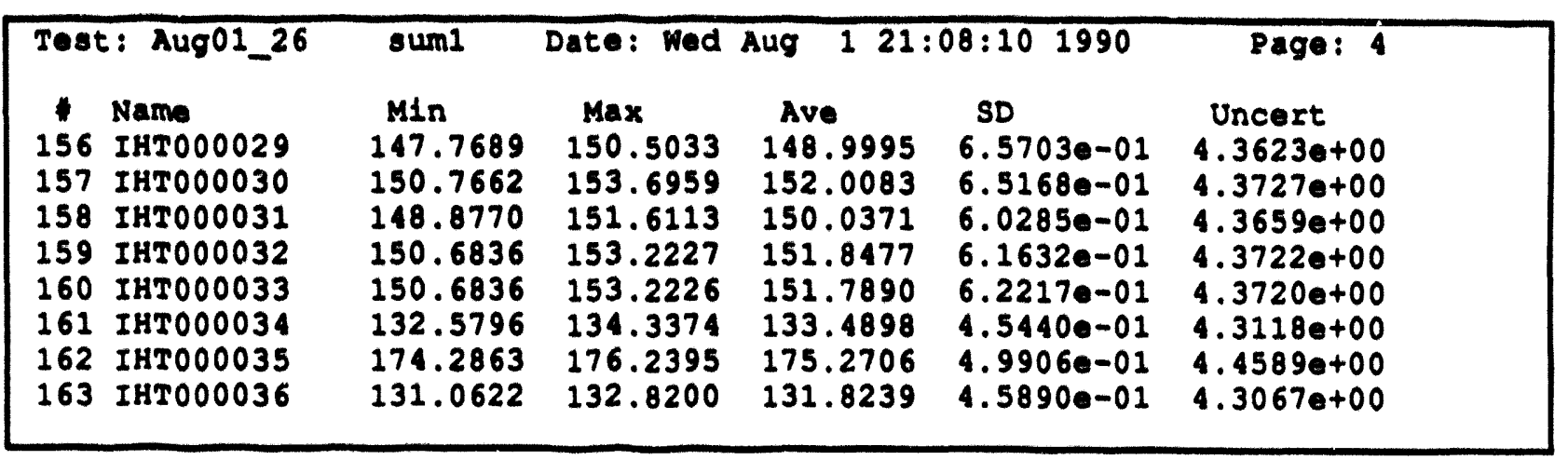




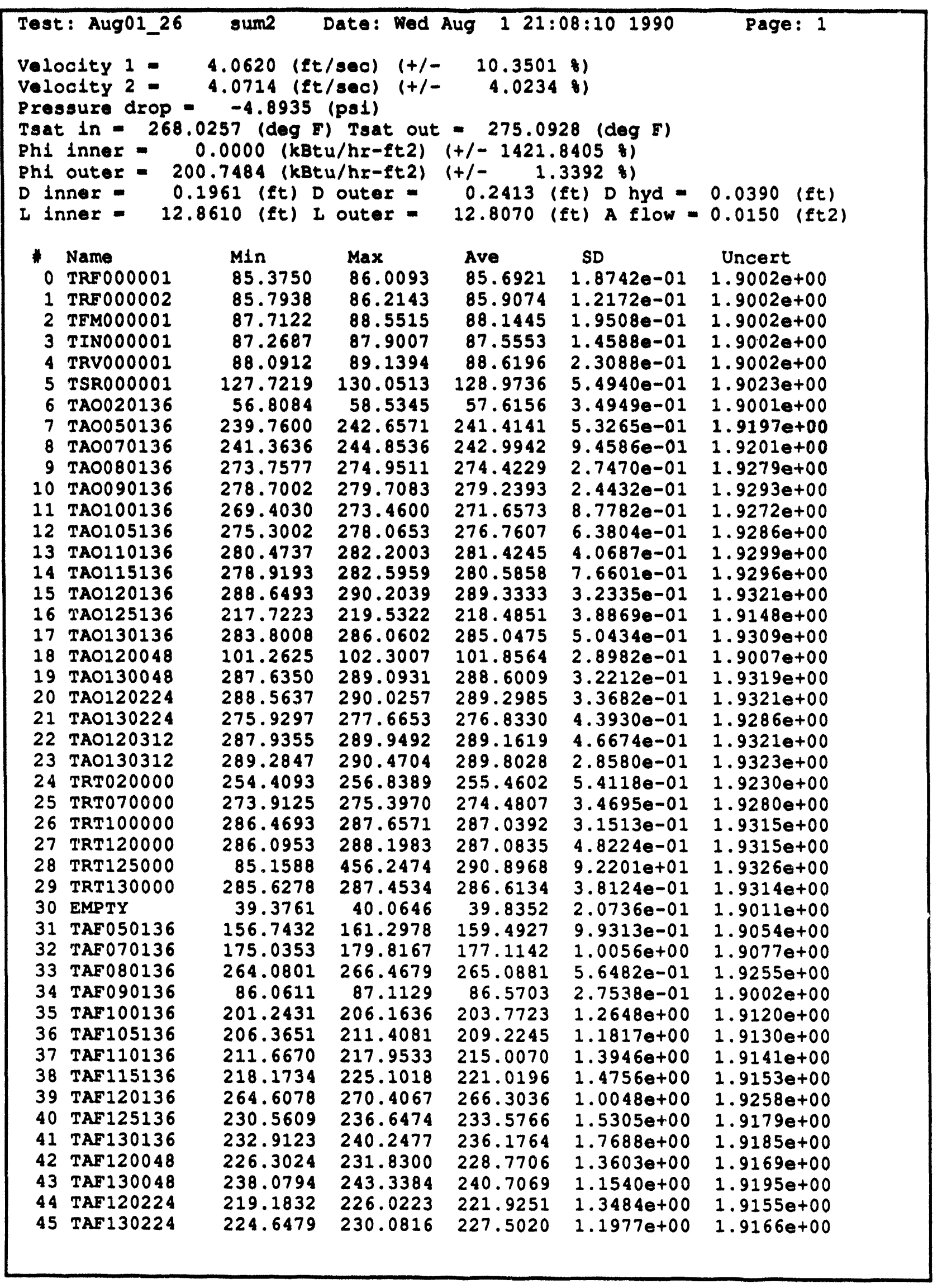




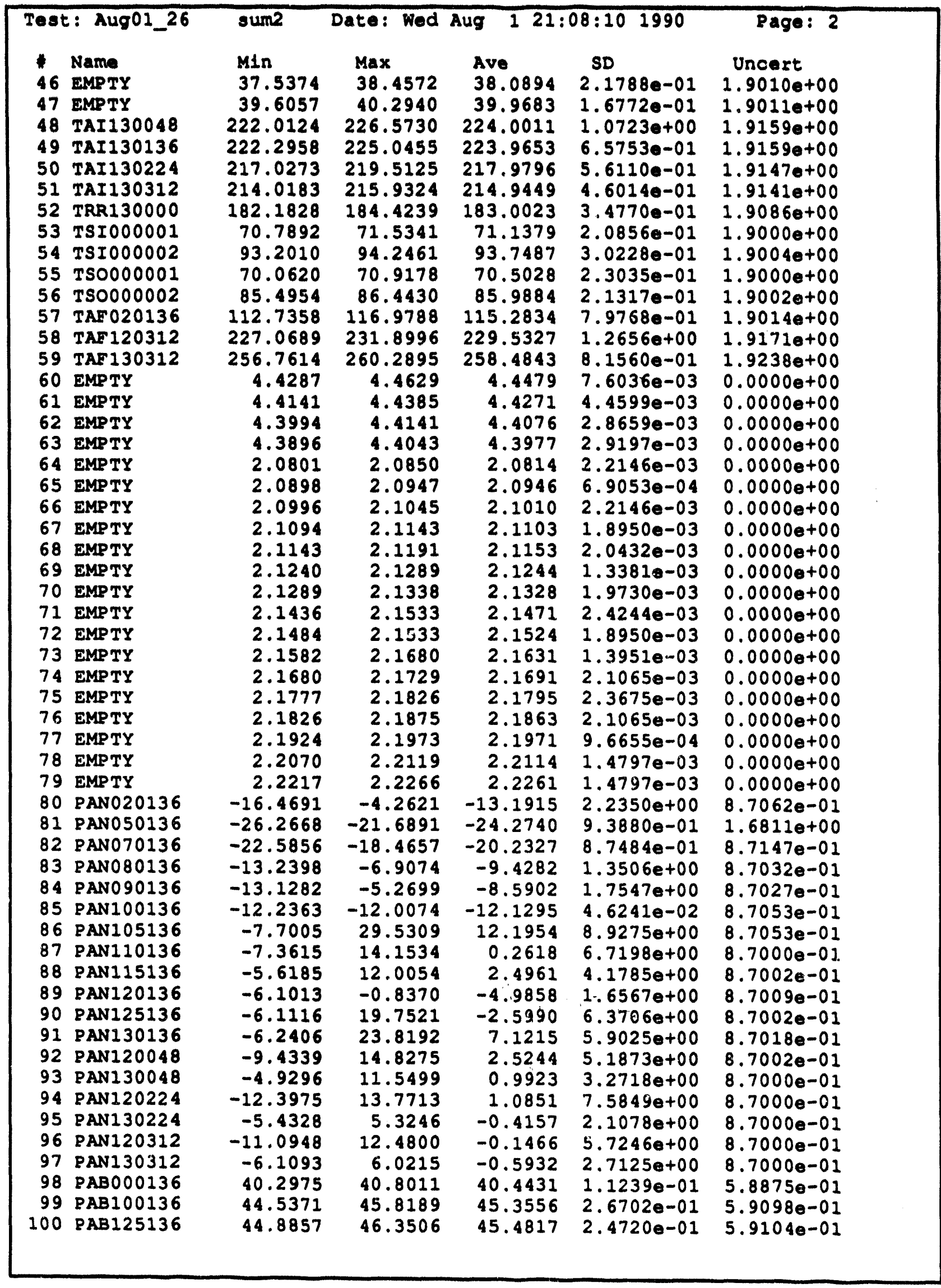




\begin{tabular}{|c|c|c|c|c|c|c|}
\hline \multicolumn{2}{|c|}{ Test: Aug01_26 } & sum2 & Date: Wed & Aug & $8: 10 \quad 1990$ & Page: 3 \\
\hline * & Name & Min & $\operatorname{Max}$ & Ave & SD & Uncert \\
\hline 101 & PINO00001 & 39.8917 & 40.6241 & 40.2140 & $1.6500 \mathrm{e}-01$ & $7.5671 e-01$ \\
\hline 102 & PRV000001 & 19.4847 & 19.6068 & 19.5970 & $3.3453 e-02$ & $7.5160 e-01$ \\
\hline 103 & PSR000001 & 44.7556 & 44.9998 & 44.8582 & $5.1496 e-02$ & $7.5834 e-01$ \\
\hline 104 & PFM000001 & 2.2553 & 2.5300 & 2.4476 & $7.2231 e-02$ & $5.0004 e-01$ \\
\hline 105 & PANO00136 & -140.5591 & -126.8262 & -135.4780 & $3.3142 e+00$ & $4.9316 e+00$ \\
\hline 106 & PFM000002 & 2.4173 & 2.5012 & 2.4592 & $2.0127 e-02$ & $2.0009 e-01$ \\
\hline 107 & ЕМРTY & -0.0049 & 0.0049 & 0.0007 & $3.8219 e-03$ & $0.0000 e+00$ \\
\hline 108 & EMPTY & -10.0000 & 9.9951 & -4.1691 & $5.3315 e+00$ & $0.0000 \mathrm{e}+00$ \\
\hline 109 & EMPTY & -0.0049 & 0.0049 & 0.0000 & $2.9594 e-03$ & $0.0000 e+00$ \\
\hline 110 & EMPTY & -10.0000 & -10.0000 & -10.0000 & $0.0000 e+00$ & $0.0000 \mathrm{e}+00$ \\
\hline 111 & EMPTY & -0.0049 & 0.0000 & -0.0001 & $6.9053 e-04$ & $0.0000 e+00$ \\
\hline 112 & VPW000001 & 145.0458 & 145.4365 & 145.2490 & $1.1133 e-01$ & $1.1898 \mathrm{e}+00$ \\
\hline 113 & VPW000002 & 168.8815 & 169.2721 & 169.1314 & $1.1864 e-01$ & $1.2984 e+00$ \\
\hline 114 & VPW000003 & 0.0526 & 0.2479 & 0.1268 & $9.5765 e-02$ & $8.1000 e-01$ \\
\hline 115 & VPW0 00004 & 148.9295 & 149.3201 & 149.1288 & $1.0052 \mathrm{e}-01$ & $1.2069 \mathrm{e}+00$ \\
\hline 116 & VPW000005 & 197.9455 & 198.3361 & 198.2110 & $1.0259 \mathrm{e}-01$ & $1.4389 \mathrm{e}+00$ \\
\hline 117 & VPW000006 & 0.1089 & 0.3042 & 0.1792 & $9.4702 e-02$ & $8.1000 e-01$ \\
\hline 118 & IPW000001 & 779.0226 & 784.8797 & 781.5995 & $1.4460 \mathrm{e}+00$ & $1.0185 e+01$ \\
\hline 119 & IPW0 00002 & 1810.2526 & 1825.8628 & 1816.9263 & $4.5819 e+00$ & $1.6481 \mathrm{e}+01$ \\
\hline 120 & IPW0 00003 & -5.0625 & 4.6875 & -0.3825 & $1.8053 e+00$ & $8.1000 e+00$ \\
\hline 121 & IPW000004 & 535.8323 & 538.2737 & 536.6719 & $6.9082 e-01$ & $4.6878 e+00$ \\
\hline 122 & IPW0 00005 & 354.3722 & 355.3487 & 354.9386 & $3.1749 e-01$ & $3.4442 e+00$ \\
\hline 123 & IPW000006 & -0.3662 & 1.0987 & 0.4834 & $3.3901 e-01$ & $2.0000 e+00$ \\
\hline 124 & EMPTY & -0.0293 & -0.0146 & -0.0195 & $3.8206 e-03$ & $0.0000 e+00$ \\
\hline 125 & EMPTY & -0.0195 & -0.0098 & -0.0138 & $3.0708 e-03$ & $0.0000 e+00$ \\
\hline 126 & EMPTY & -0.0244 & -0.0098 & -0.0158 & $3.4961 e-03$ & $0.0000 e+00$ \\
\hline 127 & EMPTY & -0.0195 & -0.0098 & -0.0149 & $2.8608 e-03$ & $0.0000 \mathrm{e}+00$ \\
\hline 128 & IHTO00001 & -1.7691 & -0.0113 & -0.7456 & $2.9676 e-01$ & $4.1000 e+00$ \\
\hline 129 & IHTO00002 & -1.6001 & -0.0376 & -0.6626 & $2.5572 e-01$ & $4.1000 e+00$ \\
\hline 130 & IHTO00003 & -1.6489 & -0.0864 & -0.7231 & $2.6707 e-01$ & $4.1000 e+00$ \\
\hline 131 & IHTO00004 & -1.3747 & -0.0075 & -0.6247 & $2.0640 e-01$ & $4.1000 \mathrm{e}+00$ \\
\hline 132 & IHTO00005 & -1.1831 & -0.2066 & -0.6792 & $1.9765 e-01$ & $4.1000 e+00$ \\
\hline 133 & IHT000006 & -1.4273 & -0.4507 & -0.9039 & $2.1043 e-01$ & $4.1000 \mathrm{e}+00$ \\
\hline 134 & IHT000007 & -1.0366 & -0.0601 & -0.6070 & $2.2668 e-01$ & $4.1000 \mathrm{e}+00$ \\
\hline 135 & IHTO00008 & -1.1343 & -0.1578 & -0.6773 & $2.3875 e-01$ & $4.1000 e+00$ \\
\hline 136 & IHTO00009 & -1.1982 & -0.2216 & -0.6786 & $2.5146 e-01$ & $4.1000 e+00$ \\
\hline 137 & IHTO00010 & -0.9240 & 1.0291 & 0.0135 & $4.2499 e-01$ & $4.1000 e+00$ \\
\hline 138 & IHT000011 & -0.4995 & 1.4536 & 0.3559 & $4.1552 e-01$ & $4.1000 e+00$ \\
\hline 139 & IHTO00012 & -0.7512 & 1.0066 & 0.0340 & $4.1851 \mathrm{e}-01$ & $4.1000 e+00$ \\
\hline 140 & IHTO00013 & 129.6311 & 131.5843 & 130.5257 & $4.3956 e-01$ & $4.3028 \mathrm{e}+00$ \\
\hline 141 & IHT000014 & 179.4922 & 181.4453 & 180.3359 & $4.5066 e-01$ & $4.4791 e+00$ \\
\hline 142 & IHTO00015 & 134.5215 & 136.2793 & 135.2519 & $4.1724 e-01$ & $4.3173 e+00$ \\
\hline 143 & IHT000016 & 125.4507 & 127.4039 & 126.3101 & $4.2682 e-01$ & $4.2902 \mathrm{e}+00$ \\
\hline 144 & IHT000017 & 148.6704 & 151.6001 & 150.2056 & $6.5198 e-01$ & $4.3665 e+00$ \\
\hline 145 & IHT000018 & 151.3897 & 153.5381 & 152.5108 & $5.7292 e-01$ & $4.3745 e+00$ \\
\hline 146 & IHT000019 & 145.9397 & 148.4788 & 147.0062 & $5.2676 e-01$ & $4.3556 e+00$ \\
\hline 147 & IHT000020 & 150.3343 & 153.2640 & 151.6586 & $5.6811 e-01$ & $4.3715 e+00$ \\
\hline 148 & IHTO00021 & -2.2198 & 0.7099 & -0.8409 & $6.0671 e-01$ & $4.1000 e+00$ \\
\hline 149 & IHTO00022 & 130.8631 & 133.0116 & 131.7577 & $4.6201 e-01$ & $4.3065 e+00$ \\
\hline 150 & IHTO00023 & 147.8215 & 149.9700 & 148.7433 & $4.3591 e-01$ & $4.3615 e+00$ \\
\hline 151 & IHTO00024 & 112.8043 & 114.9527 & 113.6753 & $4.6052 e-01$ & $4.2547 e+00$ \\
\hline 152 & IHT000025 & 147.0778 & 149.4216 & 148.1442 & $4.8361 e-01$ & $4.3594 e+00$ \\
\hline 153 & IHTO00026 & 148.6591 & 151.0028 & 149.5888 & $4.9056 e-01$ & $4.3644 \mathrm{e}+00$ \\
\hline 154 & IHTO00027 & 127.7006 & 130.0443 & 128.4194 & $4.6091 \mathrm{e}-01$ & $4.2964 e+00$ \\
\hline 155 & IHT000028 & 129.6349 & 131.9787 & 130.5802 & $6.5359 e-01$ & $4.3029 e+00$ \\
\hline
\end{tabular}




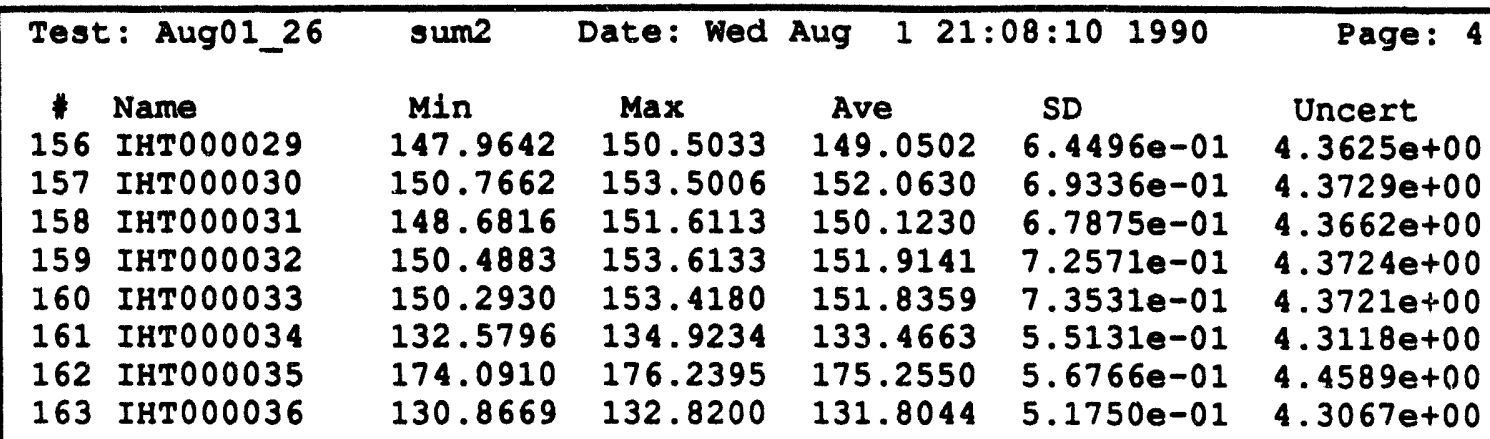




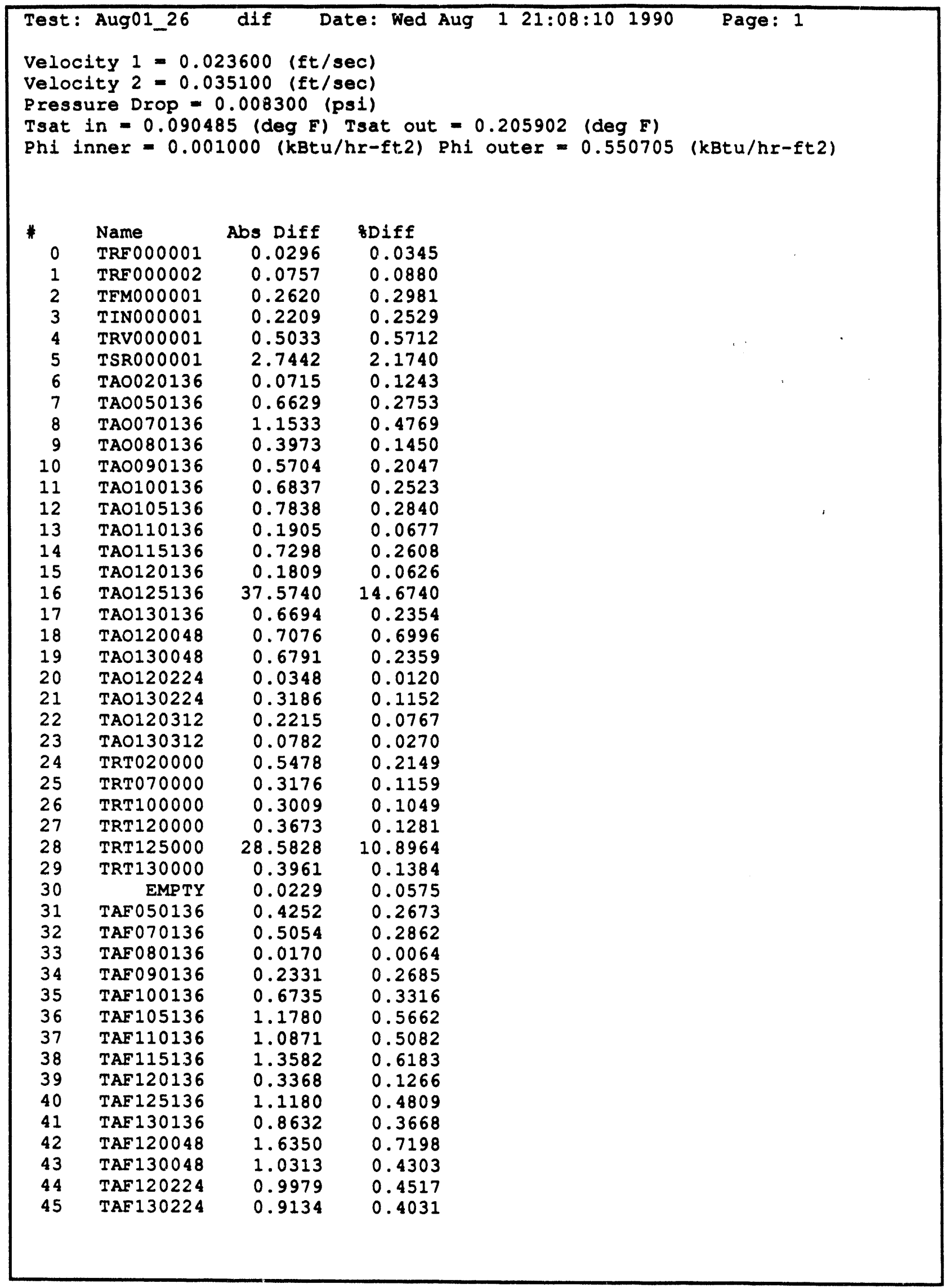




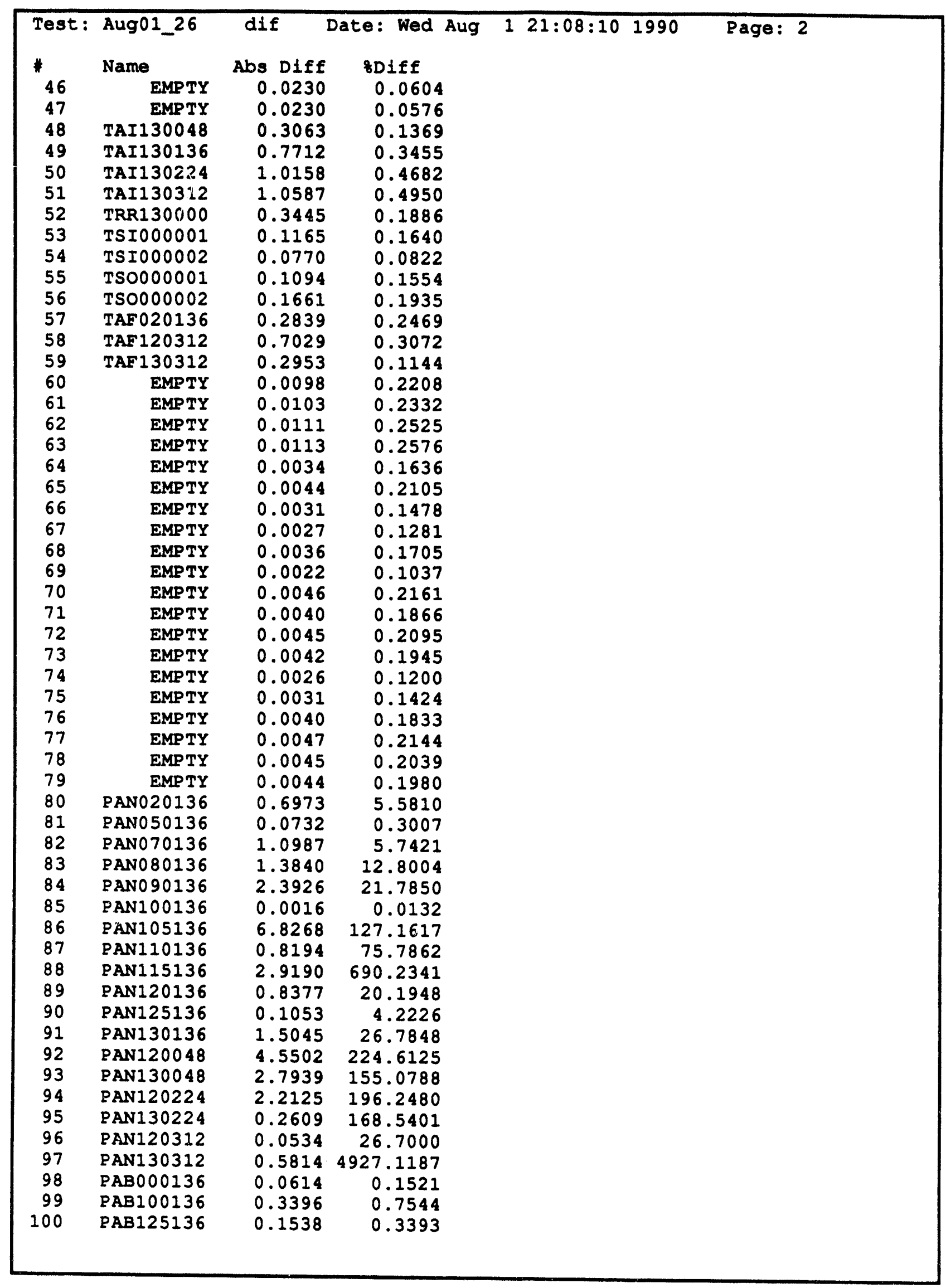




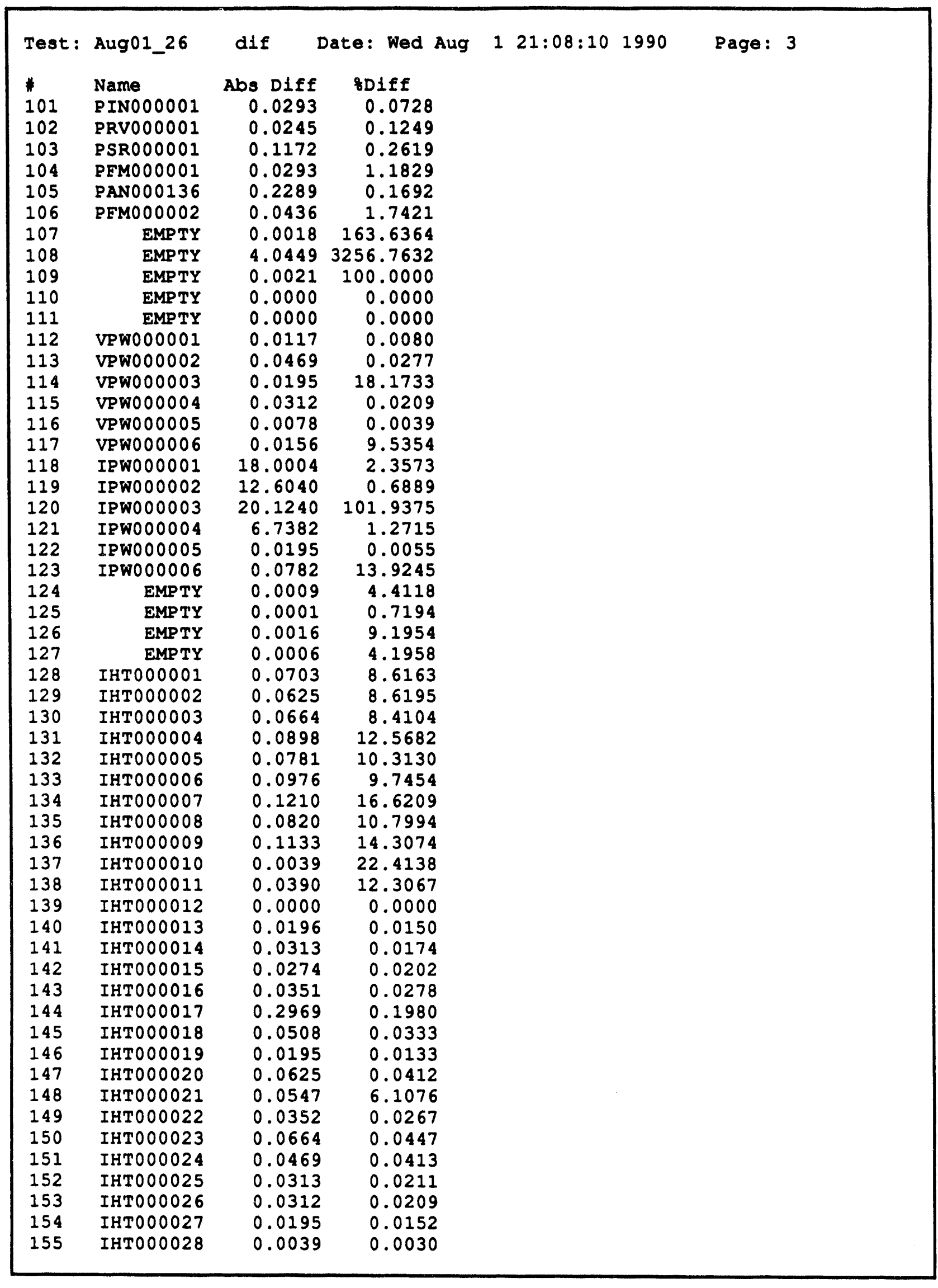




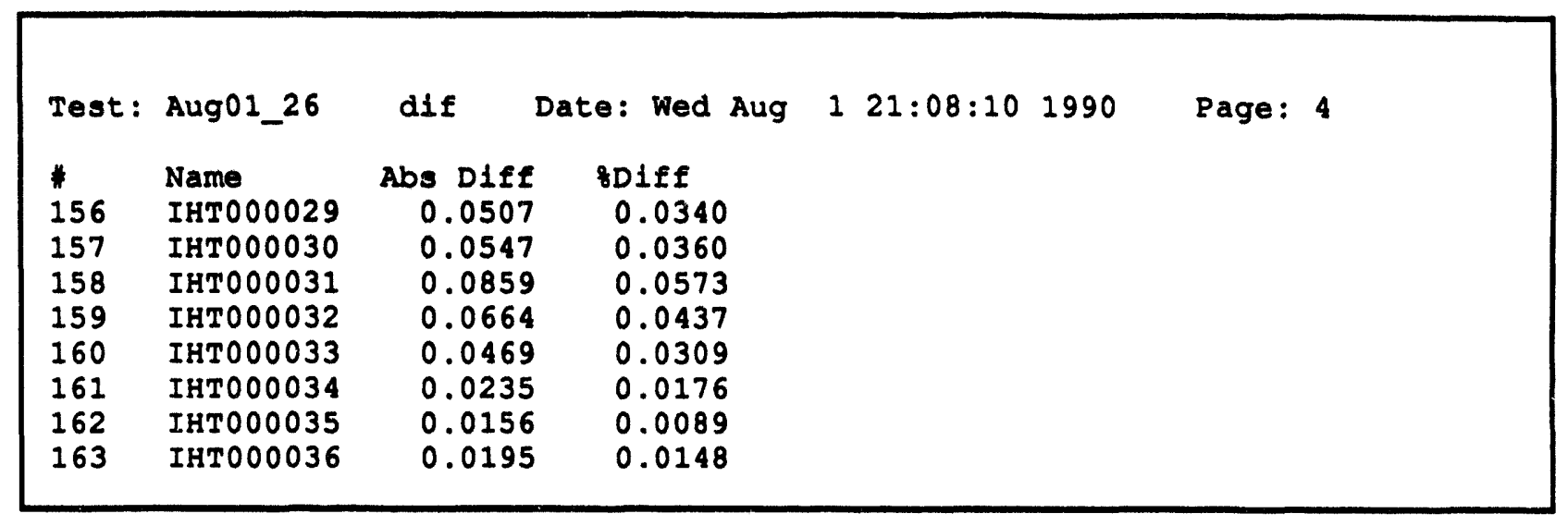




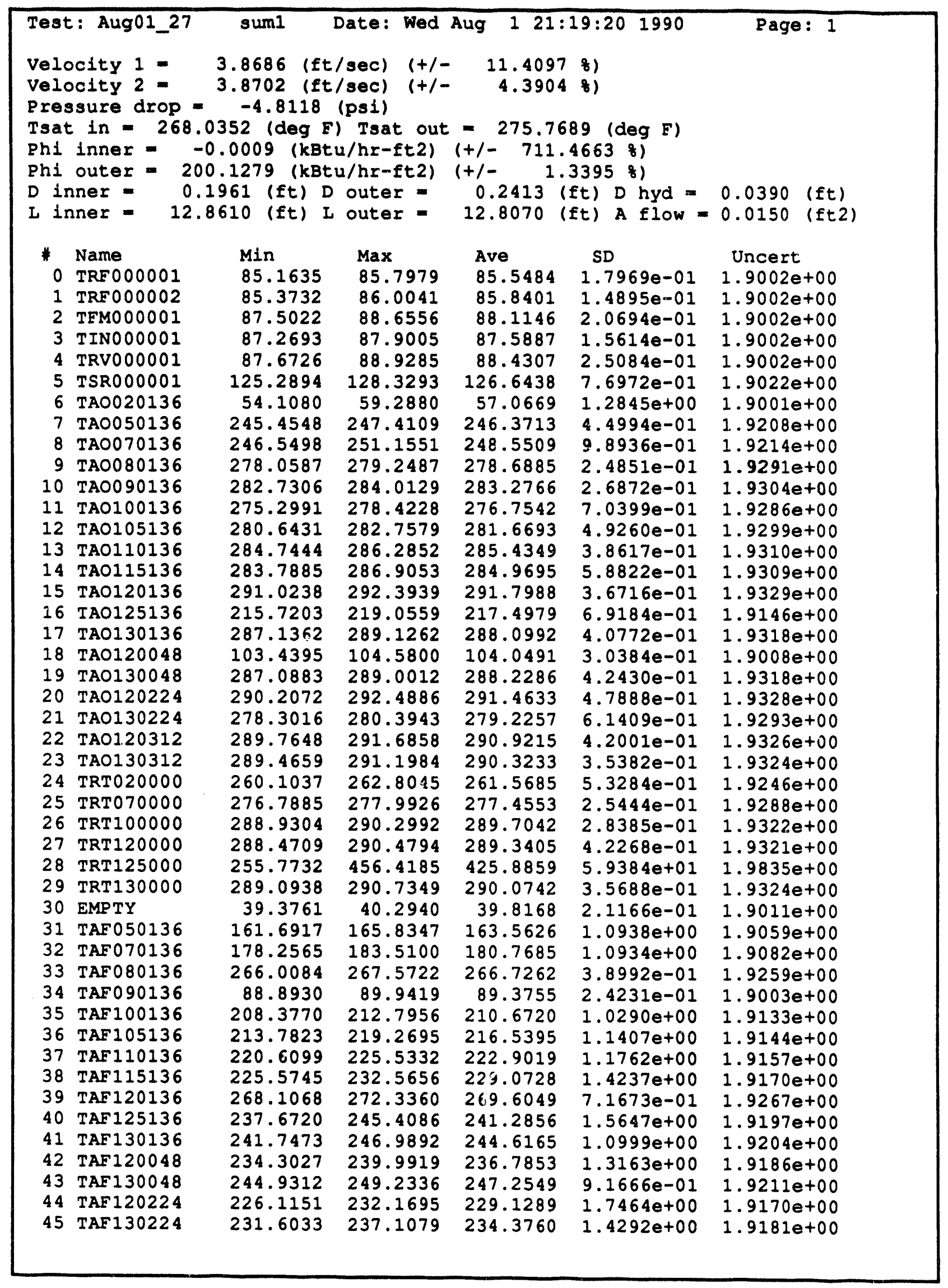




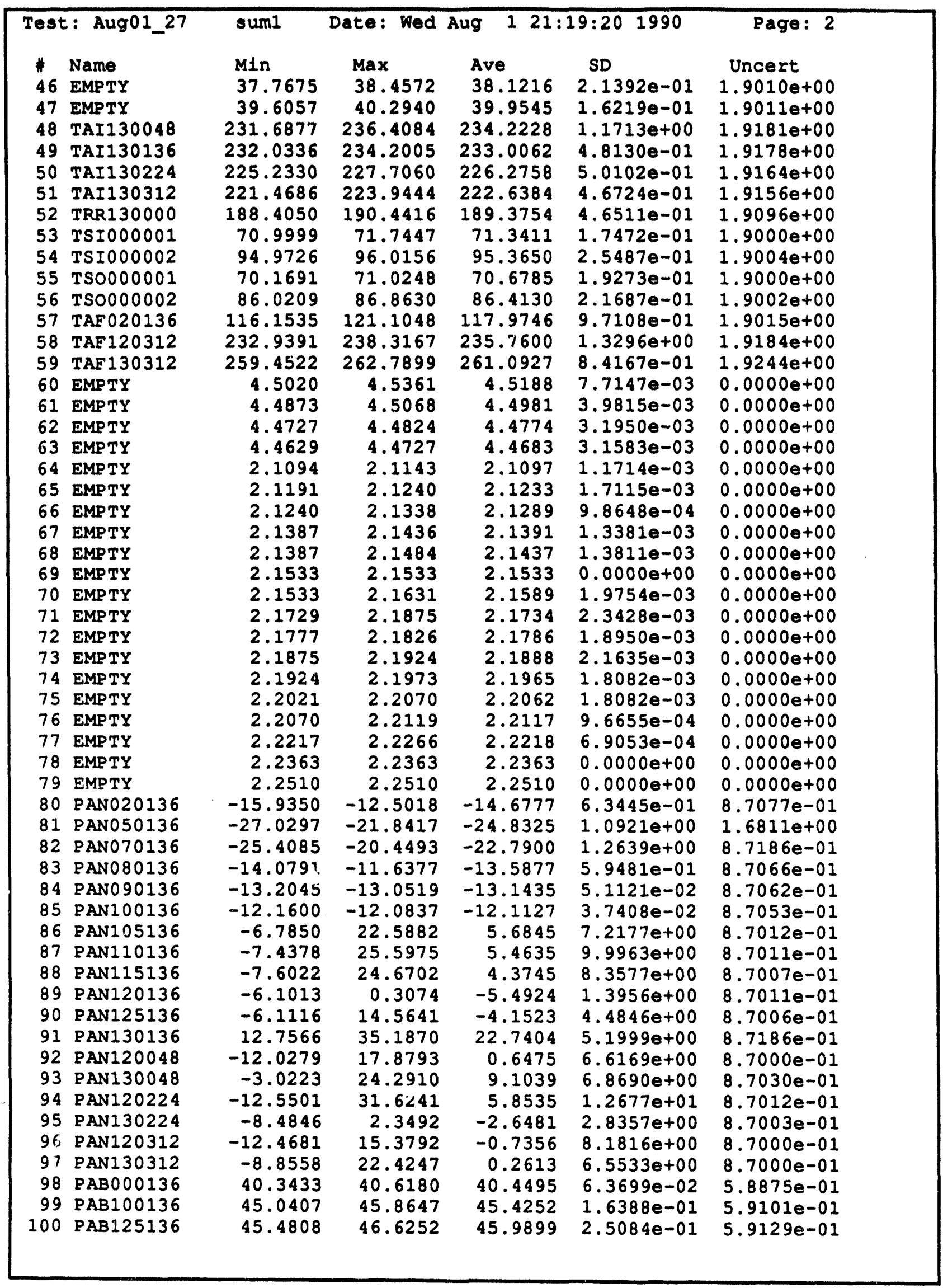




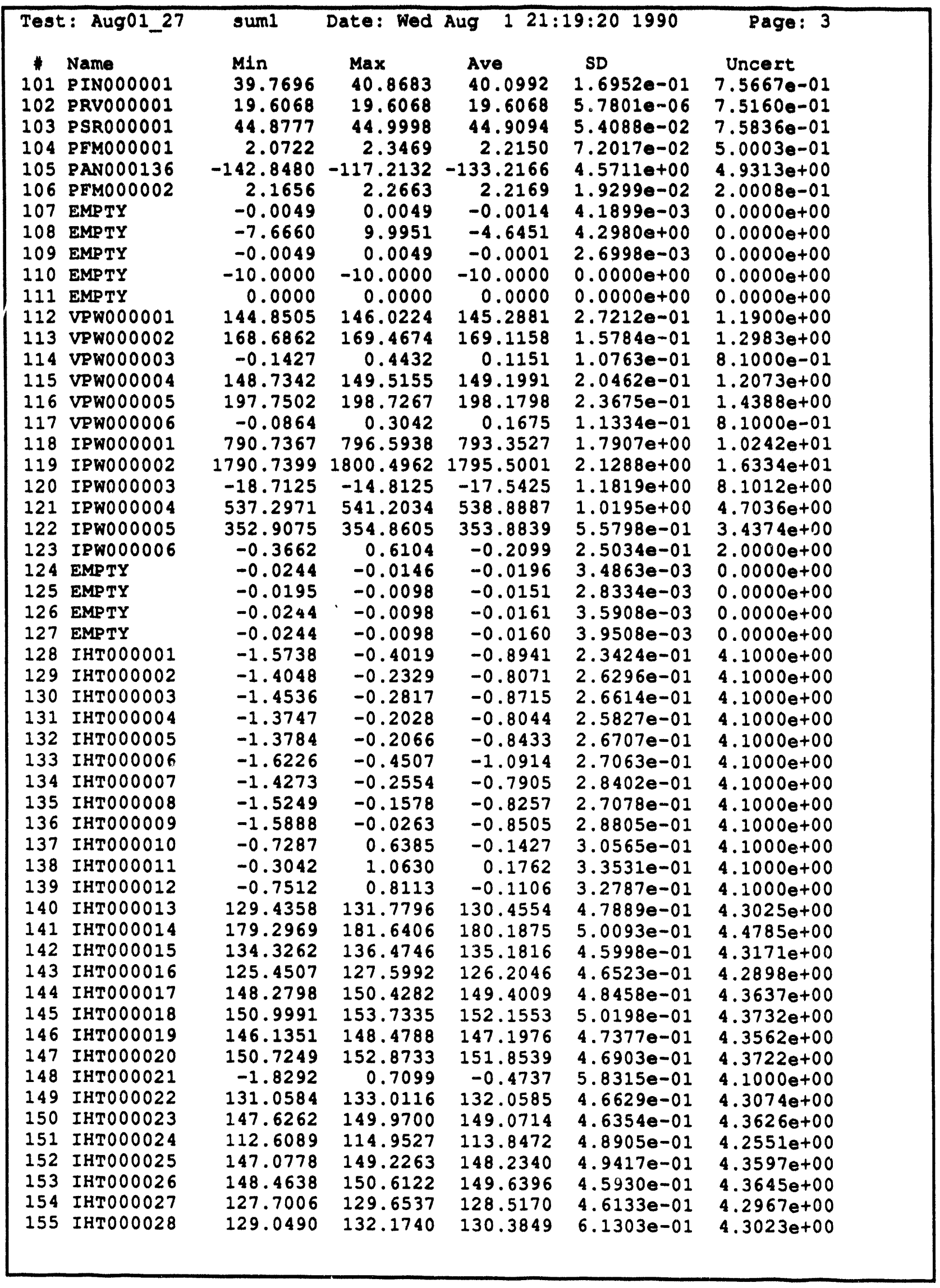




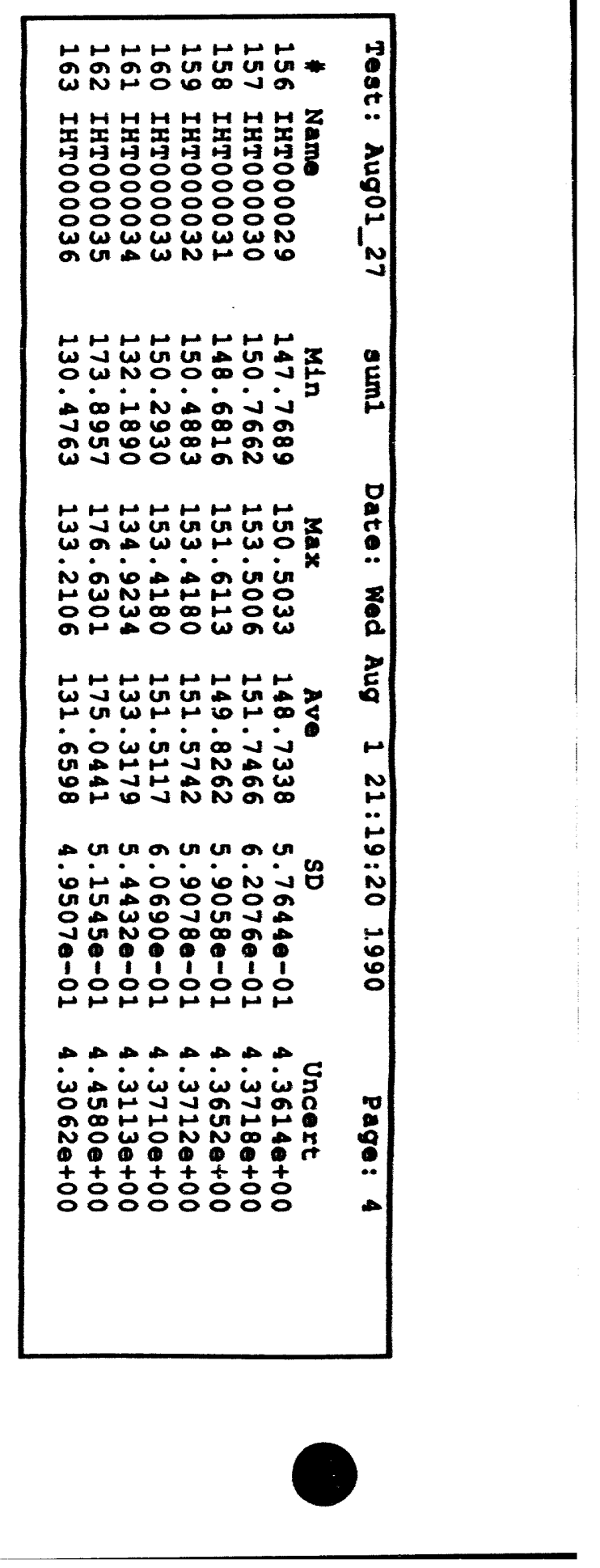




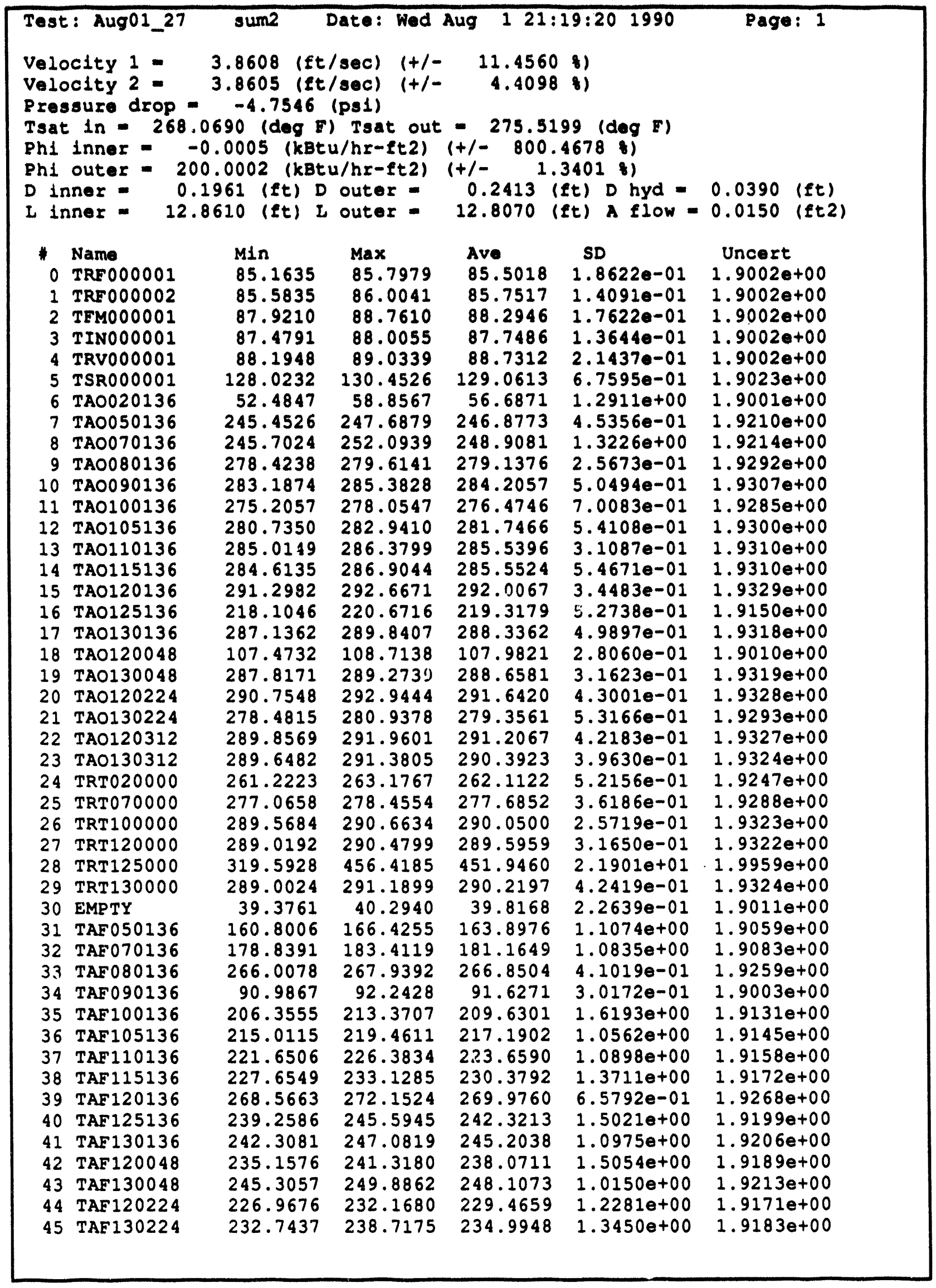




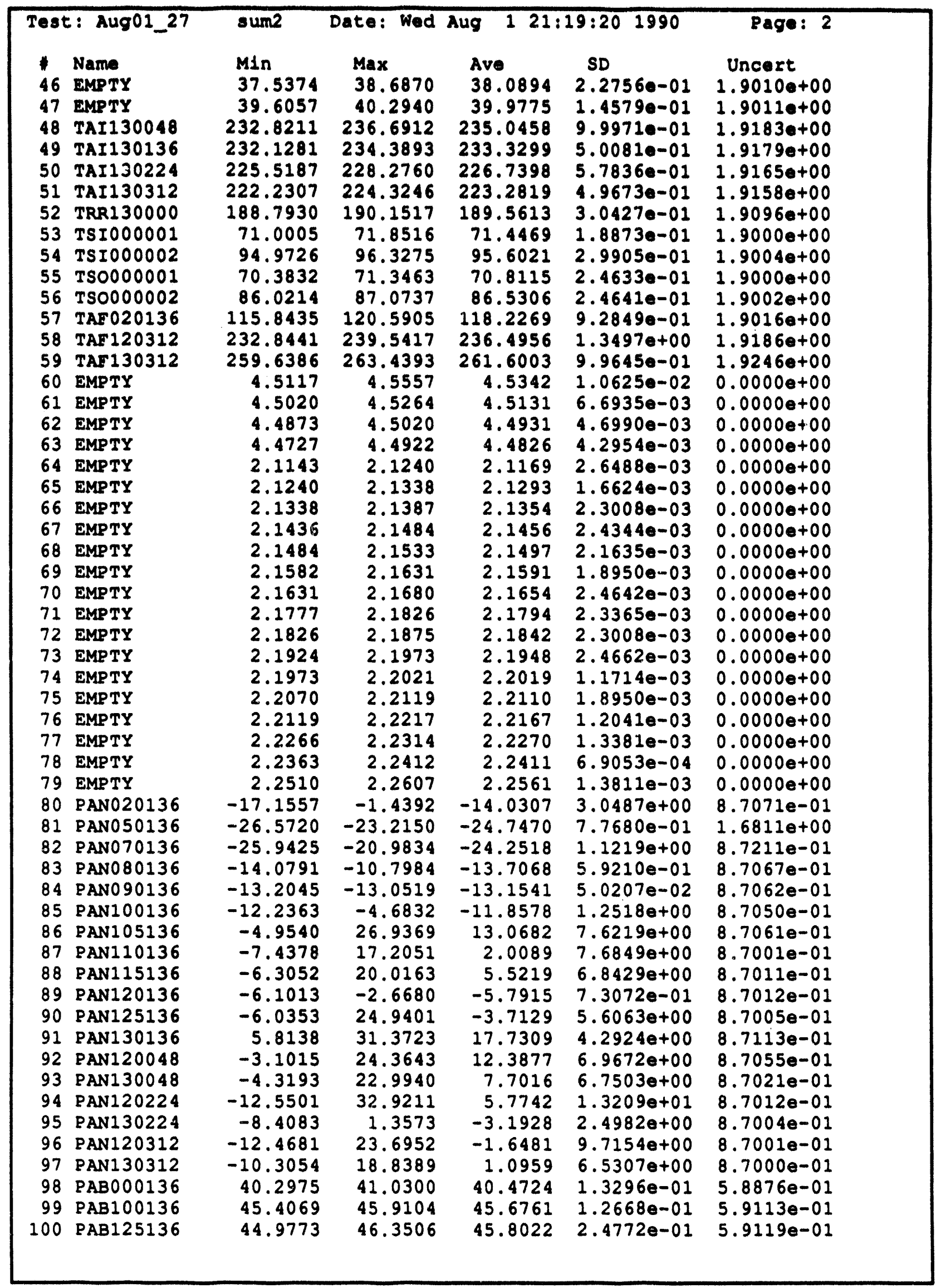




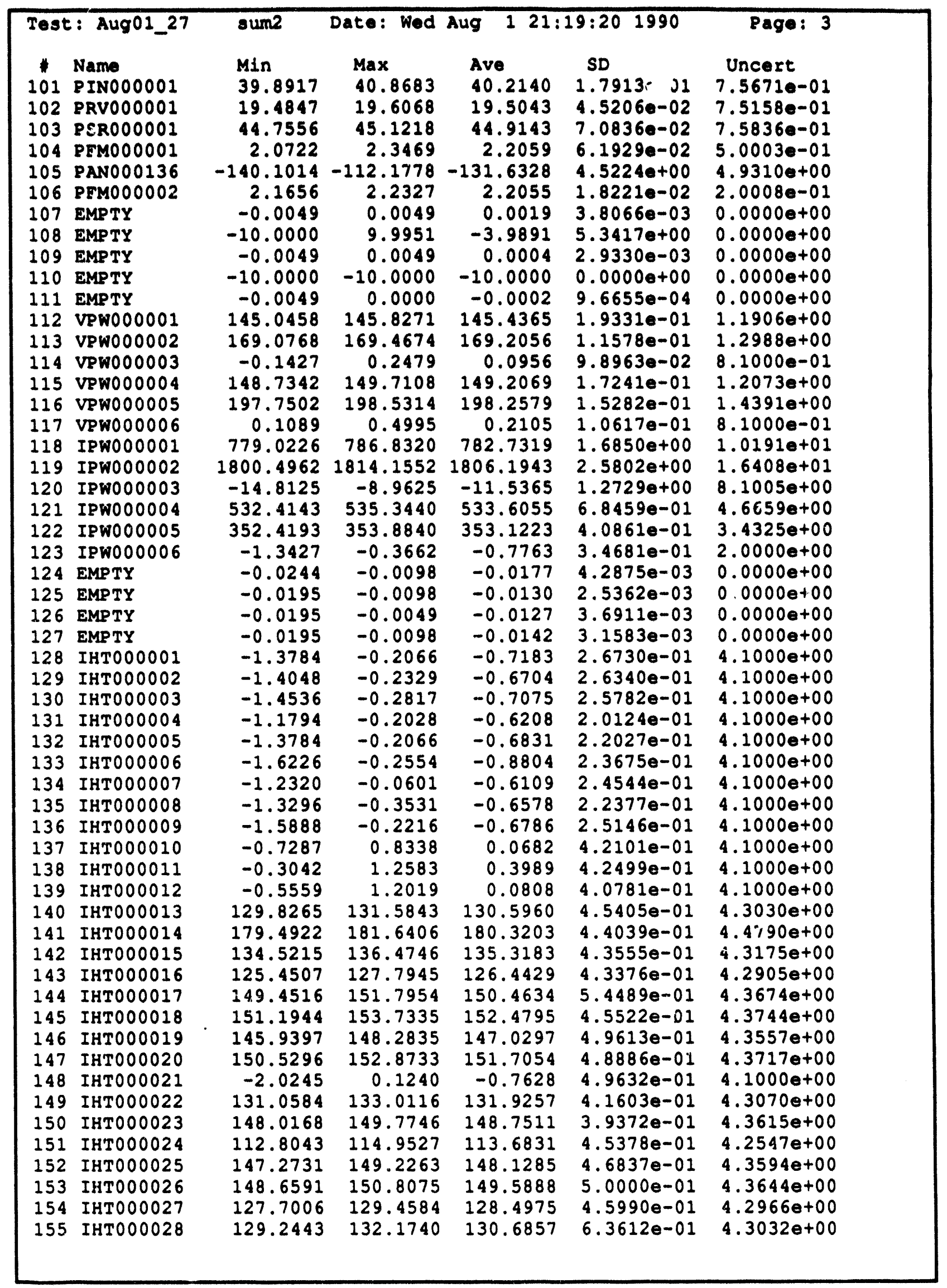




\begin{tabular}{|c|c|c|c|c|c|c|}
\hline $\begin{array}{l}1 \\
156 \\
157 \\
158 \\
159 \\
160 \\
161 \\
162 \\
163\end{array}$ & 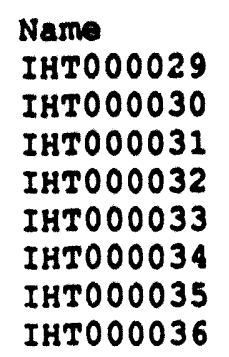 & $\begin{array}{l}\text { Min } \\
147.7689 \\
150.9615 \\
148.8770 \\
150.6836 \\
150.4883 \\
132.3843 \\
174.2863 \\
130.6716\end{array}$ & $\begin{array}{l}\operatorname{Max} \\
150.3080 \\
153.6959 \\
151.6113 \\
153.6133 \\
153.4180 \\
134.7281 \\
176.0442 \\
132.6247\end{array}$ & $\begin{array}{l}\text { Ave } \\
149.0424 \\
152.0826 \\
150.1152 \\
151.9258 \\
151.8203 \\
133.4859 \\
175.2120 \\
131.7458\end{array}$ & $\begin{array}{l}S D \\
5.7621 e-01 \\
6.1865 e-01 \\
5.9662 e-01 \\
6.2485 e-01 \\
6.2476 e-01 \\
4.8770 e-01 \\
4.9569 e-01 \\
4.9951 e-01\end{array}$ & $\begin{array}{c}\text { Uncert } \\
4.3625 e+00 \\
4.3730 e+00 \\
4.3662 e+00 \\
4.3724 e+00 \\
4.3721 e+00 \\
4.3118 e+00 \\
4.4587 e+00 \\
4.3065 e+00\end{array}$ \\
\hline
\end{tabular}




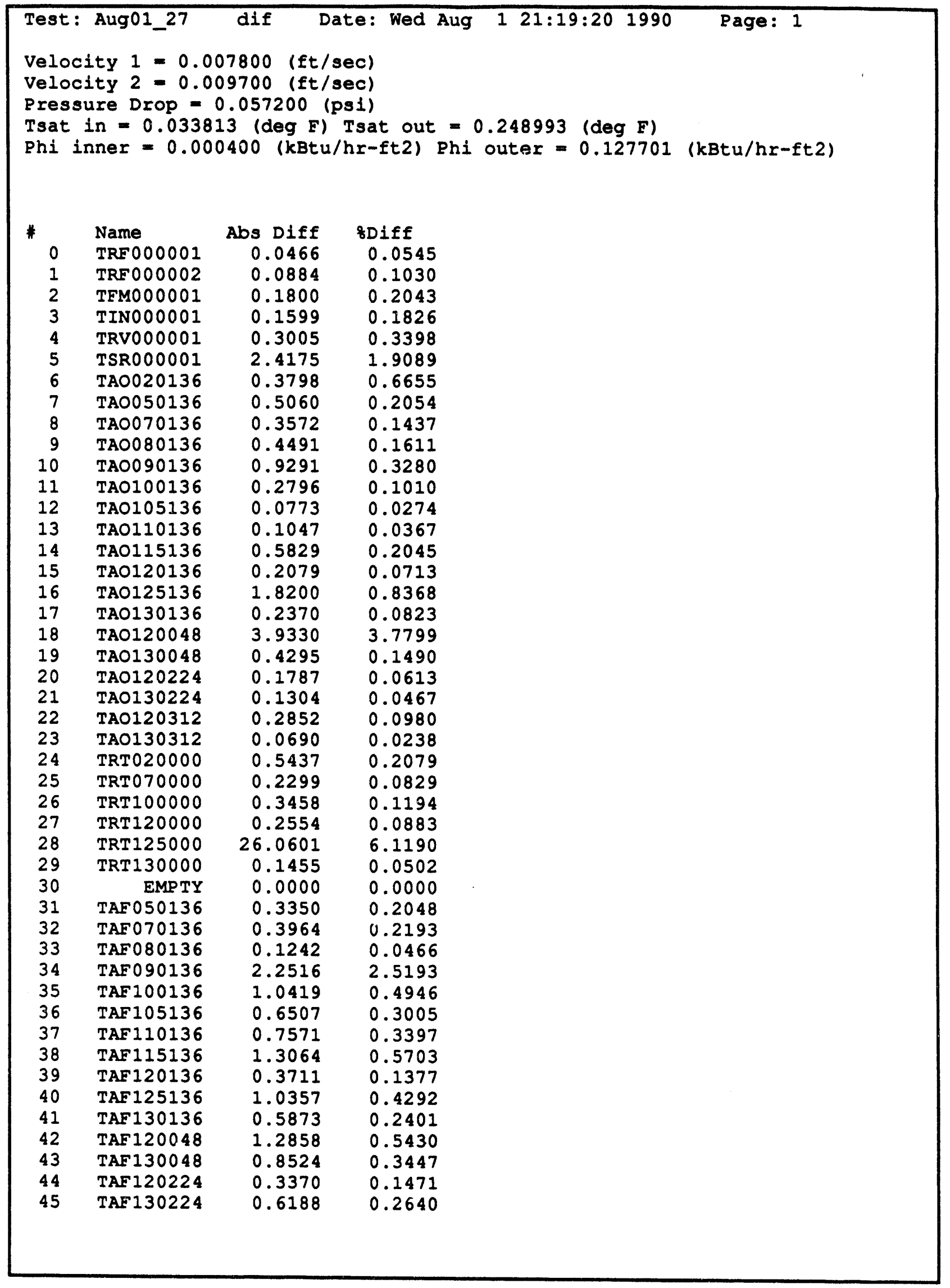




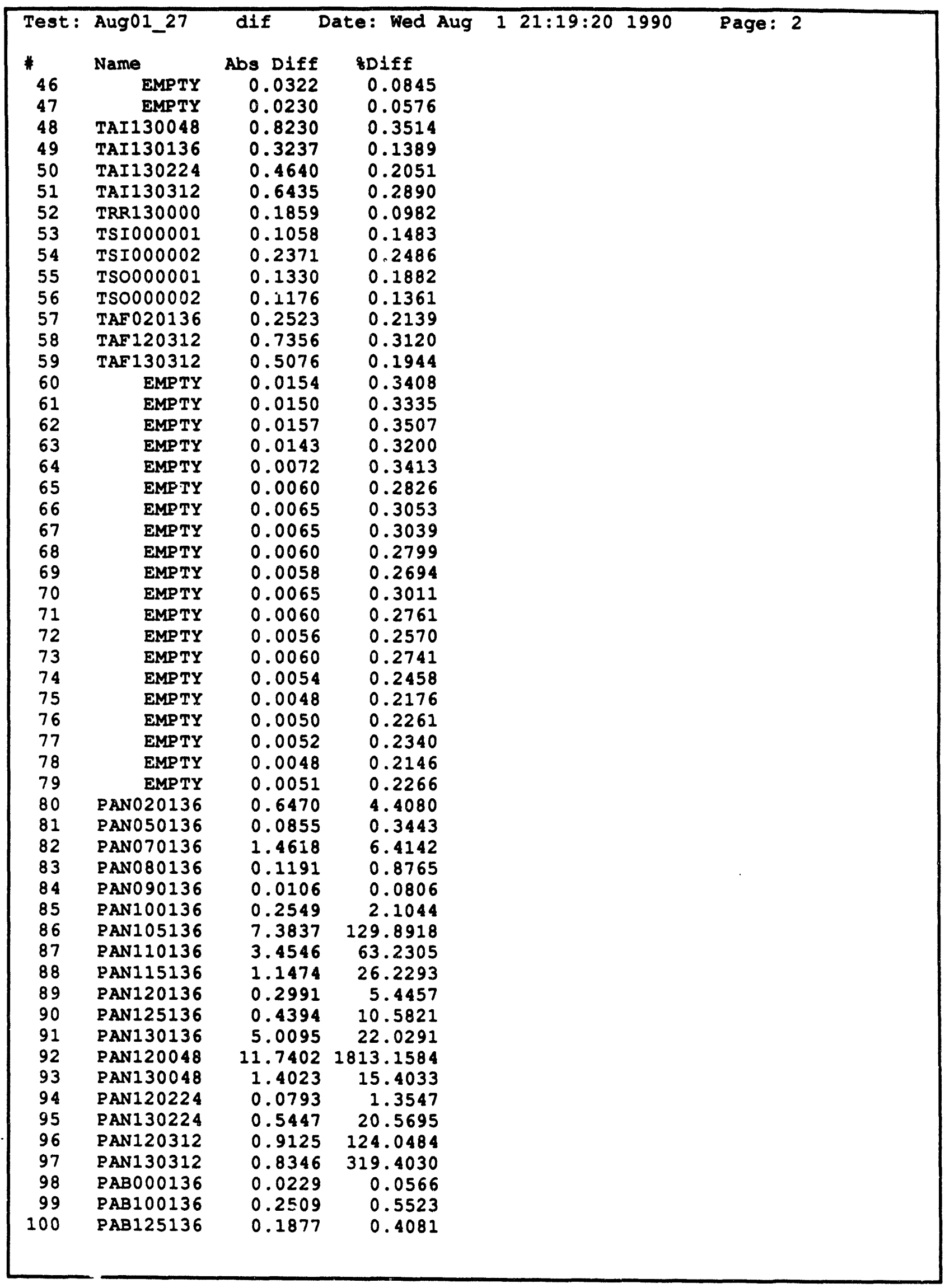




\begin{tabular}{|c|c|c|c|c|c|}
\hline Test: & Aug01_27 & dif & ate: Wed Aug & $121: 19: 201990$ & Page: 3 \\
\hline \# & Name & Abs Diff & ODiff & & \\
\hline 101 & PIN000001 & 0.1148 & 0.2863 & & \\
\hline 102 & PRV000001 & 0.1025 & 0.5228 & & \\
\hline 103 & PSR000001 & 0.0049 & 0.0109 & & \\
\hline 104 & PFM000001 & 0.0091 & 0.4108 & & \\
\hline 105 & PANO00136 & 1.5838 & 1.1889 & & \\
\hline 106 & PFM000002 & 0.0114 & 0.5142 & & \\
\hline 107 & EMPTY & 0.0033 & 235.7143 & & \\
\hline 108 & EMPTY & 0.6560 & 14.1224 & & \\
\hline 109 & EMPTY & 0.0005 & 500.0000 & & \\
\hline 110 & EMPTY & 0.0000 & 0.0000 & & \\
\hline 111 & EMPTY & 0.0002 & 100.0000 & & \\
\hline 112 & VPW000001 & 0.1484 & 0.1021 & & \\
\hline 113 & VPW0 00002 & 0.0898 & 0.0531 & & \\
\hline 114 & VPW000003 & 0.0195 & 16.9418 & & \\
\hline 115 & VRW000004 & 0.0078 & 0.0052 & & \\
\hline 116 & VPW000005 & 0.0781 & 0.0394 & & \\
\hline 117 & VPW000006 & 0.0430 & 25.6716 & & \\
\hline 118 & IPW000001 & 10.6208 & 1.3387 & & \\
\hline 119 & IPW000002 & 10.6942 & 0.5956 & & \\
\hline 120 & IPWO00003 & 6.0060 & 34.2369 & & \\
\hline 121 & IPWO00004 & 5.2831 & 0.9804 & & \\
\hline 122 & IPW000005 & 0.7616 & 0.2152 & & \\
\hline 123 & IPW000006 & 0.5664 & 269.8428 & & \\
\hline 124 & EMPTY & 0.0019 & 9.6939 & & \\
\hline 125 & EMPTY & 0.0021 & 13.9073 & & \\
\hline 126 & EMPTY & 0.0034 & 21.1180 & & \\
\hline 127 & EMPTY & 0.0018 & 11.2500 & & \\
\hline 128 & IHT000001 & 0.1758 & 19.6622 & & \\
\hline 129 & IHT 000002 & 0.1367 & 16.9372 & & \\
\hline 130 & IHT000003 & 0.1640 & 18.8181 & & \\
\hline 131 & IHT000004 & 0.1836 & 22.8245 & & \\
\hline 132 & IHT000005 & 0.1602 & 18.9968 & & \\
\hline 133 & IHT000006 & 0.2110 & 19.3330 & & \\
\hline 134 & IHTO00007 & 0.1796 & 22.7198 & & \\
\hline 135 & IHT000008 & 0.1679 & 20.3343 & & \\
\hline 136 & IHT000009 & 0.1719 & 20.2116 & & \\
\hline 137 & IHT 000010 & 0.2109 & 147.7926 & & \\
\hline 138 & IHT000011 & 0.2227 & 126.3905 & & \\
\hline 139 & IHT000012 & 0.1914 & 173.0560 & & \\
\hline 140 & IHTO00013 & 0.1406 & 0.1078 & & \\
\hline 141 & IHT000014 & 0.1328 & 0.0737 & & \\
\hline 142 & IHT000015 & 0.1367 & 0.1011 & & \\
\hline 143 & IHT000016 & 0.2383 & 0.1888 & & \\
\hline 144 & IHT000017 & 1.0625 & 0.7112 & & \\
\hline 145 & IHT000018 & 0.3242 & 0.2131 & & \\
\hline 146 & IHT000019 & 0.1679 & 0.1141 & & \\
\hline 147 & IHT000020 & 0.1485 & 0.0978 & & \\
\hline 148 & IHT000021 & 0.2891 & 61.0302 & & \\
\hline 149 & IHTO00022 & 0.1328 & 0.1006 & & \\
\hline 150 & IHT000023 & 0.3203 & 0.2149 & & \\
\hline 151 & IHT000024 & 0.1641 & 0.1441 & & \\
\hline 152 & IHT000025 & 0.1055 & 0.0712 & & \\
\hline 153 & IHTO00026 & 0.0508 & 0.0339 & & \\
\hline 154 & IHTO00027 & 0.0195 & 0.0152 & & \\
\hline 155 & IHT000028 & 0.3008 & 0.2307 & & \\
\hline
\end{tabular}




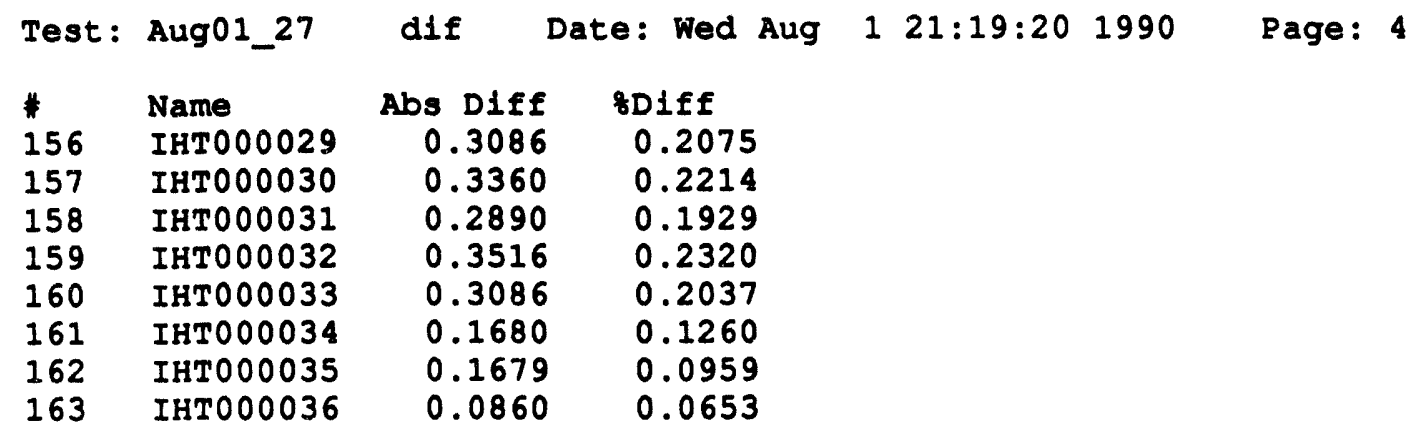




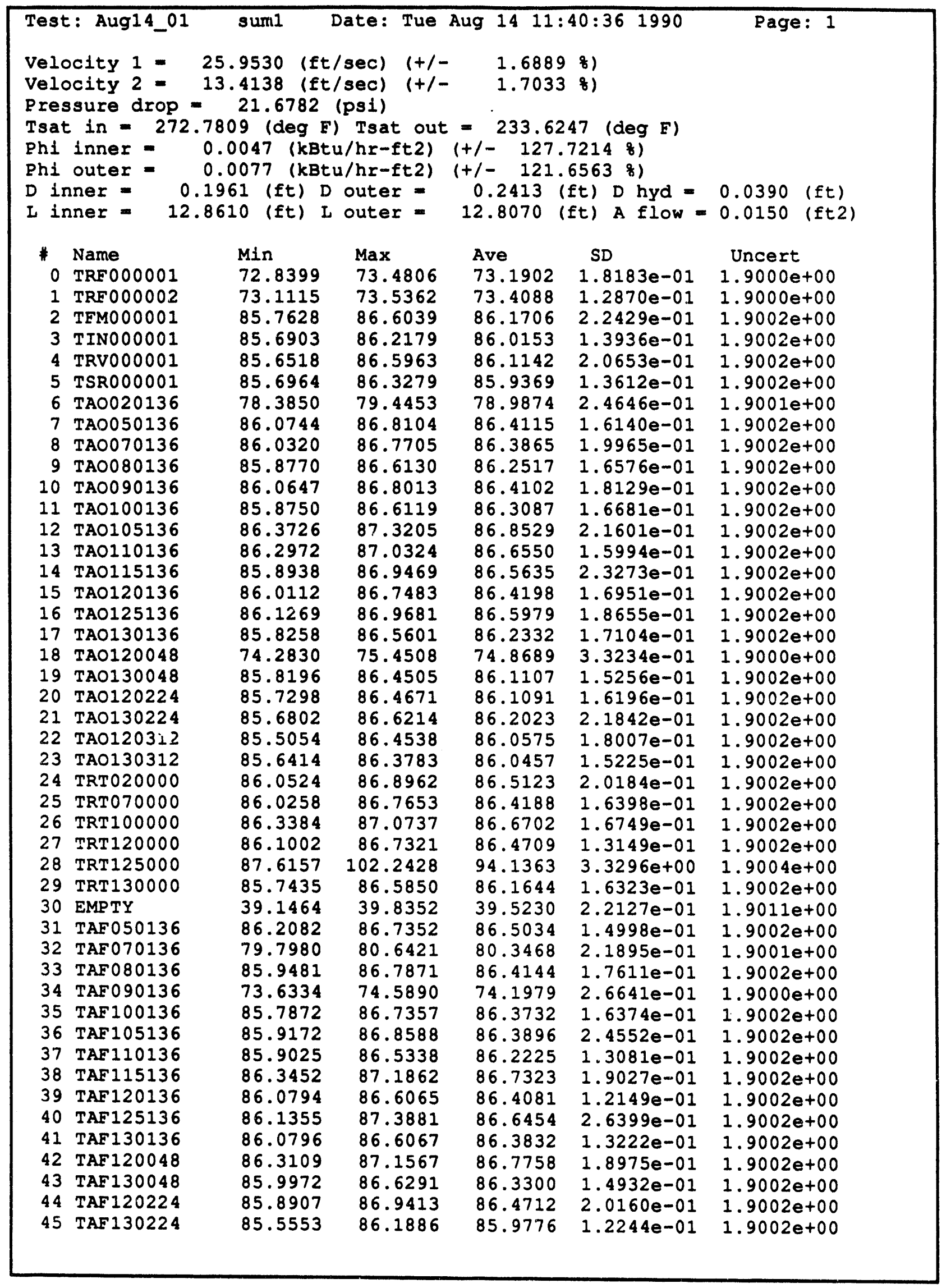




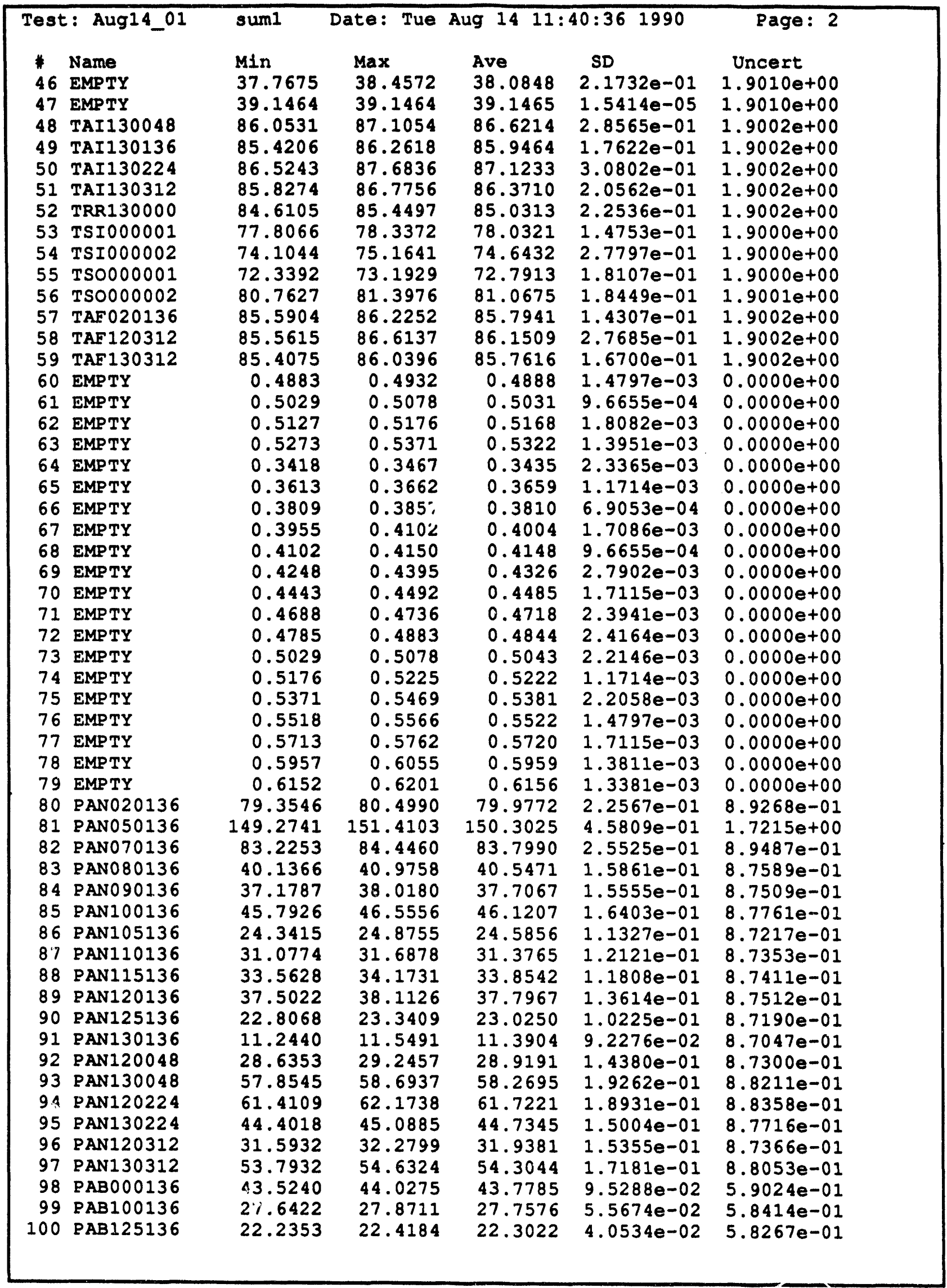




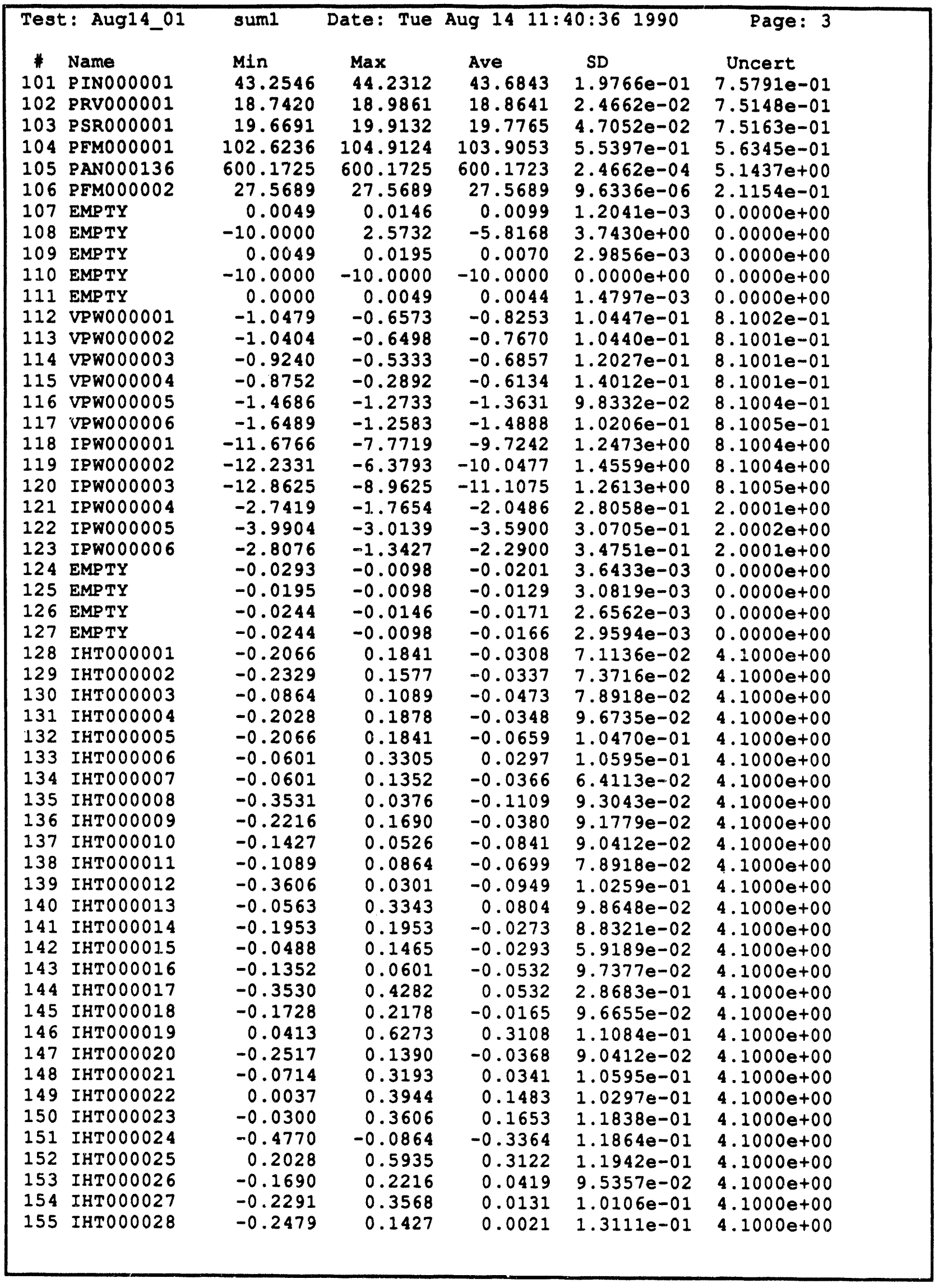




\begin{tabular}{|c|c|c|c|c|c|c|}
\hline \multicolumn{2}{|c|}{ Test: Aug14_01 } & suml & Date: Tue & \multicolumn{2}{|c|}{ ug $1411: 40: 361990$} & $\begin{array}{c}\text { Page: } \\
\text { Uncert }\end{array}$ \\
\hline $\begin{array}{c}1 \\
156 \\
157 \\
158 \\
159 \\
160 \\
161 \\
162 \\
163\end{array}$ & $\begin{array}{l}\text { Name } \\
\text { IHTOOOOO29 } \\
\text { IHTOOOOO30 } \\
\text { IHTOOOO31 } \\
\text { IHTOOOO32 } \\
\text { IHTOOOO33 } \\
\text { IHTOOOOO34 } \\
\text { IHTOOOOO35 } \\
\text { IHTOOOOO36 }\end{array}$ & $\begin{array}{l}\text { Min } \\
-0.2780 \\
-0.2103 \\
-0.3418 \\
-0.2930 \\
-0.0977 \\
-0.2329 \\
-0.1277 \\
-0.1878\end{array}$ & $\begin{array}{l}\text { Max } \\
0.1127 \\
0.3756 \\
0.2441 \\
0.0977 \\
0.0976 \\
0.1577 \\
0.2629 \\
0.2028\end{array}$ & $\begin{array}{l}\text { Ave } \\
-0.0436 \\
0.0162 \\
-0.0645 \\
-0.0469 \\
-0.0469 \\
-0.0688 \\
0.0285 \\
-0.0823\end{array}$ & $\begin{array}{l}\text { SD } \\
9.6655 \mathrm{e}-02 \\
1.0705 \mathrm{e}-01 \\
1.1897 \mathrm{e}-01 \\
9.5112 \mathrm{e}-02 \\
8.6541 \mathrm{e}-02 \\
9.9512 \mathrm{e}-02 \\
1.0440 \mathrm{e}-01 \\
1.1306 \mathrm{e}-01\end{array}$ & $\begin{array}{l}\text { Uncert } \\
4.1000 e+00 \\
4.1000 e+00 \\
4.1000 e+00 \\
4.1000 e+00 \\
4.1000 e+00 \\
4.1000 e+00 \\
4.1000 e+00 \\
4.1000 e+00\end{array}$ \\
\hline
\end{tabular}




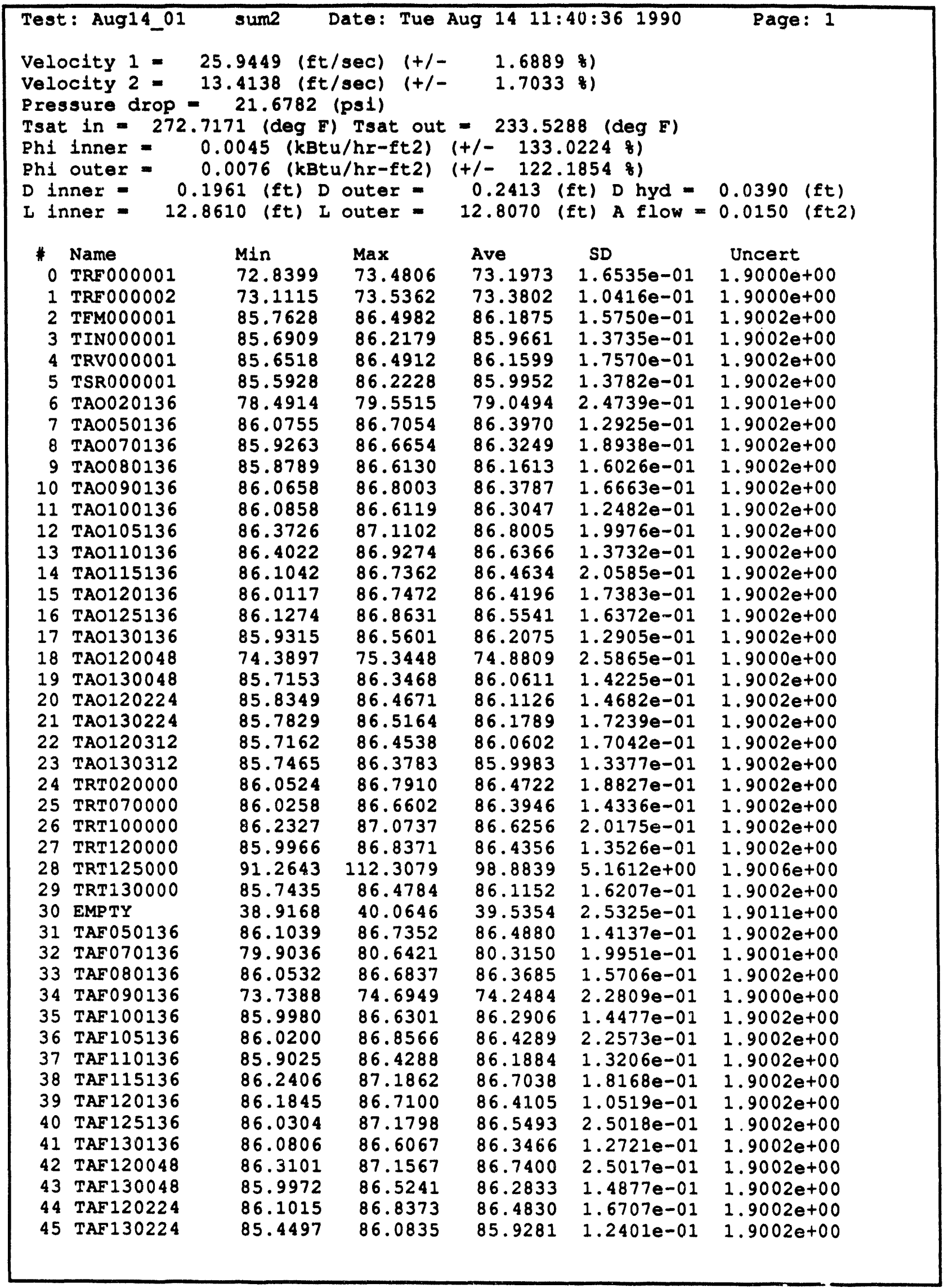




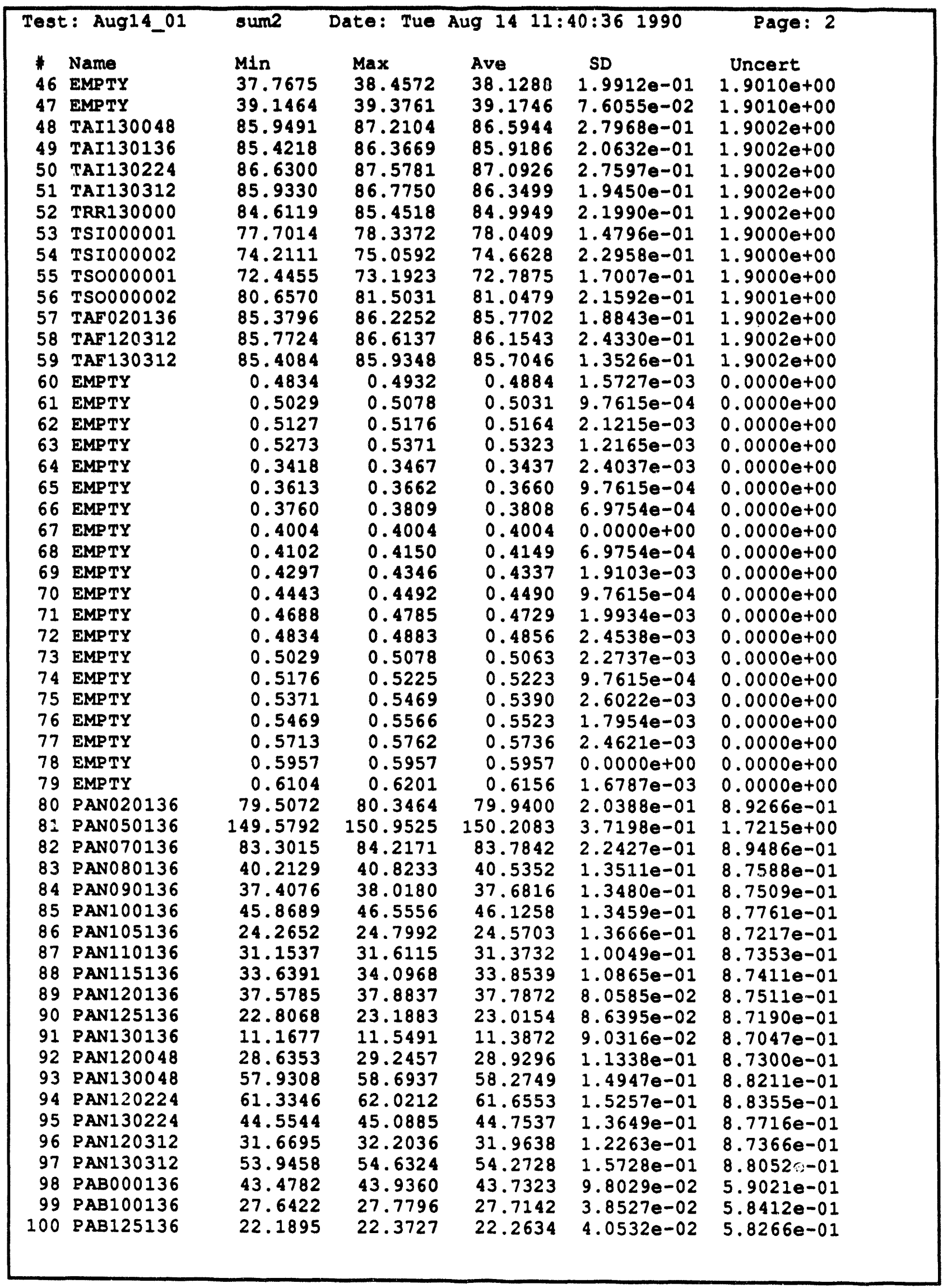




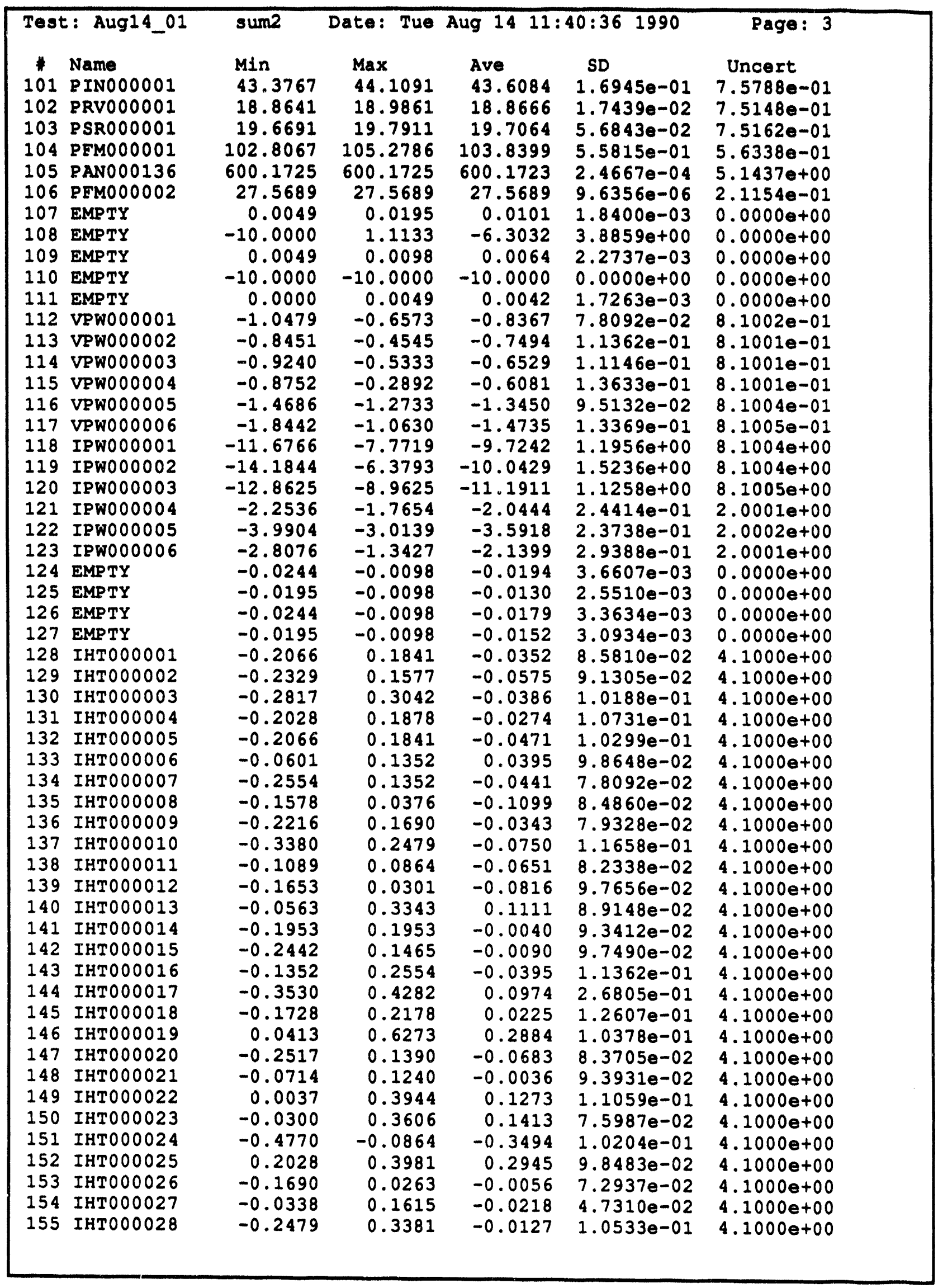




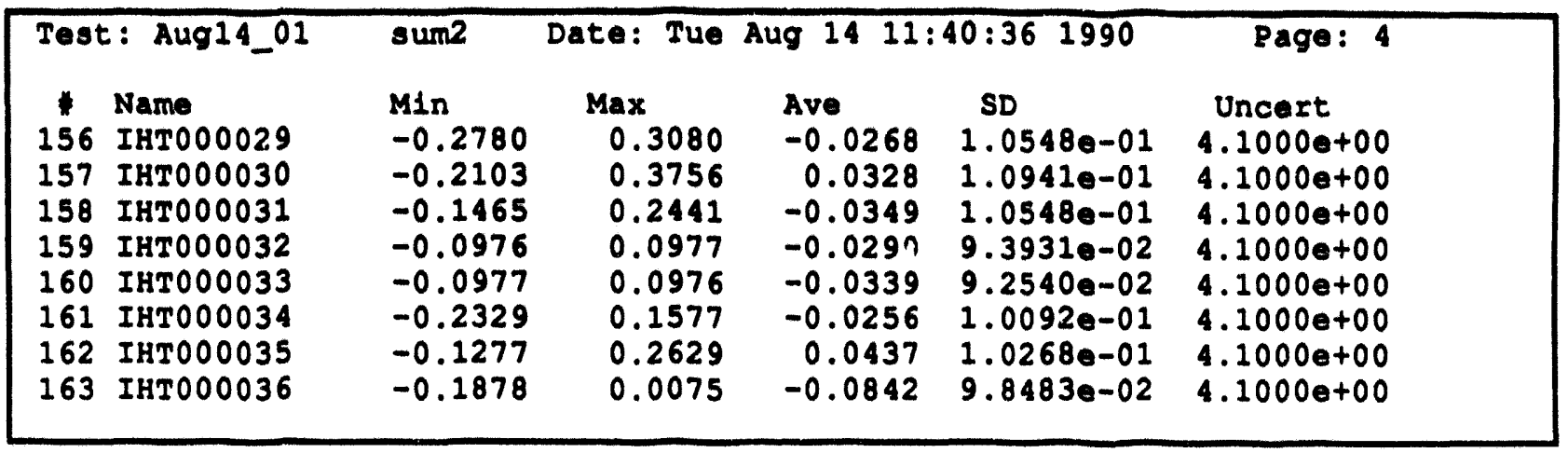




\begin{tabular}{|c|c|c|c|c|}
\hline \multicolumn{5}{|c|}{$\begin{array}{l}\text { Velocity } 1=0.008 n g 9 \text { (ft/sec) } \\
\text { Velocity } 2=0.000000 \text { (ft/sec) } \\
\text { Pressure Drop }=0.000000 \text { (psi) } \\
\text { Tsat in }=0.063812 \text { (deg F) Tsat out }=0.095901 \text { (deg F) } \\
\text { Ph1 inner }=0.000200 \text { (kBtu/hr-ft2) Phi outer }=0.000100 \text { (kBtu/hr-ft2) }\end{array}$} \\
\hline $\begin{array}{l} \\
0 \\
1 \\
2 \\
3 \\
4 \\
5 \\
6 \\
7 \\
8 \\
9 \\
10 \\
11 \\
12 \\
13 \\
14 \\
15 \\
16 \\
17 \\
18 \\
19 \\
20 \\
21 \\
22 \\
23 \\
24 \\
25 \\
26 \\
27 \\
28 \\
29 \\
30 \\
31 \\
32 \\
33 \\
34 \\
35 \\
36 \\
37 \\
38 \\
39 \\
40 \\
41 \\
42 \\
43 \\
44 \\
45\end{array}$ & $\begin{array}{l}\text { Name } \\
\text { TRE000001 } \\
\text { TRF000002 } \\
\text { TFMO00001 } \\
\text { TIN000001 } \\
\text { TRV000001 } \\
\text { TSR000001 } \\
\text { TAO020136 } \\
\text { TAO050136 } \\
\text { TAO070136 } \\
\text { TAO080136 } \\
\text { TAO090136 } \\
\text { TAO100136 } \\
\text { TAO105136 } \\
\text { TAO110136 } \\
\text { TAO115136 } \\
\text { TAO120136 } \\
\text { TAO125136 } \\
\text { TAO130136 } \\
\text { TAO120048 } \\
\text { TA0130048 } \\
\text { TAO120224 } \\
\text { TAO130224 } \\
\text { TAO120312 } \\
\text { TAO130312 } \\
\text { TRT020000 } \\
\text { TRT070000 } \\
\text { TRT100000 } \\
\text { TRT120000 } \\
\text { TRT125000 } \\
\text { TRT130000 } \\
\text { TAMPTY } \\
\text { TAF050136 } \\
\text { TAF070136 } \\
\text { TAF080136 } \\
\text { TAF090136 } \\
\text { TAF100136 } \\
\text { TAF105136 } \\
\text { TAF110136 } \\
\text { TAF115136 } \\
\text { TAF120136 } \\
\text { TAF125136 } \\
\text { TAF130136 } \\
\text { TAF120048 } \\
\text { TAF130043 } \\
\text { TAF120224 } \\
\text { TAF130224 }\end{array}$ & $\begin{array}{l}\text { Abs D1ff } \\
0.0071 \\
0.0286 \\
0.0169 \\
0.0492 \\
0.0457 \\
0.0583 \\
0.0620 \\
0.0145 \\
0.0616 \\
0.0904 \\
0.0315 \\
0.0040 \\
0.0524 \\
0.0184 \\
0.1001 \\
0.0002 \\
0.0438 \\
0.0257 \\
0.0120 \\
0.0496 \\
0.0035 \\
0.0234 \\
0.0027 \\
0.0474 \\
0.0401 \\
0.0242 \\
0.0446 \\
0.0353 \\
4.7476 \\
0.0492 \\
0.0124 \\
0.0154 \\
0.0318 \\
0.0459 \\
0.0505 \\
0.0826 \\
0.0393 \\
0.0341 \\
0.0285 \\
0.0024 \\
0.0961 \\
0.0366 \\
0.0358 \\
0.0467 \\
0.0118 \\
0.0495\end{array}$ & $\begin{array}{l}\text { PDIf } \\
0.0097 \\
0.0390 \\
0.0196 \\
0.0572 \\
0.0531 \\
0.0678 \\
0.0785 \\
0.0168 \\
0.0713 \\
0.1048 \\
0.0365 \\
0.0046 \\
0.0603 \\
0.0212 \\
0.1156 \\
0.0002 \\
0.0506 \\
0.0298 \\
0.0160 \\
0.0576 \\
0.0041 \\
0.0271 \\
0.0031 \\
0.0551 \\
0.0464 \\
0.0280 \\
0.0515 \\
0.0408 \\
5.0433 \\
0.0571 \\
0.0314 \\
0.0178 \\
0.0396 \\
0.0531 \\
0.0681 \\
0.0956 \\
0.0455 \\
0.0396 \\
0.0329 \\
0.0028 \\
0.1109 \\
0.0424 \\
0.0413 \\
0.0541 \\
0.0136 \\
0.0576\end{array}$ & \\
\hline
\end{tabular}




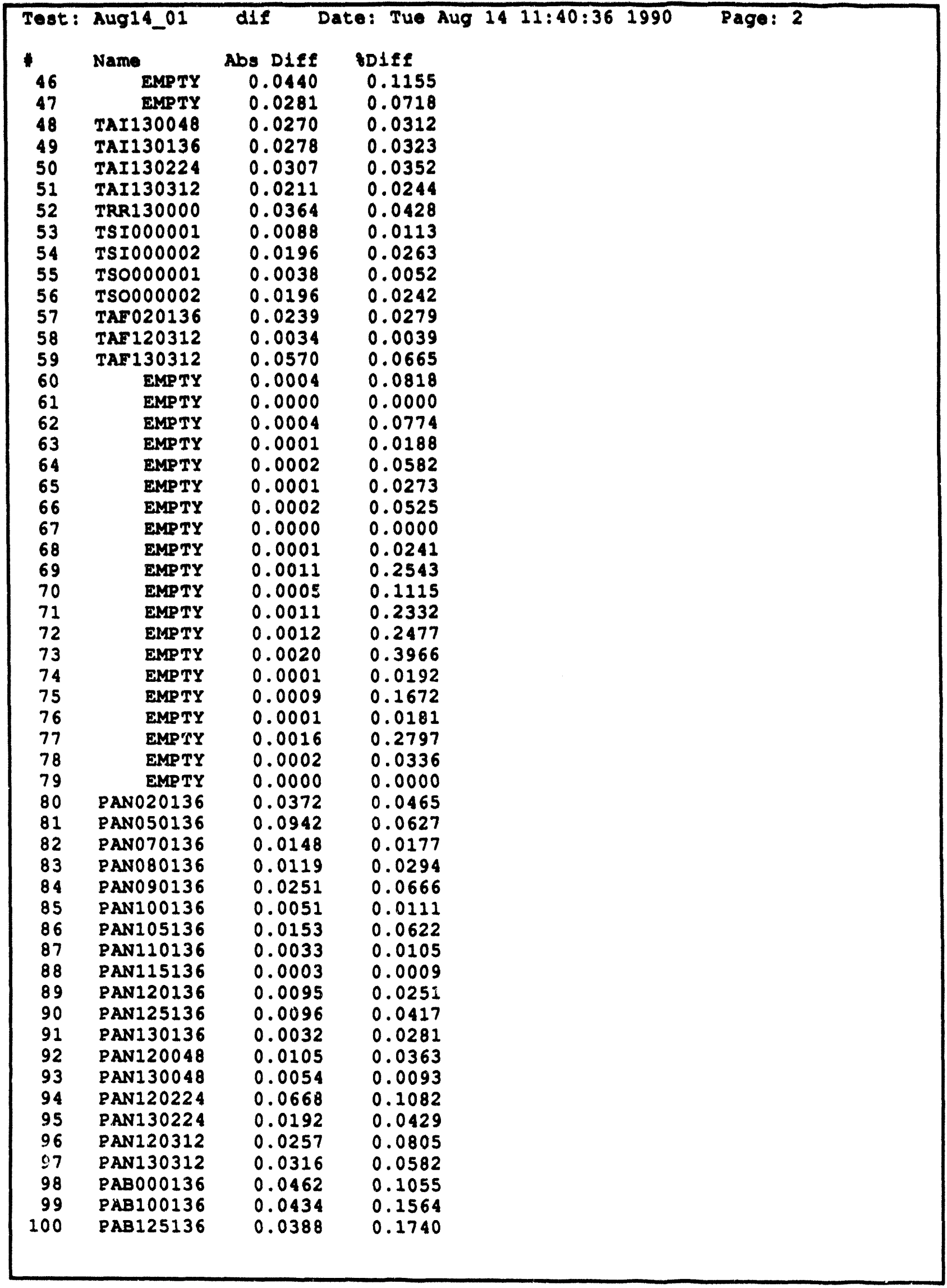



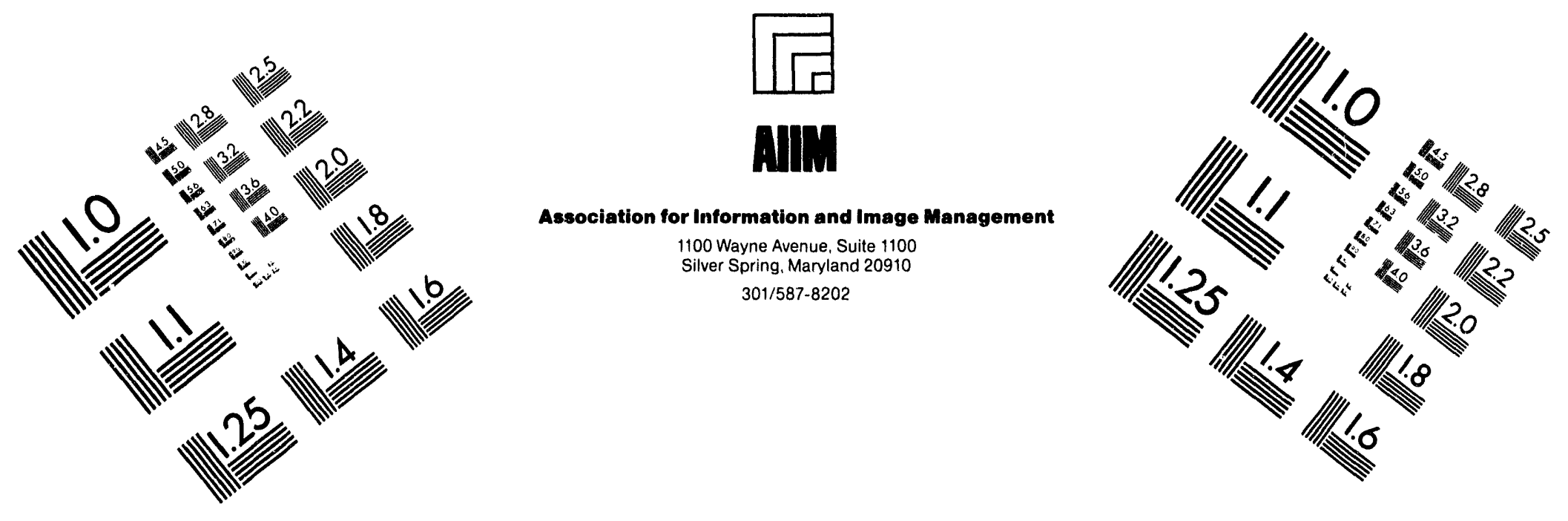

\section{Centimeter}

$\begin{array}{llllllllllllllll}1 & 2 & 3 & 4 & 5 & 6 & 7 & 8 & 9 & 10 & 11 & 12 & 13 & 14 & 15 & \mathrm{~mm}\end{array}$

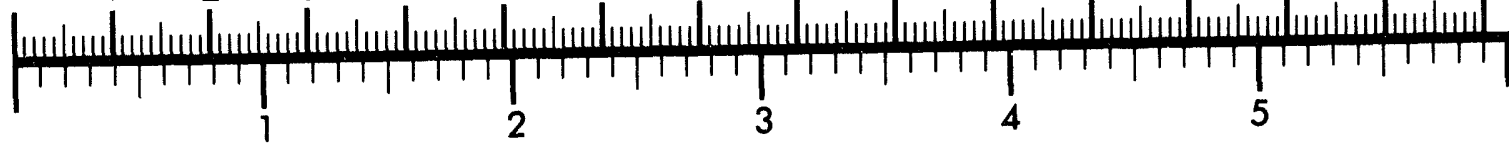
Inches

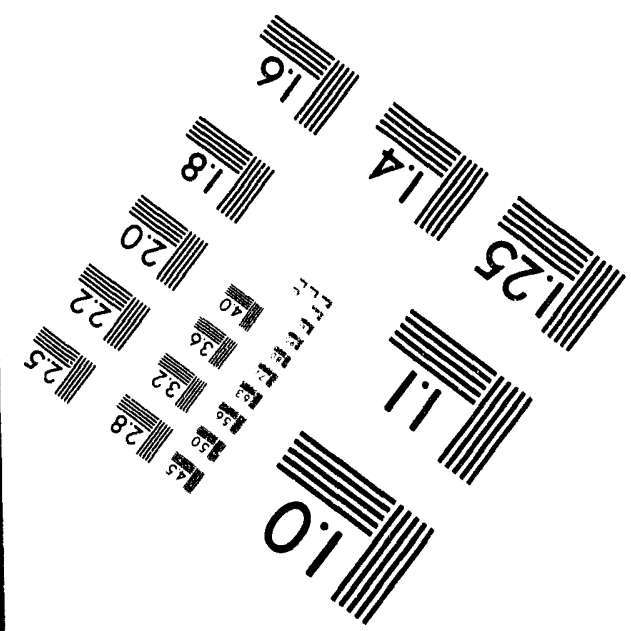

MANUFACTURED TO AIIM STANDARDS BY APPLIED IMAGE, INC.

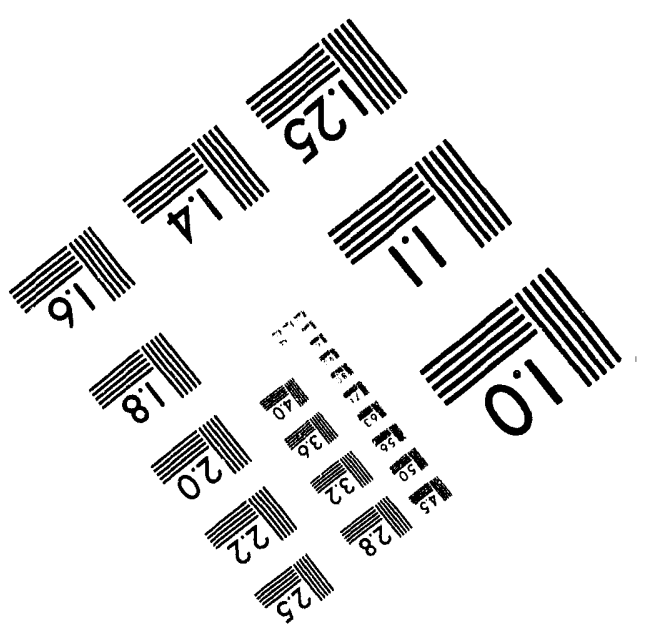



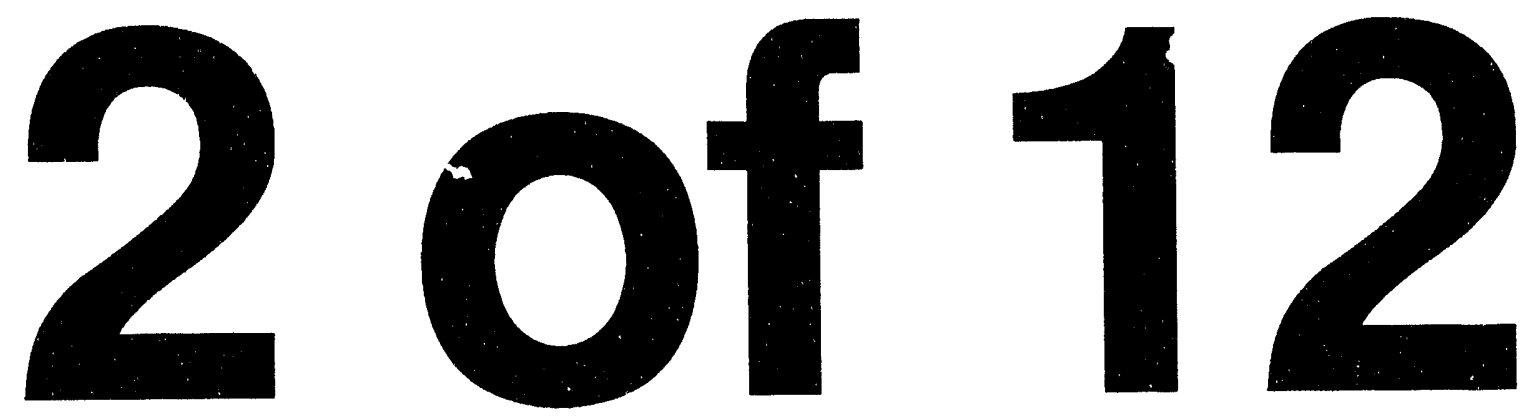


\begin{tabular}{|c|c|c|c|c|}
\hline Test: & Aug14_01 & dif & Date: Tue Aug 14 11:40:36 1990 & Page: 3 \\
\hline \# & Name & Abs Diff & zDiff & \\
\hline 101 & PIN000001 & 0.0759 & 0.1737 & \\
\hline 102 & PRV000001 & 0.0025 & 0.0133 & \\
\hline 103 & PSR000001 & 0.0701 & 0.3545 & \\
\hline 104 & PFM000001 & 0.0654 & 0.0629 & \\
\hline 105 & PAN000136 & 0.0000 & 0.0000 & \\
\hline 106 & PFM000002 & 0.0000 & 0.0000 & \\
\hline 107 & EMPTY & 0.0002 & 2.0202 & \\
\hline 108 & EMPTY & 0.4864 & 8.3620 & \\
\hline 109 & EMPTY & 0.0006 & 8.5714 & \\
\hline 110 & EMPTY & 0.0000 & 0.0000 & \\
\hline 111 & EMPTY & 0.0002 & 4.5455 & \\
\hline 112 & VPW000001 & 0.0114 & 1.3813 & \\
\hline 113 & VPW000002 & 0.0176 & 2.2947 & \\
\hline 114 & VPW000003 & 0.0328 & 4.7834 & \\
\hline 115 & VPW000004 & 0.0053 & 0.8640 & \\
\hline 116 & VPW000005 & 0.0181 & 1.3279 & \\
\hline 117 & VPW000006 & 0.0153 & 1.0277 & \\
\hline 118 & IPW000001 & 0.0000 & 0.0000 & \\
\hline 119 & IPWO 00002 & 0.0048 & 0.0478 & \\
\hline 120 & IPW000003 & 0.0836 & 0.7526 & \\
\hline 121 & IPW000004 & 0.0042 & 0.2050 & \\
\hline 122 & IPWO00005 & 0.0018 & 0.0501 & \\
\hline 123 & IPW000006 & 0.1501 & 6.5546 & \\
\hline 124 & EMPTY & 0.0007 & 3.4826 & \\
\hline 125 & EMPTY & 0.0001 & 0.7752 & \\
\hline 126 & EMPTY & 0.0008 & 4.6784 & \\
\hline 127 & EMPTY & 0.0014 & 8.4337 & \\
\hline 128 & IHT000001 & 0.0044 & 14.2857 & \\
\hline 129 & IHT000002 & 0.0238 & 70.6231 & \\
\hline 130 & IHT000003 & 0.0087 & 18.3932 & \\
\hline 131 & IHT000004 & 0.0074 & 21.2644 & \\
\hline 132 & IHT000005 & 0.0188 & 28.5281 & \\
\hline 133 & IHT000006 & 0.0098 & 32.9966 & \\
\hline 134 & IHT000007 & 0.0075 & 20.4918 & \\
\hline 135 & IHT000008 & 0.0010 & 0.9017 & \\
\hline 136 & IHT000009 & 0.0037 & 9.7368 & \\
\hline 137 & IHT 000010 & 0.0091 & 10.8204 & \\
\hline 138 & IHT000011 & 0.0048 & 6.8670 & \\
\hline 139 & IHT000012 & 0.0133 & 14.0147 & \\
\hline 140 & IHT000013 & 0.0307 & 38.1841 & \\
\hline 141 & IHTO00014 & 0.0233 & 85.3480 & \\
\hline 142 & IHTO00015 & 0.0203 & 69.2833 & \\
\hline 143 & IHT000016 & 0.0137 & 25.7519 & \\
\hline 144 & IHT000017 & 0.0442 & 83.0827 & \\
\hline 145 & IHT000018 & 0.0390 & 236.3636 & \\
\hline 146 & IHT000019 & 0.0224 & 7.2072 & \\
\hline 147 & IHT000020 & 0.0315 & 85.5978 & \\
\hline 148 & IHT000021 & 0.0377 & 110.5572 & \\
\hline 149 & IHT000022 & 0.0210 & 14.1605 & \\
\hline 150 & IHT000023 & 0.0240 & 14.5191 & \\
\hline 151 & IHT000024 & 0.0130 & 3.8645 & \\
\hline 152 & IHT000025 & 0.0177 & 5.6694 & \\
\hline 153 & IHT000026 & 0.0475 & 113.3652 & \\
\hline 154 & IHT000027 & 0.0349 & $\quad 266.4122$ & \\
\hline 155 & IHT000028 & 0.0148 & 3704.7620 & \\
\hline
\end{tabular}


Test: Aug14_01 dif Date: Tue Aug 14 11:40:36 1990 Page: 4

* Name Abs Diff \&Diff

156 IHTO00029 $0.0168 \quad 38.5321$

157 IHTOOO030 $0.0166 \quad 102.4691$

$158 \quad$ IHTOOOO31 $0.0296 \quad 45.8915$

159 IHTOO0032 $0.0170 \quad 36.2473$

160 IHTO00033 $0.0130 \quad 27.7185$

$\begin{array}{llll}161 & \text { IHTO00034 } & 0.0432 & 62.7907\end{array}$

162 IHTO00035 $0.0152 \quad 53.3333$

$\begin{array}{llll}163 & \text { IHTO00036 } & 0.0019 & 2.3086\end{array}$ 


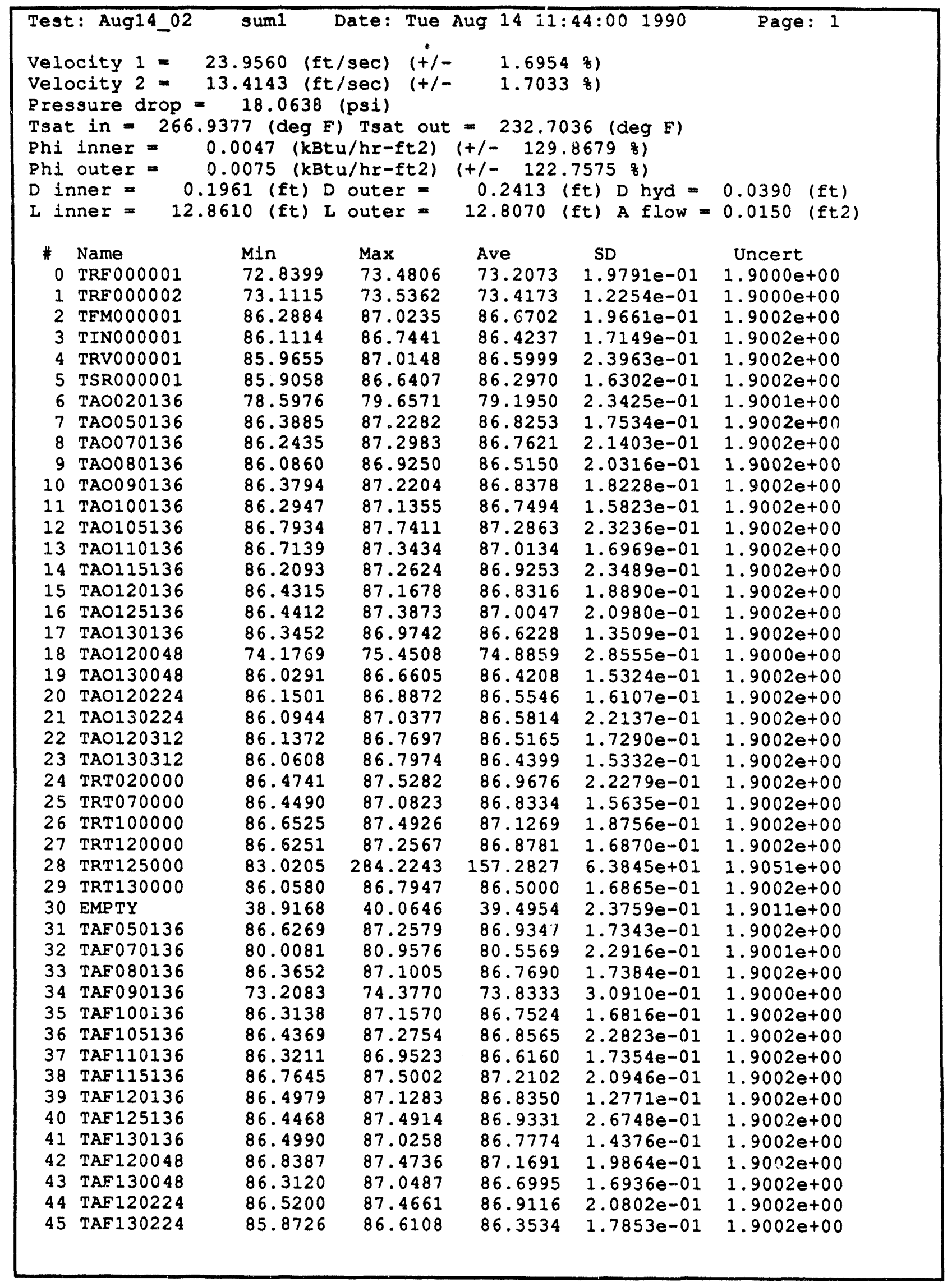




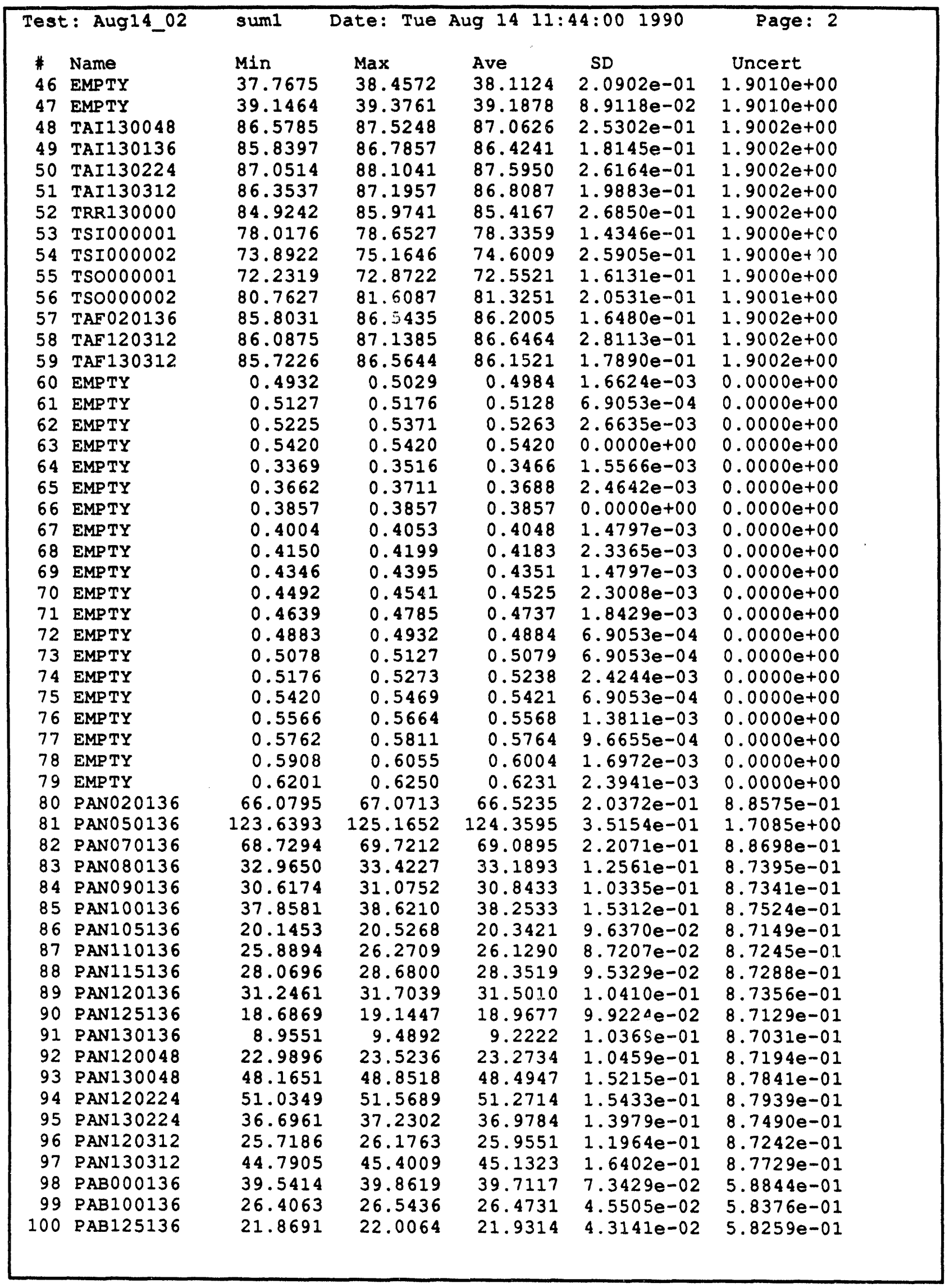




\begin{tabular}{|c|c|c|c|c|c|c|}
\hline \multicolumn{2}{|c|}{ Test: Aug14_02 } & \multirow{2}{*}{$\begin{array}{l}\text { sum1 } \\
\text { Min }\end{array}$} & \multicolumn{3}{|c|}{ Date: Tue Aug $14 \quad 11: 44: 001990$} & \multirow{2}{*}{$\begin{array}{l}\text { Page: } 3 \\
\text { Uncert }\end{array}$} \\
\hline * & Name & & $\operatorname{Max}$ & Ave & SD & \\
\hline 101 & PIN000001 & 39.1042 & 39.7146 & 39.4924 & $1.6670 \mathrm{e}-01$ & $7.5647 e-01$ \\
\hline 102 & PRV000C01 & 18.7420 & 18.8641 & 18.8152 & $6.0409 e-02$ & $7.5147 e-01$ \\
\hline 103 & PSR000001 & 19.6691 & 19.7911 & 19.7863 & $2.4164 e-02$ & $7.5163 e-01$ \\
\hline 104 & PFM000001 & 87.5173 & 89.4400 & 88.4695 & $4.3220 e-01$ & $5.4673 e-01$ \\
\hline 105 & PAN000136 & 497.1757 & 503.5844 & 500.1054 & $1.4884 \mathrm{e}+00$ & $5.0764 \mathrm{e}+00$ \\
\hline 106 & PFM000002 & 27.5689 & 27.5689 & 27.5689 & $9.6336 \mathrm{e}-06$ & $2.1154 \mathrm{e}-01$ \\
\hline 107 & EMPTY & 0.0049 & 0.0146 & 0.0097 & $1.2041 e-03$ & $0.0000 e+00$ \\
\hline 108 & EMPTY & -10.0000 & 3.0371 & -7.6607 & $2.9918 e+00$ & $0.0000 e+00$ \\
\hline 109 & EMPTY & 0.0049 & 0.0099 & 0.0054 & $1.4797 e-03$ & $0.0000 e+00$ \\
\hline 110 & EMPTY & -10.0000 & -10.0000 & -10.0000 & $0.0000 e+00$ & $0.0000 e+00$ \\
\hline 111 & EMPTY & 0.0000 & 0.0049 & 0.0041 & $1.8082 e-03$ & $0.0000 e+00$ \\
\hline 112 & VRW000001 & -1.0479 & -0.6573 & -0.7979 & $9.6976 e-02$ & $8.1001 e-01$ \\
\hline 113 & VPW0000002 & -0.8451 & -0.4545 & -0.7748 & $1.0259 e-01$ & $8.1001 e-01$ \\
\hline 114 & VPW000003 & -0.9240 & -0.5333 & -0.6779 & $1.0297 e-01$ & $8.1001 e-01$ \\
\hline 115 & VPW000004 & -0.8752 & -0.4845 & -0.6603 & $1.0625 e-01$ & $8.1001 e-01$ \\
\hline 116 & VPW000005 & -1.4686 & -1.0780 & -1.3280 & $1.0470 e-01$ & $8.1004 e-01$ \\
\hline 117 & VPW000006 & -1.6489 & -1.0630 & -1.5005 & $1.2823 e-01$ & $8.1005 e-01$ \\
\hline 118 & IPW000001 & -11.6766 & -7.7719 & -9.8413 & $1.0737 e+00$ & $8.1004 e+00$ \\
\hline 119 & IPW000002 & -12.2331 & -6.3793 & -9.5794 & $1.1668 \mathrm{e}+00$ & $8.1004 e+00$ \\
\hline 120 & IPW000003 & -12.8625 & -8.9625 & -11.1465 & $1.2851 \mathrm{e}+00$ & $8.1005 e+00$ \\
\hline 121 & IPW000004 & -2.7419 & -1.7654 & -2.0388 & $2.6396 \mathrm{e}-01$ & $2.0001 e+00$ \\
\hline 122 & IPW000005 & -3.9904 & -3.0139 & -3.6291 & $3.2431 e-01$ & $2.0002 e+00$ \\
\hline 123 & IPW000006 & -2.8076 & -1.3427 & -2.1728 & $3.8523 e-01$ & $2.0001 e+00$ \\
\hline 124 & EMPTY & -0.0244 & -0.0146 & -0.0199 & $3.2479 e-03$ & $0.0000 e+00$ \\
\hline 125 & EMPTY & -0.0195 & -0.0098 & -0.0141 & $2.5420 e-03$ & $0.0000 e+00$ \\
\hline 126 & EMPTY & -0.0244 & -0.0098 & -0.0177 & $3.1023 e-03$ & $0.0000 e+00$ \\
\hline 127 & EMPTY & -0.0244 & -0.0098 & -0.0172 & $3.7226 e-03$ & $0.0000 e+00$ \\
\hline 128 & IHT000001 & -0.2066 & 0.1841 & -0.0269 & $8.6810 e-02$ & $4.1000 e+00$ \\
\hline 129 & IHT000002 & -0.2329 & 0.1577 & -0.0571 & $9.0412 e-02$ & $4.1000 e+00$ \\
\hline 130 & IHT000003 & -0.4770 & 0.1089 & -0.0434 & $1.0654 e-01$ & $4.1000 e+00$ \\
\hline 131 & IHTO00004 & -0.2028 & 0.1878 & -0.0387 & $9.1354 e-02$ & $4.1000 e+00$ \\
\hline 132 & IHT000005 & -0.2066 & 0.1841 & -0.0542 & $9.8963 e-02$ & $4.1000 e+00$ \\
\hline 133 & IHT000006 & -0.2554 & 0.1352 & 0.0180 & $1.1161 \mathrm{e}-01$ & $4.1000 e+00$ \\
\hline 134 & IHTO00007 & -0.2554 & 0.1352 & -0.0484 & $7.2866 e-02$ & $4.1000 e+00$ \\
\hline 135 & IHTO00008 & -0.3531 & 0.0376 & -0.1109 & $9.3043 e-02$ & $4.1000 e+00$ \\
\hline 136 & IHT000009 & -0.2216 & 0.1690 & -0.0458 & $9.8648 e-02$ & $4.1000 e+00$ \\
\hline 137 & IHT000010 & -0.1427 & 0.2479 & -0.0490 & $1.0617 \mathrm{e}-01$ & $4.1000 e+00$ \\
\hline 138 & IHT000011 & -0.1089 & 0.0864 & -0.0660 & $8.1729 e-02$ & $4.1000 e+00$ \\
\hline 139 & IHT000012 & -0.3606 & 0.0301 & -0.0910 & $1.0358 e-01$ & $4.1000 e+00$ \\
\hline 140 & IHT000013 & -0.0563 & 0.3343 & 0.0687 & $1.0259 \mathrm{e}-01$ & $4.1000 e+00$ \\
\hline 141 & IHTO00014 & -0.3906 & 0.5859 & -0.0078 & $1.4743 e-01$ & $4.1000 e+00$ \\
\hline 142 & IHTO00015 & -0.4395 & 0.1465 & -0.0488 & $9.6655 e-02$ & $4.1000 e+00$ \\
\hline 143 & IHTO00016 & -0.1352 & 0.0601 & -0.0727 & $9.2034 e-02$ & $4.1000 e+00$ \\
\hline 144 & IHT000017 & -0.5484 & 0.6235 & 0.0571 & $2.9594 e-01$ & $4.1000 e+00$ \\
\hline 145 & IHTO00018 & -0.1728 & 0.2178 & -0.0283 & $1.1712 e-01$ & $4.1000 e+00$ \\
\hline 146 & IHTO00019 & 0.2366 & 0.4319 & 0.2835 & $8.4262 e-02$ & $4.1000 e+00$ \\
\hline 147 & IHT000020 & -0.2517 & 0.1390 & -0.0759 & $9.0412 e-02$ & $4.1000 e+00$ \\
\hline 148 & IHTO00021 & -0.0714 & 0.1240 & 0.0263 & $9.8648 e-02$ & $4.1000 e+00$ \\
\hline 149 & IHT000022 & 0.0037 & 0.1991 & 0.1366 & $9.2034 e-02$ & $4.1000 e+00$ \\
\hline 150 & IHT000023 & -0.0300 & 0.1653 & 0.1301 & $7.5798 e-02$ & $4.1000 e+00$ \\
\hline 151 & IHT000024 & -0.4770 & 0.1089 & -0.3207 & $1.0440 e-01$ & $4.1000 e+00$ \\
\hline 152 & IHT000025 & 0.2028 & 0.3981 & 0.3239 & $9.5764 e-02$ & $4.1000 e+00$ \\
\hline 153 & IHT000026 & -0.1690 & 0.0263 & 0.0028 & $6.4113 e-02$ & $4.1000 e+00$ \\
\hline 154 & IHTO00027 & -0.2291 & 0.1615 & 0.0014 & $9.4125 e-02$ & $4.1000 e+00$ \\
\hline 155 & IHT000028 & -0.2479 & 0.1427 & -0.0213 & $1.0705 e-01$ & $4.1000 e+00$ \\
\hline
\end{tabular}




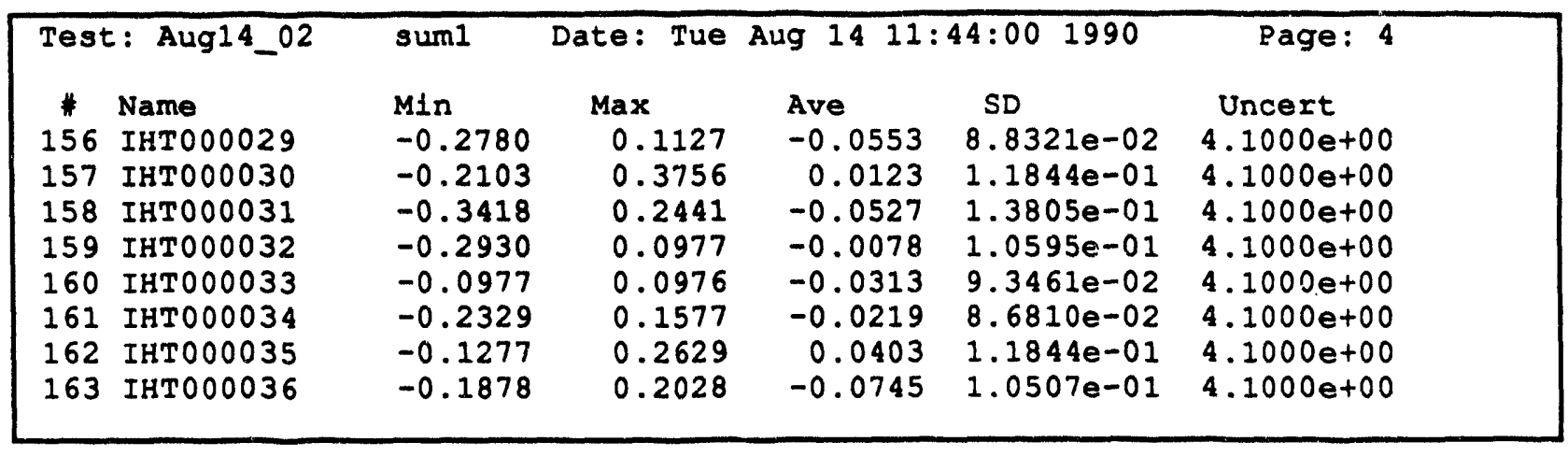




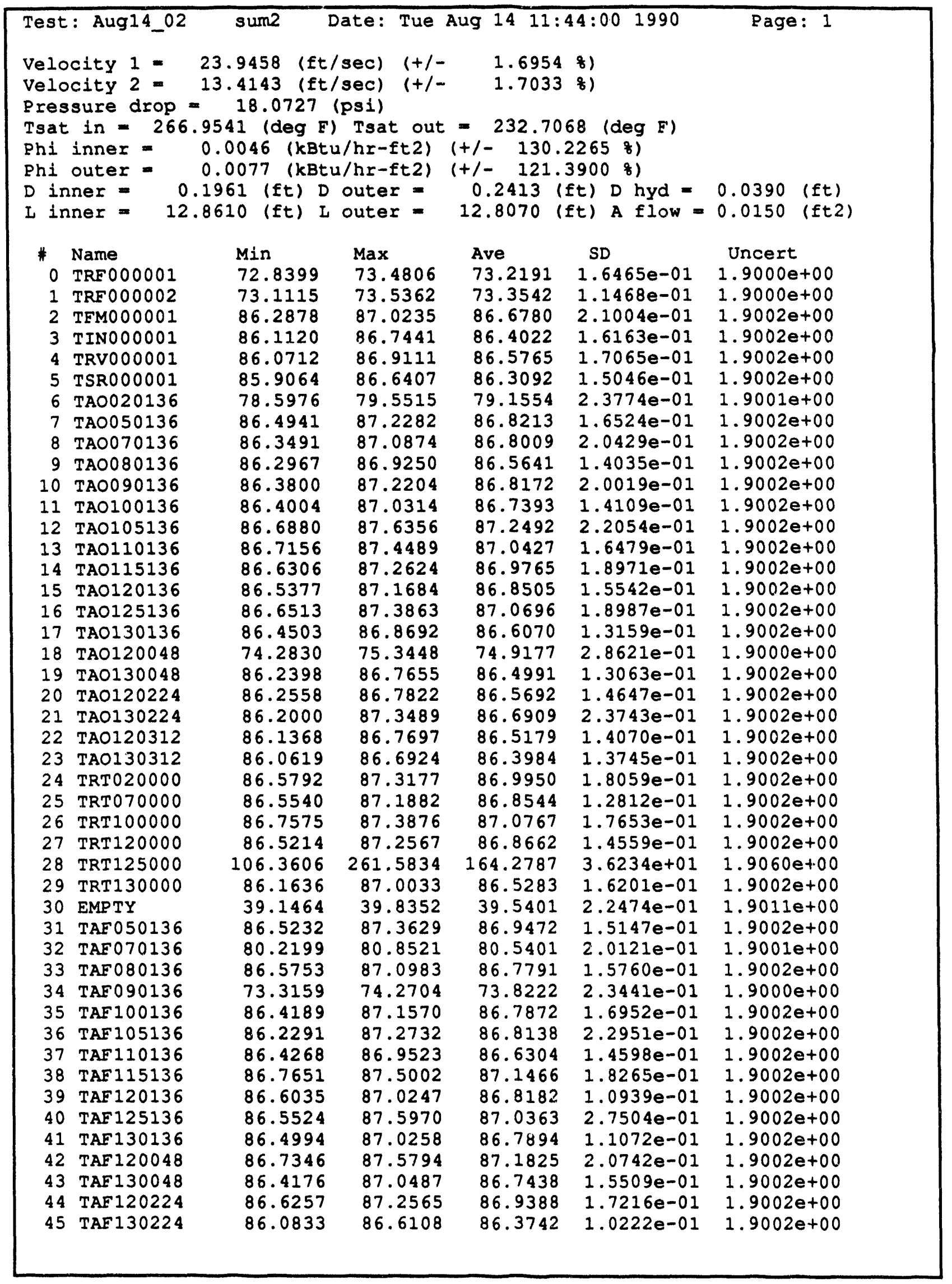




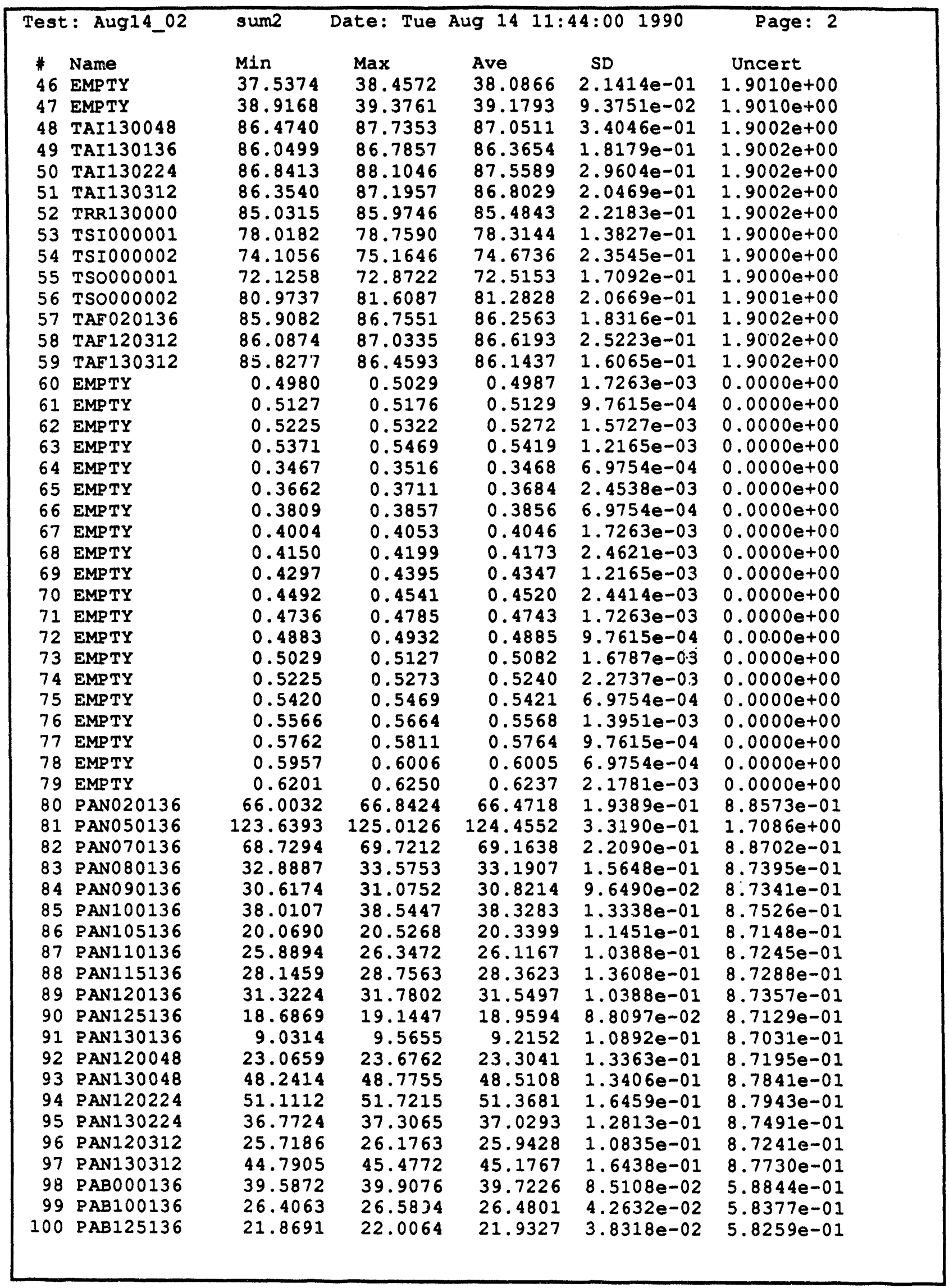




\begin{tabular}{|c|c|c|c|c|c|c|}
\hline \multicolumn{2}{|c|}{ Test: Aug14_02 } & sum2 & Date: Tue & \multicolumn{2}{|r|}{$: 001990$} & Page: 3 \\
\hline$\#$ & Name & Min & $\operatorname{Max}$ & Ave & SD & Uncert \\
\hline 101 & PIN000001 & 39.2263 & 39.7146 & 39.5128 & $1.4647 e-01$ & $7.5648 e-01$ \\
\hline 102 & PRV000001 & 18.7420 & 18.8641 & 18.8417 & $4.7758 e-02$ & $7.5148 e-01$ \\
\hline 103 & PSR000001 & 19.6691 & 19.7911 & 19.7837 & $2.9569 e-02$ & $7.5163 e-01$ \\
\hline 104 & PFMO00001 & 87.7920 & 89.2569 & 88.3936 & $3.8025 e-01$ & $5.4666 e-01$ \\
\hline 105 & PAN000136 & 497.1757 & 503.5844 & 500.3520 & $1.4488 \mathrm{e}+00$ & $5.0765 e+00$ \\
\hline 106 & PFM000002 & 27.5689 & 27.5689 & 27.5689 & $9.6356 e-06$ & $2.1154 e-01$ \\
\hline 107 & EMPTY & 0.0049 & 0.0098 & 0.0096 & $9.7615 e-04$ & $0.0000 e+00$ \\
\hline 108 & EMPTY & -10.0000 & -5.2734 & -8.1886 & $1.6252 \mathrm{e}+00$ & $0.0000 e+00$ \\
\hline 109 & EMPTY & 0.0049 & 0.0098 & 0.0060 & $2.0584 e-03$ & $0.0000 e+00$ \\
\hline 110 & EMPTY & -10.0000 & -10.0000 & -10.0000 & $0.0000 e+00$ & $0.0000 e+00$ \\
\hline 111 & EMPTY & 0.0000 & 0.0049 & 0.0039 & $1.9883 e-03$ & $0.0000 e+00$ \\
\hline 112 & VPW000001 & -1.0479 & -0.6573 & -0.8247 & $9.7656 e-02$ & $8.1002 e-01$ \\
\hline 113 & VPW000002 & -1.0404 & -0.6498 & -0.8212 & $9.4620 e-02$ & $8.1001 e-01$ \\
\hline 114 & VPW000003 & -0.9240 & -0.5333 & -0.6649 & $1.1546 e-01$ & $8.1001 e-01$ \\
\hline 115 & VPW000004 & -0.8752 & -0.4845 & -0.6639 & $1.1161 e-01$ & $8.1001 e-01$ \\
\hline 116 & VPW000005 & -1.4686 & -0.8827 & -1.3012 & $1.1960 \mathrm{e}-01$ & $8.1004 e-01$ \\
\hline 117 & VPW000006 & -1.6489 & -1.4536 & -1.5413 & $9.8153 e-02$ & $8.1005 e-01$ \\
\hline 118 & IPWO00001 & -11.6766 & -7.7719 & -10.0430 & $1.0040 e+00$ & $8.1004 e+00$ \\
\hline 119 & IPW000002 & -12.2331 & -6.3793 & -9.4456 & $1.2595 e+00$ & $8.1003 e+00$ \\
\hline 120 & IPW000003 & -12.8625 & -8.9625 & -10.9125 & $1.0531 e+00$ & $8.1005 e+00$ \\
\hline 121 & IPWO00004 & -2.7419 & -1.7654 & -2.0344 & $2.8299 e-01$ & $2.0001 e+00$ \\
\hline 122 & IPW000005 & -3.9904 & -3.0139 & -3.4922 & $3.0701 e-01$ & $2.0002 e+00$ \\
\hline 123 & IPW000006 & -3.2959 & -1.3427 & -2.2296 & $4.0698 e-01$ & $2.0001 e+00$ \\
\hline 124 & EMPTY & -0.0293 & -0.0146 & -0.0194 & $3.5224 e-03$ & $0.0000 e+00$ \\
\hline 125 & EMPTY & -0.0195 & $-0.0 \cap 98$ & -0.0139 & $2.0781 e-03$ & $0.0000 e+00$ \\
\hline 126 & EMPTY & -0.0195 & -0.0146 & -0.0171 & $2.4662 e-03$ & $0.0000 e+00$ \\
\hline 127 & EMPTY & -0.0244 & -0.0098 & -0.0159 & $2.9526 e-03$ & $0.0000 e+00$ \\
\hline 128 & IHT000001 & -0.2066 & 0.1841 & -0.0352 & $8.5810 e-02$ & $4.1000 e+00$ \\
\hline 129 & IHTO00002 & -0.2329 & 0.1577 & -0.0455 & $6.8582 e-02$ & $4.1000 e+00$ \\
\hline 130 & IHT000003 & -0.0864 & 0.1089 & -0.0306 & $8.9148 e-02$ & $4.1000 \mathrm{e}+00$ \\
\hline 131 & IHTO00004 & -0.2028 & 0.1878 & -0.0195 & $7.3601 e-02$ & $4.1000 e+00$ \\
\hline 132 & IHT000005 & -0.2066 & -0.0113 & -0.0671 & $8.9148 e-02$ & $4.1000 e+00$ \\
\hline 133 & IHT000006 & -0.2554 & 0.1352 & 0.0196 & $1.0486 e-01$ & $4.1000 e+00$ \\
\hline 134 & IHT000007 & -0.2554 & 0.1352 & -0.0521 & $6.8582 e-02$ & $4.1000 e+00$ \\
\hline 135 & IHT000008 & -0.1578 & 0.0376 & -0.0780 & $9.6990 \mathrm{e}-02$ & $4.1000 \mathrm{e}+00$ \\
\hline 136 & IHTO00009 & -0.2216 & 0.1690 & -0.0382 & $8.3705 e-02$ & $4.1000 e+00$ \\
\hline 137 & IHTO000010 & -0.3380 & 0.0526 & -0.0750 & $1.0204 e-01$ & $4.1000 e+00$ \\
\hline 138 & IHTO00011 & -0.3042 & 0.0864 & -0.0651 & $9.1482 e-02$ & $4.1000 e+00$ \\
\hline 139 & IHT000012 & -0.3606 & 0.0301 & -0.0895 & $1.0409 e-01$ & $4.1000 e+00$ \\
\hline 140 & IHTO00013 & -0.0563 & 0.3343 & 0.1031 & $8.6187 e-02$ & $4.1000 e+00$ \\
\hline 141 & IHTO00014 & -0.1953 & 0.1953 & -0.0120 & $7.3601 e-02$ & $4.1000 e+00$ \\
\hline 142 & IHTO00015 & -0.2442 & 0.3418 & -0.0209 & $8.9148 e-02$ & $4.1000 e+00$ \\
\hline 143 & IHT000016 & -0.3305 & 0.0601 & -0.0714 & $1.0076 e-01$ & $4.1000 e+00$ \\
\hline 144 & IHTO000017 & -0.3530 & 0.4282 & 0.0575 & $2.8540 e-01$ & $4.1000 \mathrm{e}+00$ \\
\hline 145 & IHTO00018 & -0.1728 & 0.2178 & -0.0094 & $9.2189 e-02$ & $4.1000 e+00$ \\
\hline 146 & IHTO00019 & 0.0413 & 0.4319 & 0.2765 & $1.0533 e-01$ & $4.1000 e+00$ \\
\hline 147 & IHTO00020 & -0.2517 & 0.1390 & -0.0723 & $8.7680 e-02$ & $4.1000 e+00$ \\
\hline 148 & IHTO00021 & -0.2667 & 0.1240 & -0.0036 & $1.0204 e-01$ & $4.1000 e+00$ \\
\hline 149 & IHT 000022 & 0.0037 & 0.1991 & 0.1393 & $9.0949 e-02$ & $4.1000 \mathrm{e}+00$ \\
\hline 150 & IHTO00023 & -0.0300 & 0.3606 & 0.1294 & $1.0299 \mathrm{e}-01$ & $4.1000 e+00$ \\
\hline 151 & IHTO00024 & -0.4770 & -0.2817 & -0.3494 & $9.3931 e-02$ & $4.1000 e+00$ \\
\hline 152 & IHTO00025 & 0.0075 & 0.3981 & 0.3025 & $1.0640 e-01$ & $4.1000 e+00$ \\
\hline 153 & IHT000026 & -0.1690 & 0.2216 & 0.0024 & $8.5810 e-02$ & $4.1000 e+00$ \\
\hline 154 & IHTO00027 & -0.0338 & 0.1615 & -0.0179 & $5.4032 e-0.2$ & $4.1000 e+00$ \\
\hline 155 & IHT000028 & -0.2479 & 0.1427 & -0.0326 & $9.9629 e-02$ & $4.1000 e+00$ \\
\hline
\end{tabular}




\begin{tabular}{|c|c|c|c|c|c|c|}
\hline \multicolumn{2}{|c|}{ Test: Aug14_02 } & sum2 & Date: Tue & Aug 141 & $4: 00 \quad 1990$ & Page: 4 \\
\hline $\begin{array}{l}* \\
156 \\
157 \\
158 \\
159 \\
160 \\
161 \\
162 \\
163\end{array}$ & 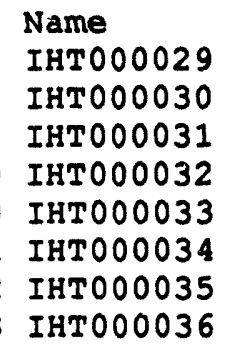 & $\begin{array}{l}\text { Min } \\
-0.0826 \\
-0.2103 \\
-0.3418 \\
-0.0976 \\
-0.2930 \\
-0.2329 \\
-0.1277 \\
-0.1878\end{array}$ & $\begin{array}{l}\text { Max } \\
0.1127 \\
0.3756 \\
0.2441 \\
0.2930 \\
0.0976 \\
0.1577 \\
0.2629 \\
0.2028\end{array}$ & $\begin{array}{l}\text { Ave } \\
-0.0508 \\
0.0288 \\
-0.0309 \\
-0.0099 \\
-0.0299 \\
-0.0296 \\
0.0238 \\
-0.0642\end{array}$ & $\begin{array}{l}\text { SD } \\
.2937 \mathrm{e}-02 \\
1.0746 \mathrm{e}-01 \\
1.2556 \mathrm{e}-01 \\
1.0594 \mathrm{e}-01 \\
1.0204 \mathrm{e}-01 \\
8.8783 \mathrm{e}-02 \\
9.1482 \mathrm{e}-02 \\
1.1755 \mathrm{e}-01\end{array}$ & $\begin{array}{c}\text { Uncert } \\
4.1000 e+00 \\
4.1000 e+00 \\
4.1000 e+00 \\
4.1000 e+00 \\
4.1000 e+00 \\
4.1000 e+00 \\
4.1000 e+00 \\
4.1000 e+00\end{array}$ \\
\hline
\end{tabular}




\begin{tabular}{|c|c|c|c|c|}
\hline \multicolumn{5}{|c|}{$\begin{array}{l}\text { Velocity } 1=0.010199 \text { (ft/sec) } \\
\text { Velocity } 2=0.000000 \text { (ft/sec) } \\
\text { Pressure Drop }=0.008900 \text { (psi) } \\
\text { Tsat in }=0.016388 \text { (deg F) Tsat out }=0.003204 \text { (deg F) } \\
\text { Phi inner }=0.000100 \text { (kBtu/hr-ft2) Phi outer }=0.000200 \text { (kBtu/hr-ft2) }\end{array}$} \\
\hline $\begin{array}{r}* \\
1 \\
2 \\
3 \\
4 \\
5 \\
6 \\
7 \\
8 \\
9 \\
10 \\
11 \\
12 \\
13 \\
14 \\
15 \\
16 \\
17 \\
18 \\
19 \\
20 \\
21 \\
22 \\
23 \\
24 \\
25 \\
26 \\
27 \\
28 \\
29 \\
30 \\
31 \\
32 \\
33 \\
34 \\
35 \\
36 \\
37 \\
38 \\
39 \\
40 \\
41 \\
42 \\
43 \\
44 \\
45\end{array}$ & 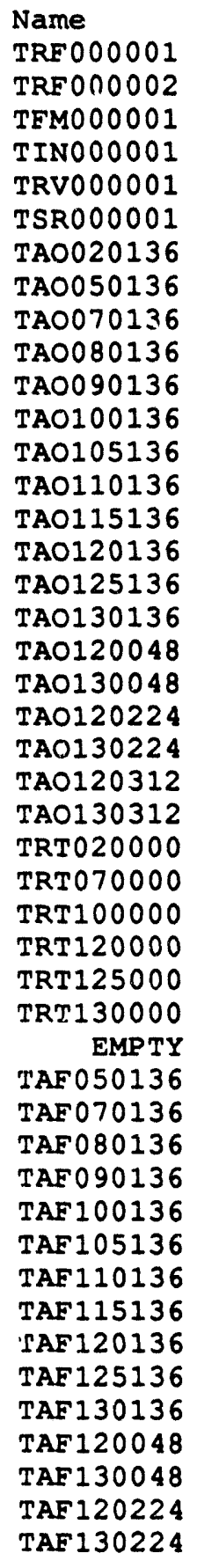 & $\begin{array}{l}\text { Abs Diff } \\
0.0118 \\
0.0631 \\
0.0078 \\
0.0215 \\
0.0234 \\
0.0122 \\
0.0396 \\
0.0040 \\
0.0388 \\
0.0491 \\
0.0206 \\
0.0101 \\
0.0371 \\
0.0293 \\
0.0512 \\
0.0189 \\
0.0649 \\
0.0158 \\
0.0318 \\
0.0783 \\
0.0146 \\
0.1095 \\
0.0014 \\
0.0415 \\
0.0274 \\
0.0210 \\
0.0502 \\
0.0119 \\
6.9960 \\
0.0283 \\
0.0447 \\
0.0125 \\
0.0168 \\
0.0101 \\
0.0111 \\
0.0348 \\
0.0427 \\
0.0144 \\
0.0636 \\
0.0168 \\
0.1032 \\
0.0120 \\
0.0134 \\
0.0443 \\
0.0272 \\
0.0208\end{array}$ & $\begin{array}{l}\text { PDDf } \\
0.0161 \\
0.0859 \\
0.0090 \\
0.0249 \\
0.0270 \\
0.0141 \\
0.0500 \\
0.0046 \\
0.0447 \\
0.0568 \\
0.0237 \\
0.0116 \\
0.0425 \\
0.0337 \\
0.0589 \\
0.0218 \\
0.0746 \\
0.0182 \\
0.0425 \\
0.0906 \\
0.0169 \\
0.1265 \\
0.0016 \\
0.0480 \\
0.0315 \\
0.024 \\
0.057\end{array}$ & \\
\hline
\end{tabular}




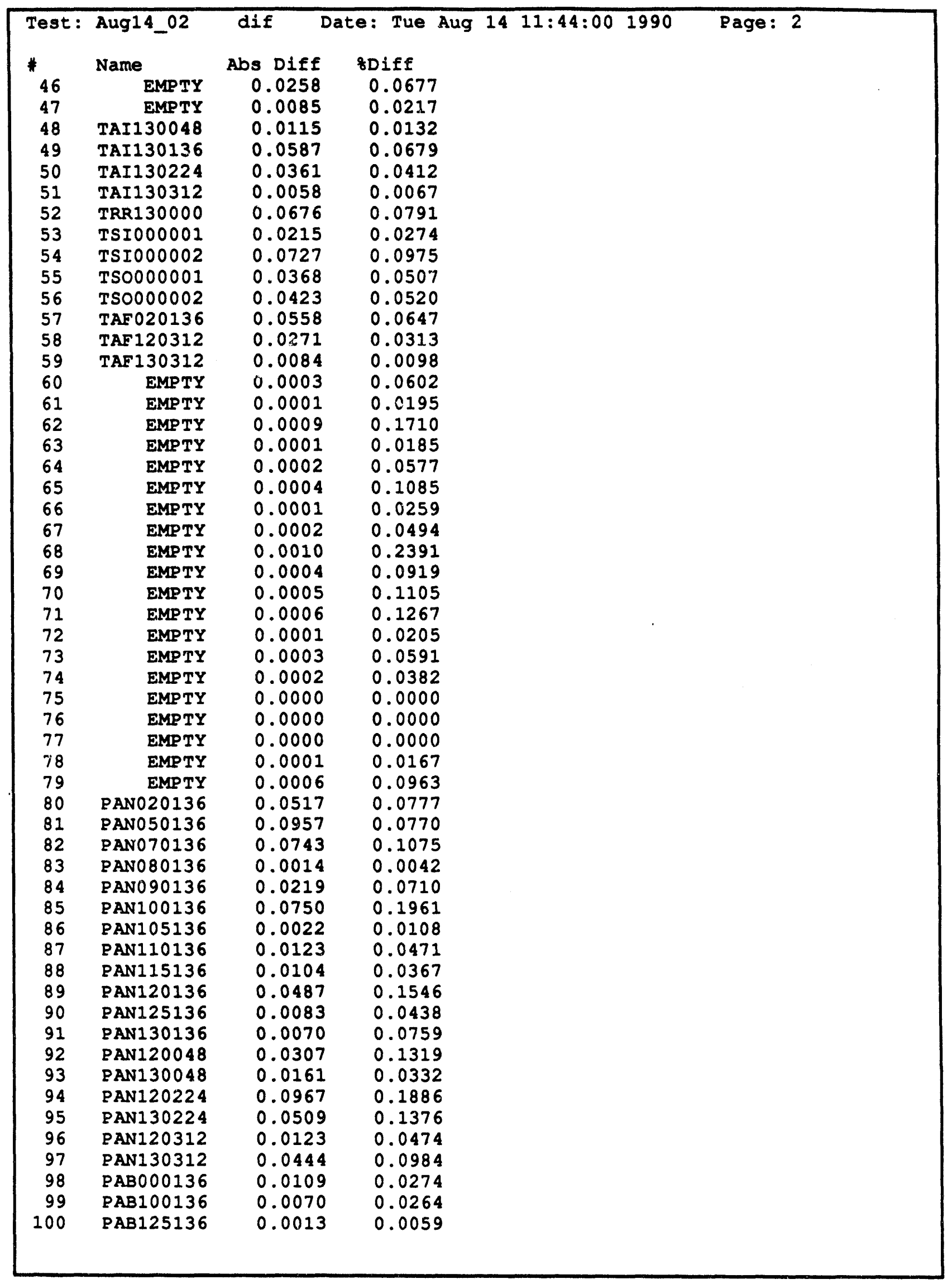




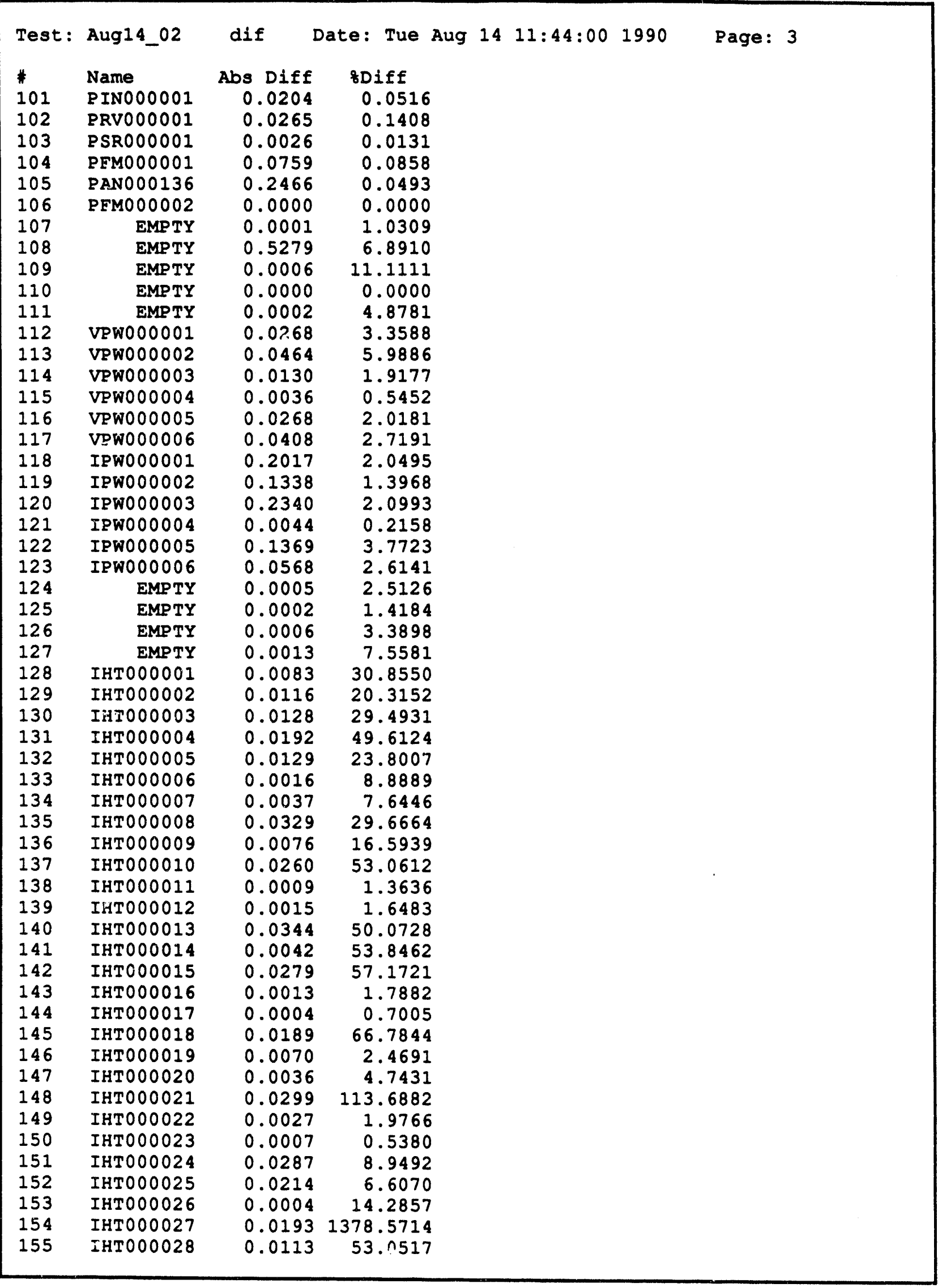




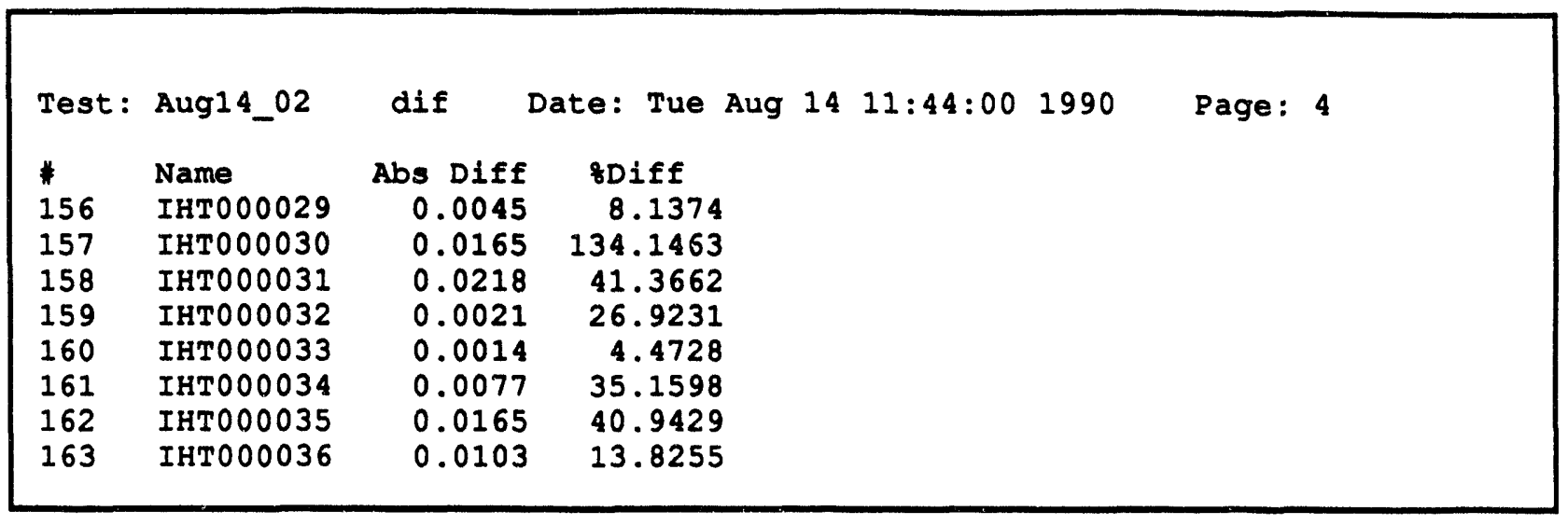




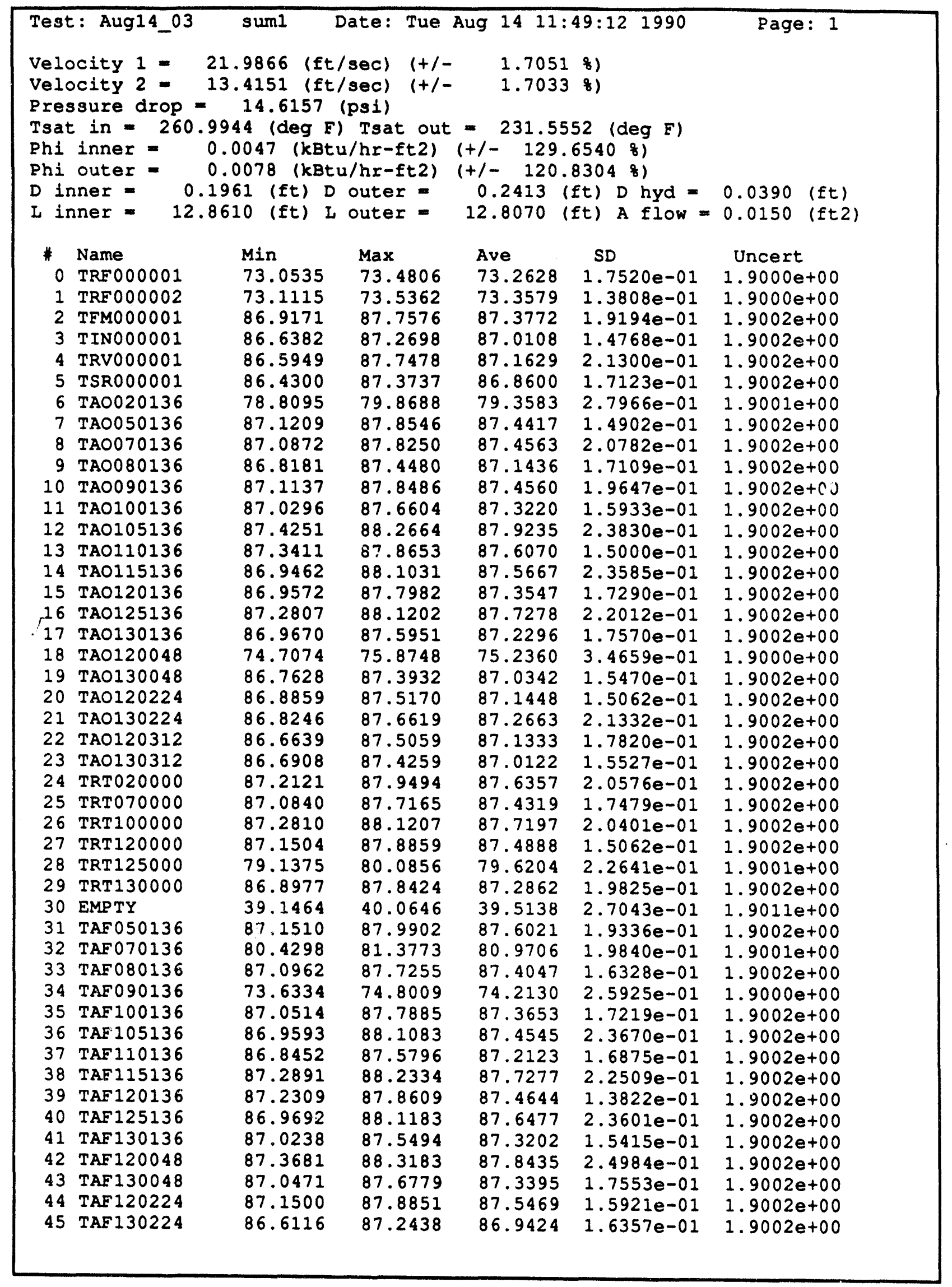




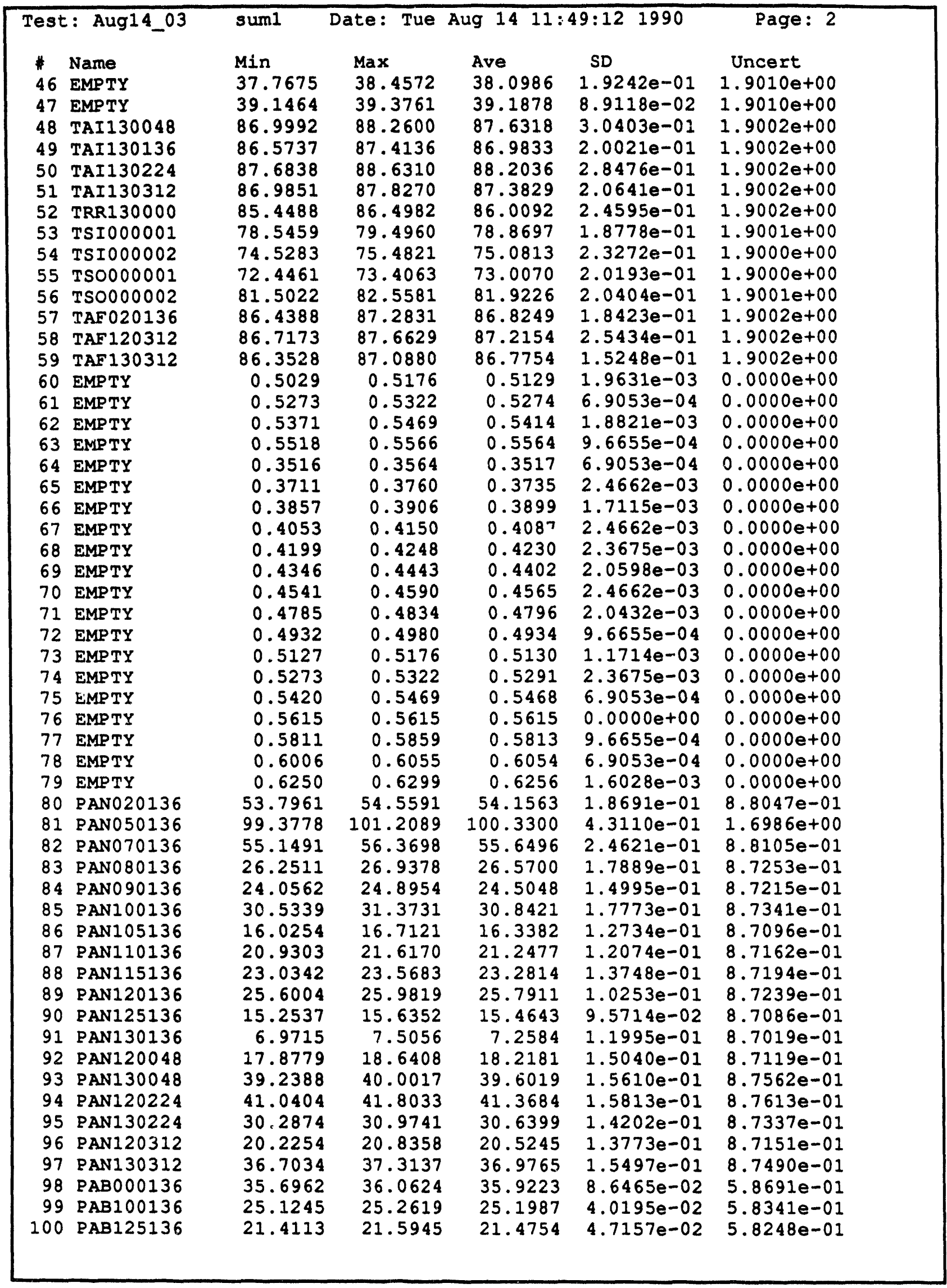




\begin{tabular}{|c|c|c|c|c|c|c|}
\hline \multicolumn{2}{|c|}{ Test: Aug14_03 } & sum1 & Date: Tue & \multicolumn{2}{|c|}{ Aug $14 \quad 11: 49: 121990$} & Page: 3 \\
\hline \# & Name & Min & $\operatorname{Max}$ & Ave & SD & Uncert \\
\hline 101 & PIN000001 & 35.4421 & 36.1745 & 35.8205 & $1.7655 \mathrm{e}-01$ & $7.5533 e-01$ \\
\hline 102 & PRV000001 & 18.8641 & 18.9861 & 18.8860 & $4.7374 e-02$ & $7.5148 e-01$ \\
\hline 103 & PSRO00001 & 19.5691 & 19.7911 & 19.7545 & $5.6508 e-02$ & $7.5162 e-01$ \\
\hline 104 & PFM000001 & 73.6929 & 75.4324 & 74.4601 & $3.7356 e-01$ & $5.3353 e-01$ \\
\hline 105 & PAN000136 & 400.1298 & 408.8273 & 404.6434 & $1.8497 \mathrm{e}+00$ & $5.0229 \mathrm{e}+00$ \\
\hline 106 & PEMO00002 & 27.5689 & 27.5689 & 27.5689 & $9.6336 e-06$ & $2.1154 e-01$ \\
\hline 107 & EMPTY & 0.0049 & 0.0098 & 0.0092 & $1.6028 e-03$ & $0.0000 e+00$ \\
\hline 108 & EMPTY & -10.0000 & 1.9531 & -6.5872 & $3.4765 e+00$ & $0.0000 e+00$ \\
\hline 109 & EMPTY & 0.0049 & 0.0098 & 0.0053 & $1.3381 e-03$ & $0.0000 e+00$ \\
\hline 110 & EMPTY & -10.0000 & -10.0000 & -10.0000 & $0.0000 e+00$ & $0.0000 e+00$ \\
\hline 111 & EMPTY & 0.0000 & 0.0195 & 0.0034 & $3.3087 e-03$ & $0.0000 e+00$ \\
\hline 112 & VPW000001 & -1.0479 & -0.6573 & -0.8331 & $9.8648 e-02$ & $8.1002 e-01$ \\
\hline 113 & VPW000002 & -1.0404 & -0.6498 & -0.7748 & $1.0259 \mathrm{e}-01$ & $8.1001 e-01$ \\
\hline 114 & VPW000003 & -0.9240 & -0.5333 & -0.6701 & $9.8648 e-02$ & $8.1001 e-01$ \\
\hline 115 & VPW000004 & -0.8752 & -0.4845 & -0.6486 & $9.9512 e-02$ & $8.1001 e-01$ \\
\hline 116 & VPW000005 & -1.6639 & -1.0780 & -1.3670 & $1.1995 e-01$ & $8.1004 e-01$ \\
\hline 117 & VPW000006 & -1.6489 & -1.2583 & -1.5005 & $1.1545 e-01$ & $8.1005 e-01$ \\
\hline 118 & IPW000001 & -11.6766 & -7.7719 & -9.6852 & $1.2151 e+00$ & $8.1004 e+00$ \\
\hline 119 & IPW000002 & $-12.233 i$ & -8.3306 & -10.2819 & $1.3075 e+00$ & $8.1004 e+00$ \\
\hline 120 & IPW000003 & -12.8625 & -8.9625 & -11.2245 & $1.2680 \mathrm{e}+00$ & $8.1005 e+00$ \\
\hline 121 & IPWO00004 & -2.7419 & -1.7654 & -2.0193 & $2.6543 e-01$ & $2.0001 e+00$ \\
\hline 122 & IPW000005 & -3.9904 & -3.0139 & -3.5607 & $3.2175 e-01$ & $2.0002 e+00$ \\
\hline 123 & IPW000006 & -2.8076 & -1.3427 & -2.2314 & $3.5141 e-01$ & $2.0001 e+00$ \\
\hline 124 & EMPTY & -0.0293 & -0.0146 & -0.0207 & $3.6326 e-03$ & $0.0000 e+00$ \\
\hline 125 & EMPTY & -0.0195 & -0.0098 & -0.0147 & $2.8744 e-03$ & $0.0000 e+00$ \\
\hline 126 & EMPTY & -0.0195 & -0.0098 & -0.0168 & $2.6396 \mathrm{e}-03$ & $0.0000 e+00$ \\
\hline 127 & EMPTY & -0.0244 & -0.0098 & -0.0177 & $3.2554 e-03$ & $0.0000 e+00$ \\
\hline 128 & IHTO00001 & -0.2066 & 0.1841 & -0.0152 & $7.3716 e-02$ & $4.1000 e+00$ \\
\hline 129 & IHTO00002 & -0.2329 & 0.1577 & -0.0493 & $9.1779 e-02$ & $4.1000 e+00$ \\
\hline 130 & IHT000003 & -0.0864 & 0.1089 & -0.0278 & $9.0412 e-02$ & $4.1000 e+00$ \\
\hline 131 & IHTO00004 & -0.2028 & 0.1878 & -0.0270 & $1.0625 e-01$ & $4.1000 e+00$ \\
\hline 132 & IHT000005 & -0.2066 & 0.1841 & -0.0425 & $1.0705 e-01$ & $4.1000 e+00$ \\
\hline 133 & IHTO00006 & -0.0601 & 0.3305 & 0.0454 & $1.0595 e-01$ & $4.1000 \mathrm{e}+00$ \\
\hline 134 & IHT000007 & -0.0601 & 0.1352 & -0.0171 & $8.1729 e-02$ & $4.1000 e+00$ \\
\hline 135 & IHTO00008 & -0.1578 & 0.0376 & -0.0679 & $9.8332 e-02$ & $4.1000 e+00$ \\
\hline 136 & IHTO00009 & -0.2216 & 0.1690 & -0.0224 & $9.2456 e-02$ & $4.1000 e+00$ \\
\hline 137 & IHTO00010 & -0.3380 & 0.0526 & -0.0959 & $1.0106 e-01$ & $4.1000 e+00$ \\
\hline 138 & IHTO00011 & -0.3042 & 0.0864 & -0.0894 & $7.1136 e-02$ & $4.1000 e+00$ \\
\hline 139 & IHTO00012 & -0.3606 & 0.0301 & -0.1028 & $1.1464 e-01$ & $4.1000 e+00$ \\
\hline 140 & IHTO00013 & -0.0563 & 0.1390 & 0.0765 & $9.2034 e-02$ & $4.1000 e+00$ \\
\hline 141 & IHTO00014 & -0.1953 & 0.1953 & -0.0234 & $7.5283 e-02$ & $4.1000 e+00$ \\
\hline 142 & IHT000015 & -0.2442 & 0.1465 & -0.0293 & $7.1136 e-02$ & $4.1000 e+00$ \\
\hline 143 & IHTO00016 & -0.1352 & 0.0601 & -0.0571 & $9.6655 e-02$ & $4.1000 e+00$ \\
\hline 144 & IHT000017 & -0.3530 & 0.6235 & 0.0571 & $2.8523 e-01$ & $4.1000 \mathrm{e}+00$ \\
\hline 145 & IHT000018 & -0.1728 & 0.4131 & 0.0069 & $1.2378 e-01$ & $4.1000 e+00$ \\
\hline 146 & IHT000019 & 0.0413 & 0.4319 & 0.2796 & $1.0654 e-01$ & $4.1000 e+00$ \\
\hline 147 & IHTO00020 & -0.2517 & 0.1390 & -0.0642 & $6.7888 e-02$ & $4.1000 e+00$ \\
\hline 148 & IHT 000021 & -0.0714 & 0.1240 & 0.0068 & $9.6655 e-02$ & $4.1000 e+00$ \\
\hline 149 & IHT000022 & 0.0037 & 0.1991 & 0.1287 & $9.4702 e-02$ & $4.1000 e+00$ \\
\hline 150 & IHT000023 & -0.2254 & 0.3606 & 0.1496 & $1.0320 \mathrm{e}-01$ & $4.1000 e+00$ \\
\hline 151 & IHTO000024 & -0.4770 & -0.2817 & -0.3793 & $9.8648 \mathrm{e}-02$ & $4.1000 e+00$ \\
\hline 152 & IHTO00025 & 0.2028 & 0.3981 & 0.2849 & $9.7377 e-02$ & $4.1000 e+00$ \\
\hline 153 & IHTO00026 & -0.1690 & 0.2216 & -0.0050 & $9.1354 e-02$ & $4.1000 e+00$ \\
\hline 154 & IHTO00027 & -0.0338 & 0.1615 & 0.0131 & $8.4262 \mathrm{e}-02$ & $4.1000 \mathrm{e}+00$ \\
\hline 155 & IHT000028 & -0.2479 & 0.1427 & 0.0021 & $1.0470 e-01$ & $4.1000 e+00$ \\
\hline
\end{tabular}




\begin{tabular}{|c|c|c|c|c|c|}
\hline Test: Aug14_03 & sum1 & Date: Tue & Aug 141 & $9: 12 \quad 1990$ & Page: 4 \\
\hline 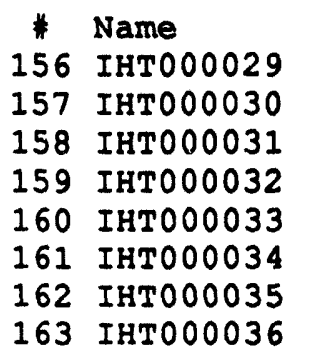 & $\begin{array}{l}\text { Min } \\
-0.2780 \\
-0.2103 \\
-0.3418 \\
-0.2930 \\
-0.0977 \\
-0.2329 \\
-0.1277 \\
-0.3831\end{array}$ & $\begin{array}{l}\operatorname{Max} \\
0.1127 \\
0.3756 \\
0.2441 \\
0.2930 \\
0.2930 \\
0.1577 \\
0.2629 \\
0.2028\end{array}$ & $\begin{array}{l}\text { Ave } \\
-0.0475 \\
0.0280 \\
-0.0566 \\
-0.0273 \\
-0.0274 \\
-0.0219 \\
0.0481 \\
-0.0316\end{array}$ & $\begin{array}{l}\text { SD } \\
9.4125 \mathrm{e}-02 \\
1.0654 \mathrm{e}-01 \\
1.2608 \mathrm{e}-01 \\
1.1679 \mathrm{e}-01 \\
1.0259 \mathrm{e}-01 \\
1.1049 \mathrm{e}-01 \\
1.2633 \mathrm{e}-01 \\
1.1838 \mathrm{e}-01\end{array}$ & $\begin{array}{l}\text { Uncert } \\
4.1000 \mathrm{e}+00 \\
4.1000 \mathrm{e}+00 \\
4.1000 \mathrm{e}+00 \\
4.1000 \mathrm{e}+00 \\
4.1000 \mathrm{e}+00 \\
4.1000 \mathrm{e}+00 \\
4.1000 \mathrm{e}+00 \\
4.1000 \mathrm{e}+00\end{array}$ \\
\hline
\end{tabular}




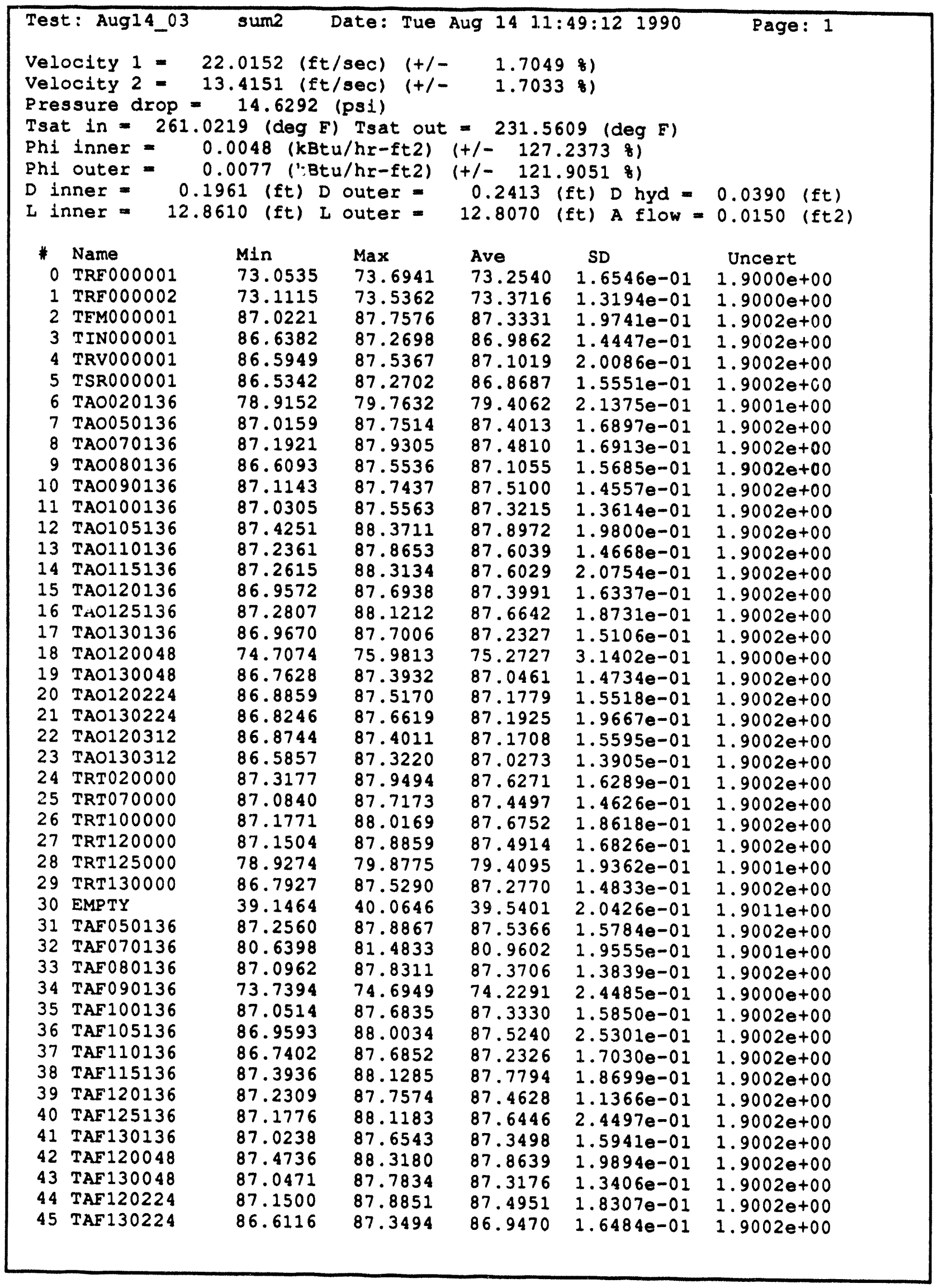




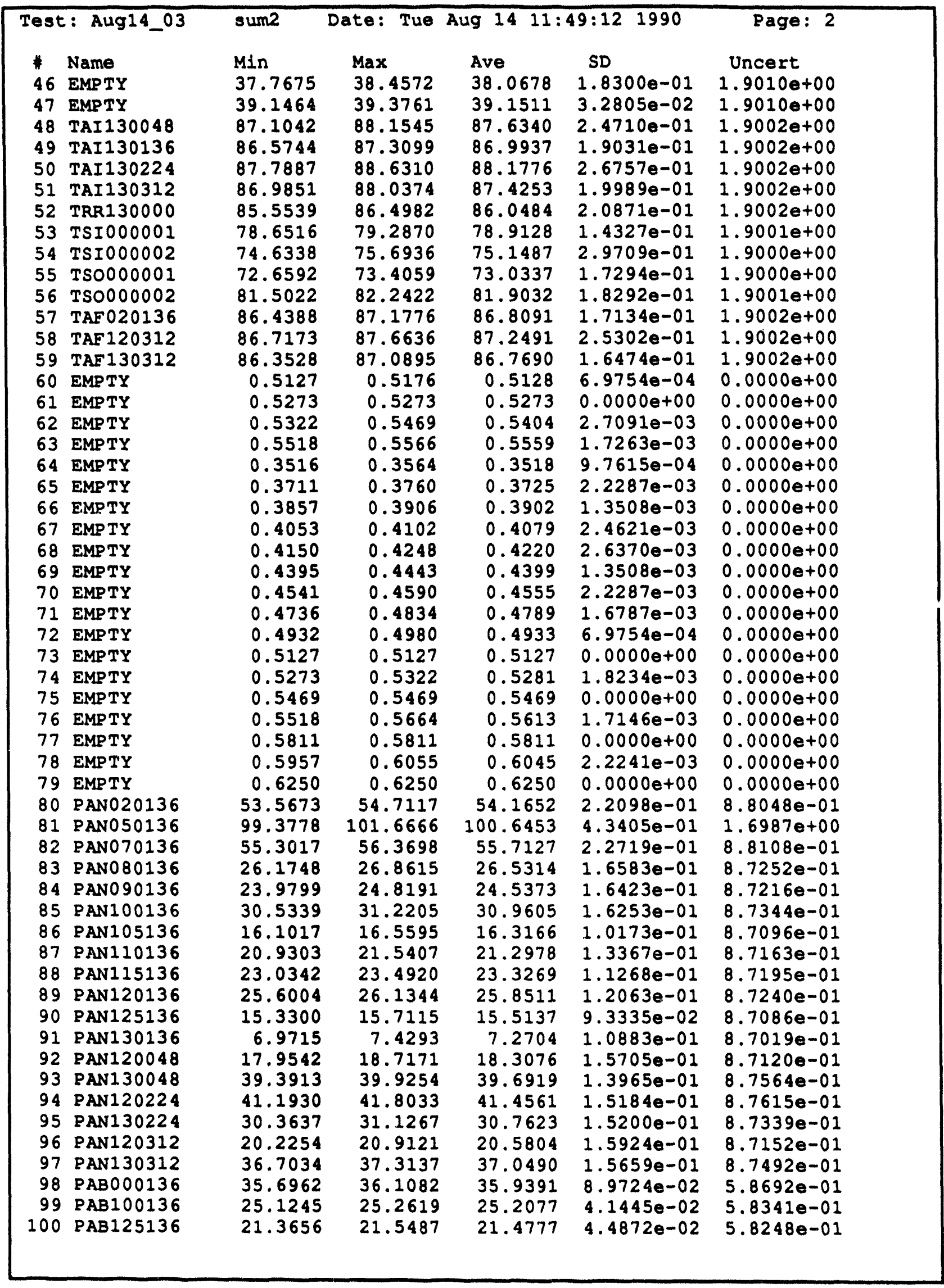




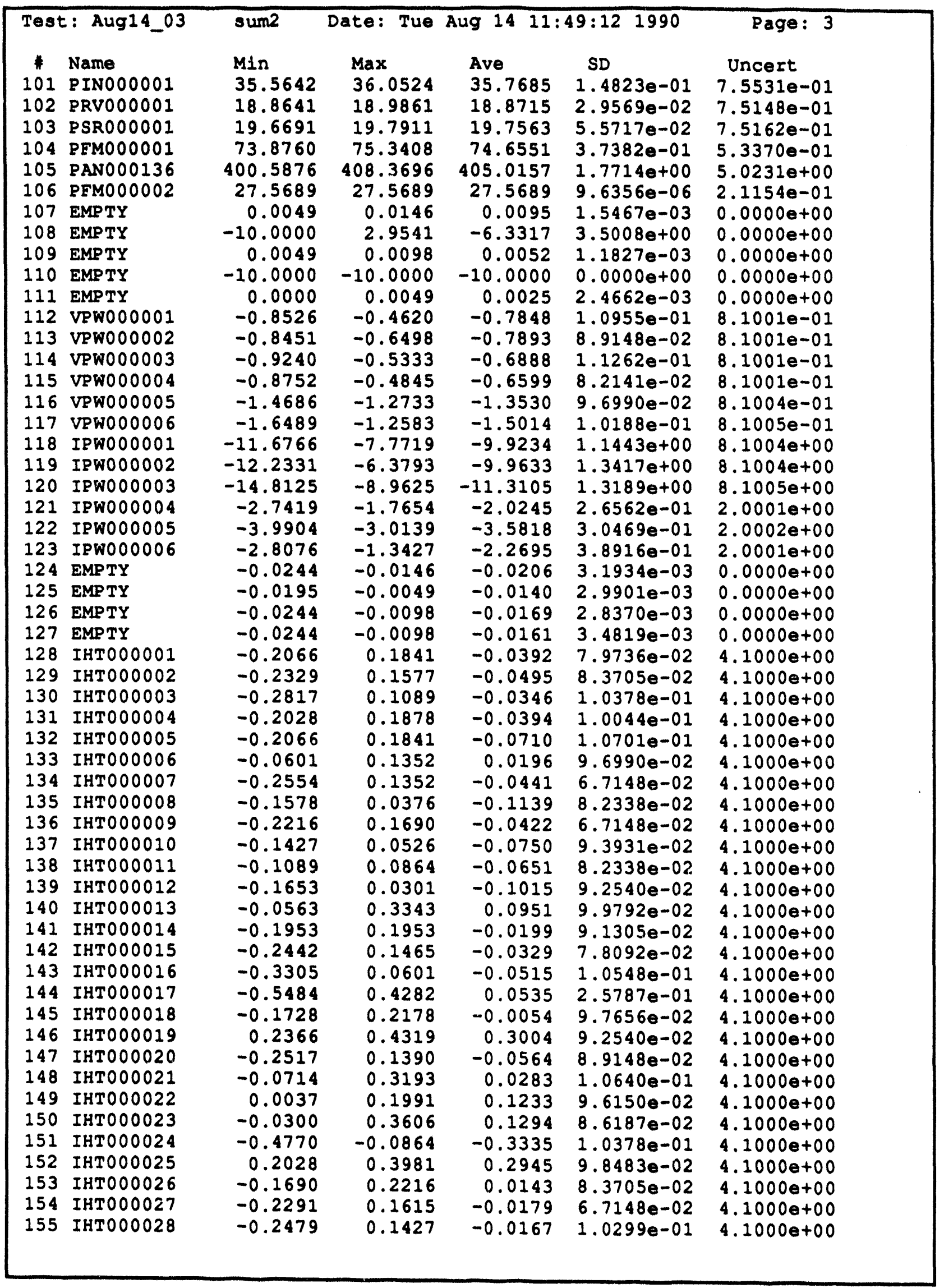




\begin{tabular}{|c|c|c|c|c|c|}
\hline 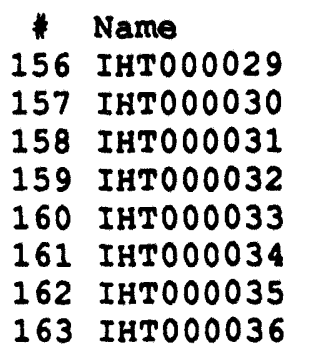 & $\begin{array}{l}\text { Min } \\
-0.2780 \\
-0.2103 \\
-0.1465 \\
-0.0976 \\
-0.0977 \\
-0.2329 \\
-0.1277 \\
-0.1878\end{array}$ & $\begin{array}{l}\operatorname{Max} \\
0.3080 \\
0.1803 \\
0.2441 \\
0.2930 \\
0.2930 \\
0.1577 \\
0.2629 \\
0.0075\end{array}$ & $\begin{array}{l}\text { Ave } \\
-0.0348 \\
0.0288 \\
-0.0349 \\
-0.0139 \\
-0.0060 \\
-0.0336 \\
0.0357 \\
-0.0682\end{array}$ & $\begin{array}{l}\text { SD } \\
1.0188 \mathrm{e}-01 \\
1.0746 \mathrm{e}-01 \\
1.1960 \mathrm{e}-01 \\
1.0548 \mathrm{e}-01 \\
1.0625 \mathrm{e}-01 \\
1.1616 \mathrm{e}-01 \\
1.0806 \mathrm{e}-01 \\
9.6150 \mathrm{e}-02\end{array}$ & $\begin{array}{l}\text { Uncert } \\
4.1000 e+00 \\
4.1000 e+00 \\
4.1000 e+00 \\
4.1000 e+00 \\
4.1000 e+00 \\
4.1000 e+00 \\
4.1000 e+00 \\
4.1000 e+00\end{array}$ \\
\hline
\end{tabular}




\begin{tabular}{|c|c|c|c|c|}
\hline \multicolumn{4}{|c|}{$\begin{array}{l}\text { Velocity } 1=0.028599 \text { (ft/sec) } \\
\text { Velocity } 2=0.000000 \text { (ft/sec) } \\
\text { Pressure Drop }=0.013500 \text { (psi) } \\
\text { Tsat in }=0.027527 \text { (deg F) Tsat out }=0.005692(\mathrm{deg} F) \\
\text { Ph1 inner }=0.000100 \text { (kBtu/hr-ft } 2) \text { Phi outer }=0.000100\end{array}$} & $(k B t u / h r-f t 2)$ \\
\hline $\begin{array}{l}* \\
1 \\
2 \\
3 \\
4 \\
5 \\
6 \\
7 \\
8 \\
9 \\
10 \\
11 \\
12 \\
13 \\
14 \\
15 \\
16 \\
17 \\
18 \\
19 \\
20 \\
21 \\
22 \\
23 \\
24 \\
25 \\
26 \\
27 \\
28 \\
29 \\
30 \\
31 \\
32 \\
33 \\
34 \\
35 \\
36 \\
37 \\
38 \\
39 \\
40 \\
41 \\
42 \\
43 \\
44 \\
45\end{array}$ & $\begin{array}{l}\text { Name } \\
\text { TRF000001 } \\
\text { TRF000002 } \\
\text { TFM000001 } \\
\text { TIN000001 } \\
\text { TRV000001 } \\
\text { TSR000001 } \\
\text { TAO020136 } \\
\text { TAO050136 } \\
\text { TAO070136 } \\
\text { TAO080136 } \\
\text { TAO090136 } \\
\text { TAO100136 } \\
\text { TAO105136 } \\
\text { TAO110136 } \\
\text { TAO115136 } \\
\text { TAO120136 } \\
\text { TAO125136 } \\
\text { TAO130136 } \\
\text { TAO120048 } \\
\text { TAO130048 } \\
\text { TAO120224 } \\
\text { TAO130224 } \\
\text { TAO120312 } \\
\text { TAO130312 } \\
\text { TRT020000 } \\
\text { TRT070000 } \\
\text { TRT100000 } \\
\text { TRT120000 } \\
\text { TRT125000 } \\
\text { TRT130000 } \\
\text { EMPTY } \\
\text { TAF050136 } \\
\text { TAF070136 } \\
\text { TAF080136 } \\
\text { TAF090136 } \\
\text { TAF100136 } \\
\text { TAF105136 } \\
\text { TAF110136 } \\
\text { TAF115136 } \\
\text { TAF120136 } \\
\text { TAF125136 } \\
\text { TAF130136 } \\
\text { TAF120048 } \\
\text { TAF130048 } \\
\text { TAF120224 } \\
\text { TAF130224 }\end{array}$ & $\begin{array}{l}\text { Abs Diff } \\
0.0088 \\
0.0137 \\
0.0441 \\
0.0246 \\
0.0610 \\
0.0087 \\
0.0479 \\
0.0404 \\
0.0247 \\
0.0381 \\
0.0540 \\
0.0005 \\
0.0263 \\
0.0031 \\
0.0362 \\
0.0444 \\
0.0636 \\
0.0031 \\
0.0367 \\
0.0119 \\
0.0331 \\
0.0738 \\
0.0375 \\
0.0151 \\
0.0086 \\
0.0178 \\
0.0445 \\
0.0026 \\
0.2109 \\
0.0092 \\
0.0263 \\
0.0655 \\
0.0104 \\
0.0341 \\
0.0161 \\
0.0323 \\
0.0695 \\
0.0203 \\
0.0517 \\
0.0016 \\
0.0031 \\
0.0296 \\
0.0204 \\
0.0219 \\
0.0518 \\
0.0046\end{array}$ & $\begin{array}{l}\text { 8Diff } \\
0.0120 \\
0.0187 \\
0.0505 \\
0.0283 \\
0.0700 \\
0.0100 \\
0.0604 \\
0.0462 \\
0.0282 \\
0.0437 \\
0.0617 \\
0.0006 \\
0.0299 \\
0.0035 \\
0.0413 \\
0.0508 \\
0.0725 \\
0.0036 \\
0.0488 \\
0.0137 \\
0.0380 \\
0.0846 \\
0.0430 \\
0.0174 \\
0.0098 \\
0.0204 \\
0.0507 \\
0.0030 \\
0.2649 \\
0.0105 \\
0.0666 \\
0.0748 \\
0.0128 \\
0.0390 \\
0.0217 \\
0.0370 \\
0.0795 \\
0.0233 \\
0.0589 \\
0.0018 \\
0.0035 \\
0.0339 \\
0.0232 \\
0.0251 \\
0.0592 \\
0.0053\end{array}$ & \\
\hline
\end{tabular}




\begin{tabular}{|c|c|c|c|}
\hline 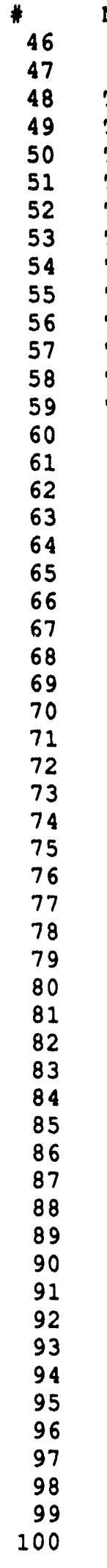 & $\begin{array}{r}\text { Name } \\
\text { EMPTY } \\
\text { EMPTY } \\
\text { TAI130048 } \\
\text { TAI130136 } \\
\text { TAI130224 } \\
\text { TAI130312 } \\
\text { TRR130000 } \\
\text { TSI000001 } \\
\text { TSI000002 } \\
\text { TSO000001 } \\
\text { TSO000002 } \\
\text { TAF020136 } \\
\text { TAF120312 } \\
\text { TAF130312 } \\
\text { EMPTY } \\
\text { EMPTY } \\
\text { EMPTY } \\
\text { EMPTY } \\
\text { EMPTY } \\
\text { EMPTY } \\
\text { EMPTY } \\
\text { EMPTY } \\
\text { EMPTY } \\
\text { EMPTY } \\
\text { EMPTY } \\
\text { EMPTY } \\
\text { EMPTY } \\
\text { EMPTY } \\
\text { EMPTY } \\
\text { EMPTY } \\
\text { EMPTY } \\
\text { EMPTY } \\
\text { EMPTY } \\
\text { EMPTY } \\
\text { PAN020136 } \\
\text { PAN050136 } \\
\text { PAN070136 } \\
\text { PAN080136 } \\
\text { PAN090136 } \\
\text { PAN100136 } \\
\text { PAN105136 } \\
\text { PAN110136 } \\
\text { PAN115136 } \\
\text { PAN120136 } \\
\text { PAN125136 } \\
\text { PAN130136 } \\
\text { PAN120048 } \\
\text { PAN130048 } \\
\text { PAN120224 } \\
\text { PAN130224 } \\
\text { PAN120312 } \\
\text { PAN130312 } \\
\text { PAB000136 } \\
\text { PAB100136 } \\
\text { PAB125136 }\end{array}$ & $\begin{array}{c}\text { Abs Diff } \\
0.0308 \\
0.0367 \\
0.0022 \\
0.0104 \\
0.0260 \\
0.0424 \\
0.0392 \\
0.0431 \\
0.0674 \\
0.0267 \\
0.0194 \\
0.0158 \\
0.0337 \\
0.0064 \\
0.0001 \\
0.0001 \\
0.0010 \\
0.0005 \\
0.0001 \\
0.0010 \\
0.0003 \\
0.0008 \\
0.0010 \\
0.0003 \\
0.0010 \\
0.0007 \\
0.0001 \\
0.0003 \\
0.0010 \\
0.0001 \\
0.0002 \\
0.0002 \\
0.0009 \\
0.0006 \\
0.0089 \\
0.3153 \\
0.0631 \\
0.0386 \\
0.0325 \\
0.1184 \\
0.0216 \\
0.0501 \\
0.0455 \\
0.0600 \\
0.0494 \\
0.0120 \\
0.0895 \\
0.0900 \\
0.0877 \\
0.1224 \\
0.0559 \\
0.0725 \\
0.0168 \\
0.0090 \\
0.0023\end{array}$ & $\begin{array}{l}\text { PDiff } \\
0.0808 \\
0.0937 \\
0.0025 \\
0.0120 \\
0.0295 \\
0.0485 \\
0.0456 \\
0.0547 \\
0.0898 \\
0.0366 \\
0.0237 \\
0.0182 \\
0.0386 \\
0.0074 \\
0.0195 \\
0.0190 \\
0.1847 \\
0.0899 \\
0.0284 \\
0.2677 \\
0.0769 \\
0.1957 \\
0.2364 \\
0.0681 \\
0.2191 \\
0.1460 \\
0.0203 \\
0.0585 \\
0.1890 \\
0.0183 \\
0.0356 \\
0.0344 \\
0.1487 \\
0.0959 \\
0.0164 \\
0.3143 \\
0.1134 \\
0.1453 \\
0.1326 \\
0.3839 \\
0.1322 \\
0.2358 \\
0.1954 \\
0.2326 \\
0.3194 \\
0.1653 \\
0.4913 \\
0.2273 \\
0.2120 \\
0.3995 \\
0.2724 \\
0.1961 \\
0.0468 \\
0.0357 \\
0.0107 \\
\end{array}$ \\
\hline
\end{tabular}




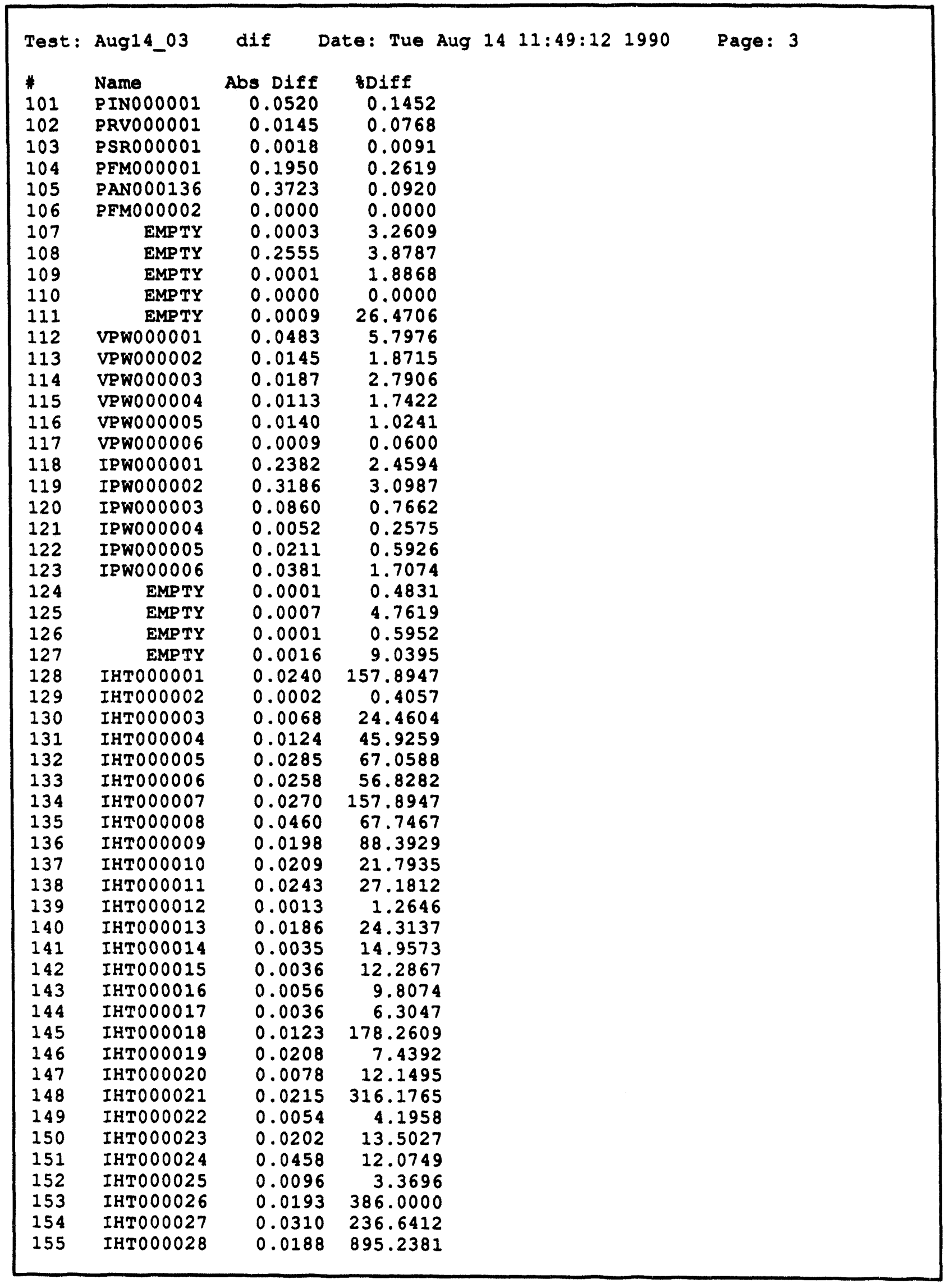




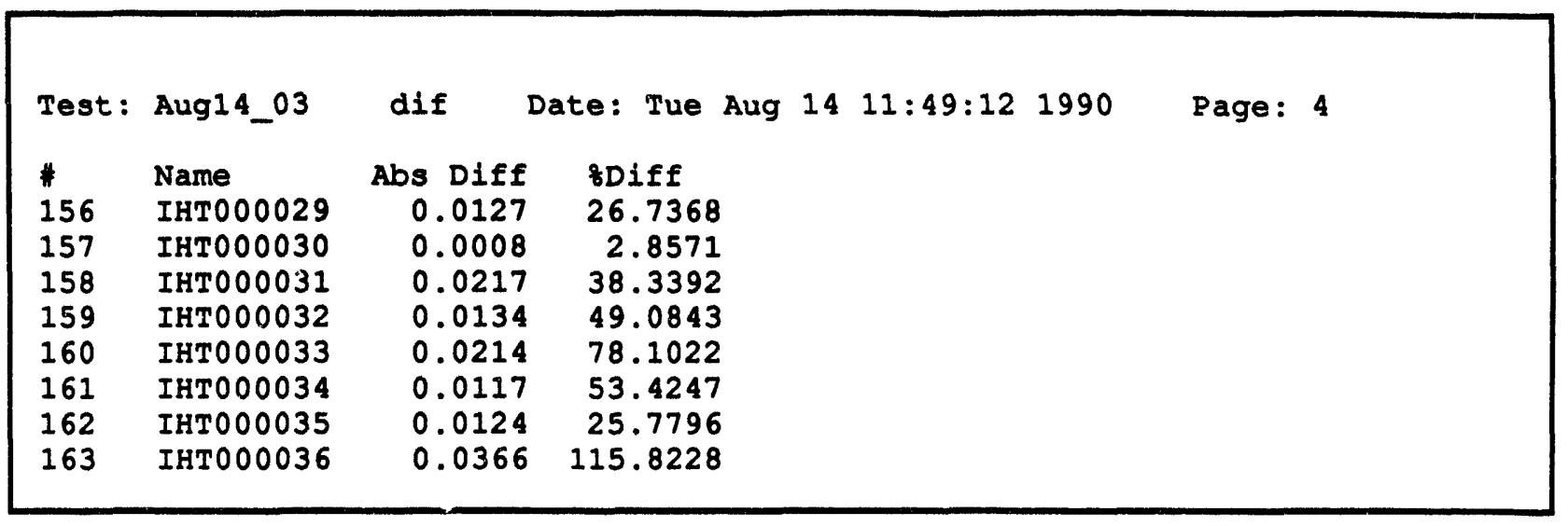




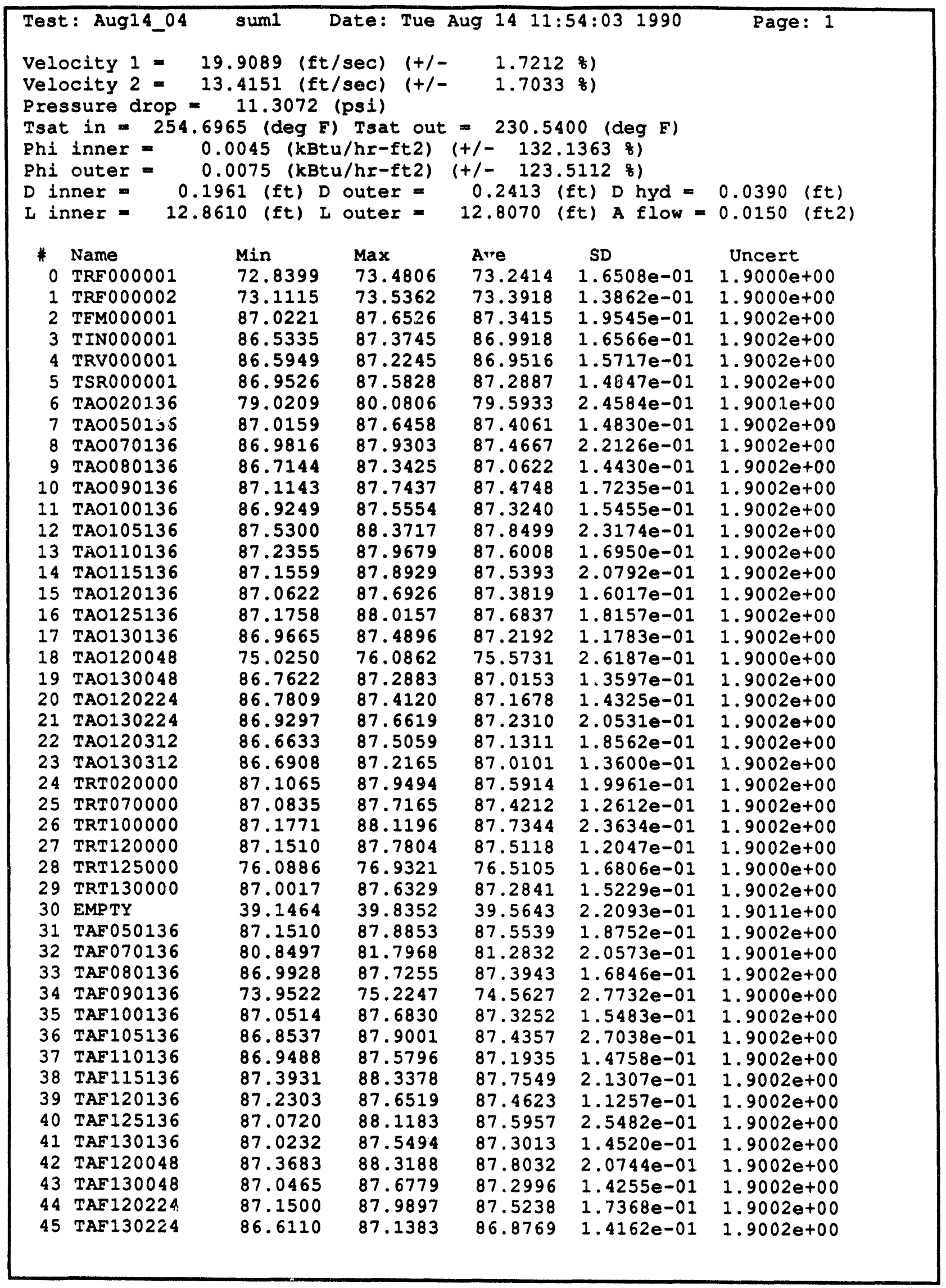




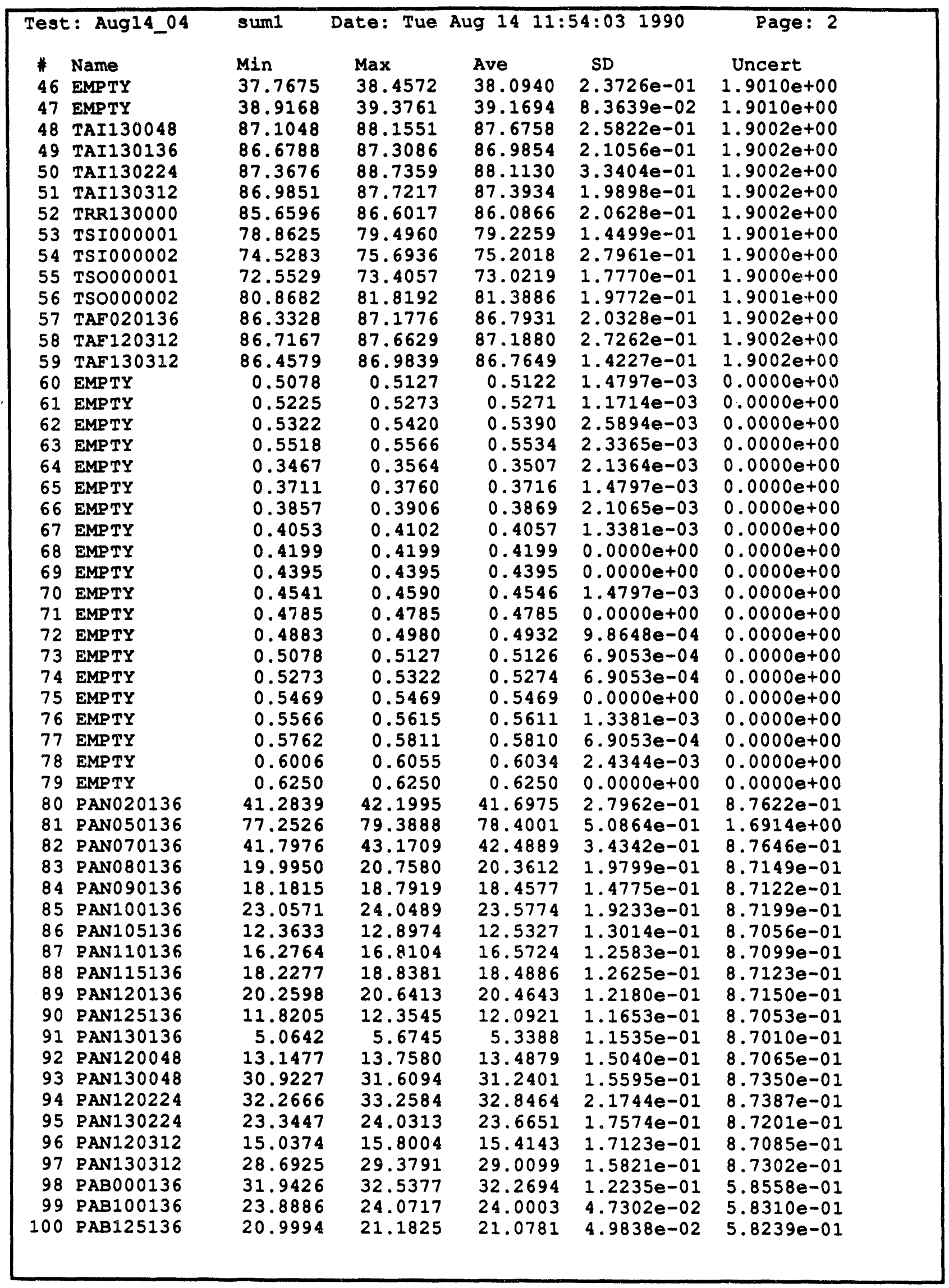




\begin{tabular}{|c|c|c|c|c|c|c|}
\hline \multicolumn{2}{|c|}{ Test: Aug14_04 } & \multirow{2}{*}{$\begin{array}{l}\text { sum1 } \\
\text { Min }\end{array}$} & Date: Tue & \multicolumn{2}{|c|}{ Aug $14 \quad 11: 54: 03 \quad 1990$} & \multirow{2}{*}{$\begin{array}{l}\text { Page: } 3 \\
\text { Uncert }\end{array}$} \\
\hline \# & Name & & $\operatorname{Max}$ & Ave & SD & \\
\hline 101 & PINO00001 & 31.6579 & 32.3903 & 32.0925 & $1.9287 e-01$ & $7.5428 e-01$ \\
\hline 102 & PRV0000001 & 18.8641 & 18.8641 & 18.8641 & $1.9267 e-06$ & $7.5148 e-01$ \\
\hline 103 & PSR000001 & 19.6691 & 19.7911 & 19.6886 & $4.5206 e-02$ & $7.5161 e-01$ \\
\hline 104 & PFMO00001 & 59.9600 & 61.7910 & 60.9982 & $3.9567 e-01$ & $5.2274 e-01$ \\
\hline 105 & PANO00136 & 306.7460 & 318.6479 & 313.0448 & $2.7256 \mathrm{e}+00$ & $4.9819 e+00$ \\
\hline 106 & PFM000002 & 27.5689 & 27.5689 & 27.5689 & $9.6336 \mathrm{e}-06$ & $2.1154 e-01$ \\
\hline 107 & EMPTY & 0.0049 & 0.0098 & 0.0091 & $1.7115 e-03$ & $0.0000 e+00$ \\
\hline 108 & EMPTY & -10.0000 & 2.0361 & -7.2907 & $3.2114 e+00$ & $0.0000 e+00$ \\
\hline 109 & EMPTY & 0.0000 & 0.0098 & 0.0050 & $1.2041 e-03$ & $0.0000 e+00$ \\
\hline 110 & EMPTY & -10.0000 & -10.0000 & -10.0000 & $0.0000 e+00$ & $0.0000 \mathrm{e}+00$ \\
\hline 111 & EMPTY & -0.0049 & 0.0049 & 0.0020 & $2.6100 e-03$ & $0.0000 e+00$ \\
\hline 112 & VPW000001 & -0.8526 & -0.6573 & -0.7784 & $9.5765 \mathrm{e}-02$ & $8.1001 e-01$ \\
\hline 113 & VPW000002 & -1.0404 & -0.6498 & -0.7943 & $1.0297 e-01$ & $8.1001 e-01$ \\
\hline 114 & VPW000003 & -0.9240 & -0.3380 & -0.6544 & $1.1765 e-01$ & $8.1001 e-01$ \\
\hline 115 & VPW000004 & -0.8752 & -0.2892 & -0.6408 & $1.4227 e-01$ & $8.1001 \mathrm{e}-01$ \\
\hline 116 & VPW 000005 & -1.4686 & -1.0780 & -1.3241 & $1.1027 e-01$ & $8.1004 e-01$ \\
\hline 117 & VPW000006 & -1.6489 & -1.2583 & -1.5083 & $1.3692 e-01$ & $8.1005 e-01$ \\
\hline 118 & IPW000001 & -11.6766 & -7.7719 & -9.7633 & $1.1493 \mathrm{e}+00$ & $8.1004 \mathrm{e}+00$ \\
\hline 119 & IPW000002 & -12.2331 & -6.3793 & -9.5794 & $1.4082 \mathrm{e}+00$ & $8.1004 e+00$ \\
\hline 120 & IPW000003 & -12.8625 & -7.0125 & -11.1075 & $1.3214 \mathrm{e}+00$ & $8.1005 e+00$ \\
\hline 121 & IPW000004 & -2.2536 & -1.7654 & -2.0388 & $2.4484 \mathrm{e}-01$ & $2.0001 \mathrm{e}+00$ \\
\hline 122 & IPW000005 & -3.9904 & -3.0139 & -3.5412 & $3.2476 e-01$ & $2.0002 e+00$ \\
\hline 123 & IPW000006 & -2.8076 & -1.3427 & -2.1728 & $3.5908 \mathrm{e}-01$ & $2.0001 e+00$ \\
\hline 124 & EMPTY & -0.0244 & -0.0146 & -0.0195 & $3.8206 e-03$ & $0.0000 \mathrm{e}+00$ \\
\hline 125 & EMPTY & -0.0195 & -0.0098 & -0.0143 & $2.7621 e-03$ & $0.0000 e+00$ \\
\hline 126 & EMF TY & -0.0195 & -0.0098 & -0.0165 & $2.9413 e-03$ & $0.0000 e+00$ \\
\hline 127 & EMPTY & -0.0244 & -0.0098 & -0.0167 & $3.2851 e-03$ & $0.0000 e+00$ \\
\hline 128 & IHTO00001 & -0.2066 & 0.1841 & -0.0659 & $9.6976 e-02$ & $4.1000 e+00$ \\
\hline 129 & IHT000002 & -0.2329 & 0.1577 & -0.0610 & $8.4997 e-02$ & $4.1000 \mathrm{e}+00$ \\
\hline 130 & IHT000003 & -0.2817 & 0.3042 & -0.0473 & $1.1161 e-01$ & $4.1000 e+00$ \\
\hline 131 & IHTO00004 & -0.2028 & 0.1878 & -0.0153 & $1.0410 e-01$ & $4.1000 e+00$ \\
\hline 132 & IHT000005 & -0.2066 & 0.1841 & -0.0620 & $1.1027 e-01$ & $4.1000 \mathrm{e}+00$ \\
\hline 133 & IHT000006 & -0.2554 & 0.3305 & 0.0102 & $1.0992 \mathrm{e}-01$ & $4.1000 \mathrm{e}+00$ \\
\hline 134 & IHT000007 & -0.2554 & 0.1352 & -0.0484 & $8.2864 e-02$ & $4.1000 \mathrm{e}+00$ \\
\hline 135 & IHT000008 & -0.1578 & 0.0376 & -0.1109 & $8.4262 e-02$ & $4.1000 e+00$ \\
\hline 136 & IHT000009 & -0.2216 & 0.1690 & -0.0302 & $8.3612 e-02$ & $4.1000 \mathrm{e}+00$ \\
\hline 137 & IHT000010 & -0.1427 & 0.0526 & -0.1037 & $7.8918 e-02$ & $4.1000 e+00$ \\
\hline 138 & IHT000011 & -0.3042 & 0.0864 & -0.0894 & $8.1347 e-02$ & $4.1000 e+00$ \\
\hline 139 & IHT000012 & -0.3606 & 0.2254 & -0.1145 & $1.1027 e-01$ & $4.1000 \mathrm{e}+00$ \\
\hline 140 & IHT000013 & -0.0563 & 0.1390 & 0.0921 & $8.4262 e-02$ & $4.1000 e+00$ \\
\hline 141 & IHTO00014 & -0.1953 & 0.1953 & -0.0156 & $9.5357 e-02$ & $4.1000 e+00$ \\
\hline 142 & IHT000015 & -0.0488 & 0.1465 & -0.0098 & $7.8918 e-02$ & $4.1000 e+00$ \\
\hline 143 & IHT000016 & -0.3305 & 0.0601 & -0.0610 & $1.0358 \mathrm{e}-01$ & $4.1000 e+00$ \\
\hline 144 & IHT000017 & -0.3530 & 0.6235 & 0.0688 & $3.4930 e-01$ & $4.1000 e+00$ \\
\hline 145 & IHT000018 & -0.1128 & 0.2178 & 0.0186 & $1.0799 e-01$ & $4.1000 \mathrm{e}+00$ \\
\hline 146 & IHT000019 & 0.0413 & 0.6273 & 0.2991 & $1.0763 e-01$ & $4.1000 e+00$ \\
\hline 147 & IHTO00020 & -0.2517 & 0.1390 & -0.0485 & $7.8523 \mathrm{e}-02$ & $4.1000 \mathrm{e}+00$ \\
\hline 148 & IHTO00021 & -0.0714 & 0.3193 & 0.0302 & $1.1327 \mathrm{e}-01$ & $4.1000 e+00$ \\
\hline 149 & IHTO00022 & -0.1916 & 0.3944 & 0.1248 & $1.3606 e-01$ & $4.1000 e+00$ \\
\hline 150 & IHTO00023 & -0.0300 & 0.3606 & 0.1379 & $9.6735 e-02$ & $4.1000 \mathrm{e}+00$ \\
\hline 151 & IHT000024 & -0.4770 & 0.1089 & -0.3129 & $1.2701 \mathrm{e}-01$ & $4.1000 \mathrm{e}+00$ \\
\hline 152 & IHTO00025 & 0.2028 & 0.5935 & 0.2966 & $1.1327 e-01$ & $4.1000 e+00$ \\
\hline 153 & IHT000026 & -0.1690 & 0.2216 & 0.0146 & $8.2864 \mathrm{e}-02$ & $4.1000 e+00$ \\
\hline 154 & IHTO00027 & -0.2291 & 0.1615 & -0.0221 & $6.1257 e-02$ & $4.1000 e+00$ \\
\hline 155 & IHT000028 & -0.0526 & 0.1427 & 0.0177 & $9.4702 e-02$ & $4.1000 e+00$ \\
\hline
\end{tabular}




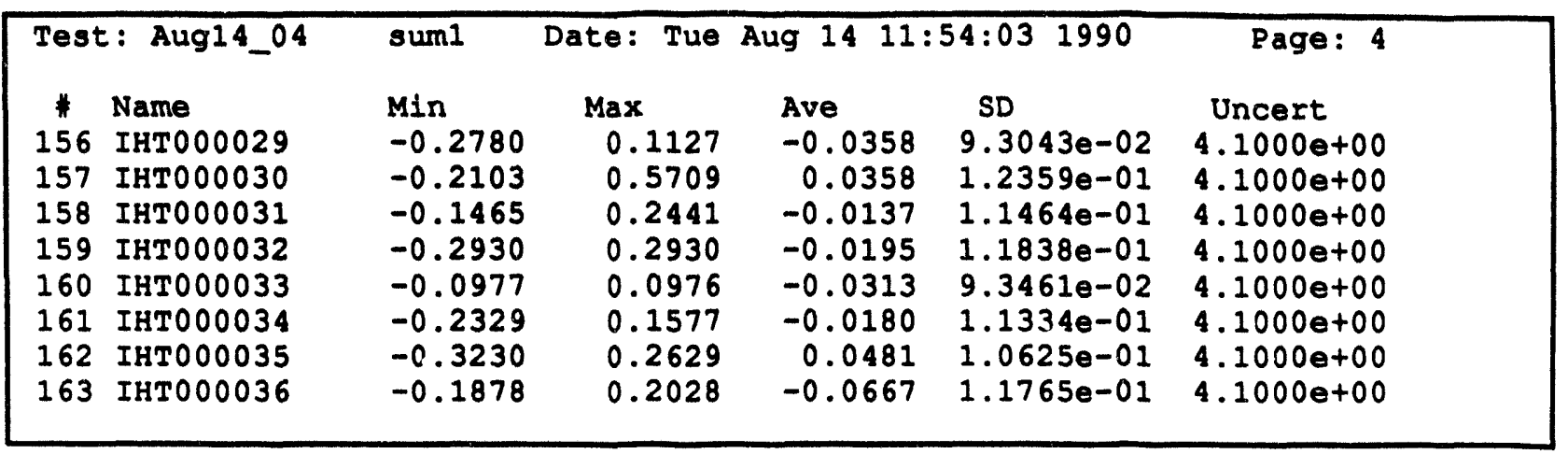




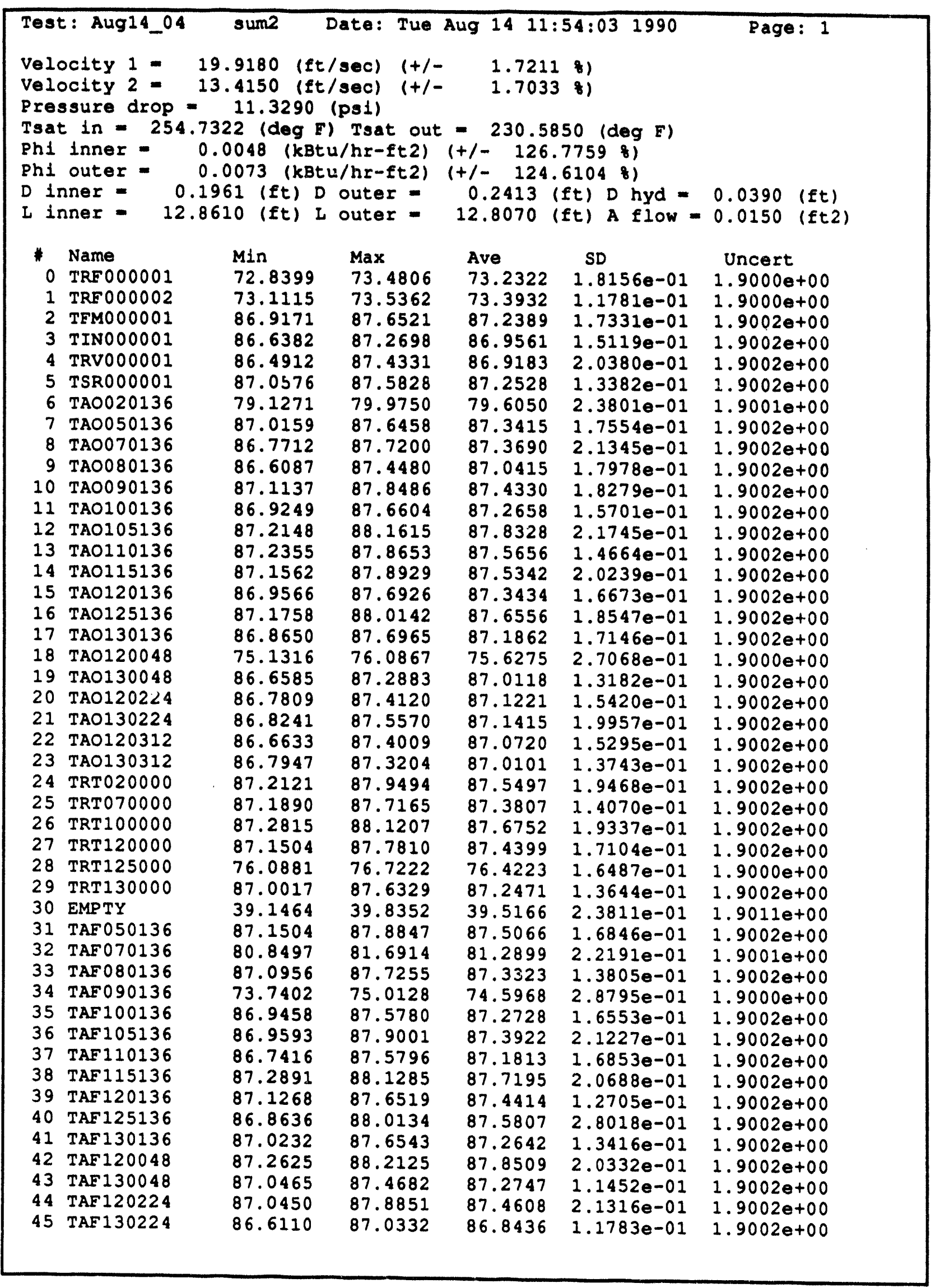




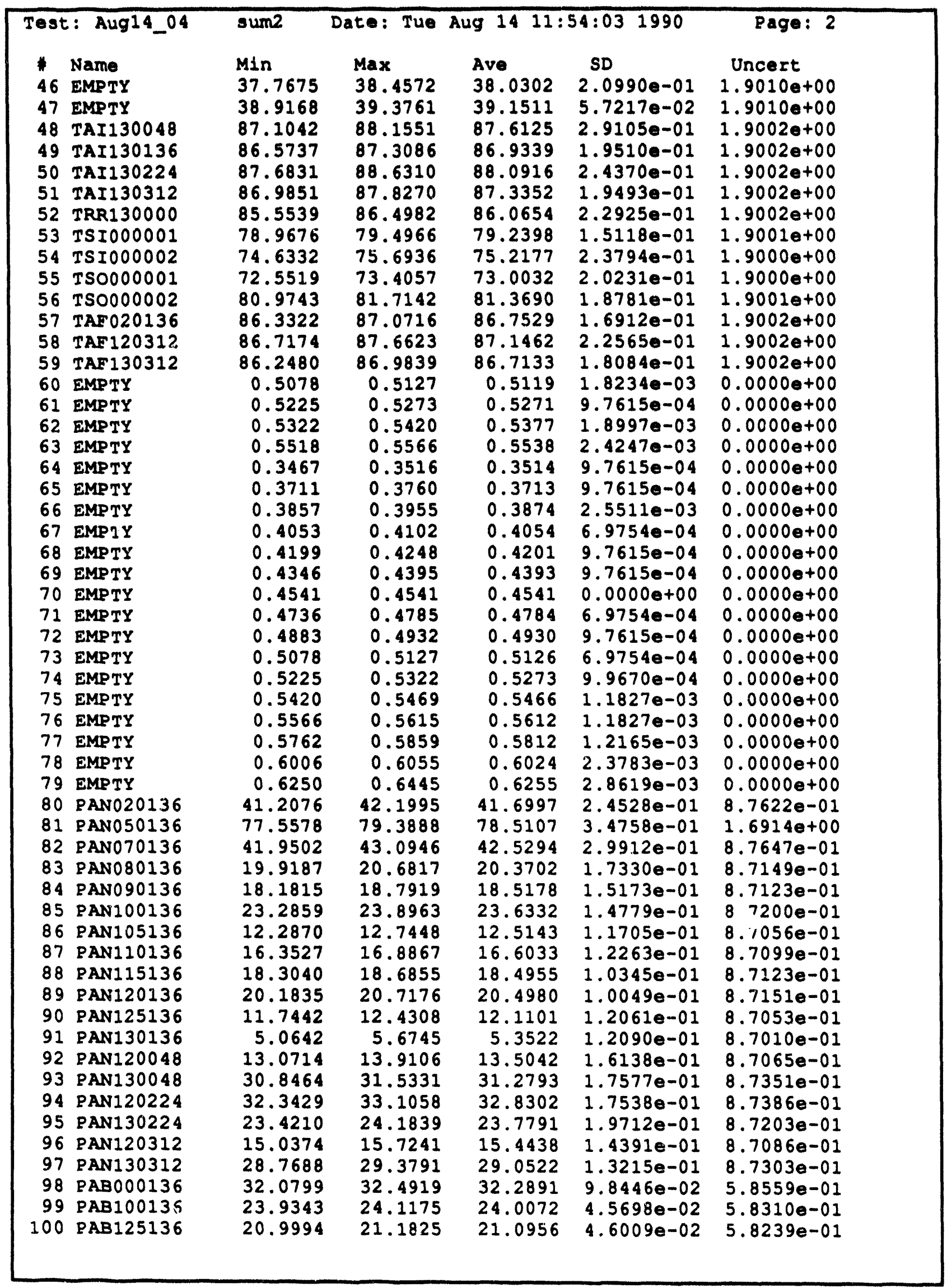




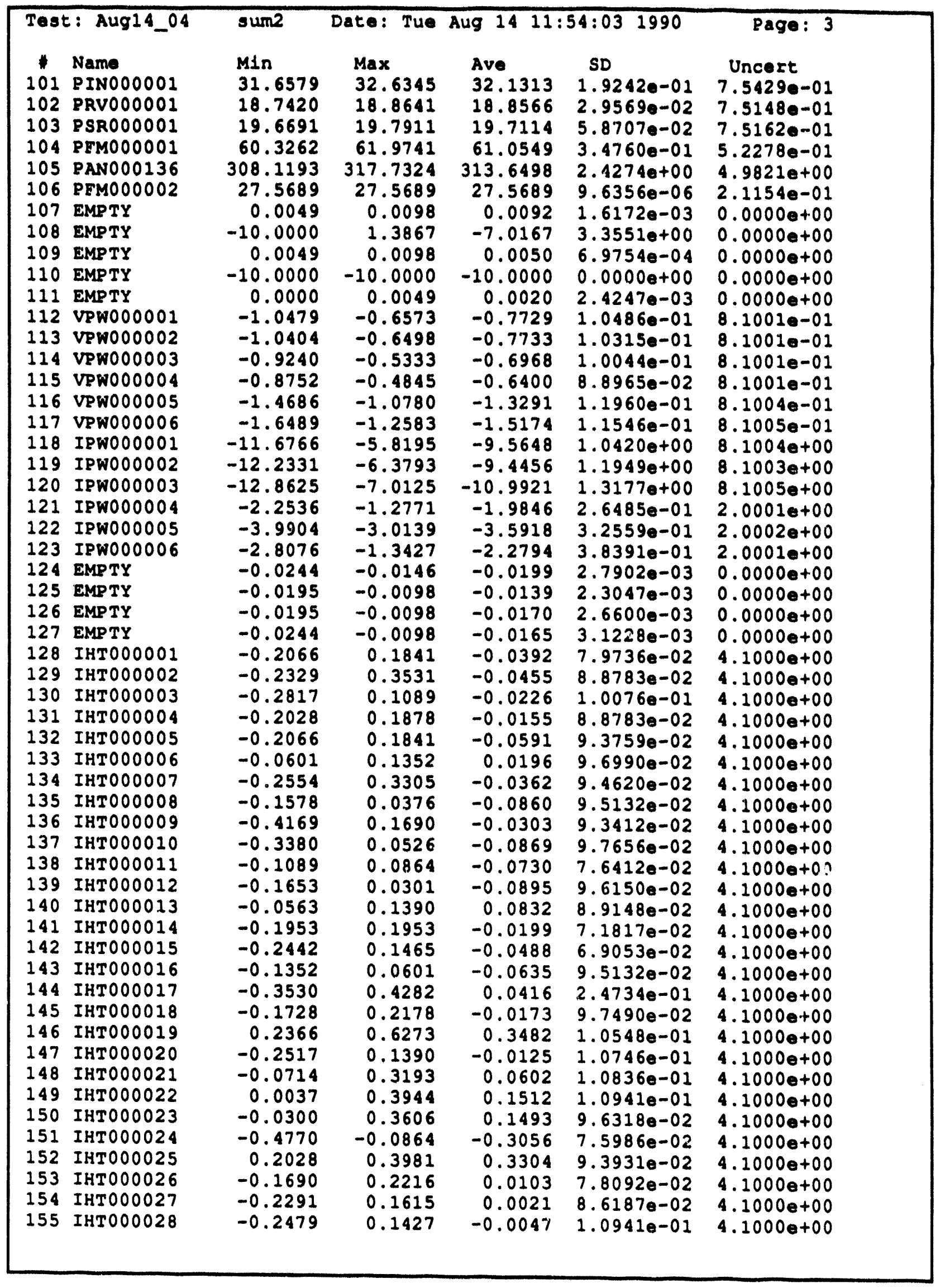




\begin{tabular}{|c|c|c|c|c|c|c|}
\hline $\begin{array}{l}1 \\
156 \\
157 \\
158 \\
159 \\
160 \\
161 \\
162 \\
163\end{array}$ & $\begin{array}{l}\text { Name } \\
\text { IHT000029 } \\
\text { IHTO00030 } \\
\text { IHTO00031 } \\
\text { IHTO00032 } \\
\text { IHTO00033 } \\
\text { IHTO0O0034 } \\
\text { IHTO00035 } \\
\text { IHTO00036 }\end{array}$ & $\begin{array}{l}M 1 n \\
-0.0826 \\
-0.2103 \\
-0.1465 \\
-0.2930 \\
-0.0977 \\
-0.2329 \\
-0.1277 \\
-0.1878\end{array}$ & $\begin{array}{l}\operatorname{Max} \\
0.1127 \\
0.1803 \\
0.2441 \\
0.2930 \\
0.2930 \\
0.1577 \\
0.2629 \\
0.2028\end{array}$ & $\begin{array}{l}\text { Ave } \\
-0.0149 \\
0.0488 \\
-0.0150 \\
-0.0139 \\
-0.0060 \\
-0.0176 \\
0.0477 \\
-0.0483\end{array}$ & $\begin{array}{l}\text { SD } \\
9.3931 e-02 \\
1.0836 e-01 \\
1.1546 e-01 \\
1.1960 e-01 \\
1.0625 e-01 \\
1.3369 e-01 \\
1.0731 e-01 \\
1.1276 e-01\end{array}$ & $\begin{array}{l}\text { Uncert } \\
4.1000 e+00 \\
4.1000 e+00 \\
4.1000 e+00 \\
4.1000 e+00 \\
4.1000 e+00 \\
4.1000 e+00 \\
4.1000 e+00 \\
4.1000 e+00\end{array}$ \\
\hline
\end{tabular}




\begin{tabular}{|c|c|c|c|c|}
\hline \multicolumn{5}{|c|}{$\begin{array}{l}\text { Velocity } 1=0.009100 \text { (ft/sec) } \\
\text { Velocity } 2=0.000100(\mathrm{ft} / \mathrm{sec}) \\
\text { Pressure Drop }=0.021800 \text { (psi) } \\
\text { Tsat in }=0.035690 \text { (deg F) Tsat out }=0.045013 \text { (deg F) } \\
\text { Ph1 1nner }=0.000300 \text { (kBtu/hr-ft2) Ph1 outer }=0.000200 \text { (kBtu/hr-ft2) }\end{array}$} \\
\hline $\begin{array}{l}0 \\
1 \\
2 \\
3 \\
4 \\
5 \\
6 \\
7 \\
8 \\
9 \\
10 \\
11 \\
12 \\
13 \\
14 \\
15 \\
16 \\
17 \\
18 \\
19 \\
20 \\
21 \\
22 \\
23 \\
24 \\
25 \\
26 \\
27 \\
28 \\
29 \\
30 \\
31 \\
32 \\
33 \\
34 \\
35 \\
36 \\
37 \\
38 \\
39 \\
40 \\
41 \\
42 \\
43 \\
44 \\
45\end{array}$ & $\begin{array}{l}\text { NATE } \\
\text { TRFO00001 } \\
\text { TRF000002 } \\
\text { TFMO00001 } \\
\text { TINO00001 } \\
\text { TRV000001 } \\
\text { TSRO00001 } \\
\text { TAO020136 } \\
\text { TAO050136 } \\
\text { TAO070136 } \\
\text { TAO080136 } \\
\text { TAO090136 } \\
\text { TAO100136 } \\
\text { TAO105136 } \\
\text { TAO110136 } \\
\text { TAO115136 } \\
\text { TAO120136 } \\
\text { TAO125136 } \\
\text { TAO130136 } \\
\text { TAO120048 } \\
\text { TAO130048 } \\
\text { TAO120224 } \\
\text { TAO130224 } \\
\text { TAO120312 } \\
\text { TAO130312 } \\
\text { TRT020000 } \\
\text { TRTO70000 } \\
\text { TRT100000 } \\
\text { TRT120000 } \\
\text { TRT125000 } \\
\text { TRT130000 } \\
\text { EMPTY } \\
\text { TAF050136 } \\
\text { TAF070136 } \\
\text { TAF080136 } \\
\text { TAF090136 } \\
\text { TAF100136 } \\
\text { TAF105136 } \\
\text { TAF110136 } \\
\text { TAF115136 } \\
\text { TAF120136 } \\
\text { TAF125136 } \\
\text { TAF130136 } \\
\text { TAF120048 } \\
\text { TAF130048 } \\
\text { TAF120224 } \\
\text { TAF130224 }\end{array}$ & $\begin{array}{c}\text { Abs D1ff } \\
0.0092 \\
0.0014 \\
0.1026 \\
0.0357 \\
0.0333 \\
0.0359 \\
0.0117 \\
0.0646 \\
0.0977 \\
0.0207 \\
0.0418 \\
0.0582 \\
0.0171 \\
0.0352 \\
0.0051 \\
0.0385 \\
0.0281 \\
0.0330 \\
0.0544 \\
0.0035 \\
0.0457 \\
0.0895 \\
0.0591 \\
0.0000 \\
0.0417 \\
0.0405 \\
0.0592 \\
0.0719 \\
0.0882 \\
0.0370 \\
0.0477 \\
0.0473 \\
0.0067 \\
0.0620 \\
0.0341 \\
0.0524 \\
0.0435 \\
0.0122 \\
0.0354 \\
0.0209 \\
0.0150 \\
0.0371 \\
0.0477 \\
0.0249 \\
0.0630 \\
0.0333\end{array}$ & $\begin{array}{l}\text { DDEf } \\
0.0126 \\
0.0019 \\
0.1175 \\
0.0410 \\
0.0383 \\
0.0411 \\
0.0147 \\
0.0739 \\
0.1117 \\
0.0238 \\
0.0478 \\
0.0666 \\
0.0195 \\
0.0402 \\
0.0058 \\
0.0441 \\
0.0320 \\
0.0378 \\
0.0720 \\
0.0040 \\
0.0524 \\
0.1026 \\
0.0678 \\
0.0000 \\
0.0476 \\
0.0463 \\
0.0675 \\
0.0822 \\
0.1153 \\
0.0424 \\
0.1206 \\
0.0540 \\
0.0082 \\
0.0709 \\
0.0457 \\
0.0600 \\
0.0498 \\
0.0140 \\
0.0403 \\
0.0239 \\
0.0171 \\
0.0425 \\
0.0543 \\
0.0285 \\
0.0720 \\
0.0383\end{array}$ & \\
\hline
\end{tabular}




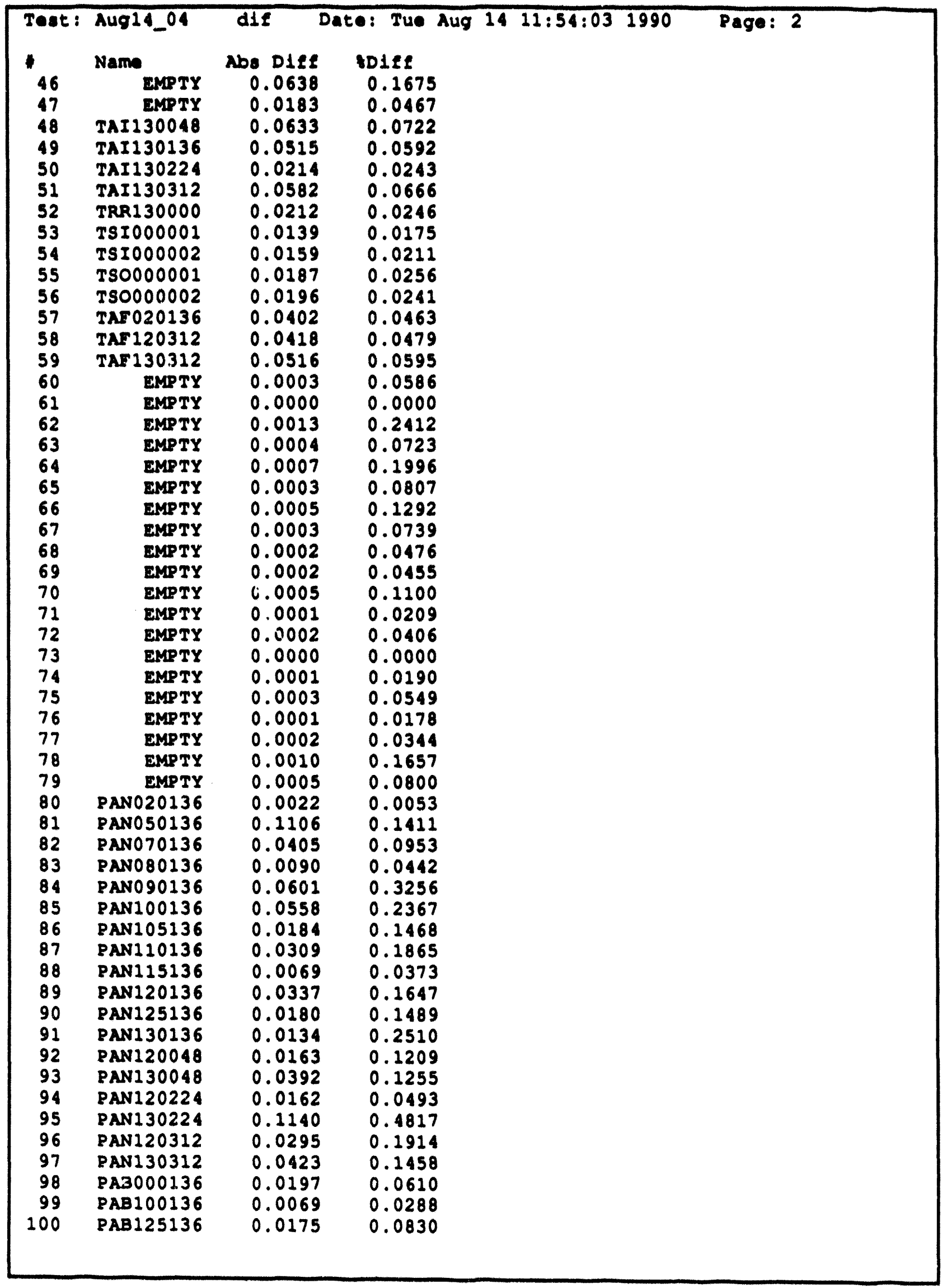




\begin{tabular}{|c|c|c|c|c|}
\hline $\begin{array}{l}\text { Test : } \\
101 \\
102 \\
103 \\
104 \\
105 \\
106 \\
107 \\
108 \\
109 \\
110 \\
111 \\
112 \\
113 \\
114 \\
115 \\
116 \\
117 \\
118 \\
119 \\
120 \\
121 \\
122 \\
123 \\
124 \\
125 \\
126 \\
127 \\
128 \\
129 \\
130 \\
131 \\
132 \\
133 \\
134 \\
135 \\
136 \\
137 \\
138 \\
139 \\
140 \\
141 \\
142 \\
143 \\
144 \\
145 \\
146 \\
147 \\
148 \\
149 \\
150 \\
151 \\
152 \\
153 \\
154 \\
155\end{array}$ & 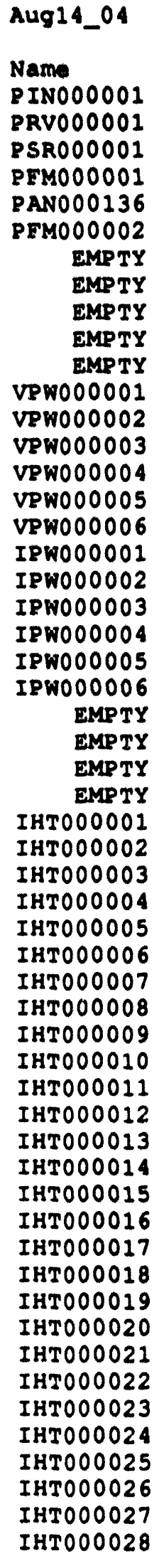 & $\begin{array}{l}\text { d1f D } \\
\text { Ab8 D1f4 } \\
0.0388 \\
0.0075 \\
0.0228 \\
0.0567 \\
0.6050 \\
0.0000 \\
0.0001 \\
0.2740 \\
0.0000 \\
0.0000 \\
0.0000 \\
0.0055 \\
0.0210 \\
0.0424 \\
0.0008 \\
0.0050 \\
0.0091 \\
0.1985 \\
0.1338 \\
0.1154 \\
0.0542 \\
0.0506 \\
0.1066 \\
0.0004 \\
0.0004 \\
0.0005 \\
0.0002 \\
0.0267 \\
0.0155 \\
0.0247 \\
0.0002 \\
0.0029 \\
0.0094 \\
0.0122 \\
0.0249 \\
0.0001 \\
0.0168 \\
0.0164 \\
0.0250 \\
0.0089 \\
0.0043 \\
0.0390 \\
0.0025 \\
0.0272 \\
0.0359 \\
0.0491 \\
0.0360 \\
0.0300 \\
0.0264 \\
0.0114 \\
0.0073 \\
0.0338 \\
0.0043 \\
0.0242 \\
0.0224\end{array}$ & 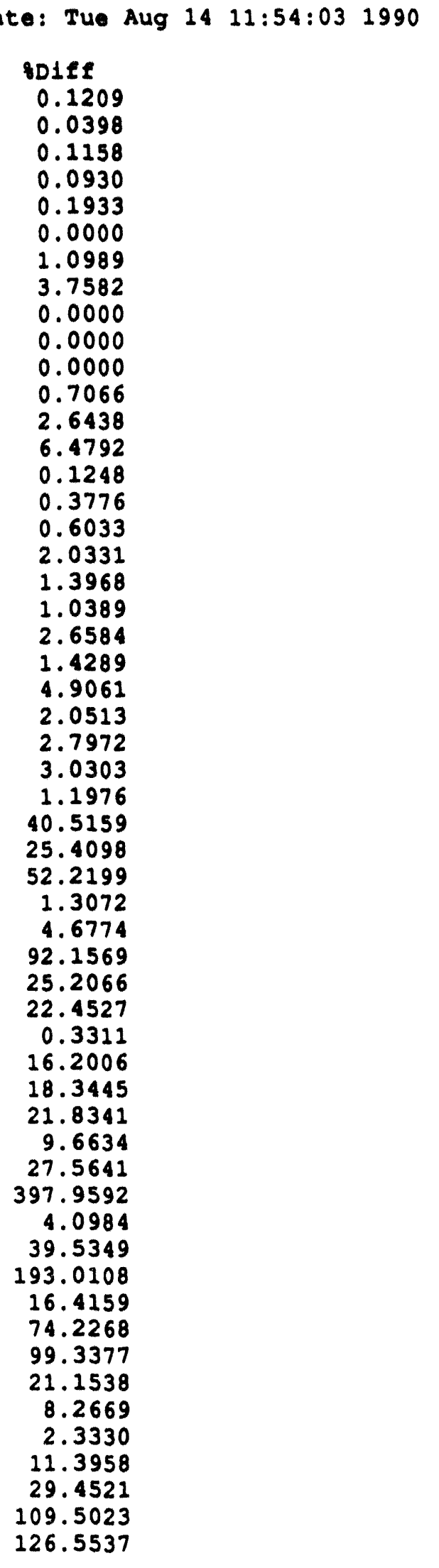 & Page: 3 \\
\hline
\end{tabular}




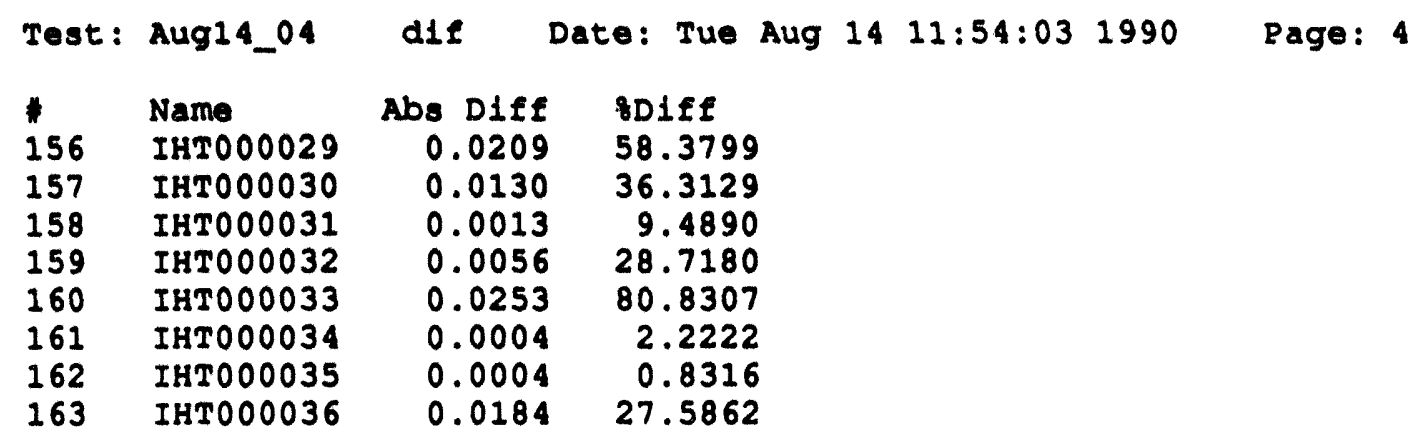




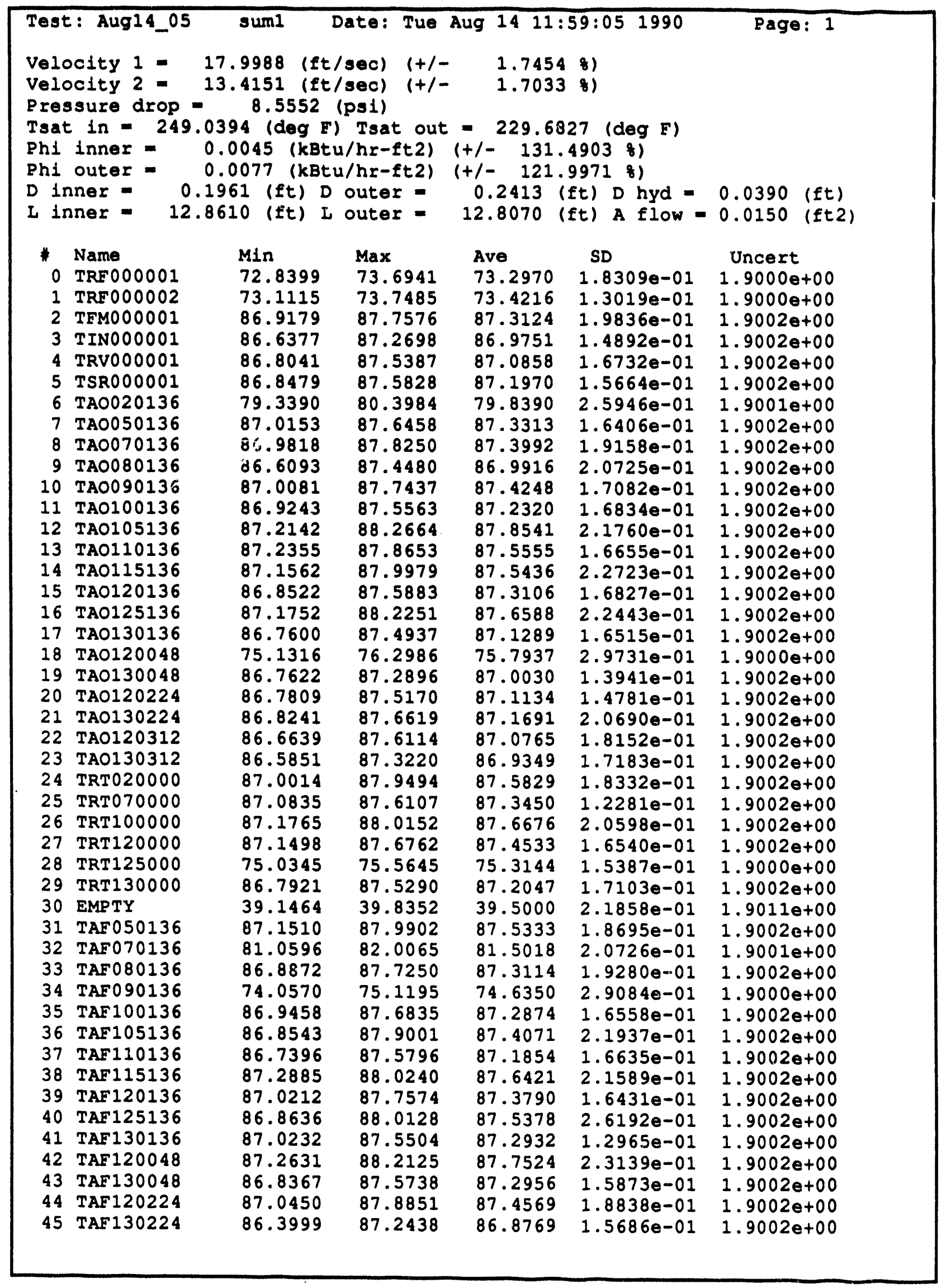




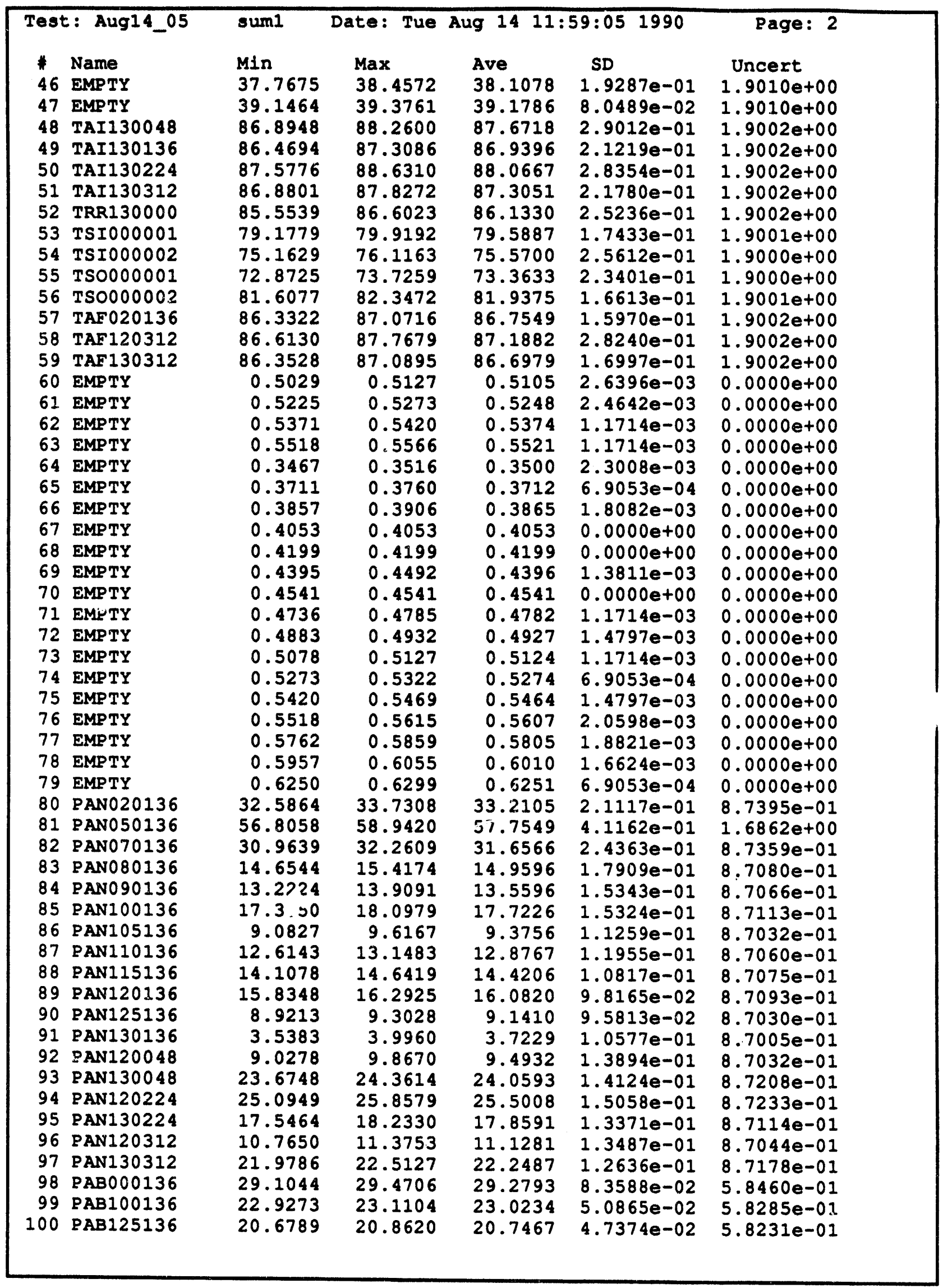




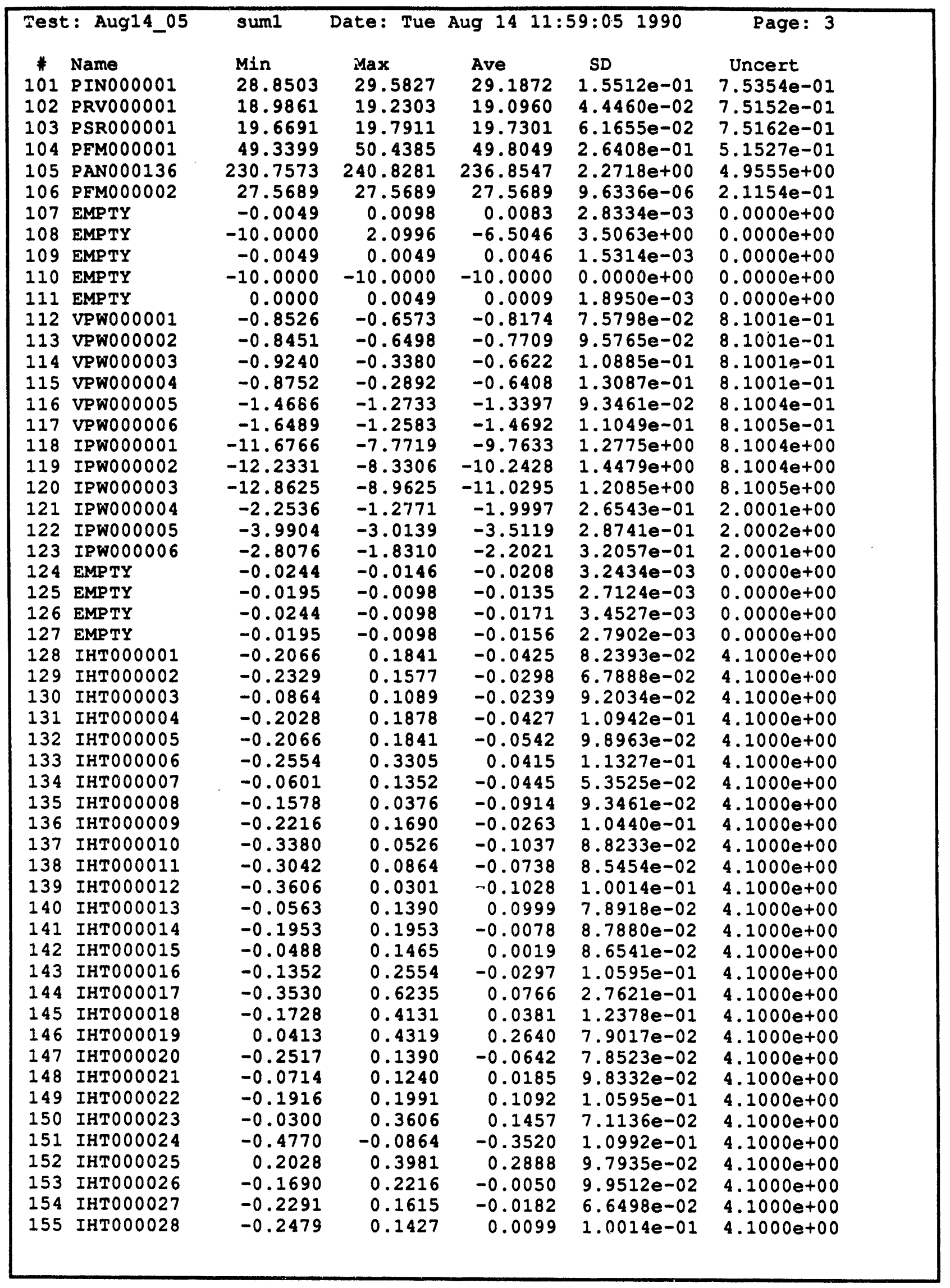




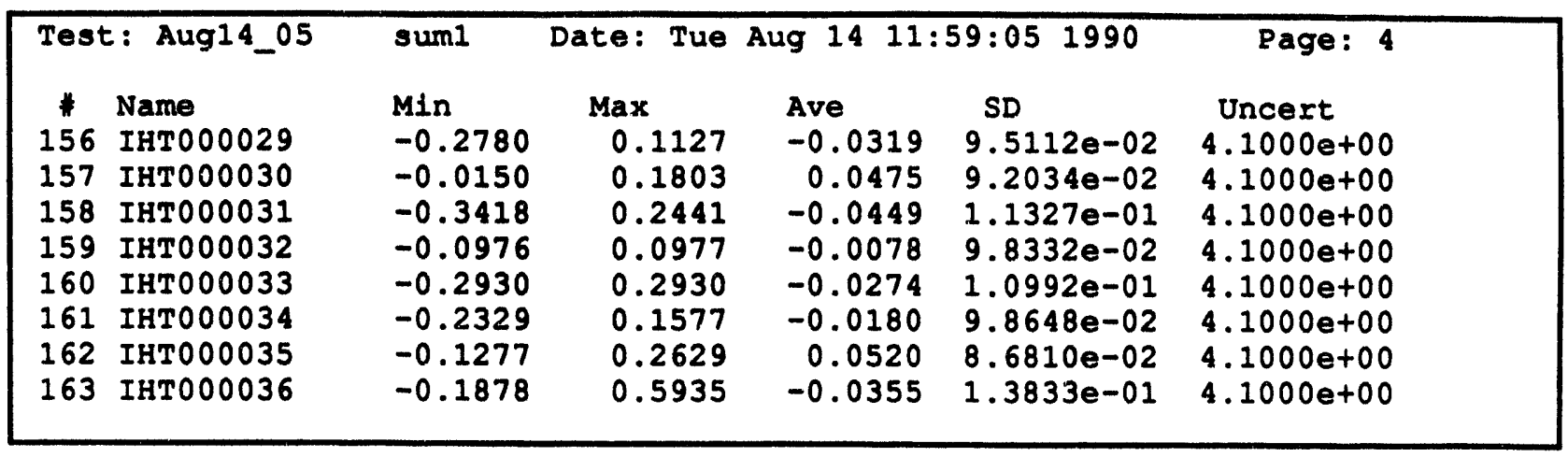




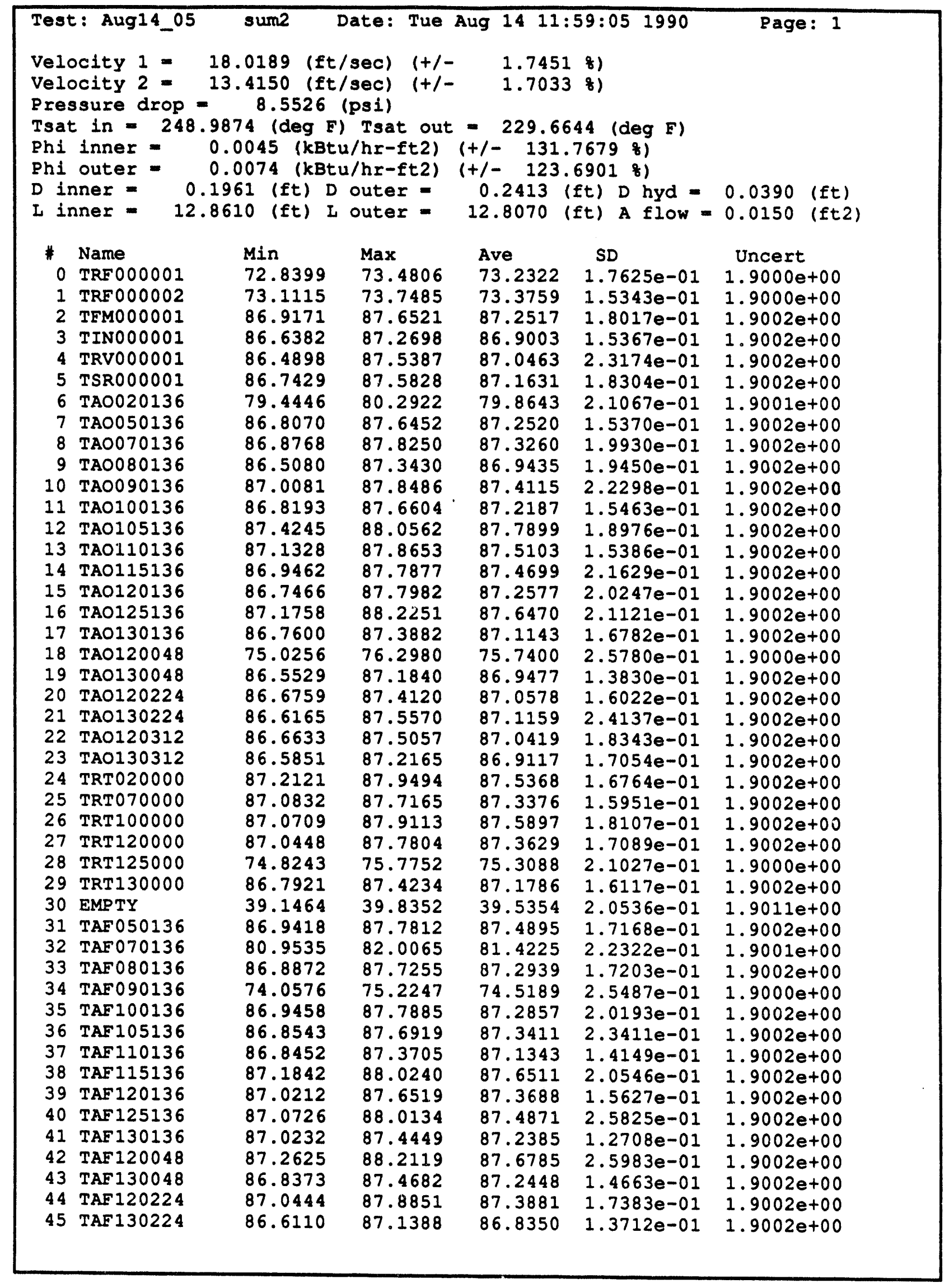




\begin{tabular}{|c|c|c|c|c|c|c|}
\hline \multicolumn{2}{|c|}{ Test: Aug14_05 } & \multicolumn{4}{|c|}{ Date: Tue Aug 14 11:59:05 1990} & Page: 2 \\
\hline \# & Name & Min & $\operatorname{Max}$ & Ave & SD & Uncert \\
\hline 46 & EMPTY & 37.5374 & 38.4572 & 38.0725 & $2.1192 e-01$ & $1.9010 \theta+00$ \\
\hline 47 & EMPTY & 39.1464 & 39.3761 & 39.1699 & $7.0231 e-02$ & $1.9010 e+00$ \\
\hline 48 & TAI130048 & 86.9987 & 88.0496 & 87.5182 & $2.7404 e-01$ & $1.9002 \mathrm{e}+00$ \\
\hline 49 & TAI 130136 & 86.3638 & 87.4136 & 86.8441 & $2.0203 e-01$ & $1.9002 e+00$ \\
\hline 50 & TAI130224 & 87.3676 & 88.5249 & 88.0015 & $3.0496 e-01$ & $1.9002 e+00$ \\
\hline 51 & TAI130312 & 86.8795 & 87.6168 & 87.2408 & $2.0260 \mathrm{e}-01$ & $1.9002 \mathrm{e}+00$ \\
\hline 52 & TRR130000 & 85.7633 & 86.4982 & 86.0910 & $1.9218 \mathrm{e}-01$ & $1.9002 \mathrm{e}+00$ \\
\hline 53 & TSI000001 & 79.1779 & 79.8130 & 79.5193 & $1.7017 e-01$ & $1.9001 e+00$ \\
\hline 54 & TSI000002 & 75.0569 & 75.9049 & 75.5284 & $2.2033 e-01$ & $1.9000 e+00$ \\
\hline 55 & TSO0000001 & 72.8725 & 73.8325 & 73.2907 & $2.1855 e-01$ & $1.9000 e+00$ \\
\hline 56 & TSO000002 & 81.2905 & 82.1362 & 81.8041 & $1.9164 \mathrm{e}-01$ & $1.9001 e+00$ \\
\hline 57 & TAF020136 & 86.3328 & 87.0716 & 86.7141 & $1.6665 e-01$ & $1.9002 \mathrm{e}+00$ \\
\hline 58 & TAF 120312 & 86.5073 & 87.5581 & 87.0606 & $2.7600 e-01$ & $1.9002 e+00$ \\
\hline 59 & TAF 130312 & 86.3522 & 86.9839 & 86.6662 & $1.5700 \mathrm{e}-01$ & $1.9002 e+00$ \\
\hline 60 & EMPTY & 0.5078 & 0.5127 & 0.5095 & $2.3483 e-03$ & $0.0000 e+00$ \\
\hline 61 & EMPTY & 0.5225 & 0.5273 & 0.5249 & $2.4662 e-03$ & $0.0000 e+00$ \\
\hline 62 & EMPTY & 0.5322 & 0.5420 & 0.5371 & $9.9670 e-04$ & $0.0000 e+00$ \\
\hline 63 & EMPTY & 0.5469 & 0.5566 & 0.5519 & $1.2165 e-03$ & $0.0000 e+00$ \\
\hline 64 & EMPTY & 0.3467 & 0.3564 & 0.3511 & $1.7954 e-03$ & $0.0000 e+00$ \\
\hline 65 & EMPTY & 0.3711 & 0.3760 & 0.3712 & $6.9754 e-04$ & $0.0000 e+00$ \\
\hline 66 & EMPTY & 0.3809 & 0.3906 & 0.3867 & $2.2241 e-03$ & $0.0000 e+00$ \\
\hline 67 & EMPTY & 0.4053 & 0.4102 & 0.4056 & $1.1827 e-03$ & $0.0000 e+00$ \\
\hline 68 & EMPTY & 0.4199 & 0.4248 & 0.4200 & $6.9754 e-04$ & $0.0000 e+00$ \\
\hline 69 & EMPTY & 0.4395 & 0.4395 & 0.4395 & $0.0000 e+00$ & $0.0000 \mathrm{e}+00$ \\
\hline 70 & EMPTY & 0.4492 & 0.4541 & 0.4540 & $6.9754 e-04$ & $0.0000 \mathrm{e}+00$ \\
\hline 71 & EMPTY & 0.4736 & 0.4785 & 0.4784 & $6.9754 e-04$ & $0.0000 e+00$ \\
\hline 72 & EMPTY & 0.4883 & 0.4980 & 0.4932 & $9.9670 \mathrm{e}-04$ & $0.0000 e+00$ \\
\hline 73 & 3 EMPTY & 0.5127 & 0.5127 & 0.5127 & $0.0000 e+00$ & $0.0000 e+00$ \\
\hline 74 & 1 EMPTY & 0.5273 & 0.5371 & 0.5275 & $1.3951 \mathrm{e}-03$ & $0.0000 e+00$ \\
\hline 75 & EMPTY & 0.5469 & 0.5469 & 0.5469 & $0.0000 e+00$ & $0.0000 e+00$ \\
\hline 76 & EMPTY & 0.5566 & 0.5615 & 0.5614 & $6.9754 e-04$ & $0.0000 e+00$ \\
\hline 77 & EMPTY & 0.5762 & 0.5811 & 0.5809 & $9.7615 e-04$ & $0.0000 \mathrm{e}+00$ \\
\hline 78 & 3 EMPTY & 0.6006 & 0.6055 & 0.6023 & $2.3483 e-03$ & $0.0000 e+00$ \\
\hline 79 & EMPTY & 0.6250 & 0.6250 & 0.6250 & $0.0000 e+00$ & $0.0000 e+00$ \\
\hline 80 & PAN020136 & 32.6627 & 33.5020 & 33.1812 & $1.8425 e-01$ & $8.7395 e-01$ \\
\hline 81 & PAN050136 & 56.6532 & 58.4843 & 57.7120 & $3.5840 \mathrm{e}-01$ & $1.6862 \mathrm{e}+00$ \\
\hline 82 & 2 PAN070136 & 30.8113 & 32.1083 & 31.6023 & $2.5097 e-01$ & $8.7358 e-01$ \\
\hline 83 & PAN0 80136 & 14.5781 & 15.2648 & 14.9238 & $1.5890 \mathrm{e}-01$ & $8.7080 e-01$ \\
\hline 84 & PAN090136 & 13.3750 & 13.9854 & 13.6132 & $1.2995 \mathrm{e}-01$ & $8.7067 e-01$ \\
\hline 85 & PAN100136 & 17.2587 & 18.2505 & 17.7601 & $1.7960 \mathrm{e}-01$ & $8.7113 e-01$ \\
\hline 86 & PAN105136 & 9.1590 & 9.6167 & 9.3754 & $1.0014 \mathrm{e}-01$ & $8.7032 e-01$ \\
\hline 87 & PAN110136 & 12.6143 & 12.9957 & 12.8525 & $9.1649 \mathrm{e}-02$ & $8.7059 e-01$ \\
\hline 88 & 3 PAN 115136 & 14.1078 & 14.7945 & 14.4628 & $1.1917 \mathrm{e}-01$ & $8.7075 e-01$ \\
\hline 89 & PAN120136 & 15.8348 & 16.2925 & 16.0605 & $1.0897 \mathrm{e}-01$ & $8.7093 e-01$ \\
\hline 90 & PAN125136 & 8.8450 & 9.3028 & 9.1159 & $1.1019 e-01$ & $8.7030 e-01$ \\
\hline 91 & PAN 130136 & 3.4620 & 3.9960 & 3.6909 & $1.3397 e-01$ & $8.7005 e-01$ \\
\hline 92 & PAN120048 & 9.1804 & 9.7144 & 9.4310 & $1.2163 e-01$ & $8.7032 e-01$ \\
\hline 93 & PAN 130048 & 23.6748 & 24.3614 & 24.0158 & $1.4282 \mathrm{e}-01$ & $8.7207 e-01$ \\
\hline 94 & PAN120224 & 25.1712 & 25.7816 & 25.4904 & $1.6457 \mathrm{e}-01$ & $8.7233 e-01$ \\
\hline 95 & PAN 130224 & 16.2494 & 18.2330 & 17.6880 & $3.8051 e-01$ & $8.7112 \mathrm{e}-01$ \\
\hline 96 & PAN120312 & 10.7650 & 11.3753 & 11.1231 & $1.4120 \mathrm{e}-01$ & $8.7044 e-01$ \\
\hline 97 & PAN130312 & 21.9023 & 22.5127 & 22.2449 & $1.5583 \mathrm{e}-01$ & $8.7178 \mathrm{e}-01$ \\
\hline 98 & PAB000136 & 29.0129 & 29.4706 & 29.2530 & $8.0668 \mathrm{e}-02$ & $5.8459 e-01$ \\
\hline 99 & PAB100136 & 22.9273 & 23.1104 & 23.0123 & $4.8553 e-02$ & $5.8285 e-01$ \\
\hline 100 & PAB125136 & 20.6789 & 20.8620 & 20.7397 & $4.3213 e-02$ & $5.8231 e-01$ \\
\hline
\end{tabular}




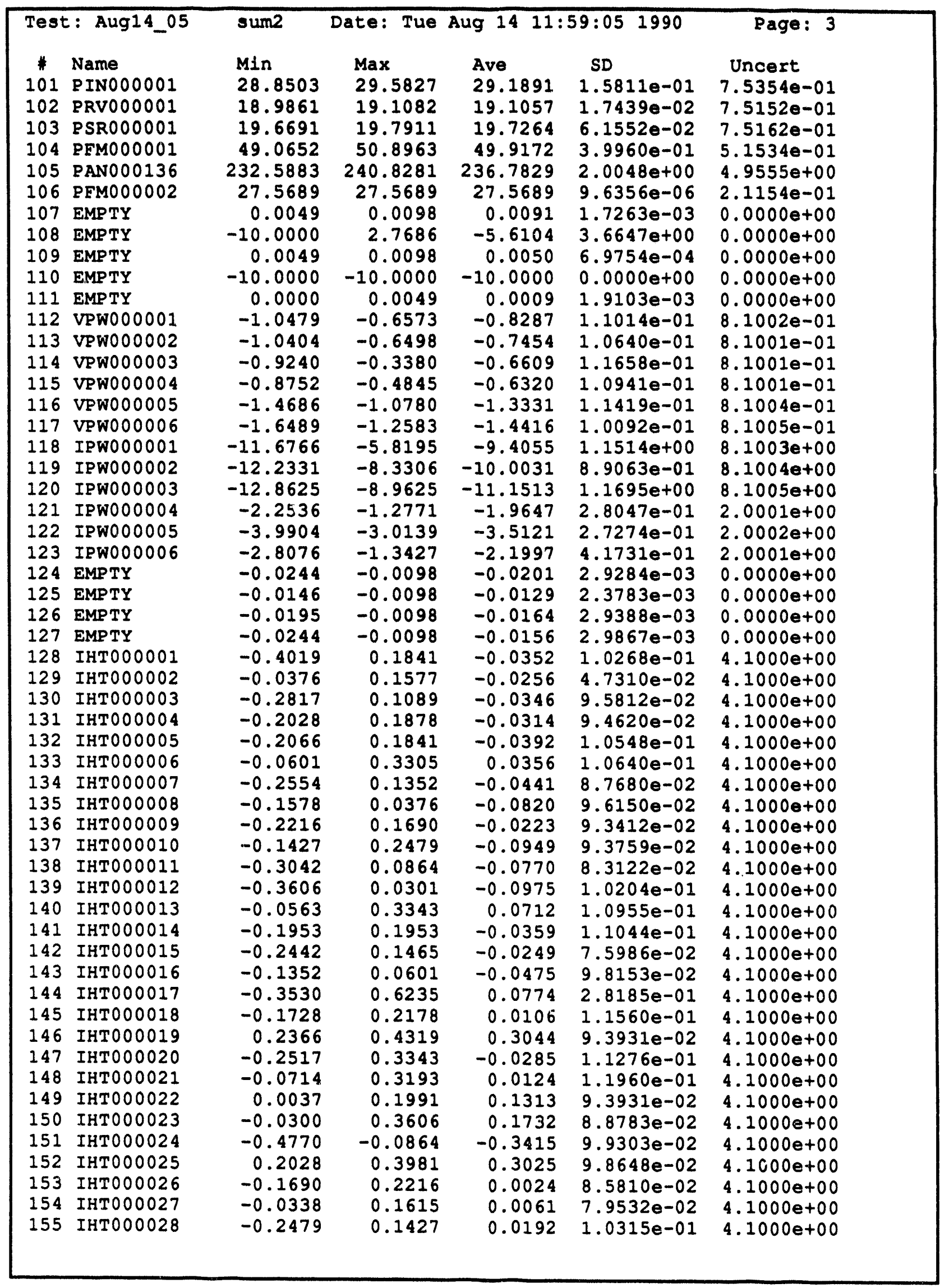




\begin{tabular}{|c|c|c|c|c|c|}
\hline $\begin{array}{cl}\text { * } & \text { Name } \\
156 & \text { IHTO000029 } \\
157 & \text { IHT000030 } \\
158 & \text { IHTO00031 } \\
159 & \text { IHTO00032 } \\
160 & \text { IHTO000033 } \\
161 & \text { IHT000034 } \\
162 & \text { IHT000035 } \\
163 & \text { IHTO00036 }\end{array}$ & $\begin{array}{l}\text { Min } \\
-0.2780 \\
-0.2103 \\
-0.1465 \\
-0.0976 \\
-0.0977 \\
-0.2329 \\
-0.1277 \\
-0.1878\end{array}$ & $\begin{array}{l}\text { Max } \\
0.1127 \\
0.3756 \\
0.2411 \\
0.2930 \\
0.0976 \\
0.1577 \\
0.2629 \\
0.2028\end{array}$ & $\begin{array}{l}\text { Ave } \\
-0.0348 \\
0.0448 \\
-0.0269 \\
-0.0139 \\
-0.0259 \\
-0.0097 \\
0.0517 \\
-0.0523\end{array}$ & $\begin{array}{l}\text { SD } \\
9.3759 \theta-02 \\
1.2095 e-01 \\
1.3112 \theta-01 \\
1.0548 \theta-01 \\
9.5132 \theta-02 \\
1.0548 e-01 \\
1.0424 e-01 \\
1.2095 e-01\end{array}$ & $\begin{array}{l}\text { Uncert } \\
4.1000 e+00 \\
4.1000 e+00 \\
4.1000 e+00 \\
4.1000 e+00 \\
4.1000 e+00 \\
4.1000 e+00 \\
4.1000 e+00 \\
4.1000 e+00\end{array}$ \\
\hline
\end{tabular}




\begin{tabular}{|c|c|c|c|c|}
\hline \multicolumn{5}{|c|}{$\begin{array}{l}\text { Velocity } 1=0.020100(\mathrm{Et} / \mathrm{sec}) \\
\text { Velocity } 2=0.000100(\mathrm{Et} / \mathrm{sec}) \\
\text { Pressure Drop }=0.002600(\mathrm{psi}) \\
\text { Tsat in }=0.052002 \text { (deg F) Tsat out }-0.018295 \text { (deg F) }\end{array}$} \\
\hline $\begin{array}{r}0 \\
1 \\
2 \\
3 \\
4 \\
5 \\
6 \\
7 \\
8 \\
9 \\
9 \\
10 \\
11 \\
12 \\
13 \\
14 \\
15 \\
16 \\
17 \\
18 \\
19 \\
20 \\
21 \\
22 \\
23 \\
24 \\
25 \\
26 \\
27 \\
28 \\
29 \\
30 \\
31 \\
32 \\
33 \\
34 \\
35 \\
36 \\
37 \\
38 \\
39 \\
40 \\
41 \\
42 \\
43 \\
44 \\
45\end{array}$ & $\begin{array}{l}\text { Name } \\
\text { TRF000001 } \\
\text { TRF000002 } \\
\text { TFMO00001 } \\
\text { TIN000001 } \\
\text { TRV000001 } \\
\text { TSR000001 } \\
\text { TAO020136 } \\
\text { TAO050136 } \\
\text { TAO070136 } \\
\text { TAO080136 } \\
\text { TAO090136 } \\
\text { TAO100136 } \\
\text { TAO105136 } \\
\text { TAO110136 } \\
\text { TAO115136 } \\
\text { TAO120136 } \\
\text { TAO125136 } \\
\text { TAO130136 } \\
\text { TAO120048 } \\
\text { TAO130048 } \\
\text { TAO120224 } \\
\text { TAO130224 } \\
\text { TAO120312 } \\
\text { TAO130312 } \\
\text { TRT020000 } \\
\text { TRT070000 } \\
\text { TRT100000 } \\
\text { TRT120000 } \\
\text { TRT125000 } \\
\text { TRT130000 } \\
\text { EMPTY } \\
\text { TAF050136 } \\
\text { TAF070136 } \\
\text { TAF080136 } \\
\text { TAF090136 } \\
\text { TAF100136 } \\
\text { TAF105136 } \\
\text { TAF110136 } \\
\text { TAF115136 } \\
\text { TAF120136 } \\
\text { TAF125136 } \\
\text { TAF130136 } \\
\text { TAF120048 } \\
\text { TAF130048 } \\
\text { TAF120224 } \\
\text { TAF130224 }\end{array}$ & $\begin{array}{l}\text { Abs Diff } \\
0.0648 \\
0.0457 \\
0.0607 \\
0.0748 \\
0.0395 \\
0.0339 \\
0.0253 \\
0.0793 \\
0.0732 \\
0.0481 \\
0.0133 \\
0.0133 \\
0.0642 \\
0.0452 \\
0.0737 \\
0.0529 \\
0.0118 \\
0.0146 \\
0.0537 \\
0.0553 \\
0.0556 \\
0.0532 \\
0.0346 \\
0.0232 \\
0.0461 \\
0.0074 \\
0.0779 \\
0.0904 \\
0.0056 \\
0.0261 \\
0.0354 \\
0.0438 \\
0.0793 \\
0.0175 \\
0.1161 \\
0.0017 \\
0.0660 \\
0.0511 \\
0.0090 \\
0.0102 \\
0.0507 \\
0.0547 \\
0.0739 \\
0.0508 \\
0.0688 \\
0.0419\end{array}$ & $\begin{array}{l}8 D \text { Dff } \\
0.0884 \\
0.0622 \\
0.0695 \\
0.0860 \\
0.0454 \\
0.0389 \\
0.0317 \\
0.0908 \\
0.0838 \\
0.0553 \\
0.0152 \\
0.0153 \\
0.0731 \\
0.0516 \\
0.0842 \\
0.0606 \\
0.0135 \\
0.0168 \\
0.0709 \\
0.0636 \\
0.0638 \\
0.0610 \\
0.0397 \\
0.0267 \\
0.0526 \\
0.0085 \\
0.0889 \\
0.1034 \\
0.0074 \\
0.0299 \\
0.0896 \\
0.0500 \\
0.0973 \\
0.0200 \\
0.1556 \\
0.0019 \\
0.0755 \\
0.0586 \\
0.0103 \\
0.0117 \\
0.0579 \\
0.0627 \\
0.0842 \\
0.0582 \\
0.0787 \\
0.0482\end{array}$ & \\
\hline
\end{tabular}




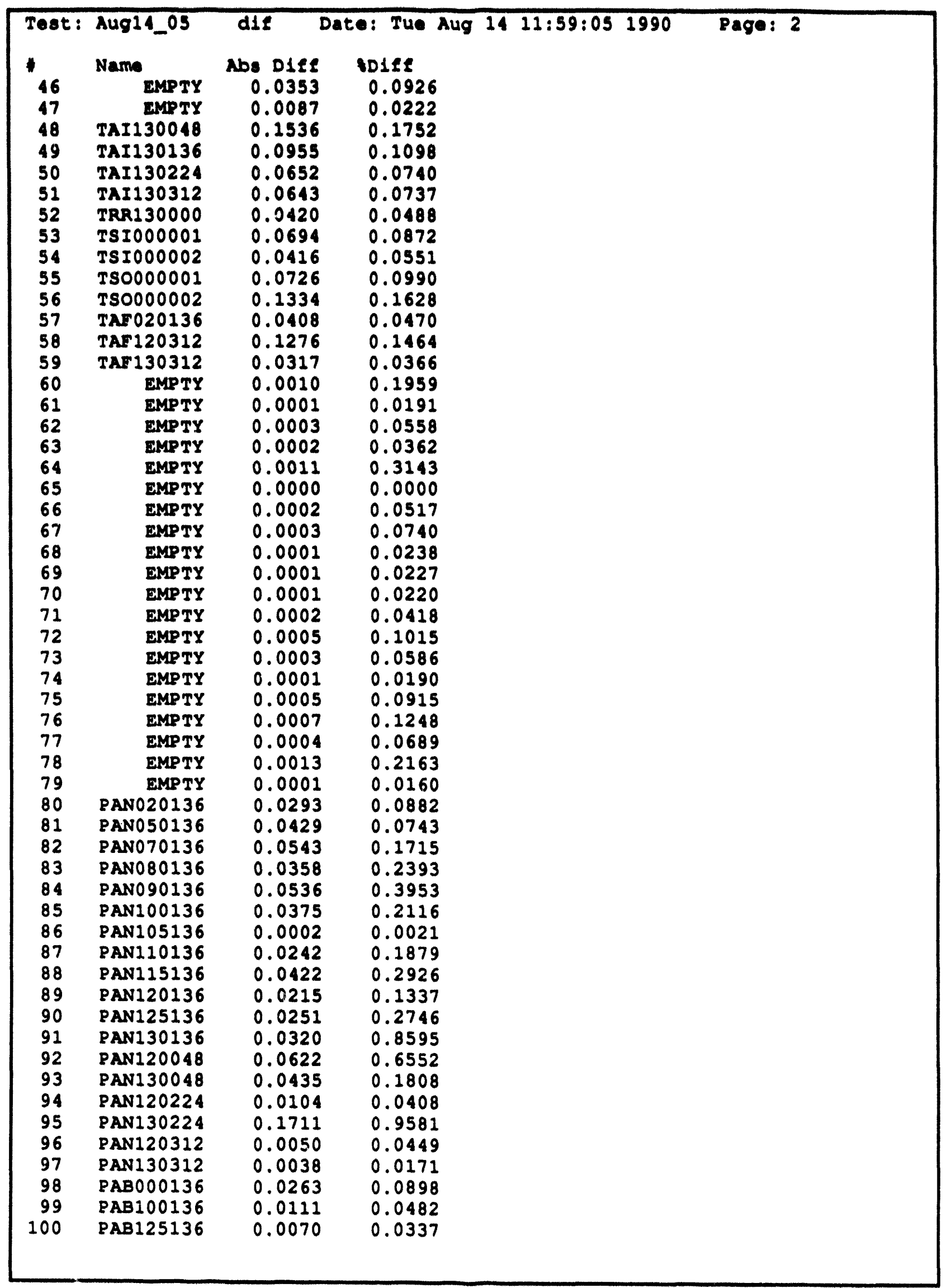




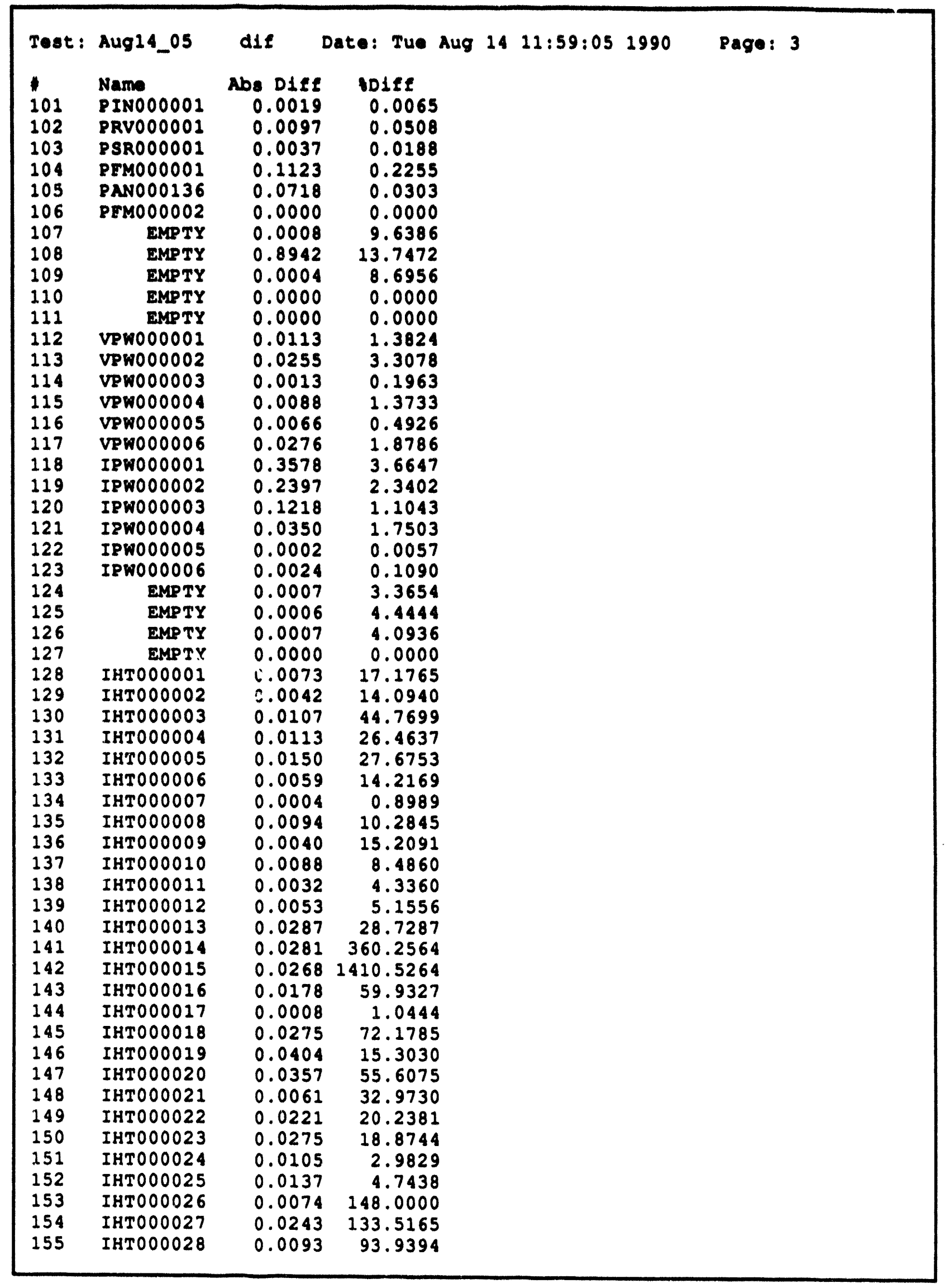




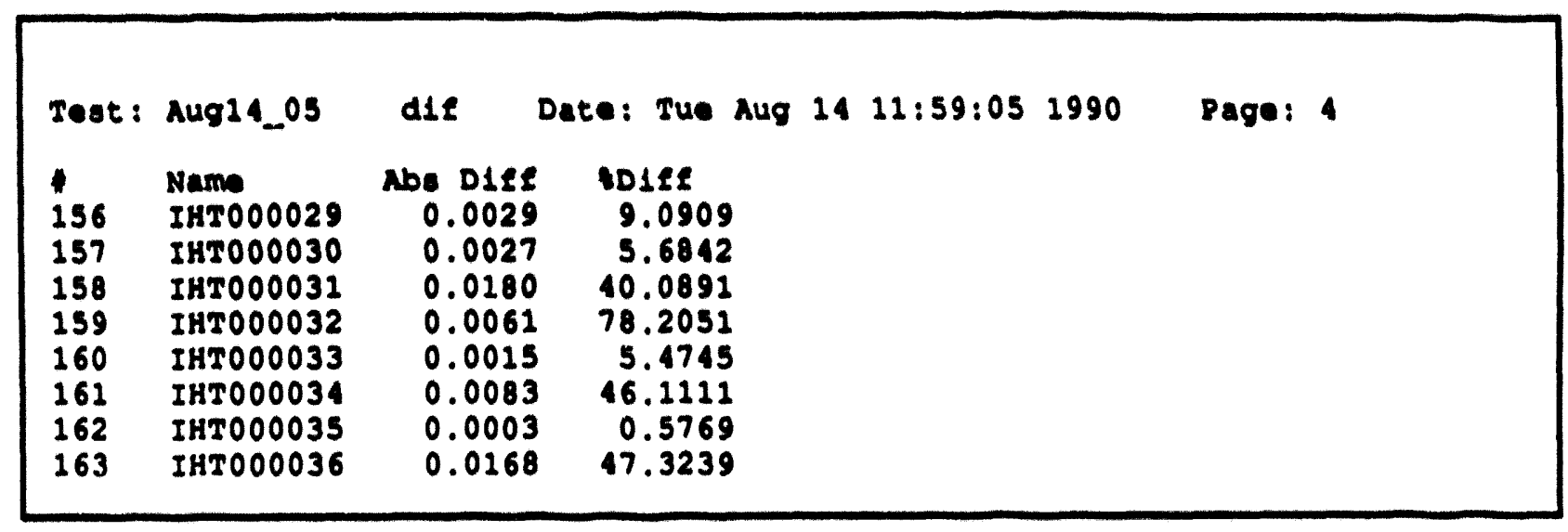




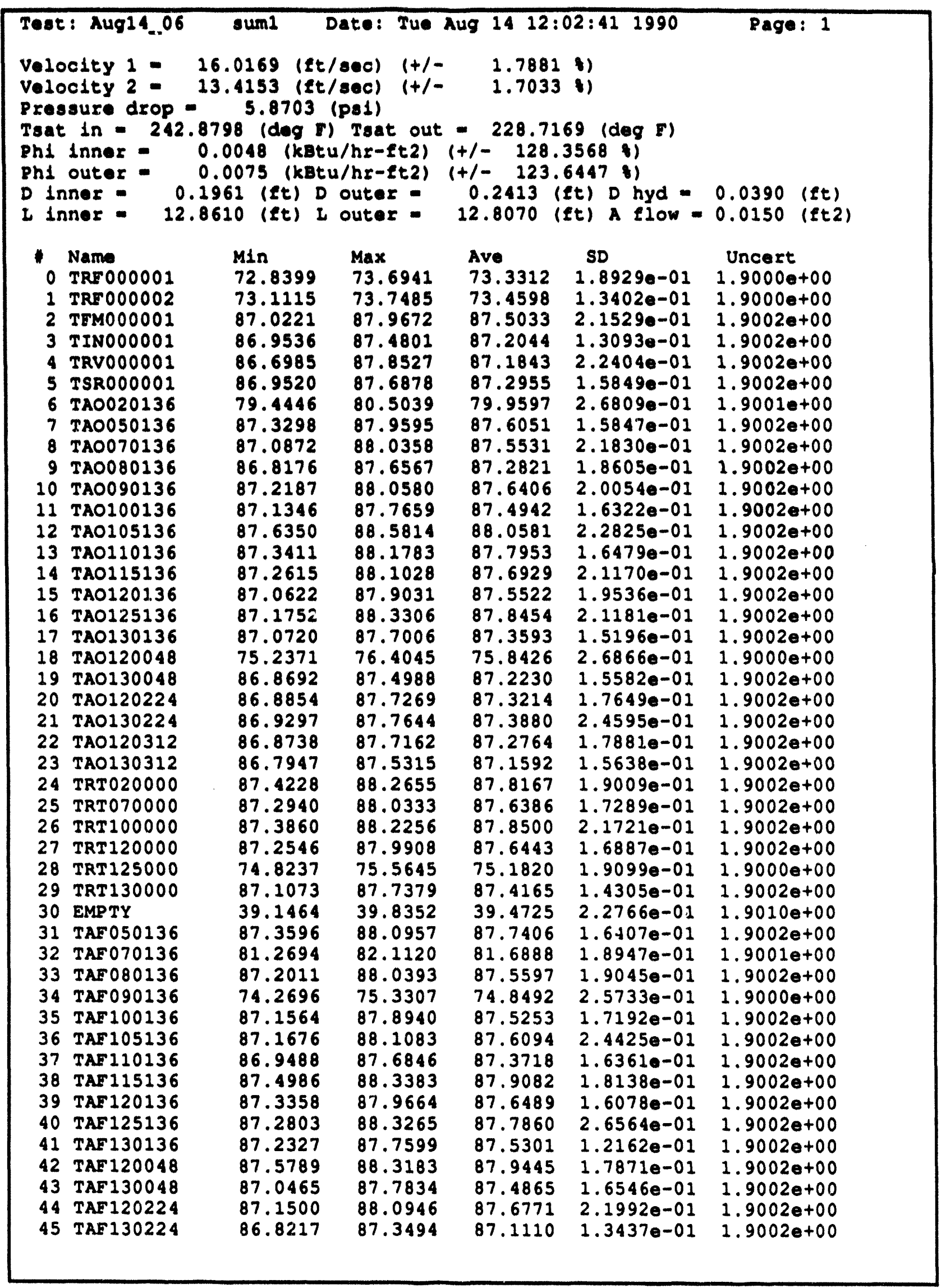




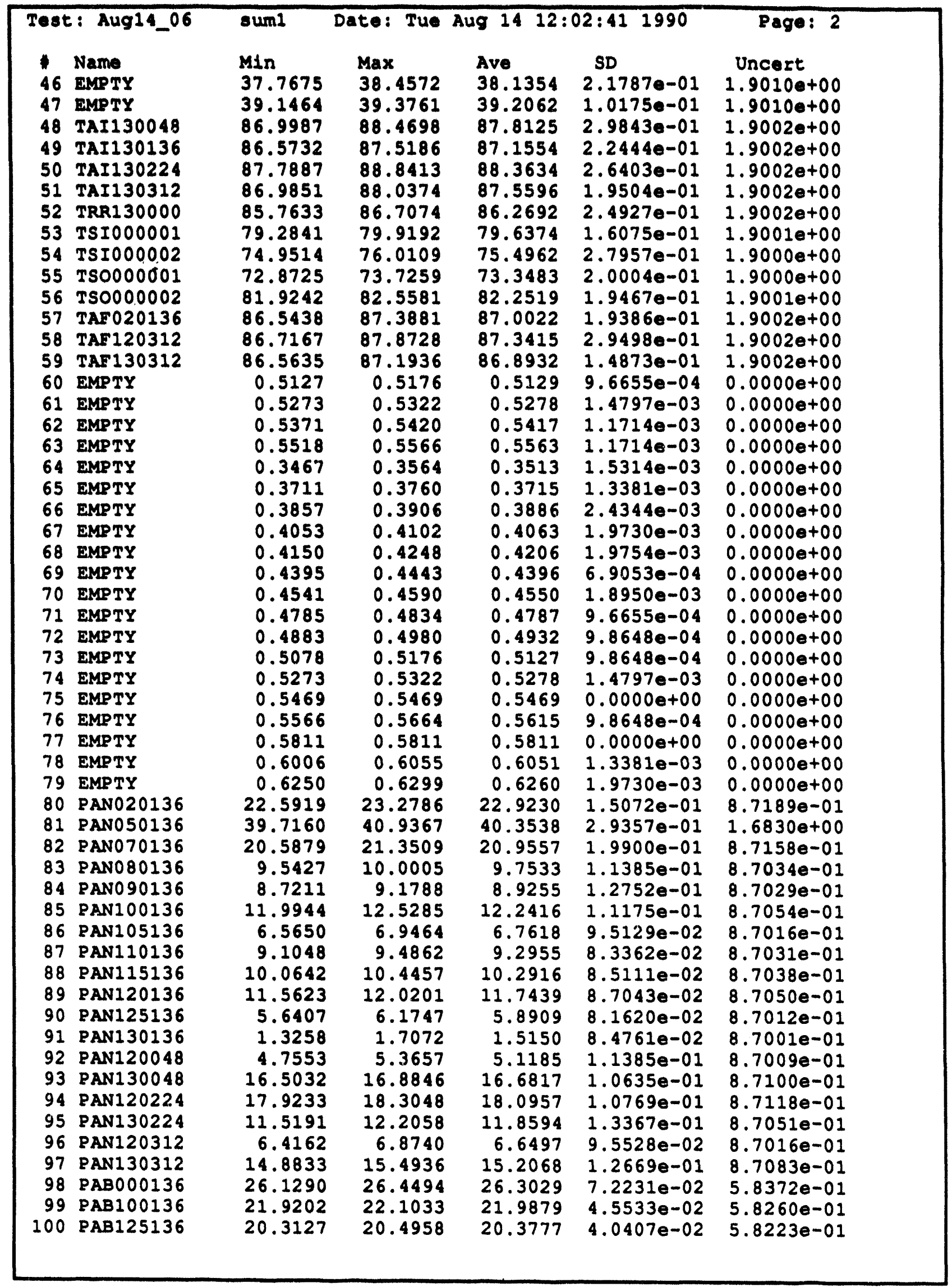




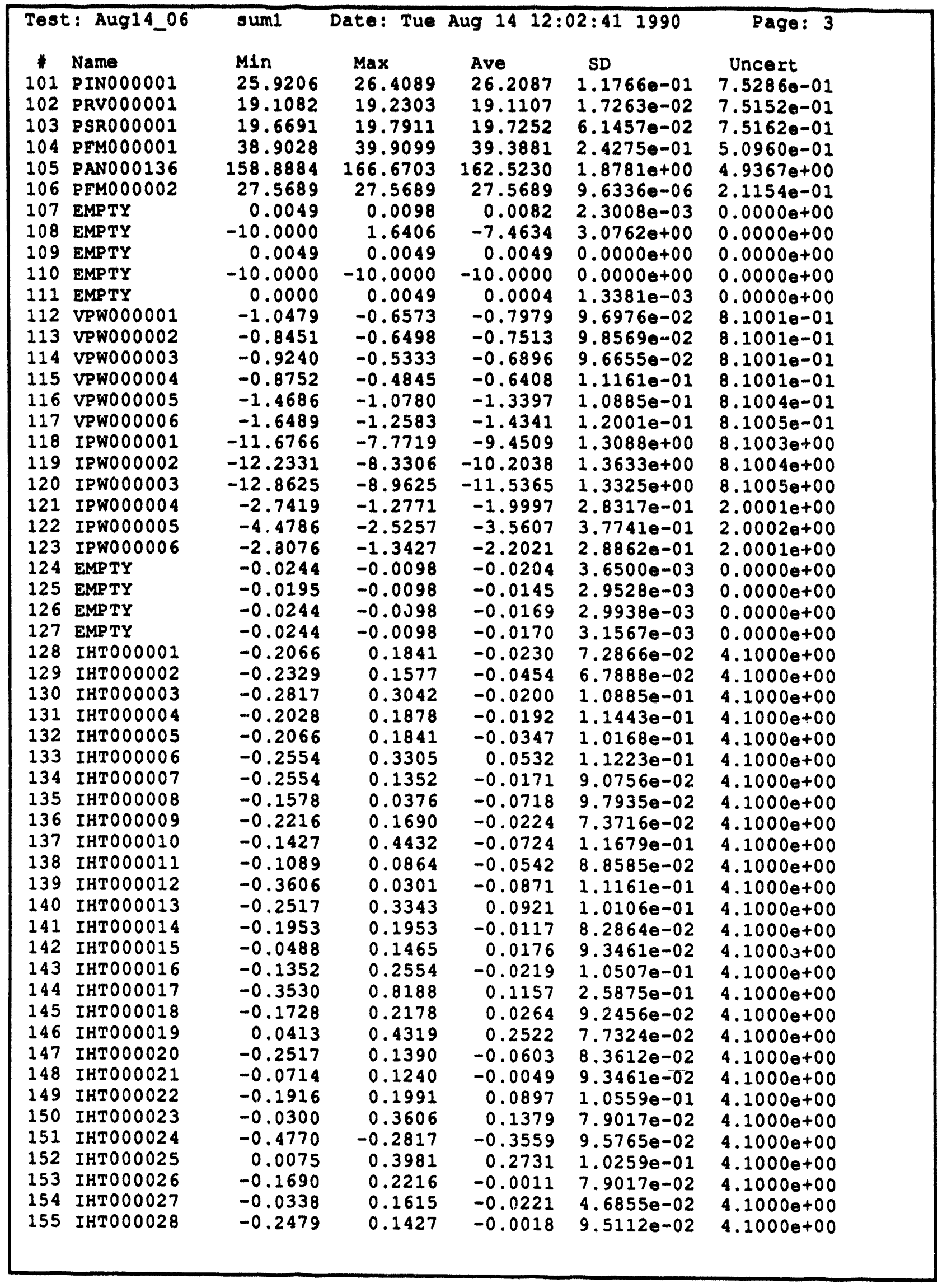




\begin{tabular}{|c|c|c|c|c|c|c|}
\hline \multicolumn{2}{|c|}{ Test: Aug14_06 } & suml & ate: Tue & ug 141 & $: 411990$ & Page: 4 \\
\hline $\begin{array}{c}* \\
156 \\
157 \\
158 \\
159 \\
160 \\
161 \\
162 \\
163\end{array}$ & $\begin{array}{l}\text { Name } \\
\text { IHTO00029 } \\
\text { IHTO00030 } \\
\text { IHTO00031 } \\
\text { IHTO00032 } \\
\text { IHTO00033 } \\
\text { IHTO00034 } \\
\text { IHTO00035 } \\
\text { IHTO00036 }\end{array}$ & $\begin{array}{l}\text { Min } \\
-0.0826 \\
-0.0150 \\
-0.1465 \\
-0.0976 \\
-0.0977 \\
-0.2329 \\
-0.1277 \\
-0.1878\end{array}$ & $\begin{array}{l}\text { Max } \\
0.1127 \\
0.1803 \\
0.2441 \\
0.2930 \\
0.2930 \\
0.1577 \\
0.2629 \\
0.0075\end{array}$ & $\begin{array}{l}\text { Ave } \\
-0.0475 \\
0.0319 \\
-0.0488 \\
-0.0390 \\
-0.0547 \\
-0.0024 \\
0.0481 \\
-0.0511\end{array}$ & $\begin{array}{l}\text { SD } \\
7.5798 e-02 \\
8.4262 e-02 \\
1.0625 e-01 \\
1.0625 e-01 \\
9.0756 e-02 \\
9.4125 e-02 \\
9.0412 e-02 \\
9.0412 e-02\end{array}$ & $\begin{array}{l}\text { Uncert } \\
4.1000 \mathrm{e}+00 \\
4.1000 \mathrm{e}+00 \\
4.1000 \mathrm{e}+00 \\
4.1000 \mathrm{e}+00 \\
4.1000 \mathrm{e}+00 \\
4.1000 \mathrm{e}+00 \\
4.1000 \mathrm{e}+00 \\
4.1000 \mathrm{e}+00\end{array}$ \\
\hline
\end{tabular}




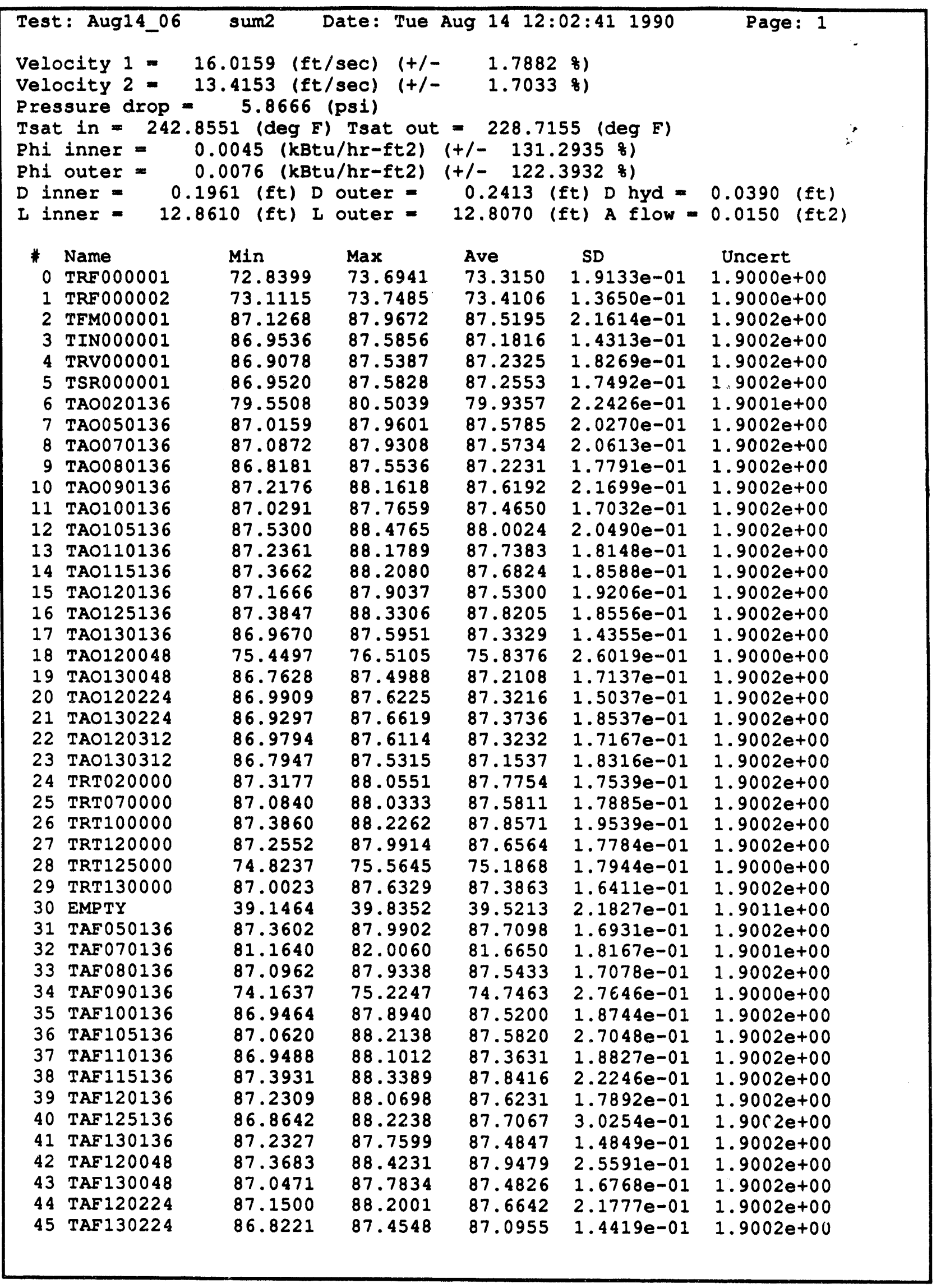




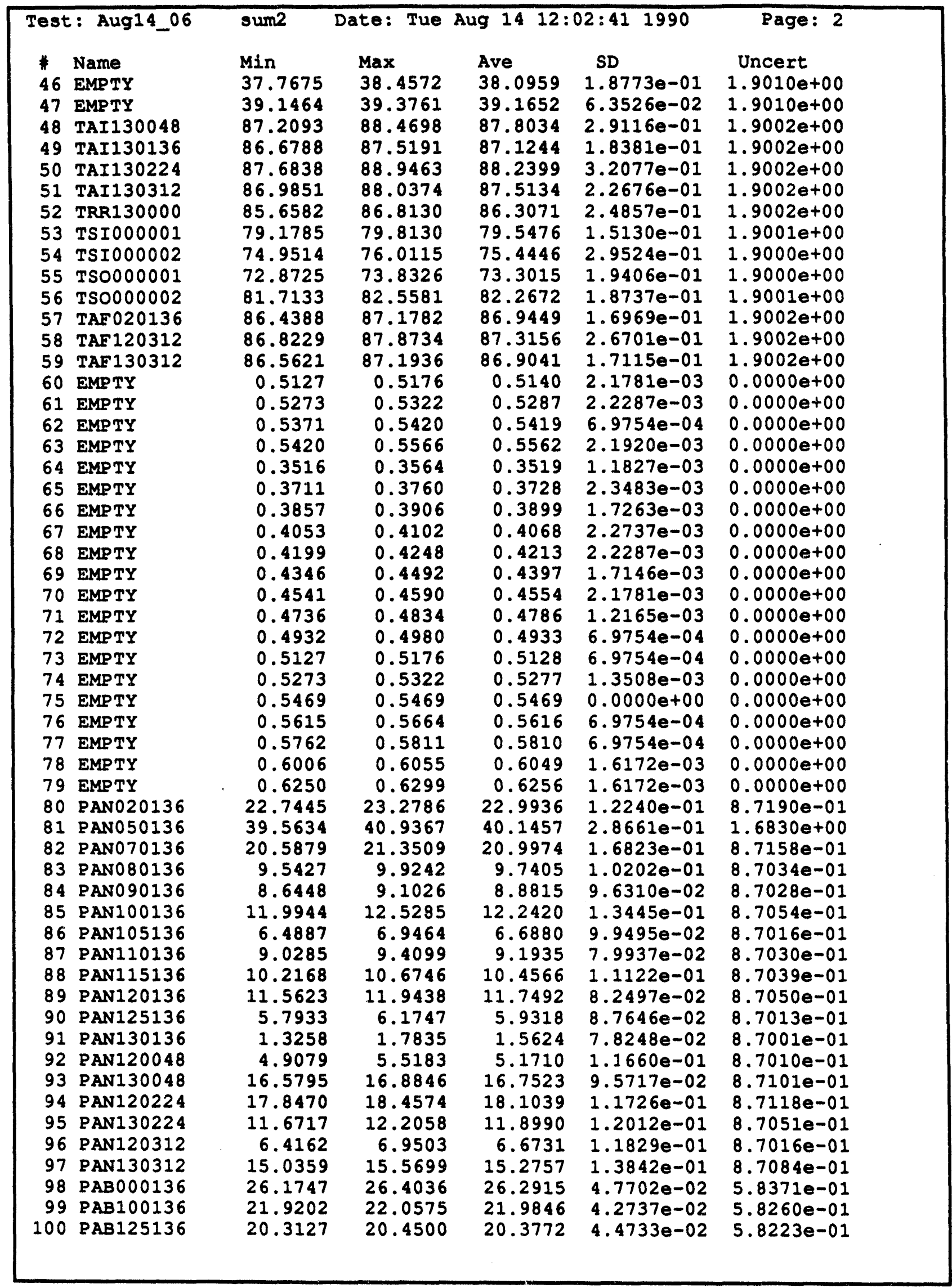




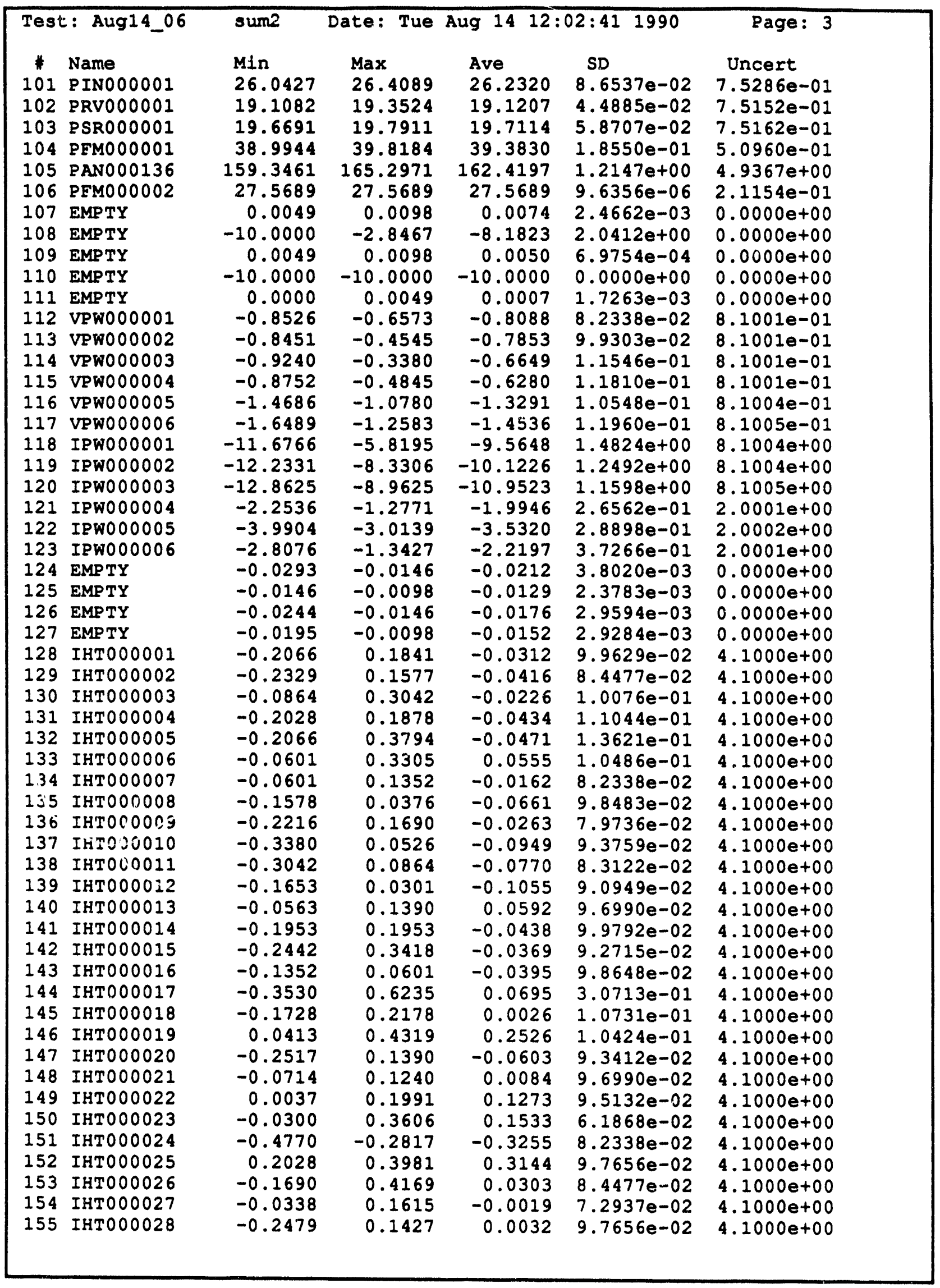




\begin{tabular}{|c|c|c|c|c|c|c|}
\hline \multicolumn{2}{|c|}{ Test: Aug14_06 } & sum2 & ate: Tue & ug 1412 & $02: 411990$ & Page: 4 \\
\hline $\begin{array}{l}* \\
156 \\
157 \\
158 \\
159 \\
160 \\
161 \\
162 \\
163\end{array}$ & $\begin{array}{l}\text { Name } \\
\text { IHTO00029 } \\
\text { IHTO00030 } \\
\text { IHTO00031 } \\
\text { IHTO00032 } \\
\text { IHTO00033 } \\
\text { IHTO00034 } \\
\text { IHTO00035 } \\
\text { IHTO00036 }\end{array}$ & $\begin{array}{l}\text { Min } \\
-0.2780 \\
-0.2103 \\
-0.1465 \\
-0.0976 \\
-0.0977 \\
-0.2329 \\
-0.1277 \\
-0.1878\end{array}$ & $\begin{array}{l}\text { Max } \\
0.1127 \\
0.1803 \\
0.0488 \\
0.0977 \\
0.0976 \\
0.1577 \\
0.2629 \\
0.2028\end{array}$ & $\begin{array}{l}\text { Ave } \\
-0.0707 \\
0.0089 \\
-0.0628 \\
-0.0418 \\
-0.0498 \\
-0.0336 \\
0.0317 \\
-0.0602\end{array}$ & $\begin{array}{l}\text { SD } \\
6.1868 e-02 \\
7.5986 e-02 \\
9.7656 e-02 \\
8.9148 e-02 \\
8.4860 e-02 \\
7.4477 e-02 \\
9.4962 e-02 \\
1.0204 e-01\end{array}$ & $\begin{array}{c}\text { Uncert } \\
4.1000 \mathrm{e}+00 \\
4.1000 \mathrm{e}+00 \\
4.1000 \mathrm{e}+00 \\
4.1000 \mathrm{e}+00 \\
4.1000 \mathrm{e}+00 \\
4.1000 \mathrm{e}+00 \\
4.1000 \mathrm{e}+00 \\
4.1000 \mathrm{e}+00\end{array}$ \\
\hline
\end{tabular}




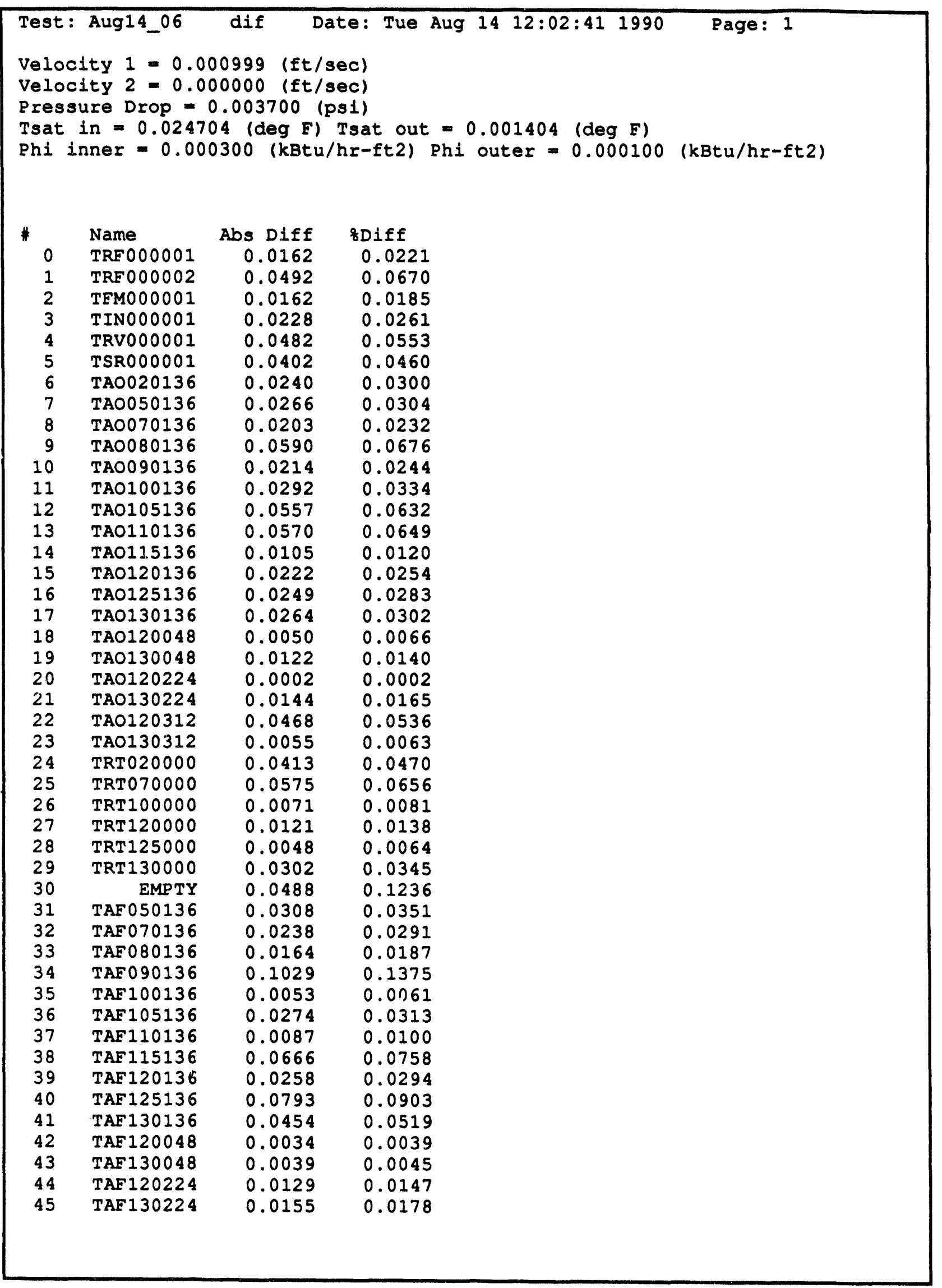




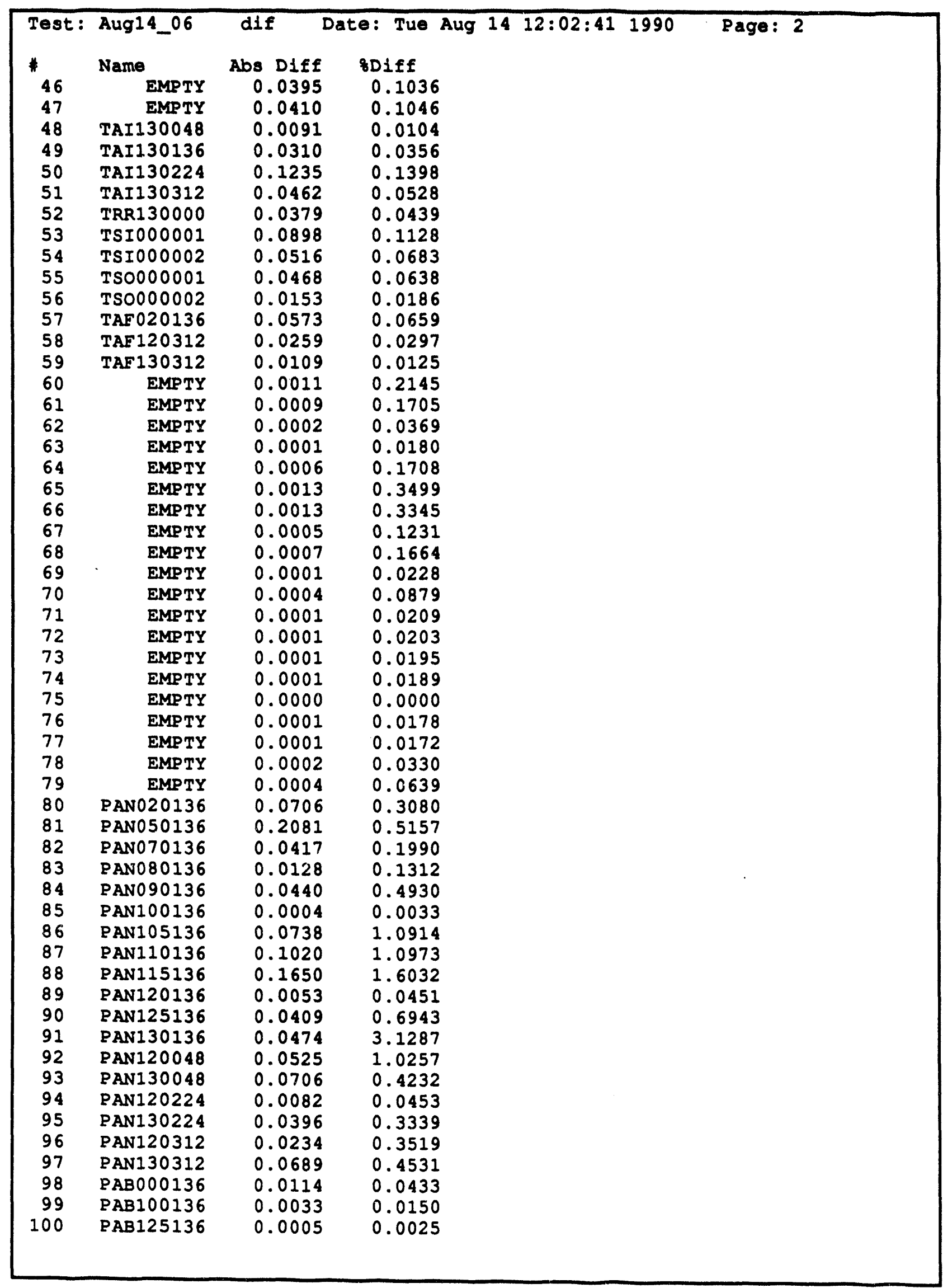




\begin{tabular}{|c|c|c|c|c|}
\hline $\begin{array}{l}\text { Test } \\
\text { 1 } \\
101 \\
102 \\
103 \\
104 \\
105 \\
106 \\
107 \\
108 \\
109 \\
110 \\
111 \\
112 \\
113 \\
114 \\
115 \\
116 \\
117 \\
118 \\
119 \\
120 \\
121 \\
122 \\
123 \\
124 \\
125 \\
126 \\
127 \\
128 \\
129 \\
130 \\
131 \\
132 \\
133 \\
134 \\
135 \\
136 \\
137 \\
138 \\
139 \\
140 \\
141 \\
142 \\
143 \\
144 \\
145 \\
146 \\
147 \\
148 \\
149 \\
150 \\
151 \\
152 \\
153 \\
154 \\
155\end{array}$ & 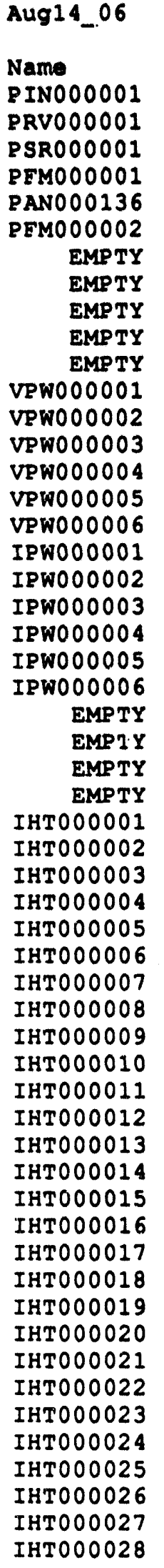 & $\begin{array}{l}\text { dif } \\
\text { Abs D1ff } \\
0.0233 \\
0.0100 \\
0.0138 \\
0.0051 \\
0.1033 \\
0.0000 \\
0.0008 \\
0.7189 \\
0.0001 \\
0.0000 \\
0.0003 \\
0.0109 \\
0.0340 \\
0.0247 \\
0.0128 \\
0.0106 \\
0.0195 \\
0.1139 \\
0.0812 \\
0.5842 \\
0.0051 \\
0.0287 \\
0.0176 \\
0.0008 \\
0.0016 \\
0.0007 \\
0.0018 \\
0.0082 \\
0.0038 \\
0.0026 \\
0.0242 \\
0.0124 \\
0.0023 \\
0.0009 \\
0.0057 \\
0.0039 \\
0.0225 \\
0.0228 \\
0.0184 \\
0.0329 \\
0.0321 \\
0.0545 \\
0.0276 \\
0.0462 \\
0.0238 \\
0.0004 \\
0.0000 \\
0.0133 \\
0.0376 \\
0.0154 \\
0.0304 \\
0.0413 \\
0.0314 \\
0.0202 \\
0.0050\end{array}$ & 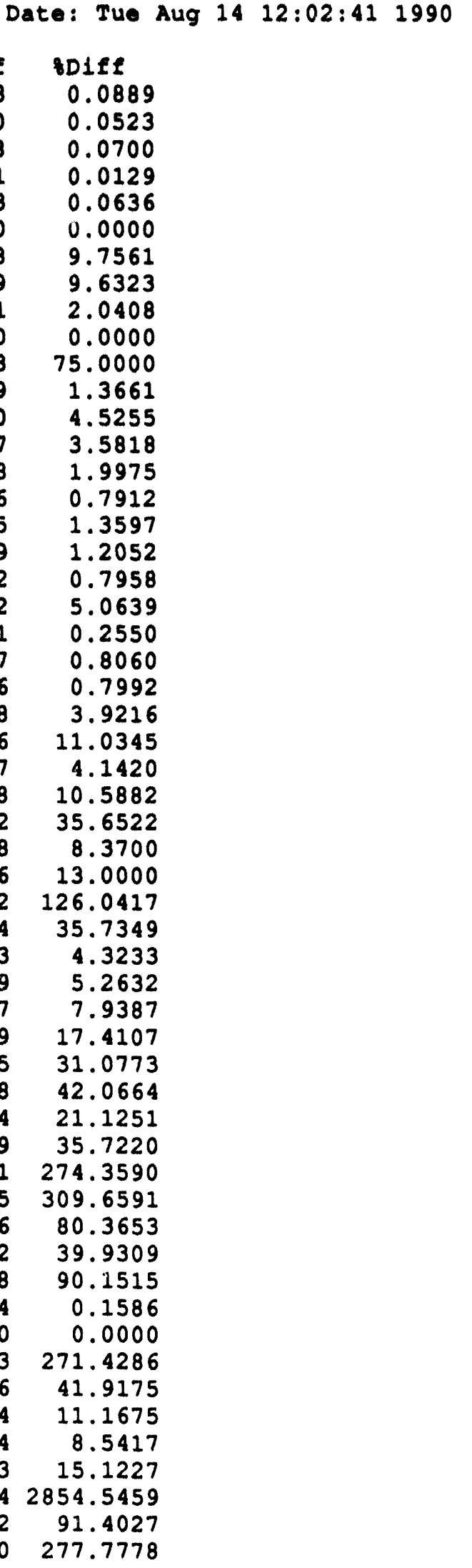 & Page: 3 \\
\hline
\end{tabular}




\begin{tabular}{|c|c|c|c|}
\hline $\begin{array}{l}1 \\
156 \\
157 \\
158 \\
159 \\
160 \\
161 \\
162 \\
163\end{array}$ & $\begin{array}{l}\text { Name } \\
\text { IHTO00029 } \\
\text { IHTO00030 } \\
\text { IHTO00031 } \\
\text { IHTO00032 } \\
\text { IHTO0O033 } \\
\text { IHTO0O0 } \\
\text { IHTO0O034 } \\
\text { IHTO00036 }\end{array}$ & $\begin{array}{c}\text { Abs D1ff } \\
0.0232 \\
0.0230 \\
0.0140 \\
0.0028 \\
0.0049 \\
0.0312 \\
0.0164 \\
0.0091\end{array}$ & $\begin{array}{r}\text { YDIEE } \\
48.8421 \\
72.1003 \\
28.6885 \\
7.1795 \\
8.9579 \\
1299.9999 \\
34.0956 \\
17.8082\end{array}$ \\
\hline
\end{tabular}




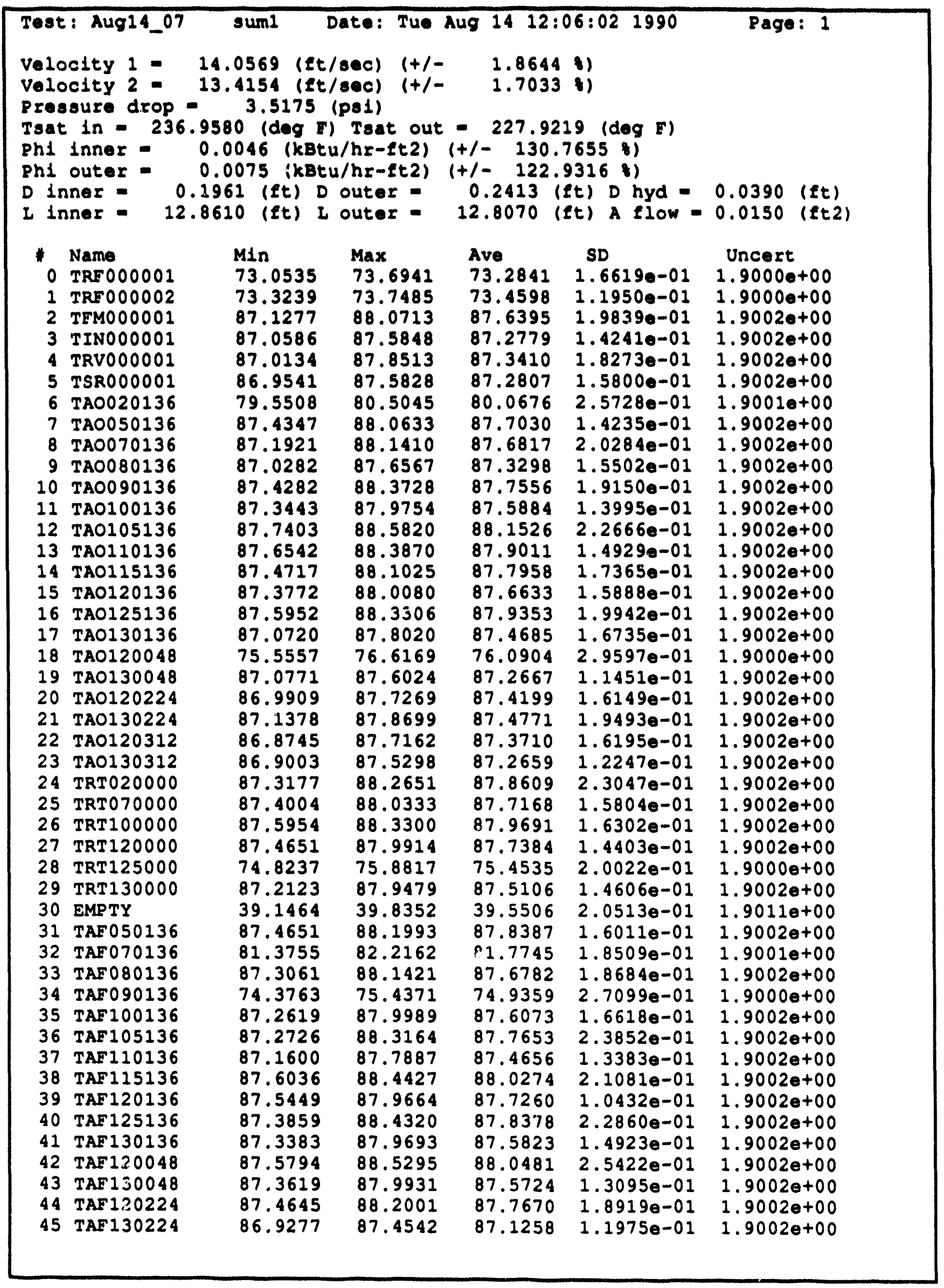




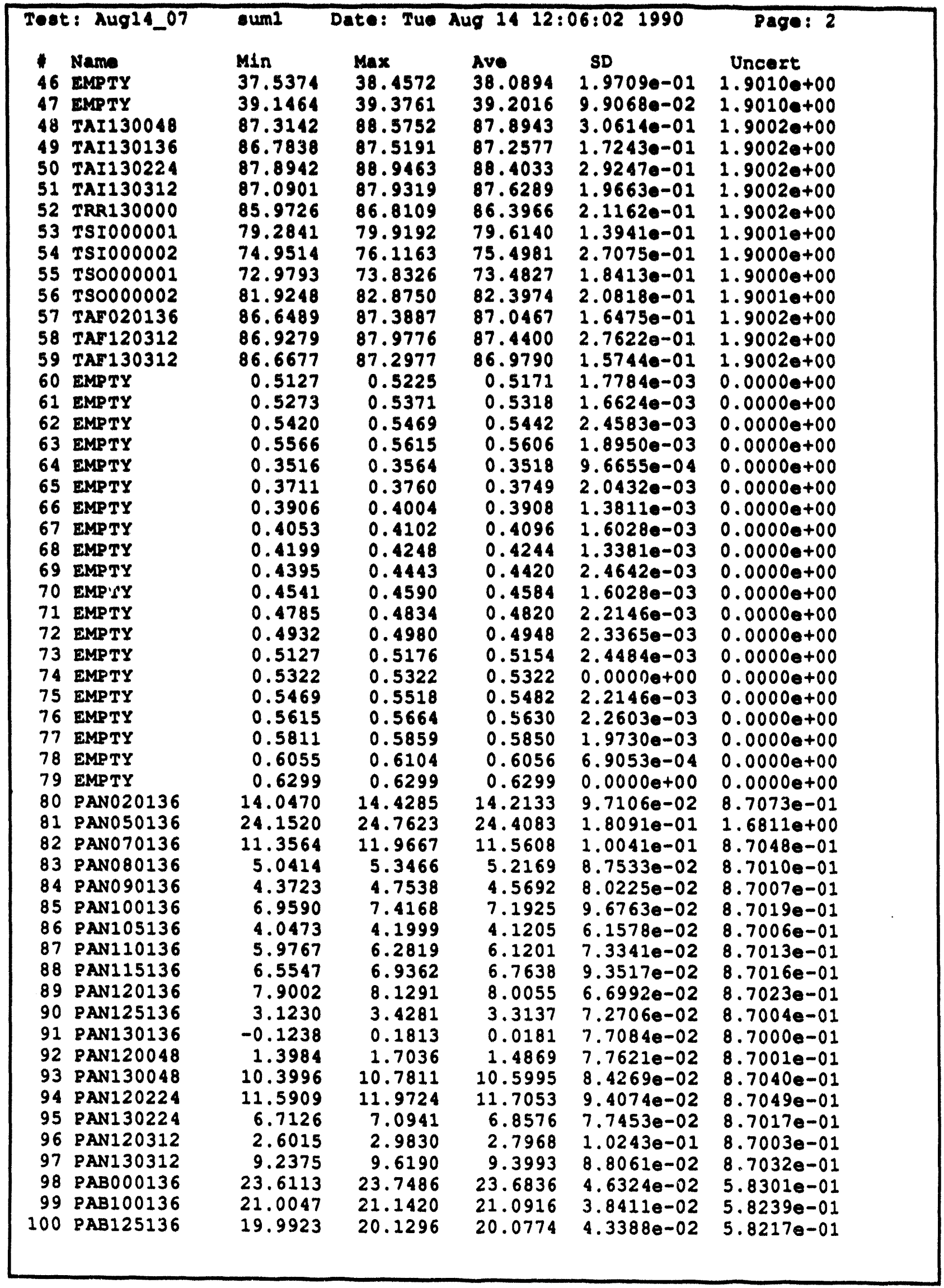




\begin{tabular}{|c|c|c|c|c|c|c|}
\hline $\begin{array}{l}1 \\
101 \\
102 \\
103 \\
104 \\
105 \\
106 \\
107 \\
108 \\
109 \\
110 \\
111 \\
112 \\
113 \\
114 \\
115 \\
116 \\
117 \\
118 \\
119 \\
120 \\
121 \\
122 \\
123 \\
124 \\
125 \\
126 \\
127 \\
128 \\
129 \\
130 \\
131 \\
132 \\
133 \\
134 \\
135 \\
136 \\
137 \\
138 \\
139 \\
140 \\
141 \\
142 \\
143 \\
144 \\
145 \\
146 \\
147 \\
148 \\
149 \\
150 \\
151 \\
152 \\
153 \\
154 \\
155\end{array}$ & 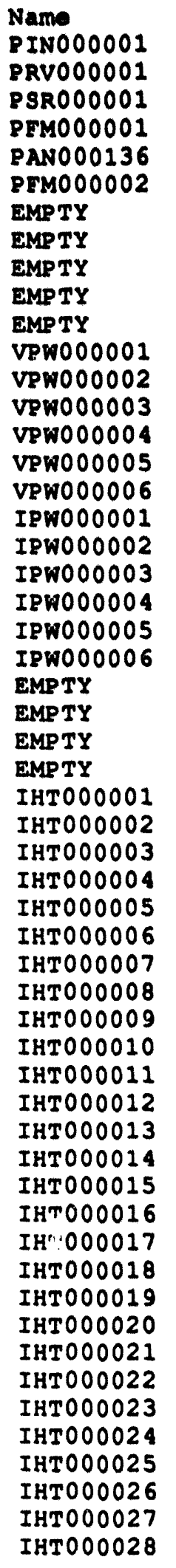 & $\begin{array}{l}\text { MIn } \\
23.4792 \\
19.1082 \\
19.6691 \\
29.9307 \\
95.7170 \\
27.5689 \\
0.0049 \\
-10.0000 \\
0.0000 \\
-10.0000 \\
0.0000 \\
-0.8526 \\
-0.8451 \\
-0.7286 \\
-0.8752 \\
-1.4686 \\
-1.6489 \\
-11.6766 \\
-12.2331 \\
-14.8125 \\
-2.2536 \\
-3.9904 \\
-2.8076 \\
-0.0293 \\
-0.0195 \\
-0.0244 \\
-0.0244 \\
-0.2066 \\
-0.2329 \\
-0.2817 \\
-0.2028 \\
-0.2066 \\
-0.0601 \\
-0.0601 \\
-0.1578 \\
-0.2216 \\
-0.1427 \\
-0.1089 \\
-0.1653 \\
-0.0563 \\
-0.1953 \\
-0.2442 \\
-0.1352 \\
-0.3530 \\
-0.1728 \\
0.0413 \\
-0.2517 \\
-0.0714 \\
0.0037 \\
-0.0300 \\
-0.8676 \\
0.2028 \\
-0.1690 \\
-0.2291 \\
-0.0526\end{array}$ & $\begin{array}{r}\text { Max } \\
23.6013 \\
19.2303 \\
19.7911 \\
30.7547 \\
99.3791 \\
27.5689 \\
0.0098 \\
0.8545 \\
0.0195 \\
-10.0000 \\
0.0000 \\
-0.4620 \\
-0.6498 \\
-0.5333 \\
-0.4845 \\
-1.0780 \\
-1.2583 \\
-7.7719 \\
-8.3306 \\
-8.9625 \\
-1.7654 \\
-3.0139 \\
-1.3427 \\
-0.0146 \\
-0.0098 \\
-0.0146 \\
-0.0098 \\
0.1841 \\
0.1577 \\
0.1089 \\
0.1878 \\
0.1841 \\
0.3305 \\
0.1352 \\
0.0376 \\
0.1690 \\
0.0526 \\
0.0864 \\
0.0301 \\
0.3343 \\
0.1953 \\
0.1465 \\
0.2554 \\
0.6235 \\
0.2178 \\
0.4319 \\
0.1390 \\
0.1240 \\
0.1991 \\
0.3606 \\
-0.2817 \\
0.3981 \\
0.2216 \\
0.1615 \\
0.3381\end{array}$ & $\begin{array}{r}\text { Ave } \\
23.5402 \\
19.1522 \\
19.6740 \\
30.2877 \\
97.3833 \\
27.5689 \\
0.0051 \\
-7.3113 \\
0.0048 \\
-10.0000 \\
0.0000 \\
-0.7940 \\
-0.7826 \\
-0.6661 \\
-0.6564 \\
-1.3241 \\
-1.4809 \\
-9.7633 \\
-9.8135 \\
-10.9905 \\
-2.0095 \\
-3.5412 \\
-2.2216 \\
-0.0207 \\
-0.0129 \\
-0.0188 \\
-0.0155 \\
-0.0191 \\
-0.0298 \\
-0.0200 \\
-0.0114 \\
-0.0269 \\
0.0688 \\
-0.0171 \\
-0.0914 \\
-0.0146 \\
-0.0880 \\
-0.0347 \\
-0.0832 \\
0.0999 \\
0.0156 \\
-0.0137 \\
-0.0297 \\
0.0649 \\
0.0030 \\
0.2288 \\
-0.0720 \\
-0.0089 \\
0.1053 \\
0.1262 \\
-0.3598 \\
0.2810 \\
0.0067 \\
-0.0143 \\
0.0334\end{array}$ & $\begin{array}{l}\text { SD } \\
6.1655 e-02 \\
5.9189 e-02 \\
2.4164 e-02 \\
1.9066 e-01 \\
7.2680 e-01 \\
9.6336 e-06 \\
9.6655 e-04 \\
1.9424 e+00 \\
2.5131 e-03 \\
0.0000 e+00 \\
0.0000 e+00 \\
9.8648 e-02 \\
9.2034 e-02 \\
9.2034 e-02 \\
1.0168 e-01 \\
9.5112 e-02 \\
1.0447 e-01 \\
1.2151 e+00 \\
1.1534 e+00 \\
1.2433 e+00 \\
2.4662 e-01 \\
3.5345 e-01 \\
3.4173 e-01 \\
4.0144 e-03 \\
2.7480 e-03 \\
3.3249 e-03 \\
3.6500 e-03 \\
7.8523 e-02 \\
8.7880 e-02 \\
1.0145 e-01 \\
1.0799 e-01 \\
1.1049 e-01 \\
1.0885 e-01 \\
8.1729 e-02 \\
9.3461 e-02 \\
9.1779 e-02 \\
8.8585 e-02 \\
9.5765 e-02 \\
9.7377 e-02 \\
8.8233 e-02 \\
6.6498 e-02 \\
8.5454 e-02 \\
1.0595 e-01 \\
2.6765 e-01 \\
1.0625 e-01 \\
6.7888 e-02 \\
6.6498 e-02 \\
9.2034 e-02 \\
9.8569 e-02 \\
8.8233 e-02 \\
1.1838 e-01 \\
9.6655 e-02 \\
8.1347 e-02 \\
7.1136 e-02 \\
1.1272 e-01\end{array}$ & $\begin{array}{l}\text { Uncert } \\
7.5231 e-01 \\
7.5153 e-01 \\
7.5161 e-01 \\
5.0570 e-01 \\
4.9260 e+00 \\
2.1154 e-01 \\
0.0000 e+00 \\
0.0000 e+00 \\
0.0000 e+00 \\
0.0000 e+00 \\
0.0000 e+00 \\
8.1001 e-01 \\
8.1001 e-01 \\
8.1001 e-01 \\
8.1001 e-01 \\
8.1004 e-01 \\
8.1005 e-01 \\
8.1004 e+00 \\
8.1004 e+00 \\
8.1005 e+00 \\
2.0001 e+00 \\
2.0002 e+00 \\
2.0001 e+00 \\
0.0000 e+00 \\
0.0000 e+00 \\
0.0000 e+00 \\
0.0000 e+00 \\
4.1000 e+00 \\
4.1000 e+00 \\
4.1000 e+00 \\
4.1000 e+00 \\
4.1000 e+00 \\
4.1000 e+00 \\
4.1000 e+00 \\
4.1000 e+00 \\
4.1000 e+00 \\
4.1000 e+00 \\
4.1000 e+00 \\
4.1000 e+00 \\
4.1000 e+00 \\
4.1000 e+00 \\
4.1000 e+00 \\
4.1000 e+00 \\
4.1000 e+00 \\
4.1000 e+00 \\
4.1000 e+00 \\
4.1000 e+00 \\
4.1000 e+00 \\
4.1000 e+00 \\
4.1000 e+00 \\
4.1000 e+00 \\
4.1000 e+00 \\
4.1000 e+00 \\
4.1000 e+00 \\
4.1000 e+00\end{array}$ \\
\hline
\end{tabular}




\begin{tabular}{|c|c|c|c|c|c|c|}
\hline $\begin{array}{l}1 \\
156 \\
157 \\
158 \\
159 \\
160 \\
161 \\
162 \\
163\end{array}$ & 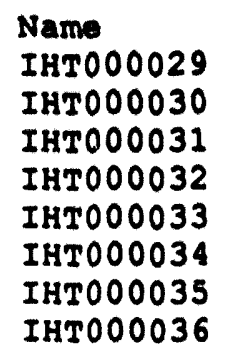 & $\begin{array}{l}M 1 n \\
-0.2780 \\
-0.2103 \\
-0.3418 \\
-0.2930 \\
-0.0977 \\
-0.2329 \\
-0.1277 \\
-0.1878\end{array}$ & $\begin{array}{l}\operatorname{Max} \\
0.3080 \\
0.3756 \\
0.2441 \\
0.2930 \\
0.2930 \\
0.1577 \\
0.2629 \\
0.2028\end{array}$ & $\begin{array}{l}\text { Ave } \\
-0.0280 \\
0.0397 \\
-0.0410 \\
-0.0273 \\
-0.0274 \\
-0.0258 \\
0.0520 \\
-0.0745\end{array}$ & $\begin{array}{l}\text { SD } \\
1.0470 e-01 \\
1.1189 e-01 \\
1.2608 e-01 \\
1.0992 e-01 \\
1.0259 e-01 \\
8.2864 e-02 \\
1.0320 e-01 \\
1.1223 e-01\end{array}$ & $\begin{array}{l}\text { Uncert } \\
4.1000 e+00 \\
4.1000 e+00 \\
4.1000 e+00 \\
4.1000 e+00 \\
4.1000 e+00 \\
4.1000 e+00 \\
4.1000 e+00 \\
4.1000 e+00\end{array}$ \\
\hline
\end{tabular}




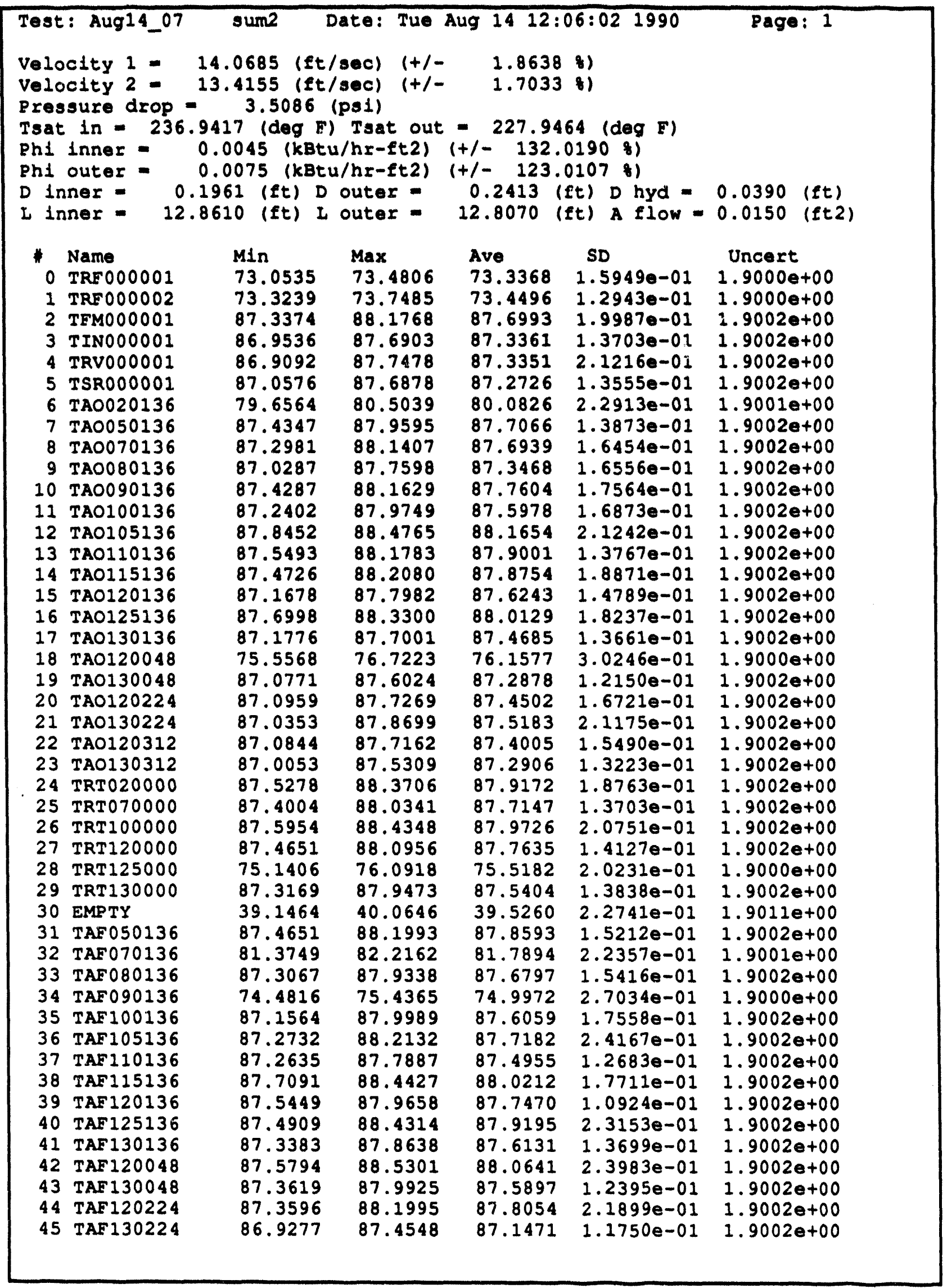




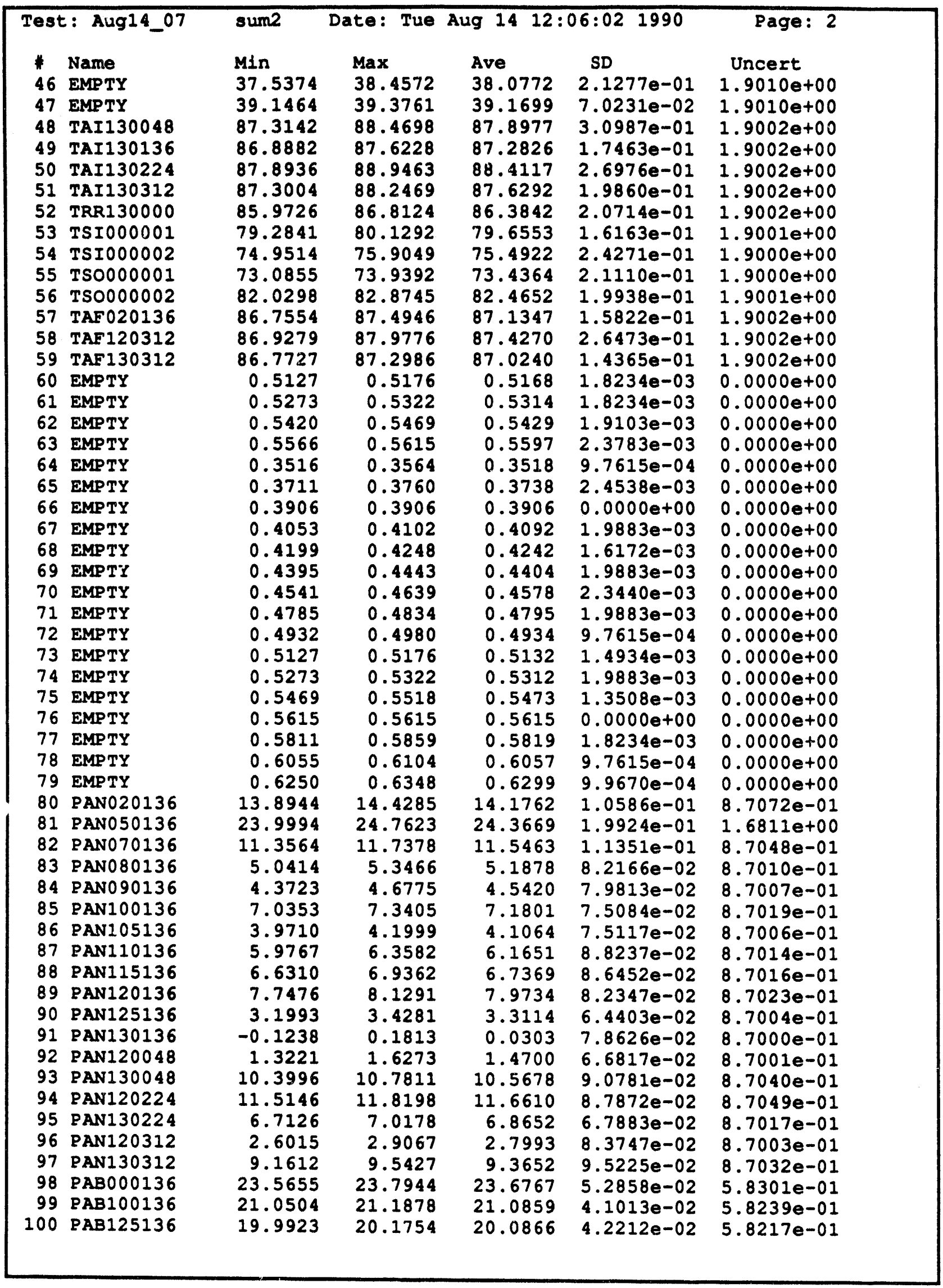




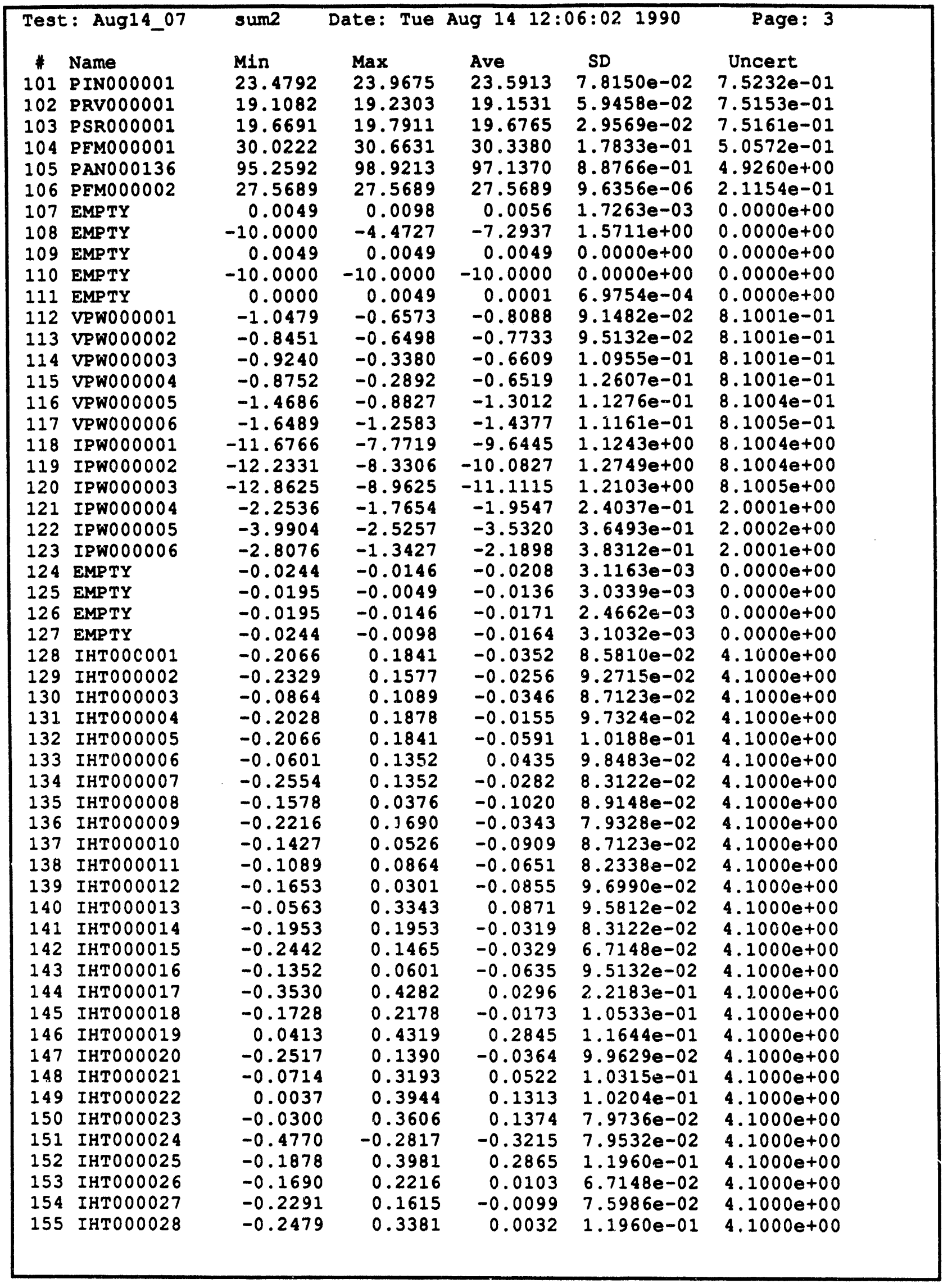




\begin{tabular}{|c|c|c|c|c|c|c|}
\hline \multicolumn{2}{|c|}{ Test: Aug14_07 } & & Date: Tue & \multicolumn{2}{|c|}{ Aug $14 \quad 12: 06: 021990$} & $\begin{array}{l}\text { Page: } 4 \\
\text { Uncert }\end{array}$ \\
\hline $\begin{array}{l}1 \\
156 \\
157 \\
158 \\
159 \\
160 \\
161 \\
162 \\
163\end{array}$ & 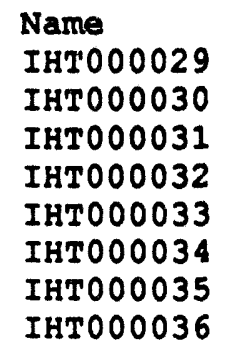 & $\begin{array}{l}\text { Min } \\
-0.2780 \\
-0.2103 \\
-0.1465 \\
-0.0976 \\
-0.0977 \\
-0.2329 \\
-0.1277 \\
-0.1878\end{array}$ & $\begin{array}{l}\operatorname{Max} \\
0.3080 \\
0.1803 \\
0.2441 \\
0.2930 \\
0.2930 \\
0.1577 \\
0.2629 \\
0.0075\end{array}$ & $\begin{array}{l}\text { Ave } \\
-0.0229 \\
0.0408 \\
-0.0030 \\
-0.0060 \\
-0.0020 \\
0.0023 \\
0.0756 \\
-0.0483\end{array}$ & $\begin{array}{l}\text { SD } \\
1.1419 \mathrm{e}-01 \\
9.7656 \mathrm{e}-02 \\
1.0378 \mathrm{e}-01 \\
1.0625 \mathrm{e}-01 \\
1.0640 \mathrm{e}-01 \\
1.1262 \mathrm{e}-01 \\
8.8783 \mathrm{e}-02 \\
8.9148 \mathrm{e}-02\end{array}$ & $\begin{array}{l}\text { Uncert } \\
4.1000 e+00 \\
4.1000 e+00 \\
4.1000 e+00 \\
4.1000 e+00 \\
4.1000 e+00 \\
4.1000 e+00 \\
4.1000 e+00 \\
4.1000 e+00\end{array}$ \\
\hline
\end{tabular}




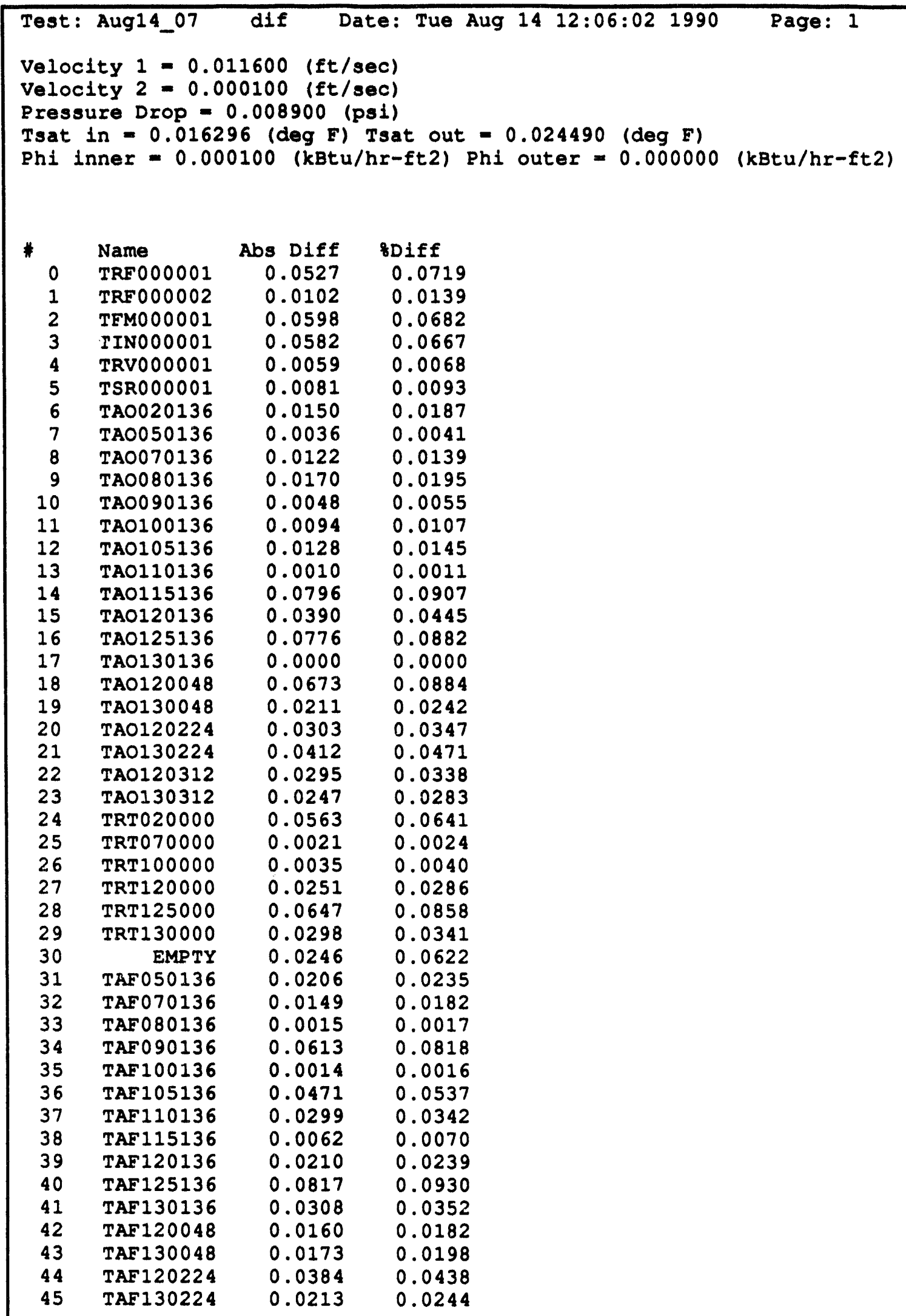




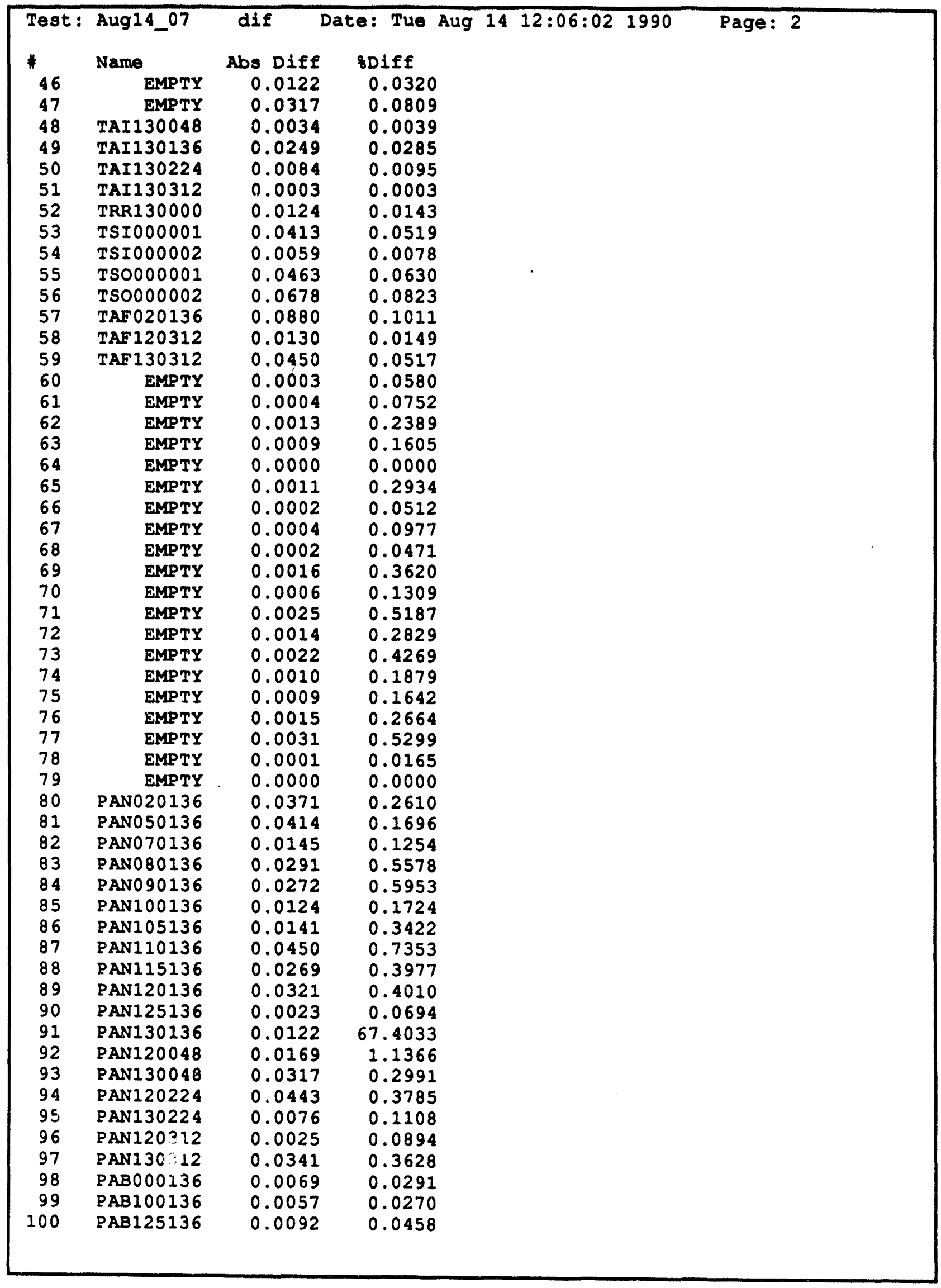




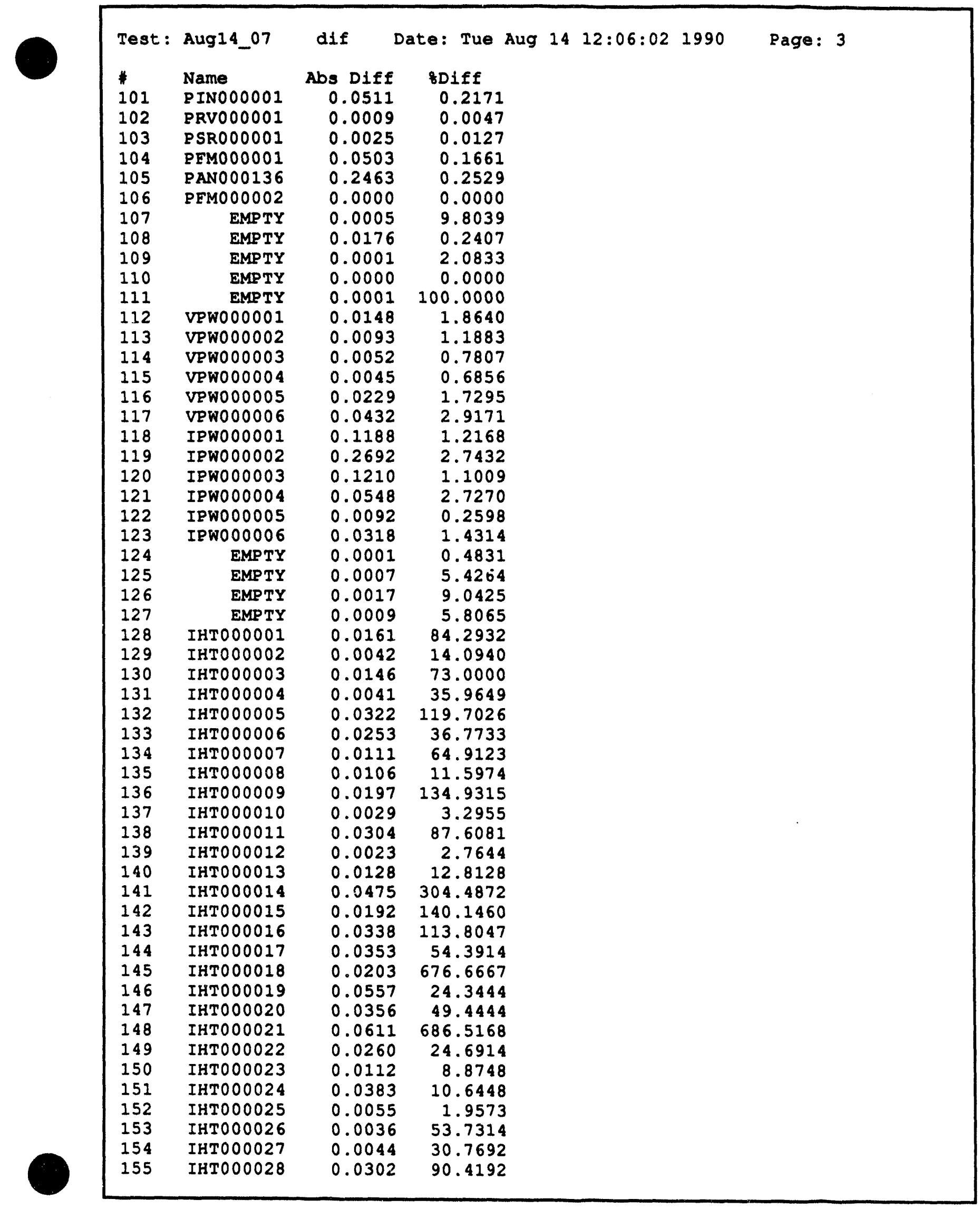


Test: Aug14_07

Name

IHT000029

IHT000030

IHT000031

IHT000032

IHT000033

IHTO00034

IHTO00035

IHT000036 dif Date: Tue Aug 14 12:06:02 1990

Page: 4

Abs Diff $8 D$ iff

$0.0051 \quad 18.2143$

$0.0011 \quad 2.7708$

$0.0380 \quad 92.6829$

0.021378 .0220

$0.0254 \quad 92.7007$

$0.0281 \quad 108.9147$

$0.0236 \quad 45.3846$

$0.0262 \quad 35.1678$ 


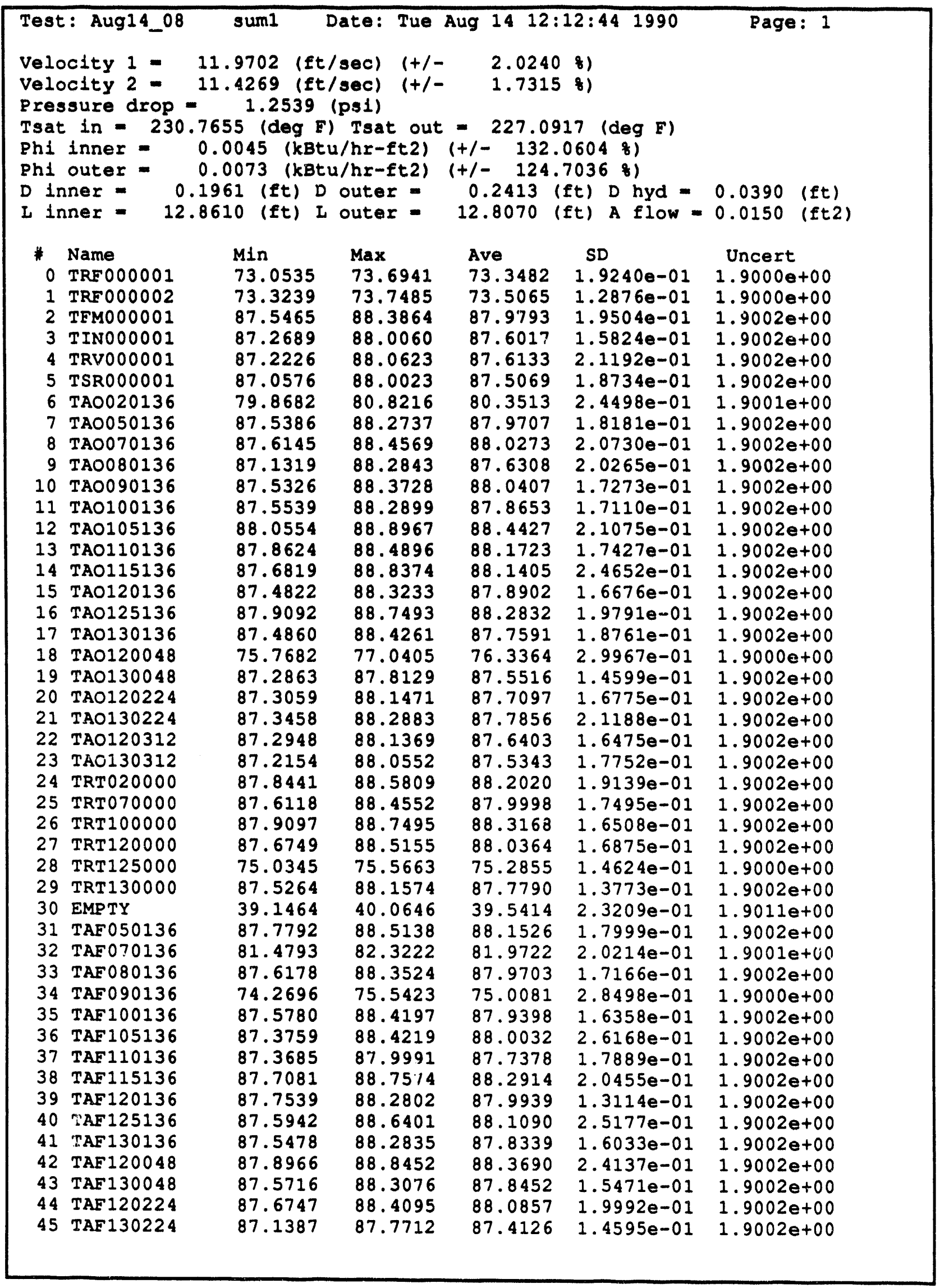




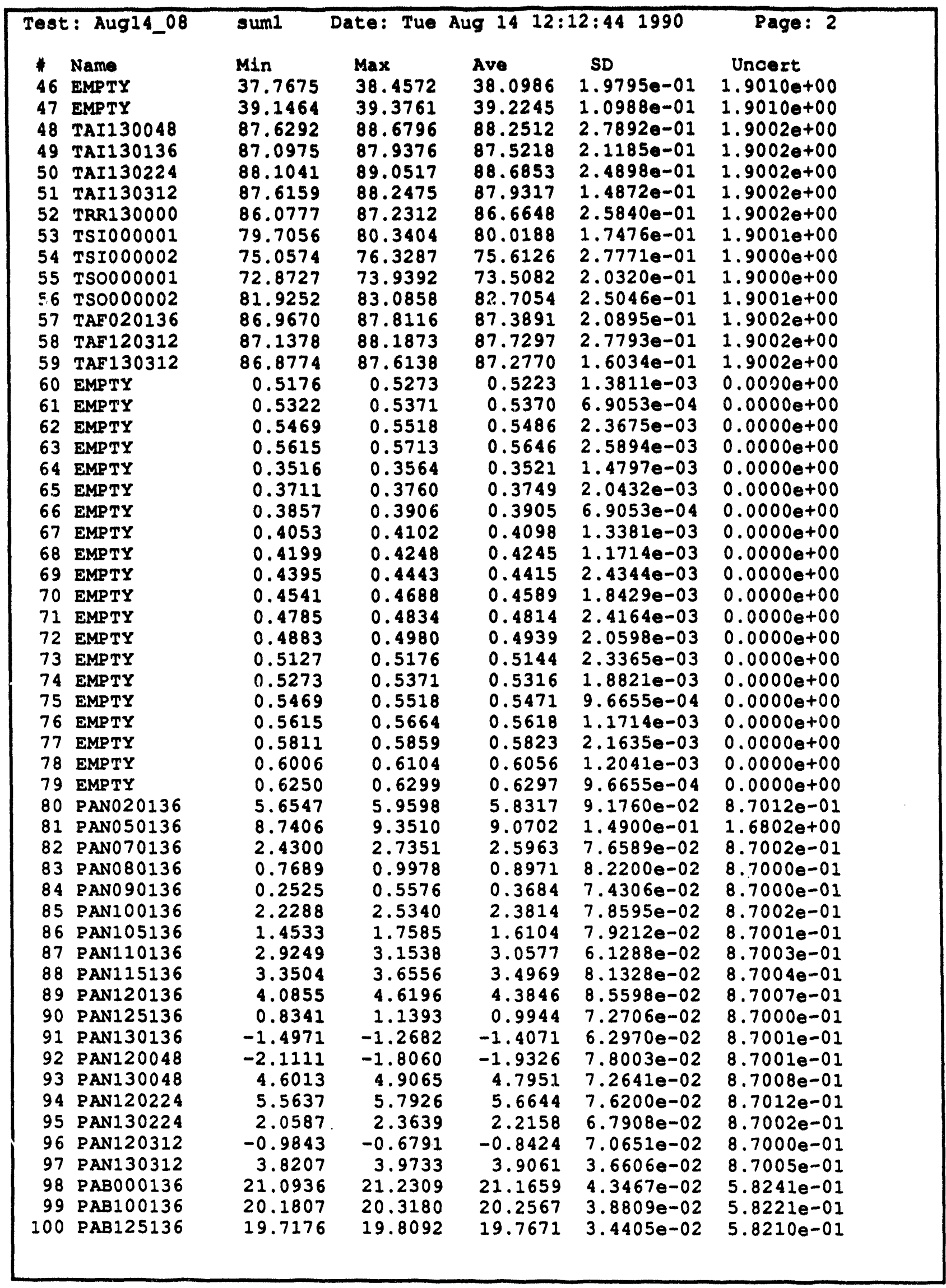




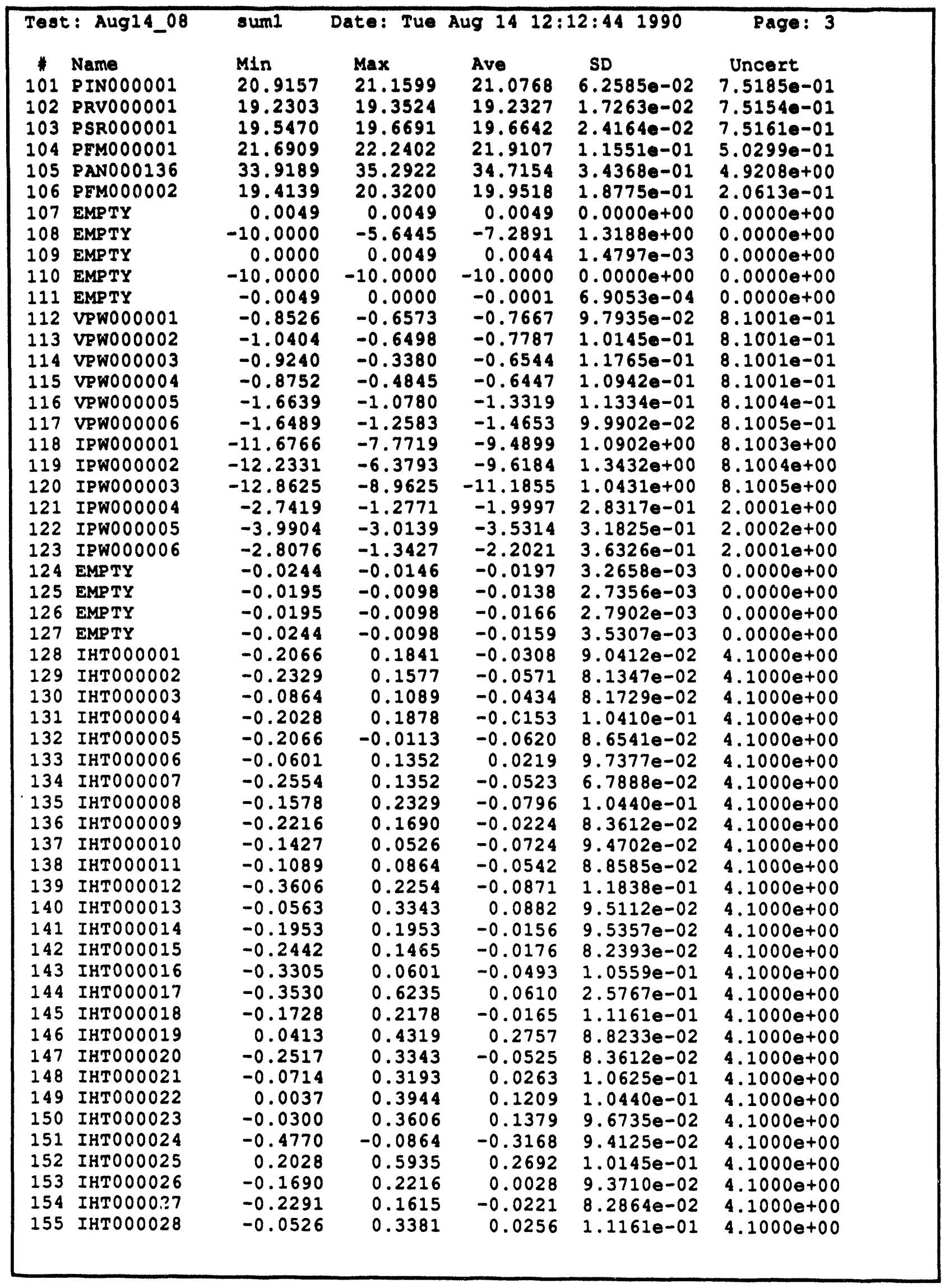




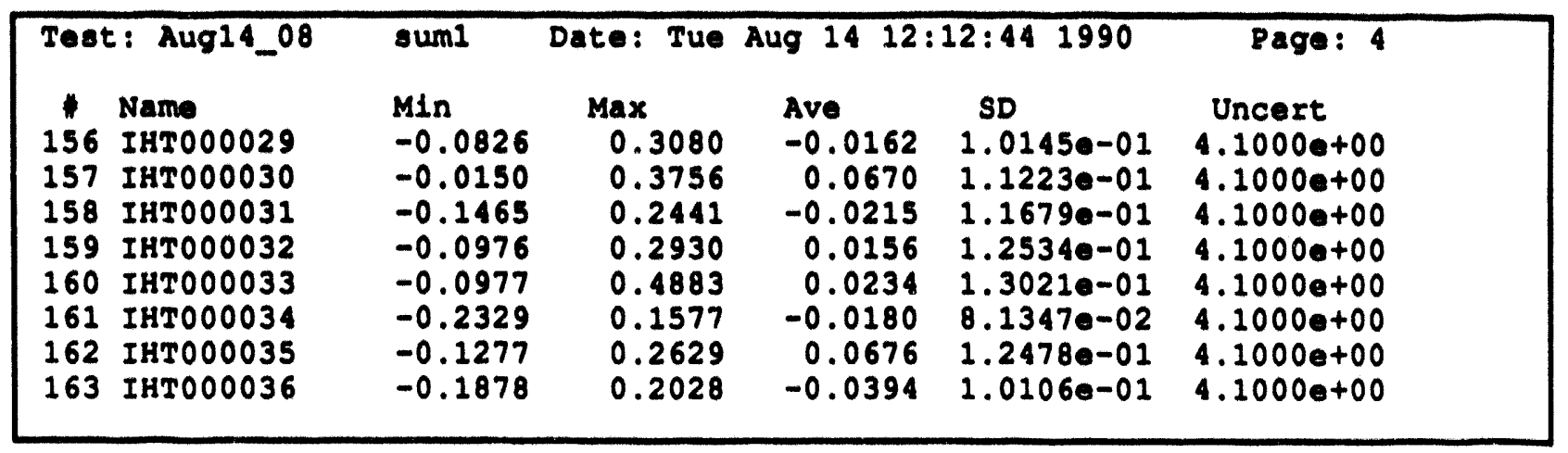




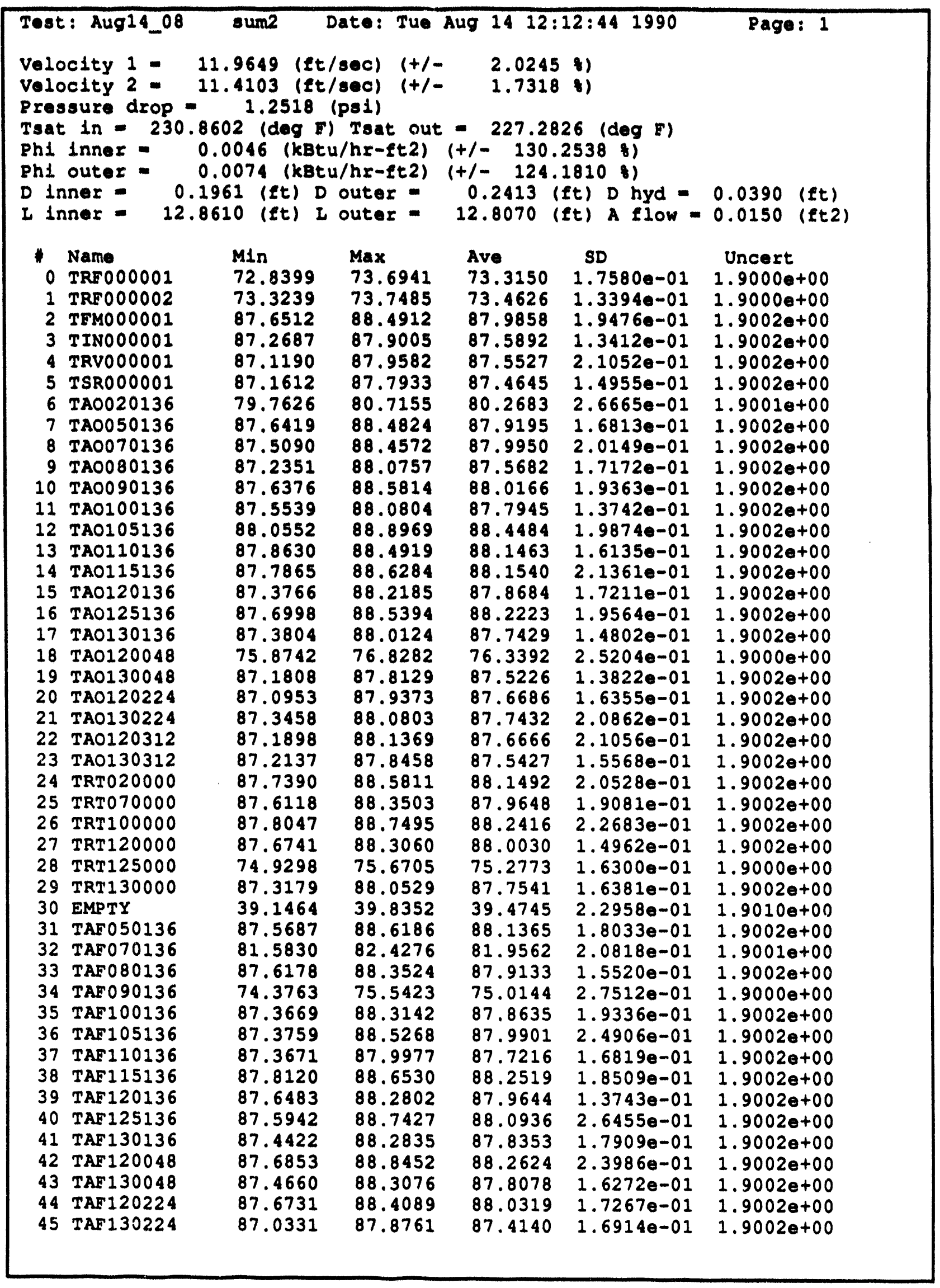




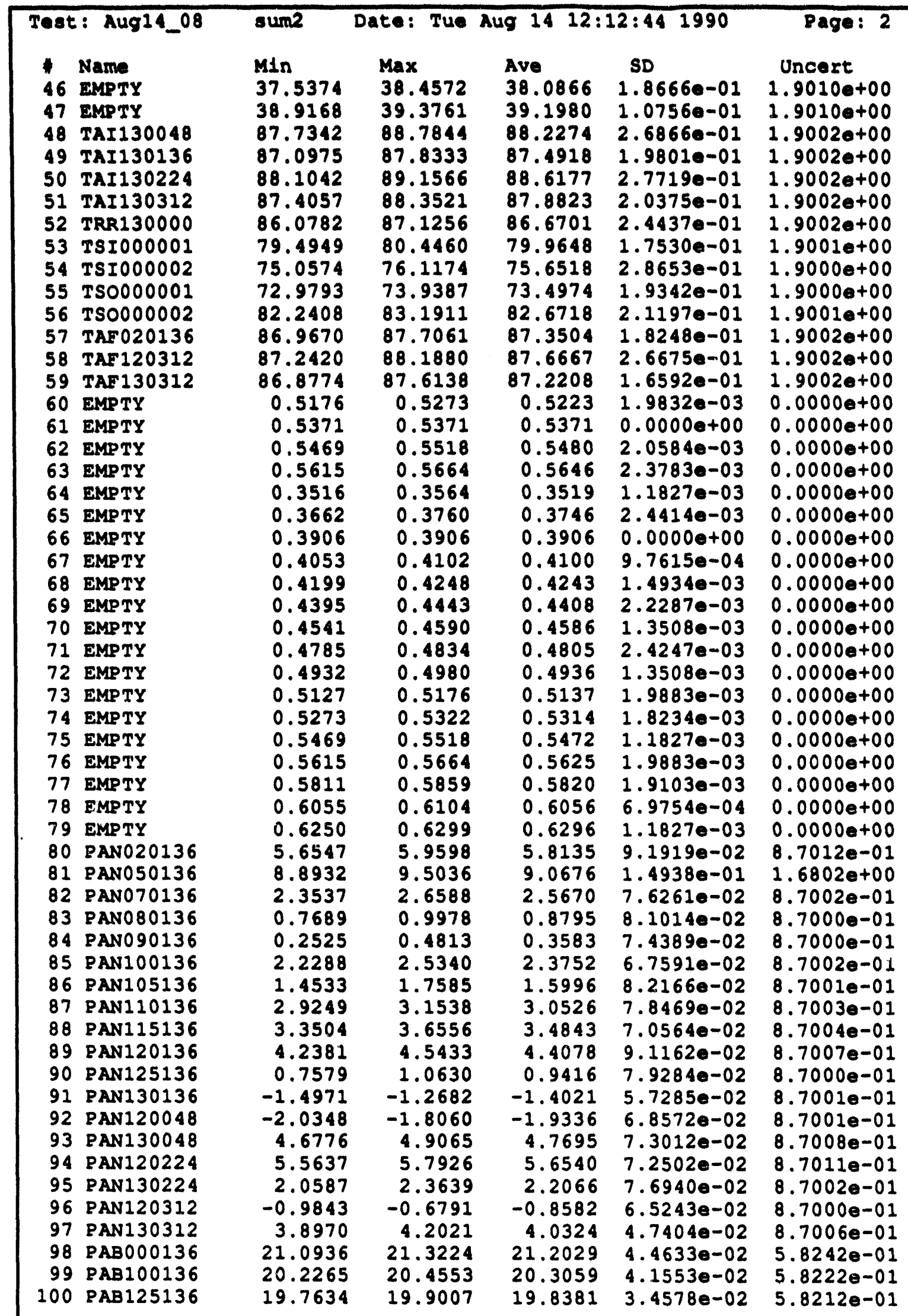




\begin{tabular}{|c|c|c|c|c|c|c|c|}
\hline $\begin{array}{l}1 \\
101 \\
102 \\
103 \\
104 \\
105 \\
106 \\
107 \\
108 \\
109 \\
110 \\
111 \\
112 \\
113 \\
114 \\
115 \\
116 \\
117 \\
118 \\
119 \\
120 \\
121 \\
122 \\
123 \\
124 \\
125 \\
126 \\
127 \\
128 \\
129 \\
130 \\
131 \\
132 \\
133 \\
134 \\
135 \\
136 \\
137 \\
138 \\
139 \\
140 \\
141 \\
142 \\
143 \\
144 \\
145 \\
146 \\
147 \\
148 \\
149 \\
150 \\
151 \\
152 \\
153 \\
154 \\
155\end{array}$ & 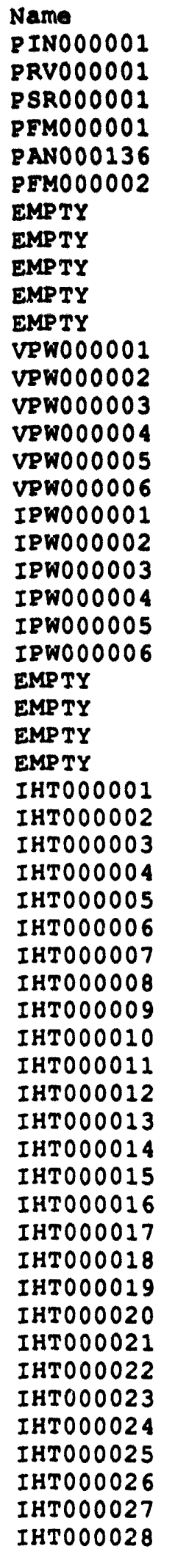 & $\begin{array}{l}\text { Min } \\
21.0378 \\
19.1082 \\
19.6691 \\
21.5994 \\
33.9189 \\
19.0280 \\
0.0049 \\
-10.0000 \\
0.0000 \\
-10.0000 \\
-0.0049 \\
-0.8526 \\
-1.0404 \\
-0.9240 \\
-0.8752 \\
-1.4686 \\
-1.6489 \\
-11.6766 \\
-12.2331 \\
-12.8625 \\
-2.2536 \\
-3.9904 \\
-2.8076 \\
-0.0293 \\
-0.0195 \\
-0.0195 \\
-0.0244 \\
-0.2066 \\
-0.2329 \\
-0.0864 \\
-0.3981 \\
-0.2066 \\
-0.2554 \\
-0.2554 \\
-0.1578 \\
-0.2216 \\
-0.3380 \\
-0.3042 \\
-0.1653 \\
-0.0563 \\
-0.1953 \\
-0.2442 \\
-0.3305 \\
-0.3530 \\
-0.1728 \\
0.0413 \\
-0.2517 \\
-0.0714 \\
0.0037 \\
-0.0300 \\
-0.4770 \\
0.2028 \\
-0.1690 \\
-0.2291 \\
-0.2479\end{array}$ & $\begin{array}{l}\text { Max } \\
21.2819 \\
19.3524 \\
19.7911 \\
22.4234 \\
35.2922 \\
20.5717 \\
0.0098 \\
-2.5293 \\
0.0049 \\
-10.0000 \\
0.0000 \\
-0.6573 \\
-0.6498 \\
-0.5333 \\
-0.4845 \\
-1.0780 \\
-1.2583 \\
-5.8195 \\
-6.3793 \\
-5.0625 \\
-1.7654 \\
-3.0139 \\
-1.3427 \\
-0.0146 \\
-0.0098 \\
-0.0098 \\
-0.0098 \\
0.1841 \\
0.1577 \\
0.1089 \\
0.3831 \\
0.1841 \\
0.3305 \\
0.1352 \\
0.0376 \\
0.1690 \\
0.2479 \\
0.0864 \\
0.0301 \\
0.1390 \\
0.1953 \\
0.1465 \\
0.0601 \\
0.6235 \\
0.2178 \\
0.4319 \\
0.1390 \\
0.3193 \\
0.1991 \\
0.3606 \\
-0.0864 \\
0.3981 \\
0.2216 \\
0.1615 \\
0.1427\end{array}$ & $\begin{array}{r}\text { Ave } \\
21.1599 \\
19.2303 \\
19.6740 \\
21.8908 \\
34.6569 \\
19.8933 \\
0.0050 \\
-7.3415 \\
0.0048 \\
-10.0000 \\
-0.0001 \\
-0.7928 \\
-0.7733 \\
-0.6768 \\
-0.6161 \\
-1.3211 \\
-1.4895 \\
-9.4453 \\
-9.9633 \\
-10.9125 \\
-1.9647 \\
-3.4822 \\
-2.1898 \\
-0.0206 \\
-0.0141 \\
-0.0164 \\
-0.0164 \\
-0.0272 \\
-0.0455 \\
-0.0186 \\
-0.0195 \\
-0.0471 \\
0.0435 \\
-0.0322 \\
-0.0980 \\
-0.0223 \\
-0.0869 \\
-0.0531 \\
-0.0736 \\
0.0871 \\
-0.0080 \\
-0.0329 \\
-0.0595 \\
0.0695 \\
-0.0054 \\
0.2805 \\
-0.0484 \\
0.0482 \\
0.1353 \\
0.1693 \\
-0.3176 \\
0.3065 \\
0.0223 \\
0.0100 \\
-0.0207\end{array}$ & $\begin{array}{l}\text { SD } \\
2.4917 e-02 \\
2.4917 e-02 \\
2.4404 e-02 \\
1.6681 e-01 \\
2.7745 e-01 \\
2.9369 e-01 \\
6.9754 e-04 \\
1.7489 e+00 \\
6.9754 e-04 \\
0.0000 e+00 \\
6.9754 e-04 \\
9.0949 e-02 \\
1.0315 e-01 \\
9.5812 e-02 \\
1.0836 e-01 \\
1.1644 e-01 \\
1.0299 e-01 \\
1.2602 e+00 \\
1.4551 e+00 \\
1.4893 e+00 \\
2.4247 e-01 \\
2.9830 e-01 \\
3.2718 e-01 \\
3.7645 e-03 \\
2.9284 e-03 \\
3.2594 e-03 \\
4.0723 e-03 \\
7.8092 e-02 \\
6.8582 e-02 \\
9.3931 e-02 \\
1.2229 e-01 \\
1.0299 e-01 \\
1.1348 e-01 \\
7.9736 e-02 \\
9.0949 e-02 \\
7.4477 e-02 \\
1.0548 e-01 \\
1.0548 e-01 \\
9.8483 e-02 \\
8.7123 e-02 \\
7.9328 e-02 \\
7.8092 e-02 \\
1.0409 e-01 \\
2.2321 e-01 \\
1.0548 e-01 \\
9.9792 e-02 \\
7.9328 e-02 \\
1.0409 e-01 \\
9.2540 e-02 \\
8.4477 e-02 \\
8.6187 e-02 \\
9.8483 e-02 \\
8.4477 e-02 \\
9.1482 e-02 \\
1.2189 e-01\end{array}$ & $\begin{array}{l}\text { Uncert } \\
7.5186 e-01 \\
7.5154 e-01 \\
7.5161 e-01 \\
5.0299 e-01 \\
4.9208 e+00 \\
2.0609 e-01 \\
0.0000 e+00 \\
0.0000 e+00 \\
0.0000 e+00 \\
0.0000 e+00 \\
0.0000 e+00 \\
8.1001 e-01 \\
8.1001 e-01 \\
8.1001 e-01 \\
8.1001 e-01 \\
8.1004 e-01 \\
3.1005 e-01 \\
8.1003 e+00 \\
8.1004 e+00 \\
8.1005 e+00 \\
2.0001 e+00 \\
2.0002 e+00 \\
2.0001 e+00 \\
0.0000 e+00 \\
0.0000 e+00 \\
0.0000 e+00 \\
0.0000 e+00 \\
4.1000 e+00 \\
4.1000 e+00 \\
4.1000 e+00 \\
4.1000 e+00 \\
4.1000 e+00 \\
4.1000 e+00 \\
4.1000 e+00 \\
4.1000 e+00 \\
4.1000 e+00 \\
4.1000 e+00 \\
4.1000 e+00 \\
4.1000 e+00 \\
4.1000 e+00 \\
4.1000 e+00 \\
4.1000 e+00 \\
4.1000 e+00 \\
4.1000 e+00 \\
4.1000 e+00 \\
4.1000 e+00 \\
4.1000 e+00 \\
4.1000 e+00 \\
4.1000 e+00 \\
4.1000 e+00 \\
4.1000 e+00 \\
4.1000 e+00 \\
4.1000 e+00 \\
4.1000 e+00 \\
4.1000 e+00\end{array}$ & $i$ \\
\hline
\end{tabular}




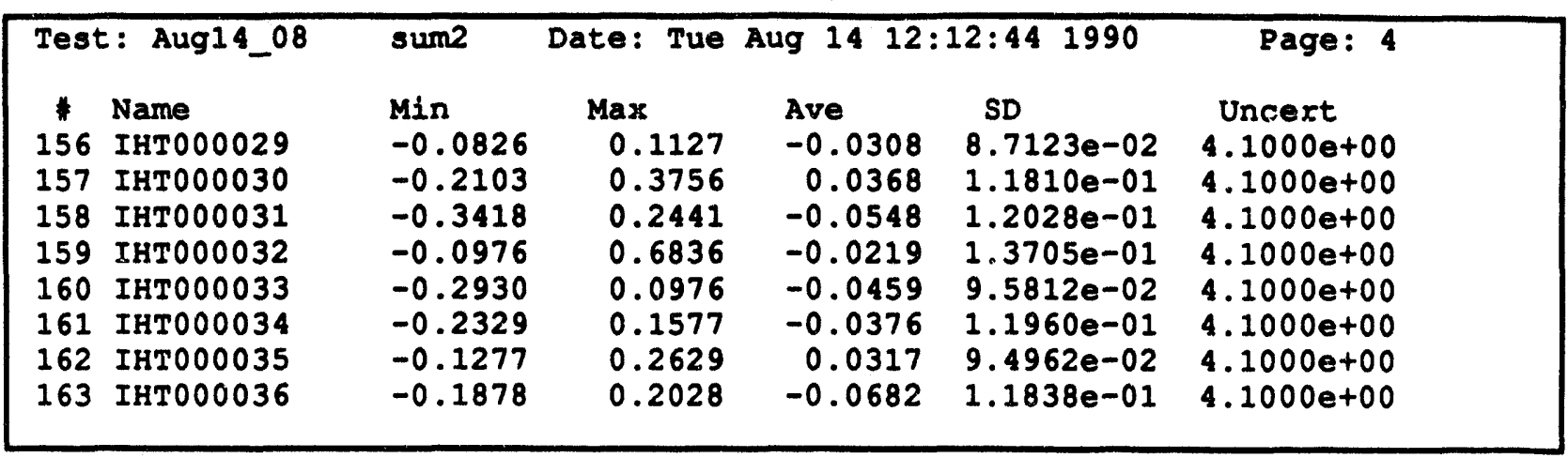




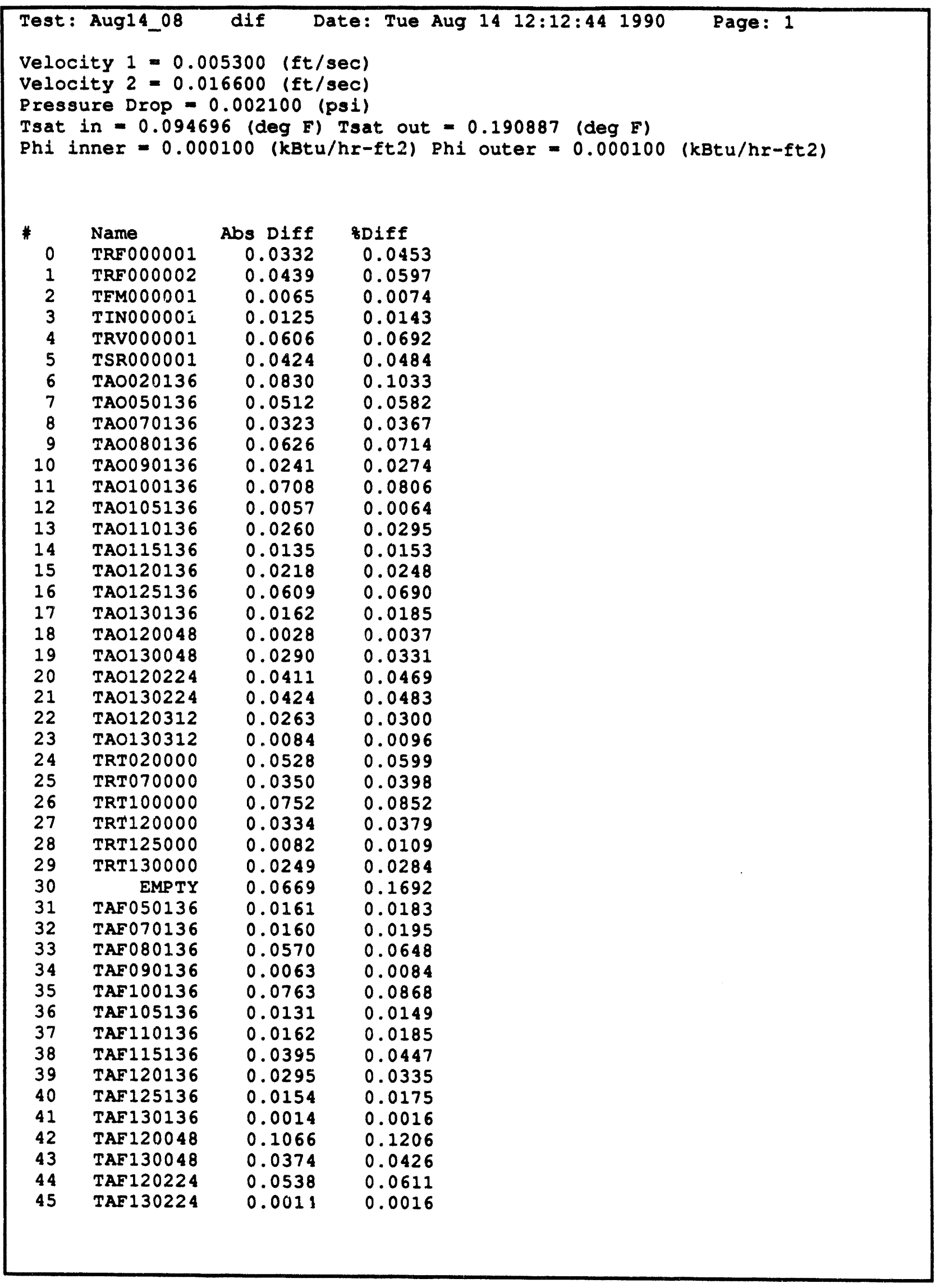




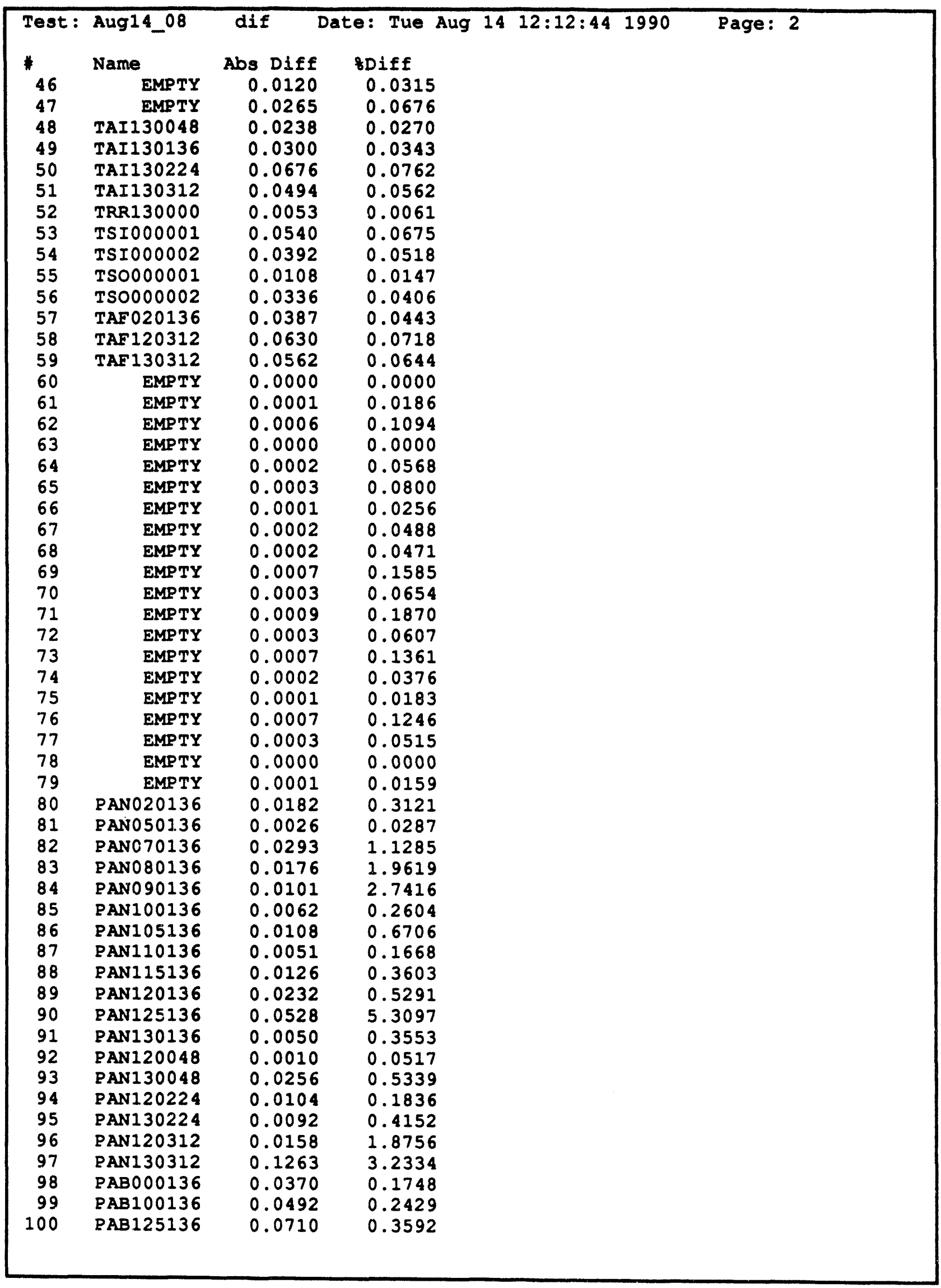




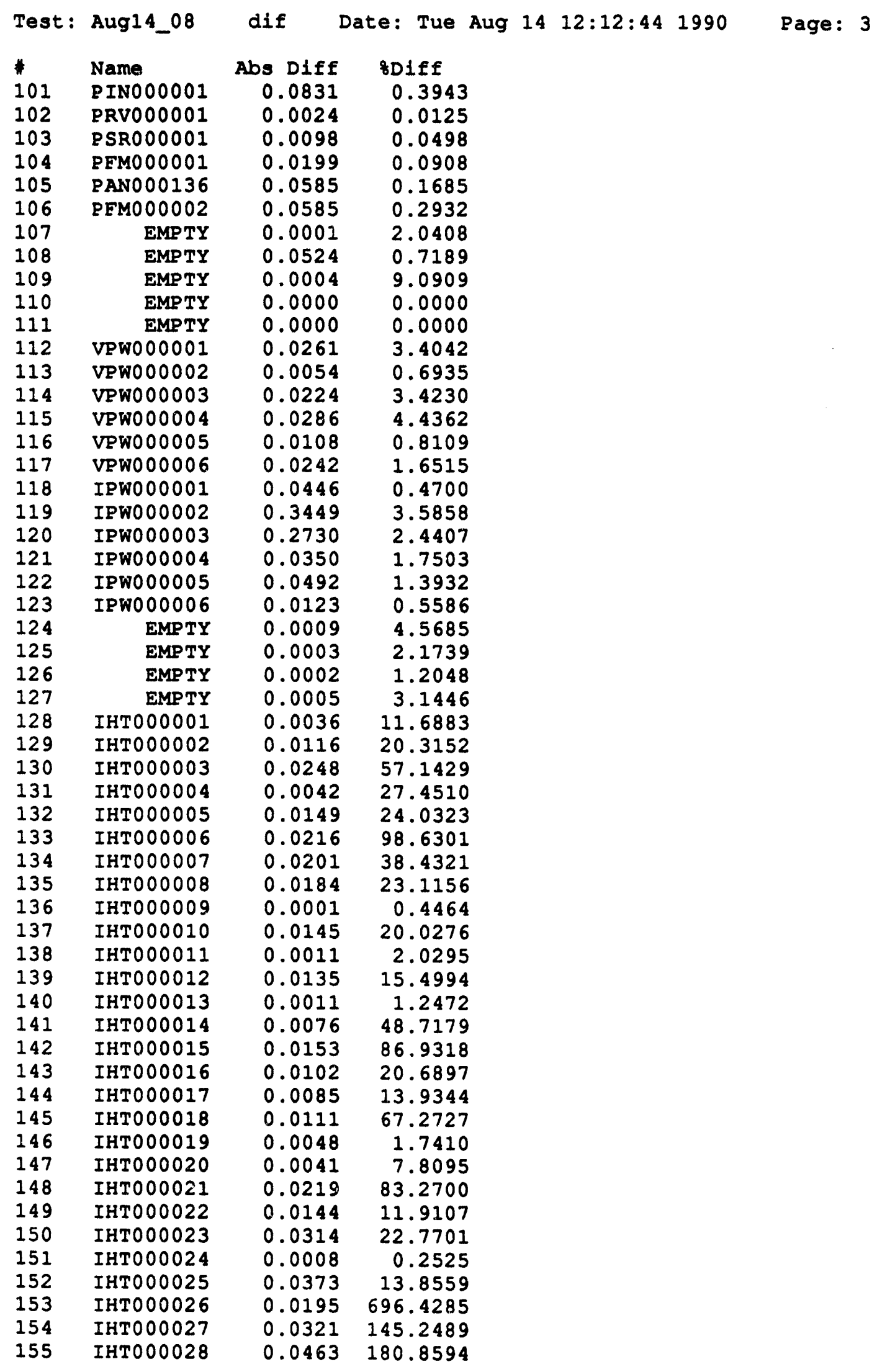

Abs Diff SDiff

\section{$0.0831 \quad 0.3943$}

$0.0024 \quad 0.0125$

$0.0098 \quad 0.0498$

$0.0199 \quad 0.0908$

$0.0585 \quad 0.1685$

$0.0585 \quad 0.2932$

$0.0001 \quad 2.0408$

$0.0524 \quad 0.7189$

$0.0004 \quad 9.0909$

$0.0000 \quad 0.0000$

$0.0000 \quad 0.0000$

$0.0261 \quad 3.4042$

$0.0054 \quad 0.6935$

$0.0224 \quad 3.4230$

$0.0286 \quad 4.4362$

$\begin{array}{ll}0.0108 & 0.8109\end{array}$

$0.0242 \quad 1.6515$

$0.0446 \quad 0.4700$

$0.3449 \quad 3.5858$

$0.2730 \quad 2.4407$

$0.0350 \quad 1.7503$

$0.0492 \quad 1.3932$

$0.0123 \quad 0.5586$

$0.0009 \quad 4.5685$

$0.0003 \quad 2.1739$

$0.0002 \quad 1.2048$

$0.0005 \quad 3.1446$

$0.0036 \quad 11.6883$

$0.0116 \quad 20.3152$

$\begin{array}{lll}\text { IHTO } 00003 & 0.0248 & 57.1429 \\ \text { IHTO00004 } & 0.0042 & 27.4510\end{array}$

IHTO00005 $0.0149 \quad 24.0323$

$\begin{array}{lll}\text { IHTO000006 } & 0.0216 & 98.6301\end{array}$

$\begin{array}{lll}\text { IHTO00007 } & 0.0201 & 38.4321\end{array}$

IHT000008 $0.0184 \quad 23.1156$

$\begin{array}{lll}\text { IHTO00009 } & 0.0001 & 0.4464\end{array}$

IHT000010 $0.0145 \quad 20.0276$

$\begin{array}{lll}\text { IHTO00011 } & 0.0011 & 2.0295\end{array}$

IHTO00012 $0.0135 \quad 15.4994$

IHTO00013 $0.0011 \quad 1.2472$

$\begin{array}{lll}\text { IHT000014 } & 0.0076 & 48.7179\end{array}$

IHT $000015 \quad 0.0153 \quad 86.9318$

$\begin{array}{lll}\text { IHTO000016 } & 0.0102 & 20.6897\end{array}$

$\begin{array}{lll}\text { IHTO00017 } & 0.0085 & 13.9344\end{array}$

$\begin{array}{lll}\text { IHT000018 } & 0.0111 & 67.2727\end{array}$

$\begin{array}{lll}\text { IHTO00019 } & 0.0048 & 1.7410\end{array}$

$\begin{array}{lll}\text { IHTO } 00020 & 0.0041 & 7.8095\end{array}$

IHTO00021 $0.0219 \quad 83.2700$

IHTO00022 $0.0144 \quad 11.9107$

IHT000023 $0.0314 \quad 22.7701$

$\begin{array}{lll}\text { IHT000024 } & 0.0008 & 0.2525\end{array}$

$\begin{array}{lll}\text { IHTO } 000025 & 0.0373 & 13.8559\end{array}$

IHT000026 $\quad 0.0195 \quad 696.4285$

$\begin{array}{lll}\text { IHTO00027 } & 0.0321 & 145.2489\end{array}$

IHTO00028

$0.0463 \quad 180.8594$ 


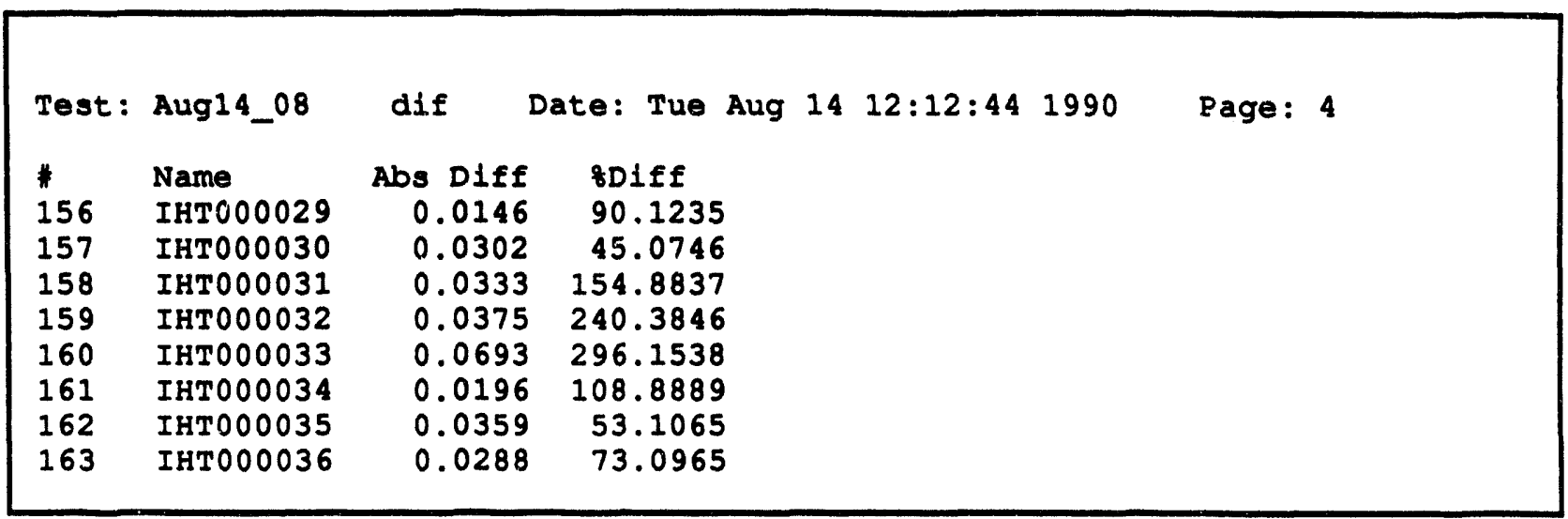




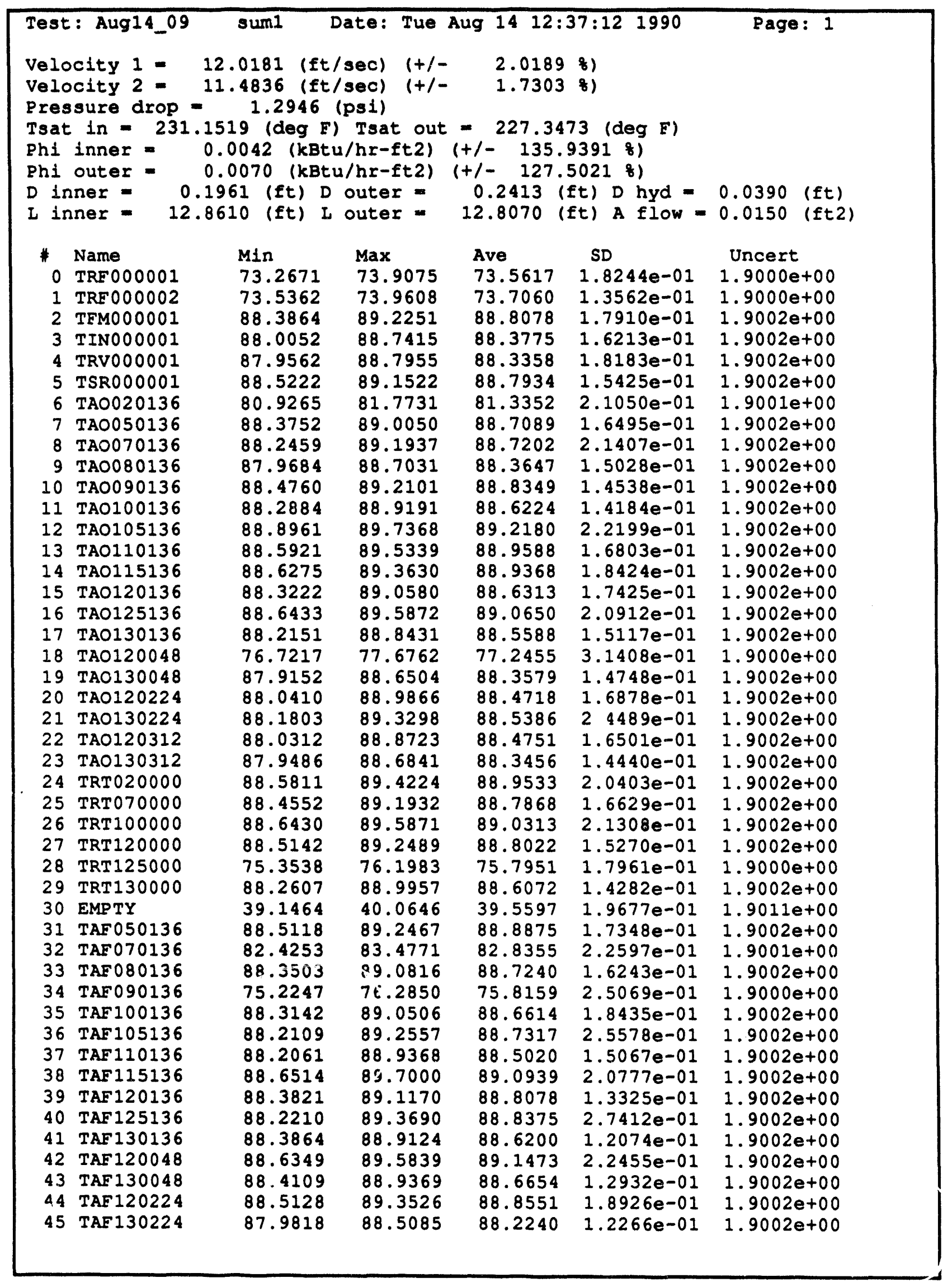




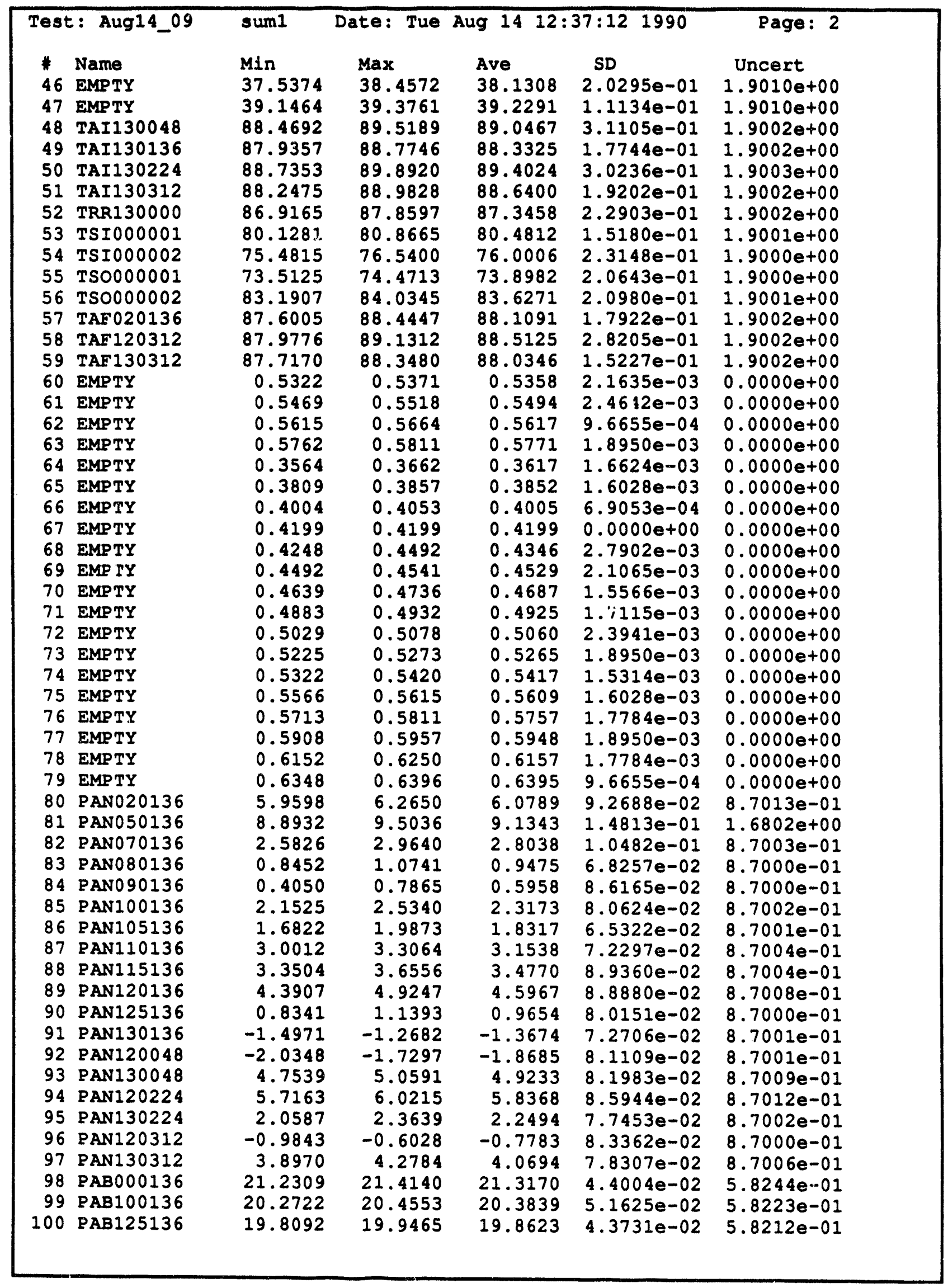




\begin{tabular}{|c|c|c|c|c|c|c|}
\hline \multicolumn{2}{|c|}{ Test: Aug14_09 } & sum 1 & Date: Tue & \multicolumn{2}{|l|}{ A } & Page: 3 \\
\hline * & Name & $\operatorname{Min}$ & $\operatorname{Max}$ & Ave & SD & Uncert \\
\hline 101 & PIN000001 & 21.1599 & 21.5261 & 21.1916 & $6.8921 e-02$ & $7.5187 e-01$ \\
\hline 102 & PRVO00001 & 18.7420 & 19.1082 & 19.0960 & $5.6508 \theta-02$ & $7.5152 e-01$ \\
\hline 103 & PSRO00001 & 19.6691 & 19.7911 & 19.7716 & $4.5206 e-02$ & $7.5163 e-01$ \\
\hline 104 & PEMO00001 & 21.7825 & 22.4234 & 22.0846 & $1.4941 e-01$ & $5.0304 e-01$ \\
\hline 105 & PAN000136 & 34.3766 & 37.1232 & 35.8415 & $5.7010 e-01$ & $4.9208 e+00$ \\
\hline 106 & PFM000002 & 18.9776 & 20.8905 & 20.1492 & $3.8125 \theta-01$ & $2.0625 e-01$ \\
\hline 107 & EMPTY & 0.0049 & 0.0098 & 0.0051 & $9.6655 e-04$ & $0.0000 e+00$ \\
\hline 108 & EMPTY & -10.0000 & -3.0615 & -7.8917 & $1.3805 e+00$ & $0.0000 e+00$ \\
\hline 109 & EMPTY & 0.0000 & 0.0049 & 0.0046 & $1.1714 \mathrm{e}-03$ & 0.0000 e +00 \\
\hline 110 & EMPTY & -10.0000 & -10.0000 & -10.0000 & $0.0000 e+00$ & $0.0000 e+00$ \\
\hline 111 & EMPTY & 0.0000 & 0.0000 & 0.0000 & $0.0000 e+00$ & $0.0000 e+00$ \\
\hline 112 & VPW000001 & -1.0479 & -0.6573 & -0.7549 & $1.0625 e-01$ & $8.1001 e-01$ \\
\hline 113 & VPW000002 & -1.0404 & -0.4545 & -0.7787 & $1.1578 \mathrm{e}-01$ & $8.1001 e-01$ \\
\hline 114 & VPW000003 & -0.9240 & -0.5333 & -0.6232 & $1.0595 e-01$ & $8.1001 e-01$ \\
\hline 115 & VPW000004 & -0.8752 & -0.4845 & -0.6291 & $9.5112 e-02$ & $8.1001 e-01$ \\
\hline 116 & VPW000005 & -1.4686 & -0.8827 & -1.2420 & $1.2072 e-01$ & $8.1003 e-01$ \\
\hline 117 & VPW000006 & -1.6489 & -1.2583 & -1.4653 & $9.1779 e-02$ & $8.1005 e-01$ \\
\hline 118 & IPW000001 & -11.6766 & -7.7719 & -9.8023 & $9.6293 e-01$ & $8.1004 e+00$ \\
\hline 119 & IPW000002 & -12.2331 & -6.3793 & -9.1111 & $1.3075 e+00$ & $8.1003 e+00$ \\
\hline 120 & IPW000003 & -12.8625 & -8.9625 & -10.5615 & $1.0190 \mathrm{e}+00$ & $8.1004 e+00$ \\
\hline 121 & IPW000004 & -2.2536 & -1.2771 & -1.8728 & $2.8403 e-01$ & $2.0001 e+00$ \\
\hline 122 & IPW000005 & -4.4786 & -3.0139 & -3.4435 & $2.8993 e-01$ & $2.0002 e+00$ \\
\hline 123 & IPW000006 & -2.8076 & -1.3427 & -2.2216 & $3.4173 e-01$ & $2.0001 e+00$ \\
\hline 124 & ЕМРTY & -0.0244 & -0.0146 & -0.0198 & $3.4751 e-03$ & $0.0000 e+00$ \\
\hline 125 & EMPTY & -0.0195 & -0.0098 & -0.0132 & $2.4662 \mathrm{e}-03$ & $0.0000 e+00$ \\
\hline 126 & EMPTY & -0.0244 & -0.0098 & -0.0165 & $3.2554 e-03$ & $0.0000 e+00$ \\
\hline 127 & EMPTY & -0.0244 & -0.0098 & -0.0156 & $3.1195 e-03$ & $0.0000 e+00$ \\
\hline 128 & IHT000001 & -0.2066 & 0.1841 & -0.0230 & $8.2864 e-02$ & $4.1000 e+00$ \\
\hline 129 & IHT 000002 & -0.2329 & 0.1577 & -0.0337 & $7.3716 e-02$ & $4.1000 e+00$ \\
\hline 130 & IHT000003 & -0.0864 & 0.3042 & -0.0122 & $1.0358 e-01$ & $4.1000 e+00$ \\
\hline 131 & IHT000004 & -0.2028 & 0.1878 & -0.0192 & $9.1779 e-02$ & $4.1000 e+00$ \\
\hline 132 & IHT000005 & -0.2066 & 0.1841 & -0.0425 & $9.9512 \mathrm{e}-02$ & $4.1000 \mathrm{e}+00$ \\
\hline 133 & IHT000006 & -0.0601 & 0.1352 & 0.0297 & $9.8332 e-02$ & $4.1000 e+00$ \\
\hline 134 & IHT000007 & -0.2554 & .0 .1352 & -0.0405 & $9.0412 e-02$ & $4.1000 e+00$ \\
\hline 135 & IHT 000008 & -0.1578 & 0.0376 & -0.0796 & $9.6655 e-02$ & $4.1000 e+00$ \\
\hline 136 & IHT000009 & -0.4169 & 0.1690 & -0.0263 & $8.8233 e-02$ & $4.1000 e+00$ \\
\hline 137 & IHT000010 & -0.1427 & $0.24,79$ & -0.0529 & $1.2608 e-01$ & $4.1000 e+00$ \\
\hline 138 & IHT000011 & -0.3042 & 0.2817 & -0.0308 & $1.1838 e-01$ & $4.1000 e+00$ \\
\hline 139 & IHT000012 & -0.1653 & 0.0301 & -0.0598 & $9.8332 e-02$ & $4.1000 e+00$ \\
\hline 140 & IHT 000013 & -0.0563 & 0.3343 & 0.0882 & $9.5112 e-02$ & $4.1000 \mathrm{e}+00$ \\
\hline 141 & IHTO00014 & -0.1953 & 0.1953 & -0.0078 & $1.0410 e-01$ & $4.1000 e+00$ \\
\hline 142 & IHT000015 & -0.2442 & 0.1465 & -0.0137 & $8.5454 e-02$ & $4.1000 e+00$ \\
\hline 143 & IHT000016 & -0.1352 & 0.0601 & -0.0415 & $9.8569 e-02$ & $4.1000 e+00$ \\
\hline 144 & IHTO00017 & -0.3530 & 0.6235 & 0.1470 & $2.7384 e-01$ & $4.1000 \mathrm{e}+00$ \\
\hline 145 & IHT000018 & -0.1728 & 0.2178 & 0.0303 & $9.6332 \theta-02$ & $4.1000 \mathrm{e}+00$ \\
\hline 146 & IHT000019 & 0.0413 & 0.4319 & 0.2249 & $1.0741 e-01$ & $4.1000 e+00$ \\
\hline 147 & IHT000020 & -0.4470 & 0.1390 & -0.0876 & $1.0705 e-01$ & $4.1000 e+00$ \\
\hline 148 & IHTO00021 & -0.0714 & 0.1240 & 0.0263 & $9.8648 e-02$ & $4.1000 e+00$ \\
\hline 149 & IHTO00022 & 0.0037 & 0.1991 & 0.1287 & $9.4702 e-02$ & $4.1000 e+00$ \\
\hline 150 & IHTO000023 & -0.0300 & 0.5559 & 0.1457 & $1.1334 e-01$ & $4.1000 e+00$ \\
\hline 151 & IHT000024 & -0.4770 & -0.0864 & -0.2817 & $7.8918 e-02$ & $4.1000 e+00$ \\
\hline 152 & IHT000025 & 0.2028 & 0.3981 & 0.2692 & $9.3461 e-02$ & $4.1000 e+00$ \\
\hline 153 & IHT 000026 & -0.1690 & 0.2216 & 0.0185 & $5.5243 e-02$ & $4.1000 e+00$ \\
\hline 154 & IHTO00027 & -0.0338 & 0.1615 & -0.0104 & $6.4113 e-02$ & $4.1000 e+00$ \\
\hline 155 & IHTO00028 & -0.2479 & 0.3381 & 0.0021 & $1.1189 \mathrm{e}-01$ & $4.1000 \mathrm{e}+00$ \\
\hline
\end{tabular}




\begin{tabular}{|c|c|c|c|c|c|c|}
\hline \multicolumn{2}{|c|}{ Test: Aug14_09 } & sum1 & Date: Tue & \multicolumn{2}{|c|}{ Aug $14 \quad 12: 37: 12 \quad 1990$} & $\begin{array}{l}\text { Page: } 4 \\
\text { Uncert }\end{array}$ \\
\hline $\begin{array}{l}\text { * } \\
156 \\
157 \\
158 \\
159 \\
160 \\
161 \\
162 \\
163\end{array}$ & $\begin{array}{l}\text { Name } \\
\text { IHTO000029 } \\
\text { IHTO00030 } \\
\text { IHTO00031 } \\
\text { IHTO00032 } \\
\text { IHTO00033 } \\
\text { IHTO00034 } \\
\text { IHTO0O035 } \\
\text { IHTO00036 }\end{array}$ & $\begin{array}{l}\text { Min } \\
-0.2780 \\
-0.2103 \\
-0.1465 \\
-0.0976 \\
-0.0977 \\
-0.2329 \\
-0.1277 \\
-0.1878\end{array}$ & $\begin{array}{l}\operatorname{Max} \\
0.3080 \\
0.3756 \\
0.2441 \\
0.2930 \\
0.2930 \\
0.1577 \\
0.2629 \\
0.0075\end{array}$ & $\begin{array}{r}\text { Ave } \\
-0.0162 \\
0.0397 \\
-0.0137 \\
-0.0039 \\
-0.0078 \\
0.0015 \\
0.0676 \\
-0.0667\end{array}$ & $\begin{array}{l}\text { SD } \\
1.0885 e-01 \\
1.0470 e-01 \\
1.2750 e-01 \\
1.1327 e-01 \\
1.0595 e-01 \\
8.8233 e-02 \\
9.6655 e-02 \\
9.5765 e-02\end{array}$ & $\begin{array}{c}\text { Uncert } \\
4.1000 \mathrm{e}+00 \\
4.1000 \mathrm{e}+00 \\
4.1000 \mathrm{e}+00 \\
4.1000 \mathrm{e}+00 \\
4.1000 \mathrm{e}+00 \\
4.1000 \mathrm{e}+00 \\
4.1000 \mathrm{e}+00 \\
4.1000 \mathrm{e}+00\end{array}$ \\
\hline
\end{tabular}




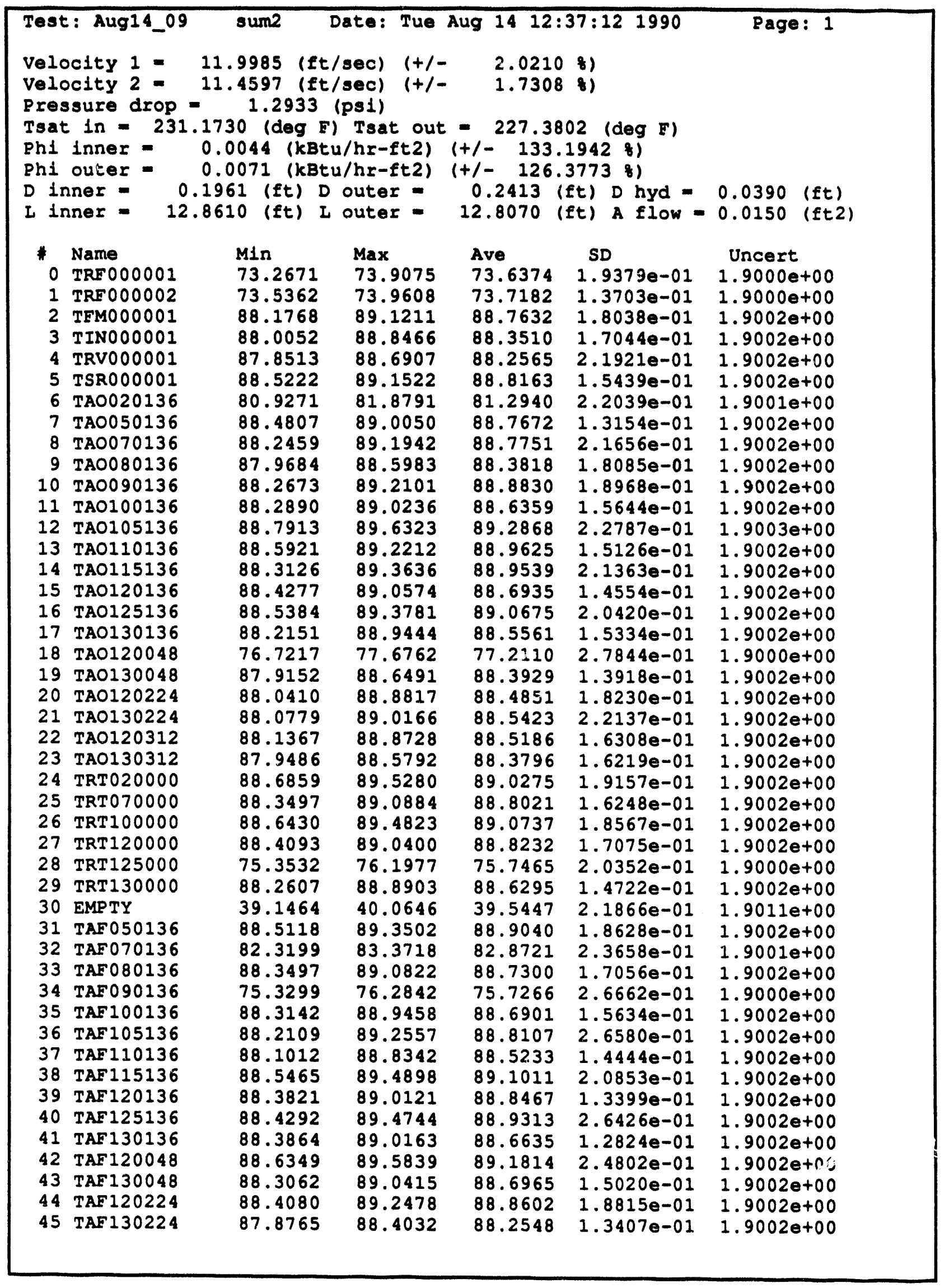




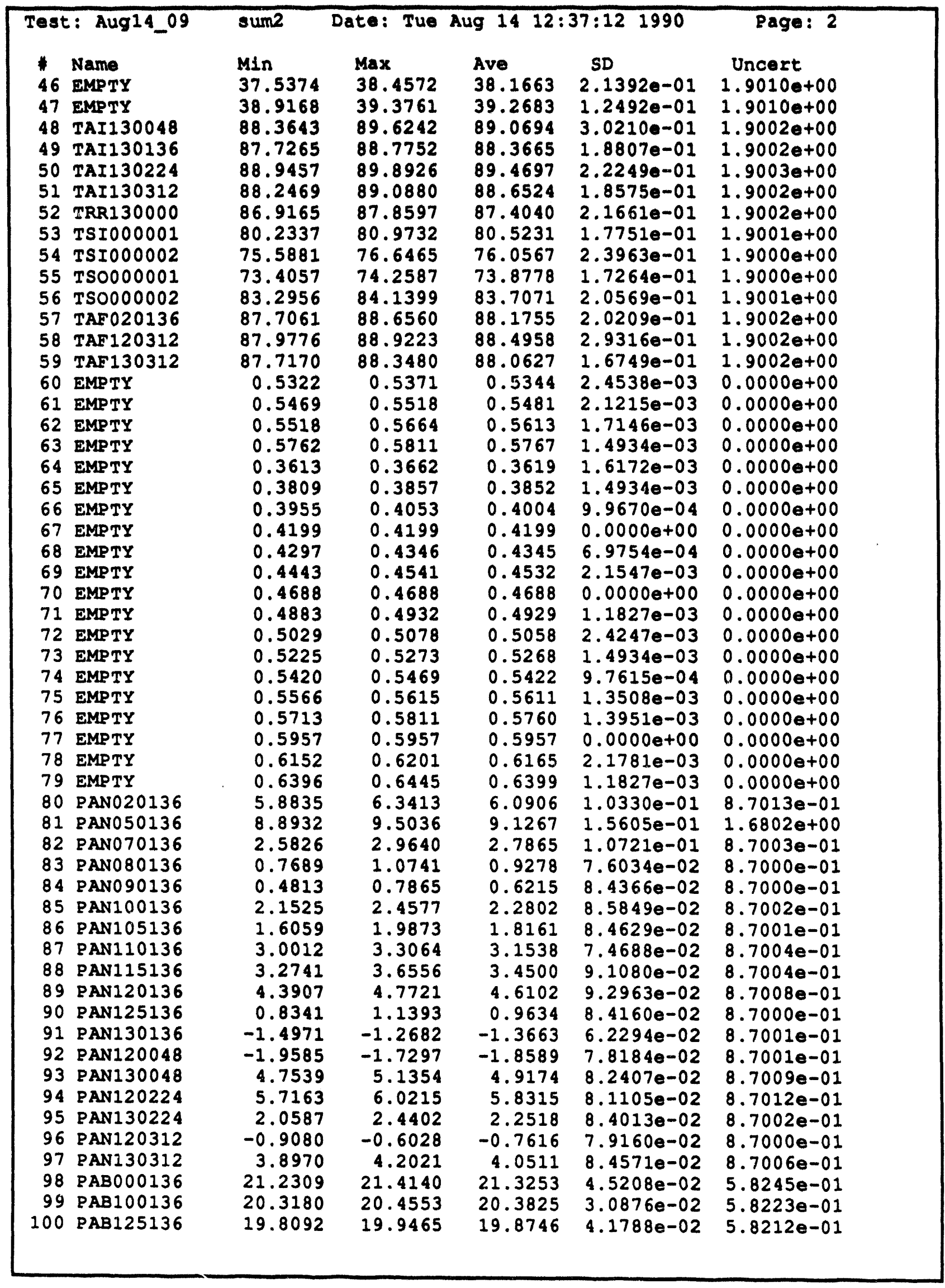




\begin{tabular}{|c|c|c|c|c|c|c|}
\hline $\begin{array}{l}1 \\
101 \\
102 \\
103 \\
104 \\
105 \\
106 \\
107 \\
108 \\
109 \\
110 \\
111 \\
112 \\
113 \\
114 \\
115 \\
116 \\
117 \\
118 \\
119 \\
120 \\
121 \\
122 \\
123 \\
124 \\
125 \\
126 \\
127 \\
128 \\
129 \\
130 \\
131 \\
132 \\
133 \\
134 \\
135 \\
136 \\
137 \\
138 \\
139 \\
140 \\
141 \\
142 \\
143 \\
144 \\
145 \\
146 \\
147 \\
148 \\
149 \\
150 \\
151 \\
152 \\
153 \\
154 \\
155\end{array}$ & 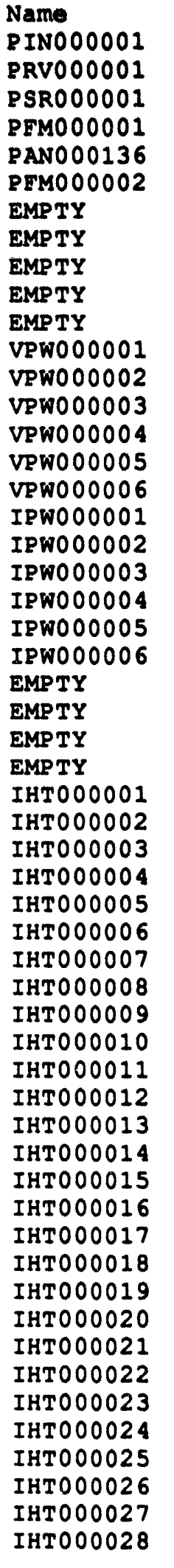 & $\begin{array}{l}\text { M1n } \\
21.1599 \\
18.9861 \\
19.6691 \\
21.6909 \\
34.8344 \\
19.2964 \\
0.0000 \\
-10.0000 \\
0.0000 \\
-10.0000 \\
0.0000 \\
-1.0479 \\
-0.8451 \\
-0.9240 \\
-0.8752 \\
-1.4686 \\
-1.6489 \\
-11.6766 \\
-12.2331 \\
-12.8625 \\
-2.2536 \\
-3.9904 \\
-2.8076 \\
-0.0244 \\
-0.0244 \\
-0.0195 \\
-0.0244 \\
-0.2066 \\
-0.2329 \\
-0.0864 \\
-0.2028 \\
-0.2066 \\
-0.0601 \\
-0.0601 \\
-0.1578 \\
-0.2216 \\
-0.1427 \\
-0.1089 \\
-0.1653 \\
-0.0563 \\
-0.1953 \\
-0.2442 \\
-0.1352 \\
-0.3530 \\
-0.1728 \\
0.0413 \\
-0.2517 \\
-0.0714 \\
0.0037 \\
-0.2254 \\
-0.4770 \\
0.2028 \\
-0.1690 \\
-0.0338 \\
-0.2479\end{array}$ & $\begin{array}{r}\text { Max } \\
21.5261 \\
19.1082 \\
19.7911 \\
22.3318 \\
36.6655 \\
20.6388 \\
0.0098 \\
-0.6348 \\
0.0049 \\
-10.0000 \\
0.0049 \\
-0.6573 \\
-0.4545 \\
-0.3380 \\
-0.4845 \\
-1.0780 \\
-1.2583 \\
-7.7719 \\
-6.3793 \\
-8.9625 \\
-1.2771 \\
-3.0139 \\
-1.3427 \\
-0.0146 \\
-0.0098 \\
-0.0098 \\
-0.0098 \\
0.5747 \\
0.1577 \\
0.1089 \\
0.1878 \\
0.1841 \\
0.1352 \\
0.1352 \\
0.0376 \\
0.1690 \\
0.0526 \\
0.0864 \\
0.0301 \\
0.3343 \\
0.1953 \\
0.1465 \\
0.2554 \\
0.6235 \\
0.2178 \\
0.4319 \\
0.1390 \\
0.3193 \\
0.1991 \\
0.3606 \\
-0.0864 \\
0.3981 \\
0.2216 \\
0.1615 \\
0.1427\end{array}$ & $\begin{array}{r}\text { Ave } \\
21.1922 \\
19.1007 \\
19.7837 \\
22.0123 \\
35.8060 \\
20.0649 \\
0.0049 \\
-7.8758 \\
0.0048 \\
-10.0000 \\
0.0001 \\
-0.7530 \\
-0.7813 \\
-0.6569 \\
-0.6440 \\
-1.2733 \\
-1.4496 \\
-9.6047 \\
-9.4058 \\
-10.8329 \\
-1.8550 \\
-3.5121 \\
-2.1599 \\
-0.0199 \\
-0.0139 \\
-0.0169 \\
-0.0157 \\
-0.0073 \\
-0.0296 \\
0.0053 \\
0.0005 \\
-0.0232 \\
0.0635 \\
-0.0162 \\
-0.0780 \\
-0.0263 \\
-0.0550 \\
-0.0531 \\
-0.0895 \\
0.0832 \\
-0.0080 \\
-0.0050 \\
-0.0196 \\
0.1412 \\
0.0026 \\
0.2486 \\
-0.0564 \\
0.0403 \\
0.1393 \\
0.1453 \\
-0.2578 \\
0.2985 \\
0.0103 \\
0.0300 \\
-0.0008\end{array}$ & $\begin{array}{l}\text { SD } \\
7.3814 e-02 \\
2.9569 e-02 \\
2.9569 e-02 \\
1.4848 e-01 \\
5.1716 e-01 \\
3.1584 e-01 \\
9.9670 e-04 \\
1.8247 e+00 \\
6.9754 e-04 \\
0.0000 e+00 \\
6.9754 e-04 \\
1.0640 e-01 \\
1.0076 e-01 \\
1.1755 e-01 \\
1.1044 e-01 \\
1.1960 e-01 \\
1.0156 e-01 \\
1.3460 e+00 \\
1.4936 e+00 \\
1.1230 e+00 \\
2.3740 e-01 \\
3.37819-01 \\
3.0538 e-01 \\
3.5710 e-03 \\
3.3574 e-03 \\
2.8370 e-03 \\
3.3453 e-03 \\
1.1616 e-01 \\
9.7323 e-02 \\
9.8483 e-02 \\
1.0517 e-01 \\
1.0092 e-01 \\
9.5132 e-02 \\
8.2338 e-02 \\
9.6990 e-02 \\
7.9736 e-02 \\
9.8153 e-02 \\
8.9148 e-02 \\
9.6150 e-02 \\
1.0548 e-01 \\
8.8783 e-02 \\
9.1482 e-02 \\
1.0486 e-01 \\
2.7362 e-01 \\
8.2141 e-02 \\
1.0851 e-01 \\
8.9148 e-02 \\
1.0548 e-01 \\
9.0949 e-02 \\
1.0731 e-01 \\
9.4620 e-02 \\
9.8648 e-02 \\
7.8092 e-02 \\
9.2540 e-02 \\
1.0378 e-01\end{array}$ & $\begin{array}{l}\text { Uncert } \\
7.5187 e-01 \\
7.5152 e-01 \\
7.5163 e-01 \\
5.0302 e-01 \\
4.9208 e+00 \\
2.0619 e-01 \\
0.0000 e+00 \\
0.0000 e+00 \\
0.0000 e+00 \\
0.0000 e+00 \\
0.0000 e+00 \\
8.1001 e-01 \\
8.1001 e-01 \\
8.1001 e-01 \\
8.1001 e-01 \\
8.1004 e-01 \\
8.1005 e-01 \\
8.1004 e+00 \\
8.1003 e+00 \\
8.1005 e+00 \\
2.0001 e+00 \\
2.0002 e+00 \\
2.0001 e+00 \\
0.0000 e+00 \\
0.0000 e+00 \\
0.0000 e+00 \\
0.0000 e+00 \\
4.1000 e+00 \\
4.1000 e+00 \\
4.1000 e+00 \\
4.1000 e+00 \\
4.1000 e+00 \\
4.1000 e+00 \\
4.1000 e+00 \\
4.1000 e+00 \\
4.1000 e+00 \\
4.1000 e+00 \\
4.1000 e+00 \\
4.1000 e+00 \\
4.1000 e+00 \\
4.1000 e+00 \\
4.1000 e+00 \\
4.1000 e+00 \\
4.1000 e+00 \\
4.1000 e+00 \\
4.1000 e+00 \\
4.1000 e+00 \\
4.1000 e+00 \\
4.1000 e+00 \\
4.1000 e+00 \\
4.1000 e+00 \\
4.1000 e+00 \\
4.1000 e+00 \\
4.1000 e+00 \\
4.1000 e+00\end{array}$ \\
\hline
\end{tabular}




\begin{tabular}{|c|c|c|c|c|c|c|}
\hline $\begin{array}{l}1 \\
156 \\
157 \\
158 \\
159 \\
160 \\
161 \\
162 \\
163\end{array}$ & 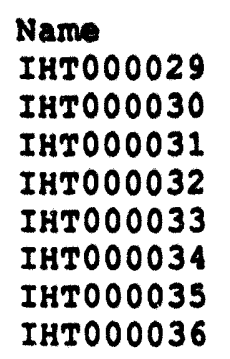 & $\begin{array}{l}M 1 n \\
-0.0826 \\
-0.2103 \\
-0.1465 \\
-0.0976 \\
-0.0977 \\
-0.2329 \\
-0.1277 \\
-0.1878\end{array}$ & $\begin{array}{l}\operatorname{Max} \\
0.3080 \\
0.3756 \\
0.2441 \\
0.2930 \\
0.2930 \\
0.3531 \\
0.2629 \\
0.2028\end{array}$ & $\begin{array}{l}\text { Ave } \\
-0.0268 \\
0.0408 \\
-0.0189 \\
0.0020 \\
-0.0020 \\
0.0063 \\
0.0317 \\
-0.0403\end{array}$ & $\begin{array}{l}\text { SD } \\
9.7656 e-02 \\
1.0548 e-01 \\
1.1658 e-01 \\
1.1362 e-01 \\
1.0640 e-01 \\
1.0746 e-01 \\
8.6187 e-02 \\
1.0941 e-01\end{array}$ & $\begin{array}{l}\text { Uncert } \\
4.1000 e+00 \\
4.1000 e+00 \\
4.1000 e+00 \\
4.1000 e+00 \\
4.1000 e+00 \\
4.1000 e+00 \\
4.1000 e+00 \\
4.1000 e+00\end{array}$ \\
\hline
\end{tabular}




\begin{tabular}{|c|c|c|c|c|}
\hline \multicolumn{5}{|c|}{$\begin{array}{l}\text { Velocity } 1=0.019600(\mathrm{ft} / \mathrm{sec}) \\
\text { Velocity } 2=0.023900(\mathrm{ft} / \mathrm{sec}) \\
\text { Pressure Drop }=0.001300 \text { (psi) }\end{array}$} \\
\hline $\begin{array}{l}0 \\
1 \\
2 \\
3 \\
4 \\
5 \\
6 \\
7 \\
8 \\
9 \\
10 \\
11 \\
12 \\
13 \\
14 \\
15 \\
16 \\
17 \\
18 \\
19 \\
20 \\
21 \\
22 \\
23 \\
24 \\
25 \\
26 \\
27 \\
28 \\
29 \\
30 \\
31 \\
32 \\
33 \\
34 \\
35 \\
36 \\
37 \\
38 \\
39 \\
40 \\
41 \\
42 \\
43 \\
44 \\
45\end{array}$ & $\begin{array}{l}\text { Name } \\
\text { TRF000001 } \\
\text { TRF000002 } \\
\text { TFM000001 } \\
\text { TIN000001 } \\
\text { TRV000001 } \\
\text { TSR000001 } \\
\text { TAO020136 } \\
\text { TAO050136 } \\
\text { TAO070136 } \\
\text { TAO080136 } \\
\text { TAO090136 } \\
\text { TAO100136 } \\
\text { TAO105136 } \\
\text { TAO110136 } \\
\text { TAO115136 } \\
\text { TAO120136 } \\
\text { TAO125136 } \\
\text { TAO130136 } \\
\text { TAO120048 } \\
\text { TAO130048 } \\
\text { TAO120224 } \\
\text { TAO130224 } \\
\text { TAO120312 } \\
\text { TAO130312 } \\
\text { TRT020000 } \\
\text { TRT070000 } \\
\text { TRT100000 } \\
\text { TRT120000 } \\
\text { TRT125000 } \\
\text { TRT130000 } \\
\text { EMPTY } \\
\text { TAF050136 } \\
\text { TAF070136 } \\
\text { TAF080136 } \\
\text { TAF090136 } \\
\text { TAF100136 } \\
\text { TAF105136 } \\
\text { TAF110136 } \\
\text { TAF115136 } \\
\text { TAF120136 } \\
\text { TAF125136 } \\
\text { TAF130136 } \\
\text { TAF120048 } \\
\text { TAF130048 } \\
\text { TAF120224 } 130224\end{array}$ & $\begin{array}{c}\text { Abs Diff } \\
0.0757 \\
0.0122 \\
0.0446 \\
0.0265 \\
0.0793 \\
0.0229 \\
0.0412 \\
0.0583 \\
0.0549 \\
0.0171 \\
0.0481 \\
0.0135 \\
0.0688 \\
0.0037 \\
0.0171 \\
0.0622 \\
0.0025 \\
0.0027 \\
0.0345 \\
0.0350 \\
0.0133 \\
0.0037 \\
0.0435 \\
0.0340 \\
0.0742 \\
0.0153 \\
0.0424 \\
0.0210 \\
0.0486 \\
0.0223 \\
0.0150 \\
0.0165 \\
0.0366 \\
0.0060 \\
0.0893 \\
0.0287 \\
0.0790 \\
0.0213 \\
0.0072 \\
0.0389 \\
0.0938 \\
0.0435 \\
0.0341 \\
0.0311 \\
0.0051 \\
0.0308\end{array}$ & $\begin{array}{l}\text { DDff } \\
0.1029 \\
0.0166 \\
0.0502 \\
0.0300 \\
0.0898 \\
0.0258 \\
0.0507 \\
0.0657 \\
0.0619 \\
0.0193 \\
0.0541 \\
0.0152 \\
0.0771 \\
0.0042 \\
0.0192 \\
0.0702 \\
0.0028 \\
0.0030 \\
0.0447 \\
0.0396 \\
0.0150 \\
0.0042 \\
0.0492 \\
0.0385 \\
0.0834 \\
0.0172 \\
0.0476 \\
0.0236 \\
0.0641 \\
0.0252 \\
0.0379 \\
0.0186 \\
0.0442 \\
0.0068 \\
0.1178 \\
0.0324 \\
0.0890 \\
0.0241 \\
0.0081 \\
0.0438 \\
0.1056 \\
0.0491 \\
0.0382 \\
0.0351 \\
0.0057 \\
0.0349\end{array}$ & \\
\hline
\end{tabular}



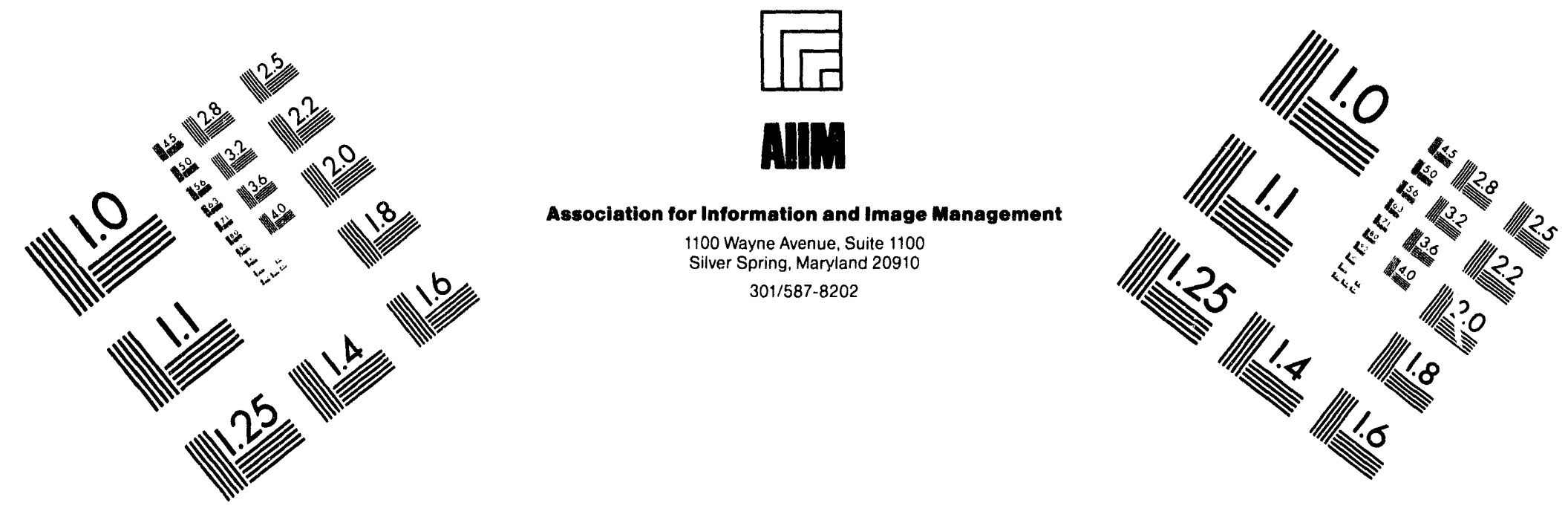

\section{Centimeter}

$\begin{array}{llllllllllllllll}1 & 2 & 3 & 4 & 5 & 6 & 7 & 8 & 9 & 10 & 11 & 12 & 13 & 14 & 15 & \mathrm{~mm}\end{array}$

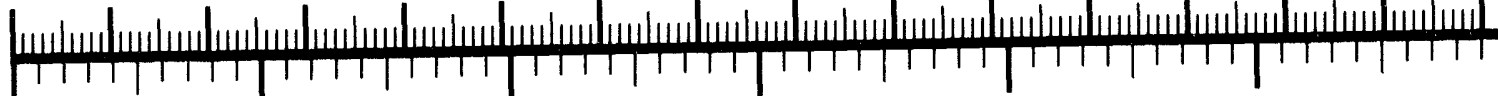

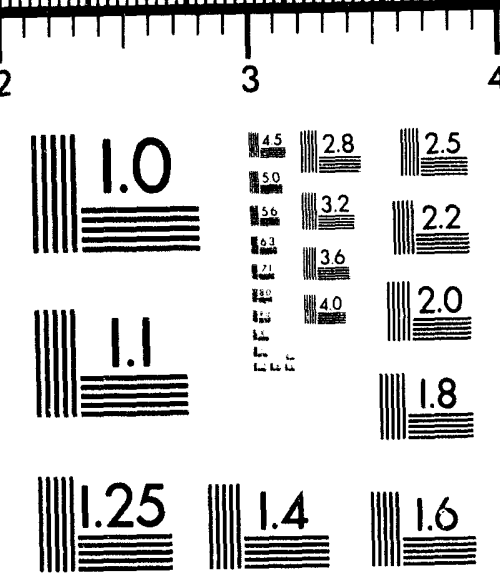

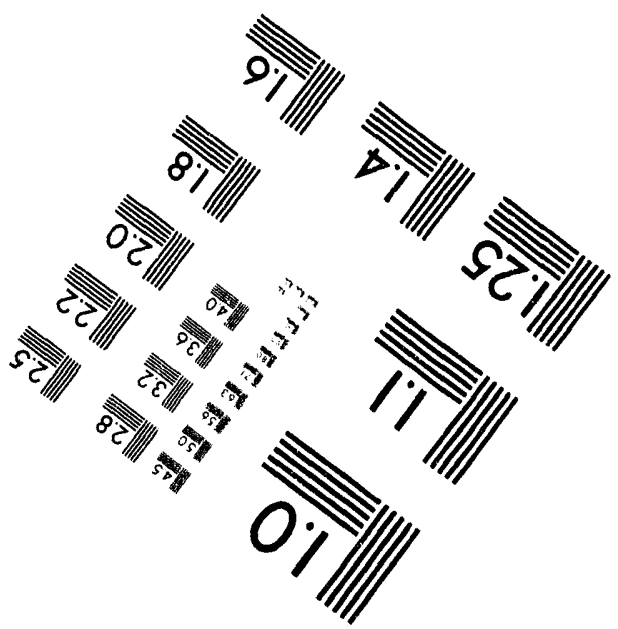

MANUFACTURED TO AIIM STANDARDS

BY APPLIED IMAGE, INC.

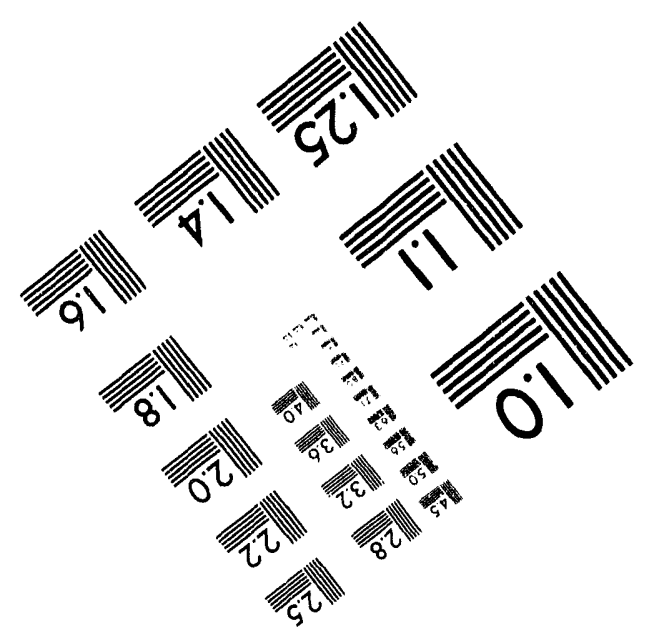




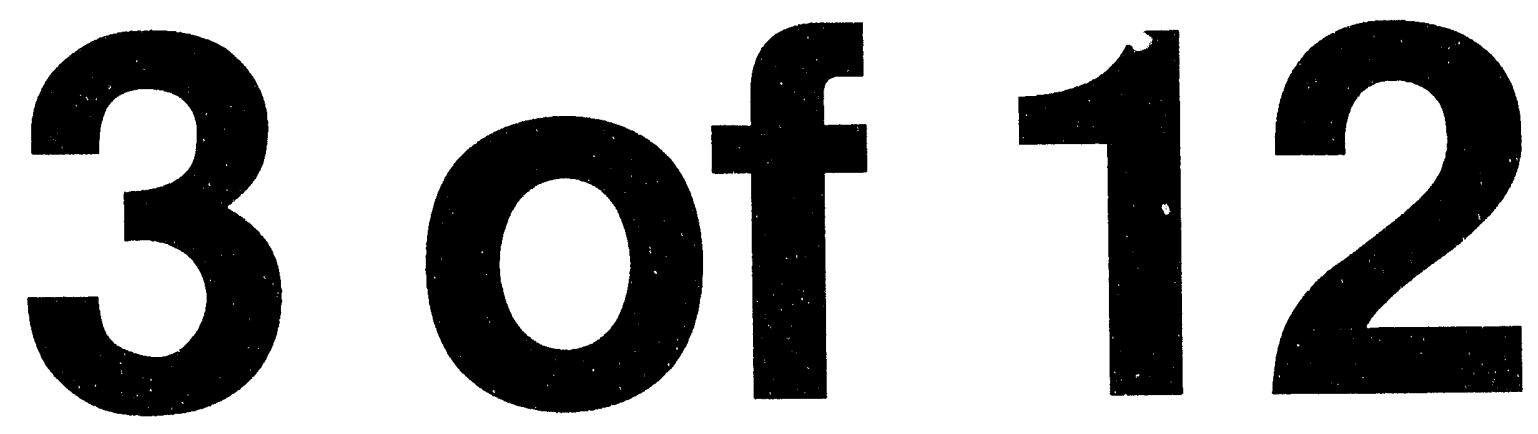




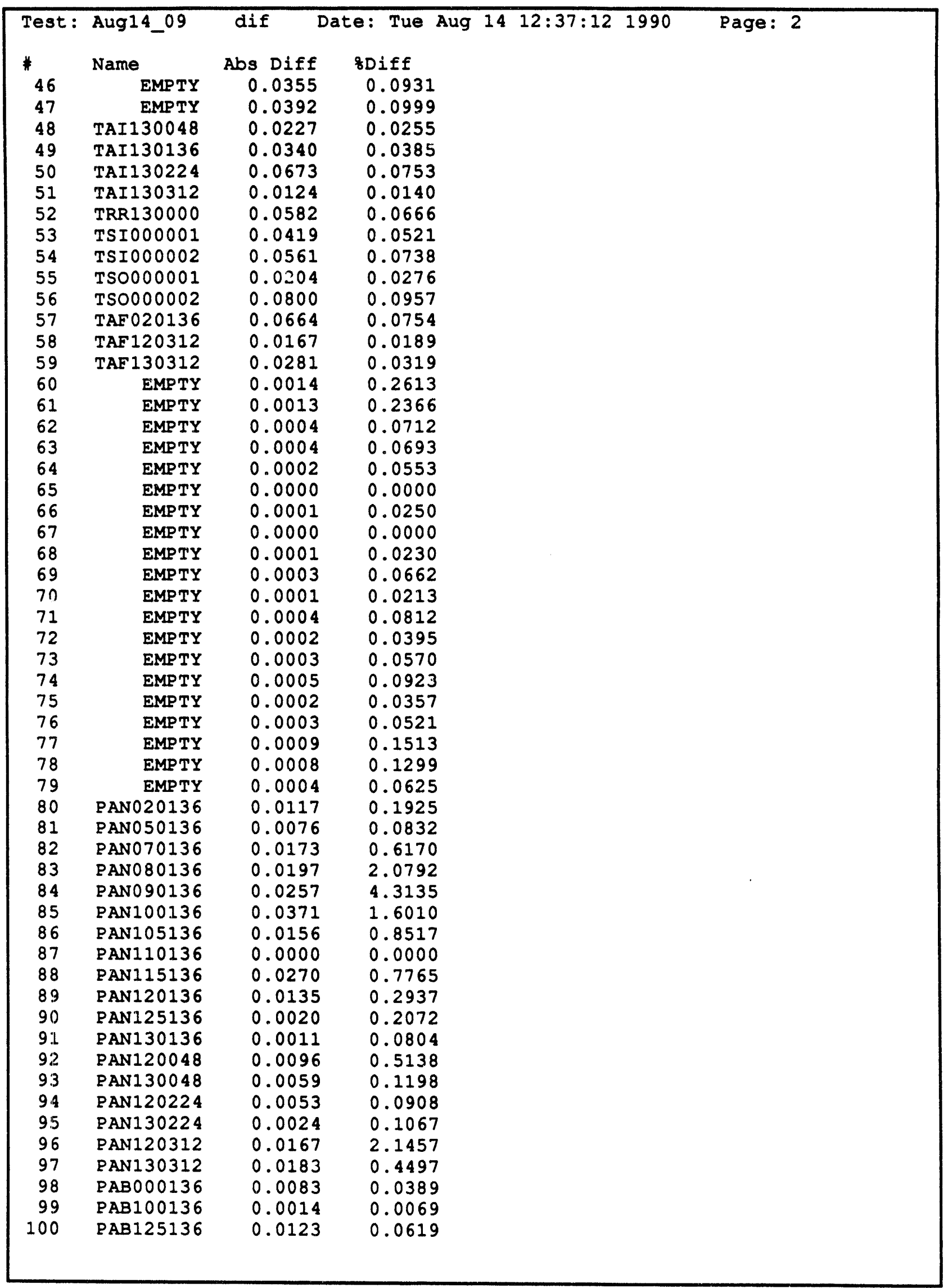




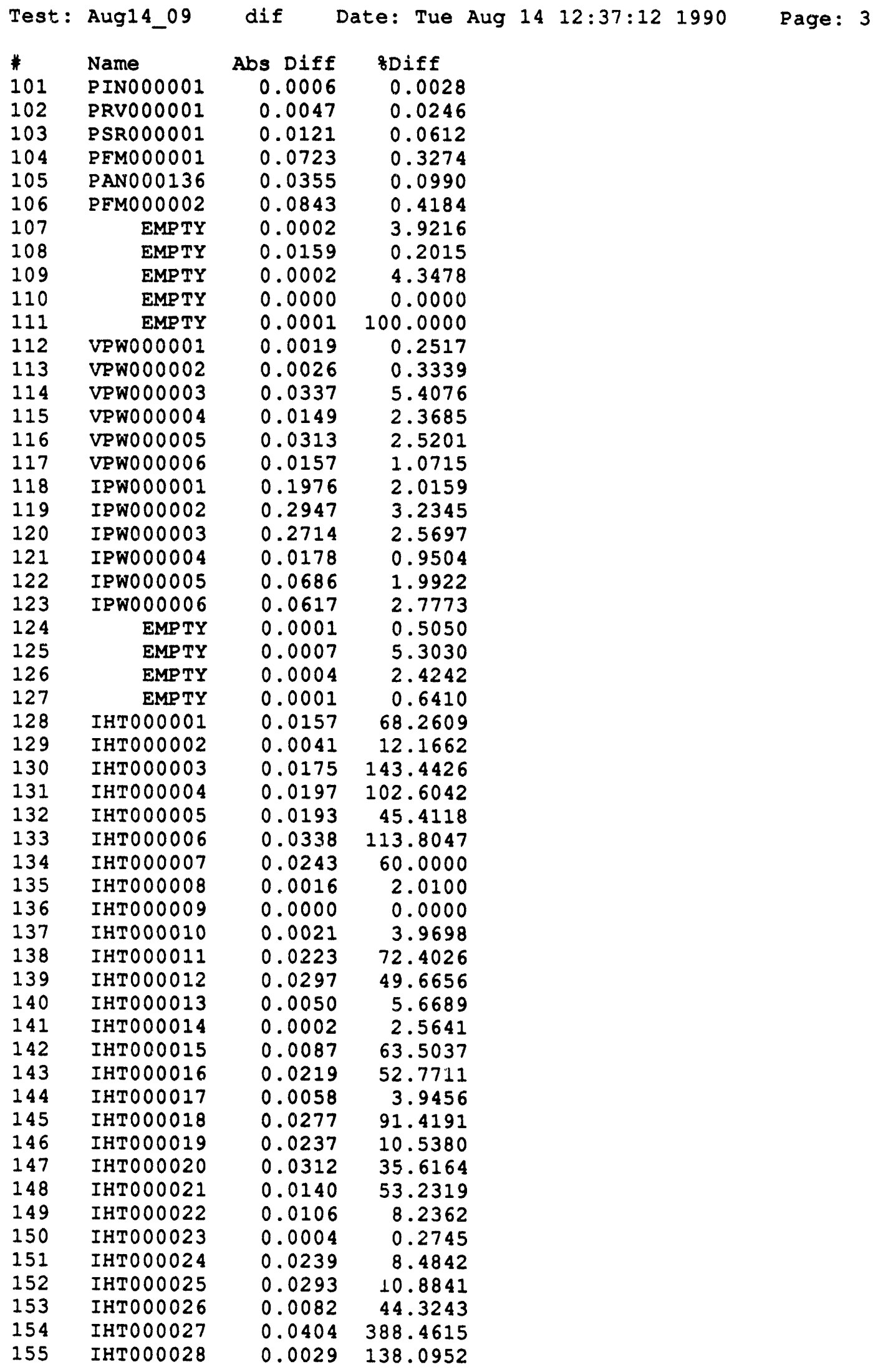

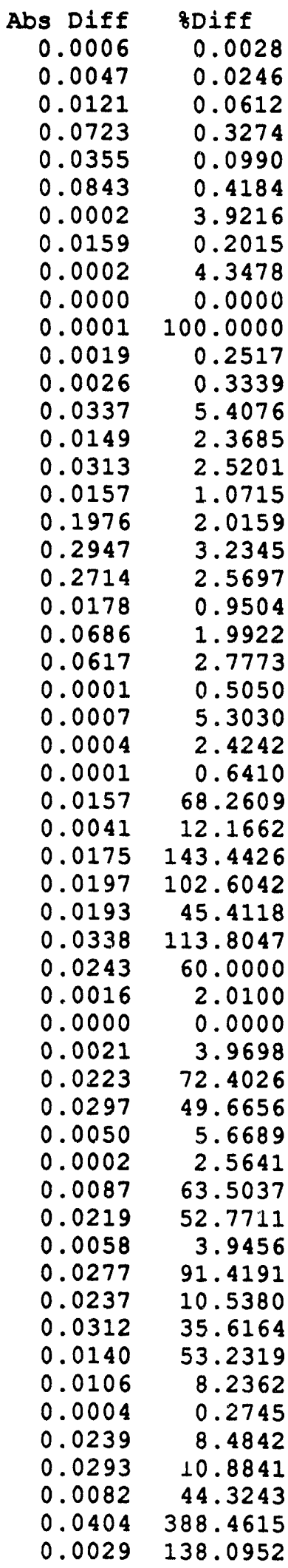


Test: Aug14_09

Name

156 IHTO00029

157 IHTO00030

158 IHTO00031

159 IHTO00032

160 IHTO00033

161 IHT000034

162 IHT000035

163 IHTO00036 dif Date: Tue Aug 14 12:37:12 1990

Page: 4
Abs Diff 8 Diff
$0.0106 \quad 65.4321$
$0.0011 \quad 2.7708$
$0.0052 \quad 37.9562$
$0.0059 \quad 151.2821$
$0.0058 \quad 74.3590$
$0.0048 \quad 320.0000$
$0.0359 \quad 53.1065$
$0.0264 \quad 39.5802$ 


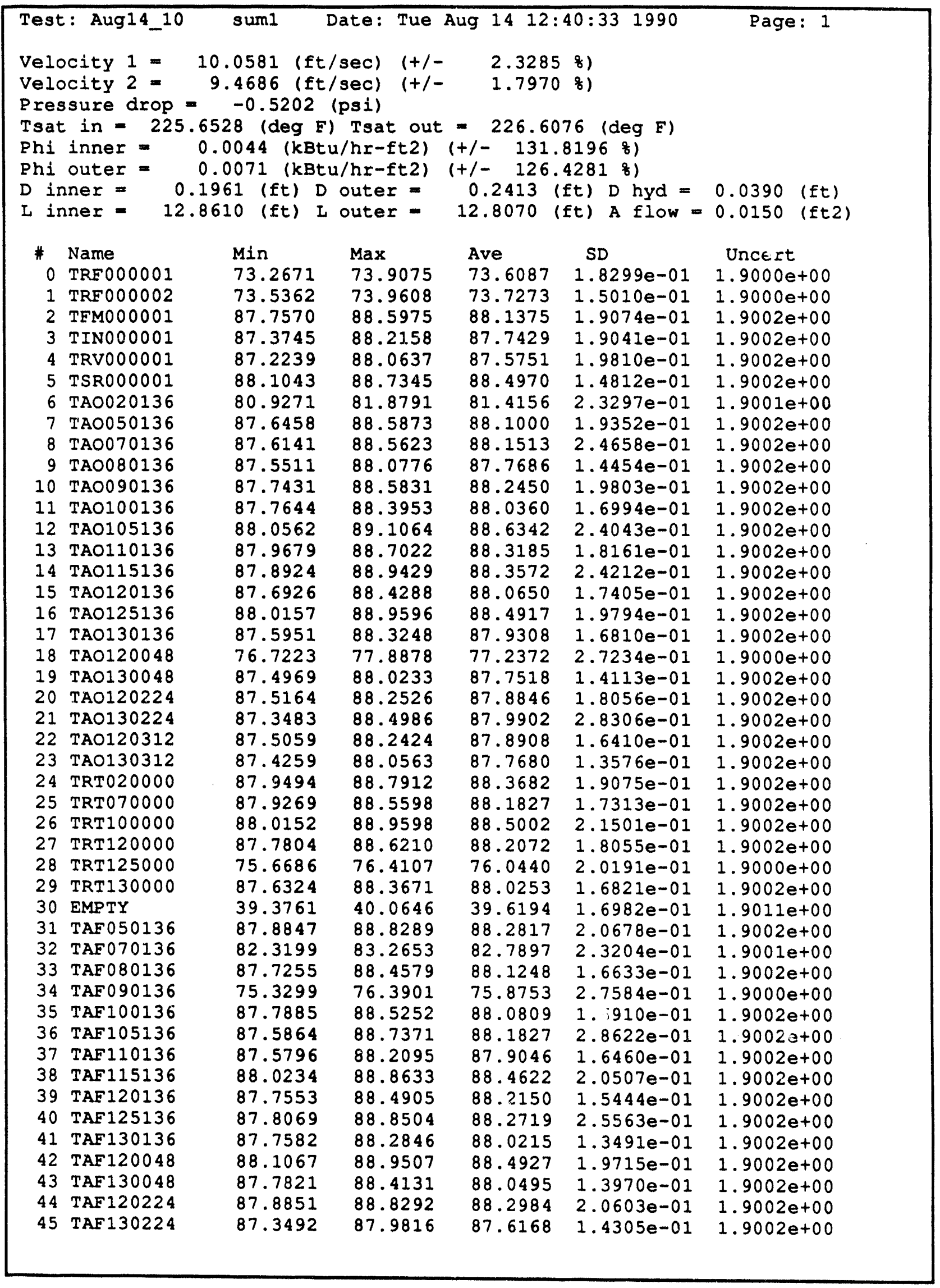




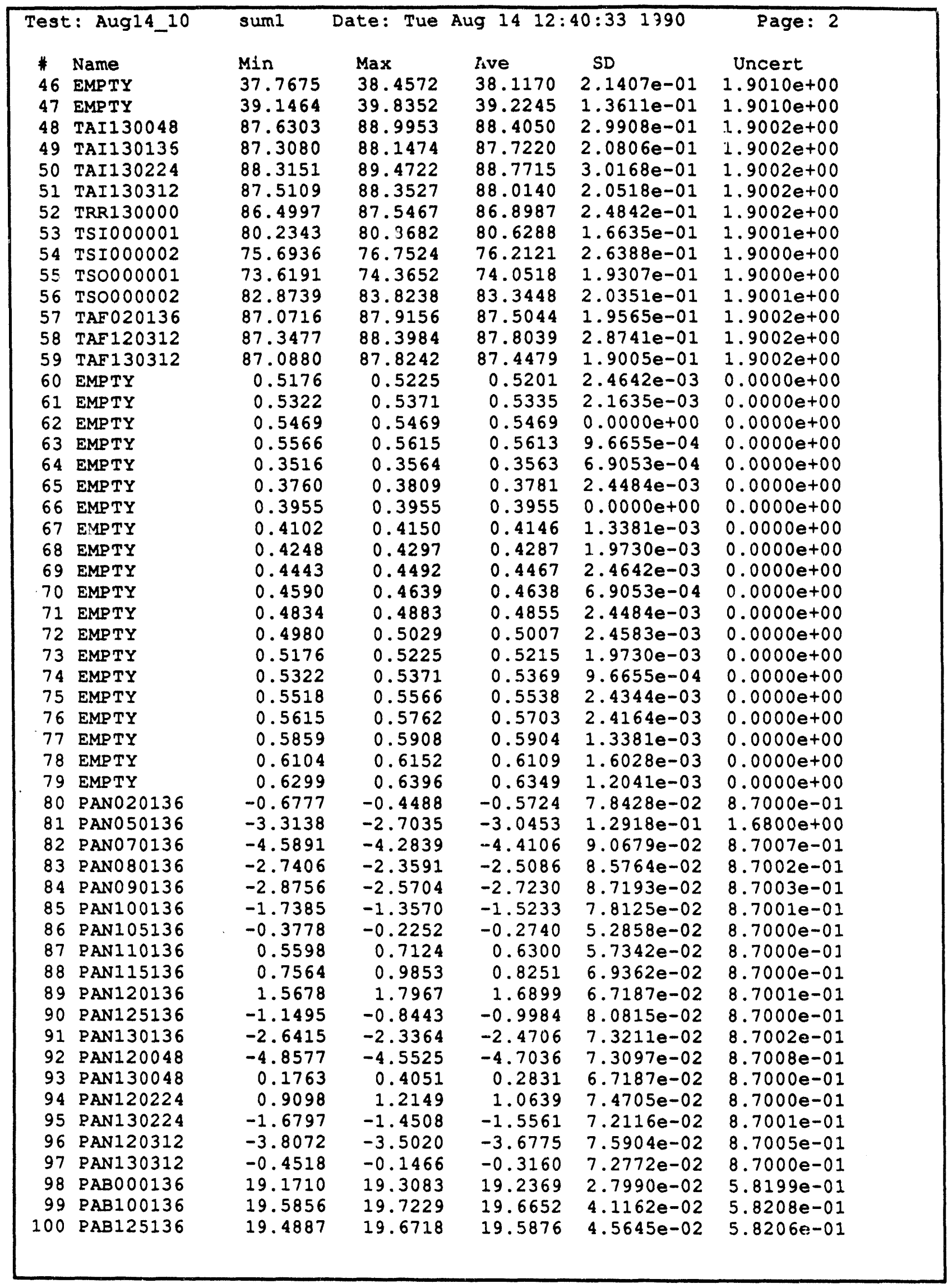




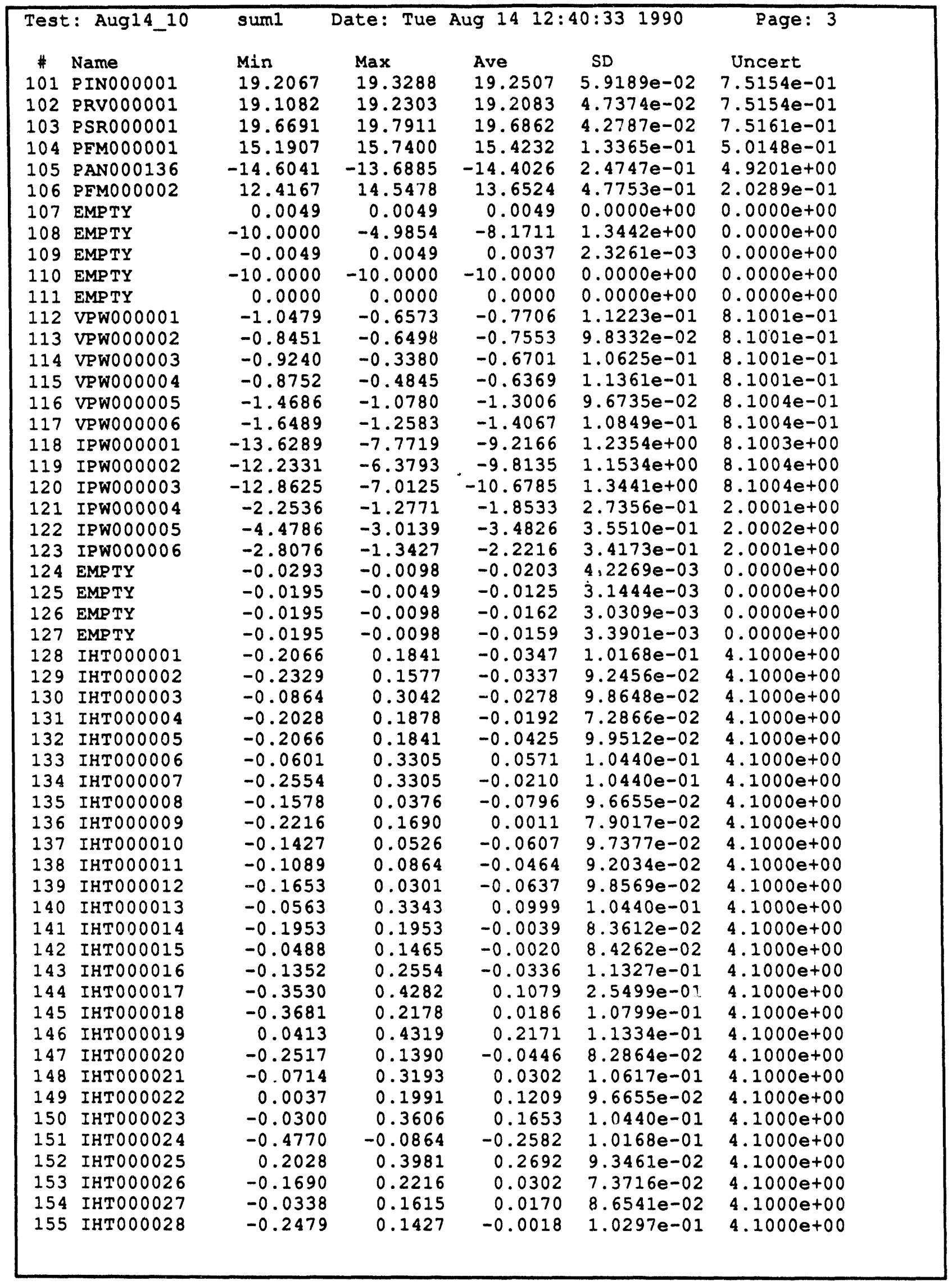




\begin{tabular}{|cllcccc|}
\hline Test: Aug14_10 & sum1 & Date: Tue Aug 14 & $12: 40: 33$ & 1990 & Page: 4 \\
\# & Name & Min & Max & Ave & SD & Uncert \\
156 & IHT000029 & -0.2780 & 0.1127 & -0.0162 & $1.0145 e-01$ & $4.1000 e+00$ \\
157 & IHTO00030 & -0.0150 & 0.1803 & 0.0436 & $9.0412 e-02$ & $4.1000 e+00$ \\
158 & IHTO00031 & -0.1465 & 0.2441 & -0.0254 & $1.1765 e-01$ & $4.1000 e+00$ \\
159 & IHT000032 & -0.0976 & 0.0977 & -0.0078 & $9.8332 e-02$ & $4.1000 e+00$ \\
160 & IHT000033 & -0.0977 & 0.0976 & 0.0039 & $9.8569 e-02$ & $4.1000 e+00$ \\
161 & IHT000034 & -0.2329 & 0.1577 & -0.0180 & $1.0625 e-01$ & $4.1000 e+00$ \\
162 & IHTO00035 & -0.1277 & 0.2629 & 0.0715 & $1.0052 e-01$ & $4.1000 e+00$ \\
163 & IHTO00036 & -0.1878 & 0.2028 & -0.0472 & $1.0470 e-01$ & $4.1000 e+00$ \\
\end{tabular}




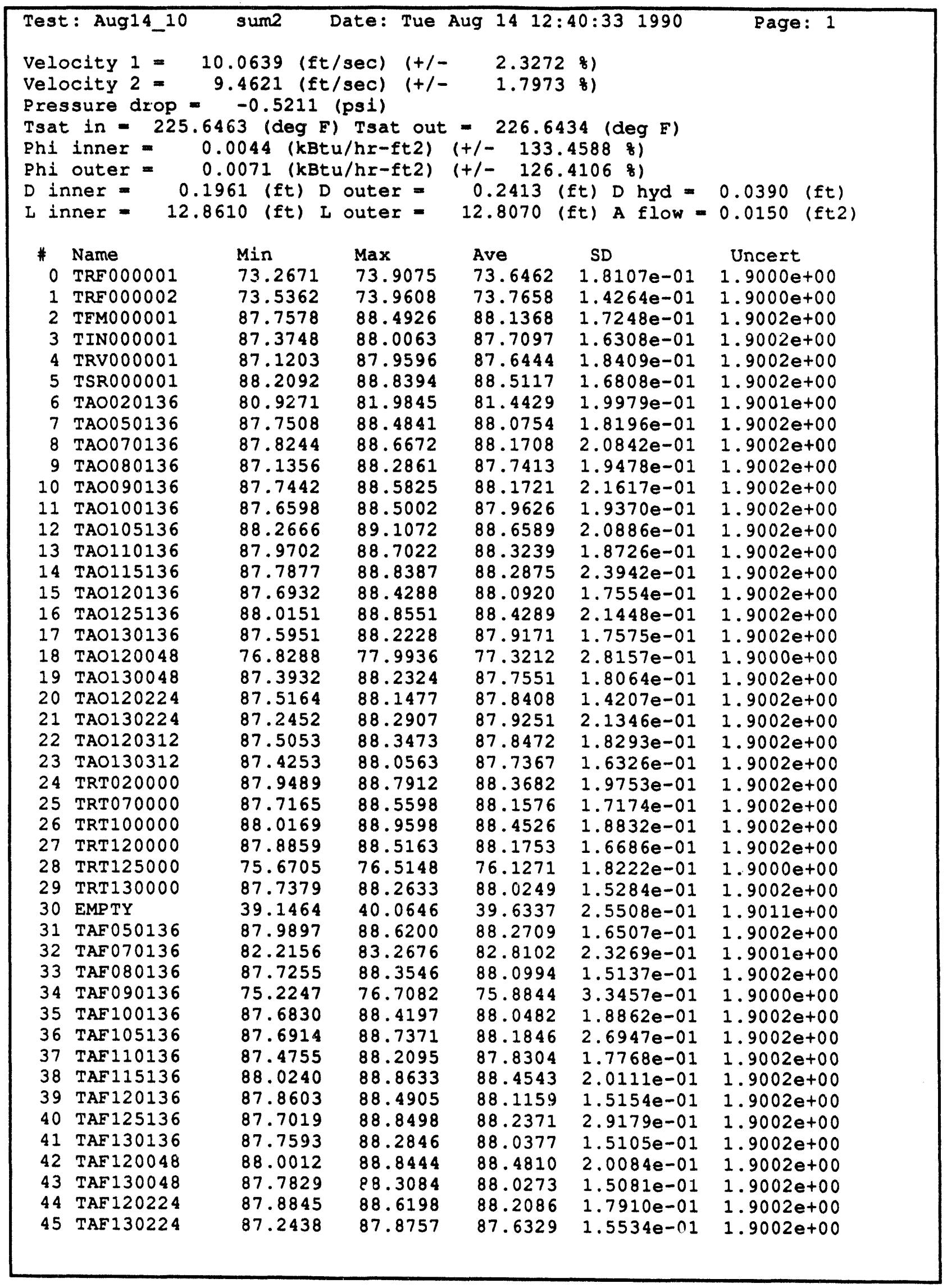




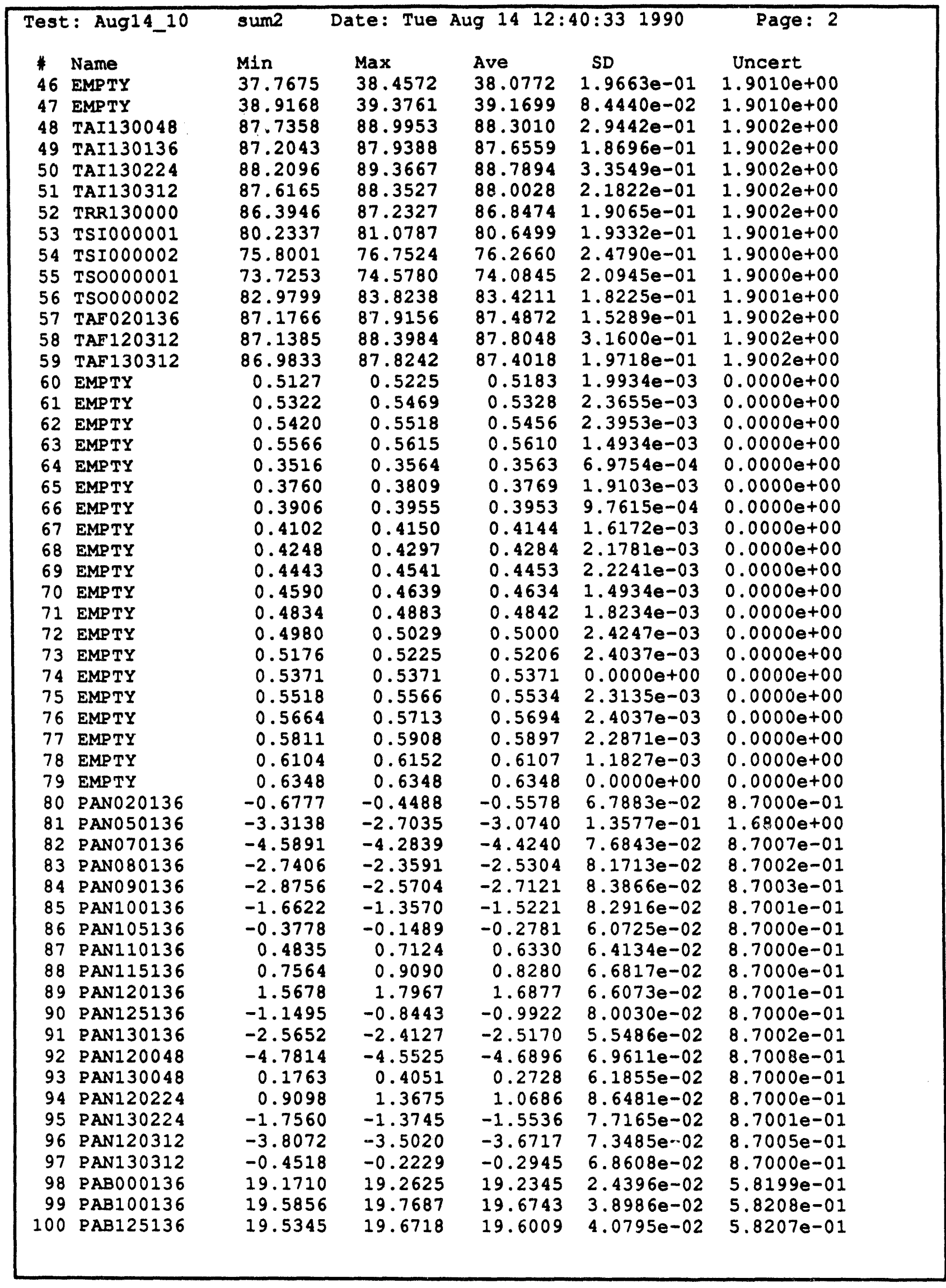




\begin{tabular}{|c|c|c|c|c|c|c|}
\hline \multicolumn{2}{|c|}{ Test: Aug14_10 } & sum2 & Date: Tue & Aug $14 \quad 12$ & $0: 331990$ & Page: 3 \\
\hline * & Name & Min & $\operatorname{Max}$ & Ave & SD & Uncert \\
\hline 101 & PIN 000001 & 19.2067 & 19.3288 & 19.2 & $3.3770 e-02$ & $7.5154 e-01$ \\
\hline 102 & PRV000001 & 18.9861 & 19.2303 & 19.1406 & $5.9882 e-02$ & $7.5152 e-01$ \\
\hline 103 & PSR000001 & 19.6691 & 19.7911 & 29.7039 & $5.5717 e-02$ & $7.5162 \mathrm{e}-01$ \\
\hline 104 & PFM000001 & 15.1907 & 15.7400 & 15.4410 & $1.2365 \mathrm{e}-01$ & $5.0149 e-01$ \\
\hline 105 & PANO00136 & -14.6041 & -14.1463 & -14.4266 & $2.2535 e-01$ & $4.9201 e+00$ \\
\hline 106 & PEMO00002 & 12.7523 & 14.4303 & 13.6334 & $4.1731 e-01$ & $2.0288 e-01$ \\
\hline 107 & EMPTY & 0.0049 & 0.0049 & 0.0049 & $0.0000 e+00$ & $0.0000 e+00$ \\
\hline 108 & EMPTY & -10.0000 & -4.6777 & -7.8987 & $1.2311 \mathrm{e}+00$ & $0.0000 e+00$ \\
\hline 109 & EMPTY & 0.0000 & 0.0049 & 0.0040 & $1.9103 e-03$ & $0.0000 e+00$ \\
\hline 110 & EMPTY & -10.0000 & -10.0000 & -10.0000 & $0.0000 e+00$ & $0.0000 e+00$ \\
\hline 111 & EMPTY & 0.0000 & 0.0049 & 0.0001 & $6.9754 e-04$ & $0.0000 e+00$ \\
\hline 112 & VPW000001 & -1.0479 & -0.4620 & -0.7769 & $1.1146 e-01$ & $8.1001 e-01$ \\
\hline 113 & VPW0 00002 & -0.8451 & -0.4545 & -0.7614 & $1.1276 e-01$ & $8.1001 e-01$ \\
\hline 114 & VPW000003 & -0.7286 & -0.5333 & -0.6489 & $9.6990 e-02$ & $8.1001 e-01$ \\
\hline 115 & VPW000004 & -0.8752 & -0.4845 & -0.6041 & $1.1838 e-01$ & $8.1001 e-01$ \\
\hline 116 & VPWO00005 & -1.4686 & -1.0780 & -1.3052 & $1.0806 e-01$ & $8.1004 e-01$ \\
\hline 117 & VPW000006 & -1.6489 & -1.2583 & -1.4496 & $9.3412 e-02$ & $8.1005 e-01$ \\
\hline 118 & IPW000001 & -11.6766 & -7.7719 & -9.4851 & $1.2995 \mathrm{e}+00$ & $8.1003 e+00$ \\
\hline 119 & IPW000002 & -12.2331 & -6.3793 & -9.4854 & $1.3161 e+00$ & $8.1003 e+00$ \\
\hline 120 & IPW000003 & -12.8625 & -8.9625 & -10.7136 & $1.0714 \mathrm{e}+00$ & $8.1004 e+00$ \\
\hline 121 & IPW000004 & -2.2536 & -1.7654 & -1.9547 & $2.4037 e-01$ & $2.0001 e+00$ \\
\hline 122 & IPW000005 & -3.9904 & -3.0139 & -3.4224 & $2.8793 e-01$ & $2.0002 e+00$ \\
\hline 123 & IPW000006 & -2.8076 & -1.8310 & -2.2097 & $3.3453 e-01$ & $2.0001 e+00$ \\
\hline 124 & EMPTY & -0.0244 & -0.0146 & -0.0192 & $3.0572 e-03$ & $0.0000 e+00$ \\
\hline 125 & EMPTY & -0.0195 & -0.0098 & -0.0133 & $2.6370 e-03$ & $0.0000 e+00$ \\
\hline 126 & EMPTY & -0.0195 & -0.0098 & -0.0164 & $2.578 \% e-03$ & $0.0000 e+00$ \\
\hline 127 & EMPTY & -0.0244 & -0.0098 & -0.0155 & $3.9459 e-03$ & $0.0000 e+00$ \\
\hline 128 & IHT000001 & -0.2066 & 0.1841 & -0.0272 & $1.0424 \mathrm{e}-01$ & $4.1000 e+00$ \\
\hline 129 & IHT000002 & -0.2329 & 0.1577 & -0.0296 & $9.7323 e-02$ & $4.1000 \mathrm{e}+00$ \\
\hline 130 & IHT000003 & -0.2817 & 0.3042 & -0.0146 & $1.1059 e-01$ & $4.1000 e+00$ \\
\hline 131 & IHT000004 & -0.2028 & 0.1878 & -0.0155 & $1.0517 e-01$ & $4.1000 e+00$ \\
\hline 132 & IHT000005 & -0.2066 & 0.1841 & -0.0431 & $1.1518 \mathrm{e}-01$ & $4.1000 e+00$ \\
\hline 133 & IHT000006 & -0.2554 & 0.3305 & 0.0555 & $1.1219 \mathrm{e}-01$ & $4.1000 e+00$ \\
\hline 134 & IHT000007 & -0.0601 & 0.1352 & -0.0083 & $8.7123 e-02$ & $4.1000 e+00$ \\
\hline 135 & IHT000008 & -0.1578 & 0.0376 & -0.0701 & $9.8153 e-02$ & $4.1000 e+00$ \\
\hline 136 & IHTO00009 & -0.2216 & 0.1690 & -0.0343 & $6.8582 e-02$ & $4.1000 e+00$ \\
\hline 137 & IHT000010 & -0.1427 & 0.0526 & -0.0550 & $9.8153 e-02$ & $4.1000 e+00$ \\
\hline 138 & IHTO00011 & -0.3042 & 0.0864 & -0.0491 & $9.9303 e-02$ & $4.1000 e+00$ \\
\hline 139 & IHTO00012 & -0.1653 & 0.0301 & -0.0656 & $9.8648 e-02$ & $4.1000 e+00$ \\
\hline 140 & IHTO00013 & -0.0563 & 0.3343 & 0.0752 & $1.0076 e-01$ & $4.1000 e+00$ \\
\hline 141 & IHTO00014 & -0.1953 & 0.1953 & 0.0000 & $7.9736 e-02$ & $4.1000 e+00$ \\
\hline 142 & IHT000015 & -0.0488 & 0.1465 & -0.0090 & $7.9532 \mathrm{e}-02$ & $4.1000 e+00$ \\
\hline 143 & IHTO00016 & -0.1352 & 0.0601 & -0.0276 & $9.8153 e-02$ & $4.1000 e+00$ \\
\hline 144 & IHTO00017 & -0.3530 & 0.4282 & 0.1173 & $2.3219 e-01$ & $4.1000 e+00$ \\
\hline 145 & IHT000018 & -0.1728 & 0.2178 & 0.0066 & $1.1161 e-01$ & $4.1000 e+00$ \\
\hline 146 & IHT000019 & 0.0413 & 0.4319 & 0.2366 & $8.9148 e-02$ & $4.1000 e+00$ \\
\hline 147 & IHT000020 & -0.2517 & 0.1390 & -0.0165 & $8.8965 e-02$ & $4.1000 e+00$ \\
\hline 148 & IHTO00021 & -0.0714 & 0.1240 & 0.0203 & $9.8483 e-02$ & $4.1000 e+00$ \\
\hline 149 & IHT000022 & 0.0037 & 0.1991 & 0.1193 & $9.6990 \mathrm{e}-02$ & $4.1000 e+00$ \\
\hline 150 & IHT000023 & -0.0300 & 0.3606 & 0.1653 & $5.6382 e-02$ & $4.1000 e+00$ \\
\hline 151 & IHTOODO24 & -0.4770 & -0.0864 & -0.2697 & $6.1868 e-02$ & $4.1000 \mathrm{e}+00$ \\
\hline 152 & IHT000025 & 0.0075 & 0.5935 & 0.2626 & $1.0701 e-01$ & $4.1000 e+00$ \\
\hline 153 & IHT00002.6 & -0.1690 & 0.2216 & -0.0056 & $8.3122 e-02$ & $4.1000 e+00$ \\
\hline 154 & IHT000027 & -0.0338 & 0.1615 & -0.0059 & $6.9053 e-02$ & $4.1000 e+00$ \\
\hline 155 & IHT000028 & -0.2479 & 0.3381 & 0.0311 & $1.1276 e-01$ & $4.1000 e+00$ \\
\hline
\end{tabular}




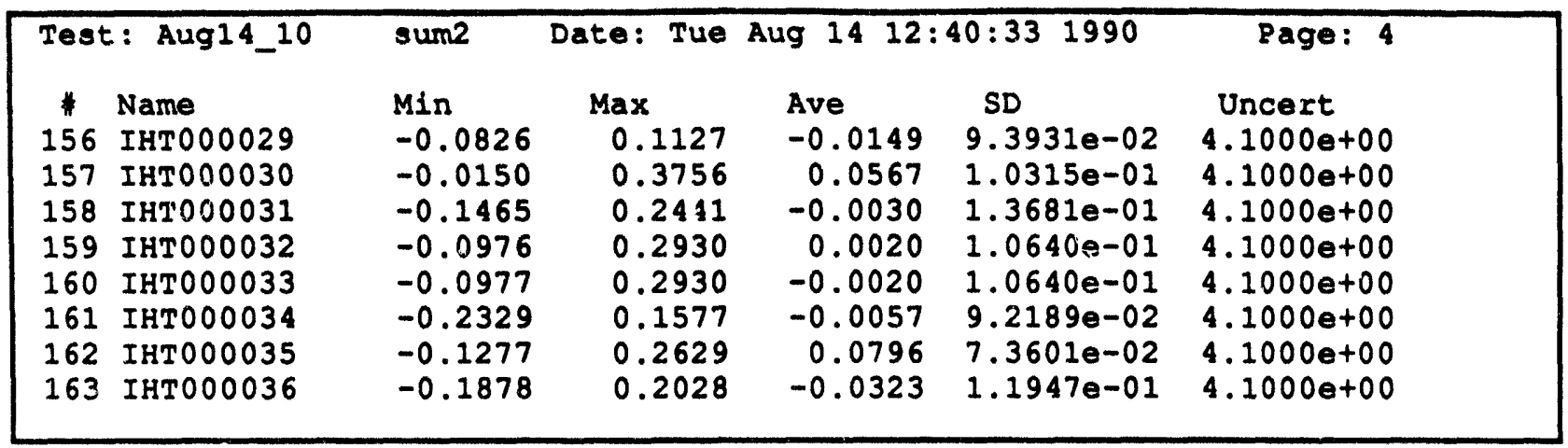




\begin{tabular}{|c|c|c|c|c|}
\hline \multicolumn{5}{|c|}{$\begin{array}{l}\text { Test: Aug14_10 dif Date: Tue Aug } 1412: 40 \\
\text { Velocity } 1=0.005800 \text { (ft/sec) } \\
\text { Velocity } 2=0.006500 \text { (ft/sec) } \\
\text { Pressure Drop }=0.000900 \text { (psi) } \\
\text { Tsat in }=0.006500 \text { (deg F) Tsat out }=0.035797\end{array}$} \\
\hline $\begin{array}{r}* \\
0 \\
1 \\
2 \\
3 \\
4 \\
5 \\
6 \\
7 \\
8 \\
9 \\
10 \\
11 \\
12 \\
13 \\
14 \\
15 \\
16 \\
17 \\
18 \\
19 \\
20 \\
21 \\
22 \\
23 \\
24 \\
25 \\
26 \\
27 \\
28 \\
29 \\
30 \\
31 \\
32 \\
33 \\
34 \\
35 \\
36 \\
37 \\
38 \\
39 \\
40 \\
41 \\
42 \\
43 \\
44 \\
45\end{array}$ & 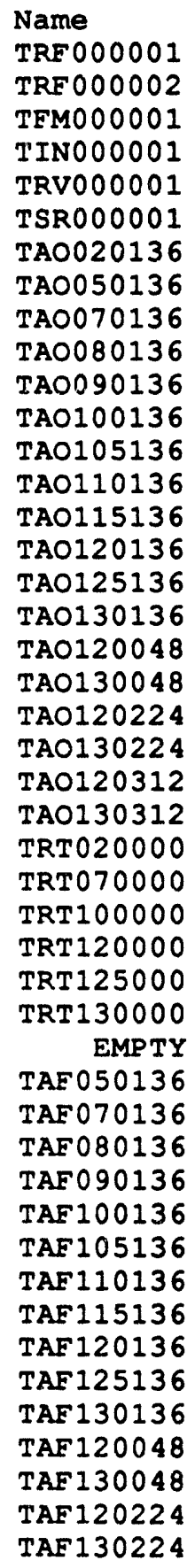 & $\begin{array}{c}\text { Abs Diff } \\
0.0375 \\
0.0385 \\
0.0007 \\
0.0332 \\
0.0693 \\
0.0147 \\
0.0273 \\
0.0246 \\
0.0195 \\
0.0273 \\
0.0729 \\
0.0734 \\
0.0247 \\
0.0054 \\
0.0697 \\
0.0270 \\
0.0628 \\
0.0137 \\
0.0840 \\
0.0033 \\
0.0438 \\
0.0651 \\
0.0436 \\
0.0313 \\
0.0000 \\
0.0251 \\
0.0476 \\
0.0319 \\
0.0831 \\
0.0004 \\
0.0143 \\
0.0108 \\
0.0205 \\
0.0254 \\
0.0091 \\
0.0327 \\
0.0019 \\
0.0742 \\
0.0079 \\
0.0991 \\
0.0348 \\
0.0162 \\
0.0117 \\
0.0222 \\
0.0898 \\
0.0161\end{array}$ & $\begin{array}{l}\text { 8DDif } \\
0.0509 \\
0.0522 \\
0.0008 \\
0.0378 \\
0.0791 \\
0.0166 \\
0.0335 \\
0.0279 \\
0.0221 \\
0.0311 \\
0.0826 \\
0.0834 \\
0.0279 \\
0.0061 \\
0.0789 \\
0.0307 \\
0.0710 \\
0.0156 \\
0.1088 \\
0.0038 \\
0.04\end{array}$ & . \\
\hline
\end{tabular}




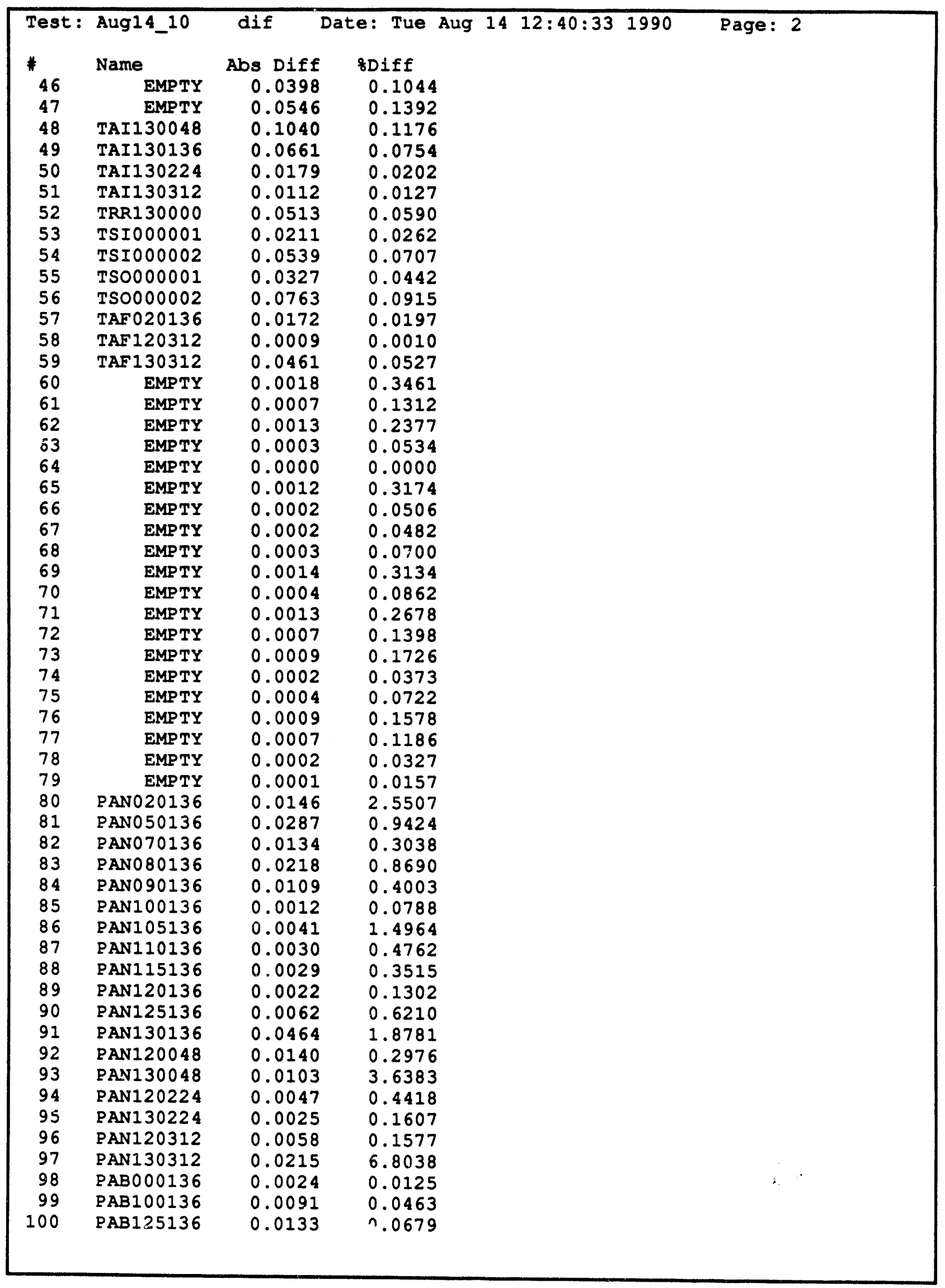




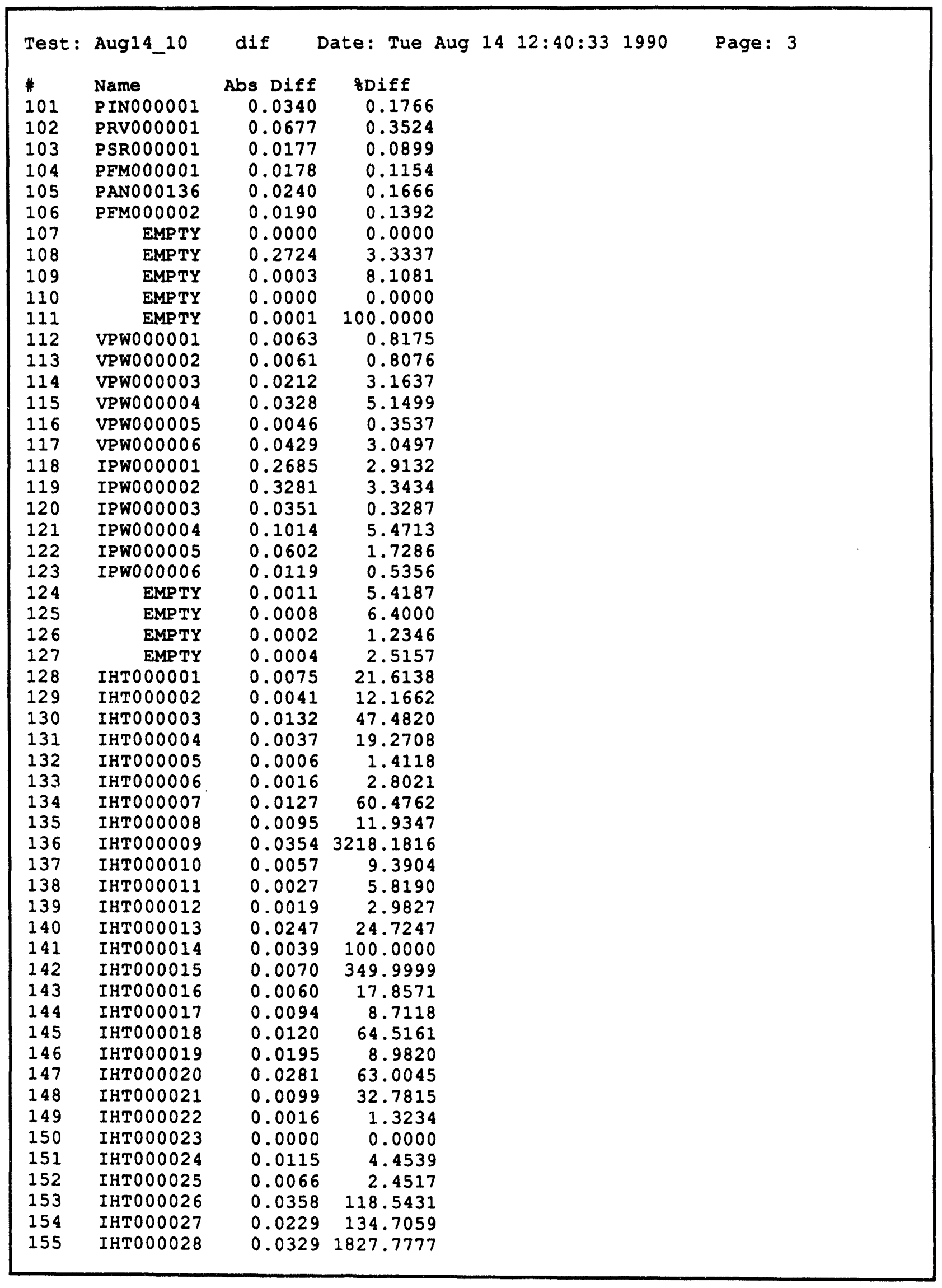




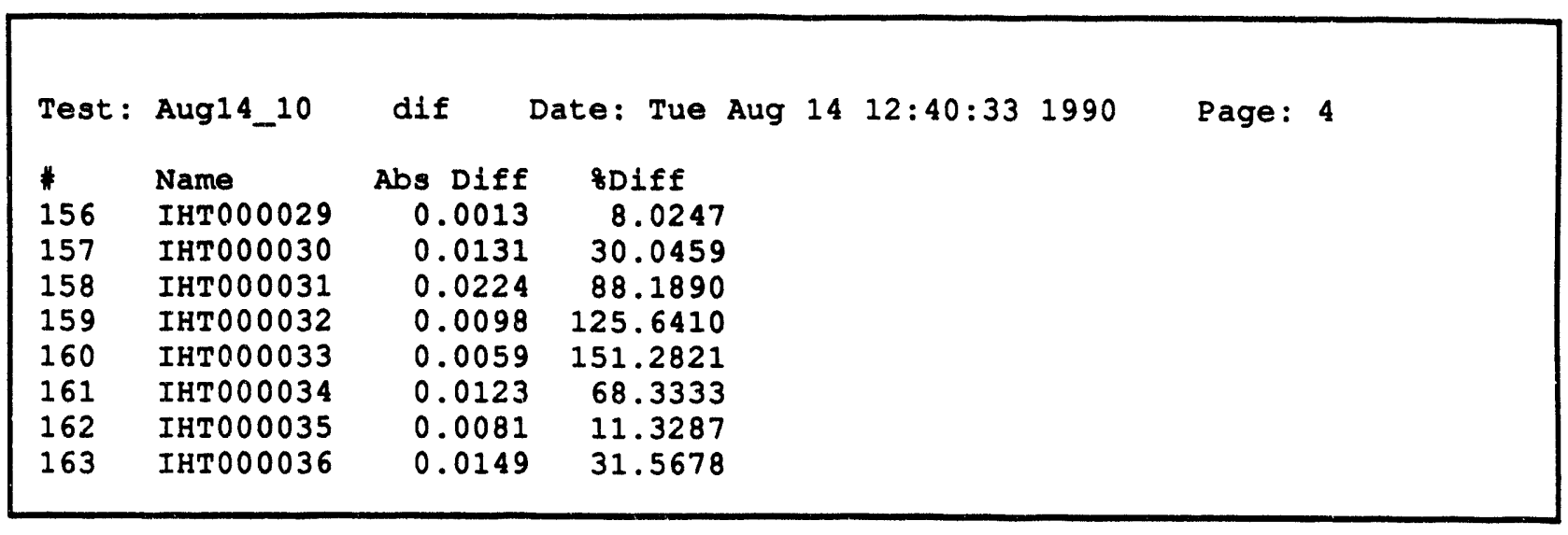




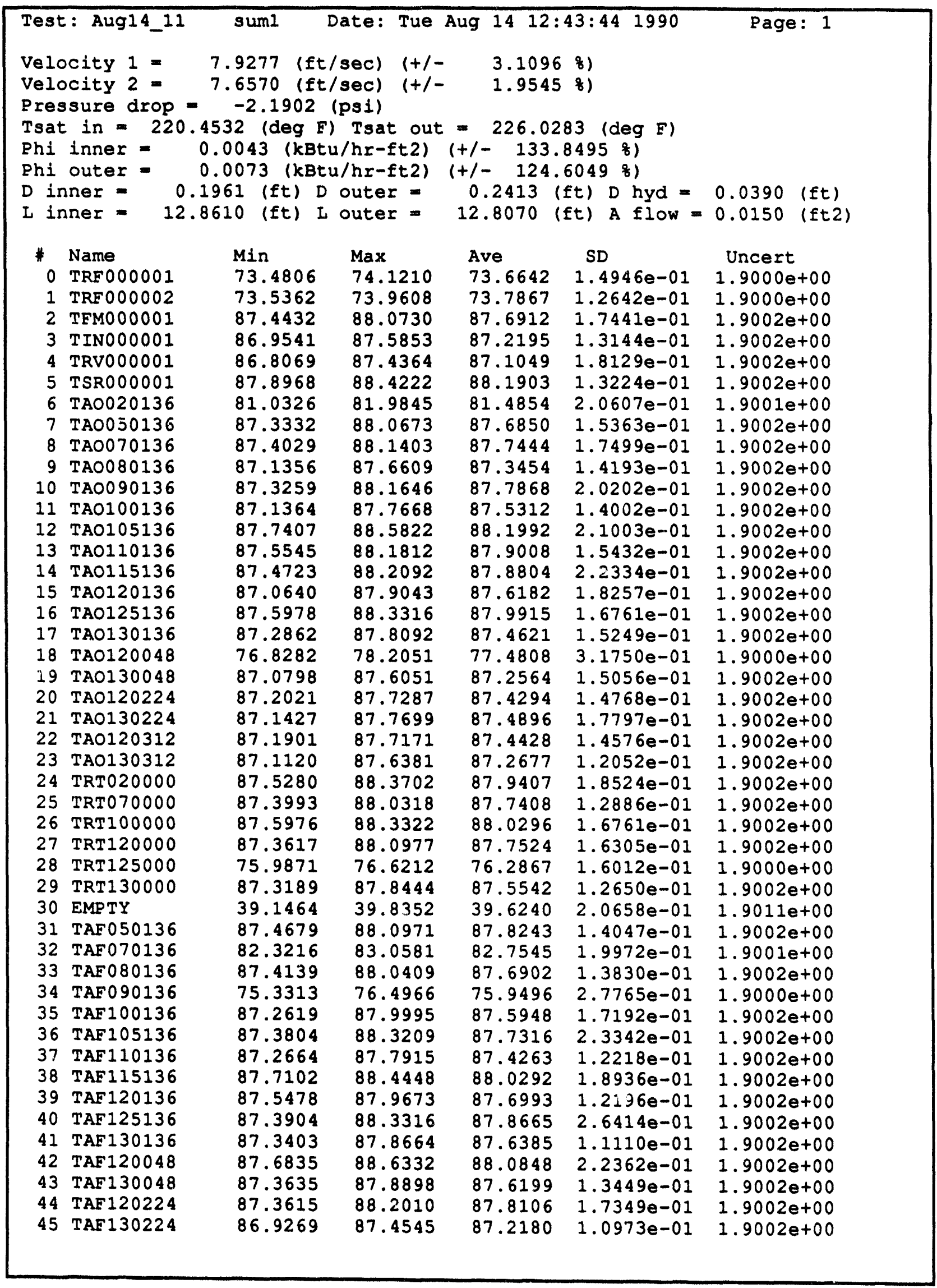




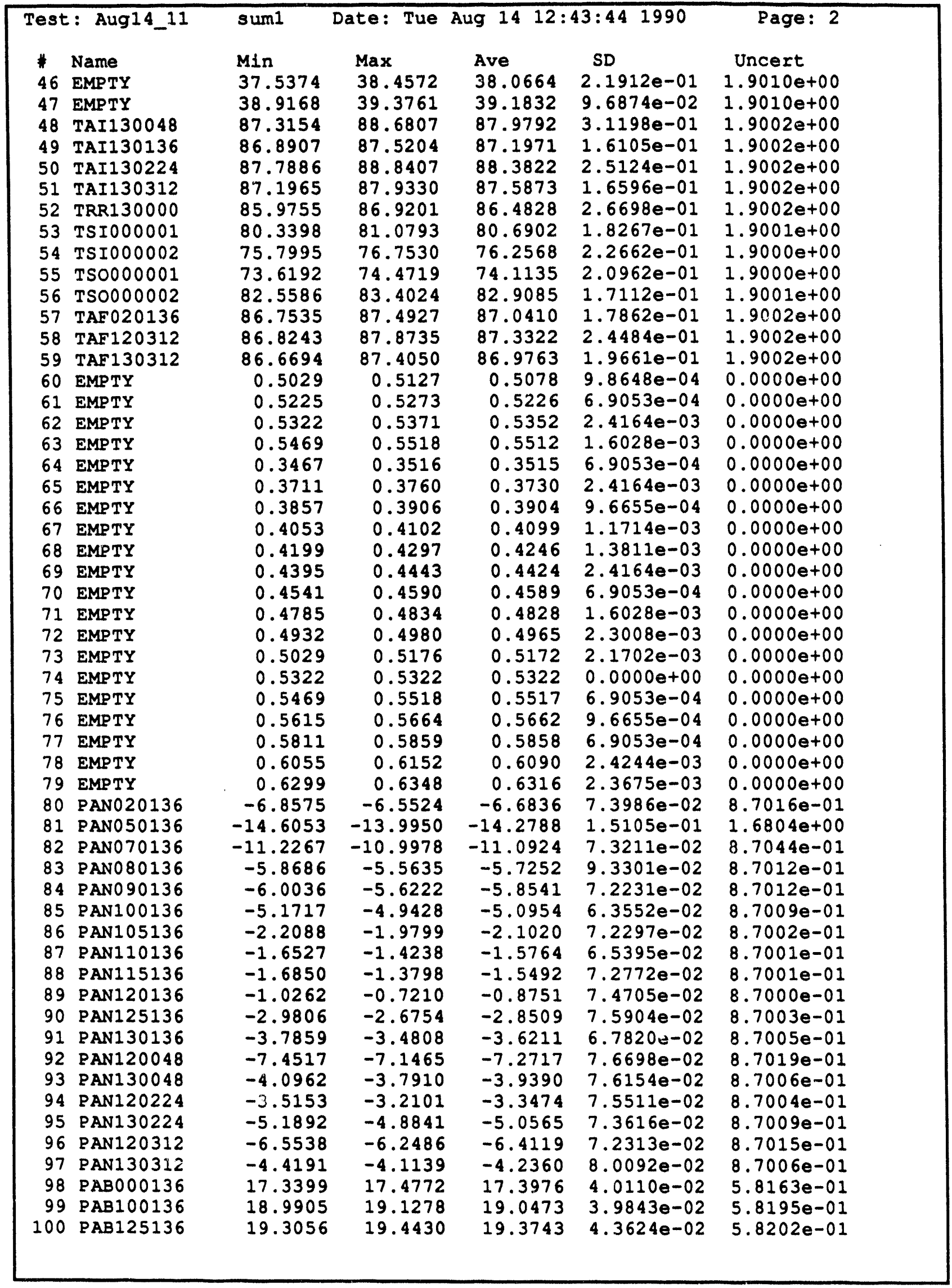




\begin{tabular}{|c|c|c|c|c|c|c|}
\hline \multicolumn{2}{|c|}{ Test: Aug14_11 } & sum1 & Date: Tue & Aug 141 & $43: 441990$ & Page: 3 \\
\hline * & Name & Min & $\operatorname{Max}$ & Ave & SD & Uncert \\
\hline 101 & PIN 000001 & 17.2536 & 17.6198 & 17.3561 & $6.2195 e-02$ & $7.5125 e-01$ \\
\hline 102 & PRV000001 & 19.1082 & 19.2303 & 19.2181 & $3.6993 e-02$ & $7.5154 e-01$ \\
\hline 103 & PSR000001 & 19.5470 & 19.7911 & 19.6740 & $3.4527 e-02$ & $7.5161 e-01$ \\
\hline 104 & PFMO00001 & 9.3313 & 9.6975 & 9.5346 & $8.9263 e-02$ & $5.0057 e-01$ \\
\hline 105 & PAN000136 & -60.8382 & -60.3804 & -60.6367 & $2.2953 e-01$ & $4.9223 e+00$ \\
\hline 106 & PFMO000002 & 8.1211 & 9.2286 & 8.8869 & $2.3263 e-01$ & $2.0123 e-01$ \\
\hline 107 & EMPTY & 0.0000 & 0.0098 & 0.0045 & $1.6624 \mathrm{e}-03$ & $0.0000 \mathrm{e}+00$ \\
\hline 108 & EMPTY & -10.0000 & -6.6064 & -7.9546 & $1.1230 e+00$ & $0.00000+00$ \\
\hline 109 & EMPTY & 0.0000 & 0.0049 & 0.0017 & $2.3365 e-03$ & $0.0000 e+00$ \\
\hline 110 & EMPTY & -10.0000 & -10.0000 & -10.0000 & $0.0000 e+00$ & $0.0000 e+00$ \\
\hline 111 & EMPTY & 0.0000 & 0.0000 & 0.0000 & $0.0000 e+00$ & $0.0000 e+00$ \\
\hline 112 & VPW000001 & -0.8526 & -0.4620 & -0.7628 & $1.1306 e-01$ & $8.1001 \mathrm{e}-01$ \\
\hline 113 & VPW000002 & -1.0404 & -0.6498 & -0.7904 & $9.6976 \mathrm{e}-02$ & $8.1001 e-01$ \\
\hline 114 & VPW000003 & -0.7286 & -0.5333 & -0.6388 & $9.8332 e-02$ & $8.1001 e-01$ \\
\hline 115 & VPW000004 & -0.8752 & -0.4845 & -0.6486 & $1.1409 e-01$ & $8.1001 \mathrm{e}-01$ \\
\hline 116 & VPW000005 & -1.4686 & -1.0780 & -1.2928 & $1.1334 \mathrm{e}-01$ & $8.1004 \mathrm{e}-01$ \\
\hline 117 & VPW000006 & -1.6489 & -1.2583 & -1.4419 & $1.2104 e-01$ & $8.1005 e-01$ \\
\hline 118 & IPW000001 & -13.6289 & -7.7719 & -9.7242 & $1.3664 \mathrm{e}+00$ & $8.1004 e+00$ \\
\hline 119 & IPW000002 & -12.2331 & -6.3793 & -9.6574 & $1.1453 e+00$ & $8.1004 \mathrm{e}+00$ \\
\hline 120 & IPW000003 & -12.8625 & -8.9625 & -10.6005 & $1.1391 e+00$ & $8.1004 \mathrm{e}+00$ \\
\hline 121 & IPW000004 & -2.2536 & -1.2771 & -1.8533 & $2.3531 e-01$ & $2.0001 e+00$ \\
\hline 122 & IPW000005 & -3.9904 & -3.0139 & -3.5509 & $3.1580 e-01$ & $2.0002 e+00$ \\
\hline 123 & IPW000006 & -2.8076 & -1.8310 & -2.2705 & $2.6562 \mathrm{e}-01$ & $2.0001 e+00$ \\
\hline 124 & EMPTY & -0.0244 & -0.0146 & -0.0197 & $3.55138-03$ & $0.0000 e+00$ \\
\hline 125 & EMPTY & -0.0195 & -0.0098 & -0.0133 & $3.2777 e-03$ & $0.0000 e+00$ \\
\hline 126 & EMPTY & -0.0195 & -0.0098 & -0.0166 & $2.7902 e-03$ & $0.0000 e+00$ \\
\hline 127 & EMPTY & -0.0244 & -0.0098 & -0.0163 & $3.2133 e-03$ & $0.0000 e+00$ \\
\hline 128 & IHT000001 & -0.2066 & 0.1841 & -0.0269 & $8.6810 e-02$ & $4.1000 e+00$ \\
\hline 129 & IHTO00002 & -0.2329 & 0.1577 & -0.0180 & $9.0412 e-02$ & $4.1000 \mathrm{e}+00$ \\
\hline 130 & IHTO00003 & -0.0864 & 0.1089 & -0.0278 & $9.0412 e-02$ & $4.1000 e+00$ \\
\hline 131 & IHTO00004 & -0.2028 & 0.3831 & 0.0198 & $1.0447 e-01$ & $4.1000 e+00$ \\
\hline 132 & IHT000005 & -0.2066 & 0.1841 & -0.0152 & $1.0052 e-01$ & $4.1000 e+00$ \\
\hline 133 & IHT000006 & -0.0601 & 0.3305 & 0.0649 & $1.0259 e-01$ & $4.1000 \mathrm{e}+00$ \\
\hline 134 & IHT000007 & -0.0601 & 0.1352 & -0.0093 & $8.6541 e-02$ & $4.1000 e+00$ \\
\hline 135 & IHT000008 & -0.1578 & 0.0376 & -0.0874 & $9.4702 e-02$ & $4.1000 e+00$ \\
\hline 136 & IHTO00009 & -0.2216 & 0.1690 & -0.0146 & $7.2866 e-02$ & $4.1000 e+00$ \\
\hline 137 & IHT000010 & -0.3380 & 0.0526 & -0.0646 & $1.1161 \mathrm{e}-01$ & $4.1000 e+00$ \\
\hline 138 & IHTO00011 & -0.3042 & 0.0864 & -0.0660 & $9.0756 e-02$ & $4.1000 e+00$ \\
\hline 139 & IHTOOOOO12 & -0.1653 & 0.0301 & -0.0832 & $9.7377 e-02$ & $4.1000 e+00$ \\
\hline 140 & IHTO00013 & -0.0563 & 0.1390 & 0.0921 & $8.4262 e-02$ & $4.1000 e+00$ \\
\hline 141 & IHTO00014 & -0.1953 & 0.1953 & -0.0039 & $9.2456 e-02$ & $4.1000 e+00$ \\
\hline 142 & IHT000015 & -0.2442 & 0.1465 & -0.0293 & $7.1136 e-02$ & $4.1000 e+00$ \\
\hline 143 & IHTO00016 & -0.1352 & 0.0601 & -0.0297 & $9.8332 e-02$ & $4.1000 e+00$ \\
\hline 144 & IHTO00017 & -0.3530 & 0.6235 & 0.1157 & $2.7052 e-01$ & $4.1000 e+00$ \\
\hline 145 & IHTO00018 & -0.1728 & 0.2178 & 0.0147 & $9.6332 \mathrm{e}-02$ & $4.1000 e+00$ \\
\hline 146 & IHTO00019 & 0.0413 & 0.4319 & 0.2210 & $8.6810 e-02$ & $4.1000 e+00$ \\
\hline 147 & IHTOOO020 & -0.2517 & 0.1390 & -0.0564 & $7.8918 e-02$ & $4.1000 e+00$ \\
\hline 148 & IHT000021 & -0.0714 & 0.1240 & -0.0049 & $9.3461 e-02$ & $4.1000 e+00$ \\
\hline 149 & IHT000022 & 0.0037 & 0.1991 & 0.1209 & $9.6655 e-02$ & $4.1000 e+00$ \\
\hline 150 & IHTO00023 & -0.0300 & 0.3606 & 0.1496 & $8.6810 e-02$ & $4.1000 e+00$ \\
\hline 151 & IHTO00024 & -0.4770 & -0.0864 & -0.2739 & $9.6332 e-02$ & $4.1000 e+00$ \\
\hline 152 & IHTO00025 & 0.2028 & 0.3981 & 0.2888 & $9.7935 e-02$ & $4.1000 e+00$ \\
\hline 153 & IHTO00026 & -0.1690 & 0.2216 & 0.0302 & $8.3612 e-02$ & $4.1000 e+00$ \\
\hline 154 & IHTO000027 & -0.0338 & 0.1615 & 0.0092 & $8.1729 e-02$ & $4.1000 \mathrm{e}+00$ \\
\hline 155 & IHT000028 & -0.0526 & 0.1427 & 0.0177 & $9.4702 e-02$ & $4.10000+00$ \\
\hline
\end{tabular}




\begin{tabular}{|c|c|c|c|c|c|c|}
\hline \multicolumn{2}{|c|}{ Test: Aug14_1.1 } & $\begin{array}{l}\text { suml } \\
\text { Min }\end{array}$ & Date: Tue & \multicolumn{2}{|c|}{ ug $14 \quad 12: 43: 44 \quad 1990$} & $\begin{array}{c}\text { Page: } 4 \\
\text { Uncert }\end{array}$ \\
\hline $\begin{array}{l}* \\
156 \\
157 \\
158 \\
159 \\
160 \\
161 \\
162 \\
163\end{array}$ & 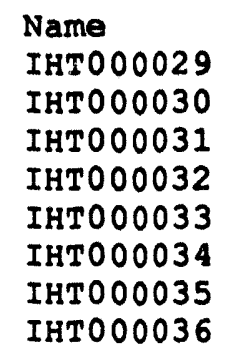 & $\begin{array}{l}\text { Min } \\
-0.2780 \\
-0.0150 \\
-0.1465 \\
-0.0976 \\
-0.0977 \\
-0.2329 \\
-0.1277 \\
-0.1878\end{array}$ & $\begin{array}{l}\text { Max } \\
0.1127 \\
0.3756 \\
0.2441 \\
0.0977 \\
0.0976 \\
0.1577 \\
0.2629 \\
0.2028\end{array}$ & $\begin{array}{l}\text { Rve } \\
-0.0436 \\
0.0397 \\
-0.0293 \\
-0.0234 \\
-0.0195 \\
-0.0141 \\
0.0559 \\
-0.0355\end{array}$ & $\begin{array}{l}\text { SD } \\
8.8233 e-02 \\
9.6976 e-02 \\
1.2478 e-01 \\
9.5765 e-02 \\
9.6655 e-02 \\
8.4997 e-02 \\
1.0741 e-01 \\
1.1361 e-01\end{array}$ & $\begin{array}{l}\text { Uncert } \\
4.1000 e+00 \\
4.1000 e+00 \\
4.1000 e+00 \\
4.1000 e+00 \\
4.1000 e+00 \\
4.1000 e+00 \\
4.1000 e+00 \\
4.1000 e+00\end{array}$ \\
\hline
\end{tabular}




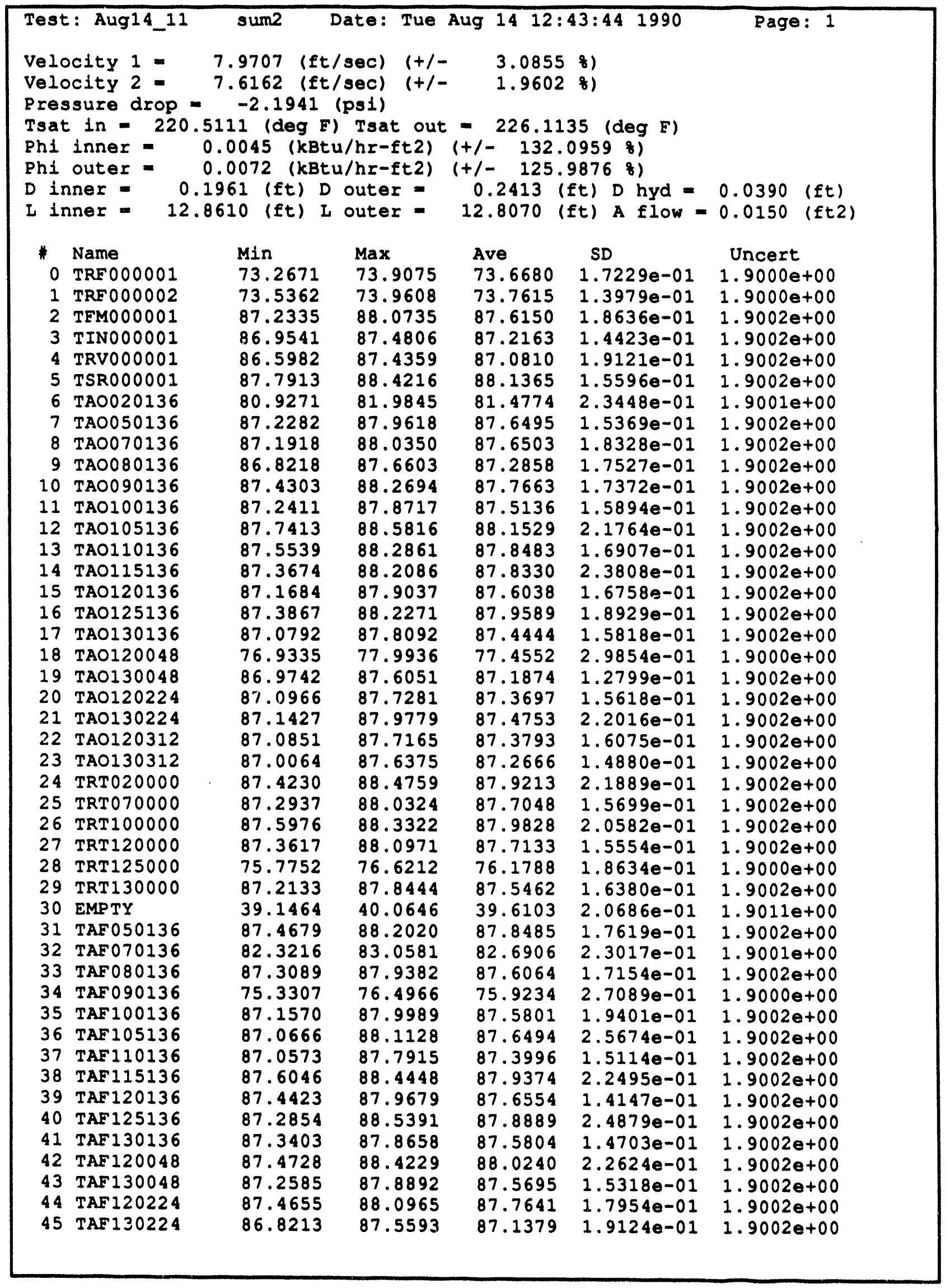




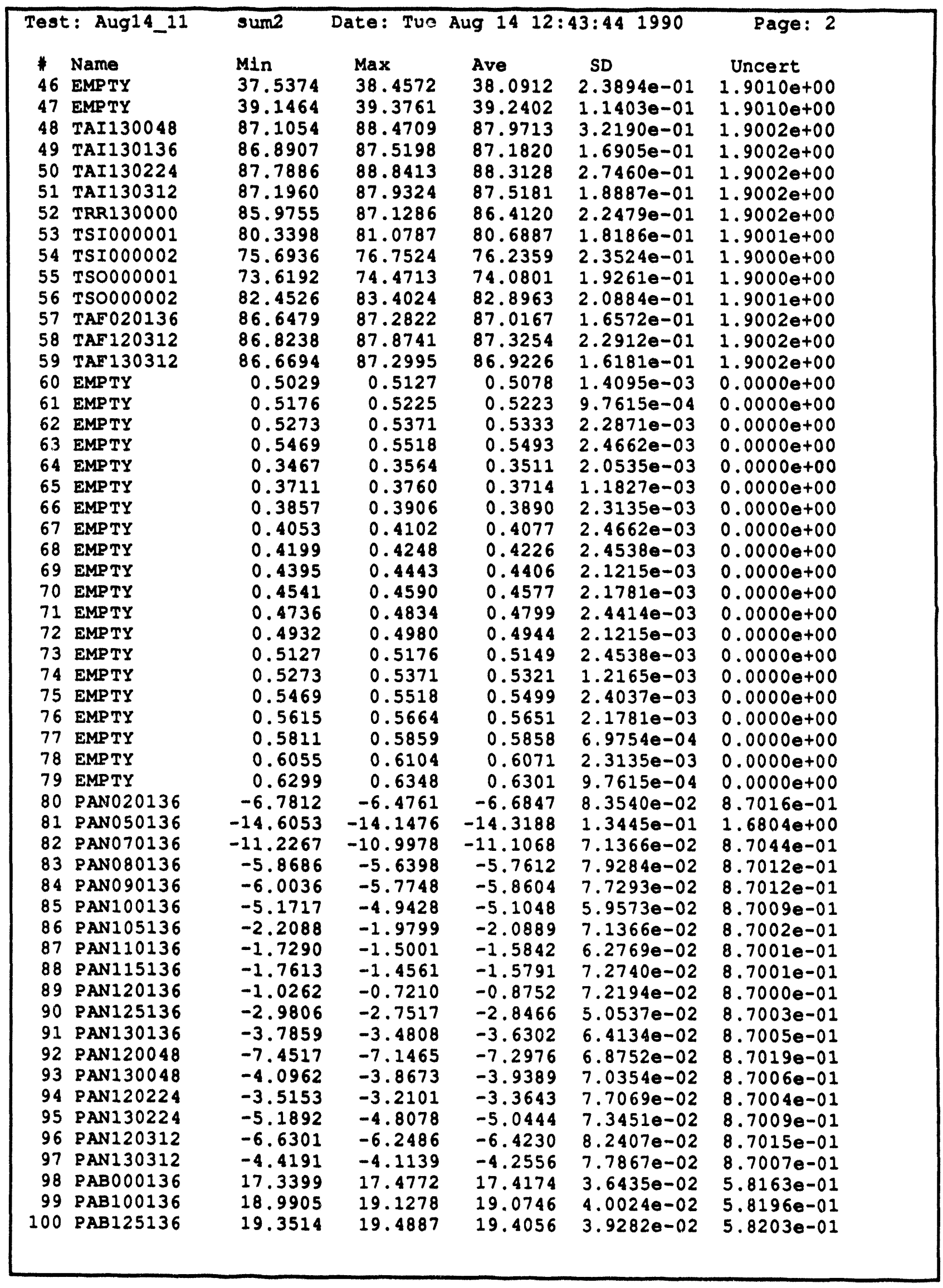




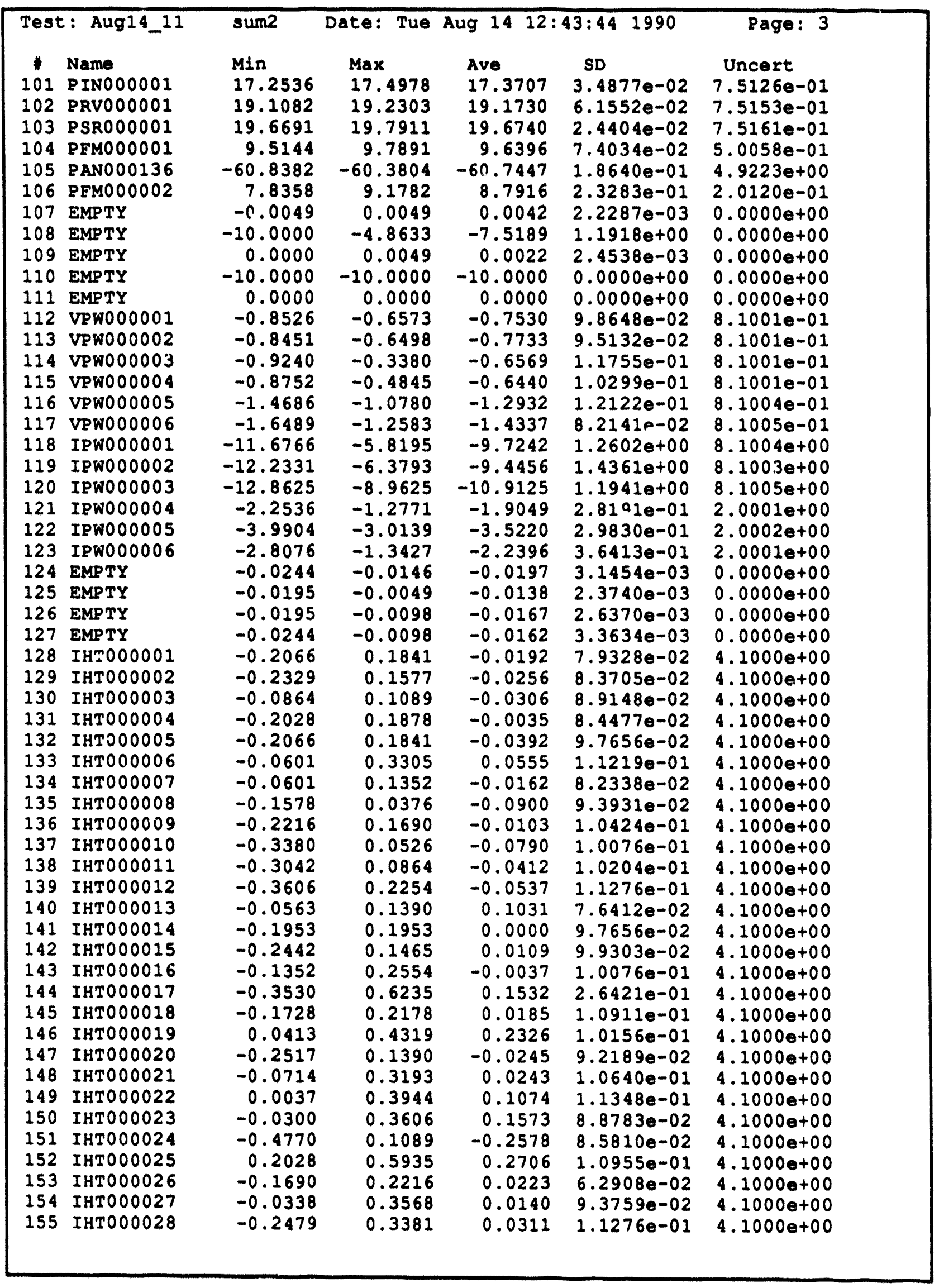




\begin{tabular}{|c|c|c|c|c|c|c|}
\hline \multicolumn{2}{|c|}{ Test: Aug14_11 } & \multicolumn{4}{|c|}{ Date: Tue Aug $14 \quad 12: 43: 44 \quad 1990$} & $\begin{array}{l}\text { Page: } 4 \\
\text { Uncert }\end{array}$ \\
\hline $\begin{array}{l}\$ \\
156 \\
157 \\
158 \\
159 \\
160 \\
161 \\
162 \\
163\end{array}$ & $\begin{array}{l}\text { Name } \\
\text { IHTOOOOO29 } \\
\text { IHTOOOOO30 } \\
\text { IHTOOOOO31 } \\
\text { IHTOOOOO32 } \\
\text { IHTOOOOO33 } \\
\text { IHTOOOOO34 } \\
\text { IHTOOOOO35 } \\
\text { IHTOOOOO36 }\end{array}$ & $\begin{array}{l}M 1 n \\
-0.0826 \\
-0.2103 \\
-0.1465 \\
-0.0976 \\
-0.0977 \\
-0.2329 \\
-0.1277 \\
-0.1878\end{array}$ & $\begin{array}{l}\text { Max } \\
0.3080 \\
0.3756 \\
0.2441 \\
0.2930 \\
0.2930 \\
0.1577 \\
0.2629 \\
0.2028\end{array}$ & $\begin{array}{l}\text { Ave } \\
-0.0149 \\
0.0607 \\
-0.0030 \\
0.0140 \\
-0.0060 \\
-0.0216 \\
0.0835 \\
-0.0523\end{array}$ & $\begin{array}{l}\text { SD } \\
1.0204 e-01 \\
1.1838 e-01 \\
1.2465 e-01 \\
1.0548 e-01 \\
1.0625 e-01 \\
8.7680 e-02 \\
9.6318 e-02 \\
1.2095 e-01\end{array}$ & $\begin{array}{l}\text { Uncert } \\
4.1000 e+00 \\
4.1000 e+00 \\
4.1000 e+00 \\
4.1000 e+00 \\
4.1000 e+00 \\
4.1000 e+00 \\
4.1000 e+00 \\
4.1000 e+00\end{array}$ \\
\hline
\end{tabular}




\begin{tabular}{|c|c|c|c|c|}
\hline \multicolumn{2}{|c|}{ Test: Aug14_11 } & \multicolumn{2}{|r|}{ Date: Tue Aug $14 \quad 12: 43: 44 \quad 1990$} & \multirow{2}{*}{$\begin{array}{c}\text { Page: } 1 \\
(k B t u / h r-f t 2)\end{array}$} \\
\hline $\begin{array}{l}\text { Veloc } \\
\text { Veloc } \\
\text { Press } \\
\text { Tsat } \\
\text { Phi }\end{array}$ & $\begin{array}{l}\text { Lty } 1=0.0 \\
\text { Lty } 2=0.0 \\
\text { ure Drop }= \\
\text { in }=0.0578 \\
\text { nner }=0.00\end{array}$ & $\begin{array}{l}3000 \text { (ft/s } \\
0800 \text { (ft/s } \\
.003900 \text { (p } \\
2 \text { (deg E) } \\
200 \text { (kBtu) }\end{array}$ & $\begin{array}{l}\text { sec) } \\
\text { sec) } \\
\text { ssi) } \\
\text { Tsat out }=0.085190 \text { (deg F) } \\
\text { (hr-ft2) Phi outer }=0.000100\end{array}$ & \\
\hline $\begin{array}{l}0 \\
1 \\
2 \\
3 \\
4 \\
5 \\
6 \\
7 \\
8 \\
9 \\
10 \\
11 \\
12 \\
13 \\
14 \\
15 \\
16 \\
17 \\
18 \\
19 \\
20 \\
21 \\
22 \\
23 \\
24 \\
25 \\
26 \\
27 \\
28 \\
29 \\
30 \\
31 \\
32 \\
33 \\
34 \\
35 \\
36 \\
37 \\
38 \\
39 \\
40 \\
41 \\
42 \\
43 \\
44 \\
45\end{array}$ & 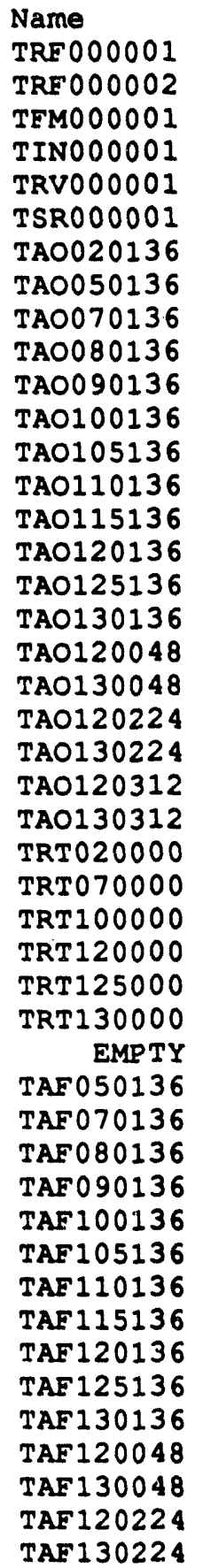 & $\begin{array}{l}\text { Abs Diff } \\
0.0038 \\
0.0252 \\
0.0762 \\
0.0032 \\
0.0239 \\
0.0538 \\
0.0080 \\
0.0355 \\
0.0941 \\
0.0596 \\
0.0205 \\
0.0176 \\
0.0463 \\
0.0525 \\
0.0474 \\
0.0144 \\
0.0326 \\
0.0177 \\
0.0256 \\
0.0690 \\
0.0597 \\
0.0143 \\
0.0635 \\
0.0011 \\
0.0194 \\
0.0360 \\
0.0468 \\
0.0391 \\
0.1079 \\
0.0080 \\
0.0137 \\
0.0242 \\
0.0639 \\
0.0838 \\
0.0262 \\
0.0147 \\
0.0822 \\
0.0267 \\
0.0918 \\
0.0439 \\
0.0224 \\
0.0581 \\
0.0608 \\
0.0504 \\
0.0465 \\
0.0801\end{array}$ & 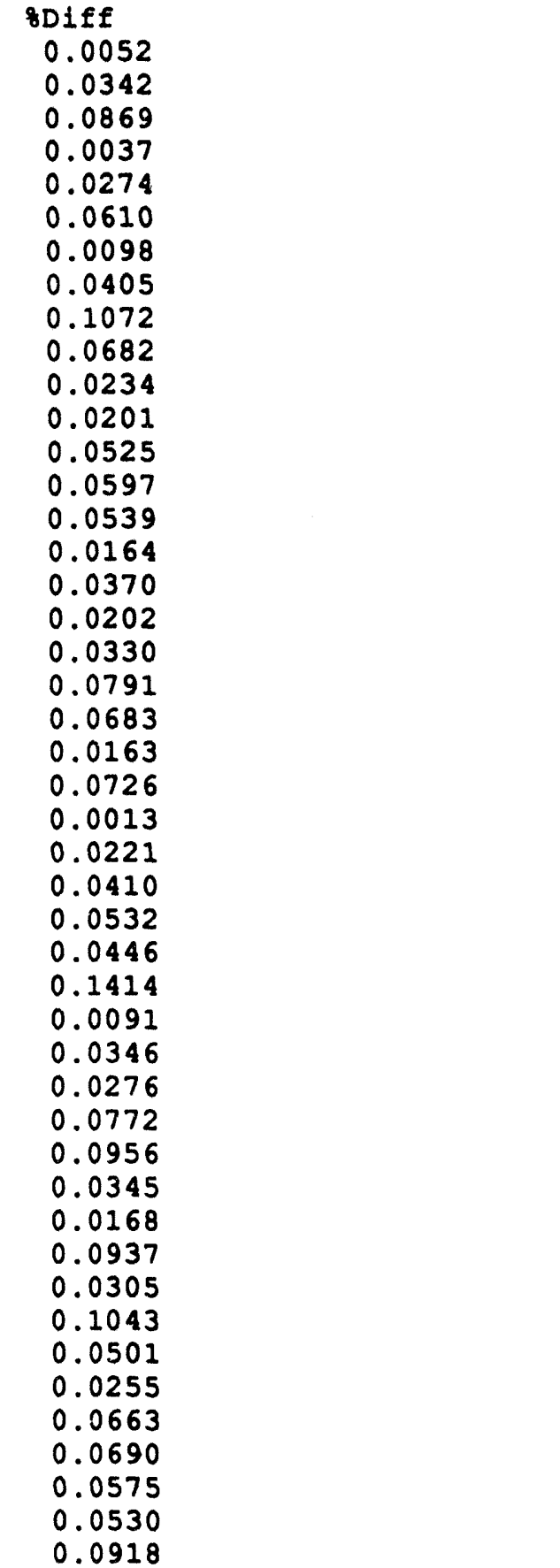 & \\
\hline
\end{tabular}




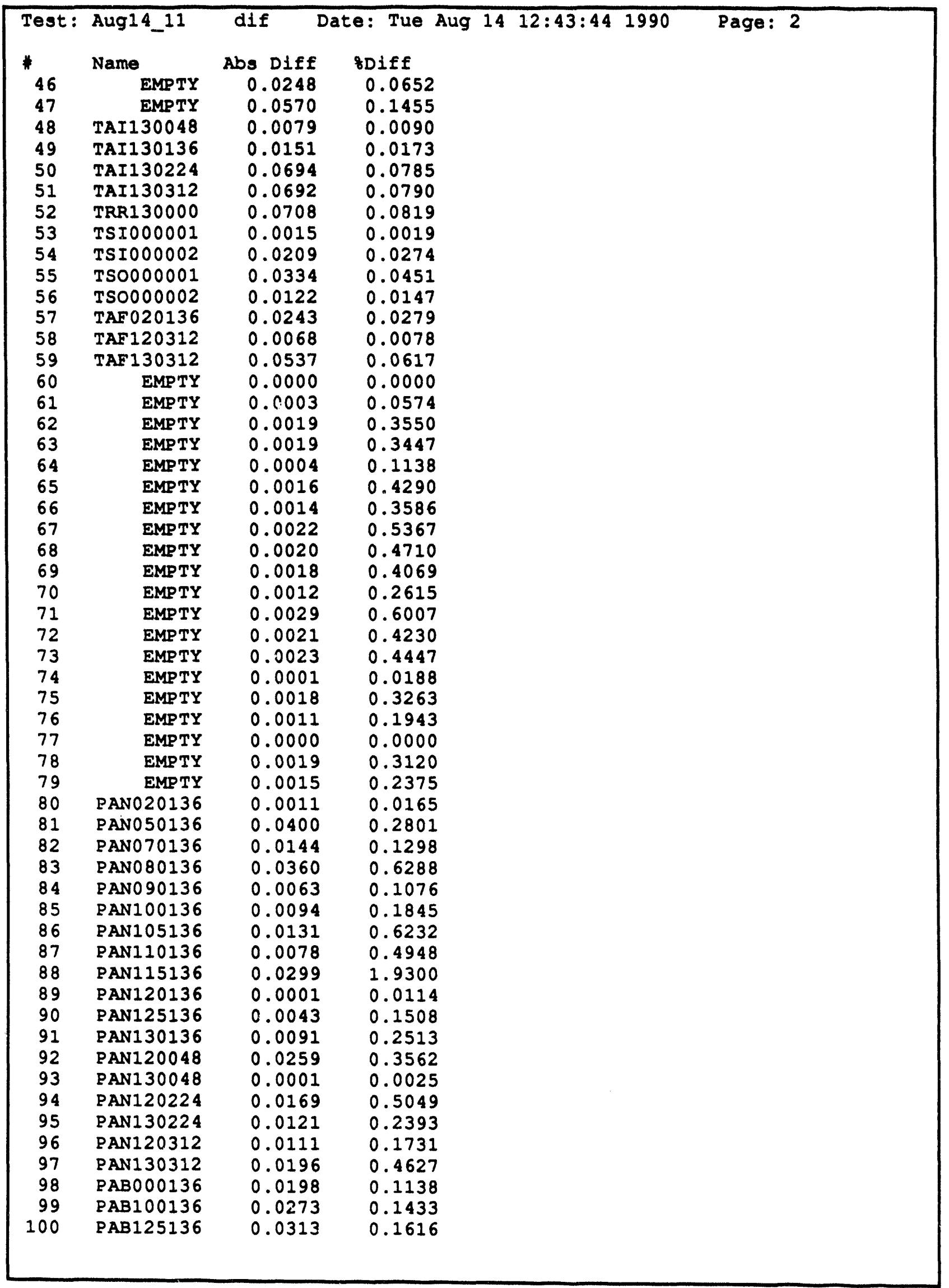




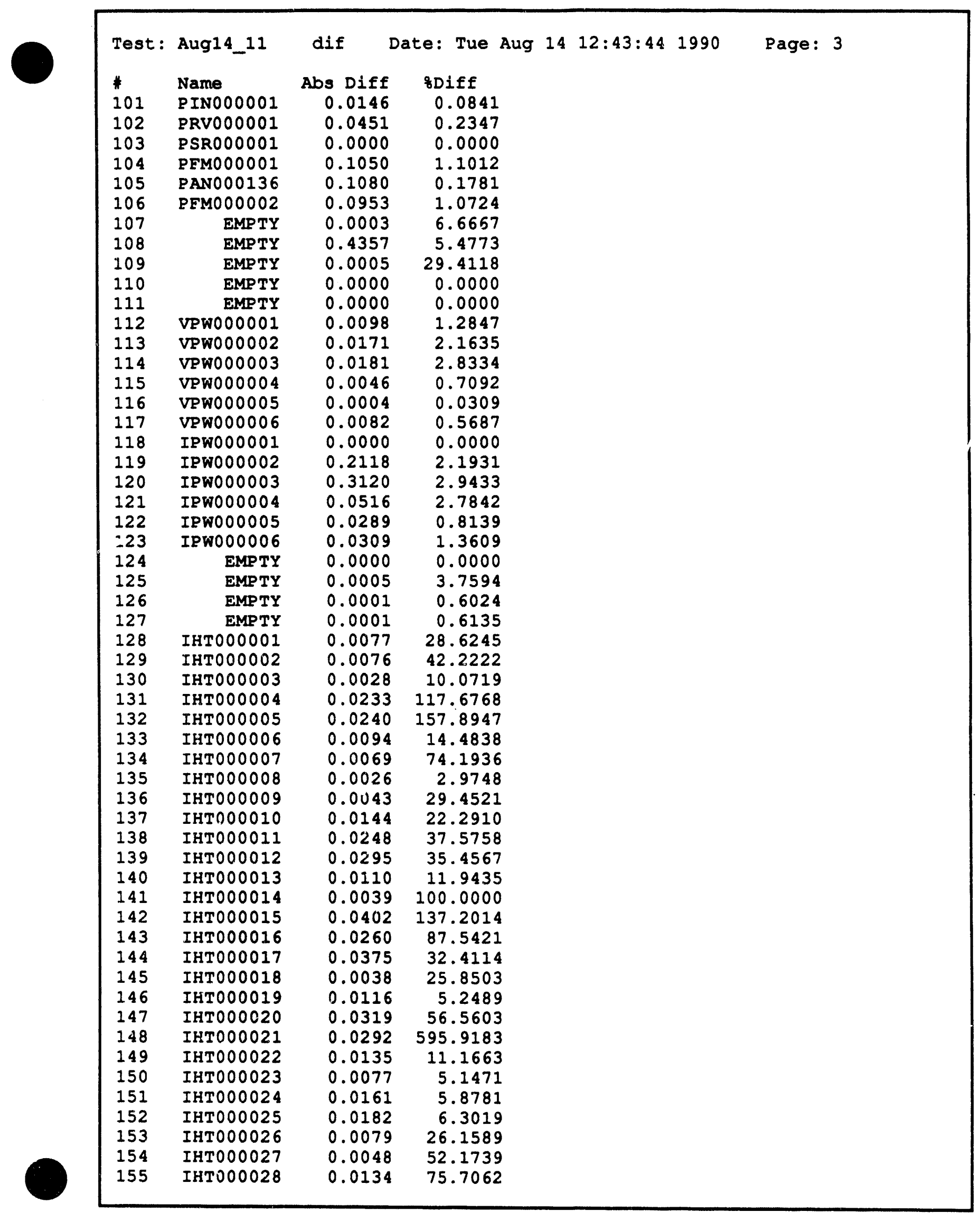




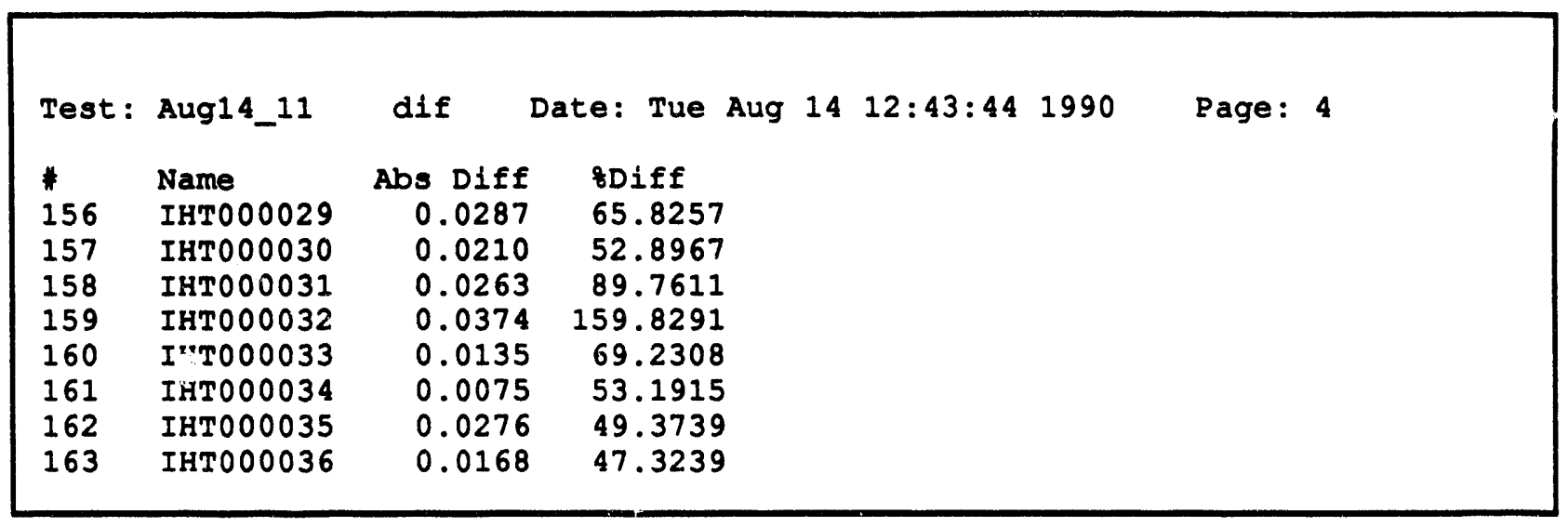




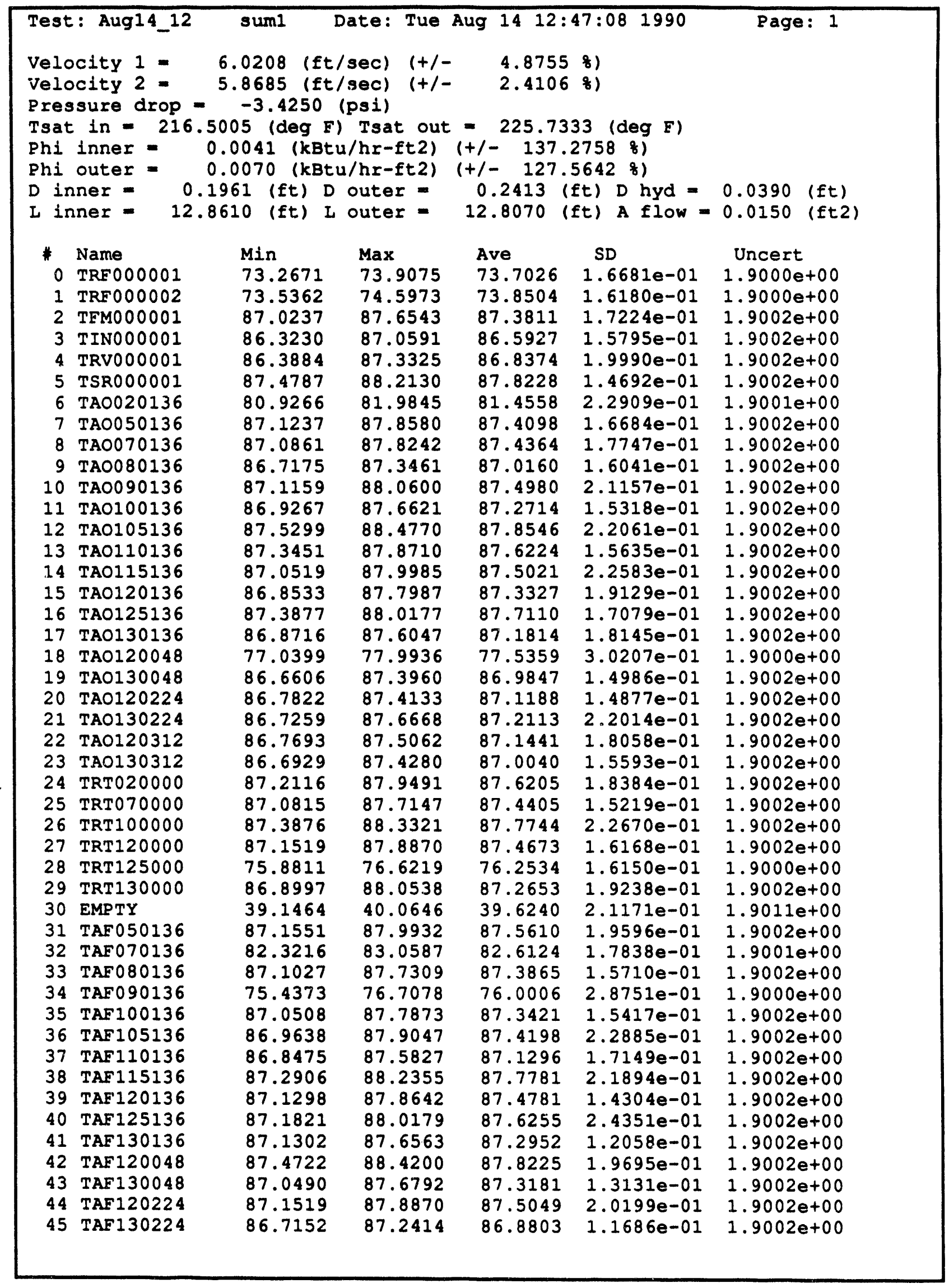




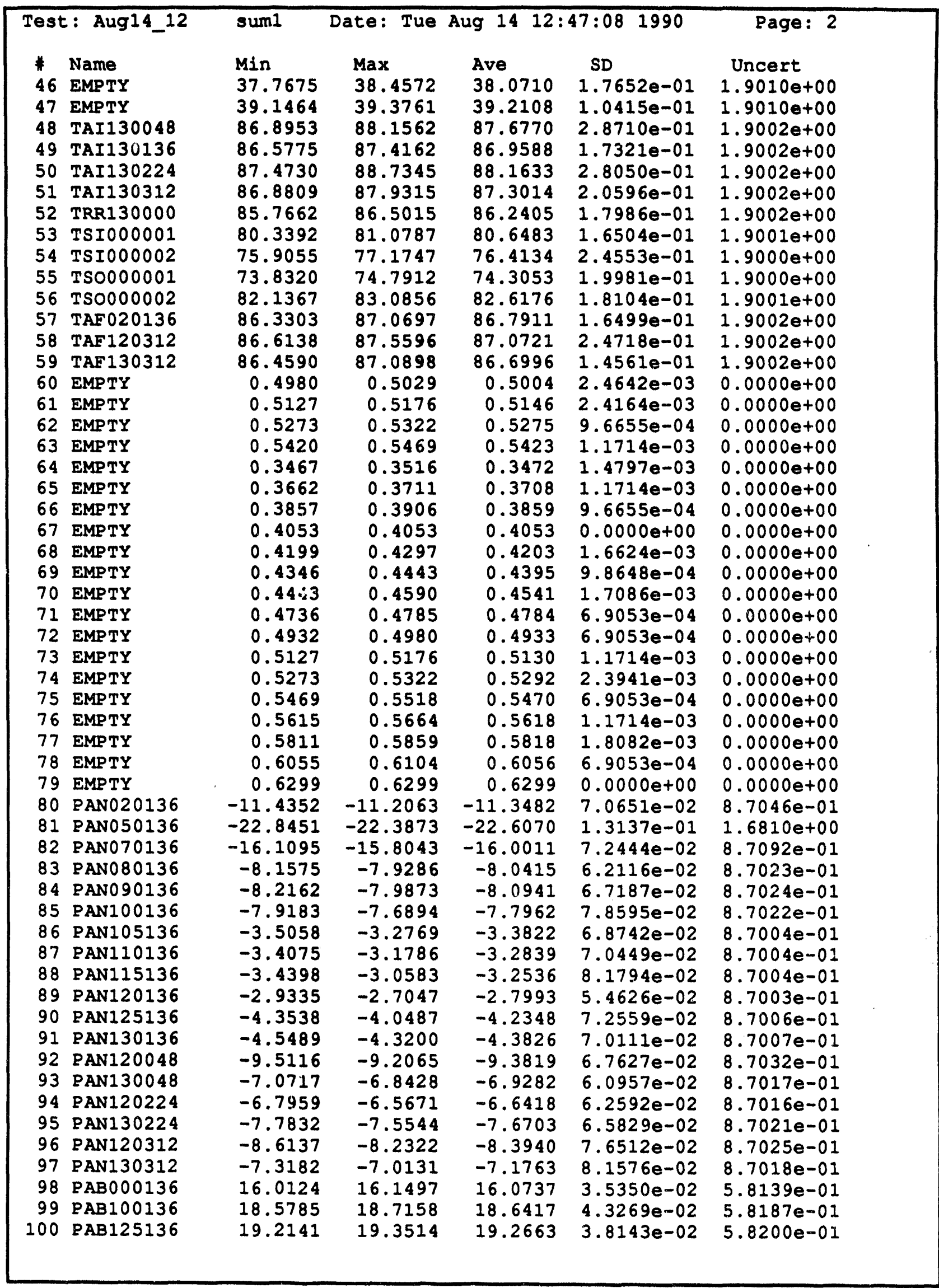




\begin{tabular}{|c|c|c|c|c|c|c|}
\hline \multicolumn{2}{|c|}{ Test: Aug14_12 } & sum1 & Date: Tue & Aug 1412 & $7: 08 \quad 1990$ & Page: 3 \\
\hline * & Name & Min & $\operatorname{Max}$ & Ave & SD & Uncert \\
\hline 101 & PINO00001 & 15.9108 & 16.1550 & 16.0329 & $2.4662 e-02$ & $7.5107 e-01$ \\
\hline 102 & PRV000001 & 19.1082 & 19.2303 & 19.2132 & $4.2787 e-02$ & $7.5154 \mathrm{e}-01$ \\
\hline 103 & PSR000001 & 19.6691 & 19.7911 & 19.6788 & $3.3453 e-02$ & $7.5161 e-01$ \\
\hline 104 & PEMO000001 & 5.3030 & 5.6692 & 5.4586 & $9.2943 e-02$ & $5.0019 e-01$ \\
\hline 105 & PANO00136 & -95.1705 & -94.7127 & -94.8225 & $1.9749 e-01$ & $4.9257 e+00$ \\
\hline 106 & PFM000002 & 5.0168 & 5.3189 & 5.1819 & $7.7850 e-02$ & $2.0042 e-01$ \\
\hline 107 & EMPTY & -0.0049 & 0.0049 & 0.0036 & $2.7568 e-03$ & $0.0000 e+00$ \\
\hline 108 & EMPTY & -10.0000 & -5.3564 & -7.3414 & $6.0239 e-01$ & $0.00000+00$ \\
\hline 109 & EMPTY & -0.0049 & 0.0049 & 0.0014 & $2.4244 e-03$ & $0.0000 e+00$ \\
\hline 110 & EMPTY & -10.0000 & -10.0000 & -10.0000 & $0.0000 e+00$ & $0.0000 e+00$ \\
\hline 111 & EMPTY & -0.0049 & 0.0000 & -0.0001 & $6.9053 e-04$ & $0.0000 e+00$ \\
\hline 112 & VPW000001 & -0.8526 & -0.6573 & -0.7549 & $9.8648 e-02$ & $8.1001 e-01$ \\
\hline 113 & TPW000002 & -1.2357 & -0.4545 & -0.7787 & $1.2232 \mathrm{e}-01$ & $8.1001 e-01$ \\
\hline 114 & VPW000003 & -0.7286 & -0.3380 & -0.6193 & $1.1272 \theta-01$ & $8.1001 e-01$ \\
\hline 115 & VPW000004 & -0.8752 & -0.2892 & -0.6056 & $1.2409 e-01$ & $8.1001 e-01$ \\
\hline 116 & VPW000005 & -1.4686 & -1.0780 & -1.2459 & $1.1168 e-01$ & $8.1003 e-01$ \\
\hline 117 & VPW000006 & -1.6499 & -1.0630 & -1.4380 & $1.1732 e-01$ & $8.1005 e-01$ \\
\hline 118 & IPWO00001 & -11.6766 & -7.7719 & -9.6852 & $1.21510+00$ & $8.1004 e+00$ \\
\hline 119 & IPW000002 & -10.2818 & -6.3793 & -9.2282 & $1.2596 \mathrm{e}+00$ & $8.1003 e+00$ \\
\hline 120 & IPW000003 & -12.8625 & -7.0125 & -10.4055 & $1.0281 e+00$ & $8.1004 e+00$ \\
\hline 121 & IPWO000004 & -2.2536 & -1.2771 & -1.8435 & $2.8523 e-01$ & $2.0001 e+00$ \\
\hline 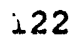 & IPW000005 & -3.9904 & -3.0139 & -3.4435 & $3.3653 e-01$ & $2.0002 e+00$ \\
\hline 123 & IPW000006 & -2.8076 & -1.3427 & -2.2021 & $3.6326 e-01$ & $2.0001 e+00$ \\
\hline 124 & EMPTY & -0.0293 & -0.0098 & -0.0202 & $4.3011 e-03$ & $0.0000 e+00$ \\
\hline 125 & EMPTY & -0.0195 & -0.0098 & -0.0135 & $2.8862 e-03$ & $0.0000 e+00$ \\
\hline 126 & EMPTY & -0.0244 & -0.0098 & -0.0163 & $3.3613 e-03$ & $0.0000 e+00$ \\
\hline 127 & EMPTY & -0.0244 & -0.0098 & -0.0160 & $3.1257 e-03$ & $0.0000 e+00$ \\
\hline 128 & IHT000001 & -0.2066 & 0.1841 & -0.0425 & $9.1354 e-02$ & $4.1000 e+00$ \\
\hline 129 & IHT000002 & -0.2329 & 0.1577 & -0.0258 & $9.1779 e-02$ & $4.1000 e+00$ \\
\hline 130 & IHT000003 & -0.0864 & 0.3042 & -0.0161 & $1.0259 e-01$ & $4.1000 e+00$ \\
\hline 131 & IHT000004 & -0.2028 & 0.1878 & 0.0042 & $9.9902 e-02$ & $4.1000 e+00$ \\
\hline 132 & IHT000005 & -0.2066 & 0.3794 & -0.0308 & $1.2633 e-01$ & $4.1000 e+00$ \\
\hline 133 & IHT000006 & -0.0601 & 0.3305 & 0.0649 & $1.0992 e-01$ & $4.1000 e+00$ \\
\hline 134 & IHT000007 & -0.0601 & 0.3305 & -0.0054 & $9.6976 e-02$ & $4.1000 e+00$ \\
\hline 135 & IHT000008 & -0.1578 & 0.0376 & -0.0445 & $9.7377 e-02$ & $4.1000 e+00$ \\
\hline 136 & IHT000009 & -0.2216 & 0.1690 & 0.0089 & $8.5454 e-02$ & $4.1000 e+00$ \\
\hline 137 & IHT000010 & -0.1427 & 0.2479 & -0.0451 & $1.1334 e-01$ & $4.1000 e+00$ \\
\hline 138 & IHT000011 & -0.1089 & 0.0864 & -0.0347 & $9.5765 e-02$ & $4.1000 e+00$ \\
\hline 139 & IHT000012 & -0.1653 & 0.2254 & -0.0481 & $1.0440 e-01$ & $4.1000 e+00$ \\
\hline 140 & IHTO00013 & -0.0563 & 0.3343 & 0.1038 & $1.0206 e-01$ & $4.1000 e+00$ \\
\hline 141 & IHTO00014 & -0.1953 & 0.1953 & 0.0156 & $8.6810 e-02$ & $4.1000 e+00$ \\
\hline 142 & IHTO00015 & -0.2442 & 0.1465 & 0.0058 & $9.6976 \mathrm{e}-02$ & $4.1000 e+00$ \\
\hline 143 & IHT000016 & -0.1352 & 0.2554 & -0.0102 & $1.0992 e-01$ & $4.1000 e+00$ \\
\hline 144 & IHT000017 & -0.3530 & 0.4282 & 0.1352 & $2.3093 e-01$ & $4.1000 e+00$ \\
\hline 145 & IHT000018 & -0.1728 & 0.2178 & 0.0186 & $1.1497 e-01$ & $4.1000 e+00$ \\
\hline 146 & IHT000019 & 0.0413 & 0.4319 & 0.2327 & $1.0052 e-01$ & $4.1000 e+00$ \\
\hline 147 & IHT 000020 & -0.2517 & 0.1390 & -0.0485 & $8.7880 e-02$ & $4.1000 e+00$ \\
\hline 148 & IHT000021 & -0.0714 & 0.3193 & 0.0341 & $1.0595 e-01$ & $4.1000 e+00$ \\
\hline 149 & IHTO00022 & 0.0037 & 0.3944 & 0.1131 & $1.0559 e-01$ & $4.1000 e+00$ \\
\hline 150 & IHT000023 & -0.0300 & 0.5559 & 0.1653 & $7.8918 \mathrm{e}-02$ & $4.1000 e+00$ \\
\hline 151 & IHT000024 & -0.4770 & -0.0864 & -0.2543 & $9.6735 e-02$ & $4.1000 \mathrm{e}+00$ \\
\hline 152 & IHTO00025 & 0.2028 & 0.7888 & 0.2692 & $1.1578 \mathrm{e}-01$ & $4.1000 e+00$ \\
\hline 153 & IHT000026 & -0.1690 & 0.2216 & 0.0224 & $7.3716 e-02$ & $4.1000 e+00$ \\
\hline 154 & IHT000027 & -0.2291 & 0.1615 & 0.0014 & $9.4125 e-02$ & $4.1000 e+00$ \\
\hline 155 & IHTO00028 & -0.0526 & 0.3381 & 0.0295 & $1.0507 e-01$ & $4.1000 e+00$ \\
\hline
\end{tabular}




\begin{tabular}{|c|c|c|c|c|c|}
\hline ast: Aug14_12 & suml & Date: Tue & ug $14 \quad 12$ & $7: 08 \quad 1990$ & Page: 4 \\
\hline 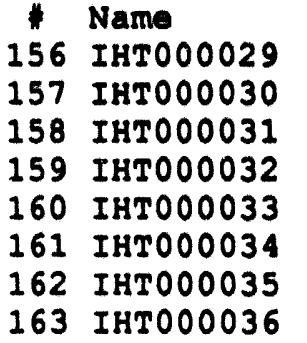 & $\begin{array}{l}\text { Min } \\
-0.0826 \\
-0.0150 \\
-0.3418 \\
-0.2930 \\
-0.0977 \\
-0.0376 \\
-0.1277 \\
-0.1878\end{array}$ & $\begin{array}{l}\text { Max } \\
0.1127 \\
0.1803 \\
0.2441 \\
0.0977 \\
0.0976 \\
0.1577 \\
0.2629 \\
0.0075\end{array}$ & $\begin{array}{r}\text { Ave } \\
-0.0201 \\
0.0475 \\
-0.0059 \\
0.0000 \\
0.0000 \\
0.0171 \\
0.0715 \\
-0.0589\end{array}$ & $\begin{array}{l}\text { SD } \\
9.2034 e-02 \\
9.2034 e-02 \\
1.1864 e-01 \\
1.0625 e-01 \\
9.8648 e-02 \\
8.8585 e-02 \\
8.3612 e-02 \\
9.3461 e-02\end{array}$ & $\begin{array}{l}\text { Uncert } \\
4.1000 e+00 \\
4.1000 e+00 \\
4.1000 e+00 \\
4.1000 e+00 \\
4.1000 e+00 \\
4.1000 \theta+00 \\
4.1000 e+00 \\
4.1000 e+00\end{array}$ \\
\hline
\end{tabular}




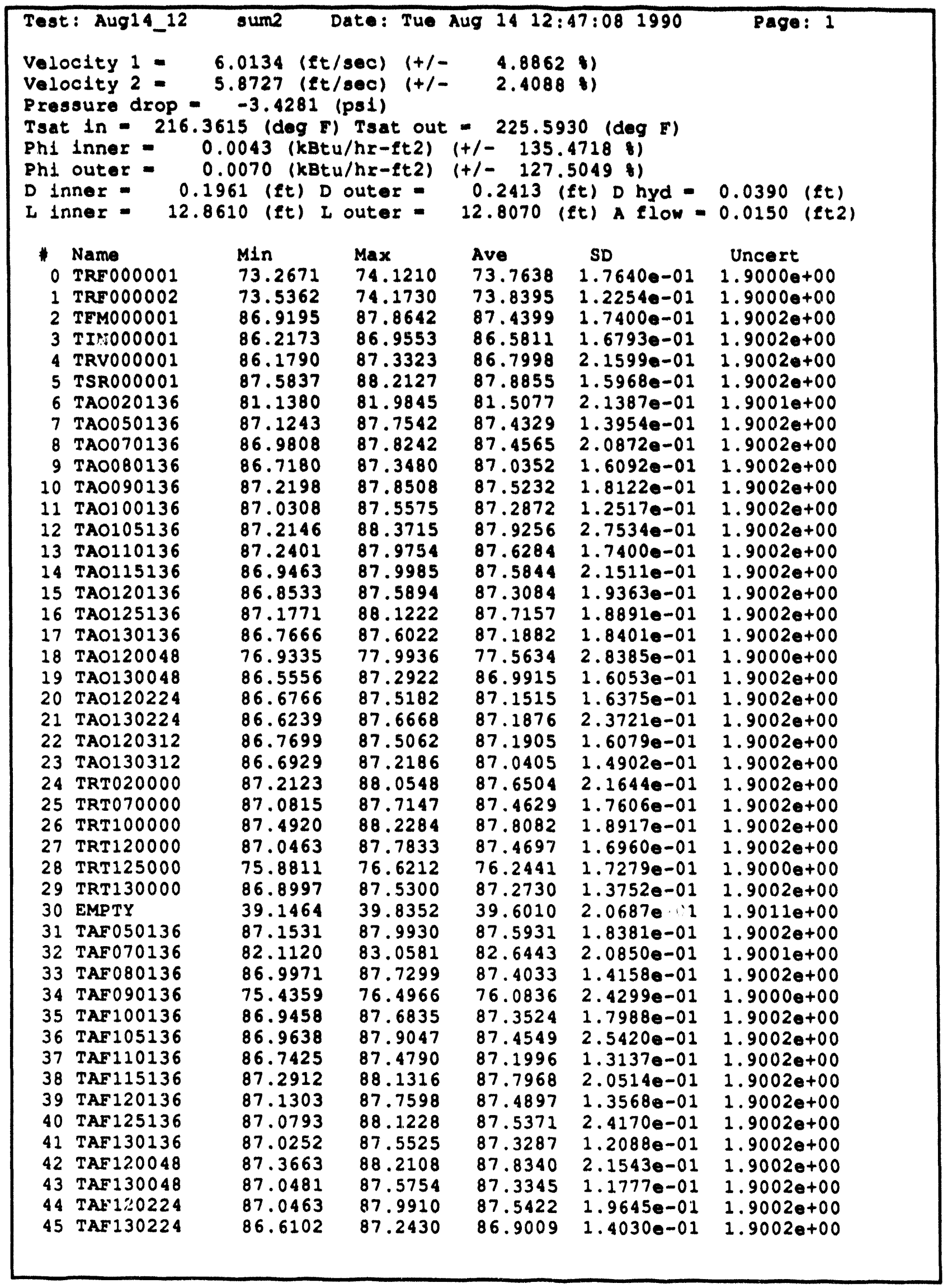




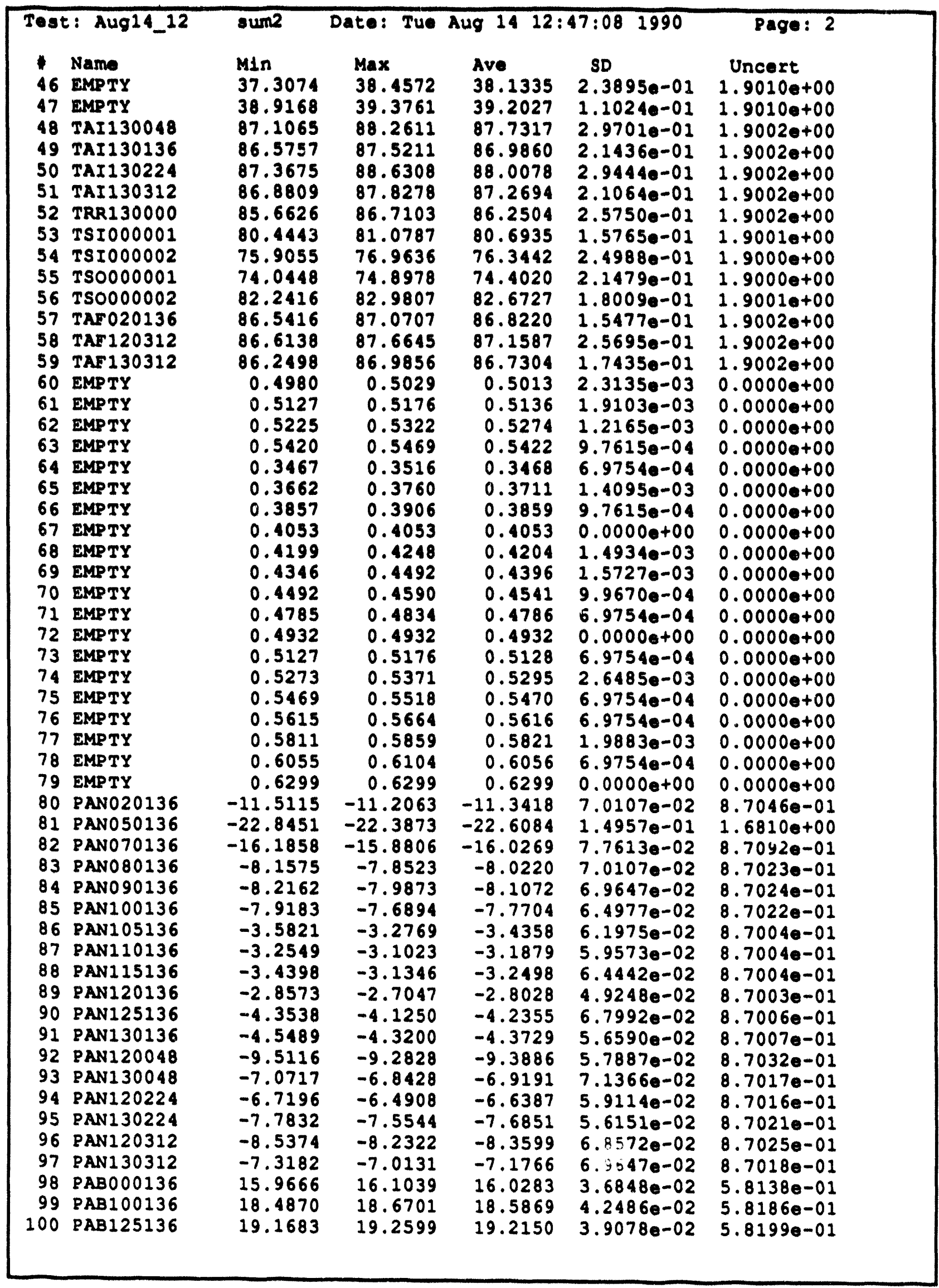




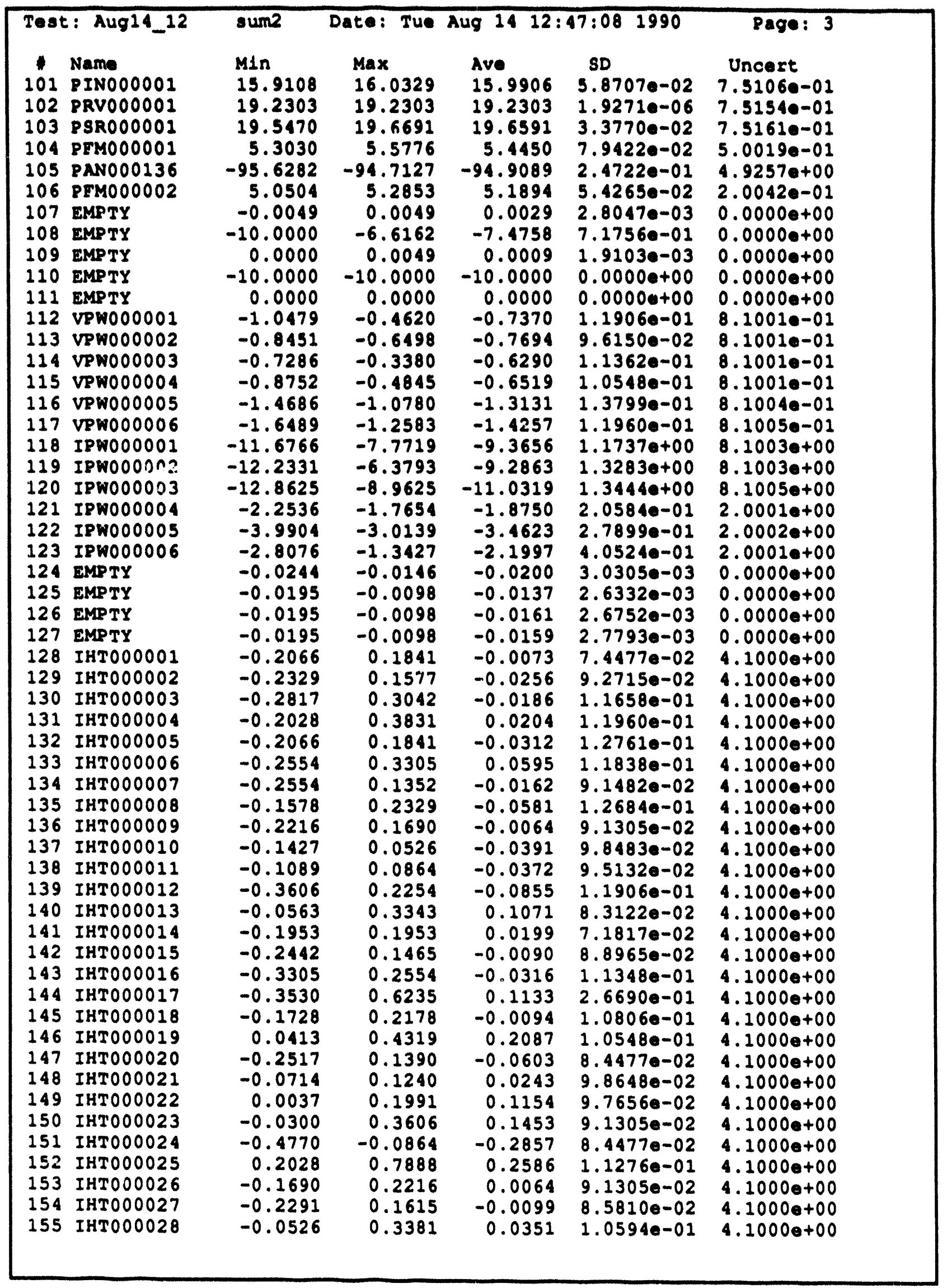




\begin{tabular}{|c|c|c|c|c|c|c|}
\hline $\begin{array}{l}1 \\
156 \\
157 \\
158 \\
159 \\
160 \\
161 \\
162 \\
163\end{array}$ & $\begin{array}{l}\text { Name } \\
\text { IHTO00029 } \\
\text { IHTO00030 } \\
\text { IHTO00031 } \\
\text { IHTO00032 } \\
\text { IHTO00033 } \\
\text { IHTO00034 } \\
\text { IHTO00035 } \\
\text { IHTO00036 }\end{array}$ & $\begin{array}{l}M 1 n \\
-0.0826 \\
-0.0130 \\
-0.1465 \\
-0.0976 \\
-0.0977 \\
-0.2329 \\
-0.1277 \\
-0.1878\end{array}$ & $\begin{array}{l}\operatorname{Max} \\
0.3080 \\
0.3756 \\
0.4394 \\
0.2930 \\
0.2930 \\
0.1577 \\
0.2629 \\
0.2028\end{array}$ & $\begin{array}{l}\text { Ave } \\
0.0011 \\
0.0647 \\
-0.0030 \\
-0.0060 \\
0.0060 \\
-0.0176 \\
0.0716 \\
-0.0523\end{array}$ & $\begin{array}{l}\text { SD } \\
1.1276 e-01 \\
1.1219 e-01 \\
1.3681 \bullet-01 \\
1.1348 \bullet-01 \\
1.1348 \bullet-01 \\
8.2141 \bullet-02 \\
1.0911 \bullet-01 \\
1.1419 \bullet-01\end{array}$ & $\begin{array}{l}\text { Uncert } \\
4.1000 \bullet+00 \\
4.1000 \bullet+00 \\
4.1000 \bullet+00 \\
4.1000 \bullet+00 \\
4.1000 e+00 \\
4.1000 e+00 \\
4.1000 \bullet+00 \\
4.1000 \bullet+00\end{array}$ \\
\hline
\end{tabular}




\begin{tabular}{|c|c|c|c|c|}
\hline \multicolumn{5}{|c|}{$\begin{array}{l}\text { Velocity } 1=0.007400(\mathrm{ft} / \mathrm{sec}) \\
\text { Velocity } 2=0.004200(\mathrm{ft} / \mathrm{sec}) \\
\text { Pressure Drop }=0.003100 \text { (psi) } \\
\text { Tsat In }=0.139008 \text { (deg F) Tsat out }=0.140305(\mathrm{deg} F) \\
\text { Phi Inner }=0.000200 \text { (kBtu/hr-ft2) Phi outer }=0.000000 \text { (kBtu/hr-ft2) }\end{array}$} \\
\hline $\begin{array}{l}0 \\
1 \\
2 \\
3 \\
4 \\
5 \\
6 \\
7 \\
8 \\
9 \\
10 \\
11 \\
12 \\
13 \\
14 \\
15 \\
16 \\
17 \\
18 \\
19 \\
20 \\
21 \\
22 \\
23 \\
24 \\
25 \\
26 \\
27 \\
28 \\
29 \\
30 \\
31 \\
32 \\
33 \\
34 \\
35 \\
36 \\
37 \\
38 \\
39 \\
40 \\
41 \\
42 \\
43 \\
44 \\
45\end{array}$ & $\begin{array}{l}\text { NAm0 } \\
\text { TRFO00001 } \\
\text { TRF000002 } \\
\text { TFMO00001 } \\
\text { TINO00001 } \\
\text { TRV000001 } \\
\text { TSRO00001 } \\
\text { TAO020136 } \\
\text { TAO050136 } \\
\text { TAO070136 } \\
\text { TAO080136 } \\
\text { TAO090136 } \\
\text { TAO100136 } \\
\text { TAO105136 } \\
\text { TAO110136 } \\
\text { TAO115136 } \\
\text { TAO120136 } \\
\text { TAO125136 } \\
\text { TAO130136 } \\
\text { TAO120048 } \\
\text { TAO130048 } \\
\text { TAO120224 } \\
\text { TAO130224 } \\
\text { TAO120312 } \\
\text { TAO130312 } \\
\text { TRT020000 } \\
\text { TRT070000 } \\
\text { TRT100000 } \\
\text { TRT120000 } \\
\text { TRT125000 } \\
\text { TRT130000 } \\
\text { EMPTY } \\
\text { TAF050136 } \\
\text { TAF070136 } \\
\text { TAF080136 } \\
\text { TAF090136 } \\
\text { TAF100136 } \\
\text { TAF105136 } \\
\text { TAF110136 } \\
\text { TAF115136 } \\
\text { TAF120136 } \\
\text { TAF125136 } \\
\text { TAF130136 } \\
\text { TAF120048 } \\
\text { TAF130048 } \\
\text { TAF120224 } \\
\text { TAF130224 }\end{array}$ & $\begin{array}{l}\text { Abs DIff } \\
0.0612 \\
0.0109 \\
0.0588 \\
0.0116 \\
0.0376 \\
0.0627 \\
0.0519 \\
0.0231 \\
0.0201 \\
0.0192 \\
0.0252 \\
0.0158 \\
0.0710 \\
0.0060 \\
0.0823 \\
0.0243 \\
0.0047 \\
0.0068 \\
0.0275 \\
0.0068 \\
0.0327 \\
0.0237 \\
0.0464 \\
0.0365 \\
0.0299 \\
0.0224 \\
0.0338 \\
0.0024 \\
0.0093 \\
0.0077 \\
0.0230 \\
0.0321 \\
0.0319 \\
0.0168 \\
0.0830 \\
0.0103 \\
0.0351 \\
0.0700 \\
0.0187 \\
0.0116 \\
0.0884 \\
0.0335 \\
0.0115 \\
0.0164 \\
0.0373 \\
0.0206\end{array}$ & $\begin{array}{l}\text { DDIf } \\
0.0830 \\
0.0148 \\
0.0673 \\
0.0134 \\
0.0433 \\
0.0714 \\
0.0637 \\
0.0264 \\
0.0230 \\
0.0221 \\
0.0288 \\
0.0181 \\
0.0808 \\
0.0069 \\
0.0941 \\
0.0278 \\
0.0054 \\
0.0078 \\
0.0355 \\
0.0078 \\
0.0375 \\
0.0272 \\
0.0532 \\
0.0420 \\
0.0341 \\
0.0256 \\
0.0385 \\
0.0027 \\
0.0122 \\
0.0088 \\
0.0580 \\
0.0367 \\
0.0386 \\
0.0192 \\
0.1092 \\
0.0118 \\
0.0402 \\
0.0803 \\
0.0213 \\
0.0133 \\
0.1009 \\
0.0384 \\
0.0131 \\
0.0188 \\
0.0426 \\
0.0237\end{array}$ & \\
\hline
\end{tabular}




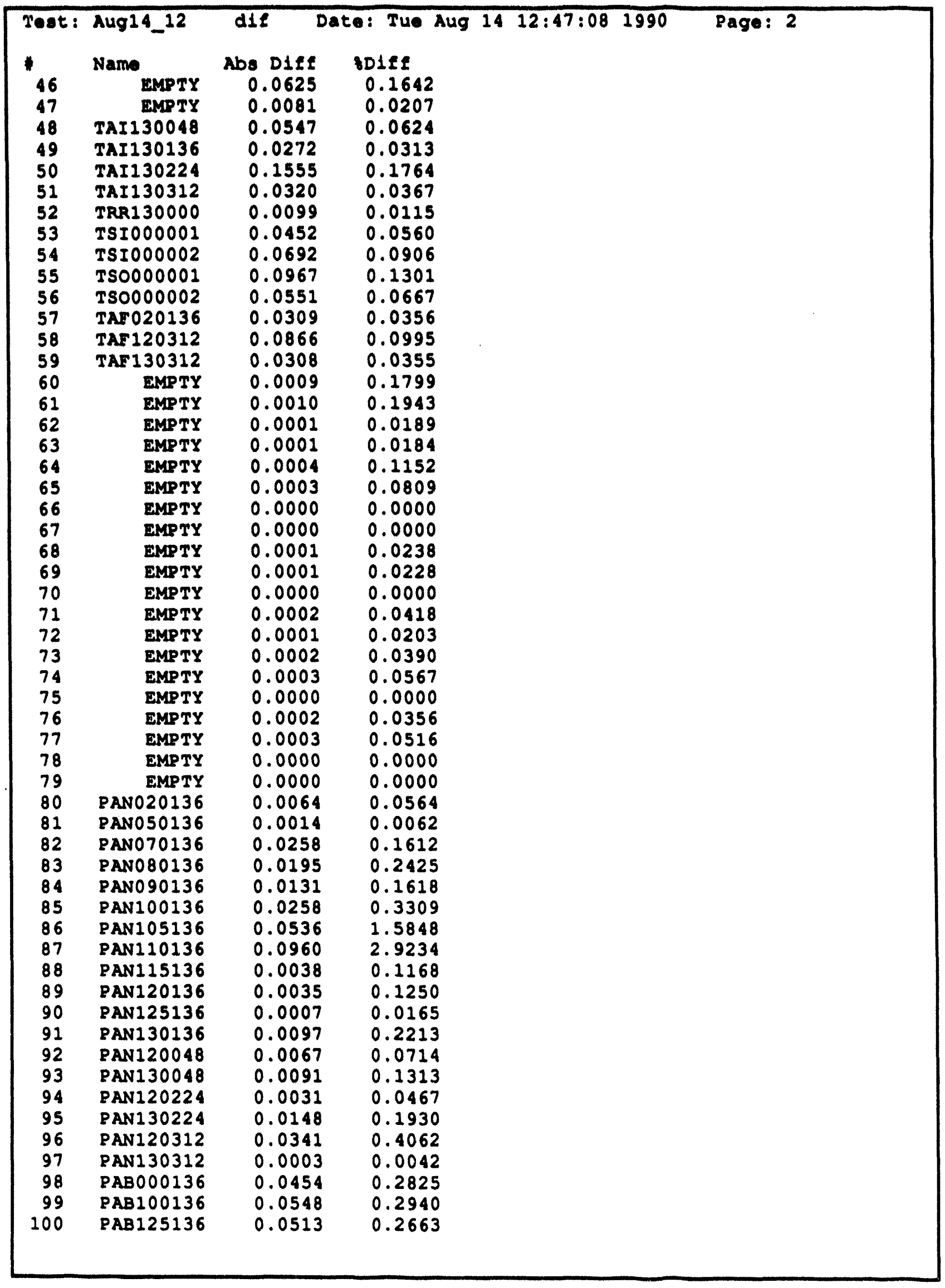




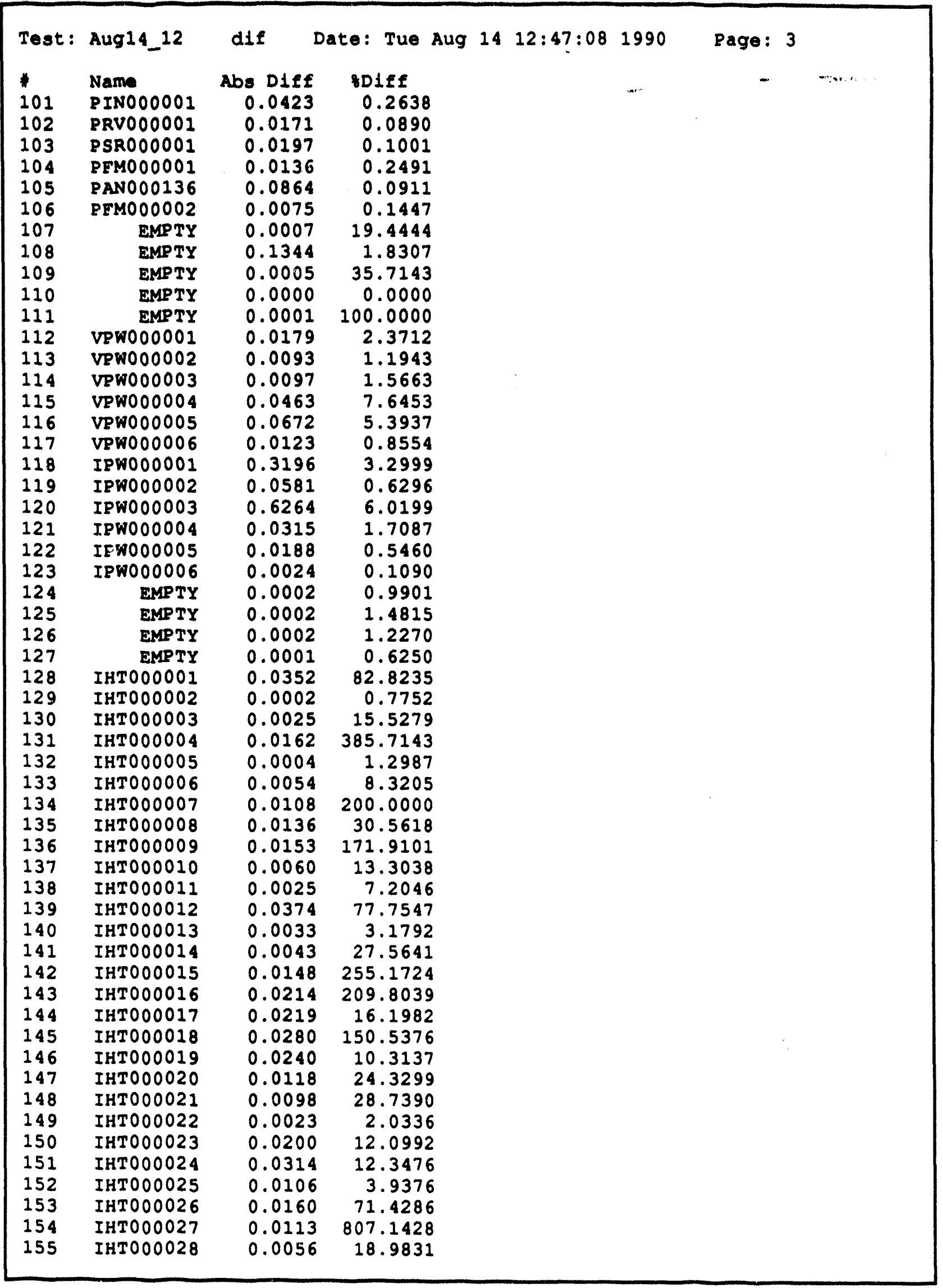




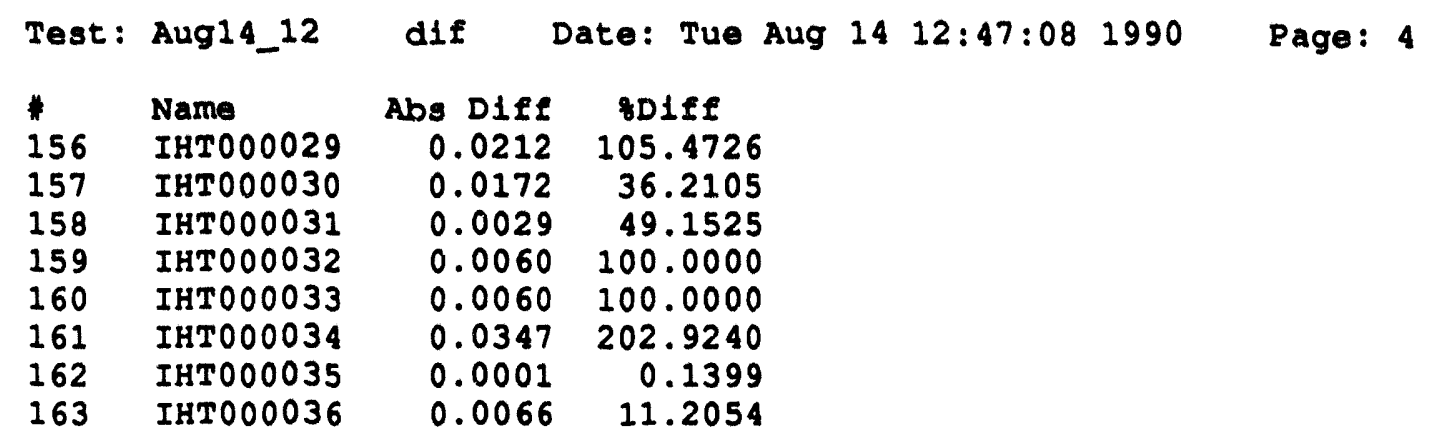




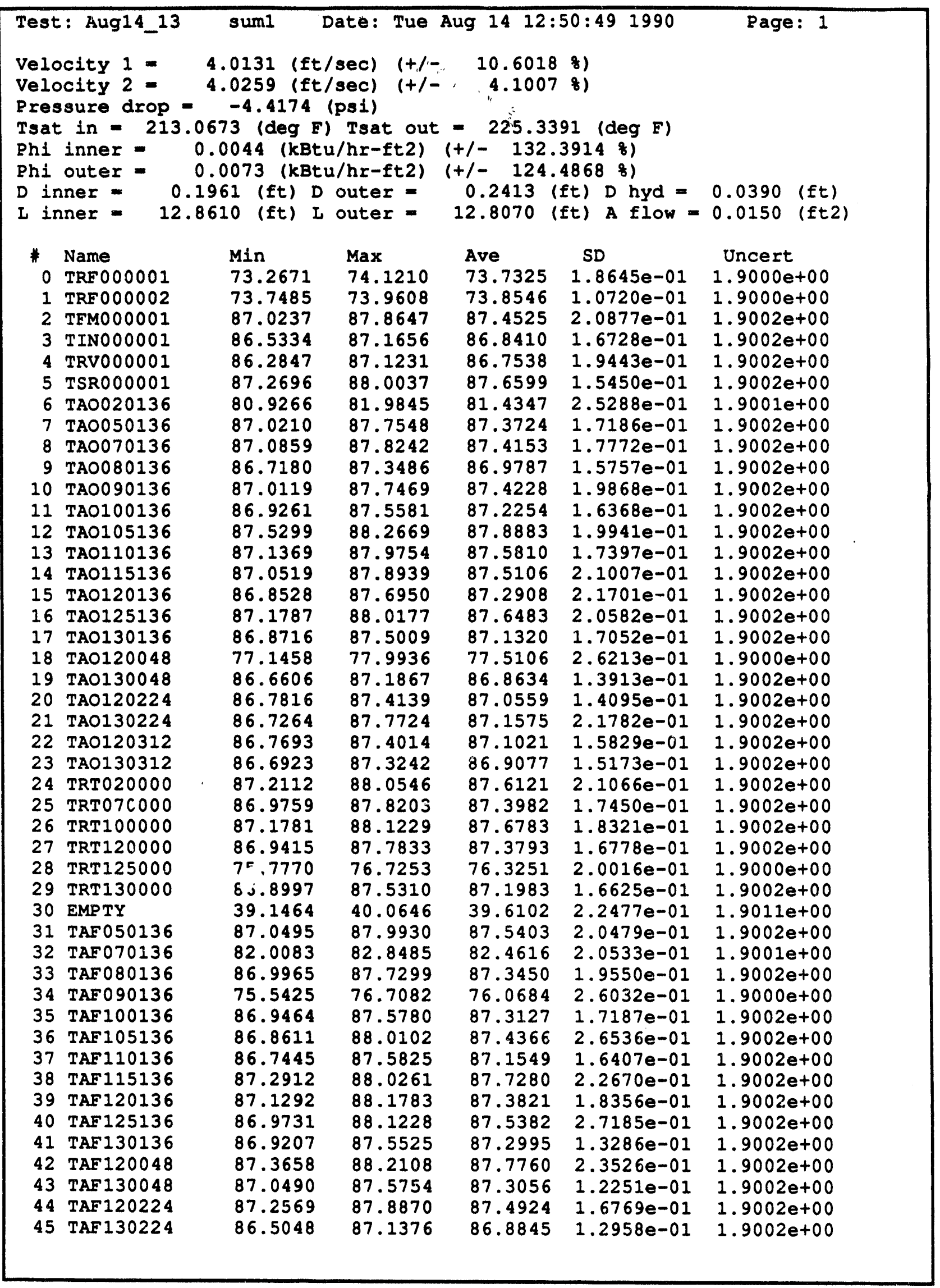




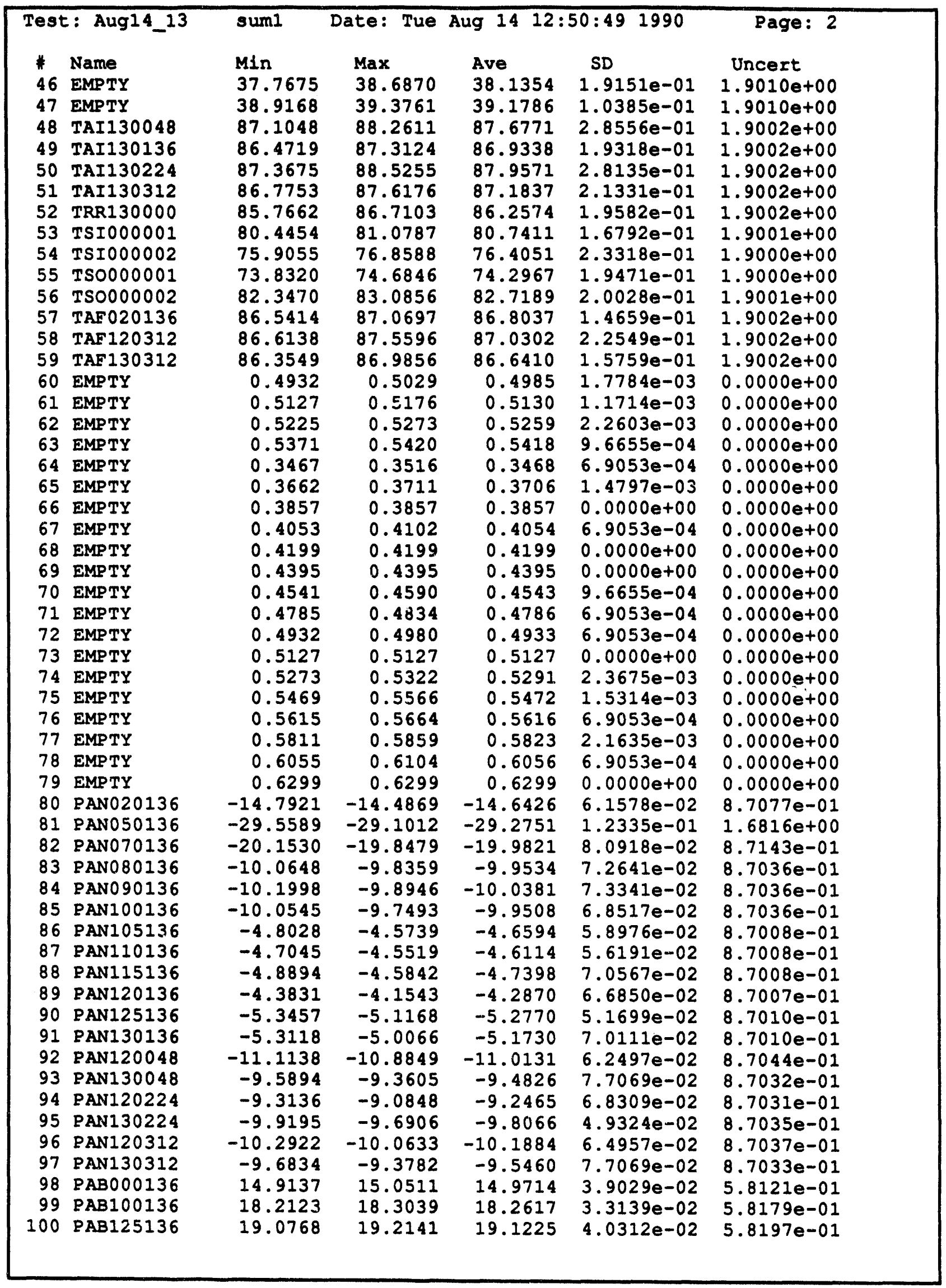




\begin{tabular}{|c|c|c|c|c|c|c|}
\hline \multicolumn{2}{|c|}{ Test: Aug14_13 } & sum1 & Date: Tue & Aug $14 \quad 12$ & $0: 491990$ & \multirow[t]{2}{*}{ Page: 3} \\
\hline$*$ & Name & Min & $\operatorname{Max}$ & Ave & SD & \\
\hline 101 & PINO00001 & 15.0563 & 15.0563 & 15.0563 & $7.7069 e-06$ & $7.5094 e-01$ \\
\hline 102 & PRV000001 & 18.9861 & 19.3524 & 19.2498 & $6.2195 e-02$ & $7.5154 e-01$ \\
\hline 103 & PSR000001 & 19.6691 & 19.7911 & 19.6813 & $3.6993 e-02$ & $7.5161 \mathrm{e}-01$ \\
\hline 104 & PEM000001 & 2.2817 & 2.5564 & 2.3880 & $5.0180 e-02$ & $5.0004 \mathrm{e}-01$ \\
\hline 105 & PANO00136 & -122.6363 & -122.1785 & -122.2976 & $2.0283 e-01$ & $4.9295 e+00$ \\
\hline 106 & PFM000002 & 2.3488 & 2.4495 & 2.4036 & $2.1119 e-02$ & $2.0009 \mathrm{e}-01$ \\
\hline 107 & EMPTY & -0.0049 & 0.0049 & 0.0014 & $3.8257 e-03$ & $0.0000 e+00$ \\
\hline 108 & EMPTY & -8.7207 & -6.0938 & -7.4315 & $3.6201 e-01$ & $0.0000 \mathrm{e}+00$ \\
\hline 109 & EMPTY & -0.0049 & 0.0049 & 0.0003 & $1.5314 e-03$ & $0.0000 \mathrm{e}+00$ \\
\hline 110 & EMPTY & -10.0000 & -10.0000 & -10.0000 & $0.0000 e+00$ & $0.0000 e+00$ \\
\hline 111 & EMPTY & 0.0000 & 0.0000 & 0.0000 & $0.0000 e+00$ & $0.0000 \mathrm{e}+00$ \\
\hline 112 & VPW000001 & -1.0479 & -0.6573 & -0.7979 & $9.6976 \mathrm{e}-02$ & $8.1001 e-01$ \\
\hline 113 & VPW000002 & -0.8451 & -0.6498 & -0.7748 & $9.4702 e-02$ & $8.1001 e-01$ \\
\hline 114 & VPW000003 & -0.9240 & -0.5333 & -0.6661 & $1.0014 \mathrm{e}-01$ & $8.1001 e-01$ \\
\hline 115 & VPW000004 & -0.6798 & -0.2892 & -0.5978 & $1.0507 e-01$ & $8.1001 e-01$ \\
\hline 116 & VPW000005 & -1.4686 & -1.0780 & -1.2967 & $1.0907 e-01$ & $8.1004 e-01$ \\
\hline 117 & VPW000006 & -1.6489 & -1.2583 & -1.4263 & $1.0447 \mathrm{e}-01$ & $8.1005 e-01$ \\
\hline 118 & IPW000001 & -11.6766 & -5.8195 & -9.2947 & $1.2653 \mathrm{e}+00$ & $8.1003 e+00$ \\
\hline 119 & IPW000002 & -12.2331 & -6.3793 & -10.1258 & $1.2366 \mathrm{e}+00$ & $8.1004 e+00$ \\
\hline 120 & IPW000003 & -12.8625 & -8.9625 & -10.5225 & $1.1143 e+00$ & $8.1004 e+00$ \\
\hline 121 & IPWOO00004 & -2.2536 & -1.2771 & -1.8728 & $2.4741 e-01$ & $2.0001 e+00$ \\
\hline 122 & IPW000005 & -4.4786 & -3.0139 & -3.4826 & $3.4112 e-01$ & $2.0002 e+00$ \\
\hline 123 & IPW000006 & -2.8076 & -1.3427 & -2.2119 & $3.7291 \mathrm{e}-01$ & $2.0001 e+00$ \\
\hline 124 & EMPTY & -0.0342 & -0.0146 & -0.0202 & $4.0685 e-03$ & $0.0000 e+00$ \\
\hline 125 & EMPTY & -0.0195 & -0.0049 & -0.0126 & $2.8058 e-03$ & $0.0000 e+00$ \\
\hline 126 & EMPTY & -0.0195 & -0.0098 & -0.0158 & $2.8862 e-03$ & $0.0000 \mathrm{e}+00$ \\
\hline 127 & EMPTY & -0.0244 & -0.0098 & -0.0160 & $3.2777 e-03$ & $0.0000 e+00$ \\
\hline 128 & IHT000001 & -0.2066 & 0.1841 & -0.0308 & $9.0412 e-02$ & $4.1000 e+00$ \\
\hline 129 & IHTO00002 & -0.2329 & 0.1577 & -0.0298 & $6.7888 e-02$ & $4.1000 e+00$ \\
\hline 130 & IHT000003 & -0.2817 & 0.1089 & -0.0239 & $1.0014 e-01$ & $4.1000 \mathrm{e}+00$ \\
\hline 131 & IHT000004 & -0.2028 & 0.1878 & -0.0231 & $9.5357 e-02$ & $4.1000 \mathrm{e}+00$ \\
\hline 132 & IHT000005 & -0.2066 & 0.1841 & -0.0347 & $1.0907 e-01$ & $4.1000 \mathrm{e}+00$ \\
\hline 133 & IHT000006 & -0.0601 & 0.1352 & 0.0297 & $9.8332 e-02$ & $4.1000 e+00$ \\
\hline 134 & IHT000007 & -0.2554 & 0.3305 & -0.0366 & $8.4997 e-02$ & $4.1000 e+00$ \\
\hline 135 & IHT000008 & -0.1578 & 0.2329 & -0.0523 & $1.0595 e-01$ & $4.1000 e+00$ \\
\hline 136 & IHT000009 & -0.2216 & 0.1690 & -0.0185 & $7.8523 e-02$ & $4.1000 e+00$ \\
\hline 137 & IHT000010 & -0.1427 & 0.2479 & -0.0724 & $1.0259 \mathrm{e}-01$ & $4.1000 e+00$ \\
\hline 138 & IHT000011 & -0.3042 & 0.0864 & -0.0699 & $8.8233 e-02$ & $4.1000 e+00$ \\
\hline 139 & IHT000012 & -0.3606 & 0.0301 & -0.0520 & $1.0507 e-01$ & $4.1000 \mathrm{e}+00$ \\
\hline 140 & IHT000013 & -0.0563 & 0.3343 & 0.0999 & $8.8233 e-02$ & $4.1000 e+00$ \\
\hline 141 & IHT000014 & -0.1953 & 0.1953 & 0.0078 & $6.7888 e-02$ & $4.1000 e+00$ \\
\hline 142 & IHT000015 & -0.2442 & 0.1465 & -0.0020 & $9.3043 e-02$ & $4.1000 \mathrm{e}+00$ \\
\hline 143 & IHT000016 & -0.1352 & 0.0601 & -0.0219 & $9.7377 e-02$ & $4.1000 e+00$ \\
\hline 144 & IHT000017 & -0.3530 & 0.6235 & 0.0688 & $2.7155 e-01$ & $4.1000 \mathrm{e}+00$ \\
\hline 145 & IHT000018 & -0.1728 & 0.2178 & 0.0421 & $9.0412 e-02$ & $4.1000 e+00$ \\
\hline 146 & IHT000019 & 0.0413 & 0.4319 & 0.2444 & $7.8523 e-02$ & $4.1000 e+00$ \\
\hline 147 & IHT000020 & -0.2517 & 0.1390 & -0.0564 & $8.8233 e-02$ & $4.1000 e+00$ \\
\hline 148 & IHT000021 & -0.0714 & 0.1240 & 0.0458 & $9.6655 e-02$ & $4.1000 e+00$ \\
\hline 149 & IHT000022 & 0.0037 & 0.3944 & 0.1405 & $1.0625 e-01$ & $4.1000 e+00$ \\
\hline 150 & IHTO00023 & -0.0300 & 0.3606 & 0.1614 & $9.2456 e-02$ & $4.1000 e+00$ \\
\hline 151 & IHT000024 & -0.4770 & -0.0864 & -0.2504 & $8.2393 e-02$ & $4.1000 \mathrm{e}+00$ \\
\hline 152 & IHT000025 & 0.2028 & 0.3981 & 0.2536 & $8.6541 \mathrm{e}-02$ & $4.1000 e+00$ \\
\hline 153 & IHTO00026 & -0.1690 & 0.2216 & 0.0224 & $7.3716 e-02$ & $4.1000 \mathrm{e}+00$ \\
\hline 154 & IHT000027 & -0.0338 & 0.1615 & 0.0170 & $8.6541 \mathrm{e}-02$ & $4.1000 e+00$ \\
\hline 155 & IHT000028 & -0.0526 & 0.1427 & 0.0295 & $9.7377 e-02$ & $4.1000 e+00$ \\
\hline
\end{tabular}




\begin{tabular}{|c|c|c|c|c|c|c|}
\hline \multicolumn{2}{|c|}{ Test: Aug14_13 } & suml & Date: Tue & \multicolumn{2}{|c|}{ Aug $14 \quad 12: 50: 49 \quad 1990$} & $\begin{array}{l}\text { Page: } 4 \\
\text { Uncert }\end{array}$ \\
\hline $\begin{array}{l}* \\
156 \\
157 \\
158 \\
159 \\
160 \\
161 \\
162 \\
163\end{array}$ & 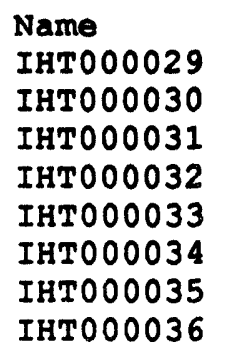 & $\begin{array}{l}\text { Min } \\
-0.2780 \\
-0.0150 \\
-0.1465 \\
-0.0976 \\
-0.0977 \\
-0.2329 \\
-0.1277 \\
-0.1878\end{array}$ & $\begin{array}{l}\text { Max } \\
0.1127 \\
0.1803 \\
0.2441 \\
0.0977 \\
0.0976 \\
0.1577 \\
0.2629 \\
0.2028\end{array}$ & $\begin{array}{l}\text { Ave } \\
-0.0319 \\
0.0670 \\
-0.0098 \\
-0.0039 \\
-0.0117 \\
-0.0063 \\
0.0559 \\
-0.0355\end{array}$ & $\begin{array}{l}\text { SD } \\
9.5112 e-02 \\
9.7377 e-02 \\
1.1334 e-01 \\
9.8569 e-02 \\
9.7935 e-02 \\
9.1354 e-02 \\
7.2866 e-02 \\
9.8963 e-02\end{array}$ & $\begin{array}{c}\text { Uncert } \\
4.1000 \mathrm{e}+00 \\
4.1000 \mathrm{e}+00 \\
4.1000 \mathrm{e}+00 \\
4.1000 \mathrm{e}+00 \\
4.1000 \mathrm{e}+00 \\
4.1000 \mathrm{e}+00 \\
4.1000 \mathrm{e}+00 \\
4.1000 \mathrm{e}+00\end{array}$ \\
\hline
\end{tabular}




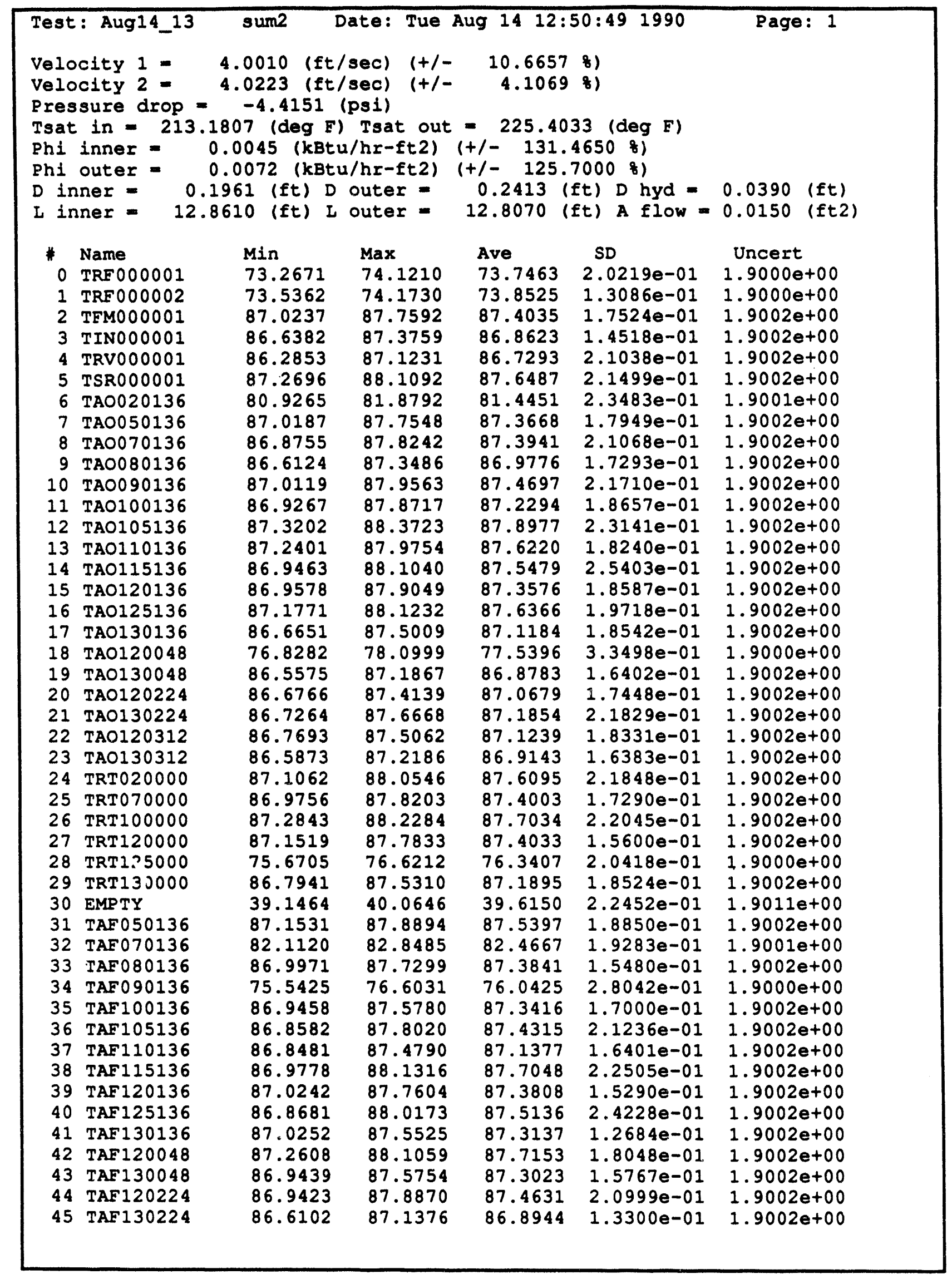




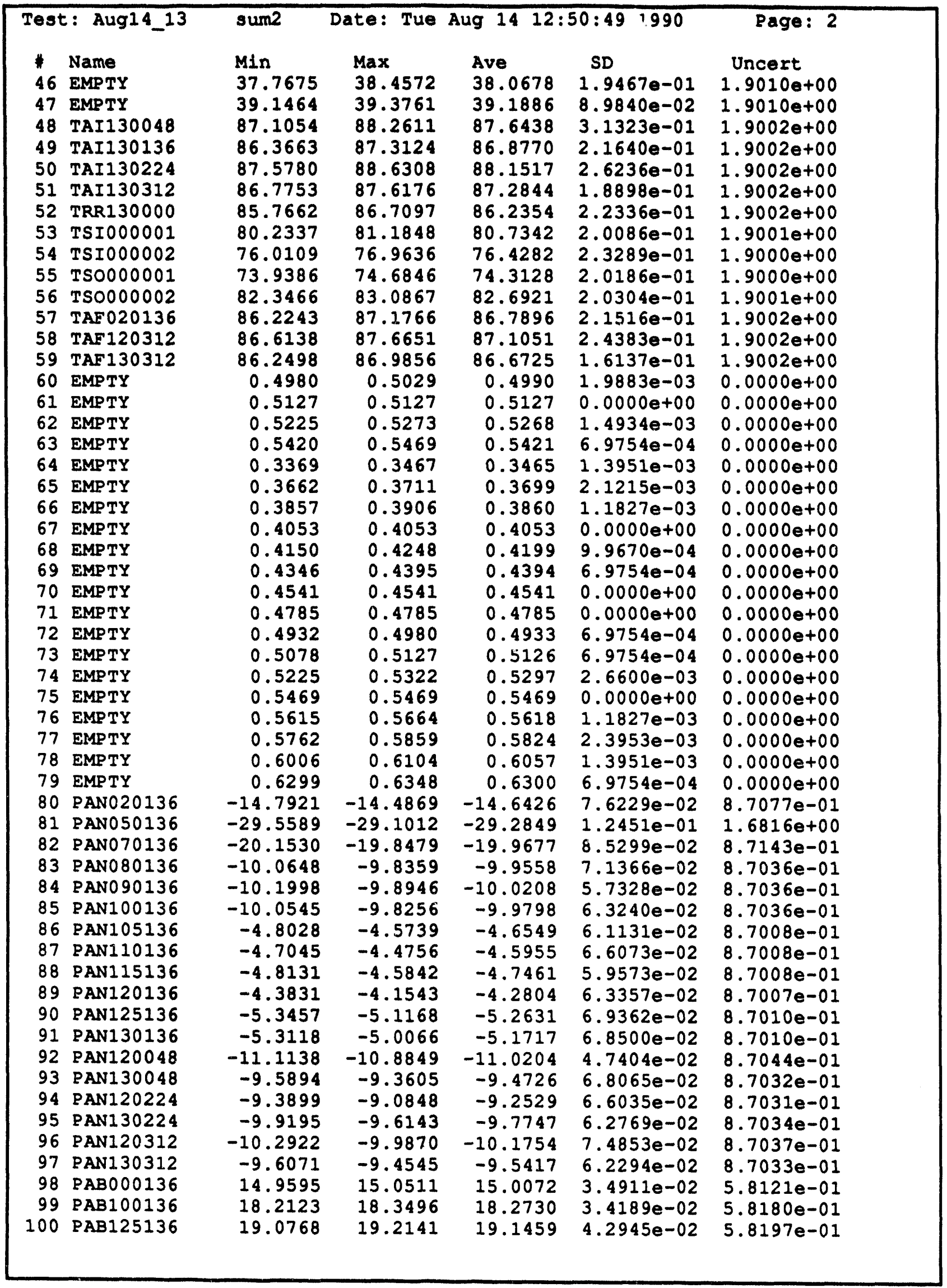




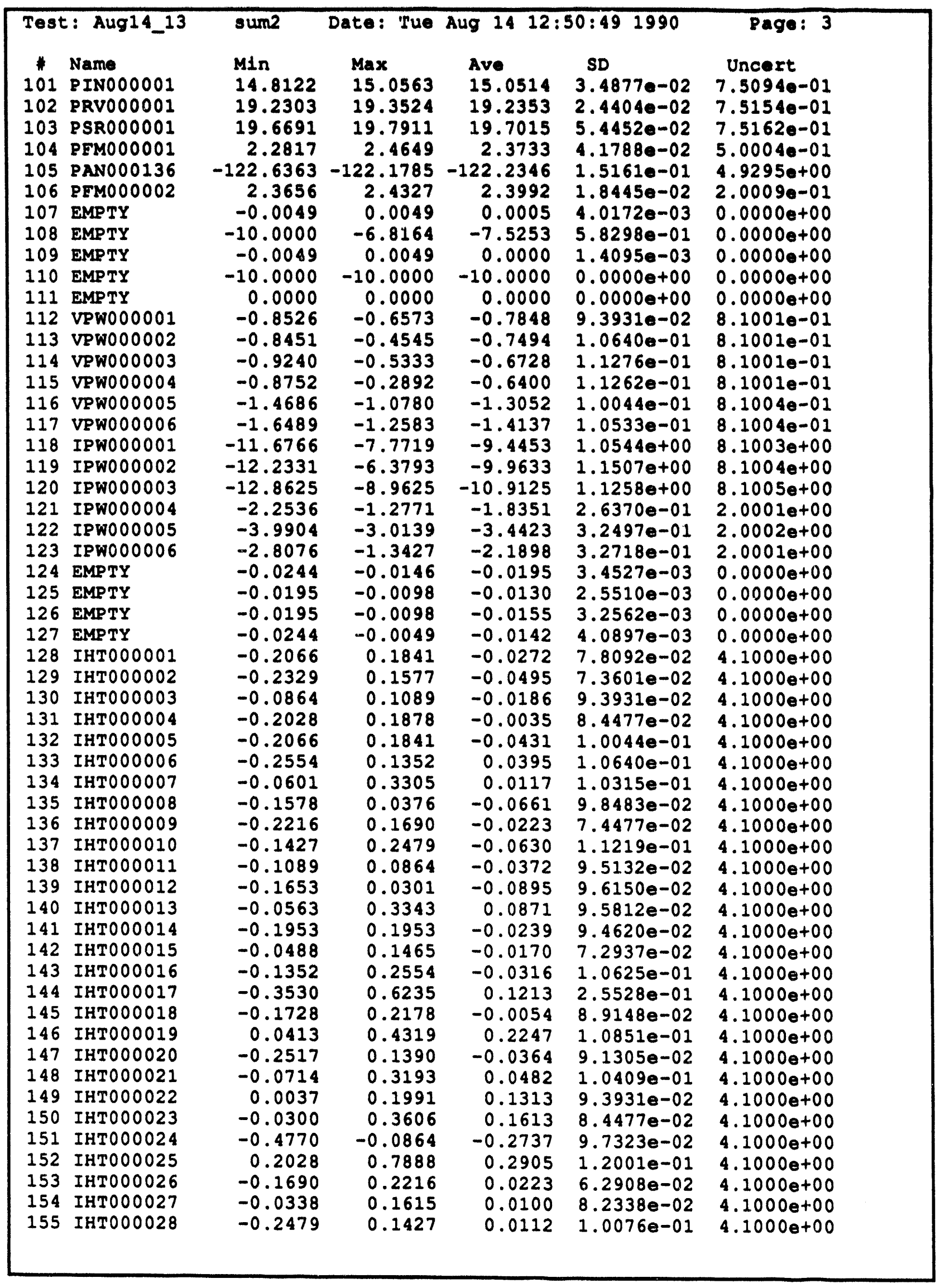




\begin{tabular}{|c|c|c|c|c|c|c|}
\hline $\begin{array}{l}1 \\
156 \\
157 \\
158 \\
159 \\
160 \\
161 \\
162 \\
163\end{array}$ & 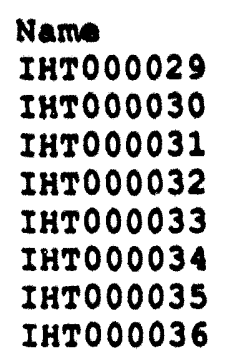 & $\begin{array}{l}\text { Min } \\
-0.0826 \\
-0.2103 \\
-0.1465 \\
-0.0976 \\
-0.0977 \\
-0.2329 \\
-0.1277 \\
-0.1878\end{array}$ & $\begin{array}{l}\text { Max } \\
0.1127 \\
0.1803 \\
0.2441 \\
0.0977 \\
0.2930 \\
0.1577 \\
0.2629 \\
0.2028\end{array}$ & $\begin{array}{r}\text { Ave } \\
-0.0268 \\
0.0448 \\
0.0010 \\
0.0020 \\
0.0179 \\
-0.0216 \\
0.0756 \\
-0.0443\end{array}$ & $\begin{array}{l}\text { SD } \\
8.9148 \bullet-02 \\
9.9303 \bullet-02 \\
1.0941 \bullet-01 \\
9.8648 \bullet-02 \\
1.0486 \bullet-01 \\
8.7680 \bullet-02 \\
6.8582 e-02 \\
1.0378 \bullet-01\end{array}$ & $\begin{array}{l}\text { Uncert } \\
4.1000 e+00 \\
4.1000 e+00 \\
4.1000 e+00 \\
4.1000 e+00 \\
4.1000 \theta+00 \\
4.1000 \theta+00 \\
4.1000 e+00 \\
4.1000 e+00\end{array}$ \\
\hline
\end{tabular}




\begin{tabular}{|c|c|c|c|c|}
\hline \multicolumn{5}{|c|}{$\begin{array}{l}\text { Velocity } 1=0.012100(\mathrm{ft} / \mathrm{sec}) \\
\text { Velocity } 2=0.003600(\mathrm{ft} / \mathrm{sec}) \\
\text { Pressure Drop }=0.002300 \text { (psi) } \\
\text { Tsat in }=0.113388 \text { (deg r) Tsat out }=0.064209 \text { (deg E) } \\
\text { Ph1 inner }=0.000100 \text { (kBtu/hr-ft2) Ph1 outer }=0.000100 \text { (kBtu/hr-ft2) }\end{array}$} \\
\hline $\begin{array}{l}0 \\
0 \\
1 \\
2 \\
3 \\
4 \\
5 \\
6 \\
7 \\
8 \\
9 \\
10 \\
11 \\
12 \\
13 \\
14 \\
15 \\
16 \\
17 \\
18 \\
19 \\
20 \\
21 \\
22 \\
23 \\
24 \\
25 \\
26 \\
27 \\
28 \\
29 \\
30 \\
31 \\
32 \\
33 \\
34 \\
35 \\
36 \\
37 \\
38 \\
39 \\
40 \\
41 \\
42 \\
43 \\
44 \\
45\end{array}$ & $\begin{array}{l}\text { NAm0 } \\
\text { TRF000001 } \\
\text { TRF000002 } \\
\text { TFMO00001 } \\
\text { TINO00001 } \\
\text { TRV000001 } \\
\text { TSR000001 } \\
\text { TAO020136 } \\
\text { TAO050136 } \\
\text { TAO070136 } \\
\text { TAO080136 } \\
\text { TAO090136 } \\
\text { TAO100136 } \\
\text { TAO105136 } \\
\text { TAO110136 } \\
\text { TAO115136 } \\
\text { TAO120136 } \\
\text { TAO125136 } \\
\text { TAO130136 } \\
\text { TAO120048 } \\
\text { TAO130048 } \\
\text { TAO120224 } \\
\text { TA0130224 } \\
\text { TAO120312 } \\
\text { TAO130312 } \\
\text { TRT020000 } \\
\text { TRT070000 } \\
\text { TRT100000 } \\
\text { TRT120000 } \\
\text { TRT125000 } \\
\text { TRT130000 } \\
\text { EMPTY } \\
\text { TAF050136 } \\
\text { TAF070136 } \\
\text { TAF080136 } \\
\text { TAF090136 } \\
\text { TAF100136 } \\
\text { TAF105136 } \\
\text { TAF110136 } \\
\text { TAF115136 } \\
\text { TAF120136 } \\
\text { TAF125136 } \\
\text { TAF130136 } \\
\text { TAF120048 } \\
\text { TAF130048 } \\
\text { TAF120224 } \\
\text { TAF130224 }\end{array}$ & $\begin{array}{c}\text { Abo D111 } \\
0.0138 \\
0.0021 \\
0.0490 \\
0.0213 \\
0.0245 \\
0.0112 \\
0.0104 \\
0.0056 \\
0.0212 \\
0.0011 \\
0.0469 \\
0.0040 \\
0.0094 \\
0.0410 \\
0.0373 \\
0.0668 \\
0.0117 \\
0.0136 \\
0.0290 \\
0.0149 \\
0.0120 \\
0.0279 \\
0.0218 \\
0.0066 \\
0.0026 \\
0.0021 \\
0.0251 \\
0.0240 \\
0.0156 \\
0.0088 \\
0.0048 \\
0.0006 \\
0.0051 \\
0.0391 \\
0.0259 \\
0.0289 \\
0.0051 \\
0.0172 \\
0.0232 \\
0.0013 \\
0.0246 \\
0.0142 \\
0.0607 \\
0.0033 \\
0.0293 \\
0.0099 \\
0.0\end{array}$ & $\begin{array}{l}\text { DIEL } \\
0.0187 \\
0.0028 \\
0.0560 \\
0.0245 \\
0.0282 \\
0.0128 \\
0.0128 \\
0.0064 \\
0.0243 \\
0.0013 \\
0.0537 \\
0.0046 \\
0.0107 \\
0.0468 \\
0.0426 \\
0.0765 \\
0.0134 \\
0.0150 \\
0.0374 \\
0.0172 \\
0.0138 \\
0.0320 \\
0.0250 \\
0.0076 \\
0.0030 \\
0.0024 \\
0.0286 \\
0.0275 \\
0.0204 \\
0.0101 \\
0.0121 \\
0.0007 \\
0.0062 \\
0.0448 \\
0.0340 \\
0.0331 \\
0.0058 \\
0.0197 \\
0.0264 \\
0.0015 \\
0.0281 \\
0.0163 \\
0.0692 \\
0.0038 \\
0.0335 \\
0.0114\end{array}$ & \\
\hline
\end{tabular}




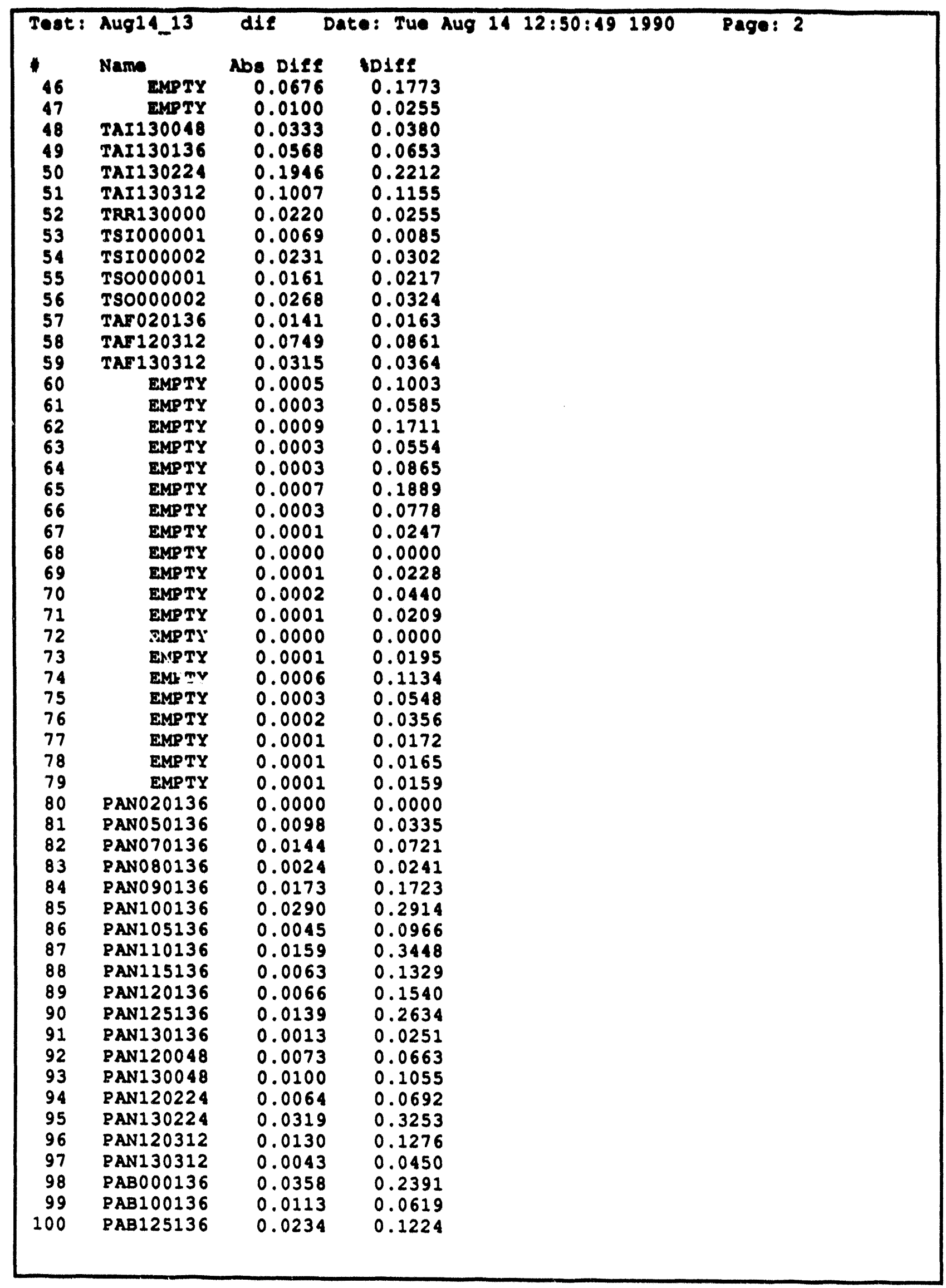




\begin{tabular}{|c|c|c|c|c|}
\hline $\begin{array}{l}\text { Test } \\
101 \\
101 \\
102 \\
103 \\
104 \\
105 \\
106 \\
107 \\
108 \\
109 \\
110 \\
111 \\
112 \\
113 \\
114 \\
115 \\
116 \\
117 \\
118 \\
119 \\
120 \\
121 \\
122 \\
123 \\
124 \\
125 \\
126 \\
127 \\
128 \\
129 \\
130 \\
131 \\
132 \\
133 \\
134 \\
135 \\
136 \\
137 \\
138 \\
139 \\
140 \\
141 \\
142 \\
143 \\
144 \\
145 \\
146 \\
147 \\
148 \\
149 \\
150 \\
151 \\
152 \\
153 \\
154 \\
155\end{array}$ & 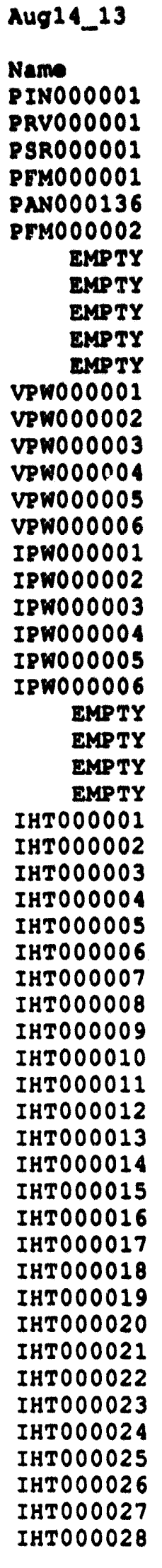 & $\begin{array}{l}\text { d1E } \\
\text { Abo D D } \\
0.0049 \\
0.0145 \\
0.0202 \\
0.0147 \\
0.0630 \\
0.0044 \\
0.0009 \\
0.0938 \\
0.0003 \\
0.0000 \\
0.0000 \\
0.0131 \\
0.0254 \\
0.0067 \\
0.0422 \\
0.0085 \\
0.0126 \\
0.1506 \\
0.1625 \\
0.3900 \\
0.0377 \\
0.0403 \\
0.0221 \\
0.0007 \\
0.0004 \\
0.0003 \\
0.0018 \\
0.0036 \\
0.0197 \\
0.0053 \\
0.0196 \\
0.0084 \\
0.0098 \\
0.0483 \\
0.0138 \\
0.0038 \\
0.0094 \\
0.0327 \\
0.0375 \\
0.0128 \\
0.0317 \\
0.0150 \\
0.0097 \\
0.0525 \\
0.0475 \\
0.0197 \\
0.0200 \\
0.0024 \\
0.0092 \\
0.0001 \\
0.0233 \\
0.0369 \\
0.0001 \\
0.0070 \\
0.0183\end{array}$ & $\begin{array}{l}\text { Tue Aug } 1412: 50: 491990 \\
\text { D1f1 } \\
0.0325 \\
0.0753 \\
0.1026 \\
0.6156 \\
0.0515 \\
0.1831 \\
64.2857 \\
1.2622 \\
100.0000 \\
0.0000 \\
0.0000 \\
1.6418 \\
3.2783 \\
1.0059 \\
7.0592 \\
0.6555 \\
0.8834 \\
1.6203 \\
1.6048 \\
3.7063 \\
2.0130 \\
1.1572 \\
0.9991 \\
3.4653 \\
3.1746 \\
1.8987 \\
11.2500 \\
11.6883 \\
66.1074 \\
22.1757 \\
84.8485 \\
24.2075 \\
32.9966 \\
131.9672 \\
26.3862 \\
20.5405 \\
12.9834 \\
46.7811 \\
72.1154 \\
12.8128 \\
406.4103 \\
750.0000 \\
44.2922 \\
76.3081 \\
112.8266 \\
8.0606 \\
35.4610 \\
5.2402 \\
6.5480 \\
0.0620 \\
9.3051 \\
14.5505 \\
0.4464 \\
41.1765 \\
62.0339 \\
\end{array}$ & Page: 3 \\
\hline
\end{tabular}


Test: Aug14_13 dif Date: Tue Aug 14 12:50:49 1990 Page: 4

- Name Abe DiEe NDIEE

156 IHTO00029 $0.0051 \quad 15.9875$

157 IHT000030 $0.0222 \quad 33.1343$

158 IHT000031 $0.0108 \quad 110.2041$

159 IHTO00032 $0.0059 \quad 151.2821$

160 IHT000033 $0.0296 \quad 252.9914$

161 IHTO00034 $0.0153 \quad 242.8571$

162 IHTO00035 $0.0197 \quad 35.2415$

$\begin{array}{llll}163 & \text { IHT000036 } & 0.0088 & 24.7887\end{array}$ 


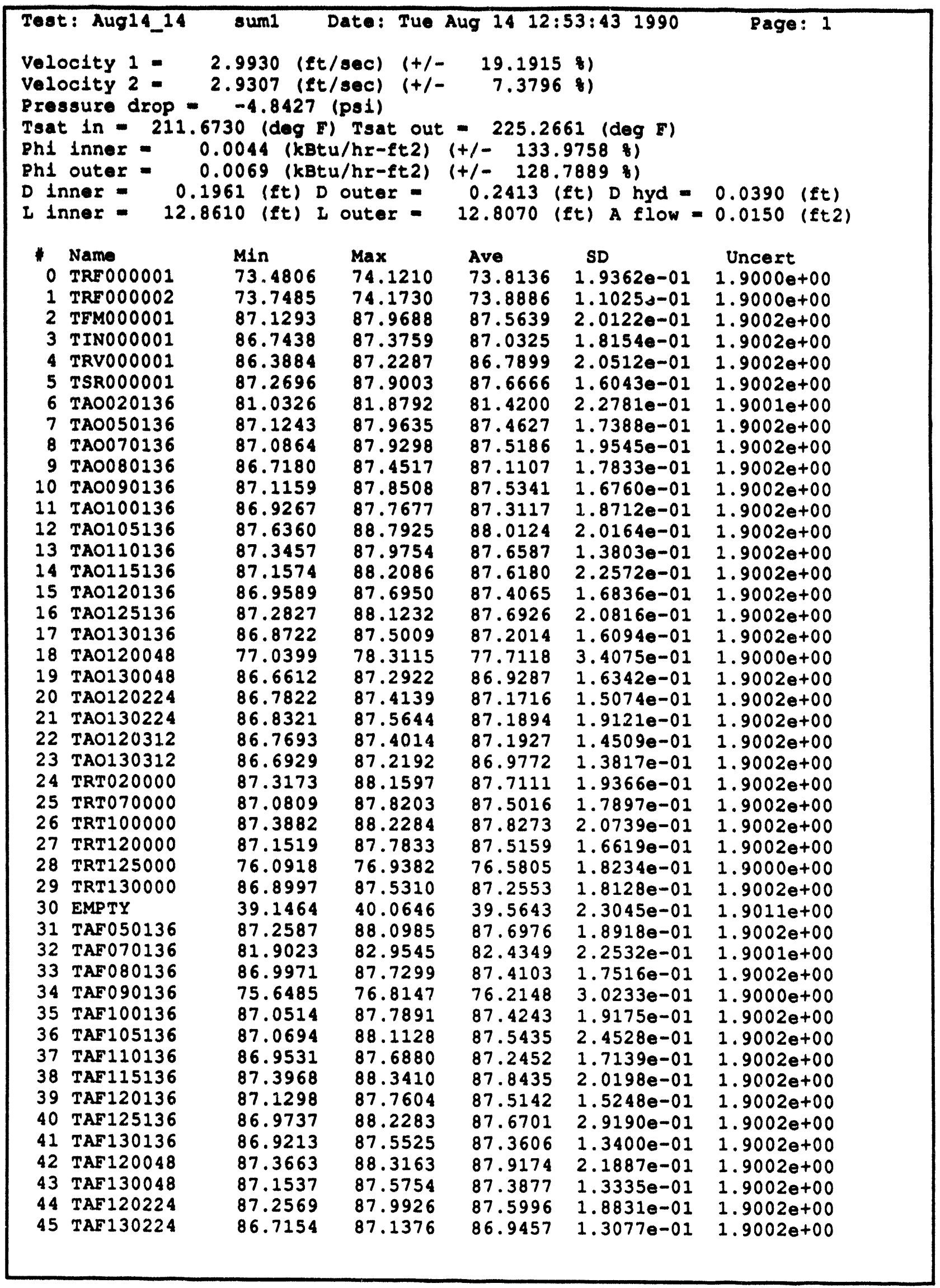




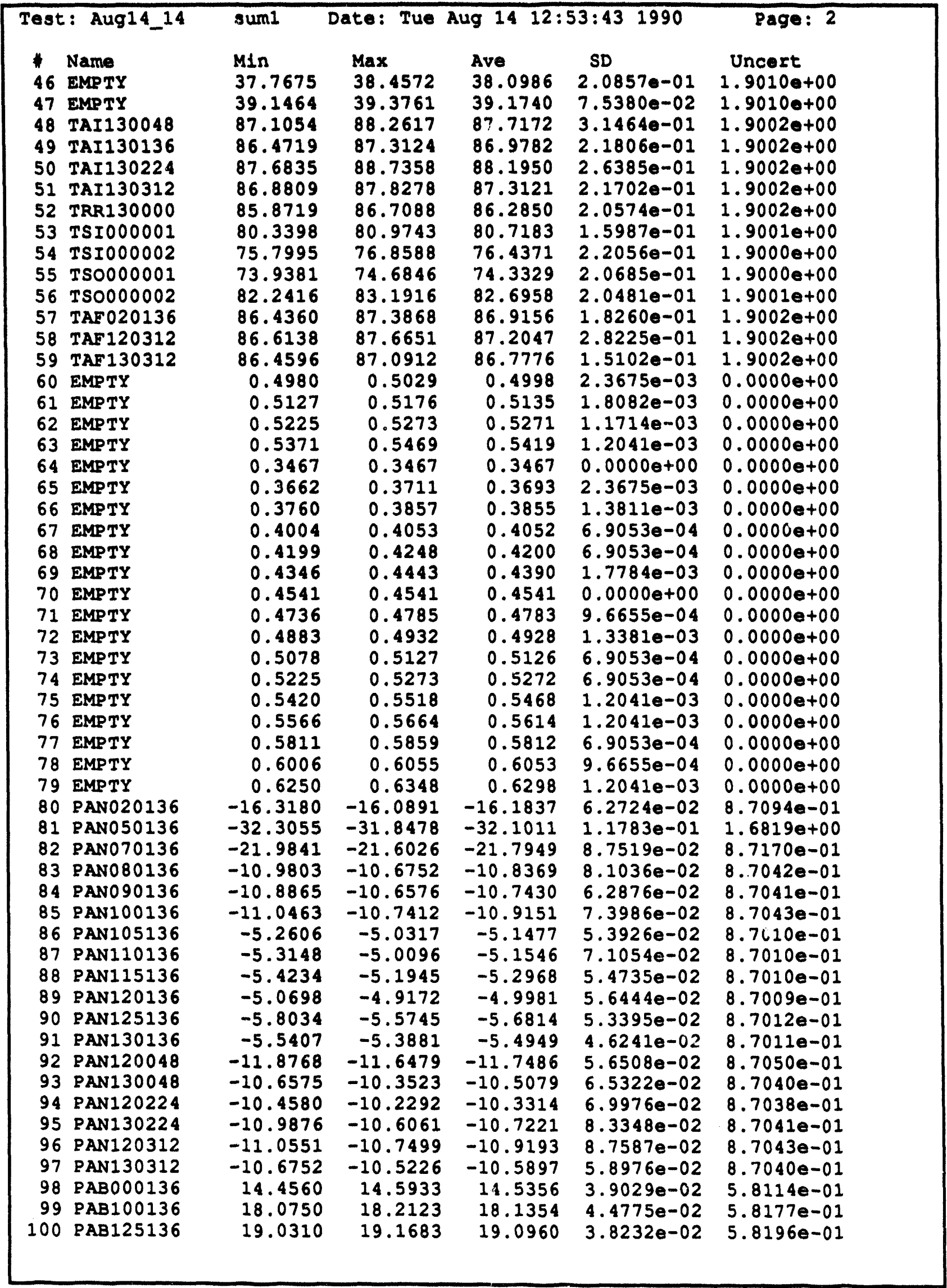




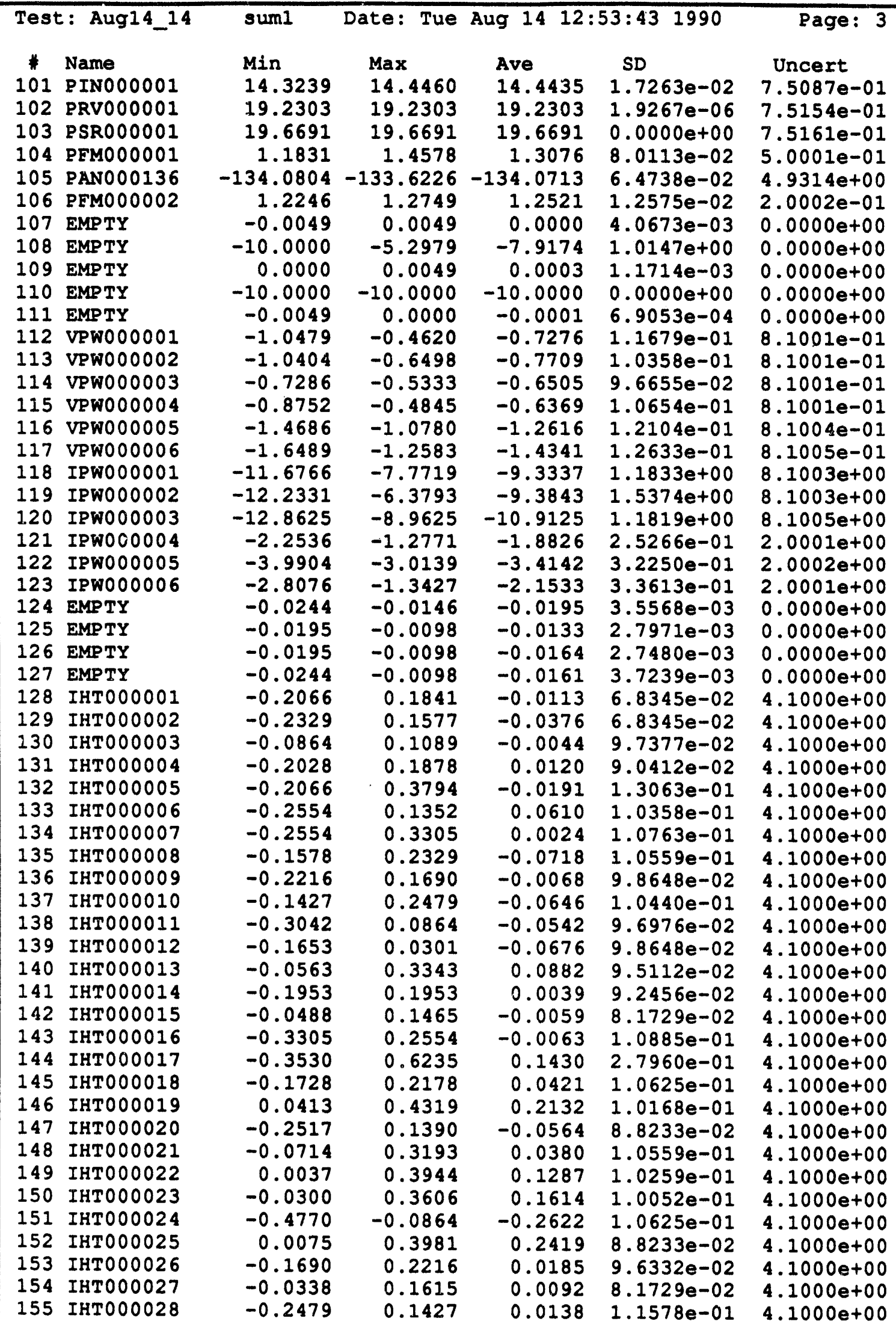




\begin{tabular}{|c|c|c|c|c|c|c|}
\hline \multicolumn{2}{|c|}{ Test: Aug14_14 } & sum1 & Date: Tue & \multicolumn{2}{|c|}{ Aug $14 \quad 12: 53: 43 \quad 1990$} & $\begin{array}{l}\text { Page: } 4 \\
\text { Uncert }\end{array}$ \\
\hline $\begin{array}{c}\# \\
156 \\
157 \\
158 \\
159 \\
160 \\
161 \\
162 \\
163\end{array}$ & $\begin{array}{l}\text { Name } \\
\text { IHTO00029 } \\
\text { IHTO00030 } \\
\text { IHTO00031 } \\
\text { IHTO00032 } \\
\text { IHTO00033 } \\
\text { IHTO00034 } \\
\text { IHTO00035 } \\
\text { IHTO00036 }\end{array}$ & $\begin{array}{l}\text { Min } \\
-0.0826 \\
-0.2103 \\
-0.1465 \\
-0.0976 \\
-0.0977 \\
-0.2329 \\
-0.1277 \\
-0.1878\end{array}$ & $\begin{array}{l}\text { Max } \\
0.1127 \\
0.3756 \\
0.2441 \\
0.2930 \\
0.2930 \\
0.1577 \\
0.2629 \\
0.0075\end{array}$ & $\begin{array}{l}\text { Ave } \\
-0.0162 \\
0.0397 \\
-0.0059 \\
0.0195 \\
0.0000 \\
-0.0102 \\
0.0676 \\
-0.0394\end{array}$ & $\begin{array}{l}S D \\
9.3461 e-02 \\
1.0470 e-01 \\
1.1189 e-01 \\
1.0440 e-01 \\
1.0625 e-01 \\
9.6735 e-02 \\
6.8345 e-02 \\
8.4262 e-02\end{array}$ & $\begin{array}{l}\text { Uncert } \\
4.1000 e+00 \\
4.1000 e+00 \\
4.1000 e+00 \\
4.1000 e+00 \\
4.1000 e+00 \\
4.1000 e+00 \\
4.1000 e+00 \\
4.1000 e+00\end{array}$ \\
\hline
\end{tabular}




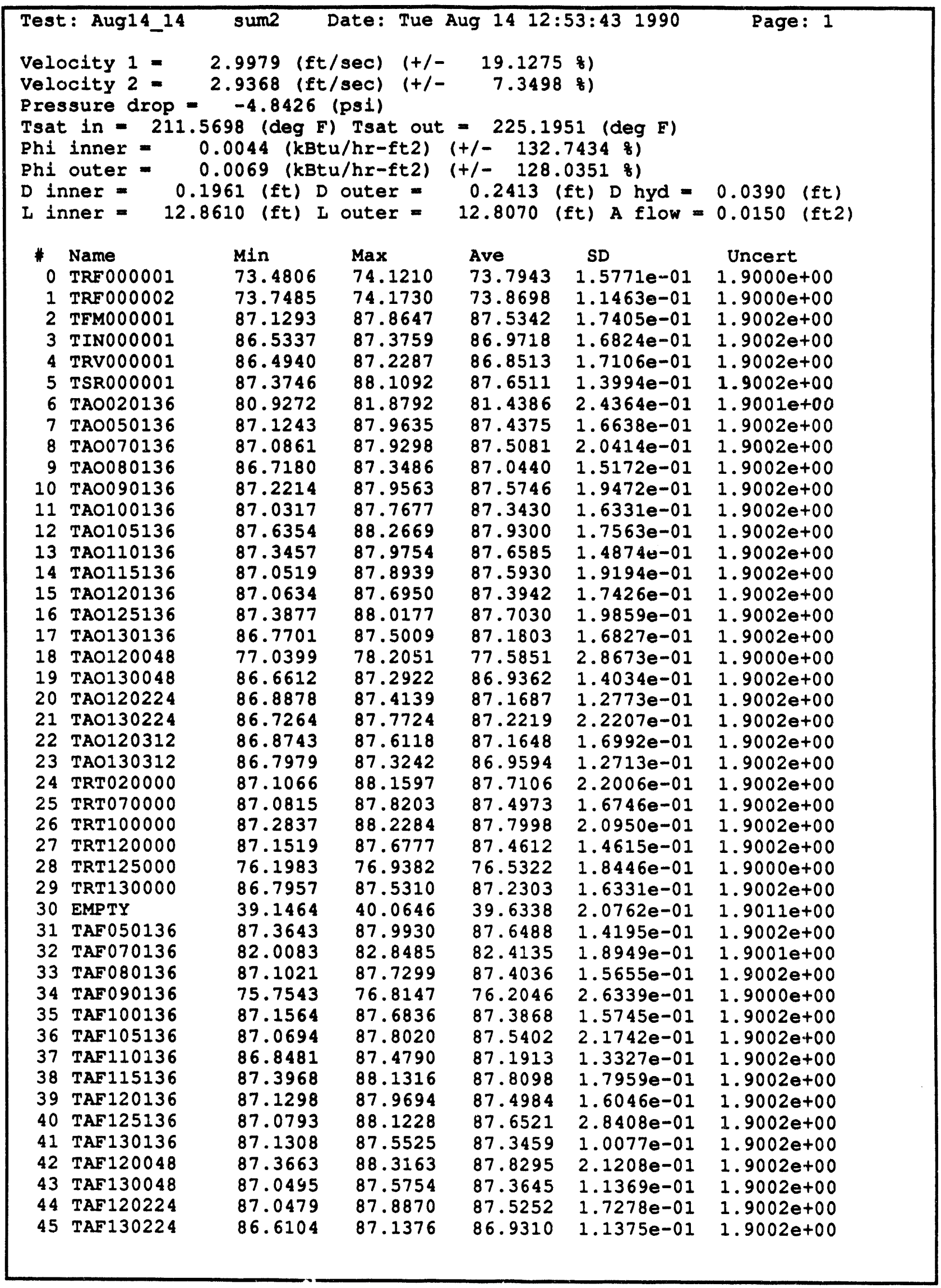




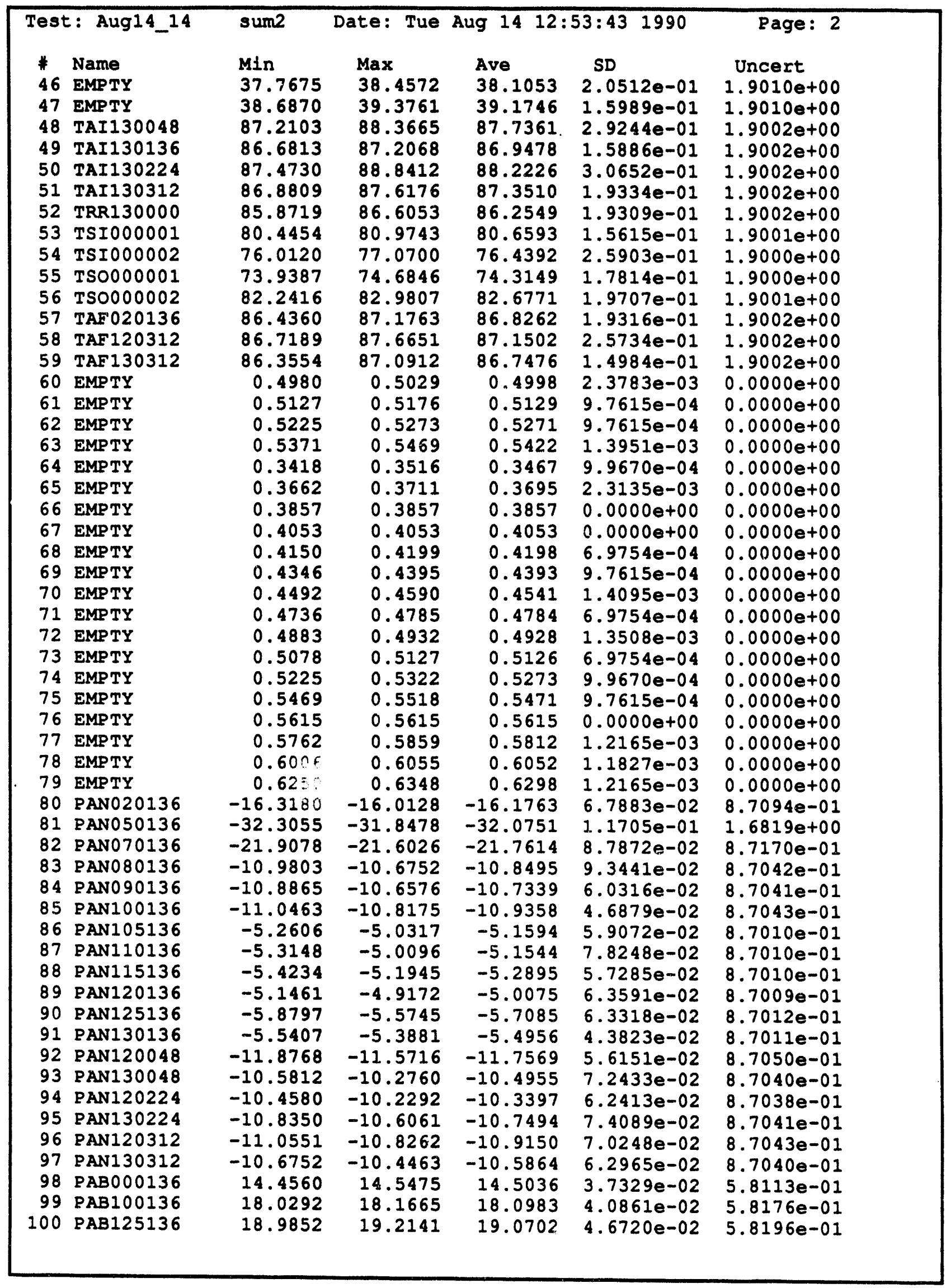




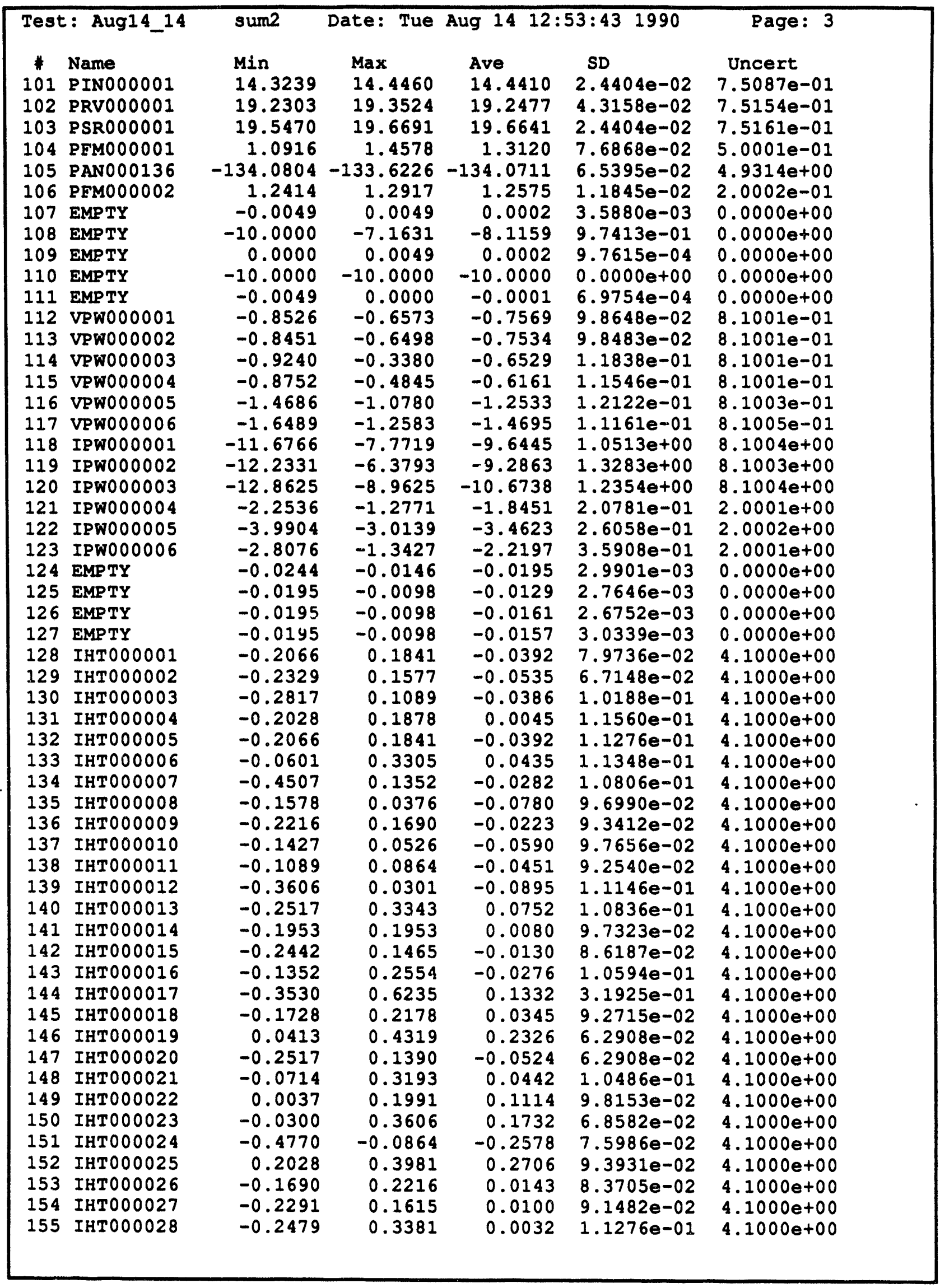




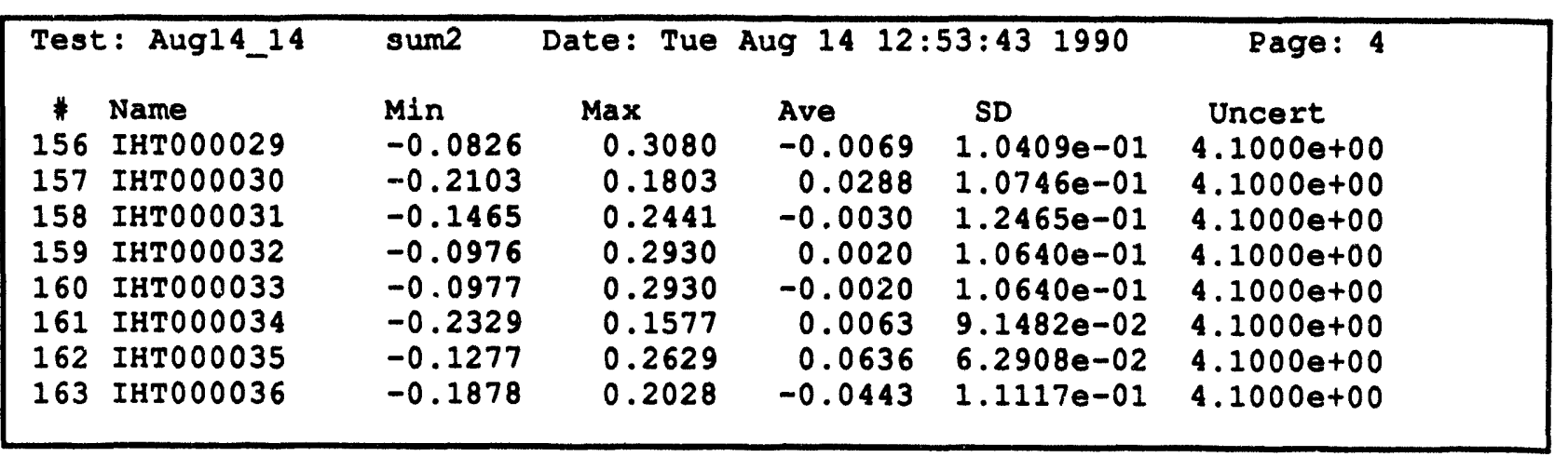




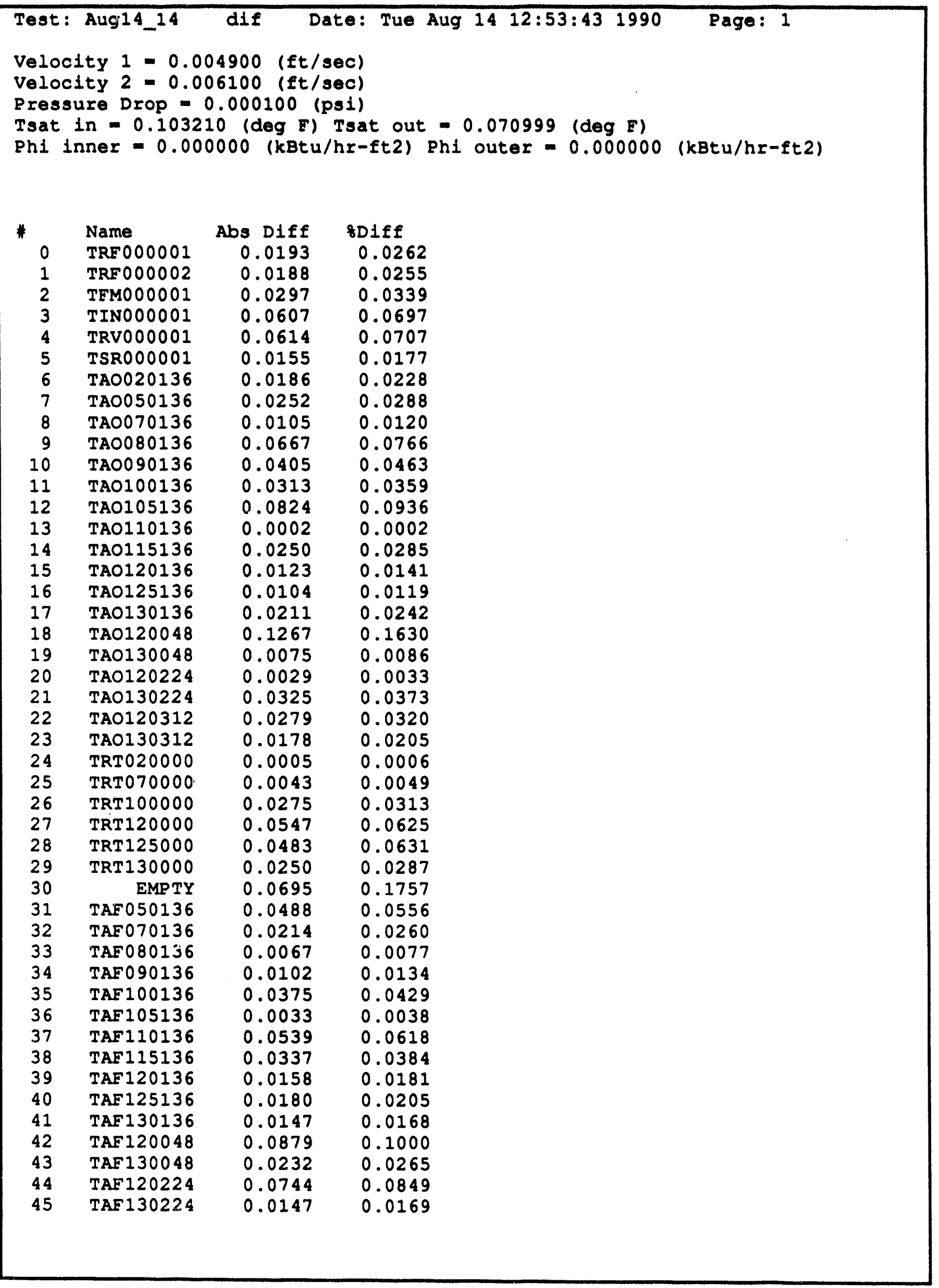




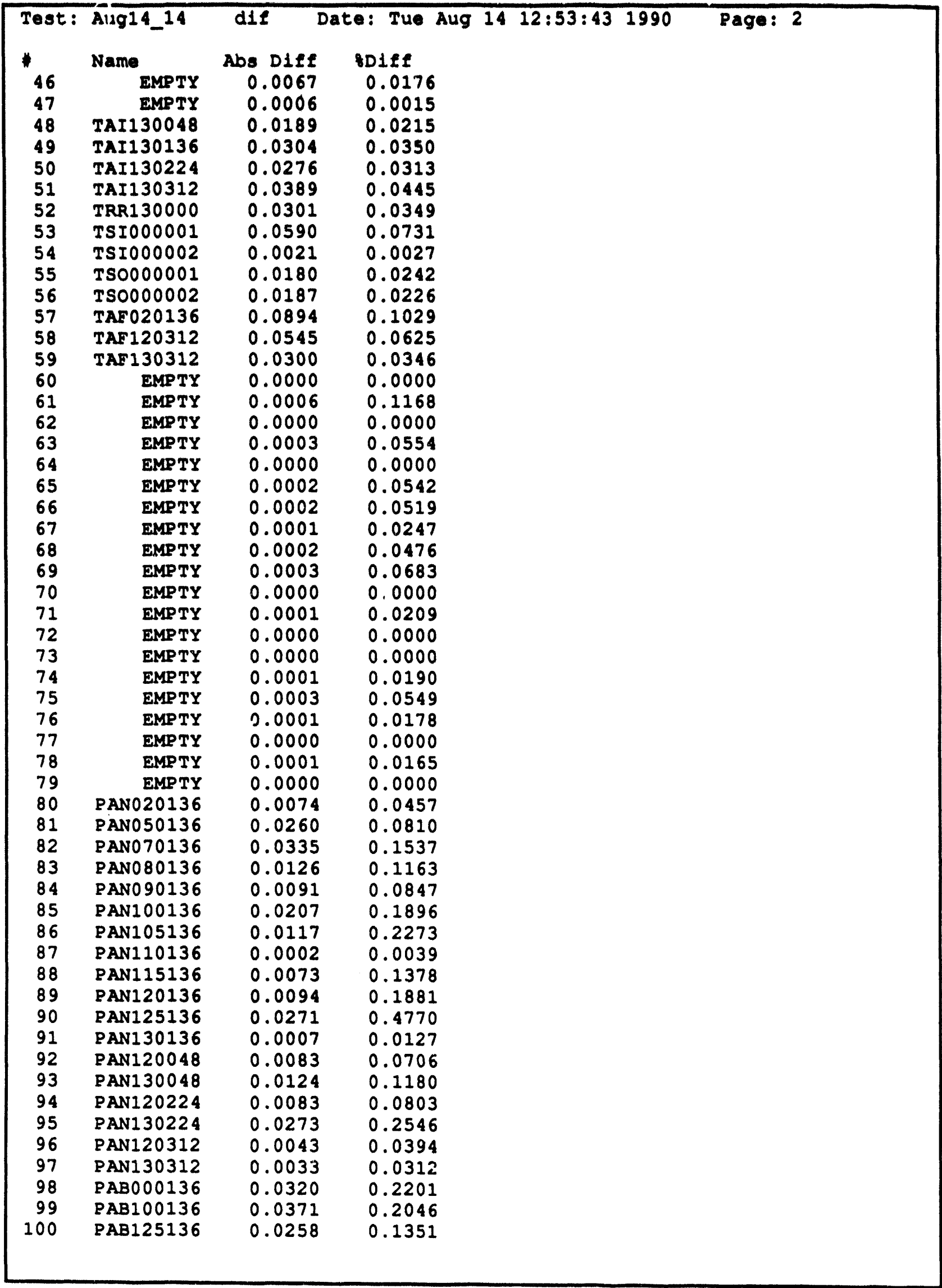




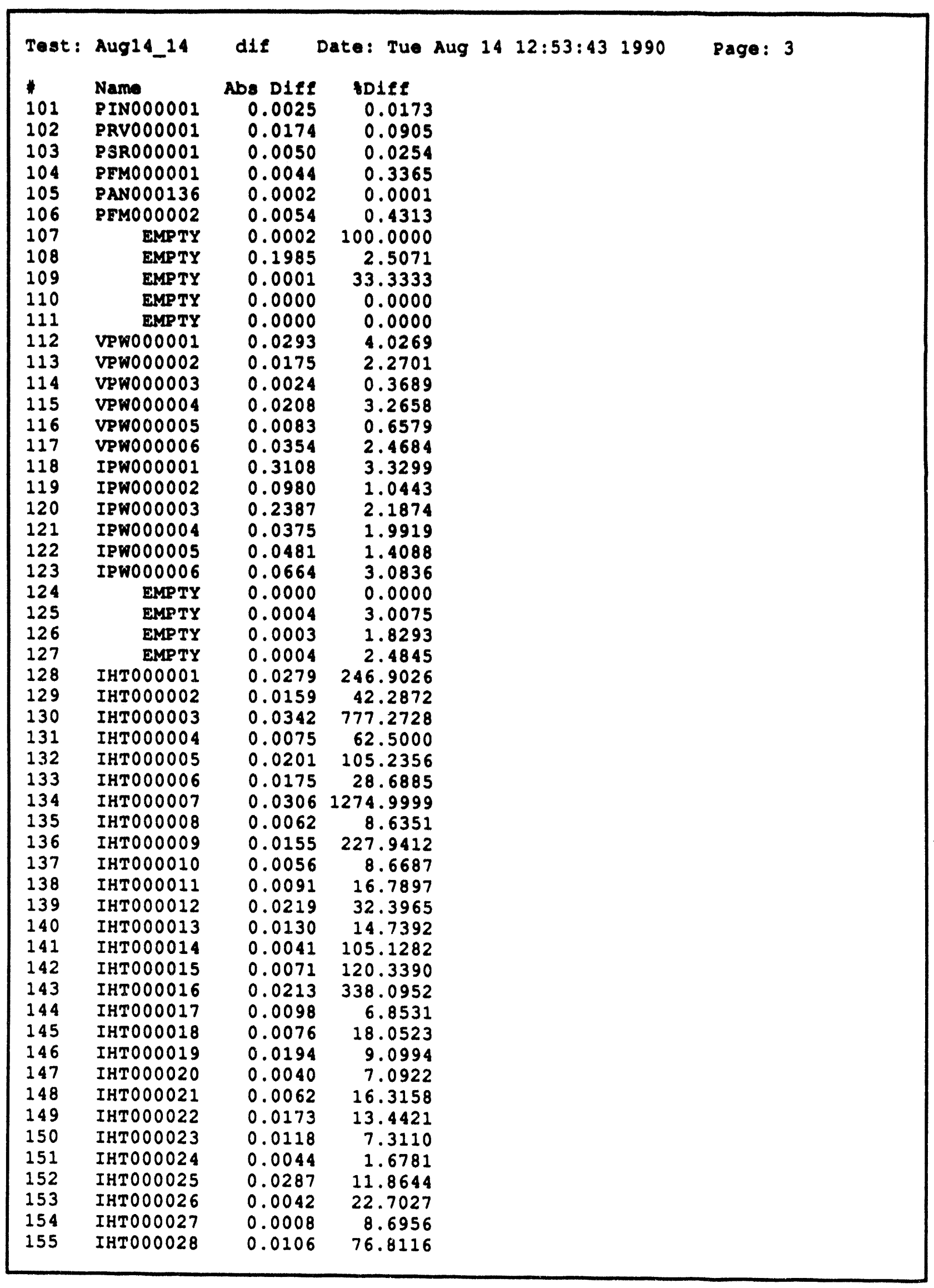




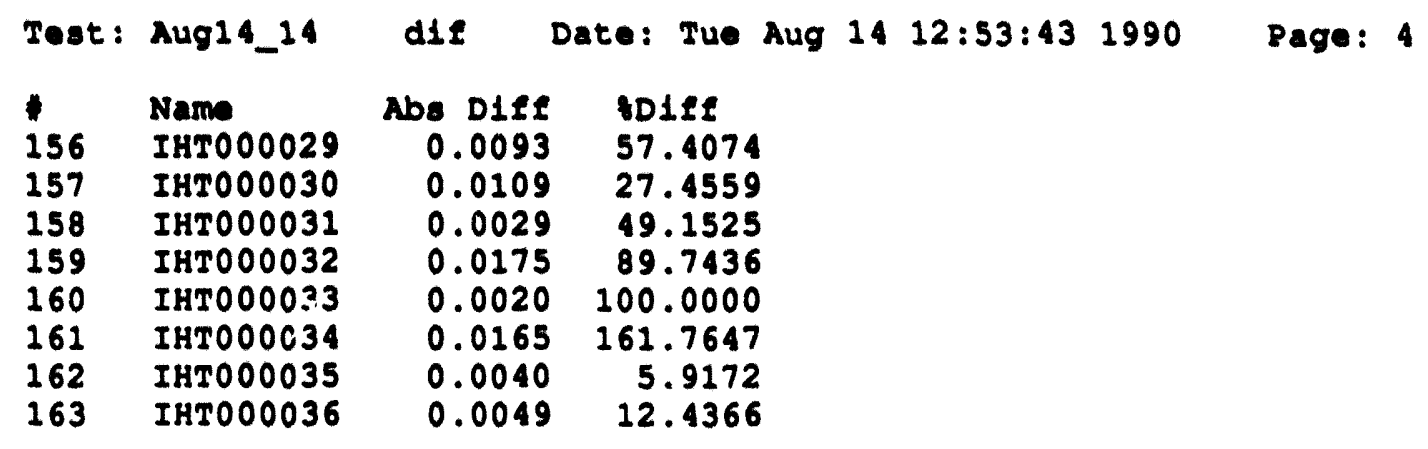




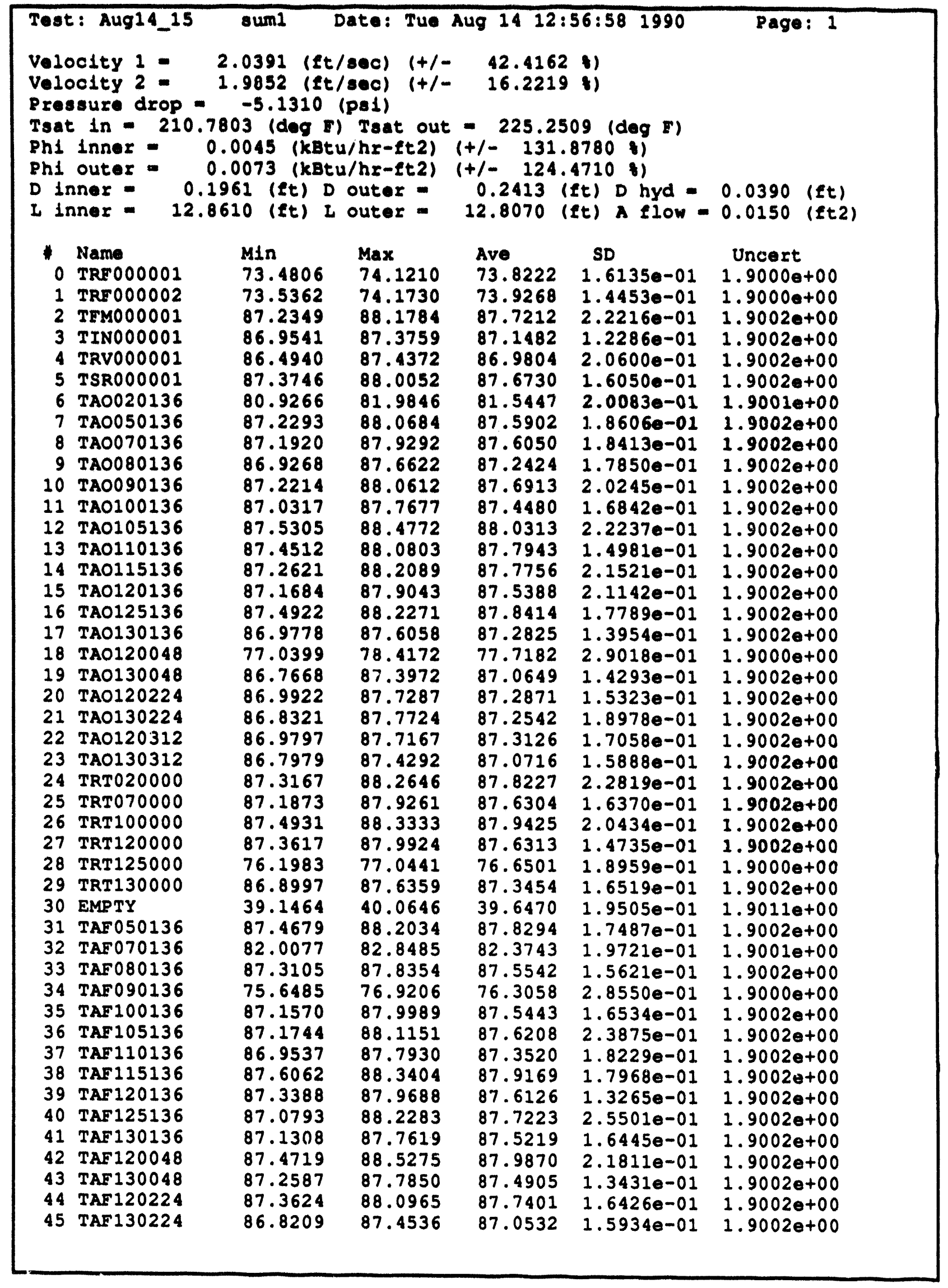




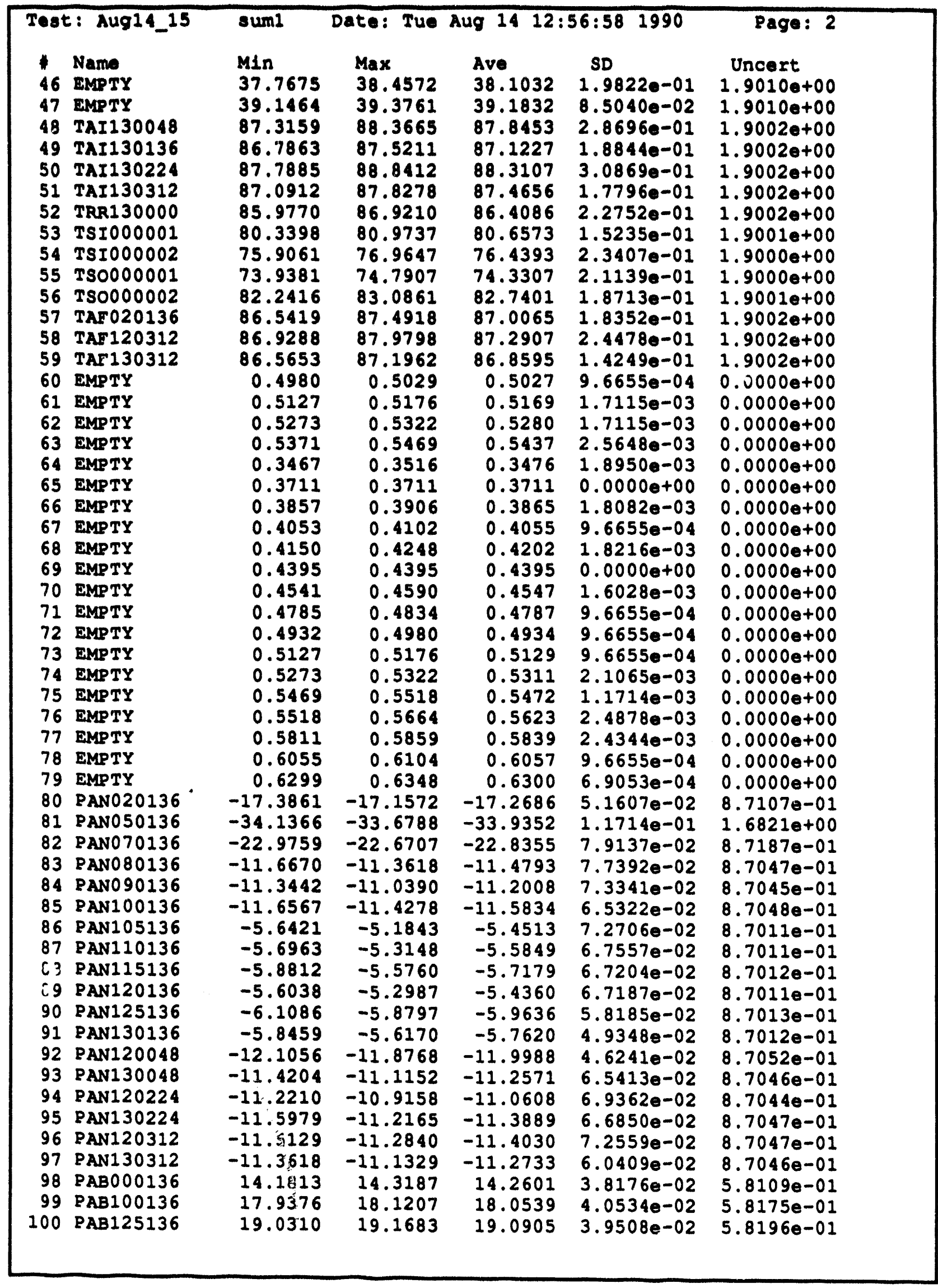




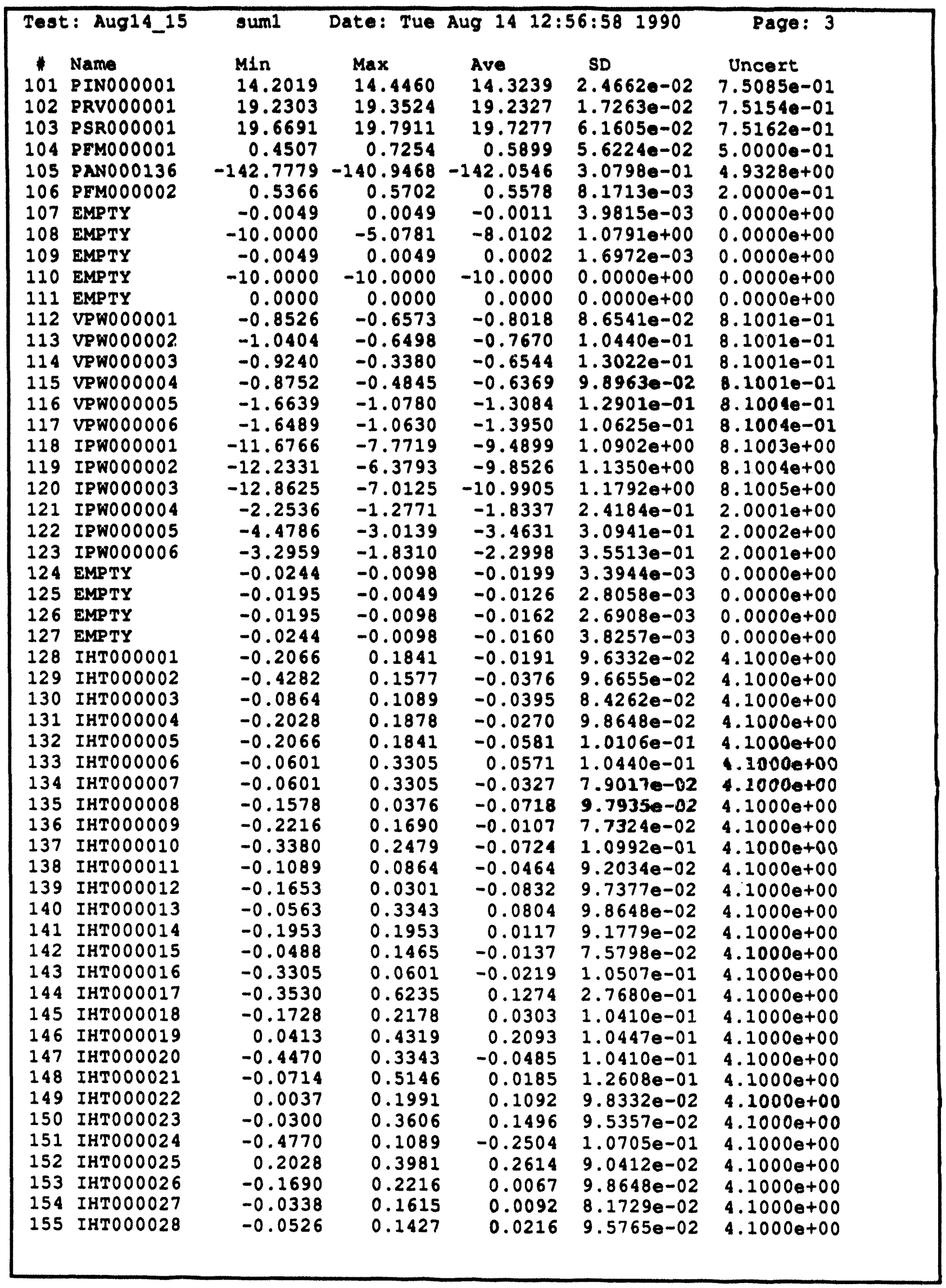




\begin{tabular}{|c|c|c|c|c|c|}
\hline 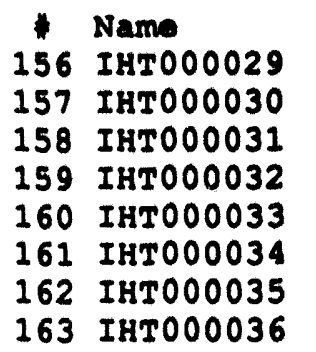 & $\begin{array}{l}\text { Min } \\
-0.2780 \\
-0.0150 \\
-0.1465 \\
-0.0976 \\
-0.0977 \\
-0.2329 \\
-0.1277 \\
-0.1878\end{array}$ & $\begin{array}{l}\text { Max } \\
0.3080 \\
0.1803 \\
0.2441 \\
0.4883 \\
0.2930 \\
0.3531 \\
0.4582 \\
0.2028\end{array}$ & $\begin{array}{l}\text { Ave } \\
-0.0240 \\
0.0631 \\
-0.0059 \\
-0.0039 \\
0.0000 \\
0.0054 \\
0.0637 \\
-0.0550\end{array}$ & $\begin{array}{l}\text { SD } \\
1.0625 e-01 \\
9.6655 e-02 \\
1.2503 e-01 \\
1.2627 e-01 \\
1.1334 e-01 \\
1.2027 e-01 \\
1.1497 e-01 \\
1.0014 e-01\end{array}$ & $\begin{array}{l}\text { Uncert } \\
4.1000 e+00 \\
4.1000 e+00 \\
4.1000 e+00 \\
4.1000 e+00 \\
4.1000 e+00 \\
4.1000 e+00 \\
4.1000 e+00 \\
4.1000 e+00\end{array}$ \\
\hline
\end{tabular}




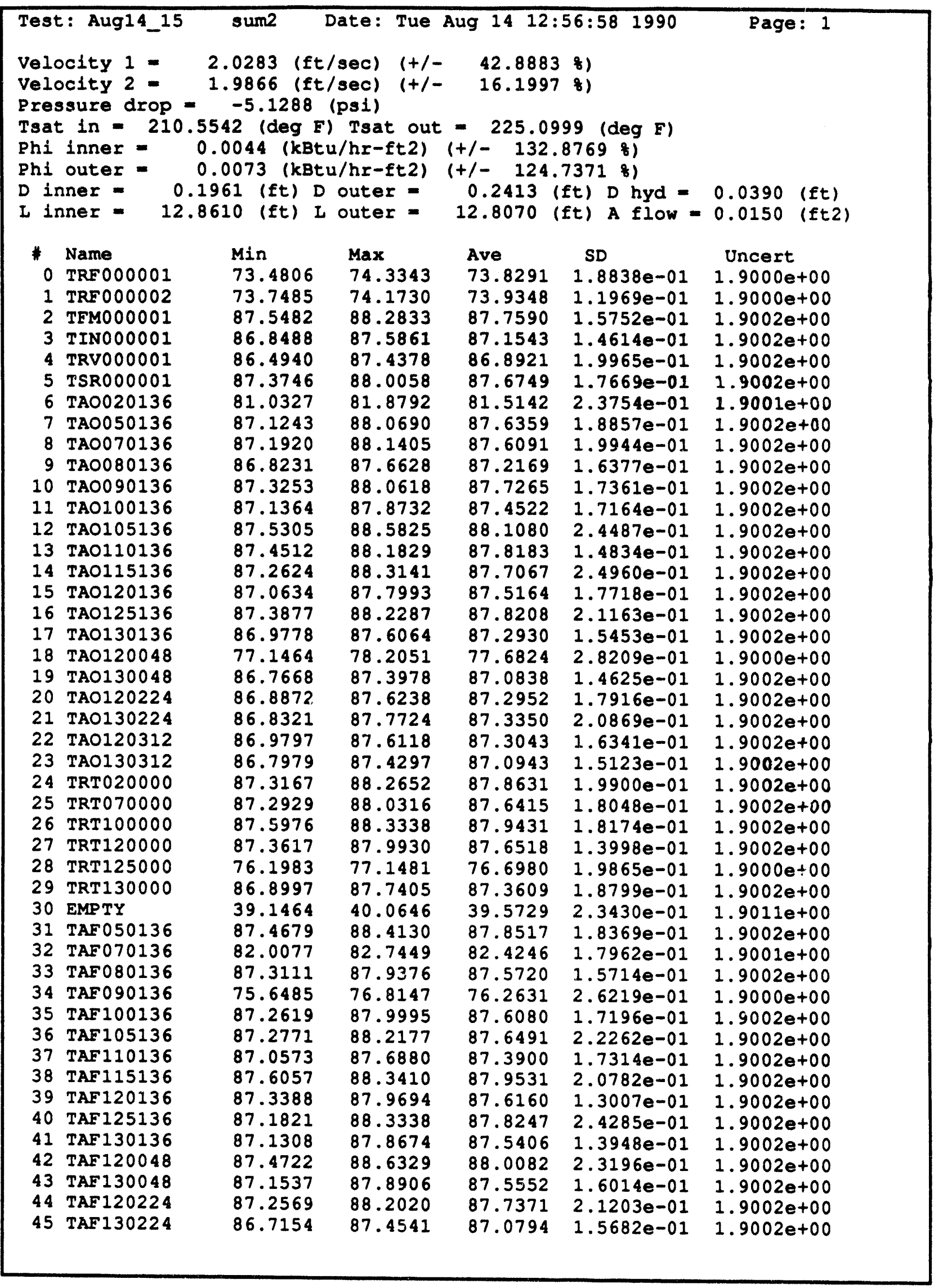




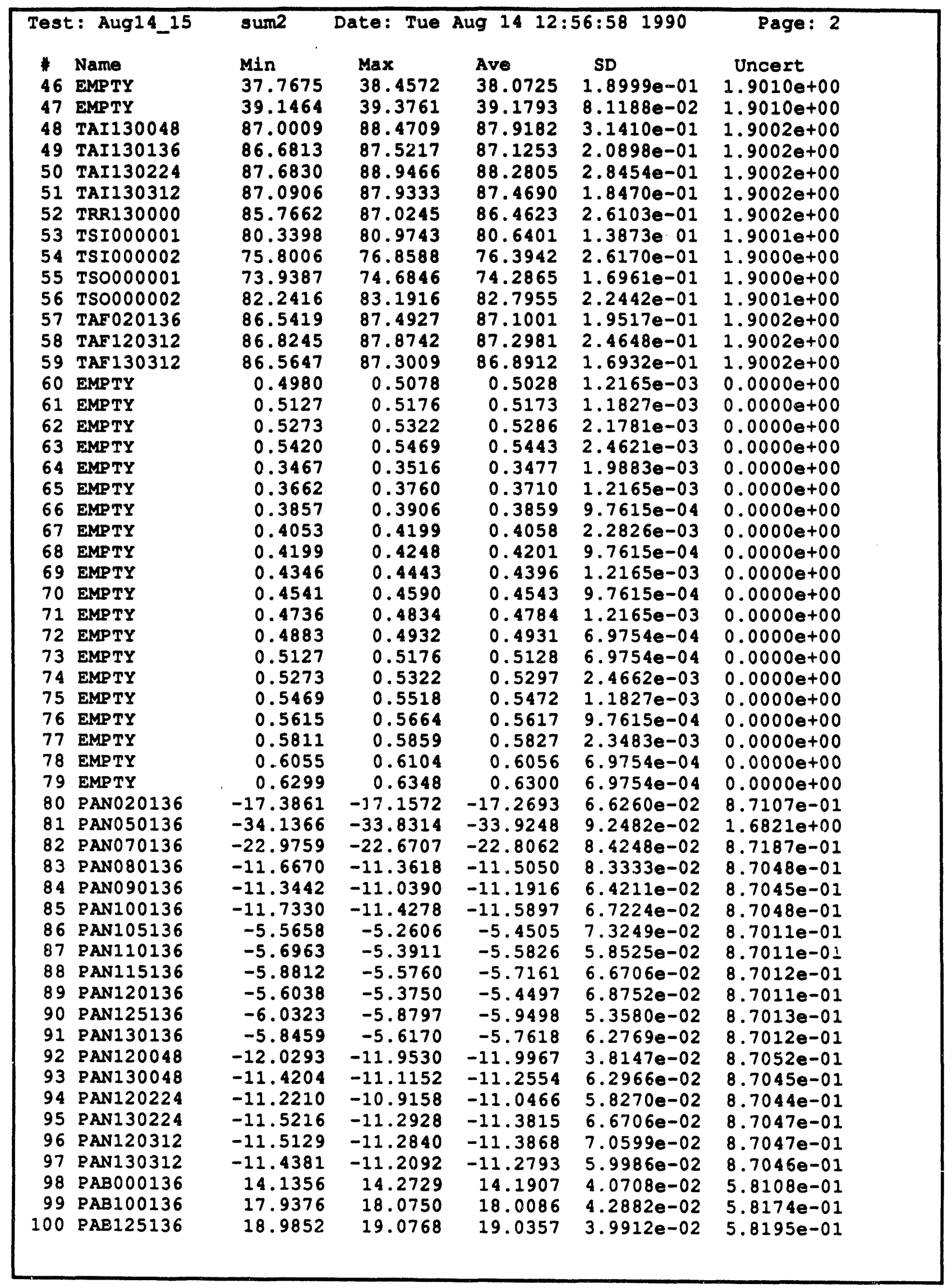




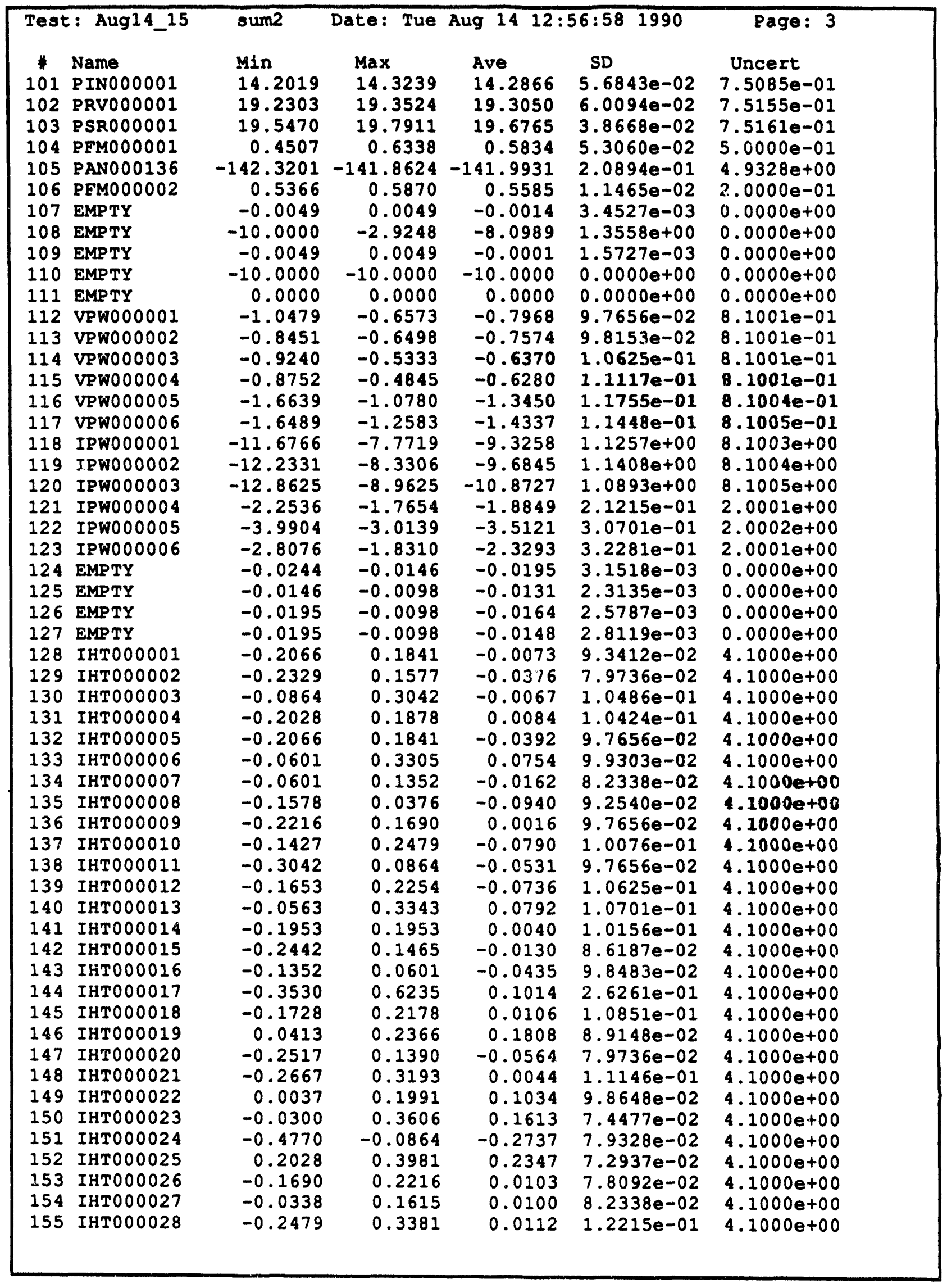




\begin{tabular}{|c|c|c|c|c|c|c|}
\hline Tes & $t:$ Aug14_15 & sum2 & ate: Tue & ug 141 & $56: 58 \quad 1990$ & Page: 4 \\
\hline $\begin{array}{l}* \\
156 \\
157 \\
158 \\
159 \\
160 \\
161 \\
162 \\
163\end{array}$ & 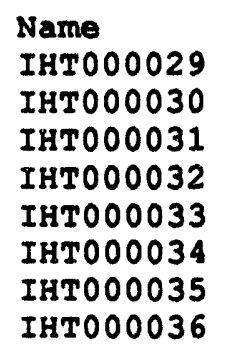 & $\begin{array}{l}\text { Min } \\
-0.2780 \\
-0.0150 \\
-0.1465 \\
-0.0976 \\
-0.0977 \\
-0.2329 \\
-0.1277 \\
-0.1878\end{array}$ & $\begin{array}{l}\text { Max } \\
0.1127 \\
0.1803 \\
0.2441 \\
0.2930 \\
0.0976 \\
0.1577 \\
0.4582 \\
0.2028\end{array}$ & $\begin{array}{l}\text { Ave } \\
-0.0109 \\
0.0647 \\
0.0090 \\
0.0020 \\
0.0020 \\
0.0143 \\
0.0875 \\
-0.0403\end{array}$ & $\begin{array}{l}\text { SD } \\
1.0315 \mathrm{e}-01 \\
9.6990 \mathrm{e}-02 \\
1.1262 \mathrm{e}-01 \\
1.0640 \mathrm{e}-01 \\
9.8648 \mathrm{e}-02 \\
1.1117 \mathrm{e}-01 \\
1.3369 \mathrm{e}-01 \\
1.1644 \mathrm{e}-01\end{array}$ & $\begin{array}{l}\text { Uncert } \\
4.1000 e+00 \\
4.1000 e+00 \\
4.1000 e+00 \\
4.1000 e+00 \\
4.1000 e+00 \\
4.1000 e+00 \\
4.1000 e+00 \\
4.1000 e+00\end{array}$ \\
\hline
\end{tabular}




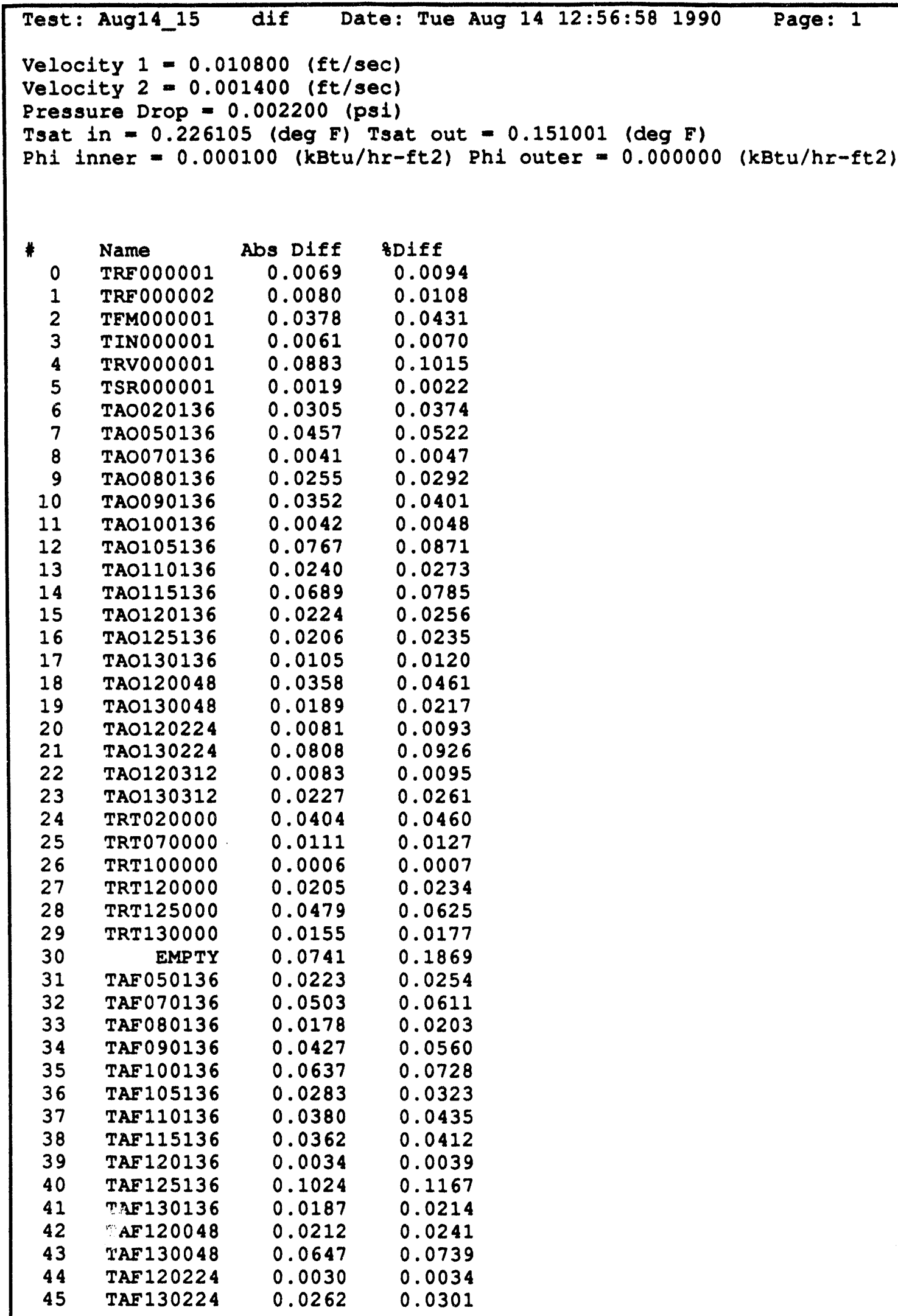




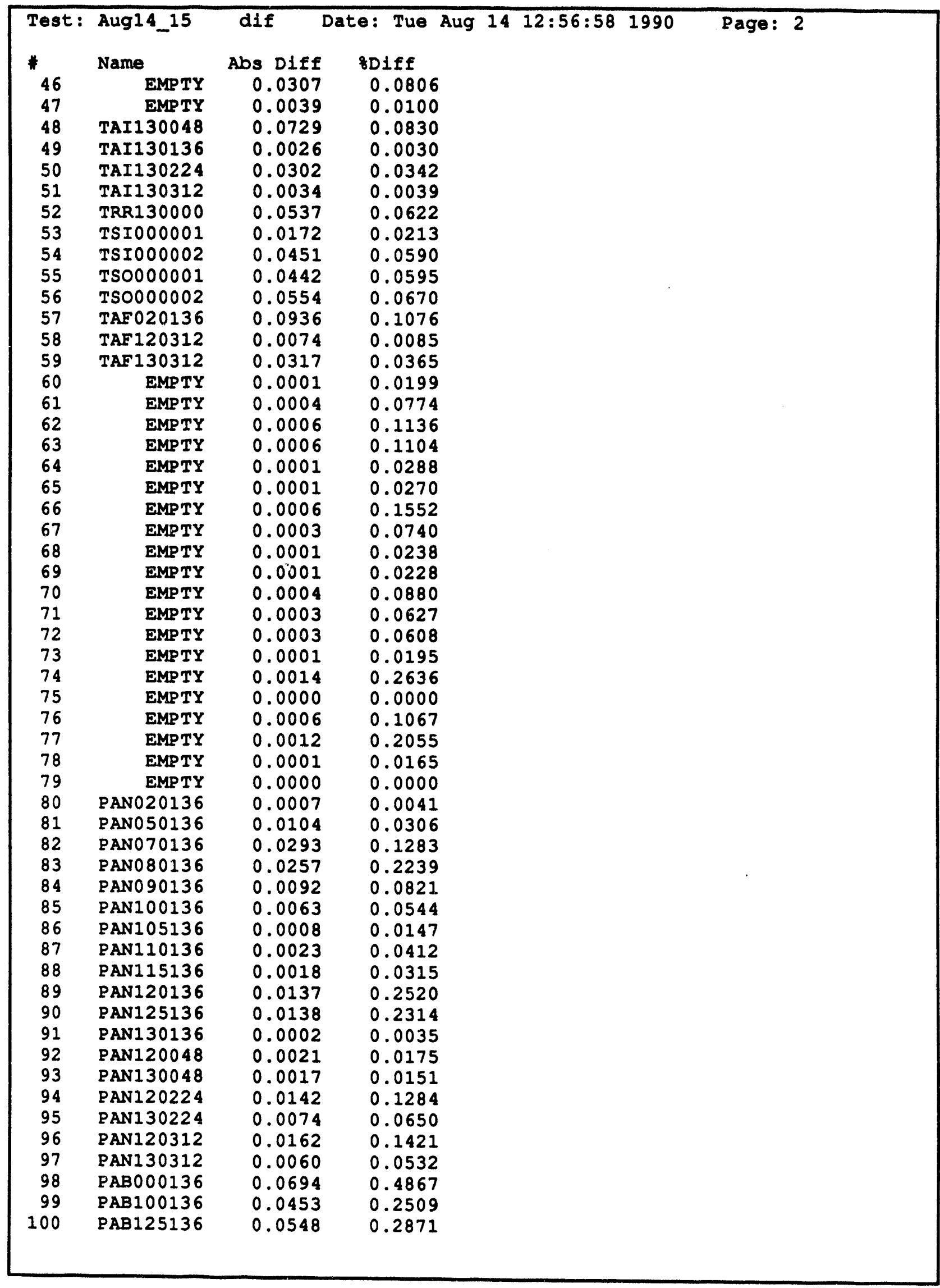




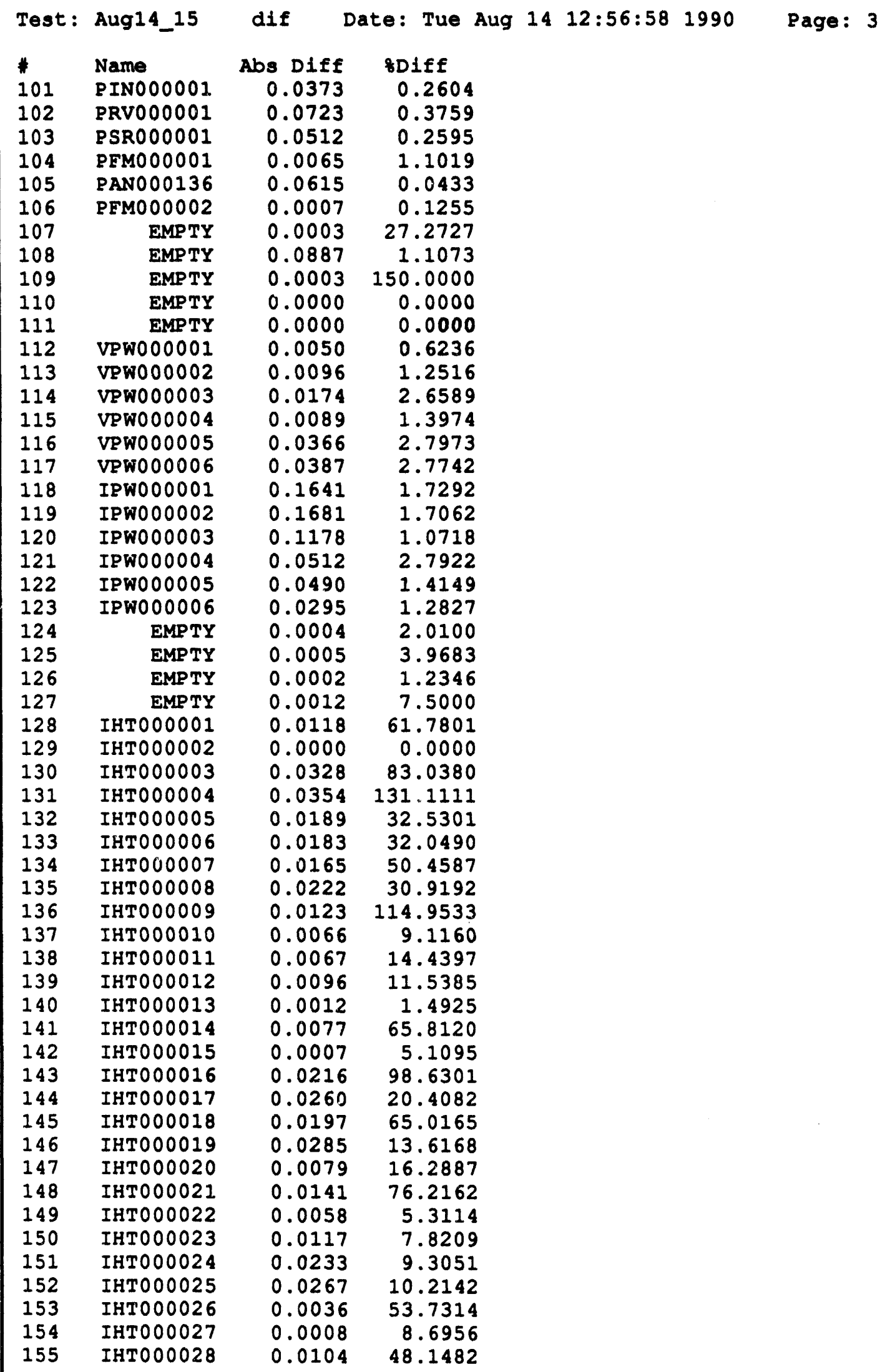




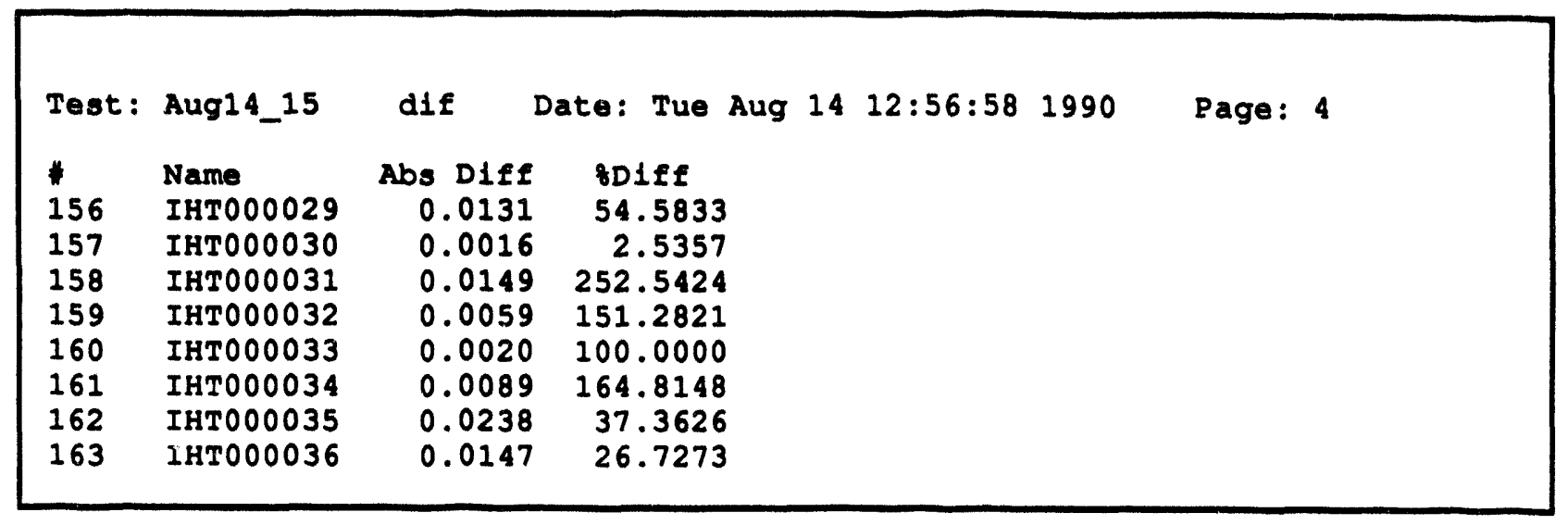




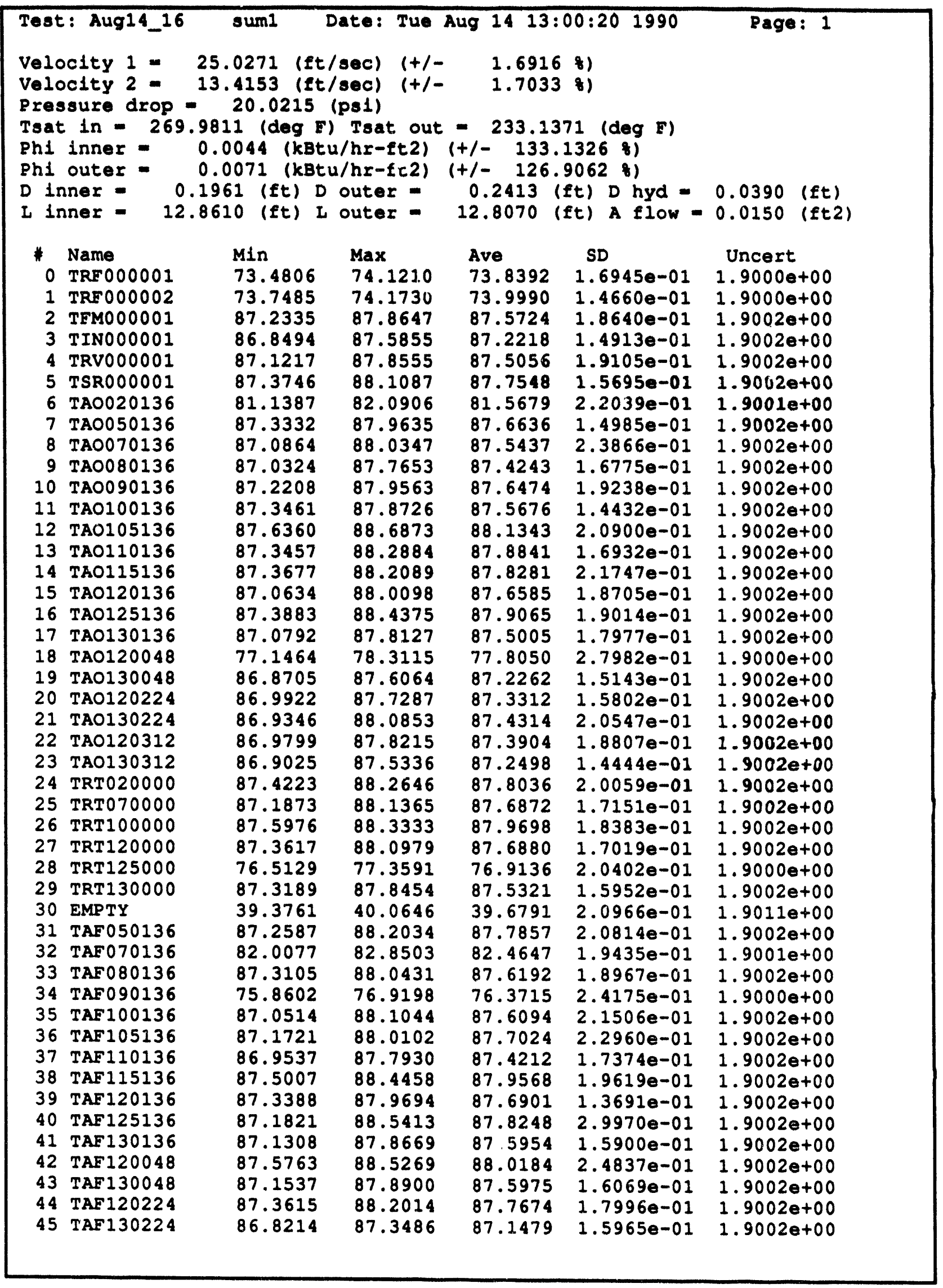




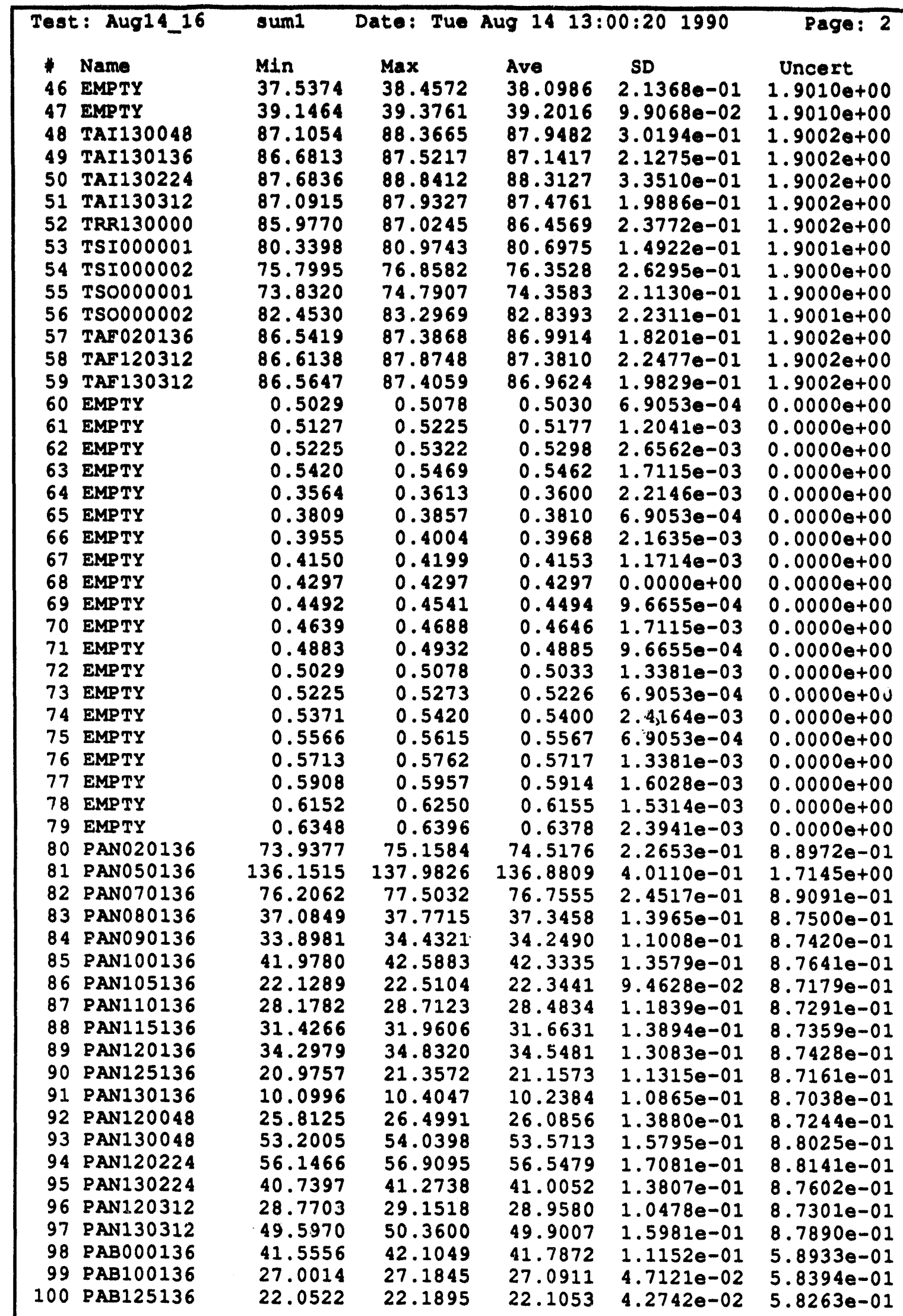




\begin{tabular}{|c|c|c|c|c|c|c|}
\hline $\begin{array}{l}1 \\
101 \\
102 \\
103 \\
104 \\
105 \\
106 \\
107 \\
108 \\
109 \\
110 \\
111 \\
112 \\
113 \\
114 \\
115 \\
116 \\
117 \\
118 \\
119 \\
120 \\
121 \\
122 \\
123 \\
124 \\
125 \\
126 \\
127 \\
128 \\
129 \\
130 \\
131 \\
132 \\
133 \\
134 \\
135 \\
136 \\
137 \\
138 \\
139 \\
140 \\
141 \\
142 \\
143 \\
144 \\
145 \\
146 \\
147 \\
148 \\
149 \\
150 \\
151 \\
152 \\
153 \\
154 \\
155\end{array}$ & 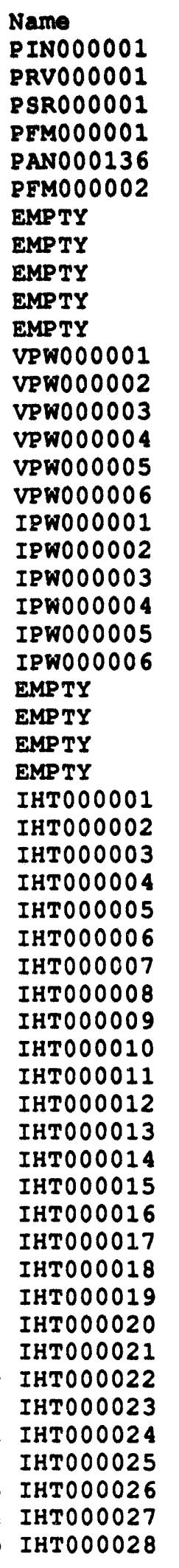 & $\begin{array}{r}\text { MIn } \\
41.4235 \\
19.1082 \\
19.6691 \\
95.6655 \\
551.1918 \\
27.5689 \\
0.0049 \\
-10.0000 \\
0.0049 \\
-10.0000 \\
0.0000 \\
-1.0479 \\
-0.8451 \\
-0.7286 \\
-0.8752 \\
-1.4686 \\
-1.6489 \\
-11.6766 \\
-12.2331 \\
-12.8625 \\
-2.2536 \\
-3.9904 \\
-2.8076 \\
-0.0293 \\
-0.0195 \\
-0.0244 \\
-0.0244 \\
-0.2066 \\
-0.2329 \\
-0.0864 \\
-0.2028 \\
-0.2066 \\
-0.0601 \\
-0.2554 \\
-0.3531 \\
-0.2216 \\
-0.1427 \\
-0.1089 \\
-0.1653 \\
-0.0563 \\
-0.1953 \\
-0.2442 \\
-0.1352 \\
-0.3530 \\
-0.03681 \\
0.0413 \\
-0.2517 \\
-0.0714 \\
0.0037 \\
-0.0300 \\
-0.4770 \\
0.0075 \\
-0.1690 \\
-0.0338 \\
-0.2479\end{array}$ & $\begin{array}{r}\text { Max } \\
42.0339 \\
19.2303 \\
19.7911 \\
97.8628 \\
559.4316 \\
27.5689 \\
0.0146 \\
-5.3027 \\
0.0098 \\
-10.0000 \\
0.0049 \\
-0.6573 \\
-0.4545 \\
-0.3380 \\
-0.2892 \\
-1.0780 \\
-1.2583 \\
-5.8195 \\
-6.3793 \\
-8.9625 \\
-1.2771 \\
-2.5257 \\
-1.3427 \\
-0.0146 \\
-0.0098 \\
-0.0098 \\
-0.0098 \\
0.1841 \\
0.1577 \\
0.1089 \\
0.3831 \\
0.1841 \\
0.3305 \\
0.1352 \\
0.0376 \\
0.1690 \\
0.0526 \\
0.0864 \\
0.0301 \\
0.1390 \\
0.1953 \\
0.1465 \\
0.2554 \\
0.6235 \\
0.2178 \\
0.4319 \\
0.1390 \\
0.3193 \\
0.1991 \\
0.3606 \\
-0.0864 \\
0.3981 \\
0.2216 \\
0.1615 \\
0.3381\end{array}$ & $\begin{array}{r}\text { Ave } \\
41.7019 \\
19.2278 \\
19.7667 \\
96.5756 \\
554.3045 \\
27.5689 \\
0.0098 \\
-8.3555 \\
0.0075 \\
-10.0000 \\
0.0046 \\
-0.7901 \\
-0.7396 \\
-0.6427 \\
-0.6134 \\
-1.3006 \\
-1.4419 \\
-9.3337 \\
-9.5794 \\
-10.9905 \\
-1.8923 \\
-3.4240 \\
-2.2509 \\
-0.0197 \\
-0.0128 \\
-0.0170 \\
-0.0159 \\
-0.0191 \\
-0.0376 \\
-0.0395 \\
-0.0192 \\
-0.0425 \\
0.0454 \\
-0.0210 \\
-0.0796 \\
-0.0185 \\
-0.0646 \\
-0.0581 \\
-0.1028 \\
0.0530 \\
-0.0117 \\
-0.0293 \\
-0.0454 \\
0.0923 \\
-0.0048 \\
0.2054 \\
-0.0525 \\
0.0576 \\
0.1287 \\
0.1535 \\
-0.2817 \\
0.2458 \\
0.0263 \\
0.0248 \\
-0.0096\end{array}$ & $\begin{array}{l}\text { SD } \\
1.5990 e-01 \\
1.7263 e-02 \\
4.9324 e-02 \\
4.5511 e-01 \\
1.7498 e+00 \\
9.6336 e-06 \\
9.8648 e-04 \\
1.0758 e+00 \\
2.4583 e-03 \\
0.0000 e+00 \\
1.1714 e-03 \\
1.0014 e-01 \\
1.0595 e-01 \\
1.0559 e-01 \\
1.1578 e-01 \\
1.0447 e-01 \\
1.0741 e-01 \\
1.3082 e+00 \\
1.2316 e+00 \\
1.0393 e+00 \\
2.5743 e-01 \\
3.9897 e-01 \\
3.4187 e-01 \\
3.6858 e-03 \\
2.9413 e-03 \\
2.4513 e-03 \\
3.3901 e-03 \\
8.7880 e-02 \\
6.8345 e-02 \\
8.4262 e-02 \\
1.2104 e-01 \\
9.1354 e-02 \\
1.0595 e-01 \\
8.8233 e-02 \\
1.0440 e-01 \\
7.8523 e-02 \\
9.6655 e-02 \\
8.6541 e-02 \\
9.2034 e-02 \\
9.7935 e-02 \\
6.1257 e-02 \\
7.1136 e-02 \\
1.0595 e-01 \\
2.7063 e-01 \\
1.1168 e-01 \\
9.1354 e-02 \\
8.3612 e-02 \\
1.0145 e-01 \\
9.4702 e-02 \\
9.1779 e-02 \\
7.8918 e-02 \\
9.0756 e-02 \\
3.9459 e-02 \\
9.0412 e-02 \\
1.0654 e-01\end{array}$ & $\begin{array}{l}\text { Uncert } \\
7.5721 e-01 \\
7.5154 e-01 \\
7.5163 e-01 \\
5.5524 e-01 \\
5.1114 e+00 \\
2.1154 e-01 \\
0.0000 e+00 \\
0.0000 e+00 \\
0.0000 e+00 \\
0.0000 e+00 \\
0.0000 e+00 \\
8.1001 e-01 \\
8.1001 e-01 \\
8.1001 e-01 \\
8.1001 e-01 \\
8.1004 e-01 \\
8.1005 e-01 \\
8.1003 e+00 \\
8.1004 e+00 \\
8.1005 e+00 \\
2.0001 e+00 \\
2.0002 e+00 \\
2.0001 e+00 \\
0.0000 e+00 \\
0.0000 e+00 \\
0.0000 e+00 \\
0.0000 e+00 \\
4.1000 e+00 \\
4.1000 e+00 \\
4.1000 e+00 \\
4.1000 e+00 \\
4.1000 e+00 \\
4.1000 e+00 \\
4.1000 e+00 \\
4.1000 e+00 \\
4.1000 e+00 \\
4.1000 e+00 \\
4.1000 e+00 \\
4.1000 e+00 \\
4.1000 e+00 \\
4.1000 e+00 \\
4.1000 e+00 \\
4.1000 e+00 \\
4.1000 e+00 \\
4.1000 e+00 \\
4.1000 e+00 \\
4.1000 e+00 \\
4.1000 e+00 \\
4.1000 e+00 \\
4.1000 e+00 \\
4.1000 e+00 \\
4.1000 e+00 \\
4.1000 e+00 \\
4.1000 e+00 \\
4.1000 e+00\end{array}$ \\
\hline
\end{tabular}




\begin{tabular}{|c|c|c|c|c|c|c|}
\hline $\begin{array}{l}1 \\
156 \\
157 \\
158 \\
159 \\
160 \\
161 \\
162 \\
163\end{array}$ & 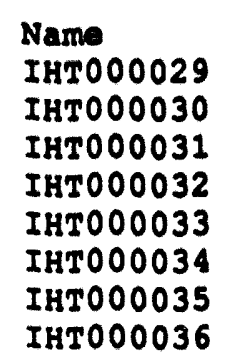 & $\begin{array}{l}\text { Min } \\
-0.0826 \\
-0.2103 \\
-0.3418 \\
-0.0976 \\
-0.0977 \\
-0.2329 \\
-0.1277 \\
-0.1878\end{array}$ & $\begin{array}{l}\text { Max } \\
0.3080 \\
0.3756 \\
0.2441 \\
0.2930 \\
0.0976 \\
0.1577 \\
0.2629 \\
0.2028\end{array}$ & $\begin{array}{l}\text { Ave } \\
-0.0240 \\
0.0280 \\
-0.0371 \\
-0.0156 \\
-0.0391 \\
0.0054 \\
0.0481 \\
-0.0394\end{array}$ & $\begin{array}{l}\text { SD } \\
9.8648 e-02 \\
9.8963 e-02 \\
1.3760 e-01 \\
1.0507 e-01 \\
9.0412 e-02 \\
9.8963 e-02 \\
9.8648 e-02 \\
1.0849 e-01\end{array}$ & $\begin{array}{l}\text { Uncert } \\
4.1000 e+00 \\
4.1000 e+00 \\
4.1000 e+00 \\
4.1000 e+00 \\
4.1000 e+00 \\
4.1000 e+00 \\
4.1000 e+00 \\
4.1000 e+00\end{array}$ \\
\hline
\end{tabular}




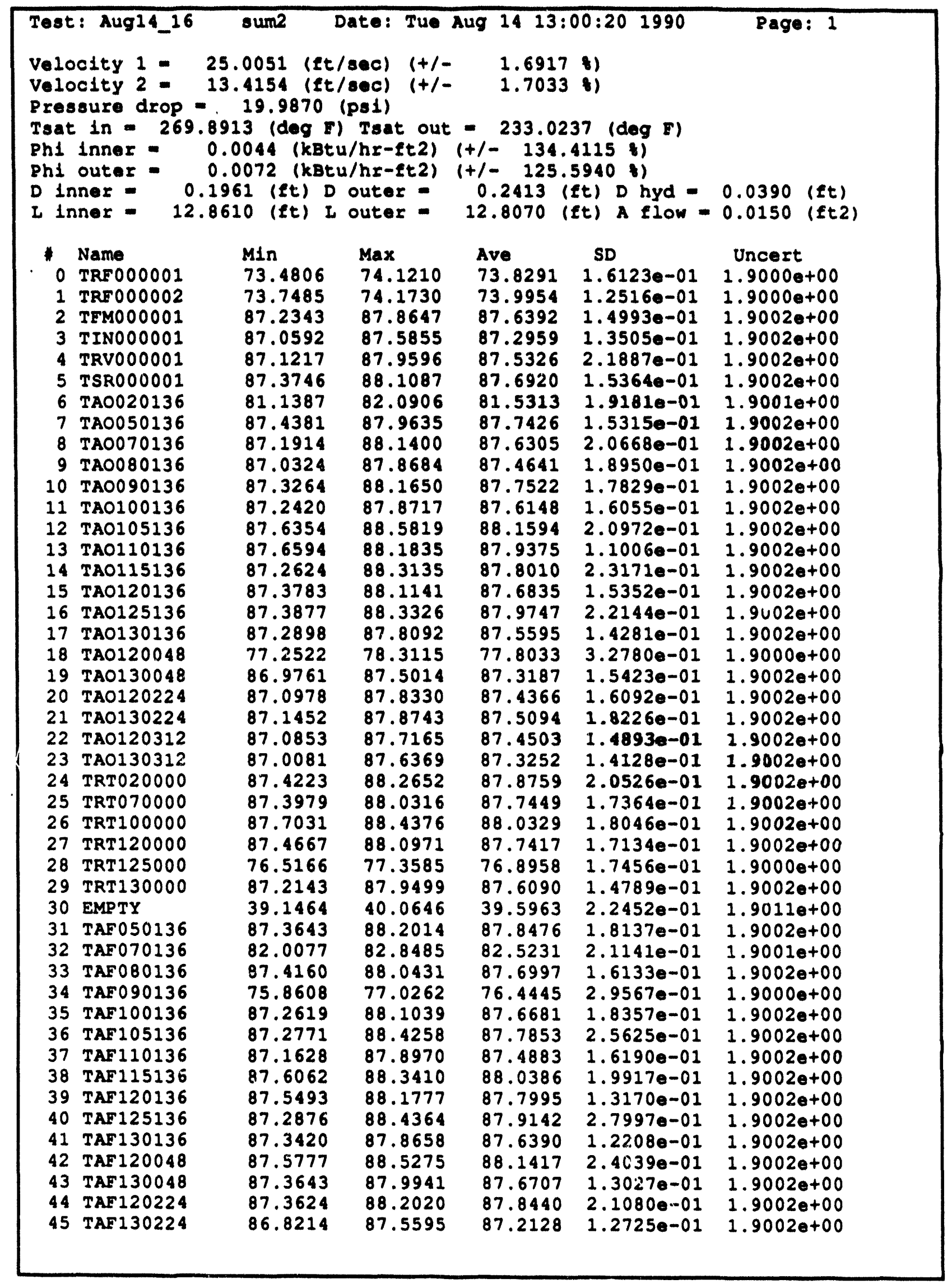




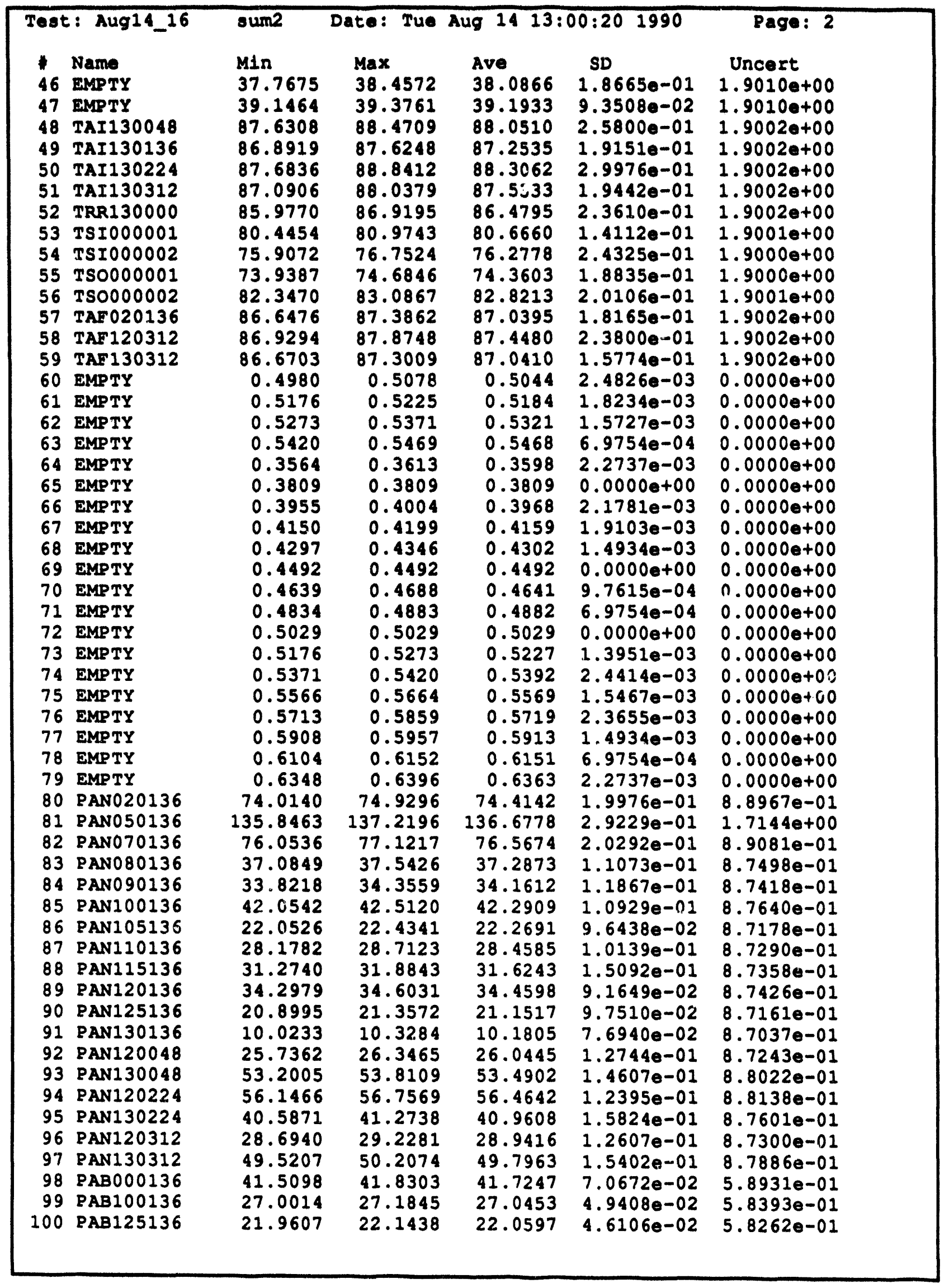




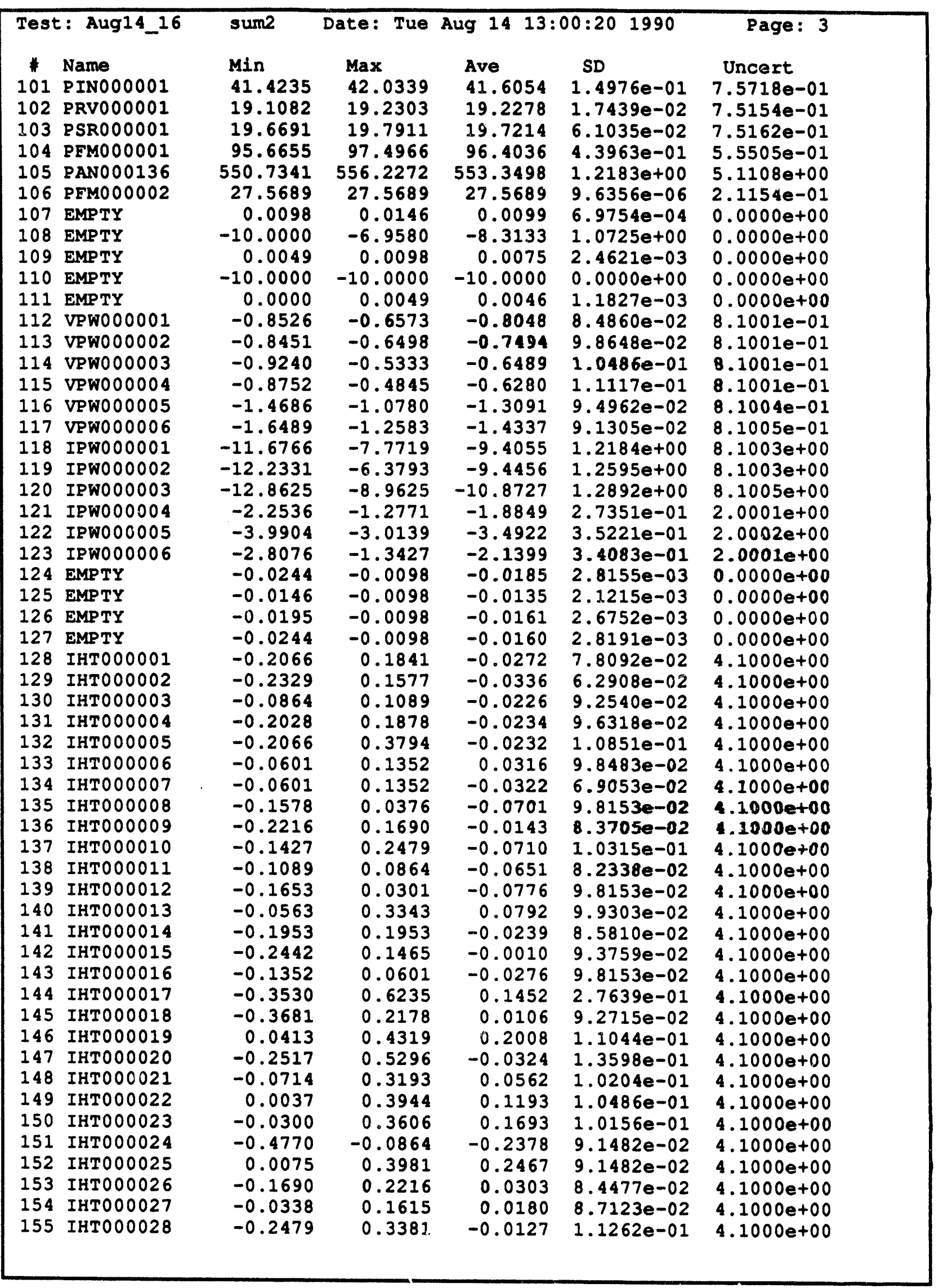




\begin{tabular}{|c|c|c|c|c|c|c|}
\hline \multicolumn{2}{|c|}{ Test: Aug14_16 } & sum2 & \multicolumn{3}{|c|}{ Date: Tue Aug $14 \quad 13: 00: 201990$} & $\begin{array}{c}\text { Page: } 4 \\
\text { Uncert }\end{array}$ \\
\hline $\begin{array}{l}* \\
156 \\
157 \\
158 \\
159 \\
160 \\
161 \\
162 \\
163\end{array}$ & 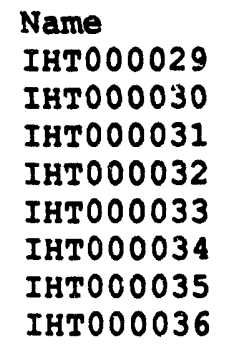 & $\begin{array}{l}\text { Min } \\
-0.0826 \\
-0.2103 \\
-0.1465 \\
-0.0976 \\
-0.0977 \\
-0.2329 \\
-0.1277 \\
-0.1878\end{array}$ & $\begin{array}{l}\text { Max } \\
0.1127 \\
0.3756 \\
0.2441 \\
0.2930 \\
0.2930 \\
0.1577 \\
0.2629 \\
0.2028\end{array}$ & $\begin{array}{l}\text { Ave } \\
-0.0308 \\
0.0328 \\
-0.0269 \\
-0.0139 \\
-0.0140 \\
-0.0176 \\
0.0556 \\
-0.0443\end{array}$ & $\begin{array}{l}\text { SD } \\
8.7123 e-02 \\
1.0188 e-01 \\
1.1146 e-01 \\
1.0548 e-01 \\
1.0548 e-01 \\
1.1448 e-01 \\
8.3705 e-02 \\
9.5812 e-02\end{array}$ & $\begin{array}{l}\text { Uncert } \\
4.1000 e+00 \\
4.1000 e+00 \\
4.1000 e+00 \\
4.1000 e+00 \\
4.1000 e+00 \\
4.1000 e+00 \\
4.1000 e+00 \\
4.1000 e+00\end{array}$ \\
\hline
\end{tabular}




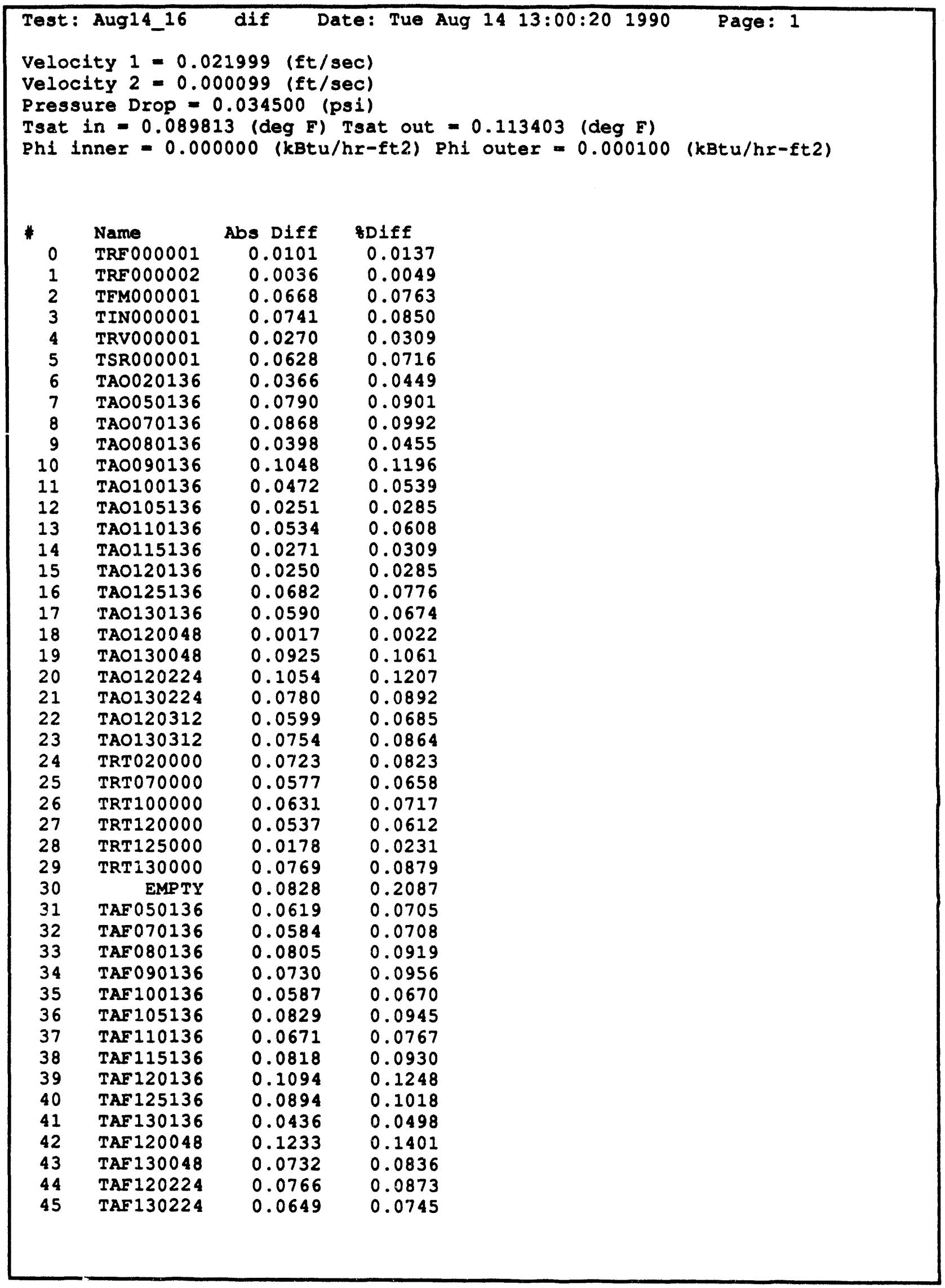




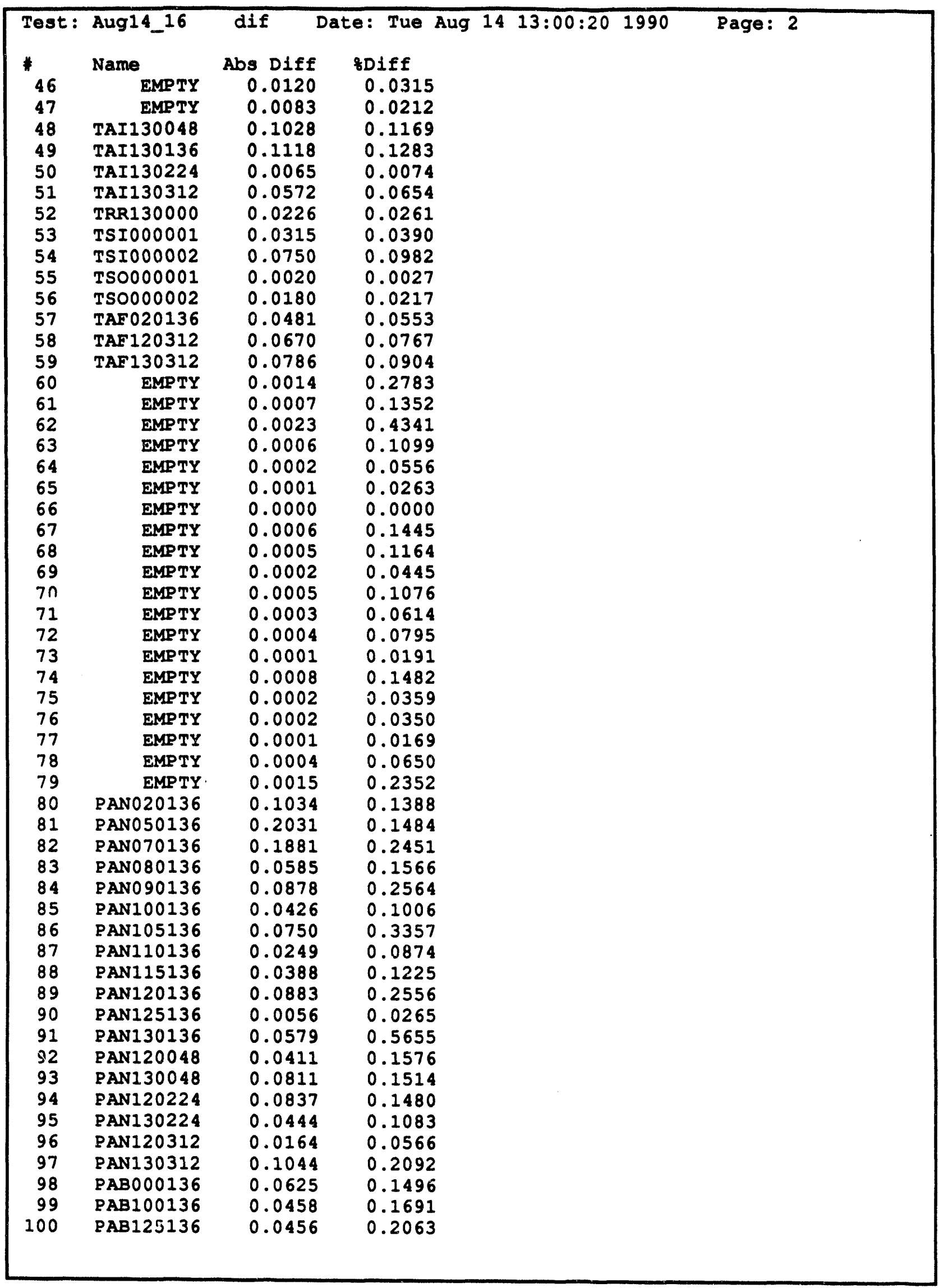




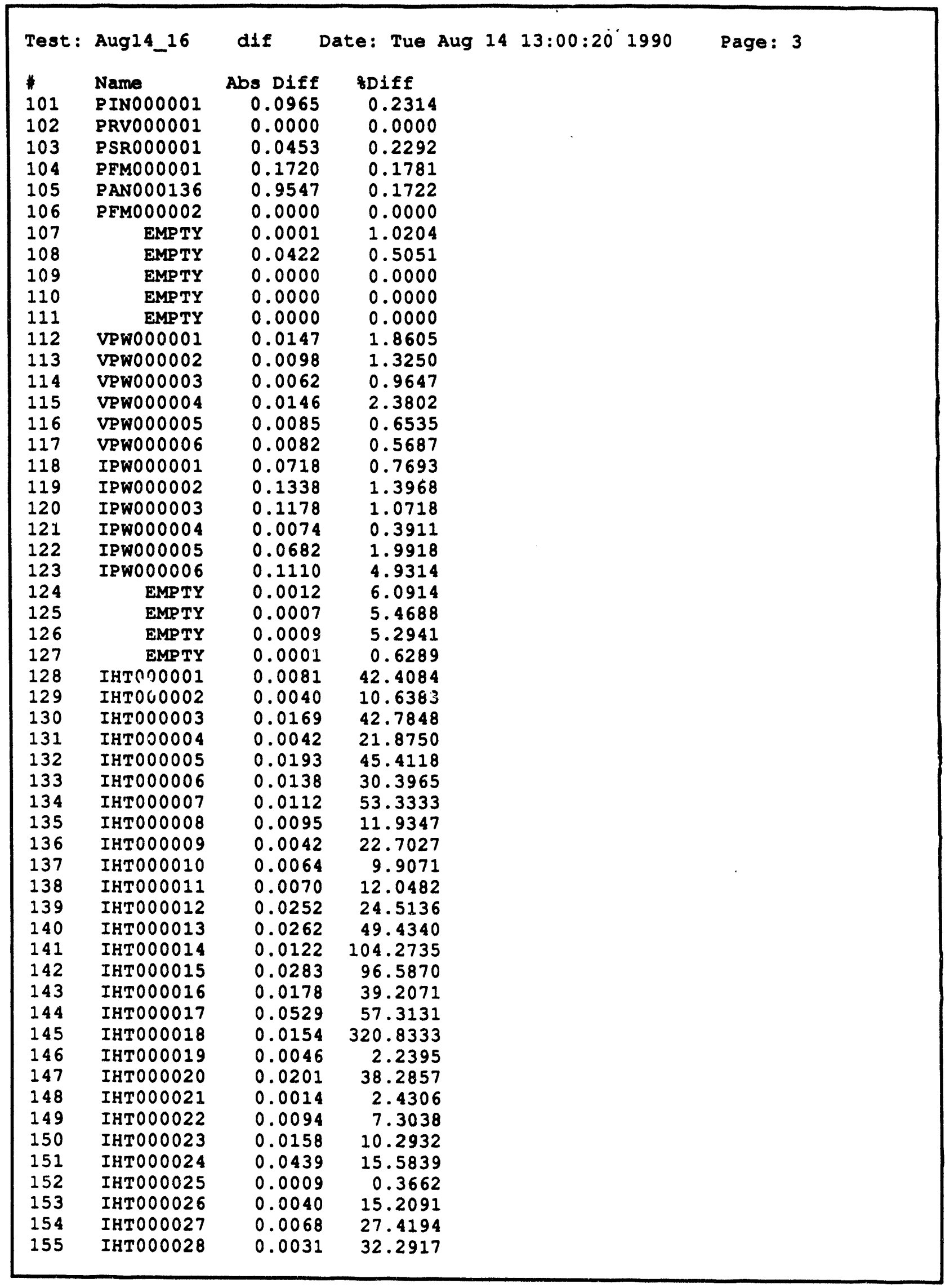


Test: Aug14_16

Name

IHTO00029

IHT000030

IHT000031

IHT000032

IHT000033

IHT000034

IHT000035

IHT000036 dif Date: Tue Aug 14 13:00:20 1990

Page: 4

\begin{tabular}{rr} 
Abs Diff & \multicolumn{1}{l}{ \&Diff } \\
0.0068 & 28.3333 \\
0.0048 & 17.1429 \\
0.0102 & 27.4933 \\
0.0017 & 10.8974 \\
0.0251 & 64.1944 \\
0.0230 & 425.9259 \\
0.0075 & 15.5925 \\
0.0049 & 12.4366
\end{tabular}




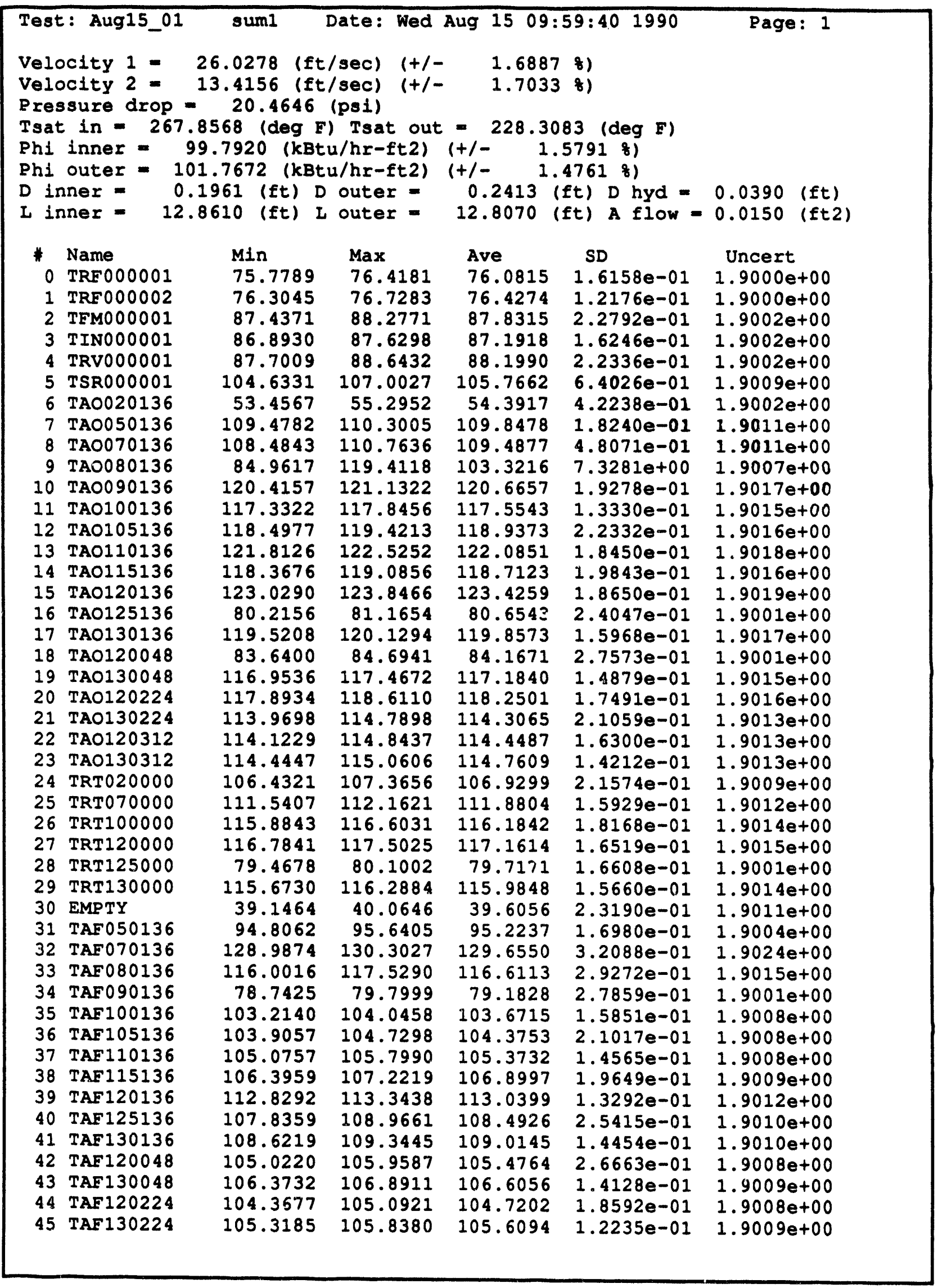




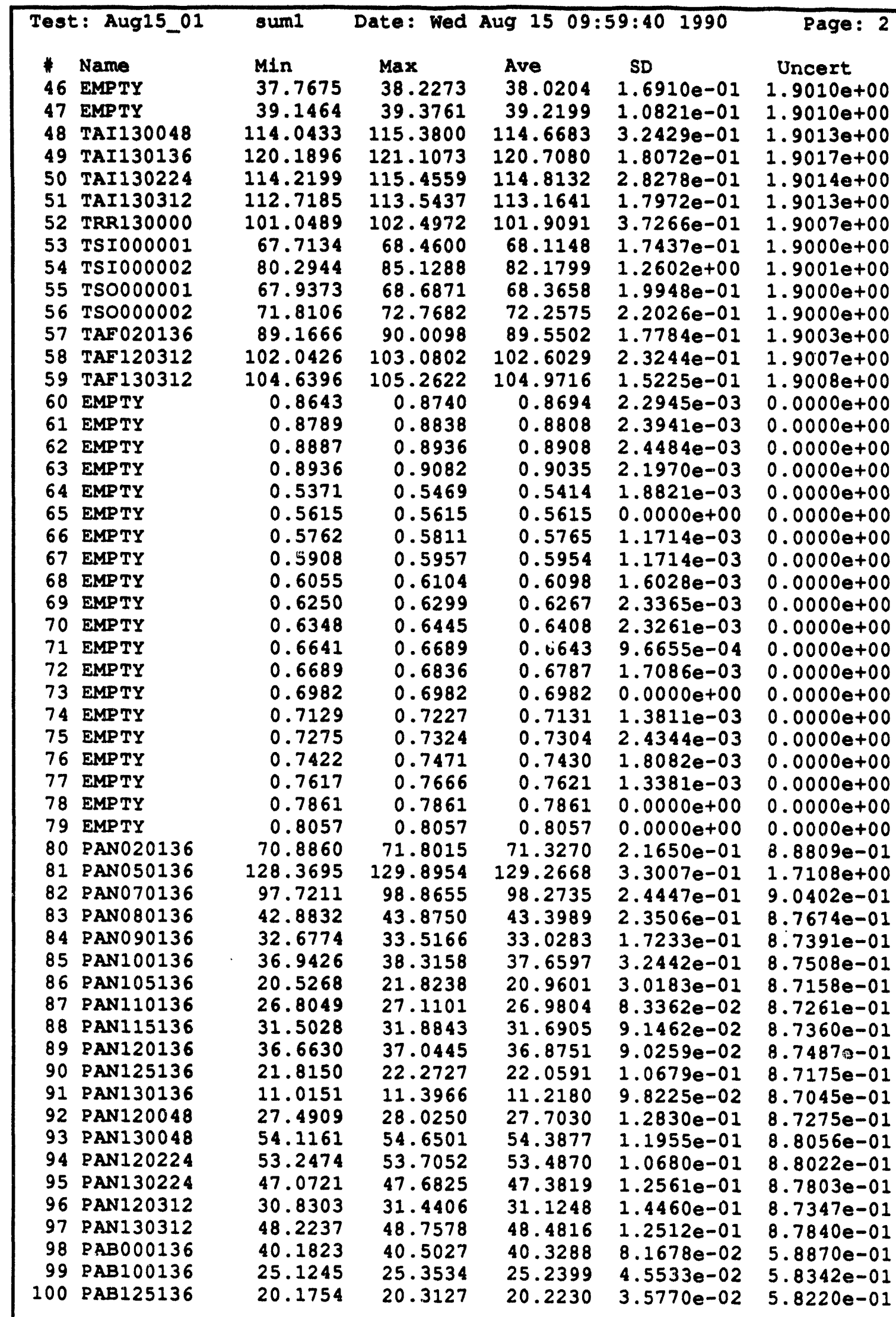




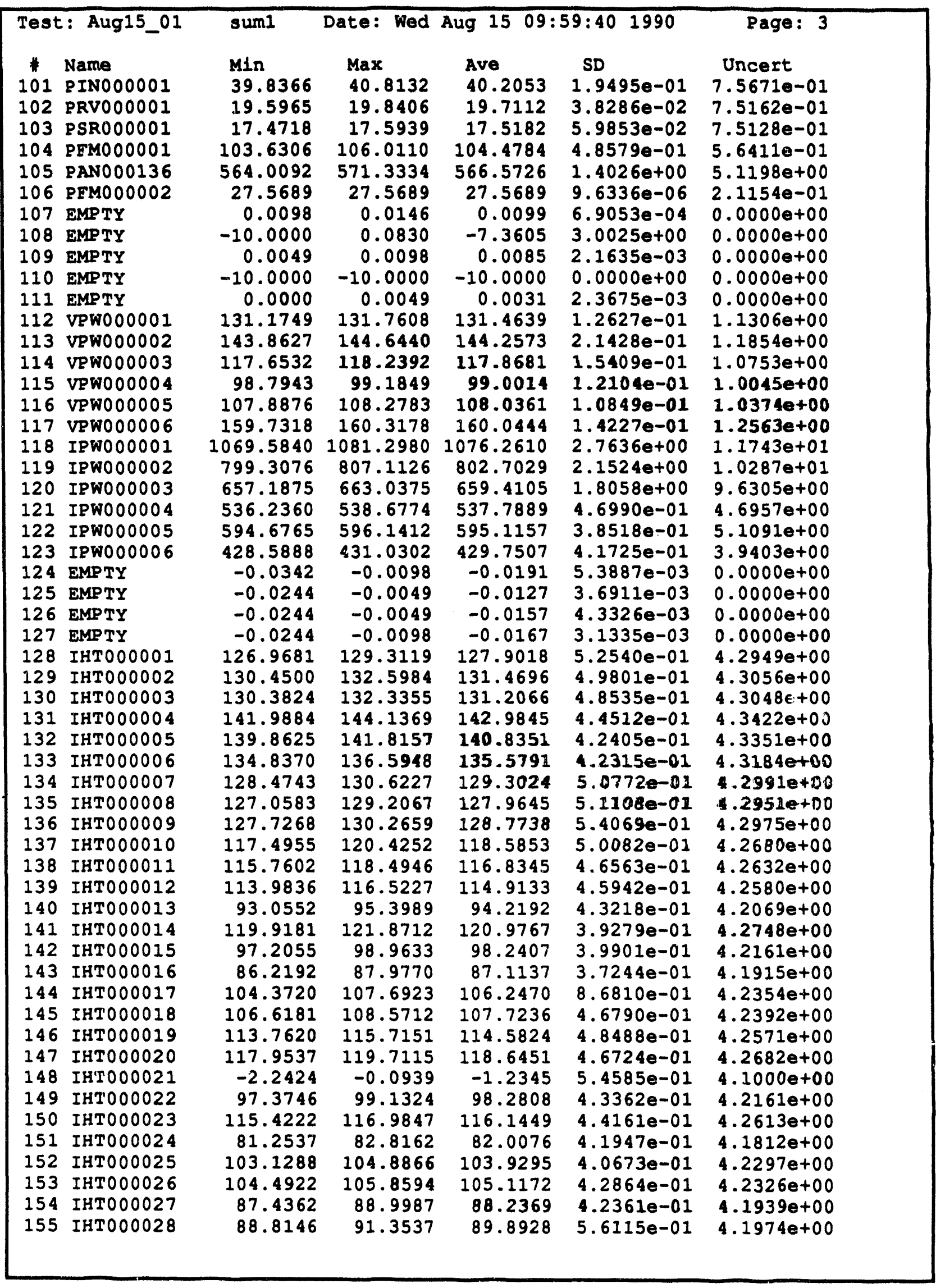




\begin{tabular}{|c|c|c|c|c|c|}
\hline Test: Aug15_01 & suml & Date: Wed & Aug 1509 & $59: 401990$ & Page: \\
\hline 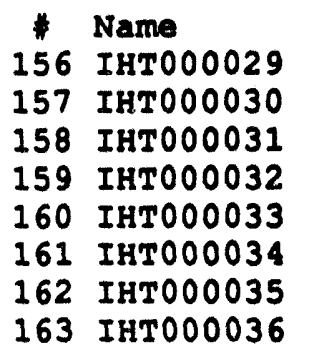 & $\begin{array}{r}\text { Min } \\
104.0302 \\
106.5955 \\
95.3500 \\
96.3792 \\
96.1689 \\
88.4428 \\
116.5978 \\
86.9479\end{array}$ & $\begin{array}{r}\text { Max } \\
106.3740 \\
108.9393 \\
97.4985 \\
98.5276 \\
98.5126 \\
90.5912 \\
118.9416 \\
89.2916\end{array}$ & $\begin{array}{r}\text { Ave } \\
105.0849 \\
107.5213 \\
96.3540 \\
97.3479 \\
97.2900 \\
89.3647 \\
117.5470 \\
87.9478\end{array}$ & $\begin{array}{l}\text { SD } \\
5.8394 e-01 \\
5.6608 e-01 \\
5.4820 e-01 \\
6.0097 e-01 \\
6.0336 e-01 \\
4.9291 e-01 \\
4.8650 e-01 \\
4.4229 e-01\end{array}$ & $\begin{array}{c}\text { Uncert } \\
4.2325 e+00 \\
4.2386 e+00 \\
4.2117 e+00 \\
4.2140 e+00 \\
4.2139 e+00 \\
4.1963 e+00 \\
4.2652 e+00 \\
4.1933 e+00\end{array}$ \\
\hline
\end{tabular}




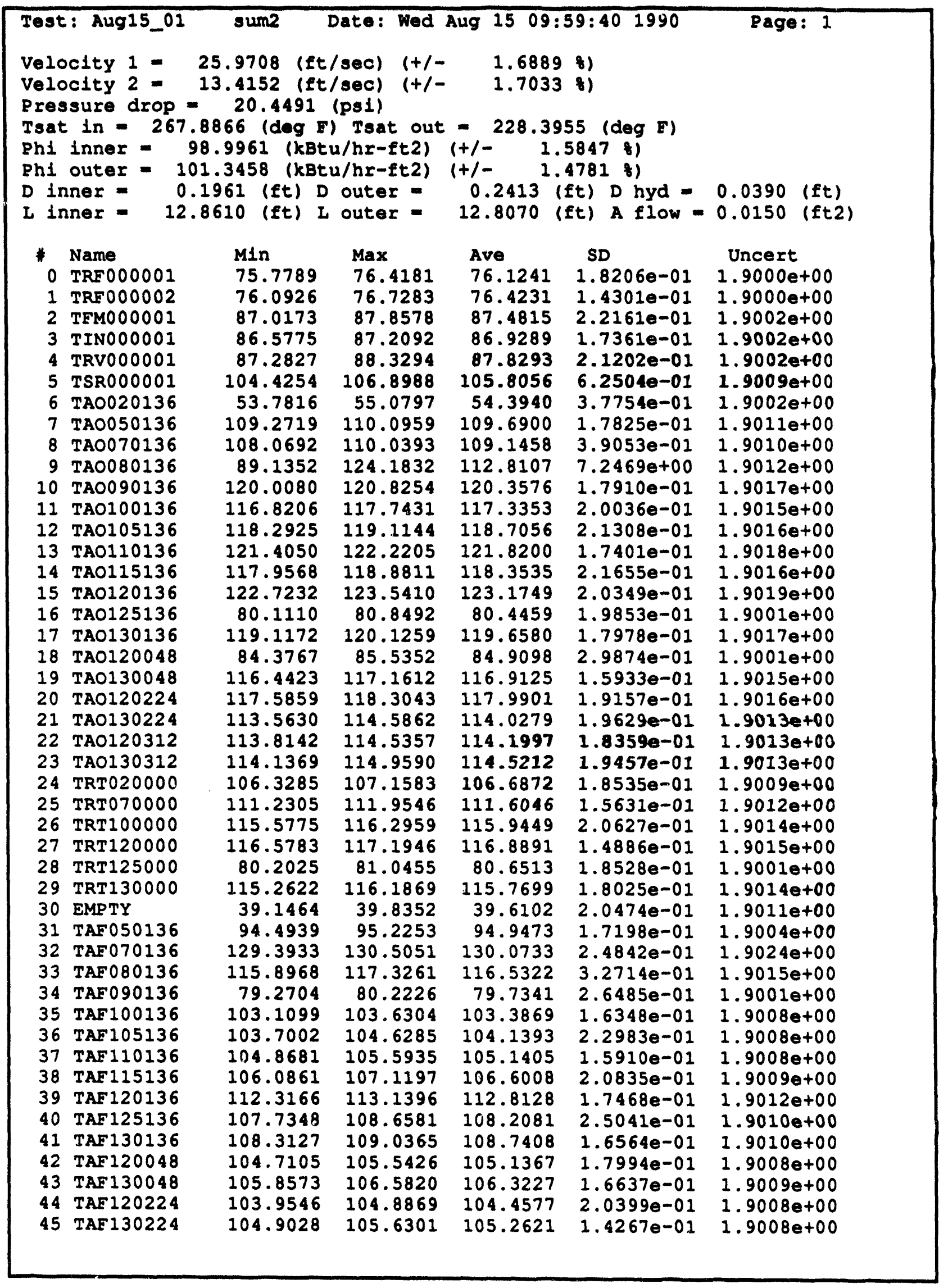




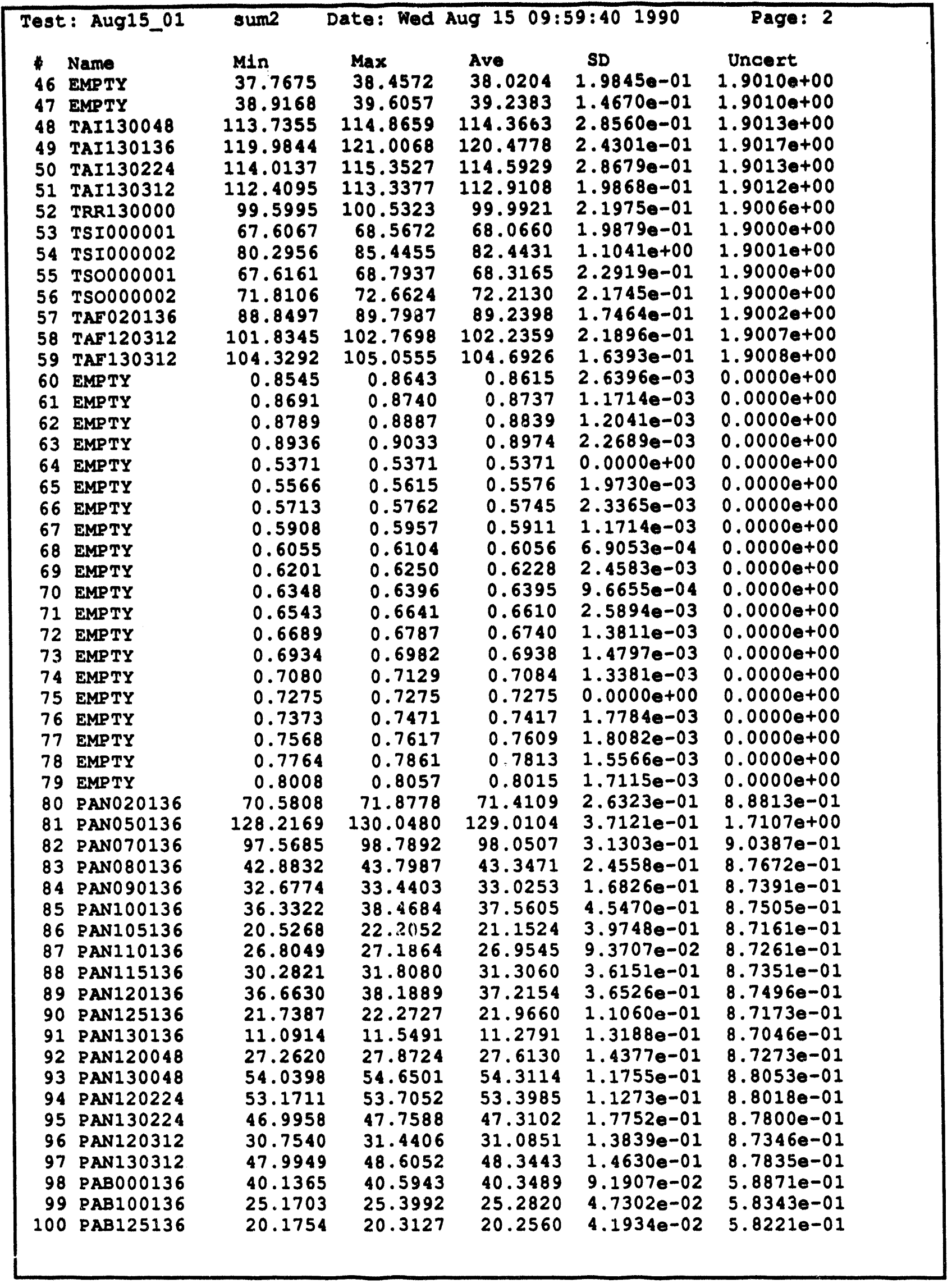




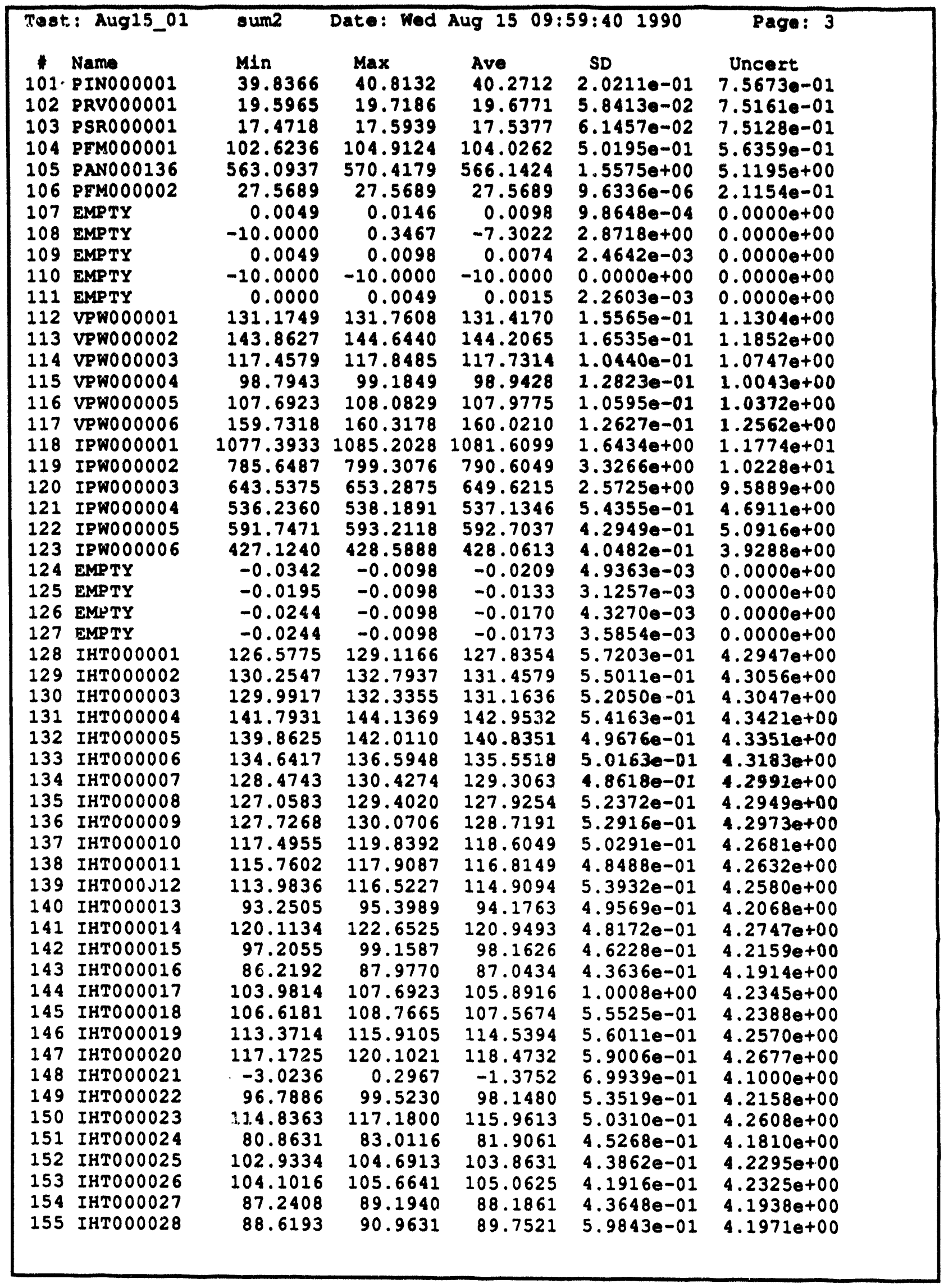




\begin{tabular}{|c|c|c|c|c|c|}
\hline 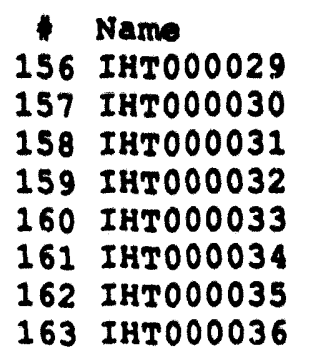 & $\begin{array}{l}\text { MIn } \\
103.8349 \\
106.4002 \\
95.1547 \\
95.9886 \\
95.9736 \\
88.2474 \\
116.5978 \\
86.7525\end{array}$ & $\begin{array}{r}\operatorname{Max} \\
106.1786 \\
108.5487 \\
97.4985 \\
98.7230 \\
98.5126 \\
90.2006 \\
118.7462 \\
88.9010\end{array}$ & $\begin{array}{r}\text { Ave } \\
104.9365 \\
107.3495 \\
96.2134 \\
97.2425 \\
97.2002 \\
89.2006 \\
117.3986 \\
87.8463\end{array}$ & $\begin{array}{l}\text { SD } \\
5.8495 e-01 \\
5.7183 e-01 \\
6.2152 e-01 \\
6.5565 e-01 \\
6.4723 e-01 \\
5.1696 e-01 \\
5.1031 e-01 \\
5.0686 \theta-01\end{array}$ & $\begin{array}{c}\text { Uncert } \\
4.2322 \theta+00 \\
4.2382 \theta+00 \\
4.2114 \theta+00 \\
4.2137 \theta+00 \\
4.2136 \theta+00 \\
4.1959 \theta+00 \\
4.2648 \theta+00 \\
4.1931 \theta+00\end{array}$ \\
\hline
\end{tabular}



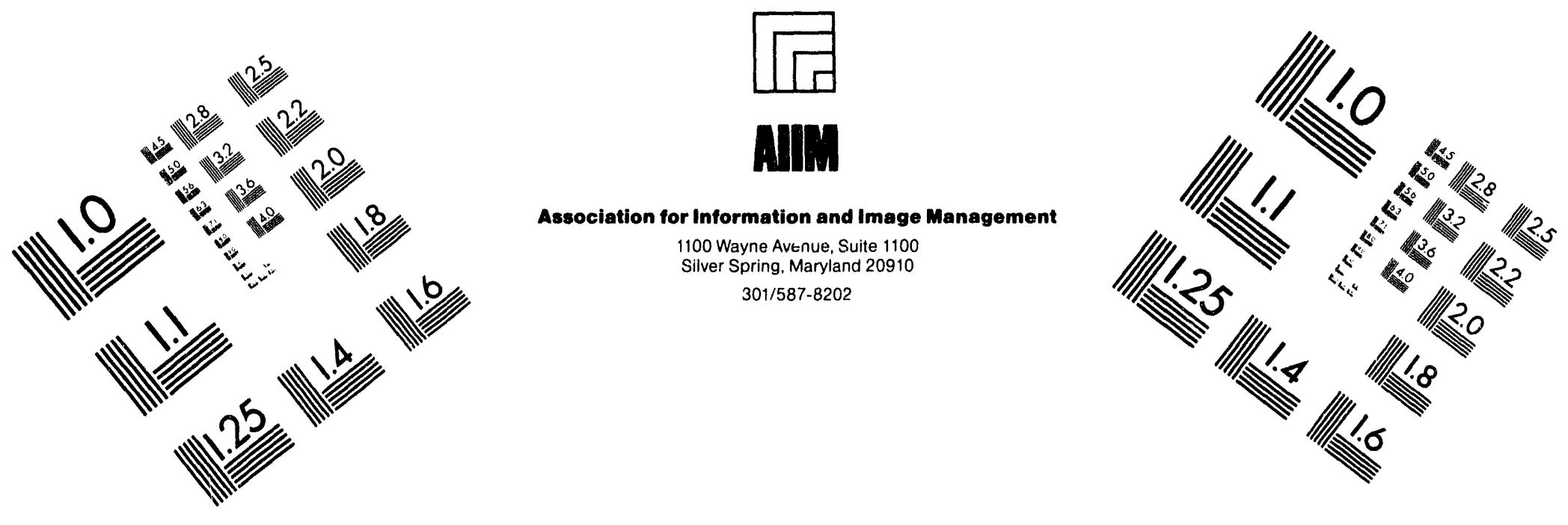

\section{Centimeter}

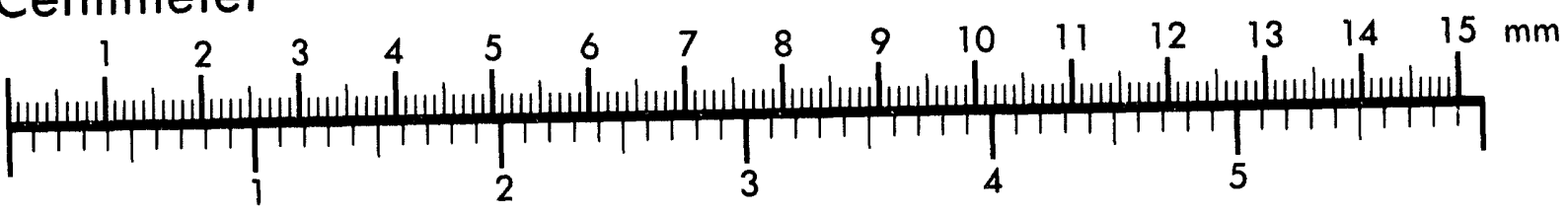
Inches
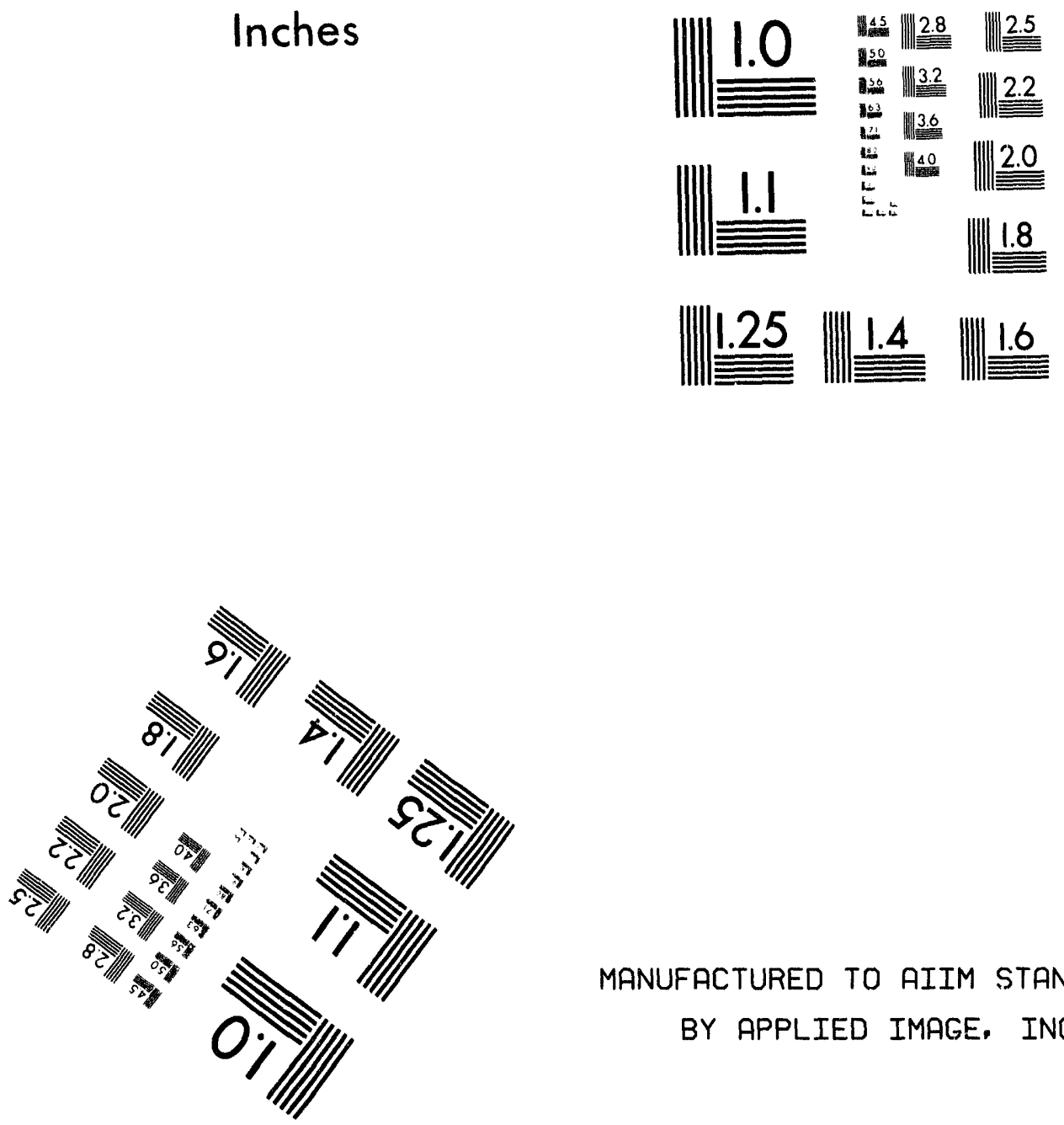

MANUFACTURED TO AIIM STANDARDS

BY APPLIED IMAGE, INC.

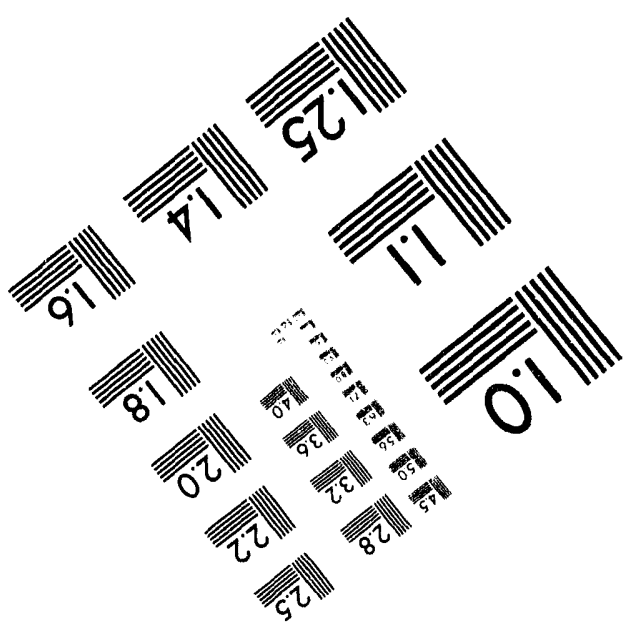



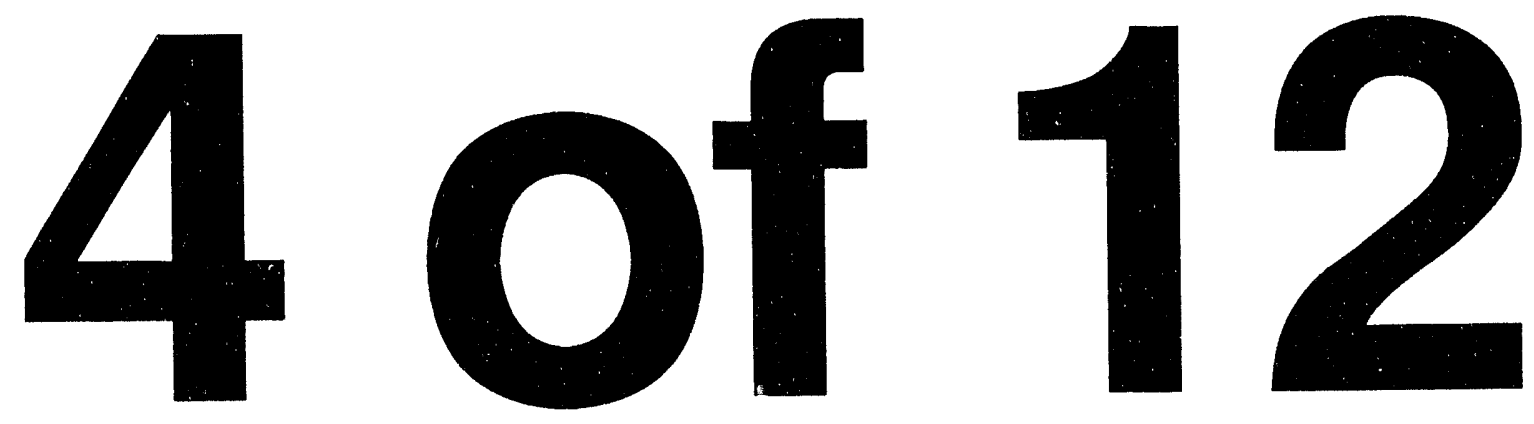


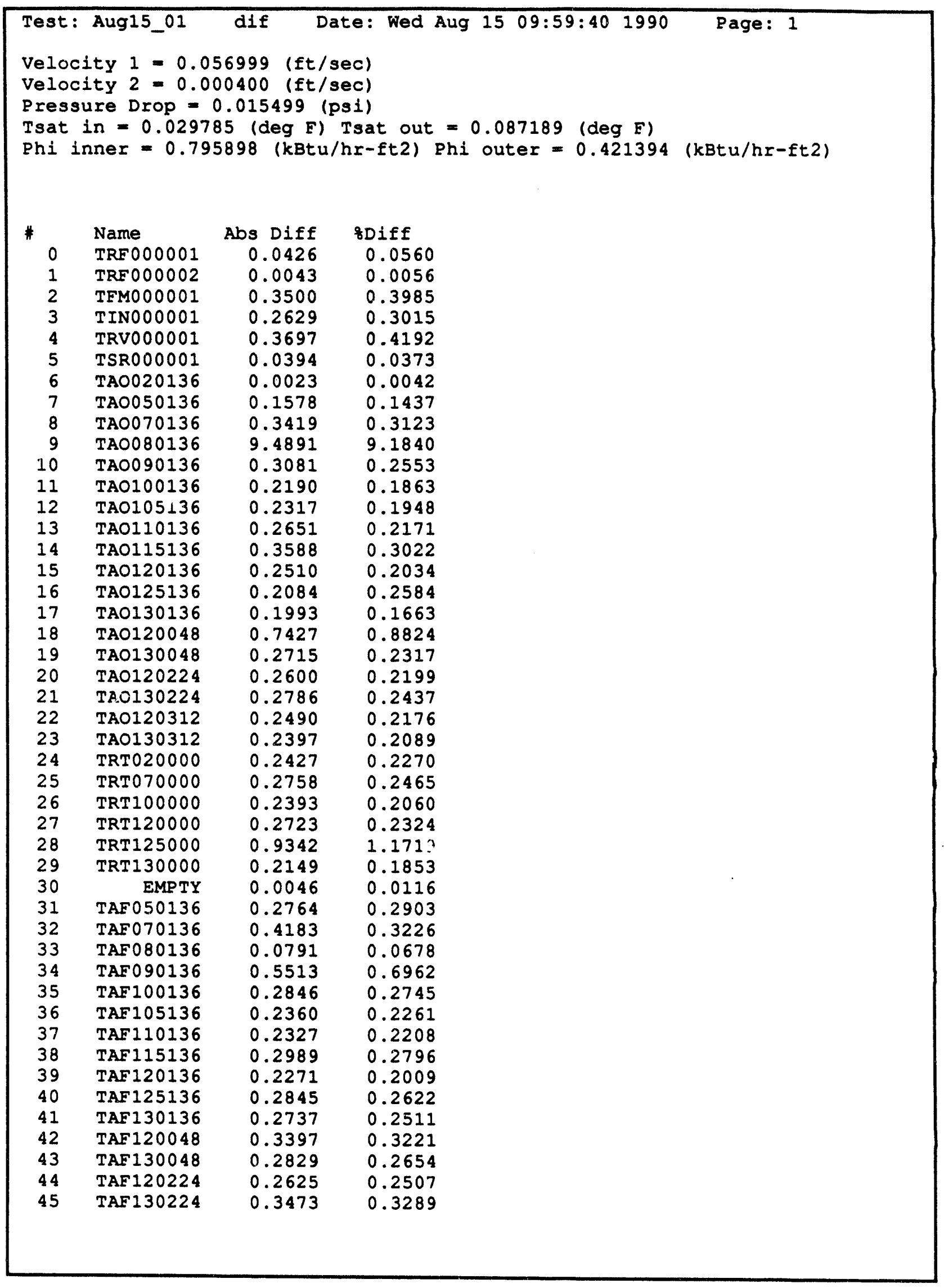




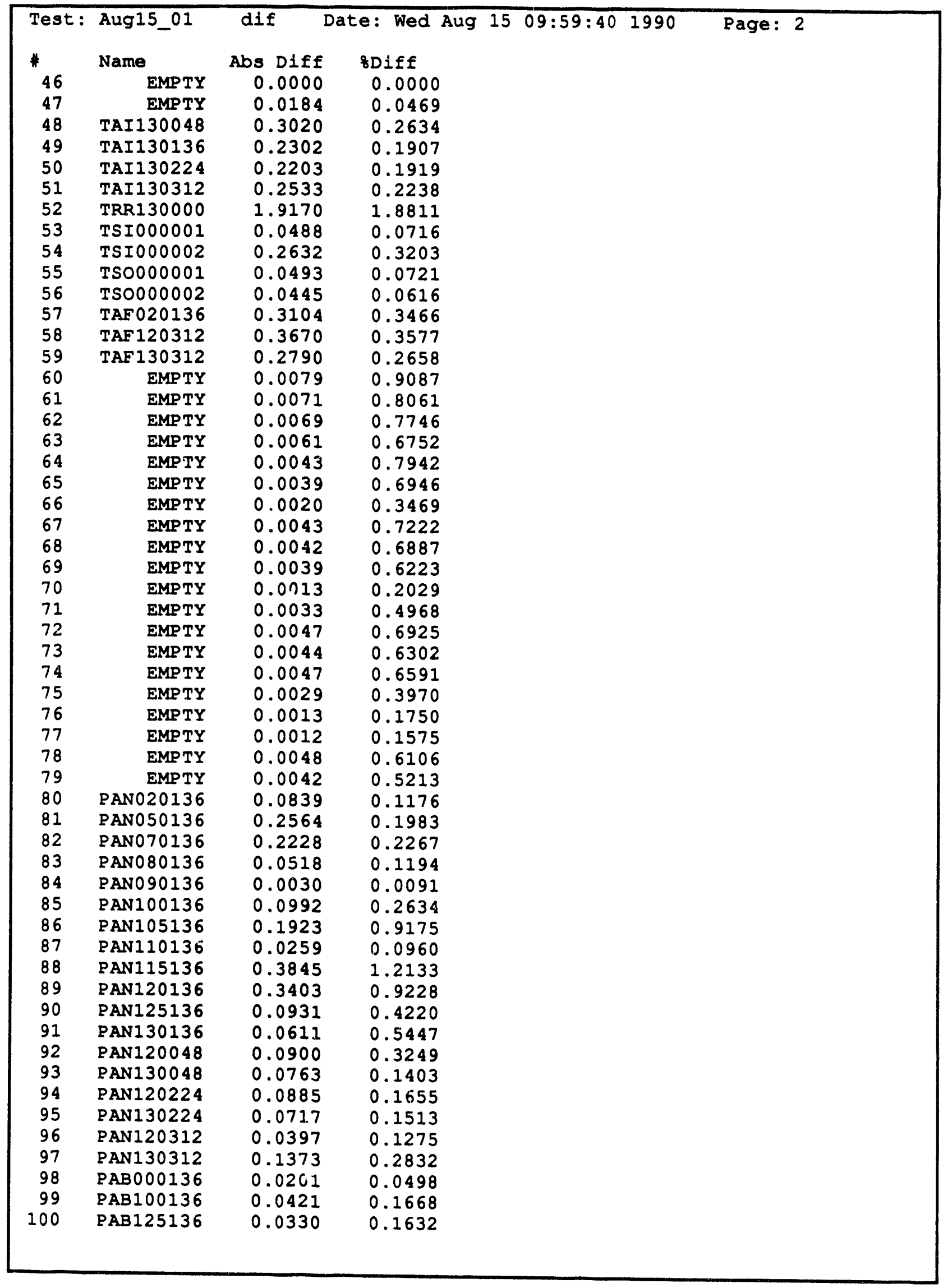




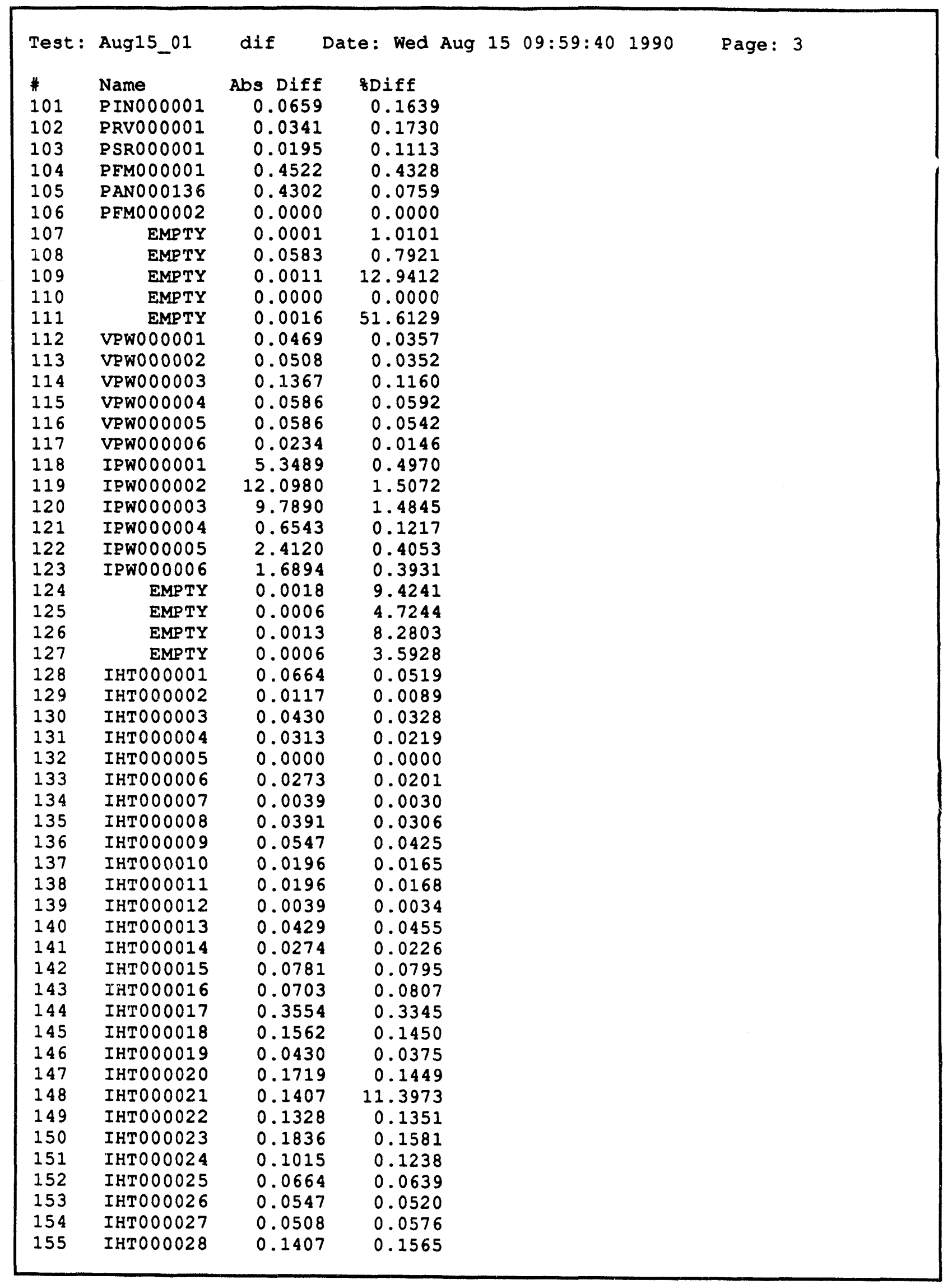


Test: Aug15_01 dif Date: Wed Aug 15 09:59:40 1990 Page: 4

* Name Abs Dirf \&Diff

156 IHTO00029 $0.1484 \quad 0.1412$

157 IHTOOOO30 $0.1718 \quad 0.1598$

158 IHTOOO031 $0.1406 \quad 0.1459$

159 IHTO00032 $0.1054 \quad 0.1083$

160 IHTO00033 $0.0898 \quad 0.0923$

161 IHT000034 $0.1641 \quad 0.1836$

162 IHTO00035 $0.1484 \quad 0.1262$

163 IHT000036 $0.1015 \quad 0.1154$ 


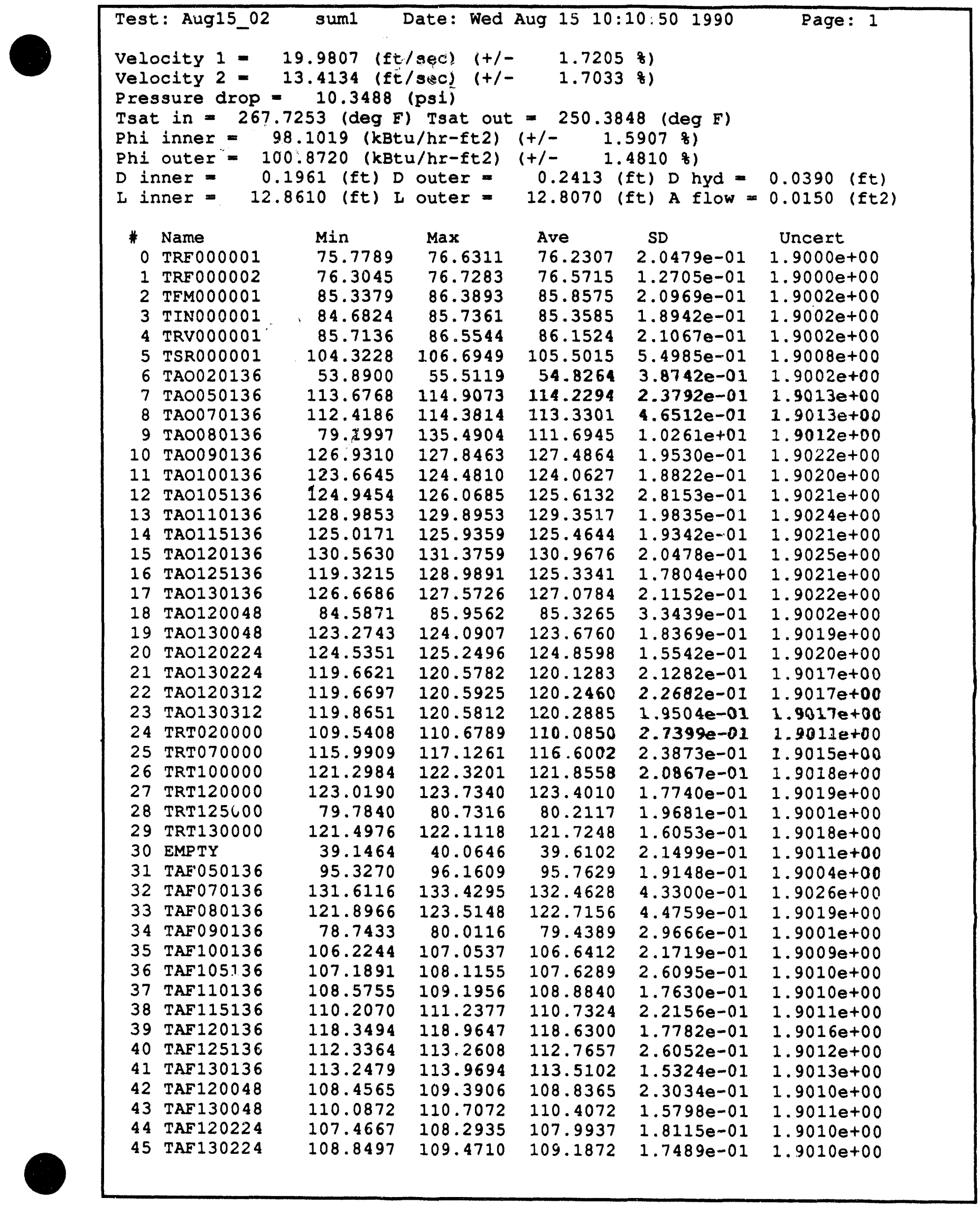




\begin{tabular}{|c|c|c|c|c|c|c|}
\hline \multicolumn{2}{|c|}{ Test: Aug15_02 } & suml & Date: Wed & \multicolumn{2}{|c|}{ Aug $15 \quad 10: 10: 501990$} & $\begin{array}{l}\text { Page: } 2 \\
\text { Uncert }\end{array}$ \\
\hline 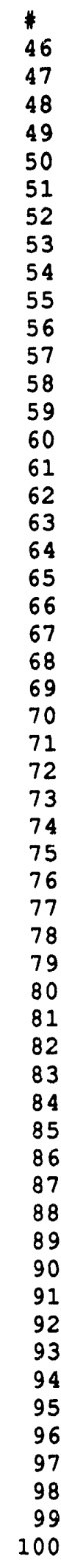 & 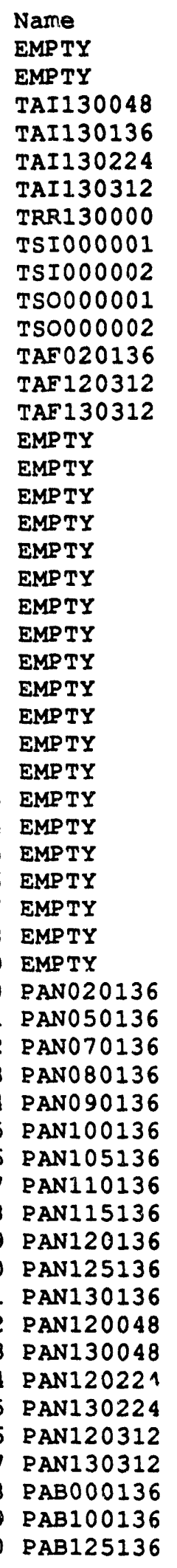 & 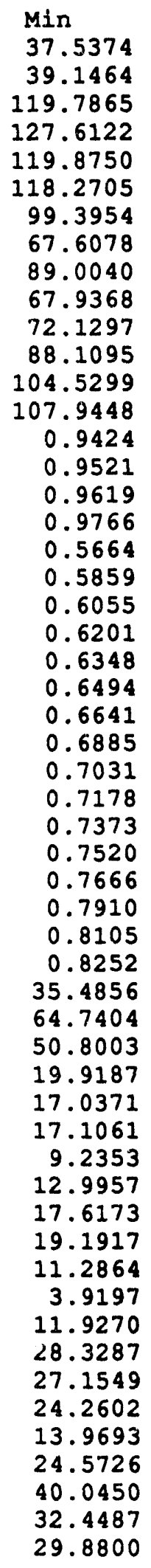 & $\begin{array}{r}\text { Max } \\
38.4572 \\
39.6057 \\
121.4239 \\
128.4261 \\
121.1062 \\
119.1942 \\
100.6367 \\
68.4612 \\
92.8677 \\
68.7938 \\
72.9818 \\
89.0591 \\
105.8770 \\
108.6681 \\
0.9521 \\
0.9619 \\
0.9717 \\
0.9814 \\
0.5713 \\
0.5957 \\
0.6104 \\
0.6250 \\
0.6396 \\
0.6592 \\
0.6738 \\
0.6934 \\
12.68 \\
29.09917 \\
27.9178 \\
24.9469 \\
14.6560 \\
25.3356 \\
40.4570 \\
32.6776 \\
30.0631 \\
0.7275 \\
0.7520 \\
0.7617 \\
0.7954 \\
0.8154 \\
0.8350 \\
36.7063 \\
66.4188 \\
51.9447 \\
20.9868 \\
17.7238 \\
17.7928 \\
13.76930 \\
11.8205 \\
130\end{array}$ & 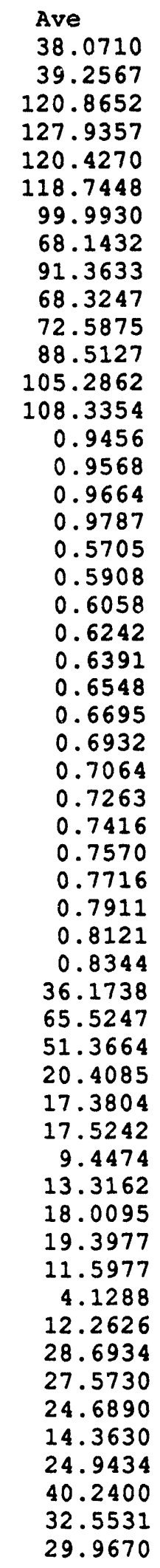 & $\begin{array}{l}\text { SD } \\
2.3886 e-01 \\
1.3317 e-01 \\
3.2568 e-01 \\
1.8437 e-01 \\
3.0593 e-01 \\
2.5219 e-01 \\
3.2462 e-01 \\
1.8226 e-01 \\
1.2064 e+00 \\
2.0284 e-01 \\
2.0093 e-01 \\
2.0061 e-01 \\
2.9687 e-01 \\
1.7003 e-01 \\
2.5362 e-03 \\
2.6025 e-03 \\
1.6624 e-03 \\
2.4484 e-03 \\
1.8082 e-03 \\
9.8648 e-04 \\
1.1714 e-03 \\
1.8082 e-03 \\
1.6028 e-03 \\
1.7784 e-03 \\
1.8821 e-03 \\
9.6655 e-04 \\
2.3008 e-03 \\
2.3778 e-03 \\
3.3656 e-03 \\
1.3811 e-03 \\
1.2041 e-03 \\
6.9053 e-04 \\
2.3008 e-03 \\
2.1249 e-03 \\
2.8567 e-01 \\
4.3379 e-01 \\
3.2077 e-01 \\
2.2181 e-01 \\
1.7250 e-01 \\
1.6475 e-01 \\
1.5203 e-01 \\
1.2617 e-01 \\
1.3873 e-01 \\
1.3011 e-01 \\
1.0206 e-01 \\
9.4779 e-02 \\
1.6744 e-01 \\
1.6835 e-01 \\
1.8576 e-01 \\
1.7702 e-01 \\
1.5595 e-01 \\
1.6313 e-01 \\
9.0995 e-02 \\
5.5520 e-02 \\
4.2632 e-02\end{array}$ & $\begin{array}{l}\text { Uncert } \\
1.9010 e+00 \\
1.9010 e+00 \\
1.9017 e+00 \\
1.9023 e+00 \\
1.9017 e+00 \\
1.9016 e+00 \\
1.9006 e+00 \\
1.9000 e+00 \\
1.9003 e+00 \\
1.9000 e+00 \\
1.9000 e+00 \\
1.9002 e+00 \\
1.9008 e+00 \\
1.9010 e+00 \\
0.0000 e+00 \\
0.0000 e+00 \\
0.0000 e+00 \\
0.0000 e+00 \\
0.0000 e+00 \\
0.0000 e+00 \\
0.0000 e+00 \\
0.0000 e+00 \\
0.0000 e+00 \\
0.0000 e+00 \\
0.0000 e+00 \\
0.0000 e+00 \\
0.0000 e+00 \\
0.0000 e+00 \\
0.0000 e+00 \\
0.0000 e+00 \\
0.0000 e+00 \\
0.0000 e+00 \\
0.0000 e+00 \\
0.0000 e+00 \\
8.7469 e-01 \\
1.6880 e+00 \\
8.7943 e-01 \\
8.7149 e-01 \\
8.7108 e-01 \\
8.7110 e-01 \\
8.7032 e-01 \\
8.7064 e-01 \\
8.7116 e-01 \\
8.7135 e-01 \\
8.7048 e-01 \\
8.7006 e-01 \\
8.7054 e-01 \\
8.7295 e-01 \\
8.7273 e-01 \\
8.7219 e-01 \\
8.7074 e-01 \\
5.7223 e-01 \\
5.8566 e-01 \\
5.8482 e-01\end{array}$ \\
\hline
\end{tabular}




\begin{tabular}{|c|c|c|c|c|c|c|}
\hline \multicolumn{2}{|c|}{ Test: Aug15_02 } & sum1 & Date: Wed & Aug 1510 : & $10: 50 \quad 1990$ & Page: 3 \\
\hline \# & Name & Min & $\operatorname{Max}$ & Ave & SD & Uncert \\
\hline 101 & PIN000001 & 39.5925 & 40.3249 & 40.0124 & $1.8150 e-01$ & $7.5664 e-01$ \\
\hline 102 & PRV000001 & 19.7186 & 19.8406 & 19.8235 & $4.2787 e-02$ & $7.5164 e-01$ \\
\hline 103 & PSR000001 & 28.2140 & 28.5802 & 28.4337 & $9.2276 e-02$ & $7.5336 e-01$ \\
\hline 104 & PEM000001 & 60.8755 & 62.0657 & 61.4559 & $2.9917 e-01$ & $5.2307 e-01$ \\
\hline 105 & PANO00136 & 281.1113 & 291.1821 & 286.5129 & $2.3813 e+00$ & $4.9719 \mathrm{e}+00$ \\
\hline 106 & PFM000002 & 27.5689 & 27.5689 & 27.5689 & $9.6336 e-06$ & $2.1154 e-01$ \\
\hline 107 & EMPTY & 0.0049 & 0.0146 & 0.0092 & $1.8821 e-03$ & $0.0000 e+00$ \\
\hline 108 & EMPTY & -10.0000 & -5.0098 & -8.2148 & $1.9022 \mathrm{e}+00$ & $0.0000 e+00$ \\
\hline 109 & EMPTY & 0.0049 & 0.0098 & 0.0051 & $9.6655 e-04$ & $0.0000 e+00$ \\
\hline 110 & EMPTY & -10.0000 & -10.0000 & -10.0000 & $0.0000 e+00$ & $0.0000 e+00$ \\
\hline 111 & EMPTY & 0.0000 & 0.0049 & 0.0005 & $1.4797 e-03$ & $0.0000 \mathrm{e}+00$ \\
\hline 112 & VPW000001 & 131.3702 & 131.7608 & 131.5303 & $1.2283 e-01$ & $1.1309 e+00$ \\
\hline 113 & VPW000002 & 144.0580 & 144.4486 & 144.3315 & $1.0440 e-01$ & $1.1858 \mathrm{e}+00$ \\
\hline 114 & VPW000003 & 117.4579 & 118.0439 & 117.7158 & $1.2750 \mathrm{e}-01$ & $1.0747 e+00$ \\
\hline 115 & VPW000004 & 98.7943 & 99.3802 & 99.0522 & $1.0763 e-01$ & $1.0046 e+00$ \\
\hline 116 & VRW000005 & 107.6923 & 108.0829 & 108.0166 & $1.0145 e-01$ & $1.0374 e+00$ \\
\hline 117 & VPW000006 & 159.7318 & 160.3178 & 160.0444 & $1.2478 e-01$ & $1.2563 e+00$ \\
\hline 118 & IPW000001 & 1077.3933 & 1089.1074 & 1082.2351 & $3.0614 e+00$ & $1.1777 e+01$ \\
\hline 119 & IPW000002 & 771.9898 & 785.6487 & 776.0096 & $3.4006 \mathrm{e}+00$ & $1.0158 e+01$ \\
\hline 120 & IPW000003 & 635.7375 & 639.6375 & 638.3503 & $1.3424 \mathrm{e}+00$ & $9.5416 \mathrm{e}+00$ \\
\hline 121 & IPW000004 & 537.2126 & 538.6774 & 537.9257 & $3.8473 e-01$ & $4.6967 e+00$ \\
\hline 122 & IPW000005 & 589.3060 & 590.2824 & 589.8530 & $3.2174 e-01$ & $5.0709 e+00$ \\
\hline 123 & IPW000006 & 425.6591 & 428.5888 & 427.1433 & $8.3682 e-01$ & $3.9226 \mathrm{e}+00$ \\
\hline 124 & EMPTY & -0.0293 & -0.0146 & -0.0229 & $3.4527 e-03$ & $0.0000 e+00$ \\
\hline 125 & EMPTY & -0.0195 & -0.0049 & -0.0131 & $3.3366 e-03$ & $0.0000 e+00$ \\
\hline 126 & EMPTY & -0.0244 & -0.0146 & -0.0187 & $3.0708 e-03$ & $0.0000 e+00$ \\
\hline 127 & EMPTY & -0.0244 & -0.0098 & -0.0163 & $3.0581 e-03$ & $0.0000 e+00$ \\
\hline 128 & IHT000001 & 126.7728 & 129.3119 & 127.8120 & $5.8052 e-01$ & $4.2946 e+00$ \\
\hline 129 & IHT000002 & 130.4500 & 132.7937 & 131.5008 & $5.3654 e-01$ & $4.3057 e+00$ \\
\hline 130 & IHT000003 & 130.3824 & 132.3355 & 131.2691 & $4.8842 e-01$ & $4.3050 e+00$ \\
\hline 131 & IHTO00004 & 141.5978 & 144.1369 & 142.9376 & $5.0841 e-01$ & $4.3420 e+00$ \\
\hline 132 & IHT000005 & 139.4719 & 141.8157 & 140.8273 & $4.9507 e-01$ & $4.3351 \mathrm{e}+00$ \\
\hline 133 & IHT000006 & 134.2511 & 136.7901 & 135.5869 & $4.9656 e-01$ & $4.3184 e+00$ \\
\hline 134 & IHT000007 & 128.2790 & 130.4274 & 129.3688 & $4.7693 e-01$ & $4.2993 e+00$ \\
\hline 135 & IHTO00008 & 127.0583 & 129.2067 & 127.9996 & $4.6969 e-01$ & $4.2952 e+00$ \\
\hline 136 & IHTO00009 & 127.9222 & 130.2659 & 128.8597 & $4.7351 e-01$ & $4.2977 \mathrm{e}+00$ \\
\hline 137 & IHTO000010 & 117.3002 & 119.6439 & 118.5306 & $5.2682 e-01$ & $4.2679 e+\infty 0$ \\
\hline 138 & IHT000011 & 115.7602 & 118.1040 & 116.7954 & $5.1788 e-01$ & $4.2631 e+20$ \\
\hline 139 & IHTO00012 & 113.7883 & 116.1321 & 114.9445 & $5.7296 e-01$ & $4.2581 \mathrm{e}+00$ \\
\hline 140 & IHTO00013 & 92.8598 & 95.3989 & 94.0981 & $5.3468 e-01$ & $4.2066 e+00$ \\
\hline 141 & IHTO00014 & 119.9181 & 122.4572 & 120.9767 & $5.4117 e-01$ & $4.2748 e+00$ \\
\hline 142 & IHTO00015 & 97.2055 & 99.3540 & 98.1352 & $5.2134 e-01$ & $4.2158 \mathrm{e}+00$ \\
\hline 143 & IHTO00016 & 86.2192 & 88.3676 & 87.0395 & $4.9126 e-01$ & $4.1914 \mathrm{e}+00$ \\
\hline 144 & IHTO00017 & 103.3954 & 106.9111 & 104.7822 & $7.8448 e-01$ & $4.2318 e+00$ \\
\hline 145 & IHT000018 & 106.4228 & 108.7665 & 107.4424 & $5.7085 e-01$ & $4.2384 e+00$ \\
\hline 146 & IHTO00019 & 113.3714 & 115.7151 & 114.4964 & $5.4613 e-01$ & $4.2569 \mathrm{e}+00$ \\
\hline 147 & IHTO00020 & 117.3678 & 119.3209 & 118.3951 & $5.3205 e-01$ & $4.2675 \mathrm{e}+00$ \\
\hline 148 & IHTO00021 & -2.8283 & -0.2892 & -1.4845 & $6.4554 e-01$ & $4.1000 e+00$ \\
\hline 149 & IHT000022 & 96.9839 & 99.1324 & 98.0152 & $5.1454 e-01$ & $4.2155 e+00$ \\
\hline 150 & IHT000023 & 115.0316 & 116.9847 & 115.9339 & $4.9582 e-01$ & $4.2608 e+00$ \\
\hline 151 & IHTO00024 & 81.0584 & 83.2069 & 81.8475 & $4.6347 e-01$ & $4.1809 \mathrm{e}+00$ \\
\hline 152 & IHTO00025 & 102.7381 & 105.2772 & 103.6717 & $4.8213 e-01$ & $4.2290 e+00$ \\
\hline 153 & IHTO00026 & 103.9063 & 106.6406 & 104.8242 & $5.1031 e-01$ & $4.2319 e+00$ \\
\hline 154 & IHTO00027 & 87.2408 & 89.7799 & 88.1314 & $5.1017 e-01$ & $4.1937 e+00$ \\
\hline 155 & IHTO00028 & 88.8146 & 90.9631 & 89.7795 & $5.1661 e-01$ & $4.1971 e+00$ \\
\hline
\end{tabular}




\begin{tabular}{|c|c|c|c|c|c|c|}
\hline Tes & : : Aug15_02 & sum1 & Date: Wed & Aug $15 \quad 10$ & $10: 501990$ & Page: 4 \\
\hline $\begin{array}{l}\# \\
156 \\
157 \\
158 \\
159 \\
160 \\
161 \\
162 \\
163\end{array}$ & $\begin{array}{l}\text { Name } \\
\text { IHTO00029 } \\
\text { IHTO00030 } \\
\text { IHTO00031 } \\
\text { IHTO0O032 } \\
\text { IHTOOOO } 033 \\
\text { IHTO00034 } \\
\text { IHTO00035 } \\
\text { IHTO00036 }\end{array}$ & $\begin{array}{r}\text { Min } \\
103.8349 \\
106.2049 \\
95.1547 \\
96.1839 \\
95.9736 \\
88.6381 \\
116.5978 \\
87.1432\end{array}$ & $\begin{array}{r}\text { Max } \\
106.1786 \\
108.5487 \\
97.4985 \\
98.3323 \\
98.3173 \\
90.5912 \\
118.5509 \\
89.0963\end{array}$ & $\begin{array}{l}\text { Ave } \\
104.8505 \\
107.2245 \\
96.1235 \\
97.1565 \\
97.1298 \\
89.3725 \\
117.4884 \\
87.9986\end{array}$ & $\begin{array}{l}\text { SD } \\
4.8004 e-01 \\
4.7726 e-01 \\
4.6682 e-01 \\
4.9676 e-01 \\
4.9417 e-01 \\
4.0349 e-01 \\
4.0464 e-01 \\
4.0219 e-01\end{array}$ & $\begin{array}{l}\text { Uncert } \\
4.2319 e+00 \\
4.2379 e+00 \\
4.2112 e+00 \\
4.2135 e+00 \\
4.2135 e+00 \\
4.1963 e+00 \\
4.2650 e+00 \\
4.1934 e+00\end{array}$ \\
\hline
\end{tabular}




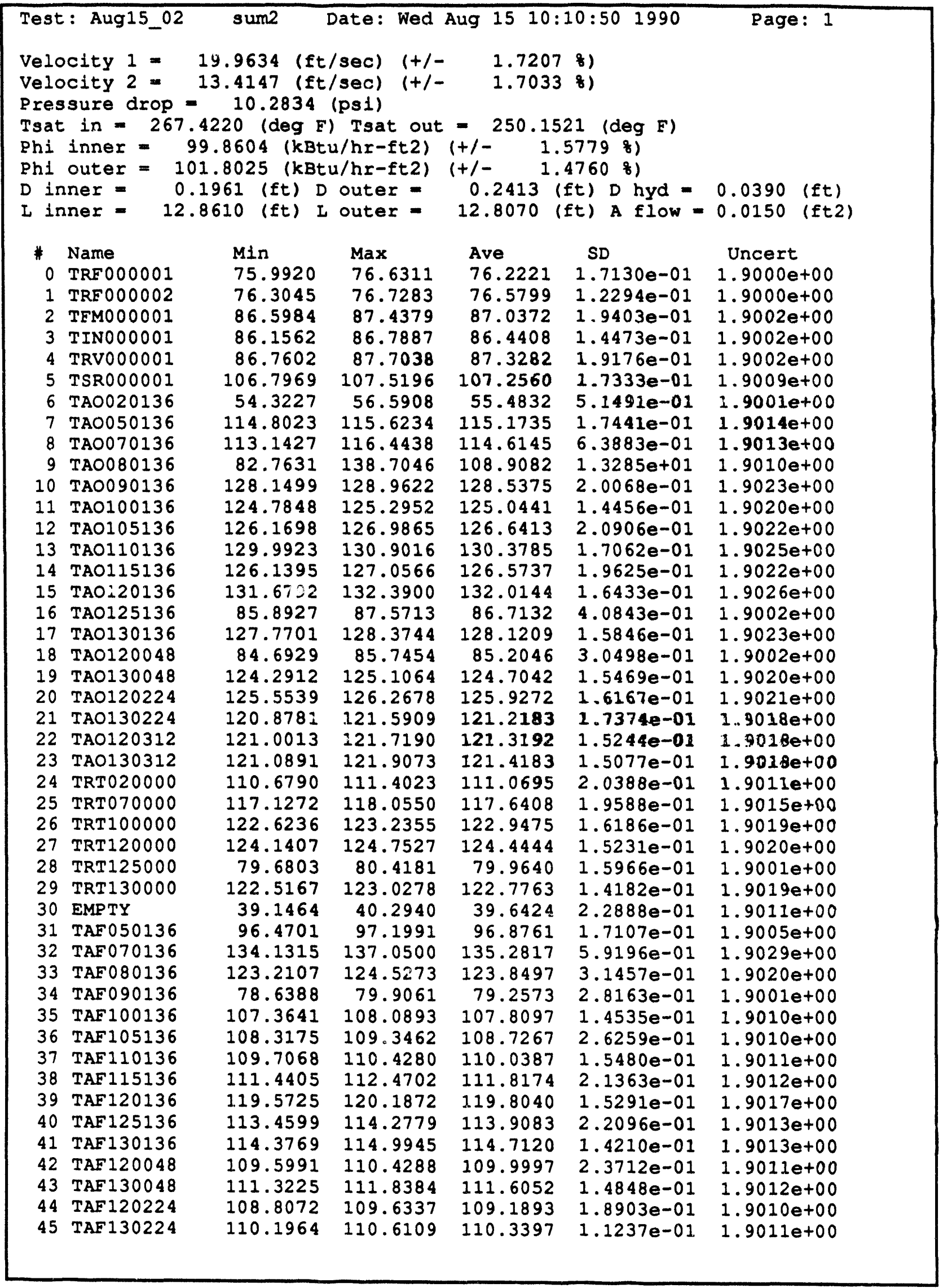




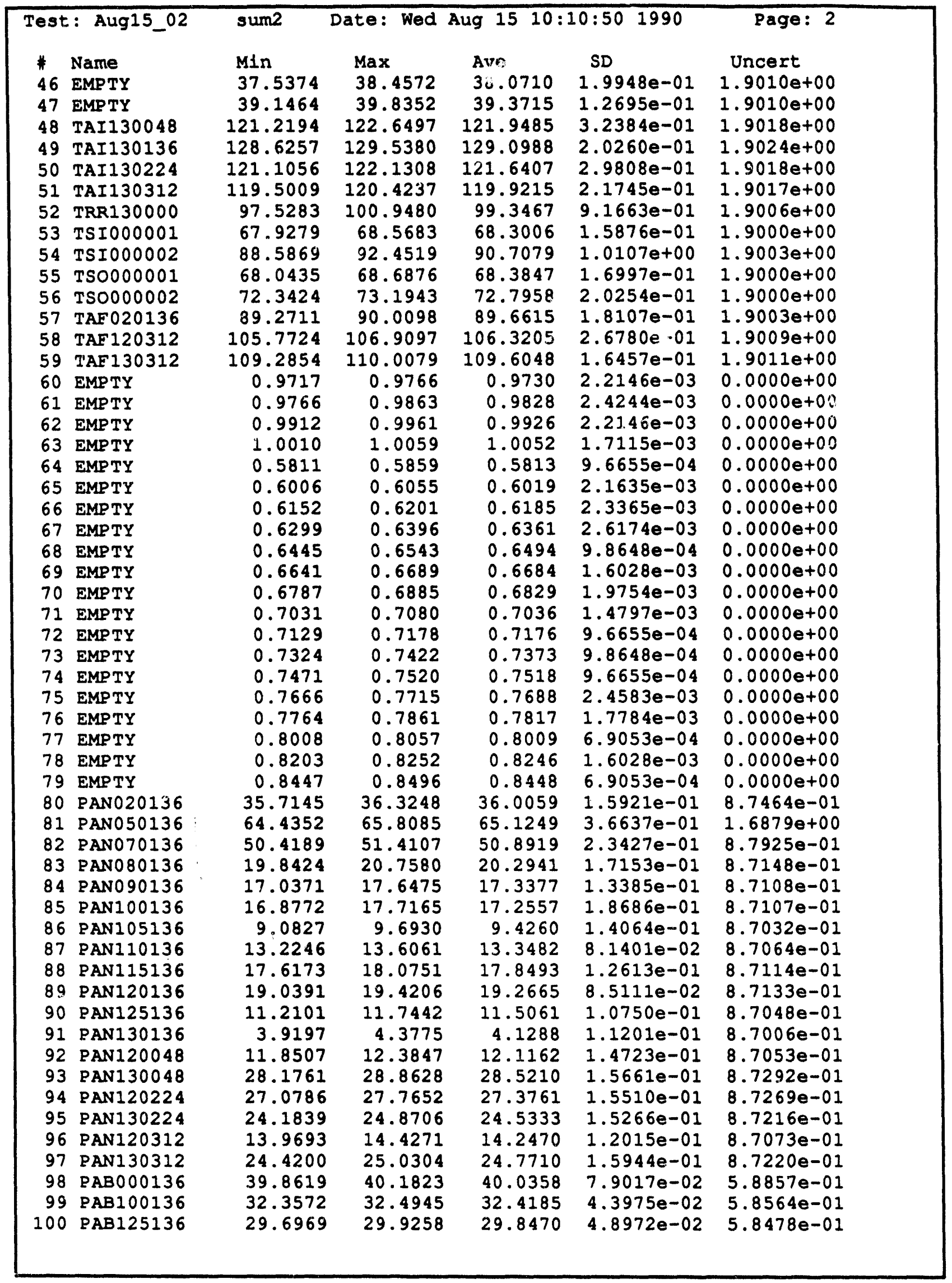




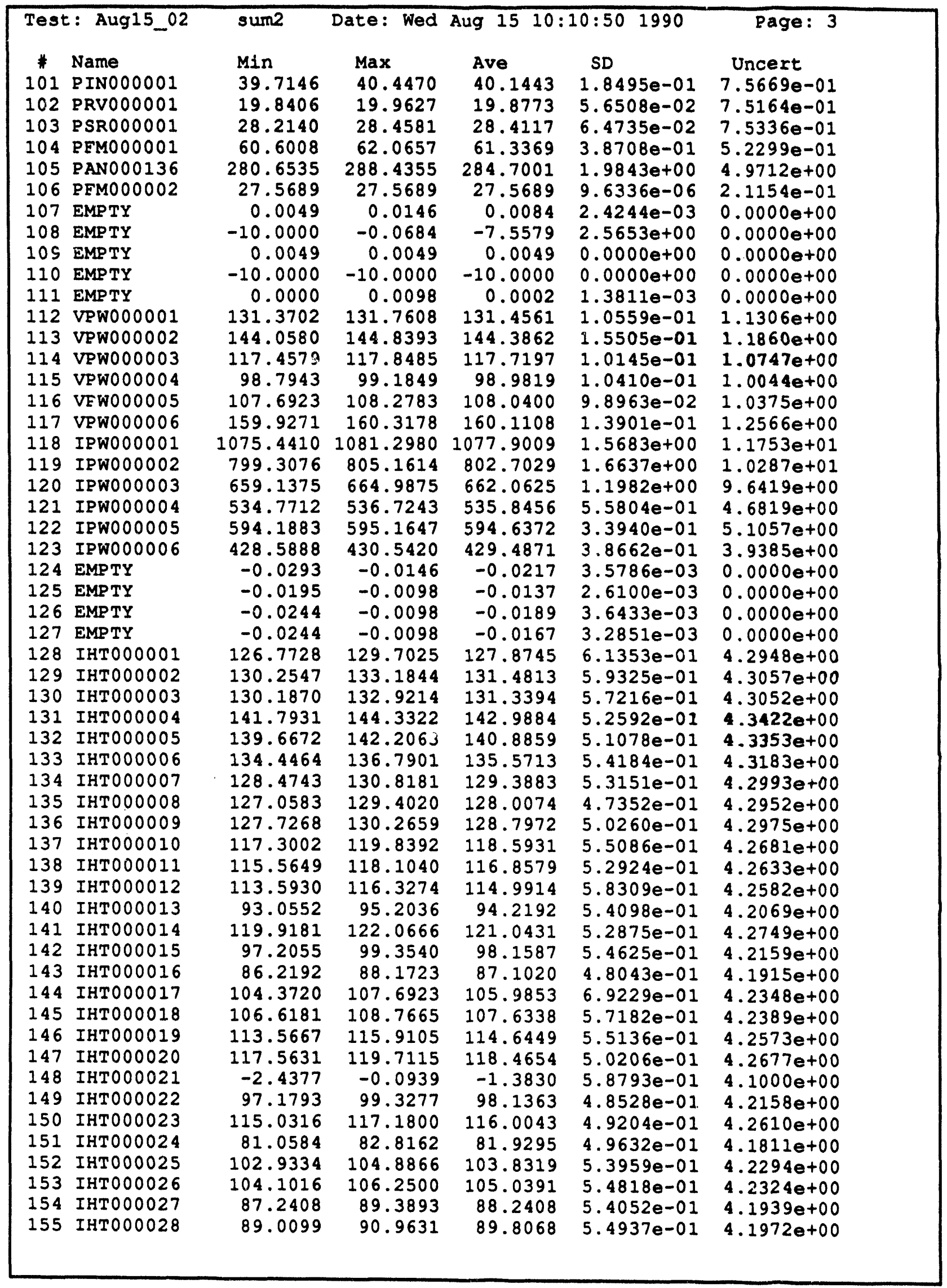




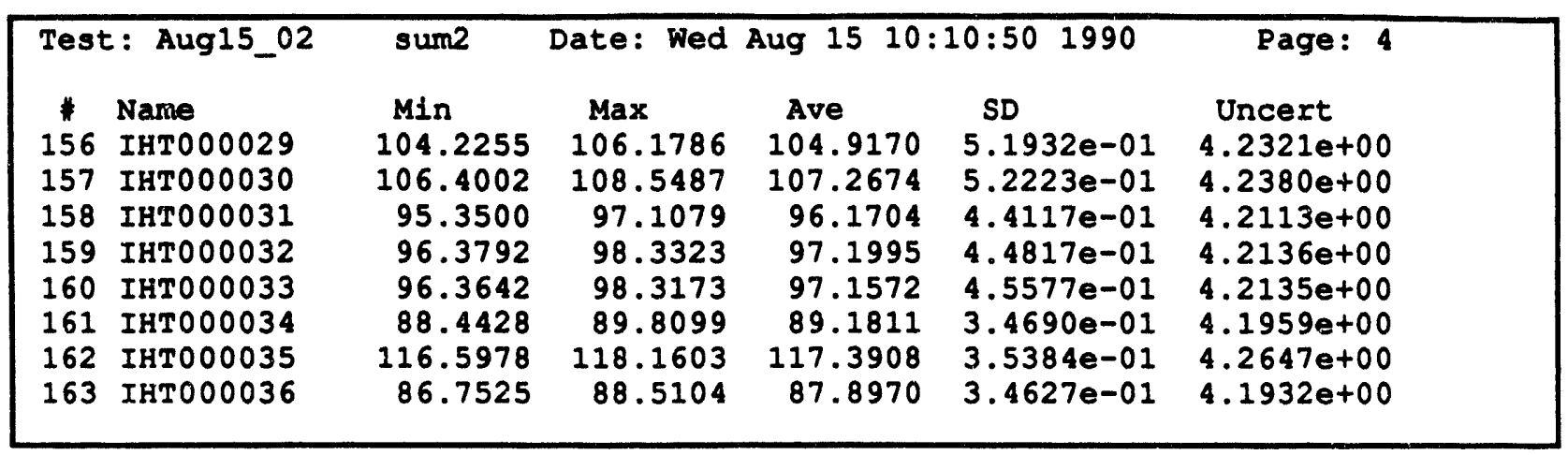




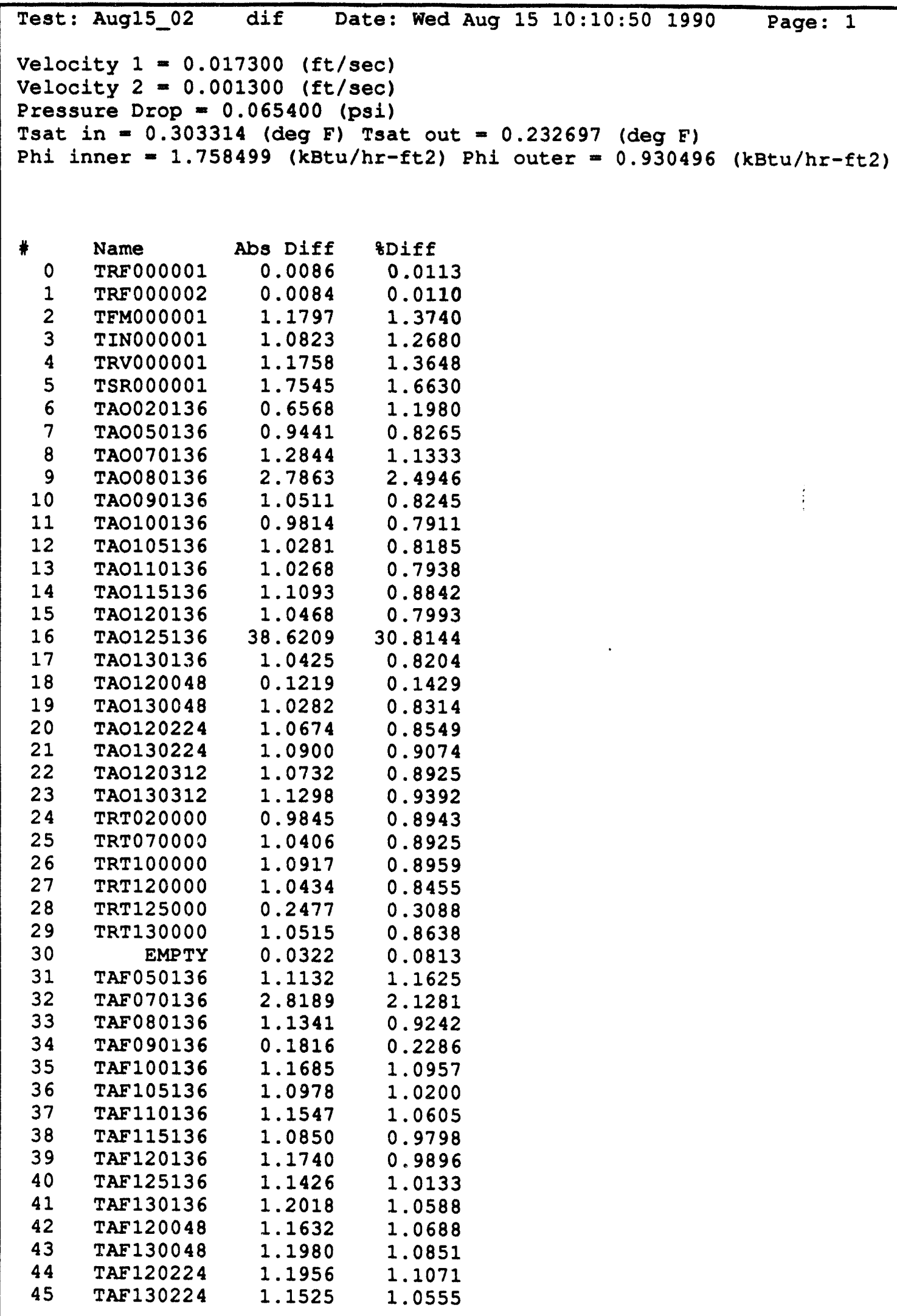




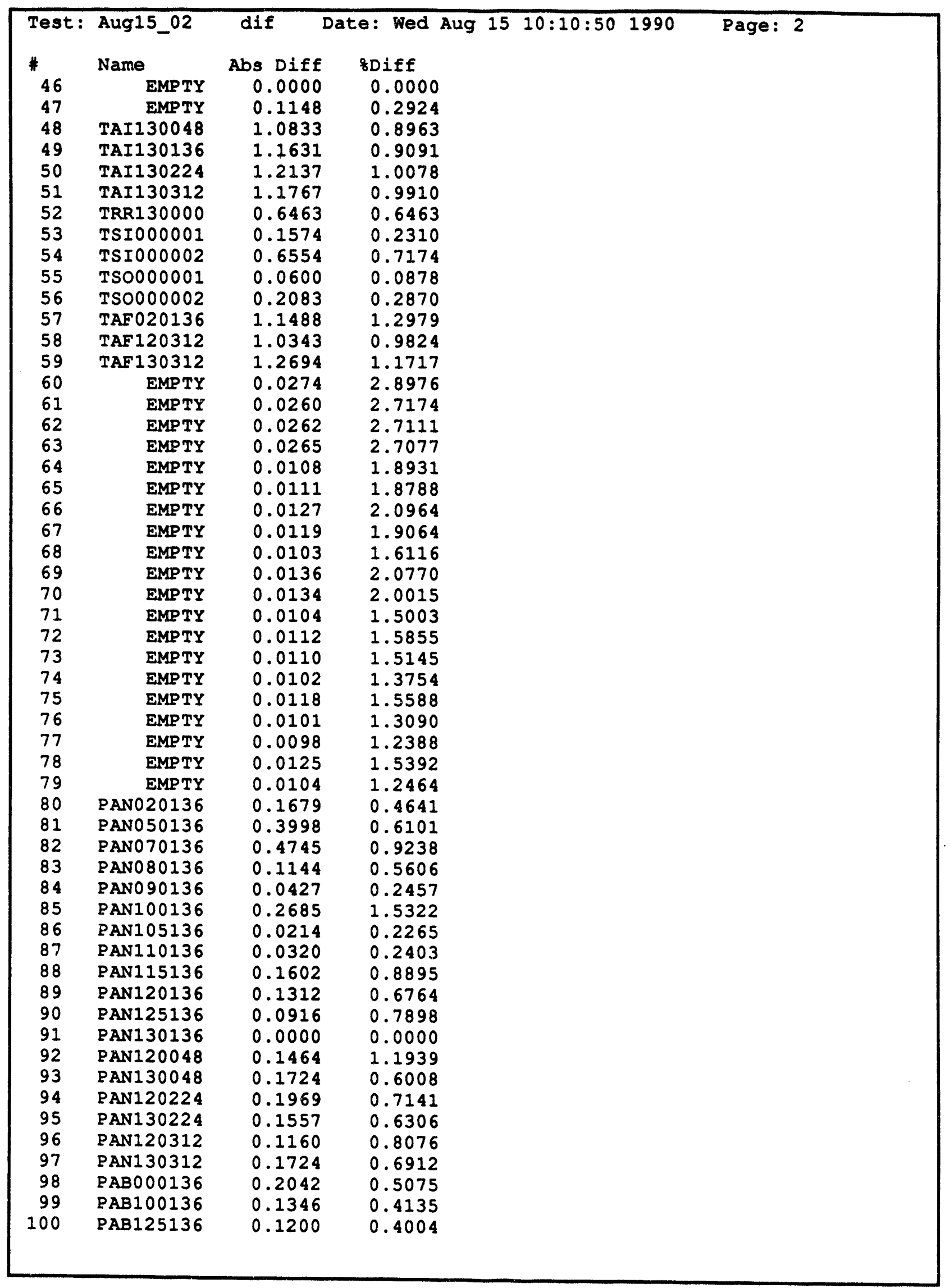




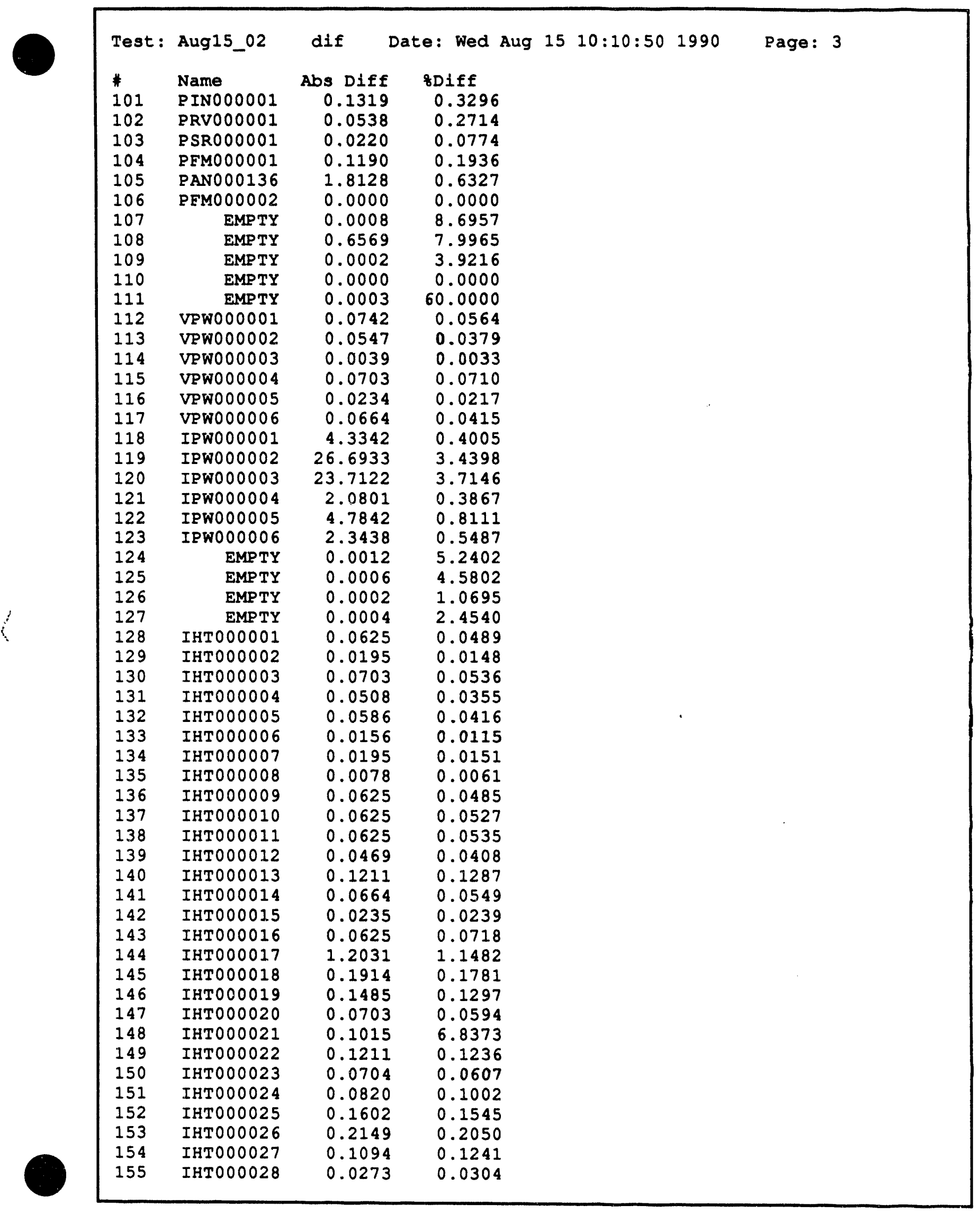




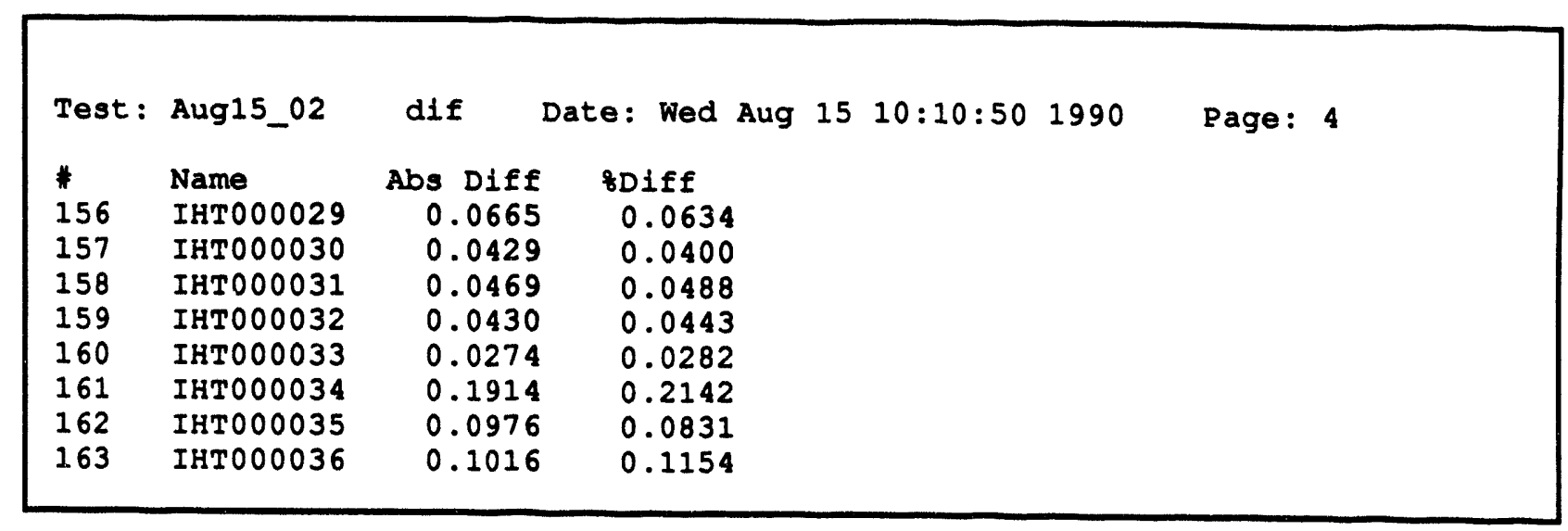




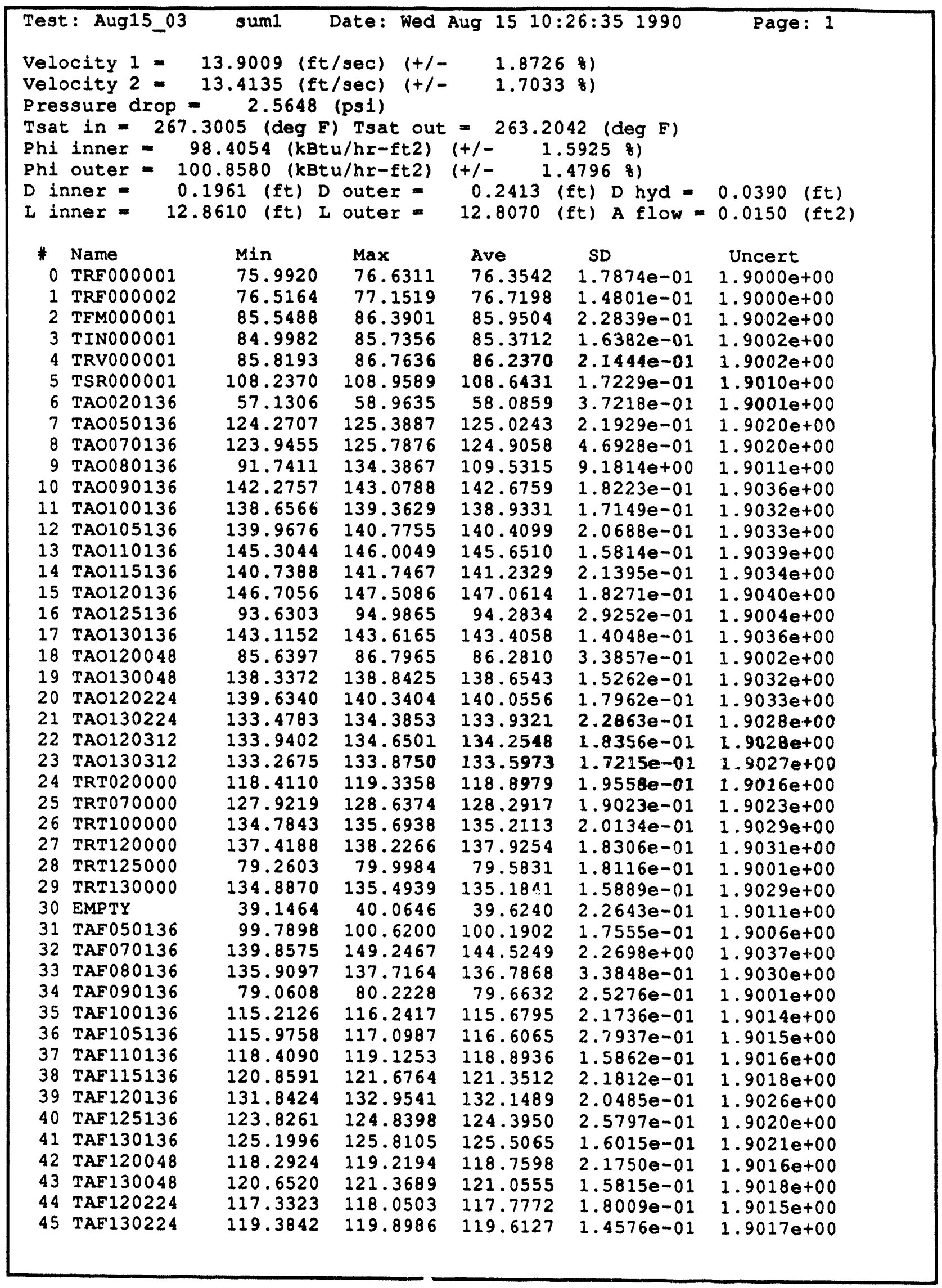




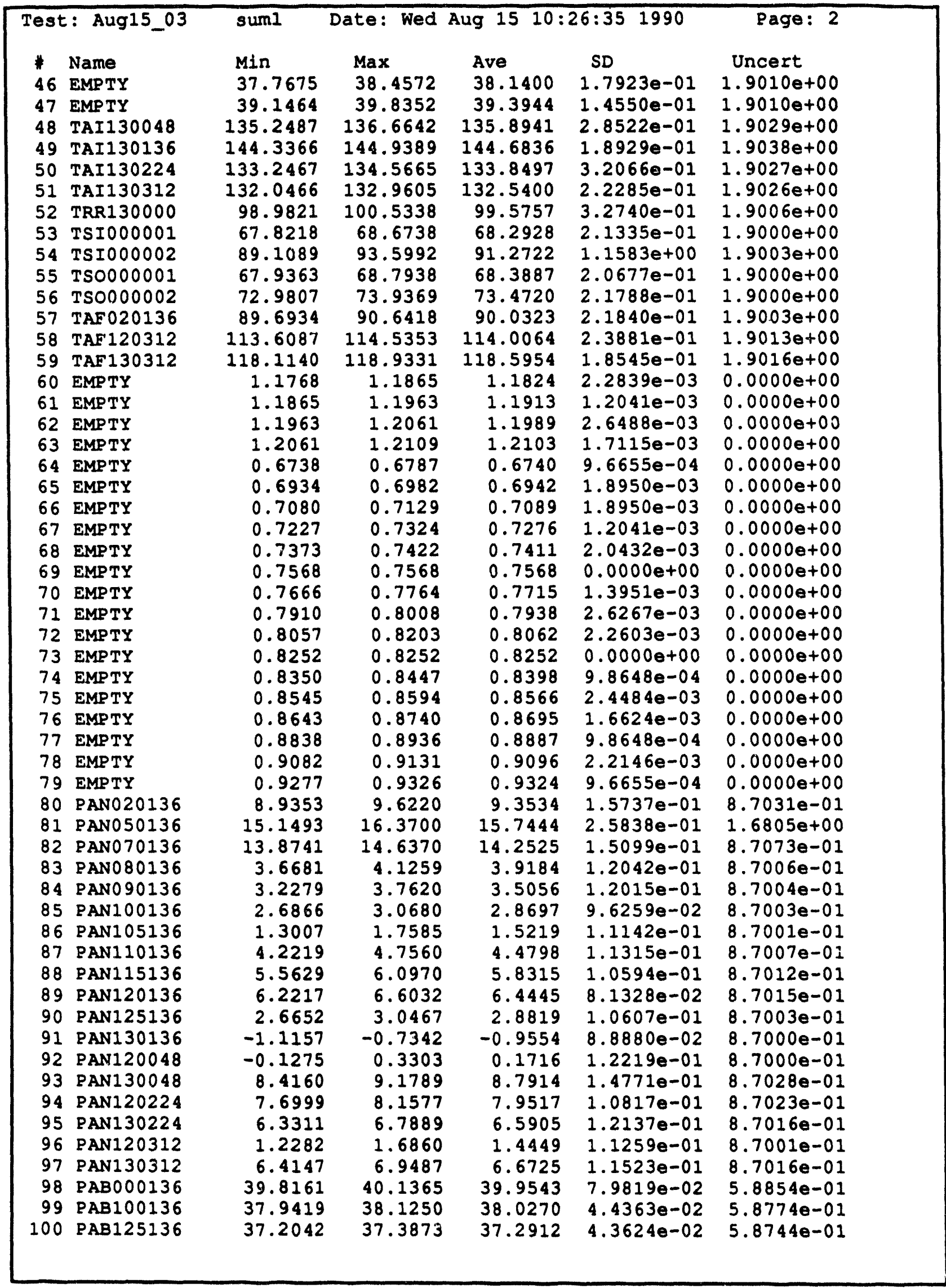




\begin{tabular}{|c|c|c|c|c|c|c|}
\hline \multicolumn{2}{|c|}{ Test: Aug15_03 } & suml & Date: Wed & Aug 151 & $6: 351990$ & Page: 3 \\
\hline 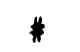 & Name & Min & $\operatorname{Max}$ & Ave & SD & Uncert \\
\hline 101 & PIN000001 & 39.4704 & 40.0808 & 39.7268 & $1.4848 e-01$ & $7.5655 e-01$ \\
\hline 102 & PRV000001 & 19.7186 & 20.2069 & 20.0408 & $8.8095 e-02$ & $7.5167 e-01$ \\
\hline 103 & PSR000001 & 36.3927 & 36.7589 & 36.6002 & $1.0243 \theta-01$ & $7.5556 e-01$ \\
\hline 104 & PEM000001 & 29.0151 & 30.1138 & 29.6230 & $2.2484 e-01$ & $5.0545 e-01$ \\
\hline 105 & PANO00136 & 67.7934 & 73.7443 & 71.0069 & $1.5898 \mathrm{e}+00$ & $4.9232 e+00$ \\
\hline 106 & PEMO00002 & 27.5689 & 27.5689 & 27.5689 & $9.6336 e-06$ & $2.1154 e-01$ \\
\hline 107 & EMPTY & 0.0049 & 0.0098 & 0.0067 & $2.3941 e-03$ & $0.0000 e+00$ \\
\hline 108 & EMPTY & -10.0000 & -0.3369 & -8.0381 & $2.3869 e+00$ & $0.0000 e+00$ \\
\hline 109 & EMPTY & 0.0000 & 0.0098 & 0.0049 & $9.8648 e-04$ & $0.0000 e+00$ \\
\hline 110 & EMPTY & -10.0000 & -10.0000 & -10.0000 & $0.0000 e+00$ & $0.0000 e+00$ \\
\hline 111 & EMPTY & 0.0000 & 0.0000 & 0.0000 & $0.0000 e+00$ & $0.0000 e+00$ \\
\hline 112 & VPW000001 & 131.3702 & 131.7608 & 131.6201 & $1.1864 e-01$ & $1.1313 e+00$ \\
\hline 113 & VPWO00002 & 144.2533 & 144.6440 & 144.4174 & $9.1354 e-02$ & $1.1861 \mathrm{e}+00$ \\
\hline 114 & VPW000003 & 117.6532 & 118.2392 & 117.8486 & $1.5784 e-01$ & $1.0752 e+00$ \\
\hline 115 & VPW0000004 & 98.9896 & 99.3802 & 99.1460 & $1.0440 e-01$ & $1.0050 e+00$ \\
\hline 116 & VPW000005 & 107.8876 & 108.2783 & 108.1338 & $1.1027 e-01$ & $1.0378 e+00$ \\
\hline 117 & VPW0000006 & 159.9271 & 160.5131 & 160.2007 & $1.1161 e-01$ & $1.2570 e+00$ \\
\hline 118 & IPW000001 & 1081.2980 & 1091.0597 & 1085.2030 & $2.7041 e+00$ & $1.1794 e+01$ \\
\hline 119 & IPW0 00002 & 773.9410 & 783.6974 & 779.5215 & $2.3983 e+00$ & $1.0175 e+01$ \\
\hline 120 & IPW000003 & 631.8375 & 639.6375 & 634.9575 & $2.0088 e+00$ & $9.5274 e+00$ \\
\hline 121 & IPW0 00004 & 534.2829 & 539.1657 & 536.5682 & $1.4446 e+00$ & $4.6870 e+00$ \\
\hline 122 & IPWO 00005 & 588.3295 & 590.2824 & 589.5308 & $5.2390 e-01$ & $5.0686 \mathrm{e}+00$ \\
\hline 123 & IPWO00006 & 425.6591 & 427.6123 & 426.5281 & $4.7607 e-01$ & $3.9184 e+00$ \\
\hline 124 & EMPTY & -0.0293 & -0.0098 & -0.0195 & $4.6270 e-03$ & $0.0000 e+00$ \\
\hline 125 & EMPTY & -0.0195 & -0.0049 & -0.0130 & $3.3613 e-03$ & $0.0000 e+00$ \\
\hline 126 & EMPTY & -0.0244 & -0.0098 & -0.0164 & $3.6593 e-03$ & $0.0000 e+00$ \\
\hline 127 & EMPTY & -0.0244 & -0.0098 & -0.0169 & $3.1521 e-03$ & $0.0000 e+00$ \\
\hline 128 & IHTO00001 & 126.7728 & 128.9213 & 127.7221 & $5.7725 e-01$ & $4.2943 e+00$ \\
\hline 129 & IHT000002 & 130.4500 & 132.5984 & 131.3602 & $5.5329 e-01$ & $4.3053 e+00$ \\
\hline 130 & IHT000003 & 130.3824 & 132.3355 & 131.2456 & $5.3972 e-01$ & $4.3049 e+00$ \\
\hline 131 & IHTO00004 & 141.9884 & 144.1369 & 142.9376 & $5.8128 e-01$ & $4.3420 e+00$ \\
\hline 132 & IHTO00005 & 140.0578 & 142.0110 & 140.8390 & $5.5524 e-01$ & $4.3352 e+00$ \\
\hline 133 & IHT000006 & 134.6417 & 136.5948 & 135.5635 & $5.5248 e-01$ & $4.3183 e+00$ \\
\hline 134 & IHTO00007 & 128.2790 & 130.6227 & 129.3493 & $5.5558 e-01$ & $4.2992 e+00$ \\
\hline 135 & IHT 000008 & 127.0583 & 129.2067 & 127.9450 & $5.2230 e-01$ & $4.2950 e+00$ \\
\hline 136 & IHT000009 & 127.9222 & 129.6800 & 128.6956 & $5.3081 e-01$ & $4.2972 e+00$ \\
\hline 137 & IHTO00010 & 117.4955 & 119.6439 & 118.6088 & $4.7062 e-01$ & $4.2681 e+00$ \\
\hline 138 & IHTO00011 & 115.5649 & 117.9087 & 116.8267 & $4.6890 e-01$ & $4.2632 e+00$ \\
\hline 139 & IHT000012 & 113.7883 & 115.9368 & 114.9679 & $4.8802 e-01$ & $4.2581 e+00$ \\
\hline 140 & IHTO00013 & 93.0552 & 95.2036 & 94.2388 & $5.2409 e-01$ & $4.2069 e+00$ \\
\hline 141 & IHT000014 & 120.1134 & 122.2619 & 121.0861 & $5.0914 e-01$ & $4.2751 e+00$ \\
\hline 142 & IHTO00015 & 97.0102 & 99.3540 & 98.2134 & $5.2698 e-01$ & $4.2160 e+00$ \\
\hline 143 & IHT000016 & 86.0239 & 88.1723 & 87.1411 & $4.7848 e-01$ & $4.1916 e+00$ \\
\hline 144 & IHT000017 & 103.7861 & 107.8876 & 105.7080 & $1.1418 \mathrm{e}+00$ & $4.2341 e+00$ \\
\hline 145 & IHT000018 & 106.4228 & 108.5712 & 107.5713 & $5.8079 e-01$ & $4.2388 e+00$ \\
\hline 146 & IHTO00019 & 113.7620 & 116.3011 & 114.6058 & $4.8398 e-01$ & $4.2572 e+00$ \\
\hline 147 & IHT000020 & 117.5631 & 119.7115 & 118.4146 & $4.7147 e-01$ & $4.2676 e+00$ \\
\hline 148 & IHT000021 & -2.4377 & 0.1014 & -1.3869 & $5.8778 e-01$ & $4.1000 e+00$ \\
\hline 149 & IHTO000022 & 97.1793 & 99.7183 & 98.0738 & $5.0700 e-01$ & $4.2157 e+00$ \\
\hline 150 & IHTO000023 & 115.0316 & 117.1800 & 115.9652 & $5.1189 e-01$ & $4.2608 \mathrm{e}+00$ \\
\hline 151 & IHTO00024 & 80.8631 & 82.8162 & 81.8826 & $4.8695 e-01$ & $4.1810 e+00$ \\
\hline 152 & IHTO00025 & 102.7381 & 105.0819 & 103.8631 & $5.4328 e-01$ & $4.2295 e+00$ \\
\hline 153 & IHTO00026 & 104.1016 & 106.0547 & 105.1094 & $4.9499 e-01$ & $4.2326 \mathrm{e}+00$ \\
\hline 154 & IHTO00027 & 87.2408 & 89.1940 & 88.2759 & $4.6228 e-01$ & $4.1940 e+00$ \\
\hline 155 & IHTO00028 & 88.8146 & 91.1584 & 89.8420 & $5.8502 e-01$ & $4.1973 \mathrm{e}+00$ \\
\hline
\end{tabular}




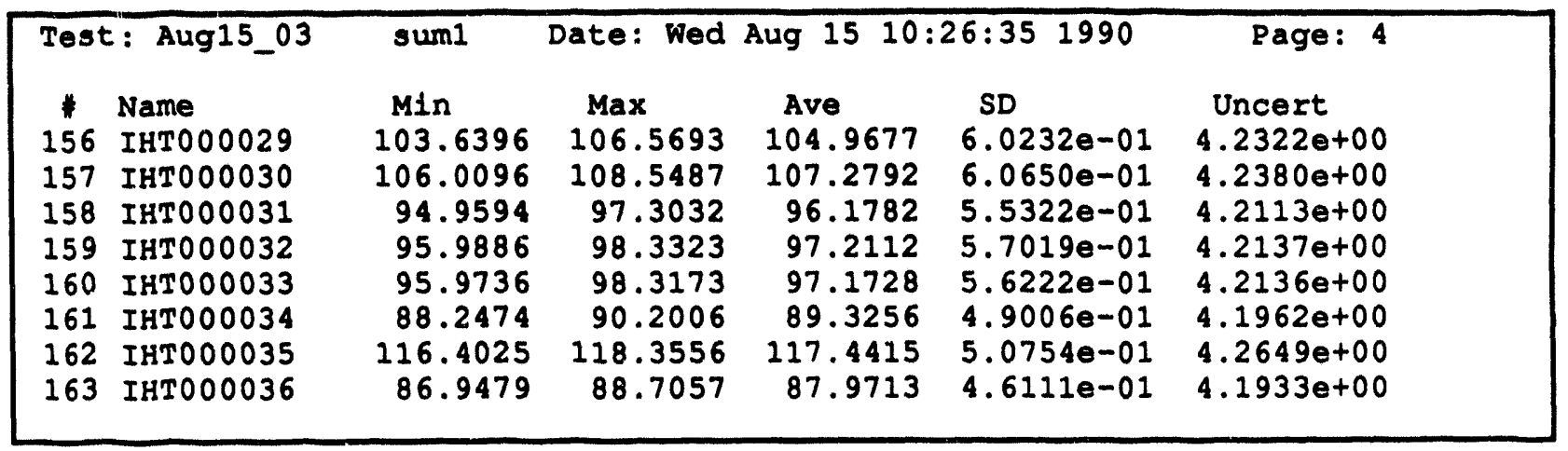




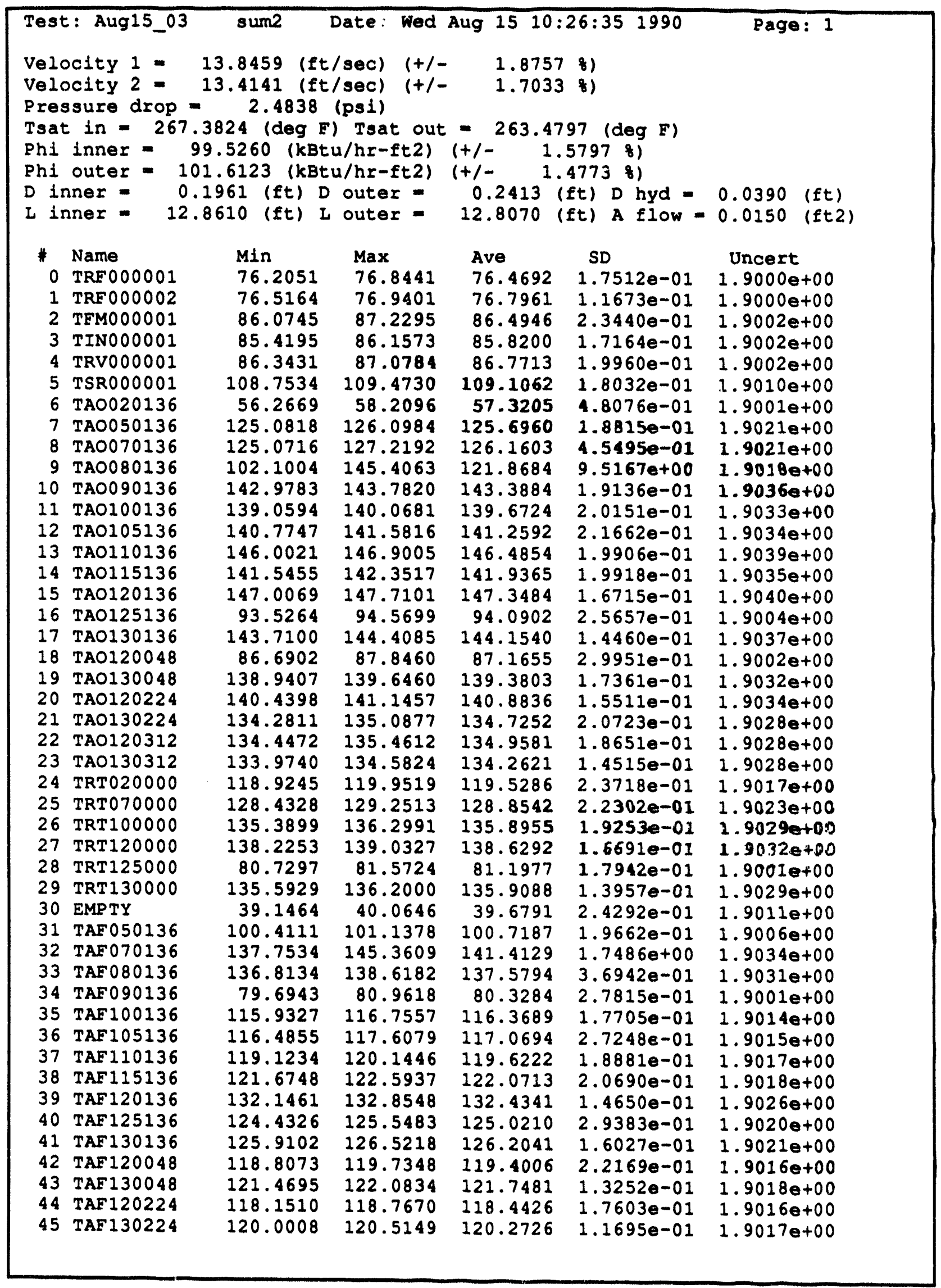




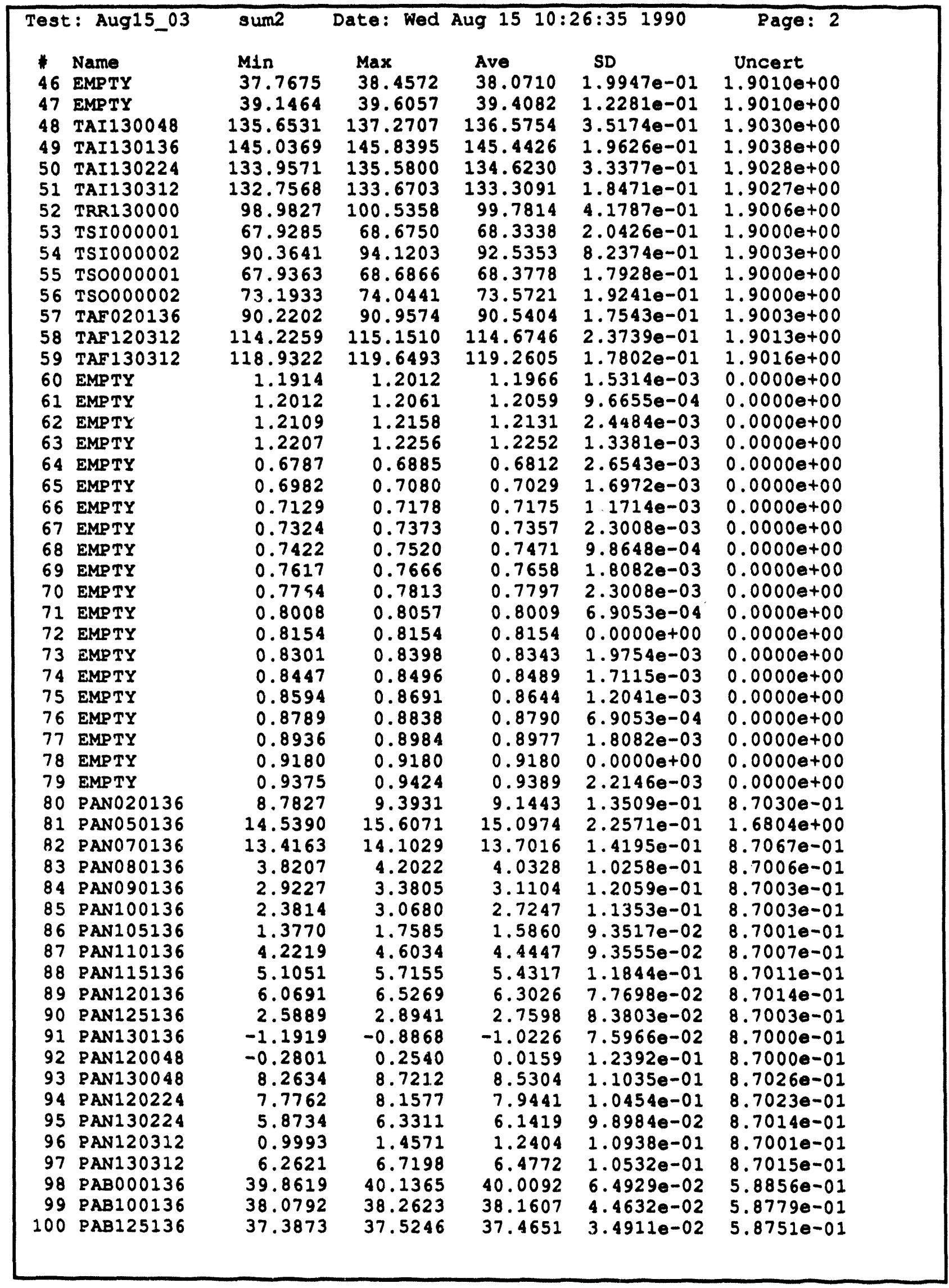




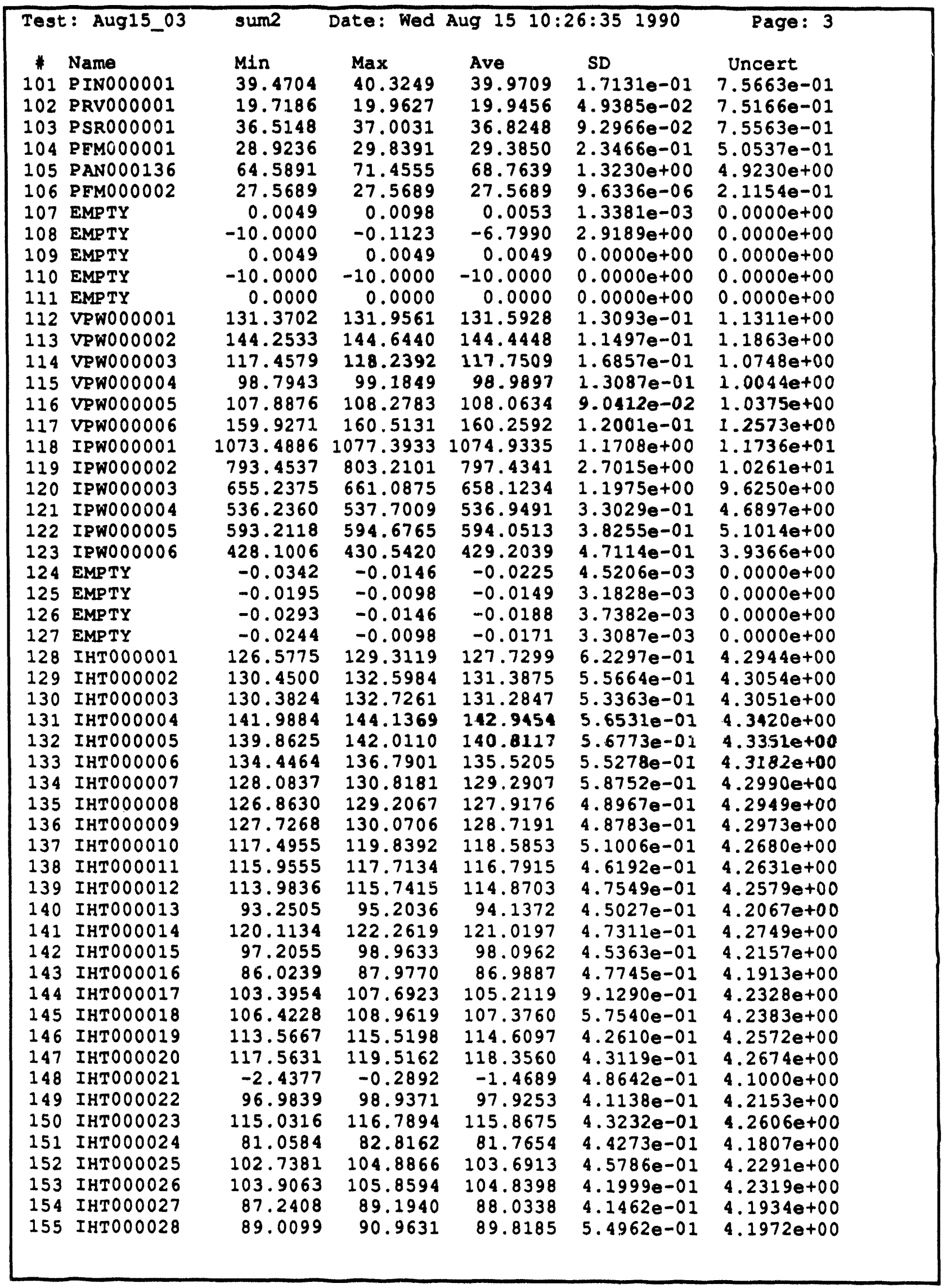




\begin{tabular}{|c|c|c|c|c|c|c|}
\hline Tes & $t:$ Aug15_03 & sum2 & Date: Wed & Aug 1510 & $26: 351990$ & Page: 4 \\
\hline $\begin{array}{l}1 \\
156 \\
157 \\
158 \\
159 \\
160 \\
161 \\
162 \\
163\end{array}$ & $\begin{array}{l}\text { Name } \\
\text { IHTO00029 } \\
\text { IHTO00030 } \\
\text { IHTO00031 } \\
\text { IHTOOOO32 } \\
\text { IHTO0O033 } \\
\text { IHTO00034 } \\
\text { IHTO00035 } \\
\text { IHTO00036 }\end{array}$ & $\begin{array}{r}\text { Min } \\
104.0302 \\
106.4002 \\
95.1547 \\
96.3792 \\
96.3642 \\
88.4428 \\
116.5978 \\
87.1432\end{array}$ & $\begin{array}{r}\text { Max } \\
106.3740 \\
108.9393 \\
97.4985 \\
98.7230 \\
98.9032 \\
90.5912 \\
118.7462 \\
89.2916\end{array}$ & $\begin{array}{r}\text { Ave } \\
104.9677 \\
107.2752 \\
96.1860 \\
97.1839 \\
97.1533 \\
89.3100 \\
117.4923 \\
87.9908\end{array}$ & $\begin{array}{l}\text { SD } \\
5.9973 e-01 \\
6.0263 e-01 \\
5.6777 e-01 \\
5.8346 e-01 \\
5.9837 e-01 \\
5.3403 e-01 \\
5.1462 e-01 \\
4.8103 e-01\end{array}$ & $\begin{array}{c}\text { Uncert } \\
4.2322 \mathrm{e}+00 \\
4.2380++00 \\
4.2113 \mathrm{e}+00 \\
4.2136 \mathrm{e}+00 \\
4.2135 \mathrm{e}+00 \\
4.1961 \mathrm{e}+00 \\
4.2650 \mathrm{e}+00 \\
4.1934 \mathrm{e}+00\end{array}$ \\
\hline
\end{tabular}




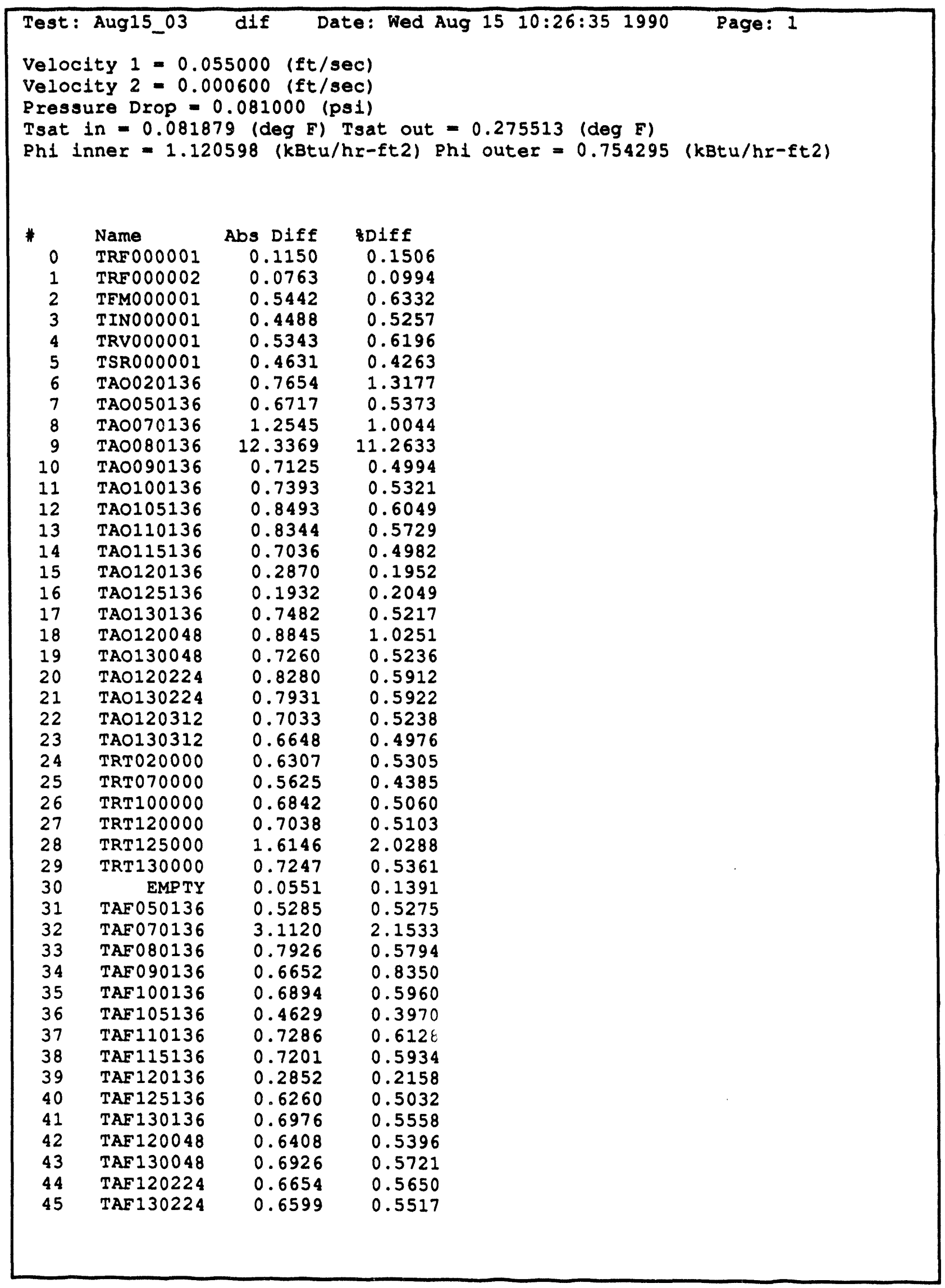




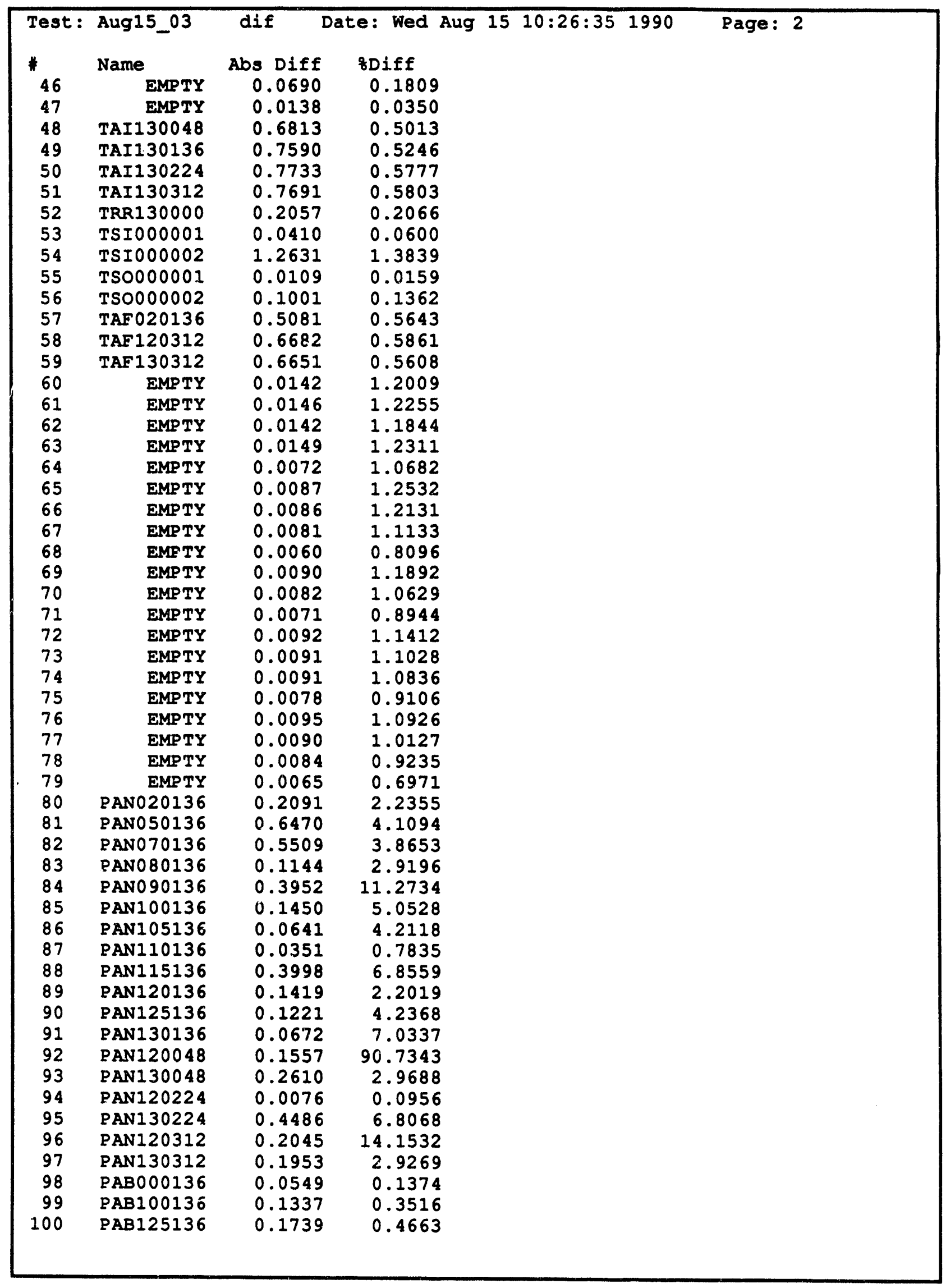




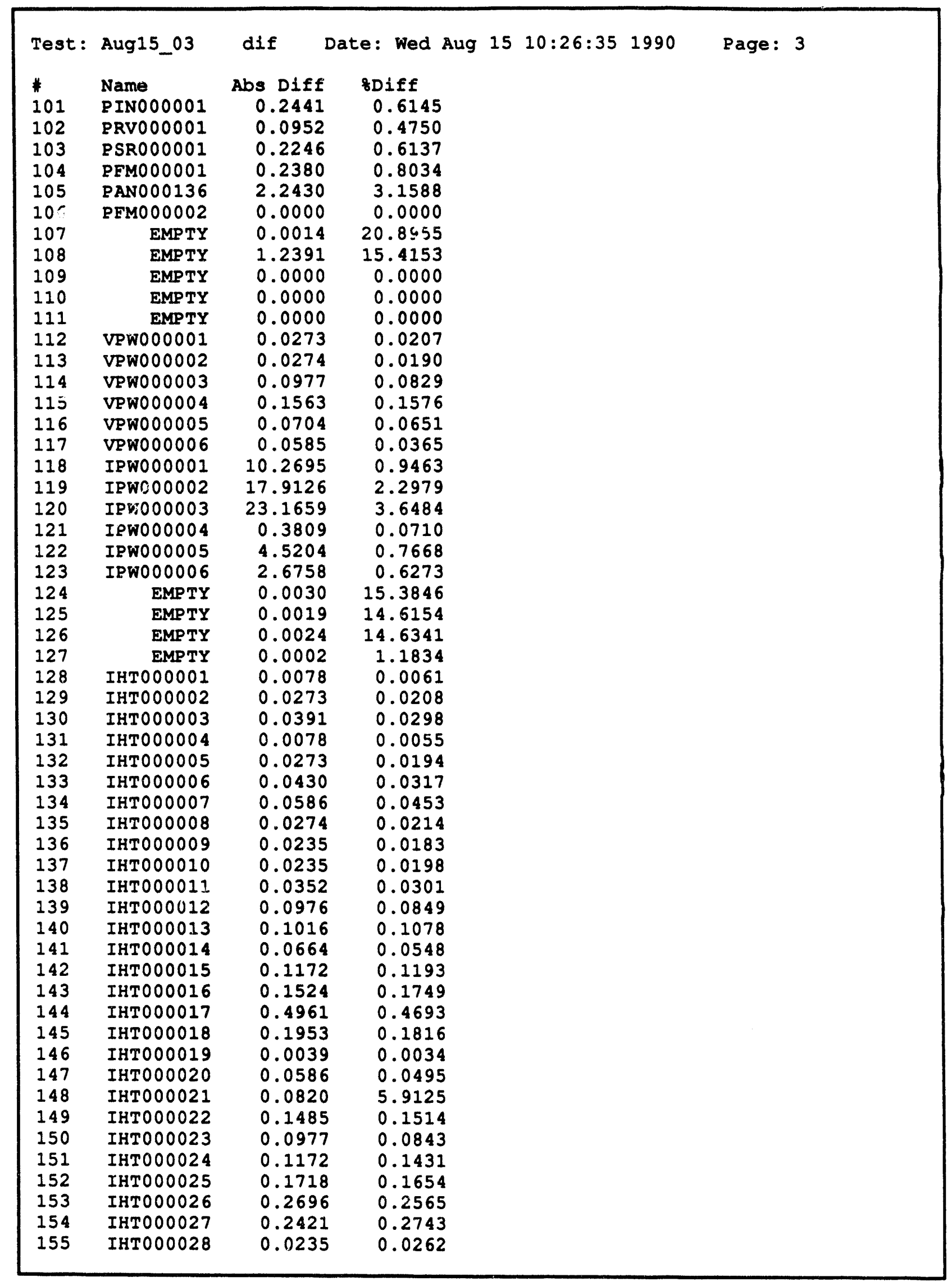


Test: Aug15_03 dif Date: Wed Aug 15 10:26:35 1990 Page: 4

$\begin{array}{ll}\text { Abs Diff } & \text { 8Diff } \\ 0.0000 & 0.0000 \\ 0.0040 & 0.0037 \\ 0.0078 & 0.0081 \\ 0.0273 & 0.0281 \\ 0.0195 & 0.0201 \\ 0.0156 & 0.0175 \\ 0.0508 & 0.0433 \\ 0.0195 & 0.0222\end{array}$

Name

IHT000029

IHTOO0030

IHT000031

IHT000032

IHTO00033

IHT000034 $\begin{array}{ll}162 & \text { IHTO00035 } \\ 163 & \text { IHTO00036 }\end{array}$

$\begin{array}{ll}162 & \text { IHTO00035 } \\ 163 & \text { IHTO00036 }\end{array}$

Abs Diff \&Diff

0.0037

0.0081

0.0281

017

0.0433

0.0508 


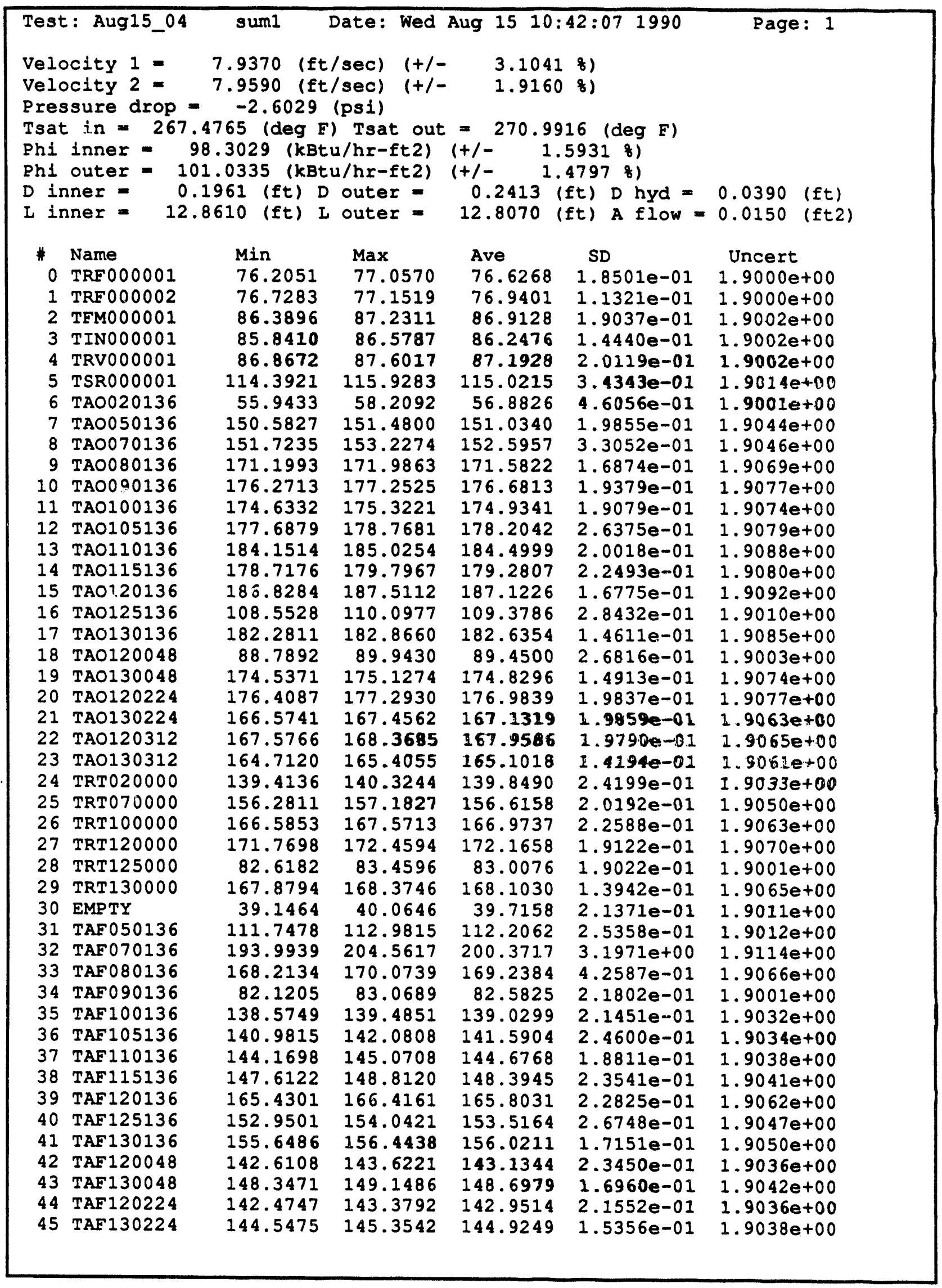




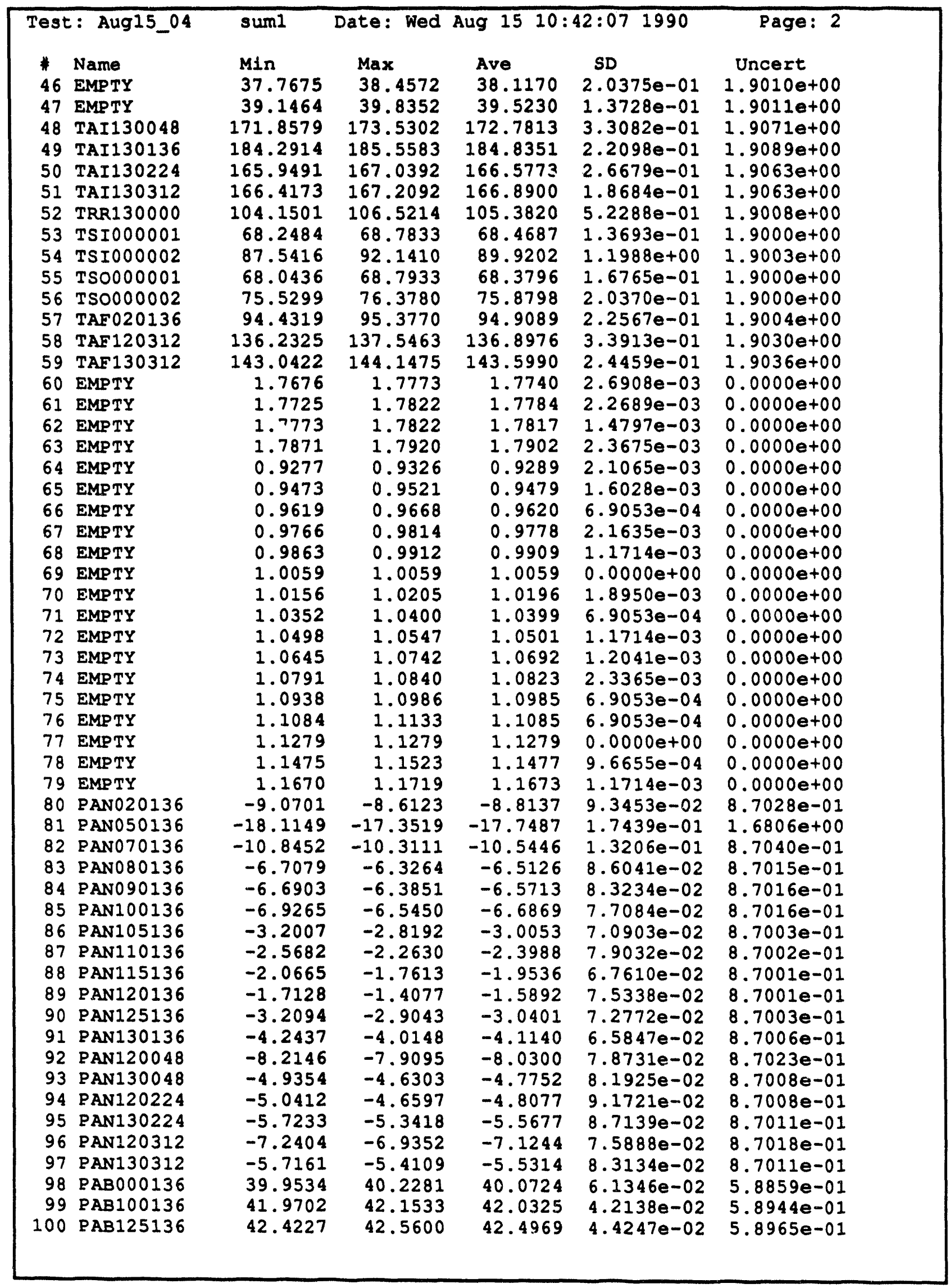




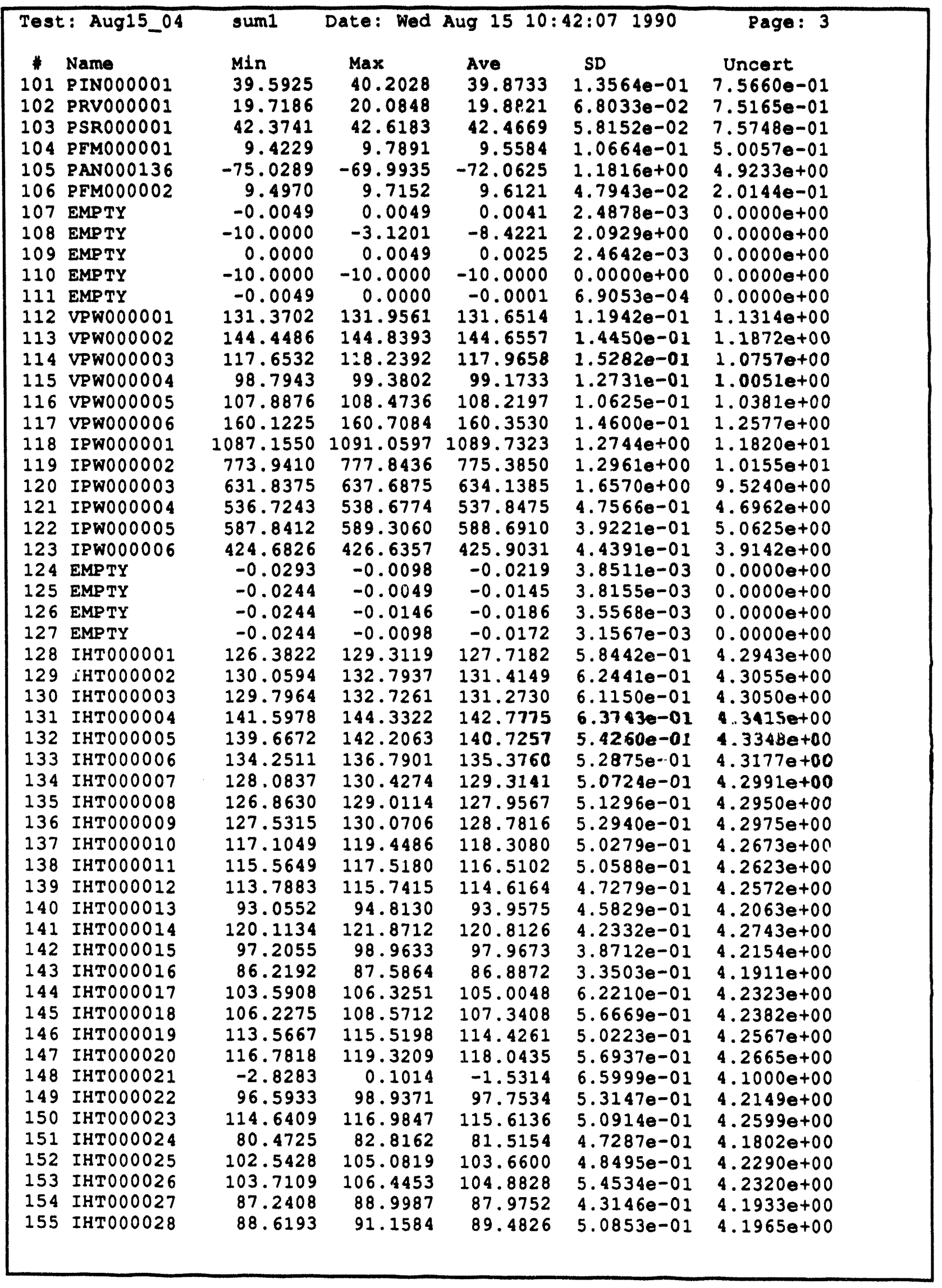




\begin{tabular}{|c|c|c|c|c|c|c|}
\hline $\begin{array}{l}1 \\
156 \\
157 \\
158 \\
159 \\
160 \\
161 \\
162 \\
163\end{array}$ & 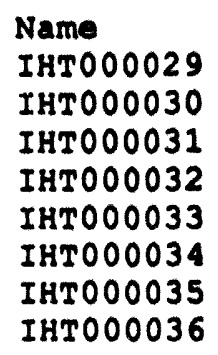 & $\begin{array}{r}\text { M1n } \\
103.8349 \\
106.0096 \\
94.9594 \\
95.7933 \\
95.7782 \\
88.2474 \\
116.2072 \\
87.1432\end{array}$ & $\begin{array}{r}\text { Max } \\
105.7880 \\
107.7674 \\
96.7172 \\
98.1370 \\
97.9267 \\
90.3959 \\
118.5509 \\
89.4869\end{array}$ & $\begin{array}{r}\text { Ave } \\
104.6826 \\
106.9627 \\
95.8384 \\
96.8948 \\
96.8369 \\
89.0756 \\
117.1954 \\
87.8111\end{array}$ & $\begin{array}{l}\text { SD } \\
5.0007 e-01 \\
4.4754 \theta-01 \\
4.6563 e-01 \\
5.0186 \theta-01 \\
4.9926 e-01 \\
4.4215 e-01 \\
4.4888 e-01 \\
4.6201 e-01\end{array}$ & $\begin{array}{c}\text { Uncert } \\
4.2315 e+00 \\
4.2372 e+00 \\
4.2105 e+00 \\
4.2129 e+00 \\
4.2128 e+00 \\
4.1956 e+00 \\
4.2642 e+00 \\
4.1930 e+00\end{array}$ \\
\hline
\end{tabular}




\begin{tabular}{|c|c|c|c|c|c|c|}
\hline \multicolumn{7}{|c|}{ 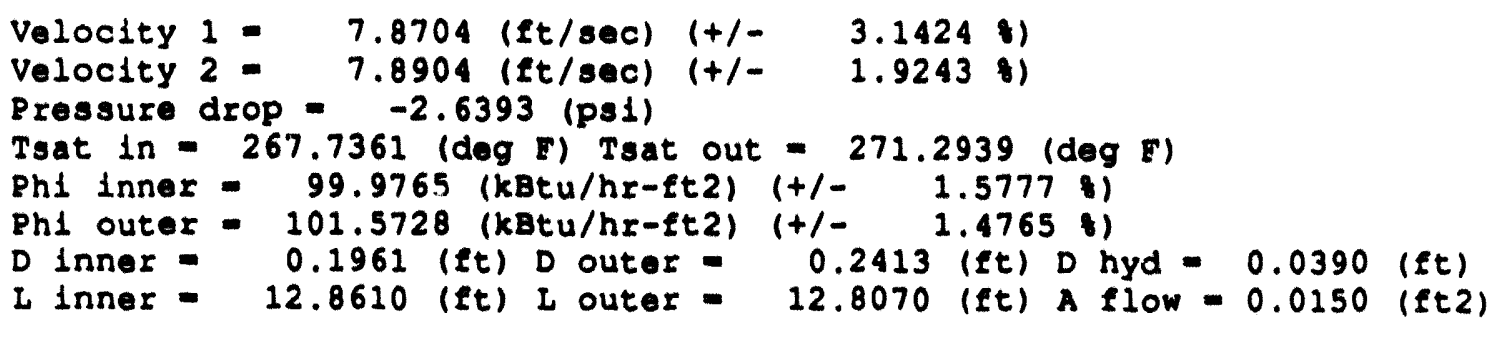 } \\
\hline $\begin{array}{l}* \\
0 \\
1 \\
2 \\
3 \\
4 \\
5 \\
6 \\
7 \\
8 \\
9 \\
10 \\
11 \\
12 \\
13 \\
14 \\
15 \\
16 \\
17 \\
18 \\
19 \\
20 \\
21 \\
22 \\
23 \\
24 \\
25 \\
26 \\
27 \\
28 \\
29 \\
30 \\
31 \\
32 \\
33 \\
34 \\
35 \\
36 \\
37 \\
38 \\
39 \\
40 \\
41 \\
42 \\
43 \\
44 \\
45\end{array}$ & 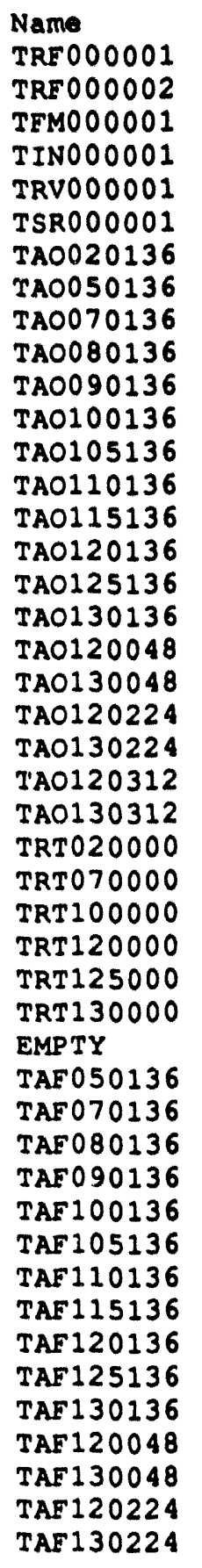 & $\begin{array}{r}\text { Min } \\
76.4181 \\
76.7283 \\
87.2295 \\
86.8937 \\
87.5997 \\
116.9478 \\
58.5320 \\
151.7753 \\
153.4276 \\
172.3736 \\
177.6413 \\
175.7120 \\
178.9633 \\
185.4093 \\
179.7959 \\
187.8991 \\
107.6246 \\
183.4362 \\
90.3606 \\
175.7112 \\
177.8792 \\
168.0401 \\
168.7627 \\
165.7973 \\
140.2231 \\
157.1829 \\
167.7666 \\
172.8511 \\
83.7704 \\
168.9625 \\
39.1464 \\
112.7733 \\
183.5484 \\
169.5836 \\
82.5418 \\
139.7878 \\
142.3787 \\
145.5696 \\
149.0117 \\
166.4141 \\
154.1397 \\
156.9399 \\
143.9257 \\
149.5476 \\
143.3783 \\
145.7579\end{array}$ & $\begin{array}{r}\text { Max } \\
77.0570 \\
77.3636 \\
88.1741 \\
87.5250 \\
88.6481 \\
117.9705 \\
60.4706 \\
152.4732 \\
154.3289 \\
172.9644 \\
178.3275 \\
176.6932 \\
179.9449 \\
186.1873 \\
180.9725 \\
188.7760 \\
108.6576 \\
184.2130 \\
91.4084 \\
176.4964 \\
178.5660 \\
168.8274 \\
169.5536 \\
166.6863 \\
141.2334 \\
158.0831 \\
168.4574 \\
173.9329 \\
84.5058 \\
169.5554 \\
40.0646 \\
113.6978 \\
201.3013 \\
171.5434 \\
83.5958 \\
140.6971 \\
143.5775 \\
146.2713 \\
150.2102 \\
167.6947 \\
155.1301 \\
157.6359 \\
144.9360 \\
150.2484 \\
144.5842 \\
146.5644\end{array}$ & $\begin{array}{r}\text { Ave } \\
76.6992 \\
77.0333 \\
87.7710 \\
87.1294 \\
88.0987 \\
117.4598 \\
59.4069 \\
152.1454 \\
153.8002 \\
172.6844 \\
177.9983 \\
176.1755 \\
179.4502 \\
185.7710 \\
180.5449 \\
188.4059 \\
108.1452 \\
183.8165 \\
90.9392 \\
176.0724 \\
178.1895 \\
168.4686 \\
169.1148 \\
166.2598 \\
140.7607 \\
157.5830 \\
168.0670 \\
173.4611 \\
84.1866 \\
169.3256 \\
39.6607 \\
113.2871 \\
194.4525 \\
170.6378 \\
83.2123 \\
140.2767 \\
142.9141 \\
145.8988 \\
149.6189 \\
167.1079 \\
154.6863 \\
157.2904 \\
144.4430 \\
149.9196 \\
143.9130 \\
146.2699\end{array}$ & $\begin{array}{l}\text { SD } \\
1.6906 e-01 \\
1.2950 e-01 \\
2.1443 e-01 \\
1.4037 e-01 \\
2.2744 \theta-01 \\
2.4861 e-01 \\
4.2388 e-01 \\
1.8324 \theta-01 \\
2.4782 e-01 \\
1.7107 e-01 \\
1.5680 e-01 \\
2.0701 e-01 \\
2.1616 e-01 \\
1.9807 e-01 \\
2.5869 e-01 \\
2.0075 e-01 \\
2.5436 e-01 \\
1.5646 e-01 \\
2.6774 e-01 \\
1.5283 e-01 \\
1.5143 e-01 \\
2.1940 e-01 \\
2.1971 e-01 \\
1.8985 e-01 \\
2.4619 e-01 \\
2.0208 e-01 \\
1.7927 e-01 \\
2.1302 e-01 \\
1.9432 e-01 \\
1.5112 e-01 \\
2.2510 e-01 \\
2.3365 e-01 \\
4.0917 e+00 \\
4.2091 e-01 \\
3.0603 e-01 \\
2.1918 e-01 \\
2.3936 e-01 \\
1.7055 e-01 \\
2.6063 e-01 \\
2.7505 e-01 \\
2.3056 e-01 \\
1.4793 e-01 \\
2.3863 e-01 \\
1.7977 e-01 \\
2.3873 e-01 \\
1.9184 e-01\end{array}$ & $\begin{array}{l}\text { Uncert } \\
1.9000 e+00 \\
1.9000 e+00 \\
1.9002 e+00 \\
1.9002 e+00 \\
1.9002 e+00 \\
1.9015 e+00 \\
1.9001 e+00 \\
1.9045 e+00 \\
1.9047 e+00 \\
1.9071 e+00 \\
1.9078 e+00 \\
1.9076 e+00 \\
1.9081 e+00 \\
1.9090 e+00 \\
1.9082 e+00 \\
1.9094 e+00 \\
1.9010 e+00 \\
1.9087 e+00 \\
1.9003 e+00 \\
1.9076 e+00 \\
1.9079 e+00 \\
1.9065 e+00 \\
1.9066 e+00 \\
1.9062 e+00 \\
1.9034 e+00 \\
1.9052 e+00 \\
1.9065 e+00 \\
1.9072 e+00 \\
1.9001 e+00 \\
1.9066 e+00 \\
1.9011 e+00 \\
1.9013 e+00 \\
1.9104 e+00 \\
1.9068 e+00 \\
1.9001 e+00 \\
1.9033 e+00 \\
1.9036 e+00 \\
1.9039 e+00 \\
1.9043 e+00 \\
1.9063 e+00 \\
1.9048 e+00 \\
1.9051 e+00 \\
1.9037 e+00 \\
1.9043 e+00 \\
1.9037 e+00 \\
1.9039 e+00\end{array}$ \\
\hline
\end{tabular}




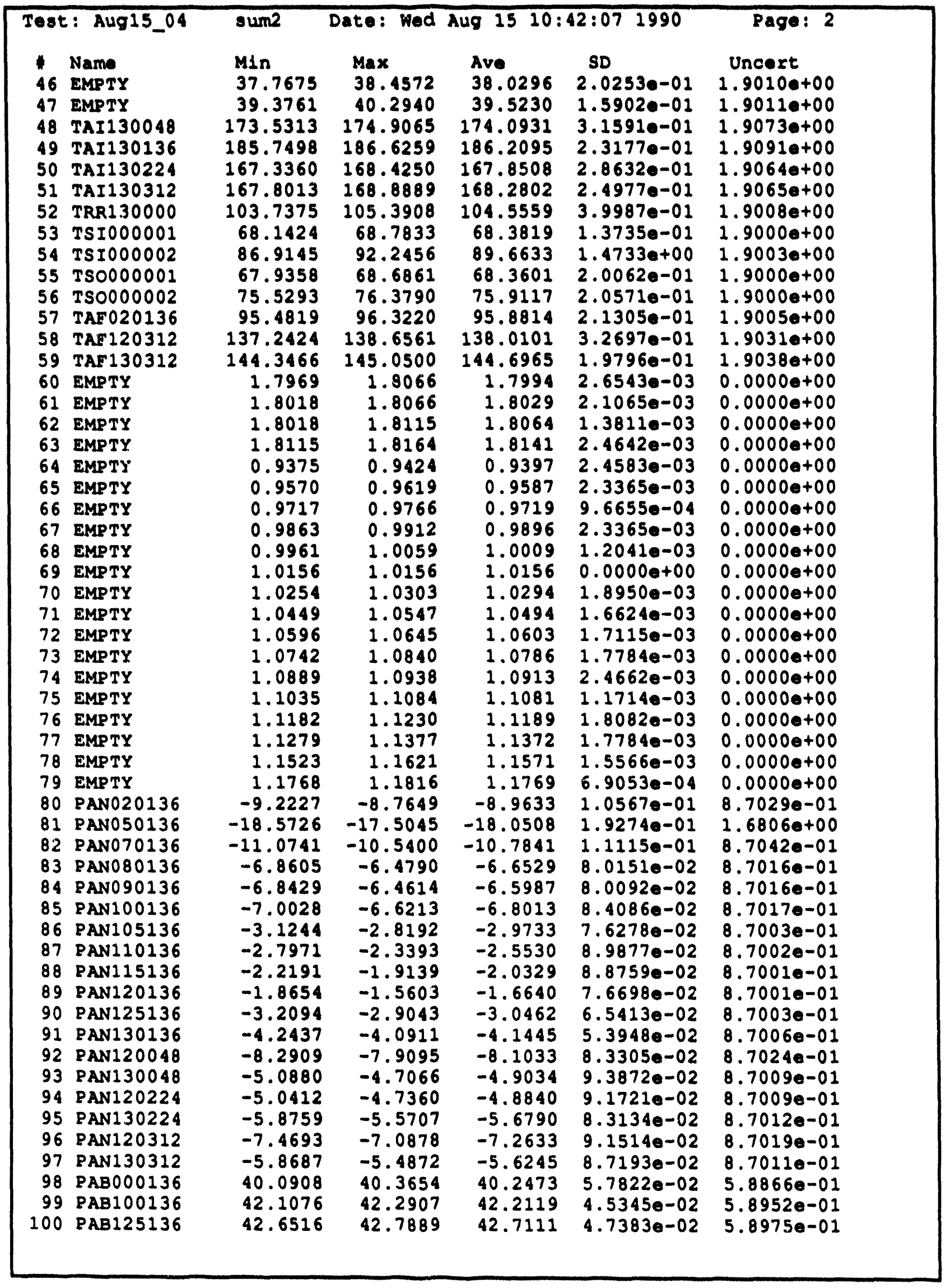




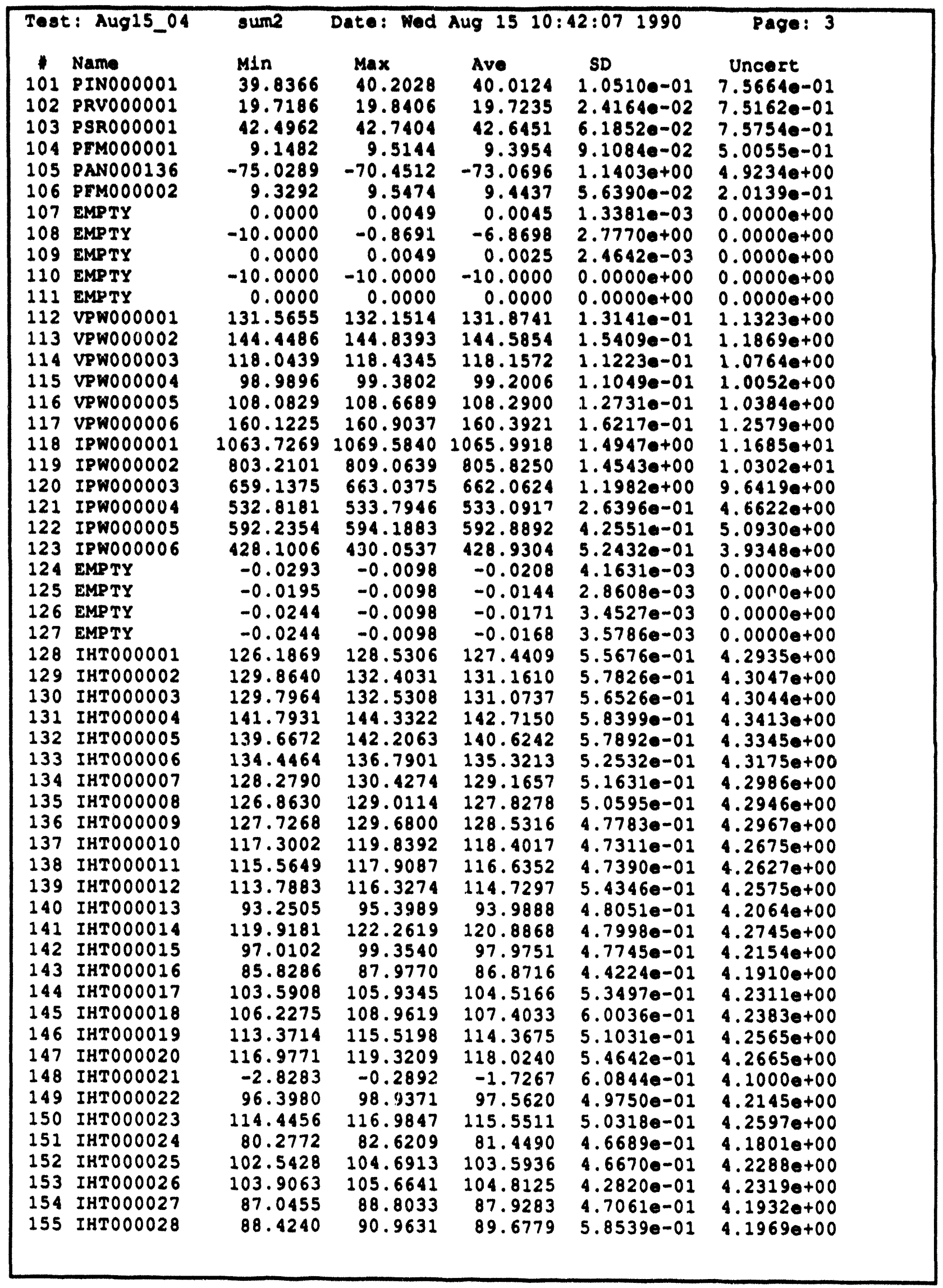




\begin{tabular}{|c|c|c|c|c|c|c|}
\hline $\begin{array}{l}11 \\
156 \\
157 \\
158 \\
159 \\
160 \\
161 \\
162 \\
163\end{array}$ & $\begin{array}{l}\text { Name } \\
\text { IHTO00029 } \\
\text { IHTO00030 } \\
\text { IHTO00031 } \\
\text { IHTO00032 } \\
\text { IHTO00033 } \\
\text { IHTO00034 } \\
\text { IHTO00035 } \\
\text { IHTO00036 }\end{array}$ & $\begin{array}{l}\text { M1n } \\
103.6396 \\
106.0096 \\
94.9594 \\
95.5980 \\
95.5829 \\
88.2474 \\
116.4025 \\
86.9479\end{array}$ & $\begin{array}{r}\operatorname{Max} \\
106.1786 \\
108.5487 \\
97.4985 \\
98.5276 \\
98.7079 \\
90.0053 \\
118.3556 \\
89.0963\end{array}$ & $\begin{array}{r}\text { Ave } \\
104.8974 \\
107.1581 \\
95.9243 \\
96.9534 \\
96.8994 \\
89.1655 \\
117.4181 \\
87.9009\end{array}$ & $\begin{array}{l}\text { SD } \\
5.6519 e-01 \\
5.5753 \bullet-01 \\
5.7779 \bullet-01 \\
6.2066 e-01 \\
6.2242 \bullet-01 \\
1.6563 \bullet-01 \\
5.0686 \bullet-01 \\
5.0322 \bullet-01\end{array}$ & $\begin{array}{c}\text { Uncert } \\
4.2321 \bullet+00 \\
4.2377 \bullet+00 \\
4.2107 \bullet+00 \\
4.2131 \bullet+00 \\
4.2130 \bullet+00 \\
4.1958 \bullet+00 \\
4.2648 \bullet+00 \\
4.1932 \bullet+00\end{array}$ \\
\hline
\end{tabular}




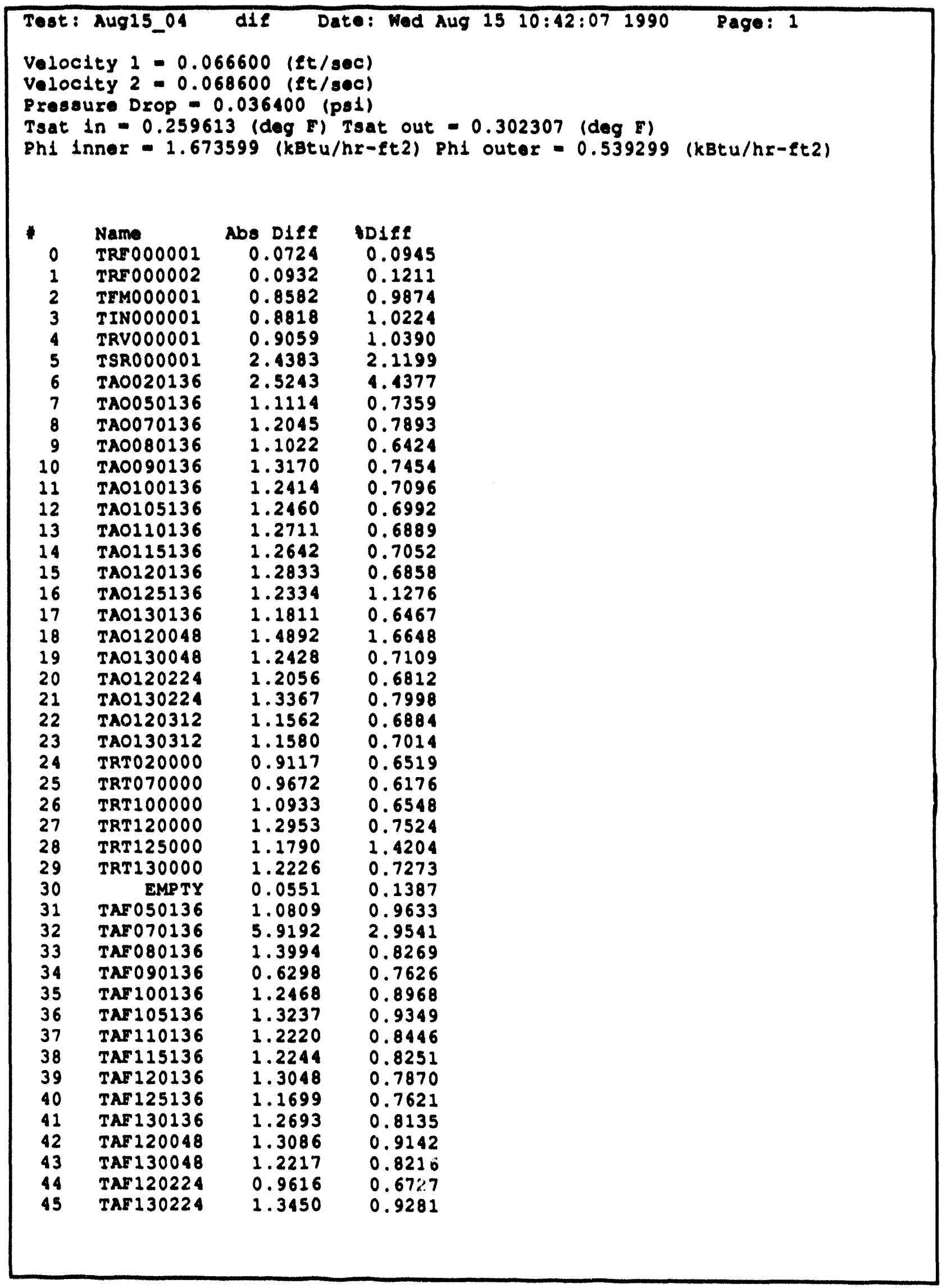




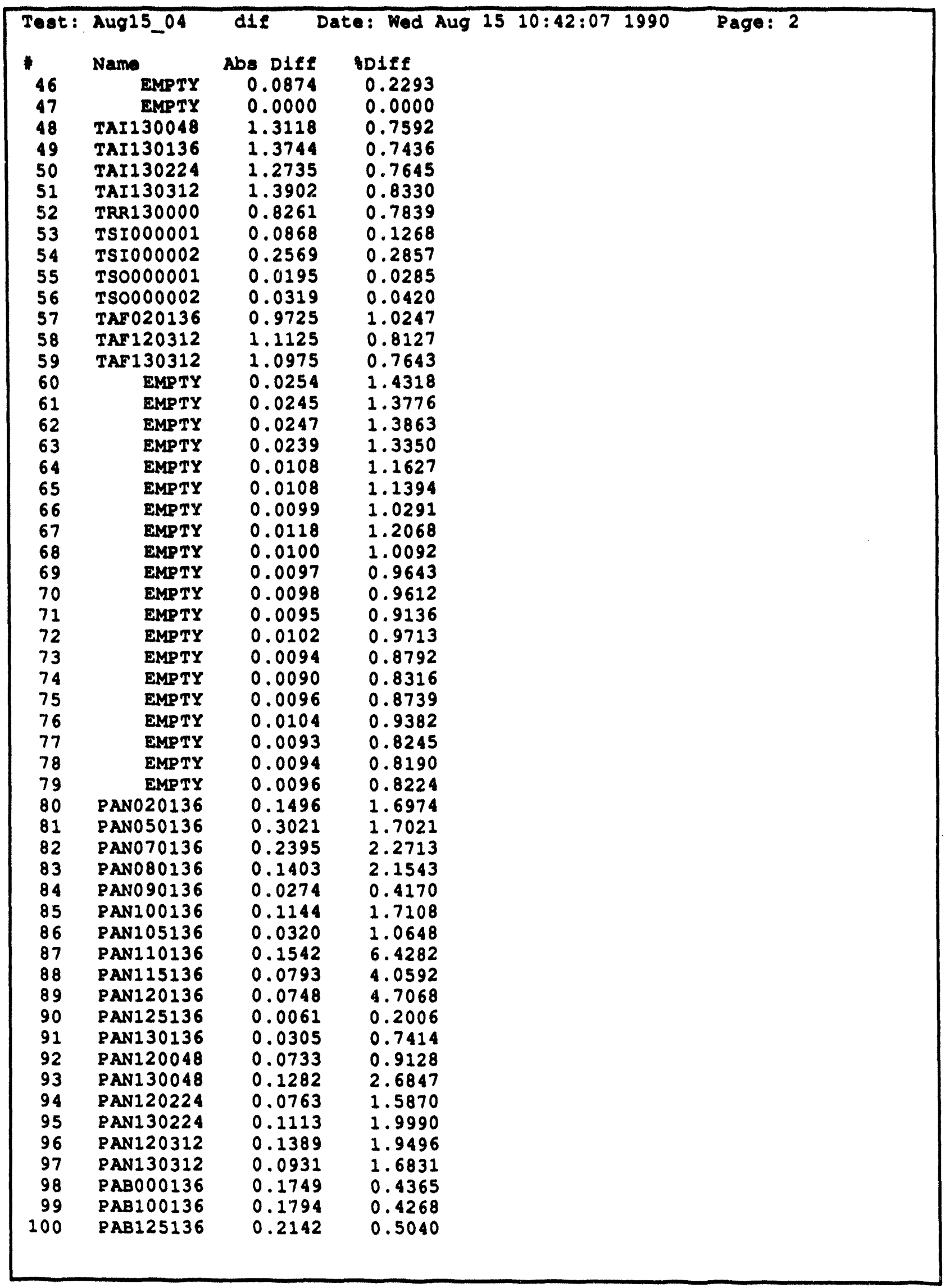




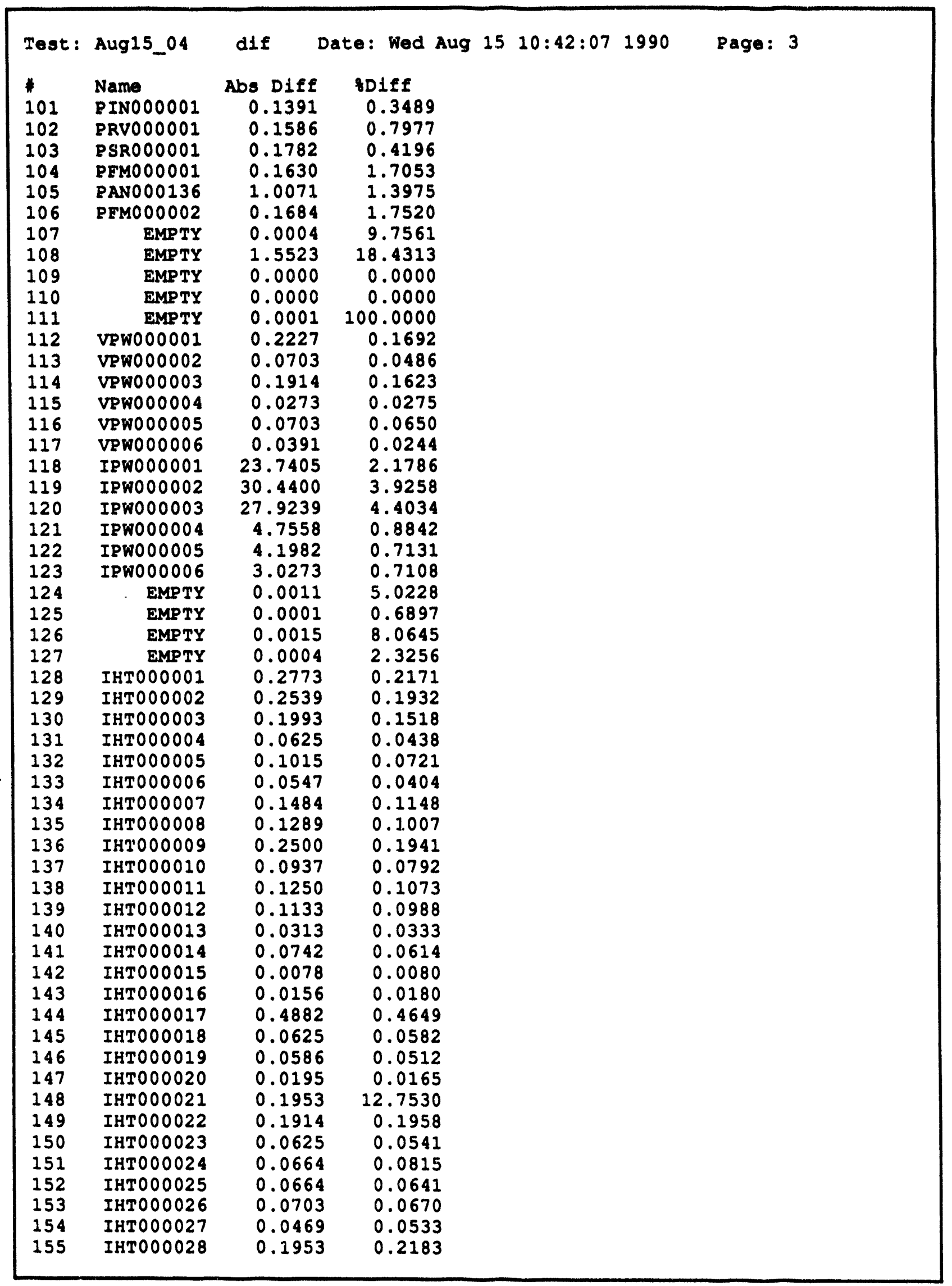




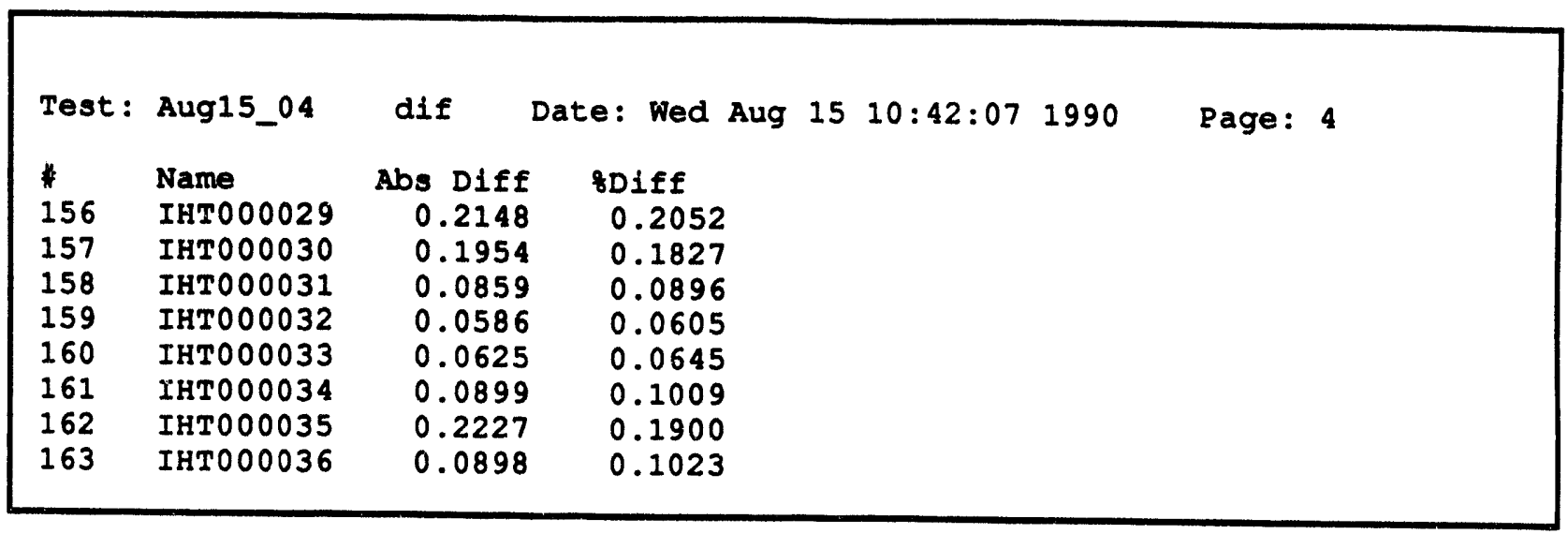




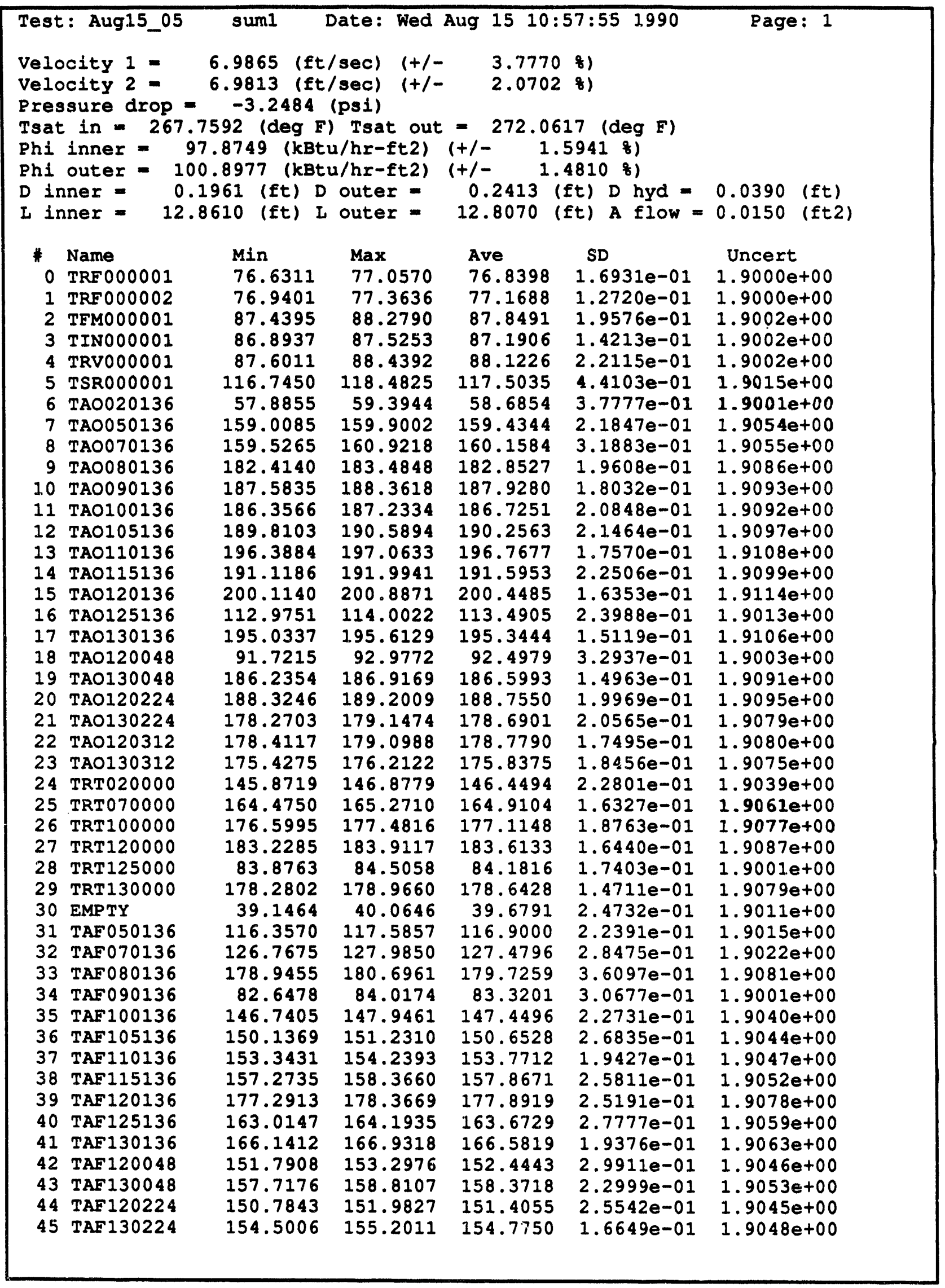




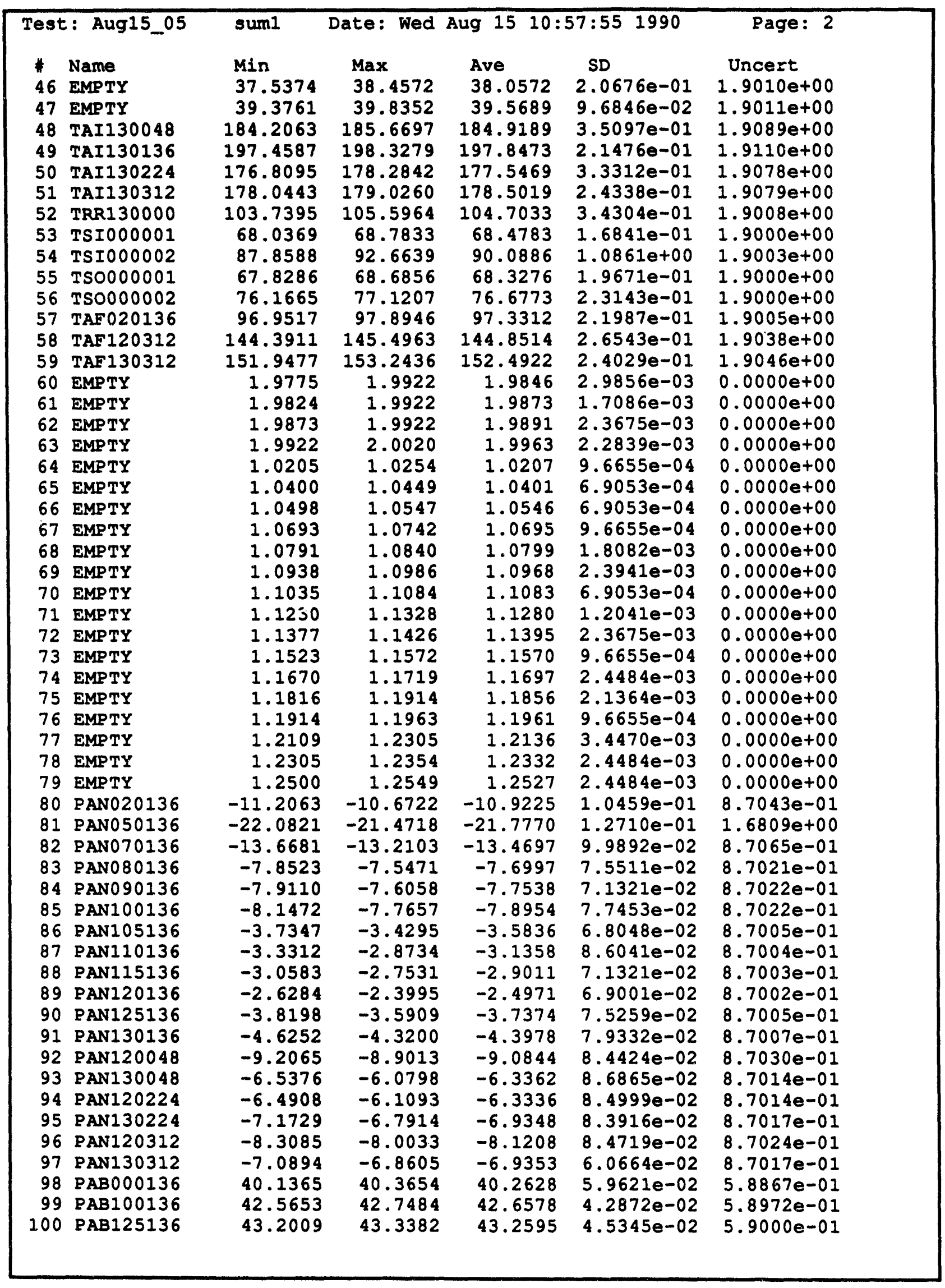




\begin{tabular}{|c|c|c|c|c|c|c|}
\hline \multicolumn{2}{|c|}{ Test: Aug15_05 } & sum1 & Date: Wed & Aug 151 & $57: 551990$ & Page: 3 \\
\hline * & Name & Min & $\operatorname{Max}$ & Ave & SD & Uncert \\
\hline 101 & PIN000001 & 39.9587 & 40.4470 & 40.1491 & $9.9140 \mathrm{e}-02$ & $7.5669 e-01$ \\
\hline 102 & PRV000001 & 19.5965 & 19.7186 & 19.6111 & $4.0071 e-02$ & $7.5160 e-01$ \\
\hline 103 & PSR000001 & 43.2286 & 43.3507 & 43.3043 & $5.9853 e-02$ & $7.5777 e-01$ \\
\hline 104 & PEM000001 & 7.2256 & 7.5918 & 7.3812 & $9.1084 e-02$ & $5.0034 e-01$ \\
\hline 105 & PANO00136 & -91.9661 & -87.8463 & -89.9336 & $9.1627 e-01$ & $4.9251 e+00$ \\
\hline 106 & PEMO00002 & 7.2821 & 7.4499 & 7.3700 & $4.0900 \mathrm{e}-02$ & $2.0085 e-01$ \\
\hline 107 & EMPTY & -0.0049 & 0.0049 & 0.0046 & $1.5314 e-03$ & $0.0000 e+00$ \\
\hline 108 & EMPTY & -10.0000 & -2.2754 & -5.3059 & $2.7837 e+00$ & $0.0000 e+00$ \\
\hline 109 & EMPTY & 0.0000 & 0.0049 & 0.0030 & $2.3941 \mathrm{e}-03$ & $0.0000 \mathrm{e}+00$ \\
\hline 110 & EMPTY & -10.0000 & -10.0000 & -10.0000 & $0.0000 e+00$ & $0.0000 e+00$ \\
\hline 111 & EMPTY & -0.0049 & 0.0098 & 0.0001 & $1.5566 \mathrm{e}-03$ & $0.0000 e+00$ \\
\hline 112 & VPWO00001 & 131.5655 & 131.9561 & 131.7725 & $1.3901 e-01$ & $1.1319 \mathrm{e}+00$ \\
\hline 113 & VPW000002 & 144.2533 & 144.6440 & 144.5034 & $1.0470 e-01$ & $1.1865 e+00$ \\
\hline 114 & VPW000003 & 117.4579 & 118.2392 & 117.8408 & $2.0105 e-01$ & $1: 0752 e+00$ \\
\hline 115 & VPWO00004 & 98.9896 & 99.3802 & 99.2163 & $1.0705 e-01$ & $1.0052 e+00$ \\
\hline 116 & VPW000005 & 108.0829 & 108.4736 & 108.2978 & $1.2633 e-01$ & $1.0384 \mathrm{e}+00$ \\
\hline 117 & VPW000006 & 160.3178 & 160.9037 & 160.4936 & $1.2001 \mathrm{e}-01$ & $1.2583 \mathrm{e}+00$ \\
\hline 118 & IPW000001 & 1083.2504 & 1089.1074 & 1085.5151 & $1.6434 \mathrm{e}+00$ & $1.1796 \mathrm{e}+01$ \\
\hline 119 & IPW000002 & 768.0872 & 773.9410 & 770.8192 & $1.3656 e+00$ & $1.0134 \mathrm{e}+01$ \\
\hline 120 & IPW000003 & 629.8875 & 635.7375 & 632.6174 & $1.7618 e+00$ & $9.5177 \mathrm{e}+00$ \\
\hline 121 & IPW000004 & 537.7009 & 539.1657 & 538.2284 & $4.2818 e-01$ & $4.6989 e+00$ \\
\hline 122 & IPW000005 & 588.3295 & 590.2824 & 589.1304 & $4.4944 e-01$ & $5.0657 e+00$ \\
\hline 123 & IPW000006 & 424.1943 & 425.6591 & 425.0632 & $3.5962 e-01$ & $3.9085 e+00$ \\
\hline 124 & EMPTY & -0.0244 & -0.0098 & -0.0173 & $3.5854 e-03$ & $0.0000 e+00$ \\
\hline 125 & EMPTY & -0.0195 & -0.0049 & -0.0116 & $2.9413 e-03$ & $0.0000 e+00$ \\
\hline 126 & EMPTY & -0.0195 & -0.0049 & -0.0132 & $3.5908 e-03$ & $0.0000 e+00$ \\
\hline 127 & EMPTY & -0.0244 & -0.0098 & -0.0168 & $3.7121 e-03$ & $0.0000 e+00$ \\
\hline 128 & IHT000001 & 126.5775 & 128.5306 & 127.6479 & $4.3627 e-01$ & $4.2941 \mathrm{e}+00$ \\
\hline 129 & IHT000002 & 130.2547 & 132.2078 & 131.2274 & $4.6436 e-01$ & $4.3049 e+00$ \\
\hline 130 & IHT000003 & 130.1870 & 132.1402 & 131.1362 & $4.6857 e-01$ & $4.3046 e+00$ \\
\hline 131 & IHTO00004 & 141.7931 & 144.1369 & 142.7931 & $5.3473 e-01$ & $4.3415 e+00$ \\
\hline 132 & IHTO000005 & 139.8625 & 141.6203 & 140.7648 & $4.8950 \mathrm{e}-01$ & $4.3349 e+00$ \\
\hline 133 & IHT000006 & 134.4464 & 136.5948 & 135.4424 & $5.0262 e-01$ & $4.3179 e+00$ \\
\hline 134 & IHT000007 & 128.4743 & 130.4274 & 129.2438 & $5.1510 e-01$ & $4.2989 e+00$ \\
\hline 135 & IHT000008 & 127.0583 & 129.2067 & 127.8981 & $5.1486 e-01$ & $4.2949 e+00$ \\
\hline 136 & IHTO00009 & 127.5315 & 129.6800 & 128.6019 & $5.1936 e-01$ & $4.2970 e+00$ \\
\hline 137 & IHTO00010 & 117.3002 & 119.8392 & 118.5502 & $5.9712 e-01$ & $4.2680 \mathrm{e}+00$ \\
\hline 138 & IHT000011 & 115.5649 & 117.9087 & 116.7173 & $6.0522 e-01$ & $4.2629 e+00$ \\
\hline 139 & IHTO00012 & 113.7883 & 116.1321 & 114.6750 & $5.9348 e-01$ & $4.2574 e+00$ \\
\hline 140 & IHTO00013 & 93.0552 & 95.3989 & 94.0317 & $5.7453 e-01$ & $4.2064 \mathrm{e}+00$ \\
\hline 141 & IHT000014 & 119.9181 & 121.8712 & 120.8673 & $5.5103 e-01$ & $4.2744 e+00$ \\
\hline 142 & IHTO00015 & 96.8149 & 99.1587 & 97.9829 & $5.4030 e-01$ & $4.2155 e+00$ \\
\hline 143 & IHT000016 & 86.0239 & 88.1723 & 86.9848 & $4.9574 e-01$ & $4.1913 e+00$ \\
\hline 144 & IHT000017 & 105.3486 & 107.4970 & 106.5517 & $5.4869 e-01$ & $4.2362 e+00$ \\
\hline 145 & IHT000018 & 106.4228 & 108.3759 & 107.4619 & $5.1060 e-01$ & $4.2385 e+00$ \\
\hline 146 & IHTO00019 & 113.5667 & 116.1058 & 114.5394 & $5.1673 e-01$ & $4.2570 e+00$ \\
\hline 147 & IHTO00020 & 117.3678 & 119.7115 & 118.1763 & $4.9444 e-01$ & $4.2669 e+00$ \\
\hline 148 & IHTO00021 & -2.2424 & 0.8827 & -1.2697 & $6.5375 e-01$ & $4.1000 e+00$ \\
\hline 149 & IHT000022 & 96.9839 & 99.7183 & 97.8511 & $4.9152 e-01$ & $4.2151 \mathrm{e}+00$ \\
\hline 150 & IHTO00023 & 114.8363 & 117.5706 & 115.7855 & $5.3816 e-01$ & $4.2604 e+00$ \\
\hline 151 & IHTO00024 & 80.8631 & 83.5975 & 81.6521 & $5.1894 e-01$ & $4.1805 e+00$ \\
\hline 152 & IHTO00025 & 102.7381 & 105.2772 & 103.7147 & $5.2348 e-01$ & $4.2291 \mathrm{e}+00$ \\
\hline 153 & IHT000026 & 103.7109 & 106.4453 & 104.9727 & $5.4277 e-01$ & $4.2322 e+00$ \\
\hline 154 & IHT000027 & 87.4362 & 89.3893 & 88.2681 & $5.1571 e-01$ & $4.1939 \mathrm{e}+00$ \\
\hline 155 & IHTO00028 & 88.8146 & 90.7677 & 89.7131 & $5.1900 e-01$ & $4.1970 e+00$ \\
\hline
\end{tabular}




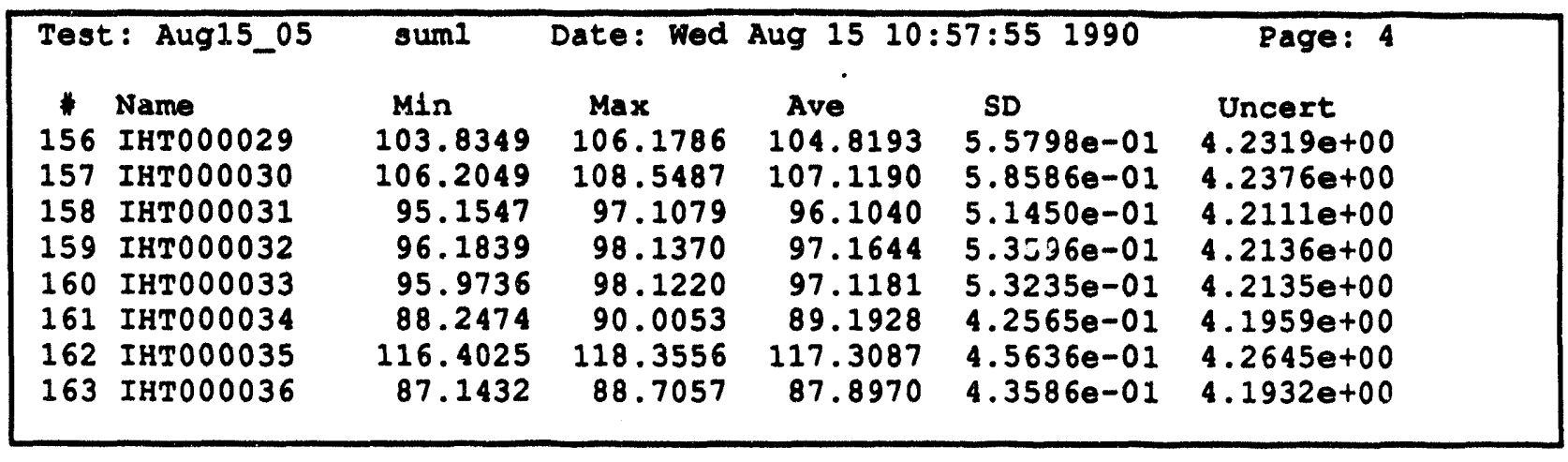




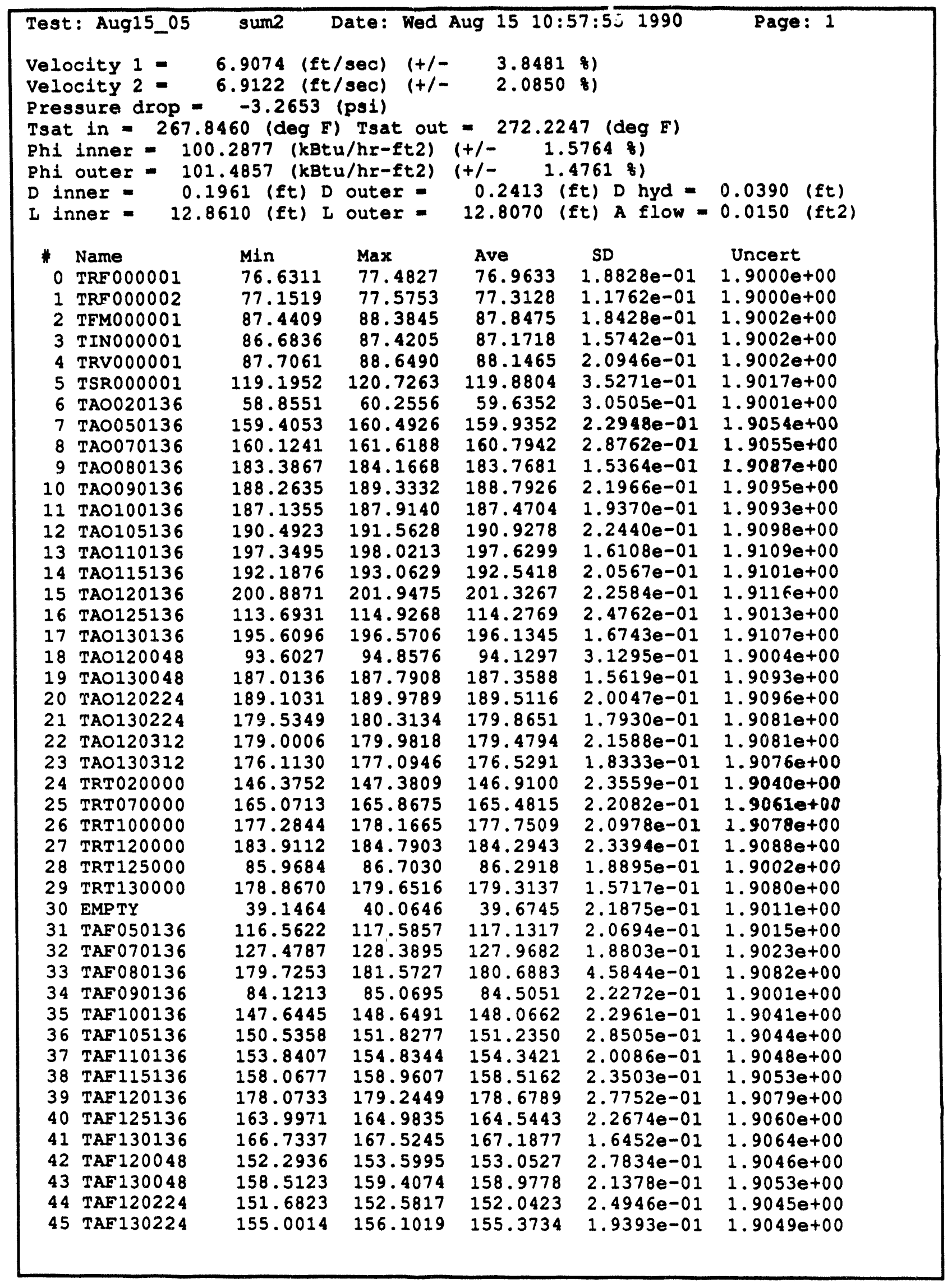




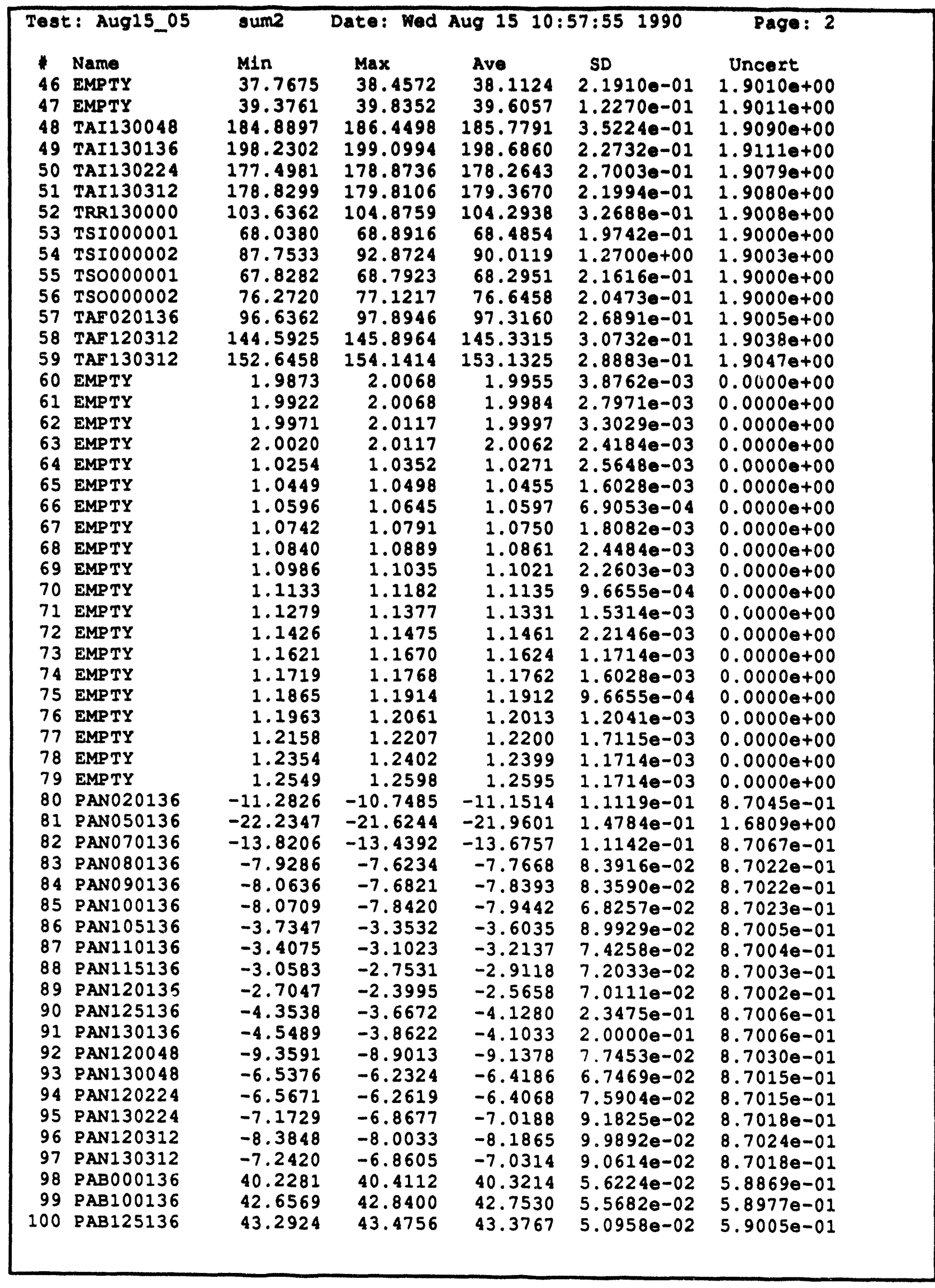




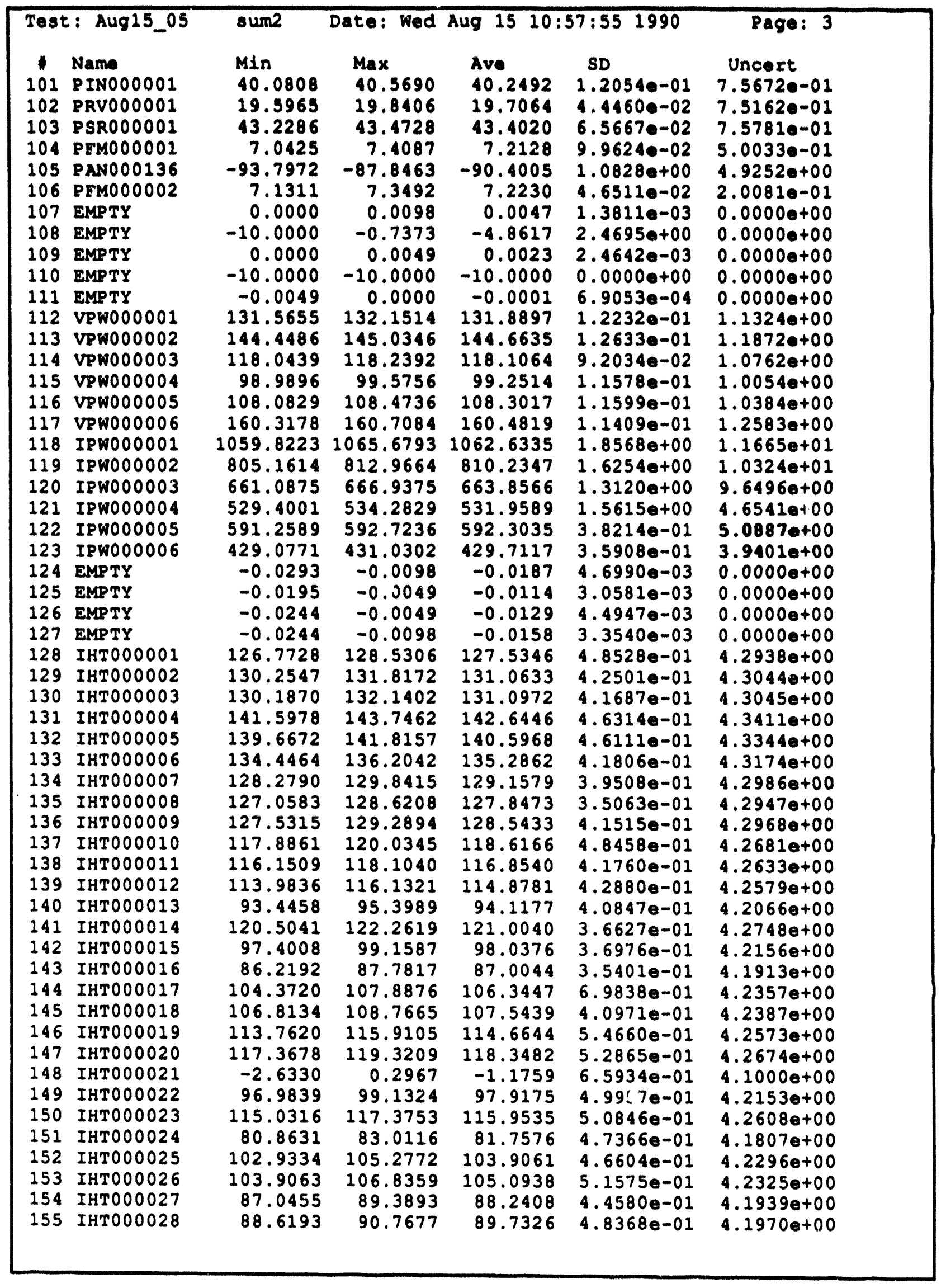




\begin{tabular}{|c|c|c|c|c|c|c|}
\hline $\begin{array}{l}1 \\
156 \\
157 \\
158 \\
159 \\
160 \\
161 \\
162 \\
163\end{array}$ & $\begin{array}{l}\text { Namb } \\
\text { IHTO00029 } \\
\text { IHTO00030 } \\
\text { IHT000031 } \\
\text { IHTO00032 } \\
\text { IHTO00033 } \\
\text { IHT000034 } \\
\text { IHT000035 } \\
\text { IHTO00036 }\end{array}$ & $\begin{array}{r}\text { Min } \\
104.0302 \\
106.4002 \\
95.1547 \\
95.9886 \\
95.9736 \\
88.4428 \\
116.5978 \\
87.1432\end{array}$ & $\begin{array}{r}\text { Max } \\
105.9833 \\
108.1580 \\
97.1079 \\
98.3323 \\
98.1220 \\
90.2006 \\
118.7462 \\
89.2916\end{array}$ & $\begin{array}{r}\text { Ave } \\
104.9521 \\
107.2752 \\
96.0688 \\
97.1097 \\
97.0634 \\
89.2045 \\
117.3868 \\
87.9048\end{array}$ & $\begin{array}{l}\text { SD } \\
4.8011 \theta-01 \\
4.72260-01 \\
4.6091 \bullet-01 \\
5.0657 \bullet-01 \\
4.5693 \theta-01 \\
4.41610-01 \\
4.46360-01 \\
4.18060-01\end{array}$ & $\begin{array}{c}\text { Uncert } \\
4.2322 e+00 \\
4.2380 \bullet+00 \\
4.2110 e+00 \\
4.2134 \theta+00 \\
4.2133 e+00 \\
4.1959 e+00 \\
4.2647 e+00 \\
4.1932 e+00\end{array}$ \\
\hline
\end{tabular}




\begin{tabular}{|c|c|c|c|}
\hline \multicolumn{4}{|c|}{$\begin{array}{l}\text { Velocity } 1=0.079100(\mathrm{ft} / \mathrm{sec}) \\
\text { Velocity } 2=0.069100(\mathrm{ft} / \mathrm{sec}) \\
\text { Pressure Drop }=0.016900 \text { (psi) } \\
\text { Tsat in }=0.086823 \text { (deg F) Tsat out }=0.162994 \text { (deg E) } \\
\text { Ph1 Inner }=2.412796 \text { (kBtu/hr-ft2) Ph1 outer }=0.588005 \text { (kBtu/hr-ft2) }\end{array}$} \\
\hline $\begin{array}{l}0 \\
0 \\
1 \\
2 \\
3 \\
4 \\
5 \\
6 \\
7 \\
8 \\
9 \\
10 \\
11 \\
12 \\
13 \\
14 \\
15 \\
16 \\
17 \\
18 \\
19 \\
20 \\
21 \\
22 \\
23 \\
24 \\
25 \\
26 \\
27 \\
28 \\
29 \\
30 \\
31 \\
32 \\
33 \\
34 \\
35 \\
36 \\
37 \\
38 \\
39 \\
40 \\
41 \\
42 \\
43 \\
44 \\
45\end{array}$ & $\begin{array}{l}\text { Name } \\
\text { TRF000001 } \\
\text { TRF000002 } \\
\text { TFMO00001 } \\
\text { TIN000001 } \\
\text { TRV000001 } \\
\text { TSR000001 } \\
\text { TAO020136 } \\
\text { TAO050136 } \\
\text { TAO070136 } \\
\text { TAO080136 } \\
\text { TAO090136 } \\
\text { TAO100136 } \\
\text { TAO105136 } \\
\text { TAO110136 } \\
\text { TAO115136 } \\
\text { TAO120136 } \\
\text { TAO125136 } \\
\text { TAO130136 } \\
\text { TAD120148 } \\
\text { TAO: } 30048 \\
\text { TAO120 } 124 \\
\text { TAO130224 } \\
\text { TAO120312 } \\
\text { TAO130312 } \\
\text { TRT020000 } \\
\text { TRT070000 } \\
\text { TRT100000 } \\
\text { TRT120000 } \\
\text { TRT125000 } \\
\text { TRT130000 } \\
\text { EMPTY } \\
\text { TAF050136 } \\
\text { TAF070136 } \\
\text { TAF080136 } \\
\text { TAF090136 } \\
\text { TAF100136 } \\
\text { TAF105136 } \\
\text { TAF110136 } \\
\text { TAF115136 } \\
\text { TAF120136 } \\
\text { TAF125136 } \\
\text { TAF130136 } \\
\text { TAF120048 } \\
\text { TAF130048 } \\
\text { TAF120224 } \\
\text { TAF130224 }\end{array}$ & $\begin{array}{c}\text { Abs D1ff } \\
0.1235 \\
0.1440 \\
0.0016 \\
0.0188 \\
0.0239 \\
2.3769 \\
0.9498 \\
0.5008 \\
0.6358 \\
0.9154 \\
0.8646 \\
0.7453 \\
0.6715 \\
0.8622 \\
0.9465 \\
0.8782 \\
0.7864 \\
0.7901 \\
1.6318 \\
0.7595 \\
0.7566 \\
1.1750 \\
0.7004 \\
0.6916 \\
0.4606 \\
0.5711 \\
0.6361 \\
0.6810 \\
2.1102 \\
0.6709 \\
0.0046 \\
0.2317 \\
0.4886 \\
0.9624 \\
1.1850 \\
0.6166 \\
0.5822 \\
0.5709 \\
0.6491 \\
0.7870 \\
0.8714 \\
0.6058 \\
0.6084 \\
0.6060 \\
0.6368 \\
0.5984\end{array}$ & $\begin{array}{l}\text { DDIf } \\
0.1607 \\
0.1866 \\
0.0018 \\
0.0216 \\
0.0271 \\
2.0228 \\
1.6185 \\
0.3141 \\
0.3970 \\
0.5006 \\
0.4601 \\
0.3991 \\
0.3529 \\
0.4382 \\
0.4940 \\
0.4381 \\
0.6929 \\
0.4045 \\
1.7641 \\
0.4070 \\
0.4008 \\
0.6576 \\
0.3918 \\
0.3933 \\
0.3145 \\
0.3463 \\
0.3591 \\
0.3709 \\
2.5067 \\
0.3756 \\
0.0116 \\
0.1982 \\
0.3833 \\
0.5355 \\
1.4222 \\
0.4182 \\
0.3865 \\
0.3713 \\
0.4112 \\
0.4424 \\
0.5324 \\
0.3637 \\
0.3991 \\
0.3826 \\
0.4206 \\
0.3866\end{array}$ \\
\hline
\end{tabular}




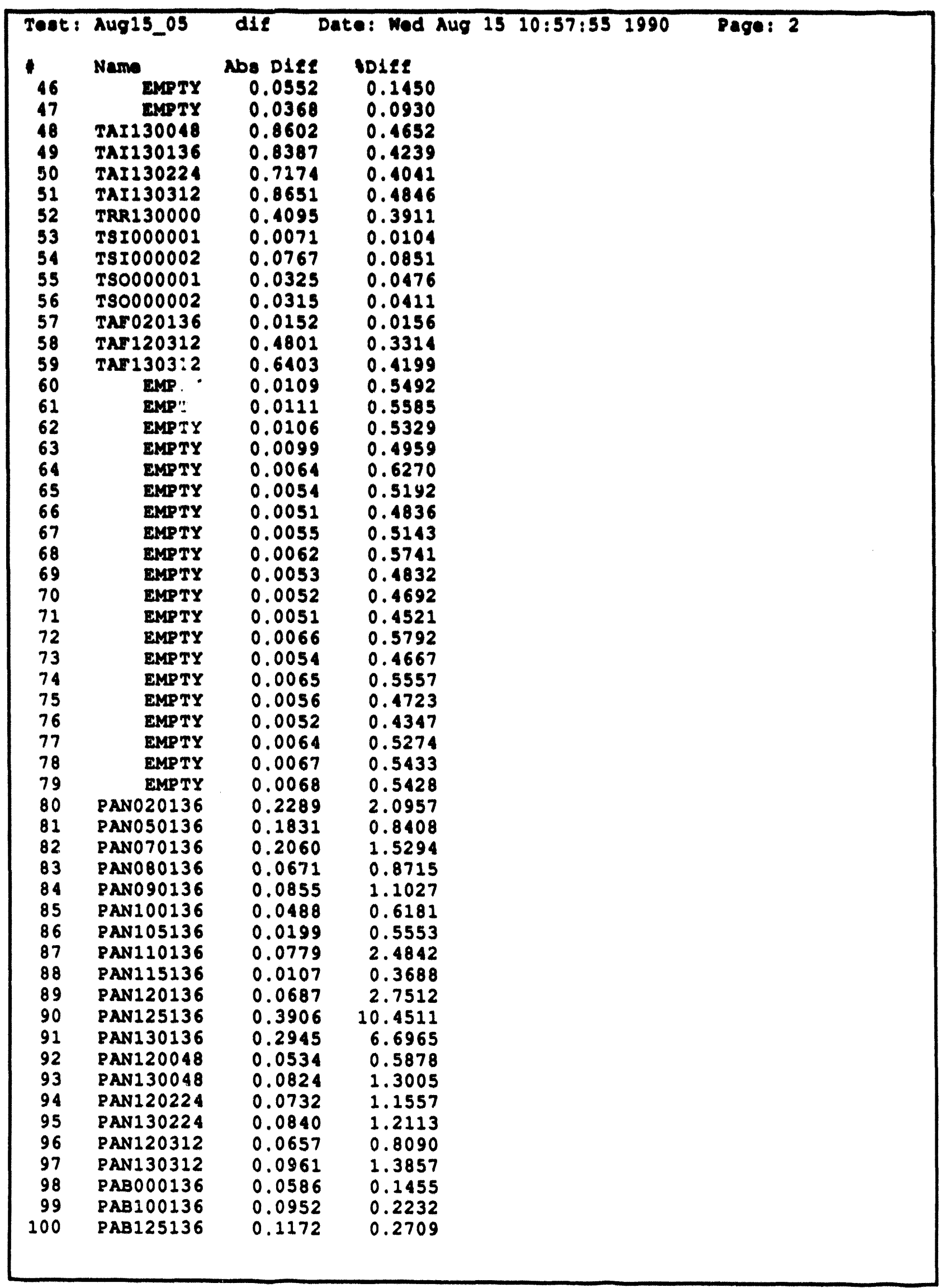




\begin{tabular}{|c|c|c|c|c|c|}
\hline $\begin{array}{l}\text { Toet } \\
101 \\
101 \\
102 \\
103 \\
104 \\
105 \\
106 \\
107 \\
108 \\
109 \\
110 \\
111 \\
112 \\
113 \\
114 \\
115 \\
116 \\
117 \\
118 \\
119 \\
120 \\
121 \\
122 \\
123 \\
124 \\
125 \\
126 \\
127 \\
128 \\
129 \\
130 \\
131 \\
132 \\
133 \\
134 \\
135 \\
136 \\
137 \\
138 \\
139 \\
140 \\
141 \\
142 \\
143 \\
144 \\
145 \\
146 \\
147 \\
148 \\
149 \\
150 \\
151 \\
152 \\
153 \\
154 \\
155\end{array}$ & 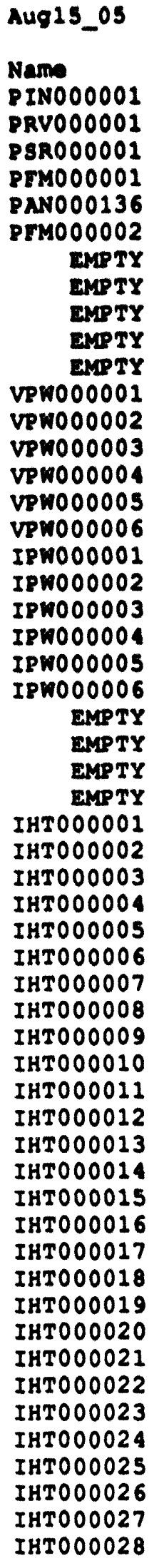 & $\begin{array}{l}\text { d1E } \\
\text { Abs D D } 182 \\
0.1001 \\
0.0953 \\
0.0977 \\
0.1681 \\
0.1669 \\
0.1470 \\
0.0001 \\
0.1442 \\
0.0007 \\
0.0000 \\
0.0002 \\
0.1172 \\
0.1601 \\
0.2656 \\
0.0351 \\
0.0039 \\
0.0117 \\
22.8816 \\
39.4155 \\
31.2393 \\
6.2695 \\
3.1732 \\
4.6485 \\
0.0014 \\
0.0002 \\
0.0003 \\
0.0010 \\
0.1133 \\
0.1641 \\
0.0390 \\
0.1485 \\
0.1680 \\
0.1562 \\
0.0859 \\
0.0508 \\
0.0586 \\
0.0664 \\
0.1367 \\
0.2031 \\
0.0860 \\
0.1367 \\
0.0547 \\
0.0196 \\
0.2070 \\
0.0820 \\
0.1250 \\
0.1719 \\
0.0938 \\
0.0664 \\
0.1680 \\
0.1055 \\
0.1914 \\
0.1211 \\
0.0273 \\
0.0195\end{array}$ & 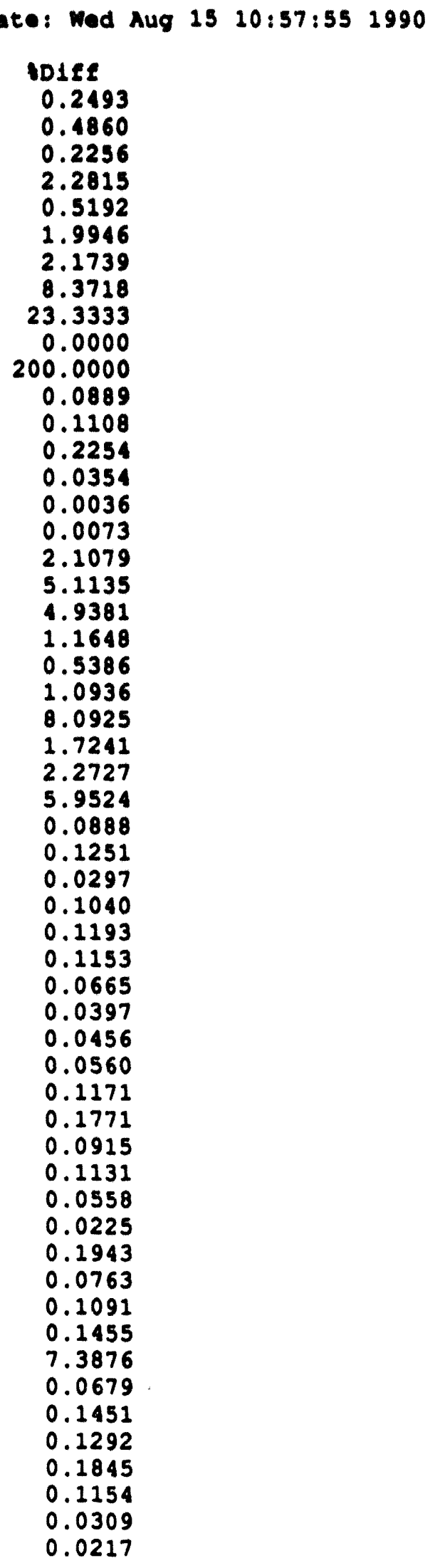 & Page: 3 & 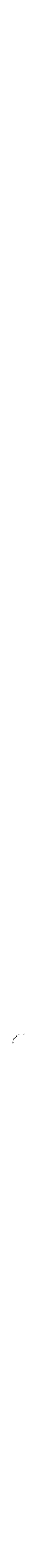 \\
\hline
\end{tabular}




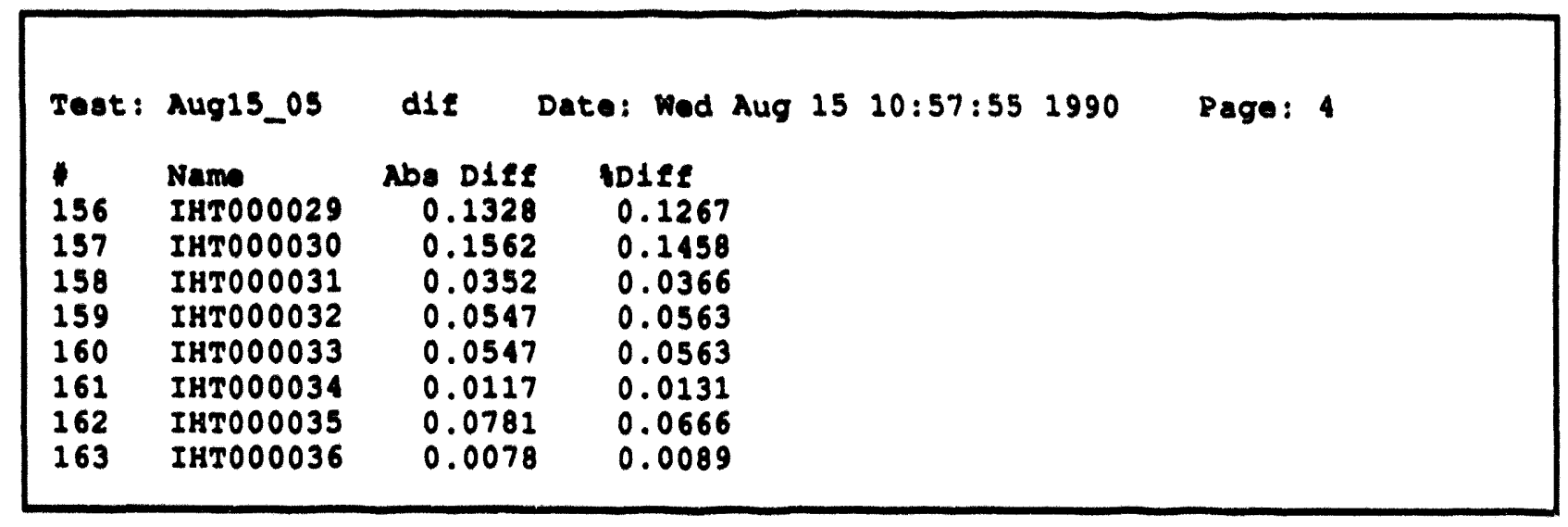




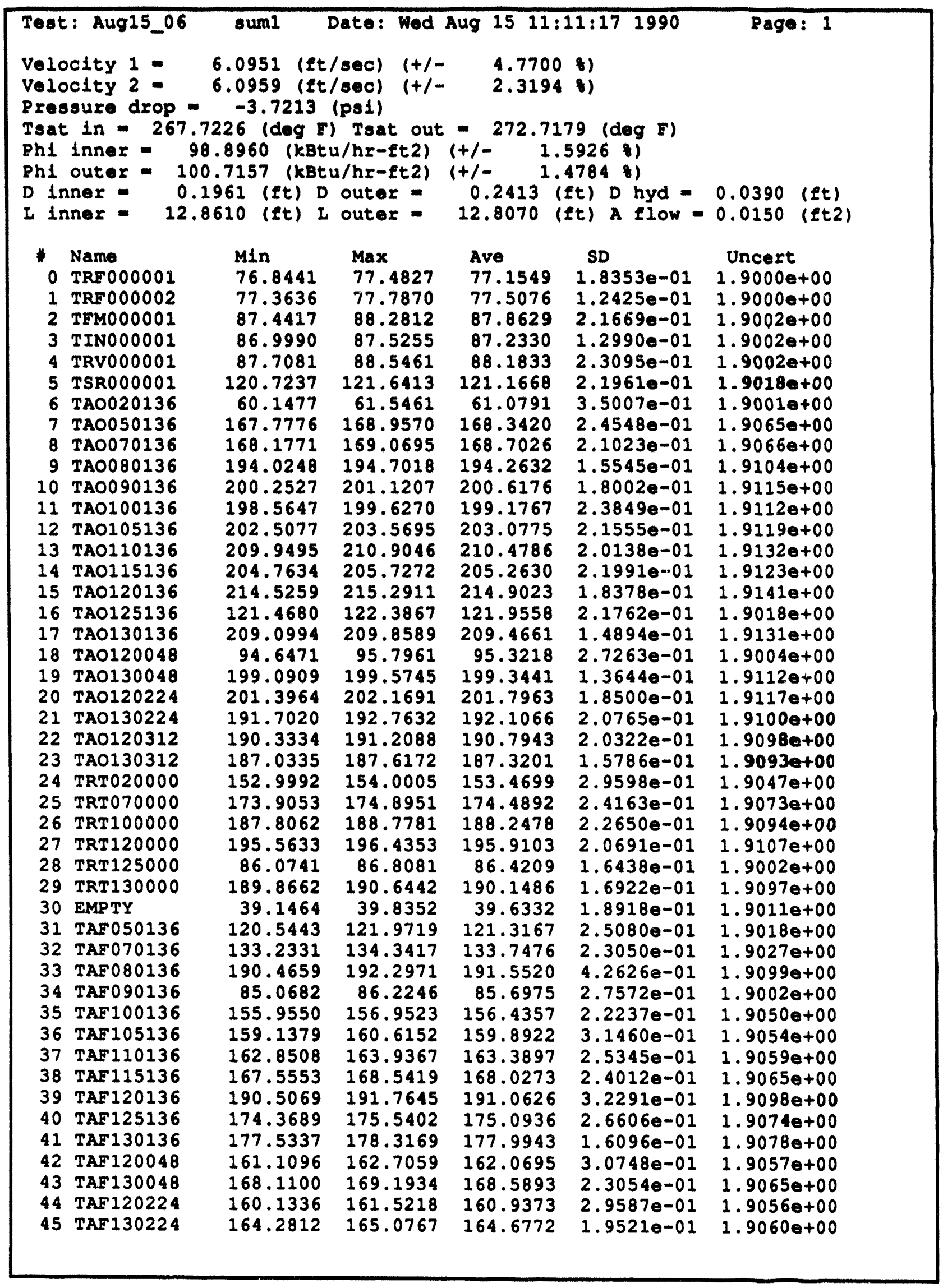




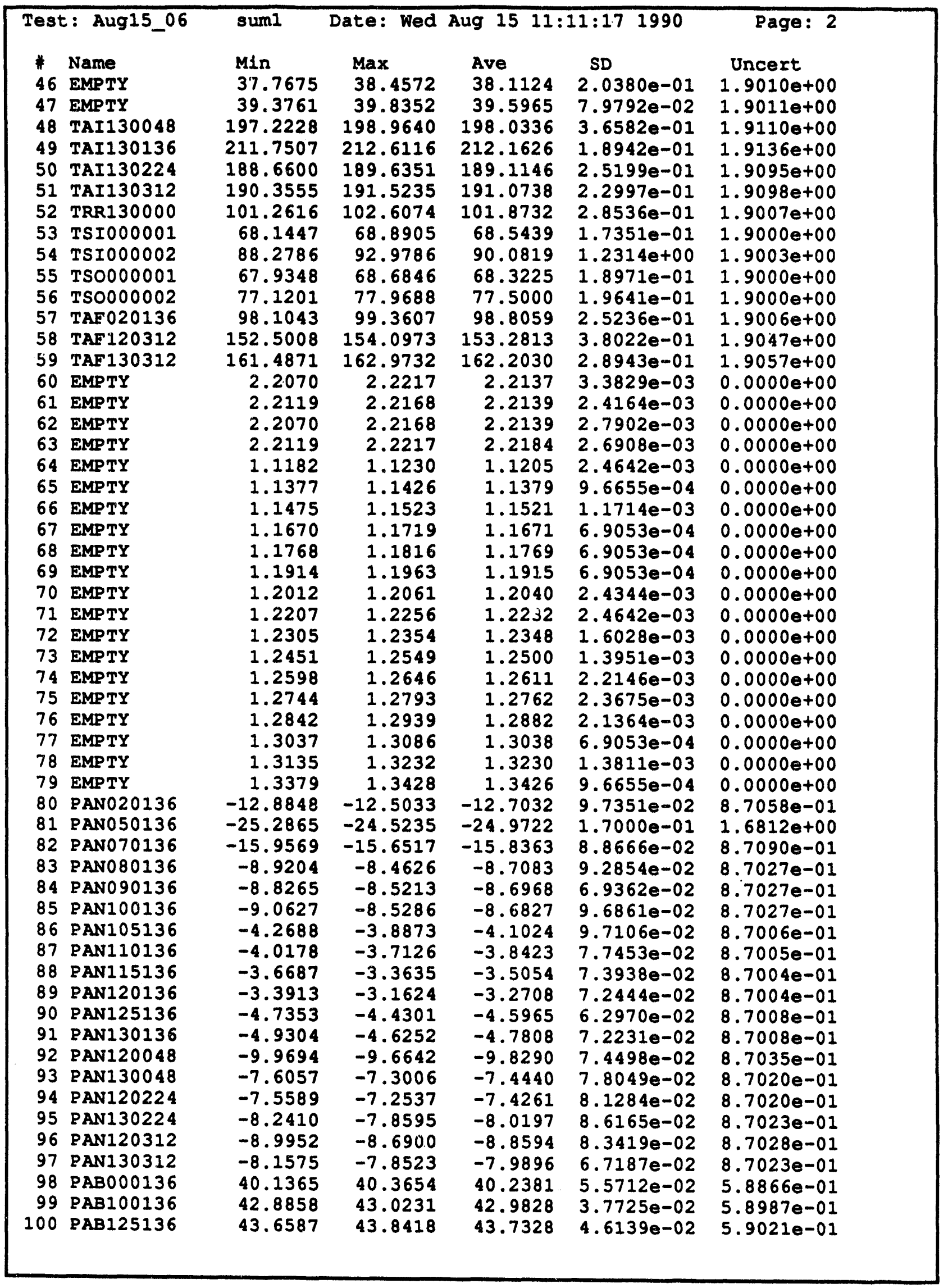




\begin{tabular}{|c|c|c|c|c|c|c|}
\hline \multicolumn{2}{|c|}{ Test: Aug15_06 } & sum1 & Date: Wed & \multicolumn{2}{|c|}{ Aug $15 \quad 11: 11: 17 \quad 1990$} & \multirow{2}{*}{$\begin{array}{l}\text { Page: } 3 \\
\text { Uncert }\end{array}$} \\
\hline \# & Name & Min & $\operatorname{Max}$ & Ave & SD & \\
\hline 101 & PINO00001. & 39.8366 & 40.2028 & 39.9831 & $6.9754 e-02$ & $7.5663 e-01$ \\
\hline 102 & PRV000001 & 19.4744 & 19.8406 & 19.5867 & $7.3325 e-02$ & $7.5160 e-01$ \\
\hline 103 & PSR000001 & 43.7169 & 43.8390 & 43.7706 & $6.1209 e-02$ & $7.5794 e-01$ \\
\hline 104 & PEM000001 & 5.3945 & 5.8523 & 5.5960 & $9.4314 \mathrm{e}-02$ & $5.0020 e-01$ \\
\hline 105 & PANO00136 & -104.3257 & -101.5792 & -103.0257 & $7.5338 e-01$ & $4.9267 e+00$ \\
\hline 106 & PEM000002 & 5.5034 & 5.6545 & 5.5974 & $2.7541 e-02$ & $2.0049 e-01$ \\
\hline 107 & EMPTY & -0.0049 & 0.0049 & 0.0039 & $2.4164 e-03$ & $0.0000 e+00$ \\
\hline 108 & EMPTY & -10.0000 & -1.9141 & -4.7001 & $1.9944 \mathrm{e}+00$ & $0.0000 e+00$ \\
\hline 109 & EMPTY & 0.0000 & 0.0049 & 0.0029 & $2.4164 e-03$ & $0.0000 e+00$ \\
\hline 110 & EMPTY & -10.0000 & -10.0000 & -10.0000 & $0.0000 e+00$ & $0.0000 e+00$ \\
\hline 111 & EMPTY & 0.0000 & 0.0000 & 0.0000 & $0.0000 e+00$ & $0.0000 e+00$ \\
\hline 112 & VRW000001 & 131.7608 & 132.1514 & 131.9287 & $1.2484 e-01$ & $1.1326 \mathrm{e}+00$ \\
\hline 113 & VPW000002 & 144.6440 & 144.8393 & 144.7495 & $9.8332 e-02$ & $1.1876 e+00$ \\
\hline 114 & VPW000003 & 117.8485 & 118.4345 & 118.0830 & $1.1161 \mathrm{e}-01$ & $1.0761 e+00$ \\
\hline 115 & VPW000004 & 98.9896 & 99.3802 & 99.1928 & $1.11330-01$ & $1.0051 \mathrm{e}+00$ \\
\hline 116 & VPW000005 & 107.8876 & 108.4736 & 108.2666 & $1.2731 e-01$ & $1.0383 e+00$ \\
\hline 117 & VPW000006 & 160.1225 & 160.7084 & 160.4702 & $1.0654 \mathrm{e}-01$ & $1.2582 e+00$ \\
\hline 118 & IPW000001 & 1075.4410 & 1085.2028 & 1079.3062 & $2.1053 e+00$ & $1.1761 \mathrm{e}+01$ \\
\hline 119 & IPW000002 & 781.7462 & 793.4537 & 788.3414 & $2.9471 e+00$ & $1.0217 \mathrm{e}+01$ \\
\hline 120 & IPW000003 & 633.7875 & 639.6375 & 636.2057 & $1.3395 e+00$ & $9.5326 e+00$ \\
\hline 121 & IPW000004 & 532.8181 & 534.7712 & 533.8729 & $6.1889 \mathrm{e}-01$ & $4.6678 e+00$ \\
\hline 122 & IPW000005 & 586.8648 & 587.8412 & 587.2947 & $3.5068 e-01$ & $5.0523 e+00$ \\
\hline 123 & IPW000006 & 423.2177 & 425.1709 & 424.2625 & $4.7320 e-01$ & $3.9030 \mathrm{e}+00$ \\
\hline 124 & EMPTY & -0.0293 & -0.0098 & -0.0180 & $4.2361 \mathrm{e}-03$ & $0.0000 \mathrm{e}+00$ \\
\hline 125 & EMPTY & -0.0195 & -0.0049 & -0.0123 & $3.9754 e-03$ & $0.0000 \mathrm{e}+00$ \\
\hline 126 & EMPTY & -0.0244 & -0.0049 & -0.0143 & $4.3940 \mathrm{e}-03$ & $0.000 \mathrm{ce}+00$ \\
\hline 127 & EMPTY & -0.0244 & -0.0098 & -0.0164 & $3.2359 e-03$ & $0.0000 \mathrm{e}+00$ \\
\hline 128 & IHT000001 & 126.3822 & 129.3119 & 127.5815 & $6.0104 e-01$ & $4.2939 e+00$ \\
\hline 129 & IHT000002 & 130.0594 & 132.5984 & 131.1297 & $5.5976 e-01$ & $4.3046 e+00$ \\
\hline 130 & IHT000003 & 129.9917 & 132.7261 & 131.1909 & $5.9058 e-01$ & $4.3048 e+00$ \\
\hline 131 & IHTO00004 & 141.4025 & 144.1369 & 142.6134 & $5.5243 e-01$ & $4.3410 \mathrm{e}+00$ \\
\hline 132 & IHTO00005 & 139.4719 & 142.0110 & 140.6007 & $5.2392 e-01$ & $4.3344 \mathrm{e}+00$ \\
\hline 133 & IHT000006 & 134.2511 & 136.7901 & 135.3096 & $4.9770 e-01$ & $4.3175 e+00$ \\
\hline 134 & IHT000007 & 128.2790 & 130.4274 & 129.2946 & $4.8648 e-01$ & $4.2990 \mathrm{e}+00$ \\
\hline 135 & IHTO00008 & 127.0583 & 128.8161 & 127.9371 & $4.5549 \mathrm{e}-01$ & $4.2950 \mathrm{e}+00$ \\
\hline 136 & IHT000009 & 127.5315 & 129.6800 & 128.6800 & $5.0477 e-01$ & $4.2972 \mathrm{e}+00$ \\
\hline 137 & IHTO00010 & 117.4955 & 119.4486 & 118.5619 & $5.1177 e-01$ & $4.2680 \mathrm{e}+00$ \\
\hline 138 & IHT000011 & 115.7602 & 117.9087 & 116.7759 & $4.7515 e-01$ & $4.2631 e+00$ \\
\hline 139 & IHT000012 & 113.9836 & 115.7415 & 114.8273 & $4.9196 e-01$ & $4.2578 e+00$ \\
\hline 140 & IHT000013 & 93.0552 & 95.0083 & 94.0630 & $4.5568 e-01$ & $4.2065 e+00$ \\
\hline 141 & IHT000014 & 120.1134 & 122.4572 & 121.0431 & $4.5602 e-01$ & $4.2749 e+00$ \\
\hline 142 & IHT000015 & 97.2055 & 99.5493 & 97.9907 & $4.6770 e-01$ & $4.2155 e+00$ \\
\hline 143 & IHT000016 & 86.0239 & 88.1723 & 86.8833 & $4.5163 e-01$ & $4.1910 \mathrm{e}+00$ \\
\hline 144 & IHT 000017 & 104.1767 & 107.8876 & 106.0166 & $9.5609 e-01$ & $4.2348 e+00$ \\
\hline 145 & IHTO00018 & 106.4228 & 108.7665 & 107.4619 & $5.4454 \mathrm{e}-01$ & $4.2385 e+00$ \\
\hline 146 & IHT000019 & 113.3714 & 115.7151 & 114.6214 & $4.9126 \mathrm{e}-01$ & $4.2572 e+00$ \\
\hline 147 & IHT000020 & 117.1725 & 119.1256 & 118.2740 & $4.8289 e-01$ & $4.2672 e+00$ \\
\hline 148 & IHT 000021 & -2.6330 & -0.0939 & -1.1447 & $5.9042 e-01$ & $4.1000 e+00$ \\
\hline 149 & IHT000022 & 96.5933 & 98.7418 & 97.8042 & $4.9284 e-01$ & $4.2150 e+00$ \\
\hline 150 & IHT000023 & 114.8363 & 116.9847 & 115.8910 & $5.3233 \mathrm{e}-01$ & $4.2606 e+00$ \\
\hline 151 & IHTO00024 & 80.4725 & 82.8162 & 81.6951 & $5.0349 e-01$ & $4.1806 \mathrm{e}+00$ \\
\hline 152 & IHTO00025 & 102.7381 & 104.6913 & 103.8436 & $5.1238 e-01$ & $4.2295 e+00$ \\
\hline 153 & IHT000026 & 103.7109 & 106.2500 & 105.0352 & $5.5564 e-01$ & $4.2324 e+00$ \\
\hline 154 & IHTO00027 & 87.0455 & 89.1940 & 88.1041 & $5.1764 e-01$ & $4.1936 \mathrm{e}+00$ \\
\hline 155 & IHT000028 & 88.6193 & 90.7677 & 89.6623 & $5.9137 e-01$ & $4.1969 e+00$ \\
\hline
\end{tabular}




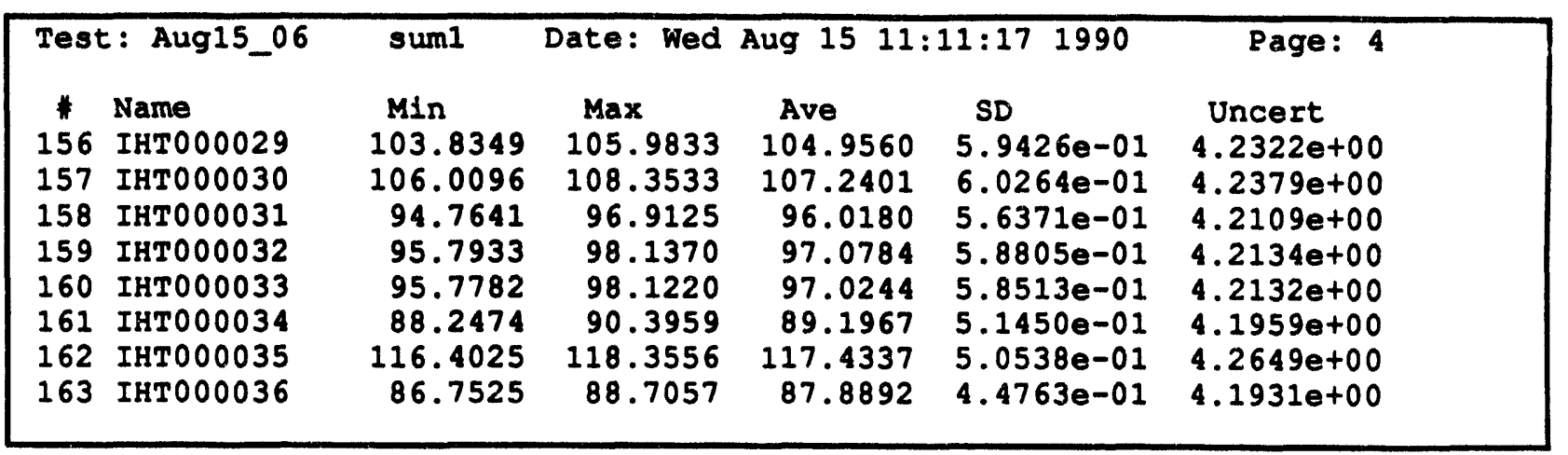




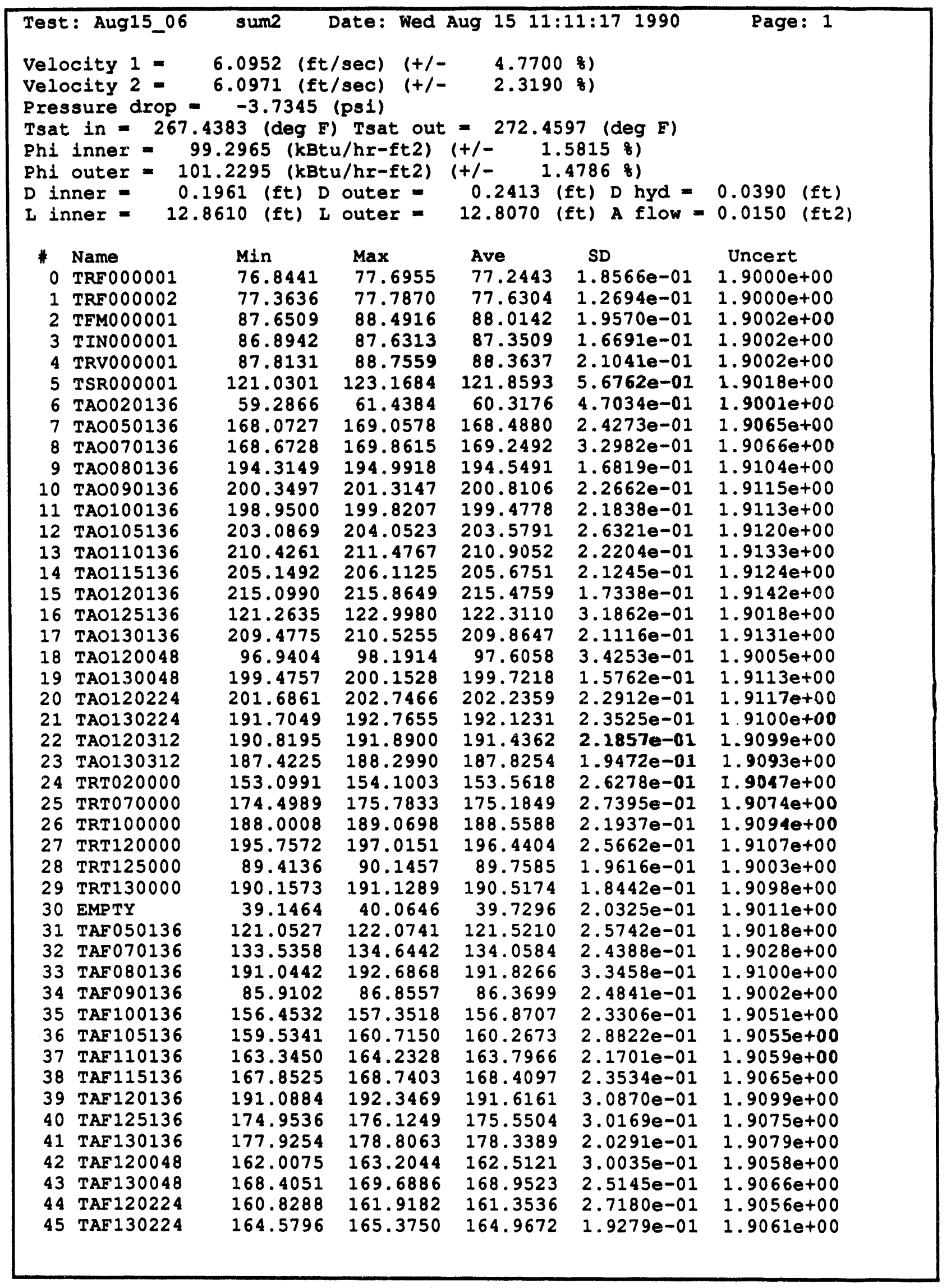




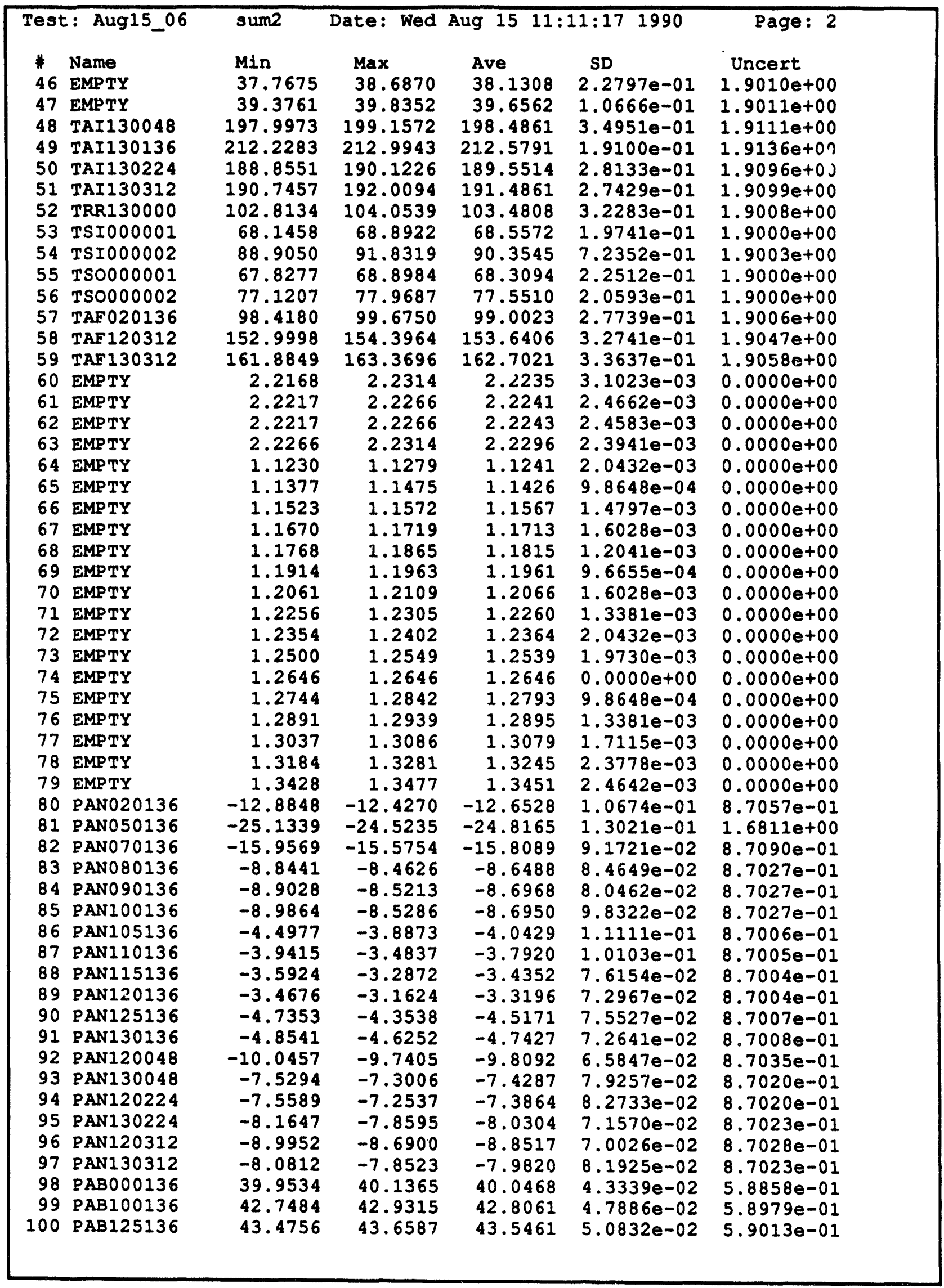




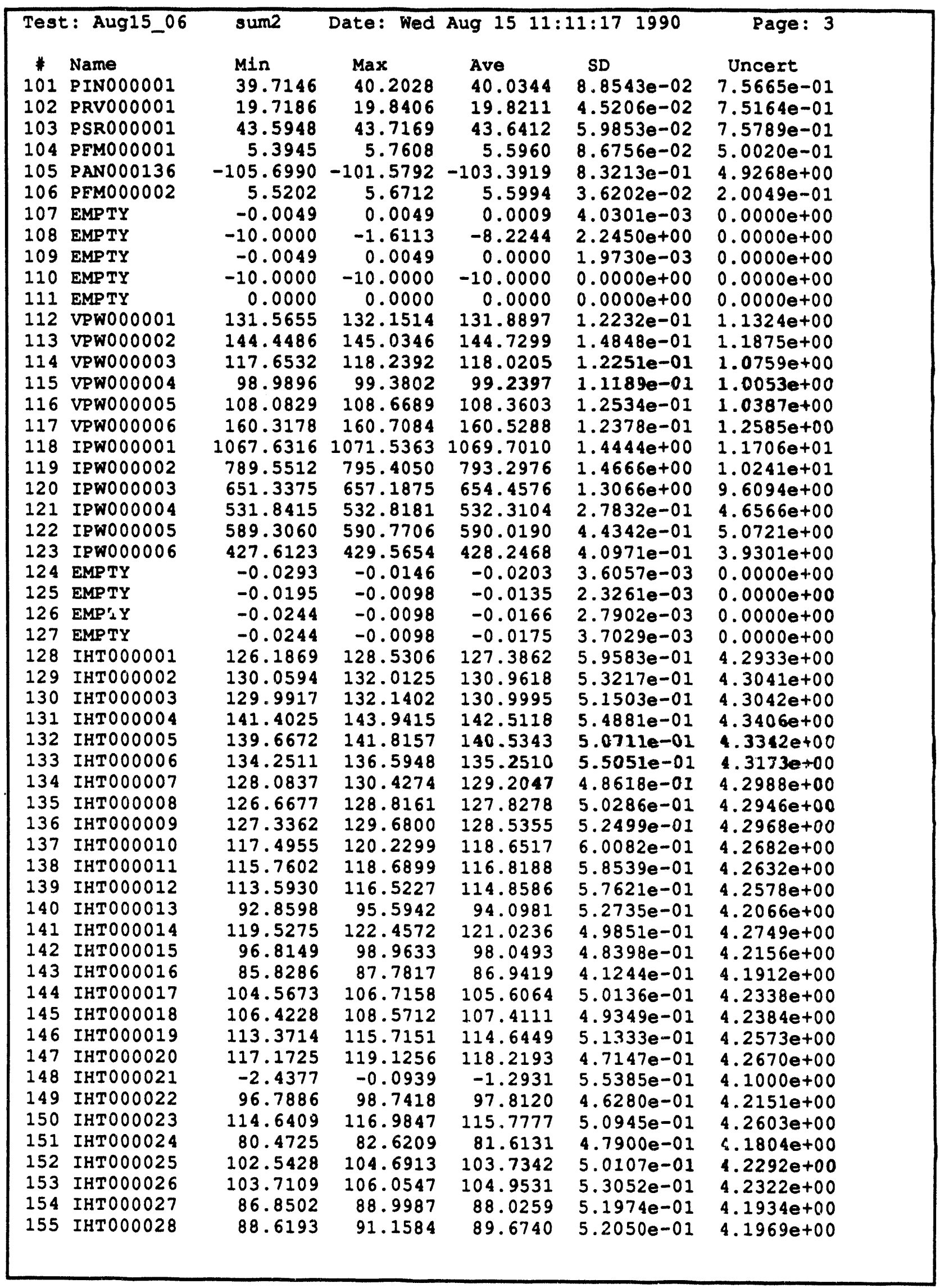




\begin{tabular}{|c|c|c|c|c|c|c|}
\hline \multicolumn{2}{|c|}{ Test: Aug15_06 } & & Date: Wed & \multicolumn{2}{|c|}{ Aug $15 \quad 11: 11: 17 \quad 1990$} & $\begin{array}{l}\text { Page: } 4 \\
\text { Uncert }\end{array}$ \\
\hline $\begin{array}{c}1 \\
156 \\
157 \\
158 \\
159 \\
160 \\
161 \\
162 \\
163\end{array}$ & 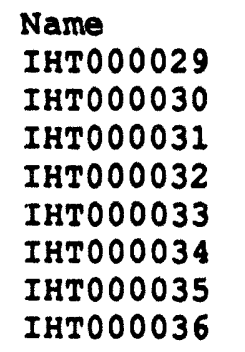 & $\begin{array}{l}\text { Min } \\
103.8349 \\
106.0096 \\
94.9594 \\
95.9886 \\
95.9736 \\
88.2474 \\
116.4025 \\
86.9479\end{array}$ & $\begin{array}{r}\text { Max } \\
106.3740 \\
108.9393 \\
97.6938 \\
98.9183 \\
98.9032 \\
90.2006 \\
117.9650 \\
88.5104\end{array}$ & $\begin{array}{r}\text { Ave } \\
104.9326 \\
107.1659 \\
96.0219 \\
97.0433 \\
96.9853 \\
89.1108 \\
117.3009 \\
87.8111\end{array}$ & $\begin{array}{l}\text { SD } \\
5.0823 e-01 \\
5.2026 e-01 \\
5.0093 e-01 \\
5.1900 e-01 \\
5.1854 e-01 \\
4.0258 e-01 \\
3.4849 e-01 \\
3.8275 e-01\end{array}$ & $\begin{array}{c}\text { Uncert } \\
4.2321 e+00 \\
4.2377 e+00 \\
4.2109 e+00 \\
4.2133 e+00 \\
4.2131 e+00 \\
4.1957 e+00 \\
4.2645 e+00 \\
4.1930 e+00\end{array}$ \\
\hline
\end{tabular}




\begin{tabular}{|c|c|c|c|c|}
\hline \multicolumn{5}{|c|}{$\begin{array}{l}\text { Velocity } 1=0.000100 \text { (ft/sec) } \\
\text { Velocity } 2=0.001200 \text { (ft/sec) } \\
\text { Pressure Drop }=0.013200 \text { (psi) } \\
\text { Tsat in }=0.284302 \text { (deg E) Tsat out }=0.258209 \text { (dea F) }\end{array}$} \\
\hline $\begin{array}{r}0 \\
1 \\
1 \\
2 \\
3 \\
4 \\
5 \\
6 \\
7 \\
8 \\
9 \\
10 \\
11 \\
12 \\
13 \\
14 \\
15 \\
16 \\
17 \\
18 \\
19 \\
20 \\
21 \\
22 \\
23 \\
24 \\
25 \\
26 \\
27 \\
28 \\
29 \\
30 \\
31 \\
32 \\
33 \\
34 \\
35 \\
36 \\
37 \\
38 \\
39 \\
40 \\
41 \\
42 \\
43 \\
44 \\
45\end{array}$ & $\begin{array}{l}\text { Name } \\
\text { TRF000001 } \\
\text { TRF000002 } \\
\text { TFMO00001 } \\
\text { TIN000001 } \\
\text { TRV000001 } \\
\text { TSR000001 } \\
\text { TAO020136 } \\
\text { TAO050136 } \\
\text { TAO070136 } \\
\text { TAO080136 } \\
\text { TAO090136 } \\
\text { TAO100136 } \\
\text { TAO105136 } \\
\text { TAO110136 } \\
\text { TAO115136 } \\
\text { TAO120136 } \\
\text { TAO125136 } \\
\text { TAO130136 } \\
\text { TAO120048 } \\
\text { TAO130048 } \\
\text { TAO120224 } \\
\text { TAO130224 } \\
\text { TAO120312 } \\
\text { TAO130312 } \\
\text { TRT020000 } \\
\text { TRT070000 } \\
\text { TRT100000 } \\
\text { TRT120000 } \\
\text { TRT125000 } \\
\text { TRT130000 } \\
\text { TAF0 EMTY } \\
\text { TAF0 } 0136 \\
\text { TAF0 } 0130136 \\
\text { TAF090136 } 100136 \\
\text { TAF110136 } \\
\text { TAF115136 } \\
\text { TAF120136 } \\
\text { TAF125136 } \\
\text { TAF130136 } \\
\text { TAF120048 } \\
\text { TAF130048 } \\
\text { TAF120224 } \\
\text { TAF130224 }\end{array}$ & $\begin{array}{l}\text { Abs Diff } \\
0.0894 \\
0.1228 \\
0.1513 \\
0.1179 \\
0.1804 \\
0.6925 \\
0.7615 \\
0.1460 \\
0.5466 \\
0.2859 \\
0.1930 \\
0.3011 \\
0.5016 \\
0.4266 \\
0.4121 \\
0.5736 \\
0.3552 \\
0.3986 \\
2.2840 \\
0.3777 \\
0.4396 \\
0.0165 \\
0.6419 \\
0.5053 \\
0.0919 \\
0.6957 \\
0.3110 \\
0.5301 \\
3.3376 \\
0.3688 \\
0.0964 \\
0.2043 \\
0.3108 \\
0.2746 \\
0.6724 \\
0.4350 \\
0.3751 \\
0.4069 \\
0.3824 \\
0.5535 \\
0.4568 \\
0.3446 \\
0.4426 \\
0.3630 \\
0.4163 \\
0.2900\end{array}$ & $\begin{array}{l}\text { PDiff } \\
0.1159 \\
0.1584 \\
0.1722 \\
0.1352 \\
0.2046 \\
0.5715 \\
1.2467 \\
0.0867 \\
0.3240 \\
0.1472 \\
0.0962 \\
0.1512 \\
0.2470 \\
0.2027 \\
0.2008 \\
0.2669 \\
0.2912 \\
0.1903 \\
2.3961 \\
0.1895 \\
0.2178 \\
0.0086 \\
0.3364 \\
0.2697 \\
0.0599 \\
0.3987 \\
0.1652 \\
0.2706 \\
3.8620 \\
0.1939 \\
0.2432 \\
0.1684 \\
0.2324 \\
0.1434 \\
0.7846 \\
0.2781 \\
0.2346 \\
0.2490 \\
0.2276 \\
0.2897 \\
0.2609 \\
0.1936 \\
0.2731 \\
0.2153 \\
0.2587 \\
0.1761\end{array}$ & \\
\hline
\end{tabular}




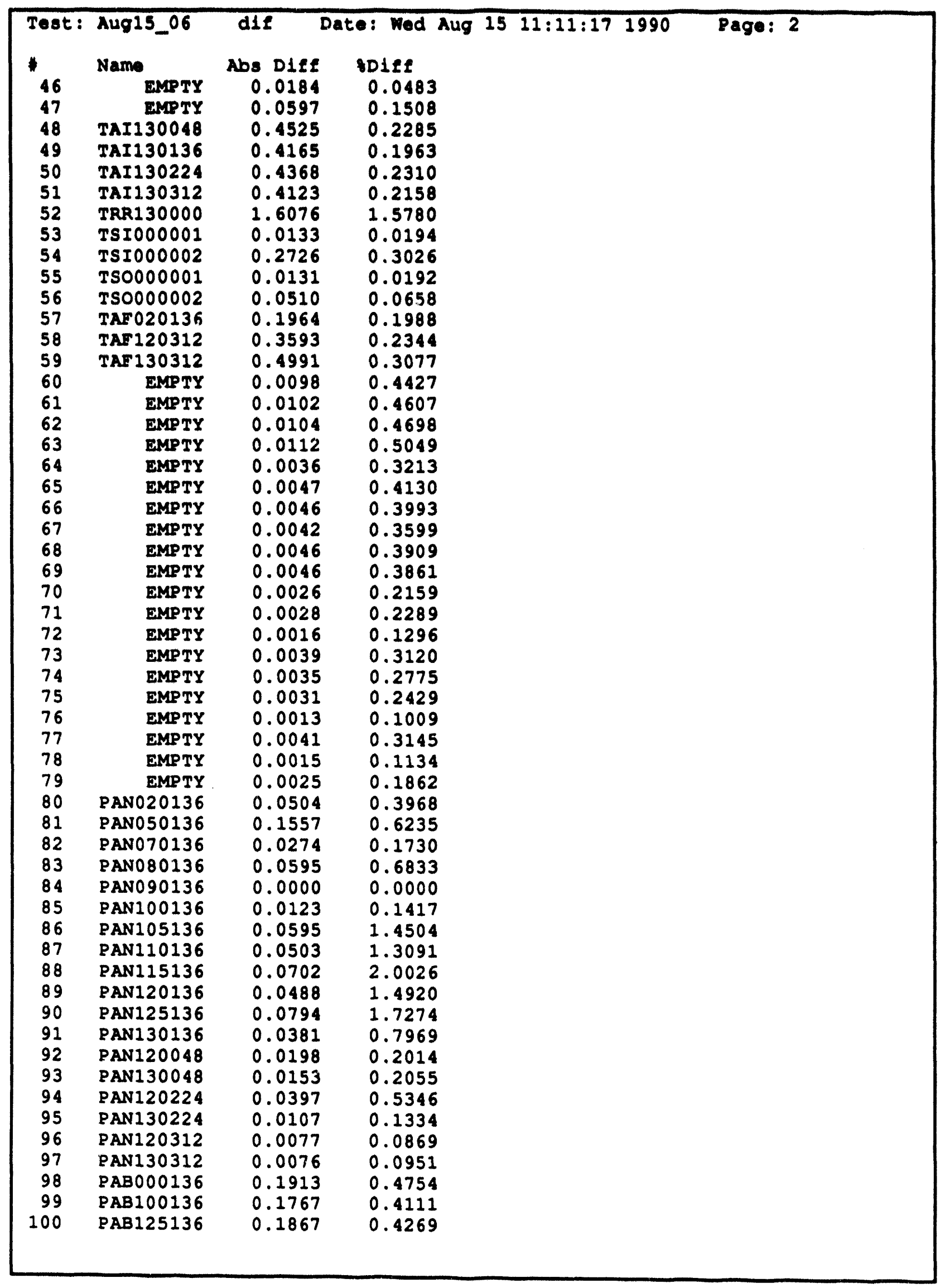




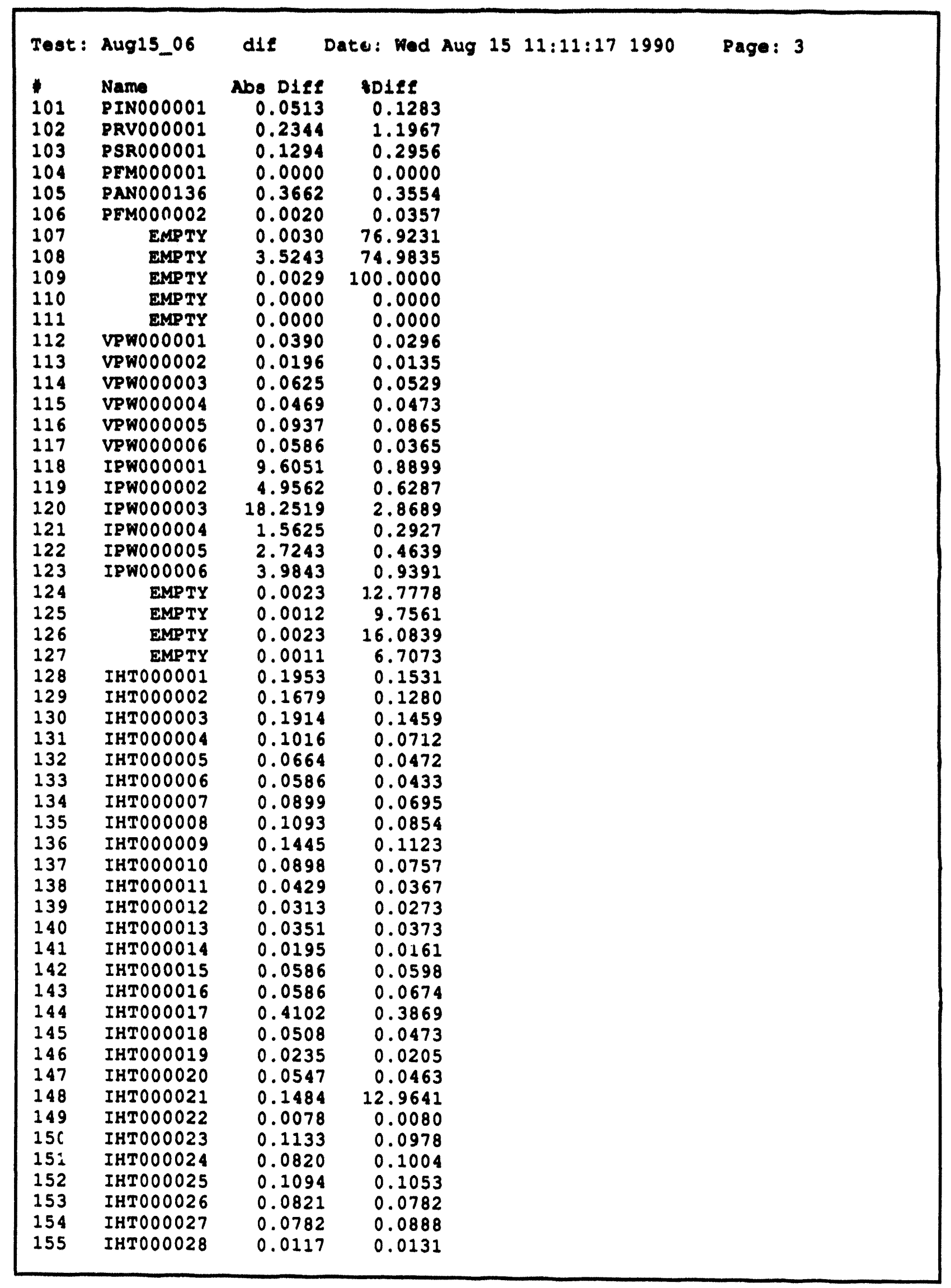




\begin{tabular}{|c|c|c|c|c|}
\hline $\begin{array}{l}\text { Test: } \\
156 \\
157 \\
158 \\
159 \\
160 \\
161 \\
162 \\
163\end{array}$ & 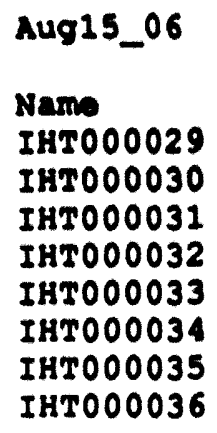 & $\begin{array}{l}\text { dif } \\
\text { Abs Diff } \\
0.0234 \\
0.0742 \\
0.0039 \\
0.0351 \\
0.0391 \\
0.0859 \\
0.1328 \\
0.0781\end{array}$ & $\begin{array}{l}\text { Date: Wed Aug } 1511: 11: 171990 \\
\text { IDIfE } \\
0.0223 \\
0.0692 \\
0.0041 \\
0.0362 \\
0.0403 \\
1 \\
0.0963 \\
0.1131 \\
0.0889\end{array}$ & Page: 4 \\
\hline
\end{tabular}




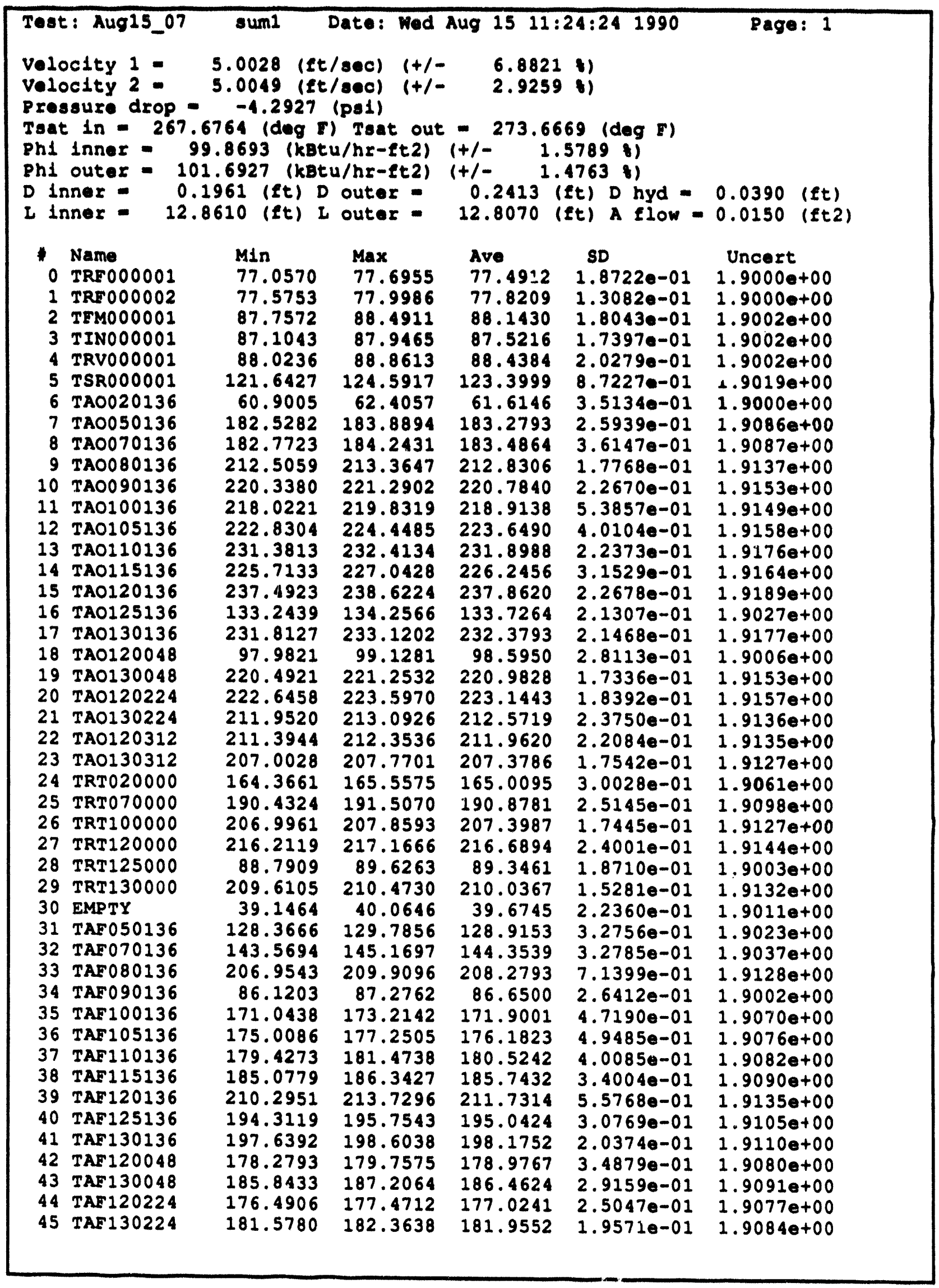




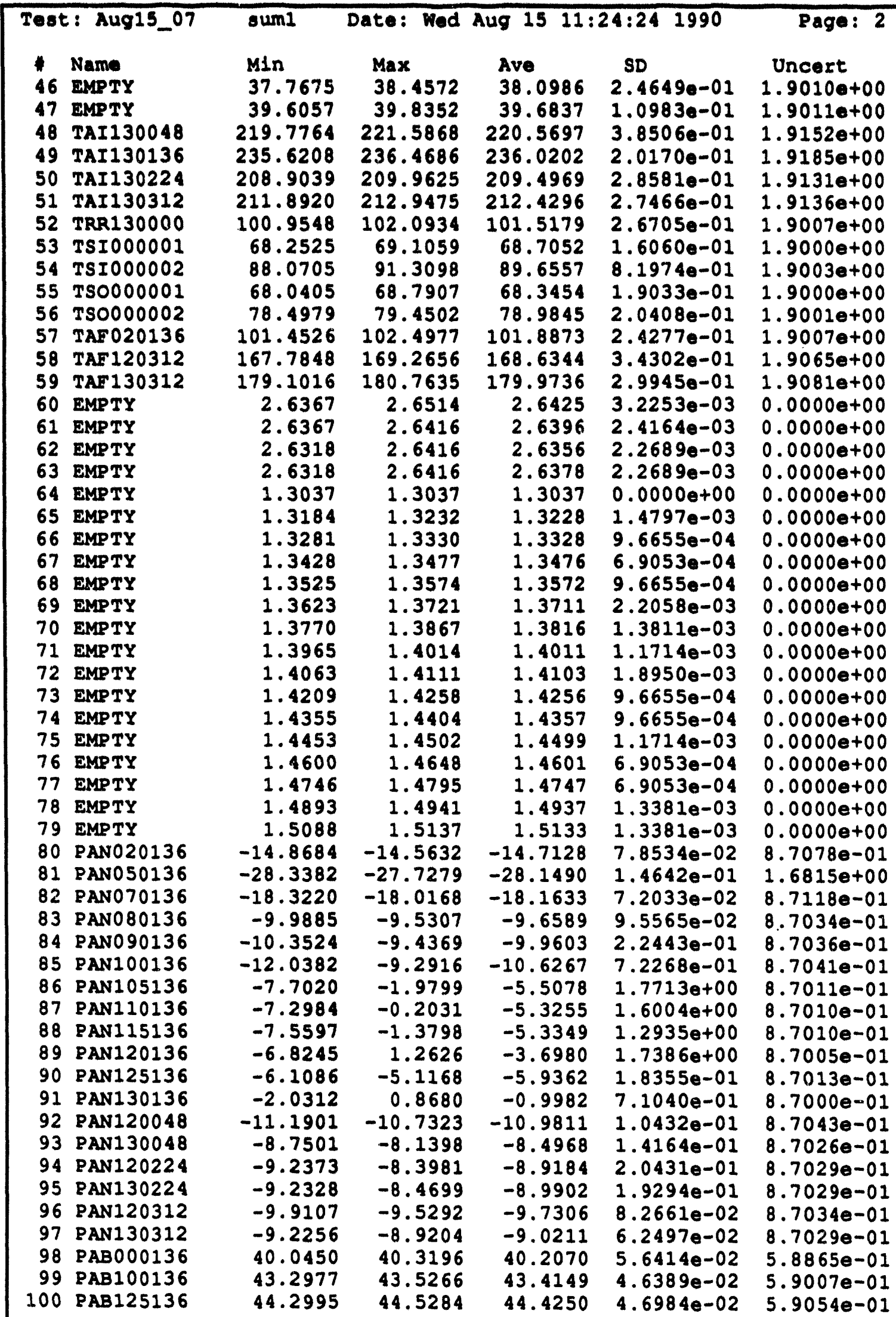




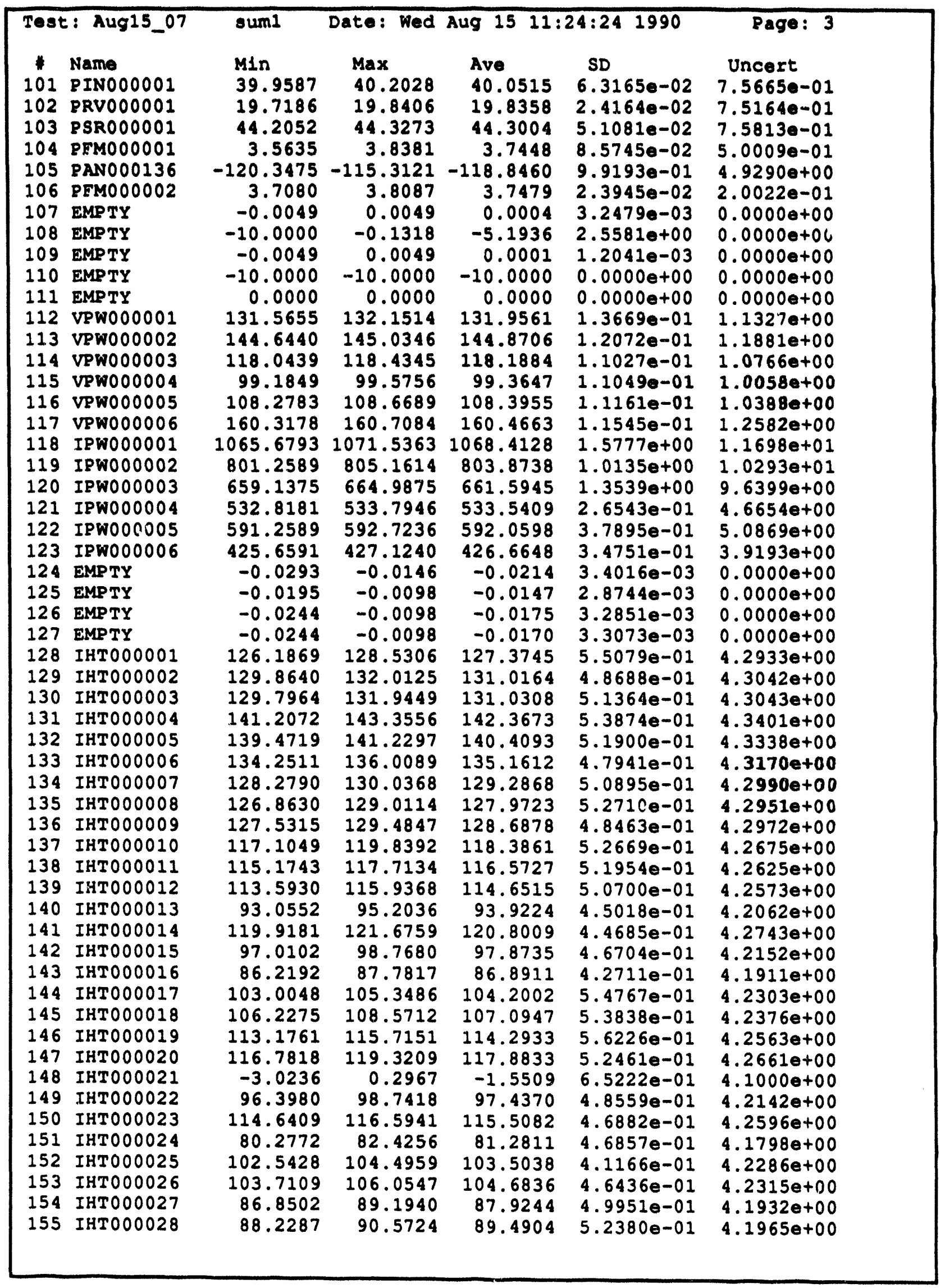




\begin{tabular}{|c|c|c|c|c|c|c|}
\hline \multicolumn{2}{|c|}{ Test: Aug15_07 } & & Date: Wed & \multicolumn{2}{|c|}{ Aug $15 \quad 11: 24: 241990$} & $\begin{array}{l}\text { Page: } 4 \\
\text { Uncert }\end{array}$ \\
\hline $\begin{array}{l}1 \\
156 \\
157 \\
158 \\
159 \\
160 \\
161 \\
162 \\
163\end{array}$ & $\begin{array}{l}\text { Name } \\
\text { IHTO00029 } \\
\text { IHTO00030 } \\
\text { IHTO00031 } \\
\text { IHTO00032 } \\
\text { IHTO00033 } \\
\text { IHTO00034 } \\
\text { IHTO00035 } \\
\text { IHTO00036 }\end{array}$ & $\begin{array}{l}\text { M1n } \\
103.4443 \\
105.8143 \\
94.7641 \\
95.5980 \\
95.5829 \\
88.2474 \\
116.2072 \\
86.9479\end{array}$ & $\begin{array}{l}\text { Max } \\
105.7880 \\
107.7674 \\
97.1079 \\
97.9417 \\
97.9267 \\
90.2006 \\
118.1603 \\
88.9010\end{array}$ & $\begin{array}{l}\text { Ave } \\
104.6045 \\
106.8651 \\
95.7329 \\
96.8128 \\
96.7588 \\
89.2436 \\
117.3283 \\
87.9361\end{array}$ & $\begin{array}{l}\text { SD } \\
5.1812 e-01 \\
5.0515 e-01 \\
5.2193 e-01 \\
5.5284 e-01 \\
5.4746 e-01 \\
4.5890 e-01 \\
4.7648 e-01 \\
4.6590 e-01\end{array}$ & $\begin{array}{l}\text { Uncert } \\
4.2313 e+00 \\
4.2370 e+00 \\
4.2103 e+00 \\
4.2128 e+00 \\
4.2126 e+00 \\
4.1960 e+00 \\
4.2646 e+00 \\
4.1932 e+00\end{array}$ \\
\hline
\end{tabular}




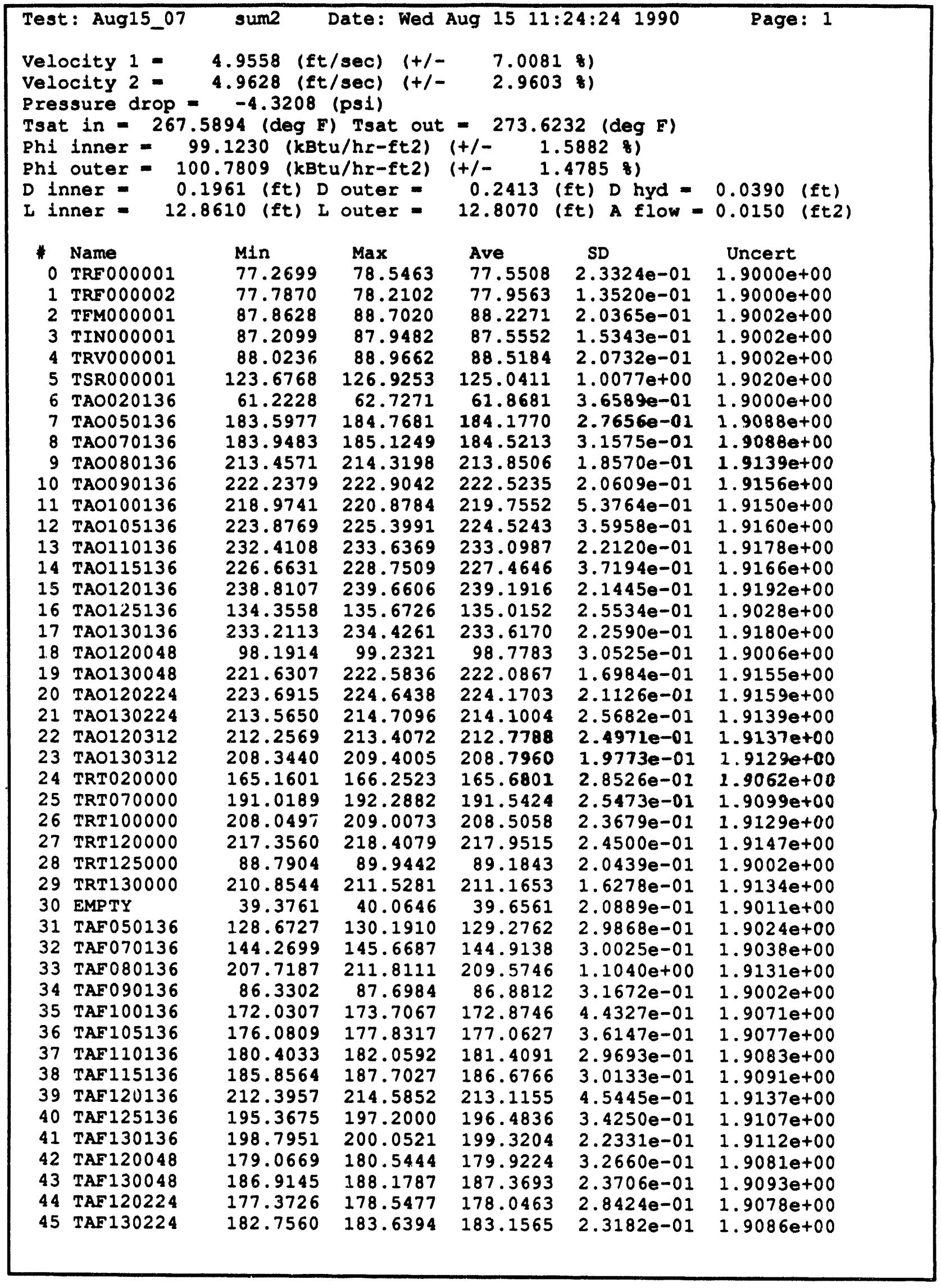




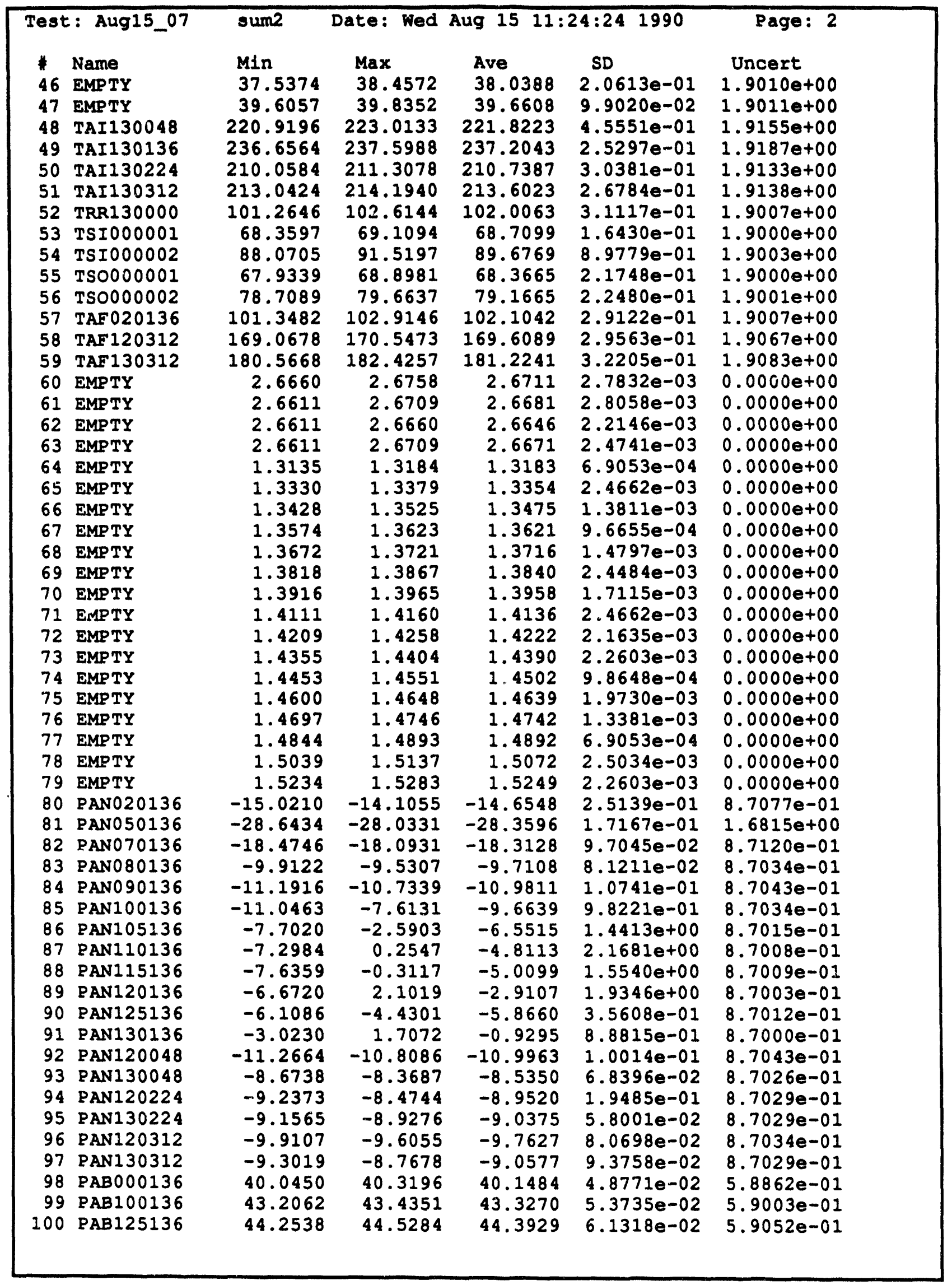




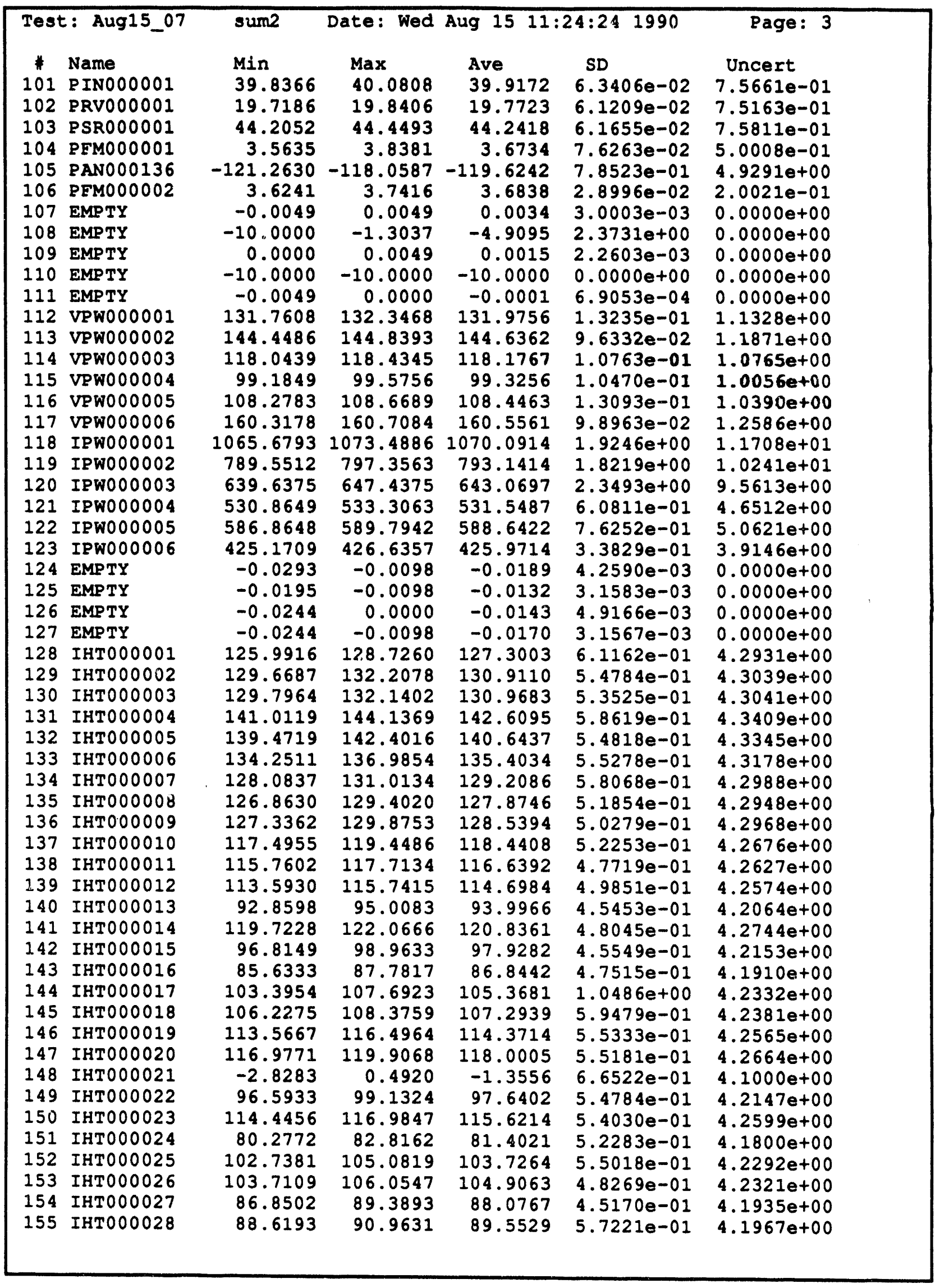




\begin{tabular}{|c|c|c|c|c|c|c|}
\hline \multicolumn{2}{|c|}{ Test: Aug15_07 } & sum2 & Date: Wed & Aug 1511 & $24: 24 \quad 1990$ & Page: 4 \\
\hline $\begin{array}{l}\not \\
156 \\
157 \\
158 \\
159 \\
160 \\
161 \\
162 \\
163\end{array}$ & $\begin{array}{l}\text { Name } \\
\text { IHTO00029 } \\
\text { IHTO00030 } \\
\text { IHTO00031 } \\
\text { IHTO00032 } \\
\text { IHTO00033 } \\
\text { IHTO00034 } \\
\text { IHTO00035 } \\
\text { IHTO00036 }\end{array}$ & $\begin{array}{l}\text { Min } \\
103.8349 \\
106.2049 \\
94.9594 \\
95.9886 \\
95.9736 \\
88.4428 \\
116.4025 \\
87.1432\end{array}$ & $\begin{array}{l}\text { Max } \\
106.3740 \\
108.5487 \\
97.6938 \\
98.5276 \\
98.5126 \\
90.3959 \\
118.1603 \\
88.7057\end{array}$ & $\begin{array}{l}\text { Ave } \\
104.7802 \\
107.1112 \\
95.9634 \\
97.0433 \\
96.9931 \\
89.2241 \\
117.3009 \\
87.8892\end{array}$ & $\begin{array}{l}\text { SD } \\
6.0407 e-01 \\
5.8362 e-01 \\
5.8395 e-01 \\
6.0746 e-01 \\
5.8032 e-01 \\
4.7351 e-01 \\
4.8488 e-01 \\
4.5795 e-01\end{array}$ & $\begin{array}{l}\text { Uncert } \\
4.2318 e+00 \\
4.2376 e+00 \\
4.2108 e+00 \\
4.2133 e+00 \\
4.2132 e+00 \\
4.1960 e+00 \\
4.2645 e+00 \\
4.1931 e+00\end{array}$ \\
\hline
\end{tabular}




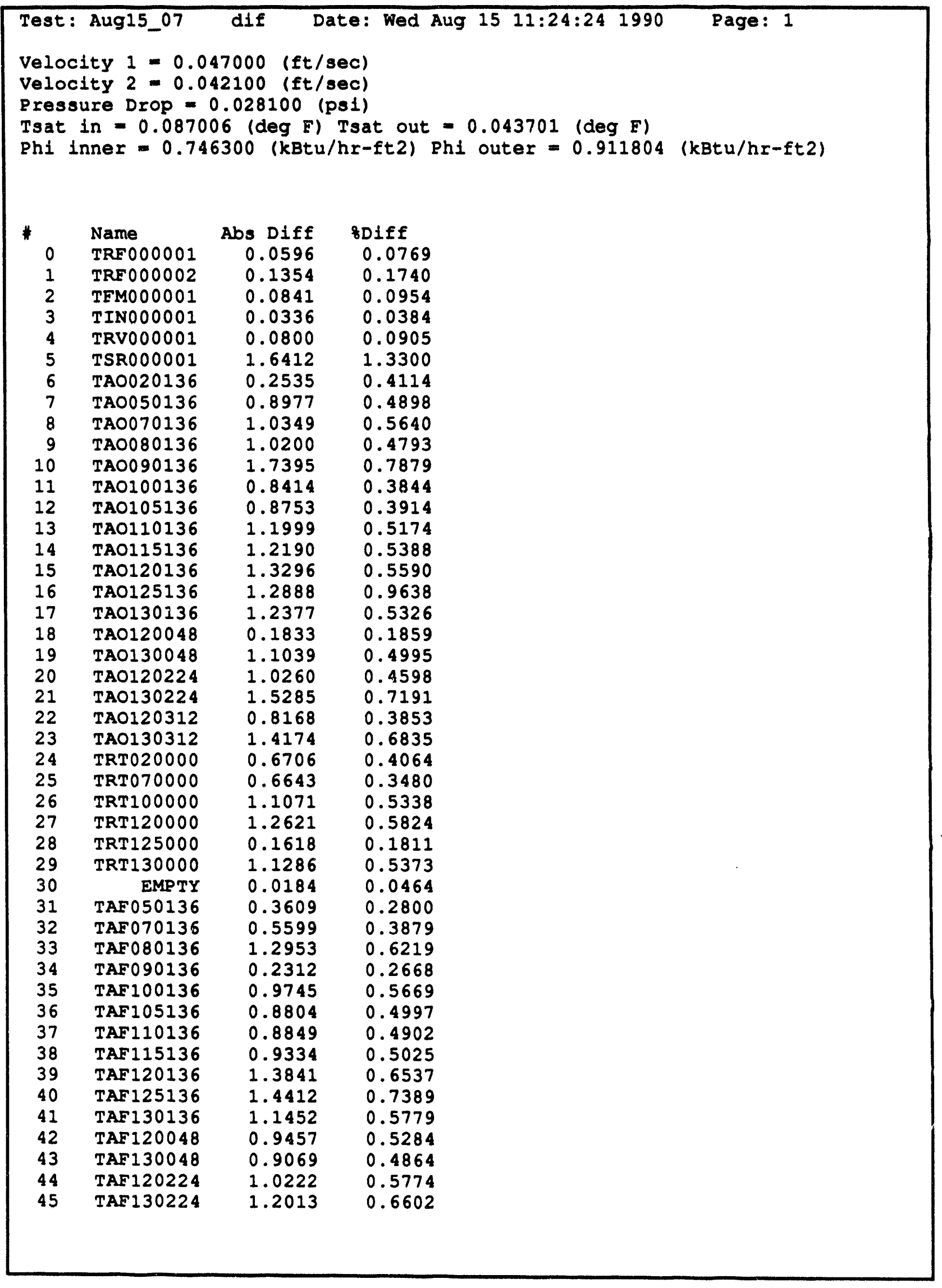




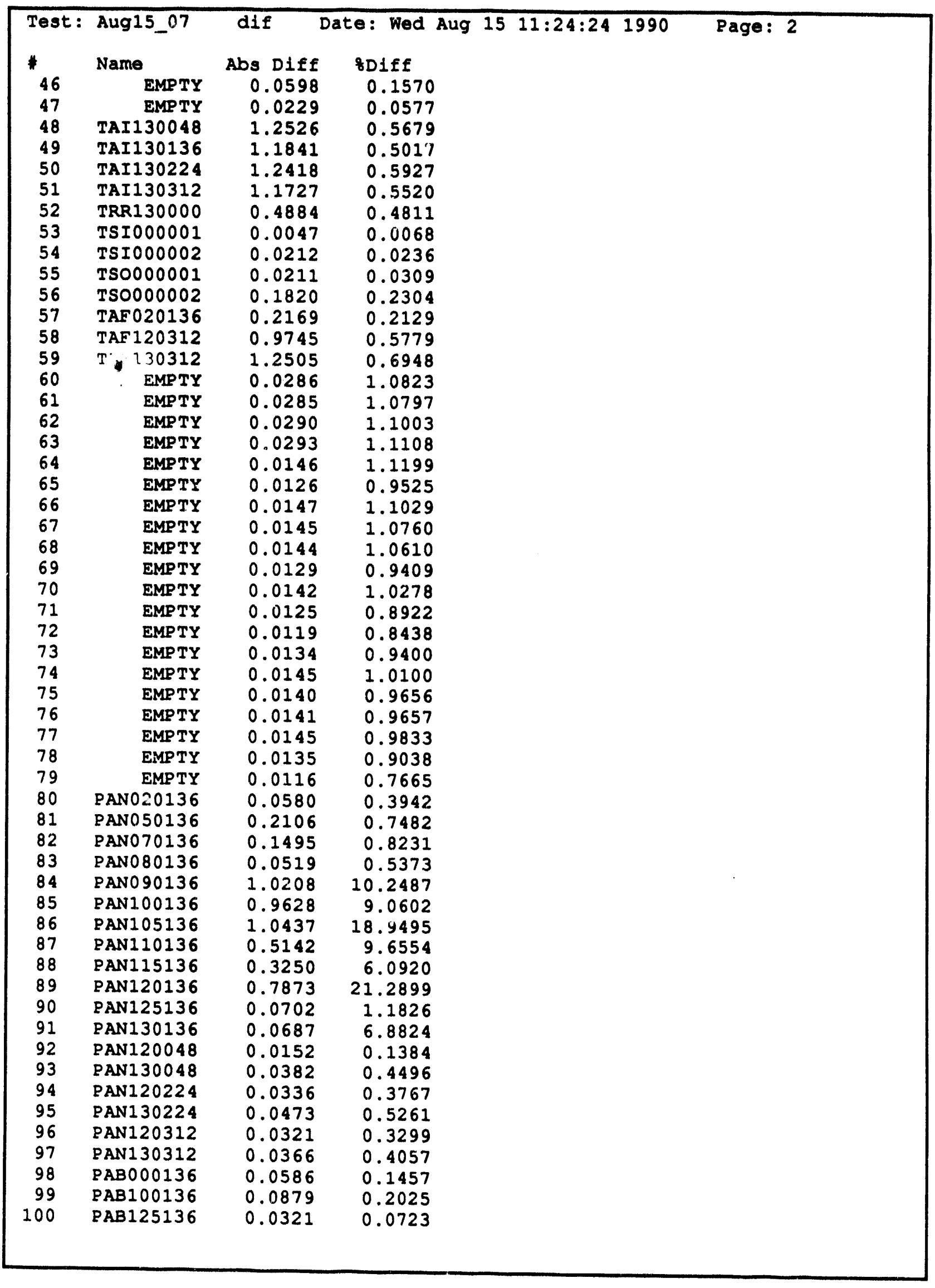




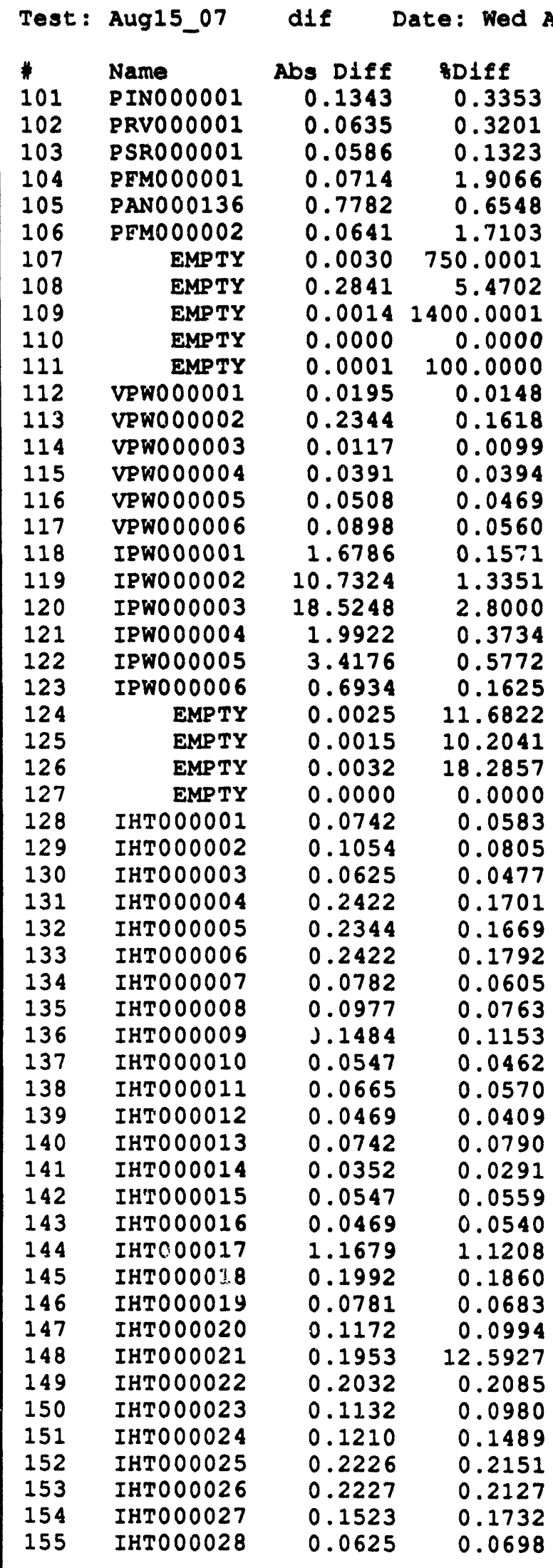




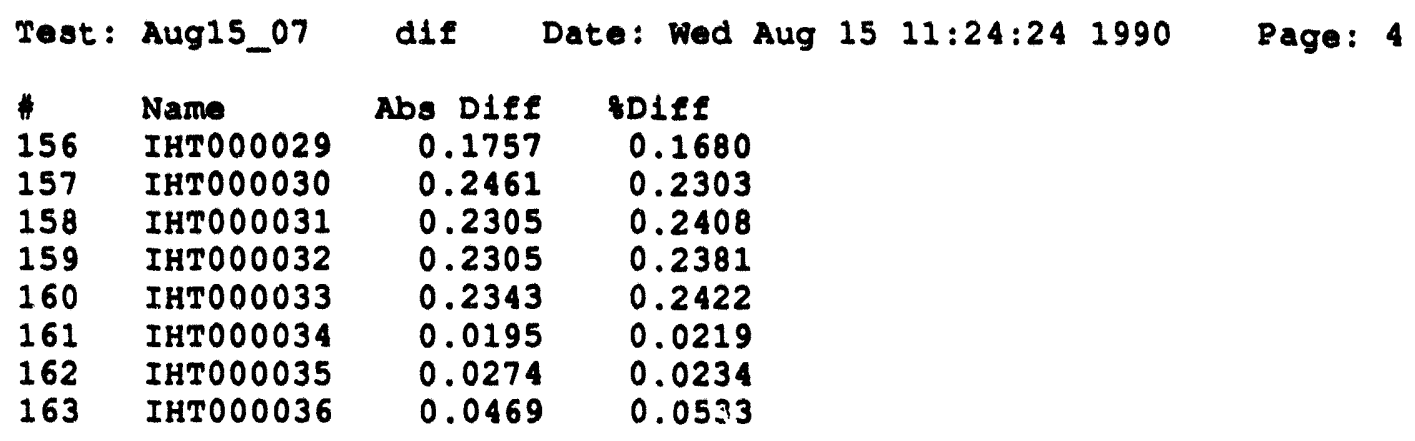




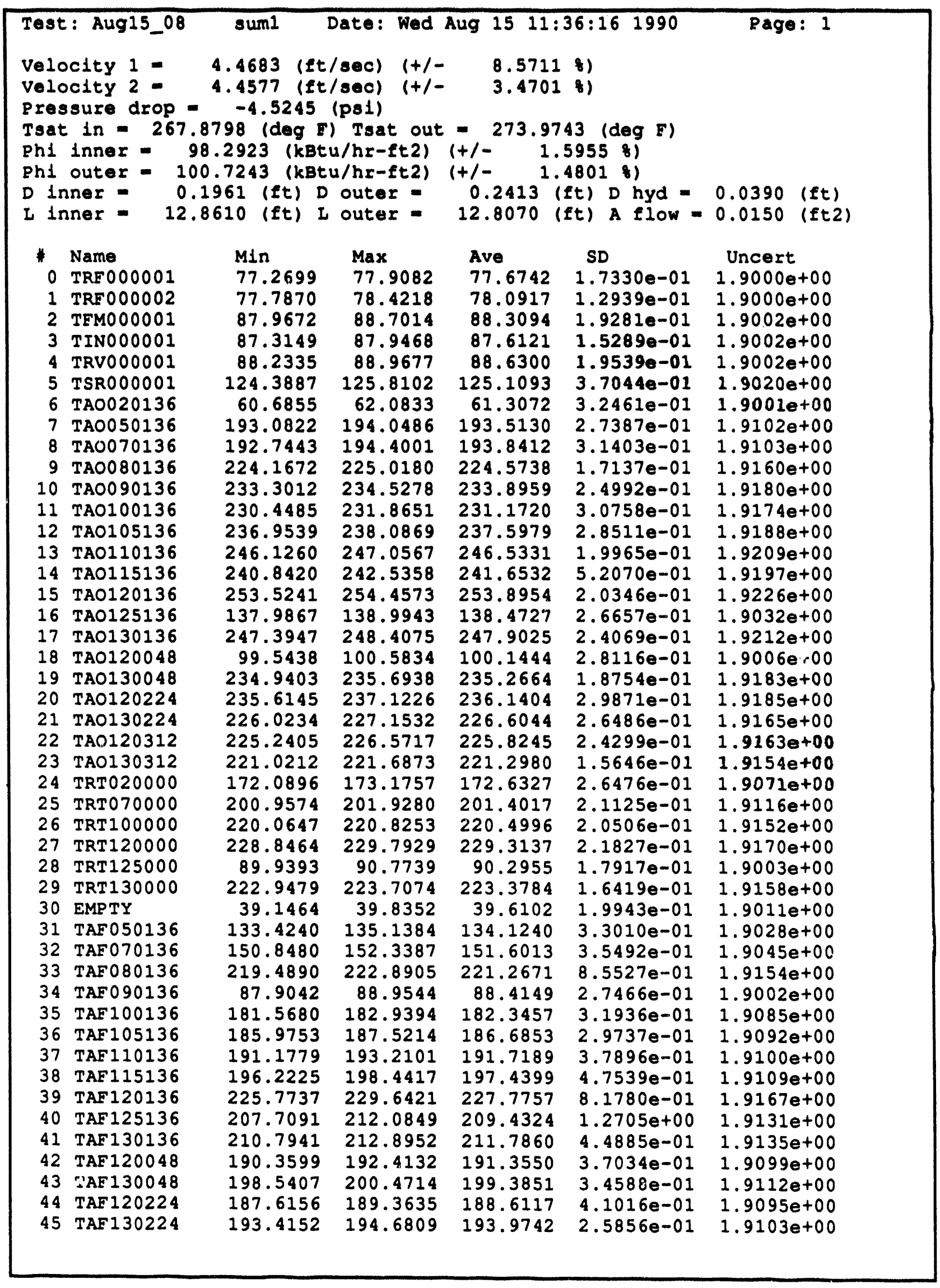




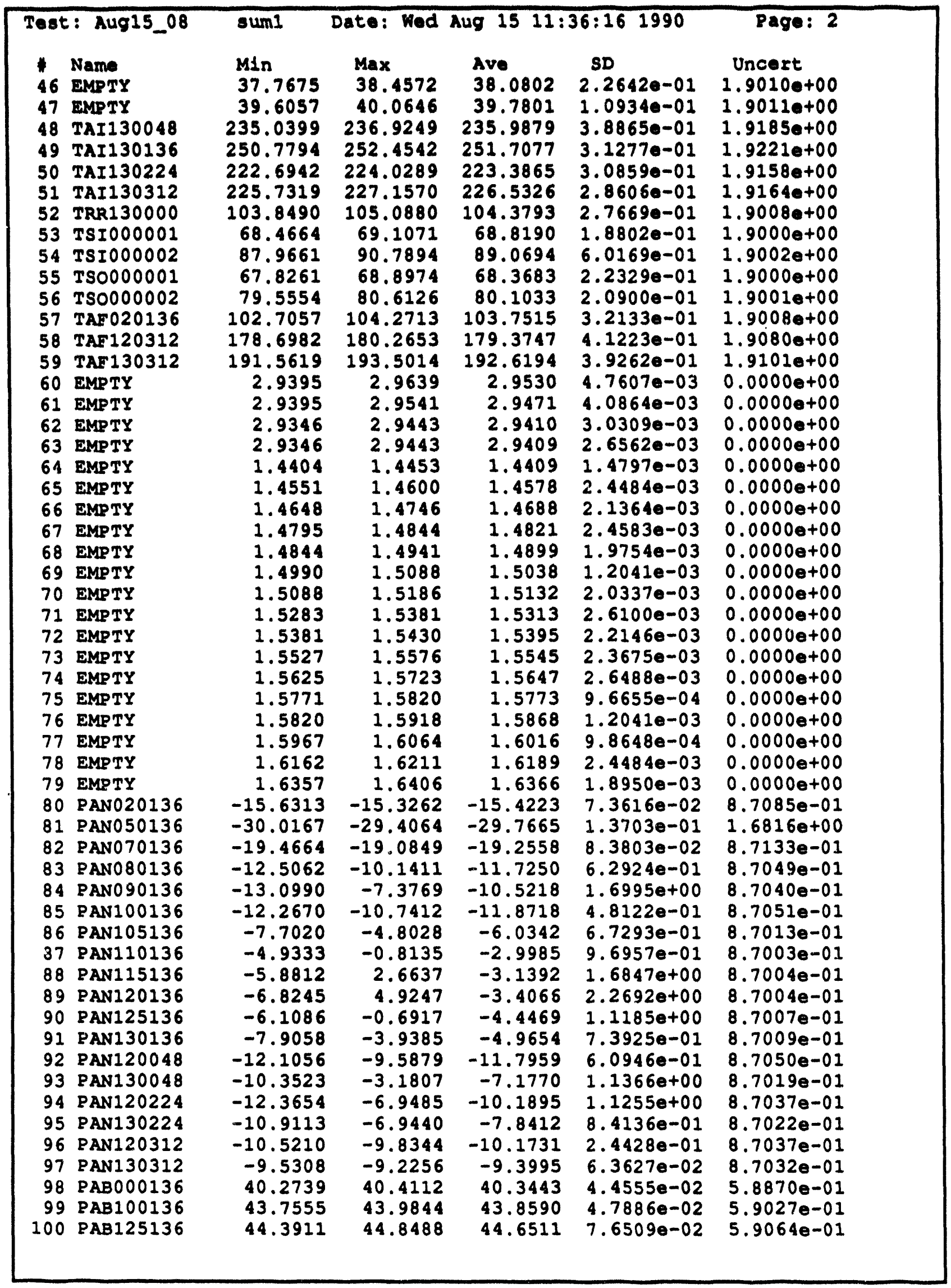




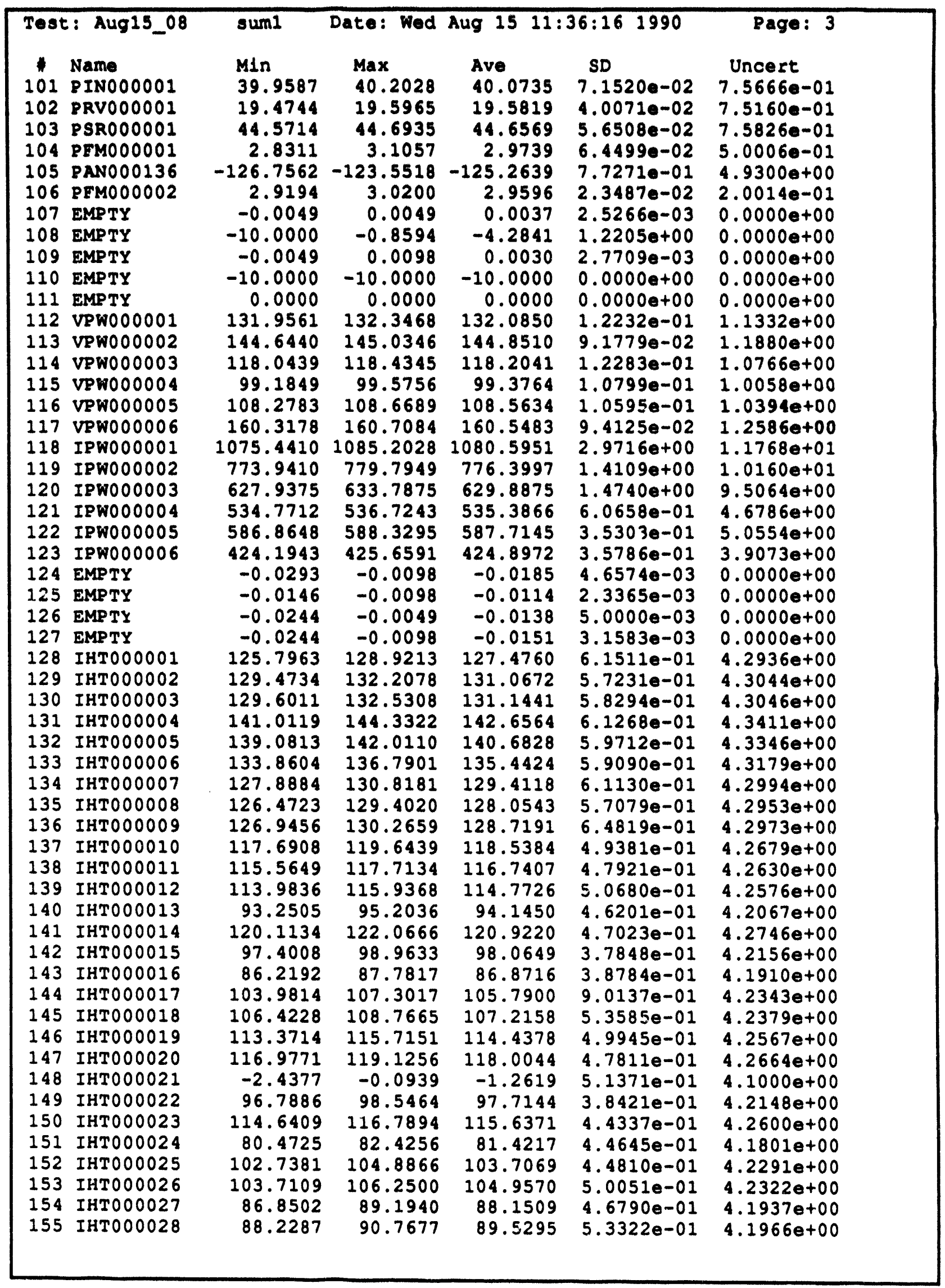




\begin{tabular}{|c|c|c|c|c|c|c|}
\hline $\begin{array}{l}1 \\
156 \\
157 \\
158 \\
159 \\
160 \\
161 \\
162 \\
163\end{array}$ & $\begin{array}{l}\text { Name } \\
\text { IHTO00029 } \\
\text { IHTO00030 } \\
\text { IHTO00031 } \\
\text { IHTO00032 } \\
\text { IHTO00033 } \\
\text { IHTO00034 } \\
\text { IHTO00035 } \\
\text { IHTO0O0 } 0036\end{array}$ & $\begin{array}{l}\text { Min } \\
103.6396 \\
105.8143 \\
94.9594 \\
95.9886 \\
95.7782 \\
88.0521 \\
116.5978 \\
87.1432\end{array}$ & $\begin{array}{r}\operatorname{Max} \\
105.7880 \\
107.9627 \\
97.1079 \\
98.3323 \\
98.5126 \\
90.0053 \\
118.1603 \\
88.9010\end{array}$ & $\begin{array}{l}\text { Ave } \\
104.7568 \\
107.0917 \\
96.0571 \\
97.1370 \\
97.0908 \\
89.1733 \\
117.3009 \\
87.8853\end{array}$ & $\begin{array}{l}\text { SD } \\
5.0229 e-01 \\
5.3410 e-01 \\
5.2924 e-01 \\
5.2592 e-01 \\
5.3385 e-01 \\
4.1161 e-01 \\
4.1760 e-01 \\
4.1946 e-01\end{array}$ & $\begin{array}{c}\text { Uncert } \\
4.2317 e+00 \\
4.2376 e+00 \\
4.2110 e+00 \\
4.2135 e+00 \\
4.2134 e+00 \\
4.1959 e+00 \\
4.2645 e+00 \\
4.1931 e+00\end{array}$ \\
\hline
\end{tabular}




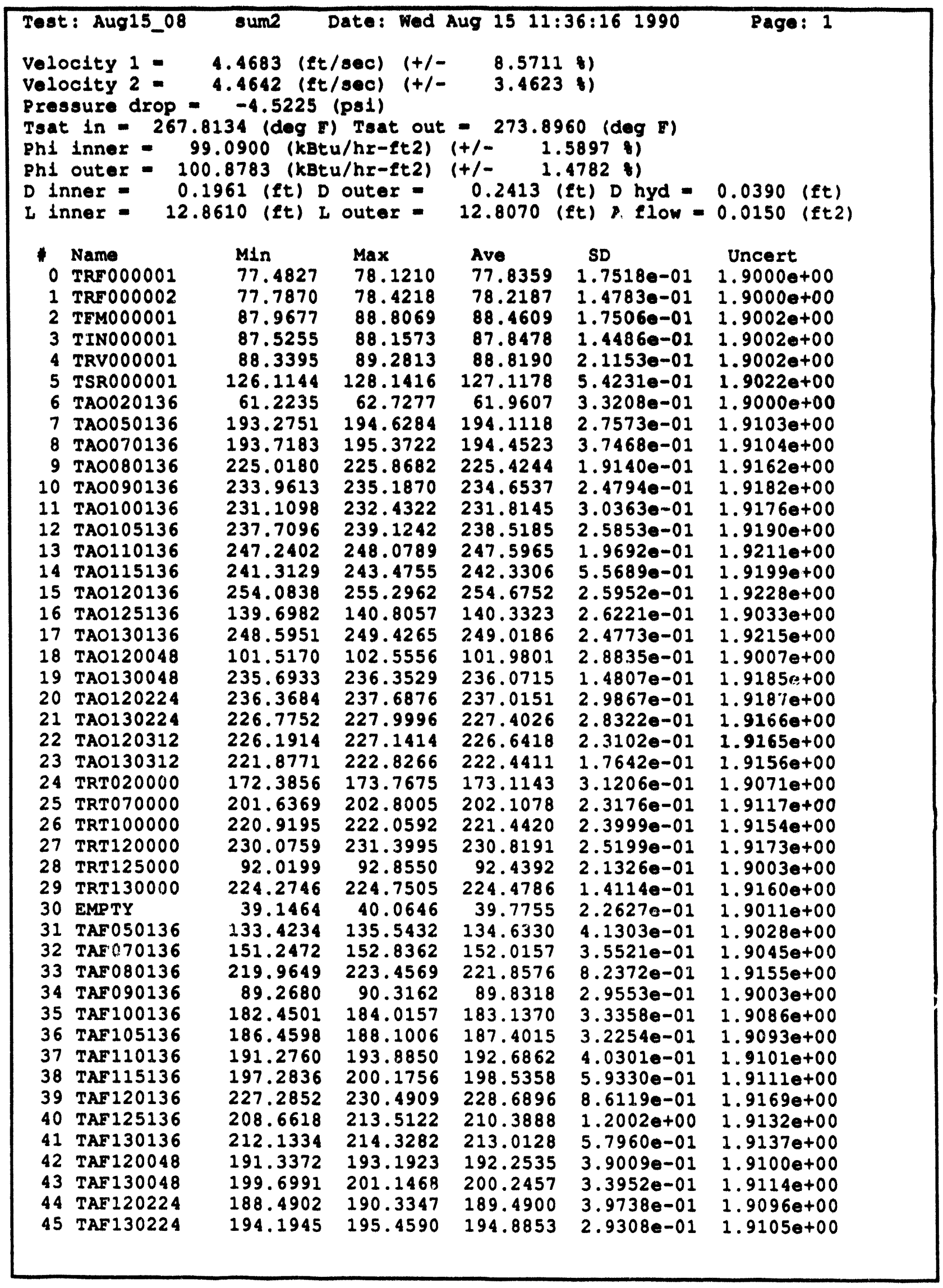




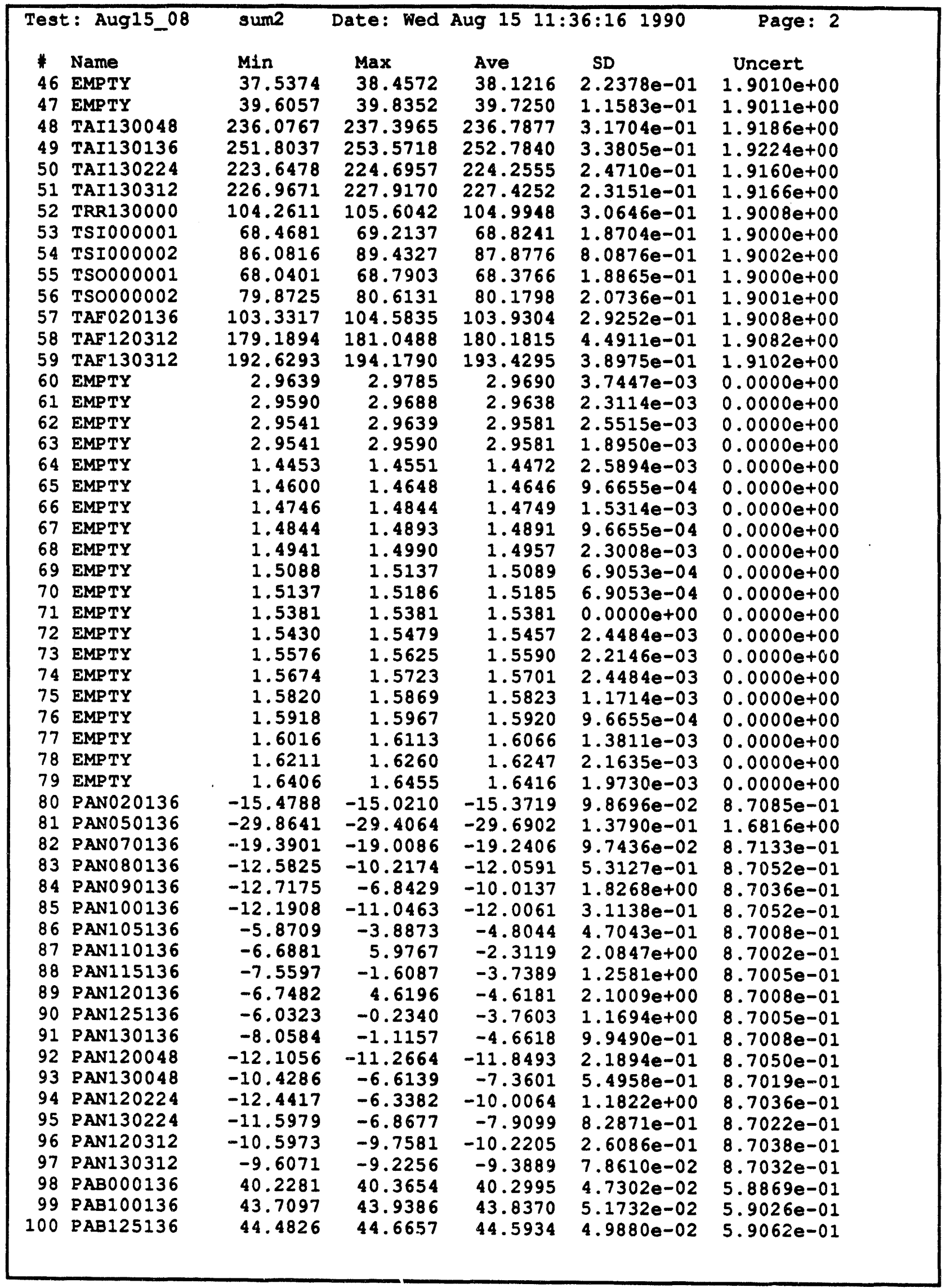




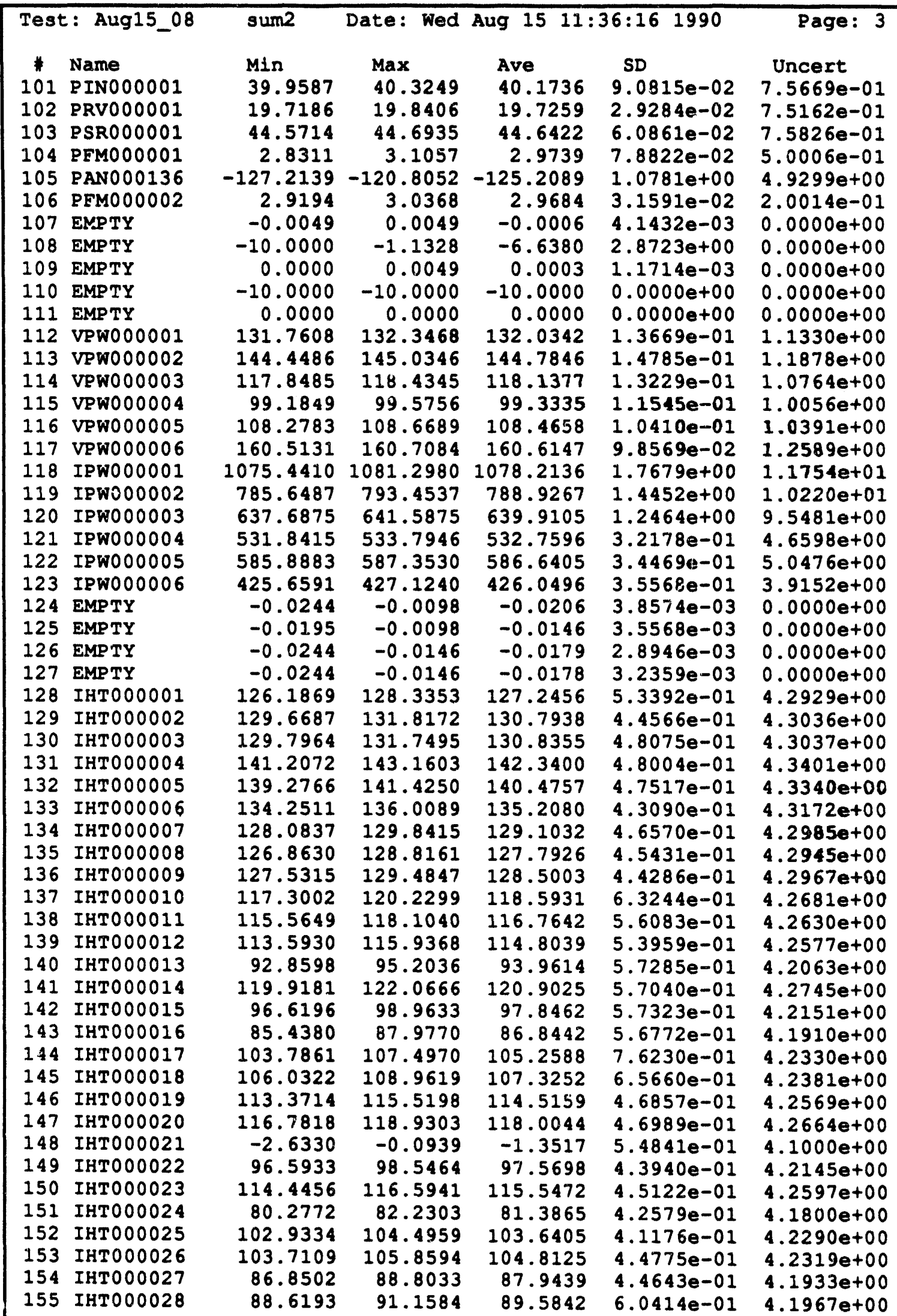




\begin{tabular}{|c|c|c|c|c|c|c|}
\hline $\begin{array}{l}* \\
156 \\
157 \\
158 \\
159 \\
160 \\
161 \\
162 \\
163\end{array}$ & 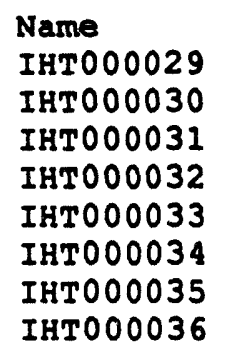 & \begin{tabular}{r}
\multicolumn{1}{c}{ Min } \\
103.8349 \\
105.8143 \\
94.7641 \\
95.7933 \\
95.7782 \\
88.0521 \\
116.4025 \\
86.7525
\end{tabular} & $\begin{array}{l}\text { Max } \\
106.3740 \\
108.7440 \\
97.3032 \\
98.3323 \\
98.3173 \\
90.2006 \\
118.3556 \\
89.0963\end{array}$ & $\begin{array}{l}\text { Ave } \\
104.7529 \\
106.9862 \\
95.7915 \\
96.8167 \\
96.8017 \\
88.9819 \\
117.1993 \\
87.7720\end{array}$ & $\begin{array}{l}\text { SD } \\
5.6806 \mathrm{e}-01 \\
5.5524 \mathrm{e}-01 \\
5.2022 \mathrm{e}-01 \\
5.2580 \mathrm{e}-01 \\
5.1533 \mathrm{e}-01 \\
4.8095 \mathrm{e}-01 \\
5.1120 \mathrm{e}-01 \\
4.9330 \mathrm{e}-01\end{array}$ & $\begin{array}{c}\text { Uncert } \\
4.2317 \mathrm{e}+00 \\
4.2373 \mathrm{e}+00 \\
4.2104 \mathrm{e}+00 \\
4.2128 \mathrm{e}+00 \\
4.2127 \mathrm{e}+00 \\
4.1954 \mathrm{e}+00 \\
4.2642 \mathrm{e}+00 \\
4.1929 \mathrm{e}+00\end{array}$ \\
\hline
\end{tabular}




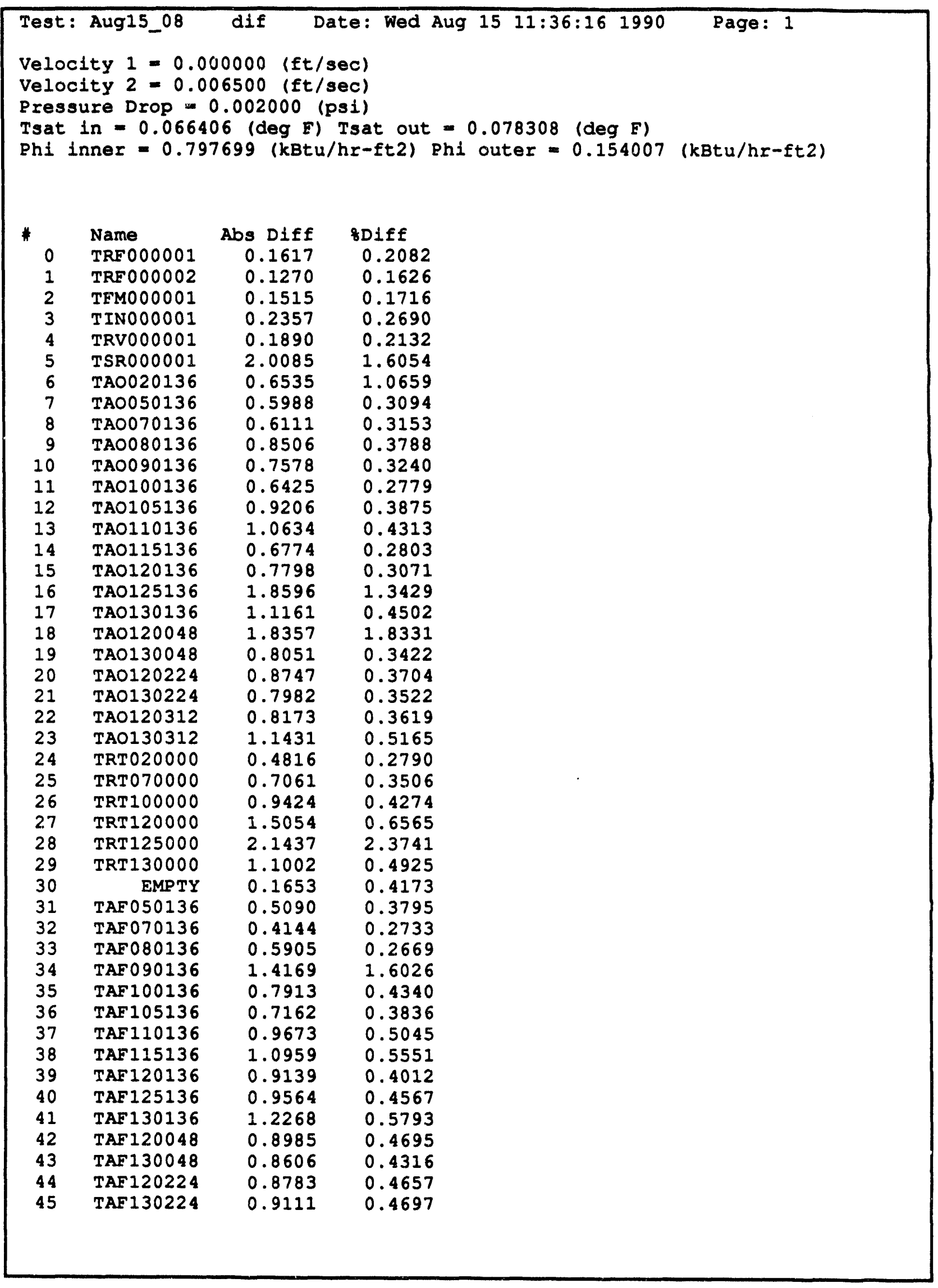




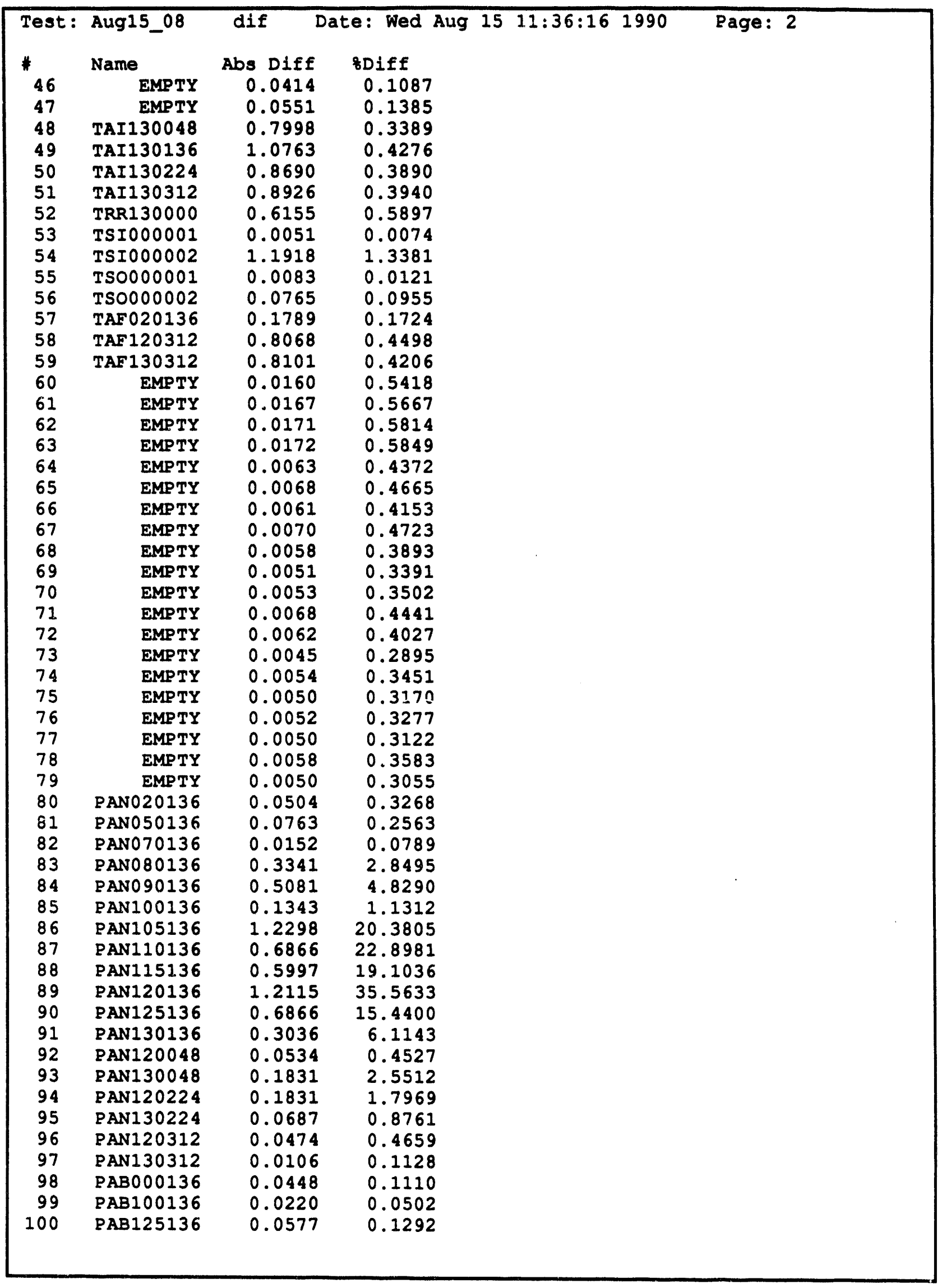




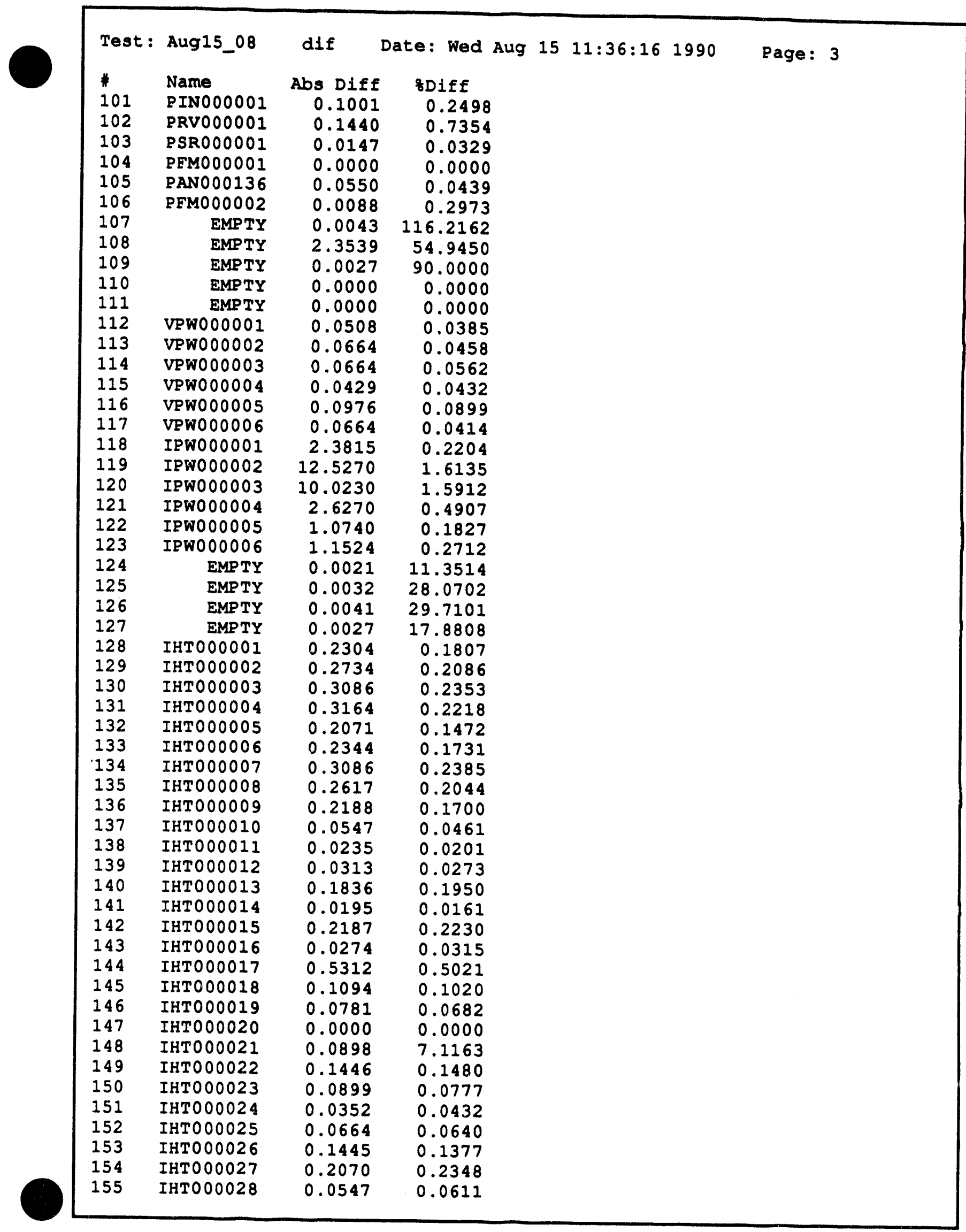




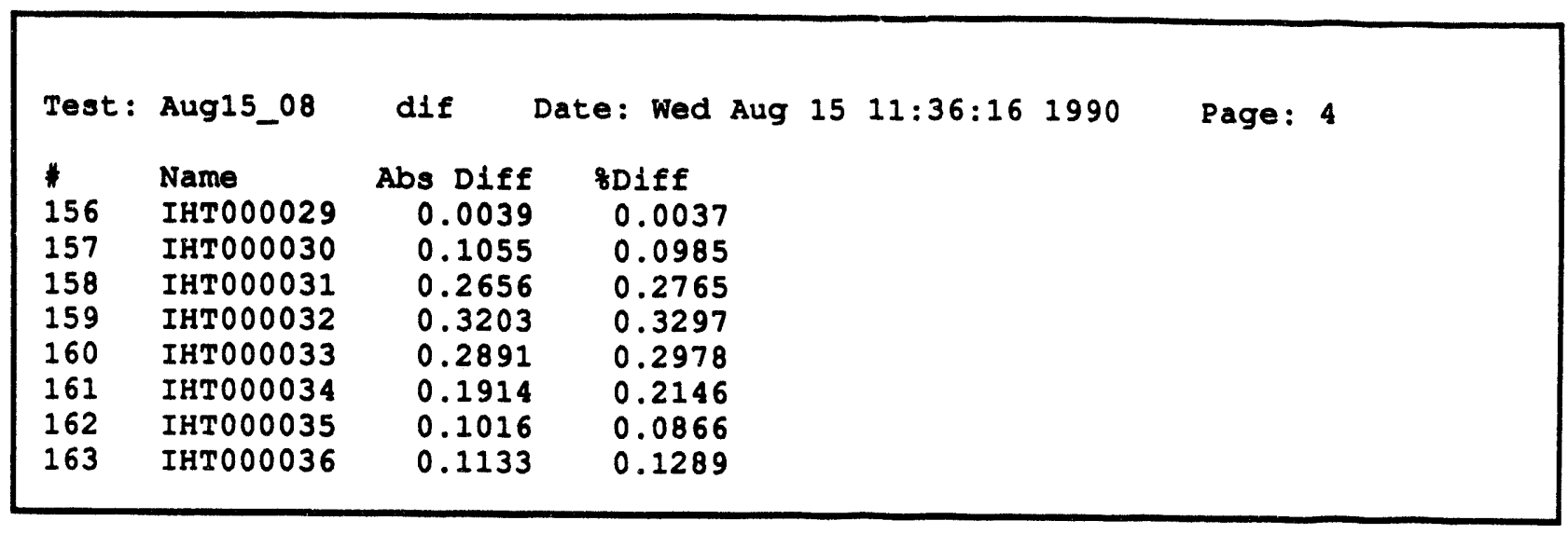




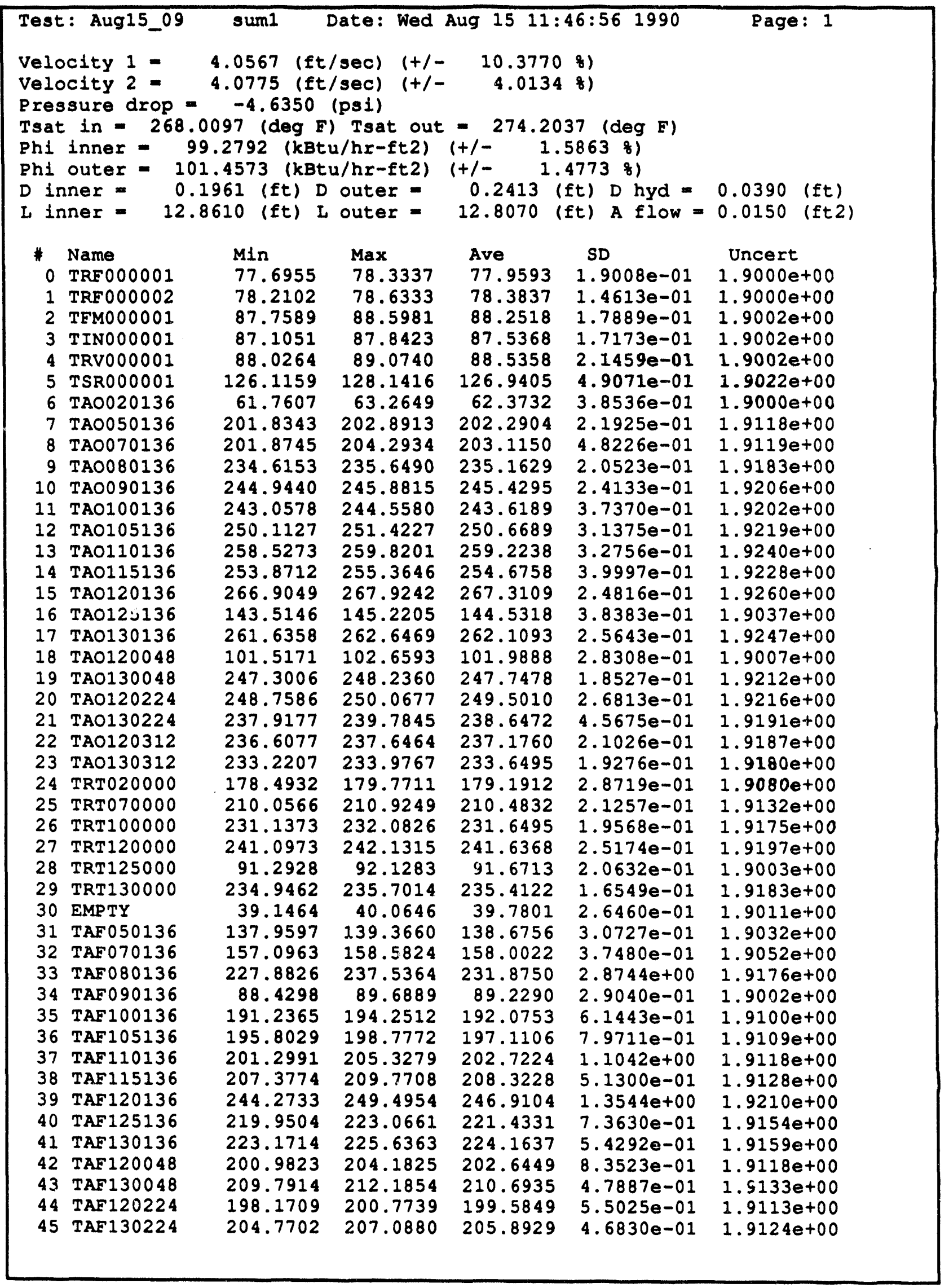




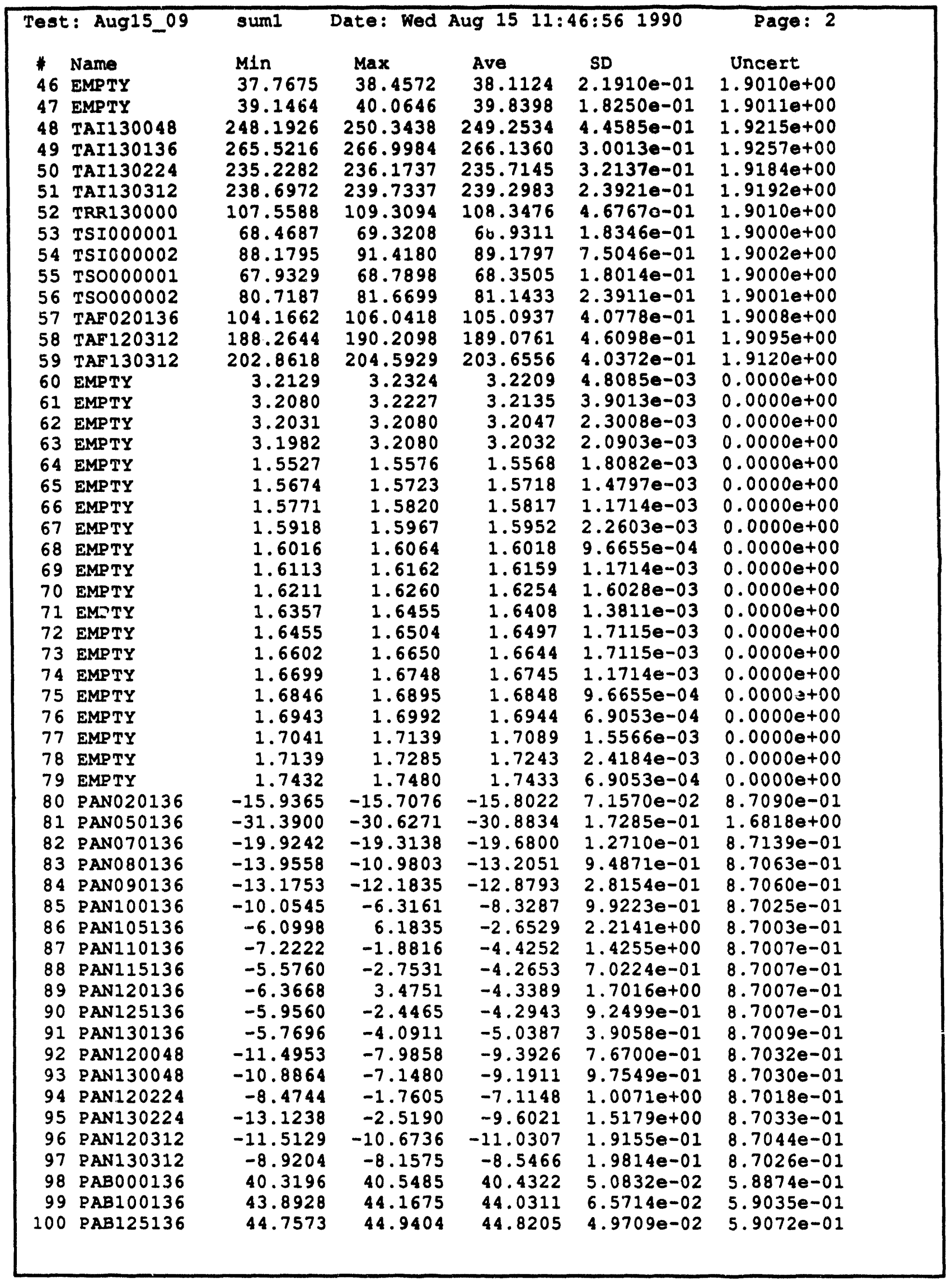




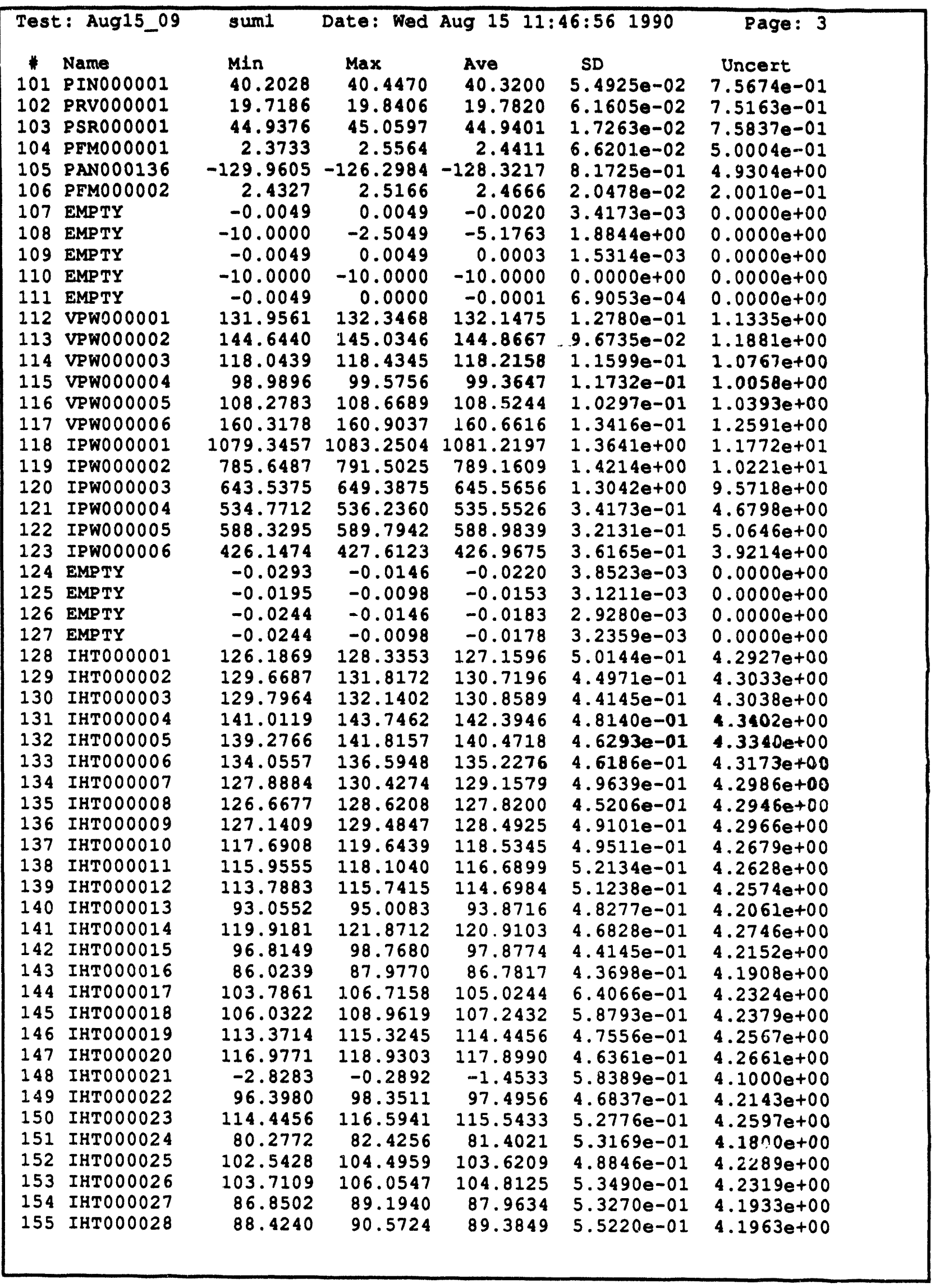




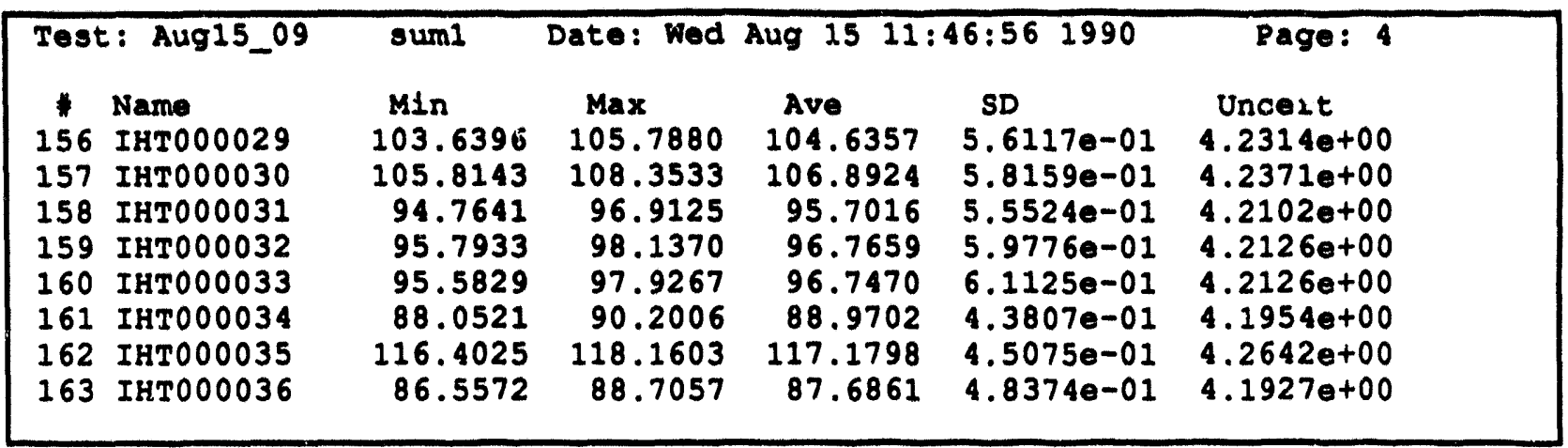




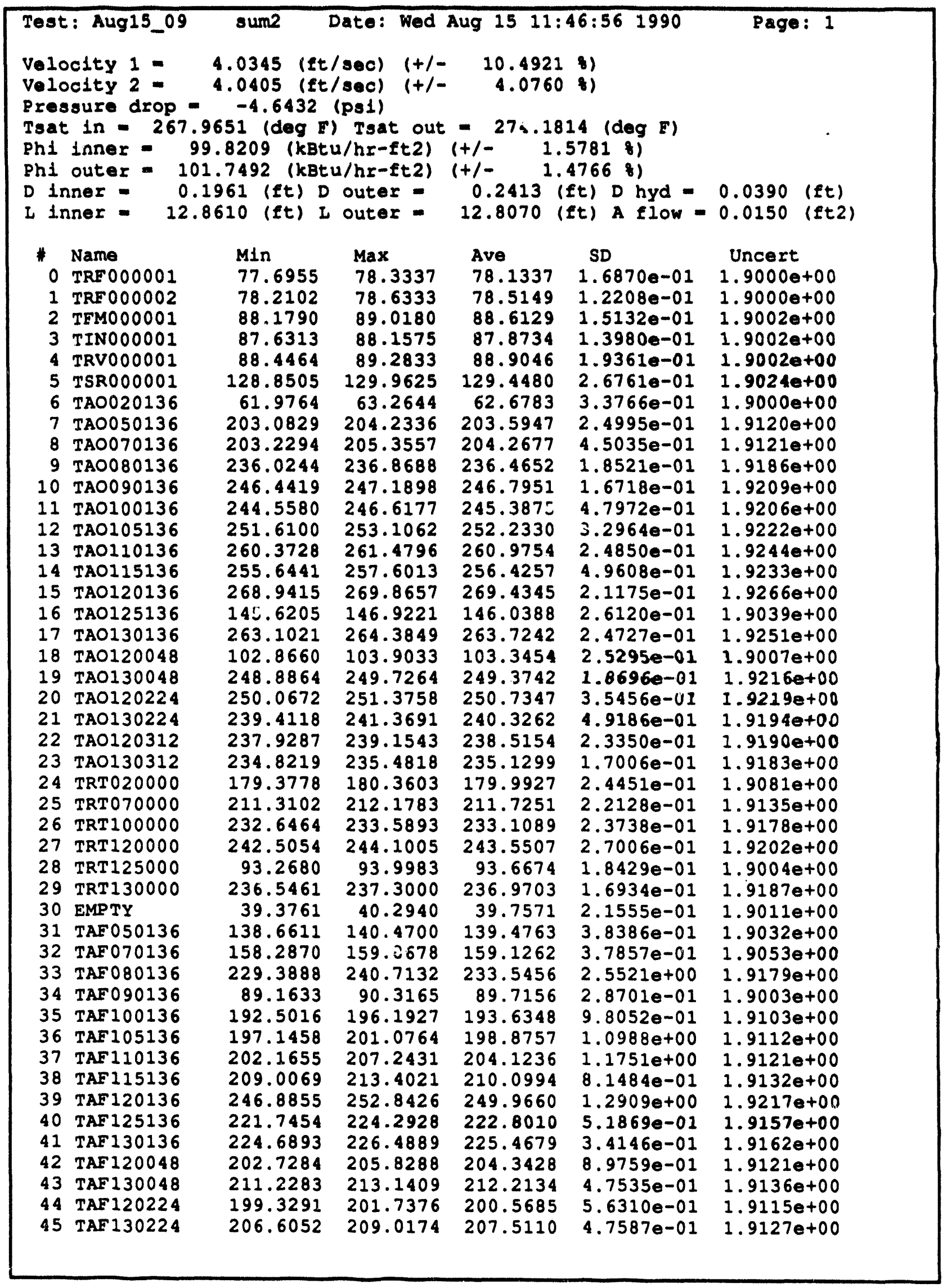




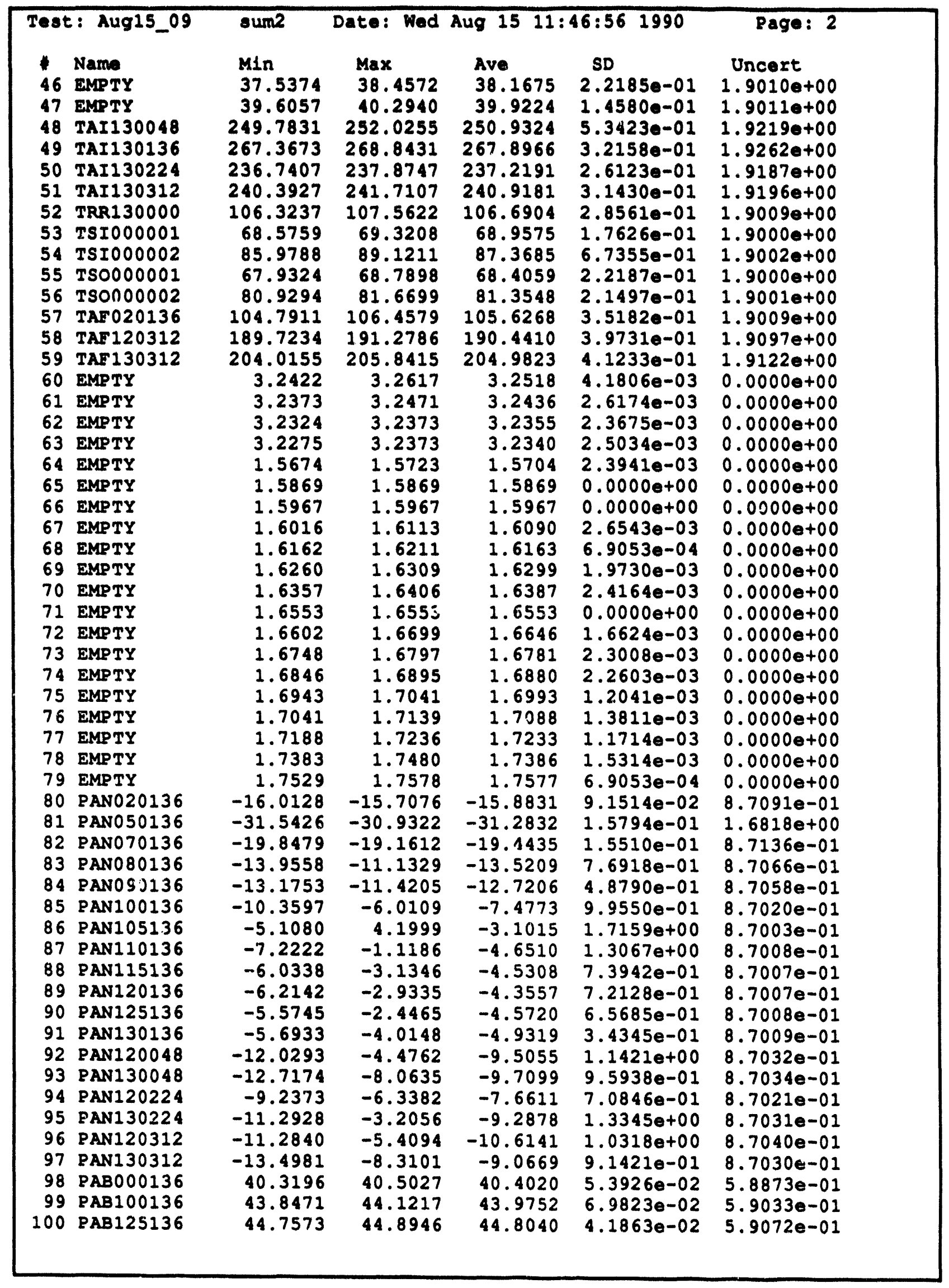




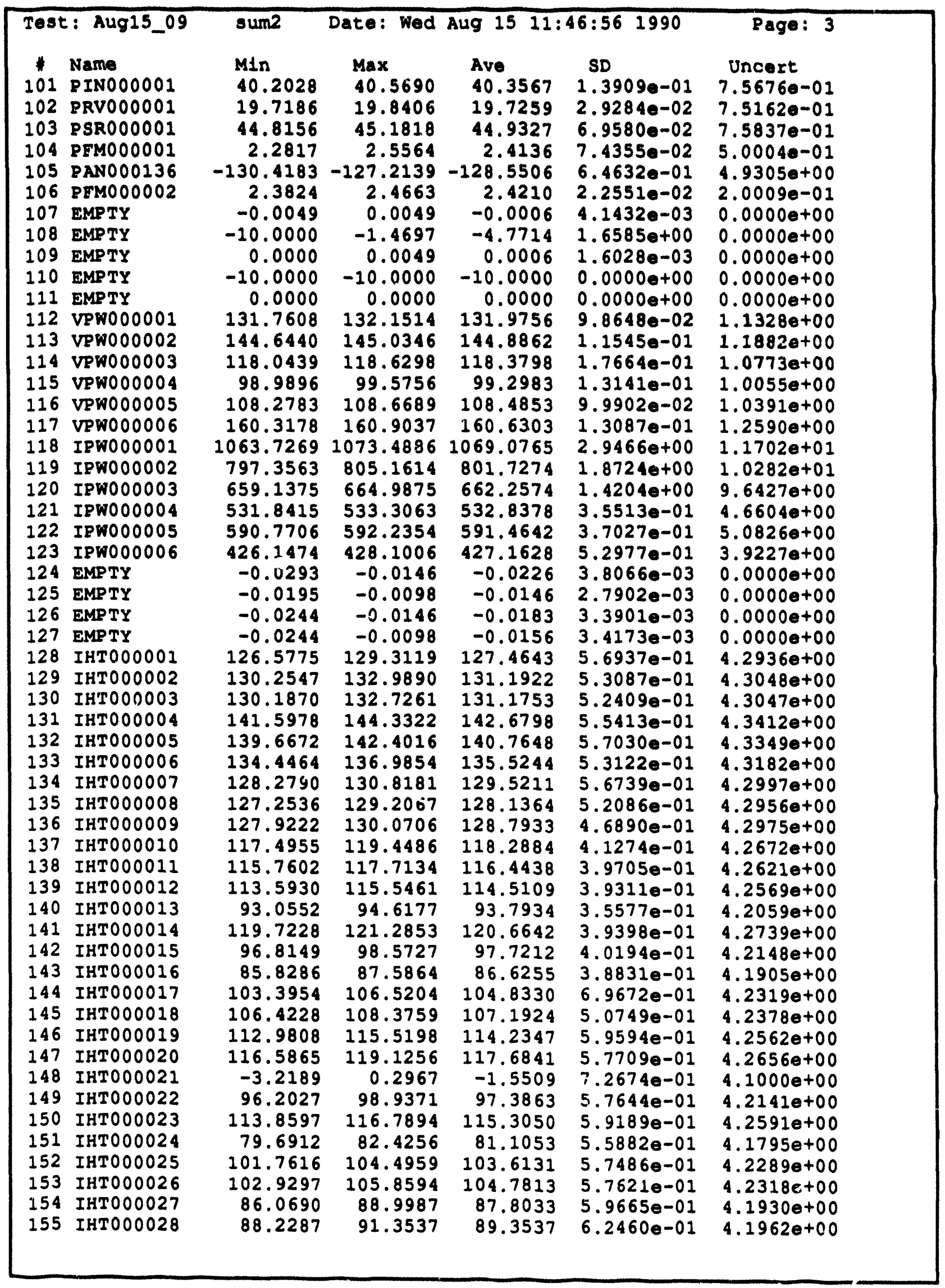



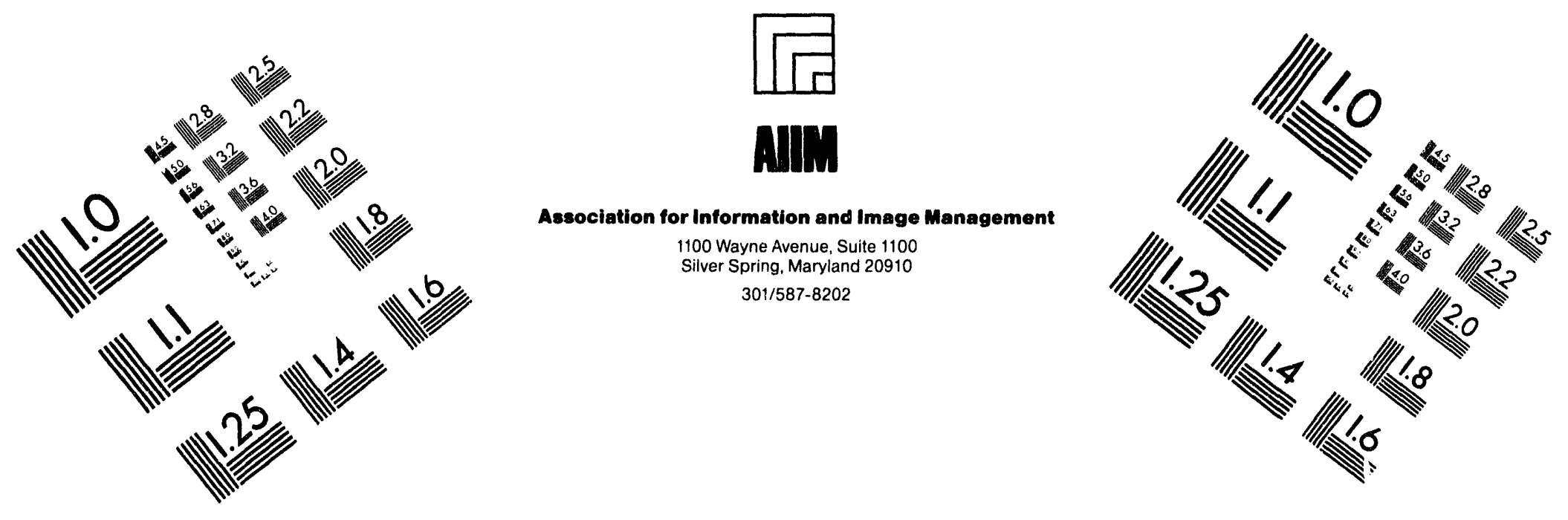

\section{Centimeter}

$\begin{array}{llllllllllllllll}1 & 2 & 3 & 4 & 5 & 6 & 7 & 8 & 9 & 10 & 11 & 12 & 13 & 14 & 15 & \mathrm{~mm}\end{array}$

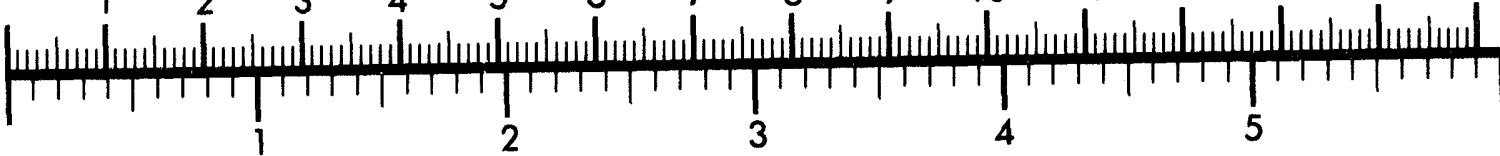

\section{Inches}
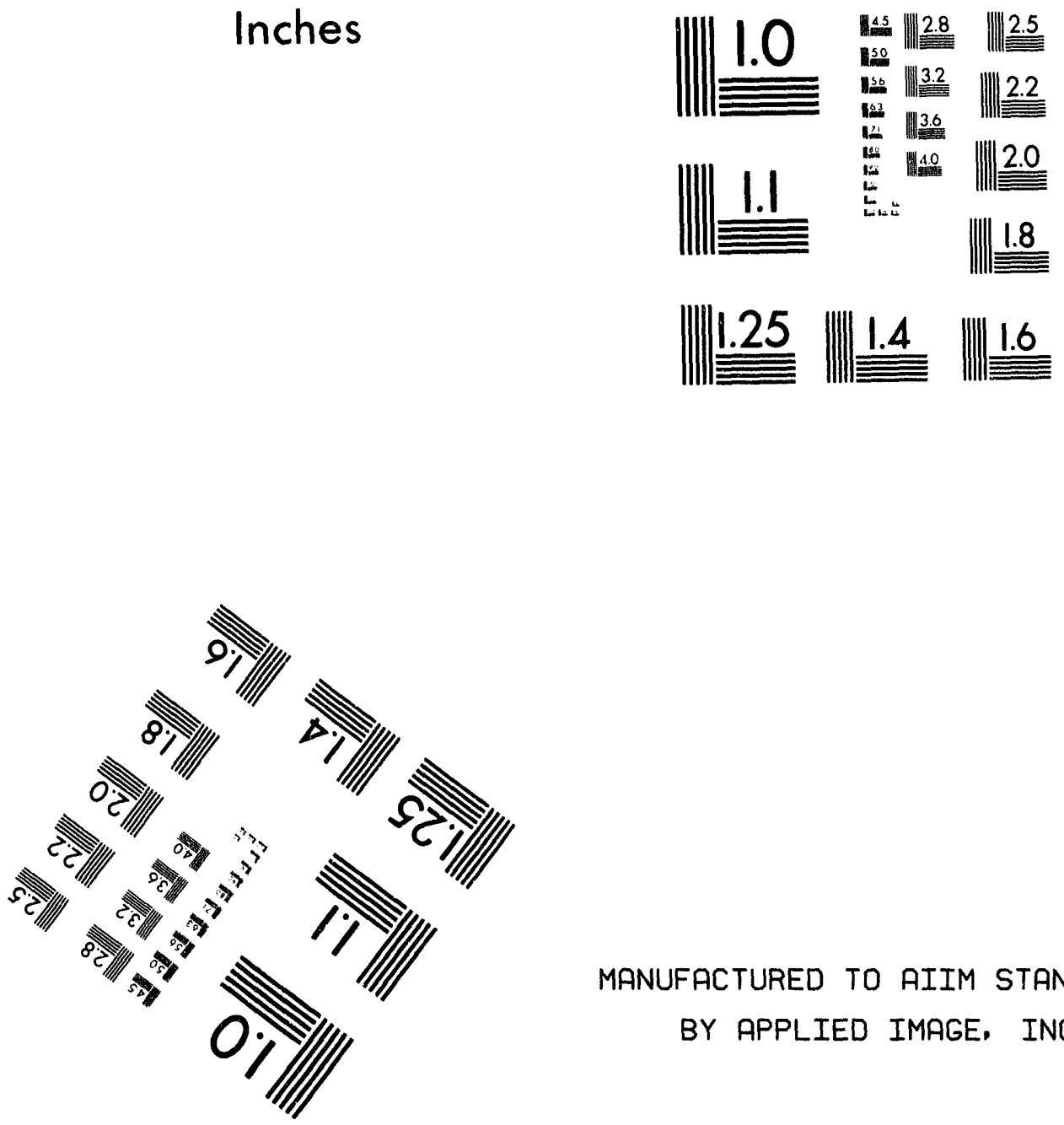

MANUFACTURED TO AIIM STANDARDS

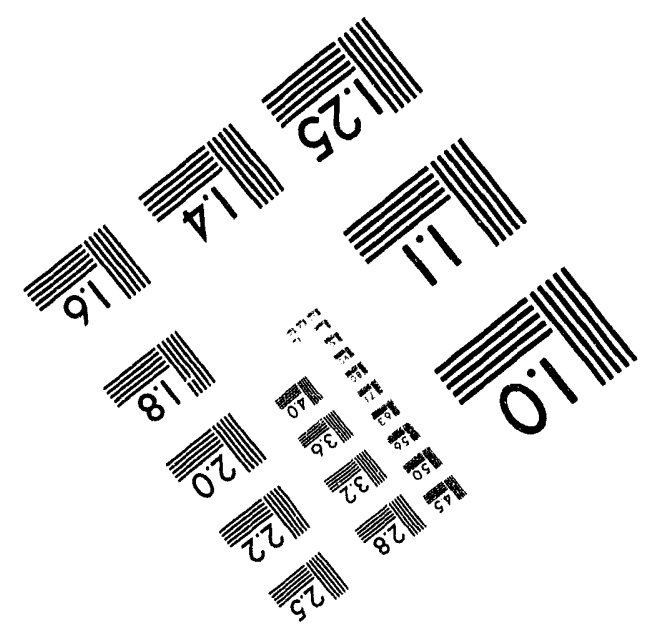



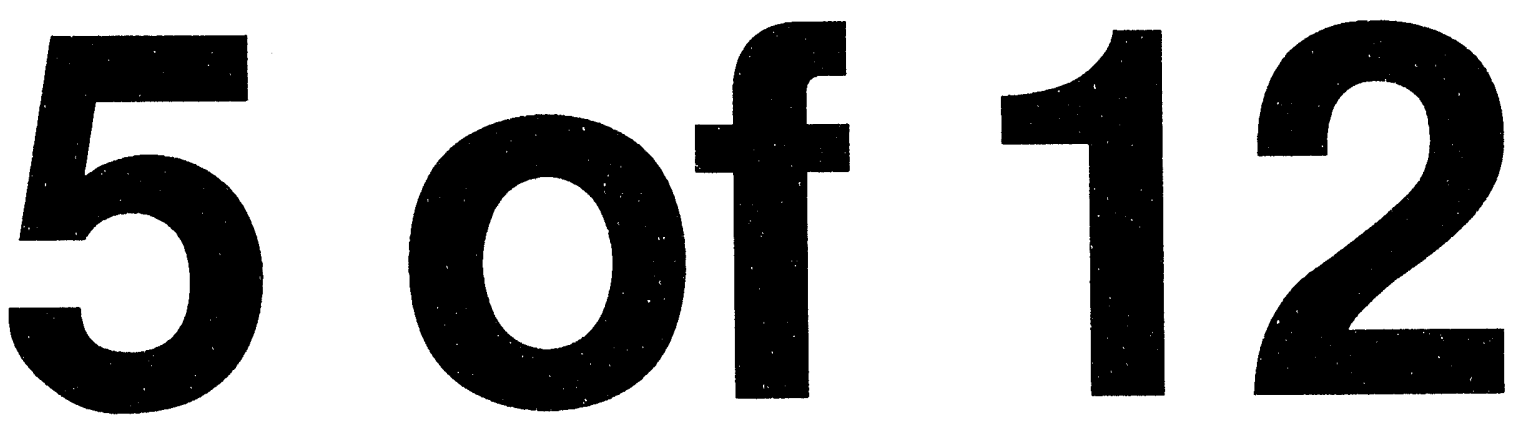


\begin{tabular}{|c|c|c|c|c|c|}
\hline Test: Aug15_09 & sum2 & Date: Wed & Aug $15 \quad 11$ & $46: 56 \quad 1990$ & Page: 4 \\
\hline 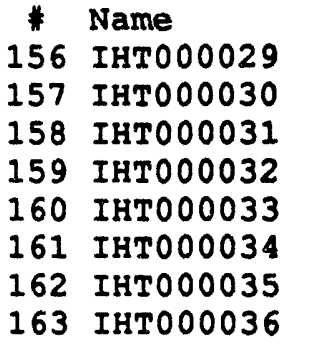 & $\begin{array}{r}\text { Min } \\
103.6396 \\
105.8143 \\
94.5688 \\
95.5980 \\
95.5829 \\
88.0521 \\
116.0119 \\
86.7525\end{array}$ & $\begin{array}{r}\text { Max } \\
106.1786 \\
108.3533 \\
96.7172 \\
97.7464 \\
97.9267 \\
90.0053 \\
118.1603 \\
88.9010\end{array}$ & \begin{tabular}{r}
\multicolumn{1}{c}{ Ave } \\
104.6709 \\
106.9667 \\
95.6469 \\
96.7151 \\
96.6767 \\
88.9350 \\
117.0431 \\
87.7017
\end{tabular} & $\begin{array}{l}\text { SD } \\
5.3093 e-01 \\
4.7882 e-01 \\
4.4335 e-01 \\
4.4475 e-01 \\
4.4817 e-01 \\
4.0480 e-01 \\
4.4300 e-01 \\
4.4118 e-01\end{array}$ & $\begin{array}{c}\text { Uncert } \\
4.2315 e+00 \\
4.2372 e+00 \\
4.2101 e+00 \\
4.2125 e+00 \\
4.2124 e+00 \\
4.1953 e+00 \\
4.2638 e+00 \\
4.1928 e+00\end{array}$ \\
\hline
\end{tabular}




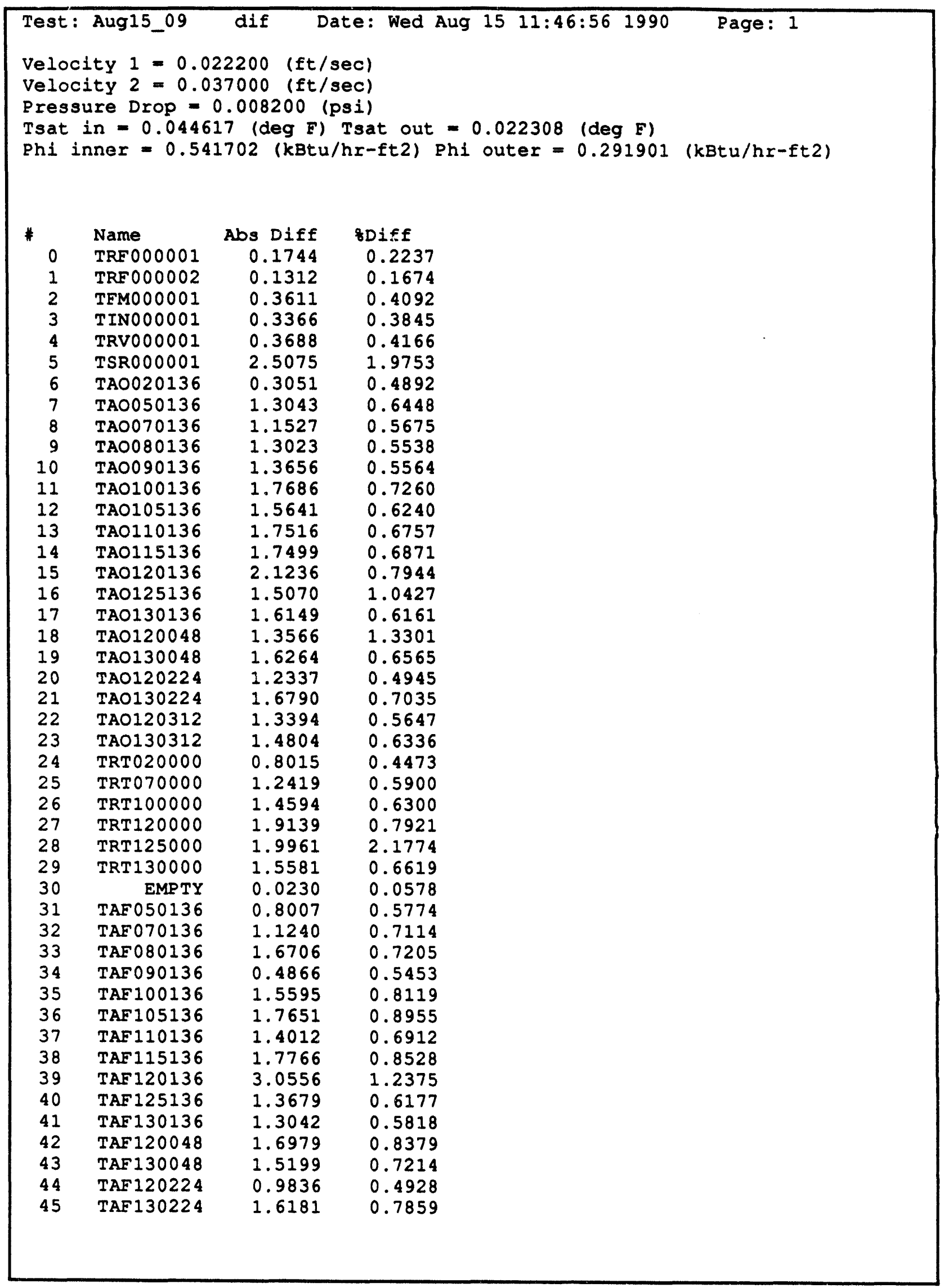




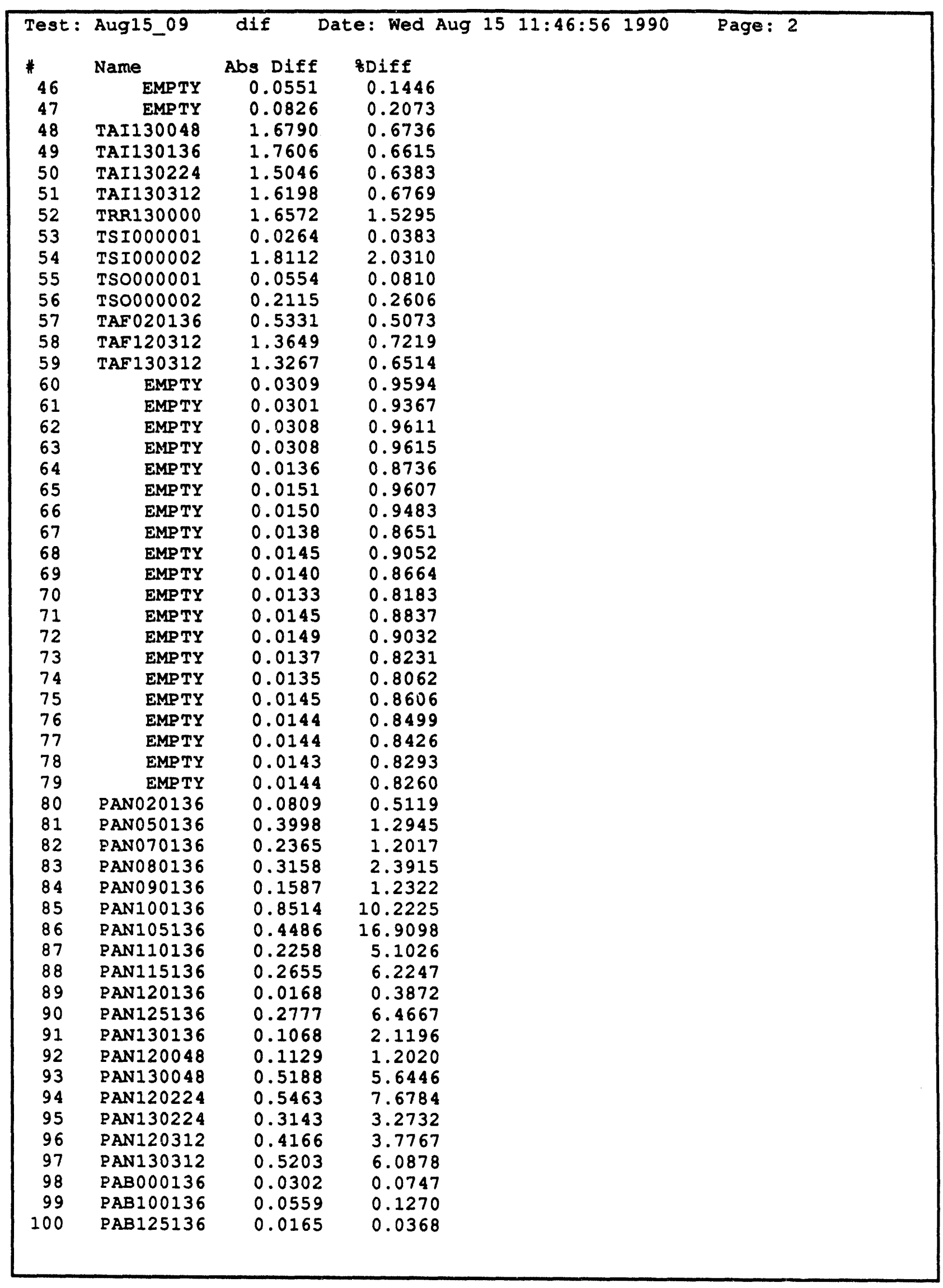




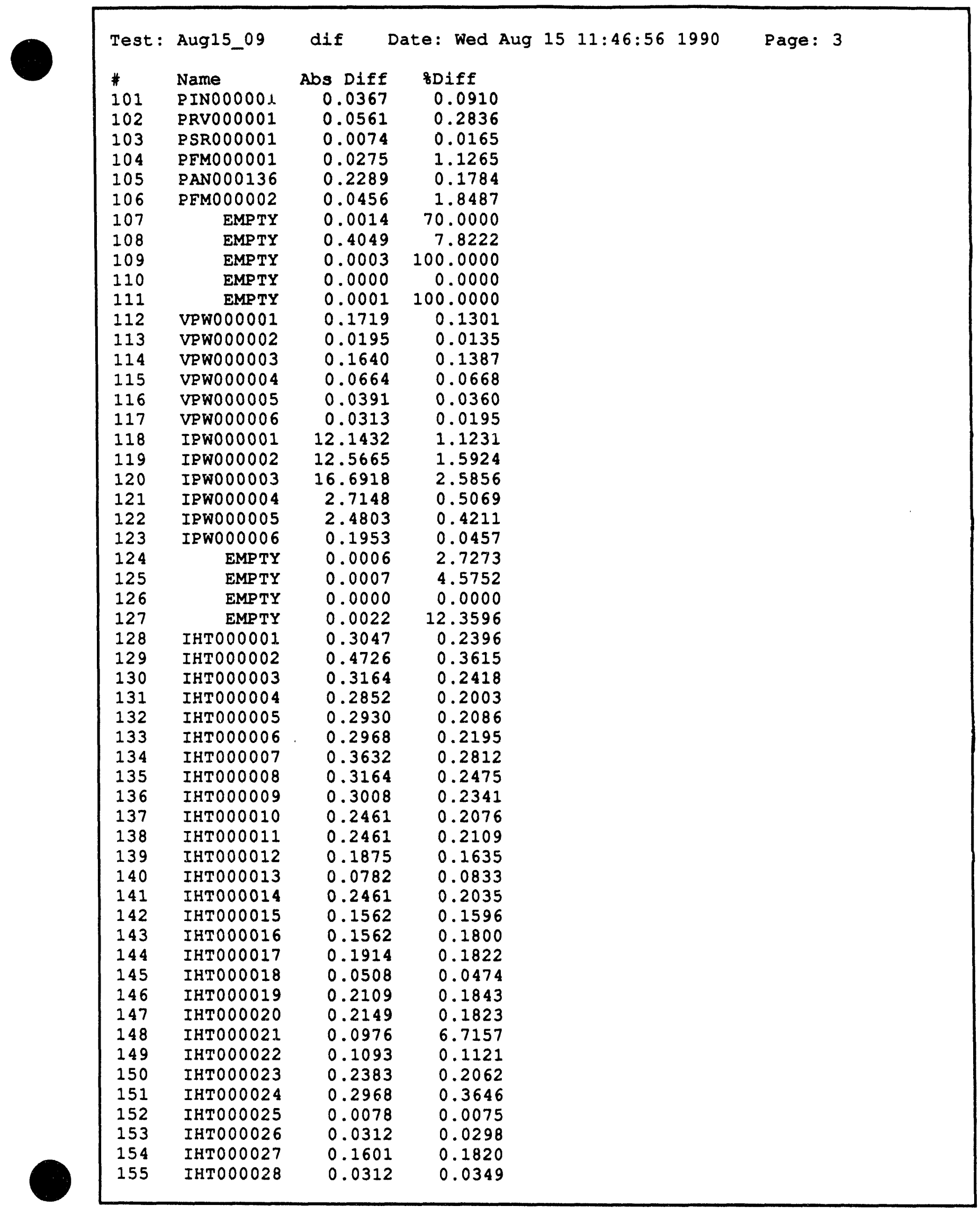




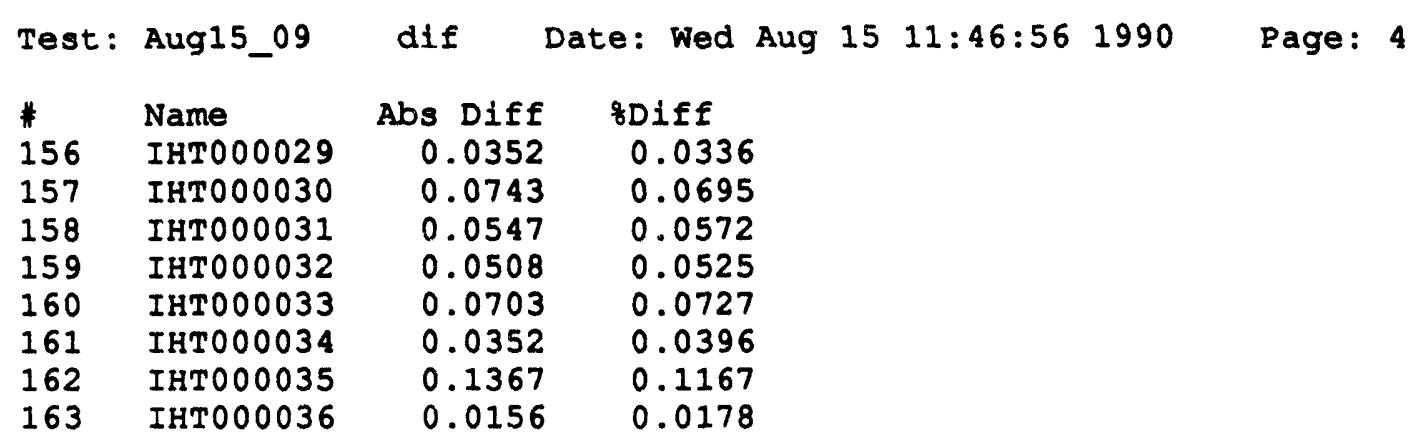




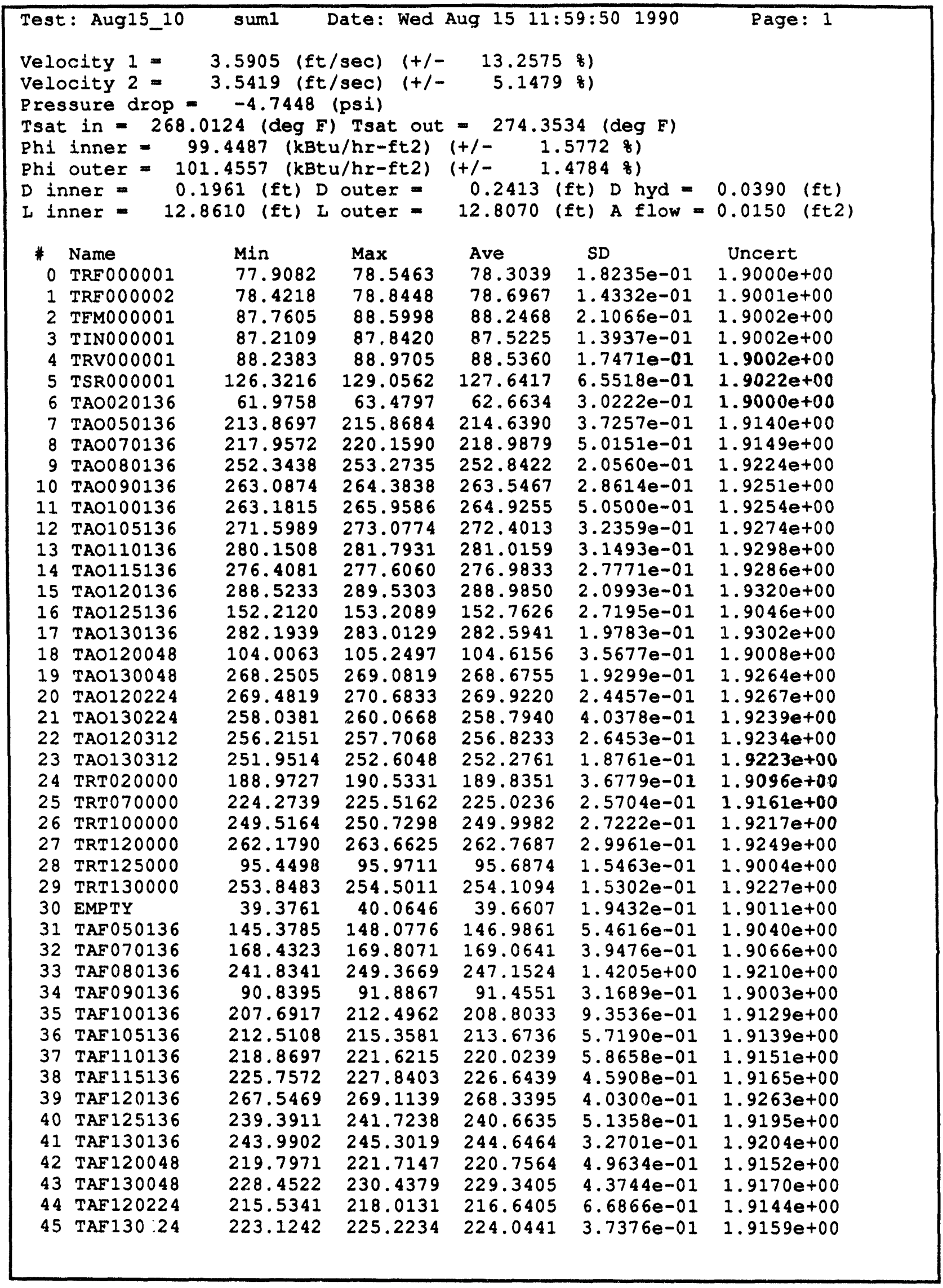




\begin{tabular}{|c|c|c|c|c|c|c|}
\hline & $t:$ Aug15_10 & suml & Date: Wed & Aug 1511 & $59: 50 \quad 1990$ & Page: 2 \\
\hline 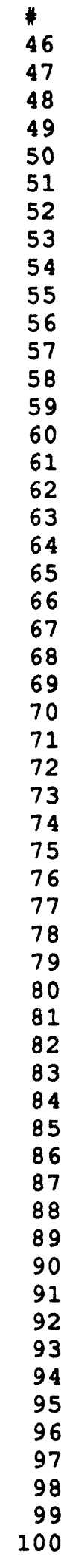 & 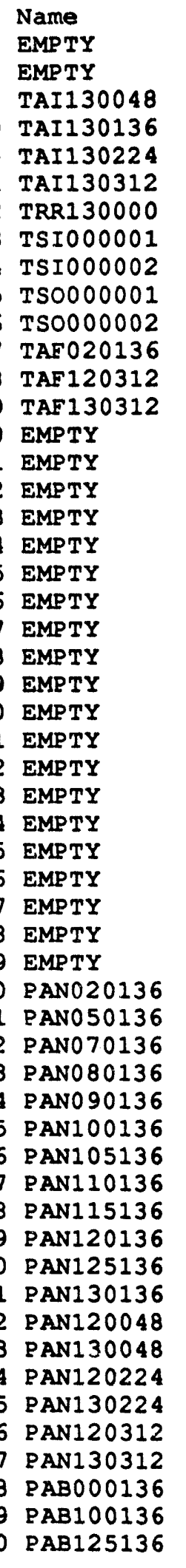 & 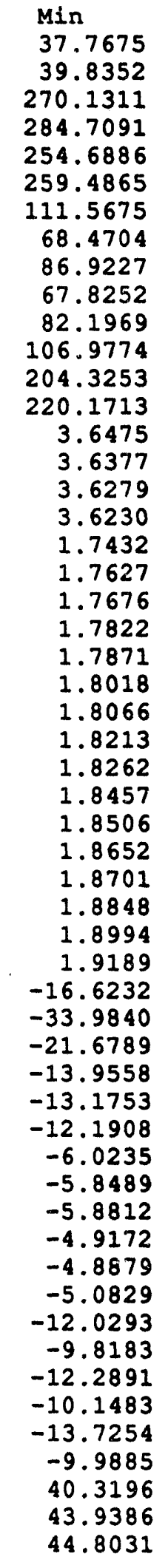 & $\begin{array}{r}\text { Max } \\
38.4572 \\
40.2940 \\
271.8861 \\
285.5331 \\
255.9033 \\
260.6967 \\
113.1067 \\
69.3231 \\
89.9567 \\
68.6817 \\
83.0415 \\
108.9535 \\
207.0171 \\
222.5474 \\
3.6719 \\
3.6523 \\
3.6426 \\
3.6377 \\
1.7578 \\
1.7773 \\
1.7725 \\
1.7871 \\
1.7920 \\
1.8066 \\
1.8164 \\
1.8262 \\
1.8359 \\
1.8506 \\
-1.8604 \\
1.8701 \\
-90.94867 \\
44.3506 \\
45.0320 \\
-9.8750 \\
1.8945 \\
1.9043\end{array}$ & 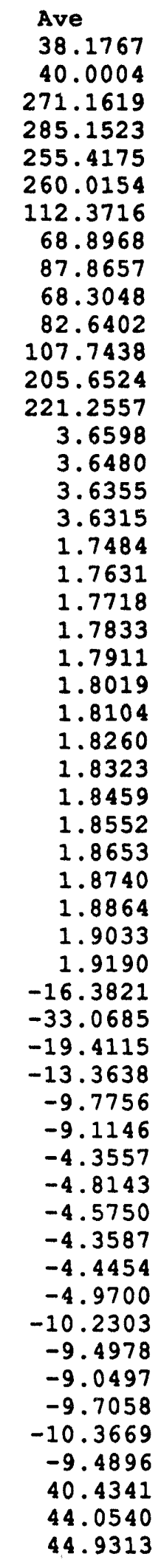 & $\begin{array}{l}\text { SD } \\
1.8747 e-01 \\
1.1393 e-01 \\
4.1808 e-01 \\
2.1423 e-01 \\
2.9662 e-01 \\
2.9392 e-01 \\
3.3136 e-01 \\
1.9844 e-01 \\
6.0196 e-01 \\
2.1993 e-01 \\
1.8596 e-01 \\
4.9445 e-01 \\
6.3557 e-01 \\
5.3628 e-01 \\
5.7724 e-03 \\
3.9013 e-03 \\
3.1444 e-03 \\
2.5743 e-03 \\
1.9331 e-03 \\
2.1702 e-03 \\
1.7115 e-03 \\
2.0432 e-03 \\
1.8950 e-03 \\
6.9053 e-04 \\
2.2689 e-03 \\
9.6655 e-04 \\
2.3778 e-03 \\
9.6655 e-04 \\
1.5314 e-03 \\
6.9053 e-04 \\
1.9730 e-03 \\
2.5362 e-03 \\
1.9730 e-03 \\
6.9053 e-04 \\
1.0938 e-01 \\
8.3633 e-01 \\
8.4214 e-01 \\
9.7015 e-01 \\
2.6665 e+00 \\
1.5930 e+00 \\
1.0201 e+00 \\
5.2659 e-01 \\
5.2512 e-01 \\
2.6761 e-01 \\
2.1579 e-01 \\
7.5888 e-02 \\
5.7427 e-01 \\
1.7095 e-01 \\
8.4131 e-01 \\
2.3629 e-01 \\
2.5756 e-01 \\
6.9155 e-02 \\
5.1492 e-02\end{array}$ & 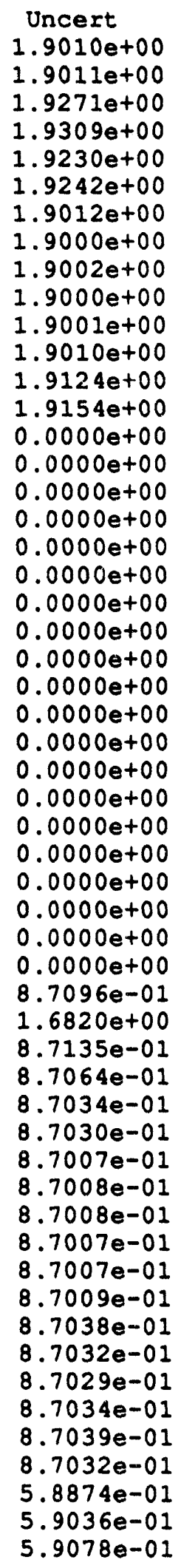 \\
\hline
\end{tabular}




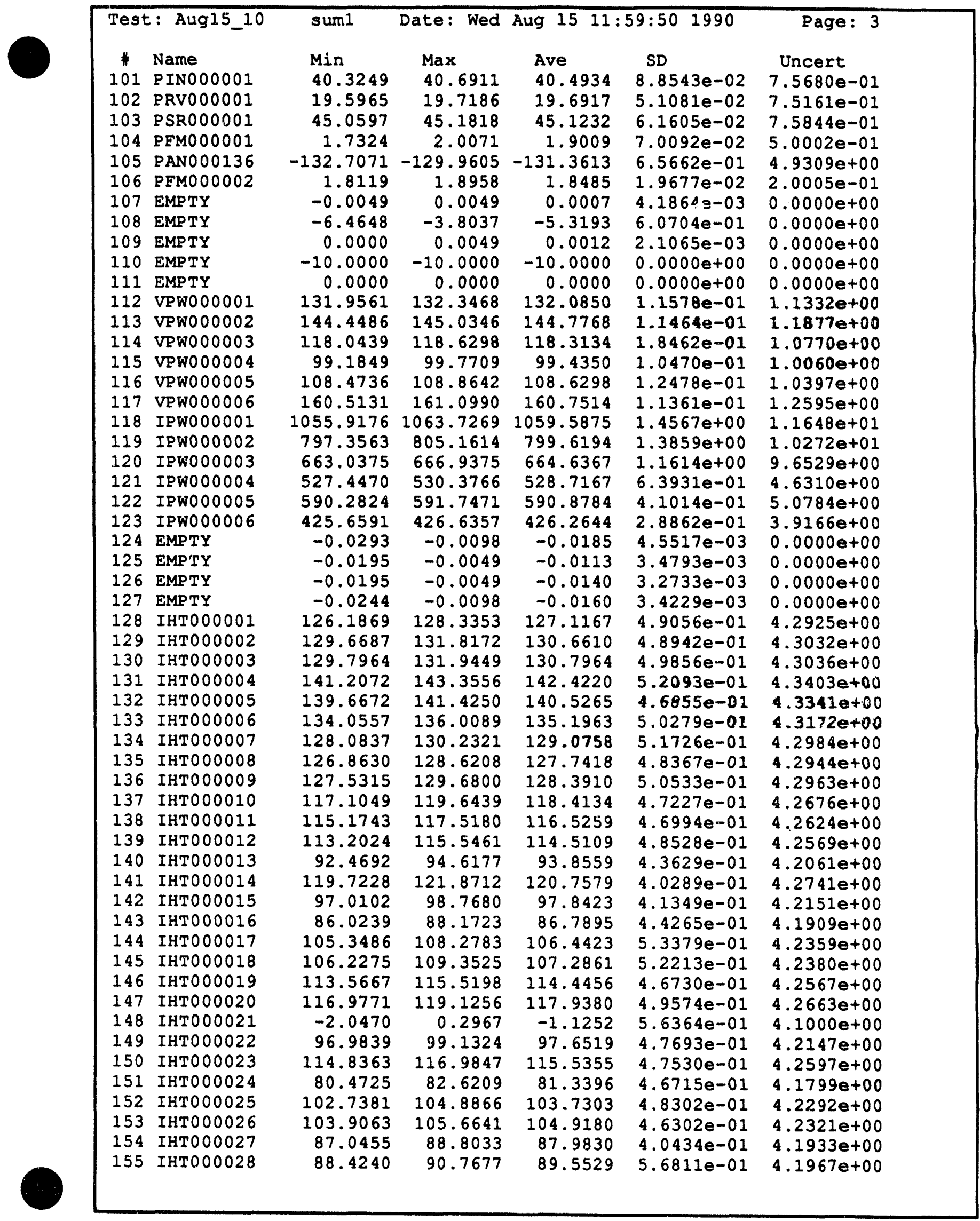




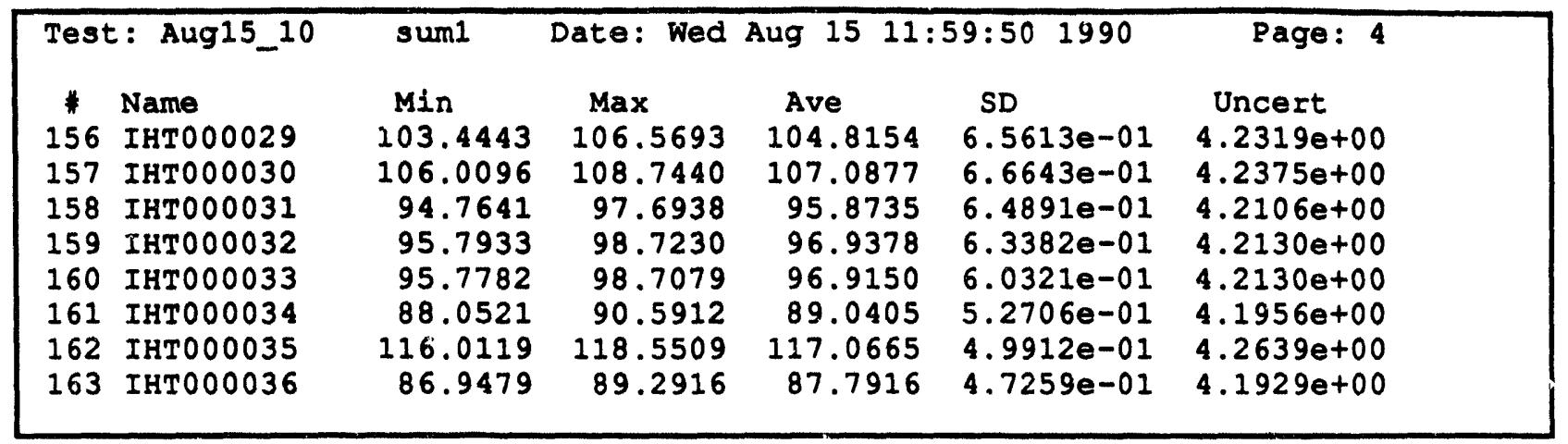




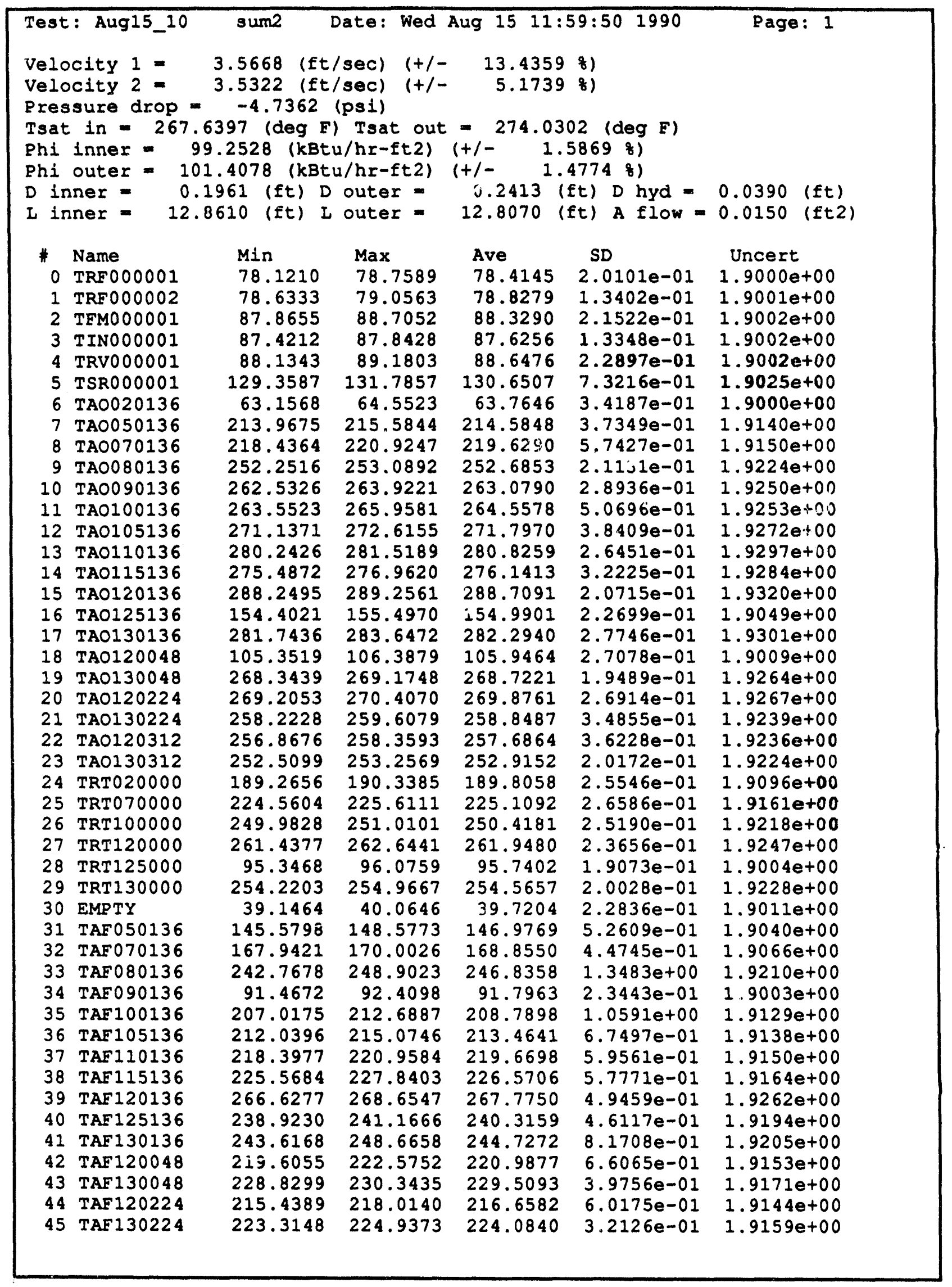




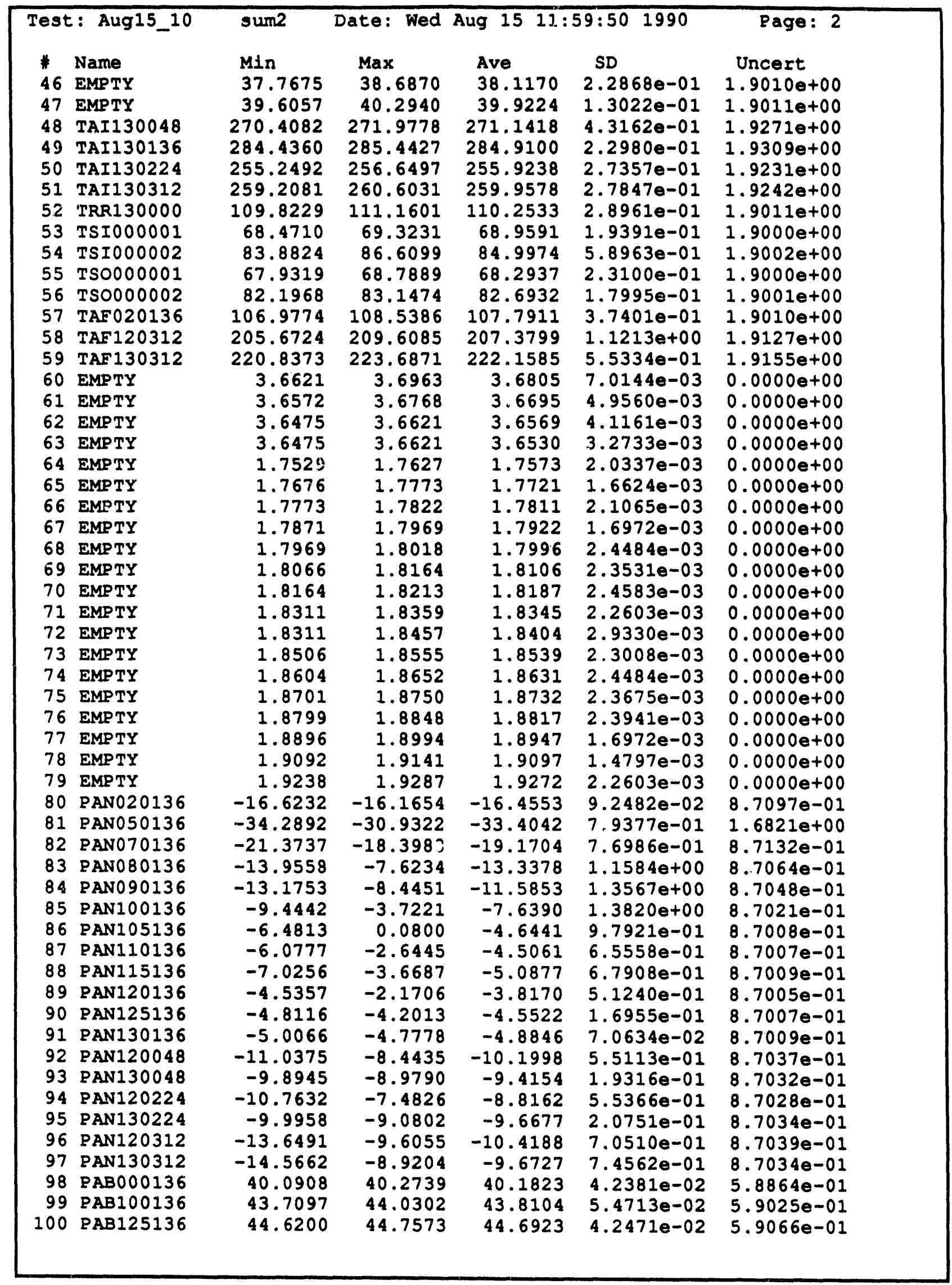




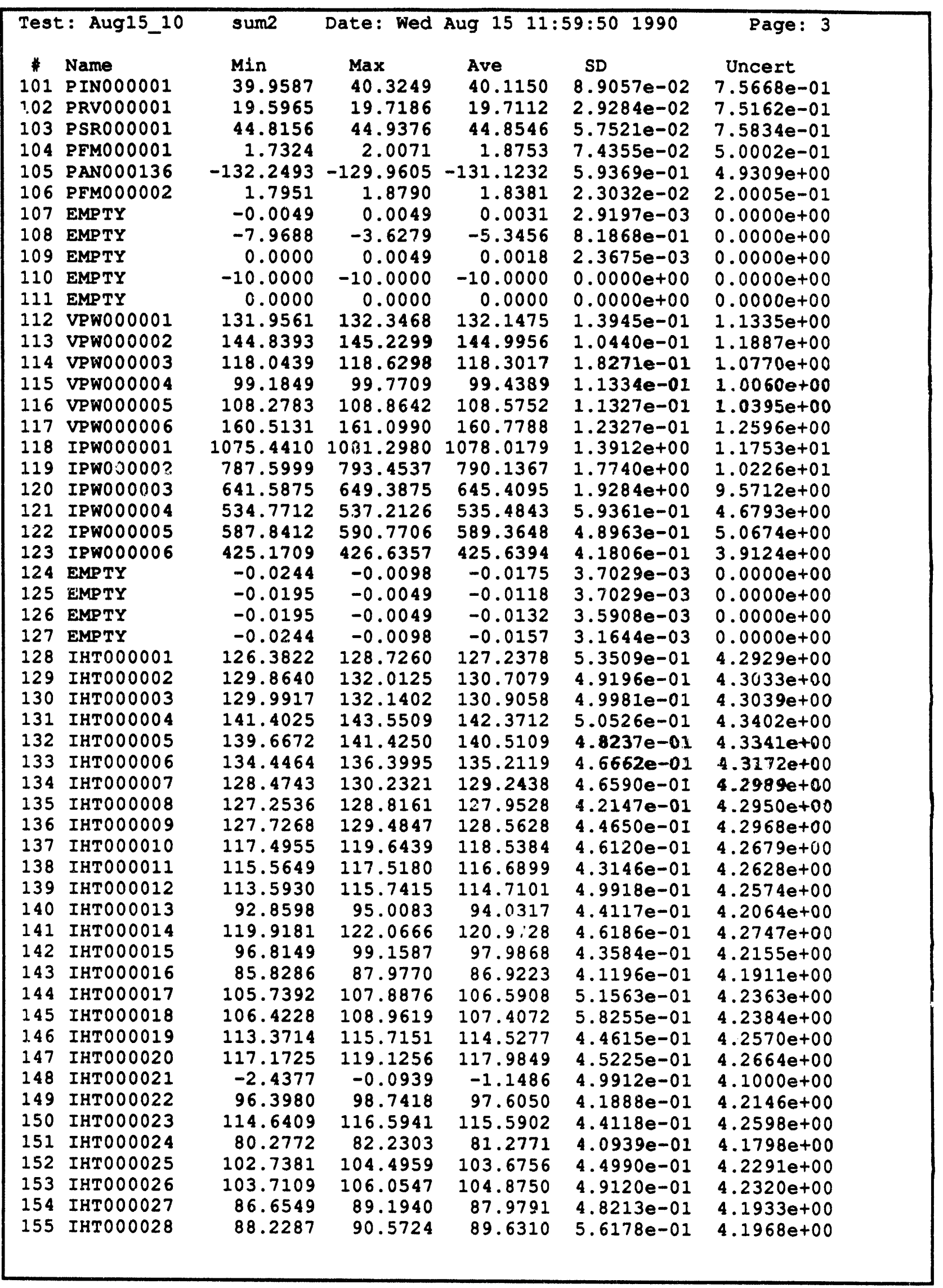




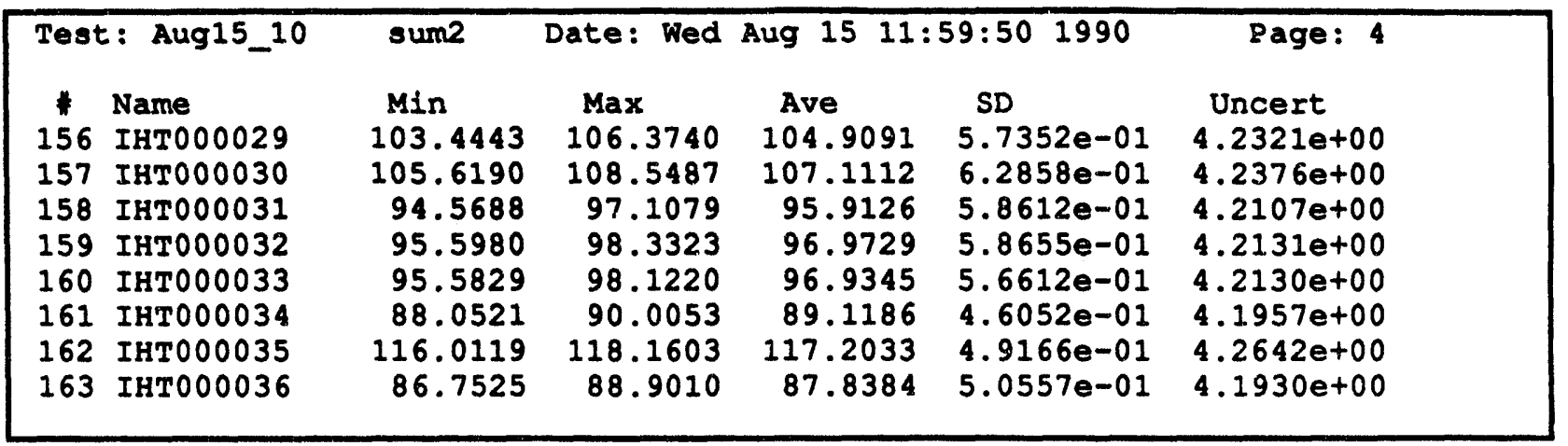




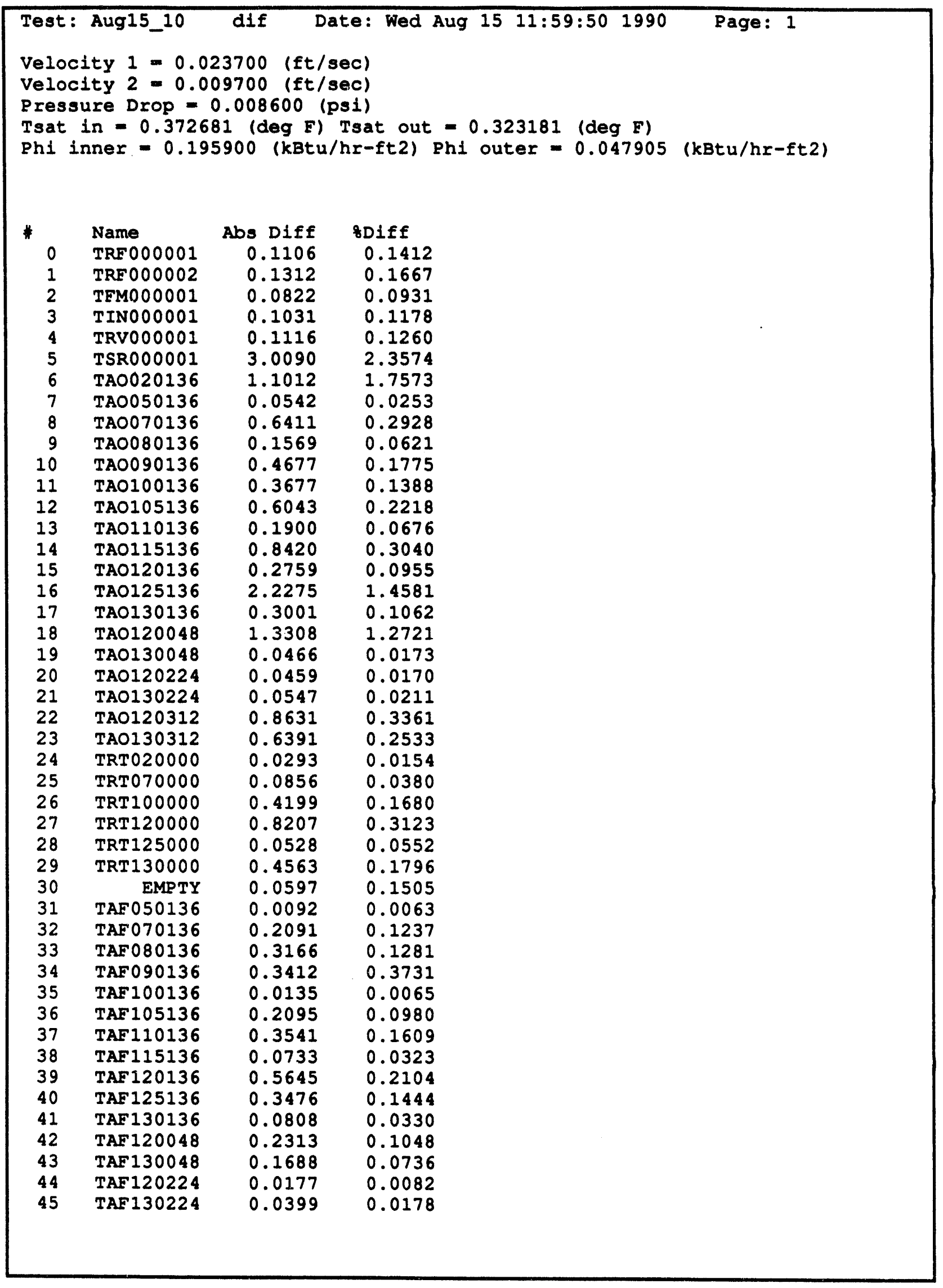




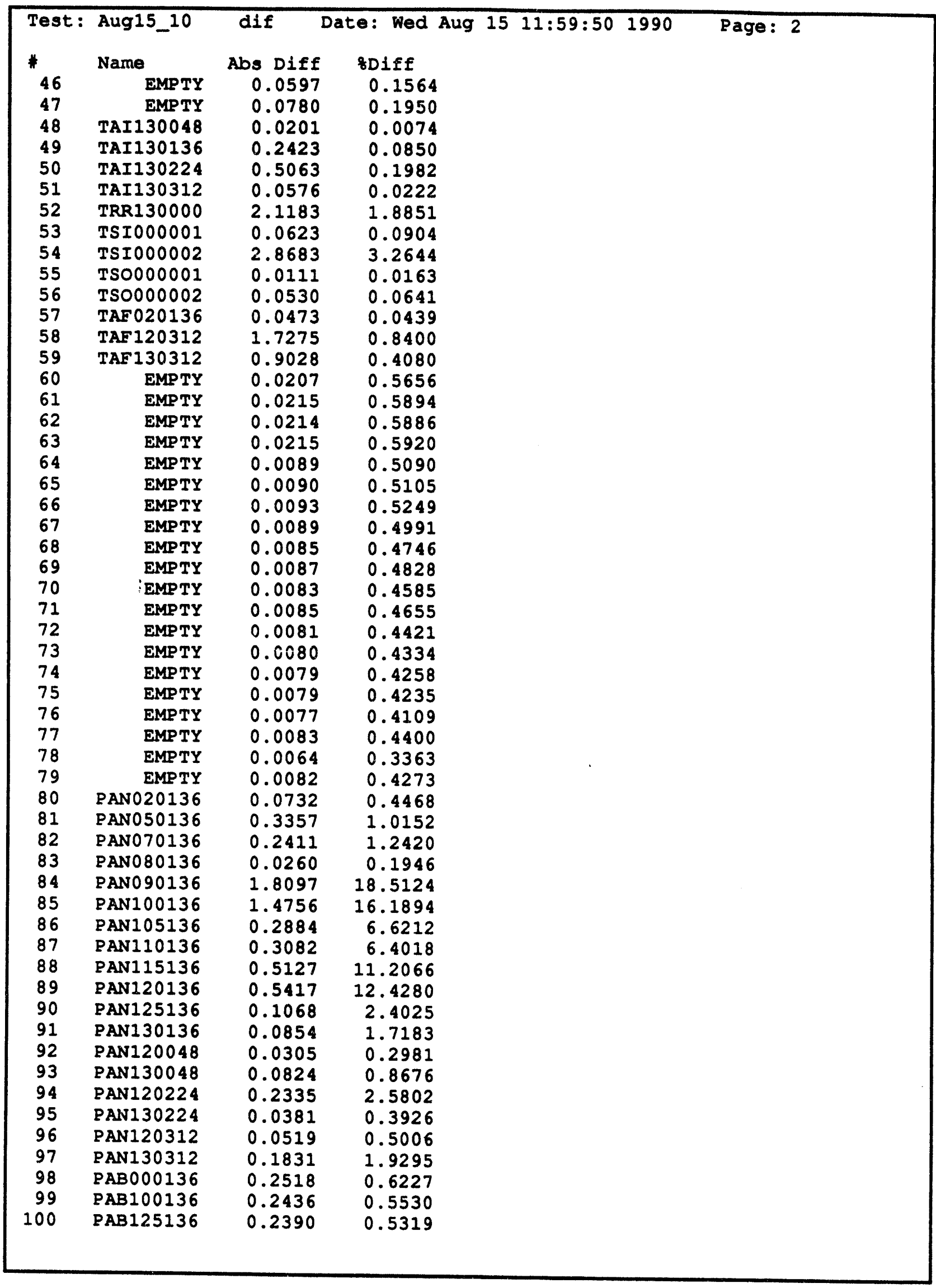




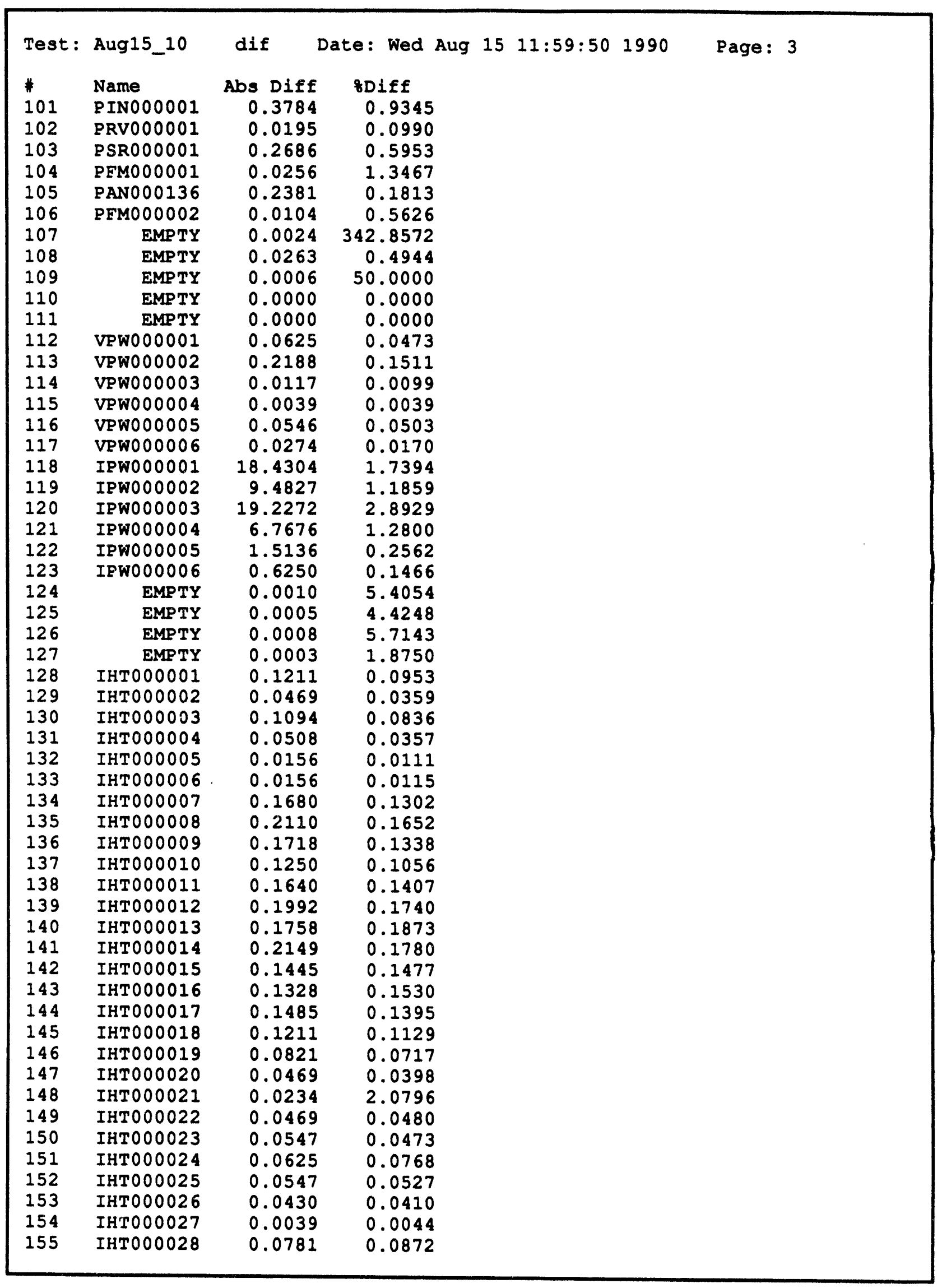




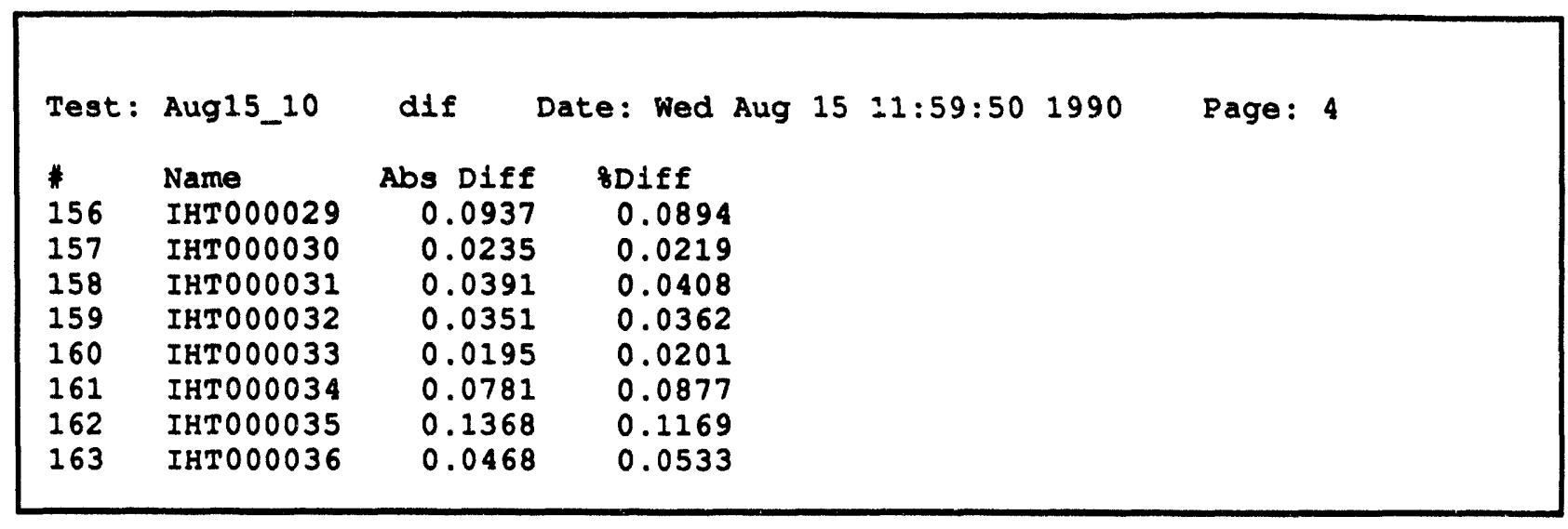




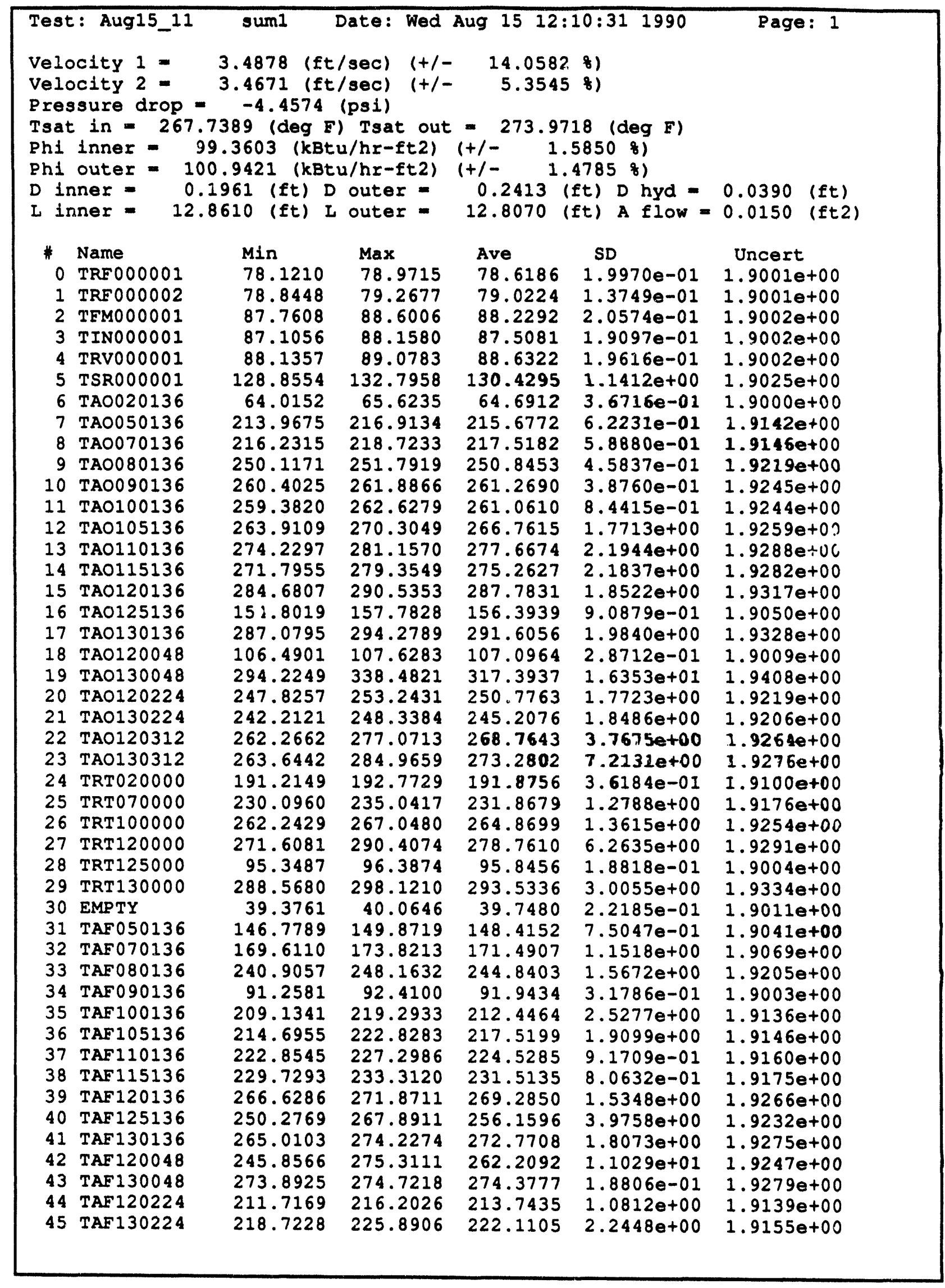




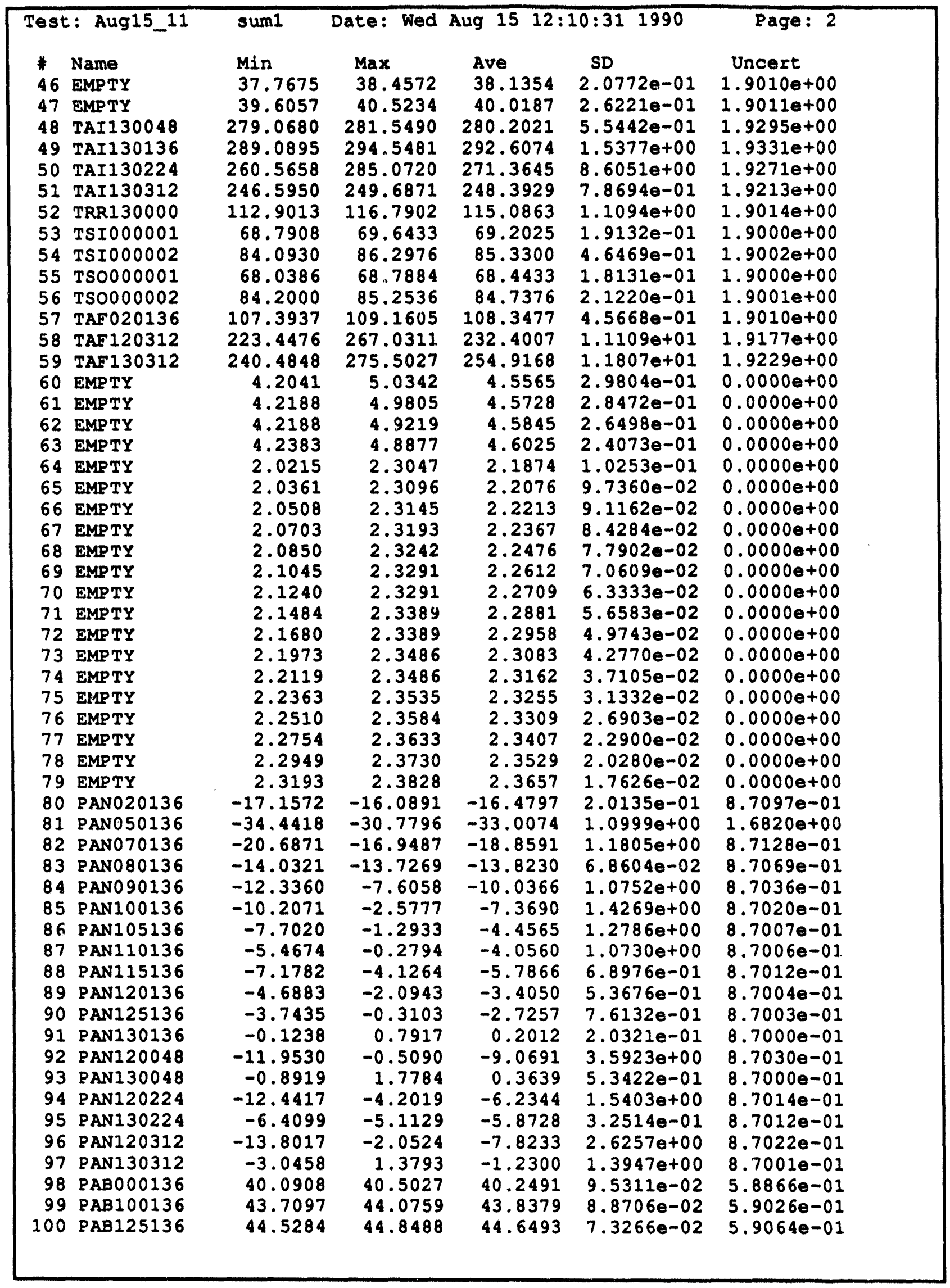




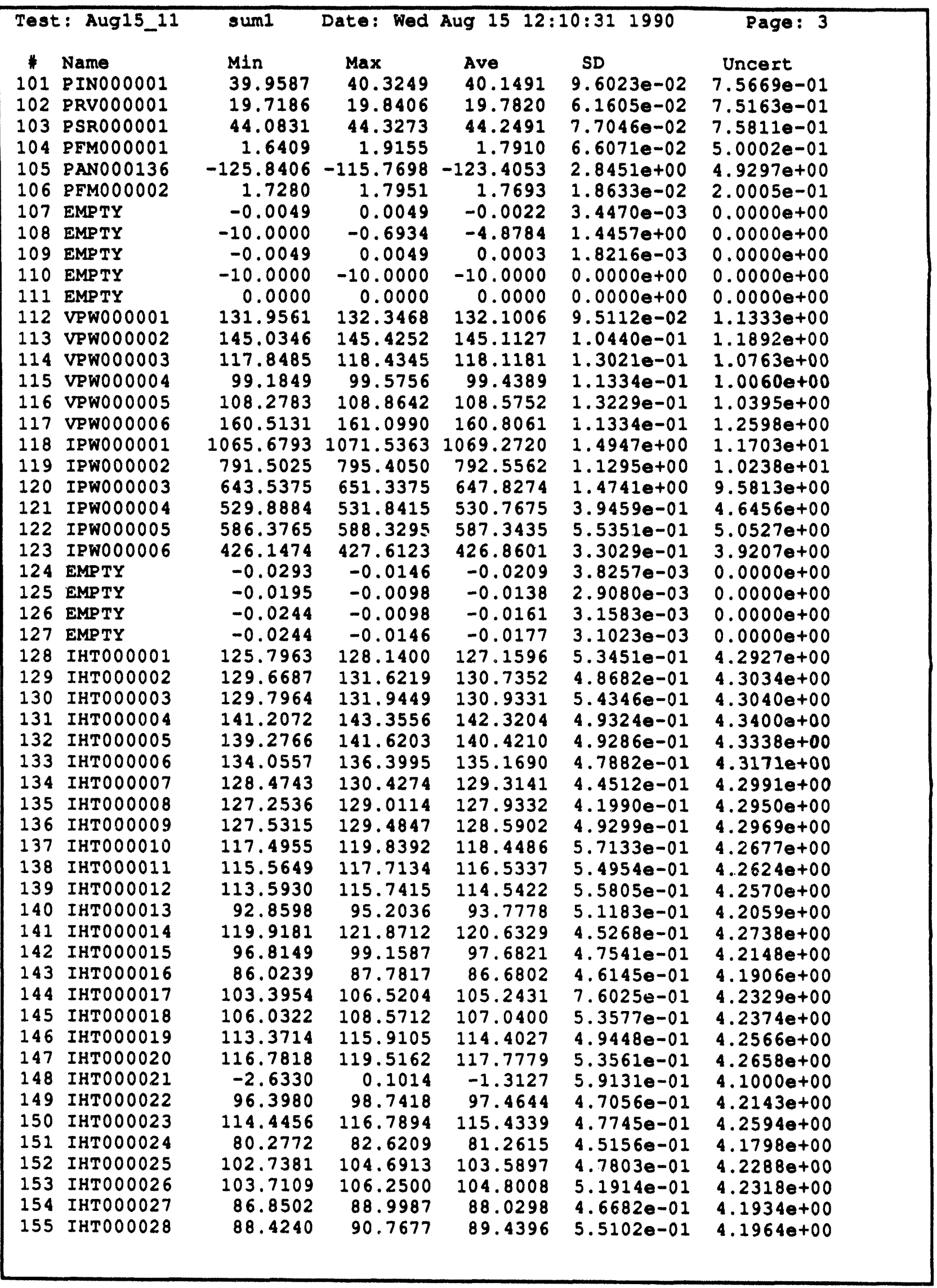




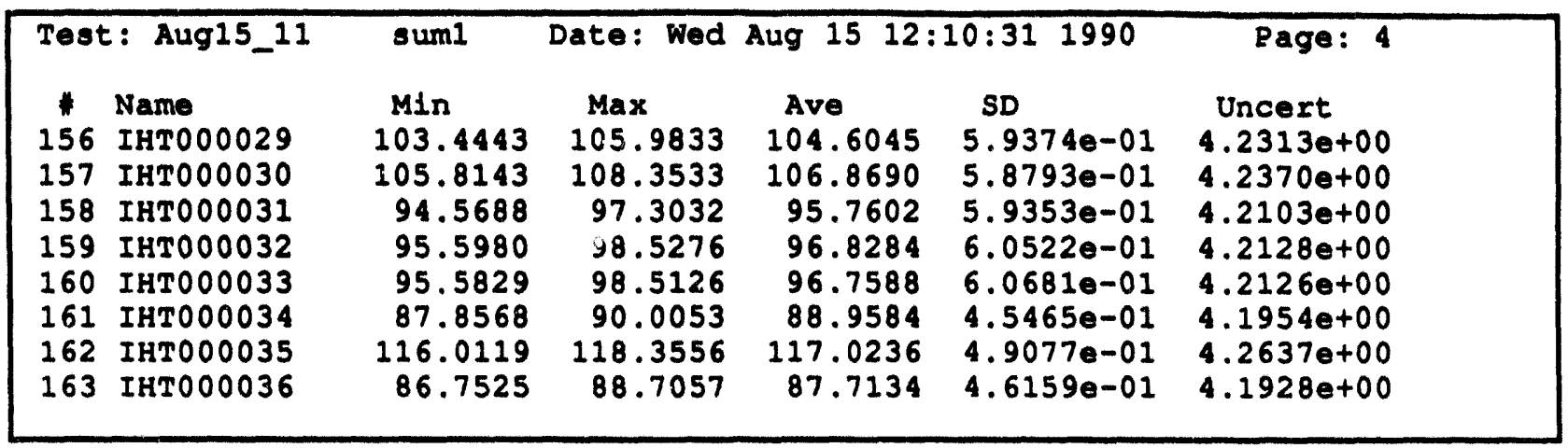




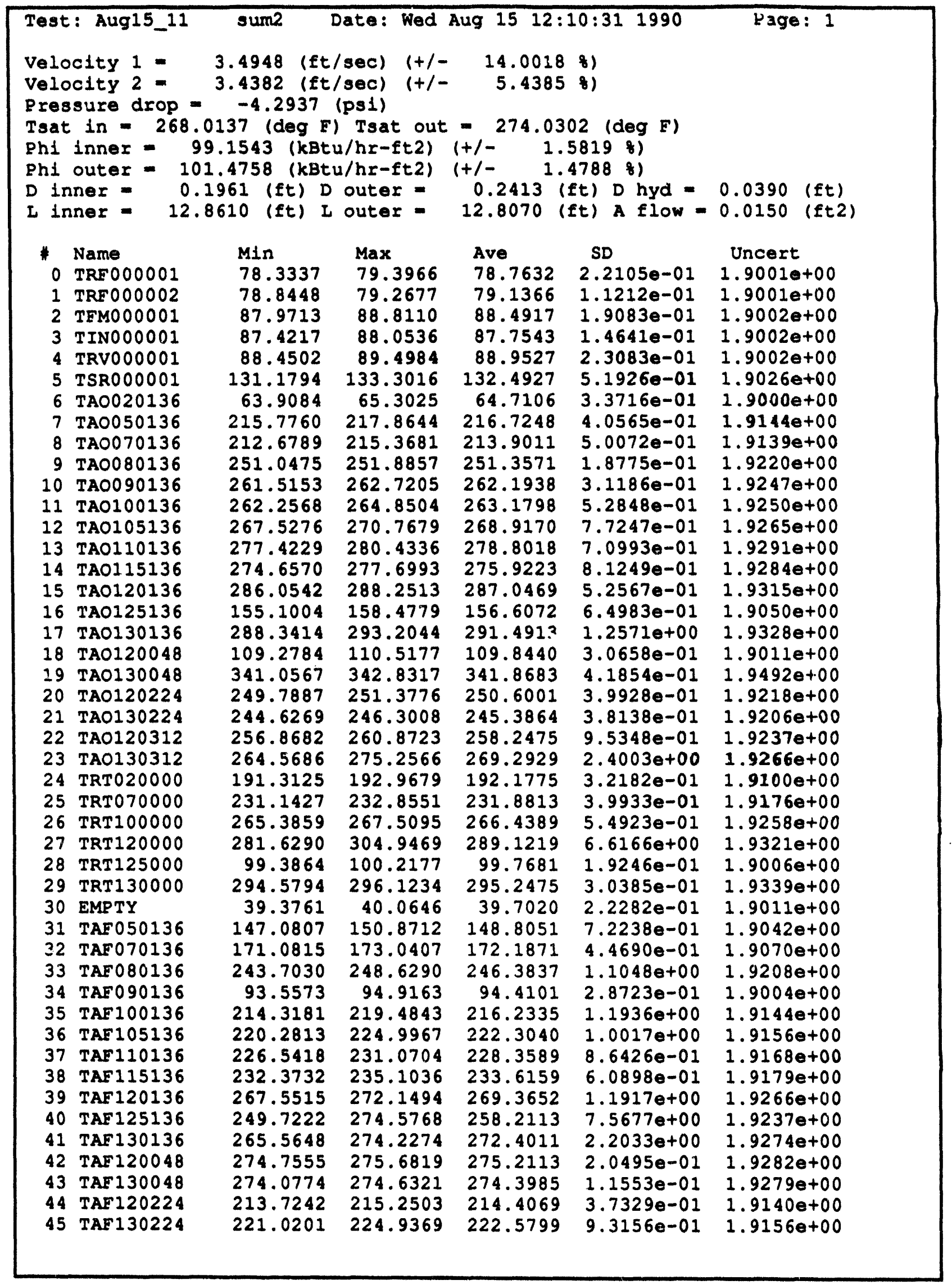




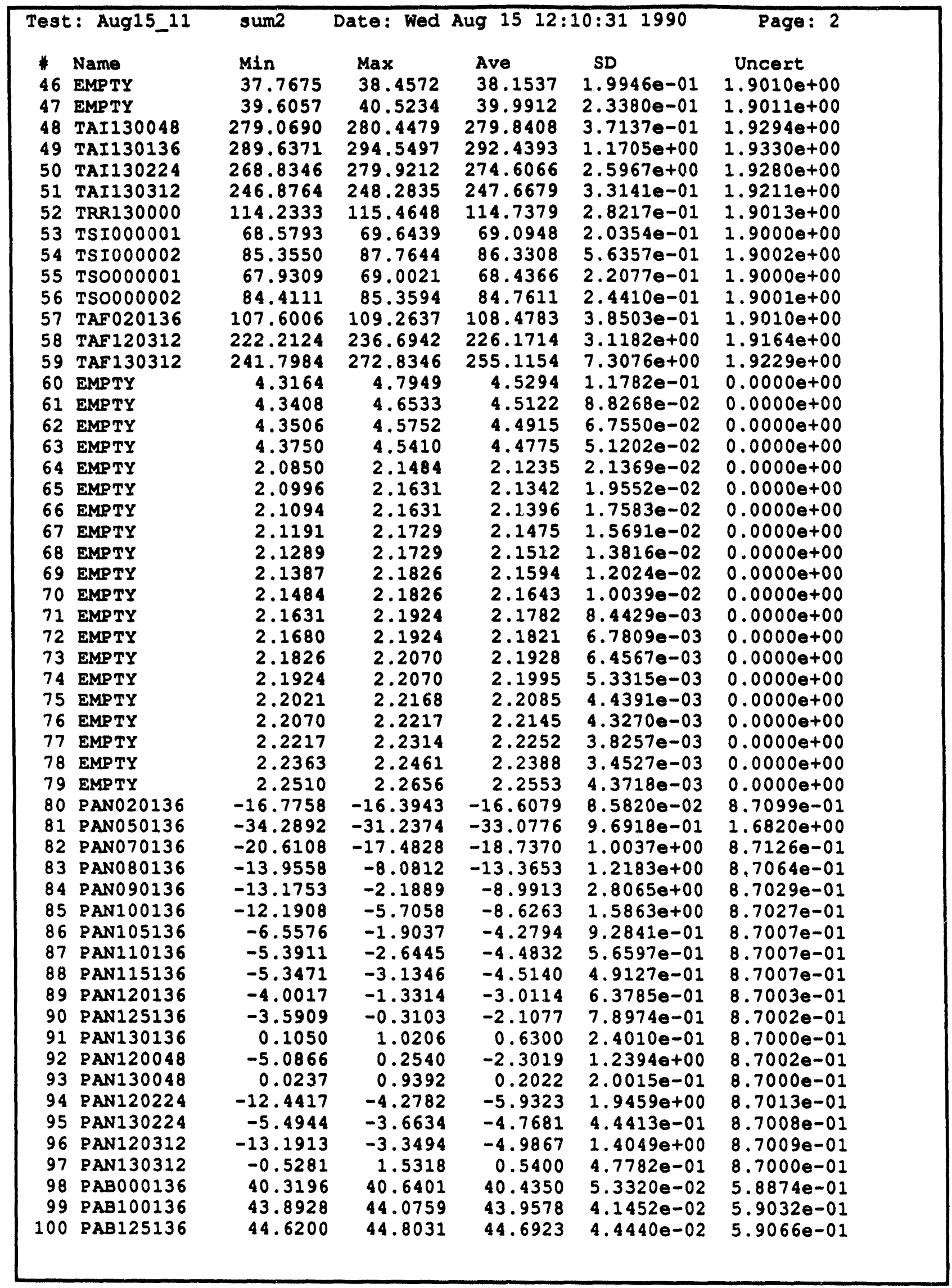




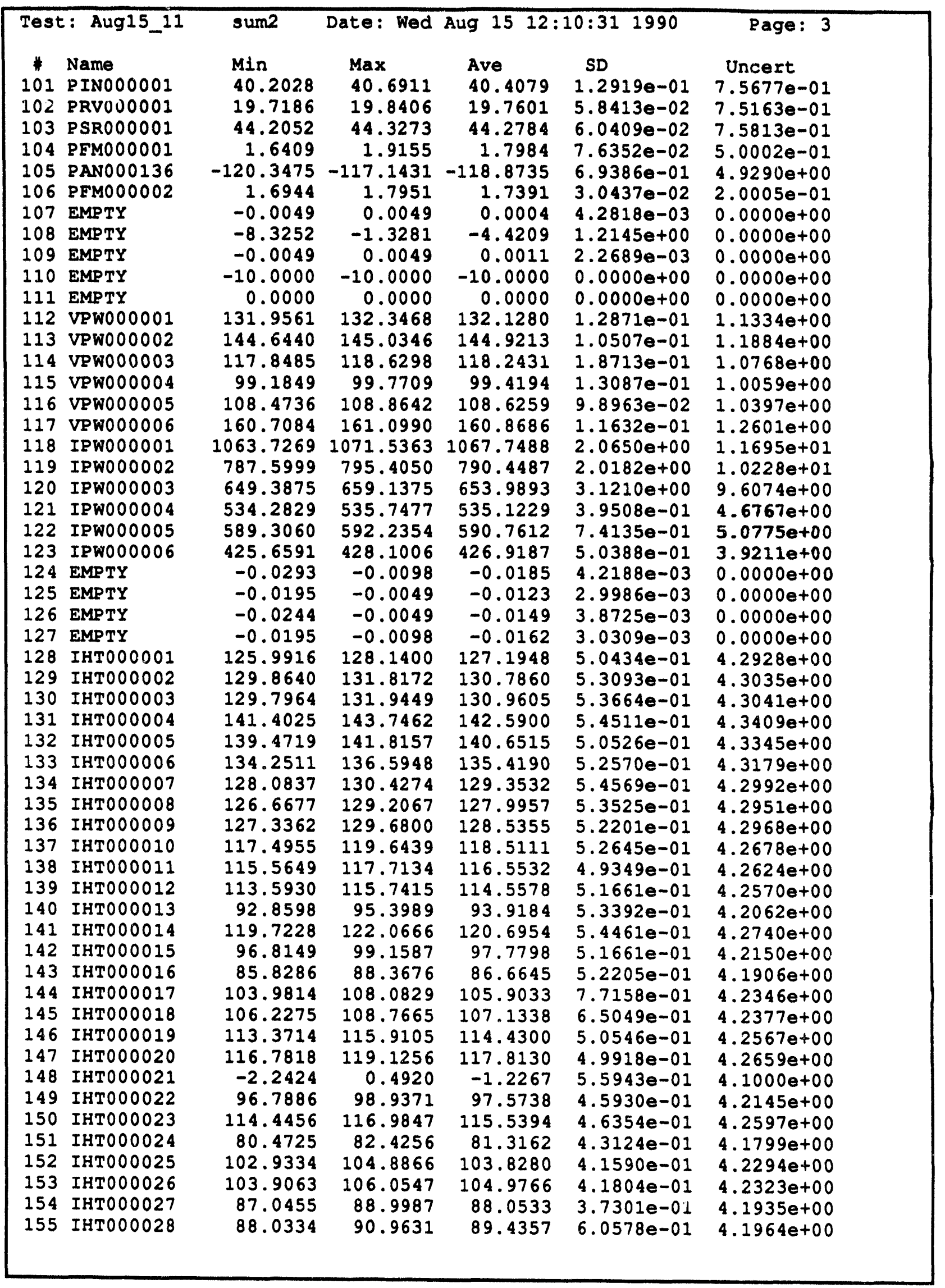




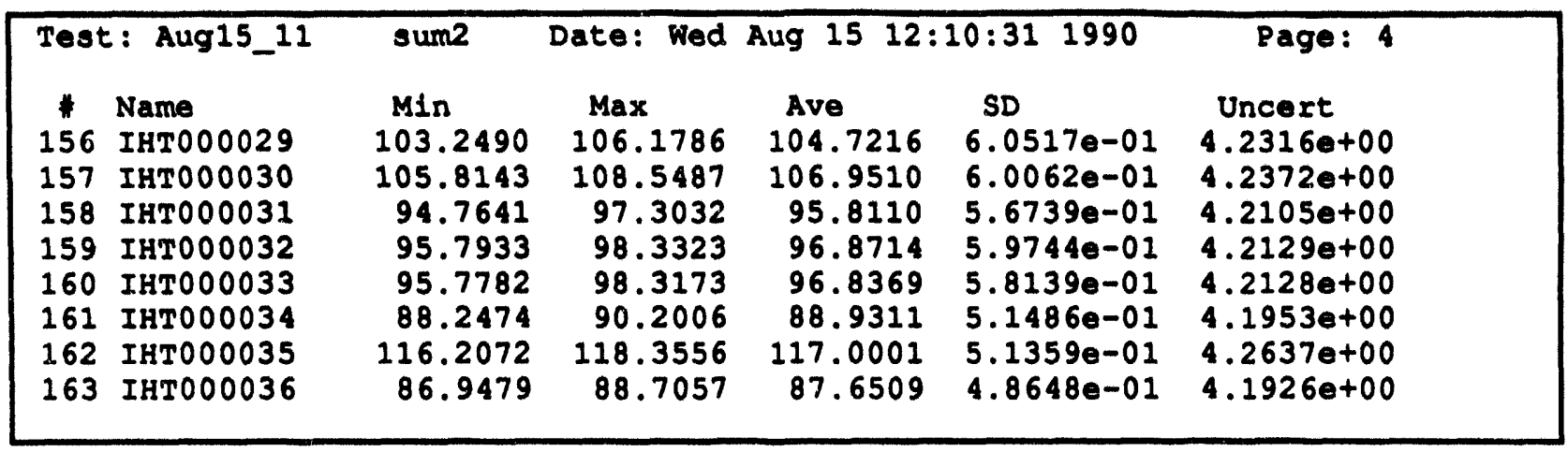




\begin{tabular}{|c|c|c|c|}
\hline \multicolumn{4}{|c|}{$\begin{array}{l}\text { Velocity } 1=0.007000 \text { (ft/sec) } \\
\text { Velocity } 2=0.028900 \text { (ft/sec) } \\
\text { Pressure Drop }=0.163700 \text { (psi) } \\
\text { Tsat in }=0.274811 \text { (deg F) Tsat out }=0.058411 \text { (deg F) } \\
\text { Phi inner }=0.206001 \text { (kBtu/hr-ft2) Phi outer }=0.533699 \text { (kBtu/hr-ft2) }\end{array}$} \\
\hline $\begin{array}{l}* \\
1 \\
1 \\
2 \\
3 \\
4 \\
5 \\
6 \\
7 \\
8 \\
9 \\
10 \\
11 \\
12 \\
13 \\
14 \\
15 \\
16 \\
17 \\
18 \\
19 \\
20 \\
21 \\
22 \\
23 \\
24 \\
25 \\
26 \\
27 \\
28 \\
29 \\
30 \\
31 \\
32 \\
33 \\
34 \\
35 \\
36 \\
37 \\
38 \\
39 \\
40 \\
41 \\
42 \\
43 \\
44 \\
45\end{array}$ & $\begin{array}{l}\text { Name } \\
\text { TRF000001 } \\
\text { TRF000002 } \\
\text { TFM000001 } \\
\text { TIN000001 } \\
\text { TRV000001 } \\
\text { TSR000001 } \\
\text { TAO020136 } \\
\text { TAO050136 } \\
\text { TAO070136 } \\
\text { TAO080136 } \\
\text { TAO090136 } \\
\text { TAO100136 } \\
\text { TAO105136 } \\
\text { TAO110136 } \\
\text { TAO115136 } \\
\text { TAO120136 } \\
\text { TAO125136 } \\
\text { TAO130136 } \\
\text { TAO120048 } \\
\text { TAO130048 } \\
\text { TAO120224 } \\
\text { TAO130224 } \\
\text { TAO120312 } \\
\text { TAO130312 } \\
\text { TRT020000 } \\
\text { TRT070000 } \\
\text { TRT100000 } \\
\text { TRT120000 } \\
\text { TRT125000 } \\
\text { TRT130000 } \\
\text { TAF0 EMPTY } \\
\text { TAF070136 } \\
\text { TAF080136 } \\
\text { TAF090136 } \\
\text { TAF100136 } \\
\text { TAF105136 } \\
\text { TAF110136 } \\
\text { TAF115136 } \\
\text { TAF120136 } \\
\text { TAF125136 } \\
\text { TAF130136 } \\
\text { TAF120048 } \\
\text { TAF130048 } \\
\text { TAF120224 } \\
\text { TAF130224 }\end{array}$ & $\begin{array}{r}\text { Abs Diff } \\
0.1446 \\
0.1142 \\
0.2625 \\
0.2462 \\
0.3205 \\
2.0632 \\
0.0194 \\
1.0476 \\
3.6171 \\
0.5118 \\
0.9248 \\
2.1188 \\
2.1555 \\
1.1344 \\
0.6596 \\
0.7362 \\
0.2133 \\
0.1143 \\
2.7476 \\
24.4746 \\
0.1762 \\
0.1788 \\
10.5168 \\
3.9873 \\
0.3019 \\
0.0134 \\
1.5690 \\
10.3609 \\
3.9225 \\
1.7139 \\
0.0460 \\
0.3899 \\
0.6964 \\
1.5434 \\
2.4667 \\
3.7871 \\
4.7841 \\
3.8304 \\
2.1024 \\
0.0802 \\
2.0517 \\
0.3697 \\
13.0021 \\
0.0208 \\
0.6634 \\
0.4694\end{array}$ & $\begin{array}{l}\text { 8Diff } \\
0.1839 \\
0.1445 \\
0.2975 \\
0.2813 \\
0.3616 \\
1.5819 \\
0.0300 \\
0.4857 \\
1.6629 \\
0.2040 \\
0.3540 \\
0.8116 \\
0.8080 \\
0.4085 \\
0.2396 \\
0.2558 \\
0.1364 \\
0.0392 \\
2.5655 \\
7.7111 \\
0.0703 \\
0.0729 \\
3.9130 \\
1.4591 \\
0.1573 \\
0.0058 \\
0.5924 \\
3.7168 \\
4.0925 \\
0.5839 \\
0.1157 \\
0.2627 \\
0.4061 \\
0.6304 \\
2.6829 \\
1.7826 \\
2.1994 \\
1.7060 \\
0.9081 \\
0.0298 \\
0.8009 \\
0.1355 \\
4.9587 \\
0.0076 \\
0.3104 \\
0.2113\end{array}$ \\
\hline
\end{tabular}




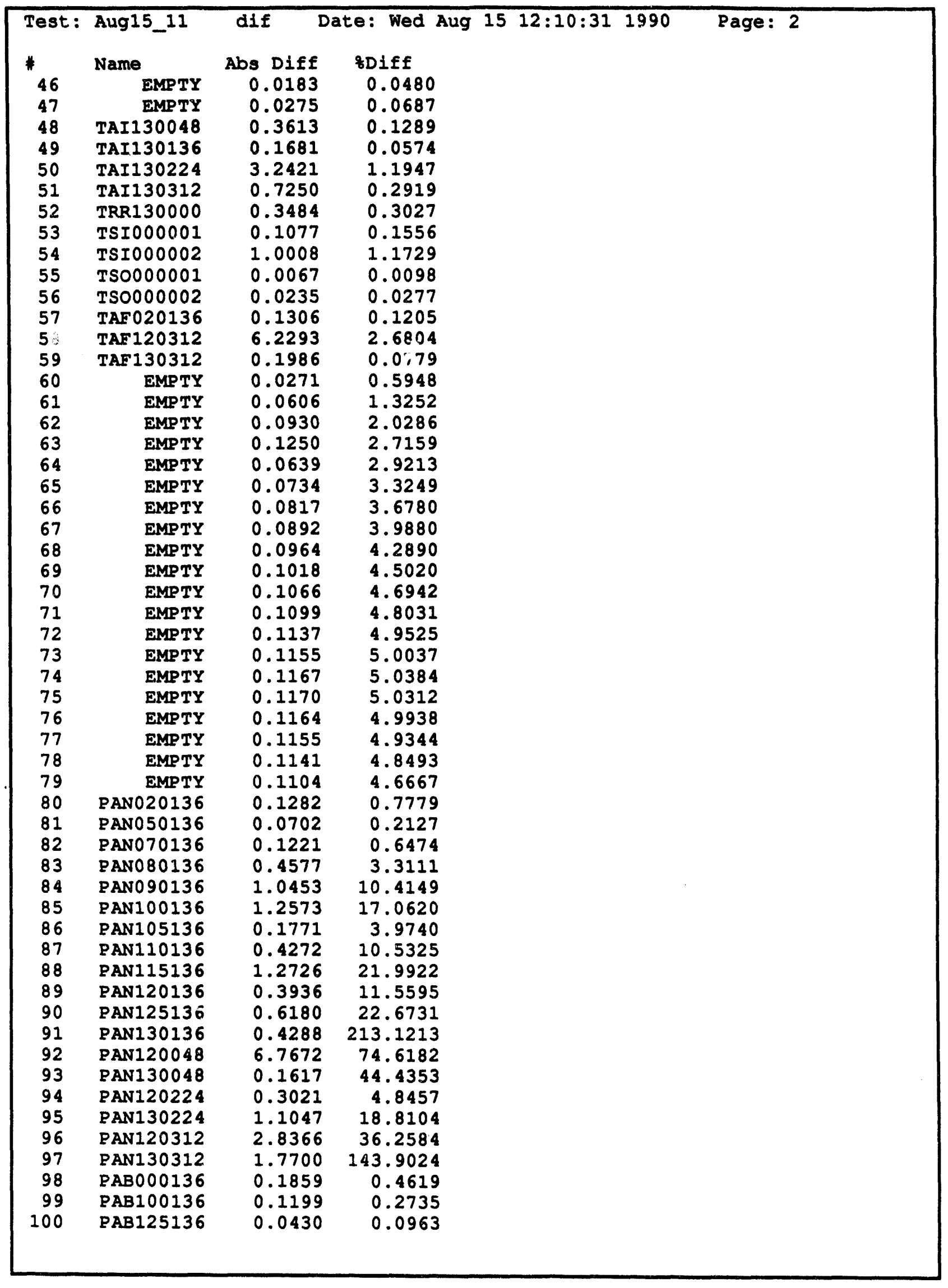


Test: Aug15_11

Name

PIN000001

PRV000001

PSR000001

PFM000001

PAN000136

PFM000002

EMPTY

EMPTY

EMPTY

EMPTY

EMPTY

VPW000001

VPW000002

VPW000003

VPW000004

VPW000005

VPW000006

IPW000001

IPWO00002

IPW000003

IPWO00004

IPW000005

IPW000006

EMPTY

EMPTY

EMPTY

EMPTY

IHT000001

IHTO0000?

IHT000003

IHT000004

IHT000005

IHT000006

IHT000007

IHT000008

IHT000009

IHT000010

IHT000011

IHT000012

IHT000013

IHT000014

IHT000015

IHT000016

IHT000017

IHT000018

IHT000019

IHT000020

IHT000021

IHT000022

IHT000023

IHTO00024

IHT000025

IHT000026

IHT000027

IHT000028 dif Date: Wed Aug 15 12:10:31 1990

Page: 3

Abs Diff

0.2588

\&Diff

0.0219

0.0293

0.0074

4.5318

0.0302

0.0026

0.4575

0.0008

0.0000

0.0000

0.0274

0.1914

0.1250

0.0195

0.0507

0.0625

1.5232

2.1075

6.1619

4.3554

3.4177

0.0586

0.0024

0.0015

0.0012

0.0015

0.0352

0.0508

0.0274

0.2696

0.2305

0.2500

0.0391

0.0625

0.0547

0.0625

0.0195

0.0156

0.1406

0.0625

0.0977

0.0157

0.6602

0.0938

0.0273

0.0351

0.0860

0.1094

0.1055

0.0547

0.2383

0.1758

0.0235

0.0039

\subsection{6}

0.1107

0.0662

0.4132

3.6723

1.7069

118.1818

9.3781

266.6667

0.0000

0.0000

0.0207

0.1319

0.1058

0.0196

0.0467

0.0389

0.1425

0.2659

0.9512

0.8206

0.5819

0.0137

11.4833

10.8696

7.4534

8.4746

0.0277

0.0389

0.0209

0.1894

0.1641

0.1850

0.0302

0.0489

0.0425

0.0528

0.0167

0.0136

0.1499

0.0518

0.1000

0.0181

0.6273

0.0876

0.0239

0.0298

6.5514

0.1122

0.0914

0.0673

0.2300

0.1678

0.0267

0.0044 


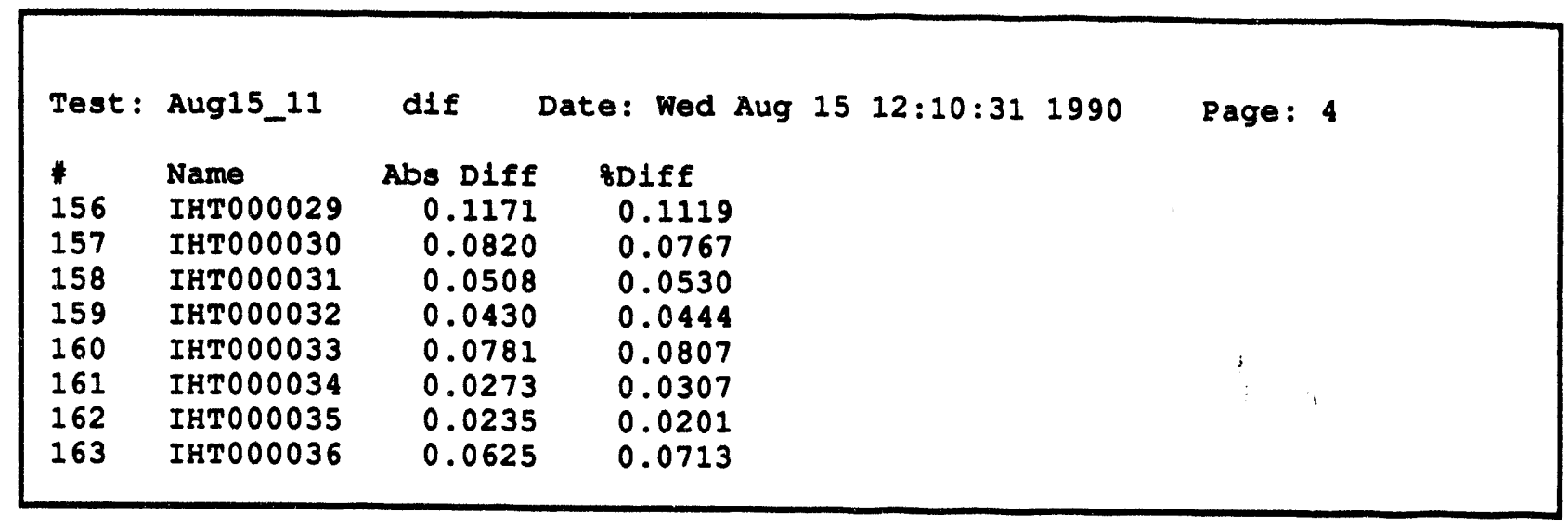




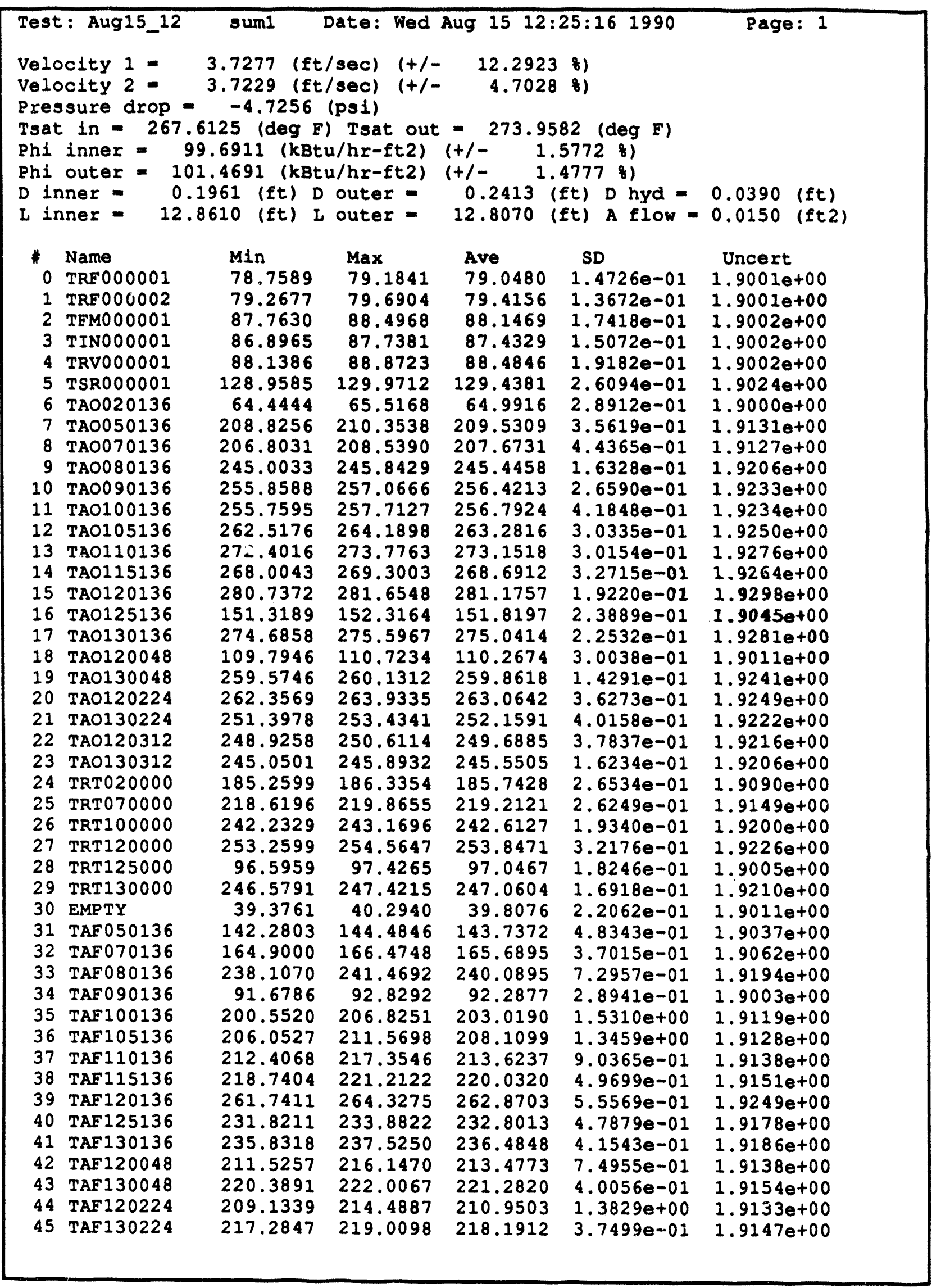




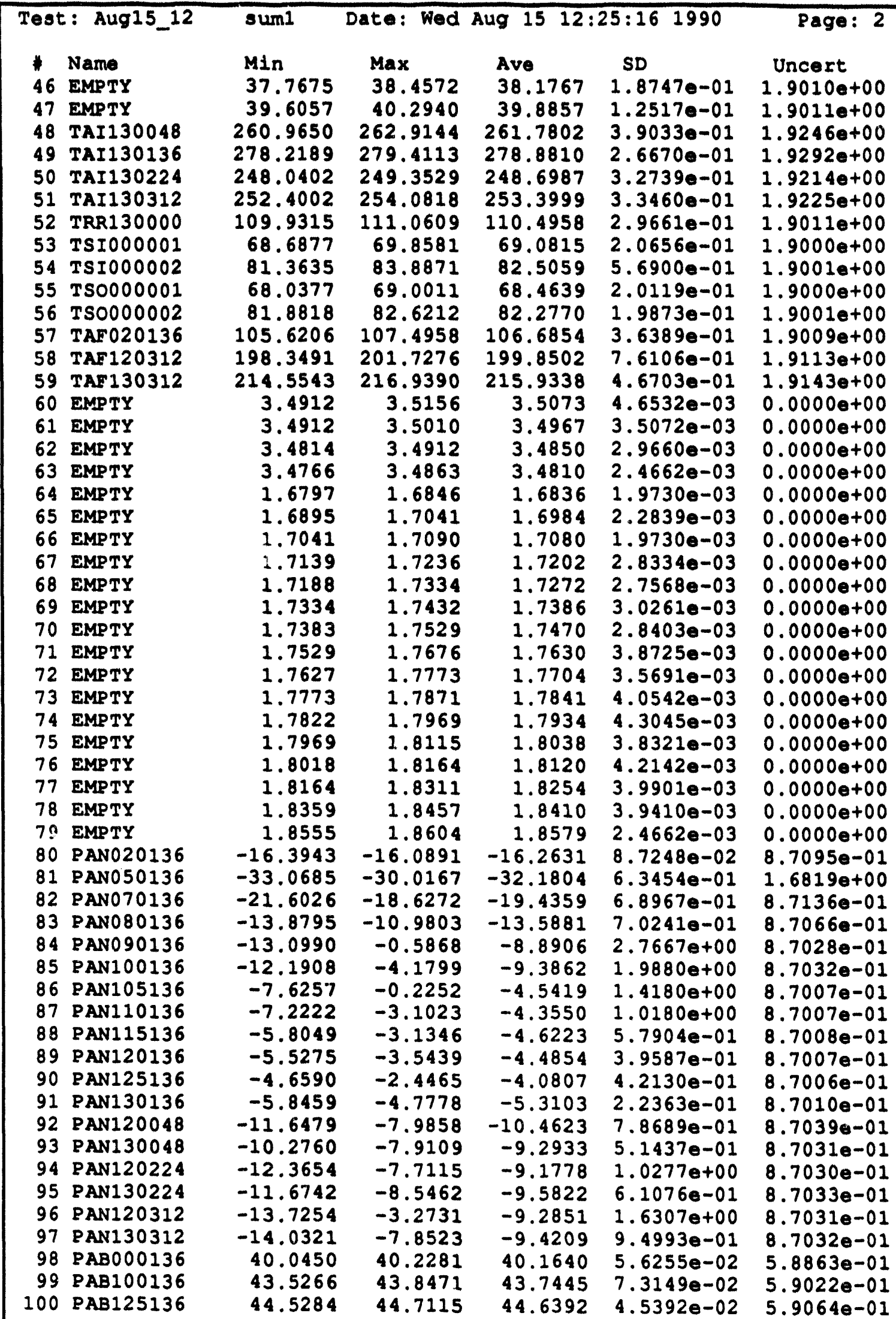




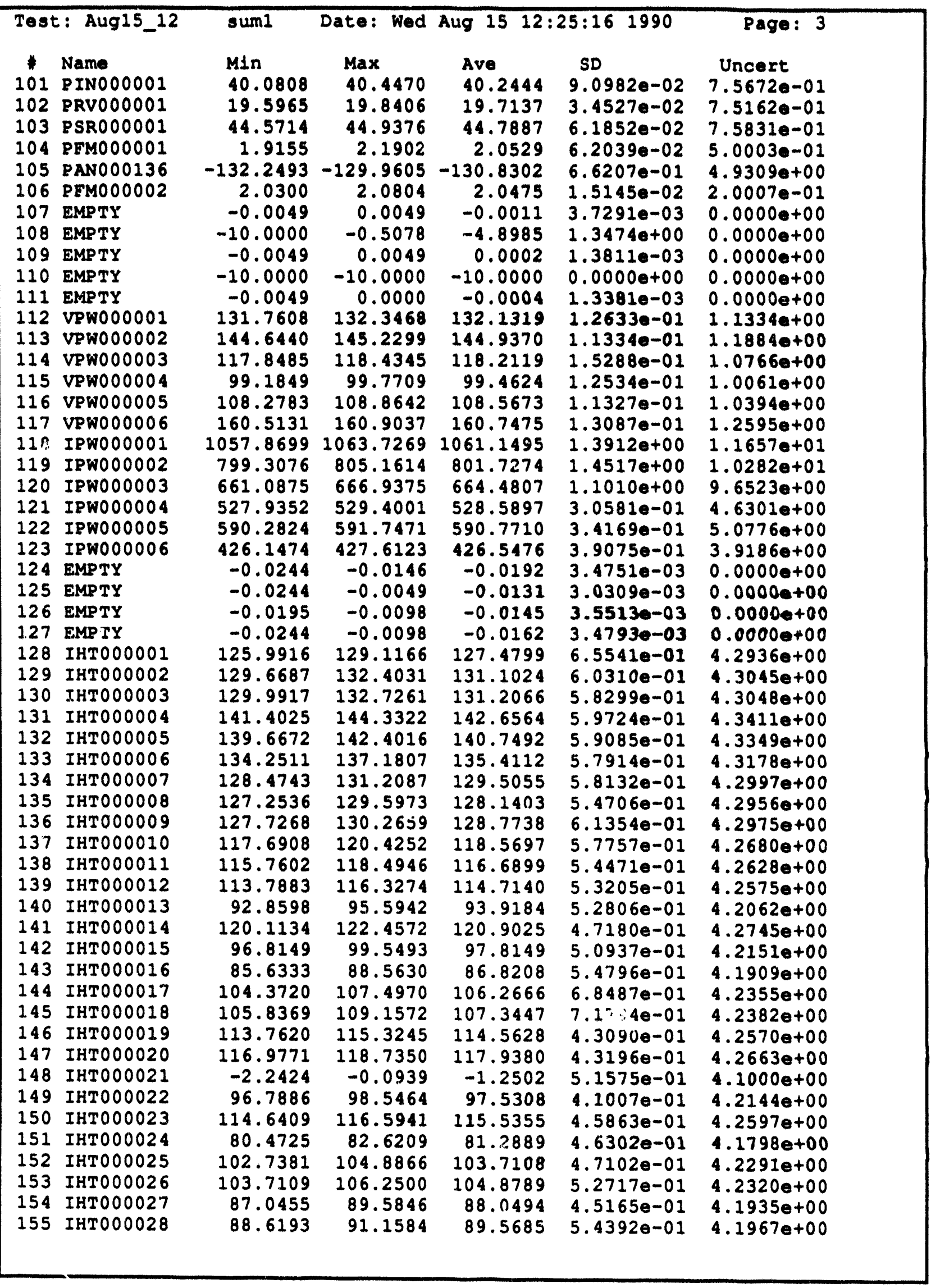




\begin{tabular}{|c|c|c|c|c|c|c|}
\hline $\begin{array}{l}1 \\
156 \\
157 \\
158 \\
159 \\
160 \\
161 \\
162 \\
163\end{array}$ & 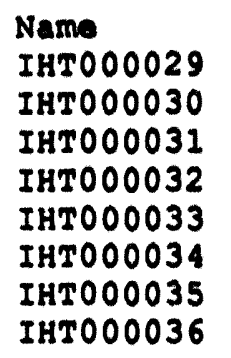 & $\begin{array}{l}\text { Min } \\
103.6396 \\
105.6190 \\
94.7641 \\
95.7933 \\
95.7782 \\
88.0521 \\
116.0119 \\
86.9479\end{array}$ & $\begin{array}{l}\text { Max } \\
106.3740 \\
108.5487 \\
97.3032 \\
98.7230 \\
98.5126 \\
90.9818 \\
118.5509 \\
89.2916\end{array}$ & $\begin{array}{r}\text { Ave } \\
104.8310 \\
107.0526 \\
95.8384 \\
96.9729 \\
96.9267 \\
89.1498 \\
117.2228 \\
87.8697\end{array}$ & $\begin{array}{l}\text { SD } \\
6.0005 e-01 \\
6.2839 e-01 \\
5.9875 e-01 \\
6.5905 e-01 \\
6.3336 e-01 \\
5.5368 \theta-01 \\
5.6221 e-01 \\
5.1605 e-01\end{array}$ & $\begin{array}{c}\text { Uncert } \\
4.2319 e+00 \\
4.2375 e+00 \\
4.2105 e+00 \\
4.2131 e+00 \\
4.2130 e+00 \\
4.1958 \theta+00 \\
4.2643 e+00 \\
4.1931 e+00\end{array}$ \\
\hline
\end{tabular}




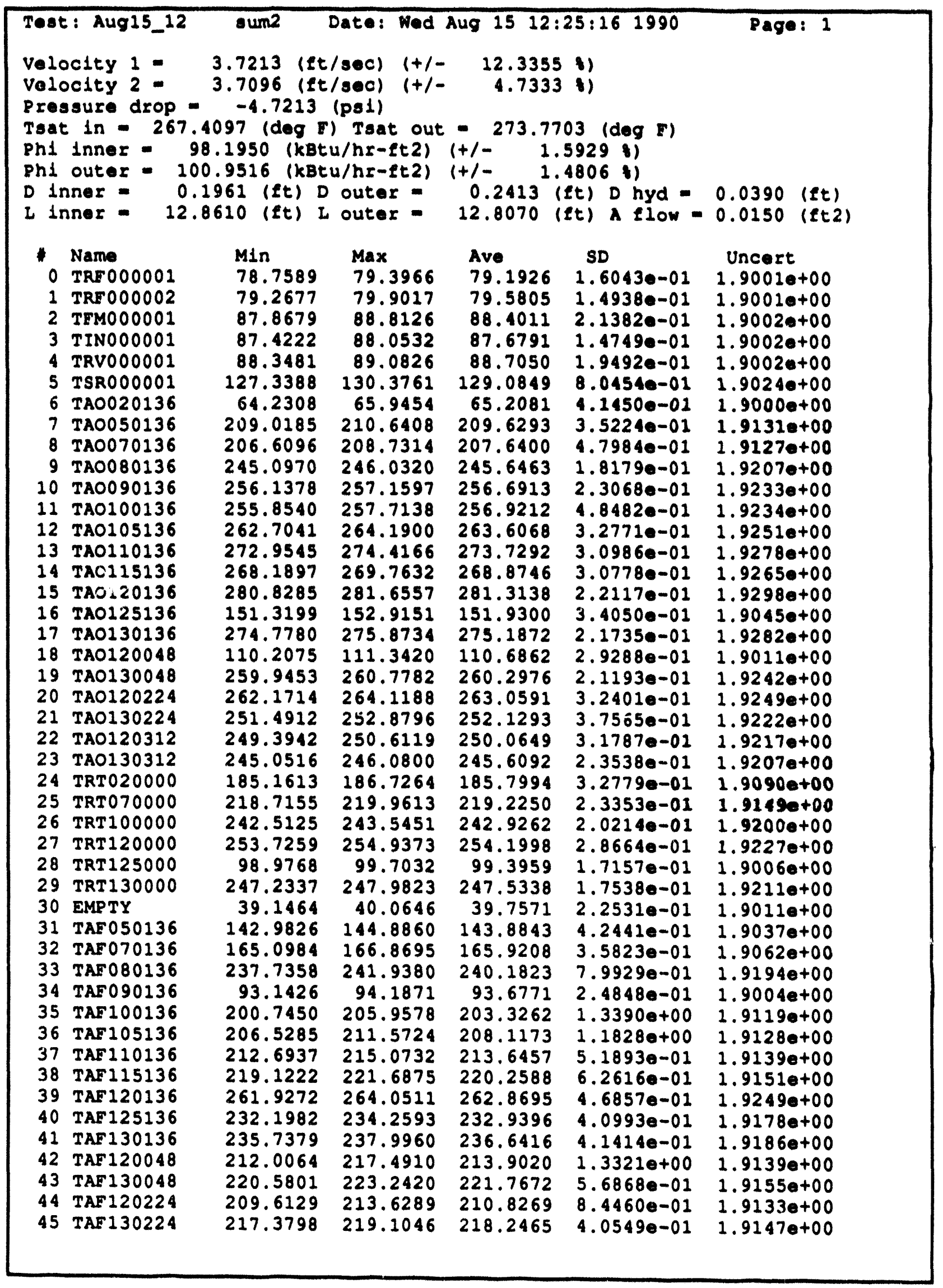




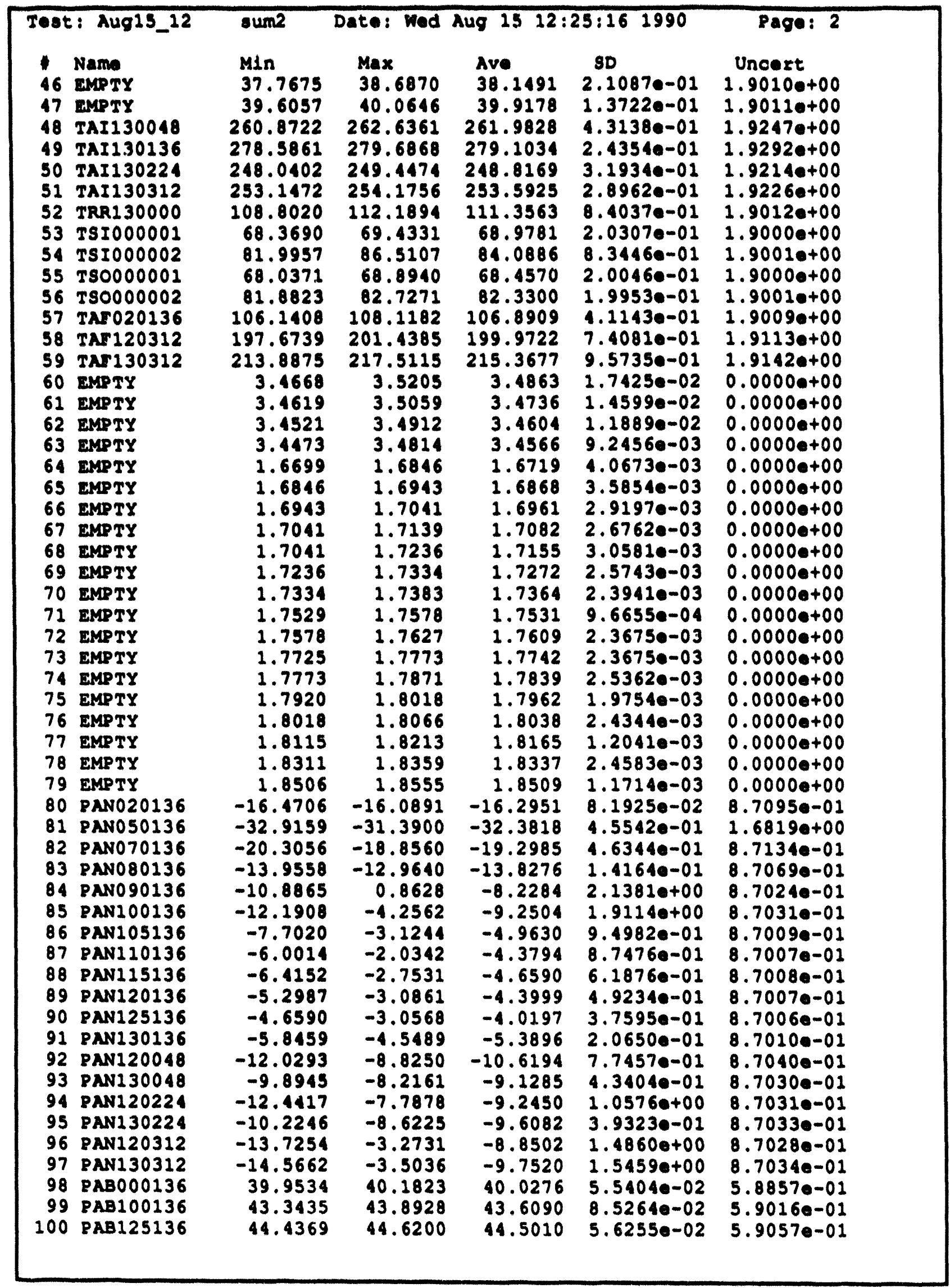




\begin{tabular}{|c|c|c|c|c|c|c|}
\hline $\begin{array}{l}1 \\
101 \\
102 \\
103 \\
104 \\
105 \\
106 \\
107 \\
108 \\
109 \\
110 \\
111 \\
112 \\
113 \\
114 \\
115 \\
116 \\
117 \\
118 \\
119 \\
120 \\
121 \\
122 \\
123 \\
124 \\
125 \\
126 \\
127 \\
128 \\
129 \\
130 \\
131 \\
132 \\
133 \\
134 \\
135 \\
136 \\
137 \\
138 \\
139 \\
140 \\
141 \\
142 \\
143 \\
144 \\
145 \\
146 \\
147 \\
148 \\
149 \\
150 \\
151 \\
152 \\
153 \\
154 \\
155\end{array}$ & 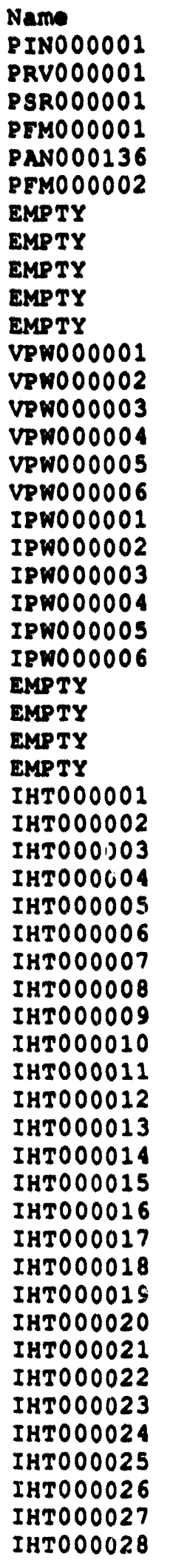 & $\begin{array}{r}\text { Min } \\
39.7146 \\
19.7186 \\
44.5714 \\
1.9155 \\
-131.7916 \\
1.9965 \\
-0.0049 \\
-9.8340 \\
-0.0049 \\
-10.0000 \\
0.0000 \\
131.9561 \\
144.6440 \\
117.8485 \\
99.1849 \\
108.2783 \\
160.5131 \\
1075.4410 \\
768.0872 \\
627.9375 \\
535.2595 \\
586.3765 \\
123.2177 \\
-0.0244 \\
-0.0193 \\
-0.0244 \\
-0.0244 \\
125.9916 \\
129.6687 \\
129.7964 \\
141.4025 \\
139.6672 \\
134.2511 \\
128.0837 \\
126.8630 \\
127.5315 \\
117.6908 \\
115.7602 \\
113.7883 \\
93.0552 \\
119.9181 \\
96.8149 \\
114.2503 \\
80.0819 \\
102.5428 \\
103.7109 \\
87.0455 \\
88.2287 \\
85.6333 \\
103.3954 \\
105.6415 \\
113.1761 \\
116.5865 \\
-3.0236 \\
136\end{array}$ & 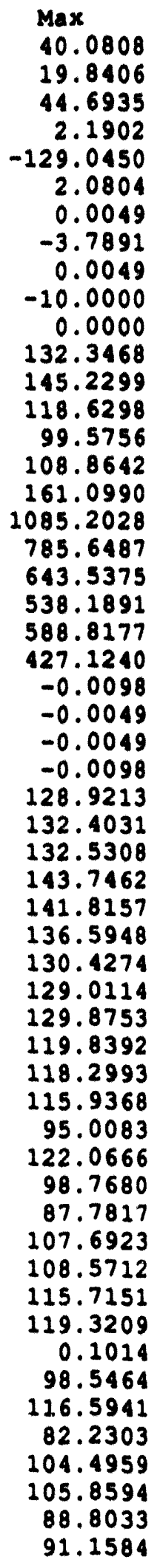 & 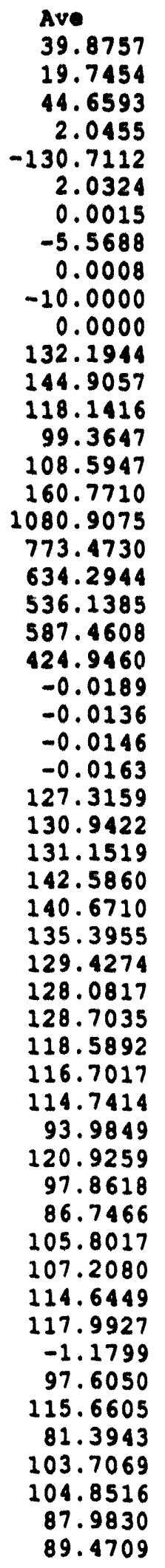 & $\begin{array}{l}\text { SD } \\
1.1419 e-01 \\
5.1081 e-02 \\
5.5366 e-02 \\
7.1851 e-02 \\
6.2561 e-01 \\
2.5143 e-02 \\
3.7239 e-03 \\
9.9408 e-01 \\
2.0598 e-03 \\
0.0000 e+00 \\
0.0000 e+00 \\
1.4385 e-01 \\
1.0885 e-01 \\
1.6857 \bullet-01 \\
1.0320 e-01 \\
1.4167 e-01 \\
1.2750 e-01 \\
2.8715 e+00 \\
5.7610 e+00 \\
3.2441 e+00 \\
7.1817 e-01 \\
5.7759 e-01 \\
9.4212 e-01 \\
4.0241 e-03 \\
3.7291 e-03 \\
4.5206 e-03 \\
3.0581 e-03 \\
6.2177 e-01 \\
6.1414 e-01 \\
5.6828 e-01 \\
5.3730 e-01 \\
5.1601 e-01 \\
5.3961 e-01 \\
4.9227 e-01 \\
4.7443 e-01 \\
5.1145 e-01 \\
4.6522 e-01 \\
5.0638 e-01 \\
4.6293 e-01 \\
4.4039 e-01 \\
4.7080 e-01 \\
4.6145 e-01 \\
4.6563 e-01 \\
1.0781 e+00 \\
6.1446 e-01 \\
5.2680 e-01 \\
5.4247 e-01 \\
6.7792 e-01 \\
5.2302 e-01 \\
4.9172 e-01 \\
4.5854 e-01 \\
5.0372 e-01 \\
5.2253 e-01 \\
4.2499 e-01 \\
6.1985 e-01\end{array}$ & $\begin{array}{c}\text { Uncert } \\
7.5660 e-01 \\
7.5162 e-01 \\
7.5826 e-01 \\
5.0003 e-01 \\
4.9308 e+00 \\
2.0006 e-01 \\
0.0000 e+00 \\
0.0000 e+00 \\
0.0000 e+00 \\
0.0000 e+00 \\
0.0000 e+00 \\
1.1337 e+00 \\
1.1883 e+00 \\
1.0764 e+00 \\
1.0058 e+00 \\
1.0395 e+00 \\
1.2596 e+00 \\
1.1770 e+01 \\
1.0146 e+01 \\
9.5247 e+00 \\
4.6840 e+00 \\
5.0535 e+00 \\
3.9077 \bullet+00 \\
0.0000 e+00 \\
0.0000 e+00 \\
0.0000 e+00 \\
0.0000 e+00 \\
4.2931 e+00 \\
4.3040 e+00 \\
4.3047 e+00 \\
4.3409 e+00 \\
4.3346 e+00 \\
4.3178 e+00 \\
4.2994 e+00 \\
4.2954 e+00 \\
4.2973 e+00 \\
4.2681 e+00 \\
4.2629 e+00 \\
4.2575 e+00 \\
4.2063 e+00 \\
4.2746 e+00 \\
4.2152 e+00 \\
4.1908 e+00 \\
4.2343 e+00 \\
4.2378 e+00 \\
4.2573 e+00 \\
4.2664 e+00 \\
4.1000 e+00 \\
4.2146 e+00 \\
4.2600 e+00 \\
4.1800 e+00 \\
4.2291 e+00 \\
4.2319 e+00 \\
4.1933 e+00 \\
4.1965 e+00\end{array}$ \\
\hline
\end{tabular}




\begin{tabular}{|c|c|c|c|c|c|c|}
\hline $\begin{array}{l}156 \\
156 \\
157 \\
158 \\
159 \\
160 \\
161 \\
162 \\
163\end{array}$ & $\begin{array}{l}\text { Nam0 } \\
\text { IHT000029 } \\
\text { IHTO00030 } \\
\text { IHTO00031 } \\
\text { IHT000032 } \\
\text { IHTO00033 } \\
\text { IHTO00034 } \\
\text { IHTO00035 } \\
\text { IHT000036 }\end{array}$ & $\begin{array}{r}\text { M1n } \\
103.4443 \\
105.6190 \\
94.5688 \\
95.9886 \\
95.9736 \\
88.0521 \\
116.4025 \\
86.9479\end{array}$ & $\begin{array}{l}\operatorname{Max} \\
106.1786 \\
108.3533 \\
97.1079 \\
98.5276 \\
98.3173 \\
90.3959 \\
118.3556 \\
88.7057\end{array}$ & $\begin{array}{r}\text { Ave } \\
104.7373 \\
106.9745 \\
95.8657 \\
96.9690 \\
96.9306 \\
89.0561 \\
117.2814 \\
87.7955\end{array}$ & $\begin{array}{l}\text { SD } \\
6.1243 e-01 \\
6.0414 e-01 \\
5.6739 e-01 \\
5.8059 e-01 \\
5.6943 e-01 \\
4.8969 e-01 \\
4.6059 e-01 \\
4.3514 e-01\end{array}$ & $\begin{array}{l}\text { Uncert } \\
4.2317 e+00 \\
4.2373 \theta+00 \\
4.2106 \theta+00 \\
4.2131 e+00 \\
4.2130 \theta+00 \\
4.1956 e+00 \\
4.2644 \theta+00 \\
4.1929 e+00\end{array}$ \\
\hline
\end{tabular}




\begin{tabular}{|c|c|c|c|c|}
\hline \multicolumn{5}{|c|}{$\begin{array}{l}\text { Velocity } 1=0.006400 \text { (ft/sec) } \\
\text { Velocity } 2=0.013300 \text { (ft/sec) } \\
\text { Pressure Drop }=0.004300 \text { (psi) } \\
\text { Tsat in }=0.202789 \text { (deg F) Tsat out }=0.187897 \text { (deg F) } \\
\text { Phi inner }=1.496101 \text { (kBtu/hr-ft2) Phi outer }=0.517502 \text { (kBtu/hr-ft2) }\end{array}$} \\
\hline $\begin{array}{l}* \\
1 \\
2 \\
2 \\
3 \\
4 \\
5 \\
6 \\
7 \\
8 \\
9 \\
10 \\
11 \\
12 \\
13 \\
14 \\
15 \\
16 \\
17 \\
18 \\
19 \\
20 \\
21 \\
22 \\
23 \\
24 \\
25 \\
26 \\
27 \\
28 \\
29 \\
30 \\
31 \\
32 \\
33 \\
34 \\
35 \\
36 \\
37 \\
38 \\
39 \\
40 \\
41 \\
42 \\
43 \\
44 \\
45\end{array}$ & $\begin{array}{l}\text { Name } \\
\text { TRF000001 } \\
\text { TRF000002 } \\
\text { TEM000001 } \\
\text { TIN000001 } \\
\text { TRV000001 } \\
\text { TSR000001 } \\
\text { TAO020136 } \\
\text { TAO050136 } \\
\text { TAO070136 } \\
\text { TAO080136 } \\
\text { TAO090136 } \\
\text { TAO100136 } \\
\text { TAO105136 } \\
\text { TAO110136 } \\
\text { TAO115136 } \\
\text { TAO120136 } \\
\text { TAO125136 } \\
\text { TAO130136 } \\
\text { TAO120048 } \\
\text { TAO130048 } \\
\text { TAO120224 } \\
\text { TAO130224 } \\
\text { TAO120312 } \\
\text { TAO130312 } \\
\text { TRT020000 } \\
\text { TRT070000 } \\
\text { TRT100000 } \\
\text { TRT120000 } \\
\text { TRT125000 } \\
\text { TRT130000 } \\
\text { TAF0 EMPTY } \\
\text { TAF0 } 0136 \\
\text { TAF080136 } \\
\text { TAF090136 } \\
\text { TAF100136 } \\
\text { TAF105136 } \\
\text { TAF110136 } \\
\text { TAF115136 } \\
\text { TAF120136 } \\
\text { TAF125136 } \\
\text { TAF130136 } \\
\text { TAF120048 } \\
\text { TAF130048 } \\
\text { TAF120224 } \\
\text { TAF130224 }\end{array}$ & $\begin{array}{l}\text { Abs Diff } \\
0.1446 \\
0.1649 \\
0.2542 \\
0.2462 \\
0.2204 \\
0.3532 \\
0.2165 \\
0.0984 \\
0.0331 \\
0.2005 \\
0.2700 \\
0.1288 \\
0.3252 \\
0.5774 \\
0.1834 \\
0.1381 \\
0.1103 \\
0.1458 \\
0.4188 \\
0.4358 \\
0.0051 \\
0.0298 \\
0.3764 \\
0.0587 \\
0.0566 \\
0.0129 \\
0.3135 \\
0.3527 \\
2.3492 \\
0.4734 \\
0.0505 \\
0.1471 \\
0.2313 \\
0.0928 \\
1.3894 \\
0.3072 \\
0.0074 \\
0.0220 \\
0.2268 \\
0.0008 \\
0.1383 \\
0.1568 \\
0.4247 \\
0.4852 \\
0.1234 \\
0.0553\end{array}$ & $\begin{array}{l}\text { 8Diff } \\
0.1829 \\
0.2076 \\
0.2884 \\
0.2816 \\
0.2491 \\
0.2729 \\
0.3331 \\
0.0470 \\
0.0159 \\
0.0817 \\
0.1053 \\
0.0502 \\
0.1235 \\
0.2114 \\
0.0683 \\
0.0491 \\
0.0726 \\
0.0530 \\
0.3798 \\
0.1677 \\
0.0019 \\
0.0118 \\
0.1507 \\
0.0239 \\
0.0305 \\
0.0059 \\
0.1292 \\
0.1389 \\
2.4207 \\
0.1916 \\
0.1269 \\
0.1023 \\
0.1396 \\
0.0387 \\
1.5055 \\
0.1513 \\
0.0036 \\
0.0103 \\
0.1031 \\
0.0003 \\
0.0594 \\
0.0663 \\
0.1989 \\
0.2193 \\
0.0585 \\
0.0254\end{array}$ & \\
\hline
\end{tabular}




\begin{tabular}{|c|c|c|c|}
\hline 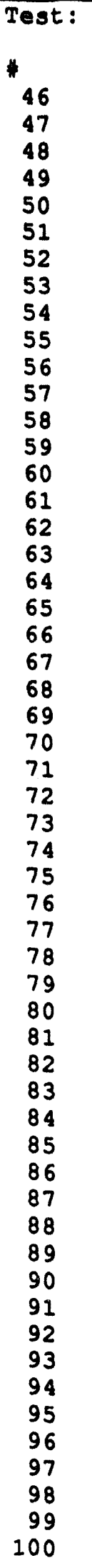 & 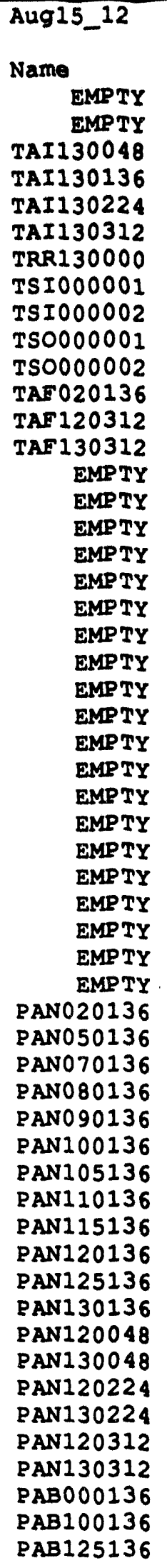 & 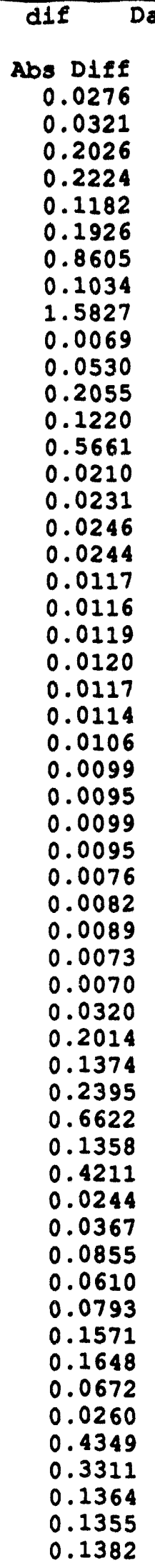 & 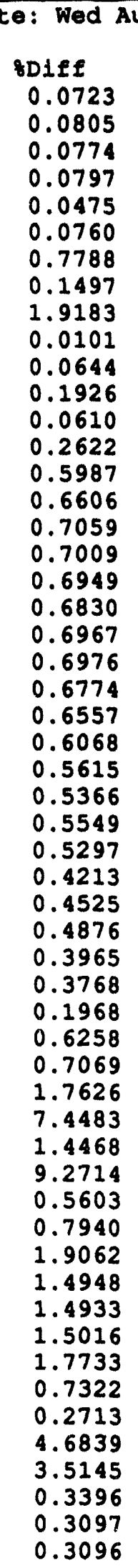 \\
\hline
\end{tabular}




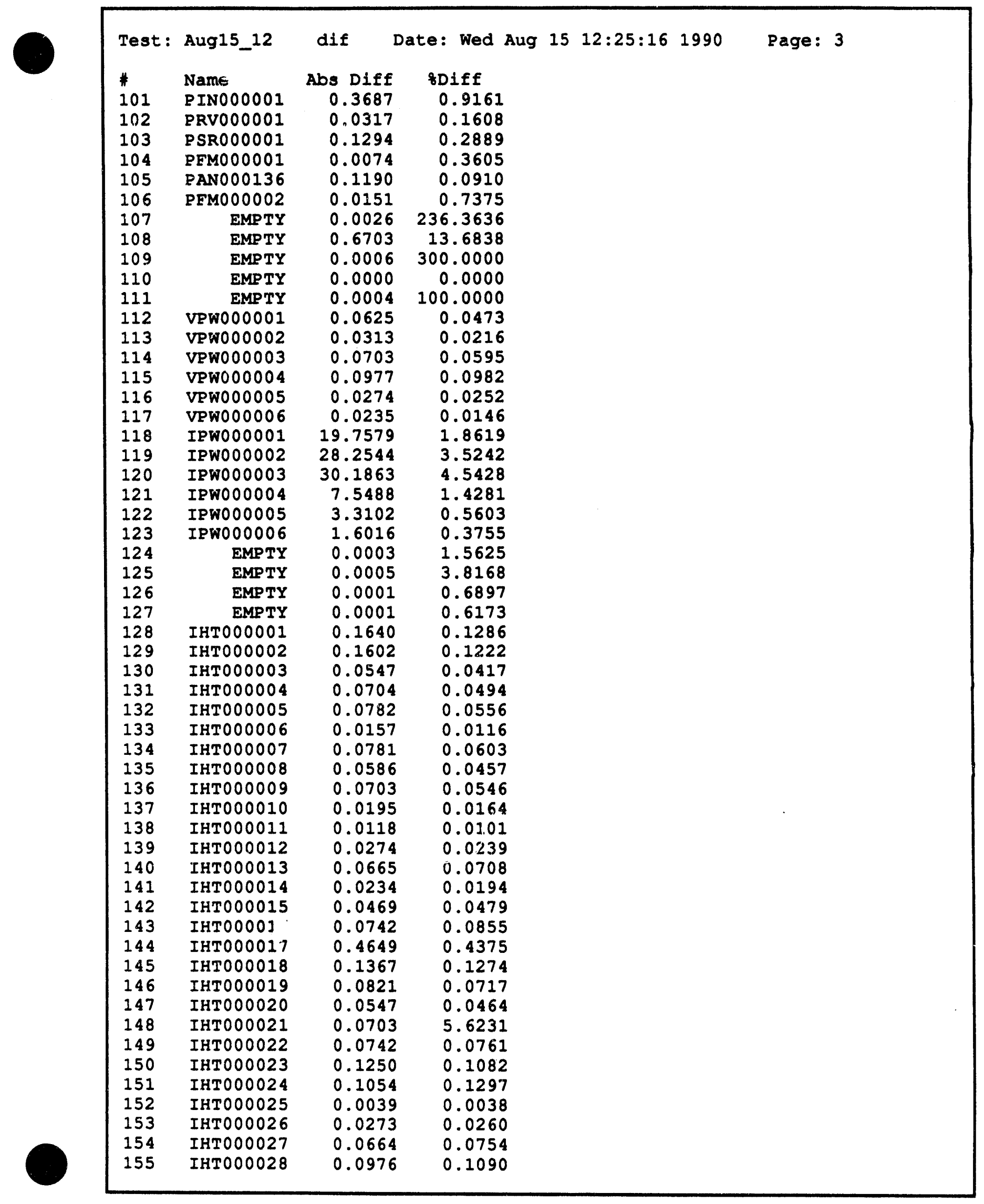


Test: Aug15_12 dif Date: Wed Aug 15 12:25:16 1990 Page: 4

\begin{tabular}{lrc} 
Name & Abs Diff & \multicolumn{1}{l}{ 8Diff } \\
IHT000029 & 0.0937 & 0.0894 \\
IHTO00030 & 0.0781 & 0.0729 \\
IHTO00031 & 0.0273 & 0.0285 \\
IHT000032 & 0.0039 & 0.0040 \\
IHT000033 & 0.0039 & 0.0040 \\
IHT000034 & 0.0937 & 0.1051 \\
IHT000035 & 0.0586 & 0.0500 \\
IHT000036 & 0.0742 & 0.0844
\end{tabular}




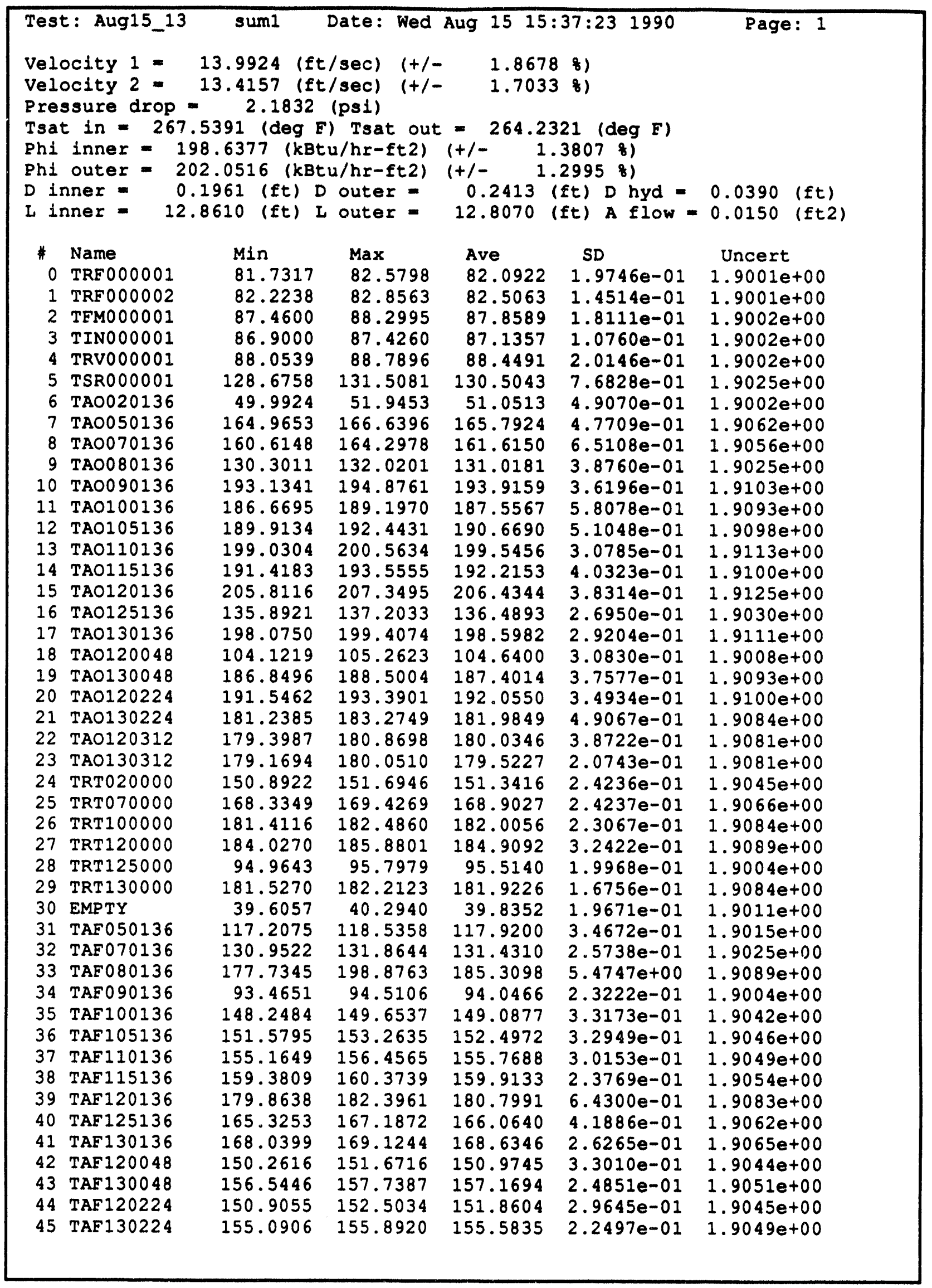




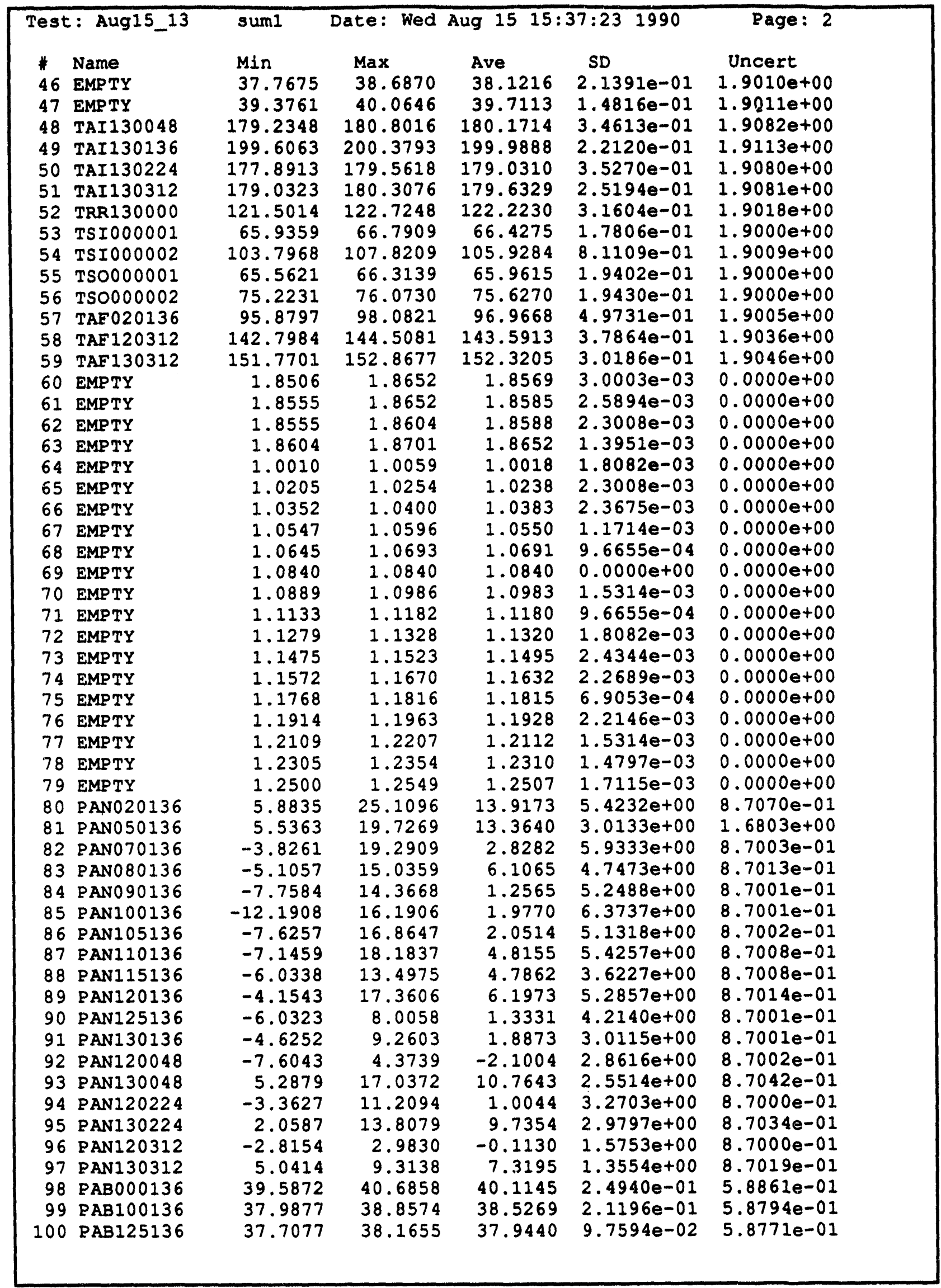




\begin{tabular}{|c|c|c|c|c|c|c|}
\hline \multicolumn{2}{|c|}{ Test: Aug15_13 } & sum1 & \multicolumn{3}{|c|}{ Date: Wed Aug $15 \quad 15: 37: 231990$} & Page: 3 \\
\hline * & Name & Min & $\operatorname{Max}$ & Ave & SD & Uncert \\
\hline 101 & PIN000001 & 39.3483 & 40.9353 & 40.0832 & $3.8562 e-01$ & $7.5666 \mathrm{e}-01$ \\
\hline 102 & PRV000001 & 19.8406 & 19.8406 & 19.8406 & $1.9267 e-06$ & $7.5164 e-01$ \\
\hline 103 & PSR000001 & 36.7589 & 37.0031 & 36.8468 & $6.5435 e-02$ & $7.5564 e-01$ \\
\hline 104 & PFM000001 & 29.6560 & 30.2969 & 30.0076 & $1.4954 e-01$ & $5.0560 e-01$ \\
\hline 105 & PANO00136 & 43.9897 & 80.1530 & 60.4418 & $8.7029 e+00$ & $4.9223 e+00$ \\
\hline 106 & PFM000002 & 27.5689 & 27.5689 & 27.5689 & $9.6336 e-06$ & $2.1154 e-01$ \\
\hline 107 & EMPTY & 0.0049 & 0.0098 & 0.0069 & $2.4344 e-03$ & $0.0000 e+00$ \\
\hline 108 & EMPTY & -10.0000 & -6.2695 & -9.9230 & $5.2749 e-01$ & $0.0000 e+00$ \\
\hline 109 & EMPTY & 0.0049 & 0.0098 & 0.0052 & $1.1714 e-03$ & $0.0000 e+00$ \\
\hline 110 & EMPTY & -10.0000 & -10.0000 & -10.0000 & $0.0000 e+00$ & $0.0000 e+00$ \\
\hline 111 & EMPTY & 0.0000 & 0.0000 & 0.0000 & $0.0000 e+00$ & $0.0000 \mathrm{e}+00$ \\
\hline 112 & VPW000001 & 187.0343 & 187.4249 & 187.2139 & $1.1732 \mathrm{e}-01$ & $1.3849 \mathrm{e}+00$ \\
\hline 113 & VPW000002 & 204.9955 & 205.3861 & 205.1908 & $1.0440 e-01$ & $1.4737 e+00$ \\
\hline 114 & VPW000003 & 167.4579 & 167.8485 & 167.6103 & $1.1361 e-01$ & $1.2913 \mathrm{e}+00$ \\
\hline 115 & VPW000004 & 140.2006 & 140.7865 & 140.5366 & $1.1189 e-01$ & $1.1692 \mathrm{e}+00$ \\
\hline 116 & VPW000005 & 153.2001 & 153.5907 & 153.4580 & $1.0763 e-01$ & $1.2263 e+00$ \\
\hline 117 & VPW000006 & 227.5053 & 228.0912 & 227.7552 & $1.1864 \mathrm{e}-01$ & $1.5886 \mathrm{e}+00$ \\
\hline 118 & IPWO000001 & 1497.1472 & 1503.0043 & 1501.2472 & $1.2628 \theta+00$ & $1.4362 e+01$ \\
\hline 119 & IPW000002 & 1119.3158 & 1127.1208 & 1123.1791 & $1.6008 e+00$ & $1.2014 e+01$ \\
\hline 120 & IPW000003 & 920.4375 & 926.2875 & 923.7133 & $1.2729 e+00$ & $1.0902 e+01$ \\
\hline 121 & IPW000004 & 745.7087 & 747.1735 & 746.2360 & $3.0945 e-01$ & $6.2253 e+00$ \\
\hline 122 & IPW000005 & 832.9339 & 834.3986 & 833.3536 & $3.2730 e-01$ & $6.8806 e+00$ \\
\hline 123 & IPW000006 & 599.4872 & 603.8818 & 602.0845 & $1.2299 e+00$ & $5.1598 e+00$ \\
\hline 124 & EMPTY & -0.0293 & -0.0146 & -0.0207 & $3.4961 \mathrm{e}-03$ & $0.0000 e+00$ \\
\hline 125 & EMPTY & -0.0195 & -0.0098 & -0.0135 & $2.8862 e-03$ & $0.0000 e+00$ \\
\hline 126 & EMPTY & -0.0195 & -0.0098 & -0.0165 & $3.4016 e-03$ & $0.0000 e+00$ \\
\hline 127 & EMPTY & -0.0244 & -0.0049 & -0.0151 & $3.3087 e-03$ & $0.0000 e+00$ \\
\hline 128 & IHT0000001 & 178.3353 & 180.8744 & 179.6049 & $6.4144 e-01$ & $4.4761 e+00$ \\
\hline 129 & IHT000002 & 183.3797 & 186.1140 & 184.6141 & $5.9639 \mathrm{e}-01$ & $4.4965 e+00$ \\
\hline 130 & IHT000003 & 184.2886 & 186.6324 & 185.4761 & $5.6750 e-01$ & $4.5000 e+00$ \\
\hline 131 & IHT000004 & 199.8009 & 202.7306 & 201.2462 & $6.1130 e-01$ & $4.5673 \mathrm{e}+00$ \\
\hline 132 & IHTO00005 & 197.0891 & 199.6282 & 198.5538 & $5.9222 e-01$ & $4.5555 e+00$ \\
\hline 133 & IHT000006 & 191.2823 & 194.0167 & 192.7354 & $5.4983 e-01$ & $4.5304 \mathrm{e}+00$ \\
\hline 134 & IHT 000007 & 181.4040 & 183.7477 & 182.5876 & $5.0595 e-01$ & $4.4882 e+00$ \\
\hline 135 & IHT000008 & 179.5973 & 181.7458 & 180.7809 & $4.6923 e-01$ & $4.4809 e+00$ \\
\hline 136 & IHT000009 & 180.4612 & 182.8050 & 181.6996 & $5.4905 e-01$ & $4.4846 \mathrm{e}+00$ \\
\hline 137 & IHT 000010 & 166.9095 & 169.8392 & 167.9643 & $6.6380 e-01$ & $4.4307 e+00$ \\
\hline 138 & IHTO00011 & 164.1977 & 166.7368 & 165.2602 & $6.2907 e-01$ & $4.4205 e+00$ \\
\hline 139 & IHTO00012 & 161.4446 & 164.1790 & 162.4445 & $6.3826 e-01$ & $4.4101 e+00$ \\
\hline 140 & IHT000013 & 133.0942 & 135.6333 & 134.0825 & $6.1815 e-01$ & $4.3137 e+00$ \\
\hline 141 & IHTO00014 & 170.3087 & 173.2384 & 171.4454 & $5.6592 e-01$ & $4.4440 e+00$ \\
\hline 142 & IHTO00015 & 137.6352 & 140.5649 & 138.8110 & $5.7790 e-01$ & $4.3286 e+00$ \\
\hline 143 & IHTO00016 & 120.9848 & 124.3051 & 122.4809 & $5.9137 e-01$ & $4.2790 \mathrm{e}+00$ \\
\hline 144 & IHTO000017 & 146.9501 & 150.8564 & 148.8135 & $7.0720 e-01$ & $4.3617 e+00$ \\
\hline 145 & IHT000018 & 149.9775 & 152.5165 & 151.1650 & $6.2991 \mathrm{e}-01$ & $4.3698 e+00$ \\
\hline 146 & IHTO00019 & 160.4417 & 163.1761 & 161.8558 & $5.2432 e-01$ & $4.4079 e+00$ \\
\hline 147 & IHTO00020 & 165.8053 & 167.9537 & 167.1412 & $4.9341 \mathrm{e}-01$ & $4.4276 e+00$ \\
\hline 148 & IHTO00021 & -2.6330 & 0.1014 & -1.2970 & $6.0019 e-01$ & $4.1000 e+00$ \\
\hline 149 & IHT0000022 & 137.2183 & 138.9761 & 138.0269 & $4.1387 e-01$ & $4.3261 e+00$ \\
\hline 150 & IHTO00023 & 162.8831 & 164.8363 & 163.7699 & $4.7385 e-01$ & $4.4150 e+00$ \\
\hline 151 & IHTO00024 & 127.7381 & 130.0819 & 128.9491 & $4.7679 e-01$ & $4.2980 e+00$ \\
\hline 152 & IHTO00025 & 145.3163 & 147.8553 & 146.5468 & $4.9007 e-01$ & $4.3540 \mathrm{e}+00$ \\
\hline 153 & IHTO00026 & 147.0703 & 149.6094 & 148.4766 & $5.1599 e-01$ & $4.3606 e+00$ \\
\hline 154 & IHT000027 & 122.9830 & 125.3268 & 124.3345 & $5.2176 \mathrm{e}-01$ & $4.2844 \mathrm{e}+00$ \\
\hline 155 & IHT000028 & 124.9474 & 127.6818 & 126.3185 & $6.0295 e-01$ & $4.2902 e+00$ \\
\hline
\end{tabular}




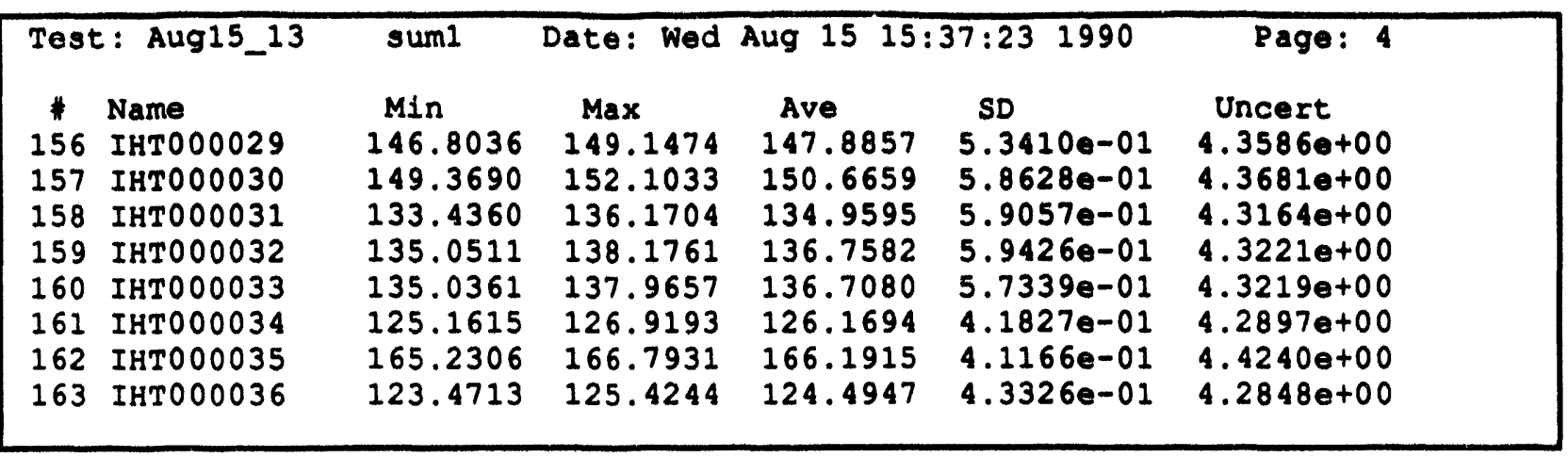




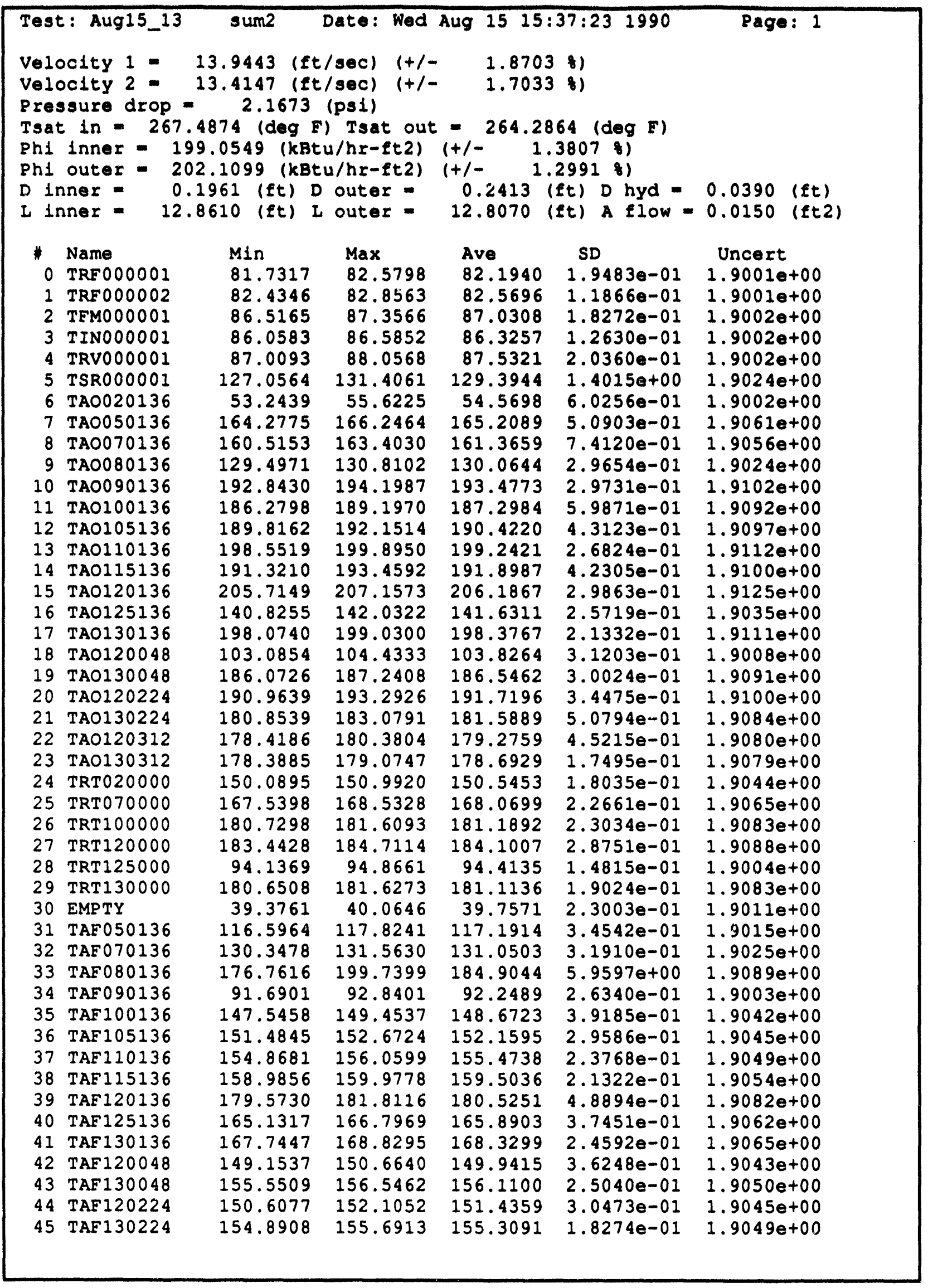




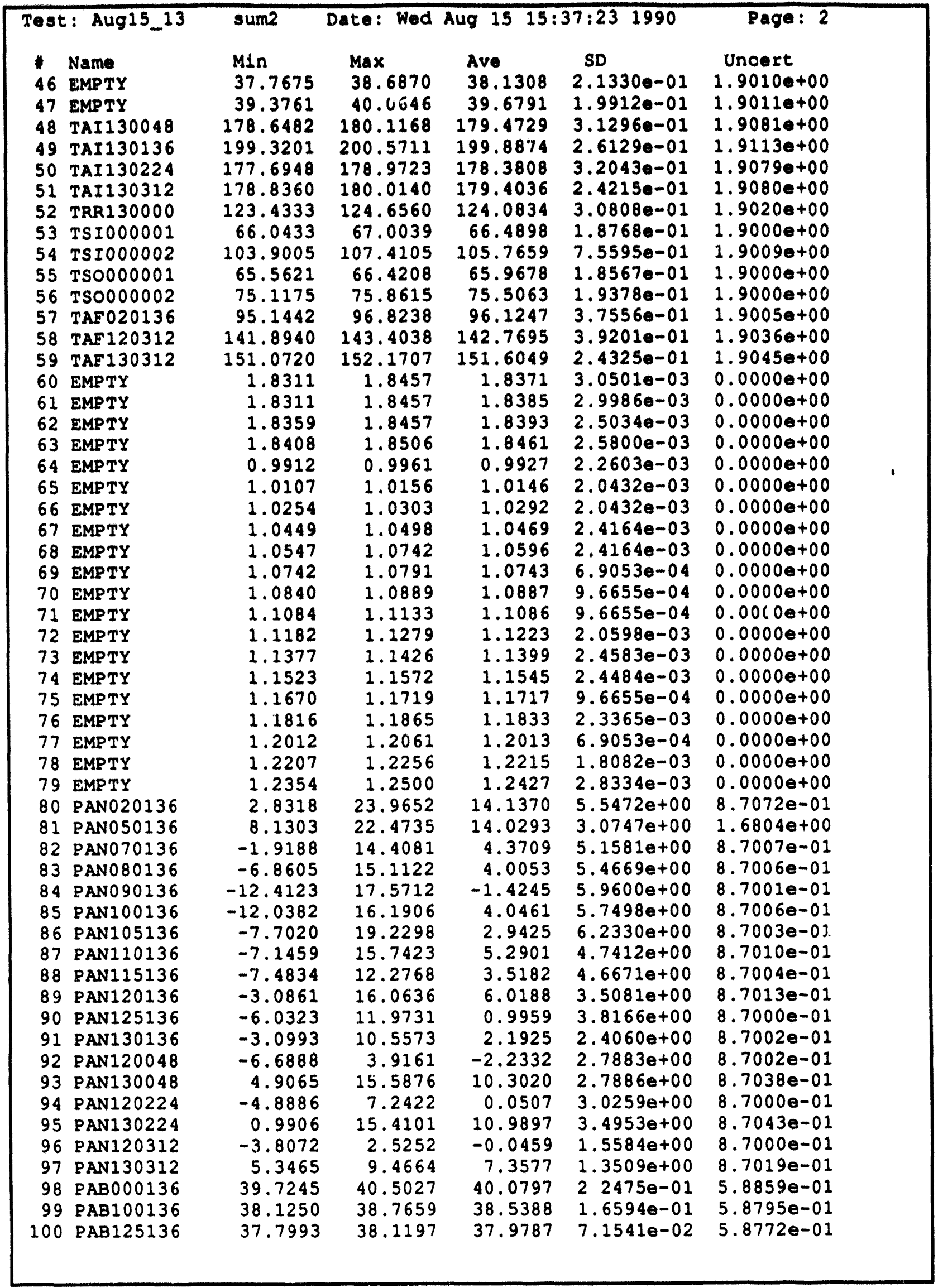




\begin{tabular}{|c|c|c|c|c|c|c|}
\hline $\begin{array}{l}1 \\
101 \\
102 \\
103 \\
104 \\
105 \\
106 \\
107 \\
108 \\
109 \\
110 \\
111 \\
112 \\
113 \\
114 \\
115 \\
116 \\
117 \\
118 \\
119 \\
120 \\
121 \\
122 \\
123 \\
124 \\
125 \\
126 \\
127 \\
128 \\
129 \\
130 \\
131 \\
132 \\
133 \\
134 \\
135 \\
136 \\
137 \\
138 \\
139 \\
140 \\
141 \\
142 \\
143 \\
144 \\
145 \\
146 \\
147 \\
148 \\
149 \\
150 \\
151 \\
152 \\
153 \\
154 \\
155\end{array}$ & 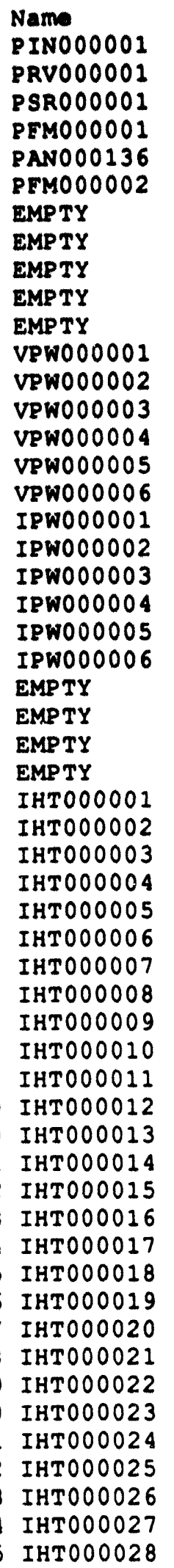 & $\begin{array}{r}\text { M1n } \\
39.3483 \\
19.8406 \\
36.6368 \\
29.3814 \\
44.9052 \\
27.5689 \\
0.0049 \\
-10.0000 \\
0.0049 \\
-10.0000 \\
0.0000 \\
186.8389 \\
204.9955 \\
167.2626 \\
140.0052 \\
153.2001 \\
227.5053 \\
1497.1472 \\
1125.1696 \\
920.4375 \\
750.5915 \\
831.9575 \\
602.4169 \\
-0.0244 \\
-0.0195 \\
-0.0195 \\
-0.0244 \\
178.7260 \\
183.5750 \\
184.6792 \\
200.1915 \\
197.4797 \\
191.8682 \\
181.4040 \\
179.7926 \\
181.0472 \\
166.5189 \\
164.0024 \\
161.0540 \\
132.7036 \\
170.1134 \\
137.4399 \\
121.3755 \\
148.5126 \\
150.3681 \\
161.0276 \\
166.0006 \\
-2.4377 \\
137.0230 \\
162.6878 \\
127.9334 \\
145.5116 \\
147.4609 \\
123.3737 \\
125.1427\end{array}$ & $\begin{array}{r}\text { Max } \\
40.6911 \\
19.9627 \\
37.0031 \\
30.4800 \\
77.4064 \\
27.5689 \\
0.0098 \\
-6.8506 \\
0.0049 \\
-10.0000 \\
0.0000 \\
187.2296 \\
205.3861 \\
167.8485 \\
140.7865 \\
153.5907 \\
227.8959 \\
1503.0043 \\
1131.0233 \\
926.2875 \\
751.5681 \\
832.9339 \\
603.3935 \\
-0.0098 \\
-0.0049 \\
-0.0098 \\
-0.0098 \\
181.0697 \\
186.3094 \\
187.2183 \\
202.9259 \\
200.0188 \\
194.4073 \\
184.1384 \\
182.1364 \\
183.1956 \\
169.6439 \\
166.5415 \\
163.5930 \\
135.2426 \\
173.0431 \\
140.5649 \\
123.9145 \\
151.4423 \\
152.9072 \\
162.3948 \\
167.7584 \\
-0.4845 \\
138.5855 \\
164.4456 \\
129.4959 \\
147.4647 \\
149.0234 \\
125.3268 \\
127.8771\end{array}$ & $\begin{array}{r}\text { Ave } \\
40.1101 \\
19.8870 \\
36.8566 \\
29.8043 \\
60.0023 \\
27.5689 \\
0.0063 \\
-9.6105 \\
0.0049 \\
-10.0000 \\
0.0000 \\
187.1319 \\
205.2299 \\
167.5595 \\
140.5053 \\
153.4463 \\
227.6966 \\
1500.0372 \\
1127.6670 \\
923.3622 \\
751.1188 \\
832.3868 \\
602.7681 \\
-0.0187 \\
-0.0129 \\
-0.0146 \\
-0.0168 \\
179.8237 \\
184.7899 \\
185.7457 \\
201.3673 \\
198.6671 \\
192.8643 \\
182.8727 \\
181.0739 \\
182.0980 \\
168.1401 \\
165.4204 \\
162.5773 \\
134.2934 \\
171.6407 \\
139.0493 \\
122.7075 \\
150.0439 \\
151.5869 \\
161.5668 \\
166.8208 \\
-1.6955 \\
137.6948 \\
163.4496 \\
128.5897 \\
146.3710 \\
148.2266 \\
124.2329 \\
126.4982\end{array}$ & $\begin{array}{l}\text { SD } \\
3.6626 e-01 \\
5.9853 e-02 \\
6.9754 e-02 \\
2.5822 e-01 \\
7.5482 e+00 \\
9.6336 e-06 \\
2.2603 e-03 \\
8.8384 e-01 \\
0.0000 e+00 \\
0.0000 e+00 \\
0.0000 e+00 \\
1.0625 e-01 \\
9.6655 e-02 \\
1.1327 e-01 \\
1.4314 e-01 \\
1.0297 e-01 \\
1.1497 e-01 \\
1.2622 e+00 \\
1.2491 e+00 \\
1.1982 e+00 \\
2.3839 e-01 \\
3.3652 e-01 \\
3.1258 e-01 \\
3.5141 e-03 \\
3.2359 e-03 \\
3.4173 e-03 \\
3.2955 e-03 \\
5.4231 e-01 \\
5.4001 e-01 \\
5.2824 e-01 \\
5.3596 e-01 \\
5.1120 e-01 \\
5.1938 e-01 \\
5.6667 e-01 \\
5.4556 e-01 \\
4.9895 e-01 \\
6.5584 e-01 \\
6.1487 e-01 \\
6.4356 e-01 \\
5.7590 e-01 \\
6.4680 e-01 \\
6.3572 e-01 \\
6.0062 e-01 \\
6.0921 e-01 \\
6.1834 e-01 \\
3.9766 e-01 \\
4.3762 e-01 \\
5.1750 e-01 \\
3.9883 e-01 \\
4.1055 e-01 \\
4.0387 e-01 \\
4.3405 e-01 \\
3.8020 e-01 \\
4.5847 e-01 \\
6.0285 e-01\end{array}$ & $\begin{array}{l}\text { Uncert } \\
7.5667 e-01 \\
7.5165 e-01 \\
7.5564 e-01 \\
5.0552 e-01 \\
4.9223 e+00 \\
2.1154 e-01 \\
0.0000 e+00 \\
0.0000 e+00 \\
0.0000 e+00 \\
0.0000 e+00 \\
0.0000 e+00 \\
1.3845 e+00 \\
1.4739 e+00 \\
1.2911 e+00 \\
1.1691 e+00 \\
1.2263 e+00 \\
1.5883 e+00 \\
1.4354 e+01 \\
1.2040 e+01 \\
1.0900 e+01 \\
6.2618 e+00 \\
6.8733 e+00 \\
5.1 .648 e+00 \\
0.0000 e+00 \\
0.0000 e+00 \\
0.0000 e+00 \\
0.0000 e+00 \\
4.4770 e+00 \\
4.4972 e+00 \\
4.5011 e+00 \\
4.5678 e+00 \\
4.5560 e+00 \\
4.5310 e+00 \\
4.4893 e+00 \\
4.4821 e+00 \\
4.4862 e+00 \\
4.4314 e+00 \\
4.4211 e+00 \\
4.4106 e+00 \\
4.3143 e+00 \\
4.4448 e+00 \\
4.3294 e+00 \\
4.2797 e+00 \\
4.3659 e+00 \\
4.3713 e+00 \\
4.4069 e+00 \\
4.4264 e+00 \\
4.1000 e+00 \\
4.3250 e+00 \\
4.4138 e+00 \\
4.2969 e+00 \\
4.3534 e+00 \\
4.3597 e+00 \\
4.2841 e+00 \\
4.2907 e+00\end{array}$ \\
\hline
\end{tabular}




\begin{tabular}{|c|c|c|c|c|c|c|}
\hline $\begin{array}{l}1 \\
156 \\
157 \\
158 \\
159 \\
160 \\
161 \\
162 \\
163\end{array}$ & $\begin{array}{l}\text { Name } \\
\text { IHTO00029 } \\
\text { IHTO00030 } \\
\text { IHTO00031 } \\
\text { IHTO00032 } \\
\text { IHTO00033 } \\
\text { IHTO00034 } \\
\text { IHTO00035 } \\
\text { IHT } 000036\end{array}$ & $\begin{array}{l}\text { Min } \\
146.6083 \\
149.3690 \\
133.8266 \\
135.6370 \\
135.6220 \\
125.1615 \\
165.2306 \\
123.4713\end{array}$ & $\begin{array}{l}\operatorname{Max} \\
149.7333 \\
152.4940 \\
136.5610 \\
138.3714 \\
138.1611 \\
127.5053 \\
167.3790 \\
125.6197\end{array}$ & $\begin{array}{l}\text { Ave } \\
148.0810 \\
150.9003 \\
135.2251 \\
137.0472 \\
136.9970 \\
126.2827 \\
166.3282 \\
124.6314\end{array}$ & $\begin{array}{l}S D \\
6.0000 e-01 \\
6.2808 e-01 \\
6.0793 e-01 \\
6.2923 e-01 \\
6.2634 e-01 \\
5.0657 e-01 \\
5.1432 e-01 \\
4.7089 e-01\end{array}$ & $\begin{array}{l}\text { Uncert } \\
4.3592 e+00 \\
4.3689 e+00 \\
4.3172 e+00 \\
4.3230 e+00 \\
4.3228 e+00 \\
4.2901 e+00 \\
4.4245 e+00 \\
4.2852 e+00\end{array}$ \\
\hline
\end{tabular}




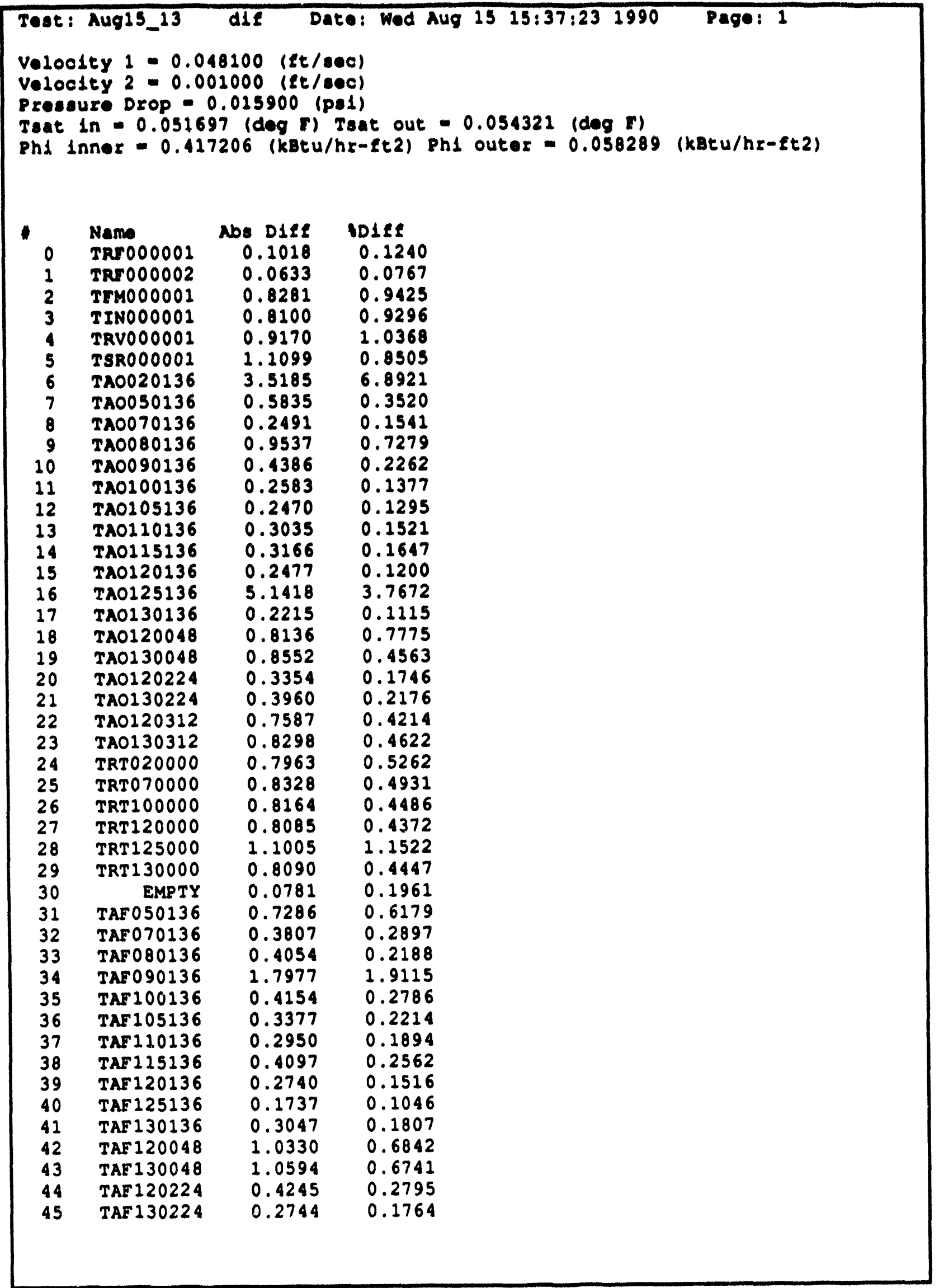




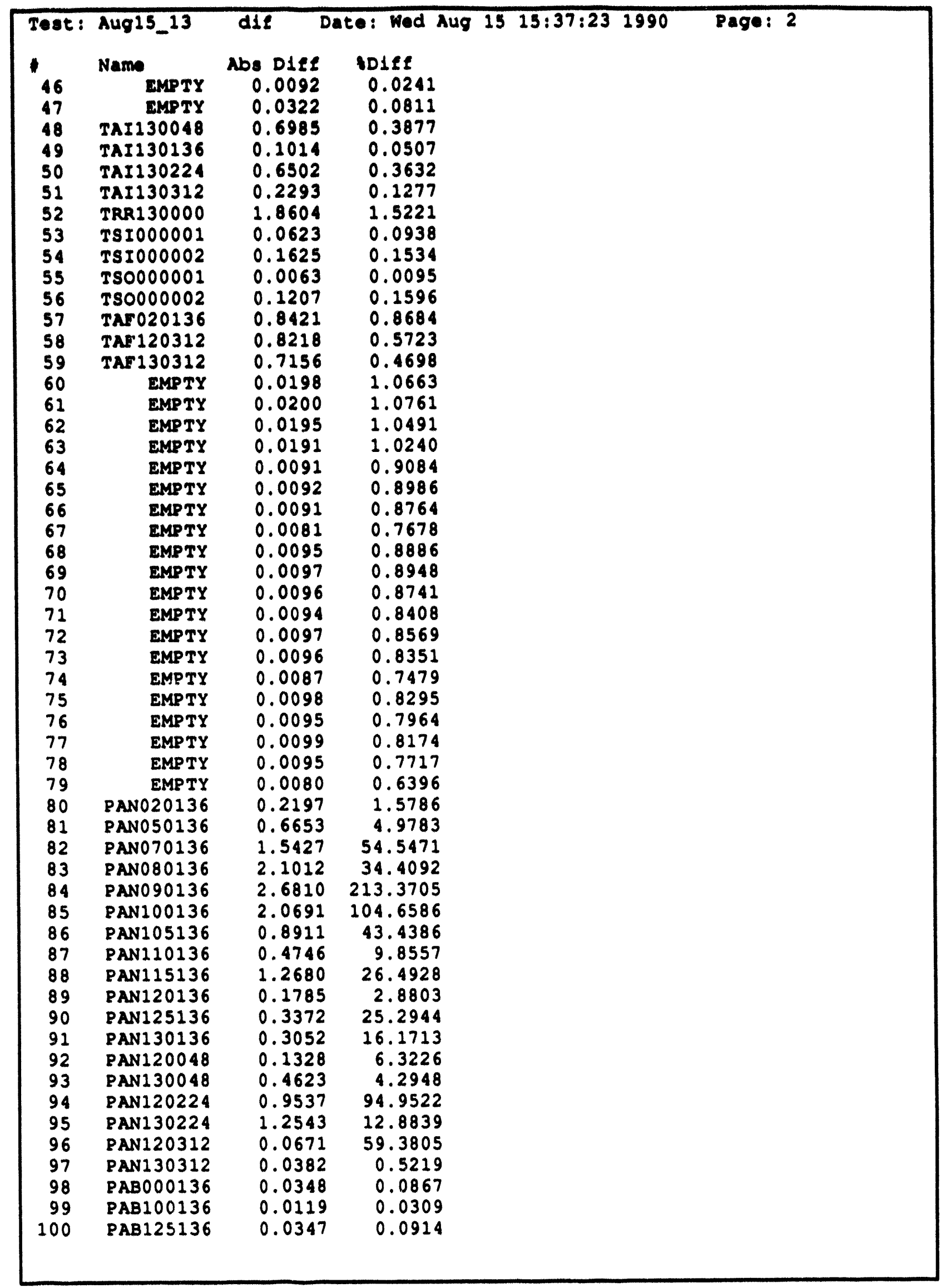




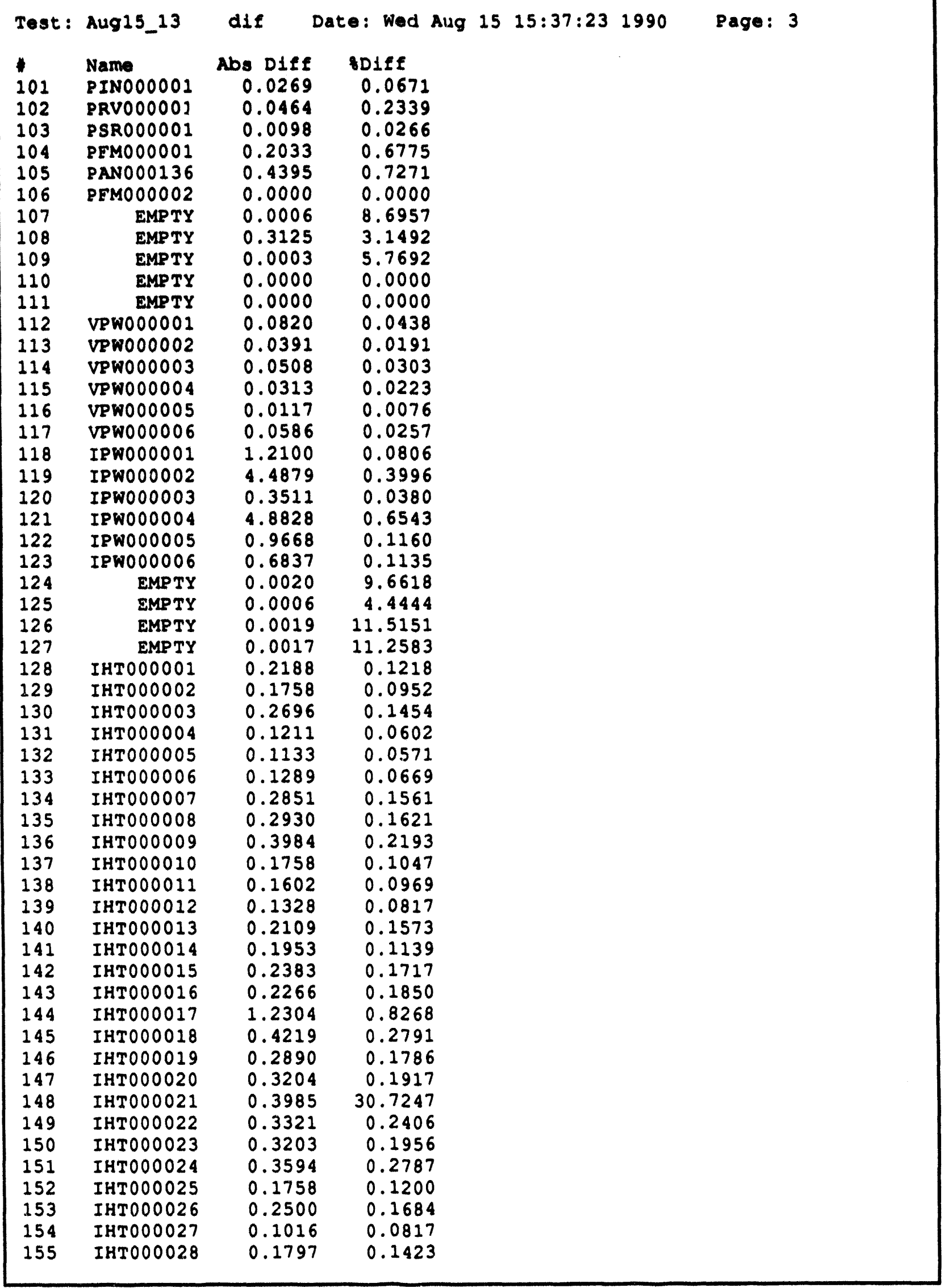


Test: Aug15_13 dif Date: Wed Aug 15 15:37:23 1990 Page: 4

* Name Abs Diff rdiff

156 IHTO0O029 $0.1953 \quad 0.1321$

157 IHT000030 $0.2344 \quad 0.1556$

$\begin{array}{llll}158 & \text { IHT000031 } & 0.2656 & 0.1968\end{array}$

$\begin{array}{llll}159 & \text { IHT000032 } & 0.2890 & 0.2113\end{array}$

160 IHT000033 $0.2890 \quad 0.2114$

161 IHT000034 $0.1133 \quad 0.0898$

162 IHT000035 $0.1367 \quad 0.0823$

$\begin{array}{llll}163 & \text { IHT000036 } & 0.1367 & 0.1098\end{array}$ 


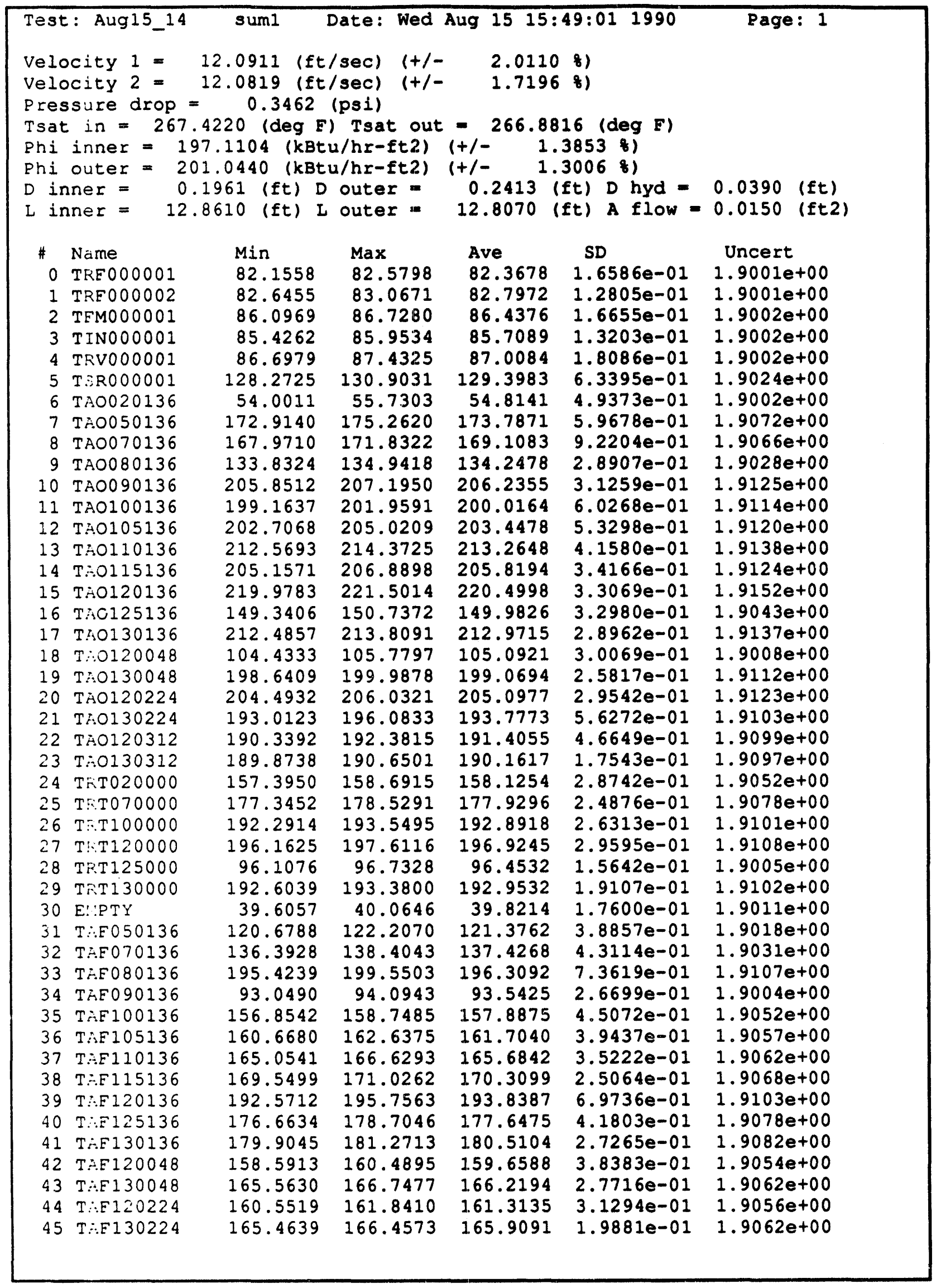




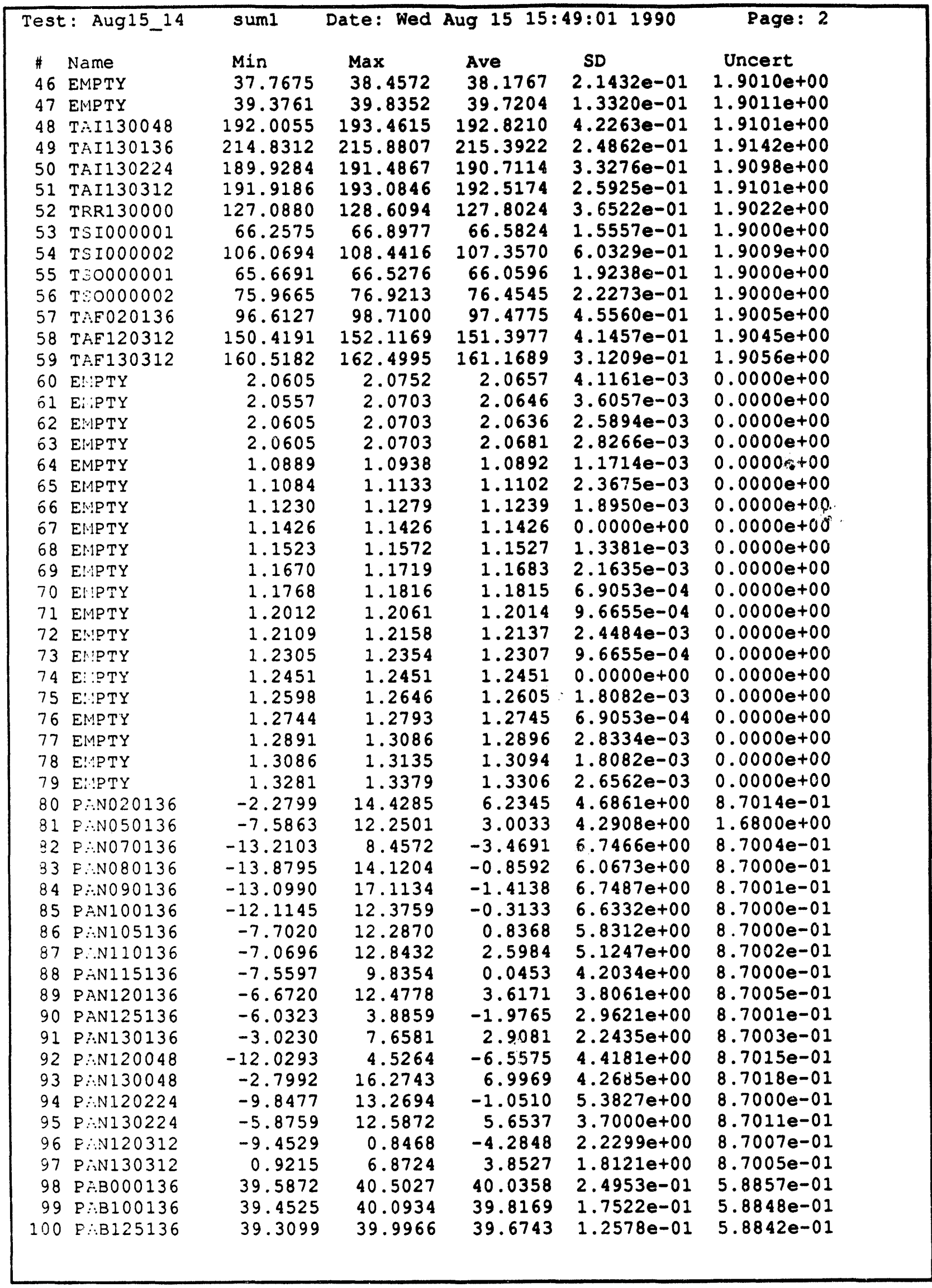




\begin{tabular}{|c|c|c|c|c|c|c|}
\hline \multicolumn{2}{|c|}{ Test: Aug15_14 } & sum1 & Date: Wed & Aug 151 & $: 011990$ & Page: 3 \\
\hline$\#$ & $\mathrm{~N}$ came & Min & Max & Ave & SD & Uncert \\
\hline 101 & PINO00001 & 39.1042 & 40.5690 & 39.8830 & $4.5601 e-01$ & $7.5660 e-01$ \\
\hline 102 & PRV000001 & 19.8406 & 19.9627 & 19.9090 & $6.1209 e-02$ & $7.5165 e-01$ \\
\hline 103 & PSR000001 & 38.5900 & 38.8341 & 38.7560 & $6.8700 e-02$ & $7.5623 e-01$ \\
\hline 104 & PrM000001 & 21.8740 & 22.7896 & 22.3648 & $1.7426 e-01$ & $5.0312 e-01$ \\
\hline 105 & PANO00136 & -2.7022 & 23.8481 & 9.5842 & $7.9912 e+00$ & $4.9201 e+00$ \\
\hline 106 & PEM000002 & 22.1154 & 22.5014 & 22.3306 & $1.0292 e-01$ & $2.0765 e-01$ \\
\hline 107 & EMPTY & -0.0049 & 0.0098 & 0.0049 & $1.7086 e-03$ & $0.0000 e+00$ \\
\hline 108 & EMIPTY & -10.0000 & -9.8926 & -9.9979 & $1.5192 e-02$ & $0.0000 e+00$ \\
\hline 109 & ENPTY & 0.0000 & 0.0098 & 0.0051 & $1.3811 e-03$ & $0.0000 e+00$ \\
\hline 110 & ENPTY & -10.0000 & -10.0000 & -10.0000 & $0.0000 e+00$ & $0.0000 e+00$ \\
\hline 111 & ENPTY & 0.0000 & 0.0049 & 0.0001 & $6.9053 e-04$ & $0.0000 \mathrm{e}+00$ \\
\hline 112 & VENO00001 & 187.0343 & 187.4249 & 187.1904 & $1.3087 e-01$ & $1.3848 e+00$ \\
\hline 113 & $V:$ N000002 & 204.9955 & 205.3861 & 205.1947 & $1.0799 \mathrm{e}-01$ & $1.4737 e+00$ \\
\hline 114 & VEW000003 & 166.8720 & 167.6532 & 167.4619 & $1.4493 e-01$ & $1.2906 \mathrm{e}+00$ \\
\hline 115 & $V=N 000004$ & 140.0052 & 140.7865 & 140.5366 & $1.3692 e-01$ & $1.1692 \mathrm{e}+00$ \\
\hline 116 & V: N000005 & 153.2001 & 153.5907 & 153.4424 & $1.2823 e-01$ & $1.2263 e+00$ \\
\hline $1: 7$ & $v: 1000006$ & 227.5053 & 228.0912 & 227.8021 & $1.1995 \mathrm{e}-01$ & $1.5888 \mathrm{e}+00$ \\
\hline 118 & Ii: $: 000001$ & 1504.9567 & 1508.8613 & 1507.6508 & $1.2404 \mathrm{e}+00$ & $1.4404 e+01$ \\
\hline 119 & IPW000002 & 1103.7056 & 1111.5106 & 1107.4131 & $1.4350 \mathrm{e}+00$ & $1.1923 e+01$ \\
\hline 120 & IF 1000003 & 902.8875 & 908.7375 & 905.3834 & $1.3090 e+00$ & $1.0806 \mathrm{e}+01$ \\
\hline 121 & IEW000004 & 751.0798 & 753.0329 & 752.0856 & $4.1161 e-01$ & $6.2691 e+00$ \\
\hline 122 & II W000005 & 824.6340 & 825.6104 & 825.2394 & $2.8858 \mathrm{e}-01$ & $6.8193 \mathrm{e}+00$ \\
\hline 123 & IFW000006 & 598.0224 & 599.4872 & 598.8716 & $3.3901 e-01$ & $5.1365 e+00$ \\
\hline 124 & EN!PTY & -0.0244 & -0.0098 & -0.0184 & $4.0144 e-03$ & $0.0000 e+00$ \\
\hline 125 & EUPTY & -0.0195 & -0.0049 & -0.0134 & $3.0897 e-03$ & $0.0000 e+00$ \\
\hline 126 & E!:PTY & -0.0195 & -0.0049 & -0.0144 & $3.0261 e-03$ & $0.0000 e+00$ \\
\hline 127 & EWPTY & -0.0244 & -0.0098 & -0.0172 & $3.5895 e-03$ & $0.0000 e+00$ \\
\hline 128 & IHTTO00001 & 178.1400 & 181.4603 & 179.5307 & $6.5867 e-01$ & $4.4758 e+00$ \\
\hline 129 & Ii:T000002 & 182.9890 & 186.1140 & 184.5009 & $6.4333 e-01$ & $4.4960 e+00$ \\
\hline 130 & I:.:000003 & 184.2886 & 187.0230 & 185.5269 & $6.0181 \mathrm{e}-01$ & $4.5002 e+00$ \\
\hline 131 & I::'T000004 & 200.1915 & 202.7306 & 201.2696 & $6.3041 e-01$ & $4.5674 e+00$ \\
\hline 132 & IHTT000005 & 197.4797 & 200.0188 & 198.6124 & $6.1889 e-01$ & $4.5557 e+00$ \\
\hline 133 & I!!T000006 & 191.6729 & 193.8214 & 192.7588 & $6.0381 e-01$ & $4.5305 e+00$ \\
\hline 134 & $I:$ I 000007 & 181.4040 & 183.7477 & 182.6188 & $5.8831 e-01$ & $4.4883 e+00$ \\
\hline 135 & $I: T 000008$ & 179.5973 & 181.7458 & 180.8278 & $5.4283 e-01$ & $4.4811 e+00$ \\
\hline 136 & I!:T000009 & 180.6565 & 183.1956 & 181.7347 & $5.5557 e-01$ & $4.4847 e+00$ \\
\hline 137 & I!:T000010 & 166.5189 & 169.4486 & 167.9331 & $6.7377 e-01$ & $4.4306 e+00$ \\
\hline 138 & I:TT000011 & 163.6118 & 166.9321 & 165.2251 & $6.5294 e-01$ & $4.4204 e+00$ \\
\hline 139 & I::T000012 & 160.6633 & 164.1790 & 162.3898 & $7.1394 e-01$ & $4.4099 \mathrm{e}+00$ \\
\hline 140 & I: T000013 & 132.5083 & 135.6333 & 134.1255 & $6.6268 \mathrm{e}-01$ & $4.3138 \mathrm{e}+00$ \\
\hline 141 & II:T000014 & 169.9181 & 173.2384 & 171.5157 & $7.0110 e-01$ & $4.4443 e+00$ \\
\hline $1 \div 2$ & I!IT000015 & 137.2446 & 140.3696 & 138.8462 & $6.5791 e-01$ & $4.3287 e+00$ \\
\hline 143 & I:T000016 & 120.9848 & 124.1098 & 122.5278 & $6.4386 e-01$ & $4.2792 e+00$ \\
\hline $1: 4$ & I::T000017 & 147.9267 & 151.8329 & 149.5986 & $7.3943 e-01$ & $4.3644 \mathrm{e}+00$ \\
\hline 145 & IH:T000018 & 149.5869 & 153.1025 & 151.2510 & $7.7849 e-01$ & $4.3701 e+00$ \\
\hline 146 & IHTO00019 & 160.8323 & 163.3714 & 161.9418 & $5.9639 e-01$ & $4.4082 e+00$ \\
\hline 147 & IH:T000020 & 166.1959 & 168.5396 & 167.1490 & $5.8745 e-01$ & $4.4276 e+00$ \\
\hline 148 & I:T000021 & -2.6330 & 0.2967 & -1.1603 & $6.8710 \mathrm{e}-01$ & $4.1000 e+00$ \\
\hline 149 & IHTT000C22 & 137.0230 & 139.3667 & 138.1128 & $5.0700 e-01$ & $4.3264 \mathrm{e}+00$ \\
\hline 150 & IHTO00023 & 162.6878 & 164.8363 & 163.7426 & $4.8327 e-01$ & $4.4149 \mathrm{e}+00$ \\
\hline 151 & IF:T000024 & 127.9334 & 129.8866 & 128.8983 & $4.3479 e-01$ & $4.2978 \mathrm{e}+00$ \\
\hline 152 & II:T J00025 & 145.5116 & 147.8553 & 146.5702 & $4.8663 e-01$ & $4.3541 e+00$ \\
\hline 153 & IHTO000026 & 147.2656 & 149.8047 & 148.4961 & $5.2977 e-01$ & $4.3606 e+00$ \\
\hline 154 & I:ST000027 & 123.1783 & 125.5221 & 124.3892 & $5.6909 e-01$ & $4.2845 e+00$ \\
\hline 155 & I!:T000028 & 125.1427 & 127.8771 & 126.3966 & $5.6647 e-01$ & $4.2904 e+00$ \\
\hline
\end{tabular}




\begin{tabular}{|c|c|c|c|c|c|c|}
\hline \multicolumn{2}{|c|}{ Test: Aug15_14 } & sum1 & Date: Wed & Aug $15 \quad 15$ & 49:01 1990 & Page: 4 \\
\hline$\#$ & Name & $\operatorname{Min}$ & $\operatorname{Max}$ & Ave & SD & Uncert \\
\hline 156 & IHTO00029 & 146.9989 & 149.5380 & 148.0146 & $5.6497 e-01$ & $4.3590 e+00$ \\
\hline 157 & IHTO000030 & 149.5643 & 152.4940 & 150.7636 & $6.1004 a-01$ & $4.3684 e+00$ \\
\hline 158 & IHT000031 & 133.8266 & 136.7563 & 135.1001 & $5.6805 e-01$ & $4.3169 e+00$ \\
\hline 159 & IHTO000032 & 135.8323 & 138.7620 & 136.9183 & $5.8148 e-01$ & $4.3226 e+00$ \\
\hline 160 & I:TO000033 & 135.8173 & 138.7470 & 136.8759 & $5.7058 e-01$ & $4.3224 e+00$ \\
\hline 10 1 & I:IT000034 & 125.1615 & 127.1146 & 126.0952 & $4.1999 e-01$ & $4.2895 e+00$ \\
\hline 162 & IHTO00035 & 165.4259 & 167.3790 & 166.1446 & $4.3484 e-01$ & $4.4238 e+00$ \\
\hline 163 & IHT000036 & 123.6666 & 125.6197 & 124.4673 & $3.8913 e-01$ & $4.2848 \mathrm{e}+00$ \\
\hline
\end{tabular}




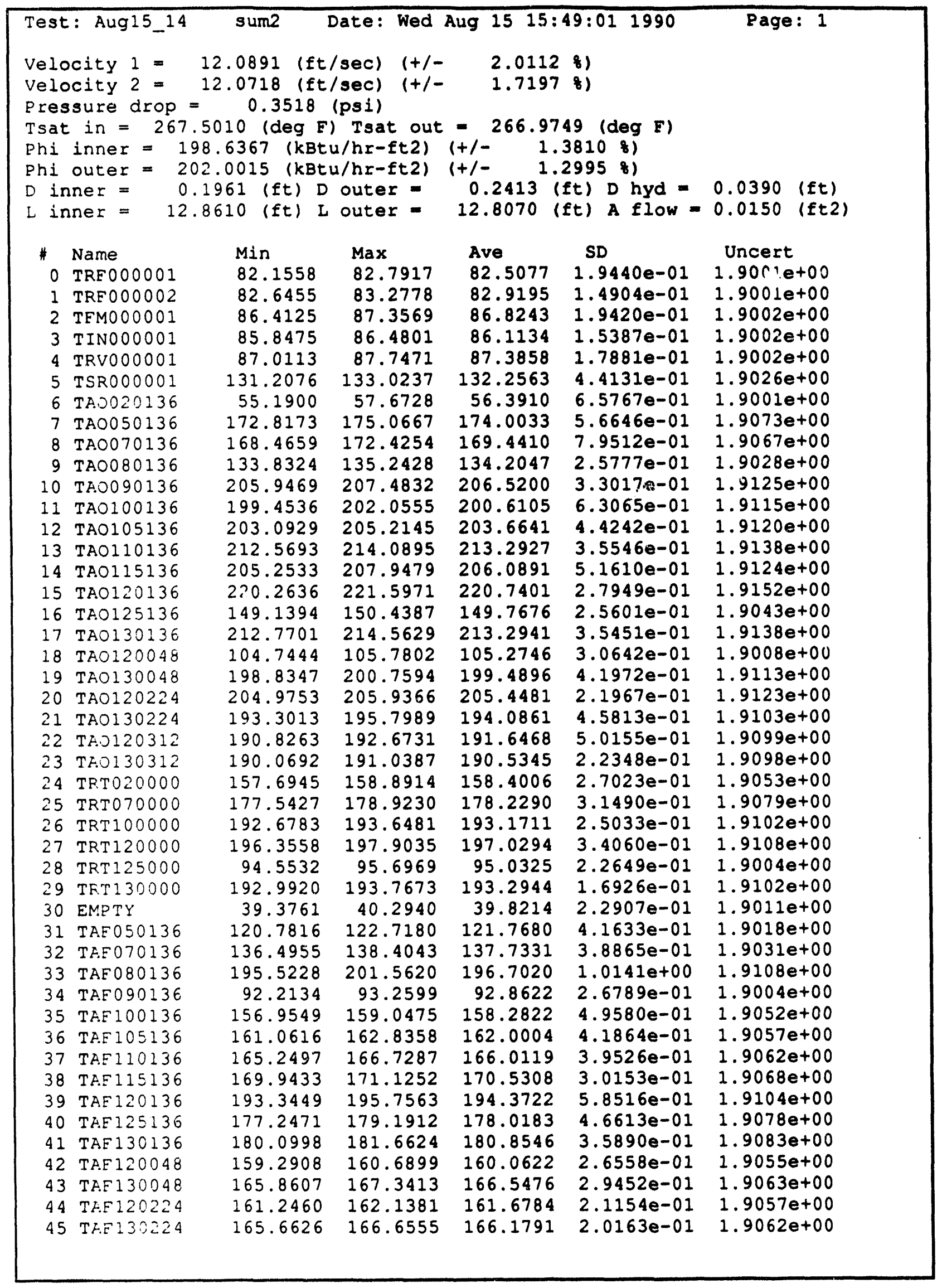




\begin{tabular}{|c|c|c|c|c|c|c|}
\hline \multicolumn{2}{|c|}{ Test: Aug15_14 } & sum2 & Date: Wed & \multicolumn{2}{|c|}{ Aug $1515: 49: 011990$} & \multirow{2}{*}{$\begin{array}{l}\text { Page: } 2 \\
\text { Uncert }\end{array}$} \\
\hline$\#$ & Name & Min & $\operatorname{Max}$ & Ave & SD & \\
\hline 46 & EMPTY & 37.7675 & 38.4572 & 38.1583 & $2.0380 \theta-01$ & $1.9010 e+00$ \\
\hline 47 & EMPTY & 39.3761 & 39.8352 & 39.6562 & $1.0666 e-01$ & $1.9011 e+00$ \\
\hline 48 & TAI 130048 & 192.5877 & 194.1400 & 193.3295 & $3.7638 \theta-01$ & $1.9102 \mathrm{e}+00$ \\
\hline 49 & TAI 130136 & 215.1178 & 216.4529 & 215.8271 & $2.6635 e-01$ & $1.9143 e+00$ \\
\hline 50 & TAI 130224 & 190.4158 & 191.5844 & 190.9997 & $2.5954 e-01$ & $1.9098 e+00$ \\
\hline 51 & TAI 130312 & 192.1137 & 193.5702 & 192.8012 & $2.6394 e-01$ & $1.9101 e+00$ \\
\hline 52 & TRR 130000 & 126.9891 & 128.6094 & 127.8438 & $4.4064 \theta-01$ & $1.9023 e+00$ \\
\hline 53 & TSI000001 & 66.1518 & 67.1124 & 66.6087 & $1.8689 e-01$ & 1.9000 e +00 \\
\hline 54 & TSI000002 & 105.0386 & 108.3395 & 106.4999 & $8.3992 e-01$ & $1.9009 e+00$ \\
\hline 55 & TSO000001 & 65.6680 & 66.6344 & 66.0507 & $2.0808 \theta-01$ & $1.9000 e+00$ \\
\hline 56 & TSO000002 & 76.0735 & 76.9212 & 76.5057 & $2.1300 e-01$ & $1.9000 e+00$ \\
\hline 57 & TAF020136 & 96.7178 & 99.0236 & 97.7245 & $4.5616 e-01$ & $1.9005 e+00$ \\
\hline 58 & TAF 120312 & 151.0182 & 152.4174 & 151.6219 & $4.0660 e-01$ & $1.9045 e+00$ \\
\hline 59 & TAF 130312 & 160.9149 & 162.6973 & 161.5259 & $3.4390 e-01$ & $1.9056 e+00$ \\
\hline 60 & EMPTY & 2.0654 & 2.0850 & 2.0713 & $5.4032 \theta-03$ & $0.0000 \mathrm{e}+00$ \\
\hline 61 & EMPTY & 2.0654 & 2.0801 & 2.0713 & $4.3000 e-03$ & $0.0000 e+00$ \\
\hline 62 & EMPTY & 2.0654 & 2.0752 & 2.0695 & $3.1752 \theta-03$ & $0.0000 \mathrm{e}+00$ \\
\hline 63 & EMPTY & 2.0703 & 2.0801 & 2.0747 & $3.1583 e-03$ & $0.0000 e+00$ \\
\hline 64 & EMPTY & 1.0889 & 1.0986 & 1.0942 & $2.2603 e-03$ & $0.0000 e+00$ \\
\hline 65 & EMPTY & 1.1084 & 1.1182 & 1.1147 & $2.4662 e-03$ & $0.0000 e+00$ \\
\hline 66 & EMP $+Y$ & 1.1279 & 1.1328 & 1.1288 & $1.8950 e-03$ & $0.0000 \mathrm{e}+00$ \\
\hline 67 & EMPTY & 1.1426 & 1.1475 & 1.1461 & $2.2146 e-03$ & $0.0000 e+00$ \\
\hline 68 & EMPTY & 1.1523 & 1.1621 & 1.1570 & $1.9631 e-03$ & $0.0000 e+00$ \\
\hline 69 & EMPTY & 1.1719 & 1.1768 & 1.1732 & $2.2146 e-03$ & $0.0000 \mathrm{e}+00$ \\
\hline 70 & EMPTY & 1.1816 & 1.1914 & 1.1862 & $1.5314 \theta-03$ & $0.0000 e+00$ \\
\hline 71 & EMPTY & 1.2061 & 1.2109 & 1.2062 & $9.6655 e-04$ & $0.0000 \mathrm{e}+00$ \\
\hline 72 & EMPTY & 1.2158 & 1.2207 & 1.2173 & $2.2603 \theta-03$ & $0.0000 e+00$ \\
\hline 73 & EMPTY & 1.2354 & 1.2402 & 1.2354 & $6.9053 e-04$ & $0.0000 e+00$ \\
\hline 74 & EMPTY & 1.2451 & 1.2500 & 1.2491 & $1.8950 e-03$ & $0.0000 e+00$ \\
\hline 75 & EMPTY & 1.2549 & 1.2695 & 1.2646 & $1.7086 e-03$ & $0.0000 e+00$ \\
\hline 76 & EMPTY & 1.2744 & 1.2793 & 1.2771 & $2.4484 e-03$ & $0.0000 e+00$ \\
\hline 77 & EMPTY & 1.2891 & 1.2988 & 1.2939 & $9.8648 e-04$ & $0.0000 e+00$ \\
\hline 78 & EMPTY & 1.3086 & 1.3184 & 1. 3138 & $1.5314 e-03$ & $0.0000 e+00$ \\
\hline 79 & EMPTY & 1.3330 & 1.3379 & 1.3333 & $1.1714 e-03$ & $0.0000 e+00$ \\
\hline 80 & PAN020136 & -6.7812 & 17.4039 & 4.6537 & $5.4969 e+00$ & $8.7008 e-01$ \\
\hline 81 & PAN0 50136 & -8.0441 & 11.4872 & 2.8843 & $5.2025 e+00$ & $1.6800 \mathrm{e}+00$ \\
\hline 82 & PAN070136 & -12.8288 & 8.6861 & -5.2742 & $5.8032 e+00$ & $8.7010 e-01$ \\
\hline 83 & PANO 80136 & -12.8877 & 13.4337 & -0.6211 & $5.5604 e+00$ & $8.7000 e-01$ \\
\hline 84 & PANO90136 & -13.1753 & 14.8246 & -1.1742 & $5.9863 e+00$ & $8.7000 e^{-01}$ \\
\hline 85 & PAN 100136 & -12.1908 & 13.6729 & 1.4460 & $6.4379 e+00$ & $8.7001 e-01$ \\
\hline 86 & PAN105136 & -7.6257 & 6.0309 & 0.0128 & $3.6451 e+00$ & $8.7000 e-01$ \\
\hline 87 & PAN 110136 & -7.2222 & 13.8350 & 2.1590 & $3.7300 e+00$ & $8.7002 e-01$ \\
\hline 88 & PAN115136 & -6.0338 & 8.3095 & 1.3255 & $3.4752 e+00$ & $8.7001 e-01$ \\
\hline 89 & PAN120136 & -4.9172 & 10.2653 & 3.3302 & $3.9280 \mathrm{e}+00$ & $8.7004 e-01$ \\
\hline 90 & PAN125136 & -6.0323 & 4.8777 & -1.0061 & $3.2625 e+00$ & $8.7000 e-01$ \\
\hline 91 & PAN 130136 & -4.7778 & 6.5900 & 1.0816 & $2.7337 \mathrm{e}+00$ & $8.7000 e-01$ \\
\hline 92 & PAN120048 & -12.0293 & 2.9243 & -7.5936 & $3.6848 \mathrm{e}+00$ & $8.7021 e-01$ \\
\hline 93 & PAN 130048 & -1.2733 & 16.5032 & 9.8656 & $3.5018 e+00$ & $8.7035 e-01$ \\
\hline 94 & PAN120224 & -10.6869 & 7.0133 & -2.1527 & $4.4493 e+00$ & $8.7002 e-01$ \\
\hline 95 & PAN130224 & -2.1375 & 11.3665 & 6.8103 & $2.8595 e+00$ & $8.7017 e-01$ \\
\hline 96 & PAN120312 & -8.3848 & -0.2214 & -4.3595 & $2.1036 e+00$ & $8.7007 e-01$ \\
\hline 97 & PAN130312 & 1.2267 & 7.4828 & 4.2861 & $2.0425 e+00$ & $8.7007 e-01$ \\
\hline 98 & PAB000136 & 39.6330 & 40.5943 & 40.0889 & $2.6190 e-01$ & $5.8860 e-01$ \\
\hline 99 & PAB100136 & 39.5441 & 40.1392 & 39.8865 & $1.6883 e-01$ & $5.8851 e-01$ \\
\hline 100 & PAB125136 & 39.5846 & 40.0881 & 39.7366 & $1.1230 \mathrm{e}-01$ & $5.8845 e-01$ \\
\hline
\end{tabular}




\begin{tabular}{|c|c|c|c|c|c|c|}
\hline \multicolumn{2}{|c|}{ Test: Aug15_14 } & sum2 & Date: Wed & Aug 15 & 011990 & Page: 3 \\
\hline$\#$ & Name & Min & $\operatorname{Max}$ & Ave & SD & Uncert \\
\hline 101 & PIN000001 & 39.1042 & 40.6911 & 40.0442 & $4.4885 e-01$ & $7.5665 e-01$ \\
\hline 102 & PRV000001 & 19.8406 & 19.9627 & 19.8724 & $5.4088 e-02$ & $7.5164 e-01$ \\
\hline 103 & PSR000001 & 38.7120 & 38.9562 & 38.8488 & $5.8569 e-02$ & $7.5626 e-01$ \\
\hline 104 & PEMOODO01 & 21.8740 & 22.7896 & 22.3556 & $1.9471 e-01$ & $5.03110-01$ \\
\hline 105 & PR:11000136 & -4.0755 & 25.2214 & 9.7398 & $8.2681 e+00$ & $4.9201 e+00$ \\
\hline 106 & PEMOCOOOO2 & 21.9812 & 22.4678 & 22.2913 & $1.0897 e-01$ & $2.0762 e-01$ \\
\hline 107 & EMPTY & 0.0049 & 0.0098 & 0.0051 & $9.6655 e-04$ & $0.0000 e+00$ \\
\hline 108 & EMPTY & -10.0000 & -8.7793 & -9.9652 & $1.8613 e-01$ & $0.0000 e+00$ \\
\hline 109 & EMPTY & 0.0000 & 0.0049 & 0.0047 & $9.66550-04$ & $0.0000 e+00$ \\
\hline 110 & EMPTY & -10.0000 & -10.0000 & -10.0000 & $0.0000 e+00$ & $0.00000+00$ \\
\hline 111 & EMPTY & 0.0000 & 0.0049 & 0.0001 & $6.9053 e-04$ & $0.0000 e+00$ \\
\hline 112 & VPWO00001 & 187.0343 & 187.4249 & 187.2178 & $1.2104 e-01$ & $1.3849 e+00$ \\
\hline 113 & VPW000002 & 204.9955 & 205.3861 & 205.1986 & $9.6332 \theta-02$ & $1.4737 e+00$ \\
\hline 114 & VPW000003 & 167.2626 & 168.0439 & 167.7470 & $1.6852 \mathrm{e}-01$ & $1.2919 \mathrm{e}+00$ \\
\hline 115 & VPWOODOOO4 & 140.3959 & 140.7865 & 140.6694 & $1.0440 e-01$ & $1.1698 \mathrm{e}+00$ \\
\hline 116 & VPWO0O005 & 153.2001 & 153.5907 & 153.4775 & $1.0507 e-01$ & $1.2264 \mathrm{e}+00$ \\
\hline 117 & VPW200j06́ & 227.7006 & 228.0912 & 227.8568 & $9.6655 e-02$ & $1.5891 e+00$ \\
\hline 118 & IFNOCSOOLI & 1495.1949 & 1503.0043 & 1500.0370 & $2.1690 e+00$ & $1.4354 e+01$ \\
\hline 119 & IPW000002 & 1117.3645 & 1131.0233 & 1123.6084 & $3.9224 e+00$ & $1.2017 e+01$ \\
\hline 120 & IPW000003 & 918.4875 & 924.3375 & 922.5824 & $1.4872 e+00$ & $1.0896 \mathrm{e}+01$ \\
\hline 121 & IPW000004 & 745.7087 & 747.1735 & 746.5485 & $3.8257 e-01$ & $6.2276 e+00$ \\
\hline 122 & IPW000005 & 831.9575 & 833.4221 & 832.7579 & $3.6588 \mathrm{e}-01$ & $6.8761 e+00$ \\
\hline 123 & IFW000006 & 598.9990 & 603.3935 & 601.7036 & $1.4337 e+00$ & $5.1571 e+00$ \\
\hline 124 & EMPTY & -0.0293 & -0.0098 & -0.0195 & $3.8206 e-03$ & $0.0000 e+00$ \\
\hline 125 & EMPTY & -0.0195 & -0.0098 & -0.0130 & $2.7213 e-03$ & $0.0000 e+00$ \\
\hline 126 & EMPTY & -0.0244 & -0.0098 & -0.0156 & $3.5568 e-03$ & $0.0000 e+00$ \\
\hline 127 & EMPTY & -0.0244 & -0.0098 & -0.0156 & $3.4173 e-03$ & $0.0000 e+00$ \\
\hline 128 & IHT000001 & 178.3353 & 181.2650 & 179.5854 & $6.8459 e-01$ & $4.4761 e+00$ \\
\hline 129 & IHTOOCOO2 & 183.3797 & 186.1140 & 184.5868 & $6.8081 e-01$ & $4.4964 e+00$ \\
\hline 130 & IHTO00003 & 184.4839 & 187.0230 & 185.6675 & $6.6076 e-01$ & $4.5008 e+00$ \\
\hline 131 & IHTDOO) 04 & 200.1915 & 202.5353 & 201.3165 & $6.6446 e-01$ & $4.5676 e+00$ \\
\hline 132 & IHT000005 & 197.4797 & 199.8235 & 198.6280 & $6.2595 e-01$ & $4.5558 \mathrm{e}+00$ \\
\hline 133 & IHT000006 & 191.6729 & 194.2120 & 192.8135 & $6.1683 e-01$ & $4.5308 e+00$ \\
\hline 134 & IHTD00007 & 181.2087 & 183.9431 & 182.6735 & $6.2046 e-01$ & $4.4885 e+00$ \\
\hline 135 & IHT0O2008 & 179.4020 & 182.1364 & 180.8942 & $5.9025 e-01$ & $4.4813 e+00$ \\
\hline 136 & IHTO00009 & 180.4612 & 183.1956 & 181.7855 & $5.9750 e-01$ & $4.4849 e+00$ \\
\hline 137 & IHTO00010 & 167.1049 & 169.8392 & 168.2143 & $6.1440 e-01$ & $4.4317 e+00$ \\
\hline 138 & IHT000011 & 164.5884 & 167.3227 & 165.4165 & $5.8999 e-01$ & $4.4211 e+00$ \\
\hline 139 & IHTO0O012 & 161.4446 & 164.5696 & 162.5304 & $6.1783 e-01$ & $4.4104 e+00$ \\
\hline 140 & IHTO00013 & 133.2895 & 136.2192 & 134.2466 & $6.1289 e-01$ & $4.3142 e+00$ \\
\hline 141 & IHTOODO & 170.6994 & 173.2384 & 171.5938 & $5.6094 e-01$ & $4.4446 \mathrm{e}+00$ \\
\hline 142 & IHTO00015 & 137.8305 & 140.7602 & 138.8970 & $5.5413 e-01$ & $4.3289 e+00$ \\
\hline 143 & IHT000016 & 121.7661 & 124.5005 & 122.6528 & $5.1177 e-01$ & $4.2795 e+00$ \\
\hline 144 & IHTO00017 & 148.1220 & 150.6611 & 149.3525 & $6.1034 e-01$ & $4.3636 \mathrm{e}+00$ \\
\hline 145 & IHT000018 & 150.5634 & 152.7119 & 151.5205 & $5.3561 e-01$ & $4.3710 e+00$ \\
\hline 146 & IHT000019 & 160.2464 & 162.5901 & 161.6801 & $5.2141 e-01$ & $4.4073 e+00$ \\
\hline 147 & IHT000020 & 165.4146 & 167.9537 & 166.7623 & $5.0107 e-01$ & $4.4262 \mathrm{e}+00$ \\
\hline $1: 8$ & IHTOCOD21 & -3.8049 & -0.6798 & -1.7033 & $7.0758 e-01$ & $4.1000 e+00$ \\
\hline 149 & IHT000022 & 136.0464 & 138.5855 & 137.5464 & $5.2562 e-01$ & $4.3246 e+00$ \\
\hline 150 & IHTOOOO23 & 162.1019 & 164.4456 & 163.3715 & $5.4140 e-01$ & $4.4135 e+00$ \\
\hline 151 & IHTOOOOO24 & 127.3475 & 129.4959 & 128.5428 & $5.0938 \theta-01$ & $4.2968 e+00$ \\
\hline 152 & IHT000025 & 145.3163 & 147.4647 & 146.4374 & $5.0503 e-01$ & $4.3537 e+00$ \\
\hline 153 & IHTO00026 & 147.2656 & 149.4141 & 148.3125 & $4.3001 e-01$ & $4.3600 \mathrm{e}+00$ \\
\hline 154 & IHT000027 & 122.9830 & 125.3268 & 124.2095 & $4.9448 e-01$ & $4.2840 e+00$ \\
\hline 155 & IHTOO:028 & 124.5568 & 128.0724 & 126.4396 & $7.8001 e-01$ & $4.2905 e+00$ \\
\hline
\end{tabular}




\begin{tabular}{|c|c|c|c|c|c|c|}
\hline \multicolumn{2}{|c|}{ Test: Aug15_14 } & sum2 & Date: Wed & Aug 151 & $19: 011990$ & Page: 4 \\
\hline $\begin{array}{l}11 \\
156 \\
161 \\
158 \\
159 \\
160 \\
161 \\
162 \\
163\end{array}$ & 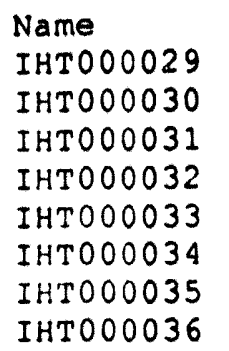 & $\begin{array}{c}\text { Min } \\
146.4130 \\
149.3690 \\
133.6313 \\
135.4417 \\
135.4267 \\
125.1615 \\
164.8400 \\
123.4713\end{array}$ & $\begin{array}{l}\operatorname{Max} \\
149.9286 \\
152.8846 \\
136.9516 \\
139.1526 \\
139.1376 \\
127.7006 \\
167.5744 \\
126.0104\end{array}$ & $\begin{array}{l}\text { Ave } \\
148.1591 \\
150.8964 \\
135.0610 \\
136.8988 \\
136.8447 \\
126.2671 \\
166.2384 \\
124.6665\end{array}$ & $\begin{array}{l}\text { SD } \\
7.6046 e-01 \\
8.0063 e-01 \\
7.6660 e-01 \\
8.0598 e-01 \\
7.7806 e-01 \\
6.0568 e-01 \\
6.2185 e-01 \\
5.9141 e-01\end{array}$ & $\begin{array}{c}\text { Uncert } \\
4.3595 e+00 \\
4.3689 \theta+00 \\
4.3167 e+00 \\
4.3225 e+00 \\
4.3223 e+00 \\
4.2900 \theta+00 \\
4.4242 \theta+00 \\
4.2853 e+00\end{array}$ \\
\hline
\end{tabular}




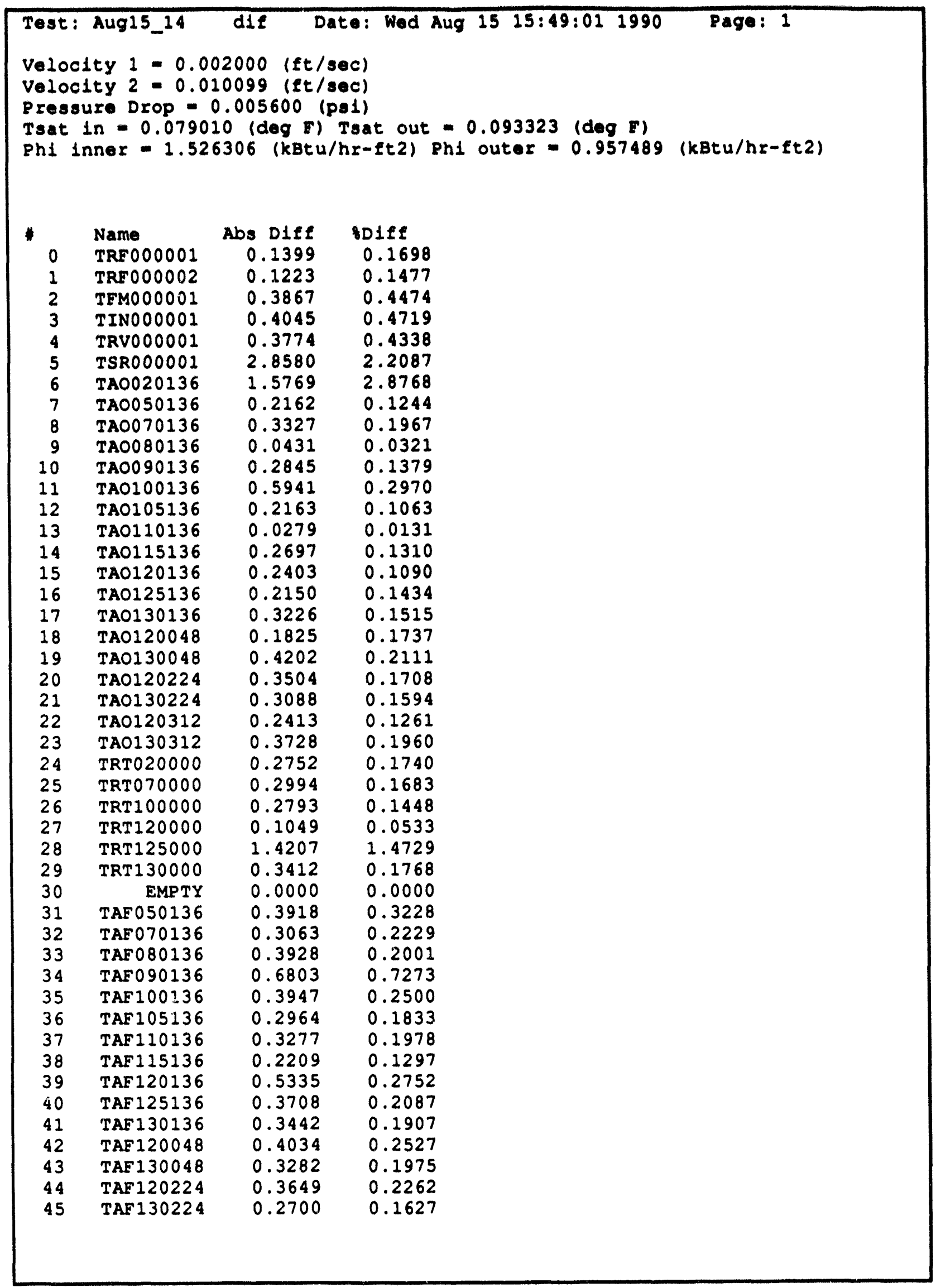




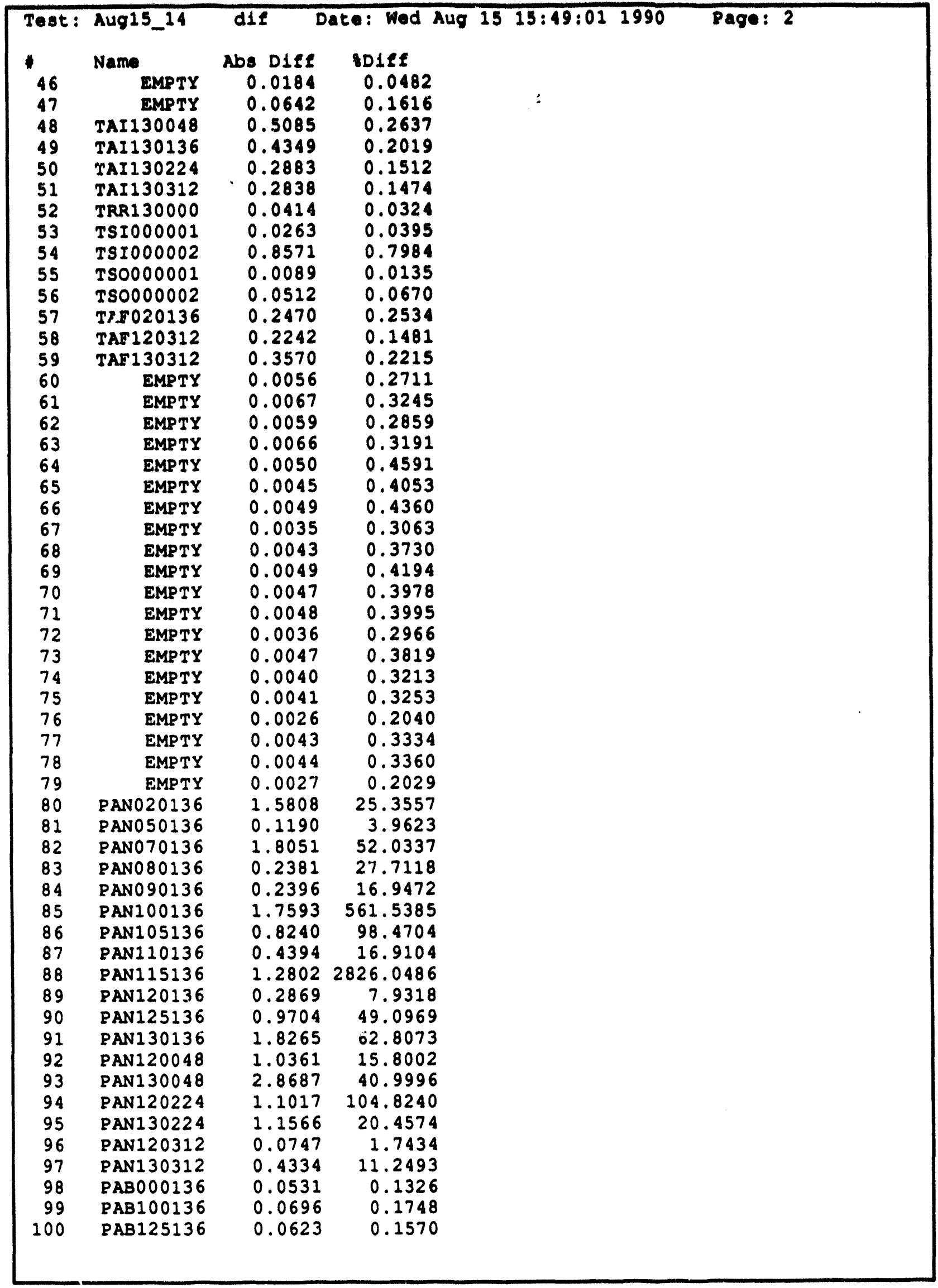




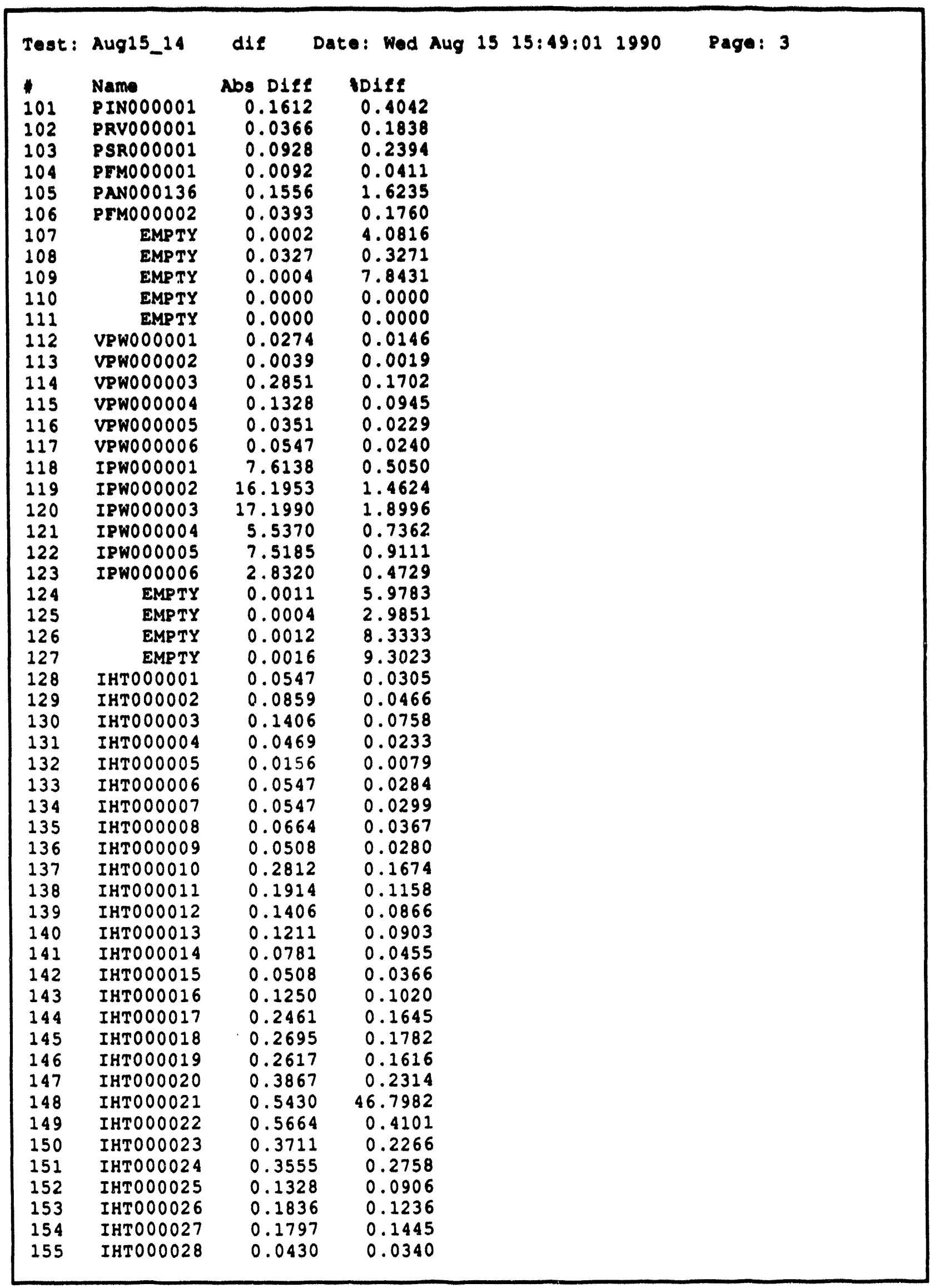




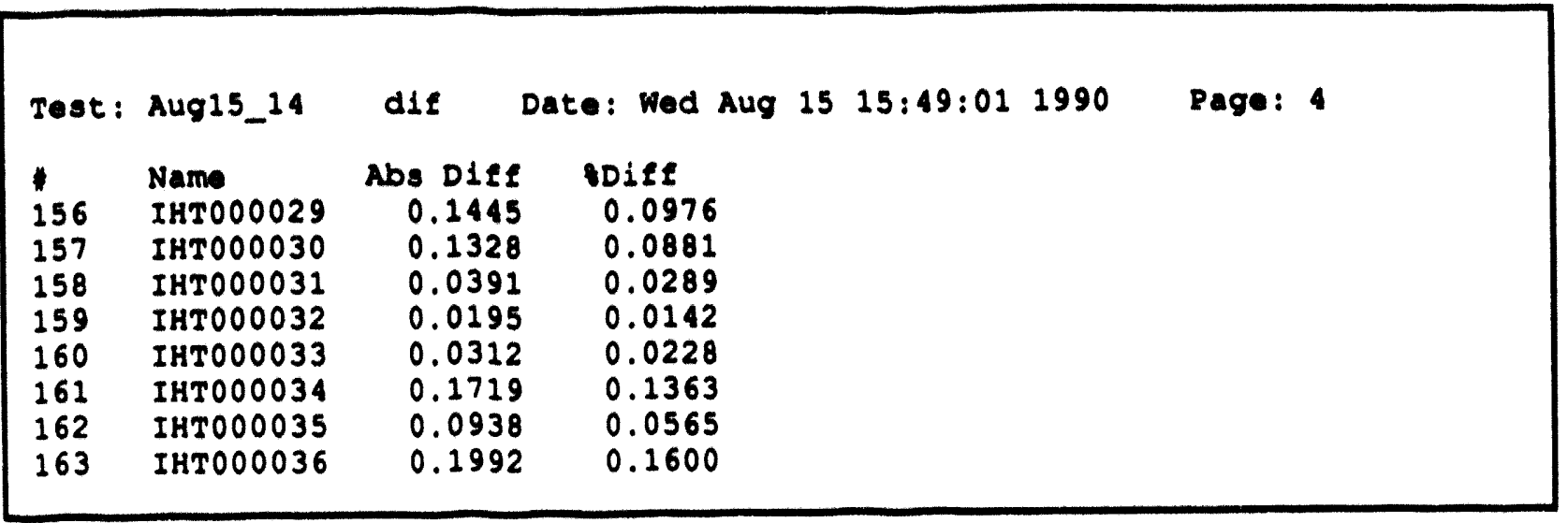




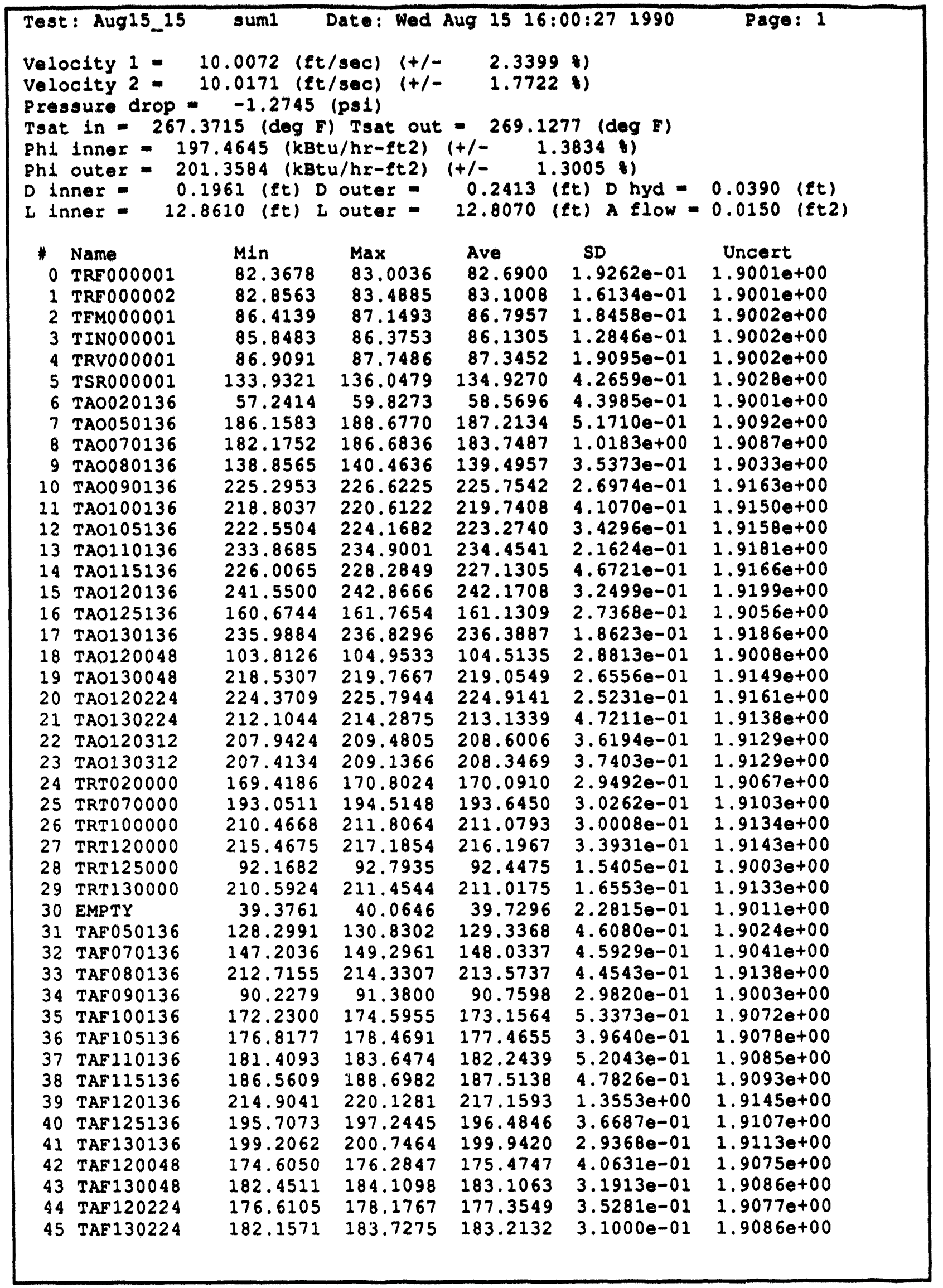




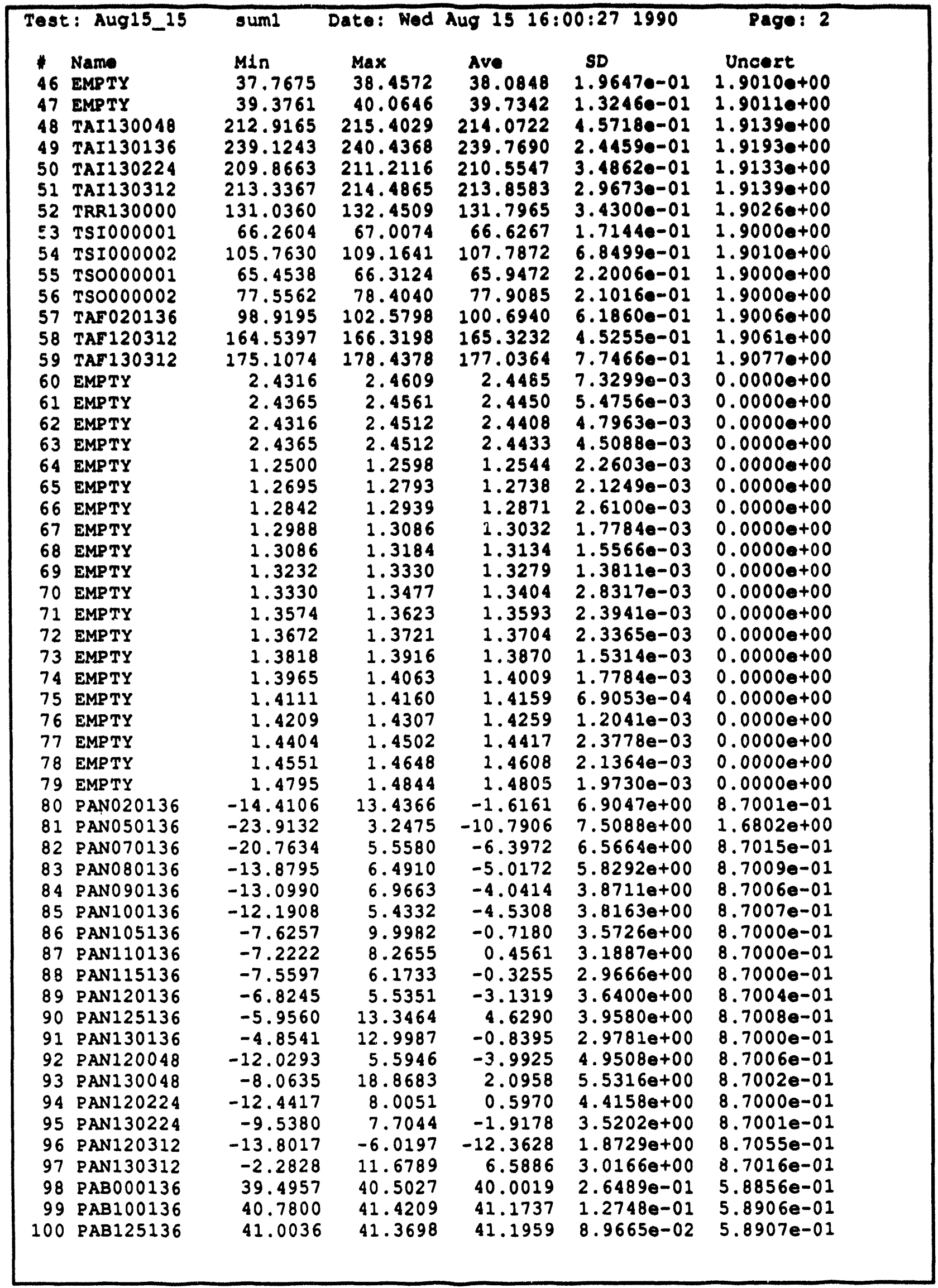




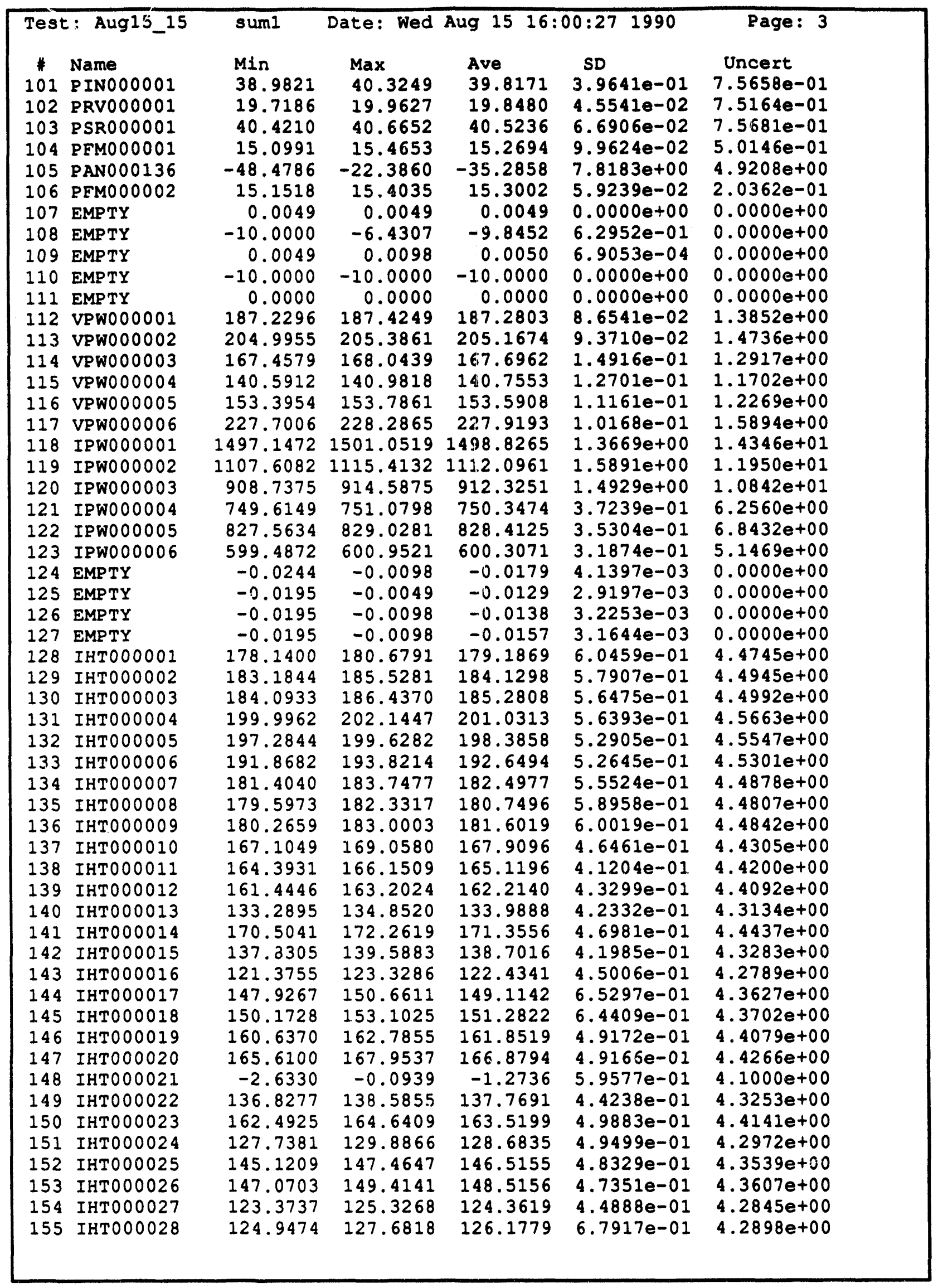




\begin{tabular}{|c|c|c|c|c|c|c|}
\hline Test & $t:$ Aug15_15 & suml & Date: Wed & Aug 1516 & $00: 271990$ & Page: 4 \\
\hline $\begin{array}{l}\not \\
156 \\
157 \\
158 \\
159 \\
160 \\
161 \\
162 \\
163\end{array}$ & 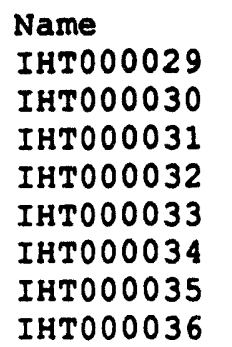 & $\begin{array}{l}\text { Min } \\
146.6083 \\
149.5643 \\
133.8266 \\
135.6370 \\
135.6220 \\
124.9662 \\
164.8400 \\
123.2760\end{array}$ & $\begin{array}{c}\text { Max } \\
149.1474 \\
151.9080 \\
136.1704 \\
138.1761 \\
138.1611 \\
127.5053 \\
167.5744 \\
125.8150\end{array}$ & $\begin{array}{l}\text { Ave } \\
147.8076 \\
150.5800 \\
134.8891 \\
136.7504 \\
136.7158 \\
126.1108 \\
166.0587 \\
124.4595\end{array}$ & $\begin{array}{l}\text { SD } \\
6.3136 e-01 \\
5.9451 e-01 \\
6.0381 e-01 \\
6.4144 e-01 \\
6.4356 e-01 \\
5.1450 e-01 \\
5.2432 e-01 \\
4.9820 e-01\end{array}$ & $\begin{array}{l}\text { Uncert } \\
4.3583 e+00 \\
4.3678 e+00 \\
4.3162 e+00 \\
4.3220 e+00 \\
4.3219 e+00 \\
4.2896 e+00 \\
4.4235 e+00 \\
4.2847 e+00\end{array}$ \\
\hline
\end{tabular}




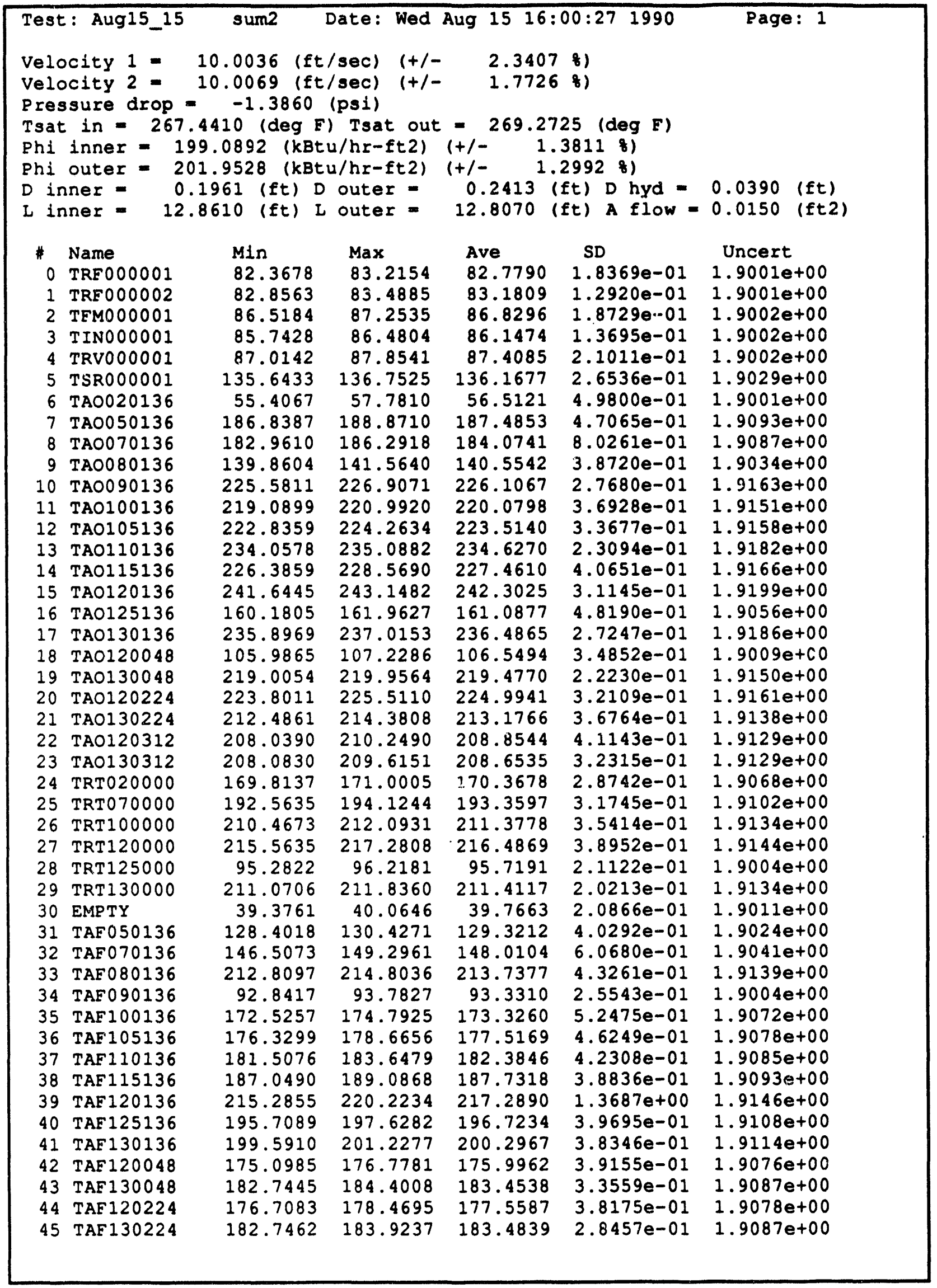




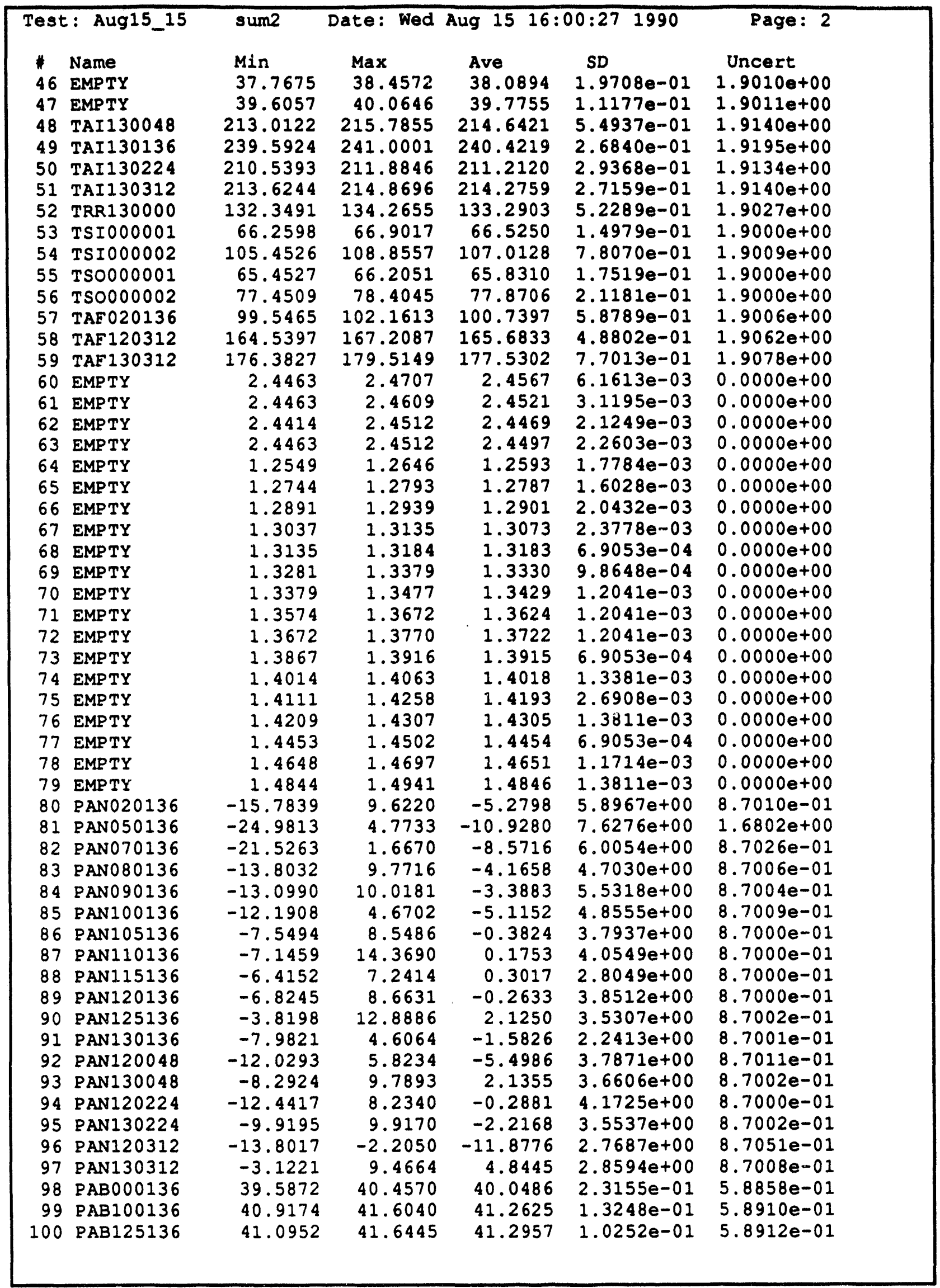




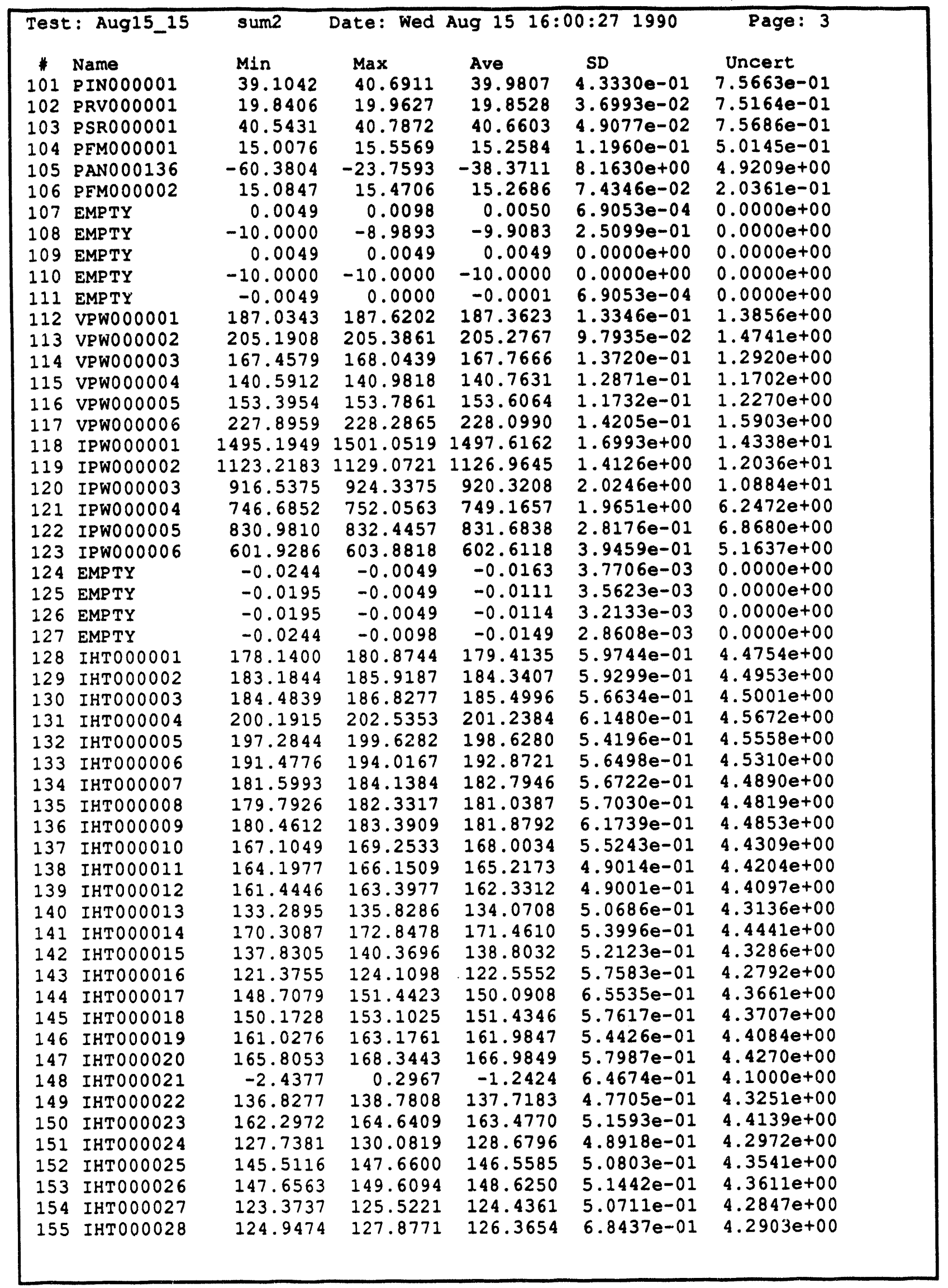




\begin{tabular}{|c|c|c|c|c|c|c|}
\hline \multicolumn{2}{|c|}{ Test: Aug15_15 } & sum2 & Date: W & ug & $: 271$ & Page: 4 \\
\hline $\begin{array}{c}* \\
156 \\
157 \\
158 \\
159 \\
160 \\
161 \\
162 \\
163\end{array}$ & 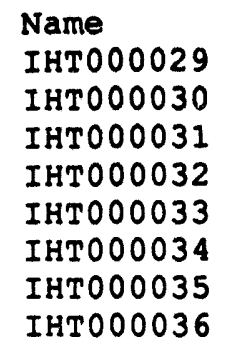 & $\begin{array}{l}\text { Min } \\
146.8036 \\
149.3690 \\
133.8266 \\
135.4417 \\
135.4267 \\
124.9662 \\
164.8400 \\
123.6666\end{array}$ & $\begin{array}{l}\text { Max } \\
149.5380 \\
152.2987 \\
136.7563 \\
138.7620 \\
138.5517 \\
127.1146 \\
167.1837 \\
125.6197\end{array}$ & $\begin{array}{l}\text { Ave } \\
148.0029 \\
150.7206 \\
135.0415 \\
136.8168 \\
136.7900 \\
126.0796 \\
166.0392 \\
124.4947\end{array}$ & $\begin{array}{l}\text { SD } \\
6.4839 e-01 \\
6.8894 e-01 \\
6.3416 e-01 \\
6.4833 e-01 \\
6.2576 e-01 \\
4.8528 e-01 \\
5.0380 e-01 \\
4.9687 e-01\end{array}$ & $\begin{array}{c}\text { Uncert } \\
4.3590 e+00 \\
4.3683 e+00 \\
4.3167 e+00 \\
4.3223 e+00 \\
4.3222 e+00 \\
4.2895 e+00 \\
4.4234 e+00 \\
4.2848 e+00\end{array}$ \\
\hline
\end{tabular}




\begin{tabular}{|c|c|c|c|}
\hline \multicolumn{4}{|c|}{$\begin{array}{l}\text { Velocity } 1=0.003600 \text { (ft/sec) } \\
\text { Velocity } 2=0.010201 \text { (ft/sec) } \\
\text { Pressure Drop }=0.111500 \text { (psi) } \\
\text { Tsat in }=0.069519 \text { (deg F) Tsat out }=0.144806 \text { (deg F) } \\
\text { Phi inner }=1.624710 \text { (kBtu/hr-ft2) Phi outer }=0.594406 \text { (kBtu/hr-ft2) }\end{array}$} \\
\hline${ }^{*} 0$ & Name & Abs Diff & \&Diff \\
\hline $\begin{array}{l}0 \\
1\end{array}$ & TRF000001 & 0.0890 & 0.1076 \\
\hline 1 & TRF 000002 & 0.0801 & 0.0964 \\
\hline 2 & TFM000001 & 0.0339 & 0.0391 \\
\hline 3 & TIN000001 & 0.0169 & 0.0196 \\
\hline 4 & TRV000001 & 0.0633 & 0.0725 \\
\hline 5 & TSRO00001 & 1.2407 & 0.9195 \\
\hline 6 & TAO020136 & 2.0575 & 3.5129 \\
\hline 7 & TA0050136 & 0.2719 & 0.1452 \\
\hline 8 & TA0070136 & 0.3254 & 0.1771 \\
\hline 9 & TAO080136 & 1.0585 & 0.7588 \\
\hline 10 & TAO090136 & 0.3525 & 0.1561 \\
\hline 11 & TA0100136 & 0.3390 & 0.1543 \\
\hline 12 & TAO105136 & 0.2400 & 0.1075 \\
\hline 13 & TA0110136 & 0.1729 & 0.0737 \\
\hline 14 & TA0115136 & 0.3305 & 0.1455 \\
\hline 15 & TA0120136 & 0.1317 & 0.0544 \\
\hline 16 & TAO125136 & 0.0432 & 0.0268 \\
\hline 17 & TA0130136 & 0.0978 & 0.0414 \\
\hline 18 & TA0120048 & 2.0359 & 1.9480 \\
\hline 19 & TAO130048 & 0.4221 & 0.1927 \\
\hline 20 & TAO120224 & 0.0800 & 0.0356 \\
\hline 21 & TA0130224 & 0.0427 & 0.0200 \\
\hline 22 & TA0120312 & 0.2538 & 0.1217 \\
\hline 23 & TAO130312 & 0.3066 & 0.1472 \\
\hline 24 & TRT020000 & 0.2768 & 0.1627 \\
\hline 25 & TRT070000 & 0.2853 & 0.1473 \\
\hline 26 & TRT 100000 & 0.2985 & 0.1414 \\
\hline 27 & TRT 120000 & 0.2902 & 0.1342 \\
\hline 28 & TRT 125000 & 3.2716 & 3.5389 \\
\hline 29 & TRT130000 & 0.3942 & 0.1868 \\
\hline 30 & EMPTY & 0.0367 & 0.0924 \\
\hline 31 & TAF050136 & 0.0156 & 0.0121 \\
\hline 32 & TAF070136 & 0.0233 & 0.0157 \\
\hline 33 & TAF080136 & 0.1640 & 0.0768 \\
\hline 34 & TAF090136 & 2.5712 & 2.8330 \\
\hline 35 & TAF 100136 & 0.1696 & 0.0979 \\
\hline 36 & TAF 105136 & 0.0514 & 0.0290 \\
\hline 37 & TAF 110136 & 0.1407 & 0.0772 \\
\hline 38 & TAF 115136 & 0.2180 & 0.1163 \\
\hline 39 & TAF 120136 & 0.1297 & 0.0597 \\
\hline 40 & TAF 125136 & 0.2388 & 0.1215 \\
\hline 41 & TAF 130136 & 0.3547 & 0.1774 \\
\hline 42 & TAF 120048 & 0.5215 & 0.2972 \\
\hline 43 & TAF 130048 & 0.3475 & 0.1898 \\
\hline 44 & TAF 120224 & 0.2038 & 0.1149 \\
\hline 45 & TAF130224 & 0.2707 & 0.1478 \\
\hline
\end{tabular}




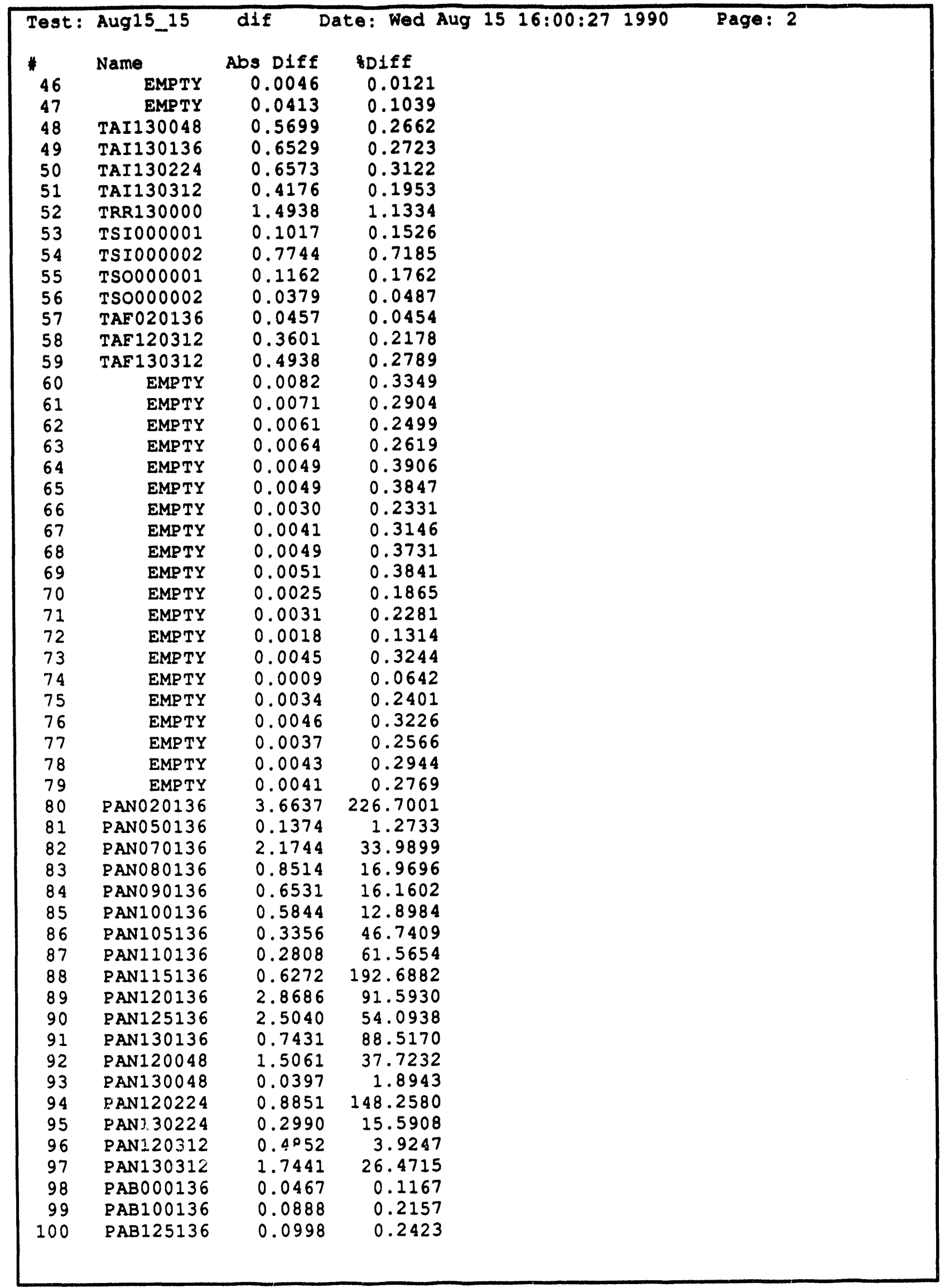




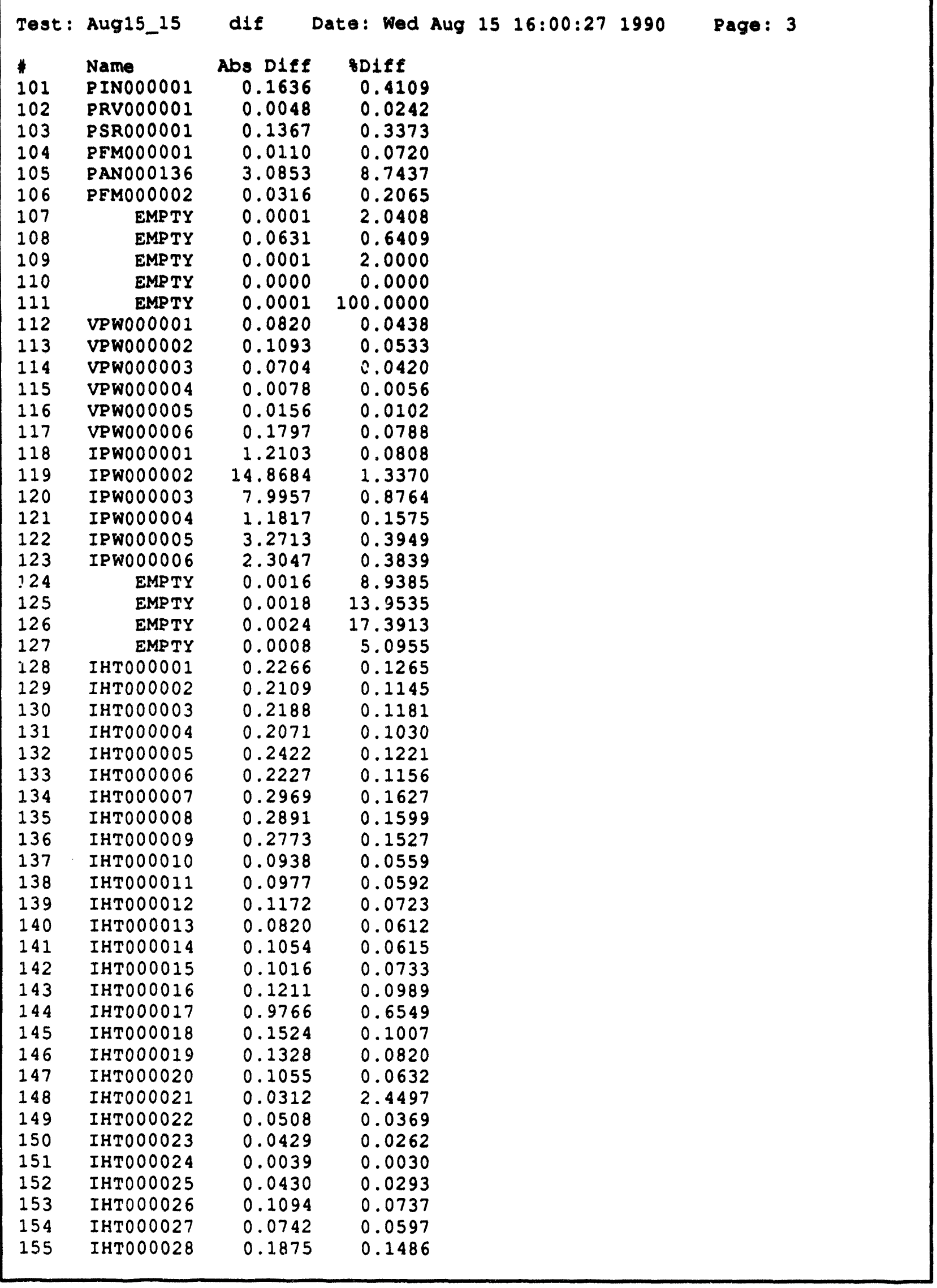




\begin{tabular}{|c|c|c|c|c|}
\hline $\begin{array}{l}\text { Test: } \\
1 \\
156 \\
157 \\
158 \\
159 \\
160 \\
161 \\
162 \\
163\end{array}$ & $\begin{array}{l}\text { Aug15_15 } \\
\text { Name } \\
\text { IHTO00029 } \\
\text { IHTO00030 } \\
\text { IHTO0O031 } \\
\text { IHTO0O032 } \\
\text { IHTO00033 } \\
\text { IHTO0O034 } \\
\text { IHTOOOO35 } \\
\text { IHTOOOO36 }\end{array}$ & $\begin{array}{r}\text { dif } \\
\text { Abs } D 1 f f \\
0.1953 \\
0.1406 \\
0.1524 \\
0.0664 \\
0.0742 \\
0.0312 \\
0.0195 \\
0.0352\end{array}$ & $\begin{array}{l}\text { Date: Wed Aug } 1516: 00: 271990 \\
\text { odff } \\
0.1321 \\
0.0934 \\
0.1130 \\
0.0486 \\
0.0543 \\
0.0247 \\
0.0117 \\
5 \\
0.0283\end{array}$ & Page: 4 \\
\hline
\end{tabular}




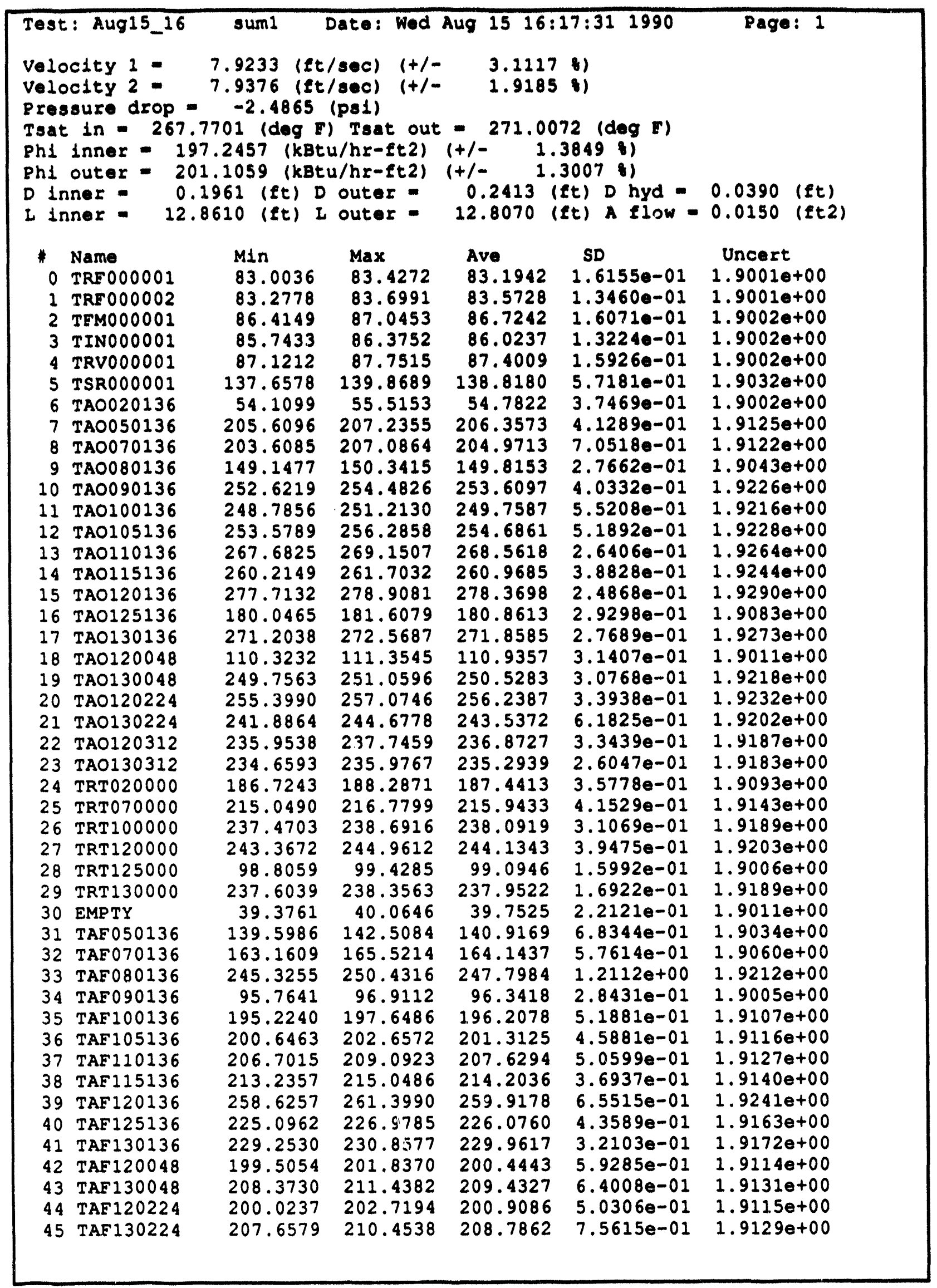




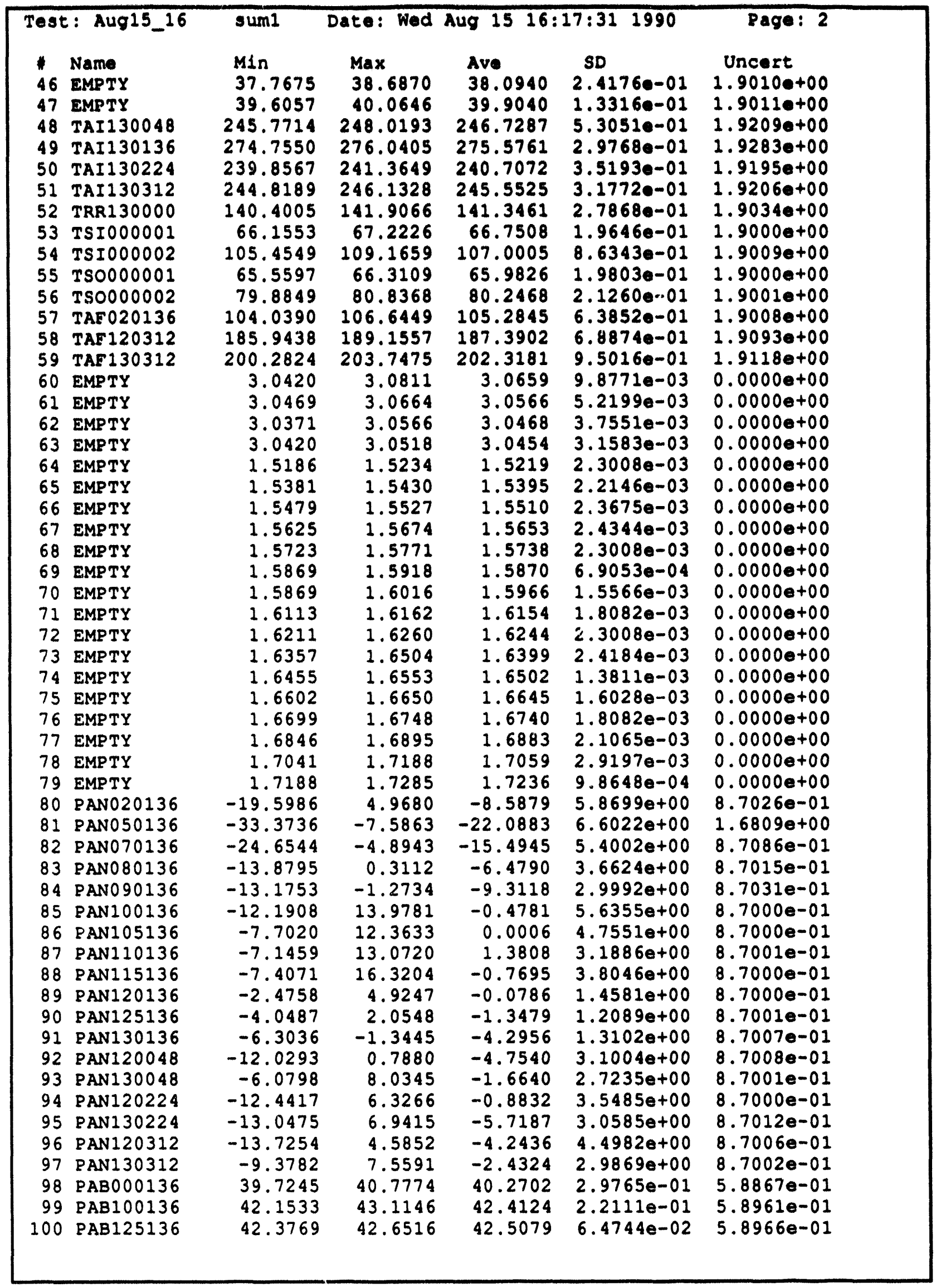




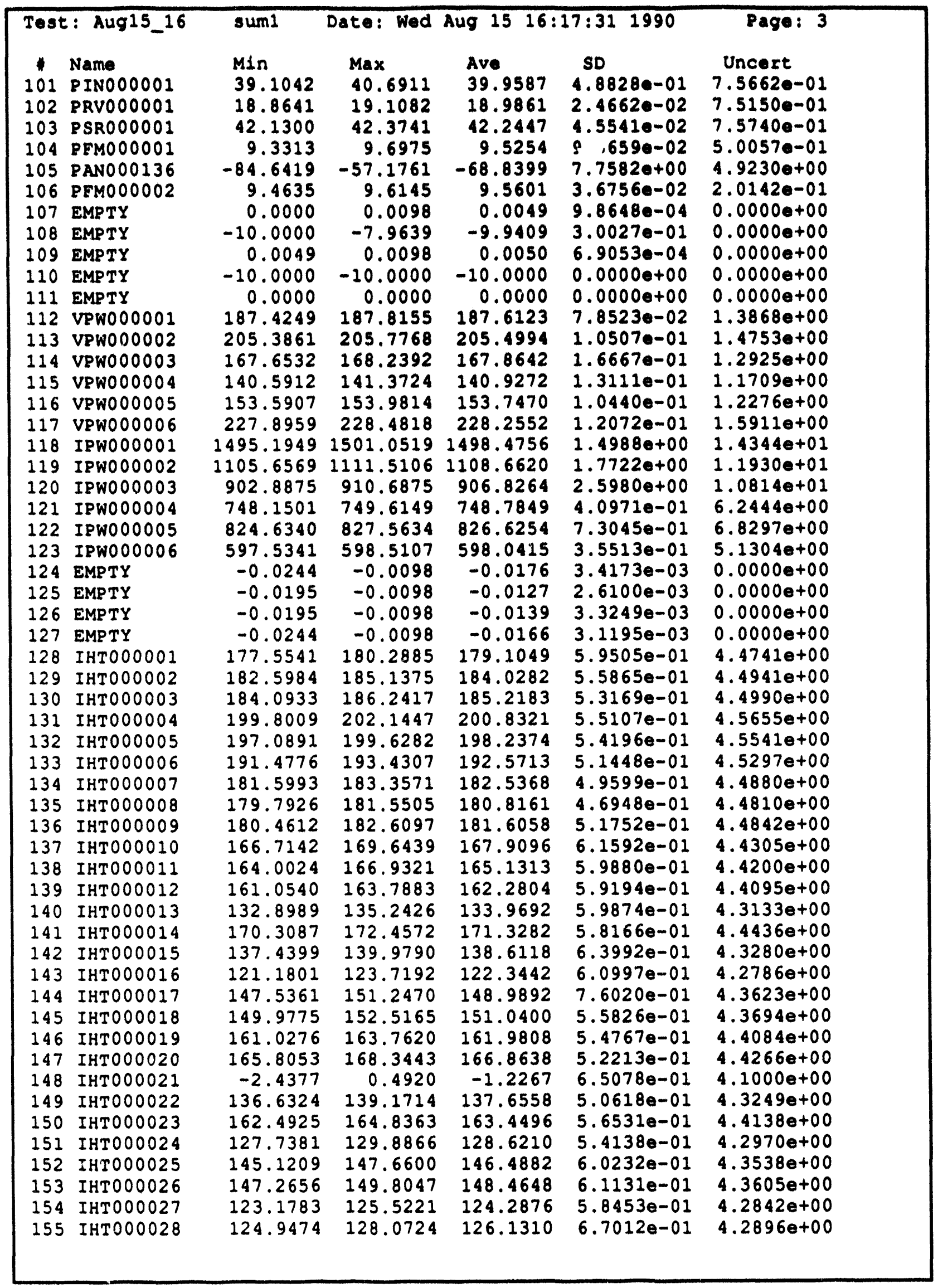




\begin{tabular}{|c|c|c|c|c|c|c|}
\hline $\begin{array}{l}1 \\
156 \\
157 \\
158 \\
159 \\
160 \\
161 \\
162 \\
163\end{array}$ & $\begin{array}{l}\text { Name } \\
\text { IHTO00029 } \\
\text { IHTO00030 } \\
\text { IHTO00031 } \\
\text { IHTO00032 } \\
\text { IHTOO0033 } \\
\text { IHTO00034 } \\
\text { IHTO00035 } \\
\text { IHTO00036 }\end{array}$ & $\begin{array}{l}\text { Min } \\
146.6083 \\
149.3690 \\
133.8266 \\
135.6370 \\
135.6220 \\
125.1615 \\
165.0353 \\
123.4713\end{array}$ & $\begin{array}{l}\operatorname{Max} \\
149.7333 \\
152.4940 \\
136.3657 \\
138.5667 \\
138.5517 \\
127.5053 \\
167.5744 \\
125.8150\end{array}$ & $\begin{array}{l}\text { Ave } \\
147.7412 \\
150.5097 \\
134.8071 \\
136.6214 \\
136.5517 \\
125.9194 \\
165.9220 \\
124.3111\end{array}$ & $\begin{array}{l}\text { SD } \\
6.2140 e-01 \\
5.9104 \theta-01 \\
5.7520 e-01 \\
5.8389 \theta-01 \\
5.9915 \theta-01 \\
4.6125 e-01 \\
4.9632 \theta-01 \\
4.8848 \theta-01\end{array}$ & $\begin{array}{c}\text { Uncert } \\
4.3581 e+00 \\
4.3675 e+00 \\
4.3159 e+00 \\
4.3216 e+00 \\
4.3214 e+00 \\
4.2890 e+00 \\
4.4230 e+00 \\
4.2843 e+00\end{array}$ \\
\hline
\end{tabular}




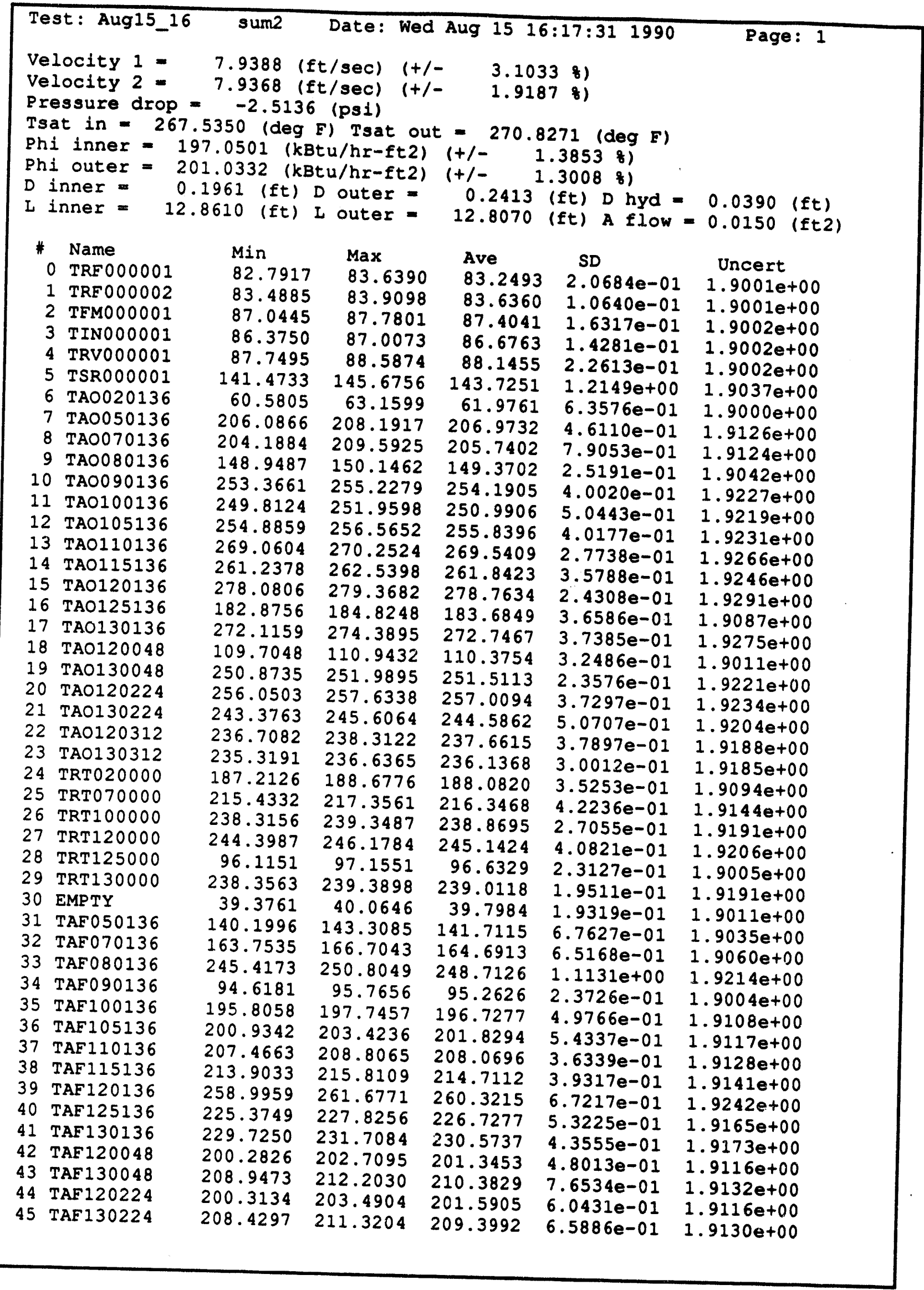




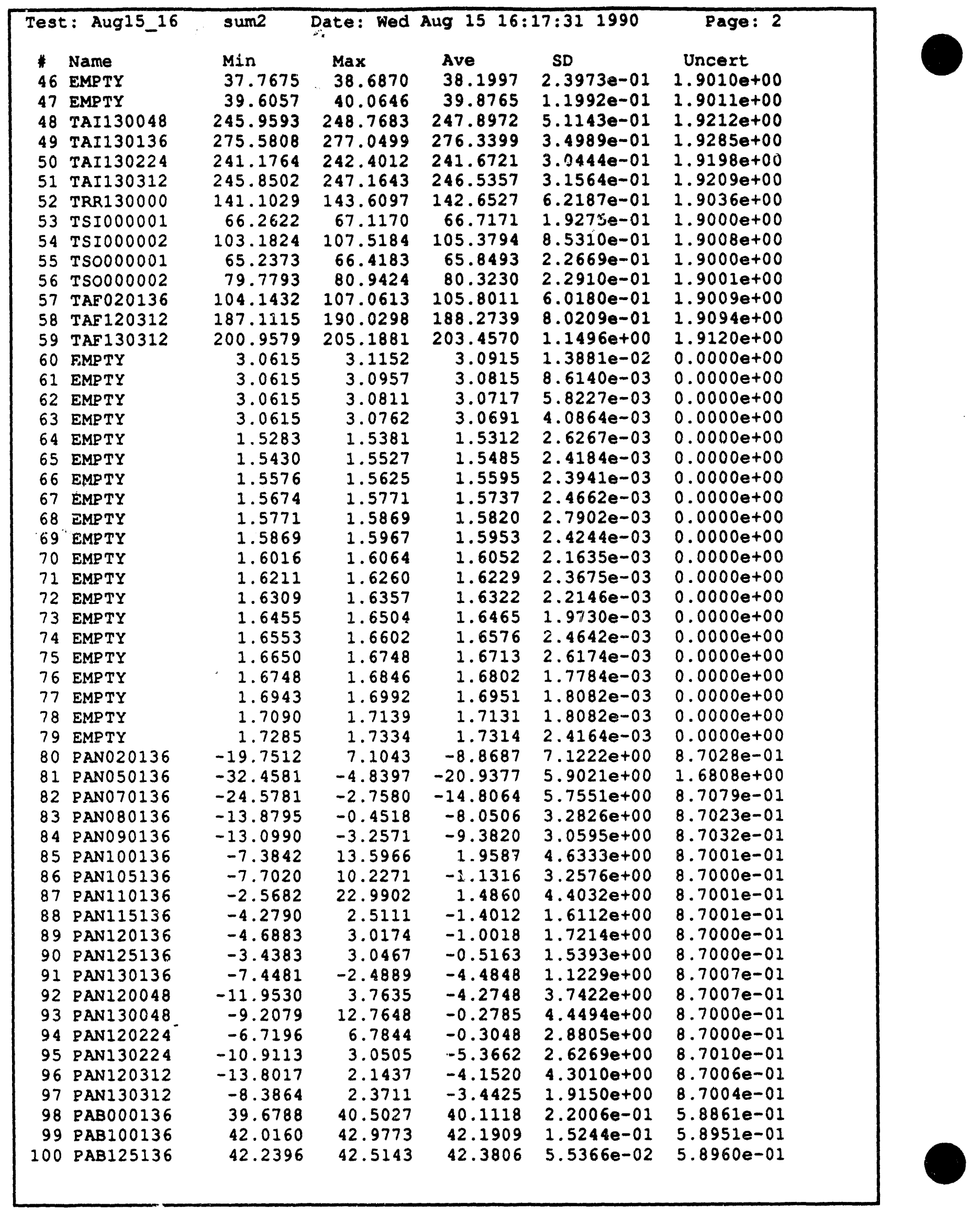




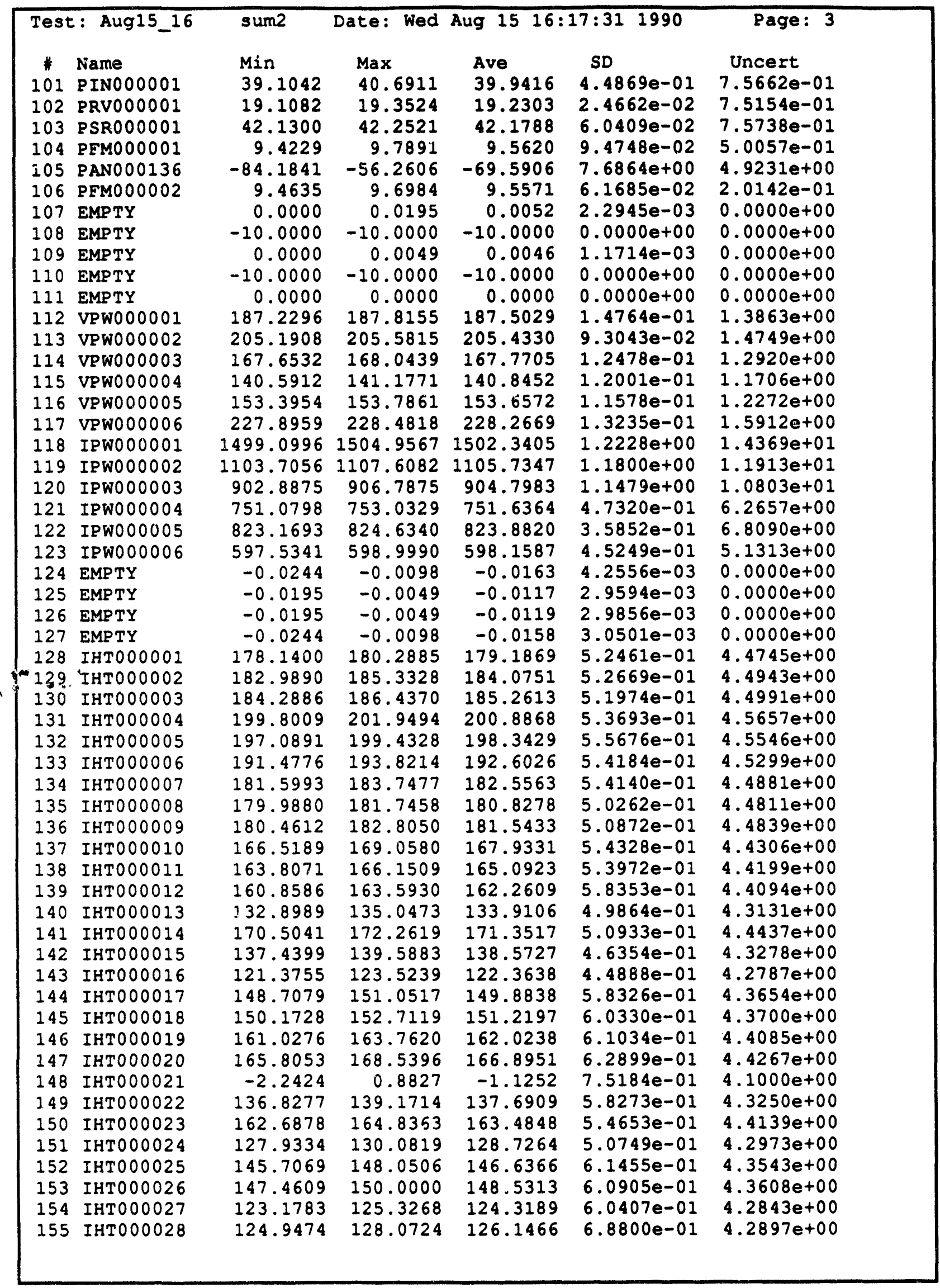




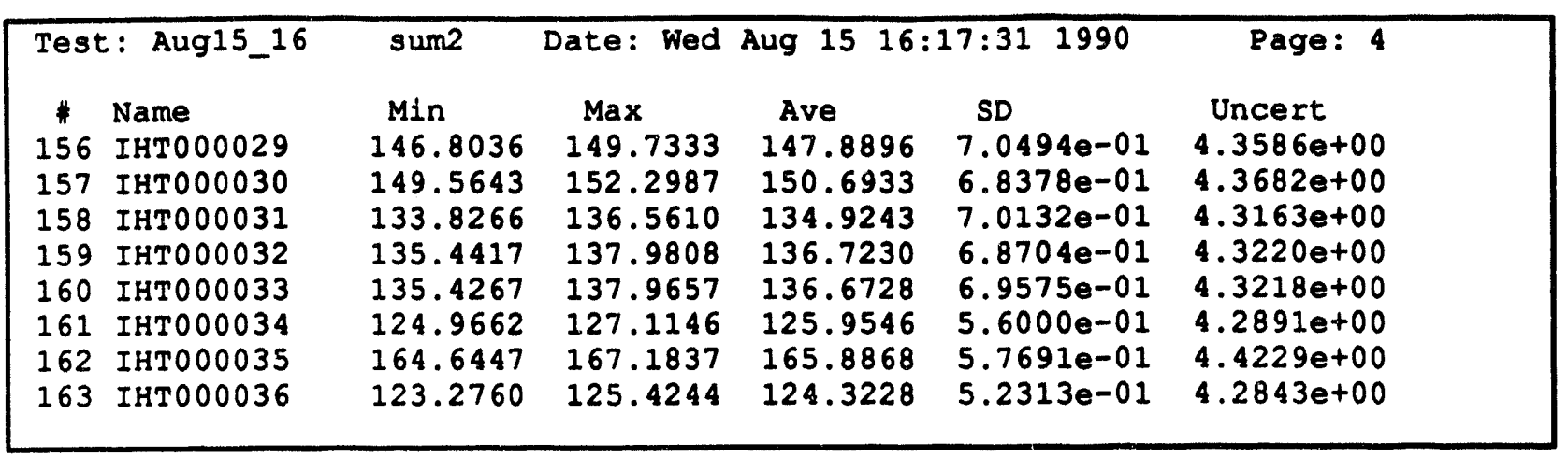




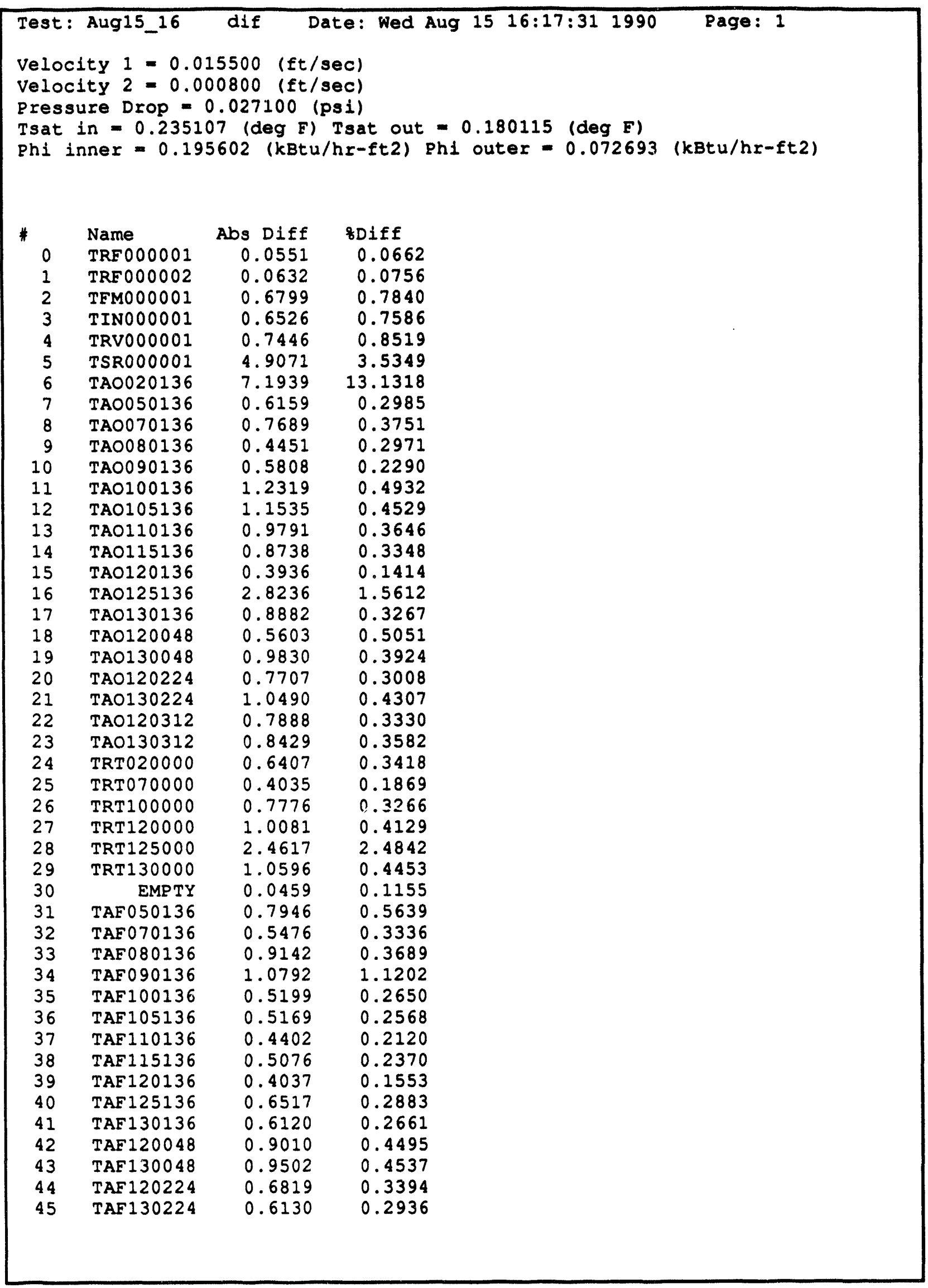




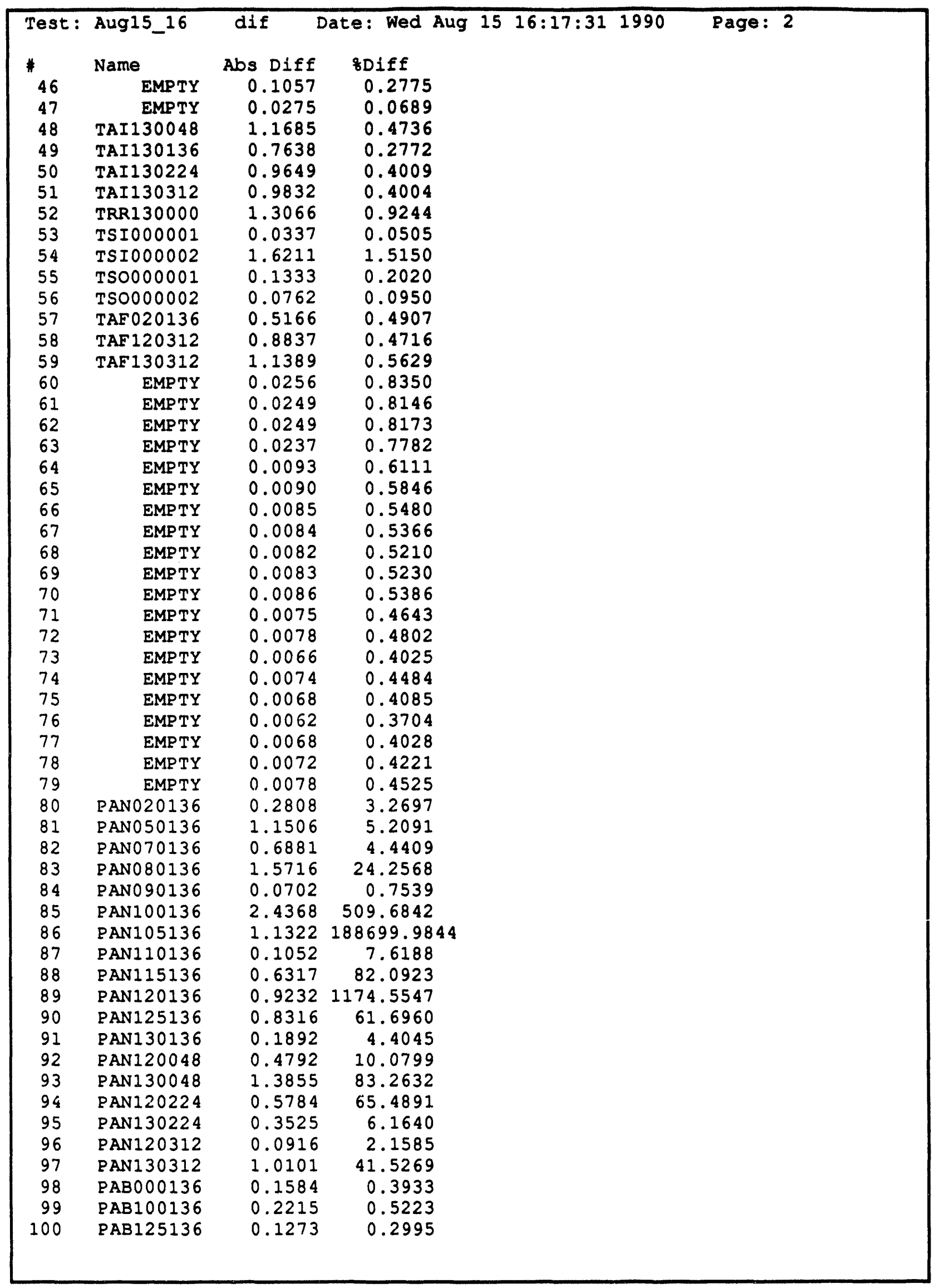




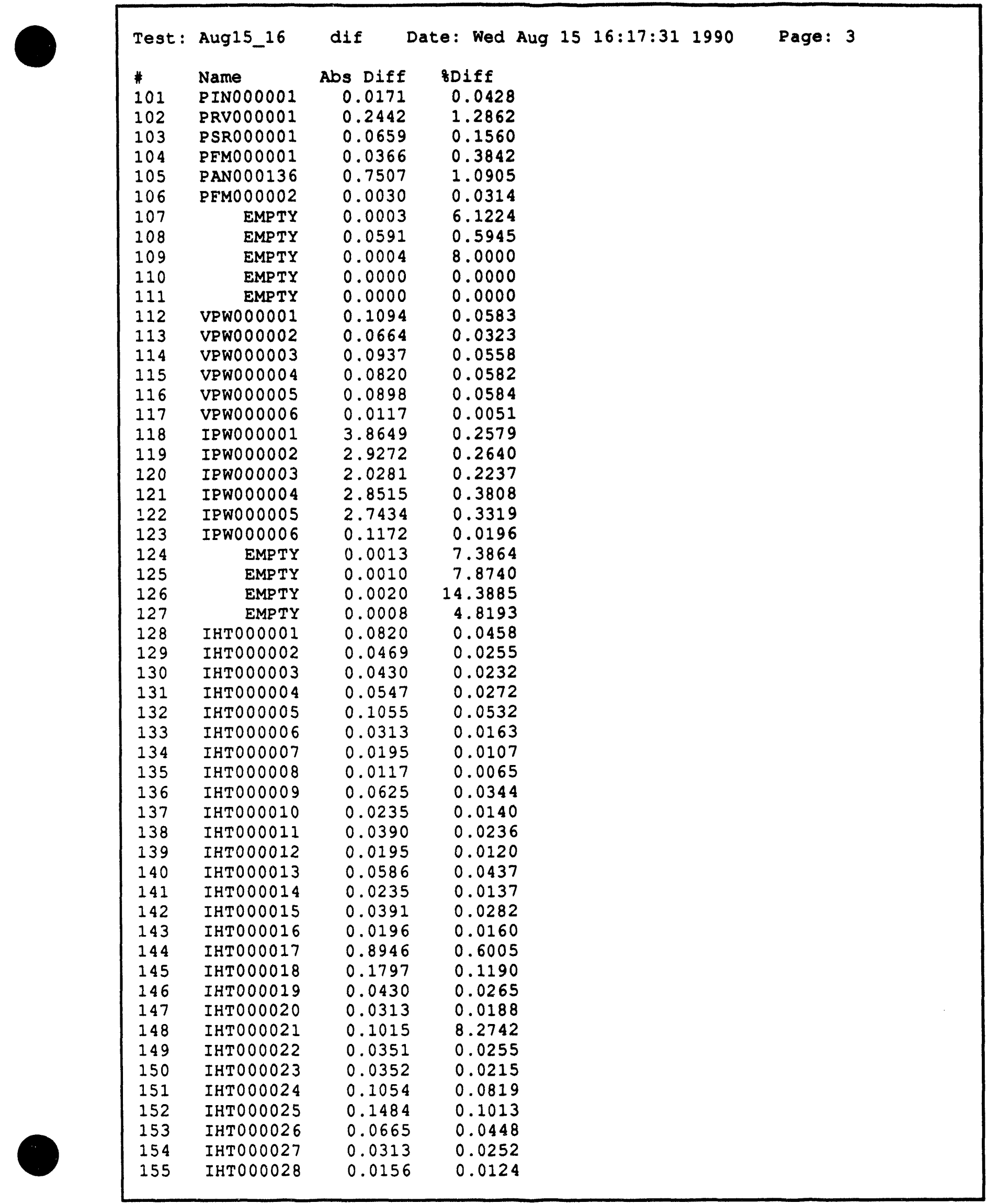




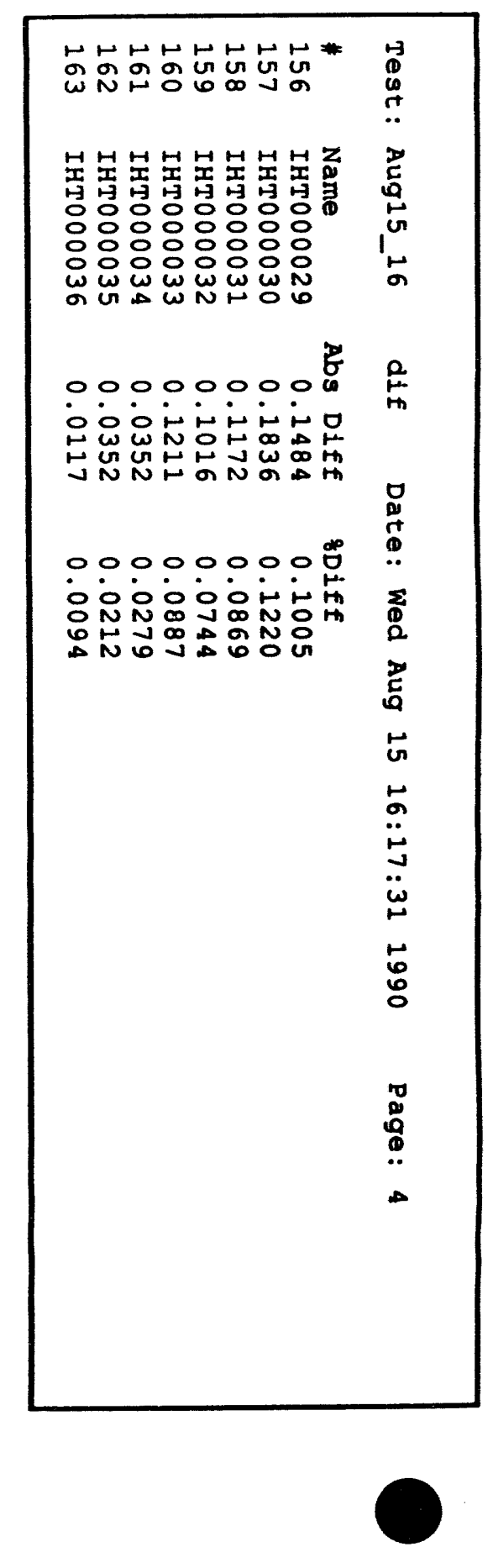




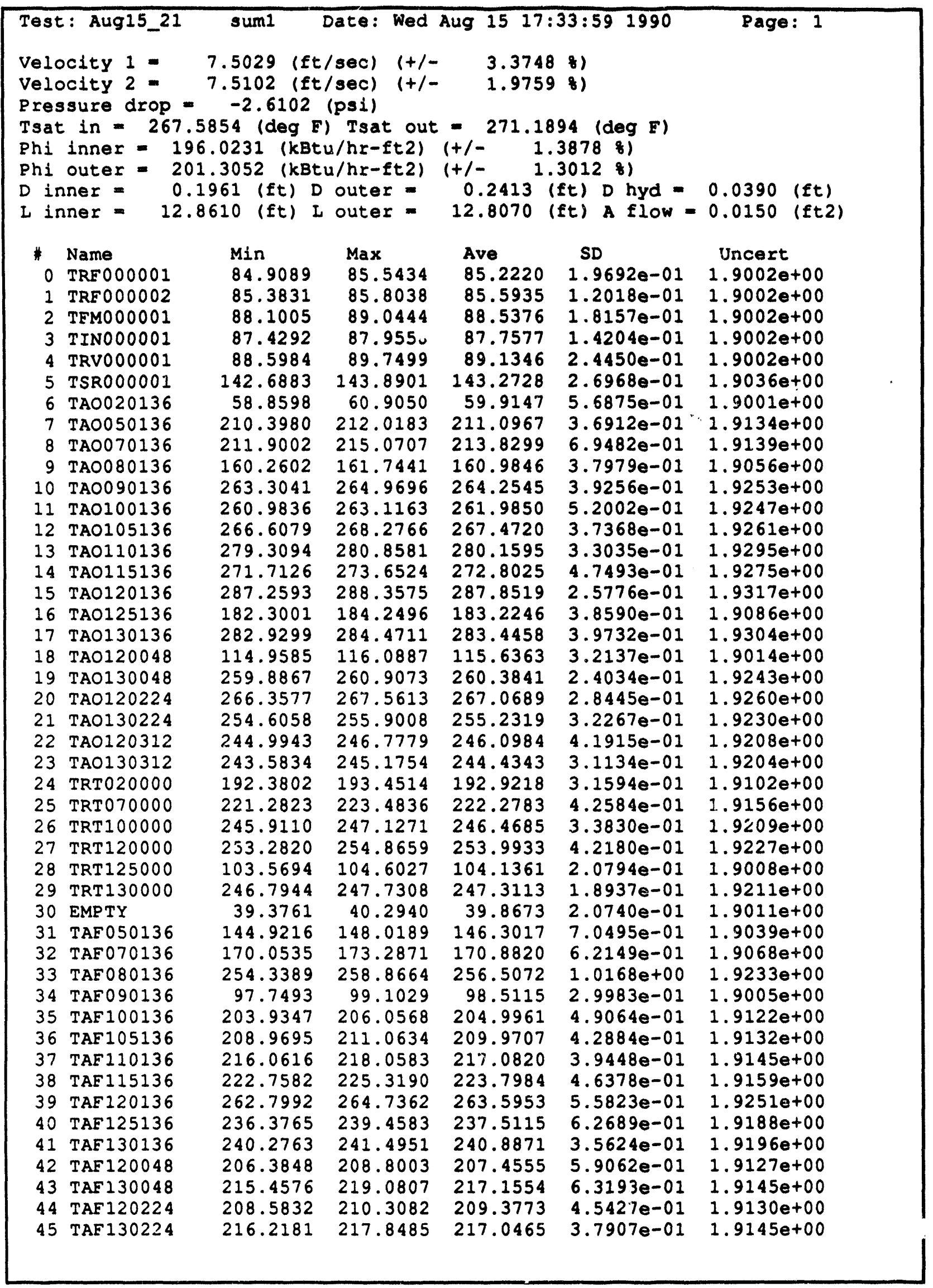




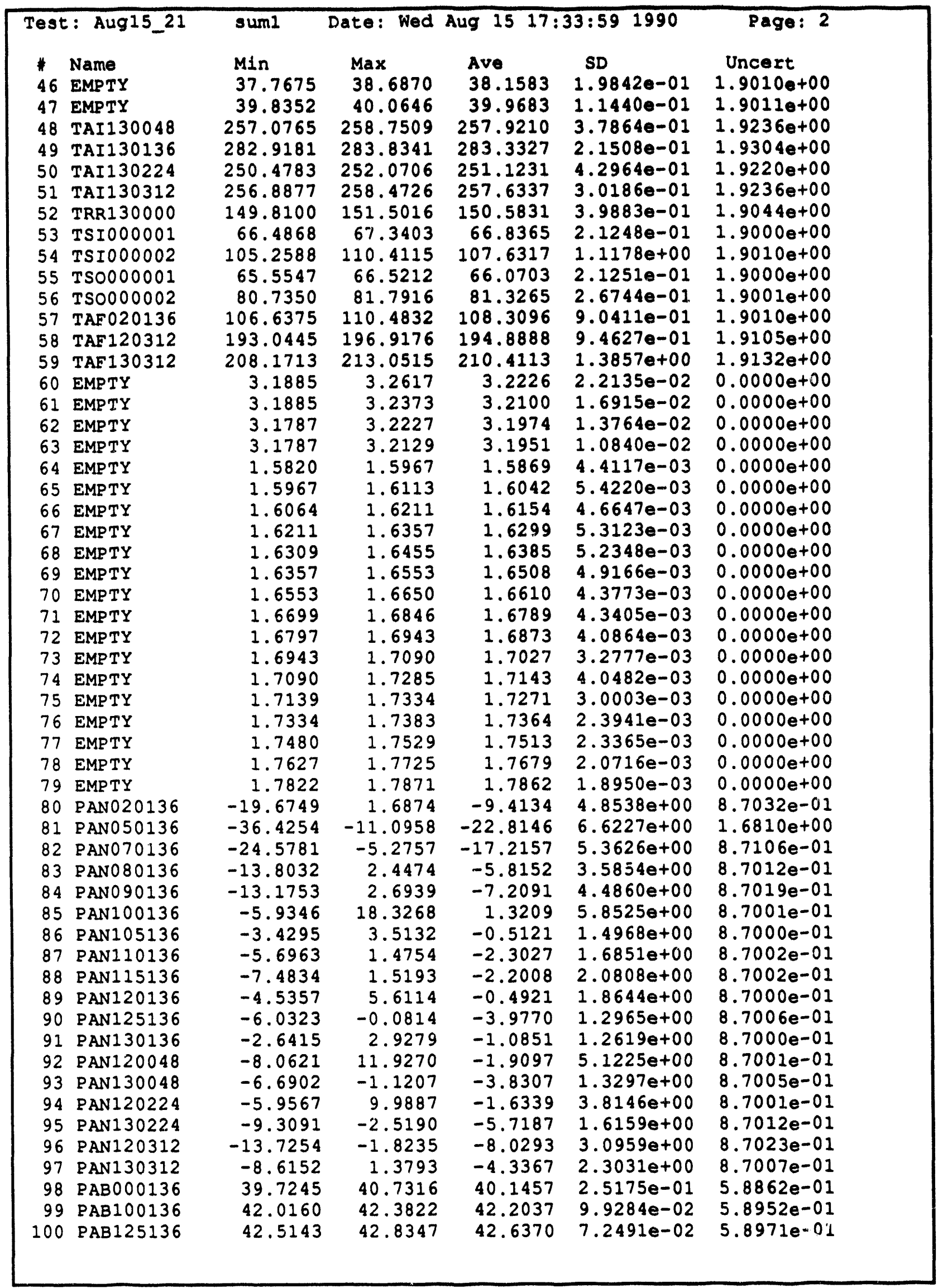




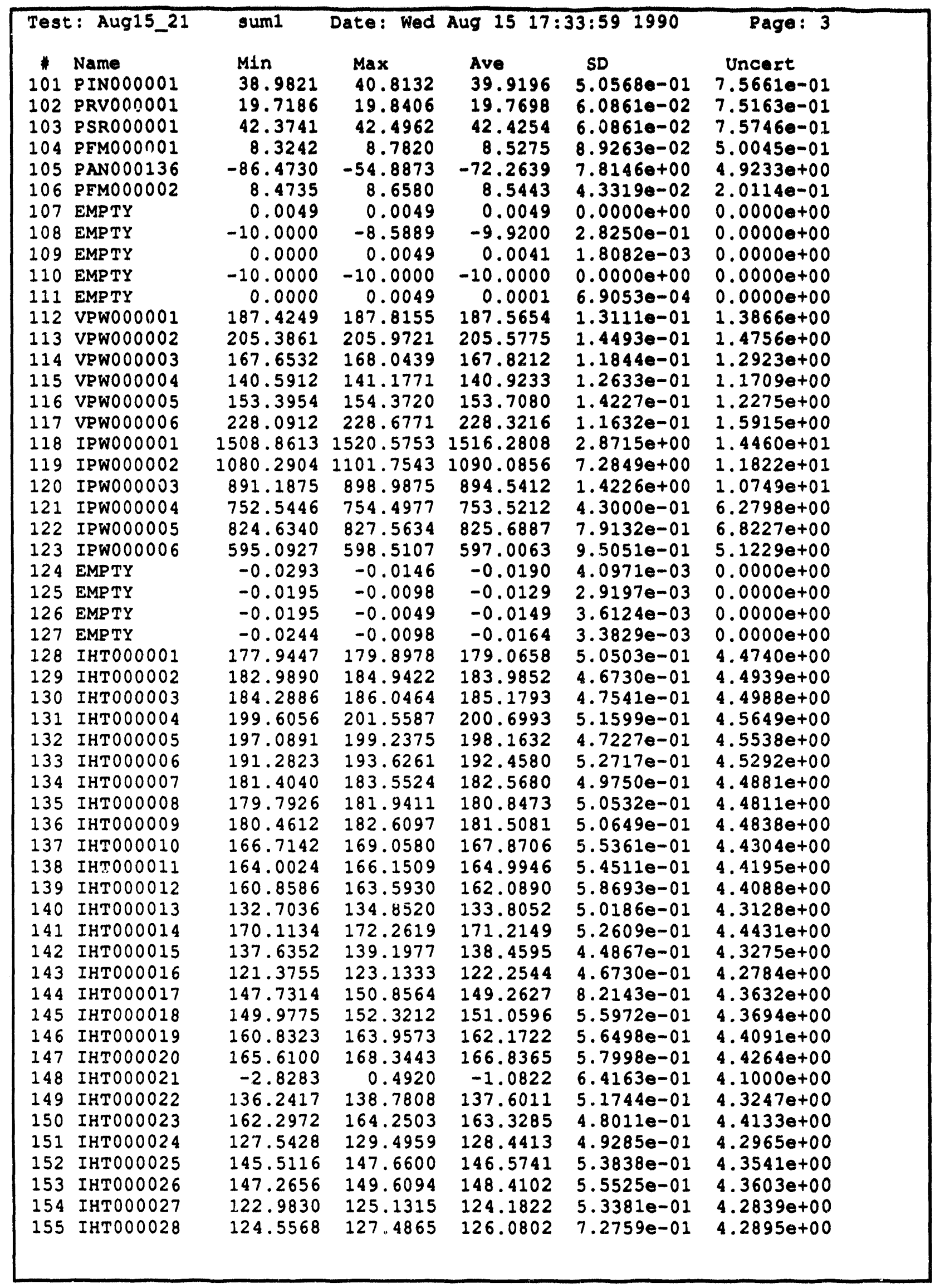




\begin{tabular}{|c|c|c|c|c|c|c|}
\hline$T \in S$ & $t:$ Aug15_21 & sum1 & Date: Wed & 49151 & $: 591990$ & Page: 4 \\
\hline $\begin{array}{l}1 \\
156 \\
157 \\
158 \\
159 \\
160 \\
161 \\
162 \\
163\end{array}$ & 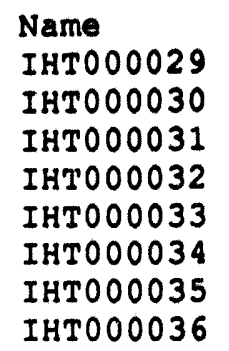 & $\begin{array}{c}\text { Min } \\
146.2177 \\
149.1737 \\
133.4360 \\
135.2464 \\
135.0361 \\
124.5756 \\
164.6447 \\
123.0807\end{array}$ & $\begin{array}{l}\text { Max } \\
149.3427 \\
152.1033 \\
136.7563 \\
138.3714 \\
138.3564 \\
126.7240 \\
166.9884 \\
125.2291\end{array}$ & $\begin{array}{l}\text { Ave } \\
147.7568 \\
150.5058 \\
134.7524 \\
136.5941 \\
136.5439 \\
125.8217 \\
165.7501 \\
124.1236\end{array}$ & $\begin{array}{l}\text { SD } \\
7.1315 e-01 \\
6.9776 e-01 \\
7.1552 e-01 \\
7.2464 \theta-01 \\
7.3296 e-01 \\
5.6894 e-01 \\
5.8342 e-01 \\
4.8103 e-01\end{array}$ & $\begin{array}{c}\text { Uncert } \\
4.3581 e+00 \\
4.3675 e+00 \\
4.3158 e+00 \\
4.3215 e+00 \\
4.3214 e+00 \\
4.2887 e+00 \\
4.4224 e+00 \\
4.2838 e+00\end{array}$ \\
\hline
\end{tabular}




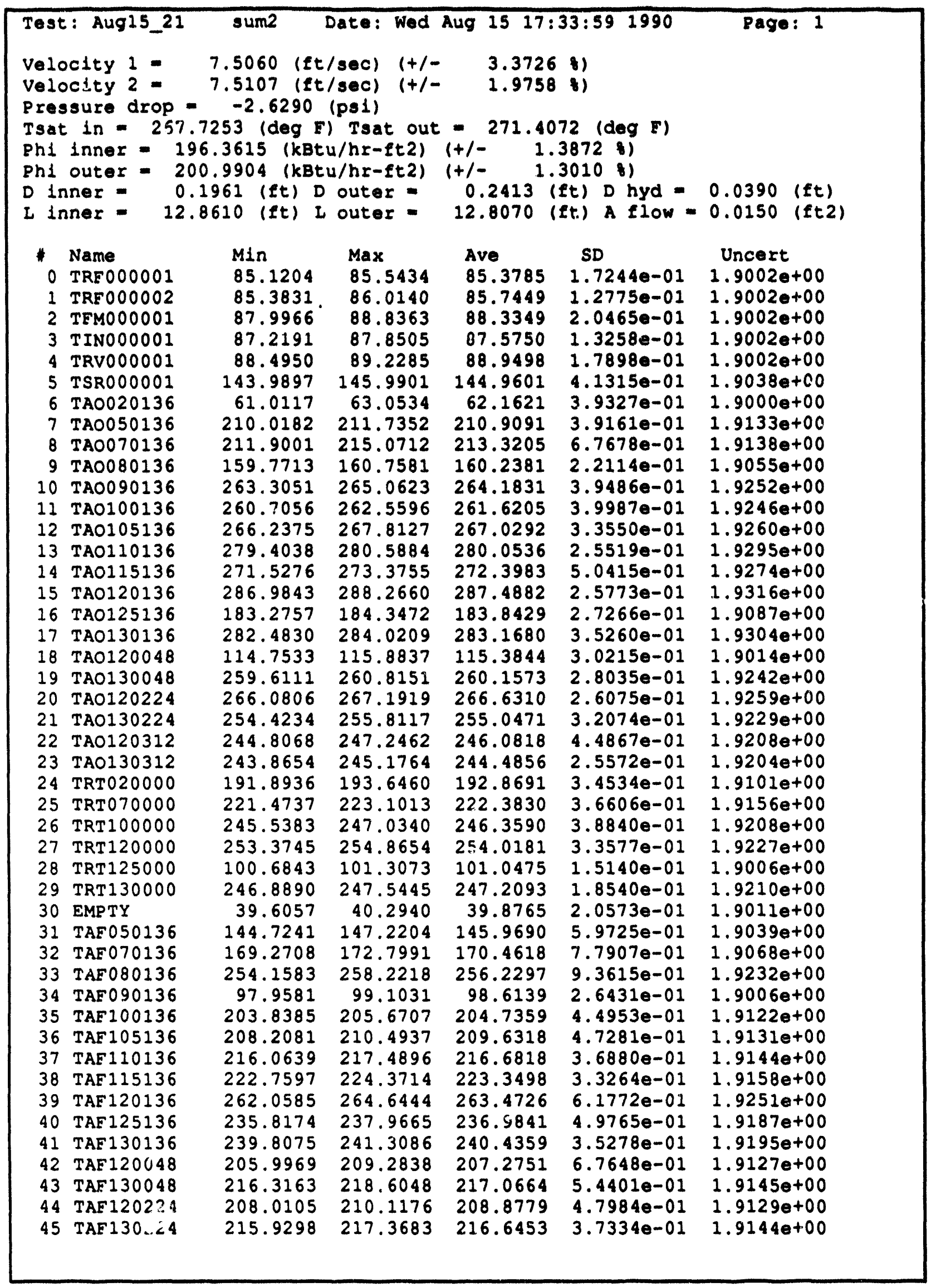




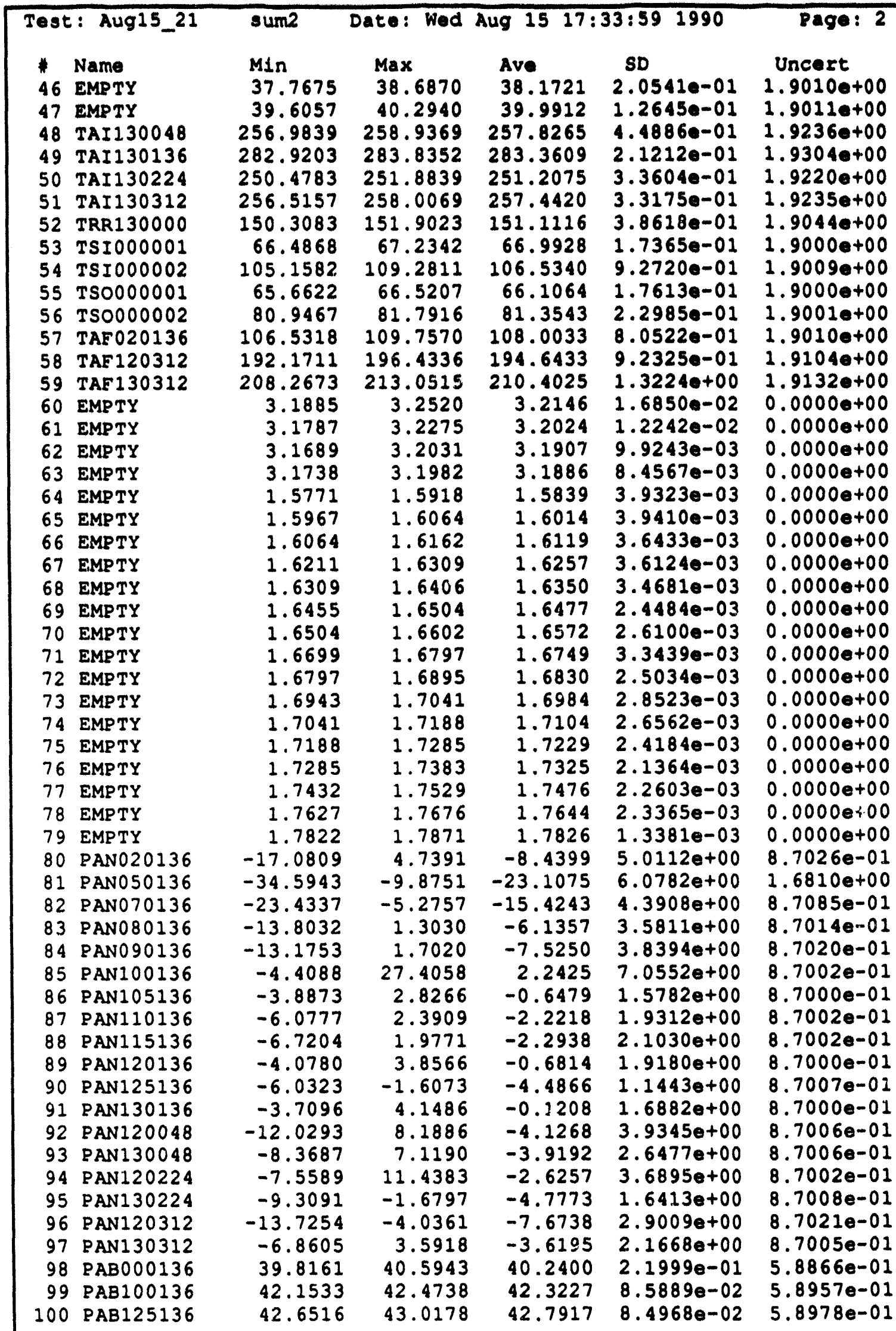



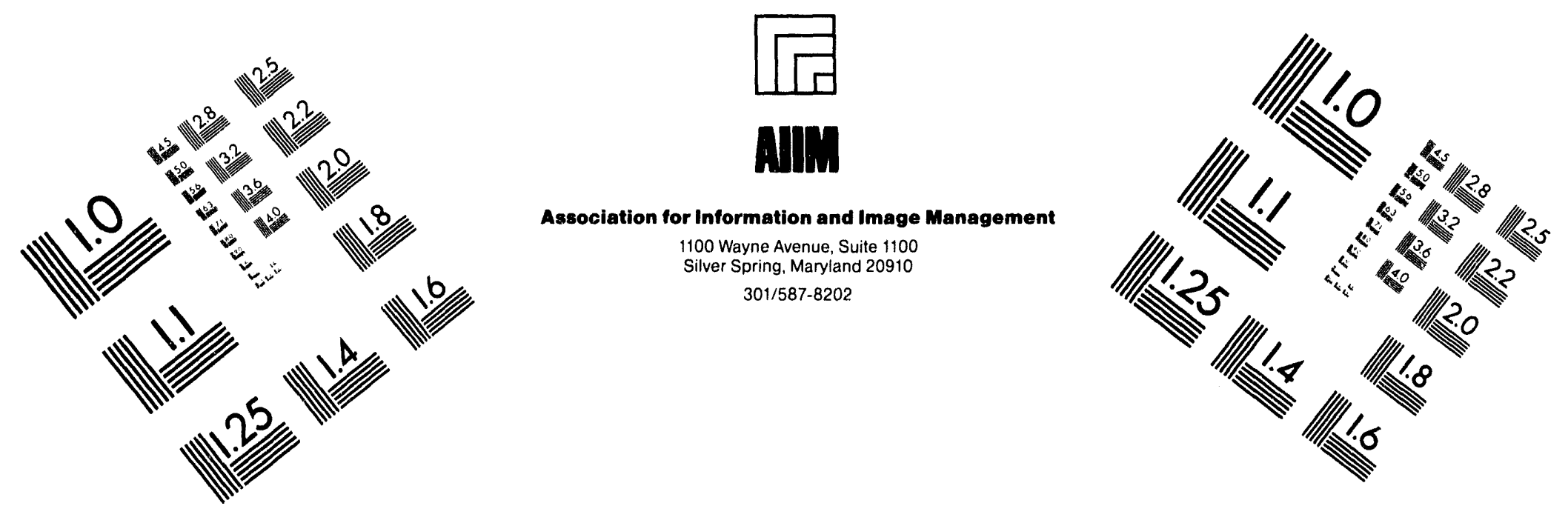

\section{Centimeter}

$\begin{array}{llllllllllllllll}1 & 2 & 3 & 4 & 5 & 6 & 7 & 8 & 9 & 10 & 11 & 12 & 13 & 14 & 15 & \mathrm{~mm}\end{array}$

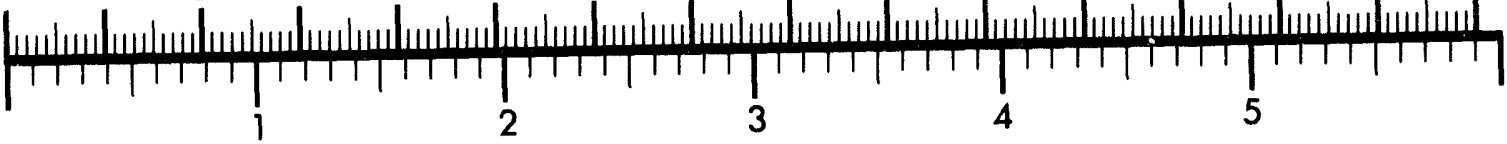
Inches
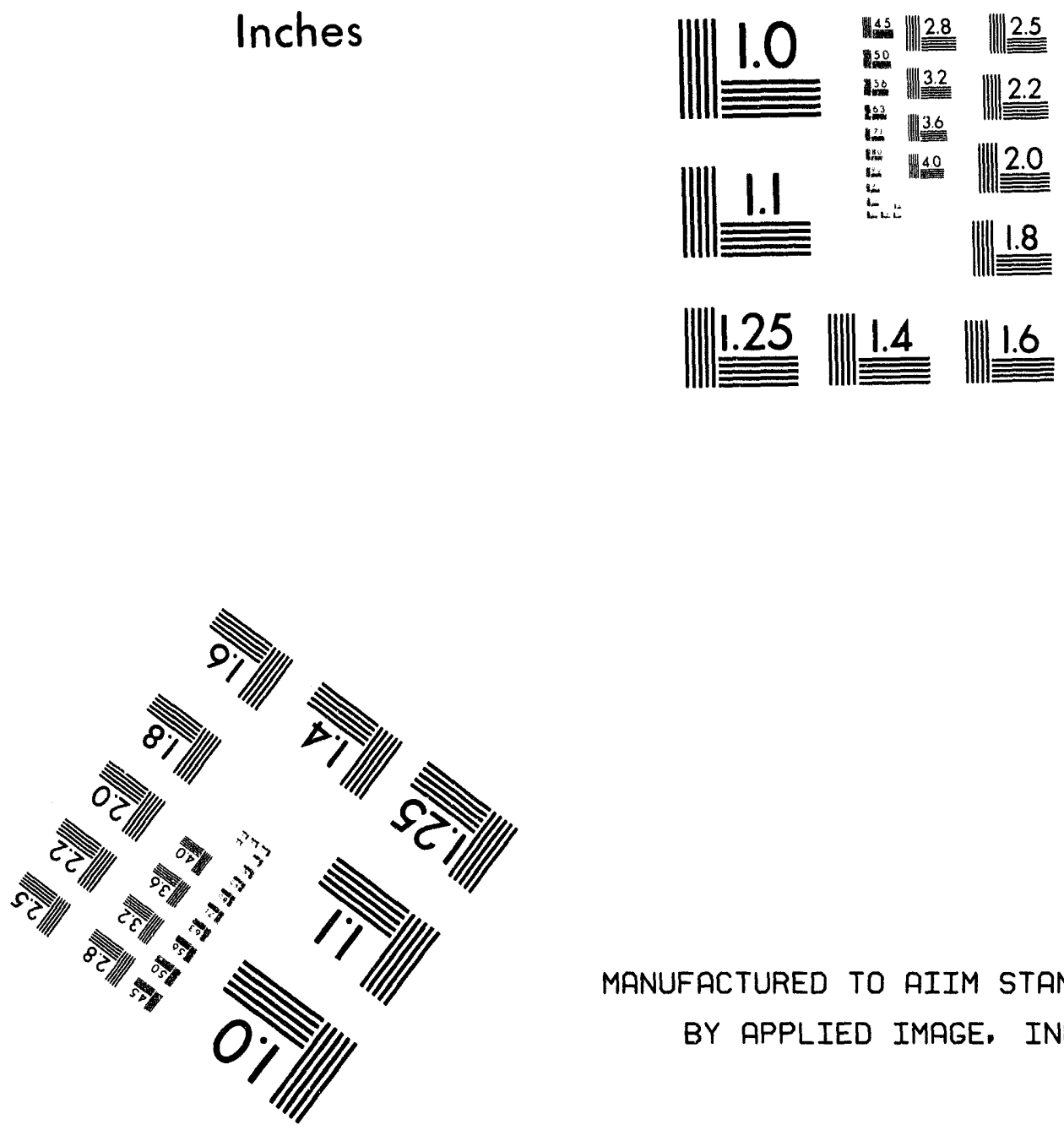

MANUFACTURED TO AIIM STANDARDS

BY APPLIED IMAGE, INC.

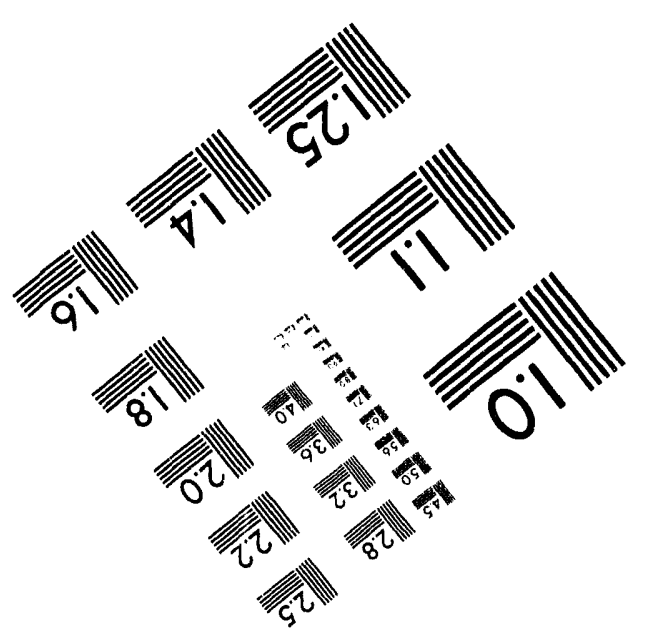




$$
\begin{aligned}
& 0 \\
& \stackrel{0}{0} \\
& \vec{N}
\end{aligned}
$$




\begin{tabular}{|c|c|c|c|c|c|c|}
\hline \multicolumn{2}{|c|}{ Test: Aug15_21 } & sum2 & \multicolumn{3}{|c|}{ Date: Wed Aug 15 17:33:59 1990} & \multirow[t]{2}{*}{ Page: 3} \\
\hline \# & Name & Min & Max & Ave & SD & \\
\hline 101 & PIN000001 & 39.2263 & 40.6911 & 40.0832 & $4.3874 e-01$ & $7.5666 \mathrm{e}-01$ \\
\hline 102 & PRV000001 & 19.7186 & 19.8406 & 19.7772 & $6.1605 e-02$ & $7.5163 e-01$ \\
\hline 103 & PSR000001 & 42.4962 & 42.6183 & 42.5646 & $6.1209 e-02$ & $7.5751 \mathrm{e}-01$ \\
\hline 104 & PFM000001 & 8.3242 & 8.7820 & 8.5348 & $8.5264 e-02$ & $5.0046 e-01$ \\
\hline 105 & PAN 000136 & -84.6419 & -59.0071 & -72.7858 & $7.3133 e+00$ & $4.9234 e+00$ \\
\hline 106 & PFM0000002 & 8.4399 & 8.6748 & 8.5456 & $4.4233 e-02$ & $2.0114 e-01$ \\
\hline 107 & EMPTY & -0.0049 & 0.0049 & 0.0042 & $2.2080 e-03$ & $0.0000 e+00$ \\
\hline 108 & EMPTY & -10.0000 & -4.8096 & -9.7228 & $9.9259 e-01$ & $0.0000 e+00$ \\
\hline 109 & EMPTY & -0.0049 & 0.0049 & 0.0033 & $2.5034 e-03$ & $0.0000 \mathrm{e}+00$ \\
\hline 110 & EMPTY & -10.0000 & -10.0000 & -10.0000 & $0.0000 e+00$ & $0.0000 e+00$ \\
\hline 111 & EMPTY & 0.0000 & 0.0000 & 0.0000 & $0.0000 e+00$ & $0.0000 \mathrm{e}+00$ \\
\hline 112 & VPW000001 & 187.4249 & 187.8155 & 187.5889 & $1.0705 e-01$ & $1.3867 e+00$ \\
\hline 113 & VPW0 00002 & 205.3861 & 205.7768 & 205.5775 & $1.1497 e-01$ & $1.4756 \mathrm{e}+00$ \\
\hline 114 & VPW000003 & 167.4579 & 168.6298 & 167.8017 & $1.7451 \mathrm{e}-01$ & $1.2922 e+00$ \\
\hline 115 & VPW000004 & 140.7865 & 141.3724 & 140.9272 & $1.3111 \mathrm{e}-01$ & $1.1709 e+00$ \\
\hline 116 & VPWO00005 & 153.5907 & 153.9814 & 153.7627 & $9.3710 e-02$ & $3.2277 e+00$ \\
\hline 117 & VPW000006 & 228.0912 & 228.6771 & 228.3216 & $1.2283 e-01$ & $1.5915 e+00$ \\
\hline 118 & IPW000001 & 1508.8613 & 1512.7660 & 1511.0474 & $1.3457 \mathrm{e}+00$ & $1.4426 \mathrm{e}+01$ \\
\hline 119 & IPW000002 & 1093.9492 & 1099.8031 & 1096.9934 & $1.3747 e+00$ & $1.1862 \mathrm{e}+01$ \\
\hline 120 & IPW000003 & 897.0375 & 900.9375 & 898.4808 & $1.2953 e+00$ & $1.0770 \mathrm{e}+01$ \\
\hline 121 & IPW000004 & 747.6618 & 749.6149 & 748.7458 & $4.1019 e-01$ & $6.2441 e+00$ \\
\hline 122 & IPWO00005 & 823.1693 & 824.6340 & 823.8430 & $3.1022 \mathrm{e}-01$ & $6.8087 e+00$ \\
\hline 123 & IPWO00006 & 595.0927 & 596.0693 & 595.6099 & $3.1828 e-01$ & $5.1127 e+00$ \\
\hline 124 & EMPTY & -0.0244 & -0.0146 & -0.0187 & $3.5141 \mathrm{e}-03$ & $0.0000 e+00$ \\
\hline .25 & EMPTY & -0.0195 & -0.0049 & -0.0133 & $3.2777 e-03$ & $0.0000 e+00$ \\
\hline 126 & EMPTY & -0.0195 & -0.0098 & -0.0149 & $3.3322 e-03$ & $0.0000 e+00$ \\
\hline 127 & EMPTY & -0.0244 & -0.0098 & -0.0173 & $3.1521 e-03$ & $0.0000 e+00$ \\
\hline 128 & IHTO00001 & 177.9447 & 180.4838 & 179.1518 & $6.2849 e-01$ & $4.4743 e+00$ \\
\hline 129 & IHTO00002 & 182.9890 & 185.5281 & 184.0985 & $5.7105 e-01$ & $4.4944 \mathrm{e}+00$ \\
\hline 130 & IHTO00003 & 184.0933 & 186.4370 & 185.2300 & $6.2227 e-01$ & $4.4990 e+00$ \\
\hline 131 & IHTOOO0004 & 199.6056 & 202.5353 & 200.7930 & $6.5773 e-01$ & $4.5653 e+00$ \\
\hline 132 & IHTO00005 & 197.0891 & 199.8235 & 198.2921 & $6.5359 e-01$ & $4.5543 e+00$ \\
\hline 133 & IHTO00006 & 191.4776 & 194.2120 & 192.5596 & $5.9479 e-01$ & $4.5297 e+00$ \\
\hline 134 & IHTO000007 & 181.4040 & 184.1384 & 182.5797 & $5.6150 e-01$ & $4.4882 e+00$ \\
\hline 135 & IHTO00008 & 179.7926 & 182.5270 & 180.8473 & $5.3815 e-01$ & $4.4811 e+00$ \\
\hline 136 & IHT000009 & 180.4612 & 183.3909 & 181.5980 & $5.5621 e-01$ & $4.4842 e+00$ \\
\hline 137 & IHTO00010 & 166.7142 & 169.4486 & 168.0151 & $5.7536 e-01$ & $4.4309 \mathrm{e}+00$ \\
\hline 138 & IHTO00011 & 164.0024 & 166.5415 & $165 \quad 0766$ & $5.5978 e-01$ & $4.4198 \mathrm{e}+00$ \\
\hline 139 & IHTO00012 & 161.0540 & 163.3977 & 162.1984 & $5.4962 e-01$ & $4.4092 e+00$ \\
\hline 140 & IHT000013 & 132.7036 & 134.8520 & 133.8677 & $5.2342 e-01$ & $4.3130 e+00$ \\
\hline 141 & IHTO00014 & 170.1134 & 172.4572 & 171.2813 & $5.2123 e-01$ & $4.4434 e+00$ \\
\hline 142 & IHTO00015 & 137.2446 & 139.7837 & 138.4868 & $5.4069 e-01$ & $4.3276 e+00$ \\
\hline 143 & IHT000016 & 120.9848 & 123.5239 & 122.2427 & $5.4983 e-01$ & $4.2784 e+00$ \\
\hline 144 & IHTO00017 & 147.5361 & 151.0517 & 149.2275 & $7.1639 e-01$ & $4.3631 e+00$ \\
\hline 145 & IHT000018 & 149.9775 & 152.5165 & 151.0439 & $6.1156 e-01$ & $4.3694 \mathrm{e}+00$ \\
\hline 146 & IHT000019 & 161.2230 & 163.7620 & 162.2621 & $6.1693 e-01$ & $4.4094 e+00$ \\
\hline 147 & IHTO00020 & 165.6100 & 168.1490 & 166.8326 & $5.9295 e-01$ & $4.4264 \mathrm{e}+00$ \\
\hline 148 & IHT000021 & -2.6330 & 0.1014 & -1.2228 & $6.7807 e-01$ & $4.1000 e+00$ \\
\hline 149 & IHT000022 & 136.4371 & 138.5855 & 137.5777 & $5.3867 e-01$ & $4.3247 e+00$ \\
\hline 150 & IHT000023 & 162.1019 & 164.4456 & 163.2582 & $5.5921 e-01$ & $4.4131 e+00$ \\
\hline 151 & IHTO00024 & 127.3475 & 129.6913 & 128.4178 & $5.4568 e-01$ & $4.2964 e+00$ \\
\hline 152 & IHT000025 & 145.3163 & 147.6600 & 146.4843 & $5.7790 e-01$ & $4.3538 \mathrm{e}+00$ \\
\hline 153 & IHT000026 & 147.2656 & 149.6094 & 148.3555 & $5.5955 e-01$ & $4.3602 e+00$ \\
\hline 154 & IHTO00027 & 123.3737 & 125.5221 & 124.2290 & $5.5086 e-01$ & $4.2841 \mathrm{e}+00$ \\
\hline 155 & IHT000028 & 124.7521 & 127.6818 & 126.0451 & $7.1233 e-01$ & $4.2894 \mathrm{e}+00$ \\
\hline
\end{tabular}




\begin{tabular}{|c|c|c|c|c|c|}
\hline Test: Aug15_21 & sum2 & Date: Wed & Aug 1517 & $33: 591990$ & Page: 4 \\
\hline Name & Min & $\operatorname{Max}$ & Ave & SD & Uncext \\
\hline 156 IHTO00029 & 146.4130 & 149.5380 & 147.7763 & $6.7944 e-01$ & $4.3582 e+00$ \\
\hline 157 IHTO000 30 & 149.3690 & 152.2987 & 150.5136 & $0.4839 e-01$ & $4.3675 e+00$ \\
\hline 158 IHT000031 & 133.4360 & 135.9751 & 134.7798 & $6.0443 e-01$ & $4.3158 e+00$ \\
\hline 159 IHTO00032 & 135.4417 & 138.1761 & 136.5902 & $6.1970 \mathrm{e}-01$ & $4.3215 e+00$ \\
\hline 160 IHT000033 & 135.2314 & 138.1611 & 136.5595 & $6.1511 e-01$ & $4.3214 e+00$ \\
\hline 161 IHTD00034 & 124.9662 & 127.1146 & 125.7358 & $5.0749 e-01$ & $4.2885 e+00$ \\
\hline 162 IHT 000035 & 164.8400 & 166.7931 & 165.7579 & $5.2977 e-01$ & $4.4224 e+00$ \\
\hline 163 IHT000036 & 123.0807 & 126.0104 & 124.1822 & $5.4926 e-01$ & $4.2839 e+00$ \\
\hline
\end{tabular}




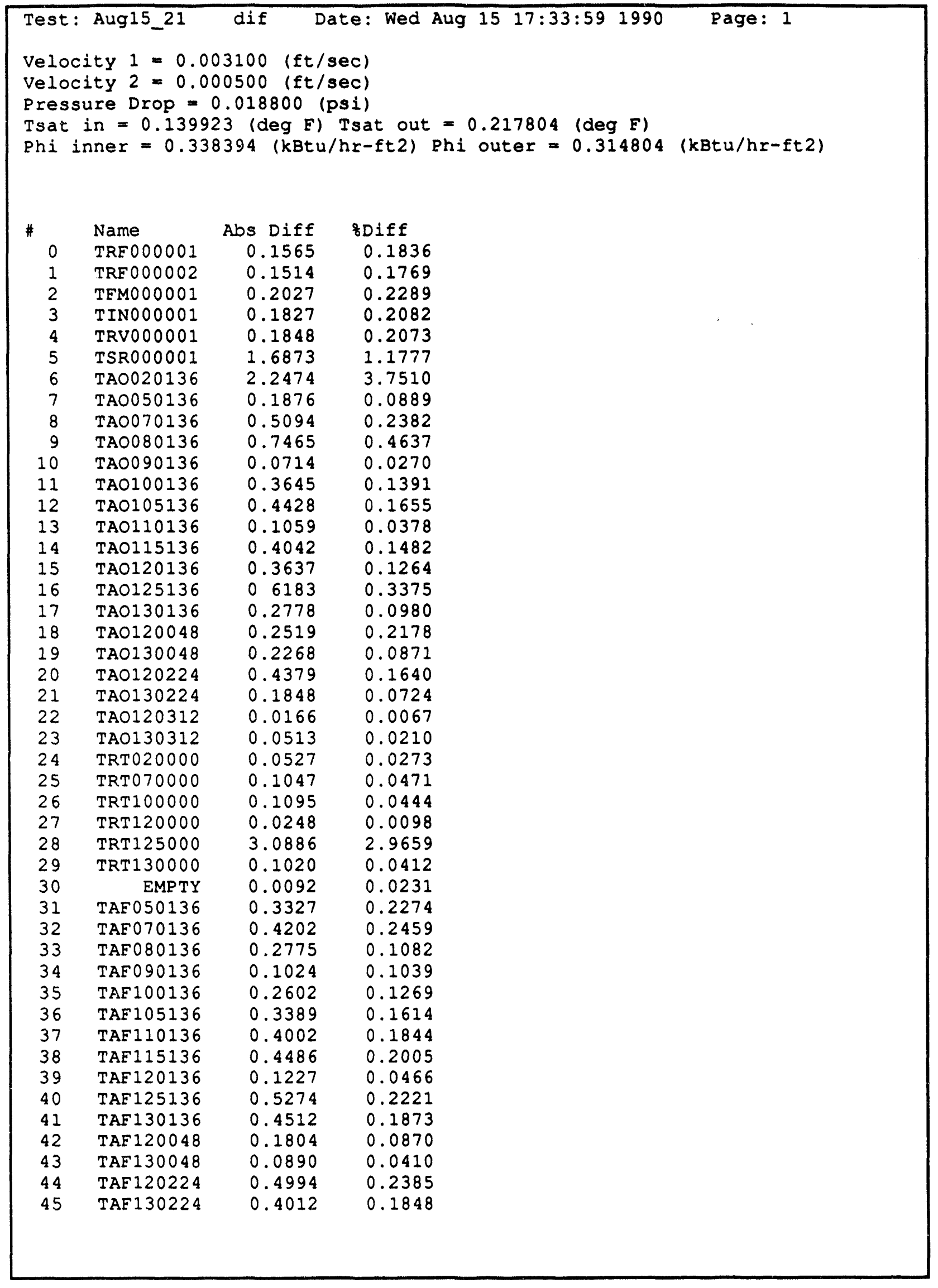




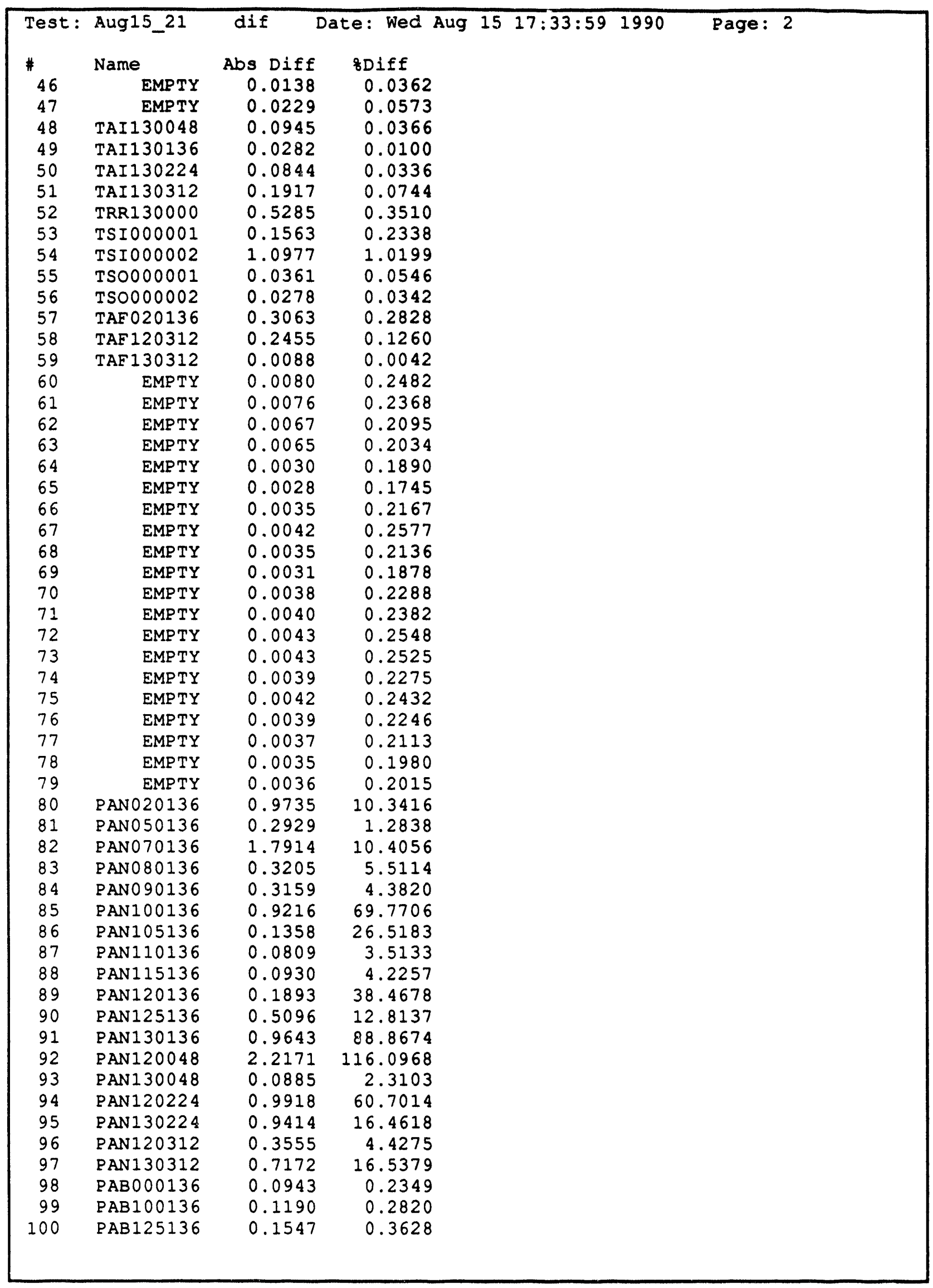




\begin{tabular}{|c|c|c|c|c|}
\hline Test: & Aug15_21. & dif & Date: Wed Aug 15 17:33:59 1990 & Page: 3 \\
\hline * & Name & Abs Diff & \&Diff & \\
\hline 101 & PIN000001 & 0.1636 & 0.4098 & \\
\hline 102 & PRV000001 & 0.0074 & 0.0374 & \\
\hline 103 & PSR000001 & 0.1392 & 0.3281 & \\
\hline 104 & PFM000001 & 0.0073 & 0.0856 & \\
\hline 105 & PANO00136 & 0.5219 & 0.7222 & \\
\hline 106 & PFMO00002 & 0.0013 & 0.0152 & \\
\hline 107 & EMPTY & 0.0007 & 14.2857 & \\
\hline 108 & EMPTY & 0.1972 & 1.9879 & \\
\hline 109 & EMPTY & 0.0008 & 19.5122 & \\
\hline 110 & EMPTY & 0.0000 & 0.0000 & \\
\hline 111 & EMPTY & 0.0001 & 100.0000 & \\
\hline 112 & VPWO00001 & 0.0235 & 0.0125 & \\
\hline 113 & VPW0 00002 & 0.0000 & 0.0000 & \\
\hline 114 & VPW000003 & 0.0195 & 0.0116 & \\
\hline 115 & VPW000004 & 0.0039 & 0.0028 & \\
\hline 116 & VPW000005 & 0.0547 & 0.0356 & \\
\hline 117 & VPW000006 & 0.0000 & 0.0000 & \\
\hline 118 & IPW000001 & 5.2334 & 0.3451 & \\
\hline 119 & IPW0 00002 & 6.9078 & 0.6337 & \\
\hline 120 & IPW000003 & 3.9396 & 0.4404 & \\
\hline 121 & IPW000004 & 4.7754 & 0.6337 & \\
\hline 122 & IPW000005 & 1.8457 & 0.2235 & \\
\hline 123 & IPW000006 & 1.3964 & 0.2339 & \\
\hline 124 & EMPTY & 0.0003 & 1.5789 & \\
\hline 125 & EMPTY & 0.0004 & 3.1008 & \\
\hline 126 & EMPTY & 0.0000 & 0.0000 & \\
\hline 127 & EMPTY & 0.0009 & 5.4878 & \\
\hline 128 & IHTO00001 & 0.0860 & 0.0480 & \\
\hline 129 & IHTO00002 & 0.1133 & 0.0616 & \\
\hline 130 & IHT000003 & 0.0507 & 0.0274 & \\
\hline 131 & IHTO00004 & 0.0937 & 0.0467 & \\
\hline 132 & IHTO00005 & 0.1289 & 0.0651 & \\
\hline 133 & IHT000006 & 0.1016 & 0.0528 & \\
\hline 134 & IHT000007 & 0.0117 & 0.0064 & \\
\hline 135 & IHT 000008 & 0.0000 & 0.0000 & \\
\hline 136 & IHTO00009 & 0.0899 & 0.0495 & \\
\hline 137 & IHTO00010 & 0.1445 & 0.0861 & \\
\hline 138 & IHT000011 & 0.0820 & 0.0497 & \\
\hline 139 & IHTO 00012 & 0.1094 & 0.0675 & \\
\hline 140 & IHTO00013 & 0.0625 & 0.0467 & \\
\hline 141 & IHTO00014 & 0.0664 & 0.0388 & \\
\hline 142 & IHTO00015 & 0.0273 & 0.0197 & \\
\hline 143 & IHT000016 & 0.0117 & 0.0096 & \\
\hline 144 & IHTO00017 & 0.0352 & 0.0236 & \\
\hline 145 & IHTO00018 & 0.0157 & 0.0104 & \\
\hline 146 & IHTO00019 & 0.0899 & 0.0554 & \\
\hline 147 & IHT000020 & 0.0039 & 0.0023 & \\
\hline 148 & IHTO00021 & 0.1406 & 12.9921 & \\
\hline 149 & IHTO00022 & 0.0234 & 0.0170 & \\
\hline 150 & IHTO00023 & 0.0703 & 0.0430 & \\
\hline 151 & IHTO00024 & 0.0235 & 0.0183 & \\
\hline 152 & IHTO00025 & 0.0898 & 0.0613 & \\
\hline 153 & IHTO00026 & 0.0547 & 0.0369 & \\
\hline 154 & IHTO00027 & 0.0468 & 0.0377 & \\
\hline 155 & IHT000028 & 0.0351 & 0.0278 & \\
\hline
\end{tabular}


Test: Aug15_21 dif Date: Wed Aug 15 17:33:59 1990 Page: 4

* Name Abs Diff \&Diff

156 IHTO00029 $0.0195 \quad 0.0132$

157 IHTOOOO30 $0.0078 \quad 0.0052$

158 IHTO00031 $0.0274 \quad 0.0203$

159 IHTO00032 $0.0039 \quad 0.0029$

160 IHTO00033 $0.0156 \quad 0.0114$

161 IHTOOOO34 $0.0859 \quad 0.0683$

$\begin{array}{llll}162 & \text { IHTOO0035 } & 0.0078 & 0.0047\end{array}$

$\begin{array}{llll}163 & \text { IHTO00036 } & 0.0586 & 0.0472\end{array}$ 


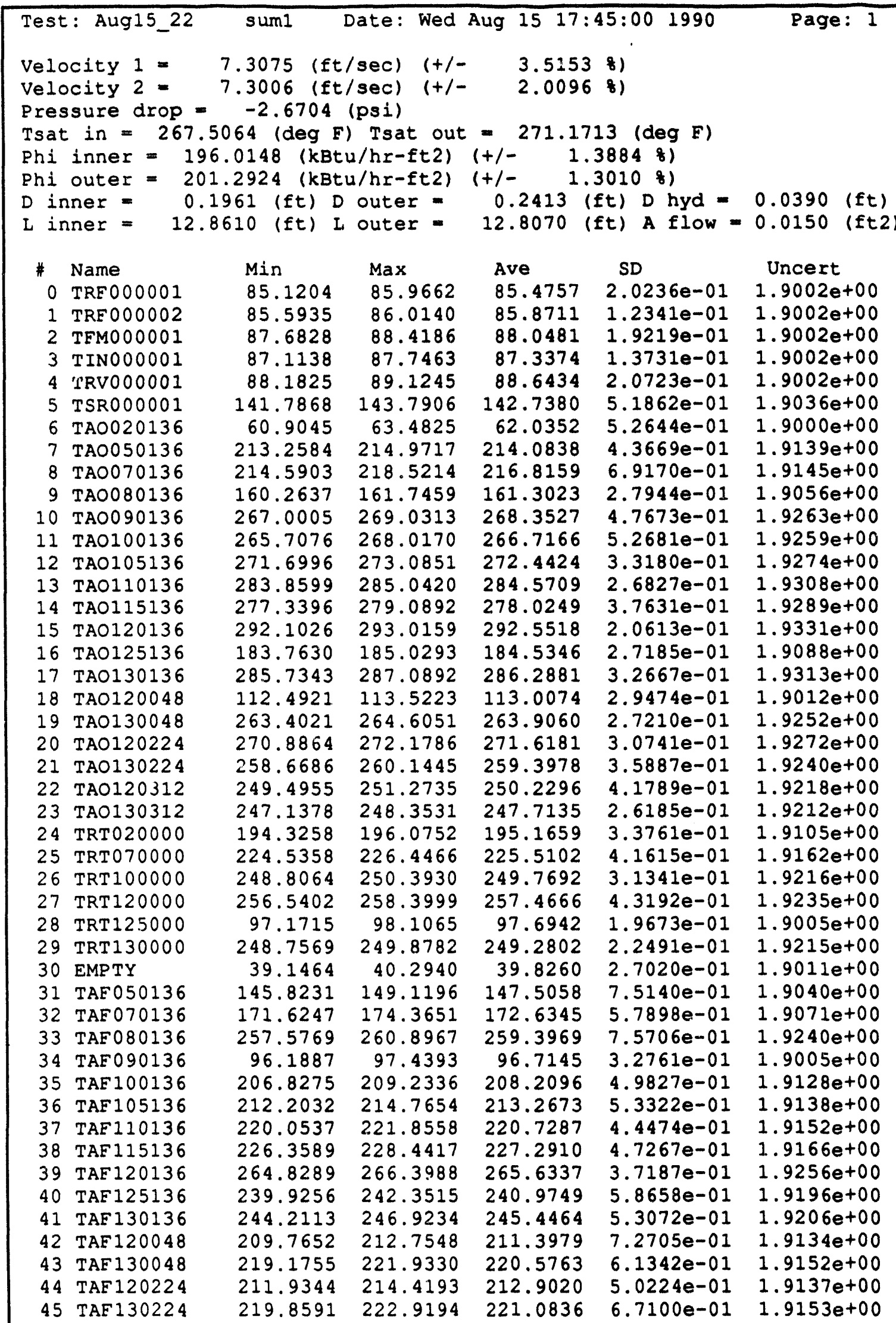




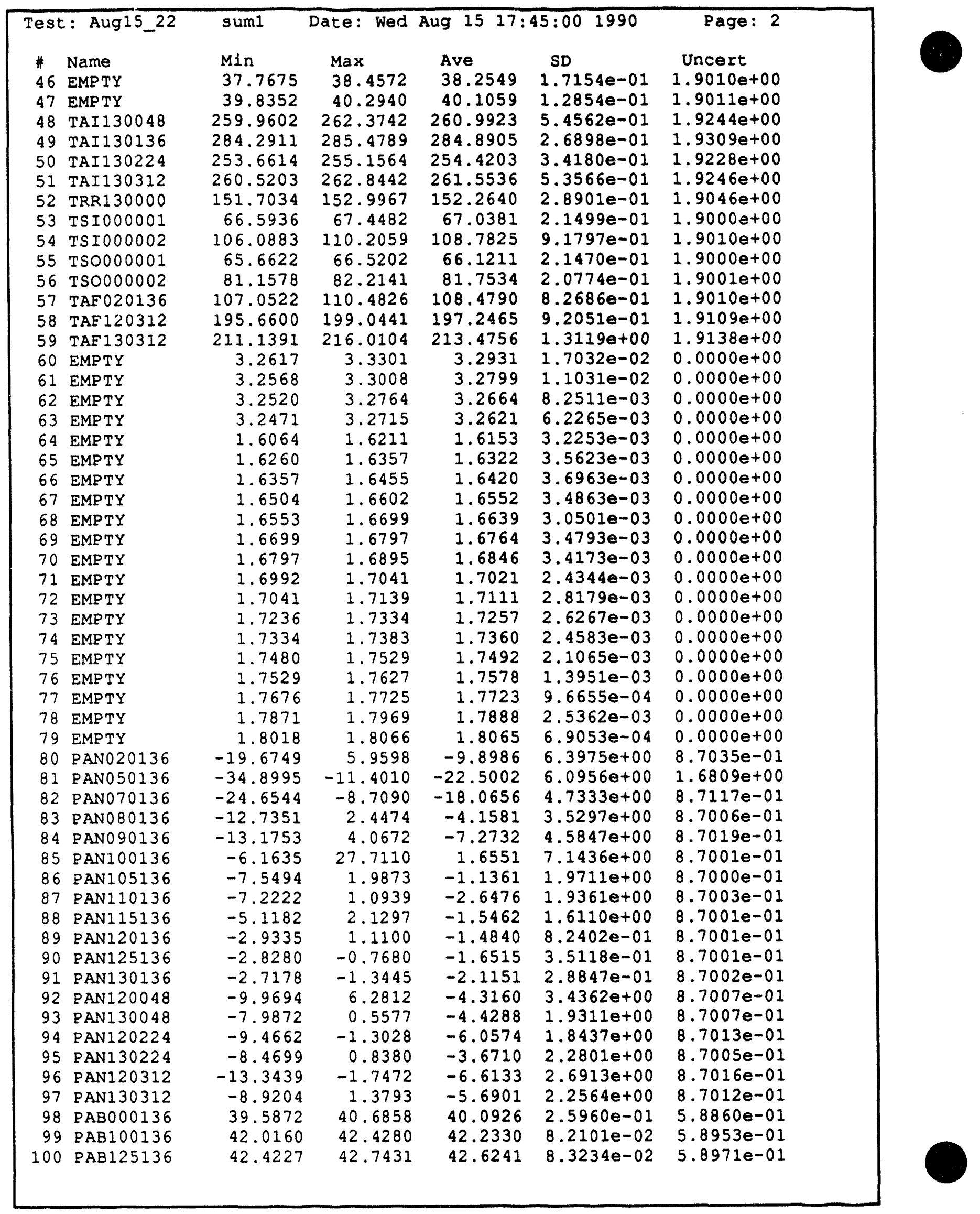




\begin{tabular}{|c|c|c|c|c|c|c|}
\hline Test & $:$ Aug15_22 & sum1 & Date: Wed & $\begin{array}{lll}\text { Aug } & 15 \quad 17\end{array}$ & $5: 001990$ & Page: 3 \\
\hline \# & Name & Min & $\operatorname{Max}$ & Ave & SD & Uncert \\
\hline 101 & PIN000001 & 38.9821 & 40.6911 & 39.8928 & $4.8903 e-01$ & $7.5660 e-01$ \\
\hline 102 & PRV000001 & 19.7186 & 19.8406 & 19.8358 & $2.4164 e-02$ & $7.5164 e-01$ \\
\hline 103 & PSR000001 & 42.3741 & 42.7404 & 42.5255 & $8.7402 e-02$ & $7.5750 e-01$ \\
\hline 104 & PFMO00001 & 7.8665 & 8.2327 & 8.0844 & $9.2279 e-02$ & $5.0041 e-01$ \\
\hline 105 & PANO00136 & -86.9307 & -59.4649 & -73.9302 & $7.1229 e+00$ & $4.9235 e+00$ \\
\hline 106 & PFM000002 & 7.9701 & 8.1547 & 8.0687 & $4.0771 e-02$ & $2.0101 e-01$ \\
\hline 107 & EMPTY & -0.0049 & 0.0049 & 0.0041 & $2.2839 e-03$ & $0.0000 e+00$ \\
\hline 108 & EMPTY & -10.0000 & -8.9209 & -9.9784 & $1.5261 e-01$ & $0.0000 \mathrm{e}+00$ \\
\hline 109 & EMPTY & 0.0000 & 0.0098 & 0.0036 & $2.3778 e-03$ & $0.0000 e+00$ \\
\hline 110 & EMPTY & -10.0000 & -10.0000 & -10.0000 & $0.0000 e+00$ & $0.0000 e+00$ \\
\hline 111 & EMPTY & -0.0049 & 0.0000 & -0.0001 & $6.9053 e-04$ & $0.0000 e+00$ \\
\hline 112 & VPW000001 & 187.2296 & 187.8155 & 187.5264 & $1.2627 e-01$ & $1.3864 \mathrm{e}+00$ \\
\hline 113 & VPW000002 & 205.3861 & 205.7768 & 205.5658 & $1.4139 e-01$ & $1.4756 \mathrm{e}+00$ \\
\hline 114 & VPW000003 & 167.4579 & 268.0439 & 167.7666 & $1.3141 e-01$ & $1.2920 \mathrm{e}+00$ \\
\hline 115 & VPWO000004 & 140.7865 & 141.1771 & 140.9585 & $1.2251 e-01$ & $1.1711 \mathrm{e}+00$ \\
\hline 116 & VPW000005 & 153.5907 & 153.9814 & 153.7627 & $1.0168 \mathrm{e}-01$ & $1.2277 e+00$ \\
\hline 117 & VPWO00006 & 228.0912 & 228.4818 & 228.3177 & $1.1409 \mathrm{e}-01$ & $1.5915 \mathrm{e}+00$ \\
\hline 118 & IPW000001 & 1518.6230 & 1522.5277 & 1520.0680 & $1.1023 e+00$ & $1.4485 \mathrm{e}+01$ \\
\hline 119 & IPW0 00002 & 1088.0955 & 1093.9492 & 1090.3977 & $1.5109 e+00$ & $1.1824 e+01$ \\
\hline 120 & IPW000003 & 889.2375 & 895.0875 & 893.0592 & $1.5238 e+00$ & $1.0742 e+01$ \\
\hline 121 & IPWO000004 & 752.5446 & 754.4977 & 753.3747 & $3.8523 e-01$ & $6.2787 e+00$ \\
\hline 122 & IPW000005 & 823.1693 & 825.1222 & 824.2336 & $3.9072 e-01$ & $6.8117 e+00$ \\
\hline 123 & IPW000006 & 594.6044 & 597.0458 & 595.7563 & $5.2903 e-01$ & $5.1138 e+00$ \\
\hline 124 & EMPTY & -0.0293 & -0.0098 & -0.0192 & $4.1161 \mathrm{e}-03$ & $0.0000 e+00$ \\
\hline 125 & EMPTY & -0.0195 & -0.0098 & -0.0136 & $3.0067 e-03$ & $0.0000 \mathrm{e}+00$ \\
\hline 126 & EMPTY & -0.0195 & -0.0098 & -0.0147 & $3.1950 e-03$ & $0.0000 e+00$ \\
\hline 127 & EMPTY & -0.0244 & -0.0098 & -0.0166 & $3.4173 e-03$ & $0.0000 e+00$ \\
\hline 128 & IHTO00001 & 177.9447 & 180.0931 & 179.0776 & $5.6221 e-01$ & $4.4740 \mathrm{e}+00$ \\
\hline 129 & IHTO00002 & 182.9890 & 184.9422 & 183.9774 & $5.4163 e-01$ & $4.4939 e+00$ \\
\hline 130 & IHT000003 & 183.8980 & 186.2417 & 185.1011 & $5.6132 e-01$ & $4.4985 e+00$ \\
\hline 131 & IHT000004 & 199.6056 & 201.7540 & 200.6876 & $5.4990 e-01$ & $4.5648 \mathrm{e}+00$ \\
\hline 132 & IHT000005 & 196.8938 & 199.2375 & 198.1866 & $5.5508 e-01$ & $4.5539 \mathrm{e}+00$ \\
\hline 133 & IHT000006 & 191.2823 & 193.4307 & 192.5166 & $5.1816 e-01$ & $4.5295 e+00$ \\
\hline 134 & IHT000007 & 181.2087 & 183.5524 & 182.5915 & $5.3502 e-01$ & $4.4882 e+00$ \\
\hline 135 & IHT000008 & 179.5973 & 181.9411 & 180.9137 & $5.4649 e-01$ & $4.4814 e+00$ \\
\hline 136 & IHT000009 & 180.4612 & 182.6097 & 181.6097 & $5.1394 e-01$ & $4.4842 e+00$ \\
\hline 137 & IHT000010 & 166.7142 & 169.0580 & 167.7807 & $5.3118 e-01$ & $4.4300 e+00$ \\
\hline 138 & IHT000011 & 163.8071 & 166.1509 & 164.9165 & $5.2710 e-01$ & $4.4192 e+00$ \\
\hline 139 & IHT000012 & 160.8586 & 163.3977 & 162.1007 & $5.4069 e-01$ & $4.4088 e+00$ \\
\hline 140 & IHT000013 & 132.7036 & 135.0473 & 133.8286 & $5.0310 e-01$ & $4.3129 e+00$ \\
\hline 141 & IHTO00014 & 169.9181 & 172.4572 & 171.2032 & $5.3392 e-01$ & $4.4431 e+00$ \\
\hline 142 & IHTO00015 & 137.2446 & 139.5883 & 138.5298 & $5.3828 e-01$ & $4.3277 e+00$ \\
\hline 143 & IHT000016 & 121.1801 & 123.3286 & 122.3403 & $5.0749 e-01$ & $4.2786 e+00$ \\
\hline 144 & IHTO00017 & 147.1454 & 151.0517 & 149.2080 & $6.8591 e-01$ & $4.3631 e+00$ \\
\hline 145 & IHT000018 & 149.3915 & 152.5165 & 151.1025 & $6.5621 e-01$ & $4.3696 e+00$ \\
\hline 146 & IHT000019 & 160.6370 & 163.1761 & 161.8168 & $6.2634 e-01$ & $4.4078 e+00$ \\
\hline 147 & IHTO00020 & 165.2193 & 167.5631 & 166.3951 & $6.0937 e-01$ & $4.4248 \mathrm{e}+00$ \\
\hline 148 & IHT000021 & -2.8283 & 0.2967 & -1.5119 & $6.9341 \mathrm{e}-01$ & $4.1000 e+00$ \\
\hline 149 & IHT000022 & 136.0464 & 138.3902 & 137.1480 & $5.2313 e-01$ & $4.3233 e+00$ \\
\hline 150 & IHT 000023 & 161.9066 & 164.0550 & 162.9301 & $5.5602 e-01$ & $4.4119 e+00$ \\
\hline 151 & IHTO00024 & 126.9569 & 129.3006 & 128.0819 & $5.4471 e-01$ & $4.2954 e+00$ \\
\hline 152 & IHT000025 & 145.1209 & 147.4647 & 146.3007 & $5.7040 e-01$ & $4.3532 e+00$ \\
\hline 153 & IHTO00026 & 147.0703 & 149.4141 & 148.1133 & $5.8873 e-01$ & $4.3593 e+00$ \\
\hline 154 & IHTO00027 & 122.9830 & 125.3268 & 124.0025 & $5.1037 e-01$ & $4.2834 \mathrm{e}+00$ \\
\hline 155 & IHT000028 & 124.7521 & 127.2912 & 126.0997 & $5.7352 e-01$ & $4.2895 e+00$ \\
\hline
\end{tabular}




\begin{tabular}{|c|c|c|c|c|c|c|}
\hline \multicolumn{2}{|c|}{ Test: Aug15_22 } & sum1 & Date: Wed & $\begin{array}{lll}\text { Aug } & 15 \quad 17\end{array}$ & $45: 00 \quad 1990$ & Page: 4 \\
\hline * & Name & Min & $\operatorname{Max}$ & Ave & SD & Uncert \\
\hline 156 & IHTO00029 & 146.6083 & 188.9521 & 147.7959 & $5.5501 e-01$ & $4.3583 e+00$ \\
\hline 157 & IHTO00030 & 149.1737 & 152.1033 & 150.537 & $5.8059 e-01$ & $4.3676 e+00$ \\
\hline 158 & IHT000031 & 133.4360 & 136.3657 & 134.8345 & $5.9759 e-01$ & 4. $3160 e+00$ \\
\hline 159 & IHTO00032 & 135.2464 & 137.9808 & 136.6410 & $6.2641 e-01$ & $4.3217 e+00$ \\
\hline 160 & IHTO000033 & 135.0361 & 137.9657 & 136.5908 & $6.3987 e-01$ & $4.3215 e+00$ \\
\hline 161 & IHTO000034 & 124.9662 & 126.9193 & 125.8647 & $5.0223 e-01$ & $4.2888 \mathrm{e}+00$ \\
\hline 162 & IHTO000035 & 164.8400 & 166.9884 & 165.7931 & $5.4909 e-01$ & $4.4225 e+00$ \\
\hline 163 & IHT000036 & 123.4713 & 125.6197 & 124.2915 & $5.0378 e-01$ & $4.2843 e+00$ \\
\hline
\end{tabular}




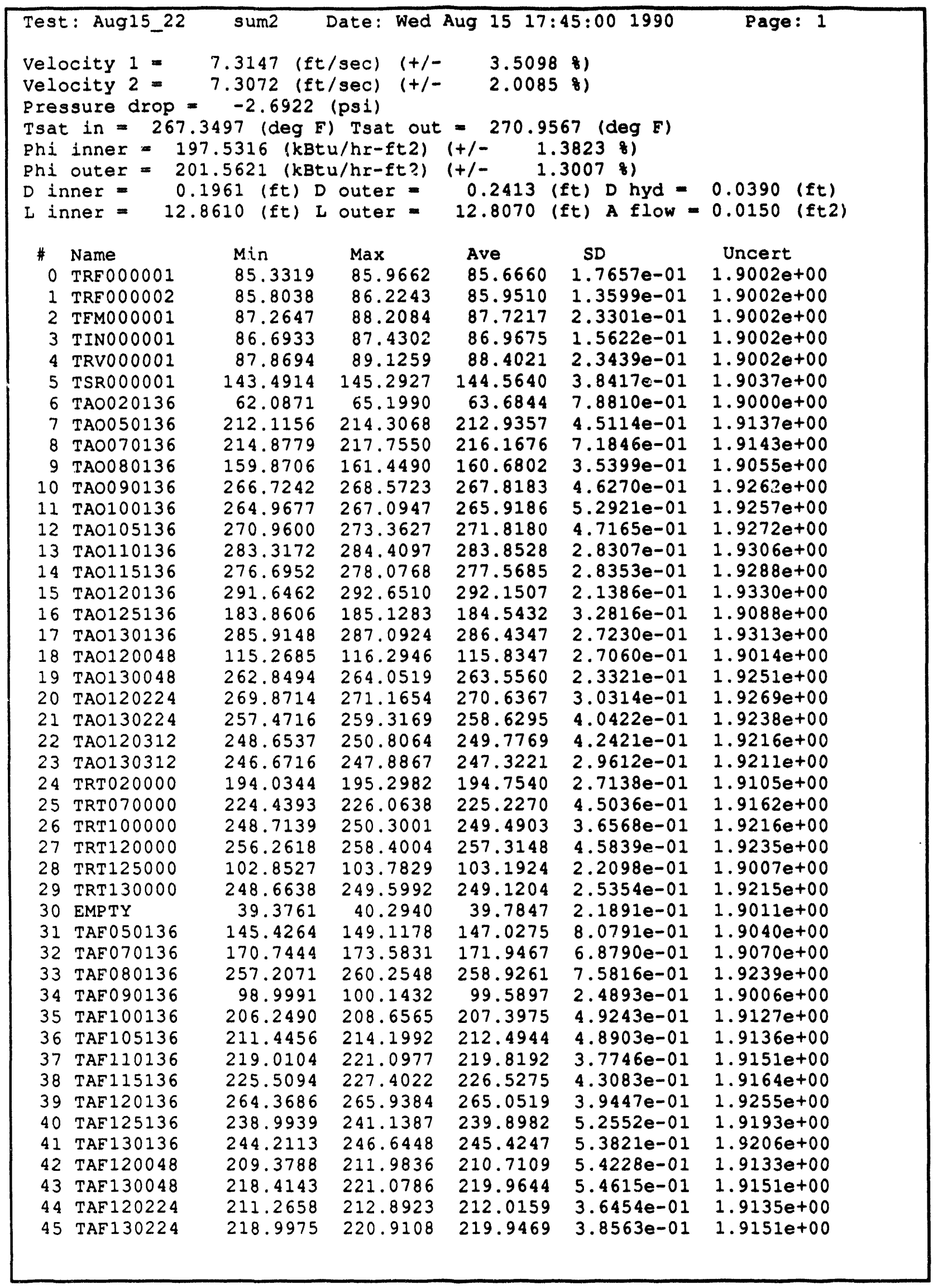




\begin{tabular}{|c|c|c|c|c|c|c|}
\hline & 2 & sum2 & ate: & log 151 & 00 & Page: \\
\hline \# & Name & Min & $\operatorname{Max}$ & Ave & SD & Uncert \\
\hline 46 & EMPTY & 37.7675 & 38.4572 & 38.1813 & $1.6747 e-01$ & $1.9010 \mathrm{e}+$ \\
\hline 47 & EMPTY & 39.8352 & 40.0646 & 39.9821 & $1.1126 \mathrm{e}$ & $1.9011 e+c$ \\
\hline 48 & TAI1 30048 & 259.3097 & 262.0031 & 260.7885 & 6.684 & 1.92 \\
\hline 49 & TAI 130136 & 284.1089 & 285.2058 & 284.8329 & $2.5496 e-01$ & $1.9308 e+00$ \\
\hline 50 & TAI130224 & 253.4743 & 254.9700 & 254.2485 & $3.4151 e-01$ & $1.9227 e+00$ \\
\hline 51 & TAI130312 & 260.2408 & 261.6363 & 260.8511 & $3.6104 e-01$ & $1.9244 \mathrm{e}+00$ \\
\hline 52 & TRR130000 & 151.3050 & 154.1895 & 152.6507 & $7.0089 e-01$ & $1.9046 e+0 c$ \\
\hline 53 & TSIO & 66.5947 & 4482 & 66.9879 & 1 & \\
\hline 54 & TSI000002 & 105.5716 & 110.5146 & 107.4938 & $1.1975 e+00$ & $1.9009 e+00$ \\
\hline 55 & TSO000001 & 65.6617 & 66.6276 & 66.1811 & $2.0724 e-01$ & $1.9000 e+00$ \\
\hline 56 & TSOOO & 81.1583 & 82.1091 & 81.6652 & 2.2273 & $1 e+00$ \\
\hline 57 & TAF020136 & 106.0108 & 110.1701 & 108.1291 & $9.7135 e-01$ & $1.9010 e+00$ \\
\hline 58 & TAE 1 & 195.2734 & 4304 & 196.8216 & 9.875 & $e+00$ \\
\hline 59 & TAF 130312 & 210.8533 & 16.2019 & 213.0751 & $007 e+00$ & $e+00$ \\
\hline 60 & EMPTY & ?.2520 & 3.3154 & 3.2809 & $1.7504 e-02$ & $0 e+00$ \\
\hline 61 & EMPTY & 3.2422 & 3.2910 & 3.2692 & 1.2718 & $e+00$ \\
\hline 62 & EMPTY & 3.2422 & 3.2715 & 3.2580 & 9.9 & $10 e+00$ \\
\hline 63 & EMPT & 3.2422 & 3.2666 & 3.2553 & 8.337 & 0. \\
\hline 64 & EMPTY & 1.6064 & 1.6211 & 1.6130 & $3.6393 e-03$ & be+00 \\
\hline 65 & EMPTY & 1.6260 & 1.6357 & 1.6309 & 3.4173 & e+oo \\
\hline 66 & EMPTY & 1.6357 & 1.6455 & 1.6409 & $3.3322 \epsilon$ & $e+00$ \\
\hline 67 & EMPTY & 1.6504 & 1.6602 & 1.6556 & 2.6853 & $0 e+00$ \\
\hline 68 & EMPTY & 1.6602 & 1.6699 & 1.6636 & 2.4662 & $0 e+00$ \\
\hline 69 & EMPTY & 1.6699 & 1.6797 & 1.6764 & 2.690 & $e+00$ \\
\hline 70 & EMPTY & 1.6797 & 1.6895 & 1.6860 & 2.466 & $e+00$ \\
\hline 71 & EMPT & 1.6992 & 1.70 & 1.7038 & 2.49 & $e+0$ \\
\hline 72 & EMPTY & 1.7090 & 1.7139 & 1.7120 & $2.3941 e-03$ & $e+00$ \\
\hline 73 & EMPTY & 1.7236 & 1.7285 & 1.7267 & 2.3941 & $e+00$ \\
\hline 74 & EMP T & 1.7334 & 1.7383 & 1.7373 & $1.9730 \mathrm{e}$ & 0 . \\
\hline 75 & EMPTY & 1.7480 & 1.7578 & 1.7507 & $2.6488 e-03$ & $e+00$ \\
\hline 76 & EMPT & 1.7 & & 1. & & $e+0 c$ \\
\hline 77 & EMPT & 1.77 & 1.78 & 1.7731 & 2.4 & +00 \\
\hline 78 & EMPTY & 1.7871 & 1.7920 & 1.7917 & $1.1^{\circ}$ & $e+00$ \\
\hline 79 & EMP? & & & 1.8 & & $.0 c_{2}$ \\
\hline 80 & PANO & -19. & 6.7 & -8.2202 & 6. & -01 \\
\hline 81 & PANO & -36.2728 & -11.8587 & -25.1186 & 5.4041 & 1.6 \\
\hline 82 & PAN & -24.5 & -9 & -18.2 & & \\
\hline 83 & PANO & -8.7 & 3. & -3.5279 & 2.9 & -01 \\
\hline 84 & PAN & -13.1 & -0.6 & -8.0 & & -01 \\
\hline 85 & PAN & -5.1 & 31.9 & 2.0 & & 8 \\
\hline 86 & PAN 1 & -4.65 & 1.68 & -1.3070 & 1. & -01 \\
\hline 87 & $\mathrm{PAI}$ & -7 & & -2 . & & \\
\hline 88 & PAN & -5.3 & 4.1 & -0.6810 & & -01 \\
\hline 89 & PAN 1 & -2.85 & 1.11 & -1.8486 & 8. & -01 \\
\hline 90 & PAI & -2.5 & -0 . & -1 & & \\
\hline 91 & PAN1 & -3.25 & -2.03 & -2.7 & & -01 \\
\hline 92 & PAN & -9.8931 & 7.12 & -5.6573 & 3. & -01 \\
\hline 93 & PAI & -8.6 & -0.4 & -4 & 1. & -0 \\
\hline 94 & PAN1 & -9.16 & & -5.9002 & $2.4655 e+00$ & $e-01$ \\
\hline 95 & PAN & 0. & -0.2 & -3.9258 & 2. & -01 \\
\hline 96 & $\mathrm{PA}$ & -13.72 & -2 & -7.2999 & 2.8 & 8. \\
\hline 97 & PAN & -9.83 & -0.6044 & -5.0264 & $1.9873 e+00$ & $8.7009 e-0$ \\
\hline 98 & & 5414 & & 873 & & \\
\hline 99 & PAB 1 & 41.9 & 42 & 42.0691 & 6.5 & \\
\hline 00 & 5136 & 42.3769 & 42.6058 & 42.4722 & $5.0519 e-02$ & 581 \\
\hline
\end{tabular}




\begin{tabular}{|c|c|c|c|c|c|c|}
\hline \multicolumn{2}{|c|}{ Test: Aug15_22 } & sum2 & Date: Wed & Aug 151 & $45: 001990$ & Page: 3 \\
\hline \# & Name & Min & $\operatorname{Max}$ & Ave & $S D$ & Uncert \\
\hline 101 & PIN 000001 & 38.9821 & 40.5690 & 39.8415 & $4.6464 e-01$ & $7.5659 e-01$ \\
\hline 102 & PRV000001 & 19.7186 & 19.8406 & 19.8089 & $5.4088 e-02$ & $7.5163 e-01$ \\
\hline 103 & PSR000001 & 42.2521 & 42.4962 & 42.3717 & $4.6072 e-02$ & $7.5744 e-01$ \\
\hline 104 & PEMO00001 & 7.9580 & 8.3242 & 8.1008 & $9.9881 e-02$ & $5.0041 e-01$ \\
\hline 105 & PANO00136 & -86.0152 & -58.5494 & -74.5344 & $7.3300 e+00$ & $4.9235 e+00$ \\
\hline 106 & PEM000002 & 8.0204 & 8.1714 & 8.0842 & $3.4572 e-02$ & $2.0102 e-01$ \\
\hline 107 & EMPTY & 0.0000 & 0.0098 & 0.0049 & $9.8648 e-04$ & $0.0000 e+00$ \\
\hline 108 & EMPTY & -10.0000 & -5.9961 & -9.3396 & $1.3124 \mathrm{e}+00$ & $0.0000 e+00$ \\
\hline 109 & EMPTY & -0.0049 & 0.0049 & 0.0044 & $1.7784 e-03$ & $0.0000 e+00$ \\
\hline 110 & EMPTY & -10.0000 & -10.0000 & -10.0000 & $0.0000 e+00$ & $0.0000 e+00$ \\
\hline 111 & EMPTY & 0.0000 & 0.0000 & 0.0000 & $0.0000 e+00$ & $0.0000 e+00$ \\
\hline 112 & VPW000001 & 187.2296 & 187.8155 & 187.6006 & $1.2633 \mathrm{e}-01$ & $1.3868 \mathrm{e}+00$ \\
\hline 113 & VPW000002 & 205.3861 & 205.7768 & 205.5267 & $1.1189 \mathrm{e}-01$ & $1.4754 \mathrm{e}+00$ \\
\hline 124 & VPWO000003 & 167.6532 & 168.2392 & 167.9189 & $1.6154 \mathrm{e}-01$ & $1.2927 e+00$ \\
\hline 115 & VPWO000004 & 140.7865 & 141.1771 & 140.9506 & $1.0705 e-01$ & $1.1710 e+00$ \\
\hline 116 & VPW000005 & 153.3954 & 153.9814 & 153.7666 & $1.1334 \mathrm{e}-01$ & $1.2277 \mathrm{e}+00$ \\
\hline 117 & VPW000006 & 228.0912 & 228.4818 & 228.3529 & $1.1578 e-01$ & $1.5916 \mathrm{e}+00$ \\
\hline 118 & IPWO00001 & 1485.4332 & 1499.0996 & 1493.4375 & $3.7471 e+00$ & $1.4311 \mathrm{e}+01$ \\
\hline 119 & IPW0 00002 & 1107.6082 & 1115.4132 & 1111.0032 & $2.3259 e+00$ & $1.1943 e+01$ \\
\hline 120 & IPW000003 & 912.6375 & 920.4375 & 916.4988 & $1.7833 e+00$ & $1.0864 e+01$ \\
\hline 121 & IPWO000004 & 747.6618 & 750.1032 & 749.1657 & $7.1026 e-01$ & $6.2472 \mathrm{e}+00$ \\
\hline 122 & IPWO00005 & 826.5869 & 830.4927 & 827.7875 & $1.0449 e+00$ & $6.8385 e+00$ \\
\hline 123 & IPW000006 & 598.9990 & 600.4638 & 599.8579 & $4.1338 e-01$ & $5.1436 \mathrm{e}+00$ \\
\hline 124 & EMPTY & -0.0244 & -0.0098 & -0.0172 & $3.9754 e-03$ & $0.0000 e+00$ \\
\hline 125 & EMPTY & -0.0195 & -0.0049 & -0.0120 & $3.1521 e-03$ & $0.0000 e+00$ \\
\hline 126 & EMPTY & -0.0195 & -0.0049 & -0.0125 & $3.1444 e-03$ & $0.0000 e+00$ \\
\hline 127 & EMPTY & -0.0244 & -0.0098 & -0.0166 & $3.5568 e-03$ & $0.0000 e+00$ \\
\hline 128 & IHTOOOOOOI & 177.5541 & 180.6791 & 179.1088 & $6.4109 e-01$ & $4.4741 e+00$ \\
\hline 129 & IHTO00002 & 182.5984 & 185.5281 & 183.9930 & $6.3871 e-01$ & $4.4939 \mathrm{e}+00$ \\
\hline 130 & IHTO00003 & 183.8980 & 186.6324 & 185.1324 & $6.0675 e-01$ & $4.4986 e+00$ \\
\hline 131 & IHTOOOOOO4 & 199.6056 & 202.1447 & 200.6993 & $6.2639 e-01$ & $4.5649 \mathrm{e}+00$ \\
\hline 132 & IHTO00005 & 196.8938 & 199.4328 & 198.2061 & $5.8798 e-01$ & $4.5540 \mathrm{e}+00$ \\
\hline 133 & IHT000006 & 191.0870 & 193.6261 & 192.4736 & $6.2297 e-01$ & $4.5293 e+00$ \\
\hline 134 & IHTO00007 & 181.0134 & 183.7477 & 182.5290 & $5.6436 e-01$ & $4.4879 e+00$ \\
\hline 135 & IHTOOOOO & 179.4020 & 181.7458 & 180.8551 & $5.5686 e-01$ & $4.4812 \mathrm{e}+00$ \\
\hline 136 & IHT000009 & 179.8753 & 182.6097 & 181.5238 & $5.8946 e-01$ & $4.4839 e+00$ \\
\hline 137 & IHTOOOOOIO & 166.7142 & 169.4486 & 167.8862 & $5.8127 e-01$ & $4.4304 e+00$ \\
\hline 138 & IHTOOOOO11 & 164.0024 & 166.1509 & 165.0259 & $5.4040 \mathrm{e}-01$ & $4.4197 e+00$ \\
\hline 139 & IHTO00012 & 160.8586 & 163.3977 & 162.0929 & $5.4310 e-01$ & $4.4088 e+00$ \\
\hline 140 & IHTO00013 & 132.7036 & 135.4380 & 133.8286 & $5.4184 e-01$ & $4.3129 e+00$ \\
\hline 141 & IHTO0O014 & 169.9181 & 172.8478 & 171.1837 & $5.2532 e-01$ & $4.4430 \mathrm{e}+00$ \\
\hline 142 & IHTOOOOLI5 & 137.6352 & 139.7837 & 138.4477 & $4.4879 e-01$ & $4.3274 e+00$ \\
\hline 143 & IHTO00016 & 122.3755 & 123.5239 & 122.2466 & $4.5883 e-01$ & $4.2784 e+00$ \\
\hline 144 & IHT 000017 & 147.7314 & 150.8564 & 149.4580 & $6.7238 e-01$ & $4.3639 e+00$ \\
\hline 145 & IHTO00018 & 149.9775 & 152.5165 & 151.1689 & $6.2297 e-01$ & $4.3698 \mathrm{e}+00$ \\
\hline 146 & IHTO00019 & 161.0276 & 162.9808 & 162.1410 & $4.5206 \mathrm{e}-01$ & $4.4090 \mathrm{e}+00$ \\
\hline 147 & IHTO000020 & 165.6100 & 167.7584 & 166.8443 & $4.9037 e-01$ & $4.4265 e+00$ \\
\hline 148 & IHT000021 & -2.2424 & 0.2967 & -0.9924 & $5.7318 e-01$ & $4.1000 e+00$ \\
\hline 149 & IHTOON022 & 136.8277 & 138.7808 & 137.6480 & $4.7021 e-01$ & $4.3249 e+00$ \\
\hline 150 & IHT000023 & 162.4925 & 164.6409 & 163.3637 & $4.6890 \mathrm{e}-01$ & $4.4135 e+00$ \\
\hline 151 & IHTOOOOO24 & 127.5428 & 129.6913 & 128.4647 & $4.6024 e-01$ & $4.2965 e+00$ \\
\hline 152 & IHTO00025 & 145.7069 & 147.6600 & 146.6561 & $4.8810 e-01$ & $4.3544 e+00$ \\
\hline 153 & IHTO00026 & 147.2656 & 149.6094 & 148.5117 & $4.9425 e-01$ & $4.3607 \mathrm{e}+00$ \\
\hline 154 & IHT 000027 & $123 .: 783$ & 124.9362 & 124.2486 & $4.1430 e-01$ & $4.2841 \mathrm{e}+00$ \\
\hline 155 & IHTOOOO28 & 124.5568 & 127.2912 & 126.0802 & $6.5078 e-01$ & $4.2895 e+00$ \\
\hline
\end{tabular}




\begin{tabular}{|c|c|c|c|c|c|c|}
\hline Tes & $t:$ Aug15_22 & sum2 & Date: Wed & Aug 1517 & $45: 00 \quad 1990$ & Page: 4 \\
\hline $\begin{array}{l}\# \\
156 \\
157 \\
158 \\
159 \\
160 \\
161 \\
162 \\
163\end{array}$ & 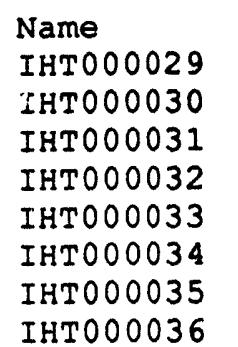 & $\begin{array}{l}\text { Min } \\
146.2177 \\
149.1737 \\
133.4360 \\
135.0511 \\
135.2314 \\
124.5756 \\
164.4494 \\
123.0807\end{array}$ & $\begin{array}{l}\text { Max } \\
149.1474 \\
151.9080 \\
136.1704 \\
137.9808 \\
137.9657 \\
126.9193 \\
166.5978 \\
125.0338\end{array}$ & $\begin{array}{l}\text { Ave } \\
147.8271 \\
150.6308 \\
134.8735 \\
136.6371 \\
136.5830 \\
125.7827 \\
165.7501 \\
124.1548\end{array}$ & $\begin{array}{l}\text { SD } \\
6.2833 e-01 \\
6.0773 e-01 \\
6.2110 e-01 \\
6.2719 e-01 \\
5.9952 e-01 \\
5.4203 e-01 \\
5.0626 e-01 \\
4.9639 e-01\end{array}$ & $\begin{array}{l}\text { Uncert } \\
4.3584 e+00 \\
4.3679 e+00 \\
4.3161 e+00 \\
4.3217 e+00 \\
4.3215 e+00 \\
4.2886 e+00 \\
4.4224 e+00 \\
4.2839 e+00\end{array}$ \\
\hline
\end{tabular}




\begin{tabular}{|c|c|c|c|}
\hline \multicolumn{4}{|c|}{$\begin{array}{l}\text { Velocity } 1=0.007200(\mathrm{ft} / \mathrm{sec}) \\
\text { Velocity } 2=0.006600(\mathrm{ft} / \mathrm{sec}) \\
\text { Pressure Drop }=0.021800(\mathrm{psi})\end{array}$} \\
\hline \# & Name & Abs Diff & ZDiff \\
\hline 0 & TRE000001 & 0.1903 & 0.2226 \\
\hline 1 & TRF000002 & 0.0799 & 0.0930 \\
\hline 2 & TFM000001 & 0.3264 & 0.3707 \\
\hline 3 & TINO00001 & 0.3699 & 0.4235 \\
\hline 4 & TRV000001 & 0.2413 & 0.2722 \\
\hline 5 & TSR000001 & 1.8260 & 1.2793 \\
\hline 6 & TAO020136 & 1.6492 & 2.6585 \\
\hline 7 & TAO050136 & 1.1481 & 0.5363 \\
\hline 8 & TA0070:36 & 0.6483 & 0.2990 \\
\hline 9 & TA0080136 & 0.6221 & 0.3857 \\
\hline 10 & TAO090136 & 0.5344 & 0.1991 \\
\hline 11 & TAO100136 & 0.7980 & 0.2992 \\
\hline 12 & TAO105136 & 0.6244 & 0.2292 \\
\hline 13 & TA0110136 & 0.7181 & 0.2523 \\
\hline 14 & TAO115136 & 0.4564 & 0.1642 \\
\hline 15 & TA0120136 & 0.4011 & 0.1371 \\
\hline 16 & TAO125135 & 0.0086 & 0.0047 \\
\hline 17 & TA0130136 & 0.1466 & 0.0512 \\
\hline 18 & TAO120048 & 2.8273 & 2.5019 \\
\hline 19 & TAO130048 & 0.3500 & 0.1326 \\
\hline 20 & TAO120224 & 0.9814 & 0.3613 \\
\hline 21 & TAO130224 & 0.7683 & 0.2962 \\
\hline 22 & TAO120312 & 0.4527 & 0.1809 \\
\hline 23 & TA0130312 & 0.3914 & 0.1580 \\
\hline 24 & TRT020000 & 0.4119 & 0.2110 \\
\hline 25 & TRT070000 & 0.2832 & 0.1256 \\
\hline 26 & TRT100000 & 0.2789 & 0.1117 \\
\hline 27 & TRT120000 & 0.1518 & 0.0590 \\
\hline 28 & TRT125000 & 5.4982 & 5.6280 \\
\hline 29 & TRT130000 & 0.1598 & 0.0641 \\
\hline 30 & EMPTY & 0.0413 & 0.1037 \\
\hline 31 & TAF0 050136 & 0.4783 & 0.3243 \\
\hline 32 & TAF 070136 & 0.6878 & 0.3984 \\
\hline 33 & TAF080136 & 0.4708 & 0.1815 \\
\hline 34 & TAF 090136 & 2.8752 & 2.9729 \\
\hline 35 & TAE 100136 & 0.8121 & 0.3900 \\
\hline 36 & TAF 105136 & 0.7729 & 0.3624 \\
\hline 37 & TAF 110136 & 0.9095 & 0.4120 \\
\hline 38 & TAF 115136 & 0.7635 & 0.3359 \\
\hline 39 & TAF 120136 & 0.5818 & 0.2190 \\
\hline 40 & TAF 125136 & 1.0767 & 0.4468 \\
\hline 41 & TAF 130136 & 0.0217 & 0.0088 \\
\hline 42 & TAF 120048 & 0.6870 & 0.3250 \\
\hline 43 & TAF 130048 & 0.6119 & 0.2774 \\
\hline 44 & TAF 120224 & 0.8861 & 0.4162 \\
\hline 45 & TAE 130224 & 1.1367 & 0.5142 \\
\hline
\end{tabular}




\begin{tabular}{|c|c|c|c|c|}
\hline Test: & Aug15_22 & dif & te: Wed Aug $15 \quad 17: 45: 001990$ & Page: 2 \\
\hline$\#$ & Name & Abs Diff & \&Diff & \\
\hline 46 & EMPTY & 0.0736 & 0.1924 & \\
\hline 47 & EMPTY & 0.1238 & 0.3087 & \\
\hline 48 & TAI 130048 & 0.2038 & 0.0781 & \\
\hline 49 & TAI130136 & 0.0576 & 0.0202 & \\
\hline 50 & TAI130224 & 0.1718 & 0.0675 & \\
\hline 51 & TAI130312 & 0.7025 & 0.2686 & \\
\hline 52 & TRR1 30000 & 0.3867 & 0.2540 & \\
\hline 53 & TSI000001 & 0.0502 & 0.0749 & \\
\hline 54 & TSI000002 & 1.2887 & 1.1847 & \\
\hline 55 & TSO000001 & 0.0600 & 0.0907 & \\
\hline 56 & TSO000002 & 0.0882 & 0.1079 & \\
\hline 57 & TAF020136 & 0.3499 & 0.3226 & \\
\hline 58 & TAF 120312 & 0.4249 & 0.2154 & \\
\hline 59 & TAF 130312 & 0.4005 & 0.1876 & \\
\hline 60 & EMP TY & 0.0122 & 0.3705 & \\
\hline 61 & EMPTY & 0.0107 & 0.3262 & \\
\hline 62 & EMPTY & 0.0084 & 0.2572 & \\
\hline 63 & EMPTY & 0.0068 & 0.2085 & \\
\hline 64 & EMPTY & 0.0023 & 0.1424 & \\
\hline 65 & EMP TY & 0.0013 & 0.0796 & \\
\hline 66 & EMPTY & 0.0011 & 0.0670 & \\
\hline 67 & EMPTY & 0.0004 & 0.0242 & \\
\hline 68 & EMPTY & 0.0003 & 0.0180 & \\
\hline 69 & EMPTY & 0.0000 & 0.0000 & \\
\hline 70 & EMPTY & 0.0014 & 0.0831 & \\
\hline 71 & EMPTY & 0.0017 & 0.0999 & \\
\hline 72 & EMPTY & 0.0009 & 0.0526 & \\
\hline 73 & EMPTY & 0.0010 & 0.0579 & \\
\hline 74 & EMPTY & 0.0013 & 0.0749 & \\
\hline 75 & EMPTY & 0.0015 & 0.0858 & \\
\hline 76 & EMPTY & 0.0012 & 0.0683 & \\
\hline 77 & EMPTY & 0.0008 & 0.0451 & \\
\hline 78 & EMPTY & 0.0029 & 0.1621 & \\
\hline 79 & EMPTY & 0.0016 & 0.0886 & \\
\hline 80 & PAN020136 & 1.6784 & 16.9559 & \\
\hline 81 & PAN050136 & 2.6184 & 11.6372 & \\
\hline 82 & PAN 070136 & 0.2213 & 1.2250 & \\
\hline 83 & PAN0 80136 & 0.6302 & 15.1560 & \\
\hline 84 & PAN090136 & 0.7629 & 10.4892 & \\
\hline 85 & PAN100136 & 0.4349 & 26.2764 & \\
\hline 86 & PAN 105136 & 0.1709 & 15.0427 & \\
\hline 87 & PAN110136 & 0.1007 & 3.8034 & \\
\hline 88 & PAN 115136 & 0.8652 & 55.9565 & \\
\hline 89 & PAN 120136 & 0.3646 & 24.5687 & \\
\hline 90 & PAN 125136 & 0.0214 & 1.2958 & \\
\hline 91 & PAN 130136 & 0.6287 & 29.7244 & \\
\hline 92 & PAN 120048 & 1.3413 & 31.0774 & \\
\hline 93 & PAN130048 & 0.3356 & 7.5777 & \\
\hline 94 & PAN 120224 & 0.1572 & 2.5952 & \\
\hline 95 & PAN 130224 & 0.2548 & 6.9409 & \\
\hline 96 & PAN 120312 & 0.6866 & 10.3821 & \\
\hline 97 & PAN 130312 & 0.6637 & 11.6641 & \\
\hline 98 & PAB000136 & 0.1053 & 0.2626 & \\
\hline 99 & $\mathrm{PAB} 100136$ & 0.1639 & 0.3881 & \\
\hline 100 & PAB125136 & 0.1519 & 0.3564 & \\
\hline
\end{tabular}




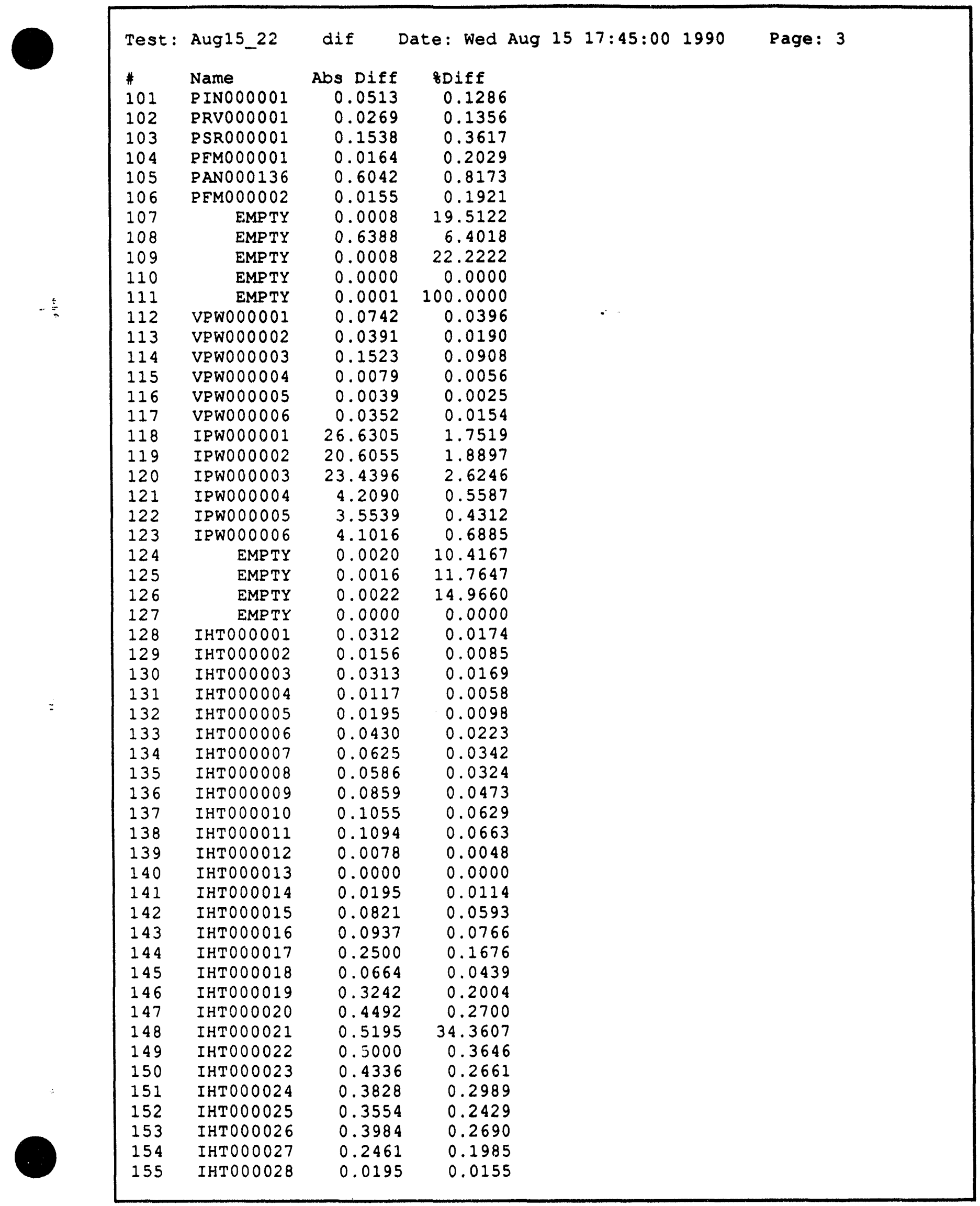




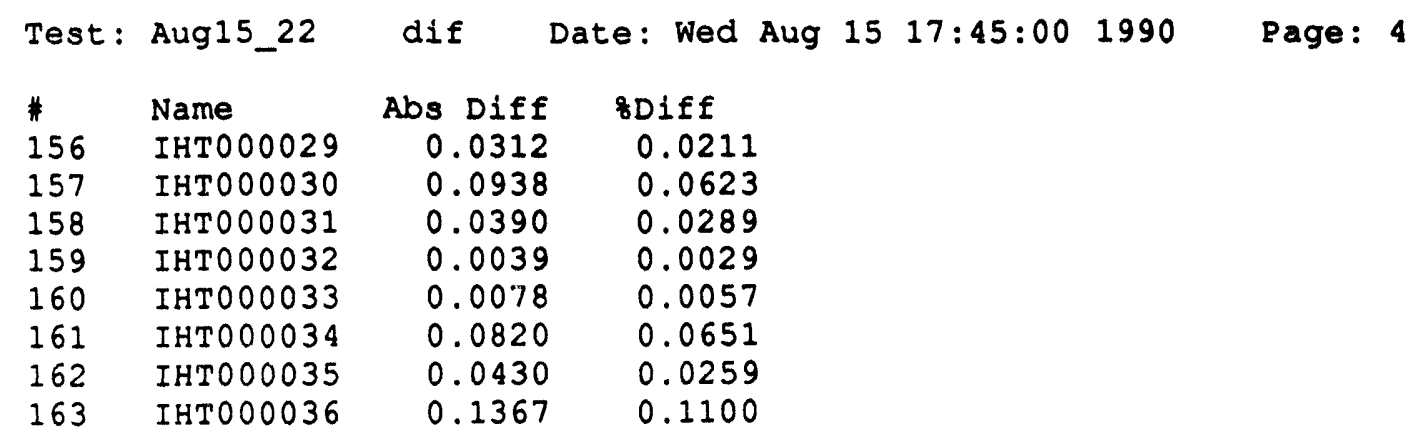




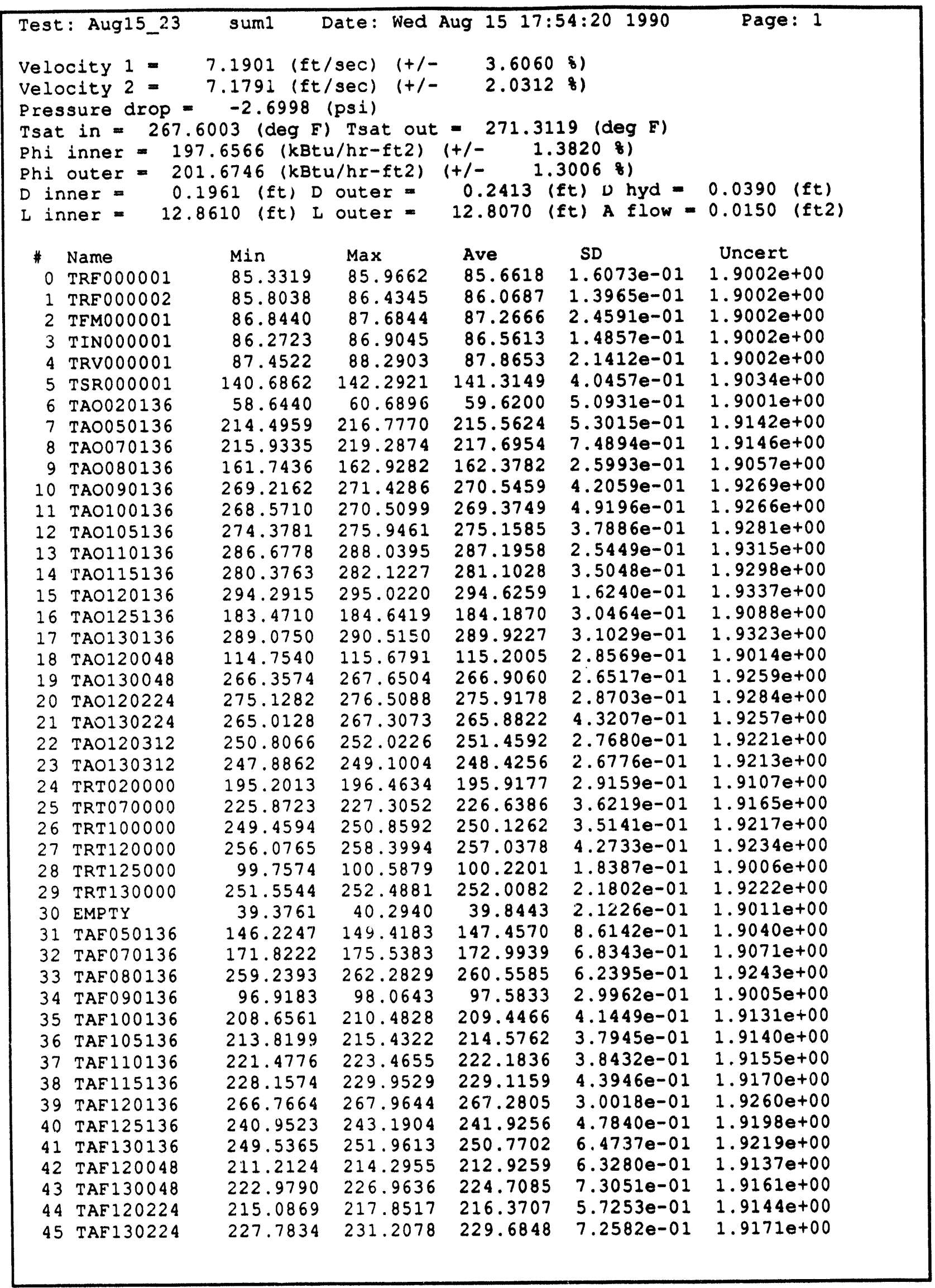




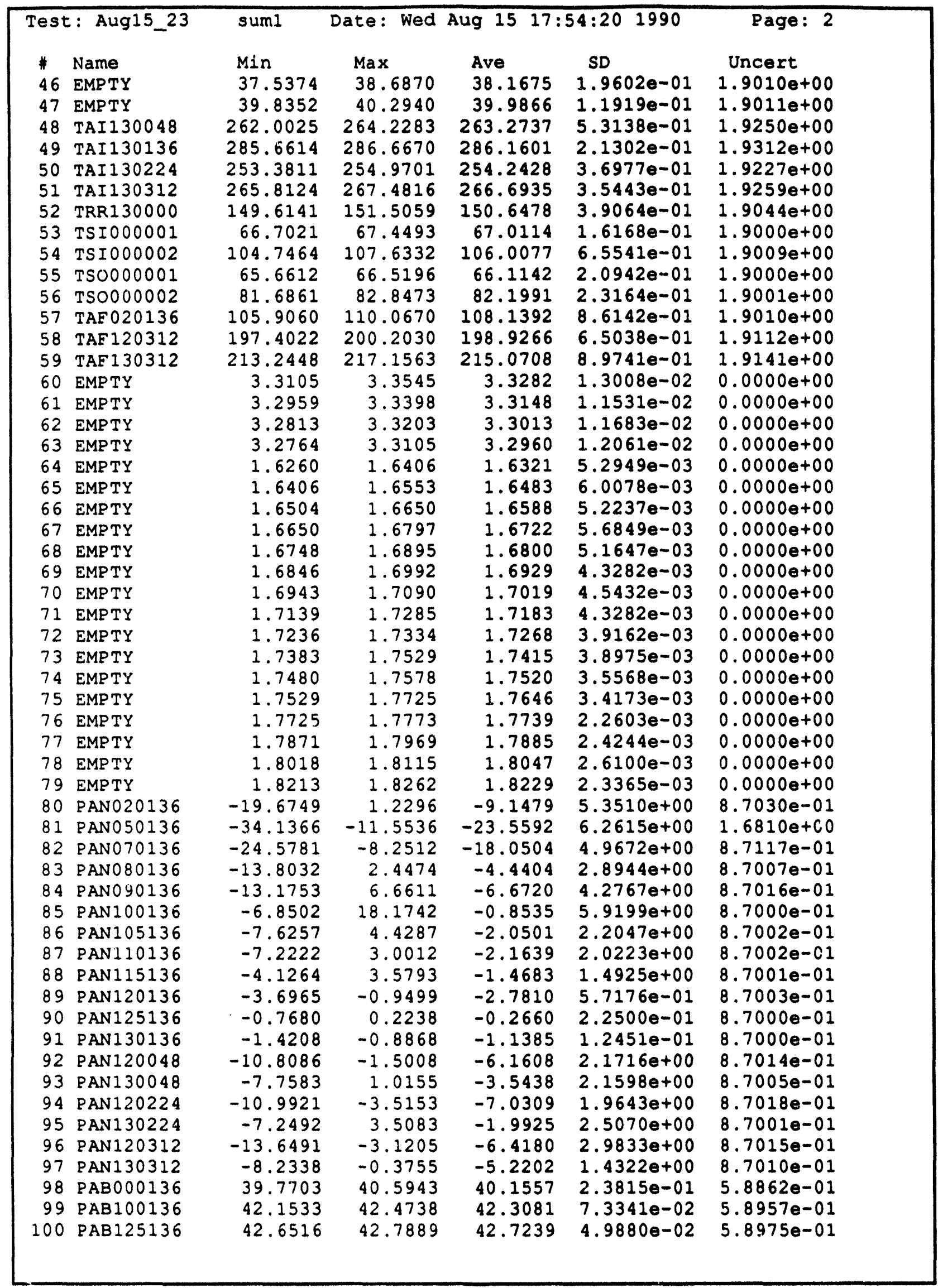




\begin{tabular}{|c|c|c|c|c|c|c|}
\hline \multicolumn{2}{|c|}{ Test: Aug15_23 } & sum 1 & Date: Wed & Aug 1517 & $4: 201990$ & Page: 3 \\
\hline$\#$ & Name & Min & $\operatorname{Max}$ & Ave & $S D$ & Uncert \\
\hline 101 & PINO00001 & 39.2263 & 40.6911 & 40.0954 & $3.9431 e-01$ & $7.5667 e-01$ \\
\hline 102 & PRV000001 & 19.5965 & 19.7186 & 19.7161 & $1.7263 e-02$ & $7.5162 e-01$ \\
\hline 103 & PSR000001 & 42.3741 & 42.6183 & 42.5133 & $5.5201 e-02$ & $7.5749 \theta-01$ \\
\hline 104 & PFM0000001 & 7.5918 & 8.0496 & 7.8243 & $9.9966 e-02$ & $5.0038 e-01$ \\
\hline 105 & PAN000136 & -87.3885 & -62.2115 & -74.7450 & $7.9303 \theta+00$ & $4.9235 e+00$ \\
\hline 106 & PFM0000002 & 7.7016 & 7.9533 & 7.8003 & $5.4939 e-02$ & $2.0095 e-01$ \\
\hline $10 \%$ & EMPTY & 0.0000 & 0.0049 & 0.0048 & $6.9053 e-04$ & $0.0000 e+00$ \\
\hline 108 & EMPTY & -10.0000 & -5.8534 & -9.4188 & $1.3494 e+00$ & $0.0000 e+00$ \\
\hline 109 & EMPTY & 0.0000 & 0.0049 & 0.0043 & $1.6028 e-03$ & $0.0000 e+00$ \\
\hline 110 & EMPTY & -10.0000 & -10.0000 & -10.0000 & $0.0000 e+00$ & $0.0000 e+00$ \\
\hline 111 & EMPTY & 0.0000 & 0.0000 & 0.0000 & $0.0000 e+00$ & $0.0000 e+00$ \\
\hline 112 & VPW000001 & 187.2296 & 187.8155 & 187.6084 & $9.9902 e-02$ & $1.38680+00$ \\
\hline 113 & VPWO 00002 & 205.3861 & 205.7768 & 205.6127 & $1.2072 e-01$ & $1.4758 e+00$ \\
\hline 114 & VPW000003 & 167.6532 & 168.2392 & 167.9345 & $1.3182 e-01$ & $1.2928 \theta+00$ \\
\hline 115 & VPWOOOOOO4 & 140.7865 & 141.1771 & 141.0210 & $1.2478 e-01$ & $1.1713 \mathrm{e}+00$ \\
\hline 116 & VPWO00005 & 153.3954 & 153.9814 & 153.8095 & $1.2251 \mathrm{e}-01$ & $1.2279 e+00$ \\
\hline 117 & VPW000006 & 228.2865 & 228.6771 & 228.4622 & $1.2633 e-01$ & $1.5922 e+00$ \\
\hline 118 & IPW000001 & 1489.3379 & 1497.1472 & 1493.8281 & $1.6850 e+00$ & $1.4314 \mathrm{e}+01$ \\
\hline 119 & IPWO00002 & 1109.5594 & 1113.4619 & 1111.0812 & $1.13500+00$ & $1.1944 \theta+01$ \\
\hline 120 & IPW000003 & 914.5875 & 920.4375 & 917.6689 & $1.3698 \mathrm{e}+00$ & $1.0870 e+01$ \\
\hline 121 & IPW0 00004 & 748.6384 & 749.6149 & 749.1364 & $3.0389 e-01$ & $6.2470 e+00$ \\
\hline 122 & IPW000005 & 827.0751 & 828.5398 & 827.5824 & $3.2654 e-01$ & $6.8370 e+00$ \\
\hline 123 & IPW000006 & 599.4872 & 600.9521 & 600.2290 & $3.4513 e-01$ & $5.1463 e+00$ \\
\hline 124 & EMPTY & -0.0244 & -0.0098 & -0.0176 & $4.1853 e-03$ & $0.0000 e+00$ \\
\hline 125 & EMPTY & -0.0146 & -0.0049 & -0.0113 & $3.0309 e-03$ & $0.0000 e+00$ \\
\hline 126 & EMPTY & -0.0195 & -0.0049 & -0.0133 & $2.9660 \mathrm{e}-03$ & $0.0000 e+00$ \\
\hline 127 & EMPTY & -0.0244 & -0.0098 & -0.0153 & $3.2733 e-03$ & $0.0000 e+00$ \\
\hline 128 & IHT000001 & 177.5541 & 180.2885 & 179.1635 & $5.8867 e-01$ & $4.4744 e+00$ \\
\hline 129 & IHTO00002 & 182.4031 & 185.1375 & 184.0048 & $5.8527 e-01$ & $4.4940 e+00$ \\
\hline 130 & IHT000003 & 183.7027 & 186.4370 & 185.2300 & $6.1471 e-01$ & $4.4990 \mathrm{e}+00$ \\
\hline 131 & IHTO00004 & 199.2150 & 202.3400 & 200.7930 & $6.2991 e-01$ & $4.5653 e+00$ \\
\hline 132 & IHTO00005 & 196.6985 & 199.8235 & 198.2882 & $6.0233 e-01$ & $4.5543 e+00$ \\
\hline 133 & IHT000006 & 191.0870 & 193.8214 & 192.5557 & $5.9220 e-01$ & $4.5297 e+00$ \\
\hline 134 & IHTO00007 & 181.0134 & 183.7477 & 182.6305 & $6.3016 e-01$ & $4.4884 e+00$ \\
\hline 135 & IHT 000008 & 179.5973 & 182.3317 & 180.9059 & $6.2795 e-01$ & $4.4814 e+00$ \\
\hline 136 & IHTO00009 & 180.2659 & 182.8050 & 181.6019 & $6.2060 e-01$ & $4.4842 e+00$ \\
\hline 137 & IHTO00010 & 166.9095 & 169.2533 & 168.0307 & $5.9818 e-01$ & $4.4310 e+00$ \\
\hline 138 & IHTO00011 & 163.8071 & 166.3462 & 165.1587 & $5.7837 e-01$ & $4.4202 e+00$ \\
\hline 139 & IHTO00012 & 161.2493 & 163.3977 & 162.2413 & $6.0726 e-01$ & $4.4093 e+00$ \\
\hline 140 & IHTO00013 & 133.0942 & 135.0473 & 134.0005 & $5.7826 e-01$ & $4.3134 e+00$ \\
\hline 141 & IHTOOO014 & 170.3087 & 172.8478 & 171.4063 & $6.0088 e-01$ & $4.4439 e+00$ \\
\hline 142 & IHTO00015 & 137.4399 & 139.9790 & 138.6509 & $5.7182 e-01$ & $4.32810+00$ \\
\hline 143 & IHTO00016 & 121.1801 & 123.7192 & 122.4341 & $5.0853 e-01$ & $4.2789 e+00$ \\
\hline 144 & IHT000017 & 148.9033 & 151.0517 & 149.9267 & $5.0155 e-01$ & $4.3655 e+00$ \\
\hline 145 & IHT 000018 & 149.9775 & 152.7119 & 151.2744 & $6.2858 e-01$ & $4.3702 \theta+00$ \\
\hline 146 & IHTO00019 & 160.6370 & 163.3714 & 162.0043 & $6.4958 e-01$ & $4.4085 e+00$ \\
\hline 147 & IHTO00020 & 165.6100 & 168.3443 & 166.6490 & $6.2818 e-01$ & $4.4257 e+00$ \\
\hline 148 & IHT000021 & -2.8283 & 0.2967 & -1.2736 & $7.6408 \mathrm{e}-01$ & $4.1000 e+00$ \\
\hline 149 & IHT0000:22 & 136.2417 & 138.5855 & 137.3316 & $5.3972 e-01$ & $4.3239 e+00$ \\
\hline 150 & IHTO00023 & 161.9066 & 164.6409 & 163.1879 & $6.0767 \theta-01$ & $4.4128 e+00$ \\
\hline 151 & IHTO00024 & 127.1522 & 129.4959 & 128.2850 & $5.7993 e-01$ & $4.29600+00$ \\
\hline 152 & IHTO00025 & 145.3163 & 148.0506 & 146.4921 & $6.3072 e-01$ & $4.3538 e+00$ \\
\hline 153 & IHTO00026 & 147.0703 & 150.0000 & 148.3008 & $6.7687 e-01$ & $4.3600 e+00$ \\
\hline 154 & IHT000027 & 122.7877 & 125.5221 & 124.0415 & $6.3022 e-01$ & $4.2835 e+00$ \\
\hline 155 & IHTO00028 & 125.1427 & 127.8771 & 126.2560 & $7.1817 e-01$ & $4.2900 \mathrm{e}+00$ \\
\hline
\end{tabular}




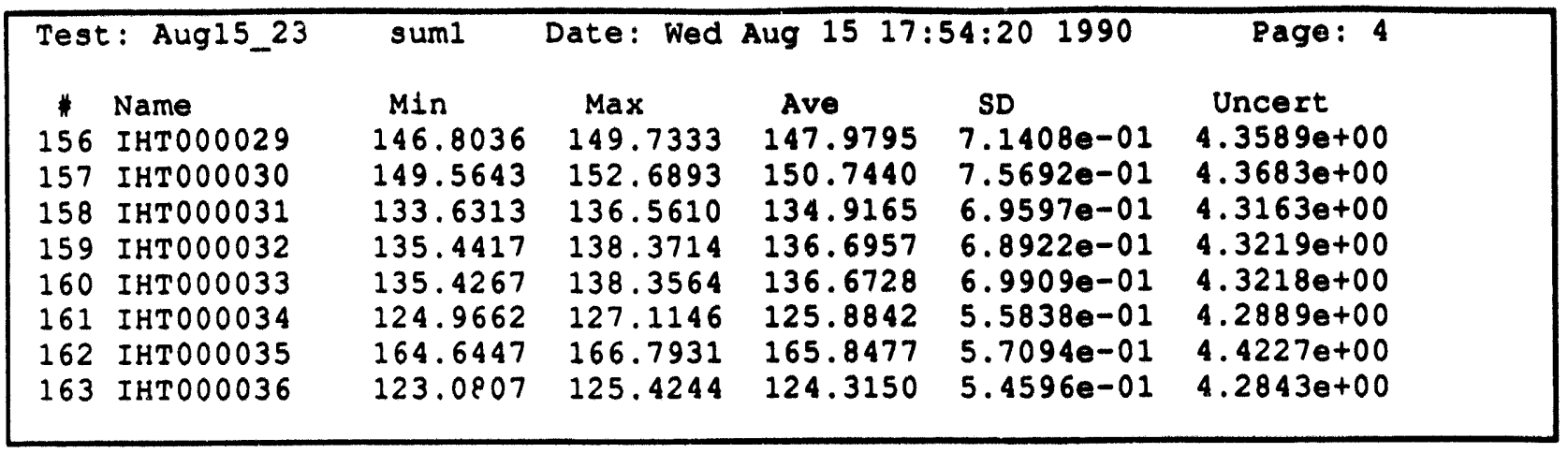




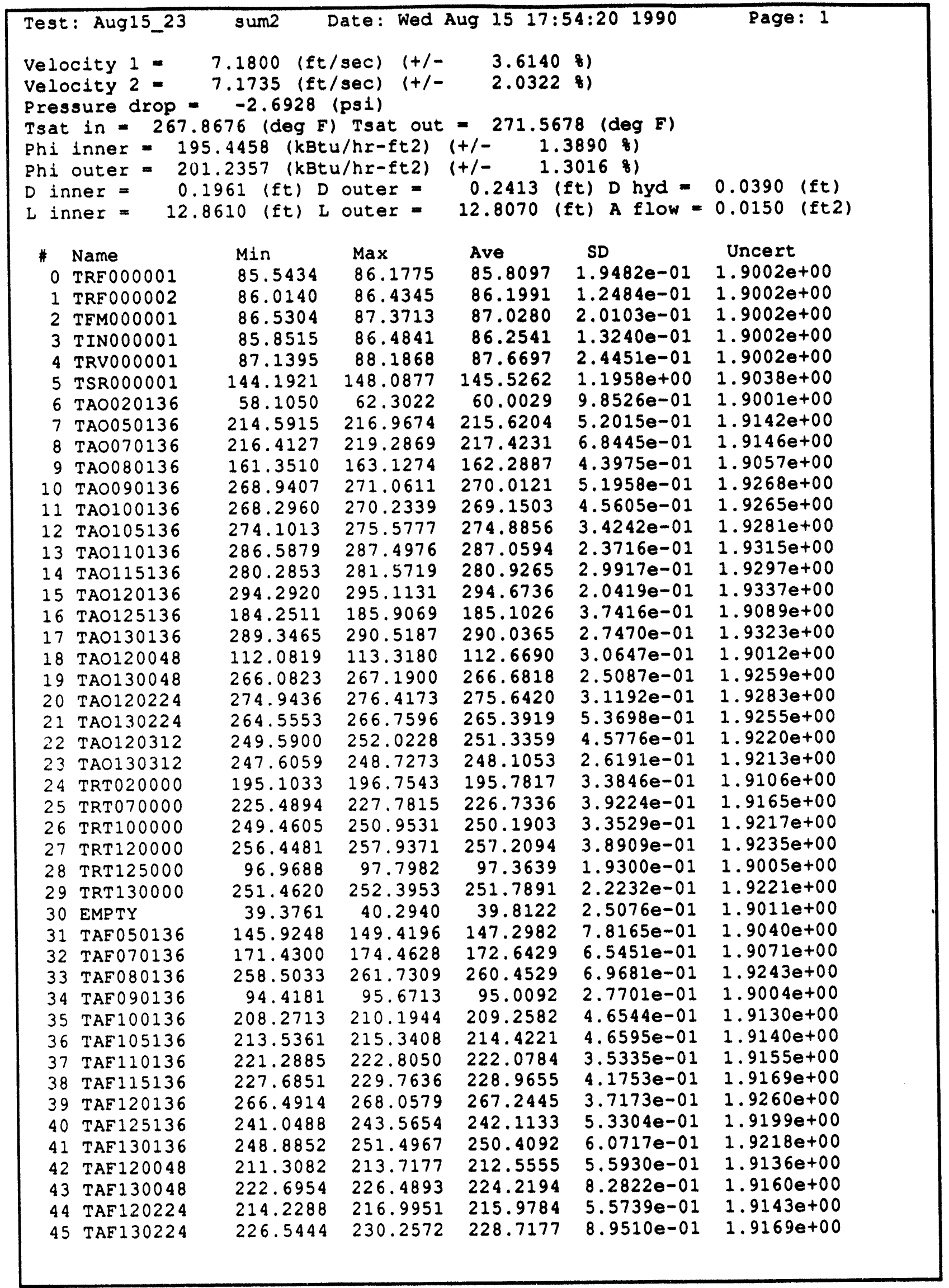




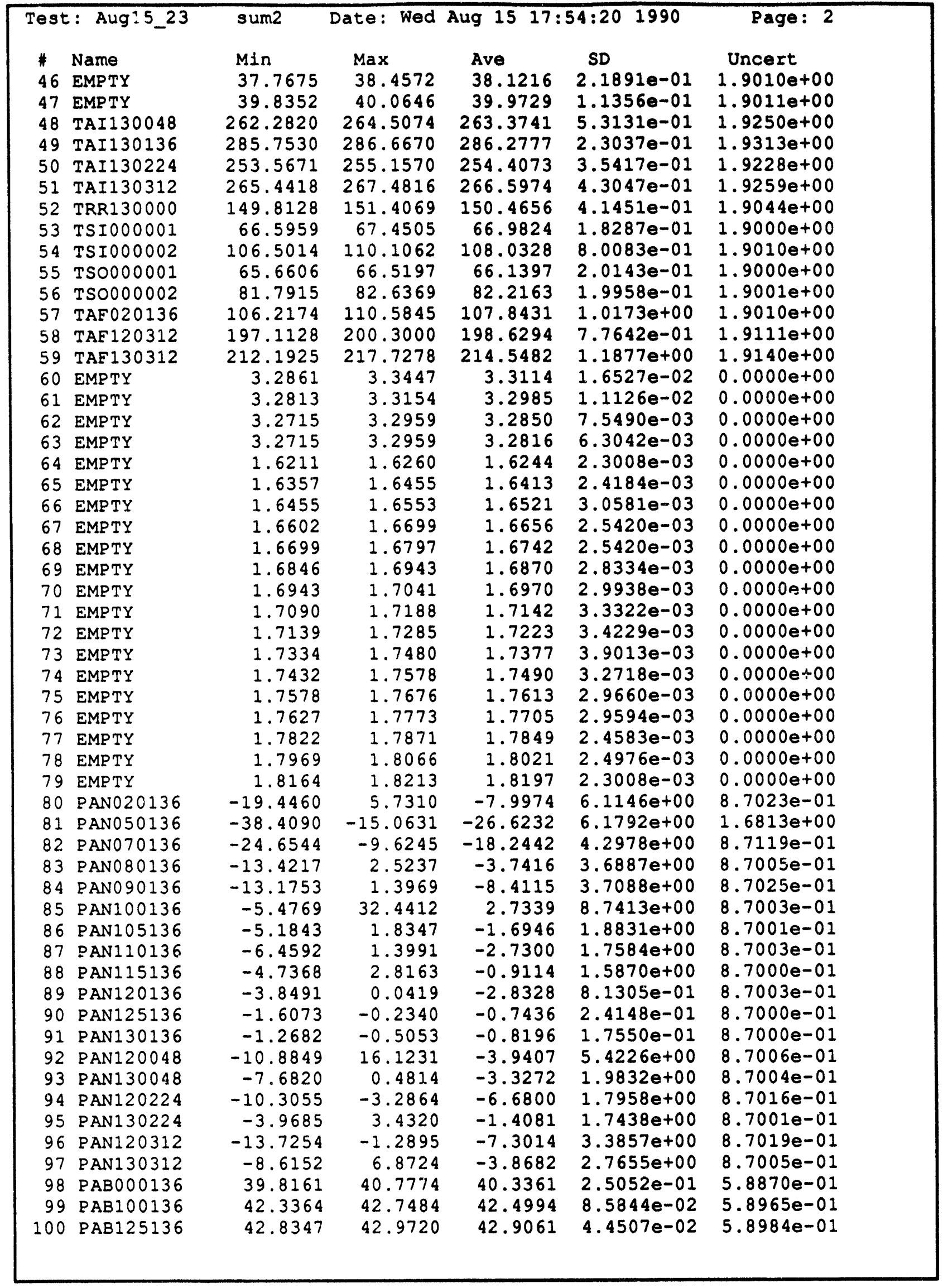




\begin{tabular}{|c|c|c|c|c|c|c|}
\hline \multicolumn{2}{|c|}{ Test: Aug15_23 } & sum2 & \multicolumn{3}{|c|}{ Date: Wed Aug $15 \quad 17: 54: 201990$} & Page: 3 \\
\hline$\#$ & Name & Min & $\operatorname{Max}$ & Ave & SD & Uncert \\
\hline 101 & PINO00001 & 39.1042 & 40.8132 & 40.0368 & $4.8060 e-01$ & $7.5665 \theta-01$ \\
\hline 102 & PRV000001 & 19.5965 & 19.8406 & 19.7186 & $2.4662 e-02$ & $7.5162 e-01$ \\
\hline 103 & PSR000001 & 42.6183 & 42.7404 & 42.6622 & $5.9189 e-02$ & $7.5755 e-01$ \\
\hline 104 & PFM000001 & 7.5003 & 7.9580 & 7.8024 & $1.0173 e-01$ & $5.0038 e-01$ \\
\hline 105 & PAN000136 & -89.6773 & -58.5494 & -74.5528 & $7.7199 e+00$ & $4.92350+00$ \\
\hline 106 & PFM000002 & 7.6680 & 7.9030 & 7.7882 & $5.3317 e-02$ & $2.0095 e-01$ \\
\hline 107 & EMPTY & 0.0049 & 0.0049 & 0.0049 & $0.0000 e+00$ & $0.0000 e+00$ \\
\hline 108 & EMPTY & -10.0000 & -6.2158 & -9.8545 & $6.2359 e-01$ & $0.0000 e+00$ \\
\hline 109 & EMPTY & 0.0000 & 0.0049 & 0.0041 & $1.8082 e-03$ & $0.0000 e+00$ \\
\hline 110 & EMPTY & -10.0000 & -10.0000 & -10.0000 & $0.0000 e+00$ & $0.0000 e+00$ \\
\hline 111 & EMPTY & 0.0000 & 0.0000 & 0.0000 & $0.0000 e+00$ & $0.0000 e+00$ \\
\hline 112 & VPW000001 & 187.4249 & 188.0108 & 187.6436 & $1.1599 e-01$ & $1.3870 e+00$ \\
\hline 113 & VPWO00002 & 205.3861 & 205.7768 & 205.6010 & $1.1334 e-01$ & $1.4758 e+00$ \\
\hline 114 & VPW000003 & 167.8485 & 168.2392 & 167.9853 & $1.0625 \theta-01$ & $1.2931 e+00$ \\
\hline 115 & VPWO00004 & 140.5912 & 141.1771 & 140.9545 & $1.2484 e-01$ & $1.1710 \mathrm{e}+00$ \\
\hline 116 & VPW000005 & 153.3954 & 153.9814 & 153.8330 & $1.3416 e-01$ & $1.2280 e+00$ \\
\hline 117 & VPW000006 & 228.0912 & 228.8725 & 228.4388 & $1.2658 \theta-01$ & $1.5921 e+00$ \\
\hline 118 & IPWO00001 & 1512.7660 & 1522.5277 & 1515.6167 & $2.0575 e+00$ & $1.4456 \mathrm{e}+01$ \\
\hline 119 & IPW0 00002 & 1080.2904 & 1090.0468 & 1085.1293 & $1.8591 e+00$ & $1.1794 e+01$ \\
\hline 120 & IPW000003 & 889.2375 & 893.1375 & 890.6027 & $1.3214 \mathrm{e}+00$ & $1.0729 \mathrm{e}+01$ \\
\hline 121 & IPWO 00004 & 754.4977 & 755.9626 & 755.1032 & $4.3629 e-01$ & $6.2917 e+00$ \\
\hline 122 & IPW0 00005 & 824.1458 & 826.5869 & 825.5030 & $5.3383 e-01$ & $6.8213 e+00$ \\
\hline 123 & IPW000006 & 594.6044 & 596.0693 & 595.1899 & $4.0674 e-01$ & $5.1097 e+00$ \\
\hline 124 & EMPTY & -0.0293 & -0.0098 & -0.0166 & $4.3000 e-03$ & $0.0000 e+00$ \\
\hline 125 & EMPTY & -0.0195 & -0.0098 & -0.0126 & $2.8058 e-03$ & $0.0000 e+00$ \\
\hline 126 & EMPTY & -0.2244 & -0.0049 & -0.0134 & $4.7114 e-03$ & $0.0000 e+00$ \\
\hline 127 & EMPTY & -0.0244 & 0.0000 & -0.0157 & $3.9815 e-03$ & $0.0000 e+00$ \\
\hline 128 & IHTO00001 & 177.7494 & 180.4838 & 179.0112 & $6.0645 e-01$ & $4.4738 e+00$ \\
\hline 129 & IHTO00002 & 182.7937 & 184.9422 & 183.9071 & $5.7352 e-01$ & $4.4936 \mathrm{e}+00$ \\
\hline 130 & IHTO00003 & 183.7027 & 186.2417 & 185.0933 & $5.9535 e-01$ & $4.4984 \mathrm{e}+00$ \\
\hline 131 & IHTO00004 & 199.6056 & 201.9494 & 200.6915 & $5.9078 e-01$ & $4.5648 e+00$ \\
\hline 132 & IHT000005 & 197.0891 & 199.2375 & 198.1085 & $5.4575 e-01$ & $4.5535 e+00$ \\
\hline 133 & IHTO00006 & 191.4776 & 193.4307 & 192.4815 & $5.4678 e-01$ & $4.5293 e+00$ \\
\hline 134 & IHT 000007 & 181.4040 & 183.7477 & 182.5368 & $5.2645 e-01$ & $4.4880 e+00$ \\
\hline 135 & IHT000008 & 179.7926 & 181.9411 & 180.8707 & $5.0876 e-01$ & $4.4812 e+00$ \\
\hline 136 & IHTO00009 & 180.4612 & 182.4144 & 181.5316 & $4.9950 e-01$ & $4.4839 e+00$ \\
\hline 137 & IHTO00010 & 166.5189 & 169.2533 & 167.8354 & $5.7833 e-01$ & $4.4302 e+00$ \\
\hline 138 & IHTO00011 & 163.6118 & 166.1509 & 164.9751 & $5.4318 e-01$ & $4.4195 e+00$ \\
\hline 139 & IHTO00012 & 160.6633 & 163.5930 & 162.0617 & $5.3577 e-01$ & $4.4087 e+00$ \\
\hline 1.40 & IHT000013 & 132.5083 & 135.2426 & 133.8325 & $5.0884 e-01$ & $4.3129 e+00$ \\
\hline 141 & IHTO00014 & 170.1134 & 172.2619 & 171.2227 & $4.9825 e-01$ & $4.4432 e+00$ \\
\hline 142 & IHT000015 & 137.6352 & 139.5883 & 138.5063 & $4.8038 e-01$ & $4.3276 \mathrm{e}+00$ \\
\hline 143 & IHT000016 & 121.3755 & 123.1333 & 122.2192 & $5.1515 e-01$ & $4.2783 e+00$ \\
\hline 144 & IHT000017 & 147.7314 & 151.4423 & 149.7314 & $8.3850 e-01$ & $4.3649 e+00$ \\
\hline 145 & IHTO00018 & 149.5869 & 152.5165 & 151.2275 & $6.0102 e-01$ & $4.3700 e+00$ \\
\hline 146 & IHTO00019 & 160.8323 & 163.5667 & 162.1097 & $6.5698 e-01$ & $4.4088 e+00$ \\
\hline 147 & IHTO00020 & 165.6100 & 168.1490 & 166701 & $6.5127 e-01$ & $4.4262 e+00$ \\
\hline 148 & IHT000021 & -2.6330 & 0.4920 & -1.0783 & $7.7017 e-01$ & $4.1000 e+00$ \\
\hline 149 & IHT000022 & 136.2417 & 138.7808 & 137.5855 & $6.2967 e-01$ & $4.3247 e+00$ \\
\hline 150 & IHTO00023 & 161.9066 & 164.4456 & 163.3090 & $6.2101 \theta-01$ & $4.4133 \mathrm{e}+00$ \\
\hline 151 & IHT000024 & 126.7616 & 129.8866 & 128.4413 & $6.0361 e-01$ & $4.2965 e+00$ \\
\hline 152 & IHT000025 & 145.1209 & 148.0506 & 146.6991 & $5.7837 e-01$ & $4.3545 e+00$ \\
\hline 153 & IHT000026 & 147.0703 & 149.8047 & 148.5586 & $5.9828 e-01$ & $4.3608 e+00$ \\
\hline 154 & IHT000027 & 122.9830 & 125.5221 & 124.2564 & $5.7756 e-01$ & $4.2842 e+00$ \\
\hline 155 & IHTO00028 & 124.7521 & 127.6818 & 126.0841 & $6.1724 e-01$ & $4.2895 e+00$ \\
\hline
\end{tabular}




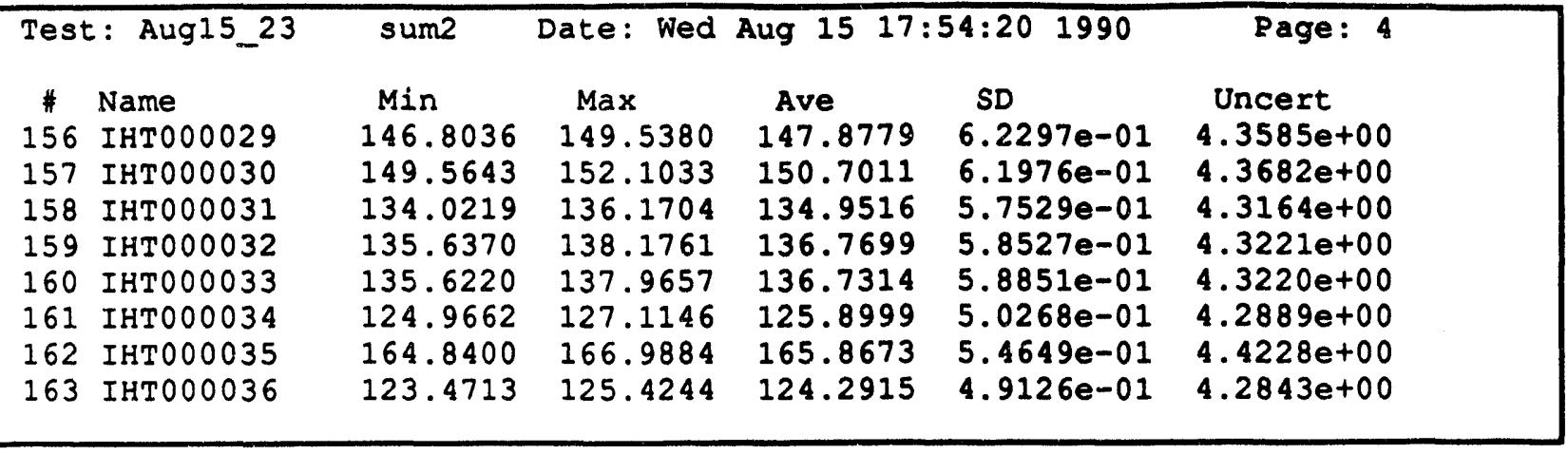




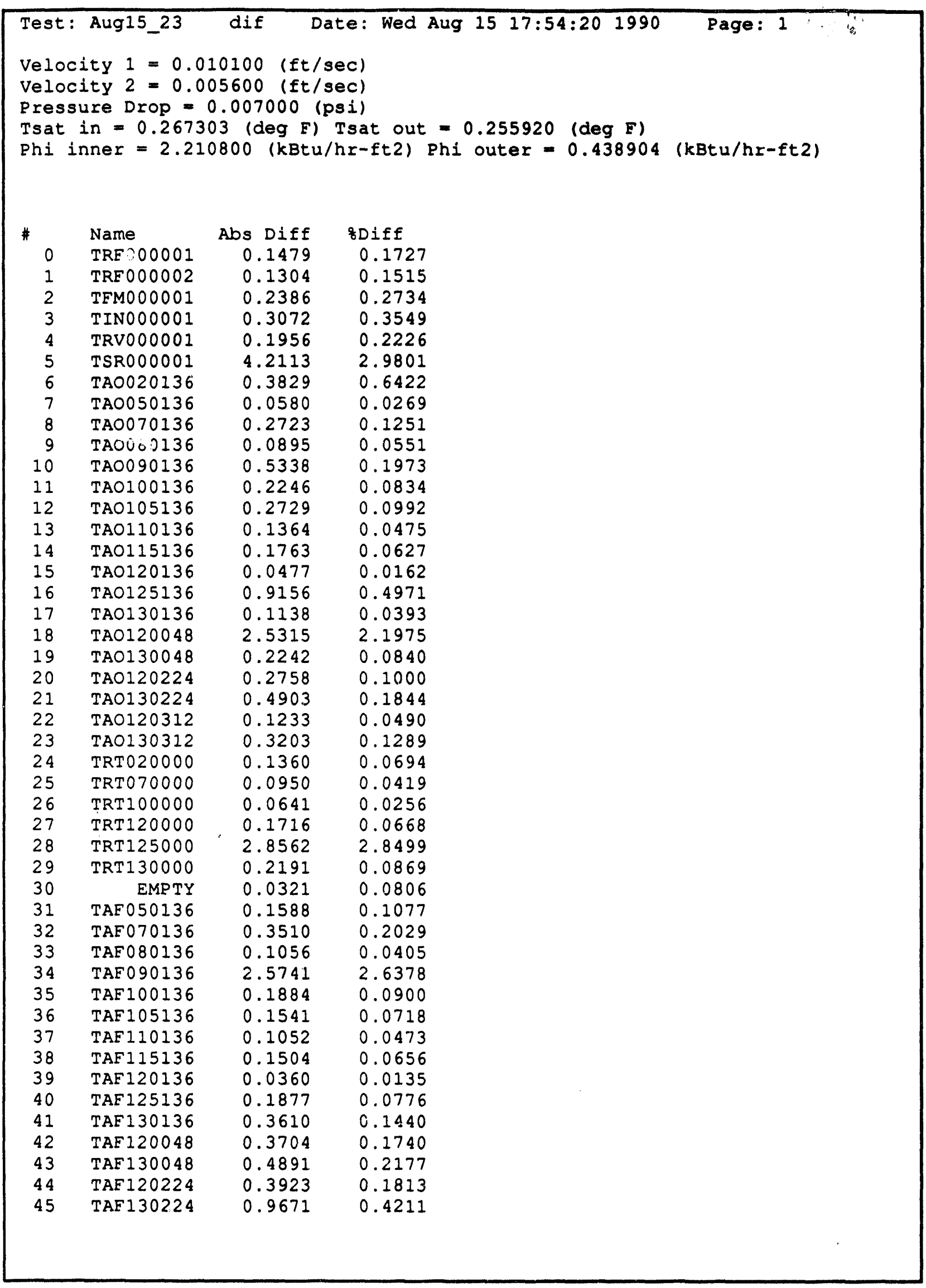




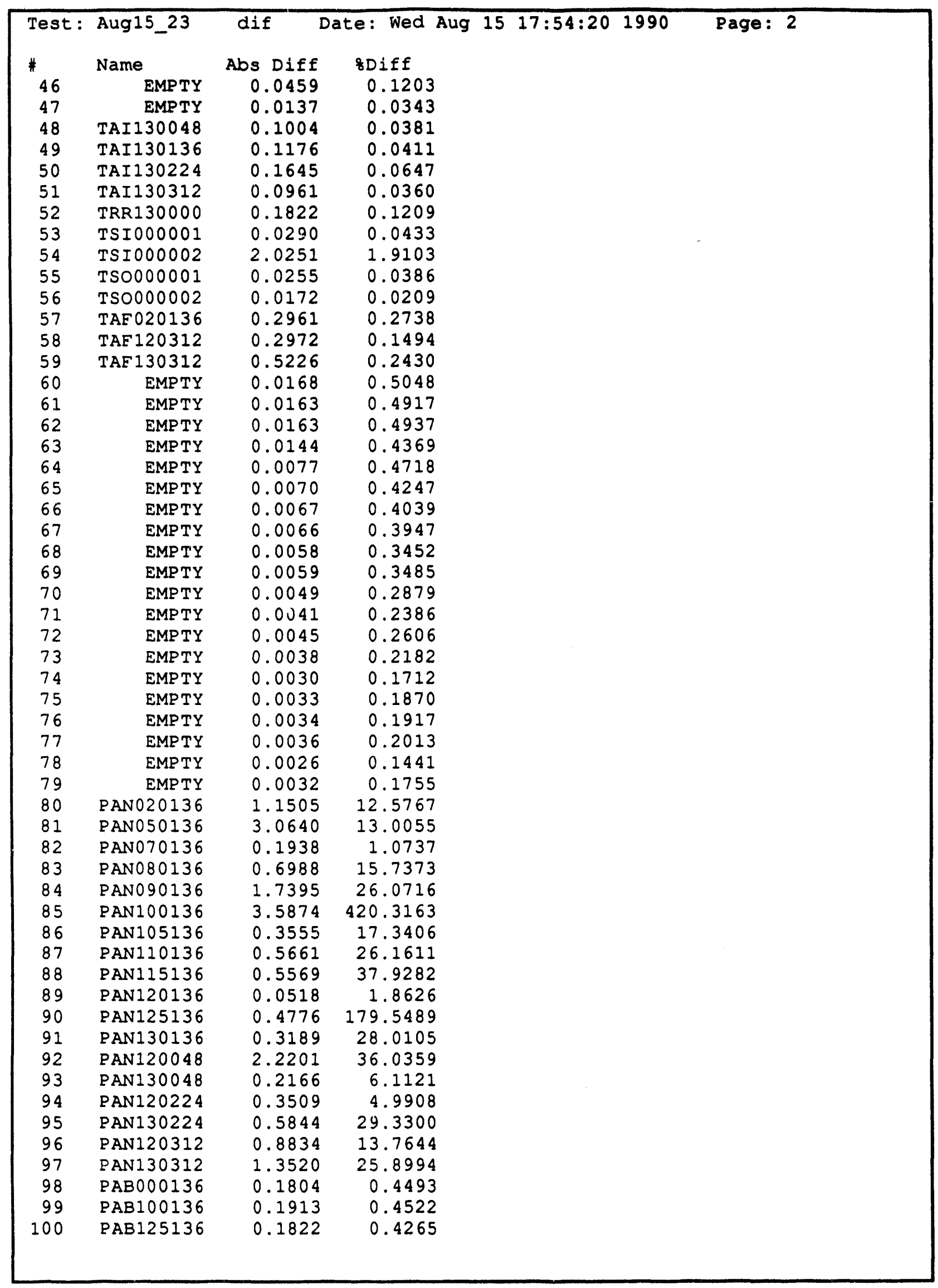




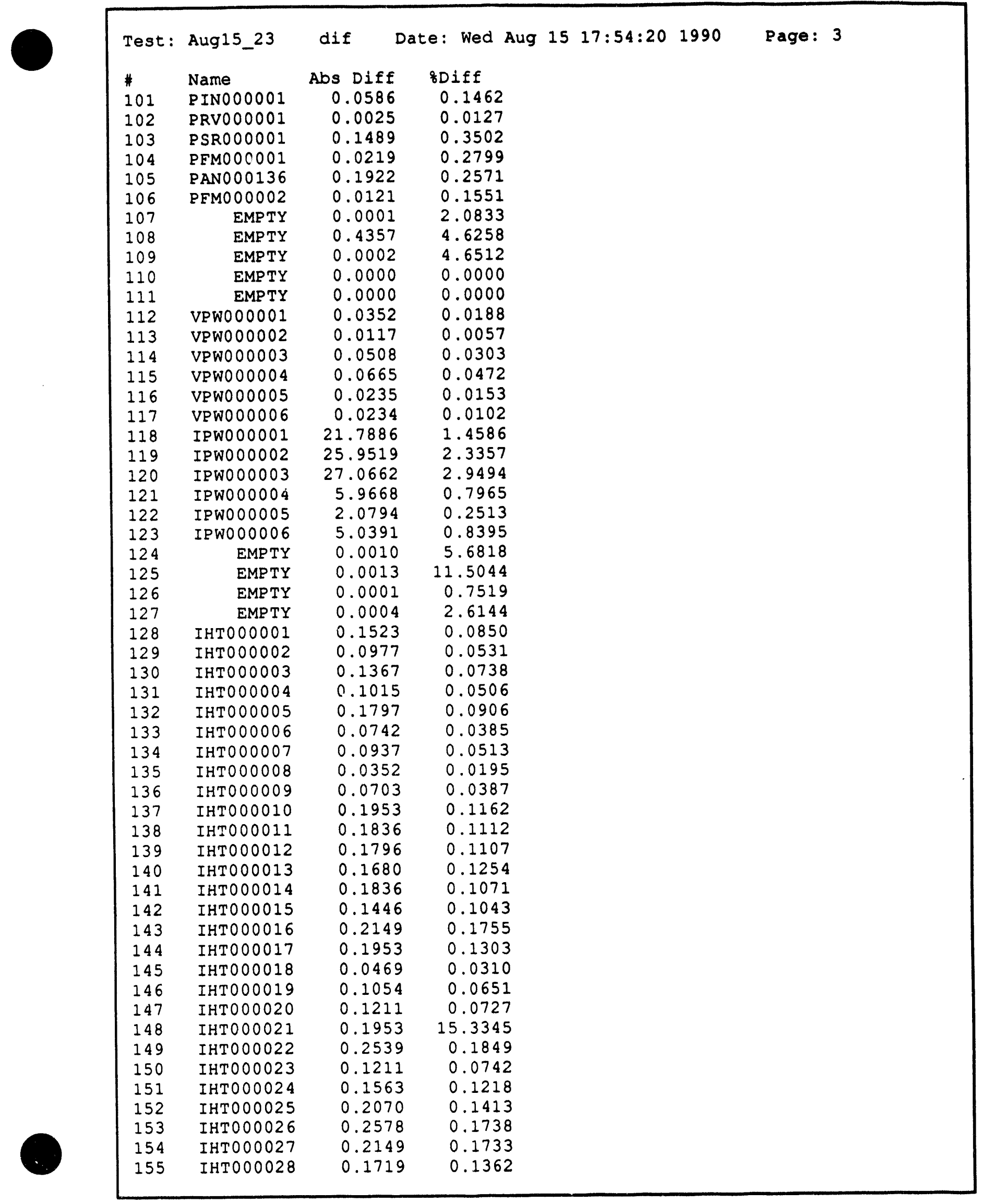




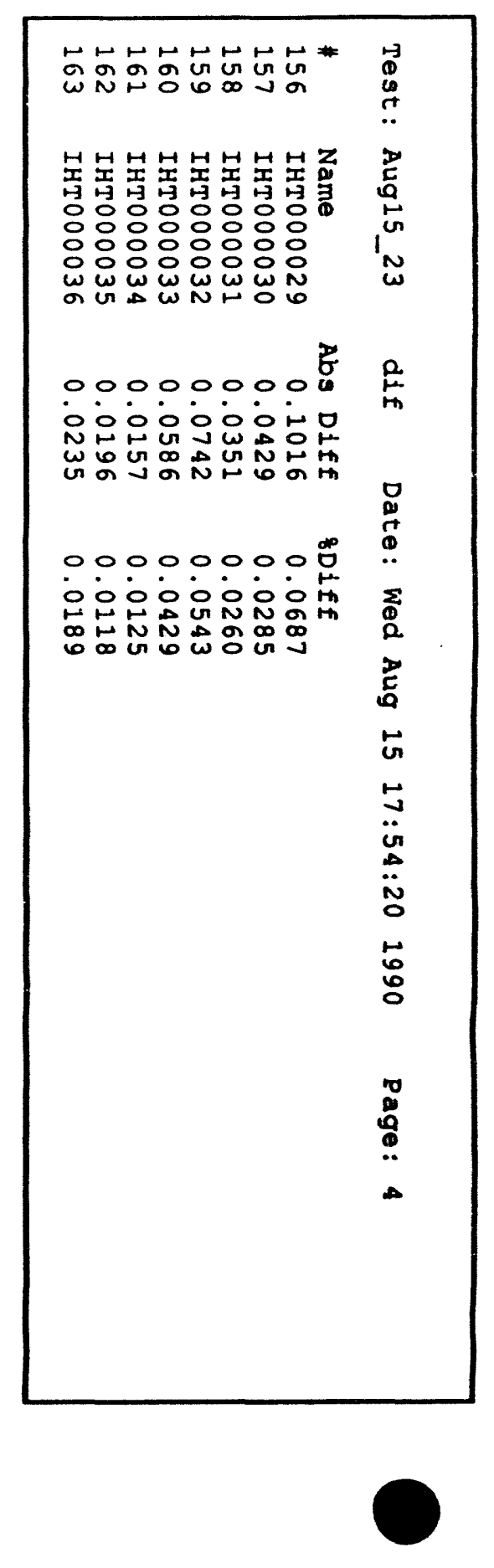




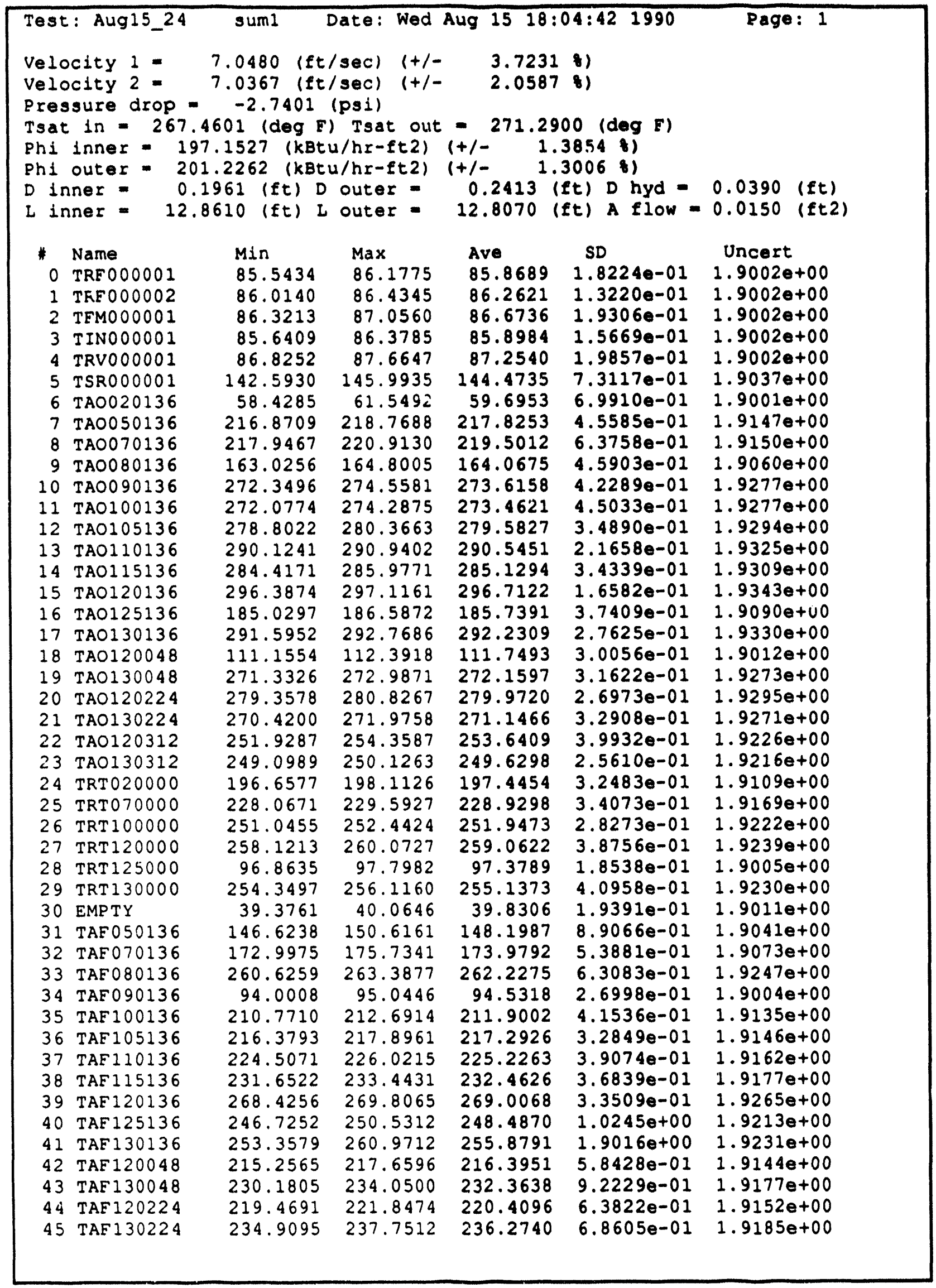




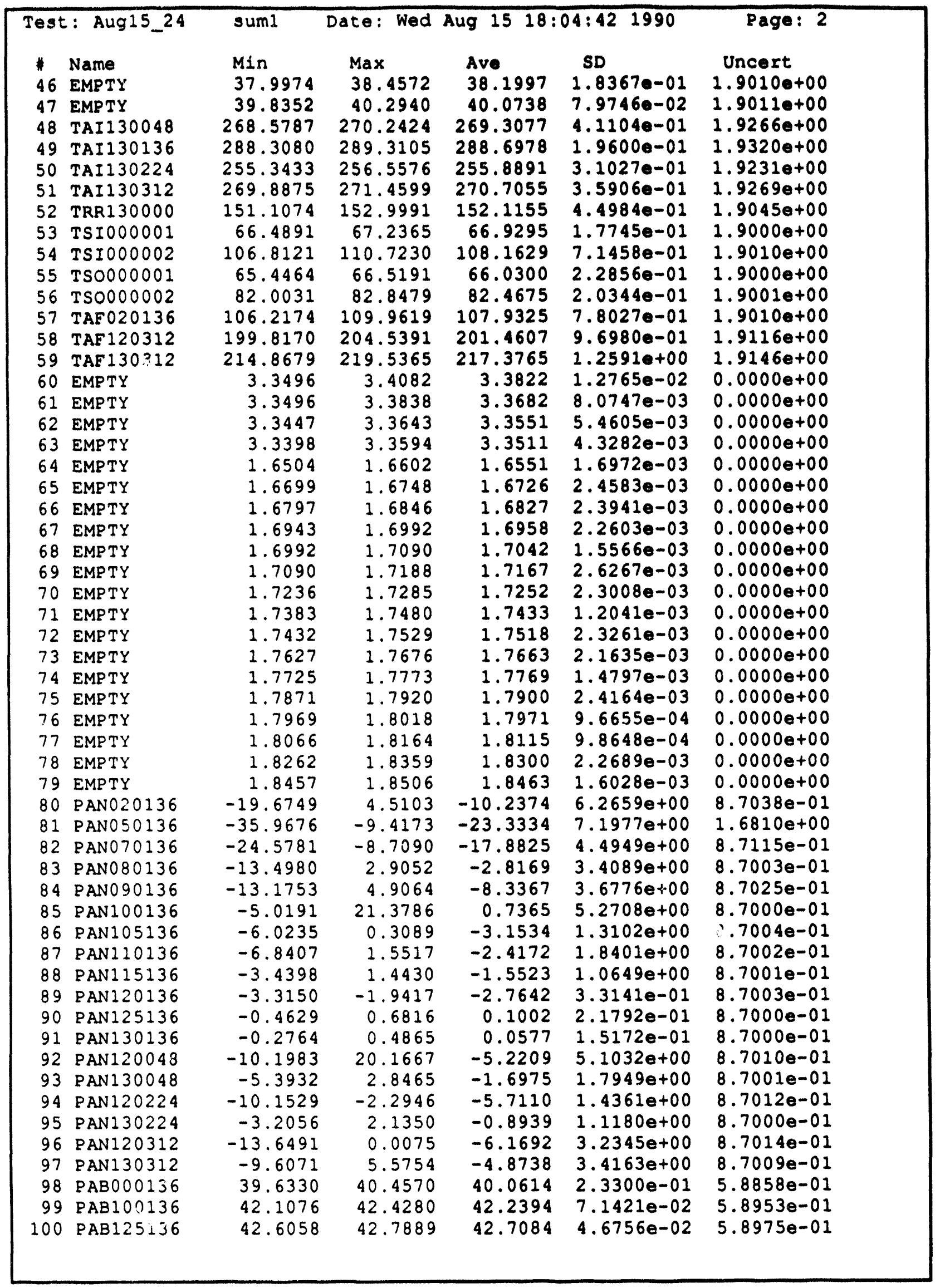




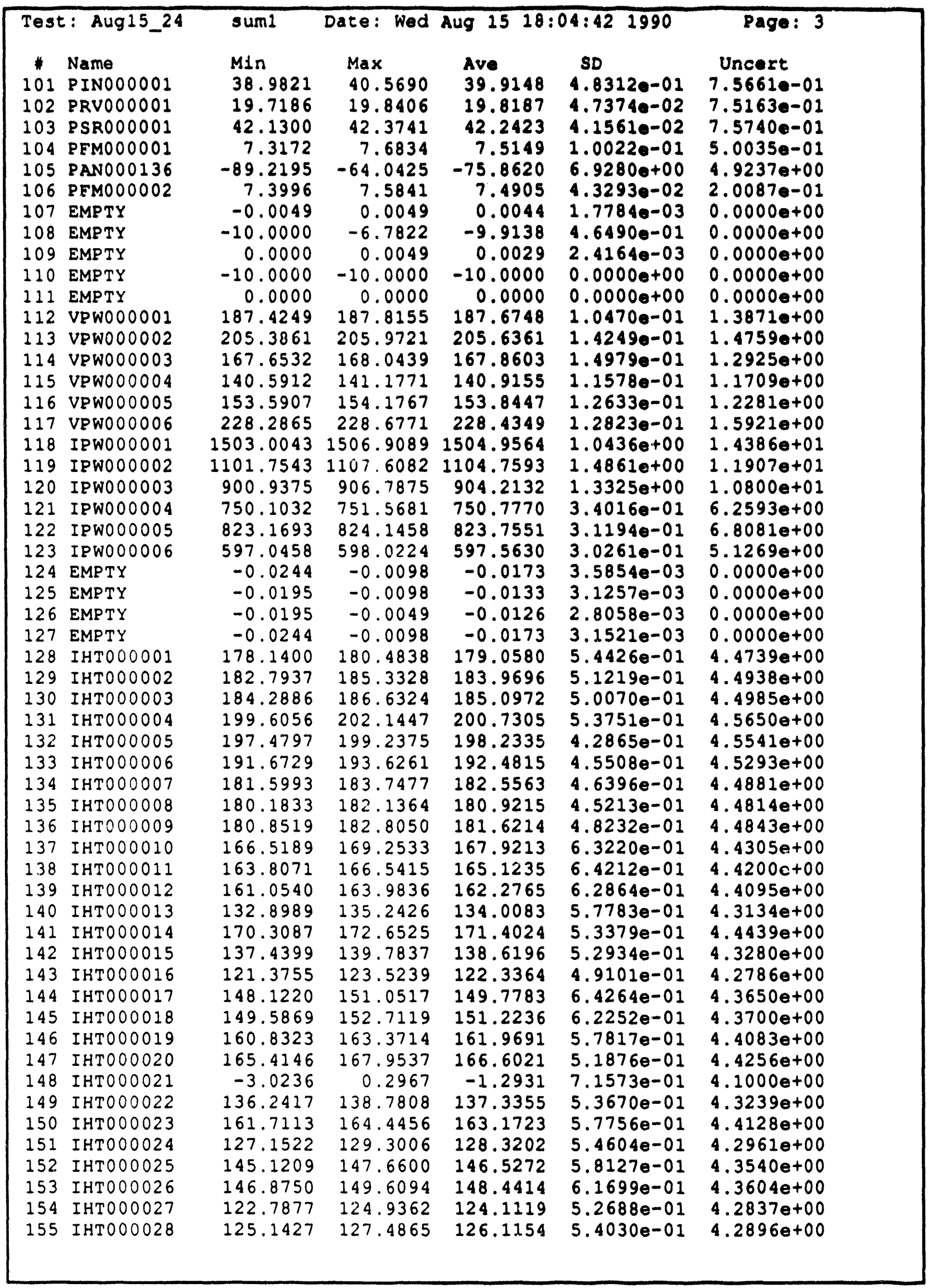




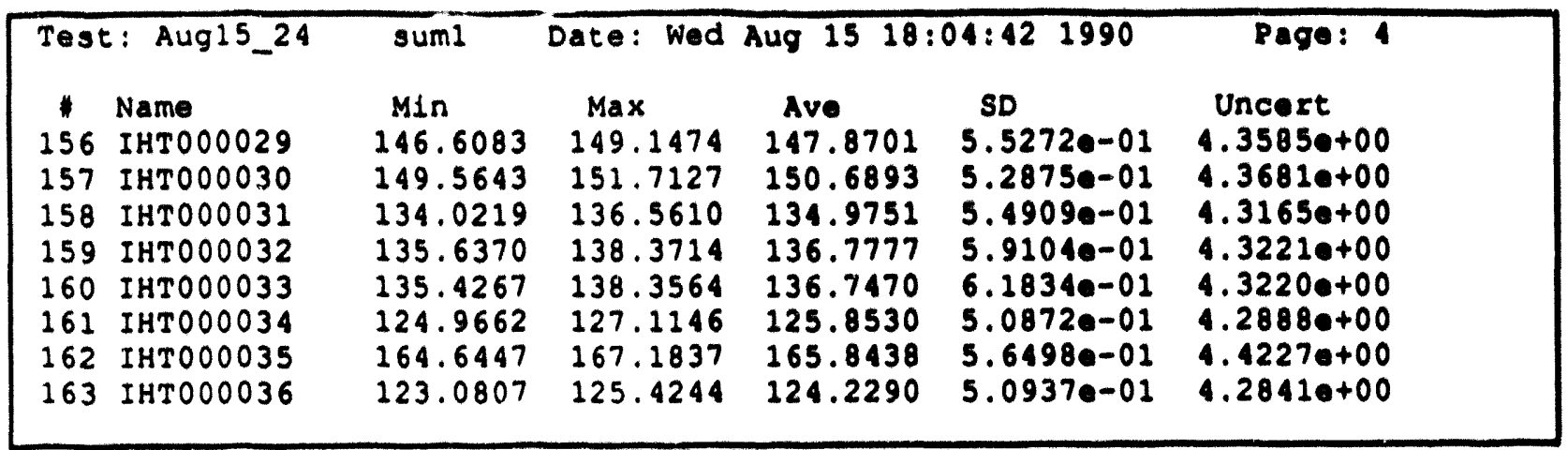




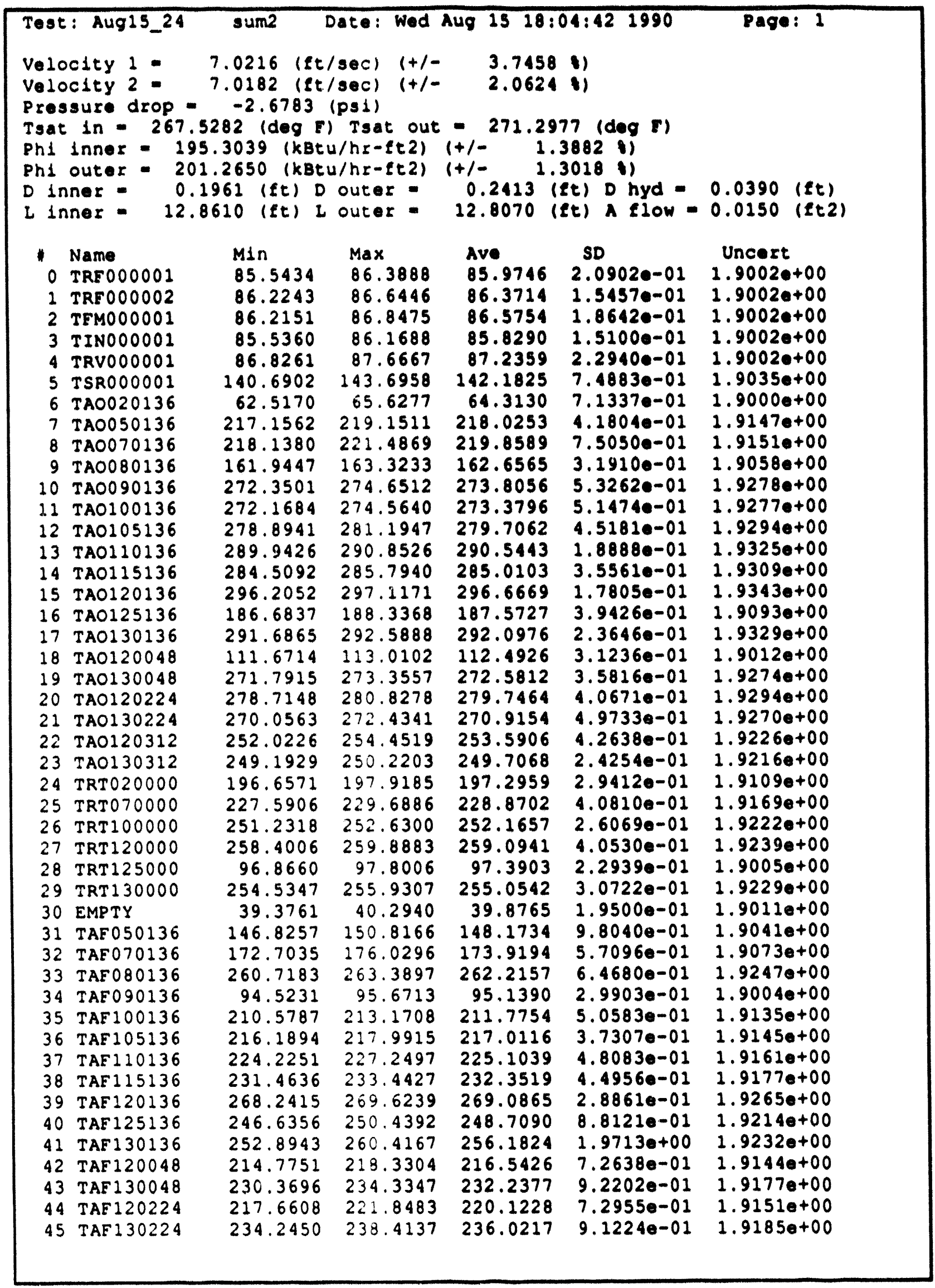




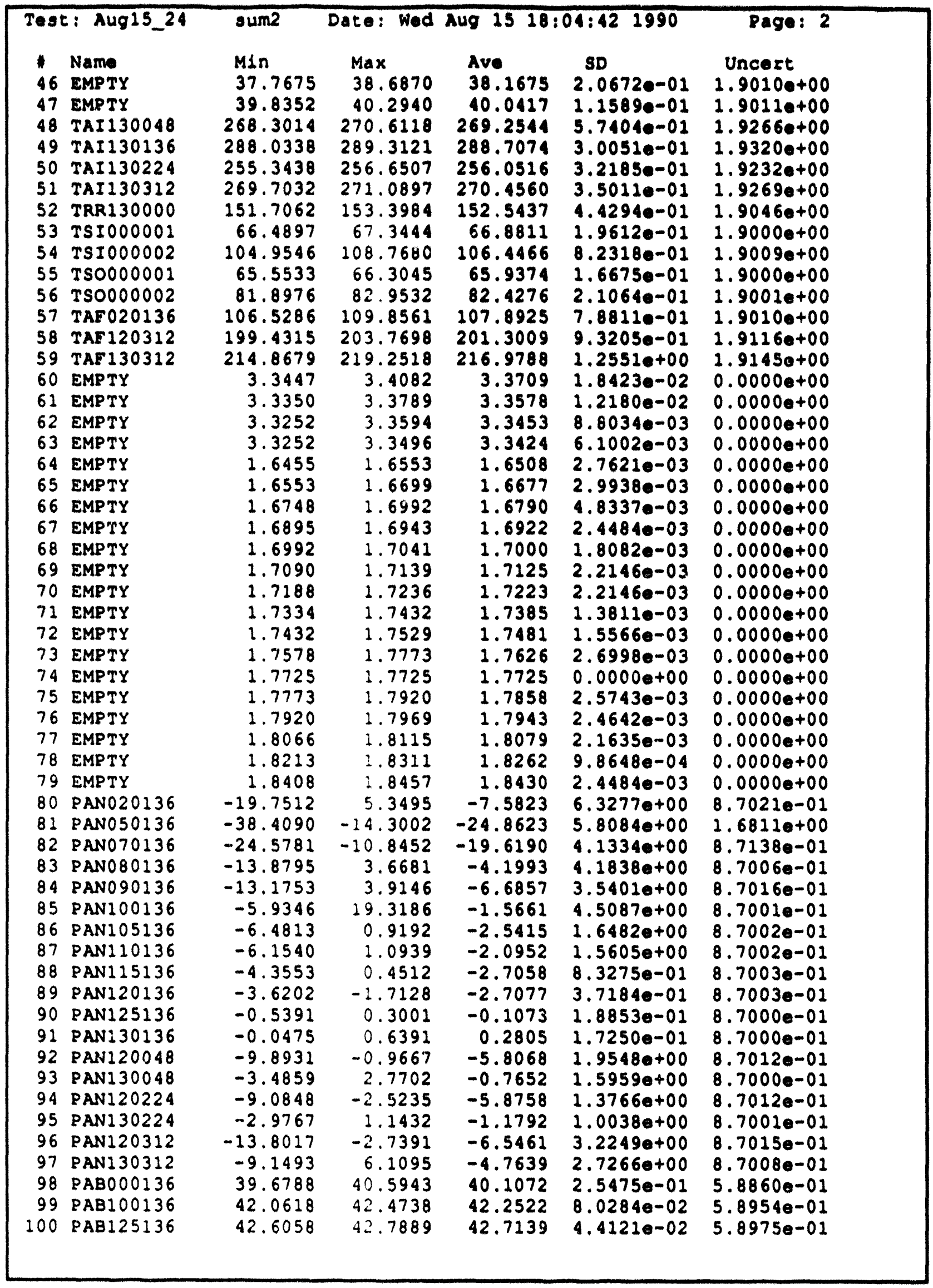




\begin{tabular}{|c|c|c|c|c|c|c|}
\hline \multicolumn{2}{|c|}{ Test: Alig15_24 } & sum2 & \multicolumn{3}{|c|}{ Date: Wed Aug $15 \quad 18: 04: 421990$ Page: 3} & \multirow{2}{*}{$\begin{array}{l}\text { Page: } 3 \\
\text { Uncert }\end{array}$} \\
\hline \multirow{2}{*}{\multicolumn{2}{|c|}{$\begin{array}{ll}\text { Name } \\
101 & \text { PIN000001 }\end{array}$}} & \multirow{5}{*}{$\begin{array}{l}\text { Min } \\
38.9821 \\
19.7186 \\
42.1300 \\
7.2256\end{array}$} & \multirow{2}{*}{$\begin{array}{l}\text { Max } \\
40.6911\end{array}$} & Ave & SD & \\
\hline & & & & 39.8806 & $5.1717 e-01$ & $7.5660 e-01$ \\
\hline 102 & PRV000001 & & 19.9627 & 19.8455 & $4.2430 e-02$ & $7.51640-01$ \\
\hline 103 & PSR000001 & & 42.3741 & 42.2399 & $4.4460--02$ & $7.5740 e-01$ \\
\hline 104 & PEMO00001 & & 7.6834 & 7.4581 & $8.71690-02$ & $5.0035 e-01$ \\
\hline 105 & PANO00136 & -89.2195 & -58.5494 & -74.1499 & $8.0118 e+00$ & $4.9235 e+00$ \\
\hline 106 & PEM000002 & 7.3660 & 7.5674 & 7.4509 & $4.9407--02$ & $2.0087 e-01$ \\
\hline 107 & EMPTY & 0.0000 & 0.0098 & 0.0047 & $1.3811 e-03$ & $0.0000 e+00$ \\
\hline 108 & EMPTY & -10.0000 & -9.3848 & -9.9788 & $9.64010-02$ & $0.00000+00$ \\
\hline 109 & EMPTY & 0.0000 & 0.0049 & 0.0037 & $2.1065 e-03$ & $0.0000 e+00$ \\
\hline 110 & EMPTY & -10.0000 & -10.0000 & -10.0000 & $0.0000 e+00$ & $0.0000 e+00$ \\
\hline 111 & EMPTY & -0.0049 & 0.0000 & -0.0001 & $6.90530-04$ & $0.00000+00$ \\
\hline 112 & VPW000001 & 187.4249 & 187.8155 & 187.6279 & $1.04100-01$ & $1.3869 e+00$ \\
\hline 113 & VPW000002 & 205.3861 & 205.7768 & 205.5892 & $9.6332 \theta-02$ & $1.4757 e+00$ \\
\hline 114 & VPWO00003 & 167.6532 & 168.0439 & 167.8447 & $1.3375 e-01$ & $1.2924 e+00$ \\
\hline 115 & VPW000004 & 140.5912 & 141.1771 & 140.9155 & $1.2853 e-01$ & $1.1709 e+00$ \\
\hline 116 & VPWO00005 & 153.5907 & 154.1767 & 153.7744 & $1.3329 e-01$ & $1.2278 e+00$ \\
\hline 117 & VPW000006 & 228.2865 & 228.6771 & 228.3919 & $1.1306 e-01$ & $1.5918 e+00$ \\
\hline 118 & IPW000001 & 1510.8136 & 1518.6230 & 1516.9059 & $2.1474 \theta+00$ & $1.4464 e+01$ \\
\hline 119 & IPW000002 & 1078.3391 & 1086.1442 & 1082.4369 & $1.4350 e+00$ & $1.1779 e+01$ \\
\hline 120 & IPWO00003 & 891.1875 & 895.0875 & 893.3713 & $1.0889 e+00$ & $1.0743 e+01$ \\
\hline 121 & IPWO00004 & 752.5446 & 754.0095 & 753.1891 & $4.34950-01$ & $6.27730+00$ \\
\hline 122 & IPWO00005 & 824.6340 & 825.6104 & 825.1516 & $3.0257 e-01$ & $6.8186 e+00$ \\
\hline 123 & IPWO00006 & 594.6044 & 598.0224 & 596.0298 & $8.7591 \mathrm{e}-01$ & $5.1158 e+00$ \\
\hline 124 & EMPTY & -0.0249 & -0.0098 & -0.0186 & $3.8206 e-03$ & $0.00000+00$ \\
\hline 125 & EMPTY & -0.0195 & -0.0098 & -0.0132 & $3.0003 e-03$ & $0.0000 e+00$ \\
\hline 126 & EMPTY & -0.0195 & -0.0098 & -0.0155 & $3.0708 \theta-03$ & $0.0000 e+00$ \\
\hline 127 & EMPTY & -0.0244 & -0.0098 & -0.0164 & $3.3829 e-03$ & $0.0000 e+00$ \\
\hline 128 & IHTO0000I & 177.7494 & 180.6791 & 179.0268 & $6.6522 e-01$ & $4.4738 e+00$ \\
\hline 129 & IHTO00002 & 182.7937 & 185.5281 & 183.9305 & $6.4921 \mathrm{e}-01$ & $4.4937 e+00$ \\
\hline 130 & IHTO00003 & 183.8980 & 186.8277 & 185.0308 & $6.2764 e-01$ & $4.4982 e+00$ \\
\hline 131 & IHTOOOOOO4 & 199.4103 & 202.1447 & 200.6680 & $6.0767 e-01$ & $4.5647 e+00$ \\
\hline 132 & IHTO00005 & 196.8938 & 199.4328 & 198.1319 & $5.8873 \theta-01$ & $4.5536 e+00$ \\
\hline 133 & IHTO00006 & 191.2823 & 193.8214 & 192.4151 & $5.7045 e-01$ & $4.5291 e+00$ \\
\hline 134 & IHTO00007 & 181.2087 & 183.7477 & 182.4743 & $5.2532 e-01$ & $4.4877 e+00$ \\
\hline 135 & IHT 000008 & 179.5973 & 181.7458 & 180.8200 & $5.1268 e-01$ & $4.4810 e+00$ \\
\hline 136 & IHTO00009 & 180.2659 & 182.8050 & 181.5511 & $5.6233 \theta-01$ & $4.48400+00$ \\
\hline 137 & IHTO00010 & 167.1049 & 169.2533 & 167.8784 & $5.3519 \theta-01$ & $4.4304 e+00$ \\
\hline 138 & IHTO00011 & 164.1977 & 166.1509 & 165.0376 & $5.0262 e-01$ & $4.4197 e+00$ \\
\hline 139 & IHTO00012 & 160.8586 & 163.3977 & 162.1710 & $5.8132 e-01$ & $4.4091 e+00$ \\
\hline 140 & IHTO00013 & 132.7036 & 135.2426 & 133.8755 & $5.0992 e-01$ & $4.3130 e+00$ \\
\hline 141 & IHTO00014 & 169.9181 & 172.6525 & 171.2852 & $5.1750 e-01$ & $4.4434 e+00$ \\
\hline 142 & IHTO00015 & 137.2446 & 139.9790 & 138.5532 & $5.2237 \theta-01$ & $4.3278 \theta+00$ \\
\hline 143 & IHT000016 & 120.9848 & 123.5239 & 122.3169 & $5.6178 \theta-01$ & $4.2786 e+00$ \\
\hline 144 & IHTO00017 & 147.3408 & 150.6611 & 149.1846 & $8.5012 e-01$ & $4.3630 e+00$ \\
\hline 145 & IHTO00018 & 149.5869 & 152.7119 & 151.1103 & $6.3992 e-01$ & $4.3696 e+00$ \\
\hline 146 & IHTO00019 & 160.6370 & 162.7855 & 161.7855 & $5.4339 e-01$ & $4.4077 e+00$ \\
\hline 147 & IHTO00020 & 165.4146 & 167.7584 & 166.4458 & $5.4396 \theta-01$ & $4.4250 e+00$ \\
\hline 148 & IHTO00021 & -2.8283 & -0.0939 & -1.4455 & $6.0598 e-01$ & $4.1000 \theta+00$ \\
\hline 149 & IHT000022 & 136.2417 & 138.1949 & 137.1636 & $4.5513 e-01$ & $4.3234 e+00$ \\
\hline 150 & IHT 000023 & 162.1019 & 163.8597 & 162.9652 & $4.8982 e-01$ & $4.4120 e+00$ \\
\hline 151 & IHTO00024 & 127.1522 & 129.4959 & 128.1288 & $4.9285 \theta-01$ & $4.2955 e+00$ \\
\hline 152 & IHTO00025 & 145.3163 & 147.4647 & 146.3749 & $4.7201 e-01$ & $4.35350+00$ \\
\hline 153 & IHT000026 & 147.0703 & 149.4141 & 148.2266 & $4.8783 e-01$ & $4.3597 e+00$ \\
\hline 154 & IHTO00027 & 122.7877 & 125.3268 & 124.0259 & $5.0007 e-01$ & $4.2835 e+00$ \\
\hline 155 & IHTO00028 & 124.7521 & 127.4865 & 125.9943 & $6.2981 \theta-01$ & $4.2892 \theta+00$ \\
\hline
\end{tabular}




\begin{tabular}{|c|c|c|c|c|c|c|}
\hline \multicolumn{2}{|c|}{ Test: Aug15_24 } & sum2 & Date: Wed & 2 & $: 4219$ & Page: 4 \\
\hline $\begin{array}{l}1 \\
156 \\
157 \\
158 \\
159 \\
160 \\
161 \\
162 \\
163\end{array}$ & 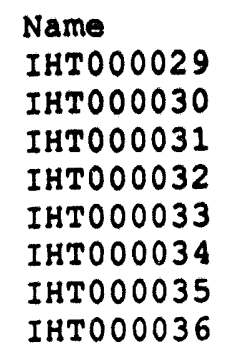 & $\begin{array}{c}\text { Min } \\
146.4130 \\
149.1737 \\
133.4360 \\
135.4417 \\
135.2314 \\
124.7709 \\
164.8400 \\
123.2760\end{array}$ & $\begin{array}{c}\text { Max } \\
149.1474 \\
151.9080 \\
136.1704 \\
137.9808 \\
137.9657 \\
127.1146 \\
167.1837 \\
125.4244\end{array}$ & $\begin{array}{l}\text { Ave } \\
147.6943 \\
150.4940 \\
134.7993 \\
136.6136 \\
136.5634 \\
125.8178 \\
165.8087 \\
124.2329\end{array}$ & $\begin{array}{l}\text { SD } \\
6.3523 e-01 \\
6.3938 e-01 \\
5.8858 e-01 \\
6.1637 e-01 \\
5.9802 e-01 \\
4.9875 e-01 \\
5.3519 e-01 \\
4.9481 e-01\end{array}$ & $\begin{array}{c}\text { Uncert } \\
4.3579 e+00 \\
4.3675 e+00 \\
4.3159 e+00 \\
4.3216 e+00 \\
4.3215 e+00 \\
4.2887 e+00 \\
4.4226 e+00 \\
4.2841 e+00\end{array}$ \\
\hline
\end{tabular}




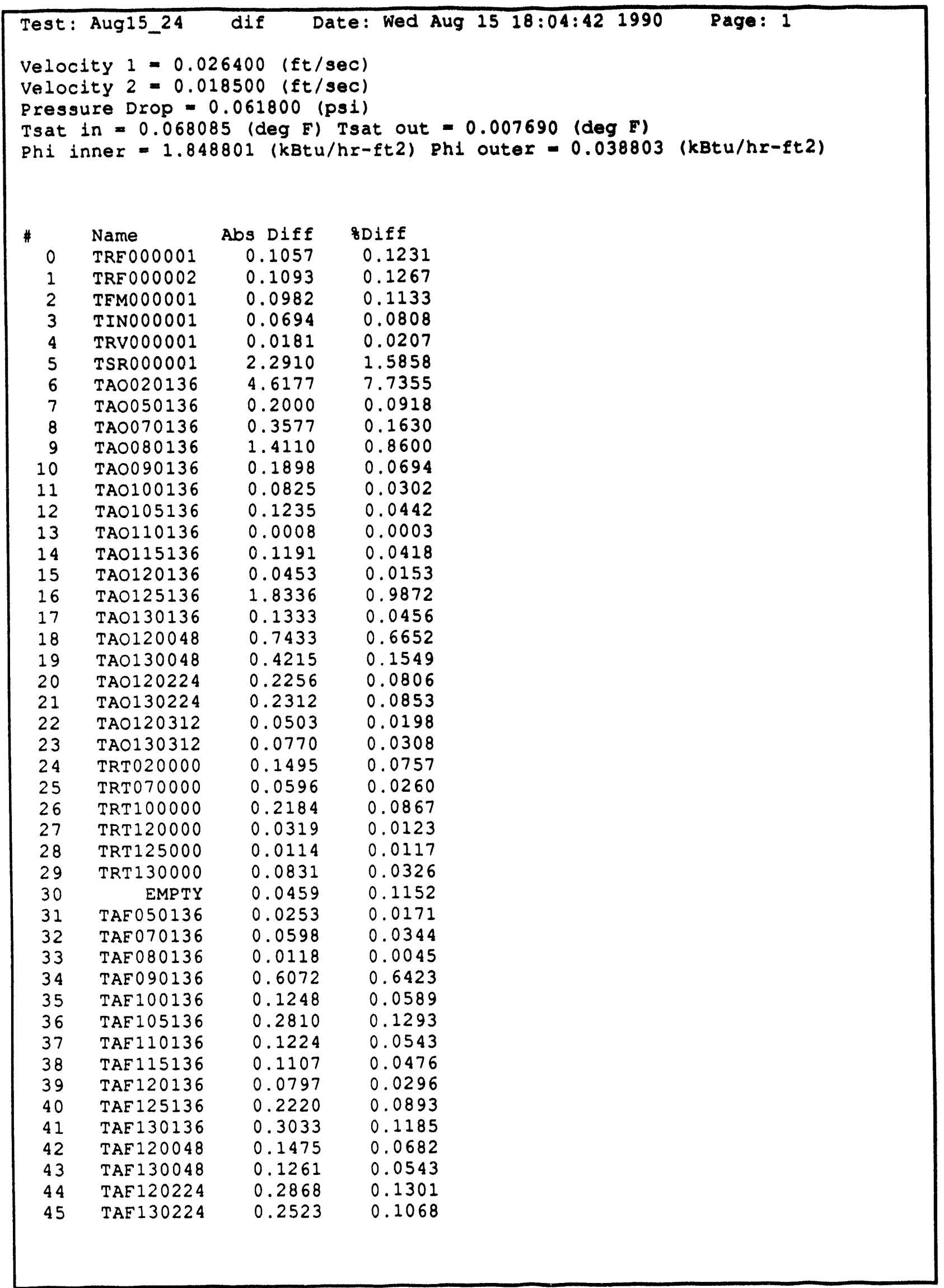




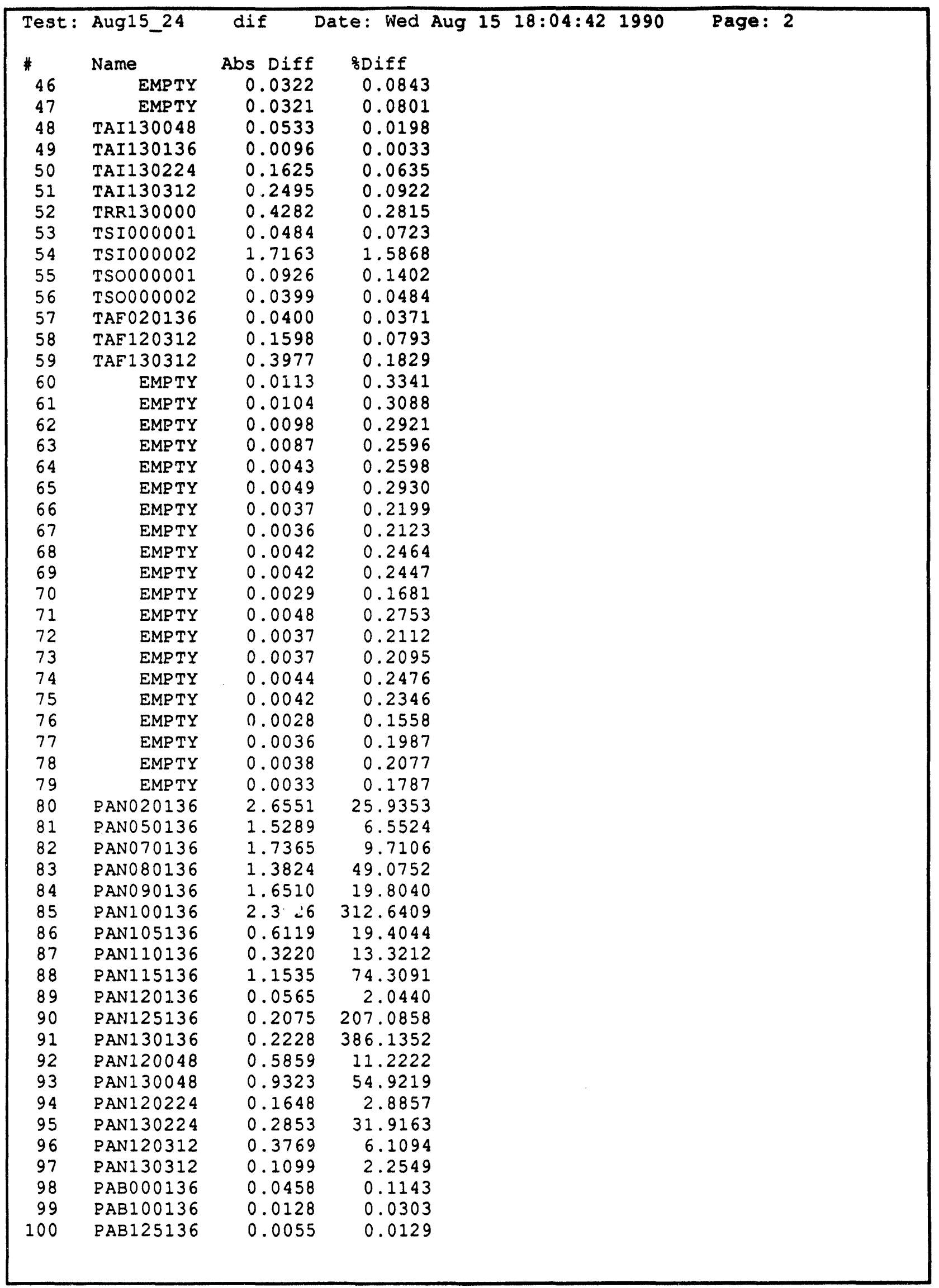


Test: Aug15_24

Name

101

102

103

104

105

106

107

108

109

110

111

112

113

114

115

116

117

118

119

120

121

122

123

124

125

126

127

128

129

130

131

132

133

134

135

136

137

138

139

140

141

142

143

144

145

146

147

148

149

150

151

152

153

154

155
PIN000001

PRV000001

PSRO00001

PEMO00001

PANO00136

PEMO00002

EMPTY

EMPTY

EMPTY

EMPTY

EMPTY

VPWO00001

VPW000002

VPW000003

VPW000004

VPW000005

IPWO00001

IPWOO0002

IPWO00003

IPWOOOOO

IPWO 00005

IPWOOOOOO6

EMPTY

EMPTY

EMPTY

EMPTY

IHTO00001

IHTOOOOO2

IHTOOOOO3

IHTOOOOO4

IHTO00005

IHTO00006

IHTO00007

IHTO00008

IHTOOOOO9

IHTO00010

IHTO00011

IHTO00012

IHTO00013

IHTO00014

IHTO00015

IHT000016

IHTO00017

IHT000018

IHTO00019

IHTO00020

IHT000021

IHTO00022

IHTOOOO23

IHT000024

IHTOOOO25

IHTO00026

IHTOOO027

IHT000028
VPWO00006
Date: Wed Aug 15 18:04:42 1990

Page: 3

Abs Diff

0.0342

0.0268

0.0024

0.0568

1.7121

0.0396

0.0003

0.0650

0.0008

0.0000

0.0001

0.0469

0.0469

0.0156

0.0000

0.0703

0.0430

11.9495

22.3224

10.8419

2. 4121

1. 3965

1.5332

0.0013

0.0001

0.0029

0.0009

0.0312

0.0391

0.0664

0.0625

0.1016

0.0664

0.0820

0.1015

0.0703

0.0429

0.0859

0.1055

0.1328

0.1172

0.0664

0.0195

0.5937

0.1133

0.1836

0.1563

0.1524

0.1719

0.2071

0.1914

0.1523

0.2148

0.0860

0.1211

\&Diff

0.0857

0.1352

0.0057

0.7558

2. 2569

0.5287

6.8182

0.6556

27.5862

0.0000

100.0000

0.0250

0.0228

0.0093

0.0000

0.0457

0.0188

0.7940

2.0206

1. 1990

0.3213

0.1695

0.2566

7.5144

0.7519

23.0159

5.2023

0.0174

0.0213

0.0359

0.0311

0.0513

0.0345

0.0449

0.0561

0.0387

0.0255

0.0520

0.0650

0.0991

0.0684

0.0479

0.0159

0.3964

0.0749

0.1134

0.0938

11.7856

0.1252

0.1269

0.1492

0.1039

0.1447

0.0693

0.0960 
Test: Aug15_24 dif Date: Wed Aug 15 18:04:42 1990 Page: 4

\begin{tabular}{llrl}
$\#$ & Name & Abs Diff & \multicolumn{1}{l}{ \&Diff } \\
156 & IHTO00029 & 0.1758 & 0.1189 \\
157 & IHTO00030 & 0.1953 & 0.1296 \\
158 & IHTO00031 & 0.1758 & 0.1302 \\
159 & IHTO00032 & 0.1641 & 0.1200 \\
160 & IHTO00033 & 0.1836 & 0.1343 \\
161 & IHTO00034 & 0.0352 & 0.0280 \\
162 & IHTO00035 & 0.0351 & 0.0212 \\
163 & IHTO00036 & 0.0039 & 0.0031
\end{tabular}




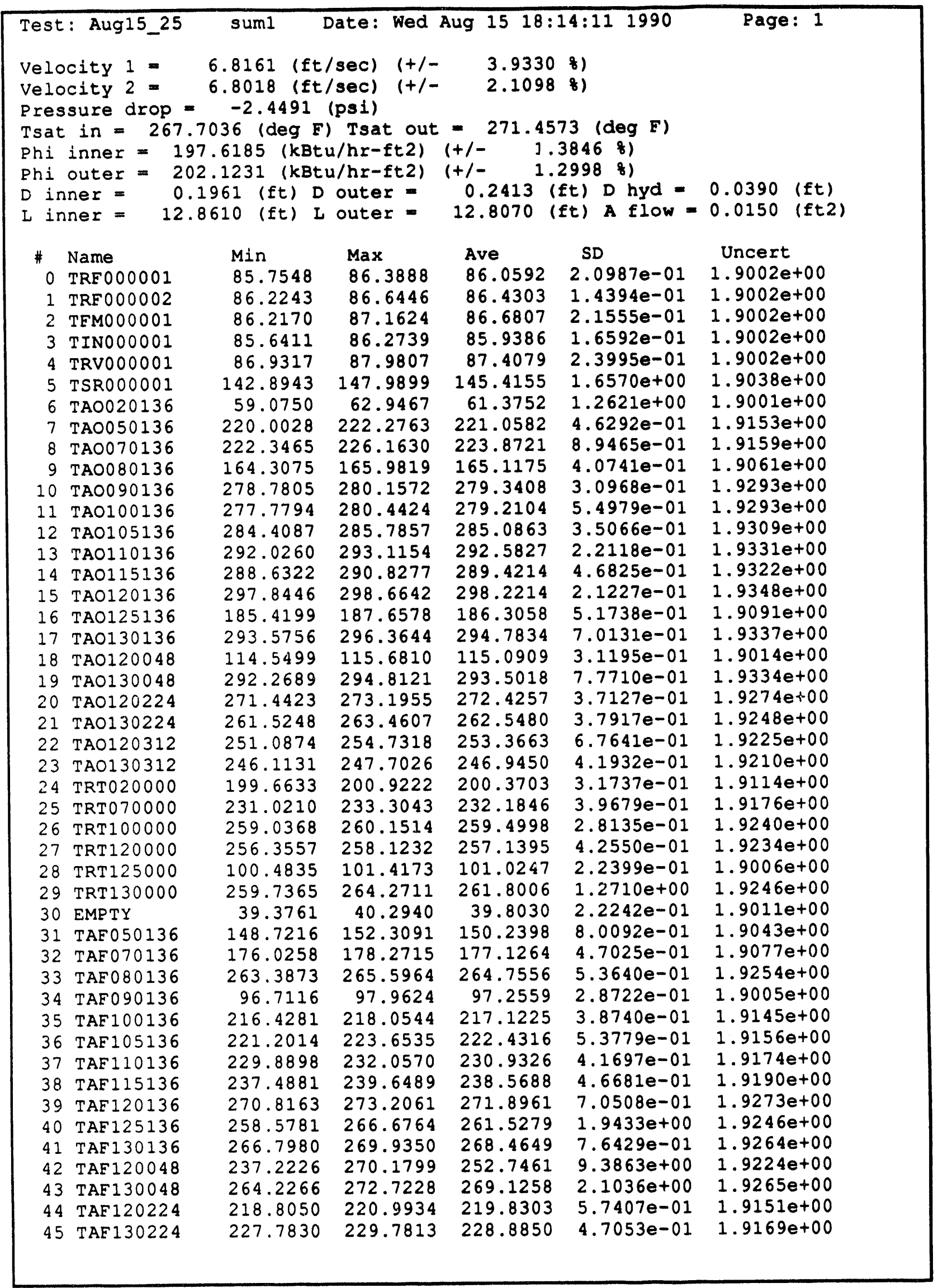




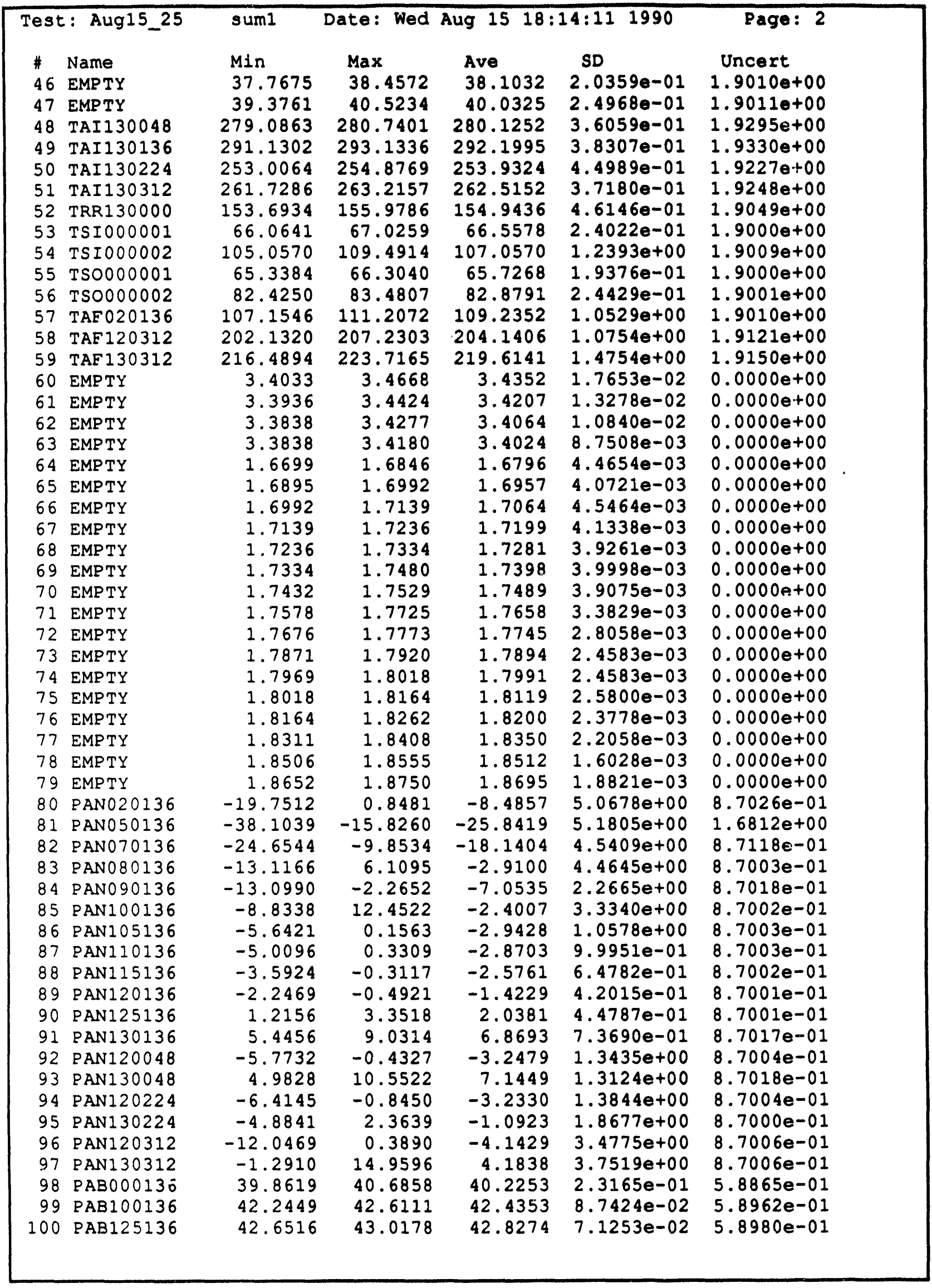




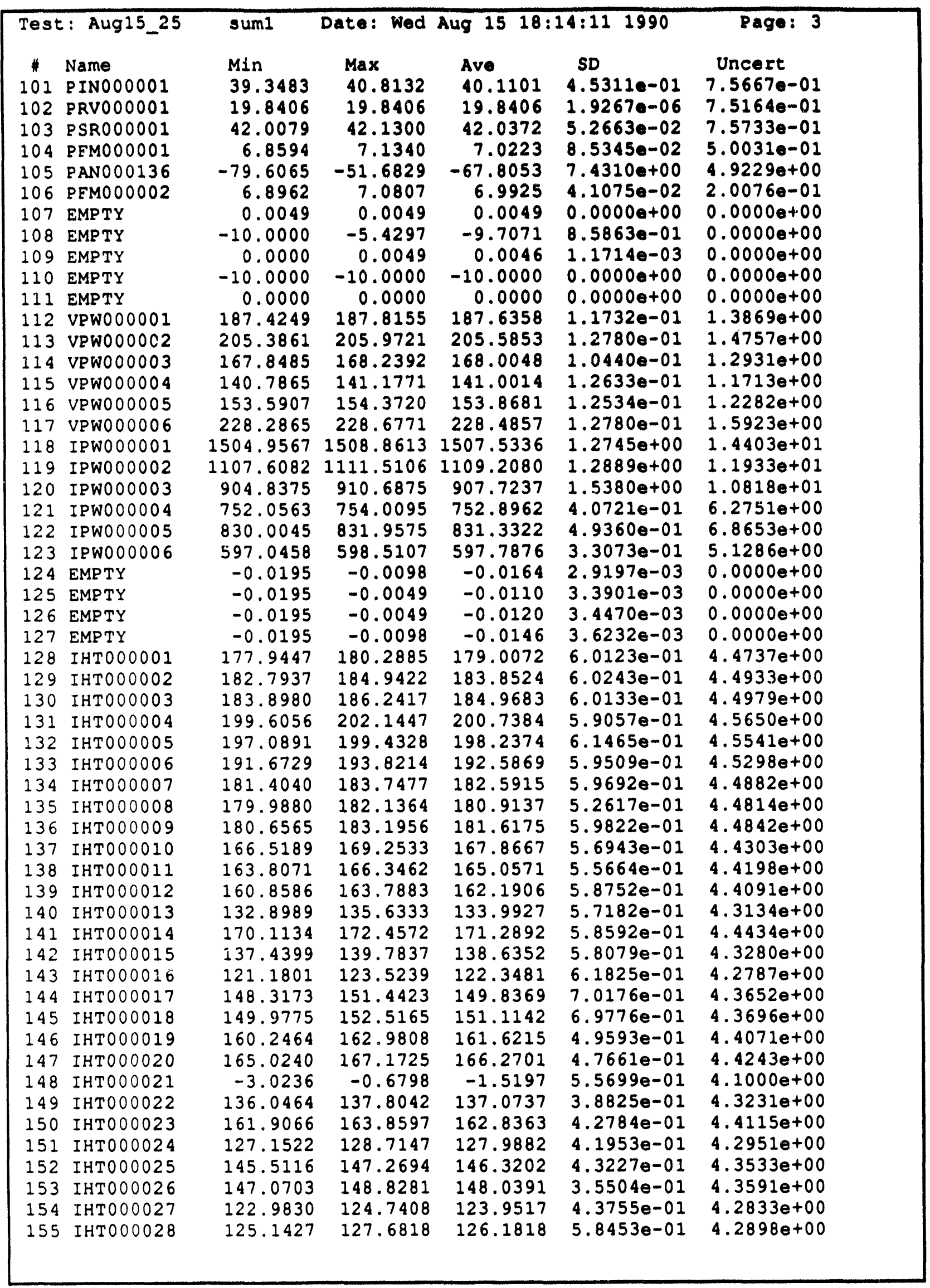




\begin{tabular}{|c|c|c|c|c|c|c|}
\hline $\begin{array}{l}\# \\
156 \\
157 \\
158 \\
159 \\
160 \\
161 \\
162 \\
163\end{array}$ & 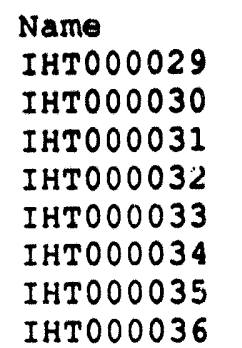 & $\begin{array}{l}\text { Min } \\
146.8036 \\
149.5643 \\
133.8266 \\
135.2464 \\
135.2314 \\
124.5756 \\
164.4494 \\
122.8854\end{array}$ & $\begin{array}{l}\text { Max } \\
149.3427 \\
152.1033 \\
136.5610 \\
138.1761 \\
138.1611 \\
126.9193 \\
166.7931 \\
125.6197\end{array}$ & $\begin{array}{l}\text { Ave } \\
147.8935 \\
150.7323 \\
134.9868 \\
136.7347 \\
136.6611 \\
125.8764 \\
165.7501 \\
124.2290\end{array}$ & $\begin{array}{l}\text { SD } \\
5.6509 e-01 \\
5.6288 e-01 \\
5.9635 e-01 \\
6.1497 e-01 \\
6.2570 e-01 \\
4.9538 e-01 \\
4.7941 e-01 \\
5.2740 e-01\end{array}$ & $\begin{array}{c}\text { Uncert } \\
4.3586 e+00 \\
4.3683 e+00 \\
4.3165 e+00 \\
4.3220 e+00 \\
4.3218 e+00 \\
4.2889 e+00 \\
4.4224 e+00 \\
4.2841 e+00\end{array}$ \\
\hline
\end{tabular}




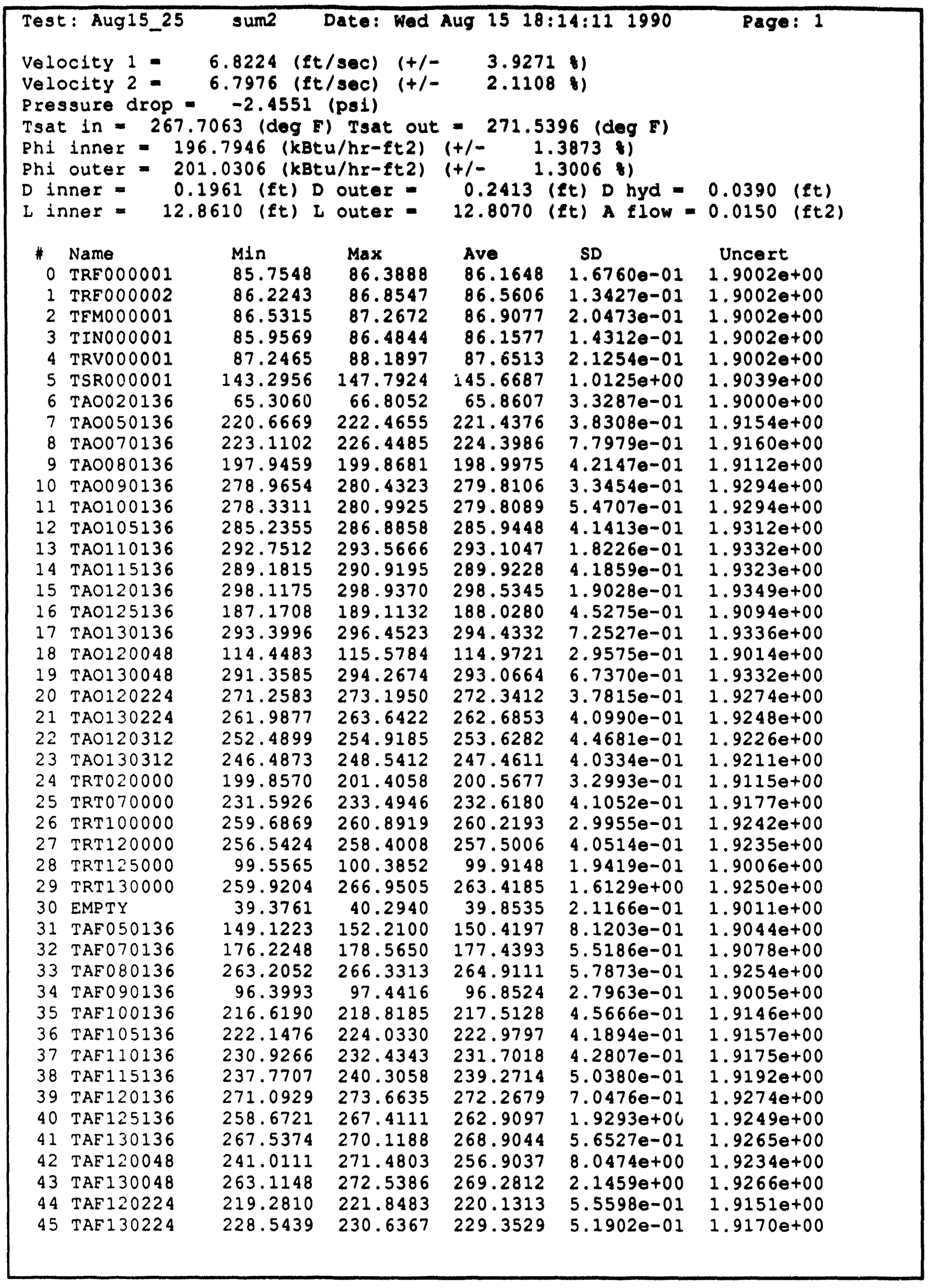




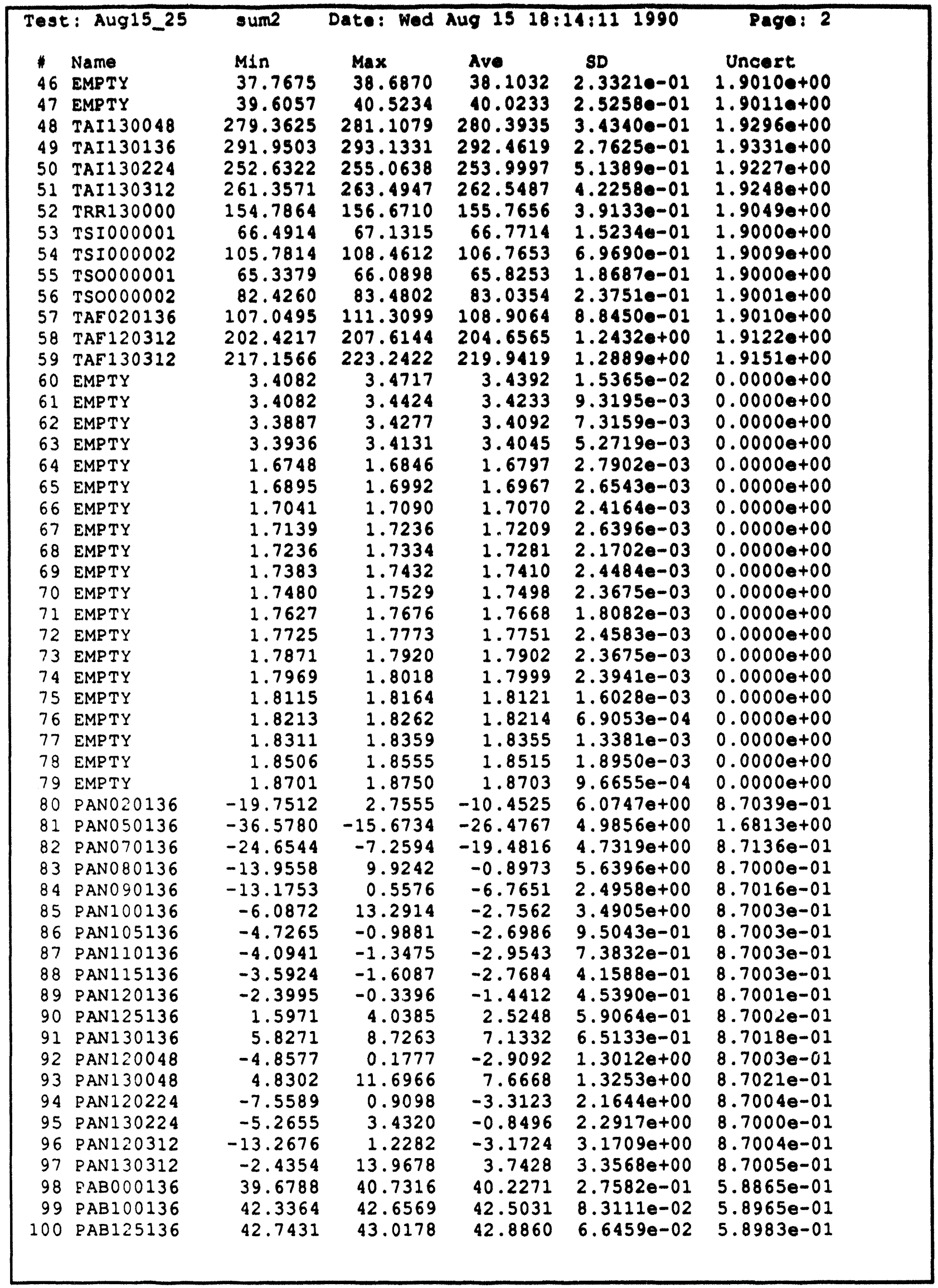




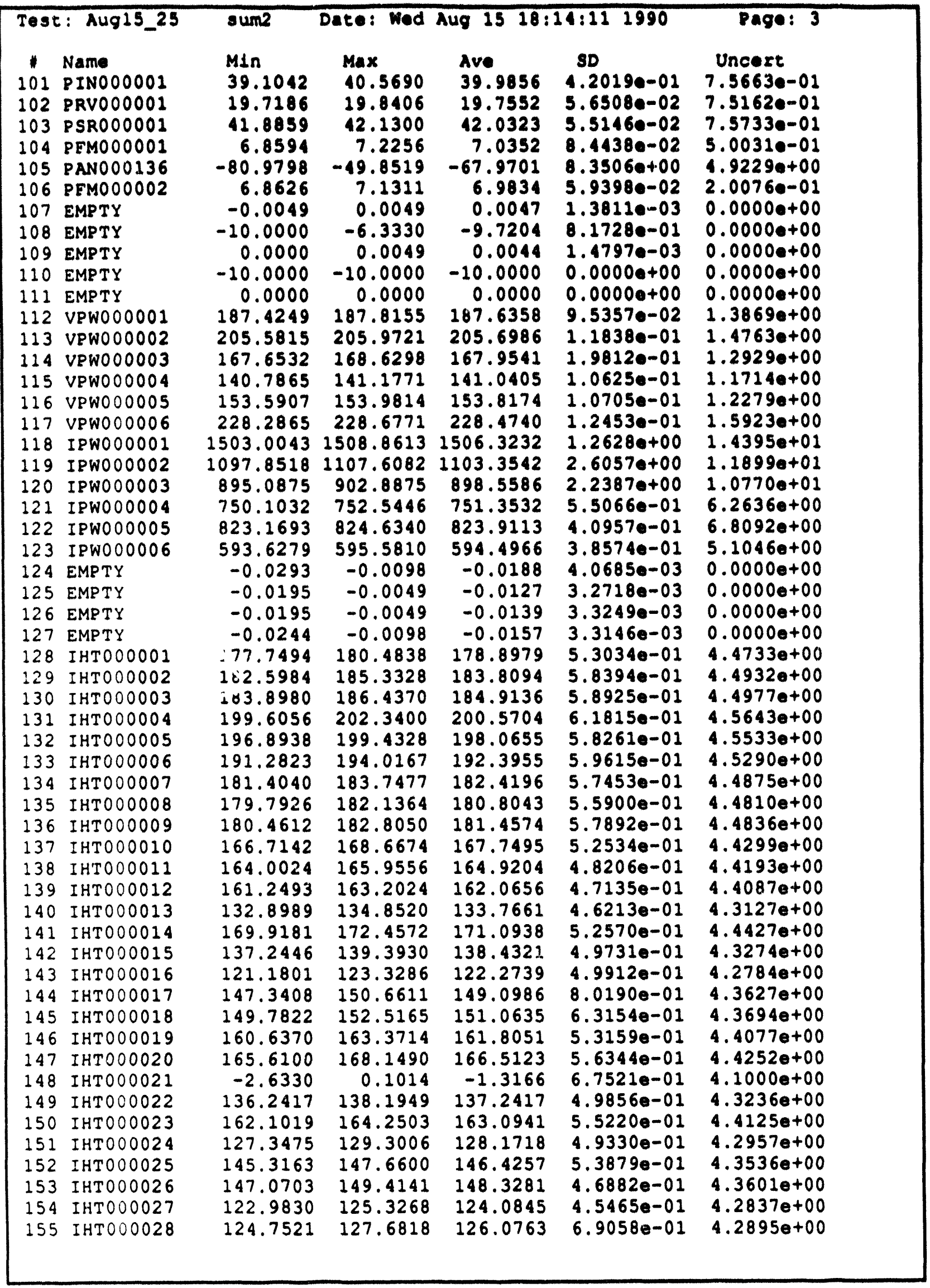




\begin{tabular}{|c|c|c|c|c|c|c|}
\hline $\begin{array}{l}156 \\
157 \\
158 \\
159 \\
160 \\
161 \\
162 \\
163\end{array}$ & $\begin{array}{l}\text { Name } \\
\text { IHTO00029 } \\
\text { IHTO00030 } \\
\text { IHTO00031 } \\
\text { IHTO00032 } \\
\text { IHTOOO033 } \\
\text { IHTO00034 } \\
\text { IHTO00035 } \\
\text { IHTO00036 }\end{array}$ & $\begin{array}{l}\text { M1n } \\
146.4130 \\
149.1737 \\
133.4360 \\
135.2464 \\
135.2314 \\
124.7709 \\
164.6447 \\
123.0807\end{array}$ & $\begin{array}{l}\operatorname{Max} \\
149.1474 \\
152.1033 \\
136.3657 \\
138.5667 \\
138.5517 \\
127.3099 \\
167.5744 \\
125.8150\end{array}$ & $\begin{array}{l}\text { Ave } \\
147.7099 \\
130.5326 \\
134.8266 \\
136.5980 \\
136.5634 \\
125.8686 \\
165.7696 \\
124.2603\end{array}$ & $\begin{array}{l}8 D \\
7.0117 e-01 \\
7.1703 e-01 \\
7.2827 \bullet-01 \\
7.6192 a-01 \\
7.5458 a-01 \\
5.9042 a-01 \\
6.2460 a-01 \\
5.8787 \bullet-01\end{array}$ & $\begin{array}{l}\text { Uncert } \\
4.3580 e+00 \\
4.3677 \theta++00 \\
4.3160 \theta+00 \\
4.3216 \theta+00 \\
4.3215 \theta+00 \\
4.2889 \theta+00 \\
4.4224 \theta++00 \\
4.2842 \theta+00\end{array}$ \\
\hline
\end{tabular}




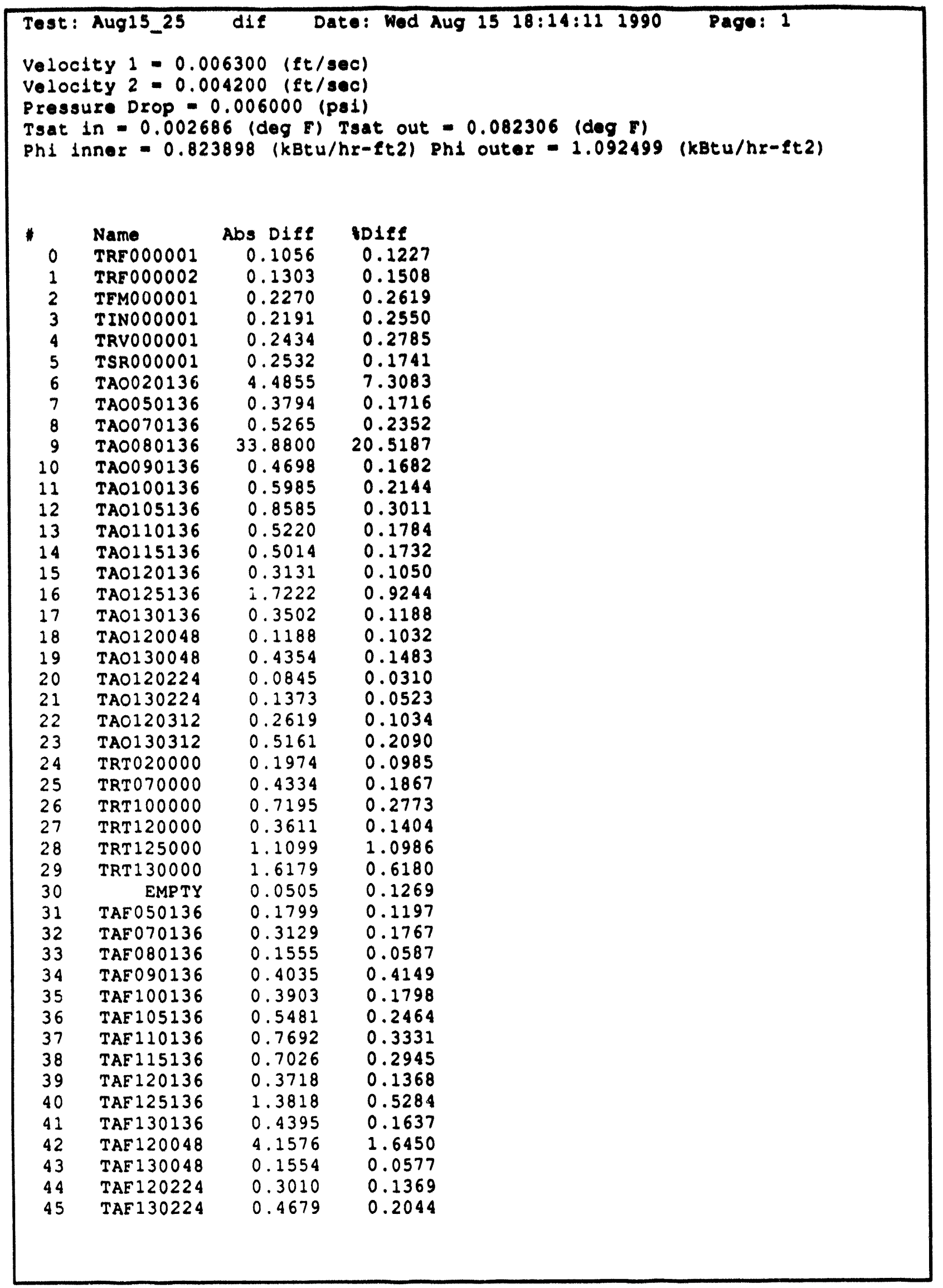




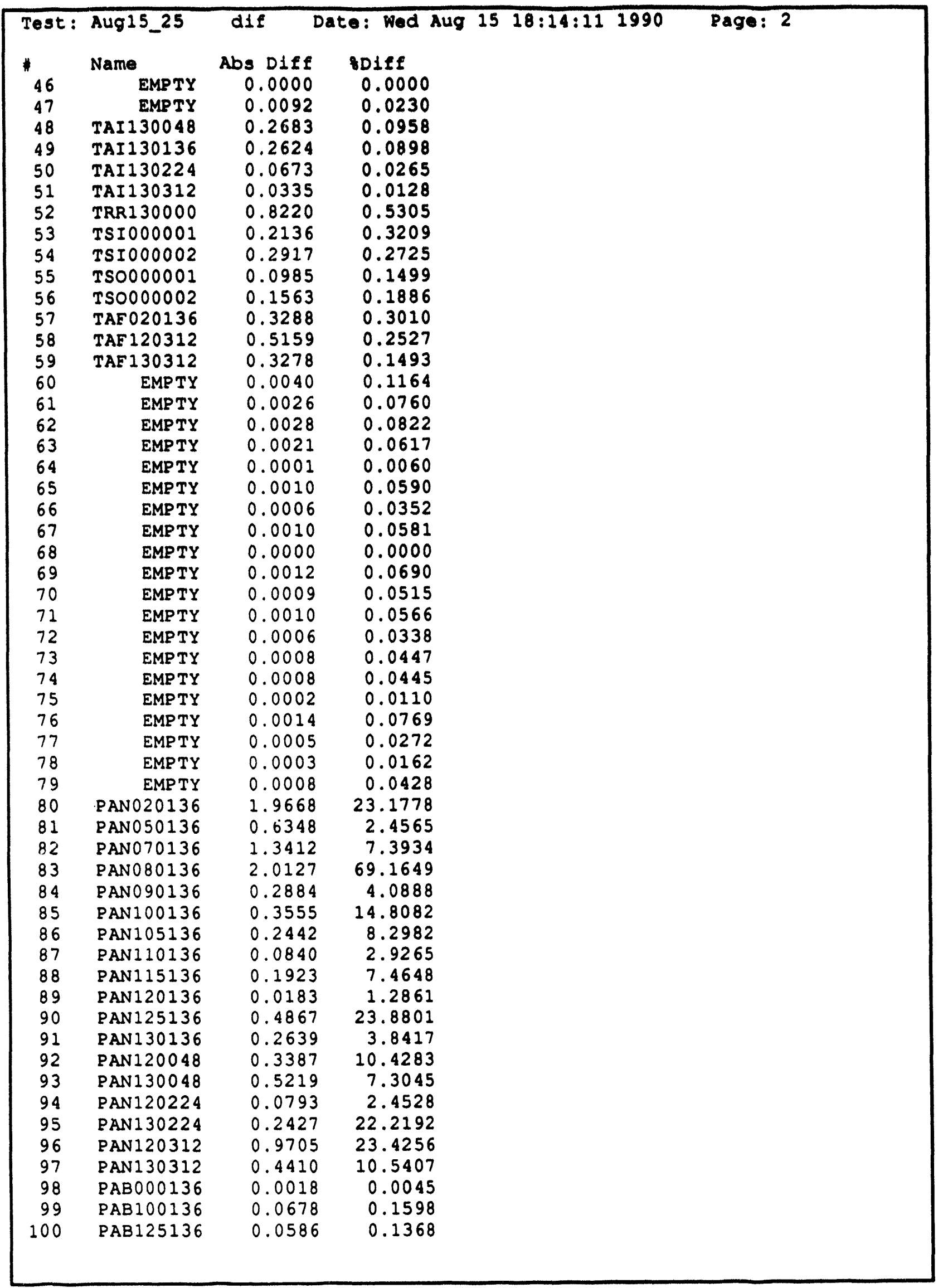




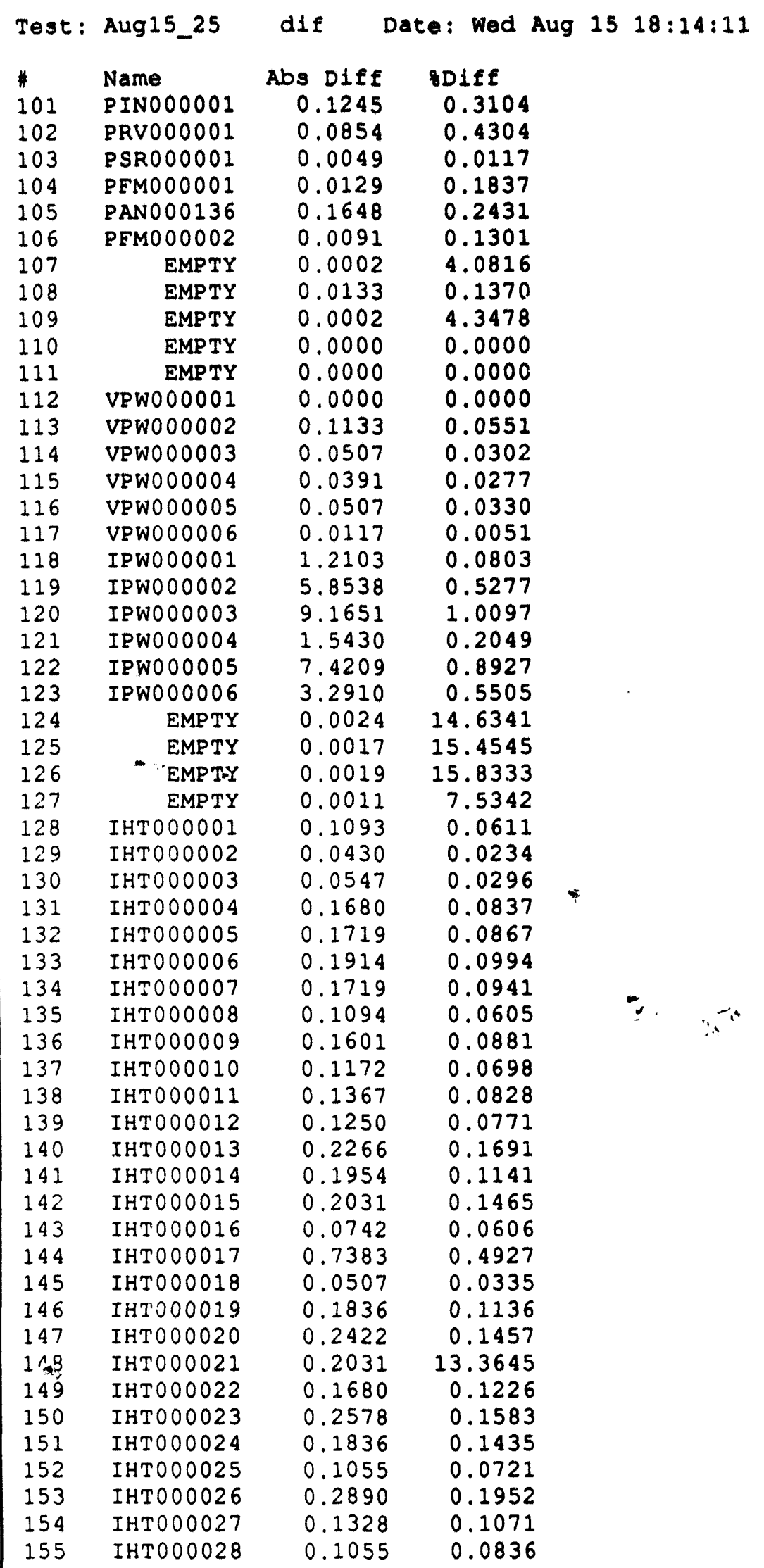




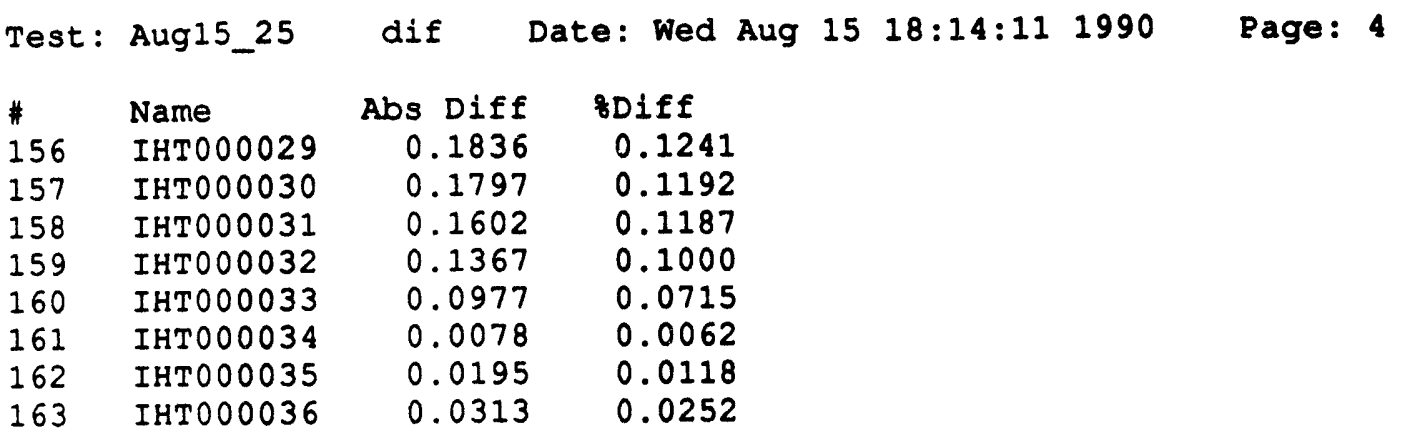




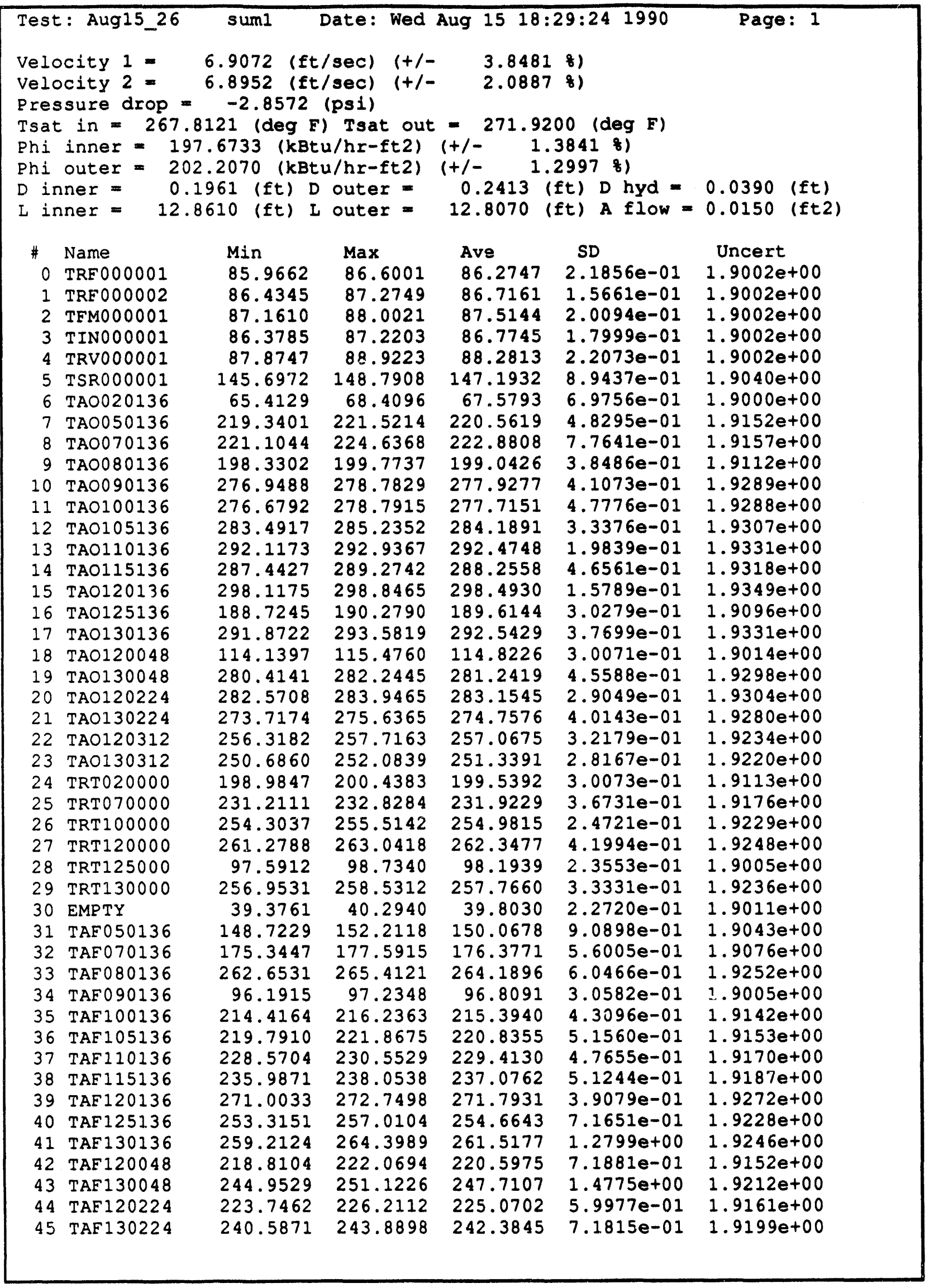




\begin{tabular}{|c|c|c|c|c|c|c|}
\hline & : : Aug & sum1 & ate: We & ug & $: 241990$ & Page: \\
\hline \# & Name & Min & $\operatorname{Max}$ & Ave & SD & Uncert \\
\hline 46 & EMPTY & 37.7675 & 38.6870 & 38.1491 & $087 e$ & \\
\hline 47 & EMPTY & 39.8352 & 40.2940 & 40.0784 & 1.0781 & \\
\hline 48 & TAI130048 & 272.7348 & 275.5009 & 274.1772 & 5.7514 & $1.9279 e+0$ \\
\hline 49 & TAI130136 & 287.2159 & 4024 & 287.7631 & $2.7112 e-01$ & $1.9317 \mathrm{e}+0$ \\
\hline 50 & TAI130224 & 256.8374 & 258.1441 & 257.5170 & $3.5117 e-01$ & $1.9235 e+00$ \\
\hline 51 & TAII & 272 & 0462 & 523 & 3.0431 & $1.92^{\circ}$ \\
\hline 52 & TRR1 & 155.0865 & 156.5724 & 155.8002 & $3.8655 e-01$ & $1.9050 e+00$ \\
\hline 53 & TSIO & 66.2784 & 67.1332 & 6251 & 1.8135 & $1.9000 e+0$ \\
\hline 54 & TSIO & 103.9224 & 107.1234 & 884 & 8.3 & 1.90 \\
\hline 55 & TSOO & 2299 & 66.3035 & 65.7411 & $2.2068 e-01$ & $1.9000 \mathrm{e}+00$ \\
\hline 56 & TSOO & 81.8980 & 83.1644 & 82.6329 & $2.4123 e-01$ & $1.9001 e+00$ \\
\hline 57 & TAFO & 529 & 110.9979 & 108.9453 & 9.46 & 1.9 \\
\hline 58 & TAF 1 & 181 & 6942 & 204.1972 & 7.079 & 1.9 \\
\hline 59 & TAF 1 & 3009 & .4850 & 219.4267 & 1.48 & $1.9150 \mathrm{e}+00$ \\
\hline 60 & EMPTY & 3.3936 & 3.4668 & 3.4232 & 1.98 & $0 e+00$ \\
\hline 61 & EMPTY & 3.3936 & 3.4375 & 3.4089 & 1.18 & $0 e+00$ \\
\hline 62 & EMPTY & 3.3887 & 3.4131 & 3.3963 & 6.9 & +00 \\
\hline 63 & EMPTY & & 3.4 & 3.3939 & & 0 . \\
\hline 64 & EMPTY & 1.6699 & 1.6846 & 1.6755 & 3.6 & 0.0 \\
\hline 65 & ЕMPTY & 1.6895 & 1.7041 & 1.6926 & 4.3 & \\
\hline 66 & EMPTY & 1.6992 & 1.7139 & 1.7034 & 4. & +00 \\
\hline 67 & EMPTY & 1.7139 & 1.7285 & 1.7172 & 4.67 & 0.0 \\
\hline 68 & EMPTY & & 1.7 & 1.7265 & 4.8 & \\
\hline 69 & EMPTY & 34 & 1.7 & 1.7385 & & +00 \\
\hline 70 & EMPTY & & & 1.7482 & 5. & +00 \\
\hline 71 & EMPTY & 1. & 1.7 & 1.7653 & $5 .(1-2)$ & +0 \\
\hline 72 & EMPTY & 1. & & 1.7737 & & +00 \\
\hline 73 & EMPTY & 1.7 & 1.7 & 1.7883 & 6.1 & +00 \\
\hline 74 & EMPTY & & & 995 & 5.3 & $+\infty 0$ \\
\hline 75 & EMPTY & & & 1.8124 & 4.6 & 0 . \\
\hline 76 & EMPTY & 1.8 & 1.8 & 1.8216 & 4.66 & +0 \\
\hline 77 & EMPI & 8 & 1. & 1.8361 & 4.2 & +00 \\
\hline 78 & EMPTY & 1.8 & & 1.8523 & 4.2 & +00 \\
\hline 79 & EMPTY & & 1.8 & 1.8709 & 4.226 & $e+00$ \\
\hline 80 & $\mathrm{PANO} 2 \mathrm{C}$ & 0 & & 16 & 5.7 & $e-01$ \\
\hline 81 & PANO & 38 & -14 . & 473 & 5.4 & $+\infty 0$ \\
\hline 82 & PANO & -24.6544 & -11 & -20.3575 & 3.99 & $9 e-0$ \\
\hline 83 & PANO & & & & 4.10 & 8. \\
\hline 84 & PANO & -12 & -0 . & -6. & 2.51 & 8. \\
\hline 85 & PAN1 & -6 & & -3.5939 & 1.927 & 8. \\
\hline 86 & PAN 1 & & & & 1.468 & \\
\hline 87 & PAN 1 & -4 & -0 . & -3.1999 & 1.038 & \\
\hline 88 & PAN1 & -3.82 & -1.5 & -2.7043 & 4.919 & 8. \\
\hline 89 & PAN1 & & -1 . & & 2.0 & \\
\hline 90 & PAN1 & -0 . & & -0.0 & 1.25 & \\
\hline 91 & PAN 1 & & & 32 & 3.2 & 8. \\
\hline 92 & PAN1 & & 0 & -6.3546 & 2.159 & \\
\hline 93 & PAN 130048 & -2.9518 & 3.10 & -0.3242 & $1.6948 \mathrm{e}+00$ & 8.7 \\
\hline 94 & PAN1 & -6.64 & , & & 1.5254 & $8.7-7$ \\
\hline 95 & PAN1 & -1 . & & -1.1258 & $5.4731 e-01$ & \\
\hline 96 & PAN120312 & -12.8862 & -0.14 & -4.7792 & $2.2475 e+00$ & 8.7 \\
\hline 97 & PAN & & $0.54 C$ & -4 & 2.3 & 8.7 \\
\hline 98 & PAB000136 & & & 40.2986 & $2.4138 e-01$ & $5.8868 \mathrm{e}-$ \\
\hline 99 & PAB100136 & 42.5196 & 42.84 & 42.6779 & $7.1762 \mathrm{e}-02$ & $5.8973 e-0$ \\
\hline 100 & PAB125136 & 43.0178 & 43.2467 & 43.1579 & $5.7317 e-02$ & $5.8995 e-c$ \\
\hline
\end{tabular}




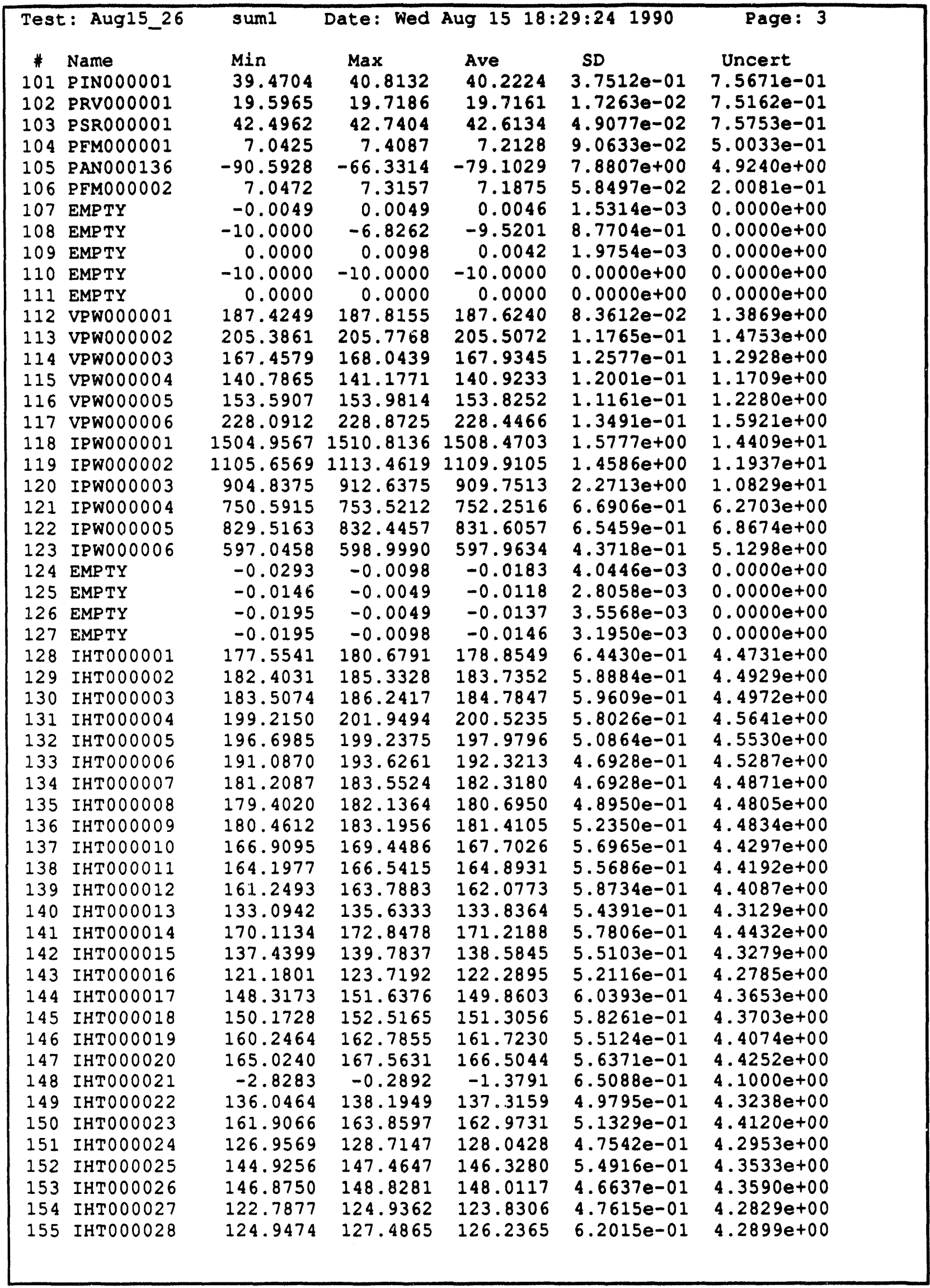




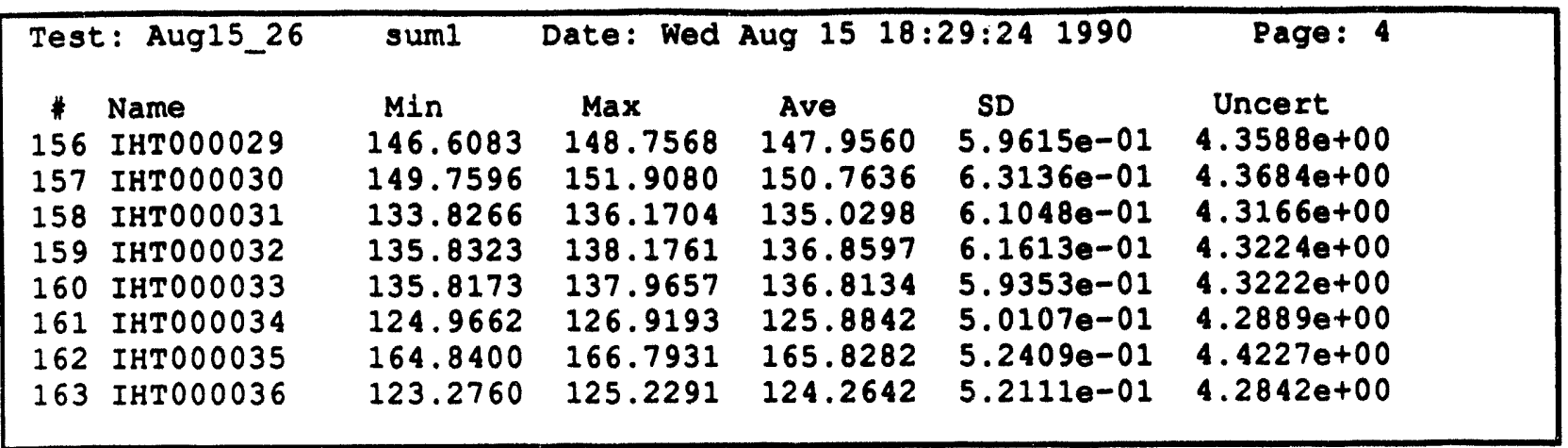




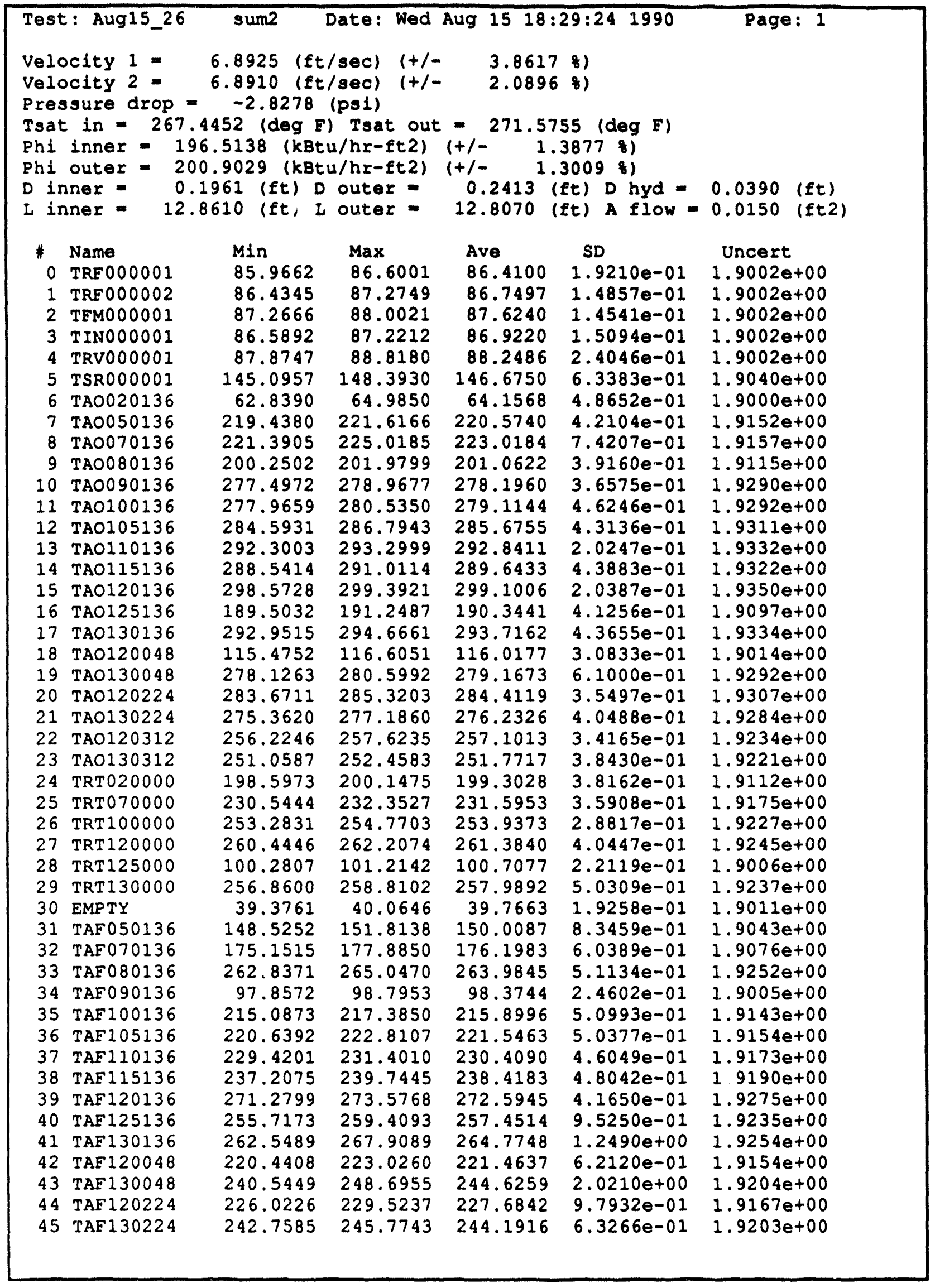




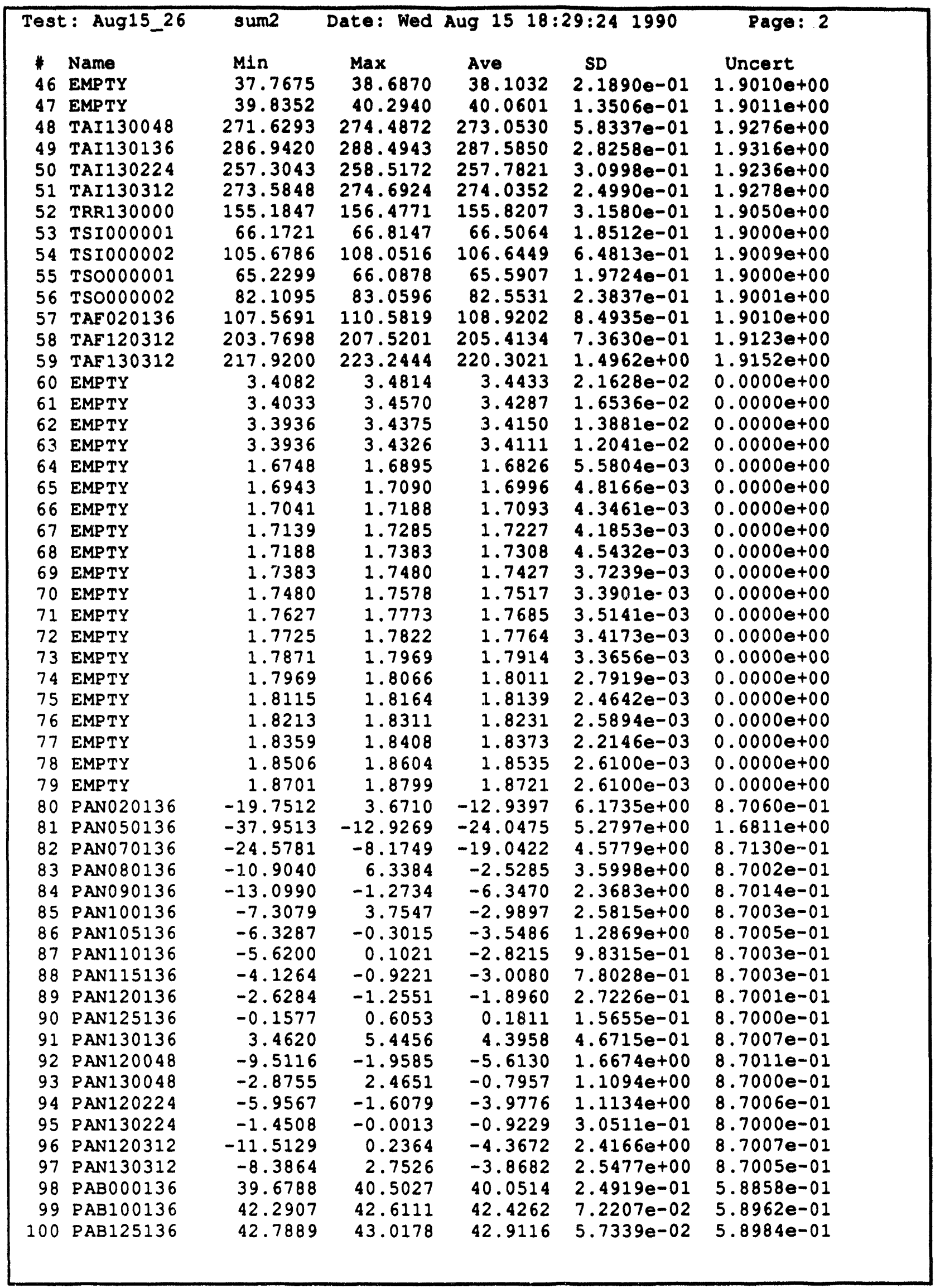




\begin{tabular}{|c|c|c|c|c|c|c|}
\hline \multicolumn{2}{|c|}{ Test: Aug15. 26} & sum2 & \multicolumn{3}{|c|}{ Date: Wed Aug $1518: 29: 241990$} & \multirow[t]{2}{*}{ Page: 3} \\
\hline * & Name & Min & Max & Ave & SD & \\
\hline 101 & PINO00001 & 38.9821 & 40.3249 & 39.7634 & $4.2286 e-01$ & $7.5656 e-01$ \\
\hline 102 & PRV000001 & 19.7186 & 19.8406 & 19.7894 & $6.0861 \theta-02$ & $7.5163 e-01$ \\
\hline 103 & PSR000001 & 42.1300 & 42.3741 & 42.2692 & $5.5201 e-02$ & $7.5741 e-01$ \\
\hline 104 & PFM000001 & 6.9509 & 7.4087 & 7.1817 & $1.0664 \theta-01$ & $5.0032 e-01$ \\
\hline 105 & PAN000136 & -91.0506 & -67.7047 & -78.2881 & $7.43700+00$ & $4.9239 e+00$ \\
\hline 106 & PFM000002 & 7.0975 & 7.2318 & 7.1784 & $3.6146 \theta-02$ & $2.0080 e-01$ \\
\hline 107 & EMPTY & 0.0049 & 0.0049 & 0.0049 & $0.0000 \theta+00$ & $0.0000 e+00$ \\
\hline 108 & EMPTY & -10.0000 & -7.2266 & -9.7926 & $6.1248 e-01$ & $0.0000 e+00$ \\
\hline 109 & EMPTY & 0.0000 & 0.0098 & 0.0049 & $9.8648 \theta-04$ & $0.0000 e+00$ \\
\hline 110 & EMPTY & -10.0000 & -10.0000 & -10.0000 & $0.0000 e+00$ & $0.0000 e+00$ \\
\hline 111 & EMPTY & 0.0000 & 0.0000 & 0.0000 & $0.00000+00$ & $0.0000 e+00$ \\
\hline 112 & VPW000001 & 187.4249 & 187.8155 & 1.87 .6201 & $7.8918 \theta-02$ & $1.3868 \mathrm{e}+00$ \\
\hline 113 & VPWO00002 & 205.3861 & 205.9721 & 205.5892 & $1.4205 \theta-01$ & $1.4757 \mathrm{e}+00$ \\
\hline 114 & VPWO00003 & 167.6532 & 168.2392 & 167.9541 & $1.1306 e-01$ & $1.2929 e+00$ \\
\hline 115 & VPW000004 & 140.7865 & 141.1771 & 140.9702 & $1.2731 \mathrm{e}-01$ & $1.1711 e+00$ \\
\hline 116 & VPW000005 & 153.5907 & 153.9814 & 153.8252 & $1.2478 e-01$ & $1.2280 e+00$ \\
\hline 117 & VPW000006 & 228.2865 & 228.6771 & 228.4935 & $1.2104 e-01$ & $1.5924 e+00$ \\
\hline 118 & IPW000001 & 1503.0043 & 1508.8613 & 1505.9719 & $1.3224 \theta+00$ & $1.4393 \mathrm{e}+01$ \\
\hline 119 & IPW000002 & 1097.8518 & 1103.7056 & 1100.6619 & $1.6330 \mathrm{e}+00$ & $1.1883 e+01$ \\
\hline 120 & IPW000003 & 895.0875 & 898.9875 & 896.5304 & $1.3539 \mathrm{e}+00$ & $1.0760 \mathrm{e}+01$ \\
\hline 121 & IPW000004 & 751.0798 & 752.5446 & 751.8708 & $3.4016 e-01$ & $6.2675 e+00$ \\
\hline 122 & IPW000005 & 823.6575 & 824.6340 & 823.9797 & $2.8945 e-01$ & $6.8098 \mathrm{e}+00$ \\
\hline 123 & IPW000006 & 594.1161 & 595.5810 & 594.6724 & $4.1864 e-01$ & $5.1059 e+00$ \\
\hline 124 & EMPTY & -0.0244 & -0.0098 & -0.0180 & $4.4599 e-03$ & $0.0000 e+00$ \\
\hline 125 & EMPTY & -0.0195 & -0.0049 & -0.0118 & $3.2851 e-03$ & $0.0000 e+00$ \\
\hline 126 & EMPTY & -0.0244 & -0.0049 & -0.0126 & $3.7029 e-03$ & $0.0000 e+00$ \\
\hline 127 & EMPTY & -0.0244 & -0.0098 & -0.0152 & $3.5072 e-03$ & $0.0000 e+00$ \\
\hline 128 & IHTO00001 & 177.9447 & 180.0931 & 179.0737 & $5.3857 e-01$ & $4.4740 e+00$ \\
\hline 129 & IHTO00002 & 182.7937 & 185.1375 & 183.9110 & $5.2946 \mathrm{e}-01$ & $4.4936 e+00$ \\
\hline 130 & IHT000003 & 183.8980 & 186.0464 & 184.9839 & $4.8834 e-01$ & $4.4980 e+00$ \\
\hline 131 & IHTO0O004 & 199.8009 & 201.7540 & 200.6407 & $4.6897 e-01$ & $4.5646 e+00$ \\
\hline 132 & IHTO00005 & 197.0891 & 199.2375 & 198.1007 & $4.8116 e-01$ & $4.5535 e+00$ \\
\hline 133 & IHT000006 & 191.4176 & 193.4307 & 192.4268 & $4.4819 e-01$ & $4.5291 e+00$ \\
\hline 134 & IHTO000007 & 181.5993 & 183.5524 & 182.3961 & $4.7816 \mathrm{e}-01$ & $4.4874 e+00$ \\
\hline 135 & IHT000008 & 179.9880 & 182.1364 & 180.7184 & $4.7648 e-01$ & $4.4806 e+00$ \\
\hline 136 & IHT000009 & 180.6565 & 182.8050 & 181.5042 & $5.4335 e-01$ & $4.4838 e+00$ \\
\hline 137 & IHTO00010 & 166.5189 & 169.0580 & 167.7221 & $6.5716 e-01$ & $4.4298 e+00$ \\
\hline 138 & IHI'000011 & 163.6118 & 166.3462 & 164.8970 & $6.5684 e-01$ & $4.4192 e+00$ \\
\hline 139 & IHTO000012 & 160.6633 & 163.5930 & 161.9757 & $6.2520 \mathrm{e}-01$ & $4.4084 e+00$ \\
\hline 140 & IHTO00013 & 132.5083 & 135.2426 & 133.7973 & $6.4113 e-01$ & $4.3128 e+00$ \\
\hline 141 & IHTO00014 & 169.9181 & 172.4572 & 171.0548 & $6.2973 e-01$ & $4.4425 e+00$ \\
\hline 142 & IHTO00015 & 137.4399 & 140.1743 & 138.4868 & $6.3105 e-01$ & $4.3276 e+00$ \\
\hline 143 & IHTO00016 & 121.1801 & 123.9145 & 122.2309 & $5.9958 e-01$ & $4.2783 e+00$ \\
\hline 144 & IHT000017 & 148.3173 & 151.4423 & 149.5478 & $7.2464 \mathrm{e}-01$ & $4.3642 e+00$ \\
\hline 145 & IHT000018 & 149.7822 & 152.7119 & 151.0049 & $6.4454 \mathrm{e}-01$ & $4.3692 e+00$ \\
\hline 146 & IHT0000I9 & 160.8323 & 162.7855 & 161.8832 & $4.0412 e-01$ & $4.4080 e+00$ \\
\hline 147 & IHTO000020 & 165.6100 & 167.5631 & 166.6138 & $4.0628 e-01$ & $4.4256 e+00$ \\
\hline 148 & IHTO00021 & -2.2424 & -0.2892 & -1.1994 & $4.0933 e-01$ & $4.1000 e+00$ \\
\hline 149 & IHT000022 & 136.6324 & 137.9996 & 137.4253 & $2.9898 e-01$ & $4.3242 e+00$ \\
\hline 150 & IHT000023 & 162.1019 & 163.8597 & 163.2387 & $3.5882 e-01$ & $4.4130 e+00$ \\
\hline 151 & IHTO00024 & 127.1522 & 129.1053 & 128.3710 & $4.0156 e-01$ & $4.2963 e+00$ \\
\hline 152 & IHT000025 & 145.1209 & 147.8553 & 146.6249 & $4.7392 \mathrm{e}-01$ & $4.3543 e+00$ \\
\hline 153 & IHT000026 & 147.0703 & 149.6094 & 148.5469 & $5.0403 e-01$ & $4.3608 e+00$ \\
\hline 154 & IHT000027 & 122.7877 & 125.5221 & 124.2173 & $4.9825 e-01$ & $4.2840 e+00$ \\
\hline 155 & IHT000028 & 124.9474 & 127.0959 & 126.1036 & $5.6887 e-01$ & $4.2895 \mathrm{e}+00$ \\
\hline
\end{tabular}




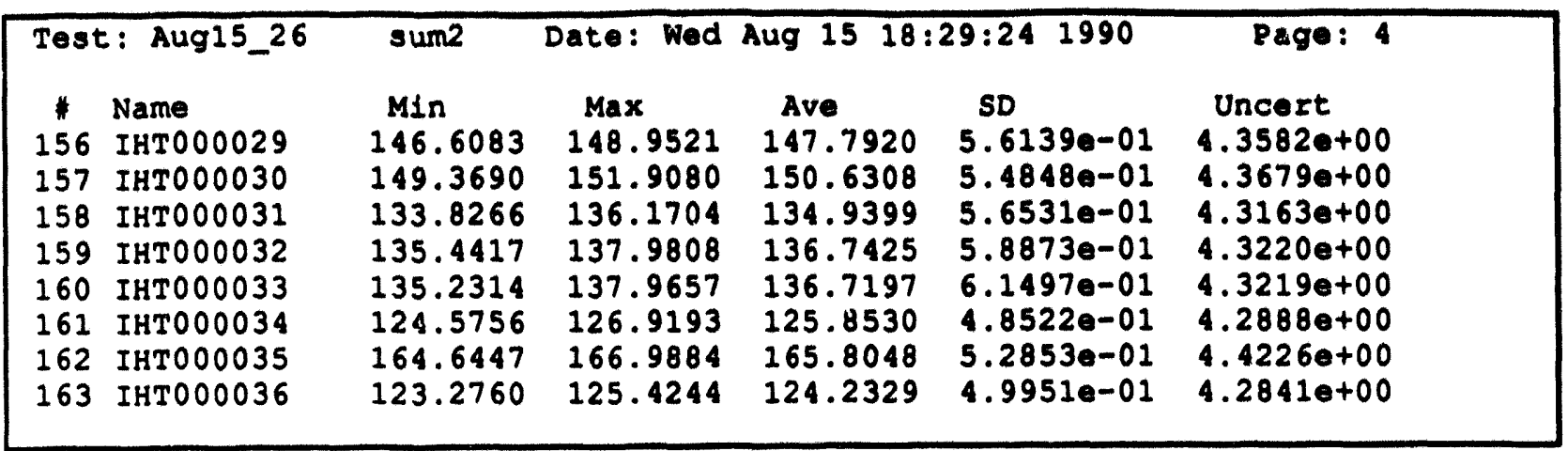




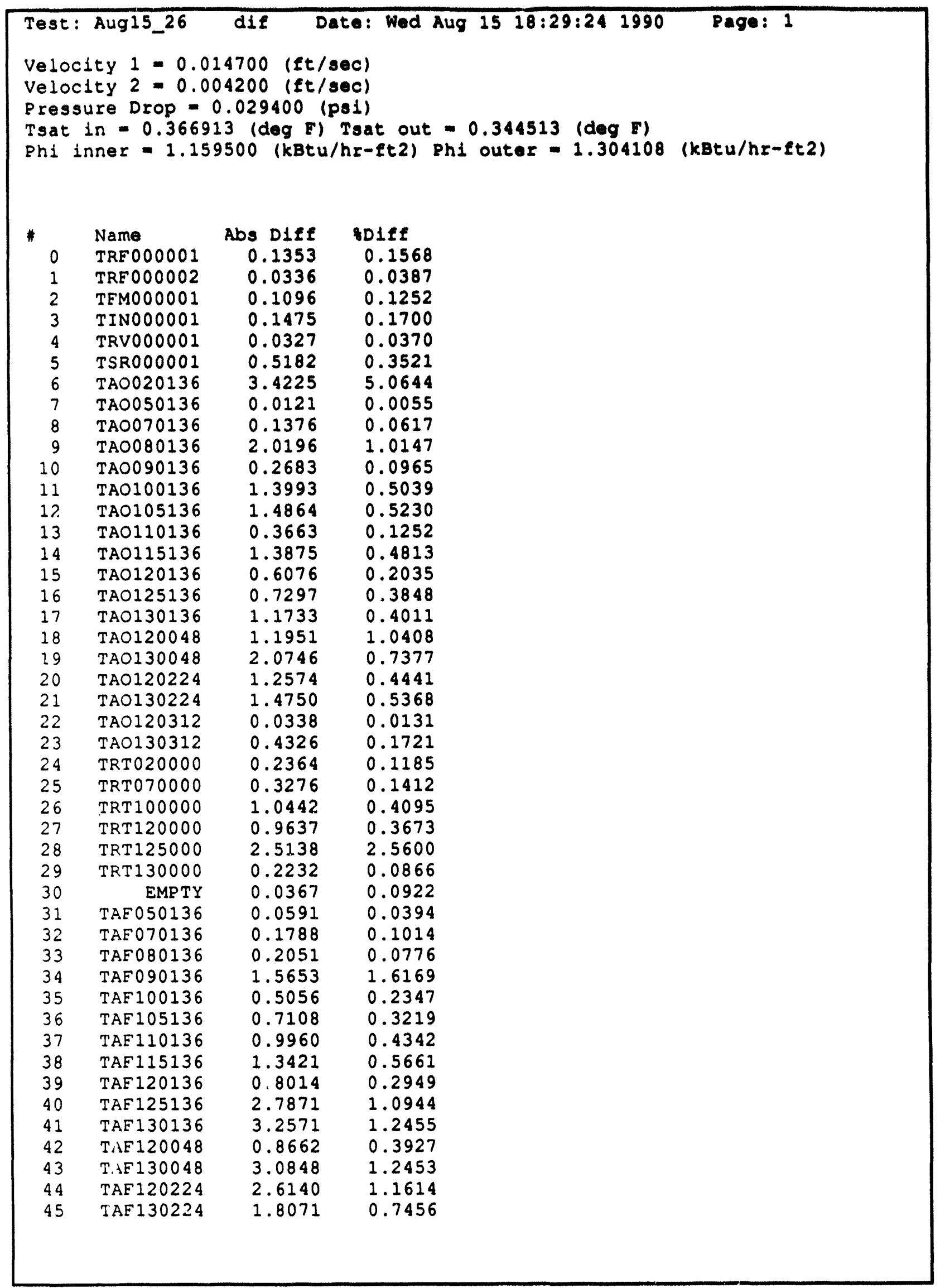




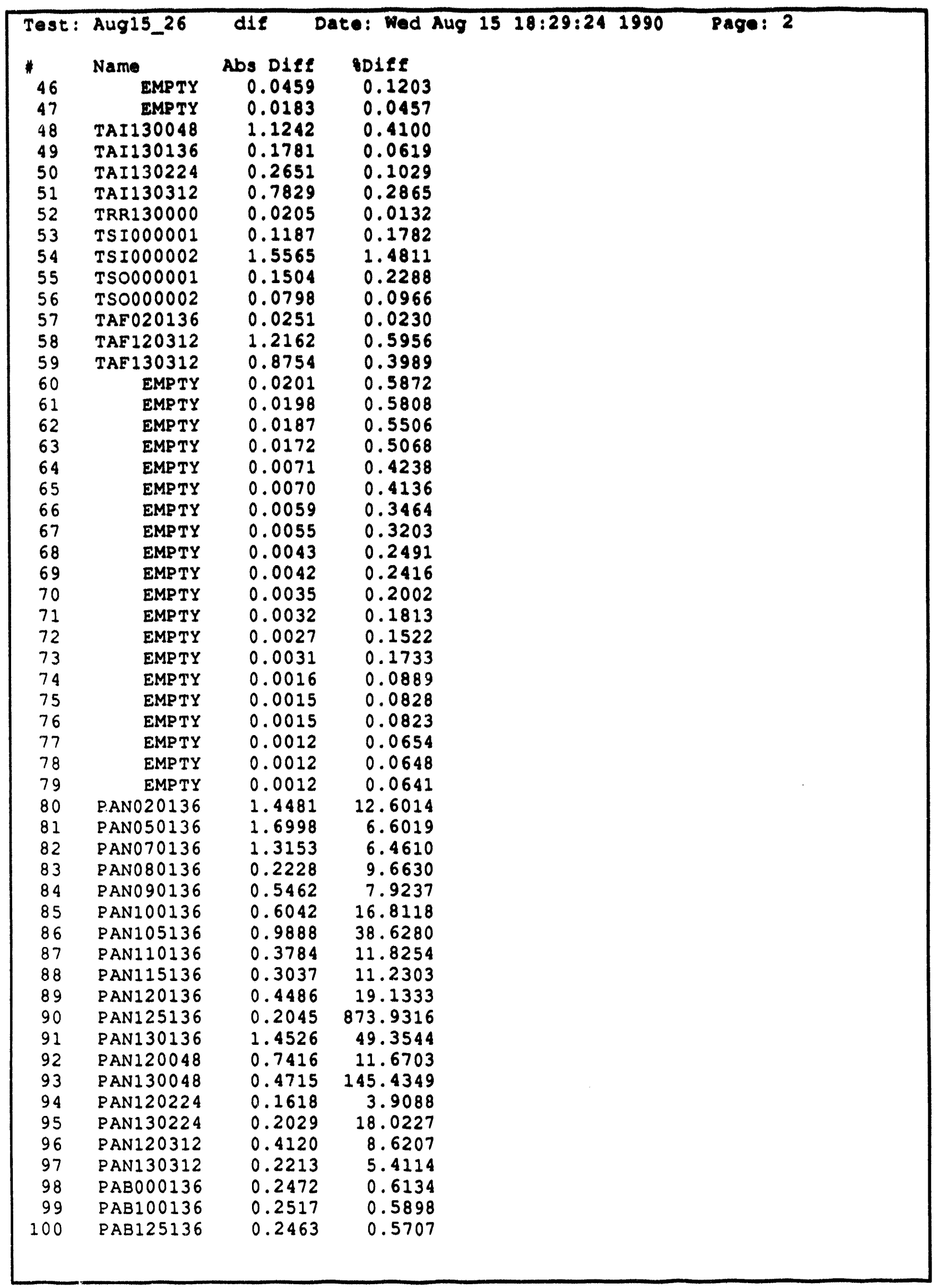




\begin{tabular}{|c|c|c|c|c|}
\hline Test: & Aug15_26 & dif & te: Wed Aug $1518: 29: 241990$ & Page: 3 \\
\hline$*$ & Name & Abs Diff & SDiff & \\
\hline 101 & PINO00001 & 0.4590 & 1.1412 & \\
\hline 102 & PRV000001 & 0.0733 & 0.3718 & \\
\hline 103 & PSR000001 & 0.3442 & 0.8077 & \\
\hline 104 & PFM000001 & 0.0311 & 0.4312 & \\
\hline $\begin{array}{l}105 \\
106\end{array}$ & $\begin{array}{l}\text { PAN000136 } \\
\text { PFMO00002 }\end{array}$ & $\begin{array}{l}0.8148 \\
0.0091\end{array}$ & $\begin{array}{l}1.0300 \\
0.1266\end{array}$ & \\
\hline 107 & EMPTY & 0.0003 & 6.5217 & \\
\hline 108 & EMPTY & 0.2725 & 2.8624 & \\
\hline 109 & EMPTY & 0.0007 & 16.6667 & \\
\hline 110 & EMPTY & 0.0000 & 0.0000 & \\
\hline 111 & EMPTY & 0.0000 & 0.0000 & \\
\hline 112 & VPW000001 & 0.0039 & 0.0021 & \\
\hline 113 & VPW000002 & 0.0820 & 0.0399 & \\
\hline 114 & VPWO00003 & 0.0196 & 0.0117 & \\
\hline 115 & VPWO 00004 & 0.0469 & 0.0333 & \\
\hline 116 & VPWO00005 & 0.0000 & 0.0000 & \\
\hline 117 & VPWO00006 & 0.0469 & 0.0205 & \\
\hline 118 & IPWO00001 & 2.4984 & 0.1656 & \\
\hline 119 & IPW000002 & 9.2487 & 0.8333 & \\
\hline 120 & IPW000003 & 13.2209 & 1.4532 & \\
\hline 121 & IPW000004 & 0.3808 & 0.0506 & \\
\hline 122 & IPWO00005 & 7.6260 & 0.9170 & \\
\hline 123 & IPWO00006 & 3.2910 & 0.5504 & \\
\hline 124 & EMPTY & 0.0003 & 1.6394 & \\
\hline 125 & EMPTY & 0.0000 & 0.0000 & \\
\hline 126 & EMPTY & 0.0011 & 8.0292 & \\
\hline 127 & EMPTY & 0.0006 & 4.1096 & \\
\hline 128 & IHTO00001 & 0.2188 & 0.1223 & \\
\hline 129 & IHTO00002 & 0.1758 & 0.0957 & \\
\hline 130 & IHTO00003 & 0.1992 & 0.1078 & \\
\hline 131 & IHTO00004 & 0.1172 & 0.0584 & \\
\hline 132 & IHT000005 & 0.1211 & 0.0612 & \\
\hline 133 & IHTO00006 & 0.1055 & 0.0549 & \\
\hline 134 & IHT000007 & 0.0781 & 0.0428 & \\
\hline 135 & IHT000008 & 0.0234 & 0.0129 & \\
\hline 136 & IHT000009 & 0.0937 & 0.0516 & \\
\hline 137 & IHTO00010 & 0.0195 & 0.0116 & \\
\hline 138 & IHTO00011 & 0.0039 & 0.0024 & \\
\hline 139 & IHT000012 & 0.1016 & 0.0627 & \\
\hline 140 & IHTO00013 & 0.0391 & 0.0292 & \\
\hline 141 & IHTOOOOOL & 0.1640 & 0.0958 & \\
\hline 142 & IHTO00015 & 0.0977 & 0.0705 & \\
\hline 143 & IHT000016 & 0.0586 & 0.0479 & \\
\hline 144 & IHTO00017 & 0.3125 & 0.2085 & \\
\hline 145 & IHTO00018 & 0.3007 & 0.1987 & \\
\hline 146 & IHTO00019 & 0.1602 & 0.0991 & \\
\hline 147 & IHTO00020 & 0.1094 & 0.0657 & \\
\hline 148 & IHTO00021 & 0.1797 & 13.0302 & \\
\hline 149 & IHTO00022 & 0.1094 & 0.0797 & \\
\hline 150 & IHTO00023 & 0.2656 & 0.1630 & \\
\hline 151 & IHTO00024 & 0.3282 & 0.2563 & \\
\hline 152 & IHTO00025 & 0.2969 & 0.2029 & \\
\hline 153 & IHT000026 & 0.5352 & 0.3616 & \\
\hline 154 & IHTO00027 & 0.3867 & 0.3123 & \\
\hline 155 & IHTO00028 & 0.1329 & 0.1053 & \\
\hline
\end{tabular}




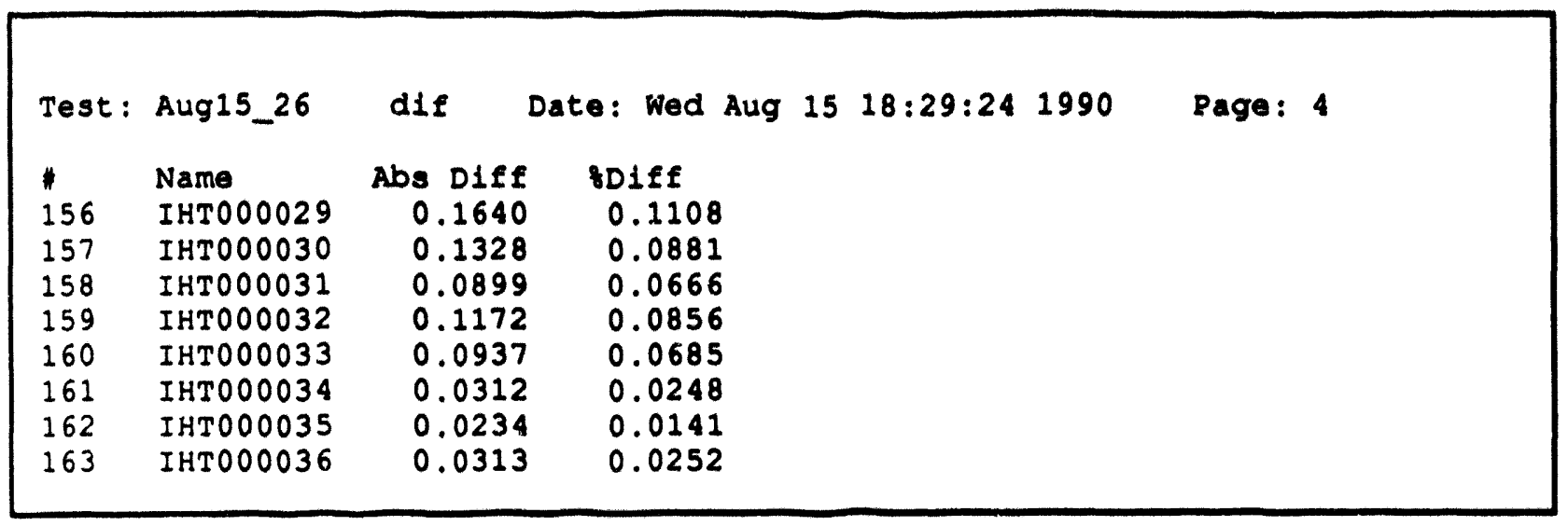




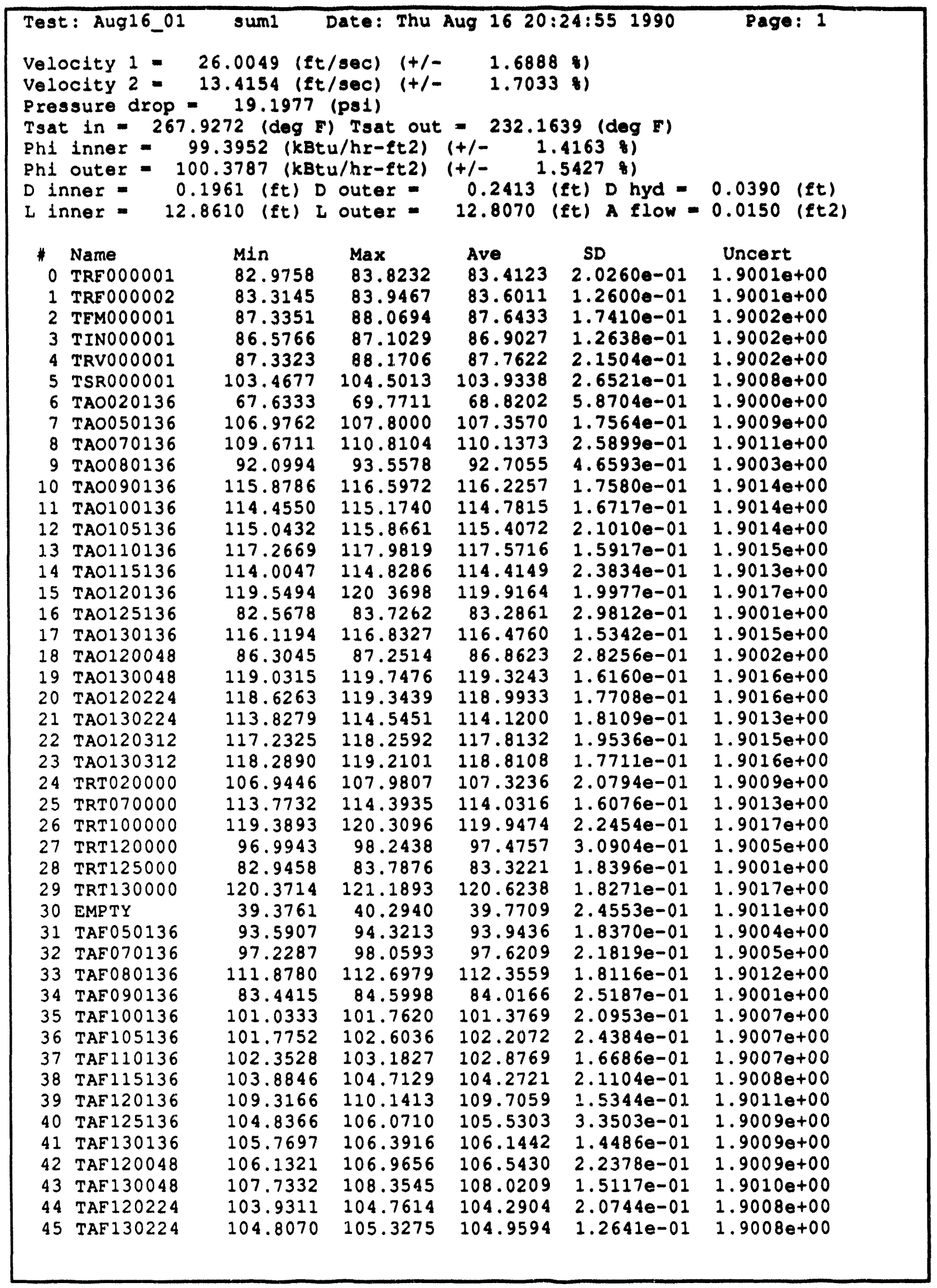




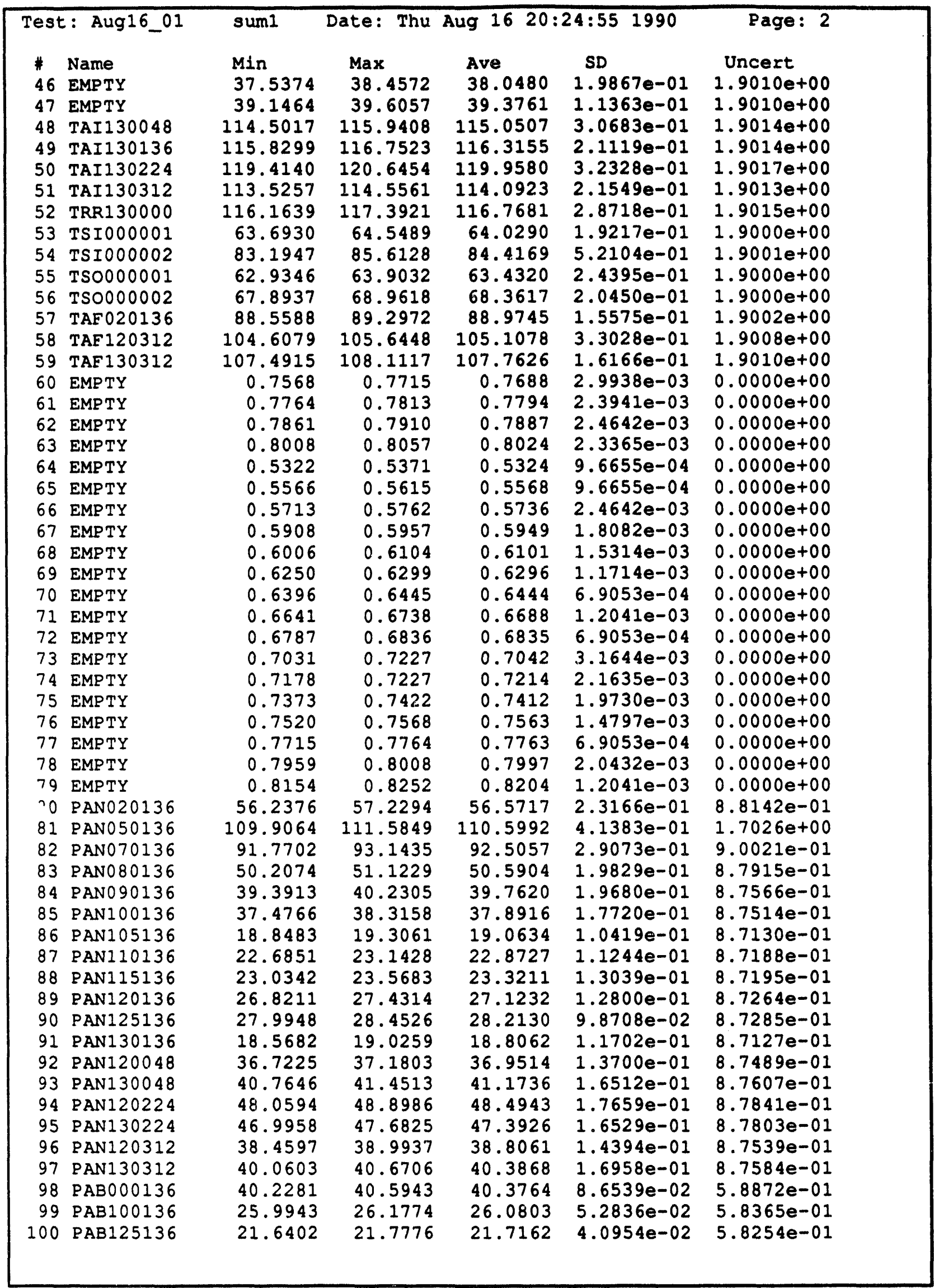




\begin{tabular}{|c|c|c|c|c|c|c|}
\hline \multicolumn{2}{|c|}{ Test: Aug16_01 } & sum 1 & \multicolumn{3}{|c|}{ Date: Thu Aug $16 \quad 20: 24: 551990$} & Page: 3 \\
\hline \# & Name & Min & Max & Ave & SD & Uncert \\
\hline 101 & PIN 000001 & 39.8366 & 40.3249 & 40.1003 & $1.2172 e-01$ & $7.5667 e-01$ \\
\hline 102 & PRV000001 & 19.5965 & 19.7186 & 19.5989 & $1.7263 e-02$ & $7.5160 e-01$ \\
\hline 103 & PSR000001 & 18.6925 & 18.8146 & 18.7902 & $4.9324 e-02$ & $7.5147 e-01$ \\
\hline 104 & PEM000001 & 103.5391 & 105.3701 & 104.2972 & $4.9667 e-01$ & $5.6390 e-01$ \\
\hline 105 & PAN000136 & 527.3881 & 535.1701 & 531.4988 & $1.7090 e+00$ & $5.0963 e+00$ \\
\hline 106 & PFM000002 & 27.5689 & 27.5689 & 27.5689 & $9.6336 e-06$ & $2.1154 \theta-01$ \\
\hline 107 & EMPTY & 0.0098 & 0.0146 & 0.0116 & $2.3941 e-03$ & $0.0000 e+00$ \\
\hline 108 & EMPTY & -10.0000 & -5.7031 & -9.6008 & $1.0282 e+00$ & $0.0000 \mathrm{e}+00$ \\
\hline 109 & EMPTY & 0.0098 & 0.0195 & 0.0100 & $1.3811 e-03$ & $0.0000 e+00$ \\
\hline 110 & EMPTY & -10.0000 & -10.0000 & -10.0000 & $0.0000 e+00$ & $0.0000 e+00$ \\
\hline 111 & EMPTY & 0.0049 & 0.0049 & 0.0049 & $0.0000 e+00$ & $0.0000 \mathrm{e}+00$ \\
\hline 112 & VPW000001 & 101.4385 & 102.0245 & 101.6416 & $1.4205 e-01$ & $1.0139 e+00$ \\
\hline 113 & VPW000002 & 118.4608 & 118.8514 & 118.6210 & $1.0942 e-01$ & $1.0783 e+00$ \\
\hline 114 & VPW000003 & 146.4506 & 146.8412 & 146.5561 & $1.1306 e-01$ & $1.1955 e+00$ \\
\hline 115 & VPW000004 & 104.2781 & 104.8640 & 104.5711 & $1.3235 e-01$ & $1.0246 \mathrm{e}+00$ \\
\hline 116 & VPW000005 & 138.8709 & 139.6522 & 139.1327 & $1.2853 e-01$ & $1.1632 e+00$ \\
\hline 117 & VPW000006 & 146.8938 & 147.6750 & 147.2491 & $1.5124 e-01$ & $1.1986 \mathrm{e}+00$ \\
\hline 118 & IPW000001 & 564.9782 & 574.7399 & 569.1172 & $2.2877 e+00$ & $9.2641 \mathrm{e}+00$ \\
\hline 119 & IPW000002 & 1275.0045 & 1288.6635 & 1282.0289 & $3.4367 e+00$ & $1.2969 e+01$ \\
\hline 120 & IPW000003 & 1308.4498 & 1316.2499 & 1311.9989 & $1.7920 e+00$ & $1.3154 \mathrm{e}+01$ \\
\hline 121 & IPWO000004 & 384.5872 & 387.0286 & 385.8665 & $6.5354 e-01$ & $3.6459 e+00$ \\
\hline 122 & IPWO000005 & 253.5616 & 254.5381 & 254.0498 & $3.1192 e-01$ & $2.8334 \mathrm{e}+00$ \\
\hline 123 & IPWO000006 & 260.7798 & 262.7329 & 261.6683 & $4.2647 e-01$ & $2.8763 e+00$ \\
\hline 124 & EMPTY & -0.0244 & -0.0098 & -0.0178 & $3.2359 e-03$ & $0.0000 \mathrm{e}+00$ \\
\hline 125 & EMPTY & -0.0195 & -0.0098 & -0.0129 & $3.0819 e-03$ & $0.0000 \mathrm{e}+00$ \\
\hline 126 & EMPTY & -0.0195 & -0.0049 & -0.0125 & $3.2955 e-03$ & $0.0000 e+00$ \\
\hline 127 & EMPTY & -0.0244 & -0.0098 & -0.0147 & $3.0389 e-03$ & $0.0000 e+00$ \\
\hline 128 & IHTOOOOOI & 129.1091 & 131.4528 & 130.0817 & $5.6150 e-01$ & $4.3014 e+00$ \\
\hline 129 & IHTO00002 & 132.5721 & 134.9158 & 133.8573 & $5.4403 e-01$ & $4.3130 e+00$ \\
\hline 130 & IHT000003 & 132.6998 & 135.0436 & 133.9146 & $5.0731 e-01$ & $4.3132 e+00$ \\
\hline 131 & IHTO00004 & 131.1861 & 133.1393 & 132.3540 & $5.1974 e-01$ & $4.3083 e+00$ \\
\hline 132 & IHT000005 & 129.6574 & 131.8059 & 130.6652 & $5.1654 e-01$ & $4.3032 e+00$ \\
\hline 133 & IHTO00006 & 124.3878 & 126.3409 & 125.4580 & $4.8043 e-01$ & $4.2877 e+00$ \\
\hline 134 & IHT000007 & 131.1899 & 133.9243 & 132.4282 & $5.0007 e-01$ & $4.3086 e+00$ \\
\hline 135 & IHT000008 & 129.9654 & 132.1139 & 130.9888 & $4.5942 e-01$ & $4.3042 e+00$ \\
\hline 136 & IHT000009 & 130.3448 & 132.8839 & 131.5049 & $4.9191 \mathrm{e}-01$ & $4.3057 e+00$ \\
\hline 137 & IHT 000010 & -39.9076 & $-38 . .498$ & -39.0638 & $5.1364 e-01$ & $4.1186 e+00$ \\
\hline 138 & IHT000011 & 130.5025 & 132.8463 & 131.4048 & $5.2776 e-01$ & $4.3054 e+00$ \\
\hline 139 & IHT000012 & 128.1175 & 130.8519 & 129.1058 & $5.1207 e-01$ & $4.2985 e+00$ \\
\hline 140 & IHT000013 & 91.4063 & 94.3359 & 92.5313 & $5.1834 e-01$ & $4.2031 e+00$ \\
\hline 141. & IHTO000014 & 127.9522 & 130.4913 & 128.8545 & $5.2481 e-01$ & $4.2977 e+00$ \\
\hline 142 & IHTO00015 & 95.2073 & 98.1370 & 96.3011 & $5.5243 e-01$ & $4.2116 \mathrm{e}+00$ \\
\hline 143 & IHTO00016 & 88.9122 & 91.8419 & 89.8771 & $5.5160 e-01$ & $4.1974 \mathrm{e}+00$ \\
\hline 144 & IHTO00017 & 106.4378 & 109.7581 & 107.8167 & $8.3557 e-01$ & $4.2394 \mathrm{e}+00$ \\
\hline 145 & IHTO00018 & 107.1590 & 111.0652 & 108.8153 & $7.7146 e-01$ & $4.2419 e+00$ \\
\hline 146 & IHTO00019 & 103.8687 & 106.2124 & 104.9351 & $5.0101 e-01$ & $4.2322 e+00$ \\
\hline 147 & IHTOOO0020 & 106.7833 & 109.3224 & 107.8771 & $5.2050 e-01$ & $4.2395 e+00$ \\
\hline 148 & IHTO00021 & -2.2273 & 0.8977 & -1.0515 & $6.5494 e-01$ & $4.1000 \mathrm{e}+00$ \\
\hline 149 & IHTO00022 & 92.8598 & 95.3989 & 93.9419 & $4.9001 e-01$ & $4.2062 e+00$ \\
\hline 150 & IHTO00023 & 104.9504 & 106.9036 & 105.8449 & $5.0546 e-01$ & $4.2344 e+00$ \\
\hline 151 & IHTO00024 & 70.1585 & 72.1116 & 71.0491 & $4.7541 e-01$ & $4.1611 \mathrm{e}+00$ \\
\hline 152 & IHTO00025 & 104.7439 & 106.8923 & 105.7907 & $5.0341 e-01$ & $4.2343 e+00$ \\
\hline 153 & IHTO00026 & 105.3260 & 107.4745 & 106.5135 & $5.1726 e-01$ & $4.2361 \mathrm{e}+00$ \\
\hline 154 & IHTO00027 & 90.3846 & 92.3377 & 91.2987 & $4.9981 \mathrm{e}-01$ & $4.2004 e+00$ \\
\hline 155 & IHTO00028 & 91.1396 & 94.2646 & 92.8310 & $5.9259 e-01$ & $4.2038 e+00$ \\
\hline
\end{tabular}




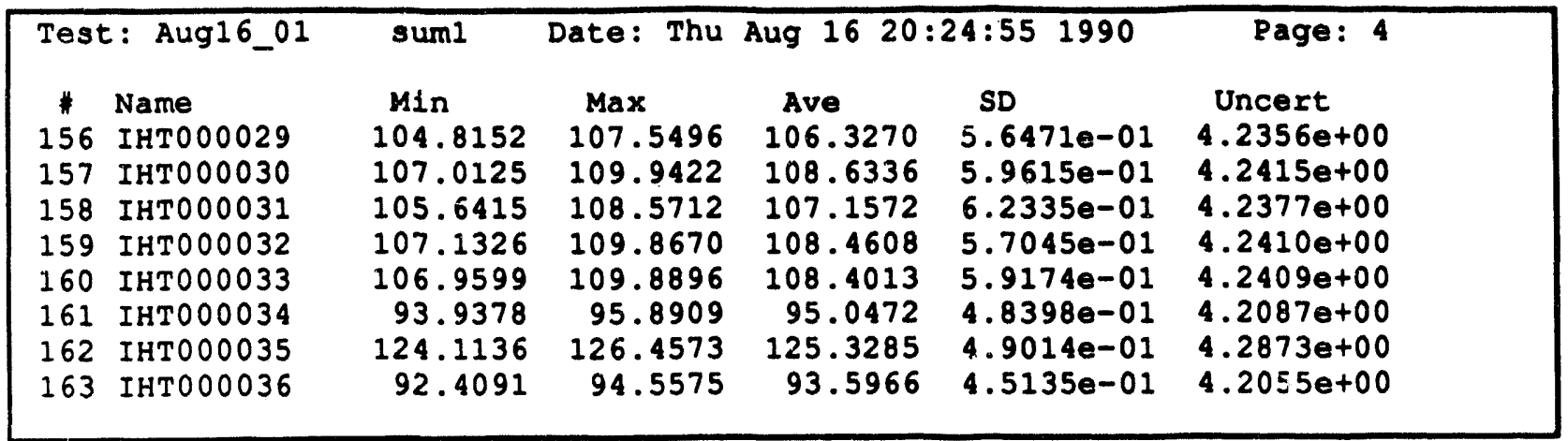




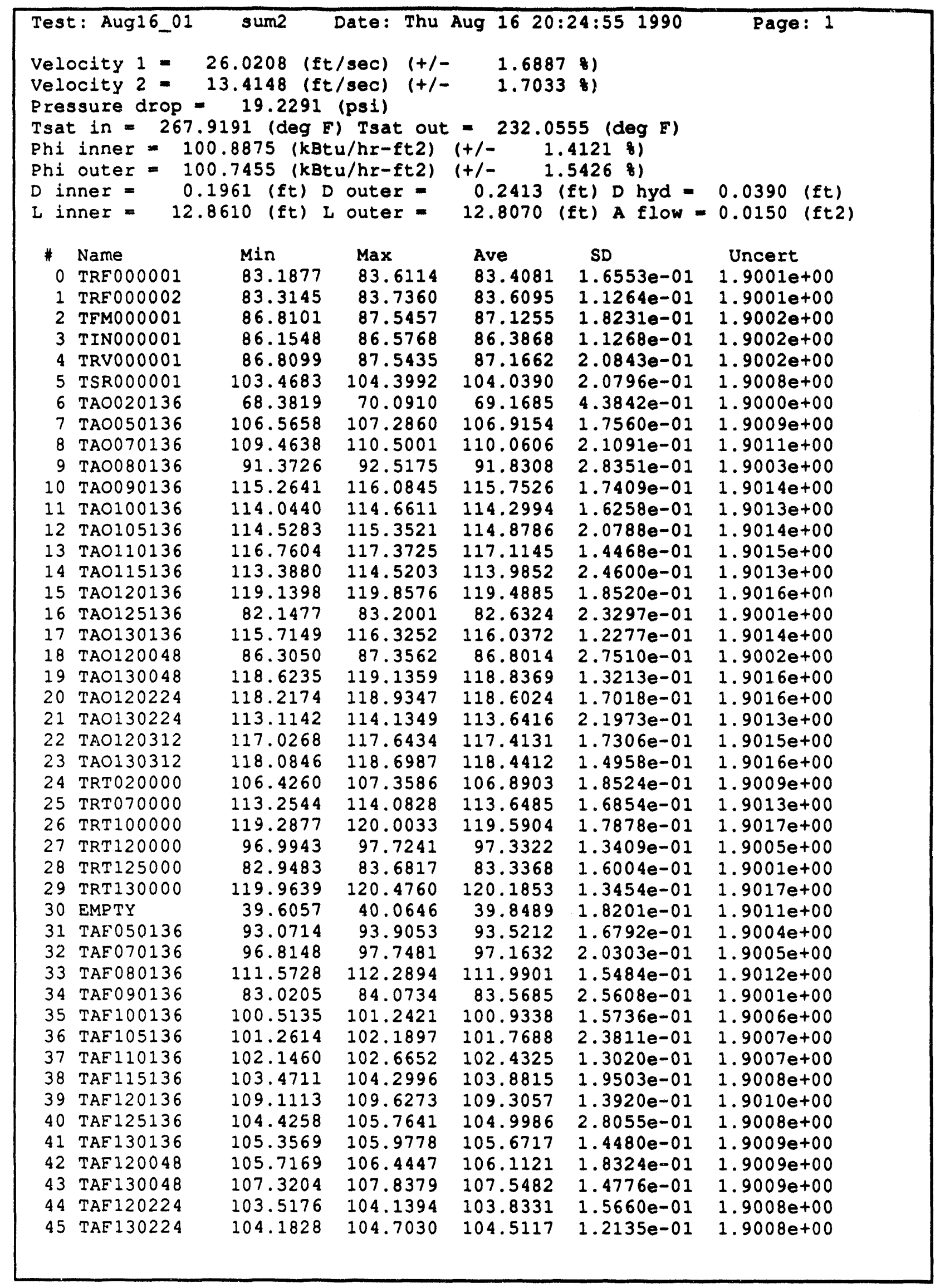




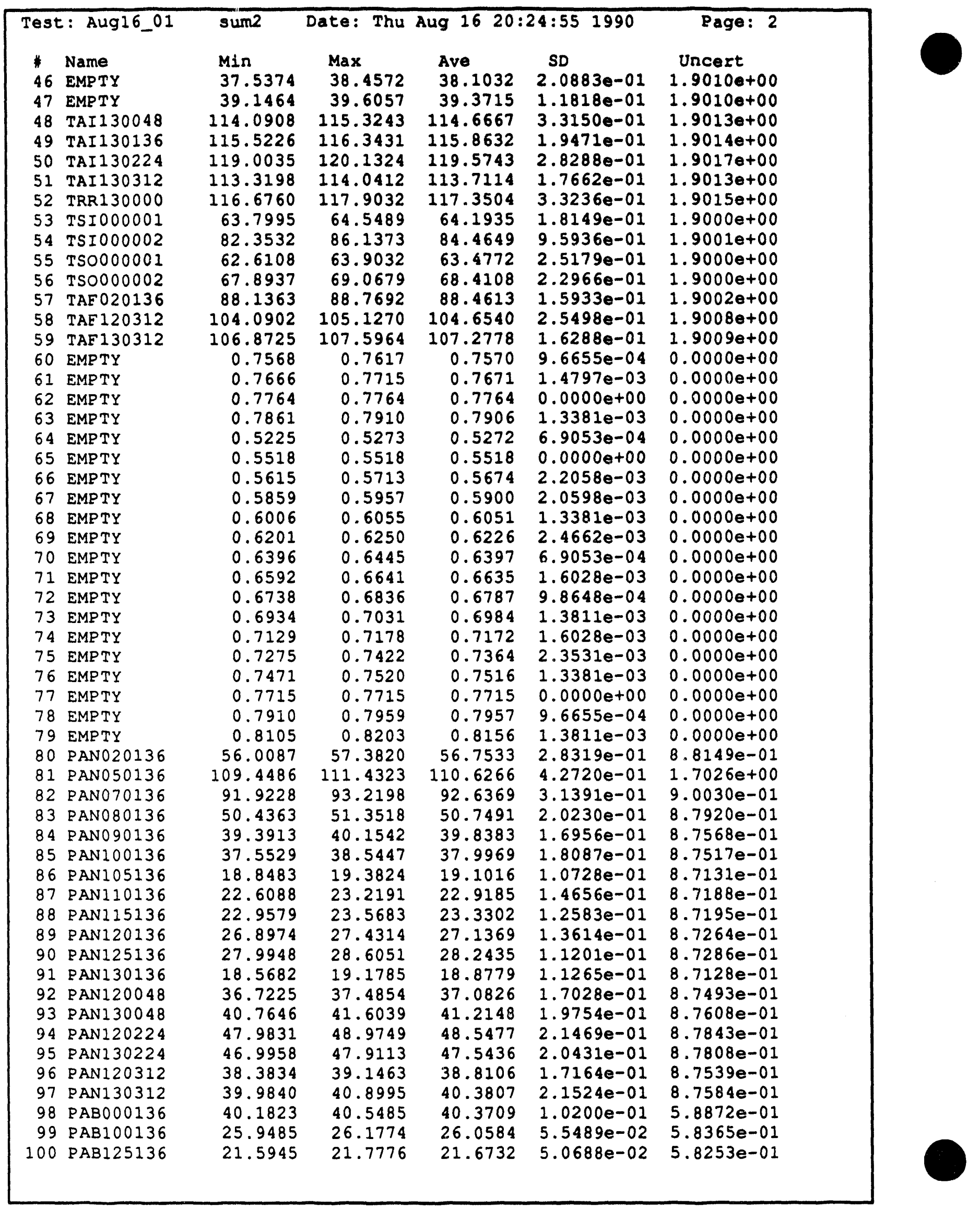




\begin{tabular}{|c|c|c|c|c|c|c|}
\hline \multicolumn{2}{|c|}{ Test: Aug16_01 } & sum2 & Date: Thu & Aug 16 & $1: 551990$ & Page: 3 \\
\hline$\#$ & Name & $\operatorname{Min}$ & $\operatorname{Max}$ & Ave & SD & Uncert \\
\hline 101 & PIN 000001 & 39.9587 & 40.5690 & 40.2370 & $1.3960 e-01$ & $7.5672 \mathrm{e}-01$ \\
\hline 102 & PRV000001 & 19.5965 & 19.7186 & 19.6575 & $6.1655 e-02$ & $7.5161 \mathrm{e}-01$ \\
\hline 103 & PSR000001 & 18.6925 & 18.8146 & 18.7267 & $5.5366 e-02$ & $7.5146 e-01$ \\
\hline 104 & PFM000001 & 103.2644 & 105.0955 & 104.4345 & $4.2392 e-01$ & $5.6406 e-01$ \\
\hline 105 & PAN 000136 & 527.8459 & 536.5434 & 532.3684 & $1.9347 \mathrm{e}+00$ & $5.0968 e+00$ \\
\hline 106 & PFM000002 & 27.5689 & 27.5689 & 27.5689 & $9.6336 e-06$ & $2.1154 e-01$ \\
\hline 107 & EMPTY & 0.0098 & 0.0146 & 0.0104 & $1.6028 e-03$ & $0.0000 e+00$ \\
\hline 108 & EMPTY & -10.0000 & -10.0000 & -10.0000 & $0.0000 e+00$ & $0.0000 e+00$ \\
\hline 109 & EMPTY & 0.0049 & 0.0146 & 0.0090 & $2.0598 e-03$ & $0.0000 e+00$ \\
\hline 110 & EMPTY & -10.0000 & -10.0000 & -10.0000 & $0.0000 e+00$ & $0.0000 e+00$ \\
\hline 111 & EMPTY & 0.0000 & 0.0049 & 0.0044 & $1.4797 e-03$ & $0.0000 e+00$ \\
\hline 112 & VPW000001 & 101.2432 & 101.8292 & 101.6260 & $1.7629 e-01$ & $1.0139 e+00$ \\
\hline 113 & VPWO00002 & 118.4608 & 118.8514 & 118.6483 & $7.8523 e-02$ & $1.0784 e+00$ \\
\hline 114 & VPWO00003 & 146.4506 & 146.8412 & 146.7124 & $1.0885 e-01$ & $1.1962 e+00$ \\
\hline 115 & VPWO000004 & 104.2781 & 104.8640 & 104.5906 & $1.3669 e-01$ & $1.0247 e+00$ \\
\hline 116 & VPWO00005 & 139.0663 & 139.4569 & 139.1991 & $1.0763 e-01$ & $1.1635 e+00$ \\
\hline 117 & VPW0000006 & 147.0891 & 147.6750 & 147.4640 & $1.5201 e-01$ & $1.1996 e+00$ \\
\hline 118 & IPWO00001 & 547.4072 & 553.2642 & 549.8670 & $1.1707 e+00$ & $9.1913 e+00$ \\
\hline 119 & IPWO 00002 & 1304.2736 & 1312.0786 & 1306.9279 & $1.5650 e+00$ & $1.3123 e+01$ \\
\hline 120 & IPW000003 & 1327.9498 & 1333.7999 & 1331.1093 & $1.1066 \mathrm{e}+00$ & $1.3274 e+01$ \\
\hline 121 & IPW000004 & 383.6106 & 384.5872 & 384.2747 & $2.7480 e-01$ & $3.6354 e+00$ \\
\hline 122 & IPWO 00005 & 254.5381 & 256.0028 & 255.2119 & $3.2551 e-01$ & $2.8399 \mathrm{e}+00$ \\
\hline 123 & IPWO00006 & 263.7095 & 265.1743 & 264.3831 & $4.3995 e-01$ & $2.8918 e+00$ \\
\hline 124 & EMPTY & -0.0293 & -0.0146 & -0.0205 & $3.1195 e-03$ & $0.0000 e+00$ \\
\hline 125 & EMPTY & -0.0195 & -0.0049 & -0.0136 & $2.8403 e-03$ & $0.0000 e+00$ \\
\hline 126 & EMPTY & -0.0244 & -0.0098 & -0.0167 & $3.1335 e-03$ & $0.0000 e+00$ \\
\hline 127 & EMPTY & -0.0195 & -0.0098 & -0.0159 & $2.5743 e-03$ & $0.0000 e+00$ \\
\hline 128 & IHTO00001 & 128.9137 & 131.6481 & 130.1012 & $6.5058 e-01$ & $4.3015 e+00$ \\
\hline 129 & IHTO00002 & 132.7674 & 135.3065 & 134.0018 & $5.9770 e-01$ & $4.3134 \mathrm{e}+00$ \\
\hline 130 & IHT000003 & 132.6998 & 135.0436 & 134.0435 & $5.9009 e-01$ & $4.3136 e+00$ \\
\hline 131 & IHTO00004 & 130.9908 & 133.7252 & i 32.2720 & $6.0895 e-01$ & $4.3081 e+00$ \\
\hline 132 & IHTOOOO005 & 129.2668 & 131.6106 & 130.6261 & $5.8122 e-01$ & $4.3031 \mathrm{e}+00$ \\
\hline 133 & IHT000006 & 123.9971 & 126.3409 & 125.4463 & $5.9069 e-01$ & $4.2876 e+00$ \\
\hline 134 & IHT000007 & 130.9946 & 133.9243 & 132.4399 & $5.8660 e-01$ & $4.3086 e+00$ \\
\hline 135 & IHTO00008 & 129.5748 & 132.1139 & 130.9380 & $5.7248 e-01$ & $4.3040 \mathrm{e}+00$ \\
\hline 136 & IHTO00009 & 129.9542 & 132.6885 & 131.4893 & $6.3382 e-01$ & $4.3057 \mathrm{e}+00$ \\
\hline 137 & IHTO00010 & -40.1029 & -37.7592 & -38.9545 & $5.3908 e-01$ & $4.1185 e+00$ \\
\hline 138 & IHTO00011 & 130.3072 & 132.4557 & 131.6001 & $5.2629 e-01$ & $4.3060 \mathrm{e}+00$ \\
\hline 139 & IHTO00012 & 127.9222 & 130.2659 & 129.2503 & $4.8327 e-01$ & $4.2989 e+00$ \\
\hline 140 & IHTO000013 & 91.4063 & 93.3594 & 92.5430 & $4.7300 e-01$ & $4.2031 e+00$ \\
\hline 141 & IHTO00014 & 127.9522 & 129.7101 & 128.8936 & $4.7791 e-01$ & $4.2978 e+00$ \\
\hline 142 & IHTOOOOO15 & 95.2073 & 96.9651 & 96.2425 & $4.4686 e-01$ & $4.2114 \mathrm{e}+00$ \\
\hline 143 & IHTO00016 & 88.7169 & 91.0607 & 89.8029 & $5.3403 e-01$ & $4.1972 e+00$ \\
\hline 144 & IHTO000017 & 105.4613 & 109.3675 & 107.4105 & $8.3937 e-01$ & $4.2384 e+00$ \\
\hline 145 & IHTO00018 & 107.1590 & 110.0886 & 108.7449 & $6.1718 e-01$ & $4.2418 e+00$ \\
\hline 146 & IHTO00019 & 103.8687 & 106.6031 & 104.9116 & $5.4621 e-01$ & $4.2321 e+00$ \\
\hline 147 & IHTOOO0020 & 106.7833 & 109.5177 & 107.7794 & $5.5278 e-01$ & $4.2393 e+00$ \\
\hline 148 & IHTO00021 & -2.8132 & 0.8977 & -1.2742 & $7.3147 e-01$ & $4.1000 e+00$ \\
\hline 149 & IHTO000022 & 92.4692 & 95.5942 & 93.8208 & $6.2744 e-01$ & $4.2060 e+00$ \\
\hline 150 & IHTO00023 & 104.5598 & 107.2942 & 105.7824 & $5.3059 e-01$ & $4.2343 e+00$ \\
\hline 151 & IHTOOO0024 & 69.7679 & 72.5023 & 71.0726 & $5.4454 e-01$ & $4.1611 \mathrm{e}+00$ \\
\hline 152 & IHTO000025 & 104.5485 & 106.8923 & 105.8024 & $5.5955 e-01$ & $4.2343 e+00$ \\
\hline 153 & IHTO00026 & 105.3260 & 107.8651 & 106.5487 & $6.1233 e-01$ & $4.2362 e+00$ \\
\hline 154 & IHT000027 & 89.9940 & 92.3377 & 91.2127 & $5.8469 e-01$ & $4.2002 \mathrm{e}+00$ \\
\hline 155 & IHTO00028 & 91.5302 & 94.2646 & 92.7568 & $6.7432 e-01$ & $4.2036 e+00$ \\
\hline
\end{tabular}




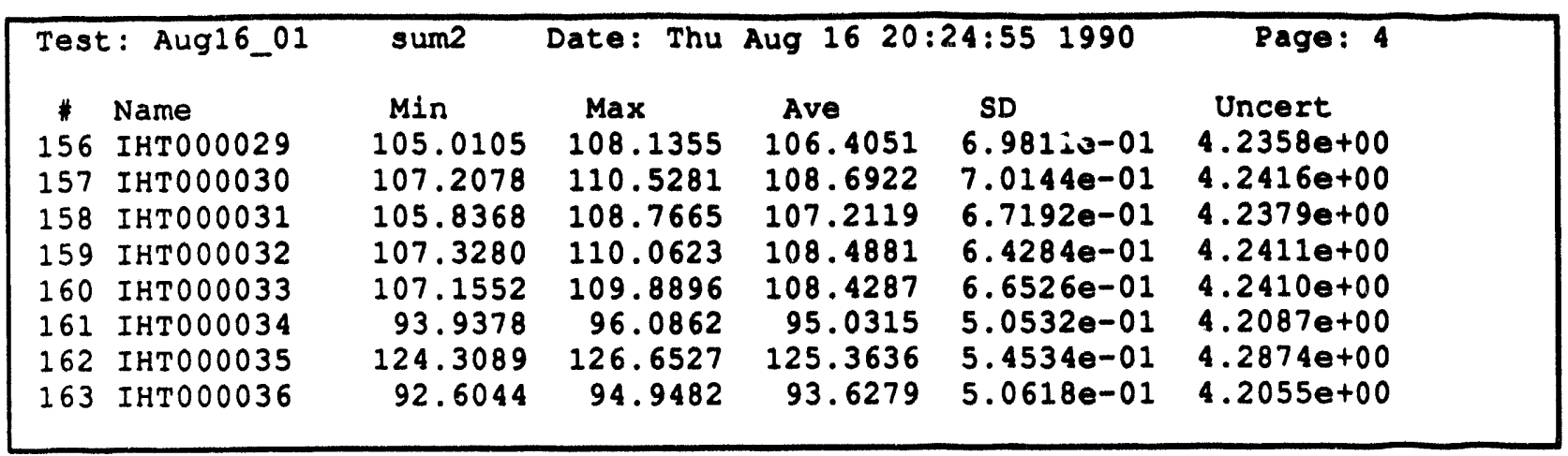




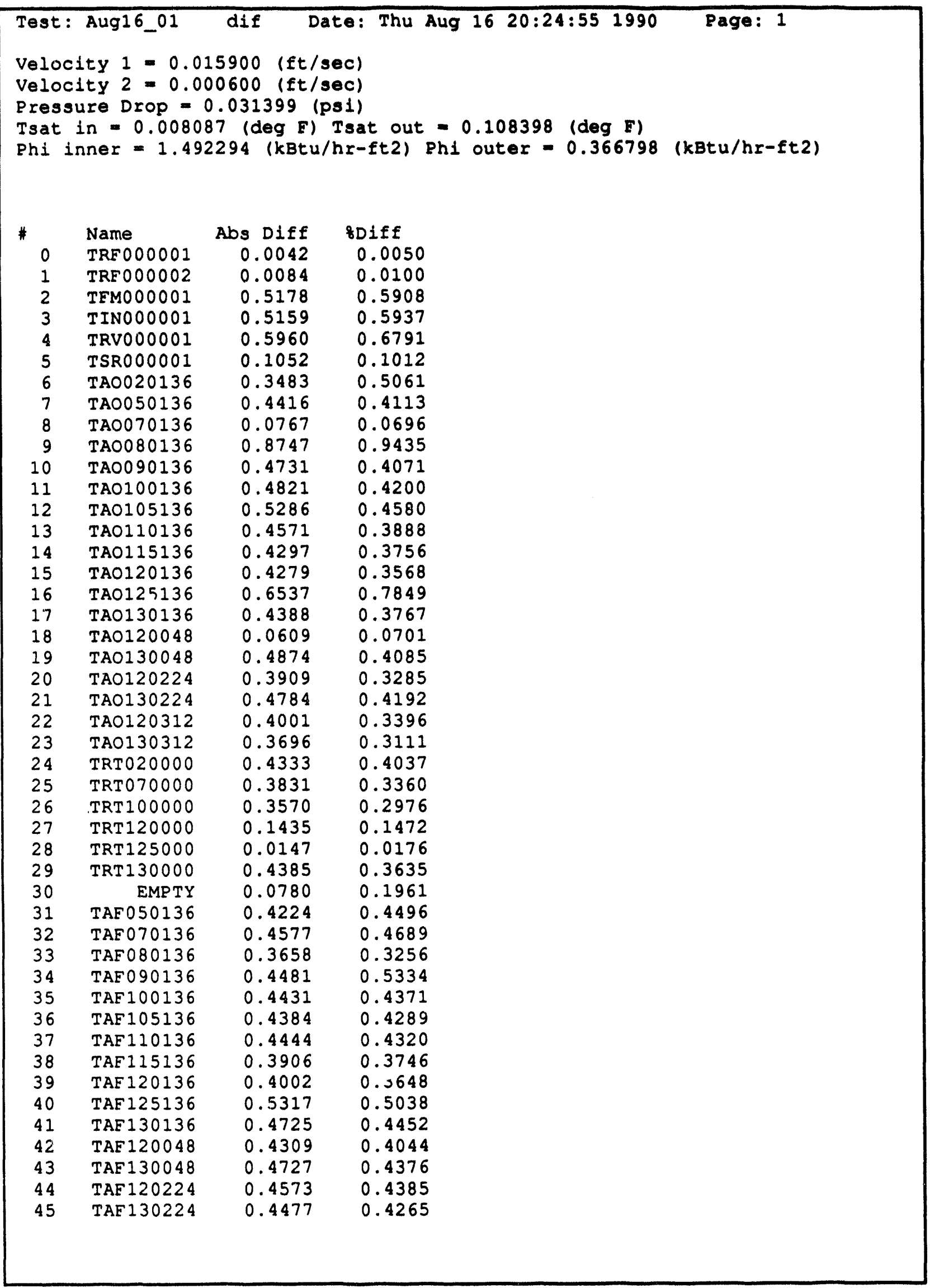




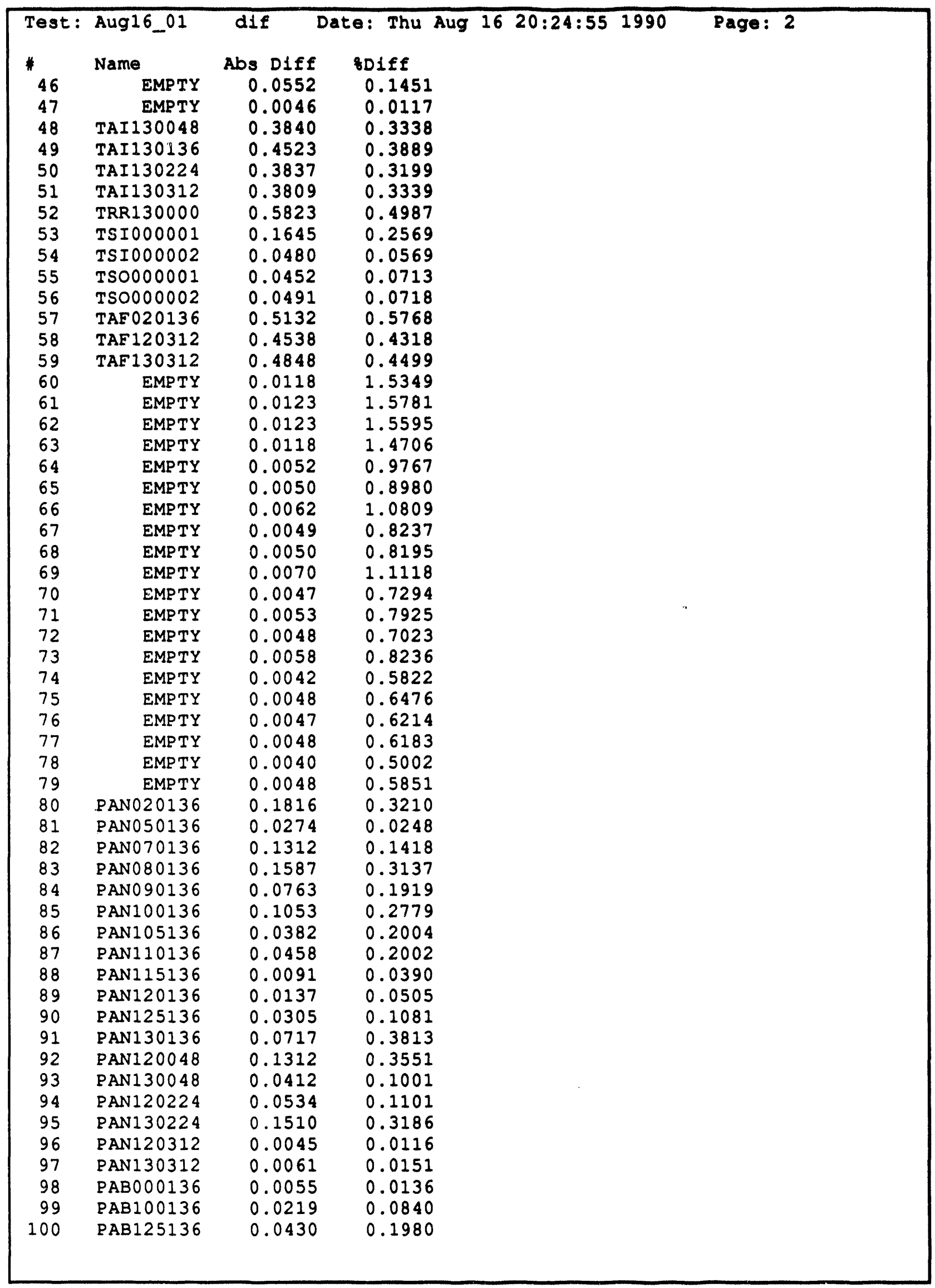




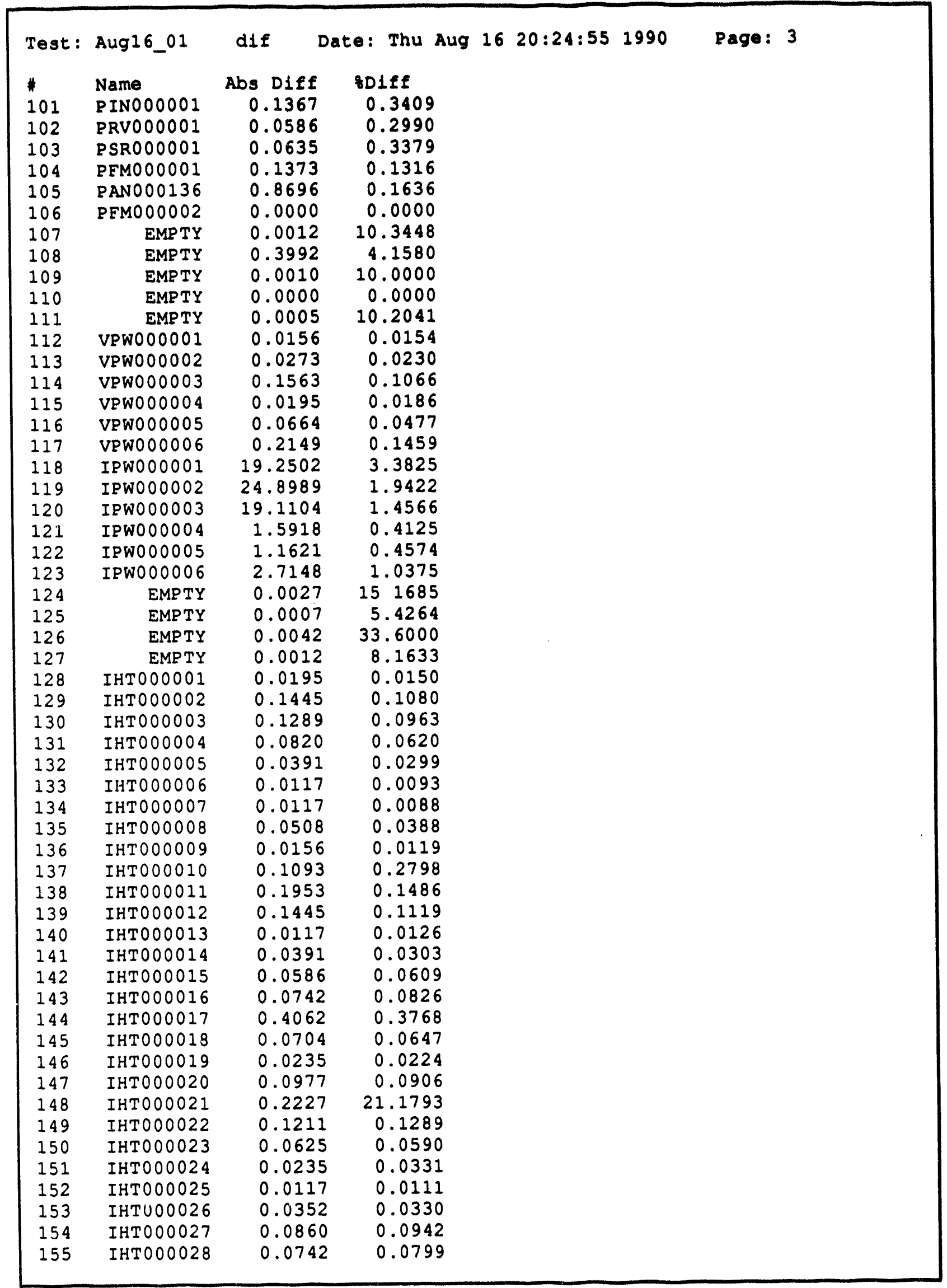




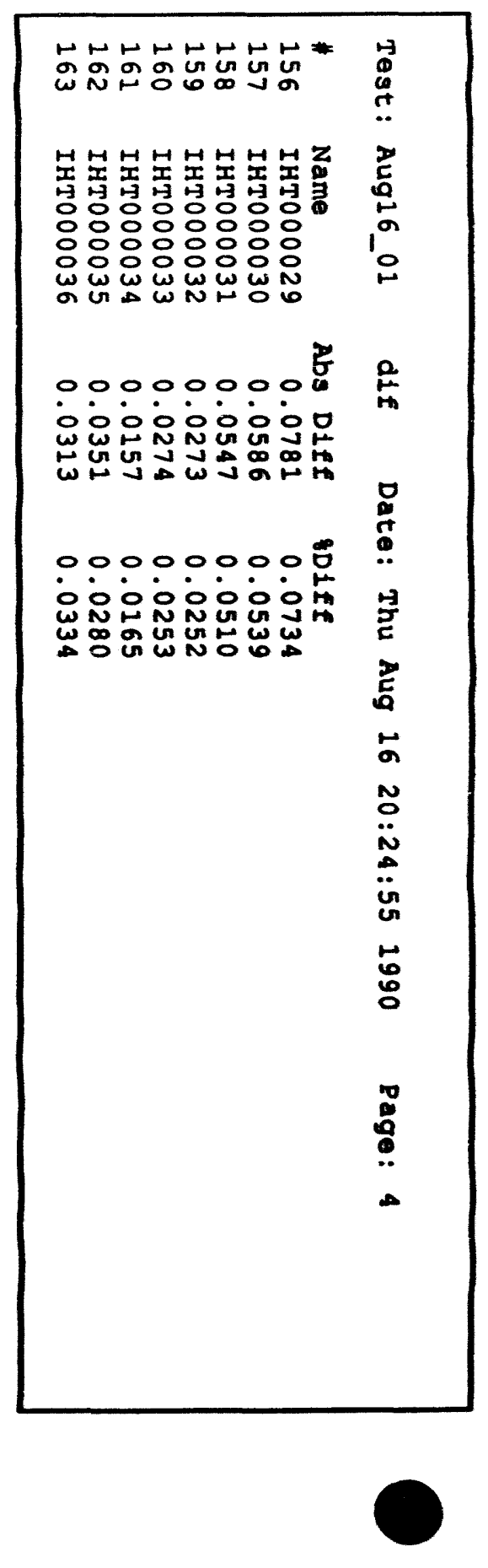




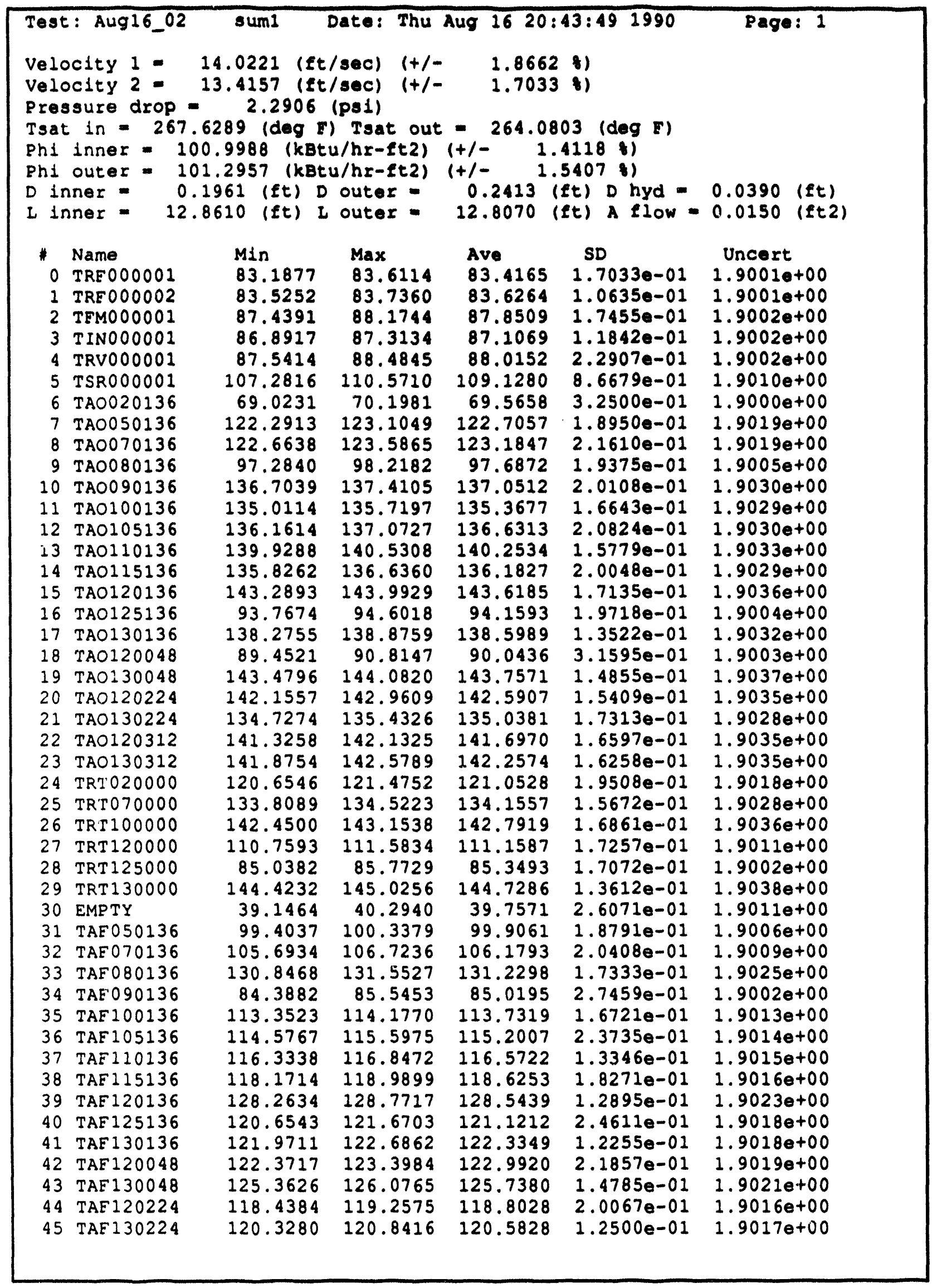




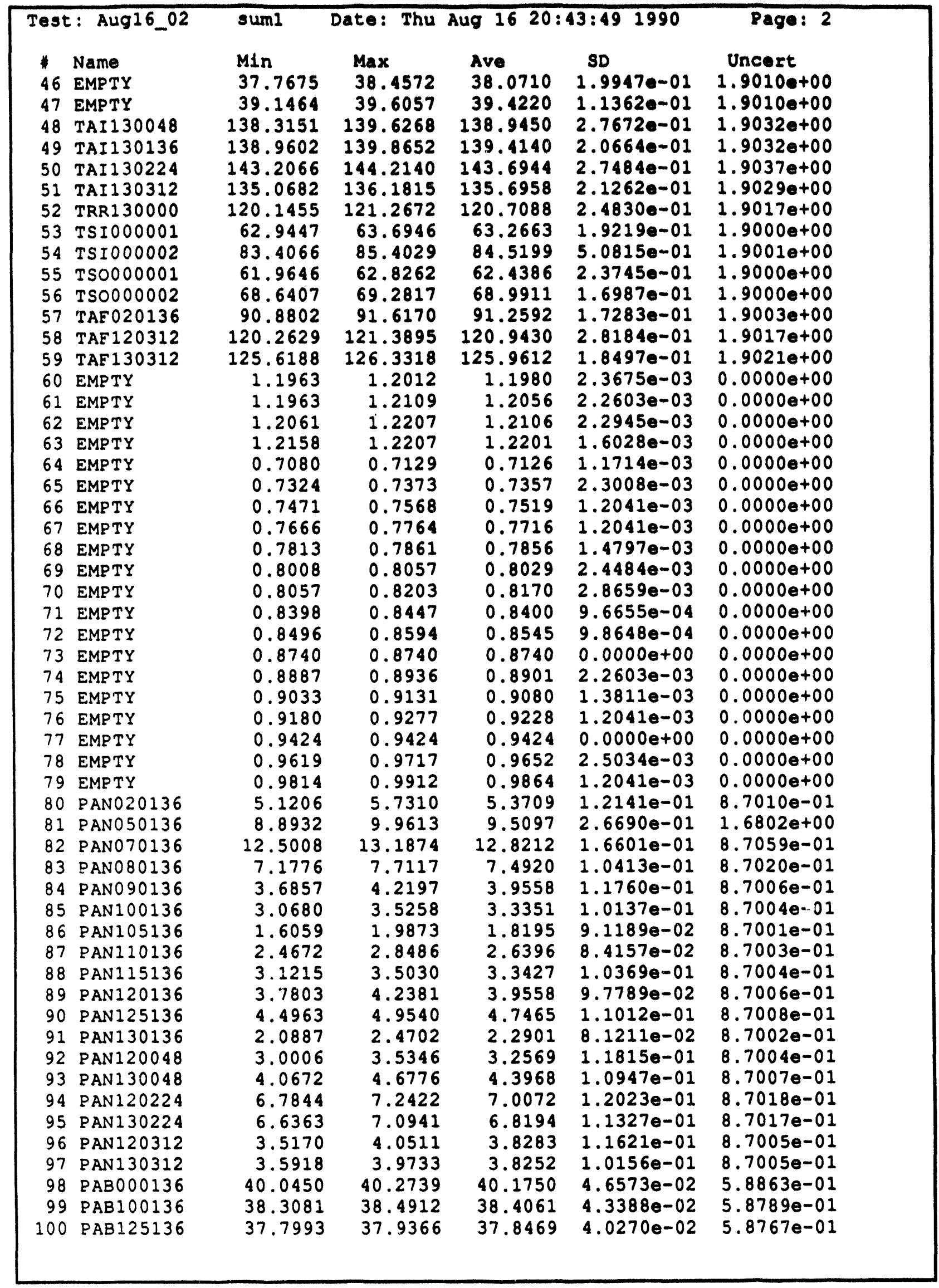




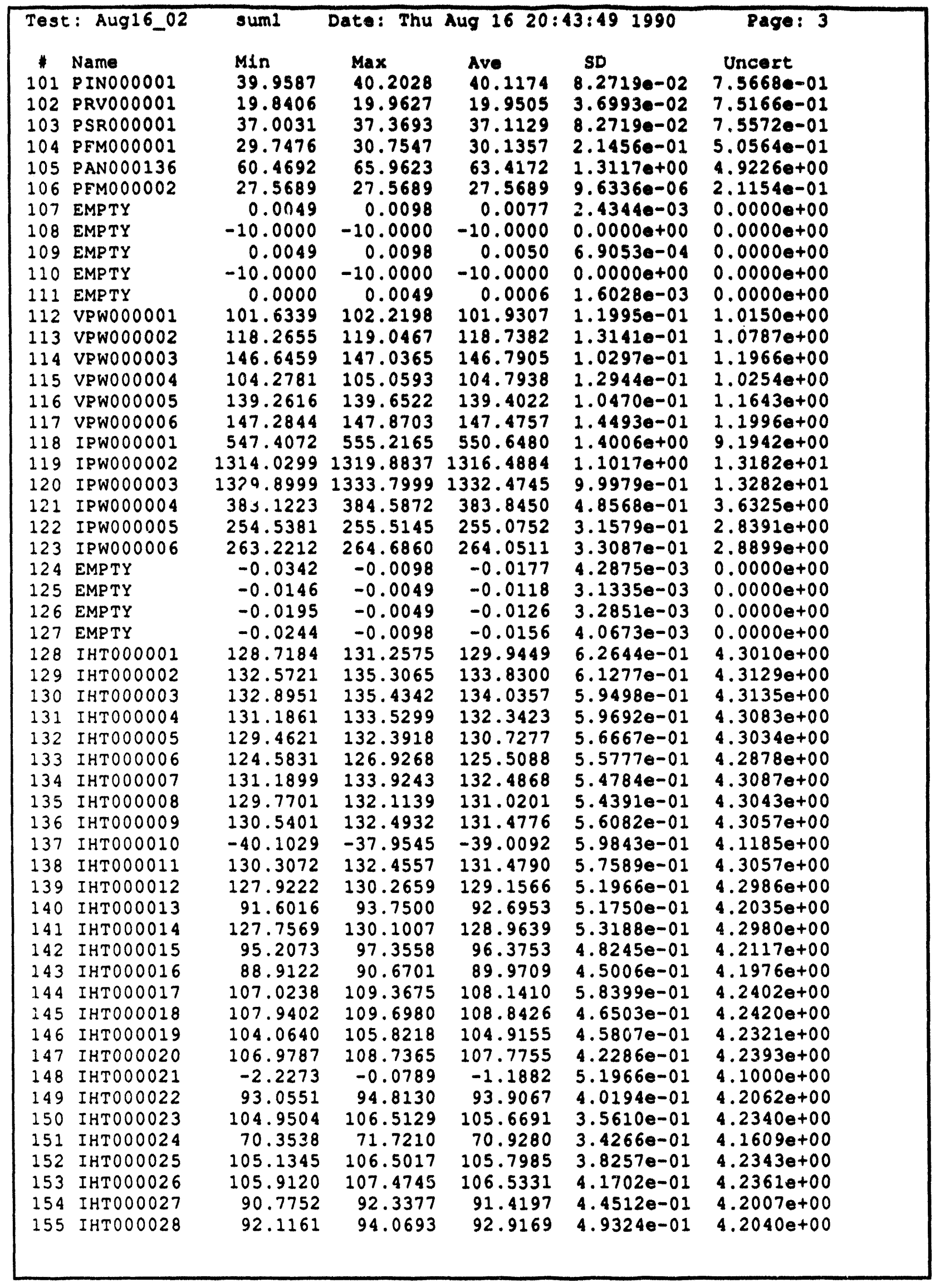




\begin{tabular}{|c|c|c|c|c|c|c|}
\hline Tes & $t:$ Aug16_02 & suml & Date: Thu & Aug 162 & $13: 491990$ & Page: 4 \\
\hline $\begin{array}{l}\# \\
156 \\
157 \\
158 \\
159 \\
160 \\
161 \\
162 \\
163\end{array}$ & $\begin{array}{l}\text { Name } \\
\text { IHTO00029 } \\
\text { IHTOOOOO30 } \\
\text { IHTO00031 } \\
\text { IHTO00032 } \\
\text { IHTO00033 } \\
\text { IHTO00034 } \\
\text { IHTO00035 } \\
\text { IHTO00036 }\end{array}$ & $\begin{array}{l}\text { Min } \\
105.4012 \\
107.5984 \\
106.0322 \\
107.5233 \\
107.5458 \\
94.3284 \\
124.5042 \\
92.9950\end{array}$ & $\begin{array}{l}\text { Max } \\
107.5496 \\
110.1375 \\
108.7665 \\
109.8670 \\
109.6943 \\
96.0862 \\
126.2620 \\
94.5575\end{array}$ & $\begin{array}{l}\text { Ave } \\
106.3934 \\
108.61510 \\
107.1377 \\
108.4725 \\
108.4209 \\
95.1253 \\
125.3285 \\
93.7138\end{array}$ & $\begin{array}{l}S D \\
4.9417 e-01 \\
4.8993 e-01 \\
5.1085 e-01 \\
5.0225 e-01 \\
4.8687 e-01 \\
4.0786 e-01 \\
4.0103 e-01 \\
4.1280 e-01\end{array}$ & $\begin{array}{l}\text { Uncert } \\
4.2358 e+00 \\
4.2415 e+00 \\
4.2377 e+00 \\
4.2411 e+00 \\
4.2409 e+00 \\
4.2089 e+00 \\
4.2873 e+00 \\
4.2057 e+00\end{array}$ \\
\hline
\end{tabular}




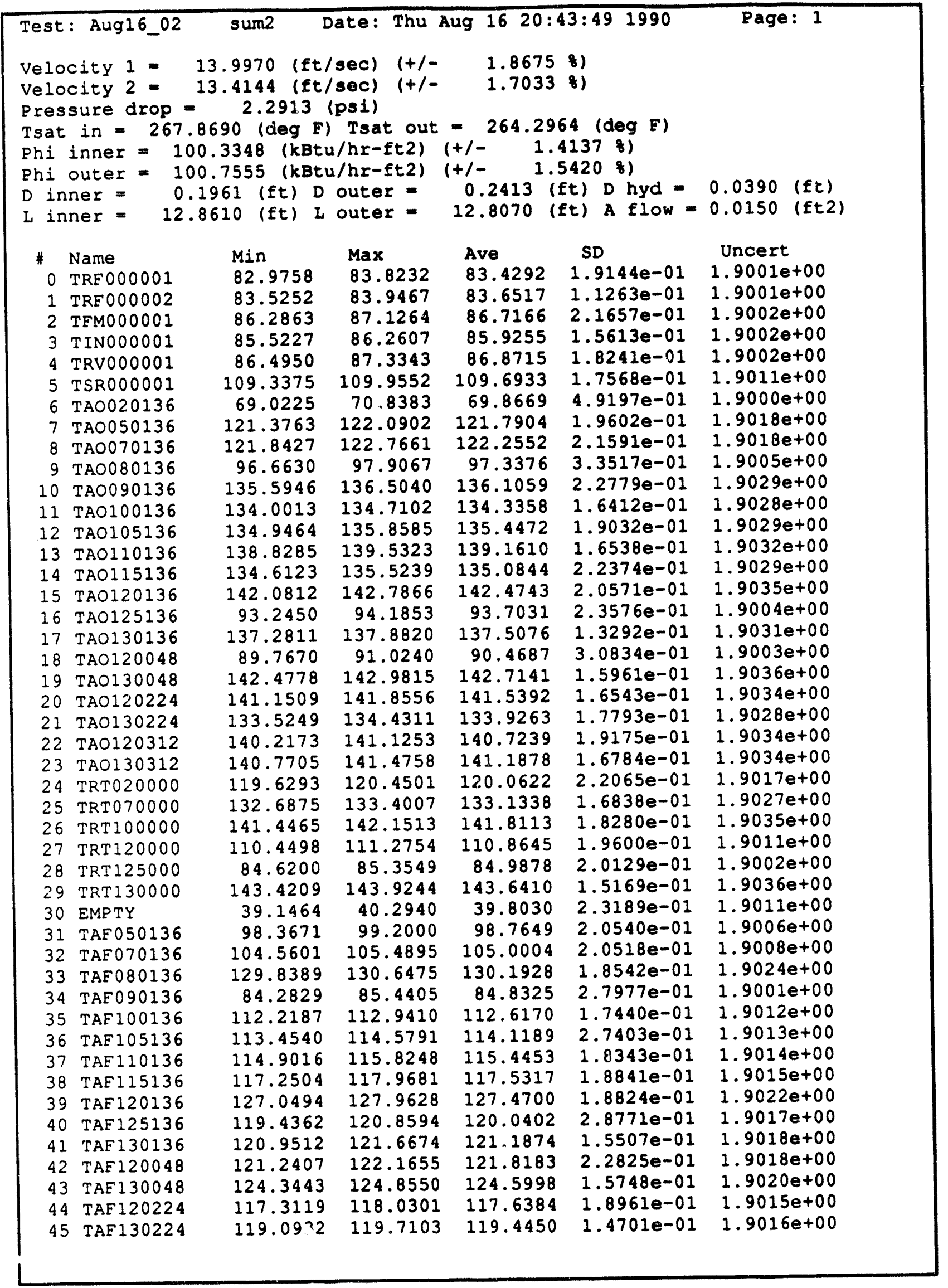




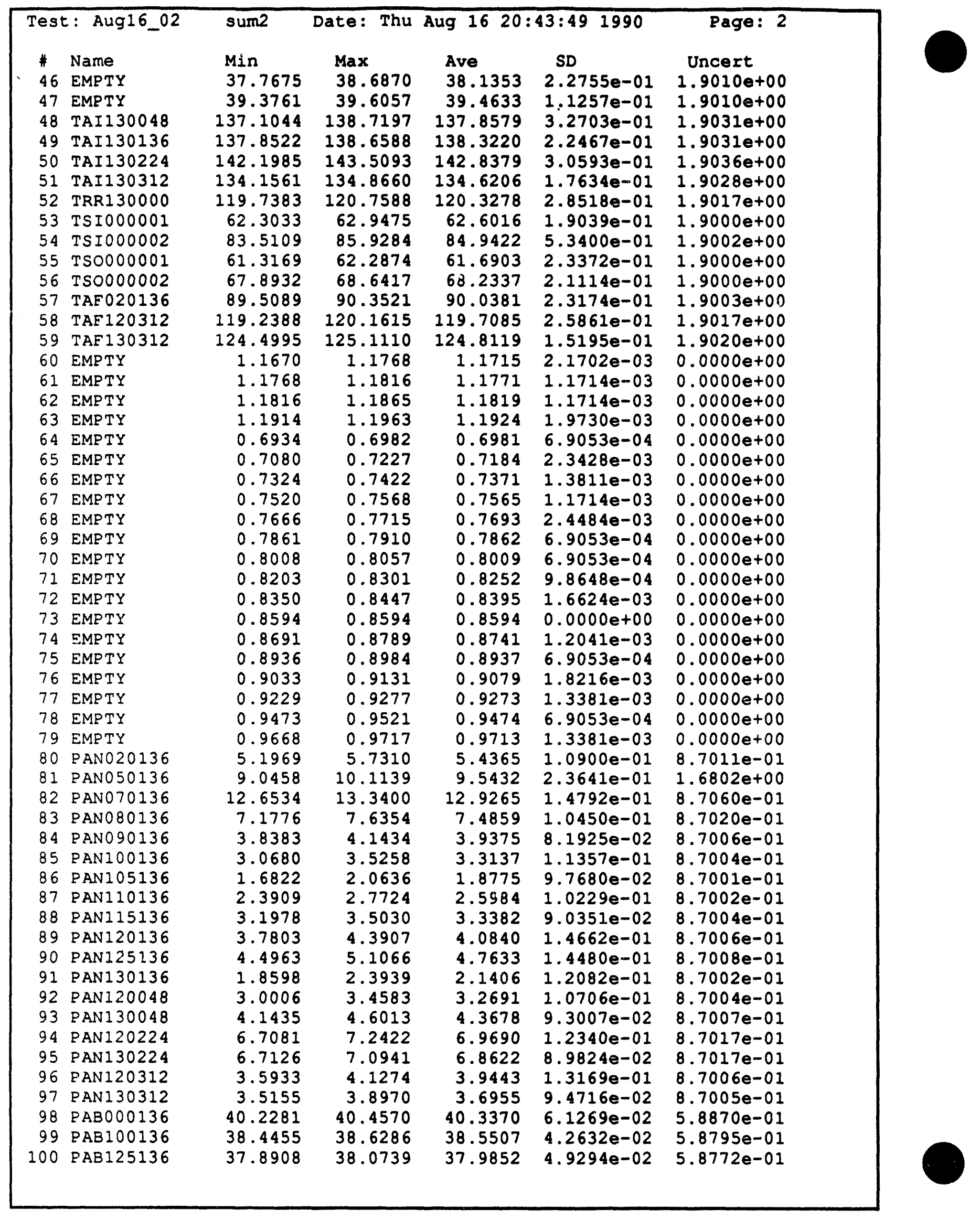




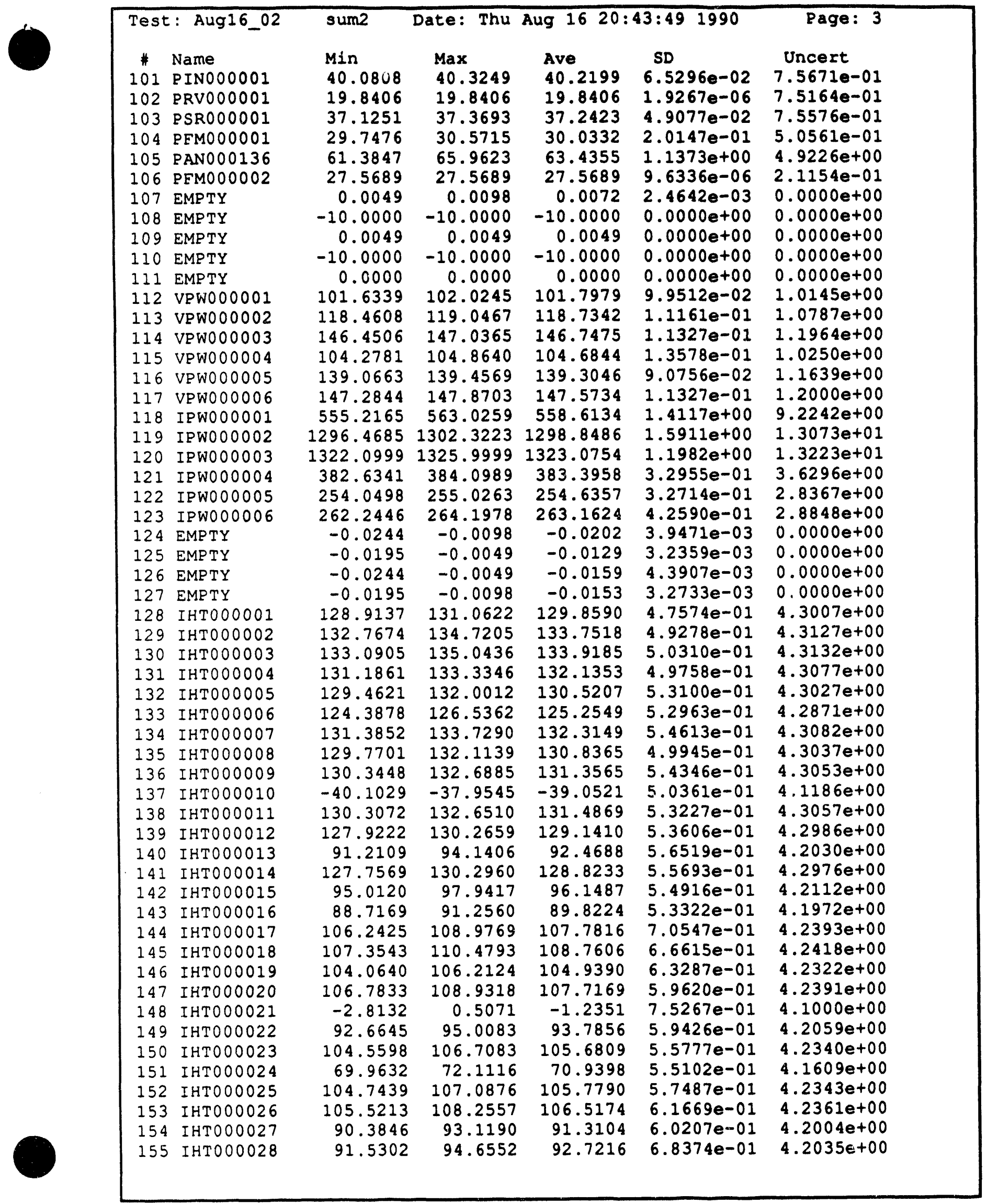




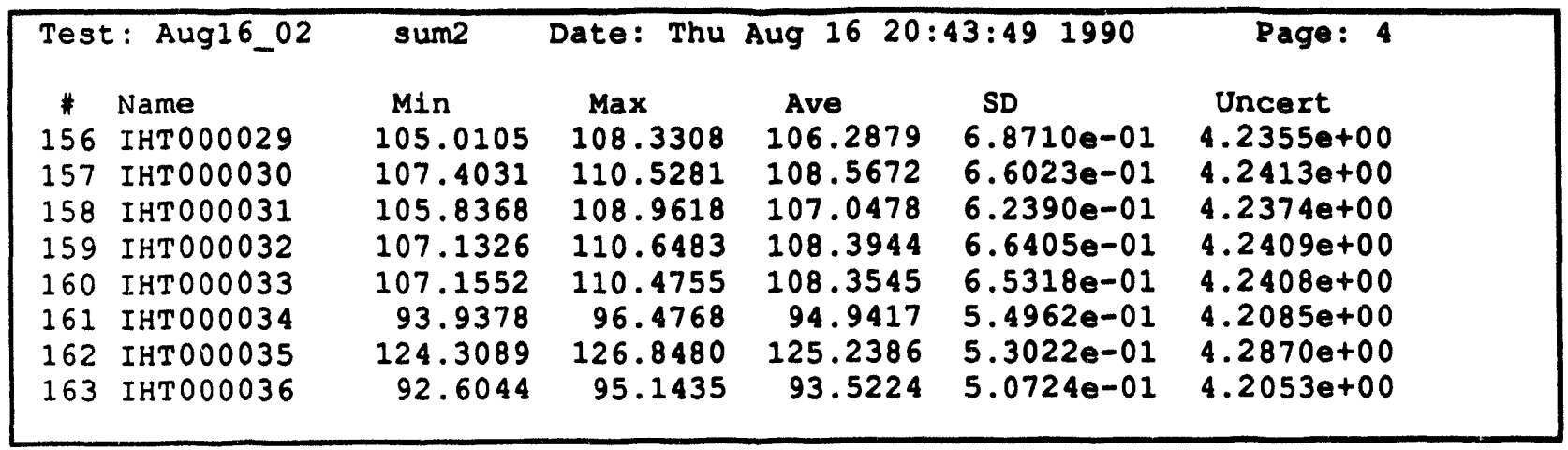




\begin{tabular}{|c|c|c|c|c|}
\hline \multicolumn{5}{|c|}{$\begin{array}{l}\text { Velocity } 1=0.025101(\mathrm{ft} / \mathrm{sec}) \\
\text { Velocity } 2=0.001300(\mathrm{ft} / \mathrm{sec}) \\
\text { Pressure Drop }=0.000700 \text { (psi) }\end{array}$} \\
\hline$\#$ & Name & Abs Diff & \&Diff & \\
\hline 0 & TRF 000001 & 0.0127 & 0.0152 & \\
\hline 1 & TRF 000002 & 0.0253 & 0.0303 & \\
\hline 2 & TFM000001 & 1.1343 & 1.2912 & \\
\hline 3 & TINO00001 & 1.1814 & 1.3563 & \\
\hline 4 & TRV000001 & 1.1437 & 1.2994 & \\
\hline 5 & TSR000001 & 0.5653 & 0.5180 & \\
\hline 6 & TAO020136 & 0.3011 & 0.4328 & \\
\hline 7 & TA0050136 & 0.9153 & 0.7459 & \\
\hline 8 & TA0070136 & 0.9295 & 0.7546 & \\
\hline 9 & TAO0 80136 & 0.3496 & 0.3579 & \\
\hline 10 & TAO090136 & 0.9453 & 0.6897 & \\
\hline 11 & TAO100136 & 1.0319 & 0.7623 & \\
\hline 12 & TAO105136 & 1.1841 & 0.8666 & \\
\hline 13 & TA0110136 & 1.0924 & $0.778 y$ & \\
\hline 14 & TAO115136 & 1.0983 & 0.8065 & \\
\hline 15 & TAO120136 & 1.1442 & 0.7967 & \\
\hline 16 & TAO125136 & 0.4562 & 0.4845 & \\
\hline 17 & TAO130136 & 1.0913 & 0.7874 & \\
\hline 18 & TAO120048 & 0.4251 & 0.4721 & \\
\hline 19 & TAO130048 & 1.0430 & 0.7255 & \\
\hline 20 & TAO120224 & 1.0515 & 0.7374 & \\
\hline 21 & TAO130224 & 1.1118 & 0.8233 & \\
\hline 22 & TAO120312 & 0.9731 & 0.6867 & \\
\hline 23 & TAO130312 & 1.0696 & 0.7519 & \\
\hline 24 & TRT020000 & 0.9906 & 0.8183 & \\
\hline 25 & TRT 070000 & 1.0219 & 0.7617 & \\
\hline 26 & TRT 100000 & 0.9806 & 0.6867 & \\
\hline 27 & TRT 120000 & 0.2942 & 0.2647 & \\
\hline 28 & TRT 125000 & 0.3615 & 0.4235 & \\
\hline 29 & TRT 130000 & 1.0876 & 0.7515 & \\
\hline 30 & EMPTY & 0.0459 & 0.1155 & \\
\hline 31 & TAF050136 & 1.1412 & 1.1423 & \\
\hline 32 & TAF070136 & 1.1789 & 1.1103 & \\
\hline 33 & TAF080136 & 1.0370 & 0.7902 & \\
\hline 34 & TAF090136 & 0.1870 & 0.2200 & \\
\hline 35 & TAF 100136 & 1.1149 & 0.9803 & \\
\hline 36 & TAF 105136 & 1.0818 & 0.9391 & \\
\hline 37 & TAF 110136 & 1.1269 & 0.9667 & \\
\hline 38 & TAF 115136 & 1.0936 & 0.9219 & \\
\hline 39 & TAF 120136 & 1.0739 & 0.8354 & \\
\hline 40 & TAF 125136 & 1.0810 & 0.8925 & \\
\hline 41 & TAF 130136 & 1.1475 & 0.9380 & \\
\hline 42 & TAF 120048 & 1.1737 & 0.9543 & \\
\hline 43 & TAE 130048 & 1.1382 & 0.9052 & \\
\hline 44 & TAE 120224 & 2.1644 & 0.9801 & \\
\hline 45 & TAF 130224 & 1.1378 & 0.9436 & \\
\hline
\end{tabular}




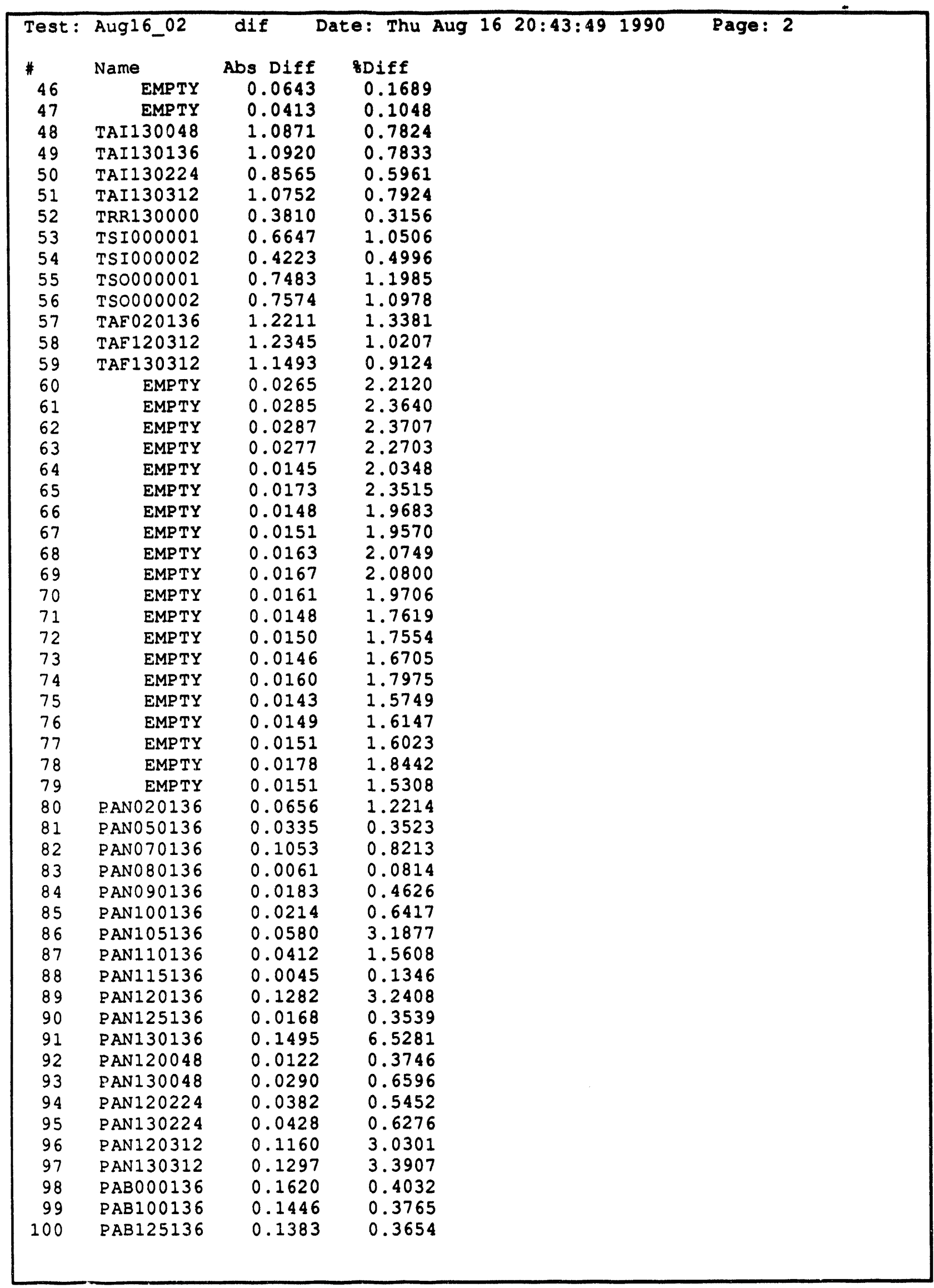




\begin{tabular}{|c|c|c|c|c|}
\hline Test: & Aug16_02 & dif & Date: Thu Aug $16 \quad 20: 43: 491990$ & Page: 3 \\
\hline \# & Name & Abs Diff & ZDiff & \\
\hline 101 & PINO00001 & 0.1025 & 0.2555 & \\
\hline 102 & PRV000001 & 0.1099 & 0.5509 & \\
\hline 103 & PSR0000001 & 0.1294 & 0.3487 & \\
\hline 104 & PFM000001 & 0.1025 & 0.3401 & \\
\hline 105 & PANO00136 & 0.0183 & 0.0289 & \\
\hline 106 & PEM000002 & 0.0000 & 0.0000 & \\
\hline 107 & EMPTY & 0.0005 & 6.4935 & \\
\hline 108 & EMPTY & 0.0000 & 0.0000 & \\
\hline 109 & EMPTY & 0.0001 & 2.0000 & \\
\hline 110 & EMPTY & 0.0000 & 0.0000 & \\
\hline 111 & ЕМРTY & 0.0006 & $6 \quad 100.0000$ & \\
\hline 112 & VPWO00001 & 0.1328 & 0.1303 & \\
\hline 113 & VRW000002 & 0.0040 & 0.0034 & \\
\hline 114 & VPW000003 & 0.0430 & 0.0293 & \\
\hline 115 & VPWO00004 & 0.1094 & 0.1044 & \\
\hline 116 & VPWO00005 & 0.0976 & 0.0700 & \\
\hline 117 & VPWO00006 & 0.0977 & 0.0662 & \\
\hline 118 & IPW000001 & 7.9654 & 1.4465 & \\
\hline 119 & IPWO 00002 & 17.6398 & 1.3399 & \\
\hline 120 & IPWO00003 & 9.3990 & 0.7054 & \\
\hline 121 & IPWO00004 & 0.4492 & 0.1170 & \\
\hline 122 & IPWO00005 & 0.4395 & 0.1723 & \\
\hline 123 & IPW000006 & 0.8887 & 0.3366 & \\
\hline 124 & EMPTY & 0.0025 & 14.1243 & \\
\hline 125 & EMPTY & 0.0011 & 9.3220 & \\
\hline 126 & EMPTY & 0.0033 & 26.1905 & \\
\hline 127 & EMPTY & 0.0003 & 1.9231 & \\
\hline 128 & IHTO00001 & 0.0859 & 0.0661 & \\
\hline 129 & IHTO00002 & 0.0782 & 0.0584 & \\
\hline 130 & IHTO00003 & 0.1172 & 0.0874 & \\
\hline 131 & IHTO00004 & 0.2070 & 0.1564 & \\
\hline 132 & IHTO000005 & 0.2070 & 0.1583 & \\
\hline 133 & IHTO00006 & 0.2539 & 0.2023 & \\
\hline 134 & IHT000007 & 0.1719 & 0.1298 & \\
\hline 135 & IHTO00008 & 0.1836 & 0.1401 & \\
\hline 136 & IHTO00009 & 0.1211 & 0.0921 & \\
\hline 137 & IHTO00010 & 0.0429 & 0.1100 & \\
\hline 138 & IHTO00011 & 0.0079 & 0.0060 & \\
\hline 139 & IHT000012 & 0.0156 & 0.0121 & \\
\hline 140 & IHTO00013 & 0.2265 & 0.2443 & \\
\hline 141 & IHTOOOOO14 & 0.1406 & 0.1090 & \\
\hline 142 & IHT000015 & 0.2266 & 0.2351 & \\
\hline 143 & IHT 000016 & 0.1485 & 0.1651 & \\
\hline 144 & IHT000017 & 0.3594 & 0.3323 & \\
\hline 145 & IHT 000018 & 0.0820 & 0.0753 & \\
\hline 146 & IHT000019 & 0.0235 & 0.0224 & \\
\hline 147 & IHTO00020 & 0.0586 & 0.0544 & \\
\hline 148 & IHTO00021 & 0.0469 & 3.9471 & \\
\hline 149 & IHTO00022 & 0.1211 & 0.1290 & \\
\hline 150 & IHT000023 & 0.0118 & 0.0112 & \\
\hline 151 & IHTO00024 & 0.0118 & 0.0166 & \\
\hline 152 & IHTO00025 & 0.0195 & 0.0184 & \\
\hline 153 & IHTO00026 & 0.0157 & 0.0147 & \\
\hline 154 & IHTO00027 & 0.1093 & 0.1196 & \\
\hline 155 & IHTO00028 & 0.1953 & 0.2102 & \\
\hline
\end{tabular}


Test: Aug16_02 dif Date: Thu Aug 16 20:43:49 1990 Page: 4

* Name Abs Diff oDiff

156 IHT000029 $0.1055 \quad 0.0992$

$\begin{array}{llll}157 & \text { IHTO0OO30 } & 0.0938 & 0.0863\end{array}$

$\begin{array}{llll}158 & \text { IHT } 000031 & 0.0899 & 0.0839\end{array}$

$\begin{array}{llll}159 & \text { IHTO00032 } & 0.0781 & 0.0720\end{array}$

$\begin{array}{llll}160 & \text { IHT } 000033 & 0.0664 & 0.0612\end{array}$

$\begin{array}{llll}161 & \text { IHTO00034 } & 0.1836 & 0.1930\end{array}$

$\begin{array}{llll}162 & \text { IHT000035 } & 0.0899 & 0.0717\end{array}$

$\begin{array}{llll}163 & \text { IHTO00036 } & 0.1914 & 0.2042\end{array}$ 


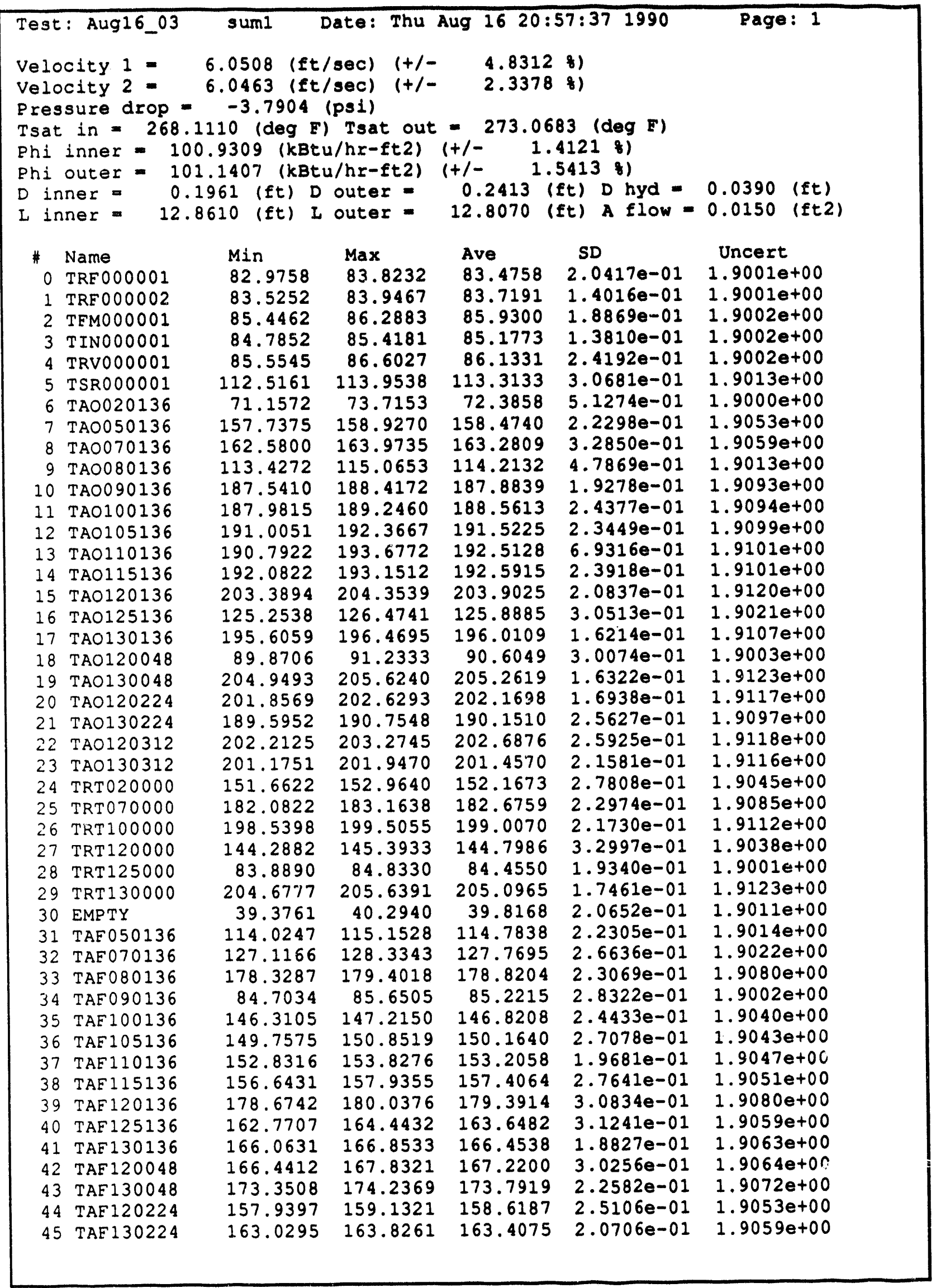




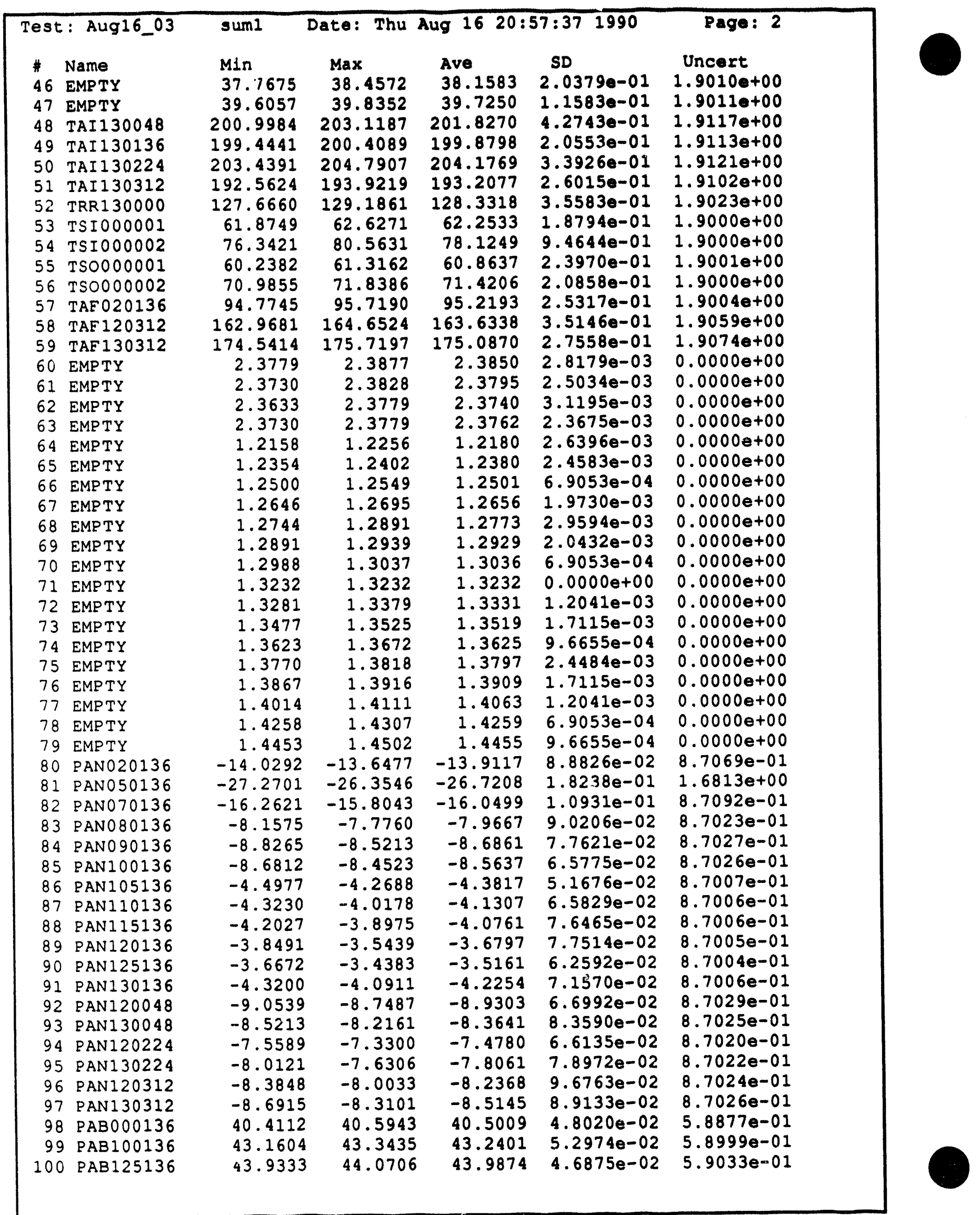




\begin{tabular}{|c|c|c|c|c|c|c|}
\hline \multicolumn{2}{|c|}{ Test: Aug16_03 } & sum 1 & Date: Thu & Aug & J & Page: 3 \\
\hline$\#$ & Name & $\operatorname{Min}$ & $\operatorname{Max}$ & Ave & SD & Uncert \\
\hline 101 & PIN 000001 & 40.2028 & 40.5690 & 40.3518 & $1.0255 e-01$ & $7.5675 e-01$ \\
\hline 102 & PRV000001 & 19.7186 & 19.9627 & 19.8406 & $4.2716 e-02$ & $7.5164 \theta-01$ \\
\hline 103 & PSR000001 & 43.9611 & 44.2052 & 44.0953 & $5.0842 e-02$ & $7.5806 e-01$ \\
\hline 104 & PEMO00001 & 5.3030 & 5.7608 & 5.5154 & $1.0696 a-01$ & $5.0019 e-01$ \\
\hline 105 & PAN000136 & -107.0723 & -103.4102 & -104.9391 & $7.7713 \theta-01$ & $4.9270 e+00$ \\
\hline 106 & PFM000002 & 5.4195 & 5.6209 & 5.5071 & $4.5896 e-02$ & $2.0047 e-01$ \\
\hline 107 & EMPTY & 0.0000 & 0.0049 & 0.0048 & $6.9053 e-04$ & $0.0000 e+00$ \\
\hline 108 & EMPTY & -10.0000 & -10.0000 & -10.0000 & $0.0000 e+00$ & $0.0000 e+00$ \\
\hline 109 & EMPTY & 0.0000 & 0.0049 & 0.0036 & $2.1635 e-03$ & $0.00000+00$ \\
\hline 110 & EMPTY & -10.0000 & -10.0000 & -10.0000 & $0.0000 \theta+00$ & $0.0000 e+00$ \\
\hline 111 & EMPTY & 0.0000 & 0.0000 & 0.0000 & $0.0000 \theta+00$ & $0.0000 e+00$ \\
\hline 112 & VPW000001 & 101.6339 & 102.2198 & 101.8877 & $1.7313 e-01$ & $1.0148 e+00$ \\
\hline 113 & VPW000002 & 118.8514 & 119.2421 & 119.0819 & $1.0942 \theta-01$ & $1.0801 e+00$ \\
\hline 114 & VPW000003 & 146.8412 & 147.4271 & 147.0991 & $1.4994 \theta-01$ & $1.1979 e+00$ \\
\hline 115 & VPWO000004 & 104.6687 & 105.0593 & 104.8367 & $1.4770 e-01$ & $1.0256 e+00$ \\
\hline 116 & VPWO00005 & 139.4569 & 139.8475 & 139.6405 & $9.9902 \theta-02$ & $1.1654 \mathrm{e}+00$ \\
\hline 117 & VPW0000006 & 147.4797 & 148.0657 & 147.7296 & $1.2503 e-01$ & $1.2007 \mathrm{e}+00$ \\
\hline 118 & IPW000001 & 545.4548 & 553.2642 & 548.9690 & $2.08710+00$ & $9.1879 e+00$ \\
\hline 119 & IPW000002 & 1304.2736 & 1319.8837 & 1311.1809 & $4.7176 e+00$ & $1.3149 e+01$ \\
\hline 120 & IPWO000003 & 1325.9999 & 1331.8499 & 1328.5347 & $1.4872 \theta+00$ & $1.3258 e+01$ \\
\hline 121 & IPW000004 & 382.1458 & 384.0989 & 383.0833 & $5.8227 \theta-01$ & $3.6275 e+00$ \\
\hline 122 & IPWO000005 & 254.0498 & 255.5145 & 254.6162 & $2.8519 e-01$ & $2.8365 e+00$ \\
\hline 123 & IPW000006 & 262.7329 & 264.6860 & 263.6702 & $4.3940 e-01$ & $2.8877 e+00$ \\
\hline 124 & EMPTY & -0.0293 & -0.0098 & -0.0192 & $4.3461 e-03$ & $0.0000 e+00$ \\
\hline 125 & EMPTY & -0.0195 & -0.0049 & -0.0126 & $2.9742 e-03$ & $0.0000 e+00$ \\
\hline 126 & EMPTY & -0.0244 & -0.0049 & -0.0145 & $3.9410 e-03$ & $0.0000 e+00$ \\
\hline 127 & EMPTY & -0.0195 & 0.0000 & -0.0142 & $3.4527 e-03$ & $0.0000 e+00$ \\
\hline 128 & IHTO00001 & 128.9137 & 131.0622 & 129.7574 & $5.4454 e-01$ & $4.3004 e+00$ \\
\hline 129 & IHTO00002 & 132.7674 & 134.9158 & 133.5956 & $5.0772 e-01$ & $4.3122 e+00$ \\
\hline 130 & IHTO00003 & 132.8951 & 135.0436 & 133.8169 & $4.5854 e-01$ & 4. $3129 e+00$ \\
\hline 131 & IHTO00004 & 131.1861 & 133.1393 & 132.0611 & $4.6057 e-01$ & $4.3074 e+00$ \\
\hline 132 & IHTO00005 & 129.4621 & 131.4153 & 130.3996 & $4.3225 e-01$ & $4.3024 e+00$ \\
\hline 133 & IHTO00006 & 124.1925 & 126.1456 & 125.2198 & $4.4957 e-01$ & $4.2870 e+00$ \\
\hline 134 & IHTO00007 & 131.1899 & 133.3384 & 132.2954 & $4.6790 e-01$ & $4.3082 e+00$ \\
\hline 135 & IHTO00008 & 129.9654 & 131.7233 & 130.7779 & $4.3290 e-01$ & $4.3035 e+00$ \\
\hline 136 & IHTO00009 & 130.3448 & 132.4932 & 131.2315 & $4.7549 e-01$ & $4.3049 e+00$ \\
\hline 137 & IHTO000010 & -40.6889 & -37.7592 & -39.1498 & $5.5893 e-01$ & $4.1186 e+00$ \\
\hline 138 & IHTO00011 & 129.7213 & 132.2603 & 131.1822 & $5.2532 e-01$ & $4.3048 \mathrm{e}+00$ \\
\hline 139 & IHTO00012 & 127.3362 & 129.8753 & 128.8206 & $5.5664 e-01$ & $4.2976 e+00$ \\
\hline 140 & IHTO00013 & 90.6250 & 93.3594 & 92.2188 & $5.1351 e-01$ & $4.2024 e+00$ \\
\hline 141 & IHTO00014 & 127.1710 & 129.3194 & 128.5850 & $4.7443 e-01$ & $4.2969 e+00$ \\
\hline 142 & IHTO00015 & 94.6214 & 96.9651 & 95.9651 & $4.8430 e-01$ & $4.2108 e+00$ \\
\hline 143 & IHTO000016 & 88.3263 & 90.6701 & 89.5177 & $4.8368 e-01$ & $4.1966 \mathrm{e}+00$ \\
\hline 144 & IHTO000017 & 105.6566 & 109.1722 & 107.2972 & $7.5696 e-01$ & $4.2381 e+00$ \\
\hline 145 & IHTO00018 & 107.1590 & 109.6980 & 108.5457 & $5.4569 e-01$ & $4.2413 e+00$ \\
\hline 146 & IHTO000019 & 103.6734 & 105.8218 & 104.7827 & $4.8878 e-01$ & $4.2318 e+00$ \\
\hline 147 & IHTO00020 & 106.7833 & 108.5412 & 107.5216 & $4.5897 e-01$ & $4.2386 e+00$ \\
\hline 148 & IHTO00021 & -2.4226 & -0.0789 & -1.3172 & $5.6028 e-01$ & $4.1000 \mathrm{e}+00$ \\
\hline 149 & IHTO00022 & 92.4692 & 94.4223 & 93.3872 & $4.2909 e-01$ & $4.2050 e+00$ \\
\hline 150 & IHTO000023 & 104.5598 & 106.7083 & 105.5441 & $4.9436 e-01$ & $4.2337 e+00$ \\
\hline 151 & IHTO00024 & 69.7679 & 71.5257 & 70.7366 & $4.4461 e-01$ & $4.1606 e+00$ \\
\hline 152 & IHTO000025 & 104.5485 & 106.5017 & 105.6305 & $4.7056 e-01$ & $4.2339 e+00$ \\
\hline 153 & IHTO000026 & 105.3260 & 107.6698 & 106.3690 & $5.2883 e-01$ & $4.2357 e+00$ \\
\hline 154 & IHTO000027 & 89.7987 & 92.5330 & 91.0057 & $5.3770 e-01$ & $4.1998 e+00$ \\
\hline 155 & IHTO00028 & 91.3349 & 93.6786 & 92.4404 & $4.8905 e-01$ & $4.2029 e+00$ \\
\hline
\end{tabular}




\begin{tabular}{|c|c|c|c|c|c|c|}
\hline $\begin{array}{l}\| \\
156 \\
157 \\
158 \\
159 \\
160 \\
161 \\
162 \\
163\end{array}$ & 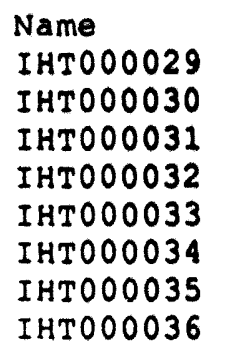 & $\begin{array}{l}\text { Min } \\
105.2058 \\
107.4031 \\
105.8368 \\
107.1326 \\
107.1552 \\
93.7425 \\
124.1136 \\
92.4091\end{array}$ & $\begin{array}{l}\operatorname{Max} \\
107.3543 \\
109.5515 \\
107.7900 \\
109.0858 \\
109.1083 \\
95.5003 \\
125.8714 \\
94.1669\end{array}$ & $\begin{array}{l}\text { Ave } \\
106.2020 \\
108.4578 \\
106.9150 \\
108.1678 \\
108.1357 \\
94.7034 \\
125.0629 \\
93.3388\end{array}$ & $\begin{array}{l}\text { SD } \\
4.7062 e-01 \\
4.4643 e-01 \\
4.2543 \theta-01 \\
4.1432 \theta-01 \\
4.2221 \theta-01 \\
4.0786 e-01 \\
3.9064 \theta-01 \\
3.8371 \theta-01\end{array}$ & $\begin{array}{c}\text { Uncert } \\
4.2353 e+00 \\
4.2410 e+00 \\
4.2371 e+00 \\
4.2403 e+00 \\
4.2402 e+00 \\
4.2080 e+00 \\
4.2865 e+00 \\
4.2049 e+00\end{array}$ \\
\hline
\end{tabular}




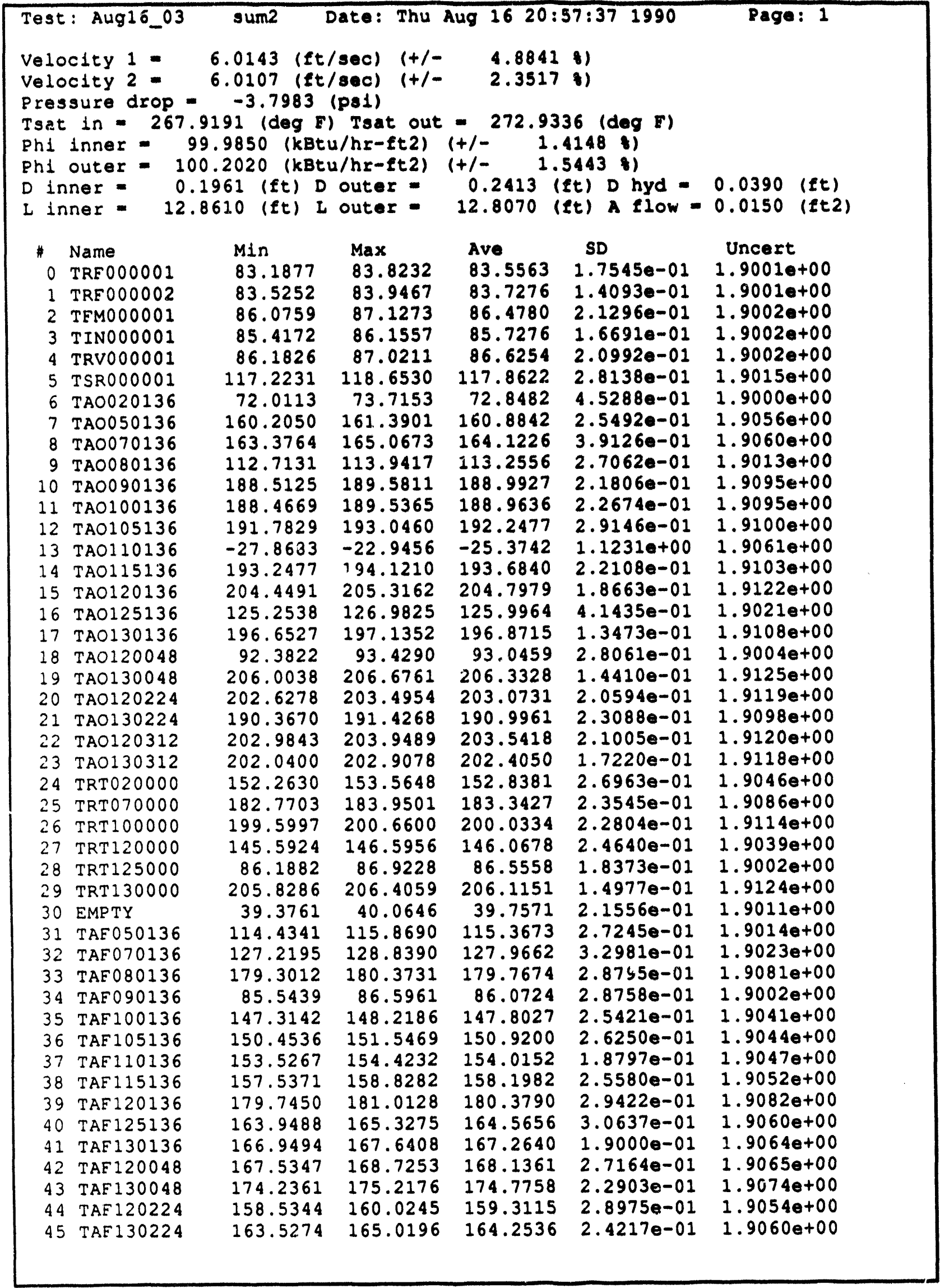



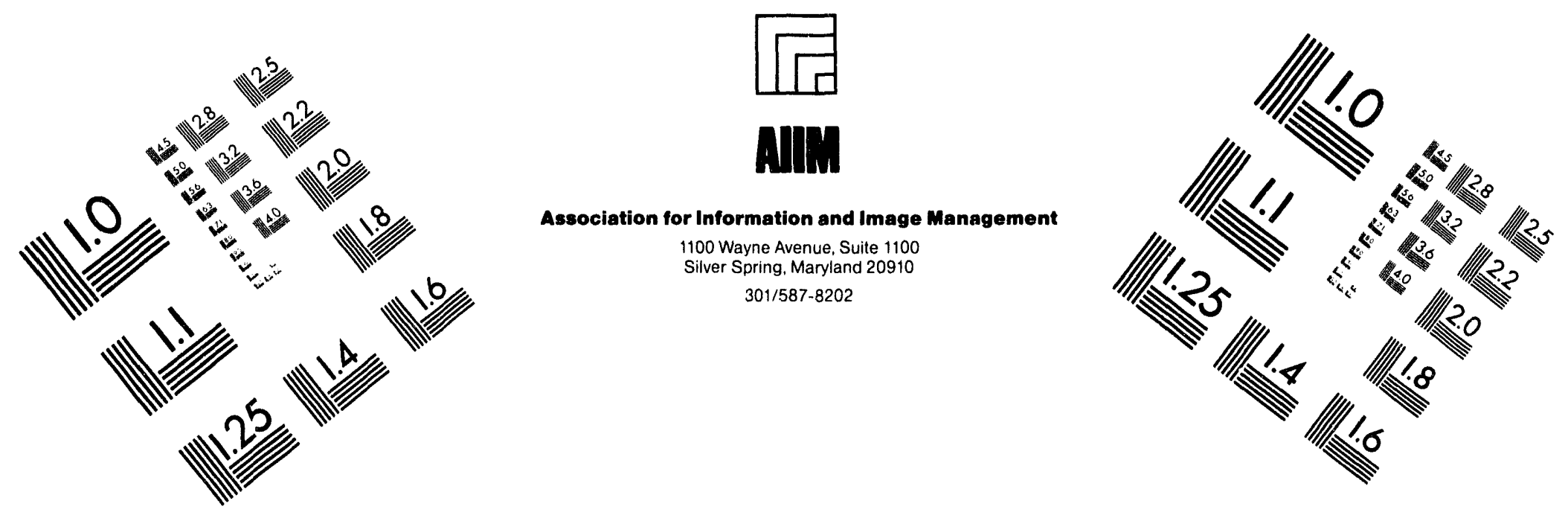

\section{Centimeter}

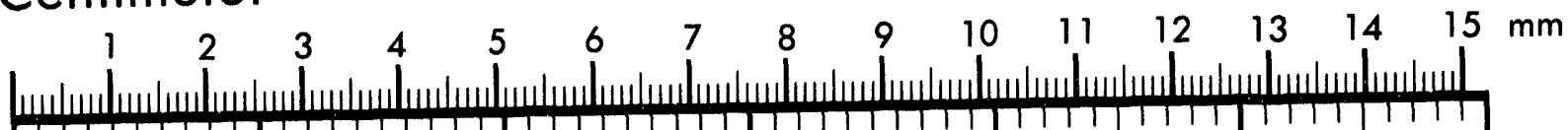
作 Inches
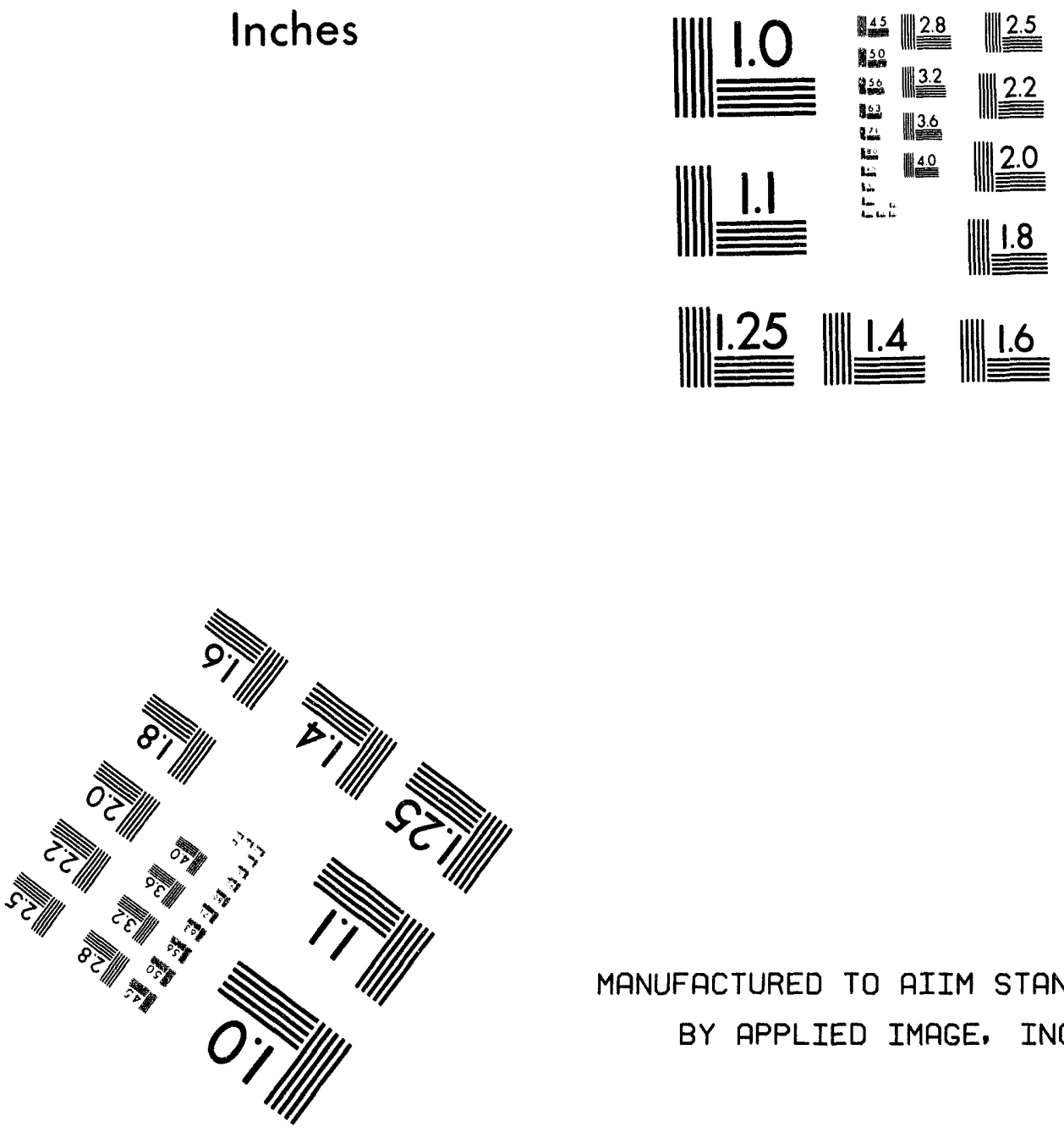

MANUFACTURED TO AIIM STANDARDS BY APPLIED IMAGE, INC.

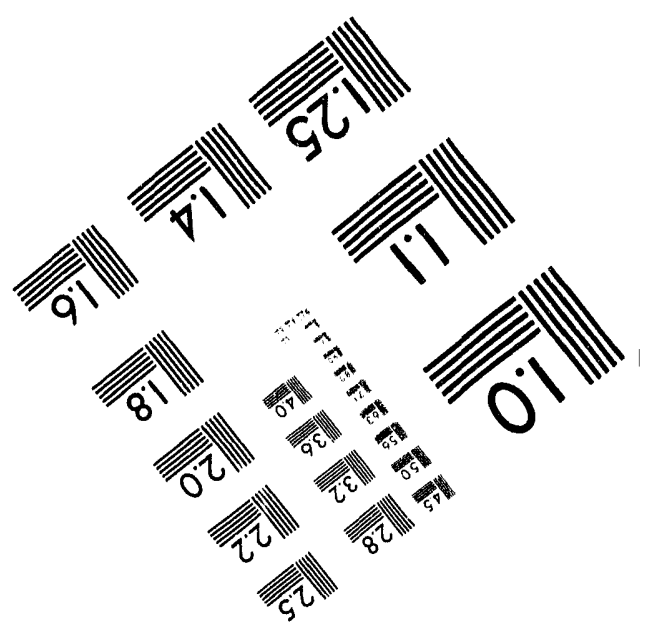



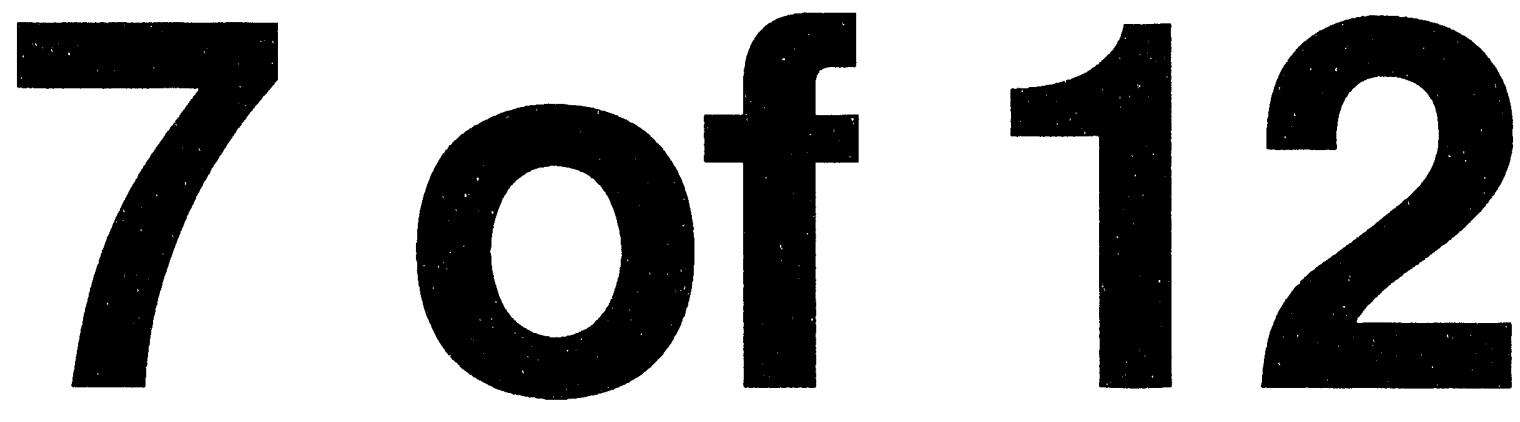


\begin{tabular}{|c|c|c|c|c|c|c|}
\hline \multicolumn{2}{|c|}{ Test: Aug16_03 } & sum2 & Date: Thu & Aug 162 & $7: 371990$ & Page: 2 \\
\hline \# & Name & Min & $\operatorname{Max}$ & Ave & SD & Uncert \\
\hline 46 & EMPTY & 37.5374 & 38.4572 & 38.0756 & $2.3045 e-01$ & $1.9010 e+00$ \\
\hline 47 & EMPTY & 39.3761 & 39.8352 & 39.6149 & $7.9786 e-02$ & $1.9011 \mathrm{e}+00$ \\
\hline 48 & TAI 130048 & 202.3476 & 203.7919 & 203.0236 & $3.2632 e-01$ & $1.9119 e+00$ \\
\hline 49 & TAI 130136 & 200.4066 & 201.2743 & 200.8558 & $1.9902 e^{-01}$ & $1.9115 e+00$ \\
\hline 50 & TAI130224 & 204.7900 & 206.0437 & 205.3864 & $3.1817 e-01$ & $1.9123 e+00$ \\
\hline 51 & TAI130312 & 193.7274 & 194.9893 & 194.2618 & $2.6977 e-01$ & $1.9104 \mathrm{e}+00$ \\
\hline 52 & TRR130000 & 121.5722 & 122.9995 & 122.3407 & $3.9056 e-01$ & $1.9018 e+00$ \\
\hline 53 & TSI000001 & 61.4474 & 62.3060 & 61.9520 & $1.9995 e-01$ & $1.9000 \mathrm{e}+00$ \\
\hline 54 & TSI000002 & 75.0709 & 78.1382 & 76.3227 & $6.3898 e-01$ & $1.9000 \mathrm{e}+00$ \\
\hline 55 & TSO000001 & 60.1296 & 60.9926 & 60.5636 & $2.1517 e-01$ & $1.9001 e+00$ \\
\hline 56 & TSC 300002 & 70.7731 & 71.7317 & 71.2398 & $2.2771 e-01$ & $1.9000 \mathrm{e}+00$ \\
\hline 57 & TAF020136 & 95.0890 & 96.0341 & 95.5196 & $2.7088 e-01$ & $1.9004 \mathrm{e}+00$ \\
\hline 58 & TAF 120312 & 163.7610 & 165.5437 & 164.5433 & $3.5859 e-01$ & $1.9060 \mathrm{e}+00$ \\
\hline 59 & $\operatorname{TAF} 130312$ & 175.4240 & 176.8951 & 176.1163 & $3.0533 e-01$ & $1.9076 \mathrm{e}+00$ \\
\hline 60 & EMPTY & 2.4023 & 2.4121 & 2.4090 & $3.2359 e-03$ & $0.0000 e+00$ \\
\hline 61 & EMPTY & 2.3975 & 2.4072 & 2.4038 & $2.6562 e-03$ & $0.0000 e+00$ \\
\hline 62 & EMPTY & 2.3926 & 2.4023 & 2.3978 & $2.2945 e-03$ & $0.0000 e+00$ \\
\hline 63 & EMPTY & 2.3975 & 2.4023 & 2.4001 & $2.4583 e-03$ & $0.0000 e+00$ \\
\hline 64 & EMPTY & 1.2256 & 1.2354 & 1.2295 & $2.2058 e-03$ & $0.0000 e+00$ \\
\hline 65 & EMPTY & 1.2451 & 1.2549 & 1.2484 & $2.5034 e-03$ & $0.0000 \mathrm{e}+00$ \\
\hline 66 & EMPTY & 1.2549 & 1.2646 & 1.2602 & $1.6624 \mathrm{e}-03$ & $0.0000 e+00$ \\
\hline 67 & EMPTY & 1.2744 & 1.2793 & 1.2771 & $2.4484 e-03$ & $0.0000 \mathrm{e}+00$ \\
\hline 68 & EMPTY & 1.2842 & 1.2891 & 1.2887 & $1.3381 e-03$ & $0.0000 \mathrm{e}+00$ \\
\hline 69 & EMPTY & 1.3037 & 1.3037 & 1.3037 & $0.0000 e+00$ & $0.0000 \mathrm{e}+00$ \\
\hline 70 & EMPTY & 1.3135 & 1.3184 & 1.3139 & $1.3381 e-03$ & $0.0000 e+00$ \\
\hline 71 & EMPTY & 1.3330 & 1.3379 & 1.3332 & $9.6655 e-04$ & $0.0000 \mathrm{e}+00$ \\
\hline 72 & EMPTY & 1.3428 & 1.3477 & 1.3436 & $1.8082 e-03$ & $0.0000 \mathrm{e}+00$ \\
\hline 73 & EMPTY & 1.3574 & 1.3623 & 1.3621 & $9.6655 e-04$ & $0.0000 \mathrm{e}+00$ \\
\hline 74 & EMPTY & 1.3721 & 1.3867 & 1.3749 & $2.9742 e-03$ & $0.0000 e+00$ \\
\hline 75 & EMPTY & 1.3867 & 1.3916 & 1.3911 & $1.4797 e-03$ & $0.0000 e+00$ \\
\hline 76 & EMPTY & 1.4014 & 1.4014 & 1.4014 & $0.0000 e+00$ & $0.0000 e+00$ \\
\hline 77 & EMPTY & 1.4160 & 1.4209 & 1.4184 & $2.4642 e-03$ & $0.0000 e+00$ \\
\hline 78 & EMPTY & 1.4307 & 1.4404 & 1.4371 & $2.5034 e-03$ & $0.0000 e+00$ \\
\hline 79 & EMPTY & 1.4551 & 1.4600 & 1.4581 & $2.3941 e-03$ & $0.0000 e+00$ \\
\hline 80 & PAN020136 & -14.1055 & -13.8003 & -13.9605 & $7.7453 e-02$ & $8.7070 e-01$ \\
\hline 81 & PAN050136 & -27.2701 & -26.5072 & -26.8612 & $1.6148 \mathrm{e}-01$ & $1.6813 \mathrm{e}+00$ \\
\hline 82 & PAN070136 & -16.3383 & -15.9569 & -16.1201 & $9.8708 e-02$ & $8.7093 e-01$ \\
\hline 83 & PAN080136 & -8.2337 & -7.8523 & -8.0278 & $9.5328 e-02$ & $8.7023 e-01$ \\
\hline 84 & PAN090136 & -8.8265 & -8.5976 & -8.7304 & $7.0314 \mathrm{e}-02$ & $8.7027 e-01$ \\
\hline 85 & PAN 100136 & -8.6812 & -8.3761 & -8.5485 & $7.5212 e-02$ & \\
\hline 86 & PAN 105136 & -4.5739 & -4.2688 & -4.4198 & $7.3097 e-02$ & $\begin{array}{l}8.7026 e-01 \\
8.7007 e-01\end{array}$ \\
\hline 87 & PAN 110136 & -4.3230 & -4.0178 & & $6.1655 e-02$ & $\begin{array}{l}8.7007 e-01 \\
8.7006 e-01\end{array}$ \\
\hline 8.8 & PAN 115136 & -4.2790 & -4.0501 & -4.1478 & $6.5467 e-02$ & $8.7006 e-01$ \\
\hline 89 & PAN 120136 & -3.9254 & -3.5439 & -3.7529 & $\begin{array}{l}0.3461 e-02 \\
7.6775 e-02\end{array}$ & $8.7006 e-01$ \\
\hline 90 & & -3.6672 & -3.4383 & $\begin{array}{l}-3.7529 \\
-3.5589\end{array}$ & $7.6775 e-02$ & $8.7005 e-01$ \\
\hline 91 & PAN 130136 & -4.3200 & -4.0911 & $\begin{array}{l}-3.5589 \\
-4.2025\end{array}$ & $6.9087 e-02$ & $8.7005 e-01$ \\
\hline 92 & PAN 120048 & -9.0539 & -8.8250 & -4.2025 & $8.6110 e-02$ & $8.7006 e-01$ \\
\hline 93 & PAN 130048 & -8.5975 & & -8.9455 & $6.9087 e-02$ & $8.7029 e-01$ \\
\hline 94 & PAN 120224 & -7.7115 & $\begin{array}{l}-8.2924 \\
-7.3300\end{array}$ & -8.4556 & $9.1202 e-02$ & $8.7026 e-01$ \\
\hline 95 & $\begin{array}{l}\text { PAN120224 } \\
\text { PAN } 130224\end{array}$ & -7.7115 & -7.3300 & -7.5467 & $9.0351 e-02$ & $8.7020 e-01$ \\
\hline 96 & & -8.0884 & -7.6306 & -7.8809 & $8.4481 e-02$ & $8.7022 e-01$ \\
\hline 97 & $\begin{array}{l}\text { PAN } 120312 \\
\text { PAN1 } 30312\end{array}$ & -8.4611 & -8.1559 & -8.3101 & $7.6278 e-02$ & $8.7025 e-01$ \\
\hline 98 & PAN1 30312 & -8.6915 & -8.3101 & -8.5832 & $8.8666 e-02$ & $8.7026 e-0 I$ \\
\hline 98 & $\mathrm{PAB} 000136$ & 40.2281 & 40.4570 & 40.3709 & $4.8621 e-02$ & $5.8872 e-01$ \\
\hline 99 & $\mathrm{PAB} 100136$ & 43.0231 & 43.2520 & 43.1256 & $5.1125 e-02$ & $5.8994 e-01$ \\
\hline 100 & PAB 125136 & 43.7960 & 43.9791 & 43.8894 & $4.7121 e-02$ & $5.9029 e-01$ \\
\hline
\end{tabular}




\begin{tabular}{|c|c|c|c|c|c|c|}
\hline \multicolumn{2}{|c|}{ Test: Aug16_03 } & sum2 & Date: Thu & Aug 1620 & $57: 37 \quad 1990$ & Page: 3 \\
\hline$\#$ & Name & Min & $\operatorname{Max}$ & Ave & $S D$ & Uncert \\
\hline 101 & PIN 000001 & 39.9587 & 40.2028 & 40.1052 & $6.0409 e-02$ & $7.5667 e-01$ \\
\hline 102 & PRV000001 & 19.4744 & 19.8406 & 19.6966 & $5.8828 e-02$ & $7.5161 \mathrm{e}-01$ \\
\hline 103 & PSR000001 & 43.8390 & 44.0831 & 43.9586 & $3.8916 e-02$ & $7.5801 e-01$ \\
\hline 104 & PFMO00001 & 5.3030 & 5.6692 & 5.4476 & $9.0783 e-02$ & $5.0019 e-01$ \\
\hline 105 & PAN 000136 & -107.0723 & -102.9525 & -105.1589 & $7.3806 e-01$ & $4.9270 \mathrm{e}+00$ \\
\hline 106 & PFM0000002 & 5.3692 & 5.5034 & 5.4410 & $3.7142 \mathrm{e}-02$ & $2.0046 e-01$ \\
\hline 107 & EMPTY & 0.0049 & 0.0049 & 0.0049 & $0.0000 e+00$ & $0.0000 e+00$ \\
\hline 108 & EMPTY & -10.0000 & -9.8047 & -9.9961 & $2.7621 e-02$ & $0.0000 e+00$ \\
\hline 109 & EMPTY & 0.0000 & 0.0049 & 0.0046 & $1.1714 e-03$ & $0.0000 e+00$ \\
\hline 110 & EMPTY & -10.0000 & -10.0000 & -10.0000 & $0.0000 e+00$ & $0.0000 e+00$ \\
\hline 111 & EMPTY & -0.0049 & 0.0000 & -0.0001 & $6.9053 e-04$ & $0.0000 e+00$ \\
\hline 112 & VPWO00001 & 101.6339 & 102.4151 & 102.0245 & $1.8924 e-01$ & $1.0153 \mathrm{e}+00$ \\
\hline 113 & VPWO00002 & 118.8514 & 119.2421 & 119.0546 & $6.7888 e-02$ & $1.0800 \mathrm{e}+00$ \\
\hline 114 & VPWO00003 & 146.8412 & 147.2318 & 147.0561 & $1.3811 e-01$ & $1.1978 \mathrm{e}+00$ \\
\hline 115 & VPWO00004 & 104.6687 & 105.2546 & 104.9344 & $1.1679 e-01$ & $1.0259 \mathrm{e}+00$ \\
\hline 116 & VPWO00005 & 139.4569 & 139.8475 & 139.6600 & $1.2453 e-01$ & $1.1655 \mathrm{e}+00$ \\
\hline 117 & VPWO000006 & 147.6750 & 148.0657 & 147.9327 & $1.1464 e-01$ & $1.2016 \mathrm{e}+00$ \\
\hline 118 & IPWO00001 & 557.1689 & 563.0259 & 559.7459 & $1.4988 \mathrm{e}+00$ & $9.2284 \mathrm{e}+00$ \\
\hline 119 & IPWO 00002 & 1275.0045 & 1290.6146 & 1279.5316 & $3.1150 e+00$ & $1.2953 \mathrm{e}+01$ \\
\hline 120 & IPW000003 & 1310.3999 & 1318.1998 & 1314.4954 & $2.0184 \mathrm{e}+00$ & $1.3170 \mathrm{e}+01$ \\
\hline 121 & IPWO000004 & 383.1223 & 384.0989 & 383.6204 & $2.8744 e-01$ & $3.6311 \mathrm{e}+00$ \\
\hline 122 & IPWO00005 & 253.0734 & 254.0498 & 253.6104 & $3.1580 e-01$ & $2.8309 \mathrm{e}+00$ \\
\hline 123 & IPW000006 & 262.2446 & 263.709 .5 & 262.8011 & $3.8219 e-01$ & $2.8828 \mathrm{e}+00$ \\
\hline 124 & EMPTY & -0.0244 & -0.0098 & -0.0179 & $3.6393 e-03$ & $0.0000 e+00$ \\
\hline 125 & EMPTY & -0.0195 & -0.0049 & -0.0122 & $3.3087 e-03$ & $0.0000 e+00$ \\
\hline 126 & EMPTY & -0.0195 & -0.0049 & -0.0131 & $3.1874 e-03$ & $0.0000 e+00$ \\
\hline 127 & EMPTY & -0.0195 & -0.0098 & -0.0144 & $3.7447 e-03$ & $0.0000 e+00$ \\
\hline 128 & IHTO000001 & 128.7184 & 130.8669 & 129.7926 & $5.2682 e-01$ & $4.3005 e+00$ \\
\hline 129 & IHT000002 & 132.5721 & 134.7205 & 133.5878 & $5.0992 e-01$ & $4.3121 e+00$ \\
\hline 130 & IHT000003 & 132.8951 & 135.0436 & 133.8716 & $5.0378 e-01$ & $4.3130 \mathrm{e}+00$ \\
\hline 131 & IHTO00004 & 131.1861 & 133.3346 & 132.1158 & $4.6280 e-01$ & $4.3076 e+00$ \\
\hline 132 & IHTO00005 & 129.4621 & 131.8059 & 130.4582 & $5.0571 e-01$ & $4.3025 e+00$ \\
\hline 133 & IHTO000006 & 124.5831 & 126.5362 & 125.3291 & $4.7954 e-01$ & $4.2873 e+00$ \\
\hline 134 & IHTO000007 & 131.5806 & 133.7290 & 132.3618 & $4.9284 e-01$ & $4.3084 e+00$ \\
\hline 135 & IHTO00008 & 129.9654 & 132.1139 & 130.8208 & $4.7497 e-01$ & $4.3037 e+00$ \\
\hline 136 & IHTOO00009 & 130.3448 & 132.4932 & 131.2432 & $5.1900 e-01$ & $4.3049 e+00$ \\
\hline 137 & IHTOOOOO10 & -40.1029 & -37.9545 & -39.1068 & $5.3416 e-01$ & $4.1186 \mathrm{e}+00$ \\
\hline 138 & IHTOOOOOII & 130.5025 & 132.4557 & 131.4751 & $4.7921 e-01$ & $4.3056 e+00$ \\
\hline 139 & IHTOOO0012 & 128.1175 & 130.2659 & 129.1253 & $5.2402 e-01$ & $4.2985 e+00$ \\
\hline 140 & IHTOO00013 & 91.4063 & 93.3594 & 92.3789 & $4.9045 e-01$ & $4.2028 e+00$ \\
\hline 141 & IHTOO00014 & 127.7569 & 129.9054 & 128.6827 & $4.8778 e-01$ & $4.2972 e+00$ \\
\hline 142 & IHTOO00015 & 95.2073 & 97.1604 & 96.0628 & $4.5488 e-01$ & $4.2110 \mathrm{e}+00$ \\
\hline 143 & IHTO00016 & 88.7169 & 90.6701 & 89.5763 & $4.8488 e-01$ & $4.1967 \mathrm{e}+00$ \\
\hline 144 & IHTO000017 & 105.4613 & 108.1956 & 106.9808 & $6.5589 e-01$ & $4.2373 e+00$ \\
\hline 145 & IHTO00018 & 107.1590 & 109.8933 & 108.4246 & $5.8426 e-01$ & $4.2409 e+00$ \\
\hline 146 & IHTOOO0019 & 103.6734 & 105.6265 & 104.6304 & $4.9324 e-01$ & $4.2314 e+00$ \\
\hline 147 & IHTO000020 & 106.5880 & 108.5412 & 107.4318 & $4.7750 e-01$ & $4.2384 e+00$ \\
\hline 148 & IHTO00021 & -2.2273 & -0.0789 & -1.3132 & $6.1944 e-01$ & $4.1000 e+00$ \\
\hline 149 & IHTO00022 & 92.4692 & 94.6176 & 93.4028 & $5.2688 e-01$ & $4.2050 e+00$ \\
\hline 150 & IHTDO00023 & 104.7551 & 106.7083 & 105.4309 & $4.4853 e-01$ & $4.2334 e+00$ \\
\hline 151 & IHTOOO0024 & 69.9632 & 71.7210 & 70.6233 & $4.2479 e-01$ & $4.1604 \mathrm{e}+00$ \\
\hline 152 & IHTO000025 & 104.7439 & 106.5017 & 105.5407 & $4.4440 e-01$ & $4.2337 e+00$ \\
\hline 153 & IHTO00026 & 105.5213 & 107.2792 & 106.2635 & $4.6017 e-01$ & $4.2355 e+00$ \\
\hline 154 & IHT000027 & 90.1893 & 92.1424 & 90.9822 & $4.6757 e-01$ & $4.1997 e+00$ \\
\hline 155 & IHTOOO0028 & 91.3349 & 94.8505 & 92.5146 & $6.6023 e-01$ & $4.2031 e+00$ \\
\hline
\end{tabular}




\begin{tabular}{|c|c|c|c|c|c|c|}
\hline \multicolumn{2}{|c|}{ Test: Aug16_03 } & sum2 & Date: Thu & Aug 1620 & $57: 37 \quad 1990$ & Page: 4 \\
\hline$\#$ & Name & Min & $\operatorname{Max}$ & Ave & SD & Uncert \\
\hline 156 & IHTO00029 & 105.0105 & 108.5262 & 106.0926 & $6.5579 e-01$ & $4.2350 \mathrm{e}+00$ \\
\hline 157 & IHTOO00030 & 107.4031 & 110.9187 & 108.3953 & $6.8894 \mathrm{e}-01$ & $4.2409 e+00$ \\
\hline 158 & IHT000031 & 105.6415 & 109.3525 & 106.8994 & $7.0824 e-01$ & $4.2371 e+00$ \\
\hline 159 & IHT000032 & 107.1326 & 110.6483 & 108.2069 & $7.3531 e-01$ & $4.2404 e+00$ \\
\hline 160 & IHT000033 & 106.9599 & 110.6708 & 108.1670 & $7.1756 \mathrm{e}-01$ & $4.2403 e+00$ \\
\hline 161 & IHT000034 & 93.9378 & 97.0628 & 94.8987 & $5.7971 e-01$ & $4.2084 e+00$ \\
\hline 162 & IHT000035 & 124.3089 & 127.2386 & 125.1644 & $5.8646 e-01$ & $4.2868 e+00$ \\
\hline 163 & IHT000036 & 92.6044 & 94.9482 & 93.4677 & $5.3392 e-01$ & $4.2052 e+00$ \\
\hline
\end{tabular}




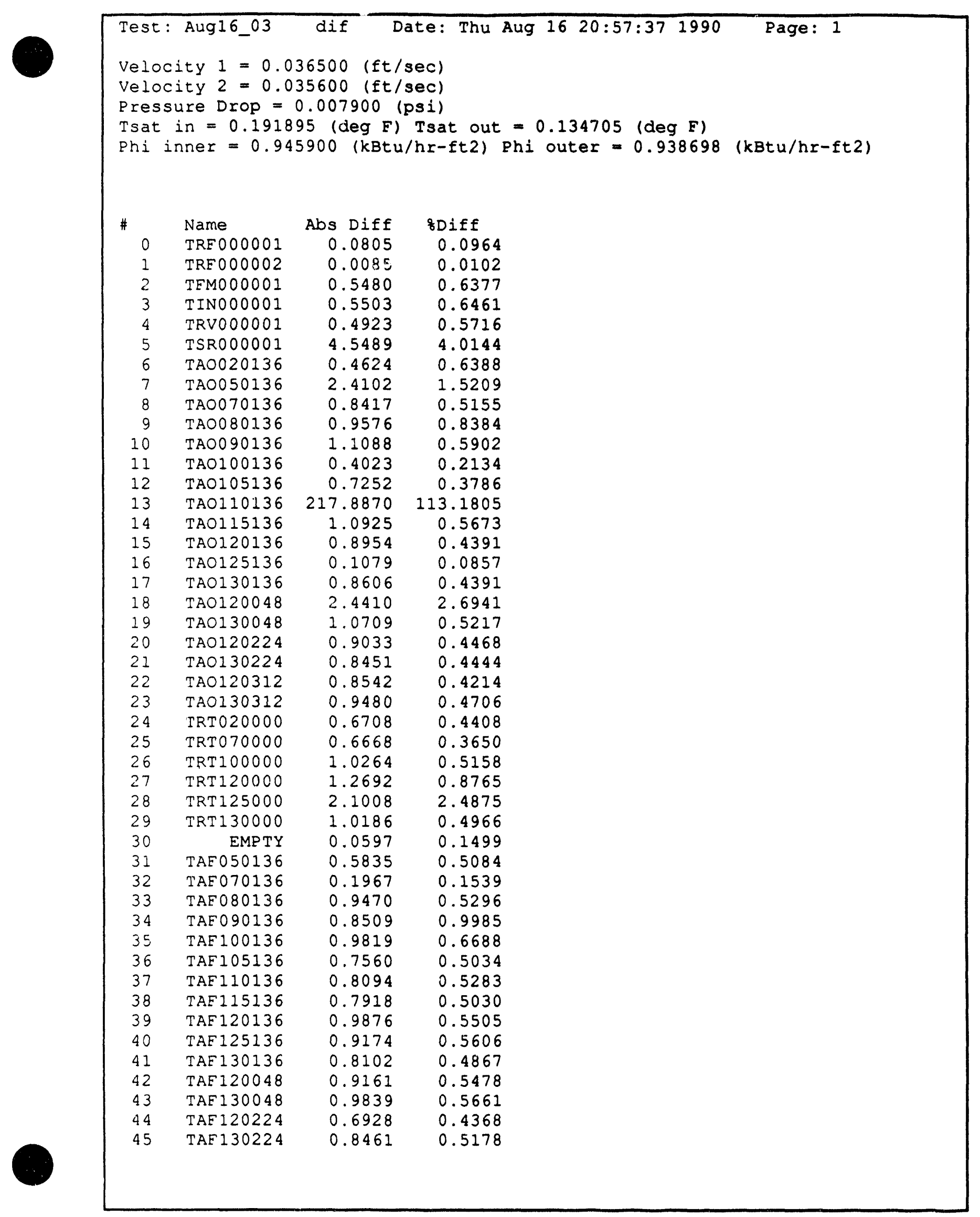




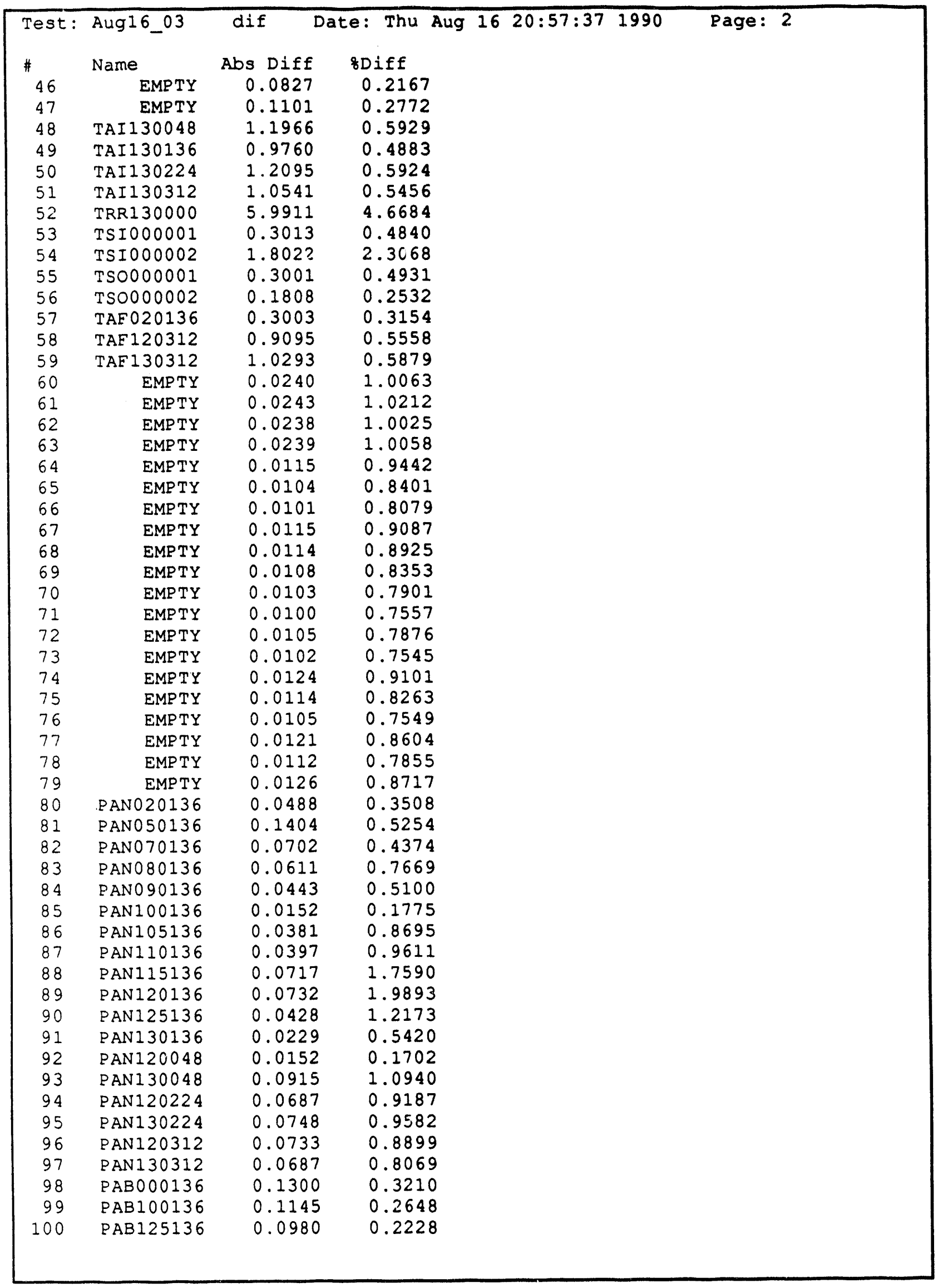




\begin{tabular}{|c|c|c|c|c|}
\hline Test: & Aug16_03 & dif & ate: Thu Aug $16 \quad 20: 57: 37 \quad 1990$ & Page: 3 \\
\hline$\#$ & Name & Abs Diff & \&Diff & \\
\hline 101 & PIN000001 & 0.2466 & 0.6111 & \\
\hline 102 & PRV000001 & 0.1440 & 0.7258 & \\
\hline 103 & PSR000001 & 0.1367 & 0.3100 & \\
\hline 104 & PFM000001 & 0.0678 & 1.2293 & \\
\hline 105 & PAN000136 & 0.2198 & 0.2095 & \\
\hline 106 & PEM000002 & 0.0661 & 1.2003 & \\
\hline 107 & EMPTY & 0.0001 & 2.0833 & \\
\hline 108 & EMPTY & 0.0039 & 0.0390 & \\
\hline 109 & EMPTY & 0.0010 & 27.7778 & \\
\hline 110 & EMPTY & 0.0000 & 0.0000 & \\
\hline 111 & EMPTY & 0.0001 & 100.0000 & \\
\hline 112 & VPWOOOOOO1 & 0.1368 & 0.1343 & \\
\hline 113 & VPW000002 & 0.0273 & 0.0229 & \\
\hline 114 & VPW000003 & 0.0430 & 0.0292 & \\
\hline 115 & VPW000004 & 0.0977 & 0.0932 & \\
\hline 116 & VPW000005 & 0.0195 & 0.0140 & \\
\hline 117 & VPW000006 & 0.2031 & 0.1375 & \\
\hline 118 & IPWO00001 & 10.7769 & 1.9631 & \\
\hline 119 & IPW000002 & 31.6493 & 2.4138 & \\
\hline 120 & IPWO00003 & 14.0393 & 1.0568 & \\
\hline 121 & IPWO00004 & 0.5371 & 0.1402 & \\
\hline 122 & IPW000005 & 1.0058 & 0.3950 & \\
\hline 123 & IPWO00006 & 0.8691 & 0.3296 & \\
\hline 124 & EMPTY & 0.0013 & 6.7708 & \\
\hline 125 & EMPTY & 0.0004 & 3.1746 & \\
\hline 126 & EMPTY & 0.0014 & 9.6552 & \\
\hline 127 & EMPTY & 0.0002 & 1.4084 & \\
\hline 128 & IHTO00001 & 0.0352 & 0.0271 & \\
\hline 129 & IHTOOOOO2 & 0.0078 & 0.0058 & \\
\hline 130 & IHTO00003 & 0.0547 & 0.0409 & \\
\hline 131 & IHTO00004 & 0.0547 & 0.0414 & \\
\hline 132 & IHTO00005 & 0.0586 & 0.0449 & \\
\hline 133 & IHTO00000 & 0.1093 & 0.0873 & \\
\hline 134 & IHTO00007 & 0.0664 & 0.0502 & \\
\hline 135 & IHT000008 & 0.0429 & 0.0328 & \\
\hline 136 & IHTO00009 & 0.0117 & 0.0089 & \\
\hline 137 & IHTOOOOIO & 0.0430 & 0.1098 & \\
\hline 138 & IHTO00011 & 0.2929 & 0.2233 & \\
\hline 139 & IHTO00012 & 0.3047 & 0.2365 & \\
\hline 140 & IHTO00013 & 0.1601 & 0.1736 & \\
\hline 141 & IHTO00014 & 0.0977 & 0.0760 & \\
\hline 142 & IHTOOOOLI5 & 0.0977 & 0.1018 & \\
\hline 143 & IHTO00016 & 0.0586 & 0.0655 & \\
\hline 144 & IHTO00017 & 0.3164 & 0.2949 & \\
\hline 145 & IHTO00018 & 0.1211 & 0.1116 & \\
\hline 146 & IHTO00019 & 0.1523 & 0.1453 & \\
\hline 147 & IHTOOOO20 & 0.0898 & 0.0835 & \\
\hline 148 & IHTO00021 & 0.0040 & 0.3037 & \\
\hline 149 & IHTO00022 & 0.0156 & 0.0167 & \\
\hline 150 & IHTO00023 & 0.1132 & 0.1073 & \\
\hline 151 & IHTO00024 & 0.1133 & 0.1602 & \\
\hline 152 & IHTO00025 & 0.0898 & 0.0850 & \\
\hline 153 & IHTO00026 & 0.1055 & 0.0992 & \\
\hline 154 & IHTO00027 & 0.0235 & 0.0258 & \\
\hline 155 & IHTO00028 & 0.0742 & 0.0803 & \\
\hline
\end{tabular}




\begin{tabular}{|c|c|c|c|c|}
\hline Test: & Aug16_03 & dif & Date: Thu Aug $16 \quad 20: 57: 371990$ & Page: 4 \\
\hline \# & Name & Abs Diff & fDiff & \\
\hline 156 & IHTO00029 & 0.1094 & 0.1030 & \\
\hline 157 & IHTOOO0030 & 0.0625 & 0.0576 & \\
\hline 158 & IHTO00031 & 0.0156 & 0.0146 & \\
\hline 159 & IHTO00032 & 0.0391 & 0.0361 & \\
\hline 160 & IHTO00033 & 0.0313 & 0.0289 & \\
\hline 161 & IHTO00034 & 0.1953 & 0.2062 & \\
\hline 162 & IHTO00035 & 0.1015 & 0.0812 & \\
\hline 163 & IHT000036 & 0.1289 & 0.1381 & \\
\hline
\end{tabular}




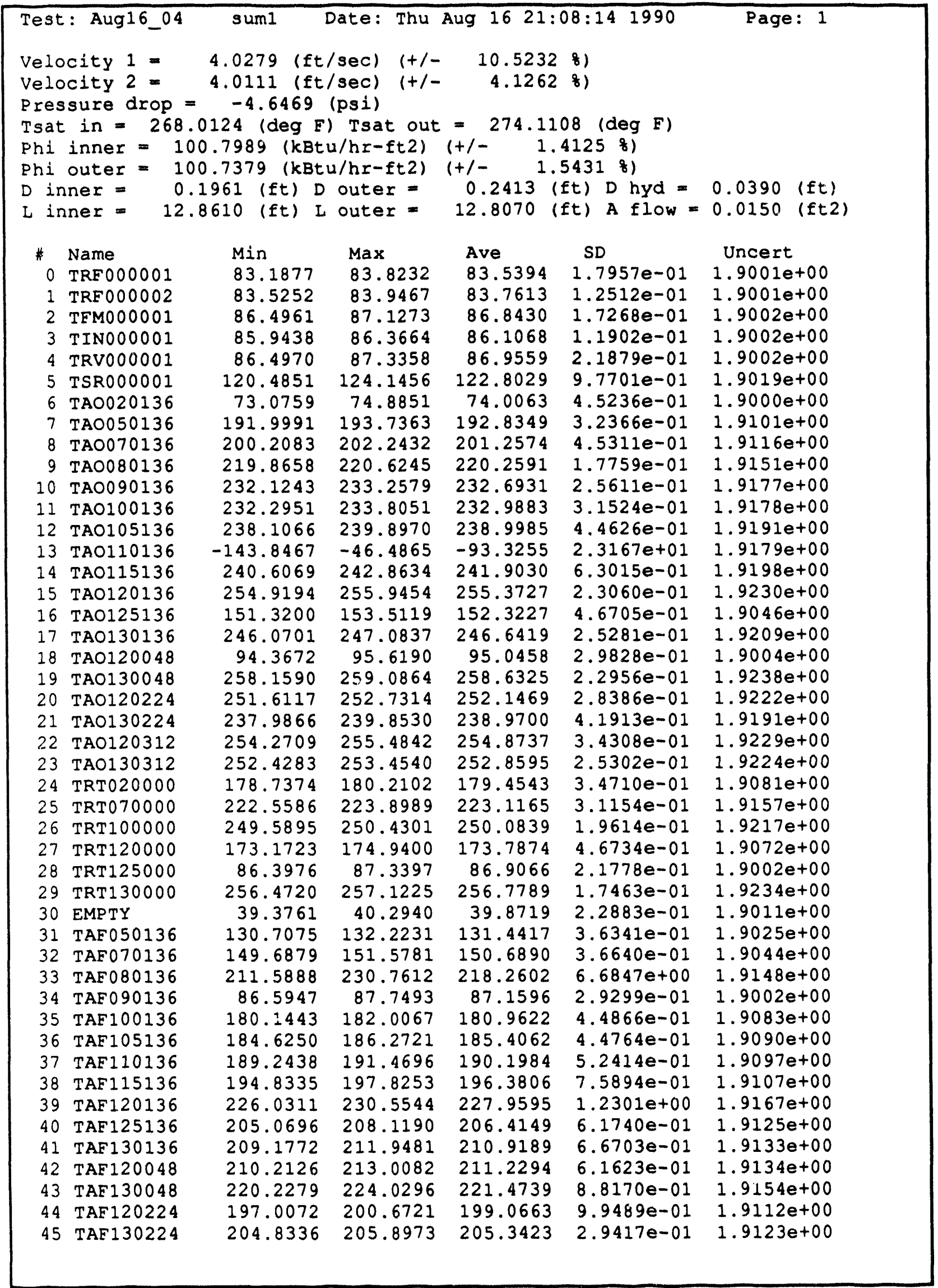




\begin{tabular}{|c|c|c|c|c|c|c|}
\hline \multicolumn{2}{|c|}{ Test: Aug16_04 } & suml & Date: Thu & \multicolumn{2}{|c|}{ Aug $16 \quad 21: 08: 141990$} & \multirow{2}{*}{$\begin{array}{l}\text { Page: } 2 \\
\text { Uncert }\end{array}$} \\
\hline$\#$ & Name & Min & $\operatorname{Max}$ & Ave & SD & \\
\hline 46 & EMPTY & 37.5374 & 38.4572 & 38.1124 & $2.1413 e-01$ & $1.9010 \mathrm{e}+00$ \\
\hline 47 & EMPTY & 39.6057 & 40.2940 & 39.8444 & $1.7932 e-01$ & $1.9011 \mathrm{e}+00$ \\
\hline 48 & TAI 130048 & 256.8550 & 258.7159 & 257.9287 & $3.6119 e-01$ & $1.9236 \mathrm{e}+00$ \\
\hline 49 & TAI 130136 & 252.3401 & 253.4581 & 252.8636 & $2.5028 e-01$ & $1.9224 \mathrm{e}+00$ \\
\hline 50 & TAI 130224 & 257.5975 & 258.7168 & 258.0737 & $2.7701 e-01$ & $1.9237 e+00$ \\
\hline 51 & TAI 130312 & 244.7746 & 246.1825 & 245.5575 & $3.4627 e-01$ & $1.9207 e+00$ \\
\hline 52 & TRR130000 & 122.6921 & 123.7108 & 123.2277 & $2.9411 e-01$ & $1.9019 \mathrm{e}+00$ \\
\hline 53 & TSI000001 & 61.9832 & 62.6271 & 62.3242 & $1.4954 \mathrm{e}-01$ & $1.9000 \mathrm{e}+00$ \\
\hline 54 & TSI000002 & 71.8921 & 75.2836 & 73.1248 & $7.5571 e-01$ & $1.9000 \mathrm{e}+00$ \\
\hline 55 & TSO000001 & 59.9136 & 60.8852 & 60.5117 & $2.0820 e-01$ & $1.9001 e+00$ \\
\hline 56 & TSO000002 & 74.3887 & 75.2383 & 74.8667 & $1.7726 \mathrm{e}-01$ & $1.9000 \mathrm{e}+00$ \\
\hline 57 & TAF020136 & 100.4366 & 102.3180 & 101.4835 & $4.4925 e-01$ & $1.9007 e+00$ \\
\hline 58 & TAF 120312 & 205.1578 & 208.8120 & 207.1643 & $1.0359 e+00$ & $1.9126 \mathrm{e}+00$ \\
\hline 59 & TAF 130312 & 220.7729 & 223.9065 & 222.6593 & $8.8555 e-01$ & $1.9156 \mathrm{e}+00$ \\
\hline 60 & EMPTY & 3.5449 & 3.5986 & 3.5729 & $1.8919 e-02$ & $0.0000 e+00$ \\
\hline 61 & EMPTY & 3.5303 & 3.5791 & 3.5535 & $1.6670 e-02$ & $0.0000 e+00$ \\
\hline 62 & EMPTY & 3.5156 & 3.5596 & 3.5352 & $1.4665 e-02$ & $0.0000 e+00$ \\
\hline 63 & EMPTY & 3.5107 & 3.5498 & 3.5280 & $1.3169 e-02$ & $0.0000 e+00$ \\
\hline 64 & EMPTY & 1.7188 & 1.7334 & 1.7260 & $4.8568 e-03$ & $0.0000 \mathrm{e}+00$ \\
\hline 65 & EMPTY & 1.7334 & 1.7529 & 1.7422 & $5.5804 e-03$ & $0.0000 \mathrm{e}+00$ \\
\hline 66 & EMPTY & 1.7432 & 1.7627 & 1.7515 & $4.3282 e-03$ & $0.0000 \mathrm{e}+00$ \\
\hline 67 & EMPTY & 1.7578 & 1.7725 & 1.7647 & $4.3101 e-03$ & $0.0000 \mathrm{e}+00$ \\
\hline 68 & EMPTY & 1.7676 & 1.7822 & 1.7717 & $4.1102 e-03$ & $0.0000 e+00$ \\
\hline 69 & EMPTY & 1.7773 & 1.7920 & 1.7840 & $4.3851 e-03$ & $0.0000 \mathrm{e}+00$ \\
\hline 70 & EMPTY & 1.7871 & 1.8018 & 1.7928 & $4.1102 e-03$ & $0.0000 \mathrm{e}+00$ \\
\hline 71 & EMPTY & 1.8018 & 1.8164 & 1.8085 & $4.7197 e-03$ & $0.0000 \mathrm{e}+00$ \\
\hline 72 & EMPTY & 1.8066 & 1.8213 & 1.8162 & $4.0626 e-03$ & $0.0000 \mathrm{e}+00$ \\
\hline 73 & EMPTY & 1.8262 & 1.8359 & 1.8313 & $3.8725 e-03$ & $0.0000 \mathrm{e}+00$ \\
\hline 74 & EMPTY & 1.8359 & 1.8506 & 1.8413 & $4.2 i 42 e-03$ & $0.0000 \mathrm{e}+00$ \\
\hline 75 & EMPTY & 1.8457 & 1.8604 & 1.8541 & $3.9508 e-03$ & $0.0000 e+00$ \\
\hline 76 & EMPTY & 1.8506 & 1.8750 & 1.8628 & $3.5908 e-03$ & $0.0000 e+00$ \\
\hline 77 & EMPTY & 1.8701 & 1.8799 & 1.8768 & $3.3829 e-03$ & $0.0000 e+00$ \\
\hline 78 & EMPTY & 1.8896 & 1.9043 & 1.8929 & $3.5030 e-03$ & $0.0000 e+00$ \\
\hline 79 & EMPTY & 1.9043 & 1.9141 & 1.9111 & $2.6100 e-03$ & $0.0000 e+00$ \\
\hline 80 & PAN020136 & -16.8520 & -16.3943 & -16.6812 & $9.5714 e-02$ & $8.7100 e-01$ \\
\hline 81 & PAN050136 & -32.0004 & -31.2374 & -31.6555 & $2.0170 e-01$ & $1.6819 e+00$ \\
\hline 82 & PAN070136 & -20.3056 & -19.6953 & -20.0416 & $1.2255 e-01$ & $8.7144 \mathrm{e}-01$ \\
\hline 83 & PAN080136 & -10.8277 & -10.2937 & -10.6278 & $1.0887 e-01$ & $8.7041 e-01$ \\
\hline 84 & PAN090136 & -12.0309 & -7.9873 & -10.7766 & $1.0435 e+00$ & $8.7042 e-01$ \\
\hline 85 & PAN100136 & -12.1908 & -10.8175 & -12.0229 & $3.1701 e-01$ & $8.7052 e-01$ \\
\hline 86 & PAN 105136 & -5.1843 & 3.9710 & -3.2495 & $1.5322 \mathrm{e}+00$ & $8.7004 e-01$ \\
\hline 87 & PAN 110136 & -7.1459 & -2.7971 & -4.3138 & $7.3851 e-01$ & $8.7007 e-01$ \\
\hline 88 & PAN115136 & -7.4834 & -1.3798 & -4.6193 & $9.2344 e-01$ & $8.7008 e-01$ \\
\hline 89 & PAN 120136 & -6.7482 & 3.1700 & -4.7814 & $1.5275 e+00$ & $8.7008 e-01$ \\
\hline 90 & PAN125136 & -6.0323 & -3.5909 & -4.6483 & $5.2813 e-01$ & $8.7008 e-01$ \\
\hline 91 & PAN130136 & -5.0829 & 2.4702 & -4.2315 & $1.1407 \mathrm{e}+00$ & $8.7006 e-01$ \\
\hline 92 & PAN 120048 & -12.0293 & -9.3591 & -10.5233 & $5.7027 e-01$ & $8.7040 e-01$ \\
\hline 93 & PAN130048 & -10.1997 & -8.0635 & -9.5711 & $3.8024 e-01$ & $8.7033 e-01$ \\
\hline 94 & PAN120224 & -12.4417 & -2.9812 & -9.2251 & $1.5121 e+00$ & $8.7031 e-01$ \\
\hline 95 & PAN130224 & -14.0393 & -8.8514 & -9.9897 & $1.1658 \mathrm{e}+00$ & $8.7036 e-01$ \\
\hline 96 & PAN 120312 & -13.7254 & -5.4856 & -9.4972 & $1.2308 \mathrm{e}+00$ & $8.7032 e-01$ \\
\hline 97 & PAN 130312 & -14.5662 & -3.6561 & -10.1121 & $1.3540 \mathrm{e}+00$ & $8.7037 e-01$ \\
\hline 98 & $\mathrm{PAB} 000136$ & 40.3196 & 40.5485 & 40.4341 & $6.0113 e-02$ & $5.8874 e-01$ \\
\hline 99 & PAB100136 & 43.8471 & 44.0302 & 43.9386 & $5.1492 \mathrm{e}^{-0} 02$ & $5.9031 e-01$ \\
\hline 100 & PAB125136 & 44.6657 & 44.8488 & 44.7518 & $4.5907 e-02$ & $5.9069 e-01$ \\
\hline
\end{tabular}




\begin{tabular}{|c|c|c|c|c|c|c|}
\hline \multicolumn{2}{|c|}{ Test: Aug16_04 } & sum 1 & Date: Thu & Aug 162 & $8: 141990$ & Page: 3 \\
\hline \# & Name & Min & $\operatorname{Max}$ & Ave & SD & Uncert \\
\hline 101 & PIN 000001 & 40.2028 & 40.5690 & 40.3371 & $9.9771 e-02$ & $7.5675 e-01$ \\
\hline 102 & PRV000001 & 19.5965 & 19.8406 & 19.7039 & $4.7052 e-02$ & $7.5162 e-01$ \\
\hline 103 & PSR000001 & 44.8156 & 45.1818 & 44.9254 & $6.1655 e-02$ & $7.5836 e-01$ \\
\hline 104 & PFMO00001 & 2.2817 & 2.5564 & 2.4063 & $8.0113 e-02$ & $5.0004 e-01$ \\
\hline 105 & PANO00136 & -130.4183 & -119.4319 & -128.6513 & $1.6099 \mathrm{e}+00$ & $4.9305 e+00$ \\
\hline 106 & PFM000002 & 2.3488 & 2.4160 & 2.3858 & $1.4777 e-02$ & $2.0009 e-01$ \\
\hline 107 & EMPTY & -0.0049 & 0.0049 & 0.0014 & $4.3045 e-03$ & $0.0000 e+00$ \\
\hline 108 & EMPTY & -10.0000 & -10.0000 & -10.0000 & $0.0000 e+00$ & $0.0000 e+00$ \\
\hline 109 & EMPTY & 0.0000 & 0.0049 & 0.0011 & $2.0432 e-03$ & $0.0000 e+00$ \\
\hline 110 & EMPTY & -10.0000 & -10.0000 & -10.0000 & $0.0000 e+00$ & $0.0000 e+00$ \\
\hline 111 & EMPTY & 0.0000 & 0.0000 & 0.0000 & $0.0000 e+00$ & $0.0000 e+00$ \\
\hline 112 & VPWO00001 & 101.6339 & 102.4151 & 102.0557 & $1.9475 e-01$ & $1.0154 e+00$ \\
\hline 113 & VPW000002 & 119.0467 & 119.2421 & 119.1522 & $9.8332 e-02$ & $1.0804 e+00$ \\
\hline 114 & VPW000003 & 146.8412 & 147.4271 & 147.1108 & $1.6690 e-01$ & $1.1980 \mathrm{e}+00$ \\
\hline 115 & VPWO00004 & 104.8640 & 105.4500 & 105.1219 & $1.2750 \mathrm{e}-01$ & $1.0266 \mathrm{e}+00$ \\
\hline 116 & VPW000005 & 139.4569 & 140.0428 & 139.7968 & $1.5185 e-01$ & $1.1660 \mathrm{e}+00$ \\
\hline 117 & VPW000006 & 147.4797 & 148.0657 & 147.8195 & $1.3560 \mathrm{e}-01$ & $1.2011 \mathrm{e}+00$ \\
\hline 118 & IPW000001 & 543.5024 & 549.3595 & 546.7433 & $1.5077 e+00$ & $9.1797 e+00$ \\
\hline 119 & IPW000002 & 1298.4198 & 1304.2736 & 1301.6586 & $1.6543 e+00$ & $1.3090 e+01$ \\
\hline 120 & IPW000003 & 1324.0499 & 1329.8999 & 1326.5068 & $1.1693 \mathrm{e}+00$ & $1.3245 e+01$ \\
\hline 121 & IPW0000004 & 381.6575 & 383.6106 & 382.7806 & $4.2142 e-01$ & $3.6255 e+00$ \\
\hline 122 & IPW000005 & 253.5616 & 254.5381 & 254.0010 & $3.4523 e-01$ & $2.8331 e+00$ \\
\hline 123 & IPW000006 & 262.7329 & 264.1978 & 263.3480 & $3.0897 e-01$ & $2.8859 e+00$ \\
\hline 124 & EMPTY & -0.0293 & -0.0098 & -0.0194 & $4.4654 e-03$ & $0.0000 e+00$ \\
\hline 125 & EMPTY & -0.0146 & 0.0000 & -0.0121 & $2.9986 e-03$ & $0.0000 e+00$ \\
\hline 126 & EMPTY & -0.0244 & -0.0098 & -0.0157 & $3.7291 e-03$ & $0.0000 e+00$ \\
\hline 127 & EMPTY & -0.0195 & -0.0098 & -0.0147 & $3.3439 e-03$ & $0.0000 \mathrm{e}+00$ \\
\hline 128 & IHT000001 & 128.5231 & 130.8669 & 129.7067 & $6.2192 e-01$ & $4.3003 e+00$ \\
\hline 129 & IHTO00002 & 132.3768 & 135.3065 & 133.4706 & $6.2764 e-01$ & $4.3118 e+00$ \\
\hline 130 & IHTO00003 & 132.5045 & 135.0436 & 133.6607 & $6.0082 e-01$ & $4.3124 e+00$ \\
\hline 131 & IHT000004 & 130.7955 & 133.9205 & 131.9283 & $6.5435 e-01$ & $4.3070 e+00$ \\
\hline 132 & IHTO00005 & 129.2668 & 132.3918 & 130.3410 & $6.2297 e-01$ & $4.3022 e+00$ \\
\hline 133 & IHT000006 & 123.9971 & 127.3175 & 125.1612 & $6.7654 e-01$ & $4.2868 \mathrm{e}+00$ \\
\hline 134 & IHT000007 & 130.9946 & 133.9243 & 132.1704 & $6.1192 e-01$ & $4.3078 e+00$ \\
\hline 135 & IHT000008 & 129.7701 & 132.6998 & 130.6529 & $6.1919 e-01$ & $4.3031 e+00$ \\
\hline 136 & IHTO00009 & 130.1495 & 132.6885 & 131.1299 & $6.3930 e-01$ & $4.3046 e+00$ \\
\hline 137 & IHTO00010 & -40.4935 & -37.9545 & -39.2084 & $6.1775 e-01$ & $4.1187 e+00$ \\
\hline 138 & IHTO00011 & 129.7213 & 132.2603 & 131.1744 & $5.9078 e-01$ & $4.3047 e+00$ \\
\hline 139 & IHTO00012 & 127.3362 & 130.0706 & 128.8831 & $5.3063 e-01$ & $4.2978 e+00$ \\
\hline 140 & IHT000013 & 91.0156 & 93.3594 & 92.1914 & $5.1673 e-01$ & $4.2024 e+00$ \\
\hline 141 & IHTO00014 & 127.1710 & 129.5147 & 128.3624 & $5.2386 e-01$ & $4.2962 e+00$ \\
\hline 142 & IHTO000015 & 94.6214 & 96.7698 & 95.9183 & $4.4775 e-01$ & $4.2107 e+00$ \\
\hline 143 & IHTO00016 & 88.1310 & 90.2794 & 89.3654 & $4.8237 e-01$ & $4.1963 e+00$ \\
\hline 144 & IHTO000017 & 105.0706 & 108.7816 & 107.2230 & $9.4824 \mathrm{e}-01$ & $4.2379 e+00$ \\
\hline 145 & IHTOOO0018 & 106.9636 & 109.3074 & 108.3582 & $6.1258 e-01$ & $4.2408 e+00$ \\
\hline 146 & IHTO00019 & 103.6734 & 105.6265 & 104.5679 & $4.8982 e-01$ & $4.2312 e+00$ \\
\hline 147 & IHTO000020 & 106.3927 & 108.3458 & 107.2091 & $5.0485 e-01$ & $4.2379 e+00$ \\
\hline 148 & IHTO00021 & -2.4226 & -0.0789 & -1.3289 & $6.2639 e-01$ & $4.1000 e+00$ \\
\hline 149 & IHTO00022 & 92.0786 & 94.6176 & 93.1997 & $5.5777 e-01$ & $4.2046 \mathrm{e}+00$ \\
\hline 150 & IHTO00023 & 104.1692 & 106.7083 & 105.2238 & $5.6359 e-01$ & $4.2329 e+00$ \\
\hline 151 & IHTO00024 & 69.5726 & 71.7210 & 70.4749 & $5.1583 e-01$ & $4.1601 e+00$ \\
\hline 152 & IHT000025 & 104.3532 & 106.6970 & 105.4391 & $4.9310 \mathrm{e}-01$ & $4.2334 e+00$ \\
\hline 153 & IHT000026 & 104.9354 & 107.0838 & 106.2049 & $4.9007 e-01$ & $4.2353 e+00$ \\
\hline 154 & IHT000027 & 89.4080 & 91.9471 & 90.9119 & $5.6943 e-01$ & $4.1996 \mathrm{e}+00$ \\
\hline 155 & IHT000028 & 91.1396 & 93.4833 & 92.2724 & $5.3233 e-01$ & $4.2025 e+00$ \\
\hline
\end{tabular}




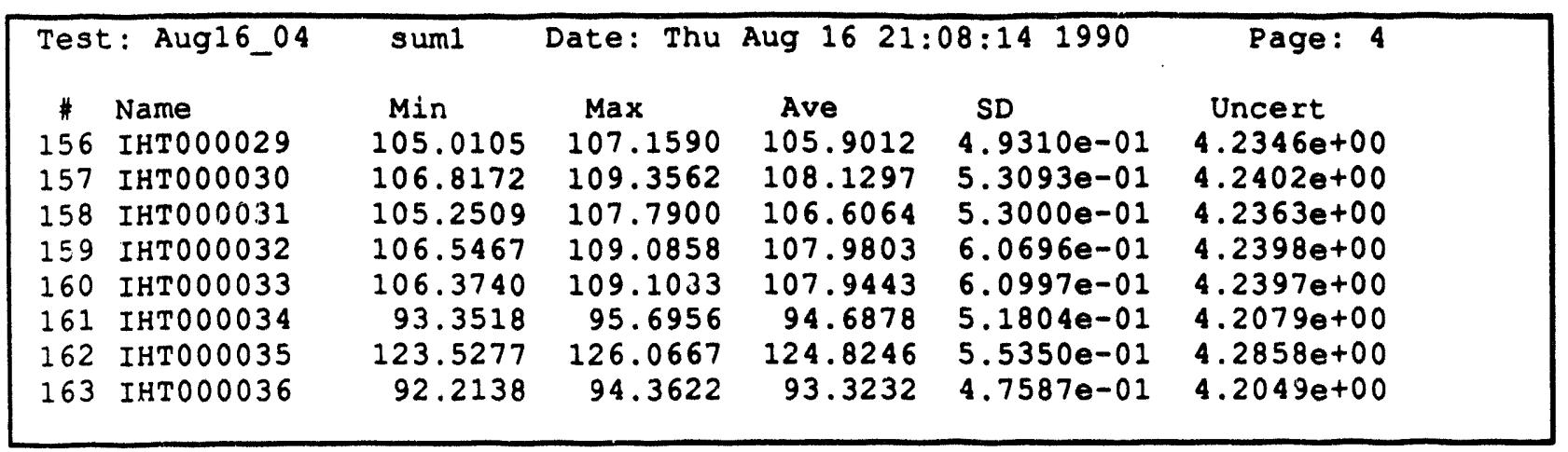




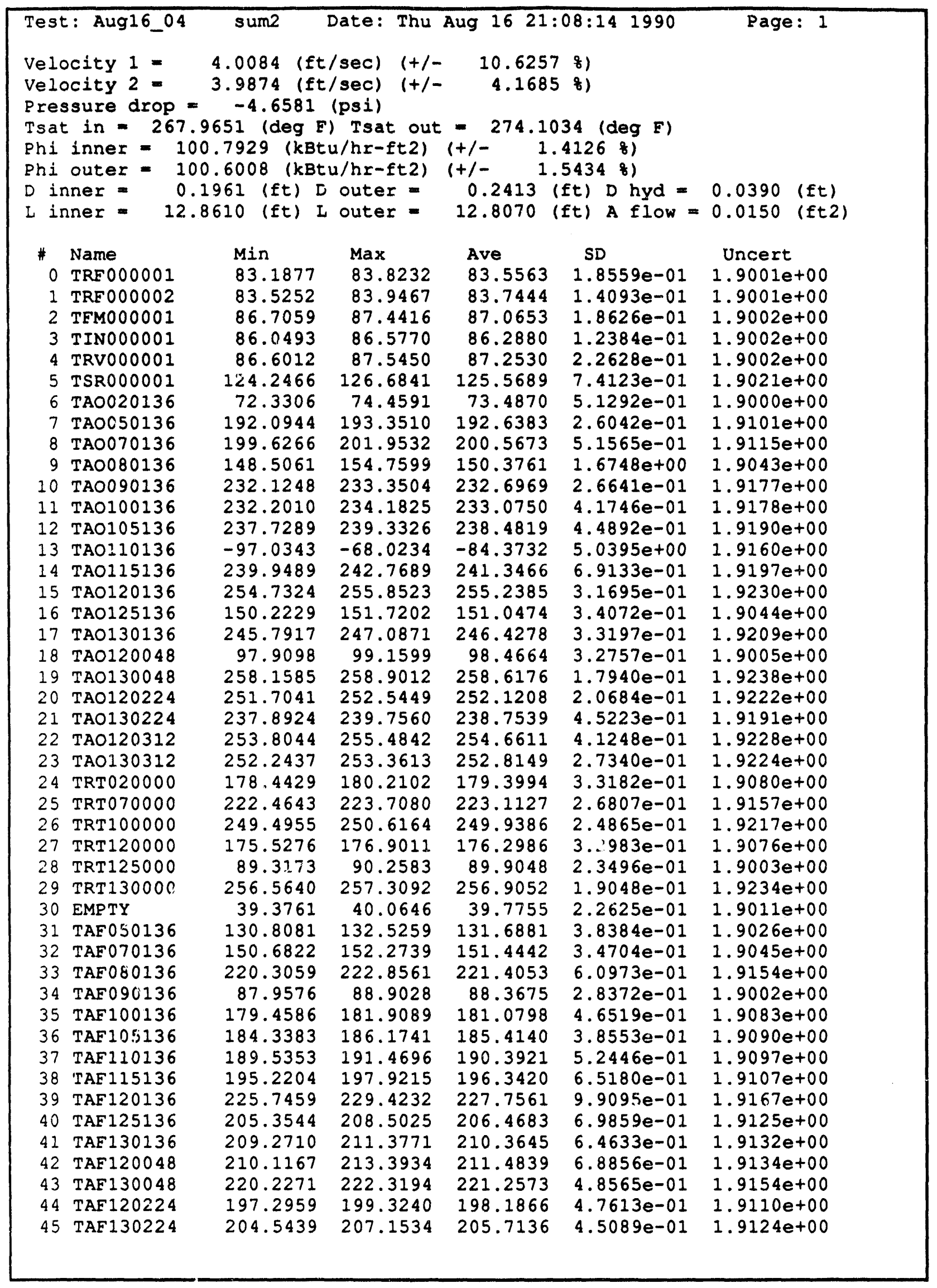




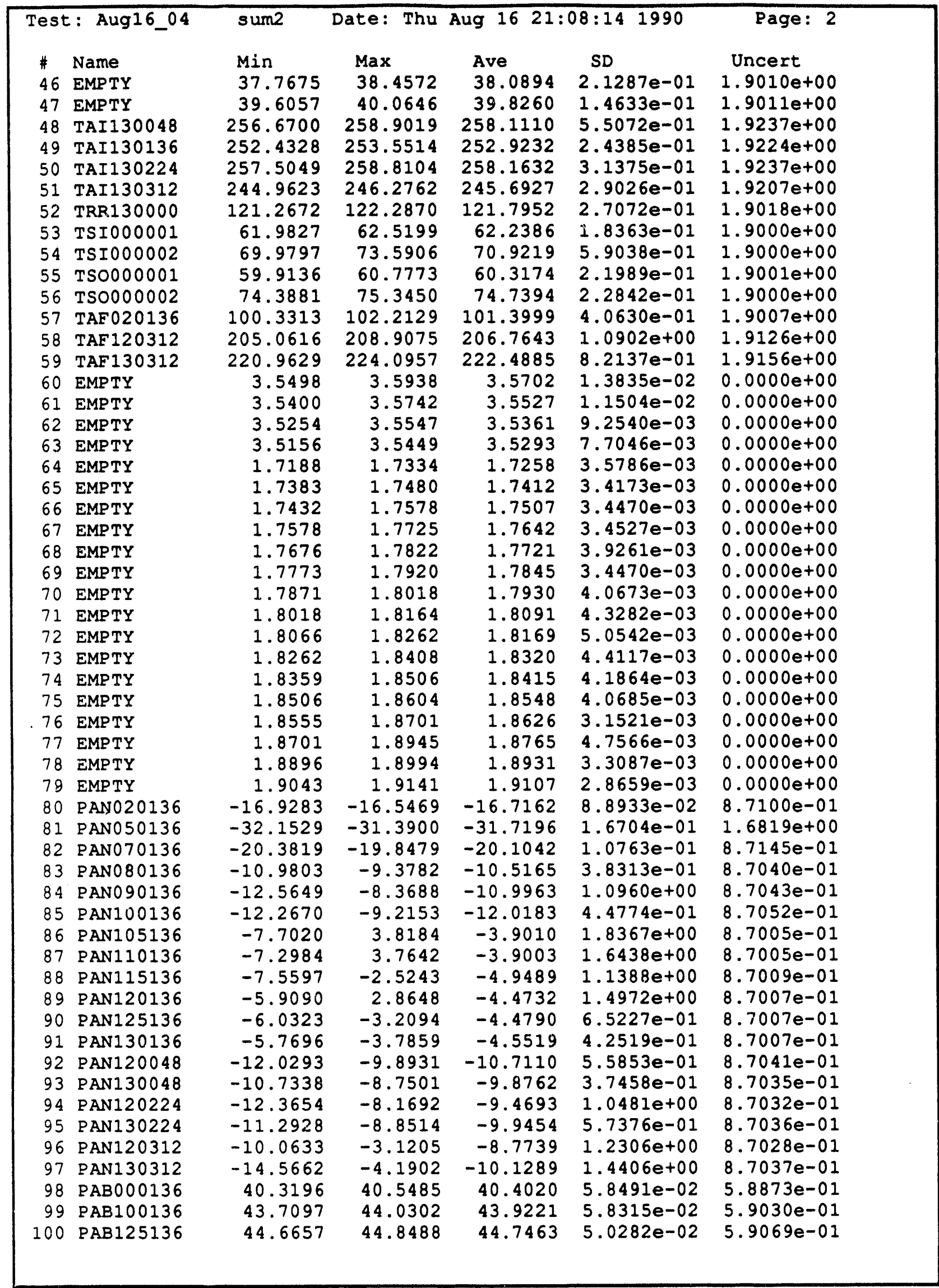




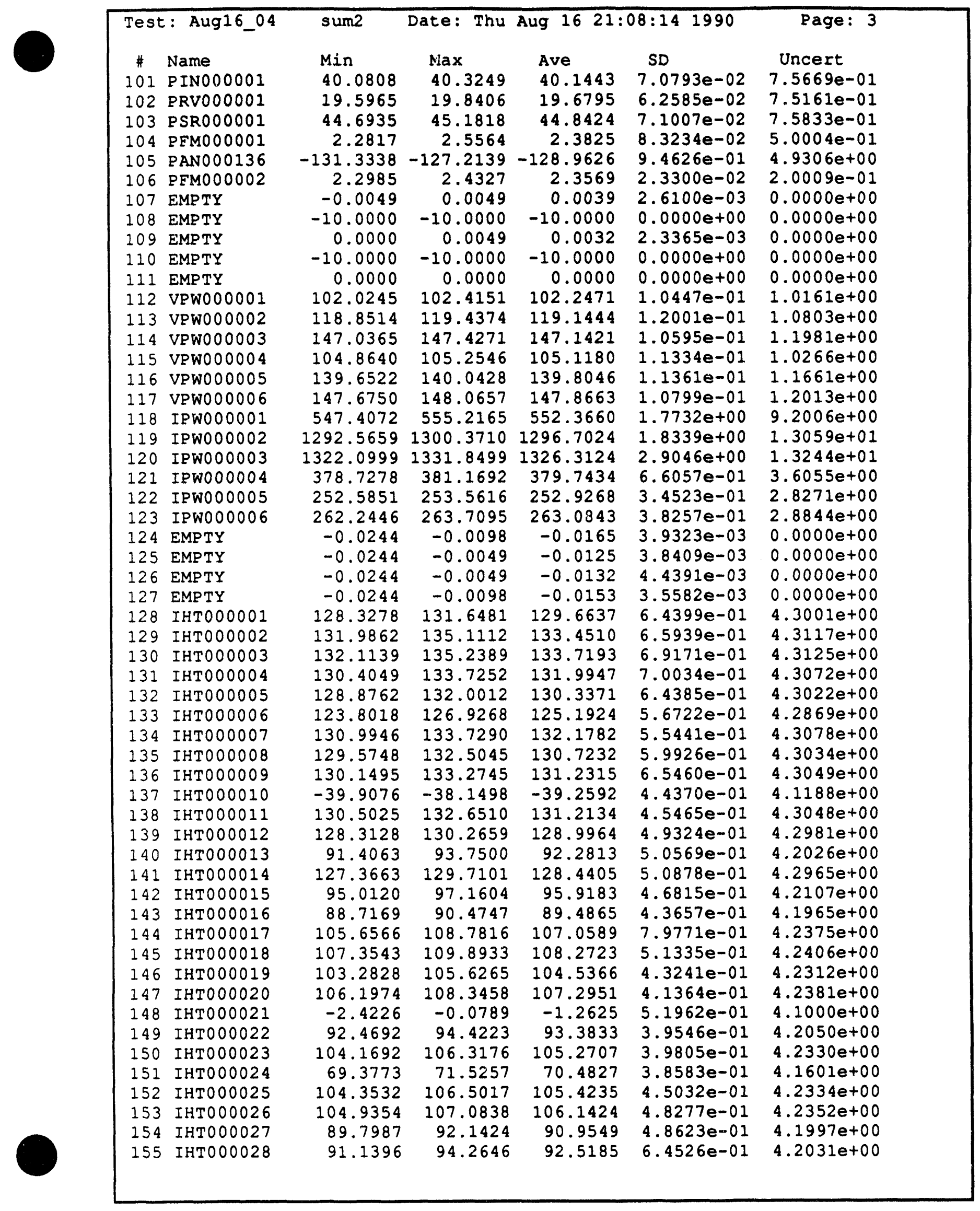




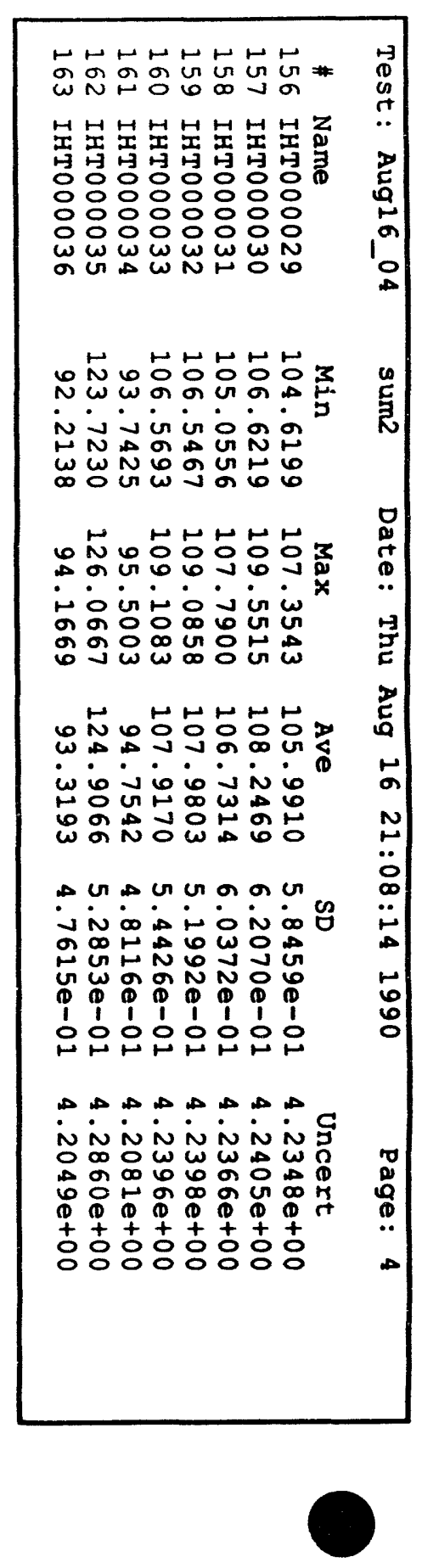




\begin{tabular}{|c|c|c|c|}
\hline \multicolumn{4}{|c|}{$\begin{array}{l}\text { Velocity } 1=0.019500(\mathrm{ft} / \mathrm{sec}) \\
\text { Velocity } 2=0.023700(\mathrm{ft} / \mathrm{sec}) \\
\text { Pressure Drop }=0.011200 \text { (psi) } \\
\text { Tsat in }=0.047302 \text { (deg F) Tsat out }=0.007416 \text { (deg F) } \\
\text { Phi inner }=0.005997 \text { (kBtu/hr-ft2) Phi outer }=0.137100 \text { (kBtu/hr-ft2) }\end{array}$} \\
\hline \# & Name & Abs Diff & gDiff \\
\hline 0 & TRF000001 & 0.0169 & 0.0202 \\
\hline 1 & TRF000002 & 0.0169 & 0.0202 \\
\hline 2 & TFM000001 & 0.2223 & 0.2560 \\
\hline 3 & TIN000001 & 0.1812 & 0.2104 \\
\hline 4 & TRV000001 & 0.2971 & 0.3417 \\
\hline 5 & TSR000001 & 2.7660 & 2.2524 \\
\hline 6 & TA0020136 & 0.5193 & 0.7017 \\
\hline 7 & TA0050136 & 0.1966 & 0.1019 \\
\hline 8 & TA0070136 & 0.6901 & 0.3429 \\
\hline 9 & TAO0 80136 & 69.8830 & 31.7276 \\
\hline 10 & TA0090136 & 0.0038 & 0.0016 \\
\hline 11 & TA0100136 & 0.0867 & 0.0372 \\
\hline 12 & TA0105136 & 0.5166 & 0.2162 \\
\hline 13 & TA0110136 & 8.9523 & 9.5926 \\
\hline 14 & TAO115136 & 0.5564 & 0.2300 \\
\hline 15 & TAO120136 & 0.1342 & 0.0526 \\
\hline 16 & TA0125136 & 1.2753 & 0.8372 \\
\hline 17 & TA0130136 & 0.2141 & 0.0868 \\
\hline 18 & TA0120048 & 3.4206 & 3.5989 \\
\hline 19 & TA0130048 & 0.0149 & 0.0058 \\
\hline 20 & TAO120224 & 0.0261 & 0.0103 \\
\hline 21 & TAO130224 & 0.2161 & 0.0904 \\
\hline 22 & TA0120312 & 0.2126 & 0.0834 \\
\hline 23 & TA0130312 & 0.0446 & 0.0176 \\
\hline 24 & TRT020000 & 0.0549 & 0.0306 \\
\hline 25 & TRT070000 & 0.0038 & 0.0017 \\
\hline 26 & TRT 100000 & 0.1453 & 0.0581 \\
\hline 27 & TRT 120000 & 2.5112 & 1.4450 \\
\hline 28 & TRT 125000 & 2.9982 & 3.4499 \\
\hline 29 & TRT 130000 & 0.1263 & 0.0492 \\
\hline 30 & EMPTY & 0.0964 & 0.2418 \\
\hline 31 & TAF050136 & 0.2464 & 0.1875 \\
\hline 32 & TAF070136 & 0.7552 & 0.5012 \\
\hline 33 & TAF080136 & 3.1451 & 1.4410 \\
\hline 34 & TAF090136 & 1.2079 & 1.3858 \\
\hline 35 & TAF 100136 & 0.1176 & 0.0650 \\
\hline 36 & TAF 105136 & 0.0078 & 0.0042 \\
\hline 37 & TAF 110136 & 0.1937 & 0.1018 \\
\hline 38 & TAF 115136 & 0.0386 & 0.0197 \\
\hline 39 & TAF 120136 & 0.2034 & 0.0892 \\
\hline 40 & TAF 125136 & 0.0534 & 0.0259 \\
\hline 41 & TAF130136 & 0.5544 & 0.2628 \\
\hline 42 & TAF 120048 & 0.2545 & 0.1205 \\
\hline 43 & TAF 130048 & 0.2166 & 0.0978 \\
\hline 44 & TAF 120224 & 0.8797 & 0.4419 \\
\hline 45 & TAF 130224 & 0.3713 & 0.1808 \\
\hline
\end{tabular}




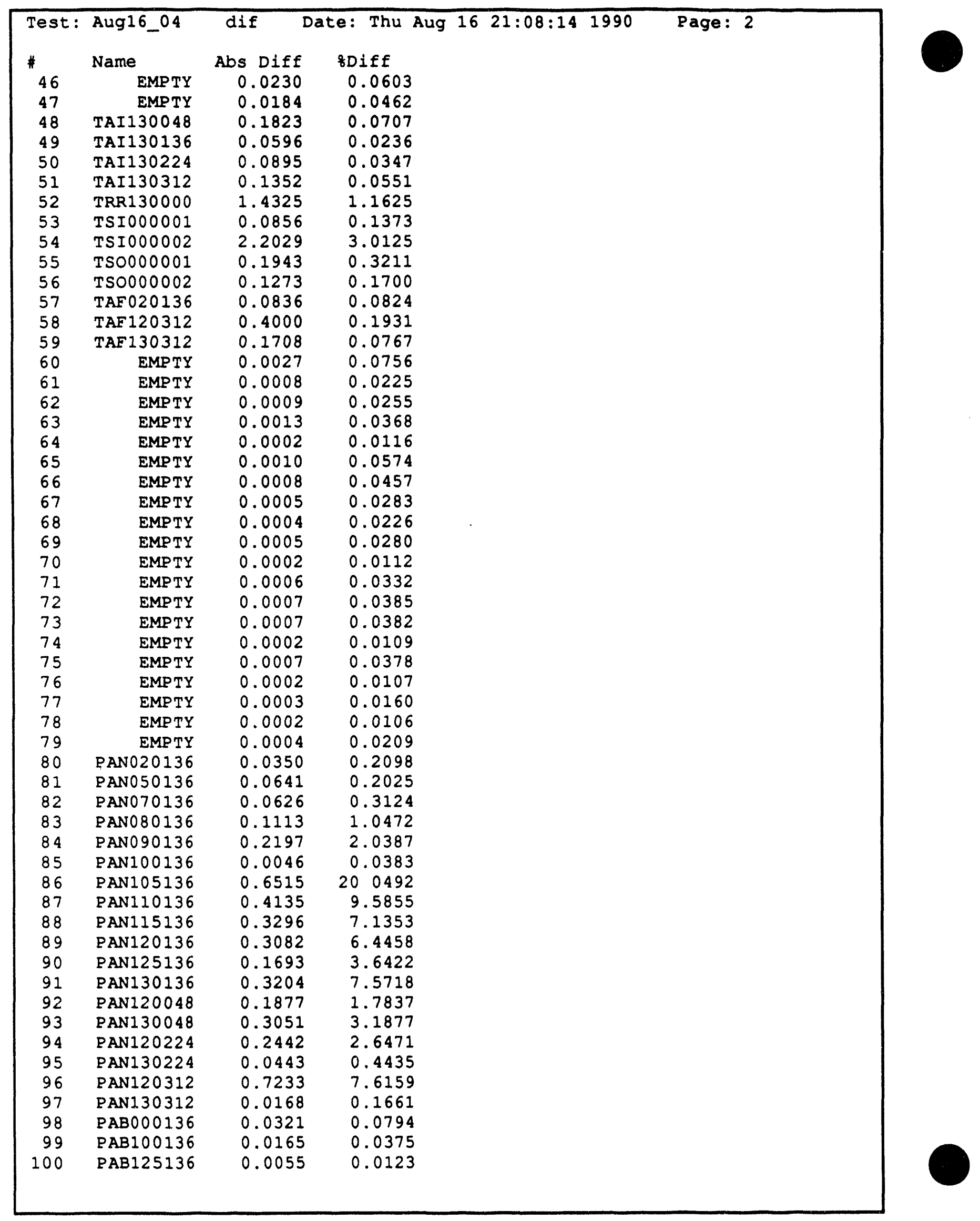




\begin{tabular}{|c|c|c|c|c|}
\hline Test: & Aug16_04 & dif & ate: Thu Aug 16 21:08:14 1990 & Page: 3 \\
\hline \# & Name & Abs Diff & gDiff & \\
\hline 101 & PINO00001 & 0.1928 & 0.4780 & \\
\hline 102 & PRV000001 & 0.0244 & 0.1238 & \\
\hline 103 & PSR000001 & 0.0830 & 0.1848 & \\
\hline 104 & PFM000001 & 0.0238 & 0.9891 & \\
\hline 105 & PAN000136 & 0.3113 & 0.2420 & \\
\hline 106 & PFM000002 & 0.0289 & 1.2113 & \\
\hline 107 & EMPTY & 0.0025 & 178.5714 & \\
\hline 108 & EMPTY & 0.0000 & 0.0000 & \\
\hline 109 & EMPTY & 0.0021 & 190.9091 & \\
\hline 110 & EMPTY & 0.0000 & 0.0000 & \\
\hline 111 & EMPTY & 0.0000 & 0.0000 & \\
\hline 112 & VPW000001 & 0.1914 & 0.1875 & \\
\hline 113 & VPW000002 & 0.0078 & 0.0065 & \\
\hline 114 & VPW000003 & 0.0313 & 0.0213 & \\
\hline 115 & VPW000004 & 0.0039 & 0.0037 & \\
\hline 116 & VPW0 00005 & 0.0078 & 0.0056 & \\
\hline 117 & VPW000006 & 0.0468 & 0.0317 & \\
\hline 118 & IPWO00001 & 5.6227 & 1.0284 & \\
\hline 119 & IPW0 00002 & 4.9562 & 0.3808 & \\
\hline 120 & IPWO 00003 & 0.1945 & 0.0147 & \\
\hline 121 & IPW000004 & 3.0372 & 0.7935 & \\
\hline 122 & IPW000005 & 1.0742 & 0.4229 & \\
\hline 123 & IPW000006 & 0.2637 & 0.1001 & \\
\hline 124 & EMPTY & 0.0029 & 14.9485 & \\
\hline 125 & EMPTY & 0.0004 & 3.3058 & \\
\hline 126 & EMPTY & 0.0025 & 15.9236 & \\
\hline 127 & EMPTY & 0.0006 & 4.0816 & \\
\hline 128 & IHT000001 & 0.0430 & 0.0332 & \\
\hline 129 & IHTO00002 & 0.0196 & 0.0147 & \\
\hline 130 & IHT000003 & 0.0586 & 0.0438 & \\
\hline 131 & IHTO00004 & 0.0664 & 0.0503 & \\
\hline 132 & IHTO00005 & 0.0039 & 0.0030 & \\
\hline 133 & IHT000006 & 0.0312 & 0.0249 & \\
\hline 134 & IHT000007 & 0.0078 & 0.0059 & \\
\hline 135 & IHTO00008 & 0.0703 & 0.0538 & \\
\hline 136 & IHTO00009 & 0.1016 & 0.0775 & \\
\hline 137 & IHTO00010 & 0.0508 & 0.1296 & \\
\hline 138 & IHTO00011 & 0.0390 & 0.0297 & \\
\hline 139 & IHT 000012 & 0.1133 & 0.0879 & \\
\hline 140 & IHTO00013 & 0.0899 & 0.0975 & \\
\hline 141 & IHTO00014 & 0.0781 & 0.0609 & \\
\hline 142 & IHT000015 & 0.0000 & 0.0000 & \\
\hline 143 & IHT000016 & 0.1211 & 0.1355 & \\
\hline 144 & IHT000017 & 0.1641 & 0.1530 & \\
\hline 145 & IHT000018 & 0.0859 & 0.0793 & \\
\hline 146 & IHTO00019 & 0.0313 & 0.0299 & \\
\hline 147 & IHTO00020 & 0.0860 & 0.0802 & \\
\hline 148 & IHTO00021 & 0.0664 & 4.9966 & \\
\hline 149 & IHTO00022 & 0.1836 & 0.1970 & \\
\hline 150 & IHTO00023 & 0.0469 & 0.0446 & \\
\hline 151 & IHT 000024 & 0.0078 & 0.0111 & \\
\hline 152 & IHTO00025 & 0.0156 & 0.0148 & \\
\hline 153 & IHT000026 & 0.0625 & 0.0588 & \\
\hline 154 & IHT000027 & 0.0430 & 0.0473 & \\
\hline 155 & IHT 000028 & 0.2461 & 0.2667 & \\
\hline
\end{tabular}




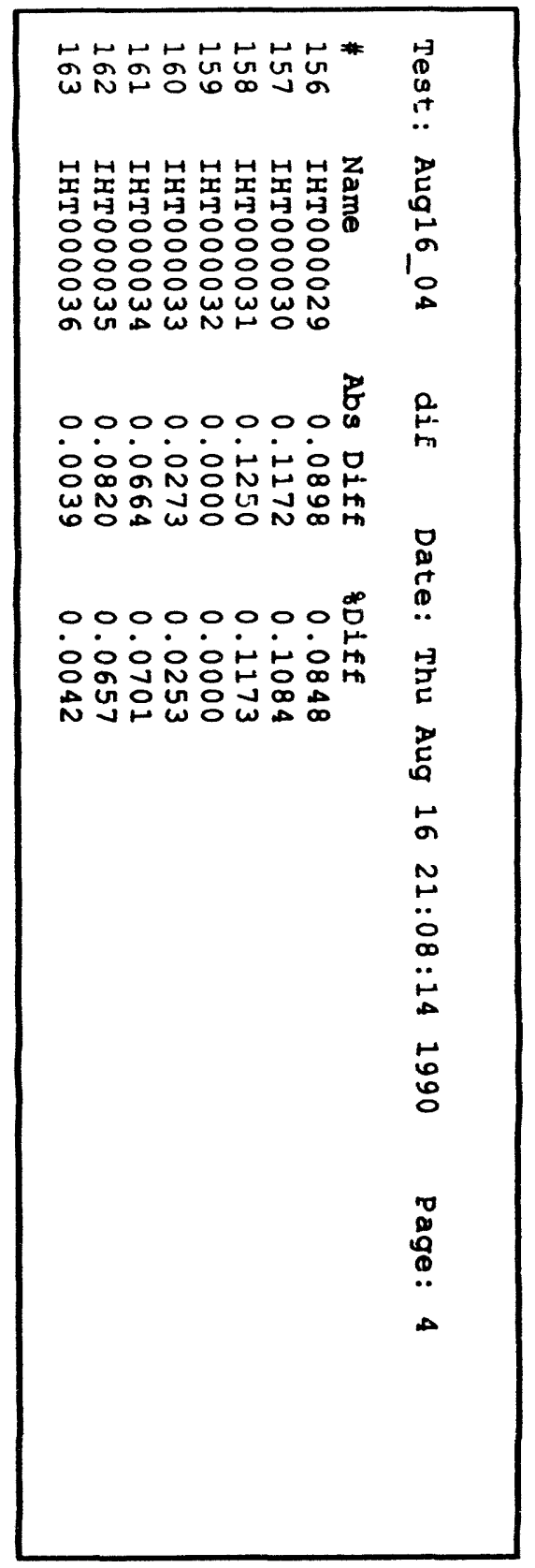




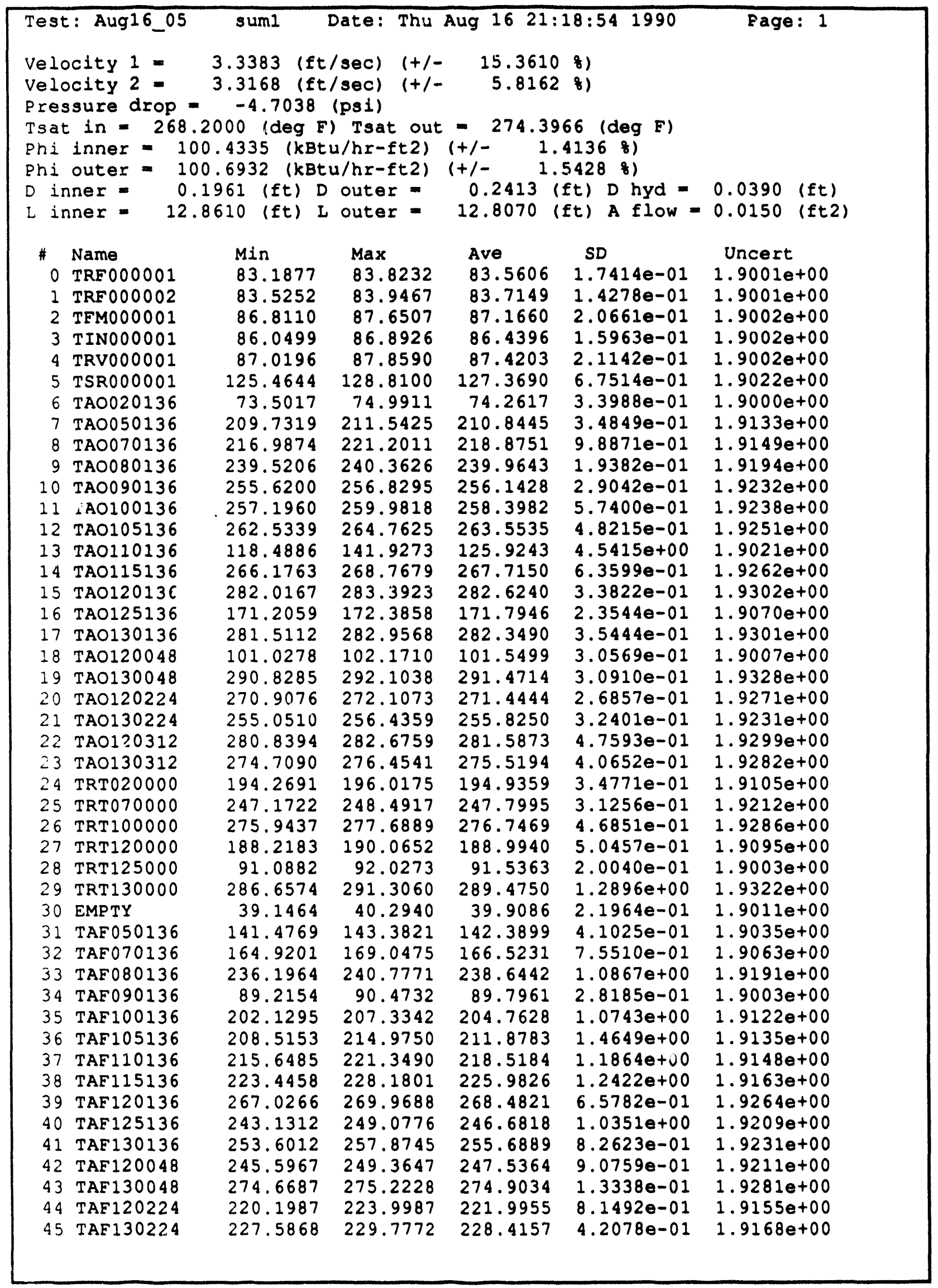




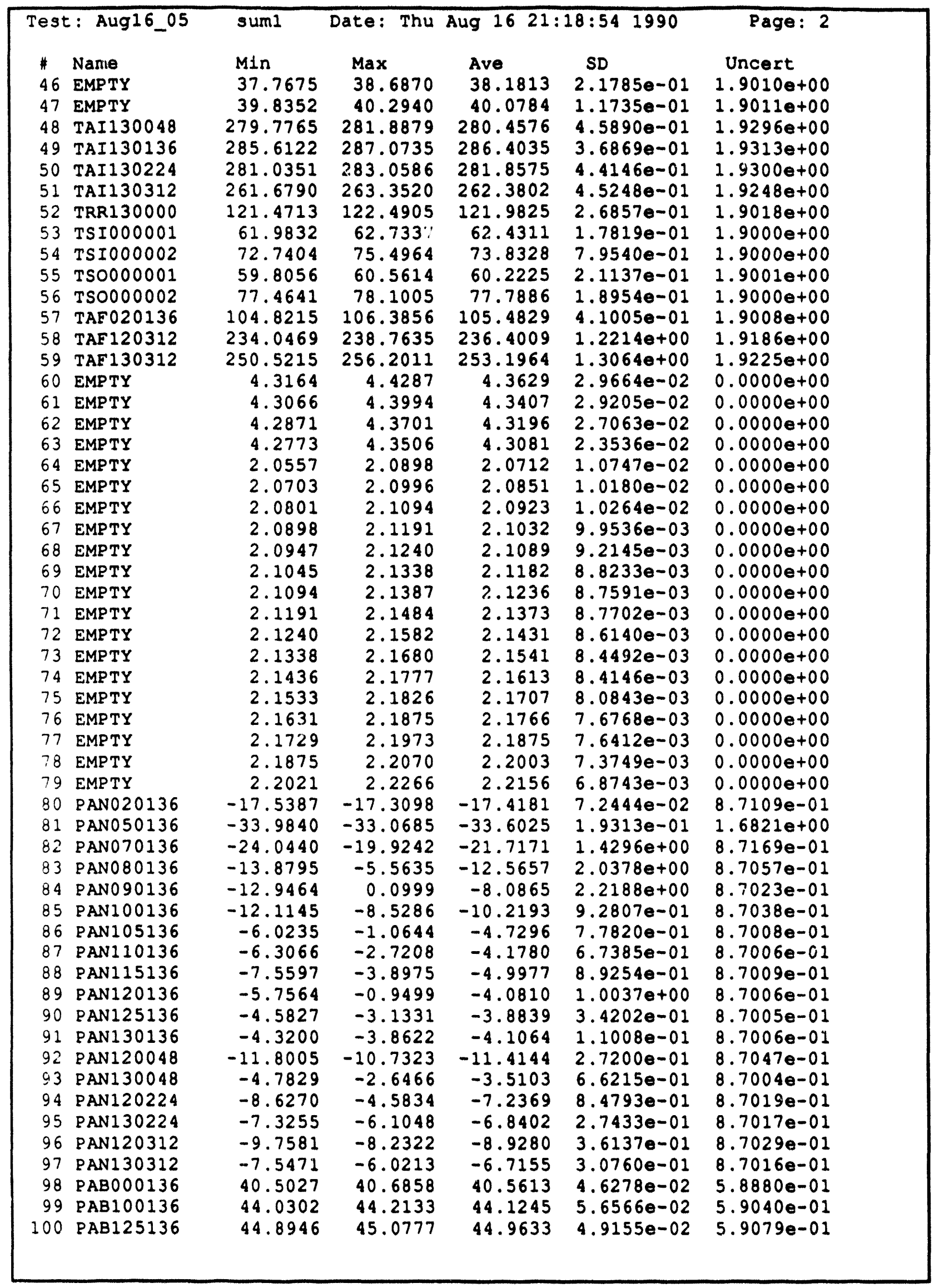




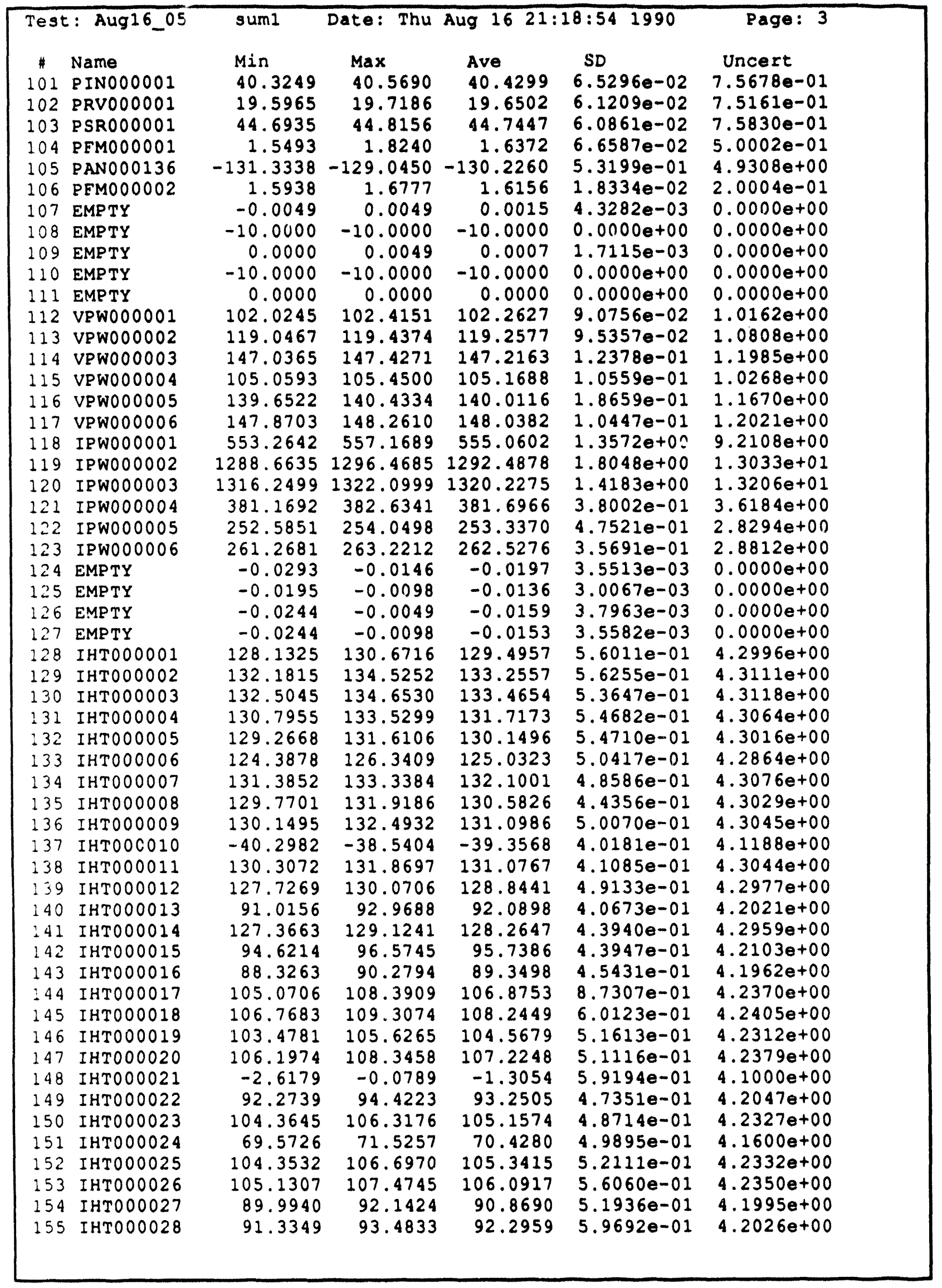




\begin{tabular}{|c|c|c|c|c|c|c|}
\hline Tes & Aug16_05 & sum I & Date: Thu & Aug 162 & $8: 541990$ & Page: 4 \\
\hline$\#$ & Name & Min & $\operatorname{Max}$ & Ave & SD & Uncert \\
\hline 156 & IHTO000029 & 104.8152 & 107.1590 & 105.8387 & $5.9524 e-01$ & $4.2344 e+00$ \\
\hline 157 & IHTO00030 & 107.0125 & 109.1609 & 108.0516 & $5.5164 e-01$ & $4.2400 e+00$ \\
\hline 158 & IHTO000031 & 1.05 .4462 & 107.5947 & 106.4853 & $5.5586 \theta-01$ & $4.2360 \mathrm{e}+00$ \\
\hline 159 & IHT 000032 & 106.7420 & 109.0858 & 107.8045 & $5.7610 e-01$ & $4.2394 e+00$ \\
\hline 160 & IHT 000033 & 106.7646 & 109.1083 & 107.7724 & $5.6409 e-01$ & $4.2393 e+00$ \\
\hline 161 & IHTO00034 & 93.7425 & 95.6956 & 94.4495 & $4.0025 e-01$ & $4.2074 e+00$ \\
\hline 162 & IHTO000035 & 123.9183 & 125.6761 & 124.6175 & $4.2332 e-01$ & $4.2852 e+00$ \\
\hline 163 & IHT000036 & 92.2138 & 93.9716 & 93.1044 & $3.9094 e-01$ & $4.2044 e+00$ \\
\hline
\end{tabular}




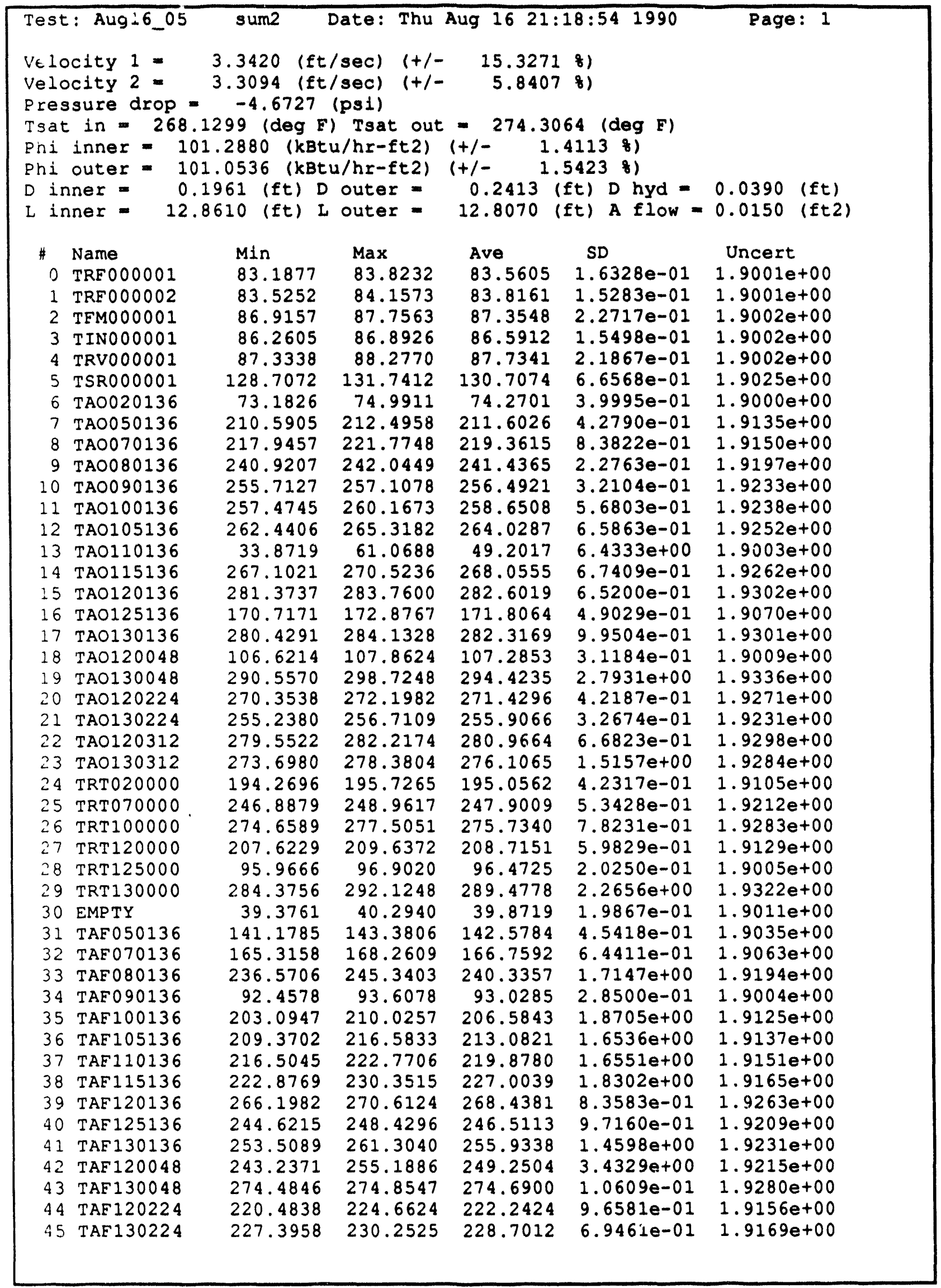




\begin{tabular}{|c|c|c|c|c|c|c|}
\hline & sum2 & Date: Thu & Aug 162 & $8: 54 \quad 1990$ & Page: 2 \\
\hline \multicolumn{2}{|c|}{$\begin{array}{l}\text { Test: Aug16_05 } \\
\# \text { Name }\end{array}$} & $\operatorname{Min}$ & $\operatorname{Max}$ & Ave & SD & Uncert \\
\hline 46 & EMPTY & 37.7675 & 38.6870 & 38.1859 & $2.0079 e-01$ & $1.9010 e+00$ \\
\hline 47 & EMPTY & 39.8352 & 40.2940 & 40.1518 & $1.2166 e-01$ & $1.9011 e+00$ \\
\hline 48 & TAI130048 & 279.1326 & 280.8776 & 280.2484 & $3.3342 e-01$ & $1.9295 e+00$ \\
\hline 49 & TAI130136 & 284.2435 & 288.0760 & 286.1295 & $1.1687 e+00$ & $1.9312 \mathrm{e}+00$ \\
\hline 50 & TAI130224 & 280.1143 & 283.1502 & 281.4526 & $7.2471 e-01$ & $1.9299 e+00$ \\
\hline 51 & TAI 130312 & 262.0506 & 263.2588 & 262.6960 & $3.40610-01$ & $1.9249 e+00$ \\
\hline 52 & TRR130000 & 121.4708 & 123.1003 & 122.2636 & $3.6603 e-01$ & $1.9018 e+00$ \\
\hline 53 & TSI000001 & 62.0905 & 62.7331 & 62.4227 & $1.6082 e-01$ & $1.9000 e+00$ \\
\hline 54 & TSI000002 & 69.2362 & 72.8477 & 71.0918 & $7.2577 \mathrm{e}-01$ & $1.9000 e+00$ \\
\hline 55 & TSO0000001 & 59.5890 & 60.5609 & 60.1833 & $2.1273 e-01$ & $1.9001 e+00$ \\
\hline 56 & TSO0000002 & 77.3582 & 78.4175 & 77.8733 & $2.3743 e-01$ & $1.9000 e+00$ \\
\hline 57 & TAF 020136 & 104.6130 & 106.8007 & 105.8993 & $4.7645 e-01$ & $1.9009 e+00$ \\
\hline 58 & TAF 120312 & 232.9134 & 241.3045 & 236.7959 & $2.3024 e+00$ & $1.9187 e+00$ \\
\hline 59 & TAF 130312 & 249.7751 & 263.8091 & 256.8539 & $4.0272 e+00$ & $1.9234 \mathrm{e}+00$ \\
\hline 60 & EMPTY & 4.2871 & 4.5850 & 4.4391 & $9.9094 e-02$ & $0.0000 e+00$ \\
\hline 61 & EMPTY & 4.2676 & 4.5361 & 4.4005 & $9.6923 e-02$ & $0.0000 e+00$ \\
\hline 62 & ЕМРTY & 4.2480 & 4.4922 & 4.3627 & $9.1881 e-02$ & $0.0000 e+00$ \\
\hline 63 & EMPTY & 4.2334 & 4.4727 & 4.3372 & $8.5527 e-02$ & $0.0000 e+00$ \\
\hline 64 & EMPTY & 2.0361 & 2.1387 & 2.0756 & $3.6856 e-02$ & $0.0000 e+00$ \\
\hline 65 & EMPTY & 2.0508 & 2.1484 & 2.0858 & $3.5026 e-02$ & $0.0000 e+00$ \\
\hline 66 & EMPTY & 2.0557 & 2.1533 & 2.0896 & $3.3087 e-02$ & $0.0000 e+00$ \\
\hline 67 & EMPTY & 2.0654 & 2.1582 & 2.0972 & $3.1105 e-02$ & $0.0000 e+00$ \\
\hline 68 & EMPTY & 2.0752 & 2.1582 & 2.1003 & $2.8489 e-02$ & $0.0000 e+00$ \\
\hline 69 & EMPTY & 2.0850 & 2.1631 & 2.1072 & $2.5840 e-02$ & $0.0000 e+00$ \\
\hline 70 & EMPTY & 2.0898 & 2.1631 & 2.1114 & $2.4024 e-02$ & $0.0000 e+00$ \\
\hline 71 & EMPTY & 2.1045 & 2.1777 & 2.1239 & $2.2462 e-02$ & $0.0000 e+00$ \\
\hline 72 & EMPTY & 2.1094 & 2.1729 & 2.1284 & $1.9312 \mathrm{e}-02$ & $0.0000 \mathrm{e}+00$ \\
\hline 73 & EMPTY & 2.1191 & 2.1826 & 2.1383 & $1.7364 e-02$ & $0.0000 e+00$ \\
\hline 74 & EMPTY & 2.1289 & 2.1826 & 2.1455 & $1.5058 e-02$ & $0.0000 e+00$ \\
\hline 75 & EMPTY & 2.1436 & 2.1924 & 2.1546 & $1.3627 e-02$ & $0.0000 e+00$ \\
\hline 76 & EMPTY & 2.1484 & 2.1924 & 2.1605 & $1.1929 e-02$ & $0.0000 e+00$ \\
\hline 77 & EMPTY & 2.1582 & 2.2021 & 2.1718 & $1.0313 e-02$ & $0.0000 e+00$ \\
\hline 78 & EMPTY & 2.1729 & 2.2119 & 2.1855 & $8.8783 e-03$ & $0.0000 e+00$ \\
\hline 79 & EMPTY & 2.1924 & 2.2266 & 2.2017 & $8.4429 e-03$ & $0.0000 e+00$ \\
\hline 80 & PAN020136 & -17.6150 & -17.2335 & -17.4212 & $8.4719 e-02$ & $8.7109 e-01$ \\
\hline 81 & PAN050136 & -33.8314 & -32.9159 & -33.2058 & $2.4517 e-01$ & $1.6820 \mathrm{e}+00$ \\
\hline 82 & PAN070136 & -24.5781 & -19.9242 & -21.8361 & $1.4476 \mathrm{e}+00$ & $8.7171 e-01$ \\
\hline 83 & PAN080136 & -13.9558 & -9.1493 & -12.7015 & $1.4386 e+00$ & $8.7058 e-01$ \\
\hline 84 & PAN090136 & -13.0990 & -6.4614 & -9.5300 & $1.4391 e+00$ & $8.7033 e-01$ \\
\hline 85 & PAN100136 & -10.8175 & -6.5450 & -9.0169 & $1.0887 e+00$ & $8.7029 e-01$ \\
\hline 86 & PAN105136 & -7.6257 & -1.0644 & -4.4091 & $1.2691 e+00$ & $8.7007 e-01$ \\
\hline 87 & PAN110136 & -6.8407 & 0.2547 & -4.2116 & $1.0358 e+00$ & $8.7006 e-01$ \\
\hline 88 & PAN115136 & -7.5597 & -2.9057 & -4.7307 & $8.6754 e-01$ & $8.7008 e-01$ \\
\hline 89 & PAN 120136 & -6.1379 & -2.7047 & -4.1863 & $7.5544 e-01$ & $8.7006 e-01$ \\
\hline 90 & PAN125136 & -4.5827 & -2.2939 & -3.9129 & $4.7089 e-01$ & $8.7006 e-01$ \\
\hline 91 & PAN 130136 & -4.3200 & -3.7096 & -3.0813 & $1.6684 e-01$ & $8.7006 e-01$ \\
\hline 92 & PAN 120048 & -11.8768 & -9.9694 & $-11.2,39$ & $4.1209 e-01$ & $8.7046 e-01$ \\
\hline 93 & PAN130048 & -4.7829 & -1.3496 & -2.4437 & $9.9816 e-01$ & $8.7002 e-01$ \\
\hline 94 & PAN 120224 & -9.0085 & -5.4989 & -7.4048 & $7.2305 e-01$ & $8.7020 e-01$ \\
\hline 95 & PAN130224 & -7.5544 & -5.7233 & -6.6617 & $4.4172 e-01$ & $8.7016 \mathrm{e}-01$ \\
\hline 96 & PAN120312 & -9.6818 & -6.0960 & -8.7556 & $5.5788 e-01$ & $8.7028 e-01$ \\
\hline 97 & PAN130312 & -7.4708 & -5.7161 & -6.5141 & $3.9274 e-01$ & $8.7015 e-01$ \\
\hline 98 & PAB 000136 & 40.4112 & 40.5943 & 40.5137 & $4.5832 e-02$ & $5.8878 e-01$ \\
\hline 99 & PAB100136 & 43.9386 & 44.1675 & 44.0732 & $5.8790 e-02$ & $5.9037 e-01$ \\
\hline 100 & PAB125136 & 44.8031 & 44.9862 & 44.8965 & $4.7121 e-02$ & $5.9076 e-01$ \\
\hline
\end{tabular}




\begin{tabular}{|c|c|c|c|c|c|c|}
\hline Test & $t:$ Aug16_05 & sum2 & Date: Thu & $\begin{array}{lll}\text { Aug } & 16 \quad 21\end{array}$ & $8: 541990$ & Page: 3 \\
\hline$\#$ & Name & Min & $\operatorname{Max}$ & Ave & SD & Uncert \\
\hline 101 & PINO000001 & 40.2028 & 40.5690 & 40.3664 & $9.0982 e-02$ & $7.5676 \mathrm{e}-01$ \\
\hline 102 & PRVO00001 & 19.5965 & 19.7186 & 19.6795 & $5.7521 e-02$ & $7.5161 \mathrm{e}-01$ \\
\hline 103 & PSR000001 & 44.5714 & 44.8156 & 44.6642 & $5.8152 e-02$ & $7.5827 e-01$ \\
\hline 104 & PFM000001 & 1.4578 & 1.7324 & 1.6409 & $7.8474 e-02$ & $5.0002 e-01$ \\
\hline 105 & PAN 000136 & -131.3338 & -127.6717 & -129.3654 & $7.4130 e-01$ & $4.9306 \mathrm{e}+00$ \\
\hline 106 & PFM000002 & 1.5602 & 1.6609 & 1.6082 & $2.0624 e-02$ & $2.0004 e-01$ \\
\hline 107 & EMPTY & -0.0049 & 0.0049 & 0.0018 & $4.0385 e-03$ & $0.0000 e+00$ \\
\hline 108 & EMPTY & -10.0000 & -9.4971 & -9.9690 & $1.0428 e-01$ & $0.0000 e+00$ \\
\hline 109 & EMPTY & -0.0049 & 0.0049 & 0.0014 & $2.7971 e-03$ & $0.0000 e+00$ \\
\hline 110 & EMPTY & -10.0000 & -10.0000 & -10.0000 & $0.0000 e+00$ & $0.0000 e+00$ \\
\hline 111 & EMPTY & 0.0000 & 0.0049 & 0.0001 & $6.9053 e-04$ & $0.0000 e+00$ \\
\hline 112 & VPW000001 & 102.2198 & 102.4151 & 102.2784 & $9.0412 e-02$ & $1.0162 \mathrm{e}+00$ \\
\hline 113 & VPW000002 & 119.0467 & 119.4374 & 119.2928 & $9.5112 e-02$ & $1.0809 e+00$ \\
\hline 114 & VPW000003 & 146.8412 & 147.6225 & 147.2241 & $1.6250 e-01$ & $1.1985 \mathrm{e}+00$ \\
\hline 115 & VPWOOOOOO4 & 105.0593 & 105.4500 & 105.2625 & $1.3063 e-01$ & $1.0271 \mathrm{e}+00$ \\
\hline 116 & VPW000005 & 139.8475 & 140.2381 & 140.0897 & $9.3043 e-02$ & $1.1673 \mathrm{e}+00$ \\
\hline 117 & VPW000006 & 148.0657 & 148.4563 & 148.1788 & $1.1223 e-01$ & $1.2027 e+00$ \\
\hline 118 & IPWO00001 & 537.6454 & 547.4072 & 542.9557 & $2.8454 e+00$ & $9.1656 e+00$ \\
\hline 119 & IPWOOO0002 & 1310.1273 & 1317.9325 & 1312.7032 & $2.2797 e+00$ & $1.3159 \mathrm{e}+01$ \\
\hline 120 & IPWOO00003 & 1325.9999 & 1335.7499 & 1331.7329 & $2.5044 \mathrm{e}+00$ & $1.3278 \mathrm{e}+01$ \\
\hline 121 & IPWO00004 & 378.2395 & 379.7044 & 378.8841 & $3.6165 e-01$ & $3.5909 e+00$ \\
\hline 122 & IPWO00005 & 253.0734 & 254.0498 & 253.6104 & $3.1580 e-01$ & $2.8309 e+00$ \\
\hline 123 & IPWO000006 & 263.7095 & 264.6860 & 264.1683 & $3.4751 e-01$ & $2.8905 e+00$ \\
\hline 124 & EMPTY & -0.0244 & -0.0098 & -0.0185 & $3.3146 e-03$ & $0.0000 \mathrm{e}+00$ \\
\hline 125 & EMPTY & -0.0244 & -0.0098 & -0.0129 & $3.2359 e-03$ & $0.0000 \mathrm{e}+00$ \\
\hline 126 & EMPTY & -0.0244 & -0.0049 & -0.0154 & $4.1102 e-03$ & $0.0000 e+00$ \\
\hline 127 & EMPTY & -0.0195 & -0.0098 & -0.0153 & $2.7919 e-03$ & $0.0000 e+00$ \\
\hline 128 & IHTO00001 & 128.3278 & 131.2575 & 129.5074 & $6.5431 e-01$ & $4.2997 e+00$ \\
\hline 129 & IHTO00002 & 132.1815 & 134.9158 & 133.2596 & $5.8692 e-01$ & $4.3111 e+00$ \\
\hline 130 & IHT000003 & 132.5045 & 134.8483 & 133.5162 & $5.6316 e-01$ & $4.3119 \mathrm{e}+00$ \\
\hline 131 & I.4T000004 & 130.7955 & 133.1393 & 131.8306 & $6.2172 e-01$ & $4.3067 e+00$ \\
\hline 132 & IHT000005 & 129.2668 & 131.6106 & 130.2589 & $5.9168 e-01$ & $4.3019 e+00$ \\
\hline 133 & IHT000006 & 123.9971 & 126.5362 & 125.1182 & $5.7698 e-01$ & $4.2867 e+00$ \\
\hline 134 & IHT000007 & 130.7993 & 133.5337 & 132.1509 & $5.9299 e-01$ & $4.3077 e+00$ \\
\hline 135 & IHT000008 & 129.1842 & 131.9186 & 130.6216 & $5.7013 e-01$ & $4.3030 \mathrm{e}+00$ \\
\hline 136 & IHT000009 & 129.7589 & 132.4932 & 131.0752 & $6.1106 e-01$ & $4.3044 e+00$ \\
\hline 137 & IHTO00010 & -40.4935 & -37.7592 & -39.2553 & $6.2342 e-01$ & $4.1187 e+00$ \\
\hline 138 & IHT000011 & 130.3072 & 133.0416 & 131.2681 & $5.8373 e-01$ & $4.3050 \mathrm{e}+00$ \\
\hline 139 & IHT000012 & 128.1175 & 130.2659 & 129.0238 & $5.7826 e-01$ & $4.2982 e+00$ \\
\hline 140 & IHTO00013 & 91.0156 & 93.7500 & 92.1641 & $5.8745 e-01$ & $4.2023 e+00$ \\
\hline 141 & IHTO000014 & 127.1710 & 129.7101 & 128.3623 & $5.7623 e-01$ & $4.2962 e+00$ \\
\hline 142 & IHTO00015 & 94.6214 & 97.1604 & 95.7854 & $5.7312 e-01$ & $4.2104 e+00$ \\
\hline 143 & IHTO00016 & 88.1310 & 90.4747 & 89.3459 & $5.3712 e-01$ & $4.1962 e+00$ \\
\hline 144 & IHTO00017 & 104.6800 & 108.9769 & 106.9144 & $9.2889 e-01$ & $4.2371 \mathrm{e}+00$ \\
\hline 145 & IHTO00018 & 107.1590 & 109.6980 & 108.2606 & $5.9420 e-01$ & $4.2405 e+00$ \\
\hline 146 & IHTO00010 & 103.4781 & 105.4312 & 104.5249 & $4.6481 e-01$ & $4.2311 e+00$ \\
\hline 147 & IHTO000020 & 106.1974 & 108.3458 & 107.2169 & $4.8854 e-01$ & $4.2379 e+00$ \\
\hline 148 & IHT000021 & -2.8132 & 0.1164 & -1.3172 & $5.9922 e-01$ & $4.1000 \mathrm{e}+00$ \\
\hline 149 & IHTO00022 & 92.0786 & 94.4223 & 93.2622 & $4.8874 e-01$ & $4.2047 e+00$ \\
\hline 150 & IHTOOO0023 & 103.9739 & 106.7083 & 105.1418 & $5.2272 e-01$ & $4.2327 e+00$ \\
\hline 151 & IHTO000024 & 69.1820 & 71.9163 & 70.3929 & $4.8488 e-01$ & $4.1600 e+00$ \\
\hline 152 & IHTO00025 & 103.9626 & 106.8923 & 105.3102 & $5.4996 \mathrm{e}-01$ & $4.2331 e+00$ \\
\hline 153 & IHTO00026 & 104.7401 & 107.6698 & 106.0721 & $5.9541 \mathrm{e}-01$ & $4.2350 e+00$ \\
\hline 154 & IHTO00027 & 89.6034 & 92.3377 & 90.8416 & $5.7671 e-01$ & $4.1994 \mathrm{e}+00$ \\
\hline 155 & IHTO00028 & 91.3349 & 93.8739 & 92.3896 & $5.9189 e-01$ & $4.2028 e+00$ \\
\hline
\end{tabular}




\begin{tabular}{|c|c|c|c|c|c|c|}
\hline Test & $\therefore$ : Aug16_05 & sum2 & Date: Thu & Aug 162 & $18: 541990$ & Page: 4 \\
\hline $\begin{array}{l}\# \\
156 \\
157 \\
158 \\
159 \\
160 \\
161 \\
162 \\
163\end{array}$ & 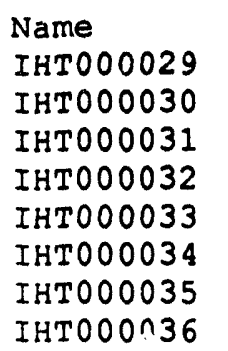 & $\begin{array}{l}\text { Min } \\
105.0105 \\
107.2078 \\
105.6415 \\
106.9373 \\
106.7646 \\
93.7425 \\
123.9183 \\
92.2138\end{array}$ & $\begin{array}{l}\text { Max } \\
107.1590 \\
109.5515 \\
107.9853 \\
109.0858 \\
109.1083 \\
95.6956 \\
126.0667 \\
94.5575\end{array}$ & $\begin{array}{l}\text { Ave } \\
105.9442 \\
108.1610 \\
106.5947 \\
107.8983 \\
107.8349 \\
94.6058 \\
124.8168 \\
93.2568\end{array}$ & $\begin{array}{l}\text { SD } \\
5.9750 e-01 \\
5.7404 e-01 \\
6.1338 e-01 \\
6.1364 e-01 \\
6.2420 e-01 \\
5.0237 e-01 \\
5.0686 e-01 \\
5.1238 e-01\end{array}$ & $\begin{array}{l}\text { Uncert } \\
4.2347 e+00 \\
4.2403 e+00 \\
4.2363 e+00 \\
4.2396 e+00 \\
4.2394 e+00 \\
4.2077 e+00 \\
4.2858 e+00 \\
4.2047 e+00\end{array}$ \\
\hline
\end{tabular}




\begin{tabular}{|c|c|c|c|c|}
\hline \multicolumn{5}{|c|}{$\begin{array}{l}\text { Velocity } 1=0.003700 \text { (ft/sec) } \\
\text { Velocity } 2=0.007400 \text { (ft/sec) } \\
\text { Pressure Drop }=0.031100 \text { (psi) } \\
\text { Tsat in }=0.070099 \text { (deg E) Tsat out }=0.090210 \text { (deg F) }\end{array}$} \\
\hline$\#$ & Name & Abs Diff & \&Diff & \\
\hline 0 & TRF000001 & 0.0001 & 0.0001 & \\
\hline 1 & TRF000002 & 0.1012 & 0.1209 & \\
\hline 2 & TEMO00001 & 0.1888 & 0.2166 & \\
\hline 3 & TIN000001 & 0.1516 & 0.1754 & \\
\hline 4 & TRV000001 & 0.3138 & 0.3590 & \\
\hline 5 & TSR000001 & 3.3384 & 2.6210 & \\
\hline 6 & TAO020136 & 0.0084 & 0.0113 & \\
\hline 7 & TAO050136 & 0.7581 & 0.3596 & \\
\hline 8 & TA0070136 & 0.4864 & 0.2222 & \\
\hline 9 & TAO080136 & 1.4722 & 0.6135 & \\
\hline 10 & TAO090136 & 0.3493 & 0.1364 & \\
\hline 11 & TA0100136 & 0.2526 & 0.0978 & \\
\hline 12 & TAO105136 & 0.4752 & 0.1803 & \\
\hline 13 & TAO110136 & 76.7226 & 60.9276 & \\
\hline 14 & TAO115136 & 0.3405 & 0.1272 & \\
\hline 15 & TAO120136 & 0.0221 & 0.0078 & \\
\hline 16 & TAO125136 & 0.0118 & 0.0069 & \\
\hline 17 & TAO130136 & 0.0321 & 0.0114 & \\
\hline 18 & TAO120048 & 5.7354 & 5.6479 & \\
\hline 19 & TAO130048 & 2.9521 & 1.0128 & \\
\hline 20 & TAO120224 & 0.0148 & 0.0055 & \\
\hline 21 & TAO130224 & 0.0816 & 0.0319 & \\
\hline 22 & TAO120312 & 0.6209 & 0.2205 & \\
\hline 23 & TA0130312 & 0.5871 & 0.2131 & \\
\hline 24 & TRT020000 & 0.1203 & 0.0617 & \\
\hline 25 & TRT070000 & 0.1014 & 0.0409 & \\
\hline 26 & TRT 100000 & 1.0129 & 0.3660 & \\
\hline 27 & TRT 120000 & 19.7211 & 10.4348 & \\
\hline 28 & TRT 125000 & 4.9362 & 5.3926 & \\
\hline 29 & TRT 130000 & 0.0028 & 0.0010 & \\
\hline 30 & EMPTY & 0.0367 & 0.0920 & \\
\hline 31 & TAF050136 & 0.1885 & 0.1324 & \\
\hline 32 & TAF070136 & 0.2361 & 0.1418 & \\
\hline 33 & TAF080136 & 1.6915 & 0.7088 & \\
\hline 34 & TAF090136 & 3.2324 & 3.5997 & \\
\hline 35 & TAF 100136 & 1.8215 & 0.8896 & \\
\hline 36 & TAF 105136 & 1.2038 & 0.5682 & \\
\hline 37 & TAF 110136 & 1.3596 & 0.6222 & \\
\hline 38 & TAF 115136 & 1.0213 & 0.4519 & \\
\hline 39 & TAF 120136 & 0.0440 & 0.0164 & \\
\hline 40 & TAE 125136 & 0.1705 & 0.0691 & \\
\hline 41 & TAF 130136 & 0.2449 & 0.0958 & \\
\hline 42 & TAF 120048 & 1.7140 & 0.6924 & \\
\hline 43 & TAF 130048 & 0.2134 & 0.0776 & \\
\hline 44 & $\operatorname{TAF} 120224$ & 0.2469 & 0.1112 & \\
\hline 45 & TAF 130224 & 0.2855 & 0.1250 & \\
\hline
\end{tabular}




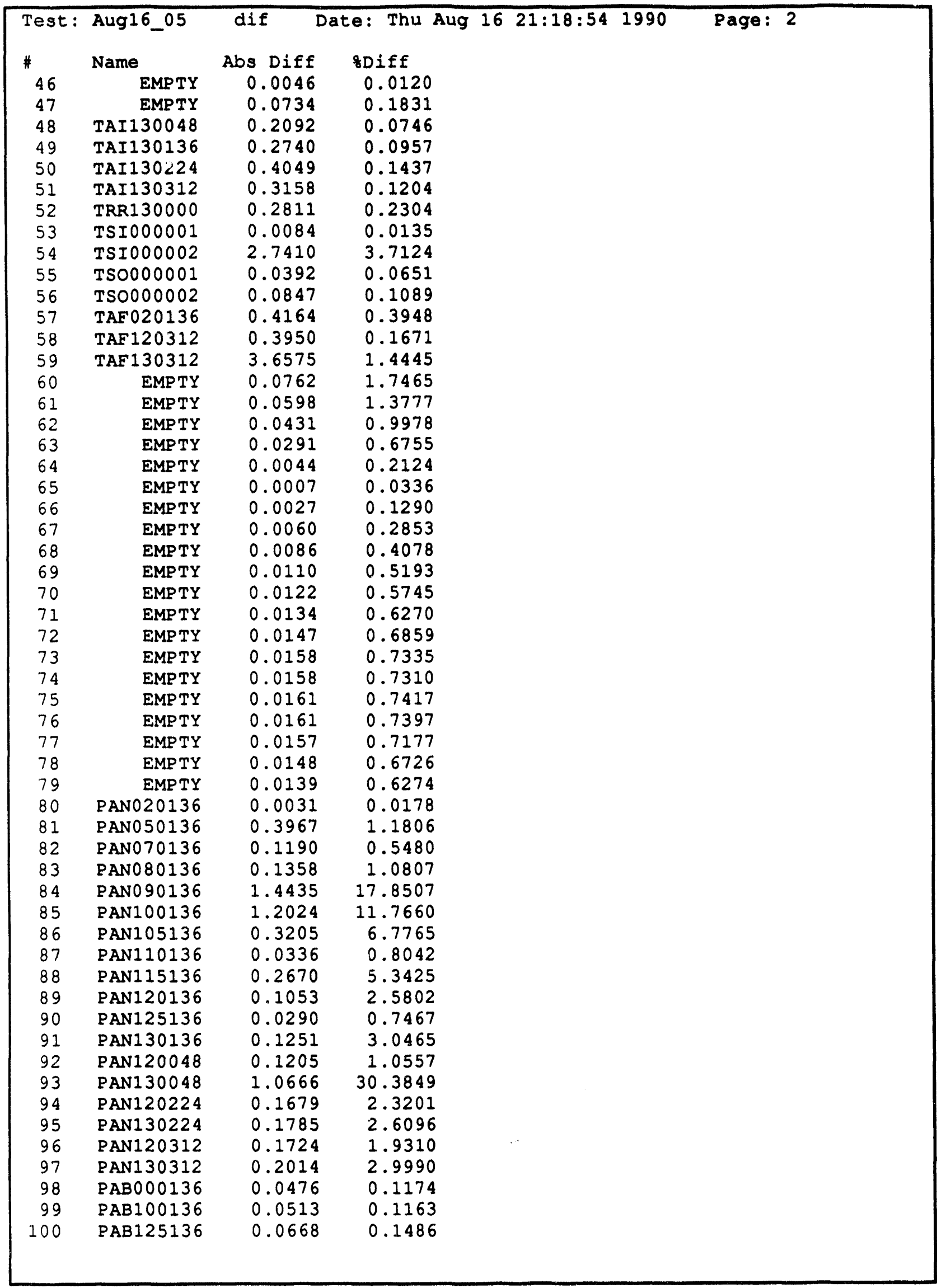




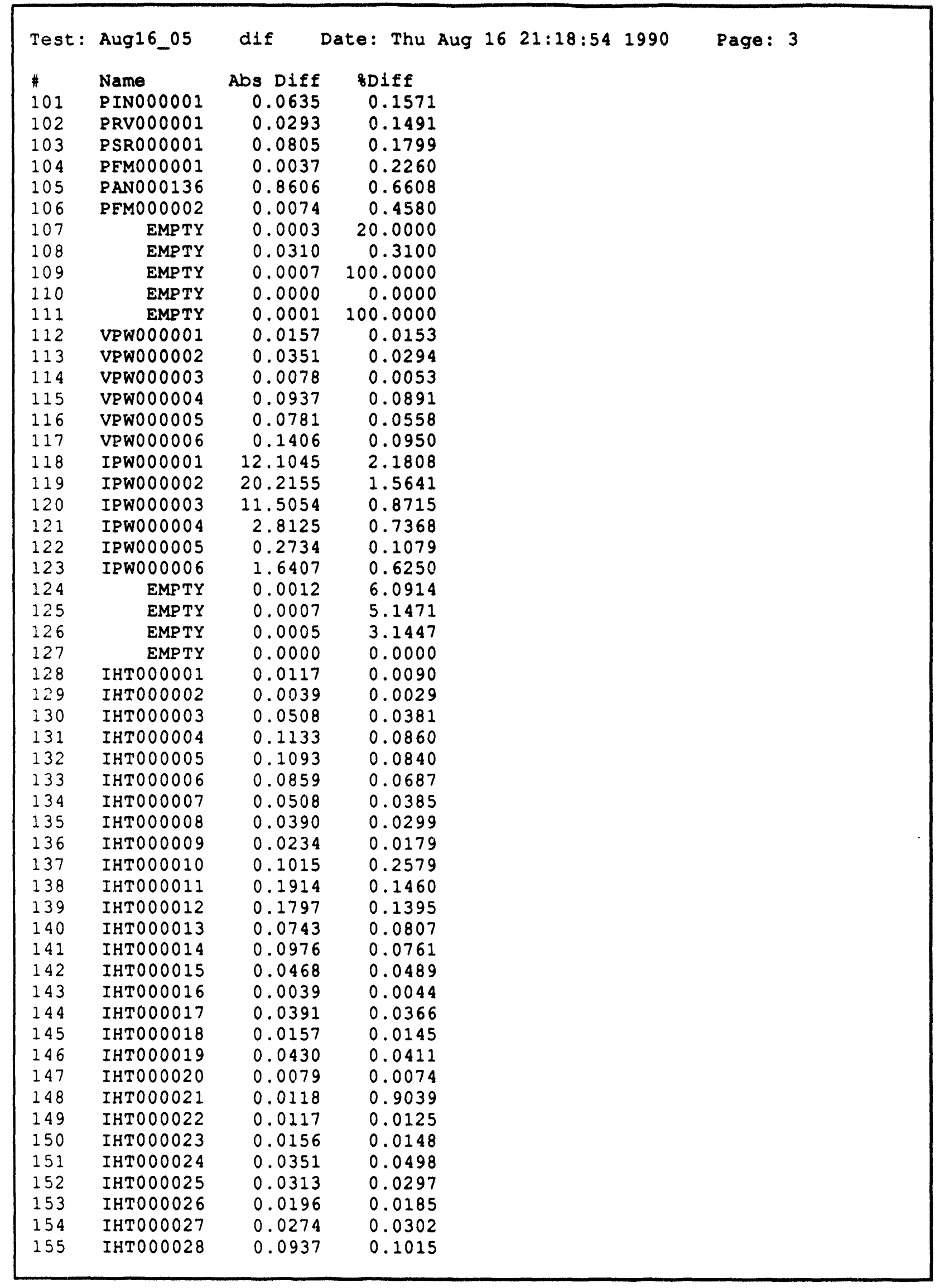




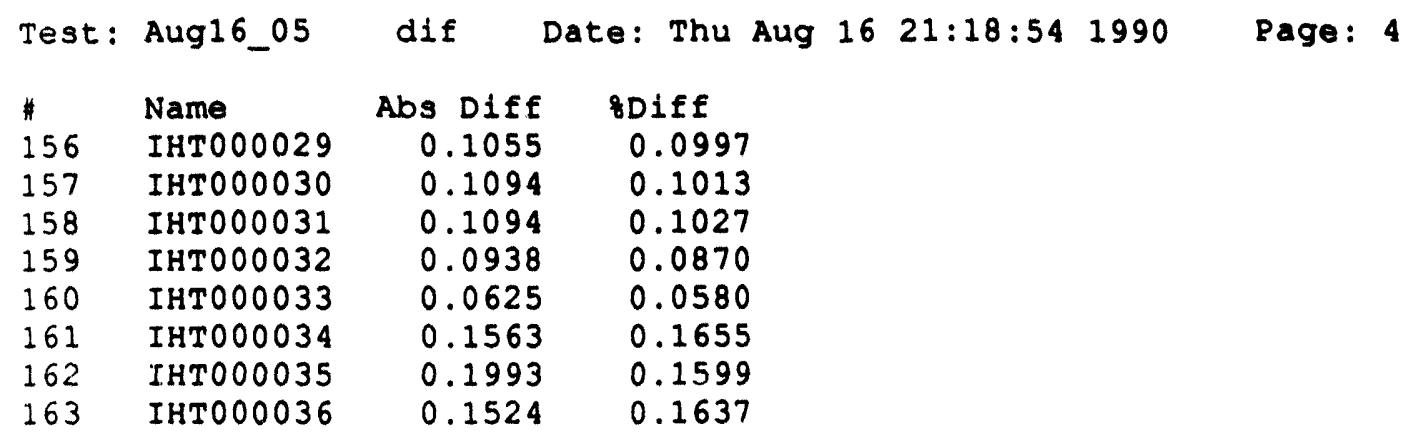




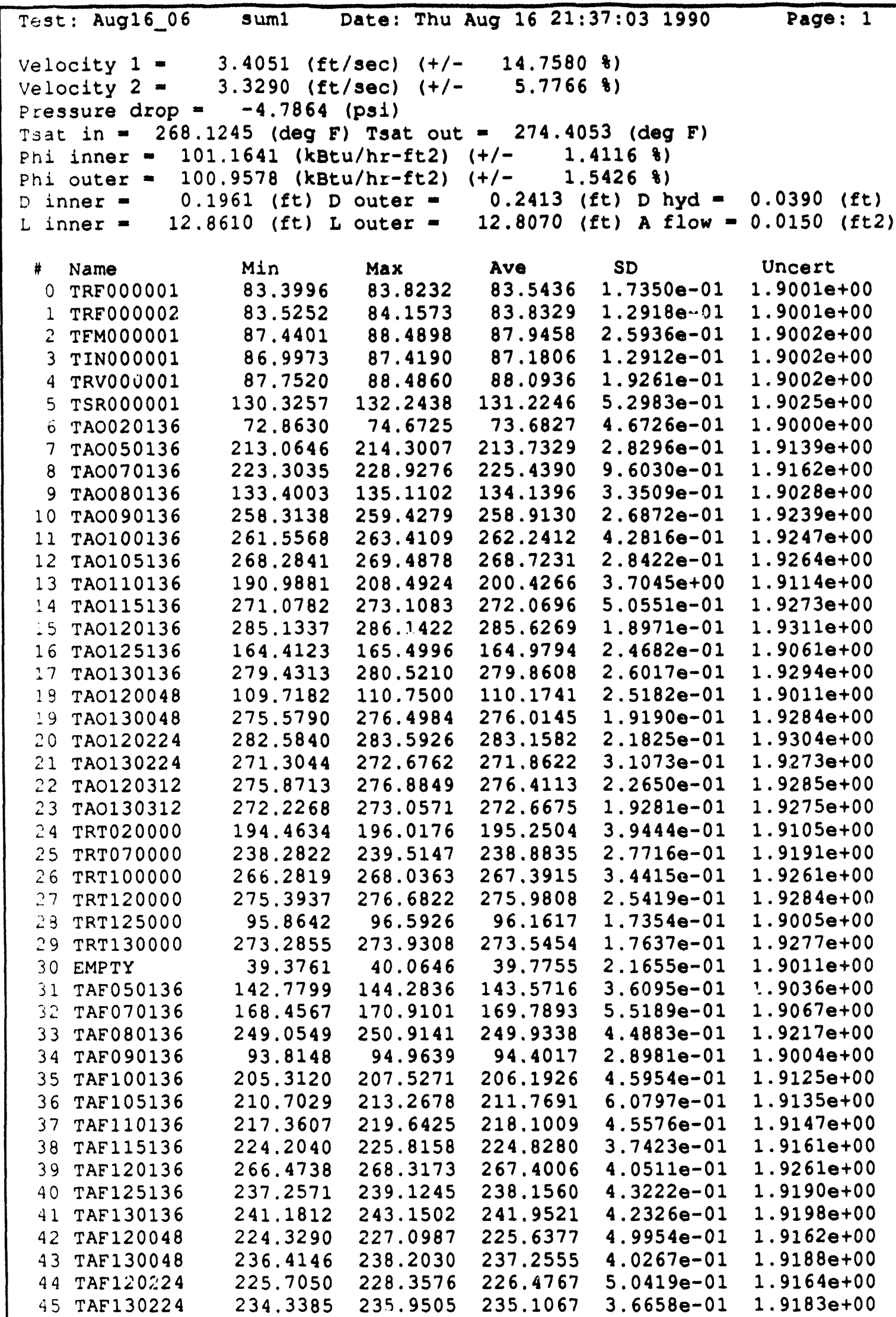




\begin{tabular}{|c|c|c|c|c|c|c|}
\hline \multicolumn{2}{|c|}{ Test: Aug16_06 } & sum1 & \multicolumn{3}{|c|}{ Date: Thu Aug $16 \quad 21: 37: 03 \quad 1990$} & Page: 2 \\
\hline$\#$ & Name & Min & Max & Ave & SD & Uncert \\
\hline 46 & EMPTY & 37.7675 & 38.4572 & 38.1308 & $1.9202 e-01$ & $1.90100+00$ \\
\hline 47 & EMPTY & 39.8352 & 40.2940 & 40.0555 & $1.0324 e-01$ & $1.9011 e+00$ \\
\hline 48 & TAI 130048 & 275.9154 & 277.1124 & 276.5065 & $2.9528 \theta-01$ & $1.92850+00$ \\
\hline 49 & TAI130136 & 284.0597 & 285.2491 & 284.5883 & $2.5699 e-01$ & $1.9308 e+00$ \\
\hline $\begin{array}{l}50 \\
51\end{array}$ & $\begin{array}{l}\text { TAI1 } 30224 \\
\text { TAI1 } 30312\end{array}$ & $\begin{array}{l}280.1147 \\
276.7604\end{array}$ & $\begin{array}{l}281.2192 \\
278.3262\end{array}$ & $\begin{array}{l}280.7129 \\
277.6429\end{array}$ & $\begin{array}{l}2.7596 e-01 \\
3.2450 e-01\end{array}$ & $\begin{array}{l}1.9297 e+00 \\
1.9288 \theta+00\end{array}$ \\
\hline 52 & TRR130000 & 84.2778 & 86.0616 & 85.2870 & $4.3681 e-01$ & $1.9002 e+00$ \\
\hline 53 & TSI000001 & 61.6625 & 62.4132 & 61.9777 & $2.1043 e-01$ & $1.9000 e+00$ \\
\hline 54 & TSI000002 & 74.8607 & 78.1377 & 76.1874 & $6.6572 e-01$ & $1.90000+00$ \\
\hline 55 & TSO000001 & 59.4815 & 60.4529 & 59.9803 & $2.07890-01$ & $1.90010+00$ \\
\hline 56 & TSO0000002 & 76.6165 & 77.4651 & 76.9199 & $1.8929 e-01$ & $1.9000 e+00$ \\
\hline 57 & TAF020136 & 104.9265 & 106.9049 & 106.0076 & $4.1804 e-01$ & $1.9009 e+00$ \\
\hline $\begin{array}{l}58 \\
59\end{array}$ & TAF 120312 & 221.4330 & 224.0974 & 222.7411 & $6.1540 e-01$ & $1.9157 e+00$ \\
\hline $\begin{array}{l}59 \\
60\end{array}$ & TAF 130312 & 239.6658 & 241.7316 & 240.8804 & $5.0391 e-01$ & $1.9196 e+00$ \\
\hline $\begin{array}{l}60 \\
61\end{array}$ & EMPTY & 4.0332 & 4.0527 & 4.0417 & $5.1078 e-03$ & $0.0000 e+00$ \\
\hline 61 & EMPTY & 4.0137 & 4.0283 & 4.0206 & $3.1335 e-03$ & $0.0000 e+00$ \\
\hline 62 & EMPTY & 3.9941 & 4.0039 & 4.0002 & $2.8862 e-03$ & $0.0000 e+00$ \\
\hline 63 & EMPTY & 3.9844 & 3.9941 & 3.9912 & $2.6100 e-03$ & $0.0000 e+00$ \\
\hline 64 & EMPTY & 1.9238 & 1.9287 & 1.9251 & $2.1635 e-03$ & $0.0000 e+00$ \\
\hline 65 & EMPTY & 1.9336 & 1.9434 & 1.9393 & $2.0598 \theta-03$ & $0.0000 e+00$ \\
\hline$\partial \epsilon$ & EMPTY & 1.9434 & 1.9531 & 1.9481 & $1.20410-03$ & $0.0000 e+00$ \\
\hline 67 & EMPTY & 1.9580 & 1.9629 & 1.9590 & $1.97300-03$ & $0.0000 e+00$ \\
\hline 68 & EMPTY & 1.9629 & 1.9678 & 1.9650 & $2.4484 e-03$ & $0.0000 e+00$ \\
\hline 69 & EMPTY & 1.9678 & 1.9775 & 1.9755 & $2.6267 e-03$ & $0.0000 e+00$ \\
\hline 70 & EMPTY & 1.9775 & 1.9873 & 1.9826 & $1.3811 e-03$ & $0.0000 e+00$ \\
\hline 71 & EMPTY & 1.9971 & 2.0020 & 1.9972 & $6.9053 e-04$ & $0.0000 e+00$ \\
\hline 72 & EMPTY & 1.9971 & 2.0068 & 2.0046 & $2.6488 e-03$ & $0.0000 e+00$ \\
\hline 73 & EMPTY & 2.0117 & 2.0166 & 2.0165 & $6.9053 e-04$ & $0.0000 e+00$ \\
\hline 74 & EMPTY & 2.0264 & 2.0264 & 2.0264 & $0.0000 e+00$ & $0.0000 e+00$ \\
\hline 75 & EMPTY & 2.0361 & 2.0410 & 2.0362 & $6.9053 e-04$ & $0.0000 e+00$ \\
\hline $7 \epsilon$ & EMPTY & 2.0410 & 2.0459 & 2.0427 & $2.3365 e-03$ & $0.0000 e+00$ \\
\hline 77 & EMPTY & 2.0557 & 2.0605 & 2.0558 & $6.9053 e-04$ & $0.0000 e+00$ \\
\hline 78 & EMPTY & 2.0703 & 2.0801 & 2.0705 & $1.3811 \mathrm{e}-03$ & $0.0000 e+00$ \\
\hline 79 & EMPTY & 2.0850 & 2.0898 & 2.0854 & $1.4797 e-03$ & $0.0000 e+00$ \\
\hline 80 & PANO20136 & -17.5387 & -17.2335 & -17.4288 & $7.0903 e-02$ & $8.7109 e-01$ \\
\hline 81 & PAN050136 & -33.3736 & -32.7633 & -33.1265 & $1.5380 e-01$ & $1.6820 \epsilon+00$ \\
\hline 82 & PAN070136 & -22.8233 & -15.5754 & -21.7979 & $1.4153 e+00$ & $8.7171 \mathrm{e}-01$ \\
\hline 83 & PAN080136 & -13.9558 & -8.9204 & -10.0694 & $9.6805 e-01$ & $8.7036 e-01$ \\
\hline 34 & PANO 90136 & -11.8783 & -3.7911 & -10.4913 & $2.0069 e+00$ & $8.7040 e-01$ \\
\hline 85 & PAN 100136 & -12.2670 & -9.1390 & -10.1979 & $1.0018 e+00$ & $8.7037 e-01$ \\
\hline 86 & PAN 105136 & -5.8709 & -4.5739 & -5.0348 & $2.3272 e-01$ & $8.7009 e-01$ \\
\hline 87 & PAN 110136 & -6.0777 & -4.3230 & -5.0218 & $3.3535 e-01$ & $8.7009 e-01$ \\
\hline 58 & PAN 115136 & -5.6523 & -3.5924 & -5.0740 & $3.2990 e-01$ & $8.7009 e-01$ \\
\hline 59 & PAN120136 & -5.0698 & -4.3068 & -4.7249 & $1.5509 \mathrm{e}-01$ & $8.7008 e-01$ \\
\hline 90 & PAN125136 & -4.9642 & -4.2775 & -4.6880 & $1.1828 e-01$ & $8.7008 e-01$ \\
\hline 91 & PAN 130136 & -5.3881 & -5.0066 & -5.2233 & $8.2084 e-02$ & $8.7010 e-01$ \\
\hline 92 & PAN120048 & -11.1138 & -9.8168 & -10.5554 & $1.9585 e-01$ & $8.7040 e-01$ \\
\hline 93 & PAN 130048 & -9.8945 & -9.4368 & -9.6931 & $1.0312 e-01$ & $8.7034 \theta-01$ \\
\hline 94 & PAN120224 & -9.5425 & -8.9322 & -9.2740 & $1.5661 e-01$ & $8.7031 e-01$ \\
\hline 95 & PAN130224 & -10.3772 & -9.9958 & -10.2063 & $8.3803 e-02$ & $8.7037 e-01$ \\
\hline 96 & PAN 120312 & -10.3685 & -8.8426 & -9.7657 & $3.3211 e-01$ & $8.7034 e-01$ \\
\hline 97 & PAN 130312 & -10.2174 & -9.3782 & -9.9382 & $1.3224 \theta-01$ & $8.7035 e-01$ \\
\hline 98 & PAB000136 & 40.4112 & 40.5943 & 40.5100 & $4.5645 e-02$ & $5.8878 e-01$ \\
\hline 99 & PAB100136 & 43.9386 & 44.1217 & 44.0228 & $4.6573 e-02$ & $5.9035 e-01$ \\
\hline 100 & PAB125136 & 44.8946 & 45.0320 & 44.9697 & $4.2138 e-02$ & $5.9080 e-01$ \\
\hline
\end{tabular}




\begin{tabular}{|c|c|c|c|c|c|c|}
\hline \multicolumn{2}{|c|}{ Test: Aug16_06 } & suml & Date: Thu & Aug 162 & 03 & Page: 3 \\
\hline 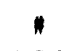 & Name & Min & $\operatorname{Max}$ & Ave & SD & Uncert \\
\hline 101 & PINO00001 & 40.2028 & 40.3249 & 40.2492 & $5.9853 e-02$ & $7.56720-01$ \\
\hline 102 & PRV000001 & 19.4744 & 19.8406 & 19.6893 & $6.3165 e-02$ & $7.51610-01$ \\
\hline 103 & PSR000001 & 45.0597 & 45.3038 & 45.1720 & $4.1561 \bullet-02$ & $7.58450-01$ \\
\hline 104 & PEMO00001 & 1.5493 & 1.8240 & 1.7050 & $6.7328 e-02$ & $5.00020-01$ \\
\hline .25 & PANO00136 & -133.6226 & -131.3338 & -132.5148 & $4.4440 e-01$ & $4.93110+00$ \\
\hline 106 & PFM000002 & 1.6105 & 1.6609 & 1.6277 & $1.2451 \bullet-02$ & $2.0004 e-01$ \\
\hline 107 & EMPTY & 0.0000 & 0.0049 & 0.0047 & $9.6655 e-04$ & $0.0000 e+00$ \\
\hline 108 & EMPTY & -10.0000 & -10.0000 & -10.0000 & $0.0000+00$ & $0.0000 e+00$ \\
\hline 109 & EMPTY & 0.0000 & 0.0049 & 0.0034 & $2.2603 e-03$ & $0.00000+00$ \\
\hline .10 & EMPTY & -10.0000 & -10.0000 & -10.0000 & $0.0000 e+00$ & $0.0000 e+00$ \\
\hline 111 & EMPTY & -0.0049 & 0.0000 & -0.0001 & $6.9053 e-04$ & $0.0000 e+00$ \\
\hline$: 12$ & VPW000001 & 102.2198 & 102.6104 & 102.3877 & $1.1168 \bullet-01$ & $1.01660+00$ \\
\hline $1: 3$ & VPWO00002 & 119.2421 & 119.6327 & 119.3788 & $1.0625 e-01$ & $1.0813 e+00$ \\
\hline$: 14$ & VPWO00003 & 147.0365 & 147.4271 & 147.2475 & $1.0320 e-01$ & $1.19860+00$ \\
\hline $2: 5$ & VPWO00004 & 104.8640 & 105.6453 & 105.1805 & $1.4167 e-01$ & $1.0268 e+00$ \\
\hline 116 & VPWO00005 & 139.6522 & 140.2381 & 139.9530 & $1.7739 e-01$ & $1.1667 e+00$ \\
\hline 117 & VPWO00006 & 147.6750 & 148.0657 & 147.8820 & $1.3329 e-01$ & $1.2014 e+00$ \\
\hline$\therefore A$ & IPW000001 & 543.5024 & 549.3595 & 545.9233 & $1.0103 e+00$ & $9.1766 e+00$ \\
\hline$\therefore$ is & IPW000002 & 1304.2736 & 1310.1273 & 1307.0446 & $1.6297 \theta+00$ & $1.3124 e+01$ \\
\hline 120 & IPW000003 & 1327.9498 & 1333.7999 & 1330.9530 & $1.2588 a+00$ & $1.3273 e+01$ \\
\hline$: 21$ & IPWO00004 & 378.2395 & 380.6809 & 379.6458 & $4.1432 e-01$ & $3.6049 e+00$ \\
\hline 122 & IPWOOOOOO5 & 252.5851 & 253.5616 & 252.9854 & $3.2250 e-01$ & $2.82740+00$ \\
\hline 123 & IPWO00006 & 262.7329 & 264.1978 & 263.3187 & $3.4173 e-01$ & $2.8857 e+00$ \\
\hline 124 & EMPTY & -0.0244 & -0.0098 & -0.0155 & $3.6500 e-03$ & $0.00000+00$ \\
\hline$: 25$ & EMPTY & -0.0146 & -0.0049 & -0.0112 & $2.6562 e-03$ & $0.00000+00$ \\
\hline$: 26$ & EMPTY & -0.0195 & -0.0049 & -0.0115 & $3.5238 e-03$ & $0.0000 e+00$ \\
\hline$: .27$ & EMPTY & -0.0195 & -0.0098 & -0.0153 & $3 .: 211 e-03$ & $0.00000+00$ \\
\hline$\therefore 28$ & IHTO00001 & 128.5231 & 130.8669 & 129.7731 & $5.1448 e-01$ & $4.3005 e+00$ \\
\hline 129 & IHTO000002 & 132.1815 & 134.5252 & 133.5135 & $4.8116 e-01$ & $4.3119 e+00$ \\
\hline$: 30$ & IHTO00003 & 132.5045 & 134.6530 & 133.7505 & $4.9895 e-01$ & $4.3126 e+00$ \\
\hline$\therefore$ & IHTO00004 & 130.6002 & 133.3346 & 131.8853 & $5.4117 e-01$ & $4.3069 e+00$ \\
\hline$\therefore 3$ & IHTO00005 & 129.2668 & 131.4153 & 130.2316 & $4.5917 e-01$ & $4.3019 e+00$ \\
\hline$: 33$ & IHT000006 & 123.9971 & 126.1456 & 125.0909 & $4.9284 \theta-01$ & $4.2866 e+00$ \\
\hline$: 34$ & IHT000007 & 130.9946 & 133.3384 & 132.1509 & $5.0662 e-01$ & $4.3077 e+00$ \\
\hline 135 & IHTO00008 & 129.7701 & 131.7233 & 130.7349 & $4.4888 \theta-01$ & $4.3034 e+00$ \\
\hline 136 & IHTO00009 & 130.1495 & 132.4932 & 131.1924 & $5.2735 e-01$ & $4.3048 \theta+00$ \\
\hline 137 & IHTO00010 & -40.2982 & -38.1498 & -39.1498 & $5.6171 e-01$ & $4.1186 \theta+00$ \\
\hline$\lcm{38}$ & IHTO00011 & 130.1119 & 132.2603 & 131.2212 & $5.6557 e-01$ & $4.30490+00$ \\
\hline 239 & IHT000012 & 127.9222 & 130.0706 & 128.9730 & $5.2332 e-01$ & $4.2981 e+00$ \\
\hline$\therefore$ is & IHT000013 & 91.0156 & 92.9688 & 92.1211 & $4.7287 e-01$ & $4.2022 \theta+00$ \\
\hline$: 41$ & IHTO00014 & 127.1710 & 129.7101 & 128.3467 & $5.09150-01$ & 4. $2962 e+00$ \\
\hline 1.42 & IHTO000015 & 94.6214 & 96.9651 & 95.8362 & $5.23910-01$ & $4.2105 e+00$ \\
\hline 143 & IHT000016 & 88.3263 & 90.6701 & 89.4826 & $4.8942 \theta-01$ & $4.1965 e+00$ \\
\hline$\therefore 44$ & IHTO00017 & 106.2425 & 108.3909 & 107.3089 & $5.8555 \theta-01$ & $4.23810+00$ \\
\hline$\therefore 45$ & IHTOOOOOI8 & 107.3543 & 109.8933 & 108.2684 & $6.2694 \theta-01$ & $4.2405 e+00$ \\
\hline 146 & IHTO00019 & 103.4781 & 105.8218 & 104.5991 & $5.4649 e-01$ & $4.2313 e+00$ \\
\hline 147 & IHTOOOOO20 & 106.1974 & 108.7365 & 107.2794 & $5.5972 e-01$ & $4.23800+00$ \\
\hline 148 & IHTO00021 & -2.4226 & 0.5071 & -1.2195 & $6.43990-01$ & $4.10000+00$ \\
\hline 149 & IHTO00022 & 92.2739 & 94.8130 & 93.3169 & $5.22900-01$ & $4.2049 e+00$ \\
\hline 150 & IHTOOOO23 & 104.1692 & 106.3176 & 105.2551 & $5.3693 \theta-01$ & $4.2329 e+00$ \\
\hline 151 & IHTO00024 & 69.3773 & 71.7210 & 70.4280 & $5.0976 e-01$ & $4.1600 e+00$ \\
\hline 152 & IHTO00025 & 104.5485 & 106.8923 & 105.3805 & $5.2469 e-01$ & $4.2333 e+00$ \\
\hline$: 53$ & IHTO00026 & 105.1307 & 107.6698 & 106.1463 & $5.7589 e-01$ & $4.2352 e+00$ \\
\hline 154 & IHTO00027 & 89.7987 & 92.1424 & 90.9119 & $5.2237 \theta-01$ & $4.1996 \theta+00$ \\
\hline 155 & IHTO00028 & 91.1396 & 93.8739 & 92.4716 & $5.7003 \theta-01$ & $4.20300+00$ \\
\hline
\end{tabular}




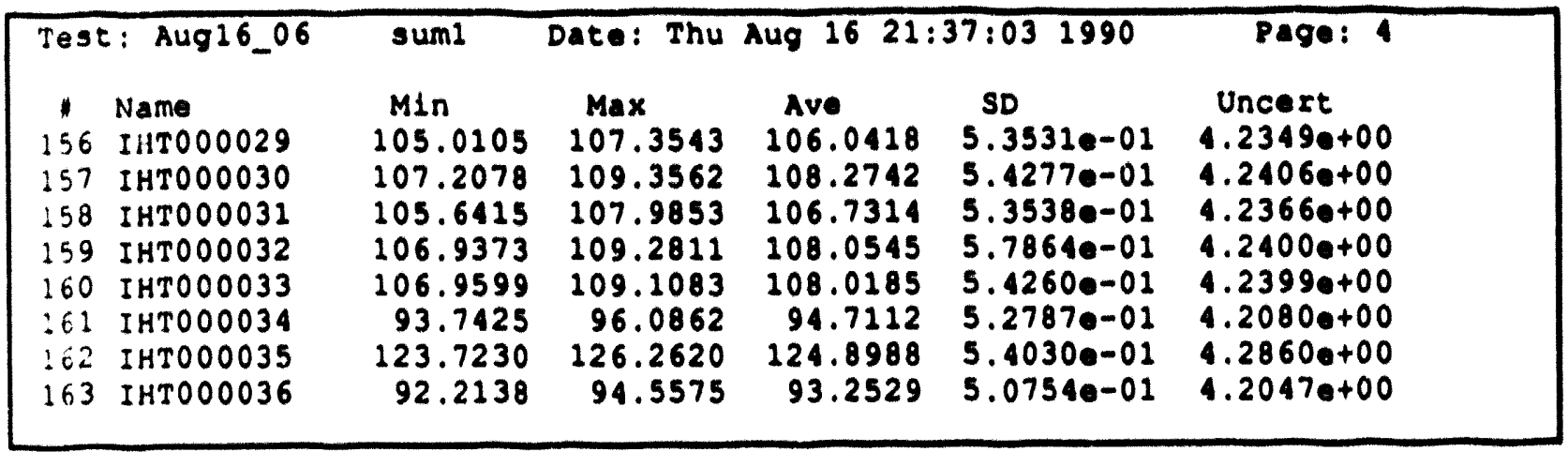




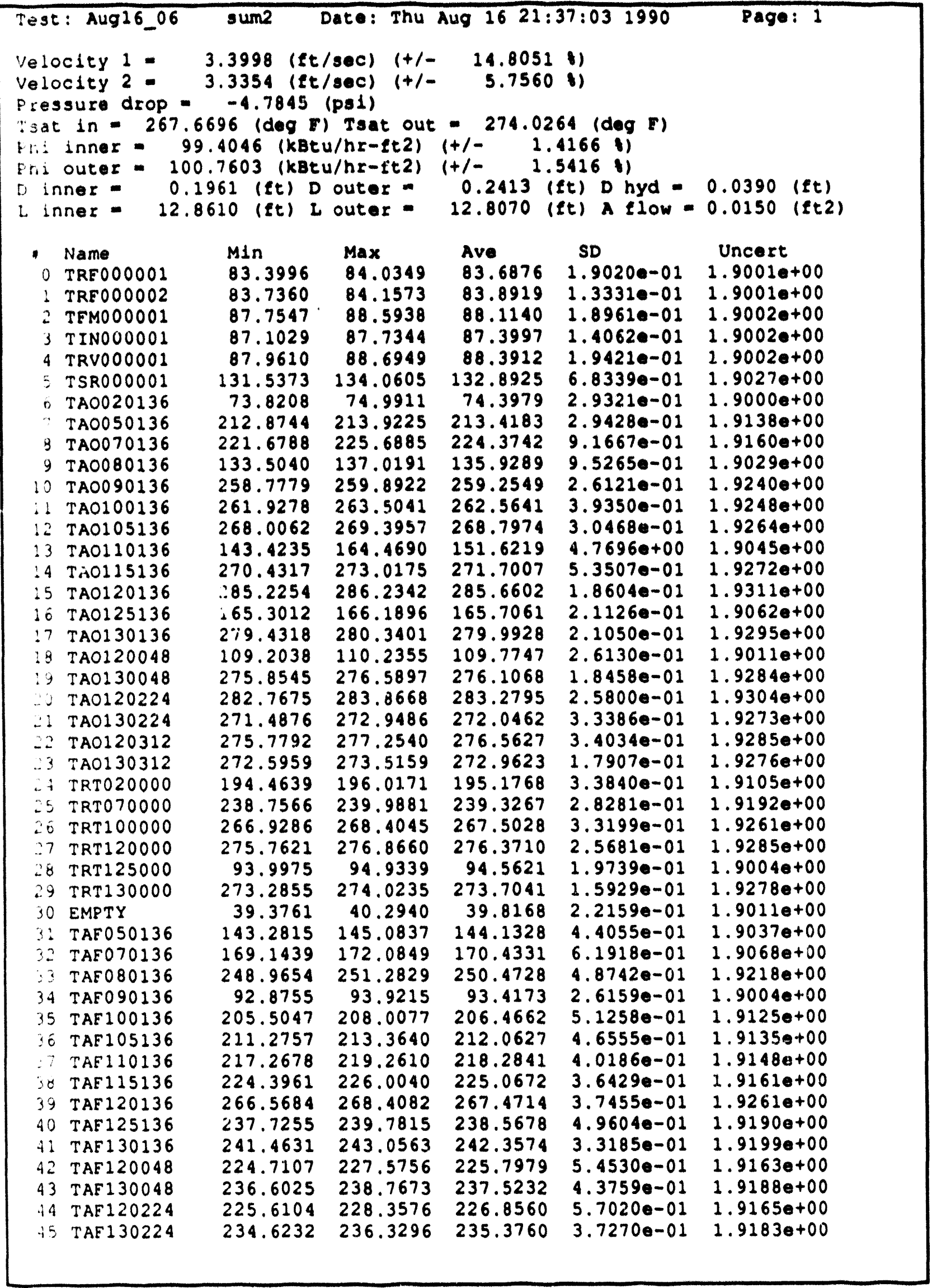




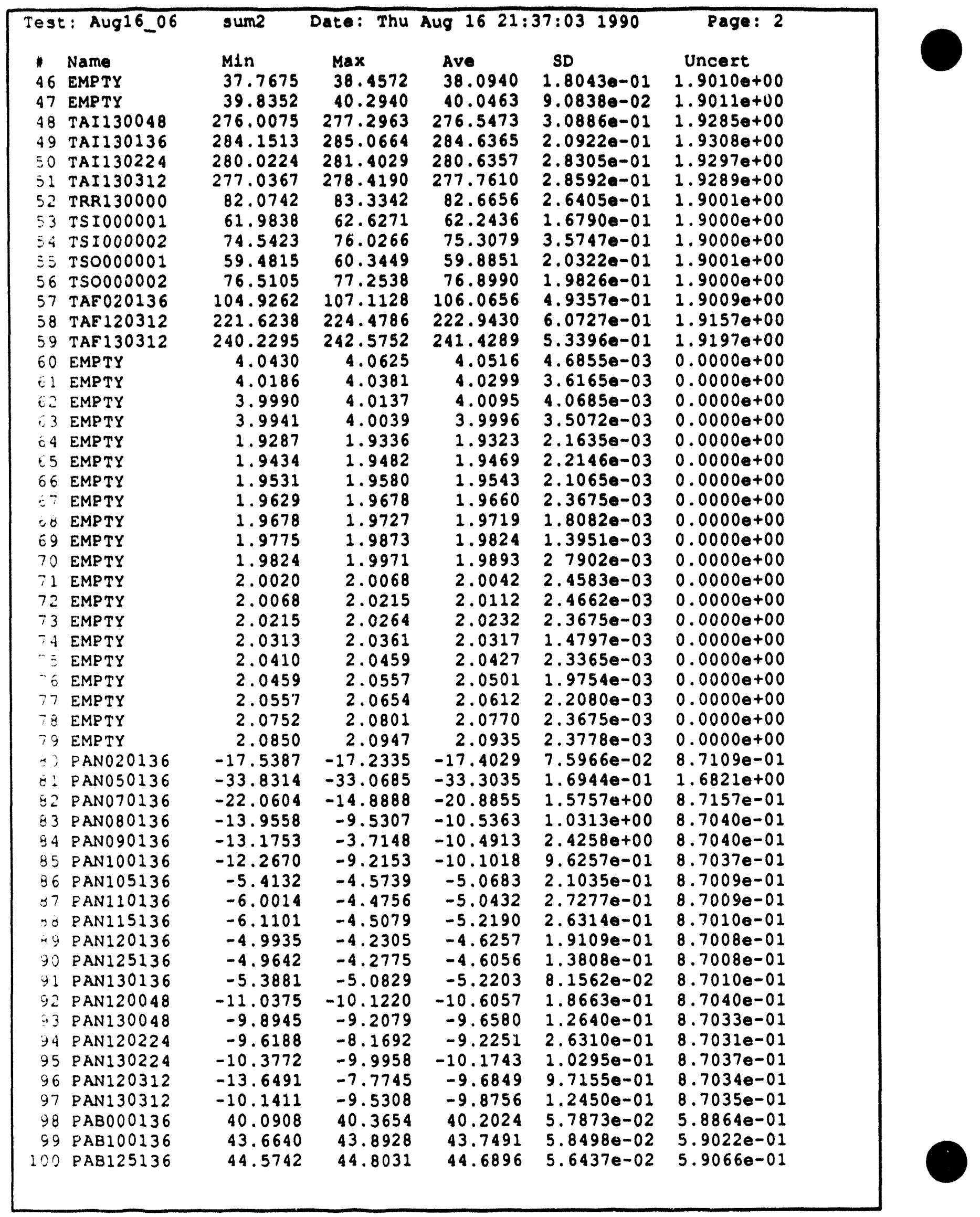




\begin{tabular}{|c|c|c|c|c|c|c|}
\hline \multicolumn{2}{|c|}{ Test: Aug16_06 } & & \multicolumn{3}{|c|}{ Date: Thu Aug $16 \quad 21: 37: 03 \quad 1990$} & \multirow{2}{*}{ Page: 3} \\
\hline$\#$ & Name & Min & $\operatorname{Max}$ & Ave & SD & \\
\hline 101 & PINO00001 & 39.8366 & 40.0808 & 39.9172 & $7.6452 e-02$ & $7.5661 e-01$ \\
\hline 102 & PRV000001 & 19.7186 & 19.8406 & 19.7259 & $2.9284 e-02$ & $7.5162 e-01$ \\
\hline 103 & PSRO00001 & 44.6935 & 44.9376 & 44.8619 & $7.7558 e-02$ & $7.5834 \theta-01$ \\
\hline 104 & PFMO000001 & 1.5493 & 1.8240 & 1.6995 & $7.5723 e-02$ & $5.0002 e-01$ \\
\hline 105 & PAN000136 & -133.6226 & -131.3338 & -132.4599 & $5.2487 \theta-01$ & $4.93110+00$ \\
\hline 106 & PEMO00002 & 1.5938 & 1.6777 & 1.6340 & $1.5535 e-02$ & $2.0004 e-01$ \\
\hline 107 & EMPTY & 0.0000 & 0.0049 & 0.0048 & $6.9053 e-04$ & $0.00000+00$ \\
\hline 108 & EMPTY & -10.0000 & -10.0000 & -10.0000 & $0.0000 e+00$ & $0.0000 e+00$ \\
\hline 109 & EMPTY & 0.0000 & 0.0049 & 0.0034 & $2.2603 \theta-03$ & $0.0000 e+00$ \\
\hline 110 & EMPTY & -10.0000 & -10.0000 & -10.0000 & $0.00000+00$ & $0.0000 e+00$ \\
\hline 111 & EMPTY & 0.0000 & 0.0000 & 0.0000 & $0.0000 e+00$ & $0.0000 e+00$ \\
\hline 112 & VPWO00001 & 102.2198 & 102.4151 & 102.2979 & $9.6655 e-02$ & $1.0163 e+00$ \\
\hline $1: 3$ & VPW000002 & 119.2421 & 119.6327 & 119.3553 & $1.1223 e-01$ & $1.0812 e+00$ \\
\hline 114 & VPW000003 & 147.0365 & 147.4271 & 147.2358 & $1.0799 e-01$ & $1.1985 e+00$ \\
\hline $\begin{array}{l}115 \\
116\end{array}$ & VPWO00004 & 105.0593 & 105.4500 & 105.2234 & $9.9512 e-02$ & $1.0270 e+00$ \\
\hline $\begin{array}{l}116 \\
117\end{array}$ & VPWO00005 & 139.6522 & 140.0428 & 139.8905 & $1.2658 \mathrm{e}-01$ & $1.1664 \mathrm{e}+00$ \\
\hline $\begin{array}{l}117 \\
118\end{array}$ & VPWO00006 & 147.2844 & 147.8703 & 147.5695 & $1.4342 \mathrm{e}-01$ & $1.2000 e+00$ \\
\hline $\begin{array}{l}118 \\
119\end{array}$ & $\begin{array}{l}\text { IPWO } 00001 \\
\text { IPWO000002 }\end{array}$ & 564.9782 & 570.8353 & 569.0781 & $1.3230 \mathrm{e}+00$ & $9.2640 \mathrm{e}+00$ \\
\hline 120 & $\begin{array}{l}\text { IPWO00002 } \\
\text { IPWO00003 }\end{array}$ & $\begin{array}{l}1275.0045 \\
1302.5999\end{array}$ & 1282.8096 & 1278.9458 & $\begin{array}{l}1.5006 e+00 \\
1.5758 e+00\end{array}$ & $1.2950 e+01$ \\
\hline 121 & IPWO00004 & $\begin{array}{r}1302.5999 \\
383.6106\end{array}$ & $\begin{array}{r}1310.3999 \\
385.0755\end{array}$ & $\begin{array}{r}1306.4998 \\
384.4310\end{array}$ & $3.6165 e-01$ & $\begin{array}{l}1.3120 e+01 \\
3.6364 e+00\end{array}$ \\
\hline 122 & IPWO 00005 & 252.5851 & 253.5616 & 252.9756 & $2.4161 e-01$ & $2.8274 \mathrm{e}+00$ \\
\hline 123 & IPW000006 & 259.3149 & 261.2681 & 260.6917 & $3.5141 e-01$ & $2.8708 e+00$ \\
\hline 124 & EMPTY & -0.0244 & -0.0098 & -0.0182 & $3.9508 e-03$ & $0.0000 e+00$ \\
\hline 125 & EMPTY & -0.0195 & -0.0049 & -0.0116 & $2.7709 e-03$ & $0.0000 e+00$ \\
\hline 126 & EMPTY & -0.0244 & -0.0049 & -0.0125 & $3.9656 e-03$ & $0.0000 e+00$ \\
\hline 127 & EMPTY & -0.0244 & -0.0098 & -0.0147 & $3.6232 e-03$ & $0.0000 e+00$ \\
\hline 128 & IHTO00001 & 128.7184 & 131.0622 & 129.7301 & $5.9016 e-01$ & $4.3003 e+00$ \\
\hline 129 & IHTO00002 & 132.3768 & 134.9158 & 133.4823 & $5.7536 e-01$ & $4.3118 e+00$ \\
\hline$: 30$ & IHTO00003 & 132.5045 & 135.0436 & 133.7115 & $5.4203 e-01$ & $4.3125 e+00$ \\
\hline 131 & IHTOOOOOO4 & 130.6002 & 132.9440 & 131.8736 & $5.4138 e-01$ & $4.3069 e+00$ \\
\hline$\div ; 2$ & IHTO00005 & 129.0715 & 131.4153 & 130.1847 & $5.1637 e-01$ & $4.3017 e+00$ \\
\hline 133 & IHTO00006 & 123.8018 & 126.3409 & 125.0635 & $5.1024 e-01$ & $4.2865 e+00$ \\
\hline 134 & IHT000007 & 130.7993 & 133.5337 & 132.0884 & $5.0223 e-01$ & 4. $3075 e+00$ \\
\hline 135 & IHTO00008 & 129.3795 & 131.7233 & 130.6529 & $4.7554 e-01$ & $4.3031 e+00$ \\
\hline$: 36$ & IHTO00009 & 129.9542 & 132.2979 & 131.1963 & $5.1864 e-01$ & $4.3048 e+00$ \\
\hline 137 & IHTO00010 & -40.1029 & -37.5639 & -39.1263 & $5.6082 e-01$ & $4.1186 e+00$ \\
\hline 138 & IHTO00011 & 129.9166 & 132.2603 & 130.9673 & $5.2481 e-01$ & $4.3041 e+00$ \\
\hline 139 & IHTO00012 & 127.7269 & 129.8753 & 128.7152 & $4.9820 e-01$ & $4.2973 e+00$ \\
\hline 140 & IHT000013 & 91.2109 & 93.1641 & 92.1797 & $4.4110 e-01$ & $4.2023 e+00$ \\
\hline 141 & IHTO00014 & 127.3663 & 129.3194 & 128.3389 & $4.5075 e-01$ & $4.2962 e+00$ \\
\hline 242 & IHTO00015 & 95.0120 & 96.7698 & 95.8909 & $4.7227 e-01$ & $4.2106 e+00$ \\
\hline 143 & IHTO00016 & 88.3263 & 90.4747 & 89.4943 & $4.8083 e-01$ & $4.1965 e+00$ \\
\hline 244 & IHTO00017 & 105.2659 & 108.3909 & 107.0003 & $7.7889 e-01$ & $4.2373 e+00$ \\
\hline$: 45$ & IHTO00018 & 106.7683 & 109.6980 & 108.2528 & $6.4958 e-01$ & $4.2405 e+00$ \\
\hline 146 & IHTO00019 & 103.4781 & 105.8218 & 104.5991 & $5.1268 e-01$ & $4.2313 e+00$ \\
\hline 147 & IHTO00020 & 106.1974 & 108.5412 & 107.2091 & $5.1703 e-01$ & $4.2379 e+00$ \\
\hline 148 & IHT 000021 & -2.4226 & 0.3118 & -1.2781 & $6.2516 e-01$ & $4.1000 e+00$ \\
\hline 149 & IHTO00022 & 92.2739 & 94.4223 & 93.2309 & $5.0724 e-01$ & $4.2047 e+00$ \\
\hline 150 & IHTO00023 & 104.1692 & 106.5129 & 105.1613 & $5.2176 e-01$ & $4.2327 e+00$ \\
\hline 151 & IHTO00024 & 69.1820 & 71.7210 & 70.3577 & $4.7431 e-01$ & $4.1599 \mathrm{e}+00$ \\
\hline 152 & IHTO00025 & 104.1579 & 106.6970 & 105.3454 & $5.1273 e-01$ & $4.2332 e+00$ \\
\hline$: 53$ & IHT000026 & 104.7401 & 107.6698 & 106.0448 & $5.1515 e-01$ & $4.2349 e+00$ \\
\hline 154 & IHT 000027 & 89.7987 & 92.5330 & 90.8612 & $5.3403 e-01$ & $4.1 .995 e+00$ \\
\hline 155 & IHTO00028 & 91.1396 & 94.4599 & 92.4326 & $6.3121 e-01$ & $4.2029 e+00$ \\
\hline
\end{tabular}




\begin{tabular}{|c|c|c|c|c|c|c|}
\hline \multicolumn{2}{|c|}{ Test: Aug16_06 } & sum2 & Date: Thu & ug 162 & $7: 031990$ & Page: 4 \\
\hline$\#$ & Name & Min & $\operatorname{Max}$ & Ave & SD & Uncert \\
\hline 156 & IHTO00029 & 104.8152 & 108.1355 & 106.0223 & $6.2352 e-01$ & $4.2349 e+00$ \\
\hline 157 & IHTO00030 & 107.2078 & 110.3328 & 108.2117 & $6.1385 e-01$ & $4.2404 \theta+00$ \\
\hline 158 & IHT000031 & 105.6415 & 109.1572 & 106.6845 & $6.0696 e-01$ & $4.2365 e+00$ \\
\hline 159 & IHT000032 & 106.9373 & 110.2576 & 108.0428 & $6.0568 e-01$ & $4.2400 e+00$ \\
\hline 160 & IHTO00033 & 106.9599 & 110.0849 & 107.9756 & $5.9189 e-01$ & $4.2398 e+00$ \\
\hline 161 & IHT000034 & 93.7425 & 95.8909 & 94.7268 & $4.6515 e-01$ & $4.2080 e+00$ \\
\hline 162 & IHT000035 & 123.9183 & 126.0667 & 124.8480 & $4.5942 e-01$ & $4.2859 e+00$ \\
\hline 163 & IHT000036 & 92.2138 & 94.3622 & 349 & 4.56 & $4.2049 e+00$ \\
\hline
\end{tabular}




\begin{tabular}{|c|c|c|c|}
\hline \multicolumn{4}{|c|}{$\begin{array}{l}\text { Velocity } 1=0.005300(\mathrm{ft} / \mathrm{sec}) \\
\text { Velocity } 2=0.006400 \text { (ft/sec) } \\
\text { Pressure Drop }=0.001900 \text { (psi) } \\
\text { Tsat in }=0.454926 \text { (deg F) Tsat out }=0.378906 \text { (deg F) } \\
\text { Phi inner }=1.759499 \text { (kBtu/hr-ft2) Phi outer }=0.197502 \text { (kBtu/hr-ft2) }\end{array}$} \\
\hline \# & Name & Abs Diff & 8Diff \\
\hline 0 & TRF 000001 & 0.1440 & 0.1724 \\
\hline 1 & TRF 000002 & 0.0590 & 0.0704 \\
\hline 2 & TEMO00001 & 0.1682 & 0.1913 \\
\hline 3 & TINO00001 & 0.2191 & 0.2513 \\
\hline 4 & TRV000001 & 0.2976 & 0.3378 \\
\hline 5 & TSR000001 & 1.6679 & 1.2710 \\
\hline 6 & TA0020136 & 0.7152 & 0.9707 \\
\hline 7 & TAO050136 & 0.3146 & 0.1472 \\
\hline 8 & TA0070136 & 1.0648 & 0.4723 \\
\hline 9 & TAO080136 & 1.7893 & 1.3339 \\
\hline 10 & TAO090136 & 0.3419 & 0.1321 \\
\hline 11 & TAO100136 & 0.3229 & 0.1231 \\
\hline 12 & TAO105136 & 0.0743 & 0.0276 \\
\hline 13 & TA0110136 & 48.8047 & 24.3504 \\
\hline 14 & TAO115136 & 0.3689 & 0.1356 \\
\hline 15 & TAO120136 & 0.0333 & 0.0117 \\
\hline 16 & TA0125136 & 0.7267 & 0.4405 \\
\hline 17 & TA0130136 & 0.1320 & 0.0472 \\
\hline 18 & TAO120048 & 0.3994 & 0.3625 \\
\hline 19 & TAO130048 & 0.0923 & 0.0334 \\
\hline 20 & TAO120224 & 0.1213 & 0.0428 \\
\hline 21 & TAO130224 & 0.1840 & 0.0677 \\
\hline 22 & TA0120312 & 0.1514 & 0.0548 \\
\hline 23 & TAO130312 & 0.2948 & 0.1081 \\
\hline 24 & TRT020000 & 0.0736 & 0.0377 \\
\hline 25 & TRT070000 & 0.4432 & 0.1855 \\
\hline 26 & TRT 100000 & 0.1113 & 0.0416 \\
\hline 27 & TRTI2C000 & 0.3902 & 0.1414 \\
\hline 28 & TRT 125000 & 1.5996 & 1.6634 \\
\hline 29 & TRT 130000 & 0.1587 & 0.0580 \\
\hline 30 & EMPTY & $0: 13$ & 0.1038 \\
\hline 31 & TAF050136 & $0 .>612$ & 0.3909 \\
\hline 32 & TAF 070136 & 0.6438 & 0.3792 \\
\hline 33 & TAF 080136 & 0.5390 & 0.2157 \\
\hline 34 & TAF090136 & 0.9844 & 1.0428 \\
\hline 35 & TAF 100136 & 0.2736 & 0.1327 \\
\hline 36 & TAF 105136 & 0.2936 & 0.1386 \\
\hline 37 & TAF 110136 & 0.1832 & 0.0840 \\
\hline 38 & TAF 115136 & 0.2392 & 0.1064 \\
\hline 39 & TAF 120136 & 0.0708 & 0.0265 \\
\hline 40 & TAF 125136 & 0.4118 & 0.1729 \\
\hline 41 & TAF 130136 & 0.4053 & 0.1675 \\
\hline 42 & TAF 120048 & 0.1602 & 0.0710 \\
\hline 43 & TAF 130048 & 0.2677 & 0.1128 \\
\hline 44 & TAF 120224 & 0.3793 & 0.1675 \\
\hline 45 & TAF 130224 & 0.2693 & 0.1145 \\
\hline
\end{tabular}




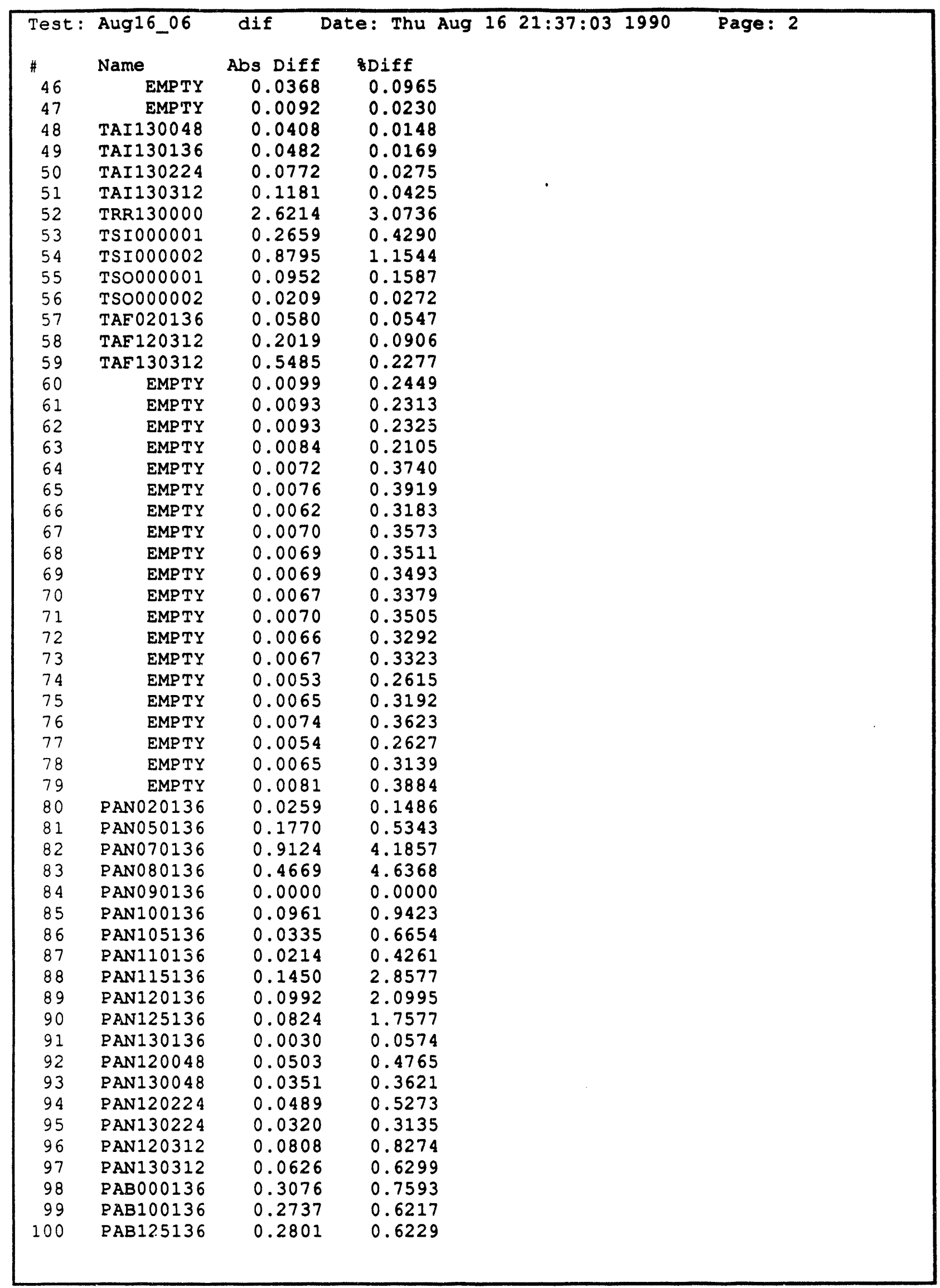




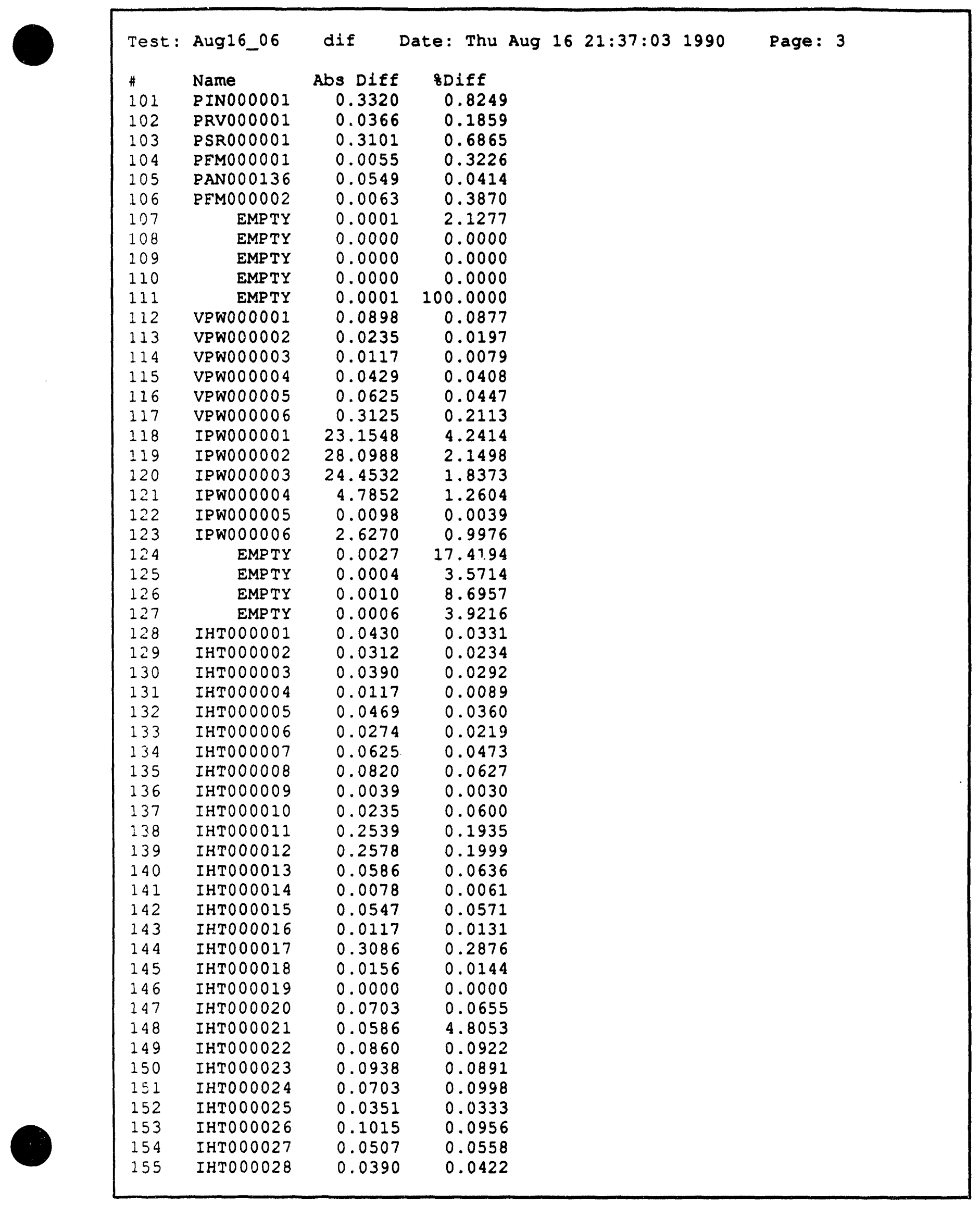


Test: Aug16_06

\# Name

156 IHTOOOO29

157 IHT000030

158 IHT000031

159 IHT000032

160 IHT000033

161 IHT000034

162 IHTOOO035

163 IHT000036 dif Date: Thu Aug 16 21:37:03 1990 Page: 4

$\begin{array}{rr}\text { Abs Diff } & \text { कDiff } \\ 0.0195 & 0.0184 \\ 0.0625 & 0.0577 \\ 0.0469 & 0.0439 \\ 0.0117 & 0.0108 \\ 0.0429 & 0.0397 \\ 0.0156 & 0.0165 \\ 0.0508 & 0.0407 \\ 0.0820 & 0.0879\end{array}$




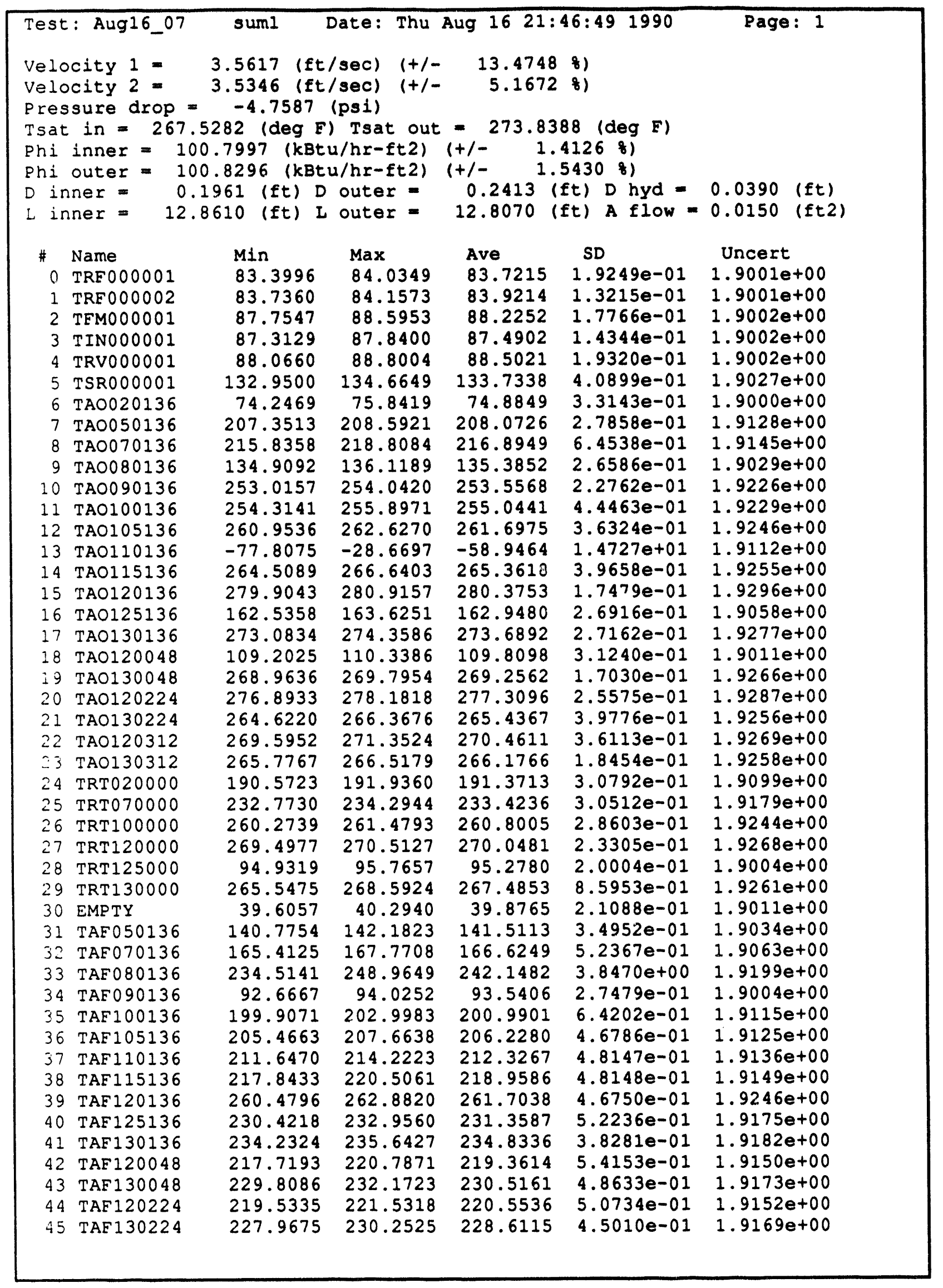




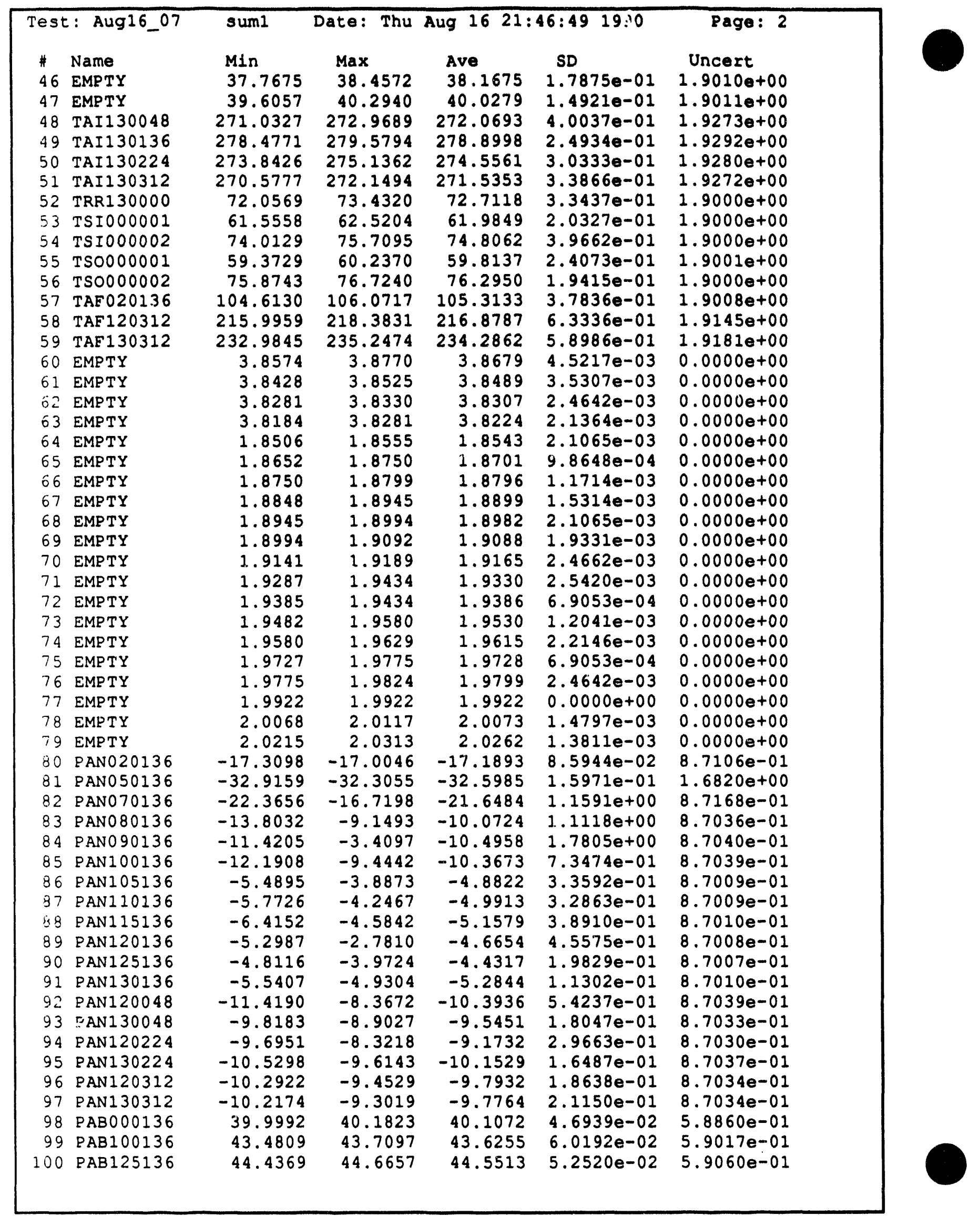




\begin{tabular}{|c|c|c|c|c|c|c|}
\hline \multicolumn{2}{|c|}{ Test: Aug16_07 } & sum1 & Date: Thu & Aug 16 & $: 491990$ & Page: \\
\hline \# & Name & Min & $\operatorname{Max}$ & Ave & SD & 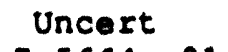 \\
\hline 101 & PIN000001 & 39.7146 & 40.0808 & 39.9978 & $9.3715 e-02$ & $7.5664 e-01$ \\
\hline 102 & PRV000001 & 19.5965 & 3406 & 19.7283 & $4.15610-02$ & $7.51620-01$ \\
\hline 103 & PSR000001 & 44.6935 & 3156 & 44.7643 & $6.0861 e-02$ & \\
\hline 104 & PFM000001 & 1.7324 & 2.0071 & 1.8698 & $7.22310-02$ & $5.0002 e-01$ \\
\hline 105 & PANO00136 & .32 .7071 & -130.4183 & -131.7458 & $5.0865 e-01$ & $4.93100+00$ \\
\hline 106 & PEMO00002 & 1.7951 & 1.8790 & 1.8408 & -02 & \\
\hline 107 & EMPTY & -0.0049 & 0.0049 & 0.0022 & $3.5854 e-03$ & $0.0000 e+00$ \\
\hline 108 & EMPTY & -10.0000 & -10.0000 & -10.0000 & $0.0000 e+00$ & $0.00000+00$ \\
\hline$: 09$ & EMPTY & -0.0049 & 0.0049 & 0.0021 & $2.6267 e-03$ & $0+00$ \\
\hline 110 & EMPTY & -10.0000 & -10.0000 & -10.0000 & $0.0000 e+00$ & $0.0000 e+00$ \\
\hline 111 & EMPTY & -0.0049 & 0.0000 & -0.0001 & 6.905 & $0 e+00$ \\
\hline 112 & VPWO00001 & 101.8292 & 102. & 102.3213 & $9 e-01$ & $1.0164 e+00$ \\
\hline 113 & VPWO00002 & 0467 & 119.6327 & 119.2967 & $1.0470 e-01$ & $1.0809 e+00$ \\
\hline 114 & VPWO00003 & 147.0365 & 147.4271 & 147.2007 & $1.0705 e-01$ & $1.1984 \theta+00$ \\
\hline 115 & VPWO00004 & 104.8640 & 105.6453 & 105.1492 & $1.3788 e-01$ & $1.0267 \mathrm{e}+00$ \\
\hline 116 & VPWO00005 & 139.4569 & 140.4334 & 139.9179 & $2.0790 e-01$ & $1.1666 \mathrm{e}+00$ \\
\hline 117 & VPWO000006 & 147.4797 & 148.0657 & 147.6749 & $1.6269 e-01$ & $1.2005 e+00$ \\
\hline 118 & IPWO000001 & 543.5024 & 551.3118 & 546.6262 & 1.673 & $9.1792 e+00$ \\
\hline 119 & IPWO 00002 & 1296.4685 & 1306.2249 & 1301.1121 & $2.2608 e+00$ & $1.3087 e+01$ \\
\hline 120 & IPW000003 & 1324.0499 & 1329.8999 & 1326.3119 & $1.4400 e+00$ & $1.3244 e+01$ \\
\hline 121 & IPWO000004 & 382.1458 & 383.1223 & 382.5755 & 3.06 & $2 e+00$ \\
\hline 122 & IPWO 00005 & 253.0734 & 381 & 253.8936 & 4.11 & $e+00$ \\
\hline 123 & IPW0000006 & 262.2446 & 263.7095 & 263.0062 & 4.086 & $39 e+00$ \\
\hline 124 & EMPTY & -0.0244 & -0.0098 & -0.0186 & 4.18 & $2+00$ \\
\hline 125 & EMPTY & -0.0195 & -0.0049 & -0.0122 & 3.00 & $0 e+00$ \\
\hline 126 & EMPTY & -0.0195 & -0.0049 & -0.0148 & $3.6858 e-03$ & $0.0000 e+00$ \\
\hline 127 & EMPTY & -0.0195 & -0.0098 & -0.0146 & 2.790 & $0 e+00$ \\
\hline 128 & IHTO00001 & 127.9372 & 131.6481 & 129.8239 & 6.668 & $5 e+00$ \\
\hline 129 & IHTO00002 & 131.5955 & 135.3065 & 133.5604 & 6.0799 & $1 e+00$ \\
\hline 130 & IHTO00003 & 131.9186 & 135.2389 & 133.7896 & -01 & +00 \\
\hline 131 & IHTOOOOOO4 & 130.0143 & 393 & 131.9400 & 6.36 & $1 e+00$ \\
\hline 132 & IHTO00005 & 128.4856 & 131.8059 & 130.2863 & $6.2302 e-01$ & $4.3020 e+00$ \\
\hline 133 & IHTO00006 & 123.2159 & 126.7315 & 125.1182 & 6.75 & $e+00$ \\
\hline 134 & IHTOO & 130 & 37 & 132.1274 & 01 & $6 e+00$ \\
\hline 135 & IHTO00008 & 129.1842 & 132.3092 & 130.6919 & 6.2644 & $4.3033 e+00$ \\
\hline$: 36$ & IHT 000009 & 129.9542 & 132.8839 & 131.2276 & & \\
\hline 237 & IHTOO & -40.2982 & -37.5639 & -39.0756 & 5.8101 & $6 e+00$ \\
\hline 138 & IHTO00011 & 129.9166 & 132.8463 & 131.0415 & 6.0175 & $4.3043 e+00$ \\
\hline 139 & IHTO00012 & 127.9222 & 130.2659 & 128.8597 & & $e+00$ \\
\hline 140 & IHTOS & 91.0156 & 17 & 92.2070 & 5.2 & $9 e+00$ \\
\hline 141 & IHTOOOOO14 & 127.1710 & 129.7101 & 128.3897 & 5.21 & $3 e+00$ \\
\hline 142 & IHTO00015 & 94.8167 & & 95.8675 & & $6 e+00$ \\
\hline 143 & IHTOO & 88.7169 & 90 . & 89.5334 & 4.6970 & $6 e+00$ \\
\hline 144 & IHTO00017 & 204.8753 & 109.3675 & 107.3402 & $1.0028 e+00$ & $4.2382 e+00$ \\
\hline 145 & IHTO00018 & 106.9636 & 109.8933 & 108.4207 & 6.6288 & $4.2409 e+00$ \\
\hline 146 & IHTO00019 & 103.4781 & 105.8218 & 104.7398 & $5.2676 e-01$ & $4.2317 e+00$ \\
\hline 147 & IHTOOOO20 & 106.0021 & 108.3458 & 107.2638 & 5.555 & $0 e+00$ \\
\hline 148 & IHTOOOO21 & -2.6179 & 0.1164 & -1.3093 & 6.7802 & $00 e+00$ \\
\hline 249 & IHTO00022 & 92.2739 & 94.6176 & 93.3169 & $5.4478 e-01$ & $4.2049 e+00$ \\
\hline 150 & IHTOOOO23 & 104.3645 & 106.7083 & 105.2316 & 5.00 & $9 e+00$ \\
\hline 151 & IHTOOO0024 & 69.5726 & 71.7210 & 70.4671 & $4.5693 e-01$ & $4.1601 e+00$ \\
\hline 152 & IHTO00025 & 104.3532 & 106.8923 & 105.4470 & $4.9756 e-01$ & $4.2334 e+00$ \\
\hline 153 & IHTO00026 & 104.7401 & 107.2792 & 106.1385 & $5.0588 e-01$ & $4.2352 e+00$ \\
\hline 154 & IHTO00027 & 89.408 & 91.9471 & 90.9315 & 4.702 & $4.1996 \mathrm{e}+00$ \\
\hline 155 & IHTOOOO28 & 91.3349 & 94.6552 & 92.4248 & $7.0596 \mathrm{e}-01$ & $4.2029 e+00$ \\
\hline
\end{tabular}




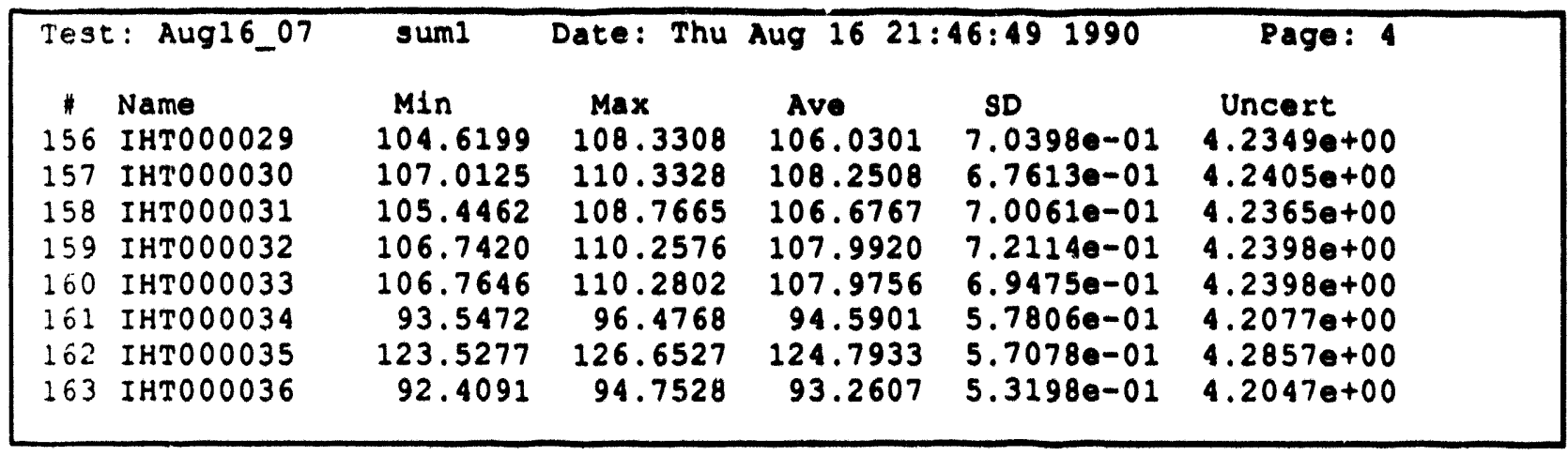




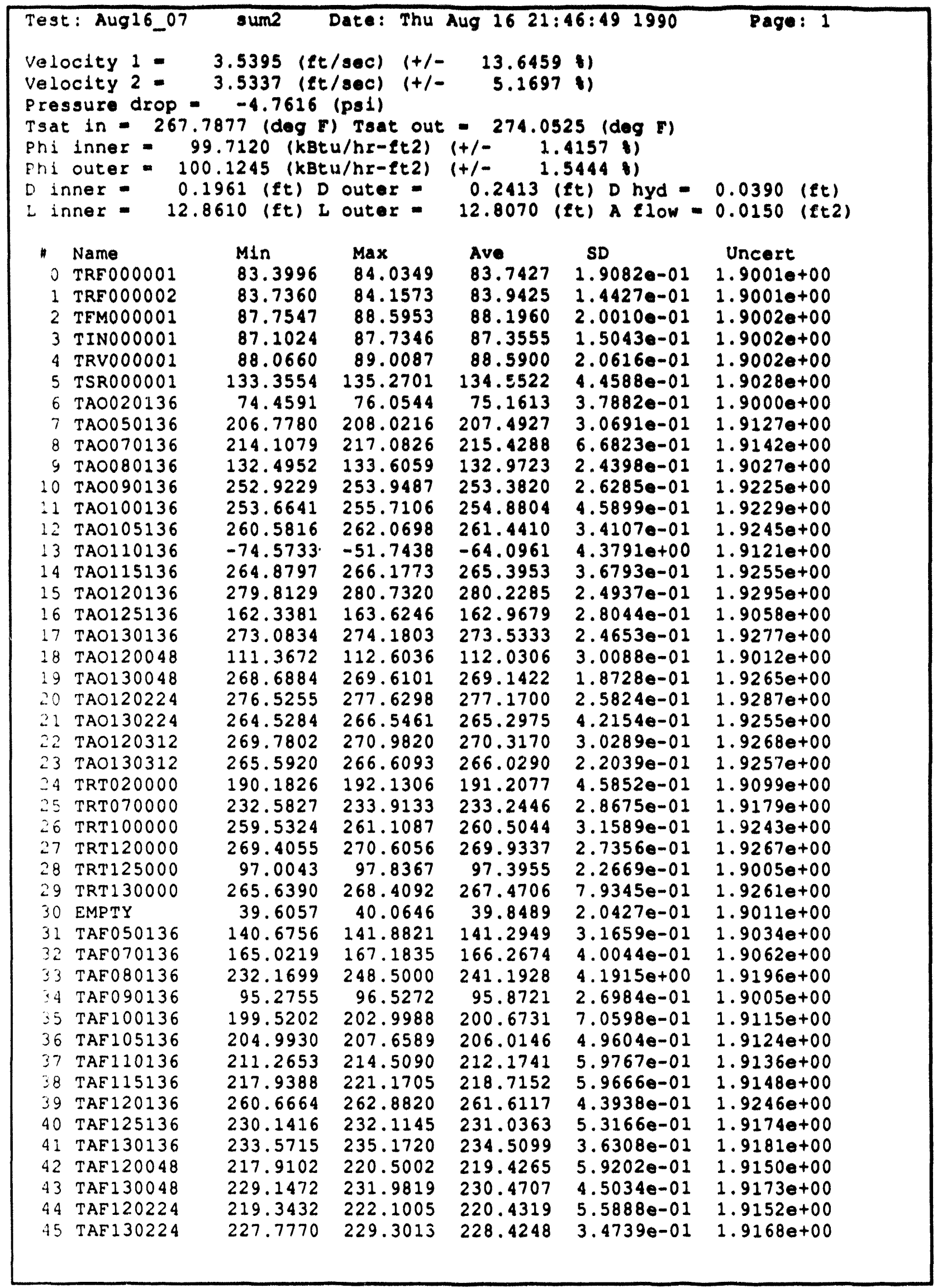




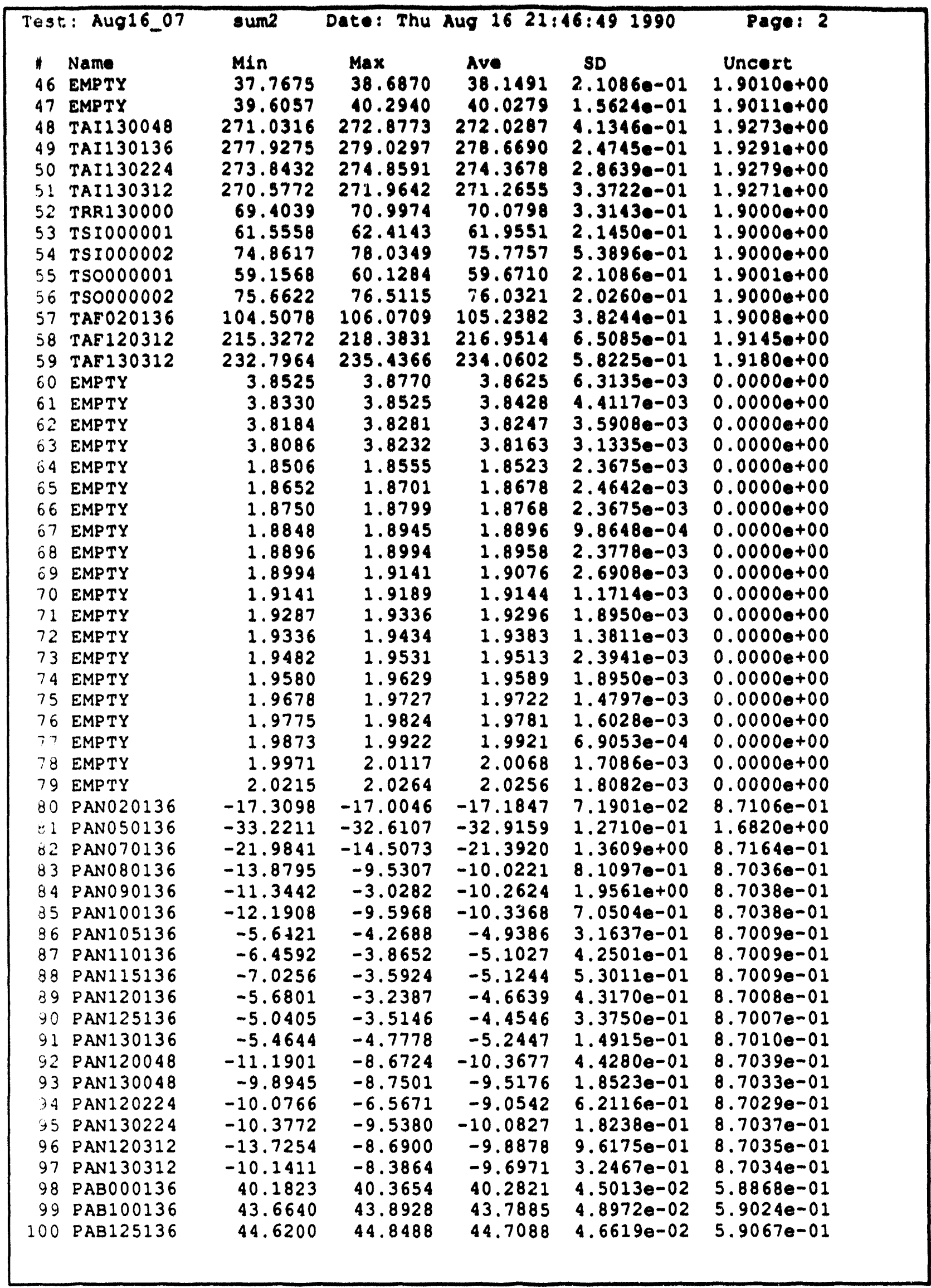




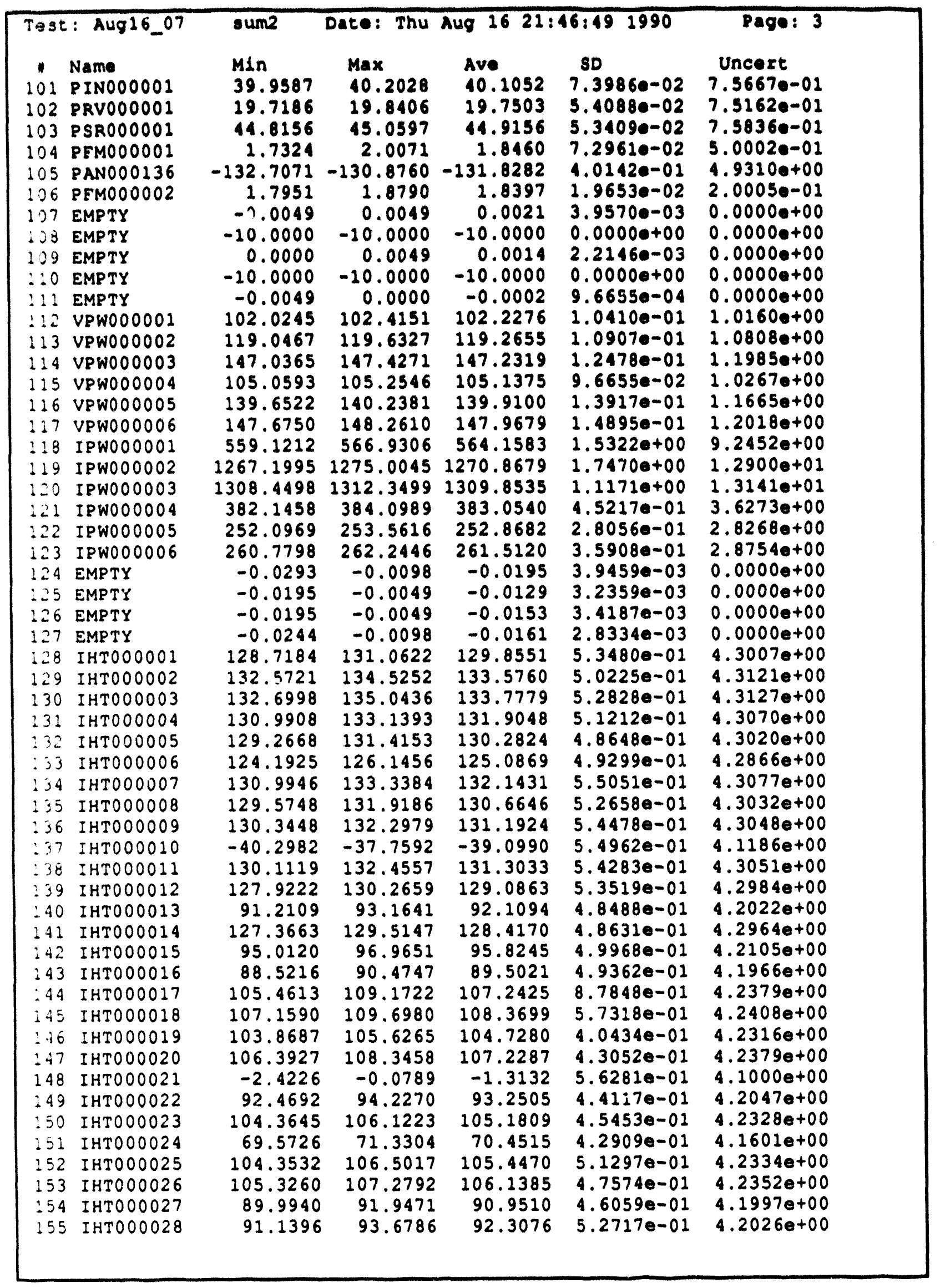




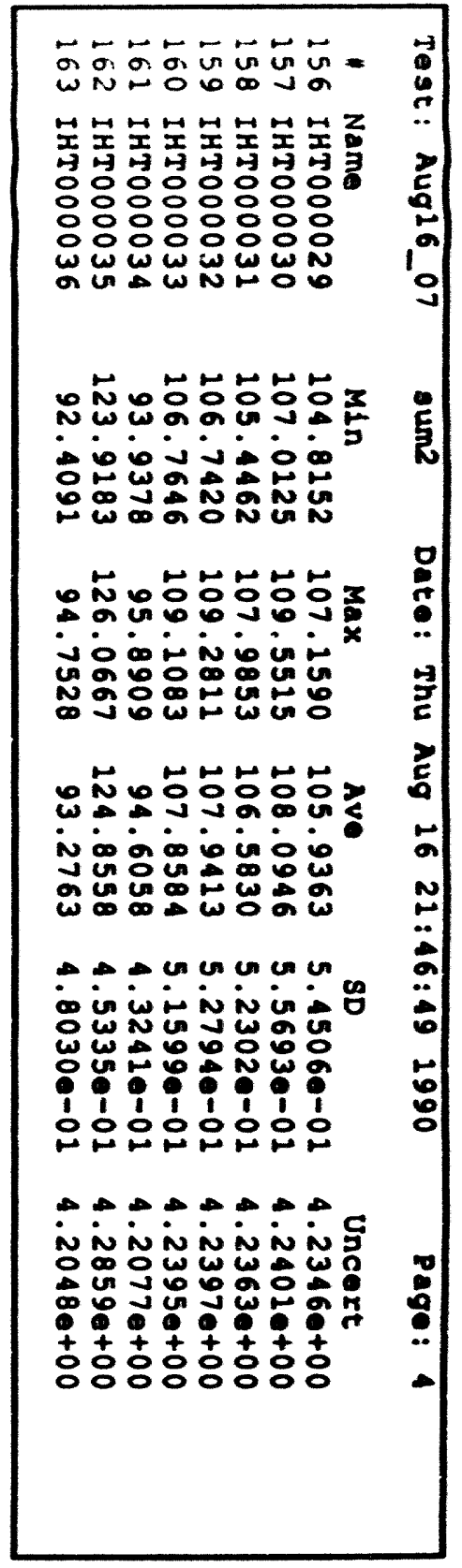




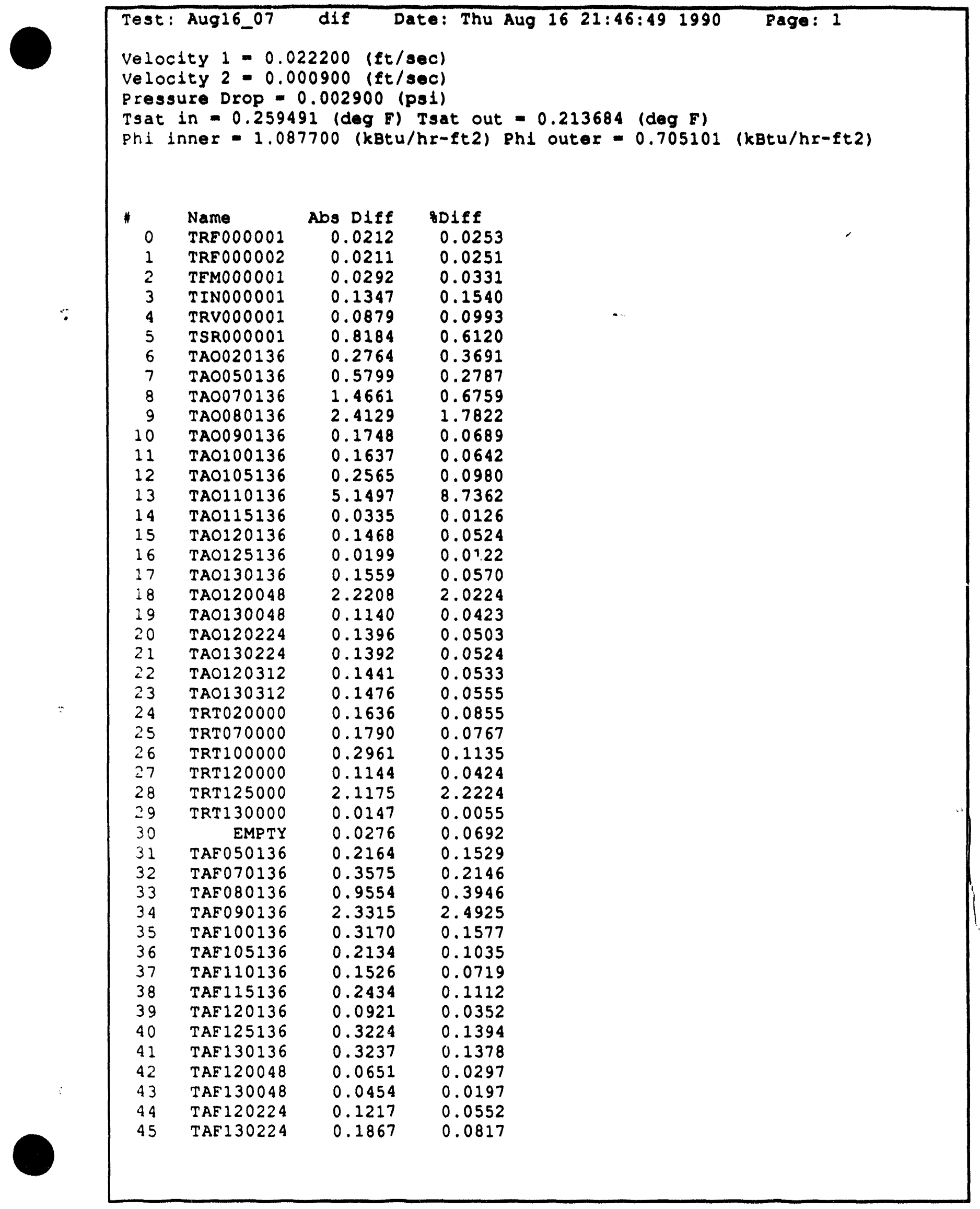




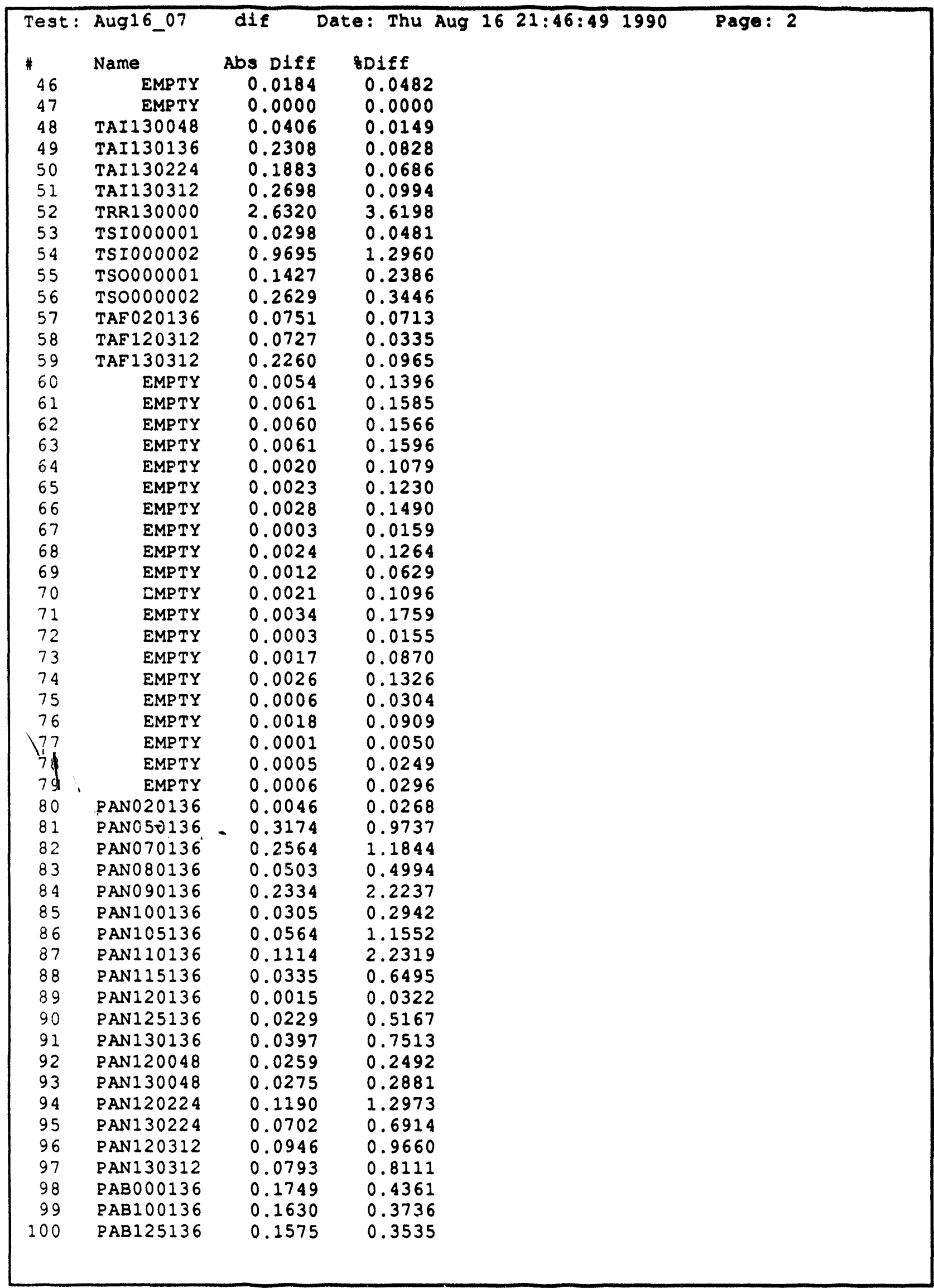




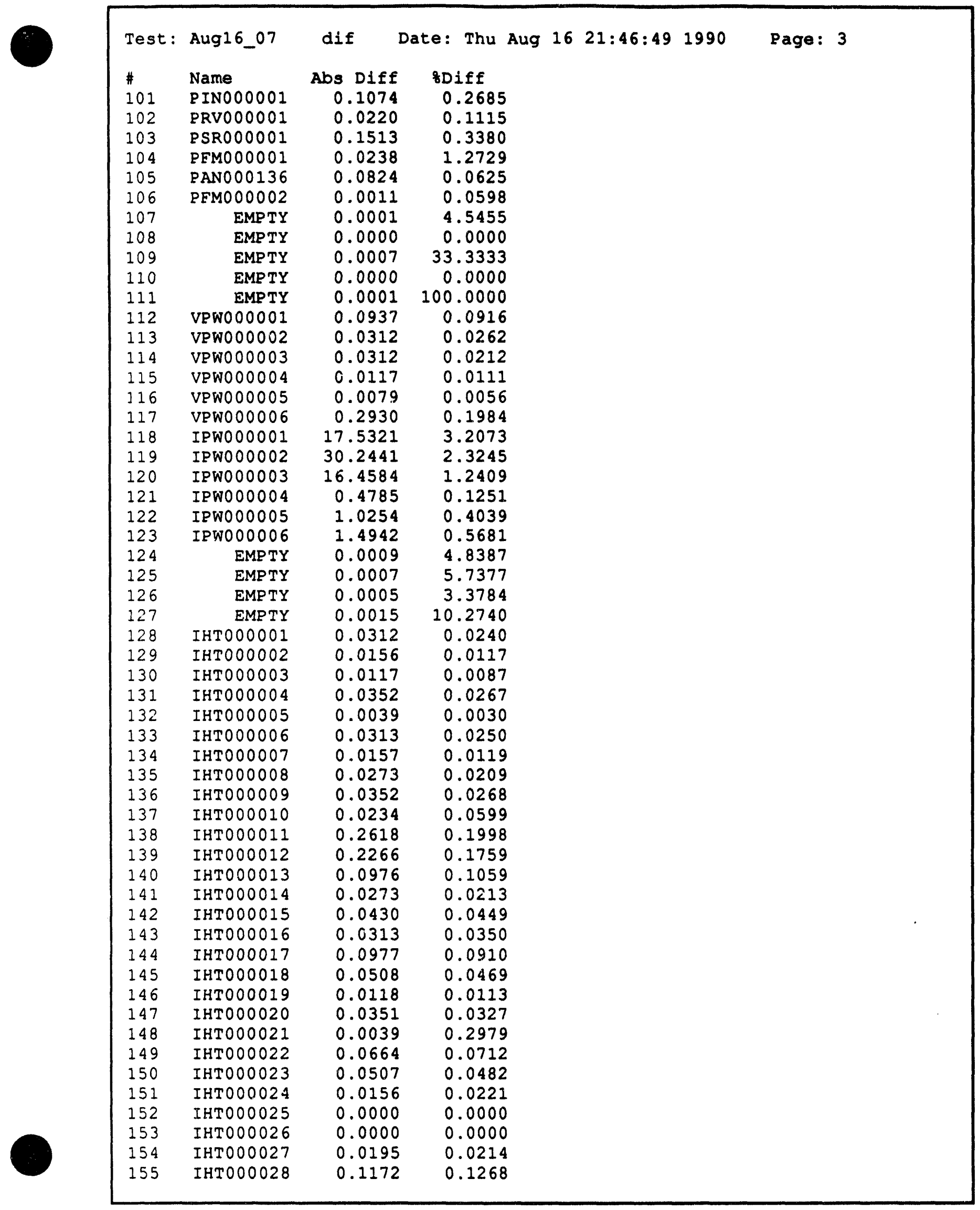




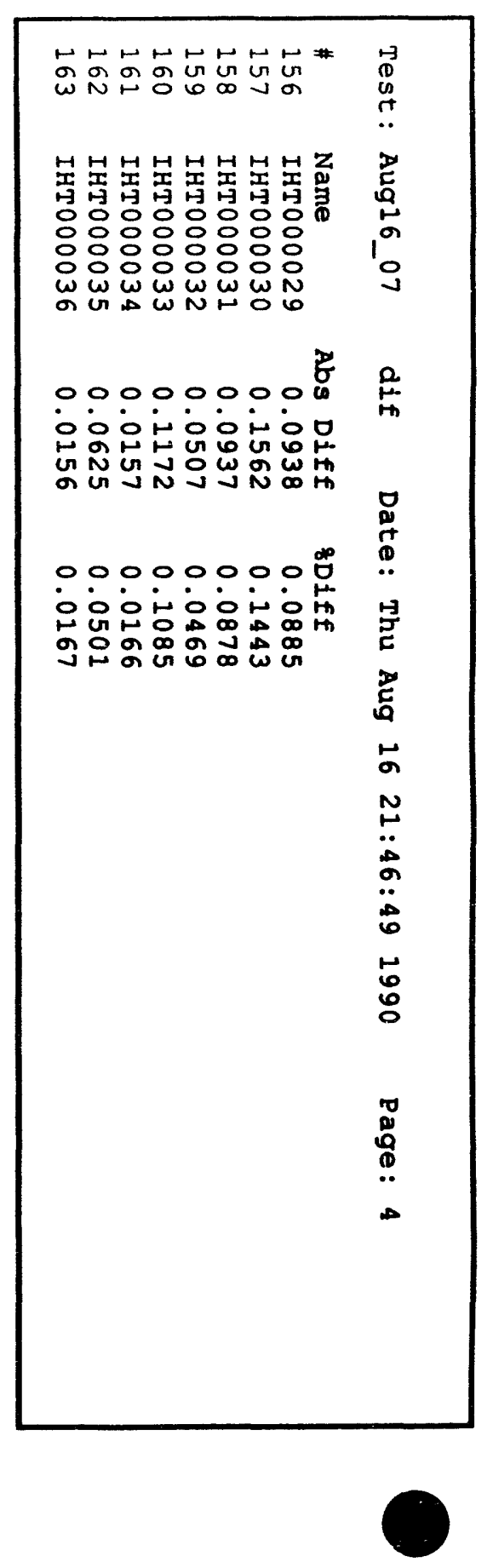




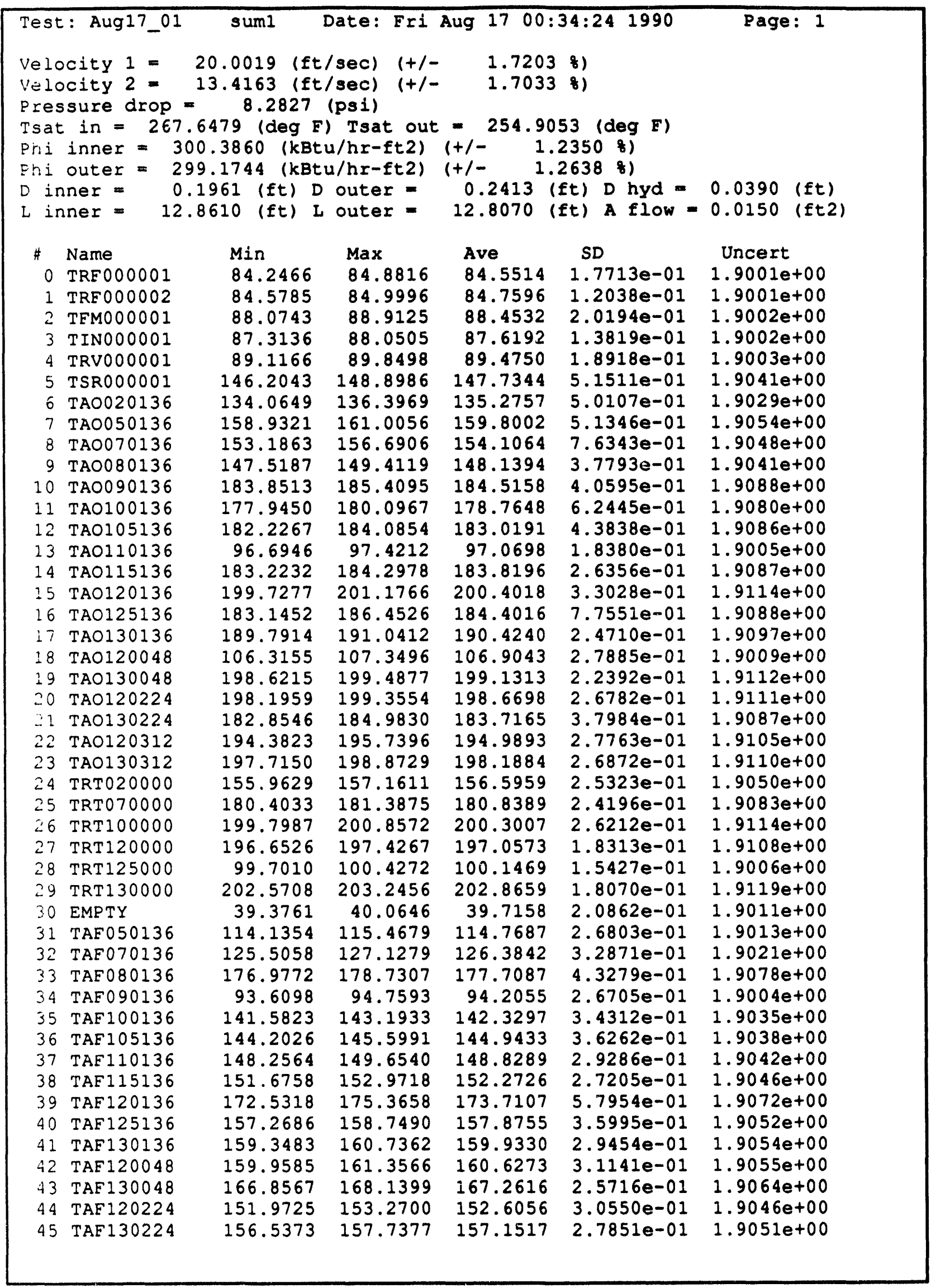




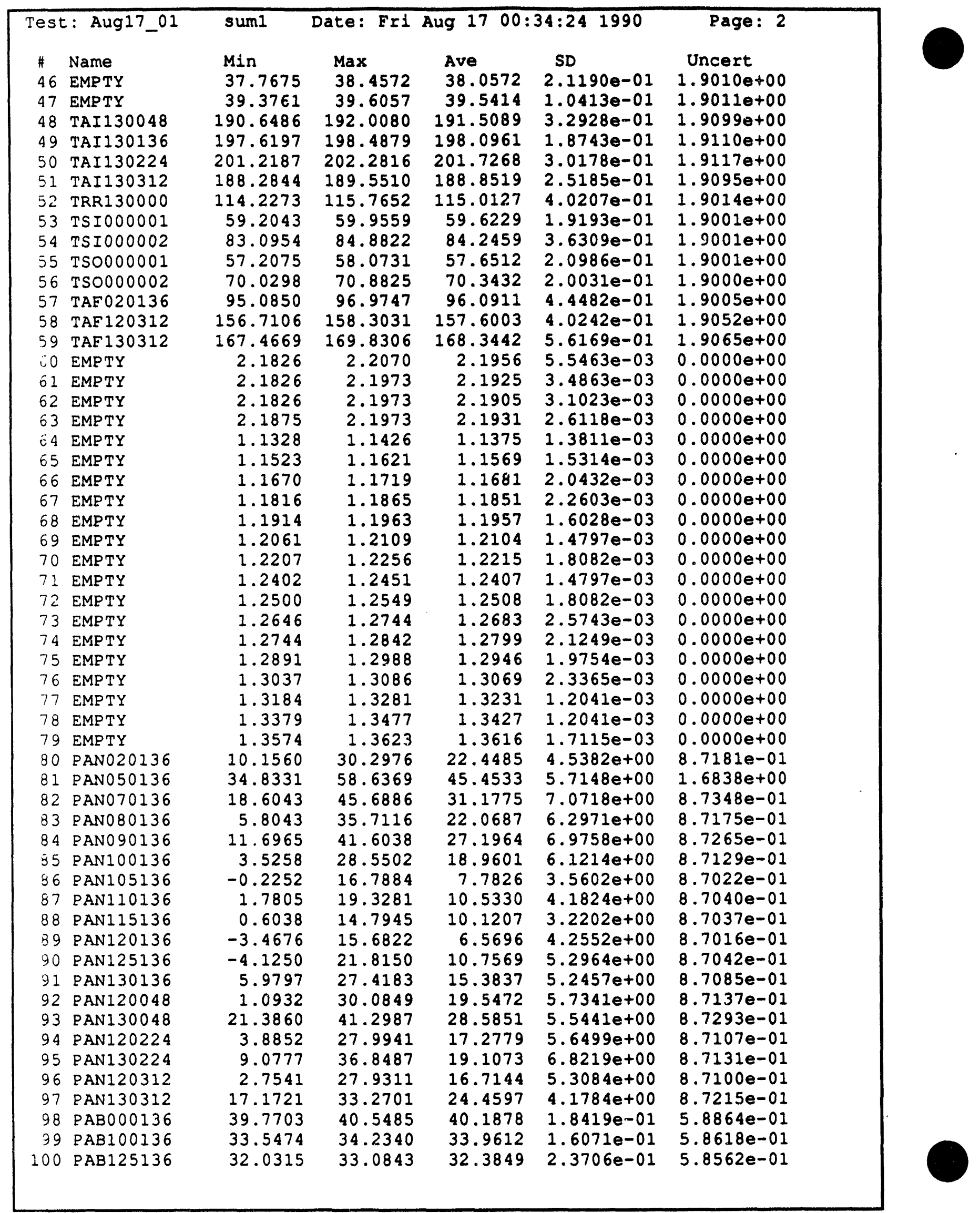




\begin{tabular}{|c|c|c|c|c|c|c|}
\hline Test & E: Aug17_01 & sum1 & Date: Fr1 & Aug 170 & $34: 241990$ & Page: 3 \\
\hline$\#$ & Name & Min & $\operatorname{Max}$ & Ave & SD & Uncert \\
\hline 101 & PINO00001 & 39.1042 & 40.8132 & 39.9831 & $3.6245 e-01$ & $7.5663 e-01$ \\
\hline 102 & PRV000001 & 19.5965 & 19.7186 & 19.6844 & $5.5366 e-02$ & $7.5161 e-01$ \\
\hline 103 & PSR000001 & 29.4347 & 29.8009 & 29.6324 & $8.1384 \theta-02$ & $7.5365 \theta-01$ \\
\hline 104 & PFM0000001 & 60.8755 & 62.0657 & 61.5603 & $2.8127 e-01$ & $5.2315 e-01$ \\
\hline 105 & PAN 000136 & 215.1933 & 242.2014 & 229.3107 & $6.4089 e+00$ & $4.9533 e+00$ \\
\hline 106 & PEMO00002 & 27.5689 & 27.5689 & 27.5689 & $9.6336 e-06$ & $2.1154 e-01$ \\
\hline 107 & EMPTY & 0.0049 & 0.0098 & 0.0096 & $9.6655 e-04$ & $0.0000 e+00$ \\
\hline 108 & EMPTY & -10.0000 & -0.5225 & -7.3491 & $1.8712 e+00$ & $0.0000 e+00$ \\
\hline 109 & EMPTY & 0.0049 & 0.0098 & 0.0052 & $1.1714 e-03$ & $0.0000 e+00$ \\
\hline 110 & EMPTY & -10.0000 & -10.0000 & -10.0000 & $0.00000+00$ & $0.0000 e+00$ \\
\hline 111 & EMPTY & 0.0000 & 0.0049 & 0.0002 & $9.6655 e-04$ & $0.0000 e+00$ \\
\hline 112 & VPW000001 & 177.0245 & 177.4151 & 177.1963 & $1.0907 e-01$ & $1.3366 e+00$ \\
\hline 113 & VPW0000002 & 206.9374 & 207.5233 & 207.2499 & $1.1161 e-01$ & $1.48400+00$ \\
\hline 114 & VPW000003 & 255.6303 & 256.2162 & 256.0482 & $1.2484 \theta-01$ & $1.7367 e+00$ \\
\hline 115 & VPW000004 & 182.0125 & 182.4031 & 182.1493 & $1.2633 e-01$ & $1.3603 e+00$ \\
\hline $1: 6$ & VPWO000005 & 242.1913 & 243.1678 & 242.6288 & $2.0336 e-01$ & $1.6659 e+00$ \\
\hline 117 & VPW000006 & 257.4407 & 257.8313 & 257.6319 & $1.2156 e-01$ & $1.7452 e+00$ \\
\hline 118 & IPWO000001 & 939.8282 & 945.6852 & 942.7570 & $1.1329 e+00$ & $1.1004 e+01$ \\
\hline 119 & IPWO000002 & 2217.4678 & 2223.3215 & 2220.0439 & $1.2738 \mathrm{e}+00$ & $1.9318 e+01$ \\
\hline$\therefore 20$ & IPW000003 & 2267.8499 & 2271.7498 & 2270.6575 & $1.2557 e+00$ & $1.9682 \mathrm{e}+01$ \\
\hline 121 & IPW000004 & 652.1653 & 653.6301 & 652.7808 & $3.5307 e-01$ & $5.5312 e+00$ \\
\hline 122 & IPW000005 & 432.7429 & 434.6958 & 433.6412 & $4.2264 e-01$ & $3.9668 \mathrm{e}+00$ \\
\hline 123 & IPW000006 & 449.7446 & 451.6978 & 450.8283 & $3.8574 \theta-01$ & $4.0847 e+00$ \\
\hline 124 & EMPTY & -0.0244 & -0.0098 & -0.0176 & $3.9459 e-03$ & $0.0000 e+00$ \\
\hline 125 & EMPTY & -0.0195 & -0.0049 & -0.0122 & $3.0003 e-03$ & $0.0000 e+00$ \\
\hline 126 & EMPTY & -0.0195 & -0.0098 & -0.0134 & $3.3901 e-03$ & $0.0000 e+00$ \\
\hline .27 & EMPTY & -0.0244 & -0.0098 & -0.0168 & $3.4400 e-03$ & $0.0000 e+00$ \\
\hline 128 & IHTO000001 & 220.7106 & 223.0544 & 221.8825 & $5.4676 e-01$ & $4.6619 e+00$ \\
\hline 229 & IHTO00002 & 227.4940 & 229.8377 & 228.4941 & $5.1394 e-01$ & $4.6937 e+00$ \\
\hline 130 & IHTOOO0003 & 229.1842 & 231.3326 & 230.0280 & $5.0600 e-01$ & $4.7012 e+00$ \\
\hline 131 & IHTOOO0004 & 224.9361 & 227.6705 & 226.0495 & $5.3123 e-01$ & $4.6819 e+00$ \\
\hline .32 & IHTO00005 & 222.4309 & 225.1653 & 223.5362 & $5.8475 e-01$ & $4.6698 \mathrm{e}+00$ \\
\hline$:: 3$ & IHT000006 & 216.3799 & 218.9190 & 217.4346 & $5.7453 e-01$ & $4.6409 e+00$ \\
\hline$: 34$ & IHTO000007 & 225.7212 & 227.6743 & 226.6898 & $4.6515 e-01$ & $4.6850 \mathrm{e}+00$ \\
\hline 235 & IHTO00008 & 223.1295 & 225.2779 & 224.1256 & $4.9639 e-01$ & $4.6726 e+00$ \\
\hline 136 & IHTO00009 & 223.7042 & 225.8526 & 224.7119 & $4.7737 e-01$ & $4.6754 e+00$ \\
\hline 137 & IHT000010 & 55.0143 & 57.3580 & 56.1314 & $5.7323 e-01$ & $4.1382 e+00$ \\
\hline$: 38$ & IHTO00011 & 224.0572 & 226.4010 & 225.1003 & $5.4905 e-01$ & $4.6773 e+00$ \\
\hline 139 & IHTO00012 & 220.1097 & 222.4534 & 221.1018 & $5.5079 e-01$ & $4.6582 e+00$ \\
\hline 140 & IHTO00013 & 258.5938 & 161.1328 & 160.0000 & $5.3379 e-01$ & $4.4011 e+00$ \\
\hline 141 & IHTO00014 & 219.9444 & 221.8976 & 220.9444 & $5.4625 e-01$ & $4.6574 \mathrm{e}+00$ \\
\hline$: 42$ & IHT000015 & 164.1526 & 166.6917 & 165.2504 & $5.5648 e-01$ & $4.4205 e+00$ \\
\hline 143 & IHT000016 & 152.7794 & 155.1232 & 153.9161 & $4.9235 e-01$ & $4.3794 e+00$ \\
\hline 144 & IHT000017 & 183.3909 & 185.7347 & 184.6371 & $5.4375 e-01$ & $4.4966 e+00$ \\
\hline$: 45$ & IHTO00018 & 184.5027 & 187.2371 & 186.2098 & $5.8235 e-01$ & $4.5030 e+00$ \\
\hline$\therefore 46$ & IHTO00019 & 179.0640 & 181.9937 & 180.6422 & $6.8554 \theta-01$ & $4.4803 e+00$ \\
\hline 147 & IHT000020 & 184.1271 & 187.0568 & 185.7677 & $6.7428 e-01$ & $4.5012 e+00$ \\
\hline 148 & IHT000021 & -3.3992 & 0.1164 & -1.4070 & $8.6541 e-01$ & $4.1000 e+00$ \\
\hline 149 & IHTO00022 & 158.8754 & 161.6098 & 160.2973 & $6.6032 e-01$ & $4.4022 e+00$ \\
\hline 150 & IHT000023 & 180.3411 & 183.4661 & 181.7472 & $6.8799 e-01$ & $4.4848 e+00$ \\
\hline 151 & IHTO000024 & 144.9632 & 147.5023 & 146.1430 & $6.4592 e-01$ & $4.3527 e+00$ \\
\hline 152 & IHT000025 & 179.5486 & 181.8923 & 180.7126 & $6.6376 e-01$ & $4.4806 e+00$ \\
\hline 153 & IHTO00026 & 181.6932 & 184.2323 & 182.9980 & $6.2570 e-01$ & $4.4899 e+00$ \\
\hline 154 & IHTO00027 & 155.0331 & 157.7674 & 156.4706 & $6.4448 e-01$ & $4.3884 e+00$ \\
\hline 155 & IHT 000028 & 156.9599 & 160.4755 & 158.8232 & $6.8369 e-01$ & $4.3969 e+00$ \\
\hline
\end{tabular}




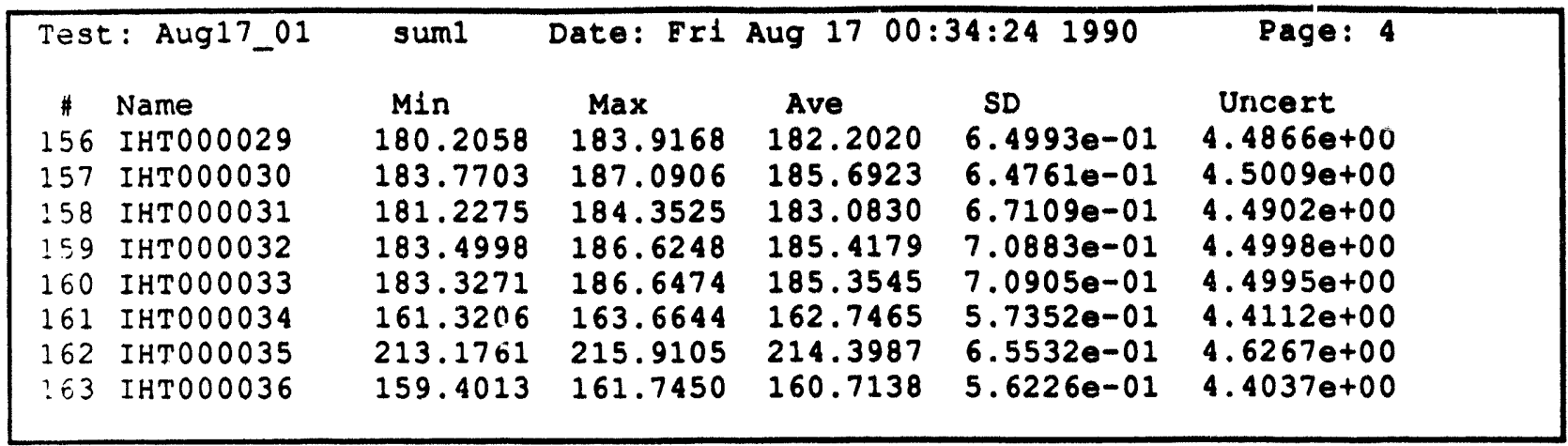




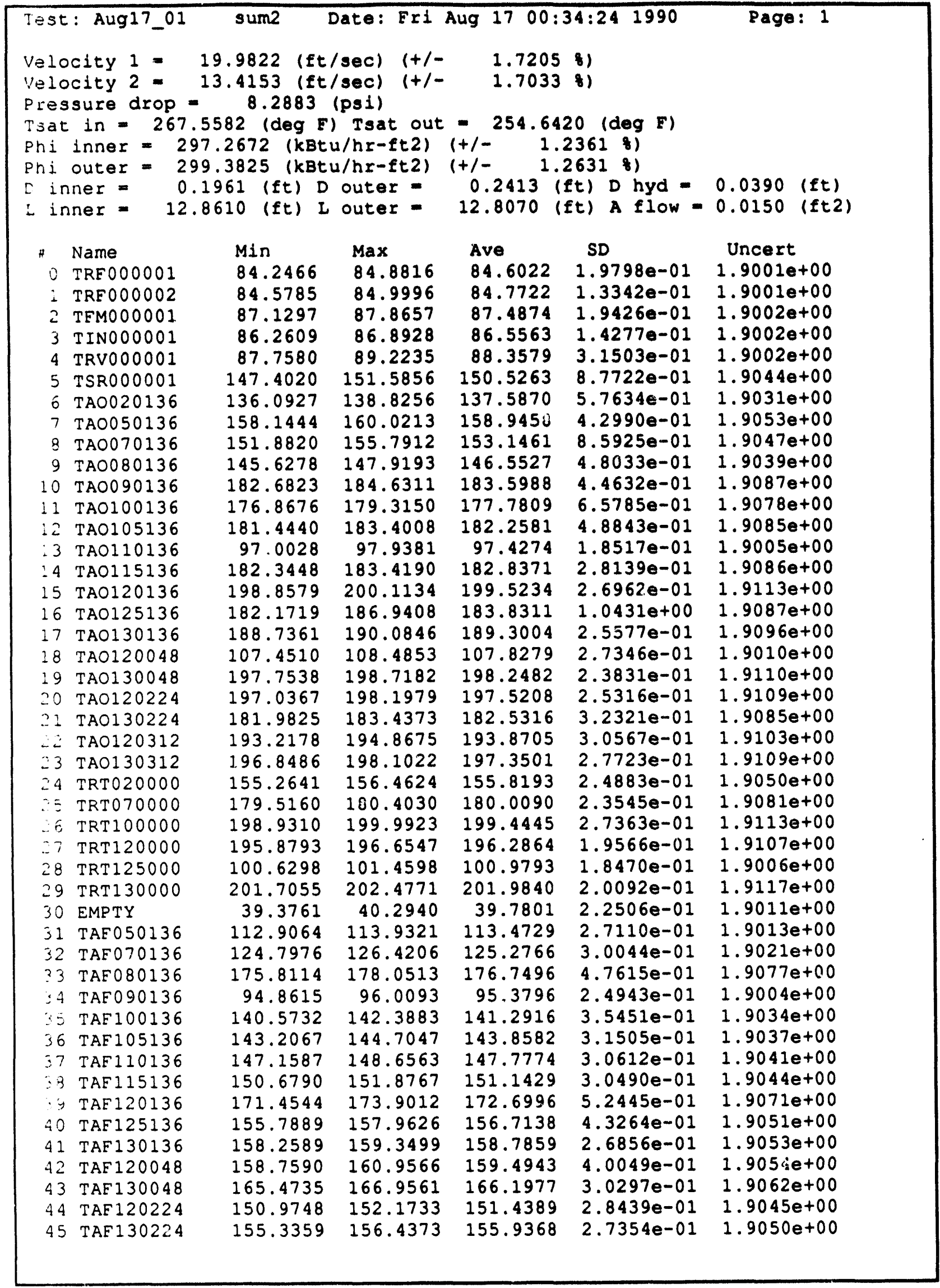




\begin{tabular}{|c|c|c|c|c|c|c|}
\hline \multicolumn{2}{|c|}{ Test: Aug1?_01 } & sum2 & Date: Eri & Aug 170 & $4: 241990$ & Page: 2 \\
\hline $\begin{array}{l}* \\
46\end{array}$ & Name & Min & $\operatorname{Max}$ & Ave & SD & Uncert \\
\hline $\begin{array}{l}46 \\
47\end{array}$ & $\begin{array}{l}\text { EMPTY } \\
\text { EMPTY }\end{array}$ & $\begin{array}{l}37.7675 \\
39.3761\end{array}$ & 38.4572 & 38.1124 & $2.0903 e-01$ & $1.90100+00$ \\
\hline 49 & TAI 130136 & $\begin{array}{l}190.0654 \\
196.6542\end{array}$ & $\begin{array}{l}191.4258 \\
197.5241\end{array}$ & & & $1.9098 \theta+00$ \\
\hline 50 & TAI 130224 & 200.1545 & $\begin{array}{l}197.5241 \\
201.4117\end{array}$ & $\begin{array}{l}197.1690 \\
200.7543\end{array}$ & $\begin{array}{l}2.0882 e-01 \\
3.1389 e-01\end{array}$ & $\begin{array}{l}1.9109 \theta+00 \\
1.9115 \theta+00\end{array}$ \\
\hline 51 & TAI 130312 & 187.3100 & 188.4805 & 187.8370 & $2.7293 e-01$ & $1.9093 e+00$ \\
\hline 52 & TRR130000 & 114.3307 & 115.8651 & 115.0477 & $3.5760 e-01$ & $1.9014 e+00$ \\
\hline $\begin{array}{l}53 \\
54\end{array}$ & TSI000001 & 59.3107 & 60.0639 & 59.6939 & $1.7534 e-01$ & $1.9001 e+00$ \\
\hline $\begin{array}{l}54 \\
55\end{array}$ & $\begin{array}{l}\text { TSI000002 } \\
\text { TSO000001 }\end{array}$ & $\begin{array}{l}83.3052 \\
57.2080\end{array}$ & 84.6732 & 84.0507 & $3.1360 e-01$ & $1.9001 e+00$ \\
\hline $\begin{array}{l}55 \\
56\end{array}$ & $\begin{array}{l}\text { TSO0000001 } \\
\text { TSO000002 }\end{array}$ & $\begin{array}{l}57.2080 \\
69.7101\end{array}$ & 58.0725 & 57.6144 & $1.9483 e-01$ & $\begin{array}{l}1.9001 e+00 \\
1.9000 e+00\end{array}$ \\
\hline $\begin{array}{l}56 \\
57\end{array}$ & $\begin{array}{l}\text { TSO0000002 } \\
\text { TAF020136 }\end{array}$ & $\begin{array}{l}69.7101 \\
94.0334\end{array}$ & $\begin{array}{l}70.6700 \\
96.0301\end{array}$ & $\begin{array}{l}70.2645 \\
94.9756\end{array}$ & $\begin{array}{l}2.1659 e-01 \\
4.8071 e-01\end{array}$ & $\begin{array}{l}1.9000 e+00 \\
1.9004 e+00\end{array}$ \\
\hline 58 & TAF 120312 & 155.6137 & 157.5071 & 156.5430 & $4.3473 e-01$ & $1.9050 e+00$ \\
\hline 59 & TAF 130312 & 166.4817 & 168.9458 & 167.3728 & $5.1252 e-01$ & $1.9064 e+00$ \\
\hline 60 & EMPTY & 2.1582 & 2.1826 & 2.1717 & $5.1788 \theta-03$ & $0.0000 e+00$ \\
\hline 61 & EMPTY & 2.1631 & 2.1777 & 2.1692 & $3.7963 e-03$ & $0.0000 e+00$ \\
\hline 62 & EMPTY & 2.1631 & 2.1729 & 2.1665 & $3.0003 e-03$ & $0.0000 e+00$ \\
\hline 63 & EMPTY & 2.1631 & 2.1777 & 2.1707 & $2.9856 e-03$ & $0.0000 e+00$ \\
\hline 64 & EMPTY & 1.1182 & 1.1328 & 1.1273 & $2.3428 e-03$ & $0.0000 e+00$ \\
\hline 65 & EMPTY & 1.1377 & 1.1523 & 1.1462 & $2.5743 e-03$ & $0.0000 e+00$ \\
\hline 66 & EMPTY & 1.1572 & 1.1719 & 1.1589 & $2.8946 e-03$ & $0.0000 e+00$ \\
\hline 67 & EMPTY & 1.1719 & 1.1768 & 1.1748 & $2.4164 e-03$ & $0.0000 e+00$ \\
\hline 68 & EMPTY & 1.1816 & 1.1914 & 1.1858 & $2.2080 e-03$ & $0.0000 e+00$ \\
\hline 69 & EMPTY & 1.1963 & 1.2012 & 1.2000 & $2.1065 e-03$ & $0.0000 e+00$ \\
\hline 70 & EMPTY & 1.2109 & 1.2158 & 1.2110 & $6.9053 e-04$ & $0.0000 e+00$ \\
\hline 71 & EMPTY & 1.2256 & 1.2354 & 1.2306 & $1.2041 e-03$ & $0.0000 e+00$ \\
\hline 72 & EMPTY & 1.2402 & 1.2451 & 1.2416 & $2.2146 e-03$ & $0.0000 e+00$ \\
\hline 73 & 3 EMPTY & 1.2549 & 1.2646 & 1.2586 & $2.3261 e-03$ & $0.0000 e+00$ \\
\hline 74 & EMPTY & 1.2646 & 1.2744 & 1.2696 & $1.2041 e-03$ & $0.0000 e+00$ \\
\hline 75 & EMPTY & 1.2842 & 1.2891 & 1.2845 & $1.1714 e-03$ & $0.0000 e+00$ \\
\hline 76 & 5 EMPTY & 1.2939 & 1.2988 & 1.2975 & $2.2146 e-03$ & $0.0000 e+00$ \\
\hline 77 & EMPTY & 1. 3135 & 1.3184 & 1.3136 & $6.9053 e-04$ & $0.0000 \mathrm{e}+00$ \\
\hline 78 & 3 EMPTY & 1.3281 & 1.3379 & 1.3328 & $1.3811 e-03$ & $0.0000 e+00$ \\
\hline 79 & EMPTY & 1.3477 & 1.3525 & 1.3524 & $6.9053 e-04$ & $0.0000 e+00$ \\
\hline 80 & PAN020136 & 14.8099 & 29.6110 & 22.8406 & $3.9454 e+00$ & $8.7187 \mathrm{e}-01$ \\
\hline 81 & PAN050136 & 35.4435 & 57.7213 & 45.6394 & $5.4254 e+00$ & $1.6839 \mathrm{e}+00$ \\
\hline 82 & 2 PAN070136 & 18.3754 & 41.8739 & 29.0581 & $7.3553 e+00$ & $8.7303 e-01$ \\
\hline 83 & 3 PAN080136 & 5.4229 & 37.1612 & 21.9970 & $7.1902 e+00$ & $8.7174 e-01$ \\
\hline 84 & 4 PAN090136 & 10.6284 & 44.0452 & 26.1146 & $8.1431 e+00$ & $8.7245 e-01$ \\
\hline 85 & 5 PAN100136 & 5.5094 & 32.4412 & 18.7129 & $7.0749 e+00$ & $8.7126 e-01$ \\
\hline 86 & 6 PAN105136 & 2.3688 & 18.3142 & 10.0348 & $3.9452 \mathrm{e}+00$ & $8.7036 e-01$ \\
\hline 87 & 7 PAN 110136 & 1.4754 & 17.9548 & 9.2314 & $3.5873 e+00$ & $8.7031 e-01$ \\
\hline 88 & B PAN115136 & -1.3798 & 15.0996 & 8.2683 & $3.2251 e+00$ & $8.7025 e-01$ \\
\hline 89 & PAN120136 & 1.2626 & 16.8266 & 8.2634 & $4.2017 e+00$ & $8.7025 e-01$ \\
\hline 90 & PAIN125136 & -2.1413 & 24.4853 & 12.0173 & $5.6909 e+00$ & $8.7052 \mathrm{e}-01$ \\
\hline 91 & PAN130136 & 5.3693 & 27.6472 & 15.3760 & $5.5505 e+00$ & $8.7085 e-01$ \\
\hline 92 & 2. PAN120048 & 6.8153 & 31.3056 & 22.6966 & $5.4645 e+00$ & $8.7185 e-01$ \\
\hline 93 & 3 PAN1 30048 & 13.3751 & 40.5358 & 24.1463 & $4.7846 e+00$ & $8.7209 e-01$ \\
\hline 94 & 4 PAN120224 & 5.5637 & 23.1876 & 15.2942 & $4.1446 e+00$ & $8.7084 e-01$ \\
\hline 95 & PAN130224 & 9.7644 & 33.4918 & 19.2370 & $5.6473 e+00$ & $8.7133 e-01$ \\
\hline 96 & 6 PAN120312 & 3.8985 & 22.8957 & 15.5684 & $4.3627 e+00$ & $8.7087 e-01$ \\
\hline 97 & 7 PAN130312 & 20.0713 & 36.1693 & 25.8559 & $3.4811 e+00$ & $8.7240 e-01$ \\
\hline 98 & $3 \mathrm{PAB} 000136$ & 39.7245 & 40.5485 & 40.1273 & $1.9066 \mathrm{e}-01$ & $5.88 \in 1 e-01$ \\
\hline 99 & 9 PAB100136 & 33.5474 & 34.1425 & 33.9025 & $1.4012 e-01$ & $5.8616 e-01$ \\
\hline 100 & 0 PAB125136 & 31.9399 & 32.9928 & 32.2393 & $2.1634 e-01$ & $5.8557 e-01$ \\
\hline
\end{tabular}




\begin{tabular}{|c|c|c|c|c|c|c|}
\hline \multicolumn{2}{|c|}{ Test: Aug17_01 } & sum2 & \multicolumn{3}{|c|}{ Date: Fr1 Aug $1700: 34: 241990$} & Page: 3 \\
\hline $\begin{array}{l}101 \\
102\end{array}$ & $\begin{array}{l}\text { PINO00001 } \\
\text { PRV000001 }\end{array}$ & $\begin{array}{l}39.1042 \\
19.4744\end{array}$ & $\begin{array}{l}40.5690 \\
19.5965\end{array}$ & $\begin{array}{l}39.8488 \\
19.5794\end{array}$ & $\begin{array}{l}3.6350 e-01 \\
4.2787 e-02\end{array}$ & $\begin{array}{l}7.56590-01 \\
7.5160 e-01\end{array}$ \\
\hline 103 & PSRO00001 & 29.4347 & 29.8009 & 29.6324 & $1.1799 \theta-01$ & $7.5365 e-01$ \\
\hline 104 & PEM000001 & 60.4177 & 62.3403 & 61.4486 & $4.3252 e-01$ & $5.2307 e-01$ \\
\hline $\begin{array}{l}106 \\
107\end{array}$ & $\begin{array}{l}\text { PEMO000002 } \\
\text { EMPTY }\end{array}$ & $\begin{array}{r}27.5689 \\
0.0049\end{array}$ & $\begin{array}{r}27.5689 \\
0.0098\end{array}$ & $\begin{array}{r}27.5689 \\
0.0096\end{array}$ & $\begin{array}{l}9.6336 e-06 \\
9.6655 e-04\end{array}$ & $2.1154 e-01$ \\
\hline 108 & EMPTY & -10.0000 & -2.4072 & $\begin{array}{r}0.0096 \\
-7.4047\end{array}$ & $\begin{array}{l}9.6655 e-04 \\
1.8599 \theta+00\end{array}$ & $\begin{array}{l}0.0000 e+00 \\
0.0000 e+00\end{array}$ \\
\hline 109 & EMPTY & 0.0049 & 0.0098 & 0.0056 & $1.7115 e-03$ & $0.00000+00$ \\
\hline $\begin{array}{l}110 \\
111\end{array}$ & EMPTY & -10.0000 & -10.0000 & -10.0000 & $0.0000 e+00$ & $0.0000 e+00$ \\
\hline $\begin{array}{l}111 \\
112\end{array}$ & $\begin{array}{l}\text { EMPTY } \\
\text { VPWO00001 }\end{array}$ & $\begin{array}{r}0.0000 \\
07\end{array}$ & 0.0049 & 0.0001 & $6.9053 e-04$ & $0.0000 e+00$ \\
\hline $\begin{array}{l}112 \\
123\end{array}$ & $\begin{array}{l}\text { VPWO00001 } \\
\text { VPWO00002 }\end{array}$ & $\begin{array}{l}177.0245 \\
207.1327\end{array}$ & 177.4151 & 177.2276 & $1.1133 e-01$ & $1.3367 e+00$ \\
\hline 114 & VPWOOO003 & $\begin{array}{l}207.1327 \\
255.8256\end{array}$ & $\begin{array}{l}207.5233 \\
256.2162\end{array}$ & $\begin{array}{l}207.2421 \\
256.0130\end{array}$ & $\begin{array}{l}1.0559 \theta-01 \\
1.1811 \mathrm{e}-01\end{array}$ & $\begin{array}{l}1.4840 e+00 \\
1.7366 e+00\end{array}$ \\
\hline 115 & VPW000004 & 181.8171 & $\begin{array}{l}256.2162 \\
182.4031\end{array}$ & 182.1571 & $1.1027 e-01$ & $1.3604 e+00$ \\
\hline 116 & VPWOOOOOS & 242.7772 & 243.9491 & 243.2499 & $2.7364 e-01$ & $1.6692 e+00$ \\
\hline 117 & VPWO00006 & 257.2454 & 257.6360 & 257.5421 & $1.0617 e-01$ & $1.7447 \mathrm{e}+00$ \\
\hline 118 & IPWO00001 & 959.3517 & 967.1610 & 963.2950 & $1.8705 e+00$ & $1.1114 e+01$ \\
\hline $\begin{array}{l}119 \\
120\end{array}$ & IPW000002 & 2194.0525 & 2201.8577 & 2199.4768 & $1.5415 e+00$ & $1.91710+01$ \\
\hline 120 & IPW000003 & 2242.4998 & 2250.2998 & 2245.8931 & $1.8398 \mathrm{e}+00$ & $1.9504 e+01$ \\
\hline $\begin{array}{l}121 \\
122\end{array}$ & IPWO00004 & 656.0715 & 658.0247 & 656.9897 & $4.5890 e-01$ & $5.5622 \mathrm{e} r 00$ \\
\hline 122 & IPW000005 & 432.7429 & 436.1605 & 434.3051 & $6.2384 \theta-01$ & $3.9714 e+00$ \\
\hline 123 & IPW000006 & 446.3149 & 448.7681 & 447.7912 & $5.0301 e-01$ & $4.0638 e+00$ \\
\hline 124 & EMPTY & -0.0293 & -0.0098 & -0.0199 & $4.0482 e-03$ & $0.0000 e+00$ \\
\hline 125 & EMPTY & -0.0195 & -0.0098 & -0.0136 & $3.1644 e-03$ & $0.0000 e+00$ \\
\hline 126 & EMPTY & -0.0195 & -0.0098 & -0.0146 & $3.4173 e-03$ & $0.0000 e+00$ \\
\hline 127 & EMPTY & -0.0244 & -0.0098 & -0.0169 & $3.3029 e-03$ & $0.0000 e+00$ \\
\hline 128 & IHT000001 & 220.1247 & 223.2497 & 221.8591 & $6.3704 e-01$ & $4.6618 e+00$ \\
\hline 129 & IHT000002 & 227.2987 & 229.4471 & 228.5175 & $5.5742 e-01$ & $4.6938 \mathrm{e}+00$ \\
\hline 130 & IHTO00003 & 228.9889 & 231.1373 & 230.1100 & $5.5075 e-01$ & $4.7016 \mathrm{e}+00$ \\
\hline 131 & IHTO00004 & 225.1315 & 227.0846 & 226.0807 & $5.5805 e-01$ & $4.6820 e+00$ \\
\hline 132 & IHTO00005 & 222.6262 & 224.5793 & 223.5714 & $5.3577 e-01$ & $4.6699 \mathrm{e}+00$ \\
\hline 133 & IHTO00006 & 216.3799 & 218.5284 & 217.5205 & $5.4727 e-01$ & $4.6413 e+00$ \\
\hline 134 & IHTO00007 & 225.7212 & 227.6743 & 226.8851 & $4.9750 e-01$ & $4.6859 \mathrm{e}+00$ \\
\hline 135 & IHT000008 & 223.3248 & 225.2779 & 224.2702 & $4.4531 e-01$ & $4.6733 e+00$ \\
\hline 136 & IHTO00009 & 223.7042 & 225.8526 & 224.7783 & $5.1486 e-01$ & $4.6757 e+00$ \\
\hline 137 & IHTO00010 & 54.4283 & 57.7486 & 56.2603 & $6.55410-01$ & $4.1384 \mathrm{e}+00$ \\
\hline 138 & IHT000011 & 223.4713 & 226.5963 & 225.1276 & $6.6291 e-01$ & $4.6774 e+00$ \\
\hline 139 & IHTO00012 & 219.5237 & 222.6487 & 221.1175 & $6.7700 e-01$ & $4.6582 \mathrm{e}+00$ \\
\hline 140 & IHTO00013 & 158.5938 & 161.3281 & 160.1211 & $6.3710 \mathrm{e}-01$ & $4.4016 e+00$ \\
\hline 141 & IHTO00014 & 219.5538 & 223.0694 & 221.5811 & $6.9686 e-01$ & $4.6605 e+00$ \\
\hline 142 & IHTOOOOOIS & 163.7620 & 166.8870 & 165.3207 & $6.0295 e-01$ & $4.4208 \mathrm{e}+00$ \\
\hline 143 & IHTO00016 & 152.5841 & 155.3185 & 153.9513 & $5.6359 \mathrm{e}-01$ & $4.3795 e+00$ \\
\hline 144 & IHTO00017 & 183.0003 & 186.1253 & 184.3753 & $7.2968 e-01$ & $4.4955 e+00$ \\
\hline 145 & IHTOOOOO18 & 184.3074 & 188.0183 & 186.1864 & $7.0132 e-01$ & $4.5029 e+00$ \\
\hline 146 & IHTOOOOOI9 & 179.4546 & 181.9937 & 180.6070 & $5.6806 e-01$ & $4.4802 e+00$ \\
\hline 147 & IHTO000020 & 184.5177 & 187.6427 & 185.6778 & $5.8449 e-01$ & $4.5008 e+00$ \\
\hline 148 & IHTO00021 & -3.0086 & 0.7024 & -1.3797 & $7.7986 e-01$ & $4.1000 e+00$ \\
\hline 149 & IHTO00022 & 159.0708 & 161.8051 & 160.4223 & $6.0598 e-01$ & $4.4027 e+00$ \\
\hline 150 & IHTOO0023 & 180.7317 & 183.2708 & 181.8019 & $5.8293 e-01$ & $4.4850 \mathrm{e}+00$ \\
\hline 151 & IHTO00024 & 144.9632 & 147.5023 & 146.1859 & $5.4076 e-01$ & $4.3528 e+00$ \\
\hline 152 & IHTO00025 & 179.5486 & 181.6970 & 180.7516 & $5.2846 e-01$ & $4.4807 e+00$ \\
\hline 153 & IHTOO0026 & 181.6932 & 184.2323 & 182.9784 & $5.9200 e-01$ & $4.4898 e+00$ \\
\hline 154 & IHTOO0027 & 155.4237 & 157.7674 & 156.5644 & $5.7637 e-01$ & $4.3888 e+00$ \\
\hline 155 & IHTOO0028 & 157.1552 & 160.2802 & 158.7724 & $6.5440 e-01$ & $4.3967 e+00$ \\
\hline
\end{tabular}




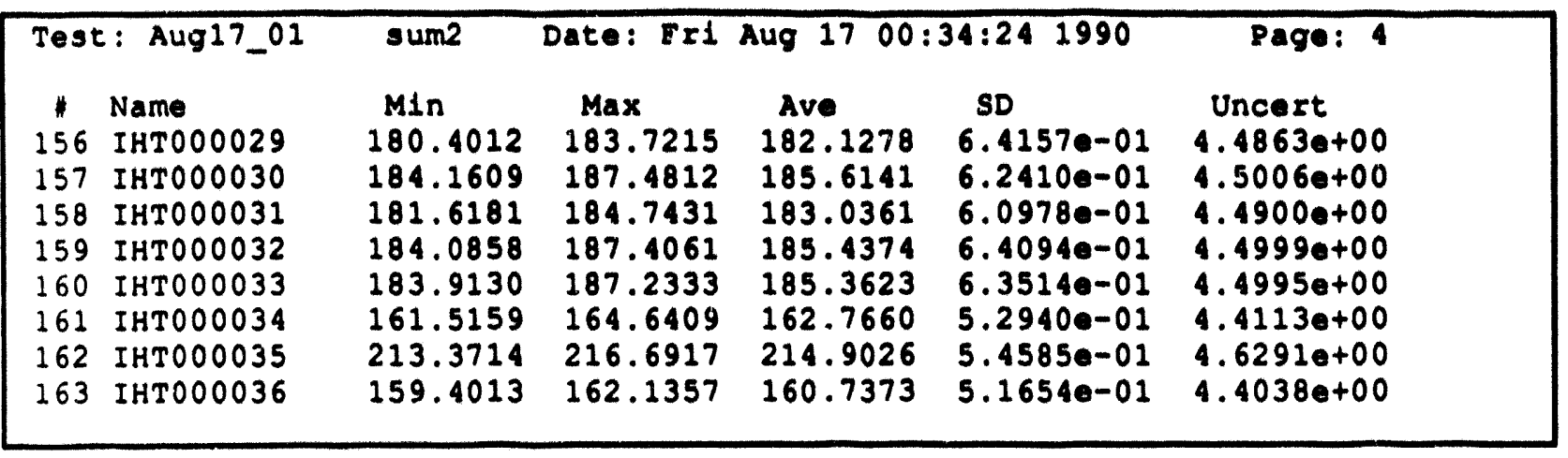




\begin{tabular}{|c|c|c|c|c|}
\hline \multicolumn{5}{|c|}{$\begin{array}{l}\text { Velocity } 1=0.019699(\mathrm{ft} / \mathrm{sec}) \\
\text { Velocity } 2=0.00099(\mathrm{ft} / \mathrm{sec}) \\
\text { Pressure Drop }=0.005600 \text { (psi) } \\
\text { Tsat in }=0.089691 \text { (deg F) Tsat out }=0.263306 \text { (deg F) }\end{array}$} \\
\hline$*$ & Name & Abs Diff & 8Diff & \\
\hline 0 & TRE000001 & 0.0508 & 0.0601 & \\
\hline 1 & TRF000002 & 0.0126 & 0.0149 & \\
\hline 2 & TEMO00001 & 0.9658 & 1.0919 & \\
\hline 3 & TINO00001 & 1.0629 & 1.2131 & \\
\hline 4 & TRV000001 & 1.1171 & 1.2485 & \\
\hline 5 & TSR000001 & 2.7919 & 1.8898 & \\
\hline 6 & TAO020136 & 2.3113 & 1.7086 & \\
\hline 7 & TAO050136 & 0.8544 & 0.5347 & \\
\hline 8 & TAO070136 & 0.9603 & 0.6231 & \\
\hline 9 & TAO080136 & 1.5867 & 1.0711 & \\
\hline$: 0$ & TAO090136 & 0.9170 & 0.4970 & \\
\hline 11 & TAO100136 & 0.9839 & 0.5504 & \\
\hline 12 & TAO105136 & 0.7610 & 0.4158 & \\
\hline 13 & TA0110136 & 0.3576 & 0.3684 & \\
\hline 14 & TAO115136 & 0.9825 & 0.5345 & \\
\hline 15 & TA0120136 & 0.8784 & 0.4383 & \\
\hline$: 6$ & TAO125136 & 0.5705 & 0.3094 & \\
\hline 17 & TAO130136 & 1.1236 & 0.5900 & \\
\hline 13 & TAO120048 & 0.9236 & 0.8640 & \\
\hline 19 & TAO130048 & 0.8831 & 0.4435 & \\
\hline 20 & TAO120224 & 1.1490 & 0.5783 & \\
\hline 21 & TAO130224 & 1.1849 & 0.6450 & \\
\hline 22 & TAO120312 & 1.1188 & 0.5738 & \\
\hline$\therefore 3$ & TAO130312 & 0.8383 & 0.4230 & \\
\hline 24 & TRT020000 & 0.7766 & 0.4959 & \\
\hline 25 & TRT070000 & 0.8299 & 0.4589 & \\
\hline 26 & TRT 100000 & 0.8562 & 0.4275 & \\
\hline 27 & TRT 120000 & 0.7709 & 0.3912 & \\
\hline 28 & TRT 125000 & 0.8324 & 0.8312 & \\
\hline 29 & TRT 130000 & 0.8819 & 0.4347 & \\
\hline 30 & EMPTY & 0.0643 & 0.1619 & \\
\hline 31 & TAE 050136 & 1.2958 & 1.1291 & \\
\hline 32 & TAF 070136 & 1.1076 & 0.8764 & \\
\hline 33 & TAF080136 & 0.9591 & 0.5397 & \\
\hline 34 & TAF090136 & 1.1741 & 1.2463 & \\
\hline 35 & TAF 100136 & 1.0381 & 0.7294 & \\
\hline 30 & TAF 105136 & 1.0851 & 0.7486 & \\
\hline 37 & TAF 110136 & 1.0515 & 0.7065 & \\
\hline 38 & TAE 115136 & 1.1297 & 0.7419 & \\
\hline 39 & TAE 120136 & 1.0111 & 0.5821 & \\
\hline 40 & TAF 125136 & 1.1617 & 0.7358 & \\
\hline 41 & TAF 130136 & 1.1471 & 0.7172 & \\
\hline 42 & TAF 120048 & 1.1330 & 0.7054 & \\
\hline 43 & TAF 130048 & 1.0639 & 0.6361 & \\
\hline 44 & TAF 120224 & 1.1667 & 0.7645 & \\
\hline 45 & TAF 130224 & 1.2149 & 0.7731 & \\
\hline
\end{tabular}




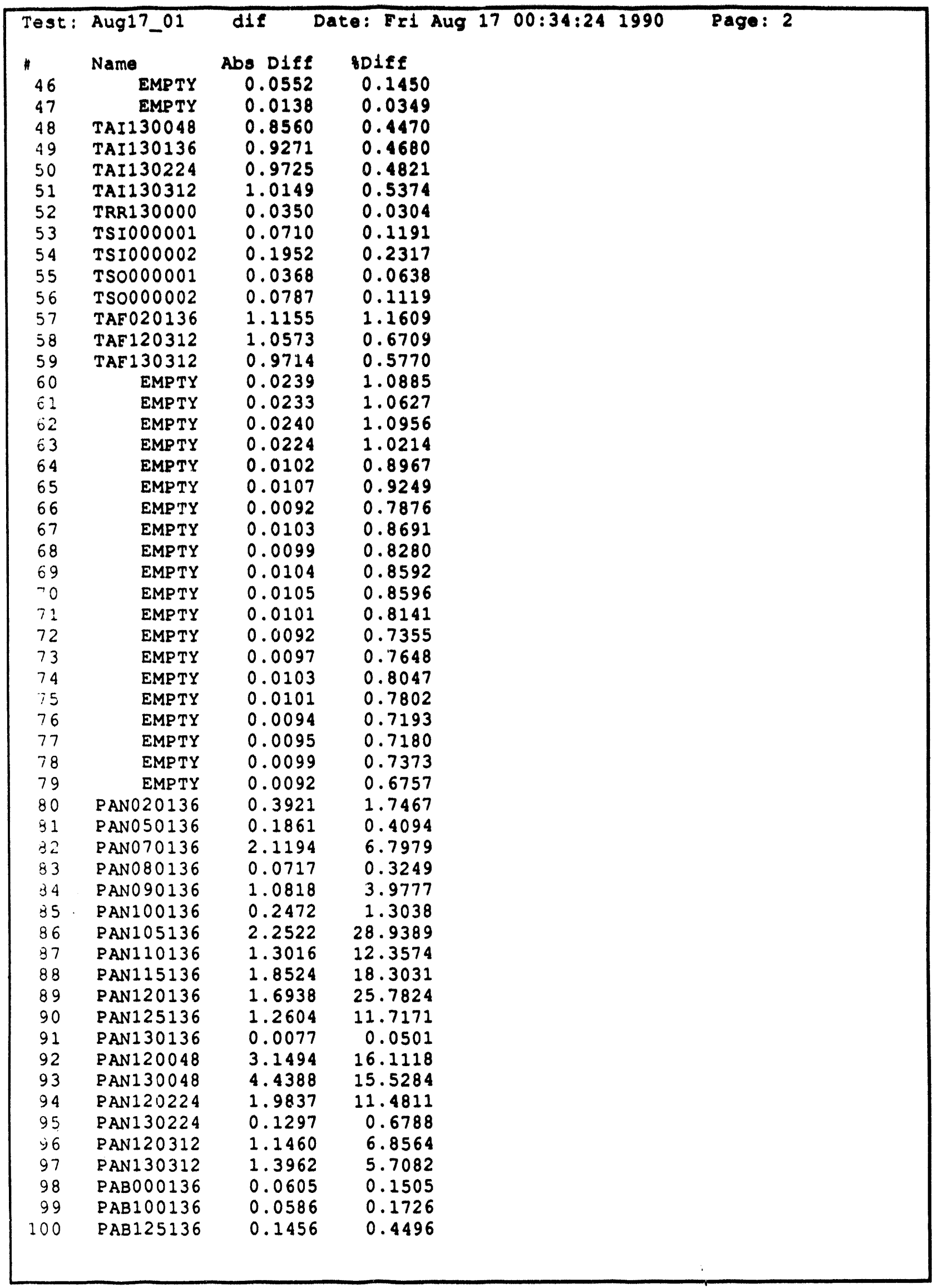




\begin{tabular}{|c|c|c|c|c|}
\hline Test: & Aug17_01 & dif & te: Fr1 Aug $17 \quad 00: 34: 241990$ & Page: 3 \\
\hline$n$ & Name & Abs Diff & SDiff & \\
\hline 101 & PIN000001 & 0.1343 & 0.3359 & \\
\hline 102 & PRV000001 & 0.1050 & 0.5334 & \\
\hline 103 & PSR000001 & 0.0000 & 0.0000 & \\
\hline 104 & PFMO00001 & 0.1117 & 0.1814 & \\
\hline 105 & PAN000136 & 0.1557 & 0.0679 & \\
\hline 106 & PFM000002 & 0.0000 & 0.0000 & \\
\hline $\begin{array}{l}107 \\
108\end{array}$ & $\begin{array}{l}\text { EMPTY } \\
\text { EMPTY }\end{array}$ & $\begin{array}{l}0.0000 \\
0.0556\end{array}$ & $\begin{array}{l}0.0000 \\
0.7566\end{array}$ & \\
\hline 109 & EMPTY & 0.0004 & 7.6923 & \\
\hline 110 & EMPTY & 0.0000 & 0.0000 & \\
\hline 111 & EMPTY & 0.0001 & 50.0000 & \\
\hline 112 & VPWO00001 & 0.0313 & 0.0177 & \\
\hline$\therefore: 3$ & VPWO00002 & 0.0078 & 0.0038 & \\
\hline 114 & VPW000003 & 0.0352 & 0.0137 & \\
\hline 115 & VPWO00004 & 0.0078 & 0.0043 & \\
\hline$\therefore: 6$ & VPW000005 & 0.6211 & 0.2560 & \\
\hline$:: 7$ & VPW000006 & 0.0898 & 0.0348 & \\
\hline 118 & IPW000001 & 20.5380 & 2.1785 & \\
\hline 119 & IPW000002 & 20.5671 & 0.9264 & \\
\hline 120 & IPWO00003 & 24.7644 & 1.0906 & \\
\hline 121 & IPWO00004 & 4.2089 & 0.6448 & \\
\hline 122 & IPWO00005 & 0.6639 & 0.1531 & \\
\hline 123 & IPW000006 & 3.0371 & 0.6737 & \\
\hline 124 & EMPTY & 0.0023 & 13.0682 & \\
\hline 125 & EMPTY & 0.0014 & 11.4754 & \\
\hline$\therefore 26$ & EMPTY & 0.0012 & 8.9552 & \\
\hline 127 & EMPTY & 0.0001 & 0.5952 & \\
\hline$: 28$ & IHTO00001 & 0.0234 & 0.0105 & \\
\hline$\therefore .29$ & IHTO00002 & 0.0234 & 0.0102 & \\
\hline$\therefore: 0$ & IHTO00003 & 0.0820 & 0.0356 & \\
\hline 131 & IHTOOO004 & 0.0312 & 0.0138 & \\
\hline$: 32$ & IHTO00005 & 0.0352 & 0.0157 & \\
\hline 133 & IHTO00006 & 0.0859 & 0.0395 & \\
\hline 134 & IHT 000007 & 0.1953 & 0.0862 & \\
\hline 235 & IHTO00008 & 0.1446 & 0.0645 & \\
\hline 136 & IHTO00009 & 0.0664 & 0.0296 & \\
\hline 137 & IHTOOOOI0 & 0.1289 & 0.2296 & \\
\hline 258 & IHTO00011 & 0.0273 & 0.0121 & \\
\hline $2: 9$ & IHTO00012 & 0.0157 & 0.0071 & \\
\hline 240 & IHTO00013 & 0.1211 & 0.0757 & \\
\hline $2+1$ & IHTO00014 & 0.6367 & 0.2882 & \\
\hline 142 & IHT000015 & 0.0703 & 0.0425 & \\
\hline$: 43$ & IHTO00016 & 0.0352 & 0.0229 & \\
\hline 144 & IHTO00017 & 0.2618 & 0.1418 & \\
\hline 245 & IHTO00018 & 0.0234 & 0.0126 & \\
\hline 246 & IHTO00019 & 0.0352 & 0.0195 & \\
\hline 147 & IHT000020 & 0.0899 & 0.0484 & \\
\hline 148 & IHTO0002.1 & 0.0273 & 1.9403 & \\
\hline 149 & IHT 000022 & 0.1250 & 0.0780 & \\
\hline $1 \equiv 0$ & IHTOOO0023 & 0.0547 & 0.0301 & \\
\hline 151 & IHTO00024 & 0.0429 & 0.0293 & \\
\hline 152 & IHTO00025 & 0.0390 & 0.0216 & \\
\hline 153 & IHT000026 & 0.0196 & 0.0107 & \\
\hline 154 & IHT 000027 & 0.0938 & 0.0600 & \\
\hline 155 & IHT000028 & 0.0508 & 0.0320 & \\
\hline
\end{tabular}




$\begin{array}{llrcr}\text { Test: Aug17_01 } & \text { dif } & \text { Date: Fr1 Aug } 1700: 34: 241990 & \text { Page: } 4 \\ & & \text { Abs Diff } & \text { 8Diff } \\ 156 & \text { IHT000029 } & 0.0742 & 0.0407 \\ 157 & \text { IHT000030 } & 0.0782 & 0.0421 \\ 158 & \text { IHT000031 } & 0.0469 & 0.0256 \\ 159 & \text { IHT000032 } & 0.0195 & 0.0105 \\ 160 & \text { IHT000033 } & 0.0078 & 0.0042 \\ 161 & \text { IHTO00034 } & 0.0195 & 0.0120 \\ 162 & \text { IHTO00035 } & 0.5039 & 0.2350 \\ 163 & \text { IHTO00036 } & 0.0235 & 0.0146\end{array}$




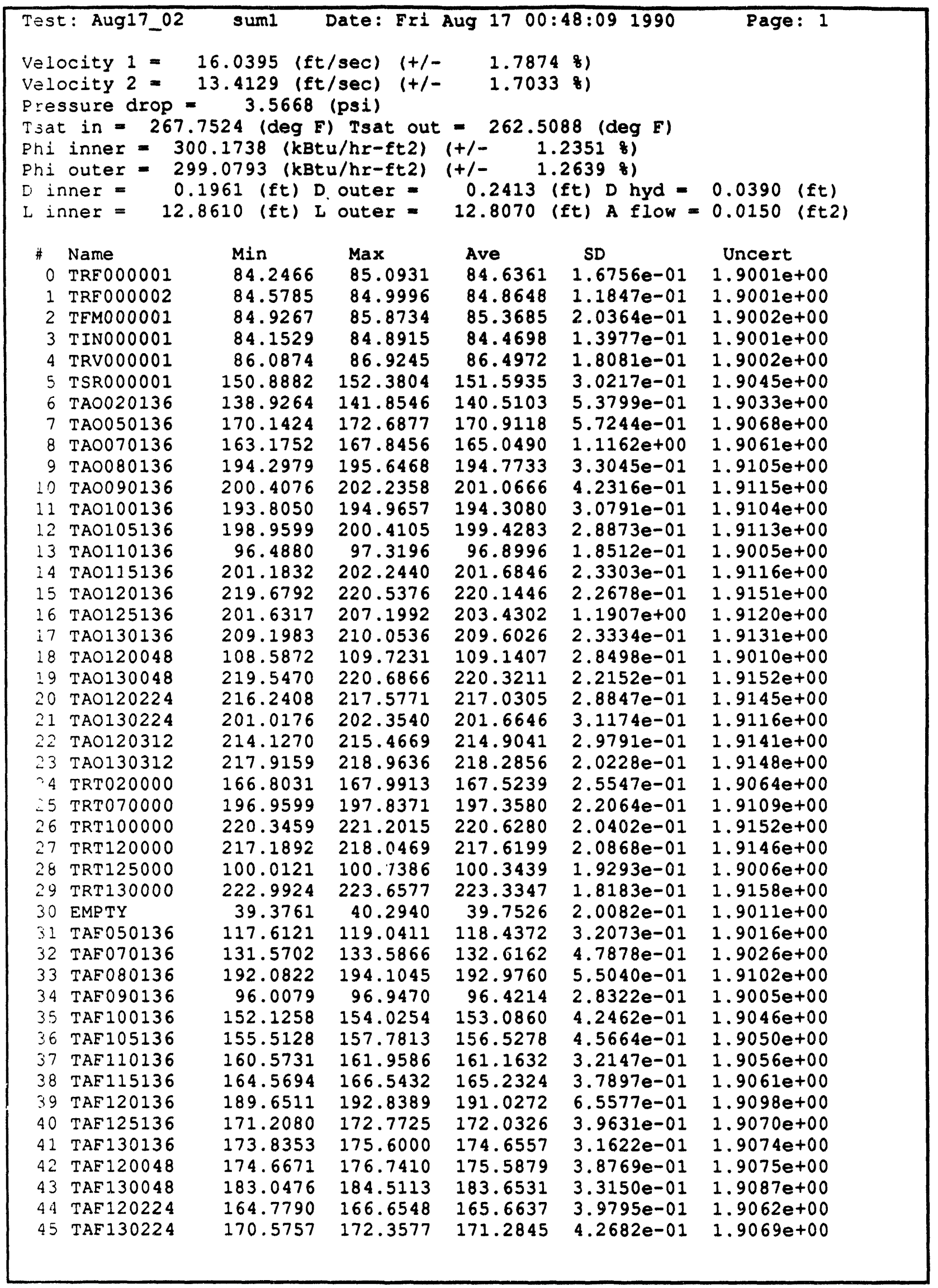




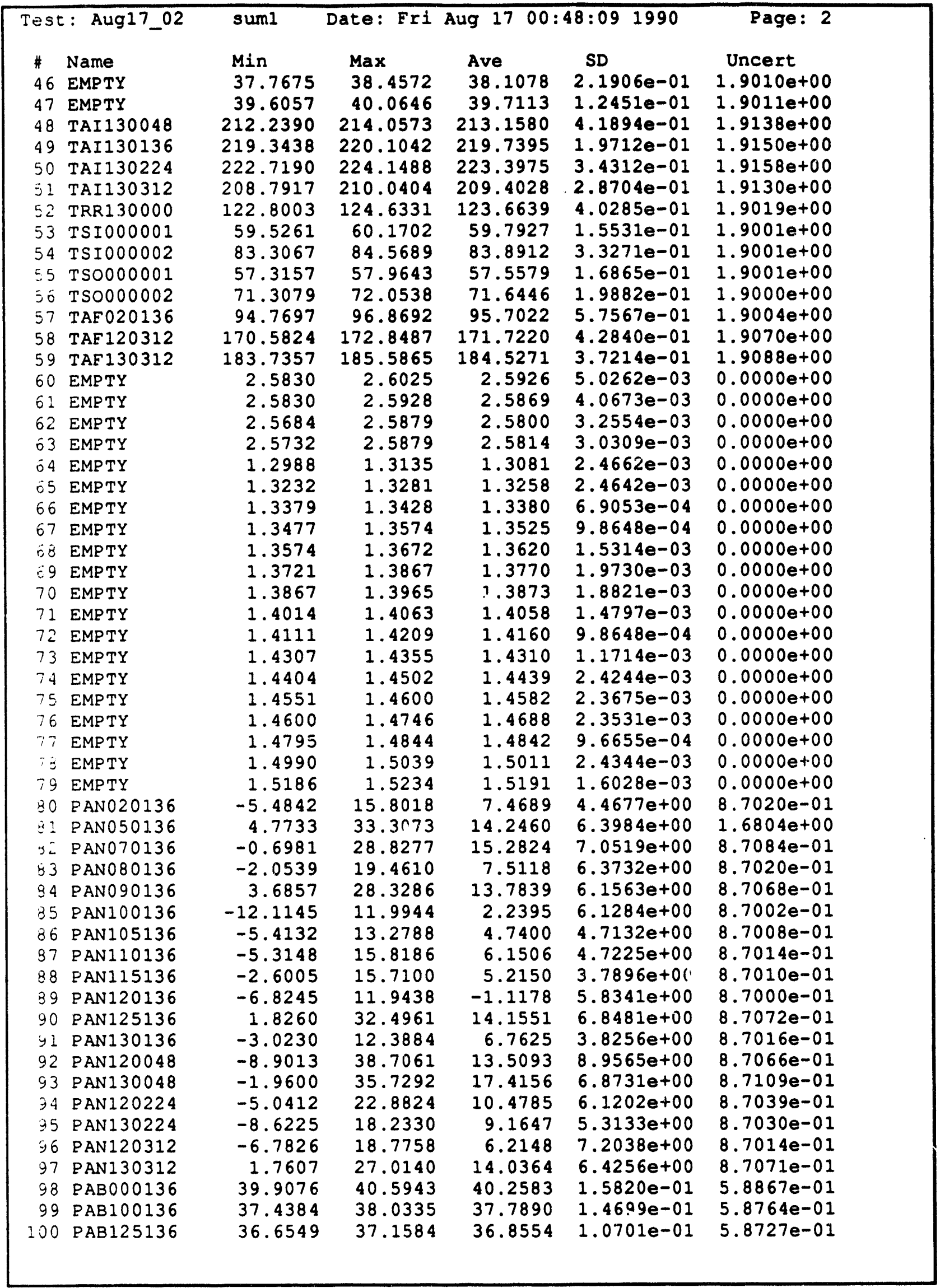




\begin{tabular}{|c|c|c|c|c|c|c|}
\hline TESt & $\therefore$ Aug17_02 & sum1 & Date: Fri & Aug 170 & $3: 091990$ & Page: 3 \\
\hline$\#$ & Name & Min & $\operatorname{Max}$ & Ave & SD & Uncert \\
\hline 101 & PIN000001 & 39.2263 & 40.8132 & 40.1247 & $3.33400-01$ & $7.5668 \mathrm{e}-01$ \\
\hline 102 & PRV000001 & 19.5965 & 19.7186 & 19.6063 & $3.3453 e-02$ & $7.5160 e-01$ \\
\hline 103 & PSR000001 & 34.9279 & 35.1720 & 35.0280 & $5.8828 e-02$ & $7.5510 \mathrm{e}-01$ \\
\hline $\begin{array}{l}104 \\
105\end{array}$ & PEM000001 & 38.8113 & 40.2761 & 39.5144 & $2.4359 e-01$ & $5.0967 e-01$ \\
\hline $\begin{array}{l}105 \\
106\end{array}$ & PAN 000136 & 82.4418 & 108.5344 & 98.7474 & $4.9076 e+00$ & $4.9262 e+00$ \\
\hline $\begin{array}{l}106 \\
107\end{array}$ & PFM000002 & 27.5689 & 27.5689 & 27.5689 & $9.6336 e-06$ & $2.1154 e-01$ \\
\hline 107 & EMPTY & 0.0049 & 0.0098 & 0.0088 & $1.9730 e-03$ & $0.0000 e+00$ \\
\hline$: 08$ & EMPTY & -10.0000 & -1.1523 & -5.8017 & $3.1244 e+00$ & $0.0000 e+00$ \\
\hline 109 & EMPTY & 0.0049 & 0.0098 & 0.0051 & $9.6655 e-04$ & $0.0000 e+00$ \\
\hline 110 & EMPTY & -10.0000 & -10.0000 & -10.0000 & $0.0000 e+00$ & $0.0000 e+00$ \\
\hline $2: 1$ & EMPTY & 0.0000 & 0.0049 & 0.0002 & $9.6655 e-04$ & $0.0000 e+00$ \\
\hline$\therefore 2$ & VPWO00001 & 177.0245 & 177.6104 & 177.3135 & $1.1995 e-01$ & $1.3371 e+00$ \\
\hline 113 & VPW000002 & 207.1327 & 207.5233 & 207.3241 & $9.2456 e-02$ & $1.4844 e+00$ \\
\hline 114 & VPW000003 & 256.0209 & 256.6068 & 256.1888 & $1.4770 e-01$ & $1.7375 e+00$ \\
\hline 115 & VPWO00004 & 182.0125 & 182.5984 & 182.2821 & $1.2409 e-01$ & $1.3610 e+00$ \\
\hline 116 & VPWO00005 & 242.5819 & 243.3631 & 242.9570 & $1.9666 \mathrm{e}-01$ & $1.6677 \mathrm{e}+00$ \\
\hline $1: 7$ & VPW000006 & 257.2454 & 258.0266 & 257.7882 & $1.5926 e-01$ & $1.7460 \mathrm{e}+00$ \\
\hline 118 & IPWO00001 & 932.0189 & 945.6852 & 940.6484 & $3.9065 e+00$ & $1.0992 e+01$ \\
\hline 119 & IPW000002 & 2215.5164 & 2225.2727 & 2218.4824 & $2.1679 e+00$ & $1.9307 e+01$ \\
\hline 120 & IPWO00003 & 2261.9998 & 2273.7000 & 2267.6157 & $2.0712 e+00$ & $1.9660 \mathrm{e}+01$ \\
\hline 121 & IPWO00004 & 651.1887 & 654.1184 & 652.4097 & $7.8454 e-01$ & $5.5285 e+00$ \\
\hline 122 & IPW000005 & 432.2547 & 435.1841 & 433.6021 & $7.4182 e-01$ & $3.9666 e+00$ \\
\hline 123 & IPW0000006 & 449.7446 & 451.2095 & 450.4279 & $4.0673 e-01$ & $4.0819 e+00$ \\
\hline 124 & EMPTY & -0.0244 & -0.0098 & -0.0185 & $3.3146 e-03$ & $0.0000 e+00$ \\
\hline$: 25$ & EMPTY & -0.0195 & -0.0098 & -0.0125 & $2.6396 e-03$ & $0.0000 e+00$ \\
\hline 126 & EMPTY & -0.0195 & -0.0049 & -0.0140 & $3.6924 e-03$ & $0.0000 e+00$ \\
\hline 127 & EMPTY & -0.0244 & -0.0098 & -0.0166 & $3.6911 \mathrm{e}-03$ & $0.0000 e+00$ \\
\hline 128 & IHTO000001 & 220.7106 & 222.8591 & 221.6521 & $5.2747 e-01$ & $4.6608 e+00$ \\
\hline 129 & IHT000002 & 227.4940 & 229.2518 & 228.2831 & $4.7508 e-01$ & $4.6927 e+00$ \\
\hline 130 & IHT000003 & 228.9889 & 231.1373 & 230.0241 & $4.6897 e-01$ & $4.7012 e+00$ \\
\hline 131 & IHTO000004 & 225.1315 & 227.0846 & 225.9518 & $4.6522 e-01$ & $4.6814 e+00$ \\
\hline 152 & IHTO000005 & 222.6262 & 224.5793 & 223.4659 & $4.4860 e-01$ & $4.6694 e+00$ \\
\hline 133 & IHTO000006 & 216.3799 & 218.5284 & 217.3760 & $4.8848 e-01$ & $4.6406 \mathrm{e}+00$ \\
\hline 134 & IHTO000007 & 225.7212 & 227.8696 & 226.6390 & $4.5720 e-01$ & $4.6847 e+00$ \\
\hline 135 & IHTO00008 & 223.1295 & 225.2779 & 224.0592 & $4.9215 e-01$ & $4.6723 e+00$ \\
\hline 136 & IHTO000009 & 223.5089 & 225.8526 & 224.4893 & $5.2865 e-01$ & $4.6743 e+00$ \\
\hline .27 & IHTO000010 & 55.2096 & 57.3580 & 56.1978 & $4.8232 e-01$ & $4.1383 e+00$ \\
\hline$: 78$ & IHTO00011 & 224.0572 & 226.4010 & 225.1510 & $5.1750 \mathrm{e}-01$ & $4.6775 e+00$ \\
\hline $1 \div 9$ & IHTO00012 & 220.1097 & 222.2581 & 221.1253 & $5.0992 e-01$ & $4.6583 e+00$ \\
\hline 140 & IHTO00013 & 159.1797 & 161.3281 & 160.1797 & $4.6961 \mathrm{e}-01$ & $4.4018 e+00$ \\
\hline 141 & IHTO000014 & 219.9444 & 222.2882 & 221.1826 & $5.3468 \mathrm{e}-01$ & $4.6586 \mathrm{e}+00$ \\
\hline $1+2$ & IHTO00015 & 164.3479 & 166.4964 & 165.3441 & $4.8848 e-01$ & $4.4208 e+00$ \\
\hline 143 & IHT000016 & 152.9747 & 155.1232 & 154.0138 & $4.8719 e-01$ & $4.3797 e+00$ \\
\hline 144 & IHTO00017 & 183.1956 & 185.3441 & 184.5003 & $5.1515 e-01$ & $4.4960 e+00$ \\
\hline 145 & IHTO00018 & 185.0886 & 187.8230 & 186.2450 & $5.3936 e-01$ & $4.5032 e+00$ \\
\hline 140 & IHTO00019 & 179.2593 & 181.6031 & 180.5992 & $6.0362 e-01$ & $4.4801 e+00$ \\
\hline 147 & IHTO00020 & 184.5177 & 186.6662 & 185.5802 & $5.7067 e-01$ & $4.5004 e+00$ \\
\hline 148 & IHTO00021 & -2.8132 & -0.0789 & -1.3601 & $6.9717 e-01$ & $4.1000 e+00$ \\
\hline 149 & IHTO00022 & 159.0708 & 161.4145 & 160.2153 & $5.5385 e-01$ & $4.4019 \mathrm{e}+00$ \\
\hline 250 & IHTO00023 & 180.3411 & 182.8801 & 181.7042 & $5.4030 e-01$ & $4.4846 e+00$ \\
\hline 151 & IHTO000024 & 144.9632 & 146.9163 & 146.1000 & $4.9235 e-01$ & $4.3525 e+00$ \\
\hline 152 & IHTO00025 & 179.5486 & 181.8923 & 180.7047 & $5.3792 e-01$ & $4.4806 \mathrm{e}+00$ \\
\hline 153 & IHTO000026 & 181.4979 & 184.2323 & 182.8495 & $5.5501 e-01$ & $4.4893 e+00$ \\
\hline 154 & IHTOO00027 & 155.2284 & 157.7674 & 156.4159 & $5.5781 \mathrm{e}-01$ & $4.3882 e+00$ \\
\hline 155 & IHT000028 & 157.1552 & 159.6942 & 158.5615 & $5.8793 e-01$ & $4.3959 e+00$ \\
\hline
\end{tabular}




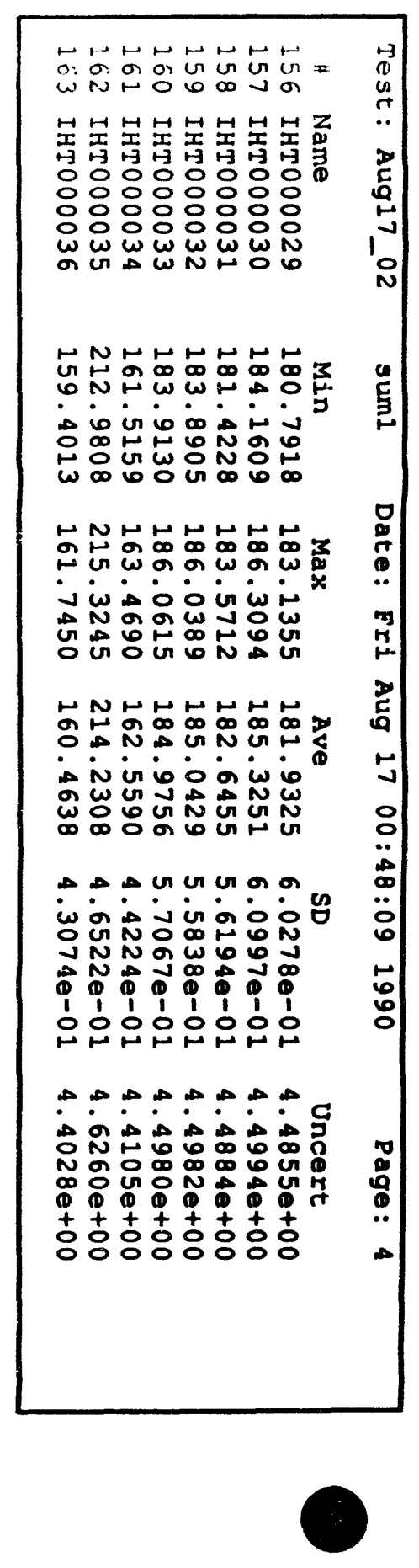




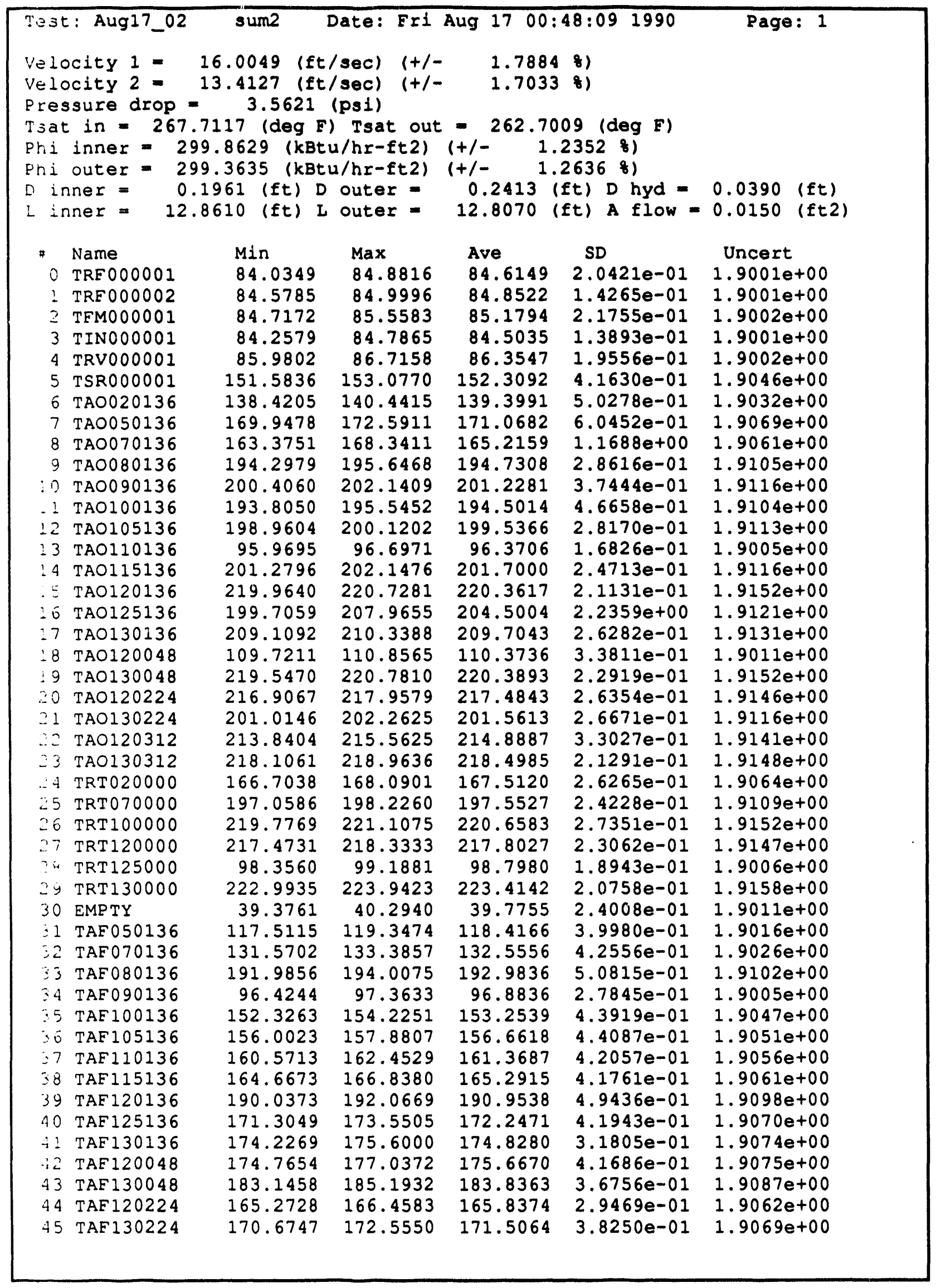




\begin{tabular}{|c|c|c|c|c|c|c|}
\hline \multicolumn{2}{|c|}{ Test: Aug17_02 } & sum2 & Date: Fri & Aug 1700 : & $48: 091990$ & Page: 2 \\
\hline 4 & Name & $\operatorname{Min}$ & $\operatorname{Max}$ & Ave & SD & Uncert \\
\hline 46 & EMPTY & 37.5374 & 38.9168 & 38.0848 & $2.4530 e-01$ & $1.9010 e+00$ \\
\hline 47 & EMPTY & 39.3761 & 39.8352 & 39.6653 & $1.2960 e-01$ & $1.9011 e+00$ \\
\hline 48 & TAI 130048 & 212.7182 & 214.4391 & 213.4907 & $3.4380 e-01$ & $1.9138 \mathrm{e}+00$ \\
\hline 49 & TAI 130136 & 219.4384 & 220.5793 & 220.0547 & $2.3927 e-01$ & $1.9151 \mathrm{e}+00$ \\
\hline 50 & TAI 130224 & 223.2902 & 224.7196 & 223.9081 & $3.3956 e-01$ & $1.9159 e+00$ \\
\hline 51 & TAI 130312 & 209.2711 & 210.3287 & 209.7369 & $2.7262 e-01$ & $1.9131 \mathrm{e}+00$ \\
\hline 52 & TRR 130000 & 123.6132 & 125.1400 & 124.2369 & $3.6624 e-01$ & $1.9020 \mathrm{e}+00$ \\
\hline 53 & TSI000001 & 59.5245 & 60.3844 & 59.9512 & $1.6949 e-01$ & $1.9001 e+00$ \\
\hline$\therefore 1$ & TSI000002 & 83.0949 & 84.4631 & 83.8953 & $3.1675 e-01$ & $1.9001 \mathrm{e}+00$ \\
\hline 55 & TSO000001 & 57.0992 & 57.8567 & 57.5449 & $1.7836 e-01$ & $1.9001 \mathrm{e}+00$ \\
\hline 50 & TSO000002 & 71.2009 & 72.0538 & 71.6446 & $2.2719 e-01$ & $1.9000 \mathrm{e}+00$ \\
\hline 37 & TAE020136 & 94.8741 & 96.5548 & 95.6435 & $4.6639 e-01$ & $1.9004 e+00$ \\
\hline$\because 8$ & TAF 120312 & 170.8772 & 173.2424 & 171.8008 & $4.8546 e-01$ & $1.9070 e+00$ \\
\hline 59 & TAF 130312 & 184.0277 & 185.3919 & 184.6906 & $3.4819 e-01$ & $1.9088 e+00$ \\
\hline 60 & EMPTY & 2.5879 & 2.6025 & 2.5961 & $3.8762 e-03$ & $0.0000 e+00$ \\
\hline 61 & EMPTY & 2.5830 & 2.5928 & 2.5897 & $2.5894 e-03$ & $0.0000 e+00$ \\
\hline 02 & EMPTY & 2.5781 & 2.5879 & 2.5829 & $1.5566 e-03$ & $0.0000 e+00$ \\
\hline 63 & EMPTY & 2.5830 & 2.5879 & 2.5834 & $1.3381 e-03$ & $0.0000 e+00$ \\
\hline 64 & EMPTY & 1.3037 & 1.3135 & 1.3086 & $9.8648 e-04$ & $0.0000 e+00$ \\
\hline 65 & EMPTY & 1.3232 & 1.3281 & 1.3270 & $2.1065 e-03$ & $0.0000 \mathrm{e}+00$ \\
\hline 86 & EMPTY & 1.3379 & 1.3428 & 1.3383 & $1.3381 e-03$ & $0.0000 e+00$ \\
\hline$\therefore 7$ & EMPTY & 1.3525 & 1.3574 & 1.3527 & $9.6655 e-04$ & $0.0000 e+00$ \\
\hline 68 & EMPTY & 1.3623 & 1.3672 & 1.3625 & $9.6655 e-04$ & $0.0000 e+00$ \\
\hline 69 & EMPTY & 1.3721 & 1.3818 & 1.3770 & $9.8648 e-04$ & $0.0000 \mathrm{e}+00$ \\
\hline 70 & EMPTY & 1.3867 & 1.3916 & 1.3871 & $1.3381 e-03$ & $0.0000 e+00$ \\
\hline : & EMPTY & 1.4063 & 1.4160 & 1.4064 & $1.3811 e-03$ & $0.0000 \mathrm{e}+00$ \\
\hline$\because 2$ & EMPTY & 1.4111 & 1.4209 & 1.4160 & $9.8648 e-04$ & $0.0000 e+00$ \\
\hline 73 & EMPTY & 1.4307 & 1.4355 & 1.4315 & $1.8950 \mathrm{e}-03$ & $0.0000 e+00$ \\
\hline$? 4$ & EMPTY & 1.4404 & 1.4453 & 1.4438 & $2.2603 e-03$ & $0.0000 \mathrm{e}+00$ \\
\hline 75 & EMPTY & 1.4551 & 1.4600 & 1.4591 & $1.8950 e-03$ & $0.0000 \mathrm{e}+00$ \\
\hline 70 & EMPTY & 1.4648 & 1.4697 & 1.4695 & $9.6655 e-04$ & $0.0000 e+00$ \\
\hline 77 & EMPTY & 1.4795 & 1.4893 & 1.4843 & $1.2041 \mathrm{e}-03$ & $0.0000 e+00$ \\
\hline 79 & EMPTY & 1.4990 & 1.5039 & 1.5021 & $2.3675 e-03$ & $0.0000 \mathrm{e}+00$ \\
\hline$\cdots$ & EMPTY & 1.5088 & 1.5234 & 1.5204 & $2.9413 e-03$ & $0.0000 e+00$ \\
\hline$\because 8$ & PANO20136 & -2.2036 & 20.5320 & 7.7039 & $5.0078 e+00$ & $8.7021 e-01$ \\
\hline$\because 1$ & PAN050136 & 4.4682 & 30.2555 & 16.4768 & $6.0422 \mathrm{e}+00$ & $1.6805 \mathrm{e}+00$ \\
\hline$x^{2}$ & PAN070136 & -1.2321 & 27.4544 & 12.8990 & $6.6623 e+00$ & $8.706 \mathrm{Ce}-01$ \\
\hline$\because 3$ & PAN080136 & -4.4190 & 21.4446 & 7.9360 & $6.5624 \mathrm{e}+00$ & $8.7023 e-01$ \\
\hline$\because 4$ & PAN090136 & 0.4050 & 26.6502 & 14.3439 & $5.2302 e+00$ & $8.7074 \mathrm{e}-01$ \\
\hline 25 & PAN100136 & -10.2834 & 13.3677 & 5.6010 & $5.1389 e+00$ & $8.7011 e-01$ \\
\hline 36 & PAN 105136 & -6.6339 & 10.9137 & 2.3535 & $4.5491 e+00$ & $8.7002 e-01$ \\
\hline 87 & PAN110136 & -6.8407 & 19.6333 & 5.3404 & $6.0853 e+00$ & $8.7010 e-01$ \\
\hline 38 & PAN 115136 & -4.3553 & 17.6173 & 6.1534 & $5.1785 e+00$ & $8.7014 e-01$ \\
\hline$\div 9$ & PAN 120136 & -6.7482 & 6.4506 & -2.1752 & $4.3506 e+00$ & $8.7002 e-01$ \\
\hline 90 & PAN 125136 & -2.7517 & 26.3163 & 11.5901 & $6.6591 e+00$ & $8.7048 e-01$ \\
\hline 91 & PAN 130136 & -0.2001 & 28.0286 & 10.3590 & $6.0589 e+00$ & $8.7039 e-01$ \\
\hline 92 & PAN120048 & -2.7215 & 25.3547 & 14.8612 & $7.2134 e+00$ & $8.7079 e-01$ \\
\hline 3 & PAN 130048 & 3.2280 & 33.0589 & 15.7143 & $7.4614 e+00$ & $8.7089 e-01$ \\
\hline 14 & PAN120224 & 2.2830 & 19.4492 & 12.6407 & $3.6234 \mathrm{e}+00$ & $8.7057 e-01$ \\
\hline 95 & PAN 130224 & -3.8160 & 18.0041 & 6.5478 & $4.7571 e+00$ & $8.7015 e-01$ \\
\hline+6 & PAN120312 & -7.0115 & 19.2336 & 7.7956 & $5.7885 e+00$ & $8.7022 e-01$ \\
\hline$\because$ & PAN 130312 & 1.9133 & 32.6598 & 14.4362 & $6.7033 e+00$ & $8.7075 e-01$ \\
\hline 98 & PAB000136 & 39.9076 & 40.6401 & 40.2308 & $1.8343 e-01$ & $5.8866 e-01$ \\
\hline 99 & PAB100136 & 37.3468 & 38.0335 & 37.6865 & $1.3563 e-01$ & $5.8760 e-01$ \\
\hline 100 & PAB125136 & 36.6549 & 38.1655 & 36.9753 & $2.6142 e-01$ & $5.8732 e-01$ \\
\hline
\end{tabular}




\begin{tabular}{|c|c|c|c|c|c|c|}
\hline \multicolumn{2}{|c|}{ Test: Aug17_02 } & sum2 & Date: Fri & Aug 1700 & $18: 091990$ & Page: 3 \\
\hline$\#$ & Name & Min & $\operatorname{Max}$ & Ave & SD & Uncert \\
\hline 201 & PIN 000001 & 39.2263 & 40.6911 & 40.0515 & $4.0790 e-01$ & $7.5665 e-01$ \\
\hline 102 & PRV000001 & 19.7186 & 19.7186 & 19.7186 & $1.9267 e-06$ & $7.5162 e-01$ \\
\hline 103 & PSR000001 & 34.8058 & 35.1720 & 35.0426 & $7.1520 e-02$ & $7.5510 e-01$ \\
\hline 104 & PFM000001 & 38.8113 & 40.0015 & 39.3441 & $2.7664 e-01$ & $5.0958 e-01$ \\
\hline 105 & PANO00136 & 80.6108 & 111.2809 & 98.6192 & $6.5529 e+00$ & $4.9262 e+00$ \\
\hline 106 & PFM000002 & 27.5689 & 27.5689 & 27.5689 & $9.6336 e-06$ & $2.1154 e-01$ \\
\hline 107 & EMPTY & 0.0049 & 0.0098 & 0.0090 & $1.8082 e-03$ & $0.0000 e+00$ \\
\hline 108 & EMPTY & -10.0000 & -0.6396 & -5.1242 & $3.1045 e+00$ & $0.0000 e+00$ \\
\hline $1: 9$ & EMPTY & 0.0049 & 0.0098 & 0.0052 & $1.1714 \mathrm{e}-03$ & $0.0000 e+00$ \\
\hline 1.2 & EMPTY & -10.0000 & -10.0000 & -10.0000 & $0.0000 e+00$ & $0.0000 e+00$ \\
\hline $1: !$ & EMPTY & -0.0049 & 0.0049 & -0.0001 & $1.2041 e-03$ & $0.0000 \mathrm{e}+00$ \\
\hline 112 & VPW000001 & 177.0245 & 177.6104 & 177.3370 & $1.2478 e-01$ & $1.3373 e+00$ \\
\hline $2: 3$ & VPWO00002 & 207.1327 & 207.5233 & 207.3866 & $9.8648 e-02$ & $1.4847 \mathrm{e}+00$ \\
\hline$\therefore ! 4$ & VPW000003 & 256.0209 & 256.6068 & 256.2786 & $1.2124 \mathrm{e}-01$ & $1.7380 \mathrm{e}+00$ \\
\hline 1.5 & VPW000004 & 182.0125 & 182.5984 & 182.3173 & $1.1272 \mathrm{e}-01$ & $1.3611 \mathrm{e}+00$ \\
\hline $1: 6$ & VPWO00005 & 242.3866 & 243.5584 & 243.0273 & $2.3689 e-01$ & $1.6680 \mathrm{e}+00$ \\
\hline $1: 7$ & VPWO000006 & 257.6360 & 258.2219 & 257.9288 & $1.3811 e-01$ & $1.7467 e+00$ \\
\hline $1: 8$ & IPW 000001 & 941.7806 & 949.5900 & 945.8805 & $1.6382 \mathrm{e}+00$ & $1.1020 e+01$ \\
\hline $1: 9$ & IPW000002 & 2211.6140 & 2221.3704 & 2217.3501 & $2.8539 \mathrm{e}+00$ & $1.9299 e+01$ \\
\hline 120 & IPW000003 & 2261.9998 & 2269.7998 & 2264.8862 & $2.1664 \mathrm{e}+00$ & $1.9641 e+01$ \\
\hline 121 & IPW000004 & 651.6770 & 654.6067 & 652.8198 & $1.0834 e+00$ & $5.5315 e+00$ \\
\hline 1.2 & IPW000005 & 431.7664 & 434.2076 & 432.8893 & $5.6016 e-01$ & $3.9617 e+00$ \\
\hline $2 \therefore 3$ & IPW000006 & 448.2798 & 450.7212 & 449.3049 & $5.3352 e-01$ & $4.0742 e+00$ \\
\hline$\therefore \therefore 4$ & EMPTY & -0.0244 & -0.0098 & -0.0175 & $3.8321 \mathrm{e}-03$ & $0.0000 e+00$ \\
\hline 125 & EMPTY & -0.0195 & -0.0049 & -0.0134 & $3.3901 e-03$ & $0.0000 e+00$ \\
\hline$\therefore \therefore$ & EMPTY & -0.0195 & -0.0049 & -0.0137 & $3.4173 e-03$ & $0.0000 e+00$ \\
\hline$\therefore \therefore 7$ & EMPTY & -0.0244 & -0.0146 & -0.0176 & $3.1195 e-03$ & $0.0000 e+00$ \\
\hline$\therefore s$ & IHTO00001 & 220.3200 & 223.2497 & 221.6013 & $5.9733 e-01$ & $4.6605 e+00$ \\
\hline $12: 9$ & IHTO000002 & 226.9080 & 230.0330 & 228.2519 & $6.3090 e-01$ & $4.6925 e+00$ \\
\hline $2: 0$ & IHTO00003 & 228.7936 & 231.5280 & 230.0592 & $6.1287 e-01$ & $4.7014 e+00$ \\
\hline 231 & IHTO00004 & 224.7408 & 227.2799 & 225.9323 & $5.9090 e-01$ & $4.6813 \mathrm{e}+00$ \\
\hline$\because 22$ & IHT000005 & 222.2356 & 224.5793 & 223.3878 & $5.6255 e-01$ & $4.6691 \mathrm{e}+00$ \\
\hline 133 & IHT000006 & 216.1846 & 218.5284 & 217.3291 & $5.3235 e-01$ & $4.6404 \mathrm{e}+00$ \\
\hline 134 & IHT0000007 & 225.3306 & 227.8696 & 226.6390 & $5.6669 \mathrm{e}-01$ & $4.6847 e+00$ \\
\hline$\therefore \vdots$ & IHTO00008 & 222.9342 & 225.2779 & 224.0749 & $5.4442 e-01$ & $4.6724 \mathrm{e}+00$ \\
\hline$: 8$ & IHTO00009 & 223.3135 & $2 \angle 5.6573$ & 224.4619 & $5.2592 e-01$ & $4.6742 \mathrm{e}+00$ \\
\hline$\therefore ?$ & IHTO000010 & 55.2096 & 57.9440 & 56.1314 & $5.7864 e-01$ & $4.1382 \mathrm{e}+00$ \\
\hline 158 & IHTO00011 & 224.2525 & 226.2057 & 225.0885 & $5.0692 e-01$ & $4.6772 e+00$ \\
\hline 139 & IHTO00012 & 220.1097 & 222.2581 & 221.1370 & $4.7484 e-01$ & $4.6583 e+00$ \\
\hline$\therefore 0$ & IHT000013 & 158.9844 & 161.5234 & 160.0977 & $5.1031 e-01$ & $4.4015 e+00$ \\
\hline$\therefore 1$ & IHTO00014 & 219.9444 & 222.4835 & 221.1826 & $6.0052 e-01$ & $4.6586 \mathrm{e}+00$ \\
\hline$i a_{2}$ & IHTO00015 & 164.1526 & 166.3011 & 165.2035 & $4.7497 e-01$ & $4.4203 e+00$ \\
\hline 1.43 & IHTO00016 & 152.7794 & 154.7326 & 153.7520 & $4.6100 \mathrm{e}-01$ & $4.3788 e+00$ \\
\hline $1 \div 4$ & IHTO00017 & 182.8050 & 185.9300 & 184.1996 & $7.7726 e-01$ & $4.4948 \mathrm{e}+00$ \\
\hline $1 i_{5}$ & IHTO00018 & 184.5027 & 187.4324 & 186.2067 & $6.6175 e-01$ & $4.5022 \mathrm{e}+00$ \\
\hline 146 & IHTO00019 & 179.4546 & 181.9937 & 180.5875 & $4.9756 e-01$ & $4.4801 e+00$ \\
\hline 147 & IHTO000020 & 184.7130 & 187.0568 & 185.6231 & $5.0007 e-01$ & $4.5006 e+00$ \\
\hline 243 & IHTO00021 & -2.6179 & 0.1164 & -1.3601 & $5.9472 e-01$ & $4.1000 e+00$ \\
\hline $1: 3$ & IHTO00022 & 159.0708 & 161.4145 & 160.2466 & $5.0914 \mathrm{e}-01$ & $4.4020 e+00$ \\
\hline $1: 0$ & IHTO00023 & 180.9270 & 183.2708 & 181.8331 & $4.9404 \mathrm{e}-01$ & $4.4851 e+00$ \\
\hline $1 \equiv 1$ & IHTO00024 & 145.3538 & 147.1116 & 146.2133 & $4.2682 e-01$ & $4.3529 e+00$ \\
\hline $1 \leq 2$ & IHTOOOOO25 & 179.9392 & 181.6970 & 180.8415 & $4.5658 e-01$ & $4.4811 e+00$ \\
\hline $1: 3$ & IHT000026 & 182.2792 & 184.2323 & 183.1386 & $4.2499 e-01$ & $4.4904 e+00$ \\
\hline $1 \div 4$ & IHTO00027 & 155.6190 & 157.7674 & 156.5448 & $4.6322 e-01$ & $4.3887 e+00$ \\
\hline 155 & IHTO00028 & 157.3505 & 160.4755 & 158.9638 & $7.1986 \mathrm{e}-01$ & $4.3974 \mathrm{e}+00$ \\
\hline
\end{tabular}




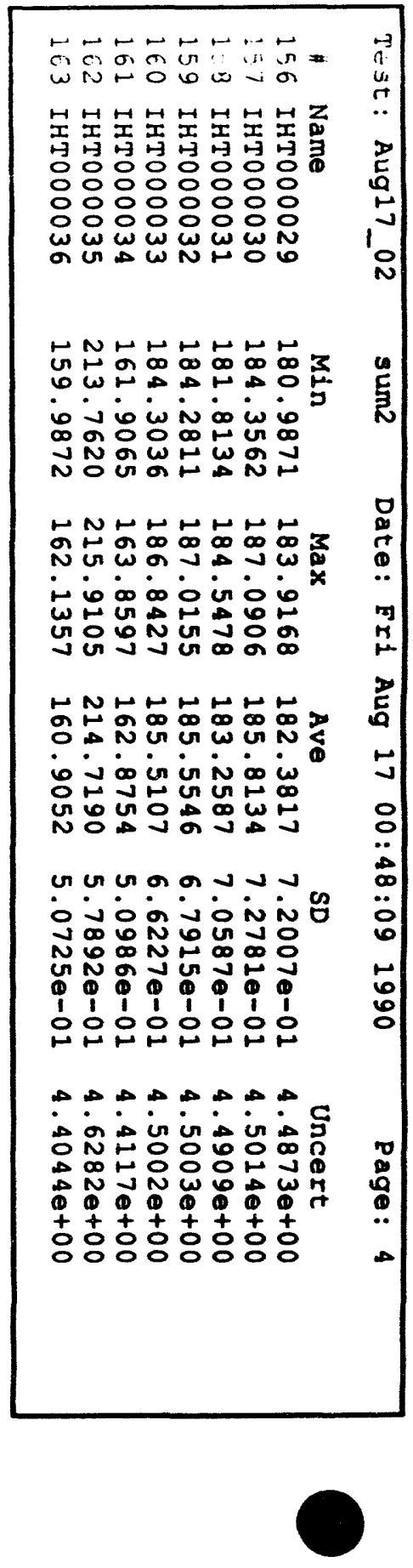




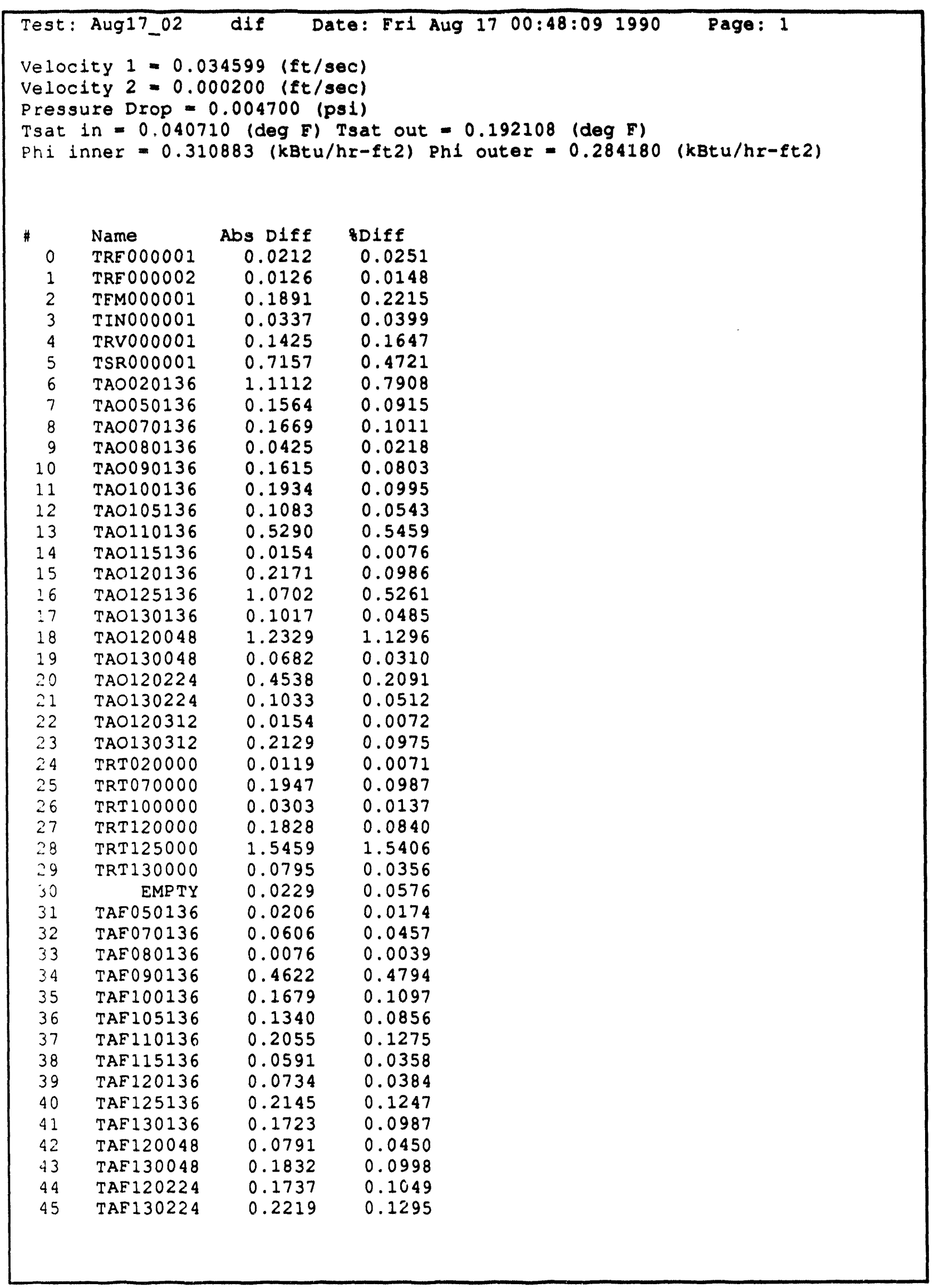




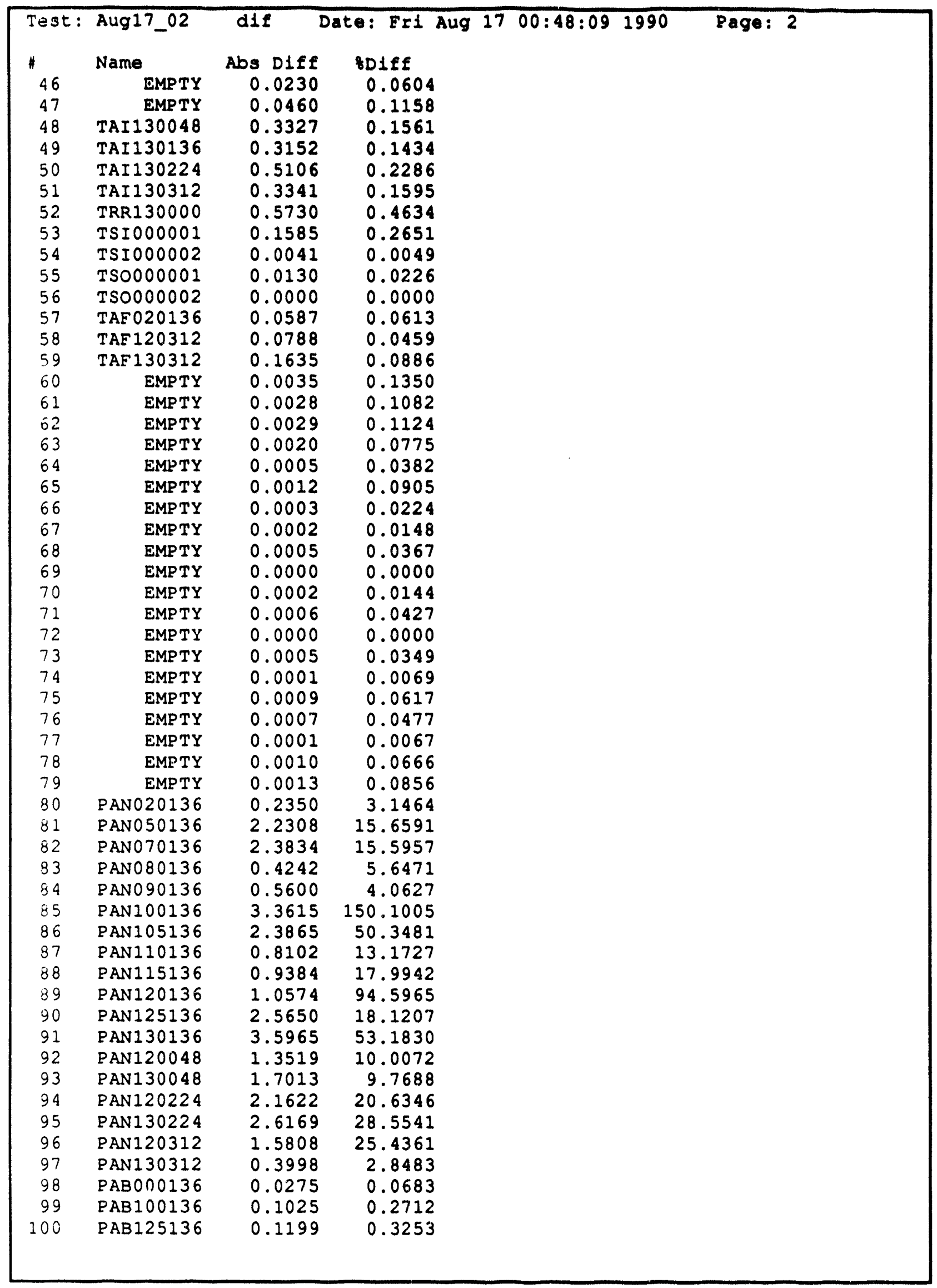




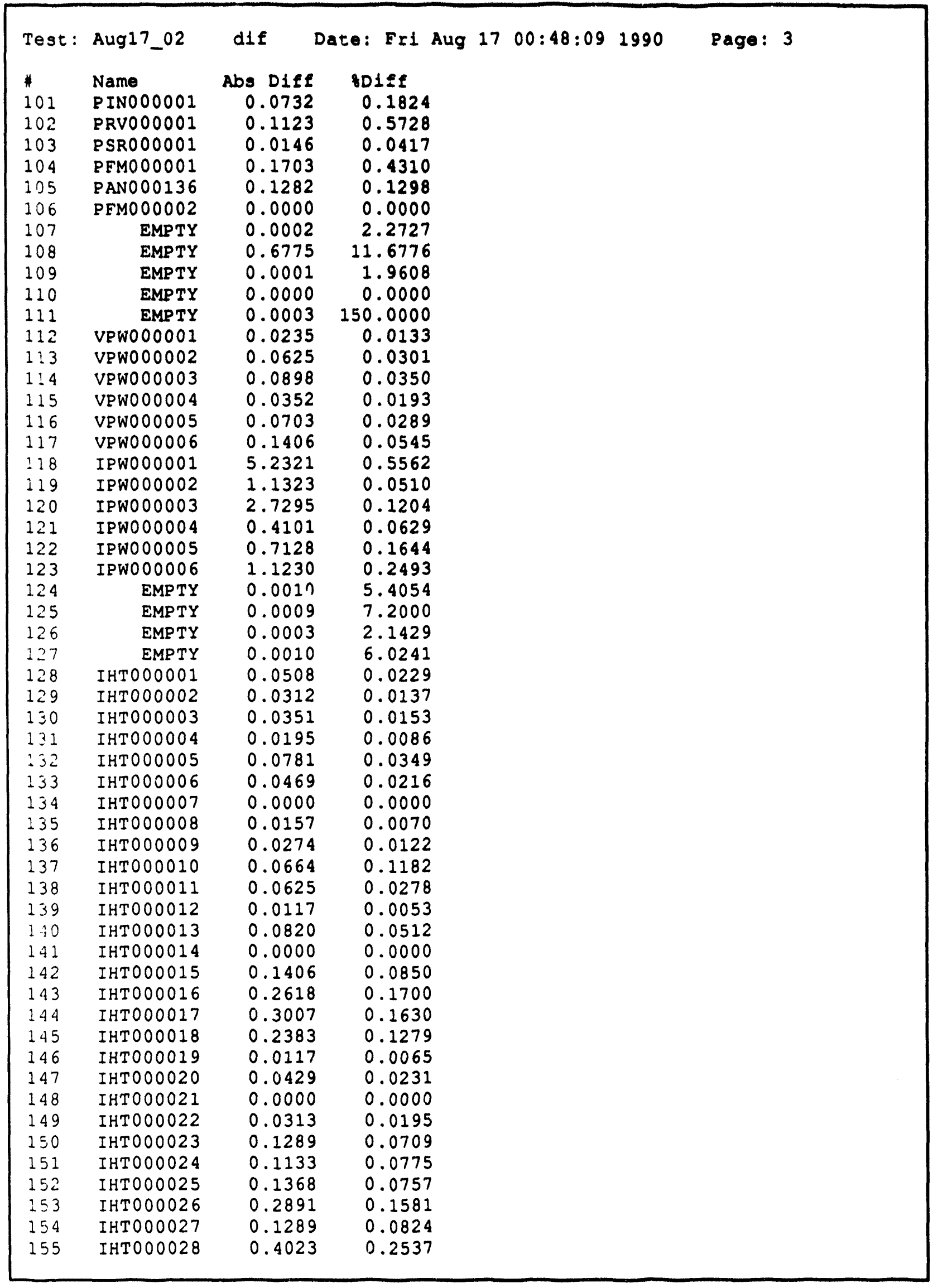




$\begin{array}{llrcr}\text { Test: Aug17_02 } & \text { dif } & \text { Date: Eri Aug } 1700: 48: 091990 & \text { Page: } 4 \\ & & \text { Abs D1ff } & \text { Diff } \\ 156 & \text { IHTO00029 } & 0.4492 & 0.2469 & \\ 157 & \text { IHTO00030 } & 0.4883 & 0.2635 \\ 158 & \text { IHTO00031 } & 0.6132 & 0.3357 \\ 159 & \text { IHTO00032 } & 0.5117 & 0.2765 \\ 160 & \text { IHTO00033 } & 0.5351 & 0.2893 \\ 161 & \text { IHTO00034 } & 0.3164 & 0.1946 \\ 162 & \text { IHTO00035 } & 0.4882 & 0.2279 \\ 163 & \text { IHTO00036 } & 0.4414 & 0.2751\end{array}$




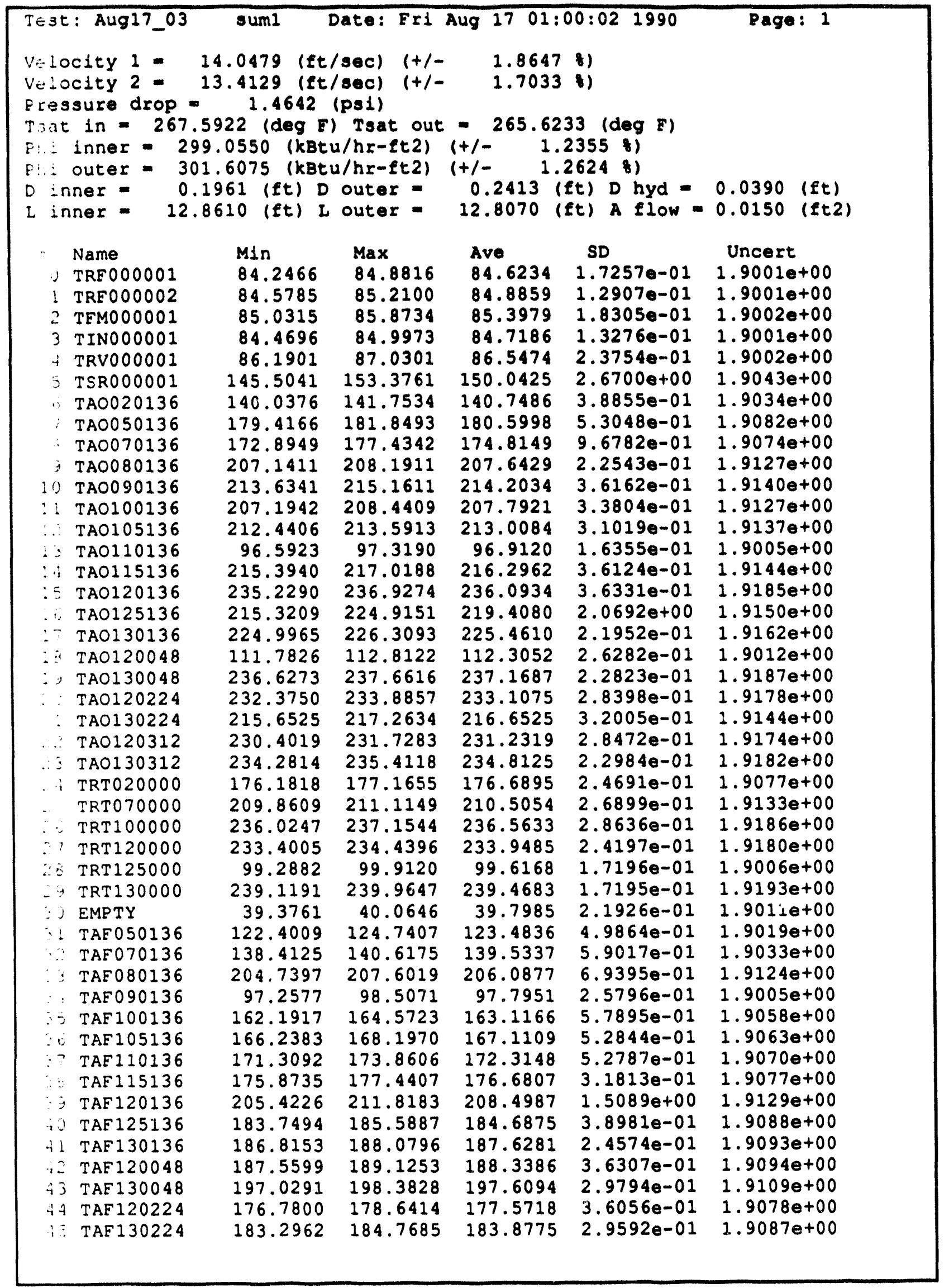




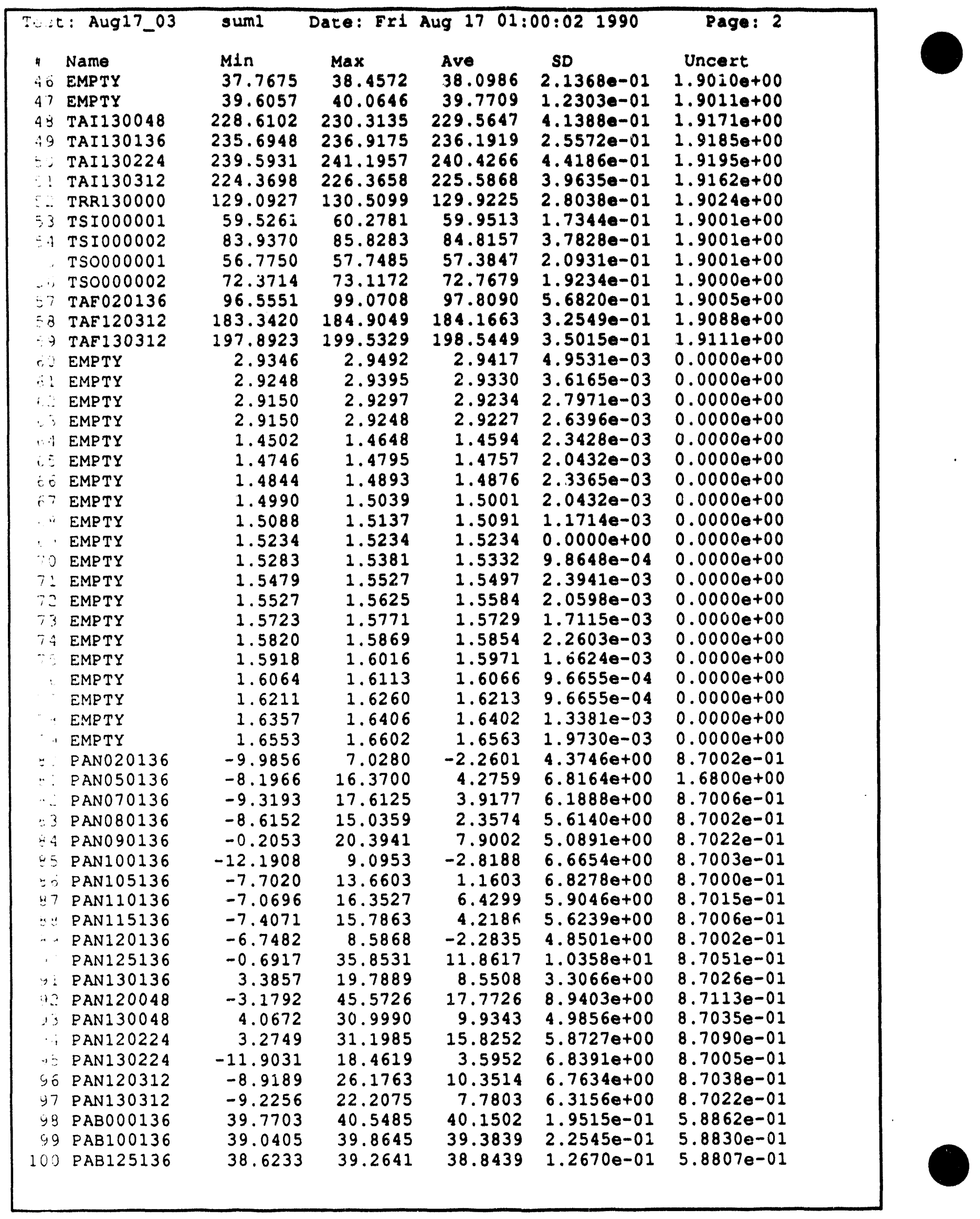




\begin{tabular}{|c|c|c|c|c|c|c|}
\hline \multicolumn{2}{|c|}{ TGSt: Aug17_03 } & suml & \multicolumn{3}{|c|}{ Date: Fri Aug 17 01:00:02 1990} & \multirow[t]{2}{*}{ Page: 3} \\
\hline 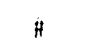 & Name & Min & $\operatorname{Max}$ & Ave & SD & \\
\hline 101 & PINO00001 & 39.2263 & 40.5690 & 39.8318 & $4.2067 e-01$ & $8 e-01$ \\
\hline 102 & PRV000001 & 19.7186 & 19.8406 & 19.7552 & $5.6508 e-02$ & $7.5162 e-01$ \\
\hline $1: 3$ & PSR0000001 & 37.1251 & 37.3693 & 37.2399 & $4.5541 e-02$ & $7.5576 e-01$ \\
\hline 104 & PFMO000001 & 29.8391 & 30.6631 & 30.2603 & $2.0346 \mathrm{e}-01$ & $5.0569 e-01$ \\
\hline $1: 5$ & PANO00136 & 29.3412 & 52.6872 & 40.5382 & $6.0829 e+00$ & $4.9210 e+00$ \\
\hline $2 \therefore \therefore$ & PEM0000002 & 27.5689 & 27.5689 & 27.5689 & $9.6336 \mathrm{e}-06$ & $2.1154 e-01$ \\
\hline $1::$ & EMPTY & 0.0049 & 0.0098 & 0.0063 & $2.2146 \mathrm{e}-03$ & $0.0000 \mathrm{e}+00$ \\
\hline $2 \cdots$ & EMPTY & -10.0000 & -0.5664 & -4.0729 & $2.1112 \mathrm{e}+00$ & $0.0000 e+00$ \\
\hline $10 y$ & EMPTY & 0.0049 & 0.0049 & 0.0049 & $0.0000 e+00$ & $0.0000 e+00$ \\
\hline $1: 0$ & EMPTY & -10.0000 & -10.0000 & -10.0000 & $0.0000 e+00$ & $0.0000 \mathrm{e}+00$ \\
\hline $1:$ & EMPTY & 0.0000 & 0.0000 & 0.0000 & $0.0000 e+00$ & $0.0000 e+00$ \\
\hline $1: 2$ & VPWO000001 & 177.2198 & 177.6104 & 177.4698 & $1.0470 e-01$ & $1.3379 e+00$ \\
\hline 113 & VPW0000002 & 207.3280 & 207.7186 & 207.4804 & $9.8963 e-02$ & $1.4852 \mathrm{e}+00$ \\
\hline 114 & VPW000003 & 256.2162 & 256.6068 & 256.3919 & $1.0625 e-01$ & $1.7386 e+00$ \\
\hline $1: 5$ & VPWO000004 & 182.0125 & 182.5984 & 182.3563 & $1.2823 e-01$ & $1.3613 e+00$ \\
\hline $1: 6$ & VPWO00005 & 242.5819 & 243.5584 & 243.0859 & $3.4420 e-01$ & $1.6683 e+00$ \\
\hline $1: 7$ & VPWO000006 & 257.6360 & 258.0266 & 257.8936 & $1.0763 e-01$ & $1.7465 \mathrm{e}+00$ \\
\hline $1: 2$ & IPW000001 & 943.7329 & 951.5423 & 948.4966 & $2.3719 e+00$ & $1.1034 \mathrm{e}+01$ \\
\hline $1: 0$ & IPWO000002 & 2235.0291 & 2244.7854 & 2238.3853 & $2.3335 e+00$ & $1.9450 \mathrm{e}+01$ \\
\hline $1 \%:$ & IPW000003 & 2252.2498 & 2261.9998 & 2256.2666 & $2.3771 e+00$ & $1.9579 \mathrm{e}+01$ \\
\hline $1 \ldots:$ & IPWO000004 & 654.6067 & 657.0481 & 655.6421 & $7.3584 e-01$ & $5.5523 e+00$ \\
\hline 122 & IPW000005 & 434.2076 & 437.1370 & 435.5940 & $6.1092 e-01$ & $3.9802 e+00$ \\
\hline 123 & IPW0000006 & 448.2798 & 450.7212 & 449.6662 & $5.6117 e-01$ & $4.0767 e+00$ \\
\hline $12 \mathrm{i}$ & EMPTY & -0.0293 & -0.0146 & -0.0206 & $3.7291 e-03$ & $0.0000 e+00$ \\
\hline $1 \leqslant 0$ & EMPTY & -0.0195 & -0.0098 & -0.0134 & $2.3778 e-03$ & $0.0000 \mathrm{e}+00$ \\
\hline 126 & EMPTY & -0.0146 & -0.0049 & -0.0132 & $2.4662 e-03$ & $0.0000 e+00$ \\
\hline 127 & EMPTY & -0.0244 & -0.0146 & -0.0179 & $3.2133 e-03$ & $0.0000 e+00$ \\
\hline 128 & IHT 000001 & 219.5387 & 222.2731 & 221.2614 & $6.4680 e-01$ & $4.6589 e+00$ \\
\hline $1: \because y$ & IHTO00002 & 226.1268 & 228.8612 & 227.8769 & $6.2385 e-01$ & $4.6907 e+00$ \\
\hline 130 & IHT000003 & 228.0123 & 230.7467 & 229.8288 & $6.4144 e-01$ & $4.7002 e+00$ \\
\hline $1: !$ & IHTO00004 & 223.9596 & 226.6940 & 225.6510 & $5.7400 e-01$ & $4.6799 \mathrm{e}+00$ \\
\hline 130 & IHTO00005 & 221.4543 & 224.1887 & 223.1378 & $6.0088 e-01$ & $4.6679 e+00$ \\
\hline$\because 3$ & IHTO000006 & 215.5987 & 218.3331 & 217.1182 & $5.8699 e-01$ & $4.6394 e+00$ \\
\hline $1 \therefore:$ & IHTO000007 & 224.9399 & 227.6743 & 226.5023 & $6.1384 e-01$ & $4.6841 \mathrm{e}+00$ \\
\hline $1 \vdots \vdots$ & IHT000008 & 222.3483 & 225.0826 & 223.9420 & $6.0019 e-01$ & $4.6717 e+00$ \\
\hline $2: 0$ & IHTO00009 & 222.7276 & 225.6573 & 224.3682 & $6.7428 e-01$ & $4.6738 e+00$ \\
\hline $1 \because 7$ & IHTO00010 & 54.6236 & 57.1627 & 56.1119 & $5.0051 e-01$ & $4.1382 \mathrm{e}+00$ \\
\hline $1 \therefore:$ & IHTO00011 & 223.8619 & 225.8150 & 225.0534 & $4.6228 e-01$ & $4.6771 e+00$ \\
\hline 139 & IHTO00012 & 220.1097 & 222.0628 & 221.1136 & $4.9601 e-01$ & $4.6582 \mathrm{e}+00$ \\
\hline $1 \div 0$ & IHTO00013 & 159.3750 & 161.5234 & 160.3125 & $4.7515 e-01$ & $4.4023 e+00$ \\
\hline 141 & IHTO000014 & 220.1397 & 222.0929 & 221.3233 & $5.4734 e-01$ & $4.6592 \mathrm{e}+00$ \\
\hline 142 & IHTO00015 & 164.1526 & 166.3011 & 165.3832 & $4.8368 e-01$ & $4.4210 \mathrm{e}+00$ \\
\hline 143 & IHTO00016 & 153.1701 & 154.9279 & 154.2481 & $4.5204 e-01$ & $4.3806 e+00$ \\
\hline 144 & IHTO00017 & 183.9769 & 186.1253 & 185.0824 & $5.1541 e-01$ & $4.4984 \mathrm{e}+00$ \\
\hline 145 & IHTO00018 & 185.4793 & 187.6277 & 186.5028 & $5.3169 e-01$ & $4.5043 e+00$ \\
\hline $1: 5$ & IHTO00019 & 179.4546 & 181.7984 & 180.4859 & $5.2799 e-01$ & $4.4797 e+00$ \\
\hline $1 \div 7$ & IHTO00020 & 184.1271 & 186.4708 & 185.5255 & $4.9812 \mathrm{e}-01$ & $4.5002 \mathrm{e}+00$ \\
\hline 196 & IHTO000021 & -3.2039 & -0.0789 & -1.4109 & $6.5991 e-01$ & $4.1000 \mathrm{e}+00$ \\
\hline $1: 9$ & IHTO000022 & 158.6801 & 161.4145 & 160.1919 & $5.5916 e-01$ & $4.4018 e+00$ \\
\hline$: \div$ & IHTO000023 & 180.3411 & 182.8801 & 181.7784 & $5.3780 e-01$ & $4.4849 e+00$ \\
\hline $1 \ldots$ & IHTO000024 & 145.1585 & 147.5023 & 146.1351 & $4.8967 e-01$ & $4.3526 \mathrm{e}+00$ \\
\hline $1=2$ & IHTO00025 & 179.9392 & 182.0876 & 180.8610 & $4.5170 e-01$ & $4.4812 e+00$ \\
\hline 153 & IHTO000026 & 181.8885 & 184.4276 & 183.1308 & $5.3490 \mathrm{e}-01$ & $4.4904 \mathrm{e}+00$ \\
\hline $1 \equiv 4$ & IHTO000027 & 155.4237 & 157.7674 & 156.4628 & $5.0136 e-01$ & $4.3884 e+00$ \\
\hline 155 & IHTO000028 & 156.9599 & 160.0849 & 158.4912 & $7.4070 e-01$ & $4.3957 e+00$ \\
\hline
\end{tabular}




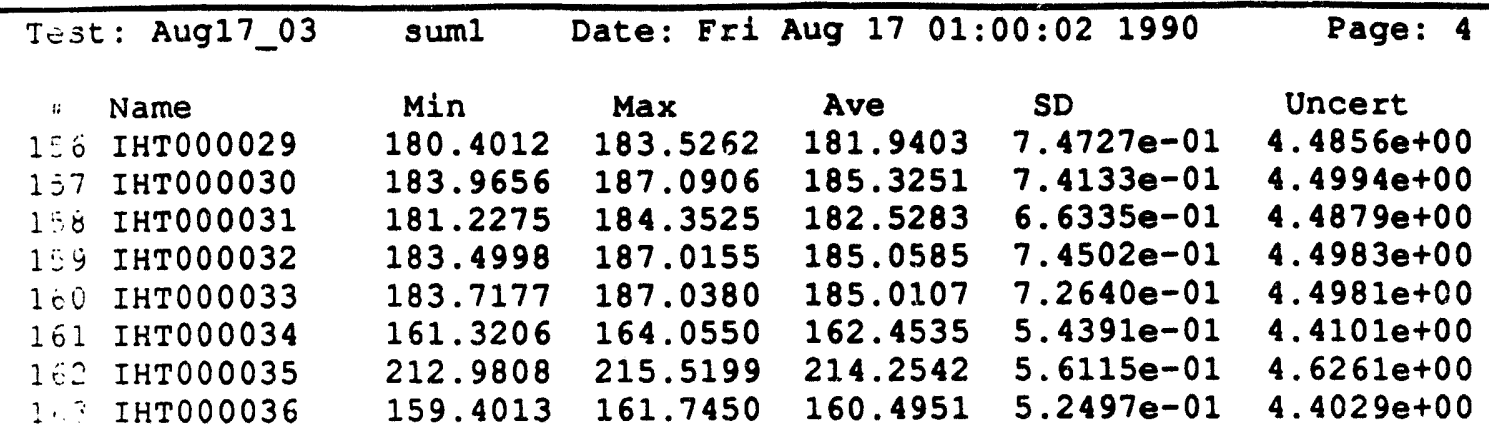




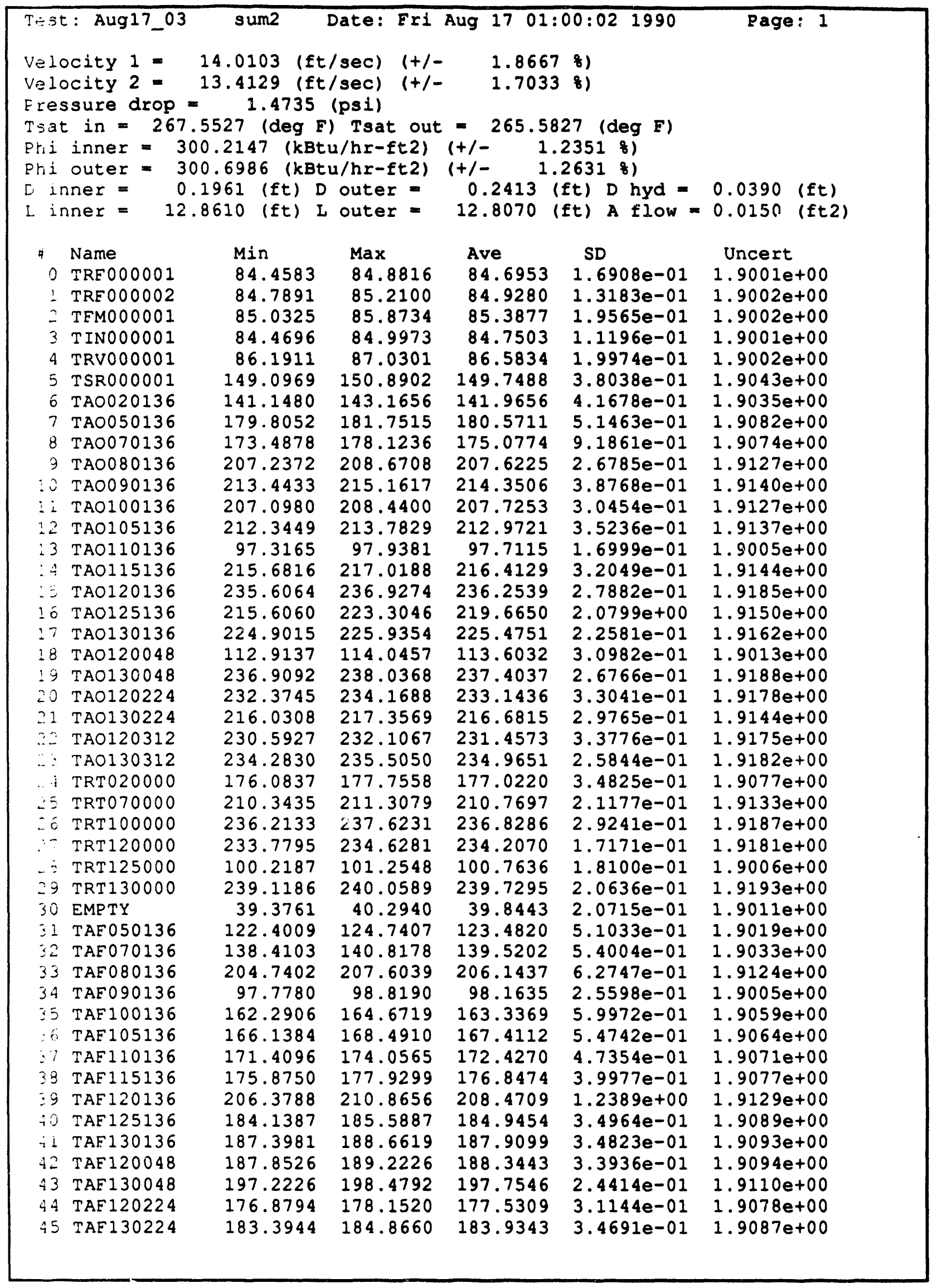




\begin{tabular}{|c|c|c|c|c|c|c|}
\hline \multicolumn{2}{|c|}{ Test: Aug17_03 } & sum2 & Date: Eri & Aug 17 01: & $00: 021990$ & Page: 2 \\
\hline म & Name & Min & $\operatorname{Max}$ & Ave & SD & Uncert \\
\hline 46 & EMPTY & 37.5374 & 38.4572 & 38.0802 & $2.1166 \mathrm{e}-01$ & $1.9010 e+00$ \\
\hline 47 & EMPTY & 39.6057 & 40.0646 & 39.8168 & $1.2126 \mathrm{e}-01$ & $1.9011 \mathrm{e}+00$ \\
\hline 48 & TAI 130048 & 229.4627 & 231.1655 & 230.2744 & $4.0375 e-01$ & $1.9172 e+00$ \\
\hline 49 & TAI 130136 & 236.2589 & 237.2944 & 236.8558 & $2.5897 e-01$ & $1.9187 e+00$ \\
\hline 50 & TAI 130224 & 240.0644 & 241.6664 & 240.9412 & $3.4698 \mathrm{e}-01$ & $1.9196 \mathrm{e}+00$ \\
\hline 51 & TAI130312 & 225.4154 & 226.7455 & 225.9992 & $2.8087 e-01$ & $1.9163 e+00$ \\
\hline 52 & TRR130000 & 129.3954 & 130.7127 & 130.1272 & $3.1377 e-01$ & $1.9024 e+00$ \\
\hline 53 & TSI000001 & 59.5261 & 60.2781 & 59.9023 & $1.7392 \mathrm{e}-01$ & $1.9001 e+00$ \\
\hline 54 & TSI000002 & 84.6732 & 86.0387 & 85.3179 & $3.2318 e-01$ & $1.9002 \mathrm{e}+00$ \\
\hline 55 & TSO000001 & 56.8827 & 57.8561 & 57.2958 & $2.2254 e-01$ & $1.9001 e+00$ \\
\hline 56 & TSO000002 & 72.2655 & 72.9046 & 72.6468 & $1.6388 e-01$ & $1.9000 e+00$ \\
\hline 57 & TAF 020136 & 96.4492 & 99.0711 & 97.8361 & $5.2369 e-01$ & $1.9005 e+00$ \\
\hline 58 & TAE 120312 & 183.2438 & 185.1977 & 184.3558 & $5.2461 e-01$ & $1.9088 e+00$ \\
\hline 59 & TAF 130312 & 197.9900 & 199.6296 & 198.7399 & $3.8450 \mathrm{e}-01$ & $1.9111 \mathrm{e}+00$ \\
\hline 60 & EMPTY & 2.9346 & 2.9541 & 2.9455 & $4.1338 e-03$ & $0.0000 e+00$ \\
\hline 61 & EMPTY & 2.9297 & 2.9395 & 2.9355 & $2.7902 e-03$ & $0.0000 e+00$ \\
\hline $\mathrm{c} 2$ & EMPTY & 2.9199 & 2.9297 & 2.9258 & $2.6100 e-03$ & $0.0000 e+00$ \\
\hline 63 & EMPTY & 2.9199 & 2.9297 & 2.9248 & $2.6100 e-03$ & $0.0000 e+00$ \\
\hline 64 & EMPTY & 1.4551 & 1.4648 & 1.4599 & $1.5566 e-03$ & $0.0000 e+00$ \\
\hline 65 & EMPTY & 1.4746 & 1.4844 & 1.4763 & $2.5362 e-03$ & $0.0000 e+00$ \\
\hline 66 & EMPTY & 1.4844 & 1.4893 & 1.4873 & $2.4164 e-03$ & $0.0000 e+00$ \\
\hline$: 7$ & EMPTY & 1.4990 & 1.5039 & 1.5007 & $2.3365 e-03$ & $0.0000 e+00$ \\
\hline 68 & EMPTY & 1.5039 & 1.5137 & 1.5089 & $1.2041 \mathrm{e}-03$ & $0.0000 \mathrm{e}+00$ \\
\hline 69 & EMPTY & 1.5186 & 1.5332 & 1.5232 & $1.9631 \mathrm{e}-03$ & $0.0000 e+00$ \\
\hline in & EMPTY & 1.5283 & 1.5332 & 1.5326 & $1.6028 e-03$ & $0.0000 e+00$ \\
\hline 71 & EMPTY & 1.5479 & 1.5527 & 1.5503 & $2.4662 e-03$ & $0.0000 e+00$ \\
\hline 72 & EMPTY & 1.5576 & 1.5625 & 1.5581 & $1.4797 e-03$ & $0.0000 e+00$ \\
\hline 73 & EMPTY & 1.5723 & 1.5771 & 1.5731 & $1.8950 e-03$ & $0.0000 e+00$ \\
\hline 74 & EMPTY & 1.5771 & 1.5869 & 1.5845 & $2.6562 e-03$ & $0.0000 e+00$ \\
\hline$\because 5$ & EMPTY & 1.5918 & 1.6016 & 1.5966 & $1.5566 e-03$ & $0.0000 \mathrm{e}+00$ \\
\hline 76 & EMPTY & 1.6016 & 1.6113 & 1.6064 & $9.8648 e-04$ & $0.0000 \mathrm{e}+00$ \\
\hline 77 & EMPTY & 1.6211 & 1.6406 & 1.6216 & $2.8334 e-03$ & $0.0000 \mathrm{e}+00$ \\
\hline$\because 9$ & EMPTY & 1.6357 & 1.6406 & 1.6398 & $1.8082 e-03$ & $0.0000 \mathrm{e}+00$ \\
\hline 79 & EMPTY & 1.6553 & 1.6602 & 1.6559 & $1.6028 e-03$ & $0.0000 e+00$ \\
\hline$\therefore$ & PAN020136 & -11.1300 & 10.8427 & 1.2311 & $5.4771 e+00$ & $8.7001 \mathrm{e}-01$ \\
\hline i? & PANO50136 & -8.3492 & 17.4381 & 2.8904 & $6.6047 e+00$ & $1.6800 \mathrm{e}+00$ \\
\hline 52 & PAN070136 & -9.9297 & 15.7814 & 5.0118 & $5.9478 e+00$ & $\begin{array}{l}1.6800 e+00 \\
8.7009 e-01\end{array}$ \\
\hline 3 & PAN080136 & -8.1575 & 12.6708 & 2.5496 & $5.0899 e+00$ & $8.7009 e-01$ \\
\hline$\cdots$ & PAN090136 & -1.9601 & 21.2333 & 9.4718 & $5.3112 e+00$ & $8.7002 e-01$ \\
\hline 35 & PAN 100136 & -12.1908 & 9.4767 & -2.8341 & $\begin{array}{l}5.3112 e+00 \\
6.5185 e+00\end{array}$ & $8.7032 e-01$ \\
\hline 36 & PAN 105136 & -7.7020 & 12.1344 & 0.3714 & $6.5185 e+00$ & $8.7003 e-01$ \\
\hline 7 & PAN1 10136 & -3.7126 & 17.5734 & 0.3714 & $6.1832 e+00$ & $8.7000 e-01$ \\
\hline$\therefore 8$ & PAN115136 & -7.5597 & 10.5220 & 7.3912 & $4.9072 e+00$ & $8.7020 \mathrm{e}-01$ \\
\hline 9 & PAN120136 & -7.0534 & & 2.9811 & $3.8153 e+00$ & $8.7003 e-01$ \\
\hline & $\begin{array}{l}\text { PAN120136 } \\
\text { PAN125136 }\end{array}$ & -0.3103 & $\begin{array}{r}6.2217 \\
25.0956\end{array}$ & -2.0409 & $4.2731 e+00$ & $8.7001 e-01$ \\
\hline 9ii & PAN125136 & & 25.0956 & 7.7021 & $6.1773 e+00$ & $8.7021 e-01$ \\
\hline $1 i$ & PAN130136 & 1.5546 & 13.6091 & 8.3204 & $3.1737 e+00$ & $8.7025 e-01$ \\
\hline$\Rightarrow 2$ & EAN120048 & -2.0348 & 41.8342 & 14.1822 & $7.8293 e+00$ & $8.7072 e-01$ \\
\hline 95 & PAN130048 & 1.8547 & 31.7619 & 9.8427 & $5.2987 e+00$ & $8.7035 e-01$ \\
\hline 94 & PAN120224 & 1.7490 & 26.9260 & 14.7037 & $5.2615 e+00$ & $8.7078 e-01$ \\
\hline 95 & PAN130224 & -13.9630 & 14.1131 & 4.1919 & $5.6151 \mathrm{e}+00$ & $8.7006 e-01$ \\
\hline$\ddot{0}$ & PAN120312 & -6.8589 & 26.1000 & 11.6759 & $7.4403 e+00$ & $8.7049 e-01$ \\
\hline 47 & PAN130312 & -3.4273 & 26.7089 & 8.4899 & $6.6004 e+00$ & $8.7026 e-01$ \\
\hline 98 & PAB000136 & 39.7703 & 40.5027 & 40.1237 & $2.0368 e-01$ & $5.8861 e-01$ \\
\hline 99 & PAB100136 & 38.7201 & 39.6356 & 39.2685 & $1.9300 e-01$ & $5.8825 e-01$ \\
\hline 100 & PAB125136 & 38.4859 & 39.3557 & 38.8174 & $1.6376 \mathrm{e}-01$ & $5.8806 e-01$ \\
\hline
\end{tabular}




\begin{tabular}{|c|c|c|c|c|c|c|}
\hline \multicolumn{2}{|c|}{ Test: Aug17_03 } & sum2 & Date: Fri & Aug 170 & $0: 021990$ & Page: 3 \\
\hline \# & Name & $\operatorname{Min}$ & $\operatorname{Max}$ & Ave & SD & Uncert \\
\hline (D) 1 & PIN000001 & 39.1042 & 40.5690 & 39.8952 & $4.3857 e-01$ & $7.5660 e-01$ \\
\hline 102 & PRV000001 & 19.7186 & 19.8406 & 19.8162 & $4.9324 \theta-02$ & $7.5163 e-01$ \\
\hline 103 & PSR000001 & 37.1251 & 37.2472 & 37.1813 & $6.1457 e-02$ & $7.5574 e-01$ \\
\hline 104 & PFM000001 & 29.6560 & 30.4800 & 30.0973 & $1.6408 e-01$ & $5.0563 e-01$ \\
\hline 105 & PANO00136 & 24.7636 & 54.9760 & 40.7945 & $6.7185 e+00$ & $4.9211 e+00$ \\
\hline 106 & PFM000002 & 27.5689 & 27.5689 & 27.5689 & $9.6336 e-06$ & $2.1154 e-01$ \\
\hline 107 & EMPTY & 0.0049 & 0.0098 & 0.0071 & $2.4583 e-03$ & $0.0000 e+00$ \\
\hline 108 & EMPTY & -9.1797 & -0.9375 & -4.6435 & $1.7796 \mathrm{e}+00$ & $0.0000 e+00$ \\
\hline 109 & EMPTY & 0.0049 & 0.0049 & 0.0049 & $0.0000 e+00$ & $0.0000 e+00$ \\
\hline $1: 0$ & EMPTY & -10.0000 & -10.0000 & -10.0000 & $0.0000 e+00$ & $0.0000 e+00$ \\
\hline 111 & EMPTY & 0.0000 & 0.0000 & 0.0000 & $0.0000 e+00$ & $0.0000 e+00$ \\
\hline $1: 2$ & VPW000001 & 177.2198 & 177.6104 & 177.3995 & $1.2378 e-01$ & $1.3376 \mathrm{e}+00$ \\
\hline $1: 3$ & VPW000002 & 207.3280 & 207.5233 & 207.3866 & $9.0412 e-02$ & $1.4847 e+00$ \\
\hline 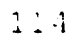 & VPW000003 & 256.2162 & 256.6068 & 256.4349 & $1.1599 \mathrm{e}-01$ & $1.7388 \mathrm{e}+00$ \\
\hline 115 & VPWO00004 & 182.2078 & 182.9890 & 182.4305 & $1.2484 e-01$ & $1.3617 e+00$ \\
\hline 116 & VPWO00005 & 242.9725 & 243.9491 & 243.3671 & $2.4481 e-01$ & $1.6698 \mathrm{e}+00$ \\
\hline 117 & VPW000006 & 257.6360 & 258.2219 & 257.9405 & $1.3760 e-01$ & $1.7468 e+00$ \\
\hline 118 & IPWO00001 & 933.9712 & 939.8282 & 936.8995 & $1.4358 \mathrm{e}+00$ & $1.0972 e+01$ \\
\hline 119 & IPW000002 & 2236.9805 & 2246.7368 & 2240.0627 & $1.6297 e+00$ & $1.9462 e+01$ \\
\hline 120 & IPW000003 & 2261.9998 & 2269.7998 & 2265.1587 & $1.8849 e+00$ & $1.9643 e+01$ \\
\hline $12 !$ & IPW000004 & 651.1887 & 653.1418 & 651.9604 & $3.7029 e-01$ & $5.5252 e+00$ \\
\hline 122 & IPW000005 & 433.2311 & 436.6487 & 435.1840 & $7.8910 e-01$ & $3.9774 \mathrm{e}+00$ \\
\hline $2: 3$ & IPW000006 & 449.7446 & 452.1860 & 450.8088 & $6.5947 e-01$ & $4.0845 e+00$ \\
\hline 124 & EMPTY & -0.0293 & -0.0098 & -0.0189 & $3.7745 e-03$ & $0.0000 e+00$ \\
\hline $12: 5$ & E:IPTY & -0.0195 & -0.0049 & -0.0126 & $3.1335 e-03$ & $0.0000 e+00$ \\
\hline 226 & EMPTY & -0.0195 & -0.0049 & -0.0146 & $4.1256 e-03$ & $0.0000 \mathrm{e}+00$ \\
\hline$\therefore .$. & EMPTY & -0.0244 & -0.0098 & -0.0161 & $3.1583 e-03$ & $0.0000 e+00$ \\
\hline 128 & IHTO00001 & 219.7341 & 222.4684 & 221.2185 & $6.4958 e-01$ & $4.6587 e+00$ \\
\hline 129 & IHTO00002 & 226.3221 & 229.0565 & 227.8730 & $6.7012 e-01$ & $4.6907 e+00$ \\
\hline 130 & IHT 000003 & 228.4030 & 230.9420 & 229.8170 & $6.3327 e-01$ & $4.7002 e+00$ \\
\hline 132 & IHTO00004 & 223.9596 & 226.8893 & 225.5924 & $6.8318 e-01$ & $4.6797 e+00$ \\
\hline 132 & IHTO00005 & 221.8449 & 224.5793 & 223.1768 & $6.5991 e-01$ & $4.6681 \mathrm{e}+00$ \\
\hline 133 & IHT 000006 & 215.7940 & 218.3331 & 217.1455 & $6.3851 e-01$ & $4.6395 e+00$ \\
\hline 234 & IHT0000007 & 225.3306 & 228.0649 & 226.5961 & $5.8426 e-01$ & $4.6845 e+00$ \\
\hline 235 & IHT000008 & 222.9342 & 225.4733 & 224.0436 & $6.1566 e-01$ & $4.6722 e+00$ \\
\hline$\therefore 8$ & IHT000009 & 223.1182 & 225.6573 & 224.3955 & $5.9216 e-01$ & $4.6739 e+00$ \\
\hline $1 \vdots 7$ & IHTO00010 & 54.8190 & 57.3580 & 56.1119 & $5.8246 e-01$ & $4.1382 e+00$ \\
\hline 138 & IHT000011 & 224.0572 & 226.2057 & 225.1198 & $5.8681 e-01$ & $4.6774 e+00$ \\
\hline $1 \vdots 9$ & IHTO00012 & 220.1097 & 222.2581 & 221.1839 & $6.1669 e-01$ & $4.6586 e+00$ \\
\hline 240 & IHTO00013 & 159.5703 & 161.7188 & 160.4023 & $5.5916 e-01$ & $4.4026 e+00$ \\
\hline $1+1$ & IHT000014 & 220.3351 & 223.0694 & 221.7256 & $6.2843 e-01$ & $4.6611 e+00$ \\
\hline 142 & IHTO00015 & 164.3479 & 167.0823 & 165.5121 & $5.7583 e-01$ & $4.4215 e+00$ \\
\hline 143 & IHT000016 & 153.3654 & 155.7091 & 154.2833 & $5.1938 e-01$ & $4.3807 e+00$ \\
\hline 144 & IHTO00017 & 183.1956 & 185.9300 & 184.6566 & $5.9483 e-01$ & $4.4966 \mathrm{e}+00$ \\
\hline 145 & IHT000018 & 185.2840 & 187.8230 & 186.4482 & $5.5096 e-01$ & $4.5040 e+00$ \\
\hline 146 & IHTO00019 & 178.8687 & 181.7984 & 180.3961 & $7.1211 e-01$ & $4.4793 e+00$ \\
\hline 147 & IHTO00020 & 183.5412 & 186.8615 & 185.3575 & $7.3742 e-01$ & $4.4995 e+00$ \\
\hline $1+8$ & IHT000021 & -3.2039 & 0.1164 & -1.5086 & $8.8712 e-01$ & $4.1000 \mathrm{e}+00$ \\
\hline $1: 9$ & IHT000022 & 158.6801 & 161.6098 & 160.0083 & $6.9699 e-01$ & $4.4012 e+00$ \\
\hline $1 \equiv 0$ & IHTO00023 & 180.5364 & 183.0754 & 181.6105 & $6.7572 e-01$ & $4.4842 e+00$ \\
\hline 151 & IHTOOO0024 & 144.9632 & 147.3070 & 146.0726 & $6.5843 e-01$ & $4.3524 \mathrm{e}+00$ \\
\hline 152 & IHTO00025 & 179.7439 & 182.4782 & 180.7985 & $6.4838 e-01$ & $4.4809 e+00$ \\
\hline $2 \equiv 3$ & IHTO00026 & 181.8885 & 184.8182 & 183.0761 & $6.4698 \mathrm{e}-01$ & $4.4902 e+00$ \\
\hline 154 & IHTO00027 & 155.2284 & 158.1581 & 156.4276 & $6.4599 \mathrm{e}-01$ & 4. $3883 e+00$ \\
\hline 155 & IHT000028 & 157.1552 & 160.0849 & 158.6591 & $6.3042 e-01$ & $4.3963 e+00$ \\
\hline
\end{tabular}




\begin{tabular}{|c|c|c|c|c|c|c|}
\hline \multicolumn{2}{|c|}{ Test: Aug17_03 } & sum2 & Date: Fri & Aug 17 & $00: 021990$ & Page: \\
\hline$\#$ & Name & Min & $\operatorname{Max}$ & Ave & SD & Uncert \\
\hline 156 & IHTO00029 & 180.2058 & 183.5262 & 182.0887 & $6.8545 e-01$ & $4.4862 \theta+00$ \\
\hline 157 & IHTO00030 & 183.3797 & 186.8953 & 185.4423 & $7.1153 e-01$ & $4.4999 e+00$ \\
\hline 158 & IHTO00031 & 180.6415 & 184.3525 & 182.7158 & $6.9909 \mathrm{e}-01$ & $4.4887 e+00$ \\
\hline 159 & IHTO00032 & 183.1092 & 186.8201 & 185.1483 & $7.7138 e-01$ & $4.4987 e+00$ \\
\hline 160 & IHTO000033 & 182.9365 & 186.8427 & 185.0928 & $7.7521 e-01$ & $4.4984 e+00$ \\
\hline 161 & IHTO00034 & 161.3206 & 164.0550 & 162.6761 & $5.8048 e-01$ & $4.4109 e+00$ \\
\hline 162 & IHT000035 & 213.1761 & 216.1058 & 214.6409 & $6.5939 e-01$ & $4.6279 e+00$ \\
\hline 163 & IHT000036 & 159.2060 & 162.1357 & 160.7138 & $5.4682 e-01$ & $4.4037 e+00$ \\
\hline
\end{tabular}




\begin{tabular}{|c|c|c|c|}
\hline $\begin{array}{l}\text { Ve:oo } \\
\text { Viloc } \\
\text { Pres } \\
\text { T:at } \\
\text { Fi.i }\end{array}$ & $\begin{array}{l}\text { ity } 1=0.0 \\
\text { ity } 2=0.0 \\
\text { ure Drop }= \\
\text { in }=0.0394 \\
\text { nner }=1.15\end{array}$ & $\begin{array}{l}37601 \text { (ft/s } \\
00000(\mathrm{ft} / \mathrm{s} \\
0.009300(\mathrm{~F} \\
90(\mathrm{deg} \mathrm{F}) \\
9698 \text { (kBtu) }\end{array}$ & $\begin{array}{l}\text { ec) } \\
\text { ec) } \\
\text { si) } \\
\text { Tsat out }=0.040588 \text { (deg F) } \\
\text { hr-ft2) Phi outer }=0.908905 \text { (kBtu/hr-ft2) }\end{array}$ \\
\hline$\#$ & Name & Abs Diff & 8Diff \\
\hline ? & TRF 000001 & 0.0719 & 0.0850 \\
\hline 1 & TRF000002 & 0.0421 & 0.0496 \\
\hline 2 & TEMO000001 & 0.0102 & 0.0119 \\
\hline 3 & TINO00001 & 0.0317 & 0.0374 \\
\hline 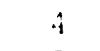 & TRV000001 & 0.0360 & 0.0416 \\
\hline$\vdots$ & TSR000001 & 0.2937 & 0.1957 \\
\hline 6 & TAO0 20136 & 1.2170 & 0.8647 \\
\hline 7 & TAO0 50136 & 0.0287 & 0.0159 \\
\hline$\therefore$ & TAO070136 & 0.2625 & 0.1502 \\
\hline 9 & TAO0080136 & 0.0204 & 0.0098 \\
\hline 10 & TAO090136 & 0.1472 & 0.0687 \\
\hline 11 & TAO100136 & 0.0668 & 0.0321 \\
\hline 22 & TA0105136 & 0.0363 & 0.0170 \\
\hline 13 & TA0110136 & 0.7995 & 0.8250 \\
\hline 14 & TAO115136 & 0.1167 & 0.0540 \\
\hline$: 5$ & TAO120136 & 0.1605 & 0.0680 \\
\hline$\therefore \bar{c}$ & TA0125136 & 0.2570 & 0.1171 \\
\hline$\therefore$ & TA0130136 & 0.0141 & 0.0063 \\
\hline$\vdots \hat{3}$ & TAO120048 & 1.2980 & 1.1558 \\
\hline$\therefore$ & TAO130048 & 0.2350 & 0.0991 \\
\hline 2 & TAO120224 & 0.0361 & 0.0155 \\
\hline$\therefore:$ & TAO130224 & 0.0290 & 0.0134 \\
\hline $2=$ & TAO120312 & 0.2254 & 0.0975 \\
\hline 23 & TAO130312 & 0.1526 & 0.0650 \\
\hline$\therefore 4$ & TRT020000 & 0.3325 & 0.1882 \\
\hline 25 & TRT070000 & 0.2643 & 0.1256 \\
\hline 20 & TRT 100000 & 0.2653 & 0.1121 \\
\hline$=7$ & TRT120000 & 0.2585 & 0.1105 \\
\hline$\because$ & TRT 125000 & 1.1468 & 1.1512 \\
\hline$\therefore$ & TRT 130000 & 0.2612 & 0.1091 \\
\hline$\therefore:$ & EMPTY & 0.0458 & 0.1151 \\
\hline$\vdots$ & TAF0 50136 & 0.0016 & 0.0013 \\
\hline$\because$ & TAF070136 & 0.0135 & 0.0097 \\
\hline$\therefore$ & TAF080136 & 0.0560 & 0.0272 \\
\hline$\cdot i$ & TAF090136 & 0.3684 & 0.3767 \\
\hline$\vdots \vdots$ & TAF 100136 & 0.2203 & 0.1351 \\
\hline$\vdots \varepsilon$ & TAF 105136 & 0.3003 & 0.1797 \\
\hline 37 & TAF 110136 & 0.1122 & 0.0651 \\
\hline ;3 & TAF 115136 & 0.1667 & 0.0944 \\
\hline 39 & TAF 120136 & 0.0278 & 0.0133 \\
\hline 40 & TAF 125136 & 0.2579 & 0.1396 \\
\hline $4 !$ & TAF 130136 & 0.2818 & 0.1502 \\
\hline$G=$ & TAF 120048 & 0.0057 & 0.0030 \\
\hline$\therefore$ & TAF 130048 & 0.1452 & 0.0735 \\
\hline 44 & TAF 120224 & 0.0409 & 0.0230 \\
\hline 45 & TAF 130224 & 0.0568 & 0.0309 \\
\hline
\end{tabular}




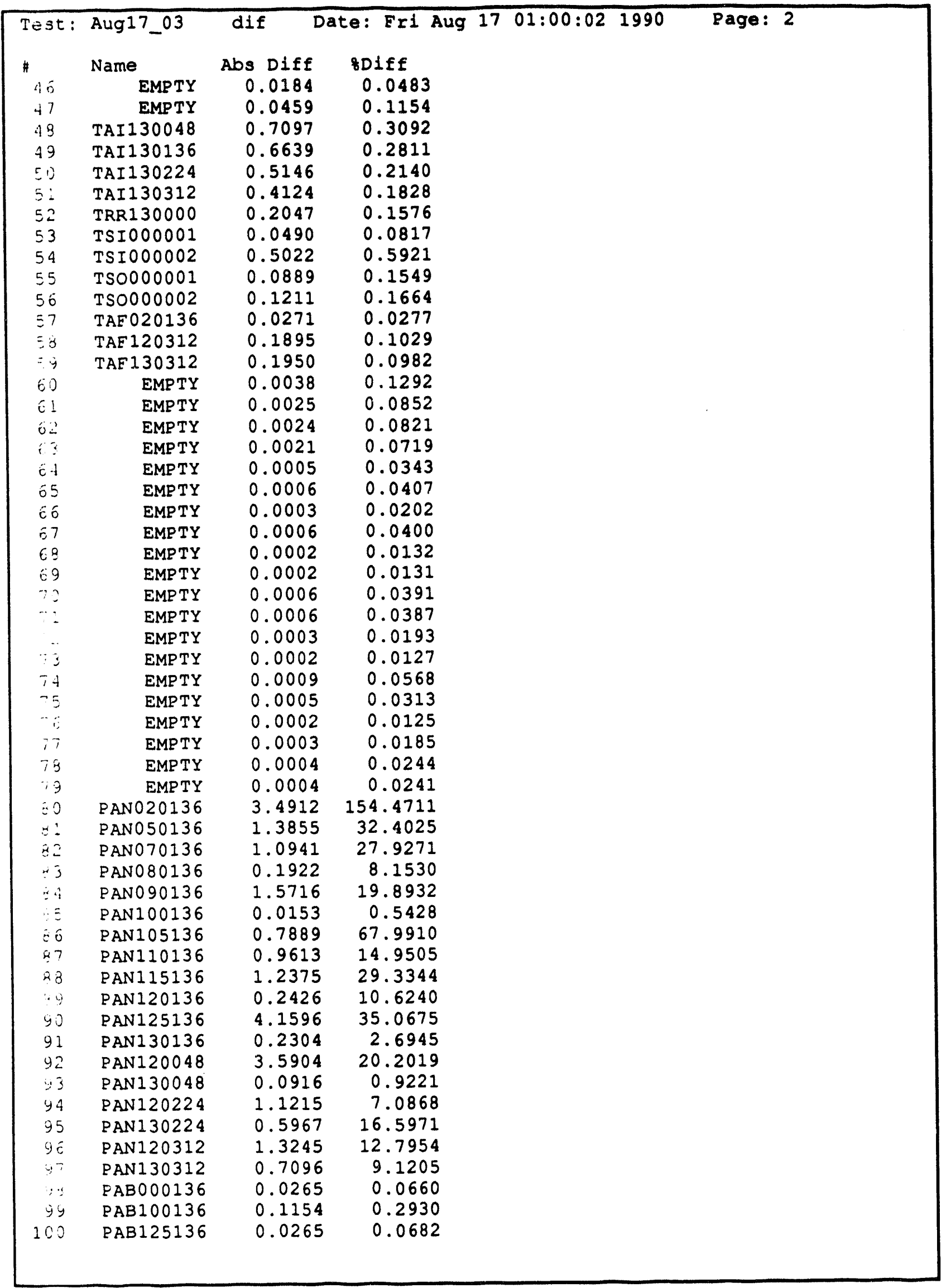




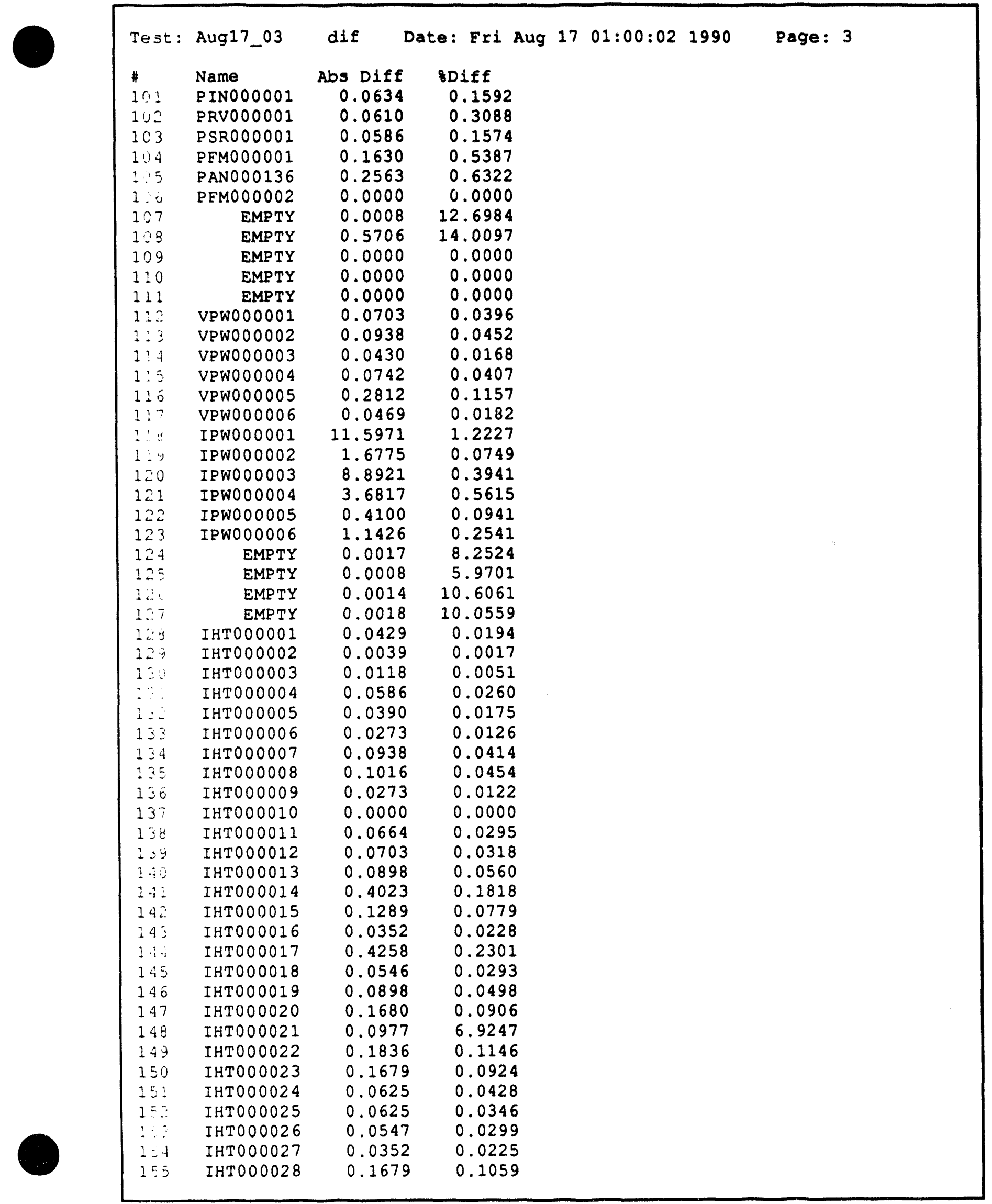


Test : Aug17_03

\# Name

$1: 6 \quad$ IHT000029

$1: ;$ IHTOOOOSO

158 IHT000031

159 IHTO00032

161 IHTO00033

: 6 I IHT000034

162 IHT000035

163 IHT000036 dif Date: Fri Aug 17 01:00:02 1990

Page: 4

Abs Diff tDiff

$0.1484 \quad 0.0816$

$0.1172 \quad 0.0632$

$0.1875 \quad 0.1027$

$0.0898 \quad 0.0485$

$0.0821 \quad 0.0444$

$0.2226 \quad 0.1370$

$0.3867 \quad 0.1805$

$0.2187 \quad 0.1363$ 


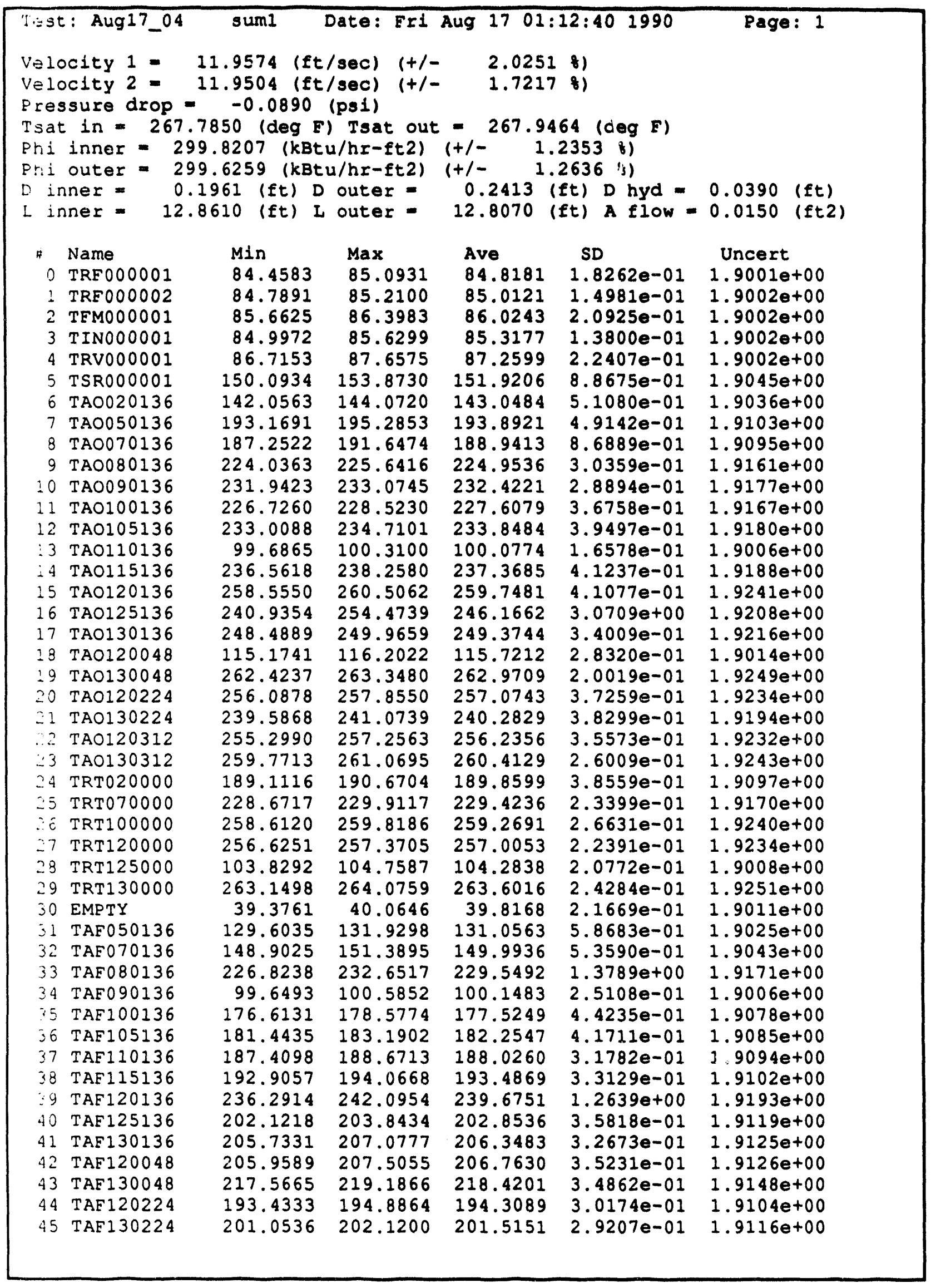




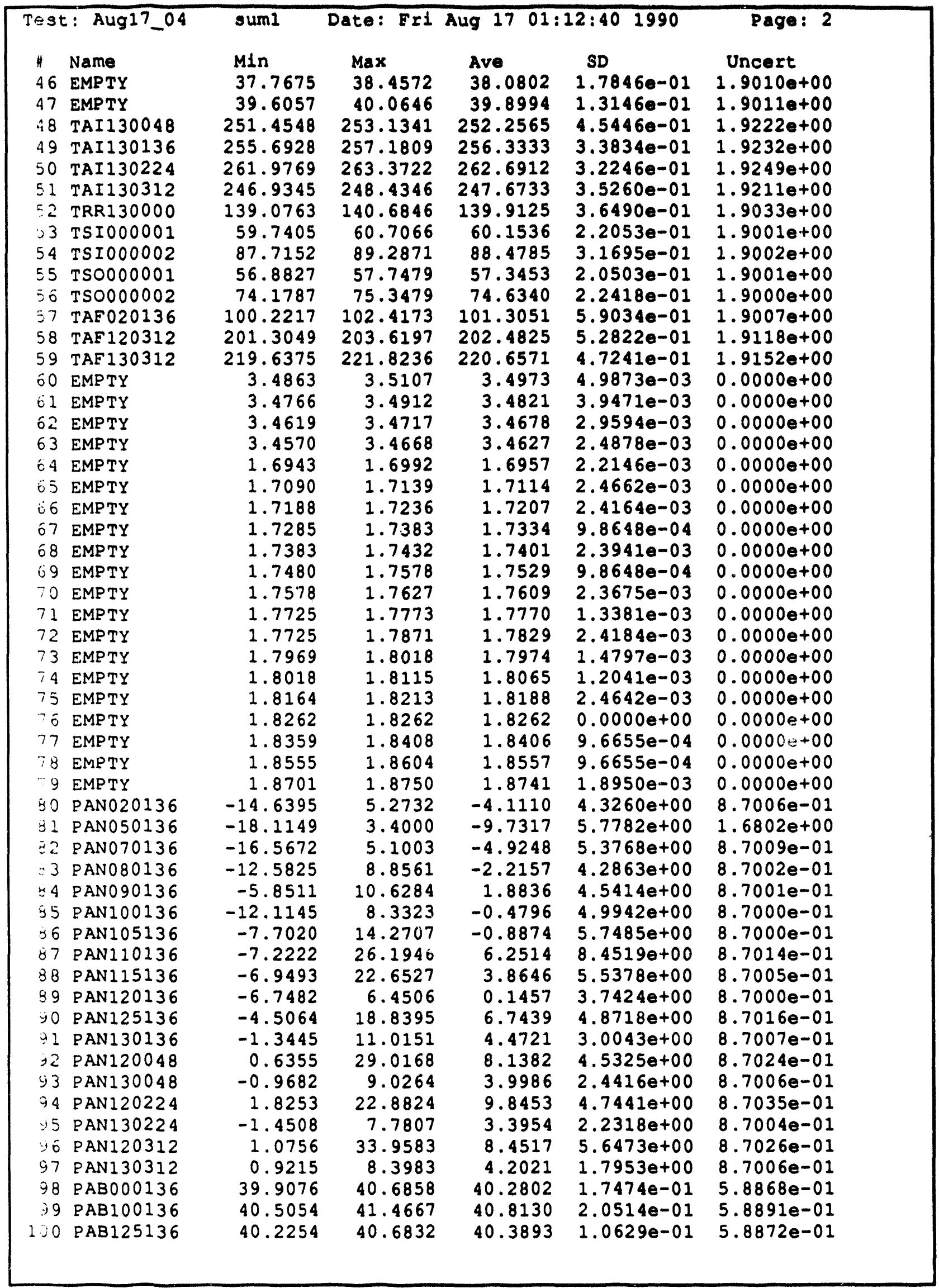




\begin{tabular}{|c|c|c|c|c|c|c|}
\hline \multicolumn{2}{|c|}{ Test: Aug17_04 } & sum 1 & Date: Ex1 & Aug 17 & $: 401$ & Page: 3 \\
\hline " & Name & $M \perp n$ & $\operatorname{Max}$ & Ave & SD & Uncert \\
\hline $\begin{array}{l}101 \\
102\end{array}$ & PINO00001 & 39.4704 & 40.9353 & 40.1589 & $4.0506 e-01$ & $7.5669--01$ \\
\hline $\begin{array}{l}104 \\
105\end{array}$ & $\begin{array}{l}\text { PFM000001 } \\
\text { PANO00136 }\end{array}$ & $\begin{array}{r}21.5078 \\
-14.6041\end{array}$ & 22.2402 & 21.8704 & $1.8024 e-01$ & $5.0298 e-01$ \\
\hline 106 & $\begin{array}{l}\text { PANO00136 } \\
\text { PEMO00002 }\end{array}$ & $\begin{array}{r}-14.6041 \\
21.6792\end{array}$ & 12.4040 & -2.4642 & $6.3424 e+00$ & $4.9200 \theta+00$ \\
\hline 107 & EMPTY & $\begin{array}{r}21.6792 \\
0.0049\end{array}$ & 22.1154 & $\begin{array}{r}21.8446 \\
0.0052\end{array}$ & $1.0283 e-01$ & $2.0732 e-01$ \\
\hline 108 & EMPTY & $\begin{array}{r}0.0049 \\
-10.0000\end{array}$ & 0.0098 & $\begin{array}{r}0.0052 \\
-4.7613\end{array}$ & $1.1714 e-03$ & $0.0000 e+00$ \\
\hline 1.9 & EMPTY & $\begin{array}{r}-10.0000 \\
0.0049\end{array}$ & $\begin{array}{r}-1.3867 \\
0.0049\end{array}$ & $\begin{array}{r}-4.7613 \\
0.0049\end{array}$ & $\begin{array}{l}1.6086 e+00 \\
0.0000 e+00\end{array}$ & $0.00000+00$ \\
\hline 110 & EMPTY & $\begin{array}{r}0.0049 \\
-10.0000\end{array}$ & $\begin{array}{r}0.0049 \\
-10.0000\end{array}$ & $\begin{array}{r}0.0049 \\
-10.0000\end{array}$ & $\begin{array}{l}0.0000 e+00 \\
0.0000 e+00\end{array}$ & $0.0000 e+00$ \\
\hline 111 & EMPTY & $\begin{array}{r}-10.00000 \\
0.0000\end{array}$ & $\begin{array}{r}-10.0000 \\
0.0049\end{array}$ & $\begin{array}{r}-10.0000 \\
0.0003\end{array}$ & $\begin{array}{l}0.0000 e+00 \\
1.1714 e-03\end{array}$ & $\begin{array}{l}0.0000 e+00 \\
0.0000 e+00\end{array}$ \\
\hline $1: 2$ & VPWO00001 & 177.2198 & $\begin{array}{r}0.0049 \\
177.6104\end{array}$ & $\begin{array}{r}0.0003 \\
177.4698\end{array}$ & $\begin{array}{l}1.1714 \theta-03 \\
1.1864 \theta-01\end{array}$ & $\begin{array}{l}0.0000 e+00 \\
1.3379 e+00\end{array}$ \\
\hline$\therefore 3$ & VPWO00002 & 207.3280 & 207.7186 & 207.5351 & $9.9902 e-02$ & $1.4855 e+00$ \\
\hline $1: 4$ & VPW000003 & 256.4115 & 256.9974 & 256.6810 & $1.1765 e-01$ & $1.74010+00$ \\
\hline 115 & VPWOOO0004 & 182.4031 & 182.9890 & 182.5204 & $1.3087 e-01$ & $1.3621 \mathrm{e}+00$ \\
\hline 116 & VPWO 00005 & 242.9725 & 213.7538 & 243.3163 & $2.0336 e-01$ & $1.6696 e+00$ \\
\hline 117 & VPWO00006 & 257.8313 & 258.4172 & 258.2257 & $1.3375 e-01$ & $1.7483 e+00$ \\
\hline $1: 8$ & IPWOOO0001 & 932.0189 & 945.6852 & 939.5156 & $3.8517 e+00$ & $1.0986 e+01$ \\
\hline 119 & IPW0 00002 & 2219.4189 & 2225.2727 & 2222.2283 & $1.2565 \mathrm{e}+00$ & $1.9334 e+01$ \\
\hline 120 & IPW000003 & 2260.0498 & 2265.8999 & 2260.9854 & $1.1975 e+00$ & $1.9613 e+01$ \\
\hline 1.21 & IPW0 00004 & 648.7473 & 654.1184 & 650.5249 & $1.8840 e+00$ & $5.5146 e+00$ \\
\hline $1 \because 2$ & IPWO00005 & 434.2076 & 436.6487 & 435.2327 & $4.8574 e-01$ & $3.9777 e+00$ \\
\hline 123 & IPW000006 & 447.7915 & 449.7446 & 448.7580 & $4.0059 \theta-01$ & $4.0704 e+00$ \\
\hline 124 & EMPTY & -0.0244 & -0.0146 & -0.0182 & $3.1257 e-03$ & $0.0000 e+00$ \\
\hline $12: 5$ & EMPTY & -0.0195 & -0.0049 & -0.0122 & $3.0003 \theta-03$ & $0.0000 e+00$ \\
\hline $12 \overline{0}$ & EMPTY & -0.0195 & -0.0049 & -0.0141 & $3.9013 e-03$ & $0.0000 e+00$ \\
\hline 127 & EMPTY & -0.0244 & -0.0098 & -0.0159 & $3.2434 e-03$ & $0.0000 e+00$ \\
\hline 128 & IHTO00001 & 219.9294 & 222.6637 & 221.2731 & $6.0828 e-01$ & $4.6590 e+00$ \\
\hline 129 & IHTOOOOOO2 & 226.5174 & 229.2518 & 227.8573 & $5.8661 e-01$ & $4.6906 \mathrm{e}+00$ \\
\hline 130 & IHTO00003 & 228.5983 & 230.9420 & 229.8717 & $5.8957 e-01$ & $4.7004 e+00$ \\
\hline 131 & IHTOOOOOO4 & 224.1549 & 226.6940 & 225.6393 & $6.3626 \mathrm{e}-01$ & $4.6799 \mathrm{e}+00$ \\
\hline 132 & IHTO00005 & 221.8449 & 224.1887 & 223.2433 & $5.8708 e-01$ & $4.6684 \mathrm{e}+00$ \\
\hline 133 & IHTO00006 & 215.7940 & 218.3331 & 217.2471 & $6.4737 e-01$ & $4.6400 e+00$ \\
\hline $1: 4$ & IHT000007 & 225.1352 & 227.8696 & 226.6820 & $6.2991 \mathrm{e}-01$ & $4.6849 \mathrm{e}+00$ \\
\hline $1: 5$ & IHT:000008 & 222.9342 & 225.2779 & 224.0905 & $5.6612 e-01$ & $4.6724 e+00$ \\
\hline $1: 6$ & IHT000009 & 222.9229 & 225.8526 & 224.3135 & $6.3336 e-01$ & $4.6735 e+00$ \\
\hline $1 \vdots 7$ & IHTO000010 & 55.0143 & 56.9674 & 55.8892 & $4.8687 \mathrm{e}-01$ & $4.1379 e+00$ \\
\hline 128 & IHTO00011 & 223.8619 & 225.6197 & 224.7604 & $5.0532 e-01$ & $4.6757 e+00$ \\
\hline$\therefore 9$ & IHTOOOOO12 & 219.7191 & 221.8675 & 220.7815 & $5.6656 e-01$ & $4.6567 \mathrm{e}+00$ \\
\hline 140 & IHTO00013 & 158.9844 & 161.1328 & 159.9141 & $5.4328 e-01$ & $4.4008 e+00$ \\
\hline 141 & IHTO00014 & 219.9444 & 222.0929 & 221.0694 & $5.0464 e-01$ & $4.6580 e+00$ \\
\hline 142 & IHTO00015 & 163.7620 & 166.1058 & 165.0433 & $5.1473 e-01$ & $4.4197 e+00$ \\
\hline 143 & IHTO00016 & 152.5341 & 154.7326 & 153.6192 & $4.9166 e-01$ & $4.3783 e+00$ \\
\hline 144 & IHTO00017 & 183.0003 & 186.7112 & 184.3011 & $7.6678 \mathrm{e}-01$ & $4.4952 e+00$ \\
\hline 145 & IHTO00018 & 184.8933 & 187.6277 & 186.0262 & $6.8912 e-01$ & $4.5023 e+00$ \\
\hline $1 \cdot i \hat{0}$ & IHTO00019 & 179.2593 & 182.1890 & 180.2750 & $5.1318 \theta-01$ & $4.4788 \mathrm{e}+00$ \\
\hline 1.7 & IHTO00020 & 183.9318 & 187.2521 & 185.2364 & $5.8586 e-01$ & $4.4990 e+00$ \\
\hline ins & IHTO00021 & -3.0086 & 0.3118 & -1.6257 & $6.8554 e-01$ & $4.1000 e+00$ \\
\hline $1 \div 9$ & IHTO00022 & 158.6801 & 161.2192 & 159.5747 & $5.2510 e-01$ & $4.3996 e+00$ \\
\hline $1 \equiv 0$ & IHT000023 & 180.3411 & 182.6848 & 181.3566 & $5.3087 e-01$ & $4.4832 e+00$ \\
\hline 151 & IHTO00024 & 1.4 .7679 & 146.9163 & 145.8578 & $4.8342 e-01$ & $4.3517 e+00$ \\
\hline 152 & IHTO00025 & 179.3532 & 182.0876 & 180.6305 & $5.4277 e-01$ & $4.4803 e+00$ \\
\hline 153 & IHTO00026 & 181.4979 & 184.4276 & 182.8339 & $6.1809 e-01$ & $4.4892 \theta+00$ \\
\hline 154 & IHTO00027 & 154.8377 & 157.3768 & 156.0995 & $5.4421 e-01$ & $4.3871 e+00$ \\
\hline 155 & IHT000028 & 156.7646 & 159.8896 & 158.6318 & $6.8591 \mathrm{e}-01$ & $4.3962 \theta+00$ \\
\hline
\end{tabular}




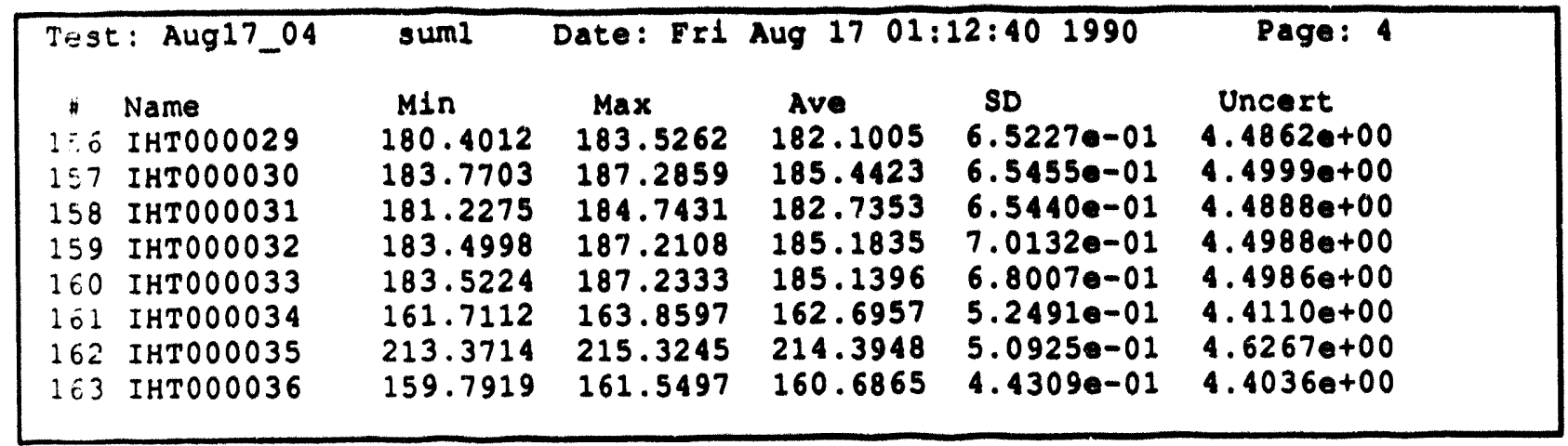



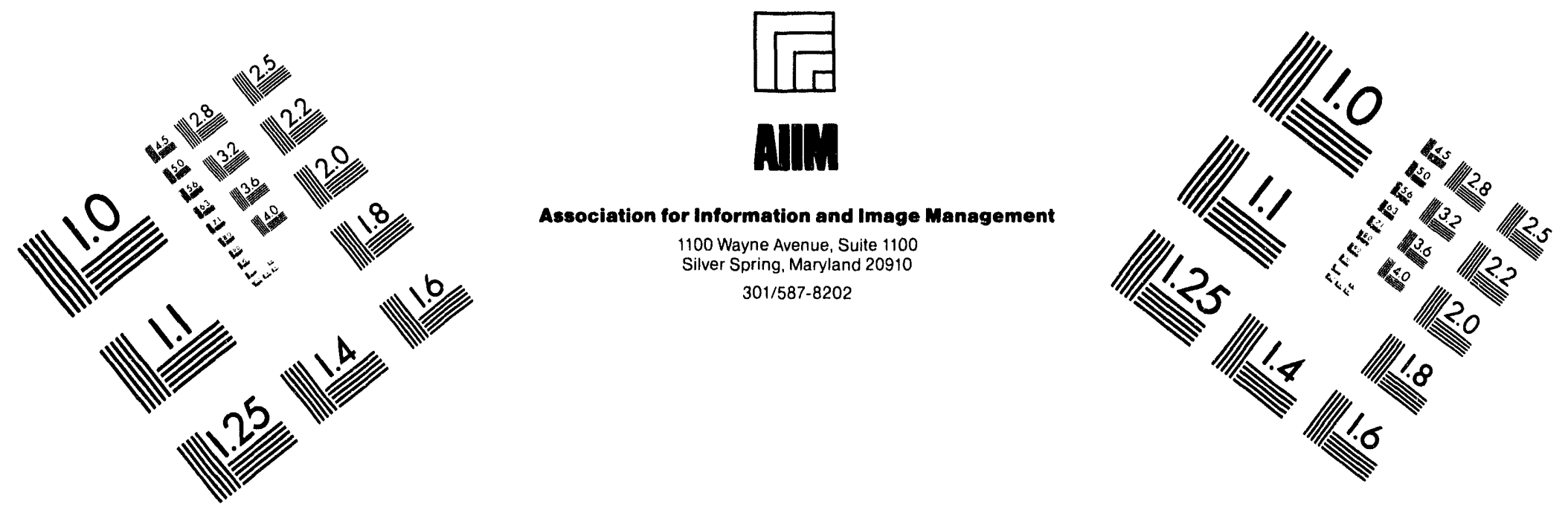

\section{Centimeter}

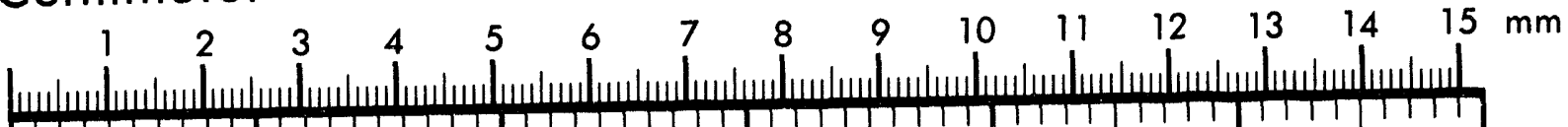

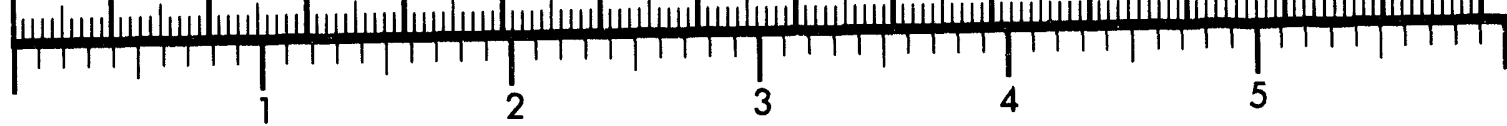
Inches

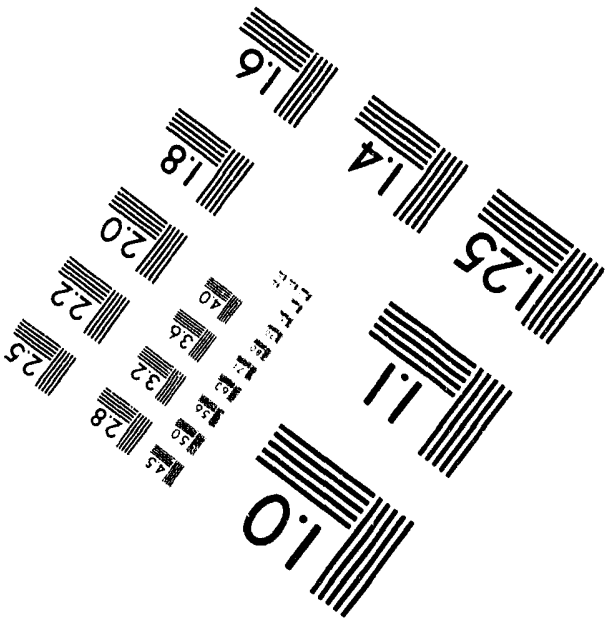

MANUFACTURED TO AIIM STANDARDS BY APPLIED IMAGE, INC.

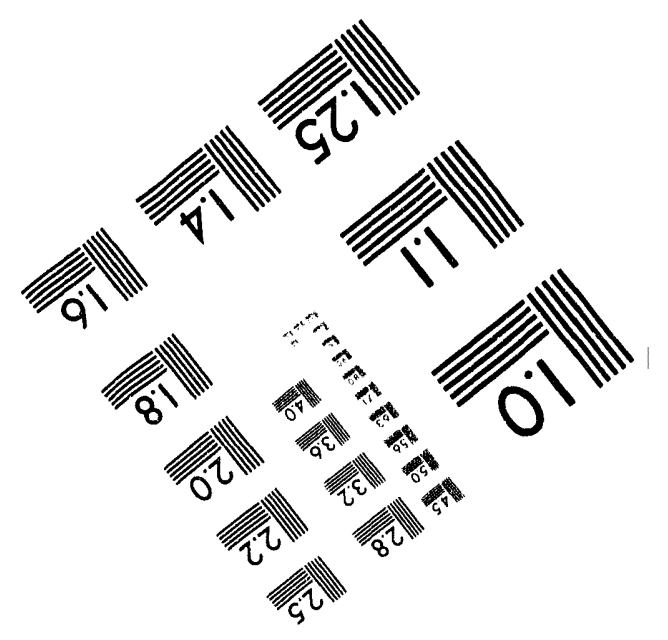



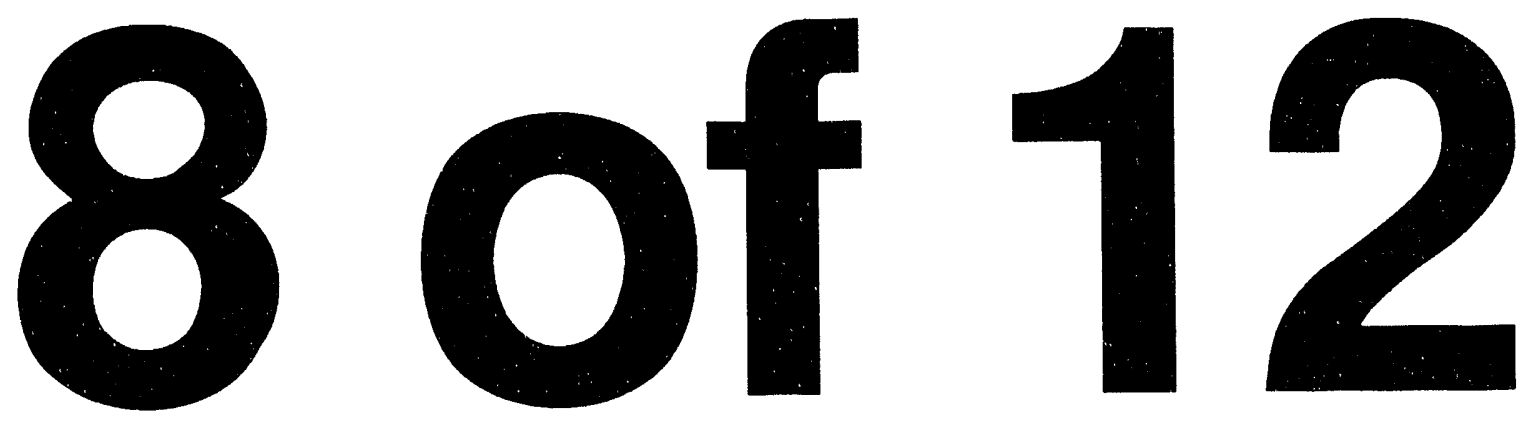


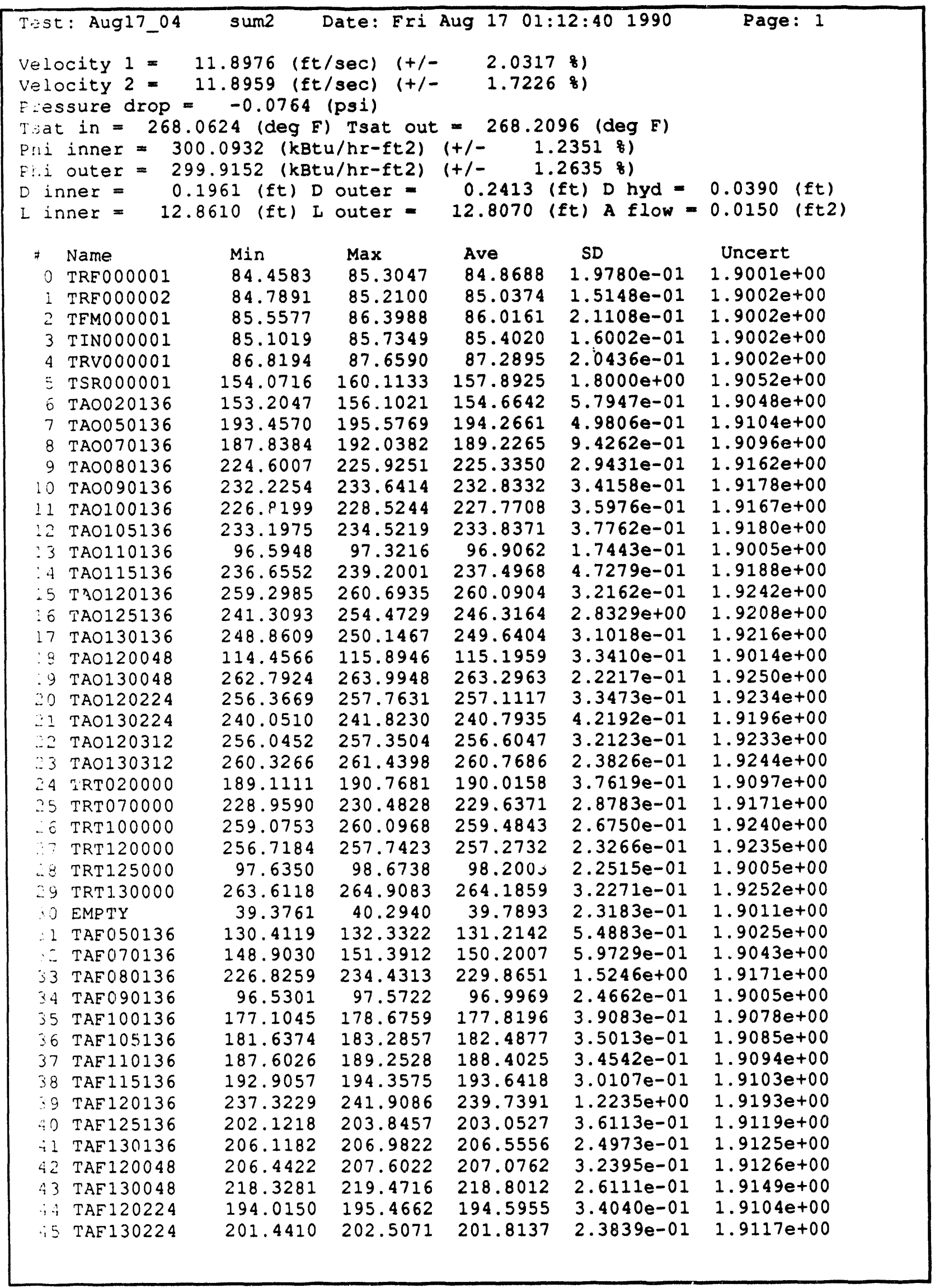




\begin{tabular}{|c|c|c|c|c|c|c|}
\hline \multicolumn{2}{|c|}{ Test: Aug17_04 } & sum2 & Date: Fri & Aug 17 01: & $12: 40 \quad 1990$ & Page: 2 \\
\hline$H$ & Name & Min & $\operatorname{Max}$ & Ave & SD & Uncert \\
\hline 46 & EMPTY & 37.7675 & 38.4572 & 38.1124 & $2.3341 e-01$ & $1.9010 e+00$ \\
\hline 47 & EMPTY & 39.6057 & 40.0646 & 39.8444 & $1.2232 e-01$ & $1.9011 e+00$ \\
\hline+18 & TAI 130048 & 252.4800 & 254.5324 & 253.5326 & $4.9993 e-01$ & $1.9226 \mathrm{e}+00$ \\
\hline$\because 9$ & TAI 130136 & 256.3436 & 257.8307 & 257.2209 & $2.8659 e-01$ & $1.9235 \mathrm{e}+00$ \\
\hline$\vdots 0$ & TAI130224 & 262.8142 & 264.4873 & 263.6617 & $3.5563 e-01$ & $1.9251 \mathrm{e}+00$ \\
\hline 51 & TAI 130312 & 247.6843 & 250.4939 & 248.9210 & $7.7928 e-01$ & $1.9214 \mathrm{e}+00$ \\
\hline 52 & TRR130000 & 135.6568 & 137.0675 & 136.4426 & $3.3655 e-01$ & $1.9030 \mathrm{e}+00$ \\
\hline 53 & TSI000001 & 59.9554 & 60.7076 & 60.3210 & $1.6389 e-01$ & $1.9001 \mathrm{e}+00$ \\
\hline 54 & TSI000002 & 85.5137 & 87.2981 & 86.3281 & $3.6028 e-01$ & $1.9002 \mathrm{e}+00$ \\
\hline 55 & TSO000001 & 56.8826 & 57.7474 & 57.3020 & $2.2023 e-01$ & $1.9001 e+00$ \\
\hline 56 & TSO000002 & 74.0730 & 74.9234 & 74.5704 & $2.0867 e-01$ & $1.9000 \mathrm{e}+00$ \\
\hline 57 & TAF020136 & 100.4301 & 103.1473 & 101.2590 & $6.3086 e-01$ & $1.9007 \mathrm{e}+00$ \\
\hline$\vdots 8$ & TAF 120312 & 201.4988 & 203.5233 & 202.5790 & $4.3216 e-01$ & $1.9118 \mathrm{e}+00$ \\
\hline$\equiv 9$ & TAE 130312 & 220.0179 & 222.2995 & 220.9766 & $4.7970 e-01$ & $1.9153 e+00$ \\
\hline$\therefore 0$ & EMPTY & 3.4961 & 3.5107 & 3.5039 & $4.4117 e-03$ & $0.0000 \mathrm{e}+00$ \\
\hline$\therefore$ & EMPTY & 3.4814 & 3.4912 & 3.4890 & $2.6488 e-03$ & $0.0000 e+00$ \\
\hline 12 & EMPTY & 3.4717 & 3.4766 & 3.4742 & $2.4642 e-03$ & $0.0000 e+00$ \\
\hline 03 & EMPTY & 3.4668 & 3.4717 & 3.4694 & $2.4583 e-03$ & $0.0000 e+00$ \\
\hline c4 & EMPTY & 1.6992 & 1.7090 & 1.6994 & $1.3811 e-03$ & $0.0000 e+00$ \\
\hline$\ddot{05}$ & EMPTY & 1.7139 & 1.7188 & 1.7144 & $1.4797 e-03$ & $0.0000 e+00$ \\
\hline 66 & EMPTY & 1.7236 & 1.7285 & 1.7238 & $9.6655 e-04$ & $0.0000 \mathrm{e}+00$ \\
\hline 67 & EMPTY & 1.7383 & 1.7432 & 1.7384 & $6.9053 e-04$ & $0.0000 \mathrm{e}+00$ \\
\hline 68 & EMPTY & 1.7432 & 1.7480 & 1.7437 & $1.4797 e-03$ & $0.0000 e+00$ \\
\hline c9 & EMPTY & 1.7529 & 1.7627 & 1.7571 & $1.9754 e-03$ & $0.0000 e+00$ \\
\hline$: 0$ & EMPTY & 1.7578 & 1.7676 & 1.7635 & $2.0598 e-03$ & $0.0000 e+00$ \\
\hline$: 1$ & EMPTY & 1.7773 & 1.7822 & 1.7817 & $1.4797 e-03$ & $0.0000 e+00$ \\
\hline 72 & EMPTY & 1.7871 & 1.7920 & 1.7874 & $1.1714 \mathrm{e}-03$ & $0.0000 \mathrm{e}+00$ \\
\hline 73 & EMPTY & 1.8018 & 1.8066 & 1.8019 & $6.9053 e-04$ & $0.0000 e+00$ \\
\hline 4 & EMPTY & 1.8066 & 1.8164 & 1.8115 & $9.8648 e-04$ & $0.0000 e+00$ \\
\hline 5 & EMPTY & 1.8213 & 1.8359 & 1.8228 & $2.8334 e-03$ & $0.0000 e+00$ \\
\hline 76 & EMPTY & 1.8311 & 1.8311 & 1.8311 & $0.0000 e+00$ & $0.0000 e+00$ \\
\hline 77 & EMPTY & 1.8408 & 1.8457 & 1.8448 & $1.8950 e-03$ & $0.0000 \mathrm{e}+00$ \\
\hline 78 & EMPTY & 1.8604 & 1.8652 & 1.8604 & $6.9053 e-04$ & $0.0000 e+00$ \\
\hline$: 9$ & EMPTY & 1.8750 & 1.8848 & 1.8787 & $2.3261 e-03$ & $0.0000 \mathrm{e}+00$ \\
\hline$\$ 0$ & PAN020136 & -17.3098 & 5.5021 & -6.6958 & $5.1628 e+00$ & $8.7016 e-01$ \\
\hline 81 & PAN050136 & -19.3356 & 7.2147 & -7.5222 & $5.9513 e+00$ & $1.6801 \mathrm{e}+00$ \\
\hline 82 & PAN070136 & -20.2293 & 5.8632 & -4.5036 & $5.8099 e+00$ & $8.7007 e-01$ \\
\hline$\therefore 3$ & PAN080136 & -13.2691 & 5.1940 & -4.7044 & $4.6198 e+00$ & $8.7008 e-01$ \\
\hline$\because 4$ & PAN090136 & -9.1317 & 13.0698 & 1.1298 & $4.6317 e+00$ & $8.7000 e-01$ \\
\hline$\because 5$ & PAN 100136 & -12.1908 & 12.3759 & -0.0631 & $6.2281 e+00$ & $8.7000 e-01$ \\
\hline 56 & PAN105136 & -7.7020 & 18.7720 & -0.9713 & $6.8043 e+00$ & $8.7000 e-01$ \\
\hline$\because 7$ & PAN110136 & -7.2222 & 21.3881 & 4.7331 & $6.1505 e+00$ & $8.7008 e-01$ \\
\hline is & PAN115136 & -4.9657 & 25.0942 & 4.8747 & $6.2385 e+00$ & $8.7009 e-01$ \\
\hline 3 & PAN120136 & -2.3995 & 6.9847 & 2.5795 & $2.4712 e+00$ & $8.7002 e-01$ \\
\hline yo & PAN 125136 & -1.0732 & 9.9894 & 4.8564 & 1.8600 e +00 & $8.7008 e-01$ \\
\hline y1 & PAN130136 & -1.4971 & 7.9633 & 3.4025 & $2.5481 e+00$ & $8.7004 e-01$ \\
\hline 32 & PAN120048 & 5.5946 & 21.7689 & 9.3116 & $2.5068 e+00$ & $8.7031 e-01$ \\
\hline 93 & PAN130048 & 0.3288 & 7.9582 & 2.9396 & $1.6646 \mathrm{e}+00$ & $8.7003 e-01$ \\
\hline 94 & PAN120224 & 1.5964 & 26.3919 & 8.5758 & $4.7972 \mathrm{e}+00$ & $8.7026 e-01$ \\
\hline$\div 5$ & PAN 130224 & -0.9931 & 8.7725 & 4.0789 & $2.2254 e+00$ & $8.7006 e-01$ \\
\hline 90 & PAN 120312 & 2.9830 & 30.6777 & 9.9913 & $5.3100 e+00$ & $8.7036 e-01$ \\
\hline$\because 7$ & PAN 130312 & -0.2992 & 9.0849 & 3.5597 & $1.9254 \mathrm{e}+00$ & $8.7005 e-01$ \\
\hline 98 & PAB000136 & 40.0908 & 40.9147 & 40.4679 & $1.8207 e-01$ & $5.8876 e-01$ \\
\hline 99 & PAB100136 & 40.7343 & 41.9702 & 41.0281 & $2.4592 e-01$ & $5.8900 e-01$ \\
\hline 100 & PAB125136 & 40.3170 & 40.8205 & 40.5679 & $1.0834 e-01$ & $5.8880 e-01$ \\
\hline
\end{tabular}




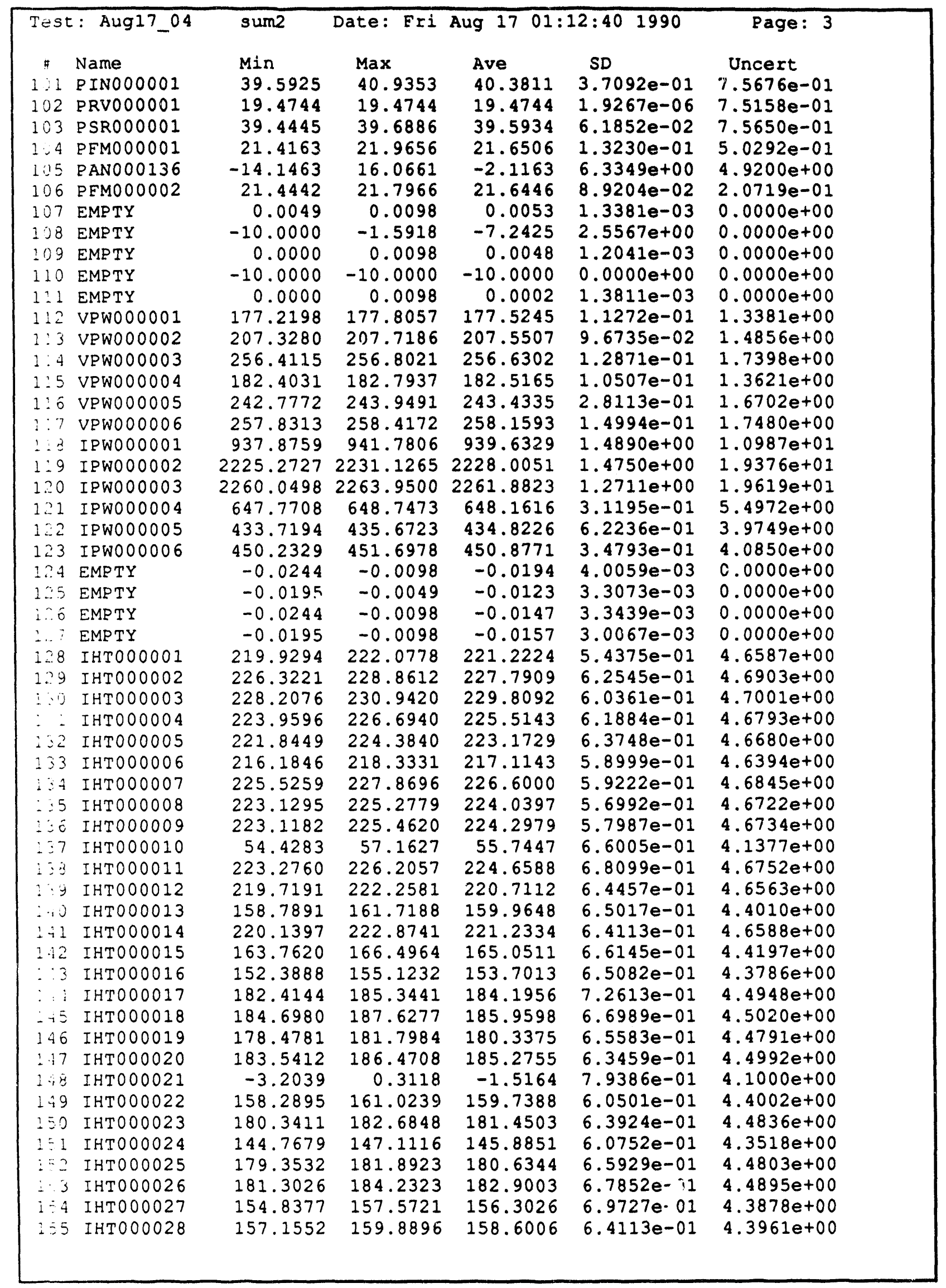




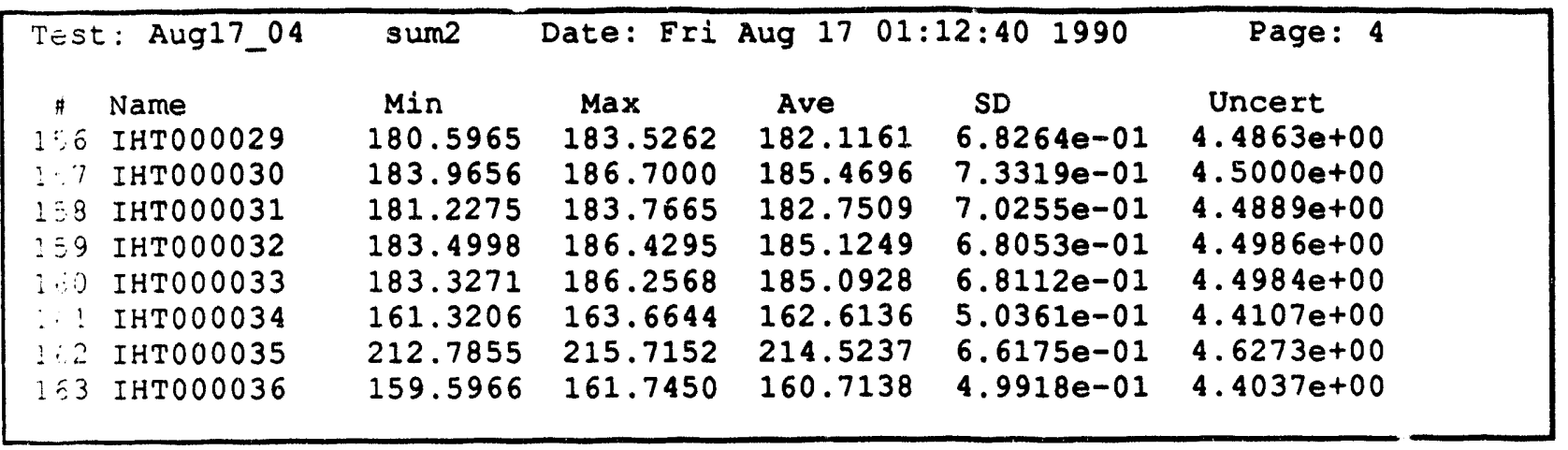




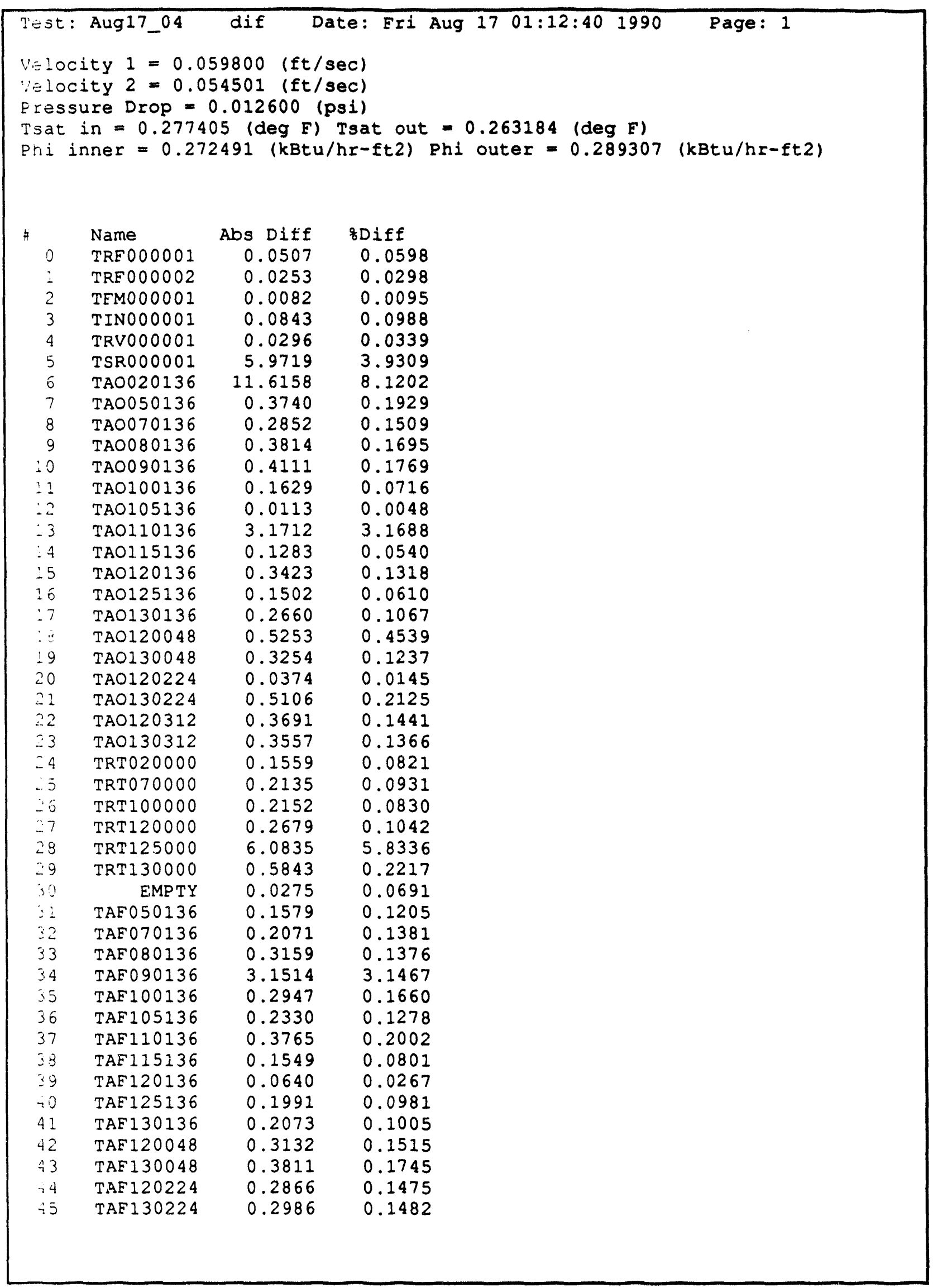




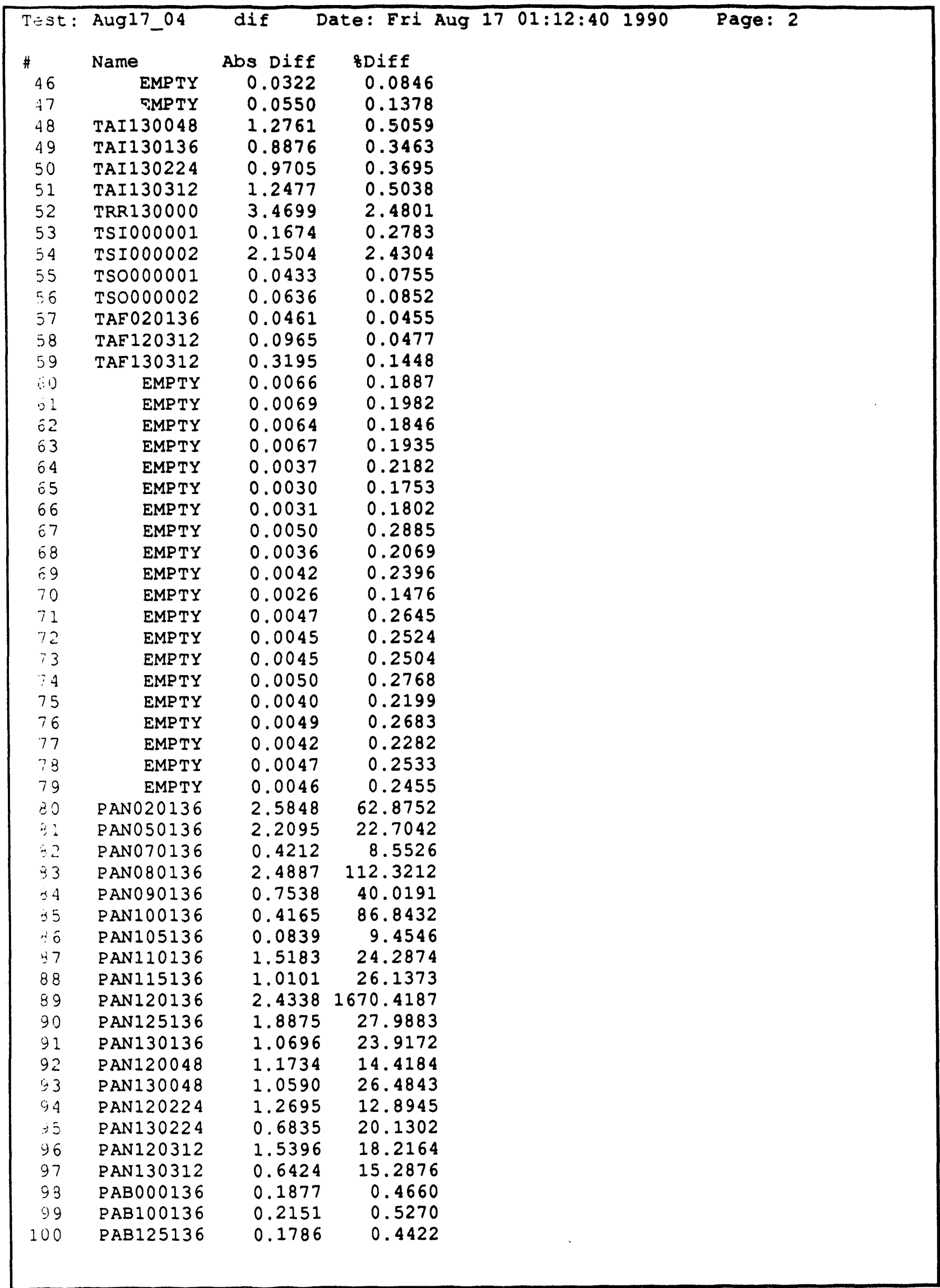




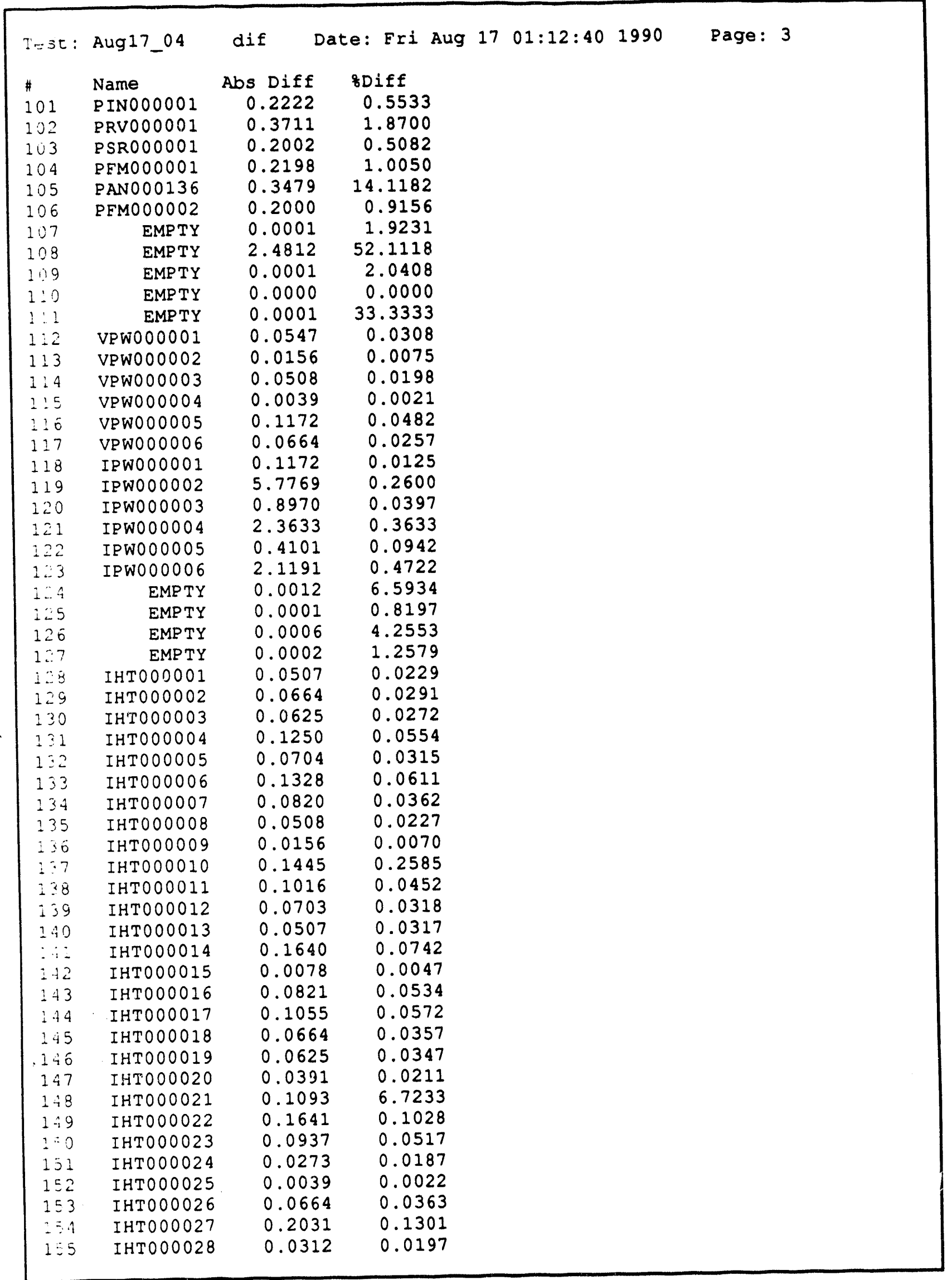




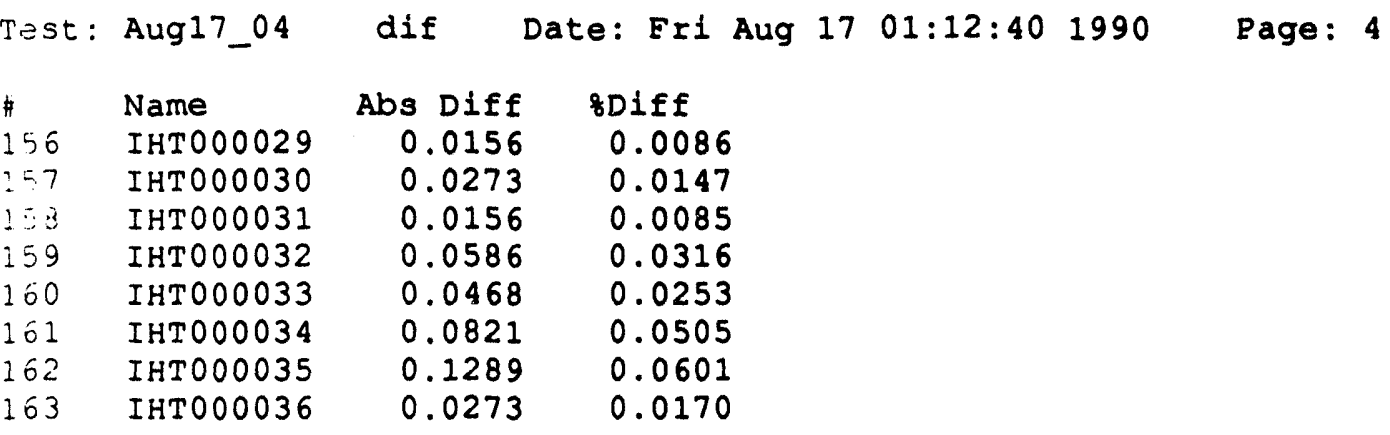




\begin{tabular}{|c|c|c|c|c|c|}
\hline 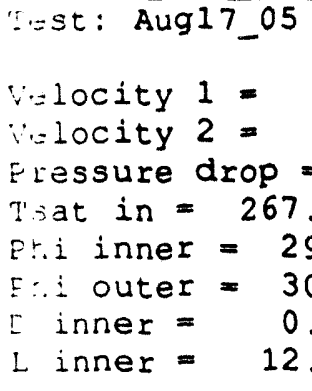 & $\begin{array}{l}5 \text { suml D } \\
11.0645 \text { (ft/ } \\
11.0614 \text { (ft/ } \\
=0-0.5401 \\
7.7212 \text { (deg E) } \\
297.4572 \text { (kBtu } \\
300.6841 \text { (kBtu } \\
0.1961 \text { (ft) D } \\
2.8610 \text { (ft) L }\end{array}$ & $\begin{array}{l}\text { Date: Fri A } \\
\text { /sec) }(+/- \\
\text { /sec) }(+/- \\
\text { (psi) } \\
\text { ) Tsat out } \\
\text { u/hr-ft2) } \\
\text { u/hr-ft2) } \\
\text { outer = } \\
\text { outer = }\end{array}$ & $\begin{array}{r}\text { Aug } 1701: \\
\begin{array}{r}2.1404 \\
1.7396\end{array} \\
=268.48 \\
1+1-\quad 1 . \\
1+1-\quad 1 . \\
0.2413 \\
12.8070\end{array}$ & $\begin{array}{l}24: 161990 \\
8) \\
8) \\
26 \text { (deg } F) \\
2362 \text { (f) } \\
2626 \text { 8) } \\
(f t) \text { D hyd = } \\
(f t) \text { A flow }\end{array}$ & $\begin{array}{ll} & \\
0.0390 \text { (ft) } \\
0.0150 \text { (ft2) }\end{array}$ \\
\hline 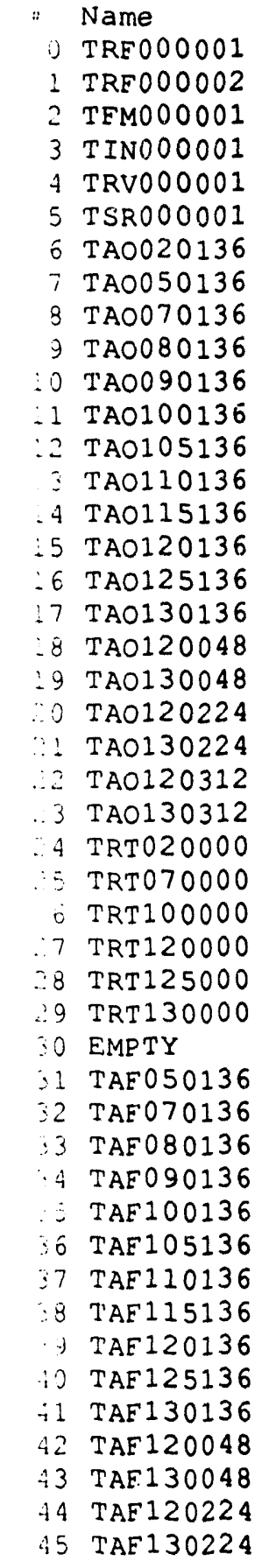 & $\begin{array}{l}\text { Min } \\
84.6700 \\
84.9996 \\
85.3478 \\
84.8915 \\
86.7153 \\
154.6671 \\
143.0645 \\
199.8967 \\
193.8893 \\
233.3536 \\
241.4397 \\
237.1051 \\
242.9100 \\
95.7684 \\
247.5558 \\
270.1425 \\
250.0977 \\
260.6143 \\
115.1755 \\
275.9554 \\
267.6803 \\
251.0035 \\
267.2848 \\
272.9703 \\
195.6292 \\
237.9929 \\
269.1491 \\
267.4709 \\
97.4300 \\
279.4417 \\
39.6057 \\
133.1395 \\
153.8714 \\
238.4521 \\
96.4258 \\
183.5735 \\
188.8900 \\
195.3435 \\
200.9141 \\
247.0382 \\
210.8939 \\
214.7220 \\
215.3133 \\
228.2046 \\
201.8329 \\
210.0448\end{array}$ & 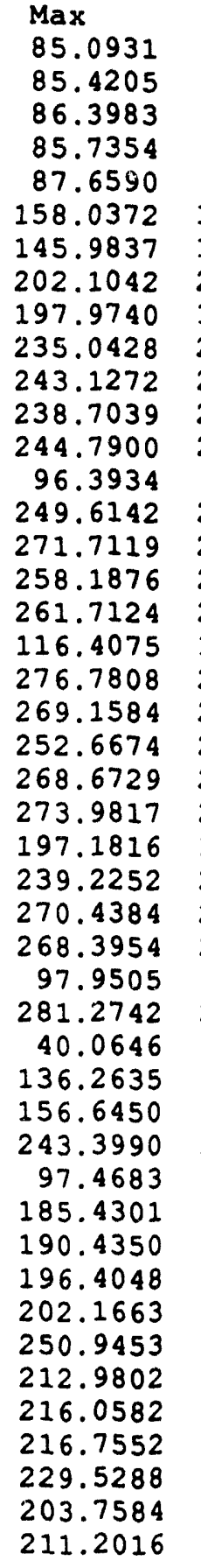 & $\begin{array}{l}\text { Ave } \\
84.9323 \\
85.1595 \\
85.9493 \\
85.3304 \\
87.1771 \\
156.2418 \\
144.2532 \\
200.9150 \\
195.7035 \\
234.2124 \\
242.5306 \\
237.8280 \\
243.8991 \\
96.0563 \\
248.7349 \\
270.9512 \\
252.7123 \\
261.0645 \\
115.7298 \\
276.3951 \\
268.3458 \\
251.8690 \\
267.9518 \\
273.4194 \\
196.3827 \\
238.6871 \\
269.7674 \\
267.9109 \\
97.6383 \\
280.1052 \\
39.8535 \\
134.6410 \\
155.1281 \\
241.4666 \\
96.9598 \\
184.4727 \\
189.5124 \\
195.8139 \\
201.4925 \\
248.9646 \\
211.7513 \\
215.3979 \\
216.1245 \\
228.8636 \\
202.7770 \\
210.5730\end{array}$ & $\begin{array}{l}\text { SD } \\
1.6861 e-01 \\
1.2440 e-01 \\
2.1022 e-01 \\
1.4353 e-01 \\
2.1378 e-01 \\
8.3576 e-01 \\
6.5250 e-01 \\
5.1723 e-01 \\
8.4802 e-01 \\
3.4050 e-01 \\
3.5046 e-01 \\
3.9141 e-01 \\
4.2497 e-01 \\
1.5657 e-01 \\
3.9788 e-01 \\
3.7711 e-01 \\
1.6586 e+00 \\
2.5473 e-01 \\
3.1116 e-01 \\
2.2550 e-01 \\
3.5860 e-01 \\
4.1436 e-01 \\
3.3656 e-01 \\
2.4918 e-01 \\
3.6332 e-01 \\
2.8552 e-01 \\
2.7871 e-01 \\
2.3365 e-01 \\
1.3446 e-01 \\
3.7885 e-01 \\
1.9026 e-01 \\
7.7978 e-01 \\
6.9632 e-01 \\
1.1146 e+00 \\
2.5653 e-01 \\
4.0323 e-01 \\
3.8405 e-01 \\
2.2773 e-01 \\
2.8445 e-01 \\
9.3018 e-01 \\
4.4276 e-01 \\
2.9525 e-01 \\
3.6436 e-01 \\
3.3342 e-01 \\
4.2373 e-01 \\
2.7617 e-01\end{array}$ & $\begin{array}{l}\text { Uncert } \\
1.9002 e+00 \\
1.9002 e+00 \\
1.9002 e+00 \\
1.9002 e+00 \\
1.9002 e+00 \\
1.9050 e+00 \\
1.9037 e+00 \\
1.9115 e+00 \\
1.9106 e+00 \\
1.9181 e+00 \\
1.9199 e+00 \\
1.9189 e+00 \\
1.9203 e+00 \\
1.9005 e+00 \\
1.9214 e+00 \\
1.9270 e+00 \\
1.9224 e+00 \\
1.9244 e+00 \\
1.9014 e+00 \\
1.9285 e+00 \\
1.9263 e+00 \\
1.9222 e+00 \\
1.9262 e+00 \\
1.9277 e+00 \\
1.9107 e+00 \\
1.9191 e+00 \\
1.9267 e+00 \\
1.9262 e+00 \\
1.9005 e+00 \\
1.9295 e+00 \\
1.9011 e+00 \\
1.9028 e+00 \\
1.9049 e+00 \\
1.9197 e+00 \\
1.9005 e+00 \\
1.9088 e+00 \\
1.9096 e+00 \\
1.9106 e+00 \\
1.9116 e+00 \\
1.9215 e+00 \\
1.9135 e+00 \\
1.9142 e+00 \\
1.9143 e+00 \\
1.9169 e+00 \\
1.9118 e+00 \\
1.9133 e+00\end{array}$ \\
\hline
\end{tabular}




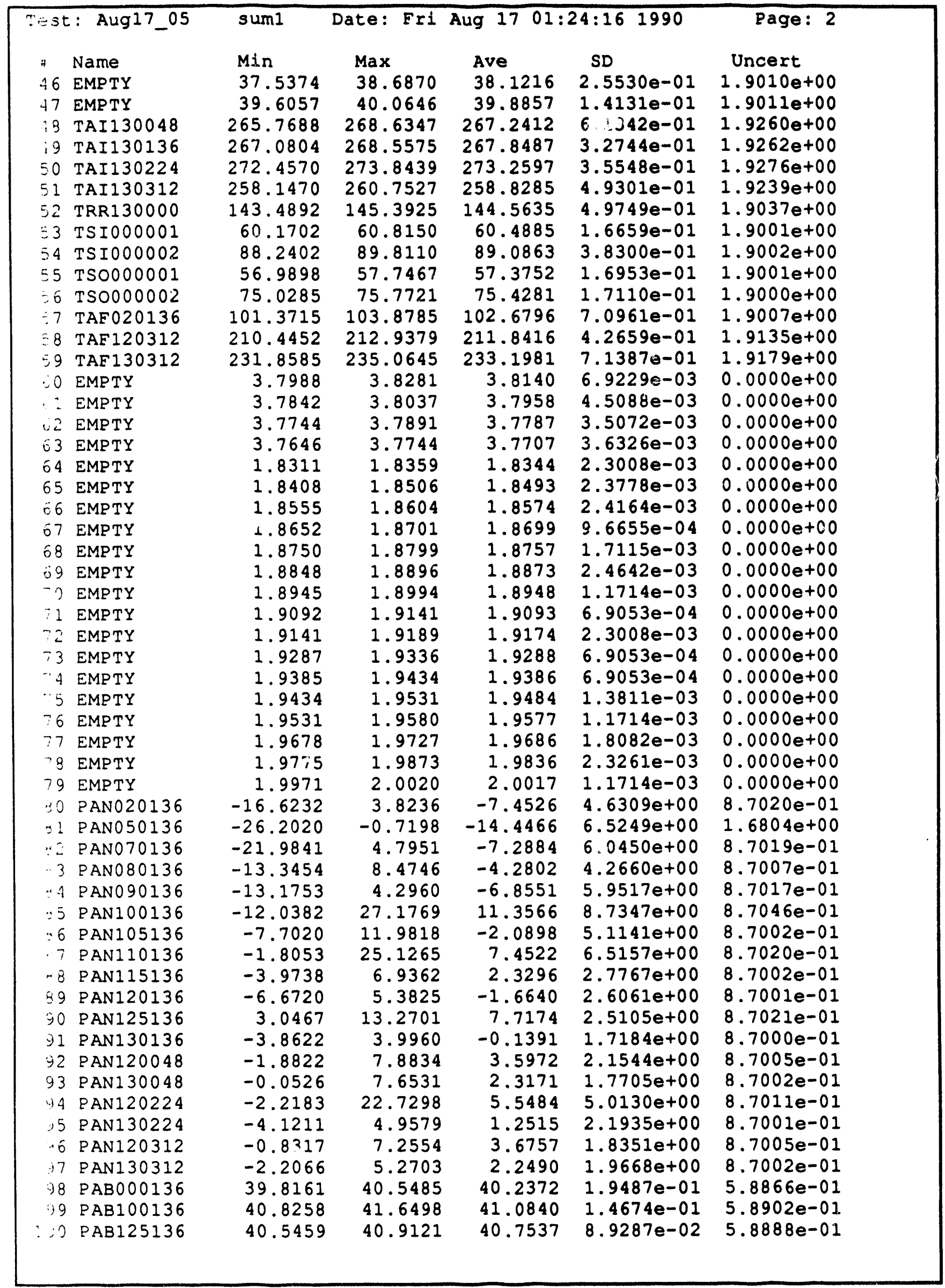




\begin{tabular}{|c|c|c|c|c|c|c|}
\hline Test & $=:$ Aug17_05 & suml & Date: Fri & Aug 17 01: & $24: 161990$ & Page: 3 \\
\hline if & Name & Min & $\operatorname{Max}$ & Ave & SD & Uncert \\
\hline 1.1 & PIN000001 & 39.2263 & 40.5690 & 40.2151 & $3.5929 \mathrm{e}-01$ & $7.5671 e-01$ \\
\hline 102 & PRV000001 & 19.4744 & 19.8406 & 19.7210 & $4.6072 \theta-02$ & $7.5162 e-01$ \\
\hline 103 & PSR000001 & 39.9327 & 40.2990 & 40.1378 & $7.9685 e-02$ & $7.5668 e-01$ \\
\hline$\therefore 4$ & PEM000001 & 18.3950 & 19.0359 & 18.7026 & $1.5226 e-01$ & $5.0218 e-01$ \\
\hline $1: 5$ & PAN000136 & -25.5904 & -3.6177 & -14.9520 & $5.4057 e+00$ & $4.9201 e+00$ \\
\hline 206 & PEM000002 & 18.4742 & 18.8769 & 18.6920 & $9.3240 e-02$ & $2.0539 e-01$ \\
\hline$: 07$ & EMPTY & -0.0049 & 0.0049 & 0.0047 & $1.3811 e-03$ & $0.0000 \mathrm{e}+00$ \\
\hline 103 & EMPTY & -10.0000 & -5.8154 & -8.8019 & $1.5204 e+00$ & $0.0000 e+00$ \\
\hline 209 & EMPTY & 0.0000 & 0.0049 & 0.0048 & $6.9053 e-04$ & $0.0000 e+00$ \\
\hline$\therefore: 0$ & EMPTY & -10.0000 & -10.0000 & -10.0000 & $0.0000 \mathrm{e}+00$ & $0.0000 \mathrm{e}+00$ \\
\hline 111 & EMPTY & 0.4000 & 0.0049 & 0.0001 & $6.9053 e-04$ & $0.0000 \mathrm{e}+00$ \\
\hline $1: 2$ & VPWO00001 & 177.2198 & 178.0011 & 177.5284 & $1.5328 e-01$ & $1.3382 \mathrm{e}+00$ \\
\hline $1: 3$ & VPW000002 & 207.1327 & 207.7186 & 207.5351 & $1.2731 e-01$ & $1.4855 e+00$ \\
\hline$\therefore: i$ & VPW000003 & 256.4115 & 256.9974 & 256.7357 & $1.0885 e-01$ & $1.7404 e+00$ \\
\hline$\because: 5$ & VPW000004 & 182.4031 & 182.7937 & 182.6571 & $1.2001 e-01$ & $1.3628 e+00$ \\
\hline $1 ! 6$ & VPW000005 & 243.3631 & 244.5350 & 243.7968 & $2.7421 e-01$ & $1.6721 e+00$ \\
\hline$\therefore 7$ & VPW000006 & 258.0266 & 258.6125 & 258.3155 & $1.1995 e-01$ & $1.7488 \mathrm{e}+00$ \\
\hline$\therefore: 9$ & IPWO00001 & 955.4470 & 959.3517 & 957.0084 & $1.2473 e+00$ & $1.1080 \mathrm{e}+01$ \\
\hline $1: 9$ & IPWO00002 & 2209.6626 & 2223.3215 & 2216.2971 & $3.5480 e+00$ & $1.9292 \mathrm{e}+01$ \\
\hline 120 & IPW000003 & 2236.6499 & 2244.4500 & 2240.3154 & $1.6546 e+00$ & $1.9464 \mathrm{e}+01$ \\
\hline$\therefore 1$ & IPW000004 & 655.0950 & 656.5598 & 655.6616 & $3.1752 e-01$ & $5.5524 \mathrm{e}+00$ \\
\hline$\because \therefore 2$ & IPW000005 & 433.7194 & 436.1605 & 434.5981 & $5.8355 e-01$ & $3.9734 \mathrm{e}+00$ \\
\hline 123 & IPW000006 & 446.8149 & 448.2798 & 447.4201 & $3.8913 e-01$ & $4.0612 e+00$ \\
\hline$\therefore \therefore 4$ & EMPTY & -0.0244 & -0.0146 & -0.0195 & $3.5568 e-03$ & $0.0000 \mathrm{e}+00$ \\
\hline 125 & EMPTY & -0.0195 & -0.0098 & -0.0131 & $2.8659 e-03$ & $0.0000 \mathrm{e}+00$ \\
\hline$\because \because 6$ & EMPTY & -0.0195 & -0.0098 & -0.0154 & $2.4878 e-03$ & $0.0000 \mathrm{e}+00$ \\
\hline$\therefore 7$ & EMPTY & -0.0195 & -0.0098 & -0.0158 & $3.0501 e-03$ & $0.0000 e+00$ \\
\hline $2: 8$ & IHT000001 & 219.5387 & 222.4684 & 221.0466 & $7.1140 e-01$ & $4.6579 e+00$ \\
\hline 1.29 & IHT000002 & 225.9315 & 228.8612 & 227.6113 & $6.7773 e-01$ & $4.6894 e+00$ \\
\hline$: 30$ & IHT000003 & 228.4030 & 231.1373 & 229.7858 & $6.5178 e-01$ & $4.7000 e+00$ \\
\hline$\therefore:$ & IHTO00004 & 224.3502 & 226.8893 & 225.4909 & $6.1556 e-01$ & $4.6792 e+00$ \\
\hline 232 & IHTO00005 & 221.6496 & 224.1887 & 223.0636 & $5.9915 e-01$ & $4.6675 e+00$ \\
\hline$: 33$ & IHT000006 & 215.7940 & 217.9424 & 216.9619 & $5.6702 e-01$ & $4.6387 e+00$ \\
\hline$: 34$ & IHTOOOOOO & 225.1352 & 227.4790 & 226.4242 & $5.1145 e-01$ & $4.6837 e+00$ \\
\hline$:: 5$ & IHT000008 & 222.5436 & 224.8873 & 223.8014 & $4.8993 e-01$ & $4.6711 e+00$ \\
\hline 136 & IHT000009 & 222.9229 & 225.0714 & 224.0791 & $5.0508 e-01$ & $4.6724 e+00$ \\
\hline 137 & IHTO00010 & 54.6236 & 56.9674 & 55.8267 & $5.5293 e-01$ & $4.1378 e+00$ \\
\hline 138 & IHT000011 & 223.6666 & 226.0103 & 224.7370 & $6.1793 e-01$ & $4.6755 e+00$ \\
\hline$:: 9$ & IHTO00012 & 219.5237 & 222.2581 & 220.7698 & $6.4584 e-01$ & $4.6566 \mathrm{e}+00$ \\
\hline 190 & IHTODO0 & 158.9844 & 161.7188 & 160.0508 & $6.6052 e-01$ & $4.4013 e+00$ \\
\hline$\therefore i 1$ & IHTU00014 & 219.9444 & 223.2647 & 221.4561 & $6.6123 e-01$ & $4.6599 e+00$ \\
\hline$: 42$ & IH'O000015 & 163.7620 & 166.4964 & 165.1644 & $5.8486 e-01$ & $4.4202 e+00$ \\
\hline$: 3$ & IHT000016 & 152.7794 & 154.9279 & 153.8810 & $5.0186 e-01$ & $4.3793 e+00$ \\
\hline$\therefore: t$ & IHTO00017 & 183.1956 & 186.5159 & 184.3363 & $6.6773 e-01$ & $4.4953 e+00$ \\
\hline$\therefore 45$ & IHTO00018 & 184.8933 & 187.8230 & 186.1278 & $6.0675 e-01$ & $4.5027 e+00$ \\
\hline$\therefore 46$ & IHTO00019 & 178.6734 & 181.9937 & 180.3609 & $7.6794 e-01$ & $4.4792 e+00$ \\
\hline 147 & IHT000020 & 183.5412 & 186.6662 & 185.2560 & $7.5725 e-01$ & $4.4991 e+00$ \\
\hline 148 & IHT000021 & -3.3992 & 0.5071 & -1.3914 & $8.8501 e-01$ & $4.1000 e+00$ \\
\hline 149 & IHTO00022 & 158.2895 & 161.0239 & 159.7973 & $6.6502 \mathrm{e}-01$ & $4.4004 e+00$ \\
\hline 150 & IHTOO0023 & 179.9504 & 182.8801 & 181.4073 & $6.7682 e-01$ & $4.4834 \mathrm{e}+00$ \\
\hline$: \vdots:$ & IHT000024 & 144.5726 & 147.5023 & 145.8851 & $6.6150 e-01$ & $4.3518 e+00$ \\
\hline$\therefore 52$ & IHT000025 & 179.1579 & 182.0876 & 180.6149 & $6.4017 e-01$ & $4.4802 e+00$ \\
\hline $2: 3$ & IHT000026 & 181.3026 & 184.2323 & 182.8886 & $7.0547 e-01$ & $4.4894 e+00$ \\
\hline 154 & IHT000027 & 154.6424 & 157.9627 & 156.2948 & $7.2888 e-01$ & $4.3878 \mathrm{e}+00$ \\
\hline$: 5$ & IHTOOOO28 & 156.7646 & 159.6942 & 158.3466 & $7.2141 e-01$ & $4.3952 e+00$ \\
\hline
\end{tabular}




\begin{tabular}{|c|c|c|c|c|c|c|}
\hline$T \leqq S$ & $t:$ Aug17_05 & suml & Date: Fri & Aug 1701 & $24: 16 \quad 1990$ & Page: 4 \\
\hline $\begin{array}{l}78 \\
156 \\
257 \\
158 \\
159 \\
\therefore \therefore 0 \\
\therefore 1 \\
102 \\
103\end{array}$ & 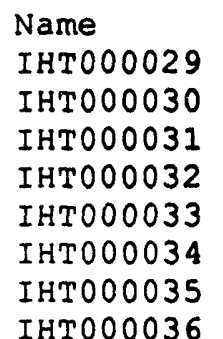 & $\begin{array}{l}\text { Min } \\
180.2058 \\
183.7703 \\
181.0322 \\
183.4998 \\
183.3271 \\
161.3206 \\
213.1761 \\
159.2060\end{array}$ & $\begin{array}{l}\text { Max } \\
183.1355 \\
186.5047 \\
183.3759 \\
185.8436 \\
185.8661 \\
163.6644 \\
215.9105 \\
161.5497\end{array}$ & $\begin{array}{l}\text { Ave } \\
181.7762 \\
185.0712 \\
182.3408 \\
184.7929 \\
184.7217 \\
162.4066 \\
214.4339 \\
160.5029\end{array}$ & $\begin{array}{l}S D \\
6.8681 e-01 \\
7.0433 e-01 \\
7.2033 e-01 \\
6.9575 e-01 \\
7.0256 e-01 \\
5.8148 e-01 \\
6.8704 e-01 \\
5.8362 e-01\end{array}$ & $\begin{array}{c}\text { Uncert } \\
4.4849 e+00 \\
4.4983 e+00 \\
4.4872 e+00 \\
4.4972 e+00 \\
4.4969 e+00 \\
4.4099 e+00 \\
4.6269 e+00 \\
4.4030 e+00\end{array}$ \\
\hline
\end{tabular}




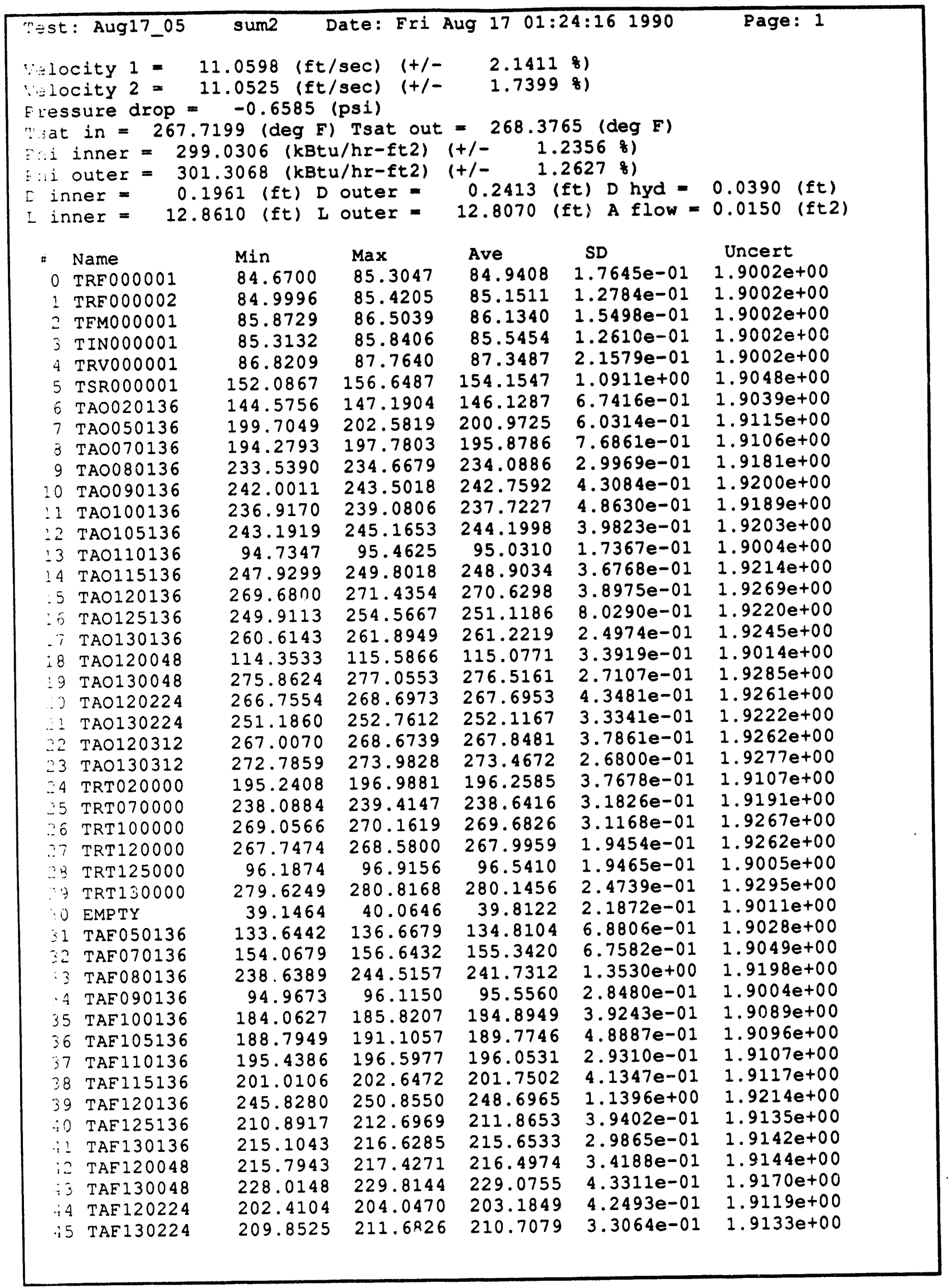




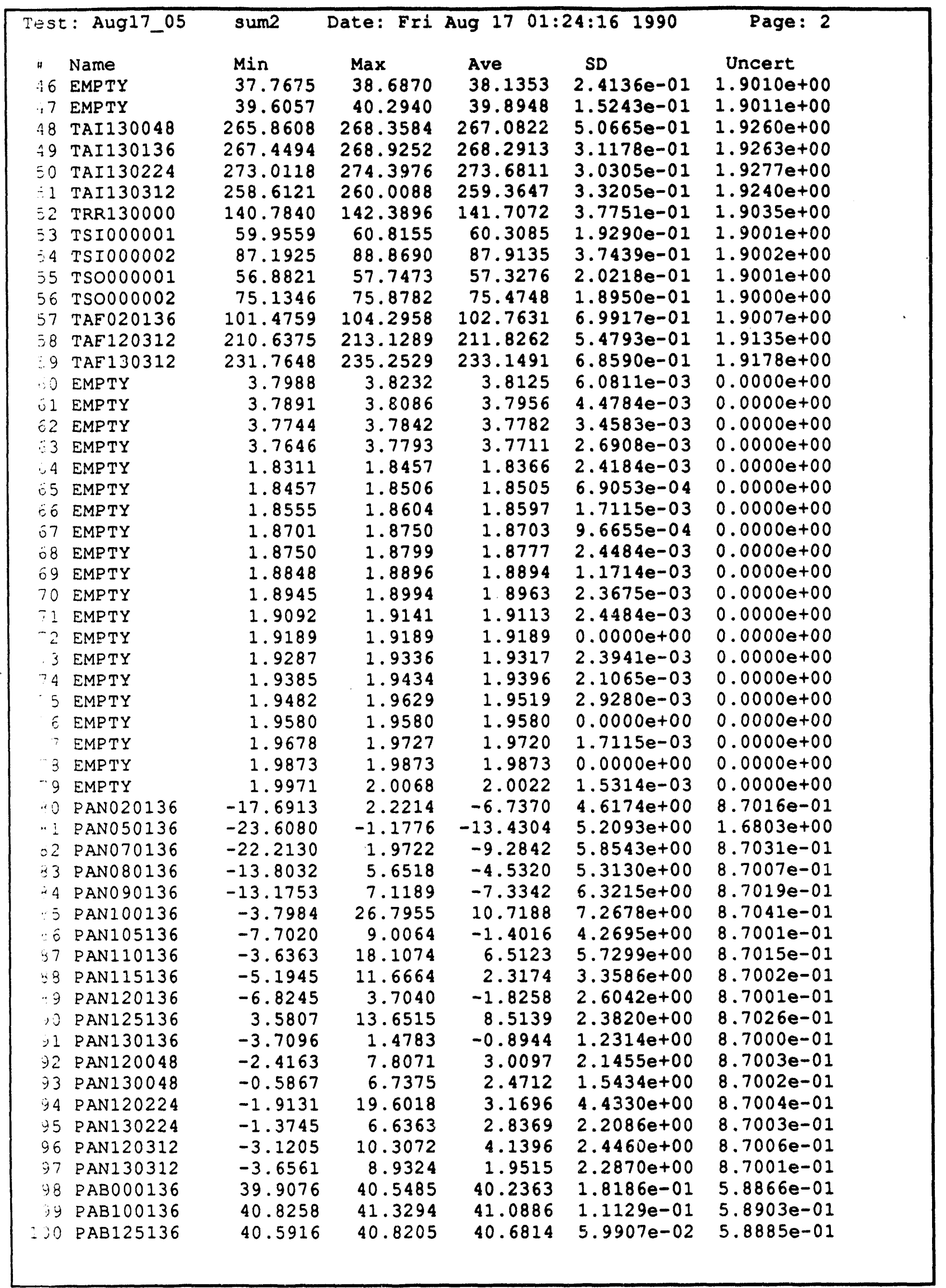




\begin{tabular}{|c|c|c|c|c|c|c|}
\hline \multicolumn{2}{|c|}{ Test: Aug17_0S } & sum2 & \multicolumn{3}{|c|}{ Date: Fri Aug $1701: 24: 161990$} & \multirow[t]{2}{*}{ Page: 3} \\
\hline$\#$ & Name & Min & $\operatorname{Max}$ & Ave & SD & \\
\hline 101 & PIN000001 & 39.2263 & 40.6911 & 39.9953 & $4.2376 e-01$ & $7.5664 \mathrm{e}-01$ \\
\hline 102 & PRV000001 & 19.5965 & 19.5965 & 19.5965 & $1.9267 e-06$ & $7.5160 e-01$ \\
\hline$: 23$ & PSR0000001 & 39.9327 & 40.1769 & 40.0768 & $5.3409 e-02$ & $7.5666 e-01$ \\
\hline $1 \div 4$ & PFMO00001 & 18.3950 & 19.0359 & 18.6862 & $1.3298 \mathrm{e}-01$ & $5.0218 e-01$ \\
\hline 105 & PANO00136 & -29.7103 & -6.3643 & -18.2296 & $6.0714 e+00$ & $4.9202 e+00$ \\
\hline 206 & PFM0000002 & 18.4742 & 18.8602 & 18.6612 & $8.7880 e-02$ & $2.0537 e-01$ \\
\hline$: 77$ & EMPTY & 0.0049 & 0.0098 & 0.0050 & $6.9053 e-04$ & $0.0000 e+00$ \\
\hline 1,8 & EMPTY & -10.0000 & -5.8789 & -7.5814 & $1.3383 e+00$ & $0.0000 e+00$ \\
\hline 209 & EMPTY & 0.0000 & 0.0049 & 0.0047 & $9.6655 e-04$ & $0.0000 e+00$ \\
\hline 110 & EMPTY & -10.0000 & -10.0000 & -10.0000 & $0.0000 \mathrm{e}+00$ & $0.0000 e+00$ \\
\hline$: 11$ & EMPTY & 0.0000 & 0.0000 & 0.0000 & $0.0000 e+00$ & $0.0000 e+00$ \\
\hline 112 & VPW0000001 & 177.4151 & 178.0011 & 177.6495 & $1.3087 e-01$ & $1.3387 e+00$ \\
\hline 113 & VPWO000002 & 207.3280 & 208.3046 & 207.5741 & $1.5690 e-01$ & $1.4857 e+00$ \\
\hline$\therefore 14$ & VPW000003 & 256.4115 & 256.9974 & 256.7591 & $1.0654 \mathrm{e}-01$ & $1.7405 e+00$ \\
\hline$\therefore \equiv$ & VPWO000004 & 182.4031 & 182.7937 & 182.5399 & $1.2001 \mathrm{e}-01$ & $1.3622 e+00$ \\
\hline$\therefore 6$ & VPWO000005 & 242.3866 & 243.5584 & 242.9882 & $3.4363 e-01$ & $1.6678 \mathrm{e}+00$ \\
\hline$:: 7$ & VPWO00006 & 258.0266 & 258.8079 & 258.3702 & $1.3984 e-01$ & $1.7491 \mathrm{e}+00$ \\
\hline$:: 8$ & IPW0 00001 & 941.7806 & 947.6376 & 944.3967 & $1.3440 e+00$ & $1.1012 e+01$ \\
\hline$:: 9$ & IPWO 00002 & 2233.0779 & 2238.9316 & 2236.7080 & $1.7634 \mathrm{e}+00$ & $1.9438 \mathrm{e}+01$ \\
\hline$\ldots 3$ & IPW0000003 & 2250.2998 & 2254.2000 & 2252.2112 & $1.4470 e+00$ & $1.9549 e+01$ \\
\hline$\therefore 1$ & IPWO000004 & 655.0950 & 656.0715 & 655.6421 & $2.7267 e-01$ & $5.5523 e+00$ \\
\hline$: 22$ & IPWO000005 & 432.7429 & 435.6723 & 434.6176 & $6.5696 \mathrm{e}-01$ & $3.9735 e+00$ \\
\hline .23 & IPWO00006 & 448.7681 & 449.7446 & 449.4416 & $2.9413 e-01$ & $4.0751 e+00$ \\
\hline 124 & EMPTY & -0.0293 & -0.0098 & -0.0191 & $4.3940 e-03$ & $0.0000 e+00$ \\
\hline .25 & EMPTY & -0.0146 & -0.0049 & -0.0118 & $2.8058 e-03$ & $0.0000 e+00$ \\
\hline 126 & EMPTY & -0.0244 & -0.0098 & -0.0168 & $3.4400 e-03$ & $0.0000 \mathrm{e}+00$ \\
\hline$\therefore: ?$ & EMPTY & -0.0195 & -0.0098 & -0.0146 & $3.1950 \mathrm{e}-03$ & $0.0000 e+00$ \\
\hline$\therefore x$ & IHTO00001 & 219.7341 & 222.4684 & 221.2575 & $6.3748 e-01$ & $4.6589 e+00$ \\
\hline$\therefore y$ & IHTO000002 & 226.1268 & 229.4471 & 227.7167 & $6.3993 e-01$ & $4.6899 e+00$ \\
\hline$\therefore 0$ & IHT000003 & 228.4030 & 231.1373 & 229.8288 & $5.9615 e-01$ & $4.7002 e+00$ \\
\hline$: 31$ & IHT 000004 & 224.3502 & 226.8893 & 225.5260 & $5.7384 e-01$ & $4.6793 e+00$ \\
\hline$: 12$ & IHTO00005 & 221.8449 & 224.5793 & 223.1300 & $5.4117 e-01$ & $4.6678 \mathrm{e}+00$ \\
\hline$\therefore 3$ & IHT000006 & 215.7940 & 218.3331 & 217.0869 & $5.4945 e-01$ & $4.6393 e+00$ \\
\hline$\therefore 4$ & IHT000007 & 225.5259 & 227.6743 & 226.6703 & $5.3381 \mathrm{e}-01$ & $4.6849 \mathrm{e}+00$ \\
\hline$: 35$ & IHTO00008 & 222.9342 & 225.2779 & 224.0866 & $5.3706 \mathrm{e}-01$ & $4.6724 e+00$ \\
\hline$\therefore \overline{0}$ & IHTO00009 & 223.1182 & 225.4620 & 224.3252 & $5.8085 e-01$ & $4.6736 \mathrm{e}+00$ \\
\hline$: 37$ & IHTO00010 & 54.2330 & 56.7721 & 55.4517 & $6.0818 e-01$ & $4.1373 e+00$ \\
\hline$: 38$ & IHTO00011 & 223.0807 & 225.8150 & 224.4206 & $6.0233 e-01$ & $4.6740 \mathrm{e}+00$ \\
\hline$: 39$ & IHTO00012 & 218.9378 & 221.6722 & 220.4339 & $5.7940 e-01$ & $4.6550 e+00$ \\
\hline 240 & IHTO000013 & 158.2031 & 160.7422 & 159.6523 & $5.5816 e-01$ & $4.3999 \mathrm{e}+00$ \\
\hline$\therefore:$ & IHTO00014 & 218.9679 & 221.8976 & 220.4404 & $7.1812 e-01$ & $4.6550 \mathrm{e}+00$ \\
\hline$\therefore: 2$ & IHTO00015 & 163.5667 & 166.4964 & 164.8363 & $5.9875 e-01$ & $4.4189 e+00$ \\
\hline$\therefore 3$ & IHTO00016 & 152.3888 & 155.1232 & 153.4747 & $5.4699 \mathrm{e}-01$ & $4.3778 e+00$ \\
\hline$\therefore 4$ & IHTO000017 & 182.4144 & 185.9300 & 184.0160 & $7.1245 e-01$ & $4.4940 e+00$ \\
\hline$: 15$ & IHTO00018 & 184.5027 & 187.4324 & 185.9598 & $6.6756 e-01$ & $4.5020 e+00$ \\
\hline$\therefore: 6$ & IHTO00019 & 178.6734 & 181.6031 & 180.2203 & $5.2473 e-01$ & $4.4786 e+00$ \\
\hline 197 & IHTOOOOO2O & 183.9318 & 186.6662 & 185.1817 & $5.7858 e-01$ & $4.4988 e+00$ \\
\hline 148 & IHT000021 & -3.0086 & 0.5071 & -1.4148 & $7.0073 e-01$ & $4.1000 \mathrm{e}+00$ \\
\hline 149 & IHT 000022 & 158.6801 & 161.2192 & 159.7856 & $5.3759 \mathrm{e}-01$ & $4.4004 \mathrm{e}+00$ \\
\hline$: 50$ & IHT000023 & 180.5364 & 182.8801 & 181.3995 & $5.0546 e-01$ & $4.4834 e+00$ \\
\hline 151 & IHTO00024 & 144.7679 & 146.9163 & 145.8266 & $4.8823 e-01$ & $4.3516 e+00$ \\
\hline 152 & IHTO000025 & 179.5486 & 182.0876 & 180.6696 & $5.4649 e-01$ & $4.4804 e+00$ \\
\hline 153 & IHTO00026 & 181.8885 & 184.4276 & 182.8769 & $5.8847 e-01$ & $4.4894 e+00$ \\
\hline$: 54$ & IHTOOOOO27 & 155.2284 & 157.9627 & 156.3261 & $6.3857 e-01$ & $4.3879 e+00$ \\
\hline$\therefore .5$ & IHTO00028 & 156.5692 & 160.0849 & 158.5537 & $7.0516 e-01$ & $4.3959 e+00$ \\
\hline
\end{tabular}




\begin{tabular}{|c|c|c|c|c|c|c|}
\hline \multicolumn{2}{|c|}{ T'est: Aug17_05 } & sum2 & Date: Fri & Aug 1701 & $24: 161990$ & Page: 4 \\
\hline & Name & Min & $\operatorname{Max}$ & Ave & SD & Uncert \\
\hline 156 & IHTO000029 & 180.0105 & 183.7215 & 181.9637 & $7.0144 e-01$ & $4.4857 e+00$ \\
\hline 157 & IHTO000030 & 183.3797 & 186.8953 & 185.3602 & $7.1791 e-01$ & $4.4995 \mathrm{e}+00$ \\
\hline 358 & IHTO00031 & 180.8368 & 184.3525 & 182.6376 & $7.0287 e-01$ & $4.4884 e+00$ \\
\hline 159 & IHTOOOOO32 & 182.9139 & 186.8201 & 184.9960 & $7.4096 \mathrm{e}-01$ & $4.4980 \mathrm{e}+00$ \\
\hline 160 & IHT000033 & 182.9365 & 186.8427 & 184.9248 & $7.3365 e-01$ & $4.4977 e+00$ \\
\hline 161 & IHTO00034 & 160.9300 & 164.2503 & 162.5043 & $5.8979 e-01$ & $4.4103 e+00$ \\
\hline 102 & IHTO000035 & 212.1995 & 215.9105 & 213.8284 & $6.0696 e-01$ & $4.6241 e+00$ \\
\hline 3.3 & IHTO00036 & 159.0107 & 161.9403 & 160.5381 & $5.3915 e-01$ & $4.4031 e+00$ \\
\hline
\end{tabular}




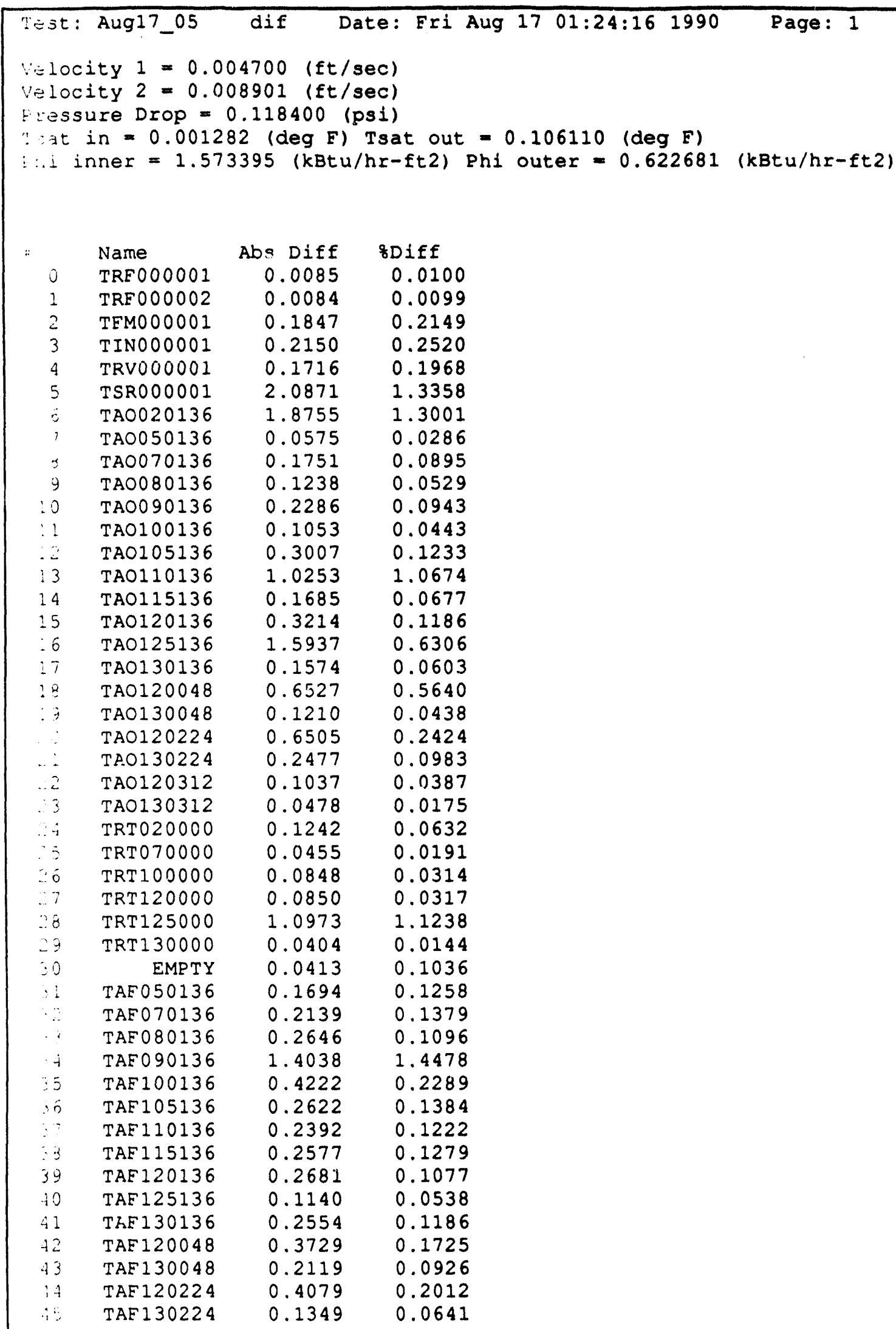




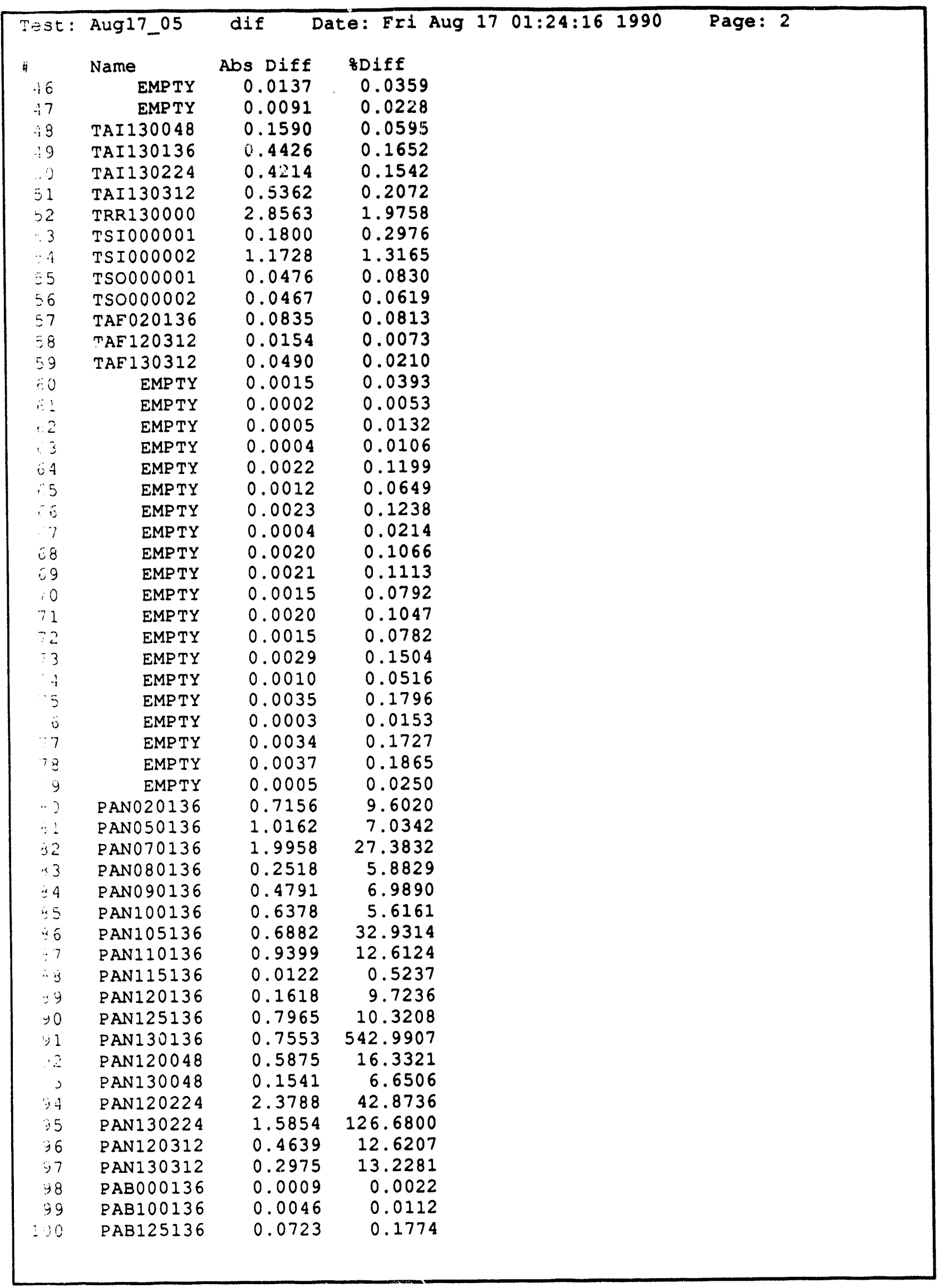




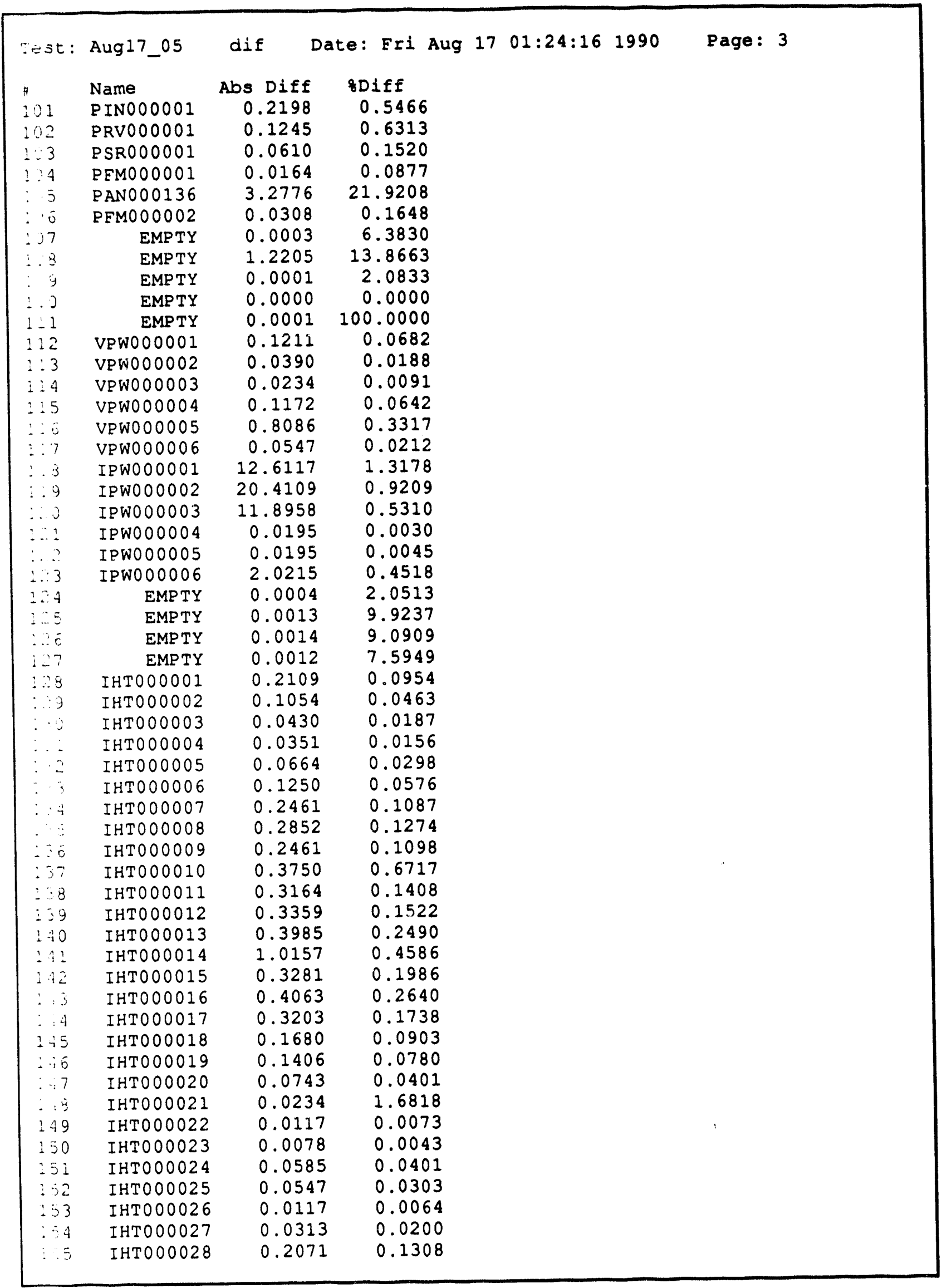




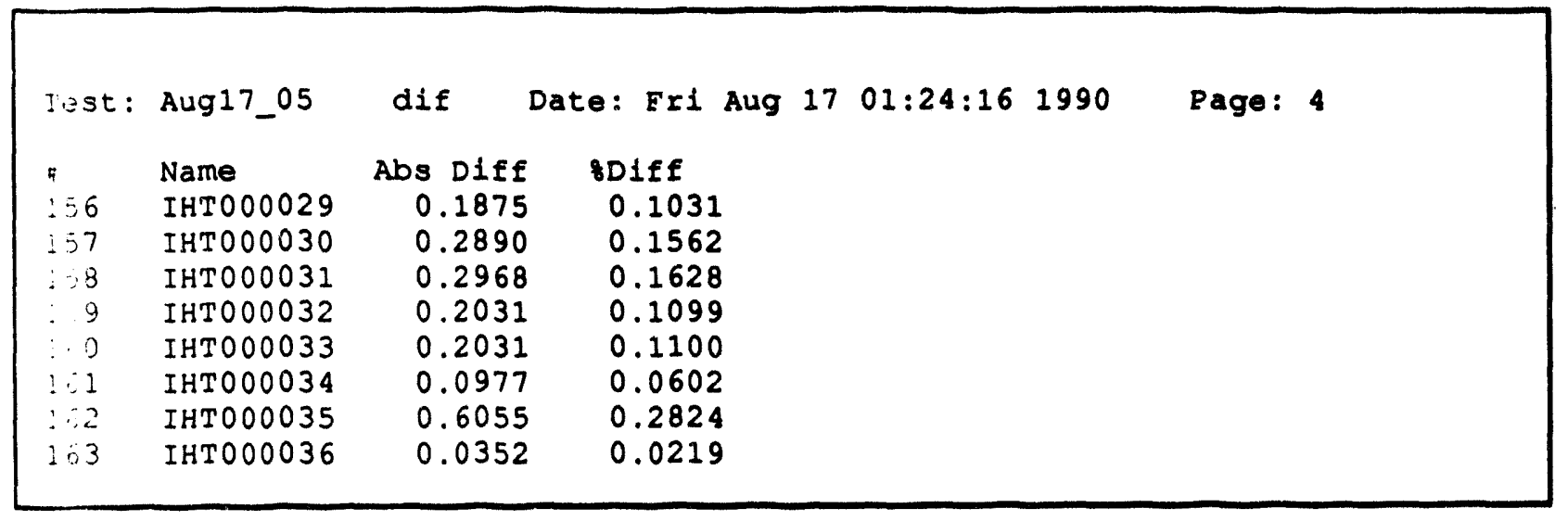




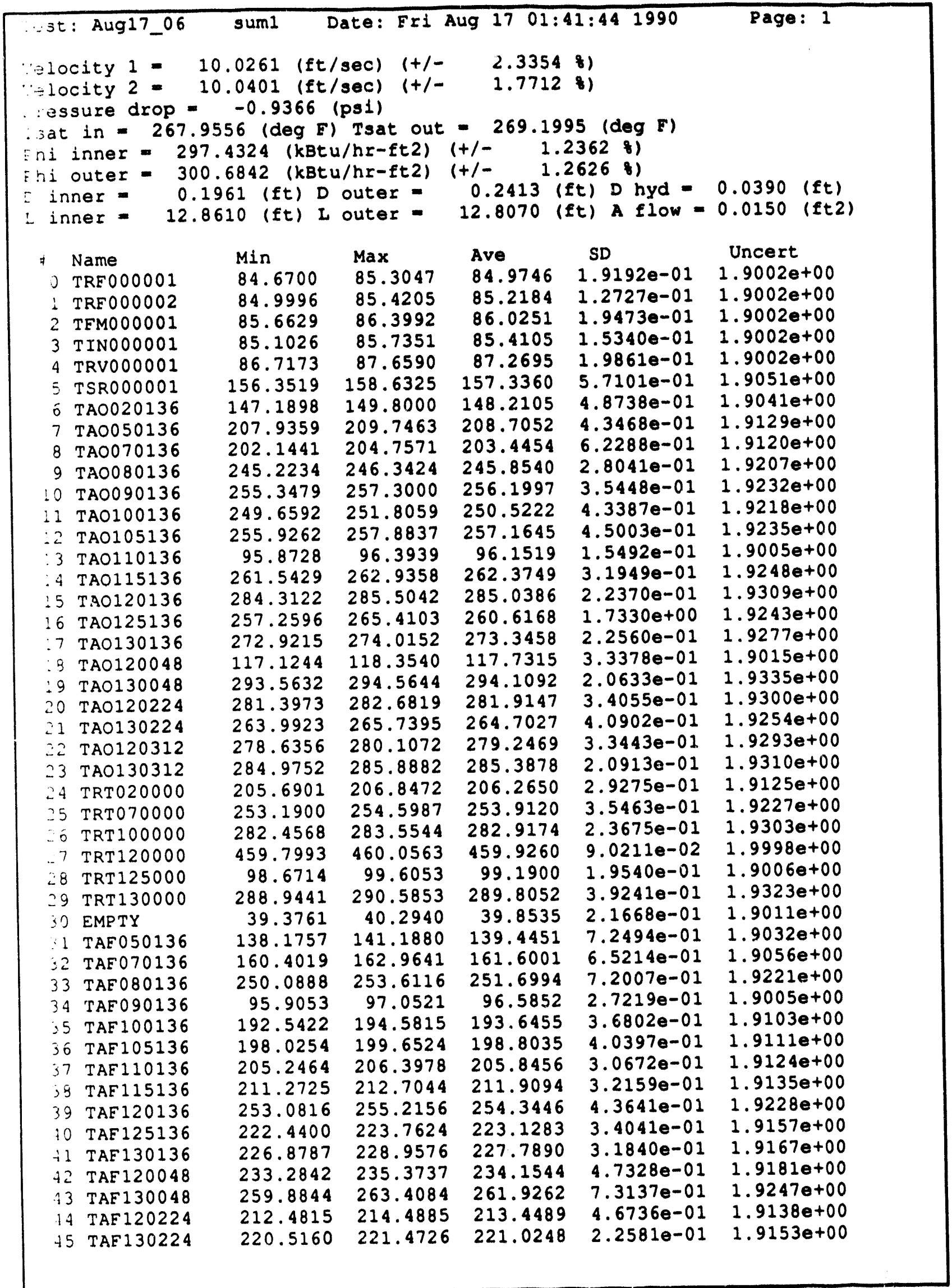




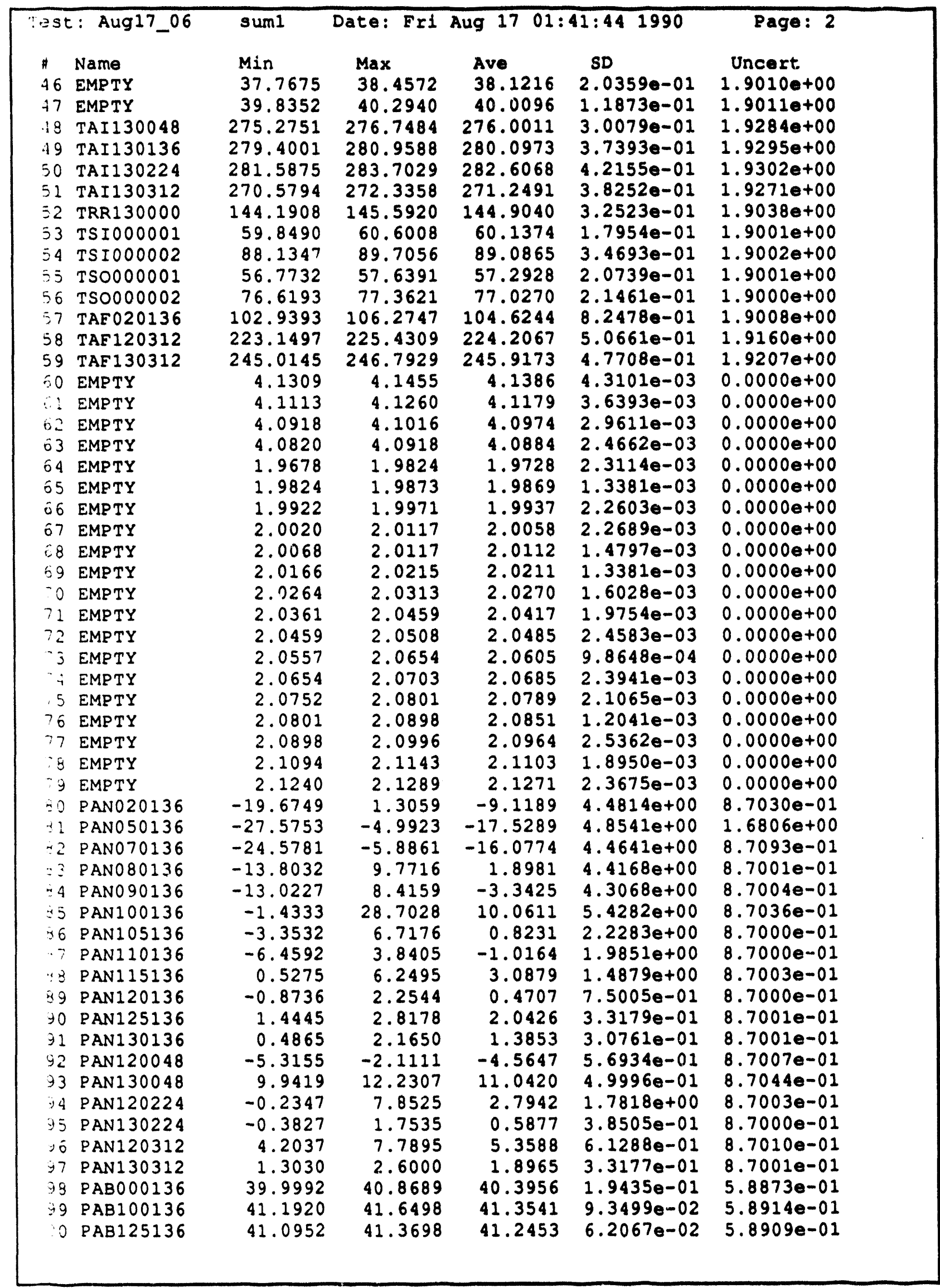




\begin{tabular}{|c|c|c|c|c|c|c|}
\hline \multicolumn{2}{|c|}{ Test: Aug17_06 } & sum1 & \multicolumn{3}{|c|}{ Date: Fri Aug $17 \quad 01: 41: 441990$} & Page: 3 \\
\hline " & Name & Min & $\operatorname{Max}$ & Ave & SD & Uncert \\
\hline$: 01$ & PIN000001 & 39.3483 & 40.8132 & 40.0979 & $3.9228 \theta-01$ & $7.5667 e-01$ \\
\hline 102 & PRV000001 & 19.5965 & 19.7186 & 19.6380 & $5.8413 e-02$ & $7.5161 e-01$ \\
\hline$: 03$ & RSR000001 & 40.6652 & 40.9093 & 40.7799 & $4.5541 \theta-02$ & $7.5690 \mathrm{e}-01$ \\
\hline 24 & $\begin{array}{l}\text { PFMOOO0001 } \\
\text { PANO00136 }\end{array}$ & 15.0991 & 15.6484 & 15.3298 & $1.1878 \theta-01$ & $5.0147 e-01$ \\
\hline $\begin{array}{l}.05 \\
: 06\end{array}$ & $\begin{array}{l}\text { PANO00136 } \\
\text { PFM000002 }\end{array}$ & $\begin{array}{r}-37.0345 \\
15.1854\end{array}$ & $\begin{array}{r}-15.0618 \\
15.5210\end{array}$ & $\begin{array}{r}-25.9291 \\
15.3730\end{array}$ & $\begin{array}{l}5.6572 e+00 \\
5.9174 \theta-02\end{array}$ & $4.9204 e+00$ \\
\hline 07 & EMPTY & $\begin{array}{r}15.1000 \\
0.0000\end{array}$ & $\begin{array}{r}15.5210 \\
0.0098\end{array}$ & $\begin{array}{r}15.3730 \\
0.0049\end{array}$ & $\begin{array}{l}5.9174 e-02 \\
9.8648 e-04\end{array}$ & $\begin{array}{l}2.0366 e-01 \\
0.0000 e+00\end{array}$ \\
\hline$\therefore 08$ & EMPTY & -10.0000 & -5.6396 & -7.5645 & $1.5653 e+00$ & $0.0000 e+00$ \\
\hline$\therefore 99$ & EMPTY & 0.0000 & 0.0049 & 0.0044 & $1.4797 \theta-03$ & $0.0000 e+00$ \\
\hline$: 10$ & EMPTY & -10.0000 & -10.0000 & -10.0000 & $0.0000 e+00$ & $0.0000 e+00$ \\
\hline$: 11$ & EMPTY & 0.0000 & 0.0049 & 0.0001 & $6.9053 e-04$ & $0.0000 e+00$ \\
\hline 12 & VPW000001 & 177.4151 & 178.0011 & 177.6768 & $1.2853 e-01$ & $1.3389 e+00$ \\
\hline$\therefore 3$ & VPW000002 & 207.5233 & 208.1092 & 207.7538 & $1.1632 \theta-01$ & $1.4866 e+00$ \\
\hline$: .4$ & VPW000003 & 256.8021 & 256.9974 & 256.8997 & $9.8648 e-02$ & $1.7413 \mathrm{e}+00$ \\
\hline.$: 5$ & VPW000004 & 182.4031 & 182.9890 & 182.7430 & $1.2359 e-01$ & $1.3632 e+00$ \\
\hline$\therefore 6$ & VPWO00005 & 242.9725 & 243.7538 & 243.2617 & $2.0594 \theta-01$ & $1.6693 \mathrm{e}+00$ \\
\hline$: 7$ & VPW000006 & 258.4172 & 259.0032 & 258.6085 & $1.4493 e-01$ & $1.7503 e+00$ \\
\hline. .8 & IPWOOO0001 & 955.4470 & 961.3040 & 957.3989 & $1.3664 e+00$ & $1.1082 \theta+01$ \\
\hline$\therefore 19$ & IPW0 00002 & 2211.6140 & 2223.3215 & 2215.6729 & $2.8383 \theta+00$ & $1.9287 e+01$ \\
\hline$: 20$ & IPWO00003 & 2236.6499 & 2240.5498 & 2238.3662 & $1.2231 e+00$ & $1.9450 e+01$ \\
\hline$\therefore 21$ & IPW000004 & 654.6067 & 656.0715 & 655.3198 & $3.4470 \theta-01$ & $5.5499 e+00$ \\
\hline$: 22$ & IPWO00005 & 432.2547 & 434.6958 & 433.2506 & $5.7481 \theta-01$ & $3.9642 e+00$ \\
\hline$: 23$ & IPW000006 & 446.3267 & 447.7915 & 447.2053 & $4.6270 e-01$ & $4.0597 e+00$ \\
\hline$: 24$ & EMPTY & -0.0244 & -0.0098 & -0.0188 & $4.0685 e-03$ & $0.0000 e+00$ \\
\hline .25 & EMPTY & -0.0195 & -0.0098 & -0.0127 & $2.9594 e-03$ & $0.0000 e+00$ \\
\hline$\therefore 6$ & EMPTY & -0.0244 & -0.0098 & -0.0154 & $3.1752 \theta-03$ & $0.0000 \mathrm{e}+00$ \\
\hline .27 & EMPTY & -0.0244 & -0.0098 & -0.0162 & $3.7486 e-03$ & $0.0000 e+00$ \\
\hline 28 & IHTO00001 & 219.9294 & 222.0778 & 220.9841 & $5.6497 e-01$ & $4.6576 e+00$ \\
\hline 29 & IHTO00002 & 226.5174 & 228.6658 & 227.5136 & $5.4996 e-01$ & $4.6889 e+00$ \\
\hline 30 & IHTO00003 & 228.4030 & 230.7467 & 229.6452 & $6.1353 e-01$ & $4.6993 e+00$ \\
\hline .31 & IHTO00004 & 223.9596 & 226.6940 & 225.4987 & $5.6859 e-01$ & $4.6792 \mathrm{e}+00$ \\
\hline .32 & IHT000005 & 221.8449 & 224.1887 & 223.0987 & $5.7330 e-01$ & $4.6677 \mathrm{e}+00$ \\
\hline$: 33$ & IHT000006 & 215.7940 & 218.3331 & 217.0713 & $5.8155 e-01$ & $4.6392 e+00$ \\
\hline$: 34$ & IHTO000007 & 225.3306 & 228.0649 & 226.6586 & $6.1130 e-01$ & $4.6848 e+00$ \\
\hline$: 35$ & IHT000008 & 222.7389 & 225.2779 & 224.0436 & $6.3435 e-01$ & $4.6722 e+00$ \\
\hline .36 & IHTO00009 & 223.3135 & 225.6573 & 224.2276 & $6.2070 e-01$ & $4.6731 \mathrm{e}+00$ \\
\hline$: 37$ & IHTOOOOOIO & 53.8424 & 57.1627 & 55.4869 & $6.3759 e-01$ & $4.1374 e+00$ \\
\hline .38 & IHTOOOOI1 & 223.0807 & 225.8150 & 224.4401 & $6.2510 e-01$ & $4.6741 e+00$ \\
\hline$\because 9$ & IHTO00012 & 219.3284 & 221.6722 & 220.5198 & $6.0264 e-01$ & $4.6554 \mathrm{e}+00$ \\
\hline 40 & IHTO00013 & 158.5938 & 160.9375 & 159.8633 & $5.5559 e-01$ & $4.4006 e+00$ \\
\hline 41 & IHTO00014 & 219.1632 & 221.5069 & 220.6162 & $6.3400 e-01$ & $4.6559 e+00$ \\
\hline .12 & IHTO00015 & 163.5667 & 165.7151 & 164.8636 & $5.4069 e-01$ & $4.4190 \mathrm{e}+00$ \\
\hline i3 & IHTO00016 & 152.3888 & 154.3419 & 153.4747 & $5.3403 e-01$ & $4.3778 e+00$ \\
\hline .14 & IHTO000017 & 182.8050 & 185.9300 & 184.2113 & $7.0033 e-01$ & $4.4948 e+00$ \\
\hline$: 45$ & IHTO00018 & 184.5027 & 187.0418 & 185.8466 & $7.1315 e-01$ & $4.5015 e+00$ \\
\hline$: 46$ & IHTO00019 & 179.0640 & 181.2124 & 180.0445 & $5.3451 e-01$ & $4.4779 e+00$ \\
\hline$: 47$ & IHTO00020 & 184.1271 & 186.2755 & 185.1583 & $5.5389 e-01$ & $4.4987 e+00$ \\
\hline 48 & IHTOOOOO21 & -2.8132 & -0.0789 & -1.5203 & $6.5303 e-01$ & $4.1000 e+00$ \\
\hline$\therefore 49$ & IHTO00022 & 158.4848 & 160.6333 & 159.5161 & $4.9448 e-01$ & $4.3994 e+00$ \\
\hline .50 & IHTOO00023 & 180.3411 & 182.4895 & 181.2706 & $5.0925 e-01$ & $4.4828 e+00$ \\
\hline 51 & IHTO00024 & 144.7679 & 146.9163 & 145.7445 & $4.9912 e-01$ & $4.3513 e+00$ \\
\hline .2 & IHTO00025 & 179.7439 & 181.6970 & 180.4977 & $4.9128 e-01$ & $4.4797 e+00$ \\
\hline 53 & IHTO00026 & 181.8885 & 183.8417 & 182.7440 & $5.2924 e-01$ & $4.4888 e+00$ \\
\hline$: 54$ & IHT 000027 & 155.0331 & . 157.1815 & 156.1151 & $5.3701 e-01$ & $4.3872 e+00$ \\
\hline 5 & IHTO00028 & 156.7646 & 159.6942 & 158.4912 & $6.5716 e-01$ & $4.3957 e+00$ \\
\hline
\end{tabular}




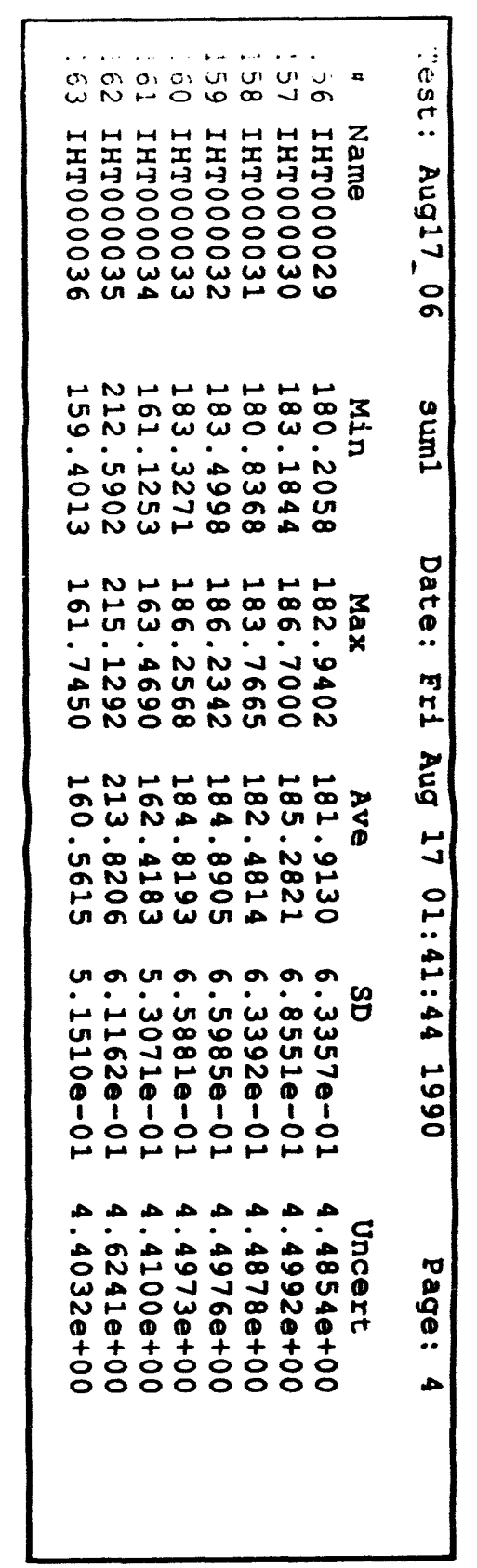




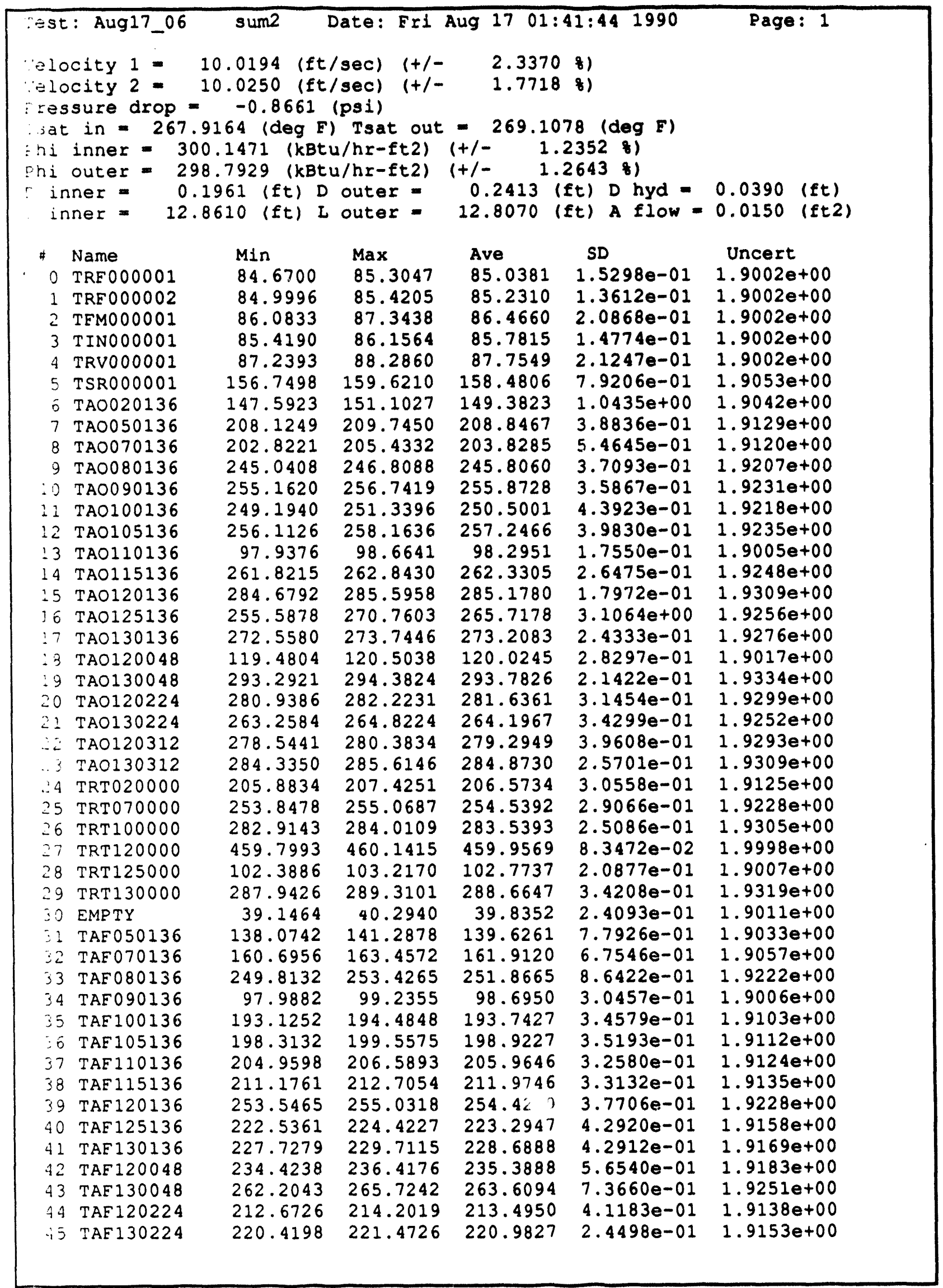




\begin{tabular}{|c|c|c|c|c|c|c|}
\hline \multicolumn{2}{|c|}{ '.est: Aug17_06 } & sum2 & Date: Eri & Aug 1701 & $41: 441990$ & Page: 2 \\
\hline \# & Name & Min & $\operatorname{Max}$ & Ave & SD & Uncert \\
\hline 46 & EMPTY & 37.7675 & 38.4572 & 38.1308 & $2.0295 e-01$ & $1.9010 \mathrm{e}+00$ \\
\hline 47 & EMPTY & 39.8352 & 40.2940 & 40.0050 & $1.6591 e-01$ & $1.9011 e+00$ \\
\hline 48 & TAI 130048 & 275.5518 & 276.4726 & 276.0454 & $2.5678 e-01$ & $1.9284 e+00$ \\
\hline 49 & TAI 130136 & 280.3165 & 281.8744 & 280.9766 & $3.5855 e-01$ & $1.9298 \mathrm{e}+00$ \\
\hline 50 & TAI 130224 & 282.2320 & 283.7024 & 283.0261 & $2.8817 e-01$ & $1.9303 e+00$ \\
\hline 51 & TAI 130312 & 270.0254 & 271.5972 & 270.8795 & $3.7890 e-01$ & $1.9270 e+00$ \\
\hline 52 & TRR 130000 & 144.5911 & 146.8924 & 145.7961 & $5.3599 e-01$ & $1.9039 e+00$ \\
\hline 53 & TSI000001 & 59.2065 & 59.9587 & 59.6747 & $1.6907 e-01$ & $1.9001 e+00$ \\
\hline 54 & TSI000002 & 87.8218 & 90.6475 & 89.4759 & $6.3779 e-01$ & $1.9003 e+00$ \\
\hline 55 & TSO000001 & 56.7731 & 57.5303 & 57.1109 & $1.9131 e-01$ & $1.9001 e+00$ \\
\hline 56 & TSO000002 & 76.5144 & 77.3626 & 76.9805 & $2.1631 e-01$ & $1.9000 \mathrm{e}+00$ \\
\hline 57 & TAF020136 & 103.4600 & 106.5874 & 104.9955 & $8.4078 e-01$ & $1.9008 e+00$ \\
\hline 58 & TAF 120312 & 223.6243 & 226.4754 & 225.1001 & $6.9371 e-01$ & $1.9161 \mathrm{e}+00$ \\
\hline 59 & TAF 130312 & 245.4839 & 247.4474 & 246.2207 & $4.1171 e-01$ & $1.9208 e+00$ \\
\hline 60 & EMPTY & 4.1357 & 4.1553 & 4.1447 & $4.8688 e-03$ & $0.0000 e+00$ \\
\hline$\because 1$ & EMPTY & 4.1211 & 4.1309 & 4.1237 & $2.6488 e-03$ & $0.0000 \mathrm{e}+00$ \\
\hline 62 & EMPTY & 4.0967 & 4.1064 & 4.1030 & $2.4662 e-03$ & $0.0000 e+00$ \\
\hline 63 & EMPTY & 4.0918 & 4.1016 & 4.0938 & $2.7902 e-03$ & $0.0000 e+00$ \\
\hline 64 & EMPTY & 1.9727 & 1.9824 & 1.9735 & $2.1364 e-03$ & $0.0000 e+00$ \\
\hline$\ddot{0} 5$ & EMPTY & 1.9824 & 1.9873 & 1.9872 & $6.9053 e-04$ & $0.0000 e+00$ \\
\hline 66 & EMPTY & 1.9922 & 1.9971 & 1.9950 & $2.4344 e-03$ & $0.0000 e+00$ \\
\hline 67 & EMPTY & 2.0020 & 2.0117 & 2.0065 & $1.8216 e-03$ & $0.0000 \mathrm{e}+00$ \\
\hline 68 & EMPTY & 2.0068 & 2.0117 & 2.0116 & $6.9053 e-04$ & $0.0000 e+00$ \\
\hline 69 & EMPTY & 2.0166 & 2.0215 & 2.0213 & $9.6655 e-04$ & $0.0000 e+00$ \\
\hline 70 & EMPTY & 2.0264 & 2.0313 & 2.0280 & $2.3365 e-03$ & $0.0000 e+00$ \\
\hline 71 & EMPTY & 2.0410 & 2.0459 & 2.0434 & $2.4642 e-03$ & $0.0000 \mathrm{e}+00$ \\
\hline 72 & EMPTY & 2.0459 & 2.0508 & 2.0492 & $2.3008 e-03$ & $0.0000 \mathrm{e}+00$ \\
\hline 73 & EMPTY & 2.0605 & 2.0654 & 2.0610 & $1.4797 e-03$ & $0.0000 \mathrm{e}+00$ \\
\hline 74 & EMPTY & 2.0654 & 2.0752 & 2.0700 & $1.8216 e-03$ & $0.0000 e+00$ \\
\hline$: 5$ & EMPTY & 2.0752 & 2.0801 & 2.0800 & $6.9053 e-04$ & $0.0000 e+00$ \\
\hline 76 & EMPTY & 2.0850 & 2.0898 & 2.0853 & $1.1714 e-03$ & $0.0000 e+00$ \\
\hline 77 & EMPTY & 2.0947 & 2.0996 & 2.0983 & $2.1635 e-03$ & $0.0000 e+00$ \\
\hline 78 & EMPTY & 2.1094 & 2.1191 & 2.1118 & $2.6562 e-03$ & $0.0000 e+00$ \\
\hline-9 & EMPTY & 2.1240 & 2.1387 & 2.1289 & $1.7086 e-03$ & $0.0000 e+00$ \\
\hline 80 & PAN020136 & -16.9283 & 1.2296 & -7.9089 & $4.4314 e+00$ & $8.7022 e-01$ \\
\hline 81 & PANO50136 & -29.4064 & -7.5863 & -19.2684 & $5.2066 e+00$ & $1.6807 e+00$ \\
\hline 32 & PAN070136 & -24.6544 & -7.6408 & -17.6384 & $4.9971 e+00$ & $8.7112 e-01$ \\
\hline 83 & PAN080136 & -9.2256 & 14.3493 & 3.8161 & $5.5073 e+00$ & $8.7005 e-01$ \\
\hline 84 & PAN090136 & -13.1753 & 12.3069 & -3.8430 & $6.4814 e+00$ & $8.7005 e-01$ \\
\hline 85 & PAN 100136 & -0.1363 & 34.5774 & 12.4003 & $7.9830 \mathrm{e}+00$ & $8.7055 e-01$ \\
\hline 26 & PAN 105136 & -6.2524 & 7.1753 & 1.5158 & $2.6743 e+00$ & $8.7001 e-01$ \\
\hline 57 & PAN 110136 & -6.3066 & 5.0612 & -1.1080 & $2.3205 e+00$ & $8.7000 e-01$ \\
\hline$\because z$ & PAN 115136 & -0.9984 & 7.7754 & 2.5935 & $2.0355 e+00$ & $8.7002 e-01$ \\
\hline$\dot{9}$ & PAN 120136 & -0.7210 & 1.8730 & 0.6813 & $5.9356 e-01$ & $8.7000 e-01$ \\
\hline 90 & PAN125136 & 1.5208 & 3.0467 & 2.1281 & $3.3839 e-01$ & $8.7002 e-01$ \\
\hline 9 & PAN130136 & 1.3258 & 3.0042 & 1.9910 & $3.6509 e-01$ & $8.7001 e-01$ \\
\hline 32 & PAN 120048 & -5.7732 & -4.4762 & -5.0942 & $3.2524 e-01$ & $8.7009 e-01$ \\
\hline 93 & PAN 130048 & 12.9936 & 15.4350 & 14.4173 & $5.9330 e-01$ & $8.7075 e-01$ \\
\hline 94 & PAN 120224 & 0.2994 & 6.0215 & 2.4799 & $1.4643 e+00$ & $8.7002 e-01$ \\
\hline 35 & PAN 130224 & 0.3802 & 2.9742 & 1.3583 & $5.2806 e-01$ & $8.7001 e-01$ \\
\hline 96 & PAN 120312 & 5.0429 & 8.4761 & 6.6420 & $8.2430 \mathrm{e}-01$ & $8.7016 e-01$ \\
\hline 97 & PAN 130312 & 1.9896 & 4.1258 & 2.9250 & $4.8518 e-01$ & $8.7003 e-01$ \\
\hline 98 & PAB000136 & 39.9076 & 40.7774 & 40.3690 & $2.2763 e-01$ & $5.8872 e-01$ \\
\hline 95 & PAB 100136 & 41.0547 & 41.5125 & 41.3120 & $1.0661 \mathrm{e}-01$ & $5.8912 e-01$ \\
\hline$: 20$ & PAB 125136 & 41.0494 & 41.3241 & 41.1822 & $6.2211 e-02$ & $5.8907 e-01$ \\
\hline
\end{tabular}




\begin{tabular}{|c|c|c|c|c|c|c|}
\hline \multicolumn{2}{|c|}{ :est: Aug17_06 } & sum2 & \multicolumn{3}{|c|}{ Date: Fri Aug 17 01:41:441990 } & \multirow[t]{2}{*}{ Page: 3} \\
\hline$\#$ & Name & Min & $\operatorname{Max}$ & Ave & SD & \\
\hline$: 01$ & PIN000001 & 39.3483 & 40.9353 & 40.0759 & $4.6070 \mathrm{e}-01$ & $7.5666 \mathrm{e}-01$ \\
\hline 102 & PRV000001 & 19.4744 & 19.7186 & 19.6258 & $5.8152 e-02$ & $7.5160 e-01$ \\
\hline$: 03$ & PSR000001 & 40.5431 & 40.7872 & 40.6383 & $7.5168 e-02$ & $7.5685 e-01$ \\
\hline$\because 4$ & PFM000001 & 15.0991 & 15.5569 & 15.3079 & $1.2820 \mathrm{e}-01$ & $5.0146 e-01$ \\
\hline$\therefore 5$ & PANO00136 & -34.7457 & -10.4842 & -23.9791 & $7.1429 e+00$ & $4.9204 e+00$ \\
\hline .06 & PFM000002 & 15.1518 & 15.5042 & 15.3253 & $8.4656 e-02$ & $2.0364 e-01$ \\
\hline$: 07$ & EMPTY & 0.0049 & 0.0098 & 0.0051 & $9.6655 e-04$ & $0.0000 e+00$ \\
\hline 08 & EMPTY & -10.0000 & -0.9668 & -7.5366 & $1.9877 e+00$ & $0.0000 e+00$ \\
\hline$: 29$ & EMPTY & 0.0000 & 0.0049 & 0.0047 & $9.6655 e-04$ & $0.0000 \mathrm{e}+00$ \\
\hline$: 10$ & EMPTY & -10.0000 & -10.0000 & -10.0000 & $0.0000 e+00$ & $0.0000 e+00$ \\
\hline$: 11$ & EMPTY & 0.0000 & 0.0000 & 0.0000 & $0.0000 \mathrm{e}+00$ & $0.0000 e+00$ \\
\hline .12 & VPWO00001 & 177.4151 & 178.0011 & 177.6846 & $1.6217 \mathrm{e}-01$ & $1.3389 \mathrm{e}+00$ \\
\hline$: 13$ & VPW000002 & 207.5233 & 207.9139 & 207.7109 & $1.1133 e-01$ & $1.4864 e+00$ \\
\hline$\vdots ! 4$ & VPW000003 & 256.8021 & 257.7787 & 256.9896 & $1.5764 e-01$ & $1.7417 \mathrm{e}+00$ \\
\hline$\therefore 25$ & VPW000004 & 182.5984 & 182.7937 & 182.6805 & $9.7377 e-02$ & $1.3629 e+00$ \\
\hline .16 & VPW000005 & 242.7772 & 244.3397 & 243.6210 & $4.1842 e-01$ & $1.6712 \mathrm{e}+00$ \\
\hline$: \because 7$ & VPW000006 & 258.2219 & 259.0032 & 258.5694 & $1.7774 e-01$ & $1.7501 e+00$ \\
\hline$: 18$ & IPWO00001 & 930.0665 & 939.8282 & 933.9318 & $2.7181 \mathrm{e}+00$ & $1.0957 \mathrm{e}+01$ \\
\hline$: 19$ & IPWO00002 & 2211.6140 & 2219.4189 & 2214.6184 & $1.8155 e+00$ & $1.9280 e+01$ \\
\hline 120 & IPWO00003 & 2256.1499 & 2263.9500 & 2260.1279 & $2.0072 e+00$ & $1.9606 \mathrm{e}+01$ \\
\hline$: 21$ & IPW0000004 & 649.2356 & 650.7004 & 650.0366 & $2.7480 e-01$ & $5.5110 \mathrm{e}+00$ \\
\hline$\therefore 2$ & IPW000005 & 431.2782 & 434.2076 & 433.0261 & $8.7721 e-01$ & $3.9627 \mathrm{e}+00$ \\
\hline$: 23$ & IPW000006 & 448.2798 & 450.2329 & 449.2463 & $4.6782 e-01$ & $4.0738 e+00$ \\
\hline$: 24$ & EMPTY & -0.0293 & -0.0098 & -0.0189 & $4.1432 e-03$ & $0.0000 e+00$ \\
\hline$: 25$ & EMPTY & -0.0146 & -0.0098 & -0.0129 & $2.3675 e-03$ & $0.0000 e+00$ \\
\hline$: 26$ & EMPTY & -0.0195 & -0.0049 & -0.0136 & $3.3146 e-03$ & $0.0000 e+00$ \\
\hline$: 27$ & EMPTY & -0.0244 & -0.0098 & -0.0170 & $3.3073 e-03$ & $0.0000 \mathrm{e}+00$ \\
\hline$: 28$ & IHTO000001 & 219.5387 & 222.4684 & 221.0974 & $6.1699 e-01$ & $4.6582 e+00$ \\
\hline$: 29$ & IHTO000002 & 225.7362 & 228.8612 & 227.5136 & $5.7892 e-01$ & $4.6889 \mathrm{e}+00$ \\
\hline$: 0$ & IHT000003 & 228.2076 & 230.5514 & 229.5319 & $5.4289 e-01$ & $4.6988 e+00$ \\
\hline 11 & IHTO000004 & 223.9596 & 226.6940 & 225.3112 & $5.7160 e-01$ & $4.6783 e+00$ \\
\hline.$\vdots 2$ & IHT000005 & 221.6496 & 223.9934 & 222.9112 & $5.5413 e-01$ & $4.6668 \mathrm{e}+00$ \\
\hline$:: 3$ & IHT000006 & 215.5987 & 217.9424 & 216.9268 & $5.6359 \mathrm{e}-01$ & $4.6385 \mathrm{e}+00$ \\
\hline .34 & IHTO00007 & 225.3306 & 227.6743 & 226.4671 & $5.9932 e-01$ & $4.6839 \mathrm{e}+00$ \\
\hline 5 & IHT000008 & 222.7389 & 225.0826 & 223.9030 & $5.7312 e-01$ & $4.6715 e+00$ \\
\hline$\therefore 6$ & IHTO00009 & 222.7276 & 225.6573 & 224.0479 & $6.4785 e-01$ & $4.6722 e+00$ \\
\hline.$\$ 7$ & IHTO00010 & 54.0377 & 56.7721 & 55.5025 & $5.7079 e-01$ & $4.1374 e+00$ \\
\hline$: 38$ & IHTO00011 & 223.0807 & 225.6197 & 224.3971 & $5.7292 e-01$ & $4.6739 e+00$ \\
\hline$\therefore 39$ & IHT000012 & 219.1331 & 221.8675 & 220.4026 & $5.8958 e-01$ & $4.6549 \mathrm{e}+00$ \\
\hline$\therefore 0$ & IHT000013 & 158.78 .91 & 161.3281 & 159.7070 & $5.6943 e-01$ & $4.4001 e+00$ \\
\hline$\therefore 4$ & IHTO00014 & 219.5538 & 222.6788 & 220.7373 & $5.9896 e-01$ & $4.6564 \mathrm{e}+00$ \\
\hline$: 42$ & IHTO00015 & 163.5667 & 166.3011 & 164.7191 & $5.4140 e-01$ & $4.4185 e+00$ \\
\hline$\therefore 4$ & IHT000016 & 151.9982 & 154.5372 & 153.4122 & $5.2432 e-01$ & $4.3776 e+00$ \\
\hline$\because 4$ & IHT000017 & 182.6097 & 185.7347 & 184.3441 & $6.9770 e-01$ & $4.4954 \mathrm{e}+00$ \\
\hline$\therefore 45$ & IHTO00018 & 183.9168 & 187.0418 & 185.8739 & $7.0420 e-01$ & $4.5017 e+00$ \\
\hline 146 & IHTO00019 & 178.2827 & 181.4077 & 179.9898 & $6.9789 e-01$ & $4.4777 e+00$ \\
\hline$\therefore 7$ & IHTO00020 & 183.5412 & 186.4708 & 185.1075 & $6.7944 e-01$ & $4.4985 e+00$ \\
\hline .83 & IHTO00021 & -3.3992 & -0.0789 & -1.6257 & $7.3169 e-01$ & $4.1000 e+00$ \\
\hline . 99 & IHT000022 & 158.2895 & 160.6333 & 159.4028 & $5.6393 e-01$ & $4.3990 \mathrm{e}+00$ \\
\hline$: 50$ & IHTO00023 & 179.9504 & 182.2942 & 181.0988 & $4.9227 e-01$ & $4.4822 e+00$ \\
\hline$: 51$ & IHTO00024 & 144.3773 & 146.7210 & 145.5531 & $5.2421 \mathrm{e}-01$ & $4.3507 e+00$ \\
\hline$\therefore 52$ & IHT000025 & 179.3532 & 181.6970 & 180.3922 & $5.4310 e-01$ & $4.4793 e+00$ \\
\hline$: 53$ & IHT000026 & 181.4979 & 184.4276 & 182.6581 & $6.2566 e-01$ & $4.4885 e+00$ \\
\hline$: 54$ & IHTO00027 & 154.8377 & 157.9627 & 156.0605 & $6.9901 e-01$ & $4.3870 e+00$ \\
\hline$: 55$ & IHT000028 & 157.1552 & 160.4755 & 158.6513 & $7.5139 e-01$ & $4.3963 e+00$ \\
\hline
\end{tabular}




\begin{tabular}{|c|c|c|c|c|c|}
\hline$\because$ st: Aug17_06 & sum2 & Date: Fri & Aug 1701 & $11: 44 \quad 1990$ & Page: 4 \\
\hline \# Name & Min & $\operatorname{Max}$ & Ave & SD & Uncert \\
\hline 156 IHTO00029 & 180.7918 & 183.7215 & 182.0809 & $7.0144 e-01$ & $4.4861 e+00$ \\
\hline 157 IHTO000030 & 184.1609 & 187.0906 & 185.4696 & $7.3319 e-01$ & $4.5000 e+00$ \\
\hline$: 58$ IHT000031 & 181.2275 & 184.1572 & 182.7236 & $7.2931 e-01$ & $4.4887 e+00$ \\
\hline :59 IHTO00032 & 183.3045 & 186.4295 & 185.0429 & $6.6877 e-01$ & $4.4982 e+00$ \\
\hline $100 \quad$ IHTO000033 & 183.1318 & 186.2568 & 184.9951 & $6.7221 e-01$ & $4.4980 e+00$ \\
\hline 161 IHTO000034 & 161.1253 & 163.6644 & 162.5472 & $5.4109 e-01$ & $4.4105 e+00$ \\
\hline$\because 2$ IHT000035 & 212.9808 & 215.7152 & 214.2815 & $6.3456 e-01$ & $4.6262 e+00$ \\
\hline$: 63$ IHT000036 & 159.4013 & 161.7450 & 160.6240 & $4.7484 e-01$ & $4.4034 e+00$ \\
\hline
\end{tabular}




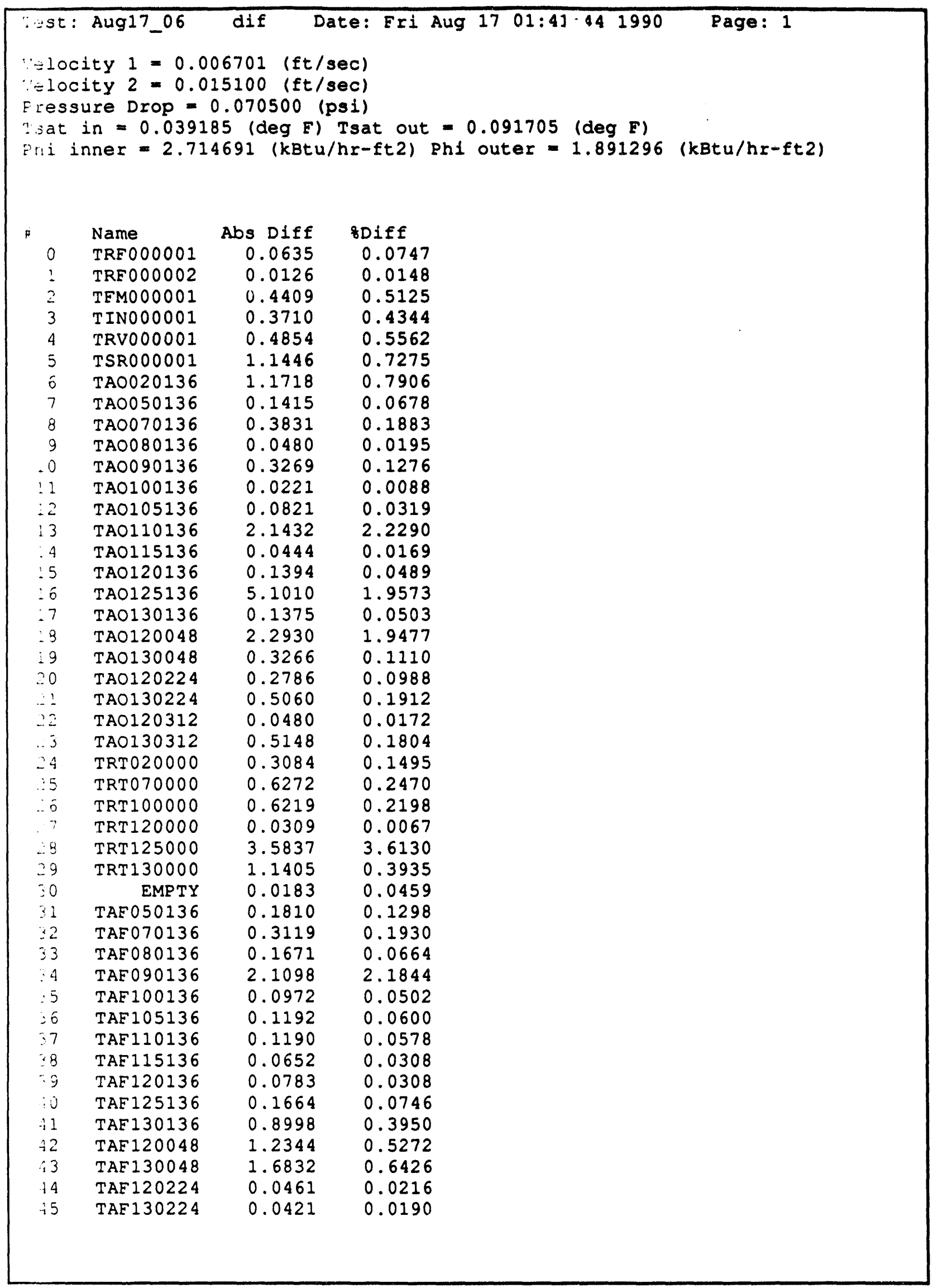




\begin{tabular}{|c|c|c|c|c|}
\hline$\therefore$ st: & Aug 17_06 & dif & ate: Fri Aug $1701: 41: 441990$ & Page: 2 \\
\hline$\sharp$ & Name & Abs Diff & 8Diff & \\
\hline 46 & EMPTY & 0.0092 & 0.0241 & \\
\hline 47 & EMPTY & 0.0046 & 0.0115 & \\
\hline 48 & TAI130048 & 0.0443 & 0.0161 & \\
\hline 49 & TAI 130136 & 0.8793 & 0.3139 & \\
\hline 50 & TAI130224 & 0.4193 & 0.1484 & \\
\hline$=1$ & TAI 130312 & 0.3696 & 0.1363 & \\
\hline$\because 2$ & TRR130000 & 0.8921 & 0.6156 & \\
\hline 33 & TSI000001 & 0.4627 & 0.7694 & \\
\hline 34 & TSI000002 & 0.3894 & 0.4371 & \\
\hline$\equiv 5$ & TSO000001 & 0.1819 & 0.3175 & \\
\hline 0 & TSO000002 & 0.0465 & 0.0604 & \\
\hline 57 & TAF020136 & 0.3711 & 0.3547 & \\
\hline 58 & TAF 120312 & 0.8934 & 0.3985 & \\
\hline 59 & TAE 130312 & 0.3034 & 0.1234 & \\
\hline 60 & EMPTY & 0.0061 & 0.1474 & \\
\hline 01 & EMPTY & 0.0058 & 0.1409 & \\
\hline 62 & EMPTY & 0.0056 & 0.1367 & \\
\hline 13 & EMPTY & 0.0054 & 0.1321 & \\
\hline$\therefore A$ & EMPTY & 0.0007 & 0.0355 & \\
\hline$\because 5$ & EMPTY & 0.0003 & 0.0151 & \\
\hline 06 & EMPTY & 0.0013 & 0.0652 & \\
\hline 67 & EMPTY & 0.0007 & 0.0349 & \\
\hline 38 & EMPTY & 0.0004 & 0.0199 & \\
\hline 89 & EMPTY & 0.0002 & 0.0099 & \\
\hline 70 & EMPTY & 0.0010 & 0.0493 & \\
\hline 71 & EMPTY & 0.0017 & 0.0833 & \\
\hline 72 & EMPTY & 0.0007 & 0.0342 & \\
\hline 73 & EMPTY & 0.0005 & 0.0243 & \\
\hline 74 & EMPTY & 0.0015 & 0.0725 & \\
\hline 75 & EMPTY & 0.0011 & 0.0529 & \\
\hline 76 & EMPTY & 0.0002 & 0.0096 & \\
\hline-7 & EMPTY & 0.0019 & 0.0906 & \\
\hline$\cdots$ & EMPTY & 0.0015 & 0.0711 & \\
\hline 79 & EMPTY & 0.0018 & 0.0846 & \\
\hline$\$ 0$ & PAN020136 & 1.2100 & 13.2691 & \\
\hline$\because 1$ & PANO50136 & 1.7395 & 9.9236 & \\
\hline 92 & PAN070136 & 1.5610 & 9.7093 & \\
\hline 53 & PAN080136 & 1.9180 & 101.0484 & \\
\hline 84 & PAN090136 & 0.5005 & 14.9738 & \\
\hline 35 & PAN100136 & 2.3392 & 23.2499 & \\
\hline 36 & PAN105136 & 0.6927 & 84.1575 & \\
\hline 97 & PAN110136 & 0.0916 & 9.0122 & \\
\hline 98 & PAN115136 & 0.4944 & 16.0109 & \\
\hline$\because 9$ & PAN120136 & 0.2106 & 44.7419 & \\
\hline, 0 & PAN125136 & 0.0855 & 4.1858 & \\
\hline 41 & PAN130136 & 0.6057 & 43.7234 & \\
\hline$j 2$ & PAN120048 & 0.5295 & 11.5999 & \\
\hline 93 & PAN130048 & 3.3753 & 30.5678 & \\
\hline 94 & PAN 120224 & 0.3143 & 11.2483 & \\
\hline 5 & PAN1 30224 & 0.7706 & 131.1213 & \\
\hline 36 & PAN120312 & 1.2832 & 23.9457 & \\
\hline 97 & PAN 130312 & 1.0285 & 54.2315 & \\
\hline 38 & PABO000136 & 0.0266 & 0.0658 & \\
\hline 99 & PAB100136 & 0.0421 & 0.1018 & \\
\hline 100 & PAB125136 & 0.0631 & 0.1530 & \\
\hline
\end{tabular}




\begin{tabular}{|c|c|c|c|c|}
\hline$\because=3 t:$ & Aug17_06 & dif & ate: Fri Aug 17 01:41:44 1990 & Page: 3 \\
\hline$"$ & Name & Abs Diff & FDiff & \\
\hline 101 & PINO00001 & 0.0220 & 0.0549 & \\
\hline$: 32$ & PRV000001 & 0.0122 & 0.0621 & \\
\hline 103 & PSROOOOOOI & 0.1416 & 0.3472 & \\
\hline$i \supset 4$ & PFM000001 & 0.0219 & 0.1429 & \\
\hline 105 & PAN000136 & 1.9500 & 7.5205 & \\
\hline$\therefore 06$ & PEM000002 & 0.0477 & 0.3103 & \\
\hline$: 7$ & EMPTY & 0.0002 & 4.0816 & \\
\hline .98 & EMPTY & 0.0279 & 0.3688 & \\
\hline$\therefore 99$ & EMPTY & 0.0003 & 6.8182 & \\
\hline$:: 0$ & EMPTY & 0.0000 & 0.0000 & \\
\hline$\therefore 1$ & EMPTY & 0.0001 & 100.0000 & \\
\hline$:: 2$ & VPW000001 & 0.0078 & 0.0044 & \\
\hline$:: 3$ & VPWO00002 & 0.0429 & 0.0206 & \\
\hline$\therefore: 4$ & VPW000003 & 0.0899 & 0.0350 & \\
\hline$:: 5$ & VPW000004 & 0.0625 & 0.0342 & \\
\hline$:: 6$ & VPW000005 & 0.3593 & 0.1477 & \\
\hline 127 & VPW000006 & 0.0391 & 0.0151 & \\
\hline$\therefore: B$ & IPW000001 & 23.4671 & 2.4511 & \\
\hline$\therefore: 9$ & IPW000002 & 1.0544 & 0.0476 & \\
\hline$\therefore 0$ & IPW000003 & 21.7617 & 0.9722 & \\
\hline 221 & IPW000004 & 5.2832 & 0.8062 & \\
\hline 1.22 & IPW000005 & 0.2245 & 0.0518 & \\
\hline 2.23 & IPW000006 & 2.0410 & 0.4564 & \\
\hline$? .4$ & EMPTY & 0.0001 & 0.5319 & \\
\hline .25 & EMPTY & 0.0002 & 1.5748 & \\
\hline$: 26$ & EMPTY & 0.0018 & 11.6883 & \\
\hline$\therefore .7$ & EMPTY & 0.0008 & 4.9383 & \\
\hline$: 28$ & IHTO00001 & 0.1133 & 0.0513 & \\
\hline$\because 29$ & IHTO00002 & 0.0000 & 0.0000 & \\
\hline$: 30$ & IHTO00003 & 0.1133 & 0.0493 & \\
\hline$\because 1$ & IHTOOOOO4 & 0.1875 & 0.0831 & \\
\hline .32 & IHTO00005 & 0.1875 & 0.0840 & \\
\hline 13 & IHT000006 & 0.1445 & 0.0666 & \\
\hline$\therefore 4$ & IHT000007 & 0.1915 & 0.0845 & \\
\hline$: 25$ & IHTO00008 & 0.1406 & 0.0628 & \\
\hline$\therefore 6$ & IHTO00009 & 0.1797 & 0.0801 & \\
\hline$\therefore 7$ & IHTO00010 & 0.0156 & 0.0281 & \\
\hline$\therefore 3$ & IHTO00011 & 0.0430 & 0.0192 & \\
\hline$\because 9$ & IHTO00012 & 0.1172 & 0.0531 & \\
\hline$: 40$ & IHTO00013 & 0.1563 & 0.0978 & \\
\hline .1 & IHTO00014 & 0.1211 & 0.0549 & \\
\hline$: 42$ & IHTO00015 & 0.1445 & 0.0876 & \\
\hline$: 43$ & IHTO00016 & 0.0625 & 0.0407 & \\
\hline$\therefore 4$ & IHTO00017 & 0.1328 & 0.0721 & \\
\hline$\therefore 45$ & IHT000018 & 0.0273 & 0.0147 & \\
\hline .16 & IHTO00019 & 0.0547 & 0.0304 & \\
\hline$\therefore: 7$ & IHTO00020 & 0.0508 & 0.0274 & \\
\hline$: 48$ & IHTO00021 & 0.1054 & 6.9328 & \\
\hline$\therefore 9$ & IHT000022 & 0.1133 & 0.0710 & \\
\hline 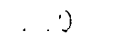 & IHT000023 & 0.1718 & 0.0948 & \\
\hline .51 & IHTO00024 & 0.1914 & 0.1313 & \\
\hline$: 52$ & IHT000025 & 0.1055 & 0.0584 & \\
\hline$: 53$ & IHT 000026 & 0.0859 & 0.0470 & \\
\hline$: 34$ & IHTO00027 & 0.0546 & 0.0350 & \\
\hline$\therefore \lesssim 5$ & IHTO00028 & 0.1601 & 0.1010 & \\
\hline
\end{tabular}




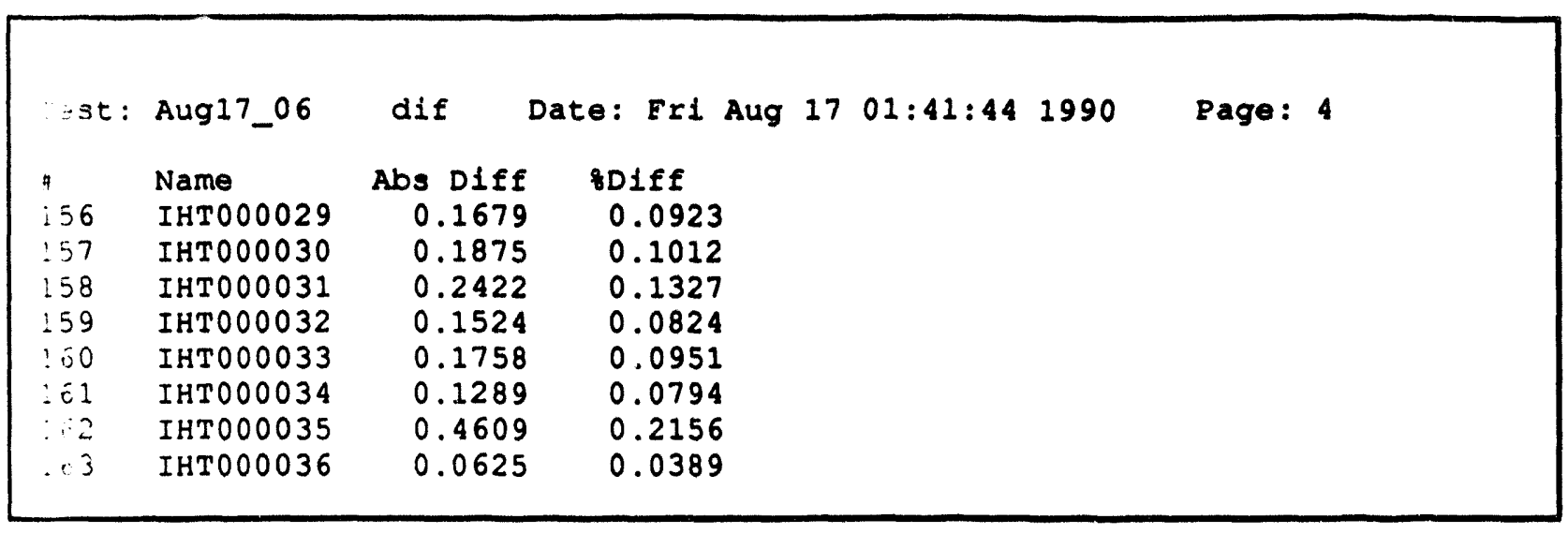




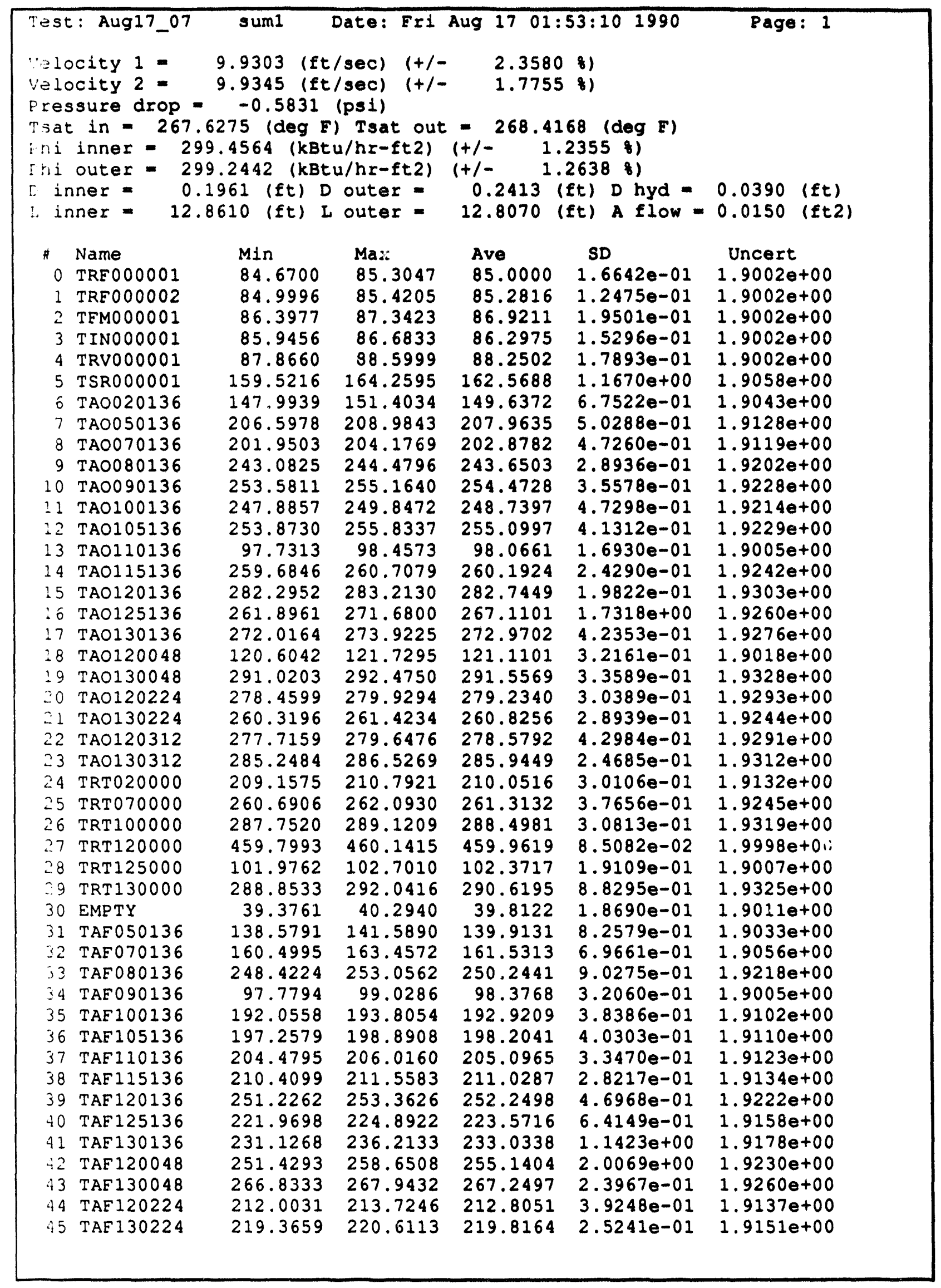




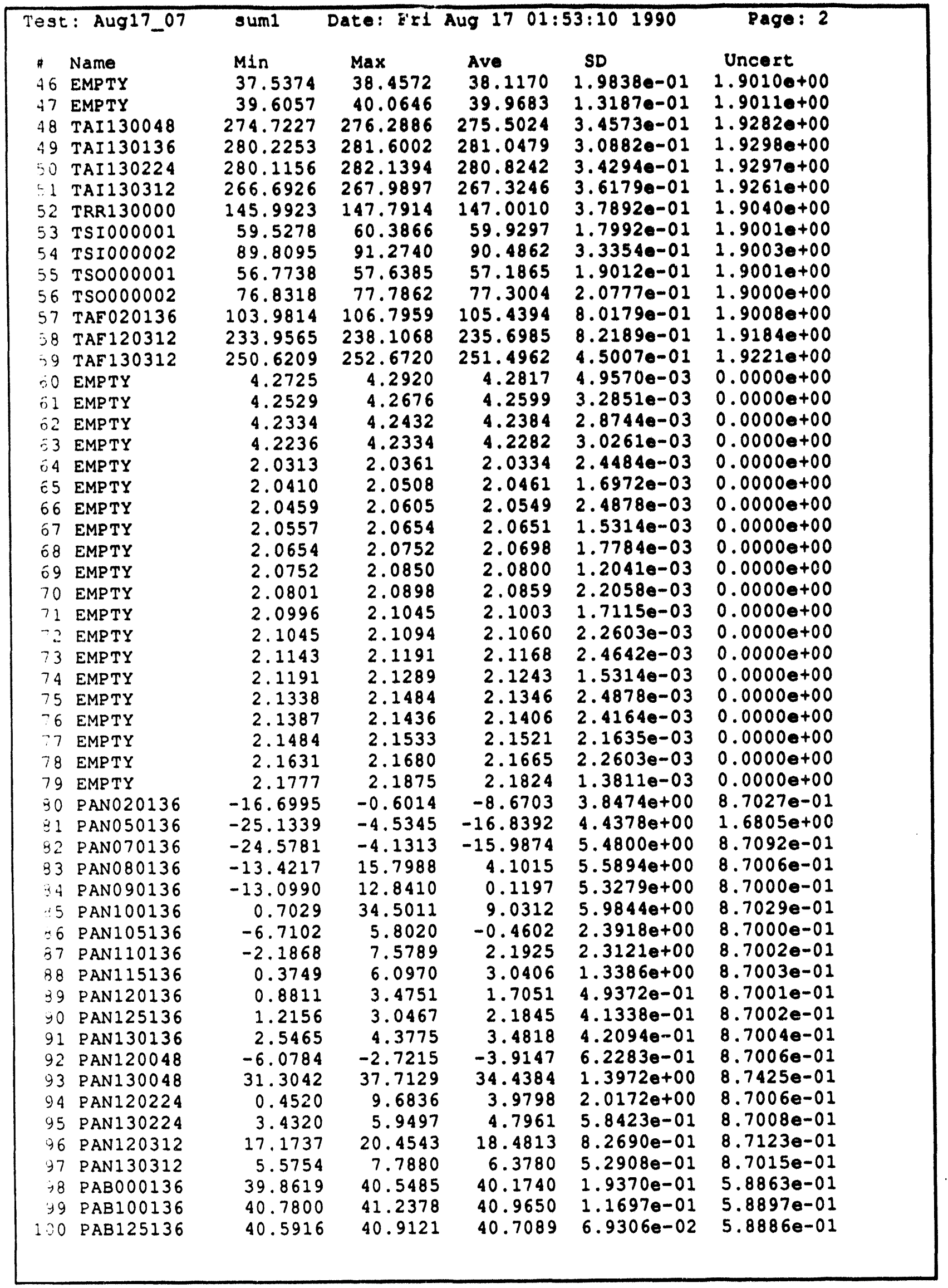




\begin{tabular}{|c|c|c|c|c|c|c|}
\hline \multicolumn{2}{|c|}{ Test: Aug17_07 } & sum 1 & Date: FE1 & Aug 170 & $3: 101990$ & Page: 3 \\
\hline " & Name & Min & $\operatorname{Max}$ & Ave & 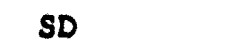 & VIC \\
\hline 201 & PINO00001 & 39.1042 & 40.5690 & 39.8293 & $3.8477 e-01$ & $7.5658 e-01$ \\
\hline 122 & PRV000001 & 19.5965 & 19.8406 & 19.7137 & $4.2430 e-02$ & $7.5162 e-01$ \\
\hline 13 & PSR000001 & 39.5665 & 39.9327 & 39.7521 & $7.49660-02$ & $7.5656 e-01$ \\
\hline 104 & PEM000001 & 14.8245 & 15.2822 & 15.0332 & $1.0792 e-01$ & $5.01410-01$ \\
\hline$: 05$ & PANO00136 & -26.0482 & -4.0755 & -16.1422 & $5.87740+00$ & $4.9202 e+00$ \\
\hline$: 26$ & PFMO00002 & 14.9169 & 15.1854 & 15.0461 & $7.3435 \bullet-02$ & $2.03510-01$ \\
\hline$: 27$ & EMPTY & 0.0049 & 0.0049 & 0.0049 & $0.0000 e+00$ & $0.0000 \theta+00$ \\
\hline 108 & EMPTY & -10.0000 & -0.9180 & -7.7484 & $1.7912 e+00$ & $0.00000+00$ \\
\hline$: 09$ & EMPTY & 0.0000 & 0.0049 & 0.0045 & $1.3381 e-03$ & $0.0000 e+00$ \\
\hline$: 10$ & EMPTX & -10.0000 & -10.0000 & -10.0000 & $0.0000 e+00$ & $0.0000 e+00$ \\
\hline$a: 1$ & EMPTY & -0.0049 & 0.0000 & -0.0001 & $6.9053 e-04$ & $0.0000 e+00$ \\
\hline $1: 2$ & VPWOO0001 & 177.4151 & 178.0011 & 177.6846 & $1.2409 e-01$ & $1.3389 e+00$ \\
\hline 113 & VPWOOOOOO2 & 207.5233 & 207.9139 & 207.7460 & $1.0447 e-01$ & $1.4865 e+00$ \\
\hline 114 & VPW000003 & 256.8021 & 257.1927 & 256.9349 & $1.2124 e-01$ & $1.7415 \bullet+00$ \\
\hline$:: 5$ & VPWO00004 & 182.5984 & 183.1843 & 182.7782 & $1.3578 e-01$ & $1.36340+00$ \\
\hline$\therefore: 6$ & VPWOOOOOO5 & 242.9725 & 244.1444 & 243.5117 & $3.19500-01$ & $1.6706 e+00$ \\
\hline .17 & VPWO00006 & 258.4172 & 259.0032 & 258.6632 & $1.2974 \theta-01$ & $1.7506 e+00$ \\
\hline $1: 8$ & IPWO00001 & 939.8282 & 949.5900 & 944.5140 & $2.08710+00$ & $1.10130+01$ \\
\hline 119 & IPWO00002 & 2205.7600 & 2219.4189 & 2212.0825 & $3.9137 e+00$ & $1.9261 e+01$ \\
\hline 120 & IPWO00003 & 2252.2498 & 2260.0498 & 2256.2280 & $2.2958 \bullet+00$ & $1.9578 e+01$ \\
\hline 121 & IPWO000004 & 650.2122 & 653.1418 & 651.6284 & $1.1302 \theta+00$ & $5.5227 e+00$ \\
\hline 122 & IPWO00005 & 429.8135 & 432.2547 & 431.1609 & $6.8736 \theta-01$ & $3.9499 e+00$ \\
\hline$: 23$ & IPWO000006 & 446.8149 & 447.7915 & 447.2346 & $2.6118 \theta-01$ & $4.05990+00$ \\
\hline$\therefore 24$ & EMPTY & -0.0244 & -0.0098 & -0.0187 & $3.3728 \theta-03$ & $0.0000 e+00$ \\
\hline$: 25$ & EMPTY & -0.0195 & -0.0049 & -0.0120 & $3.3029 \theta-03$ & $0.0000 e+00$ \\
\hline 126 & EMPTY & -0.0244 & -0.0098 & -0.0139 & $3.0180 e-03$ & $0.0000 e+00$ \\
\hline 127 & EMPTY & -0.0244 & -0.0098 & -0.0169 & $3.4470-03$ & $0.00000+00$ \\
\hline$\therefore 8$ & IHTO000001 & 218.9528 & 221.8825 & 220.7888 & $5.7318 \theta-01$ & $4.6567 e+00$ \\
\hline$\therefore 99$ & IHTOOOOOO2 & 225.5408 & 228.4705 & 227.2636 & $5.7817 e-01$ & $4.6877 e+00$ \\
\hline$\therefore: 0$ & IHTO00003 & 227.8170 & 230.3561 & 229.3561 & $5.87450-01$ & $4.6979 e+00$ \\
\hline 131 & IHT000004 & 223.7643 & 226.4986 & 225.2448 & $6.19000-01$ & $4.67800+00$ \\
\hline$: 32$ & IHTO00005 & 221.4543 & 224.1887 & 222.9229 & $5.9874 e-01$ & $4.6668 e+00$ \\
\hline$: 33$ & IHTO000006 & 215.4034 & 218.5284 & 216.9932 & $6.4357 e-01$ & $4.6388 e+00$ \\
\hline$: 34$ & IHT000007 & 225.1352 & 227.8696 & 226.5257 & $6.0956 e-01$ & $4.6842 \theta+00$ \\
\hline$:: 5$ & IHTO00008 & 222.7389 & 225.4733 & 223.9538 & $5.8299 e-01$ & $4.6718 e+00$ \\
\hline$:: 6$ & IHTO00009 & 222.3370 & 225.4620 & 224.0791 & $6.3606 e-01$ & $4.6724 e+00$ \\
\hline$: \div 7$ & IHTO00010 & 53.6471 & 56.5768 & 55.2525 & $5.4717 \theta-01$ & $4.13710+00$ \\
\hline$: \leq 8$ & IHT000011 & 222.2994 & 225.8150 & 224.1003 & $6.1039 e-01$ & $4.6725 e+00$ \\
\hline$: 39$ & IHTO00012 & 218.1566 & 221.6722 & 220.1565 & $6.3204 \theta-01$ & $4.6537 e+00$ \\
\hline$: 40$ & IHTO00013 & 157.8125 & 161.1328 & 159.5586 & $6.0414 \theta-01$ & $4.39958+00$ \\
\hline$\therefore 2$ & IHTO00014 & 218.5772 & 222.0929 & 220.4444 & $6.8021 e-01$ & $4.6551 e+00$ \\
\hline$:: 2$ & IHT000015 & 163.1761 & 165.7151 & 164.6488 & $5.9085 e-01$ & $4.4182 \theta+00$ \\
\hline$: 43$ & IHTO00016 & 151.9982 & 154.7326 & 153.3106 & $5.6364 e-01$ & $4.3773 e+00$ \\
\hline$\therefore 44$ & IHTO00017 & 182.2191 & 185.3441 & 184.0589 & 6.801 & $4.4942 \theta+00$ \\
\hline$\therefore 45$ & IHTO00018 & 184.1121 & 187.4324 & 185.8505 & $6.9615 \theta-01$ & $4.5016 e+00$ \\
\hline 146 & IHT000019 & 178.8687 & 181.2124 & 179.9507 & $4.8038 \theta-01$ & $4.4775 e+00$ \\
\hline 147 & IHTO000020 & 183.9318 & 186.0802 & 185.0333 & 5.049 & $4.4982 e+00$ \\
\hline$: 48$ & IHTO00021 & -3.2039 & -0.2742 & -1.6961 & 6.350 & $4.10000+00$ \\
\hline$\therefore 19$ & IHTO00022 & 158.2895 & 160.4379 & 159.3950 & $4.7778 e-01$ & $4.3989 e+00$ \\
\hline$: 50$ & IHTO00023 & 179.9504 & 182.2942 & 181.1261 & $5.1522 e-01$ & $4.4823 e+00$ \\
\hline 151 & IHTO00024 & 144.7679 & 146.7210 & 145.6156 & $4.6456 \theta-01$ & $4.3509 e+00$ \\
\hline$: 52$ & IHTO00025 & 179.1579 & 181.5017 & 180.4274 & $4.9795 e-01$ & $4.4794 e+00$ \\
\hline 153 & IHT 000026 & 181.4979 & 184.0370 & 182.6581 & $5.3000 e-01$ & $4.4885 e+00$ \\
\hline$:>4$ & IHTO00027 & 154.8377 & 157.3768 & 156.0097 & $5.1599 e-01$ & $4.3868 e+00$ \\
\hline$\therefore 5$ & IHT000028 & 157.5458 & 160.0849 & 158.8271 & $6.0510 e-01$ & $4.3969 \theta+00$ \\
\hline
\end{tabular}




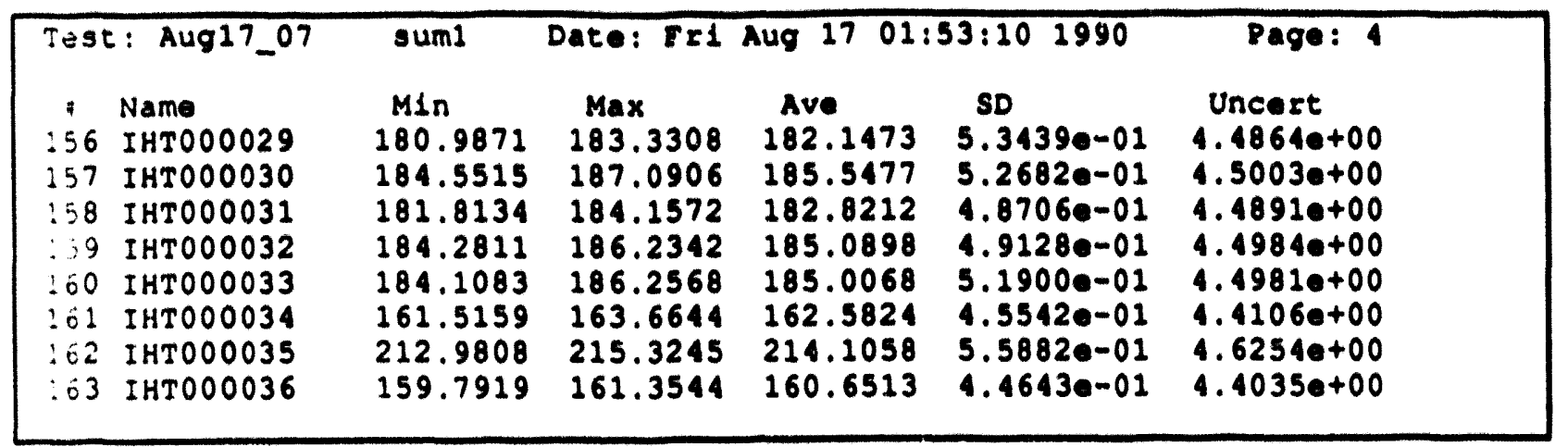




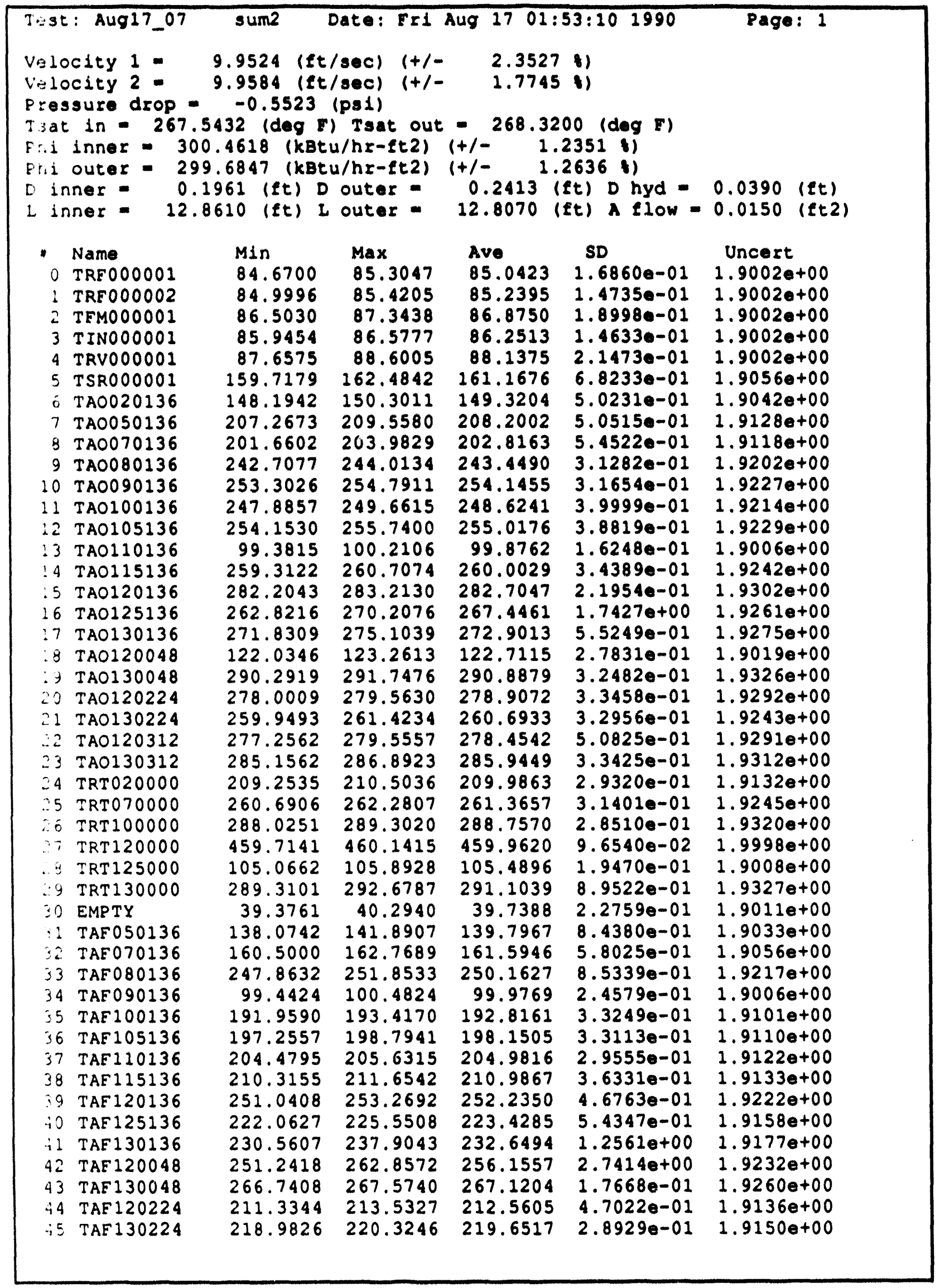




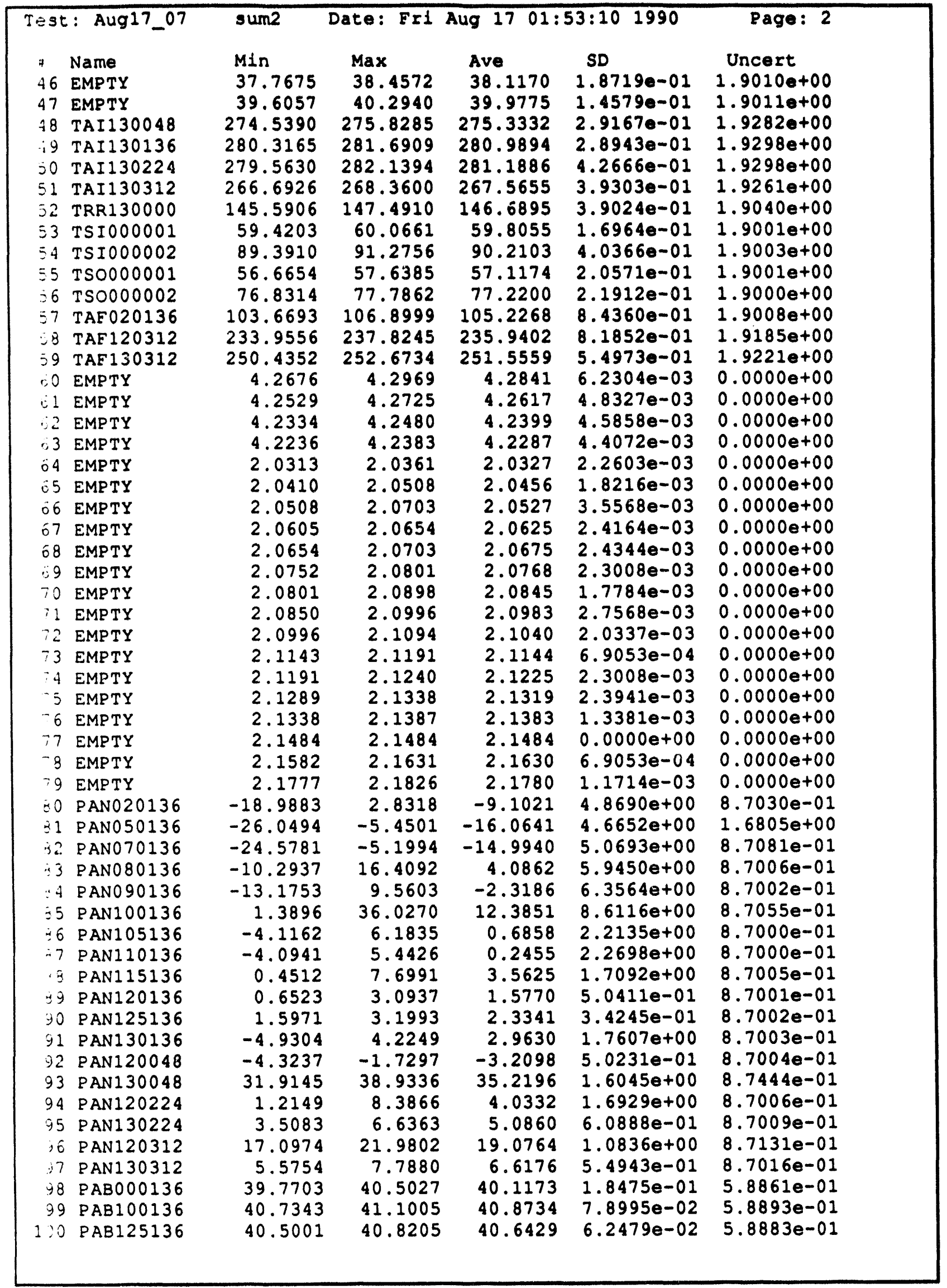




\begin{tabular}{|c|c|c|c|c|c|c|}
\hline \multicolumn{2}{|c|}{ TESt: Aug17_07 } & sum2 & \multicolumn{3}{|c|}{ Date: Fr1 Aug 17 01:53:10 1990} & Page: 3 \\
\hline 4 & Name & Min & $\operatorname{Max}$ & Ave & SD & Uncert \\
\hline 121 & PINO00001 & 39.2263 & 40.6911 & 39.9514 & $3.3671 e-01$ & $7.5662 e-01$ \\
\hline 12 & PRV000001 & 19.5965 & 19.8406 & 19.7235 & $3.4527 \bullet-02$ & $7.5162 e-01$ \\
\hline $1: 3$ & PSR000001 & 39.5665 & 39.8107 & 39.6764 & $5.0842 e-02$ & $7.5653 e-01$ \\
\hline 1.4 & PFMO00001 & 14.8245 & 15.2822 & 15.1010 & $1.1173 e-01$ & $5.0142 e-01$ \\
\hline 15 & PANO00136 & -32.9146 & 2.7910 & -15.2907 & $6.4963 e+00$ & $4.9201 e+00$ \\
\hline $1 ; 6$ & PEM000002 & 14.9169 & 15.2525 & 15.1193 & $7.8368 e-02$ & $2.0354 e-01$ \\
\hline 127 & EMPTY & 0.0049 & 0.0049 & 0.0049 & $0.0000 e+00$ & $0.0000 e+00$ \\
\hline 198 & EMPTY & -10.0000 & -0.5322 & -5.0618 & $2.91290+00$ & $0.0000 e+00$ \\
\hline 129 & EMPTY & 0.0000 & 0.0049 & 0.0045 & $1.3381 e-03$ & $0.0000 e+00$ \\
\hline $1: 0$ & EMPTY & -10.0000 & -10.0000 & -10.0000 & $0.0000 e+00$ & $0.0000 e+00$ \\
\hline 111 & EMPTY & 0.0000 & 0.0049 & 0.0001 & $6.9053 e-04$ & $0.0000 e+00$ \\
\hline $1: 2$ & VPW000001 & 177.4151 & 178.0011 & 177.7120 & $1.2627 e-01$ & $1.3390 e+00$ \\
\hline $1: 3$ & VPW000002 & 207.5233 & 207.9139 & 207.7187 & $7.8918 \theta-02$ & $1.4864 e+00$ \\
\hline $2: 4$ & VPW000003 & 256.8021 & 257.1927 & 256.9896 & $1.0410 e-01$ & $1.7417 e+00$ \\
\hline $1: 5$ & VPWO00004 & 182.5984 & 182.9890 & 182.7743 & $9.8648 e-02$ & $1.3634 e+00$ \\
\hline $1: 6$ & VPW000005 & 243.1678 & 244.5350 & 243.8476 & $3.6856 e-01$ & $1.6723 e+00$ \\
\hline $1: 7$ & VPWO00006 & 258.4172 & 258.8079 & 258.6358 & $1.0907 e-01$ & $1.7505 e+00$ \\
\hline $1: 8$ & IPW000001 & 939.8282 & 945.6852 & 942.7178 & $1.3224 \mathrm{e}+00$ & $1.1003 e+01$ \\
\hline $1: 9$ & IPW000002 & 2215.5164 & 2219.4189 & 2217.3901 & $1.4192 \mathrm{e}+00$ & $1.9299 \theta+01$ \\
\hline 120 & IPW000003 & 2261.9998 & 2265.8999 & 2263.2876 & $1.1561 \mathrm{e}+00$ & $1.9629 e+01$ \\
\hline 121 & IPWO000004 & 650.7004 & 652.1653 & 651.5796 & $3.6911 e-01$ & $5.5224 e+00$ \\
\hline 122 & IPWO00005 & 431.2782 & 434.2076 & 432.6842 & $6.4410 e-01$ & $3.9603 e+00$ \\
\hline 123 & IPW000006 & 447.7915 & 449.7446 & 448.8166 & $3.7239 e-01$ & $4.0708 \mathrm{e}+00$ \\
\hline 124 & EMPTY & -0.0293 & -0.0098 & -0.0178 & $3.7899 e-03$ & $0.0000 e+00$ \\
\hline $1 \therefore 5$ & EMPTY & -0.0195 & -0.0049 & -0.0124 & $2.8266 e-03$ & $0.0000 e+00$ \\
\hline 1.26 & EMPTY & -0.0244 & -0.0049 & -0.0138 & $3.6500 e-03$ & $0.0000 e+00$ \\
\hline $1 . .7$ & EMPTY & -0.0244 & -0.0098 & -0.0169 & $2.8266 e-03$ & $0.0000 e+00$ \\
\hline 128 & IHTO00001 & 219.5387 & 221.6872 & 220.7497 & $5.5943 e-01$ & $4.6565 e+00$ \\
\hline $1: 9$ & IHT000002 & 225.9315 & 228.2752 & 227.2441 & $5.6914 e-01$ & $4.6876 \mathrm{e}+00$ \\
\hline $1: 0$ & IHT000003 & 228.0123 & 230.1608 & 229.2897 & $5.1024 e-01$ & $4.6976 e+00$ \\
\hline$\because:$ & IHTO00004 & 223.9596 & 226.4986 & 225.1706 & $5.3525 e-01$ & $4.6776 \mathrm{e}+00$ \\
\hline 232 & IHTO00005 & 221.4543 & 223.7981 & 222.7706 & $5.0503 e-01$ & $4.6661 \mathrm{e}+00$ \\
\hline 233 & IHTO00006 & 215.5987 & 217.7471 & 216.8213 & $4.8937 \theta-01$ & $4.6380 e+00$ \\
\hline $2: 4$ & IHTOOOOOO & 224.7446 & 227.4790 & 226.3343 & $5.0841 e-01$ & $4.6832 e+00$ \\
\hline 255 & IHTO00008 & 222.1529 & 224.6920 & 223.7624 & $5.2283 e-01$ & $4.6709 e+00$ \\
\hline $2: 6$ & IHTO00009 & 222.1417 & 225.2667 & 223.8955 & $5.7384 e-01$ & $4.6715 e+00$ \\
\hline 137 & IHTO00010 & 54.4283 & 56.5768 & 55.5299 & $5.0649 e-01$ & $4.1374 e+00$ \\
\hline$\therefore 3$ & IHTO00011 & 223.2760 & 225.6197 & 224.3463 & $5.2384 e-01$ & $4.6737 e+00$ \\
\hline$\therefore: 9$ & IHTO00012 & 219.3284 & 221.4769 & 220.3792 & $4.9895 e-01$ & $4.6547 e+00$ \\
\hline$:: 0$ & IHTO00013 & 158.9844 & 160.9375 & 159.8203 & $4.5684 e-01$ & $4.4005 e+00$ \\
\hline $1 \therefore 1$ & IHTO000014 & 219.7491 & 222.2882 & 220.9873 & $5.8475 e-01$ & $4.6576 \mathrm{e}+00$ \\
\hline 142 & IHTO00015 & 163.9573 & 165.7151 & 164.8910 & $4.7234 e-01$ & $4.4192 e+00$ \\
\hline$\therefore 3$ & IHT000016 & 152.3888 & 154.7326 & 153.5997 & $5.3670 e-01$ & $4.3783 e+00$ \\
\hline$\therefore 14$ & IHTO00017 & 183.1956 & 185.3441 & 184.2816 & $5.6519 e-01$ & $4.4951 e+00$ \\
\hline $1: 5$ & IHT000018 & 184.5027 & 187.2371 & 185.8973 & $5.6636 e-01$ & $4.5018 e+00$ \\
\hline 146 & IHTO00019 & 178.8687 & 181.0171 & 179.9937 & $5.7529 e-01$ & $4.4777 e+00$ \\
\hline 147 & IHT000020 & 183.9318 & 186.0802 & 185.1661 & $5.3005 \theta-01$ & $4.4987 e+00$ \\
\hline$: 48$ & IHT000021 & -3.2039 & -0.2742 & -1.4851 & $6.6380 e-01$ & $4.1000 e+00$ \\
\hline 149 & IHT000022 & 158.4848 & 160.4379 & 159.6723 & $5.2916 e-01$ & $4.3999 e+00$ \\
\hline 150 & IHTO00023 & 180.3411 & 182.6848 & 181.2823 & $5.3770 e-01$ & $4.4829 e+00$ \\
\hline $1: 1$ & IHTO00024 & 144.7679 & 147.1116 & 145.7133 & $4.9183 e-01$ & $4.3512 e+00$ \\
\hline $2: 2$ & IHTO00025 & 179.3532 & 181.6970 & 180.5797 & $5.3676 e-01$ & $4.4801 e+00$ \\
\hline $1: 3$ & IHTO00026 & 181.6932 & 183.8417 & 182.8026 & $5.5725 e-01$ & $4.48918+00$ \\
\hline $1: 4$ & IHTO000027 & 155.0331 & 157.3768 & 156.1737 & $5.8041 e-01$ & $4.3874 e+00$ \\
\hline 155 & IHTO00028 & 157.3505 & 160.2802 & 158.7334 & $6.0982 e-01$ & $4.3965 e+00$ \\
\hline
\end{tabular}




\begin{tabular}{|c|c|c|c|c|c|c|}
\hline \multicolumn{2}{|c|}{ Test: Aug17_07 } & sum2 & Date: Fri & Aug 1701 & $53: 10 \quad 1990$ & Page: 4 \\
\hline$\$$ & Name & Min & $\operatorname{Max}$ & Ave & SD & Uncert \\
\hline 156 & IHTO000029 & 180.5965 & 183.5262 & 182.0575 & $6.0004 e-01$ & $4.4860 \mathrm{e}+00$ \\
\hline 157 & IHTO00030 & 184.1609 & 186.7000 & 185.4930 & $5.3770 e-01$ & $4.5001 e+00$ \\
\hline 158 & IHTO000031 & 181.6181 & 183.7665 & 182.7353 & $5.1151 e-01$ & $4.4888 e+00$ \\
\hline 159 & IHT 000032 & 183.6951 & 186.2342 & 185.0155 & $5.7394 e-01$ & $4.4981 e+00$ \\
\hline 160 & IHTO000033 & 183.7177 & 186.2568 & 184.9795 & $5.5833 e-01$ & $4.4980 e+00$ \\
\hline 161 & IHTO000034 & 161.5159 & 163.6644 & 162.5277 & $4.1888 \mathrm{e}-01$ & $4.4104 e+00$ \\
\hline 102 & IHTO000035 & 213.1761 & 215.5199 & 214.3479 & $5.5102 e-01$ & $4.6265 e+00$ \\
\hline 163 & IHT 000036 & 159.7919 & 161.7450 & 160.5771 & $4.4554 e-01$ & $4.4032 e+00$ \\
\hline
\end{tabular}




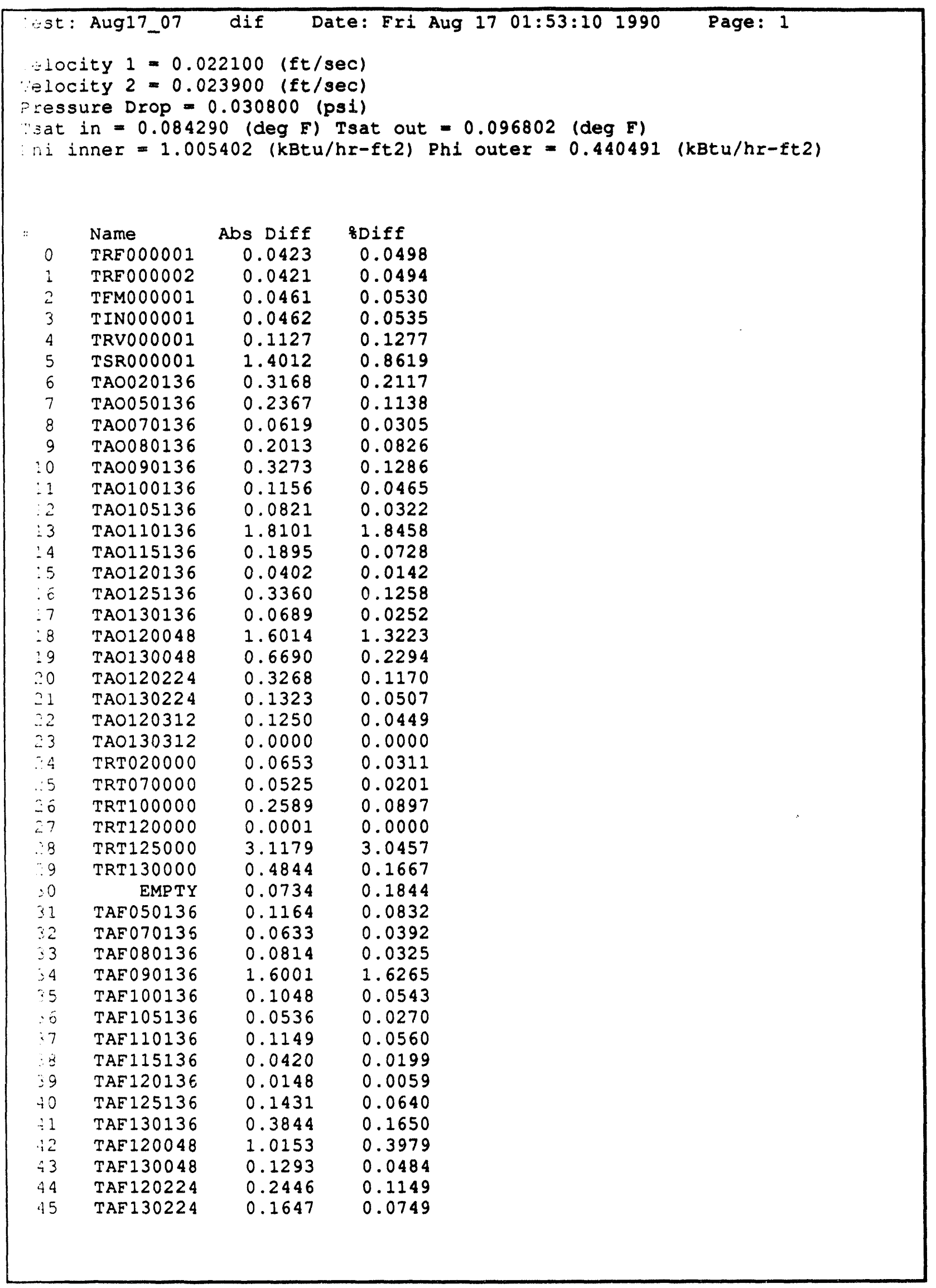




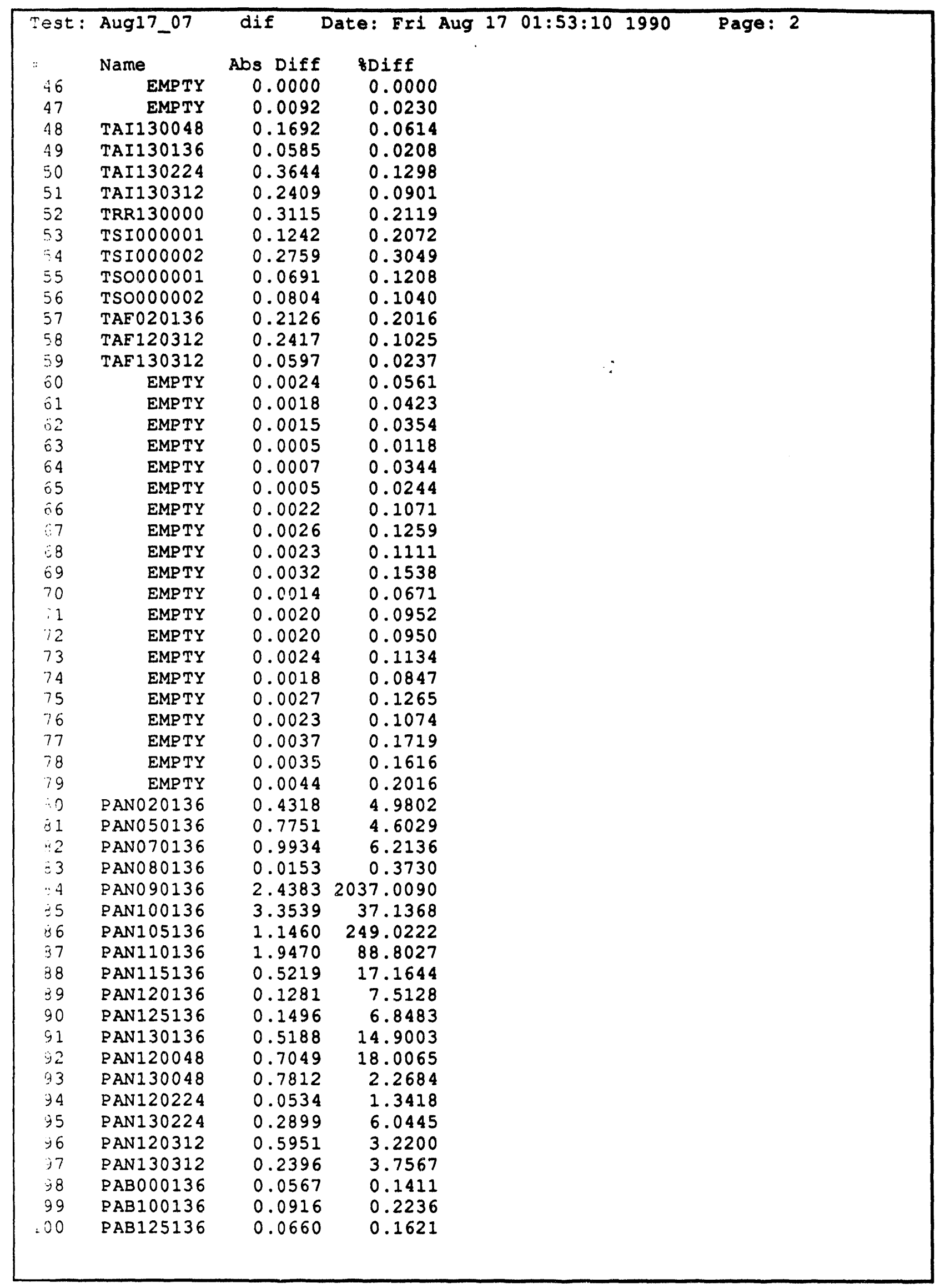




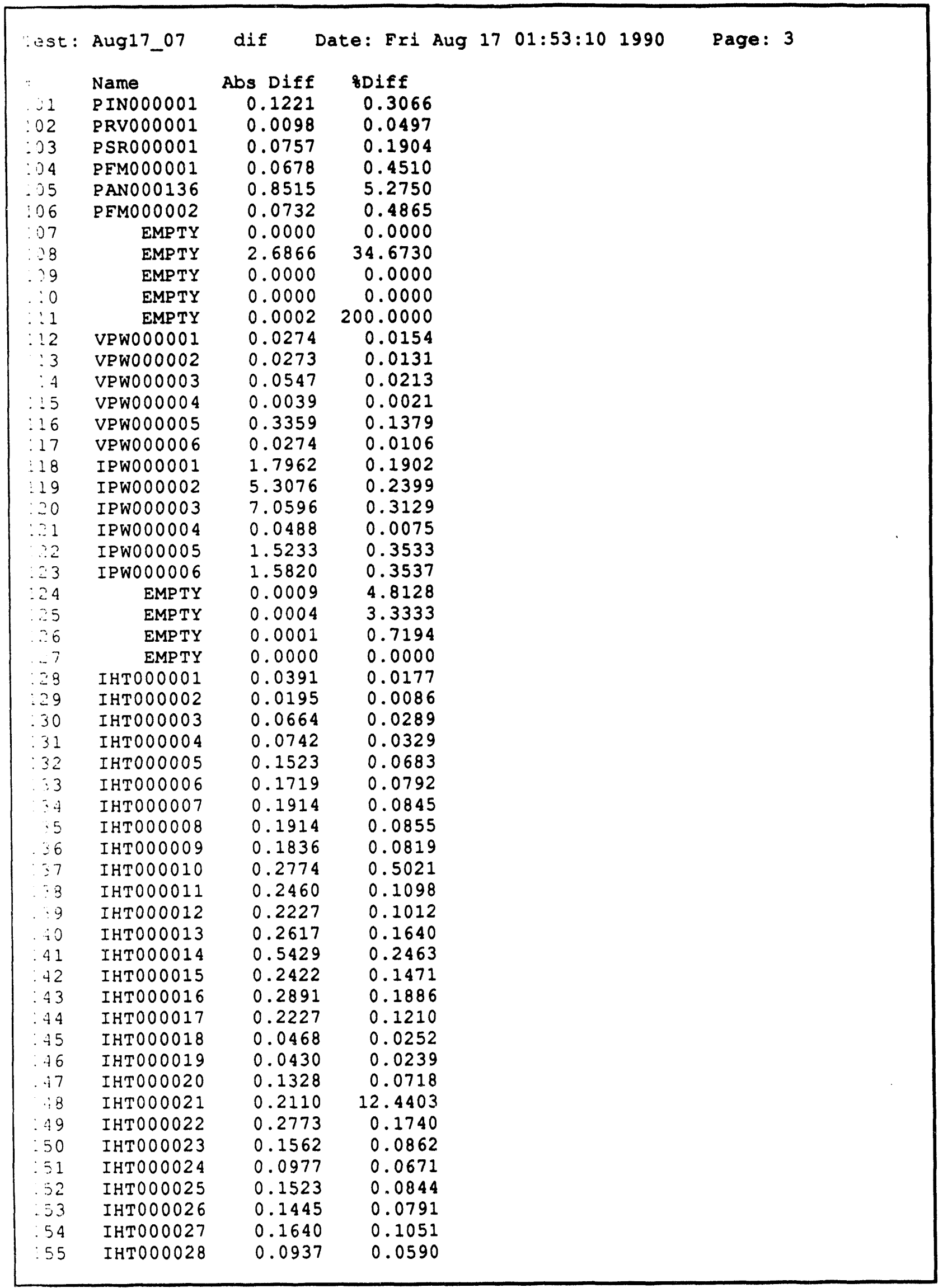




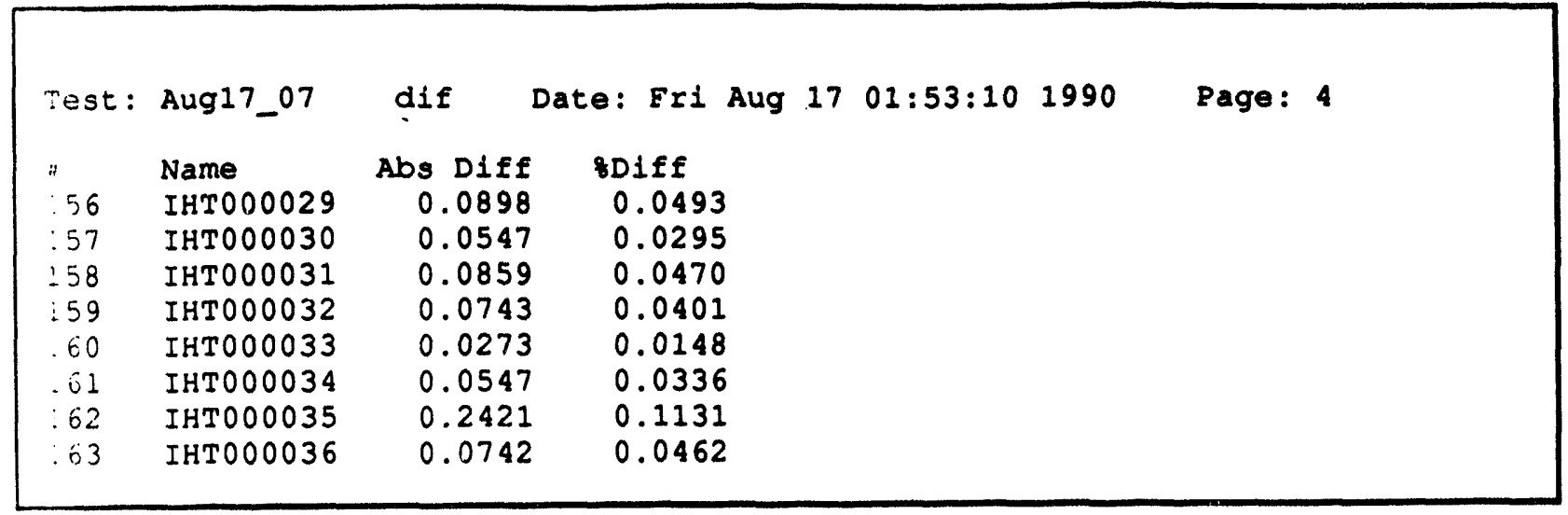




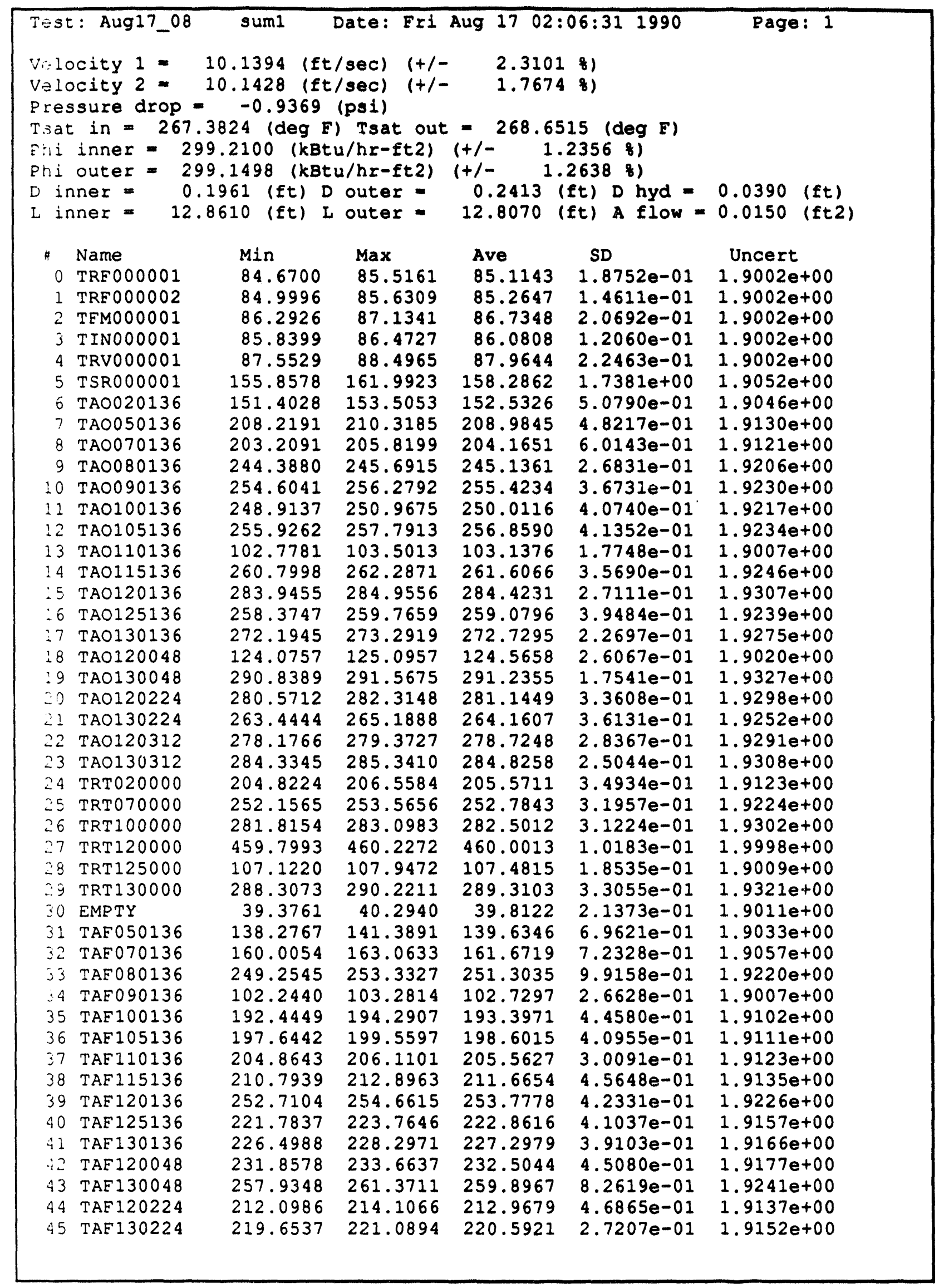




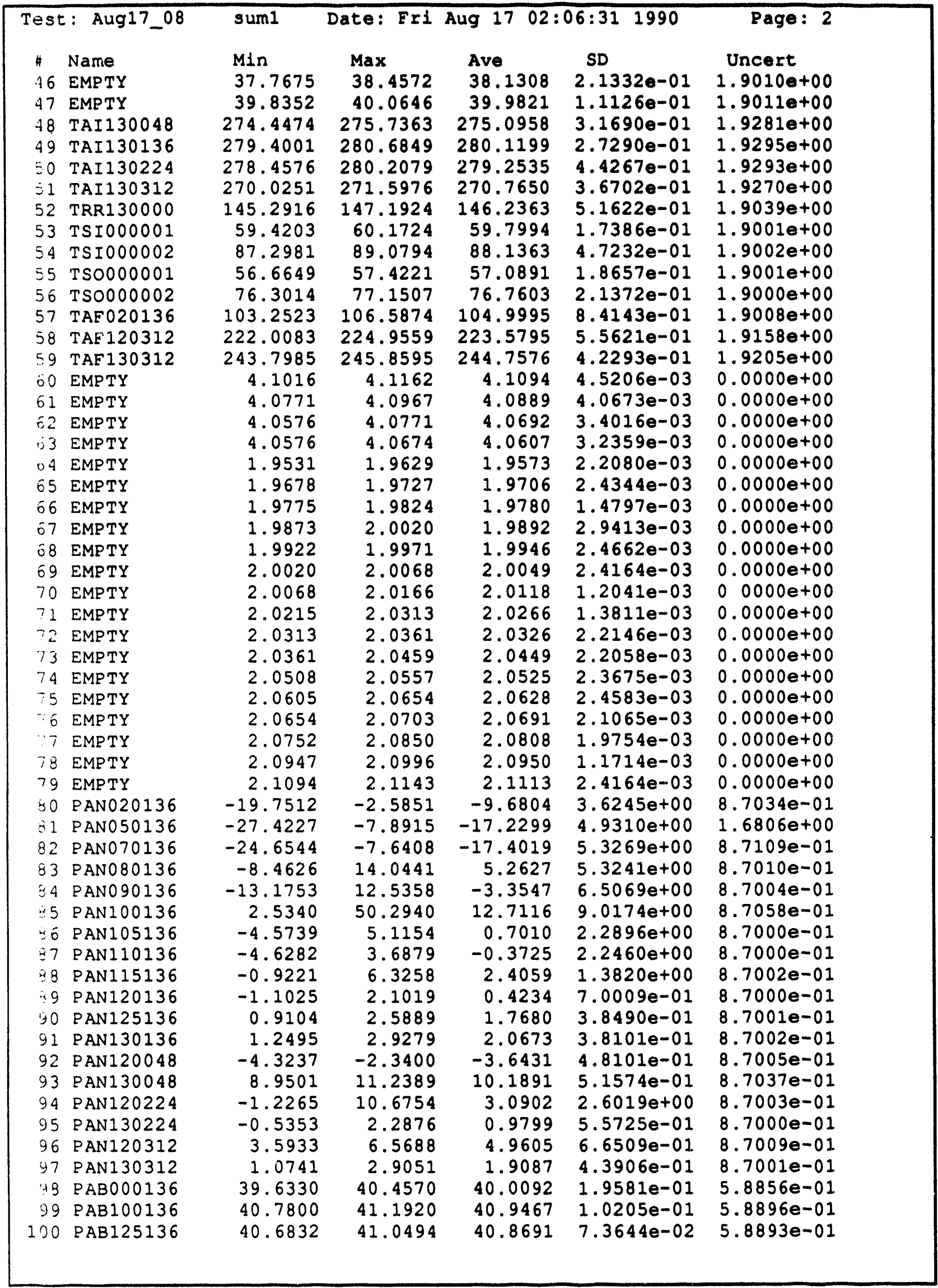




\begin{tabular}{|c|c|c|c|c|c|c|}
\hline \multicolumn{2}{|c|}{ Test: Aug17_08 } & sum 1 & Date: Er1 & Aug 170 & $: 311990$ & Page: 3 \\
\hline \# & Name & Min & $\operatorname{Max}$ & Ave & SD & Uncert \\
\hline 101 & PIN000001 & 38.9821 & 40.3249 & 39.6755 & $4.33830-01$ & $7.5653 e-01$ \\
\hline $\begin{array}{l}102 \\
103\end{array}$ & PRV000001 & 19.5965 & 19.7186 & 19.7064 & $3.6993 e-02$ & $7.5162 e-01$ \\
\hline 103 & PSR000001 & 40.2990 & 40.5431 & 40.3722 & $7.3986 e-02$ & $7.5676 e-01$ \\
\hline 104 & PFM000001 & 15.4653 & 16.0147 & 15.6796 & $1.2628 \theta-01$ & $5.0153 \theta-01$ \\
\hline $\begin{array}{l}105 \\
106\end{array}$ & RAN000136 & -33.8301 & -12.7730 & -25.9383 & $5.5755 e+00$ & $4.9204 e+00$ \\
\hline $\begin{array}{l}106 \\
107\end{array}$ & PEM000002 & 15.5713 & 15.9405 & 15.6901 & $8.07110-02$ & $2.0381 e-01$ \\
\hline $\begin{array}{l}107 \\
108\end{array}$ & EMPTY & 0.0000 & 0.0098 & 0.0049 & $9.8648 \theta-04$ & $0.0000 e+00$ \\
\hline 108 & EMPTY & -7.1143 & -0.8838 & -4.7468 & $1.4059 \theta+00$ & $0.0000 e+00$ \\
\hline 109 & EMPTY & 0.0000 & 0.0049 & 0.0047 & $9.6655 \theta-04$ & $0.0000 e+00$ \\
\hline $1: 0$ & EMPTY & -10.0000 & -10.0000 & -10.0000 & $0.0000 e+00$ & $0.0000 e+00$ \\
\hline $1: 1$ & EMPTY & 0.0000 & 0.0000 & 0.0000 & $0.0000 \theta+00$ & $0.0000 e+00$ \\
\hline 1.2 & VPW000001 & 177.4151 & 178.0011 & 177.6573 & $1.2823 e-01$ & $1.3388 e+00$ \\
\hline $\begin{array}{l}113 \\
114\end{array}$ & VPWO00002 & 207.5233 & 207.9139 & 207.7148 & $1.0052 e-01$ & $1.4864 \theta+00$ \\
\hline 114 & VPW000003 & 256.8021 & 257.1927 & 256.9857 & $1.1443 e-01$ & $1.7417 e+00$ \\
\hline $1: 5$ & VPW000004 & 182.4031 & 182.9890 & 182.6805 & $1.2534 \theta-01$ & $1.3629 e+00$ \\
\hline $1: 0$ & VPW000005 & 243.1678 & 244.9256 & 243.8359 & $4.3061 e-01$ & $1.6723 e+00$ \\
\hline $1: 7$ & VPW000006 & 258.2219 & 259.0032 & 258.6593 & $1.5565 \theta-01$ & $1.7506 e+00$ \\
\hline $1: 8$ & IPWO00001 & 939.8282 & 951.5423 & 945.1385 & $3.4168 e+00$ & $1.1016 \mathrm{e}+01$ \\
\hline 119 & IPW000002 & 2205.7600 & 2213.5652 & 2208.3357 & $1.5490 e+00$ & $1.9235 e+01$ \\
\hline 120 & IPW000003 & 2250.2998 & 2258.0999 & 2254.0044 & $2.5608 \mathrm{e}+00$ & $1.9562 e+01$ \\
\hline 121 & IPWO00004 & 652.1653 & 653.6301 & 652.7417 & $3.0708 e-01$ & $5.5309 e+00$ \\
\hline 122 & IPW000005 & 430.7900 & 434.2076 & 432.0398 & $9.3138 e-01$ & $3.9559 e+00$ \\
\hline 1.23 & IPW000006 & 444.8618 & 447.7915 & 446.7951 & $8.0723 \theta-01$ & $4.0569 e+00$ \\
\hline $1 \therefore 4$ & EMPTY & -0.0244 & -0.0098 & -0.0186 & $3.6911 e-03$ & $0.0000 e+00$ \\
\hline $1: 5$ & EMPTY & -0.0195 & -0.0049 & -0.0124 & $2.9938 e-03$ & $0.0000 e+00$ \\
\hline 126 & EMPTY & -0.0195 & -0.0049 & -0.0148 & $3.4116 e-03$ & $0.0000 e+00$ \\
\hline 1.27 & EMPTY & -0.0244 & -0.0098 & -0.0171 & $3.3087 e-03$ & $0.0000 e+00$ \\
\hline 228 & IHTO000001 & 219.5387 & 222.6637 & 220.9997 & $6.8030 e-01$ & $4.6577 \mathrm{e}+00$ \\
\hline 129 & IHTO00002 & 225.7362 & 229.0565 & 227.4159 & $6.6028 e-01$ & $4.6885 e+00$ \\
\hline 150 & IHTO00003 & 227.8170 & 231.1373 & 229.4147 & $6.9440 e-01$ & $4.6982 e+00$ \\
\hline 131 & IHTO00004 & 223.5690 & 226.8893 & 225.2096 & $7.3927 e-01$ & $4.6778 \mathrm{e}+00$ \\
\hline $1: 2$ & IHTO00005 & 221.4543 & 224.9699 & 222.8136 & $7.2433 e-01$ & $4.6663 \mathrm{e}+00$ \\
\hline $1: 3$ & IHTO000006 & 215.5987 & 218.7237 & 216.9229 & $7.2468 e-01$ & $4.6385 e+00$ \\
\hline $1 \lesssim 4$ & IHT000007 & 225.1352 & 228.0649 & 226.4281 & $6.7530 e-01$ & $4.6837 e+00$ \\
\hline 135 & IHTO00008 & 222.3483 & 225.0826 & 223.8561 & $6.0495 e-01$ & $4.6713 e+00$ \\
\hline 156 & IHTO00009 & 222.3370 & 225.0714 & 224.0166 & $6.2390 e-01$ & $4.6721 \mathrm{e}+00$ \\
\hline 137 & IHTO00010 & 54.2330 & 56.7721 & 55.5767 & $6.0314 e-01$ & $4.1375 e+00$ \\
\hline $1: 8$ & IHT000011 & 222.8853 & 226.0103 & 224.4323 & $6.6713 e-01$ & $4.6741 e+00$ \\
\hline $1: 9$ & IHTO00012 & 218.9378 & 222.2581 & 220.4339 & $7.0101 e-01$ & $4.6550 e+00$ \\
\hline 140 & IHTO00013 & 158.3984 & 161.7188 & 159.7930 & $6.6264 e-01$ & $4.4004 e+00$ \\
\hline $1: 1$ & IHTO00014 & 219.1632 & 222.8741 & 221.0381 & $7.5593 e-01$ & $4.6579 \mathrm{e}+00$ \\
\hline 1.12 & IHTO00015 & 163.3714 & 166.4964 & 164.8715 & $6.1566 e-01$ & $4.4191 e+00$ \\
\hline 143 & IHTO00016 & 152.1935 & 155.1232 & 153.4669 & $5.4568 \theta-01$ & $4.3778 e+00$ \\
\hline $1+4$ & IHT 000017 & 182.6097 & 186.1253 & 184.2699 & $6.3534 e-01$ & $4.4951 e+00$ \\
\hline $1+5$ & IHTO00018 & 184.3074 & 187.6277 & 185.9598 & $6.5579 e-01$ & $4.5020 e+00$ \\
\hline 140 & IHTO0001.9 & 179.0640 & 181.7984 & 180.2515 & $6.4094 e-01$ & $4.4787 e+00$ \\
\hline 147 & IHT000020 & 184.1271 & 186.6662 & 185.3341 & $6.0578 e-01$ & $4.4994 e+00$ \\
\hline 148 & IHT000021 & -2.8132 & 0.3118 & -1.3640 & $7.3831 e-01$ & $4.1000 e+00$ \\
\hline 149 & IHTO00022 & 158.4848 & 161.0239 & 159.6997 & $5.7085 e-01$ & $4.4000 e+00$ \\
\hline 150 & IHT000023 & 180.3411 & 182.4895 & 181.3683 & $5.2320 e-01$ & $4.4832 e+00$ \\
\hline 151 & IHTO00024 & 144.5726 & 147.1116 & 145.8461 & $5.2086 e-01$ & $4.3517 e+00$ \\
\hline 152 & IHTO00025 & 179.3532 & 181.8923 & 180.7126 & $5.4954 e-01$ & $4.4806 e+00$ \\
\hline 153 & IHTO00026 & 181.6932 & 184.0370 & 182.9784 & $5.3828 e-01$ & $4.4898 e+00$ \\
\hline $1 \equiv 4$ & IHT000027 & 155.0331 & 157.5721 & 156.2675 & $5.6143 e-01$ & $4.3877 e+00$ \\
\hline 1.5 & IHTOOOO28 & 157.1552 & 160.0849 & 158.3935 & $6.5508 e-01$ & $4.3953 e+00$ \\
\hline
\end{tabular}




\begin{tabular}{|c|c|c|c|c|c|}
\hline Test: Aug17_08 & sum1 & Date: Fr1 & Aug 170 & $6: 311990$ & Page: 4 \\
\hline 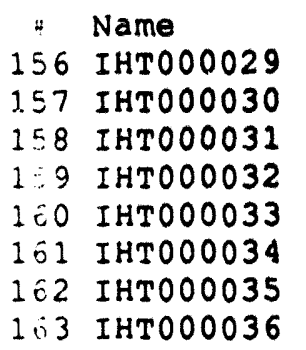 & $\begin{array}{l}\text { Min } \\
180.5965 \\
183.9656 \\
181.2275 \\
183.3045 \\
183.3271 \\
161.3206 \\
212.9808 \\
159.2060\end{array}$ & $\begin{array}{l}\operatorname{Max} \\
183.3308 \\
186.5047 \\
183.5712 \\
186.0389 \\
185.8661 \\
163.2737 \\
216.1058 \\
161.7450\end{array}$ & $\begin{array}{l}\text { Ave } \\
181.8192 \\
185.2040 \\
182.3564 \\
184.7851 \\
184.7295 \\
162.3051 \\
214.2073 \\
160.3974\end{array}$ & $\begin{array}{l}\text { SD } \\
6.5174 e-01 \\
6.7383 e-01 \\
6.5826 e-01 \\
7.0043 e-01 \\
6.9103 e-01 \\
5.1593 e-01 \\
7.0811 e-01 \\
5.9353 e-01\end{array}$ & $\begin{array}{c}\text { Uncert } \\
4.4851 e++00 \\
4.4989 e+00 \\
4.4872 e+00 \\
4.4972 \theta+00 \\
4.4969 e+00 \\
4.4096 e+00 \\
4.6258 e+00 \\
4.4026 e++00\end{array}$ \\
\hline
\end{tabular}




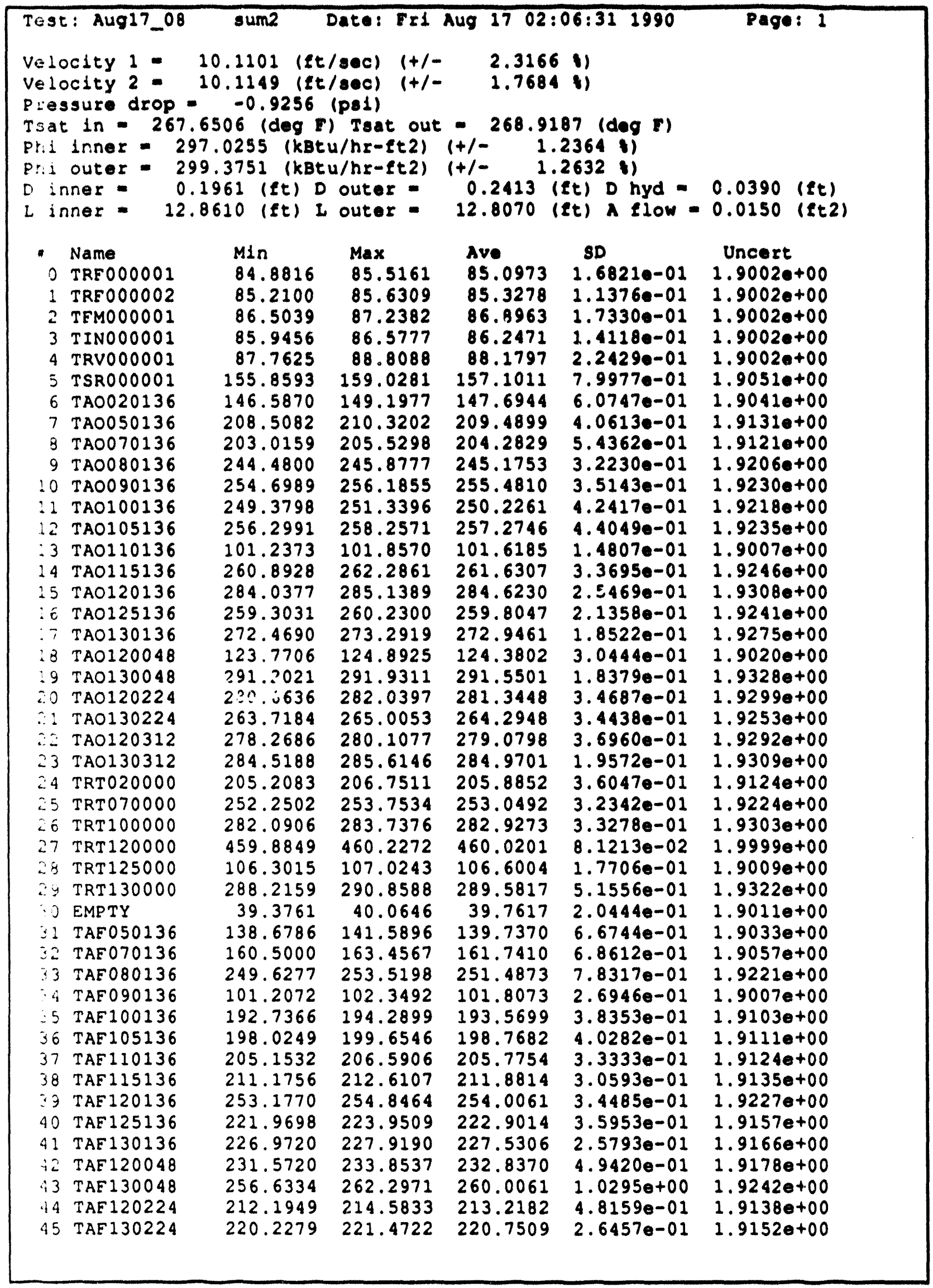




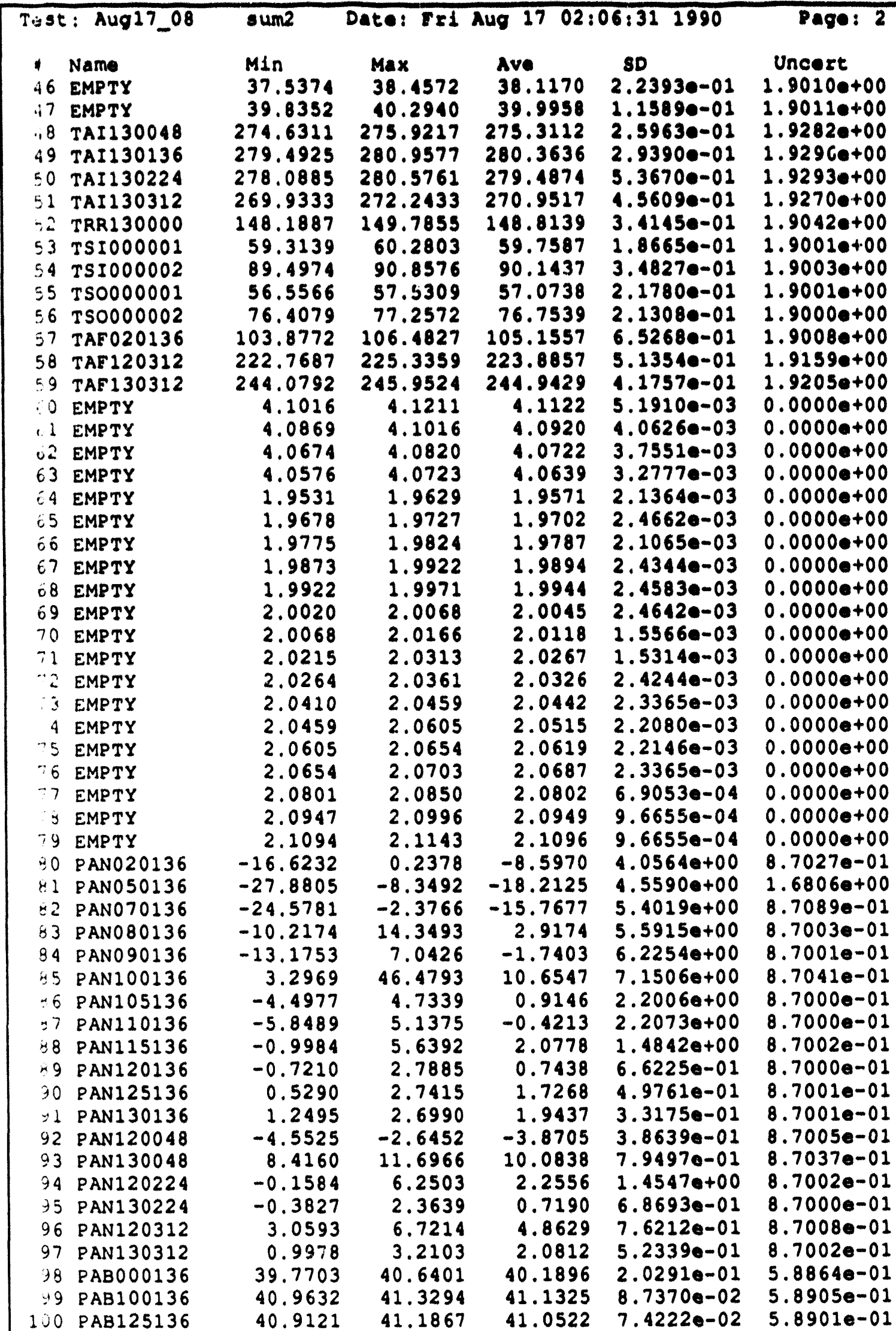




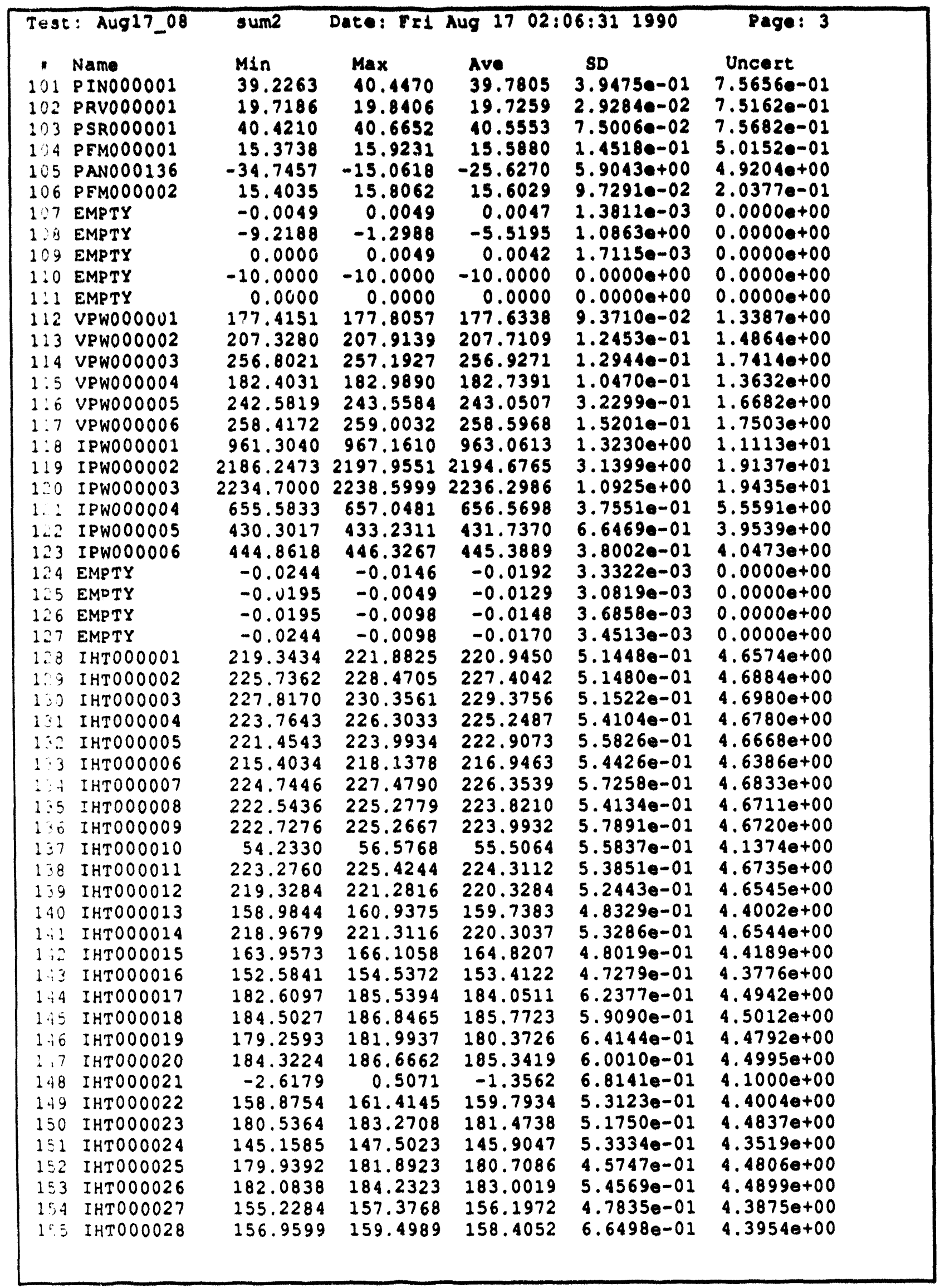




\begin{tabular}{|c|c|c|c|c|c|c|}
\hline \multicolumn{2}{|c|}{ Tegt: Aug17_08 } & sum2 & Date: Fr1 & Aug 1702 & $6: 311990$ & Page: 4 \\
\hline $\begin{array}{l}11 \\
156 \\
157 \\
158 \\
159 \\
100 \\
161 \\
162 \\
163\end{array}$ & 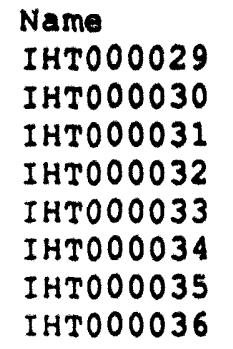 & $\begin{array}{l}M 1 n \\
180.4012 \\
183.7703 \\
180.8368 \\
183.3045 \\
183.1318 \\
160.9300 \\
211.6136 \\
159.2060\end{array}$ & $\begin{array}{l}\operatorname{Max} \\
183.3308 \\
186.7000 \\
183.9618 \\
186.4295 \\
186.4521 \\
163.8597 \\
215.1292 \\
161.7450\end{array}$ & $\begin{array}{l}\text { Ave } \\
181.9091 \\
185.2626 \\
182.4501 \\
184.9218 \\
184.8623 \\
162.4652 \\
213.6761 \\
160.4599\end{array}$ & $\begin{array}{l}\text { SD } \\
6.8691 e-01 \\
6.9448 e-01 \\
7.1986 e-01 \\
7.2442 e-01 \\
7.1573 e-01 \\
6.3012 e-01 \\
7.2454 e-01 \\
6.1141 e-01\end{array}$ & $\begin{array}{c}\text { Uncert } \\
4.4854 e+00 \\
4.4991 e+00 \\
4.4876 e+00 \\
4.4977 e+00 \\
4.4975 e+00 \\
4.4102 \theta+00 \\
4.6234 e+00 \\
4.4028 e+00\end{array}$ \\
\hline
\end{tabular}




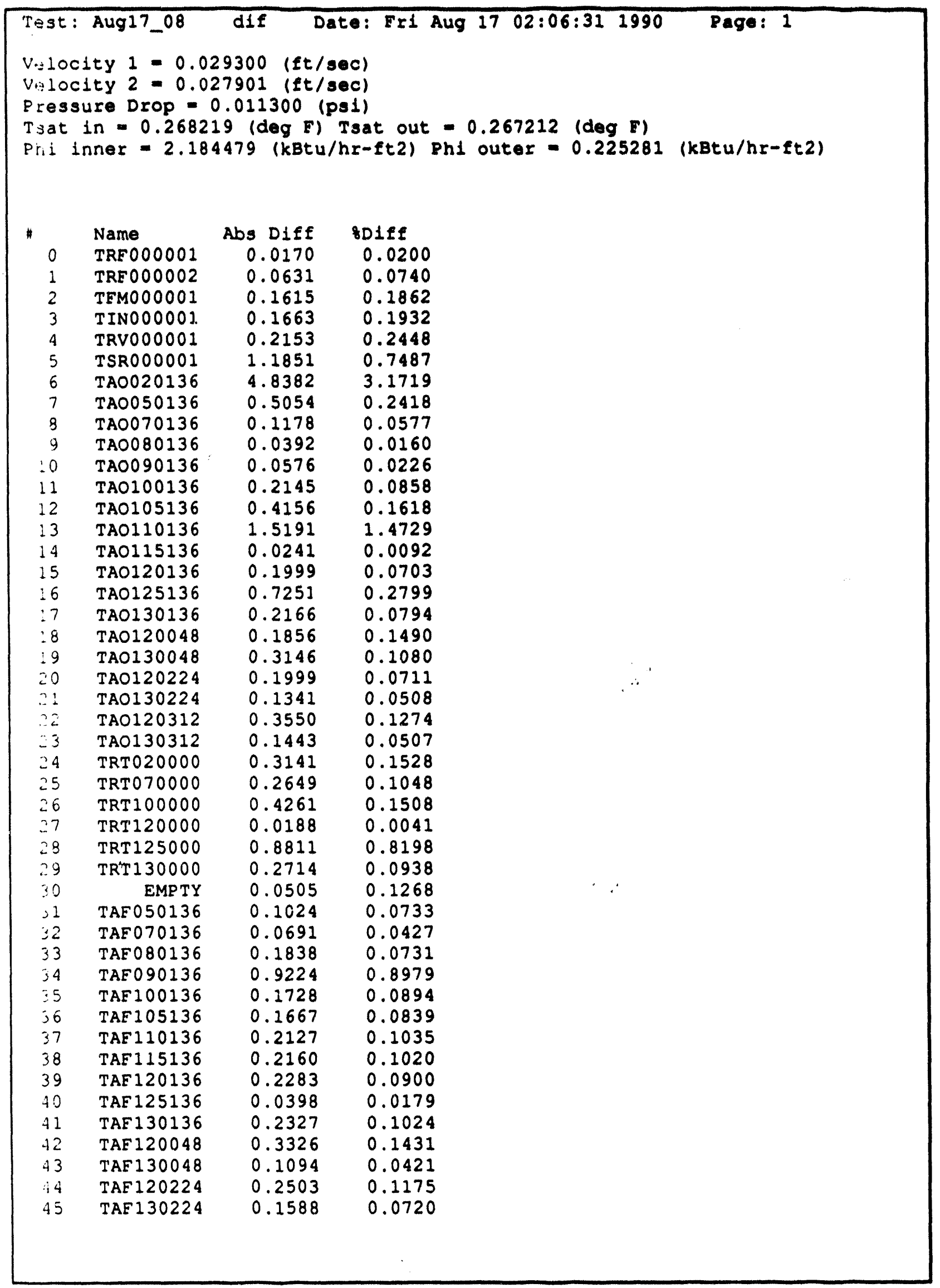




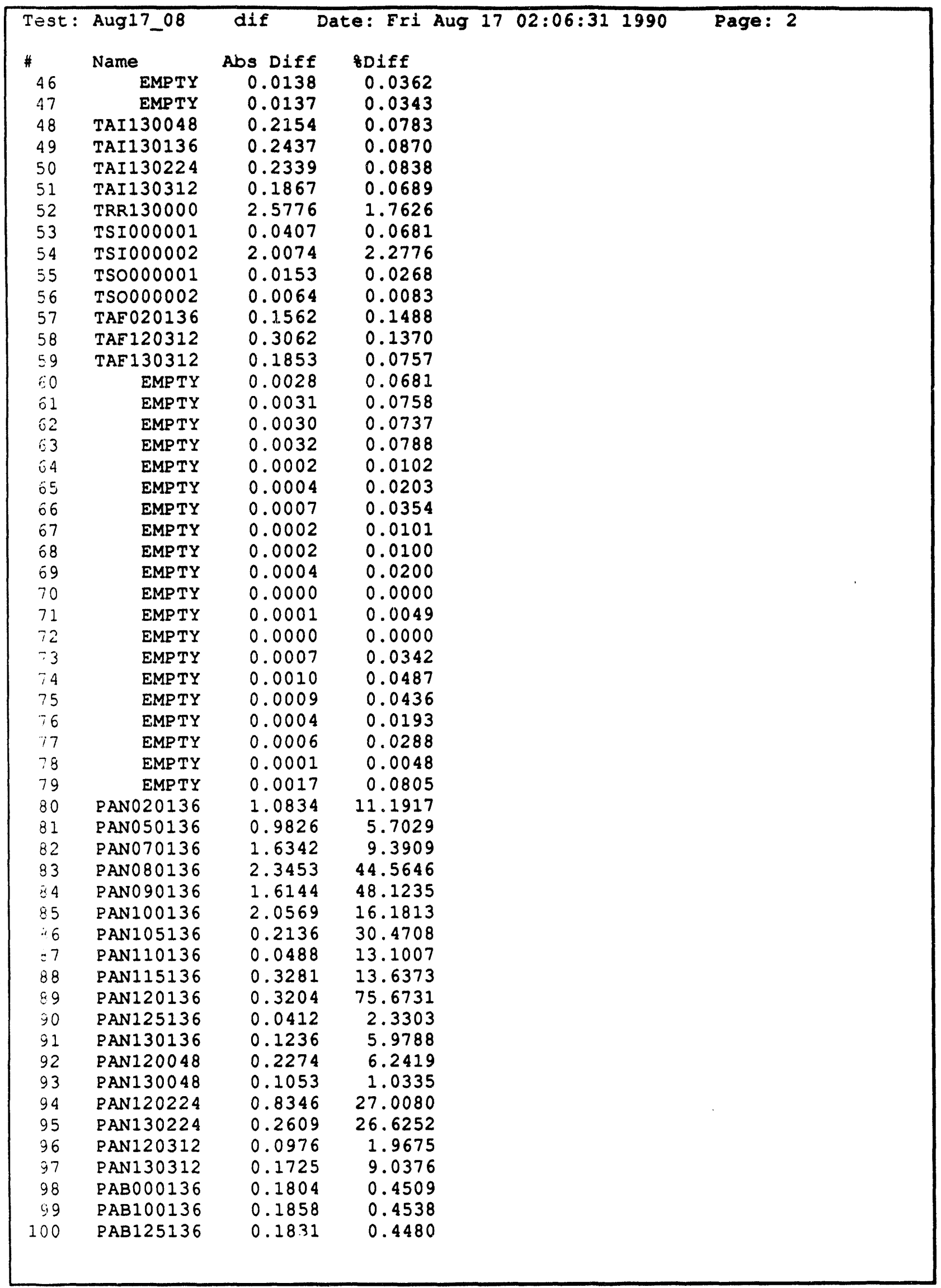




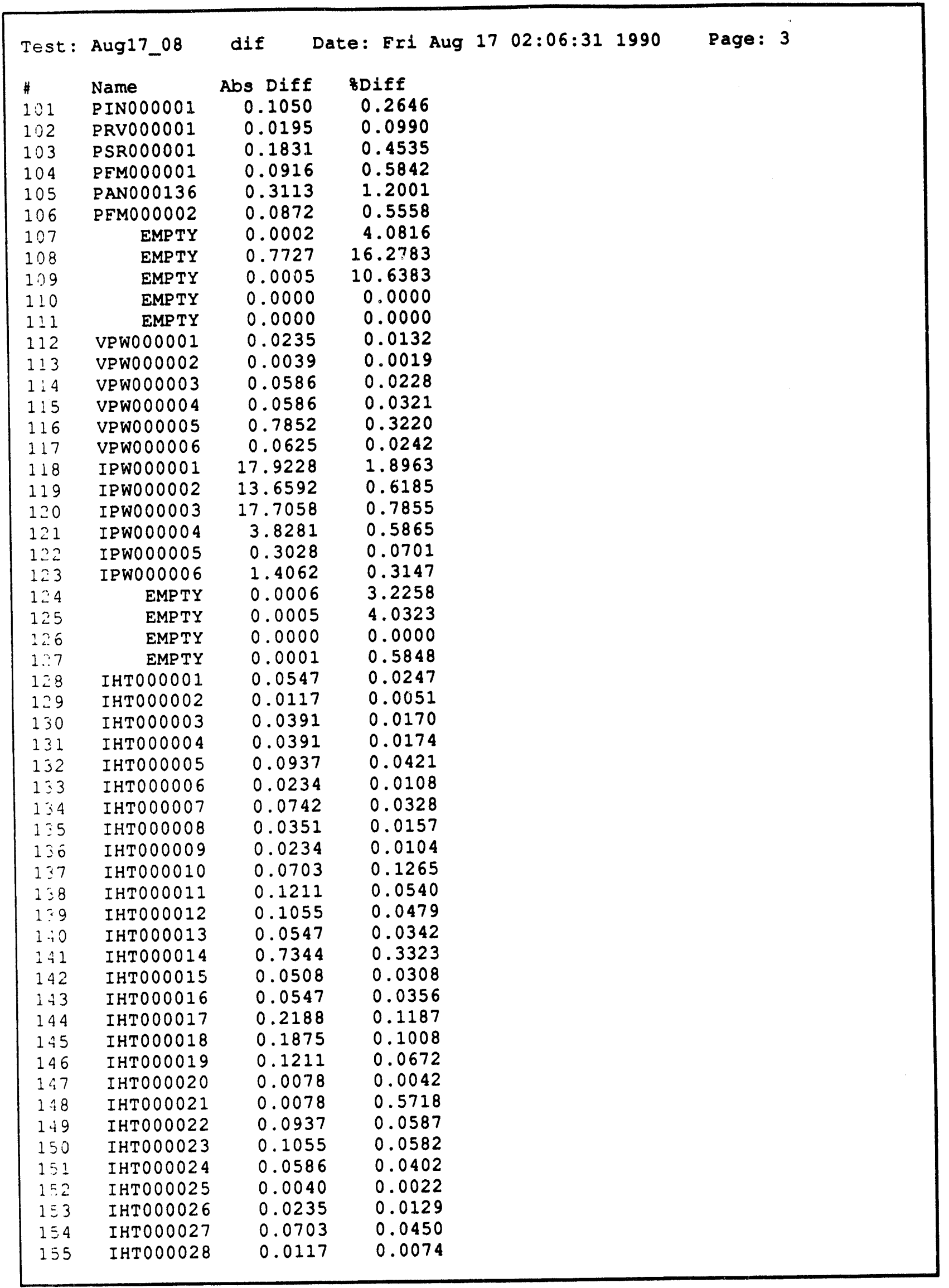




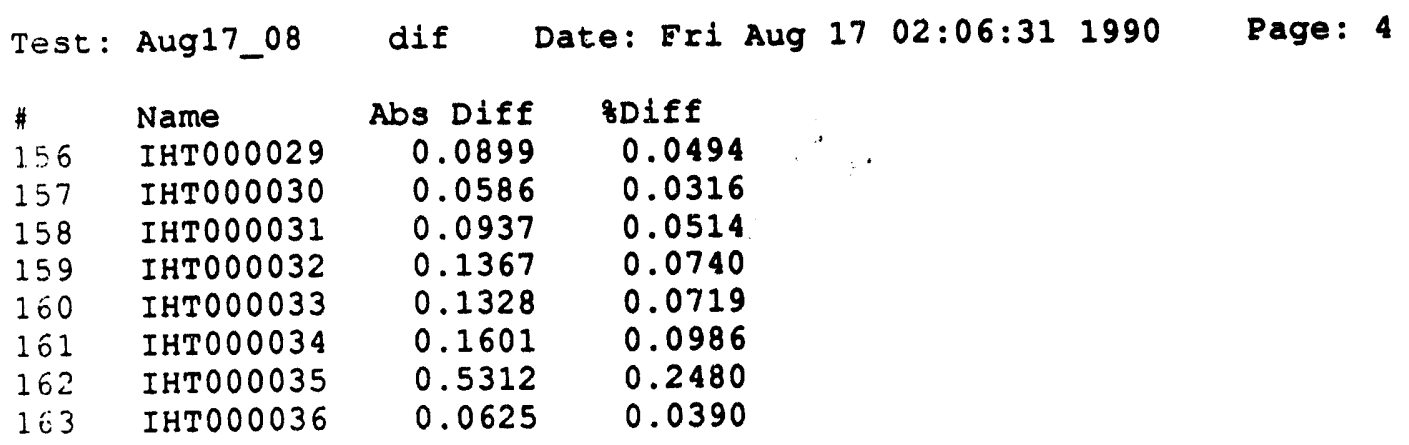




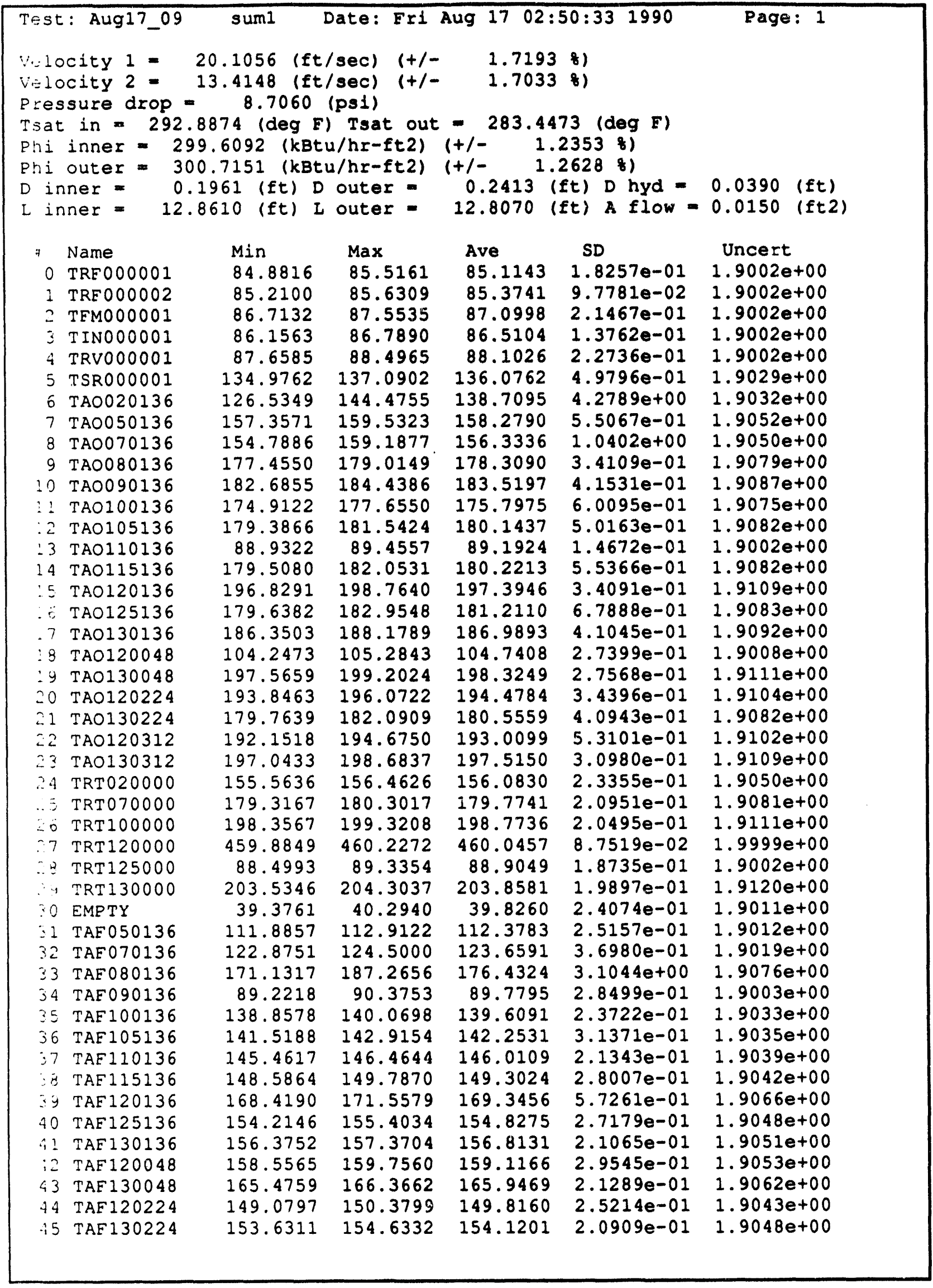




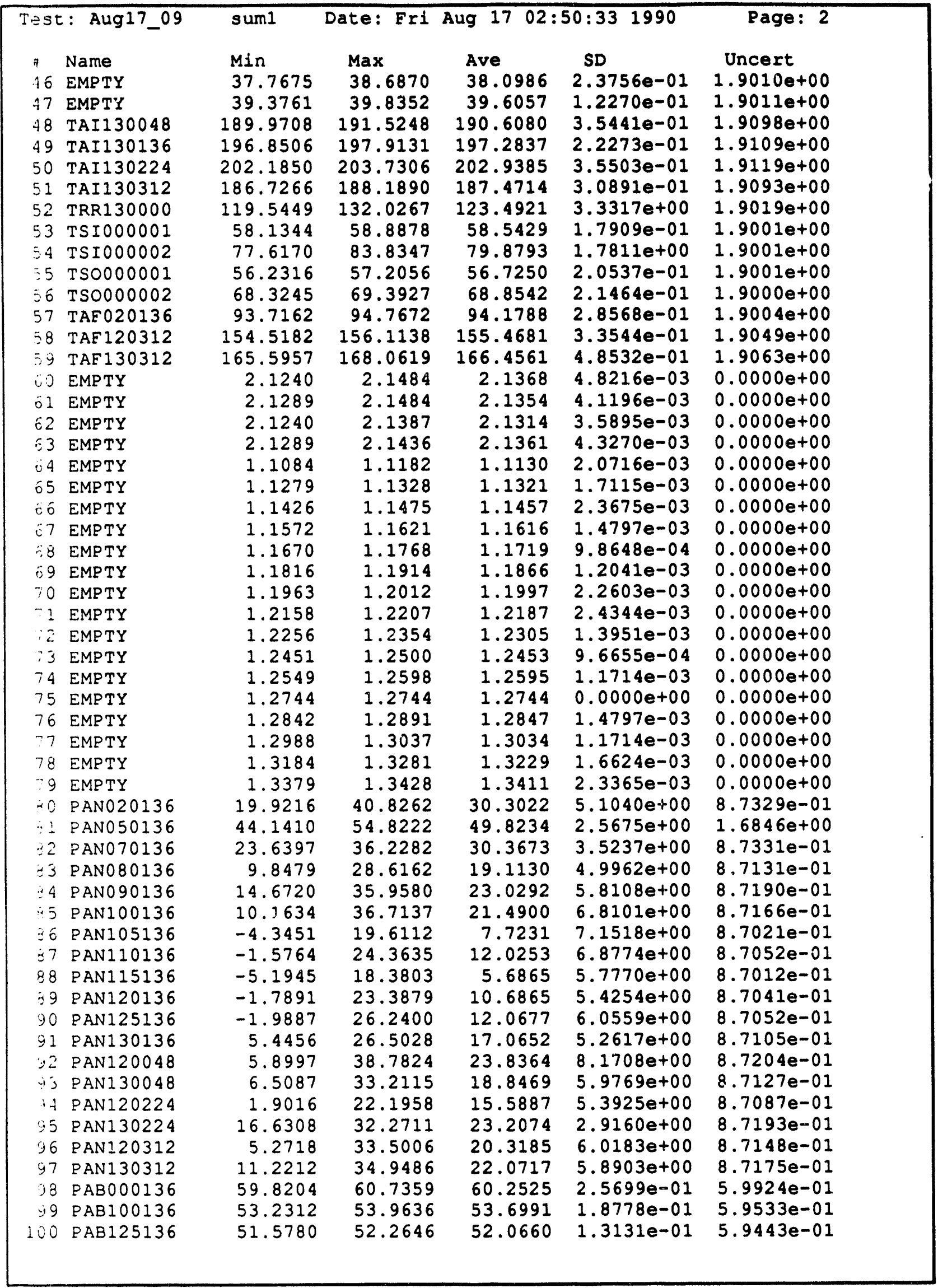




\begin{tabular}{|c|c|c|c|c|c|c|}
\hline \multicolumn{2}{|c|}{ T:St: Alug17_09 } & sum1 & Date: Fri & \multicolumn{2}{|c|}{ Aug $1702: 50: 33 \quad 1990$} & \multirow[t]{2}{*}{ Page: 3} \\
\hline \# & Name & Min & $\operatorname{Max}$ & Ave & SD & \\
\hline 121 & PINO00001 & 59.1237 & 60.8327 & 60.0881 & $4.4409 e-01$ & $7.6490 e-01$ \\
\hline 1.12 & PRV000001 & 19.8406 & 20.0848 & 19.9603 & $3.0104 e-02$ & $7.5166 e-01$ \\
\hline $1: 3$ & PSR000001 & 49.4542 & 49.6984 & 49.5763 & $8.1794 e-02$ & $7.6017 e-01$ \\
\hline 104 & PFMO00001 & 60.9671 & 63.1643 & 62.2177 & $5.2041 e-01$ & $5.2364 e-01$ \\
\hline 105 & PANO00136 & 227.0952 & 259.5964 & 241.0294 & $7.4757 e+00$ & $4.9568 e+00$ \\
\hline $1 j 6$ & PFMO00002 & 27.5689 & 27.5689 & 27.5689 & $9.6336 e-06$ & $2.1154 e-01$ \\
\hline 107 & EMPTY & 0.0049 & 0.0098 & 0.0097 & $6.9053 e-04$ & $0.0000 e+00$ \\
\hline 108 & EMPTY & -10.0000 & -0.2832 & -7.6616 & $2.3933 e+00$ & $0.0000 e+00$ \\
\hline 109 & EMPTY & 0.0049 & 0.0098 & 0.0058 & $1.8950 e-03$ & $0.0000 e+00$ \\
\hline $1: 0$ & EMPTY & -10.0000 & -10.0000 & -10.0000 & $0.0000 e+00$ & $0.0000 e+00$ \\
\hline $1: 1$ & EMPTY & 0.0000 & 0.0049 & 0.0003 & $1.1714 e-03$ & $0.0000 e+00$ \\
\hline $1: 2$ & VPW000001 & 176.8292 & 177.4151 & 177.1338 & $1.1942 e-01$ & $1.3363 e+00$ \\
\hline 113 & VPW000002 & 206.9374 & 207.1327 & 207.0155 & $9.6655 e-02$ & $1.4829 e+00$ \\
\hline $1: 4$ & VPW000003 & 256.0209 & 256.4115 & 256.2278 & $1.2731 \mathrm{e}-01$ & $1.7377 e+00$ \\
\hline$:: 5$ & VPWO00004 & 182.0125 & 182.4031 & 182.1610 & $1.1545 e-01$ & $1.3604 e+00$ \\
\hline$\therefore 0$ & VPWO00005 & 242.5819 & 242.9725 & 242.8124 & $1.2283 e-01$ & $1.6669 \mathrm{e}+00$ \\
\hline $1: 7$ & VPW000006 & 257.4407 & 258.0266 & 257.7686 & $1.3917 \mathrm{e}-01$ & $1.7459 e+00$ \\
\hline 118 & IPWO00001 & 933.9712 & 955.4470 & 947.5984 & $5.5570 e+00$ & $1.1030 \mathrm{e}+01$ \\
\hline 119 & IPW000002 & 2225.2727 & 2242.8342 & 2235.1858 & $6.3177 e+00$ & $1.9427 e+01$ \\
\hline $1: 0$ & IPW000003 & 2256.1499 & 2267.8499 & 2263.2090 & $3.2935 e+00$ & $1.9629 e+01$ \\
\hline 121 & IPWO00004 & 650.2122 & 656.0715 & 654.3922 & $2.0977 e+00$ & $5.5431 e+00$ \\
\hline 122 & IPW000005 & 435.6723 & 436.6487 & 436.0724 & $2.9077 e-01$ & $3.9834 e+00$ \\
\hline $1: 3$ & IPW000006 & 448.7681 & 450.2329 & 449.4123 & $4.1196 e-01$ & $4.0749 e+00$ \\
\hline 1.24 & EMPTY & -0.0244 & -0.0098 & -0.0191 & $4.0482 e-03$ & $0.0000 e+00$ \\
\hline 1.25 & EMPTY & -0.0195 & -0.0098 & -0.0129 & $2.9197 e-03$ & $0.0000 e+00$ \\
\hline 1.26 & EMPTY & -0.0244 & -0.0049 & -0.0157 & $3.9815 e-03$ & $0.0000 e+00$ \\
\hline 127 & EMPTY & -0.0244 & -0.0098 & -0.0161 & $3.1583 e-03$ & $0.0000 e+00$ \\
\hline$\therefore 8$ & IHTO00001 & 220.1247 & 223.2497 & 221.6677 & $6.8260 e-01$ & $4.6609 e+00$ \\
\hline$\therefore .9$ & IHTO00002 & 226.7127 & 229.4471 & 228.2011 & $6.4100 e-01$ & $4.6923 e+00$ \\
\hline $1 \geq 0$ & IHTO00003 & 228.7936 & 231.5280 & 230.2858 & $6.2485 e-01$ & $4.7025 e+00$ \\
\hline 131 & IHT 000004 & 224.5455 & 227.6705 & 226.2174 & $6.5455 e-01$ & $4.6827 e+00$ \\
\hline $1: 2$ & IHTO00005 & 222.2356 & 225.1653 & 223.7393 & $6.7225 e-01$ & $4.6708 e+00$ \\
\hline$\therefore: 3$ & IHT000006 & 216.3799 & 219.3096 & 217.7197 & $6.5674 e-01$ & $4.6422 e+00$ \\
\hline 134 & IHT 000007 & 225.7212 & 228.8462 & 227.2367 & $6.4665 e-01$ & $4.6876 e+00$ \\
\hline 135 & IHTO00008 & 223.1295 & 226.2545 & 224.6139 & $6.0746 e-01$ & $4.6749 e+00$ \\
\hline 120 & IHTO00009 & 223.3135 & 226.8292 & 224.7002 & $6.7572 e-01$ & $4.6754 \mathrm{e}+00$ \\
\hline$:: 7$ & IHTO00010 & 55.0143 & 57.3580 & 56.1353 & $5.3059 e-01$ & $4.1383 e+00$ \\
\hline $2: 3$ & IHTO00011 & 224.0572 & 226.2057 & 225.1081 & $4.9267 e-01$ & $4.6773 e+00$ \\
\hline$: 39$ & IHTO00012 & 220.1097 & 222.4534 & 221.1800 & $4.7881 e-01$ & $4.6585 e+00$ \\
\hline$\therefore 0$ & IHTO00013 & 159.5703 & 161.7188 & 160.4766 & $4.9562 e-01$ & $4.4029 e+00$ \\
\hline$:: 1$ & IHTO00014 & 220.7257 & 223.0694 & 221.6983 & $5.4461 e-01$ & $4.6610 \mathrm{e}+00$ \\
\hline$: i 2$ & IHTO00015 & 164.5433 & 167.0823 & 165.5472 & $5.4535 e-01$ & $4.4216 e+00$ \\
\hline$\therefore 43$ & IHT000016 & 153.1701 & 155.3185 & 154.1622 & $4.9259 e-01$ & $4.3803 e+00$ \\
\hline$\div 44$ & IHTO000017 & 183.3909 & 186.3206 & 184.6605 & $6.1669 e-01$ & $4.4967 e+00$ \\
\hline 145 & IHTO00018 & 185.4793 & 187.6277 & 186.4325 & $5.3034 e-01$ & $4.5040 e+00$ \\
\hline 16 & IHTO00019 & 179.4546 & 181.7984 & 180.5992 & $5.6083 e-01$ & $4.4801 e+00$ \\
\hline $2+7$ & IHTO00020 & 184.5177 & 186.8615 & 185.7364 & $5.5181 e-01$ & $4.5012 e+00$ \\
\hline 1.18 & IHT 000021 & -3.0086 & 0.3118 & -1.4851 & $6.7080 e-01$ & $4.1000 e+00$ \\
\hline $1: 9$ & IHTO00022 & 158.8754 & 161.8051 & 160.0864 & $5.4104 e-01$ & $4.4015 e+00$ \\
\hline$\because \vdots$ & IHTOOOOO23 & 180.5364 & 183.6614 & 181.7863 & $5.4818 e-01$ & $4.4849 e+00$ \\
\hline$\because \vdots 1$ & IHTOOOO 4 & 145.3538 & 148.0882 & 146.2719 & $5.2977 e-01$ & $4.3531 e+00$ \\
\hline $1 \equiv 2$ & IHTO00025 & 180.3298 & 182.8689 & 181.1032 & $5.9183 e-01$ & $4.4822 e+00$ \\
\hline $1: 3$ & IHTO00026 & 182.0838 & 184.8182 & 183.1894 & $5.7400 e-01$ & $4.4906 e+00$ \\
\hline$\because 1$ & IHTO00027 & 155.4237 & 158.3534 & 156.6151 & $5.9615 e-01$ & $4.3889 e+00$ \\
\hline$\therefore 5$ & IHTO00028 & 157.5458 & 160.6708 & 158.8076 & $6.1664 e-01$ & $4.3968 e+00$ \\
\hline
\end{tabular}




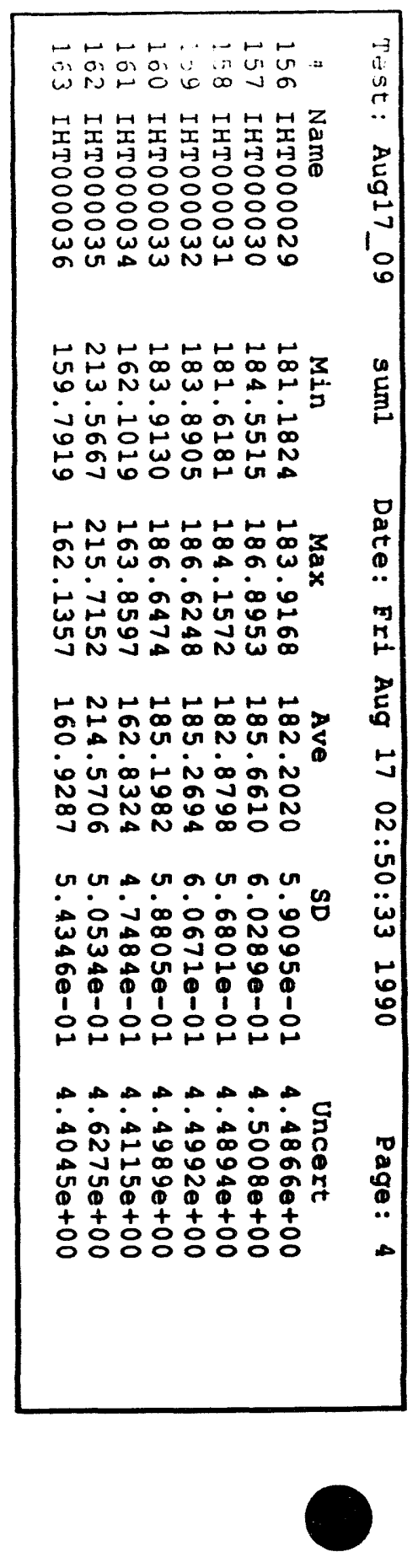




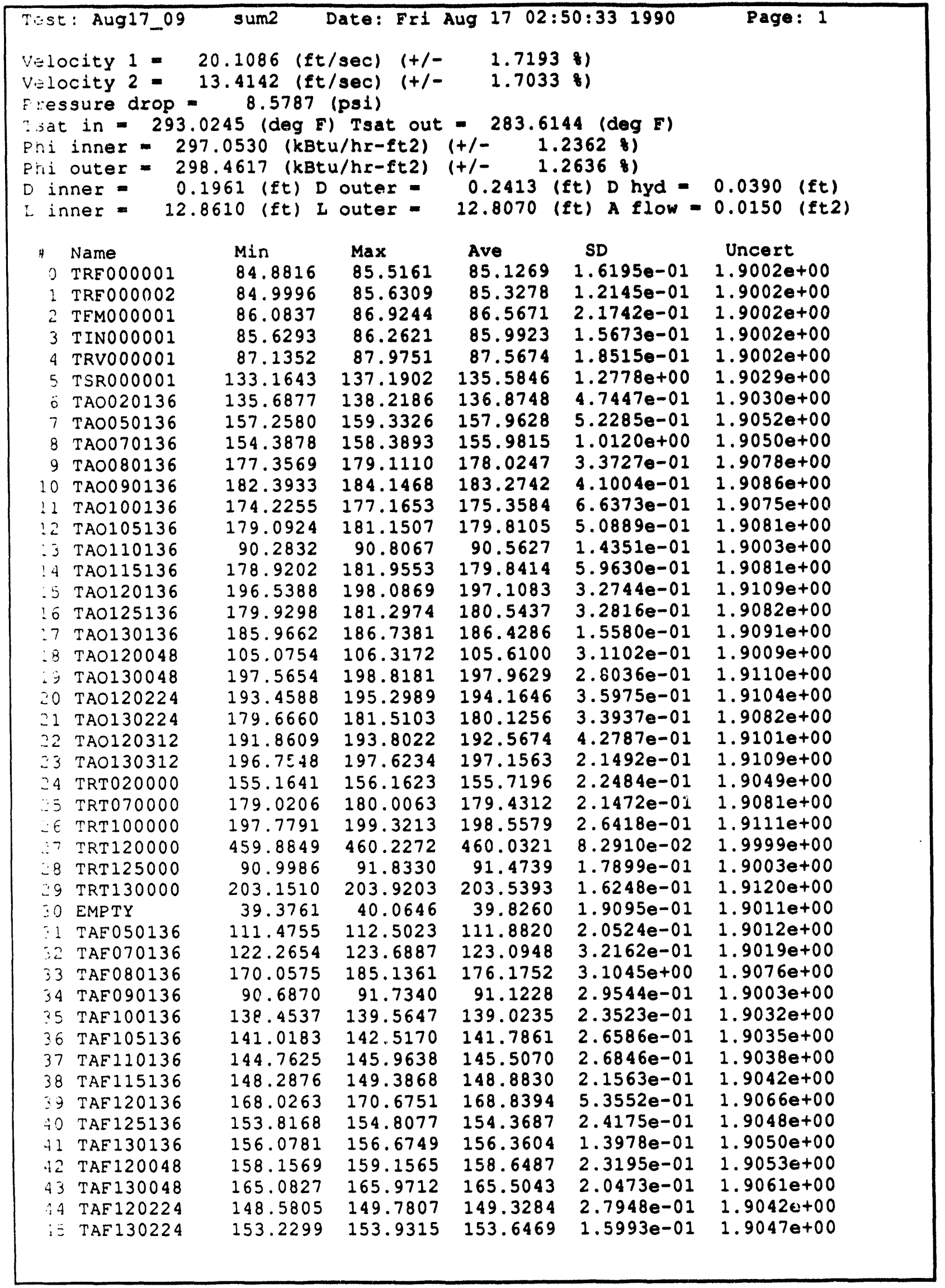




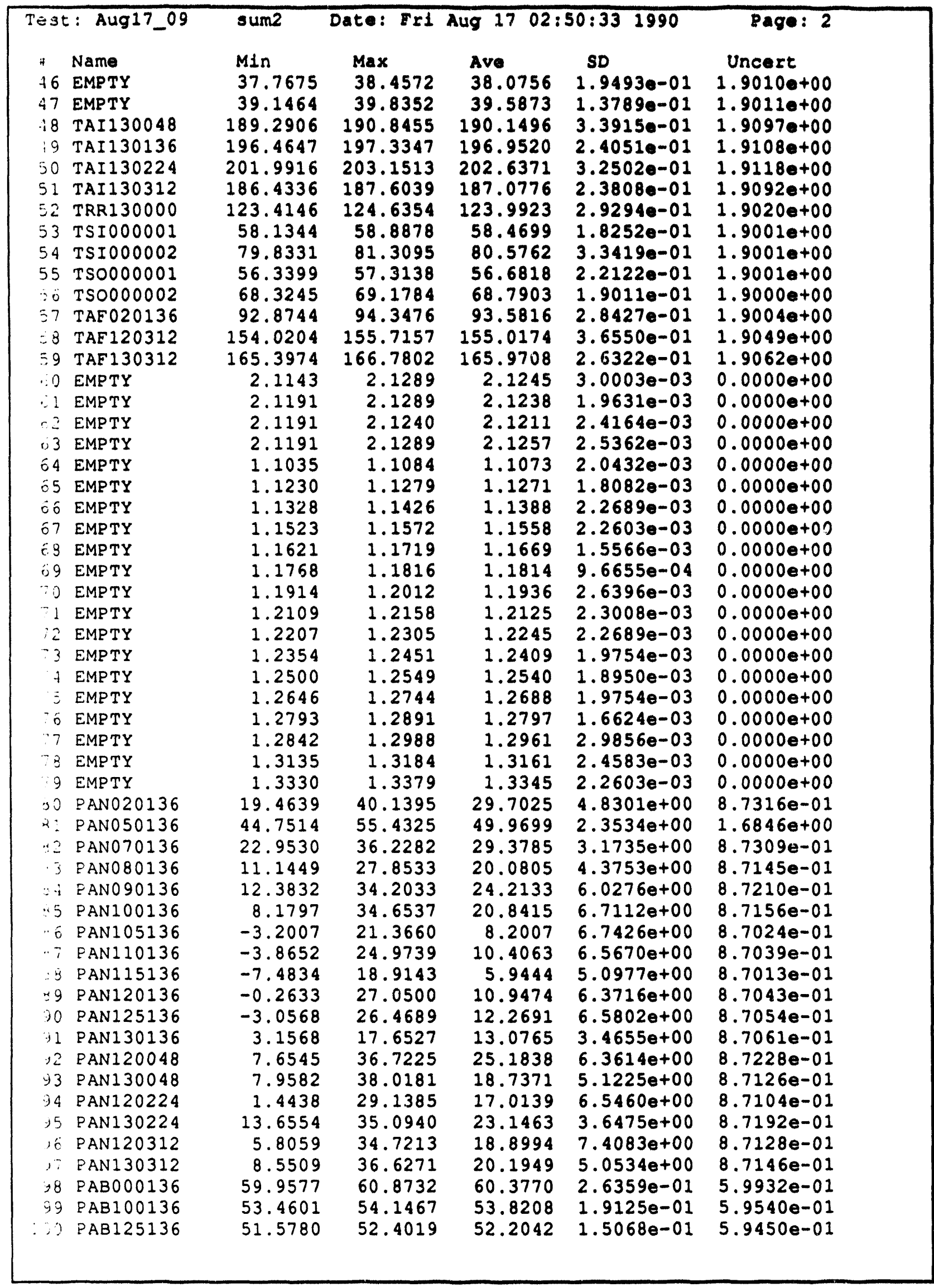




\begin{tabular}{|c|c|c|c|c|c|c|}
\hline \multicolumn{2}{|c|}{ Test: Aug17_09 } & sum2 & ate: $F$ & a 110 & 33 & Page: \\
\hline 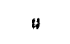 & & Wat & $\max$ & ave & & Unce it \\
\hline & 00001 & 3679 & & $-\infty$ & & \\
\hline$\therefore 12$ & 000001 & 4744 & & 1210 & 4.607 & \\
\hline & PSR000001 & 763 & & & & \\
\hline 14 & PFMC & 079 & & & & \\
\hline & PAN & 3063 & 257.3076 & 5047 & & \\
\hline 106 & PFMO & & & & & \\
\hline 127 & EMPTY & 049 & & & & \\
\hline$: 08$ & EMPTY & -10 & & & 2.440 & \\
\hline 129 & EMPTY & & & & 2. & \\
\hline$\therefore: 0$ & EMPTY & & -1 & & $0.00 c$ & \\
\hline $1: 2$ & EMPTY & 00 & & & 1.171 & +0 \\
\hline $1: 2$ & 1001 & & & & & \\
\hline $1: 3$ & VPh & & & & & 1.48 \\
\hline$\therefore 1$ & VPV & & & 25 & & \\
\hline 115 & VPV & & 4031 & & & \\
\hline$\therefore 6$ & VPV & & 9725 & 24 & & +0 \\
\hline$:: 7$ & 06 & & 25 & & & +00 \\
\hline j & & & & & & \\
\hline$\therefore: 9$ & IPh & 218 & 2190 & 218 & & 1. \\
\hline$: 20$ & IPH & 499 & 224 & 22 & 1. & 1. \\
\hline$\therefore 1$ & & & & & & \\
\hline 122 & IPW & 16 & & & & 3. \\
\hline$\therefore \therefore 3$ & IPW & & 44 & & & 4. \\
\hline $2: 24$ & EMP & & -0 & -0.0201 & & 0 \\
\hline & EME & & & -0.0 & & 0 \\
\hline & EMI & -0 . & -0 & -0 . & & 0. \\
\hline & & & -0 & -0.0170 & & 0 \\
\hline$\therefore$. is & It! & & 22 & & & 4. \\
\hline$\therefore \quad 3$ & & & & & & 4. \\
\hline$\therefore 0$ & & & & & & \\
\hline$\therefore 1$ & IH & & & & & 4. \\
\hline$\therefore 2$ & & & & & & 4 \\
\hline$\therefore: 3$ & IH & & & & & \\
\hline$: 34$ & 07 & & & & & 4. \\
\hline$\therefore 5$ & $\mathrm{IH}^{\prime}$ & & & & & 4 \\
\hline$: 26$ & & & & & & \\
\hline $1: 7$ & & & & & & 4 \\
\hline$: 7 \mathrm{j}$ & & & & & & \\
\hline$:$ : y & & & & & & \\
\hline$\ldots j$ & & & & & & 4. \\
\hline$:: 1$ & & & & & & 4. \\
\hline$\because 2$ & & & & & & \\
\hline .13 & & & & & & \\
\hline$\because i a_{1}-x$ & & & & & & \\
\hline 1.15 & & & & & & 4.5 \\
\hline$: 46$ & $\mathrm{IH}^{\prime}$ & & 182 & & & 4.4 \\
\hline$\therefore 47$ & & & 18 & & & 4. \\
\hline$\therefore 3$ & & & & & & \\
\hline$: 49$ & IHI & 8 & 161.6 & 160.1216 & 5.8 & $4.4016 e+0$ \\
\hline 150 & IH? & & 183.46 & 181.75 & & 4.4 \\
\hline & IHT & & & & & 4. \\
\hline & IHTC & 179. & 182.2829 & 181.0915 & 6.37 & $4.4821 e+0$ \\
\hline$\because j$ & & & & & & \\
\hline $1 \div 4$ & IH? & & & & & \\
\hline $1 \div 5$ & IHTO00028 & 57.5458 & 160.6708 & .0302 & .01 & $4.39^{\prime}$ \\
\hline
\end{tabular}




\begin{tabular}{|c|c|c|c|c|c|c|}
\hline $\begin{array}{l}14 \\
156 \\
\vdots 7 \\
158 \\
159 \\
100 \\
\vdots 1 \\
\vdots 2 \\
\vdots 03\end{array}$ & $\begin{array}{l}\text { Name } \\
\text { IHTO00029 } \\
\text { IHTO00030 } \\
\text { IHTO00031 } \\
\text { IHTOOOO32 } \\
\text { IHTOOO033 } \\
\text { IHTOOO034 } \\
\text { IHTO00035 } \\
\text { IHTOOOO36 }\end{array}$ & $\begin{array}{l}\operatorname{Min} \\
180.9871 \\
184.5515 \\
181.8134 \\
184.0858 \\
183.9130 \\
161.7112 \\
213.3714 \\
159.7919\end{array}$ & $\begin{array}{l}\operatorname{Max} \\
184.3074 \\
187.8719 \\
184.9384 \\
187.4061 \\
187.2333 \\
164.4456 \\
215.9105 \\
161.9403\end{array}$ & $\begin{array}{l}\text { Ave } \\
182.4247 \\
185.8954 \\
183.1142 \\
185.5312 \\
185.4521 \\
162.9222 \\
214.6409 \\
160.9560\end{array}$ & $\begin{array}{l}80 \\
7.9288 e-01 \\
7.9961 e-01 \\
7.7886 e-01 \\
8.3984 e-01 \\
8.2264 e-01 \\
6.6380 e-01 \\
6.3165 e-01 \\
5.7718 e-01\end{array}$ & $\begin{array}{c}\text { Uncert } \\
4.4875 \bullet+00 \\
4.5017 \bullet+00 \\
4.4903 e+00 \\
4.5002 e++00 \\
4.4999 e+00 \\
4.4118 e+00 \\
4.6279 e+00 \\
4.4046 e+00\end{array}$ \\
\hline
\end{tabular}




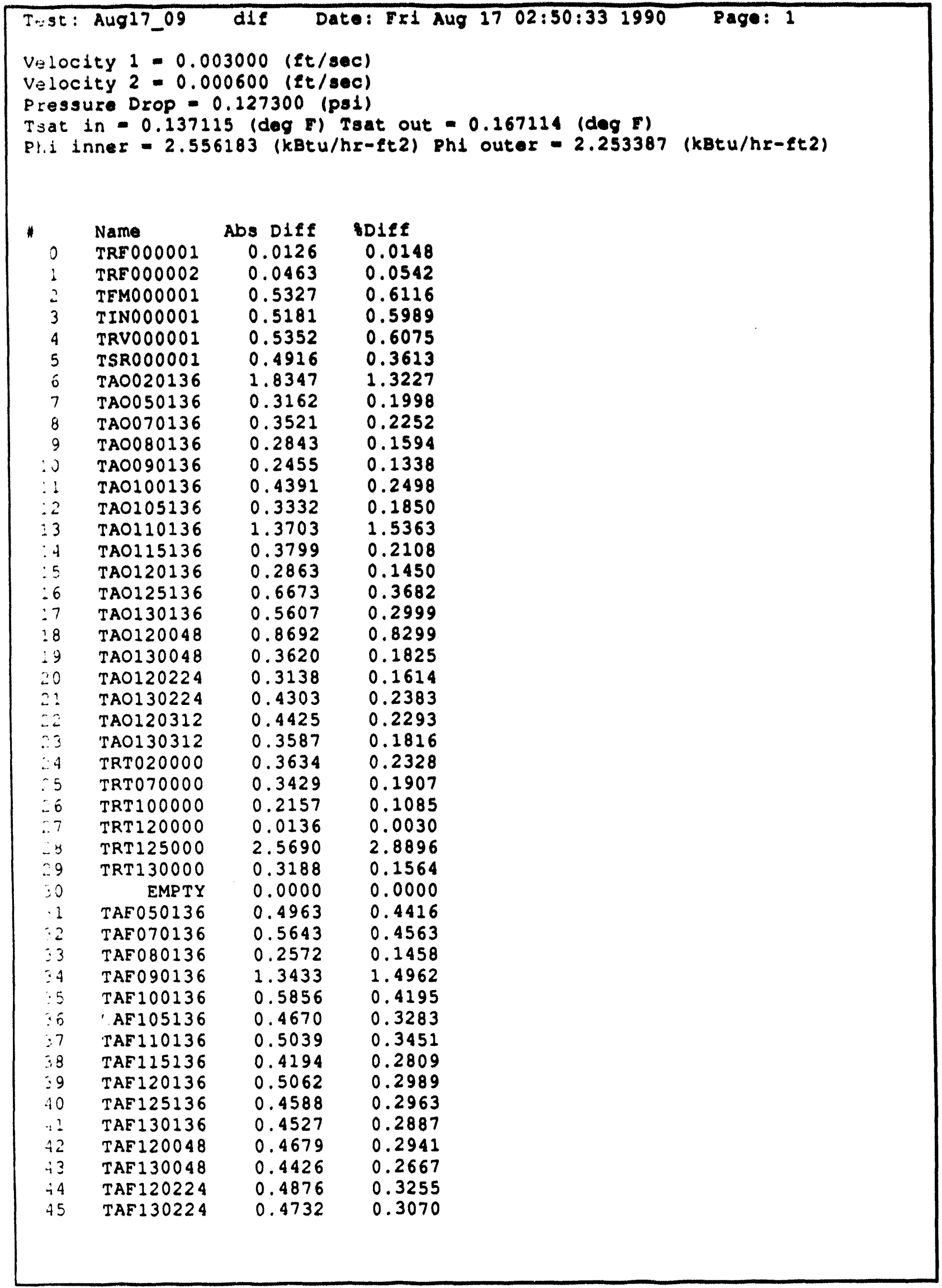




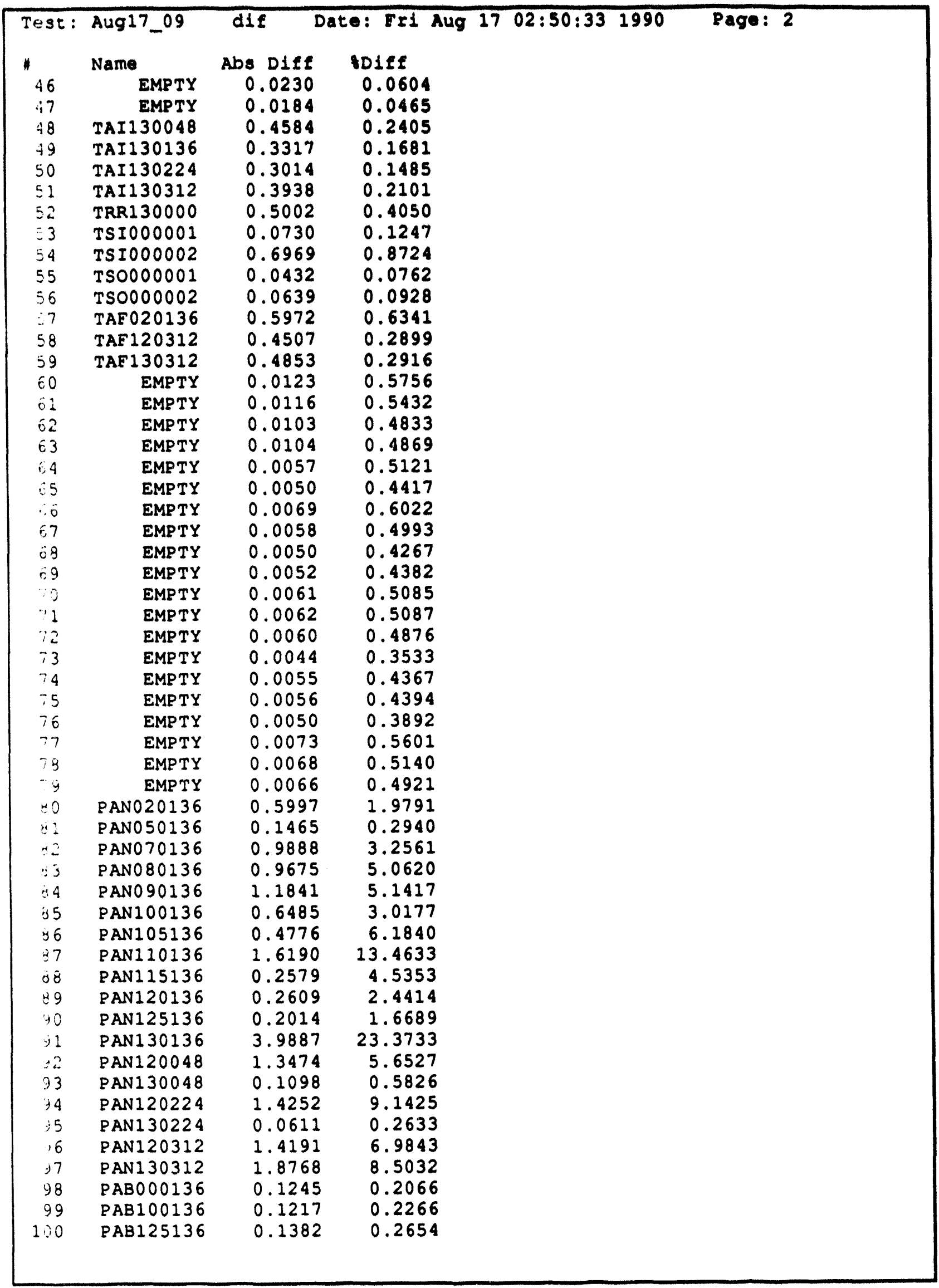




\begin{tabular}{|c|c|c|c|c|}
\hline Test : & Aug17_09 & dif & te: Fr1 Aug $1702: 50: 331990$ & Page: 3 \\
\hline$*$ & Name & Abs Diff & SDIfE & \\
\hline 101 & PINO00001 & 0.0342 & 0.0569 & \\
\hline 102 & PRVO00001 & 0.2393 & 1.1989 & \\
\hline 103 & PSR000001 & 0.0757 & 0.1527 & \\
\hline 104 & PFMO00001 & 0.0238 & 0.0383 & \\
\hline 105 & PANO00136 & 3.5247 & 1.4624 & \\
\hline $\begin{array}{l}106 \\
107\end{array}$ & $\begin{array}{r}\text { PFMO00002 } \\
\text { EMPTY }\end{array}$ & $\begin{array}{l}0.0000 \\
0.0001\end{array}$ & $\begin{array}{l}0.0000 \\
1.0309\end{array}$ & \\
\hline $1: 8$ & EMPTY & 2.6436 & 34.5045 & \\
\hline 1.9 & EMPTY & 0.0002 & 3.4483 & \\
\hline $1: 0$ & EMPTY & 0.0000 & 0.0000 & \\
\hline $1: 1$ & EMPTY & 0.0000 & 0.0000 & \\
\hline $1: 2$ & VPW000001 & 0.0391 & 0.0221 & \\
\hline $1: 3$ & VPW000002 & 0.0547 & 0.0264 & \\
\hline $1: 4$ & VPW000003 & 0.0625 & 0.0244 & \\
\hline 115 & VPW000004 & 0.0195 & 0.0107 & \\
\hline $1: 6$ & VPW0 00005 & 0.0312 & 0.0128 & \\
\hline $1: 7$ & VPW000006 & 0.0977 & 0.0379 & \\
\hline $1: 8$ & IPWO00001 & 16.0486 & 1.6936 & \\
\hline $1: 9$ & IPWO00002 & 47.2610 & 2.1144 & \\
\hline $1: 2$ & IPWO00003 & 22.1130 & 0.9771 & \\
\hline $1 \because:$ & IPWOOO0004 & 3.3983 & 0.5193 & \\
\hline $\begin{array}{l}1: 2 \\
1=3\end{array}$ & $\begin{array}{l}\text { IPW000005 } \\
\text { IPWO00006 }\end{array}$ & $\begin{array}{l}1.0155 \\
0.6738\end{array}$ & $\begin{array}{l}0.2329 \\
0.1499\end{array}$ & \\
\hline $\begin{array}{l}123 \\
1 \therefore 4\end{array}$ & EMPTY & 0.0010 & 5.2356 & \\
\hline $1: 5$ & EMPTY & 0.0001 & 0.7752 & \\
\hline $2 \therefore 6$ & EMPTY & 0.0001 & 0.6369 & \\
\hline 1.7 & EMPTY & 0.0009 & 5.5901 & \\
\hline $1 \div 3$ & IHTO00001 & 0.3008 & 0.1357 & \\
\hline $1=9$ & IHTO00002 & 0.2813 & 0.1233 & \\
\hline 130 & IHTO00003 & 0.3203 & 0.1391 & \\
\hline $1 \div 1$ & IHTO00004 & 0.3632 & 0.1606 & \\
\hline$\therefore \%$ & IHTO00005 & 0.3632 & 0.1623 & \\
\hline$: \because 3$ & IHTO00006 & 0.3750 & 0.1722 & \\
\hline$\therefore i$ & IHTO00007 & 0.3555 & 0.1564 & \\
\hline $1: \Sigma$ & IHTO00008 & 0.2929 & 0.1304 & \\
\hline $1: 0^{-}$ & IHTO00009 & 0.1641 & 0.0730 & \\
\hline $1: 7$ & IHTO00010 & 0.1523 & 0.2713 & \\
\hline i.s & IHTO00011 & 0.0938 & 0.0417 & \\
\hline $1: 3$ & IHTO00012 & 0.0938 & $0.0 / 224$ & \\
\hline $1 ; 0$ & IHTO00013 & 0.2227 & 0.1388 & \\
\hline $1+1$ & IHTOOOOO14 & 0.2735 & 0.1234 & \\
\hline $1+2$ & IHTO00015 & 0.2539 & 0.1534 & \\
\hline $1: 3$ & IHTO00016 & 0.2227 & 0.1445 & \\
\hline 1.14 & IHTO000017 & 0.2344 & 0.1269 & \\
\hline 1.5 & IHTO00018 & 0.0274 & 0.0147 & \\
\hline 1.6 & IHTO00019 & 0.0156 & 0.0086 & \\
\hline 2.7 & IHTO00020 & 0.0196 & 0.0105 & \\
\hline $1: 8$ & IHTO00021 & 0.0196 & 1.3198 & \\
\hline 1.99 & IHTO00022 & 0.0352 & 0.0220 & \\
\hline $1=0$ & IHTO00023 & 0.0313 & 0.0172 & \\
\hline $1: 2$ & IHTO00024 & 0.0274 & 0.0187 & \\
\hline $1=2$ & IHTO00025 & 0.0117 & 0.0065 & \\
\hline 153 & IHTO00026 & 0.0273 & 0.0149 & \\
\hline $2 \vdots 4$ & IHT 000027 & 0.0312 & 0.0199 & \\
\hline $1: 5$ & IHT000028 & 0.2226 & 0.1402 & \\
\hline
\end{tabular}




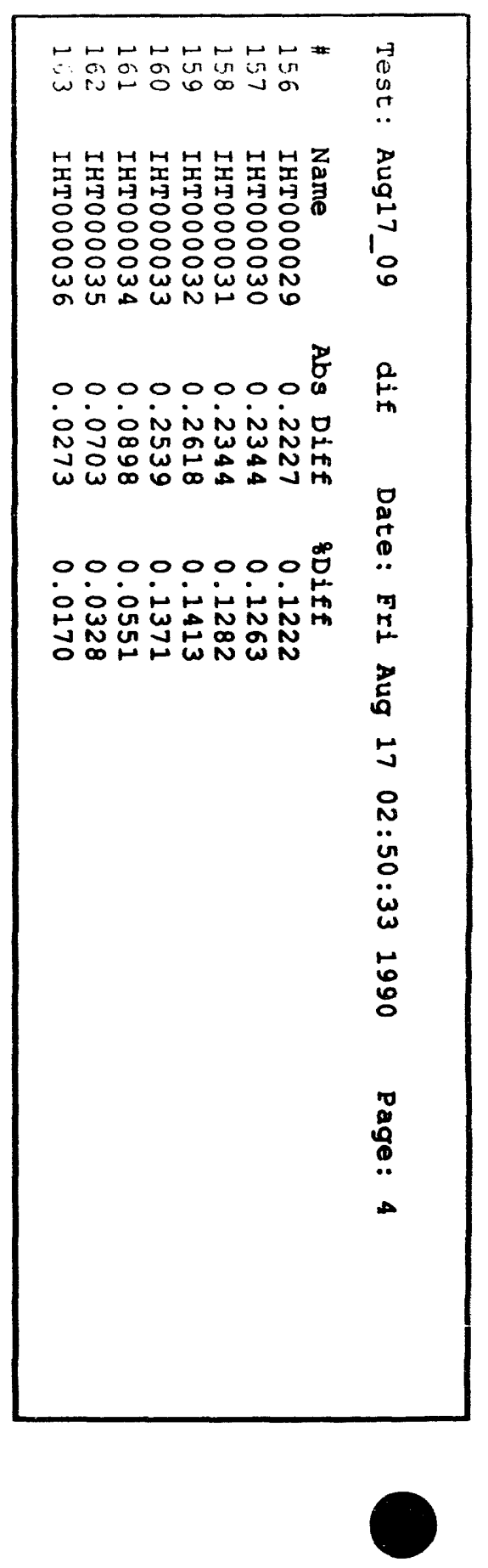




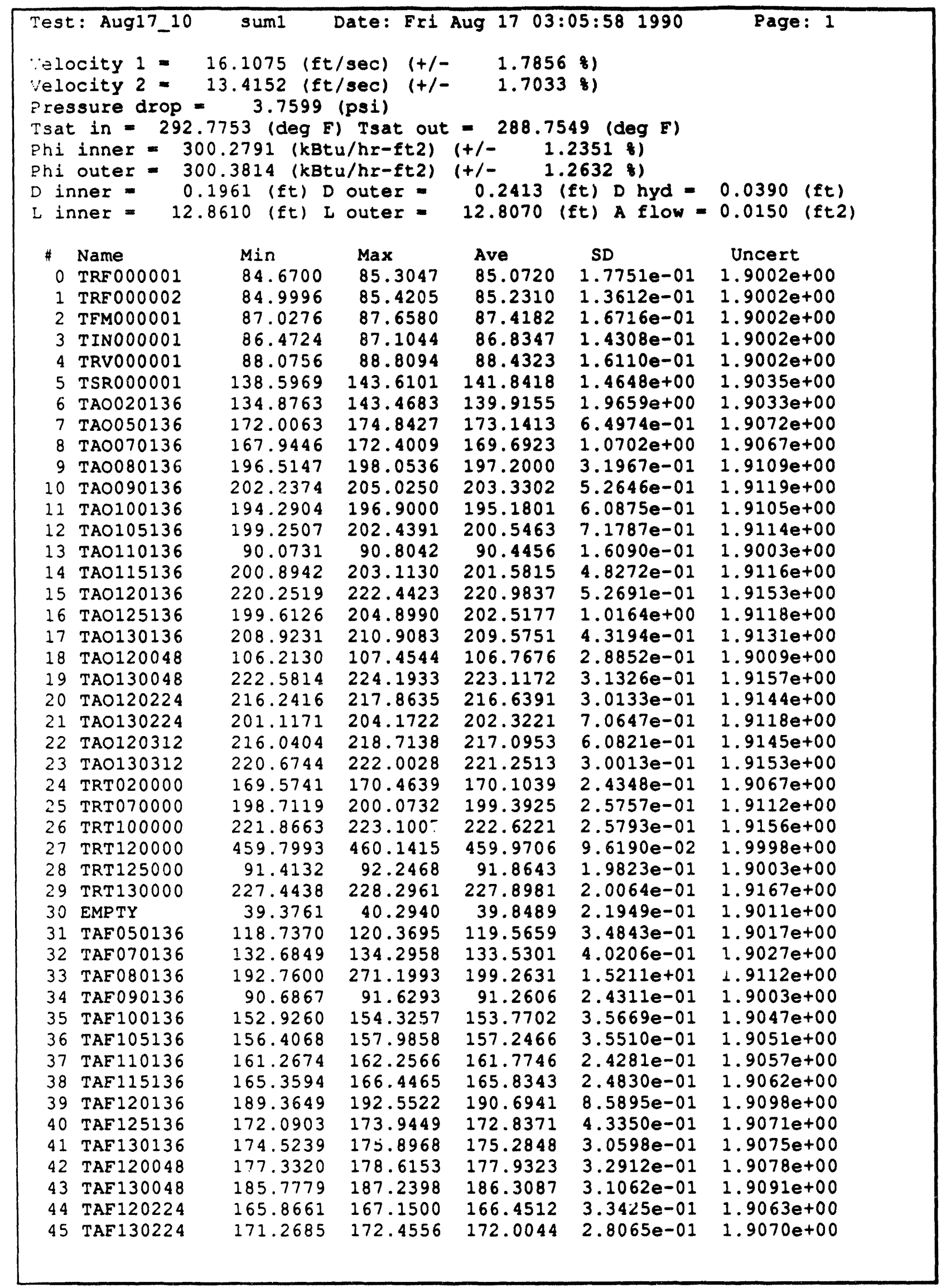




\begin{tabular}{|c|c|c|c|c|c|c|}
\hline \multicolumn{2}{|c|}{ Test: Aug17_10 } & sum 1 & Date: Eri & Aug 1703 & $05: 58 \quad 1990$ & Page: 2 \\
\hline$\#$ & Name & Min & $\operatorname{Max}$ & Ave & SD & Uncert \\
\hline 46 & EMPTY & 37.7675 & 38.6870 & 38.1262 & $1.9793 e-01$ & $1.9010 \mathrm{e}+00$ \\
\hline 47 & EMPTY & 39.1464 & 41.4403 & 39.6882 & $3.5223 e-01$ & $1.9011 e+00$ \\
\hline 48 & TAI 130048 & 214.5348 & 216.1599 & 215.3191 & $3.7620 e-01$ & $1.9142 e+00$ \\
\hline 49 & TAI 130136 & 221.6236 & 222.6693 & 222.0349 & $2.4592 e-01$ & $1.9155 e+00$ \\
\hline 50 & TAI 130224 & 226.7175 & 228.2380 & 227.4282 & $3.3104 e-01$ & $1.9166 e+00$ \\
\hline 51 & TAI 130312 & 210.8089 & 211.9595 & 211.3459 & $2.6435 e-01$ & $1.9134 e+00$ \\
\hline 52 & TRR130000 & 132.6326 & 136.5647 & 134.6464 & $1.2137 e+00$ & $1.9028 e+00$ \\
\hline 53 & TSI000001 & 58.7775 & 59.3156 & 59.0917 & $1.5326 e-01$ & $1.9001 e+00$ \\
\hline 54 & TSI000002 & 80.1487 & 82.3628 & 81.2669 & $6.3437 e-01$ & $1.9001 e+00$ \\
\hline 55 & TSO000001 & 56.3405 & 57.0979 & 56.8207 & $2.0054 e-01$ & $1.9001 e+00$ \\
\hline 56 & TSO000002 & 70.1373 & 71.2034 & 70.7173 & $2.2710 e-01$ & $1.9000 e+00$ \\
\hline 57 & TAF020136 & 96.0293 & 98.0215 & 97.0778 & $4.2843 e-01$ & $1.9005 e+00$ \\
\hline 58 & TAF 120312 & 172.7513 & 175.1113 & 173.8650 & $4.7512 e-01$ & $1.9073 e+00$ \\
\hline 59 & TAF 130312 & 185.9776 & 188.6018 & 186.9070 & $5.7770 e-01$ & $1.9092 e+00$ \\
\hline 60 & EMPTY & 2.6318 & 2.6514 & 2.6427 & $5.6917 e-03$ & $0.0000 e+00$ \\
\hline 61 & EMPTY & 2.6318 & 2.6416 & 2.6366 & $2.8744 e-03$ & $0.0000 e+00$ \\
\hline 62 & EMPTY & 2.6270 & 2.6367 & 2.6302 & $2.5362 e-03$ & $0.0000 e+00$ \\
\hline 63 & EMPTY & 2.6270 & 2.6367 & 2.6319 & $2.3114 e-03$ & $0.0000 e+00$ \\
\hline 64 & EMPTY & 1.3232 & 1.3330 & 1.3285 & $1.6624 \mathrm{e}-03$ & $0.0000 e+00$ \\
\hline 65 & EMPTY & 1.3428 & 1.3477 & 1.3466 & $2.0432 e-03$ & $0.0000 e+00$ \\
\hline 66 & EMPTY & 1.3525 & 1.3623 & 1.3578 & $1.6624 e-03$ & $0.0000 e+00$ \\
\hline 67 & EMPTY & 1.3672 & 1.3770 & 1.3727 & $1.8821 e-03$ & $0.0000 e+00$ \\
\hline 68 & EMPTY & 1.3818 & 1.3867 & 1.3824 & $1.6028 e-03$ & $0.0000 e+00$ \\
\hline 69 & EMPTY & 1.3965 & 1.4014 & 1.3966 & $6.9053 e-04$ & $0.0000 e+00$ \\
\hline 70 & EMPTY & 1.4063 & 1.4111 & 1.4070 & $1.8082 e-03$ & $0.0000 e+00$ \\
\hline 71 & EMPTY & 1.4209 & 1.4258 & 1.4257 & $6.9053 e-04$ & $0.0000 e+00$ \\
\hline 72 & EMPTY & 1.4355 & 1.4355 & 1.4355 & $0.0000 \mathrm{e}+00$ & $0.0000 e+00$ \\
\hline 73 & EMPTY & 1.4502 & 1.4551 & 1.4507 & $1.4797 e-03$ & $0.0000 e+00$ \\
\hline 74 & EMPTY & 1.4600 & 1.4648 & 1.4627 & $2.4484 e-03$ & $0.0000 e+00$ \\
\hline 75 & EMPTY & 1.4746 & 1.4795 & 1.4765 & $2.3941 e-03$ & $0.0000 e+00$ \\
\hline 76 & EMPTY & 1.4844 & 1.4893 & 1.4863 & $2.4164 e-03$ & $0.0000 e+00$ \\
\hline 77 & EMPTY & 1.4990 & 1.5088 & 1.5024 & $2.4662 e-03$ & $0.0000 e+00$ \\
\hline 78 & EMPTY & 1.5186 & 1.5234 & 1.5198 & $2.1635 e-03$ & $0.0000 e+00$ \\
\hline 79 & EMPTY & 1.5381 & 1.5430 & 1.5382 & $6.9053 e-04$ & $0.0000 e+00$ \\
\hline 80 & PAN020136 & 2.2977 & 28.5429 & 14.6100 & $6.0893 e+00$ & $8.7077 e-01$ \\
\hline 81 & PAN0 50136 & 11.1820 & 26.7460 & 19.7086 & $4.0839 e+00$ & $1.6807 e+00$ \\
\hline 82 & PAN070136 & 0.0649 & 21.3509 & 8.3702 & $5.2844 e+00$ & $8.7025 e-01$ \\
\hline 83 & PAN080136 & -5.0294 & 21.7498 & 7.4889 & $7.0095 e+00$ & $8.7020 e-01$ \\
\hline 84 & PAN090136 & -1.9601 & 27.8709 & 12.3847 & $7.3780 \mathrm{e}+00$ & $8.7055 e-01$ \\
\hline 85 & PAN 100136 & -3.9510 & 24.2015 & 9.8414 & $6.7310 \mathrm{e}+00$ & $8.7035 e-01$ \\
\hline 86 & PAN 105136 & -6.6339 & 18.6194 & 5.8005 & $5.9554 e+00$ & $8.7012 e-01$ \\
\hline 87 & PAN 110136 & -7.2222 & 18.8704 & 4.8094 & $6.9745 e+00$ & $8.7008 e-01$ \\
\hline 88 & PAN 115136 & -7.5597 & 12.8871 & 1.0463 & $4.4349 e+00$ & $8.7000 e-01$ \\
\hline 89 & PAN 120136 & -6.6720 & 18.6576 & 8.1489 & $5.2622 e+00$ & $8.7024 e-01$ \\
\hline 90 & PAN 125136 & -5.9560 & 16.0929 & 3.3976 & $5.3526 e+00$ & $8.7004 e-01$ \\
\hline 91 & PAN130136 & 2.2413 & 17.7290 & 9.4785 & $2.9785 e+00$ & $8.7032 e-01$ \\
\hline 92 & PAN 120048 & -2.4163 & 21.7689 & 8.9896 & $4.7941 \mathrm{e}+00$ & $8.7029 e-01$ \\
\hline 93 & PAN 130048 & 1.5495 & 16.5032 & 9.4033 & $2.7742 e+00$ & $8.7032 e-01$ \\
\hline 94 & PAN 120224 & -5.3463 & 14.4901 & 5.7376 & $5.1308 e+00$ & $8.7012 e-01$ \\
\hline 95 & PAN130224 & 5.0341 & 22.4292 & 13.0374 & $4.1486 \theta+00$ & $8.7061 e-01$ \\
\hline 96 & PAN 120312 & -1.3658 & 21.5224 & 11.6484 & $5.9165 \mathrm{e}+00$ & $8.7049 e-01$ \\
\hline 97 & PAN 130312 & -0.6044 & 20.7579 & 8.3068 & $4.9780 \mathrm{e}+00$ & $8.7025 e-01$ \\
\hline 98 & PAB000136 & 59.4999 & 60.6443 & 60.1509 & $2.8010 \mathrm{e}-01$ & $5.9918 e-01$ \\
\hline 99 & PAB 100136 & 56.7560 & 57.5342 & 57.3017 & $1.8470 \mathrm{e}-01$ & $5.9743 e-01$ \\
\hline 100 & PAB 125136 & 55.9725 & 56.7965 & 56.5758 & $1.4198 \mathrm{e}-01$ & $5.9700 e-01$ \\
\hline
\end{tabular}




\begin{tabular}{|c|c|c|c|c|c|c|}
\hline \multicolumn{2}{|c|}{ Test: Aug17_10 } & sum 1 & Date: Fri & ug 17 & $: 581990$ & Page: 3 \\
\hline \# & Name & Min & $\operatorname{Max}$ & Ave & SD & Uncert \\
\hline 101 & PINO000001 & 58.8796 & 60.7106 & 59.9319 & $5.0052 e-01$ & $7.6482 \mathrm{e}-01$ \\
\hline 102 & PRV000001 & 19.8406 & 19.9627 & 19.9334 & $5.2663 e-02$ & $7.5165 e-01$ \\
\hline 103 & PSR0000001 & 54.5812 & 54.9474 & 54.7862 & $7.9685 e-02$ & $7.6240 \mathrm{e}-01$ \\
\hline 104 & PFMO00001 & 39.3606 & 40.4592 & 39.8385 & $2.4419 e-01$ & $5.0982 e-01$ \\
\hline 105 & PANO00136 & 86.1039 & 122.7250 & 104.0941 & $9.0293 e+00$ & $4.9269 e+00$ \\
\hline 106 & PFM000002 & 27.5689 & 27.5689 & 27.5689 & $9.6336 \mathrm{e}-06$ & $2.1154 e-01$ \\
\hline 107 & EMPTY & -0.0049 & 0.0098 & 0.0084 & $2.7971 e-03$ & $0.0000 \mathrm{e}+00$ \\
\hline 108 & EMPTY & -10.0000 & -0.2588 & -4.5475 & $2.8882 \mathrm{e}+00$ & $0.0000 e+00$ \\
\hline 109 & EMPTY & 0.0049 & 0.0195 & 0.0052 & $2.0716 e-03$ & $0.0000 e+00$ \\
\hline 110 & EMPTY & -10.0000 & -10.0000 & -10.0000 & $0.0000 e+00$ & $0.0000 e+00$ \\
\hline 111 & EMPTY & 0.0000 & 0.0000 & 0.0000 & $0.0000 e+00$ & $0.0000 e+00$ \\
\hline 112 & VRW000001 & 177.0245 & 177.8057 & 177.4151 & $1.6741 e-01$ & $1.3376 \mathrm{e}+00$ \\
\hline 113 & VPWO00002 & 206.9374 & 207.5233 & 207.2382 & $1.2608 \mathrm{e}-01$ & $1.4840 e+00$ \\
\hline 114 & VPW000003 & 256.2162 & 256.6068 & 256.3880 & $1.0907 e-01$ & $1.7385 e+00$ \\
\hline 115 & VPW000004 & 182.0125 & 182.5984 & 182.3016 & $1.3229 e-01$ & $1.3611 e+00$ \\
\hline 116 & VPW000005 & 242.7772 & 243.1678 & 243.0273 & $1.1189 e-01$ & $1.6680 \mathrm{e}+00$ \\
\hline 117 & VPW000006 & 257.8313 & 258.4172 & 258.0772 & $1.4123 e-01$ & $1.7475 e+00$ \\
\hline 118 & IPWO00001 & 935.9235 & 943.7329 & 940.5310 & $1.4631 \mathrm{e}+00$ & $1.0992 e+01$ \\
\hline 119 & IPW0 00002 & 2229.1753 & 2248.6880 & 2237.3708 & $5.8737 e+00$ & $1.9443 e+01$ \\
\hline 120 & IPW000003 & 2260.0498 & 2267.8499 & 2265.1980 & $2.2202 e+00$ & $1.9643 e+01$ \\
\hline 121 & IPWO00004 & 648.2590 & 652.1653 & 649.5776 & $9.0547 e-01$ & $5.5076 e+00$ \\
\hline 122 & IPW000005 & 434.6958 & 435.6723 & 435.1644 & $3.5510 e-01$ & $3.9772 e+00$ \\
\hline 123 & IPW000006 & 450.7212 & 452.1860 & 451.5217 & $3.3829 e-01$ & $4.0895 \mathrm{e}+00$ \\
\hline 124 & EMPTY & -0.0293 & -0.0146 & -0.0195 & $3.8206 e-03$ & $0.0000 e+00$ \\
\hline 125 & EMPTY & -0.0195 & $-0: 0049$ & -0.0130 & $3.2133 e-03$ & $0.0000 e+00$ \\
\hline 126 & EMPTY & -0.0195 & -0.0098 & -0.0153 & $3.5582 e-03$ & $0.0000 \mathrm{e}+00$ \\
\hline 127 & EMPTY & -0.0244 & -0.0098 & -0.0150 & $3.2479 e-03$ & $0.0000 e+00$ \\
\hline 128 & IHT000001 & 219.7341 & 222.8591 & 221.2497 & $7.4718 e-01$ & $4.6589 e+00$ \\
\hline 129 & IHTO00002 & 226.3221 & 228.8612 & 227.7206 & $6.5478 e-01$ & $4.6900 e+00$ \\
\hline 130 & IHT 000003 & 228.4030 & 230.9420 & 229.6920 & $6.7543 e-01$ & $4.6996 e+00$ \\
\hline 131 & IHTO00004 & 224.1549 & 226.6940 & 225.5807 & $6.2046 e-01$ & $4.6796 e+00$ \\
\hline 132 & IHTO000005 & 221.8449 & 224.5793 & 223.1261 & $6.1783 e-01$ & $4.6678 e+00$ \\
\hline 133 & IHT000006 & 215.7940 & 218.5284 & 217.1260 & $6.5991 \mathrm{e}-01$ & $4.6394 e+00$ \\
\hline 134 & IHT 000007 & 224.9399 & 227.8696 & 226.6039 & $6.5226 e-01$ & $4.6845 e+00$ \\
\hline 135 & IHT000008 & 222.3483 & 225.4733 & 224.0514 & $7.1359 e-01$ & $4.6722 e+00$ \\
\hline 136 & IHT000009 & 222.7276 & 225.4620 & 224.2080 & $6.6038 e-01$ & $4.6730 \mathrm{e}+00$ \\
\hline 137 & IHTO00010 & 54.4283 & 57.1627 & 55.9635 & $6.3505 e-01$ & $4.1380 e+00$ \\
\hline 138 & IHTO00011 & 223.2760 & 226.0103 & 224.9010 & $6.5011 \mathrm{e}-01$ & $4.6763 e+00$ \\
\hline 139 & IHTO00012 & 219.7191 & 222.0628 & 220.9417 & $5.9818 e-01$ & $4.6574 e+00$ \\
\hline 140 & IHTO00013 & 158.9844 & 161.3281 & 160.1094 & $5.5742 e-01$ & $4.4015 e+00$ \\
\hline 141 & IHTO00014 & 220.1397 & 222.4835 & 221.2647 & $4.9215 e-01$ & $4.6589 e+00$ \\
\hline 142 & IHTO00015 & 164.3479 & 166.3011 & 165.1801 & $5.2171 \mathrm{e}-01$ & $4.4202 e+00$ \\
\hline 143 & IHT000016 & 152.7794 & 155.3185 & 153.9317 & $5.7622 e-01$ & $4.3794 \mathrm{e}+00$ \\
\hline 144 & IHT000017 & 183.1956 & 186.1253 & 184.5589 & $7.2918 e-01$ & $4.4962 e+00$ \\
\hline 145 & IHTO00018 & 185.2840 & 188.0183 & 186.5028 & $6.6563 e-01$ & $4.5043 e+00$ \\
\hline 146 & IHTO00019 & 179.4546 & 181.7984 & 180.6304 & $5.7655 e-01$ & $4.4803 e+00$ \\
\hline 147 & IHT000020 & 184.7130 & 186.8615 & 185.8380 & $4.9056 \mathrm{e}-01$ & $4.5015 e+00$ \\
\hline 148 & IHT000021 & -2.6179 & 0.1164 & -1.2547 & $6.3195 e-01$ & $4.1000 e+00$ \\
\hline 149 & IHTO00022 & 159.4614 & 161.4145 & 160.4341 & $4.8083 e-01$ & $4.4027 e+00$ \\
\hline 150 & IHTO00023 & 180.9270 & 182.8801 & 181.9034 & $5.1750 e-01$ & $4.4854 \mathrm{e}+00$ \\
\hline 151 & IHTO00024 & 145.1585 & 147.3070 & 146.3578 & $4.9128 \mathrm{e}-01$ & $4.3534 \mathrm{e}+00$ \\
\hline 152 & IHT000025 & 180.3298 & 182.2829 & 181.2516 & $5.2056 e-01$ & $4.4828 e+00$ \\
\hline 153 & IHT000026 & 182.6698 & 184.8182 & 183.5019 & $5.2765 e-01$ & $4.4919 e+00$ \\
\hline 154 & IHT 000027 & 156.0096 & 158.3534 & 156.9667 & $5.7487 e-01$ & $4.3902 e+00$ \\
\hline 155 & IHT000028 & 157.1552 & 160.2802 & 158.7724 & $7.1250 e-01$ & $4.3967 e+00$ \\
\hline
\end{tabular}




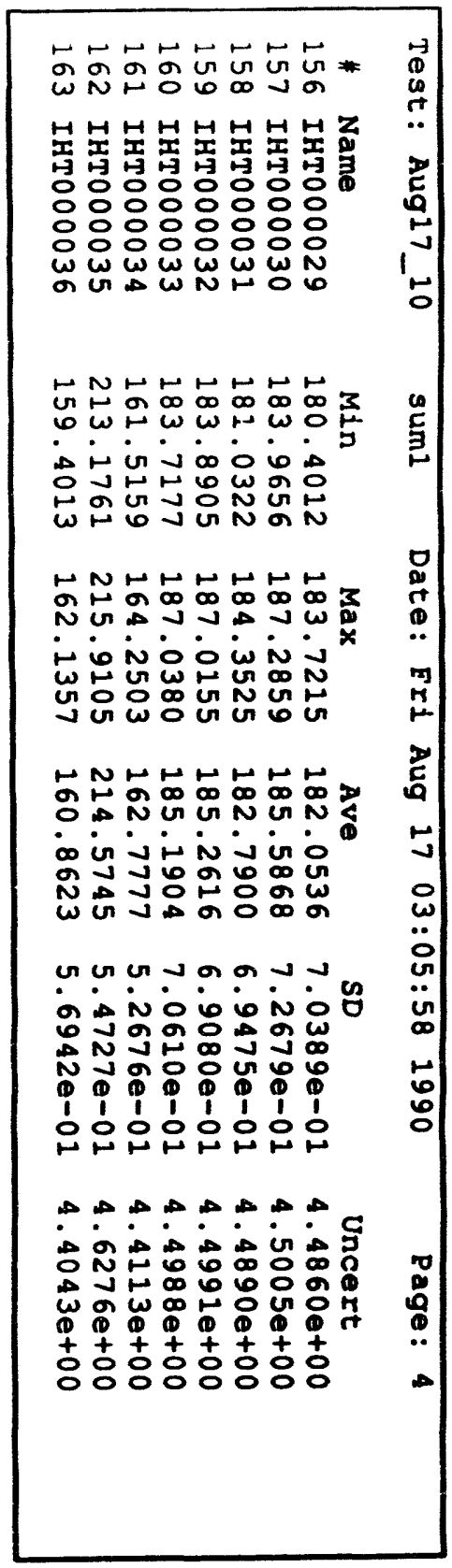




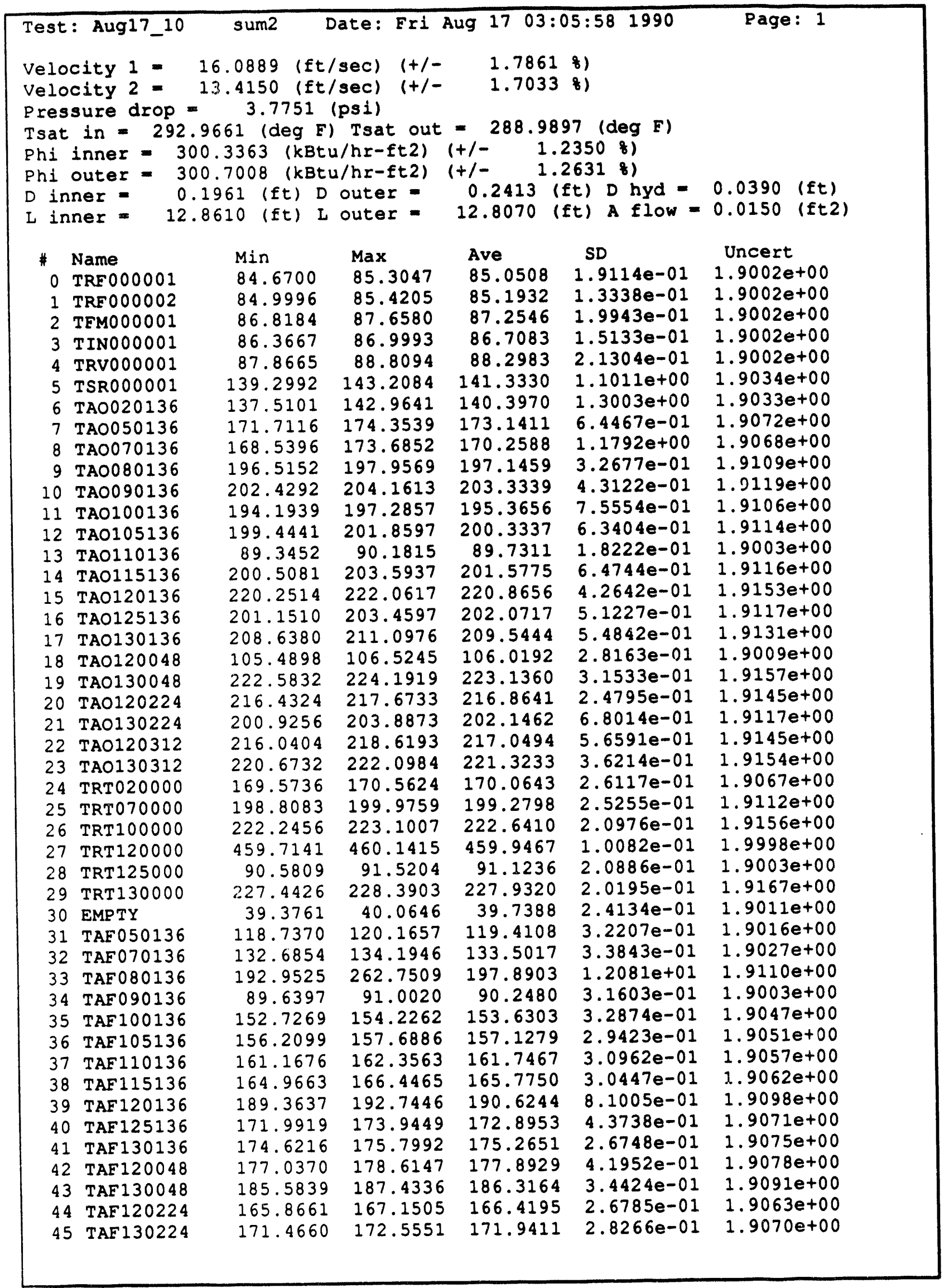




\begin{tabular}{|c|c|c|c|c|c|c|}
\hline \multicolumn{2}{|c|}{ Test: Aug17_10 } & sum2 & Date: Fri & \multicolumn{2}{|c|}{ Aug $17 \quad 03: 05: 58 \quad 1990$} & Page: 2 \\
\hline$\#$ & Name & Min & $\operatorname{Max}$ & Ave & SD & Uncert \\
\hline 46 & EMPTY & 37.5374 & 38.6870 & 38.1307 & $2.3266 \theta-01$ & $1.9010 e+00$ \\
\hline 47 & EMPTY & 39.3761 & 40.9819 & 39.6883 & $2.8858 e-01$ & $1.9011 e+00$ \\
\hline 48 & TAI 130048 & 214.6309 & 216.3508 & 215.3152 & $4.2871 e-01$ & $1.9142 \mathrm{e}+00$ \\
\hline 49 & TAI130136 & 221.6241 & 222.5737 & 222.0575 & $2.4127 e-01$ & $1.9155 e+00$ \\
\hline 50 & TAI 130224 & 226.9070 & 228.2380 & 227.5042 & $3.3750 e-01$ & $1.9166 \mathrm{e}+00$ \\
\hline 51 & TAI130312 & 210.9044 & 212.0555 & 211.4379 & $2.7619 e-01$ & $1.9134 \mathrm{e}+00$ \\
\hline 52 & TRR 130000 & 134.3472 & 137.4712 & 136.2261 & $7.8456 e-01$ & $1.9030 e+00$ \\
\hline 53 & TSI000001 & 58.4549 & 59.1012 & 58.7956 & $1.8607 e-01$ & $1.9001 \mathrm{e}+00$ \\
\hline 54 & TSI000002 & 80.1492 & 82.7837 & 81.5870 & $5.8481 e-01$ & $1.9001 e+00$ \\
\hline 55 & TSO000001 & 56.3405 & 57.2057 & 56.8382 & $1.9917 \mathrm{e}-01$ & $1.9001 e+00$ \\
\hline 56 & TSO000002 & 70.2434 & 71.2034 & 70.6959 & $2.0152 e-01$ & $1.9000 e+00$ \\
\hline 57 & TAF 020136 & 96.2379 & 97.9160 & 96.9562 & $3.7081 e-01$ & $1.9005 e+00$ \\
\hline 58 & TAF 120312 & 172.7508 & 175.0140 & 173.7823 & $5.1855 e-01$ & $1.9072 e+00$ \\
\hline 59 & TAF 130312 & 185.9781 & 188.7955 & 186.9729 & $7.0749 e-01$ & $1.9092 e+00$ \\
\hline 60 & EMPTY & 2.6367 & 2.6611 & 2.6449 & $7.9189 e-03$ & $0.0000 e+00$ \\
\hline 61 & EMPTY & 2.6270 & 2.6514 & 2.6379 & $5.6290 e-03$ & $0.0000 e+00$ \\
\hline 62 & EMPTY & 2.6221 & 2.6416 & 2.6320 & $4.2954 e-03$ & $0.0000 e+00$ \\
\hline 63 & EMPTY & 2.6270 & 2.6367 & 2.6327 & $3.7809 e-03$ & $0.0000 e+00$ \\
\hline 64 & EMPTY & 1.3232 & 1.3330 & 1.3277 & $2.7621 e-03$ & $0.0000 e+00$ \\
\hline 65 & EMPTY & 1.3428 & 1.3525 & 1.3454 & $2.6488 e-03$ & $0.0000 \mathrm{e}+00$ \\
\hline 66 & EMPTY & 1.3525 & 1.3672 & 1.3582 & $3.1752 e-03$ & $0.0000 \mathrm{e}+00$ \\
\hline 67 & EMPTY & 1.3672 & 1.3770 & 1.3723 & $1.9631 e-03$ & $0.0000 e+00$ \\
\hline 68 & EMPTY & 1.3770 & 1.3867 & 1.3818 & $1.9730 \mathrm{e}-03$ & $0.0000 e+00$ \\
\hline 69 & EMPTY & 1.3916 & 1.4014 & 1.3955 & $2.2058 e-03$ & $0.0000 e+00$ \\
\hline 70 & EMPTY & 1.4014 & 1.4111 & 1.4066 & $2.1702 e-03$ & $0.0000 \mathrm{e}+00$ \\
\hline 71 & EMPTY & 1.4209 & 1.4307 & 1.4249 & $2.1364 e-03$ & $0.0000 e+00$ \\
\hline 72 & EMPTY & 1.4307 & 1.4355 & 1.4350 & $1.6028 e-03$ & $0.0000 \mathrm{e}+00$ \\
\hline 73 & EMPTY & 1.4453 & 1.4551 & 1.4503 & $1.2041 e-03$ & $0.0000 e+00$ \\
\hline 74 & EMPTY & 1.4600 & 1.4648 & 1.4621 & $2.4484 e-03$ & $0.0000 e+00$ \\
\hline 75 & EMPTY & 1.4746 & 1.4795 & 1.4756 & $1.9730 e-03$ & $0.0000 e+00$ \\
\hline 76 & EMPTY & 1.4844 & 1.4941 & 1.4853 & $2.3531 e-03$ & $0.0000 \mathrm{e}+00$ \\
\hline 77 & EMPTY & 1.4990 & 1.5039 & 1.5009 & $2.3941 e-03$ & $0.0000 \mathrm{e}+00$ \\
\hline 78 & EMPTY & 1.5186 & 1.5234 & 1.5190 & $1.4797 e-03$ & $0.0000 e+00$ \\
\hline 79 & EMPTY & 1.5332 & 1.5381 & 1.5380 & $6.9053 e-04$ & $0.0000 e+00$ \\
\hline 80 & PAN020136 & 3.0607 & 27.7799 & 14.8389 & $6.5543 e+00$ & $8.7079 e-01$ \\
\hline 81 & PAN050136 & 13.4708 & 27.3563 & 20.3983 & $3.6098 \mathrm{e}+00$ & $1.6808 e+00$ \\
\hline 82 & PAN070136 & -1.6136 & 21.1220 & 7.9933 & $5.7738 e+00$ & $8.7023 e-01$ \\
\hline 83 & PAN080136 & -4.8005 & 17.7062 & 7.1502 & $5.6212 \mathrm{e}+00$ & $8.7018 e-01$ \\
\hline 84 & PAN090136 & -3.7911 & 28.4049 & 13.1187 & $8.0792 e+00$ & $8.7062 e-01$ \\
\hline 85 & PAN100136 & -7.1553 & 22.2178 & 9.0937 & $7.4231 e+00$ & $8.7030 e-01$ \\
\hline 86 & PAN105136 & -7.7020 & 22.1371 & 3.6582 & $6.4029 e+00$ & $8.7005 e-01$ \\
\hline 87 & PAN 110136 & -7.1459 & 18.5652 & 5.2946 & $5.7901 e+00$ & $8.7010 e-01$ \\
\hline 88 & PAN115136 & -7.4071 & 10.5983 & 1.7299 & $4.2729 e+00$ & $8.7001 e-01$ \\
\hline 89 & PAN120136 & -6.6720 & 19.1154 & 7.1678 & $5.7358 e+00$ & $8.7018 e-01$ \\
\hline 90 & PAN 125136 & -6.0323 & 11.4390 & 3.1993 & $5.4946 e+00$ & $8.7004 e-01$ \\
\hline 91 & PAN130136 & 1.6309 & 20.6281 & 8.9415 & $3.9152 \mathrm{e}+00$ & $8.7029 e-01$ \\
\hline 92 & PAN120048 & -2.4926 & 21.6926 & 11.9458 & $5.4911 e+00$ & $8.7051 e-01$ \\
\hline 93 & PAN 130048 & 3.6858 & 20.3179 & 9.1851 & $3.5529 e+00$ & $8.7030 e-01$ \\
\hline 94 & PAN 120224 & -9.0848 & 17.3892 & 4.1629 & $6.7260 \mathrm{e}+00$ & $8.7006 e-01$ \\
\hline 95 & PAN130224 & 6.7126 & 24.6417 & 12.8375 & $4.2014 \mathrm{e}+00$ & $8.7059 e-01$ \\
\hline 96 & PAN120312 & -0.4502 & 21.9039 & 10.1332 & $6.0047 e+00$ & $8.7037 e-01$ \\
\hline 97 & PAN 130312 & 1.7607 & 21.9786 & 9.3047 & $4.6010 \mathrm{e}+00$ & $8.7031 e-01$ \\
\hline 98 & РАB000136 & 59.7746 & 60.8732 & 60.3239 & $2.6305 e-01$ & $5.9929 e-01$ \\
\hline 99 & PAB100136 & 56.9391 & 57.6715 & 57.3969 & $2.0742 e-01$ & $5.9749 e-01$ \\
\hline 100 & PAB125136 & 56.3845 & 57.0711 & 56.7809 & $1.4611 \mathrm{e}-01$ & $5.9712 e-01$ \\
\hline
\end{tabular}




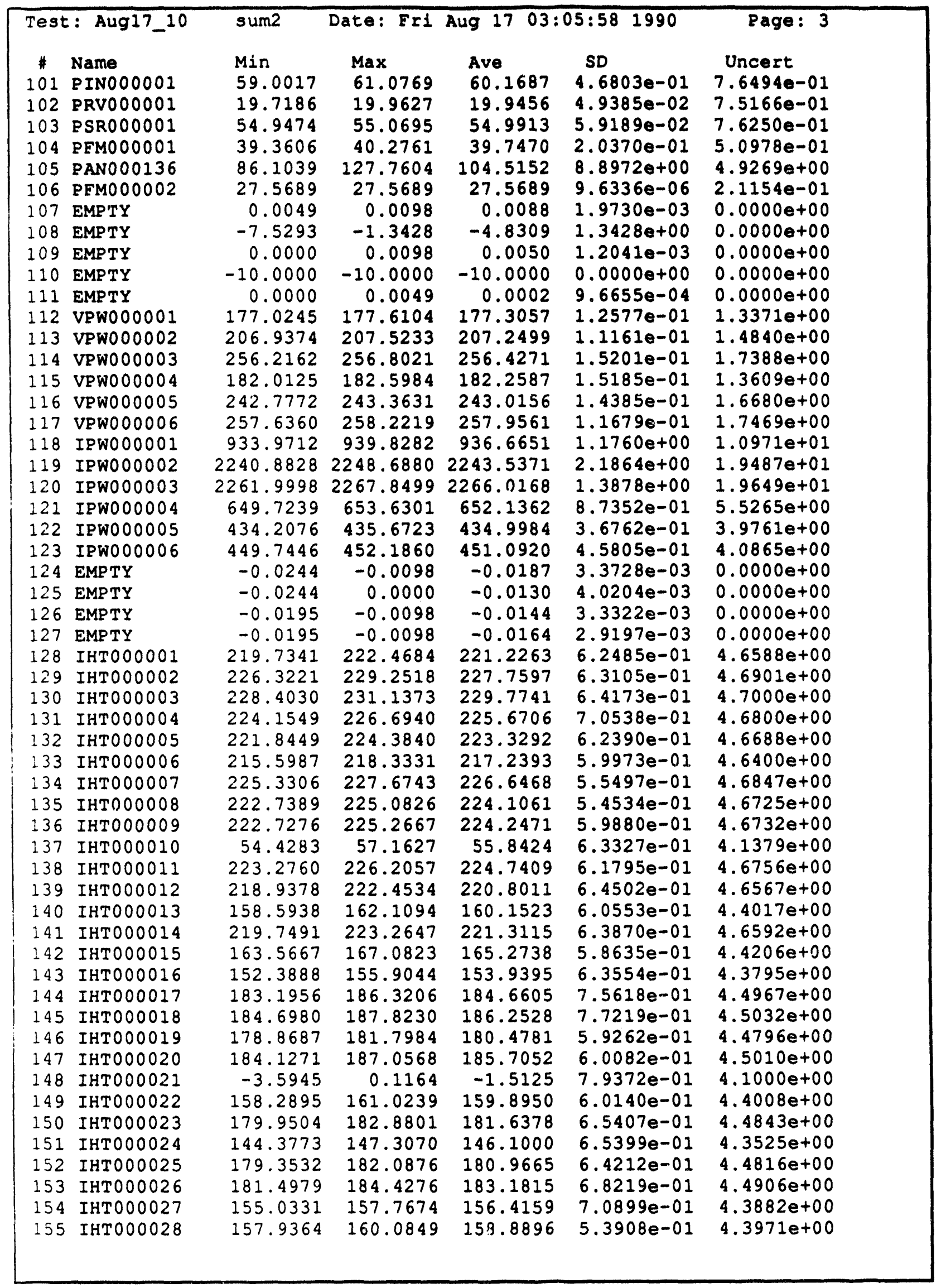




\begin{tabular}{|c|c|c|c|c|c|}
\hline Test: Aug17_10 & sum2 & Date: Fri & Aug 170 & 581990 & Page: 4 \\
\hline 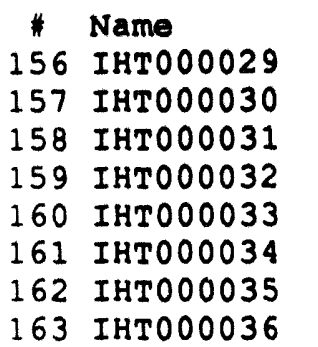 & $\begin{array}{l}\text { Min } \\
181.3777 \\
184.7469 \\
182.0087 \\
184.4764 \\
184.3036 \\
162.1019 \\
213.7620 \\
159.9872\end{array}$ & $\begin{array}{l}\operatorname{Max} \\
183.9168 \\
187.2859 \\
184.5478 \\
186.6248 \\
186.4521 \\
163.6644 \\
215.5199 \\
161.9403\end{array}$ & $\begin{array}{l}\text { Ave } \\
182.3075 \\
185.8016 \\
182.9658 \\
185.3593 \\
185.2764 \\
162.7308 \\
214.4612 \\
160.8662\end{array}$ & $\begin{array}{l}S D \\
5.4756 e-01 \\
4.9756 e-01 \\
5.4283 e-01 \\
5.7486 e-01 \\
5.6288 \theta-01 \\
4.5556 e-01 \\
4.6704 \theta-01 \\
4.4337 e-01\end{array}$ & $\begin{array}{c}\text { Uncert } \\
4.4870 e+00 \\
4.5014 e+00 \\
4.4897 e+00 \\
4.4995 e+00 \\
4.4992 e+00 \\
4.4111 e+00 \\
4.6270 e+00 \\
4.4043 e+00\end{array}$ \\
\hline
\end{tabular}




\begin{tabular}{|c|c|c|c|}
\hline \multicolumn{4}{|c|}{$\begin{array}{l}\because \text { locity } 1=0.018600(\mathrm{ft} / \mathrm{sec}) \\
\because \text { locity } 2=0.000200(\mathrm{ft} / \mathrm{sec}) \\
\because \text { assure Drop }=0.015200 \text { (psi) } \\
\text { it in }=0.190796 \text { (deg F) Tsat out }=0.234772 \text { (deg F) } \\
\vdots \text { inner }=0.057190 \text { (kBtu/hr-ft2) Ph1 outer }=0.319397 \quad(\mathrm{kBtu} / \mathrm{hr}-\mathrm{ft} 2)\end{array}$} \\
\hline & Name & Abs Diff & ODIfE \\
\hline $\begin{array}{l}\text { 1) } \\
1\end{array}$ & TRE000001 & 0.0212 & 0.0249 \\
\hline$\frac{1}{2}$ & TRF000002 & 0.0378 & 0.0444 \\
\hline $\begin{array}{l}2 \\
3\end{array}$ & TFM000001 & 0.1636 & 0.1871 \\
\hline 3 & TINO00001 & 0.1264 & 0.1456 \\
\hline $\begin{array}{l}4 \\
5\end{array}$ & TRV000001 & 0.1340 & 0.1515 \\
\hline 5 & TSR000001 & 0.5088 & 0.3587 \\
\hline$\therefore$ & TAO020136 & 0.4815 & 0.3441 \\
\hline$\because$ & TA0050136 & 0.0002 & 0.0001 \\
\hline 5 & TA0070136 & 0.5665 & 0.3338 \\
\hline 9 & TA0080136 & 0.0541 & 0.0274 \\
\hline$: 0$ & TAO090136 & 0.0037 & 0.0018 \\
\hline$i$ & TA0100136 & 0.1855 & 0.0950 \\
\hline$\therefore$ & TA0105136 & 0.2126 & 0.1060 \\
\hline$: 3$ & TAO110136 & 0.7145 & 0.7900 \\
\hline$: 4$ & TA0115136 & 0.0040 & 0.0020 \\
\hline$: 5$ & TA0120136 & 0.1181 & 0.0534 \\
\hline$: \overline{0}$ & TA0125136 & 0.4460 & 0.2202 \\
\hline .7 & TA0130136 & 0.0307 & 0.0146 \\
\hline$: 9$ & TAO120048 & 0.7484 & 0.7010 \\
\hline$: 3$ & TAO130048 & 0.0188 & 0.0084 \\
\hline$\therefore$ & TAO120224 & 0.2250 & 0.1039 \\
\hline : & TAO130224 & 0.1759 & 0.0869 \\
\hline$\therefore$ & TAO120312 & 0.0459 & 0.0211 \\
\hline : & TA0130312 & 0.0720 & 0.0325 \\
\hline$\therefore i$ & TRT020000 & 0.0396 & 0.0233 \\
\hline & TRT070000 & 0.1127 & 0.0565 \\
\hline$\therefore$ & TRT 100000 & 0.0189 & 0.0085 \\
\hline$\therefore 7$ & TRT120000 & 0.0239 & 0.0052 \\
\hline 28 & TRT 125000 & 0.7407 & 0.8063 \\
\hline$\therefore 9$ & TRT 130000 & 0.0339 & 0.0149 \\
\hline$\therefore$ & EMPTY & 0.1101 & 0.2763 \\
\hline$\therefore$ & TAF050136 & 0.1551 & 0.1297 \\
\hline$\therefore 2$ & TAF070136 & 0.0284 & 0.0213 \\
\hline$\because$ & TAF080136 & 1.3728 & 0.6889 \\
\hline$\therefore i$ & TAF090136 & 1.0126 & 1.1096 \\
\hline$\equiv$ & TAF 100136 & 0.1399 & 0.0910 \\
\hline 6 & TAE 105136 & 0.1187 & 0.0755 \\
\hline & TAE 110136 & 0.0279 & 0.0172 \\
\hline " & TAF 115136 & 0.0593 & 0.0358 \\
\hline$\therefore y$ & TAF 120136 & 0.0697 & 0.0366 \\
\hline 10 & TAF 125136 & 0.0582 & 0.0337 \\
\hline$i:$ & TAE 130136 & 0.0197 & 0.0112 \\
\hline$\therefore 2$ & TAF 120048 & 0.0394 & 0.0221 \\
\hline ij & TAE 130048 & 0.0077 & 0.0041 \\
\hline$\therefore$ & TAF 120224 & 0.0317 & 0.0190 \\
\hline$\vdots$ & TAF 130224 & 0.0633 & 0.0368 \\
\hline
\end{tabular}




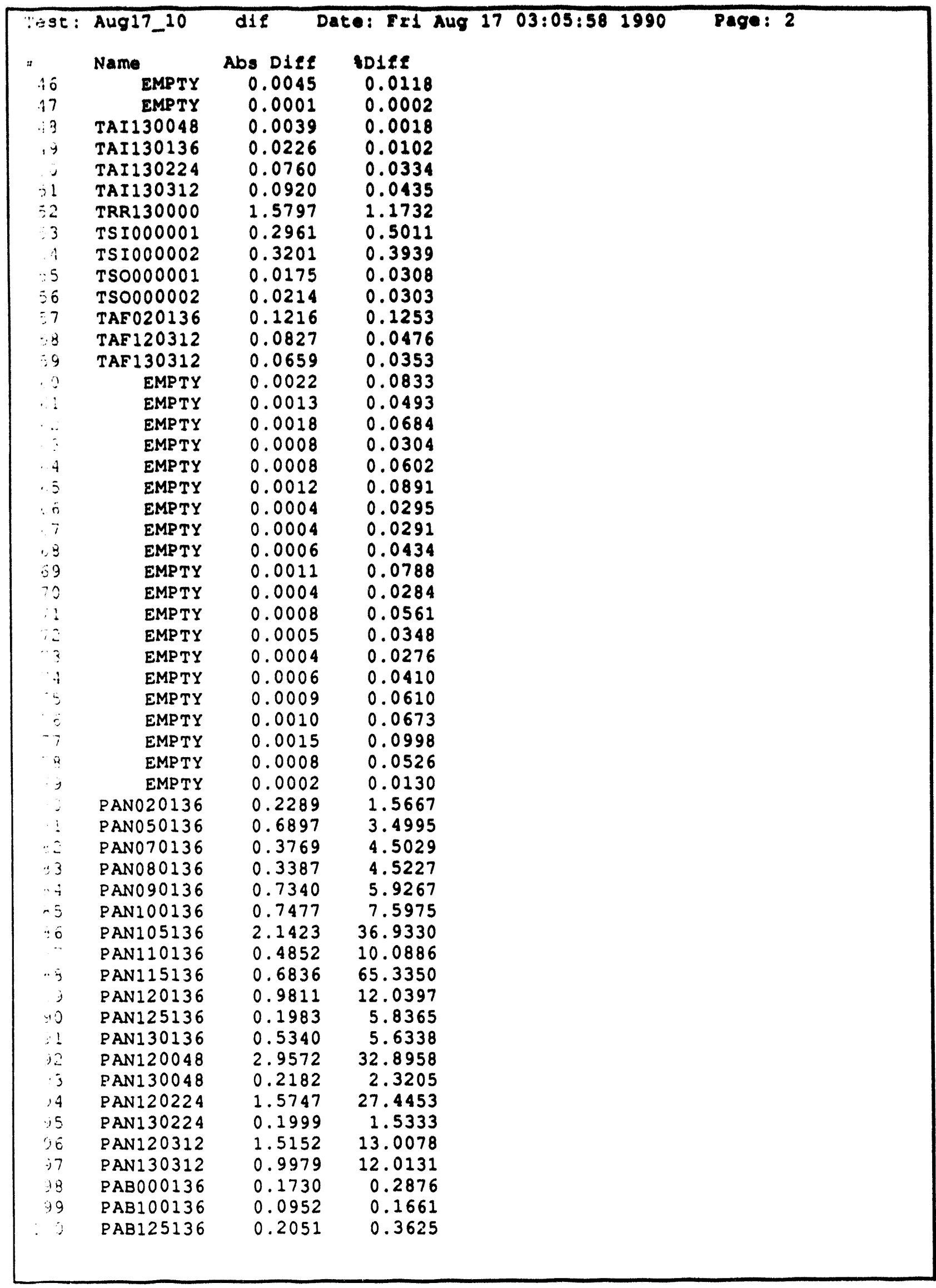




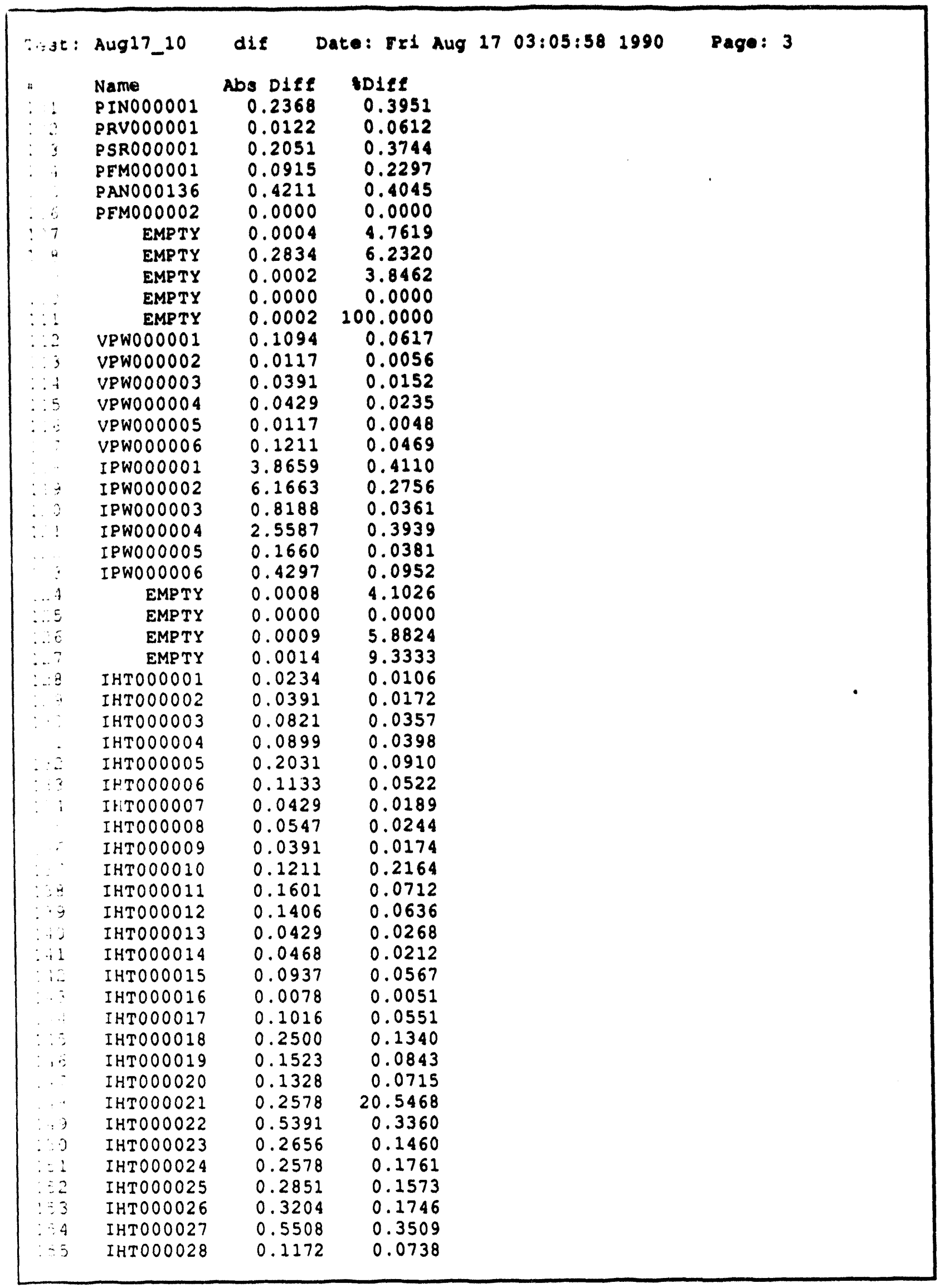




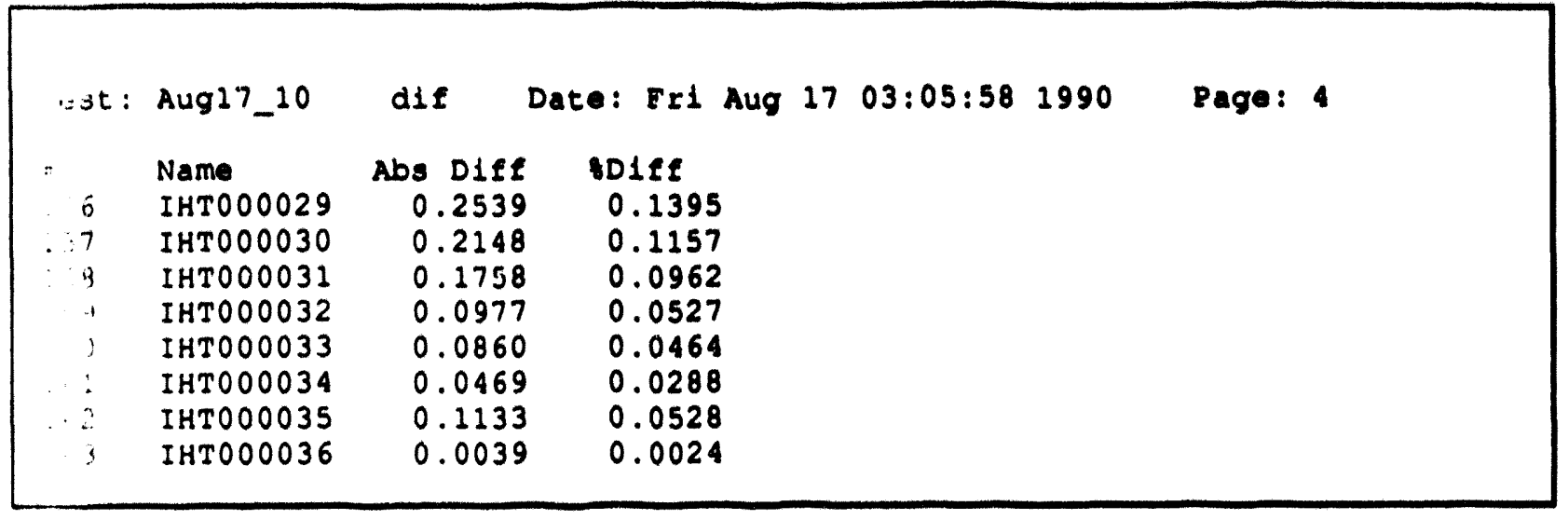




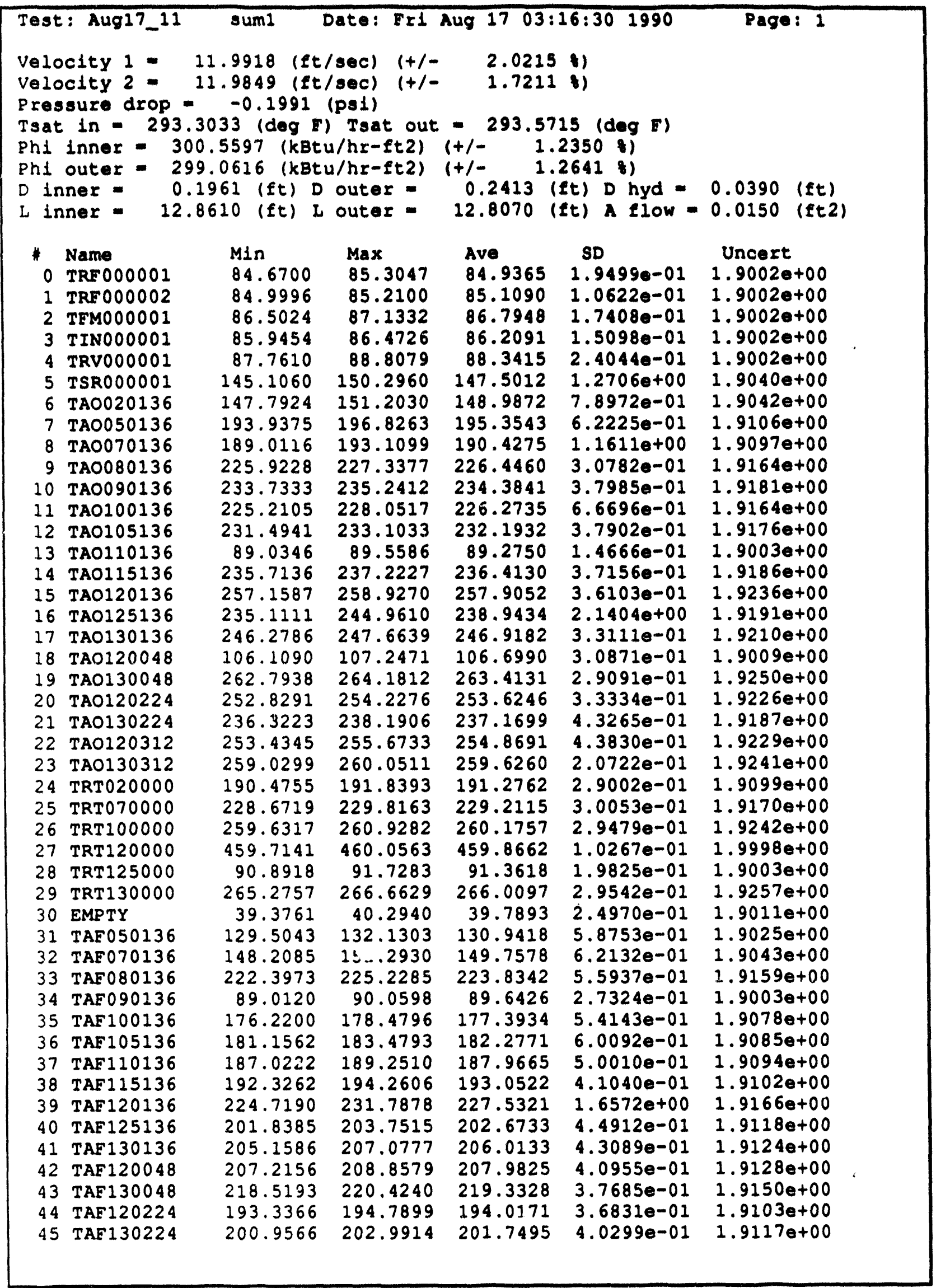




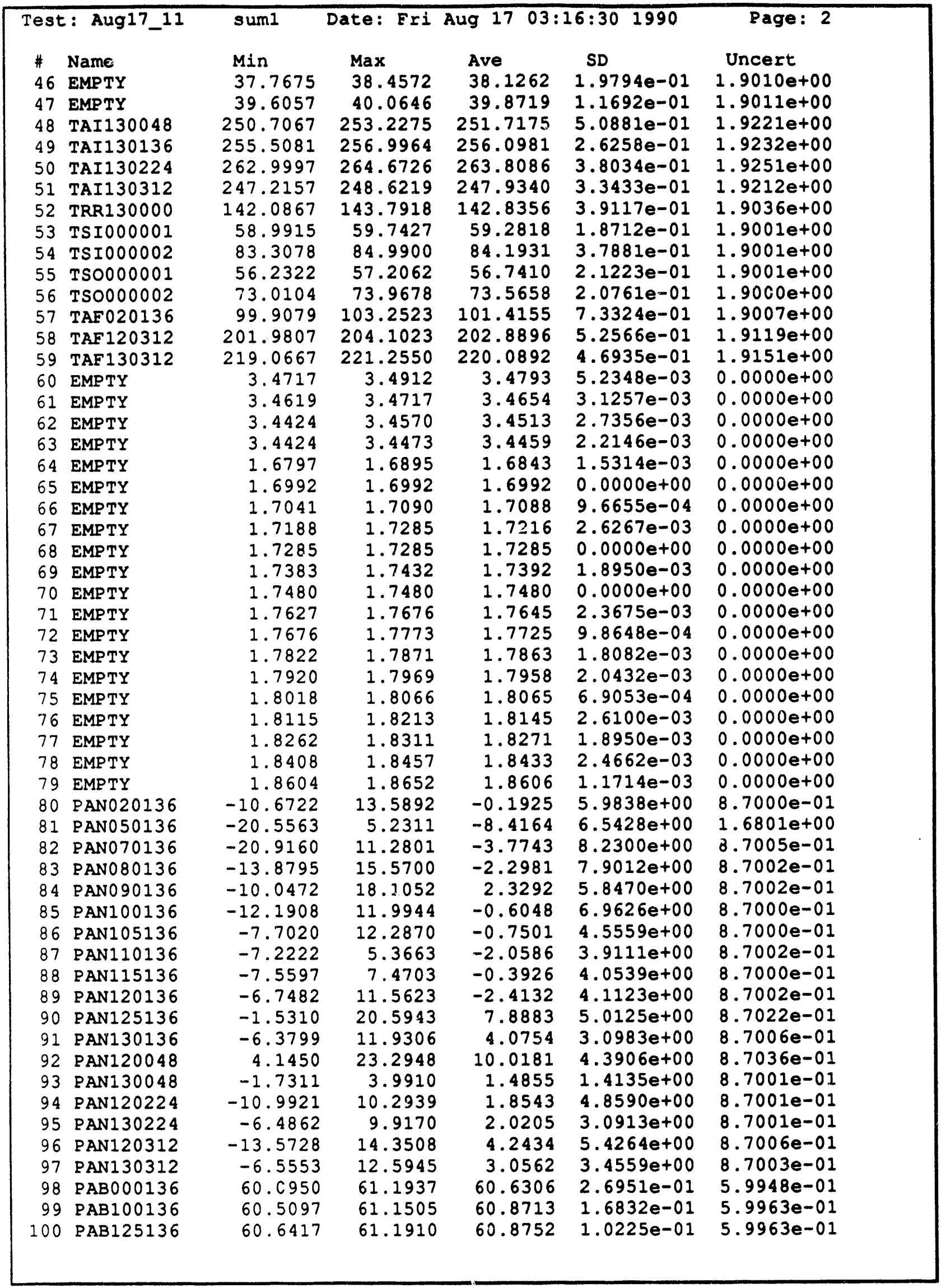




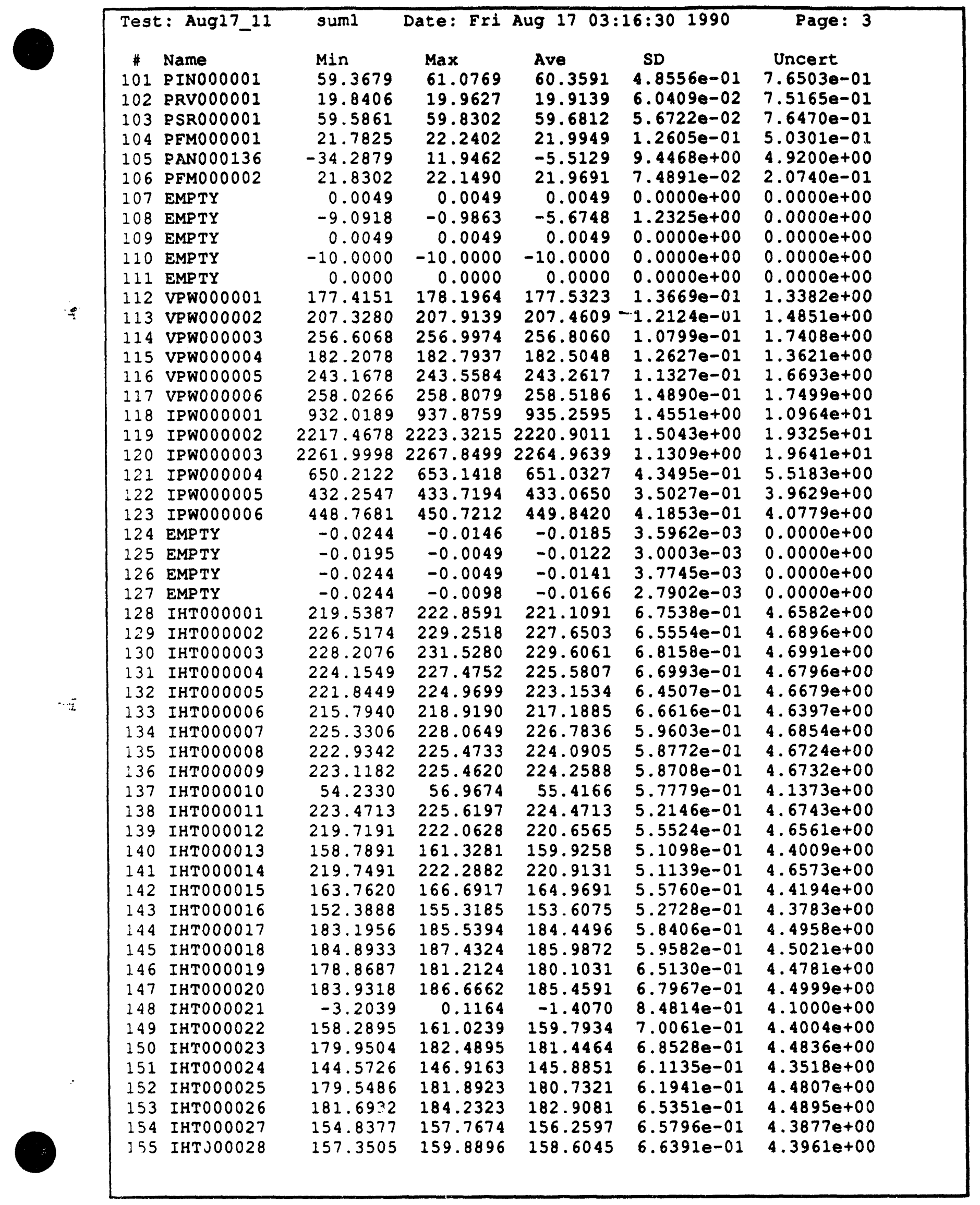




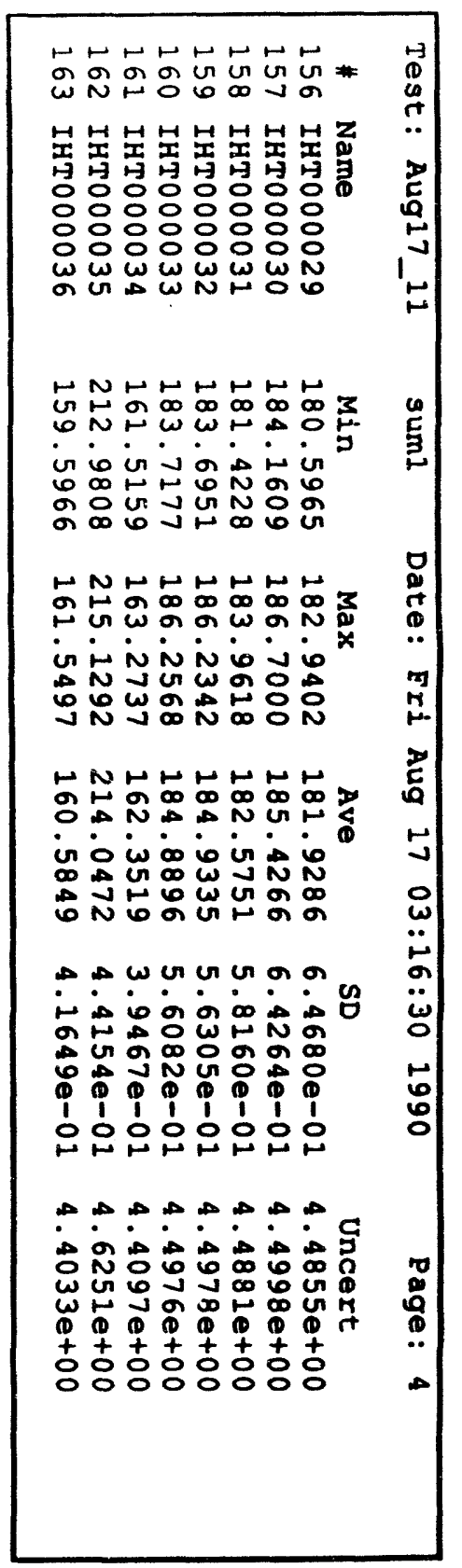




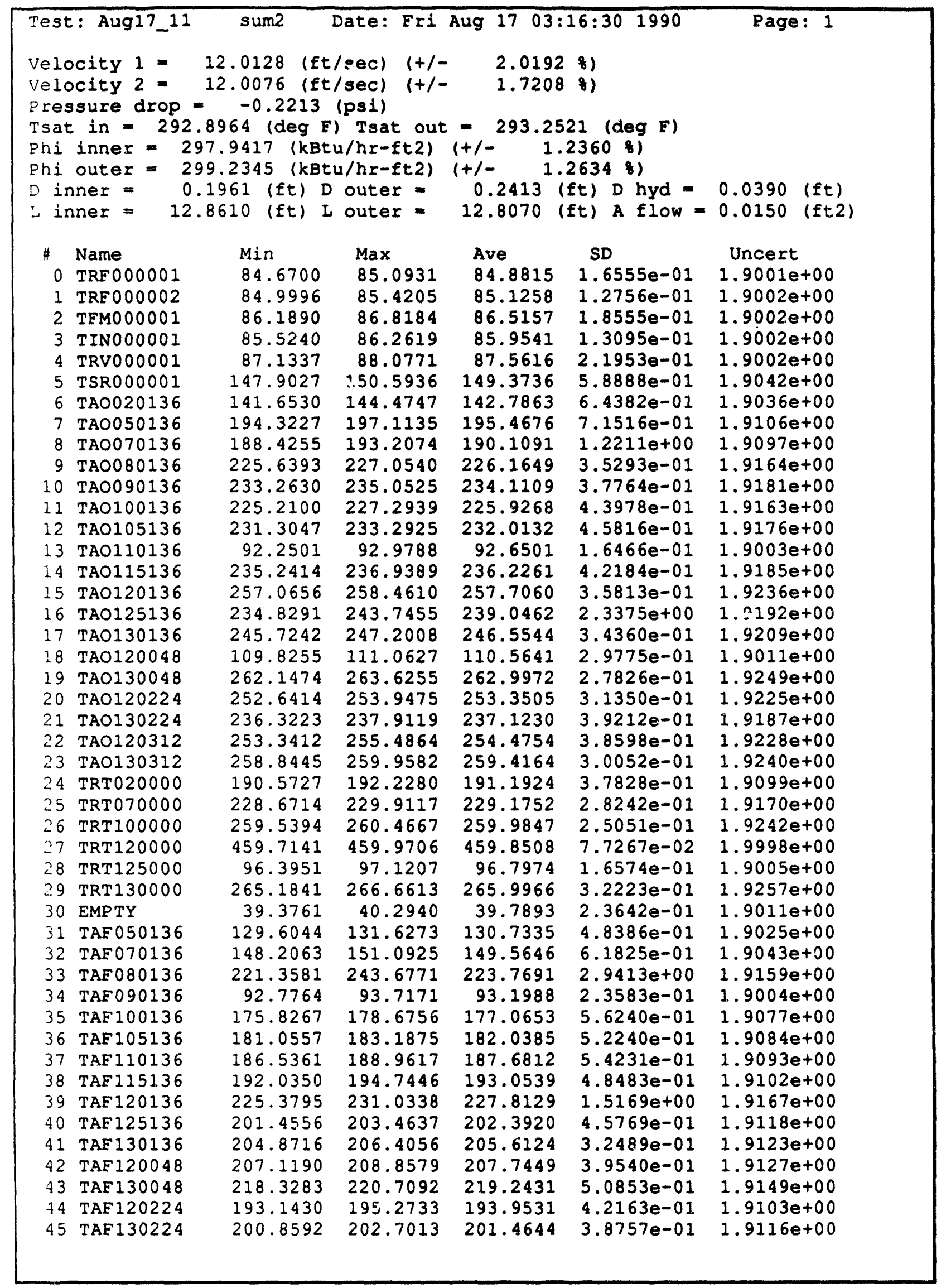




\begin{tabular}{|c|c|c|c|c|c|c|}
\hline \multicolumn{2}{|c|}{ Test: Aug17_11 } & sum2 & Date: Fri & Aug 1703 & $16: 30 \quad 1990$ & Page: 2 \\
\hline$\#$ & Name & Min & $\operatorname{Max}$ & Ave & SD & Uncert \\
\hline 46 & EMPTY & 37.7675 & 38.4572 & 38.1216 & $2.0358 e-01$ & $1.9010 e+00$ \\
\hline 47 & EMPTY & 39.6057 & 40.5234 & 39.8122 & $1.4842 e-01$ & $1.9011 e+00$ \\
\hline 48 & TAI130048 & 250.7078 & 252.5734 & 251.7155 & $4.1648 e-01$ & $1.9221 e+00$ \\
\hline 49 & TAI130136 & 255.6949 & 256.6236 & 256.1592 & $2.3360 \mathrm{e}-01$ & $1.9232 e+00$ \\
\hline 50 & TAI130224 & 263.1863 & 264.4868 & 263.7919 & $2.9995 e-01$ & $1.9251 e+00$ \\
\hline 51 & TAI130312 & 247.4031 & 248.8092 & 248.0070 & $3.1167 e-01$ & $1.9212 e+00$ \\
\hline 52 & TRR130000 & 145.8914 & 148.1892 & 146.9222 & $4.6604 e-01$ & $1.9040 \mathrm{e}+00$ \\
\hline 53 & TSI000001 & 58.7775 & 59.5289 & 59.1637 & $2.0001 e-01$ & $1.9001 e+00$ \\
\hline 54 & TSI000002 & 84.6742 & 86.2495 & 85.4846 & $3.8301 e-01$ & $1.9002 \mathrm{e}+00$ \\
\hline 55 & TSO000001 & 56.3400 & 57.0980 & 56.6998 & $1.7141 e-01$ & $1.9001 e+00$ \\
\hline 56 & TSO0000002 & 73.2230 & 74.1802 & 73.6995 & $2.1930 e-01$ & $1.9000 e+00$ \\
\hline 57 & TAF020136 & 99.5924 & 103.0430 & 101.2085 & $7.1853 e-01$ & $1.9007 e+00$ \\
\hline 58 & TAF 120312 & 201.9807 & 204.1982 & 202.8645 & $4.7606 e-01$ & $1.9119 e+00$ \\
\hline 59 & TAF 130312 & 218.7820 & 221.3493 & 220.1136 & $5.8422 e-01$ & $1.9151 e+00$ \\
\hline 60 & EMPTY & 3.4668 & 3.4961 & 3.4808 & $7.0455 e-03$ & $0.0000 e+00$ \\
\hline 61 & EMPTY & 3.4570 & 3.4766 & 3.4668 & $4.8327 e-03$ & $0.0000 e+00$ \\
\hline 62 & EMPTY & 3.4473 & 3.4570 & 3.4523 & $3.5513 e-03$ & $0.0000 e+00$ \\
\hline 63 & EMPTY & 3.4424 & 3.4521 & 3.4475 & $3.4116 e-03$ & $0.0000 e+00$ \\
\hline 64 & EMPTY & 1.6797 & 1.6846 & 1.6832 & $2.2146 e-03$ & $0.0000 \mathrm{e}+00$ \\
\hline 65 & EMPTY & 1.6943 & 1.6992 & 1.6987 & $1.4797 e-03$ & $0.0000 e+00$ \\
\hline 66 & EMPTY & 1.7041 & 1.7139 & 1.7082 & $2.0598 e-03$ & $0.0000 e+00$ \\
\hline 67 & EMPTY & 1.7188 & 1.7236 & 1.7206 & $2.3941 e-03$ & $0.0000 e+00$ \\
\hline 68 & EMPTY & 1.7236 & 1.7334 & 1.7274 & $2.4741 \mathrm{e}-03$ & $0.0000 e+00$ \\
\hline 69 & EMPTY & 1.7383 & 1.7432 & 1.7387 & $1.3381 e-03$ & $0.0000 e+00$ \\
\hline 70 & EMPTY & 1.7432 & 1.7529 & 1.7478 & $1.5314 \mathrm{e}-03$ & $0.0000 e+00$ \\
\hline 71 & EMPTY & 1.7627 & 1.7676 & 1.7631 & $1.3381 e-03$ & $0.0000 e+00$ \\
\hline 72 & EMPTY & 1.7676 & 1.7725 & 1.7716 & $1.8950 \mathrm{e}-03$ & $0.0000 e+00$ \\
\hline 73 & EMPTY & 1.7822 & 2.7871 & 1.7842 & $2.4164 e-03$ & $0.0000 e+00$ \\
\hline 74 & EMPTY & 1.7920 & 1.7969 & 1.7934 & $2.2146 e-03$ & $0.0000 e+00$ \\
\hline 75 & EMPTY & 1.8018 & 1.8066 & 1.8055 & $2.1065 e-03$ & $0.0000 e+00$ \\
\hline 76 & EMPTY & 1.8115 & 1.8164 & 1.8123 & $1.8082 e-03$ & $0.0000 e+00$ \\
\hline 77 & EMPTY & 1.8213 & 1.8311 & 1.8262 & $9.8648 e-04$ & $0.0000 e+00$ \\
\hline 78 & EMPTY & 1.8408 & 1.8457 & 1.8411 & $1.1714 e-03$ & $0.0000 e+00$ \\
\hline 79 & EMPTY & 1.8555 & 1.8604 & 1.8596 & $1.8082 e-03$ & $0.0000 e+00$ \\
\hline 80 & PAN020136 & -10.3671 & 10.9952 & -0.6335 & $5.4737 e+00$ & $8.7000 e-01$ \\
\hline 81 & PAN050136 & -17.8097 & 0.5009 & -7.6351 & $5.2985 e+00$ & $1.6801 e+00$ \\
\hline 82 & PAN070136 & -22.5944 & 6.9313 & -6.4171 & $7.9282 e+00$ & $8.7015 e-01$ \\
\hline 83 & PANO 80136 & -13.8795 & 13.4337 & -0.8866 & $7.4070 \mathrm{e}+00$ & $8.7000 e-01$ \\
\hline 84 & PANO90136 & -11.0390 & 21.0807 & 3.1287 & $7.3820 e+00$ & $8.7004 e-01$ \\
\hline 85 & PAN100136 & -12.1908 & 10.2397 & -1.0762 & $6.2072 e+00$ & $8.7000 e-01$ \\
\hline 36 & PAN105136 & -7.7020 & 10.7611 & 0.3775 & $5.0964 \mathrm{e}+00$ & $8.7000 e-01$ \\
\hline 37 & PAN110136 & -7.2222 & 13.3009 & -0.3420 & $4.7271 e+00$ & $8.7000 e-01$ \\
\hline 88 & PAN115136 & -7.5597 & 5.2577 & -1.9673 & $3.5405 e+00$ & $8.7001 e-01$ \\
\hline 89 & PAN120136 & -6.8245 & 9.7312 & -1.9158 & $4.4673 e+00$ & $8.7001 \mathrm{e}-01$ \\
\hline 90 & PAN125136 & -6.0323 & 22.7305 & 6.7179 & $4.9777 e+00$ & $8.7016 e-01$ \\
\hline 91 & PAN130136 & -0.7342 & 17.1949 & 5.6974 & $3.5142 e+00$ & $8.7012 e-01$ \\
\hline 92 & PAN120048 & 2.4665 & 20.6245 & 9.8334 & $3.8131 \mathrm{e}+00$ & $8.7035 e-01$ \\
\hline 93 & PAN130048 & -8.1398 & 10.3996 & 2.1446 & $3.0698 e+00$ & $8.7002 e-01$ \\
\hline 94 & PAN120224 & -12.2891 & 7.9288 & -0.5948 & $4.4868 \mathrm{e}+00$ & $8.7000 \mathrm{e}-01$ \\
\hline 95 & PAN130224 & -3.5108 & 12.2821 & 4.3170 & $3.7987 e+00$ & $8.7007 e-01$ \\
\hline 96 & PAN120312 & -3.7309 & 15.7241 & 5.3527 & $4.6525 e+00$ & $8.7010 e-01$ \\
\hline 97 & PAN130312 & -8.9204 & 15.2648 & 2.6244 & $3.7043 e+00$ & $8.7002 e-01$ \\
\hline 98 & PAB 000136 & 59.8204 & 60.8274 & 60.2607 & $2.6287 e-01$ & $5.9925 e-01$ \\
\hline 99 & PAB100136 & 60.0061 & 60.8759 & 60.5554 & $2.0050 \mathrm{e}-01$ & $5.9943 e-01$ \\
\hline$: 00$ & PAB125136 & 60.3670 & 61.3283 & 60.5840 & $1.6897 e-01$ & $5.9945 e-01$ \\
\hline
\end{tabular}




\begin{tabular}{|c|c|c|c|c|c|c|}
\hline Test & $t:$ Aug17_11 & sum2 & Date: Fri & Aug 170 & $6: 30 \quad 1990$ & Page: 3 \\
\hline \# & Name & Min & $\operatorname{Max}$ & Ave & SD & Uncert \\
\hline$\therefore 1$ & PIN000001 & 59.2458 & 60.5886 & 59.9587 & $4.3166 e-01$ & $7.6483 e-01$ \\
\hline 102 & PRV000001 & 19.7186 & 19.8406 & 19.7283 & $3.3453 e-02$ & $7.5162 e-01$ \\
\hline 103 & PSR000001 & 59.2198 & 59.3419 & 59.3053 & $5.6508 e-02$ & $7.6451 e-01$ \\
\hline 104 & PFMO00001 & 21.6909 & 22.4234 & 22.0736 & $1.7122 \theta-01$ & $5.0304 e-01$ \\
\hline$: 05$ & PAN000136 & -21.4705 & 7.8264 & -6.1263 & $7.9331 e+00$ & $4.9200 e+00$ \\
\hline 106 & PFM000002 & 21.8470 & 22.2832 & 22.0544 & $9.7180 e-02$ & $2.0746 e-01$ \\
\hline 107 & EMPTY & 0.0000 & 0.0098 & 0.0050 & $1.2041 e-03$ & $0.0000 e+00$ \\
\hline 108 & EMPTY & -10.0000 & -4.3994 & -6.3346 & $7.7476 e-01$ & $0.0000 e+00$ \\
\hline 109 & EMPTY & 0.0000 & 0.0098 & 0.0050 & $1.2041 e-03$ & $0.0000 e+00$ \\
\hline 110 & EMPTY & -10.0000 & -10.0000 & -10.0000 & $0.0000 e+00$ & $0.0000 e+00$ \\
\hline 111 & EMPTY & 0.0000 & 0.0000 & 0.0000 & $0.0000 e+00$ & $0.0000 e+00$ \\
\hline 112 & VPW000001 & 177.2198 & 178.0011 & 177.6182 & $1.6722 e-01$ & $1.3386 e+00$ \\
\hline 113 & VPWO00002 & 207.3280 & 207.7186 & 207.5195 & $1.0052 e-01$ & $1.4854 e+00$ \\
\hline 114 & VPWOO0003 & 256.6068 & 256.9974 & 256.7943 & $1.1133 e-01$ & $1.7407 e+00$ \\
\hline 115 & VPW000004 & 182.4031 & 182.7937 & 182.5790 & $1.1334 \mathrm{e}-01$ & $1.3624 e+00$ \\
\hline 116 & VPW000005 & 243.5584 & 244.1444 & 243.8632 & $1.4314 \mathrm{e}-01$ & $1.6724 e+00$ \\
\hline 117 & VPW000006 & 258.2219 & 258.8079 & 258.5421 & $1.2944 e-01$ & $1.7500 e+00$ \\
\hline$\therefore 28$ & IPW0 00001 & 953.4946 & 963.2563 & 957.0869 & $2.2438 \mathrm{e}+00$ & $1.1081 e+01$ \\
\hline 119 & IPW0 00002 & 2196.0037 & 2205.7600 & 2199.5154 & $2.1592 e+00$ & $1.9171 e+01$ \\
\hline 120 & IPW000003 & 2238.5999 & 2248.3499 & 2244.5276 & $2.6416 \mathrm{e}+00$ & $1.9494 e+01$ \\
\hline 121 & IPW000004 & 650.7004 & 656.0715 & 653.8647 & $1.5066 \mathrm{e}+00$ & $5.5392 e+00$ \\
\hline 122 & IPW000005 & 432.7429 & 433.7194 & 433.1334 & $3.2715 e-01$ & $3.9634 e+00$ \\
\hline 223 & IPW000006 & 445.3501 & 447.7915 & 446.6877 & $4.7114 \mathrm{e}-01$ & $4.0562 e+00$ \\
\hline 124 & EMPTY & -0.0293 & -0.0146 & -0.0189 & $3.7745 e-03$ & $0.0000 e+00$ \\
\hline .25 & EMPTY & -0.0195 & -0.0049 & -0.0134 & $3.0897 e-03$ & $0.0000 e+00$ \\
\hline 126 & EMPTY & -0.0195 & -0.0049 & -0.0141 & $3.6433 e-03$ & $0.0000 e+00$ \\
\hline$\therefore 7$ & EMPTY & -0.0244 & -0.0098 & -0.0169 & $3.5854 e-03$ & $0.0000 e+00$ \\
\hline 128 & IHTO00001 & 219.3434 & 222.6637 & 220.8747 & $6.4157 e-01$ & $4.6571 e+00$ \\
\hline 129 & IHTO00002 & 226.1268 & 228.8612 & 227.3964 & $5.9090 e-01$ & $4.6884 \mathrm{e}+00$ \\
\hline$: 30$ & IHTO00003 & 228.2076 & 230.7467 & 229.3170 & $5.9770 e-01$ & $4.6977 e+00$ \\
\hline$: 31$ & IHTOOOOOU & 223.7643 & 226.4986 & 225.3620 & $6.30^{\circ} 6 e-01$ & $4.6785 e+00$ \\
\hline$\div 32$ & IHTO00005 & 221.4543 & 223.9934 & 222.9386 & $5.7045 e-01$ & $4.6669 e+00$ \\
\hline 133 & IHT000006 & 215.5987 & 217.9424 & 216.9541 & $5.7644 e-01$ & $4.6386 e+00$ \\
\hline$\therefore 34$ & IHT000007 & 225.3306 & 227.2837 & 226.3265 & $5.1183 e-01$ & $4.6832 \mathrm{e}+00$ \\
\hline$: 35$ & IHTO00008 & 222.7389 & 224.6920 & 223.7936 & $5.2199 \mathrm{e}-01$ & $4.6710 \mathrm{e}+00$ \\
\hline 136 & IHT000009 & 222.9229 & 225.0714 & 223.9268 & $5.4392 e-01$ & $4.6717 e+00$ \\
\hline$: 37$ & IHT000010 & 54.0377 & 56.3815 & 55.5221 & $5.6221 e-01$ & $4.1374 \mathrm{e}+00$ \\
\hline 138 & IHTO00011 & 223.2760 & 225.6197 & 224.6901 & $5.7934 e-01$ & $4.6753 e+00$ \\
\hline$: 39$ & IHTO00012 & 219.7191 & 221.6722 & 220.7464 & $5.4076 \mathrm{e}-01$ & $4.6565 e+00$ \\
\hline$\therefore 40$ & IHTO00013 & 158.9844 & 161.1328 & 160.0703 & $5.6931 e-01$ & $4.4014 e+00$ \\
\hline 141 & IHTO00014 & 220.1397 & 222.8741 & 221.5303 & $6.1592 e-01$ & $4.6602 e+00$ \\
\hline 142 & IHT000015 & 163.7620 & 166.3011 & 165.1410 & $5.8449 e-01$ & $4.4201 e+00$ \\
\hline$: 43$ & IHTO00016 & 152.5841 & 154.7326 & 153.8536 & $5.2682 e-01$ & $4.3792 e+00$ \\
\hline$\therefore 44$ & IHTO00017 & 182.6097 & 185.7347 & 184.2269 & $7.2656 e-01$ & $4.4949 e+00$ \\
\hline 145 & IHT000018 & 184.5027 & 187.4324 & 186.0184 & $7.4927 e-01$ & $4.5023 e+00$ \\
\hline 146 & IHTOO0019 & 178.8687 & 181.6031 & 180.2125 & $6.5154 e-01$ & $4.4786 e+00$ \\
\hline 147 & IHTO00020 & 184.1271 & 186.8615 & 185.4981 & $6.8173 e-01$ & $4.5001 e+00$ \\
\hline 148 & IHTO00021 & -3.0086 & 0.1164 & -1.4890 & $7.6238 e-01$ & $4.1000 e+00$ \\
\hline 149 & IHTO00022 & 158.6801 & 161.0239 & 159.8013 & $5.7563 e-01$ & $4.4004 e+00$ \\
\hline 150 & IHTOOOO23 & 180.1458 & 182.8801 & 181.3605 & $6.2675 e-01$ & $4.4832 e+00$ \\
\hline 151 & IHTO00024 & 144.7679 & 147.3070 & 145.8461 & $6.1667 e-01$ & $4.3517 e+00$ \\
\hline 152 & IHTO00025 & 179.7439 & 182.2829 & 180.7243 & $6.1319 e-01$ & $4.4806 e+00$ \\
\hline 153 & IHT000026 & 181.6932 & 184.4276 & 182.8573 & $5.9707 e-01$ & $4.4893 e+00$ \\
\hline 154 & IHT000027 & 155.2284 & 157.9627 & 156.2440 & $6.1384 e-01$ & $4.3876 e+00$ \\
\hline$: 55$ & IHT000028 & 156.9599 & 160.0849 & 158.7177 & $7.3504 e-01$ & $4.3965 e+00$ \\
\hline
\end{tabular}




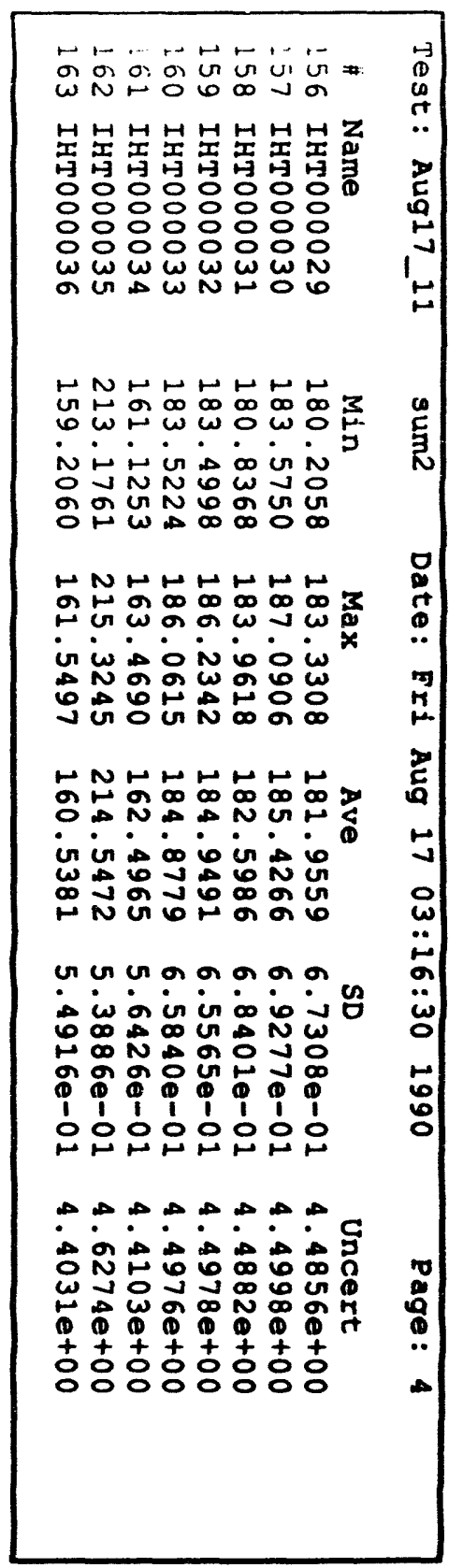




\begin{tabular}{|c|c|c|c|c|}
\hline \multicolumn{5}{|c|}{$\begin{array}{l}\text { Velocity } 1=0.021000 \text { (ft/sec) } \\
\text { Velocity } 2=0.022699 \text { (ft/sec) } \\
\text { Pressure Drop }=0.022200 \text { (psi) }\end{array}$} \\
\hline$\#$ & Name & Abs Diff & \&Diff & \\
\hline 0 & TRF000001 & 0.0550 & 0.0648 & \\
\hline 1 & TRF 000002 & 0.0168 & 0.0197 & \\
\hline 2 & TFM000001 & 0.2791 & 0.3216 & \\
\hline 3 & TINO0000I & 0.2550 & 0.2958 & \\
\hline 4 & TRV000001 & 0.7799 & 0.8828 & \\
\hline 5 & TSR000001 & 1.8724 & 1.2694 & \\
\hline 6 & TAO020136 & 6.2009 & 4.1620 & \\
\hline 7 & TAO050136 & 0.1133 & 0.0580 & \\
\hline 8 & TA0070136 & 0.3184 & 0.1672 & \\
\hline 9 & TAO080136 & 0.2811 & 0.1241 & \\
\hline 10 & TAO090136 & 0.2732 & 0.1166 & \\
\hline 11 & TAO100136 & 0.3467 & 0.1532 & \\
\hline 12 & TAO105136 & 0.1800 & 0.0775 & \\
\hline 13 & TA0110136 & 3.3751 & 3.7806 & \\
\hline 14 & TAO115136 & 0.1869 & 0.0791 & \\
\hline 15 & TAO120136 & 0.1992 & 0.0772 & \\
\hline 16 & TAO125136 & 0.1028 & 0.0430 & \\
\hline 17 & TA0130136 & 0.3638 & 0.1473 & \\
\hline 18 & TAO120048 & 3.8651 & 3.6224 & \\
\hline 19 & TAO130048 & 0.4159 & 0.1579 & \\
\hline 20 & TAO120224 & 0.2741 & 0.1081 & \\
\hline 21 & TAO130224 & 0.0469 & 0.0198 & \\
\hline 22 & TA0120312 & 0.3937 & 0.1545 & \\
\hline 23 & TAO130312 & 0.2096 & 0.0807 & \\
\hline 24 & TRT020000 & 0.0838 & 0.0438 & \\
\hline 25 & TRT070000 & 0.0363 & 0.0158 & \\
\hline 26 & TRT 100000 & 0.1910 & 0.0734 & \\
\hline 27 & TRT120000 & 0.0154 & 0.0034 & \\
\hline 28 & TRT 125000 & 5.4356 & 5.9495 & \\
\hline 29 & TRT130000 & 0.0131 & 0.0049 & \\
\hline 30 & EMPTY & 0.0000 & 0.0000 & \\
\hline 31 & TAF050136 & 0.2083 & 0.1591 & \\
\hline 32 & TAF070136 & 0.1932 & 0.1290 & \\
\hline 33 & TAF0 80136 & 0.0651 & 0.0291 & \\
\hline 34 & TAF090136 & 3.5562 & 3.9671 & \\
\hline 35 & TAF 100136 & 0.3281 & 0.1850 & \\
\hline 36 & TAF 105136 & 0.2386 & 0.1309 & \\
\hline 37 & TAF 110136 & 0.2853 & 0.1518 & \\
\hline 38 & TAF 115136 & 0.0017 & 0.0009 & \\
\hline 39 & TAF120136 & 0.2808 & 0.1234 & \\
\hline 40 & TAF 125136 & 0.2813 & 0.1388 & \\
\hline 41 & TAF 130136 & 0.4009 & 0.1946 & \\
\hline 42 & TAF 120048 & 0.2376 & 0.1142 & \\
\hline 43 & TAF 130048 & 0.0897 & 0.0409 & \\
\hline 44 & TAF 120224 & 0.0640 & 0.0330 & \\
\hline 45 & TAF 130224 & 0.2851 & 0.1413 & \\
\hline
\end{tabular}




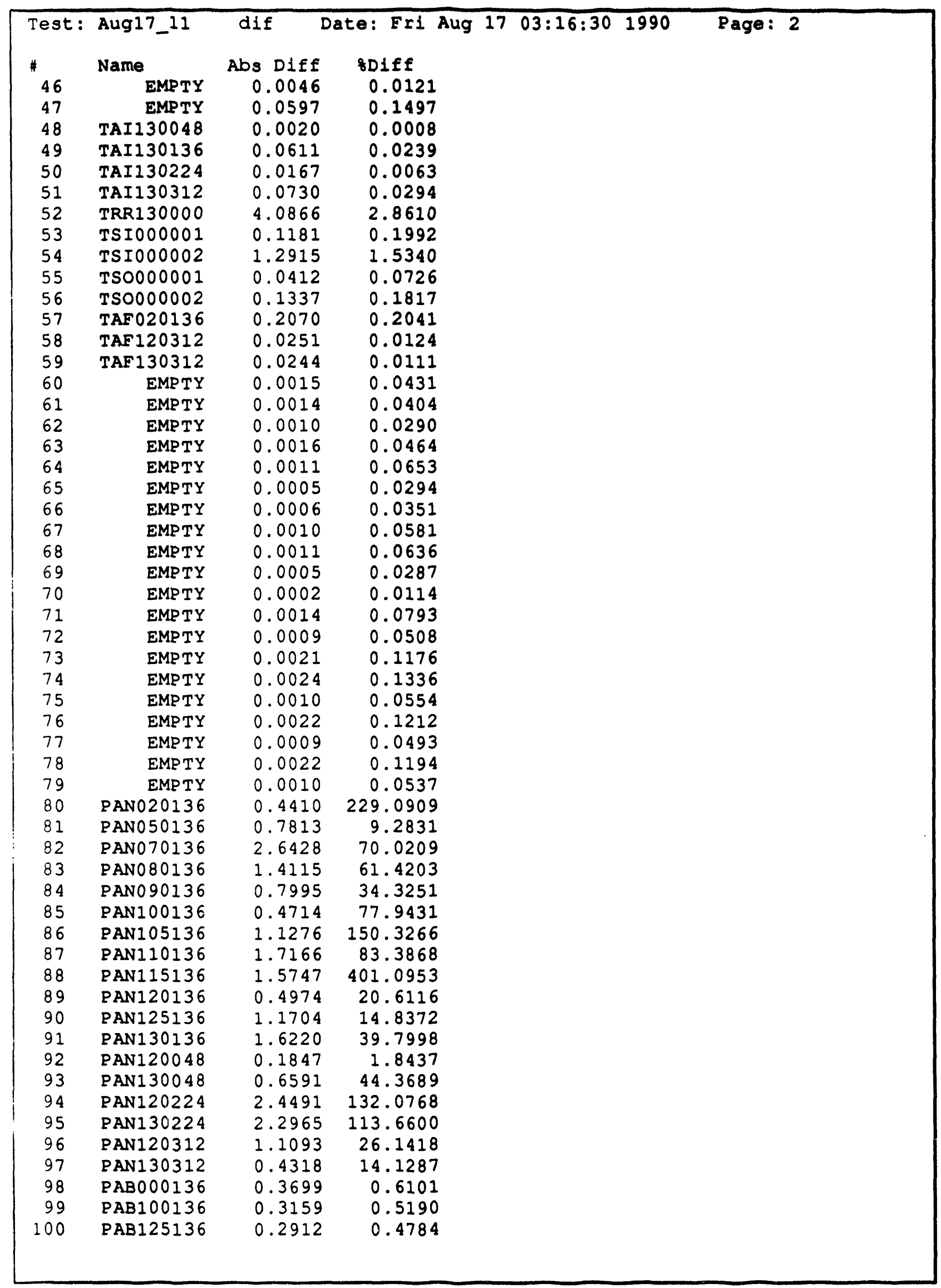




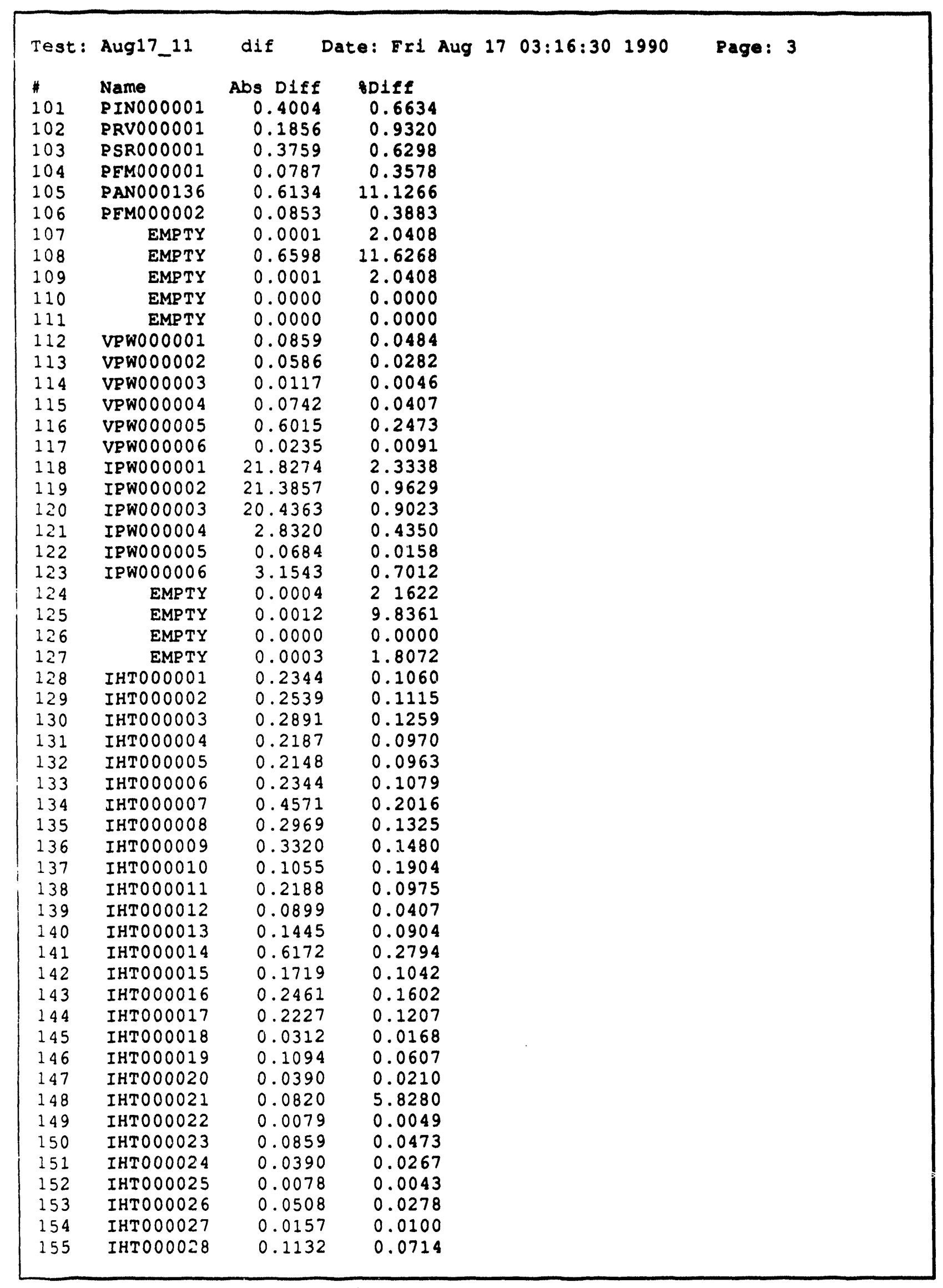




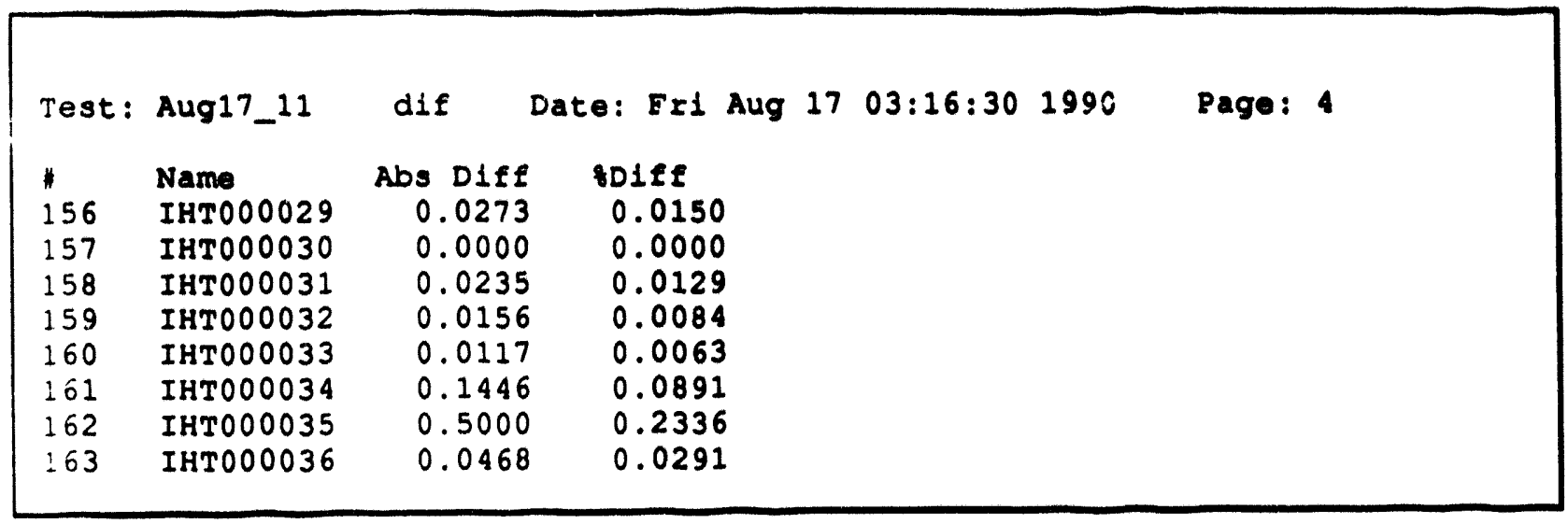




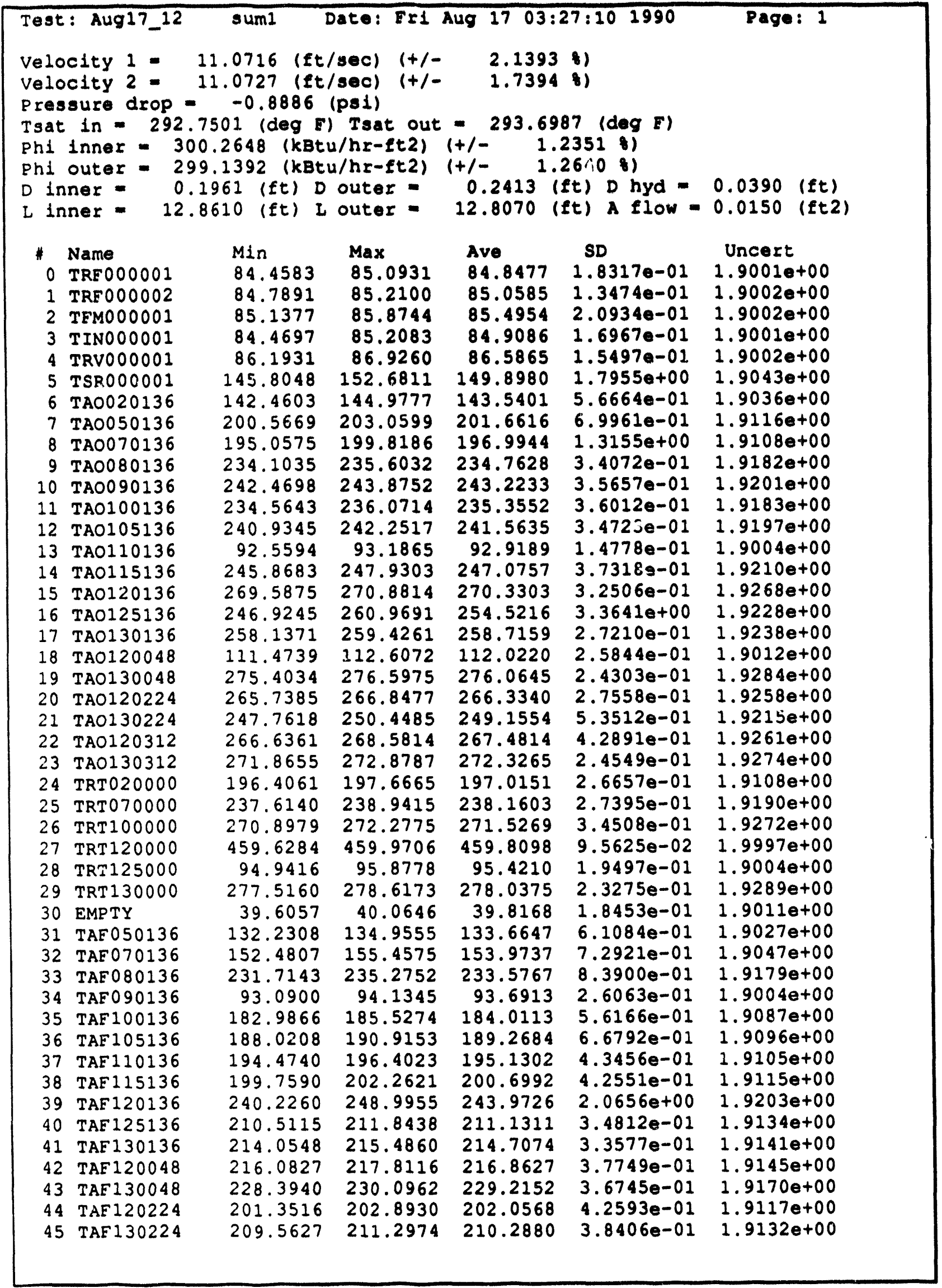




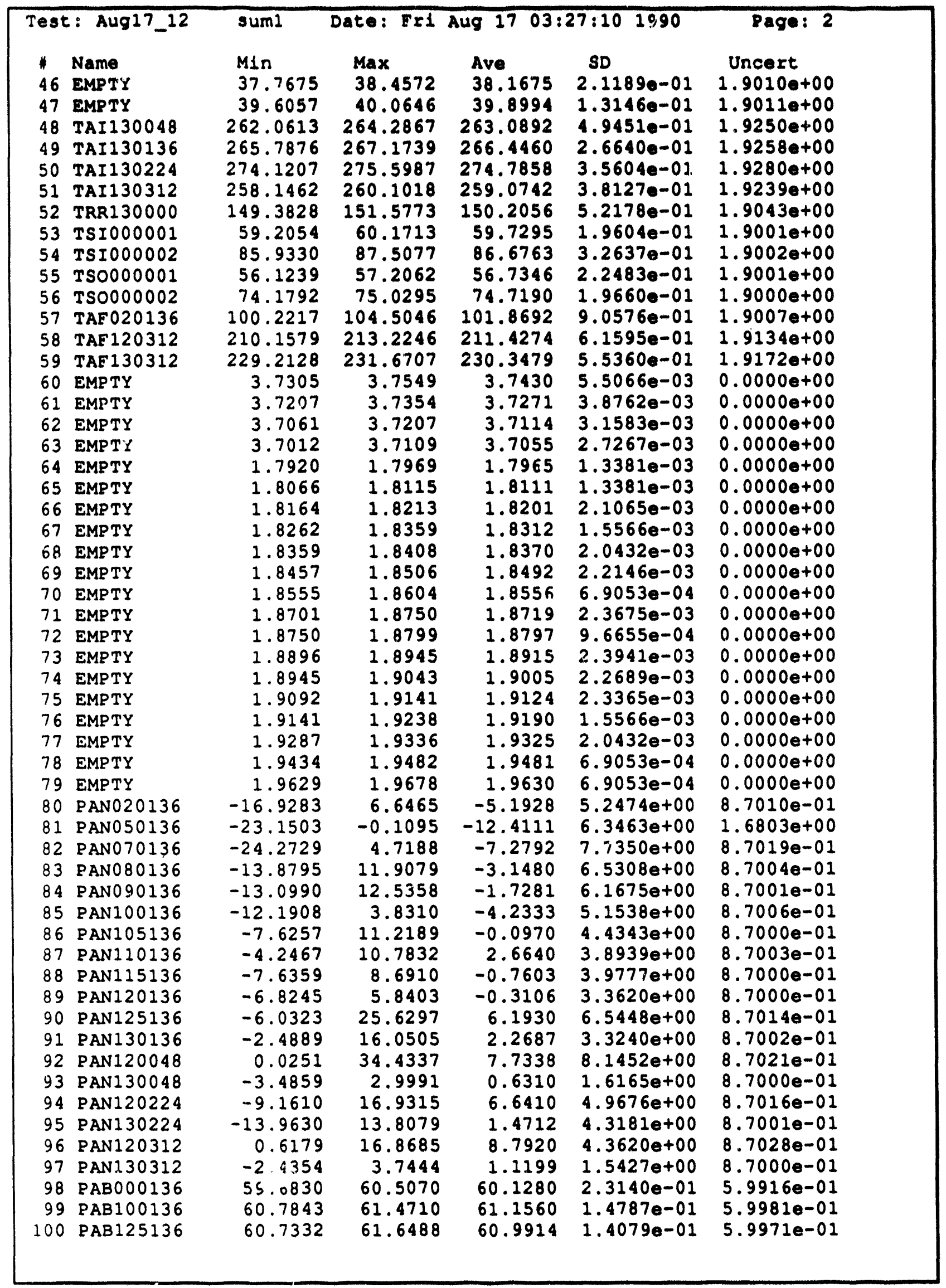




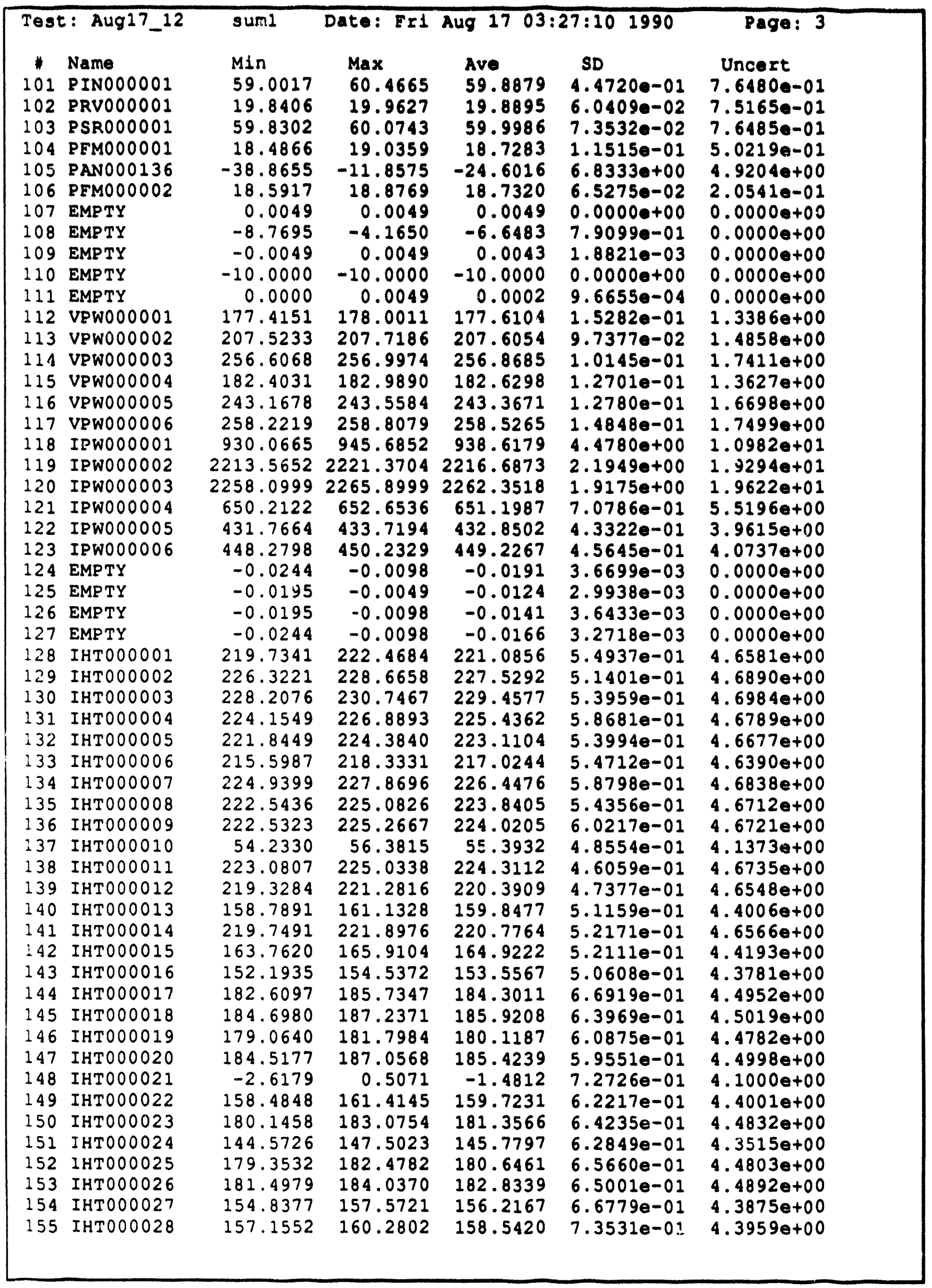



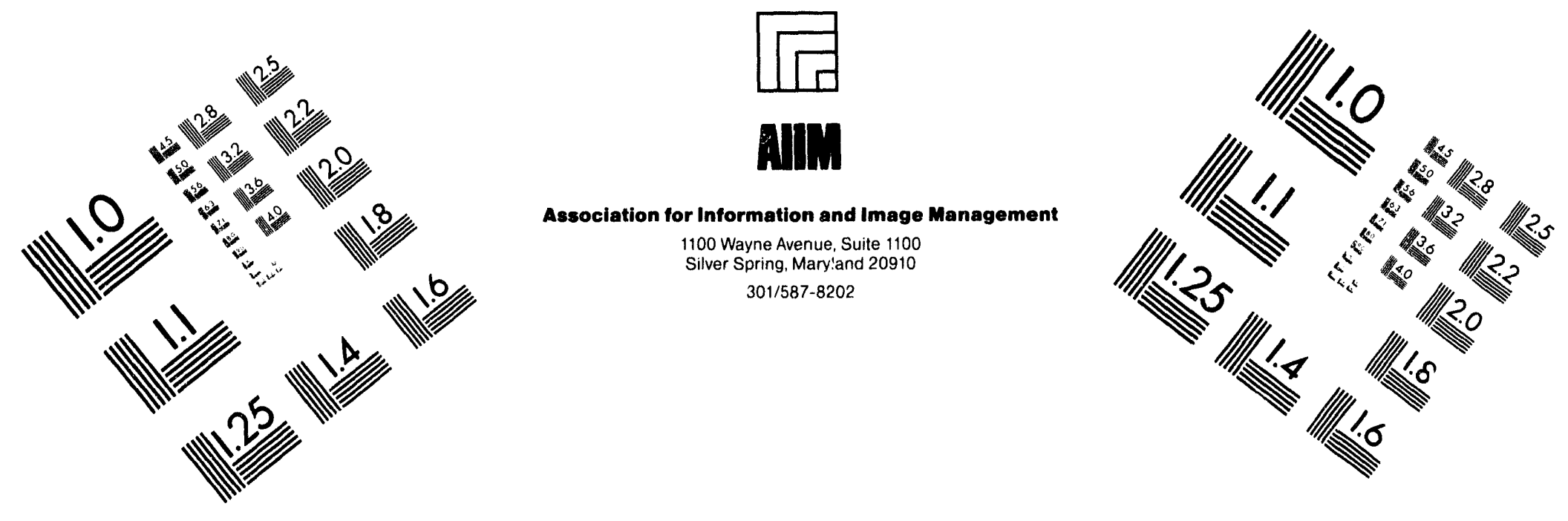

\section{Centimeter}

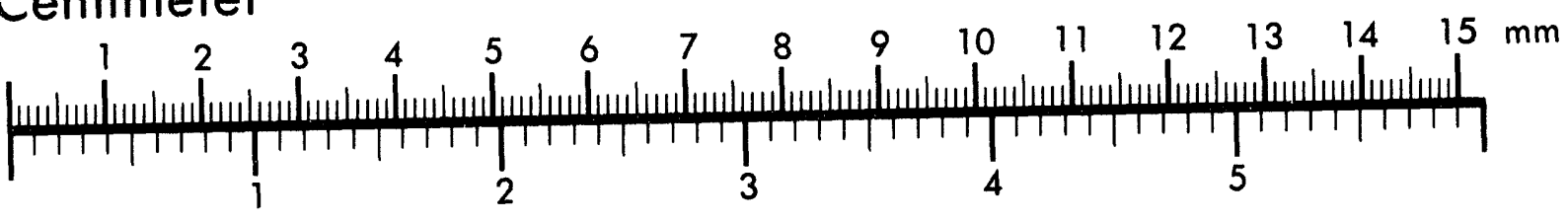
Inches
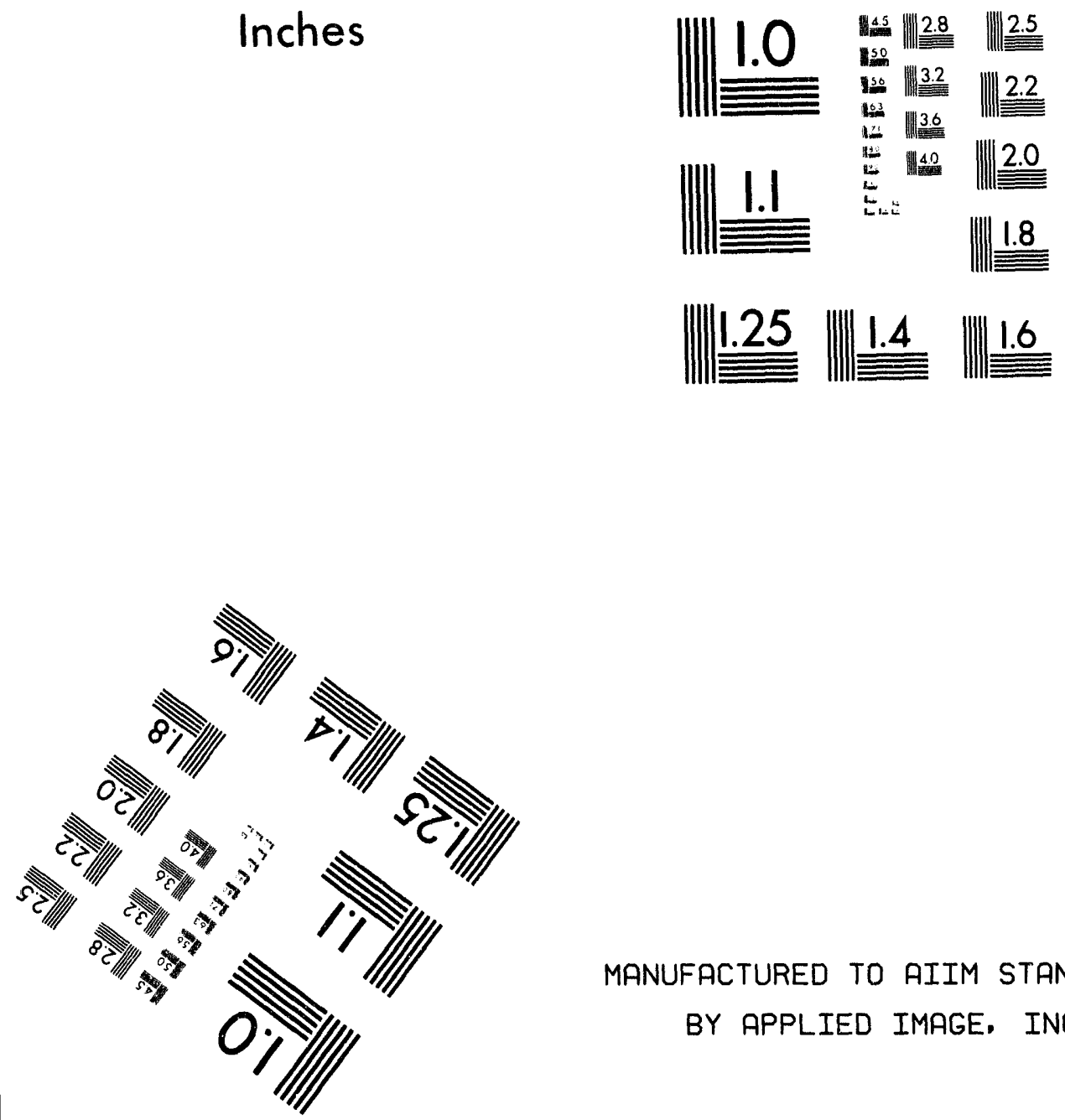

MANUFACTURED TO AIIM STANDARDS BY APPLIED IMAGE, INC.

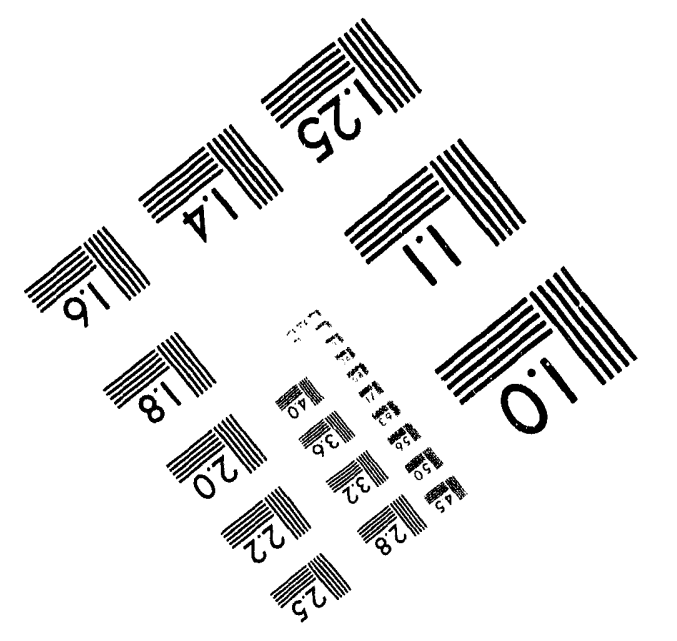



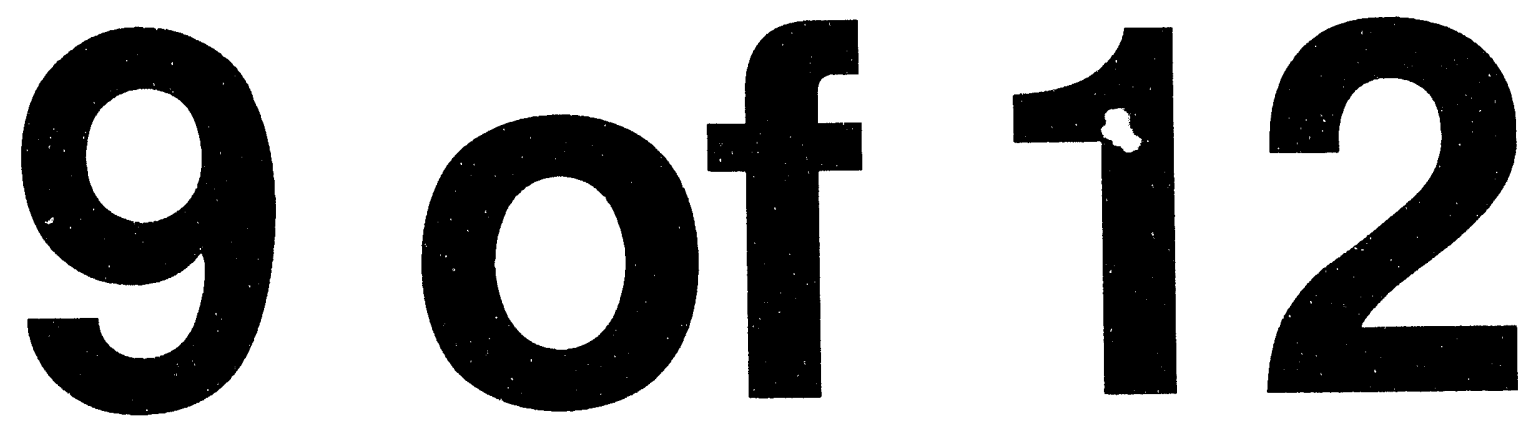


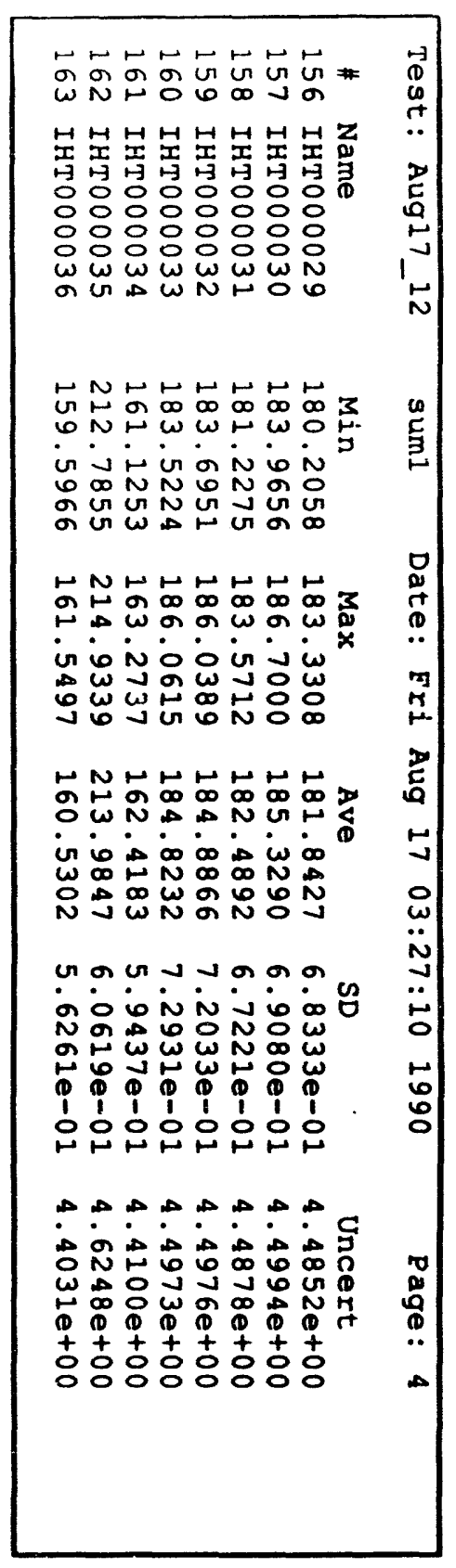




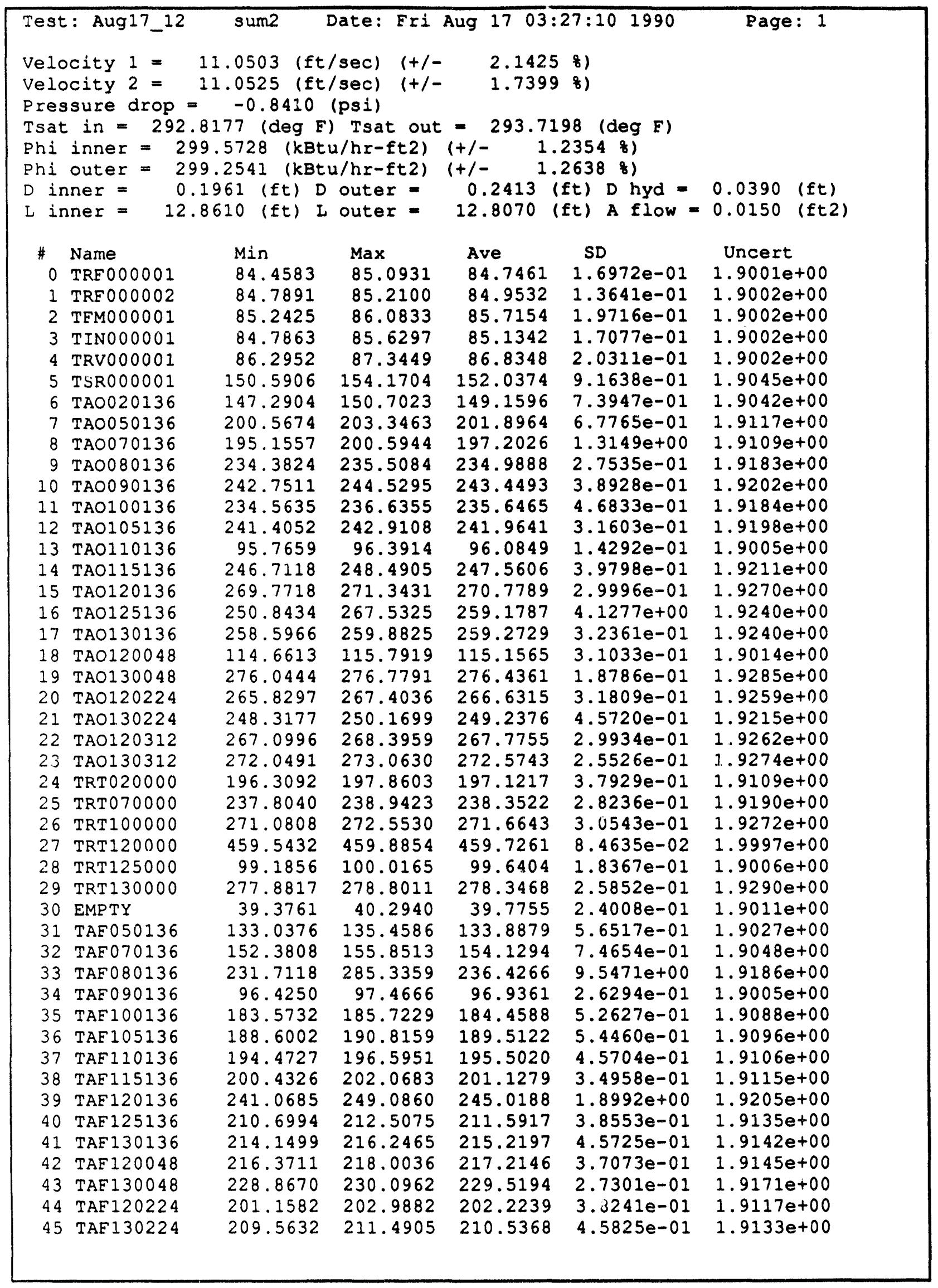




\begin{tabular}{|c|c|c|c|c|c|c|}
\hline \multicolumn{2}{|c|}{ Test: Aug17_12 } & \multirow{2}{*}{$\begin{array}{l}\text { sum2 } \\
\text { Min }\end{array}$} & \multicolumn{3}{|c|}{ Date: Fri Aug $17 \quad 03: 27: 101990$} & \multirow{2}{*}{$\begin{array}{l}\text { Page: } 2 \\
\text { Uncert }\end{array}$} \\
\hline$\#$ & Name & & $\operatorname{Max}$ & Ave & SD & \\
\hline 46 & EMPTY & 37.7675 & 38.4572 & 38.0664 & $2.1413 e-01$ & $1.9010 e+00$ \\
\hline 47 & EMPTY & 39.3761 & 40.7527 & 39.8811 & $2.2231 e-01$ & $1.9011 \mathrm{e}+00$ \\
\hline 48 & TAI 130048 & 262.7111 & 264.6573 & 263.6135 & $4.2653 e-01$ & $1.9251 \mathrm{e}+00$ \\
\hline 49 & TAI 130136 & 266.0649 & 267.7258 & 267.0858 & $2.7060 e-01$ & $1.9260 \mathrm{e}+00$ \\
\hline 50 & TAI130224 & 274.5822 & 276.1518 & 275.2013 & $3.7130 e-01$ & $1.9282 \mathrm{e}+00$ \\
\hline 51 & TAI1 30312 & 258.1465 & 260.1018 & 259.4852 & $4.0891 e-01$ & $1.9240 \mathrm{e}+00$ \\
\hline 52 & TRR1 30000 & 148.1873 & 149.6846 & 148.9552 & $3.2763 e-01$ & $1.9042 \mathrm{e}+00$ \\
\hline 53 & TSI000001 & 59.2054 & 60.1713 & 59.7054 & $2.1426 e-01$ & $1.9001 e+00$ \\
\hline 54 & TSI000002 & 84.3573 & 85.5147 & 84.8729 & $2.8961 e-01$ & $1.9001 \mathrm{e}+00$ \\
\hline 55 & TSO000001 & 56.3406 & 57.2063 & 56.7501 & $2.1121 e-01$ & $1.9001 \epsilon+00$ \\
\hline 56 & TSO000002 & 74.1792 & 75.1351 & 74.5977 & $2.2140 e-01$ & $1.9000 \mathrm{e}+00$ \\
\hline 57 & TAF020136 & 100.2225 & 104.9217 & 102.1559 & $8.9882 e-01$ & $1.9007 e+00$ \\
\hline 58 & TAF 120312 & 210.7329 & 213.5119 & 211.8622 & $5.3548 e-01$ & $1.9135 e+00$ \\
\hline 59 & TAF 130312 & 229.5922 & 231.7639 & 230.7081 & $5.7303 e-01$ & $1.9173 \mathrm{e}+\mathrm{CO}$ \\
\hline 60 & EMPTY & 3.7451 & 3.7646 & 3.7550 & $5.7088 e-03$ & $0.0000 e+00$ \\
\hline 61 & EMPTY & 3.7305 & 3.7451 & 3.7395 & $3.7382 e-03$ & $0.0000 e+00$ \\
\hline 62 & EMPTY & 3.7158 & 3.7305 & 3.7240 & $3.9998 e-03$ & $0.0000 e+00$ \\
\hline 63 & EMPTY & 3.7061 & 3.7256 & 3.7173 & $3.4527 e-03$ & $0.0000 \mathrm{e}+00$ \\
\hline 64 & EMPTY & 1.7969 & 1.8018 & 1.8012 & $1.6028 e-03$ & $0.0000 e+00$ \\
\hline 65 & EMPTY & 1.8115 & 1.8213 & 1.8160 & $1.6624 e-03$ & $0.0000 e+00$ \\
\hline 66 & EMPTY & 1.8213 & 1.8262 & 1.8242 & $2.4164 e-03$ & $0.0000 e+00$ \\
\hline 67 & EMPTY & 1.8311 & 1.8408 & 1.8359 & $1.3951 e-03$ & $0.0000 e+00$ \\
\hline 68 & EMPTY & 1.8408 & 1.8457 & 1.8415 & $1.7115 \mathrm{e}-03$ & $0.0000 e+00$ \\
\hline 69 & EMPTY & 1.8506 & 1.8555 & 1.8536 & $2.3941 e-03$ & $0.0000 \mathrm{e}+00$ \\
\hline 70 & EMPTY & 1.8604 & 1.8604 & 1.8604 & $0.0000 e+00$ & $0.0000 e+00$ \\
\hline 71 & EMPTY & 1.8701 & 1.8799 & 1.8765 & $2.4662 e-03$ & $0.0000 e+00$ \\
\hline 72 & EMPTY & 1.8799 & 1.8848 & 1.8846 & $9.6655 e-04$ & $0.0000 e+00$ \\
\hline 73 & EMPTY & 1.8945 & 1.8994 & 1.8958 & $2.1635 e-03$ & $0.0000 e+00$ \\
\hline 74 & EMPTY & 1.9043 & 1.9092 & 1.9048 & $1.4797 e-03$ & $0.0000 e+00$ \\
\hline 75 & EMPTY & 1.9141 & 1.9189 & 1.9153 & $2.1635 e-03$ & $0.0000 e+00$ \\
\hline 76 & EMPTY & 1.9189 & 1.9238 & 1.9235 & $1.1714 e-03$ & $0.0000 \mathrm{e}+00$ \\
\hline 77 & EMPTY & 1.9336 & 1.9385 & 1.9354 & $2.3675 e-03$ & $0.0000 \mathrm{e}+00$ \\
\hline 78 & EMPTY & 1.9482 & 1.9531 & 1.9514 & $2.3675 e-03$ & $0.0000 \mathrm{e}+00$ \\
\hline 79 & EMPTY & 1.9678 & 1.9727 & 1.9679 & $6.9053 e-04$ & $0.0000 e+00$ \\
\hline 80 & PAlv020136 & -14.4869 & 9.3931 & -4.3200 & $5.9027 e+00$ & $8.7007 e-01$ \\
\hline 81 & PAN0 50136 & -25.1339 & 1.5690 & -13.3572 & $8.3249 \mathrm{e}+00$ & $1.6803 \mathrm{e}+00$ \\
\hline 82 & PAN0 70136 & -24.0440 & 7.0839 & -7.8239 & $8.2055 e+00$ & $8.7022 e-01$ \\
\hline 83 & PAN0 80136 & -13.8032 & 9.3902 & -3.7492 & $5.7949 e+00$ & $8.7005 e-01$ \\
\hline 84 & PAN090136 & -12.4886 & 15.2061 & 0.3623 & $5.3690 e+00$ & $8.7000 e-01$ \\
\hline 85 & PAN 100136 & -12.1908 & 8.2560 & -3.9632 & $5.7437 e+00$ & $8.7006 e-01$ \\
\hline 86 & PAN105136 & -7.7020 & 8.4723 & -2.3233 & $4.4134 e+00$ & $8.7002 e-01$ \\
\hline 87 & PAN110136 & -5.1622 & 16.2001 & 3.3003 & $5.0733 e+00$ & $8.7004 e-01$ \\
\hline 88 & PAN1 15136 & -7.5597 & 6.9362 & 0.4253 & $3.7356 \mathrm{e}+00$ & $8.7000 e-01$ \\
\hline 89 & PAN120136 & -6.8245 & 14.1563 & 0.8155 & $5.4876 e+00$ & $8.7000 e-01$ \\
\hline 90 & PAN125136 & -1.4547 & 18.9158 & 5.3217 & $4.3514 \mathrm{e}+00$ & $8.7010 \mathrm{e}-01$ \\
\hline 91 & PAN130136 & -6.9903 & 6.7426 & 2.2108 & $2.5564 e+00$ & $8.7002 e-01$ \\
\hline 92 & PAN 120048 & -0.5853 & 22.6081 & 6.1408 & $4.6532 e+00$ & $8.7014 \mathrm{e}-01$ \\
\hline 93 & PAN1 30048 & -3.5621 & 4.9828 & 0.1671 & $1.9715 e+00$ & $8.7000 e-01$ \\
\hline 94 & PAN120224 & -5.8041 & 19.4492 & 6.0535 & $4.7533 e+00$ & $8.7013 e-01$ \\
\hline 95 & PAN1 30224 & -6.1048 & 6.6363 & 1.3217 & $2.6887 e+00$ & $8.7001 e-01$ \\
\hline 96 & PAN120312 & 2.0675 & 19.0047 & 7.7620 & $3.6286 \mathrm{e}+00$ & $8.7022 e-01$ \\
\hline 97 & PAN1 30312 & -3.1221 & 4.0495 & 0.7002 & $1.6941 \mathrm{e}+00$ & $8.7000 e-01$ \\
\hline 98 & PAB000136 & 59.7288 & 60.7359 & 60.1893 & $2.4909 e-01$ & $5.9920 e-01$ \\
\hline 99 & PAB100136 & 60.6928 & 61.5625 & 61.1606 & $1.8130 \mathrm{e}-01$ & $5.9982 e-01$ \\
\hline 100 & PAB125136 & 60.7790 & 61.2826 & 61.0107 & $9.5400 e-02$ & $5.9972 e-01$ \\
\hline
\end{tabular}




\begin{tabular}{|c|c|c|c|c|c|c|}
\hline \multicolumn{2}{|c|}{ Test: Aug17_12 } & sum2 & Date: Fri & Aug 170 & $7: 10 \quad 1990$ & Page: 3 \\
\hline \# & Name & Min & $\operatorname{Max}$ & Ave & SD & Uncert \\
\hline 101 & PIN 000001 & 58.8796 & 60.7106 & 59.9441 & $4.4735 e-01$ & $7.6483 e-01$ \\
\hline 102 & PRV000001 & 19.7186 & 19.8406 & 19.7357 & $4.2787 e-02$ & $7.5162 e-01$ \\
\hline 103 & PSR000001 & 59.8302 & 60.1964 & 60.0352 & $6.7269 e-02$ & $7.6487 e-01$ \\
\hline 104 & PEMO00001 & 18.3035 & 19.0359 & 18.6550 & $1.4370 e-01$ & $5.0217 e-01$ \\
\hline 105 & PAN000136 & -43.4432 & -4.0755 & -23.2833 & $7.8653 e+00$ & $4.9203 e+00$ \\
\hline 106 & PFM000002 & 18.5078 & 18.8769 & 18.6625 & $8.0458 e-02$ & $2.0537 e-01$ \\
\hline 107 & EMPTY & 0.0049 & 0.0098 & 0.0052 & $1.1714 e-03$ & $0.0000 e+00$ \\
\hline 108 & EMPTY & -9.8145 & -5.0830 & -6.9828 & $9.3770 e-01$ & $0.0000 e+00$ \\
\hline 109 & EMPTY & 0.0000 & 0.0098 & 0.0049 & $9.8648 e-04$ & $0.0000 e+00$ \\
\hline 110 & EMPTY & -10.0000 & -10.0000 & -10.0000 & $0.0000 e+00$ & $0.00 v 0 e+00$ \\
\hline 111 & EMPTY & 0.0000 & 0.0049 & 0.0001 & $6.9053 e-04$ & $0.0: 00 e+00$ \\
\hline 112 & VPWO00001 & 177.4151 & 178.0011 & 177.6924 & $1.3141 e-01$ & $1.3389 \mathrm{e}+00$ \\
\hline 113 & VPWO00002 & 207.3280 & 207.7186 & 207.5702 & $9.3043 e-02$ & $1.4857 e+00$ \\
\hline 114 & VPW000003 & 256.6068 & 257.1927 & 256.8919 & $1.1975 e-01$ & $1.7412 \mathrm{e}+00$ \\
\hline 115 & VPW000004 & 182.4031 & 182.7937 & 182.6063 & $1.2453 e-01$ & $1.3625 e+00$ \\
\hline 116 & VPWO00005 & 243.3631 & 243.7538 & 243.4492 & $1.0559 \mathrm{e}-01$ & $1.6702 \mathrm{e}+00$ \\
\hline 117 & VPW000006 & 258.2219 & 258.8079 & 258.4249 & $1.0410 e-01$ & $1.7494 \mathrm{e}+00$ \\
\hline 118 & IPW000001 & 941.7806 & 947.6376 & 944.8655 & $1.4271 e+00$ & $1.1015 e+01$ \\
\hline 119 & IPW000002 & 2207.7114 & 2219.4189 & 2213.4878 & $3.5251 e+00$ & $1.9271 \mathrm{e}+01$ \\
\hline 120 & IPW000003 & 2254.2000 & 2261.9998 & 2257.9443 & $1.9235 e+00$ & $1.9591 e+01$ \\
\hline 121 & IPW000004 & 650.7004 & 654.1184 & 652.2241 & $1.2886 e+00$ & $5.5271 e+00$ \\
\hline 122 & IPWO 00005 & 430.7900 & 432.2547 & 431.5124 & $3.4509 \mathrm{e}-01$ & $3.9523 e+00$ \\
\hline 123 & IPW000006 & 446.3267 & 448.2798 & 447.3615 & $4.1432 e-01$ & $4.0608 e+00$ \\
\hline 124 & EMPTY & -0.0244 & -0.0098 & -0.0180 & $3.7486 e-03$ & $0.0000 e+00$ \\
\hline 125 & EMPTY & -0.0146 & -0.0049 & -0.0125 & $2.8179 e-03$ & $0.0000 e+00$ \\
\hline 126 & EMPTY & -0.0195 & -0.0049 & -0.0142 & $3.4527 e-03$ & $0.0000 e+00$ \\
\hline 127 & EMPTY & -0.0244 & -0.0098 & -0.0176 & $3.5568 e-03$ & $0.0000 e+00$ \\
\hline 128 & IHTO00001 & 219.5387 & 222.2731 & 221.0661 & $6.0963 e-01$ & $4.6580 e+00$ \\
\hline 129 & IHT000002 & 225.9315 & 228.4705 & 227.4823 & $5.9111 e-01$ & $4.6888 e+00$ \\
\hline 130 & IHTO00003 & 227.8170 & 230.3561 & 229.3522 & $5.6773 e-01$ & $4.6979 e+00$ \\
\hline 131 & IHT000004 & 223.5690 & 226.4986 & 225.3190 & $6.1125 e-01$ & $4.6783 e+00$ \\
\hline 132 & IHT000005 & 221.2590 & 223.7981 & 222.9425 & $5.3509 e-01$ & $4.6669 e+00$ \\
\hline 133 & IHT000006 & 215.2081 & 218.1378 & 216.9932 & $5.2794 e-01$ & $4.6388 \mathrm{e}+00$ \\
\hline 134 & IHT000007 & 224.5493 & 227.8696 & 226.4203 & $5.4260 e-01$ & $4.6837 e+00$ \\
\hline 135 & IHTO00008 & 222.1529 & 225.2779 & 223.8092 & $5.2828 e-01$ & $4.6711 e+00$ \\
\hline 136 & IHT000009 & 222.7276 & 225.4620 & 223.9736 & $5.3944 e-01$ & $4.6719 e+00$ \\
\hline 137 & IHTO00010 & 53.8424 & 56.5768 & 55.4166 & $5.8449 e-01$ & $4.1373 e+00$ \\
\hline 138 & IHTO00011 & 222.6900 & 225.6197 & 224.2682 & $6.0340 e-01$ & $4.6733 e+00$ \\
\hline 139 & IHTO00012 & 218.9378 & 222.0628 & 220.3831 & $6.3011 e-01$ & $4.6548 e+00$ \\
\hline 140 & IHTO00013 & 158.5938 & 161.1328 & 159.8281 & $5.3589 e-01$ & $4.4005 e+00$ \\
\hline 141 & IHT000014 & 219.7491 & 222.2882 & 220.7608 & $5.3041 e-01$ & $4.6566 \mathrm{e}+00$ \\
\hline 142 & IHT000015 & 163.5667 & 166.3011 & 164.9105 & $5.5893 e-01$ & $4.4192 e+00$ \\
\hline 143 & IHT000016 & 152.5841 & 154.9279 & 153.6388 & $5.1145 e-01$ & $4.3784 e+00$ \\
\hline 144 & IHTO00017 & 182.8050 & 185.7347 & 184.2269 & $6.8122 e-01$ & $4.4949 e+00$ \\
\hline 145 & IHT000018 & 184.5027 & 187.2371 & 185.9169 & $6.8295 e-01$ & $4.5018 e+00$ \\
\hline 146 & IHTO00019 & 178.8687 & 181.2124 & 180.0875 & $6.5264 e-01$ & $4.4781 e+00$ \\
\hline 147 & IHTO00020 & 184.3224 & 186.6662 & 185.3575 & t. $.4748 e-01$ & $4.4995 e+00$ \\
\hline 148 & IHT000021 & -2.81 .32 & -0.0789 & -1.5984 & $7.8749 e-01$ & $4.1000 e+00$ \\
\hline 149 & IHTO00022 & 158.6801 & 161.0239 & 159.6919 & $6.4439 e-01$ & $4.4000 e+00$ \\
\hline 150 & IHTO00023 & 180.1458 & 182.4895 & 181.2198 & $6.0906 e-01$ & $4.4826 e+00$ \\
\hline 151 & IHTO00024 & 144.5726 & 146.9163 & 145.6430 & $6.0004 e-01$ & $4.3510 \mathrm{e}+00$ \\
\hline 152 & IHTO00025 & 179.3532 & 181.6970 & 180.5524 & $6.1385 e-01$ & $4.4799 e+00$ \\
\hline 153 & IHT000026 & 181.4979 & 183.8417 & 182.6815 & $6.3554 \mathrm{e}-01$ & $4.4886 e+00$ \\
\hline 154 & IHTO00027 & 154.8377 & 157.9627 & 156.1112 & $7.0833 e-01$ & $4.3871 e+00$ \\
\hline 155 & IHTO00028 & 157.1552 & 160.2802 & 158.7177 & $7.0587 e-01$ & $4.3965 e+00$ \\
\hline
\end{tabular}




\begin{tabular}{|c|c|c|c|c|c|c|}
\hline \multicolumn{2}{|c|}{ Test: Aug17_12 } & sum2 & Date: Fri & Aug 1703 & $27: 10 \quad 1990$ & Page: 4 \\
\hline \# & Name & Min & Max & Ave & SD & Uncert \\
\hline 156 & IHTO000029 & 180.4012 & 183.5262 & 181.8973 & $7.2288 e-01$ & $4.4854 e+00$ \\
\hline 157 & IHTO00030 & 183.9656 & 186.7000 & 185.3251 & $6.5312 \mathrm{e}-01$ & $4.4994 e+00$ \\
\hline 158 & IHT 000031 & 181.2275 & 183.7665 & 182.4697 & $6.5763 e-01$ & $4.4877 e+00$ \\
\hline 159 & IHTOOOO032 & 183.4998 & 186.0389 & 184.8632 & $6.8628 \mathrm{e}-01$ & $4.4975 e+00$ \\
\hline 160 & IHT 000033 & 183.3271 & 186.0615 & 184.7842 & $6.8027 e-01$ & $4.4972 e+00$ \\
\hline 161 & IHTO00034 & 161.3206 & 163.6644 & 162.4457 & $5.37519-01$ & $4.4101 e+00$ \\
\hline 162 & IHTO00035 & 212.7855 & 215.1292 & 214.0745 & $5.8394 e-01$ & $4.6252 e+00$ \\
\hline 163 & IHTO000036 & 159.2060 & 161.5497 & 160.4756 & $5.3996 \mathrm{e}-01$ & $4.4029 e+00$ \\
\hline
\end{tabular}




\begin{tabular}{|c|c|c|c|}
\hline \multicolumn{4}{|c|}{$\begin{array}{l}\text { Velocity } 1=0.021300(\mathrm{ft} / \mathrm{sec}) \\
\text { Velocity } 2=0.020200(\mathrm{ft} / \mathrm{sec}) \\
\text { Pressure Drop }=0.047600(\mathrm{psi}) \\
\text { Tsat in }=0.067596(\mathrm{deg} \mathrm{F}) \mathrm{Tsat} \text { out }=0.021088 \text { (deg F) } \\
\text { Phi inner }=0.691986(\mathrm{kBtu} / \mathrm{hr}-\mathrm{ft} 2) \text { Phi outer }=0.114899(\mathrm{kBtu} / \mathrm{hr}-\mathrm{ft} 2)\end{array}$} \\
\hline \# & Name & Abs Diff & 8Diff \\
\hline 0 & TRF000001 & 0.1016 & 0.1197 \\
\hline 1 & TRF 000002 & 0.1053 & 0.1238 \\
\hline 2 & TEMO00001 & 0.2200 & 0.2573 \\
\hline 3 & TIN000001 & 0.2256 & 0.2657 \\
\hline 4 & TRV000001 & 0.2483 & 0.2868 \\
\hline 5 & TSR000001 & 2.1394 & 1.4272 \\
\hline 6 & TAO020136 & 5.6195 & 3.9149 \\
\hline 7 & TA0050136 & 0.2348 & 0.1164 \\
\hline 8 & TA0070136 & 0.2082 & 0.1057 \\
\hline 9 & TAO0 80136 & 0.2260 & 0.0963 \\
\hline 10 & TAO090136 & 0.2260 & 0.0929 \\
\hline 11 & TAO100136 & 0.2913 & 0.1238 \\
\hline 12 & TAO105136 & 0.4006 & 0.1658 \\
\hline 13 & TAO110136 & 3.1660 & 3.4073 \\
\hline 14 & TAO115136 & 0.4849 & 0.1963 \\
\hline 15 & TA0120136 & 0.4486 & 0.1659 \\
\hline 16 & TAO125136 & 4.6571 & 1.8297 \\
\hline 17 & TA0130136 & 0.5570 & 0.2153 \\
\hline 18 & TAO120048 & 3.1345 & 2.7981 \\
\hline 19 & TAO130048 & 0.3716 & 0.1346 \\
\hline 20 & TAO120224 & 0.2975 & 0.1117 \\
\hline 21 & TAO130224 & 0.0822 & 0.0330 \\
\hline 22 & TAO120312 & 0.2941 & 0.1100 \\
\hline 23 & TA0130312 & 0.2478 & 0.0910 \\
\hline 24 & TRT020000 & 0.1066 & 0.0541 \\
\hline 25 & TRT070000 & 0.1919 & 0.0806 \\
\hline 26 & TRT $10 C 000$ & 0.1374 & 0.0506 \\
\hline 27 & TRT120000 & 0.0837 & 0.0182 \\
\hline 28 & TRT 125000 & 4.2194 & 4.4219 \\
\hline 29 & TRT1 130000 & 0.3093 & 0.1112 \\
\hline 30 & EMPTY & 0.0413 & 0.1037 \\
\hline 31 & TAE050136 & 0.2232 & 0.1670 \\
\hline 32 & TAF 070136 & 0.1557 & 0.1011 \\
\hline 33 & TAF080136 & 2.8499 & 1.2201 \\
\hline 34 & TAF090136 & 3.2448 & 3.4633 \\
\hline 35 & TAF 100136 & 0.4475 & 0.2432 \\
\hline 36 & TAF 105136 & 0.2438 & 0.1288 \\
\hline 37 & TAF 110136 & 0.3718 & 0.1905 \\
\hline 38 & TAE 115136 & 0.4287 & 0.2136 \\
\hline 39 & TAF 120136 & 1.0462 & 0.4288 \\
\hline 40 & TAF 125136 & 0.4606 & 0.2182 \\
\hline 41 & TAF 130135 & 0.5123 & 0.2386 \\
\hline 42 & TAF $1200 \cdot 3$ & 0.3519 & 0.1623 \\
\hline 43 & TAF 130048 & 0.3042 & 0.1327 \\
\hline 44 & TAF 120224 & 0.1671 & 0.0827 \\
\hline 45 & TAF 130224 & 0.2488 & 0.1183 \\
\hline
\end{tabular}




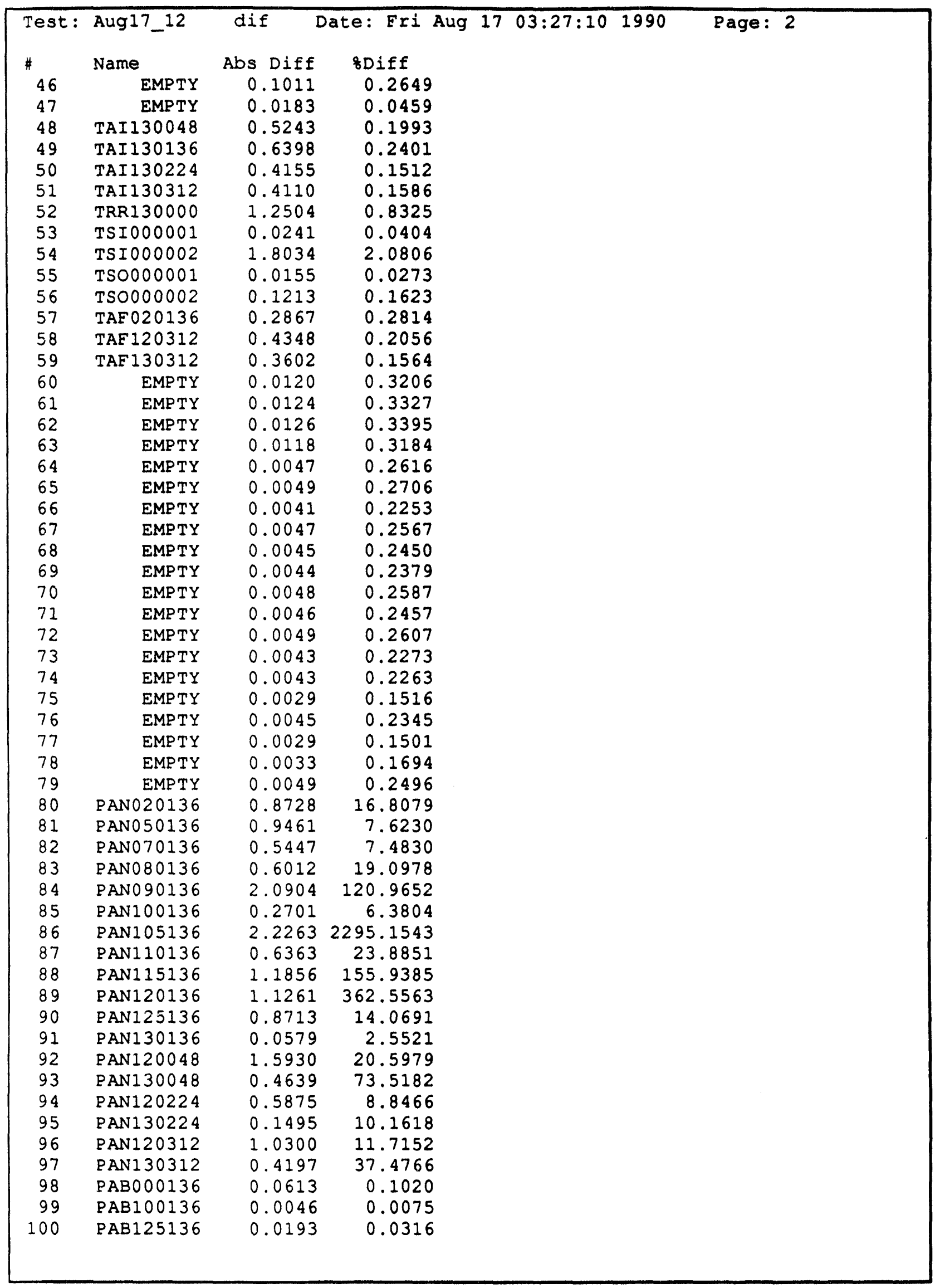




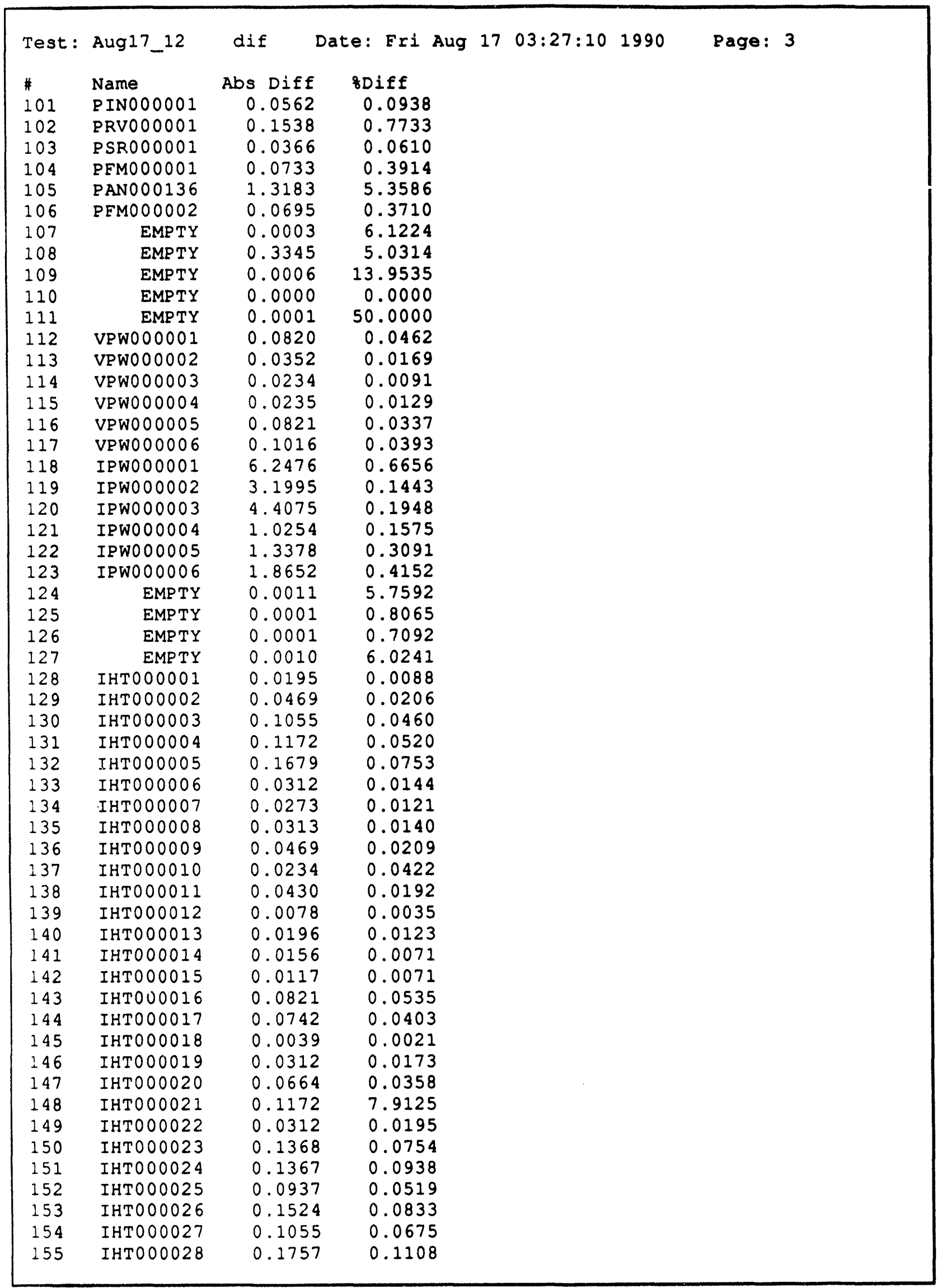




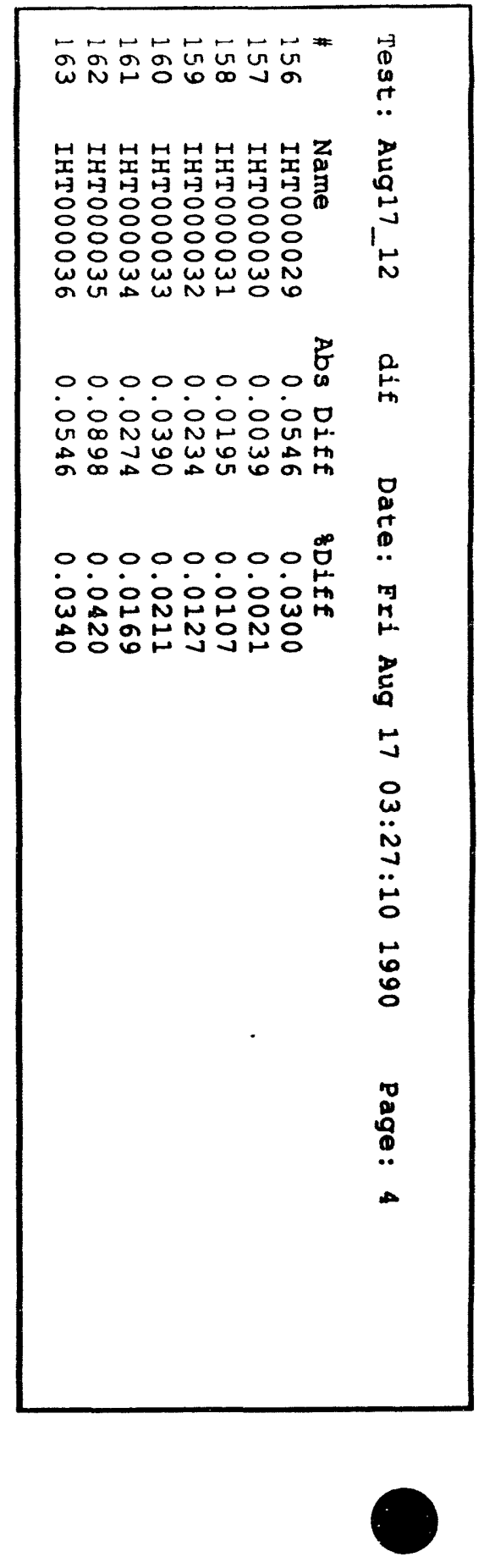




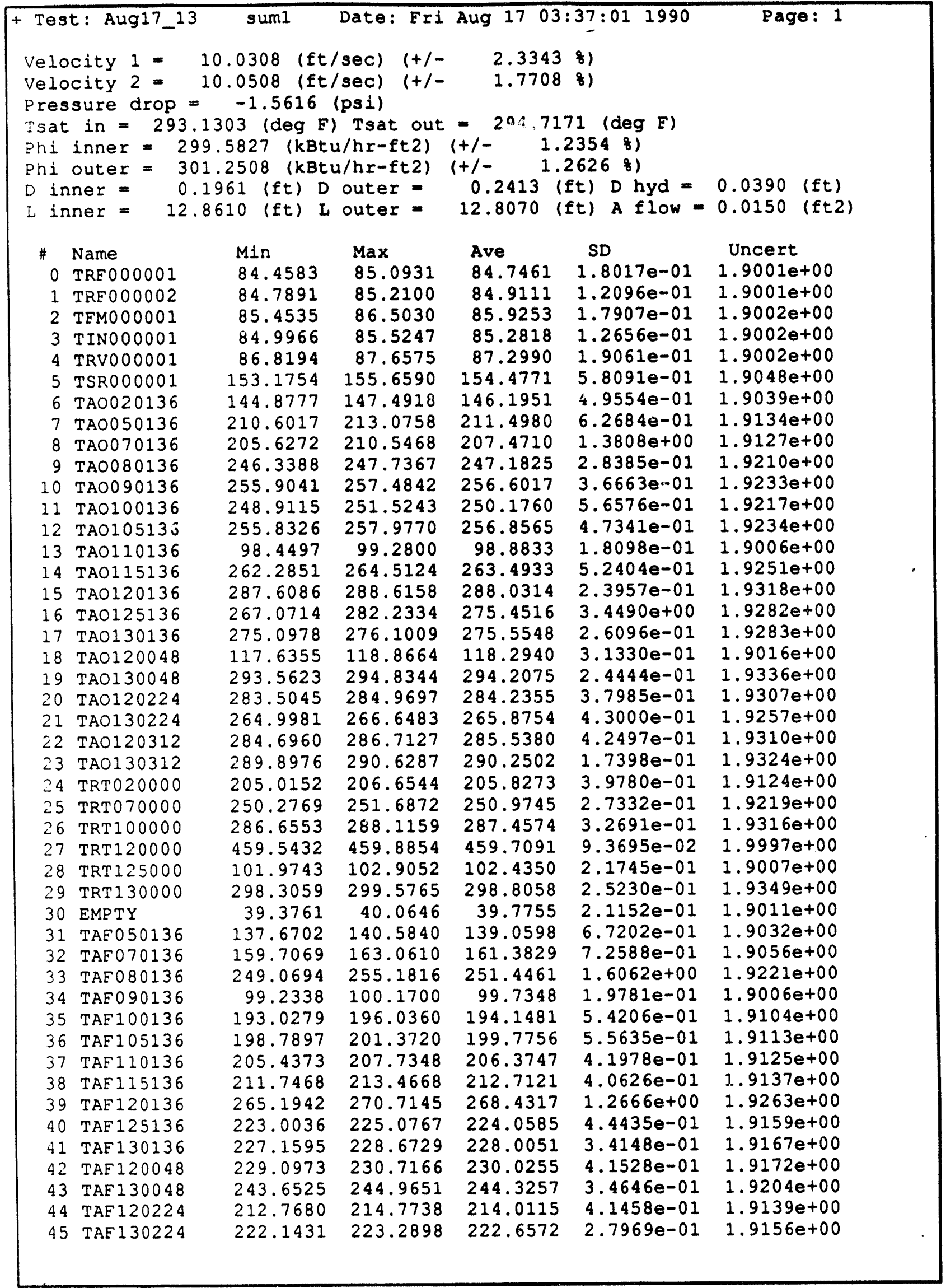




\begin{tabular}{|c|c|c|c|c|c|c|}
\hline \multicolumn{2}{|c|}{ Test: Aug17_13 } & suml & Date: Eri & Aug 1703 & $7: 011990$ & Page: 2 \\
\hline \# & Name & Min & $\operatorname{Max}$ & Ave & SD & Uncert \\
\hline 46 & EMPTY & 37.7675 & 38.4572 & 38.1124 & $2.1911 e-01$ & $1.9010 \mathrm{e}+00$ \\
\hline 47 & EMPTY & 39.8352 & 40.0646 & 39.9545 & $1.1580 e-01$ & $1.90110+00$ \\
\hline 48 & TAII30048 & 278.7697 & 280.6984 & 279.7267 & $4.4026 e-01$ & $1.9294 e+00$ \\
\hline 49 & TAI 130136 & 280.4980 & 281.9640 & 281.3246 & $3.3483 e-01$ & $1.9299 e+00$ \\
\hline 50 & TAI 130224 & 289.3906 & 290.9468 & 290.1796 & $4.1016 e-01$ & $1.9324 \mathrm{e}+00$ \\
\hline 51 & TAI 130312 & 274.0890 & 275.3797 & 274.7735 & $3.0685 e-01$ & $1.9280 \mathrm{e}+00$ \\
\hline 52 & TRR 130000 & 150.5790 & 151.9740 & 151.2586 & $3.6811 e-01$ & $1.9044 \mathrm{e}+00$ \\
\hline 53 & TSI000001 & 59.3117 & 60.0639 & 59.6710 & $1.8235 e-01$ & $1.9001 e+00$ \\
\hline 54 & TSI000002 & 86.0376 & 87.8218 & 86.9966 & $3.2905 e-01$ & $1.9002 \mathrm{e}+00$ \\
\hline$\equiv 5$ & TSO000001 & 56.2328 & 57.0985 & 56.6635 & $2.1368 e-01$ & $1.9001 e+00$ \\
\hline 56 & TSO000002 & 75.4524 & 76.1964 & 75.8244 & $1.9099 e-01$ & $1.9000 e+00$ \\
\hline 57 & TAF020136 & 102.3129 & 105.9634 & 103.9643 & $7.8423 e-01$ & $1.9008 \mathrm{e}+00$ \\
\hline 58 & TAF 120312 & 223.7194 & 226.4744 & 225.0482 & $5.9892 e-01$ & $1.9161 \mathrm{e}+00$ \\
\hline 59 & TAF 130312 & 245.6706 & 248.0058 & 246.6626 & $5.1091 e-01$ & $1.9209 e+00$ \\
\hline 60 & EMPTY & 4.1504 & 4.1797 & 4.1652 & $5.9156 e-03$ & $0.0000 e+00$ \\
\hline 61 & EMPTY & 4.1357 & 4.1504 & 4.1446 & $3.3728 e-03$ & $0.0000 \mathrm{e}+00$ \\
\hline 62 & EMPTY & 4.1211 & 4.1406 & 4.1247 & $3.5307 e-03$ & $0.0000 e+00$ \\
\hline 63 & EMPTY & 4.1113 & 4.1211 & 4.1156 & $2.1249 e-03$ & $0.0000 e+00$ \\
\hline 64 & EMPTY & 1.9775 & 1.9824 & 1.9781 & $1.6028 e-03$ & $0.0000 e+00$ \\
\hline 65 & EMPTY & 1.9873 & 1.9922 & 1.9921 & $6.9053 e-04$ & $0.0000 e+00$ \\
\hline 66 & EMPTY & 1.9971 & 2.0020 & 1.9987 & $2.3365 e-03$ & $0.0000 e+00$ \\
\hline 67 & EMPTY & 2.0068 & 2.0117 & 2.0103 & $2.2603 e-03$ & $0.0000 e+00$ \\
\hline 68 & EMPTY & 2.0117 & 2.0166 & 2.0156 & $1.9730 \mathrm{e}-03$ & $0.0000 e+00$ \\
\hline 69 & EMPTY & 2.0215 & 2.0264 & 2.0253 & $2.0432 e-03$ & $0.0000 e+00$ \\
\hline 70 & EMPTY & 2.0313 & 2.0410 & 2.0315 & $1.5314 e-03$ & $0.000 n e+00$ \\
\hline 71 & EMPTY & 2.0459 & 2.0508 & 2.0461 & $9.6655 e-04$ & $0.0000 e+00$ \\
\hline 72 & EMPTY & 2.0508 & 2.0557 & 2.0514 & $1.6028 e-03$ & $0.0000 e+00$ \\
\hline 73 & EMPTY & 2.0605 & 2.0654 & 2.0639 & $2.3008 e-03$ & $0.0000 e+00$ \\
\hline 74 & EMPTY & 2.0703 & 2.0752 & 2.0705 & $9.6655 e-04$ & $0.0000 e+00$ \\
\hline 75 & EMPTY & 2.0752 & 2.0898 & 2.0803 & $1.9631 e-03$ & $0.0000 e+00$ \\
\hline 76 & EMPTY & 2.0850 & 2.0947 & 2.0869 & $2.6100 e-03$ & $0.0000 e+00$ \\
\hline 77 & EMPTY & 2.0947 & 2.1045 & 2.0990 & $1.8821 e-03$ & $0.0000 e+00$ \\
\hline 78 & EMPTY & 2.1094 & 2.1143 & 2.1135 & $1.8082 e-03$ & $0.0000 e+00$ \\
\hline 79 & EMPTY & 2.1289 & 2.1338 & 2.1290 & $6.9053 e-04$ & $0.0000 \mathrm{e}+00$ \\
\hline 30 & PAN020136 & -17.8439 & 5.2732 & -7.8494 & $5.3039 e+00$ & $8.7022 e-01$ \\
\hline 81 & PAN 050136 & -29.7115 & -3.3138 & -18.5940 & $6.6532 e+00$ & $1.6806 \mathrm{e}+00$ \\
\hline 82 & PAN070136 & -24.5018 & 1.1330 & -10.9032 & $6.5250 e+00$ & $8.7043 e-01$ \\
\hline 83 & PAN080136 & -13.9558 & 4.8125 & -7.8553 & $5.3358 e+00$ & $8.7022 e-01$ \\
\hline 84 & PAN090136 & -11.9546 & 4.5249 & -3.1808 & $3.9480 \mathrm{e}+00$ & $8.70049-01$ \\
\hline 85 & PAN 100136 & -12.1908 & 7.4931 & -2.6037 & $5.0459 e+00$ & $8.7002 e-01$ \\
\hline 86 & PAN105136 & -7.7020 & 13.8129 & -3.1045 & $5.2044 e+00$ & $8.7003 e-01$ \\
\hline 87 & PAN 110136 & -1.8053 & 35.7313 & 10.0981 & $7.8756 e+00$ & $8.7037 e-01$ \\
\hline 88 & PAN115136 & -5.8049 & 6.4784 & 0.3200 & $3.0344 \mathrm{e}+00$ & $8.7000 e-01$ \\
\hline 59 & PAN120136 & -5.9853 & 4.8484 & 1.3267 & $2.2437 e+00$ & $8.7001 e-01$ \\
\hline 90 & PAN125136 & -4.2775 & 4.5725 & 0.6663 & $2.1030 \mathrm{e}+00$ & $8.7000 e-01$ \\
\hline 91 & PAN130136 & -4.6252 & 4.6827 & -0.3390 & $2.2577 e+00$ & $8.7000 e-01$ \\
\hline 92 & PAN120048 & -1.6534 & 5.1368 & 1.8668 & $1.8439 \mathrm{e}+00$ & $8.7001 e-01$ \\
\hline 93 & PAN 130048 & -3.9436 & 2.0073 & -1.4763 & $1.4396 \mathrm{e}+00$ & $8.7001 e-01$ \\
\hline 94 & PAN120224 & -0.3109 & 17.6181 & 3.7662 & $3.8345 \mathrm{e}+00$ & $8.7005 e-01$ \\
\hline 95 & PAN130224 & -3.2056 & 4.1186 & -0.5674 & $1.4954 \mathrm{e}+00$ & $8.7000 e-01$ \\
\hline 96 & PAN 120312 & -3.5020 & 28.2363 & 2.4428 & $5.5589 \mathrm{e}+00$ & $8.7002 e-01$ \\
\hline 97 & PAN130312 & -6.4027 & 2.6763 & -1.8602 & $2.1460 e+00$ & $8.7001 e-01$ \\
\hline 98 & PAB000136 & 60.0950 & 60.8732 & 60.4731 & $2.1705 e-01$ & $5.9938 e-01$ \\
\hline 99 & PAB100136 & 61.6541 & 62.9358 & 62.0615 & $2.4085 e-01$ & $6.0039 e-01$ \\
\hline 100 & $\mathrm{PAB} 125136$ & 61.7861 & 62.1523 & 61.9271 & $9.0065 e-02$ & $6.0031 e-01$ \\
\hline
\end{tabular}




\begin{tabular}{|c|c|c|c|c|c|c|}
\hline \multicolumn{2}{|c|}{ Test: Aug17_13 } & sum1 & Date: Fri & \multicolumn{2}{|c|}{ Aug $17 \quad 03: 37: 011990$} & \multirow{2}{*}{$\begin{array}{l}\text { Page: } 3 \\
\text { Uncert }\end{array}$} \\
\hline$\#$ & Name & Min & $\operatorname{Max}$ & Ave & SD & \\
\hline 101 & PINO00001 & 59.6120 & 60.9548 & 60.3689 & $4.5273 e-01$ & $7.6503 e-01$ \\
\hline 202 & PRV000001 & 19.7186 & 19.9627 & 19.8406 & $3.4877 e-02$ & $7.5164 e-01$ \\
\hline 103 & PSR000001 & 61.1730 & 61.4171 & 61.2462 & $6.5249 e-02$ & $7.6547 e-01$ \\
\hline 104 & PEMOOO0001 & 14.9160 & 15.6484 & 15.3445 & $1.6589 e-01$ & $5.0147 e-01$ \\
\hline 105 & PANO00136 & -59.0071 & -29.2525 & -43.2326 & $7.7785 e+00$ & $4.9212 e+00$ \\
\hline 106 & PEMOOO0002 & 15.1183 & 15.5881 & 15.4065 & $9.9801 e-02$ & $2.0368 e-01$ \\
\hline$: 07$ & EMPTY & 0.0049 & 0.0049 & 0.0049 & $0.0000 e+00$ & $0.0000 e+00$ \\
\hline 108 & EMPTY & -10.0000 & -5.9131 & -8.3900 & $1.3728 e+00$ & $0.0000 e+00$ \\
\hline 209 & EMPTY & 0.0000 & 0.0049 & 0.0043 & $1.6028 e-03$ & $0.0000 e+00$ \\
\hline$\therefore 10$ & EMPTY & -10.0000 & -10.0000 & -10.0000 & $0.0000 e+00$ & $0.0000 e+00$ \\
\hline 211 & EMPTY & 0.0000 & 0.0000 & 0.0000 & $0.0000 \mathrm{e}+00$ & $0.0000 \mathrm{e}+00$ \\
\hline 112 & VPWOOOOOI & 177.4151 & 178.0011 & 177.6690 & $1.3811 e-01$ & $1.3388 e+00$ \\
\hline 113 & VPW000002 & 207.5233 & 208.1092 & 207.7460 & $1.1844 e^{-01}$ & $1.4865 e+00$ \\
\hline 114 & VPW000003 & 256.8021 & 257.3881 & 257.1028 & $1.1975 e-01$ & $1.7423 \mathrm{e}+00$ \\
\hline 215 & VPW000004 & 182.4031 & 182.9890 & 182.7352 & $1.2001 e-01$ & $1.3632 \mathrm{e}+00$ \\
\hline 116 & VPW000005 & 243.3631 & 243.7538 & 243.5468 & $1.2104 e-01$ & $1.6708 e+00$ \\
\hline 117 & VPW000006 & 258.4172 & 258.8079 & 258.6476 & $1.2283 e-01$ & $1.7506 e+00$ \\
\hline 118 & TPWO000001 & 943.7329 & 949.5900 & 946.9344 & $1.5659 \mathrm{e}+00$ & $1.1026 e+01$ \\
\hline $1: 9$ & IPW0 00002 & 2221.3704 & 2236.9805 & 2231.7903 & $4.1833 e+00$ & $1.9403 e+01$ \\
\hline 120 & IPW0 00003 & 2248.3499 & 2260.0498 & 2255.2532 & $3.0569 \mathrm{e}+00$ & $1.9571 \mathrm{e}+01$ \\
\hline 121 & IPWOODOO4 & 651.6770 & 654.6067 & 653.6206 & $4.6782 e-01$ & $5.5374 e+00$ \\
\hline 122 & IPW0 00005 & 434.2076 & 435.1841 & 434.6470 & $3.7235 e-01$ & $3.9737 e+00$ \\
\hline$: 23$ & IPW000006 & 446.8149 & 449.2563 & 447.8986 & $4.6574 e-01$ & $4.0645 e+00$ \\
\hline$i 24$ & EMPTY & -0.0293 & -0.0146 & -0.0211 & $3.9998 e-03$ & $0.0000 \mathrm{e}+00$ \\
\hline 125 & EMPTY & -0.0195 & -0.0098 & -0.0131 & $2.6908 e-03$ & $0.0000 e+00$ \\
\hline 126 & EMPTY & -0.0244 & -0.0098 & -0.0162 & $3.3366 e-03$ & $0.0000 e+00$ \\
\hline 127 & EMPTY & -0.0244 & -0.0098 & -0.0152 & $3.5072 e-03$ & $0.0000 e+00$ \\
\hline$\therefore 28$ & IHTO00001 & 219.3434 & 222.0778 & 220.7185 & $6.5192 e-01$ & $4.6564 e+00$ \\
\hline 129 & IHTO00002 & 226.1268 & 228.4705 & 227.1425 & $6.3011 e-01$ & $4.6871 \mathrm{e}+00$ \\
\hline 130 & IHTO00003 & 227.6217 & 230.5514 & 229.0319 & $7.0948 e-01$ & $4.6963 e+00$ \\
\hline$: 31$ & IHTO00004 & 223.7643 & 226.4986 & 225.0104 & $7.4230 e-01$ & $4.6769 e+00$ \\
\hline$: 32$ & IHTOOOOOO5 & 221.2590 & 223.7981 & 222.5128 & $6.0501 e-01$ & $4.6649 e+00$ \\
\hline$: 33$ & IHTO00006 & 215.4034 & 217.9424 & 216.6651 & $6.4502 e-01$ & $4.6373 e+00$ \\
\hline 134 & IHTO00007 & 225.1352 & 227.2837 & 226.0882 & $5.7944 e-01$ & $4.6820 e+00$ \\
\hline 135 & IHTOOOOOOB & 222.5436 & 224.6920 & 223.4850 & $5.8752 e-01$ & $4.6695 e+00$ \\
\hline$: 36$ & IHTO00009 & 222.5323 & 225.0714 & 223.6455 & $6.5821 e-01$ & $4.6703 e+00$ \\
\hline$: 37$ & IHTOO0010 & 54.0377 & 56.3815 & 55.4244 & $5.5419 e-01$ & $4.1373 e+00$ \\
\hline 138 & IHTO00011 & 222.6900 & 225.6197 & 224.2487 & $5.0295 e-01$ & $4.6732 e+00$ \\
\hline 139 & IHTO00012 & 218.7425 & 221.8675 & 220.3362 & $6.5121 e-01$ & $4.6545 e+00$ \\
\hline$\$ 40$ & IHTO00013 & 158.3984 & 161.5234 & 159.7422 & $6.5035 e-01$ & $4.4002 e+00$ \\
\hline 141 & IHTO00014 & 219.3585 & 222.4835 & 220.6826 & $6.2051 e-01$ & $4.6562 e+00$ \\
\hline 142 & IHTOOOOI5 & 163.5667 & 166.3011 & $164.87 ? 5$ & $5.8052 e-01$ & $4.4191 e+00$ \\
\hline 143 & IHTO00016 & 152.3888 & 154.9279 & 153.5255 & $6.0062 e-01$ & $4.3780 e+00$ \\
\hline 144 & IHTO00017 & 182.4144 & 185.3441 & 183.8910 & $6.4496 e-01$ & $4.4935 e+00$ \\
\hline 145 & IHTO00018 & 184.5027 & 188.0183 & 185.8075 & $6.9187 e-01$ & $4.5014 e+00$ \\
\hline 146 & IHTO00019 & 178.8687 & 181.0171 & 180.1461 & $5.9609 e-01$ & $4.4783 e+00$ \\
\hline 147 & IHTO00020 & 183.9318 & 186.4708 & 185.3692 & $6.0330 e-01$ & $4.4996 \mathrm{e}+00$ \\
\hline 148 & IHT000021 & -3.2039 & 0.1164 & -1.4695 & $7.3783 e-01$ & $4.1000 e+00$ \\
\hline 149 & IHTO00022 & 158.2895 & 160.8286 & 159.6606 & $5.7924 e-01$ & $4.3999 e+00$ \\
\hline 150 & IHTO00023 & 179.7551 & 182.4895 & 181.2823 & $5.9802 e-01$ & $4.4829 e+00$ \\
\hline 151 & IHTO00024 & 144.3773 & 146.9163 & 145.7016 & $5.7221 e-01$ & $4.3512 e+00$ \\
\hline 152 & IHTOO0025 & 179.1579 & 181.8923 & 180.6071 & $5.6784 e-01$ & $4.4802 \mathrm{e}+00$ \\
\hline 153 & IHTO00026 & 181.1073 & 184.0370 & 182.7558 & $6.0123 e-01$ & $4.4889 \mathrm{e}+00$ \\
\hline 154 & IHTO00027 & 154.4471 & 157.1815 & 156.0722 & $5.4881 e^{-01}$ & $4.3870 e+00$ \\
\hline 155 & IHTO00028 & 156.7646 & 159.6942 & 158.5693 & $6.6329 e-01$ & $4.3960 \mathrm{e}+00$ \\
\hline
\end{tabular}




\begin{tabular}{|c|c|c|c|c|c|c|}
\hline \multicolumn{2}{|c|}{ Test: Aug17_13 } & suml & Date: Fri & Aug 170 & $37: 01 \quad 1990$ & Page: 4 \\
\hline \# & Name & Min & $\operatorname{Max}$ & Ave & SD & Uncert \\
\hline 156 & IHTO000029 & 180.2058 & 183.3308 & 181.8700 & $6.9948 e-01$ & $4.4853 e+00$ \\
\hline 157 & IHT000030 & 183.9656 & 186.5047 & 185.2665 & $6.3700 e-01$ & $4.4992 e+00$ \\
\hline 158 & IHT000031 & 181.0322 & 183.7665 & 182.3720 & $6.3505 e-01$ & $4.4873 e+00$ \\
\hline 159 & IHTO00032 & 183.3045 & 186.2342 & 184.7148 & $6.7461 e-01$ & $4.4969 e+00$ \\
\hline 160 & IHT 000033 & 183.1318 & 186.2568 & 184.6592 & $6.7507 e-01$ & $4.4967 e+00$ \\
\hline 161 & IHT 000034 & 160.9300 & 163.6644 & 162.2933 & $5.7655 e-01$ & $4.4095 e+00$ \\
\hline 162 & IHT 000035 & 212.7855 & 214.9339 & 213.8870 & $5.8761 e-01$ & $4.6244 e+00$ \\
\hline 163 & IHT 000036 & 159.2060 & 161.3544 & 160.3779 & $5.4104 e-01$ & $4.4025 e+00$ \\
\hline
\end{tabular}




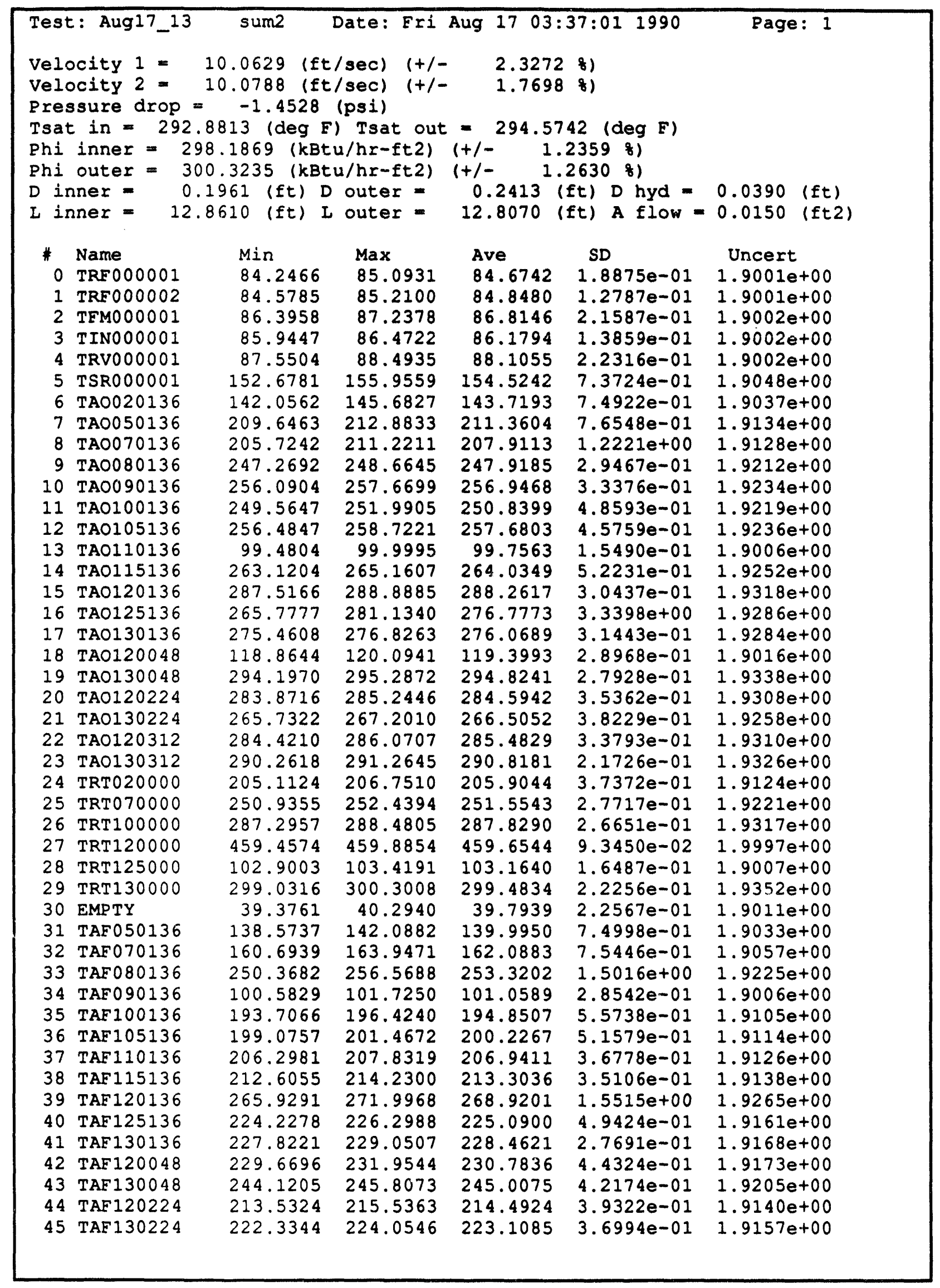




\begin{tabular}{|c|c|c|c|c|c|c|}
\hline \multicolumn{2}{|c|}{ Test: Aug17_13 } & sum2 & \multicolumn{3}{|c|}{ Date: Fri Aug $1703: 37: 011990$} & Page: 2 \\
\hline * & Name & Min & $\operatorname{Max}$ & Ave & SD & Uncert \\
\hline 46 & EMPTY & 37.5374 & 38.4572 & 38.1124 & $1.9293 e-01$ & $1.9010 e+00$ \\
\hline 47 & EMPTY & 39.6057 & 40.2940 & 39.9407 & $1.5522 e-01$ & $1.9011 e+00$ \\
\hline 48 & TAI130048 & 279.5956 & 281.5236 & 280.6923 & $4.4714 e-01$ & $1.9297 \mathrm{e}+00$ \\
\hline 49 & TAI 130136 & 281.1386 & 282.4211 & 281.8331 & $2.8605 e-01$ & $1.9300 \mathrm{e}+00$ \\
\hline 50 & TAI 130224 & 289.7569 & 291.8619 & 290.6410 & $4.0633 e-01$ & $1.9325 e+00$ \\
\hline 51 & TAI130312 & 274.1804 & 275.5649 & 275.0132 & $3.0143 e-01$ & $1.9281 \mathrm{e}+00$ \\
\hline 52 & TRR130000 & 152.4723 & 154.5571 & 153.5538 & $4.5028 e-01$ & $1.9047 \mathrm{e}+00$ \\
\hline 53 & TSI000001 & 58.9893 & 59.8496 & 59.4520 & $1.7274 e-01$ & $1.9001 e+00$ \\
\hline 54 & TSI000002 & 87.4001 & 88.6586 & 87.9856 & $3.3130 e-01$ & $1.9002 \mathrm{e}+00$ \\
\hline 55 & TSO000001 & 56.0161 & 56.9903 & 56.5208 & $2.2788 e-01$ & $1.9001 e+00$ \\
\hline 56 & TSO000002 & 75.3464 & 76.3019 & 75.8666 & $2.3424 e-01$ & $1.9000 \mathrm{e}+00$ \\
\hline 57 & TAF020136 & 103.3577 & 106.3803 & 104.9949 & $7.5258 e-01$ & $1.9008 e+00$ \\
\hline 58 & TAF 120312 & 224.2894 & 226.4749 & 225.5307 & $5.1968 e-01$ & $1.9162 \mathrm{e}+00$ \\
\hline 59 & TAF 130312 & 246.6050 & 248.7544 & 247.4662 & $5.2263 e-01$ & $1.9211 e+00$ \\
\hline 60 & EMPTY & 4.1748 & 4.2041 & 4.1888 & $7.0455 e-03$ & $0.0000 e+00$ \\
\hline 61 & EMPTY & 4.1553 & 4.1748 & 4.1674 & $5.4247 e-03$ & $0.0000 \mathrm{e}+00$ \\
\hline 62 & EMPTY & 4.1406 & 4.1602 & 4.1481 & $5.0503 e-03$ & $0.0000 e+00$ \\
\hline 63 & EMPTY & 4.1260 & 4.1455 & 4.1382 & $4.5474 e-03$ & $0.0000 \mathrm{e}+00$ \\
\hline 64 & EMPTY & 1.9824 & 1.9922 & 1.9875 & $2.1970 e-03$ & $0.0000 e+00$ \\
\hline 65 & EMPTY & 1.9971 & 2.0068 & 2.0005 & $2.6562 e-03$ & $0.0000 e+00$ \\
\hline 66 & EMPTY & 2.0068 & 2.0117 & 2.0082 & $2.2146 e-03$ & $0.0000 e+00$ \\
\hline 67 & EMPTY & 2.0166 & 2.0215 & 2.0180 & $2.2146 e-03$ & $0.0000 e+00$ \\
\hline 68 & EMPTY & 2.02 .15 & 2.0264 & 2.0232 & $2.3675 e-03$ & $0.0000 e+00$ \\
\hline 69 & EMPTY & 2.0313 & 2.0361 & 2.0325 & $2.1635 e-03$ & $0.0000 e+00$ \\
\hline 70 & $E M P r_{1} Y$ & 2.0361 & 2.0410 & 2.0395 & $2.3008 e-03$ & $0.0000 \mathrm{e}+00$ \\
\hline 71 & EMPTY & 2.0508 & 2.0557 & 2.0549 & $1.8082 e-03$ & $0.0000 e+00$ \\
\hline 72 & EMPTY & 2.0557 & 2.0605 & 2.0600 & $1.6028 e-03$ & $0.0000 e+00$ \\
\hline 73 & EMPTY & 2.0703 & 2.0752 & 2.0705 & $9.6655 e-04$ & $0.0000 e+00$ \\
\hline 74 & EMPTY & 2.0752 & 2.0850 & 2.0789 & $2.3261 e-03$ & $0.0000 \mathrm{e}+00$ \\
\hline 75 & EMPTY & 2.0850 & 2.0898 & 2.0889 & $1.9730 e-03$ & $0.0000 e+00$ \\
\hline 76 & EMPTY & 2.0947 & 2.0947 & 2.0947 & $0.0000 e+00$ & $0.0000 \mathrm{e}+00$ \\
\hline 77 & EMPTY & 2.1045 & 2.1094 & 2.1057 & $2.1065 e-03$ & $0.0000 e+00$ \\
\hline 78 & EMPTY & 2.1191 & 2.1240 & 2.1198 & $1.7115 e-03$ & $0.0000 \mathrm{e}+00$ \\
\hline 79 & EMPTY & 2.1240 & 2.1484 & 2.1361 & $3.4513 e-03$ & $0.0000 \mathrm{e}+00$ \\
\hline 80 & PAN020136 & -17.8439 & 3.1370 & -7.7273 & $5.5537 e+00$ & $8.7021 e-01$ \\
\hline 81 & PAN050136 & -29.5589 & -0.4147 & -17.0284 & $6.1901 e+00$ & $1.6805 e+00$ \\
\hline 82 & PAN070136 & -23.2811 & 1.2856 & -10.5461 & $6.4130 e+00$ & $8.7040 e-01$ \\
\hline 83 & PAN080136 & -13.8795 & 2.9815 & -7.5837 & $4.9282 e+00$ & $8.7021 e-01$ \\
\hline 84 & PAN090136 & -8.6739 & 10.2470 & -1.6518 & $4.6004 e+00$ & $8.7001 e-01$ \\
\hline 85 & PAN100136 & -12.1908 & 4.9754 & -2.7791 & $4.8680 e+00$ & $8.7003 e-01$ \\
\hline 86 & PAN105136 & -7.7020 & 10.8374 & -1.3009 & $4.6756 e+00$ & $8.7001 e-01$ \\
\hline 87 & PAN110136 & -1.2712 & 26.9575 & 4.5164 & $5.5771 \mathrm{e}+00$ & $8.7007 e-01$ \\
\hline 88 & PAN115136 & -5.1945 & 12.2768 & 0.1689 & $3.5790 \mathrm{e}+00$ & $8.7000 e-01$ \\
\hline 89 & PAN120136 & -4.9172 & 4.3144 & 0.0206 & $2.1797 e+00$ & $8.7000 e-01$ \\
\hline 90 & PAN125136 & -6.1086 & 2.4363 & -3.6336 & $1.9399 \mathrm{e}+00$ & $8.7005 e-01$ \\
\hline 91 & PAN 130136 & 1.4020 & 10.8625 & 5.9812 & $2.0200 e+00$ & $8.7013 e-01$ \\
\hline 92 & PAN 120048 & -4.9340 & 4.8316 & 0.9299 & $2.1688 \mathrm{e}+00$ & $8.7000 e-01$ \\
\hline 93 & PAN 130048 & -3.8673 & 4.4487 & -0.9910 & $1.7536 \mathrm{e}+00$ & $8.7000 e-01$ \\
\hline 94 & PAN120224 & -4.8123 & 29.5963 & 4.0363 & $6.7675 e+00$ & $8.7006 e-01$ \\
\hline 95 & PAN130224 & -4.7315 & 3.8897 & -0.6559 & $2.3586 e+00$ & $8.7000 e-01$ \\
\hline 96 & PAN120312 & -5.1042 & 4.3563 & 0.3356 & $1.9070 e+00$ & $8.7000 e-01$ \\
\hline 97 & PAN130312 & -7.2420 & 3.7444 & -0.7539 & $2.4079 e+00$ & $8.7000 e-01$ \\
\hline 98 & $\mathrm{PAB} 000136$ & 59.7288 & 60.7359 & 60.2470 & $2.4266 \mathrm{e}-01$ & $5.9924 e-01$ \\
\hline 99 & PAB100136 & 61.3794 & 62.1118 & 61.6614 & $1.6319 \mathrm{e}-01$ & $6.0014 e-01$ \\
\hline 100 & PAB125136 & 61.6030 & 62.0150 & 61.7953 & $8.7736 e-02$ & $6.0022 e-01$ \\
\hline
\end{tabular}




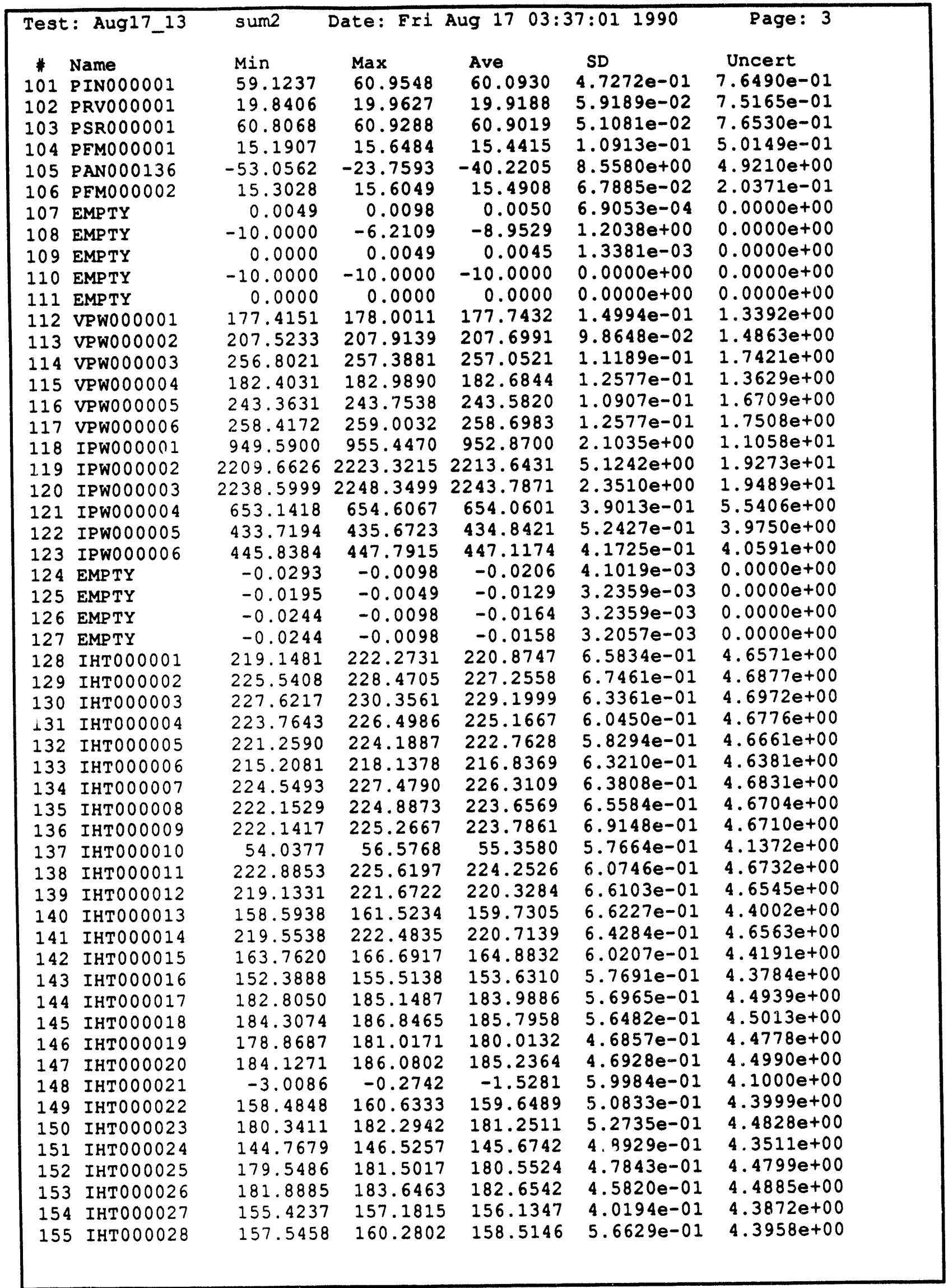




\begin{tabular}{|c|c|c|c|c|c|c|}
\hline $\begin{array}{l}\# \\
156 \\
157 \\
158 \\
159 \\
160 \\
161 \\
162 \\
163\end{array}$ & 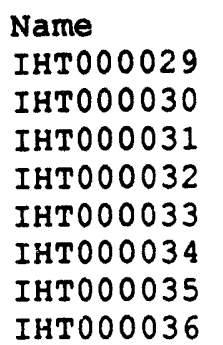 & $\begin{array}{l}\text { Min } \\
180.7918 \\
183.9656 \\
181.2275 \\
183.3045 \\
183.3271 \\
160.9300 \\
212.7855 \\
159.2060\end{array}$ & $\begin{array}{l}\operatorname{Max} \\
183.3308 \\
186.8953 \\
183.9618 \\
186.4295 \\
186.4521 \\
163.2737 \\
215.1292 \\
161.3544\end{array}$ & $\begin{array}{l}\text { Ave } \\
181.7802 \\
185.2001 \\
182.3642 \\
184.7226 \\
184.6826 \\
162.2855 \\
213.8675 \\
160.3506\end{array}$ & $\begin{array}{l}\text { SD } \\
5.8582 e-01 \\
6.1313 e-01 \\
6.4076 e-01 \\
6.7751 e-01 \\
6.7244 e-01 \\
5.2261 e-01 \\
5.3701 e-01 \\
5.2201 e-01\end{array}$ & $\begin{array}{c}\text { Uncert } \\
4.4849 \mathrm{e}+00 \\
4.4989 \mathrm{e}+00 \\
4.4873 \mathrm{e}+00 \\
4.4969 \mathrm{e}+00 \\
4.4968 \mathrm{e}+00 \\
4.4095 \mathrm{e}+00 \\
4.6243 \mathrm{e}+00 \\
4.4024 \mathrm{e}+00\end{array}$ \\
\hline
\end{tabular}




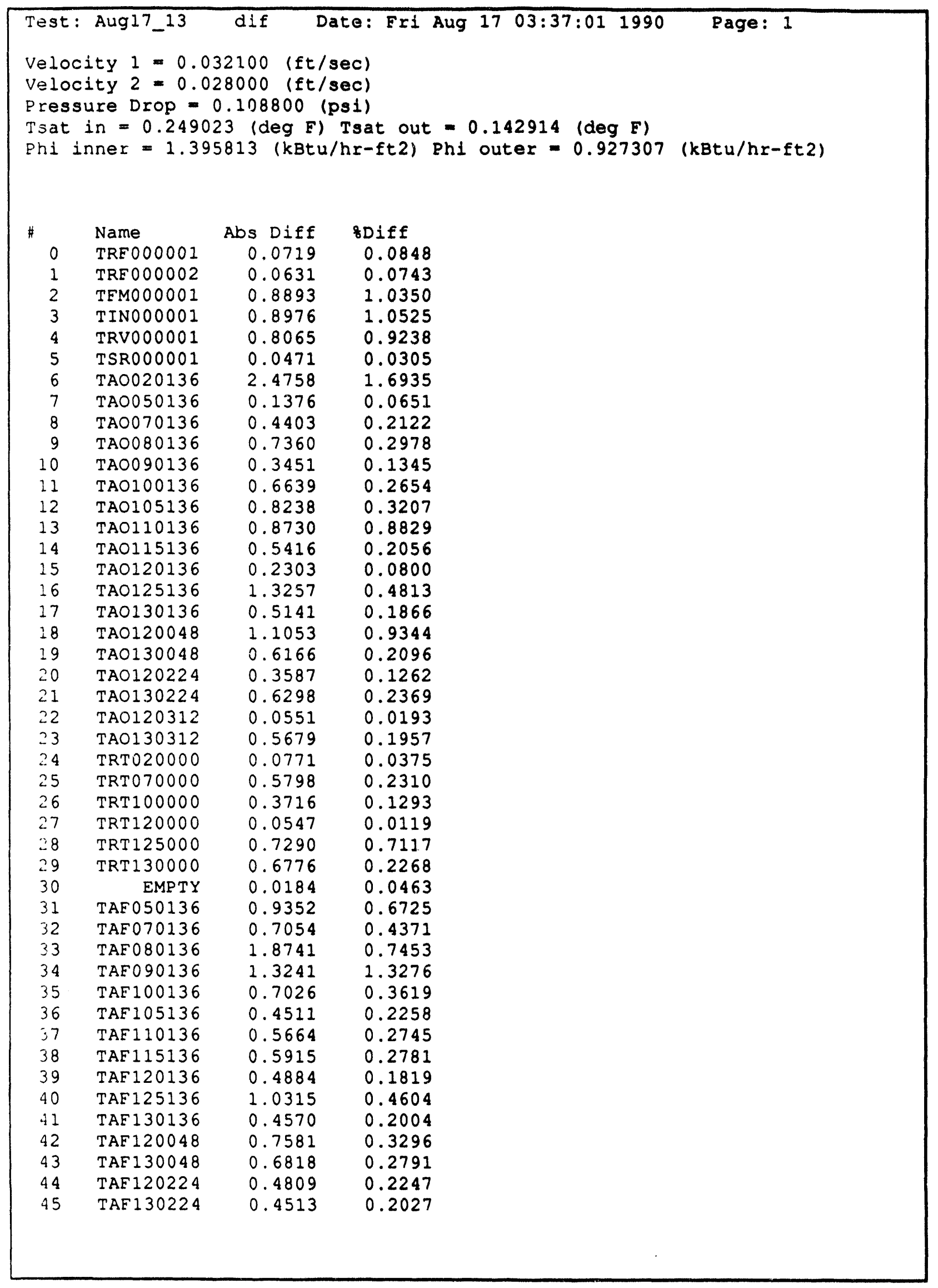




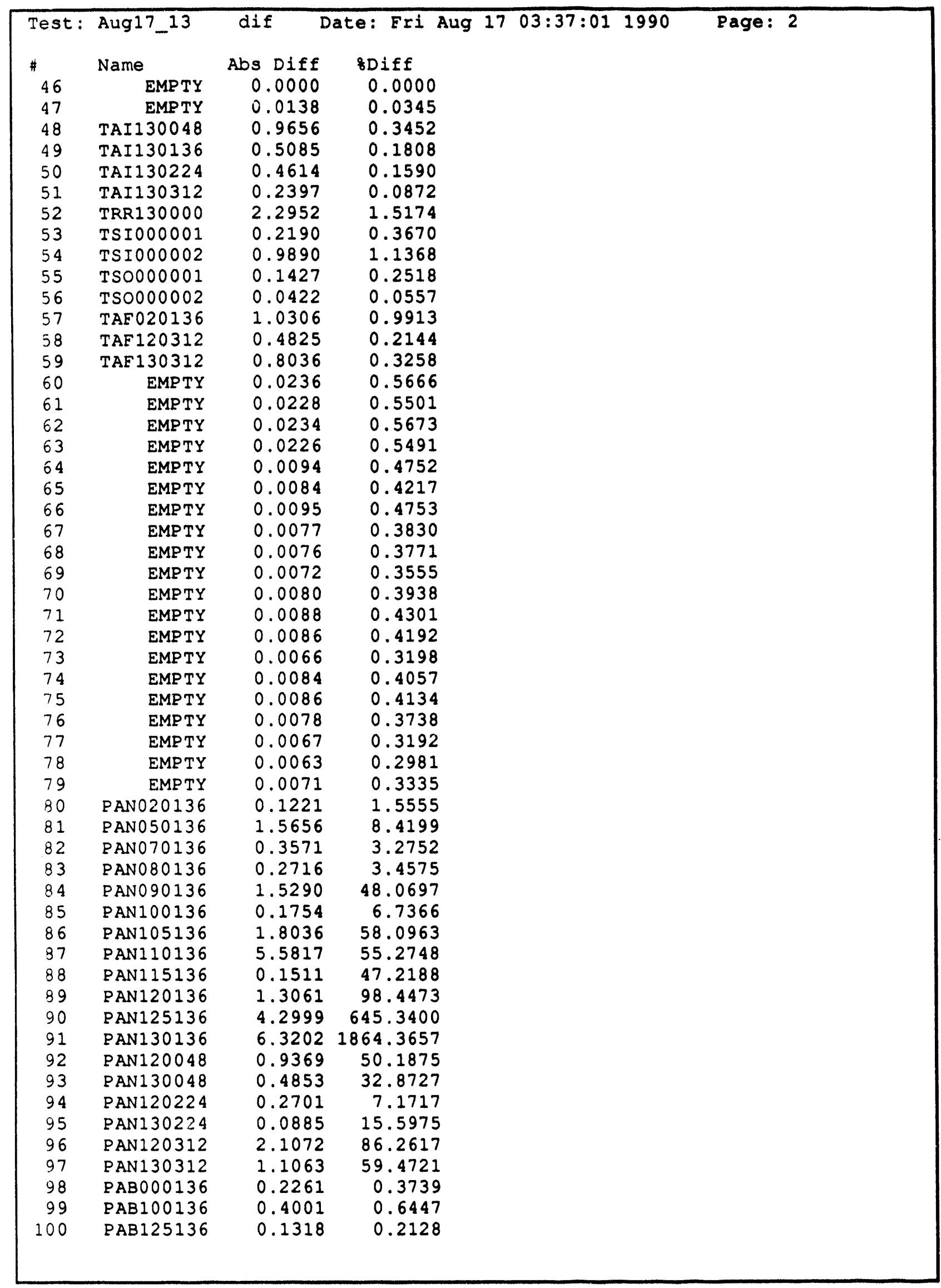




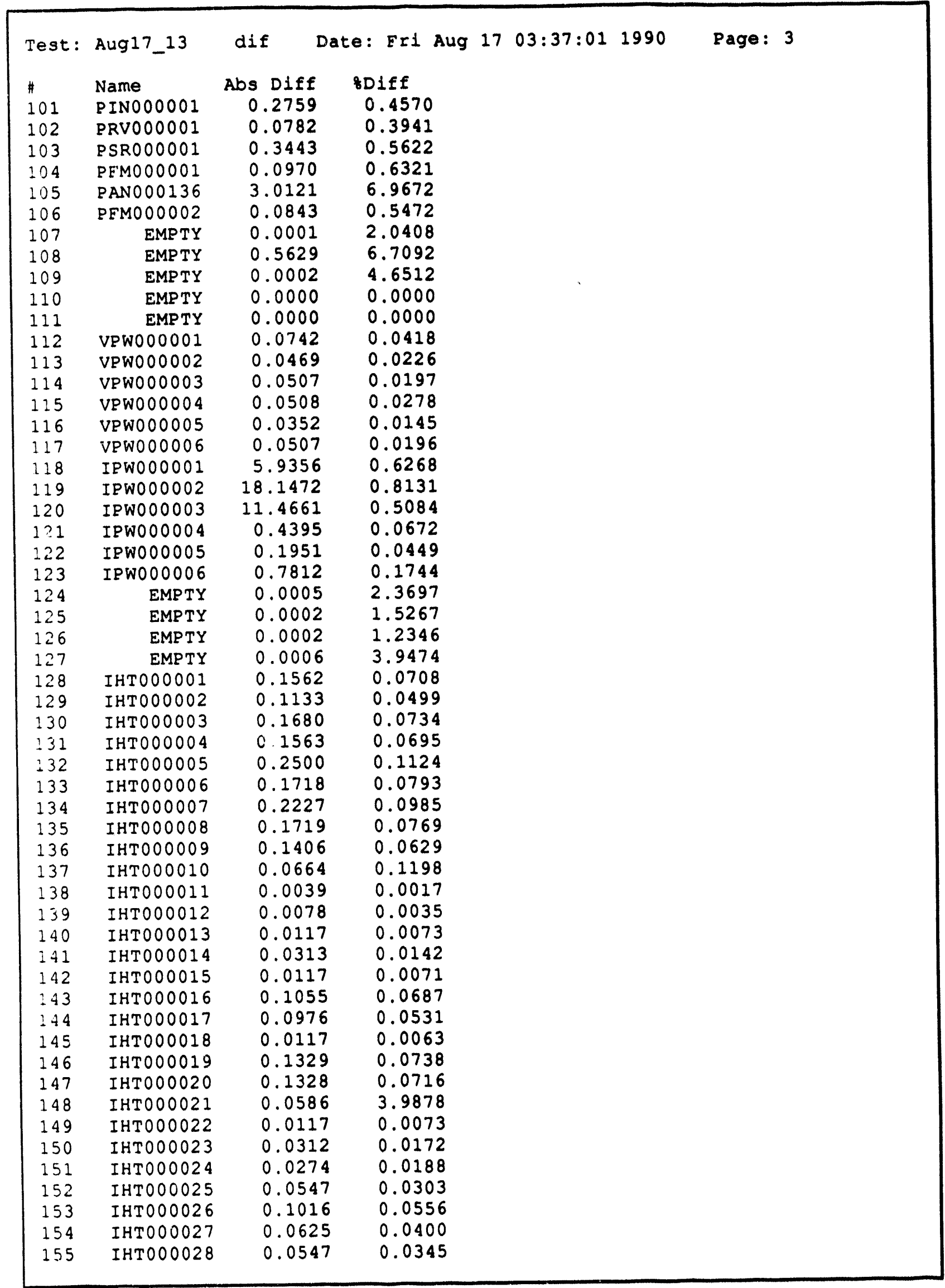




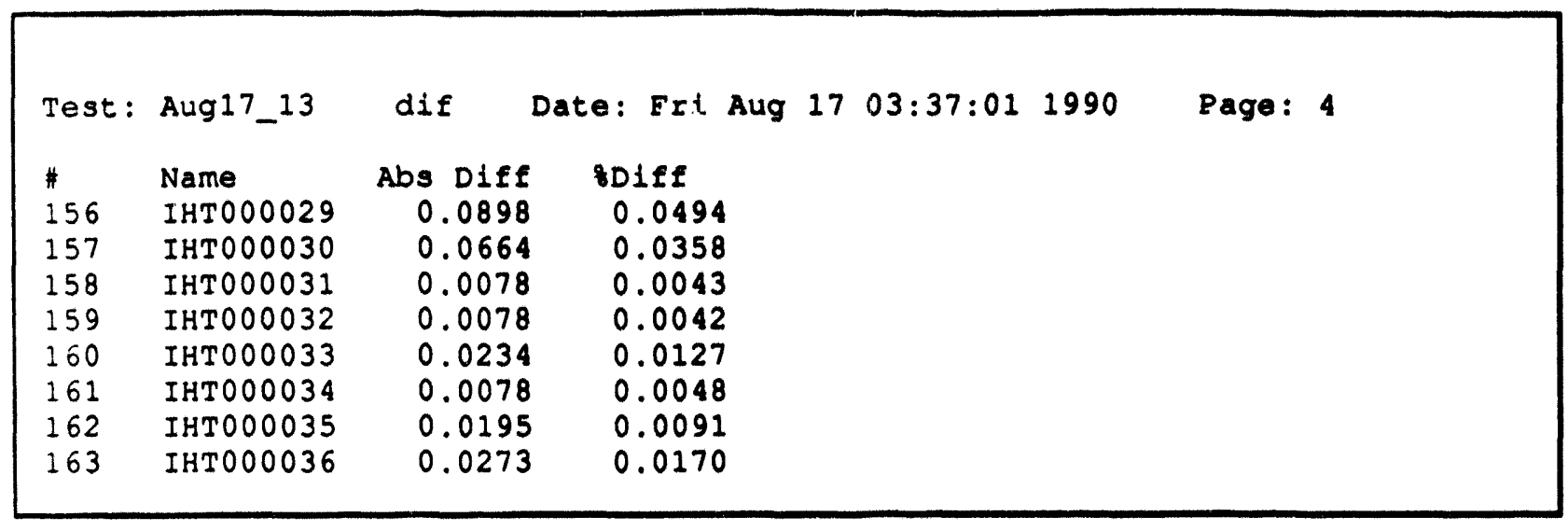




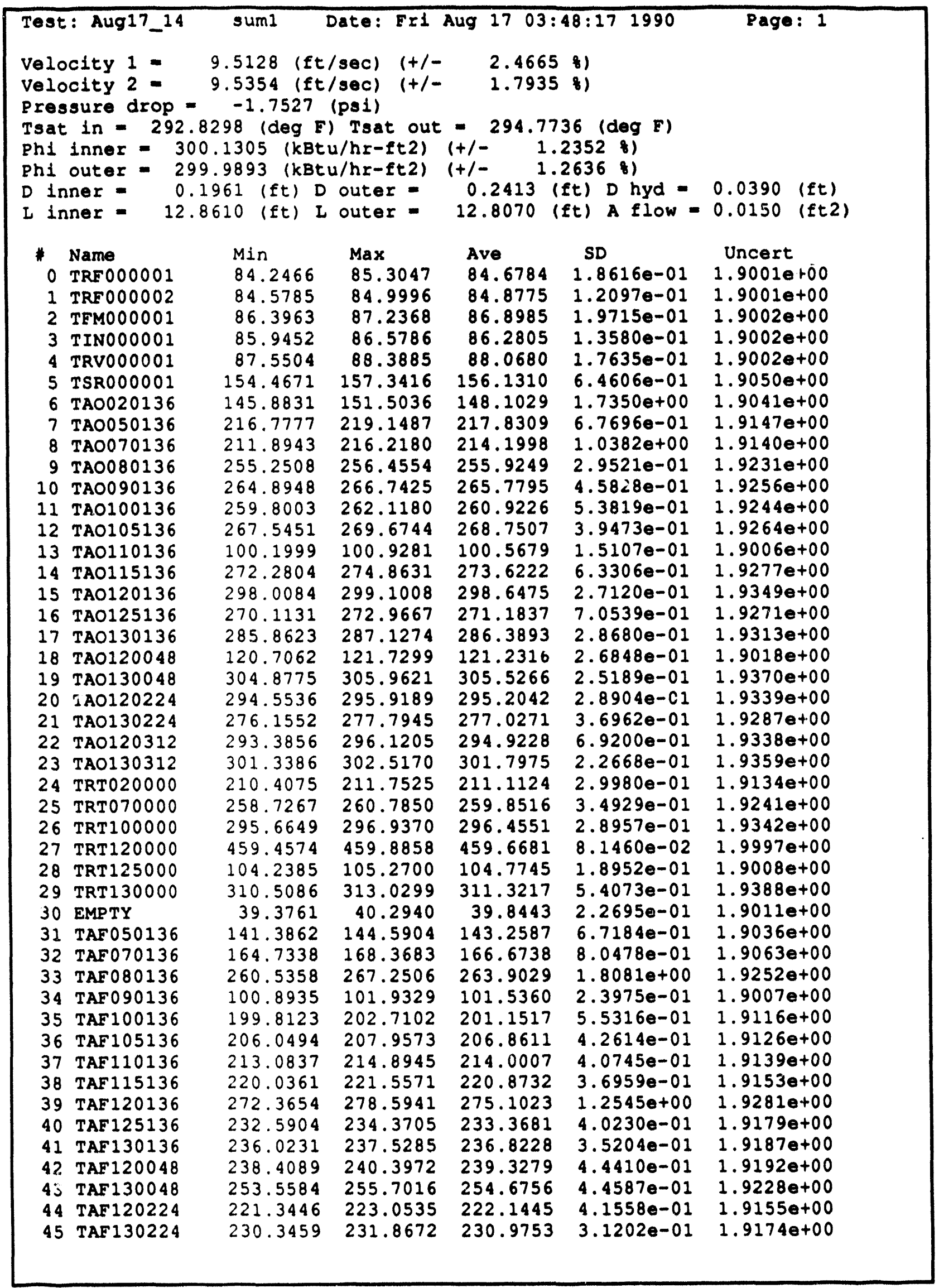




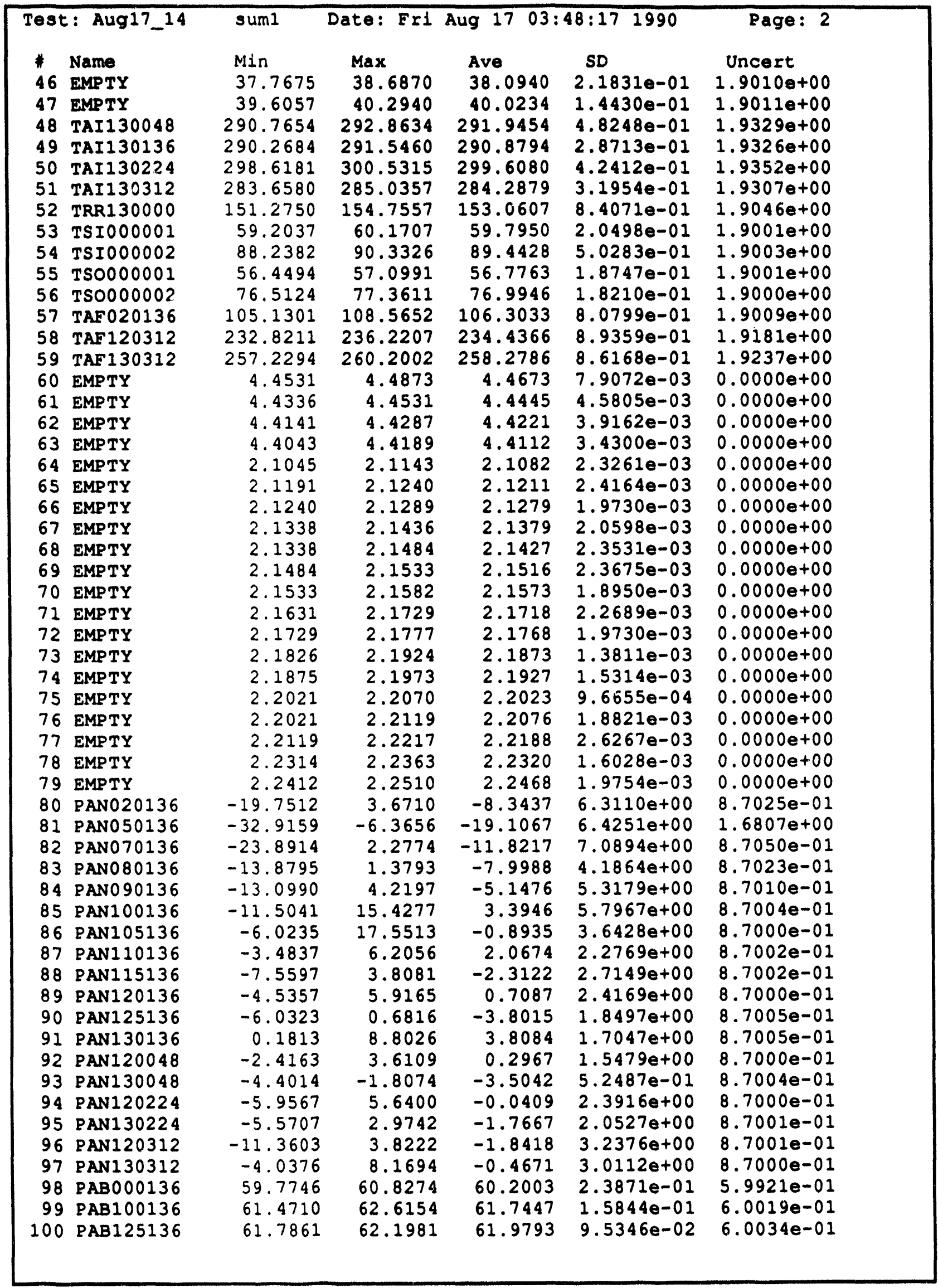




\begin{tabular}{|c|c|c|c|c|c|c|}
\hline \multicolumn{2}{|c|}{ Test: Aug17_14 } & sum1 & \multicolumn{3}{|c|}{ Date: Fr1 Aug $17 \quad 03: 48: 17 \quad 1990$} & Page: 3 \\
\hline \# & Name & Min & Max & Ave & SD & Uncert \\
\hline 101 & PIN000001 & 59.2458 & 60.8327 & 60.0198 & $4.5247 e-01$ & $7.6486 e-01$ \\
\hline 102 & PRV000001 & 19.8406 & 20.0848 & 19.8968 & $6.6221 e-02$ & $7.5165 e-01$ \\
\hline 103 & PSR000001 & 61.1730 & 61.2950 & 61.2608 & $5.5366 e-02$ & $7.6548 \mathrm{e}-01$ \\
\hline 104 & PFM000001 & 13.4512 & 14.0005 & 13.7844 & $1.4057 e-01$ & $5.0119 e-01$ \\
\hline 105 & PAN000136 & -64.5003 & -31.0836 & -48.5243 & $7.8208 e+00$ & $4.9215 e+00$ \\
\hline 106 & PFM000002 & 13.6416 & 13.9940 & 13.8507 & $9.1293 \theta-02$ & $2.0298 e-01$ \\
\hline 107 & EMPTY & 0.0000 & 0.0049 & 0.0047 & $9.6655 e-04$ & $0.0000 \mathrm{e}+00$ \\
\hline 108 & EMPTY & -10.0000 & -6.1328 & -8.3979 & $1.2825 e+00$ & $0.0000 e+00$ \\
\hline 109 & EMPTY & 0.0000 & 0.0049 & 0.0039 & $1.9730 e-03$ & $0.0000 e+00$ \\
\hline 110 & EMPTY & -10.0000 & -10.0000 & -10.0000 & $0.0000 e+00$ & $0.0000 e+00$ \\
\hline 111 & EMPTY & 0.0000 & 0.0000 & 0.0000 & $0.0000 e+00$ & $0.0000 \mathrm{e}+00$ \\
\hline 112 & VPW000001 & 177.4151 & 178.0011 & 177.6924 & $1.1897 e-01$ & $1.3389 e+00$ \\
\hline 113 & VPW000002 & 207.5233 & 207.9139 & 207.7812 & $1.0014 e-01$ & $1.4867 e+00$ \\
\hline 114 & VPW000003 & 256.8021 & 257.7787 & 257.1380 & $1.4785 e-01$ & $1.7425 e+00$ \\
\hline 115 & VPW000004 & 182.0125 & 182.9890 & 182.7587 & $1.6120 e-01$ & $1.3633 e+00$ \\
\hline 116 & VPW000005 & 243.3631 & 244.1444 & 243.7187 & $1.6120 e-01$ & $1.6717 \mathrm{e}+00$ \\
\hline 117 & VPW000006 & 258.6125 & 259.1985 & 258.8741 & $1.4557 e-01$ & $1.7518 \mathrm{e}+00$ \\
\hline 118 & IPW000001 & 930.0665 & 945.6852 & 935.0645 & $2.5611 e+00$ & $1.0963 e+01$ \\
\hline 119 & IPW0 00002 & 2217.4678 & 2240.8828 & 2228.9021 & $7.2690 e+00$ & $1.9382 e+01$ \\
\hline 120 & IPW000003 & 2248.3499 & 2261.9998 & 2257.9045 & $2.7647 e+00$ & $1.9590 \mathrm{e}+01$ \\
\hline 122 & IPWO 00004 & 646.7942 & 653.6301 & 648.5425 & $1.5694 e+00$ & $5.5000 \mathrm{e}+00$ \\
\hline 122 & IPW000005 & 433.2311 & 435.1841 & 434.0611 & $5.6877 e-01$ & $3.9697 e+00$ \\
\hline 123 & IPW000006 & 447.3032 & 450.7212 & 449.4807 & $1.1257 \mathrm{e}+00$ & $4.0754 e+00$ \\
\hline 124 & EMPTY & -0.0293 & -0.0098 & -0.0188 & $3.8219 e-03$ & $0.0000 e+00$ \\
\hline 125 & EMPTY & -0.0195 & -0.0098 & -0.0126 & $2.8058 e-03$ & $0.0000 e+00$ \\
\hline 126 & EMPTY & -0.0195 & -0.0098 & -0.0154 & $3.1752 e-03$ & $0.0000 e+00$ \\
\hline 127 & EMPTY & -0.0244 & -0.0098 & -0.0159 & $3.0897 e-03$ & $0.0000 \mathrm{e}+00$ \\
\hline 128 & IHT000001 & 219.7341 & 222.2731 & 220.8747 & $6.0019 e-01$ & $4.6571 e+00$ \\
\hline 129 & IHT 000002 & 226.3221 & 228.6658 & 227.2792 & $5.6117 e-01$ & $4.6878 \mathrm{e}+00$ \\
\hline 130 & IHT000003 & 228.2076 & 230.7467 & 229.2194 & $5.9411 e-01$ & $4.6972 \mathrm{e}+00$ \\
\hline 131 & IHTO00004 & 223.7643 & 226.6940 & 225.1549 & $6.5273 e-01$ & $4.6775 e+00$ \\
\hline 132 & IHT000005 & 221.6496 & 224.3840 & 222.8643 & $5.8566 e-01$ & $4.6666 \mathrm{e}+00$ \\
\hline 133 & IHT000006 & 215.7940 & 218.5284 & 216.8369 & $5.7400 e-01$ & $4.6381 \mathrm{e}+00$ \\
\hline 134 & IHTO000007 & 225.3306 & 228.0649 & 226.2836 & $5.7540 e-01$ & $4.6830 \mathrm{e}+00$ \\
\hline 135 & IHT000008 & 222.7389 & 225.4733 & 223.6881 & $5.7455 e-01$ & $4.6705 e+00$ \\
\hline 136 & IHT000009 & 222.7276 & 225.4620 & 223.8994 & $6.3381 e-01$ & $4.6715 e+00$ \\
\hline 137 & IHTO00010 & 53.2565 & 57.1627 & 55.2057 & $6.6789 e-01$ & $4.1370 \mathrm{e}+00$ \\
\hline 138 & IHTO00011 & 222.2994 & 226.0103 & 224.0495 & $6.6843 e-01$ & $4.6722 e+00$ \\
\hline 139 & IHT000012 & 218.7425 & 222.2581 & 220.1682 & $6.6527 e-01$ & $4.6538 \mathrm{e}+00$ \\
\hline 140 & IHTO00013 & 158.3984 & 161.5234 & 159.6484 & $6.7428 e-01$ & $4.3999 e+00$ \\
\hline 141 & IHTO00014 & 219.1632 & 222.2882 & 220.6201 & $6.6639 e-01$ & $4.6559 \mathrm{e}+00$ \\
\hline 142 & IHTO00015 & 163.1761 & 166.4964 & 164.7738 & $6.2227 e-01$ & $4.4187 e+00$ \\
\hline 143 & IHT000016 & 151.8029 & 155.1232 & 153.3849 & $6.4507 e-01$ & $4.3775 e+00$ \\
\hline 144 & IHT000017 & 182.6097 & 185.3441 & 184.0160 & $6.5910 e-01$ & $4.4940 e+00$ \\
\hline 145 & IHT000018 & 183.9168 & 187.4324 & 185.7489 & $6.1370 e-01$ & $4.5011 e+00$ \\
\hline 146 & IHT000019 & 178.6734 & 181.2124 & 179.9312 & $5.5686 e-01$ & $4.4774 e+00$ \\
\hline 147 & IHTO000020 & 183.9318 & 186.2755 & 185.1544 & $5.5357 e-01$ & $4.4987 e+00$ \\
\hline 148 & IHTO00021 & -3.2039 & 0.1164 & -1.6375 & $6.9865 e-01$ & $4.1000 e+00$ \\
\hline 149 & IHTO00022 & 158.0942 & 160.6333 & 159.4419 & $5.4712 e-01$ & $4.3991 e+00$ \\
\hline 150 & IHT000023 & 179.9504 & 182.2942 & 181.0948 & $5.6636 e-01$ & $4.4821 e+00$ \\
\hline 151 & IHTO000024 & 144.3773 & 146.5257 & 145.5687 & $4.9639 e-01$ & $4.3507 e+00$ \\
\hline 152 & IHTO00025 & 179.3532 & 181.5017 & 180.4899 & $5.3770 e-01$ & $4.4797 e+00$ \\
\hline 153 & IHT000026 & 181.6932 & 183.6463 & 182.7128 & $4.8535 e-01$ & $4.4887 e+00$ \\
\hline 154 & IHTO00027 & 154.6424 & 157.1815 & 155.9628 & $5.8202 e-01$ & $4.3866 \mathrm{e}+00$ \\
\hline 155 & IHT000028 & 157.1552 & 159.8896 & 158.4131 & $7.4989 e-01$ & $4.3954 e+00$ \\
\hline
\end{tabular}




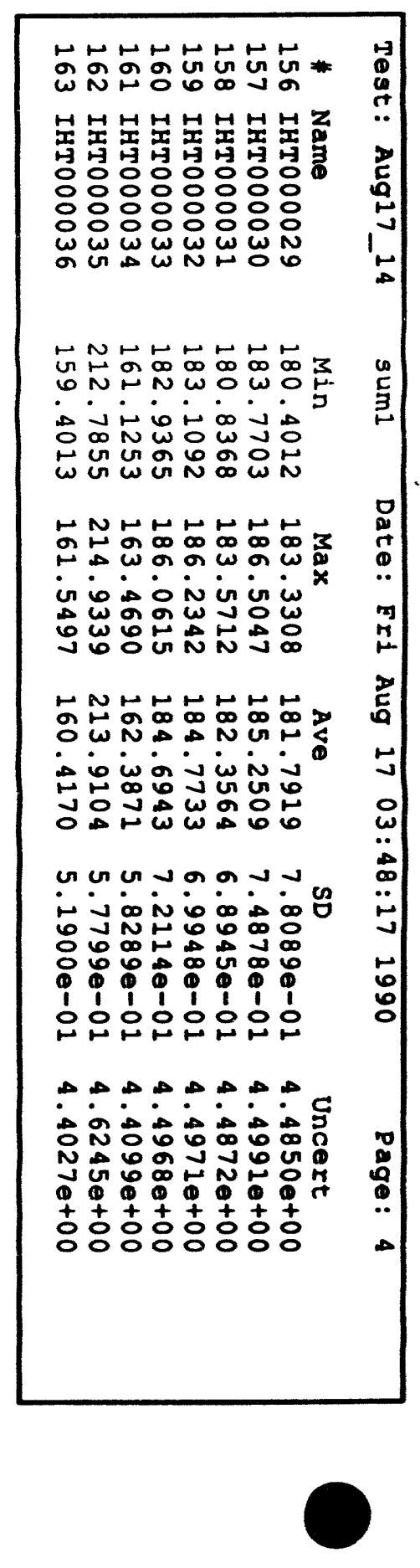




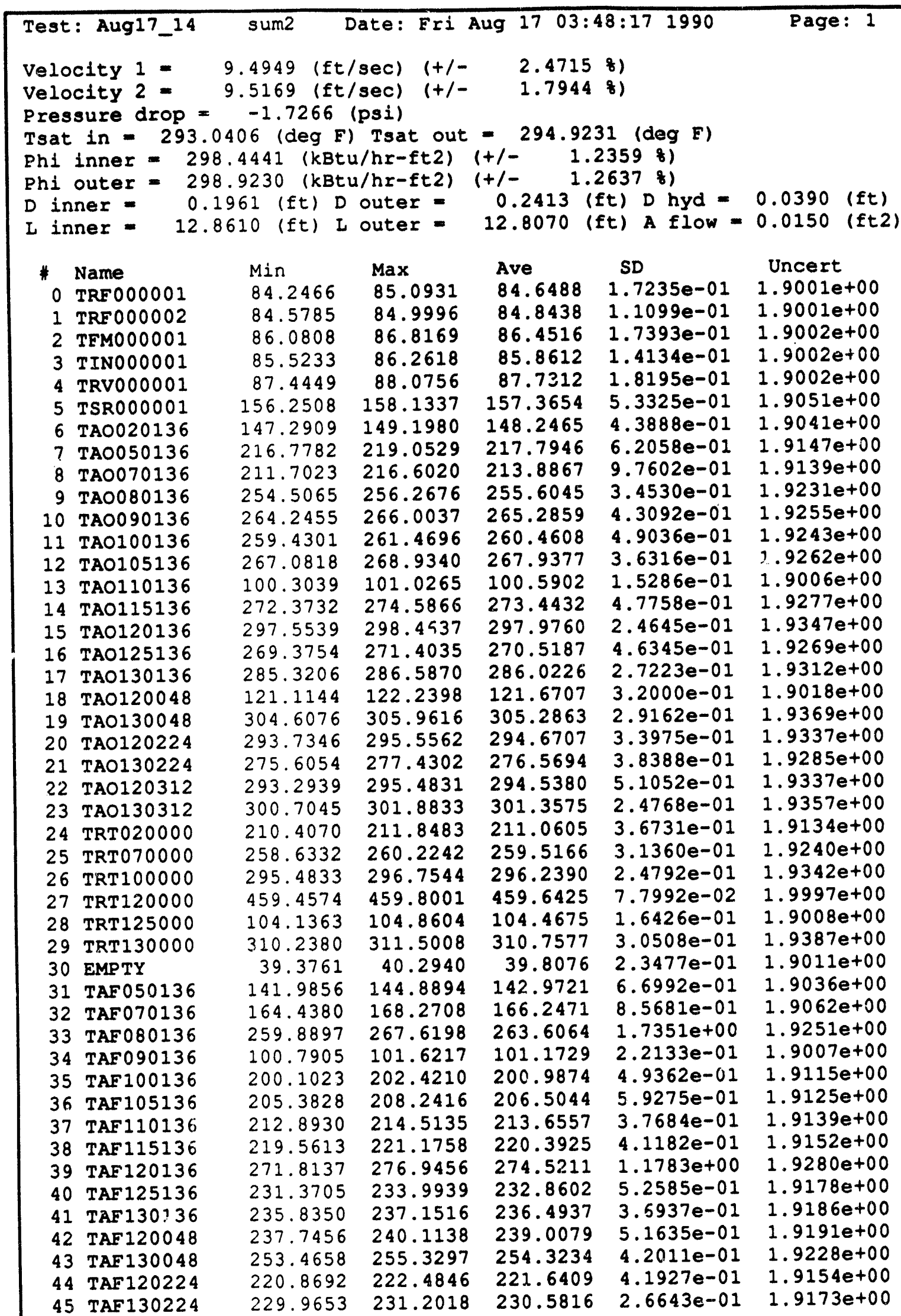




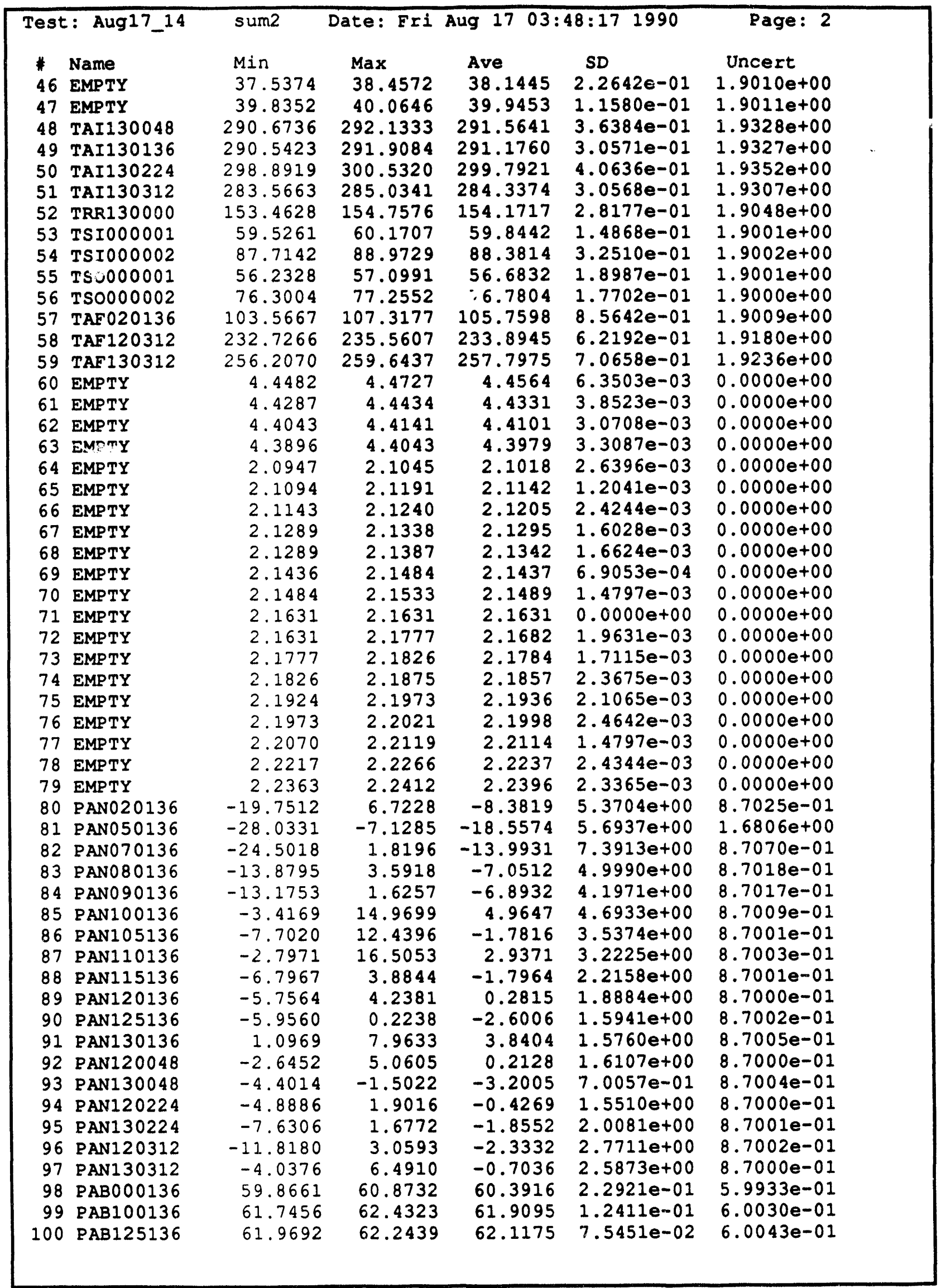




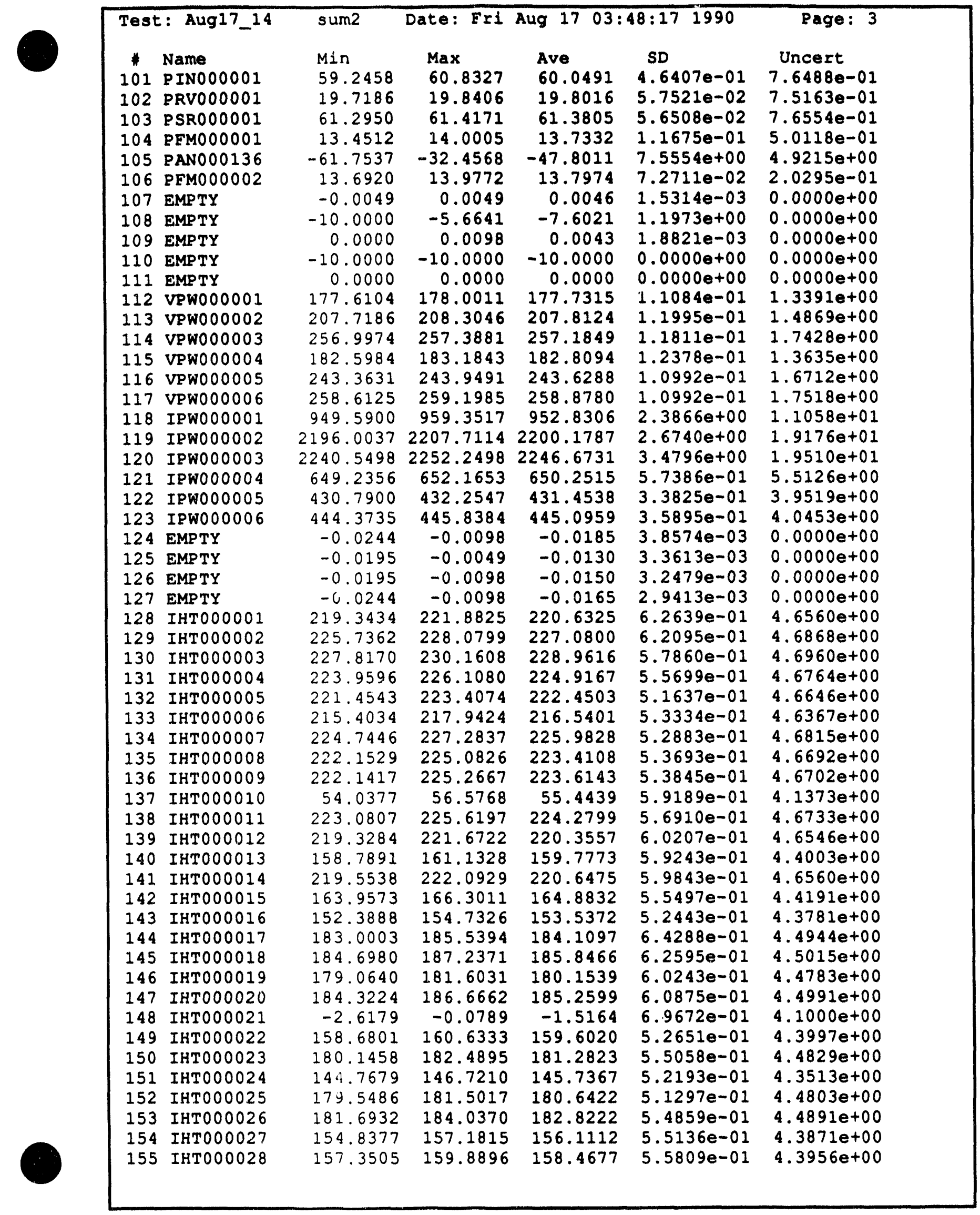




\begin{tabular}{|c|c|c|c|c|c|}
\hline Test: Aug17_14 & sum2 & Date: Fri & Aug 170 & $8: 171990$ & Page: \\
\hline 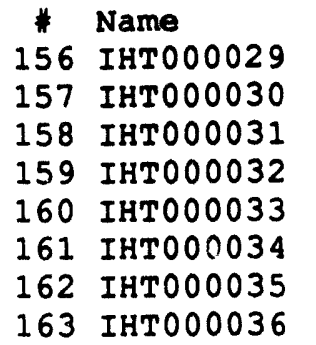 & $\begin{array}{l}\text { Min } \\
180.5965 \\
184.1609 \\
181.2275 \\
183.3045 \\
183.1318 \\
161.3206 \\
212.9808 \\
159.5966\end{array}$ & $\begin{array}{l}\operatorname{Max} \\
183.1355 \\
186.5047 \\
183.5712 \\
186.4295 \\
186.4521 \\
163.6644 \\
215.5199 \\
162.1357\end{array}$ & $\begin{array}{l}\text { Ave } \\
181.8309 \\
185.2587 \\
182.4033 \\
184.8163 \\
184.7568 \\
162.3832 \\
213.8401 \\
160.4404\end{array}$ & $\begin{array}{l}\text { SD } \\
5.8052 e-01 \\
6.1497 e-01 \\
6.5731 e-01 \\
6.5055 e-01 \\
6.8623 e-01 \\
5.4556 e-01 \\
5.2497 e-01 \\
5.1966 e-01\end{array}$ & $\begin{array}{c}\text { Uncert } \\
4.4851 e+00 \\
4.4991 e+00 \\
4.4874 e+00 \\
4.4973 e+00 \\
4.4971 e+00 \\
4.4099 e+00 \\
4.6241 e+00 \\
4.4027 e+00\end{array}$ \\
\hline
\end{tabular}




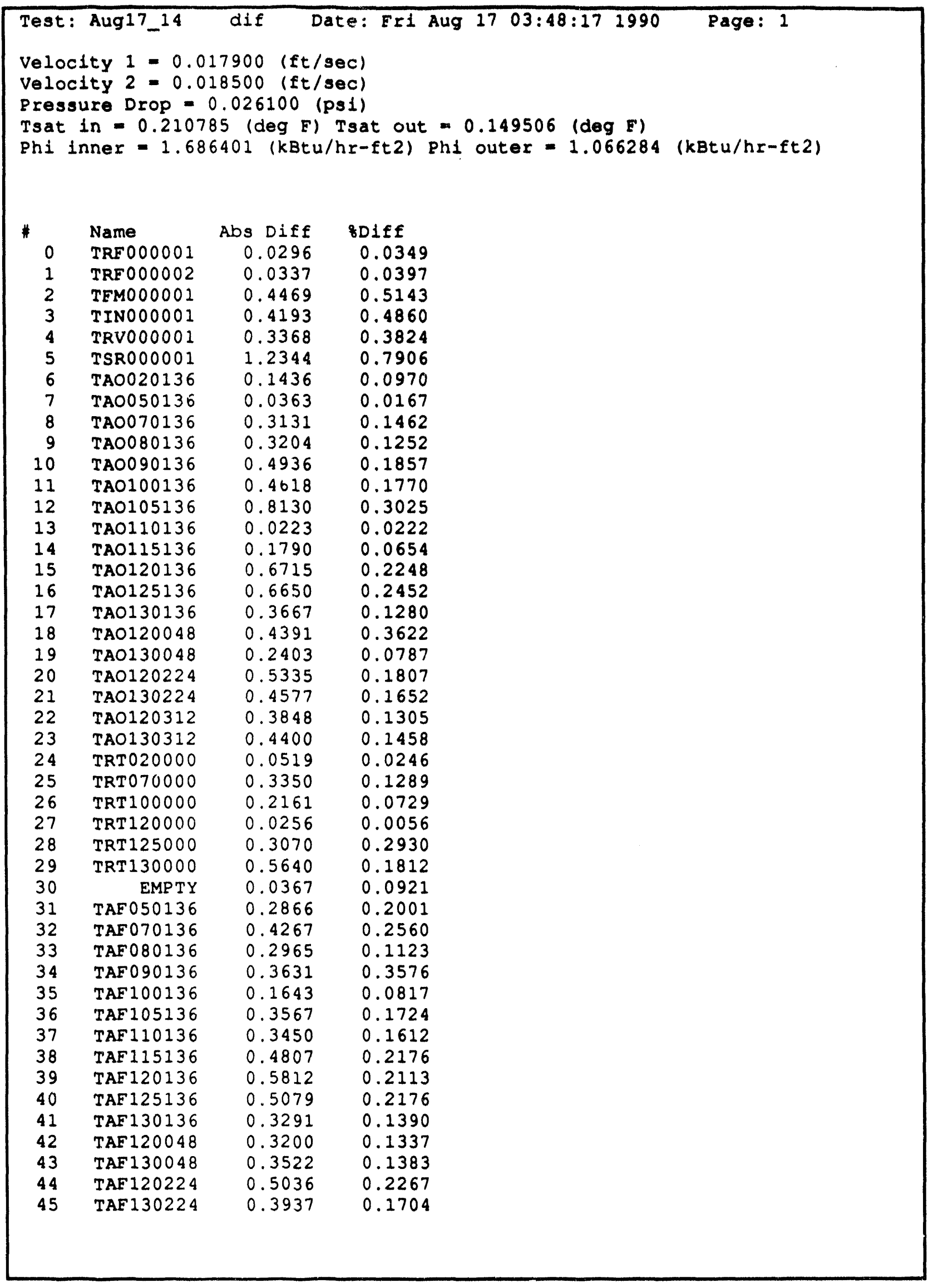




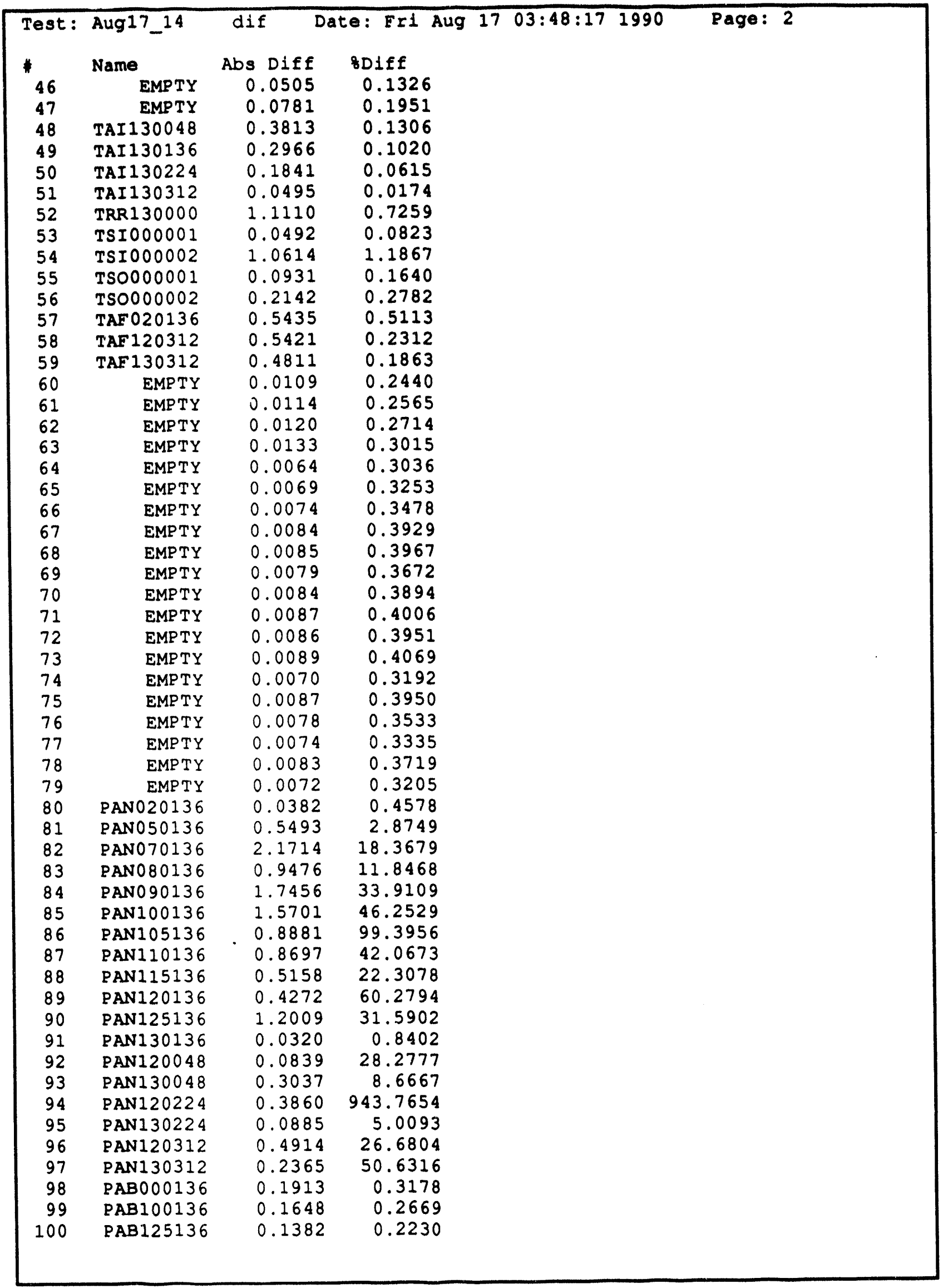




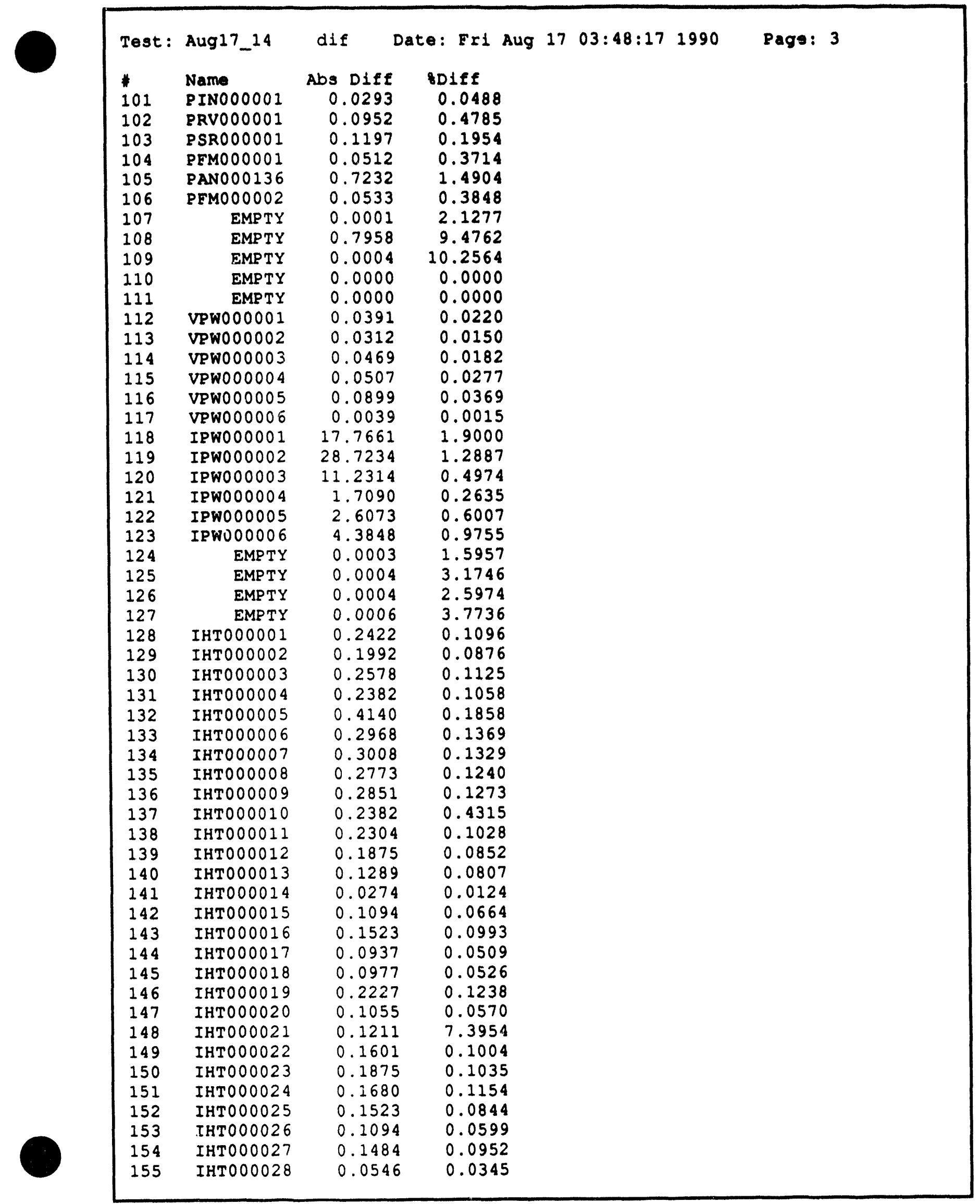




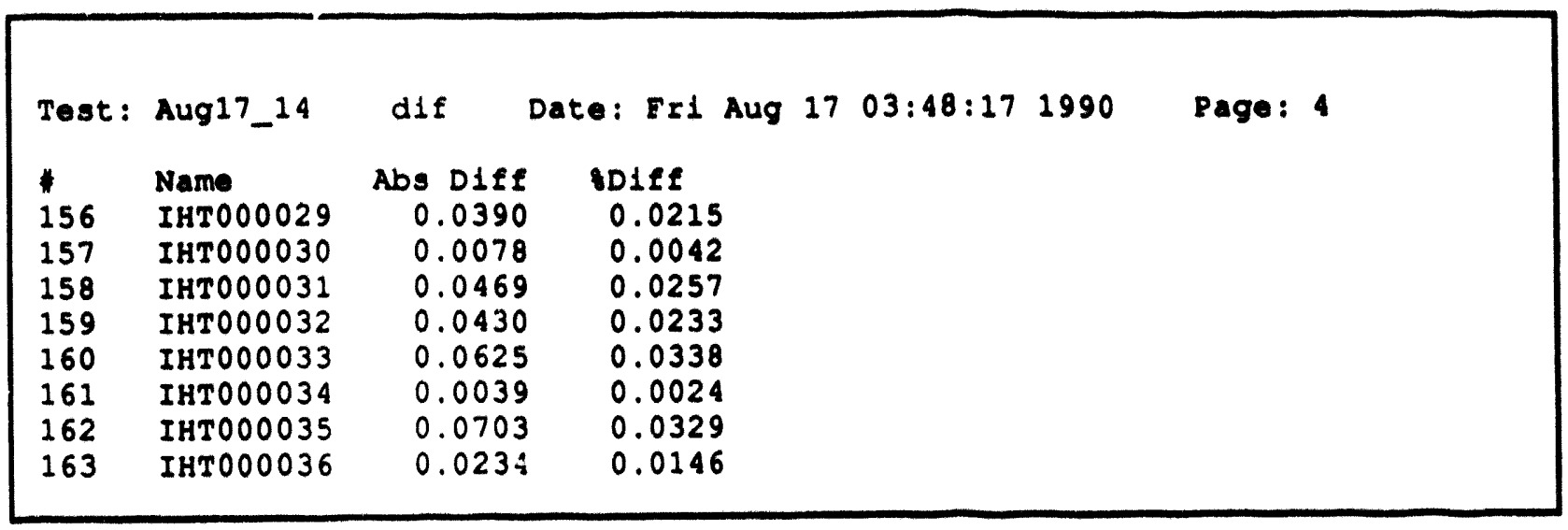




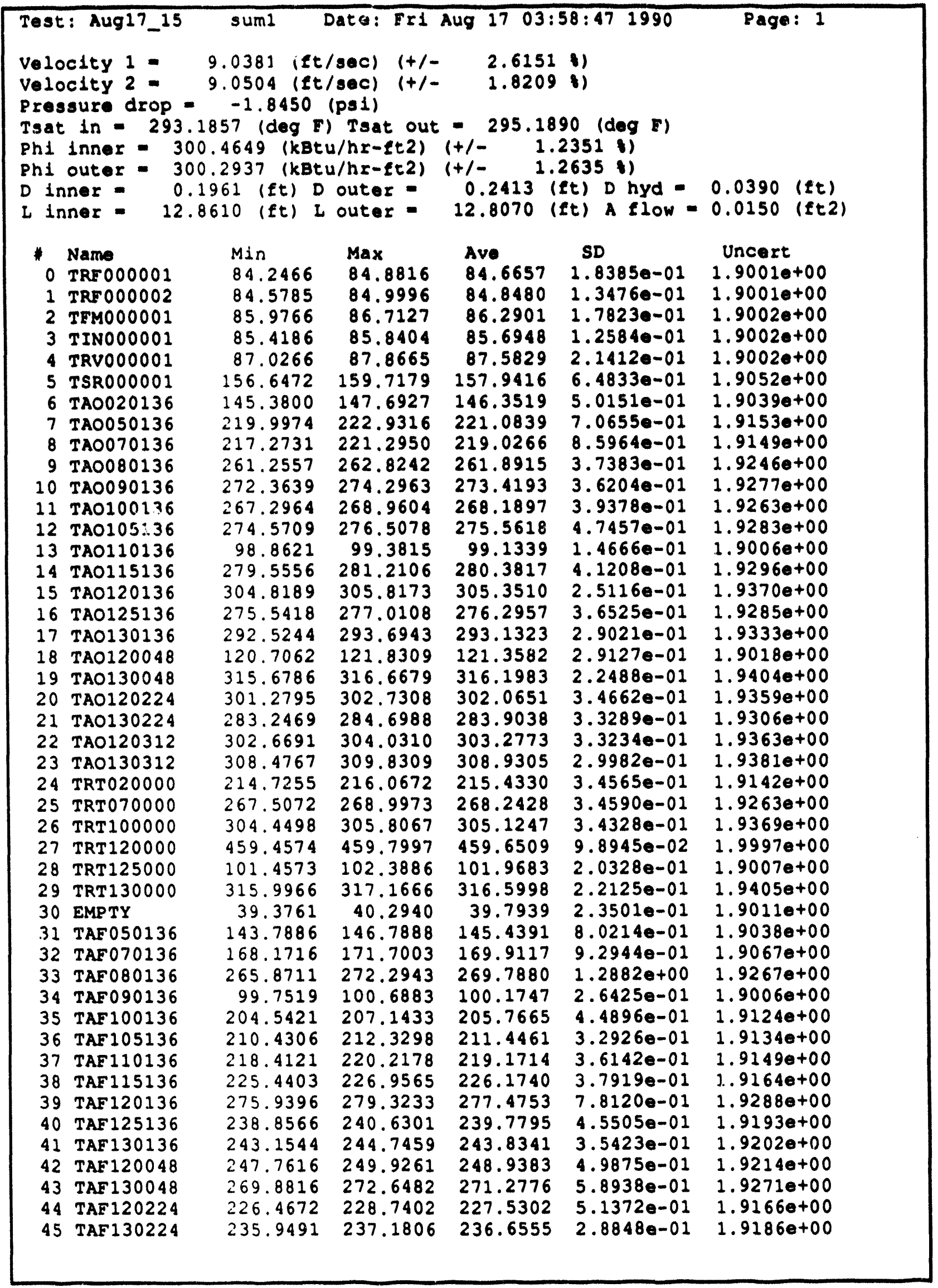




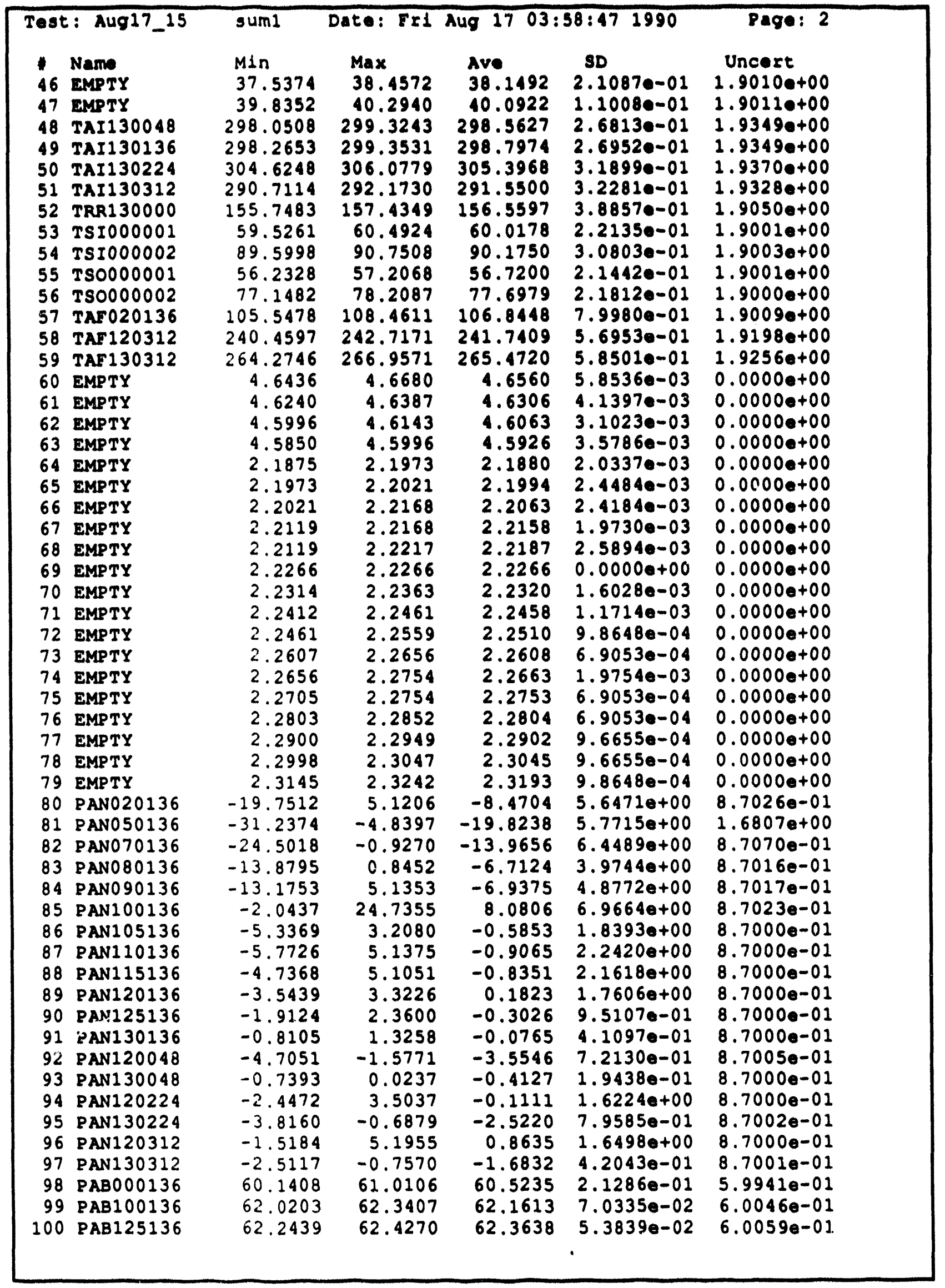




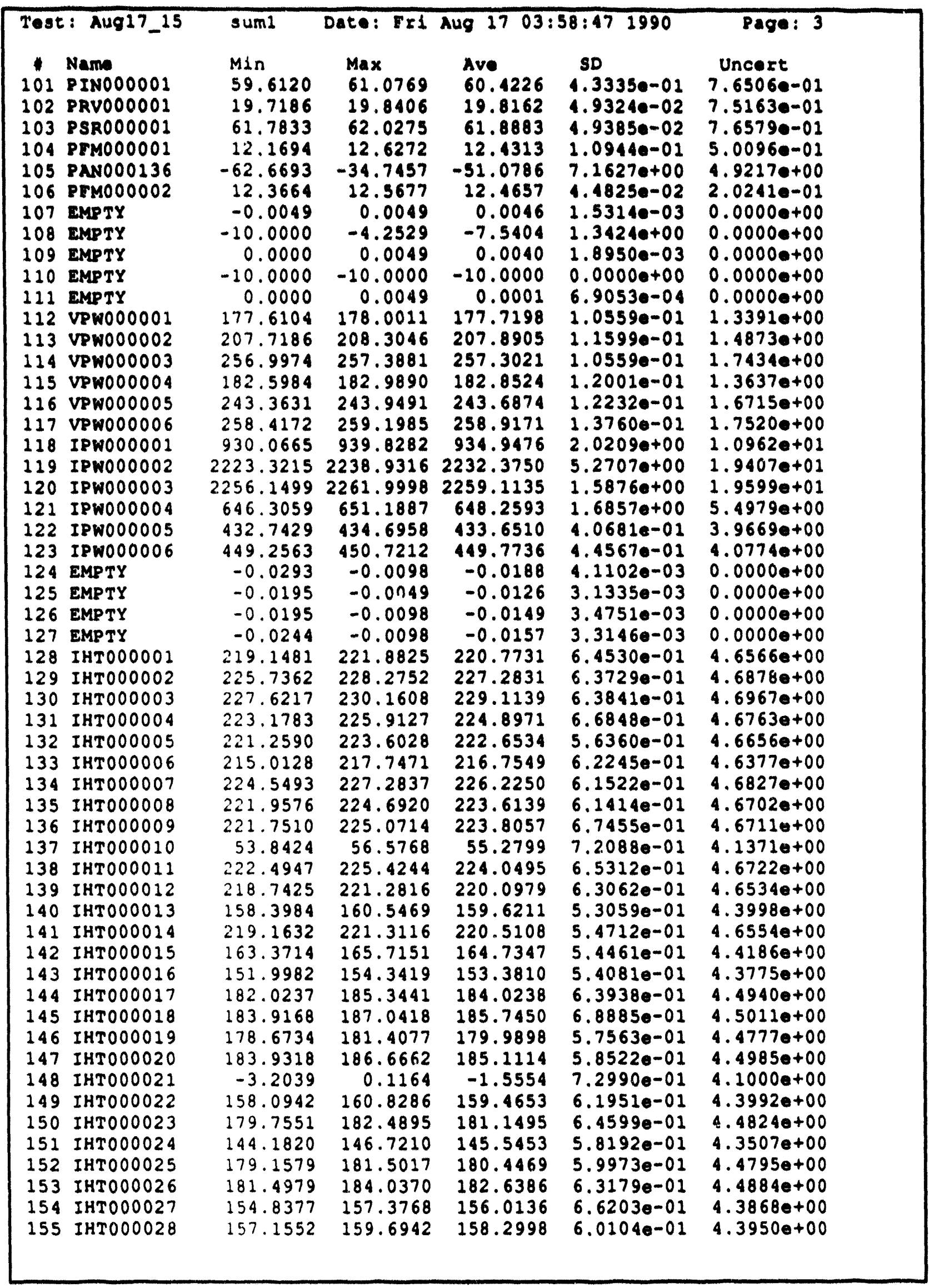




\begin{tabular}{|c|c|c|c|c|c|c|}
\hline \multicolumn{2}{|c|}{ Test: $\operatorname{Aug}_{17} 15$} & sum1 & \multicolumn{3}{|c|}{ Date: Fr1 Aug $17 \quad 03: 58: 47 \quad 1990$} & Page: 4 \\
\hline $\begin{array}{l}1 \\
156 \\
157 \\
158 \\
159 \\
160 \\
161 \\
162 \\
163\end{array}$ & $\begin{array}{l}\text { Name } \\
\text { IHTOOOO29 } \\
\text { IHTOOOOO30 } \\
\text { IHTOOOO31 } \\
\text { IHTOOOOO32 } \\
\text { IHTOOOO33 } \\
\text { IHTOOOOO34 } \\
\text { IHTOOOOO35 } \\
\text { IHTOOOO36 }\end{array}$ & $\begin{array}{l}M 1 n \\
180.5965 \\
183.7703 \\
181.0322 \\
183.3045 \\
183.3271 \\
161.1253 \\
212.5902 \\
159.0107\end{array}$ & $\begin{array}{l}\operatorname{Max} \\
182.7449 \\
186.5047 \\
183.5712 \\
186.2342 \\
186.2568 \\
163.4690 \\
214.9339 \\
161.3544\end{array}$ & $\begin{array}{l}\text { Ave } \\
181.7177 \\
185.1415 \\
182.3134 \\
184.7226 \\
184.6670 \\
162.3324 \\
213.8128 \\
160.3857\end{array}$ & $\begin{array}{l}\text { SD } \\
5.9818 \bullet-01 \\
6.1699 e-01 \\
6.2907 e-01 \\
6.5413 e-01 \\
6.6029 e-01 \\
5.5199 e-01 \\
5.6471 e-01 \\
5.3519 e-01\end{array}$ & $\begin{array}{l}\text { Uncert } \\
4.4847 e+00 \\
4.4986 e+00 \\
4.4871 e+00 \\
4.4969 e+00 \\
4.4967 e+00 \\
4.4097 e+00 \\
4.6240 e+00 \\
4.4025 e+00\end{array}$ \\
\hline
\end{tabular}




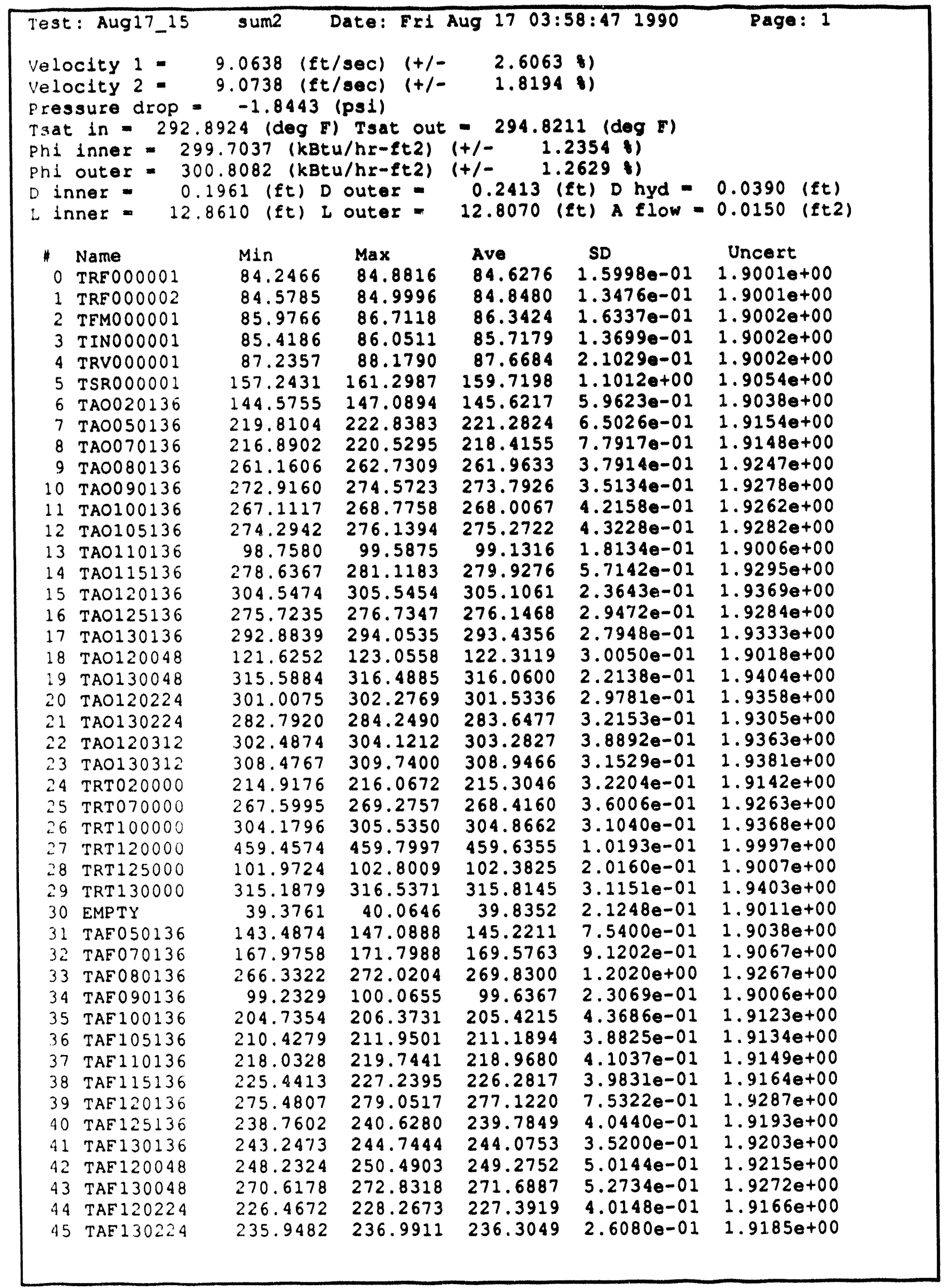




\begin{tabular}{|c|c|c|c|c|c|c|}
\hline \multicolumn{2}{|c|}{ Test: Aug17_15 } & sum2 & \multicolumn{3}{|c|}{ Date: Fri Aug $17 \quad 03: 58: 47 \quad 1990$} & \multirow{2}{*}{$\begin{array}{l}\text { Page: } 2 \\
\text { Uncert }\end{array}$} \\
\hline$\#$ & Name & Min & Max & Ave & SD & \\
\hline 46 & EMPTY & 37.7675 & 38.4572 & 38.0940 & $2.2799 e-01$ & $1.9010 e+00$ \\
\hline 47 & EMPTY & 39.8352 & 40.2940 & 40.0280 & $1.0732 \theta-01$ & $1.90110+00$ \\
\hline 48 & TAI 130048 & 297.6877 & 299.0516 & 298.3681 & $3.3437 e-01$ & $1.9348 e+00$ \\
\hline 49 & TAI 130136 & 298.8997 & 300.3517 & 299.6728 & $3.0665 e-01$ & $1.9352 e+00$ \\
\hline 50 & TAI 130224 & 304.0793 & 305.8964 & 305.2024 & $4.1840 e-01$ & $1.9369 \mathrm{e}+00$ \\
\hline 51 & TAI 130312 & 290.2536 & 292.0821 & 290.8572 & $3.7856 e-01$ & $1.9326 e+00$ \\
\hline 52 & TRR130000 & 156.9370 & 158.9196 & 157.8817 & $5.0002 e-01$ & $1.9052 \mathrm{e}+00$ \\
\hline 53 & TSI000001 & 59.6331 & 60.4913 & 60.0241 & $1.6769 e-01$ & $1.90010+00$ \\
\hline 54 & TSI000002 & 90.0182 & 91.2735 & 90.4948 & $2.7815 e-J 1$ & $1.9003 e+00$ \\
\hline 55 & TSO000001 & 56.3406 & 56.9909 & 56.6962 & $2.0279 e-01$ & $1.90010+00$ \\
\hline 56 & TSO000002 & 77.2547 & 78.2087 & 77.6576 & $2.1731 e-01$ & $1.9000 \mathrm{e}+00$ \\
\hline 57 & TAF020136 & 104.9217 & 108.1501 & 106.5513 & $7.6962 e-01$ & $1.9009 \mathrm{e}+00$ \\
\hline 58 & TAF 120312 & 240.5538 & 243.1863 & 241.6450 & $6.1440 e-01$ & $1.9197 \mathrm{e}+00$ \\
\hline 59 & TAF 130312 & 263.9968 & 266.5867 & 265.4885 & $5.8209 e-01$ & $1.9256 e+00$ \\
\hline 60 & EMPTY & 4.6436 & 4.6729 & 4.6568 & $7.1844 e-03$ & $0.0000 e+00$ \\
\hline 61 & EMPTY & 4.6191 & 4.6436 & 4.6320 & $5.1975 e-03$ & $0.0000 e+00$ \\
\hline 62 & EMPTY & 4.5996 & 4.6143 & 4.6090 & $3.3944 e-03$ & $0.0000 e+00$ \\
\hline 63 & EMPTY & 4.5898 & 4.5996 & 4.5965 & $2.9197 e-03$ & $0.0000 e+00$ \\
\hline 64 & EMPTY & 2.1875 & 2.1924 & 2.1896 & $2.4344 \mathrm{e}-03$ & $0.0000 e+00$ \\
\hline 65 & EMPTY & 2.1973 & 2.2070 & 2.2020 & $1.3811 e-03$ & $0.0000 e+00$ \\
\hline 66 & EMPTY & 2.2070 & 2.2119 & 2.2075 & $1.4797 e-03$ & $0.0000 e+00$ \\
\hline 67 & EMPTY & 2.2168 & 2.2217 & 2.2174 & $1.6028 e-03$ & $0.0000 e+00$ \\
\hline 68 & EMPTY & 2.2168 & 2.2266 & 2.2214 & $1.5314 \theta-03$ & $0.0000 e+00$ \\
\hline 69 & EMPTY & 2.2266 & 2.2314 & 2.2303 & $2.1065 e-03$ & $0.0000 e+00$ \\
\hline 70 & EMPTY & 2.2314 & 2.2412 & 2.2361 & $1.3811 e-03$ & $0.0000 e+00$ \\
\hline 71 & EMPTY & 2.2412 & 2.2559 & 2.2489 & $2.9742 e-03$ & $0.0000 e+00$ \\
\hline 72 & EMPTY & 2.2510 & 2.2607 & 2.2540 & $2.7709 e-03$ & $0.0000 e+00$ \\
\hline 73 & EMPTY & 2.2607 & 2.2705 & 2.2640 & $2.5362 e-03$ & $0.0000 e+00$ \\
\hline 74 & EMPTY & 2.2656 & 2.2754 & 2.2705 & $1.3951 e-03$ & $0.0000 e+00$ \\
\hline 75 & EMPTY & 2.2754 & 2.2803 & 2.2790 & $2.1635 e-03$ & $0.0000 e+00$ \\
\hline 76 & EMPTY & 2.2803 & 2.2900 & 2.2843 & $2.3531 e-03$ & $0.0000 \theta+00$ \\
\hline 77 & EMPTY & 2.2900 & 2.2998 & 2.2950 & $1.8429 e-03$ & $0.0000 e+00$ \\
\hline 78 & EMPTY & 2.3047 & 2.3096 & 2.3080 & $2.3008 e-03$ & $0.0000 e+00$ \\
\hline 79 & EMPTY & 2.3193 & 2.3291 & 2.3229 & $2.4244 \theta-03$ & $0.0000 e+00$ \\
\hline 80 & PAN020136 & -19.7512 & 5.5021 & -9.8330 & $6.7401 e+00$ & $8.7035 e-01$ \\
\hline 81 & PAN050136 & -29.4064 & -3.3138 & -17.7425 & $6.6763 e+00$ & $1.6806 e+00$ \\
\hline 82 & PAN070136 & -24.5781 & -1.4610 & -16.1201 & $5.7629 e+00$ & $8.7093 e-01$ \\
\hline 53 & PAN080136 & -13.8795 & 5.4229 & -2.9496 & $3.7724 e+00$ & $8.7003 e-01$ \\
\hline 84 & PANO90136 & -13.0990 & 2.5413 & -6.5621 & $4.2832 e+00$ & $8.7015 e^{* 01}$ \\
\hline 85 & PAN 100136 & -2.8066 & 18.4031 & 5.2073 & $4.9626 e+00$ & $8.7010 e-01$ \\
\hline 96 & PAN 105136 & -4.4214 & 2.8266 & -0.8401 & $1.4711 e+00$ & $8.7000 e-01$ \\
\hline 87 & PAN110136 & -3.7889 & 3.3064 & 0.0502 & $1.8894 e+00$ & $8.7000 e-01$ \\
\hline 38 & PAN115136 & -7.5597 & 2.9689 & -2.3518 & $2.8243 e+00$ & $8.7002 e-01$ \\
\hline 89 & PAN120136 & -4.8409 & 6.0691 & 1.0551 & $2.9070 e+00$ & $8.7000 e-01$ \\
\hline 90 & PAN 125136 & -1.3784 & 2.1311 & -0.0753 & $9.7276 e-01$ & $8.7000 e-01$ \\
\hline 91 & PAN 130136 & -1.0394 & 0.4865 & -0.2261 & $3.3826 e-01$ & $8.7000 e-01$ \\
\hline 92 & PAN 120048 & -4.3999 & -1.1193 & -3.4188 & $6.4277 e-01$ & $8.7004 e-01$ \\
\hline 93 & PAN 130048 & -0.9682 & 0.1763 & -0.4860 & $2.8648 e-01$ & $8.7000 e-01$ \\
\hline 94 & PAN120224 & -5.0412 & 4.0378 & -0.8374 & $2.07810+00$ & $8.7000 e-01$ \\
\hline 95 & PAN130224 & -3.0530 & 0.8380 & -1.3532 & $9.9643 e-01$ & $8.7001 e-01$ \\
\hline 96 & PAN 120312 & -1.8998 & 4.3563 & 0.9230 & $1.5771 \mathrm{e}+00$ & $8.7000 e-01$ \\
\hline 97 & PAN 130312 & -2.0540 & -0.6044 & -1.4085 & $3.1859 \mathrm{e}-01$ & $8.7001 e-01$ \\
\hline 98 & PAB 000136 & 59.6830 & 60.7359 & 60.2571 & $2.9612 e-01$ & $5.9924 e-01$ \\
\hline 99 & PAB100136 & 61.6541 & 62.0203 & 61.8363 & $8.5510 e-02$ & $6.0025 e-01$ \\
\hline 100 & PAB125136 & 61.9234 & 62.1523 & 62.0232 & $5.9714 e-02$ & $6.0037 e-01$ \\
\hline
\end{tabular}




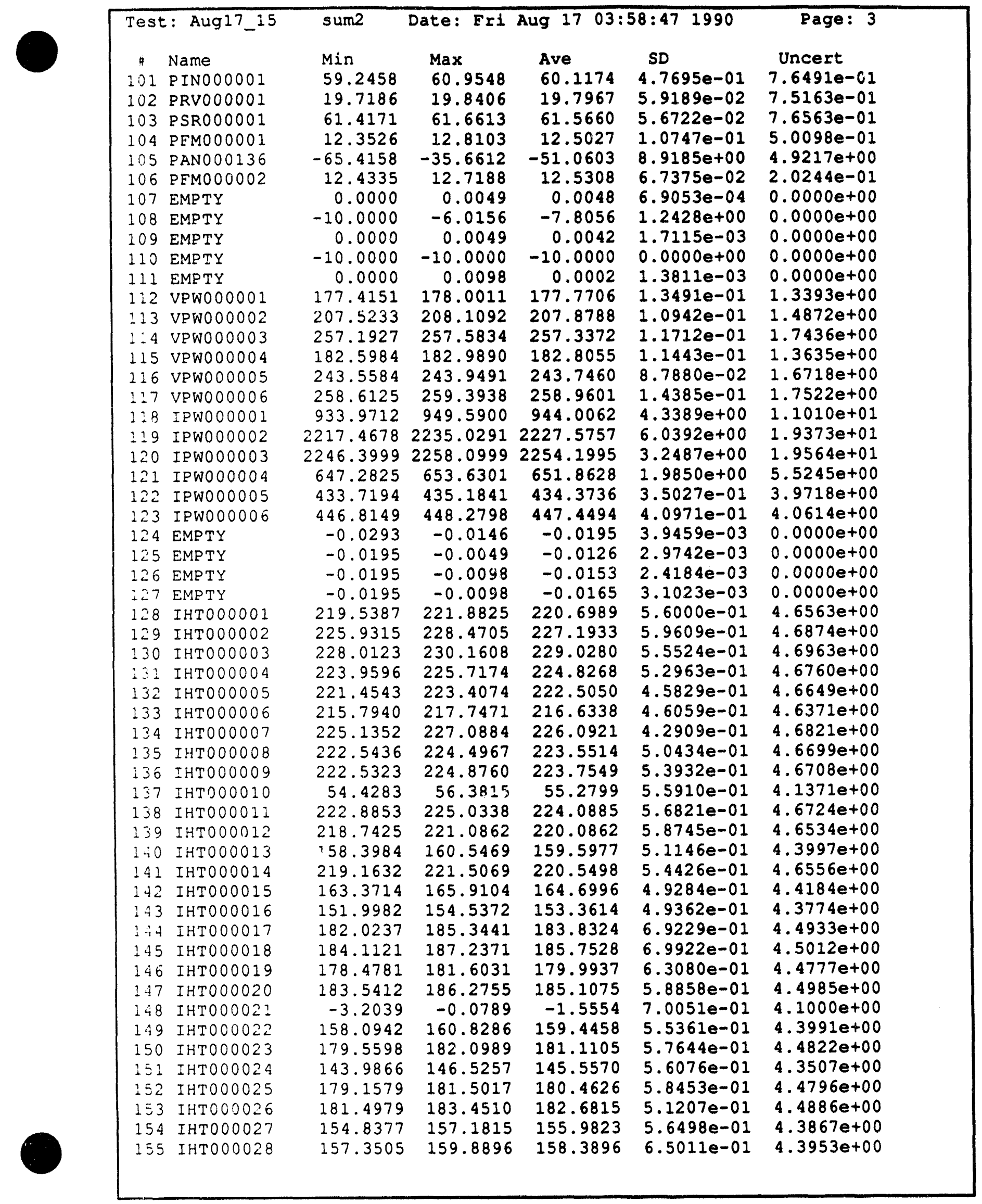




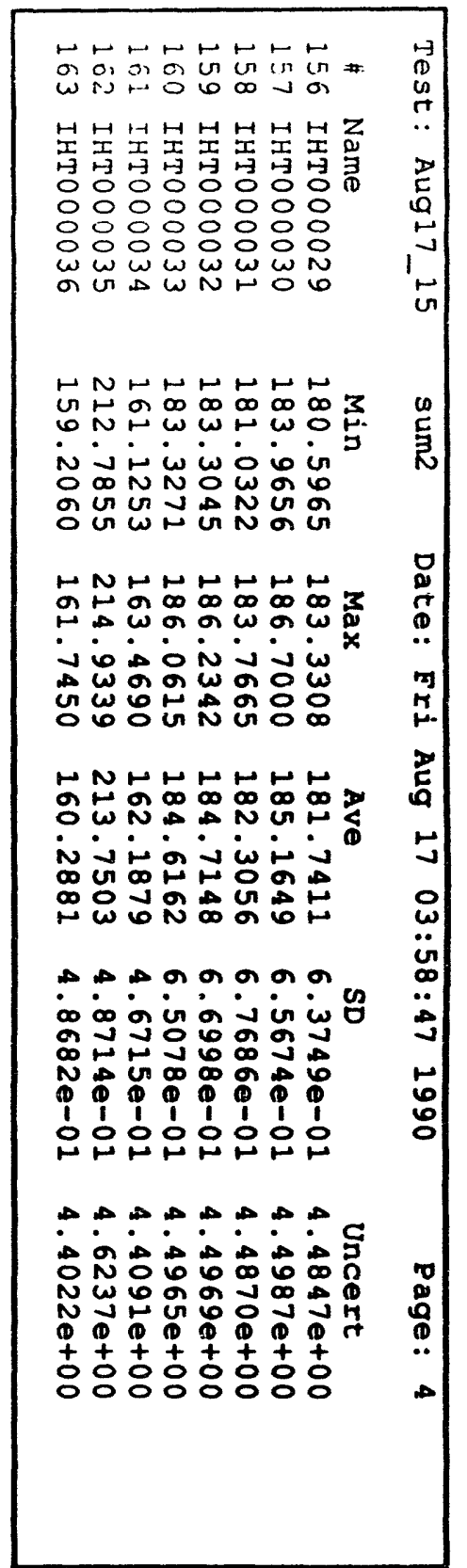




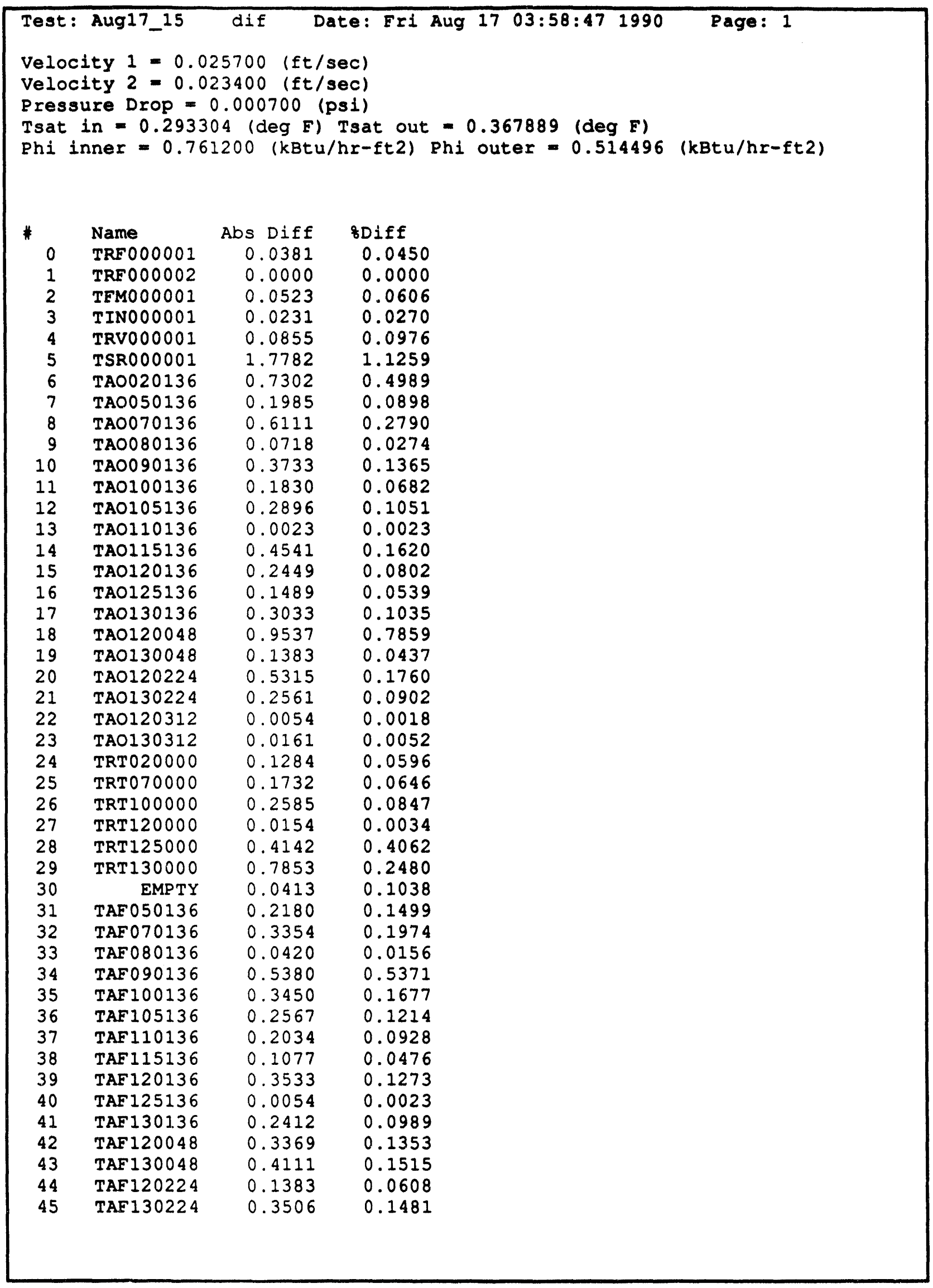




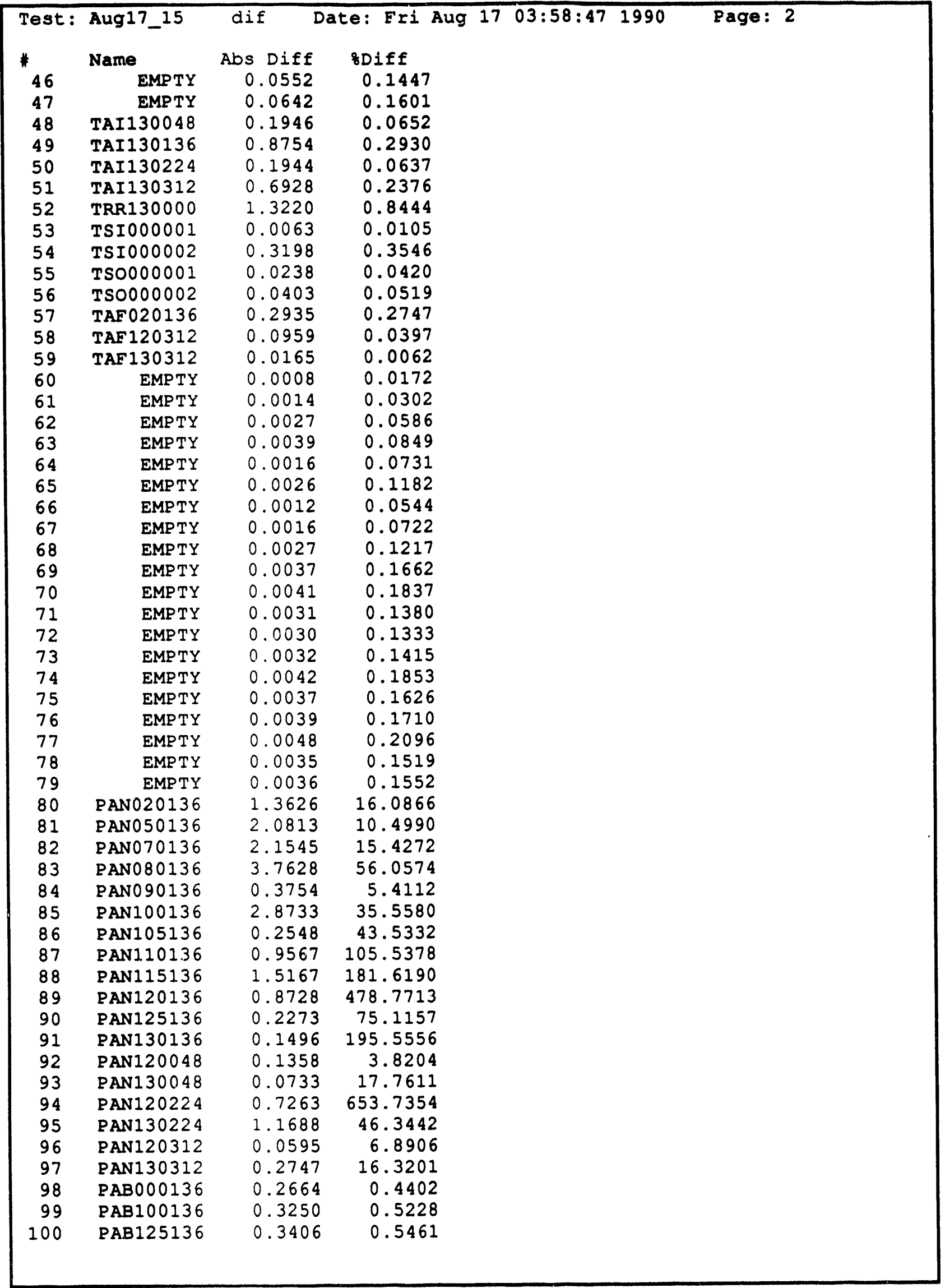




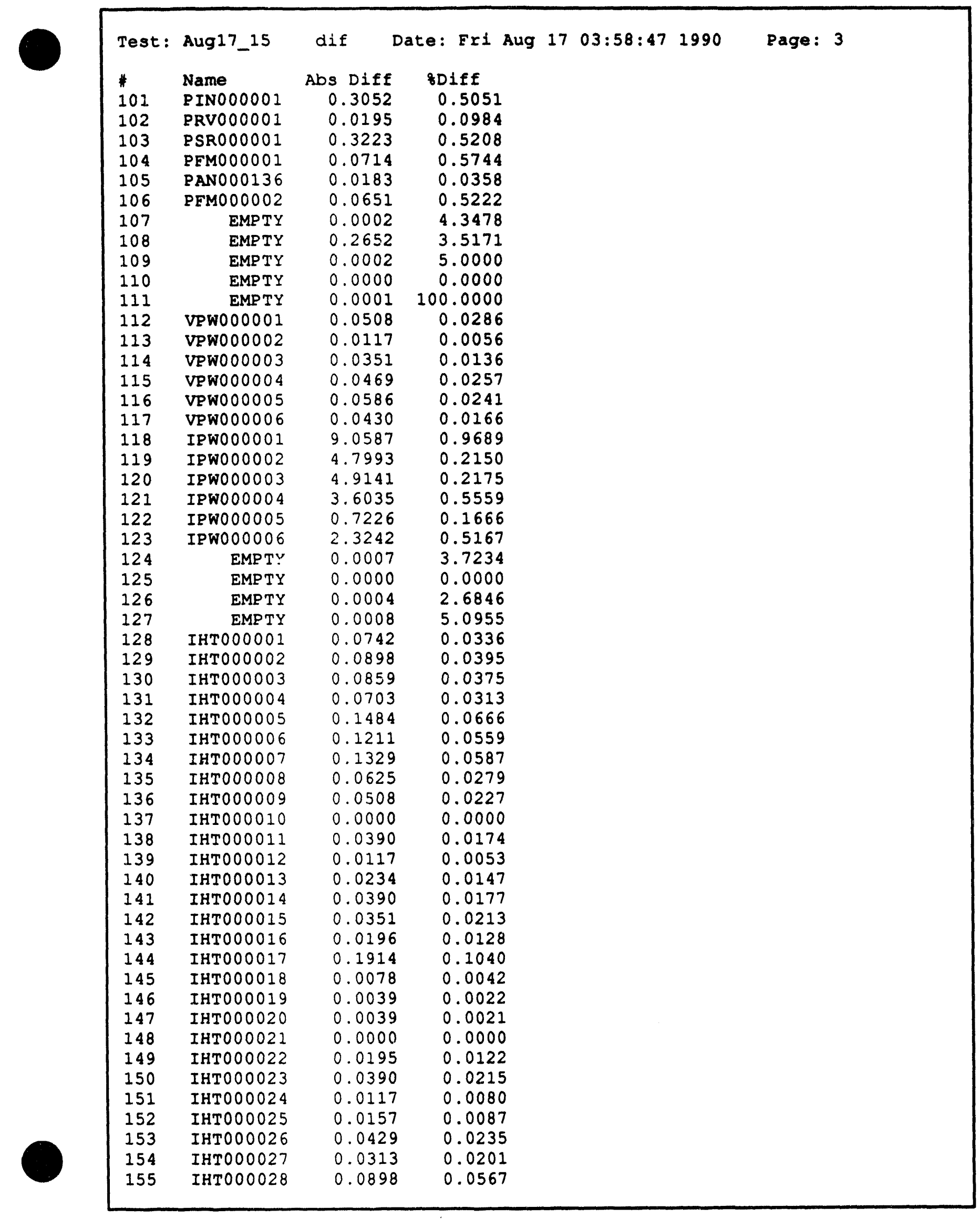




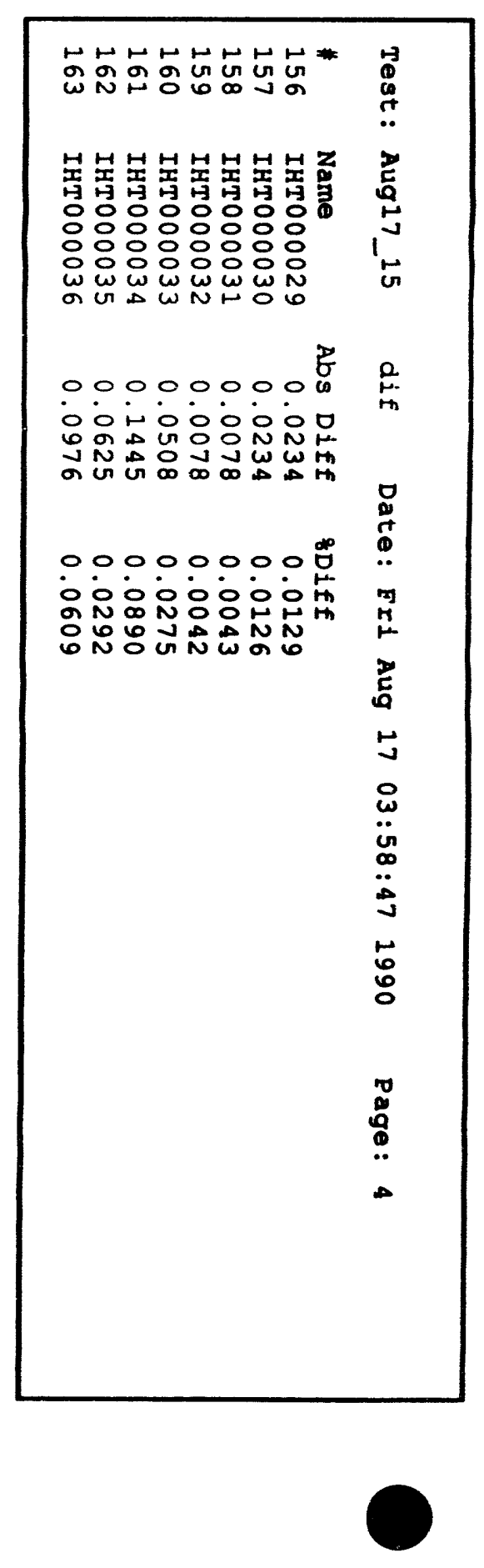




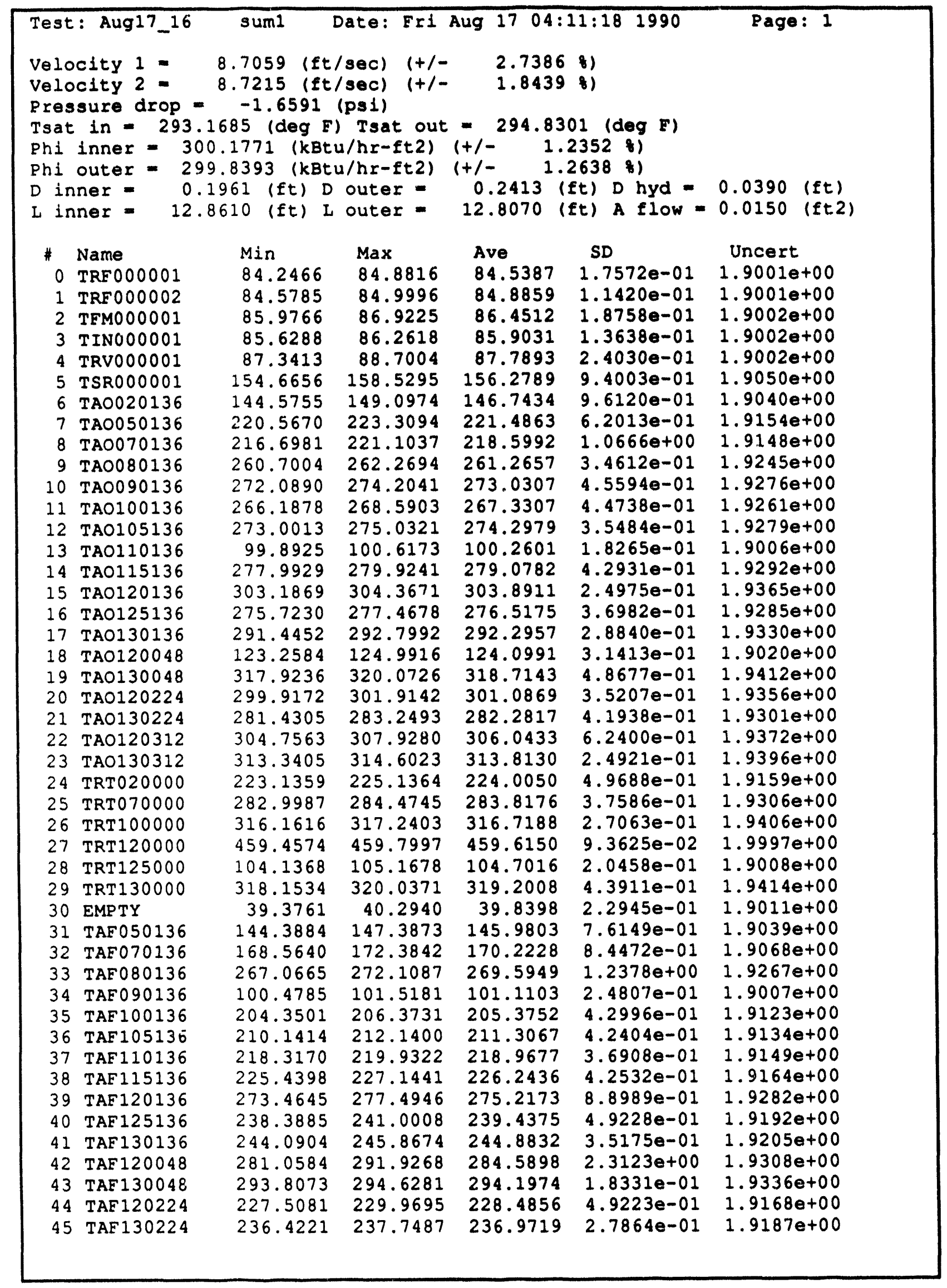




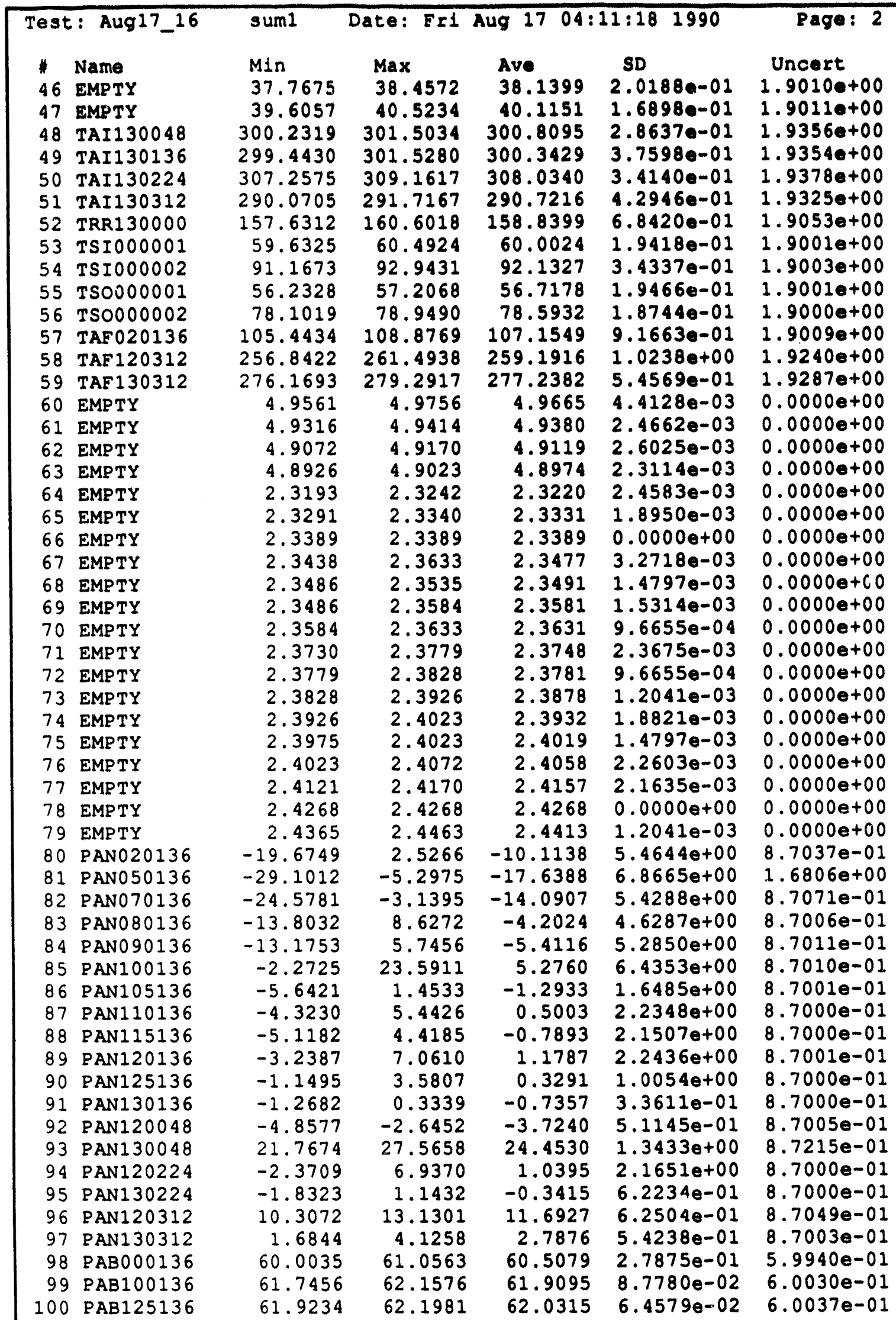




\begin{tabular}{|c|c|c|c|c|c|c|}
\hline \multicolumn{2}{|c|}{ Test: Aug17_16 } & suml & \multicolumn{3}{|c|}{ Date: Er1 Aug $1704: 11: 181990$} & Page: 3 \\
\hline 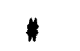 & Name & Min & $\operatorname{Max}$ & Ave & SD & Uncert \\
\hline 101 & PINO00001 & 59.1237 & 61.0769 & 60.2810 & $5.2155 \bullet-01$ & $7.64990-01$ \\
\hline 102 & PRV000001 & 19.7186 & 19.8406 & 19.8382 & $1.7263 \theta-02$ & $7.51640-01$ \\
\hline 103 & PSR000001 & 61.0509 & 61.2950 & 61.2169 & $6.41210-02$ & $7.6546 e-01$ \\
\hline 104 & PEM000001 & 11.1624 & 11.8032 & 11.5249 & $1.25390-01$ & $5.0083 e-01$ \\
\hline 105 & PANO00136 & -58.5494 & -28.7947 & -45.9334 & $7.6365 e+00$ & $4.9213 e+00$ \\
\hline 106 & PEMOOOOO2 & 11.3932 & 11.7959 & 11.5667 & $8.39750-02$ & $2.02080-01$ \\
\hline 107 & EMPTY & -0.0049 & 0.0049 & 0.0046 & $1.53140-03$ & $0.0000 e+00$ \\
\hline 108 & EMPTY & -10.0000 & -6.2451 & -9.6560 & $8.2967 e-01$ & $0.00000+00$ \\
\hline 109 & EMPTY & 0.0000 & 0.0049 & 0.0037 & $2.1065 e-03$ & $0.00000+00$ \\
\hline 110 & EMPTY & -10.0000 & -10.0000 & -10.0000 & $0.0000 e+00$ & $0.0000 e+00$ \\
\hline 111 & EMPTY & 0.0000 & 0.0000 & 0.0000 & $0.0000 e+00$ & $0.0000 e+00$ \\
\hline 112 & VPW000001 & 177.6104 & 178.1964 & 177.8409 & $1.4600 e-01$ & $1.3397 e+00$ \\
\hline 113 & VPW000002 & 207.7186 & 208.1092 & 207.8593 & $1.1189 \bullet-01$ & $1.48710+00$ \\
\hline 114 & VPW000003 & 256.8021 & 257.5834 & 257.2786 & $1.25770-01$ & $1.7433 e+00$ \\
\hline 115 & VPWOOOOOOA & 182.5984 & 183.1843 & 182.9032 & $1.1942 e-01$ & $1.3640 e+00$ \\
\hline 116 & VPWO00005 & 243.5584 & 244.1444 & 243.8828 & $1.2853 e-01$ & $1.6725 e+00$ \\
\hline 117 & VPWO00006 & 258.4172 & 259.1985 & 258.9093 & $1.4358 e-01$ & $1.75190+00$ \\
\hline 118 & IPWO00001 & 928.1141 & 937.8759 & 934.5960 & $2.47710+00$ & $1.09600+01$ \\
\hline 119 & IPWO00002 & 2209.6626 & 2236.9805 & 2225.5459 & $9.7045 a+00$ & $1.93580+01$ \\
\hline 120 & IPW000003 & 2252.2498 & 2261.9998 & 2258.0220 & $2.55190+00$ & $1.9591 e+01$ \\
\hline 121 & IPWO 00004 & 648.7473 & 651.1887 & 649.8315 & $5.4292 e-01$ & $5.5095 e+00$ \\
\hline 122 & IPWO00005 & 431.7664 & 433.7194 & 432.5865 & $6.0355 e-01$ & $3.9597 e+00$ \\
\hline 123 & IPW000006 & 447.7915 & 449.2563 & 448.4943 & $3.7121 e-01$ & $4.0686 e+00$ \\
\hline 124 & EMPTY & -0.0293 & -0.0146 & -0.0196 & $3.4863 e-03$ & $0.0000 e+00$ \\
\hline 125 & EMPTY & -0.0195 & -0.0049 & -0.0127 & $2.9594 e-03$ & $0.0000 e+00$ \\
\hline 126 & EMPTY & -0.0244 & -0.0098 & -0.0150 & $3.8002 e-03$ & $0.0000 e+00$ \\
\hline 127 & EMPTY & -0.0244 & -0.0098 & -0.0167 & $3.43000-03$ & $0.0000 e+00$ \\
\hline 128 & IHTO0000I & 218.3669 & 221.6872 & 220.2458 & $7.3598 e-01$ & $4.6541 e+00$ \\
\hline 129 & IHTO00002 & 225.1502 & 228.2752 & 226.7402 & $6.6616 e-01$ & $4.6852 e+00$ \\
\hline 130 & IHT 000003 & 227.0358 & 230.1608 & 228.6530 & $6.6385 e-01$ & $4.6945 e+00$ \\
\hline 131 & IHTO00004 & 223.1783 & 226.3033 & 224.5690 & $6.5749 \theta-01$ & $4.6747 e+00$ \\
\hline 132 & IHTO00005 & 221.0637 & 224.1887 & 222.3058 & $6.21100-01$ & $4.6639 e+00$ \\
\hline 133 & IHT000006 & 215.4034 & 218.5284 & 216.5205 & $5.9718 e-01$ & $4.6366 e+00$ \\
\hline 134 & IHTO000007 & 224.7446 & 227.8696 & 225.9984 & $6.1268 \mathrm{e}-01$ & $4.6816 e+00$ \\
\hline 135 & IHT 000008 & 222.1529 & 225.4733 & 223.3835 & $5.4426 e-01$ & $4.6690 e+00$ \\
\hline 136 & IHTO00009 & 222.1417 & 225.6573 & 223.4893 & $5.9875 e-01$ & $4.6696 e+00$ \\
\hline 137 & IHT000010 & 54.0377 & 55.9908 & 55.0924 & $4.2682 e-01$ & $4.1368 e+00$ \\
\hline 138 & IHT000011 & 222.6900 & 225.2291 & 223.8893 & $5.1902 e-01$ & $4.6715 e+00$ \\
\hline 139 & IHTO00012 & 218.9378 & 221.2816 & 219.8753 & $5.1297 e-01$ & $4.6524 e+00$ \\
\hline 140 & IHT000013 & 158.5938 & 160.9375 & 159.5586 & $5.4592 \theta-01$ & $4.3995 e+00$ \\
\hline 141 & IHTO00014 & 219.5538 & 221.7022 & 220.4990 & $5.2104 \theta-01$ & $4.6553 e+00$ \\
\hline 142 & IHTO00015 & 163.7620 & 166.4964 & 164.7269 & $5.5300 \theta-01$ & $4.4185 e+00$ \\
\hline 143 & IHT000016 & 152.5841 & 155.3185 & 153.4552 & 5.855 & $4.3778 e+00$ \\
\hline 144 & IHTO00017 & 182.6097 & 185.3441 & 183.8988 & $6.64 ?$ & $4.4935 e+00$ \\
\hline 145 & IHTO00018 & 184.6980 & 187.0418 & 185.6864 & $6.8164 e-01$ & $4.5009 e+00$ \\
\hline 146 & IHTO00019 & 178.8687 & 181.0171 & 179.9859 & $5.1151 e-01$ & $4.4777 e+00$ \\
\hline 147 & IHTO00020 & 183.9318 & 186.2755 & 185.0958 & $4.7344 e-01$ & $4.4984 \theta+00$ \\
\hline 148 & IHTO00021 & -2.8132 & -0.2742 & -1.4617 & $5.9036 \theta-01$ & $4.10000+00$ \\
\hline 149 & IHT000022 & 158.2895 & 160.8286 & 159.6372 & $5.8294 e-01$ & $4.3998 e+00$ \\
\hline 150 & IHTO00023 & 180.1458 & 182.4895 & 181.2003 & $5.4104 e-01$ & $4.4826 e+00$ \\
\hline 151 & IHTO00024 & 144.5726 & 146.5257 & 145.5883 & $4.7679 e-01$ & $4.3508 e+00$ \\
\hline 152 & IHTO00025 & 179.5486 & 181.6970 & 180.5563 & $5.4299 e-01$ & $4.4800 e+00$ \\
\hline 153 & IHT000026 & 181.6932 & 183.8417 & 182.7089 & $5.0532 e-01$ & $4.4887 e+00$ \\
\hline 154 & IHTO00027 & 155.0331 & 157.5721 & 156.0565 & $5.9524 e-01$ & $4.3870 e+00$ \\
\hline 155 & IHTO00028 & 157.3505 & 159.8896 & 158.4052 & $5.9712 e-01$ & $4.3954 e+00$ \\
\hline
\end{tabular}




\begin{tabular}{|c|c|c|c|c|c|c|}
\hline $\begin{array}{l}1 \\
156 \\
157 \\
158 \\
159 \\
160 \\
161 \\
162 \\
163\end{array}$ & 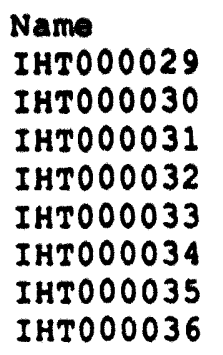 & $\begin{array}{l}\text { Min } \\
180.4012 \\
183.7703 \\
180.8368 \\
183.3045 \\
183.3271 \\
161.1253 \\
212.5902 \\
159.2060\end{array}$ & $\begin{array}{l}\operatorname{Max} \\
183.1355 \\
186.7000 \\
183.7665 \\
186.4295 \\
186.2568 \\
163.4690 \\
215.1292 \\
161.1591\end{array}$ & $\begin{array}{l}\text { Ave } \\
181.6044 \\
185.0009 \\
182.1376 \\
184.5546 \\
184.5303 \\
162.2230 \\
213.7386 \\
160.2607\end{array}$ & $\begin{array}{l}\text { SD } \\
5.6821 \theta-01 \\
5.9875 e-01 \\
5.9922 e-01 \\
6.4113 \theta-01 \\
6.1556 \theta-01 \\
5.1583 e-01 \\
5.5614 \theta-01 \\
4.7186 \theta-01\end{array}$ & $\begin{array}{c}\text { Uncert } \\
4.4842 \bullet+00 \\
4.4981 \bullet+00 \\
4.48640+00 \\
4.4962 \theta+00 \\
4.4961 \theta+00 \\
4.4093 e+00 \\
4.6237 \bullet+00 \\
4.40210+00\end{array}$ \\
\hline
\end{tabular}




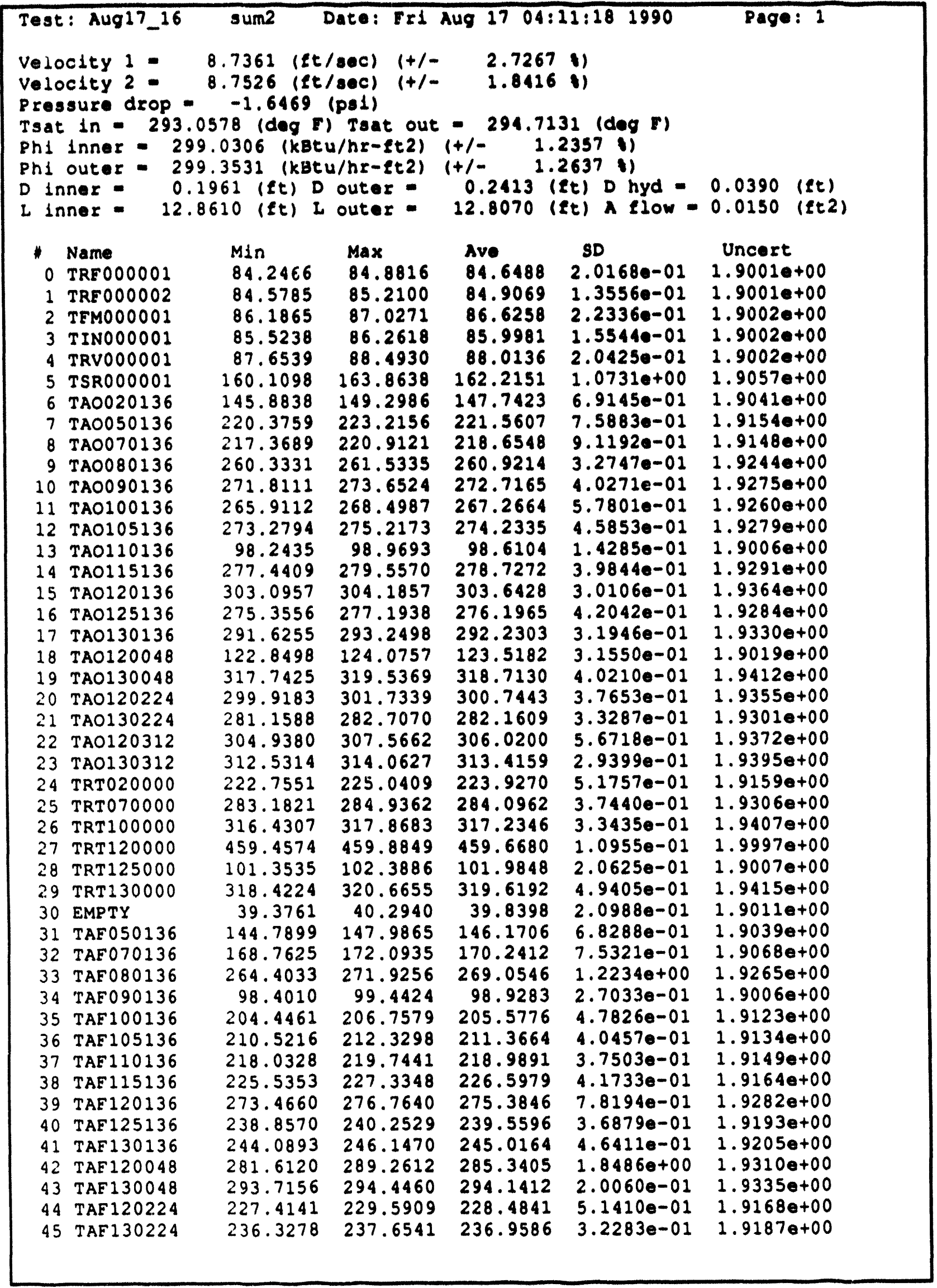




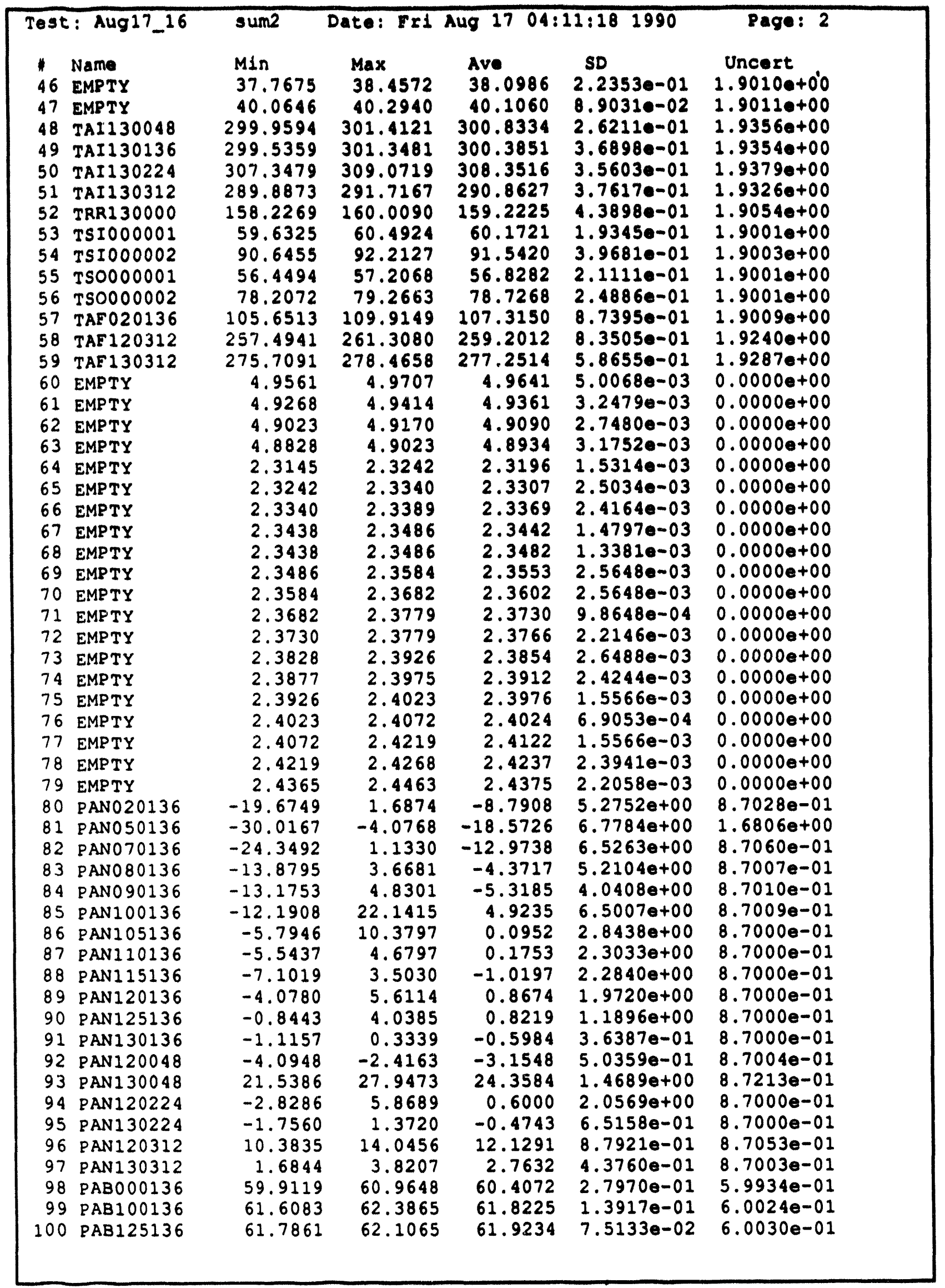




\begin{tabular}{|c|c|c|c|c|c|c|}
\hline \multicolumn{2}{|c|}{ Test: Aug17_16 } & sum2 & \multicolumn{3}{|c|}{ Date: Fri Aug $17 \quad 04: 11: 18 \quad 1990$} & Page: 3 \\
\hline$*$ & Name & Min & $\operatorname{Max}$ & Ave & SD & Uncert \\
\hline 101 & PINO00001 & 59.1237 & 60.9548 & 60.0881 & $5.1333 e-01$ & $7.6490 e-01$ \\
\hline $\begin{array}{l}103 \\
104\end{array}$ & $\begin{array}{l}\text { PSR0000001 } \\
\text { PFM0000001 }\end{array}$ & $\begin{array}{l}60.9288 \\
11.3455\end{array}$ & $\begin{array}{l}61.1730 \\
11.8948\end{array}$ & $\begin{array}{l}61.0777 \\
11.6055\end{array}$ & $\begin{array}{l}5.6722 e-02 \\
1.2995 e-01\end{array}$ & $\begin{array}{l}7.6539 e-01 \\
5.0084 e-01\end{array}$ \\
\hline $\begin{array}{l}104 \\
105\end{array}$ & $\begin{array}{l}\text { PEMO00001 } \\
\text { PANO00136 }\end{array}$ & $\begin{array}{r}11.3455 \\
-58.0916\end{array}$ & $\begin{array}{r}11.8948 \\
-29.7103\end{array}$ & $\begin{array}{r}11.6055 \\
-45.5946\end{array}$ & $7.3866 e+00$ & $\begin{array}{l}5.0084 e-01 \\
4.9213 e+00\end{array}$ \\
\hline 106 & PFMO00002 & 11.5274 & 11.8462 & 11.6499 & $7.6951 e-02$ & $2.0211 e-01$ \\
\hline 107 & EMPTY & -0.0049 & 0.0098 & 0.0048 & $1.5566 e-03$ & $0.00000+00$ \\
\hline 108 & EMPTY & -8.9209 & -5.9717 & -6.7899 & $7.1054 \theta-01$ & $0.0000 e+00$ \\
\hline 109 & EMPTY & 0.0000 & 0.0049 & 0.0045 & $1.33810-03$ & $0.0000 e+00$ \\
\hline 110 & EMPTY & -10.0000 & -10.0000 & -10.0000 & $0.0000 e+00$ & $0.0000 e+00$ \\
\hline 111 & EMPTY & -0.0049 & 0.0000 & -0.0001 & $6.9053 e-04$ & $0.0000 e+00$ \\
\hline 112 & VPWO00001 & 177.6104 & 178.1964 & 177.7979 & $1.4743 e-01$ & $1.3395 e+00$ \\
\hline 113 & VPWO00002 & 207.7186 & 208.1092 & 207.9062 & $1.2453 e-01$ & $1.4873 e+00$ \\
\hline 114 & VPWO00003 & 256.9974 & 257.5834 & 257.3841 & $1.2780 e-01$ & $1.7438 e+00$ \\
\hline 115 & VPWO000004 & 182.7937 & 183.1843 & 182.9462 & $1.2658 \mathrm{e}-01$ & $1.3642 e+00$ \\
\hline 116 & VPWO00005 & 243.5584 & 244.1444 & 243.8281 & $1.2409 \theta-01$ & $1.6722 \mathrm{e}+00$ \\
\hline 117 & VPWO00006 & 258.8079 & 259.3938 & 259.0655 & $1.3917 e-01$ & $1.7528 e+00$ \\
\hline 118 & IPWO00001 & 939.8282 & 953.4946 & 947.3644 & $3.6367 e+00$ & $1.1028 e+01$ \\
\hline 119 & IPW000002 & 2205.7600 & 2211.6140 & 2207.5166 & $1.4350 e+00$ & $1.9229 e+01$ \\
\hline 120 & IPWO000003 & 2244.4500 & 2254.2000 & 2249.6755 & $1.7811 e+00$ & $1.9531 e+01$ \\
\hline 121 & IPW000004 & 651.1887 & 652.6536 & 652.0972 & $3.9471 \theta-01$ & $5.5262 e+00$ \\
\hline 122 & IPW000005 & 430.3017 & 431.7664 & 430.9949 & $3.8316 e-01$ & $3.9488 e+00$ \\
\hline 123 & IPW000006 & 443.3970 & 447.3032 & 445.3205 & $1.2298 \theta+00$ & $4.0468 e+00$ \\
\hline 124 & EMPTY & -0.0244 & -0.0146 & -0.0186 & $3.8206 e-03$ & $0.0000 e+00$ \\
\hline 125 & EMPTY & -0.0195 & -0.0098 & -0.0137 & $3.2718 e-03$ & $0.0000 e+00$ \\
\hline 126 & EMPTY & -0.0195 & -0.0098 & -0.0142 & $3.0003 e-03$ & $0.0000 e+00$ \\
\hline 127 & EMPTY & -0.0244 & -0.0098 & -0.0179 & $3.6393 e-03$ & $0.0000 e+00$ \\
\hline 128 & IHTO00001 & 218.9528 & 221.4919 & 220.4841 & $5.5714 e-01$ & $4.6552 \mathrm{e}+00$ \\
\hline 129 & IHTOOOOO2 & 225.5408 & 228.2752 & 226.9902 & $5.6233 e-01$ & $4.6864 e+00$ \\
\hline 130 & IHT000003 & 227.6217 & 229.9655 & 228.8327 & $5.6359 e-01$ & $4.6954 \mathrm{e}+00$ \\
\hline 131 & IHTO00004 & 222.9830 & 226.3033 & 224.7253 & $6.3238 e-01$ & $4.6755 e+00$ \\
\hline 132 & IHTO00005 & 221.0637 & 223.9934 & 222.5753 & $6.9229 e-01$ & $4.6652 \mathrm{e}+00$ \\
\hline 133 & IHT000006 & 215.2081 & 218.1378 & 216.6807 & $7.0610 \mathrm{e}-01$ & $4.6374 e+00$ \\
\hline 134 & IHT000007 & 224.7446 & 227.4790 & 226.1898 & $7.1136 e-01$ & $4.6825 e+00$ \\
\hline 135 & IHTO00008 & 222.1529 & 224.8873 & 223.5241 & $7.2382 e-01$ & $4.6697 e+00$ \\
\hline 136 & IHT 000009 & 222.3370 & 225.0714 & 223.6768 & $7.0145 e-01$ & $4.6705 e+00$ \\
\hline 137 & IHTO000010 & 54.2330 & 56.7721 & 55.1080 & $6.0777 e-01$ & $4.1369 e+00$ \\
\hline 138 & IHTO00011 & 223.0807 & 225.6197 & 224.0456 & $5.7237 e-01$ & $4.6722 e+00$ \\
\hline 139 & IHTO000012 & 219.3284 & 221.6722 & 220.0550 & $5.4253 e-01$ & $4.6532 e+00$ \\
\hline 140 & IHTO00013 & 158.7891 & 161.1328 & 159.6055 & $5.1250 e-01$ & $4.3997 e+00$ \\
\hline 141 & IHTO00014 & 219.3585 & 221.3116 & 220.4053 & $4.8450 e-01$ & $4.6549 e+00$ \\
\hline 142 & IHTO00015 & 163.3714 & 165.5198 & 164.6254 & $4.7201 e-01$ & $4.4182 e+00$ \\
\hline 143 & IHTO000016 & 152.1935 & 154.1466 & 153.3028 & $4.9196 e-01$ & $4.3772 e+00$ \\
\hline 144 & IHT000017 & 182.8050 & 185.3441 & 183.9886 & $6.4405 e-01$ & $4.4939 e+00$ \\
\hline 145 & IHTO00018 & 184.5027 & 187.0418 & 185.7059 & $6.4641 e-01$ & $4.5010 \mathrm{e}+00$ \\
\hline 146 & IHTO00019 & 178.8687 & 181.4077 & 179.9195 & $6.3857 e-01$ & $4.4774 e+00$ \\
\hline 147 & IHT 000020 & 183.9318 & 186.6662 & 185.0802 & $6.1338 e-01$ & $4.4984 e+00$ \\
\hline 148 & IHTO00021 & -2.8132 & 0.1164 & -1.5789 & $6.8962 \mathrm{e}-01$ & $4.1000 e+00$ \\
\hline 149 & IHTO 00022 & 158.2895 & 160.8286 & 159.4809 & $5.65310-01$ & $4.3993 e+00$ \\
\hline 150 & IHTOO0023 & 179.9504 & 182.6848 & 181.1808 & $5.8826 e-01$ & $4.4825 e+00$ \\
\hline 151 & IHTO00024 & 144.3773 & 146.9163 & 145.5805 & $5.4585 e-01$ & $4.3508 e+00$ \\
\hline 152 & IHTOO0025 & 179.3532 & 181.8923 & 180.5016 & $5.6996 \mathrm{e}-01$ & $4.4797 e+00$ \\
\hline 153 & IHTO00026 & 181.4979 & 183.8417 & 182.6776 & $6.0613 e-01$ & $4.4886 e+00$ \\
\hline 154 & IHTO00027 & 155.0331 & 157.3768 & 156.0136 & $6.1699 e-01$ & $4.3868 e+00$ \\
\hline 155 & IHTOOOO28 & 156.9599 & 159.6942 & 158.2998 & $7.3080 e-01$ & $4.3950 \mathrm{e}+00$ \\
\hline
\end{tabular}




\begin{tabular}{|c|c|c|c|c|c|c|}
\hline Test & $\therefore$ : Aug17_16 & sum2 & Date: Fri & Aug $1704:$ & $11: 18 \quad 1990$ & Page: 4 \\
\hline $\begin{array}{c}1 \\
156 \\
157 \\
158 \\
159 \\
160 \\
161 \\
162 \\
163\end{array}$ & $\begin{array}{l}\text { Name } \\
\text { IHTO00029 } \\
\text { IHTO00030 } \\
\text { IHTO00031 } \\
\text { IHTO00032 } \\
\text { IHTO00033 } \\
\text { IHTOOOO34 } \\
\text { IHTOOOO35 } \\
\text { IHTOOOO36 }\end{array}$ & $\begin{array}{l}\text { Min } \\
180.4012 \\
183.7703 \\
181.0322 \\
183.3045 \\
183.1318 \\
160.9300 \\
212.3949 \\
158.8153\end{array}$ & $\begin{array}{l}\operatorname{Max} \\
182.9402 \\
186.3094 \\
183.3759 \\
186.0389 \\
185.6708 \\
163.0784 \\
214.5433 \\
161.3544\end{array}$ & $\begin{array}{l}\text { Ave } \\
181.5966 \\
184.9891 \\
182.1025 \\
184.5273 \\
184.4404 \\
162.0824 \\
213.5706 \\
160.1513\end{array}$ & $\begin{array}{l}\text { SD } \\
6.8759 e-01 \\
6.6680 e-01 \\
6.3410 e-01 \\
6.7290 e-01 \\
6.9838 \theta-01 \\
5.2977 e-01 \\
5.5452 e-01 \\
5.8041 e-01\end{array}$ & $\begin{array}{c}\text { Uncert } \\
4.4842 e+00 \\
4.4980 e+00 \\
4.4862 e+00 \\
4.4961 e+00 \\
4.4958 e+00 \\
4.4087 e+00 \\
4.6229 e+00 \\
4.4017 e+00\end{array}$ \\
\hline
\end{tabular}




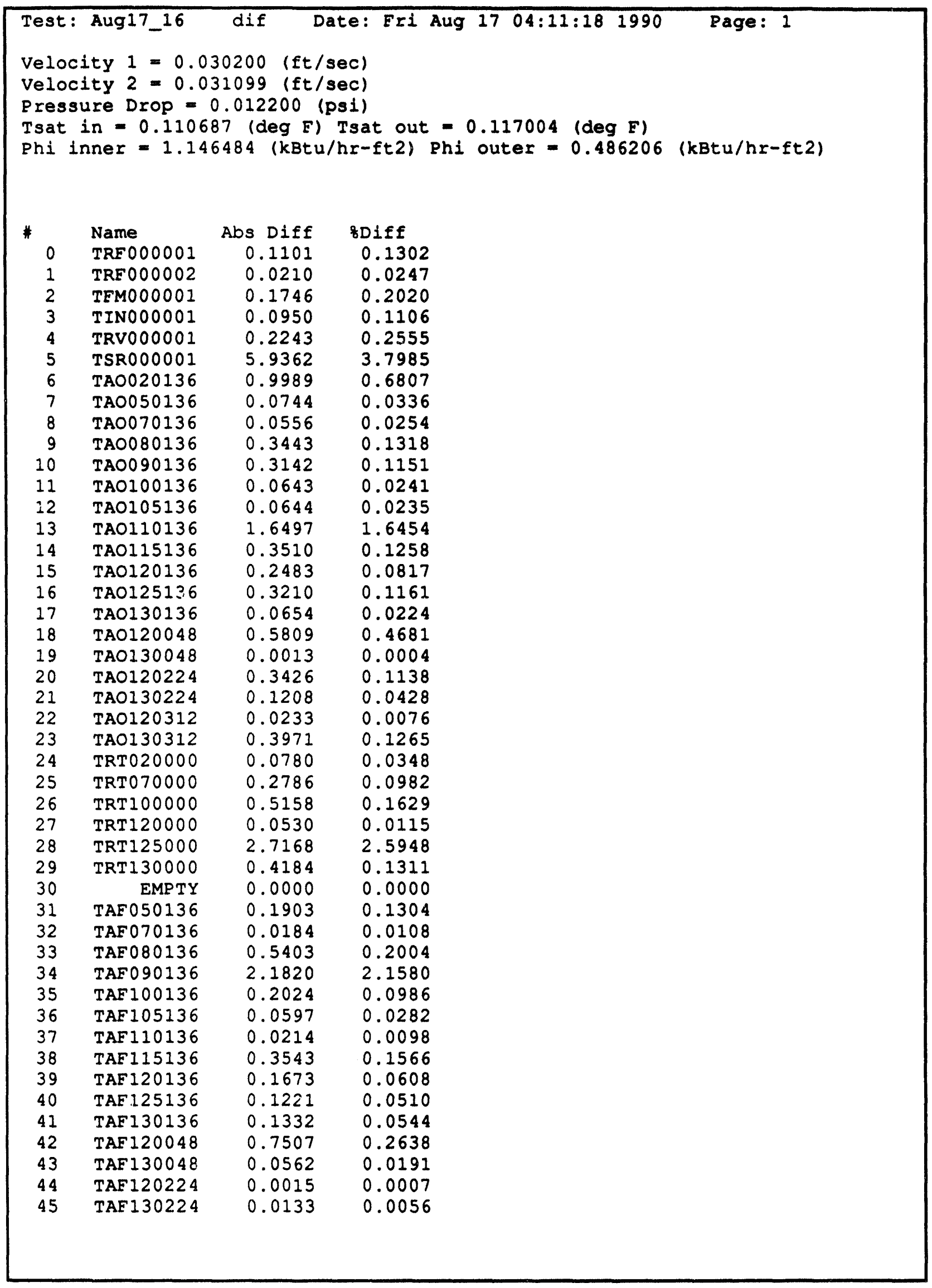




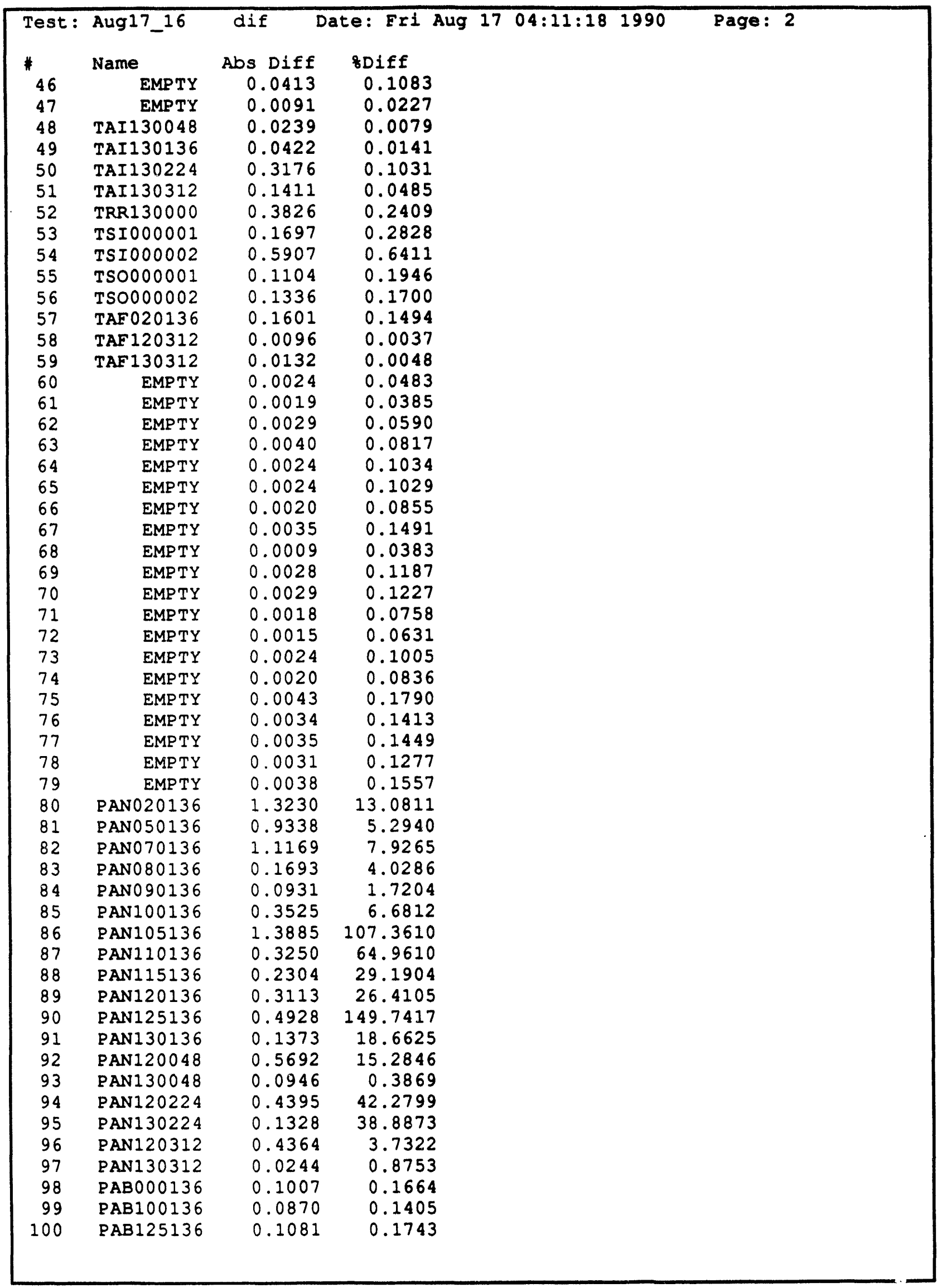




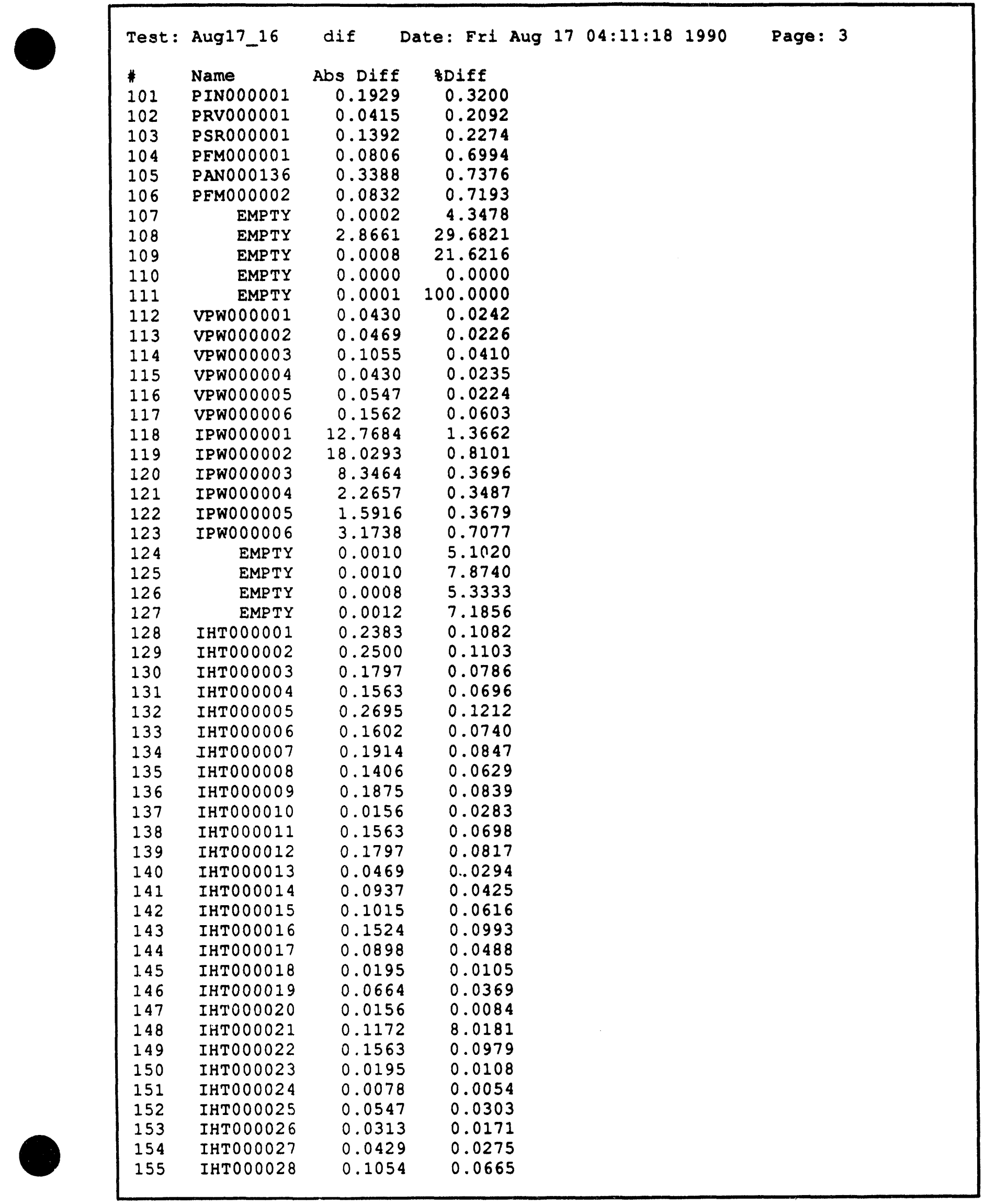




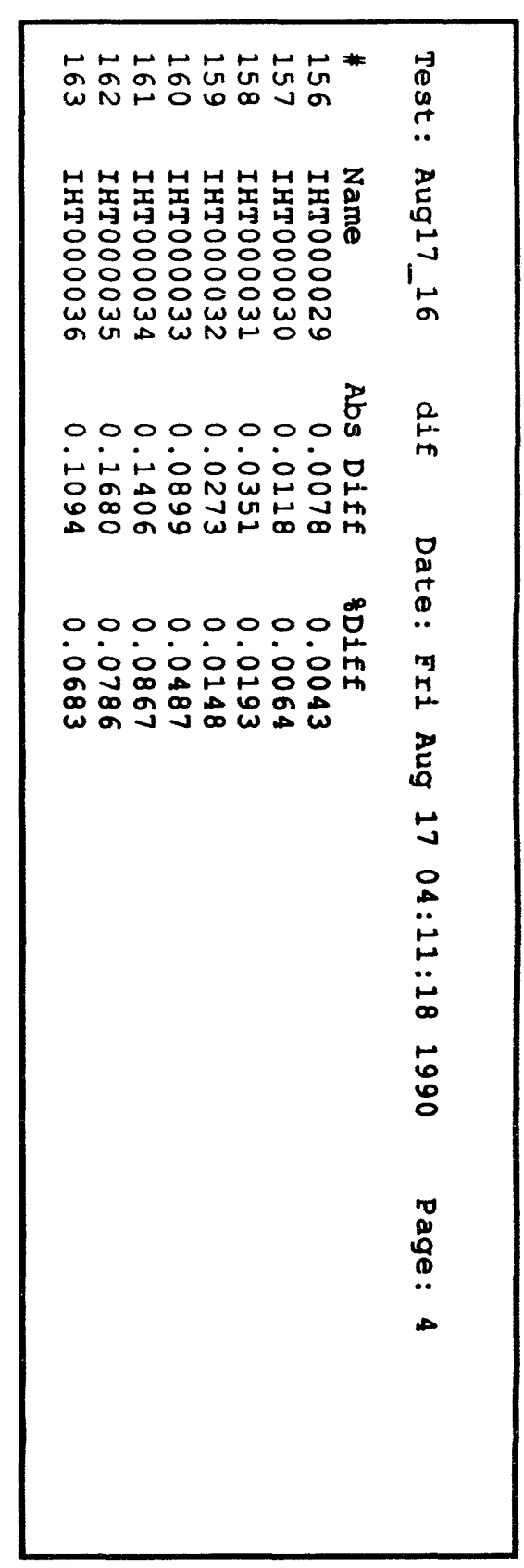




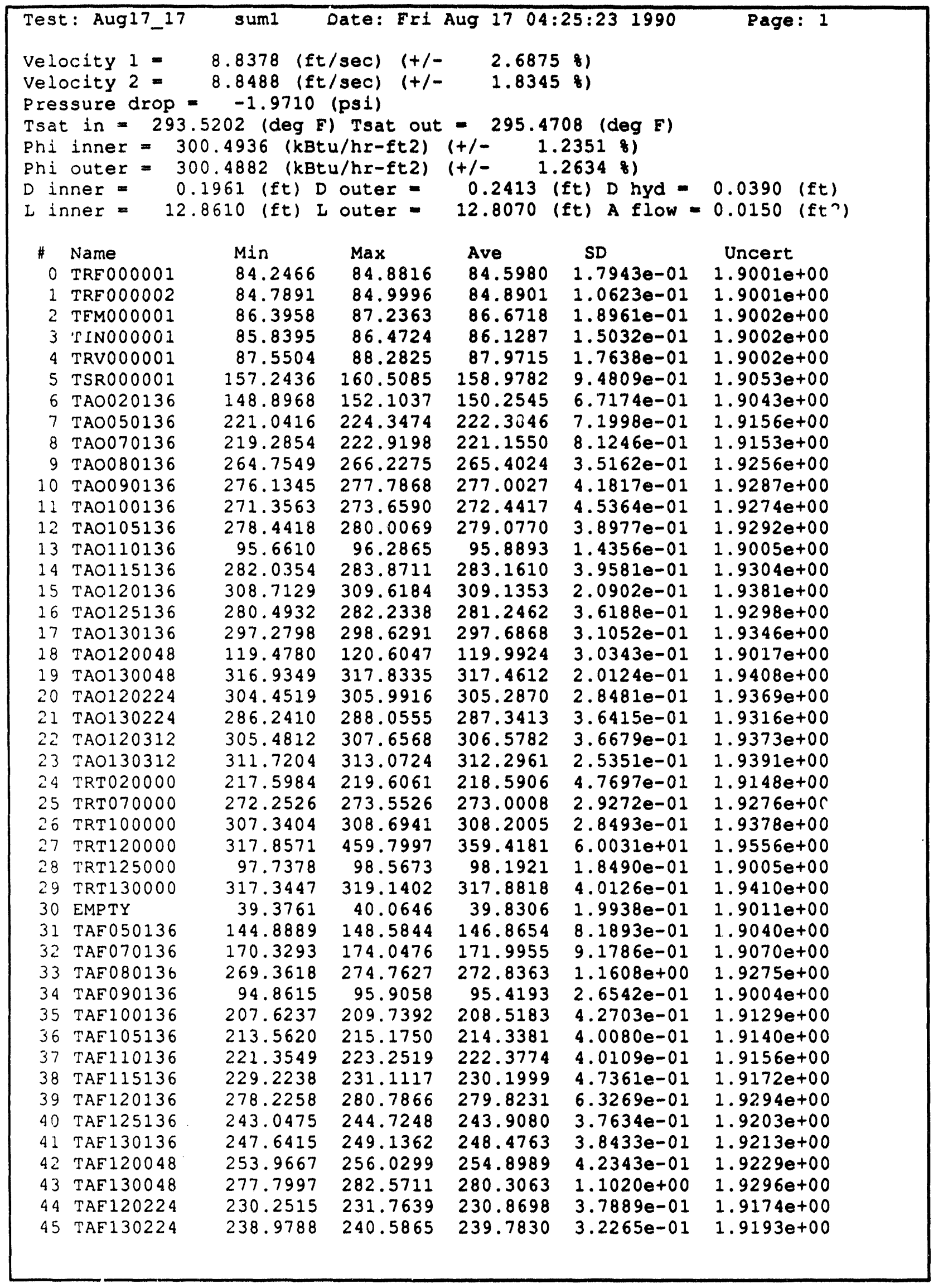




\begin{tabular}{|c|c|c|c|c|c|c|}
\hline \multicolumn{2}{|c|}{ Test: Aug17_17 } & sum1 & te: & 28 & 23 & Page: \\
\hline \# & Name & Min & $\operatorname{Max}$ & Ave & SD & Uncerc \\
\hline 46 & EMPTY & 37.7675 & 38.4572 & 38.1354 & $1.9151 \mathrm{e}-01$ & $1.9010 e+00$ \\
\hline 47 & EMPTY & 39.8352 & 40.2940 & 40.0417 & $1.2481 \mathrm{e}-01$ & $1.9011 e+00$ \\
\hline 48 & TAI1 30048 & 299.9601 & 301.1409 & 300.5392 & $3.0483 e-01$ & $1.9355 e+00$ \\
\hline 49 & TAI130136 & 304.2408 & 305.3268 & 304.7169 & $3.0795 e-01$ & $1.9368 e+00$ \\
\hline 50 & TAI130224 & 307.8922 & 308.9811 & 308.3406 & $2.3992 \mathrm{e}-01$ & $1.9379 e+00$ \\
\hline 51 & TAI1 30312 & 295.0021 & 3705 & 295.6498 & $3.2401 e-01$ & $1.9340 \mathrm{e}+00$ \\
\hline 52 & TRR130000 & 157.9294 & 160.5039 & 159.3054 & $5.6886 e-01$ & $1.9054 e+00$ \\
\hline 53 & TSI000001 & $6 \mathrm{~J} .0611$ & 60.7066 & 60.3305 & $1.4908 e-01$ & $1.9001 e+00$ \\
\hline 54 & TSI000002 & 90.5402 & 91 & 91.5753 & $3.4249 e-01$ & $1.9003 e+00$ \\
\hline 55 & TSO0000001 & 56.4495 & 57.2068 & 56.8412 & $1.7750 e-01$ & $1.9001 e+00$ \\
\hline 56 & TSO0000002 & 77.7838 & 78.7379 & 78.2737 & $2.2028 e-01$ & $1.9000 e+00$ \\
\hline 57 & TAF020136 & 105.7561 & $109 .^{\circ}$ & 107.4941 & $8.0663 e-01$ & $1.9009 e+00$ \\
\hline 58 & TAF 120312 & 243.8432 & 246.8458 & 245.2568 & $5.6202 e-01$ & $1.9206 e+00$ \\
\hline 59 & TAF 130312 & 268.1560 & 270.5553 & 269.5129 & $5.3938 e-01$ & $1.9266 e+00$ \\
\hline 60 & EMPTY & 4.7510 & 4.7754 & 4.7624 & $6.1297 e-03$ & $0.0000 e+00$ \\
\hline 61 & EMPTY & 4.7266 & 4.7412 & 4.7359 & $4.0482 e-03$ & $0.0000 e+00$ \\
\hline 62 & EMPTY & 4.7070 & 4.7168 & 4.7108 & $3.3146 e-03$ & $0.0000 e+00$ \\
\hline 63 & EMPTY & 4.6924 & 4.7021 & 4.6973 & $3.1195 e-03$ & $0.0000 e+00$ \\
\hline 64 & EMPTY & 2.2217 & 2.2363 & 2.2324 & $2.7902 e-03$ & $0.0000 e+00$ \\
\hline 65 & EMPTY & 2.2412 & 2.2461 & 2.2459 & $9.6655 e-04$ & $0.0000 e+00$ \\
\hline 66 & EMPTY & 2.2510 & 2.2559 & 2.2512 & $9.6655 e-04$ & $0.0000 e+00$ \\
\hline 67 & EMPTY & 2.2559 & 2.2607 & 2.2604 & 1.171 & $0.0000 e+00$ \\
\hline 68 & EMPTY & 2.2607 & 2.2705 & 2.2639 & $2.5648 e-03$ & $0.0000 e+00$ \\
\hline 69 & EMPTY & 2.2656 & 2.2754 & 2.2707 & $1.3811 e-03$ & $0.0000 e+00$ \\
\hline 70 & EMPTY & 2.2754 & 2.2852 & 2.2759 & 1.778 & $0.0000 e+00$ \\
\hline 71 & EMPTY & 2.2900 & 2.2949 & 2.2902 & $9.6655 e-04$ & $0.0000 e+00$ \\
\hline 72 & EMPTY & 2.2900 & 2.2949 & 2.2944 & $1.4797 e-03$ & $0.0000 e+00$ \\
\hline 73 & EMPTY & 2.2998 & 2.3096 & 2.3046 & 1.20 & $0.0000 e+00$ \\
\hline 74 & EMPTY & 2.3047 & 2.3145 & 2.3096 & 9.8648 & $0.0000 e+00$ \\
\hline 75 & EMPTY & 2.3145 & 2.3193 & 2.3187 & 1.6028 & $0.0000 e+00$ \\
\hline 76 & EMPTY & 2.3193 & 3242 & 2.3237 & 1.47 & De+00 \\
\hline 77 & EMPTY & 2.3340 & 2.3438 & 2.3342 & $1.3811 e-03$ & $0 e+00$ \\
\hline 78 & EMPTY & 2.3389 & 2.3486 & 2.3463 & $2.6543 e-03$ & $0.0000 e+00$ \\
\hline 79 & EMPTY & 2.3584 & 2.3633 & 2.3602 & 2.36 & $0 e+00$ \\
\hline 80 & PAN020136 & -19.7512 & 1.4585 & -9.8376 & $5.6769 e+00$ & -01 \\
\hline 81 & PAN050136 & -31.0848 & -7.7389 & -18.9053 & $4.7415 e+00$ & $1.6807 e+00$ \\
\hline 82 & PAN070136 & -24.5781 & -6.8779 & -16.1980 & 4.87 & $4 e-01$ \\
\hline 83 & PAN080136 & -12.5825 & 4.1259 & -4.4465 & 4.25 & $7 e-01$ \\
\hline 84 & PAN090136 & -13.1753 & 9.2551 & -6.4660 & $5.0210 e+00$ & $8.7015 e-01$ \\
\hline 85 & FAN100136 & -2.5777 & 25.5748 & 6.1351 & 5.81 & $e-01$ \\
\hline 80 & PAN105136 & -5.03 & 4.1236 & -1.3345 & $2.2344 e+00$ & $e-01$ \\
\hline 87 & PAN 110136 & -6.38 & 3.4590 & -1.0942 & $2.5575 e+00$ & $00 e-01$ \\
\hline 88 & PAN1 15136 & -6.6441 & 2.2823 & -1.4897 & $1.9565 e+00$ & $1 e-01$ \\
\hline 89 & PAN120136 & -1.1788 & 4.3907 & 1.4366 & $1.3473 e+00$ & $1 e-01$ \\
\hline 90 & PAN125136 & -1.6073 & 0.3764 & -0.6627 & $5.1466 e-01$ & $8.7000 e-01$ \\
\hline 91 & PAN1 30136 & -1.8023 & -0.4290 & -1.1019 & $3.0931 e-01$ & $8.7000 e-01$ \\
\hline 92 & PAN120048 & -5.1629 & -1.9585 & -4.4732 & $5.5745 e-01$ & $8.7007 e-01$ \\
\hline 93 & PAN 130048 & 1.6258 & 2.6177 & 2.1340 & 2.199 & 8.7002 \\
\hline 94 & PAN120224 & -3.0575 & 4.5719 & 0.5741 & $1.8208 e+00$ & $8.7000 e-01$ \\
\hline 95 & PAN130224 & -2.4427 & 0.7617 & -1.3745 & $6.0684 e-01$ & $8.7001 e-01$ \\
\hline 96 & PAN 120312 & -0.2214 & 5.2718 & 1.3945 & $1.2514 e+00$ & $8.7001 e-01$ \\
\hline 97 & PAN130312 & -1.9014 & -0.3755 & -0.9889 & $3.3236 e-01$ & $8.7000 e-01$ \\
\hline 98 & PAB000136 & 60.3239 & 61.4225 & 60.8283 & $2.5837 e-01$ & $5.9960 e-01$ \\
\hline 99 & PAB100136 & 62.2034 & 62.6154 & 62.4103 & $8.4882 e-02$ & $6.0062 e-01$ \\
\hline 100 & PAB125136 & 62.5185 & 62.7474 & 62.6256 & $6.1770 \mathrm{e}-02$ & $6.0076 e-01$ \\
\hline
\end{tabular}




\begin{tabular}{|c|c|c|c|c|c|c|}
\hline \multicolumn{2}{|c|}{ Test: Aug17_17 } & sum1 & Date: Fri & Aug 170 & $25: 231990$ & Page: 3 \\
\hline \# & Name & Min & $\operatorname{Max}$ & Ave & SD & Uncert \\
\hline 101 & PINO00001 & 59.7341 & 61.4431 & 60.6057 & $4.8391 e-01$ & $7.6515 e-01$ \\
\hline 102 & PRV0000001 & 19.5965 & 19.8406 & 19.7210 & $3.0104 e-02$ & $7.5162 \mathrm{e}-01$ \\
\hline 103 & PSR000001 & 61.9054 & 62.2716 & 62.0396 & $7.5006 e-02$ & $7.6587 e-01$ \\
\hline 104 & PFMO000001 & 11.7117 & 12.0779 & 11.8801 & $9.3146 \theta-02$ & $5.0088 e-01$ \\
\hline 105 & PANO00136 & -69.5357 & -38.8655 & -54.5668 & $7.9418 \mathrm{e}+00$ & $4.9219 e+00$ \\
\hline 106 & PEM000002 & 11.8294 & 11.9972 & 11.9100 & $4.8181 e-02$ & $2.0220 \mathrm{e}-01$ \\
\hline 107 & ЕMPTY & 0.0000 & 0.0049 & 0.0047 & $9.6655 e-04$ & $0.0000 e+00$ \\
\hline 108 & EMPTY & -9.2480 & -5.8838 & -6.8034 & $7.8351 e-01$ & $0.0000 e+00$ \\
\hline 109 & EMPTY & 0.0000 & 0.0049 & 0.0039 & $1.9730 e-03$ & $0.0000 e+00$ \\
\hline 110 & EMPTY & -10.0000 & -10.0000 & -10.0000 & $0.0000 e+00$ & $0.0000 e+00$ \\
\hline 111 & EMPTY & -0.0049 & 0.0000 & -0.0001 & $6.9053 e-04$ & $0.0000 e+00$ \\
\hline 112 & VPW000001 & 177.6104 & 178.0011 & 177.7784 & $1.1168 e-01$ & $1.3394 \mathrm{e}+00$ \\
\hline 113 & VPWO000002 & 207.7186 & 208.3046 & 207.9101 & $1.2156 e-01$ & $1.4874 e+00$ \\
\hline 114 & VPW000003 & 257.1927 & 257.5834 & 257.4153 & $1.0447 e-01$ & $1.7440 \mathrm{e}+00$ \\
\hline 115 & VPW0000004 & 182.5984 & 182.9890 & 182.8407 & $1.0106 e-01$ & $1.3637 \mathrm{e}+00$ \\
\hline 110 & YPWO000005 & 243.7538 & 244.1444 & 243.8359 & $1.0507 e-01$ & $1.6723 e+00$ \\
\hline 117 & VPW000006 & 258.8079 & 259.3938 & 258.9796 & $1.2251 e-01$ & $1.7523 e+00$ \\
\hline 118 & IPWO000001 & 930.0665 & 939.8282 & 934.6739 & $2.0404 e+00$ & $1.0960 \mathrm{e}+01$ \\
\hline 119 & IPWO000002 & 2219.4189 & 2238.9316 & 2233.5857 & $5.9107 e+00$ & $1.9416 \mathrm{e}+01$ \\
\hline 120 & IPWO00003 & 2254.2000 & 2263.9500 & 2259.0745 & $2.5908 e+00$ & $1.9599 \mathrm{e}+01$ \\
\hline 121 & IPWO000004 & 649.2356 & 651.1887 & 650.2124 & $4.9324 e-01$ & $5.5123 e+00$ \\
\hline 122 & IPWO000005 & 431.7664 & 434.2076 & 432.9869 & $8.2674 e-01$ & $3.9624 \mathrm{e}+00$ \\
\hline 123 & IPW000006 & 447.7915 & 450.2329 & 448.9728 & $4.6364 e-01$ & $4.0719 \theta+00$ \\
\hline 124 & EMPTY & -0.0293 & -0.0098 & -0.0190 & $3.8523 e-03$ & $0.0000 e+00$ \\
\hline 125 & EMPTY & -0.0195 & -0.0049 & -0.0125 & $2.8179 e-03$ & $0.0000 e+00$ \\
\hline 126 & EMPTY & -0.0195 & -0.0098 & -0.0148 & $3.4116 e-03$ & $0.0000 e+00$ \\
\hline 127 & EMPTY & -0.0244 & -0.0098 & -0.0170 & $2.9986 e-03$ & $0.0000 e+00$ \\
\hline 128 & IHTO000001 & 218.7575 & 221.8825 & 220.5466 & $7.1394 e-01$ & $4.6555 \mathrm{e}+00$ \\
\hline 123 & IHTO00002 & 225.3455 & 228.4705 & 227.0488 & $7.0481 e-01$ & $4.6867 e+00$ \\
\hline 130 & IHTO00003 & 227.2311 & 230.1608 & 228.8561 & $7.3232 e-01$ & $4.6955 \mathrm{e}+00$ \\
\hline 131 & IHTO00004 & 222.7877 & 225.9127 & 224.7018 & $7.5283 e-01$ & $4.6754 e+00$ \\
\hline 132 & IHTO00005 & 220.6731 & 223.9934 & 222.4308 & $7.1572 e-01$ & $4.6645 e+00$ \\
\hline 133 & :HT000006 & 214.8174 & 218.1378 & 216.5518 & $6.9993 e-01$ & $4.6368 e+00$ \\
\hline 134 & $=\mathrm{HT} 000007$ & 224.3540 & 227.4790 & 226.0570 & $7.1031 e-01$ & $4.6819 \mathrm{e}+00$ \\
\hline 135 & IHT000008 & 221.7623 & 224.6920 & 223.4420 & $6.5673 e-01$ & $4.6693 e+00$ \\
\hline 136 & IHT000009 & 221.9464 & 225.0714 & 223.5518 & $6.9619 e-01$ & $4.6699 \mathrm{e}+00$ \\
\hline 137 & IHT000010 & 53.4518 & 56.3815 & 54.9557 & $6.5703 e-01$ & $4.1367 e+00$ \\
\hline 138 & IHTO00011 & 222.2994 & 225.0338 & 223.8854 & $6.2843 e-01$ & $4.6715 e+00$ \\
\hline 139 & IHTO00012 & 218.5472 & 221.2816 & 220.0315 & $6.2639 \mathrm{e}-01$ & $4.6531 \mathrm{e}+00$ \\
\hline 140 & IHTO00013 & 158.0078 & 160.5469 & 159.6289 & $6.1034 e-01$ & $4.3998 e+00$ \\
\hline 141 & IHTO00014 & 218.9679 & 221.7022 & 220.5850 & $5.7459 e-01$ & $4.6557 \mathrm{e}+00$ \\
\hline 142 & IHTOOOOO15 & 163.3714 & 165.7151 & 164.7308 & $5.6215 e-01$ & $4.4186 \mathrm{e}+00$ \\
\hline 143 & IHTO00016 & 151.9982 & 154.5372 & 153.4669 & $5.2975 e-01$ & $4.3778 e+00$ \\
\hline 144 & IHTO00017 & 182.0237 & 185.9300 & 183.8792 & $6.6410 e-01$ & $4.4935 e+00$ \\
\hline 145 & IHTO00018 & 184.1121 & 187.6277 & 185.7020 & $6.5318 e-01$ & $4.5009 e+00$ \\
\hline 146 & IHTOOOOI9 & 178.6734 & 181.2124 & 179.8570 & $6.5007 e-01$ & $4.4771 e+00$ \\
\hline 147 & IHTO00020 & 183.7365 & 186.4708 & 185.0411 & $6.5130 e-01$ & $4.4982 e+00$ \\
\hline 148 & IHTO00021 & -3.2039 & 0.1164 & -1.5906 & $7.7506 e-01$ & $4.1000 \mathrm{e}+00$ \\
\hline 149 & IHTO00022 & 158.0942 & 160.6333 & 159.4106 & $5.9948 e-01$ & $4.3990 e+00$ \\
\hline 150 & IHTO00023 & 179.7551 & 182.2942 & 181.0831 & $5.6634 e-01$ & $4.4821 e+00$ \\
\hline 151 & IHTO00024 & 144.3773 & 146.7210 & 145.4789 & $5.7421 e-01$ & $4.3504 e+00$ \\
\hline 152 & IHTO00025 & 179.1579 & 182.0876 & 180.4899 & $6.2227 e-01$ & $4.4797 e+00$ \\
\hline 153 & IHTO00026 & 181.3026 & 184.0370 & 182.6698 & $5.9189 \mathrm{e}-01$ & $4.4885 e+00$ \\
\hline 154 & IHTO00027 & 154.8377 & 157.1815 & 155.9706 & $5.2940 e-01$ & $4.3866 \mathrm{e}+00$ \\
\hline 155 & IHTO00028 & 156.9599 & 159.8896 & 158.3193 & $6.9133 e-01$ & $4.3951 e+00$ \\
\hline
\end{tabular}




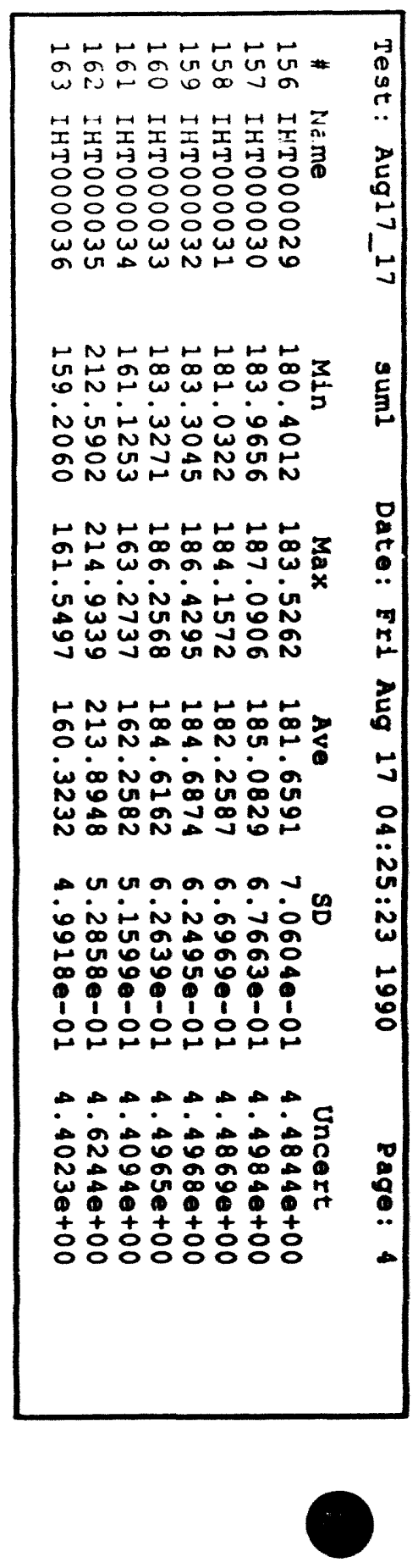




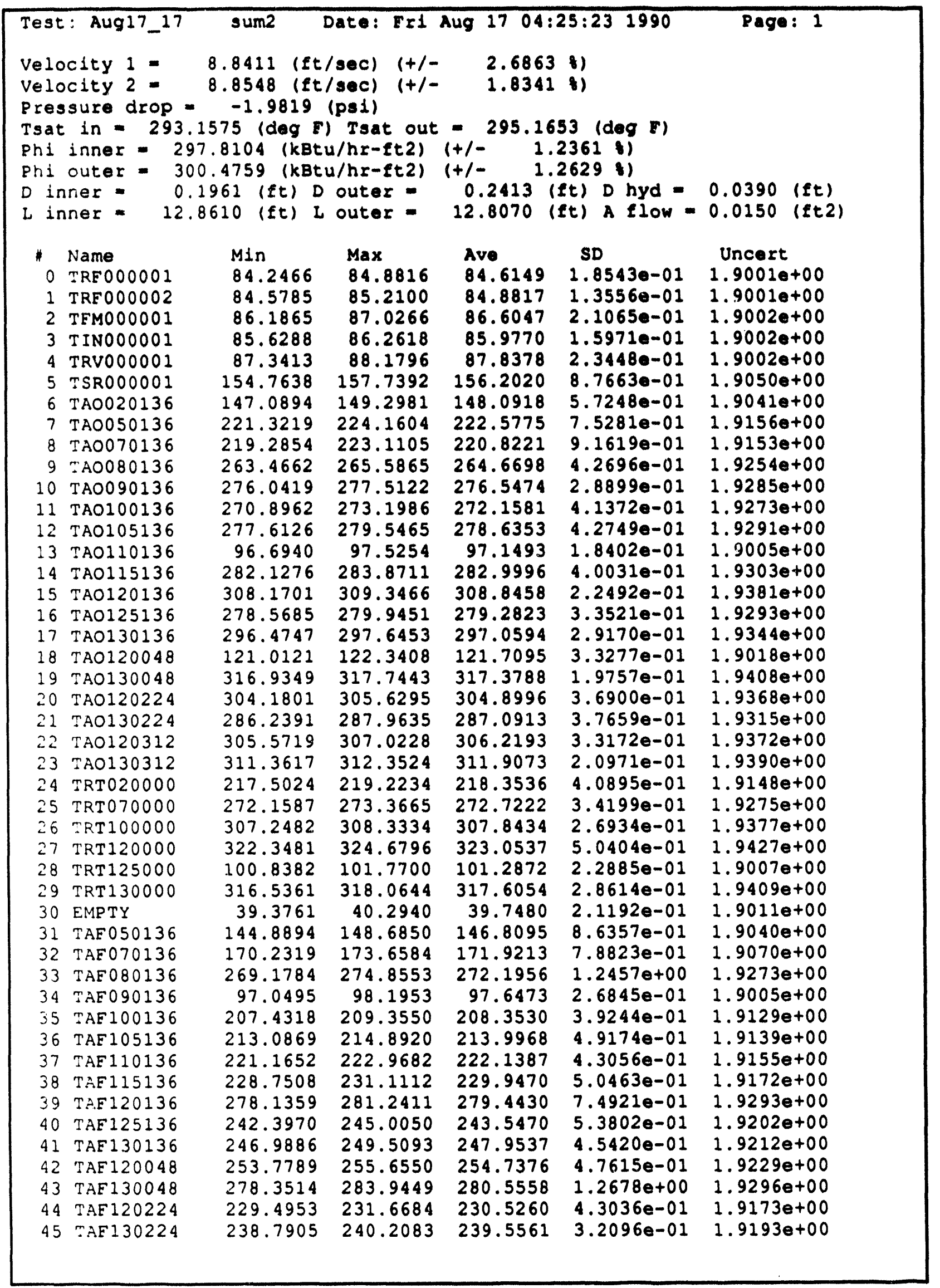




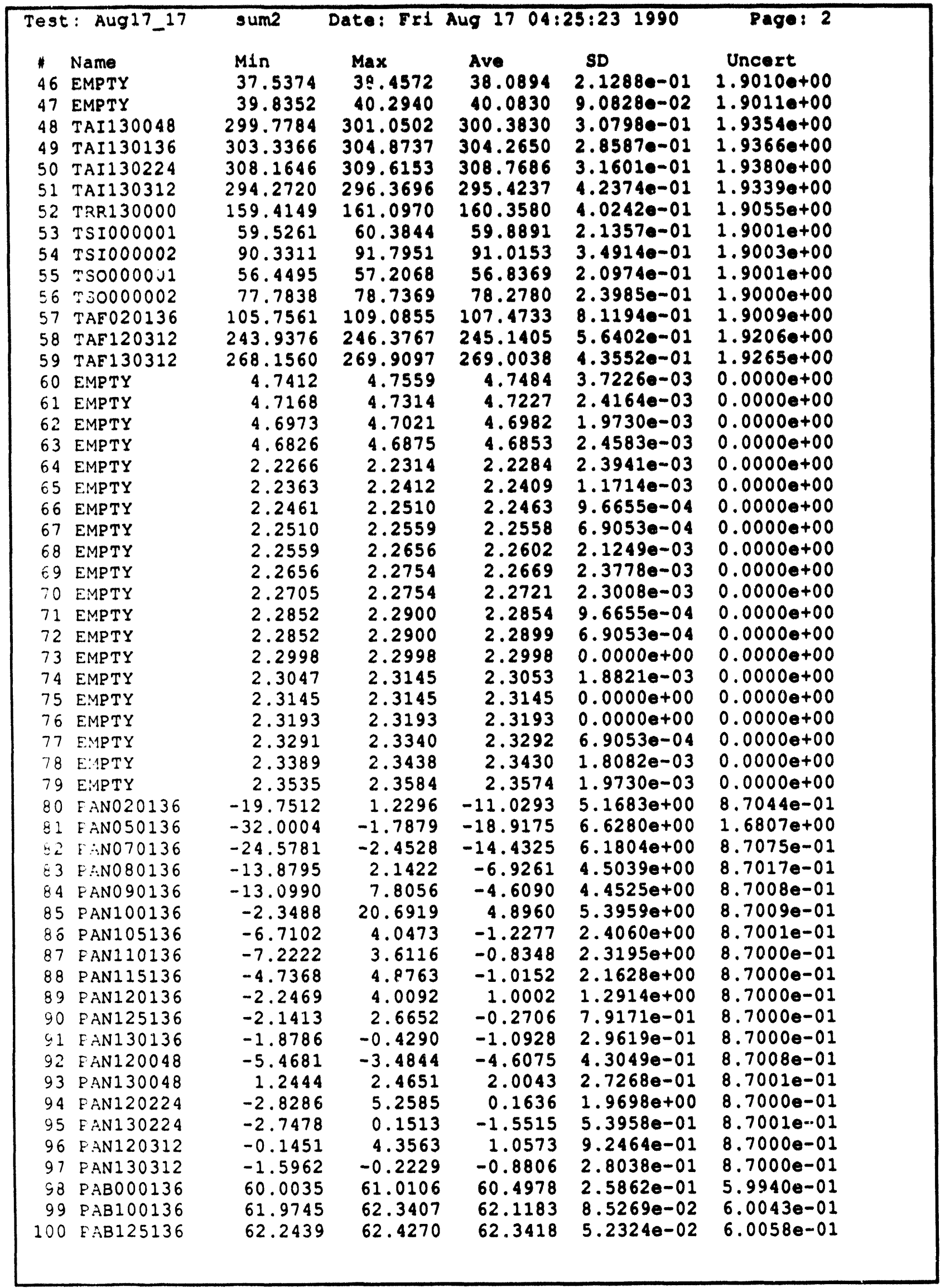




\begin{tabular}{|c|c|c|c|c|c|c|}
\hline \multicolumn{2}{|c|}{ Test: Aug17_17 } & sum2 & \multicolumn{3}{|c|}{ Date: Fr1 Aug $17 \quad 04: 25: 23 \quad 1990$} & Page: 3 \\
\hline " & Name & Min & $\operatorname{Max}$ & Ave & SD & 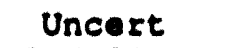 \\
\hline 101 & PINO00001 & 59.4899 & 61.0769 & 60.2883 & $4.6218 e-01$ & $7.6499 e-01$ \\
\hline 102 & PRV0000001 & 19.7186 & 19.7186 & 19.7186 & $1.9267 e-06$ & $7.5162 e-01$ \\
\hline 103 & PSR000001 & 61.6613 & 61.7833 & 61.7638 & $1.5206 e-02$ & $7.6573 e-01$ \\
\hline 104 & FEMO00001 & 11.6201 & 12.1694 & 11.8893 & $1.3262 e-01$ & $5.0088 e-01$ \\
\hline 105 & PANO00136 & -67.2469 & -36.1190 & -54.8689 & $8.0741 e+00$ & $4.9219 e+00$ \\
\hline 106 & PFMO000002 & 11.7959 & 12.0476 & 11.9264 & $6.34480-02$ & $2.0221 e-01$ \\
\hline 107 & EMPTY & -0.0049 & 0.0049 & 0.0045 & $1.93310-03$ & $0.0000 e+00$ \\
\hline 108 & E:IPTY & -10.0000 & -3.9697 & -6.4374 & $9.5973 e-01$ & $0.0000 e+00$ \\
\hline 109 & EMPTY & 0.0000 & 0.0049 & 0.0041 & $1.8082 e-03$ & $0.0000 e+00$ \\
\hline 110 & EMPTY & -10.0000 & -10.0000 & -10.0000 & $0.0000 e+00$ & $0.0000 e+00$ \\
\hline 111 & EMPTY & -0.0049 & 0.0000 & -0.0001 & $6.9053 e-04$ & $0.0000 e+00$ \\
\hline $1: 2$ & VPW000001 & 177.6104 & 178.0011 & 177.7706 & 1.2283 & $1.3393 e+00$ \\
\hline 113 & VPW000002 & 207.7186 & 208.1092 & 207.8671 & $9.3043 e-02$ & $1.48710+00$ \\
\hline 114 & VPW000003 & 256.9974 & 257.5834 & 257.3177 & $1.2327 e-01$ & $1.7435 e+00$ \\
\hline 115 & VPW000004 & 182.5984 & 183.1843 & 182.8446 & $1.4123 e-01$ & $1.3637 e+00$ \\
\hline 116 & VPWO00005 & 243.3631 & 243.9491 & 243.7695 & $1.41390-01$ & $1.67190+00$ \\
\hline 117 & $V=w 000006$ & 258.8079 & 259.1985 & 259.0108 & $1.1133 \theta-01$ & $1.7525 e+00$ \\
\hline 118 & I?W000001 & 949.5900 & 957.3993 & 952.0110 & $2.0328 \theta+00$ & $1.1053 e+01$ \\
\hline 119 & IPWO00002 & 2209.6626 & 2221.3704 & 2215.3999 & $3.64320+00$ & $1.9285 e+01$ \\
\hline 120 & $\because P W 000003$ & 2234.7000 & 2240.5498 & 2238.4443 & $1.2970 e+00$ & $1.9451 e+01$ \\
\hline 121 & $: P W 000004$ & 652.6536 & 655.5833 & 653.8843 & $9.2666 e-01$ & $5.5393 e+00$ \\
\hline 122 & IPW000005 & 433.2311 & 434.2076 & 433.4850 & $3.3069 e-01$ & $3.9658 e+00$ \\
\hline 123 & IPW000006 & 445.3501 & 447.3032 & 446.2092 & $4.1338 a-01$ & $4.0529 e+00$ \\
\hline 124 & EMPTY & -0.0244 & -0.0146 & -0.0189 & $3.2178 e-03$ & $0.0000 e+00$ \\
\hline 125 & E:!PTY & -0.0195 & -0.0098 & -0.0127 & $2.6100 e-03$ & $0.0000 e+00$ \\
\hline 126 & ENIPTY & -0.0195 & -0.0098 & -0.0151 & $3.4527 e-03$ & $0.0000 e+00$ \\
\hline 127 & EMPTY & -0.0244 & -0.0098 & -0.0162 & $3.3366 \theta-03$ & $0.0000 e+00$ \\
\hline 128 & IHTO00001 & 218.7575 & 221.8825 & 220.5427 & $6.3749 e-01$ & $4.6555 e+00$ \\
\hline 129 & IHT000002 & 225.3455 & 228.4705 & 226.9902 & $6.5802 e-01$ & $4.6864 \theta+00$ \\
\hline 130 & IHTO000003 & 227.2311 & 230.1608 & 228.7936 & $6.6145 e-01$ & $4.6952 e+00$ \\
\hline 131 & :HTOOOOOO4 & 223.3736 & 225.9127 & 224.6901 & $6.2616 e-01$ & $4.6753 e+00$ \\
\hline 132 & $I: 4 T 000005$ & 220.8684 & 223.9934 & 222.5831 & $6.3784 e-01$ & $4.6652 e+00$ \\
\hline 133 & $1: T 000006$ & 215.4034 & 217.9424 & 216.6494 & $6.0475 e-01$ & $4.6372 \mathrm{e}+00$ \\
\hline 134 & $\therefore T 000007$ & 224.7446 & 227.4790 & 226.0882 & $5.6722 e-01$ & $4.68200+00$ \\
\hline 135 & IHTO00008 & 221.9576 & 224.8873 & 223.4850 & $5.6454 e-01$ & $4.6695 e+00$ \\
\hline 136 & IHTO00009 & 222.1417 & 225.0714 & 223.6143 & $5.5131 e-01$ & $4.6702 e+00$ \\
\hline 137 & ZHT000010 & 53.8424 & 55.9908 & 54.8814 & $5.1666 e-01$ & $4.1366 e+00$ \\
\hline 138 & $\therefore T 000011$ & 222.6900 & 224.8385 & 223.8503 & $5.0287 e-01$ & $4.6713 e+00$ \\
\hline 139 & $\therefore \mathrm{HT} 000012$ & 218.5472 & 221.2816 & 219.9729 & $5.4854 e-01$ & $4.6528 e+00$ \\
\hline 140 & IHTO00013 & 158.2031 & 160.7422 & 159.5430 & $5.4678 e-01$ & $4.3995 e+00$ \\
\hline 141 & IHTO000014 & 219.1632 & 221.8976 & 220.4326 & $5.5838 e-01$ & $4.6550 \theta+00$ \\
\hline 142 & IHTO00015 & 163.3714 & 166.4964 & 164.7152 & $5.8079 e-01$ & $4.4185 \theta+00$ \\
\hline 143 & IHTO000016 & 151.9982 & 154.7326 & 153.3536 & $5.5300 e-01$ & $4.3774 e+00$ \\
\hline 144 & I:TT000017 & 182.4144 & 185.3441 & 184.1292 & $6.2302 e-01$ & $4.4945 e+00$ \\
\hline 145 & :1!T000018 & 184.1121 & 187.2371 & 185.7059 & $7.1829 \theta-01$ & $4.5010 \theta+00$ \\
\hline 146 & IHTOOOOI9 & 178.6734 & 181.0171 & 179.8843 & $5.6909 \theta-01$ & $4.4773 e+00$ \\
\hline 147 & :1T000020 & 183.9318 & 186.2755 & 185.0802 & $5.1996 e-01$ & $4.4984 \theta+00$ \\
\hline 148 & IHTO00021 & -2.6179 & 0.1164 & -1.5007 & $6.1262 e-01$ & $4.1000 e+00$ \\
\hline 149 & IHTO00022 & 158.4848 & 160.6333 & 159.4770 & $5.2621 e-01$ & $4.3992 e+00$ \\
\hline 150 & IHTO00023 & 180.1458 & 182.0989 & 181.1027 & $4.6730 e-01$ & $4.4822 e+00$ \\
\hline 151 & IHTO00024 & 144.7679 & 146.5257 & 145.5219 & $4.6018 e-01$ & $4.3506 e+00$ \\
\hline 152 & IHTO00025 & 179.7439 & 181.3064 & 180.4782 & $4.26010-01$ & $4.4796 \theta+00$ \\
\hline 153 & IHTO00026 & 181.8885 & 183.6463 & 182.6386 & $4.0503 \theta-01$ & $4.4884 e+00$ \\
\hline 154 & IHT000027 & 155.0331 & 156.7909 & 155.9589 & $4.4957 e-01$ & $4.3866 e+00$ \\
\hline 155 & IHT000028 & 156.9599 & 159.4989 & 158.4248 & $5.7892 e-01$ & $4.3954 e+00$ \\
\hline
\end{tabular}




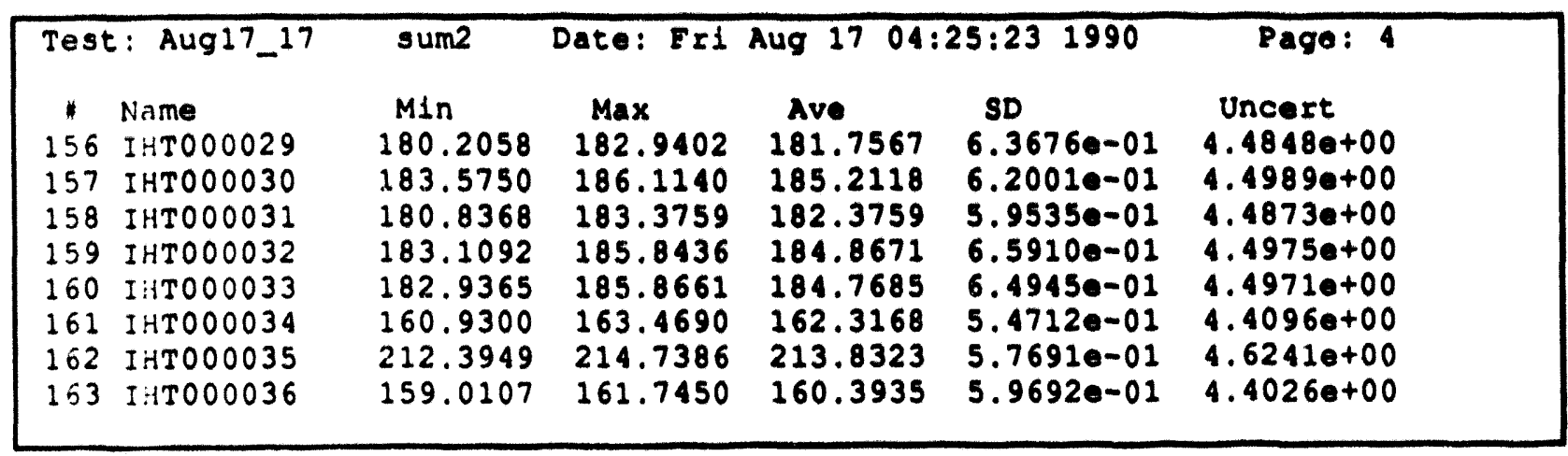




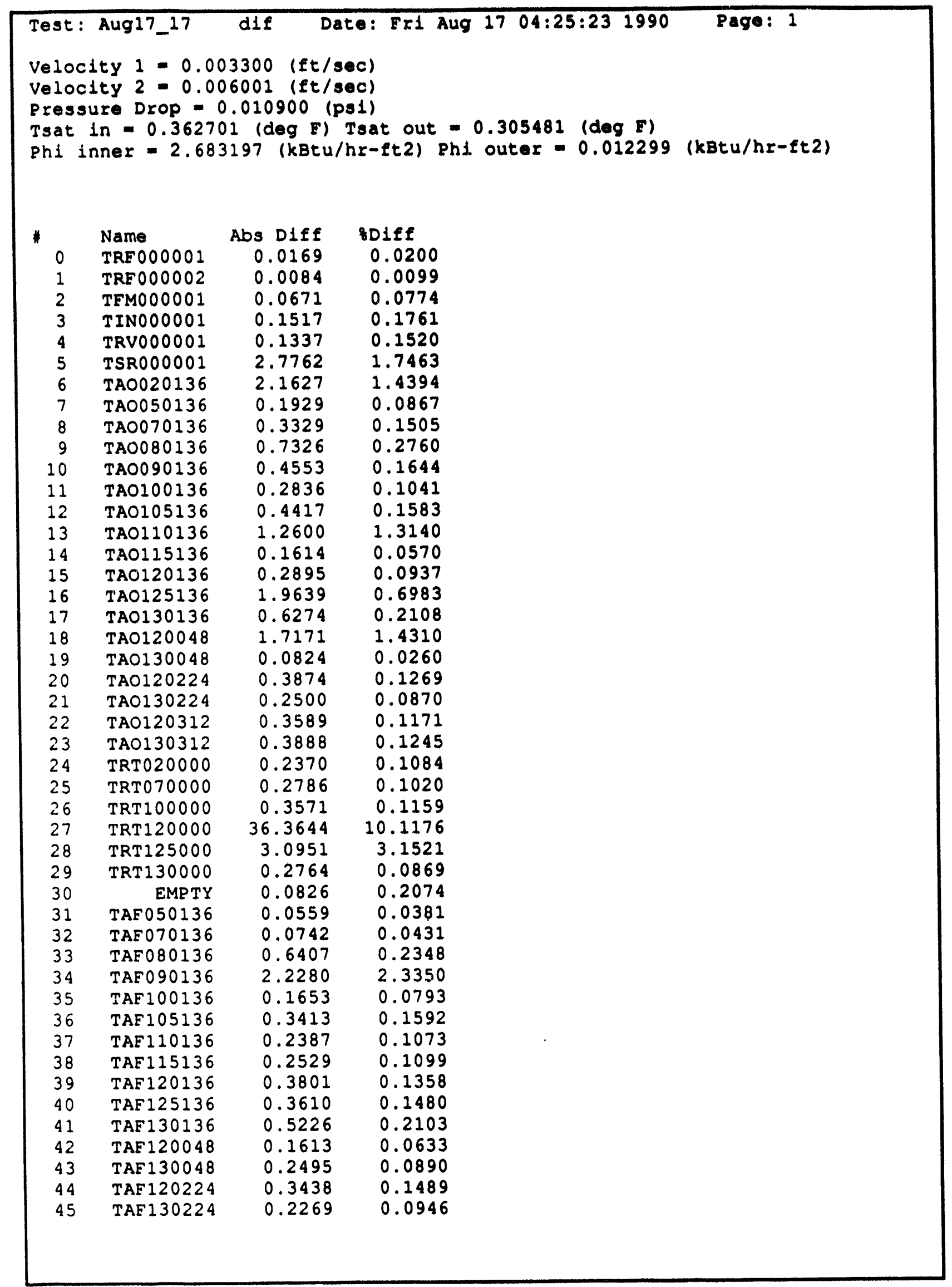




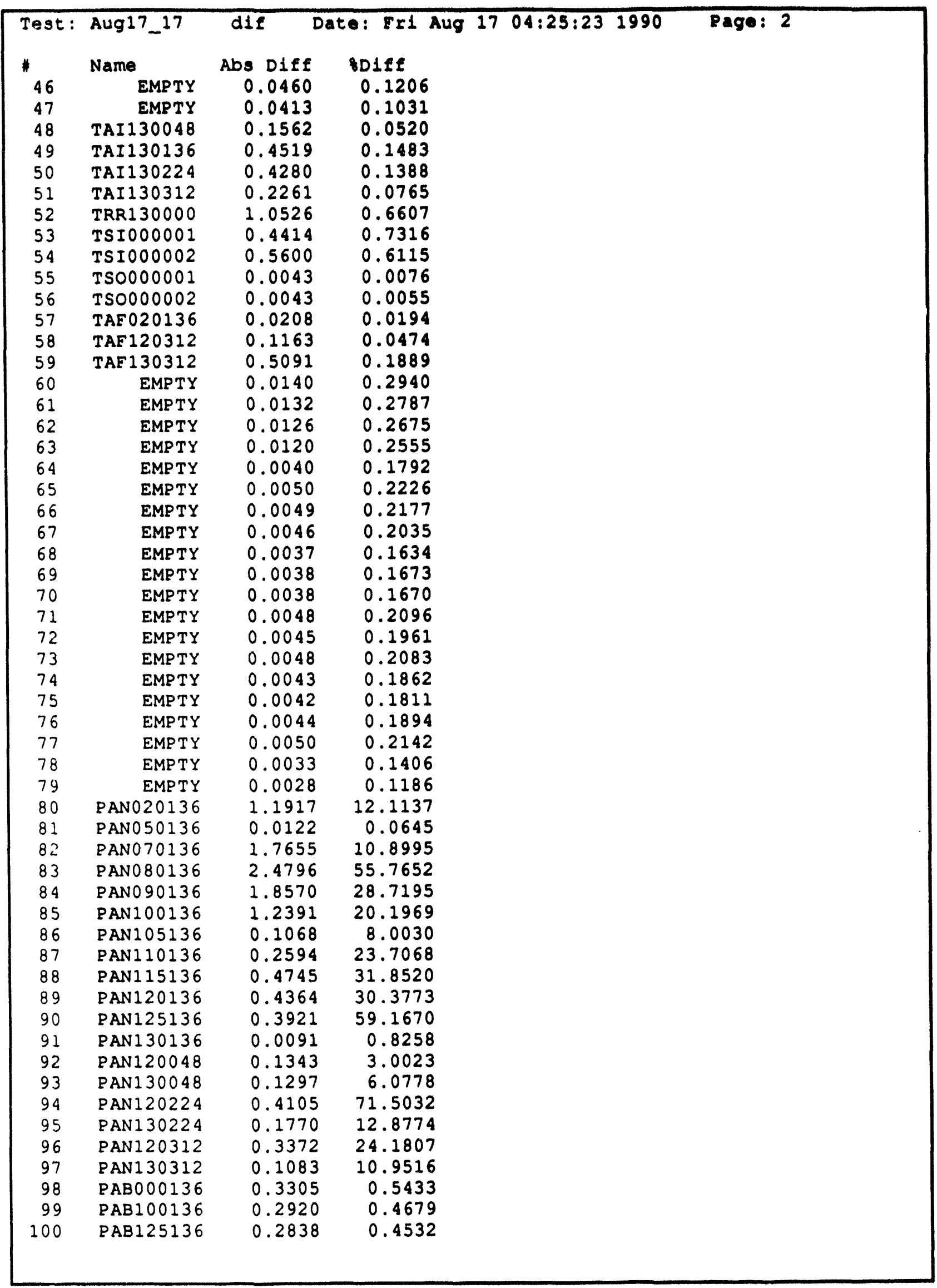




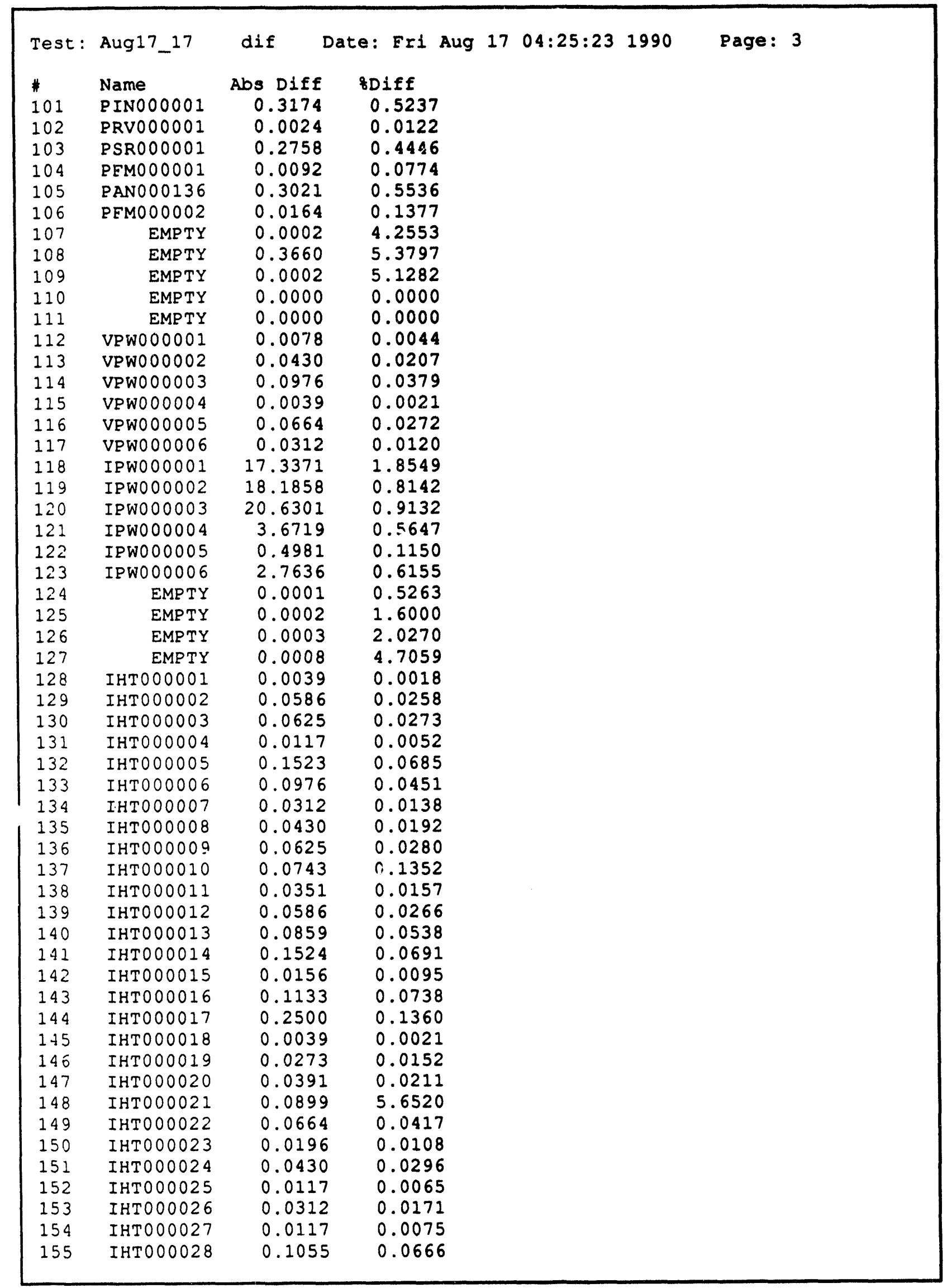




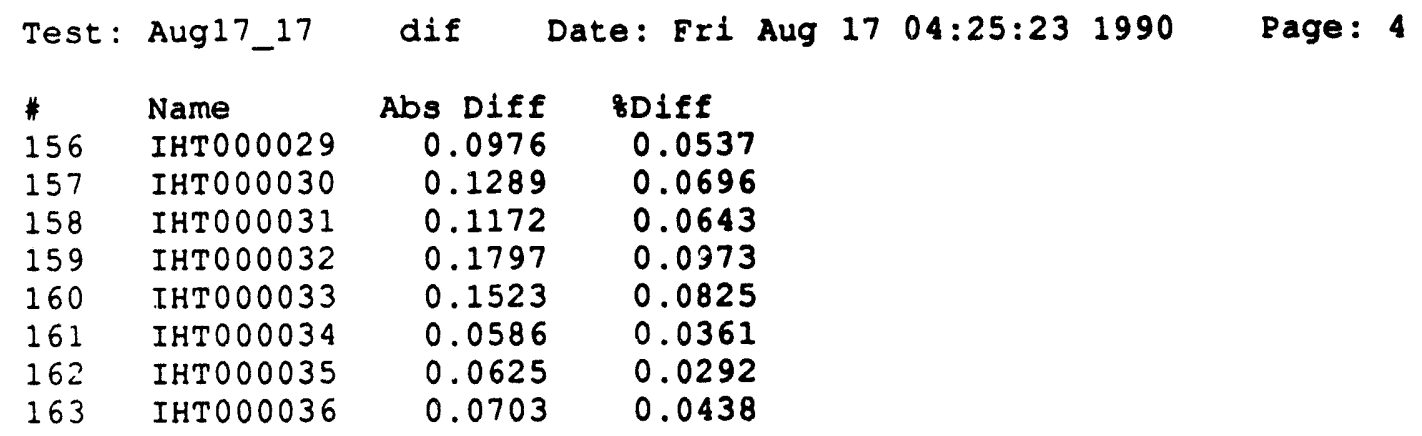




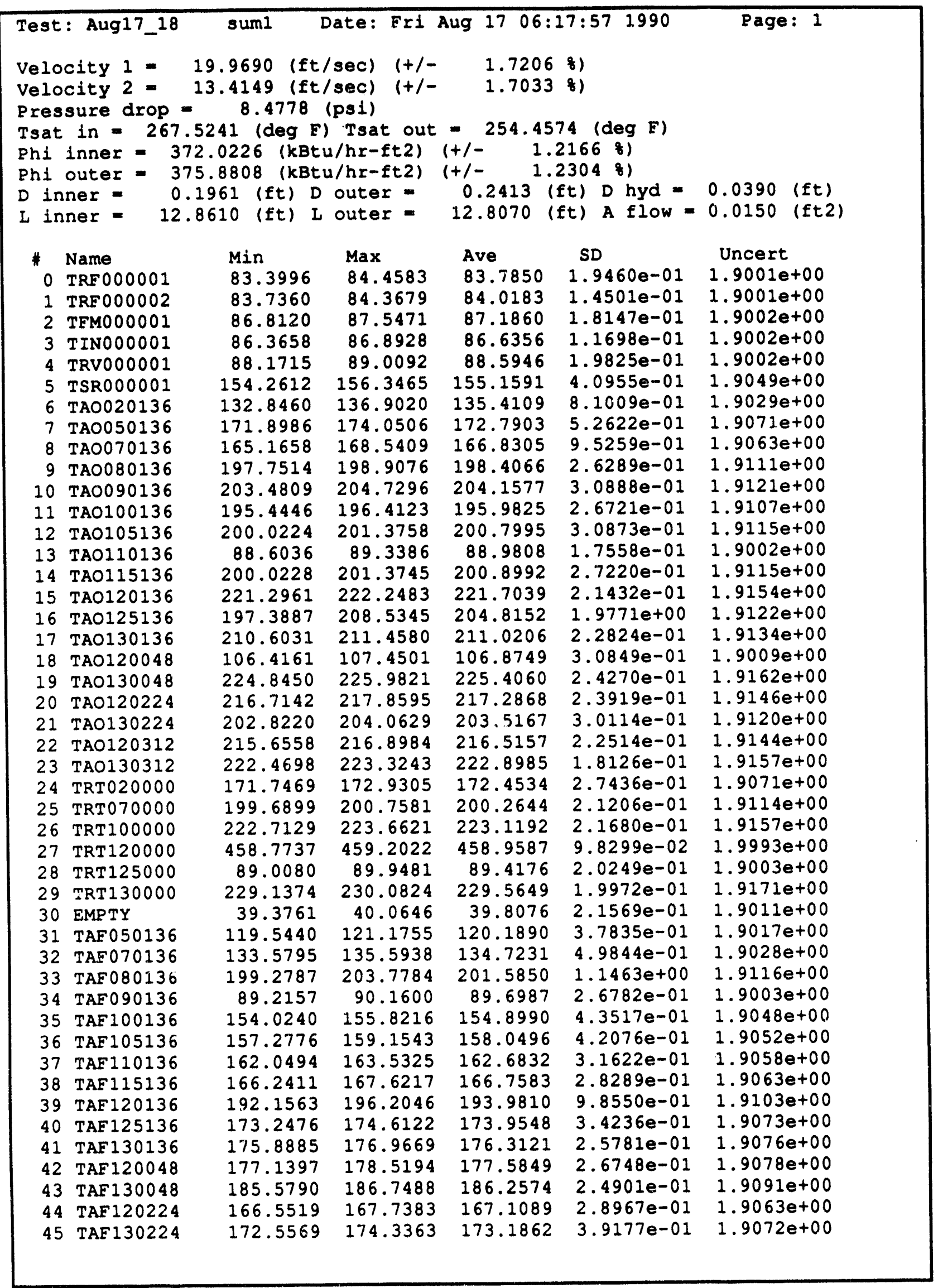




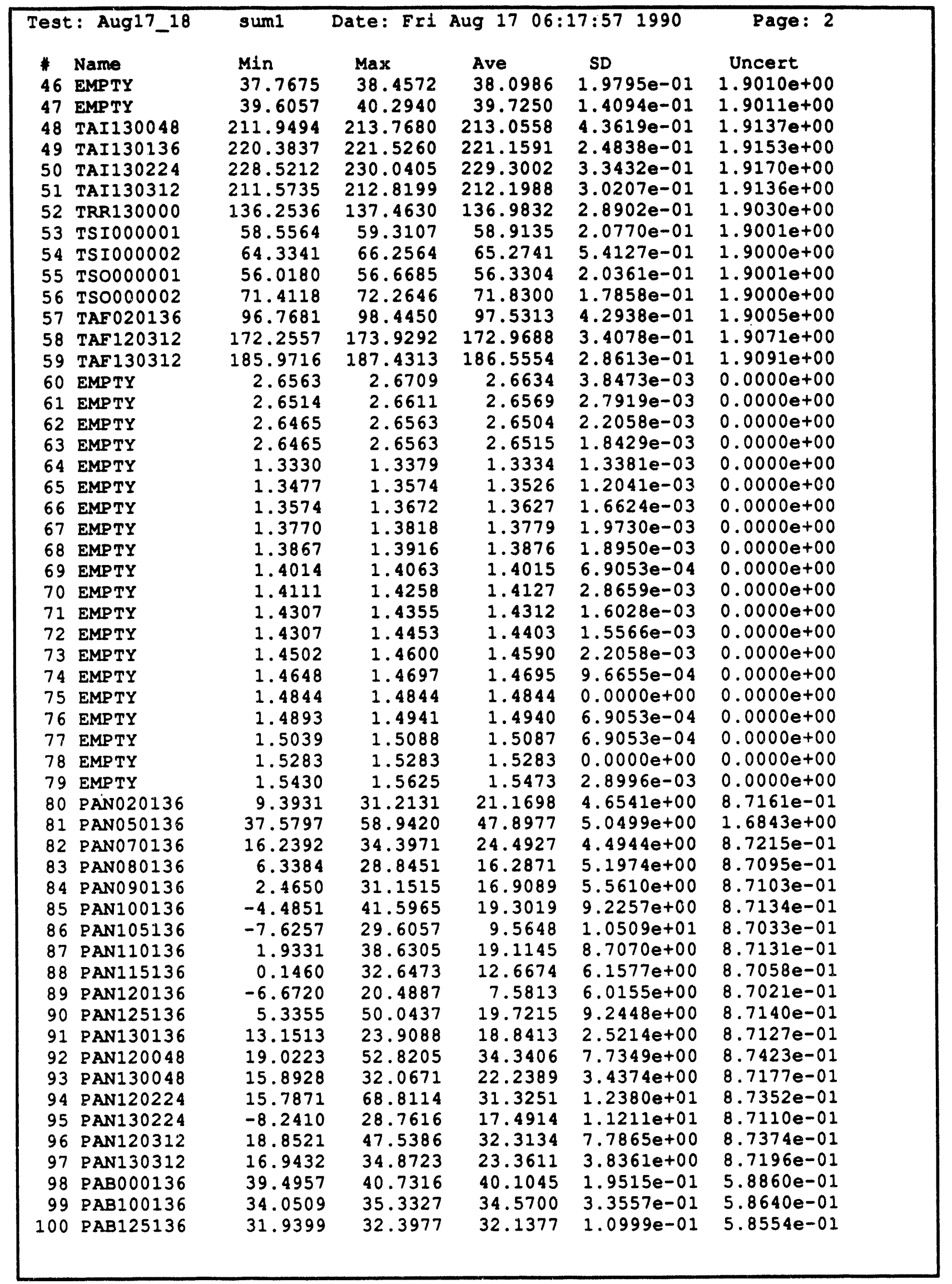




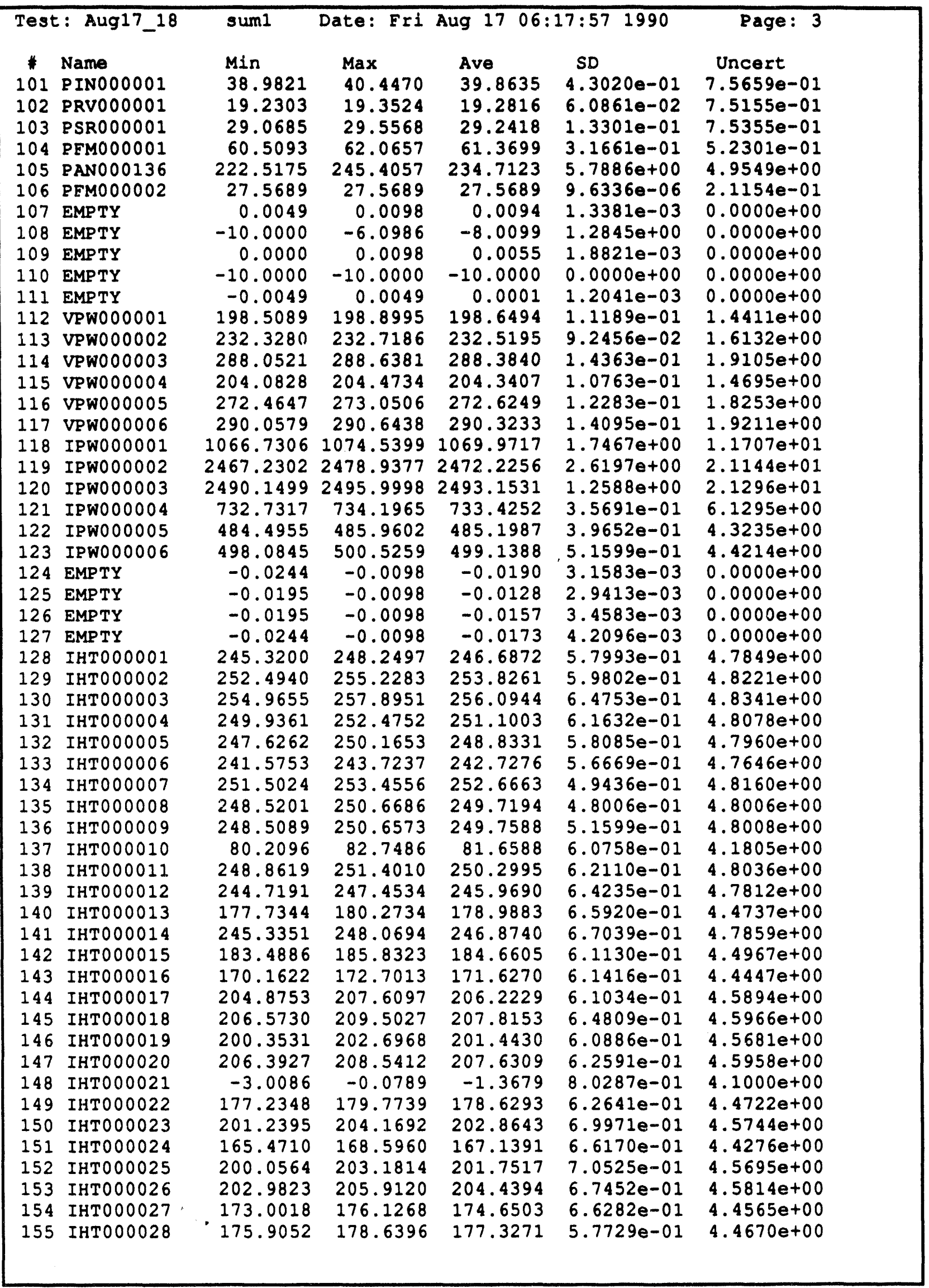




\begin{tabular}{|c|c|c|c|c|c|c|}
\hline \multicolumn{2}{|c|}{ Test: Aug17_18 } & sum 1 & Date: Fri & Aug 170 & $7: 57 \quad 1990$ & Page: 4 \\
\hline $\begin{array}{c}\# \\
156 \\
157 \\
158 \\
159 \\
160 \\
161 \\
162 \\
163\end{array}$ & 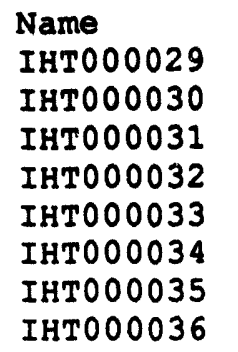 & $\begin{array}{l}\text { Min } \\
202.0808 \\
206.0359 \\
202.9072 \\
205.5701 \\
205.3974 \\
180.6565 \\
238.1761 \\
178.5419\end{array}$ & $\begin{array}{l}\text { Max } \\
204.6199 \\
208.5750 \\
205.2509 \\
207.7186 \\
207.7411 \\
182.6097 \\
240.3245 \\
180.6903\end{array}$ & $\begin{array}{l}\text { Ave } \\
203.3036 \\
207.2001 \\
204.0596 \\
206.7070 \\
206.6084 \\
181.7621 \\
239.2815 \\
179.6279\end{array}$ & $\begin{array}{l}\text { SD } \\
6.1739 \mathrm{e}-01 \\
5.8389 \mathrm{e}-01 \\
5.5137 \mathrm{e}-01 \\
5.3770 \mathrm{e}-01 \\
5.6772 \mathrm{e}-01 \\
4.4923 \mathrm{e}-01 \\
4.5611 \mathrm{e}-01 \\
4.6715 \mathrm{e}-01\end{array}$ & $\begin{array}{c}\text { Uncert } \\
4.5764 e+00 \\
4.5938 e+00 \\
4.5797 e+00 \\
4.5916 e+00 \\
4.5912 e+00 \\
4.4848 e+00 \\
4.7472 e+00 \\
4.4762 e+00\end{array}$ \\
\hline
\end{tabular}




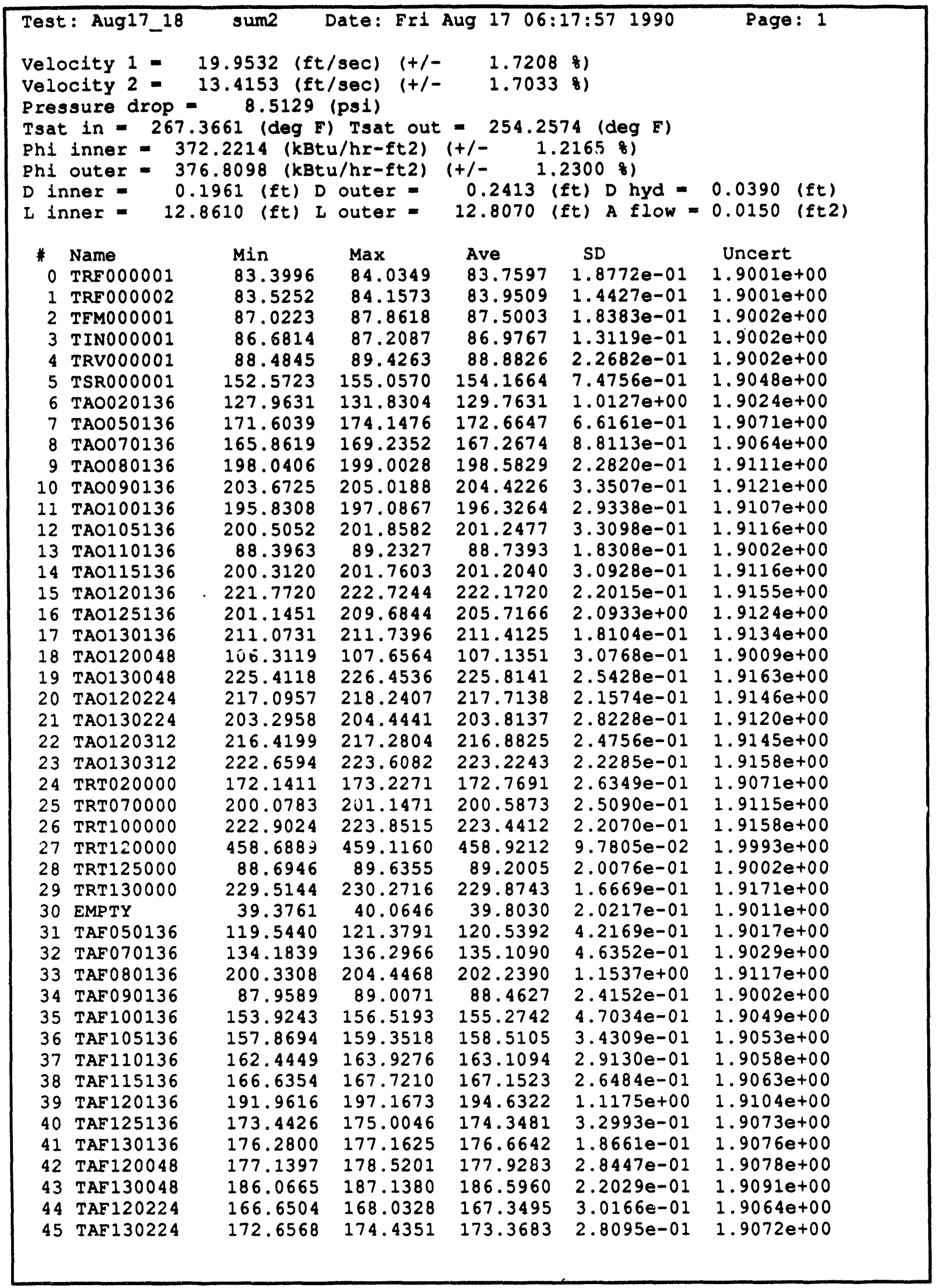




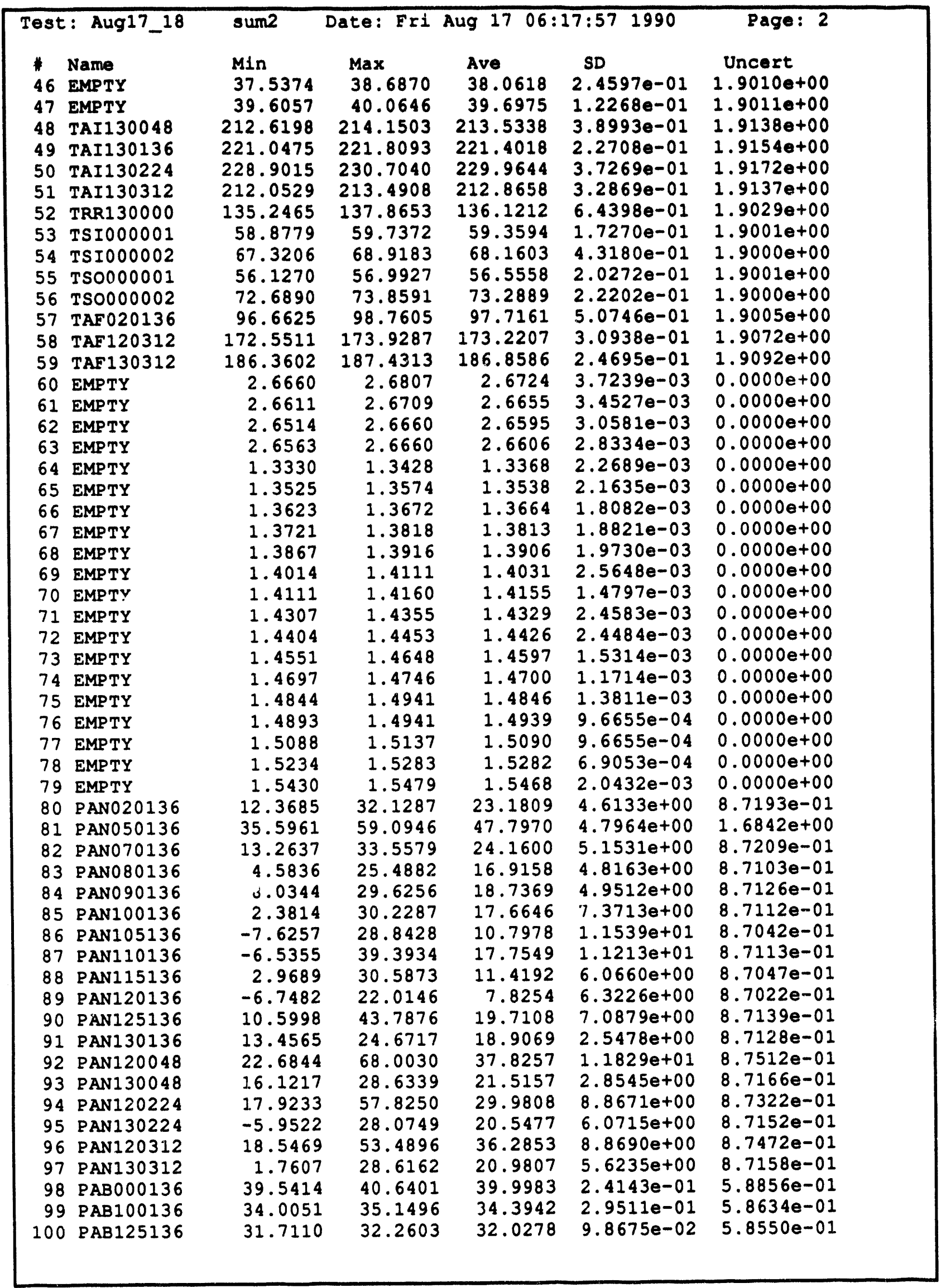




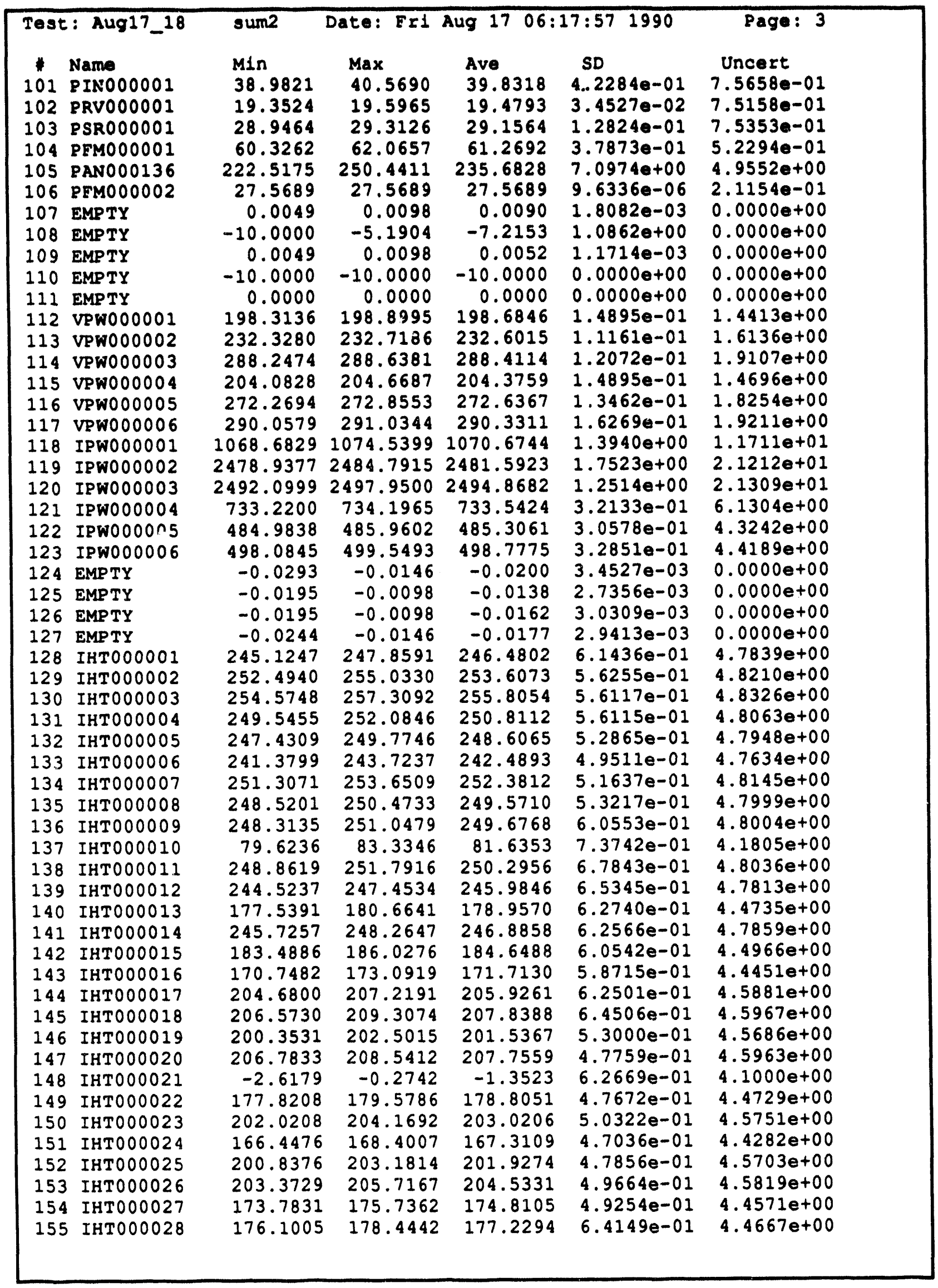




\begin{tabular}{|c|c|c|c|c|c|c|}
\hline $\begin{array}{l}1 \\
156 \\
157 \\
158 \\
159 \\
160 \\
161 \\
162 \\
163\end{array}$ & 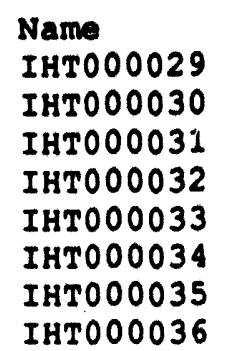 & $\begin{array}{l}\text { Min } \\
201.8855 \\
205.6453 \\
202.7118 \\
205.3748 \\
205.3974 \\
180.8519 \\
238.1761 \\
178.5419\end{array}$ & $\begin{array}{l}\text { Max } \\
204.6199 \\
208.5750 \\
205.4462 \\
208.3045 \\
208.1318 \\
183.0003 \\
240.5199 \\
180.8857\end{array}$ & $\begin{array}{l}\text { Ave } \\
203.2294 \\
207.1649 \\
204.0088 \\
206.6327 \\
206.5810 \\
181.6996 \\
239.2971 \\
179.5771\end{array}$ & $\begin{array}{l}\text { SD } \\
6.6103 e-01 \\
6.9843 e-01 \\
7.3267 e-01 \\
7.4363 e-01 \\
7.2803 e-01 \\
5.5749 e-01 \\
5.7156 e-01 \\
5.3706 e-01\end{array}$ & $\begin{array}{c}\text { Uncert } \\
4.5760 e+00 \\
4.5937 e+00 \\
4.5795 e+00 \\
4.5913 e+00 \\
4.5910 \theta+00 \\
4.4846 e+00 \\
4.7472 \theta+00 \\
4.4760 e+00\end{array}$ \\
\hline
\end{tabular}




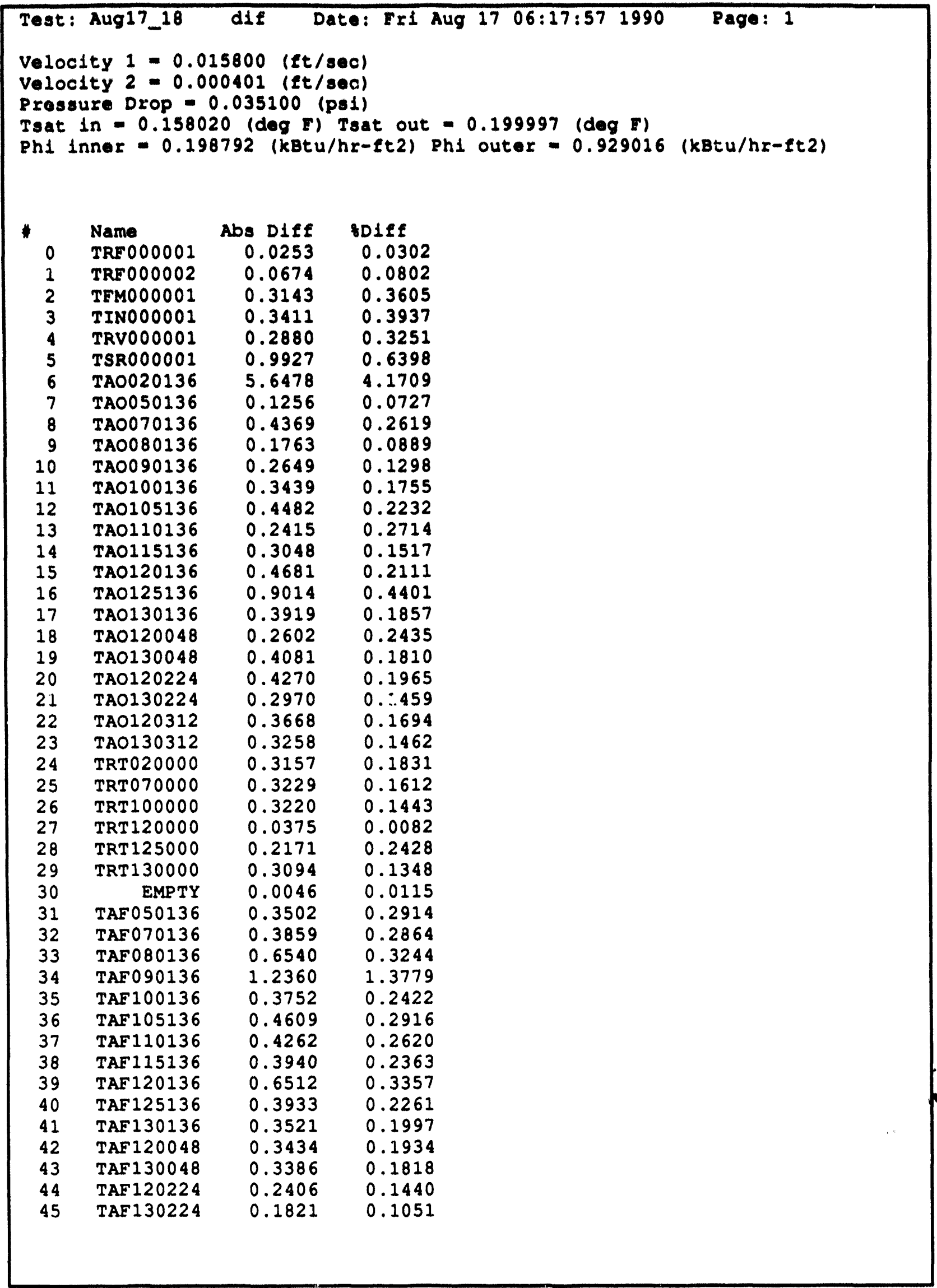




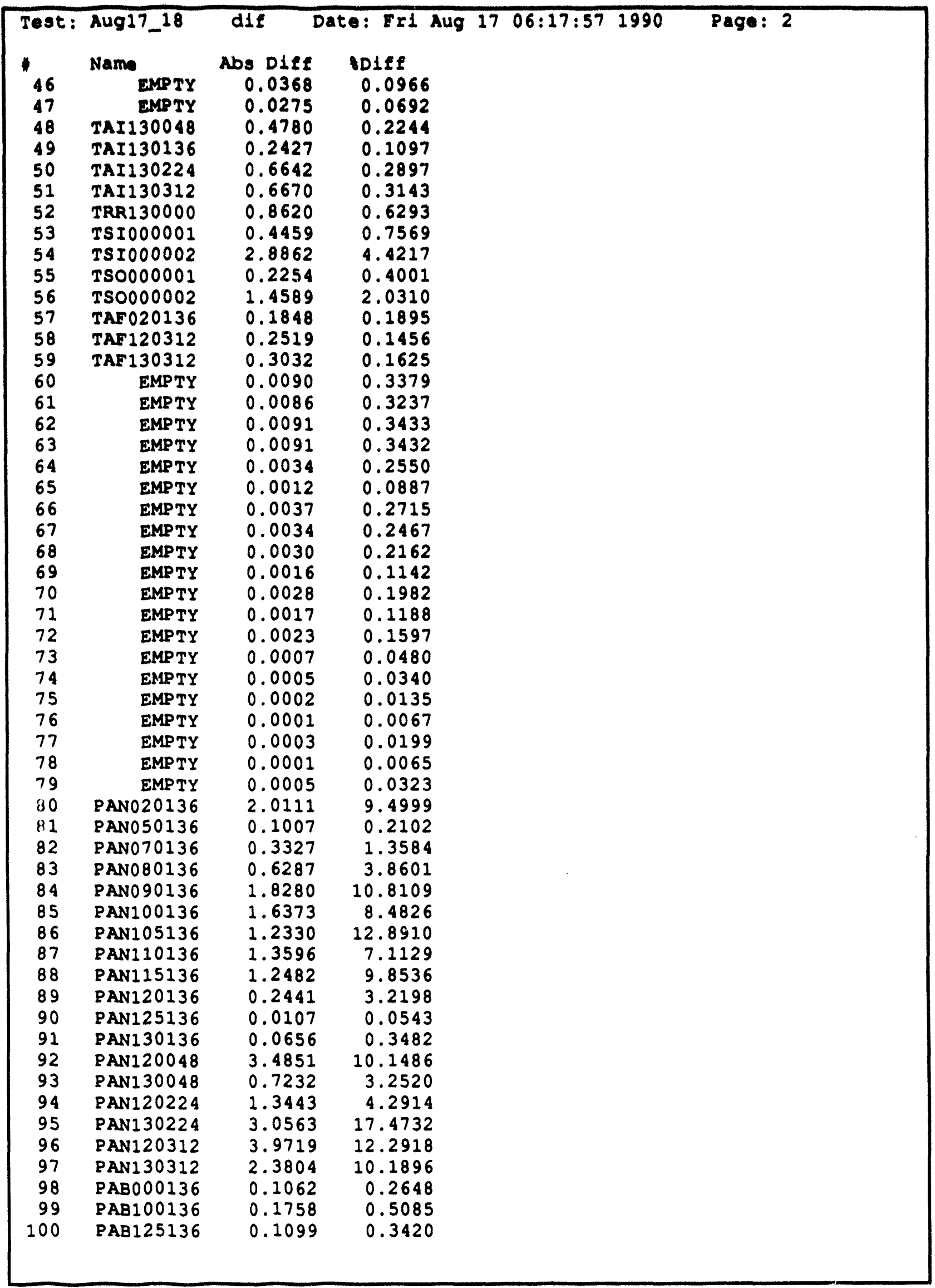




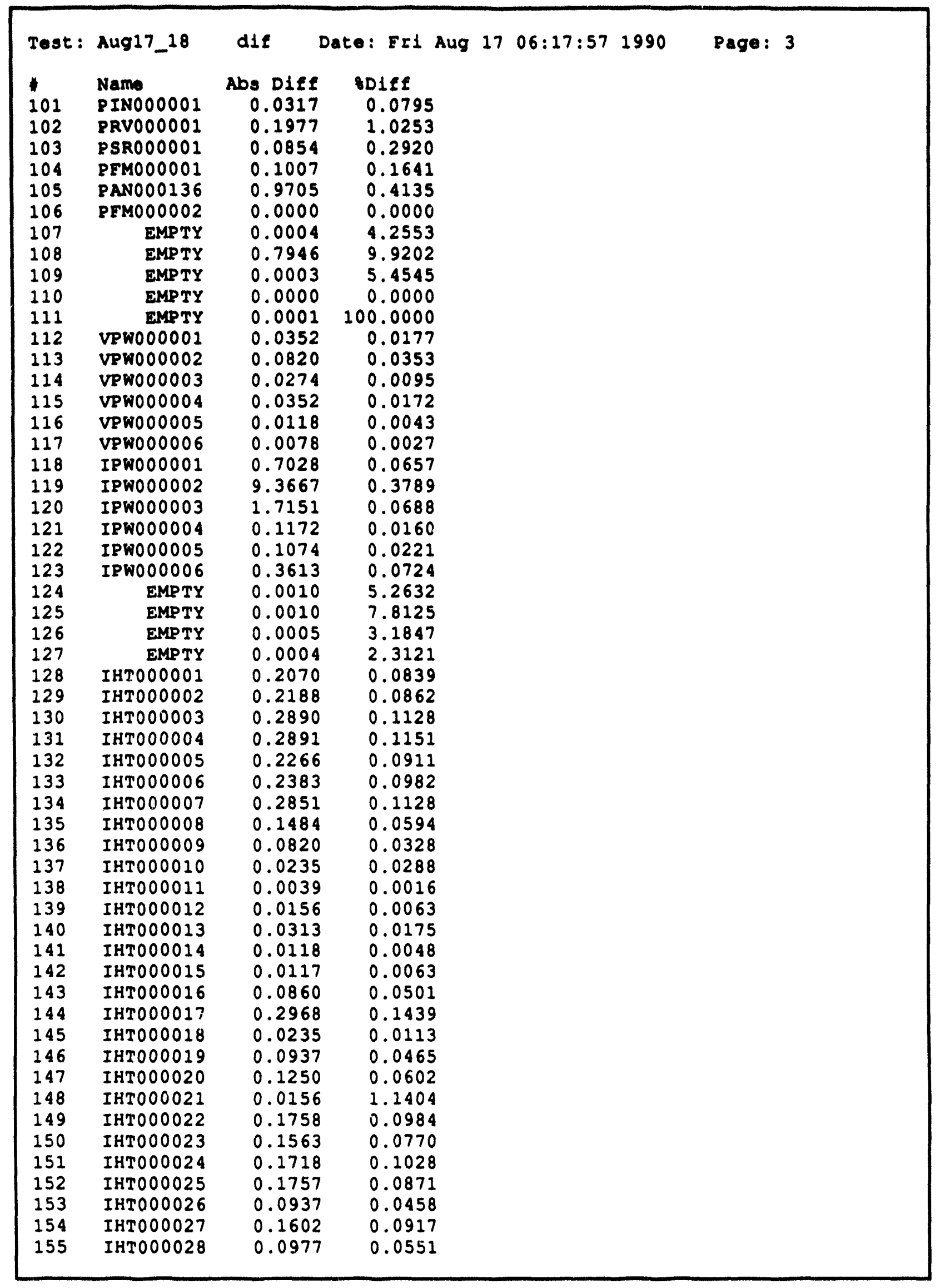




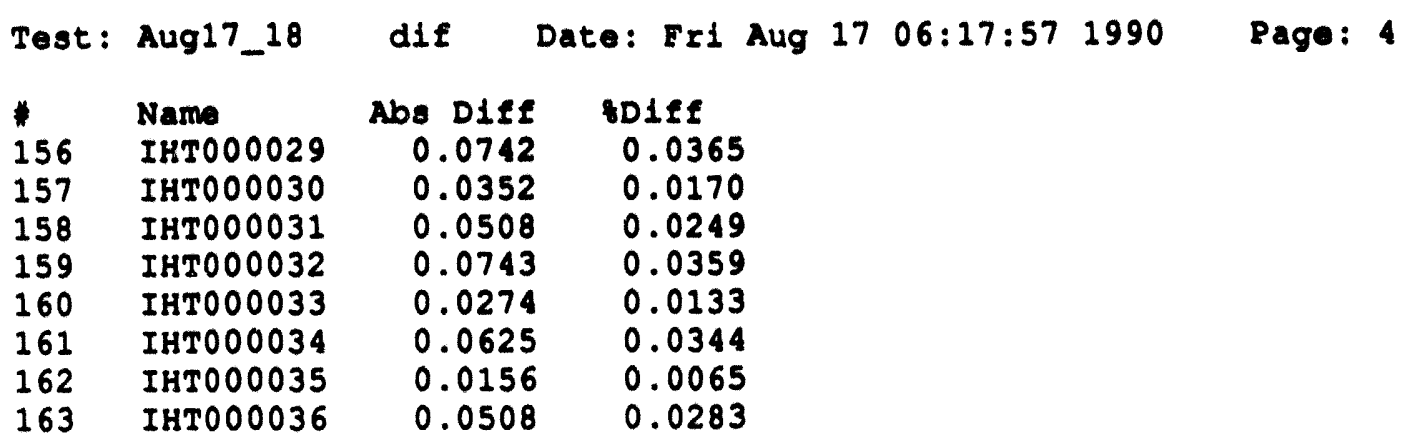




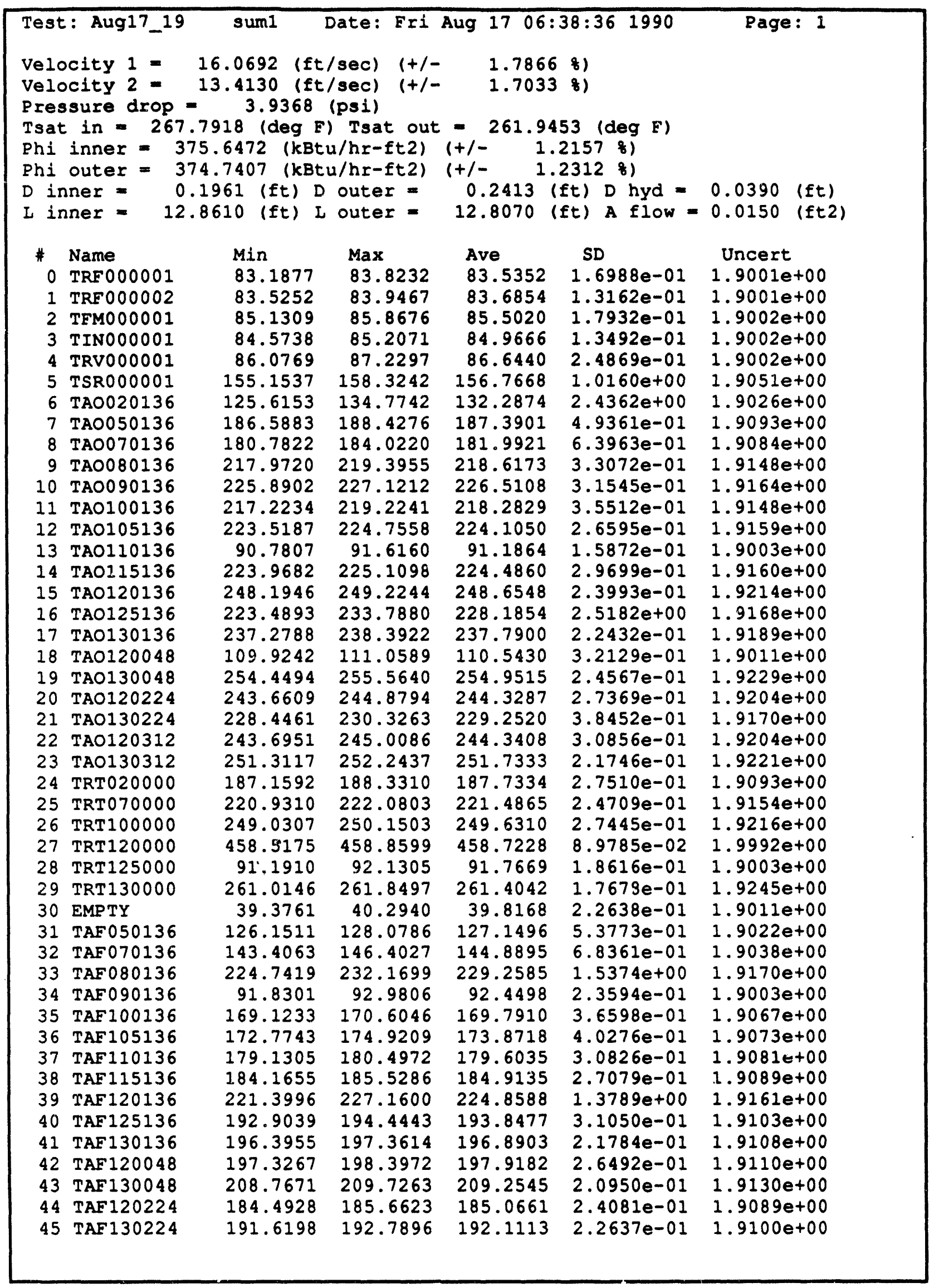




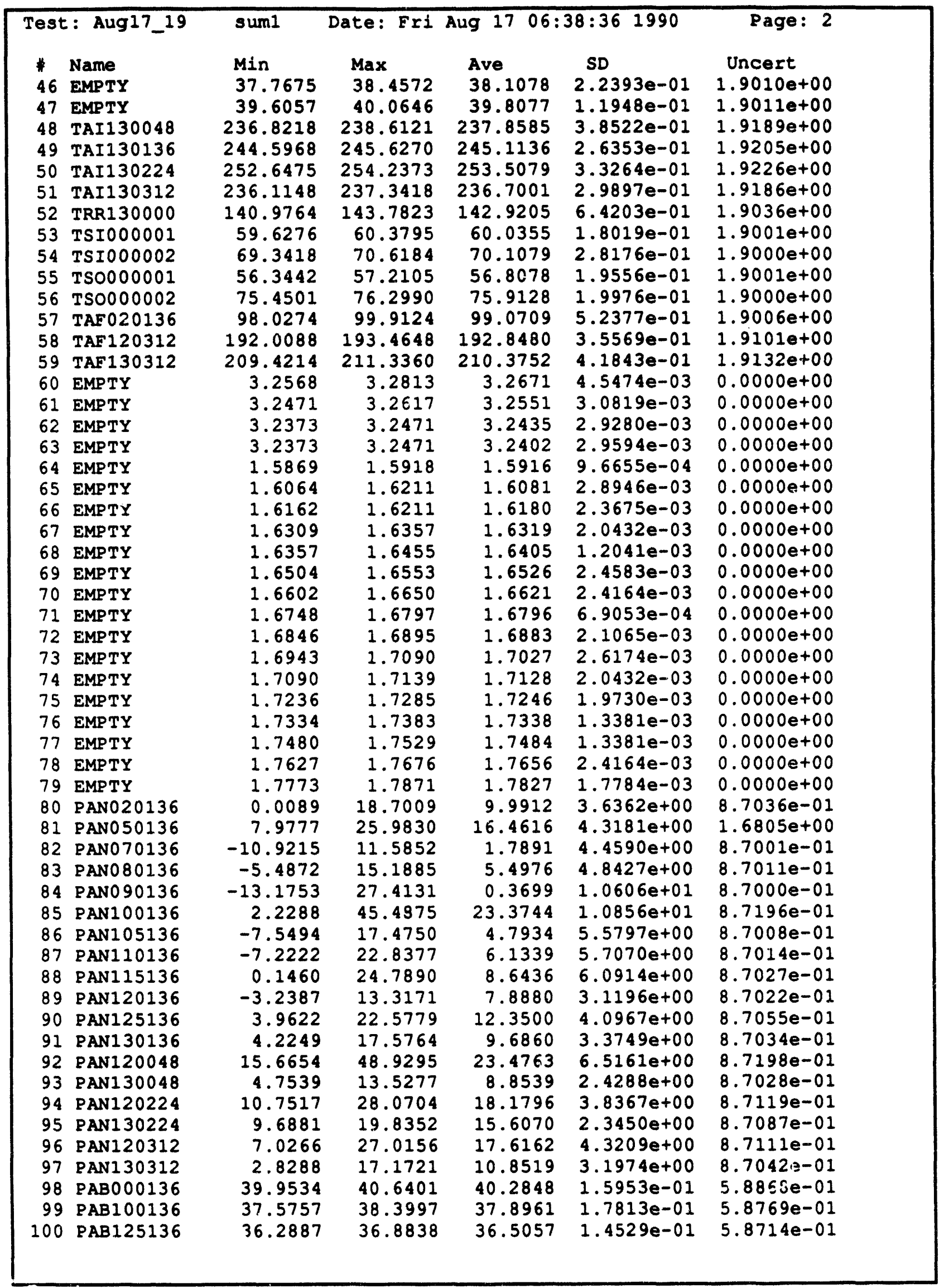




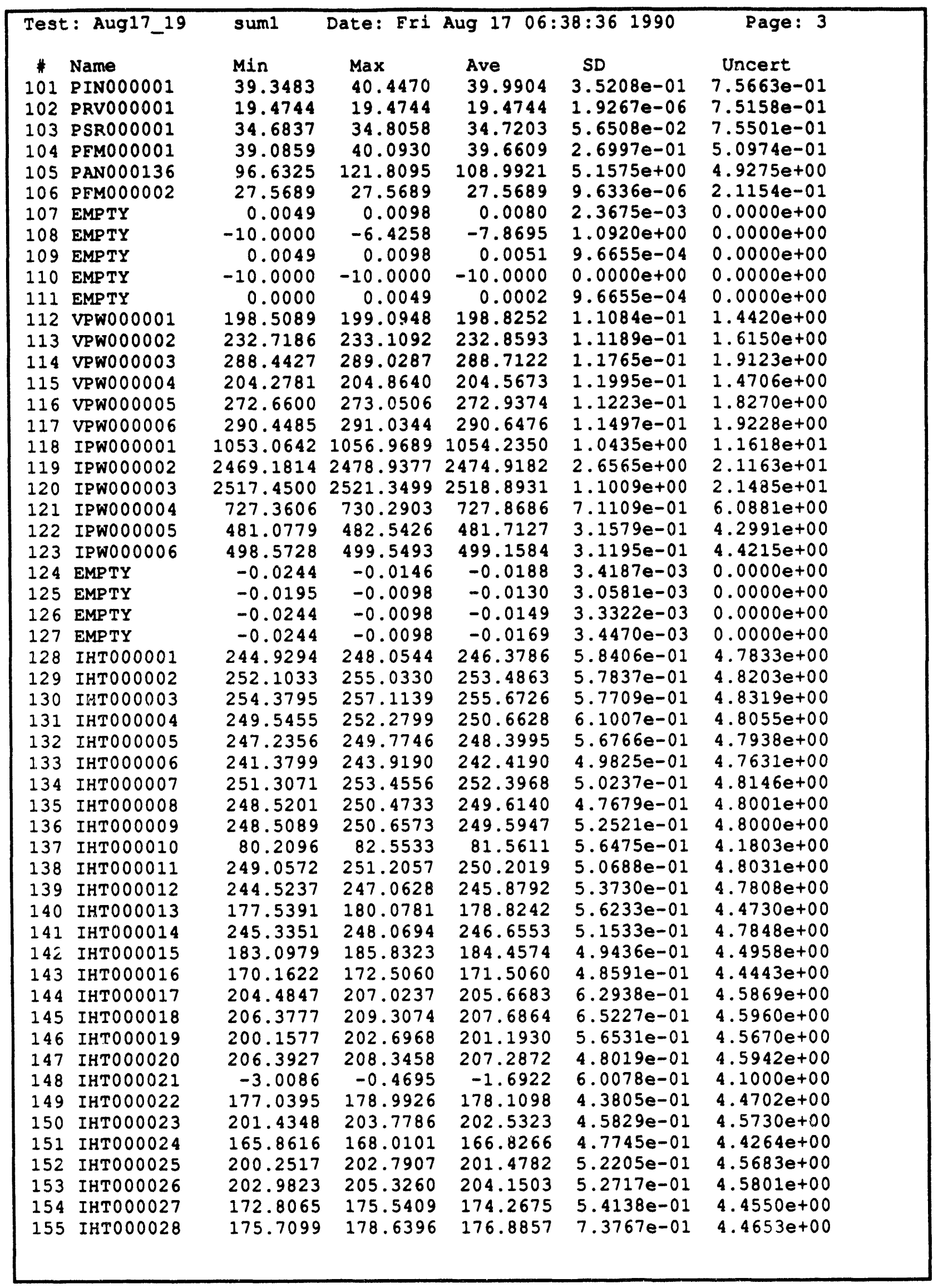




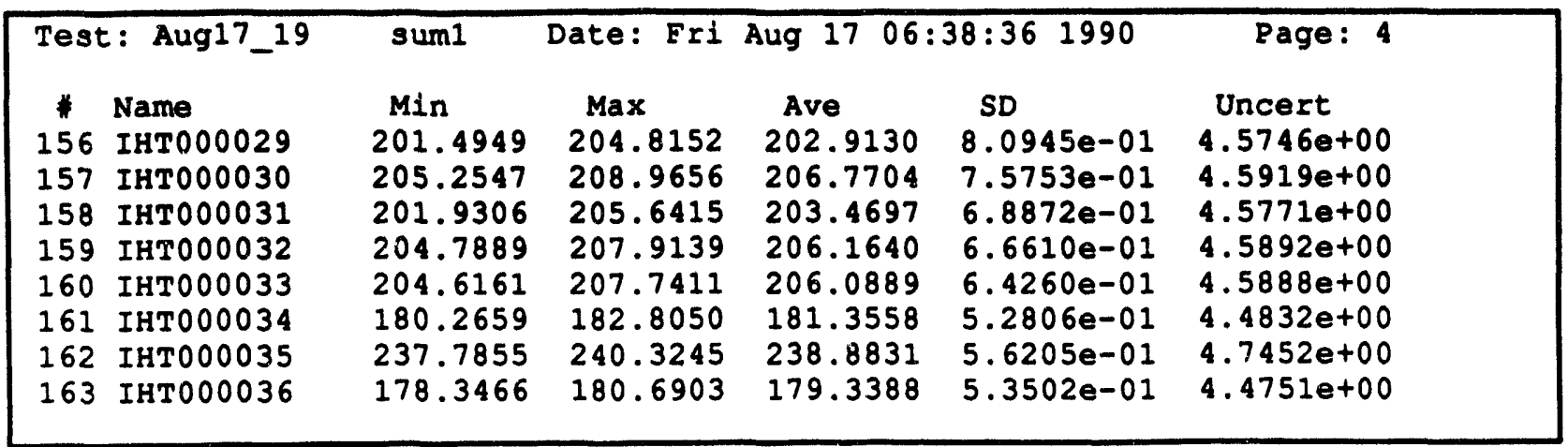




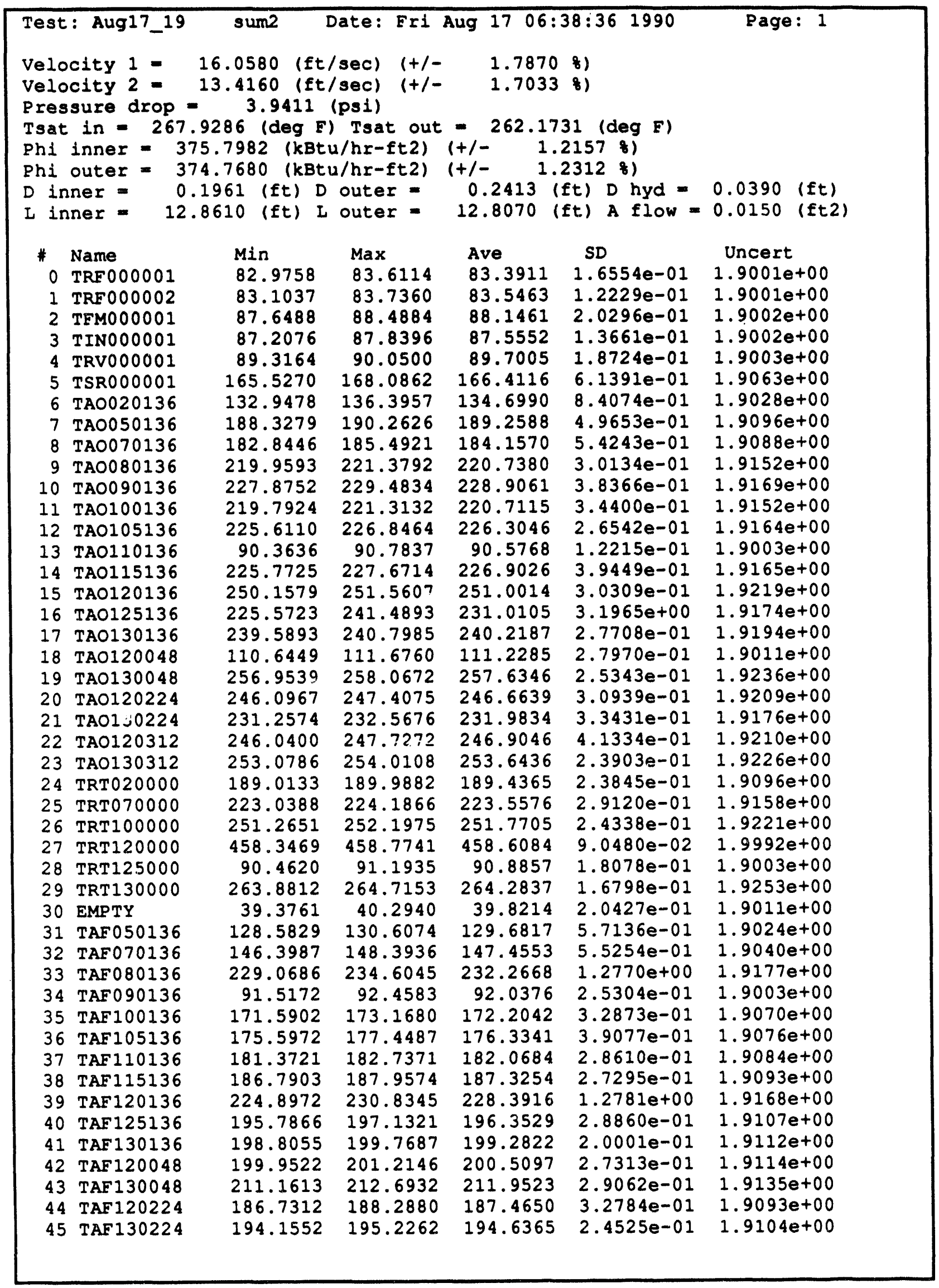




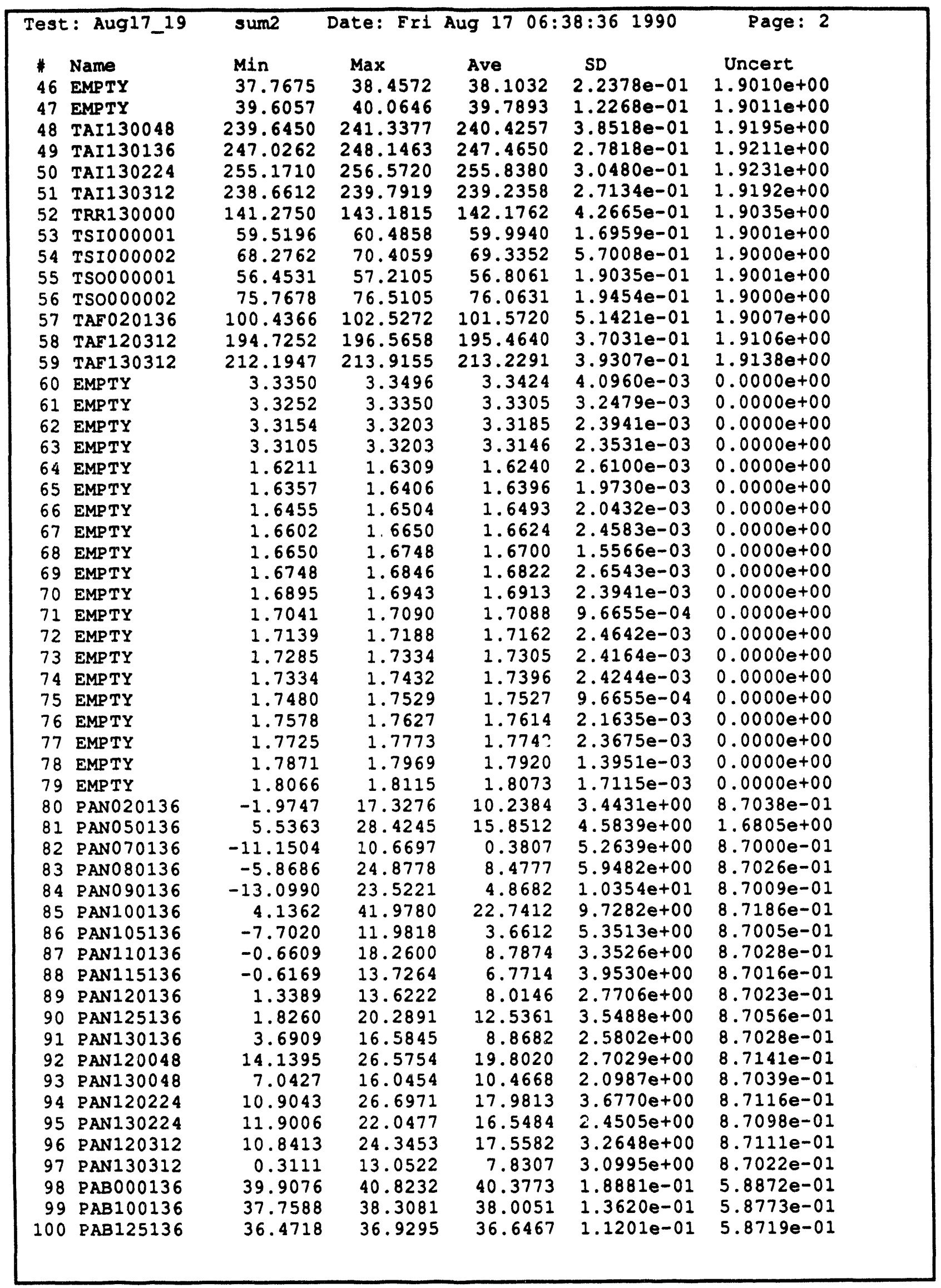




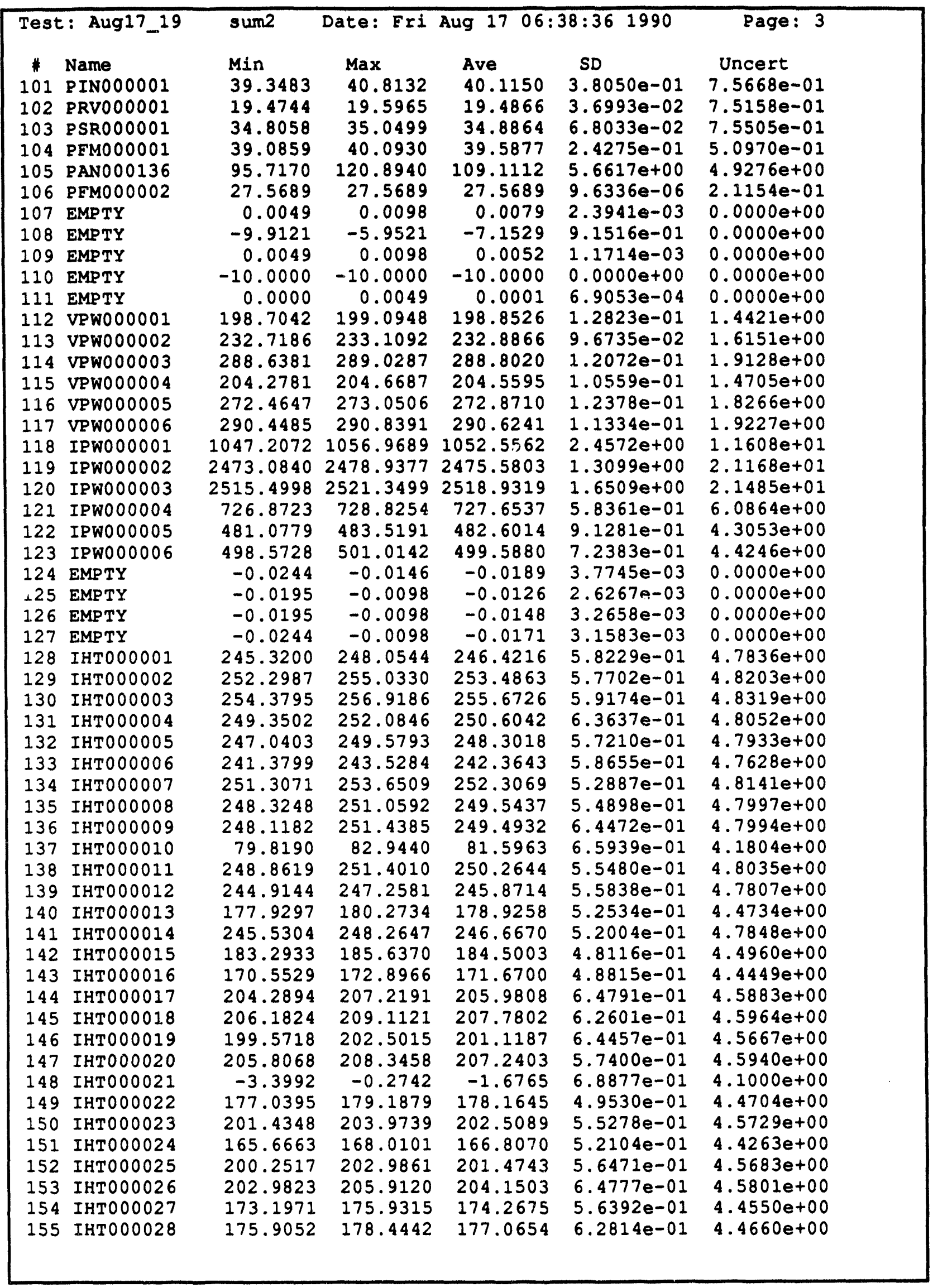




\begin{tabular}{|ccccccc|}
\hline Test: Aug17_19 & sum2 & Date: Fri Aug $1706: 38: 36$ & 1990 & Page: 4 \\
* Name & Min & Max & Ave & SD & Uncert \\
156 & IHT000029 & 202.0808 & 204.4246 & 203.0146 & $6.4270 e-01$ & $4.5751 e+00$ \\
157 & IHTO00030 & 205.6453 & 208.1844 & 206.8798 & $6.2195 e-01$ & $4.5924 e+00$ \\
158 & IHTO00031 & 202.5165 & 205.2509 & 203.6767 & $6.2814 e-01$ & $4.5780 e+00$ \\
159 & IHTO00032 & 204.9842 & 207.7186 & 206.3632 & $6.4647 e-01$ & $4.5901 e+00$ \\
160 & IHT000033 & 205.0068 & 207.7411 & 206.3076 & $6.4671 e-01$ & $4.5898 e+00$ \\
161 & IHT000034 & 180.4612 & 182.4144 & 181.4769 & $4.5677 e-01$ & $4.4837 e+00$ \\
162 & IHT000035 & 237.7855 & 240.1292 & 238.9456 & $4.7089 e-01$ & $4.7455 e+00$ \\
163 & IHTO00036 & 178.1513 & 180.4950 & 179.3584 & $5.0175 e-01$ & $4.4751 e+00$ \\
\hline
\end{tabular}




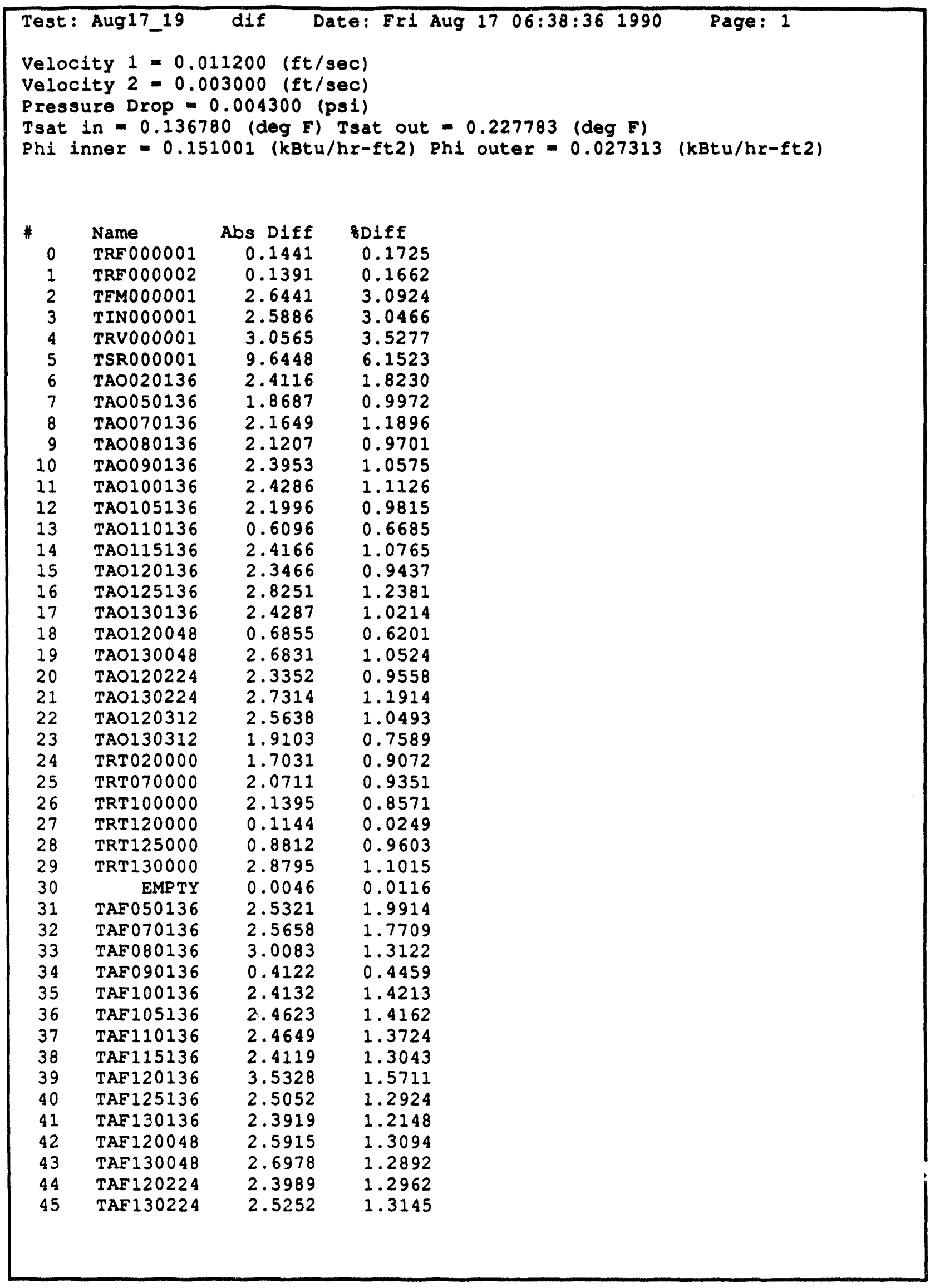




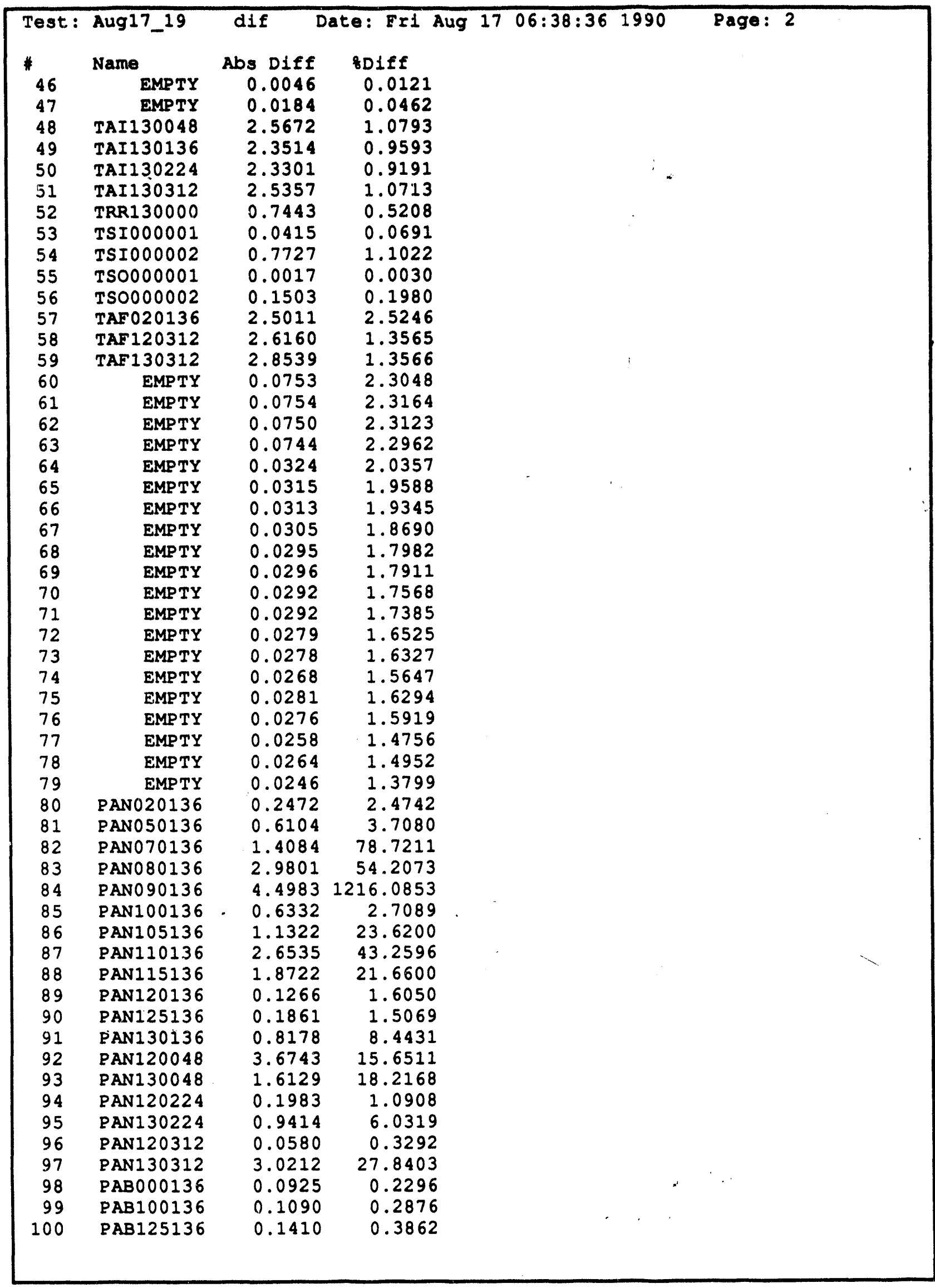




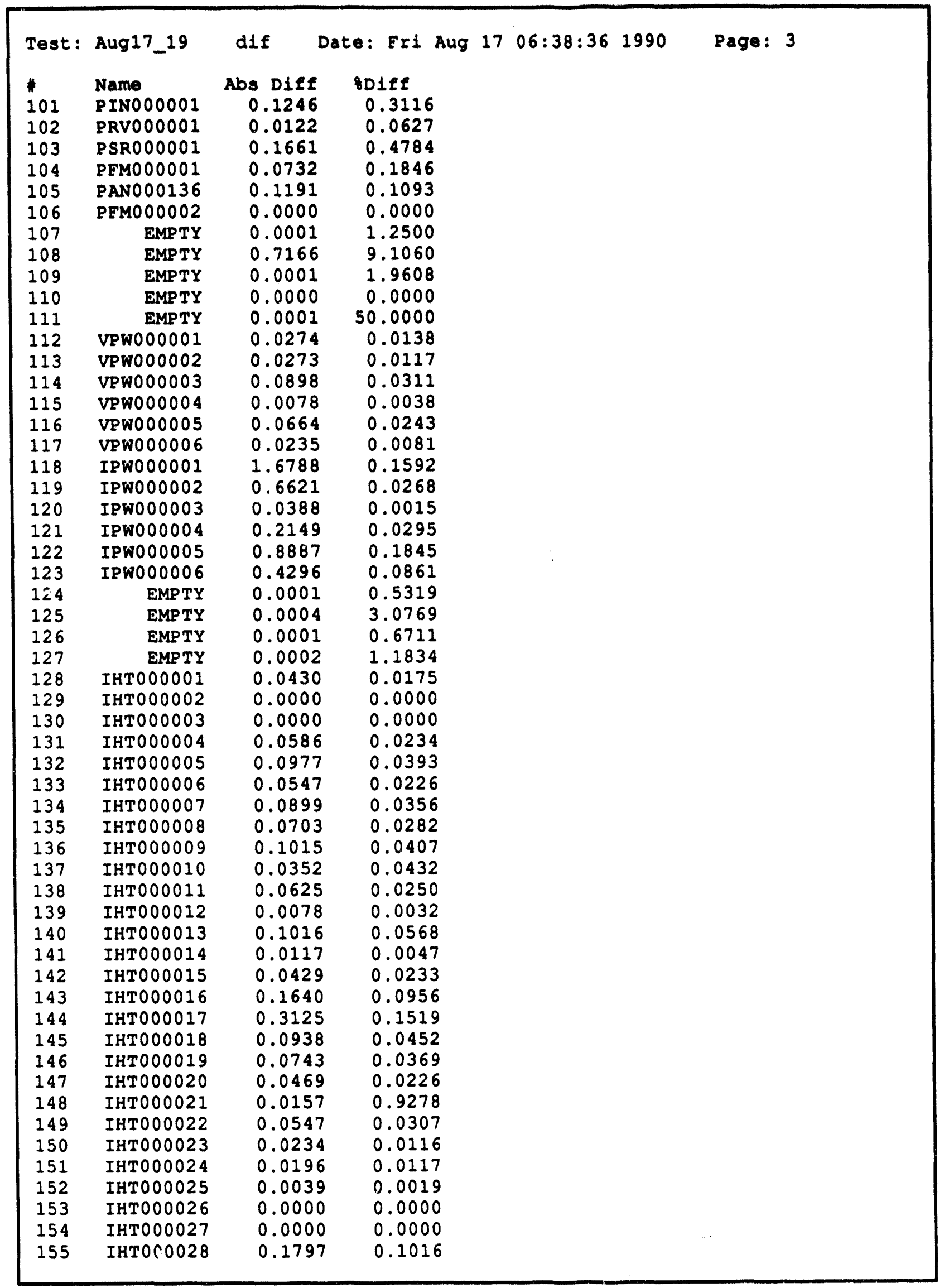


Test : Aug17_19

Name

156

157

158

159

160

161

162

163
IHT000029 IHTO00030 IHT000031 IHT000032 IHT000033 IHT000034 IHT000035 IHT000036 dif Date: Fri Aug $1706: 38: 361990$

Page: 4

Abs Diff BDiff
0.1016
0.0501
0.1094
0.0529
0.2070
0.1017
0.1992
0.2187
0.0966
0.1211
0.0625
0.1061
0.0668
0.0262
0.0196
0.0109 


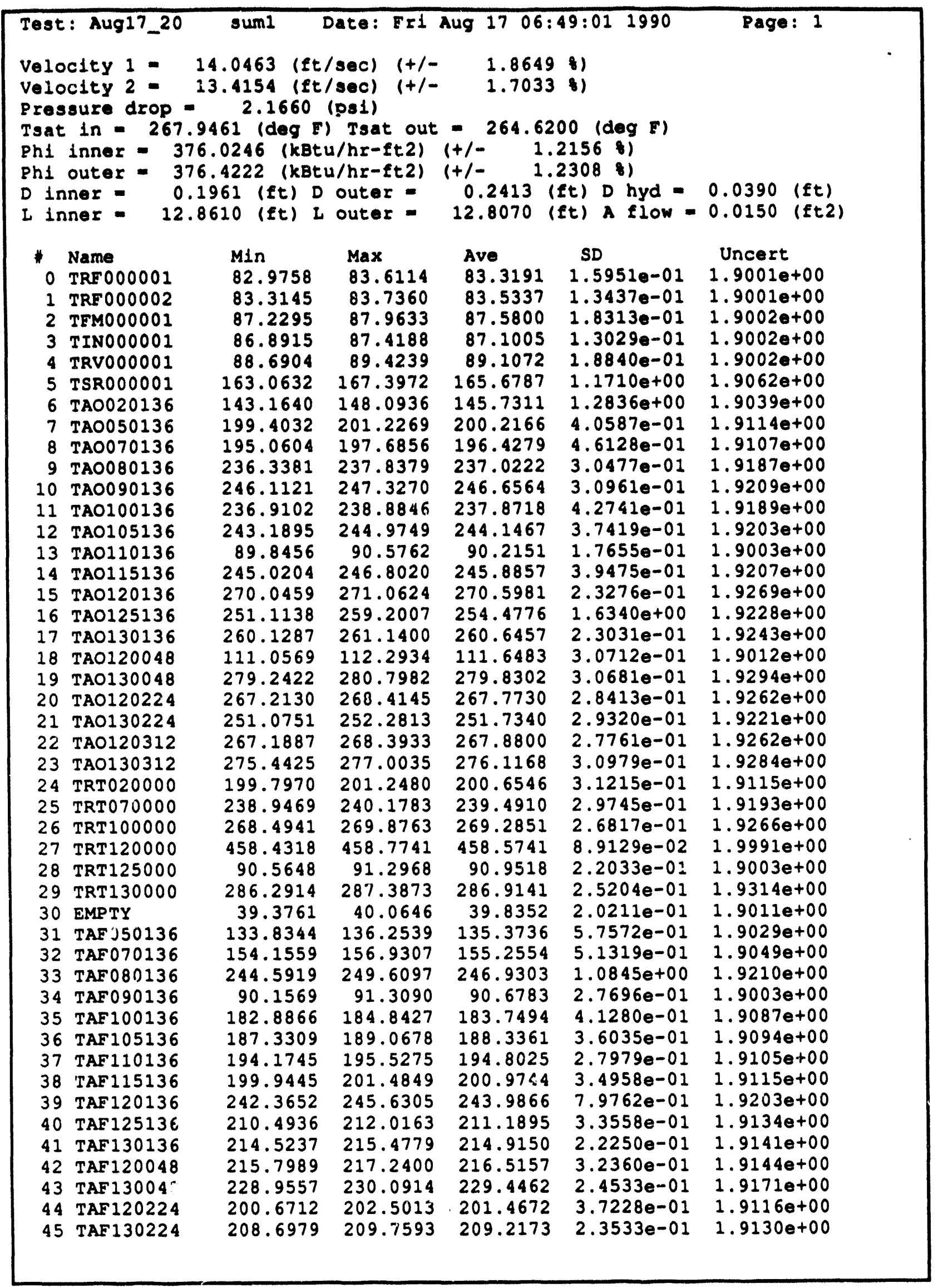




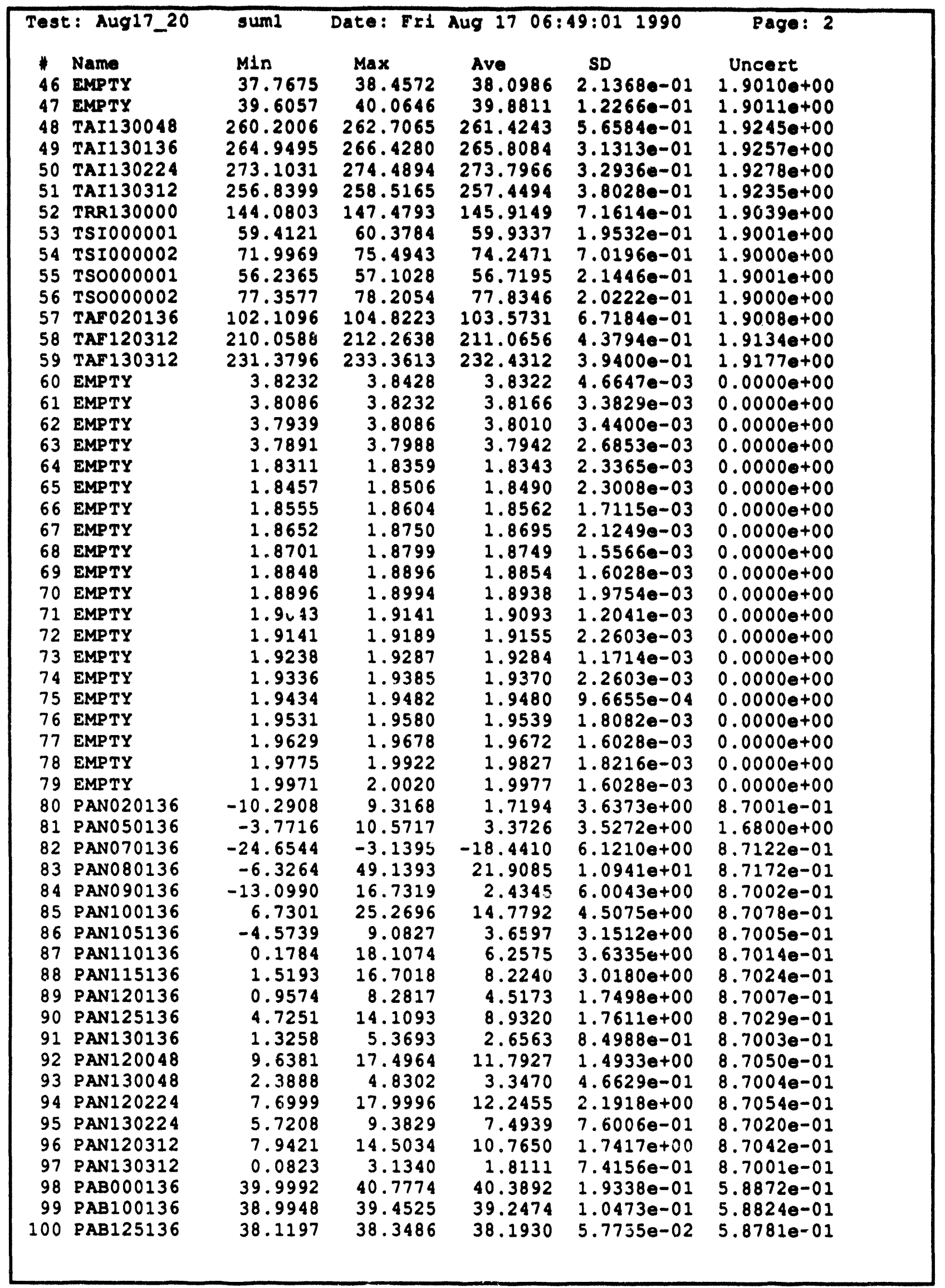



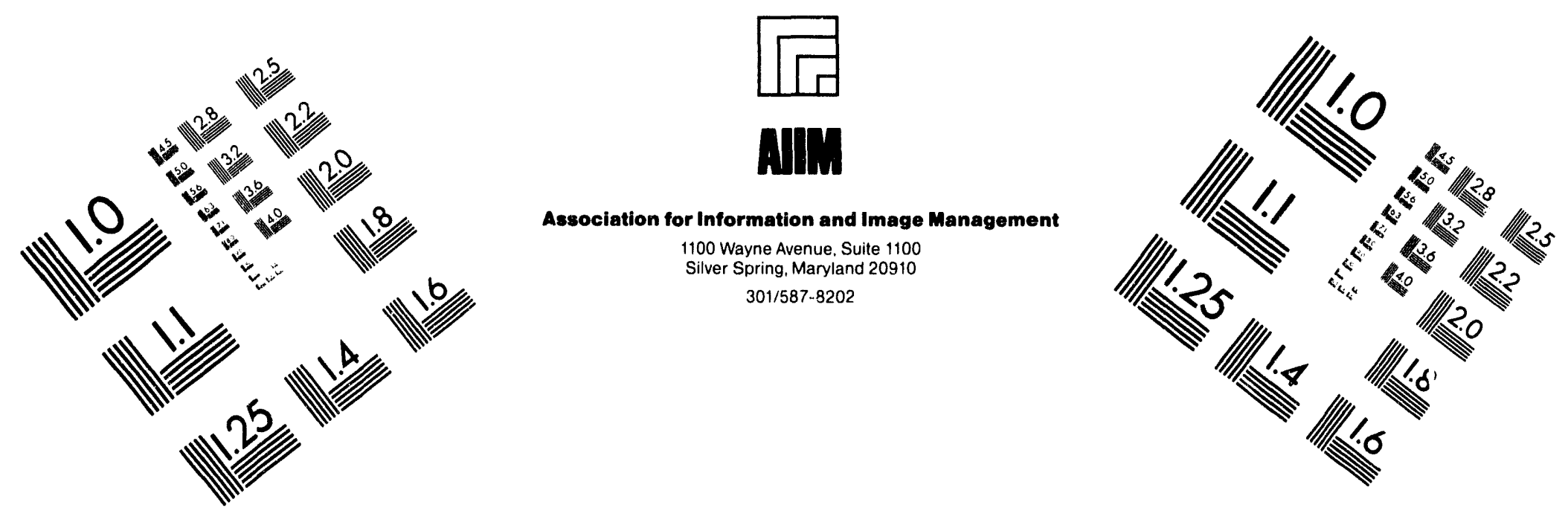

\section{Centimeter}

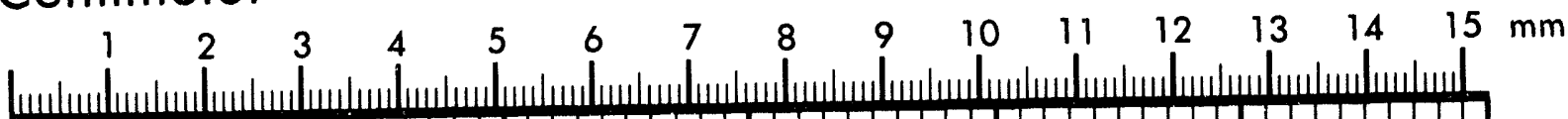

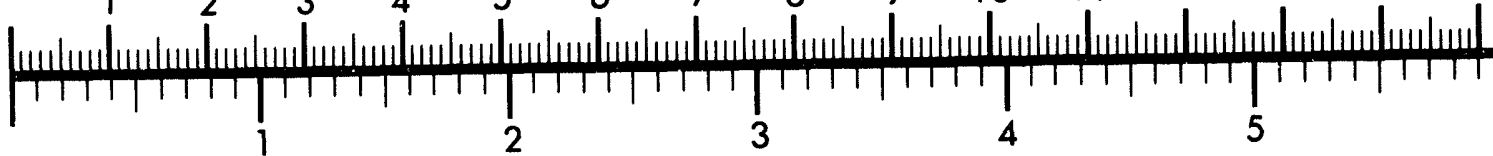
Inches
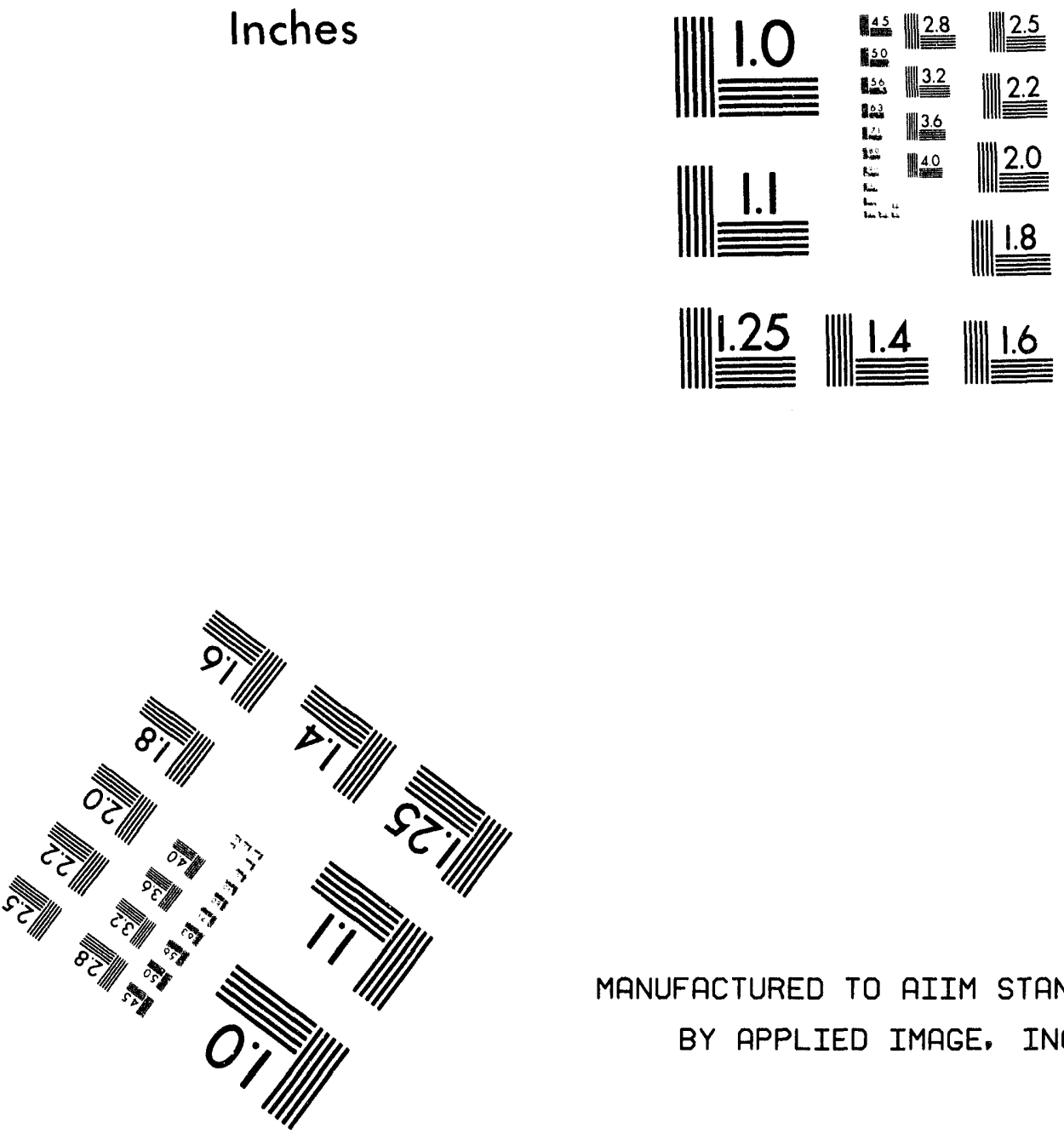

MANUFACTURED TO AIIM STANDARDS

BY APPLIED IMAGE, INC.

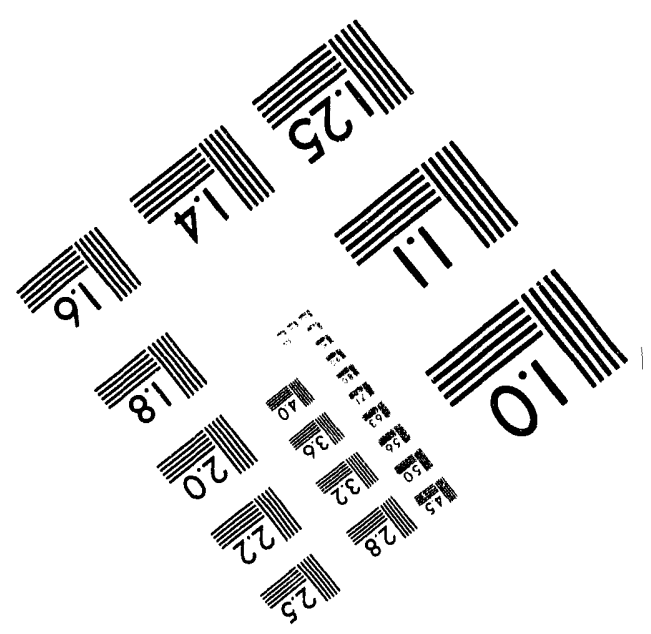



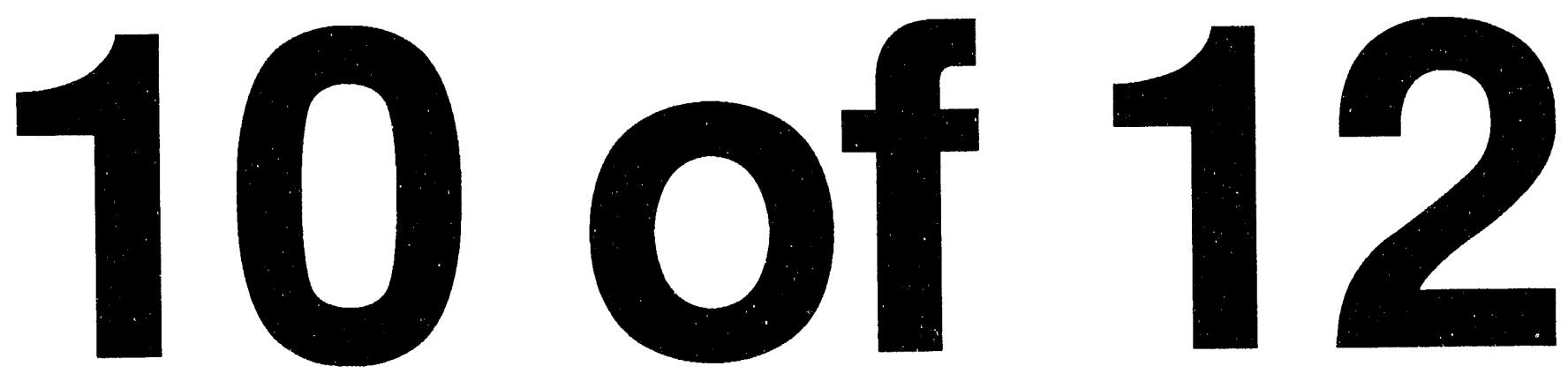


\begin{tabular}{|c|c|c|c|c|c|c|}
\hline \multicolumn{2}{|c|}{ Test: Aug17_20 } & sum1 & \multicolumn{3}{|c|}{ Date: Fri Aug $17 \quad 06: 49: 01 \quad 1990$} & Page: 3 \\
\hline \# & Name & $\operatorname{Min}$ & Max & Ave & SD & Uncert \\
\hline $\begin{array}{l}102 \\
103\end{array}$ & $\begin{array}{l}\text { PRV000001 } \\
\text { PSR000001 }\end{array}$ & $\begin{array}{l}19.7186 \\
37.0031\end{array}$ & $\begin{array}{l}19.9627 \\
37.3693\end{array}$ & 19.8406 & $\begin{array}{l}3.4877 e-02 \\
7.2155 e-02\end{array}$ & $\begin{array}{l}7.5164 e-01 \\
7.5573 e-01\end{array}$ \\
\hline 104 & $\begin{array}{l}\text { PSR000001 } \\
\text { PFMO00001 }\end{array}$ & $\begin{array}{l}37.0031 \\
29.8391\end{array}$ & $\begin{array}{l}37.3693 \\
30.6631\end{array}$ & $\begin{array}{l}37.1544 \\
30.2419\end{array}$ & $1.6018 e-01$ & $\begin{array}{l}7.5573 e-01 \\
5.0568 e-01\end{array}$ \\
\hline 105 & PAN000136 & 49.9406 & 71.4555 & 59.9657 & $4.4720 e+00$ & $4.9223 e+00$ \\
\hline $\begin{array}{l}106 \\
107\end{array}$ & PFM000002 & 27.5689 & 27.5689 & 27.5689 & $9.6336 e-06$ & $2.1154 e-01$ \\
\hline $\begin{array}{l}107 \\
108\end{array}$ & EMPTY & 0.0049 & 0.0098 & 0.0061 & $2.1065 e-03$ & $0.0000 e+00$ \\
\hline $\begin{array}{l}108 \\
109\end{array}$ & EMPTY & -9.8535 & -5.9717 & -7.3329 & $9.7179 e-01$ & $0.0000 e+00$ \\
\hline $\begin{array}{l}109 \\
110\end{array}$ & EMPTY & -0.0049 & 0.0049 & 0.0045 & $1.6624 e-03$ & $0.0000 e+00$ \\
\hline $\begin{array}{l}110 \\
111\end{array}$ & EMPTY & -10.0000 & -10.0000 & -10.0000 & $0.0000 e+00$ & $0.0000 e+00$ \\
\hline $\begin{array}{l}111 \\
1 \geq 2\end{array}$ & $\begin{array}{l}\text { EMPTY } \\
\text { VPWO00001 }\end{array}$ & 0.0000 & 0.0049 & 0.0001 & $6.9053 e-04$ & $0.0000 e+00$ \\
\hline $\begin{array}{l}112 \\
113\end{array}$ & $\begin{array}{l}\text { VPWO } 00001 \\
\text { VPW000002 }\end{array}$ & $\begin{array}{l}198.7042 \\
232.9139\end{array}$ & $\begin{array}{l}199.2901 \\
233.3046\end{array}$ & $\begin{array}{l}198.8994 \\
233.0273\end{array}$ & $1.3087 e-01$ & $1.4423 e+00$ \\
\hline 114 & VPW000003 & 288.6381 & $\begin{array}{l}233.3046 \\
289.2240\end{array}$ & $\begin{array}{l}233.0273 \\
288.9504\end{array}$ & $\begin{array}{l}1.0507 e-01 \\
1.2478 e-01\end{array}$ & $\begin{array}{l}1.6158 \mathrm{e}+00 \\
1.9136 \mathrm{e}+00\end{array}$ \\
\hline 115 & VPWO00004 & 204.4734 & 205.0593 & $\begin{array}{l}288.9504 \\
204.731 .4\end{array}$ & $1.5505 e-01$ & $1.4714 \mathrm{e}+00$ \\
\hline 116 & VPW000005 & 272.6600 & 273.6366 & 273.0898 & $2.0120 e-01$ & $1.8278 \mathrm{e}+00$ \\
\hline $\begin{array}{l}117 \\
118\end{array}$ & VPW000006 & 290.6438 & 291.2297 & 290.8390 & $1.6269 e-01$ & $1.9239 \mathrm{e}+00$ \\
\hline $\begin{array}{l}118 \\
119\end{array}$ & IPW000001 & 1039.3977 & 1049.1594 & 1044.6689 & $2.3084 \mathrm{e}+00$ & $1.1564 \mathrm{e}+01$ \\
\hline $\begin{array}{l}119 \\
120\end{array}$ & IPW000002 & 2492.5967 & 2502.3530 & 2498.3713 & $2.2287 e+00$ & $2.1335 e+01$ \\
\hline $\begin{array}{l}120 \\
121\end{array}$ & $\begin{array}{l}\text { IPWOOO0003 } \\
\text { IPWOOOO004 }\end{array}$ & 2513.5498 & 2519.3999 & 2517.0215 & $1.4363 e+00$ & $2.1471 e+01$ \\
\hline 123 & $\begin{array}{l}\text { IPW000005 } \\
\text { IPW000006 }\end{array}$ & $\begin{array}{l}483.5191 \\
501.0142\end{array}$ & $\begin{array}{l}485.4720 \\
502.4790\end{array}$ & 484.4858 & $4.1251 \mathrm{e}-01$ & $4.3185 e+00$ \\
\hline 124 & EMPTY & $\begin{array}{r}-0.0244 \\
-0.024\end{array}$ & $\begin{array}{r}502.4790 \\
-0.0098\end{array}$ & 501.6388 & $3.6963 e-01$ & $4.4390 e+00$ \\
\hline 125 & EMPTY & -0.0195 & $\begin{array}{l}-0.0098 \\
-0.004 ?\end{array}$ & $\begin{array}{l}-0.0188 \\
-0.0130\end{array}$ & $3.9901 e-03$ & $0.0000 e+00$ \\
\hline 126 & EMPTY & -0.0195 & $\begin{array}{l}-0.004 \Omega \\
-0.0098\end{array}$ & $\begin{array}{l}-0.0130 \\
-0.0151\end{array}$ & $2.7213 e-03$ & $0.0000 e+00$ \\
\hline 127 & EMPTY & -0.0244 & $\begin{array}{l}-0.0098 \\
-0.0098\end{array}$ & $\begin{array}{l}-0.0151 \\
-0.0173\end{array}$ & $3.1583 e-03$ & $0.0000 e+00$ \\
\hline 128 & IHT000001 & 244.1481 & 247.6637 & $\begin{array}{r}-0.0173 \\
246.0466\end{array}$ & $3.7186 e-03$ & $0.0000 e+00$ \\
\hline 129 & IHT000002 & 251.5174 & 254.6424 & $\begin{array}{l}246.0466 \\
253.0995\end{array}$ & $7.7227 e-01$ & $4.7816 e+00$ \\
\hline 130 & IHT000003 & 253.7936 & 256.7233 & $\begin{array}{l}253.0995 \\
255.3679\end{array}$ & $7.1599 \mathrm{e}-01$ & $4.8183 e+00$ \\
\hline 131 & IHTOOOOOO4 & 248.7643 & 251.6940 & $\begin{array}{l}255.3679 \\
250.2956\end{array}$ & $7.7062 e-01$ & $4.8302 \mathrm{e}+00$ \\
\hline 132 & IHTO00005 & 246.6496 & 249.3840 & $\begin{array}{l}250.2956 \\
247.9971\end{array}$ & $7.7961 e-01$ & $4.8036 e+00$ \\
\hline 133 & IHTO00006 & 240.5987 & 243.3331 & $\begin{array}{l}247.9971 \\
242.0674\end{array}$ & $6.9391 e-01$ & $4.7917 e+00$ \\
\hline 134 & IHT000007 & 250.1352 & 253.0649 & $\begin{array}{l}242.0674 \\
251.9866\end{array}$ & $7.4997 e-01$ & $4.7613 e+00$ \\
\hline 135 & IHT000008 & 247.5436 & 250.2779 & $\begin{array}{l}251.9866 \\
249.2194\end{array}$ & $7.3741 e-01$ & $4.8125 e+00$ \\
\hline 136 & IHT000009 & 247.5323 & 250.4620 & $\begin{array}{l}249.2194 \\
249.1963\end{array}$ & $7.2339 e-01$ & $4.7980 e+00$ \\
\hline 137 & IHT000010 & 79.6236 & 82.9440 & $\begin{array}{r}249.1963 \\
81.2916\end{array}$ & $7.5308 e-01$ & $4.7979 e+00$ \\
\hline 138 & IHT000011 & 248.6666 & 251.5963 & $\begin{array}{r}81.2916 \\
250.0378\end{array}$ & $6.8596 e-01$ & $4.1798 e+00$ \\
\hline 139 & IHTO000012 & 244.3284 & 247.0628 & $\begin{array}{l}250.0378 \\
245.6331\end{array}$ & $6.2700 e-01$ & $4.8023 e+00$ \\
\hline 140 & IHTO00013 & 177.5391 & 180.0781 & $\begin{array}{l}245.6331 \\
178.7109\end{array}$ & $5.0754 e-01$ & $4.7795 e+00$ \\
\hline 141 & IHTOOOOOL & 245.3351 & 247.4835 & $\begin{array}{l}178.7109 \\
246.3623\end{array}$ & $5.0068 e-01$ & $4.4726 \mathrm{e}+00$ \\
\hline 142 & IHT000015 & 183.2933 & 185.6370 & $\begin{array}{l}246.3623 \\
184.3089\end{array}$ & $4.5985 e-01$ & $4.7832 e+00$ \\
\hline 143 & IHT000016 & 170.1622 & 172.5060 & $\begin{array}{l}184.3089 \\
171.2755\end{array}$ & $4.9599 e-01$ & $4.4952 e+00$ \\
\hline 1.44 & IHT000017 & 203.7034 & 207.2191 & $\begin{array}{l}171.2755 \\
205.5276\end{array}$ & $4.9795 e-01$ & $4.4434 \mathrm{e}+00$ \\
\hline 1.45 & IHT000018 & 205.9871 & 209.1121 & $\begin{array}{l}205.5276 \\
207.3857\end{array}$ & $6.2091 \mathrm{e}-01$ & $4.5863 e+00$ \\
\hline 146 & IHT000019 & 199.9624 & 202.6968 & $\begin{array}{l}207.3857 \\
201.1852\end{array}$ & $6.7469 e-01$ & $4.5947 e+00$ \\
\hline .147 & IHTO00020 & 206.0021 & 208.3458 & $\begin{array}{l}201.1852 \\
207.3419\end{array}$ & $5.8635 e-01$ & $4.5670 e+00$ \\
\hline 148 & IHTO00021 & -3.0086 & -0.0789 & $\begin{array}{r}207.3419 \\
-1.3484\end{array}$ & $5.6910 e-01$ & $4.5945 e+00$ \\
\hline 149 & IHTO00022 & 177.4301 & 179.3833 & $\begin{array}{r}-1.3484 \\
178.3637\end{array}$ & $7.0061 e-01$ & $4.1000 e+00$ \\
\hline 150 & IHTO00023 & 201.6301 & 203.7786 & $\begin{array}{l}178.3637 \\
202.7276\end{array}$ & $5.1944 e-01$ & $4.4712 e+00$ \\
\hline 151 & IHTO00024 & 165.6663 & 168.0101 & $\begin{array}{l}202.7276 \\
166.9828\end{array}$ & $5.5508 e-01$ & $4.5738 e+00$ \\
\hline 152 & IHTO00025 & 200.2517 & 202.7907 & $\begin{array}{l}166.9828 \\
201.5954\end{array}$ & $5.4363 e-01$ & $4.4270 e+00$ \\
\hline 153 & IHT000026 & 202.9823 & 205.7167 & $\begin{array}{l}201.5954 \\
204.2675\end{array}$ & $5.6996 \mathrm{e}-01$ & $4.5688 \mathrm{e}+00$ \\
\hline 154 & IHT000027 & 173.0018 & 175.5409 & 204.2675 & $5.9724 e-01$ & $4.5807 e+00$ \\
\hline 155 & IHT000028 & 175.3192 & $\begin{array}{l}175.5409 \\
178.2489\end{array}$ & 174.3651 & $5.7248 e-01$ & $4.4554 \mathrm{e}+00$ \\
\hline & & & & 176.7802 & $7.1598 e-01$ & $4.4649 e+00$ \\
\hline
\end{tabular}




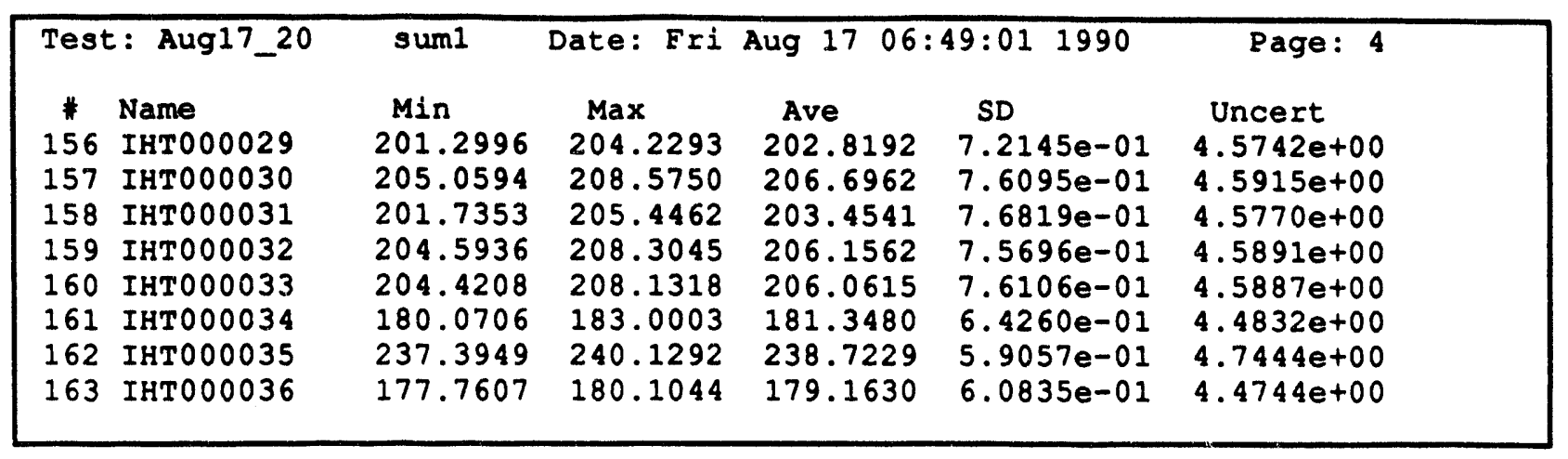




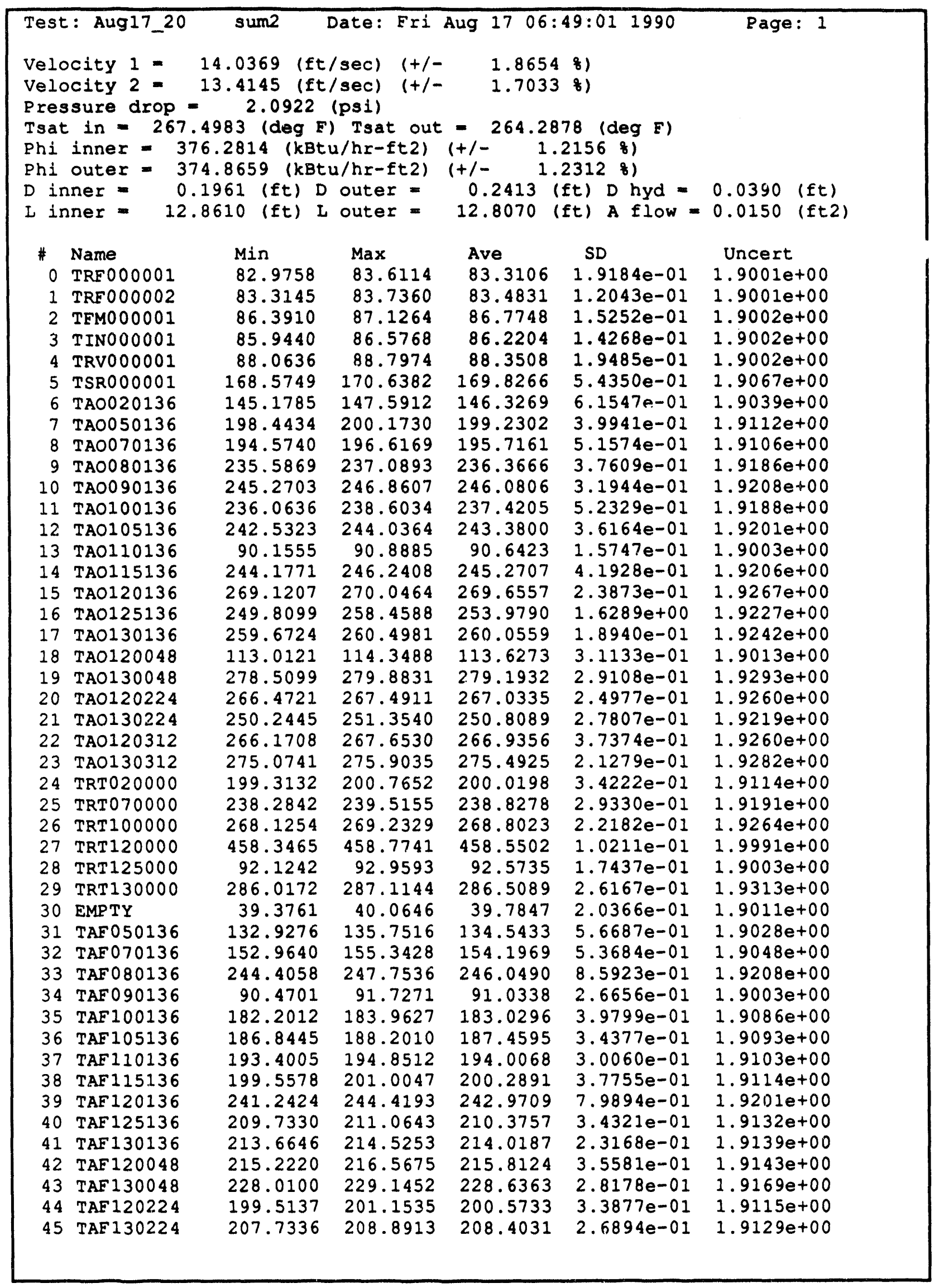




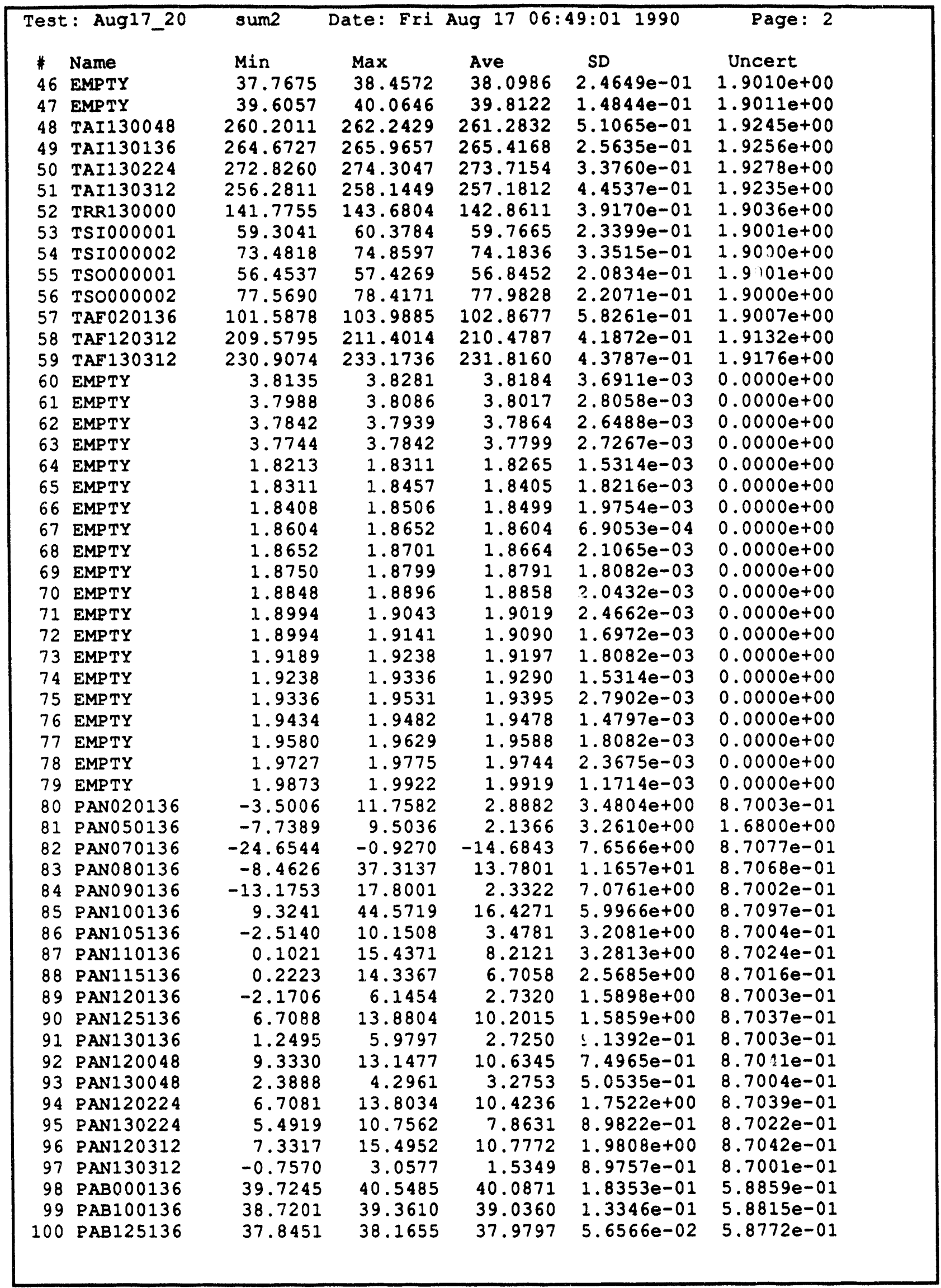




\begin{tabular}{|c|c|c|c|c|c|c|}
\hline \multicolumn{2}{|c|}{ Test: Aug17_20 } & sum2 & Date: Fri & \multicolumn{2}{|c|}{ Aug $17 \quad 06: 49: 01 \quad 1990$} & Page: 3 \\
\hline \# & Name & Min & $\operatorname{Max}$ & Ave & SD & Uncert \\
\hline 101 & PIN000001 & 39.2263 & 40.4470 & 39.8391 & $3.6869 e-01$ & $7.5658 e-01$ \\
\hline 102 & PRV000001 & 19.3524 & 19.4744 & 19.4232 & $6.0861 e-02$ & $7.5157 e-01$ \\
\hline 103 & PSR000001 & 36.8810 & 37.0031 & 36.9323 & $6.0861 e-02$ & $7.5566 \mathrm{e}-01$ \\
\hline 104 & PFM000001 & 29.8391 & 30.5715 & 30.2053 & $2.0762 e-01$ & $5.0567 e-01$ \\
\hline 105 & PANO00136 & 48.1096 & 73.2866 & 57.9240 & $5.3752 e+00$ & $4.9221 e+00$ \\
\hline 106 & PEM000002 & 27.5689 & 27.5689 & 27.5689 & $9.633 \mathrm{Ge}-06$ & $2.1154 e-01$ \\
\hline 107 & EMPTY & 0.0049 & 0.0098 & 0.0064 & $2.3008 e-03$ & $0.0000 e+00$ \\
\hline 108 & EMPTY & -10.0000 & -6.2451 & -8.1308 & $1.2266 \mathrm{e}+00$ & $0.0000 e+00$ \\
\hline 109 & EMPTY & 0.0049 & 0.0098 & 0.0050 & $6.9053 e-04$ & $0.0000 e+00$ \\
\hline 110 & EMPTY & -10.0000 & -10.0000 & -10.0000 & $0.0000 e+00$ & $0.0000 e+00$ \\
\hline 111 & EMPTY & 0.0000 & 0.0098 & 0.0003 & $1.5314 \mathrm{e}-03$ & $0.0000 e+00$ \\
\hline 112 & VPW000001 & 198.7042 & 199.2901 & 199.0205 & $1.4167 e-01$ & $1.4429 e+00$ \\
\hline 113 & VPW000002 & 232.9139 & 233.3046 & 233.0234 & $1.0559 \mathrm{e}-01$ & $1.6158 \mathrm{e}+00$ \\
\hline 114 & VPW000003 & 288.6381 & 289.2240 & 289.0051 & $1.1599 \mathrm{e}-01$ & $1.9139 e+00$ \\
\hline 115 & VPW000004 & 204.4734 & 204.8640 & 204.6689 & $1.1838 \mathrm{e}-01$ & $1.4711 e+00$ \\
\hline 116 & VPW000005 & 272.8553 & 273.2459 & 273.1640 & $1.1897 e-01$ & $1.8282 \mathrm{e}+00$ \\
\hline 117 & VPW000006 & 290.6438 & 291.2297 & 290.9015 & $1.3917 e-01$ & $1.9242 e+00$ \\
\hline 118 & IPWO00001 & 1047.2072 & 1053.0642 & 1050.0188 & $1.4309 e+00$ & $1.1594 \mathrm{e}+01$ \\
\hline 119 & IPW000002 & 2471.1328 & 2478.9377 & 2475.1135 & $1.8889 e+00$ & $2.1165 e+01$ \\
\hline 120 & IPW0 00003 & 2517.4500 & 2523.2998 & 2520.0237 & $1.3895 e+00$ & $2.1493 e+01$ \\
\hline 121 & IPWOO00004 & 727.3606 & 729.3137 & 728.1029 & $4.4381 e-01$ & $6.0898 \mathrm{e}+00$ \\
\hline 122 & IPW000005 & 482.0544 & 483.5191 & 482.8358 & $3.1192 e-01$ & $4.3069 e+00$ \\
\hline 123 & IPW000006 & 499.5493 & 500.5259 & 500.1252 & $3.0708 e-01$ & $4.4284 \mathrm{e}+00$ \\
\hline 124 & EMPTY & -0.0244 & -0.0098 & -0.0196 & $3.7551 e-03$ & $0.0000 e+00$ \\
\hline 125 & EMPTY & -0.0195 & -0.0098 & -0.0125 & $2.8179 e-03$ & $0.0000 e+00$ \\
\hline 126 & EMPTY & -0.0195 & -0.0098 & -0.0148 & $3.1133 e-03$ & $0.0000 e+00$ \\
\hline 127 & EMPTY & -0.0244 & -0.0098 & -0.0168 & $3.4400 e-03$ & $0.0000 e+00$ \\
\hline 128 & IHT000001 & 245.1247 & 247.2731 & 246.2927 & $4.4379 e-01$ & $4.7829 e+00$ \\
\hline 129 & IHTO00002 & 252.4940 & 254.0565 & 253.3847 & $4.3968 e-01$ & $4.8198 e+00$ \\
\hline 130 & IHTO00003 & 254.7701 & 256.7233 & 255.7624 & $4.7325 e-01$ & $4.8323 e+00$ \\
\hline 131 & IHT000004 & 249.7408 & 251.6940 & 250.6745 & $4.6737 e-01$ & $4.8056 e+00$ \\
\hline 132 & IHT000005 & 247.6262 & 249.5793 & 248.3917 & $4.4440 e-01$ & $4.7937 e+00$ \\
\hline 133 & IHT000006 & 241.5753 & 243.3331 & 242.4072 & $4.0971 e-01$ & $4.7630 e+00$ \\
\hline 134 & IHT000007 & 251.5024 & 253.2602 & 252.4671 & $3.8945 e-01$ & $4.8150 e+00$ \\
\hline 135 & IHT000008 & 248.7154 & 250.2779 & 249.5241 & $3.9461 \mathrm{e}-01$ & $4.7996 e+00$ \\
\hline 136 & IHTO00009 & 248.3135 & 250.2667 & 249.4072 & $4.1385 e-01$ & $4.7990 e+00$ \\
\hline 137 & IHTO00010 & 80.0143 & 82.3580 & 81.2291 & $5.1794 e-01$ & $4.1797 e+00$ \\
\hline 138 & IHTO00011 & 248.8619 & 251.2057 & 250.0378 & $4.9989 e-01$ & $4.8023 e+00$ \\
\hline 139 & IHT00001Z & 244.5237 & 246.6722 & 245.6643 & $5.5574 e-01$ & $4.7797 e+00$ \\
\hline 140 & IHT000013 & 177.5391 & 179.8828 & 178.7227 & $5.4449 e-01$ & $4.4726 e+00$ \\
\hline 141 & IHTO00014 & 245.5304 & 247.8741 & 246.4990 & $5.1139 e-01$ & $4.7839 e+00$ \\
\hline 142 & IHT000015 & 183.0979 & 185.6370 & 184.3480 & $4.9599 e-01$ & $4.4954 e+00$ \\
\hline 143 & IHTO00016 & 170.3576 & 172.5060 & 171.4747 & $4.8172 e-01$ & $4.4441 e+00$ \\
\hline 144 & IHTO00017 & 204.8753 & 207.0237 & 205.8089 & $5.8299 e-01$ & $4.5876 e+00$ \\
\hline 145 & IHT000018 & 206.5730 & 208.5261 & 207.4560 & $5.3122 e-01$ & $4.5950 \mathrm{e}+00$ \\
\hline 146 & IHTO00019 & 199.1812 & 201.9156 & 200.7437 & $6.3626 e-01$ & $4.5651 e+00$ \\
\hline 147 & IHT 000020 & 205.2208 & 208.1505 & 206.8653 & $6.0630 e-01$ & $4.5923 e+00$ \\
\hline 148 & IHTO00021 & -3.2039 & -0.2742 & -1.8718 & $7.1321 e-01$ & $4.1000 e+00$ \\
\hline 149 & IHTO00022 & 177.0395 & 178.9926 & 177.9458 & $4.9875 e-01$ & $4.4695 e+00$ \\
\hline 150 & IHT000023 & 201.2395 & 203.3879 & 202.2589 & $5.4859 e-01$ & $4.5717 e+00$ \\
\hline 151 & IHT000024 & 165.6663 & 167.6195 & 166.5609 & $5.1310 e-01$ & $4.4254 e+00$ \\
\hline 152 & IHTO00025 & 200.2517 & 202.4001 & 201.2517 & $5.4196 \mathrm{e}-01$ & $4.5673 e+00$ \\
\hline 153 & IHT000026 & 202.9823 & 204.9354 & 203.9628 & $5.1067 e-01$ & $4.5793 e+00$ \\
\hline 154 & IHT 000027 & 173.1971 & 175.5409 & 174.2284 & $5.7864 e-01$ & $4.4548 e+00$ \\
\hline 155 & IHT000028 & 175.1239 & 178.8349 & 176.9248 & $6.7461 e-01$ & $4.4654 e+00$ \\
\hline
\end{tabular}




\begin{tabular}{|c|c|c|c|c|c|c|}
\hline \multicolumn{2}{|c|}{ Test: Aug17_20 } & sum2 & Date: Fri & Aug 1706 & $9: 011990$ & Page: 4 \\
\hline \# & Name & Min & $\operatorname{Max}$ & Ave & SD & Uncert \\
\hline 156 & IHTO00029 & 201.2996 & 204.8152 & 202.8661 & $6.4051 e-01$ & $4.5744 e+00$ \\
\hline 157 & IHTO00030 & 205.4500 & 208.7703 & 206.7392 & $6.2015 e-01$ & $4.5917 e+00$ \\
\hline 158 & IHT000031 & 202.1259 & 205.4462 & 203.5752 & $5.9984 e-01$ & $4.5776 e+00$ \\
\hline 159 & IHT000032 & 204.7889 & 207.9139 & 206.2577 & $5.9613 e-01$ & $4.5896 e+00$ \\
\hline 160 & IHTO00033 & 204.6161 & 207.9365 & 206.1865 & $6.0356 e-01$ & $4.5893 e+00$ \\
\hline 161 & IHTO00034 & 180.4612 & 183.0003 & 181.4574 & $5.1788 e-01$ & $4.4836 e+00$ \\
\hline 162 & IHTO00035 & 237.7855 & 240.5199 & 238.9417 & $5.5642 e-01$ & $4.7455 e+00$ \\
\hline 163 & IHTO000036 & 178.3466 & 180.8857 & 179.3271 & $6.0809 e-01$ & $4.4750 \mathrm{e}+00$ \\
\hline
\end{tabular}




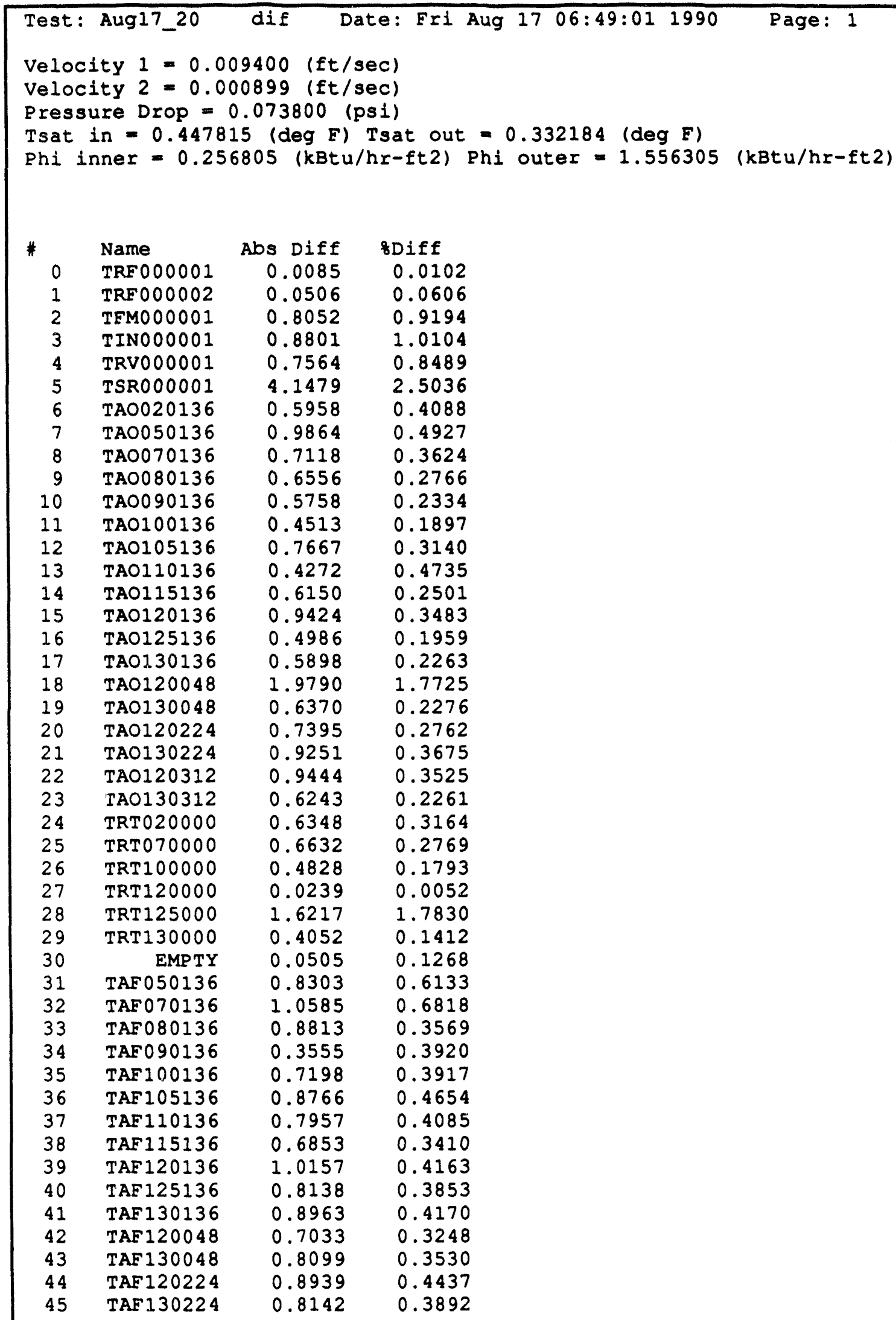




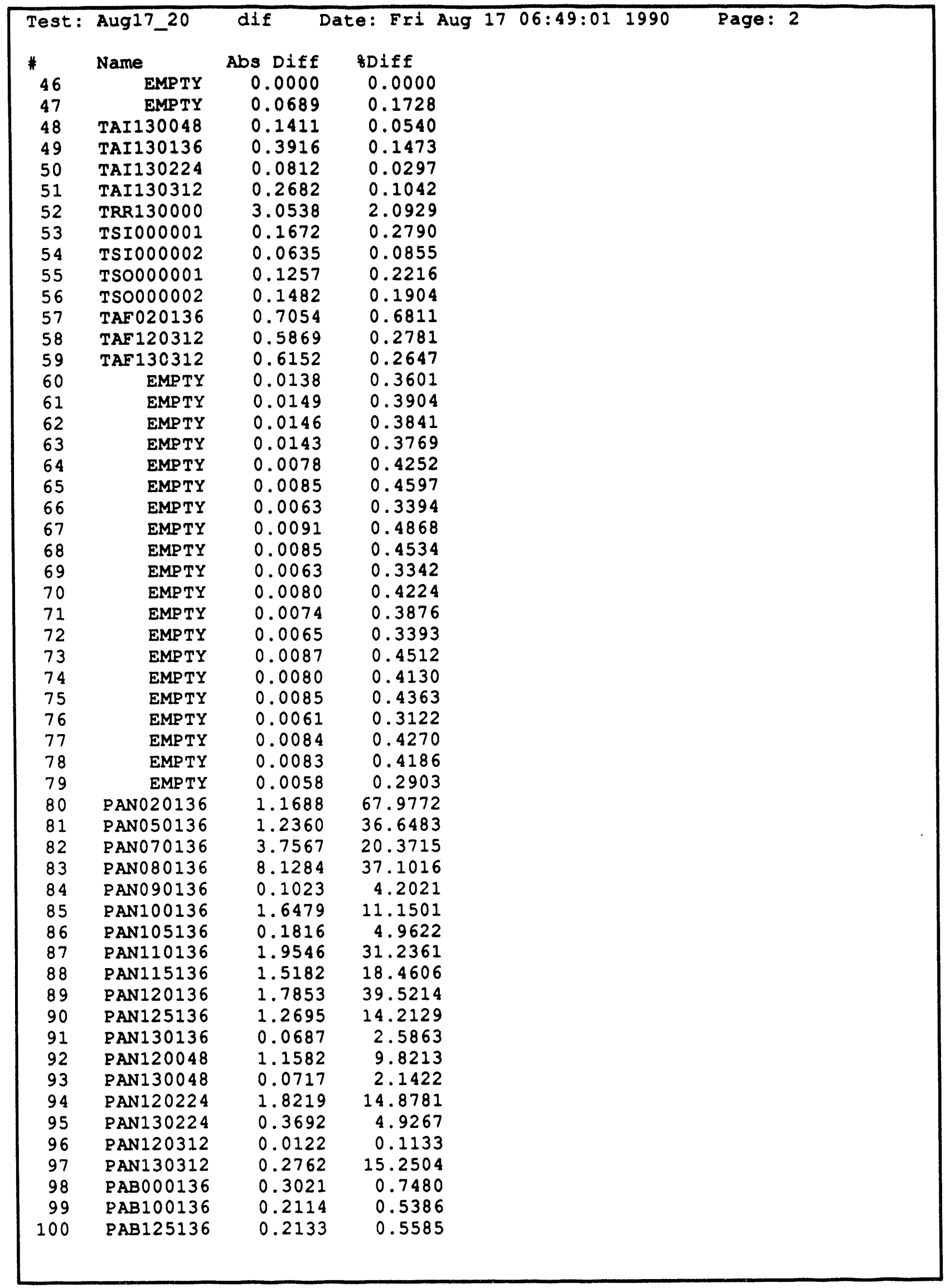




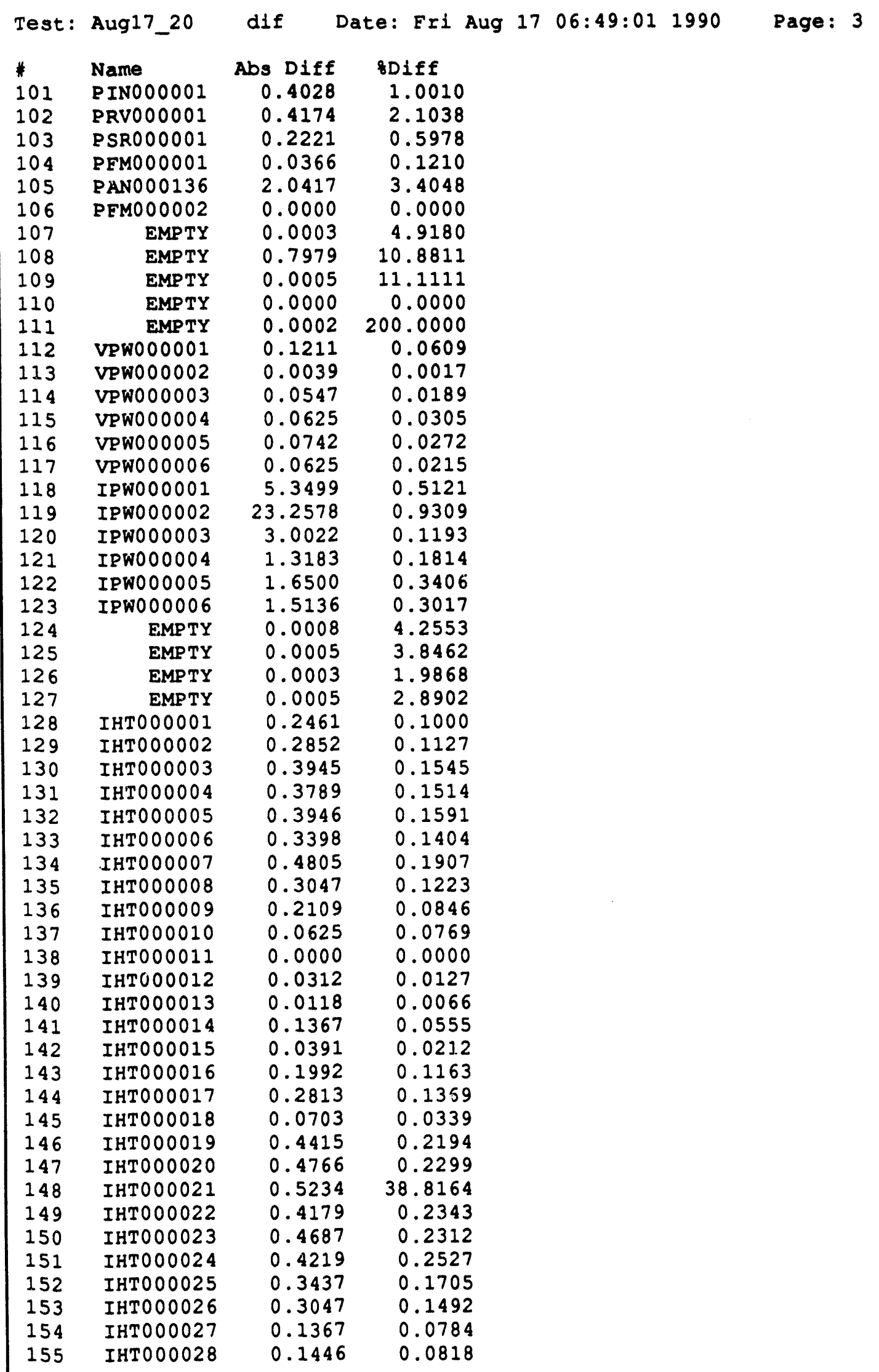




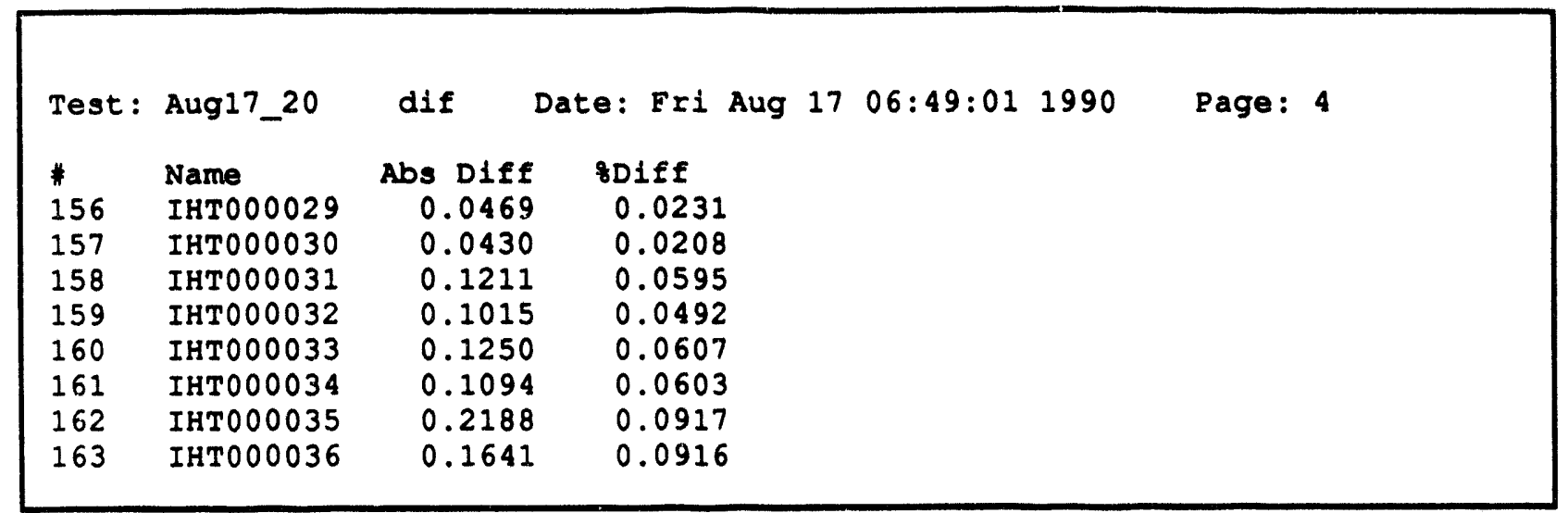




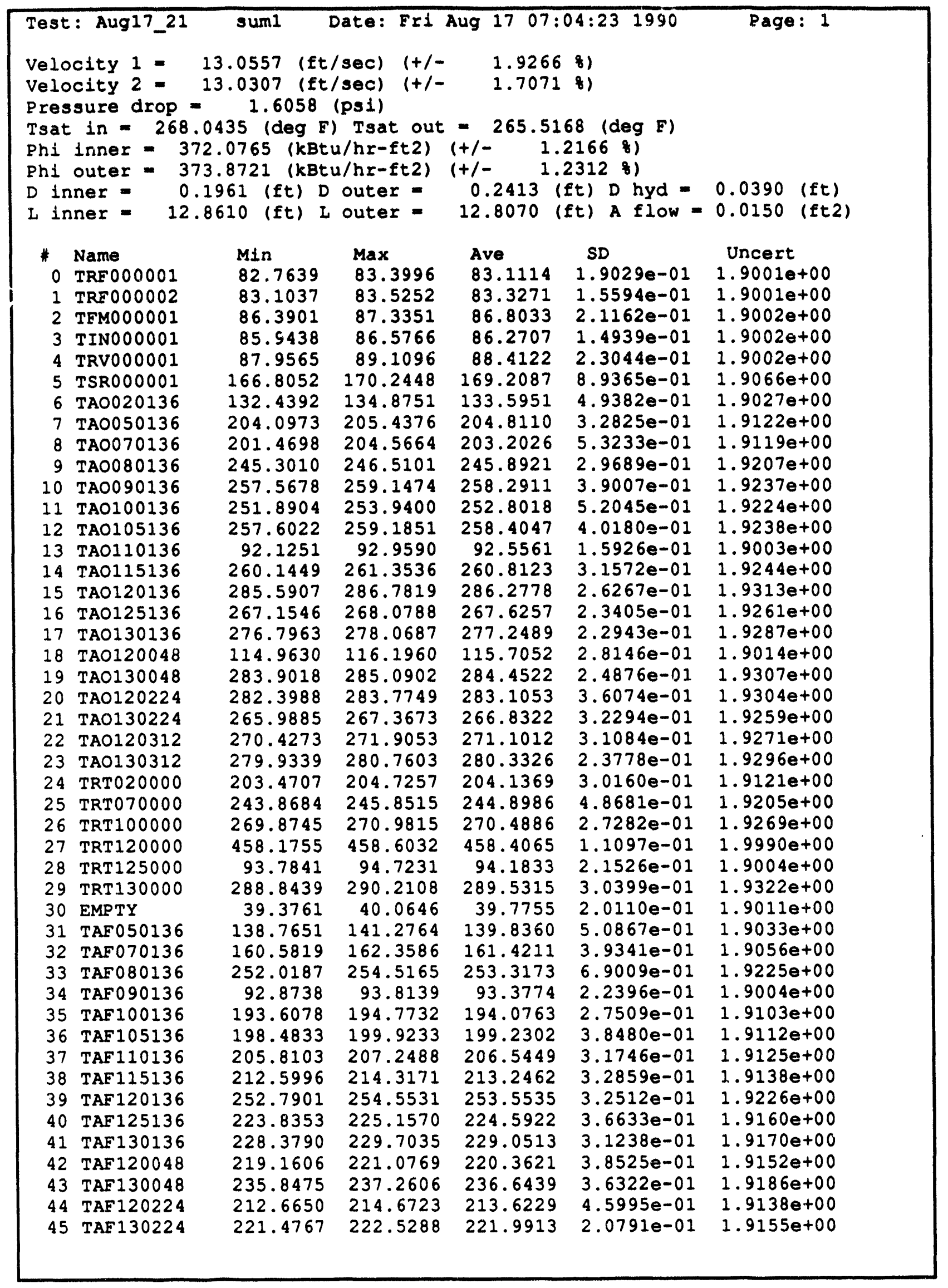




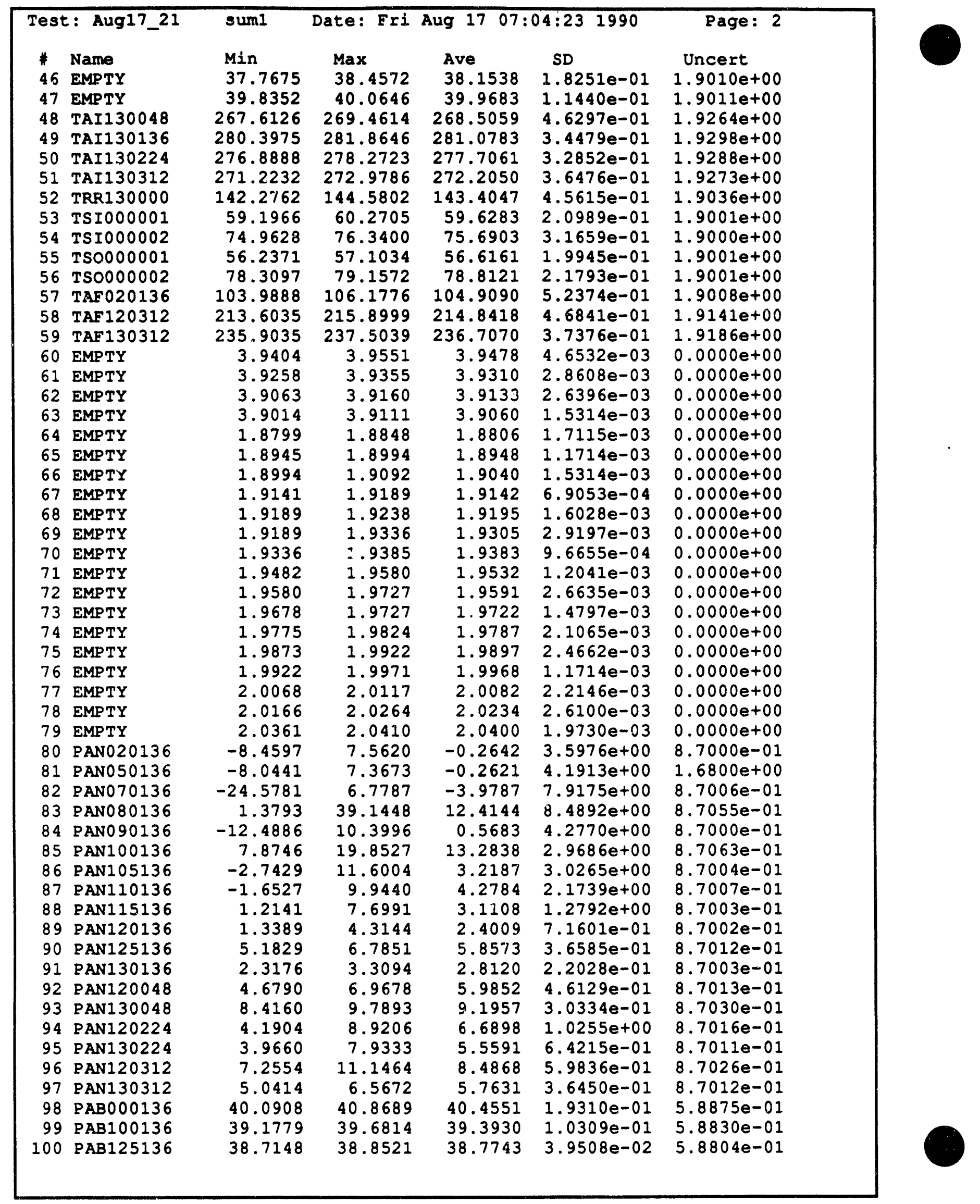




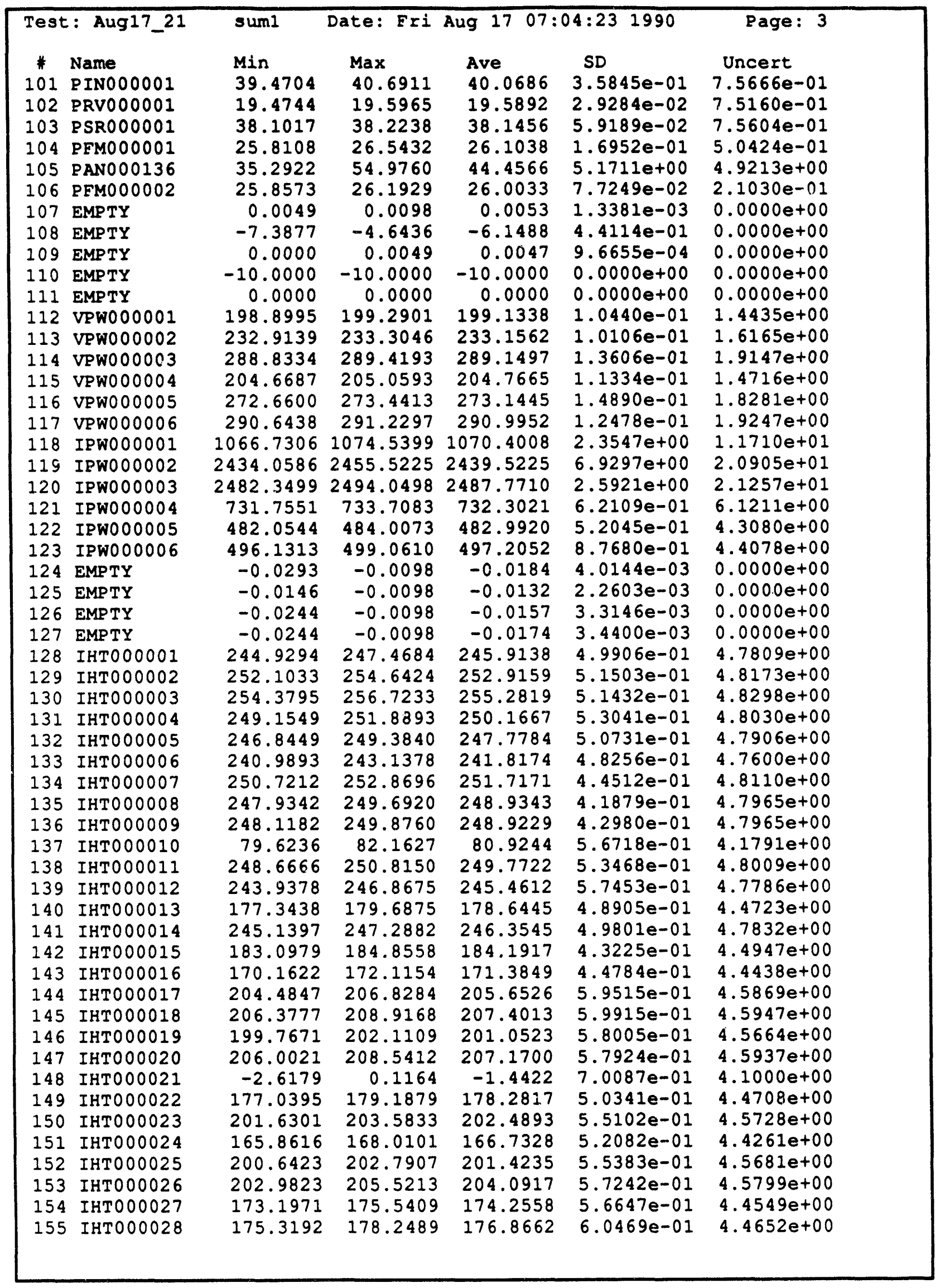




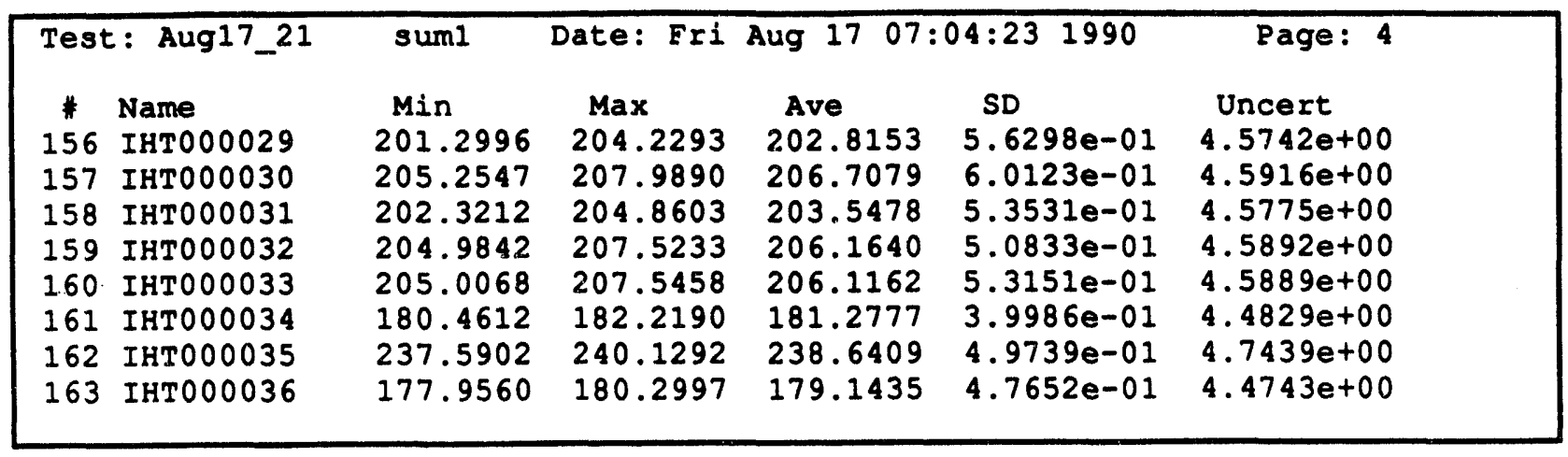




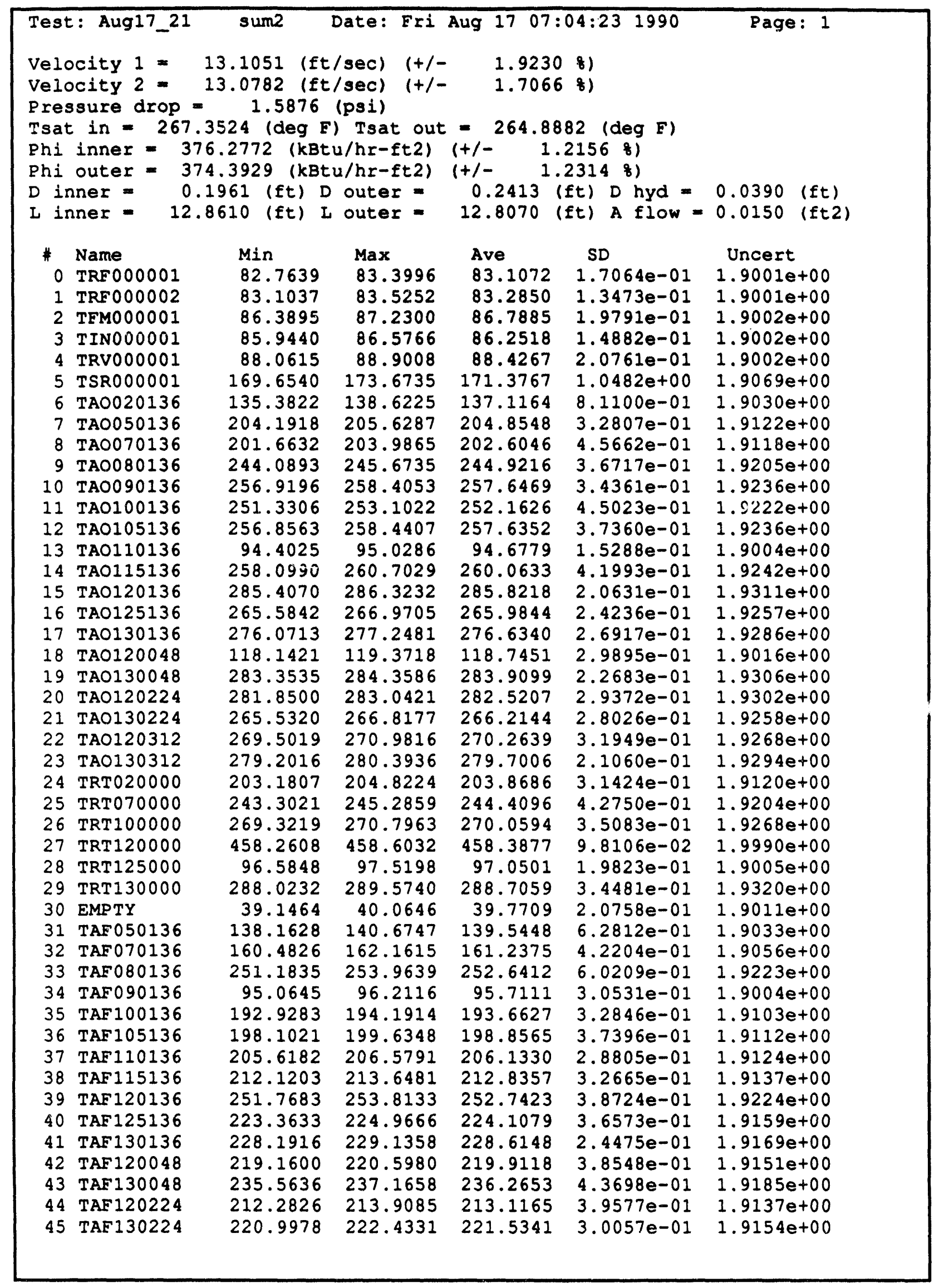




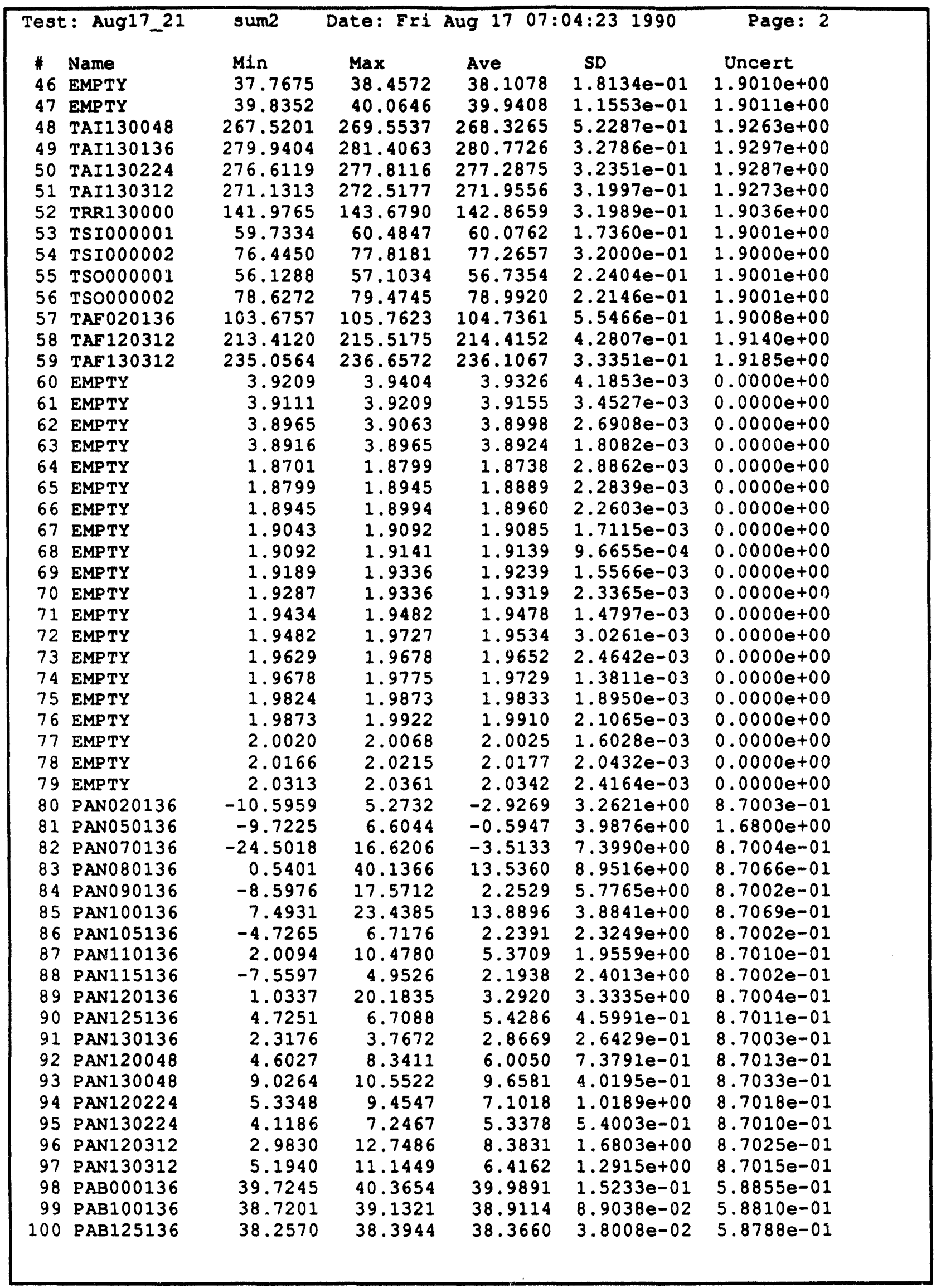




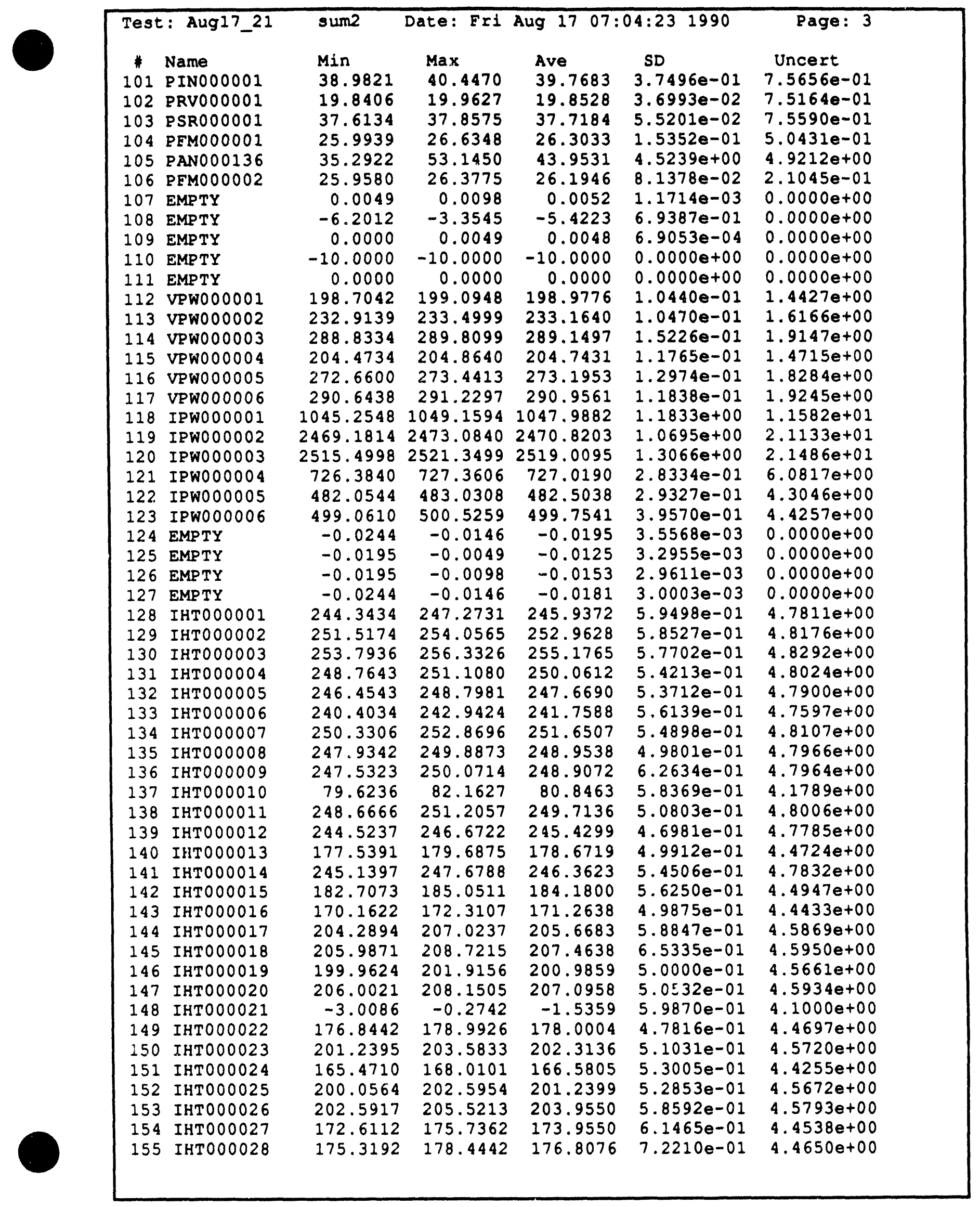




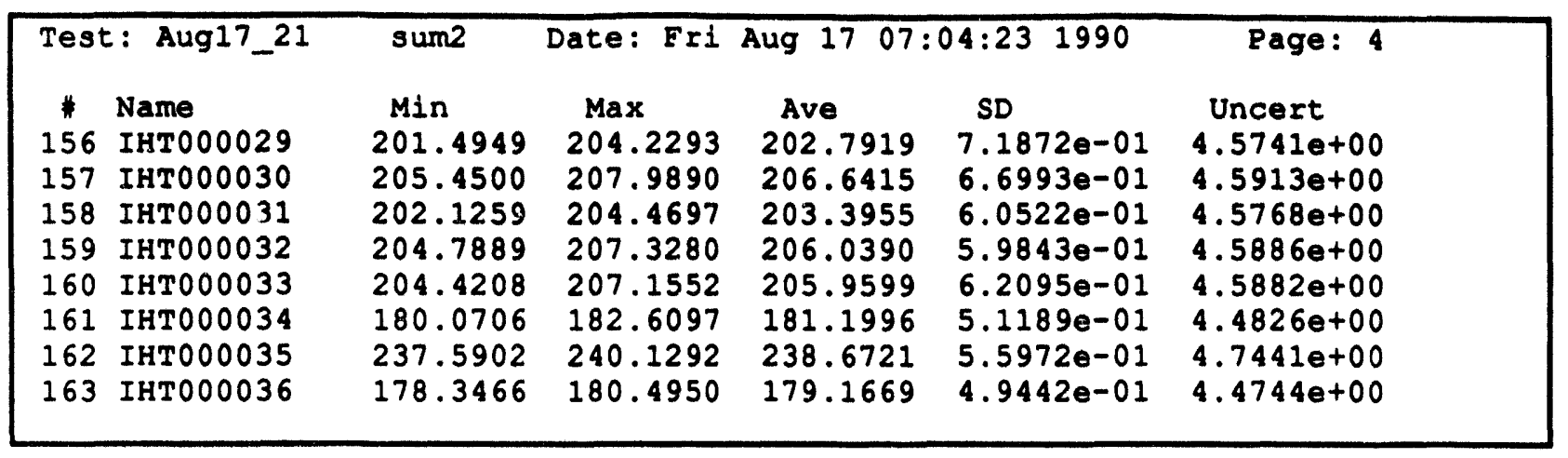




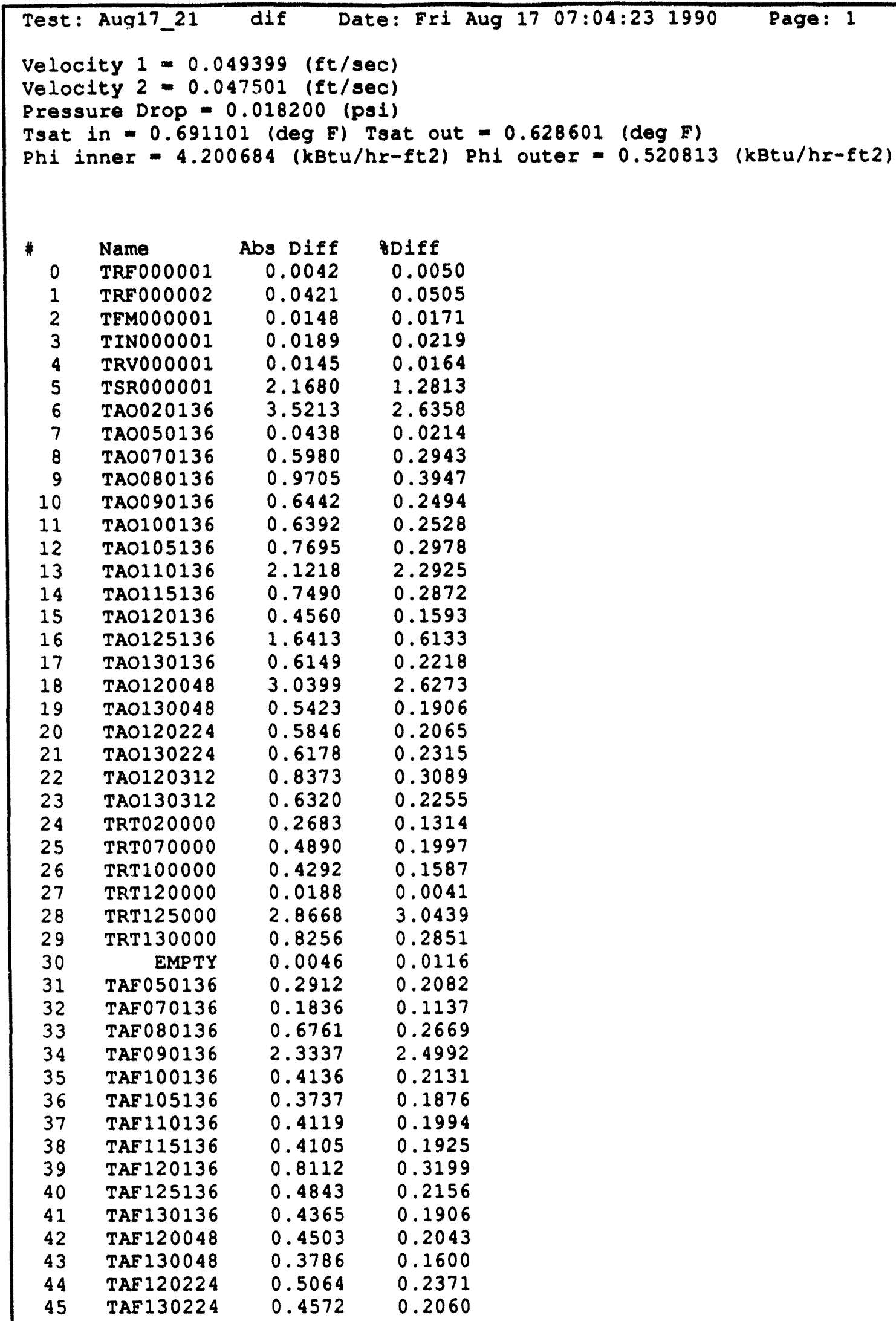




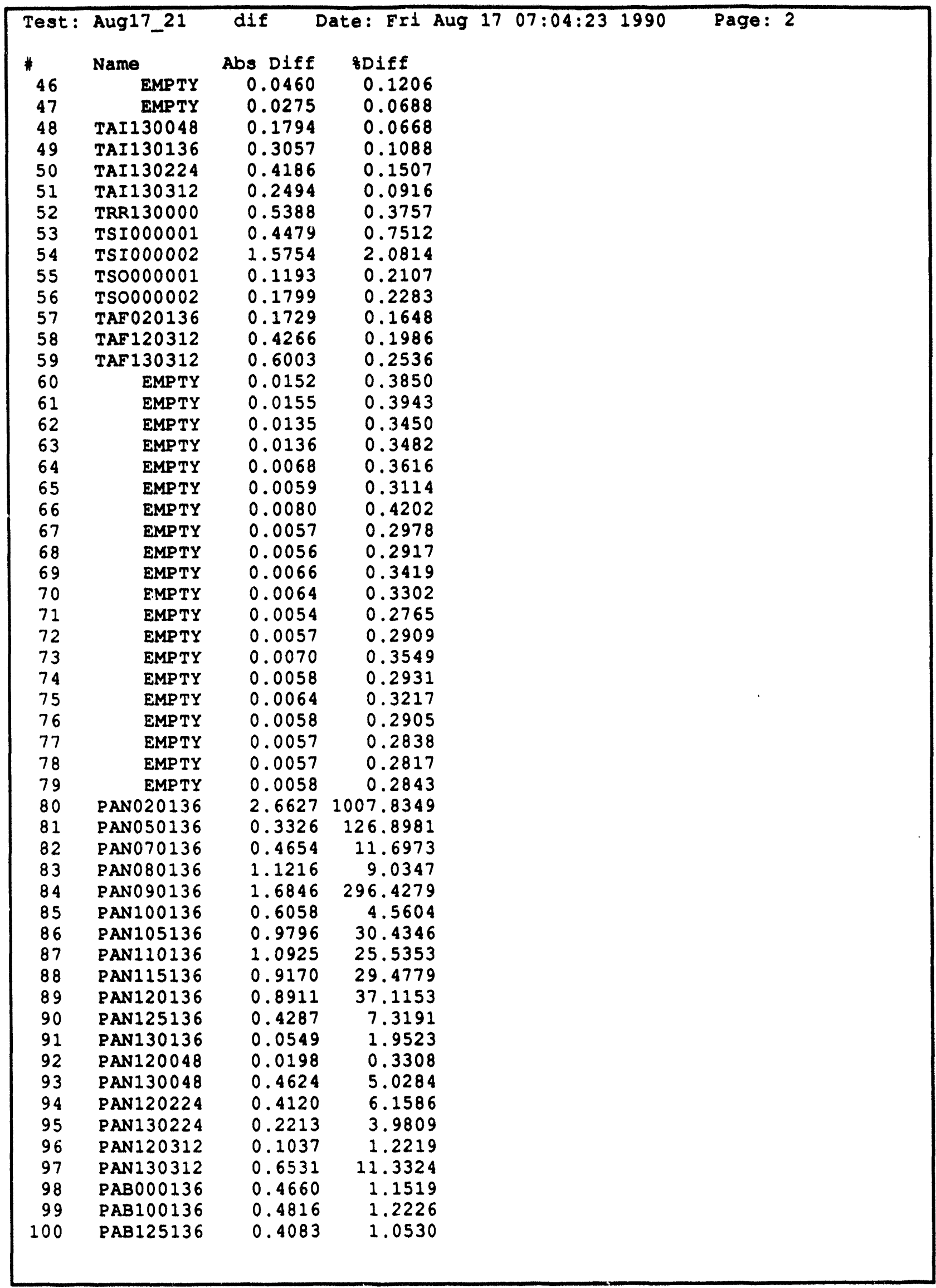




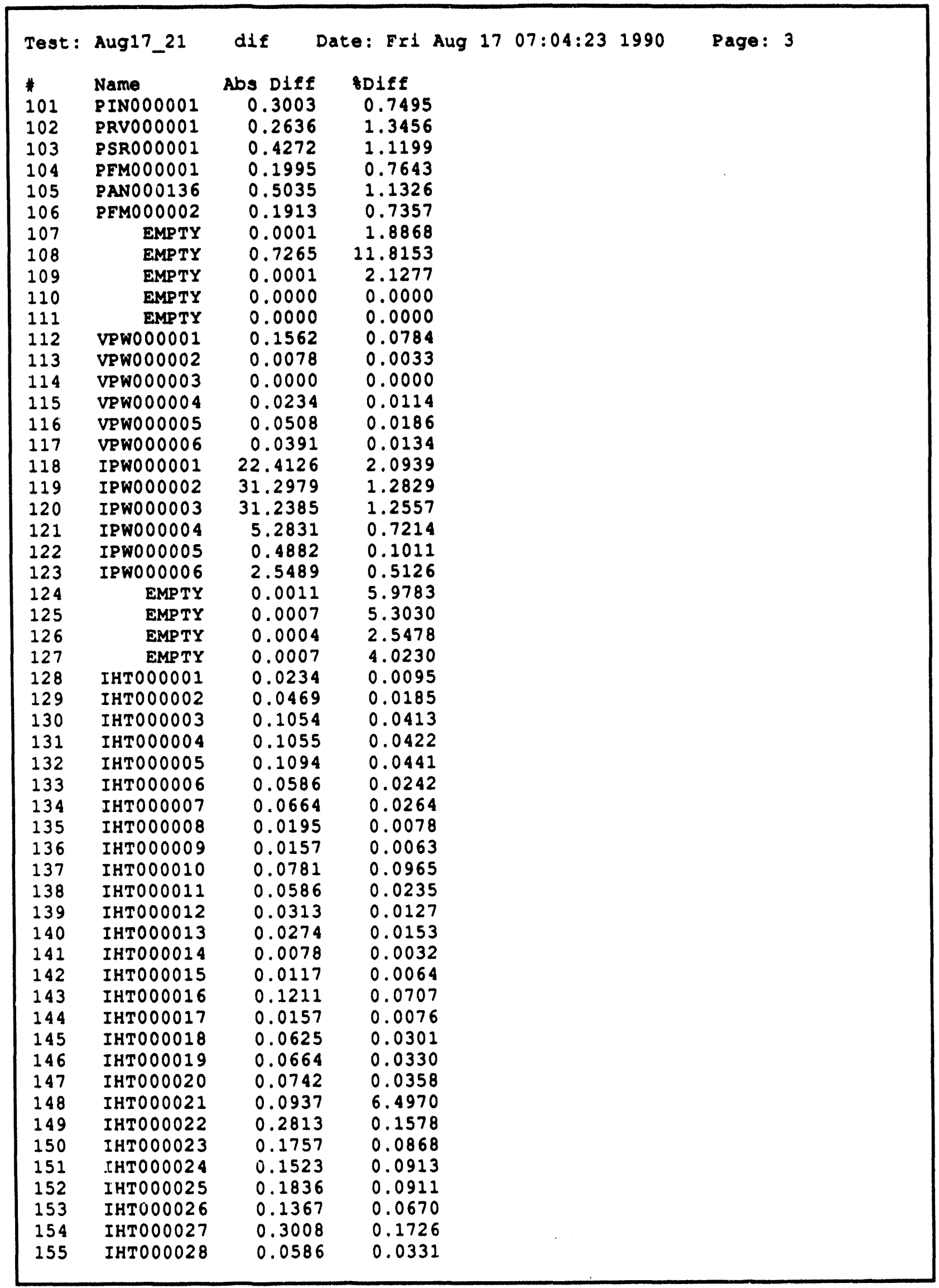




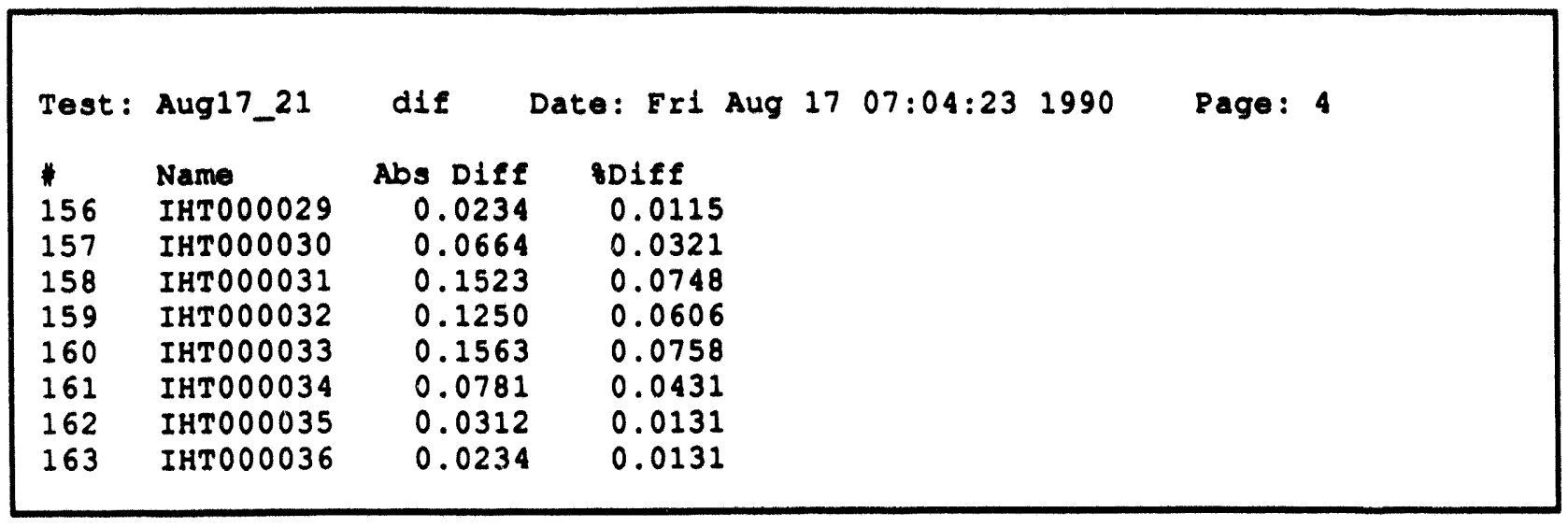




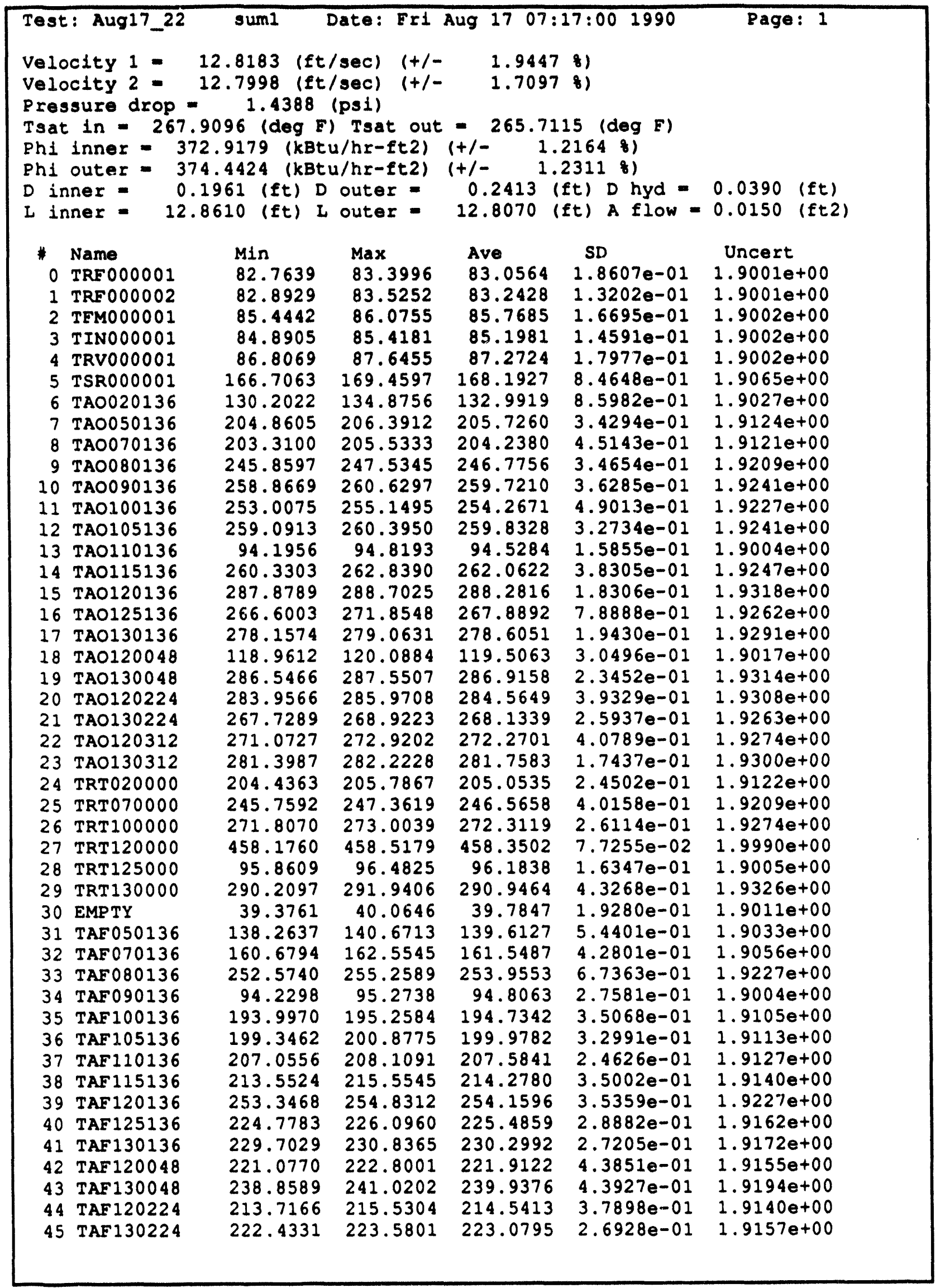




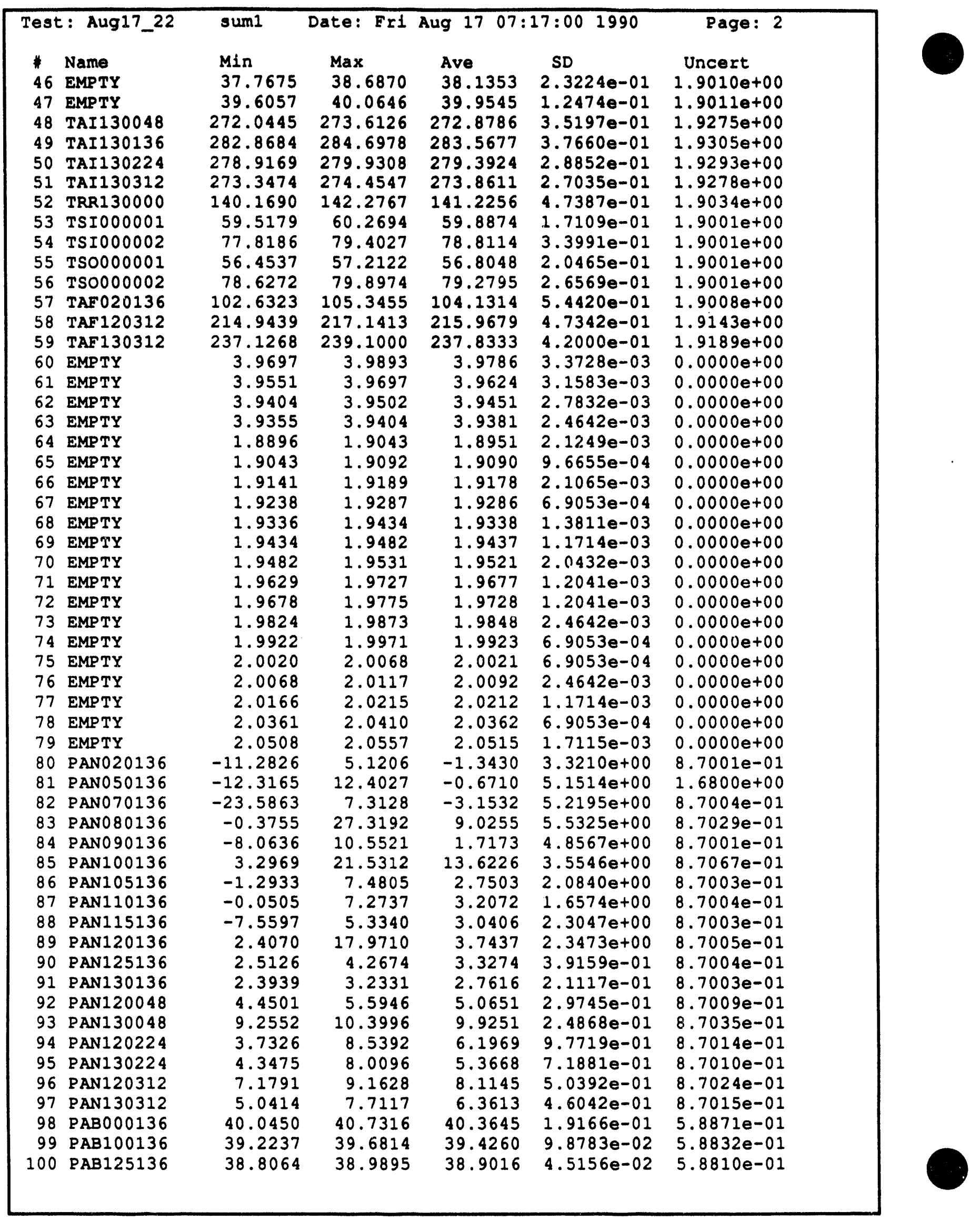




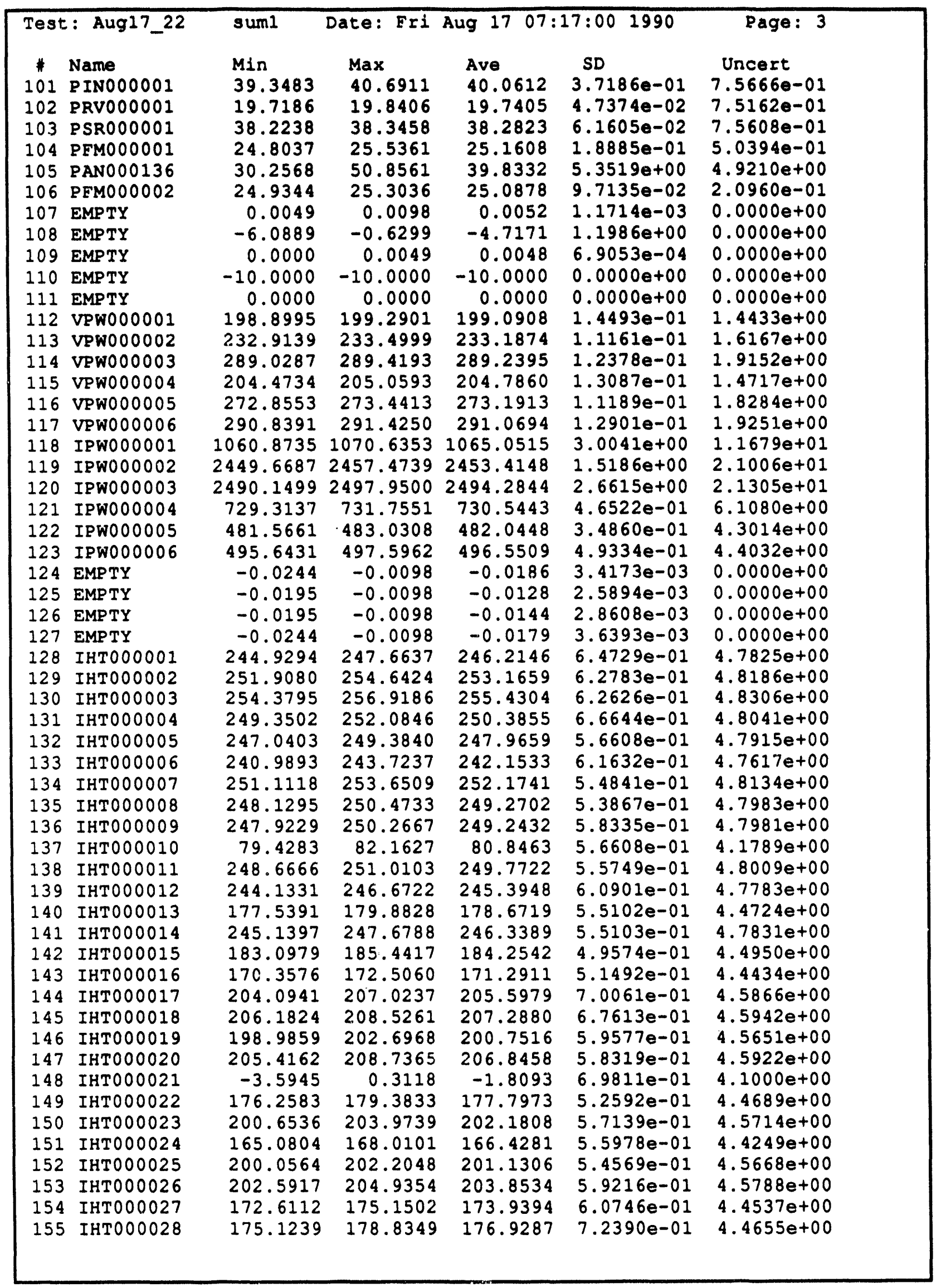




\begin{tabular}{|c|c|c|c|c|c|}
\hline Test: Aug17_22 & sum1 & Date: Fri & Aug 1707 & $17: 001990$ & Page: 4 \\
\hline 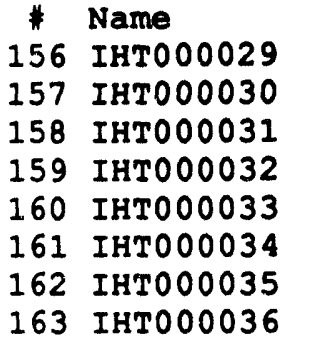 & $\begin{array}{l}\text { Min } \\
201.1043 \\
204.8640 \\
201.5400 \\
204.3983 \\
204.4208 \\
180.0706 \\
237.3949 \\
178.1513\end{array}$ & $\begin{array}{l}\operatorname{Max} \\
204.6199 \\
208.5750 \\
205.4462 \\
208.3045 \\
208.3271 \\
183.0003 \\
240.3245 \\
180.8857\end{array}$ & $\begin{array}{l}\text { Ave } \\
202.9091 \\
206.7470 \\
203.5166 \\
206.1445 \\
206.0928 \\
181.3363 \\
238.7386 \\
179.2607\end{array}$ & $\begin{array}{c}\text { SD } \\
7.4614 \mathrm{e}-01 \\
7.9190 \mathrm{e}-01 \\
7.3466 \mathrm{e}-01 \\
7.4075 \mathrm{e}-01 \\
7.3521 \mathrm{e}-01 \\
6.2669 \mathrm{e}-01 \\
6.4795 \mathrm{e}-01 \\
6.0803 \mathrm{e}-01\end{array}$ & $\begin{array}{c}\text { Uncert } \\
4.5746 e+00 \\
4.5918 \mathrm{e}+00 \\
4.5773 \mathrm{e}+00 \\
4.5891 \mathrm{e}+00 \\
4.5888 \mathrm{e}+00 \\
4.4831 \mathrm{e}+00 \\
4.7444 \mathrm{e}+00 \\
4.4748 \mathrm{e}+00\end{array}$ \\
\hline
\end{tabular}




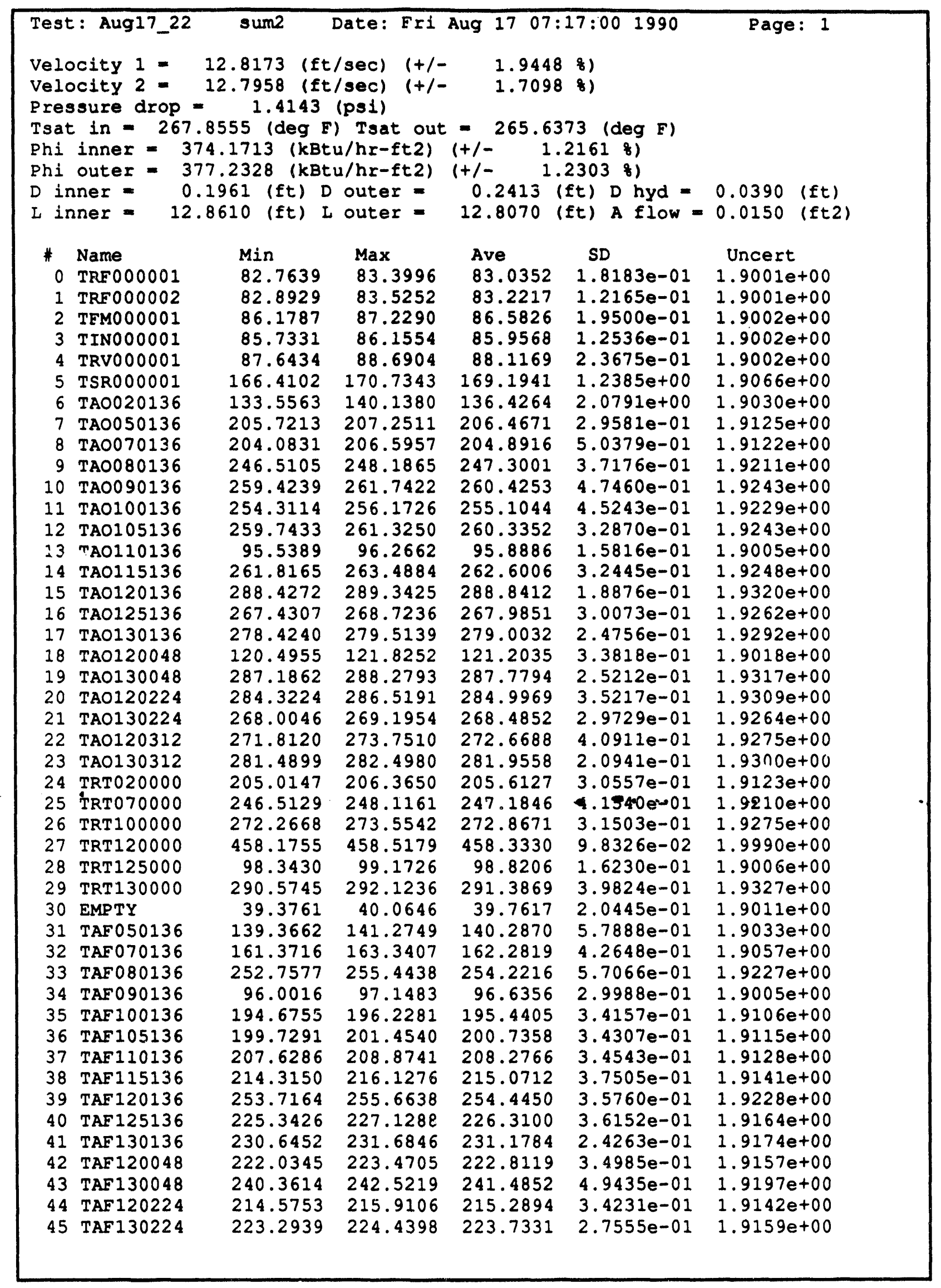




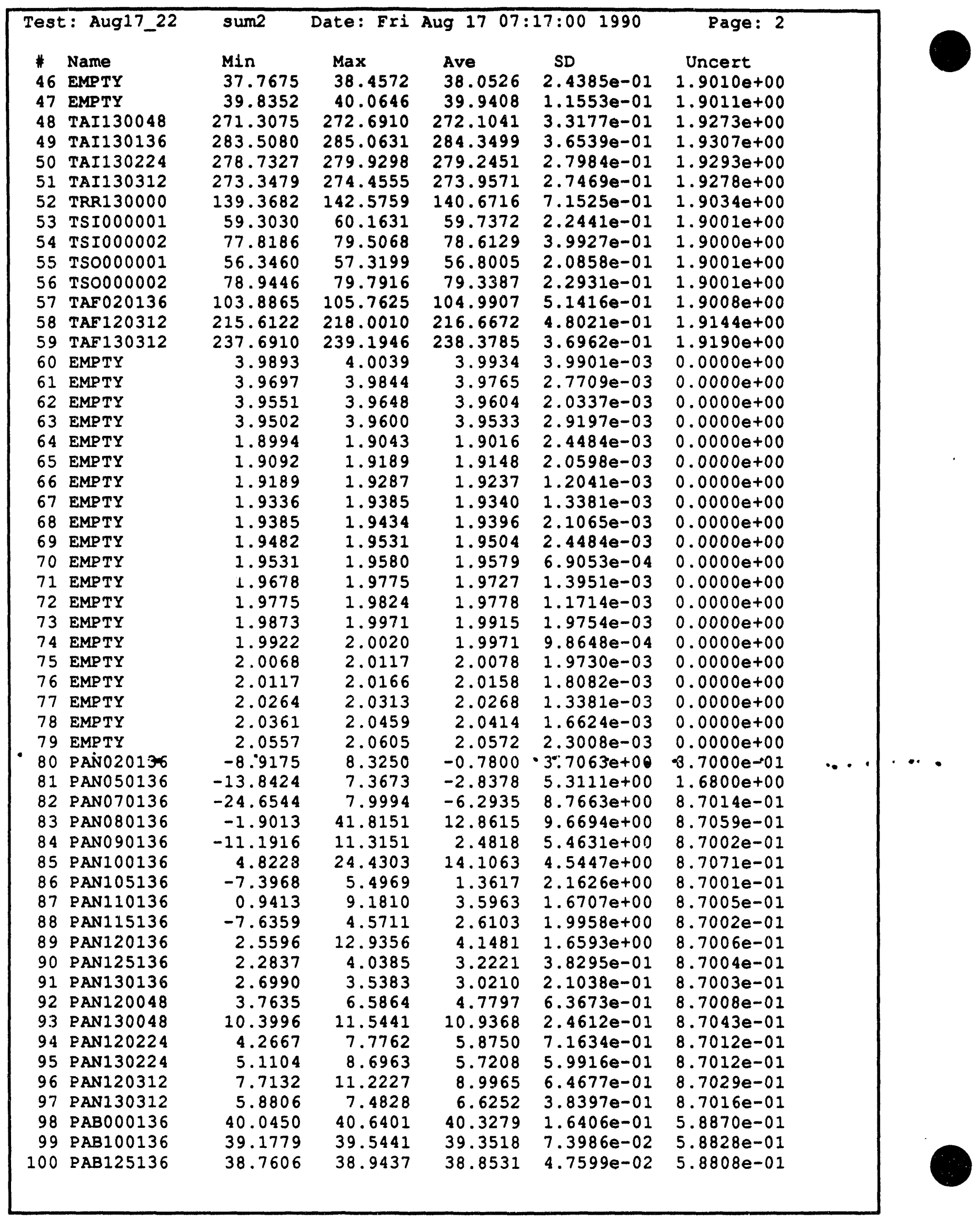




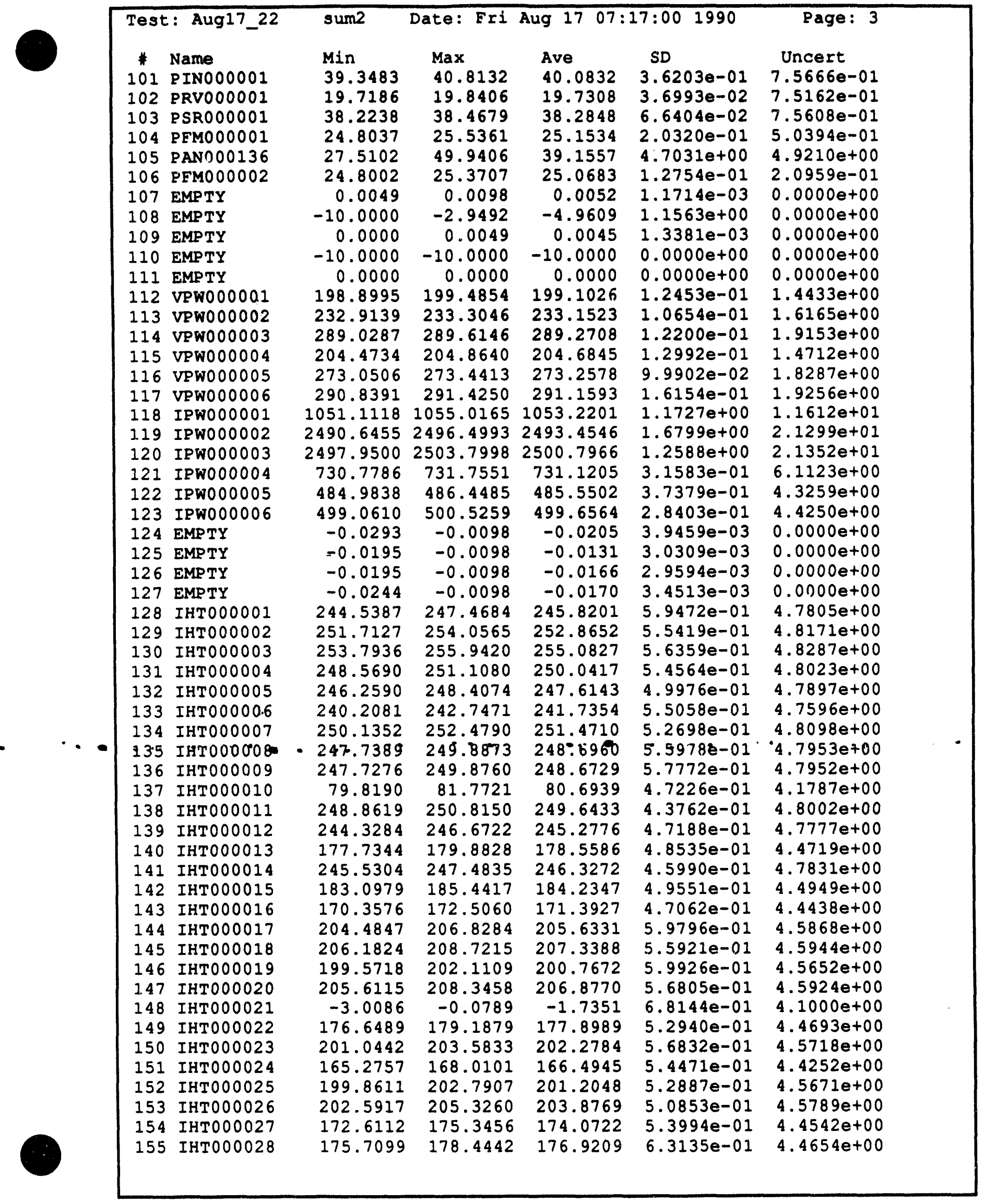




\begin{tabular}{|c|c|c|c|c|c|c|}
\hline \multicolumn{2}{|c|}{ Test: Aug17_22 } & sum2 & Date: Fri & Aug 170 & $1: 001990$ & Page: 4 \\
\hline $\begin{array}{c}* \\
156 \\
157 \\
158 \\
159 \\
160 \\
161 \\
162 \\
163\end{array}$ & $\begin{array}{l}\text { Name } \\
\text { IHTO00029 } \\
\text { IHTO00030 } \\
\text { IHTO00031 } \\
\text { IHTO00032 } \\
\text { IHTO00033 } \\
\text { IHTO00034 } \\
\text { IHTO00035 } \\
\text { IHTO00036 }\end{array}$ & $\begin{array}{l}\text { Min } \\
201.4949 \\
205.4500 \\
202.1259 \\
204.7889 \\
204.8115 \\
180.2659 \\
237.5902 \\
178.1513\end{array}$ & $\begin{array}{l}\operatorname{Max} \\
204.4246 \\
207.9890 \\
204.6650 \\
207.5233 \\
207.3505 \\
182.6097 \\
240.1292 \\
180.2997\end{array}$ & $\begin{array}{l}\text { Ave } \\
202.8036 \\
206.6415 \\
203.5127 \\
206.1562 \\
206.0693 \\
181.2542 \\
238.7073 \\
179.1552\end{array}$ & $\begin{array}{l}\text { SD } \\
7.0061 e-01 \\
6.3901 e-01 \\
6.2795 e-01 \\
6.6731 e-01 \\
6.6399 e-01 \\
5.3147 e-01 \\
5.9194 e-01 \\
5.2646 e-01\end{array}$ & $\begin{array}{c}\text { Uncert } \\
4.5742 e+00 \\
4.5913 e+00 \\
4.5773 e+00 \\
4.5891 e+00 \\
4.5887 e+00 \\
4.4828 e+00 \\
4.7443 e+00 \\
4.4743 e+00\end{array}$ \\
\hline
\end{tabular}




\begin{tabular}{|c|c|c|c|c|c|}
\hline \multicolumn{6}{|c|}{$\begin{array}{l}\text { Velocity } 1=0.001000 \text { (ft/sec) } \\
\text { Velocity } 2=0.004000 \text { (ft/sec) } \\
\text { Pressure Drop }=0.024500 \text { (psi) } \\
\text { Tsat in }=0.054108 \text { (deg F) Tsat out }=0.074188 \text { (deg F) } \\
\text { Ph1 inner }=1.253387 \text { (kBtu/hr-ft2) Ph1 outer }=2.790375 \text { (kBtu/hr-ft2) }\end{array}$} \\
\hline * & Name & Abs $D \perp f f$ & $8 D f f$ & & \\
\hline 0 & TRF000001 & 0.0212 & 0.0255 & & \\
\hline 1 & TRF 000002 & 0.0211 & 0.0253 & & \\
\hline 2 & TFM000001 & 0.8141 & 0.9492 & & \\
\hline 3 & TINO00001 & 0.7587 & 0.8905 & & \\
\hline 4 & TRV000001 & 0.8445 & 9677 & & \\
\hline 5 & TSR000001 & 1.0014 & 0.5954 & & \\
\hline 6 & TAO020136 & 3.4345 & 2.5825 & & \\
\hline 7 & TAO050136 & 0.7411 & 0.3602 & & \\
\hline 8 & TA0070136 & 0.6536 & 0.3200 & & \\
\hline 9 & TAO0880136 & 0.5245 & 0.2125 & & \\
\hline 10 & TAO090136 & 0.7043 & 0.2712 & & \\
\hline 11 & TAO100136 & 0.8373 & 0.3293 & & \\
\hline 12 & TAO105136 & 0.5024 & 0.1934 & & \\
\hline 13 & TA0110136 & 1.3602 & 1.4389 & & \\
\hline 14 & TAO115136 & 0.5384 & 0.2054 & & \\
\hline 15 & TAO120136 & 0.5596 & 0.1941 & & \\
\hline 16 & TAO125136 & 0.0959 & 0.0358 & & \\
\hline 17 & TAO130136 & 0.3981 & 0.1429 & & \\
\hline 18 & TAO120048 & 1.6972 & 1.4202 & & \\
\hline 19 & TAO130048 & 0.8636 & 0.3010 & & \\
\hline 20 & TAO120224 & 0.4320 & 0.1518 & & \\
\hline 21 & TAO130224 & 0.3513 & 0.1310 & & \\
\hline 22 & TA0120312 & 0.3987 & 0.1464 & & \\
\hline 23 & TAO130312 & 0.1975 & 0.0701 & & \\
\hline 24 & TRT020000 & 0.5592 & 6. 2727 & & \\
\hline 25 & TRT070Q00 & .0 .6188$. & 0.2510 & • & \\
\hline 26 & TRT 100000 & 0.5552 & 0.2039 & 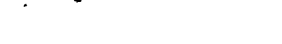 & $\cdot$ \\
\hline 27 & TRT 120000 & 0.0172 & 0.0037 & & \\
\hline 28 & TRT 125000 & 2.6368 & 2.7414 & & \\
\hline 29 & TRT 130000 & 0.4405 & 0.1514 & & \\
\hline 30 & EMPTY & 0.0230 & 0.0578 & & \\
\hline 31 & TAF050136 & 0.6743 & 0.4830 & & \\
\hline 32 & TAF070136 & 0.7332 & 0.4539 & & \\
\hline 33 & TAF080136 & 0.2663 & 0.1049 & & \\
\hline 34 & TAF090136 & 1.8293 & 1.9295 & & \\
\hline 35 & TAF 100136 & 0.7063 & 0.3627 & & \\
\hline 36 & TAF 105136 & 0.7576 & 0.3788 & & \\
\hline 37 & TAF 110136 & 0.6925 & 0.3336 & & \\
\hline 38 & TAF 115136 & 0.7932 & 0.3702 & & \\
\hline 39 & TAF 120136 & 0.2854 & 0.1123 & & \\
\hline 40 & TAF 125136 & 0.8241 & 0.3655 & & \\
\hline 41 & TAF 130136 & 0.8792 & 0.3818 & & \\
\hline 42 & TAF 120048 & 0.8997 & 0.4054 & & \\
\hline 43 & TAF 130048 & 1.5476 & 0.6450 & & \\
\hline 44 & TAF 120224 & 0.7481 & 0.3487 & & \\
\hline 45 & TAF 130224 & 0.6536 & 0.2930 & & \\
\hline
\end{tabular}




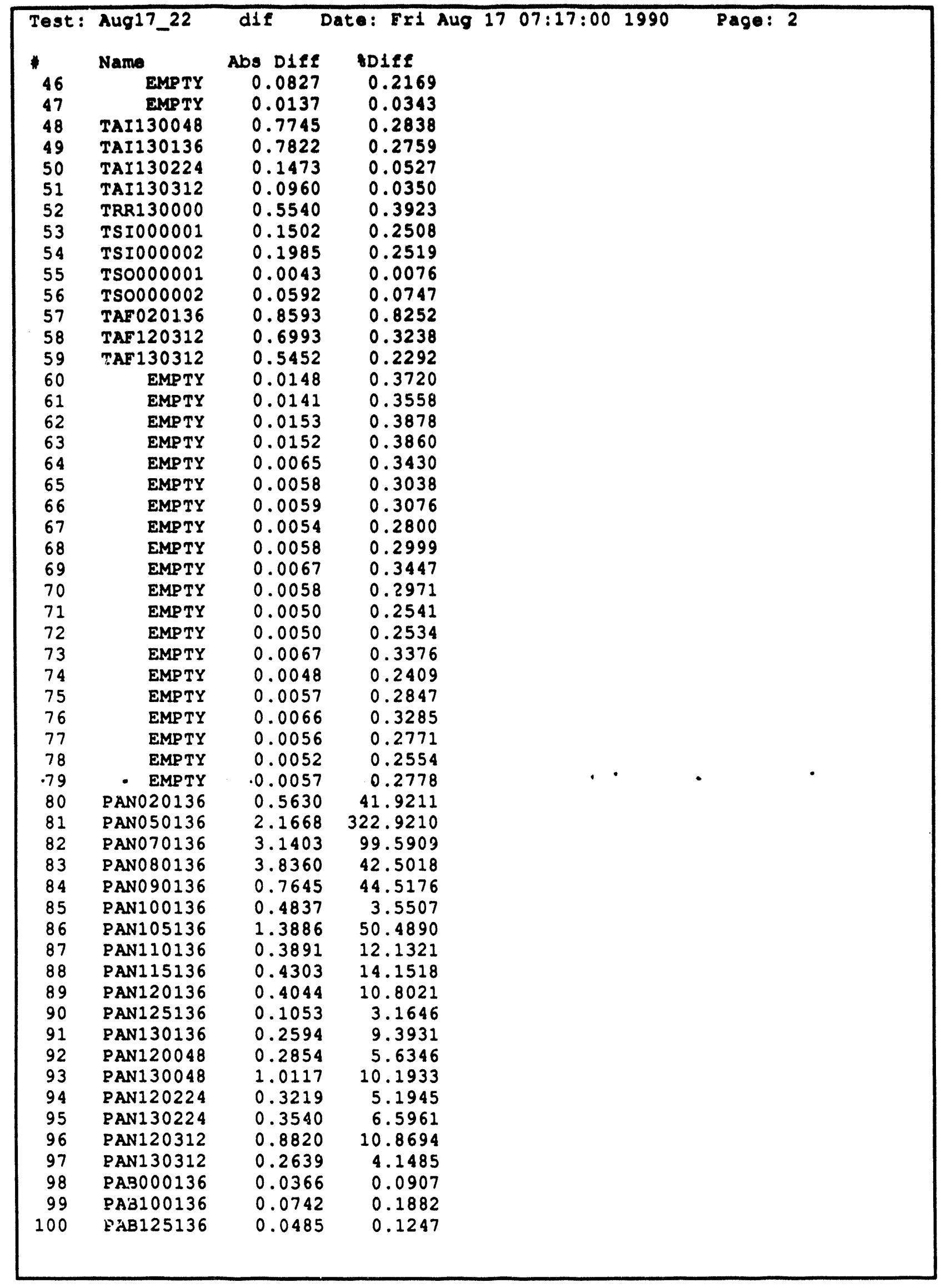




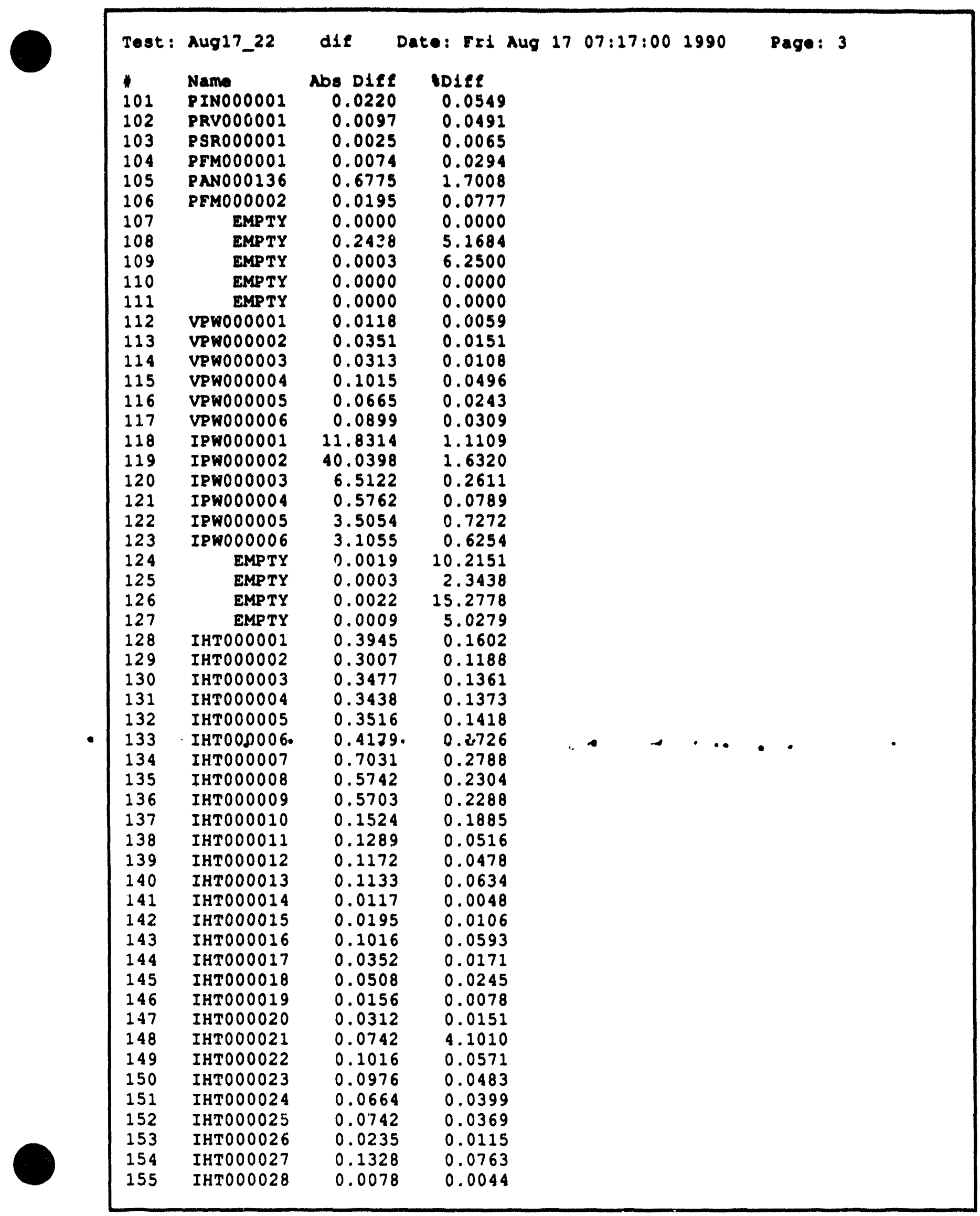




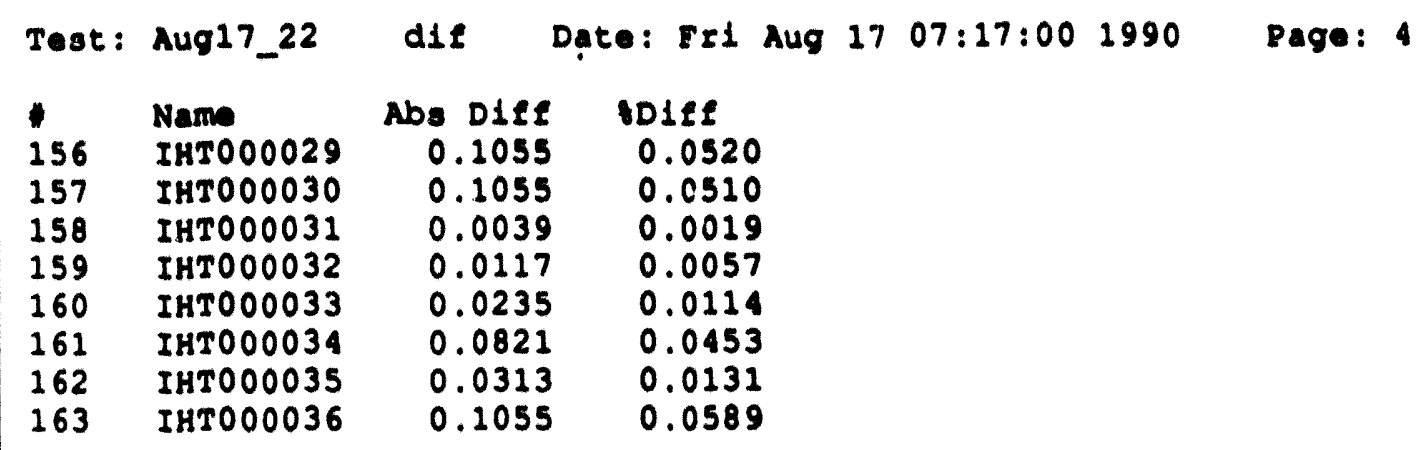




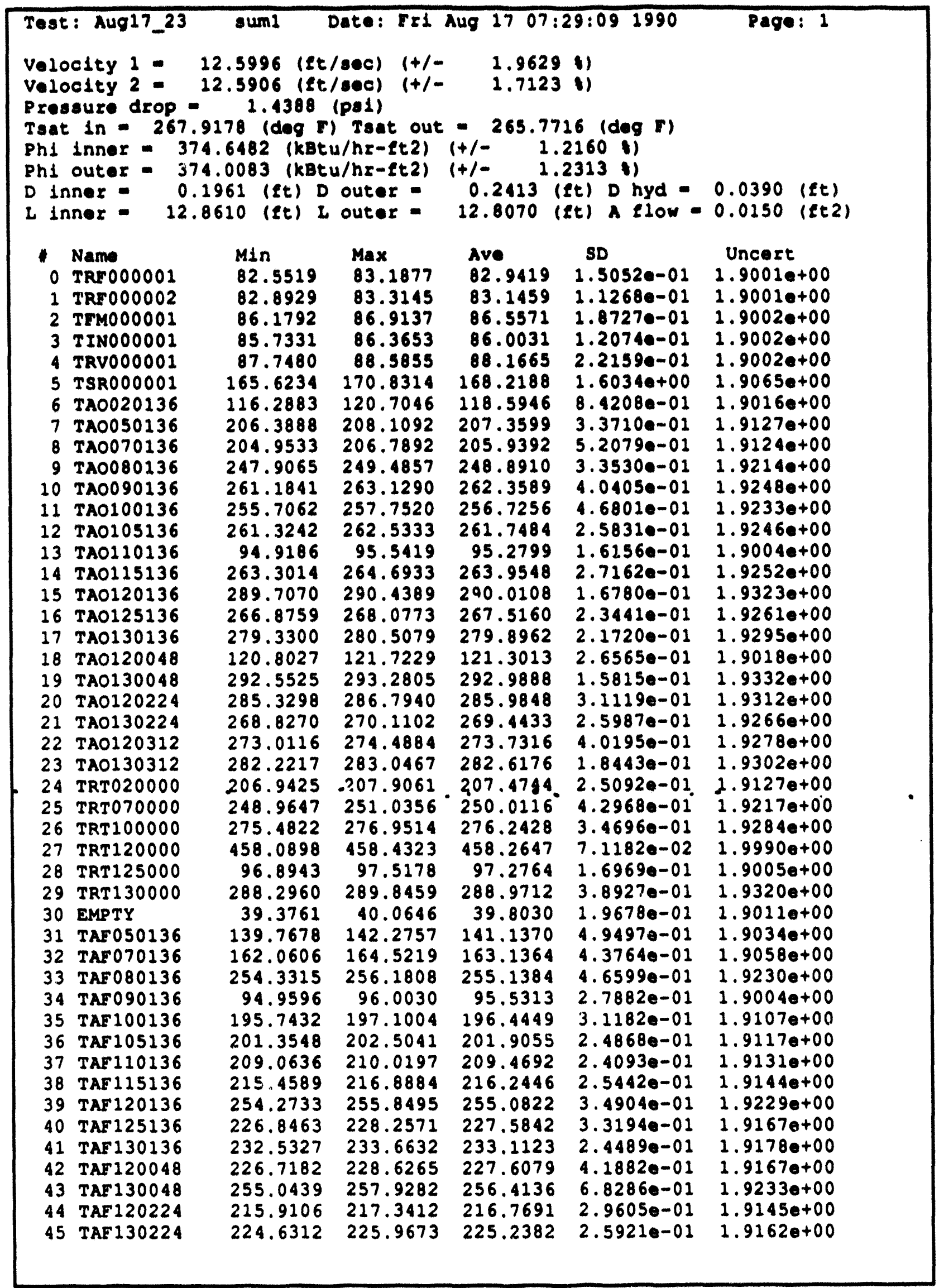




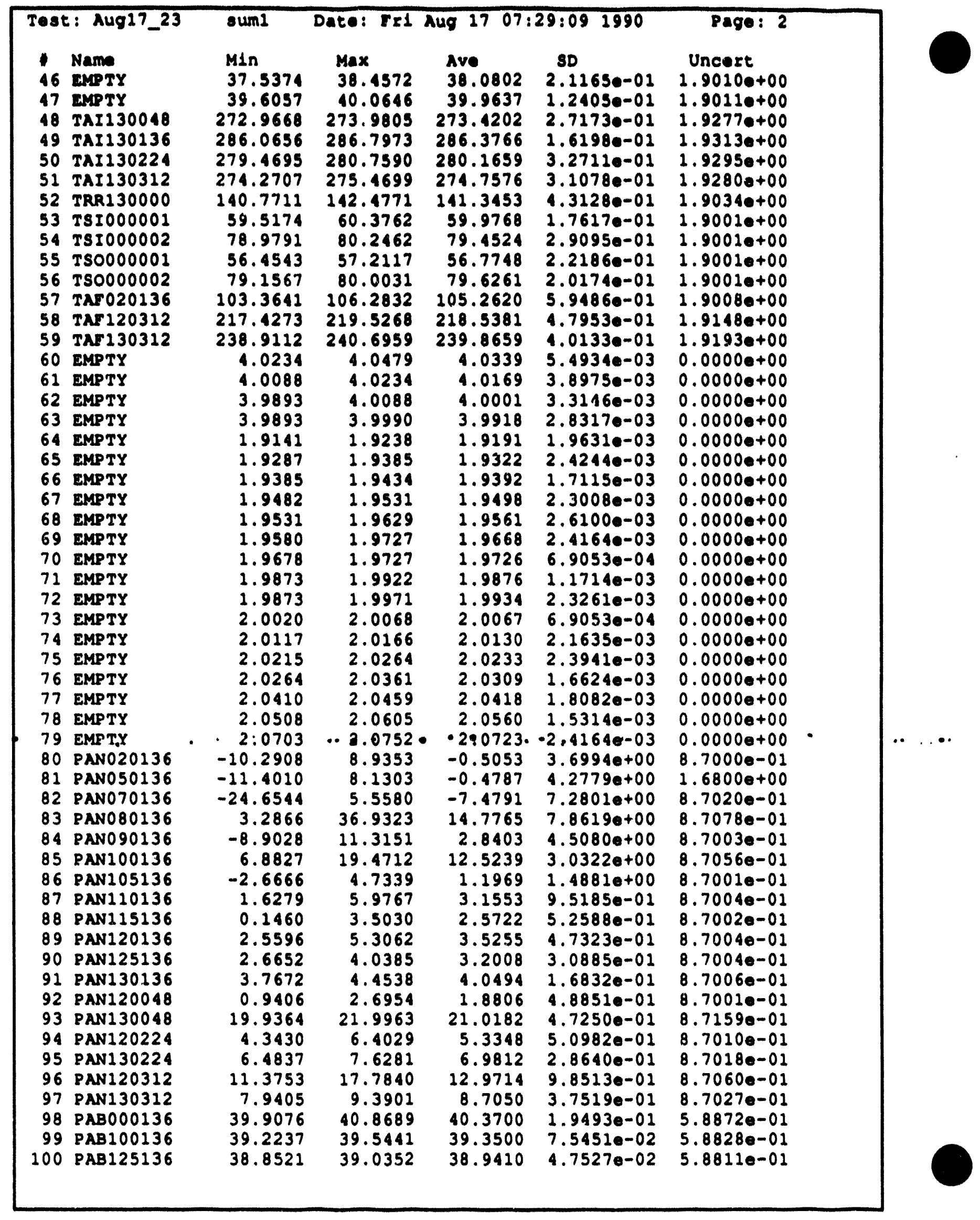




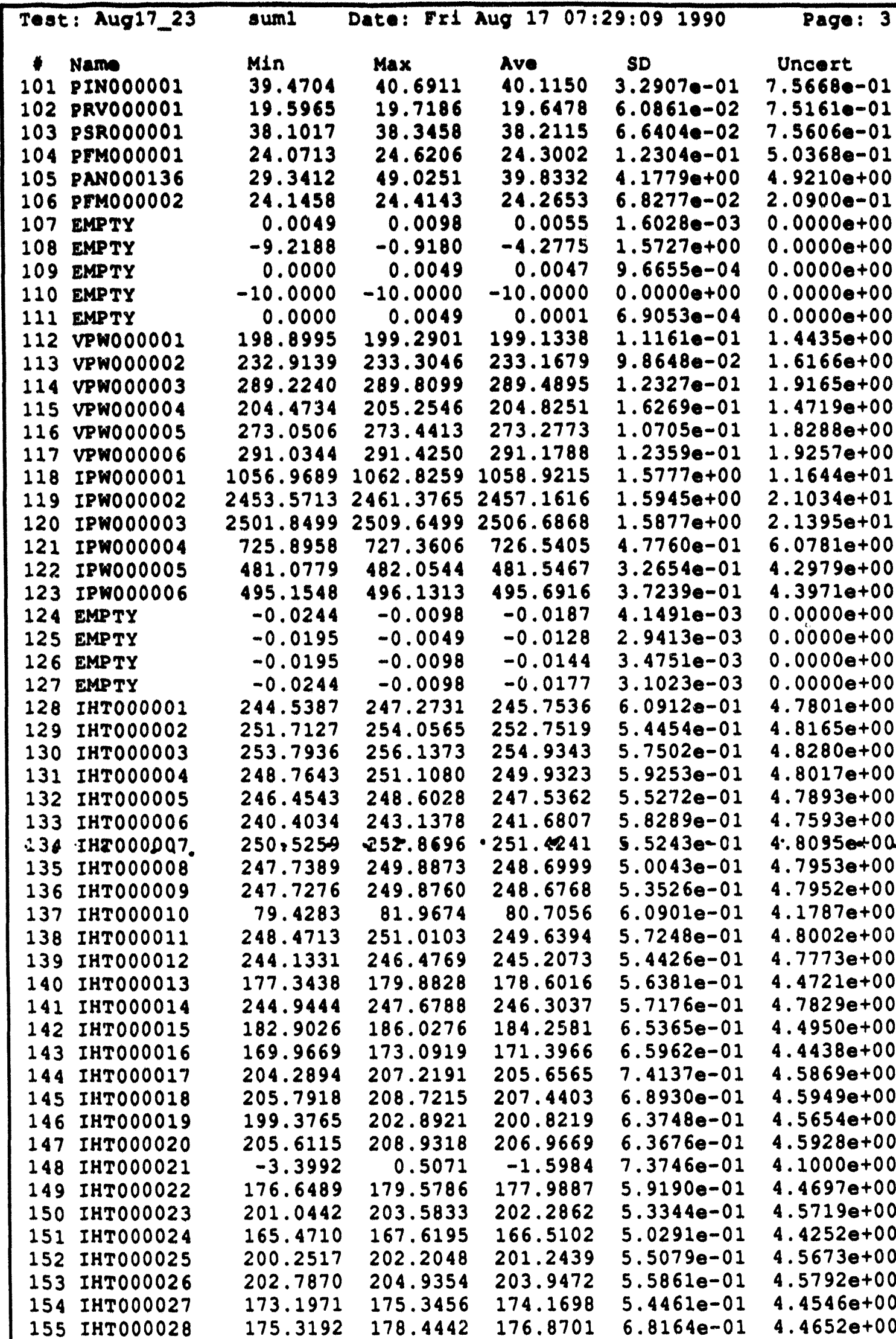




\begin{tabular}{|c|c|c|c|c|c|c|}
\hline Tes & $t:$ Aug17_23 & suml & Date: Fr1 & Aug 1707 & $: 091990$ & Page: 4 \\
\hline $\begin{array}{l}1 \\
156 \\
157 \\
158 \\
159 \\
160 \\
161 \\
162 \\
163\end{array}$ & $\begin{array}{l}\text { Name } \\
\text { IHTO00029 } \\
\text { IHTO00030 } \\
\text { IHTO00031 } \\
\text { IHTO00032 } \\
\text { IHTOOOO33 } \\
\text { IHTOOOO34 } \\
\text { IHTO00035 } \\
\text { IHTO00036 }\end{array}$ & $\begin{array}{l}\text { Min } \\
201.2996 \\
204.8640 \\
201.9306 \\
204.5936 \\
204.4208 \\
180.2659 \\
237.5902 \\
178.1513\end{array}$ & $\begin{array}{l}\text { Max } \\
204.2293 \\
208.1844 \\
205.2509 \\
207.7186 \\
207.5458 \\
182.6097 \\
239.9339 \\
180.4950\end{array}$ & $\begin{array}{l}\text { Ave } \\
202.7880 \\
206.6376 \\
203.4580 \\
206.0624 \\
205.9873 \\
181.2894 \\
238.7464 \\
179.2334\end{array}$ & $\begin{array}{l}\text { SD } \\
6.0988 e-01 \\
6.3361 e-01 \\
6.3343 e-01 \\
6.1667 e-01 \\
6.0166 e-01 \\
4.8417 e-01 \\
5.2473 e-01 \\
5.2528 \theta-01\end{array}$ & $\begin{array}{c}\text { Uncert } \\
4.5741 e+00 \\
4.5913 e+00 \\
4.5771 e+00 \\
4.5887 e+00 \\
4.5884 e+00 \\
4.4829 e+00 \\
4.7445 \theta+00 \\
4.4746 e+00\end{array}$ \\
\hline
\end{tabular}




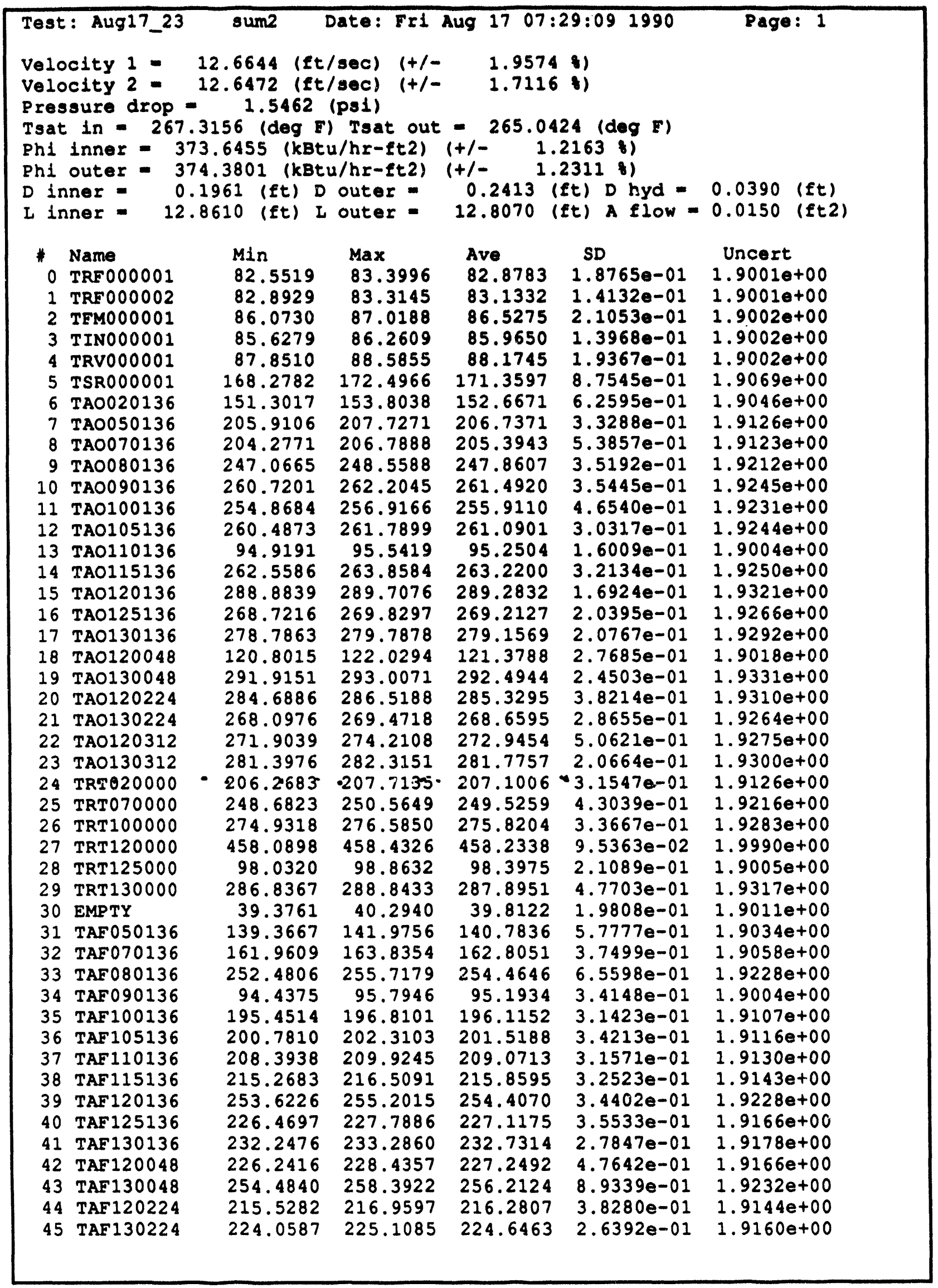




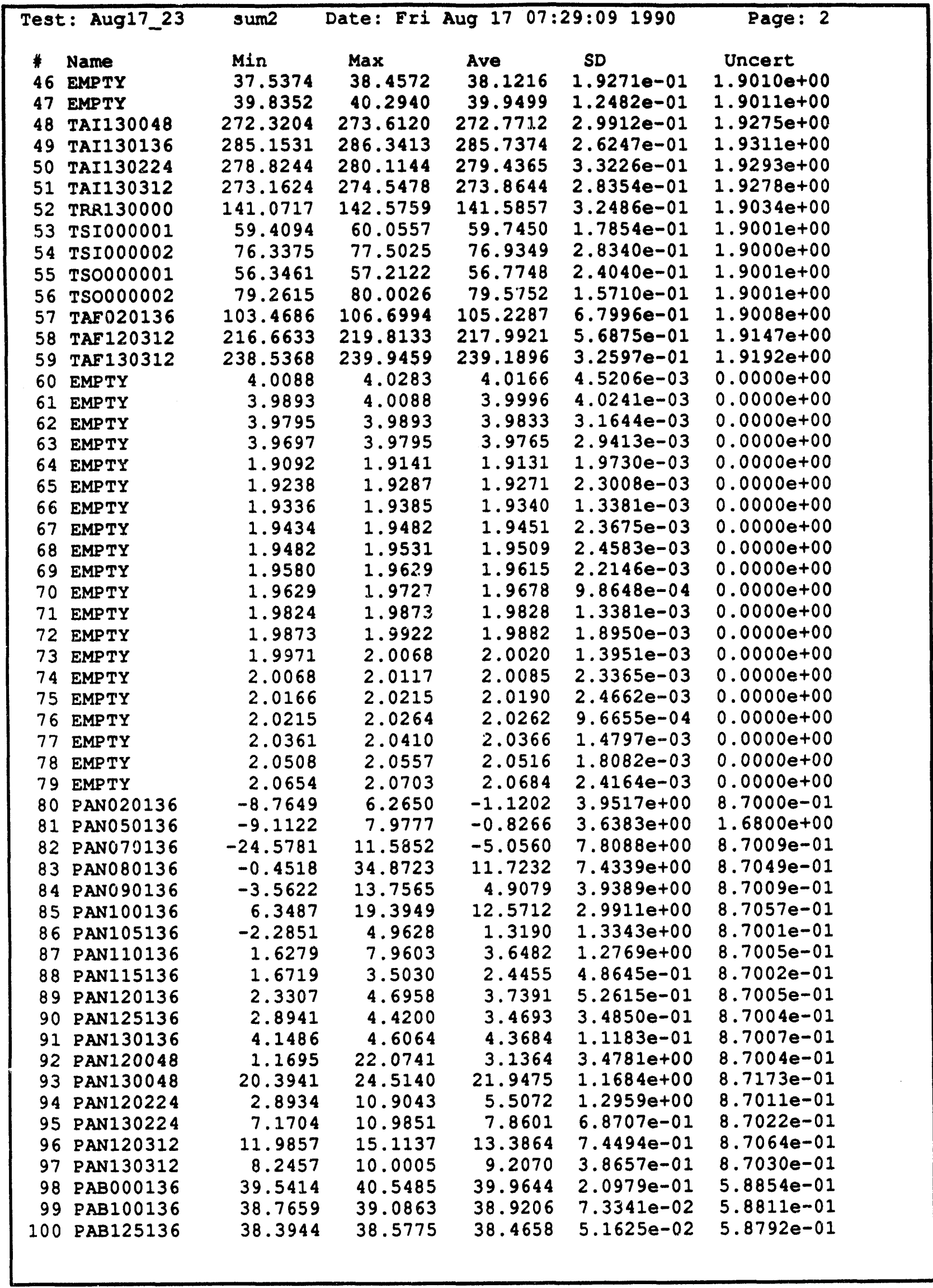




\begin{tabular}{|c|c|c|c|c|c|c|}
\hline \multicolumn{2}{|c|}{ Test: Aug17_23 } & sum2 & Date: Fri & Aug 170 & $9: 091990$ & Page: \\
\hline$*$ & Name & Min & $\operatorname{Max}$ & Ave & SD & Uncert \\
\hline 101 & PINO00001 & 38.8601 & 40.5690 & 39.6364 & $4.0879 e-01$ & $7.5652 e-01$ \\
\hline 102 & PRV000001 & 19.7186 & 19.8406 & 19.8333 & $2.9284 e-02$ & $7.5164 e-01$ \\
\hline 103 & PSR000001 & 37.6134 & 37.8575 & 37.7379 & $3.8916 \mathrm{e}-02$ & $7.5591 e-01$ \\
\hline 104 & PEMO000001 & 24.1629 & 24.9868 & 24.5529 & $2.1635 e-01$ & $5.0375 e-01$ \\
\hline 105 & PANO00136 & 33.0034 & 54.9760 & 42.8087 & $4.8287 e+00$ & $4.9212 e+00$ \\
\hline 106 & PFM000002 & 24.2800 & 24.7834 & 24.4857 & $1.3774 e-01$ & $2.0916 e-01$ \\
\hline 107 & EMPTY & 0.0049 & 0.0098 & 0.0050 & $6.9053 e-04$ & $0.0000 e+00$ \\
\hline 108 & EMPTY & -10.0000 & -0.3516 & -5.3314 & $2.9847 e+00$ & $0.0000 e+00$ \\
\hline 109 & EMPTY & 0.0049 & 0.0049 & 0.0049 & $0.0000 e+00$ & $0.0000 \mathrm{e}+00$ \\
\hline 110 & EMPTY & -10.0000 & -10.0000 & -10.0000 & $0.0000 e+00$ & $0.0000 e+00$ \\
\hline 111 & EMPTY & -0.0049 & 0.0000 & -0.0001 & $6.9053 e-04$ & $0.0000 e+00$ \\
\hline 112 & VPW000001 & 198.8995 & 199.2901 & 199.1104 & $1.1732 e-01$ & $1.4434 e+00$ \\
\hline 113 & VPW000002 & 233.1092 & 233.6952 & 233.2773 & $1.1844 e-01$ & $1.6171 \mathrm{e}+00$ \\
\hline 114 & VPW000003 & 289.2240 & 289.6146 & 289.4192 & $1.3669 e-01$ & $1.9161 e+00$ \\
\hline 115 & VPW000004 & 204.6687 & 205.0593 & 204.8173 & $1.0106 e-01$ & $1.4718 \mathrm{e}+00$ \\
\hline 116 & VPW000005 & 273.0506 & 273.4413 & 273.2421 & $1.0799 e-01$ & $1.8286 e+00$ \\
\hline 117 & VPW000006 & 291.0344 & 291.4250 & 291.1749 & $1.0470 e-01$ & $1.9257 e+00$ \\
\hline 118 & IPW000001 & 1062.8259 & 1068.6829 & 1065.0121 & $1.2246 e+00$ & $1.1679 e+01$ \\
\hline 119 & IPW000002 & 2453.5713 & 2457.4739 & 2455.4446 & $1.3633 e+00$ & $2.1021 e+01$ \\
\hline 120 & IPW000003 & 2495.9998 & 2501.8499 & 2499.1980 & $1.2308 e+00$ & $2.1341 e+01$ \\
\hline 121 & IPW000004 & 725.8958 & 727.3606 & 726.6870 & $3.5417 e-01$ & $6.0792 e+00$ \\
\hline 122 & IPW000005 & 480.5897 & 482.0544 & 481.5272 & $3.7997 e-01$ & $4.2978 e+00$ \\
\hline 123 & IPW0000006 & 495.1548 & 496.6196 & 495.7502 & $3.4583 e-01$ & $4.3975 e+00$ \\
\hline 124 & EMPTY & -0.0244 & -0.0098 & -0.0198 & $4.3461 e-03$ & $0.0000 e+00$ \\
\hline $1 ? 5$ & EMPTY & -0.0195 & -0.0049 & -0.0132 & $3.0003 e-03$ & $0.0000 e+00$ \\
\hline 126 & EMPTY & -0.0195 & -0.0098 & -0.0153 & $3.2733 e-03$ & $0.0000 e+00$ \\
\hline 127 & EMPTY & -0.0244 & -0.0098 & -0.0174 & $3.4400 e-03$ & $0.0000 e+00$ \\
\hline 128 & IHTO00001 & 244.7341 & 247.0778 & 246.0388 & $5.8983 e-01$ & $4.7816 e+00$ \\
\hline 129 & IHTO000002 & 251.9080 & 254.0565 & 252.9980 & $5.2953 e-01$ & $4.8178 e+00$ \\
\hline 130 & IHTO000003 & 254.1842 & 256.3326 & 255.2819 & $5.3217 e-01$ & $4.8298 e+00$ \\
\hline 131 & IHT000004 & 249.3502 & 251.3033 & 250.2722 & $5.6640 e-01$ & $4.8035 e+00$ \\
\hline 132 & IHTO00005 & 246.8449 & 248.9934 & 247.7979 & $5.0012 e-01$ & $4.7907 e+00$ \\
\hline 133 & IHT 000006 & 240.7940 & 243.1378 & 241.8955 & $5.3198 e-01$ & $4.7604 e+00$ \\
\hline 134 & IHT000007 & 250.7212 & 252.8696 & 251.8265 & $5.4621 e-01$ & $4.8116 e+00$ \\
\hline 135 & IHT000008 & 248.1295 & 249.8873 & 248.9069 & $4.8083 e-01$ & $4.7964 e+00$ \\
\hline 136 & IHT000009 & 247.9229 & 249.8760 & 248.9229 & $4.9227 e-01$ & $4.7965 e+00$ \\
\hline 137 & IHT000010 & 79.0377 & 81.9674 & 80.3345 & $6.3596 e-01$ & $4.1780 e+00$ \\
\hline 138 & IHT000011 & 248.0807 & 250.8150 & 249.4089 & $5.9712 e-01$ & $4.7990 e+00$ \\
\hline 139 & IHT000012 & 243.5472 & 246.4769 & 245.0628 & $6.3694 \mathrm{e}-01$ & $4.7766 e+00$ \\
\hline 140 & IHT000013 & 176.7578 & 179.8828 & 178.4414 & $6.6602 e-01$ & $4.4715 e+00$ \\
\hline 141 & IHTO00014 & 244.5538 & 247.2882 & 246.1709 & $6.4602 e-01$ & $4.7823 e+00$ \\
\hline 142 & IHTO00015 & 182.7073 & 185.4417 & 184.0824 & $5.8522 e-01$ & $4.4943 e+00$ \\
\hline 143 & IHT000016 & 169.7716 & 172.5060 & 171.2364 & $5.7892 e-01$ & $4.4432 e+00$ \\
\hline 144 & IHTO00017 & 204.2894 & 206.8284 & 205.4261 & $5.9541 e-01$ & $4.5858 e+00$ \\
\hline 145 & IHT000018 & 206.1824 & 208.7215 & 207.3466 & $5.9577 e-01$ & $4.5945 e+00$ \\
\hline 146 & IHTO00019 & 199.7671 & 202.3062 & 200.8922 & $5.5462 e-01$ & $4.5657 e+00$ \\
\hline 147 & IHTO00020 & 206.0021 & 207.9552 & 207.0255 & $5.3461 e-01$ & $4.5930 e+00$ \\
\hline 148 & IHTO00021 & -3.2039 & -0.6648 & -1.6062 & $6.4197 e-01$ & $4.1000 e+00$ \\
\hline 149 & IHT000022 & 176.8442 & 179.1879 & 178.1293 & $5.2806 e-01$ & $4.4702 e+00$ \\
\hline 150 & IHTO00023 & 201.0442 & 203.3879 & 202.4268 & $5.8373 e-01$ & $4.5725 e+00$ \\
\hline 151 & IHT000024 & 165.2757 & 167.8148 & 166.6391 & $5.3305 e-01$ & $4.4257 e+00$ \\
\hline 152 & IHTO00025 & 200.0564 & 202.7907 & 201.3181 & $5.4277 e-01$ & $4.5676 e+00$ \\
\hline 153 & IHT000026 & 202.5917 & 205.1307 & 204.0214 & $5.6832 e-01$ & $4.5796 e+00$ \\
\hline 154 & IHT000027 & 172.8065 & 175.5409 & 174.1737 & $5.8793 e-01$ & $4.4546 e+00$ \\
\hline 155 & IHT000028 & 175.1239 & 178.6396 & 176.7841 & $7.1925 e-01$ & $4.4649 e+00$ \\
\hline
\end{tabular}




\begin{tabular}{|c|c|c|c|c|c|c|}
\hline $\begin{array}{c}* \\
156 \\
157 \\
158 \\
159 \\
160 \\
161 \\
162 \\
163\end{array}$ & $\begin{array}{l}\text { Name } \\
\text { IHTO00029 } \\
\text { IHTO00030 } \\
\text { IHTO0O031 } \\
\text { IHTO00032 } \\
\text { IHTO00033 } \\
\text { IHTO0O034 } \\
\text { IHTO00035 } \\
\text { IHTO00036 }\end{array}$ & $\begin{array}{l}\text { Min } \\
201.1043 \\
205.0594 \\
201.9306 \\
204.3983 \\
204.2255 \\
179.8753 \\
237.1995 \\
177.7607\end{array}$ & $\begin{array}{l}\text { Max } \\
204.6199 \\
208.3797 \\
205.0556 \\
207.9139 \\
207.7411 \\
182.6097 \\
240.3245 \\
180.4950\end{array}$ & $\begin{array}{l}\text { Ave } \\
202.7255 \\
206.5595 \\
203.4033 \\
206.0624 \\
205.9951 \\
181.2699 \\
238.7073 \\
179.1435\end{array}$ & $\begin{array}{l}\text { SD } \\
7.4686 \mathrm{e}-01 \\
7.6355 \mathrm{e}-01 \\
7.3420 \mathrm{e}-01 \\
7.9727 \mathrm{e}-01 \\
7.7966 \mathrm{e}-01 \\
5.8262 \mathrm{e}-01 \\
6.0880 \mathrm{e}-01 \\
5.4796 \mathrm{e}-01\end{array}$ & $\begin{array}{c}\text { Uncert } \\
4.5738 e+00 \\
4.5909 e+00 \\
4.5768 e+00 \\
4.5887 e+00 \\
4.5884 e+00 \\
4.4828 e+00 \\
4.7443 e+00 \\
4.4743 e+00\end{array}$ \\
\hline
\end{tabular}




\begin{tabular}{|c|c|c|c|c|}
\hline \multicolumn{5}{|c|}{$\begin{array}{l}\text { Velocity } 1=0.064800 \text { (ft/sec) } \\
\text { Velocity } 2=0.056600 \text { (ft/sec) } \\
\text { Pressure Drop }=0.107400 \text { (psi) } \\
\text { Tsat in }=0.602173 \text { (deg F) Tsat out }=0.729218 \text { (deg E) } \\
\text { Phi inner }=1.002686 \text { (kBtu/hr-ft2) Phi outer }=0.371796 \text { (kBtu/hr-ft2) }\end{array}$} \\
\hline $\begin{array}{r}0 \\
0 \\
1 \\
2 \\
3 \\
4 \\
5 \\
6 \\
7 \\
8 \\
9 \\
10 \\
11 \\
12 \\
13 \\
14 \\
15 \\
16 \\
17 \\
18 \\
19 \\
20 \\
21 \\
22 \\
23 \\
24 \\
25 \\
26 \\
27 \\
28 \\
29 \\
30 \\
31 \\
32 \\
33 \\
34 \\
35 \\
36 \\
37 \\
38 \\
39 \\
40 \\
41 \\
42 \\
43 \\
44 \\
45\end{array}$ & $\begin{array}{l}\text { Name } \\
\text { TRF000001 } \\
\text { TRF000002 } \\
\text { TFM000001 } \\
\text { TIN000001 } \\
\text { TRV000001 } \\
\text { TSR000001 } \\
\text { TAO020136 } \\
\text { TAO0501136 } \\
\text { TAO070136 } \\
\text { TAO080136 } \\
\text { TAO090136 } \\
\text { TAO100136 } \\
\text { TAO105136 } \\
\text { TAO110136 } \\
\text { TAO115136 } \\
\text { TAO120136 } \\
\text { TAO125136 } \\
\text { TAO130136 } \\
\text { TAO120048 } \\
\text { TAO130048 } \\
\text { TAO120224 } \\
\text { TAO130224 } \\
\text { TAO120312 } \\
\text { TAO130312 } \\
\text { TRT020000 } \\
\text { TRT070000 } \\
\text { TRT100000 } \\
\text { TRT120000 } \\
\text { TRT125000 } \\
\text { TRT130000 } \\
\text { EMPTY } \\
\text { TAF050136 } \\
\text { TAF070136 } \\
\text { TAF080136 } \\
\text { TAF090136 } \\
\text { TAF100136 } \\
\text { TAF105136 } \\
\text { TAF110136 } \\
\text { TAF115136 } \\
\text { TAF120136 } \\
\text { TAF125136 } \\
\text { TAF130136 } \\
\text { TAF120048 } \\
\text { TAF130048 } \\
\text { TAF120224 } \\
\text { TAF130224 }\end{array}$ & $\begin{array}{r}\text { Abs Diff } \\
0.0636 \\
0.0127 \\
0.0296 \\
0.0381 \\
0.0080 \\
3.1409 \\
34.0725 \\
0.6228 \\
0.5449 \\
1.0303 \\
0.8669 \\
0.8146 \\
0.6583 \\
0.0295 \\
0.7348 \\
0.7276 \\
1.6967 \\
0.7393 \\
0.0775 \\
0.4944 \\
0.6553 \\
0.7838 \\
0.7862 \\
0.8419 \\
0.3738 \\
0.4857 \\
0.4224 \\
0.0309 \\
1.1211 \\
1.0761 \\
0.0092 \\
0.3534 \\
0.3313 \\
0.6738 \\
0.3379 \\
0.3297 \\
0.3867 \\
0.3979 \\
0.3851 \\
0.6752 \\
0.4667 \\
0.3809 \\
0.3587 \\
0.2012 \\
0.4884 \\
0.5919\end{array}$ & $\begin{array}{l}\text { 8Diff } \\
0.0767 \\
0.0153 \\
0.0342 \\
0.0443 \\
0.0091 \\
1.8672 \\
28.7302 \\
0.3003 \\
0.2646 \\
0.4140 \\
0.3304 \\
0.3173 \\
0.2515 \\
0.0310 \\
0.2784 \\
0.2509 \\
0.6342 \\
0.2641 \\
0.0639 \\
0.1687 \\
0.2291 \\
0.2909 \\
0.2872 \\
0.2979 \\
0.1802 \\
0.1943 \\
0.1529 \\
0.0067 \\
1.1525 \\
0.3724 \\
0.0231 \\
0.2504 \\
0.2031 \\
0.2641 \\
0.3537 \\
0.1678 \\
0.1915 \\
0.1900 \\
0.1781 \\
0.2647 \\
0.2051 \\
0.1634 \\
0.1576 \\
0.0785 \\
0.2253 \\
0.2628\end{array}$ & \\
\hline
\end{tabular}




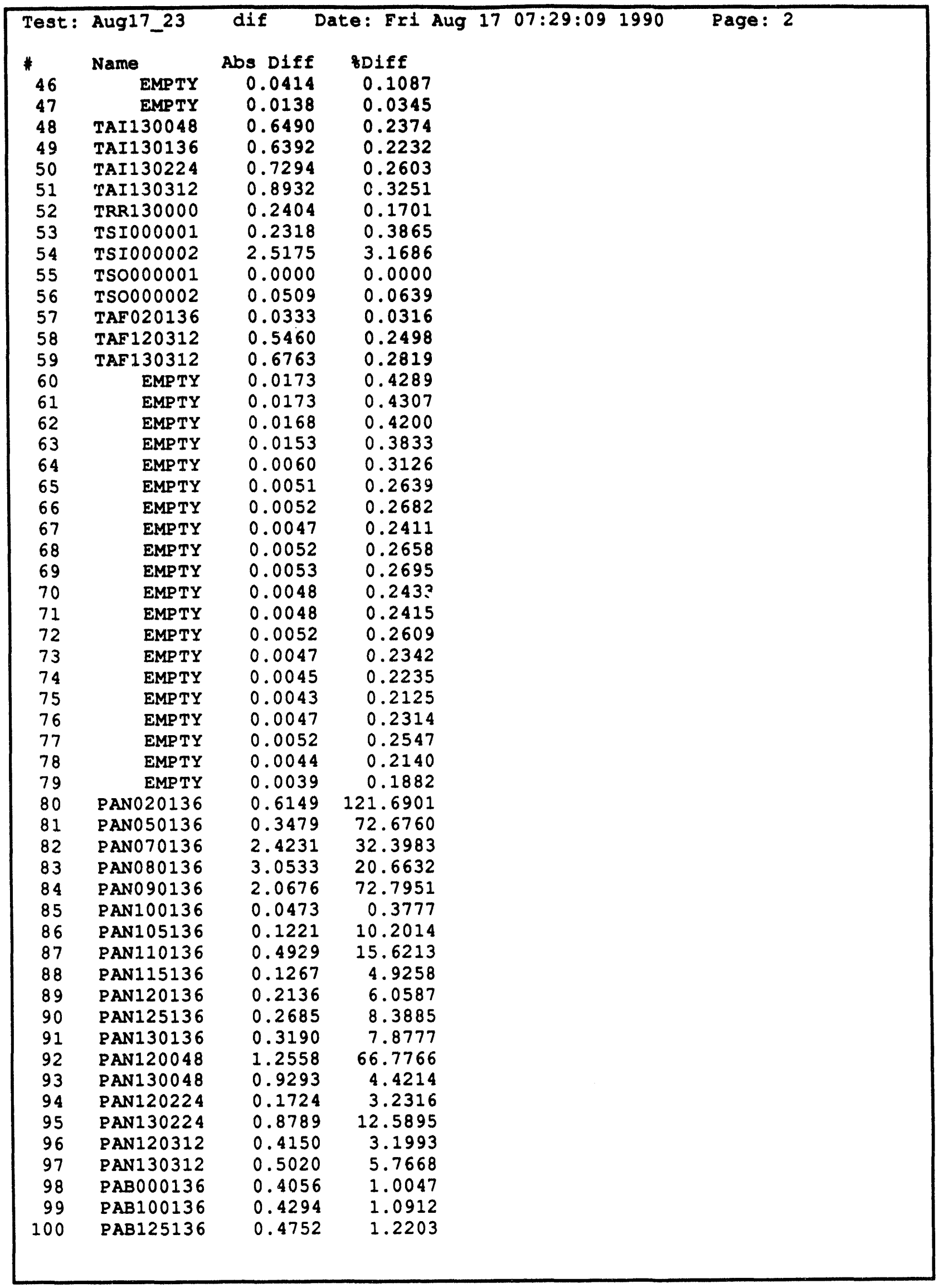




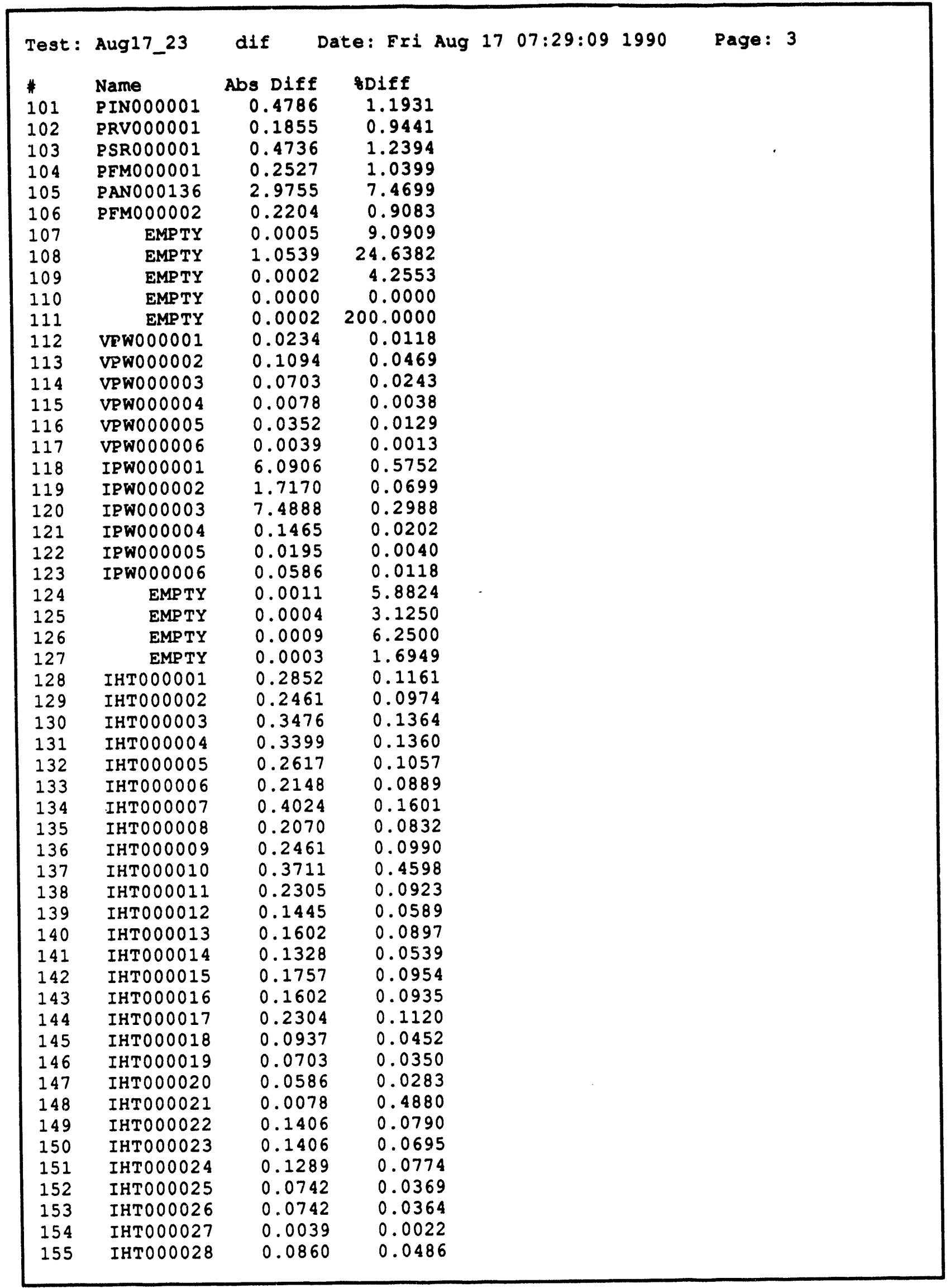




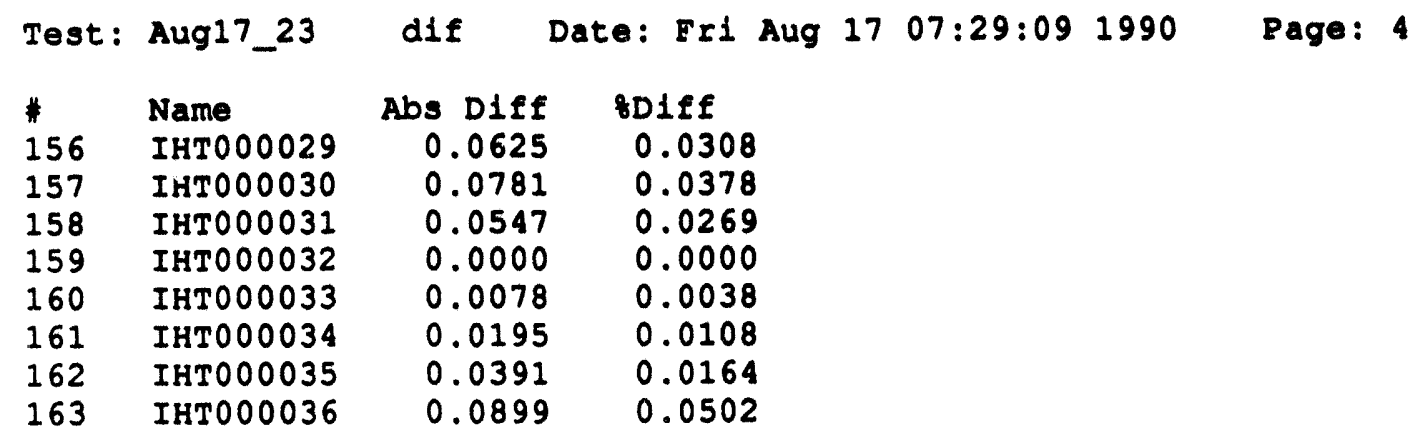




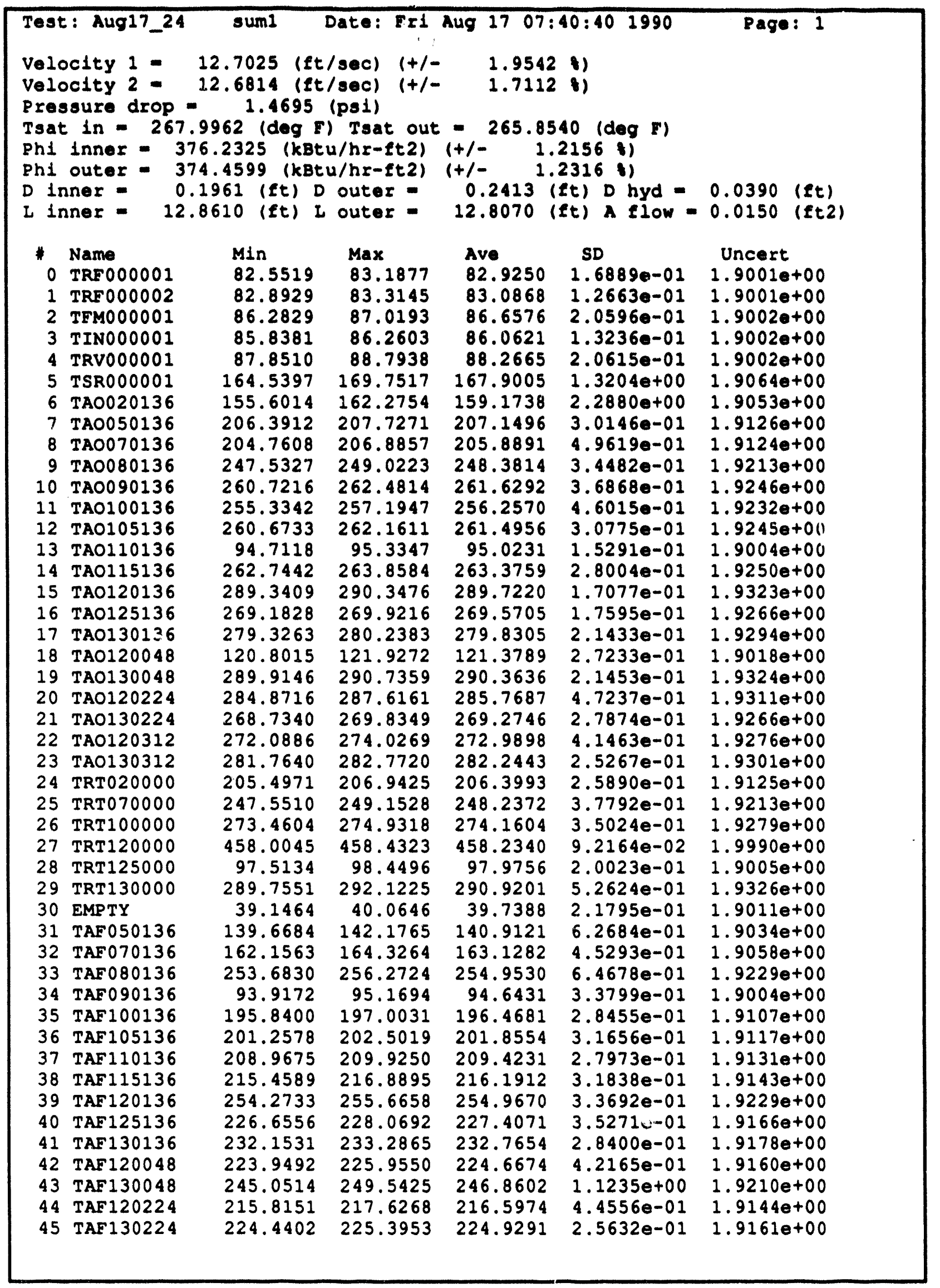




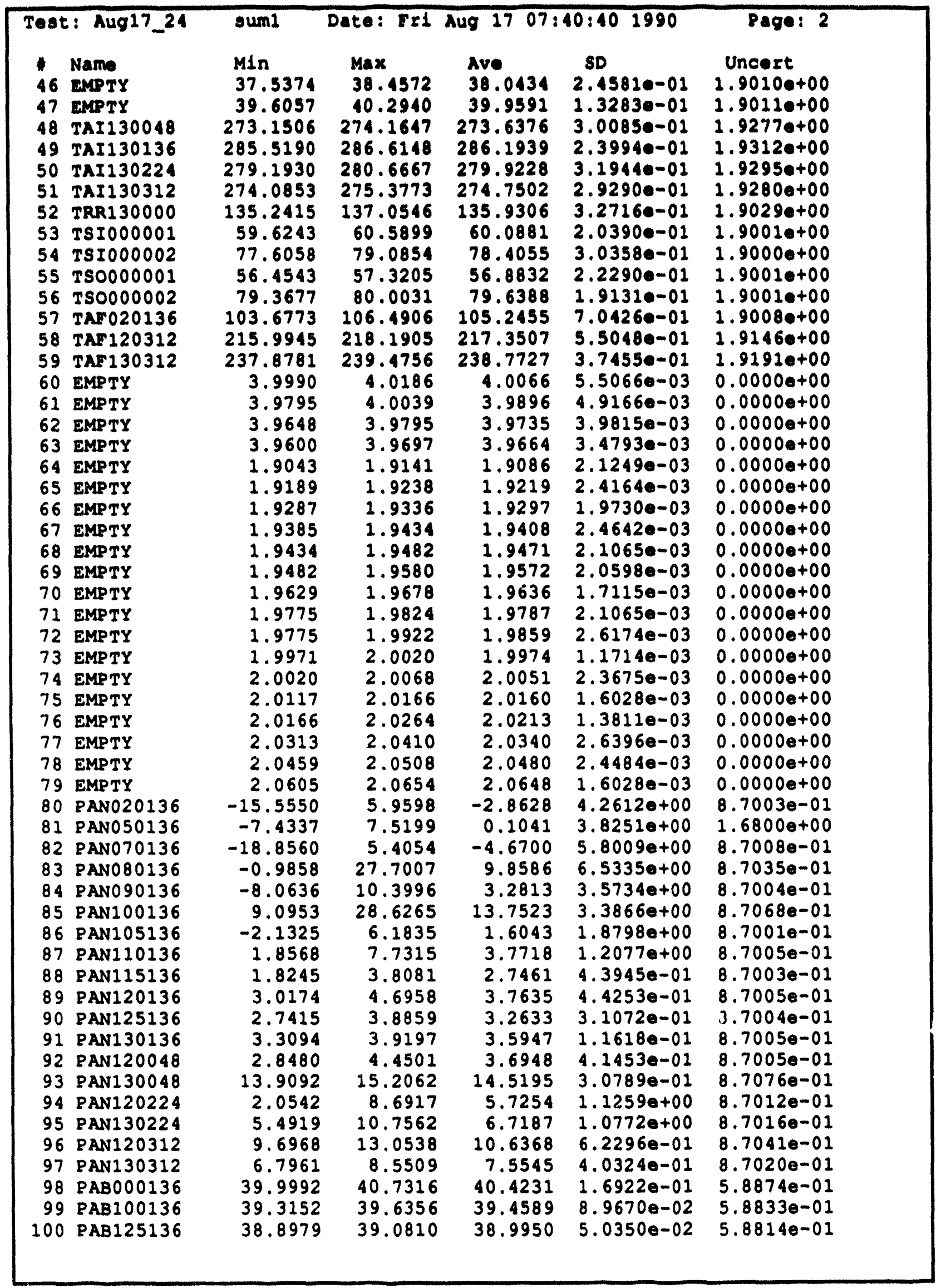




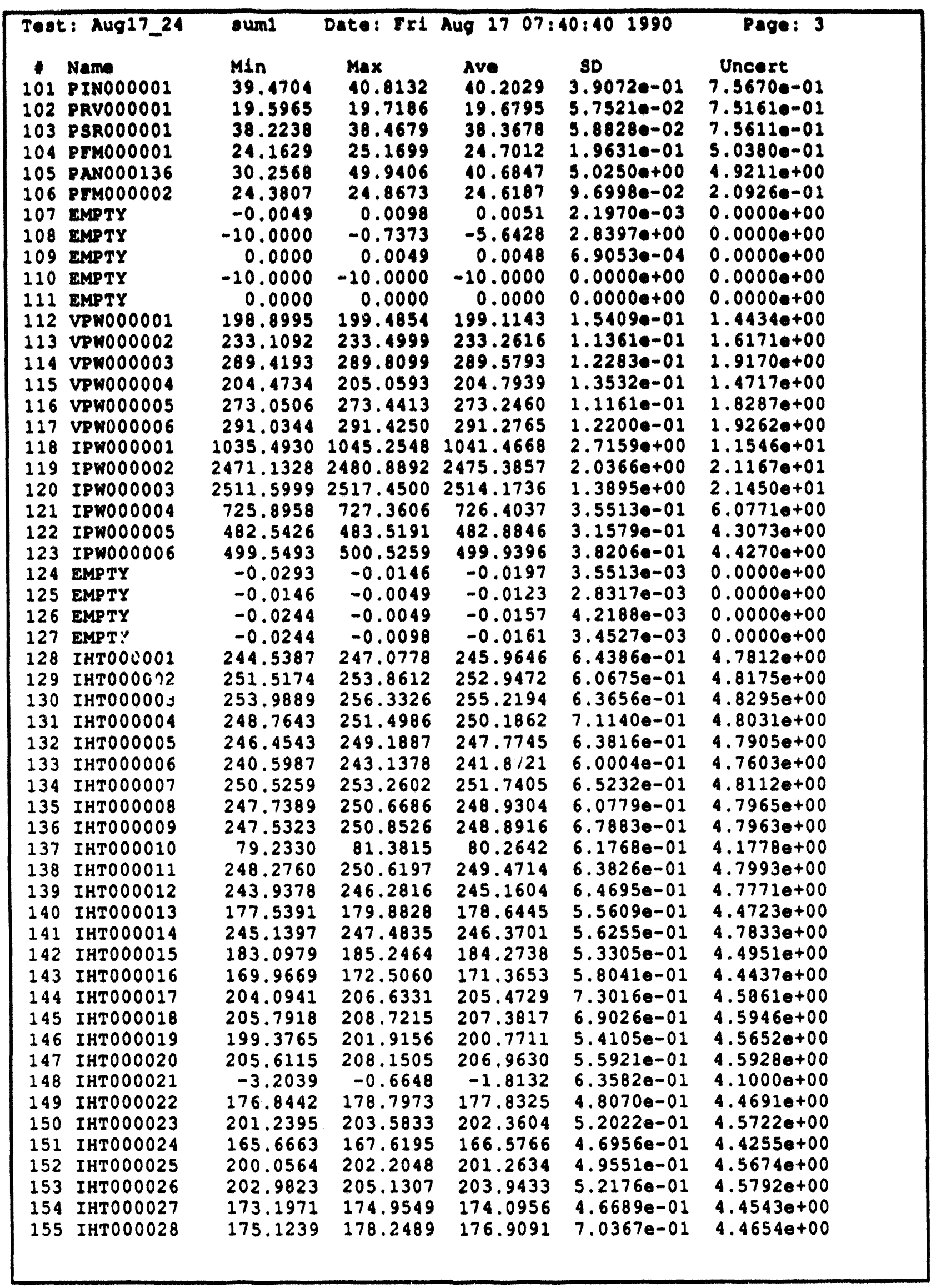




\begin{tabular}{|c|c|c|c|c|c|c|}
\hline $\begin{array}{l}15 \\
156 \\
157 \\
158 \\
159 \\
160 \\
161 \\
162 \\
163\end{array}$ & $\begin{array}{l}\text { Name } \\
\text { IHTO00029 } \\
\text { IHTO00030 } \\
\text { IHTO00031 } \\
\text { IHTO00032 } \\
\text { IHTO0O033 } \\
\text { IHTO00034 } \\
\text { IHTO00035 } \\
\text { IHTOODOO36 }\end{array}$ & $\begin{array}{l}\text { Min } \\
201.1043 \\
205.0594 \\
201.7353 \\
204.7889 \\
204.6161 \\
179.8753 \\
237.1995 \\
177.5653\end{array}$ & $\begin{array}{l}\operatorname{Max} \\
204.2293 \\
208.1844 \\
205.0556 \\
207.9139 \\
207.9365 \\
182.8050 \\
240.5199 \\
181.0810\end{array}$ & $\begin{array}{l}\text { Ave } \\
202.8778 \\
206.7860 \\
203.5713 \\
206.2187 \\
206.1474 \\
181.3714 \\
238.8128 \\
179.3232\end{array}$ & $\begin{array}{l}\text { SD } \\
6.8668 a-01 \\
7.2045 a-01 \\
7.20060-01 \\
6.8395 a-01 \\
7.0956 a-01 \\
5.6305 a-01 \\
6.1106 a-01 \\
5.9320 a-01\end{array}$ & $\begin{array}{l}\text { Uncert } \\
4.5745 \bullet++00 \\
4.5920 \bullet+00 \\
4.5776 \bullet+00 \\
4.58940+00 \\
4.5891 \bullet+00 \\
4.4833 \bullet+00 \\
4.7448 \bullet+00 \\
4.4750 \bullet+00\end{array}$ \\
\hline
\end{tabular}




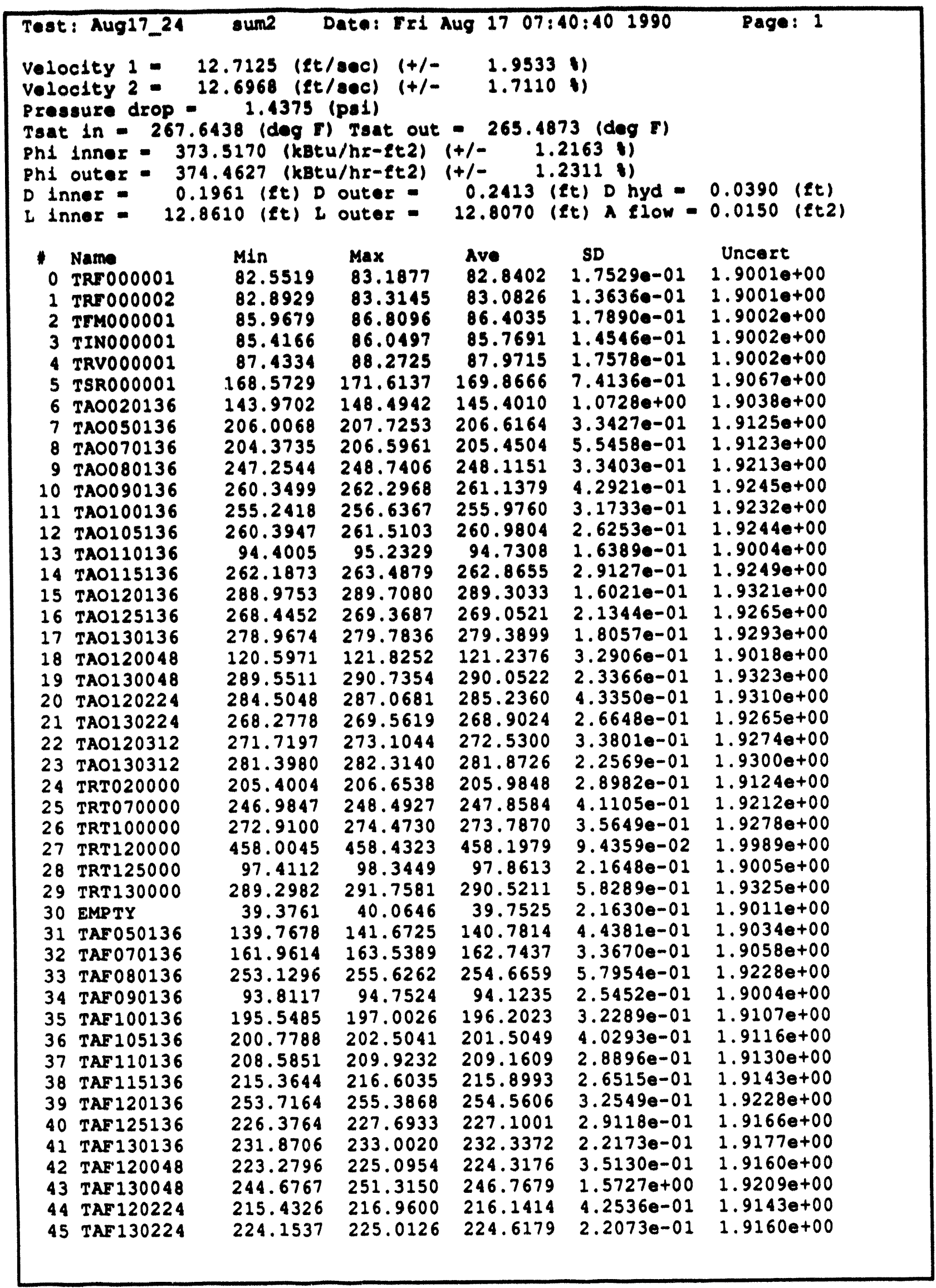




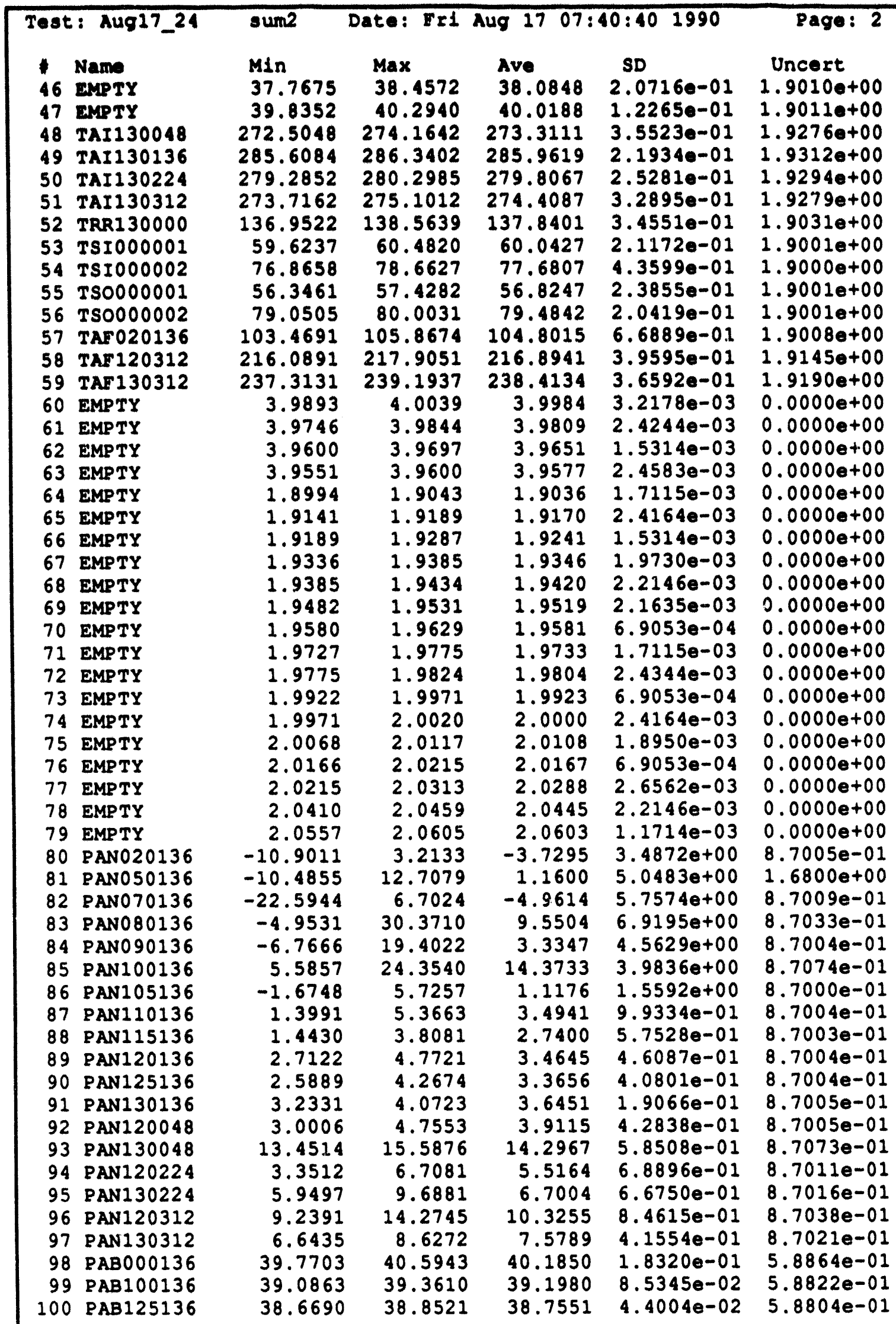




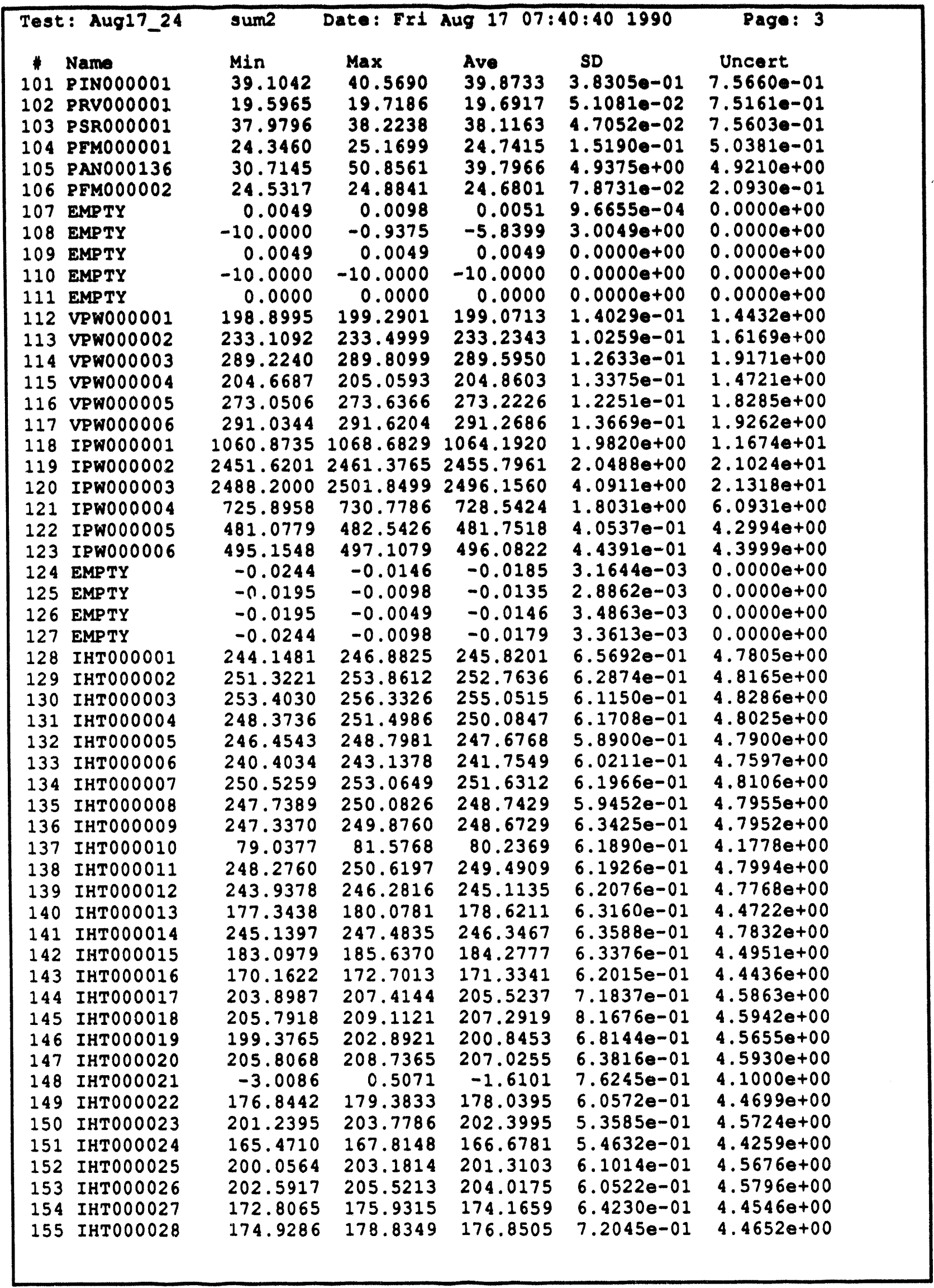




\begin{tabular}{|c|c|c|c|c|c|c|}
\hline \multicolumn{2}{|c|}{ Test: Aug17_24 } & sum2 & Date: Fr1 & Aug 1707 & $: 401990$ & Page: 4 \\
\hline $\begin{array}{l}* \\
156 \\
157 \\
158 \\
159 \\
160 \\
161 \\
162 \\
163\end{array}$ & 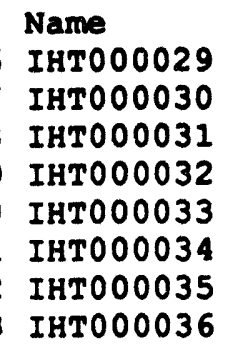 & $\begin{array}{l}\text { Min } \\
200.9090 \\
205.0594 \\
202.1259 \\
204.7889 \\
204.6161 \\
180.4612 \\
237.5902 \\
178.1513\end{array}$ & $\begin{array}{l}\text { Max } \\
204.2293 \\
208.1844 \\
204.8603 \\
207.7186 \\
207.7411 \\
182.4144 \\
239.9339 \\
180.2997\end{array}$ & $\begin{array}{l}\text { Ave } \\
202.8192 \\
206.6923 \\
203.4658 \\
206.0937 \\
206.0420 \\
181.3011 \\
238.6448 \\
179.1826\end{array}$ & $\begin{array}{l}\text { SD } \\
6.8378 \mathrm{e}-01 \\
6.9672 \mathrm{e}-01 \\
6.9252 \mathrm{e}-01 \\
7.2054 \mathrm{e}-01 \\
7.0834 \mathrm{e}-01 \\
5.5978 \mathrm{e}-01 \\
5.5804 \mathrm{e}-01 \\
5.3676 \mathrm{e}-01\end{array}$ & $\begin{array}{c}\text { Uncert } \\
4.5742 e+00 \\
4.5915 e+00 \\
4.5771 e+00 \\
4.5888 e+00 \\
4.5886 e+00 \\
4.4830 e+00 \\
4.7440 e+00 \\
4.4744 e+00\end{array}$ \\
\hline
\end{tabular}




\begin{tabular}{|c|c|c|c|c|}
\hline $\begin{array}{l}\text { Velo } \\
\text { Velo } \\
\text { Pres } \\
\text { Tsat } \\
\text { Phi }\end{array}$ & $\begin{array}{l}\text { ity } 1=0.0 \\
\text { ity } 2=0.0 \\
\text { ure Drop }= \\
\text { in }=0.3523 \\
\text { nner }=2.71\end{array}$ & $\begin{array}{l}09999 \text { (ft) } \\
15400 \text { (ft) } \\
0.032000 \\
86 \text { (deg F) } \\
5515 \text { (kBt) }\end{array}$ & $\begin{array}{l}\text { ec) } \\
\text { ec) } \\
\text { si) } \\
\text { Tsat out }=0.366699(\operatorname{deg} F) \\
\text { hr-ft2) Phi outer }=0.002808\end{array}$ & $(k B t u / h r-f t 2)$ \\
\hline * & Name & Abs Diff & 8Diff & \\
\hline 0 & TRF 000001 & 0.0848 & 0.1023 & \\
\hline 1 & TRF000002 & 0.0042 & 0.0051 & \\
\hline 2 & TFM000001 & 0.2541 & 0.2932 & \\
\hline 3 & TINO00001 & 0.2930 & 0.3405 & \\
\hline 4 & TRV000001 & 0.2950 & 0.3342 & \\
\hline 5 & TSR000001 & 1.9661 & 1.1710 & \\
\hline 6 & TA0020136 & 13.7728 & 8.6527 & \\
\hline 7 & TAO050136 & 0.5332 & 0.2574 & \\
\hline 8 & TA0070136 & 0.4387 & 0.2131 & \\
\hline 9 & TAO080136 & 0.2663 & 0.1072 & \\
\hline 10 & TAO090136 & 0.4913 & 0.1878 & \\
\hline 11 & TA0100136 & 0.2810 & 0.1097 & \\
\hline 12 & TA0105136 & 0.5152 & 0.1970 & \\
\hline 13 & TA0110136 & 0.2923 & 0.3076 & \\
\hline 14 & TAO115136 & 0.5104 & 0.1938 & \\
\hline 15 & TA0120136 & 0.4187 & 0.1445 & \\
\hline 16 & TA0125136 & 0.5184 & 0.1923 & \\
\hline 17 & TAO130136 & 0.4406 & 0.1575 & \\
\hline 18 & TAO120048 & 0.1413 & 0.1164 & \\
\hline 19 & TAO130048 & 0.3114 & 0.1072 & \\
\hline 20 & TAO120224 & 0.5327 & 0.1864 & \\
\hline 21 & TAO130224 & 0.3722 & 0.1382 & \\
\hline 22 & TAO120312 & 0.4598 & 0.1684 & \\
\hline 23 & TAO130312 & 0.3717 & 0.1317 & \\
\hline 24 & TRT020000 & 0.4145 & 0.2008 & \\
\hline 25 & TRT070000 & 0.3788 & 0.1526 & \\
\hline 26 & TRT 100000 & 0.3734 & 0.1362 & \\
\hline 27 & TRT 120000 & 0.0361 & 0.0079 & \\
\hline 28 & TRT125000 & 0.1143 & 0.1167 & \\
\hline 29 & TRT130000 & 0.3990 & 0.1372 & \\
\hline 30 & EMPTY & 0.0137 & 0.0345 & \\
\hline 31 & TAF050136 & 0.1307 & 0.0927 & \\
\hline 32 & TAF070136 & 0.3845 & 0.2357 & \\
\hline 33 & TAF080136 & 0.2871 & 0.1126 & \\
\hline 34 & TAF090136 & 0.5196 & 0.5490 & \\
\hline 35 & TAF 100136 & 0.2658 & 0.1353 & \\
\hline 36 & TAF 105136 & 0.3505 & 0.1736 & \\
\hline 37 & TAF 110136 & 0.2622 & 0.1252 & \\
\hline 38 & TAF 115136 & 0.2919 & 0.1350 & \\
\hline 39 & TAF 120136 & 0.4064 & 0.1594 & \\
\hline 40 & TAF 125136 & 0.3070 & 0.1350 & \\
\hline 41 & TAF 130136 & 0.4282 & 0.1840 & \\
\hline 42 & TAF 120048 & 0.3498 & 0.1557 & \\
\hline 43 & TAF 130048 & 0.0923 & 0.0374 & \\
\hline 44 & TAF 120224 & 0.4560 & 0.2105 & \\
\hline 45 & TAF 130224 & 0.3112 & 0.1383 & \\
\hline
\end{tabular}




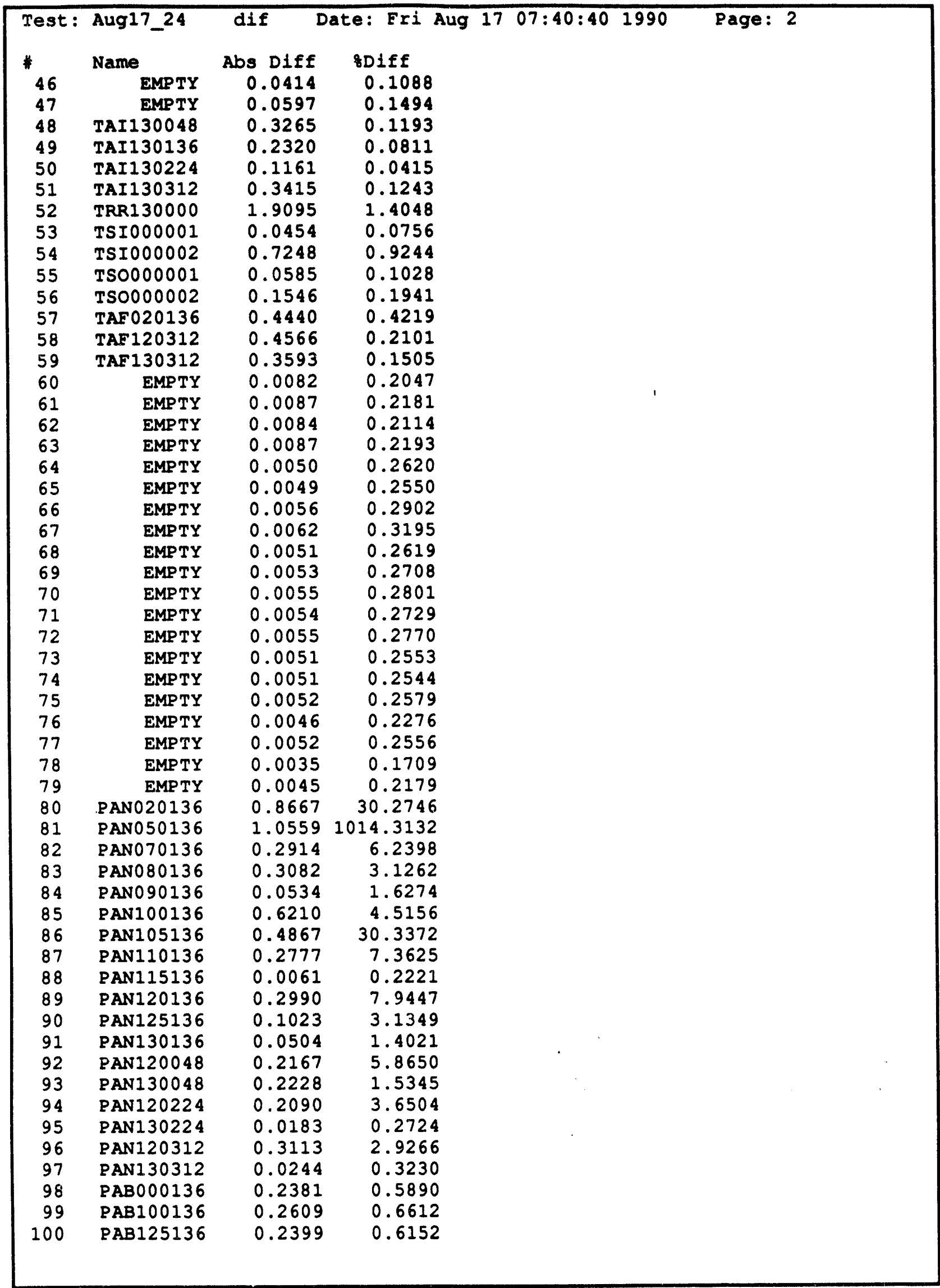




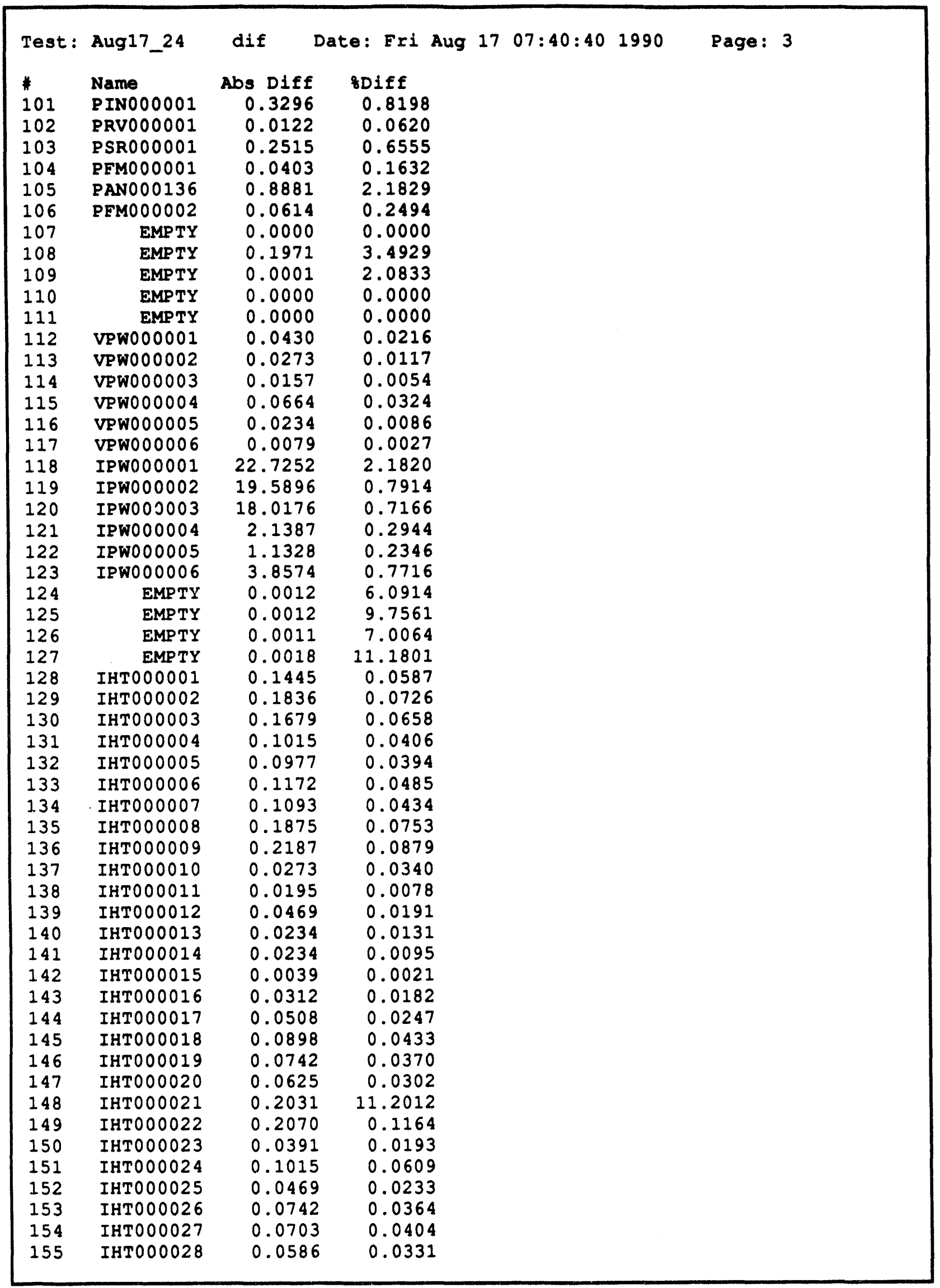


Test: Aug17_24 dif Date: Fri Aug 17 07:40:40 1990 Page: 4

* Name Abs Diff eDiff

$\begin{array}{llll}156 & \text { IHT000029 } & 0.0586 & 0.0289\end{array}$

$\begin{array}{llll}157 & \text { IHT000030 } & 0.0937 & 0.0453\end{array}$

$\begin{array}{llll}158 & \text { IHT000031 } & 0.1055 & 0.0518\end{array}$

$\begin{array}{llll}159 & \text { IHT000032 } & 0.1250 & 0.0606\end{array}$

160 IHT000033 $0.1054 \quad 0.0511$

$\begin{array}{llll}161 & \text { IHTO00034 } & 0.0703 & 0.0388\end{array}$

162 IHT000035 $0.1680 \quad 0.0703$

163 IHT000036 $0.1406 \quad 0.0784$ 


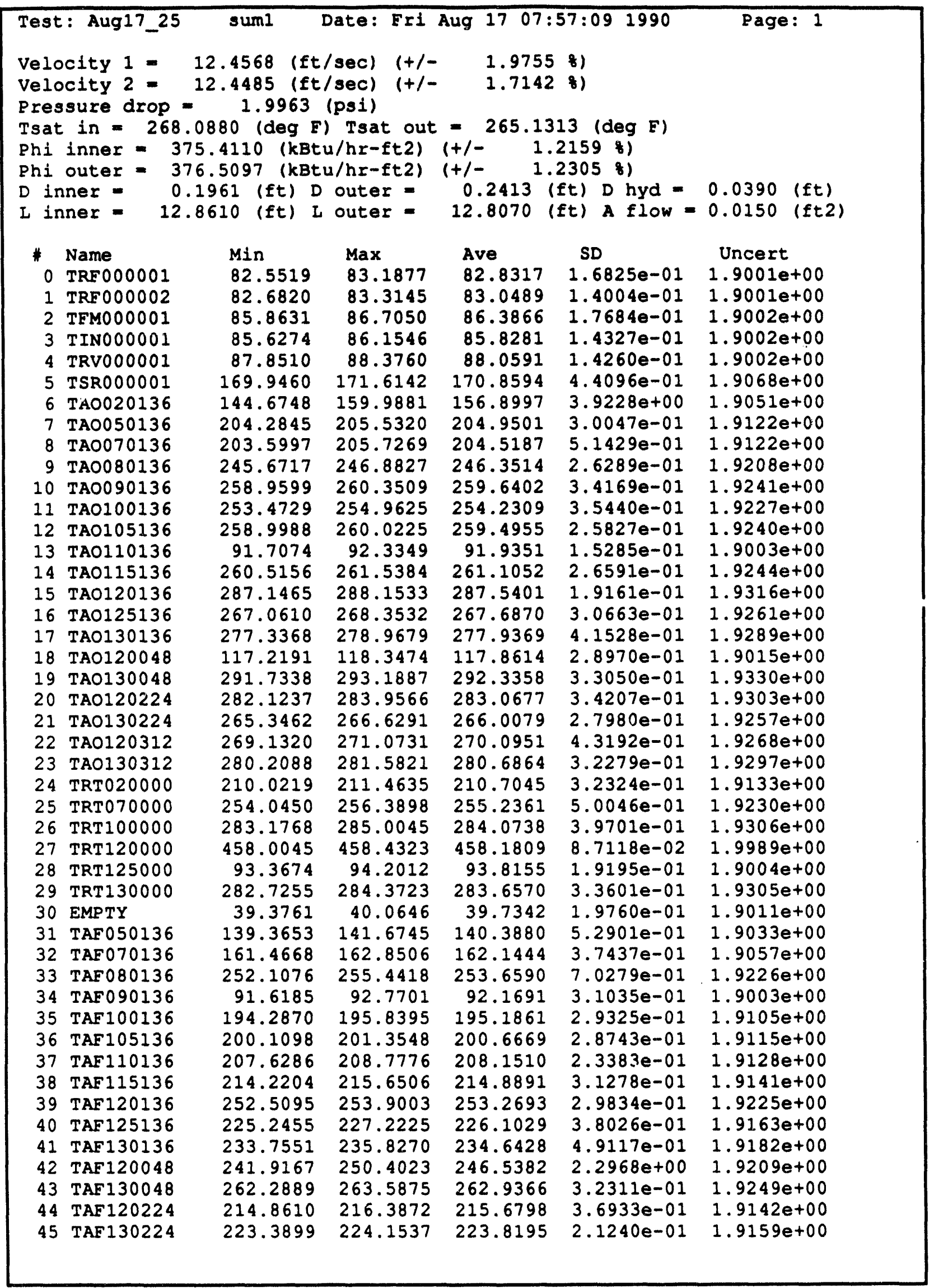




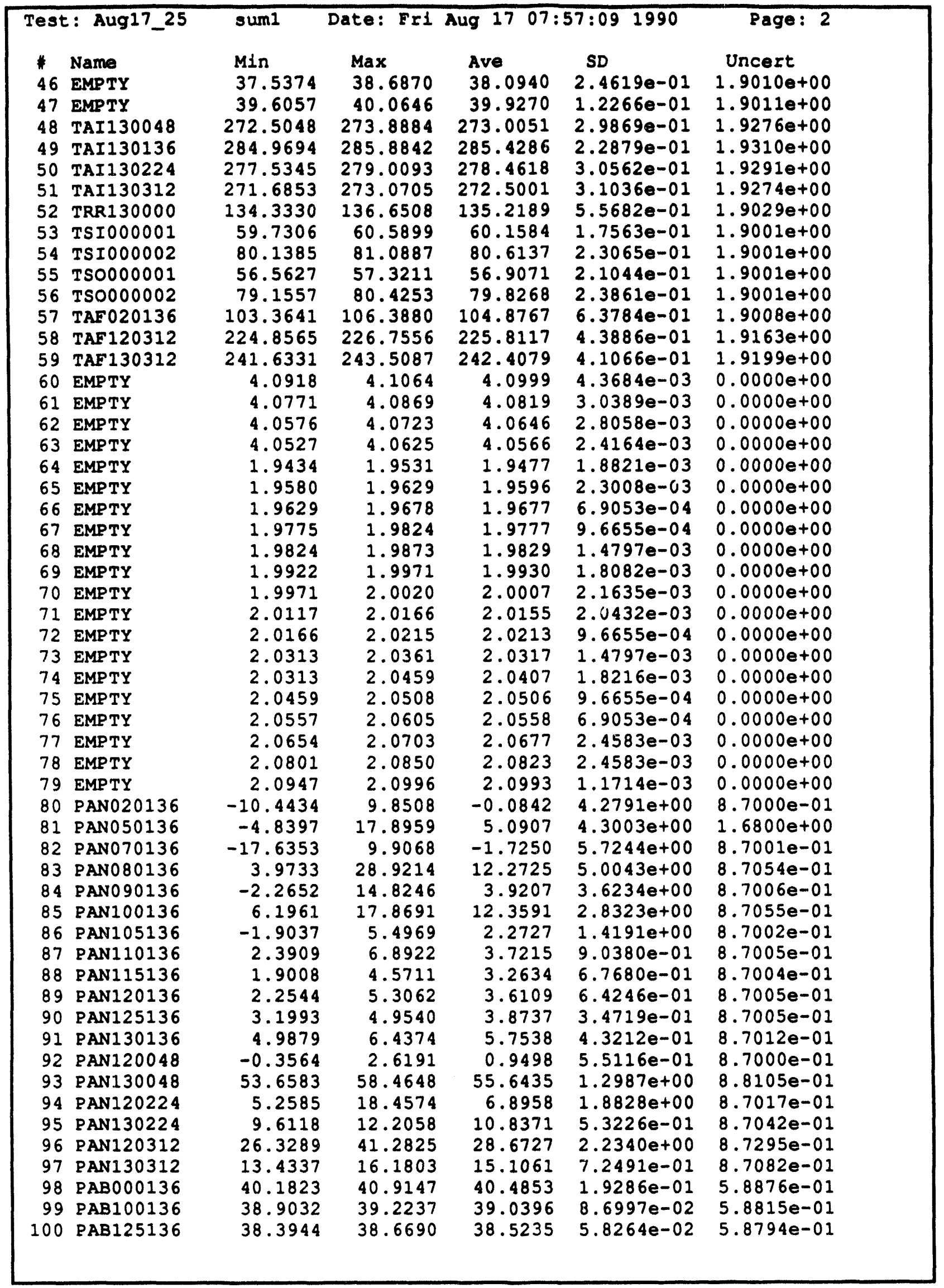




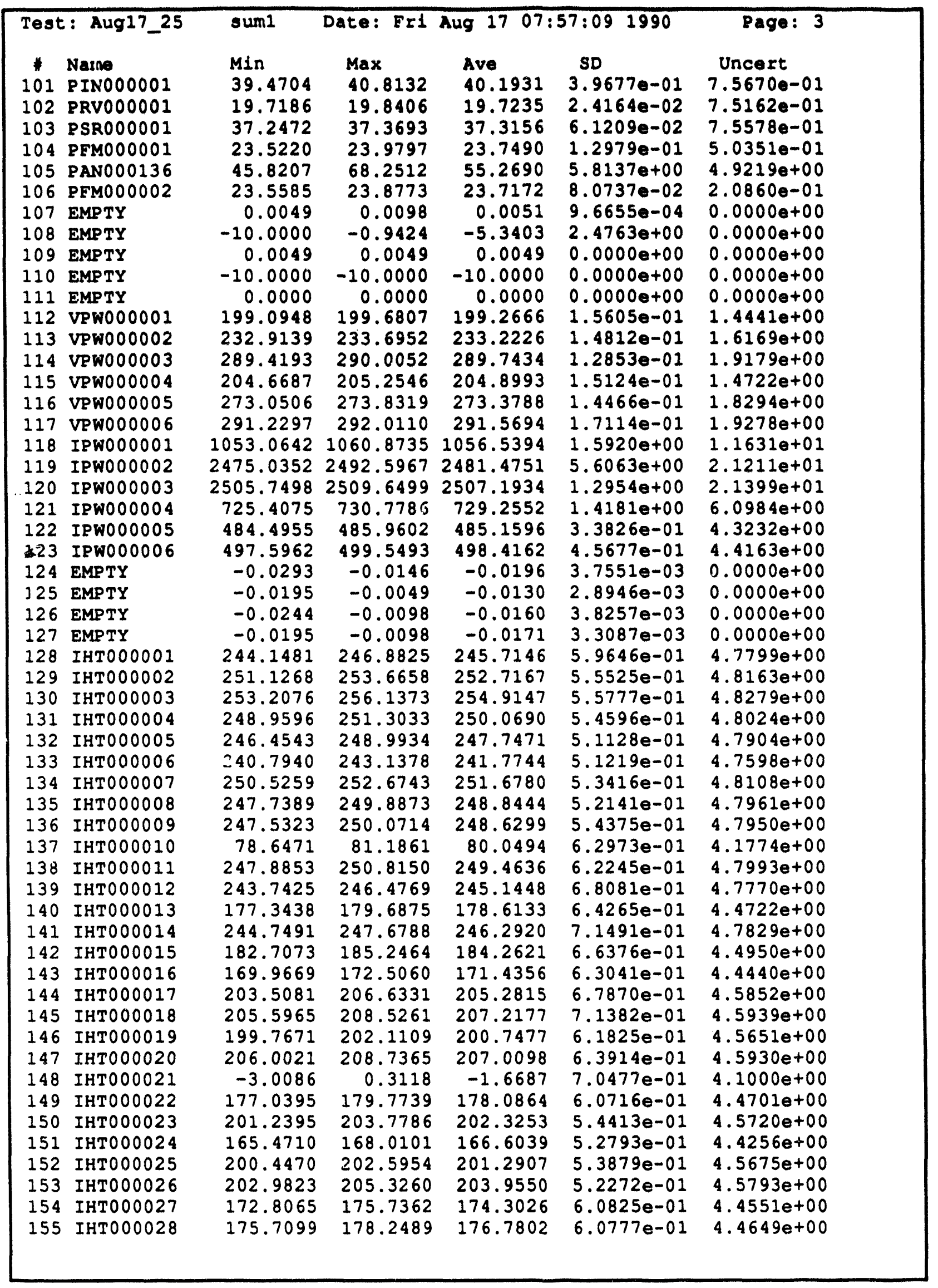




\begin{tabular}{|c|c|c|c|c|c|c|}
\hline $\begin{array}{l}1 \\
156 \\
157 \\
158 \\
159 \\
160 \\
161 \\
162 \\
163\end{array}$ & $\begin{array}{l}\text { Nam0 } \\
\text { IHTO00029 } \\
\text { IHTO00030 } \\
\text { IHTO0O031 } \\
\text { IHTO00032 } \\
\text { IHTO00033 } \\
\text { IHTO00034 } \\
\text { IHTO00035 } \\
\text { IHTO00036 }\end{array}$ & $\begin{array}{l}\text { Min } \\
201.2996 \\
205.0594 \\
201.9306 \\
204.3983 \\
204.4208 \\
179.8753 \\
237.1995 \\
177.7607\end{array}$ & $\begin{array}{l}\operatorname{Max} \\
204.0340 \\
207.7937 \\
204.6650 \\
207.3280 \\
207.3505 \\
182.2190 \\
239.9339 \\
180.2997\end{array}$ & $\begin{array}{l}\text { Ave } \\
202.5732 \\
206.5243 \\
203.3056 \\
205.9335 \\
205.8896 \\
181.1878 \\
238.5471 \\
179.0888\end{array}$ & $\begin{array}{l}\text { SD } \\
6.2170 e-01 \\
6.2297 e-01 \\
6.0227 e-01 \\
6.1131 e-01 \\
6.2170 \bullet-01 \\
5.2205 e-01 \\
5.3270 e-01 \\
4.6855 e-01\end{array}$ & $\begin{array}{c}\text { Uncert } \\
4.5731 e+00 \\
4.5908 e+00 \\
4.5764 \theta+00 \\
4.5881 e+00 \\
4.5879 e+00 \\
4.4825 e+00 \\
4.7435 e+00 \\
4.4741 e+00\end{array}$ \\
\hline
\end{tabular}




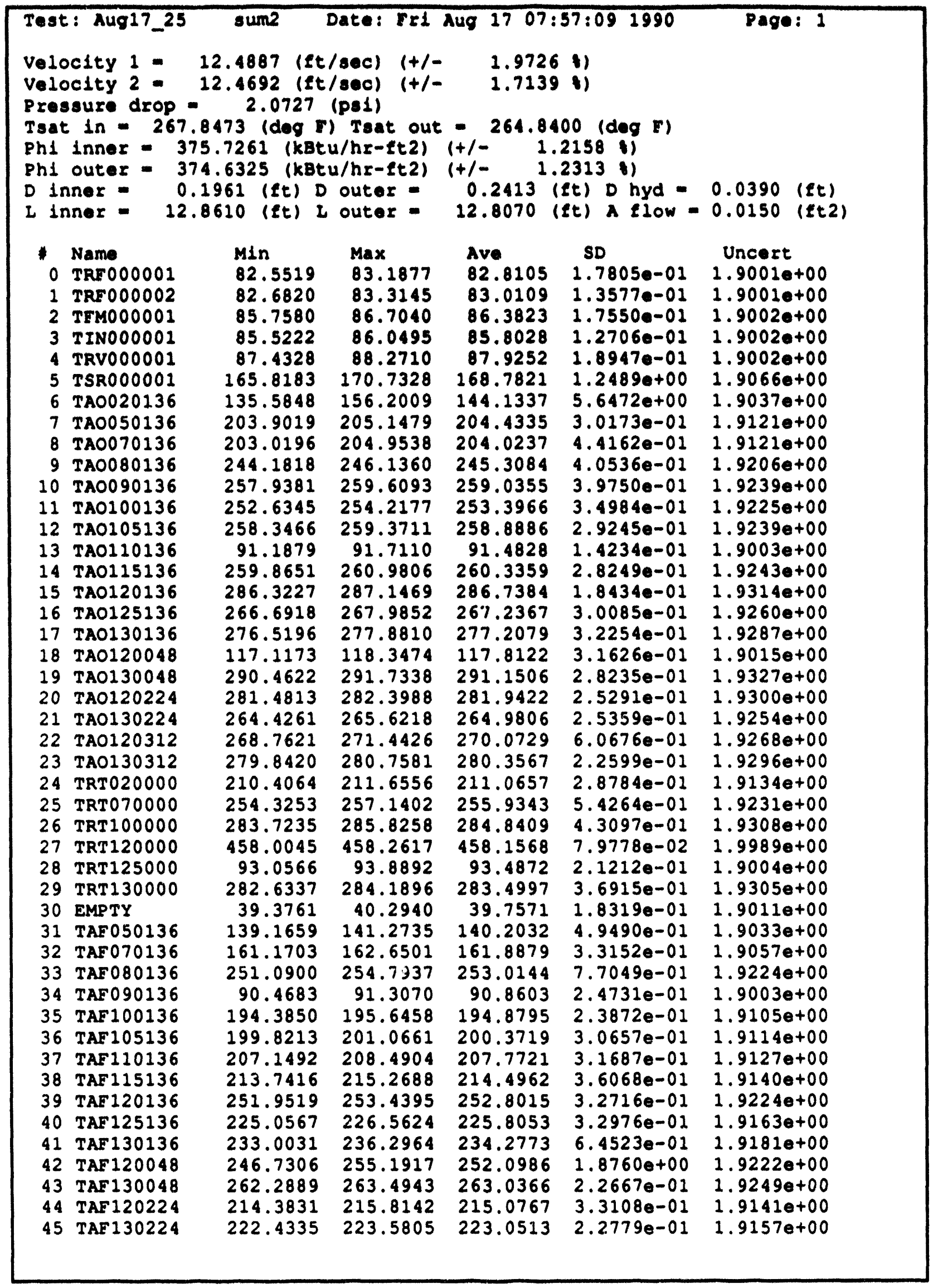




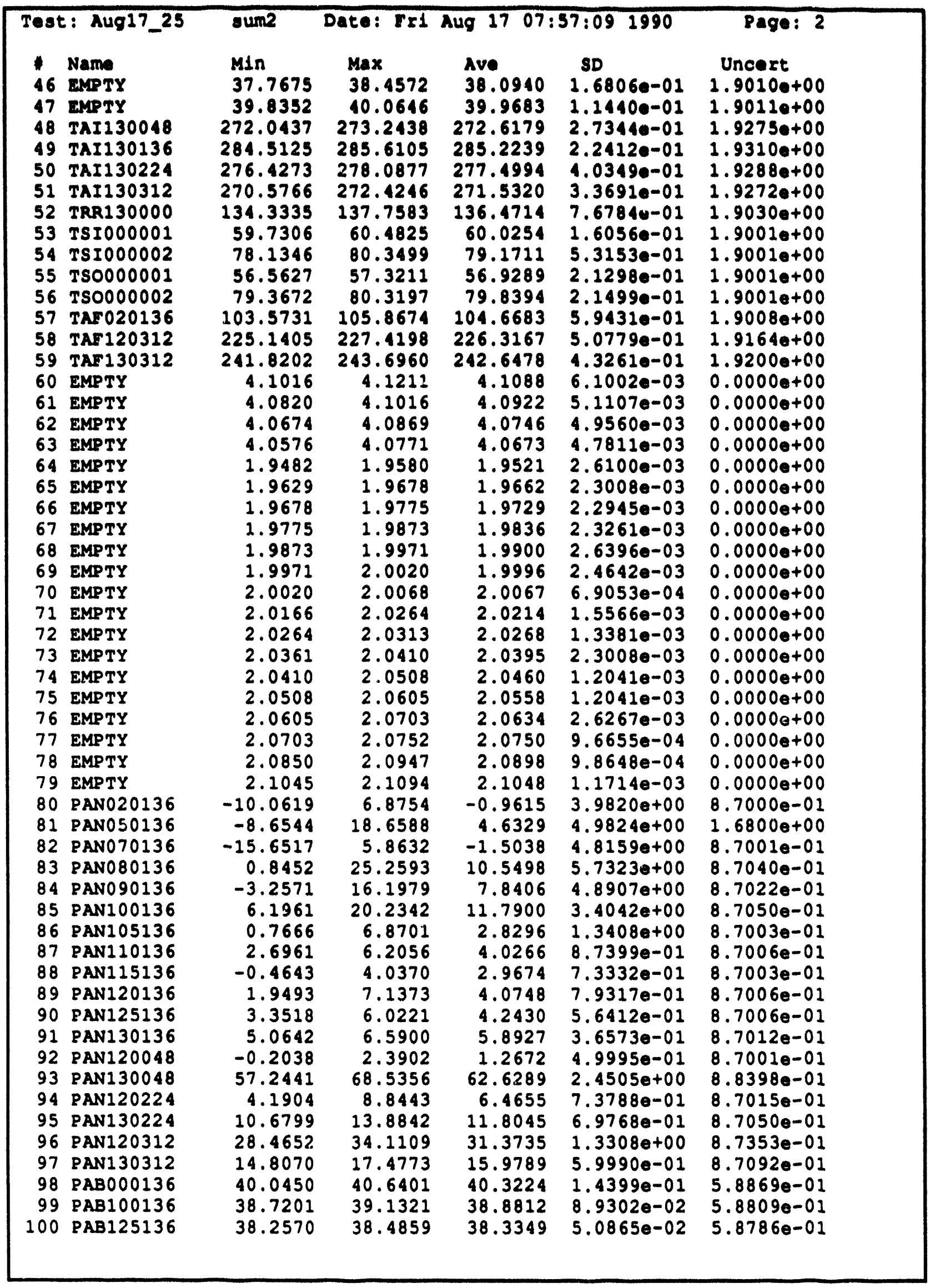




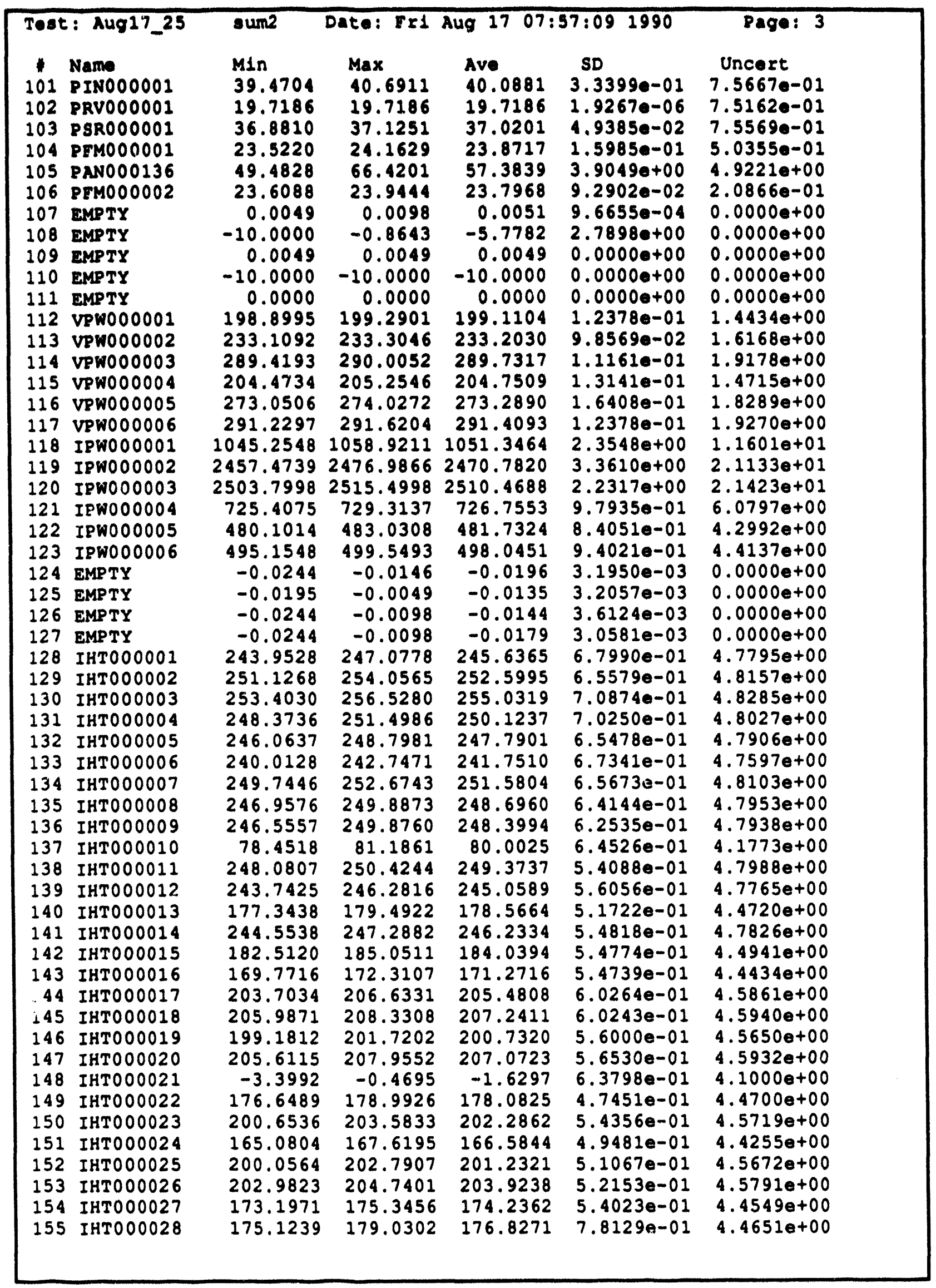




\begin{tabular}{|c|c|c|c|c|c|c|}
\hline $\begin{array}{l}1 \\
156 \\
157 \\
158 \\
159 \\
160 \\
161 \\
162 \\
163\end{array}$ & $\begin{array}{l}\text { Name } \\
\text { IHTO00029 } \\
\text { IHTO00030 } \\
\text { IHTO00031 } \\
\text { IHTO00032 } \\
\text { IHTO00033 } \\
\text { IHTO00034 } \\
\text { IHTO00035 } \\
\text { IHTO00036 }\end{array}$ & $\begin{array}{l}\text { Min } \\
201.1043 \\
204.8640 \\
201.7353 \\
204.2030 \\
204.0302 \\
179.6800 \\
237.0042 \\
177.7607\end{array}$ & $\begin{array}{l}\operatorname{Max} \\
204.6199 \\
208.5750 \\
205.4462 \\
207.5233 \\
207.5458 \\
182.4144 \\
239.5433 \\
180.1044\end{array}$ & $\begin{array}{l}\text { Ave } \\
202.6708 \\
206.6376 \\
203.4228 \\
205.9960 \\
205.9287 \\
181.1449 \\
238.5003 \\
179.0185\end{array}$ & $\begin{array}{l}\text { SD } \\
7.8571 e-01 \\
8.1523 e-01 \\
8.1036 e-01 \\
7.8591 e-01 \\
8.0871 e-01 \\
5.9745 e-01 \\
5.9400 e-01 \\
5.4841 e-01\end{array}$ & $\begin{array}{l}\text { Uncert } \\
4.5736 e+00 \\
4.5913 e+00 \\
4.5769 e+00 \\
4.5884 \theta+00 \\
4.5881 \theta+00 \\
4.4823 e+00 \\
4.7432 e+00 \\
4.4738 \theta+00\end{array}$ \\
\hline
\end{tabular}




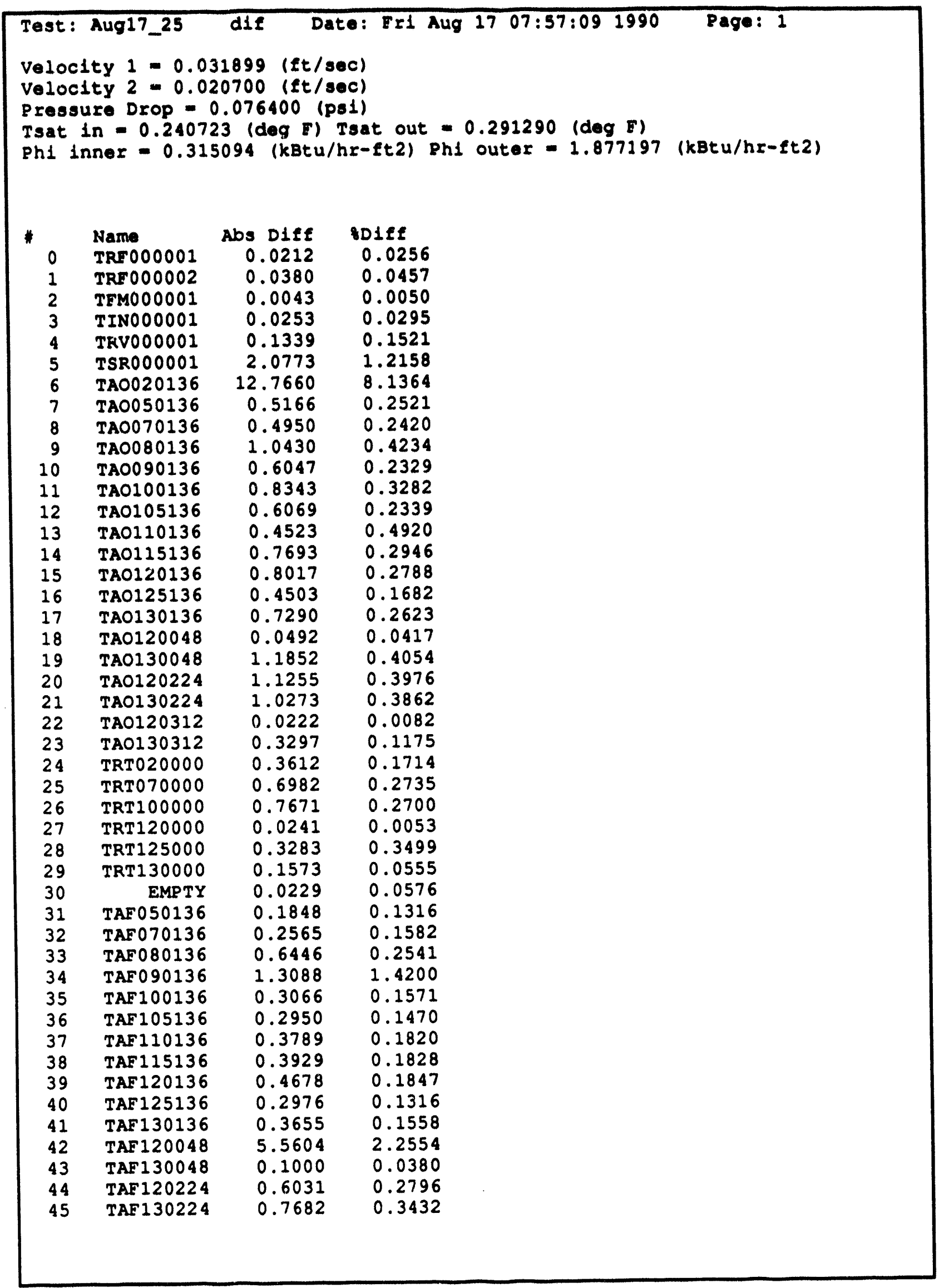




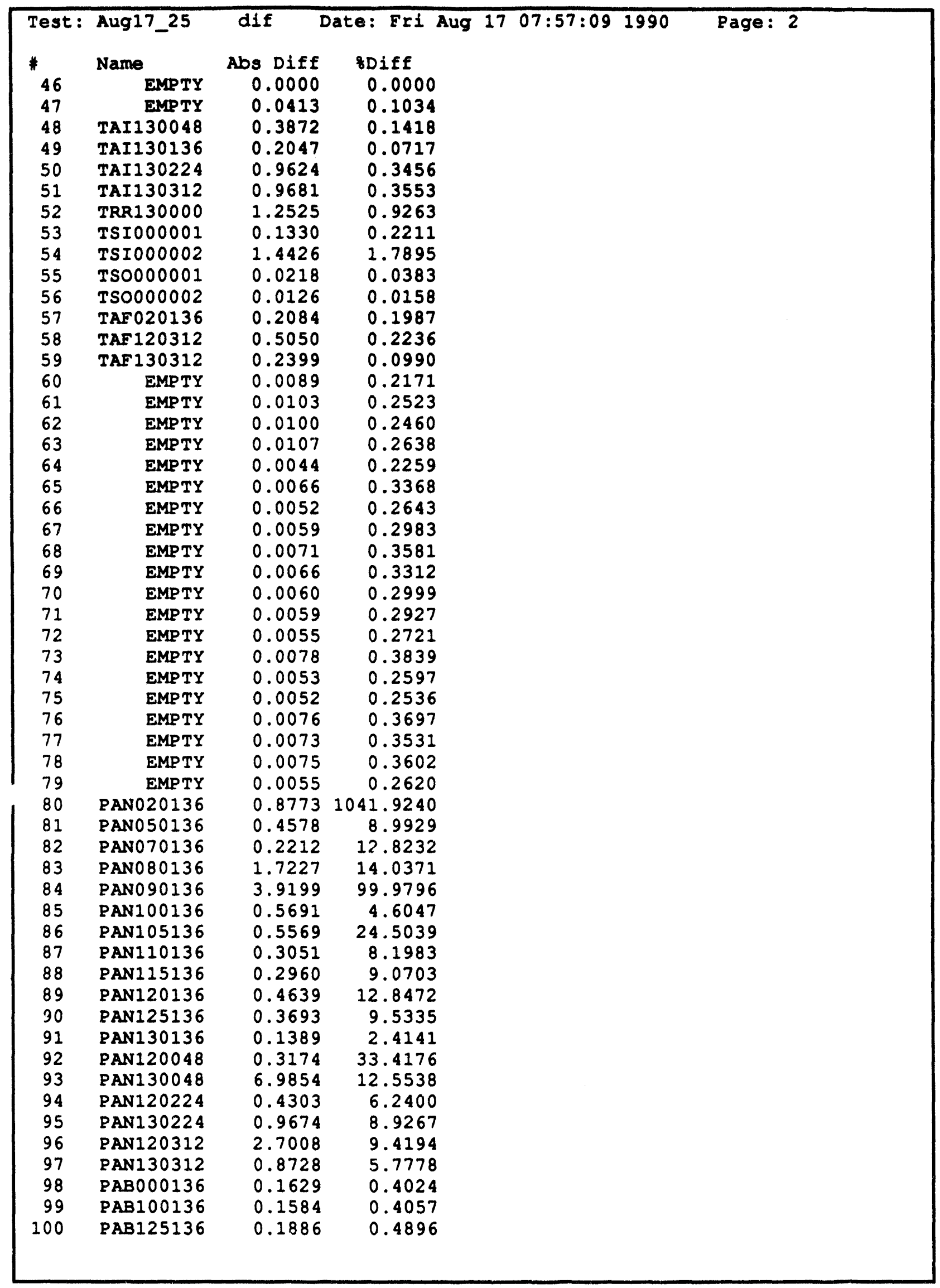




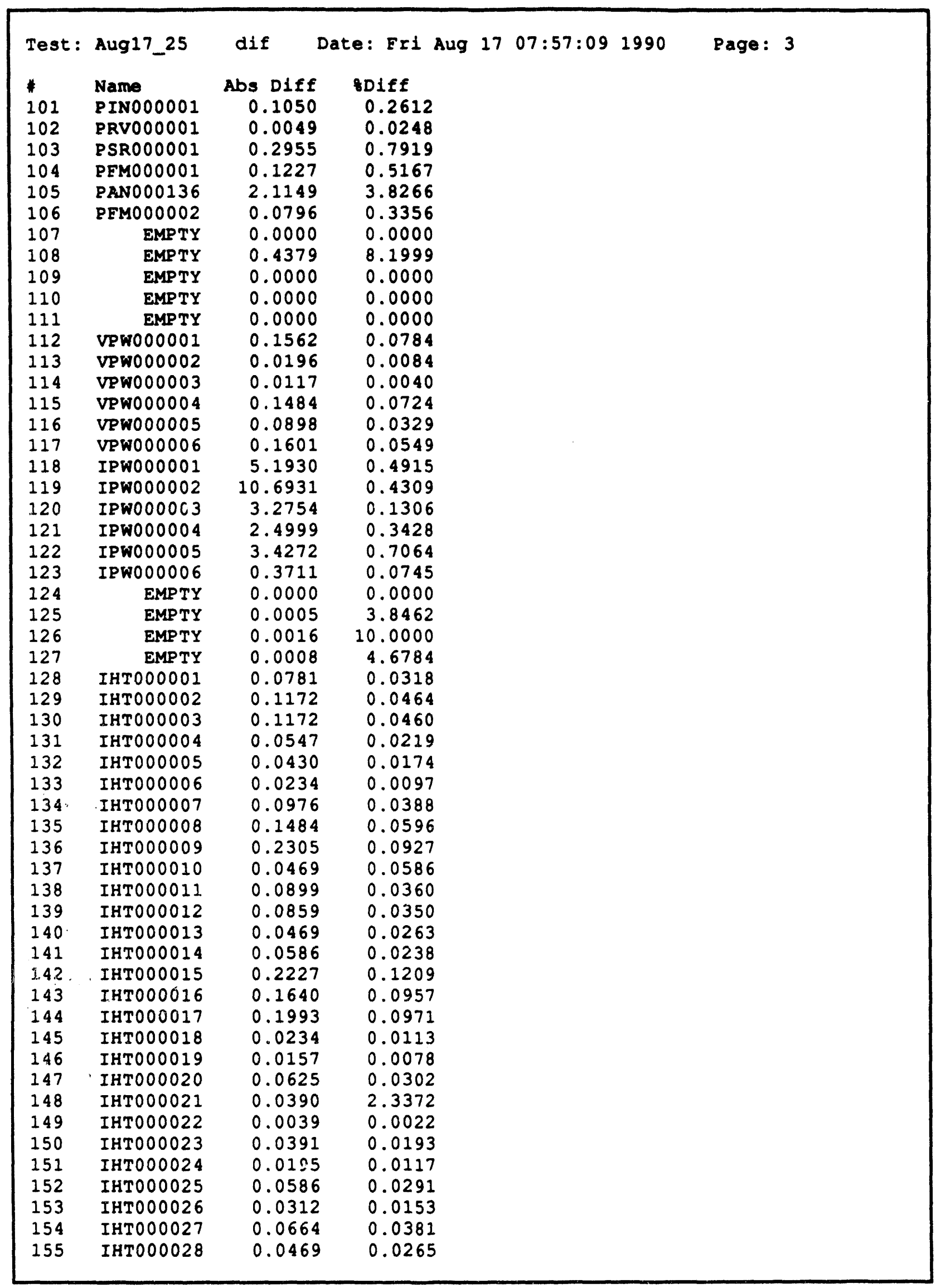




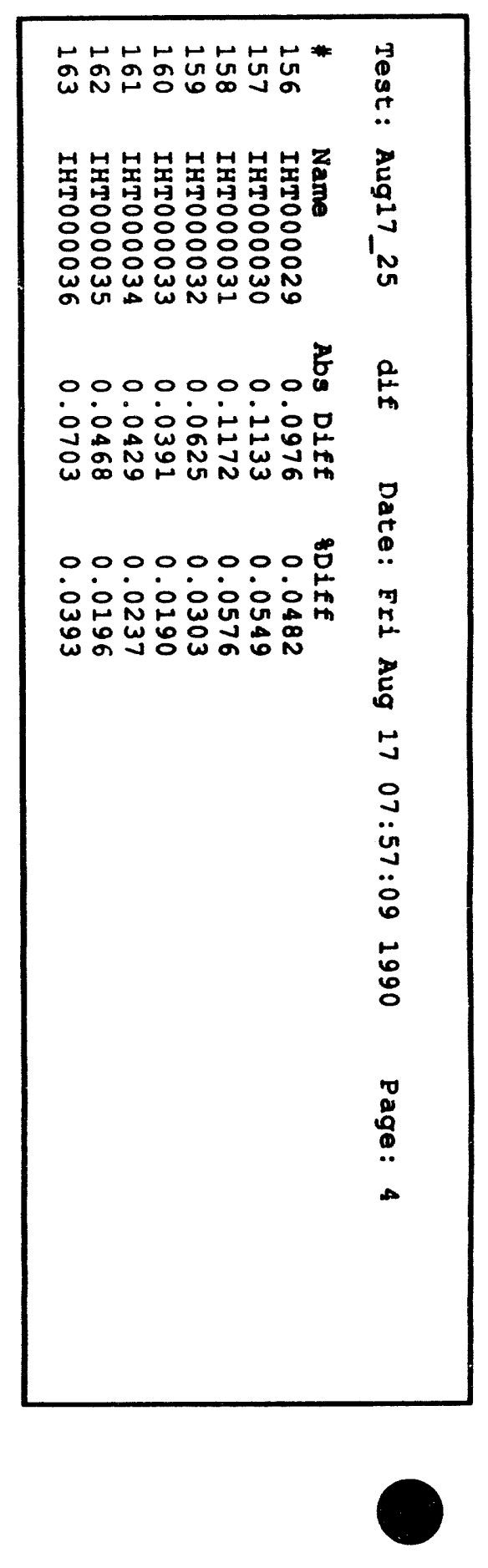




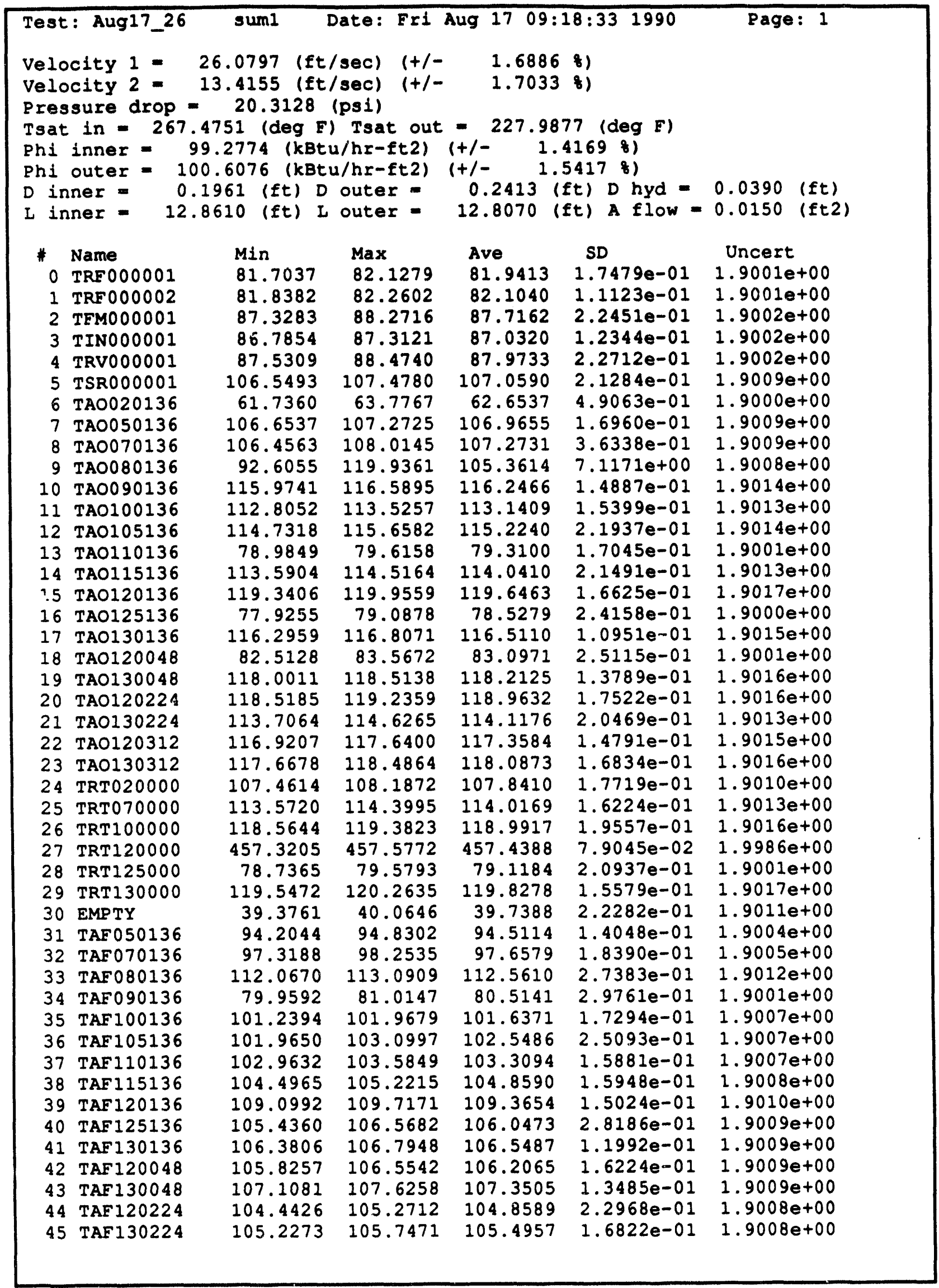




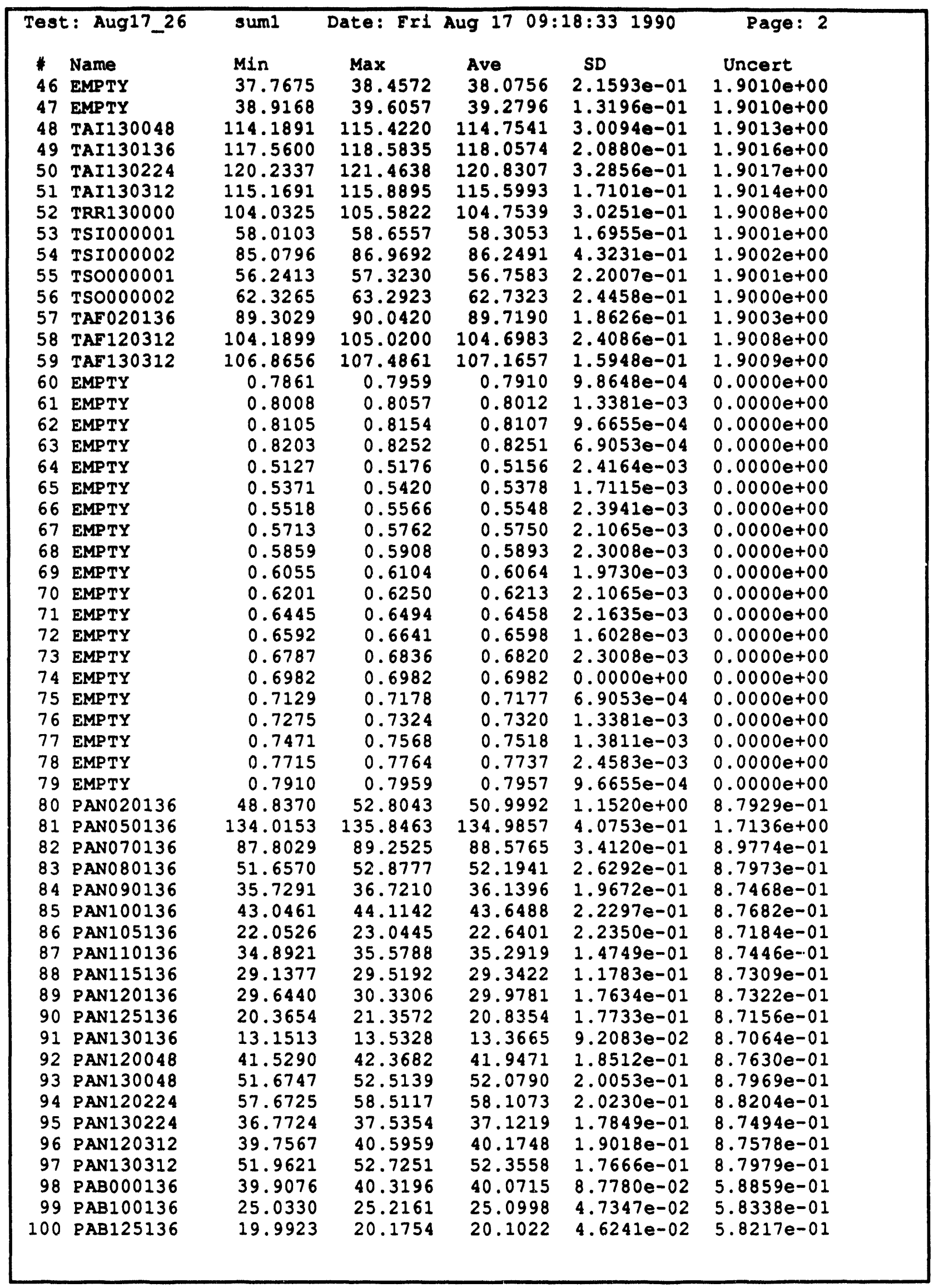




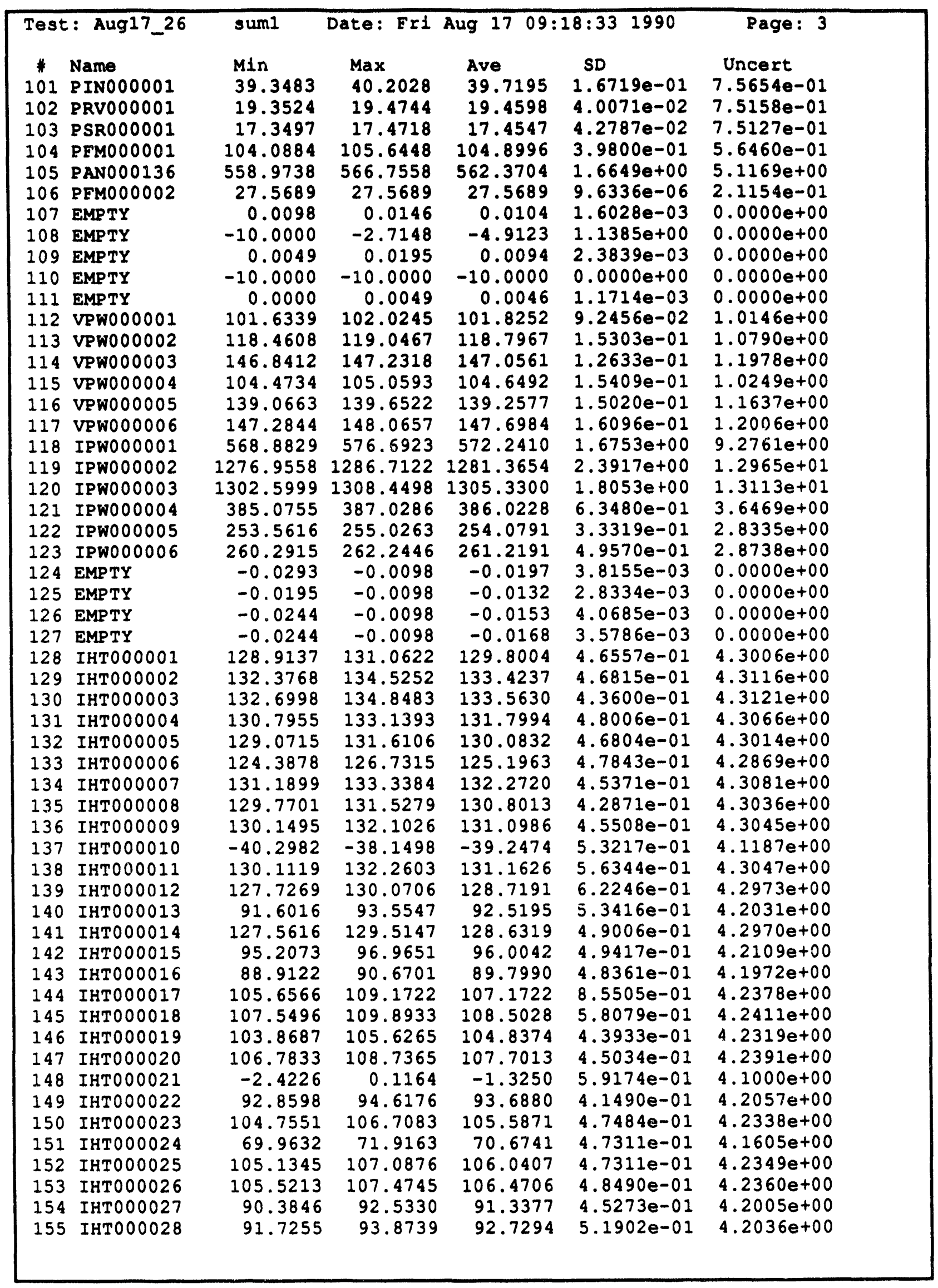




\begin{tabular}{|c|c|c|c|c|c|c|}
\hline \multicolumn{2}{|c|}{ Test: Aug17_26 } & suml & Date: Fri & Aug 1709 & $18: 331990$ & Page: 4 \\
\hline $\begin{array}{l}\# \\
156 \\
157 \\
158 \\
159 \\
160 \\
161 \\
162 \\
163\end{array}$ & $\begin{array}{l}\text { Name } \\
\text { IHTO0O029 } \\
\text { IHTO0O030 } \\
\text { IHTOOO031 } \\
\text { IHTOOO032 } \\
\text { IHTOOOO033 } \\
\text { IHTOOO034 } \\
\text { IHTOOOO035 } \\
\text { IHTOOO036 }\end{array}$ & $\begin{array}{l}\text { Min } \\
104.8152 \\
107.2078 \\
105.6415 \\
107.1326 \\
106.9599 \\
93.9378 \\
124.5042 \\
92.7997\end{array}$ & $\begin{array}{l}\text { Max } \\
107.1590 \\
109.5515 \\
108.1806 \\
109.8670 \\
109.6943 \\
96.2815 \\
126.6527 \\
95.3388\end{array}$ & $\begin{array}{l}\text { Ave } \\
106.1395 \\
108.4891 \\
106.8877 \\
108.2225 \\
108.2021 \\
94.9300 \\
125.1683 \\
93.5771\end{array}$ & $\begin{array}{l}\text { SD } \\
5.4432 e-01 \\
5.4413 e-01 \\
5.6344 e-01 \\
5.6647 e-01 \\
5.5350 e-01 \\
4.4963 e-01 \\
4.2682 e-01 \\
4.6770 e-01\end{array}$ & $\begin{array}{c}\text { Uncert } \\
4.2352 e+00 \\
4.2411 e+00 \\
4.2370 e+00 \\
4.2404 e+00 \\
4.2404 e+00 \\
4.2085 e+00 \\
4.2868 e+00 \\
4.2054 e+00\end{array}$ \\
\hline
\end{tabular}




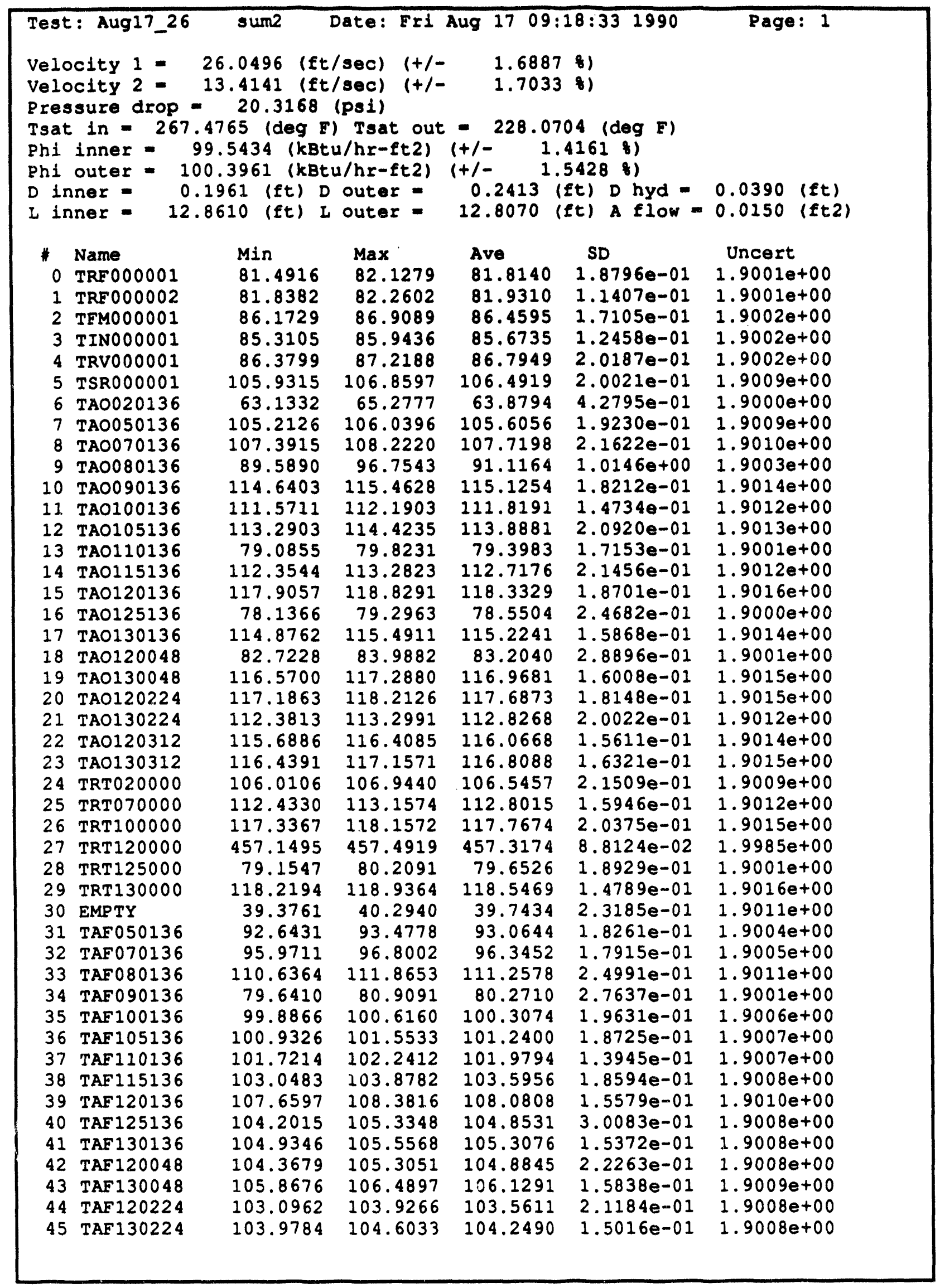




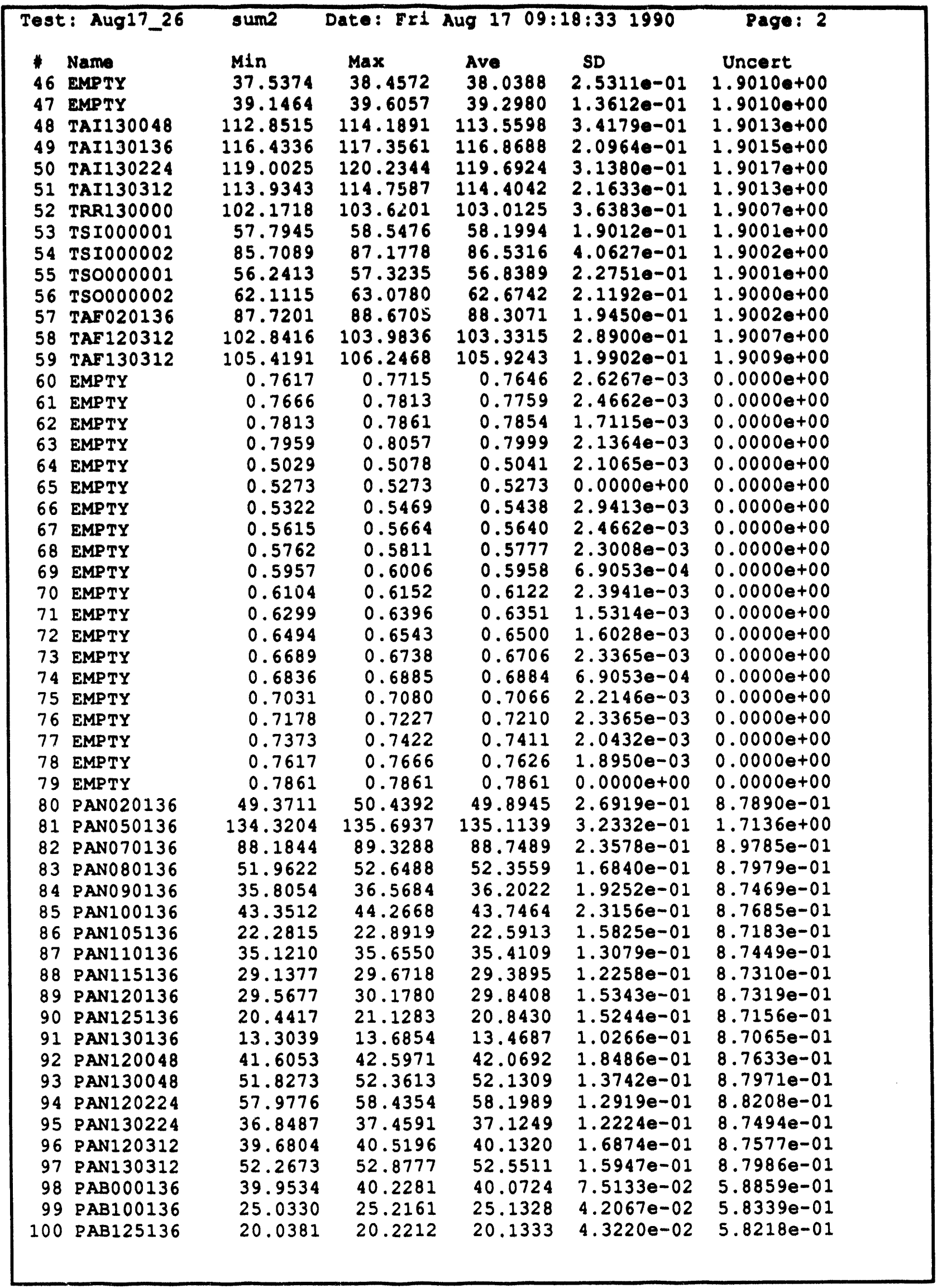




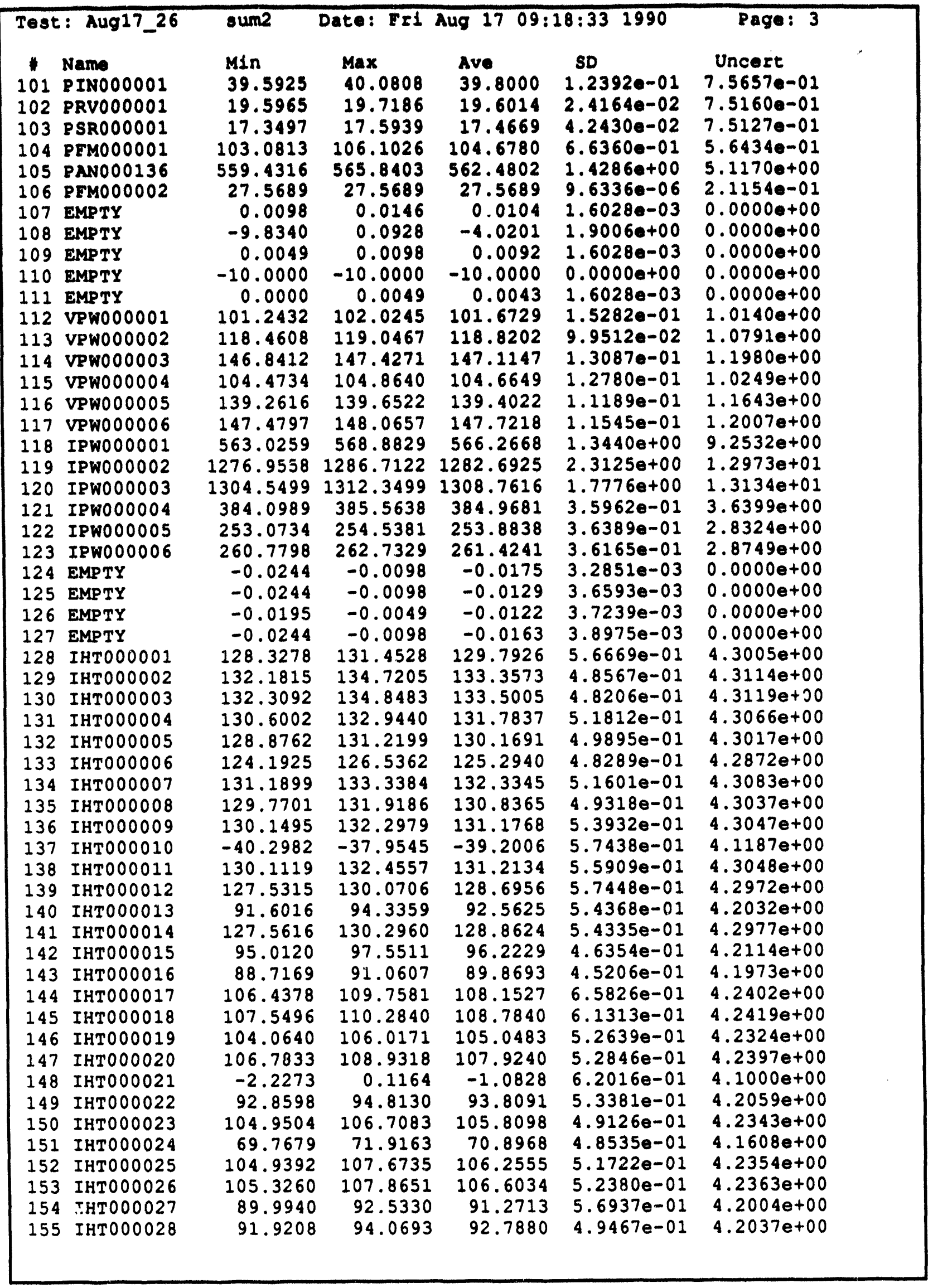




\begin{tabular}{|c|c|c|c|c|c|c|}
\hline $\begin{array}{l}1 \\
156 \\
157 \\
158 \\
159 \\
160 \\
161 \\
162 \\
163\end{array}$ & $\begin{array}{l}\text { Name } \\
\text { IHTO00029 } \\
\text { IHTO00030 } \\
\text { IHTO00031 } \\
\text { IHTO00032 } \\
\text { IHTO00033 } \\
\text { IHTO00034 } \\
\text { IHTO00035 } \\
\text { IHTO00036 }\end{array}$ & $\begin{array}{l}\text { MIn } \\
105.1012 \\
107.5984 \\
105.8368 \\
107.3280 \\
107.3505 \\
94.1331 \\
124.5042 \\
92.6044\end{array}$ & $\begin{array}{l}\text { Max } \\
107.5496 \\
110.3328 \\
108.7665 \\
110.2576 \\
110.0849 \\
96.0862 \\
126.6527 \\
94.5575\end{array}$ & $\begin{array}{l}\text { Ave } \\
106.3504 \\
108.6844 \\
107.0088 \\
108.3280 \\
108.2803 \\
94.9495 \\
125.2621 \\
93.5380\end{array}$ & $\begin{array}{l}\text { SD } \\
4.9601 e-01 \\
5.5546 e-01 \\
5.5943 e-01 \\
5.78100-01 \\
5.8867 e-01 \\
4.71350-01 \\
4.77830-01 \\
4.19990-01\end{array}$ & $\begin{array}{c}\text { Uncert } \\
4.2357 \theta+00 \\
4.2416 \theta+00 \\
4.2373 \theta+00 \\
4.2407 \theta+00 \\
4.2406 \theta+00 \\
4.2085 \theta+00 \\
4.2871 \theta+00 \\
4.2053 \theta+00\end{array}$ \\
\hline
\end{tabular}




\begin{tabular}{|c|c|c|c|}
\hline \multicolumn{4}{|c|}{$\begin{array}{l}\text { Velocity } 1=0.030100(\mathrm{ft} / \mathrm{sec}) \\
\text { velocity } 2=0.001400(\mathrm{ft} / \mathrm{sec}) \\
\text { Pressure Drop }=0.004000 \text { (psi) } \\
\text { Tsat in }=0.002404 \text { (deg F) Tsat out }=0.082703 \text { (deg F) } \\
\text { Phl inner }=0.266006 \text { (kBtu/hr-ft2) Ph1 outer }=0.211494 \text { (kBtu/hr-tt2) }\end{array}$} \\
\hline $\begin{array}{l}0 \\
1 \\
2 \\
3 \\
4 \\
5 \\
6 \\
7 \\
8 \\
9 \\
10 \\
11 \\
12 \\
13 \\
14 \\
15 \\
16 \\
17 \\
18 \\
19 \\
20 \\
21 \\
22 \\
23 \\
24 \\
25 \\
26 \\
27 \\
28 \\
29 \\
30 \\
31 \\
32 \\
33 \\
34 \\
35 \\
36 \\
37 \\
38 \\
39 \\
40 \\
41 \\
42 \\
43 \\
44 \\
45\end{array}$ & $\begin{array}{l}\text { Name } \\
\text { TRFO00001 } \\
\text { TRF000002 } \\
\text { TEMO00001 } \\
\text { TIN000001 } \\
\text { TRV000001 } \\
\text { TSR000001 } \\
\text { TAO020136 } \\
\text { TAO050136 } \\
\text { TAO070136 } \\
\text { TAO080136 } \\
\text { TAO090136 } \\
\text { TAO100136 } \\
\text { TAO105136 } \\
\text { TAO110136 } \\
\text { TAO115136 } \\
\text { TAO120136 } \\
\text { TAO125136 } \\
\text { TAO130136 } \\
\text { TAO120048 } \\
\text { TAO130048 } \\
\text { TAO120224 } \\
\text { TAO130224 } \\
\text { TAO120312 } \\
\text { TAO130312 } \\
\text { TRT020000 } \\
\text { TRT070000 } \\
\text { TRT100000 } \\
\text { TRT120000 } \\
\text { TRT125000 } \\
\text { TRT130000 } \\
\text { TAMPTY } \\
\text { TAF050136 } \\
\text { TAF070136 } \\
\text { TAF080136 } \\
\text { TAF090136 } \\
\text { TAF100136 } \\
\text { TAF105136 } \\
\text { TAF110136 } \\
\text { TAF115136 } \\
\text { TAF120136 } \\
\text { TAF125136 } \\
\text { TAF130136 } \\
\text { TAF120048 } \\
\text { TAF130048 } \\
\text { TAF120224 } \\
\text { TAF130224 }\end{array}$ & $\begin{array}{l}\text { Abs D1ff } \\
0.1273 \\
0.1730 \\
1.2567 \\
1.3585 \\
1.1784 \\
0.5671 \\
1.2257 \\
1.3599 \\
0.4467 \\
14.2450 \\
1.1212 \\
1.3218 \\
1.3359 \\
0.0883 \\
1.3234 \\
1.3134 \\
0.0225 \\
1.2869 \\
0.1069 \\
1.2444 \\
1.2759 \\
1.2908 \\
1.2916 \\
1.2785 \\
1.2953 \\
1.2154 \\
1.2243 \\
0.1214 \\
0.5342 \\
1.2809 \\
0.0046 \\
1.4470 \\
1.3127 \\
1.3032 \\
0.2431 \\
1.3297 \\
1.3086 \\
1.3300 \\
1.2634 \\
1.2846 \\
1.1942 \\
1.2411 \\
1.3220 \\
1.2214 \\
1.2978 \\
1.2467\end{array}$ & $\begin{array}{l}\text { DDEf } \\
0.1554 \\
0.2107 \\
1.4327 \\
1.5609 \\
1.3395 \\
0.5297 \\
1.9563 \\
1.2713 \\
0.4164 \\
13.5201 \\
0.9645 \\
1.1683 \\
1.1594 \\
0.1113 \\
1.1605 \\
1.0977 \\
0.0287 \\
1.1045 \\
0.1286 \\
1.0527 \\
1.0725 \\
1.1311 \\
1.1006 \\
1.0827 \\
1.2011 \\
1.0660 \\
1.0289 \\
0.0265 \\
0.6752 \\
1.0689 \\
0.0116 \\
1.5310 \\
1.3442 \\
1.1578 \\
0.3019 \\
1.3083 \\
1.2761 \\
1.2874 \\
1.2049 \\
1.1746 \\
1.1261 \\
1.1648 \\
1.2447 \\
1.1378 \\
1.2377 \\
1.1818\end{array}$ \\
\hline
\end{tabular}




\begin{tabular}{|c|c|c|c|}
\hline 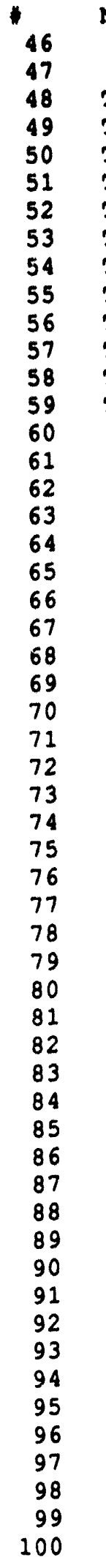 & $\begin{array}{r}\text { NATE } \\
\text { EMPTY } \\
\text { EMPTY } \\
\text { TAI130048 } \\
\text { TAI130136 } \\
\text { TAI130224 } \\
\text { TAI130312 } \\
\text { TRR130000 } \\
\text { TSI000001 } \\
\text { TSI000002 } \\
\text { TSO000001 } \\
\text { TSO000002 } \\
\text { TAF020136 } \\
\text { TAF120312 } \\
\text { TAF130312 } \\
\text { EMPTY } \\
\text { EMPTY } \\
\text { EMPTY } \\
\text { EMPTY } \\
\text { EMPTY } \\
\text { EMPTY } \\
\text { EMPTY } \\
\text { EMPTY } \\
\text { EMPTY } \\
\text { EMPTY } \\
\text { EMPTY } \\
\text { EMPTY } \\
\text { EMPTY } \\
\text { EMPTY } \\
\text { EMPTY } \\
\text { EMPTY } \\
\text { EMPTY } \\
\text { EMPTY } \\
\text { EMPTY } \\
\text { EMFTY } \\
\text { PAN130224 } \\
\text { PAN120312 } \\
\text { PAN130312 } \\
\text { PAB1000136 } \\
\text { PAB125136 } \\
\text { PAN020136 } \\
\text { PAN050136 } \\
\text { PAN070136 } \\
\text { PAN080136 } \\
\text { PAN090136 } \\
\text { PAN100136 } \\
\text { PAN105136 } \\
\text { PAN110136 } \\
\text { PAN115136 } \\
\text { PAN120136 } \\
\text { PAN125136 } \\
\text { PAN130136 } \\
\text { PAN } 120048 \\
\text { PAT }\end{array}$ & $\begin{array}{l}\text { Abs D114 } \\
0.0368 \\
0.0184 \\
1.1943 \\
1.1886 \\
1.1383 \\
1.1951 \\
1.7414 \\
0.1059 \\
0.2825 \\
0.0806 \\
0.0581 \\
1.4119 \\
1.3668 \\
1.2414 \\
0.0264 \\
0.0253 \\
0.0253 \\
0.0252 \\
0.0115 \\
0.0105 \\
0.0110 \\
0.0110 \\
0.0116 \\
0.0106 \\
0.0091 \\
0.0107 \\
0.0098 \\
0.0114 \\
0.0098 \\
0.0111 \\
0.0110 \\
0.0107 \\
0.0111 \\
0.0096 \\
1.1047 \\
0.1282 \\
0.1724 \\
0.1618 \\
0.0626 \\
0.0976 \\
0.0488 \\
0.1190 \\
0.0473 \\
0.1373 \\
0.0076 \\
0.1022 \\
0.1221 \\
0.0519 \\
0.0916 \\
0.0030 \\
0.0428 \\
0.1953 \\
0.0009 \\
0.0330 \\
0.0311\end{array}$ & 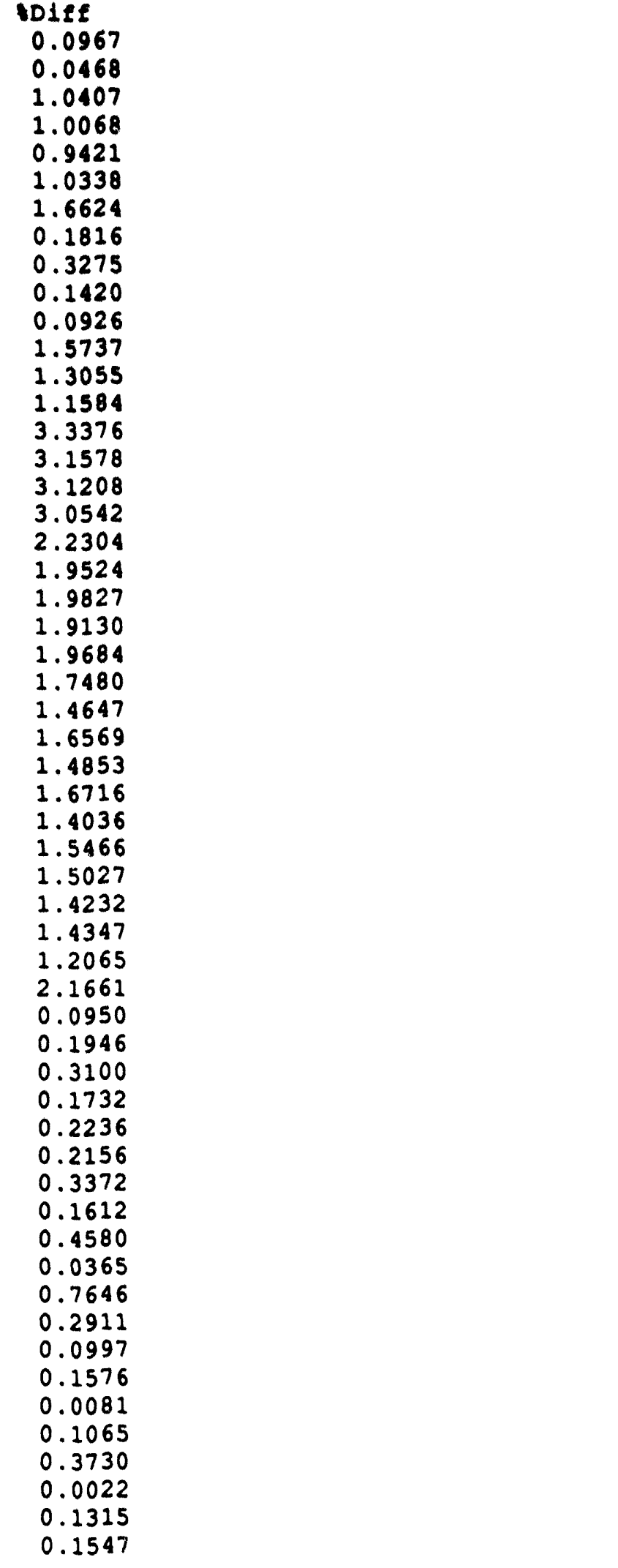 \\
\hline
\end{tabular}




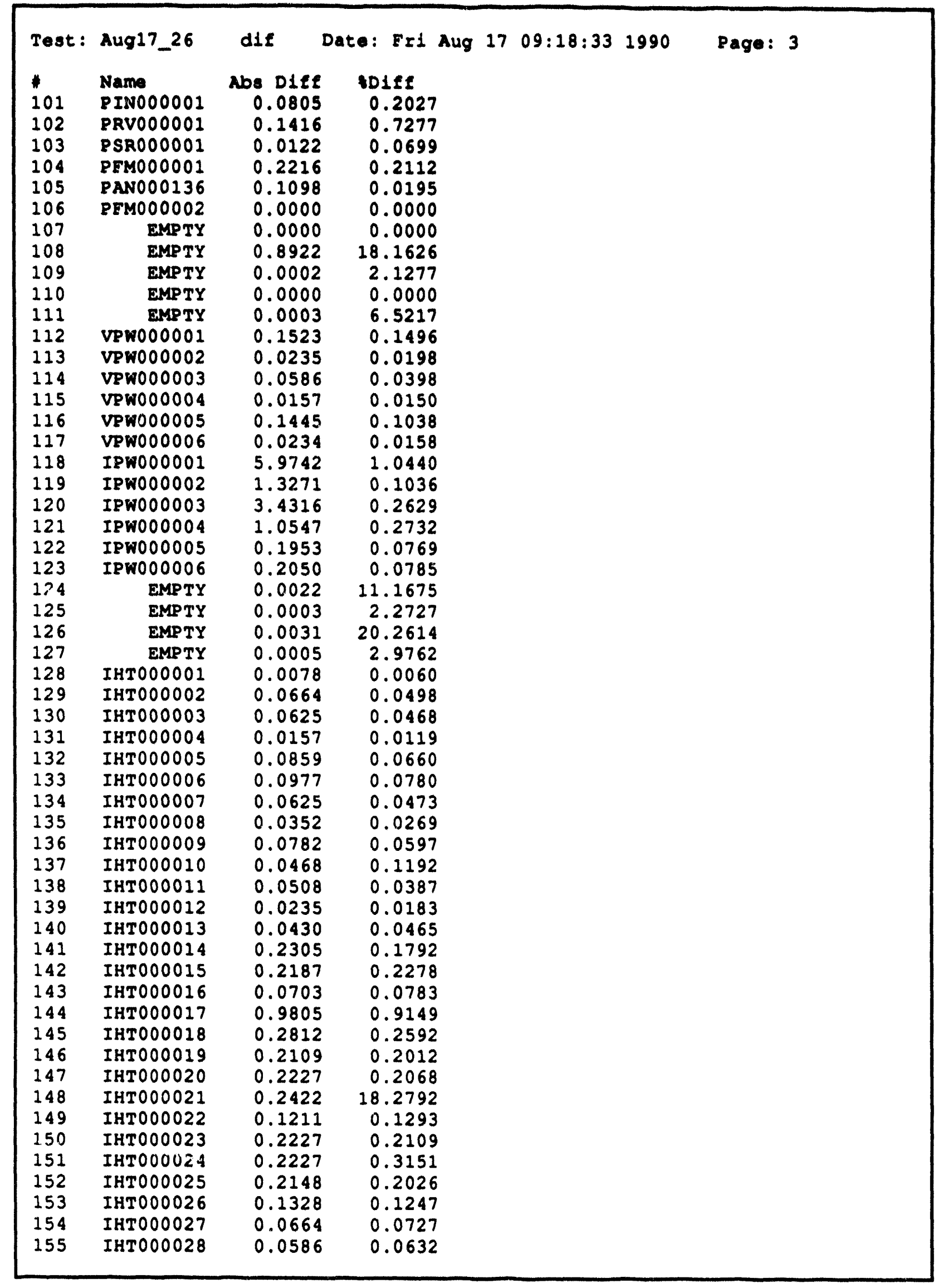


Test: Aug17_26 dif Date: Eri Aug 17 09:18:33 1990 Page: 4

Name Abs Diff \&Diff

IHTO00029 $0.2109 \quad 0.1987$

IHTO00030 $0.1953 \quad 0.1800$

IHT000031 $0.1211 \quad 0.1133$

$\begin{array}{lll}\text { IHTO } 000032 & 0.1055 & 0.0975\end{array}$

IHT000033 $0.0782 \quad 0.0723$

IHTO00034 $0.0195 \quad 0.0205$

$\begin{array}{llll}162 & \text { IHTOOOO35 } & 0.0938 & 0.0749\end{array}$

163 IHT000036 $0.0391 \quad 0.0418$ 


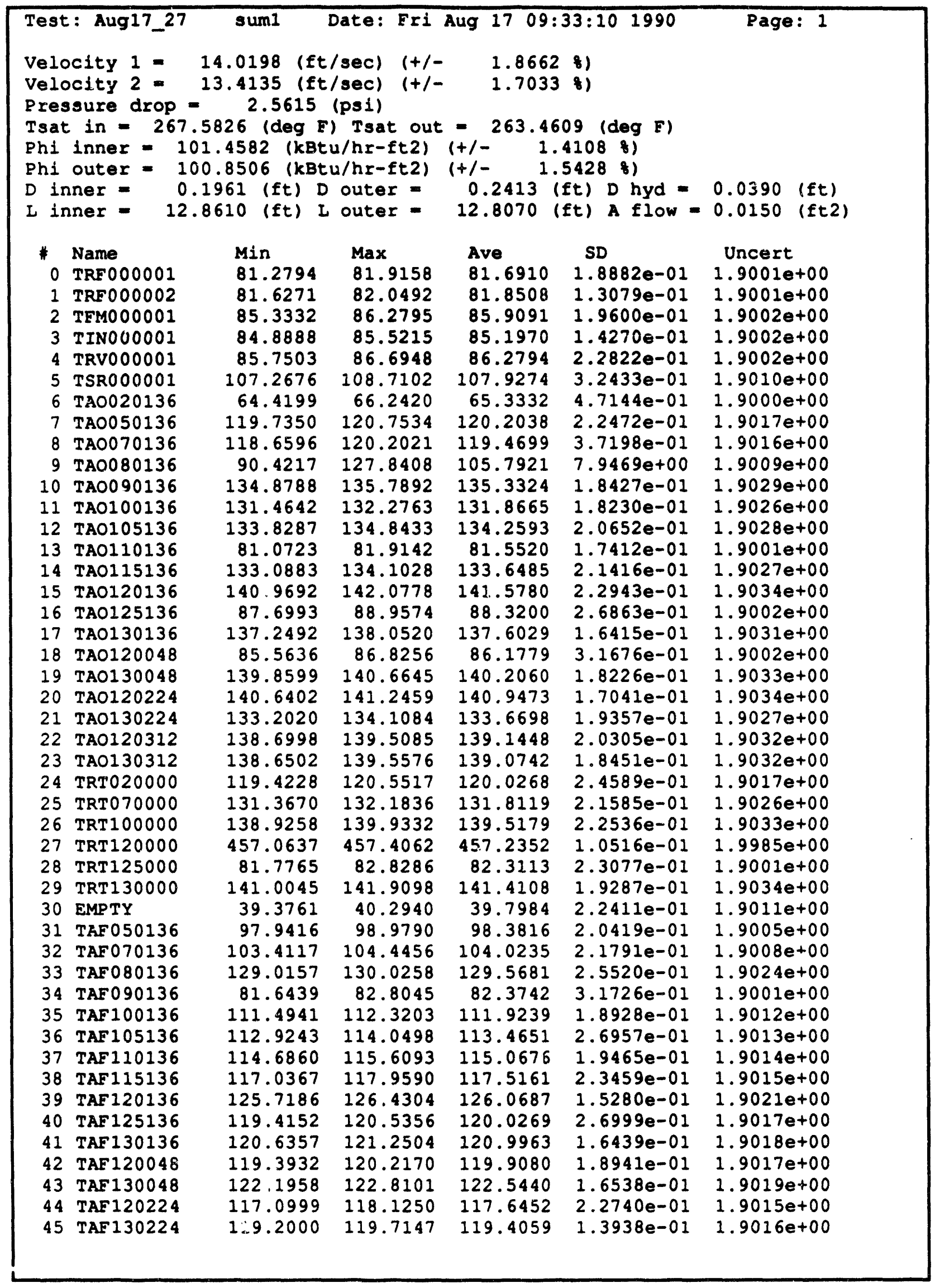




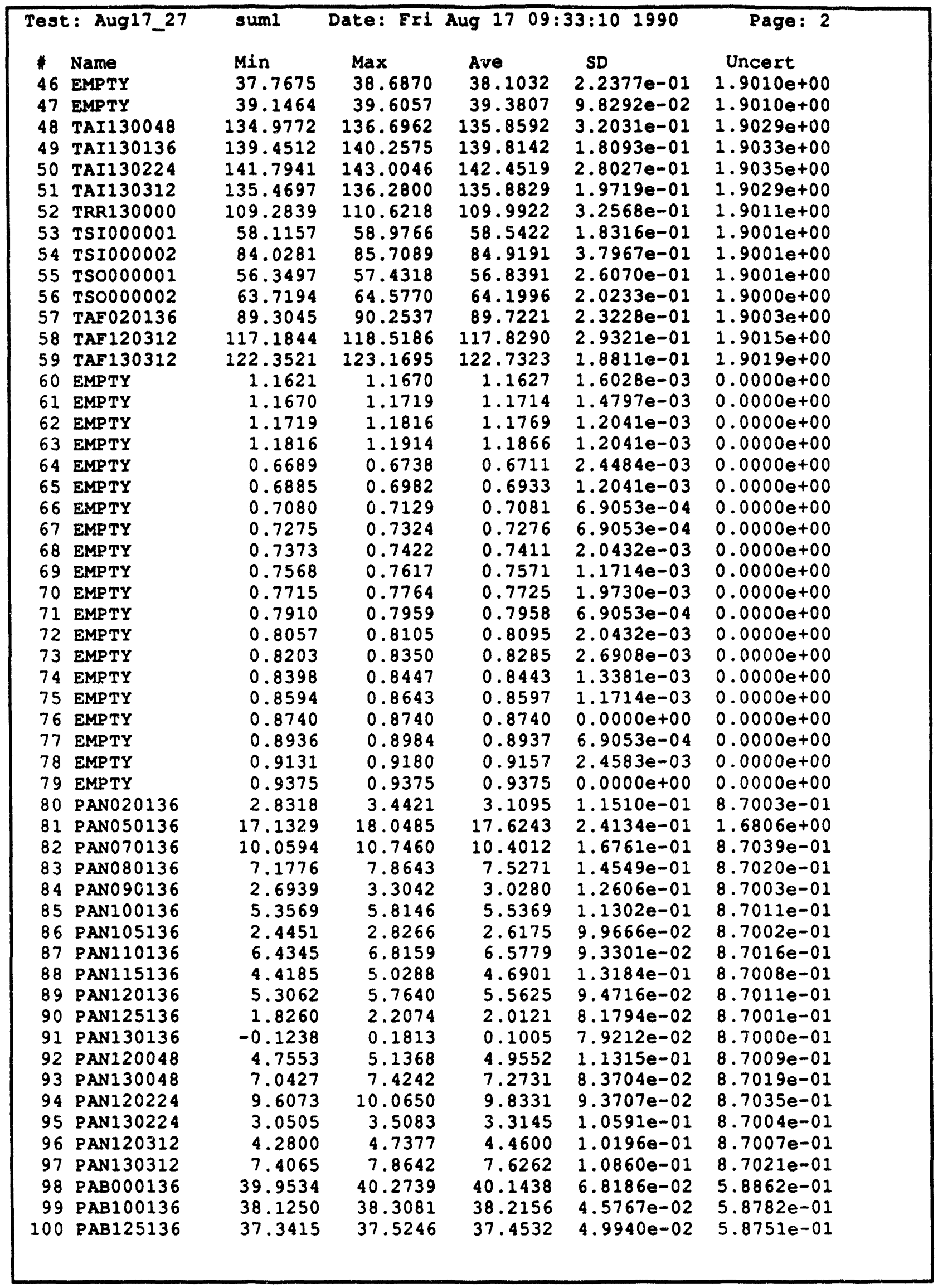




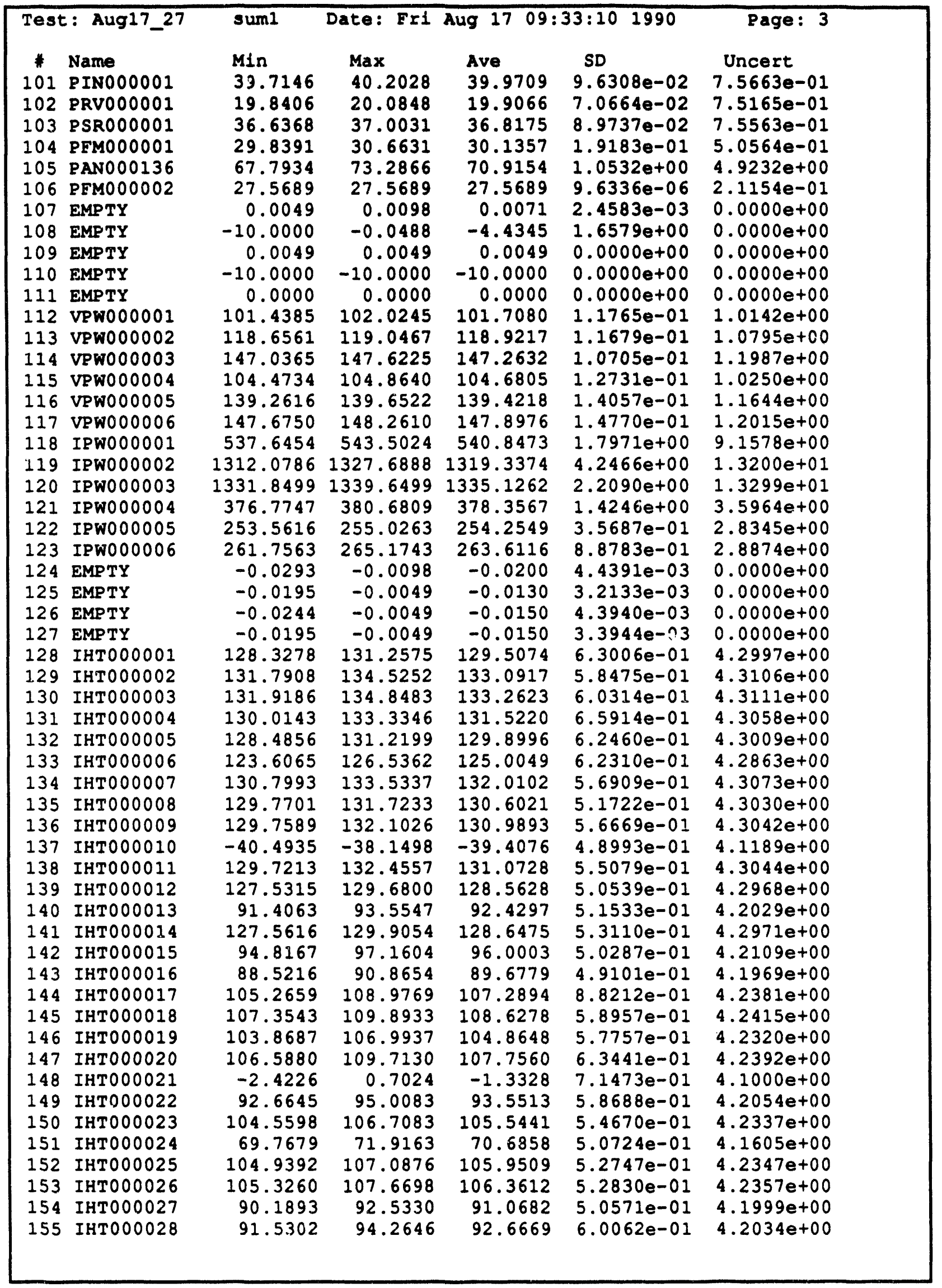




\begin{tabular}{|c|c|c|c|c|c|c|}
\hline $\begin{array}{l}1 \\
156 \\
157 \\
158 \\
159 \\
160 \\
161 \\
162 \\
163\end{array}$ & 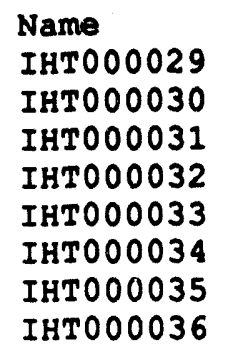 & $\begin{array}{l}\text { Min } \\
105.0105 \\
107.4031 \\
105.6415 \\
107.1326 \\
106.9599 \\
93.9378 \\
124.1136 \\
92.4091\end{array}$ & $\begin{array}{l}\text { Max } \\
107.9402 \\
109.9422 \\
108.1806 \\
109.4764 \\
109.4990 \\
95.6956 \\
126.0667 \\
94.3622\end{array}$ & $\begin{array}{l}\text { Ave } \\
106.1981 \\
108.5438 \\
106.9111 \\
108.1874 \\
108.1201 \\
94.8245 \\
125.1058 \\
93.4794\end{array}$ & $\begin{array}{l}\text { SD } \\
5.9561 e-01 \\
5.5011 e-01 \\
5.5699 e-01 \\
5.4818 e-01 \\
5.5018 e-01 \\
4.5713 e-01 \\
4.5990 e-01 \\
4.6057 e-01\end{array}$ & $\begin{array}{c}\text { Uncert } \\
4.2353 \mathrm{e}+00 \\
4.2412 \mathrm{e}+00 \\
4.2371 \mathrm{e}+00 \\
4.2403 \mathrm{e}+00 \\
4.2402 \mathrm{e}+00 \\
4.2082 \mathrm{e}+00 \\
4.2866 \mathrm{e}+00 \\
4.2052 \mathrm{e}+00\end{array}$ \\
\hline
\end{tabular}




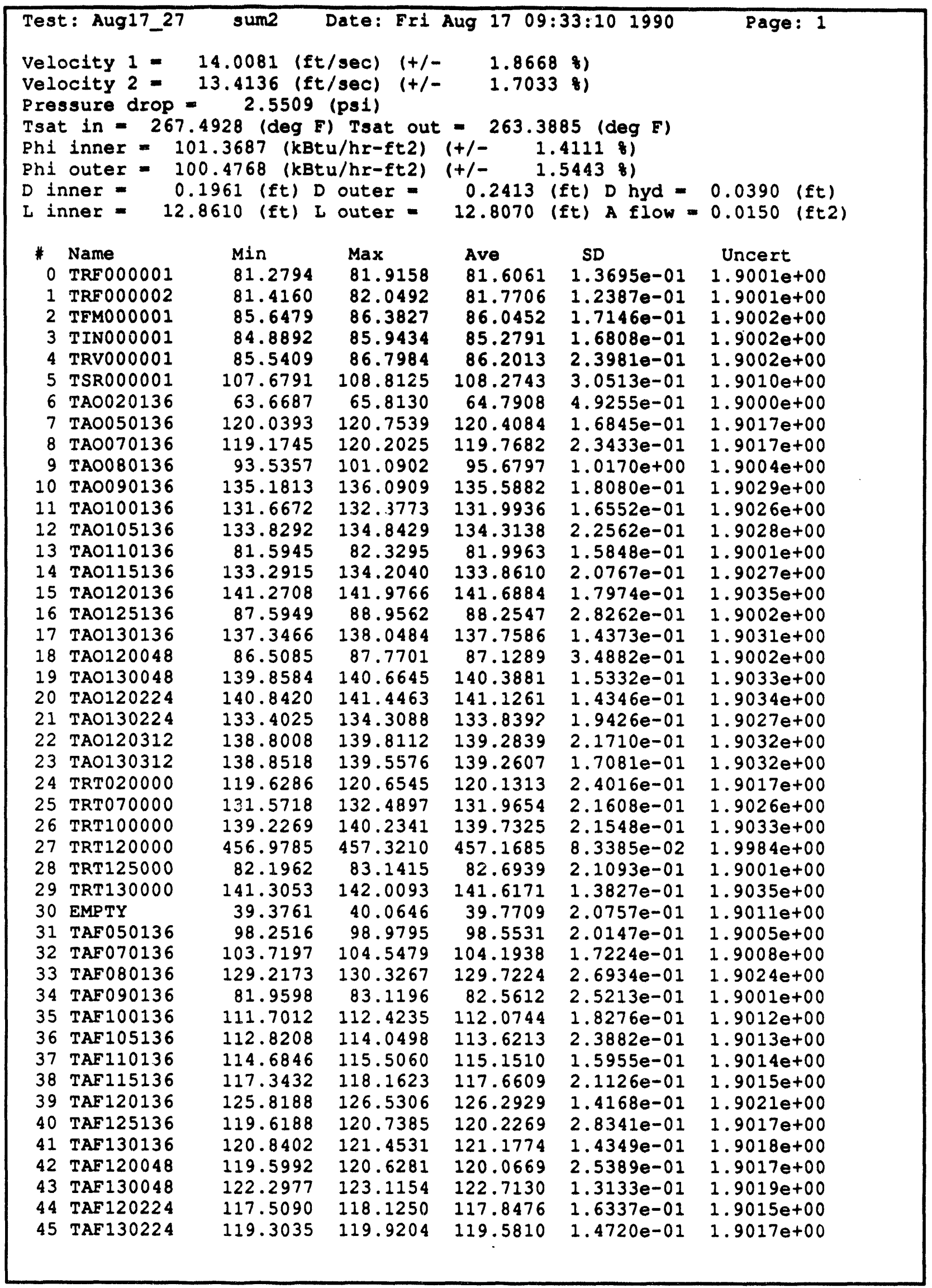




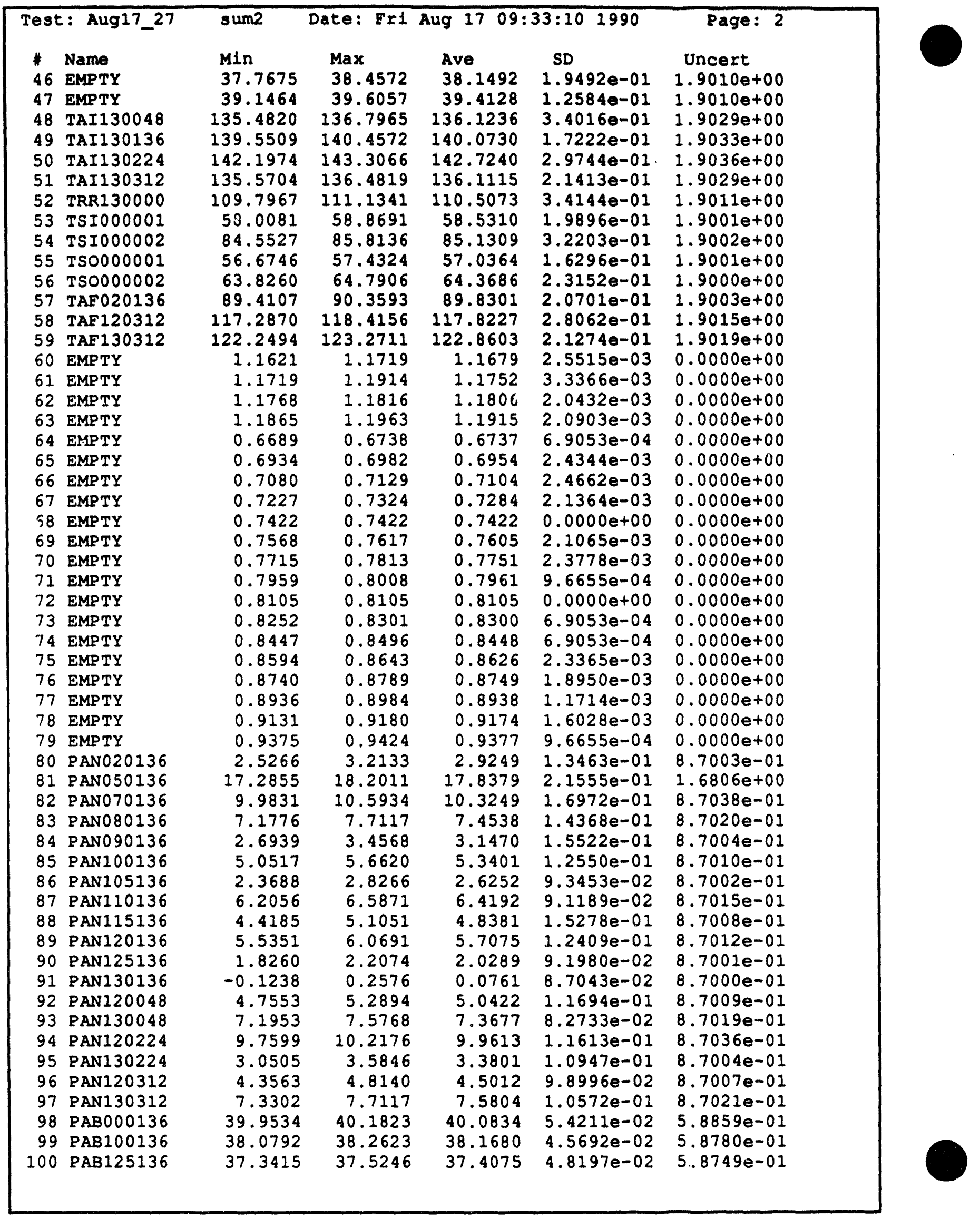




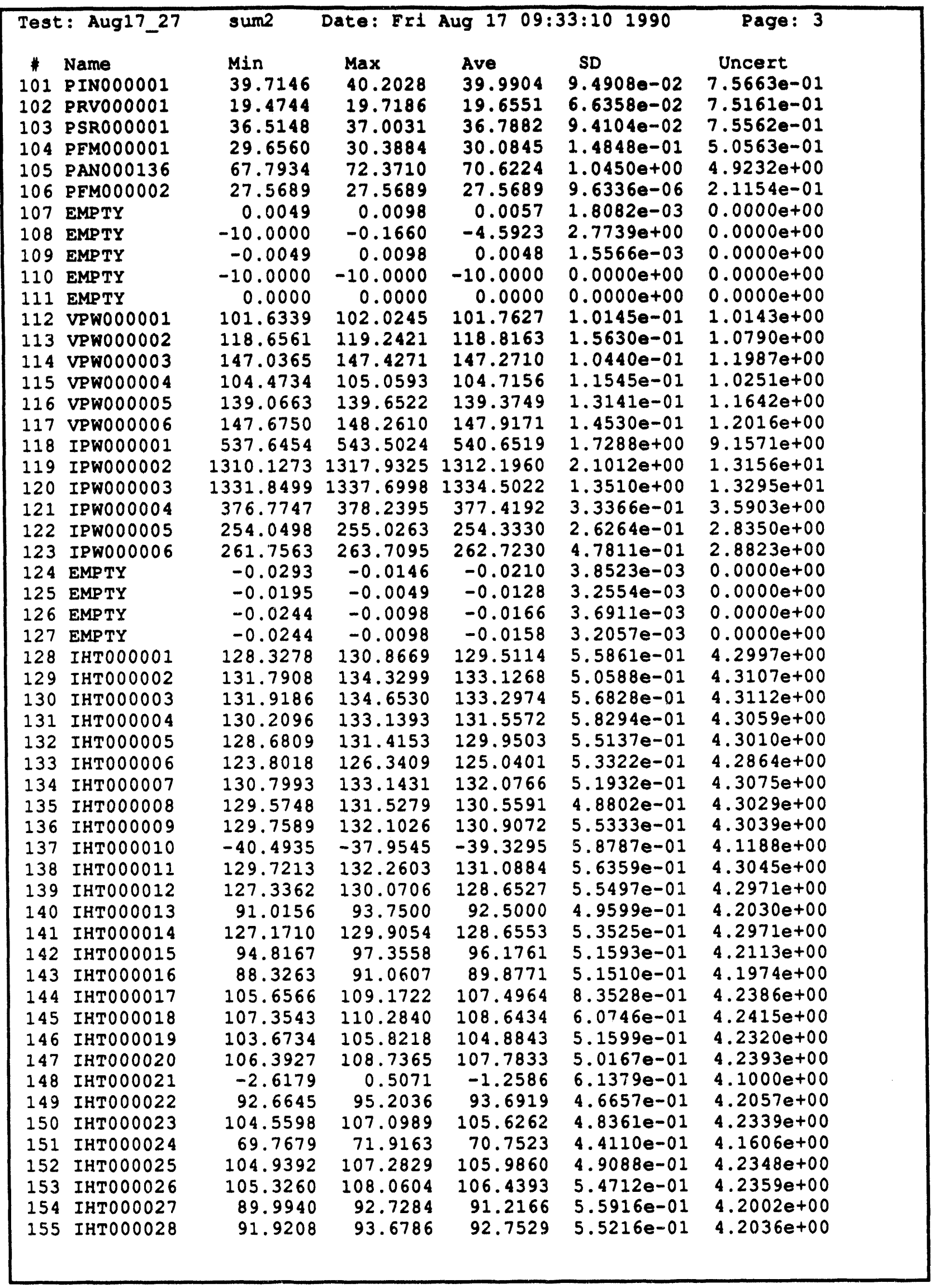




\begin{tabular}{|c|c|c|c|c|c|c|}
\hline $\begin{array}{c}* \\
156 \\
157 \\
158 \\
159 \\
160 \\
161 \\
162 \\
163\end{array}$ & $\begin{array}{l}\text { Name } \\
\text { IHTO00029 } \\
\text { IHT000030 } \\
\text { IHT000031 } \\
\text { IHTO00032 } \\
\text { IHTO00033 } \\
\text { IHTO00034 } \\
\text { IHT000035 } \\
\text { IHT000036 }\end{array}$ & $\begin{array}{r}\text { Min } \\
105.2058 \\
107.5984 \\
105.8368 \\
107.1326 \\
106.9599 \\
93.9378 \\
124.3089 \\
92.7997\end{array}$ & $\begin{array}{l}\text { Max } \\
107.5496 \\
109.9422 \\
108.1806 \\
109.4764 \\
109.6943 \\
96.0862 \\
126.0667 \\
94.3622\end{array}$ & $\begin{array}{r}\text { Ave } \\
106.2371 \\
108.6688 \\
106.9736 \\
108.2616 \\
108.2178 \\
94.9456 \\
125.1605 \\
93.5693\end{array}$ & $\begin{array}{l}\text { SD } \\
5.4539 e-01 \\
5.5558 e-01 \\
5.4489 e-01 \\
5.5704 e-01 \\
5.7203 e-01 \\
4.9341 e-01 \\
5.2014 e-01 \\
4.6086 e-01\end{array}$ & $\begin{array}{c}\text { Uncert } \\
4.2354 e+00 \\
4.2416 e+00 \\
4.2373 e+00 \\
4.2405 e+00 \\
4.2404 e+00 \\
4.2085 e+00 \\
4.2868 \theta+00 \\
4.2054 e+00\end{array}$ \\
\hline
\end{tabular}




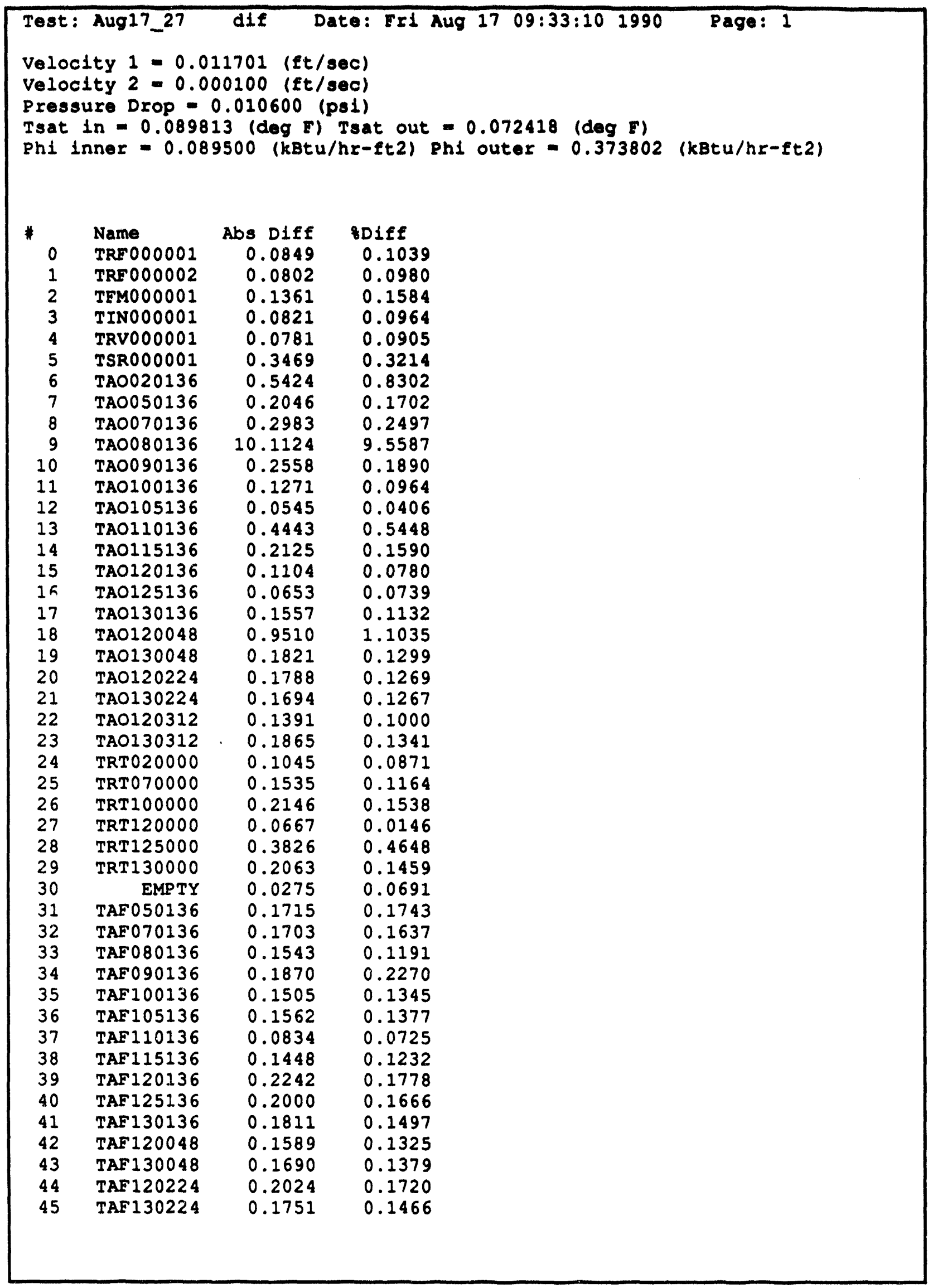




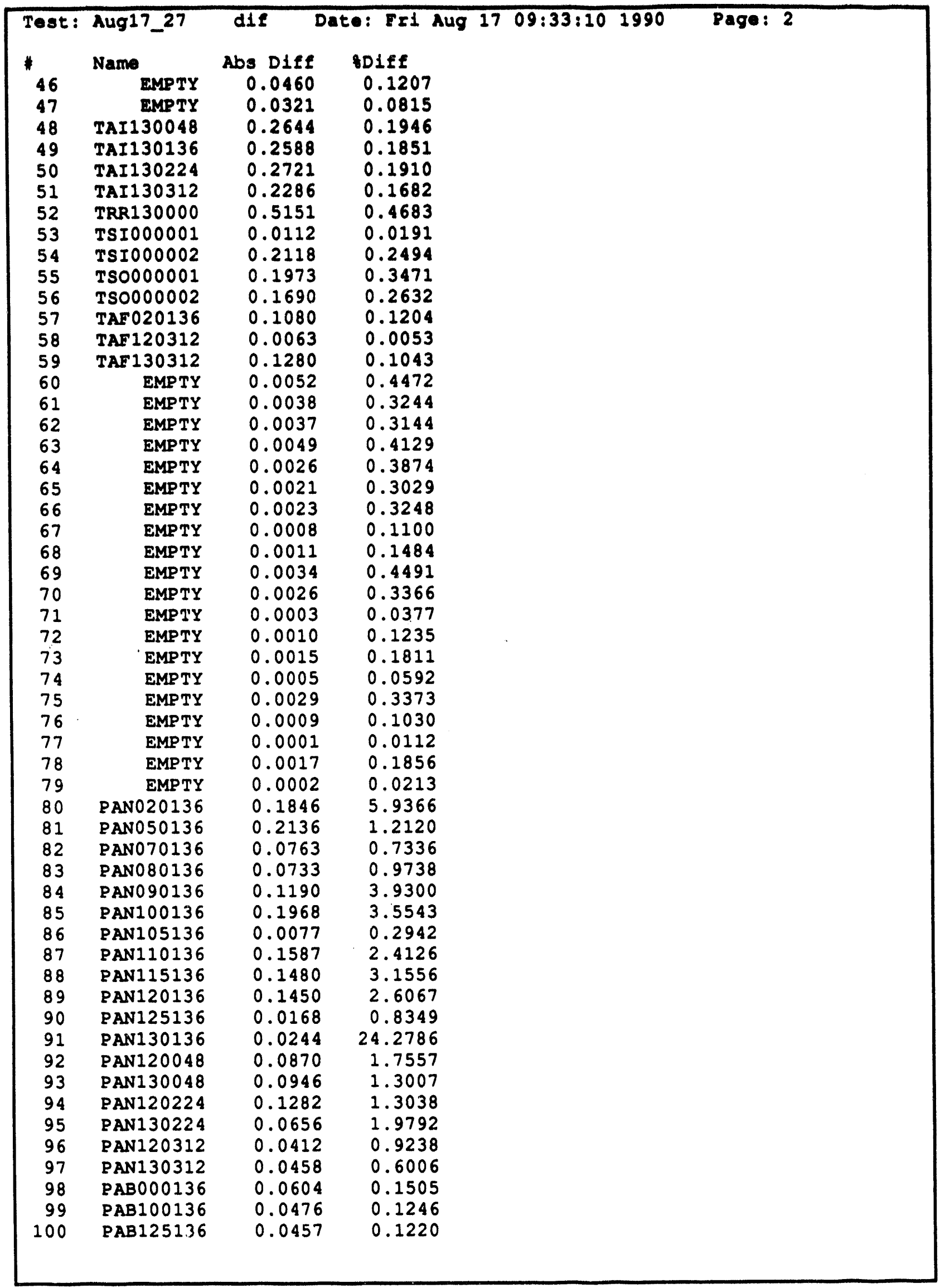




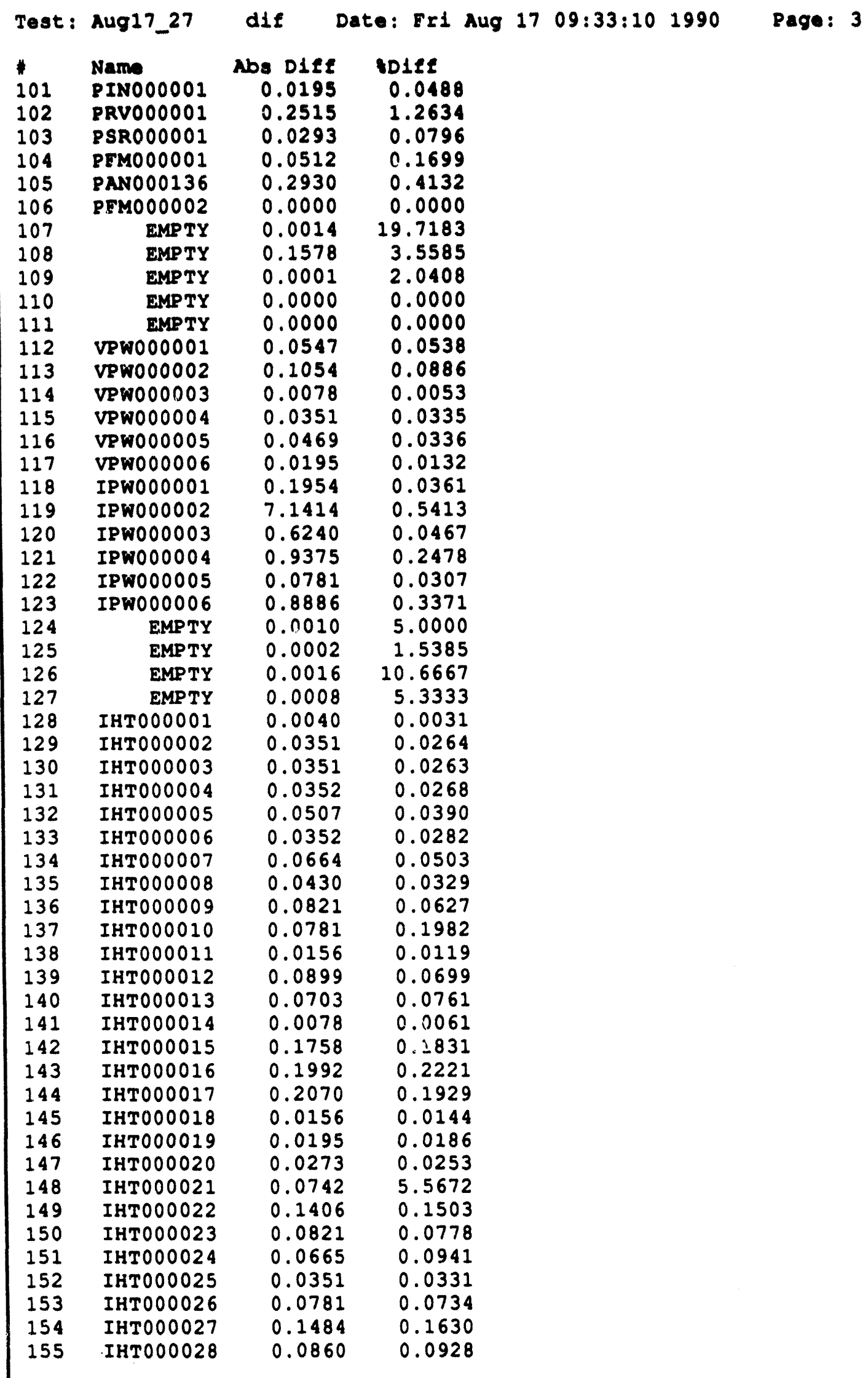


Test: Kug17_27 dif Date: Fr1 Aug 17 09:33:10 1990 Page: 4

Name Abs Dife tDiff

$\begin{array}{lll}\text { IHT } 000029 & 0.0390 & 0.0367\end{array}$

$\begin{array}{lll}\text { IHTO00030 } & 0.1250 & 0.1152\end{array}$

$\begin{array}{lll}\text { IHTO00031 } & 0.0625 & 0.0585\end{array}$

IHT000032 $0.0742 \quad 0.0686$

$\begin{array}{lll}\text { IHTO00033 } & 0.0977 & 0.0904\end{array}$

$\begin{array}{lll}\text { IHTOO00034 } & 0.1211 & 0.1277\end{array}$

$\begin{array}{llll}162 & \text { IHTO00035 } & 0.0547 & 0.0437 \\ 163 & \text { IHTO00036 } & 0.0899 & 0.0962\end{array}$ 


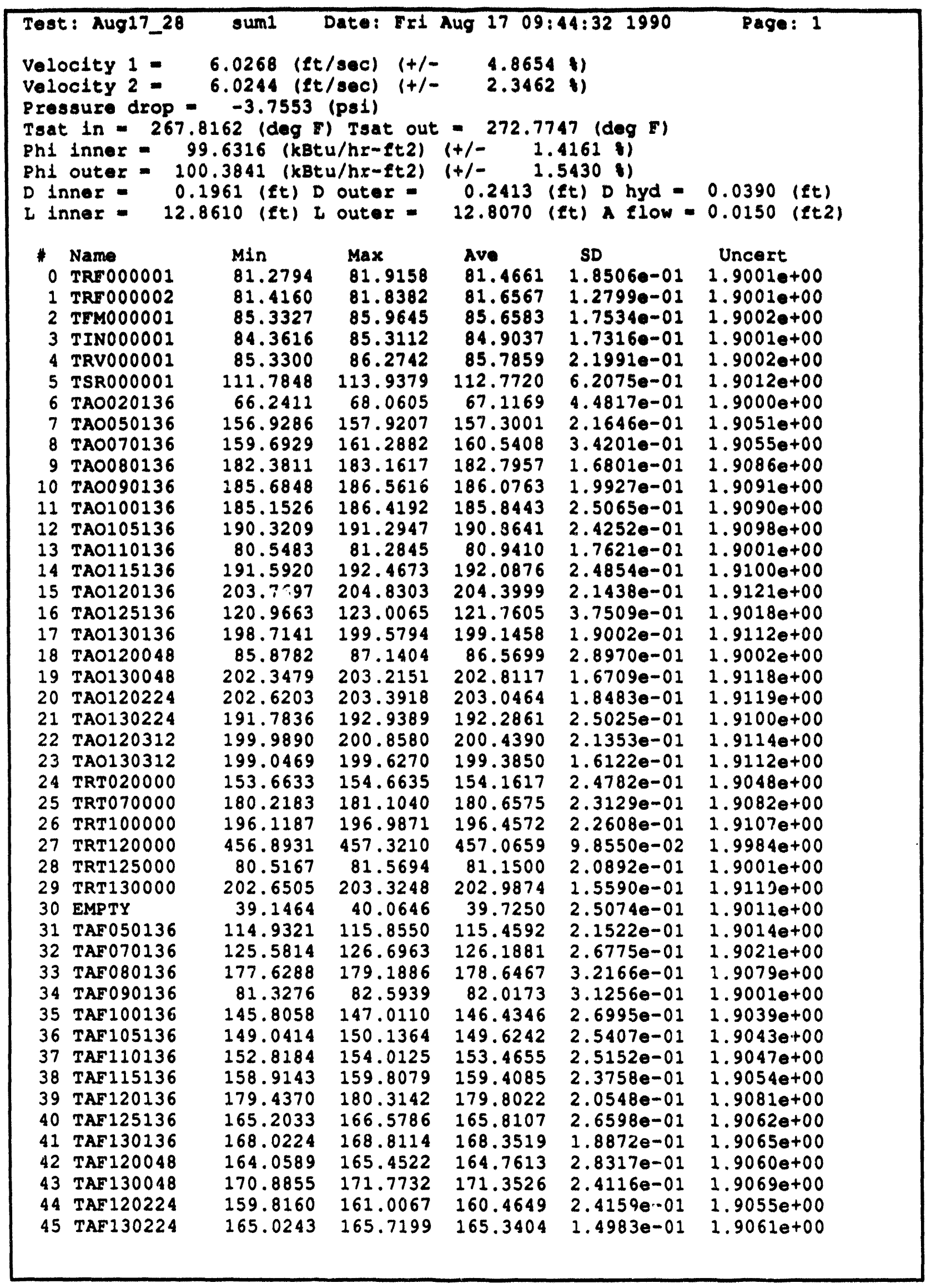



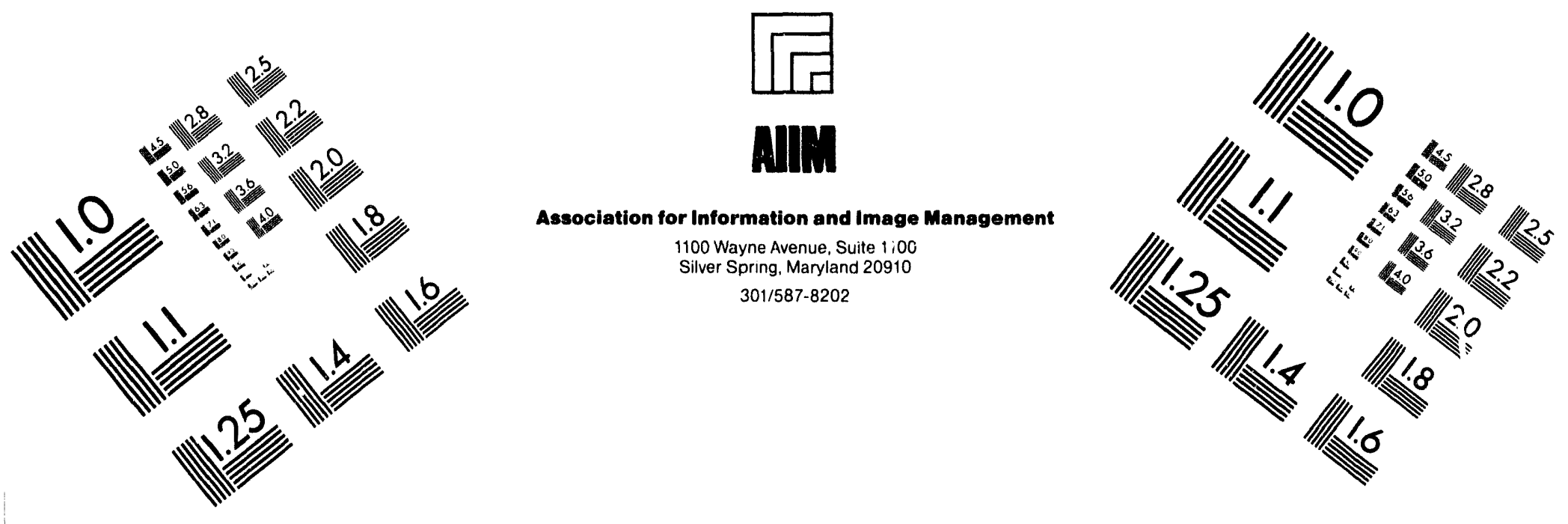

\section{Centimeter}

$\begin{array}{llllllllllllllll}1 & 2 & 3 & 4 & 5 & 6 & 7 & 8 & 9 & 10 & 11 & 12 & 13 & 14 & 15 & \mathrm{~mm}\end{array}$

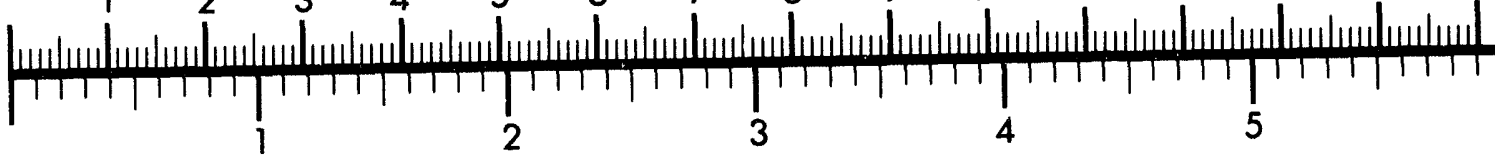
Inches

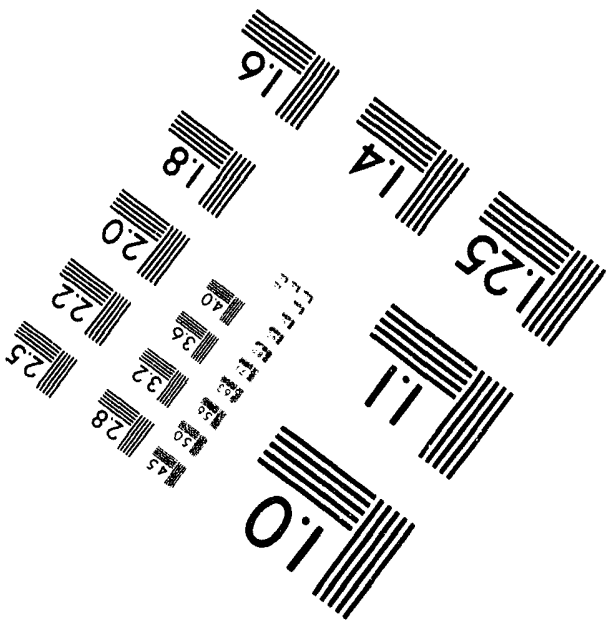

MANUFACTURED TO AIIM STANDARDS

BY APPLIED IMAGE, INC.

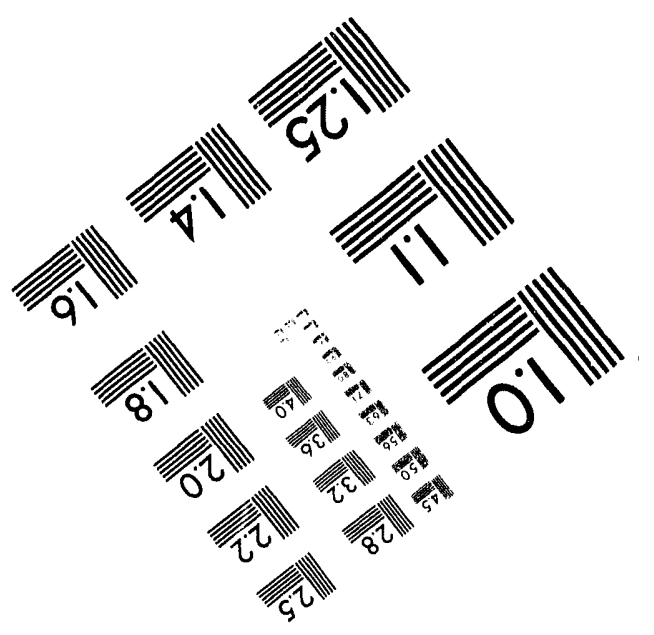



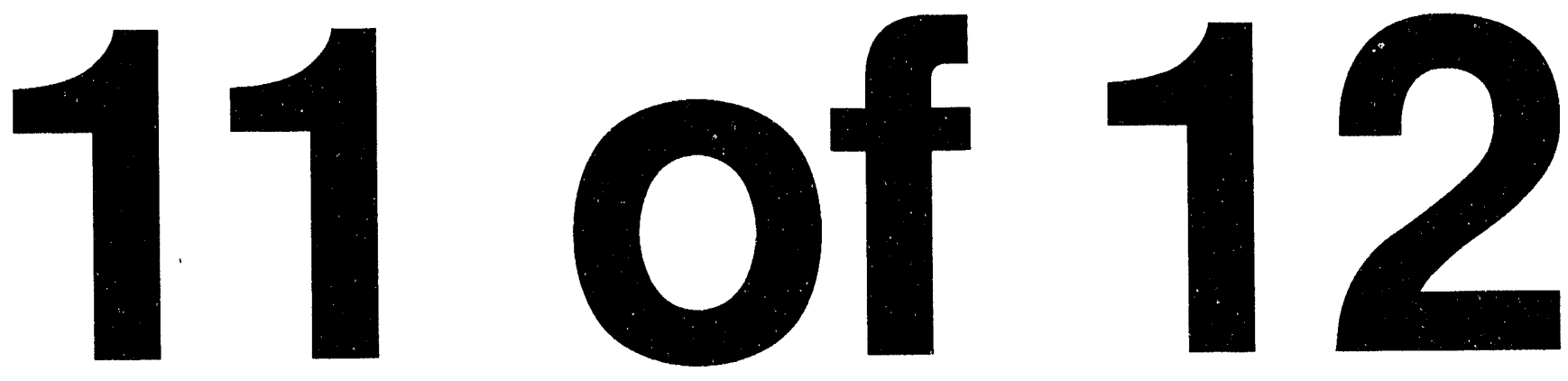


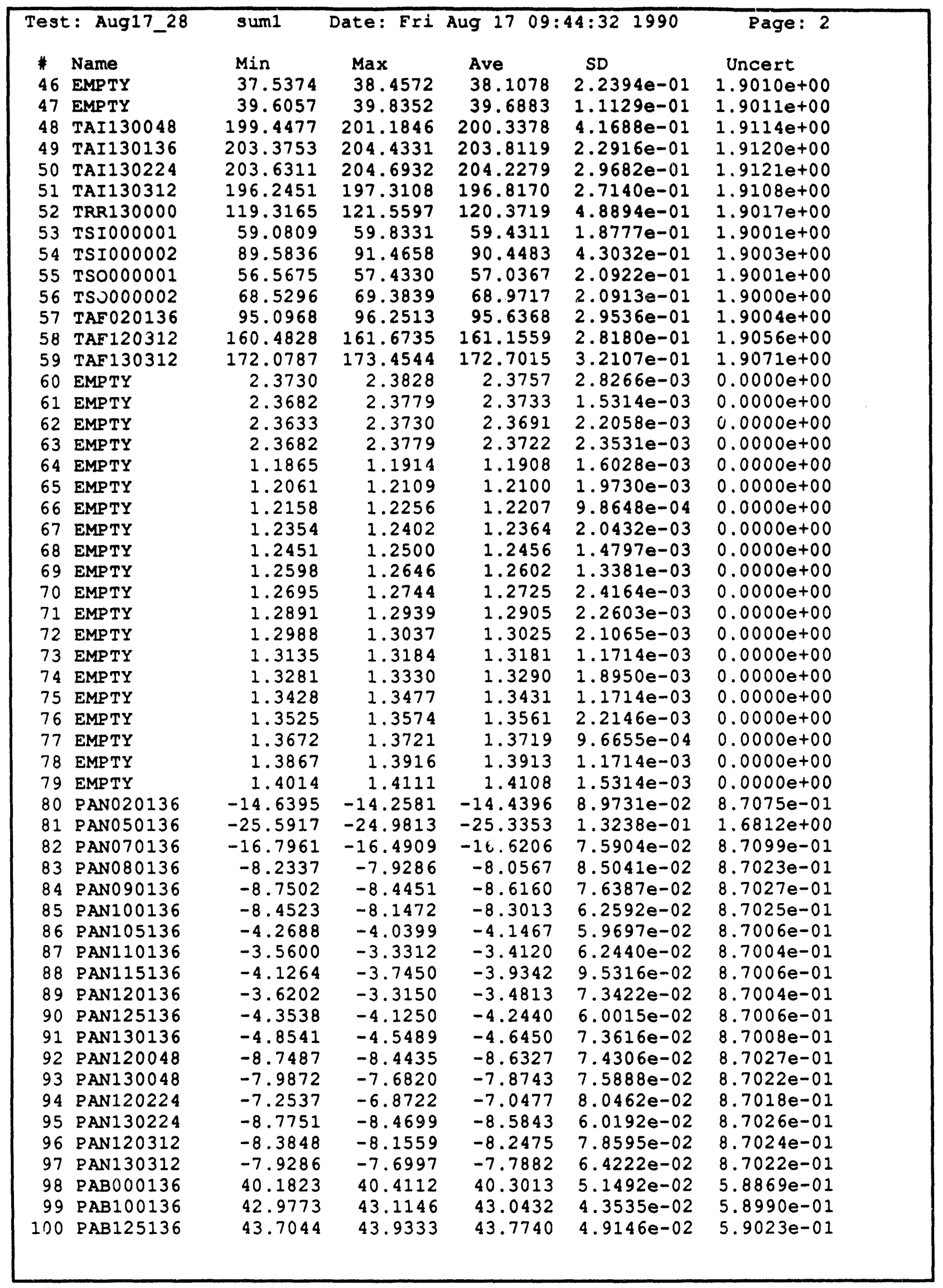




\begin{tabular}{|c|c|c|c|c|c|c|}
\hline \multicolumn{2}{|c|}{ Test: Aug17_28 } & sum 1 & Date: Fri & Aug 1709 & $4: 32 \quad 1990$ & Rage: 3 \\
\hline \# & Name & Min & $\operatorname{Max}$ & Ave & SD & Uncert \\
\hline 101 & PIN 000001 & 39.8366 & 40.2028 & 40.0173 & $8.2682 e-02$ & $7.5664 e-01$ \\
\hline 102 & PRV000001 & 19.7186 & 19.9627 & 19.8406 & $3.4877 e-02$ & $7.5164 e-01$ \\
\hline 103 & PSR0000001 & 43.7169 & 43.9611 & 43.8512 & $5.6508 e-02$ & $7.5797 e-01$ \\
\hline 104 & PEMO00001 & 5.3030 & 5.5776 & 5.4714 & $8.3460 e-02$ & $5.0019 e-01$ \\
\hline 105 & PAN 000136 & -104.7835 & -102.4947 & -103.9687 & $5.5712 e-01$ & $4.9269 e+00$ \\
\hline 106 & PFM000002 & 5.4028 & 5.5538 & 5.4669 & $3.1926 \mathrm{e}-02$ & $2.0047 e-01$ \\
\hline 107 & EMPTY & -0.0049 & 0.0049 & 0.0040 & $2.5515 e-03$ & $0.0000 e+00$ \\
\hline 108 & EMPTY & -10.0000 & -0.7910 & -6.1399 & $2.9540 \mathrm{e}+00$ & $0.0000 e+00$ \\
\hline 109 & EMPTY & 0.0000 & 0.0049 & 0.0021 & $2.4344 e-03$ & $0.0000 e+00$ \\
\hline 110 & EMPTY & -10.0000 & -10.0000 & -10.0000 & $0.0000 e+00$ & $0.0000 e+00$ \\
\hline 111 & EMPTY & 0.0000 & 0.0000 & 0.0000 & $0.0000 e+00$ & $0.0000 e+00$ \\
\hline 112 & VPW000001 & 101.6339 & 102.0245 & 101.8760 & $1.2200 \mathrm{e}-01$ & $1.0148 e+00$ \\
\hline 113 & VPW000002 & 119.0467 & 119.4374 & 119.1288 & $1.0507 e-01$ & $1.0803 e+00$ \\
\hline 114 & VPW000003 & 147.2318 & 147.6225 & 147.4311 & $1.2156 e-01$ & $1.1994 \mathrm{e}+00$ \\
\hline 115 & VPW000004 & 104.6687 & 105.0593 & 104.9149 & $1.2974 \mathrm{e}-01$ & $1.0258 e+00$ \\
\hline 116 & VPW000005 & 139.4569 & 140.0428 & 139.7030 & $1.2974 \mathrm{e}-01$ & $1.1656 e+00$ \\
\hline 117 & VPW000006 & 148.0657 & 148.6516 & 148.3468 & $1.3760 e-01$ & $1.2035 e+00$ \\
\hline 118 & IPWO00001 & 559.1212 & 566.9306 & 564.0022 & $1.9424 \mathrm{e}+00$ & $9.2446 \mathrm{e}+00$ \\
\hline 119 & IPW000002 & 1276.9558 & 1294.5172 & 1282.6923 & $4.5533 e+00$ & $1.2973 e+01$ \\
\hline 120 & IPW000003 & 1302.5999 & 1312.3499 & 1306.7336 & $2.8035 e+00$ & $1.3122 e+01$ \\
\hline 121 & IPW000004 & 379.7044 & 386.5403 & 381.4329 & $1.1846 \mathrm{e}+00$ & $3.6166 \mathrm{e}+00$ \\
\hline 122 & IPW000005 & 252.5851 & 253.5616 & 253.0537 & $3.4112 e-01$ & $2.8278 e+00$ \\
\hline 123 & IPW000006 & 260.2915 & 261.7563 & 260.9261 & $3.9766 e-01$ & $2.8721 e+00$ \\
\hline 124 & ЕMPTY & -0.0244 & -0.0098 & -0.0190 & $4.2142 e-03$ & $0.0000 e+00$ \\
\hline 125 & EMPTY & -0.0195 & -0.0049 & -0.0131 & $3.3366 e-03$ & $0.0000 e+00$ \\
\hline 126 & EMPTY & -0.0244 & -0.0049 & -0.0141 & $4.6938 e-03$ & $0.0000 e+00$ \\
\hline 127 & EMPTY & -0.0244 & -0.0098 & -0.0162 & $3.6165 e-03$ & $0.0000 e+00$ \\
\hline 128 & IHT000001 & 128.1325 & 130.6716 & 129.2692 & $6.0963 e-01$ & $4.2990 e+00$ \\
\hline 129 & IHT000002 & 131.7908 & 134.1346 & 132.8768 & $5.5265 e-01$ & $4.3099 e+00$ \\
\hline 130 & IHT000003 & 131.9186 & 134.2623 & 133.1333 & $5.8963 e-01$ & $4.3107 e+00$ \\
\hline 131 & IHT000004 & 130.2096 & 132.7486 & 131.4009 & $5.7487 e-01$ & $4.3054 e+00$ \\
\hline 132 & IHT000005 & 128.6809 & 131.0246 & 129.7628 & $5.1329 e-01$ & $4.3004 e+00$ \\
\hline 133 & IHT000006 & 123.8018 & 126.3409 & 124.8018 & $5.0631 e-01$ & $4.2857 e+00$ \\
\hline 134 & IHT000007 & 130.7993 & 133.1431 & 131.7524 & $5.0477 e-01$ & $4.3065 e+00$ \\
\hline 135 & IHT000008 & 129.3795 & 131.7233 & 130.3052 & $5.2765 e-01$ & $4.3021 e+00$ \\
\hline 136 & IHT000009 & 129.5635 & 132.2979 & 130.7002 & $5.3188 e-01$ & $4.3033 e+00$ \\
\hline 137 & IHT000010 & -41.0795 & -38.7357 & -39.6771 & $5.5900 \mathrm{e}-01$ & $4.1192 \mathrm{e}+00$ \\
\hline 138 & IHT000011 & 129.7213 & 132.0650 & 131.0181 & $5.3198 e-01$ & $4.3043 e+00$ \\
\hline 139 & IHT 000012 & 127.3362 & 129.6800 & 128.5472 & $5.0068 e-01$ & $4.2968 e+00$ \\
\hline 140 & IHTO00013 & 91.0156 & 93.3594 & 92.1953 & $4.8802 e-01$ & $4.2024 \mathrm{e}+00$ \\
\hline 141 & IHT000014 & 127.3663 & 129.5147 & 128.3624 & $4.6564 e-01$ & $4.2962 \mathrm{e}+00$ \\
\hline 142 & IHT000015 & 94.8167 & 96.7698 & 95.8440 & $4.4784 e-01$ & $4.2105 e+00$ \\
\hline 143 & IHT000016 & 88.3263 & 90.4747 & 89.4435 & $4.7028 e-01$ & $4.1964 e+00$ \\
\hline 144 & IHT000017 & 105.2659 & 108.9769 & 107.1527 & $9.6300 e-01$ & $4.2377 e+00$ \\
\hline 145 & IHT000018 & 106.9636 & 109.6980 & 108.4168 & $5.6104 e-01$ & $4.2409 \mathrm{e}+00$ \\
\hline 146 & IHT000019 & 103.4781 & 106.2124 & 104.6069 & $4.9645 e-01$ & $4.2313 e+00$ \\
\hline 147 & IHTO00020 & 106.5880 & 108.9318 & 107.4826 & $5.2213 e-01$ & $4.2385 e+00$ \\
\hline 148 & IHT000021 & -2.6179 & 0.1164 & -1.2859 & $6.1850 e-01$ & $4.1000 e+00$ \\
\hline 149 & IHT000022 & 92.4692 & 94.4223 & 93.3716 & $4.8148 e-01$ & $4.2050 e+00$ \\
\hline 150 & IHTO00023 & 104.5598 & 106.5129 & 105.3684 & $4.3764 \mathrm{e}-01$ & $4.2332 e+00$ \\
\hline 151 & IHT000024 & 69.7679 & 71.5257 & 70.4944 & $4.2506 e-01$ & $4.1602 e+00$ \\
\hline 152 & IHT000025 & 105.1345 & 106.6970 & 105.7751 & $4.1953 e-01$ & $4.2342 \mathrm{e}+00$ \\
\hline 153 & IHT000026 & 105.5213 & 137.0838 & 106.2401 & $4.3304 e-01$ & $4.2354 e+00$ \\
\hline 154 & IHT000027 & 89.9940 & 91.7518 & 90.9002 & $4.0387 e-01$ & $4.1996 e+00$ \\
\hline 155 & IHT000028 & 91.5302 & 93.4833 & 92.3779 & $5.8075 e-01$ & $4.2028 e+00$ \\
\hline
\end{tabular}




\begin{tabular}{|c|c|c|c|c|c|c|}
\hline \multicolumn{2}{|c|}{ Test: Aug17_28 } & sum 1 & Date: Fri & Aug 1709 & $14: 32 \quad 1990$ & Page: 4 \\
\hline$\#$ & Name & Min & Max & Ave & SD & Uncert \\
\hline 156 & IHTO000029 & 105.0105 & 107.3543 & 105.9324 & $6.0237 e-01$ & $4.2346 e+00$ \\
\hline 157 & IHT 000030 & 107.4031 & 109.5515 & 108.2938 & $5.4270 e-01$ & $4.2406 e+00$ \\
\hline 158 & IHTO000031 & 105.8368 & 107.9853 & 106.6689 & $5.2171 e-01$ & $4.2365 e+00$ \\
\hline 159 & IHTO000032 & 107.1326 & 109.0858 & 107.9530 & $4.7679 e-01$ & $4.2397 e+00$ \\
\hline 160 & IHT 000033 & 107.1552 & 109.1083 & 107.9053 & $4.6414 e-01$ & $4.2396 \mathrm{e}+00$ \\
\hline 161 & IHTO000034 & 93.7425 & 95.5003 & 94.6683 & $4.1349 e-01$ & $4.2079 e+00$ \\
\hline 162 & IHTO000035 & 123.9183 & 125.6761 & 124.8988 & $4.2221 e-01$ & $4.2860 e+00$ \\
\hline 163 & IHT000036 & 92.4091 & 94.3622 & 93.3271 & $4.3270 e-01$ & $4.2049 e+00$ \\
\hline
\end{tabular}




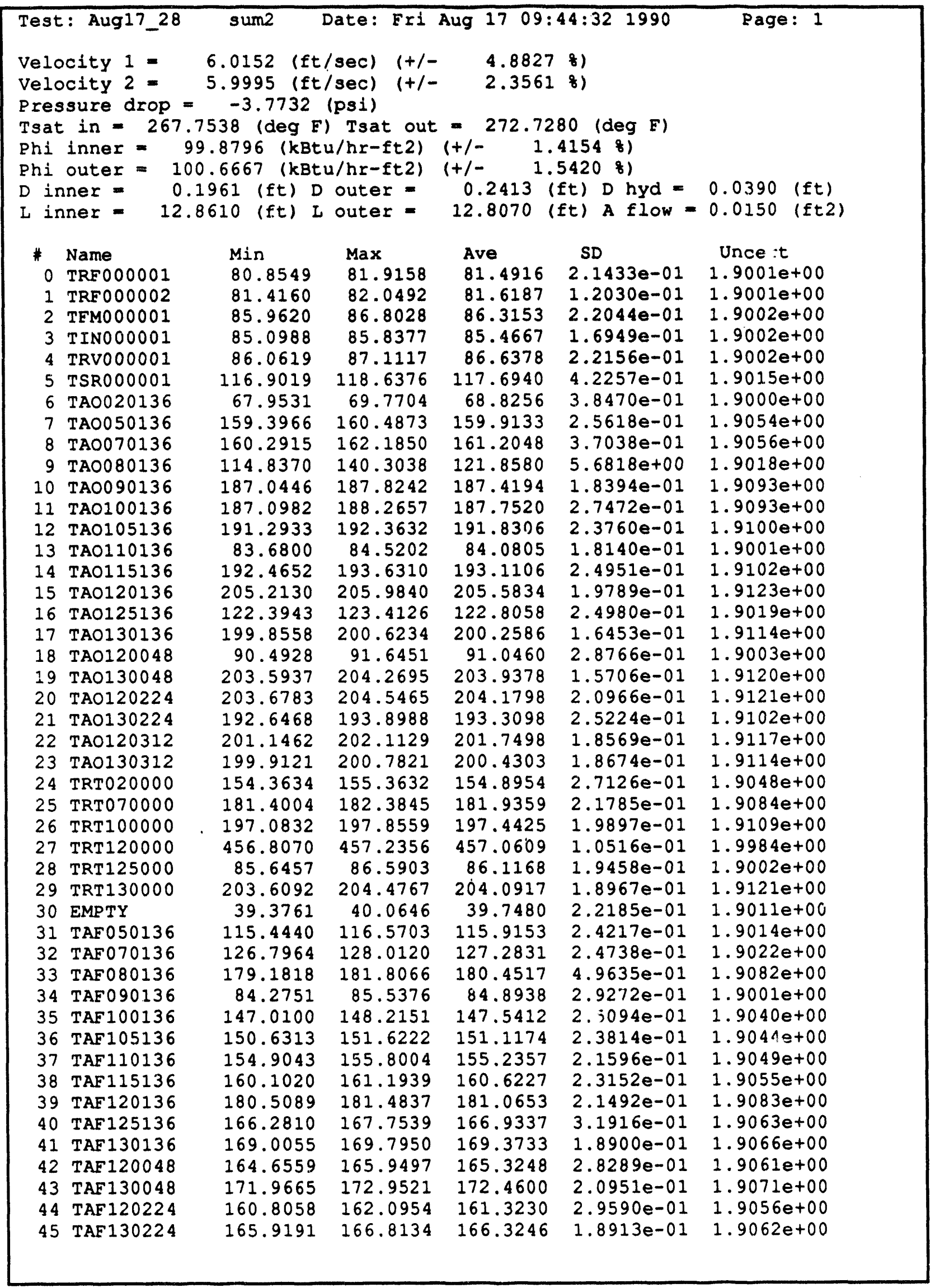




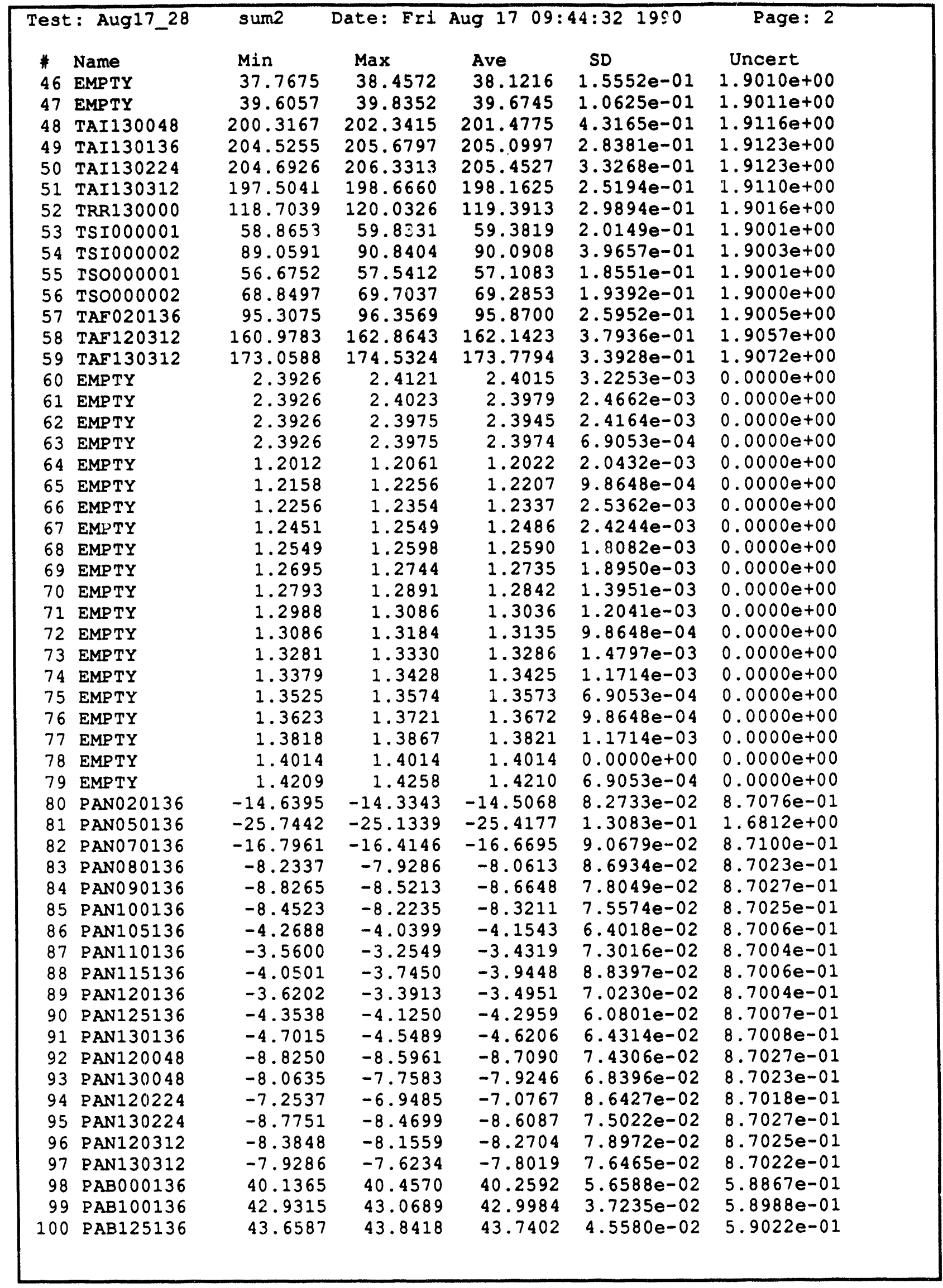




\begin{tabular}{|c|c|c|c|c|c|c|}
\hline \multicolumn{2}{|c|}{ Test: Aug17_28 } & $\operatorname{sum} 2$ & Date: Fri & Aug 1709 & $4: 321990$ & Page: 3 \\
\hline * & Name & Min & $\operatorname{Max}$ & Ave & SD & Uncert \\
\hline 101 & PIN 000001 & 39.8366 & 40.2028 & 40.0027 & $7.2993 e-02$ & $7.5664 \mathrm{e}-01$ \\
\hline 102 & PRV000001 & 19.5965 & 19.8406 & 19.7332 & $5.3123 e-02$ & $7.5162 e-01$ \\
\hline 103 & PSR000001 & 43.7169 & 43.9611 & 43.8195 & $6.2195 e-02$ & $7.5796 e-01$ \\
\hline 104 & PFM000001 & 5.2114 & 5.6692 & 5.4495 & $9.9606 e-02$ & $5.0019 e-01$ \\
\hline 105 & PAN 000136 & -105.6990 & -102.9525 & -104.4631 & $6.9362 e-01$ & $4.9269 e+00$ \\
\hline 106 & PFM000002 & 5.3356 & 5.4867 & 5.4206 & $3.4138 e-02$ & $2.0046 e-01$ \\
\hline 107 & EMPTY & -0.0049 & 0.0049 & 0.0038 & $2.6635 e-03$ & $0.0000 e+00$ \\
\hline 108 & EMPTY & -10.0000 & -0.2783 & -4.1710 & $2.5505 e+00$ & $0.0000 e+00$ \\
\hline 109 & EMPTY & 0.0000 & 0.0049 & 0.0021 & $2.4484 e-03$ & $0.0000 \mathrm{e}+00$ \\
\hline 110 & EMPTY & -10.0000 & -10.0000 & -10.0000 & $0.0000 e+00$ & $0.0000 e+00$ \\
\hline 111 & EMPTY & -0.0049 & 0.0000 & -0.0001 & $6.9053 e-04$ & $0.0000 \mathrm{e}+00$ \\
\hline 112 & VPWO00001 & 101.8292 & 102.2198 & 102.0010 & $1.0168 e-01$ & $1.0152 \mathrm{e}+00$ \\
\hline 113 & VPWO00002 & 118.8514 & 119.4374 & 119.1757 & $1.3445 e-01$ & $1.0805 e+00$ \\
\hline 114 & VPW000003 & 147.2318 & 147.8178 & 147.5288 & $1.1995 e-01$ & $1.1998 \mathrm{e}+00$ \\
\hline 115 & VPW000004 & 104.6687 & 105.2546 & 104.9266 & $1.2750 \mathrm{e}-01$ & $1.0259 e+00$ \\
\hline 116 & VPW000005 & 139.4569 & 140.0428 & 139.7460 & $1.2627 e-01$ & $1.1658 \mathrm{e}+00$ \\
\hline 117 & VPW000006 & 148.0657 & 148.6516 & 148.2804 & $1.4363 e-01$ & $1.2032 \mathrm{e}+00$ \\
\hline 118 & IPW000001 & 559.1212 & 564.9782 & 563.4163 & $1.6263 e+00$ & $9.2424 e+00$ \\
\hline 119 & IPW0 00002 & 1280.8583 & 1296.4685 & 1289.4048 & $3.9597 e+00$ & $1.3014 \mathrm{e}+01$ \\
\hline 120 & IPW000003 & 1306.4999 & 1314.2999 & 1310.1267 & $1.9304 e+00$ & $1.3143 e+01$ \\
\hline 121 & IPW000004 & 379.7044 & 381.6575 & 380.5345 & $5.3352 e-01$ & $3.6107 e+00$ \\
\hline 122 & IPWO00005 & 252.5851 & 254.0498 & 253.1416 & $3.6920 e-01$ & $2.8283 e+00$ \\
\hline 123 & IPW000006 & 259.8032 & 261.7563 & 260.6917 & $5.0964 e-01$ & $2.8708 e+00$ \\
\hline 124 & EMPTY & -0.0293 & -0.0098 & -0.0193 & $4.2954 e-03$ & $0.0000 e+00$ \\
\hline 125 & EMPTY & -0.0195 & -0.0049 & -0.0127 & $3.4173 e-03$ & $0.0000 e+00$ \\
\hline 126 & EMPTY & -0.0244 & -0.0098 & -0.0154 & $3.0180 e-03$ & $0.0000 e+00$ \\
\hline 127 & EMPTY & -0.0244 & -0.0098 & -0.0158 & $3.2057 e-03$ & $0.0000 e+00$ \\
\hline 128 & IHTO00001 & 127.9372 & 130.4762 & 129.3746 & $5.9288 e-01$ & $4.2993 e+00$ \\
\hline 129 & IHTO00002 & 131.7908 & 134.1346 & 132.9784 & $5.3356 e-01$ & $4.3103 e+00$ \\
\hline 130 & IHTO00003 & 132.1139 & 134.2623 & 133.1607 & $5.0803 e-01$ & $4.3108 e+00$ \\
\hline 131 & IHTO00004 & 130.4049 & 132.7486 & 131.4556 & $5.3363 e-01$ & $4.3056 e+00$ \\
\hline 132 & IHT000005 & 128.8762 & 130.6340 & 129.9074 & $4.5342 e-01$ & $4.3009 e+00$ \\
\hline 133 & IHT000006 & 123.8018 & 125.5596 & 124.8877 & $4.5191 e-01$ & $4.2860 e+00$ \\
\hline 134 & IHT000007 & 130.7993 & 132.7524 & 131.9048 & $4.7941 e-01$ & $4.3070 e+00$ \\
\hline 135 & IHT000008 & 129.3795 & 131.1373 & 130.3794 & $4.4754 e-01$ & $4.3023 e+00$ \\
\hline 136 & IHT000009 & 129.7589 & 131.5167 & 130.7588 & $4.7783 e-01$ & $4.3035 e+00$ \\
\hline 137 & IHTO00010 & -40.6889 & -38.3451 & -39.5365 & $5.4140 e-01$ & $4.1190 e+00$ \\
\hline 138 & IHTO00011 & 129.9166 & 132.2603 & 131.0298 & $5.3123 e-01$ & $4.3043 e+00$ \\
\hline 139 & IHT000012 & 127.5315 & 130.0706 & 128.6097 & $5.2975 e-01$ & $4.2970 e+00$ \\
\hline 140 & IHT000013 & 91.2109 & 93.3594 & 92.2773 & $5.1329 e-01$ & $4.2026 e+00$ \\
\hline 141 & IHTO00014 & 127.5616 & 130.1007 & 128.4561 & $5.3100 e-01$ & $4.2965 e+00$ \\
\hline 142 & IHTO00015 & 95.0120 & 97.5511 & 95.8675 & $5.1734 e-01$ & $4.2106 e+00$ \\
\hline 143 & IHTO00016 & 88.7169 & 91.2560 & 89.5763 & $5.0992 e-01$ & $4.1967 e+00$ \\
\hline 144 & IHTO00017 & 105.2659 & 108.9769 & 107.1253 & $8.7279 e-01$ & $4.2376 e+00$ \\
\hline 145 & IHTO00018 & 107.3543 & 109.6980 & 108.3309 & $5.4960 e-01$ & $4.2407 e+00$ \\
\hline 146 & IHT000019 & 103.4781 & 106.0171 & 104.7202 & $5.2014 e-01$ & $4.2316 \mathrm{e}+00$ \\
\hline 147 & IHTO00020 & 106.3927 & 108.9318 & 107.5529 & $5.0132 e-01$ & $4.2387 e+00$ \\
\hline 148 & IHTO00021 & -2.6179 & 0.5071 & -1.2390 & $6.2814 e-01$ & $4.1000 e+00$ \\
\hline 149 & IHTO00022 & 92.4692 & 95.0083 & 93.5239 & $5.1297 e-01$ & $4.2053 e+00$ \\
\hline 150 & IHTO00023 & 104.3645 & 106.7083 & 105.4699 & $4.9064 e-01$ & $4.2335 e+00$ \\
\hline 151 & IHTO00024 & 69.5726 & 71.5257 & 70.6155 & $4.7287 e-01$ & $4.1604 e+00$ \\
\hline 152 & IHTOOOOO25 & 104.7439 & 106.6970 & 105.8259 & $4.4853 e-01$ & $4.2344 e+00$ \\
\hline 153 & IHTO00026 & 105.3260 & 107.0838 & 106.2713 & $4.2565 e-01$ & $4.2355 e+00$ \\
\hline 154 & IHTO00027 & 90.1893 & 91.7518 & 91.0760 & $4.2537 e-01$ & $4.1999 e+00$ \\
\hline 155 & IHTO00028 & 91.3349 & 93.6786 & 92.4990 & $5.4385 e-01$ & $4.2030 \mathrm{e}+00$ \\
\hline
\end{tabular}




\begin{tabular}{|c|c|c|c|c|c|}
\hline Test: Aug17_28 & sum2 & Date: Fri & Aug 170 & $44: 321990$ & Page: 4 \\
\hline $\begin{array}{cl}\text { \# } & \text { Name } \\
156 & \text { IHT000029 } \\
157 & \text { IHT000030 } \\
158 & \text { IHT000031 } \\
159 & \text { IHT000032 } \\
160 & \text { IHT000033 } \\
161 & \text { IHT } 000034 \\
162 & \text { IHT000035 } \\
163 & \text { IHTO00036 }\end{array}$ & $\begin{array}{l}\text { Min } \\
104.8152 \\
107.0125 \\
105.4462 \\
106.5467 \\
106.5693 \\
93.7425 \\
123.9183 \\
92.2138\end{array}$ & \begin{tabular}{l}
\multicolumn{1}{c}{ Max } \\
107.7449 \\
109.7469 \\
108.1806 \\
109.2811 \\
109.3036 \\
95.8909 \\
126.2620 \\
94.5575
\end{tabular} & $\begin{array}{l}\text { Ave } \\
105.9832 \\
108.3289 \\
106.7002 \\
107.9725 \\
107.9443 \\
94.6331 \\
124.9144 \\
93.2763\end{array}$ & $\begin{array}{l}\text { SD } \\
6.1951 e-01 \\
6.6123 e-01 \\
6.8809 e-01 \\
6.8714 e-01 \\
6.6258 e-01 \\
5.5686 e-01 \\
6.3042 e-01 \\
5.7475 e-01\end{array}$ & $\begin{array}{c}\text { Uncert } \\
4.2348 e+00 \\
4.2407 e+00 \\
4.2366 e+00 \\
4.2398 e+00 \\
4.2397 e+00 \\
4.2078 e+00 \\
4.2861 e+00 \\
4.2048 e+00\end{array}$ \\
\hline
\end{tabular}




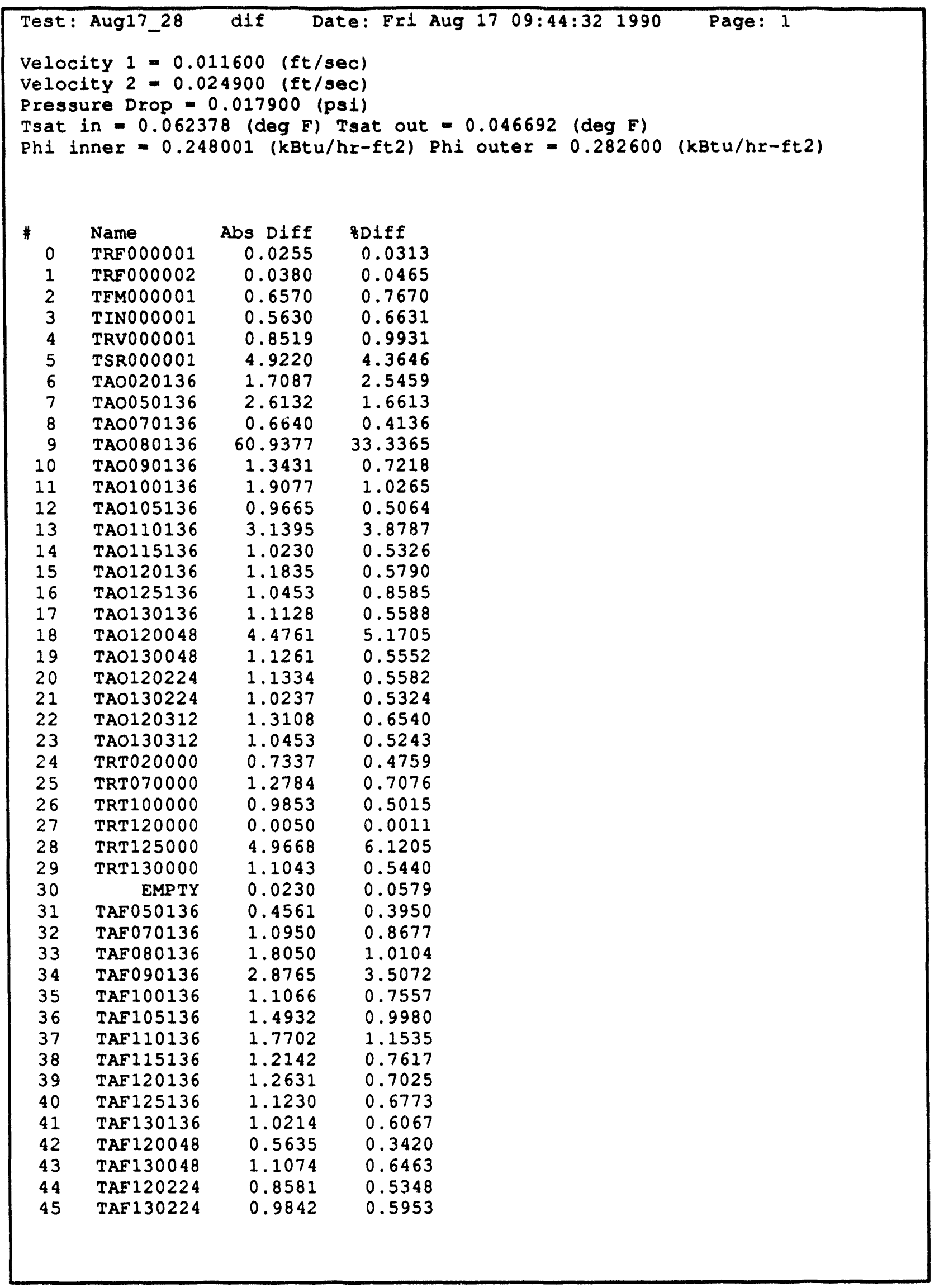




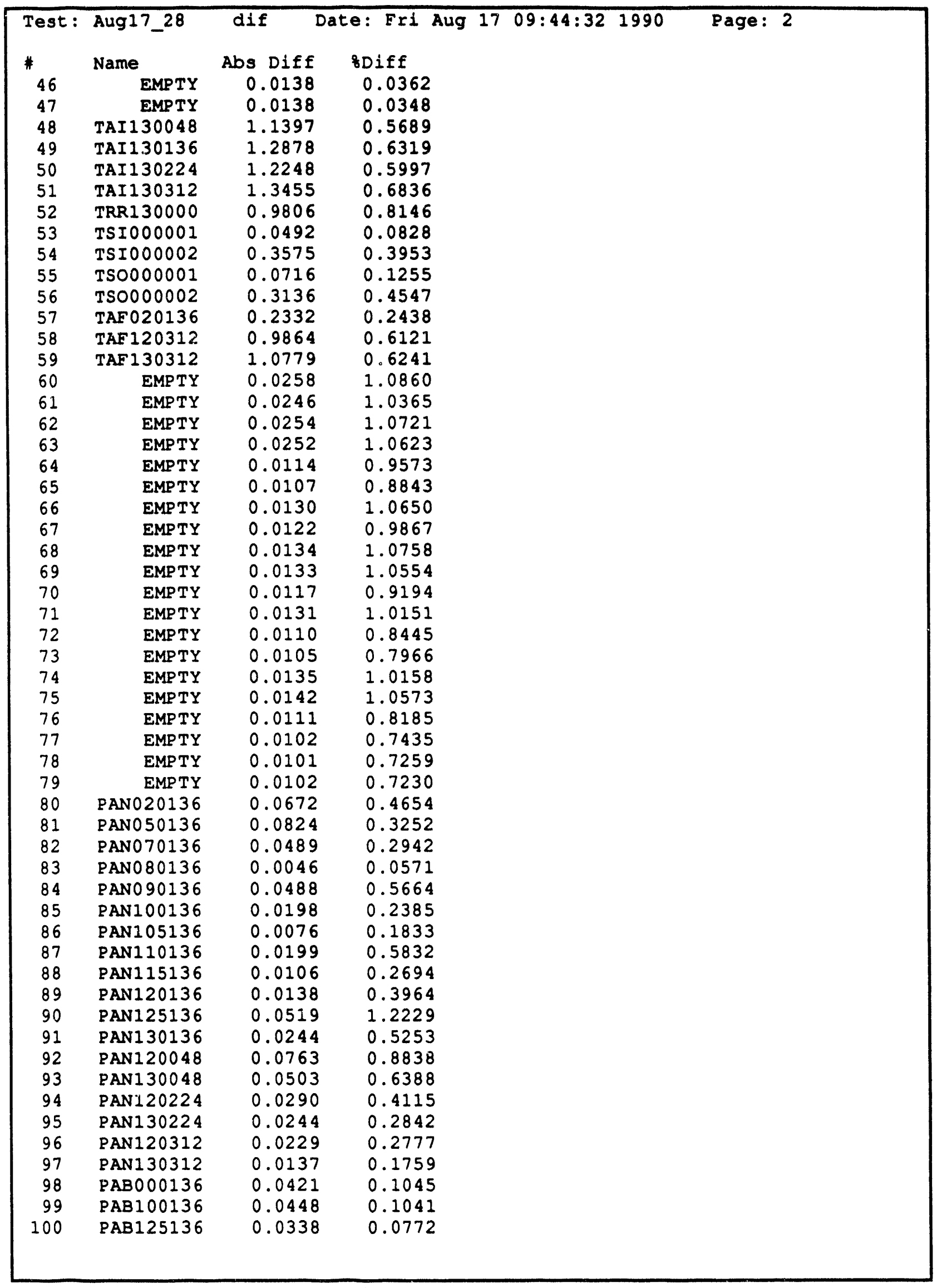




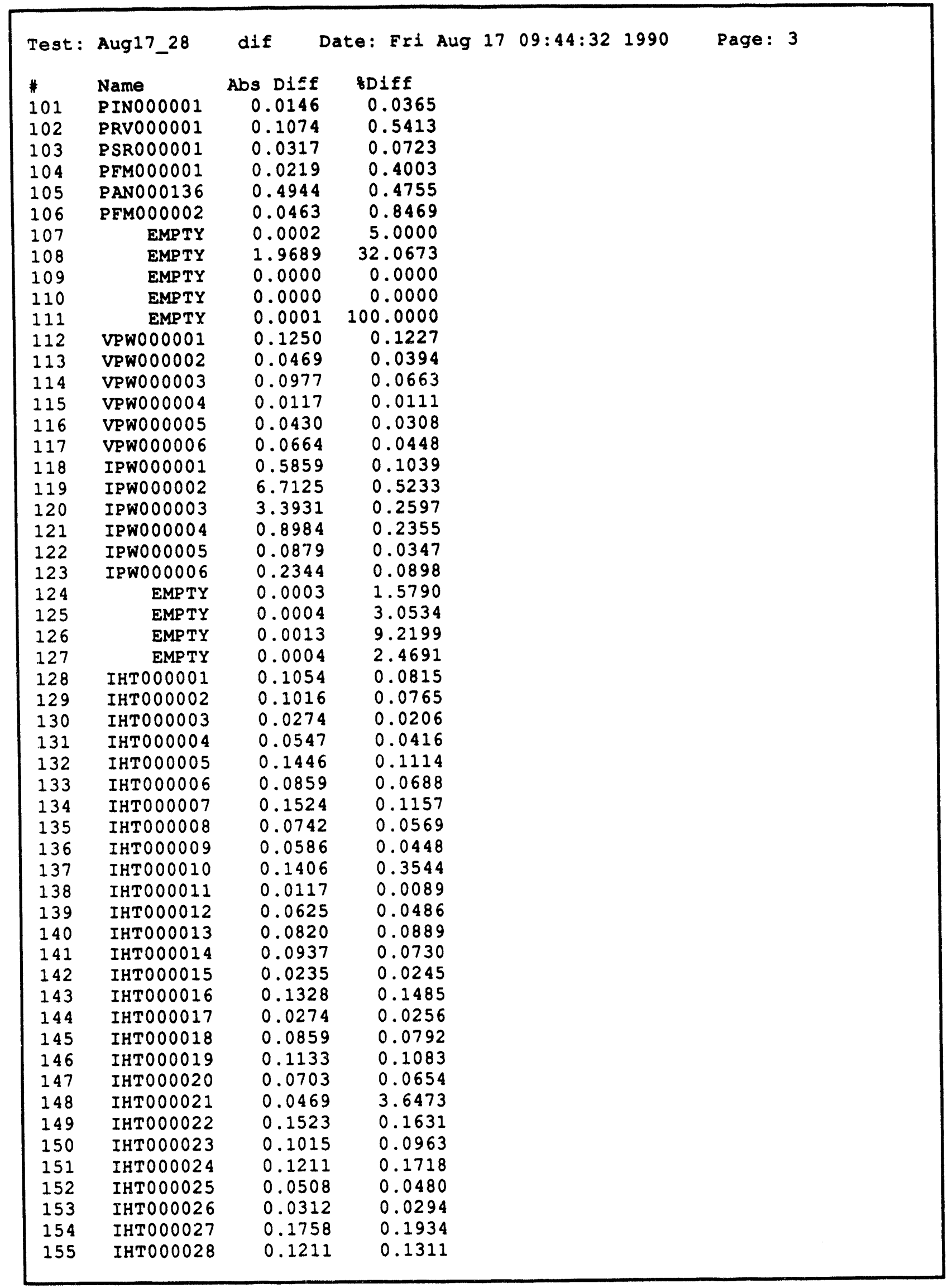




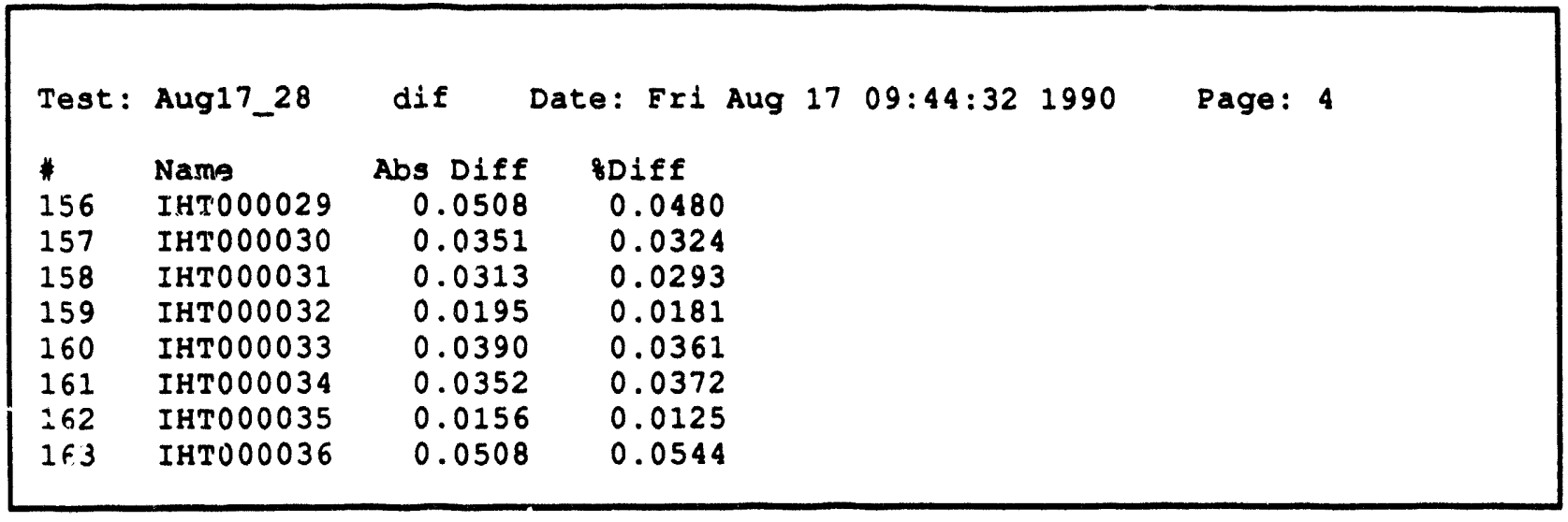




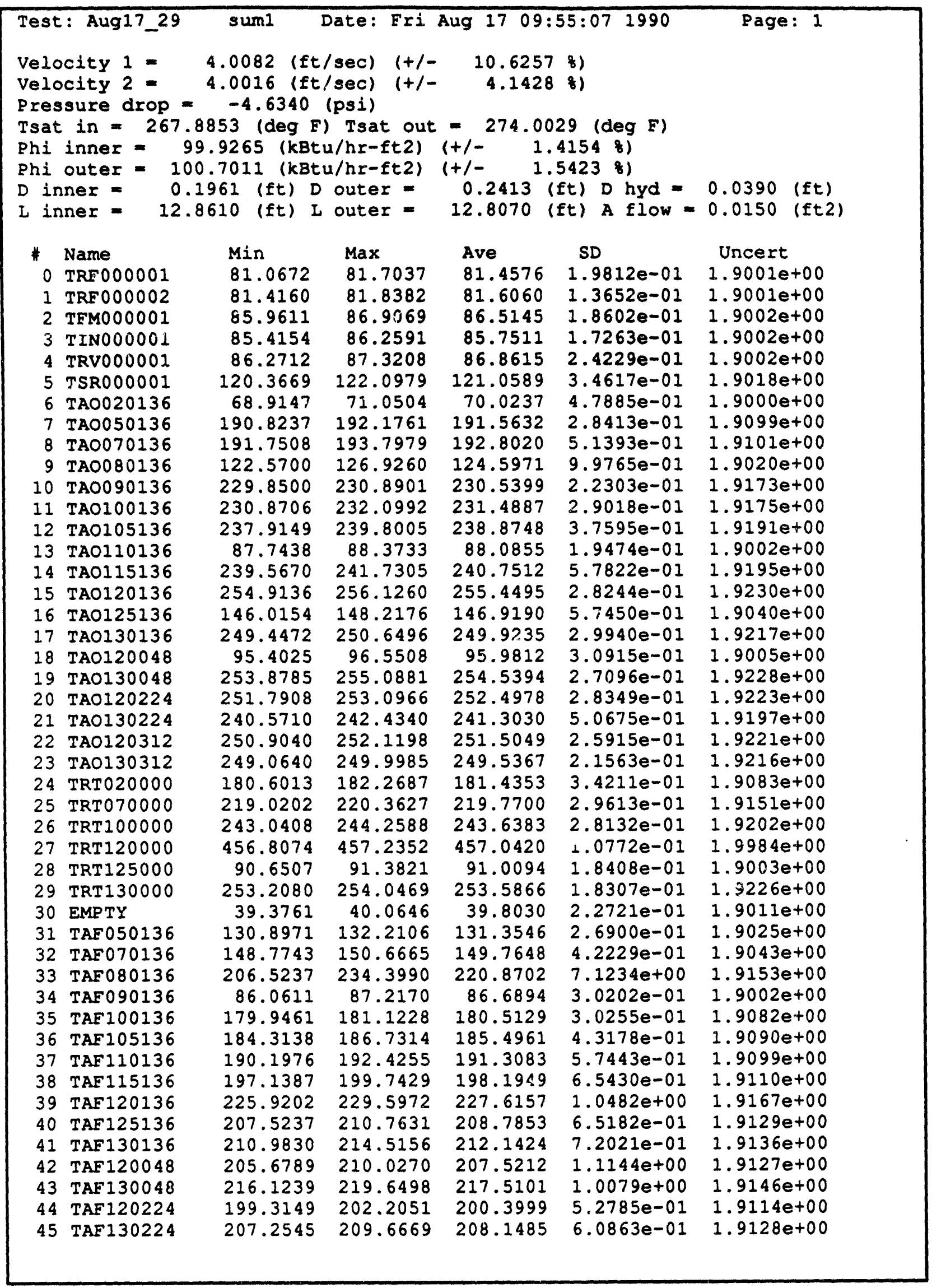




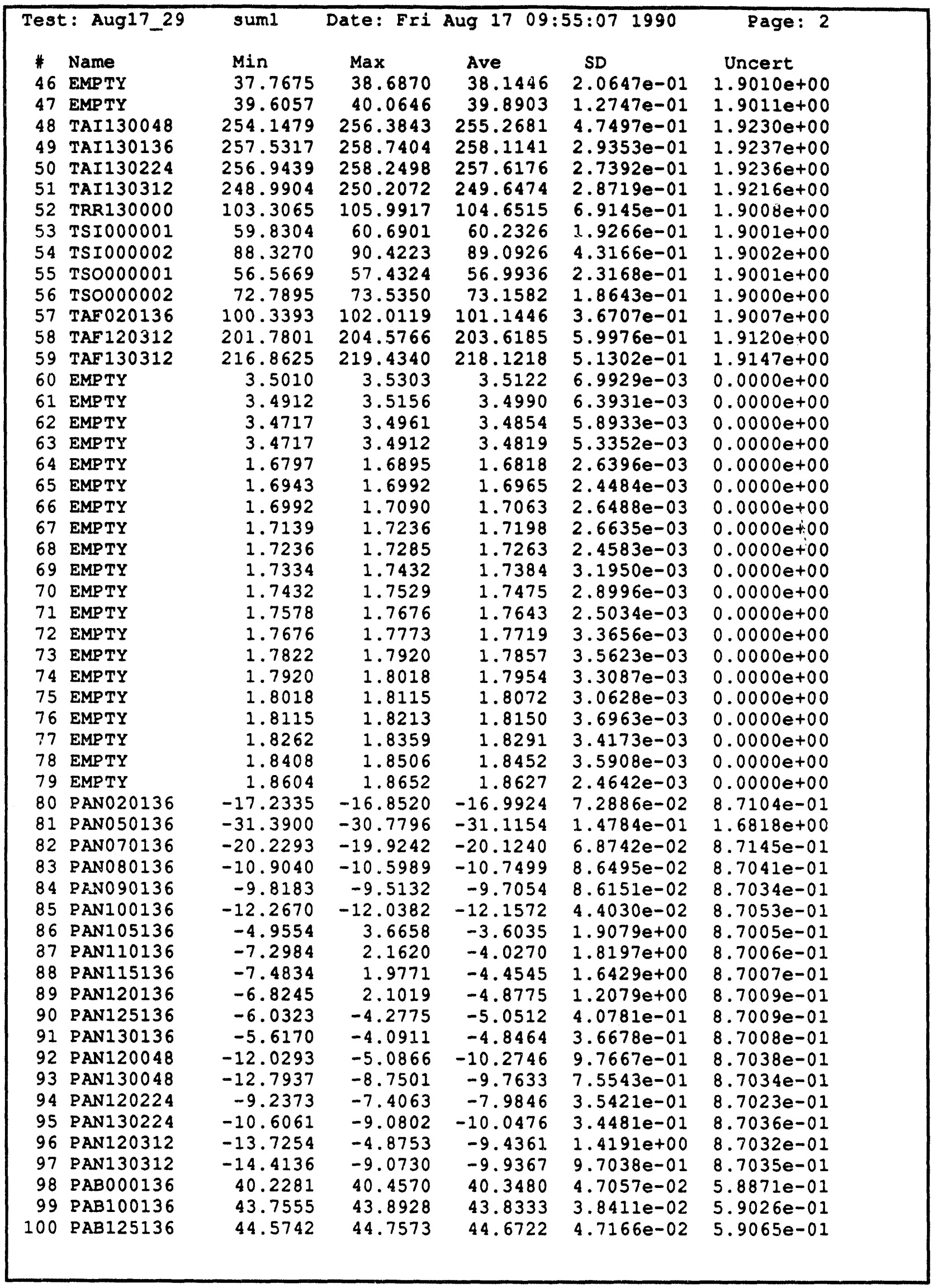




\begin{tabular}{|c|c|c|c|c|c|c|}
\hline & $t:$ Aug17_29 & sum I & Date: Fri & Aug 170 & $55: 07 \quad 1990$ & Page: 3 \\
\hline F & Name & Min & $\operatorname{Max}$ & Ave & SD & Uncert \\
\hline 101 & PINO000001 & 39.9587 & 40.2028 & 40.0930 & $7.8957 e-02$ & $7.5667 e-01$ \\
\hline 102 & PRV000001 & 19.3524 & 19.5965 & 19.5037 & $6.3165 e-02$ & $7.5158 e-01$ \\
\hline 103 & PSR0000001 & 44.5714 & 44.8156 & 44.7765 & $6.2585 e-02$ & $7.5831 \mathrm{e}-01$ \\
\hline 104 & PFMO000001 & 2.2817 & 2.5564 & 2.3825 & $6.9823 e-02$ & $5.0004 e-01$ \\
\hline 105 & PANO00136 & -129.5027 & -126.7562 & -128.2943 & $6.7175 e-01$ & $4.9304 e+00$ \\
\hline 106 & PFM000002 & 2.3153 & 2.4327 & 2.3744 & $2.4267 e-02$ & $2.0009 e-01$ \\
\hline 107 & EMPTY & -0.0049 & 0.0049 & 0.0022 & $3.8473 e-03$ & $0.0000 e+00$ \\
\hline 108 & EMPTY & -10.0000 & -0.0732 & -4.2046 & $2.9686 e+00$ & $0.0000 e+00$ \\
\hline 109 & EMPTY & -0.0049 & 0.0049 & 0.0006 & $2.1249 e-03$ & $0.0000 e+00$ \\
\hline 110 & EMPTY & -10.0000 & -10.0000 & -10.0000 & $0.0000 e+00$ & $0.0000 e+00$ \\
\hline 111 & EMPTY & 0.0000 & 0.0000 & 0.0000 & $0.0000 e+00$ & $0.0000 e+00$ \\
\hline 112 & VPW000001 & 101.8292 & 102.4151 & 102.1573 & $1.4466 e-01$ & $1.0158 e+00$ \\
\hline 113 & VPW000002 & 119.0467 & 119.6327 & 119.2967 & $1.4249 \mathrm{e}-01$ & $1.0809 e+00$ \\
\hline 114 & VPW000003 & 147.4271 & 148.0131 & 147.7280 & $1.3211 e-01$ & $1.2007 \mathrm{e}+00$ \\
\hline 115 & VPW000004 & 104.8640 & 105.4500 & 105.1219 & $1.6944 e-01$ & $1.0266 \mathrm{e}+00$ \\
\hline 116 & VPW000005 & 139.6522 & 140.2381 & 140.0155 & $1.4770 e-01$ & $1.1670 e+00$ \\
\hline 117 & VPW000006 & 148.2610 & 148.6516 & 148.4601 & $1.3375 e-01$ & $1.2040 \mathrm{e}+00$ \\
\hline 118 & IPW000001 & 551.3118 & 564.9782 & 559.8239 & $3.3413 e+00$ & $9.2287 e+00$ \\
\hline 119 & IPW0000002 & 1286.7122 & 1302.3223 & 1291.4341 & $3.8441 e+00$ & $1.3027 e+01$ \\
\hline 120 & IPW000003 & 1302.5999 & 1316.2499 & 1309.1913 & $3.3635 e+00$ & $1.3137 e+01$ \\
\hline 121 & IPW000004 & 378.2395 & 381.1692 & 379.5970 & $7.5959 e-01$ & $3.6046 e+00$ \\
\hline 122 & IPW000005 & 252.0969 & 253.0734 & 252.6729 & $3.2250 \mathrm{e}-01$ & $2.8257 \mathrm{e}+00$ \\
\hline 123 & IPW000006 & 259.3149 & 261.2681 & 260.2816 & $6.1991 e-01$ & $2.8685 e+00$ \\
\hline 124 & EMPTY & -0.0244 & -0.0098 & -0.0188 & $4.1102 e-03$ & $0.0000 e+00$ \\
\hline 125 & EMPTY & -0.0195 & -0.0049 & -0.0121 & $3.4513 e-03$ & $0.0000 e+00$ \\
\hline 126 & EMPTY & -0.0244 & -0.0049 & -0.0138 & $4.1491 e-03$ & $0.0000 e+00$ \\
\hline 127 & EMPTY & -0.0244 & -0.0098 & -0.0159 & $3.5307 e-03$ & $0.0000 e+00$ \\
\hline 128 & IHT000001 & 127.9372 & 130.6716 & 129.0778 & $5.3140 e-01$ & $4.2984 e+00$ \\
\hline 129 & IHT000002 & 131.5955 & 133.9393 & 132.6190 & $4.6948 e-01$ & $4.3092 e+00$ \\
\hline 130 & IHT000003 & 131.7233 & 134.0670 & 132.8521 & $5.0268 e-01$ & $4.3099 e+00$ \\
\hline 131 & IHTOOOOOO4 & 130.0143 & 132.5533 & 131.1587 & $4.8650 e-01$ & $4.3047 e+00$ \\
\hline 132 & IHT000005 & 128.2903 & 130.6340 & 129.4894 & $4.7188 e-01$ & $4.2996 e+00$ \\
\hline 133 & IHTO00006 & 123.6065 & 125.7550 & 124.5909 & $5.0062 e-01$ & $4.2851 e+00$ \\
\hline 134 & IHT000007 & 130.7993 & 132.5571 & 131.6548 & $4.6670 e-01$ & $4.3062 e+00$ \\
\hline 135 & IHT000008 & 129.1842 & 131.1373 & 130.1529 & $4.7014 e-01$ & $4.3016 e+00$ \\
\hline 136 & IHT000009 & 129.5635 & 131.3214 & 130.5244 & $4.7325 e-01$ & $4.3028 e+00$ \\
\hline 137 & IHT000010 & -41.0795 & -38.5404 & -39.8490 & $5.4712 e-01$ & $4.1193 e+00$ \\
\hline 138 & IHTO00011 & 129.5260 & 131.8697 & 130.6119 & $5.1321 e-01$ & $4.3030 e+00$ \\
\hline 139 & IHT 000012 & 127.1409 & 129.4847 & 128.2464 & $5.4905 e-01$ & $4.2959 e+00$ \\
\hline 140 & IHTOC0013 & 91.0156 & 93.3594 & 92.1289 & $5.8294 e-01$ & $4.2022 e+00$ \\
\hline 141 & IHTO00014 & 127.1710 & 129.5147 & 128.2647 & $5.7182 e-01$ & $4.2959 \mathrm{e}+00$ \\
\hline 142 & IHTO00015 & 94.6214 & 96.7698 & 95.7347 & $5.3706 e-01$ & $4.2103 e+00$ \\
\hline 143 & IHTO00016 & 88.5216 & 90.6701 & 89.4904 & $5.3664 e-01$ & $4.1965 \mathrm{e}+00$ \\
\hline 144 & IHT000017 & 105.0706 & 108.7816 & 107.1175 & $9.3230 e-01$ & $4.2376 e+00$ \\
\hline 145 & IHT000018 & 106.9636 & 109.8933 & 108.2137 & $6.1511 \mathrm{e}-01$ & $4.2404 \mathrm{e}+00$ \\
\hline 146 & IHTO00019 & 103.6734 & 105.6265 & 104.5054 & $4.6154 e-01$ & $4.2311 \mathrm{e}+00$ \\
\hline 147 & IHTO00020 & 106.3927 & 108.7365 & 107.3341 & $5.2153 e-01$ & $4.2382 e+00$ \\
\hline 148 & IHTO00021 & -2.6179 & 0.1164 & -1.3015 & $6.4935 e-01$ & $4.1000 e+00$ \\
\hline 149 & IHTO00022 & 92.2739 & 94.4223 & 93.3286 & $4.9126 e-01$ & $4.2049 e+00$ \\
\hline 150 & IHT000023 & 104.3645 & 106.3176 & 105.2277 & $4.6704 e-01$ & $4.2329 e+00$ \\
\hline 151 & IHTO00024 & 69.5726 & 71.7210 & 70.4007 & $4.6615 e-01$ & $4.1600 e+00$ \\
\hline 152 & IHTO00025 & 104.7439 & 106.6970 & 105.6384 & $4.6704 \mathrm{e}-01$ & $4.2339 e+00$ \\
\hline 153 & IHTO00026 & 104.9354 & 107.4745 & 106.0799 & $5.4105 e-01$ & $4.2350 e+00$ \\
\hline 154 & IHTO00027 & 89.9940 & 92.1424 & 90.9158 & $5.1303 e-01$ & $4.1996 \mathrm{e}+00$ \\
\hline 155 & IHTO00028 & 91.1396 & 93.8739 & 92.4130 & $5.1029 e-01$ & $4.2029 e+00$ \\
\hline
\end{tabular}




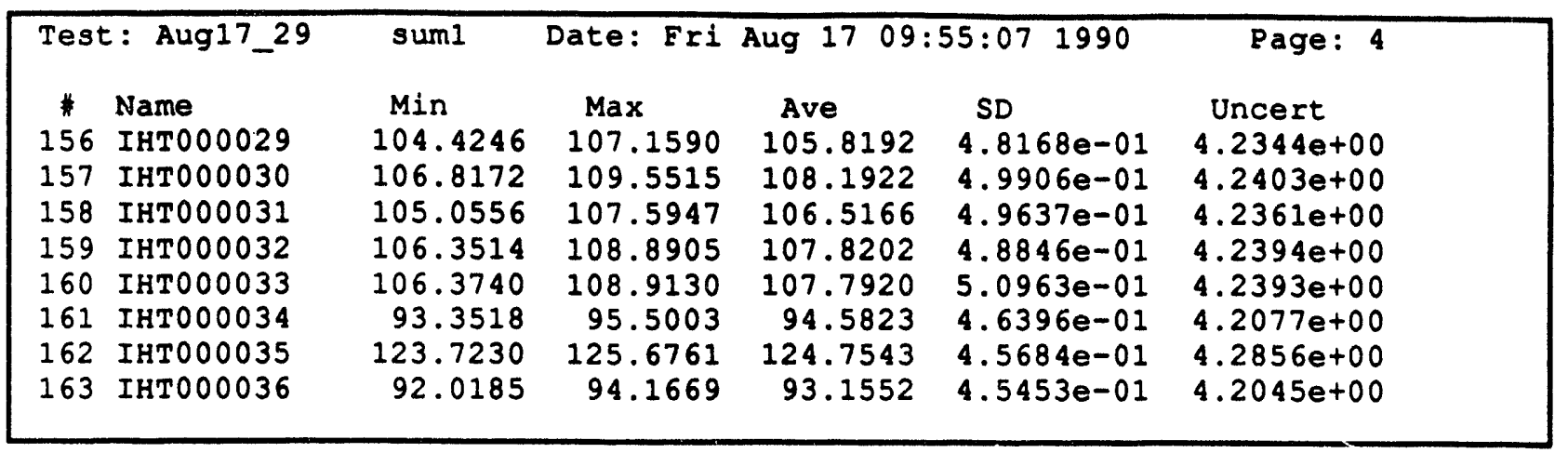




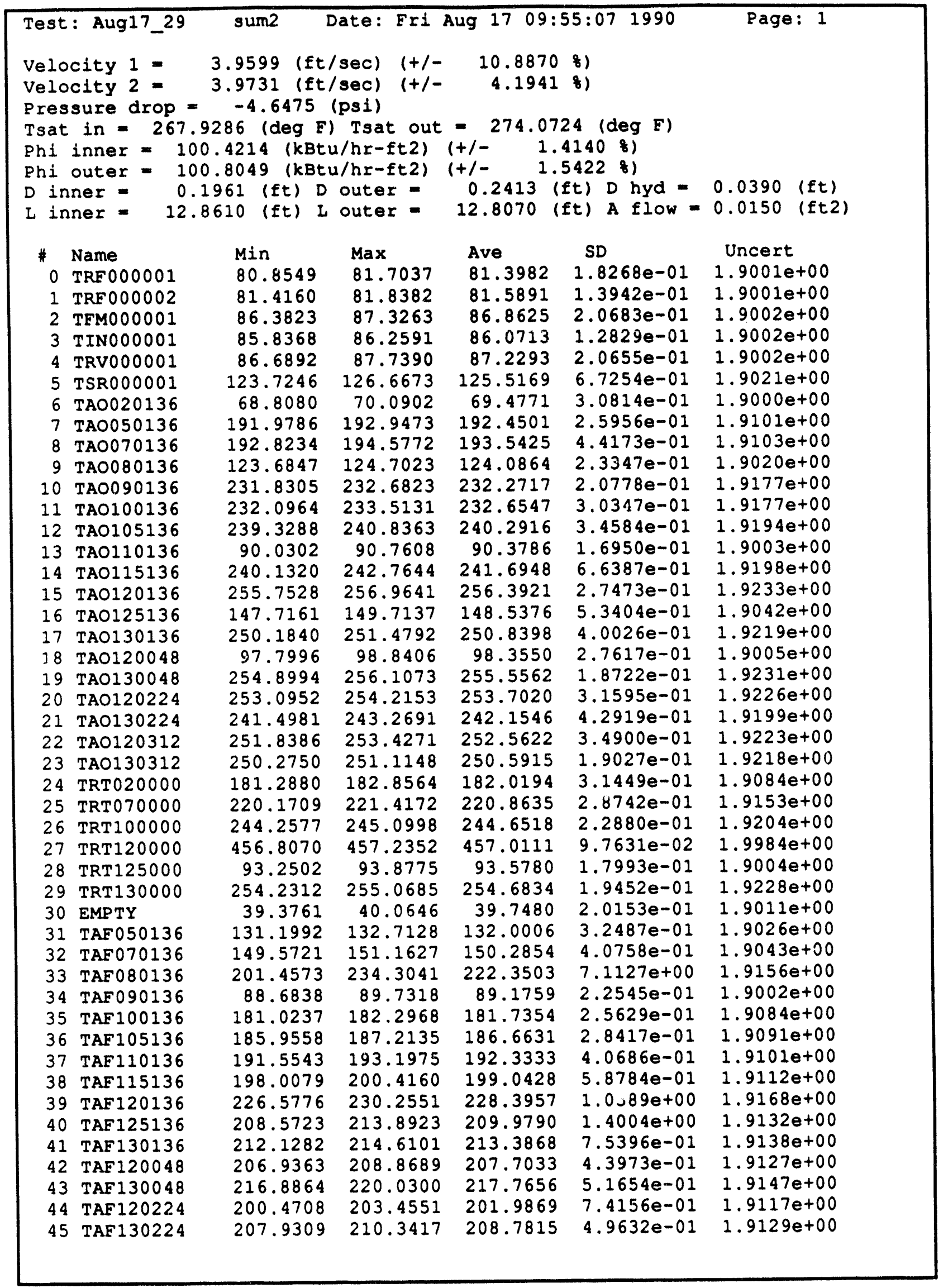




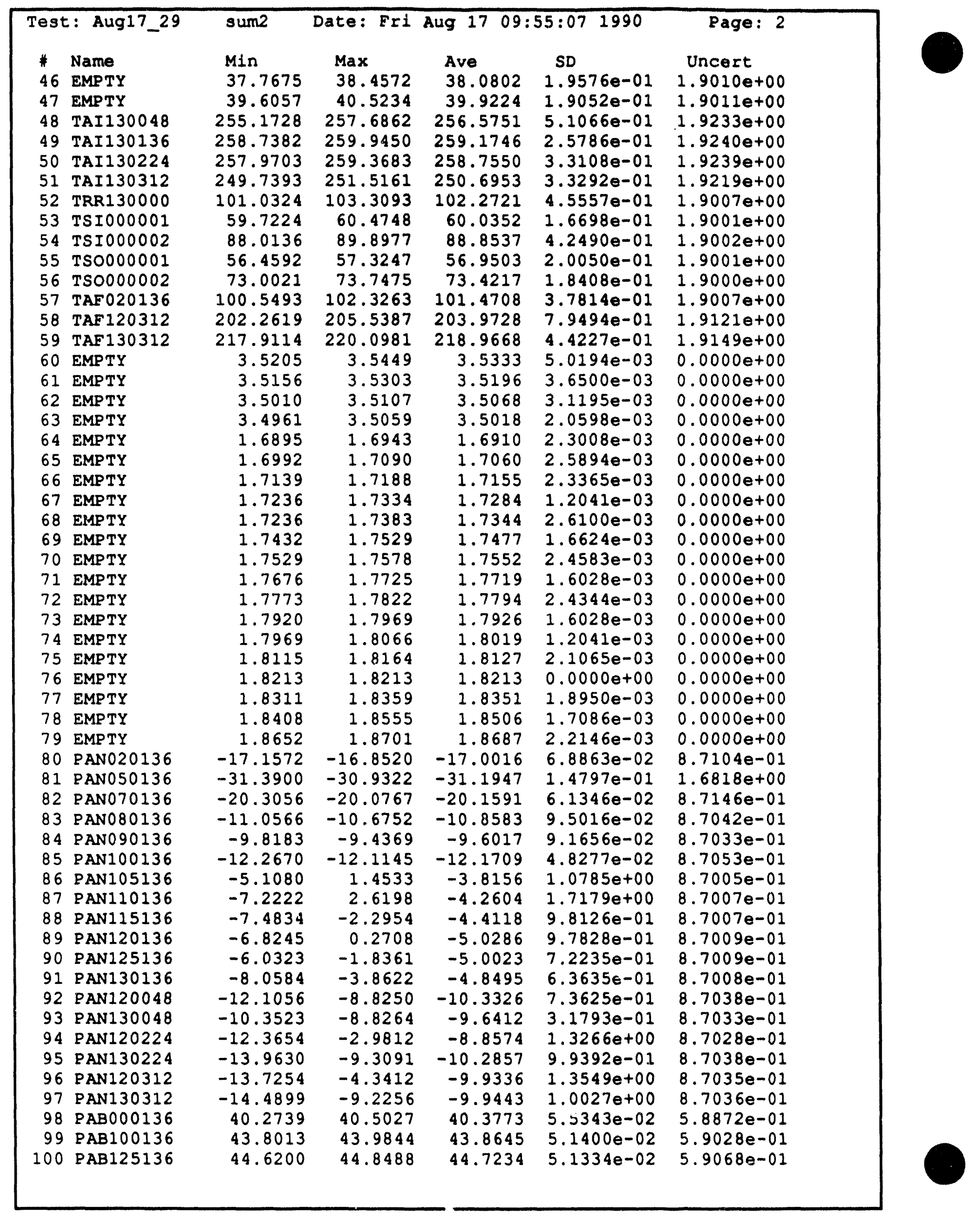




\begin{tabular}{|c|c|c|c|c|c|c|}
\hline \multicolumn{2}{|c|}{ Test: Aug17_29 } & sum2 & Date: Fri & Aug 17 & $5: 07 \quad 1990$ & Page: 3 \\
\hline \# & Name & Min & $\operatorname{Max}$ & Ave & SD & Uncert \\
\hline 101 & PINO00001 & 39.9587 & 40.3249 & 40.1101 & $9.0815 e-02$ & $7.5667 e-01$ \\
\hline 102 & PRV000001 & 19.4744 & 19.7186 & 19.5745 & $5.3409 e-02$ & $7.5159 e-01$ \\
\hline 103 & PSR000001 & 44.6935 & 44.9376 & 44.8253 & $4.8327 e-02$ & $7.5833 e-01$ \\
\hline 104 & PEM000001 & 2.1902 & 2.4649 & 2.3239 & $7.6732 e-02$ & $5.0003 e-01$ \\
\hline 105 & PANO00136 & -129.9605 & -127.6717 & -128.6696 & $5.5250 \theta-01$ & $4.9305 e+00$ \\
\hline 106 & PEM000002 & 2.2985 & 2.3992 & 2.3398 & $2.1762 e-02$ & $2.0009 \theta-01$ \\
\hline 107 & EMPTY & -0.0049 & 0.0049 & 0.0008 & $3.9901 e-03$ & $0.0000 e+00$ \\
\hline 108 & EMPTY & -10.0000 & -0.3516 & -6.3205 & $3.1572 \theta+00$ & $0.00000+00$ \\
\hline 109 & EMPTY & -0.0049 & 0.0049 & 0.0007 & $2.2080 \theta-03$ & $0.0000 e+00$ \\
\hline 110 & EMPTY & -10.0000 & -10.0000 & -10.0000 & $0.00000+00$ & $0.0000 e+00$ \\
\hline 111 & EMPTY & -0.0049 & 0.0000 & -0.0002 & $9.6655 \theta-04$ & $0.0000 e+00$ \\
\hline 112 & VPW000001 & 101.8292 & 102.6104 & 102.2627 & $1.7774 e-01$ & $1.0162 e+00$ \\
\hline 113 & VPWO00002 & 119.0467 & 119.4374 & 119.3007 & $1.0625 e-01$ & $1.0810 e+00$ \\
\hline 114 & VPW000003 & 147.6225 & 148.0131 & 147.7827 & $1.2283 e-01$ & $1.2010 e+00$ \\
\hline 115 & VPW000004 & 104.8640 & 105.4500 & 105.1375 & $1.3087 e-01$ & $1.0267 e+00$ \\
\hline 116 & VPW000005 & 139.8475 & 140.2381 & 140.0077 & $1.2283 e-01$ & $1.1670 e+00$ \\
\hline 117 & VPWO00006 & 148.2610 & 148.8469 & 148.5382 & $1.3141 e-01$ & $1.2043 e+00$ \\
\hline 118 & IPW000001 & 553.2642 & 561.0735 & 556.6221 & $1.6282 e+00$ & $9.2166 e+00$ \\
\hline 119 & IPW000002 & 1292.5659 & 1300.3710 & 1296.4684 & $1.5268 e+00$ & $1.3058 e+01$ \\
\hline 120 & IPW000003 & 1310.3999 & 1322.0999 & 1315.1189 & $2.9241 e+00$ & $1.3174 e+01$ \\
\hline 121 & IPWO00004 & 377.7513 & 379.2161 & 378.4348 & $3.2718 e-01$ & $3.5969 e+00$ \\
\hline 122 & IPW000005 & 252.0969 & 254.0498 & 253.2198 & $4.3277 e-01$ & $2.8287 e+00$ \\
\hline 123 & IPW000006 & 260.2915 & 262.2446 & 261.5023 & $5.0532 \theta-01$ & $2.8754 e+00$ \\
\hline 124 & EMPTY & -0.0244 & -0.0146 & -0.0197 & $3.2658 \theta-03$ & $0.0000 e+00$ \\
\hline 125 & EMPTY & -0.01 .95 & -0.0049 & -0.0143 & $3.0945 e-03$ & $0.0000 e+00$ \\
\hline 126 & EMPTY & -0.0244 & -0.0098 & -0.0166 & $3.69110-03$ & $0.0000 e+00$ \\
\hline 127 & EMPTY & -0.0244 & -0.0098 & -0.0163 & $3.0581 e-03$ & $0.0000 e+00$ \\
\hline 128 & IHTO00001 & 128.1325 & 130.2809 & 129.0817 & $5.6773 e-01$ & $4.2984 e+00$ \\
\hline 129 & IHT000002 & 131.7908 & 133.5487 & 132.6542 & $4.8982 e-01$ & $4.3093 e+00$ \\
\hline 130 & IHT000003 & 132.1139 & 133.8717 & 132.8716 & $4.9543 e-01$ & $4.3099 e+00$ \\
\hline 131 & IHTO00004 & 130.0143 & 132.3580 & 131.1001 & $5.0864 e-01$ & $4.3045 e+00$ \\
\hline 132 & IHT000005 & 128.2903 & 130.4387 & 129.4738 & $4.9033 e-01$ & $4.2996 e+00$ \\
\hline 133 & IHT000006 & 123.4112 & 125.7550 & 124.6104 & $5.0994 e-01$ & $4.2852 e+00$ \\
\hline 134 & IHTO00007 & 130.4087 & 132.9477 & 131.6821 & $5.7891 e-01$ & $4.3063 e+00$ \\
\hline 135 & IHT000008 & 128.9889 & 131.3326 & 130.1919 & $5.3432 e-01$ & $4.3017 e+00$ \\
\hline 136 & IHTO00009 & 129.1729 & 131.5167 & 130.5635 & $5.8877 e-01$ & $4.3029 e+00$ \\
\hline 137 & IHT000010 & -40.4935 & -38.3451 & -39.8373 & $5.4784 e-01$ & $4.1193 e+00$ \\
\hline 138 & IHT000011 & 129.9166 & 132.0650 & 130.6353 & $5.4310 e-01$ & $4.3031 e+00$ \\
\hline 139 & IHTO00012 & 127.5315 & 129.6800 & 128.3285 & $5.4225 e-01$ & $4.2961 e+00$ \\
\hline 140 & IHT000013 & 91.4063 & 93.5547 & 92.1836 & $5.4174 e-01$ & $4.2024 \mathrm{e}+00$ \\
\hline 141 & IHT000014 & 127.3663 & 129.9054 & 128.3038 & $5.5243 e-01$ & $4.2961 e+00$ \\
\hline 142 & IHT000015 & 95.0120 & 97.5511 & 95.8245 & $5.2698 e-01$ & $4.2105 e+00$ \\
\hline 143 & IHT000016 & 88.7169 & 91.2560 & 89.6740 & $5.3851 e-01$ & $4.1969 e+00$ \\
\hline 144 & IHT000017 & 105.0706 & 107.8050 & 106.4613 & $6.2345 e-01$ & $4.2360 e+00$ \\
\hline 145 & IHT000018 & 107.3543 & 109.5027 & 108.1785 & $5.3712 e-01$ & $4.2403 e+00$ \\
\hline 146 & IHT000019 & 103.4781 & 105.8218 & 104.3882 & $5.0472 e-01$ & $4.2308 e+00$ \\
\hline 147 & IHT000020 & 106.3927 & 108.3458 & 107.1857 & $4.7254 e-01$ & $4.2378 e+00$ \\
\hline 148 & IHT000021 & -2.6179 & -0.0789 & -1.4968 & $5.7563 e-01$ & $4.1000 \mathrm{e}+00$ \\
\hline 149 & IHT000022 & 92.4692 & 94.2270 & 93.3130 & $4.5922 e-01$ & $4.2048 e+00$ \\
\hline 150 & IHTO00023 & 104.3645 & 106.3176 & 105.1027 & $4.5727 e-01$ & $4.2326 e+00$ \\
\hline 151 & IHT000024 & 69.3773 & 71.5257 & 70.2718 & $4.4132 \theta-01$ & $4.1598 e+00$ \\
\hline 152 & IHT000025 & 104.5485 & 106.5017 & 105.4665 & $4.6059 e-01$ & $4.2335 e+00$ \\
\hline 153 & IHT000026 & 104.9354 & 107.2792 & 105.8573 & $5.2946 \theta-01$ & $4.2345 e+00$ \\
\hline 154 & IHT000027 & 89.7987 & 92.1424 & 90.8572 & $5.1462 e-01$ & $4.1995 e+00$ \\
\hline 155 & IHT000028 & 91.3349 & 94.0693 & 92.4443 & $6.2570 e-01$ & $4.2029 e+00$ \\
\hline
\end{tabular}




\begin{tabular}{|c|c|c|c|c|c|c|}
\hline Tes & $t:$ Aug17_29 & sum2 & Date: Fri & Aug 170 & $5: 071990$ & Page: 4 \\
\hline $\begin{array}{l}1 \\
156 \\
157 \\
158 \\
159 \\
160 \\
161 \\
162 \\
163\end{array}$ & $\begin{array}{l}\text { Name } \\
\text { IHTO00029 } \\
\text { IHTOOOO30 } \\
\text { IHTO00031 } \\
\text { IHTO00032 } \\
\text { IHTO00033 } \\
\text { IHTO00034 } \\
\text { IHTO00035 } \\
\text { IHTO00036 }\end{array}$ & $\begin{array}{l}\text { Min } \\
104.6199 \\
106.8172 \\
105.4462 \\
106.7420 \\
106.7646 \\
93.7425 \\
123.9183 \\
92.4091\end{array}$ & $\begin{array}{l}\text { Max } \\
107.1590 \\
109.5515 \\
108.1806 \\
109.2811 \\
109.3036 \\
95.8909 \\
125.8714 \\
94.1669\end{array}$ & $\begin{array}{r}\text { Ave } \\
105.7567 \\
108.0672 \\
106.4502 \\
107.7420 \\
107.7060 \\
94.6331 \\
124.7933 \\
93.2490\end{array}$ & $\begin{array}{l}\text { SD } \\
5.9541 e-01 \\
5.6634 e-01 \\
5.9583 e-01 \\
5.7404 e-01 \\
6.0707 e-01 \\
4.9310 e-01 \\
4.9006 e-01 \\
4.9007 e-01\end{array}$ & $\begin{array}{c}\text { Uncert } \\
4.2342 e+00 \\
4.2400 e+00 \\
4.2359 e+00 \\
4.2392 e+00 \\
4.2391 e+00 \\
4.2078 e+00 \\
4.2857 e+00 \\
4.2047 e+00\end{array}$ \\
\hline
\end{tabular}




\begin{tabular}{|c|c|c|c|c|}
\hline \multicolumn{5}{|c|}{$\begin{array}{l}\text { Velocity } 1=0.048300 \text { (ft/sec) } \\
\text { Velocity } 2=0.028500 \text { (ft/sec) } \\
\text { Pressure Drop }=0.013500 \text { (psi) } \\
\text { Tsat in }=0.043274 \text { (deg F) Tsat out }=0.069489 \text { (deg F) } \\
\text { Pht inner }=0.494904 \text { (kBtu/hr-ft2) Phi outer }=0.103798 \text { (kBtu/hr-ft2) }\end{array}$} \\
\hline \# & Name & Abs Diff & \&DAff & \\
\hline 0 & TRF000001 & 0.0594 & 0.0729 & \\
\hline 1 & TRF 000002 & 0.0169 & 0.0207 & \\
\hline 2 & TEM000001 & 0.3480 & 0.4022 & \\
\hline 3 & TINO00001 & 0.3202 & 0.3734 & \\
\hline 4 & TRV000001 & 0.3678 & 0.4234 & \\
\hline 5 & TSR000001 & 4.4580 & 3.6825 & \\
\hline 6 & TA0020136 & 0.5466 & 0.7806 & \\
\hline 7 & TAO050136 & 0.8869 & 0.4630 & \\
\hline 8 & TAO070136 & 0.7405 & 0.3841 & \\
\hline 9 & TAO080136 & 0.5107 & 0.4099 & \\
\hline 10 & TAO090136 & 1.7318 & 0.7512 & \\
\hline 11 & TA0100136 & 1.1660 & 0.5037 & \\
\hline 12 & TAO105136 & 1.4168 & 0.5931 & \\
\hline 13 & TAO110136 & 2.2931 & 2.6033 & \\
\hline 14 & TA0115136 & 0.9436 & 0.3919 & \\
\hline 15 & TA0120136 & 0.9426 & 0.3690 & \\
\hline 16 & TAO125136 & 1.6186 & 1.1017 & \\
\hline 17 & TA0130136 & 0.9163 & 0.3666 & \\
\hline 18 & TAO120048 & 2.3738 & 2.4732 & \\
\hline 19 & TAO130048 & 1.0168 & 0.3995 & \\
\hline 20 & TAO120224 & 1.2042 & 0.4769 & \\
\hline 21 & TAO130224 & 0.8516 & 0.3529 & \\
\hline 22 & TA0120312 & 1.0573 & 0.4204 & \\
\hline 23 & TAO130312 & 1.0548 & 0.4227 & \\
\hline 24 & TRT020000 & 0.5841 & 0.3219 & \\
\hline 25 & TRT070000 & 1.0935 & 0.4976 & \\
\hline 26 & TRT 100000 & 1.0135 & 0.4160 & \\
\hline 27 & TRT 120000 & 0.0309 & 0.0068 & \\
\hline 28 & TRT 125000 & 2.5686 & 2.8223 & \\
\hline 29 & TRT 130000 & 1.0968 & 0.4325 & \\
\hline 30 & EMPTY & 0.0550 & 0.1382 & \\
\hline 31 & TAF050136 & 0.6460 & 0.4918 & \\
\hline 32 & TAF070136 & 0.5206 & 0.3476 & \\
\hline 33 & TAF080136 & 1.4801 & 0.6701 & \\
\hline 34 & TAF090136 & 2.4865 & 2.8683 & \\
\hline 35 & TAF 100136 & 1.2225 & 0.6772 & \\
\hline 36 & TAF 105136 & 1.1670 & 0.6291 & \\
\hline 37 & TAF 110136 & 1.0250 & 0.5358 & \\
\hline 38 & TAF 115136 & 0.8479 & 0.4278 & \\
\hline 39 & TAF 120136 & 0.7800 & 0.3427 & \\
\hline 40 & TAF 125136 & 1.1937 & 0.5717 & \\
\hline 41 & TAF 130136 & 1.2444 & 0.5866 & \\
\hline 42 & TAF 120048 & 0.1821 & 0.0877 & \\
\hline 43 & TAF 130048 & 0.2555 & 0.1175 & \\
\hline 44 & TAF 120224 & 1.5870 & 0.7919 & \\
\hline 45 & TAF 130224 & 0.6330 & 0.3041 & \\
\hline
\end{tabular}




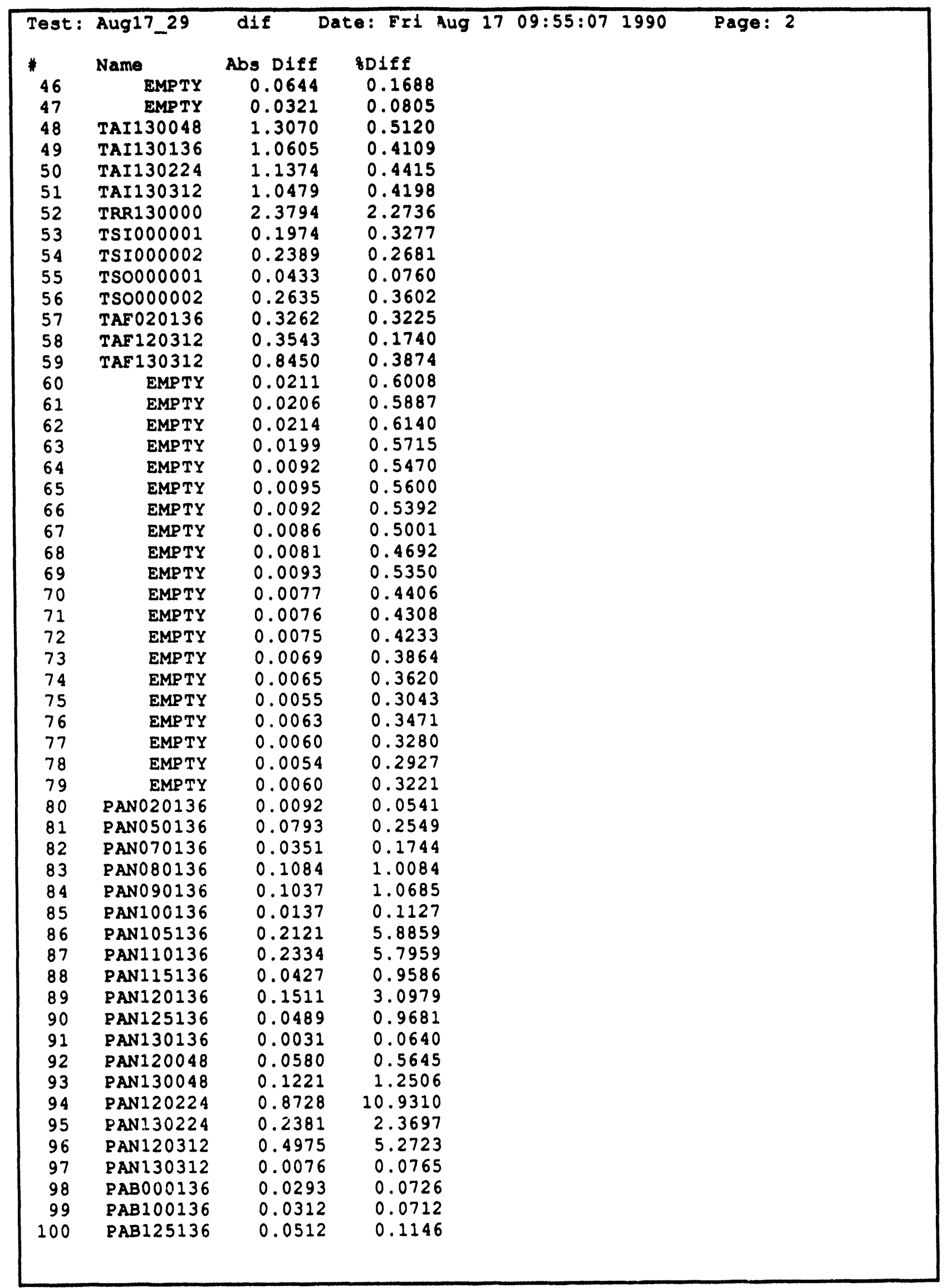




\begin{tabular}{|c|c|c|c|c|}
\hline Test: & Aug17_29 & dif & ate: Fri Aug 17 09:55:07 1990 & Page: 3 \\
\hline$*$ & Name & Abs Diff & 8Diff & \\
\hline 101 & PINO00001 & 0.0171 & 0.0427 & \\
\hline 102 & PRV000001 & 0.0708 & 0.3630 & \\
\hline 103 & PSR000001 & 0.0488 & 0.1090 & \\
\hline $\begin{array}{l}104 \\
105\end{array}$ & $\begin{array}{l}\text { PEMO00001 } \\
\text { PANO00136 }\end{array}$ & 0.0586 & $\begin{array}{l}2.4596 \\
0.2925\end{array}$ & \\
\hline 106 & $\begin{array}{l}\text { PANO00136 } \\
\text { PFM000002 }\end{array}$ & $\begin{array}{l}0.3753 \\
0.0346\end{array}$ & $\begin{array}{l}0.2925 \\
1.4572\end{array}$ & \\
\hline 107 & EMPTY & 0.0014 & 63.6364 & \\
\hline 108 & EMPTY & 2.1159 & 50.3235 & \\
\hline 109 & EMPTY & 0.0001 & 16.6667 & \\
\hline 110 & EMPTY & 0.0000 & 0.0000 & \\
\hline 111 & EMPTY & 0.0002 & 100.0000 & \\
\hline 112 & VPW000001 & 0.1054 & 0.1032 & \\
\hline 113 & VPWO00002 & 0.0040 & 0.0034 & \\
\hline 114 & VPWO00003 & 0.0547 & 0.0370 & \\
\hline 115 & VPW000004 & 0.0156 & 0.0148 & \\
\hline 116 & VPWO00005 & 0.0078 & 0.0056 & \\
\hline 117 & VPW000006 & 0.0781 & 0.0526 & \\
\hline 118 & IPW000001 & 3.2018 & 0.5719 & \\
\hline 119 & IPW000002 & 5.0343 & 0.3898 & \\
\hline 120 & IPW000003 & 5.9276 & 0.4528 & \\
\hline 121 & IPW000004 & 1.1622 & 0.3062 & \\
\hline 122 & IPW000005 & 0.5469 & 0.2164 & \\
\hline 123 & IPW000006 & 1.2207 & 0.4690 & \\
\hline 124 & EMPTY & 0.0009 & 4.7872 & \\
\hline 125 & EMPTY & 0.0022 & 18.1818 & \\
\hline 126 & EMPTY & 0.0028 & 20.2899 & \\
\hline 127 & EMPTY & 0.0004 & 2.5157 & \\
\hline 128 & IHTO00001 & 0.0039 & 0.0030 & \\
\hline 129 & IHTO00002 & 0.0352 & 0.0265 & \\
\hline 130 & IHTO00003 & 0.0195 & 0.0147 & \\
\hline 131 & IHTO00004 & 0.0586 & 0.0447 & \\
\hline 132 & IHT000005 & 0.0156 & 0.0120 & \\
\hline 133 & IHT000006 & 0.0195 & 0.0157 & \\
\hline 134 & IHT000007 & 0.0273 & 0.0207 & \\
\hline 135 & IHT000008 & 0.0390 & 0.0300 & \\
\hline 136 & IHTO00009 & 0.0391 & 0.0300 & \\
\hline 137 & IHTO00010 & 0.0117 & 0.0294 & \\
\hline 138 & IHTO00011 & 0.0234 & 0.0179 & \\
\hline 139 & IHTO00012 & 0.0821 & 0.0640 & \\
\hline 140 & IHTO00013 & 0.0547 & 0.0594 & \\
\hline 141 & IHTO00014 & 0.0391 & 0.0305 & \\
\hline 142 & IHTO00015 & 0.0898 & 0.0938 & \\
\hline 143 & IHT000016 & 0.1836 & 0.2052 & \\
\hline 144 & IHTO00017 & 0.6562 & 0.6126 & \\
\hline 145 & IHT000018 & 0.0352 & 0.0325 & \\
\hline 146 & IHTO00019 & 0.1172 & 0.1121 & \\
\hline 147 & IHTO0O020 & 0.1484 & 0.1383 & \\
\hline 148 & IHTO00021 & 0.1953 & 15.0058 & \\
\hline 149 & IHT000022 & 0.0156 & 0.0167 & \\
\hline 150 & IHTOOO023 & 0.1250 & 0.1188 & \\
\hline 151 & IHTO00024 & 0.1289 & 0.1831 & \\
\hline 152 & IHTO00025 & 0.1719 & 0.1627 & \\
\hline 153 & IHT000026 & 0.2226 & 0.2098 & \\
\hline 154 & IHT000027 & 0.0586 & 0.0645 & \\
\hline 155 & IHTO00028 & 0.0313 & 0.0339 & \\
\hline
\end{tabular}




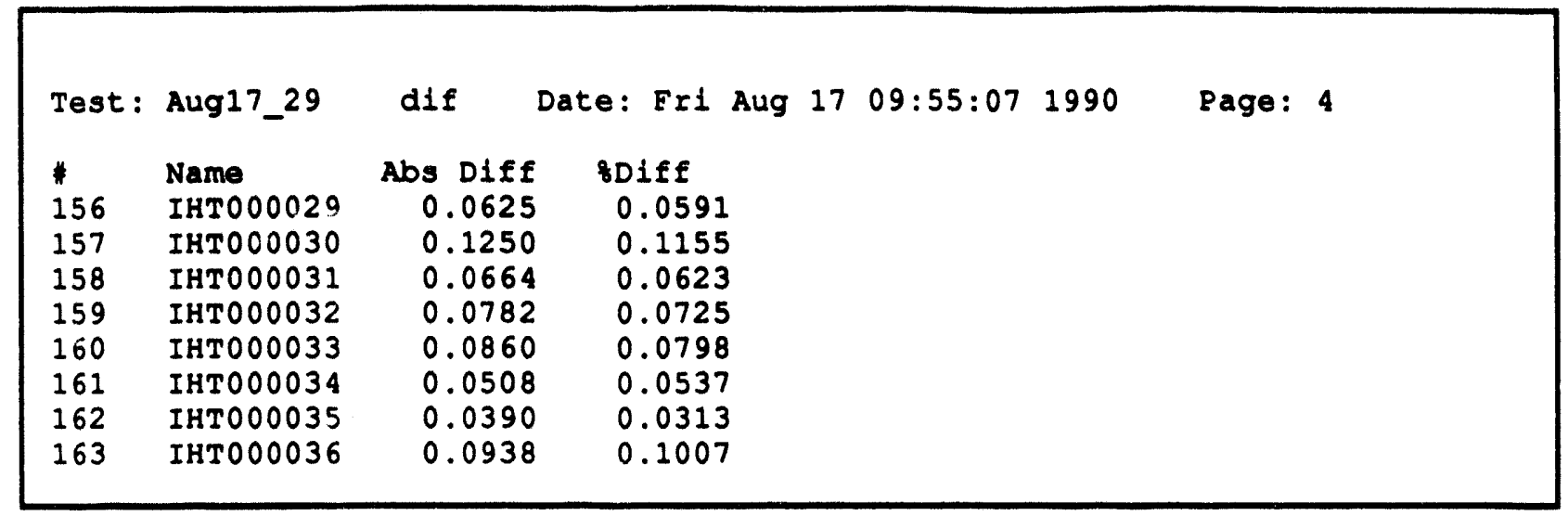




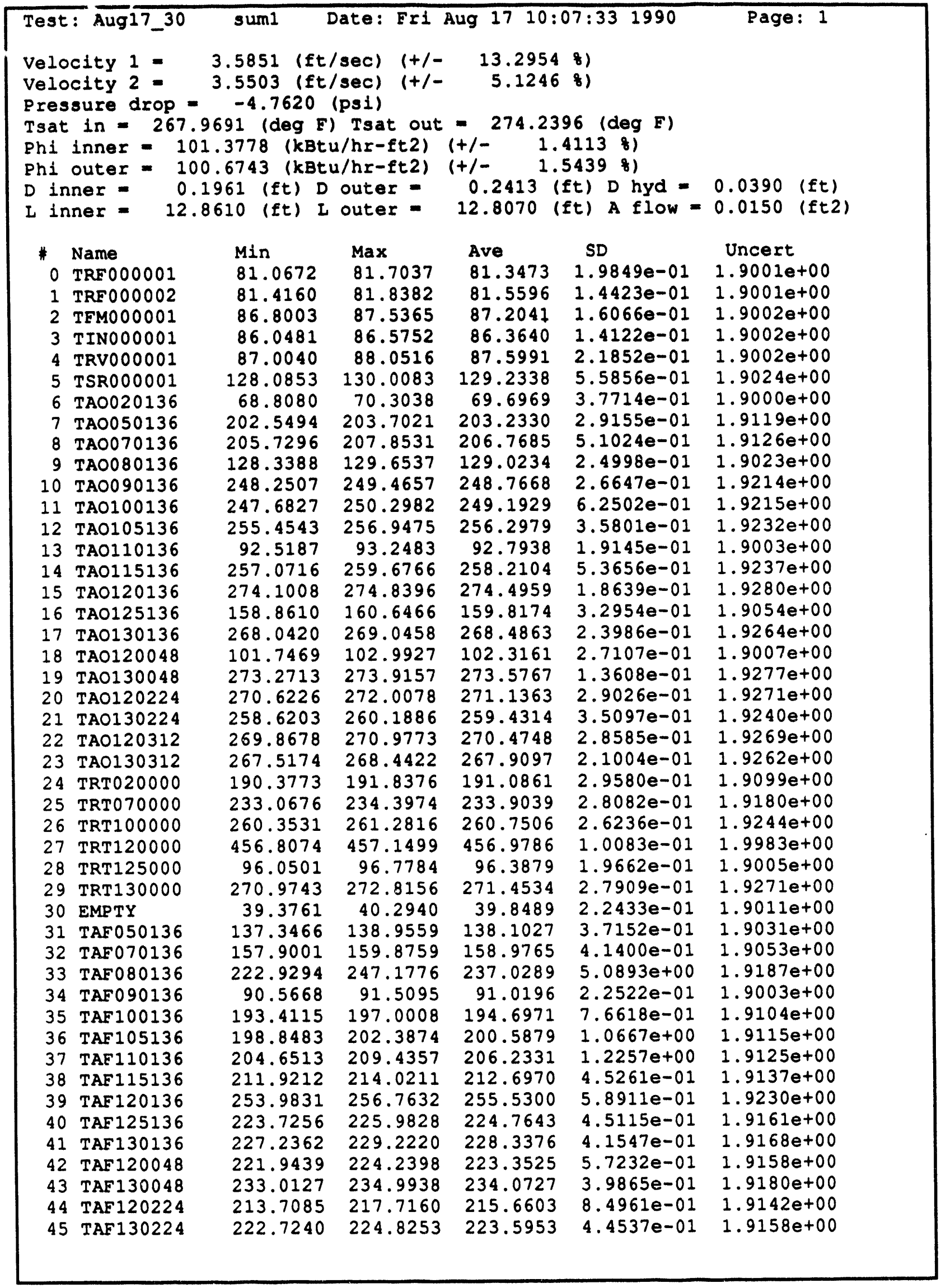




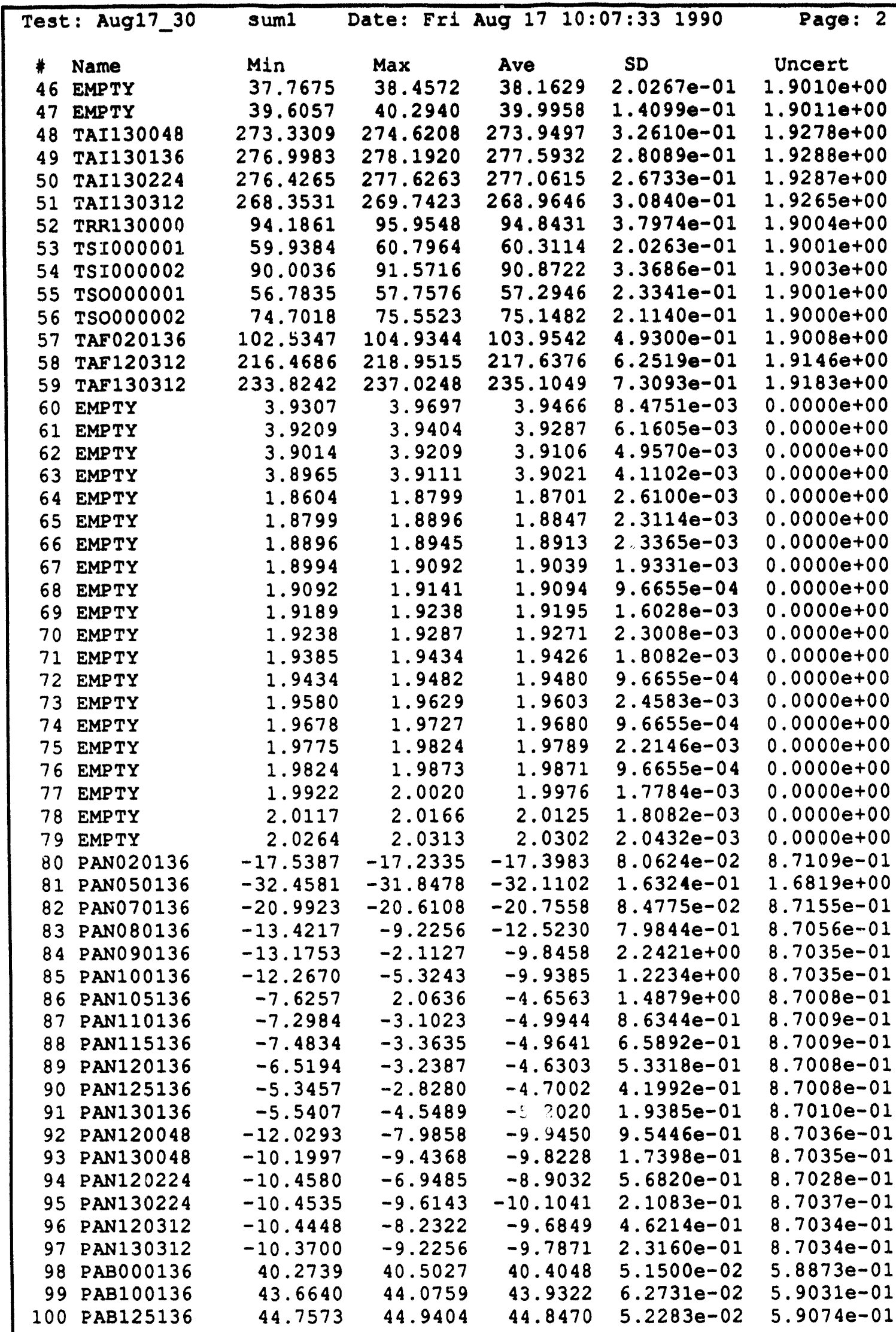




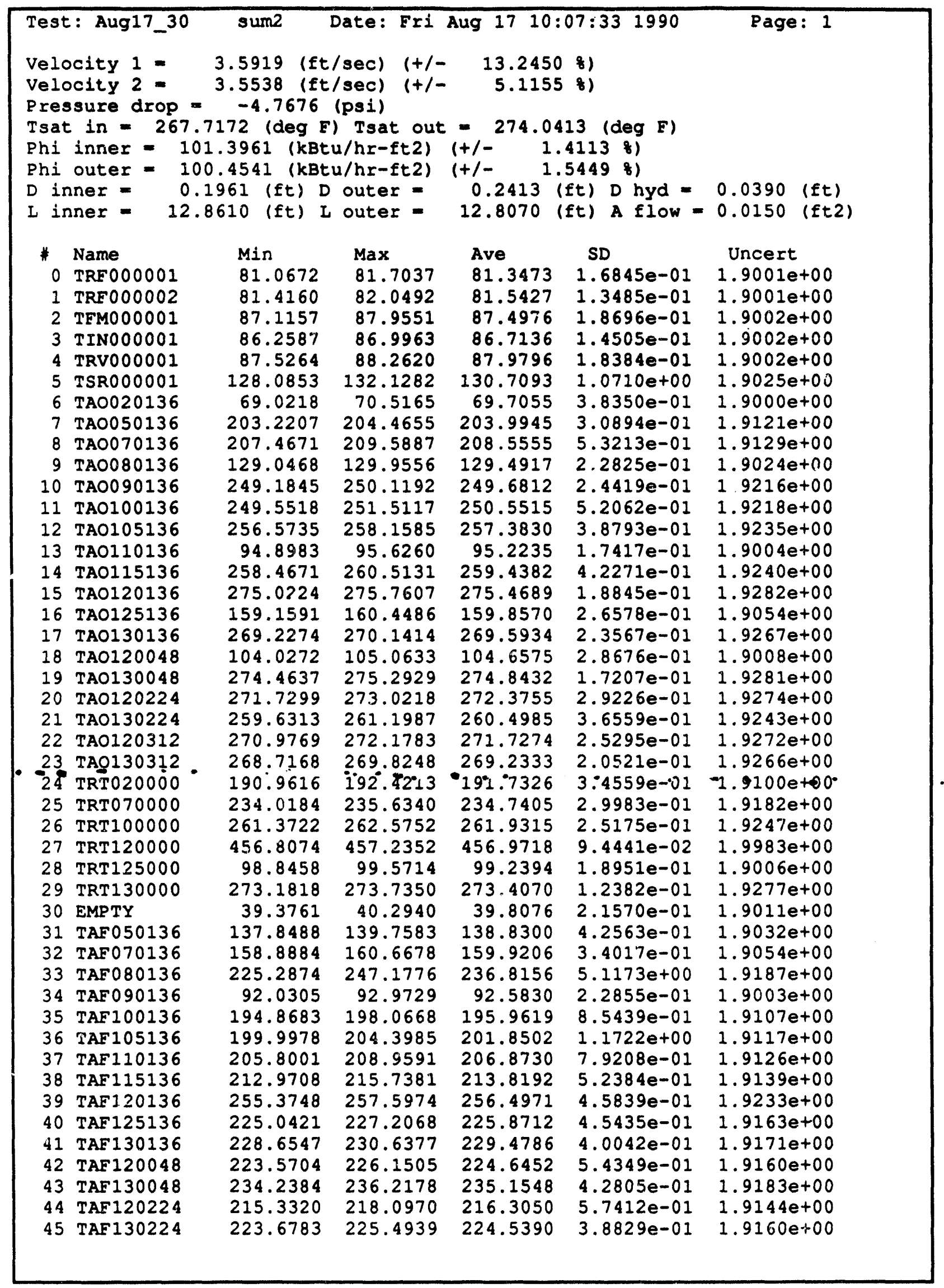




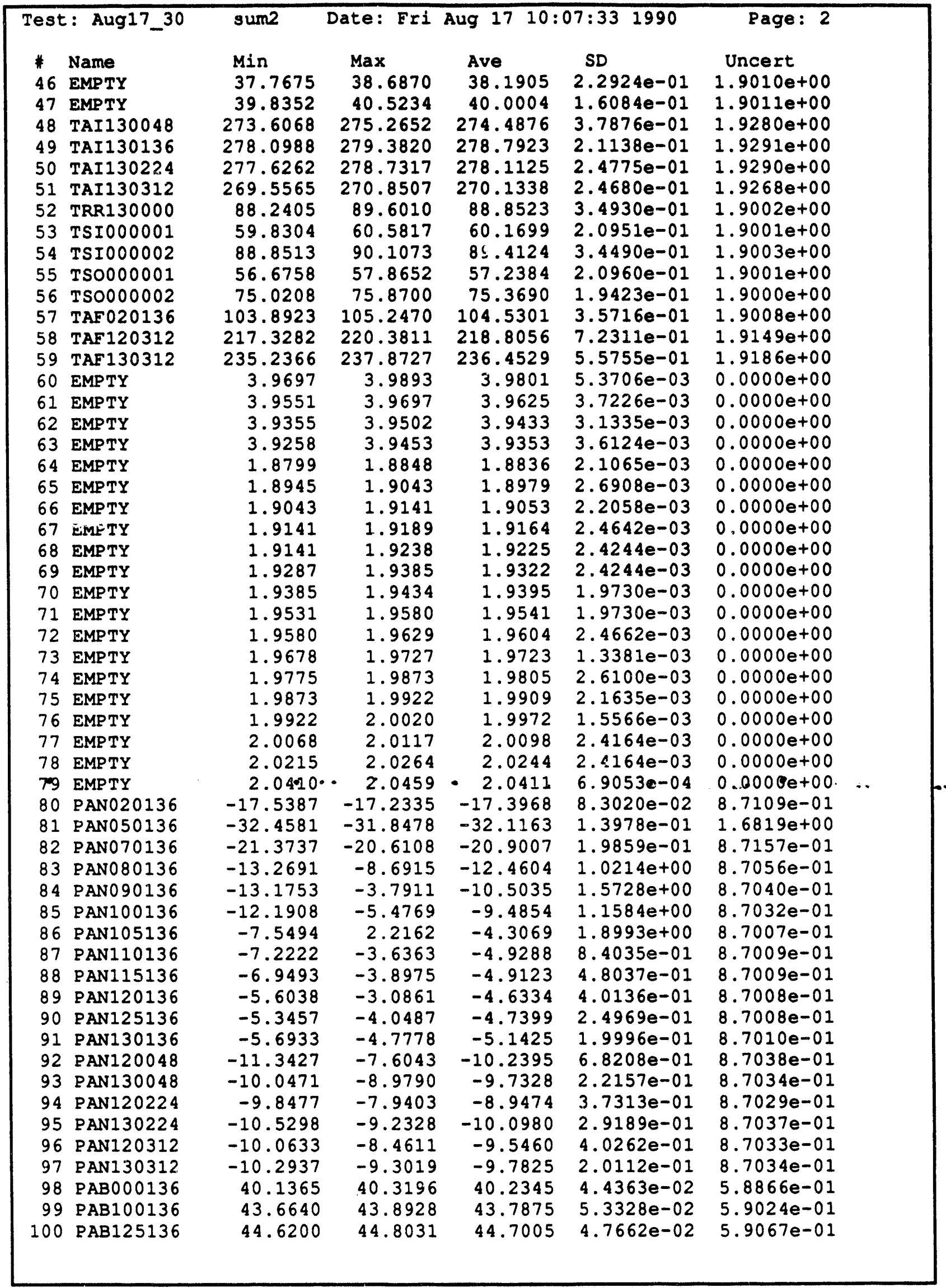




\begin{tabular}{|c|c|c|c|c|c|c|c|}
\hline \multicolumn{2}{|c|}{ Test: Aug17_30 } & sum I & Date: Fri & Aug 171 & $7: 331990$ & Page: 3 & \\
\hline * & Name & Min & $\operatorname{Max}$ & Ave & SD & Uncert & \\
\hline 101 & PINO00001 & 40.2028 & 40.4470 & 40.3078 & $8.8954 e-02$ & $7.5674 e-01$ & \\
\hline 102 & PRV000001 & 19.5965 & 19.8406 & 19.7332 & $4.7052 e-02$ & $7.5162 \mathrm{e}-01$ & \\
\hline 103 & PSR000001 & 44.9376 & 45.1818 & 45.0548 & $6.0207 e-02$ & $7.5841 e-01$ & \\
\hline 104 & PFMO000001 & 1.7324 & 2.0071 & 1.8954 & $6.4843 e-02$ & $5.0002 e-01$ & \\
\hline 105 & PANO00136 & -133.1649 & -130.8760 & -131.8373 & $4.5542 e-01$ & $4.9310 \mathrm{e}+00$ & \\
\hline 106 & PEMO00002 & 1.8119 & 1.9126 & 1.8579 & $2.8927 e-02$ & $2.0005 e-01$ & \\
\hline 107 & EMPTY & -0.0049 & 0.0049 & 0.0011 & $4.1019 e-03$ & $0.0000 e+00$ & \\
\hline 108 & EMPTY & -10.0000 & -0.2246 & -6.8460 & $2.7527 e+00$ & $0.0000 e+00$ & \\
\hline 109 & EMPTY & -0.0049 & 0.0049 & 0.0012 & $2.3261 e-03$ & $0.0000 e+00$ & \\
\hline 110 & EMPTY & -10.0000 & -10.0000 & -10.0000 & $0.0000 e+00$ & $0.0000 e+00$ & \\
\hline 111 & EMPTY & 0.0000 & 0.0000 & 0.0000 & $0.0000 e+00$ & $0.0000 e+00$ & \\
\hline 112 & VPW000001 & 102.0245 & 102.4151 & 102.1612 & $1.2001 \mathrm{e}-01$ & $1.0158 e+00$ & \\
\hline 113 & VPW0 00002 & 119.0467 & 119.4374 & 119.2967 & $1.0470 e-01$ & $1.0809 e+00$ & \\
\hline 114 & VPW000003 & 147.6225 & 148.0131 & 147.8764 & $1.2001 e-01$ & $1.2014 \mathrm{e}+00$ & \\
\hline 115 & VPW000004 & 104.8640 & 105.4500 & 105.2000 & $1.3692 \mathrm{e}-01$ & $1.0269 e+00$ & \\
\hline 116 & VPW000005 & 139.6522 & 140.4334 & 140.0429 & $1.4764 \mathrm{e}-01$ & $1.1671 \mathrm{e}+00$ & \\
\hline 117 & VRW000006 & 148.4563 & 148.8469 & 148.6749 & $9.3710 e-02$ & $1.2049 e+00$ & \\
\hline 118 & IPW000001 & 533.7407 & 547.4072 & 539.5587 & $3.3582 e+00$ & $9.1531 e+00$ & \\
\hline 119 & IPW000002 & 1304.2736 & 1315.9812 & 1308.9957 & $3.9439 \mathrm{e}+00$ & $1.3136 e+01$ & \\
\hline 120 & IPW000003 & 1324.0499 & 1331.8499 & 1328.7686 & $1.7658 \mathrm{e}+00$ & $1.3259 \mathrm{e}+01$ & \\
\hline 121 & IPW000004 & 374.8216 & 380.1927 & 377.3216 & $1.8207 e+00$ & $3.5896 \mathrm{e}+00$ & \\
\hline 122 & IPW000005 & 252.5851 & 254.0498 & 253.3858 & $3.5235 e-01$ & $2.8297 e+00$ & \\
\hline 123 & IPW000006 & 261.2681 & 262.7329 & 261.7952 & $3.3944 e-01$ & $2.8770 e+00$ & \\
\hline 124 & EMPTY & -0.0244 & -0.0098 & -0.0184 & $3.7642 e-03$ & $0.0000 e+00$ & \\
\hline 125 & EMPTY & -0.0195 & -0.0049 & -0.0123 & $3.3073 e-03$ & $0.0000 e+00$ & \\
\hline 126 & EMPTY & -0.0195 & 0.0000 & -0.0151 & $4.0971 e-03$ & $0.0000 e+00$ & \\
\hline 127 & EMPTY & -0.0244 & -0.0098 & -0.0153 & $3.5582 e-03$ & $0.0000 e+00$ & \\
\hline 128 & IHTO000001 & 128.1325 & 130.4762 & 129.4020 & $5.0724 e-01$ & $4.2994 e+00$ & \\
\hline 129 & IHT 000002 & 131.2049 & 133.9393 & 133.0839 & $5.1128 e-01$ & $4.3106 e+00$ & \\
\hline 130 & IHT000003 & 131.9186 & 134.2623 & 133.1529 & $5.0291 e-01$ & $4.3108 e+00$ & \\
\hline 131 & IHT000004 & 130.0143 & 132.3580 & 131.3462 & $5.4489 e-01$ & $4.3053 e+00$ & \\
\hline 132 & IHTO00005 & 128.6809 & 130.6340 & 129.7160 & $4.8848 e-01$ & $4.3003 e+00$ & \\
\hline 133 & IHT000006 & 123.8018 & 125.9503 & 124.7784 & $4.3405 e-01$ & $4.2857 e+00$ & \\
\hline 134 & IHTO00007 & 131.1899 & 132.9477 & 131.8110 & $4.6302 e-01$ & $4.3067 e+00$ & \\
\hline $135^{\circ}$ & IHTO00008 & 129.7701 & 131.3326 & - $130: 3638$ & $4: 2674 e-02$ & $4.3023 e+00$ & - \\
\hline 136 & IHT000009 & 129.7589 & 131.5167 & 130.6924 & $4.3457 e-01$ & $4.3033 e+00$ & \\
\hline 137 & IHT000010 & -41.2748 & -38.9310 & -40.0209 & $5.0392 e-01$ & $4.1195 e+00$ & \\
\hline 138 & IHT000011 & 129.5260 & 131.2838 & 130.5064 & $4.7921 e-01$ & $4.3027 e+00$ & \\
\hline 139 & IHTO00012 & 127.3362 & 129.0941 & 128.2464 & $4.7122 e-01$ & $4.2959 e+00$ & \\
\hline 140 & IHTO000013 & 91.0156 & 92.9688 & 92.0547 & $4.6428 e-01$ & $4.2021 e+00$ & \\
\hline 141 & IHT000014 & 127.3663 & 129.1241 & 128.1280 & $4.5890 e-01$ & $4.2955 e+00$ & \\
\hline 142 & IHT000015 & 94.8167 & 96.3792 & 95.6644 & $4.2974 e-01$ & $4.2101 e+00$ & \\
\hline 143 & IHT000016 & 88.5216 & 90.0841 & 89.3263 & $4.1505 e-01$ & $4.1962 e+00$ & \\
\hline 144 & IHTO000017 & 105.0706 & 108.5863 & 107.0433 & $7.6945 e-01$ & $4.2374 e+00$ & \\
\hline 145 & IHT000018 & 106.9636 & 109.3074 & 108.1434 & $5.3809 e-01$ & $4.2402 e+00$ & \\
\hline 146 & IHT000019 & 103.4781 & 105.4312 & 104.4155 & $5.0686 e-01$ & $4.2309 e+00$ & \\
\hline 147 & IHT 000020 & 106.1974 & 108.3458 & 107.1935 & $5.2386 e-01$ & $4.2378 e+00$ & \\
\hline 148 & IHTO00021 & -2.4226 & -0.0789 & -1.3757 & $6.2734 e-01$ & $4.1000 e+00$ & \\
\hline 149 & IHT000022 & 92.4692 & 94.4223 & 93.2153 & $5.0175 e-01$ & $4.2046 e+00$ & \\
\hline 150 & IHT000023 & 104.3645 & 106.1223 & 105.1730 & $4.8329 e-01$ & $4.2327 e+00$ & \\
\hline 151 & IHTO00024 & 69.5726 & 71.3304 & 70.2874 & $4.6624 e-01$ & $4.1598 e+00$ & \\
\hline 152 & IHTO00025 & 104.7439 & 106.5017 & 105.5251 & $4.8808 e-01$ & $4.2336 e+00$ & \\
\hline 153 & IHT000026 & 105.1307 & 106.8885 & 105.9432 & $4.1453 e-01$ & $4.2347 e+00$ & \\
\hline 154 & IHT000027 & 89.9940 & 91.9471 & 90.8455 & $4.0194 e-01$ & $4.1994 \mathrm{e}+00$ & \\
\hline 155 & IHT000028 & 91.1396 & 93.2880 & 92.2373 & $4.4273 e-01$ & $4.2025 e+00$ & \\
\hline
\end{tabular}




\begin{tabular}{|c|c|c|c|c|c|c|}
\hline $\mathrm{Te}$ & $t:$ Aug17_30 & sum 1 & Date: Fri & Aug 171 & $7: 33 \quad 1990$ & Page: 4 \\
\hline $\begin{array}{l}\# \\
156 \\
157 \\
158 \\
159 \\
160 \\
161 \\
162 \\
163\end{array}$ & 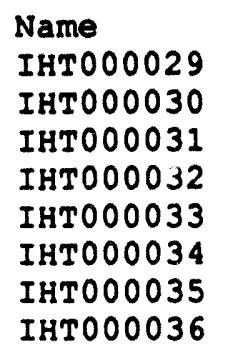 & $\begin{array}{l}\text { Min } \\
104.4246 \\
106.8172 \\
105.2509 \\
106.3514 \\
106.1786 \\
93.3518 \\
123.5277 \\
92.2138\end{array}$ & $\begin{array}{l}\text { Max } \\
106.5730 \\
109.1609 \\
107.7900 \\
109.0858 \\
109.1083 \\
95.8909 \\
126.0667 \\
94.9482\end{array}$ & $\begin{array}{l}\text { Ave } \\
105.7137 \\
108.0711 \\
106.4306 \\
107.7342 \\
107.6670 \\
94.5589 \\
124.6644 \\
93.1904\end{array}$ & $\begin{array}{l}\text { SD } \\
4.3045 e-01 \\
4.8982 e-01 \\
4.8961 e-01 \\
5.3792 e-01 \\
5.3654 e-01 \\
4.6470 e-01 \\
4.9864 e-01 \\
4.5677 e-01\end{array}$ & $\begin{array}{c}\text { Uncert } \\
4.2341 e+00 \\
4.2400 e+00 \\
4.2359 e+00 \\
4.2392 e+00 \\
4.2390 e+00 \\
4.2076 e+00 \\
4.2853 e+00 \\
4.2046 e+00\end{array}$ \\
\hline
\end{tabular}




\begin{tabular}{|c|c|c|c|c|c|c|}
\hline \multicolumn{2}{|c|}{ Test: Aug17_30 } & sum2 & Date: Fri & \multicolumn{2}{|c|}{ Aug $17 \quad 10: 07: 33 \quad 1990$} & Page: 3 \\
\hline * & Name & Min & $\operatorname{Max}$ & Ave & SD & Uncert \\
\hline 101 & PIN000001 & 40.0808 & 40.3249 & 40.2126 & $4.1561 e-02$ & $7.5671 e-01$ \\
\hline 102 & PRV000001 & 19.7186 & 19.9627 & 19.7991 & $6.3406 e-02$ & $7.5163 e-01$ \\
\hline 103 & PSR000001 & 44.8156 & 45.0597 & 44.8937 & $6.4121 e-02$ & $7.5835 e-01$ \\
\hline 104 & PFM000001 & 1.7324 & 2.0071 & 1.9027 & $6.9232 e-02$ & $5.0002 e-01$ \\
\hline 105 & PANO00136 & -133.1649 & -130.8760 & -131.9930 & $4.4507 e-01$ & $4.93110+00$ \\
\hline 106 & PEM000002 & 1.8287 & 1.8958 & 1.8616 & $1.9755 e-02$ & $2.0005 e-01$ \\
\hline 107 & EMPTY & -0.0049 & 0.0049 & -0.0019 & $3.9323 e-03$ & $0.0000 e+00$ \\
\hline 108 & ЕMPTY & -10.0000 & -0.4980 & -6.7120 & $2.4310 e+00$ & $0.0000 e+00$ \\
\hline 109 & EMPTY & -0.0049 & 0.0049 & 0.0001 & $1.2041 \mathrm{e}-03$ & $0.0000 e+00$ \\
\hline 110 & EMPTY & -10.0000 & -10.0000 & -10.0000 & $0.0000 e+00$ & $0.0000 e+00$ \\
\hline 111 & EMPTY & -0.0049 & 0.0000 & -0.0001 & $6.9053 e-04$ & $0.0000 e+00$ \\
\hline 112 & VPW000001 & 102.0245 & 102.2198 & 102.1416 & $9.6655 e-02$ & $1.0157 \mathrm{e}+00$ \\
\hline 113 & VPW000002 & 119.2421 & 119.4374 & 119.3514 & $9.7935 e-02$ & $1.0812 e+00$ \\
\hline 114 & VPW000003 & 147.6225 & 148.2084 & 147.8452 & $1.1844 e-01$ & $1.2012 \mathrm{e}+00$ \\
\hline 115 & VPW000004 & 104.6687 & 105.2546 & 105.0633 & $1.3945 e-01$ & $1.0264 e+00$ \\
\hline 116 & VPW000005 & 139.8475 & 140.4334 & 140.0780 & $1.3491 \mathrm{e}-01$ & $1.1673 e+00$ \\
\hline 117 & VPW000006 & 148.2610 & 148.8469 & 148.6163 & $1.2901 \mathrm{e}-01$ & $1.2047 e+00$ \\
\hline 118 & IPW000001 & 535.6931 & 539.5978 & 537.7625 & $1.2726 e+00$ & $9.1465 \mathrm{e}+00$ \\
\hline 119 & IPW0 00002 & 1304.2736 & 1308.1761 & 1306.6154 & $1.3075 e+00$ & $1.3121 e+01$ \\
\hline 120 & IPW000003 & 1327.9498 & 1331.8499 & 1329.5486 & $1.0190 e+00$ & $1.3264 \mathrm{e}+01$ \\
\hline 121 & IPWO00004 & 374.8216 & 376.2864 & 375.5149 & $2.8058 \theta-01$ & $3.5778 e+00$ \\
\hline 222 & IPW0 00005 & 252.5851 & 254.0498 & 253.4737 & $3.5138 \mathrm{e}-01$ & $2.8302 e+00$ \\
\hline 123 & IPW000006 & 260.7798 & 262.7329 & 261.6878 & $3.8219 e-01$ & $2.8764 e+00$ \\
\hline 124 & EMPTY & -0.0293 & -0.0098 & -0.0192 & $4.2327 e-03$ & $0.0000 e+00$ \\
\hline 125 & EMPTY & -0.0195 & -0.0098 & -0.0135 & $2.8862 e-03$ & $0.0000 \mathrm{e}+00$ \\
\hline 126 & EMPTY & -0.0195 & -0.0098 & -0.0147 & $3.1950 e-03$ & $0.0000 \mathrm{e}+00$ \\
\hline 127 & EMPTY & -0.0244 & -0.0098 & -0.0159 & $3.9224 \mathrm{e}-03$ & $0.0000 e+00$ \\
\hline 128 & IHTO00001 & 128.3278 & 130.8669 & 129.1989 & $4.5713 e-01$ & $4.2987 e+00$ \\
\hline 129 & IHT000002 & 131.9862 & 133.7440 & 132.7870 & $4.0673 e-01$ & $4.3097 e+00$ \\
\hline 130 & IHT000003 & 132.1139 & 134.2623 & 132.9693 & $4.1176 e-01$ & $4.3102 e+00$ \\
\hline 131 & IHTO00004 & 130.0143 & 132.1627 & 131.1783 & $4.2123 e-01$ & $4.3047 e+00$ \\
\hline 132 & IHT000005 & 128.4856 & 130.6340 & 129.5753 & $4.4659 e-01$ & $4.2999 e+00$ \\
\hline 133 & IHT000006 & 123.6065 & 125.5596 & 124.6416 & $4.4337 e-01$ & $4.2853 e+00$ \\
\hline 134 & IHT000007 & 130.6040 & 132.7524 & 131.6626 & $4.8180 \mathrm{e}-01$ & $4.3062 e+00$ \\
\hline 135 & IAT 000008 & $129.18^{\circ} 42$ & $130^{\circ} .9420^{\circ}$ & $130^{\circ} .1021$ & $-4.2909 e^{\circ}-01$ & $4.3015 \dot{\theta}+00$ \\
\hline 136 & IHTO00009 & 129.1729 & 131.5167 & 130.4072 & $4.8559 e-01$ & $4.3024 e+00$ \\
\hline 137 & IHTO00010 & -40.8842 & -38.3451 & -39.8607 & $5.5039 e-01$ & $4.1193 e+00$ \\
\hline 138 & IHTO00011 & 129.5260 & 131.8697 & 130.6197 & $5.3670 e-01$ & $4.3030 e+00$ \\
\hline 139 & IHTO00012 & 126.9456 & 129.4847 & 128.3324 & $5.8161 e-01$ & $4.2962 e+00$ \\
\hline 140 & IHTO00013 & 90.6250 & 93.1641 & 92.0898 & $5.6669 \mathrm{e}-01$ & $4.2021 e+00$ \\
\hline 141 & IHTO00014 & 126.7804 & 129.3194 & 128.3116 & $5.6547 e-01$ & $4.2961 \mathrm{e}+00$ \\
\hline 142 & IHTO00015 & 94.4261 & 96.7698 & 95.7034 & $5.4706 e-01$ & $4.2102 e+00$ \\
\hline 143 & IHT000016 & 88.3263 & 90.8654 & 89.4904 & $5.2934 \mathrm{e}-01$ & $4.1965 \mathrm{e}+00$ \\
\hline 144 & IHT000017 & 106.4378 & 108.5863 & 107.6214 & $5.1055 e-01$ & $4.2389 e+00$ \\
\hline 145 & IHT000018 & 107.5496 & 109.5027 & 108.3465 & $4.8942 e-01$ & $4.2407 e+00$ \\
\hline 146 & IHTO00019 & 103.2828 & 105.6265 & 104.6577 & $4.5841 \mathrm{e}-01$ & $4.2315 e+00$ \\
\hline 147 & IHTO00020 & 106.0021 & 108.3458 & 107.3419 & $4.4645 e-01$ & $4.2382 e+00$ \\
\hline 148 & IHTO00021 & -2.8132 & -0.0789 & -1.2429 & $5.0986 \mathrm{e}-01$ & $4.1000 e+00$ \\
\hline 149 & IHTO00022 & 91.8833 & 94.2270 & 93.3364 & $4.5874 e-01$ & $4.2049 e+00$ \\
\hline 150 & IHT 000023 & 103.7786 & 106.5129 & 105.2707 & $4.5636 e-01$ & $4.2330 e+00$ \\
\hline 151 & IHTO00024 & 69.1820 & 71.7210 & 70.4632 & $4.1227 e-01$ & $4.1601 e+00$ \\
\hline 152 & IHT 000025 & 104.3532 & 106.8923 & 105.7087 & $4.4365 e-01$ & $4.2341 \mathrm{e}+00$ \\
\hline 153 & IHTO00026 & 105.1307 & 107.0838 & 106.1424 & $4.2989 \mathrm{e}-01$ & $4.2352 e+00$ \\
\hline 154 & IHTO00027 & 89.9940 & 91.7518 & 90.9276 & $3.9713 e-01$ & $4.1996 \mathrm{e}+00$ \\
\hline 155 & IHTO00028 & 91.3349 & 93.6786 & 92.3584 & $4.9844 e-01$ & $4.2027 e+00$ \\
\hline
\end{tabular}




\begin{tabular}{|c|c|c|c|c|c|c|}
\hline $\begin{array}{l}* \\
156 \\
157 \\
158 \\
159 \\
160 \\
161 \\
162 \\
163\end{array}$ & $\begin{array}{l}\text { Name } \\
\text { IHTO00029 } \\
\text { IHTO00030 } \\
\text { IHTO00031 } \\
\text { IHTO00032 } \\
\text { IHTO00033 } \\
\text { IHTO00034 } \\
\text { IHTO00035 } \\
\text { IHTO00036 }\end{array}$ & $\begin{array}{l}\text { Min } \\
104.6199 \\
106.8172 \\
105.2509 \\
106.5467 \\
106.5693 \\
93.3518 \\
123.7230 \\
92.2138\end{array}$ & $\begin{array}{l}\text { Max } \\
107.3543 \\
109.3562 \\
107.9853 \\
109.4764 \\
109.3036 \\
95.6956 \\
126.0667 \\
94.3622\end{array}$ & $\begin{array}{l}\text { Ave } \\
105.8856 \\
108.2000 \\
106.5908 \\
107.9100 \\
107.8310 \\
94.4495 \\
124.6449 \\
93.0341\end{array}$ & $\begin{array}{l}\text { SD } \\
5.4994 \theta-01 \\
5.4796 e-01 \\
5.3672 \theta-01 \\
5.8059 \theta-01 \\
5.6663 \theta-01 \\
4.9582 \theta-01 \\
5.1756 e-01 \\
4.7842 \theta-01\end{array}$ & $\begin{array}{c}\text { Uncert } \\
4.2345 e+00 \\
4.2404 \theta+00 \\
4.2363 e+00 \\
4.2396 e+00 \\
4.2394 \theta+00 \\
4.2074 e+00 \\
4.2853 \theta+00 \\
4.2042 e+00\end{array}$ \\
\hline
\end{tabular}




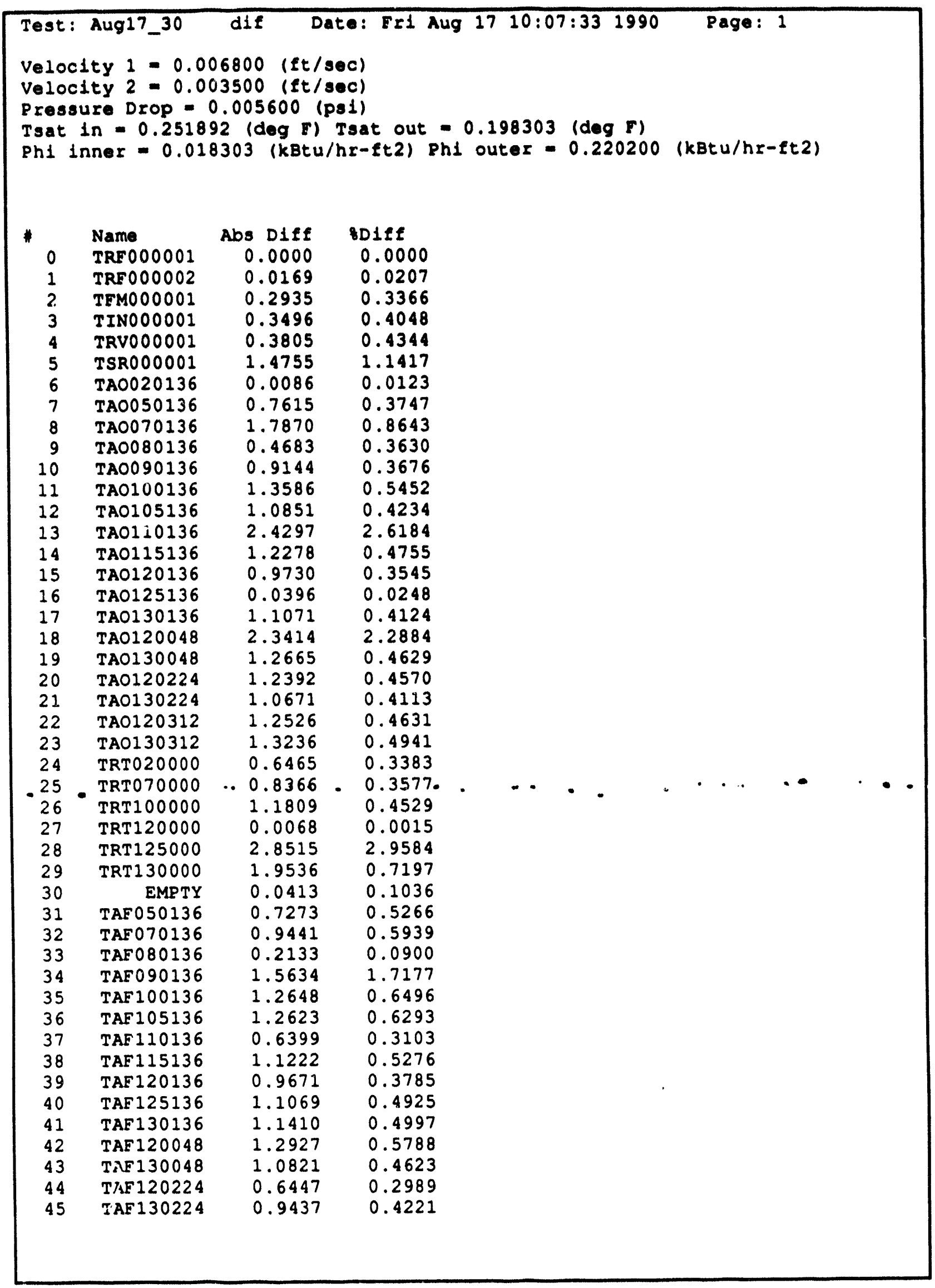




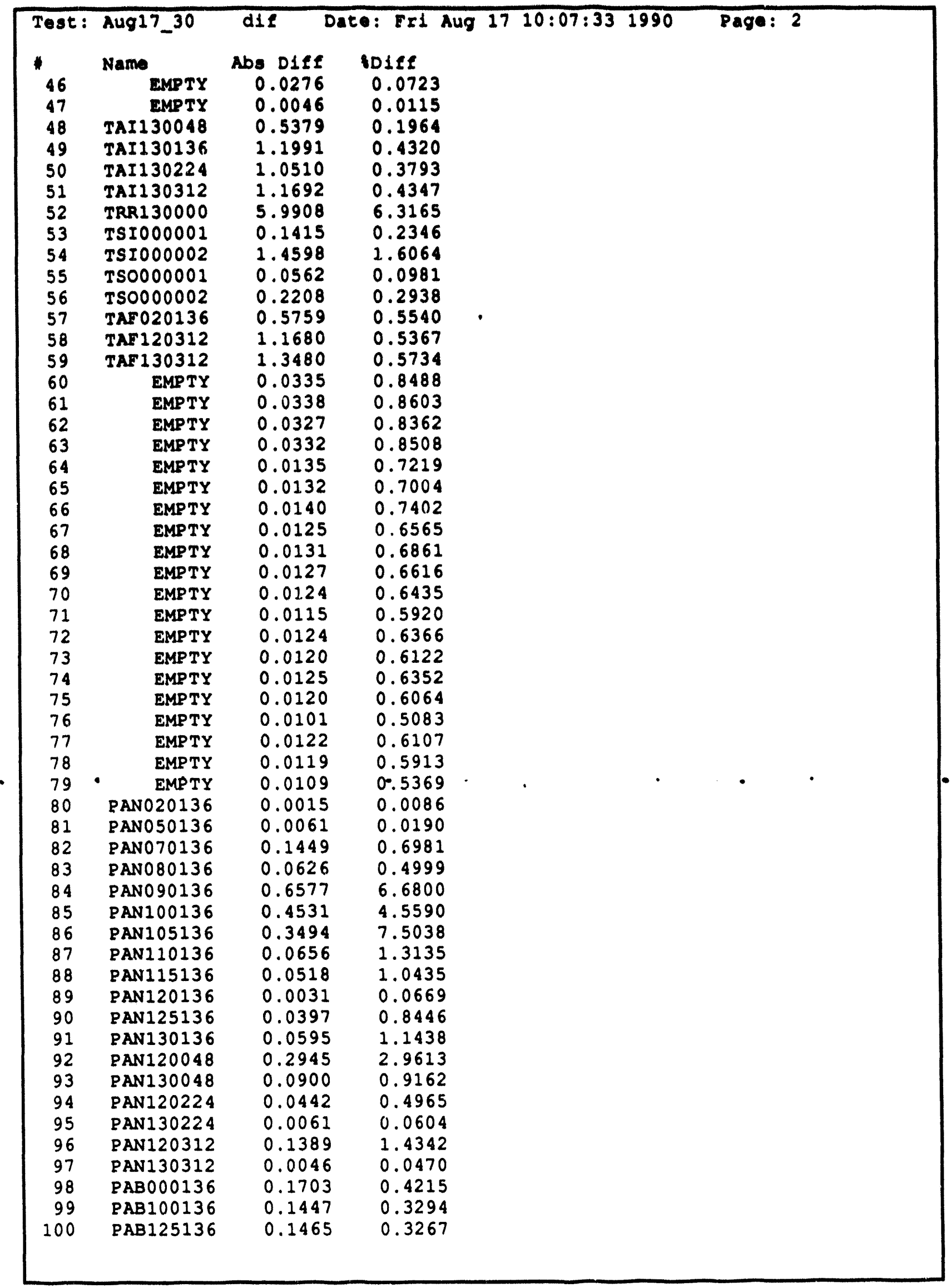




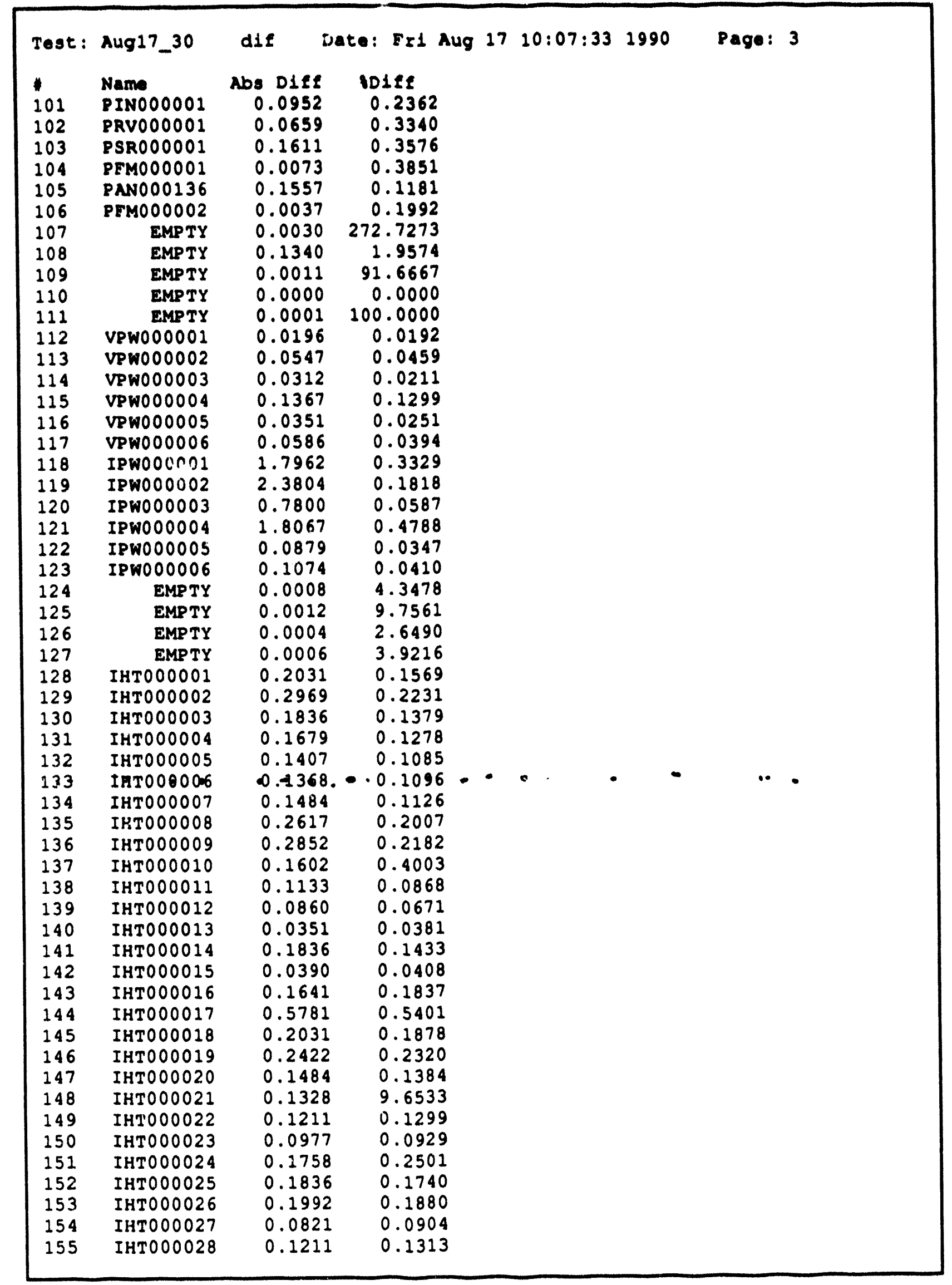




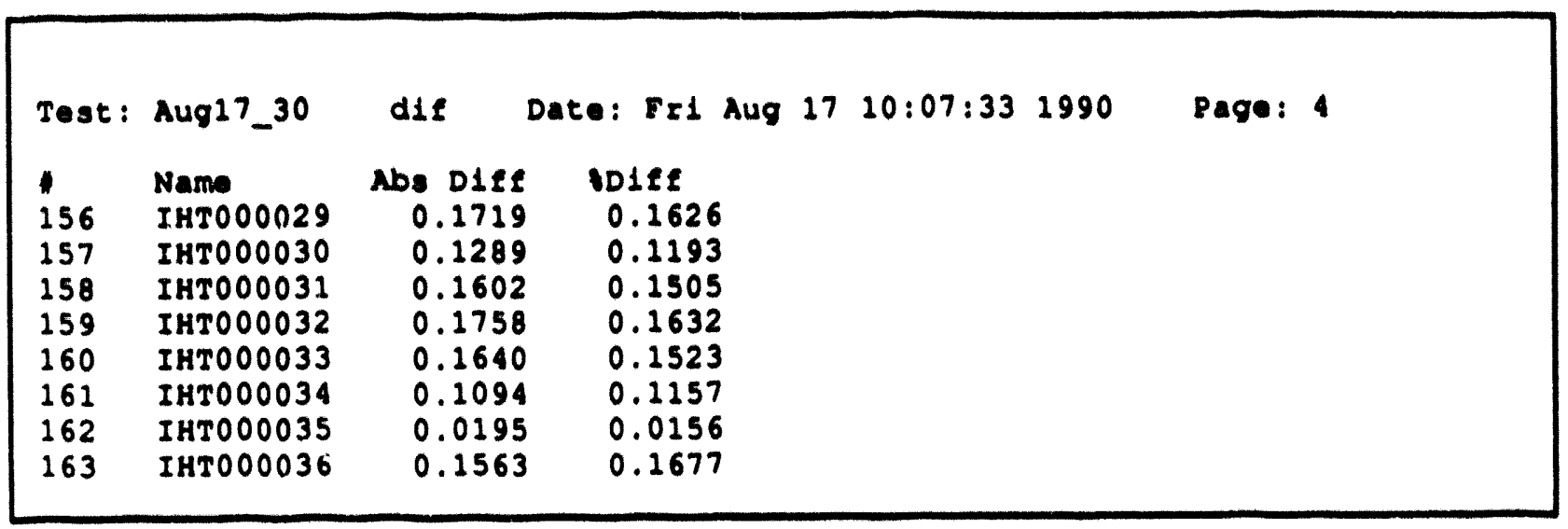




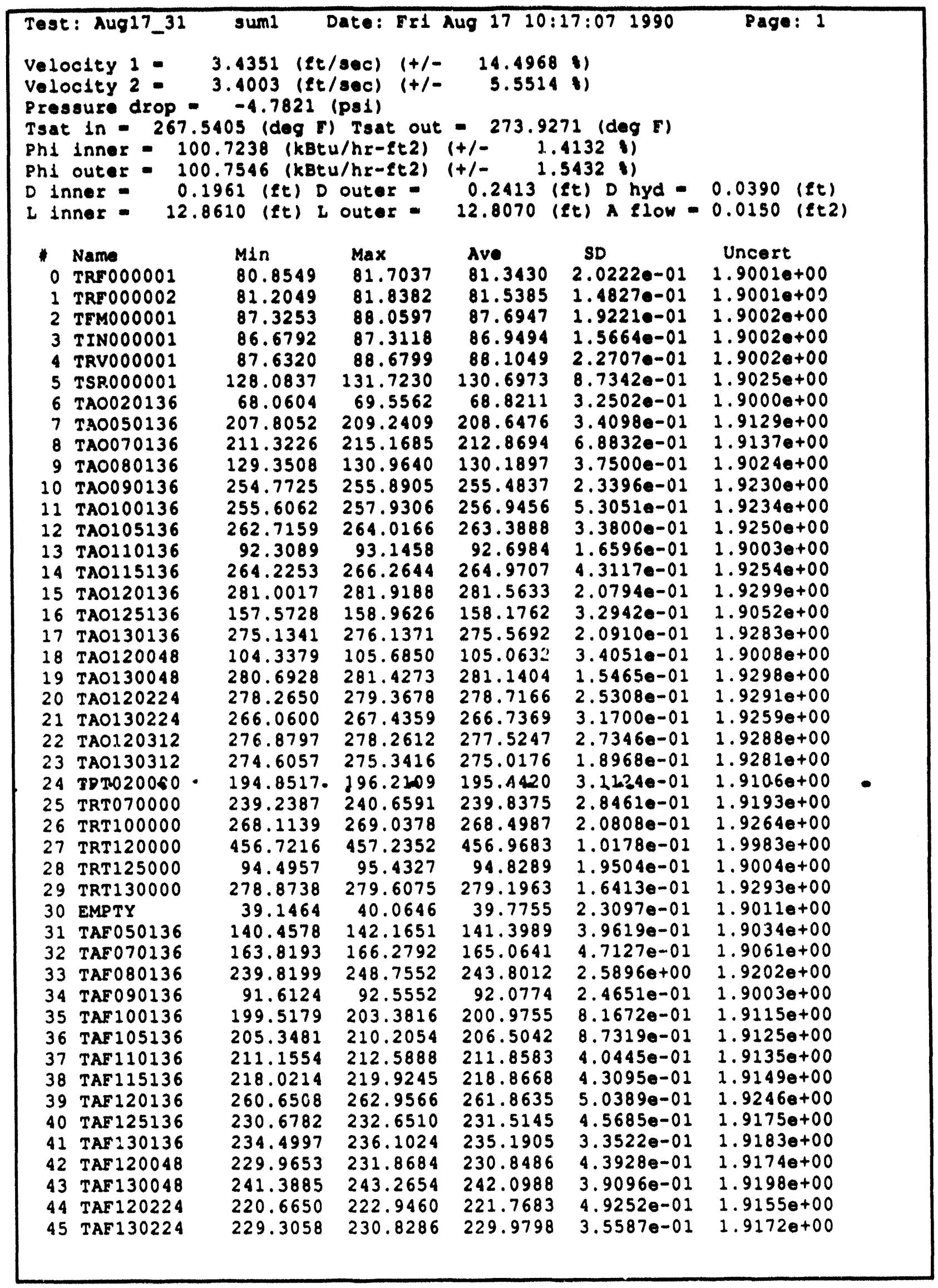




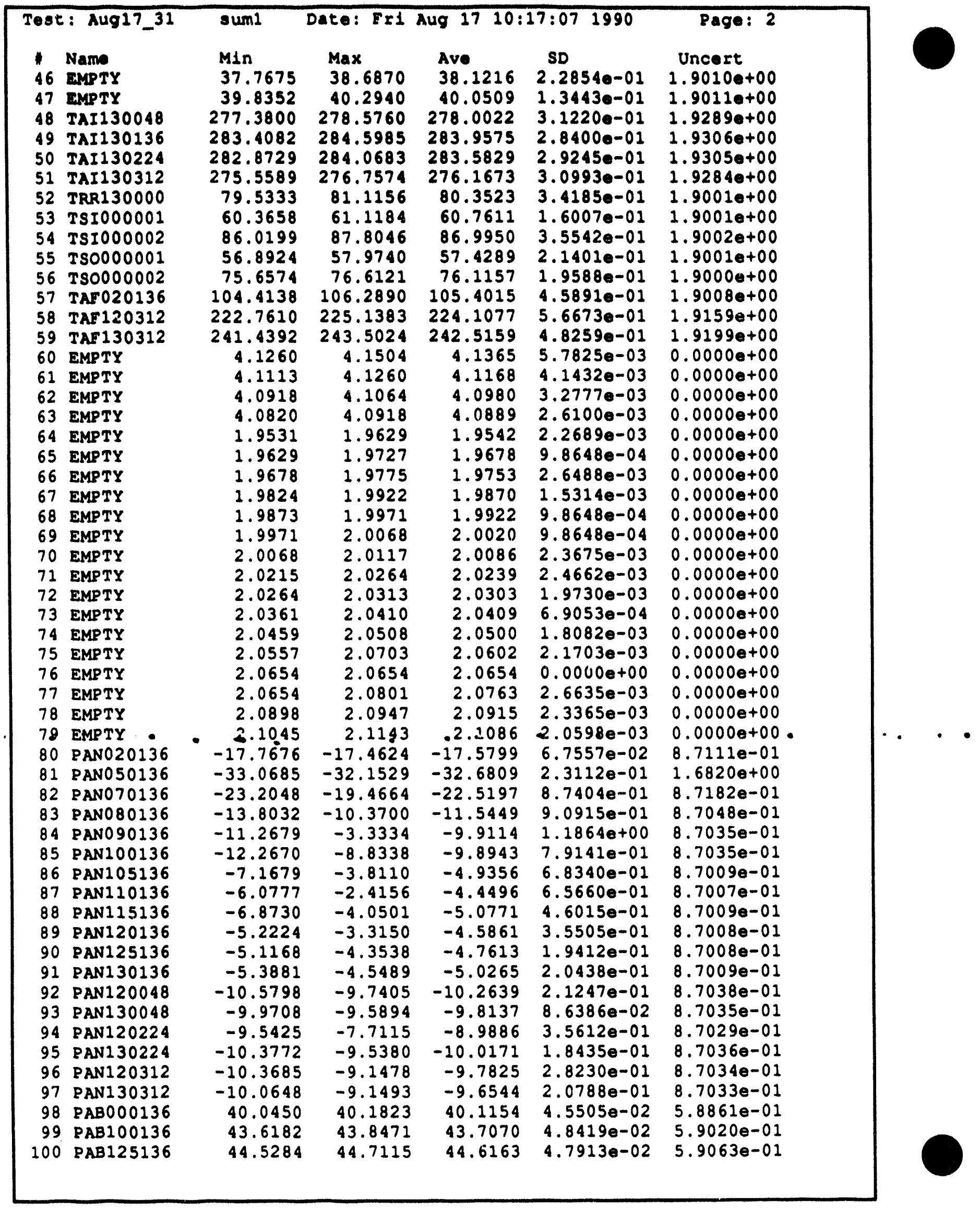




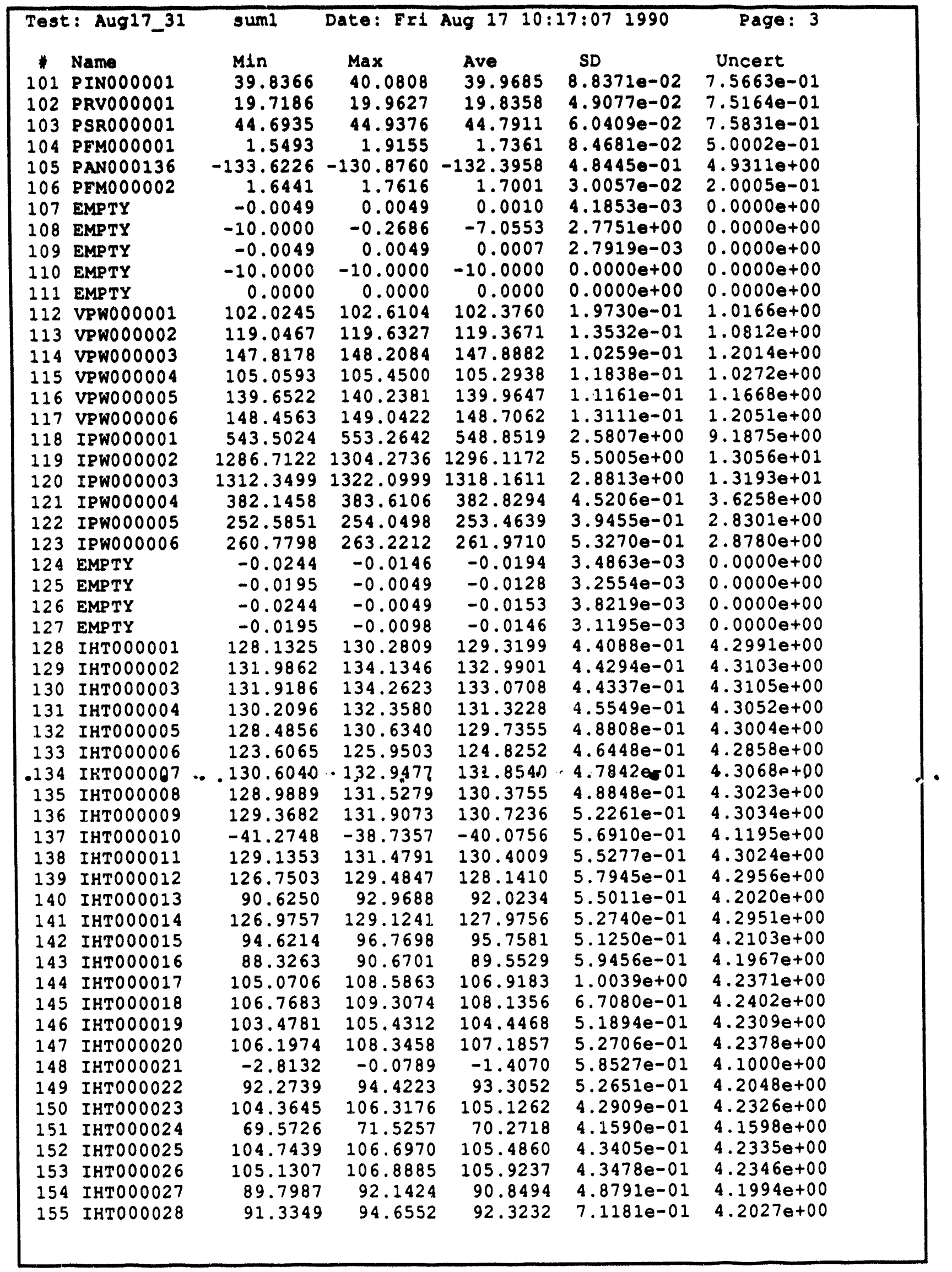




\begin{tabular}{|c|c|c|c|c|c|c|}
\hline \multicolumn{2}{|c|}{ Test: Aug17_31 } & suml & Date: Fr1 & Aug 1710 & $: 07 \quad 1990$ & Page: 4 \\
\hline $\begin{array}{c}* \\
156 \\
157 \\
158 \\
159 \\
160 \\
161 \\
162 \\
163\end{array}$ & 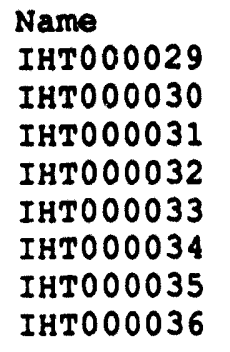 & $\begin{array}{l}\text { Min } \\
104.8152 \\
107.0125 \\
105.2509 \\
106.5467 \\
106.5693 \\
93.7425 \\
123.7230 \\
92.2138\end{array}$ & $\begin{array}{l}\text { Max } \\
107.9402 \\
109.7469 \\
108.1806 \\
109.6717 \\
109.6943 \\
95.8909 \\
125.6761 \\
94.3622\end{array}$ & $\begin{array}{l}\text { Ave } \\
105.7879 \\
108.0321 \\
106.3877 \\
107.7420 \\
107.7021 \\
94.5589 \\
124.4730 \\
93.1396\end{array}$ & $\begin{array}{l}\text { SD } \\
6.9080 e-01 \\
6.9283 e-01 \\
6.5160 e-01 \\
6.6338 e-01 \\
6.4477 e-01 \\
5.2302 e-01 \\
5.1503 e-01 \\
4.5645 e-01\end{array}$ & $\begin{array}{l}\text { Uncert } \\
4.2343 e+00 \\
4.2399 e+00 \\
4.2358 e+00 \\
4.2392 e+00 \\
4.2391 e+00 \\
4.2076 e+00 \\
4.2848 e+00 \\
4.2045 e+00\end{array}$ \\
\hline
\end{tabular}




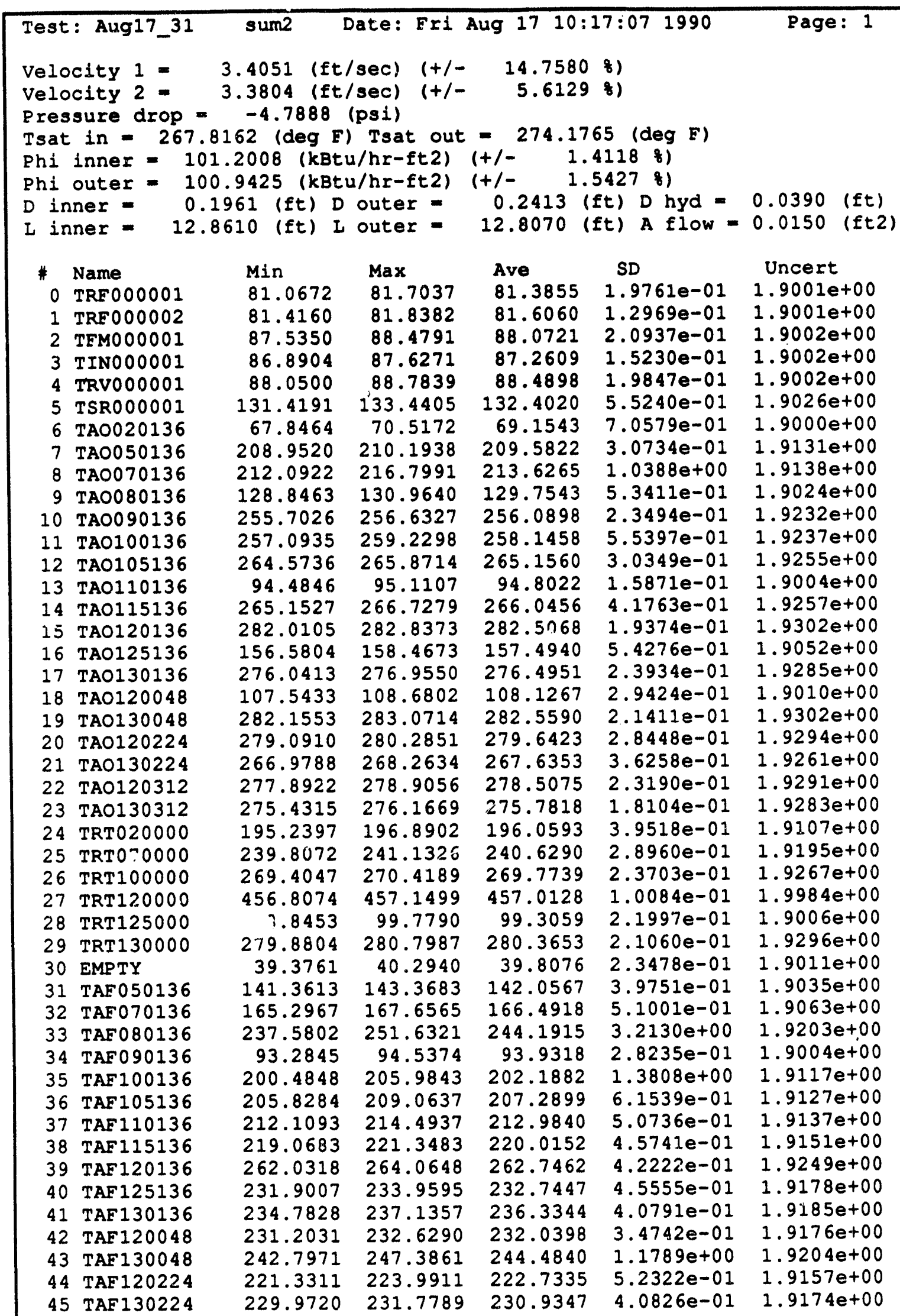




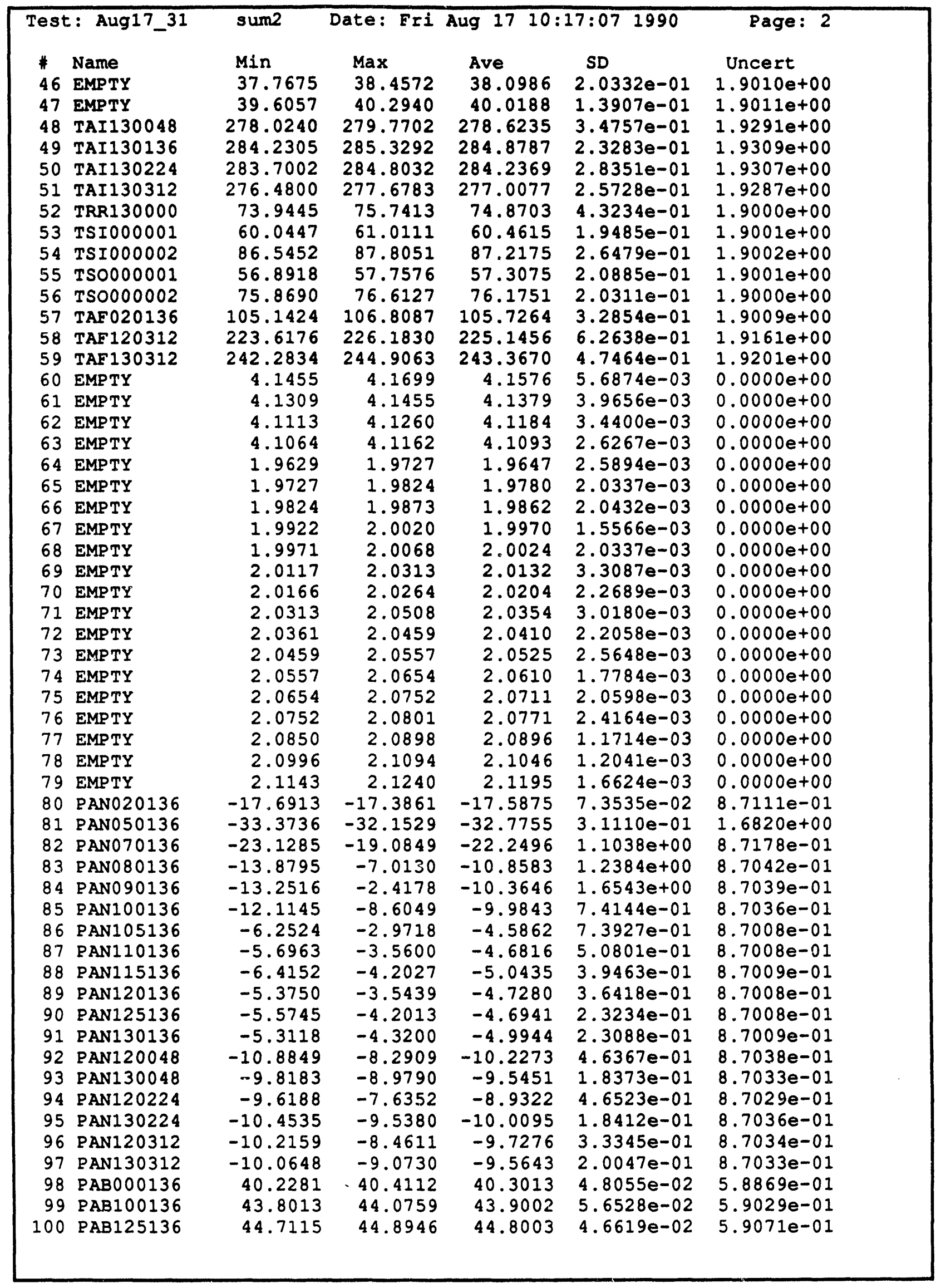




\begin{tabular}{|c|c|c|c|c|c|c|}
\hline \multicolumn{2}{|c|}{ Test: Aug17_31 } & sum2 & Date: Fri & Aug 171 & $7: 07 \quad 1990$ & Page: 3 \\
\hline$\$$ & Name & Min & $\operatorname{Max}$ & Ave & SD & Uncert \\
\hline 101 & PIN 000001 & 39.9587 & 40.3249 & 40.1345 & $8.9465 e-02$ & $7.5668 \mathrm{e}-01$ \\
\hline 102 & PRV000001 & 19.5965 & 19.8406 & 19.7405 & $5.8828 e-02$ & $7.5162 e-01$ \\
\hline 103 & PSR000001 & 44.9376 & 45.0597 & 44.9938 & $6.1457 e-02$ & $7.5839 e-01$ \\
\hline 104 & PFM000001 & 1.5493 & 2.0071 & 1.7050 & $8.3234 e-02$ & $5.0002 e-01$ \\
\hline 105 & PAN000136 & -133.6226 & -131.3338 & -132.5789 & $4.9838 e-01$ & $4.9312 e+00$ \\
\hline 106 & PFM000002 & 1.6273 & 1.7280 & 1.6797 & $2.4124 e-02$ & $2.0004 e-01$ \\
\hline 107 & EMPTY & -0.0049 & 0.0049 & 0.0016 & $4.2361 e-03$ & $0.0000 e+00$ \\
\hline 108 & EMPTY & -10.0000 & -1.9727 & -7.8683 & $2.1729 e+00$ & $0.0000 e+00$ \\
\hline 109 & EMPTY & -0.0049 & 0.0049 & 0.0006 & $1.8821 e-03$ & $0.0000 e+00$ \\
\hline 110 & EMPTY & -10.0000 & -10.0000 & -10.0000 & $0.0000 e+00$ & $0.0000 e+00$ \\
\hline 111 & EMPTY & -0.0049 & 0.0000 & -0.0001 & $6.9053 e-04$ & $0.0000 e+00$ \\
\hline 112 & VPW000001 & 102.0245 & 102.6104 & 102.3682 & $1.7451 e-01$ & $1.0165 \mathrm{e}+00$ \\
\hline 113 & VPW000002 & 119.2421 & 119.6327 & 119.4491 & $1.2104 e-01$ & $1.0816 e+00$ \\
\hline 114 & VPW000003 & 147.6225 & 148.0131 & 147.9077 & $1.0595 e-01$ & $1.2015 \mathrm{e}+00$ \\
\hline 115 & VPW000004 & 104.8640 & 105.4500 & 105.1688 & $1.4314 e-01$ & $1.0268 \mathrm{e}+00$ \\
\hline 116 & VRW000005 & 139.8475 & 140.2381 & 140.0429 & $1.3087 e-01$ & $1.1671 \mathrm{e}+00$ \\
\hline 117 & VRW000006 & 148.2610 & 148.6516 & 148.5148 & $1.2633 e-01$ & $1.2042 e+00$ \\
\hline 118 & IPW000001 & 537.6454 & 549.3595 & 544.2834 & $2.9252 e+00$ & $9.1705 e+00$ \\
\hline 119 & IPW000002 & 1292.5659 & 1319.8837 & 1308.0981 & $8.6814 e+00$ & $1.3130 \mathrm{e}+01$ \\
\hline 120 & IPW000003 & 1322.0999 & 1331.8499 & 1325.6880 & $3.1110 e+00$ & $1.3240 \mathrm{e}+01$ \\
\hline 121 & IPW000004 & 377.7513 & 379.7044 & 378.4251 & $4.1725 e-01$ & $3.5969 \mathrm{e}+00$ \\
\hline 122 & IPW000005 & 252.0969 & 253.5616 & 253.1807 & $4.1015 e-01$ & $2.8285 e+00$ \\
\hline 123 & IPW000006 & 261.2681 & 263.2212 & 262.0980 & $4.9570 e-01$ & $2.8788 e+00$ \\
\hline 124 & EMPTY & -0.0244 & -0.0098 & -0.0189 & $3.9013 e-03$ & $0.0000 \mathrm{e}+00$ \\
\hline 125 & EMPTY & -0.0195 & -0.0049 & -0.0133 & $3.1257 e-03$ & $0.0000 e+00$ \\
\hline 126 & EMPTY & -0.0244 & -0.0049 & -0.0137 & $3.6911 \mathrm{e}-03$ & $0.0000 e+00$ \\
\hline 127 & EMPTY & -0.0244 & -0.0098 & -0.0158 & $3.8913 e-03$ & $0.0000 e+00$ \\
\hline 128 & IHTO00001 & 127.7419 & 130.6716 & 128.9410 & $6.0619 e-01$ & $4.2980 \mathrm{e}+00$ \\
\hline 129 & IHT000002 & 131.5955 & 133.9393 & 132.5370 & $5.0638 e-01$ & $4.3089 e+00$ \\
\hline 130 & IHT000003 & 131.7233 & 134.0670 & 132.7935 & $5.1181 e-01$ & $4.3097 e+00$ \\
\hline 131 & IHT000004 & 129.8190 & 132.1627 & 131.0415 & $5.0810 e-01$ & $4.3043 e+00$ \\
\hline 132 & IHT000005 & 128.2903 & 130.8293 & 129.4347 & $5.0994 \mathrm{e}-01$ & $4.2995 \mathrm{e}+00$ \\
\hline 133 & IHT000006 & 123.2159 & 126.1456 & 124.5010 & $5.5396 e-01$ & $4.2849 e+00$ \\
\hline 134 & IHT000007 & 130.4087 & 133.3384 & 131.5415 & $5.8261 e-01$ & $4.3058 e+00$ \\
\hline 135 & IHTO00008 & 129.1842 & 131.7233 & 130.0552 & $5.3845 e-01$ & $4.3013 e+00$ \\
\hline 136 & IHTO00009 & 129.3682 & 131.9073 & 130.3994 & $5.5948 e-01$ & $4.3024 e+00$ \\
\hline 137 & IHTO000010 & -41.0795 & -38.5404 & -39.8959 & $5.2853 e-01$ & $4.1194 e+00$ \\
\hline 138 & IHT000011 & 129.5260 & 131.6744 & 130.4517 & $5.0349 e-01$ & $4.3025 e+00$ \\
\hline 139 & IHTO00012 & 127.1409 & 129.2894 & 128.1761 & $5.4283 e-01$ & $4.2957 e+00$ \\
\hline 140 & IHT000013 & 91.2109 & 93.3594 & 92.1484 & $5.2940 \mathrm{e}-01$ & $4.2023 e+00$ \\
\hline 143 & IHTO00014 & 127.3663 & 129.3194 & 128.2022 & $5.1455 e-01$ & $4.2958 \mathrm{e}+00$ \\
\hline 142 & IHTO00015 & 94.8167 & 96.5745 & 95.7308 & $4.8398 e-01$ & $4.2103 e+00$ \\
\hline 143 & IHT000016 & 88.7169 & 90.4747 & 89.5646 & $4.7941 e-01$ & $4.1967 e+00$ \\
\hline 144 & IHT000017 & 105.4613 & 108.7816 & 107.0980 & $8.7958 e-01$ & $4.2376 e+00$ \\
\hline 145 & IHTO000018 & 107.3543 & 109.6980 & 108.2723 & $6.0135 e-01$ & $4.2406 \mathrm{e}+00$ \\
\hline $14 \epsilon$ & IHTO00019 & 103.2828 & 105.8218 & 104.6538 & $5.5312 e-01$ & $4.2315 e+00$ \\
\hline 147 & IHTO00020 & 106.0021 & 108.9318 & 107.3302 & $5.4391 \mathrm{e}-01$ & $4.2382 e+00$ \\
\hline $14 \varepsilon$ & IHTO00021 & -2.4226 & 0.3118 & -1.2937 & $6.3047 e-01$ & $4.1000 \mathrm{e}+00$ \\
\hline 149 & IHT000022 & 92.4692 & 94.8130 & 93.3716 & $5.0361 e-01$ & $4.2050 e+00$ \\
\hline 150 & IHTO00023 & 104.1692 & 106.9036 & 105.2512 & $5.0410 e-01$ & $4.2329 e+00$ \\
\hline 15. & IHT000024 & 69.3773 & 72.1116 & 70.3890 & $4.8759 e-01$ & $4.1600 e+00$ \\
\hline 152 & IHT000025 & 104.5485 & 107.4782 & 105.6305 & $5.1024 e-01$ & $4.2339 \mathrm{e}+00$ \\
\hline 153 & IHT000026 & 105.1307 & 107.4745 & 106.0917 & $4.8942 e-01$ & $4.2350 \mathrm{e}+00$ \\
\hline 154 & IHTO00027 & 89.7987 & 92.7284 & 90.9705 & $5.2793 e-01$ & $4.1997 e+00$ \\
\hline 155 & IHTO00028 & 91.1396 & 93.8739 & 92.4443 & $6.9187 e-01$ & $4.2029 e+00$ \\
\hline
\end{tabular}




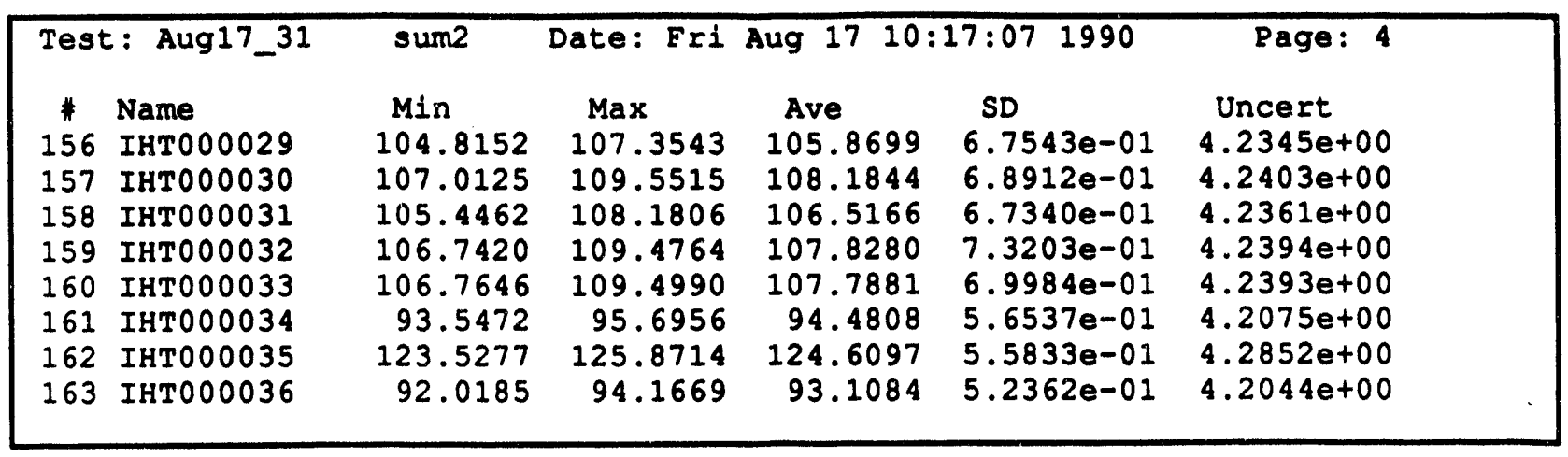




\begin{tabular}{|c|c|c|c|c|}
\hline \multicolumn{5}{|c|}{$\begin{array}{l}\text { Test: Aug17_31 dif Date: Fri Aug } 1710: 17: 071990 \text { Page: } 1 \\
\text { Velocity } 1=0.030000 \text { (ft/sec) } \\
\text { Velocity } 2=0.019900 \text { (ft/sec) } \\
\text { Pressure Drop }=0.006700 \text { (psi) } \\
\text { Tsat in }=0.275696 \text { (deg F) Tsat out }=0.249420 \text { (deg F) } \\
\text { Phi inner }=0.476997 \text { (kBtu/hr-ft2) Phi outer }=0.187897 \text { (kBtu/hr-ft2) }\end{array}$} \\
\hline $\begin{array}{r}\text { \# } \\
1 \\
2 \\
3 \\
4 \\
5 \\
6 \\
7 \\
8 \\
9 \\
10 \\
11 \\
12 \\
13 \\
14 \\
15 \\
16 \\
17 \\
18 \\
19 \\
20 \\
21 \\
22 \\
23 \\
24 \\
25 \\
26 \\
27 \\
28 \\
29 \\
30 \\
31 \\
32 \\
33 \\
34 \\
35 \\
36 \\
37 \\
38 \\
39 \\
40 \\
41 \\
42 \\
43 \\
44 \\
45\end{array}$ & 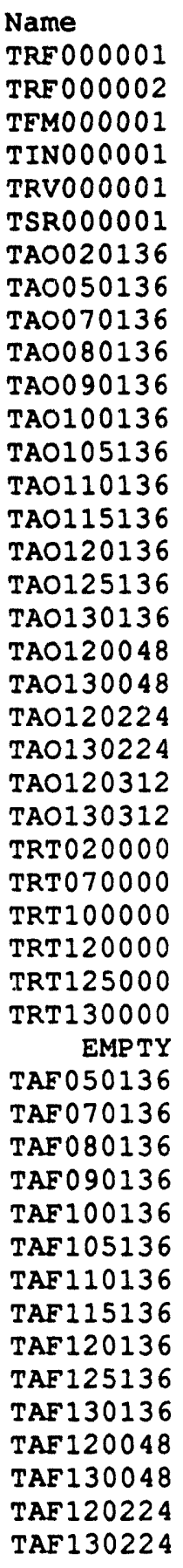 & $\begin{array}{l}\text { Abs Diff } \\
0.0425 \\
0.0675 \\
0.3774 \\
0.3115 \\
0.3849 \\
1.7047 \\
0.3332 \\
0.9346 \\
0.7571 \\
0.4354 \\
0.6061 \\
1.2002 \\
1.7672 \\
2.1038 \\
1.0749 \\
0.9435 \\
0.6822 \\
0.9259 \\
3.0635 \\
1.4186 \\
0.9257 \\
0.8984 \\
0.9828 \\
0.7642 \\
0.6173 \\
0.7915 \\
1.2752 \\
0.0445 \\
4.4770 \\
1.1690 \\
0.0321 \\
0.6578 \\
1.4277 \\
0.3903 \\
1.854 \\
0.95 \\
1.2127 \\
0.7857 \\
1.1257 \\
1.1484 \\
0.8827 \\
1.2302 \\
1.1439 \\
1.1912 \\
2.3852 \\
0.9652 \\
0.954\end{array}$ & $\begin{array}{l}\text { 8Diff } \\
0.0522 \\
0.0828 \\
0.4304 \\
0.3583 \\
0.4369 \\
1.3043 \\
0.4842 \\
0.4479 \\
0.3557 \\
0.3344 \\
0.2372 \\
0.4671 \\
0.6710 \\
2.2695 \\
0.4057 \\
0.3351 \\
0.4313 \\
0.3360 \\
2.9159 \\
0.5046 \\
0.3321 \\
0.3368 \\
0.3541 \\
0.2779 \\
0.3158 \\
0.3300 \\
0.4749 \\
0.0097 \\
4.7211 \\
0.4187 \\
0.0807 \\
0.4652 \\
0.8649 \\
0.1601 \\
2.0140 \\
0.6034 \\
0.3805 \\
0.5313 \\
0.5247 \\
0.3371 \\
0.5314 \\
0.4864 \\
0.5160 \\
0.9852 \\
0.4352 \\
0.4152\end{array}$ & \\
\hline
\end{tabular}




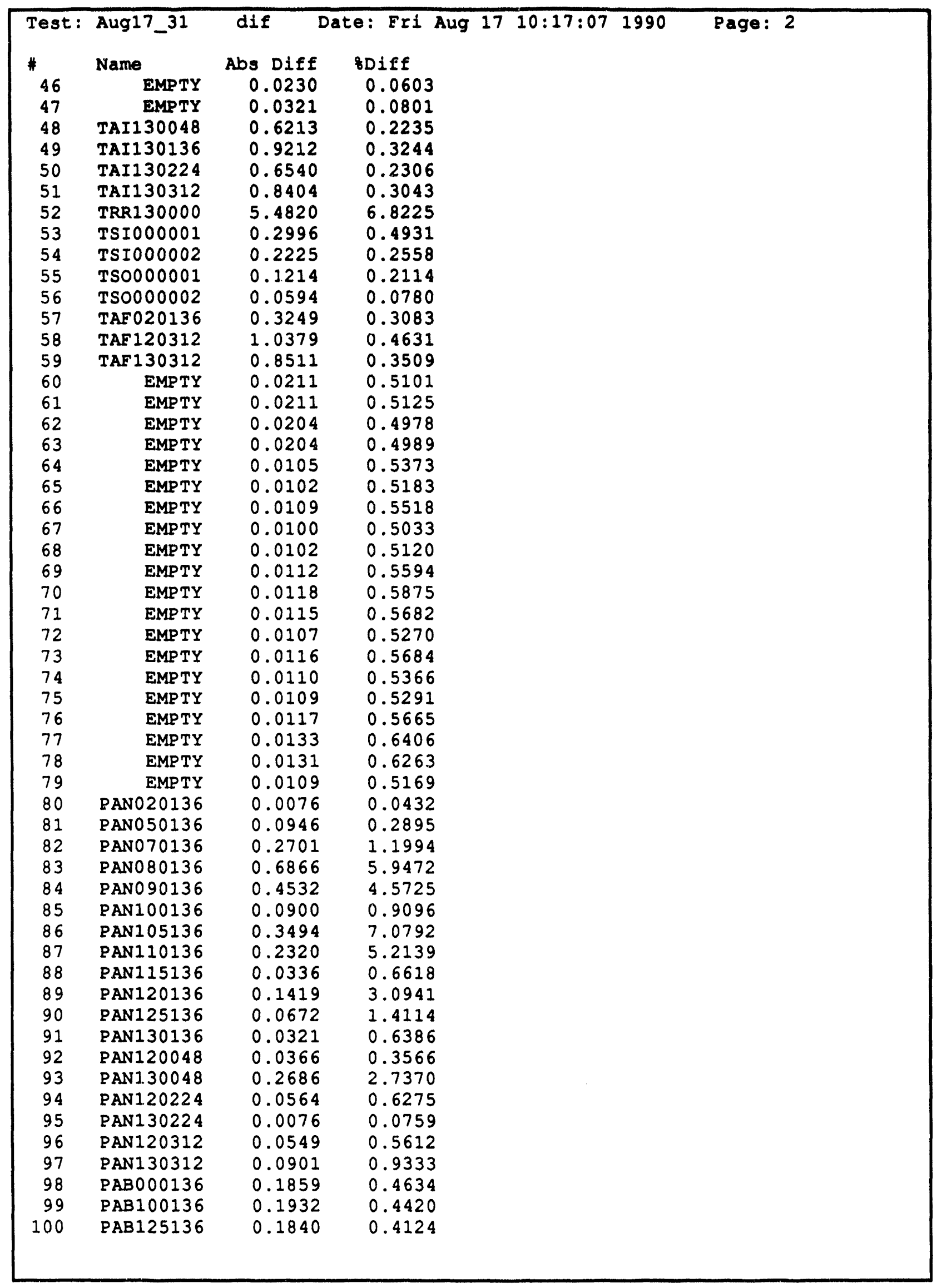




\begin{tabular}{|c|c|c|c|c|}
\hline Test: & Aug17_31 & dif & Date: Fri Aug $17 \quad 10: 17: 071990$ & Page: 3 \\
\hline \# & Name & Abs Diff & SDiff & \\
\hline 101 & PIN000001 & 0.1660 & 0.4153 & \\
\hline 102 & PRV000001 & 0.0953 & 0.4804 & \\
\hline 103 & PSR000001 & 0.2027 & 0.4525 & \\
\hline 104 & PFM000001 & 0.0311 & 1.7914 & \\
\hline 105 & PANO00136 & 0.1831 & 0.1383 & \\
\hline 106 & PFMO00002 & 0.0204 & 1.1999 & \\
\hline 107 & EMPTY & 0.0006 & 60.0000 & \\
\hline 108 & EMPTY & 0.8130 & 11.5232 & \\
\hline 109 & EMPTY & 0.0001 & 14.2857 & \\
\hline 110 & EMPTY & 0.0000 & 0.0000 & \\
\hline 111 & EMPTY & 0.0001 & 100.0000 & \\
\hline 112 & VPW000001 & 0.0078 & 0.0076 & \\
\hline 113 & VPW000002 & 0.0820 & 0.0687 & \\
\hline 114 & VPW000003 & 0.0195 & 0.0132 & \\
\hline 115 & VPW000004 & 0.1250 & 0.1187 & \\
\hline 116 & VPW000005 & 0.0782 & 0.0559 & \\
\hline 117 & VPW000006 & 0.1914 & 0.1287 & \\
\hline 118 & IPW000001 & 4.5685 & 0.8324 & \\
\hline 119 & IPW000002 & 11.9810 & 0.9244 & \\
\hline 120 & IPW000003 & 7.5269 & 0.5710 & \\
\hline 121 & IPWO00004 & 4.4043 & 1.1505 & \\
\hline 122 & IPW000005 & 0.2832 & 0.1117 & \\
\hline 123 & IPW000006 & 0.1270 & 0.0485 & \\
\hline 124 & EMPTY & 0.0005 & 2.5773 & \\
\hline 125 & EMPTY & 0.0005 & 3.9063 & \\
\hline 126 & EMPTY & 0.0016 & 10.4575 & \\
\hline 127 & EMPTY & 0.0012 & 8.2192 & \\
\hline 128 & IHTO00001 & 0.3789 & 0.2930 & \\
\hline 129 & IHTO00002 & 0.4531 & 0.3407 & \\
\hline 130 & IHT000003 & 0.2773 & 0.2084 & \\
\hline 131 & IHTO00004 & 0.2813 & 0.2142 & \\
\hline 132 & IHT000005 & 0.3008 & 0.2319 & \\
\hline 133 & IHT000006 & 0.3242 & 0.2597 & \\
\hline 134 & IHT000007 & 0.3125 & 0.2370 & \\
\hline 135 & IHT000008 & 0.3203 & 0.2457 & \\
\hline 136 & IHT000009 & 0.3242 & 0.2480 & \\
\hline 137 & IHT000010 & 0.1797 & 0.4484 & \\
\hline 138 & IHT000011 & 0.0508 & 0.0390 & \\
\hline 139 & IHT000012 & 0.0351 & 0.0274 & \\
\hline 140 & IHT000013 & 0.1250 & 0.1358 & \\
\hline 141 & IHTO00014 & 0.2266 & 0.1771 & \\
\hline 142 & IHTO00015 & 0.0273 & 0.0285 & \\
\hline 143 & IHT000016 & 0.0117 & 0.0131 & \\
\hline 144 & IHT000017 & 0.1797 & 0.1681 & \\
\hline 145 & IHT000018 & 0.1367 & 0.1264 & \\
\hline 146 & IHTO00019 & 0.2070 & 0.1982 & \\
\hline 147 & IHT000020 & 0.1445 & 0.1348 & \\
\hline 148 & IHT000021 & 0.1133 & 8.0526 & \\
\hline 149 & IHTO00022 & 0.0664 & 0.0712 & \\
\hline 150 & IHT000023 & 0.1250 & 0.1189 & \\
\hline 151 & IHT000024 & 0.1172 & 0.1668 & \\
\hline 152 & IHT000025 & 0.1445 & 0.1370 & \\
\hline 153 & IHT000026 & 0.1680 & 0.1586 & \\
\hline 154 & IHTO00027 & 0.1211 & 0.1333 & \\
\hline 155 & IHT000028 & 0.1211 & 0.1312 & \\
\hline
\end{tabular}


Test: Aug17_31 dif Date: Fr1 Aug 17 10:17:07 1990 Page: 4

* Name Abs Diff idiff

156 IHT000029 $0.0820 \quad 0.0775$

157 IHTO00030 $0.1523 \quad 0.1410$

$\begin{array}{llll}158 & \text { IHT000031 } & 0.1289 & 0.1212\end{array}$

$\begin{array}{llll}159 & \text { IHT000032 } & 0.0860 & 0.0798\end{array}$

$\begin{array}{llll}160 & \text { IHT000033 } & 0.0860 & 0.0798\end{array}$

$\begin{array}{llll}161 & \text { IHT000034 } & 0.0781 & 0.0826\end{array}$

$\begin{array}{llll}162 & \text { IHTO00035 } & 0.1367 & 0.1098\end{array}$

$\begin{array}{llll}163 & \text { IHT000036 } & 0.0312 & 0.0335\end{array}$ 


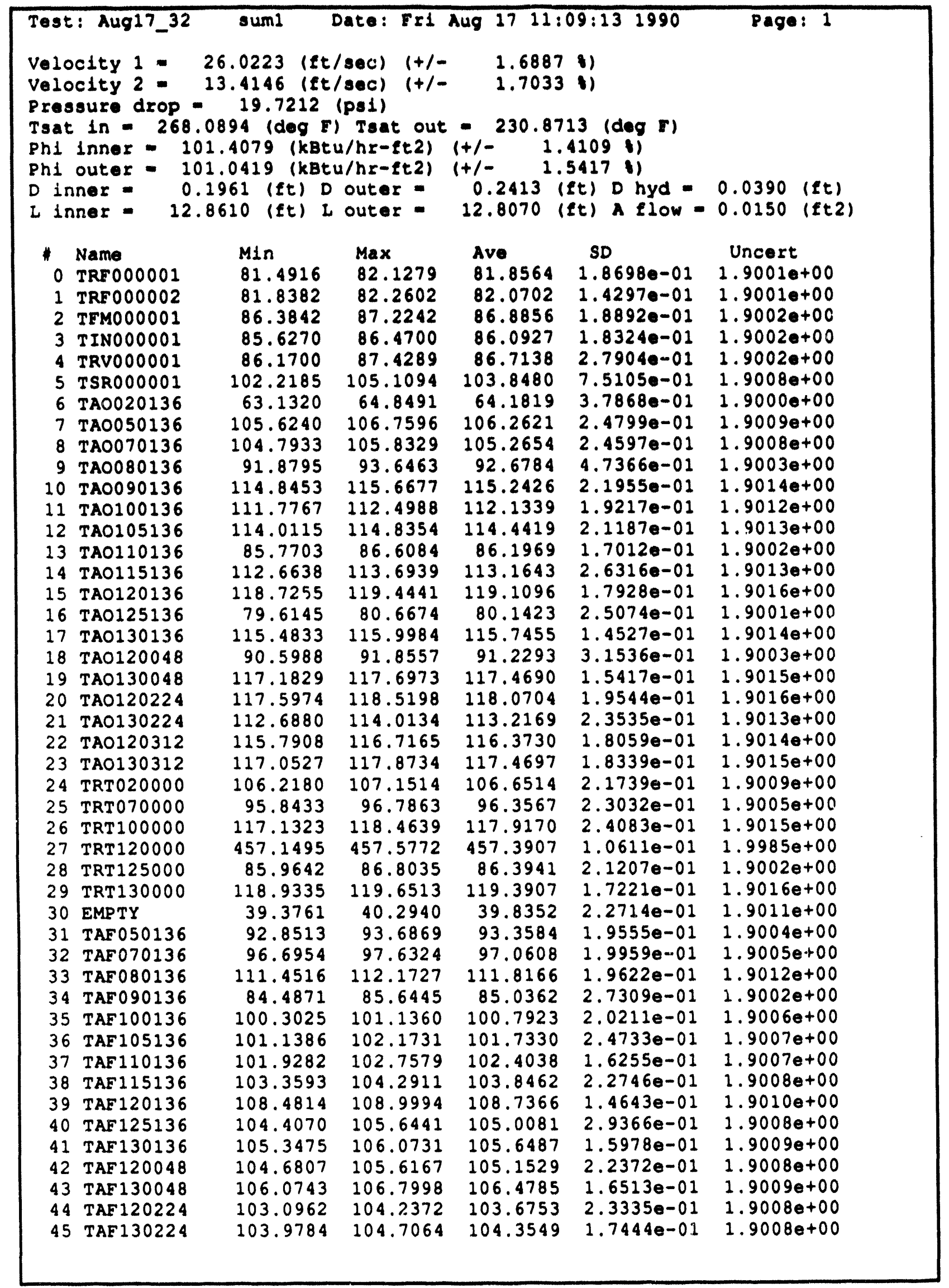




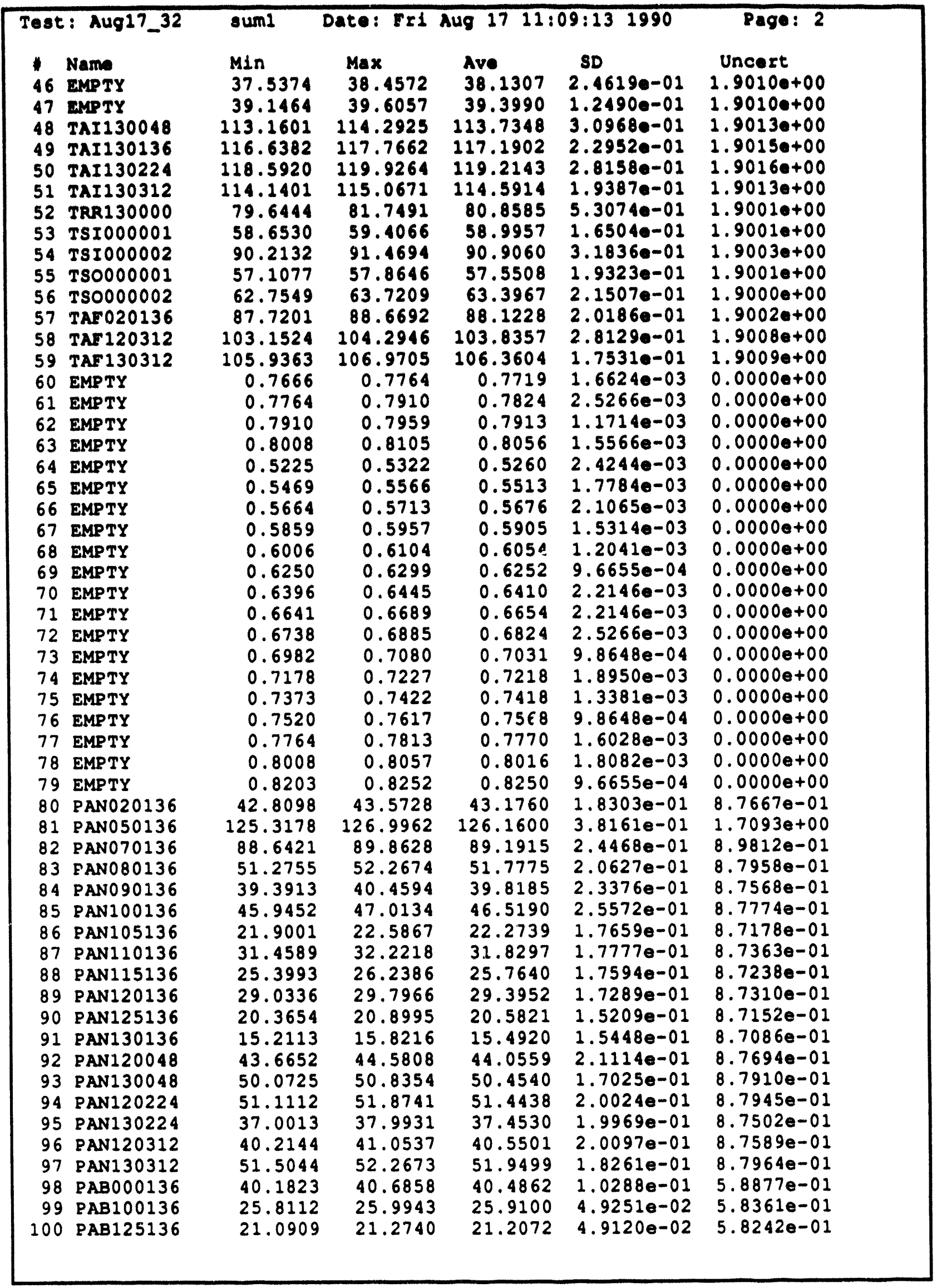




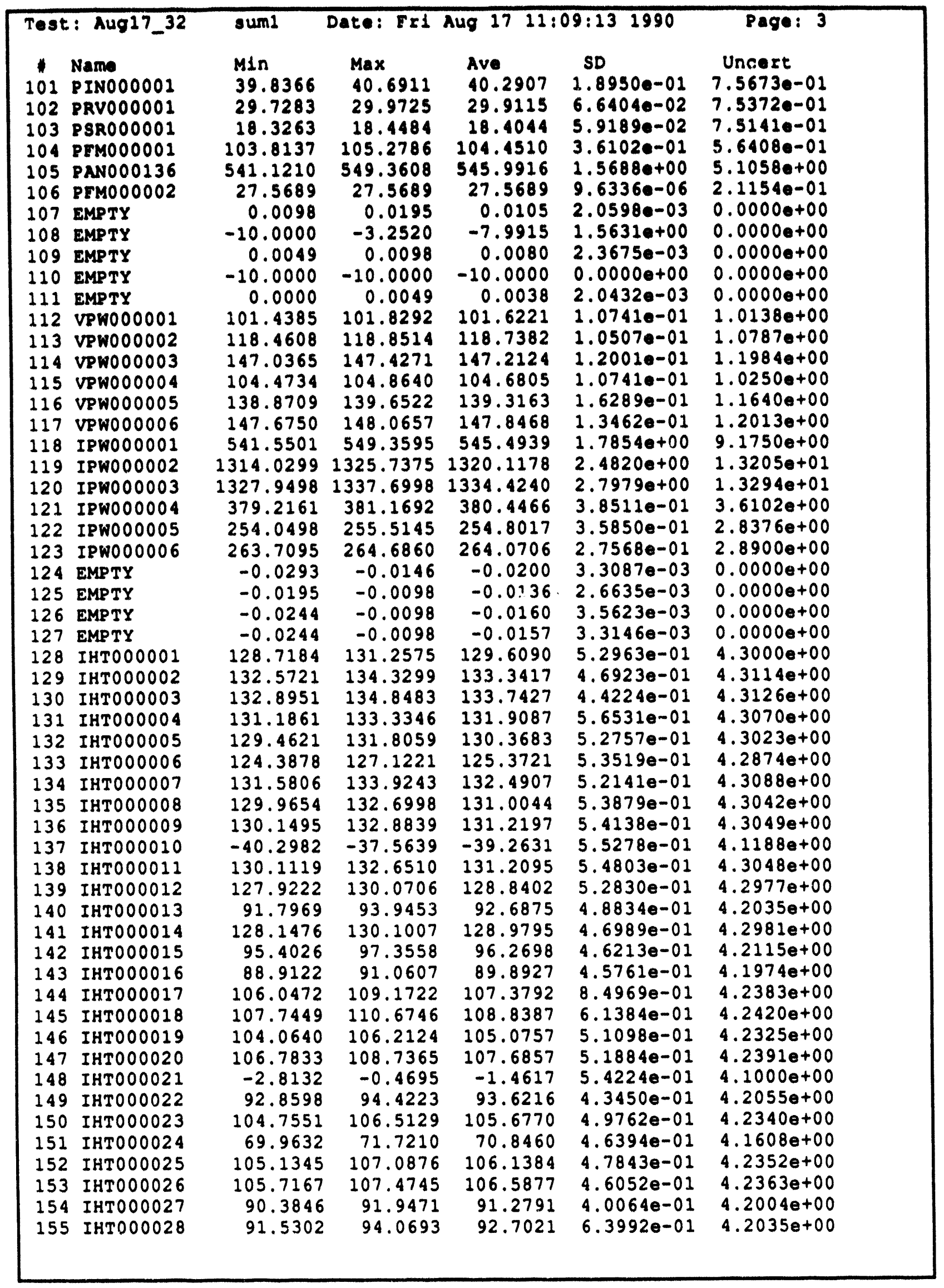




\begin{tabular}{|c|c|c|c|c|c|c|}
\hline $\begin{array}{l}1 \\
156 \\
157 \\
158 \\
159 \\
160 \\
161 \\
162 \\
163\end{array}$ & $\begin{array}{l}\text { Name } \\
\text { IHTOOOOO29 } \\
\text { IHTOOOOO30 } \\
\text { IHTOOOOO31 } \\
\text { IHTOOOOO32 } \\
\text { IHTOOOOO33 } \\
\text { IHTOOOOO34 } \\
\text { IHTOOOOO35 } \\
\text { IHTOOOOO36 }\end{array}$ & $\begin{array}{l}\text { Min } \\
105.0105 \\
107.4031 \\
105.8368 \\
107.3280 \\
107.3505 \\
94.1331 \\
124.3089 \\
92.7997\end{array}$ & $\begin{array}{l}\text { Max } \\
107.5496 \\
109.9422 \\
108.3759 \\
109.6717 \\
109.6943 \\
96.0862 \\
126.4573 \\
94.5575\end{array}$ & $\begin{array}{l}\text { Ave } \\
106.3192 \\
108.6492 \\
106.9814 \\
108.2889 \\
108.2373 \\
95.0081 \\
125.3168 \\
93.6630\end{array}$ & $\begin{array}{l}\text { SD } \\
6.2670 e-01 \\
6.2377 e-01 \\
5.8529 e-01 \\
5.8639 e-01 \\
5.9479 e-01 \\
4.2175 e-01 \\
4.7737 e-01 \\
4.4484 e-01\end{array}$ & $\begin{array}{c}\text { Uncert } \\
4.2356 e+00 \\
4.2415 e+00 \\
4.2373 e+00 \\
4.2406 e+00 \\
4.2405 e+00 \\
4.2086 \theta+00 \\
4.2872 e+00 \\
4.2056 e+00\end{array}$ \\
\hline
\end{tabular}




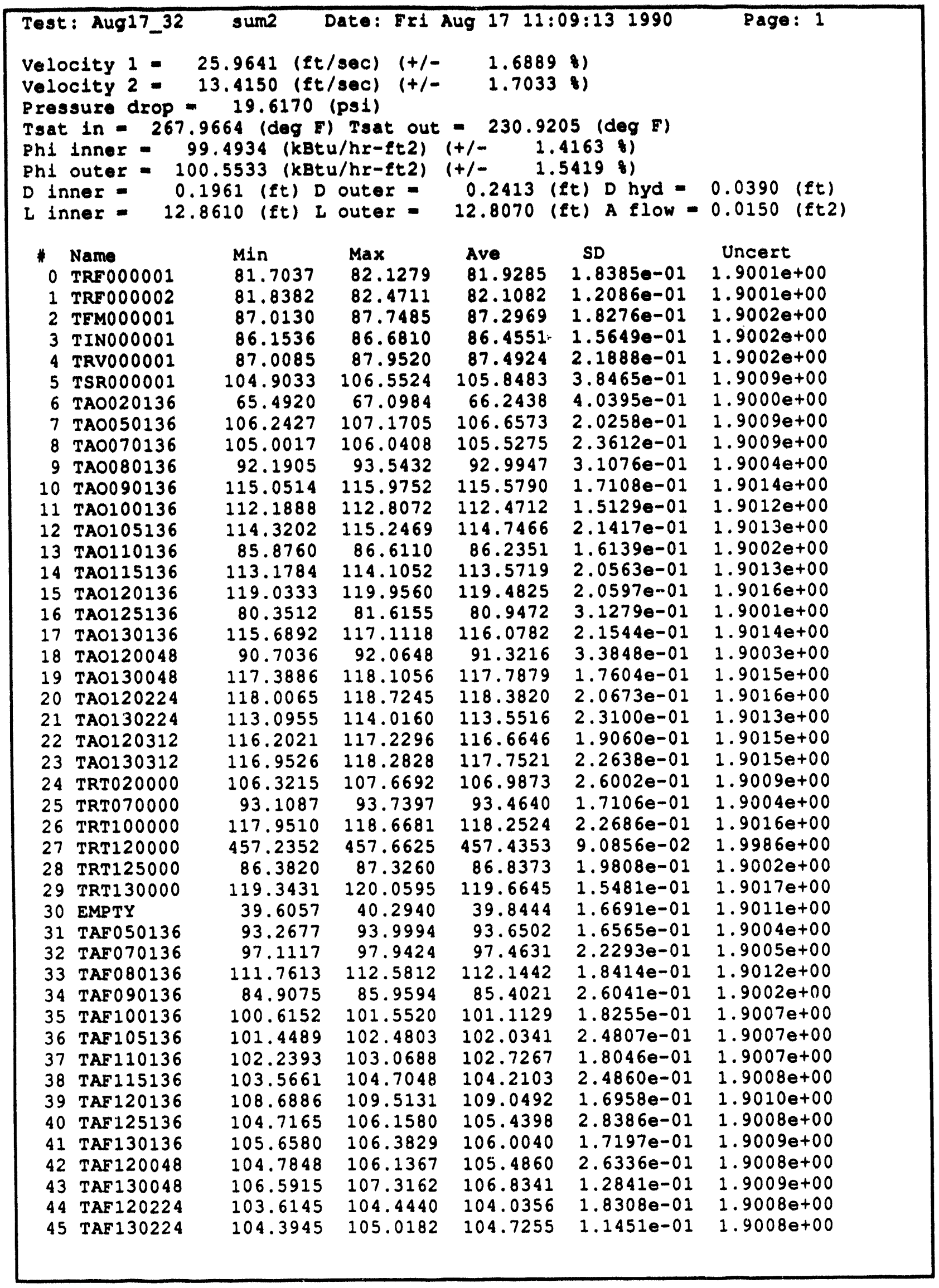




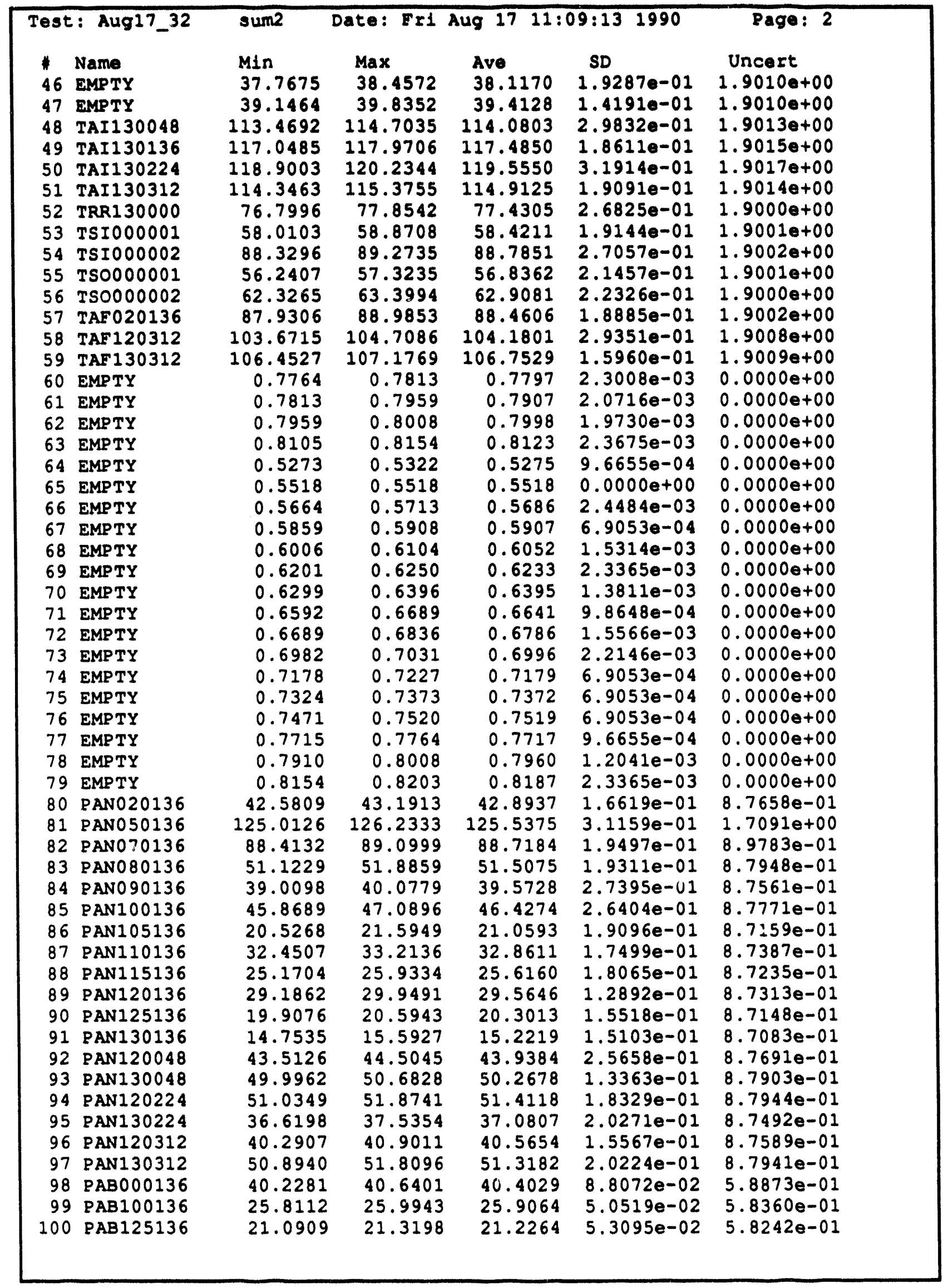




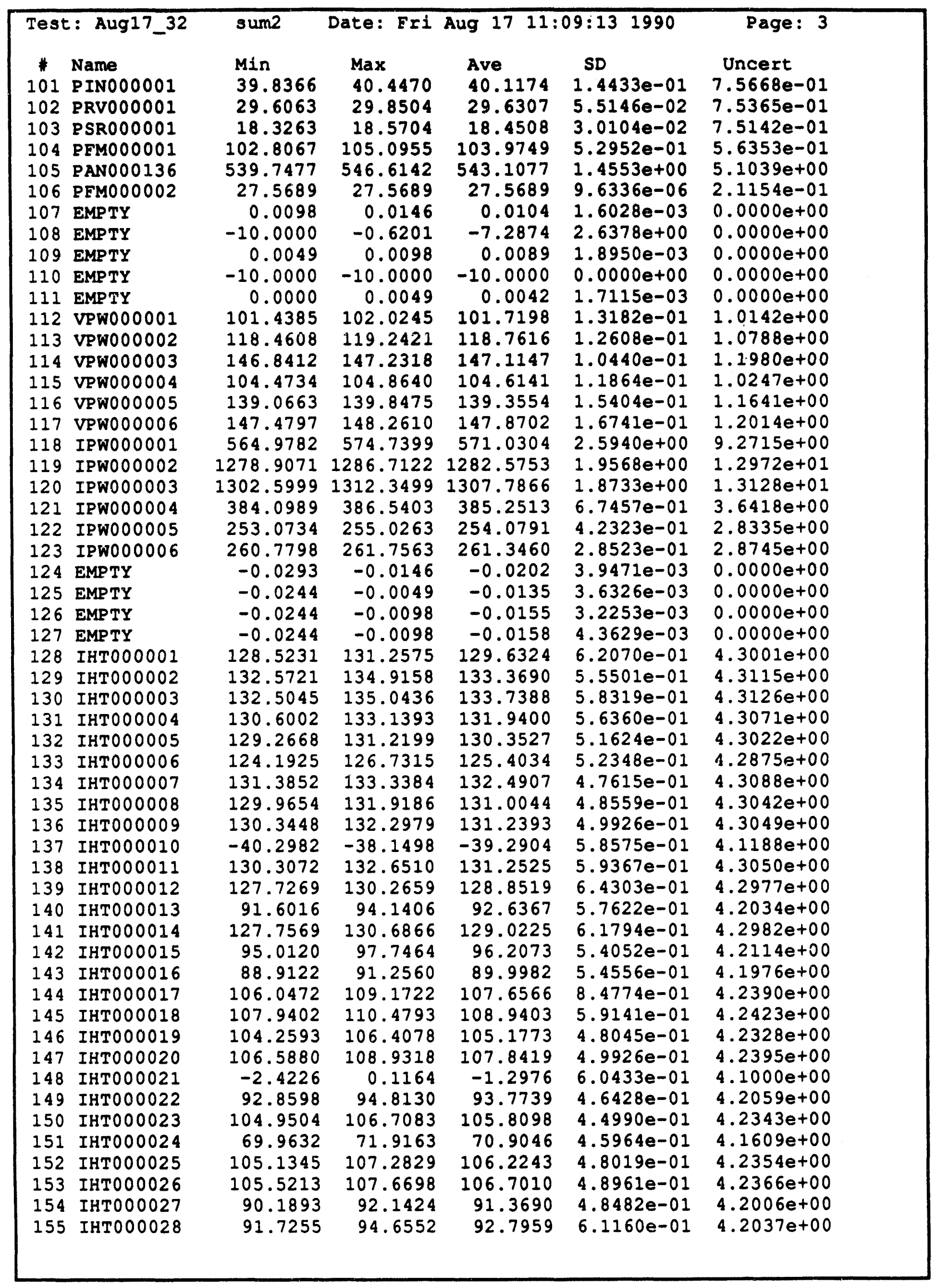




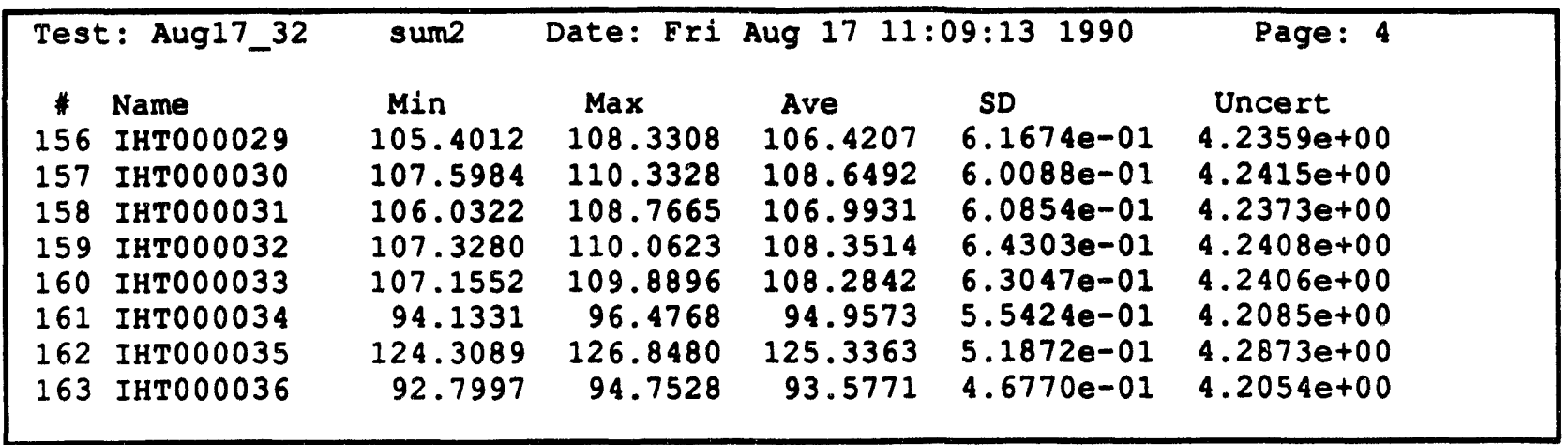




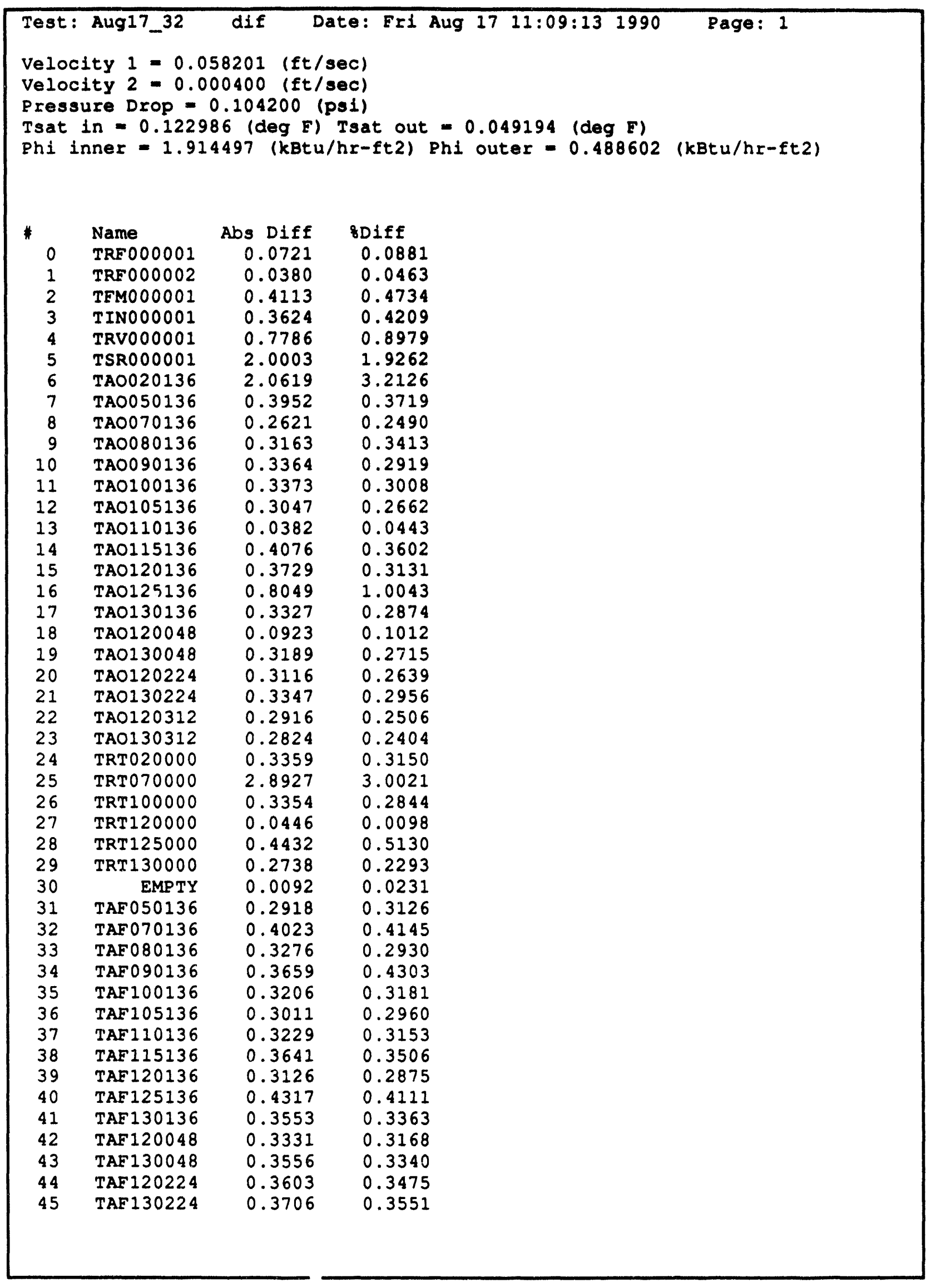




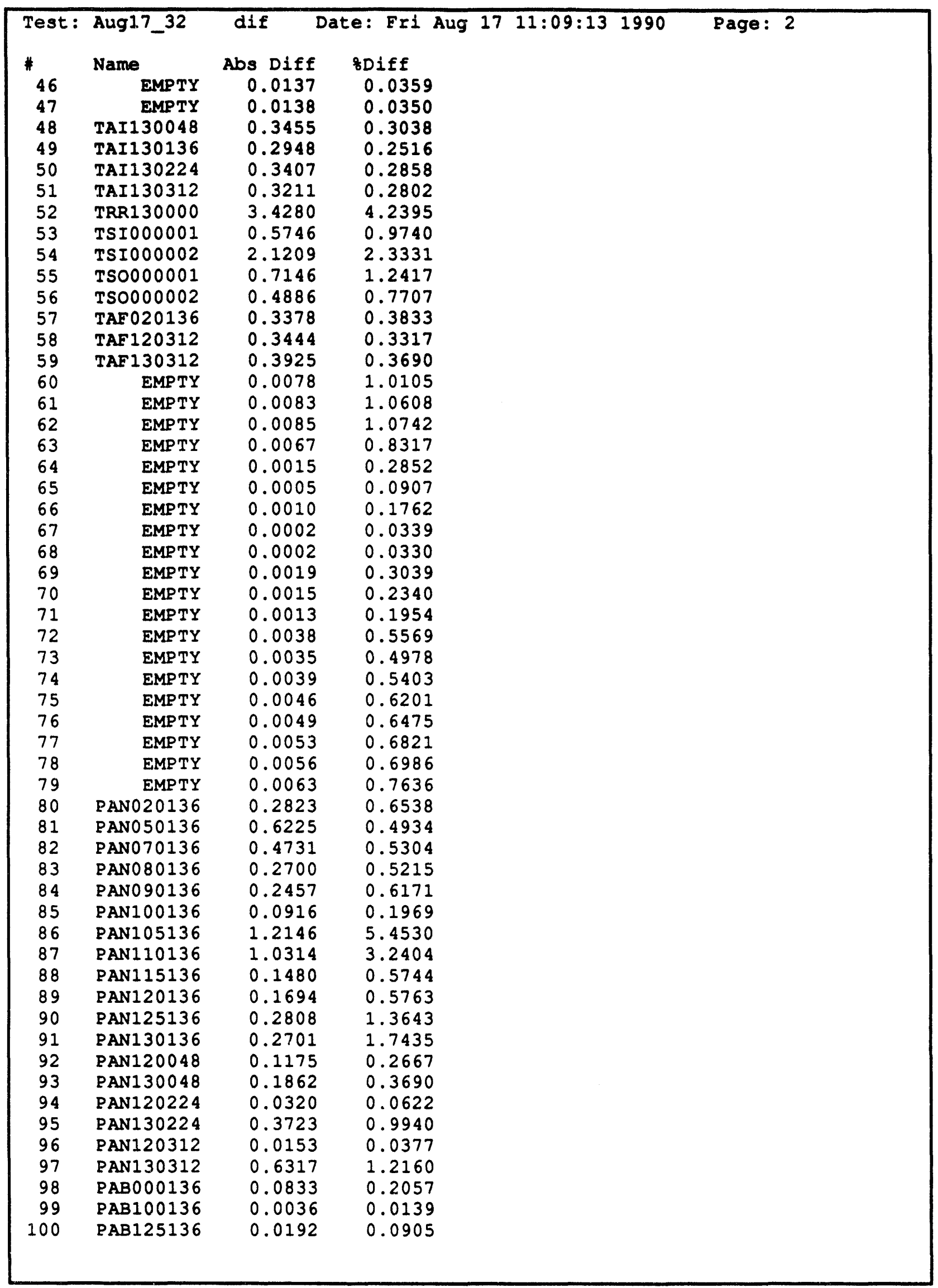




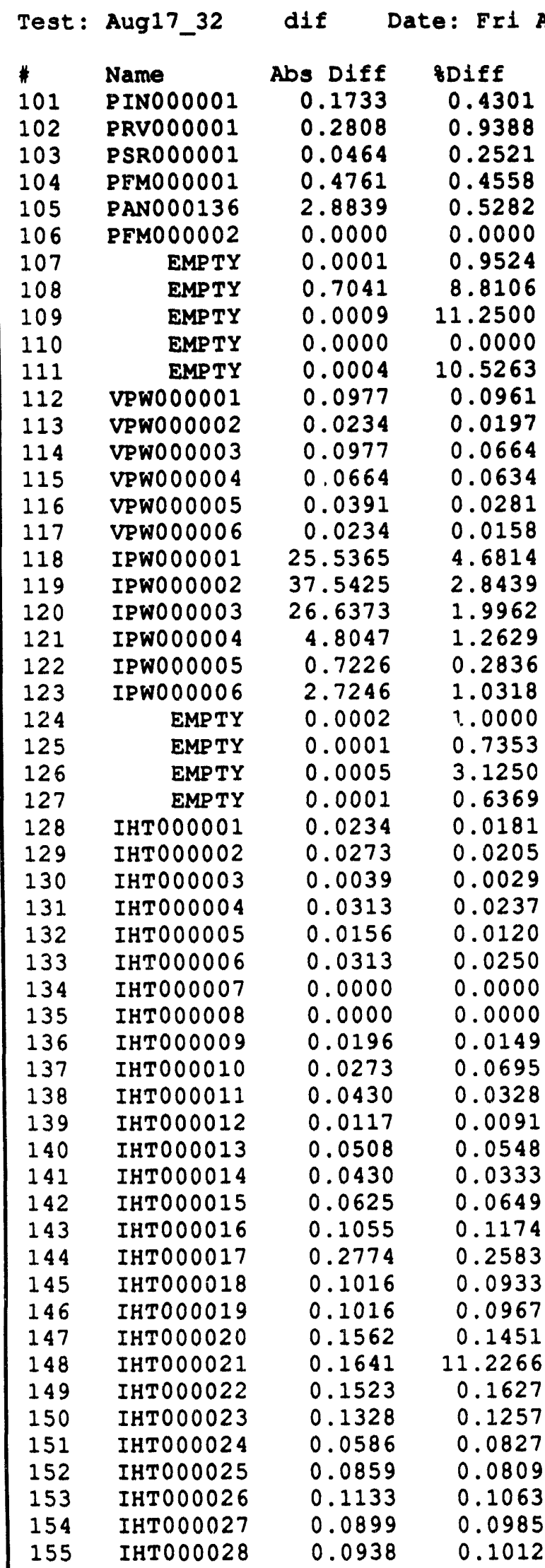

Page: 3 


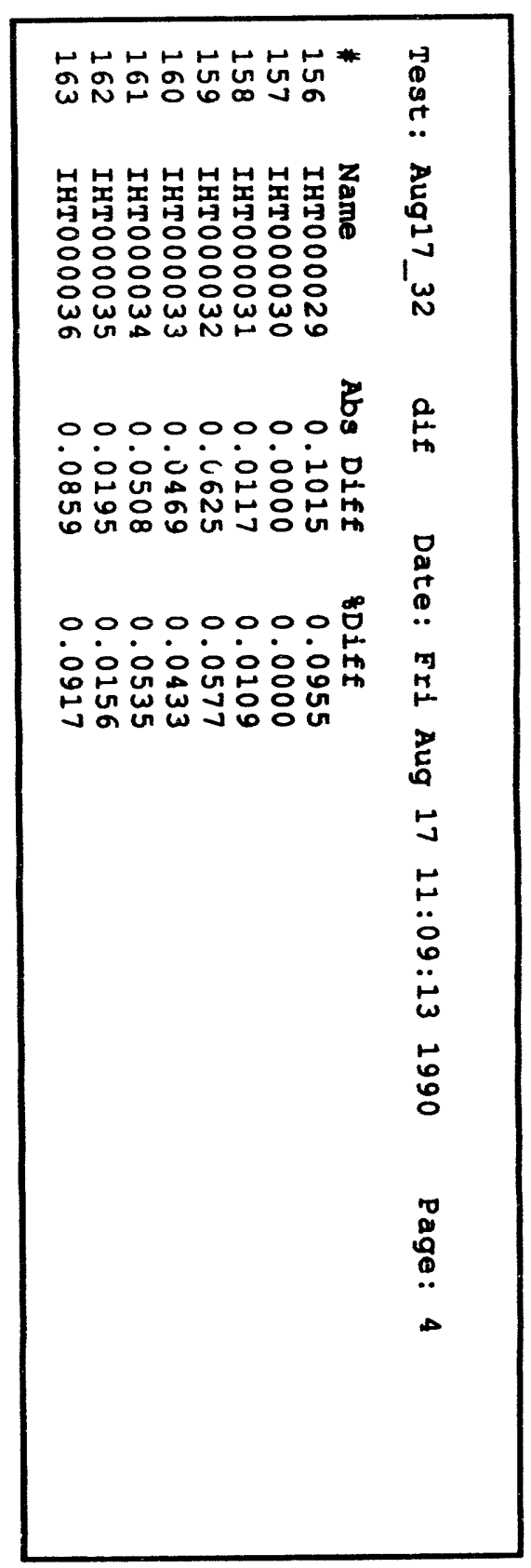




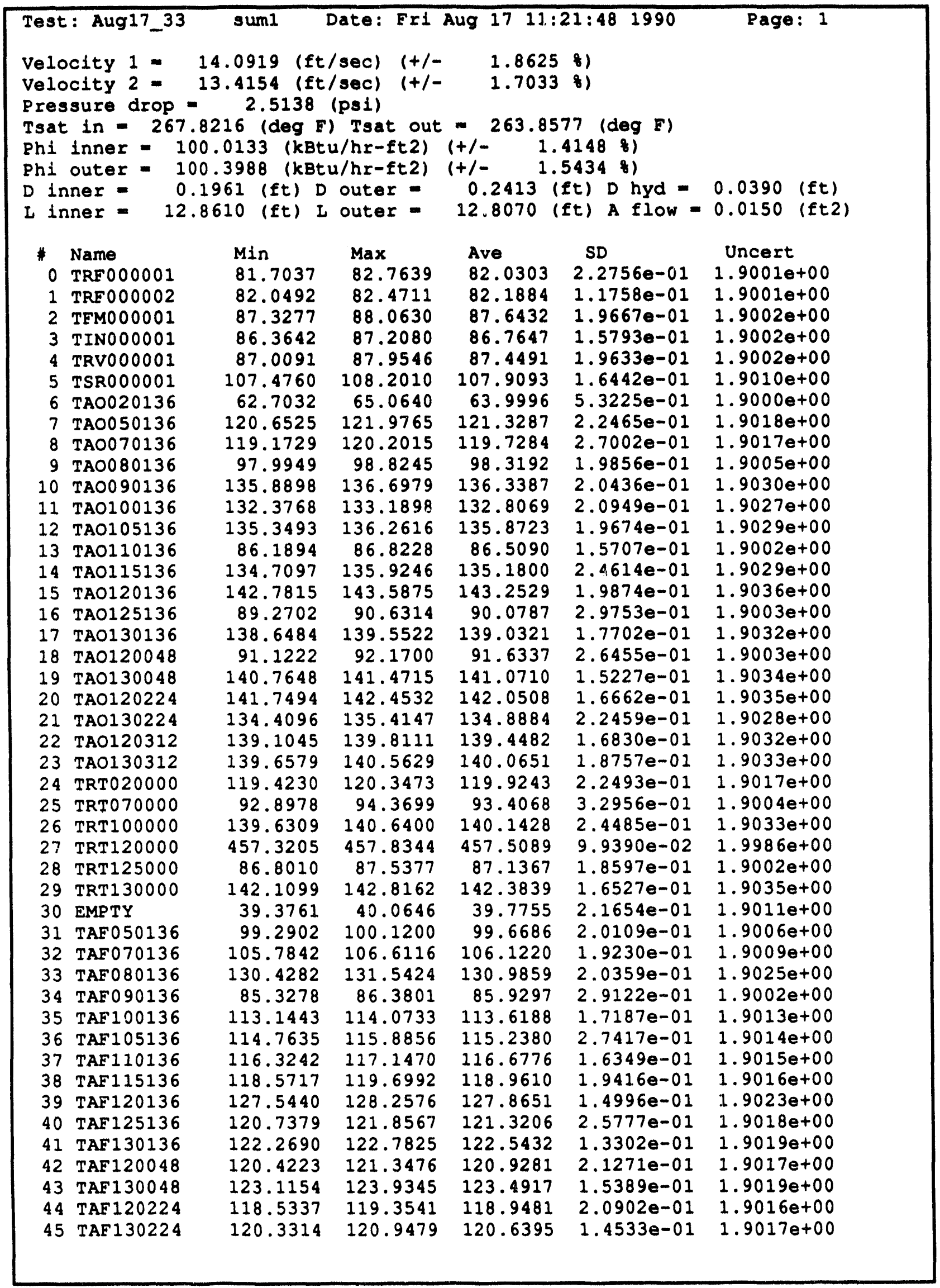




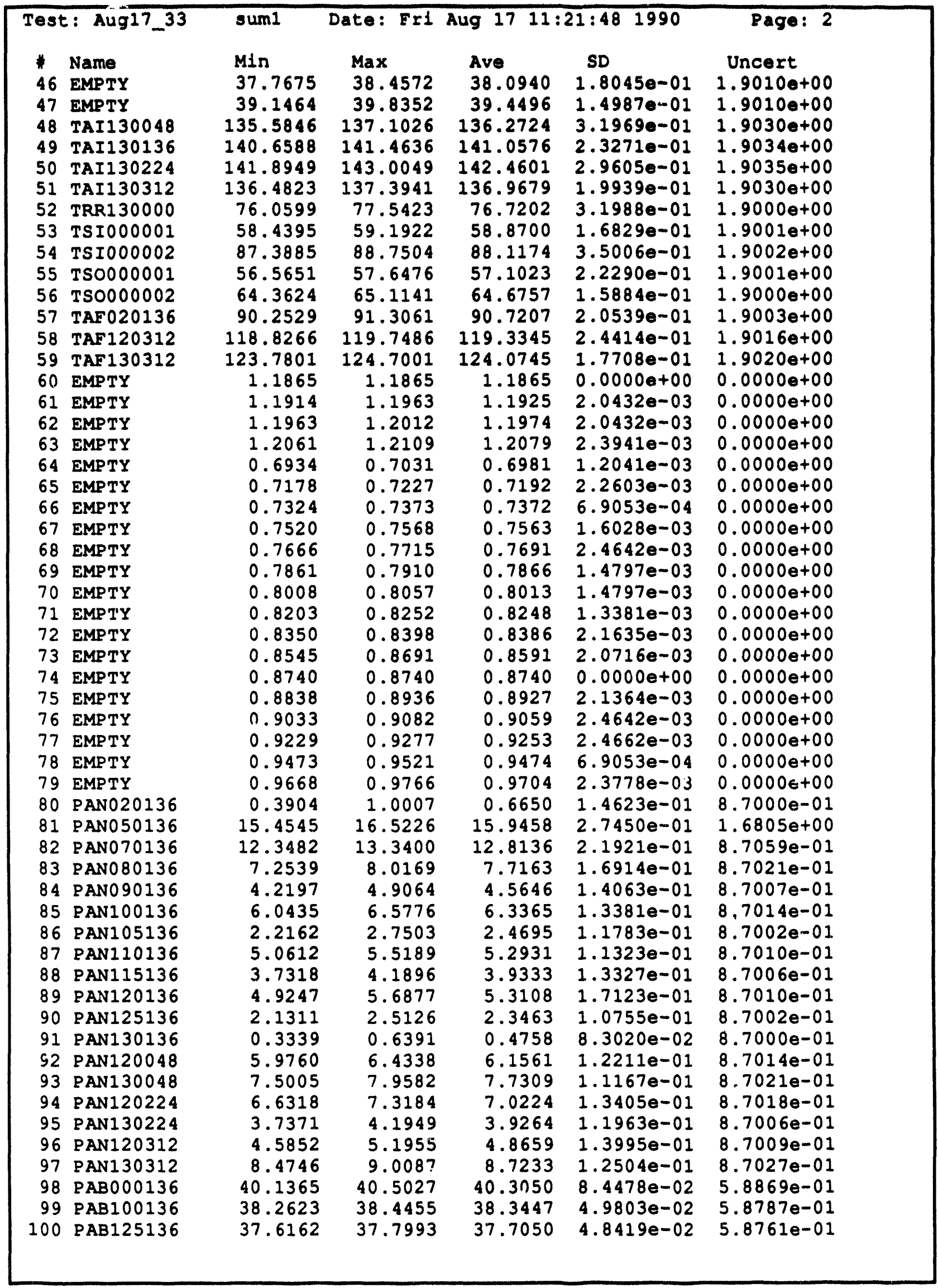




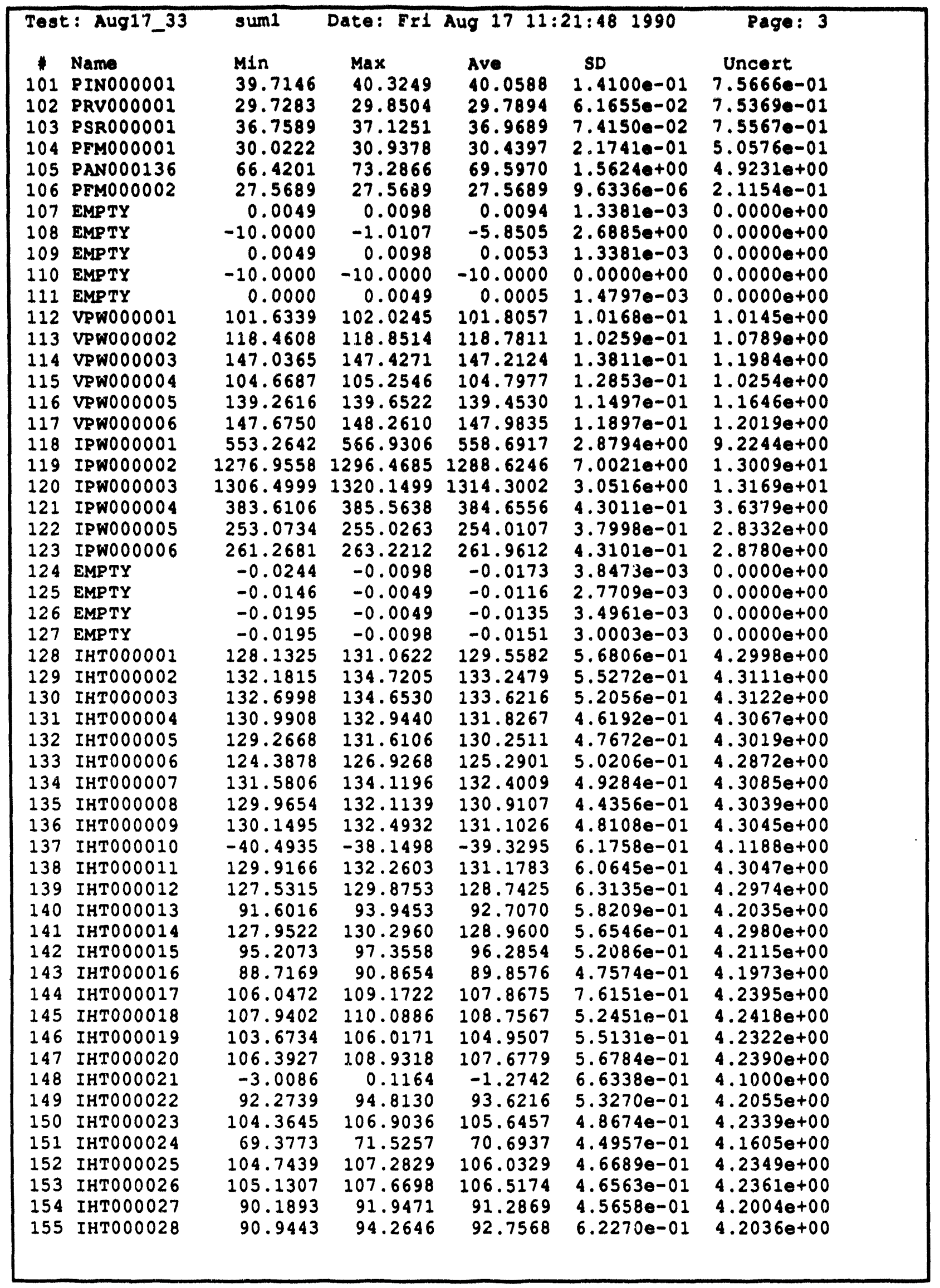




\begin{tabular}{|c|c|c|c|c|c|c|}
\hline $\begin{array}{l}1 \\
156 \\
157 \\
158 \\
159 \\
160 \\
161 \\
162 \\
163\end{array}$ & 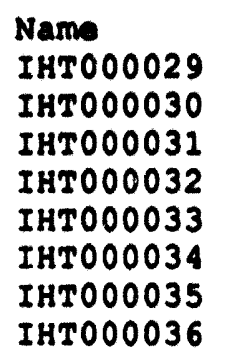 & $\begin{array}{l}\text { Min } \\
104.6199 \\
107.0125 \\
105.4462 \\
106.9373 \\
106.9599 \\
93.9378 \\
124.1136 \\
92.6044\end{array}$ & $\begin{array}{l}\operatorname{Max} \\
107.7449 \\
109.7469 \\
108.1806 \\
109.4764 \\
109.4990 \\
96.0862 \\
126.4573 \\
94.9482\end{array}$ & $\begin{array}{r}\text { Ave } \\
106.2723 \\
108.5672 \\
106.8955 \\
108.2459 \\
108.1982 \\
95.1018 \\
125.3011 \\
93.7060\end{array}$ & $\begin{array}{l}\text { SD } \\
5.7617 e-01 \\
5.8255 e-01 \\
5.7465 e-01 \\
5.6669 e-01 \\
5.6718 e-01 \\
5.0833 e-01 \\
5.0353 e-01 \\
5.3344 e-01\end{array}$ & $\begin{array}{l}\text { Uncert } \\
4.2355 e+00 \\
4.2413 e+00 \\
4.2371 e+00 \\
4.2405 e+00 \\
4.2404 e+00 \\
4.2089 e+00 \\
4.2872 e+00 \\
4.2057 e+00\end{array}$ \\
\hline
\end{tabular}




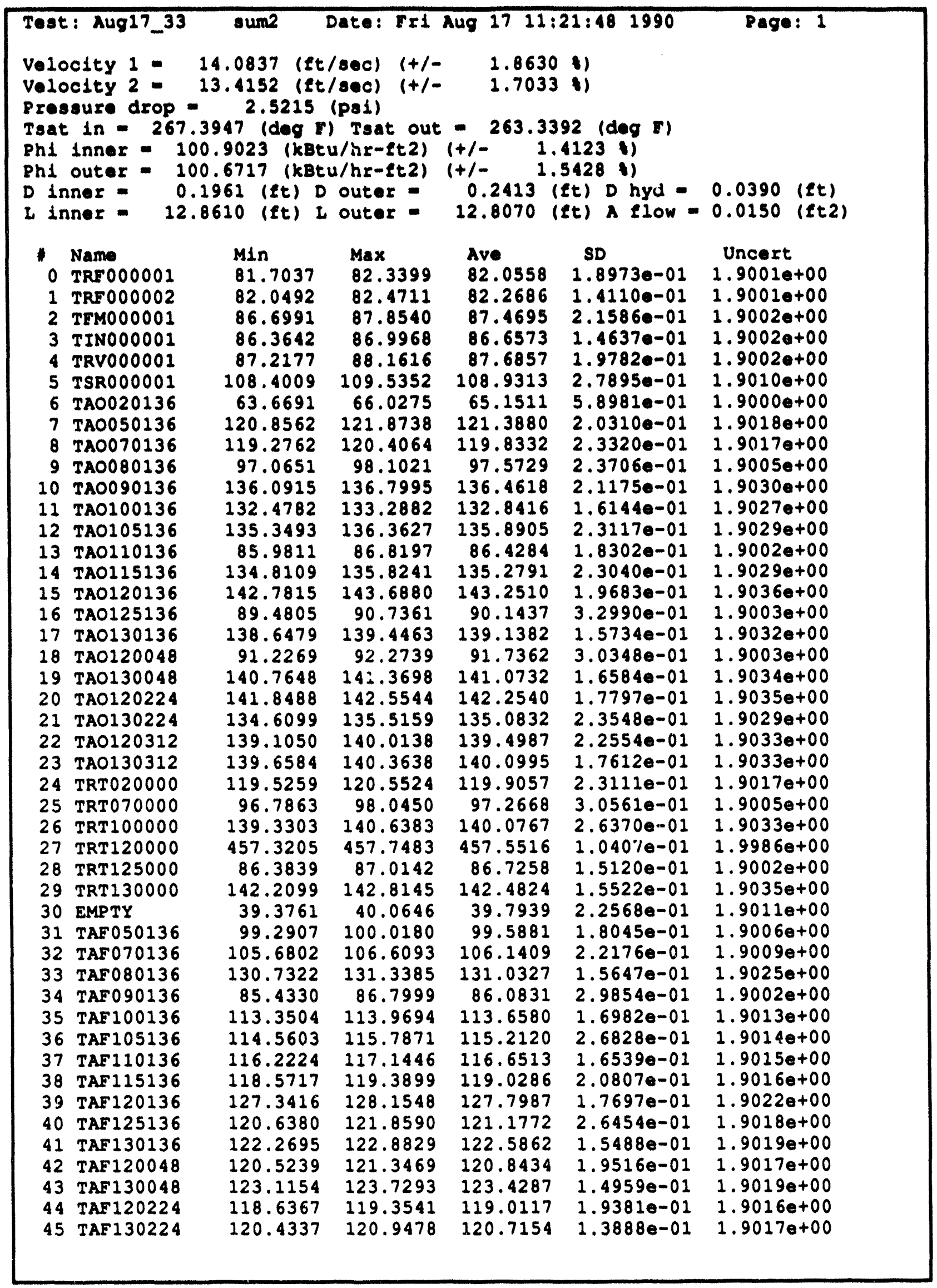




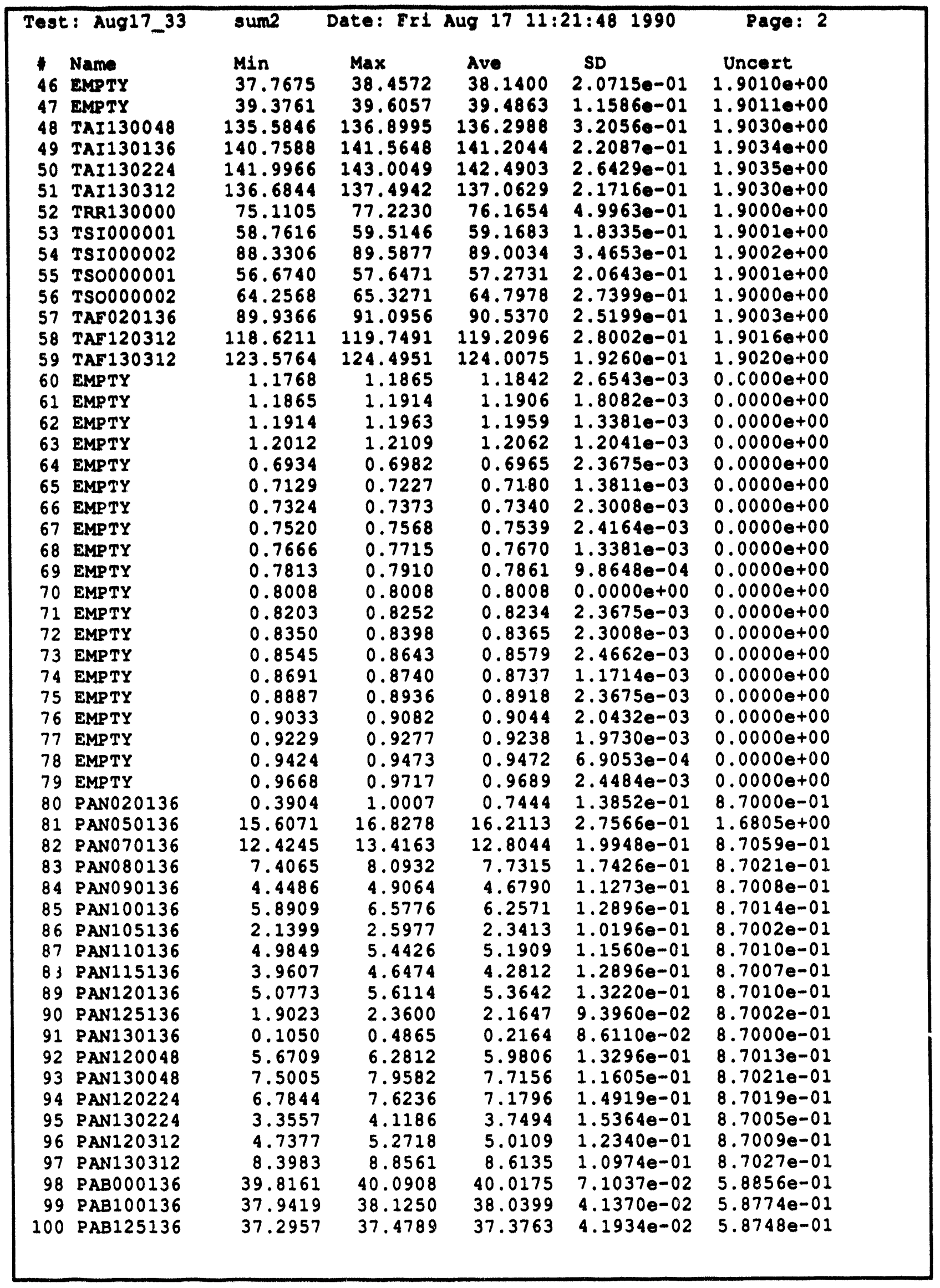




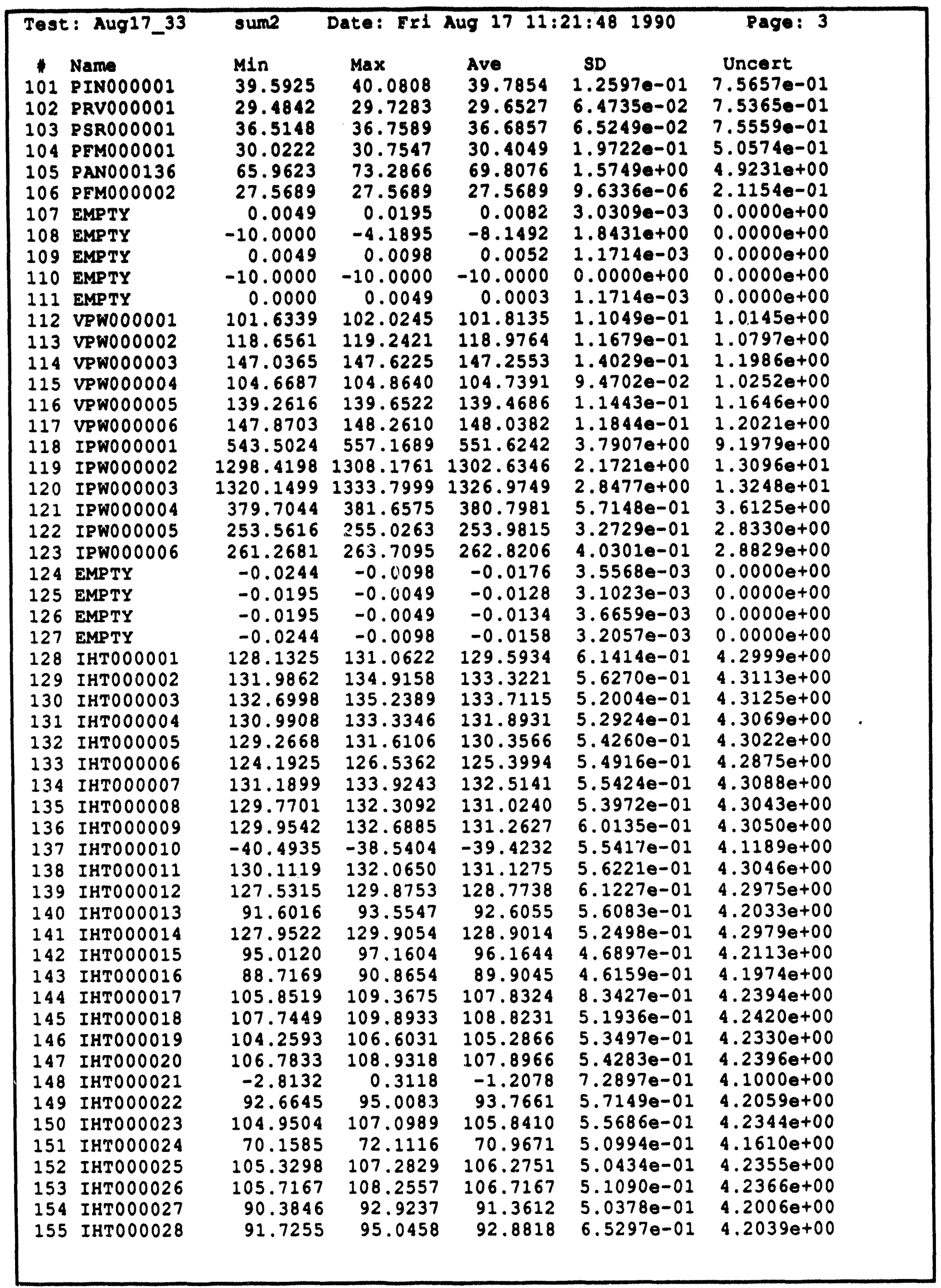




\begin{tabular}{|c|c|c|c|c|c|c|}
\hline $\begin{array}{c}1 \\
156 \\
157 \\
158 \\
159 \\
160 \\
161 \\
162 \\
163\end{array}$ & 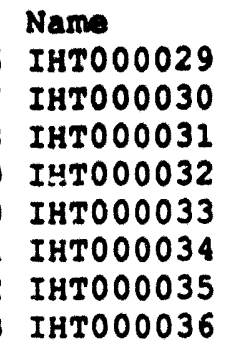 & $\begin{array}{l}\text { M1n } \\
105.4012 \\
107.7937 \\
106.2275 \\
107.5233 \\
107.5458 \\
94.1331 \\
124.5042 \\
92.9950\end{array}$ & $\begin{array}{l}\operatorname{Max} \\
108.5262 \\
110.1375 \\
108.3759 \\
110.0623 \\
110.0849 \\
96.4768 \\
126.8480 \\
95.1435\end{array}$ & $\begin{array}{r}\text { Ave } \\
106.4988 \\
108.7938 \\
107.1181 \\
108.5350 \\
108.4756 \\
95.0823 \\
125.3910 \\
93.7568\end{array}$ & $\begin{array}{l}\text { SD } \\
6.3978 e-01 \\
5.8079 e-01 \\
5.2372 e-01 \\
6.1218 e-01 \\
6.1328 e-01 \\
5.1450 e-01 \\
5.7482 e-01 \\
5.2087 e-01\end{array}$ & $\begin{array}{c}\text { Uncert } \\
4.2361 e+00 \\
4.2419 e+00 \\
4.2376 e+00 \\
4.2412 e+00 \\
4.2411 e+00 \\
4.2088 e+00 \\
4.2875 e+00 \\
4.2058 e+00\end{array}$ \\
\hline
\end{tabular}




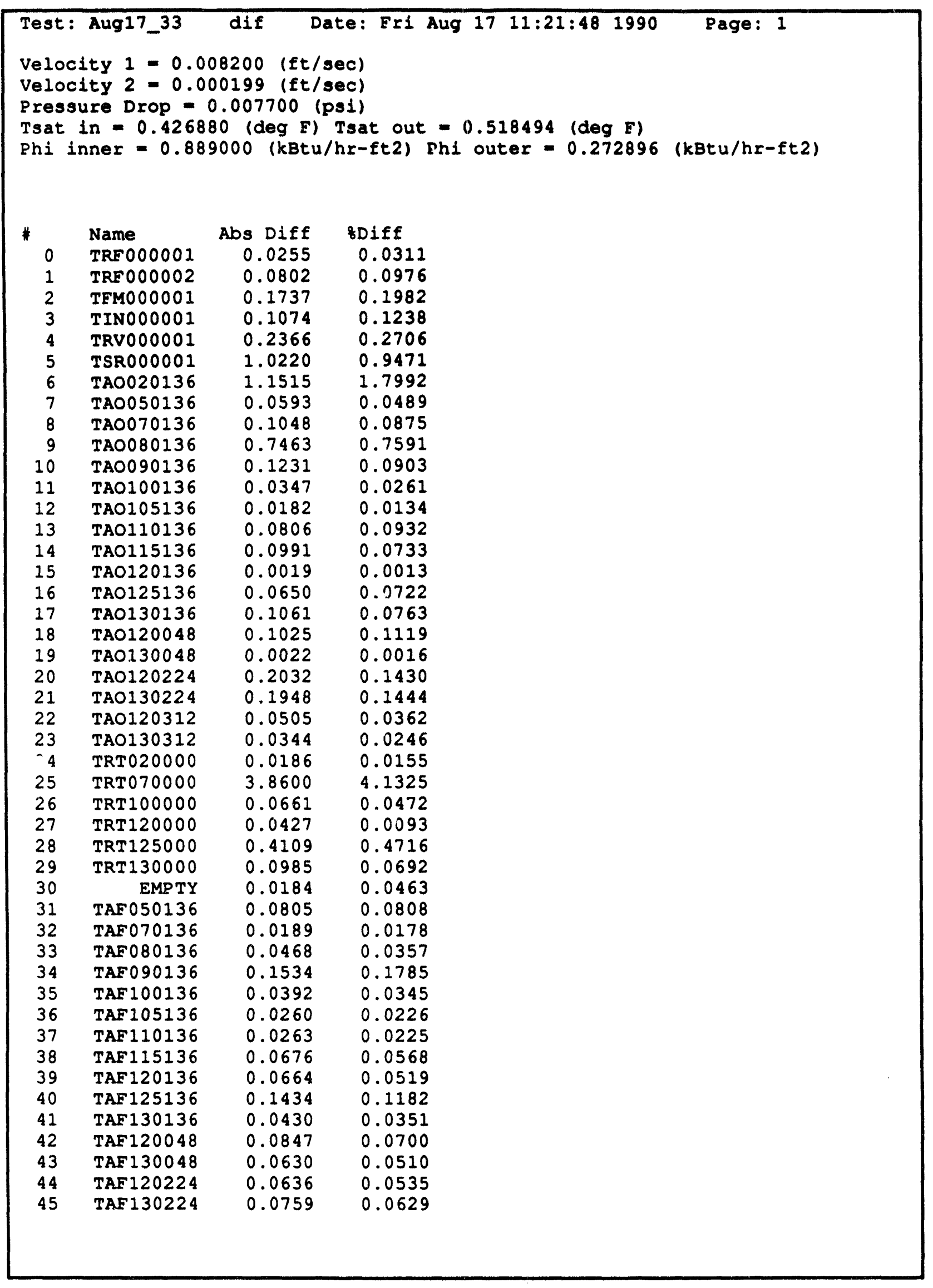




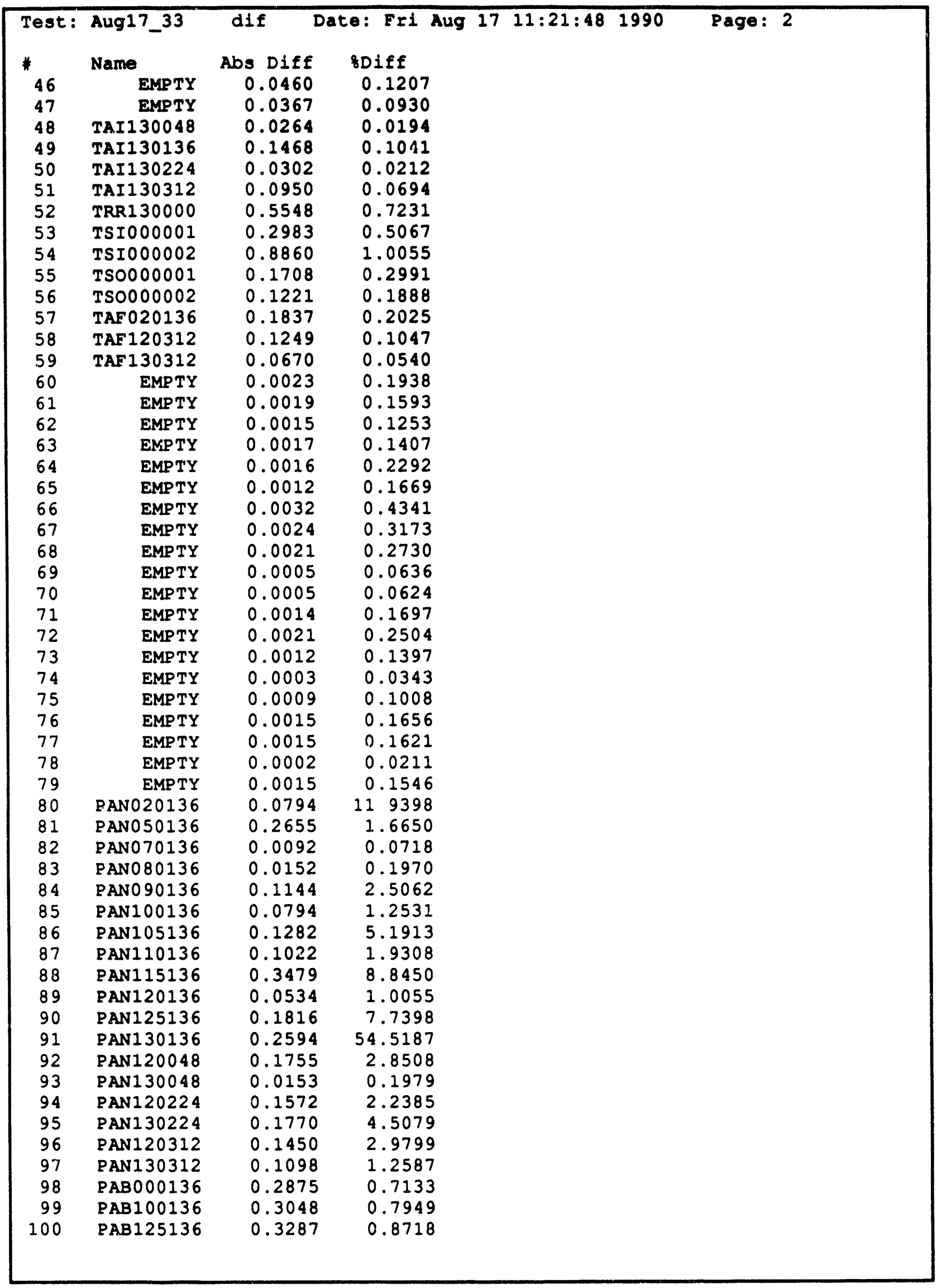




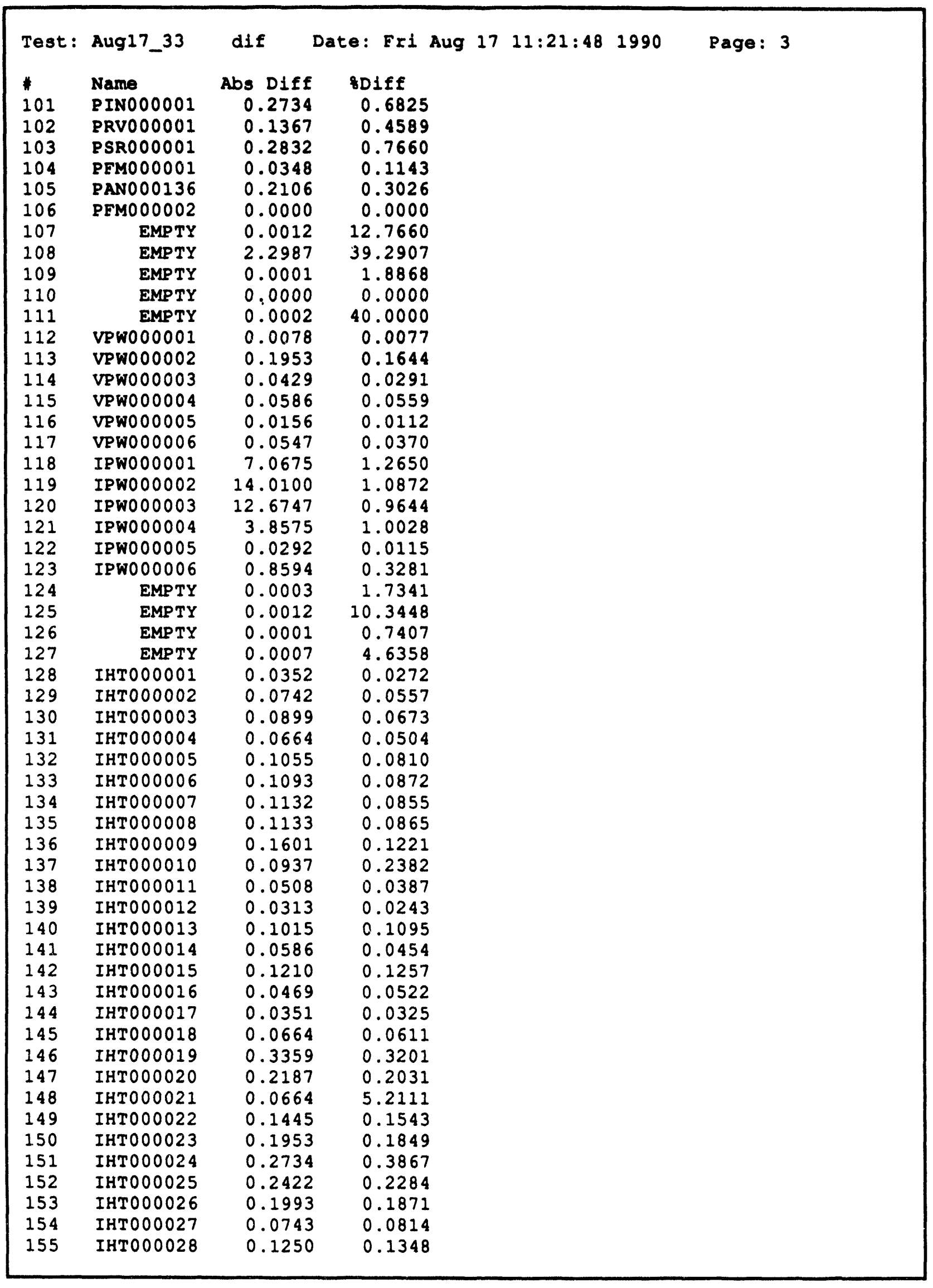




$\begin{array}{llrcrl}\text { Test: } & \text { Aug17_33 } & \text { dif } & \text { Date: Fri Aug 17 11:21:48 } 1990 & \text { Page: } 4 \\ \# & \text { Name } & \text { Abs Diff } & \text { 8Diff } & & \\ 156 & \text { IHT000029 } & 0.2265 & 0.2131 & & \\ 157 & \text { IHT000030 } & 0.2266 & 0.2087 & & \\ 158 & \text { IHTO00031 } & 0.2226 & 0.2082 & & \\ 159 & \text { IHTO00032 } & 0.2891 & 0.2671 & & \\ 160 & \text { IHT000033 } & 0.2774 & 0.2564 & & \\ 161 & \text { IHT000034 } & 0.0195 & 0.0205 & & \\ 162 & \text { IHTO00035 } & 0.0899 & 0.0717 & & \\ 163 & \text { IHT000036 } & 0.0508 & 0.0542 & & \end{array}$




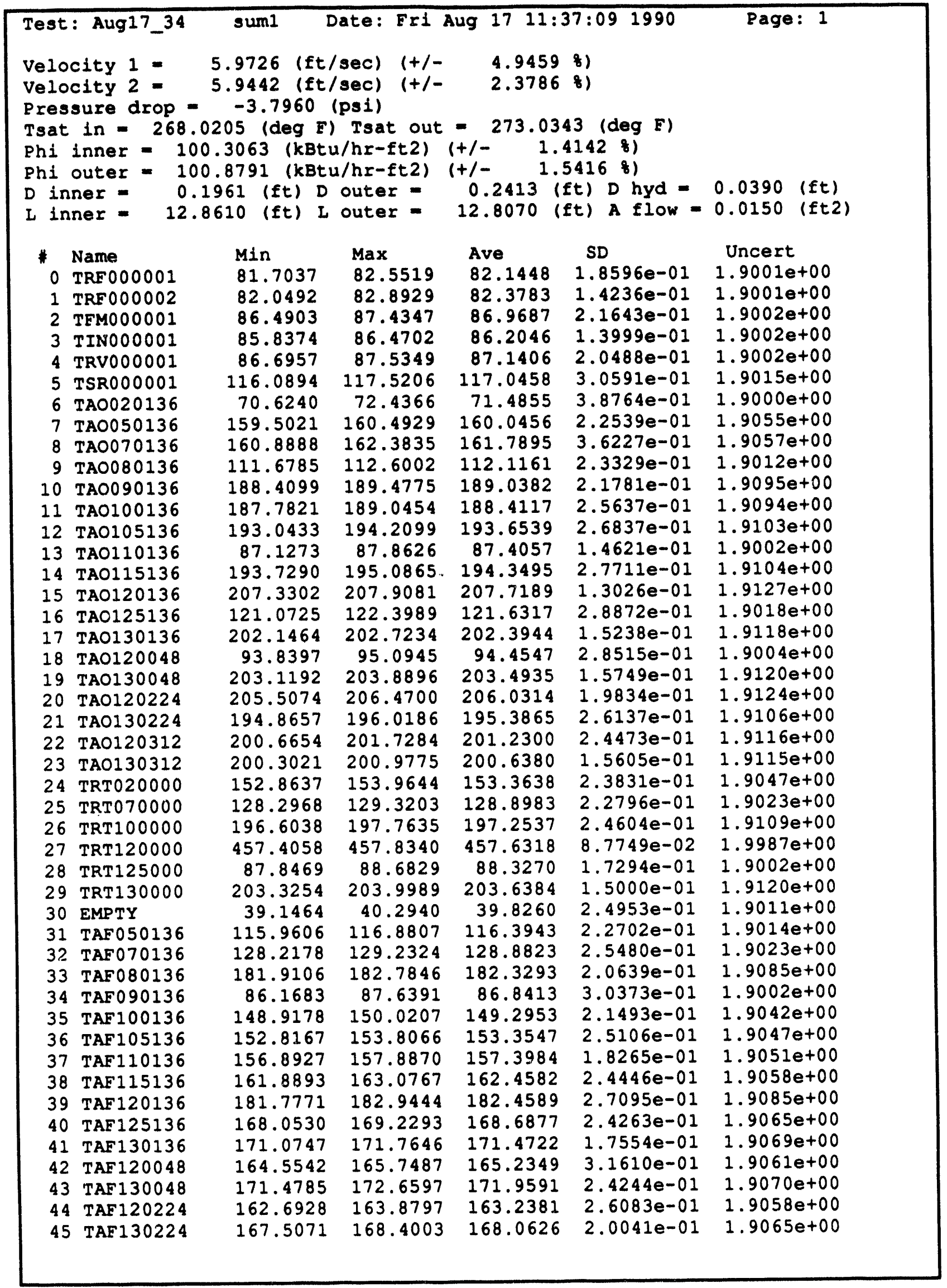




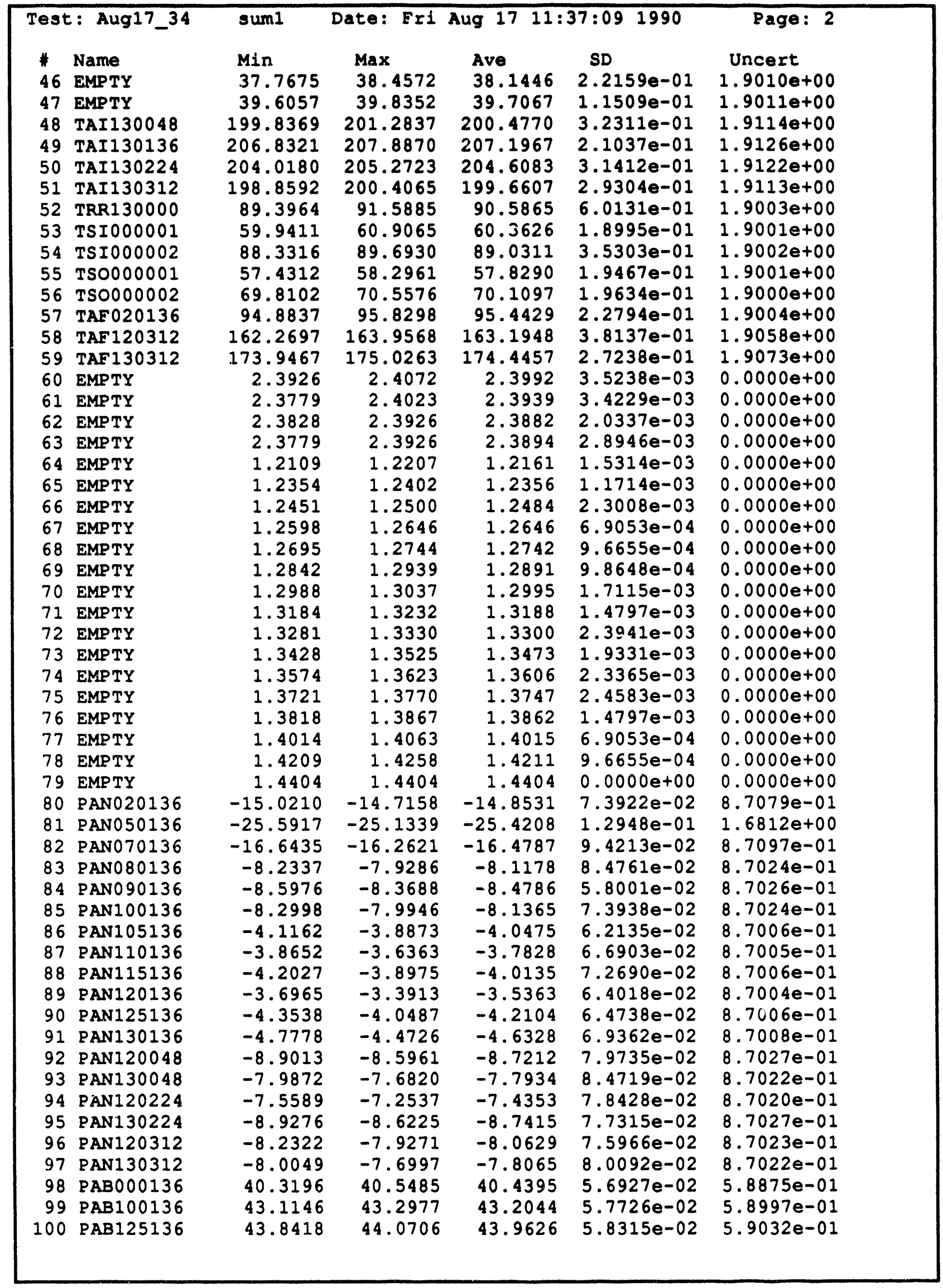




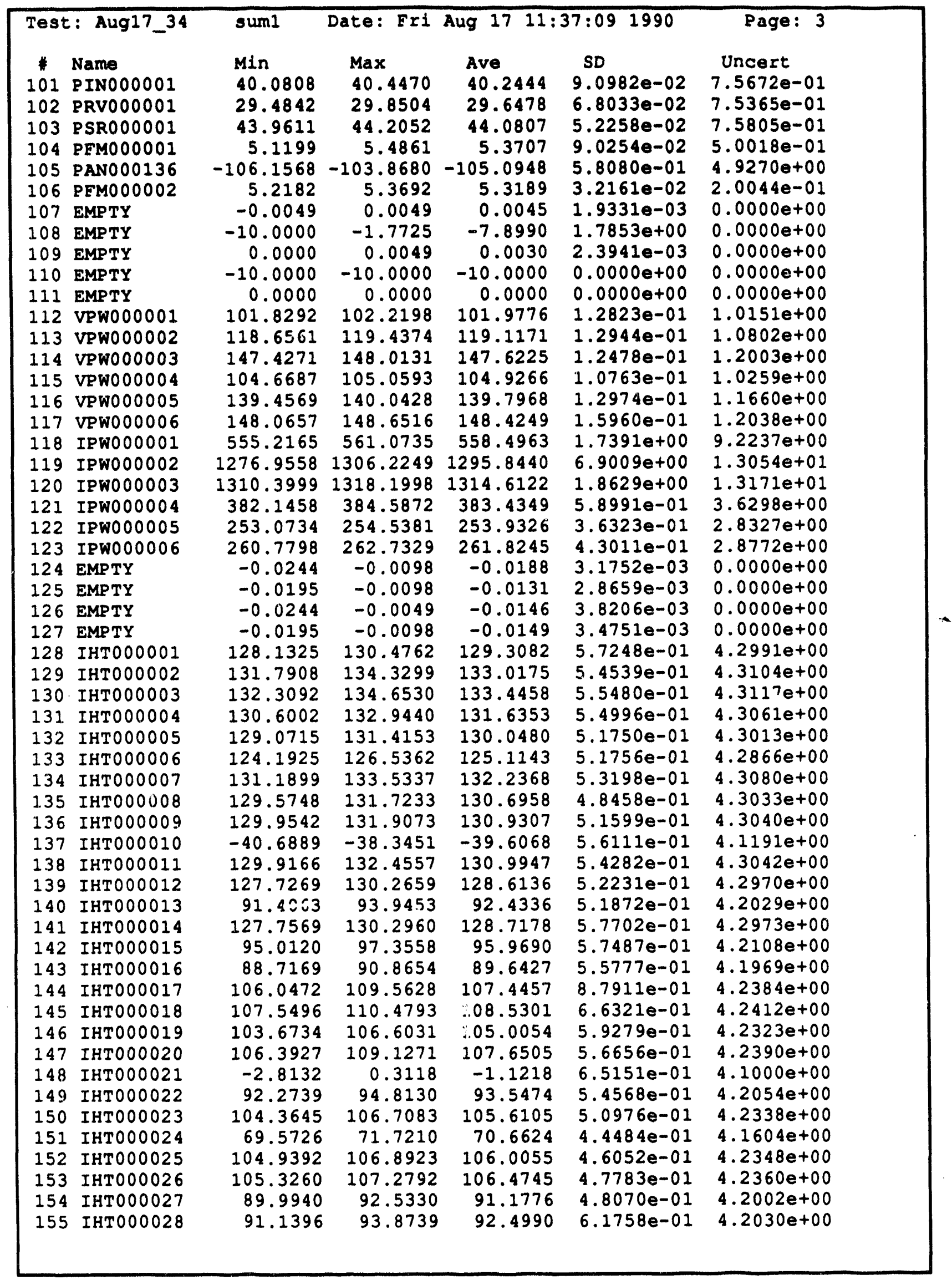




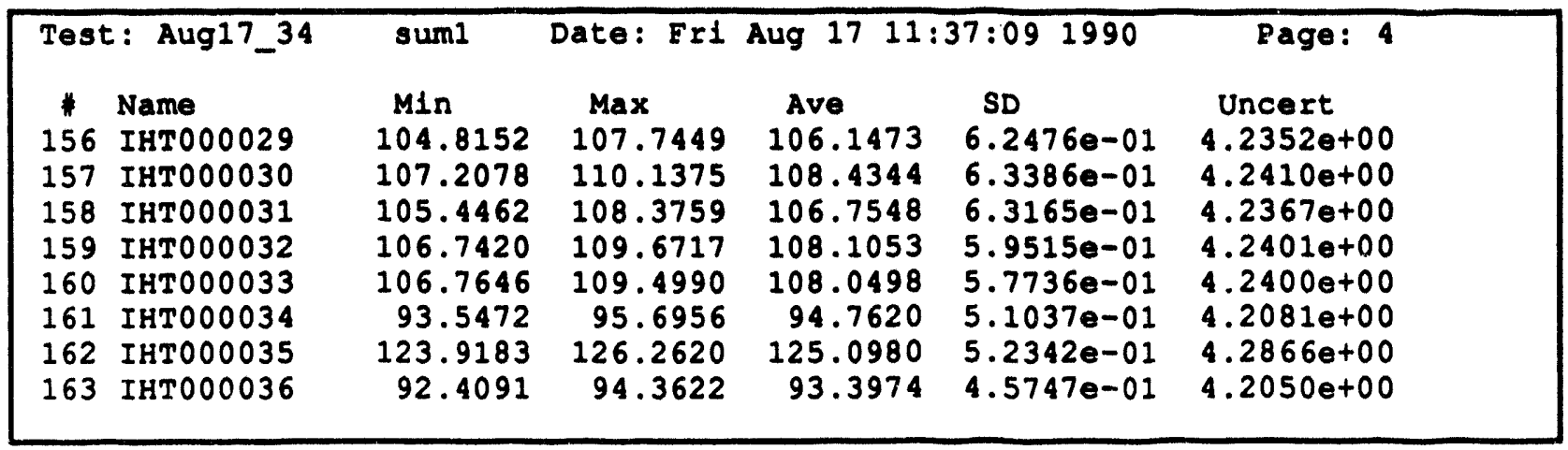




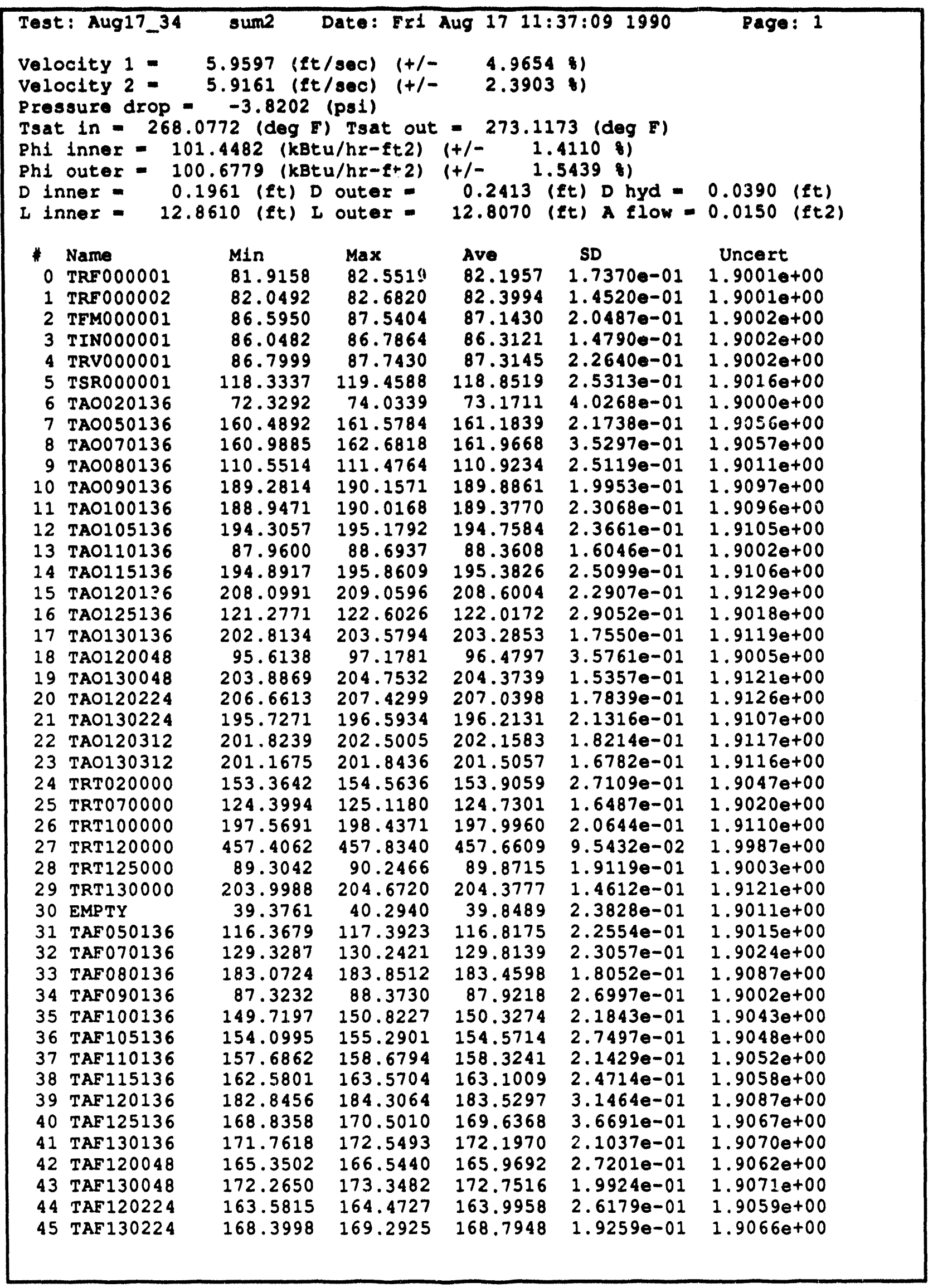




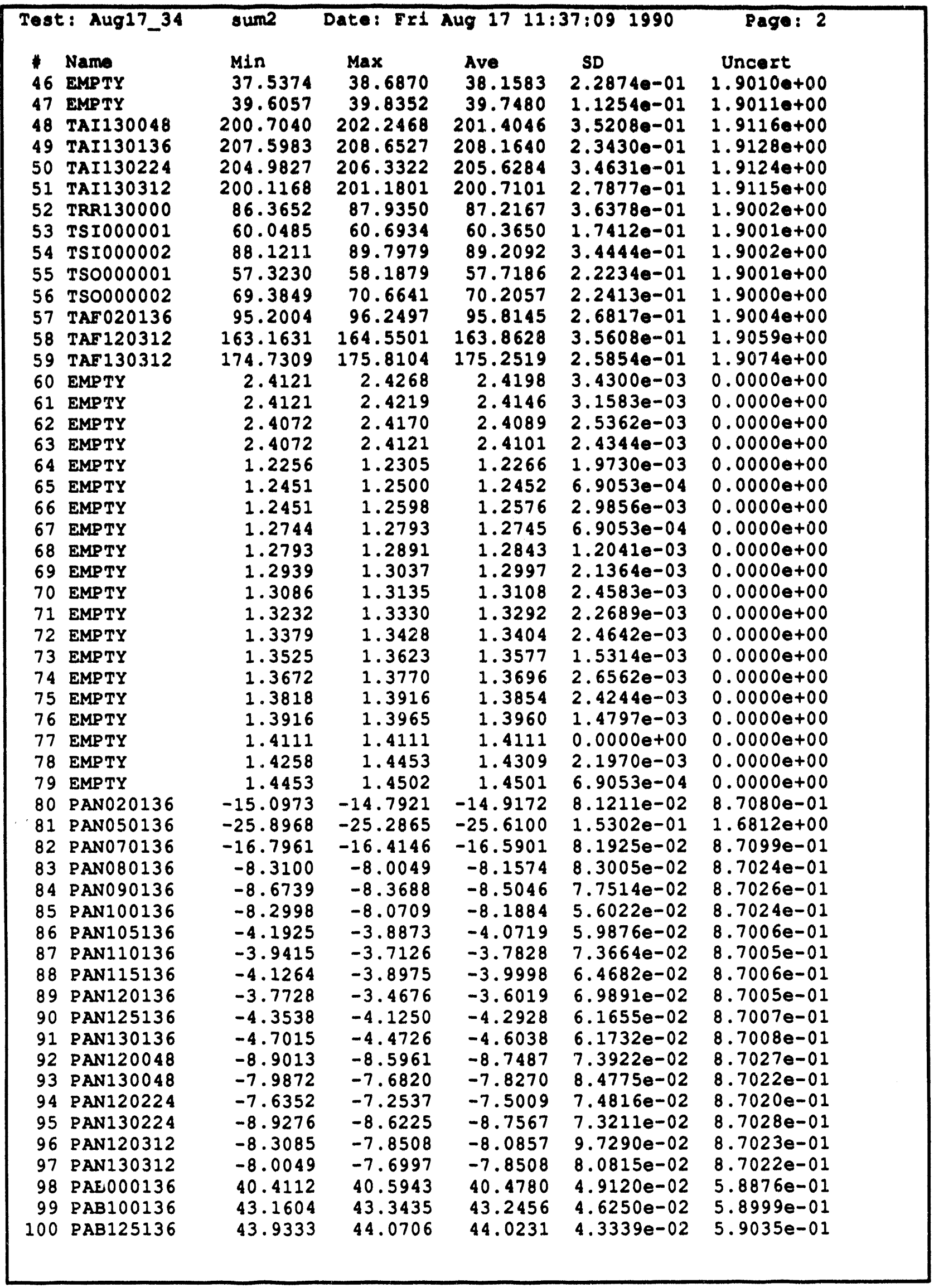




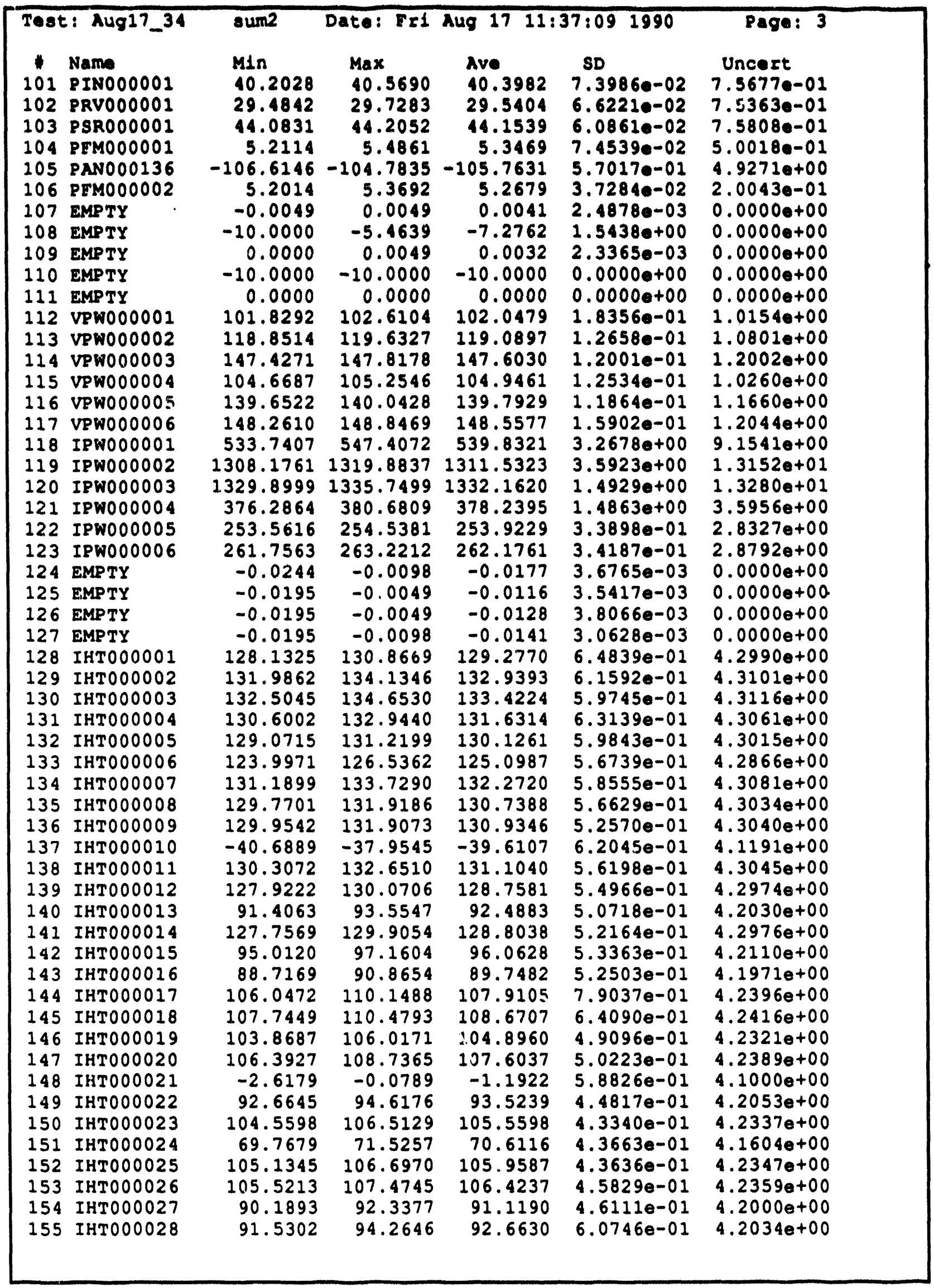




\begin{tabular}{|c|c|c|c|c|c|c|}
\hline $\begin{array}{l}1 \\
156 \\
157 \\
158 \\
159 \\
160 \\
161 \\
162 \\
163\end{array}$ & $\begin{array}{l}\text { Name } \\
\text { IHTO0O029 } \\
\text { IHTO0O030 } \\
\text { IHTO0O031 } \\
\text { IHTO00032 } \\
\text { IHTO00033 } \\
\text { IHTO00034 } \\
\text { IHTO00035 } \\
\text { IHTO0O036 }\end{array}$ & $\begin{array}{l}\text { Min } \\
105.2058 \\
107.4031 \\
105.6415 \\
106.9373 \\
106.9599 \\
93.7425 \\
124.3089 \\
92.6044\end{array}$ & $\begin{array}{l}\text { Max } \\
108.1355 \\
110.5281 \\
108.1806 \\
109.4764 \\
109.3036 \\
96.0862 \\
126.4573 \\
94.7528\end{array}$ & $\begin{array}{r}\text { Ave } \\
106.2527 \\
108.5594 \\
106.8330 \\
108.1131 \\
108.0693 \\
94.8089 \\
125.1019 \\
93.4599\end{array}$ & $\begin{array}{l}\text { SD } \\
6.2858 e-01 \\
6.3484 e-01 \\
5.8826 e-01 \\
5.6702 e-01 \\
5.4023 e-01 \\
4.3621 e-01 \\
4.4888 e-01 \\
4.7661 e-01\end{array}$ & $\begin{array}{c}\text { Uncert } \\
4.2354 e+00 \\
4.2413 e+00 \\
4.2369 e+00 \\
4.2401 e+00 \\
4.2400 e+00 \\
4.2082 e+00 \\
4.2866 e+00 \\
4.2052 e+00\end{array}$ \\
\hline
\end{tabular}




\begin{tabular}{|c|c|c|c|}
\hline \multicolumn{4}{|c|}{$\begin{array}{l}\text { Velocity } 1=0.012900(\mathrm{ft} / \mathrm{sec}) \\
\text { Velocity } 2=0.028100(\mathrm{ft} / \mathrm{sec}) \\
\text { Pressure Drop }=0.024200 \text { (psi) } \\
\text { Tsat in }=0.056702 \text { (deg F) Tsat out }=0.083008 \text { (deg F) } \\
\text { Ph1 inner }=1.141899 \text { (kBtu/hr-ft2) Ph1 outer }=0.201195 \text { (kBtu/hr-ft2) }\end{array}$} \\
\hline $\begin{array}{l}0 \\
1 \\
2 \\
3 \\
4 \\
5 \\
6 \\
7 \\
8 \\
9 \\
10 \\
11 \\
12 \\
13 \\
14 \\
15 \\
16 \\
17 \\
18 \\
19 \\
20 \\
21 \\
22 \\
23 \\
24 \\
25 \\
26 \\
27 \\
28 \\
29 \\
30 \\
31 \\
32 \\
33 \\
34 \\
35 \\
36 \\
37 \\
38 \\
39 \\
40 \\
41 \\
42 \\
43 \\
44 \\
45\end{array}$ & $\begin{array}{l}\text { Name } \\
\text { TRF000001 } \\
\text { TRF000002 } \\
\text { TEM000001 } \\
\text { TIN000001 } \\
\text { TRV000001 } \\
\text { TSR000011 } \\
\text { TAO020136 } \\
\text { TAO050136 } \\
\text { TAO070136 } \\
\text { TAO080136 } \\
\text { TAO090136 } \\
\text { TAO100136 } \\
\text { TAO105136 } \\
\text { TAO110136 } \\
\text { TAO115136 } \\
\text { TAO120136 } \\
\text { TAO125136 } \\
\text { TAO130136 } \\
\text { TAO120048 } \\
\text { TAO130048 } \\
\text { TAO120224 } \\
\text { TAO130224 } \\
\text { TAO120312 } \\
\text { TAO130312 } \\
\text { TRT020000 } \\
\text { TRT070000 } \\
\text { TRT100000 } \\
\text { TRT120000 } \\
\text { TRT125000 } \\
\text { TRT130000 } \\
\text { EMPTY } \\
\text { TAF050136 } \\
\text { TAF070136 } \\
\text { TAF080136 } \\
\text { TAF090136 } \\
\text { TAF100136 } \\
\text { TAF105136 } \\
\text { TAF110136 } \\
\text { TAF115136 } \\
\text { TAF120136 } \\
\text { TAF125136 } \\
\text { TAF130136 } \\
\text { TAF120048 } \\
\text { TAF130048 } \\
\text { TAF120224 } \\
\text { TAF130224 }\end{array}$ & $\begin{array}{l}\text { Abs D1Ef } \\
0.0509 \\
0.0211 \\
0.1743 \\
0.1075 \\
0.1739 \\
1.8061 \\
1.6856 \\
1.1383 \\
0.1773 \\
1.1927 \\
0.8479 \\
0.9653 \\
1.1045 \\
0.9551 \\
1.0331 \\
0.8815 \\
0.3855 \\
0.8909 \\
2.0250 \\
0.8804 \\
1.0084 \\
0.8266 \\
0.9283 \\
0.8677 \\
0.5421 \\
4.1682 \\
0.7423 \\
0.0291 \\
1.5445 \\
0.7393 \\
0.0229 \\
0.4232 \\
0.9316 \\
1.1305 \\
1.0805 \\
1.0321 \\
1.2167 \\
0.9257 \\
0.6427 \\
1.0708 \\
0.9491 \\
0.7248 \\
0.7343 \\
0.7925 \\
0.7577 \\
0.7322\end{array}$ & $\begin{array}{l}\text { DIEA } \\
0.0620 \\
0.0256 \\
0.2004 \\
0.1247 \\
0.1996 \\
1.5431 \\
2.3580 \\
0.7112 \\
0.1096 \\
1.0638 \\
0.4485 \\
0.5123 \\
0.5704 \\
1.0927 \\
0.5316 \\
0.4244 \\
0.3169 \\
0.4402 \\
2.1439 \\
0.4326 \\
0.4894 \\
0.4231 \\
0.4613 \\
0.4325 \\
0.3535 \\
3.2337 \\
0.3763 \\
0.0064 \\
1.7486 \\
0.3630 \\
0.0575 \\
0.3636 \\
0.7228 \\
0.6200 \\
1.2442 \\
0.6913 \\
0.7934 \\
0.5881 \\
0.3956 \\
0.5869 \\
0.5626 \\
0.4227 \\
0.4444 \\
0.4609 \\
0.4642 \\
0.4357\end{array}$ \\
\hline
\end{tabular}




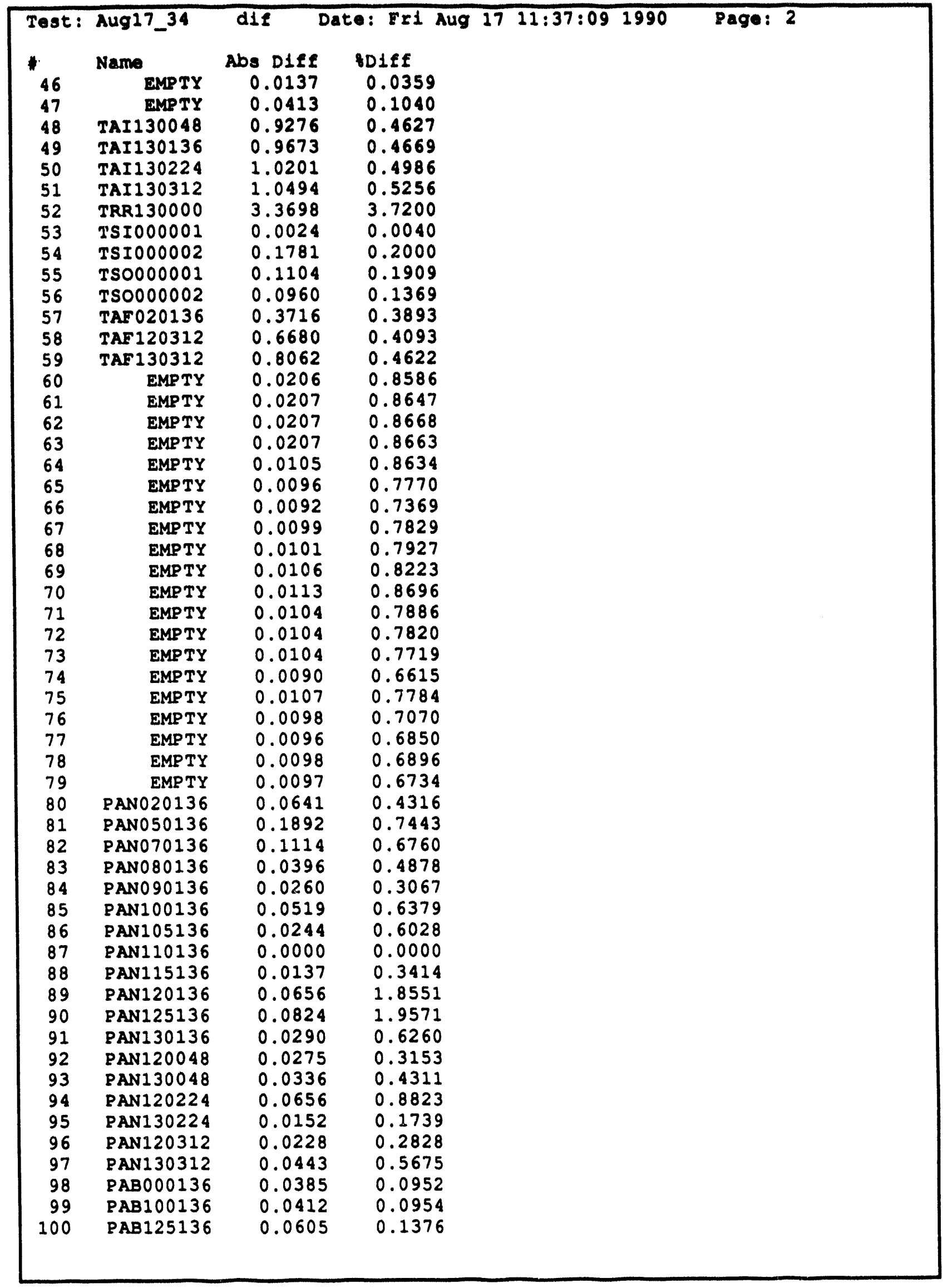




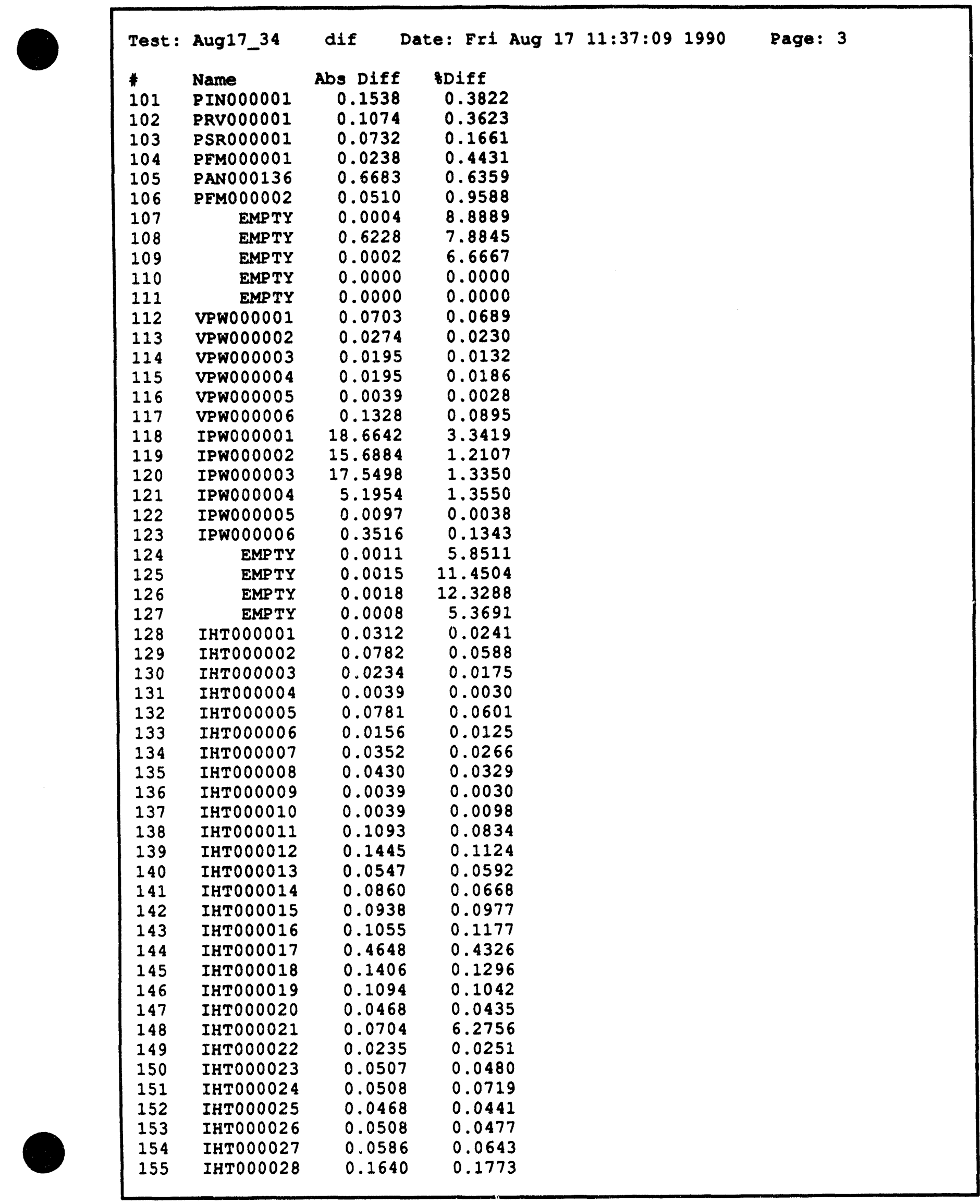


Test: Aug17_34

* Name

156 IHTO00029

157 IHTOOO030

158 IHT000031

159 IHTO00032

160 IHTO00033

161 IHT000034

162 IHT000035

163 IHT000036 dif Date: Fri Aug. 17 11:37:09 1990 Page: 4

Abs Diff Diff

0.10540 .0993

$0.1250 \quad 0.1153$

$0.0782 \quad 0.0733$

$0.0078 \quad 0.0072$

$0.0195 \quad 0.0180$

$0.0469 \quad 0.0495$

$0.0039 \quad 0.0031$

$0.0625 \quad 0.0669$ 


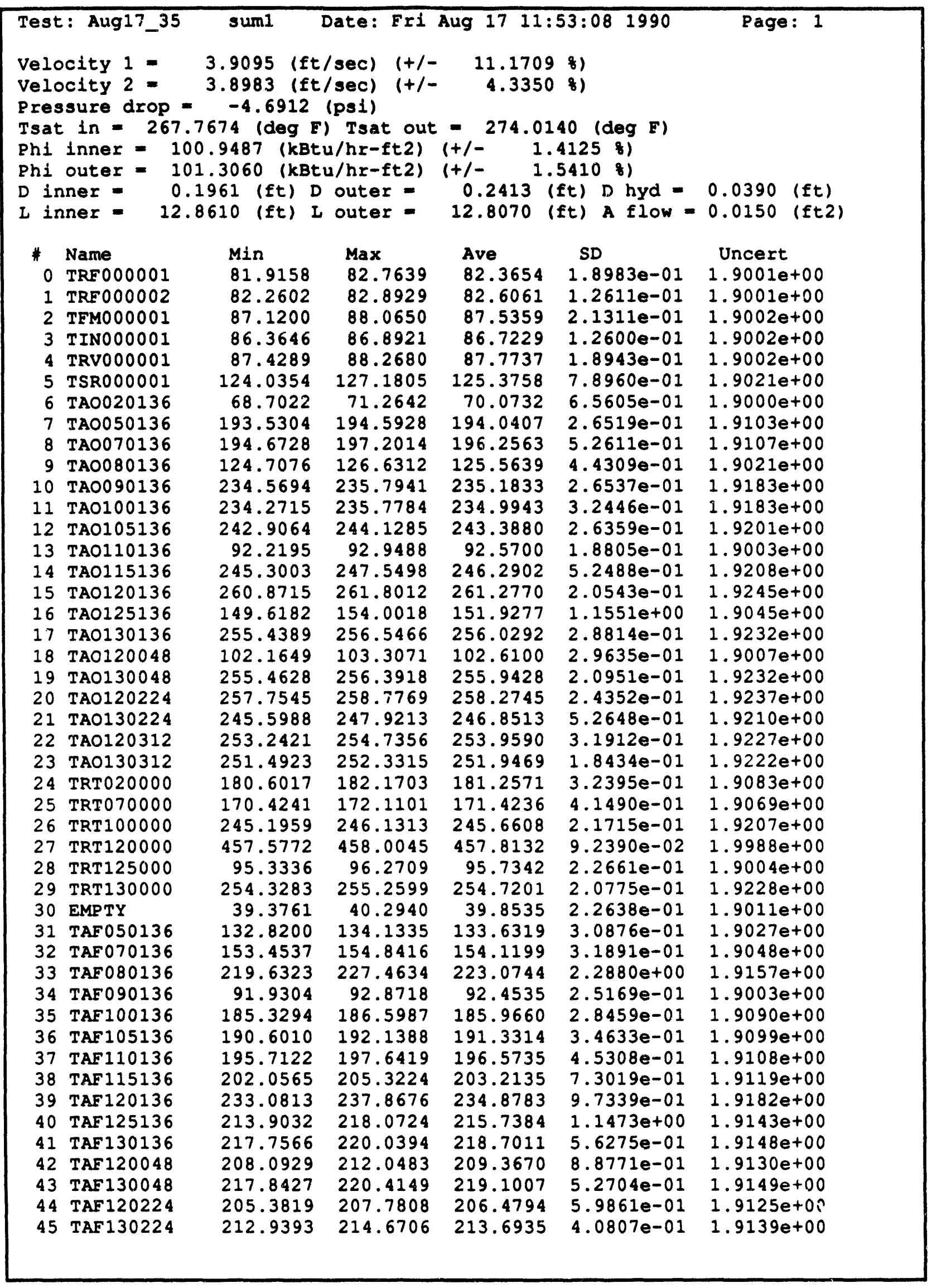




\begin{tabular}{|c|c|c|c|c|c|c|}
\hline \multicolumn{2}{|c|}{ Test: Aug17_35 } & suml & \multicolumn{3}{|c|}{ Date: Fri Aug 17 11:53:08 1} & Page: 2 \\
\hline 47 & EMPTY & $\begin{array}{l}37.5374 \\
39.8352\end{array}$ & $\begin{array}{l}38.6870 \\
40.2940\end{array}$ & $\begin{array}{l}38.2181 \\
39.9499\end{array}$ & $\begin{array}{l}2.2256 e-01 \\
1.4841 e-01\end{array}$ & $\begin{array}{l}1.9010 e+00 \\
1.9011 e+00\end{array}$ \\
\hline 48 & TAI 130048 & 254.2438 & 257.6899 & 256.5500 & $\begin{array}{l}1.4841 e-01 \\
6.6482 e-01\end{array}$ & $\begin{array}{l}1.9011 e+00 \\
1.9233 e+00\end{array}$ \\
\hline $\begin{array}{l}49 \\
50\end{array}$ & TAI 130136 & 263.1893 & 264.8530 & 264.1389 & $3.0762 e-01$ & $1.9252 e+00$ \\
\hline $\begin{array}{l}52 \\
53\end{array}$ & $\begin{array}{l}\text { TRR130000 } \\
\text { TSI000001 }\end{array}$ & $\begin{array}{l}72.8933 \\
60.2650\end{array}$ & $\begin{array}{l}75.5353 \\
61.1228\end{array}$ & $\begin{array}{l}74.2132 \\
60.6765\end{array}$ & $5.5474 e-01$ & $1.9000 e+00$ \\
\hline 54 & TSI000002 & 88.1226 & $\begin{array}{l}61.1228 \\
89.9027\end{array}$ & $\begin{array}{l}60.6765 \\
89.1326\end{array}$ & $\begin{array}{l}1.9429 e-01 \\
4.3755 e-01\end{array}$ & $\begin{array}{l}1.9001 e+00 \\
1.9002 e+00\end{array}$ \\
\hline 55 & TSO000001 & 57.1053 & 57.9710 & $\begin{array}{l}89.1326 \\
57.4518\end{array}$ & $\begin{array}{l}4.3755 e-01 \\
2.2709 e-01\end{array}$ & \\
\hline 56 & TSO0000002 & 73.8542 & 74.7047 & 74.2843 & $2.0138 e-01$ & $\begin{array}{l}1.9001 e+00 \\
1.9000 e+00\end{array}$ \\
\hline $\begin{array}{l}57 \\
58\end{array}$ & TAF020136 & 101.6945 & 103.1572 & 102.4388 & $3.6035 e-01$ & $1.9007 e+00$ \\
\hline 58 & TAF 120312 & 204.7705 & 208.0404 & 206.4177 & $8.6774 e-01$ & $1.9125 e+00$ \\
\hline 59 & TAF 130312 & 219.7223 & 223.0479 & 221.6384 & $7.0578 e-01$ & $1.9154 e+00$ \\
\hline 60 & EMPTY & 3.5547 & 3.5889 & 3.5742 & $7.7675 e-03$ & $0.0000 e+00$ \\
\hline 61 & EMPTY & 3.5449 & 3.5645 & 3.5566 & $5.3123 e-03$ & $0.0000 e+00$ \\
\hline 62 & EMPTY & 3.5303 & 3.5498 & 3.5400 & $4.3000 e-03$ & $0.0000 \mathrm{e}+00$ \\
\hline 63 & EMPTY & 3.5254 & 3.5400 & 3.5326 & $3.7226 e-03$ & $0.0000 e+00$ \\
\hline 64 & EMPTY & 1.7188 & 1.7236 & 1.7219 & $2.3675 e-03$ & $0.0000 e+00$ \\
\hline 65 & EMPTY & 1.7334 & 1.7432 & 1.7373 & $2.2058 e-03$ & $0.0000 e+00$ \\
\hline 66 & EMPTY & 1.7432 & 1.7480 & 1.7466 & $2.2603 e-03$ & $0.0000 \mathrm{e}+00$ \\
\hline 67 & EMPTY & 1.7578 & 1.7627 & 1.7597 & $2.3941 e-03$ & $0.0000 e+00$ \\
\hline 68 & EMPTY & 1.7627 & 1.7676 & 1.7661 & $2.2603 e-03$ & $0.0000 \mathrm{e}+00$ \\
\hline 69 & EMPTY & 1.7725 & 1.7871 & 1.7783 & $2.4164 e-03$ & $0.0000 \mathrm{e}+00$ \\
\hline 70 & EMPTY & 1.7822 & 1.7871 & 1.7859 & $2.1065 e-03$ & $0.0000 e+00$ \\
\hline 71 & EMPTY & 1.7969 & 1.8066 & 1.8023 & $1.8821 e-03$ & $0.0000 e+00$ \\
\hline 72 & EMPTY & 1.8066 & 1.8164 & 1.8102 & $2.4244 e-03$ & $0.0000 e+00$ \\
\hline 73 & EMPTY & 1.8213 & 1.8262 & 1.8234 & $2.4484 e-03$ & $0.0000 e+00$ \\
\hline 74 & EMPTY & 1.8262 & 1.8359 & 1.8332 & $2.6396 e-03$ & $0.0000 e+00$ \\
\hline 75 & EMPTY & 1.8408 & 1.8506 & 1.8456 & $1.2041 e-03$ & $0.0000 e+00$ \\
\hline 76 & EMPTY & 1.8506 & 1.8604 & 1.8532 & $2.6488 e-03$ & $0.0000 e+00$ \\
\hline 77 & EMPTY & 1.8506 & 1.8701 & 1.8658 & $2.8996 \mathrm{e}-03$ & $0.0000 e+00$ \\
\hline 78 & EMPTY & 1.8799 & 1.8848 & 1.8839 & $1.8950 \mathrm{e}-03$ & $0.0000 e+00$ \\
\hline 79 & EMPTY & 1.8994 & 1.9043 & 1.9005 & $2.0432 e-03$ & $0.0000 e+00$ \\
\hline 80 & PANO20136 & -17.3098 & -17.0046 & -17.1664 & $6.2876 e-02$ & $8.7106 e-01$ \\
\hline 81 & PAN050136 & -31.6952 & -31.0848 & -31.3503 & $1.4397 e-01$ & $1.6818 \mathrm{e}+00$ \\
\hline 82 & PAN070136 & -20.3819 & -20.0767 & -20.2034 & $8.5278 e-02$ & $8.7146 \mathrm{e}-01$ \\
\hline 83 & 3 PAN080136 & -12.5062 & -10.0648 & -11.6822 & $8.2316 e-01$ & $8.7049 e-01$ \\
\hline 84 & PAN090136 & -10.5050 & -7.9873 & -8.9837 & $7.7811 \mathrm{e}-01$ & $8.7029 e-01$ \\
\hline 85 & PAN 100136 & -12.2670 & -12.0382 & -12.1526 & $4.4272 e-02$ & $8.7053 e-01$ \\
\hline 86 & 5 PAN 105136 & -6.9391 & 2.5214 & -4.8990 & $1.6930 \mathrm{e}+00$ & $8.7009 e-01$ \\
\hline 87 & PAN 110136 & -7.2984 & 6.2056 & -3.2335 & $2.0081 e+00$ & $8.7004 e-01$ \\
\hline 88 & PAN1 15136 & -7.4834 & 1.3667 & -2.6585 & $1.6222 \mathrm{e}+00$ & $8.7003 e-01$ \\
\hline 89 & PAN120136 & -6.6720 & 0.4997 & -3.6538 & $1.2901 \mathrm{e}+00$ & $8.7005 e-01$ \\
\hline 90 & PAN125136 & -6.0323 & -2.8280 & -4.6987 & $7.0622 e-01$ & $8.7008 e-01$ \\
\hline 91 & PAN130136 & -6.4562 & -3.7096 & -4.8937 & $6.8226 e-01$ & $8.7009 e-01$ \\
\hline 92 & PAN 120048 & -12.0293 & -4.5525 & -7.2488 & $1.6749 \mathrm{e}+00$ & $8.7019 e-01$ \\
\hline 93 & PAN 130048 & -11.8019 & -8.2161 & -9.6046 & $7.7253 e-01$ & $8.7033 e-01$ \\
\hline 94 & PAN120224 & -7.7115 & -3.7442 & -5.4013 & $8.7601 e-01$ & $8.7010 e-01$ \\
\hline 95 & PAN130224 & -12.2846 & -8.3173 & -10.3315 & $9.8286 e-01$ & $8.7038 e-01$ \\
\hline 96 & PAN120312 & -11.2840 & -1.2895 & -6.9307 & $1.5774 \mathrm{e}+00$ & $8.7017 e-01$ \\
\hline 97 & PAN130312 & -14.4899 & -7.8523 & -9.9519 & $1.1305 \mathrm{e}+00$ & $8.7036 \mathrm{e}-01$ \\
\hline 98 & PAB 000136 & 40.1823 & 40.3654 & 40.2683 & $5.1192 e-02$ & $5.8867 e-01$ \\
\hline 99 & $\mathrm{PAB} 100136$ & 43.8471 & 44.0302 & 43.9084 & $5.3616 e-02$ & $5.9030 e-01$ \\
\hline 100 & $\mathrm{PAB} 125136$ & 44.5742 & 44.8031 & 44.6804 & $5.5056 e-02$ & $5.9066 e-01$ \\
\hline
\end{tabular}




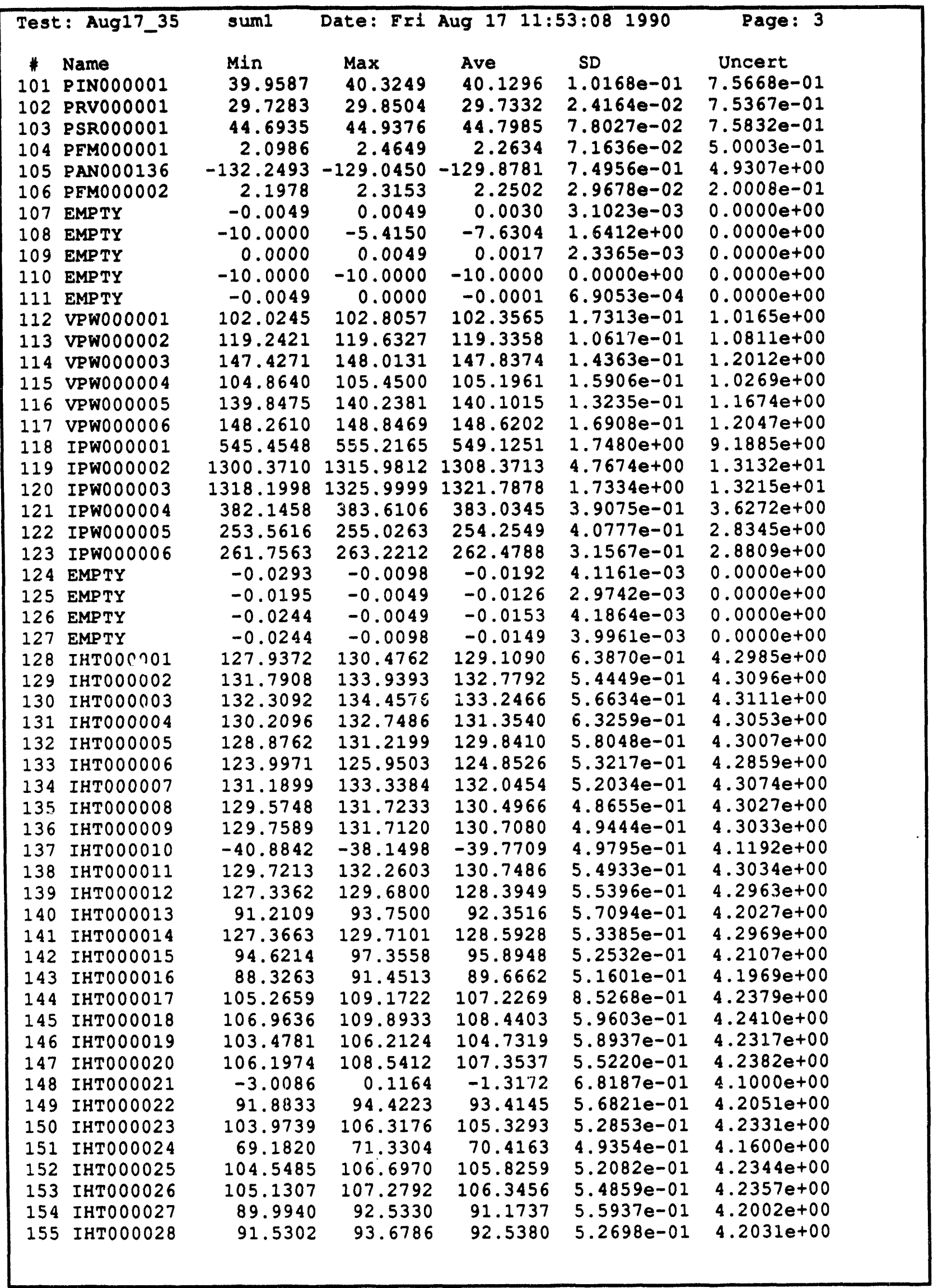




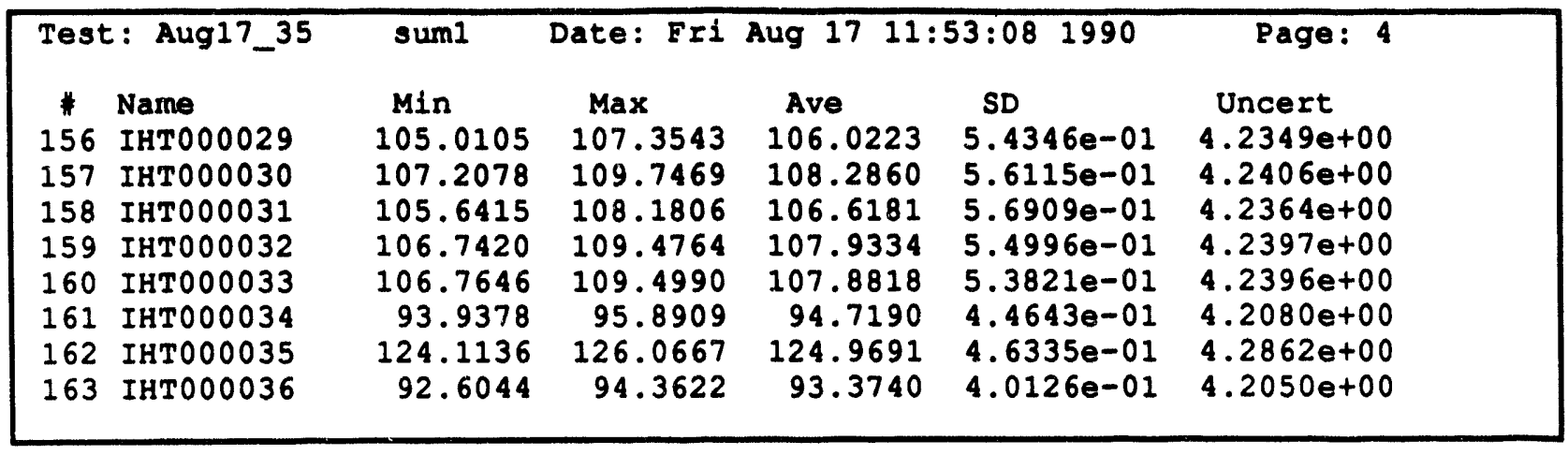




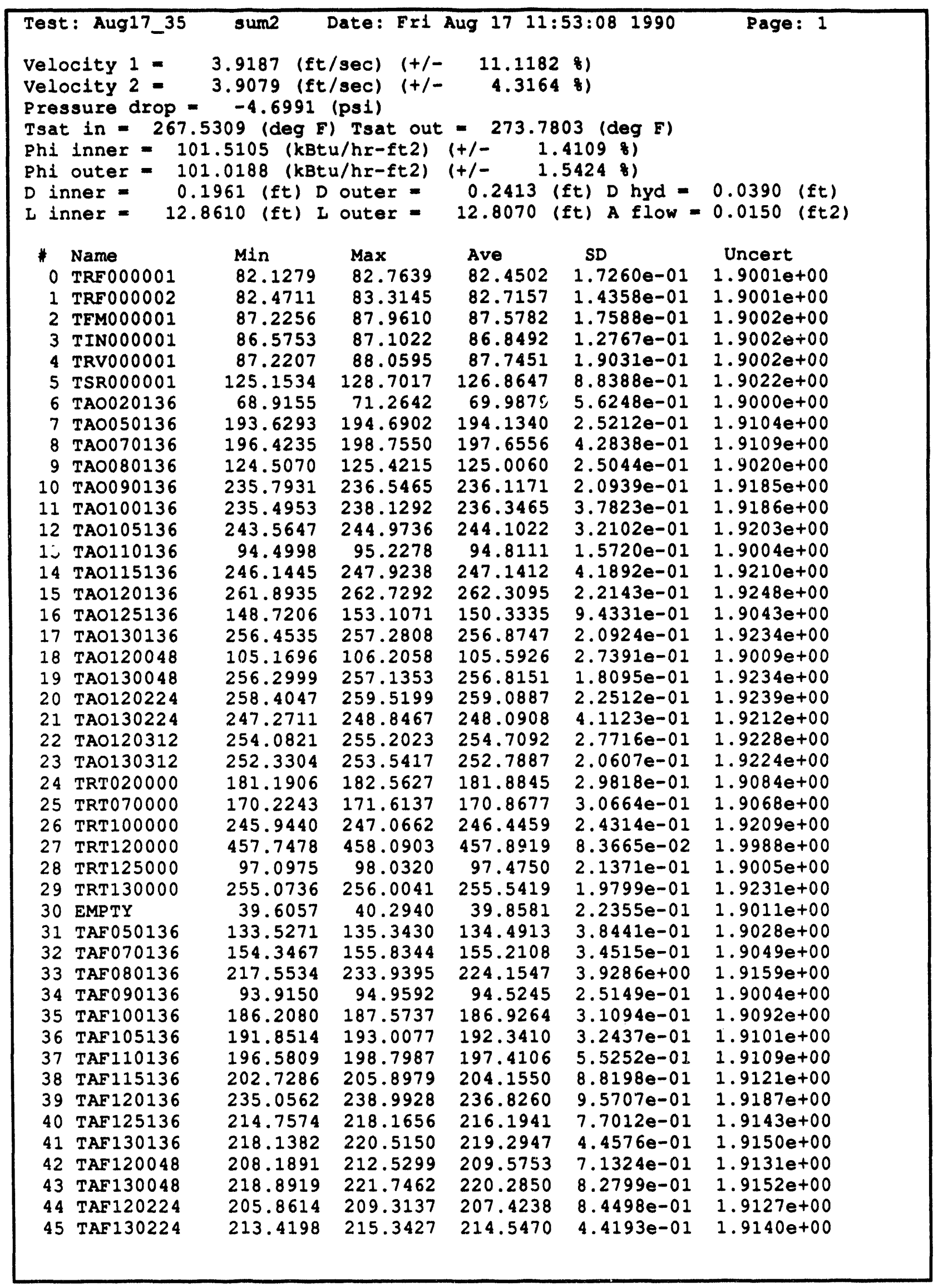




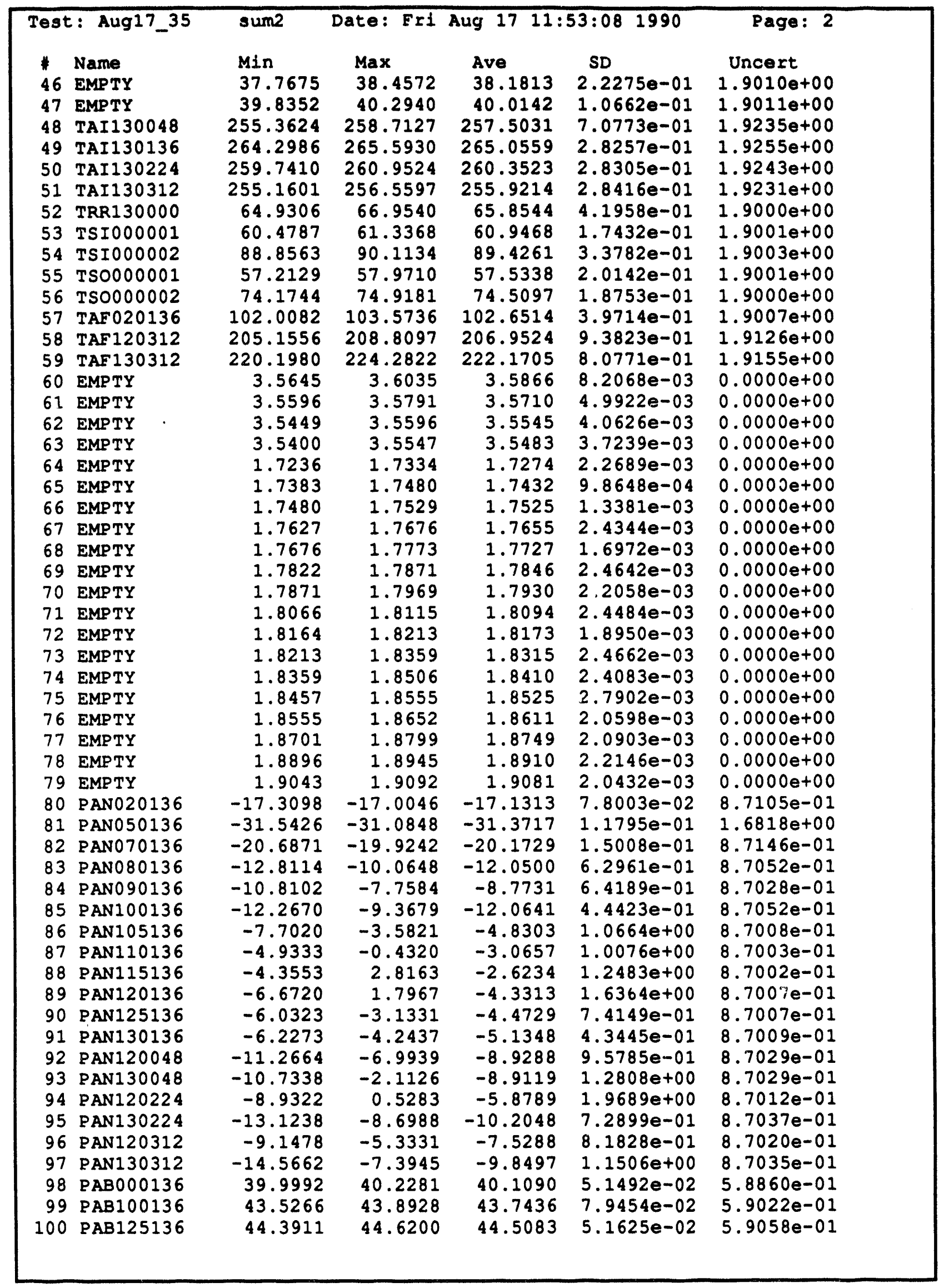




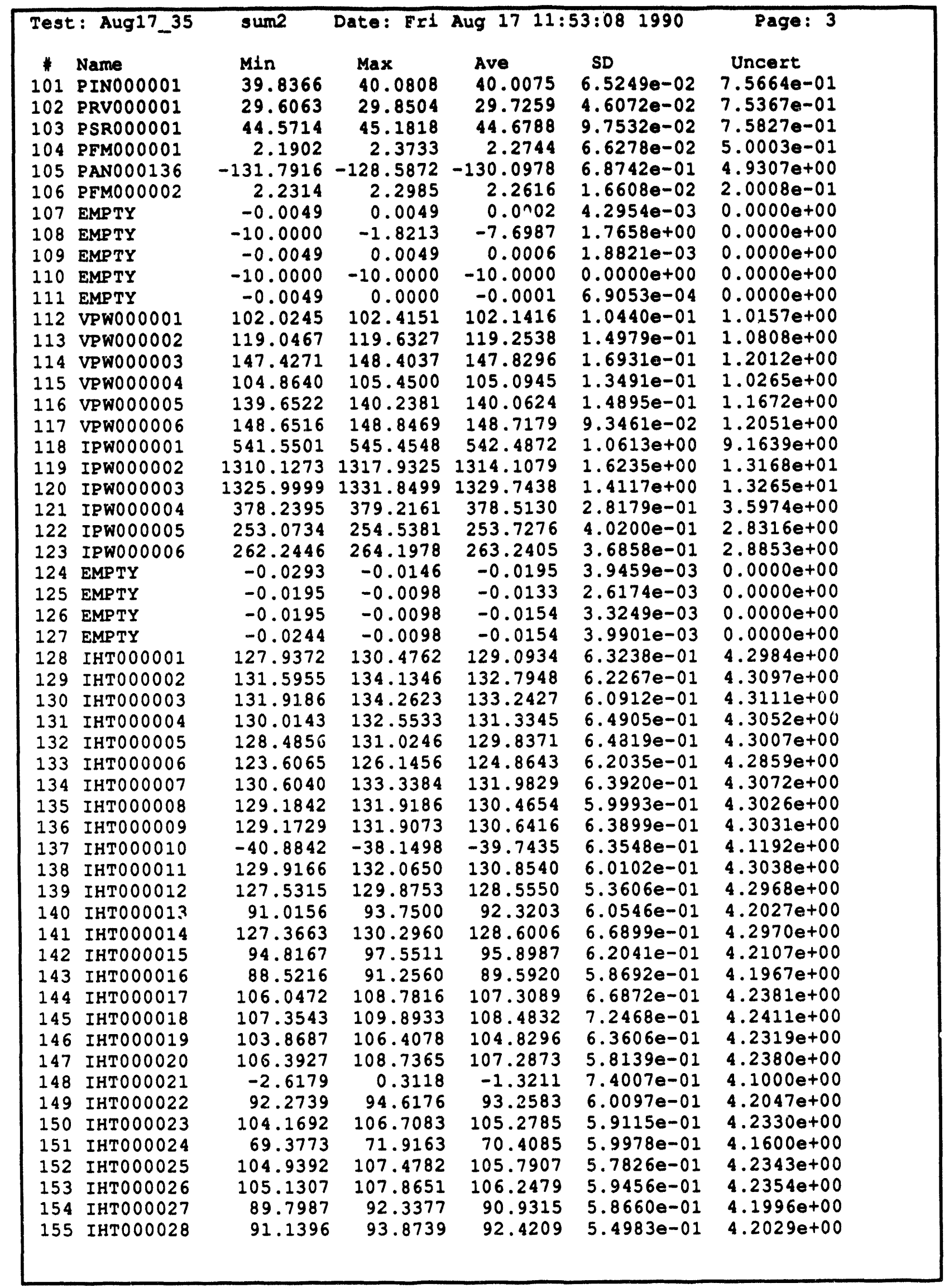




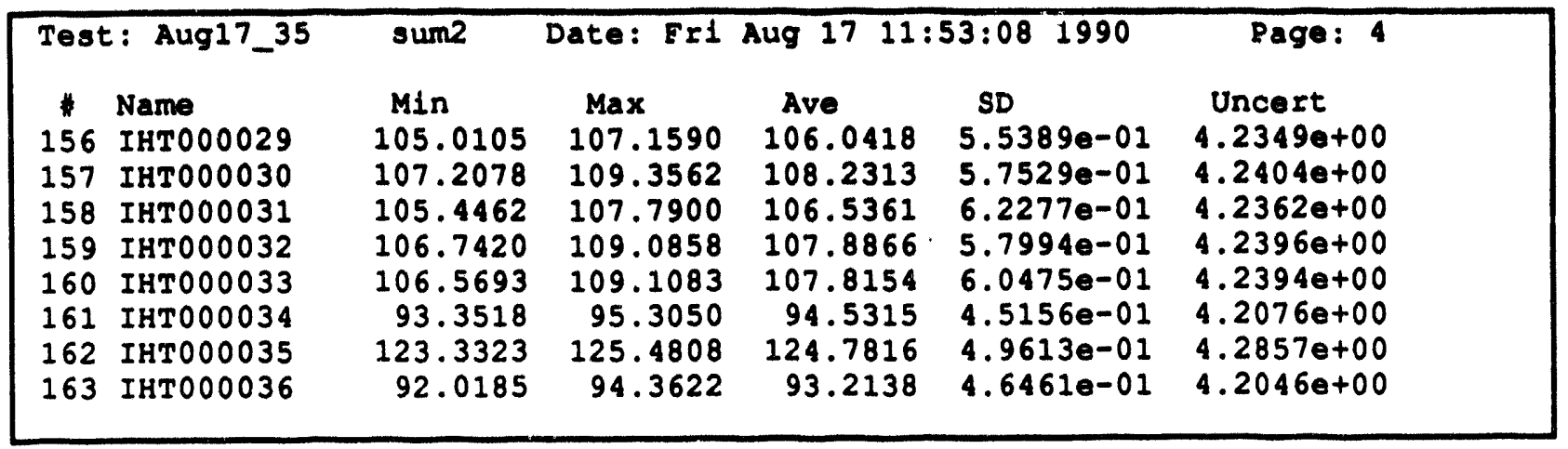




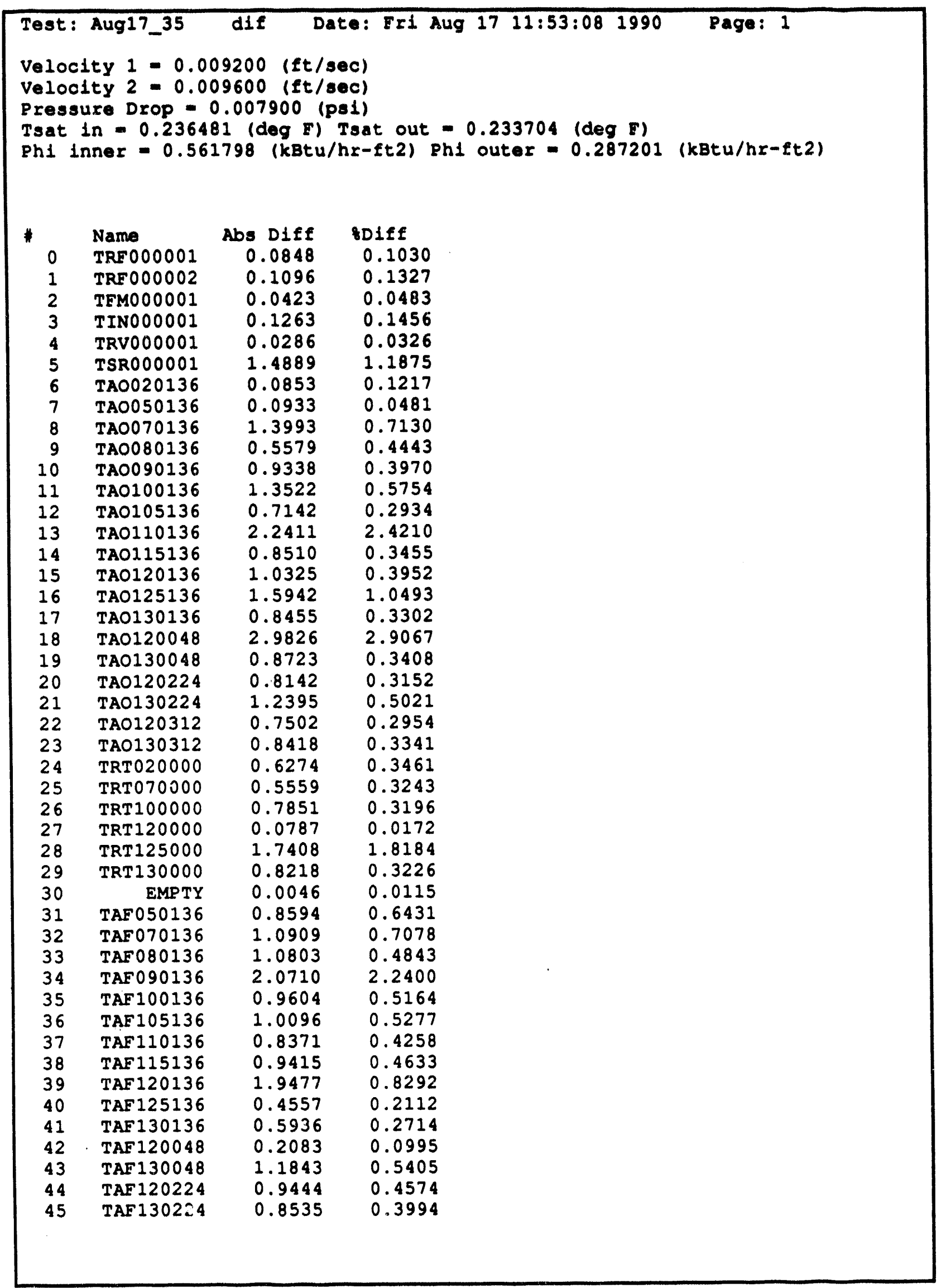




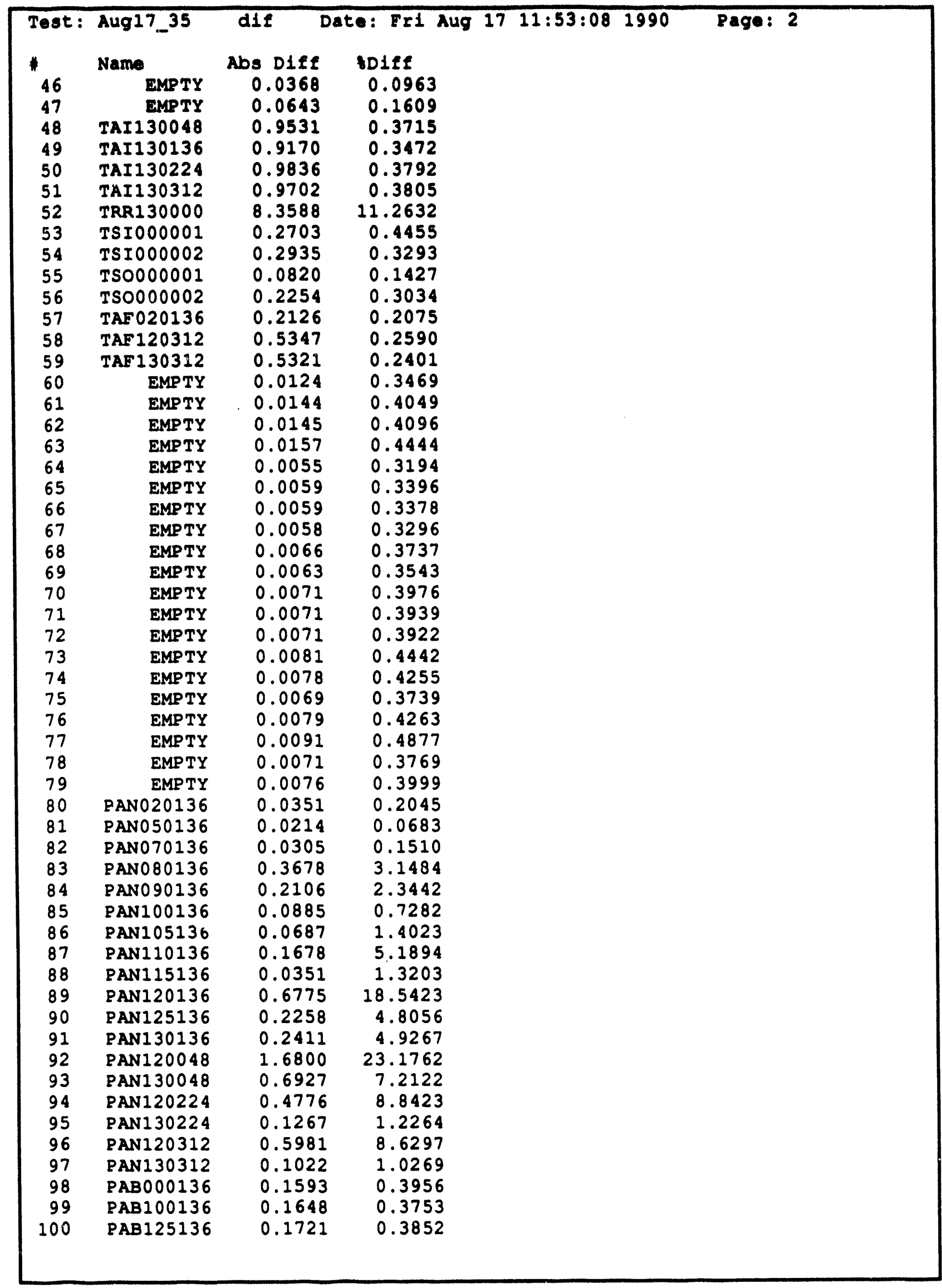




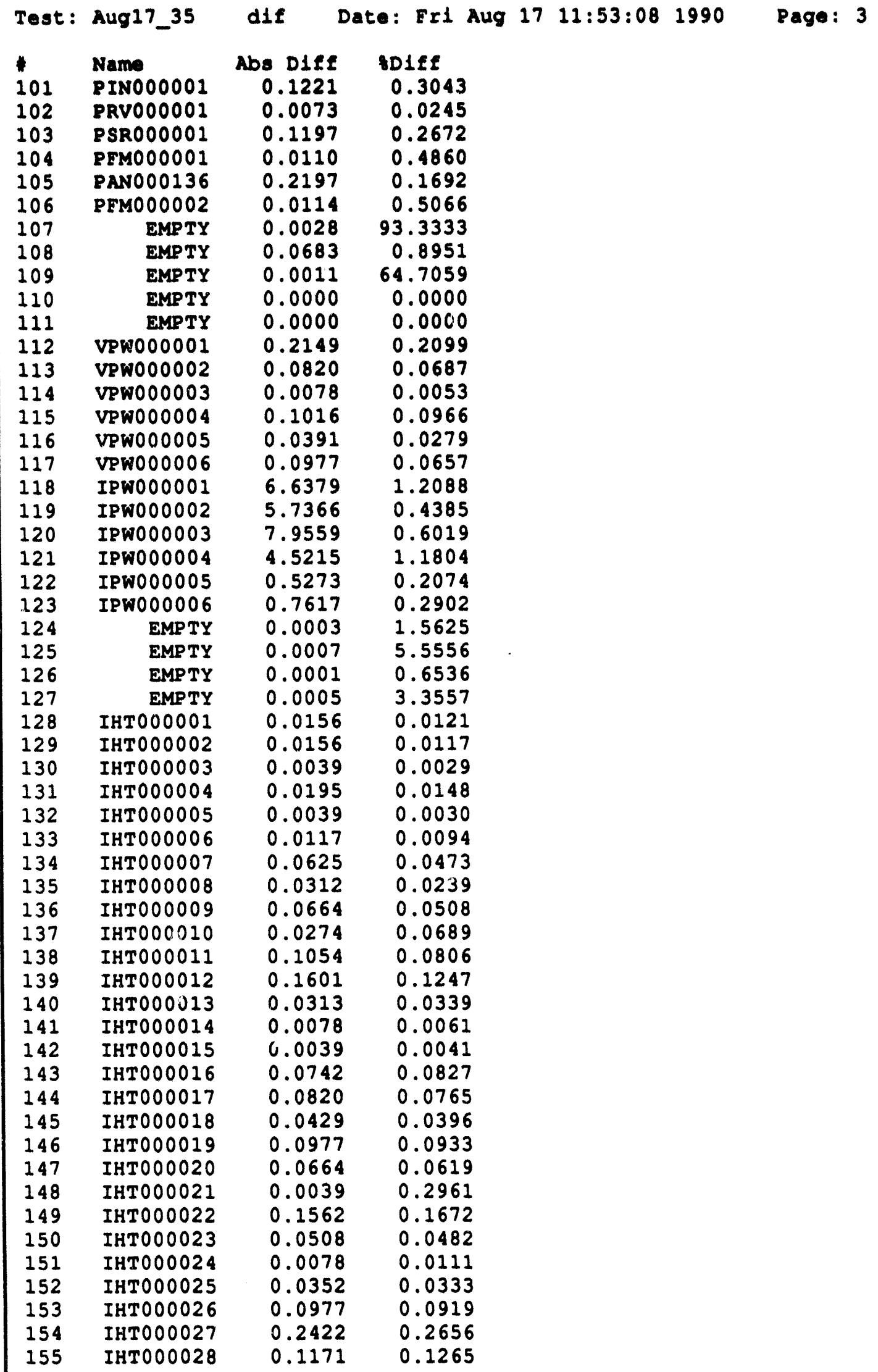


Test: Aug17_35 dff Date: Fr1 Aug 17 11:53:08 1990 Page: 4

* Name Abs Diff SDIfE

156 IHTO00029 $0.0195 \quad 0.0184$

157 IHTO00030 $0.0547 \quad 0.0505$

158 IHT000031 $0.0820 \quad 0.0769$

159 IHTO00032 $0.0468 \quad 0.0434$

160 IHTO00033 $0.0664 \quad 0.0615$

161 IHTO00034 $0.1875 \quad 0.1980$

162 IHTO00035 $0.1875 \quad 0.1500$

163 IHT000036 $0.1602 \quad 0.1716$ 

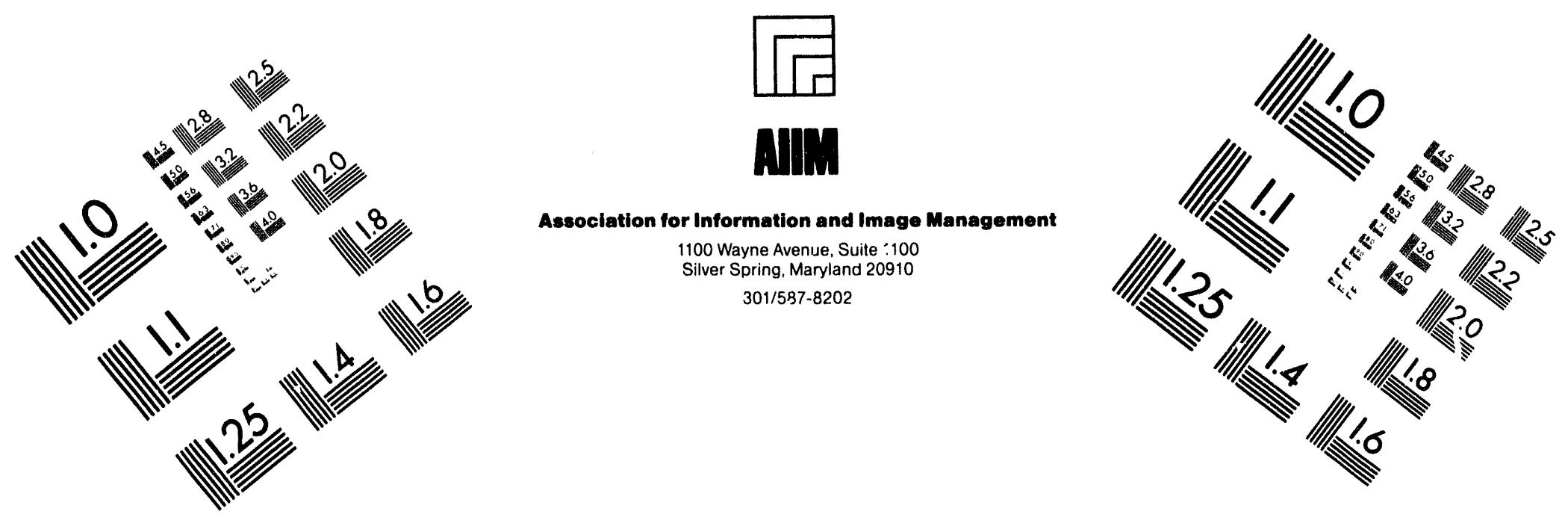

\section{Centimeter}

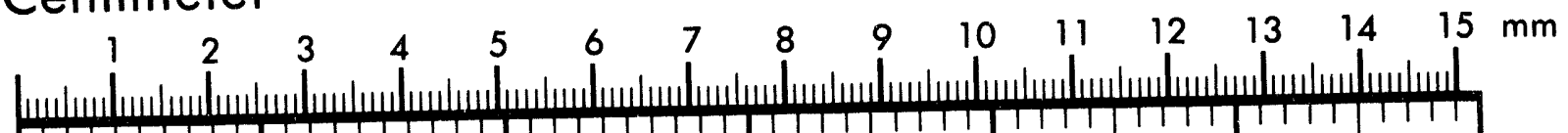

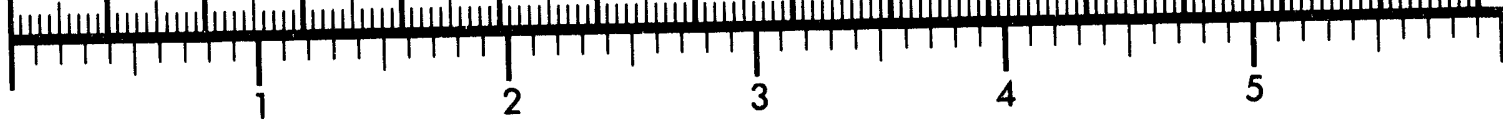
Inches
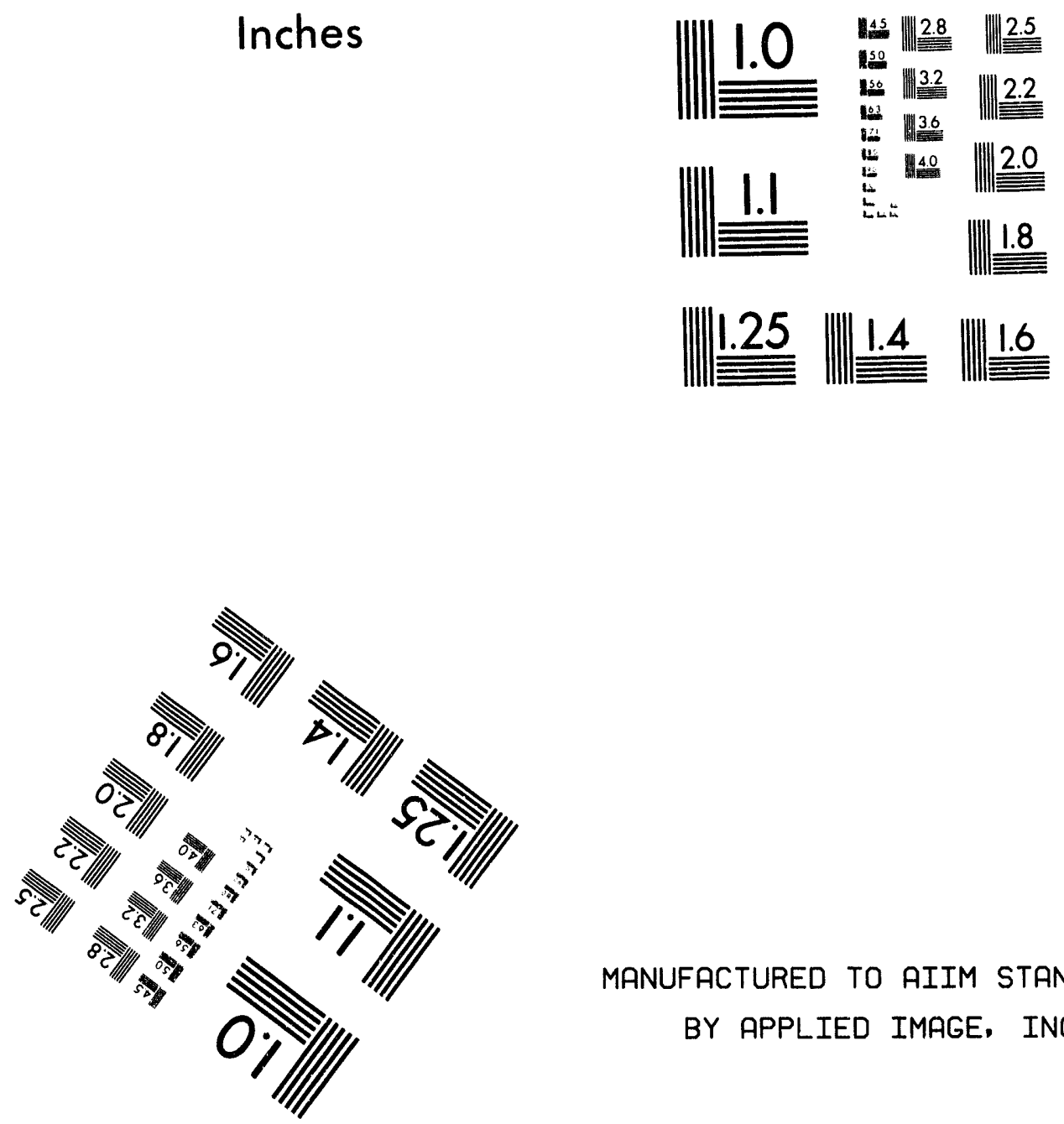

MANUFACTURED TO AIIM STANDARDS

BY APPLIED IMAGE, INC.

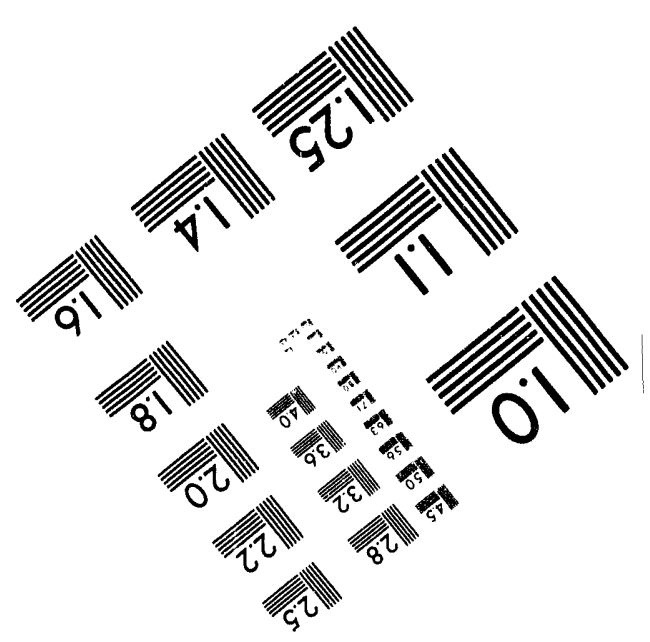



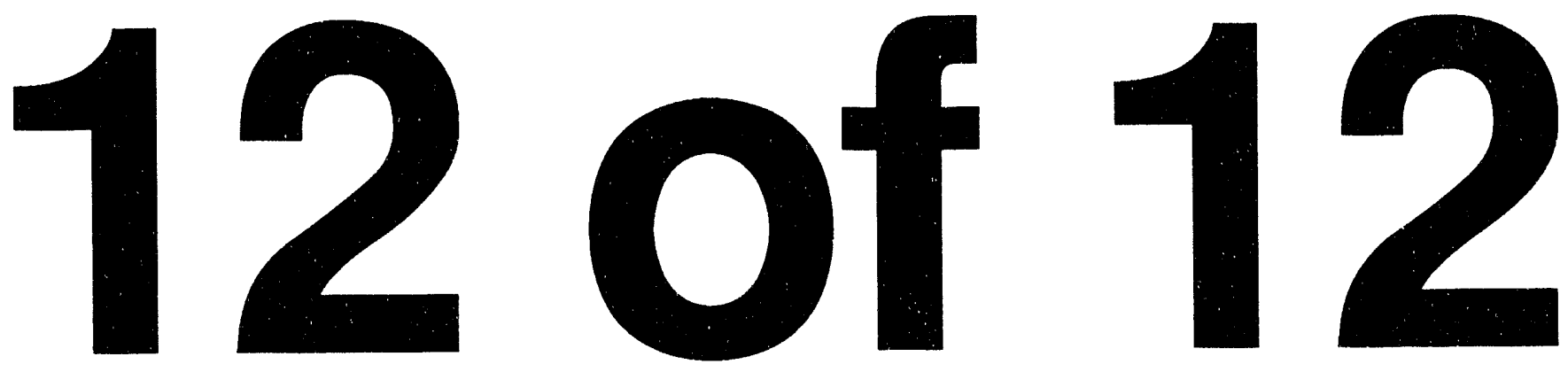


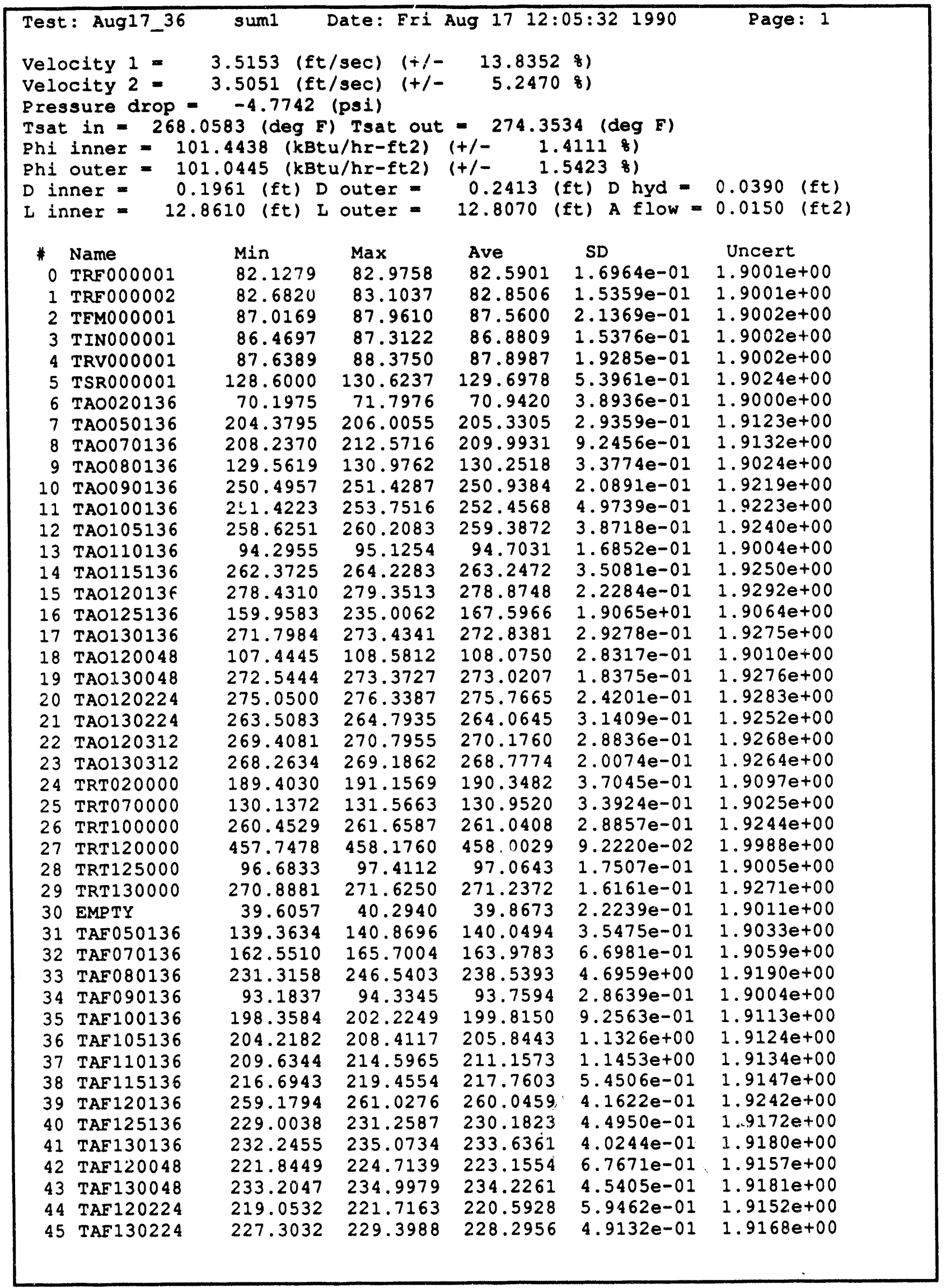




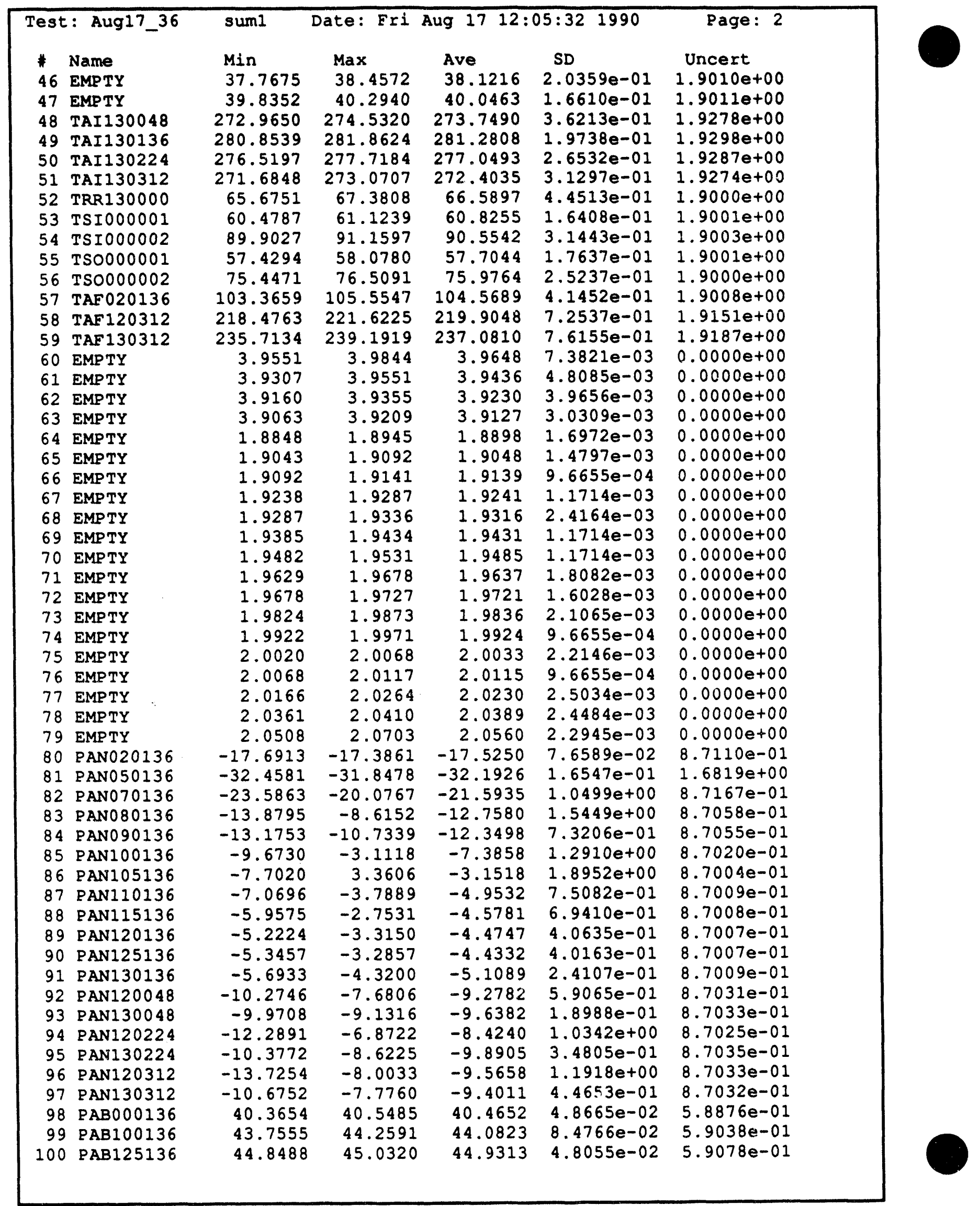




\begin{tabular}{|c|c|c|c|c|c|c|}
\hline \multicolumn{2}{|c|}{ Test: Aug17_36 } & sum 1 & Date: Fri & \multicolumn{2}{|c|}{ Aug $17 \quad 12: 05: 321990$} & Page: 3 \\
\hline \# & Name & Min & $\operatorname{Max}$ & Ave & SD & Uncert \\
\hline 101 & PIN000001 & 40.2028 & 40.4470 & 40.3249 & $6.9754 e-02$ & $7.5675 e-01$ \\
\hline 102 & PRV000001 & 29.4842 & 29.6063 & 29.5404 & $6.1457 e-02$ & $7.5363 e-01$ \\
\hline 103 & PSR000001 & 45.0597 & 45.1818 & 45.1280 & $6.1209 \mathrm{e}-02$ & $7.5844 \mathrm{e}-01$ \\
\hline 104 & PEMO000001 & 1.7324 & 1.9155 & 1.8203 & $7.1541 e-02$ & $5.0002 e-01$ \\
\hline 105 & PAN000136 & -133.1649 & -130.8760 & -132.1761 & $5.8021 e-01$ & $4.9311 e+00$ \\
\hline 106 & PFM000002 & 1.7783 & 1.8455 & 1.8095 & $1.8259 \mathrm{e}-02$ & $2.0005 e-01$ \\
\hline 107 & EMPTY & -0.0049 & 0.0049 & 0.0018 & $4.1573 e-03$ & $0.0000 e+00$ \\
\hline 108 & EMPTY & -10.0000 & -4.9805 & -7.8282 & $1.6146 \mathrm{e}+00$ & $0.0000 e+00$ \\
\hline 109 & EMPTY & 0.0000 & 0.0098 & 0.0007 & $1.9754 \mathrm{e}-03$ & $0.0000 e+00$ \\
\hline 110 & EMPTY & -10.0000 & -10.0000 & -10.0000 & $0.0000 e+00$ & $0.0000 e+00$ \\
\hline 111 & EMPTY & 0.0000 & 0.0000 & 0.0000 & $0.0000 e+00$ & $0.0000 e+00$ \\
\hline 112 & VPW000001 & 102.0245 & 102.4151 & 102.2666 & $1.0106 \mathrm{e}-01$ & $1.0162 e+00$ \\
\hline 113 & VPW000002 & 119.0467 & 119.6327 & 119.3475 & $1.1975 e-01$ & $1.0811 e+00$ \\
\hline 114 & VPW000003 & 147.8178 & 148.2084 & 147.9467 & $1.0145 e-01$ & $1.2017 \mathrm{e}+00$ \\
\hline 115 & VPW000004 & 104.8640 & 105.4500 & 105.2078 & $1.2823 e-01$ & $1.0269 e+00$ \\
\hline 116 & VPW000005 & 139.6522 & 140.2381 & 139.9140 & $1.2853 e-01$ & $1.1665 \mathrm{e}+00$ \\
\hline 117 & VPW000006 & 148.4563 & 148.8469 & 148.6241 & $1.1168 \mathrm{e}-01$ & $1.2047 e+00$ \\
\hline 118 & IPWO00001 & 539.5978 & 547.4072 & 543.1121 & $1.5276 \mathrm{e}+00$ & $9.1662 e+00$ \\
\hline 119 & IPW000002 & 1310.1273 & 1315.9812 & 1313.0933 & $1.9005 \mathrm{e}+00$ & $1.3161 e+01$ \\
\hline 120 & IPW000003 & 1325.9999 & 1331.8499 & 1328.3784 & $1.7303 e+00$ & $1.3257 e+01$ \\
\hline 121 & IPW000004 & 377.7513 & 379.2161 & 378.4446 & $3.1335 e-01$ & $3.5970 e+00$ \\
\hline 122 & IPW000005 & 252.5851 & 253.5616 & 253.3076 & $2.6541 e-01$ & $2.8292 e+00$ \\
\hline 123 & IPW000006 & 261.7563 & 263.2212 & 262.6741 & $3.7745 e-01$ & $2.8820 e+00$ \\
\hline 124 & EMPTY & -0.0244 & -0.0098 & -0.0190 & $3.4527 e-03$ & $0.0000 e+00$ \\
\hline 125 & EMPTY & -0.0195 & -0.0098 & -0.0140 & $2.9611 \mathrm{e}-03$ & $0.0000 e+00$ \\
\hline 126 & EMPTY & -0.0244 & -0.0098 & -0.0163 & $2.8946 e-03$ & $0.0000 e+00$ \\
\hline 127 & EMPTY & -0.0195 & -0.0098 & -0.0155 & $2.9080 e-03$ & $0.0000 e+00$ \\
\hline 128 & IHTO00001 & 127.7419 & 130.4762 & 129.0660 & $5.8699 e-01$ & $4.2983 e+00$ \\
\hline 129 & IHT 000002 & 131.5955 & 134.3299 & 132.7323 & $5.4346 e-01$ & $4.3095 e+00$ \\
\hline 130 & IHTO00003 & 132.1139 & 134.6530 & 133.1607 & $5.4213 e-01$ & $4.3108 e+00$ \\
\hline 131 & IH'TOO0004 & 130.2096 & 132.5533 & 131.2134 & $4.9914 e-01$ & $4.3048 e+00$ \\
\hline 132 & IHTO00005 & 128.4856 & 131.4153 & 129.7550 & $5.5419 e-01$ & $4.3004 e+00$ \\
\hline 133 & IHT000006 & 123.6065 & 125.9503 & 124.7354 & $5.6399 \mathrm{e}-01$ & $4.2855 e+00$ \\
\hline 134 & IHT000007 & 130.6040 & 133.5337 & 131.9126 & $5.5278 e-01$ & $4.3070 e+00$ \\
\hline 135 & IHT000008 & 129.3795 & 131.7233 & 130.4185 & $5.2562 e-01$ & $4.3024 e+00$ \\
\hline 136 & IHT000009 & 129.5635 & 131.9073 & 130.6416 & $5.1936 e-01$ & $4.3031 e+00$ \\
\hline 137 & IHT000010 & -41.2748 & -38.5404 & -39.8178 & $5.2971 e-01$ & $4.1193 e+00$ \\
\hline 138 & IHTO00011 & 129.5260 & 131.6744 & 130.6744 & $4.7946 e-01$ & $4.3032 e+00$ \\
\hline 139 & IHT000012 & 127.1409 & 129.2894 & 128.3714 & $4.2361 \mathrm{e}-01$ & $4.2963 e+00$ \\
\hline 140 & IHT000013 & 90.8203 & 92.9688 & 92.2109 & $4.5445 e-01$ & $4.2024 e+00$ \\
\hline 141 & IHTO00014 & 127.3663 & 128.9288 & 128.2920 & $3.8624 e-01$ & $4.2960 e+00$ \\
\hline 142 & IHT000015 & 94.8167 & 96.1839 & 95.7386 & $3.5081 e-01$ & $4.2103 e+00$ \\
\hline 143 & IHT000016 & 88.7169 & 90.0841 & 89.4904 & $3.4391 e-01$ & $4.1965 \mathrm{e}+00$ \\
\hline 144 & IHT000017 & 105.6566 & 108.3909 & 106.9417 & $6.4124 e-01$ & $4.2372 e+00$ \\
\hline 145 & IHT000018 & 107.1590 & 109.5027 & 108.3387 & $5.1442 e-01$ & $4.2407 e+00$ \\
\hline 146 & IHT000019 & 103.4781 & 105.6265 & 104.6890 & $4.6354 e-01$ & $4.2315 e+00$ \\
\hline 147 & IHTO000020 & 106.0021 & 108.1505 & 107.2091 & $4.6970 e-01$ & $4.2379 e+00$ \\
\hline 148 & IHT 000021 & -2.8132 & -0.2742 & -1.4070 & $5.4391 e-01$ & $4.1000 e+00$ \\
\hline 149 & IHT000022 & 92.2739 & 93.8364 & 93.1 .684 & $3.9674 \mathrm{e}-01$ & $4.2045 e+00$ \\
\hline 150 & IHTO00023 & 104.1692 & 106.1223 & 105.1809 & $3.9791 \mathrm{e}-01$ & $4.2328 e+00$ \\
\hline 151 & IHTO00024 & 69.3773 & 71.1351 & 70.2874 & $3.7973 e-01$ & $4.1598 e+00$ \\
\hline 152 & IHT000025 & 104.5485 & 106.6970 & 105.6774 & $4.3097 e-01$ & $4.2340 e+00$ \\
\hline 153 & IHT000026 & 105.1307 & 107.0838 & 106.1385 & $3.9922 e-01$ & $4.2352 e+00$ \\
\hline 154 & IHTO000027 & 89.7987 & 91.5565 & 90.8612 & $4.0271 e-01$ & $4.1995 \mathrm{e}+00$ \\
\hline 155 & IHTO00028 & 90.5536 & 94.0693 & 92.3310 & $6.8374 e-01$ & $4.2027 e+00$ \\
\hline
\end{tabular}




\begin{tabular}{|c|c|c|c|c|c|c|}
\hline \multicolumn{2}{|c|}{ Test: Aug17_36 } & sum 1 & Date: Fri & Aug 1712 & $5: 321990$ & Page: 4 \\
\hline & Name & Min & $\operatorname{Max}$ & Ave & SD & Uncert \\
\hline 156 & IHT 000029 & 104.4246 & 107.7449 & 105.9246 & $6.5843 e-01$ & $4.2346 e+00$ \\
\hline 157 & IHT000030 & 106.6219 & 110.1375 & 108.1492 & $6.4680 e-01$ & $4.2402 \mathrm{e}+00$ \\
\hline 158 & IHT000031 & 105.0556 & 108.3759 & 106.4150 & $6.3987 e-01$ & $4.2358 \mathrm{e}+00$ \\
\hline 159 & IHT000032 & 106.3514 & 109.4764 & 107.7303 & $6.1815 e-01$ & $4.2392 \mathrm{e}+00$ \\
\hline 160 & IHT000033 & 106.3740 & 109.4990 & 107.6904 & $6.1360 e-01$ & $4.2391 \mathrm{e}+00$ \\
\hline 161 & IHTO00034 & 93.5472 & 95.8909 & 94.5120 & $5.0287 e-01$ & $4.2075 e+00$ \\
\hline 162 & IHTO000035 & 123.5277 & 125.6761 & 124.6410 & $5.0878 e-01$ & $4.2853 e+00$ \\
\hline 163 & IHT000036 & 92.2138 & 94.1669 & 93.2255 & $5.0175 e-01$ & $4.2047 e+00$ \\
\hline
\end{tabular}




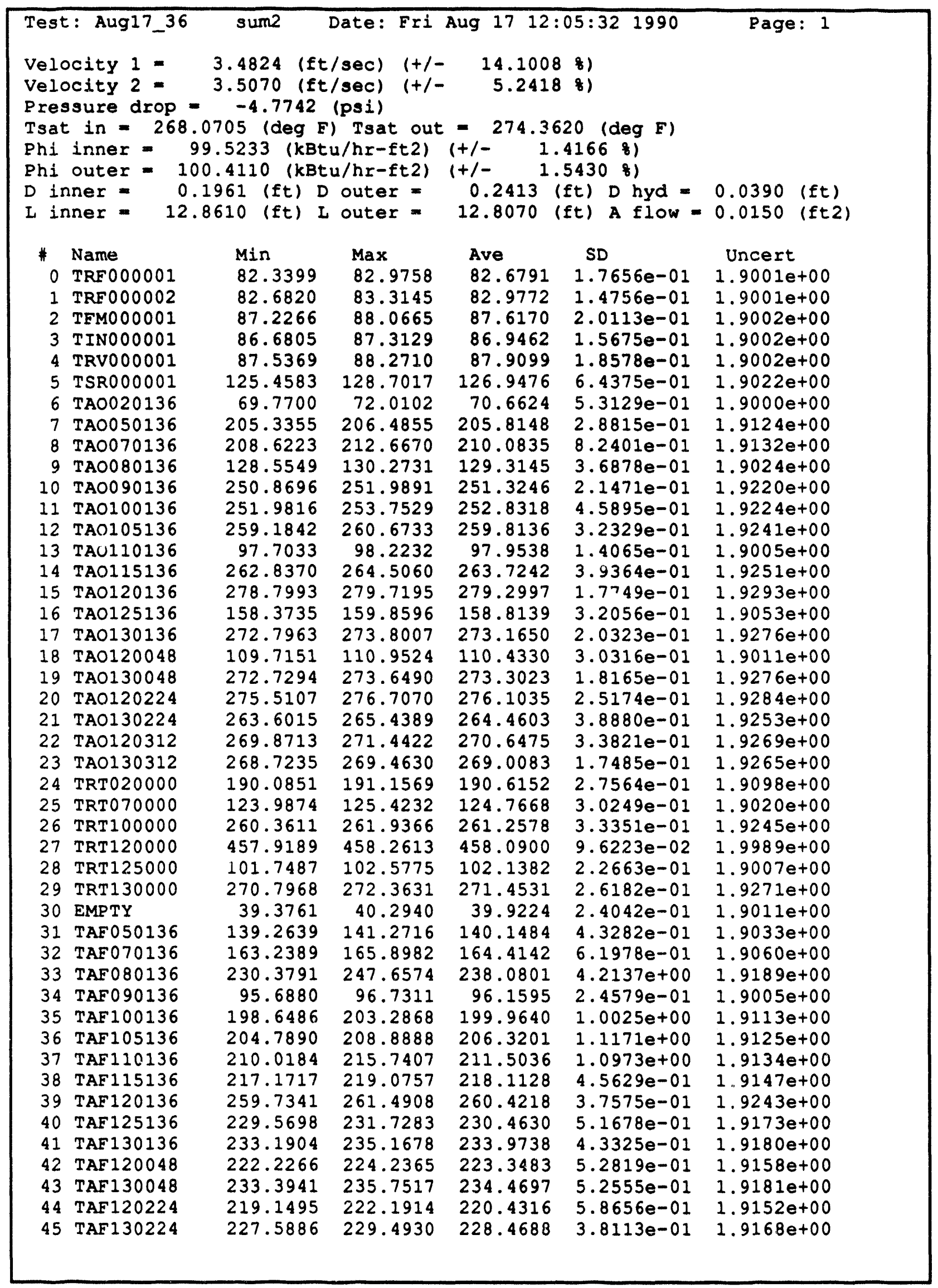




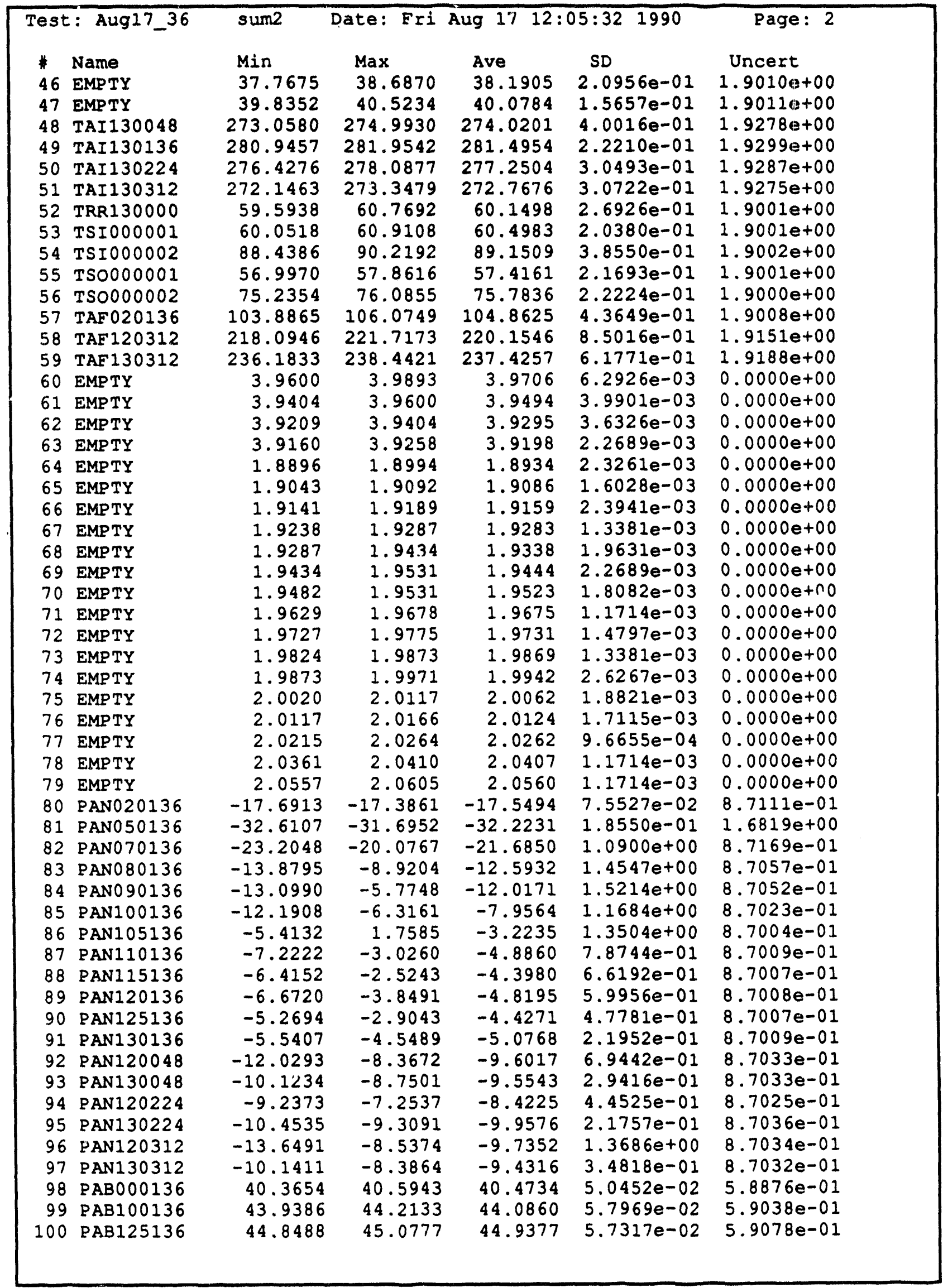




\begin{tabular}{|c|c|c|c|c|c|c|}
\hline \multicolumn{2}{|c|}{ Test: Aug17_36 } & sum2 & Date: Fri & \multicolumn{2}{|r|}{$5: 32$} & \multirow{2}{*}{$\begin{array}{l}\text { Page: } 3 \\
\text { Uncert }\end{array}$} \\
\hline * & Name & Min & $\operatorname{Max}$ & Ave & SD & \\
\hline 101 & PIN000001 & 40.2028 & 40.3249 & 40.2321 & $5.2663 e-02$ & $7.56710-01$ \\
\hline 102 & PRV000001 & 29.3621 & 29.6063 & 29.4842 & $2.4662 e-02$ & $7.5361 \mathrm{e}-01$ \\
\hline 103 & PSR000001 & 45.0597 & 45.1818 & 45.1036 & $5.9189 e-02$ & $7.5843 e-01$ \\
\hline 104 & PFM000001 & 1.7324 & 1.9155 & 1.7855 & $6.9429 e-02$ & $5.0002 e-01$ \\
\hline 105 & PANO00136 & -133.6226 & -130.4183 & -132.1761 & $6.9429 e-01$ & $4.9311 e+00$ \\
\hline 106 & PFM000002 & 1.7783 & 1.8622 & 1.8116 & $1.9323 e-02$ & $2.0005 e-01$ \\
\hline 107 & EMPTY & -0.0049 & 0.0049 & 0.0012 & $4.0144 e-03$ & $0.0000 e+00$ \\
\hline 108 & EMPTY & -10.0000 & -3.0518 & -7.3724 & $2.0140 e+00$ & $0.0000 \mathrm{e}+00$ \\
\hline 109 & EMPTY & -0.0049 & 0.0049 & 0.0008 & $2.0598 e-03$ & $0.0000 e+00$ \\
\hline 110 & EMPTY & -10.0000 & -10.0000 & -10.0000 & $0.0000 e+00$ & $0.0000 e+00$ \\
\hline 111 & EMPTY & 0.0000 & 0.0098 & 0.0002 & $1.3811 e-03$ & $0.0000 e+00$ \\
\hline 112 & VPW000001 & 102.0245 & 102.4151 & 102.1963 & $8.4997 e-02$ & $1.0159 \mathrm{e}+00$ \\
\hline 113 & VPW000002 & 119.0467 & 119.6327 & 119.3046 & $1.1464 \mathrm{e}-01$ & $1.0810 \mathrm{e}+00$ \\
\hline 114 & VPW000003 & 147.6225 & 148.4037 & 147.8803 & $1.4466 e-01$ & $1.2014 e+00$ \\
\hline 115 & VPW000004 & 104.8640 & 105.4500 & 105.1570 & $1.1334 \mathrm{e}-01$ & $1.0267 e+00$ \\
\hline 116 & VPW000005 & 139.8475 & 140.4334 & 140.0975 & $1.2503 e-01$ & $1.1673 \mathrm{e}+00$ \\
\hline 117 & VPW0000006 & 148.4563 & 148.8469 & 148.6281 & $1.1599 e-01$ & $1.2047 e+00$ \\
\hline 118 & IPWO00001 & 563.0259 & 570.8353 & 568.1408 & $1.4700 e+00$ & $9.2604 \mathrm{e}+00$ \\
\hline 119 & IPW000002 & 1269.1508 & 1276.9558 & 1273.2483 & $1.6373 e+00$ & $1.2915 \mathrm{e}+01$ \\
\hline 120 & IPW000003 & 1298.6998 & 1304.5499 & 1301.1182 & $1.4507 e+00$ & $1.3087 e+01$ \\
\hline 121 & IPW000004 & 383.1223 & 384.0989 & 383.3958 & $3.1444 \mathrm{e}-01$ & $3.6296 \mathrm{e}+00$ \\
\hline 122 & IPW000005 & 252.0969 & 253.5616 & 252.8780 & $2.9591 \mathrm{e}-01$ & $2.8268 e+00$ \\
\hline 123 & IPW000006 & 259.8032 & 261.2681 & 260.3792 & $3.9075 e-01$ & $2.8690 \mathrm{e}+00$ \\
\hline 124 & EMPTY & -0.0293 & -0.0098 & -0.0197 & $3.8155 e-03$ & $0.0000 e+00$ \\
\hline 125 & EMPTY & -0.0195 & -0.0098 & -0.0130 & $3.0581 e-03$ & $0.0000 e+00$ \\
\hline 126 & EMPTY & -0.0195 & -0.0098 & -0.0161 & $3.4527 e-03$ & $0.0000 \mathrm{e}+00$ \\
\hline 127 & EMPTY & -0.0244 & -0.0098 & -0.0157 & $3.0067 e-03$ & $0.0000 e+00$ \\
\hline 128 & IHT000001 & 127.9372 & 130.4762 & 129.1246 & $6.5654 e-01$ & $4.2985 e+00$ \\
\hline 129 & IHT000002 & 131.5955 & 133.7440 & 132.8221 & $5.9978 e-01$ & $4.3098 e+00$ \\
\hline 130 & IHTO00003 & 132.1139 & 134.6530 & 133.2466 & $5.9189 e-01$ & $4.3111 e+00$ \\
\hline 131 & IHT000004 & 130.2096 & 132.5533 & 131.3345 & $5.9262 e-01$ & $4.3052 e+00$ \\
\hline 132 & IHT000005 & 128.8762 & 130.6340 & 129.8293 & $4.9856 e-01$ & $4.3006 e+00$ \\
\hline 133 & IHT000006 & 123.8018 & 125.7550 & 124.8096 & $4.9968 e-01$ & $4.2858 \mathrm{e}+00$ \\
\hline 134 & IHT000007 & 130.9946 & 133.3384 & 131.9907 & $4.8528 e-01$ & $4.3072 \mathrm{e}+00$ \\
\hline 135 & IHT000008 & 129.3795 & 131.9186 & 130.4224 & $4.8746 e-01$ & $4.3024 e+00$ \\
\hline 136 & IHT000009 & 129.7589 & 132.2979 & 130.6338 & $4.8527 e-01$ & $4.3031 e+00$ \\
\hline 137 & IHT000010 & -40.6889 & -38.7357 & -39.7709 & $4.8688 e-01$ & $4.1192 e+00$ \\
\hline 138 & IHT000011 & 129.7213 & 131.8697 & 130.7837 & $5.0093 e-01$ & $4.3035 e+00$ \\
\hline 139 & IHT000012 & 127.5315 & 129.6800 & 128.4261 & $5.0082 e-01$ & $4.2964 e+00$ \\
\hline 140 & IHTO00013 & 91.4063 & 93.3594 & 92.2969 & $4.8030 e-01$ & $4.2026 e+00$ \\
\hline 141 & IHTO00014 & 127.7569 & 129.7101 & 128.5694 & $4.7900 \mathrm{e}-01$ & $4.2969 e+00$ \\
\hline 142 & IHTO00015 & 95.0120 & 96.9651 & 95.8401 & $4.4740 e-01$ & $4.2105 e+00$ \\
\hline 143 & IHT000016 & 88.7169 & 90.6701 & 89.5334 & $4.6470 \mathrm{e}-01$ & $4.1966 e+00$ \\
\hline 144 & IHT000017 & 105.4613 & 109.5628 & 107.1996 & $8.3356 e-01$ & $4.2378 e+00$ \\
\hline 145 & IHT000018 & 107.3543 & 110.0886 & 108.3582 & $6.1131 e-01$ & $4.2408 e+00$ \\
\hline 146 & IHT000019 & 103.6734 & 106.4078 & 104.7358 & $6.1150 e-01$ & $4.2317 \mathrm{e}+00$ \\
\hline 147 & IHTO00020 & 106.1974 & 108.7365 & 107.2248 & $6.1233 e-01$ & $4.2379 e+00$ \\
\hline 148 & IHTO00021 & -2.6179 & 0.1164 & -1.2664 & $7.0348 e-01$ & $4.1000 \mathrm{e}+00$ \\
\hline 149 & IHTOOO0022 & 92.0786 & 94.4223 & 93.2700 & $5.7757 e-01$ & $4.2048 e+00$ \\
\hline 150 & IHT000023 & 104.1692 & 106.3176 & 105.2824 & $5.5699 e-01$ & $4.2330 e+00$ \\
\hline 151 & IHTO00024 & 69.3773 & 71.5257 & 70.3655 & $5.2111 e-01$ & $4.1599 e+00$ \\
\hline 152 & IHTO00025 & 104.7439 & 107.0876 & 105.7555 & $5.6316 \mathrm{e}-01$ & $4.2342 e+00$ \\
\hline 153 & IHT000026 & 105.3260 & 107.4745 & 106.2440 & $5.1486 e-01$ & $4.2354 e+00$ \\
\hline 154 & IHT000027 & 90.1893 & 91.9471 & 91.0447 & $4.9895 e-01$ & $4.1999 e+00$ \\
\hline 155 & IHT000028 & 91.1396 & 93.2880 & 92.1669 & $5.1116 e-01$ & $4.2023 e+00$ \\
\hline
\end{tabular}




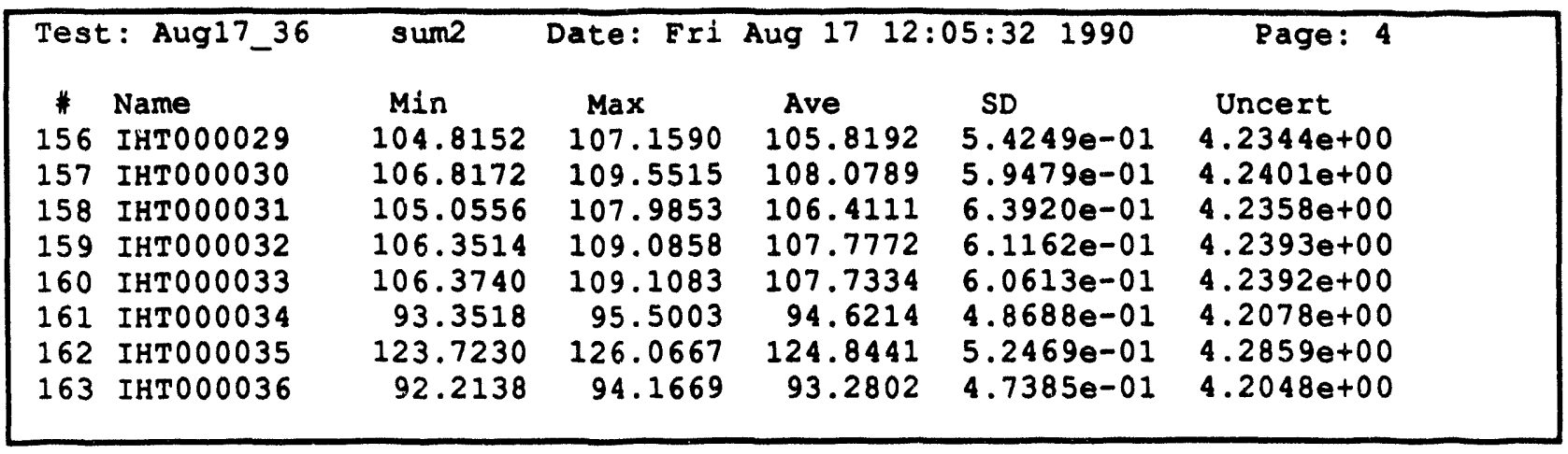




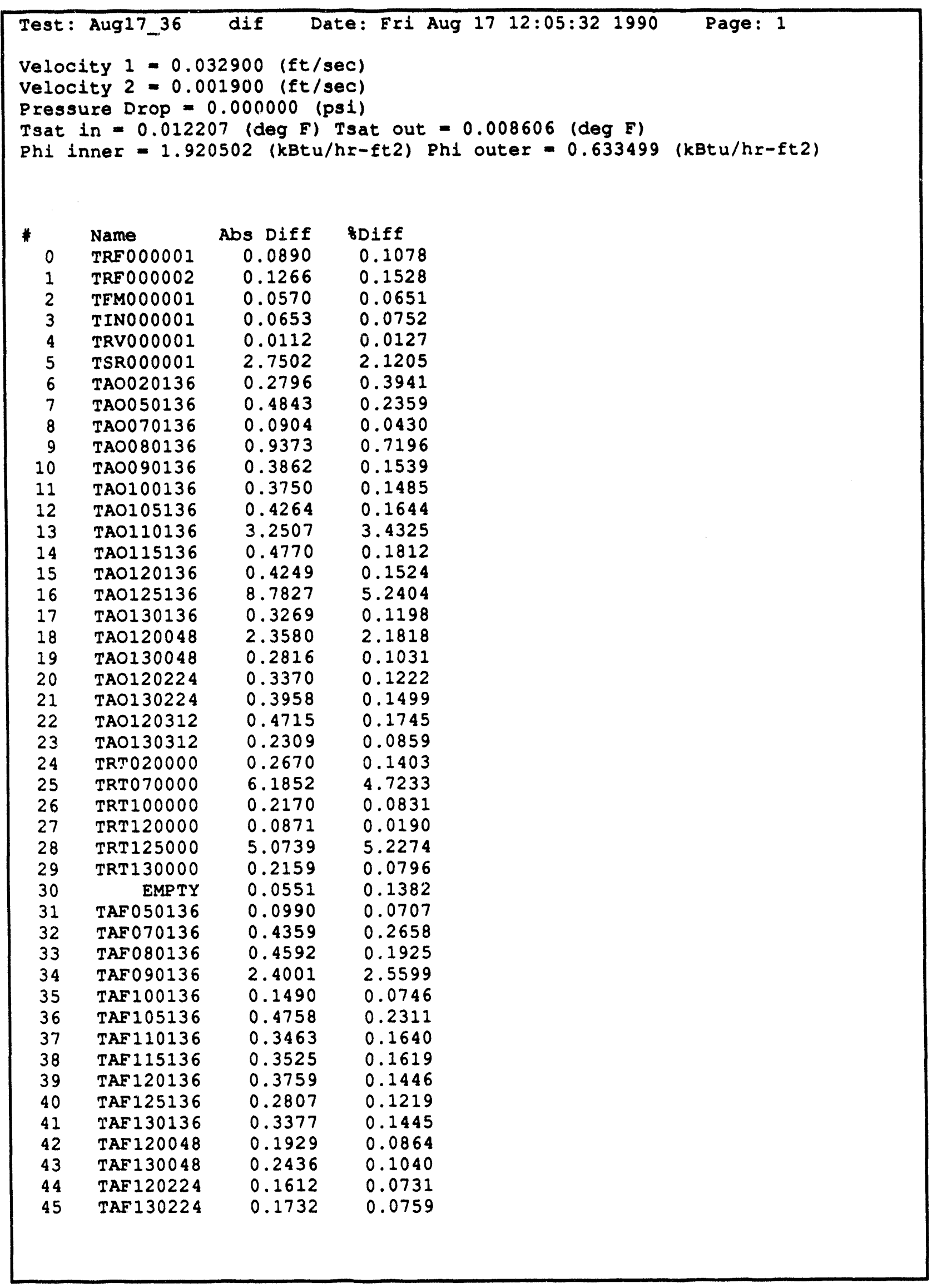




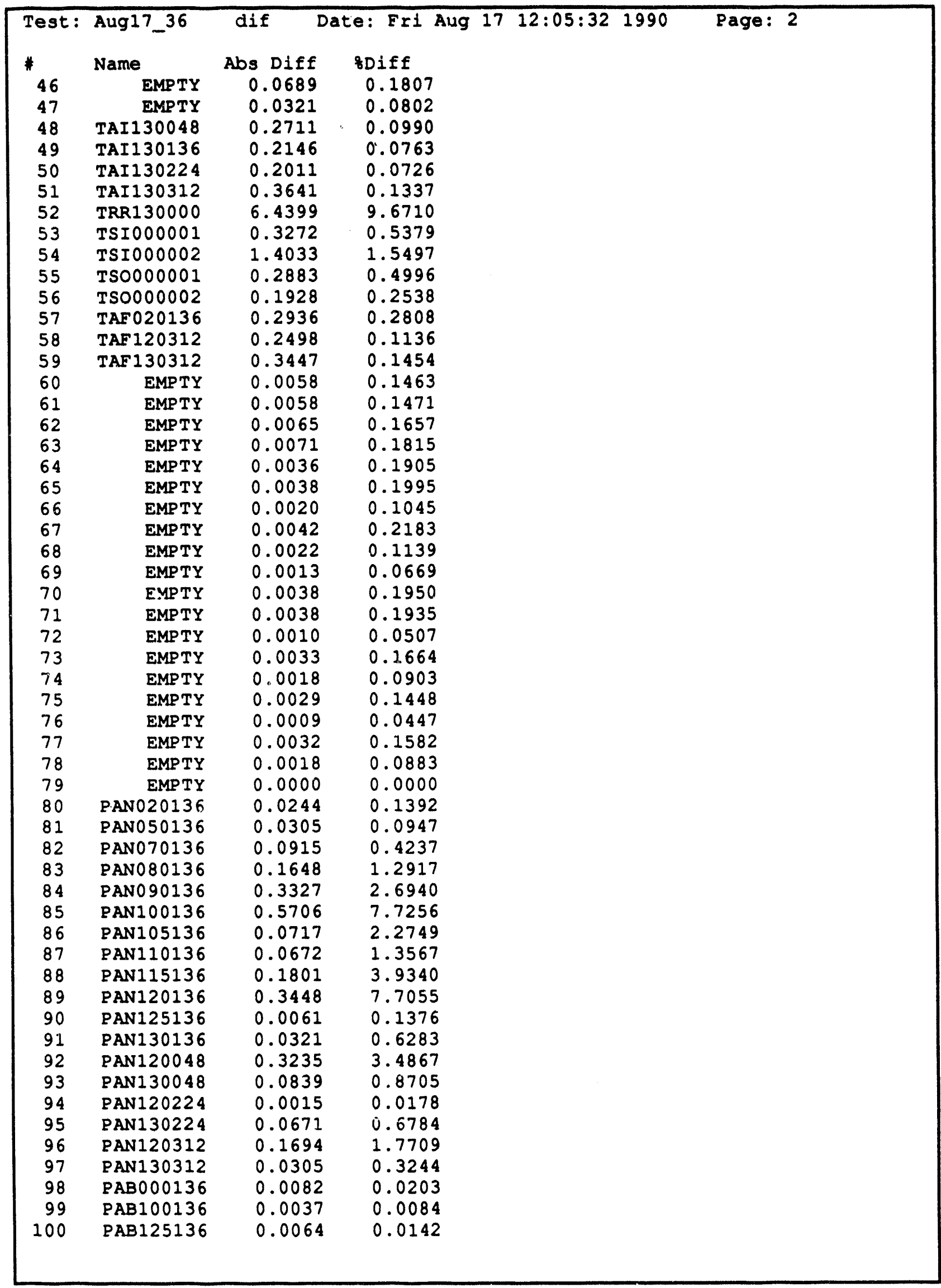




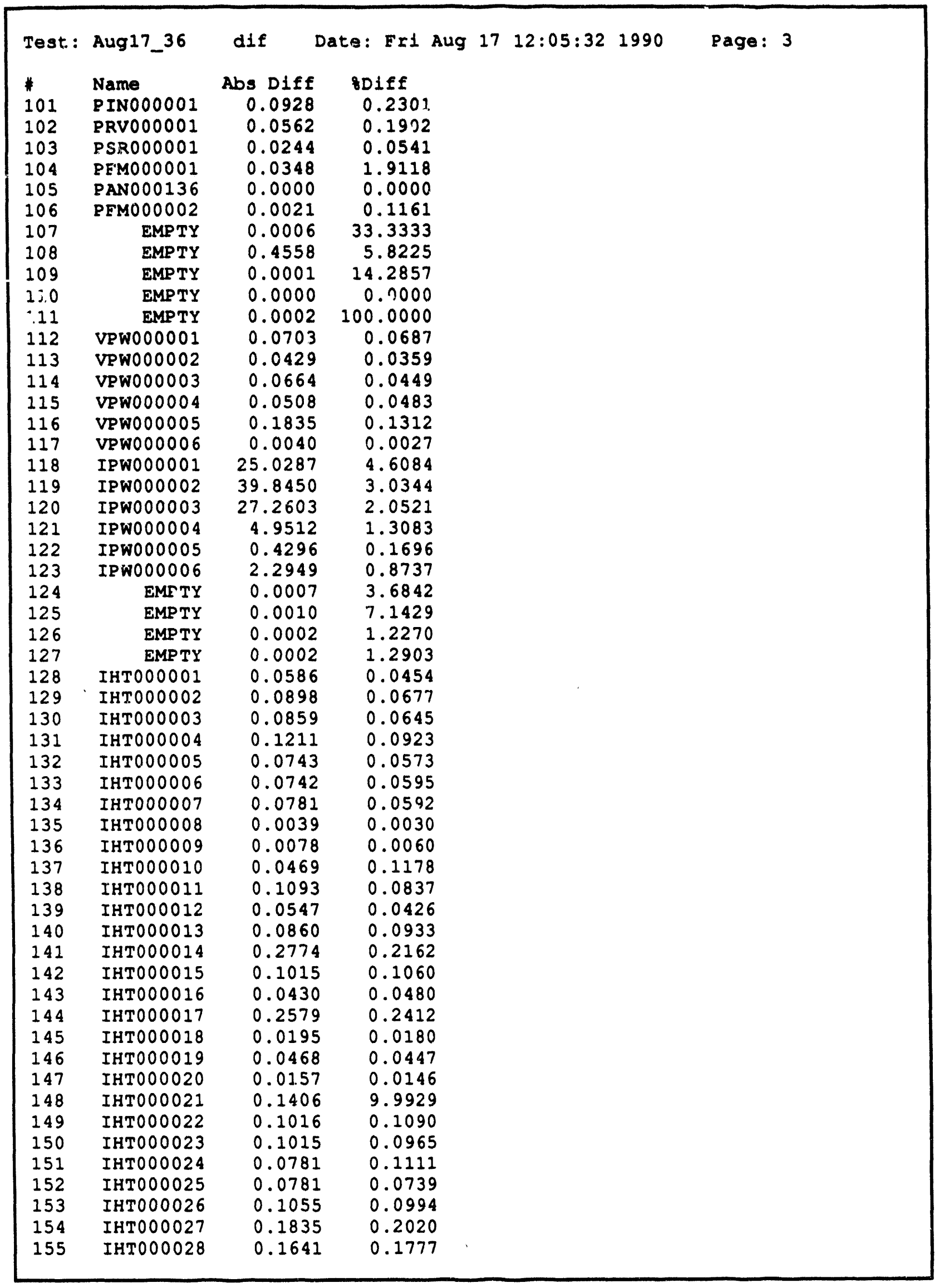


Test : Aug17_36

* Name

156 IHTO00029

157 IHTO00030

158 IHT000031

159 IHTO00032

160 IHT000033

161 IHT000034

162 IHTO00035

163 IHT000036 dif Date: Fri Aug 17 12:05:32 1990

Page : 4

\section{\&Diff}

0.0995

0.0650

0.0037

0.0435

0.0399

0.1158

0.1629

0.0587 


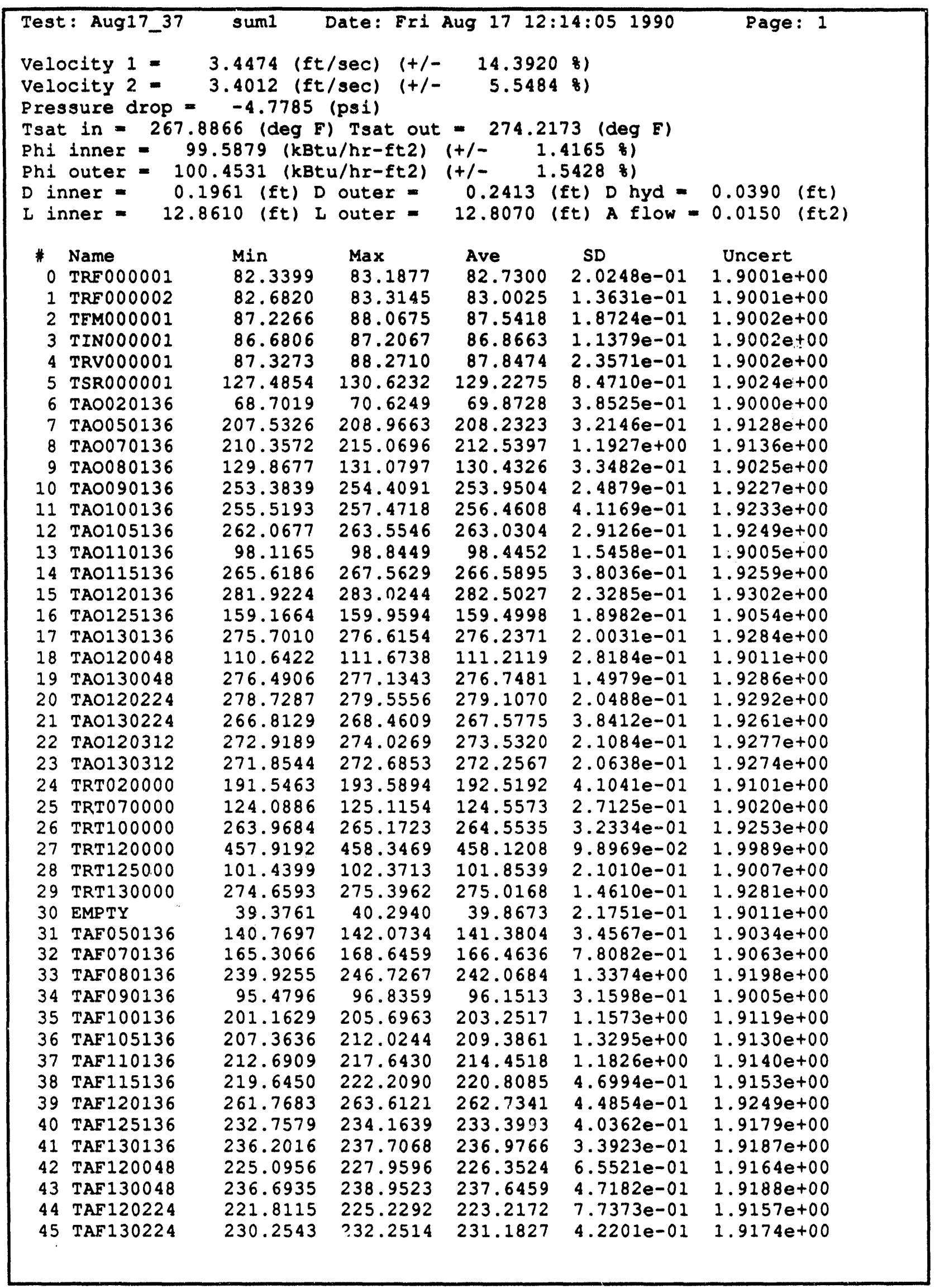




\begin{tabular}{|c|c|c|c|c|c|c|}
\hline \multicolumn{2}{|c|}{ Test: Aug17_37 } & suml & Date: Fri & ug & $: 051990$ & Page: \\
\hline \# & Name & Min & $\operatorname{Max}$ & Ave & SD & Uncert \\
\hline 46 & EMPTY & 37.5374 & 38.6870 & 38.1445 & $2.2641 e-01$ & $1.9010 e+00$ \\
\hline 47 & EMPTY & 39.8352 & 40.5234 & 40.1289 & $1.6083 e-01$ & $1.9011 e+00$ \\
\hline 48 & TAI130048 & 275.1760 & 276.5574 & 275.7587 & $3.5612 e-01$ & $1.9283 \mathrm{e}+00$ \\
\hline 49 & TAI130136 & 283.7815 & 284.8794 & 2242 & $2.4409 e-01$ & $1.9307 e+00$ \\
\hline 50 & TAI130224 & 279.5613 & 281.1266 & 280.2946 & $3.4999 \mathrm{e}-01$ & $1.9296 e+00$ \\
\hline 51 & TAI130312 & 275.2847 & 276.4834 & 275.8083 & $3.3246 e-01$ & $1.9283 \mathrm{e}+00$ \\
\hline 52 & TRR130000 & 61.4099 & 63.2278 & 62.3854 & $4.2496 \mathrm{e}-01$ & $1.9000 \mathrm{e}+00$ \\
\hline 53 & TSI000001 & 60.2661 & 61.0187 & 60.6656 & $1.7359 \mathrm{e}-01$ & $1.9001 e+00$ \\
\hline 54 & TSI000002 & 88.2291 & 89.3824 & 88.7995 & $2.9578 e-01$ & $1.9002 e+00$ \\
\hline 55 & TSO000001 & 804 & 57.8616 & 57.4680 & $2.3404 e-01$ & $1.9001 e+00$ \\
\hline 56 & TSO000002 & 75.7658 & 76.7215 & 76.2248 & $2.1278 e-01$ & $1.9000 e+00$ \\
\hline 57 & TAF020136 & 104.0954 & 106.6997 & 105.3669 & $5.0777 e-01$ & $1.9008 \mathrm{e}+00$ \\
\hline 58 & TAF120312 & 221.0511 & 224.0011 & 222.4595 & $6.2507 e-01$ & $1.9156 \mathrm{e}+00$ \\
\hline 59 & TAF 130312 & 39.0049 & 241.6340 & 240.5184 & $5.7593 e-01$ & $1.9195 e+00$ \\
\hline 60 & EMPTY & 4.0381 & 4.0625 & 4.0485 & $5.5812 e-03$ & $0.0000 e+00$ \\
\hline 61 & EMPTY & 4.0234 & 4.0332 & 4.0271 & $3.3540 e-03$ & $0.0000 e+00$ \\
\hline 62 & EMPTY & 3.9 & 4.0137 & 4.0059 & -03 & $0.0000 \mathrm{e}+00$ \\
\hline 63 & EMPTY & 3.9893 & 4.0039 & 3.9957 & 2.865 & $0 e+00$ \\
\hline 64 & ЕMPTY & 1.9238 & 1.9336 & 1.9285 & $1.6972 \mathrm{e}$ & $0 e+00$ \\
\hline 65 & EMPTY & 1.9434 & 1.9531 & 1.9438 & 1.778 & $0.0000 \mathrm{e}+00$ \\
\hline 66 & EMPTY & 1.9482 & 1.9531 & 1.9518 & $2.2146 e-03$ & $0.0000 \mathrm{e}+00$ \\
\hline 67 & EMPTY & 1.9629 & 1.9678 & 1.9630 & 6.9053 & $0.0000 e+00$ \\
\hline 68 & EMPTY & 1.9678 & 1.9727 & 1.9687 & 1.895 & $0.0000 e+00$ \\
\hline 69 & EMPTY & 1.9775 & 1.9824 & 1.9800 & 2.4662 & $0.0000 e+00$ \\
\hline 70 & EMPTY & 1.9873 & 1.9873 & 1.9873 & 0.0000 & $0.0000 e+00$ \\
\hline 71 & EMPTY & 1.9971 & 2.0117 & 2.0022 & 1.8216 & $0.0000 e+00$ \\
\hline 72 & EMPTY & 2.0068 & 2.0117 & 2.0090 & $2.4484 e-03$ & $0.0000 e+00$ \\
\hline 73 & EMPTY & 2.0215 & 2.0264 & 2.0216 & 6.9053 & $0.0000 e+00$ \\
\hline 74 & EMPTY & 2.0264 & 2.0313 & 2.0309 & 1.338 & $0.0000 e+00$ \\
\hline 75 & EMPTY & 61 & 2.0459 & 2.0410 & 9.864 & $0 e+00$ \\
\hline 76 & EMPTY & 2.0410 & 2.0557 & 2.0474 & 2.6562 & $0.0000 e+00$ \\
\hline 77 & ЕMPTY & 2.0557 & 2.0654 & 2.0605 & 1.3951 & $0.0000 e+00$ \\
\hline 8 & EMPTY & 2.0 & 2.0898 & 2.0756 & 2.1703 & $0.0000 e+00$ \\
\hline 79 & EMPTY & 2.0850 & 2.0947 & 2.0911 & 2.3778 & $0.0000 \mathrm{e}+00$ \\
\hline 80 & PAN020136 & -17.9202 & -17.5387 & -17.6821 & 8.9399 & $8.7112 e-01$ \\
\hline 1 & PAN0 50136 & -32.9159 & -32.1529 & -32.4185 & 1.711 & $0 \mathrm{e}+00$ \\
\hline 82 & PAN070136 & -23.8152 & -20.3819 & -22.0 & 1.2273 & $5 e-01$ \\
\hline 83 & PAN080136 & -13.9558 & -11.3618 & -13.2478 & 7.6170 & $3 e-01$ \\
\hline 84 & PAN090136 & -12.1072 & -7.0718 & -10.2822 & 1.0499 & $e-01$ \\
\hline 85 & PAN100136 & -12.1908 & -5.3 & -8.6 & 1.419 & $7 e-01$ \\
\hline 86 & PAN105136 & -7.4731 & 3.3606 & -3.8308 & $1.7058 \mathrm{e}+00$ & $5 e-01$ \\
\hline 87 & PAN11 & -7.29 & & & 1.116 & -01 \\
\hline 88 & PAN1 15136 & -7.55 & -2 . & -4.7 & 9.7318 & -01 \\
\hline 89 & PAN 120136 & -5.2224 & -3.0861 & -4.5266 & $4.4236 e-01$ & $8.7007 e-01$ \\
\hline 90 & PAN125136 & -5.0405 & -4.12 & -4.5156 & $2.1942 e-01$ & $7 e-01$ \\
\hline 91 & PAN 130136 & -5.3118 & -4.62 & -5.0 & $1.6673 \mathrm{e}$ & $9 e-01$ \\
\hline 92 & PAN120048 & -10.3509 & -7.4517 & -9.5956 & $6.2578 e-01$ & $8.7033 e-01$ \\
\hline 93 & PAN130048 & -9.8945 & -9.20 & -9.57 & $1.6698 e-01$ & $8.7033 e-01$ \\
\hline 94 & PAN120224 & -12.2891 & -6.33 & -8.4393 & 1.09996 & $8.7026 e-01$ \\
\hline 95 & PAN130224 & -10.3772 & -9.2328 & -9.8798 & $2.3591 e-01$ & $8.7035 e-01$ \\
\hline 96 & PAN120312 & -9.91 & -7.3930 & & $4.6676 e-01$ & $8.7029 e-01$ \\
\hline 97 & PAN130312 & -9.9122 & -9.2256 & -9.5842 & $1.6902 e-01$ & $8.7033 e-01$ \\
\hline 98 & $\mathrm{PAB} 000136$ & 40.2739 & 40.4112 & 40.3489 & $4.6019 e-02$ & $5.8871 e-01$ \\
\hline 99 & PAB100136 & & 44.0759 & & $6.2725 \mathrm{e}$ & $31 e-01$ \\
\hline 100 & PAB125136 & 44.7573 & 44.9404 & 44.8306 & $4.4353 e-02$ & $5.9073 e-01$ \\
\hline
\end{tabular}




\begin{tabular}{|c|c|c|c|c|c|c|}
\hline \multicolumn{2}{|c|}{ Test: Aug17_37 } & sum1 & Date: Fri & \multicolumn{2}{|r|}{990} & Page: 3 \\
\hline \# & Name & Min & $\operatorname{Max}$ & Ave & SD & Uncert \\
\hline 101 & PINO00001 & 39.8366 & 40.2028 & 40.0710 & $1.0417 e-01$ & $7.5666 e-01$ \\
\hline 102 & PRVO00001 & 29.3621 & 29.6063 & 29.4964 & $4.4460 \mathrm{e}-02$ & $7.5362 e-01$ \\
\hline 103 & PSR000001 & 44.8156 & 45.0597 & 45.0011 & $6.6358 e-02$ & $7.5839 e-01$ \\
\hline 104 & PFM000001 & 1.5493 & 1.9155 & 1.7489 & $7.5564 e-02$ & $5.0002 e-01$ \\
\hline 105 & PAN000136 & -133.1649 & -131.3338 & -132.2951 & $4.4593 e-01$ & $4.9311 e+00$ \\
\hline 106 & PFM000002 & 1.6609 & 1.7448 & 1.7011 & $1.6608 e-02$ & $2.0005 e-01$ \\
\hline 107 & EMPTY & -0.0049 & 0.0049 & 0.0034 & $2.6562 e-03$ & $0.0000 e+00$ \\
\hline 108 & EMPTY & -10.0000 & -1.7383 & -8.0690 & $1.9107 \mathrm{e}+00$ & $0.0000 e+00$ \\
\hline 109 & EMPTY & 0.0000 & 0.0049 & 0.0016 & $2.3008 e-03$ & $0.0000 e+00$ \\
\hline 110 & EMPTY & -10.0000 & -10.0000 & -10.0000 & $0.0000 e+00$ & $0.0000 e+00$ \\
\hline 111 & EMPTY & 0.0000 & 0.0000 & 0.0000 & $0.0000 e+00$ & $0.0000 e+00$ \\
\hline 112 & VPW000001 & 102.0245 & 102.4151 & 102.1729 & $1.0849 e-01$ & $1.0158 e+00$ \\
\hline 113 & VPW000002 & 119.0467 & 119.4374 & 119.2889 & $1.0849 e-01$ & $1.0809 e+00$ \\
\hline 114 & VPW000003 & 147.6225 & 148.2084 & 147.9272 & $1.1272 e-01$ & $1.2016 \mathrm{e}+00$ \\
\hline 115 & VPW000004 & 104.6687 & 105.4500 & 105.2078 & $1.4530 \mathrm{e}-01$ & $1.0269 e+00$ \\
\hline 116 & VPW000005 & 139.8475 & 140.4334 & 140.1561 & $1.3141 \mathrm{e}-01$ & $1.1676 e+00$ \\
\hline 117 & VPW000006 & 148.4563 & 149.0422 & 148.7491 & $1.4363 e-01$ & $1.2053 e+00$ \\
\hline 118 & IPW000001 & 563.0259 & 570.8353 & 567.9457 & $2.0205 e+00$ & $9.2597 e+00$ \\
\hline 119 & IPW000002 & 1269.1508 & 1278.9071 & 1273.9122 & $1.7700 e+00$ & $1.2919 e+01$ \\
\hline 120 & IPW000003 & 1298.6998 & 1304.5499 & 1301.6249 & $1.8161 \mathrm{e}+00$ & $1.309 j e+01$ \\
\hline 121 & IPW000004 & 383.1223 & 385.0755 & 383.8060 & $6.1605 e-01$ & $3.6323 e+00$ \\
\hline 122 & IPW000005 & 252.0959 & 253.5616 & 252.9952 & $3.1749 e-01$ & $2.8275 e+00$ \\
\hline 123 & IPW000006 & 259.3149 & 261.2681 & 260.2620 & $5.0696 e-01$ & $2.8683 e+00$ \\
\hline 124 & EMPTY & -0.0293 & -0.0098 & -0.0188 & $4.4512 e-03$ & $0.0000 e+00$ \\
\hline 125 & EMPTY & -0.0195 & -0.0098 & -0.0130 & $3.0581 e-03$ & $0.0000 e+00$ \\
\hline 126 & EMPTY & -0.0244 & -0.0049 & -0.0147 & $4.1256 e-03$ & $0.0000 e+00$ \\
\hline 127 & EMPTY & -0.0195 & -0.0049 & -0.0148 & $3.9410 e-03$ & $0.0000 e+00$ \\
\hline 128 & IHT000001 & 127.7419 & 130.6716 & 129.0660 & $6.1547 e-01$ & $4.2983 e+00$ \\
\hline 129 & IHT000002 & 131.5955 & 134.1346 & 132.7010 & $6.0695 e-01$ & $4.3094 \mathrm{e}+00$ \\
\hline 130 & IHT000003 & 132.1139 & 134.6530 & 133.2427 & $6.0912 \mathrm{e}-01$ & $4.3111 e+00$ \\
\hline 131 & IHT 000004 & 130.2096 & 132.7486 & 131.3267 & $6.3263 e-01$ & $4.3052 e+00$ \\
\hline 132 & IHT000005 & 128.8762 & 131.2199 & 129.8097 & $5.7493 e-01$ & $4.3006 e+00$ \\
\hline 133 & IHT000006 & 123.8018 & 126.1456 & 124.8018 & $5.8079 e-01$ & $4.2857 e+00$ \\
\hline 134 & IHTO000007 & 131.1899 & 133.3384 & 131.9946 & $5.4052 e-01$ & $4.3072 e+00$ \\
\hline 135 & IHT000008 & 129.5748 & 131.7233 & 130.4615 & $4.8842 e-01$ & $4.3026 e+00$ \\
\hline 136 & IHT000009 & 129.7589 & 132.1026 & 130.6768 & $5.1938 e-01$ & $4.3032 e+00$ \\
\hline 137 & IHT000010 & -40.6889 & -38.3451 & -39.7592 & $5.6160 e-01$ & $4.1192 e+00$ \\
\hline 138 & IHTO00011 & 129.9166 & 132.2603 & 130.8111 & $5.4403 e-01$ & $4.3036 e+00$ \\
\hline 139 & IHT000012 & 127.3362 & 129.4847 & 128.4417 & $4.8746 e-01$ & $4.2965 e+00$ \\
\hline 140 & IHT000013 & 91.2109 & 93.3594 & 92.2617 & $4.4971 \mathrm{e}-01$ & $4.2025 e+00$ \\
\hline 141 & IHTO00014 & 127.5616 & 129.7101 & 128.5459 & $4.6179 e-01$ & $4.2968 \mathrm{e}+00$ \\
\hline 142 & IHT000015 & 95.0120 & 96.9651 & 95.8440 & $4.4083 e-01$ & $4.2105 e+00$ \\
\hline 143 & IHT000016 & 88.5216 & 90.4747 & 89.4279 & $4.0771 e-01$ & $4.1964 e+00$ \\
\hline 144 & IHT000017 & 105.4613 & 109.3675 & 107.1683 & $8.3781 e-01$ & $4.2377 e+00$ \\
\hline 145 & IHT000018 & 107.1590 & 109.6980 & 108.2762 & $5.5529 e-01$ & $4.2406 \mathrm{e}+00$ \\
\hline 146 & IHT000019 & 103.4781 & 106.0171 & 104.7007 & $5.5357 e-01$ & $4.2316 e+00$ \\
\hline 147 & IHT000020 & 106.1974 & 108.5412 & 107.2169 & $5.9227 e-01$ & $4.2379 e+00$ \\
\hline 148 & IHTO00021 & -2.6179 & 0.1164 & -1.2781 & $6.4959 e-01$ & $4.1000 e+00$ \\
\hline 149 & IHT000022 & 92.0786 & 94.6176 & 93.1841 & $5.1992 e-01$ & $4.2046 \mathrm{e}+00$ \\
\hline 150 & IHT000023 & 104.3645 & 106.5129 & 105.2355 & $5.0872 e-01$ & $4.2329 e+00$ \\
\hline 151 & IHT000024 & 69.3773 & 71.5257 & 70.3108 & $4.3636 e-01$ & $4.1599 e+00$ \\
\hline 152 & IHT000025 & 104.7439 & 107.0876 & 105.7243 & $4.7595 e-01$ & $4.2341 \mathrm{e}+00$ \\
\hline 153 & IHT000026 & 105.1307 & 107.2792 & 106.2127 & $4.9318 e-01$ & $4.2353 e+00$ \\
\hline 154 & IHTO00027 & 89.7987 & 91.9471 & 90.8729 & $4.5720 \mathrm{e}-01$ & $4.1995 e+00$ \\
\hline 155 & IHT000028 & 90.9443 & 93.6786 & 92.2138 & $6.1289 e-01$ & $4.2024 e+00$ \\
\hline
\end{tabular}




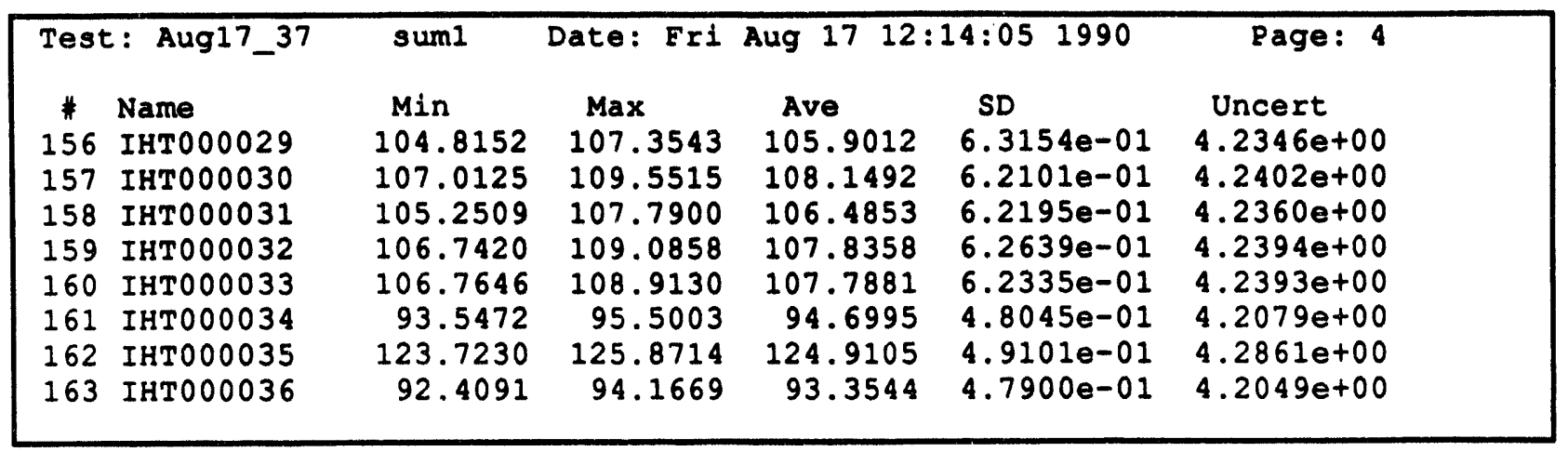




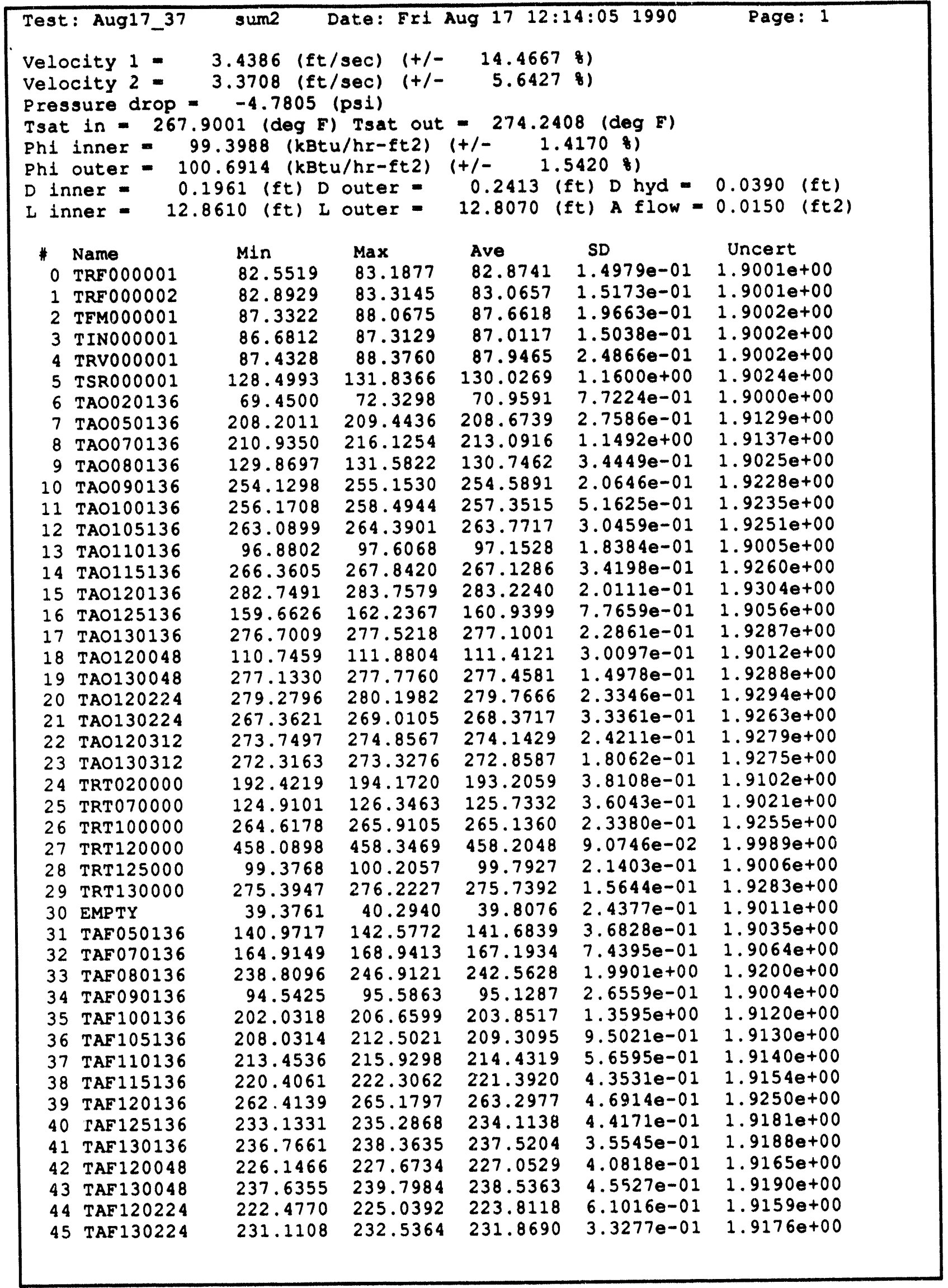




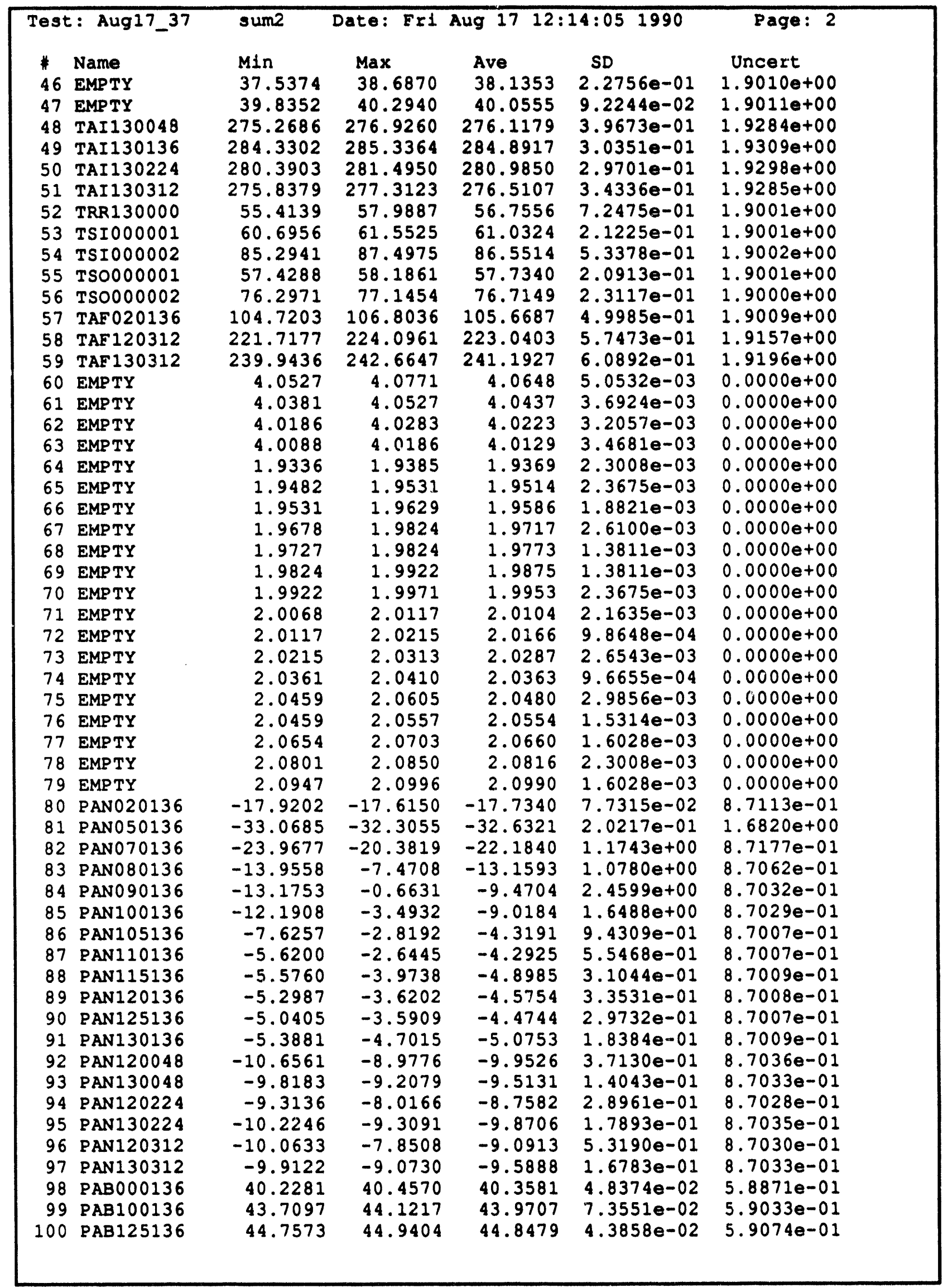




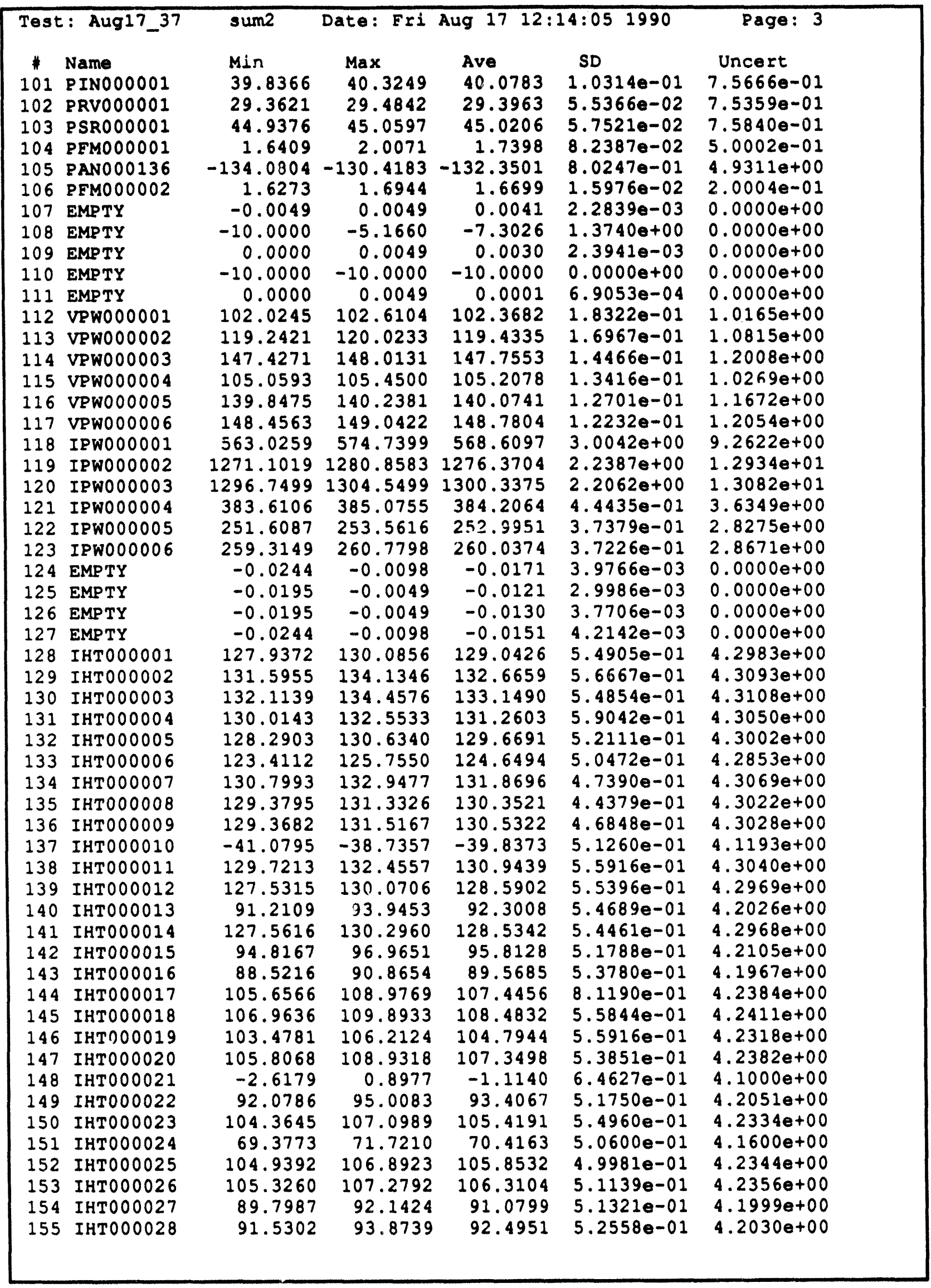




\begin{tabular}{|c|c|c|c|c|c|c|}
\hline \multicolumn{2}{|c|}{ Test: Aug17_37 } & sum2 & Date: $F x$ & Aug 171 & $: 051$ & Page: 4 \\
\hline $\begin{array}{c}* \\
156 \\
157 \\
158 \\
159 \\
160 \\
161 \\
162 \\
163\end{array}$ & 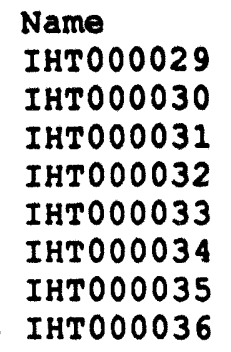 & $\begin{array}{l}\text { Min } \\
105.2058 \\
107.4031 \\
105.6415 \\
107.1326 \\
107.1552 \\
93.9378 \\
124.1136 \\
92.6044\end{array}$ & $\begin{array}{l}\text { Max } \\
107.7449 \\
109.7469 \\
108.1806 \\
109.4764 \\
109.3036 \\
95.5003 \\
125.8714 \\
94.3622\end{array}$ & $\begin{array}{l}\text { Ave } \\
106.0652 \\
108.3055 \\
106.6611 \\
107.9959 \\
107.9599 \\
94.7151 \\
124.9340 \\
93.3935\end{array}$ & $\begin{array}{l}\text { SD } \\
5.3815 e-01 \\
4.7661 e-01 \\
5.0884 \theta-01 \\
5.3828 \theta-01 \\
5.1996 e-01 \\
4.2952 e-01 \\
4.2499 e-01 \\
4.4110 e-01\end{array}$ & $\begin{array}{c}\text { Uncert } \\
4.2350 e+00 \\
4.2406 e+00 \\
4.2365 e+00 \\
4.2398 \theta+00 \\
4.2398 \theta+00 \\
4.2080 e+00 \\
4.2861 e+00 \\
4.2050 e+00\end{array}$ \\
\hline
\end{tabular}




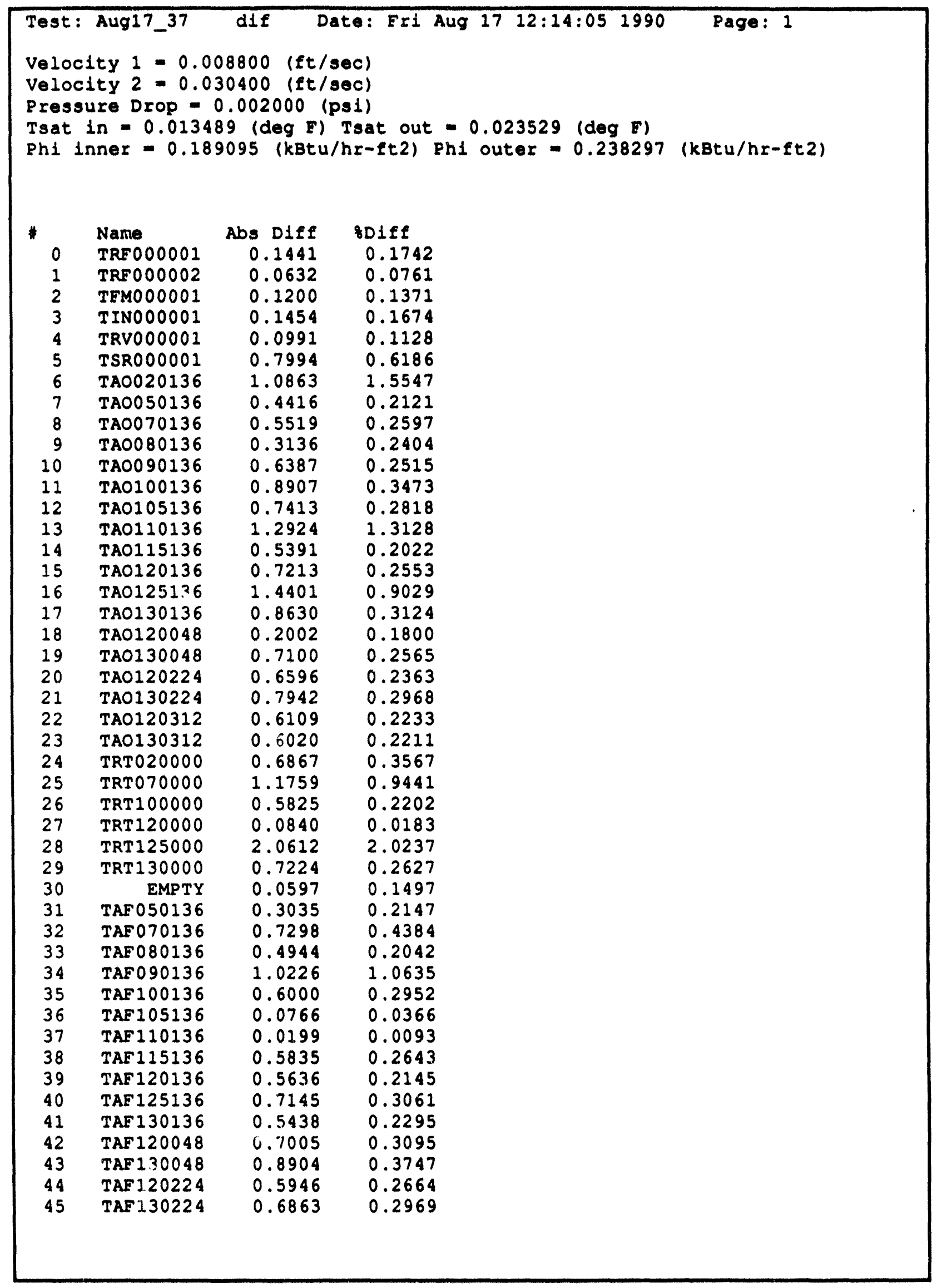




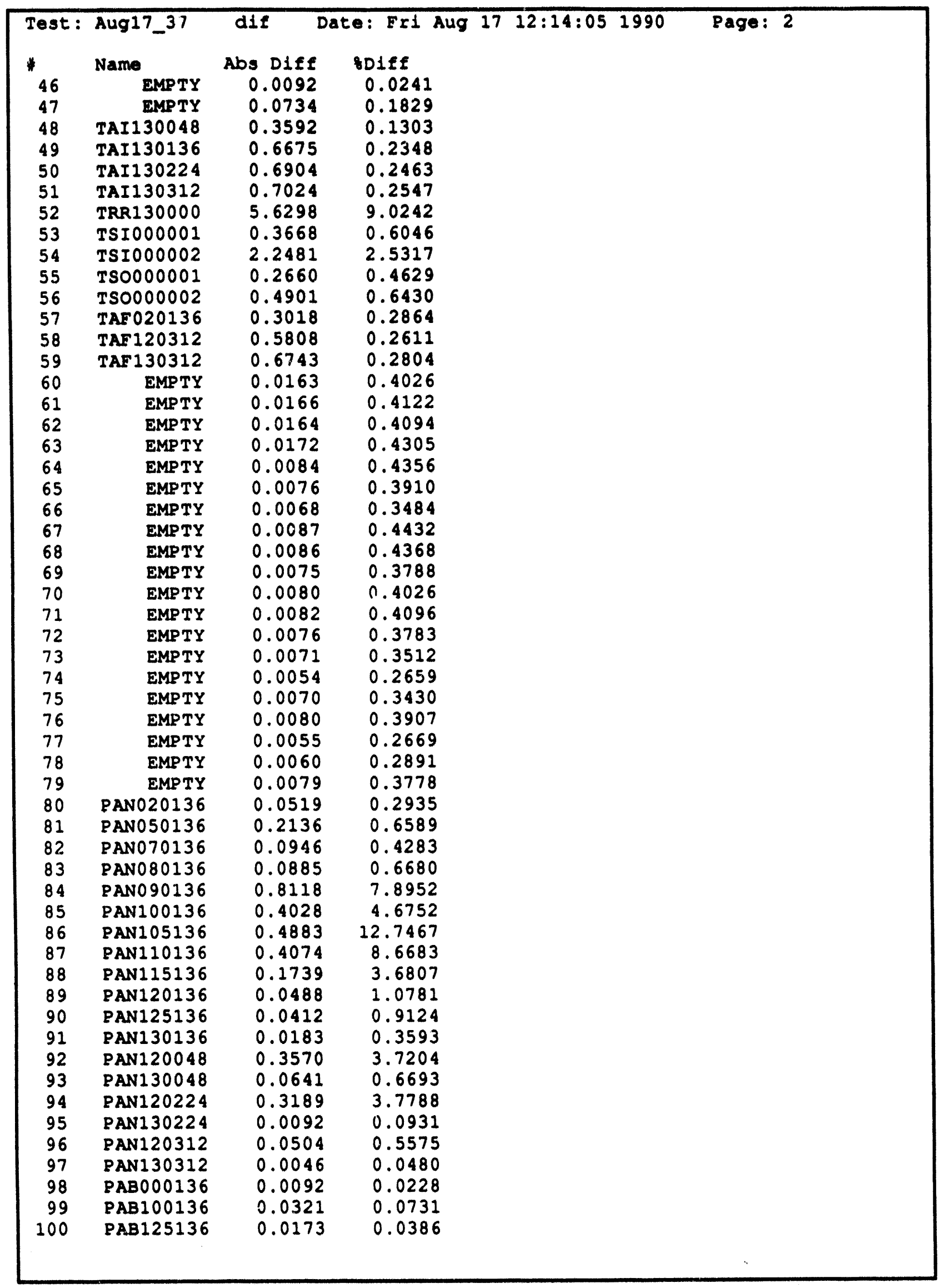




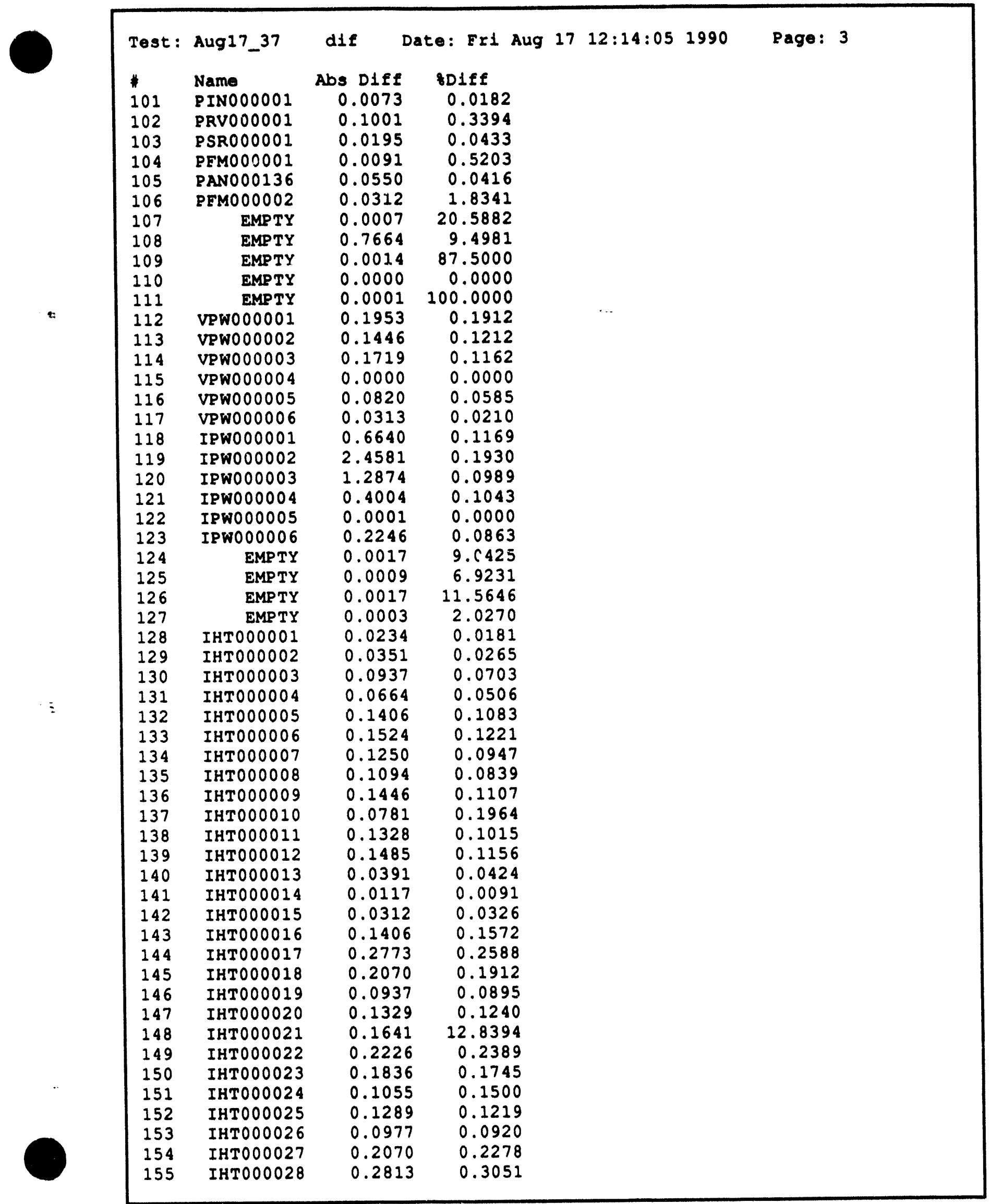




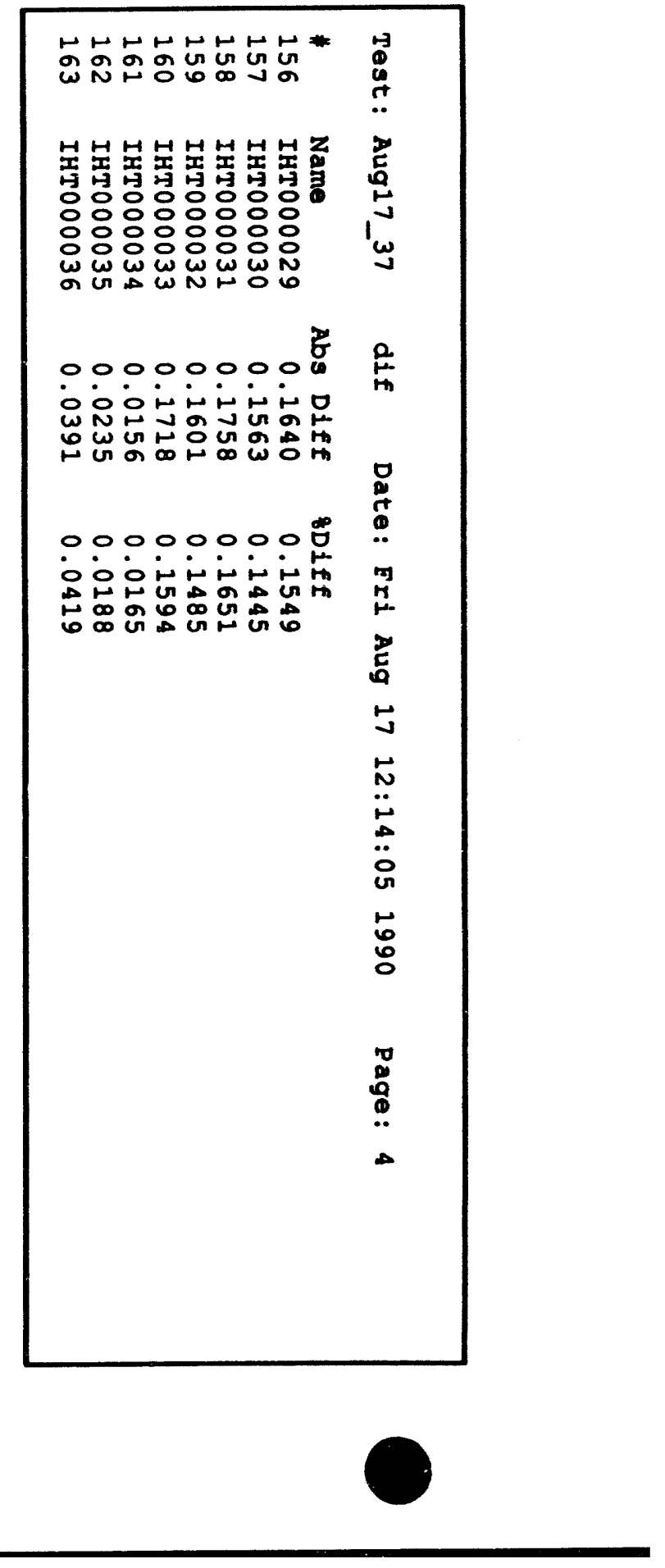



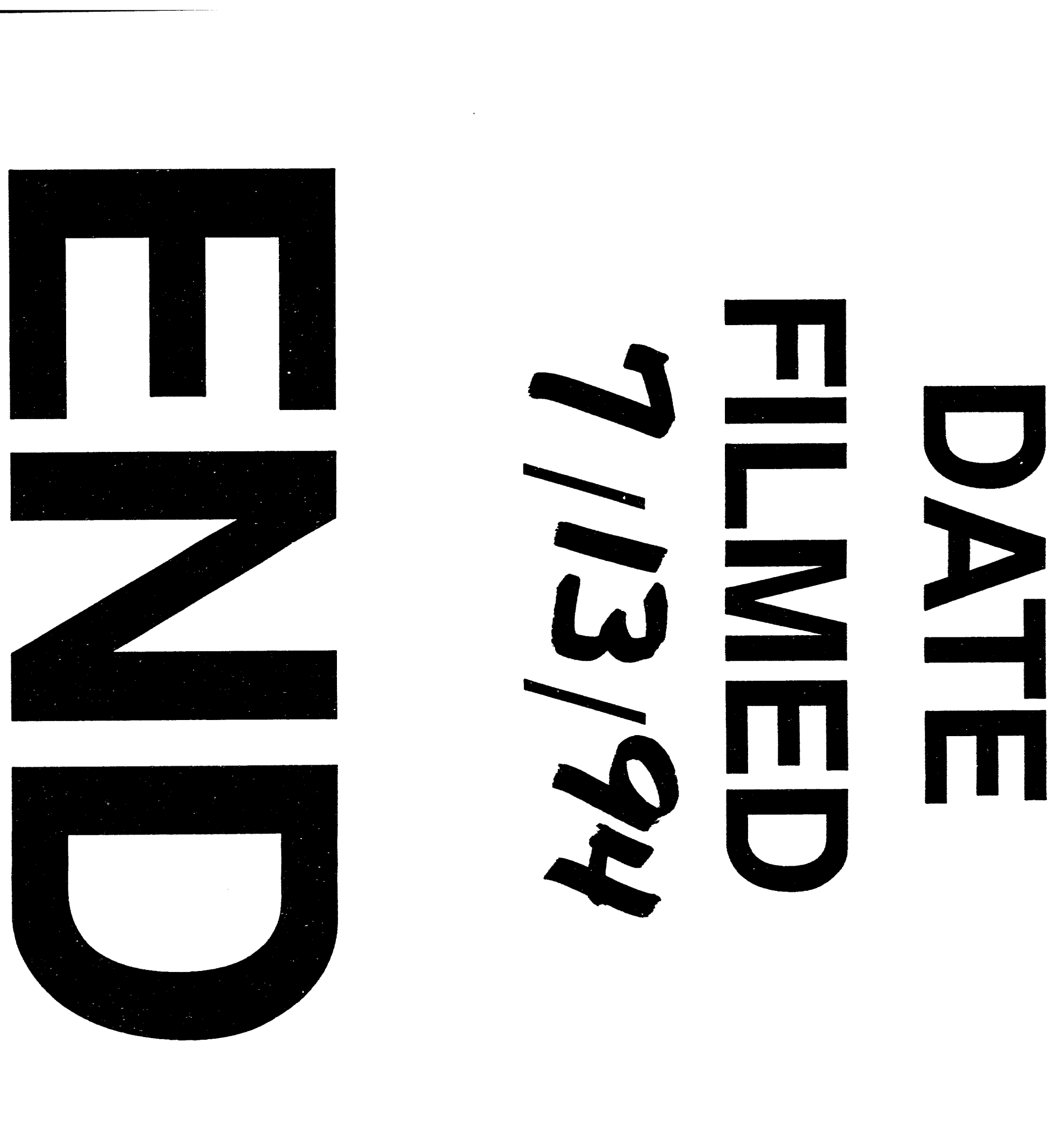

$<$

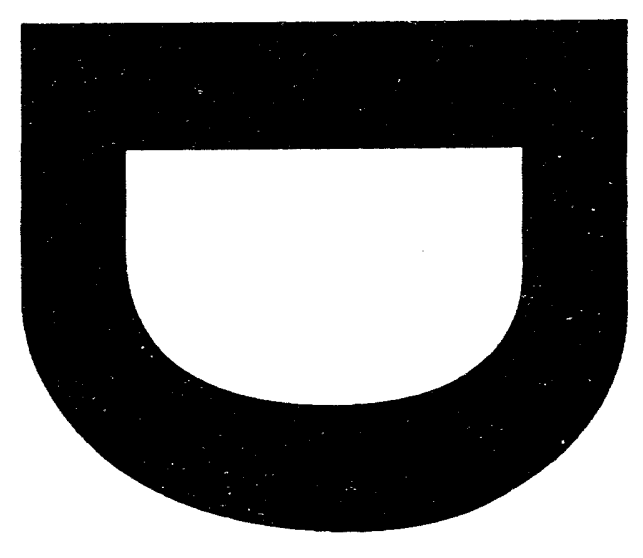

